CHPRC-01552

Revision 0

\title{
MCNP5 Calculations Replicating ARH-600 Nitrate Data
}

Prepared for the U.S. Department of Energy

Assistant Secretary for Environmental Management

Contractor for the U.S. Department of Energy

under Contract DE-AC06-08RL14788

CIM2MMHILL

Plateau Remediation Company

P.O. Box 1600

Richland, Washington 99352

Approved for Public Release;

Further Dissemination Unlimited 
CHPRC-01552

Revision 0

\section{MCNP5 Calculations Replicating ARH-600 Nitrate Data}

Document Type: TR

S. H. Finfrock

CH2M HILL Plateau Remediation Company

Date Published

October 2011

Prepared for the U.S. Department of Energy

Assistant Secretary for Environmental Management

Contractor for the U.S. Department of Energy

under Contract DE-AC06-08RL14788

\section{GH:HMHILL}

Plateau Remediation Company

P.O. Box 1600

Richland, Washington

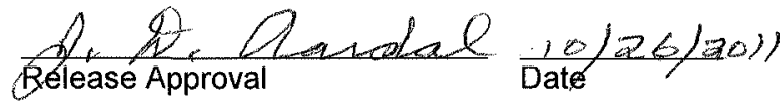


CHPRC-01552

Revision 0

TRADEMARK DISCLAIMER

Reference herein to any specific commercial product, process,

or service by trade name, trademark, manufacturer, or

otherwise, does not necessarily constitute or imply its

endorsement, recommendation, or favoring by the United

States Government or any agency thereof or its contractors or

subcontractors.

This report has been reproduced from the best available copy.

Printed in the United States of America 


\title{
MCNP5 Calculations Replicating ARH-600 Nitrate Data
}

\author{
August 2011 \\ Prepared by: MB Murphy and SH Finfrock \\ for \\ CH2M HILL Plateau Remediation Company \\ Richland, Washington
}


CHPRC-01552, Revision 0

This page is intentionally left blank. 


\section{TABLE OF CONTENTS}

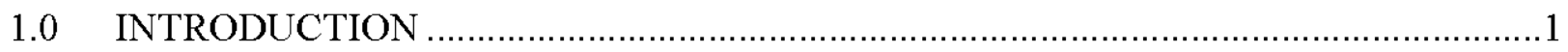

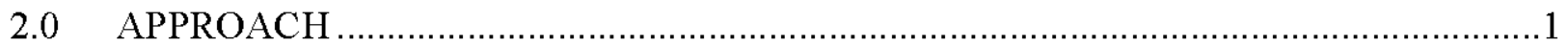

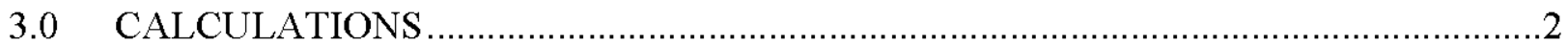

3.1 CALCULATION OF MOLARITIES FOR THE FISSILE SOLUTION .....................2

3.2 CALCULATIONS OF ATOM FRACTIONS FOR THE FISSILE SOLUTION ...........3

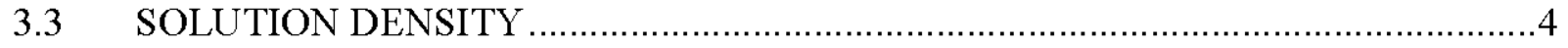

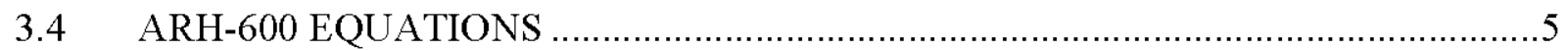

3.5 LIST OF MCNP5 CROSS-SECTION LIBRARIES USED IN MODEL....................5

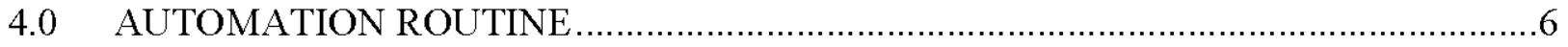

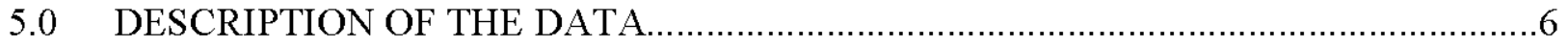

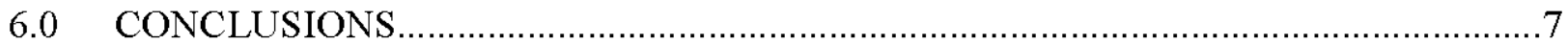

APPENDIX A - INDEPENDENT REVIEW COMMENTS AND CHECKLIST................. A-1

APPENDIX B - CALCULATIONS AND DATA …....................................................... B-i

APPENDIX C - CRITVIEW COMPATIBLE DATA FILES ............................................

APPENDIX D - TEMPLATE FILES FOR CASES ................................................... D-1

APPENDIX E - EXAMPLE INPUT FILES ...............................................................

APPENDIX F - EXAMPLE INPUT FILES .................................................................. F-1 
CHPRC-01552, Revision 0

This page is intentionally left blank. 


\subsection{INTRODUCTION}

This report serves to extend the previous document: "MCNP Calculations Replicating ARH-600 Data" by replicating the nitrate curves found in ARH-600. This report includes the MCNP models used, the calculated critical dimension for each analyzed parameter set, and the resulting data libraries for use with the CritView code. As with the ARH-600 data, this report is not meant to replace the analysis of the fissile systems by qualified criticality personnel. The MCNP data is presented without accounting for the statistical uncertainty (although this is typically less than 0.001 ) or bias and, as such, the application of a reasonable safety margin is required.

The data that follows pertains to the uranyl nitrate and plutonium nitrate spheres, infinite cylinders, and infinite slabs of varying isotopic composition, reflector thickness, and molarity. Each of the cases was modeled in MCNP (version 5.1.40), using the ENDF/B-VI cross section set. Given a molarity, isotopic composition, and reflector thickness, the fissile concentration and diameter (or thicknesses in the case of the slab geometries) were varied. The diameter for which $\mathrm{k}$-effective equals 1.00 for a given concentration could then be calculated and graphed. These graphs are included in this report. The pages that follow describe the regions modeled, formulas for calculating the various parameters, a list of cross-sections used in the calculations, a description of the automation routine and data, and finally the data output.

The data of most interest are the critical dimensions of the various systems analyzed. This is presented graphically, and in table format, in Appendix B. Appendix C provides a text listing of the same data in a format that is compatible with the CritView code. Appendices D and $\mathrm{E}$ provide listing of example Template files and MCNP input files (these are discussed further in Section 4). Appendix $\mathrm{F}$ is a complete listing of all of the output data (i.e., all of the analyzed dimensions and the resulting $\mathrm{k}_{\text {eff }}$ values).

\subsection{APPROACH}

Each nuclear system model consists of two regions - the fissile region and the reflector region (in some cases the reflector has a zero thickness and is effectively absent). The outside edge of the reflector region (or fissile region in the cases without a reflector) was treated as a vacuum boundary. For each region, the material composition, density (which was calculated), and geometry were specified.

For the fissile region, the material was listed as a uranium/plutonium salt-water mixture (for more information on material composition, see the calculations section below). The density of the region was taken as the density calculated by SCALE 6 (ORNL/TM-2005/38 Version 6, Vol. III) (which includes a module for calculating the density of a solution). The geometry was either a sphere or infinite cylinder centered at the origin with radius rd, or an infinite slab symmetric about $\mathrm{y}=0$ with boundaries of $\mathrm{y}= \pm \mathrm{rd}$. 
For the reflector region, the material composition was listed as pure light water $\left({ }^{1} \mathrm{H}\right.$ and ${ }^{16} \mathrm{O}$ ) and the density was assumed to simply be $1.0 \mathrm{~g} / \mathrm{cc}$. Material fractions were calculated as shown below in the calculations section. The geometry of this second region was a sphere or cylindrical shell concentric with the fissile region with radius between $\mathrm{rd}$ and $\mathrm{rd}+\mathrm{rw}$, or two slabs located between either $\mathrm{y}=\mathrm{rd}$ and $\mathrm{y}=\mathrm{rd}+\mathrm{rw}$, or $\mathrm{y}=-\mathrm{rd}$ and $\mathrm{y}=-\mathrm{rd}-\mathrm{rw}$.

When running a model of a nuclear system in MCNP, the density of the regions described above must be taken into consideration, as well as the atomic weights of the materials in the mixture. The calculations for determining these values can be found in the next section. Also provided in the next section are the MCNP cross-section identifiers used in the calculations.

\subsection{CALCULATiONS}

\subsection{CALCULATION OF MOLARITIES FOR THE FISSILE SOLUTION}

The MCNP models require the density of each material, and the atom fraction (or weight fraction) of each isotope in that material. The parametric analyses discussed in this document start from a specific material (with a fixed molarity of excess acid). A range of arbitrary fissile concentrations is then selected (based on the expected range of the curve and the degree of nonlinearity). The density of the material $\left(\rho_{\text {sol }}\right)$ at each concentration is calculated (see Section 3.3). From this information, it is possible to calculate the molarity of the fissile compound, and of water, in the solution.

$\underline{\text { Calculation of Molarities }(\mathrm{g} / \mathrm{L})}$

Fissile salt:

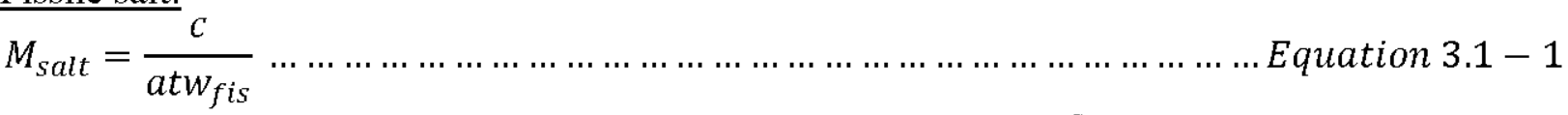

$$
\begin{aligned}
& \text { - } c=\text { Concentration of plutonium or uranium }\left[\frac{g}{L}\right] \\
& \text { - } \text { atw }_{\text {fis }}=\text { Atomic weight of plutonium or uranium }\left[\frac{g}{m o l}\right] \\
& M_{H 2 O}=\frac{\rho_{\text {sol }}-M_{\text {salt }} * a t w_{\text {salt }}-M_{\text {acid }} * \text { atw } \text { acid }}{a t w_{\text {water }}} \ldots \ldots \ldots \ldots \ldots \ldots \ldots \ldots \ldots \text { Equation } 3.1-2
\end{aligned}
$$

Atomic Weights Used in Calculations

Table 1. Atomic Weights of both Elements and Compounds used in the calculations Atomic weight in amu

\begin{tabular}{|c|c|c|c|}
\hline \multicolumn{2}{|c|}{ Elements } & \multicolumn{2}{c|}{ Compounds } \\
\hline & Atomic & & Atomic \\
Element & Weight & Compound & Weight \\
\hline
\end{tabular}


CHPRC-01550, Revision 0

\begin{tabular}{|l|r|c|r|}
\hline Hydrogen & 1.0079 & $\mathrm{H}_{2} \mathrm{O}$ & 18.0152 \\
\hline Oxygen & 15.9994 & $\mathrm{HNO}_{3}$ & 63.0128 \\
\hline Nitrogen & 14.0067 & $\mathrm{NO}_{3}$ & 62.0049 \\
\hline${ }^{239} \mathrm{Pu}$ & 239.0522 & & \\
\hline${ }^{240} \mathrm{Pu}$ & 240.0538 & & \\
\hline${ }^{235} \mathrm{U}$ & 235.0439 & & \\
\hline${ }^{238} \mathrm{U}$ & 238.0508 & & \\
\hline
\end{tabular}

Note that, for the mixtures of different fissile isotopes, the atomic weight was calculated using a weighted average.

\subsection{CALCULATIONS OF ATOM FRACTIONS FOR THE FISSILE SOLUTION}

Once the molarities of the components are known, it is possible to calculate the atom fractions of the various isotopes included in the solution. Note that MCNP normalizes all atom fractions to 100 percent. For simplicity, therefore, the "atom fraction" for MCNP is taken to be the molarity of the isotope in question - MCNP will normalize them relative to the total. Also note that MCNP5 can take weight fraction or atomic weights as the parameter on the material card. Atomic weights were chosen for the nitrates to simplify the calculations. In the below calculations, the fissile salt formula is: $\mathrm{X}\left(\mathrm{NO}_{3}\right)_{y}$.

Atomic Fraction of Fissile Material:

$A F F S=M_{\text {fissile }} *$ ena Equation $3.2-1$

- $A F F S=$ Atomic Fraction of Fissile Isotope

- $M_{\text {fissile }}=$ Molarity of fissile isotope.

- ena $=$ enrichment of the Uranium or Plutonium

Atomic Fraction of Fertile Material: $A F F=M_{\text {fissile }} *(1-$ ena $)$ Equation $3.2-2$

- $A F F=$ Atomic Fraction of Fertile Isotope

Atomic Fraction of Nitrogen in Acid:

$A F N=y * M_{\text {salt }}+M_{\text {acid }}$ Equation $3.2-3$

- $A F N=$ Atomic Fraction of Nitrogen

Atomic Fraction of Oxygen in Acid:

$A F O A=3 y * M_{\text {salt }}+3 * M_{\text {acid }}$ Equation $3.2-4$

- $A F O A=$ Atomic Fraction of Oxygen in Acid

Atomic Fraction of Oxygen in Water:

$A F O=M_{H 2 O}$ Equation $3.2-5$ 
CHPRC-01550, Revision 0

- $A F O=$ Atomic Fraction of Oxygen in Water

Atomic Fraction of Hydrogen:

$A F H=2 * M_{\text {water }}+M_{\text {acid }}$

Equation $3.2-6$

- $A F H=$ Atomic Fraction of Hydrogen

* Note that the molarity of the acid appears due to the free hydrogen ion arising out of the dissociation of the $\mathrm{HNO}_{3}$ in the solution.

\subsection{SOLUTION DENSITY}

In order to accurately model the nitrate solutions, it is necessary to know the density of the solution. Even when the concentration of the fissile material has been arbitrarily set (as is done in this report) the reactivity is heavily affected by the solution density due to the moderation and absorption properties of the water in the solution.

Calculating the density of a nitrate solution (based on only fissile concentration and acid molarity) is a difficult proposition at best. It is further complicated by the fact that, as the solution approaches and exceeds the solubility limit, the material becomes a mixture that may consist of some combination of solution, precipitate, and polymers. Such a mixture can be sufficiently heterogeneous that it cannot be modeled adequately using MCNP.

In the original ARH-600 analyses, the density for each case was determined via an equation (see Section 3.4) that was determined empirically. This method was extended across the entire range of concentrations of interest, in many cases extending far beyond the solubility limit of the fissile component. It is difficult to determine if the data in this range is even approximately accurate, much less bounding.

In recent years there have been analytical solutions presented to solve the density problem. Some of these are discussed in Section M7.A.1 of the SCALE 6 manual (ORNL/TM2005/38 Version 6, Vol. III). One of these, the Pitzer method, has been implemented by the SCALE6 code.

For the purposes of the analyses in this document, the solution density of all cases of interest were determined using the Pitzer method as implemented by the SCALE 6 code. It is important to note that this method does not provide a density value for fissile concentrations beyond the solubility limit. This document, therefore, does not present any data beyond that point (which is different for every curve). In many cases, therefore, the curves presented in this document end at a much lower concentration than the corresponding ARH-600 curves. In some cases (particularly for low enriched uranium) the solubility limit is so low that the resulting curves are of little value. 


\subsection{ARH-600 EQUATIONS}

The ARH-600 document provides the equations for calculating $\mathrm{H} / \mathrm{X}$ and density for both the plutonium and uranium solutions. These equations are empirical in nature and may not account for polymerization or precipitation and, therefore, may not be reliable beyond the saturation point of the solution. For that reason these equations were not used in this analysis but are included for completeness.

$\underline{\text { ARH-600 formula for calculating } \mathrm{H} / \mathrm{X} \text { of a nitrate solution }}$

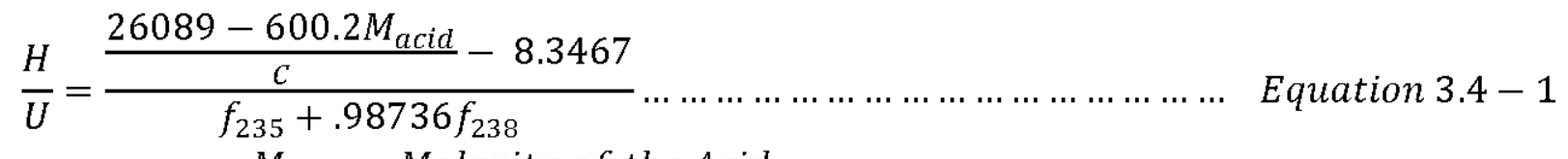

$$
\begin{aligned}
& \text { - } M_{\text {acid }}=\text { Molarity of the Acid, } \\
& \text { - } f_{235}=\text { weight fraction of Uranium 235, and } \\
& \text { - } f_{238}=\text { weight fraction of Uranium } 238 . \\
& \begin{aligned}
& \frac{H}{P u}=\frac{\frac{26535-638.5 M_{\text {acid }}}{c}-9.605}{f_{239}+.99583 f_{240}} \ldots \ldots \ldots \ldots \ldots \ldots \ldots \ldots \ldots \ldots \ldots \ldots \ldots \ldots \ldots \ldots \\
& \\
& \bullet \quad f_{239}=\text { weight fraction of Plutonium } 239, \text { and } \\
& \quad f_{240}=\text { weight fraction of Plutonium } 240 .
\end{aligned}
\end{aligned}
$$

The above equations are taken from the ARH600 handbook in Sections II.D.3-1 and II.C.3-3, respectively.

Once the molarity of the salt (see Section 3.1), the molarity of the acid, and the H/X are known, then the molarity of hydrogen, the molarity of the water, and the density of the solution can be determined as follows:

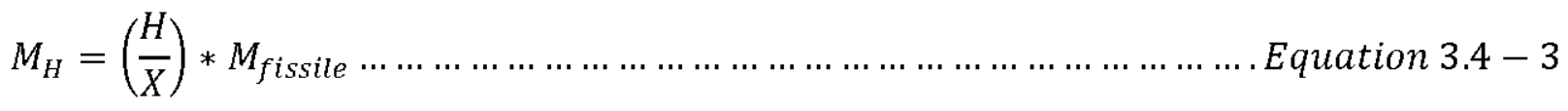

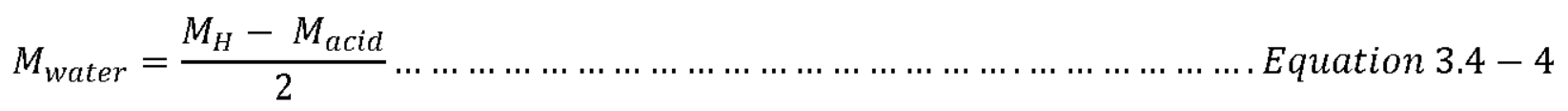

$$
\begin{aligned}
& \rho_{\text {sol }}=M_{\text {salt }} * a t w_{\text {salt }}+M_{\text {water }} * \text { atw } w_{\text {water }}+M_{\text {acid }} * \text { atw } w_{\text {acid }} \ldots \ldots \ldots \ldots \text { Equation } 3.4-5
\end{aligned}
$$

\subsection{LIST OF MCNP5 CROSS-SECTION LIBRARIES USED IN MODEL}

- $1001.66 \mathrm{c}-$ The cross section from the endf66 library modeling ${ }^{1} \mathrm{H}$.

- $7014.66 \mathrm{c}$ - The cross section from the endf66 library modeling ${ }^{14} \mathrm{~N}$.

- $8016.66 \mathrm{c}$ - The cross section from the endf66 library modeling ${ }^{16} \mathrm{O}$. 
- $92235.66 \mathrm{c}$ - The cross section from the endf66 library modeling ${ }^{235} \mathrm{U}$.

- $92238.66 \mathrm{c}$ - The cross section from the endf66 library modeling ${ }^{238} \mathrm{U}$.

- $94239.66 \mathrm{c}$ - The cross section from the endf66 library modeling ${ }^{239} \mathrm{Pu}$.

- $94240.66 \mathrm{c}$ - The cross section from the endf66 library modeling ${ }^{240} \mathrm{Pu}$.

- Lwtr.60t- $\mathrm{S}(\alpha, \beta)$ cross section from the endf6.3 library modeling $\mathrm{H}_{2} \mathrm{O}$.

\subsection{AUTOMATION ROUTINE}

The various systems modeled for this handbook were categorized as a set of specific values for five parameters: fissile material, excess acid molarity, geometry, enrichment, and reflector thickness. For each such set, a range of concentrations were considered, and for each concentration, a range of diameters (or thicknesses in the case of slabs) was analyzed with MCNP. From the resulting library of data, the diameter for which $k_{\text {eff }}$ equals one for a given concentration was calculated using a linear interpolation for each data set. Many thousands of individual cases were required to produce these results for the ARH-600 conditions analyzed. In order to create, run, and evaluate all of these cases in an efficient manner, an automation routine was developed. The automation routine consists of batch files written in disk operating system (DOS) script language, executable files written in Fortran, and a routine written with Perl called "worm" (worm was written and distributed by Tom Jones). The batch files were named buildwormset-nitrate, run-set, and extract-all. Buildwormset-nitrate was used to create the various input files for each molarity, isotopic composition, and reflector width. Run-set was used to submit these sets to a queue, the queue being a list of files for which the computers assigned to the calculations would run. All MCNP calculations were conducted on computers for which MCNP verification test cases have been successfully run. Once an MCNP run was completed, local queuing software pulled the name of the next file out of the queue to run. Finally, once all of the sets were run, the extract-all batch file took the large amount of data output and consolidated it into a critview output file.

\subsection{DESCRIPTION OF THE DATA}

The data contained in this manual are arranged such that for each parameter set, there is a graph of the critical diameter vs. concentration in Appendix B. This same data is also presented as a table (also in Appendix B), and as a text listing, in Appendix C, that is in a format compatible with the CritView (version 1.02) electronic handbook code. Appendix D presents the template files that were used by the worm script to produce the MCNP input files for that set. A sample input file for each case, produced by the worm script, is shown in Appendix E. Appendix $\mathrm{F}$ lists all of the raw data results (i.e., the dimensions for cases where $\mathrm{k}$ ranged from approximately .93 to 1.01$)$.

The graphs of the data in Appendix B are provided for visual reference and comparison to the ARH-600 data (note that a few of the cases analyzed in this report do not have corresponding ARH-600 graphs). In this analysis "full water reflection" (as indicated by ARH600 ) was equated to 10 inches of water reflection (for the MCNP data). The precise thickness used in the original ARH-600 calculations is not known, however, 10 inches was demonstrated 
as a conservative value to use in these calculations. The graphs are titled with the molarity, fissile isotope percent, and reflector width. If there is no fissile isotope percent, it shall be taken as 100 percent.

The tables in Appendix B have six columns which are intended to give a summary of all of the cases run for the specific parameter set. The first column contains the concentrations evaluated for the given set. The second column contains the density of the salt water solution (as calculated using above formulas or Scale 6) in $\mathrm{g} / \mathrm{cc}$. The third and fourth columns contain the first and last diameter, in inches, analyzed for each concentration. The fifth column consists of the step size, in inches, between each analyzed diameter. The sixth and arguably most important column, contains the interpolated diameter for which keff $=1$. It should be noted that at times there is only "--" shown. This signifies that the data for $\mathrm{K}_{\text {eff }}$ was not calculated either because

$\mathrm{k}_{\mathrm{eff}}=1$ was impossible or was only possible with a diameter greater than the maximum evaluated (typically 999.99 inches).

\subsection{CONCLUSIONS}

This report documents a series of parametric studies that reproduce much of the plutonium and uranium nitrate data presented in ARH-600. This peer reviewed data has been presented in a series of curves that are equivalent to those in ARH-600. The data has also been formatted into an electronic file that can be read by the CritView electronic database file, allowing the data to be presented by that software. 
CHPRC-01550, Revision 0

This page is intentionally left blank. 
CHPRC-01552, Revision 0

APPENDIX A - INDEPENDENT REVIEW COMMENTS AND CHECKLIST 
CHPRC-01552, Revision 0

This page is intentionally left blank. 
CHPRC-01552, Revision 0

\title{
CHPRC REVIEW CHECKLIST
}

\author{
Document Reviewed: \\ CHPRC-01552, Revision 0, Appendix B - Calculations and Data
}

cope of Review

Main text and Appendix B Note: The use of this data in Critview was not reviewed.

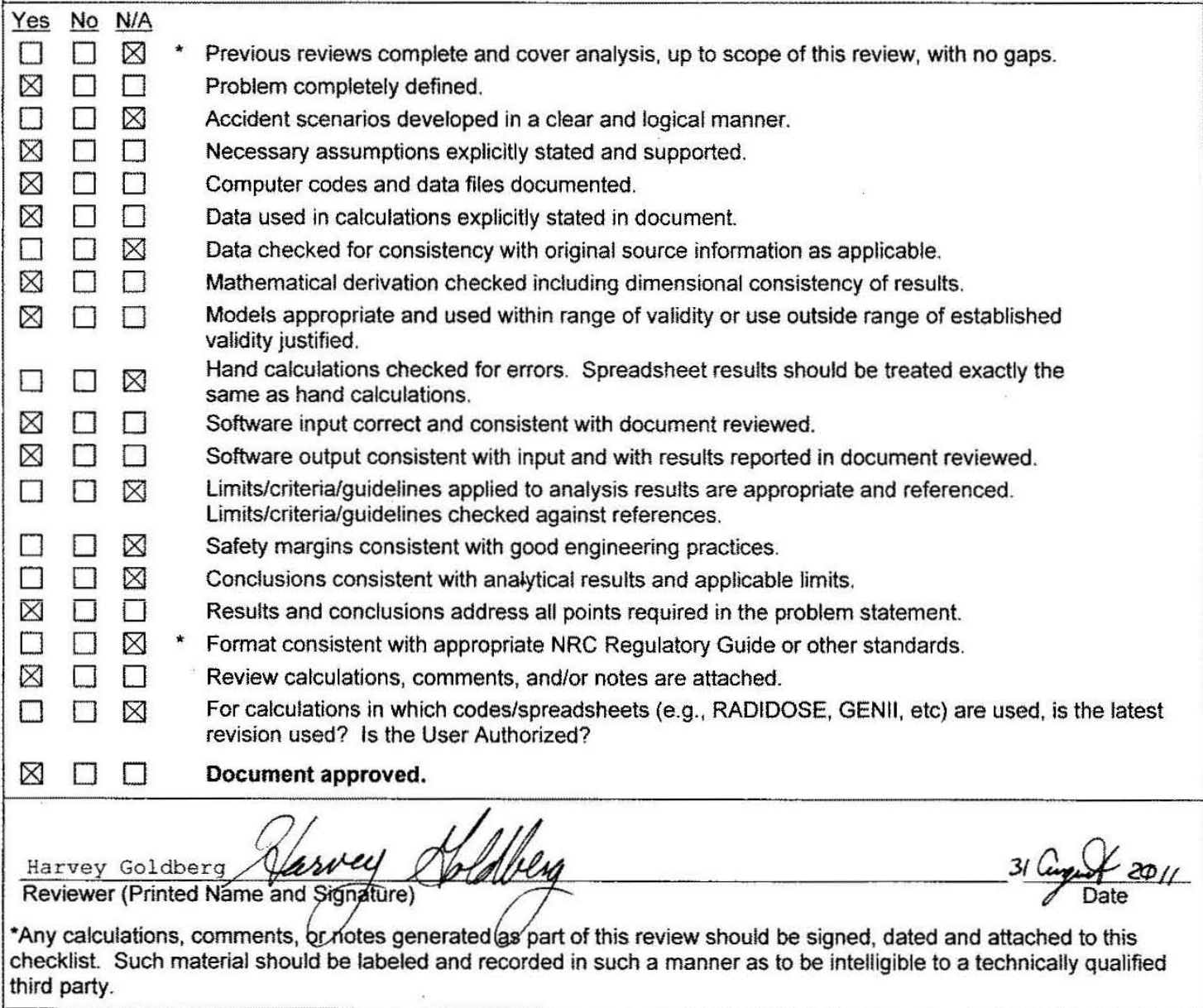




\section{CHPRC-01552, Revision 0}

\section{Comments:}

The MCNP5 input and output decks were checked. For each graph random output data were checked against the graphs. The ARH-600 curves on the graphs were spot checked for general shape, but not for numerical values. CritView was not run so, although the graphs are CriView products, this reviewer did not produce test graphs from the output data to check if the results were identical to those published.
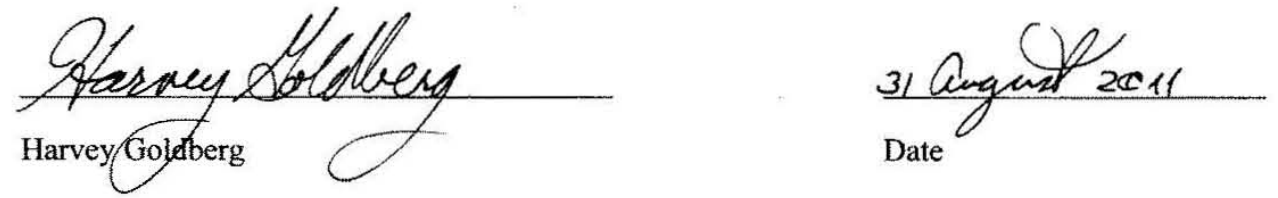
CHPRC-01552, Revision 0

APPENDIX B - CALCULATIONS AND DATA

B-i 
CHPRC-01552, Revision 0

This page is intentionally left blank.

B-ii 


\section{TABLE OF CONTENTS}

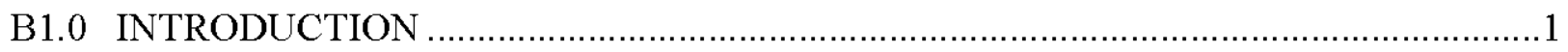

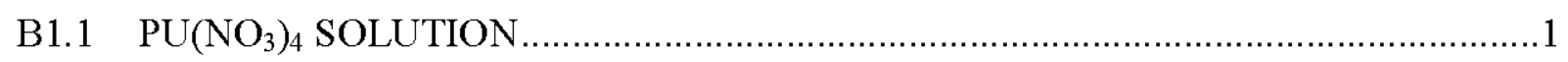

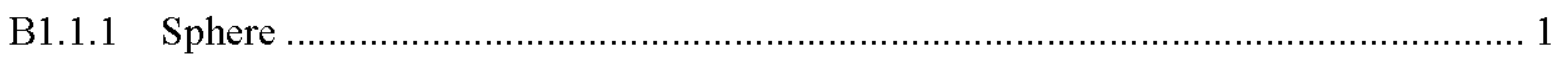

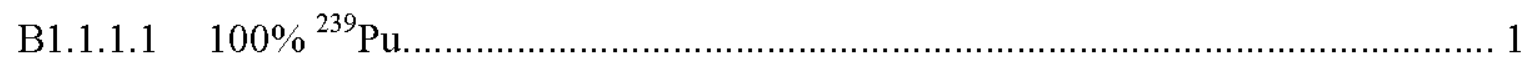

B1.1.1.1.1 0 Molar Excess Acid ............................................................................... 1

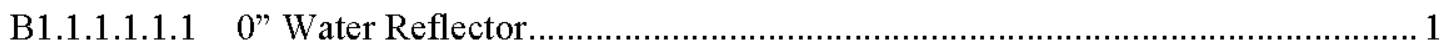

B1.1.1.1.1.2 1" Water Reflector................................................................................ 4

B1.1.1.1.1.3 10" Water Reflector............................................................................ 7

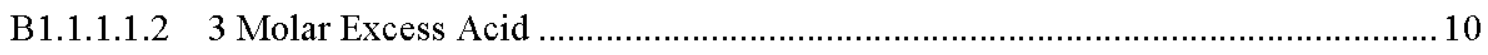

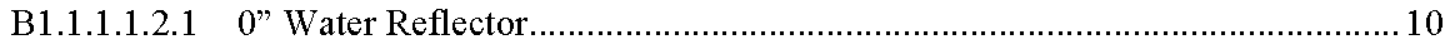

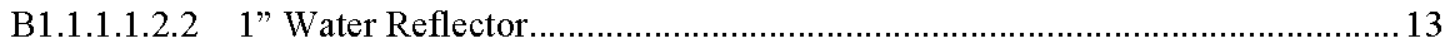

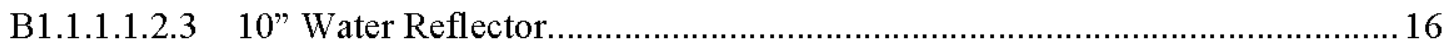

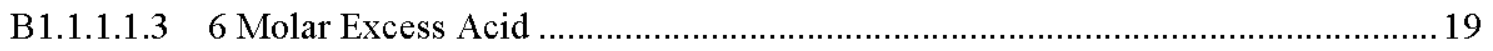

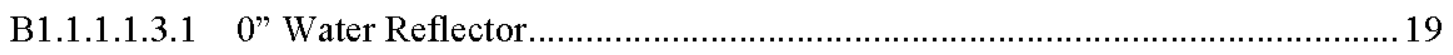

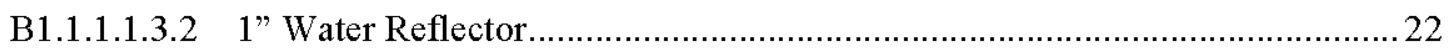

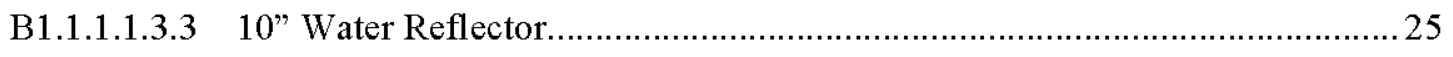

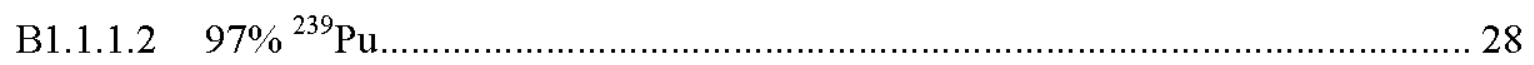

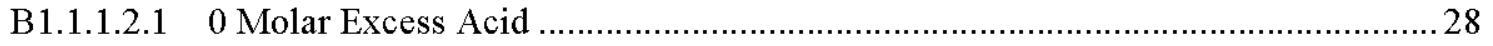

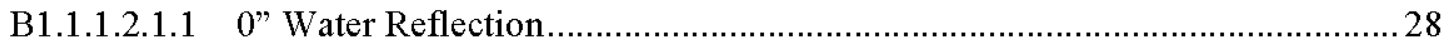

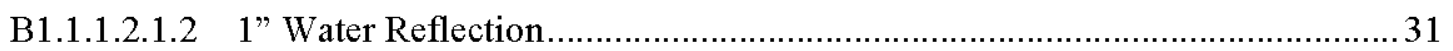

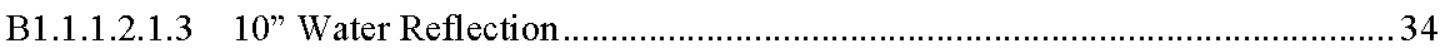

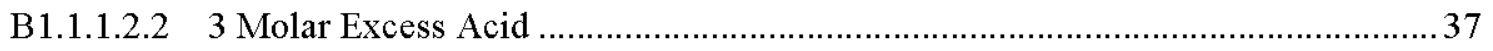

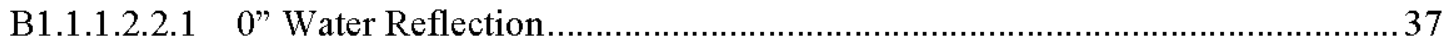

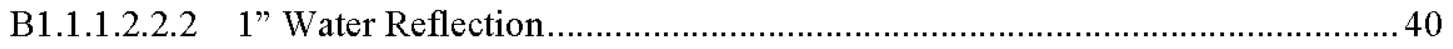

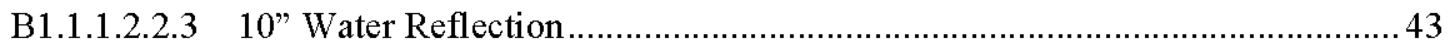

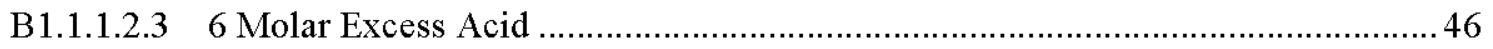

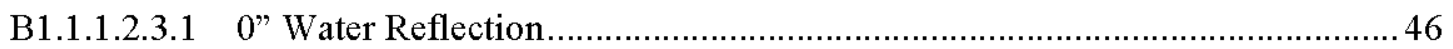

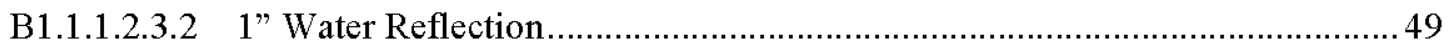

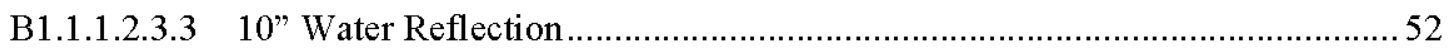

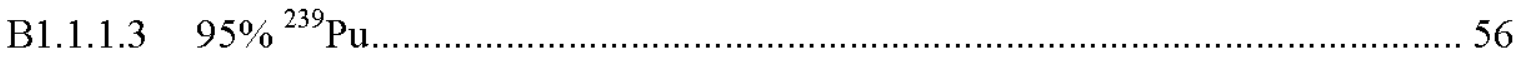

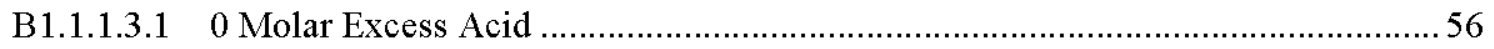




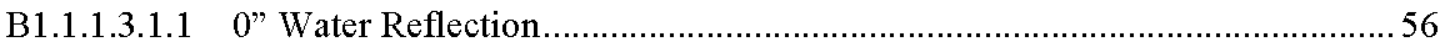

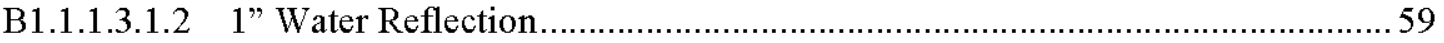

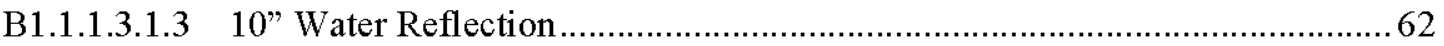

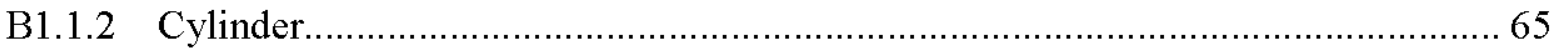

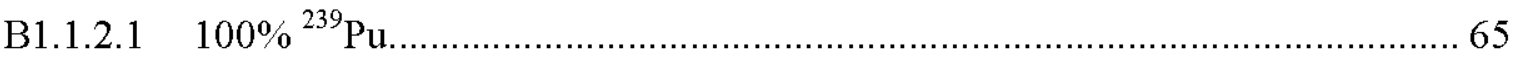

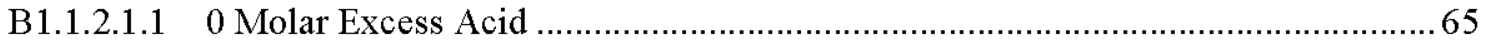

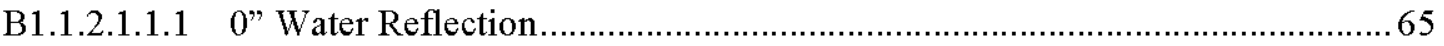

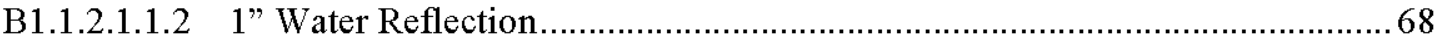

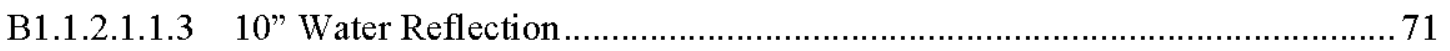

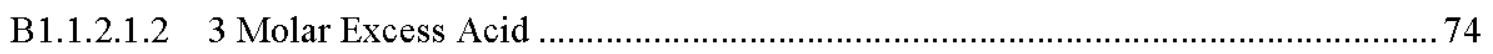

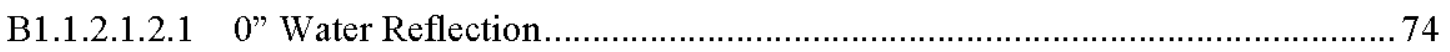

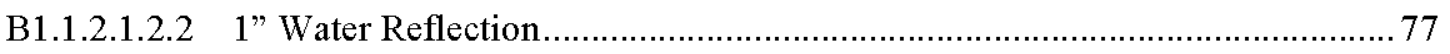

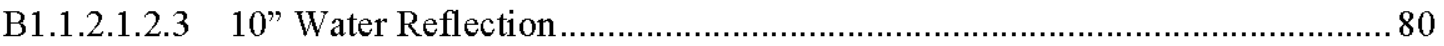

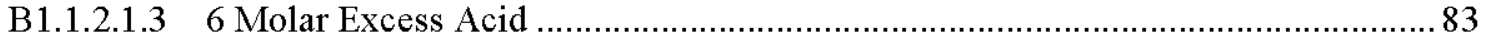

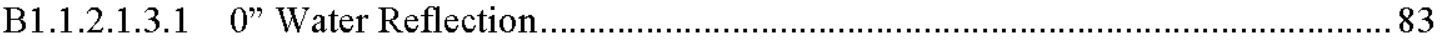

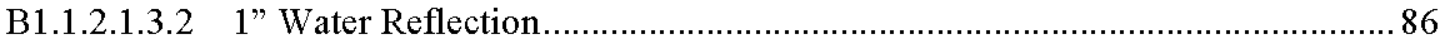

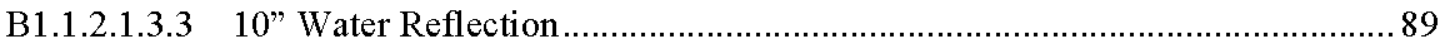

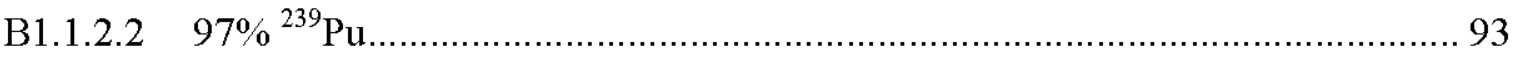

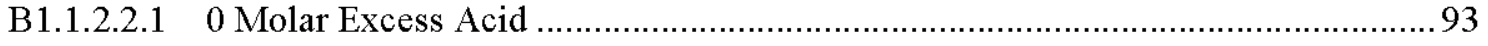

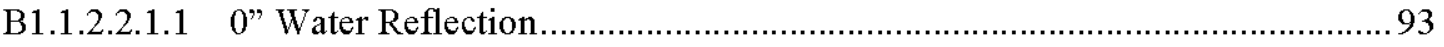

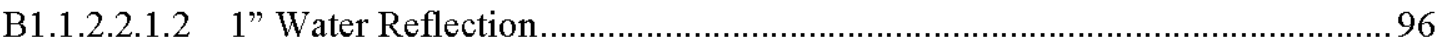

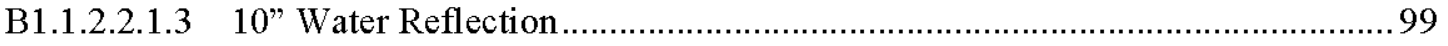

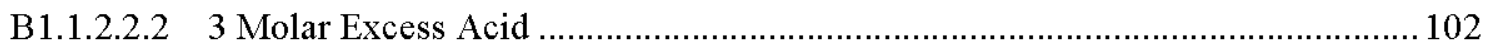

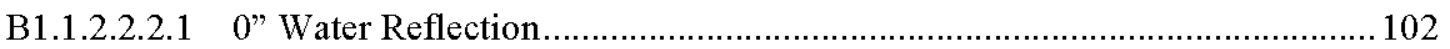

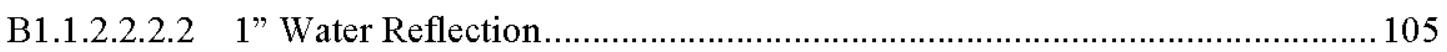

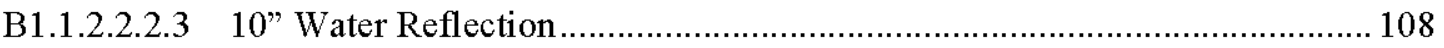

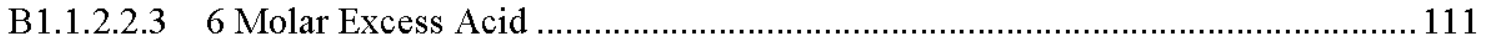

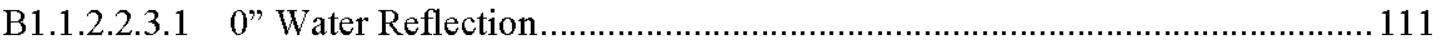

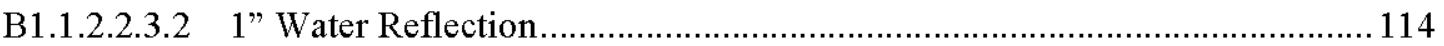

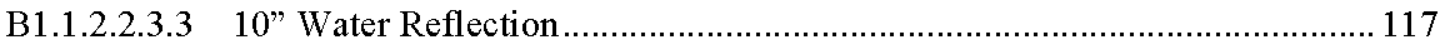

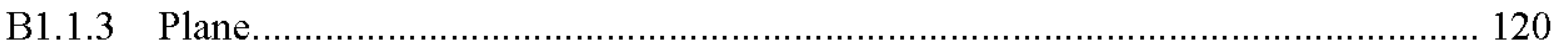

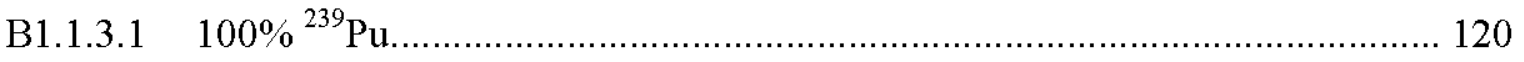

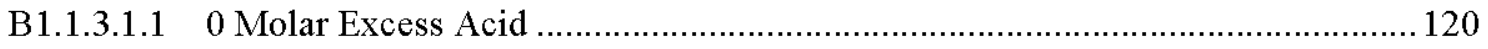




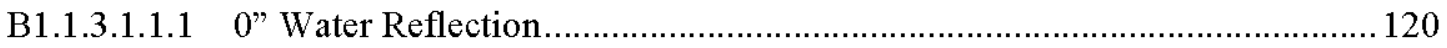

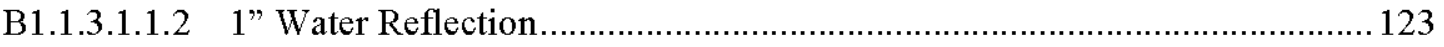

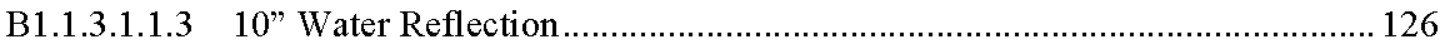

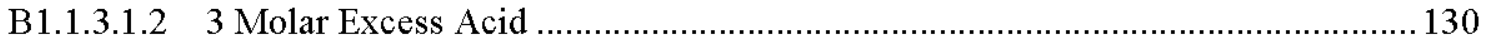

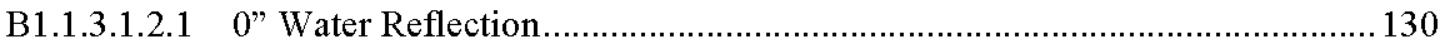

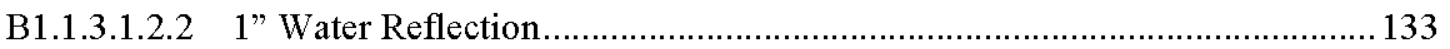

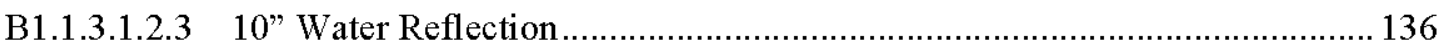

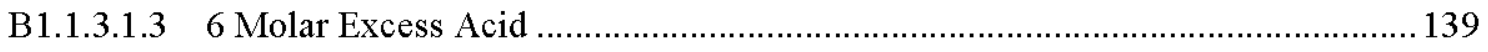

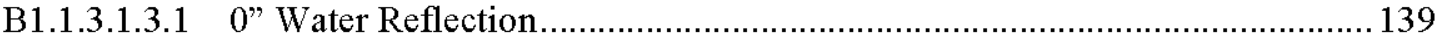

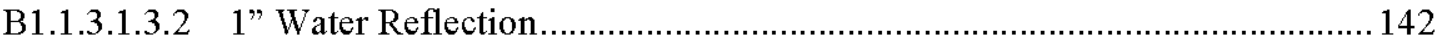

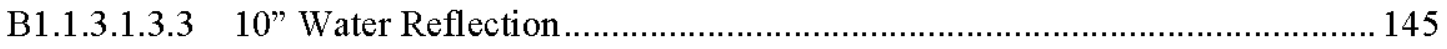

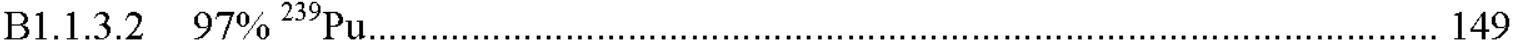

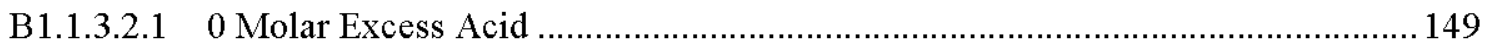

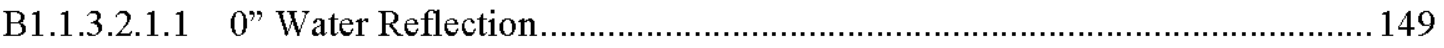

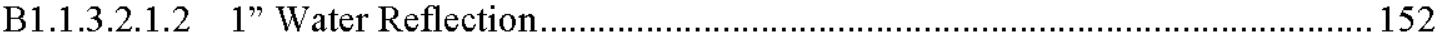

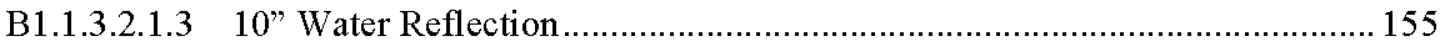

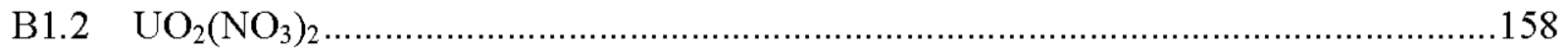

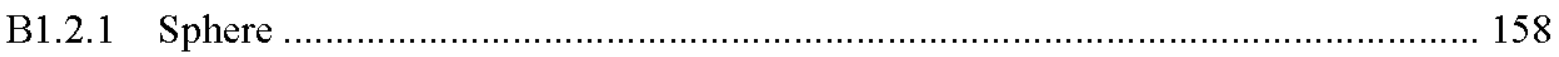

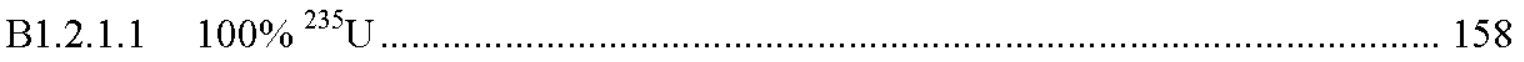

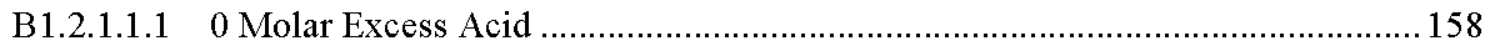

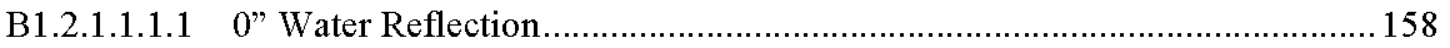

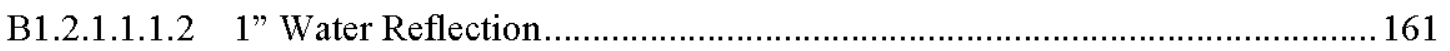

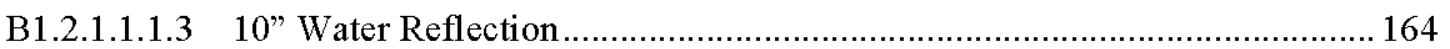

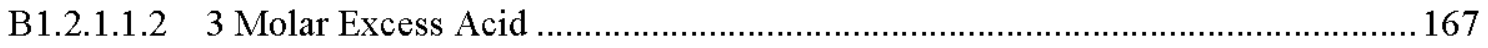

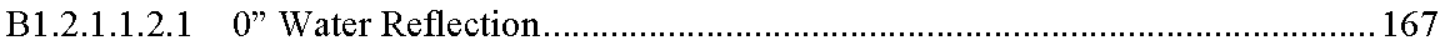

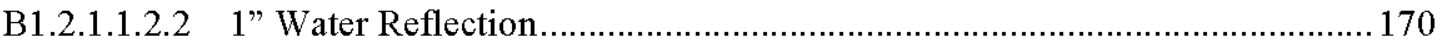

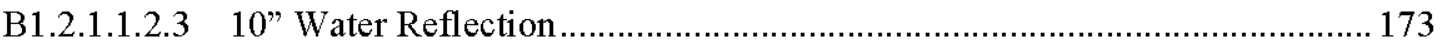

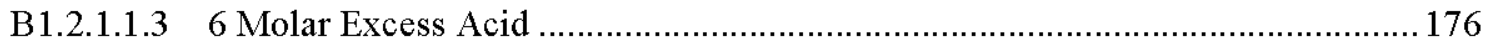

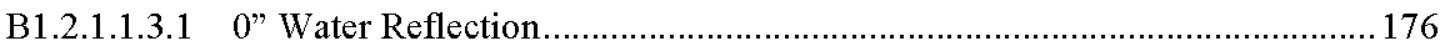

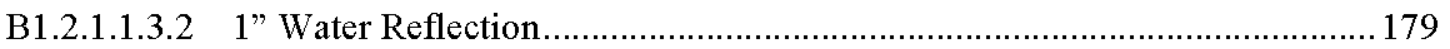

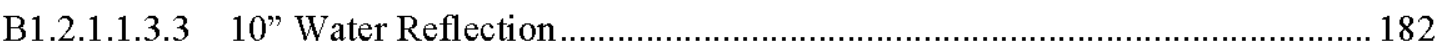

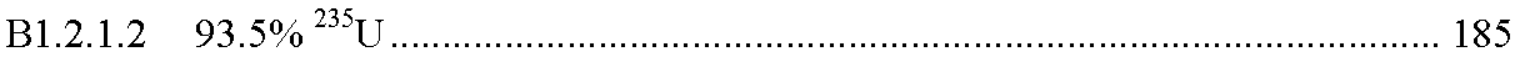

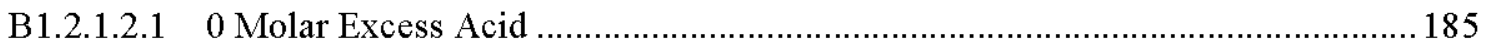




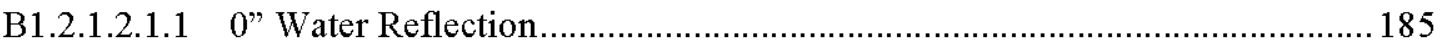

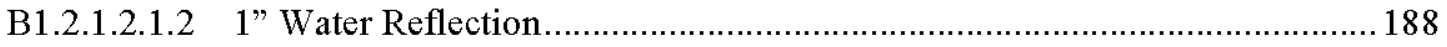

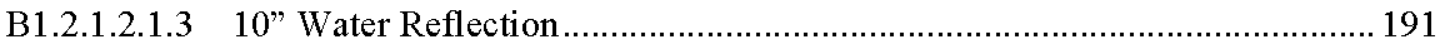

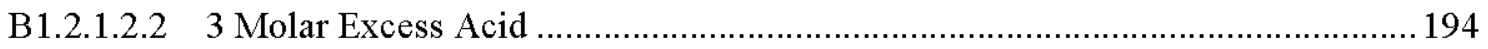

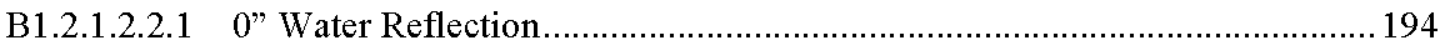

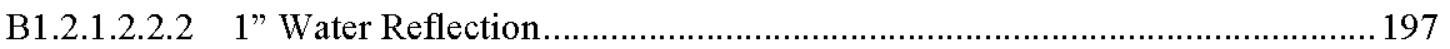

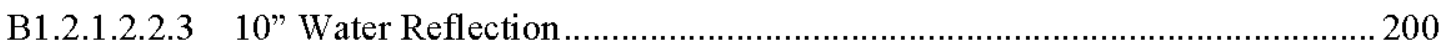

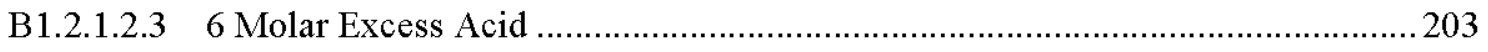

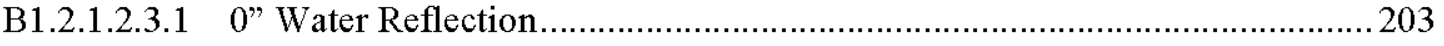

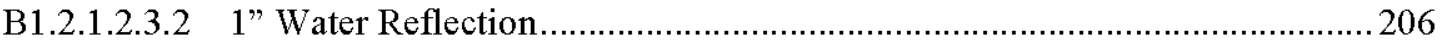

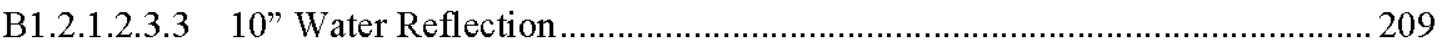

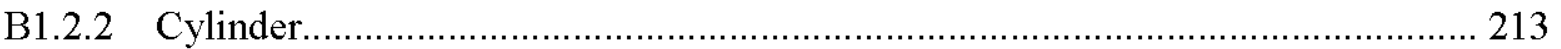

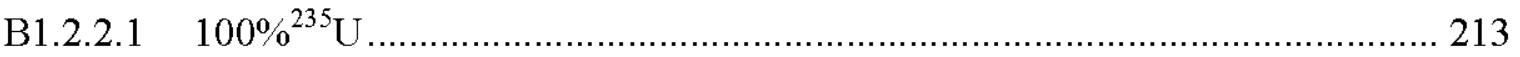

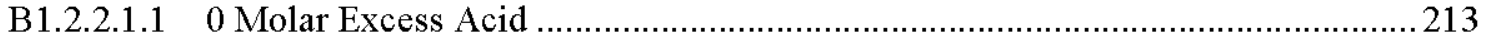

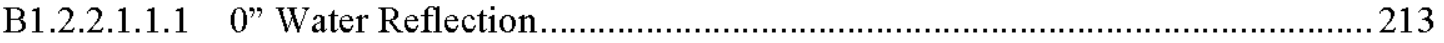

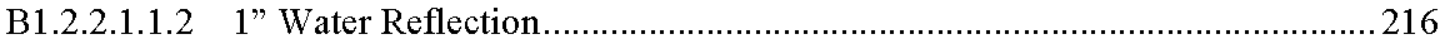

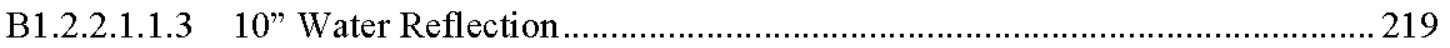

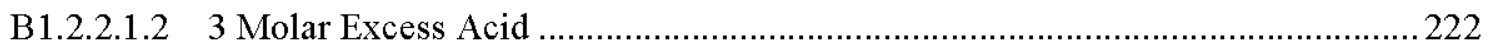

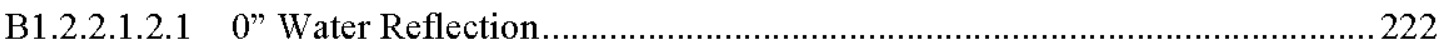

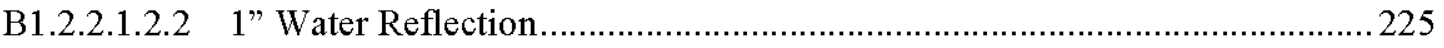

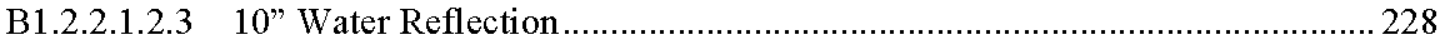

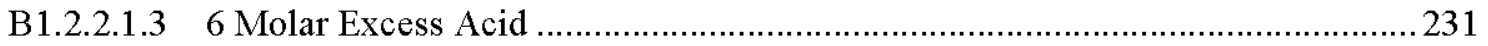

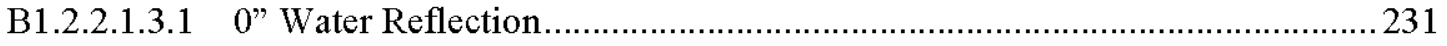

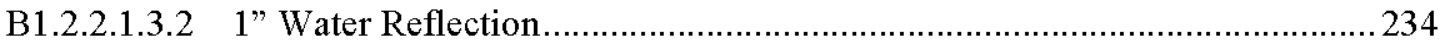

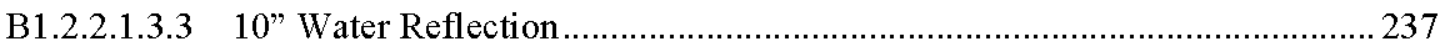

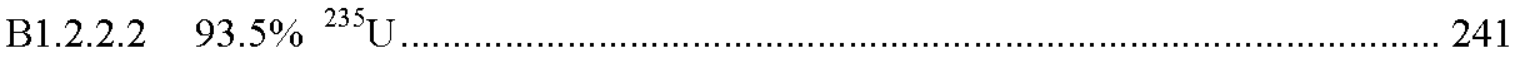

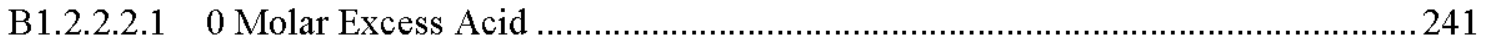

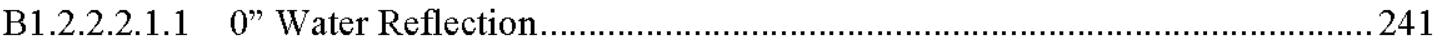

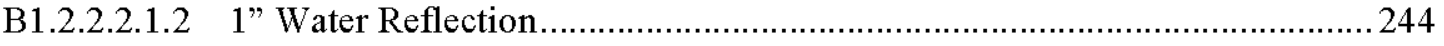

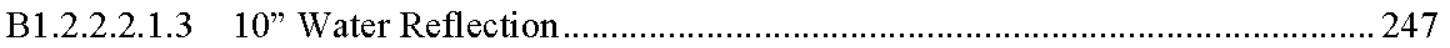

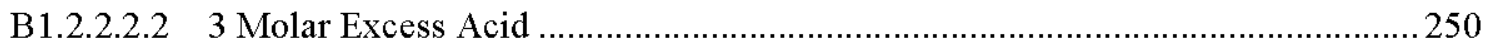

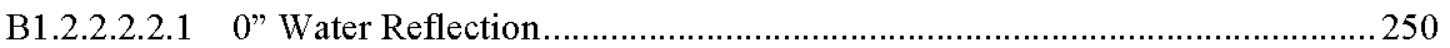

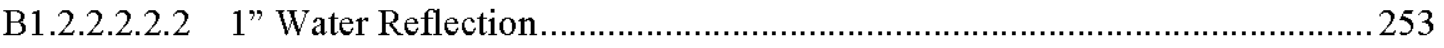




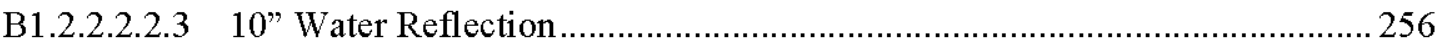

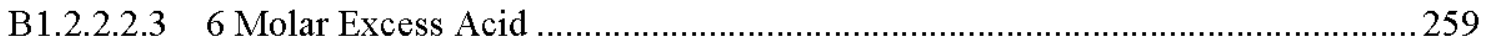

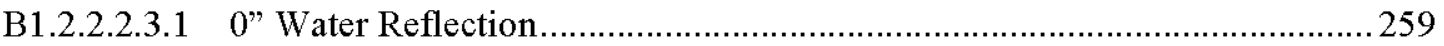

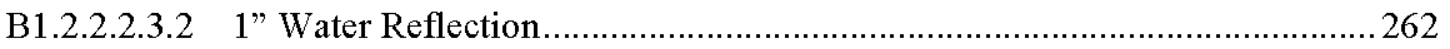

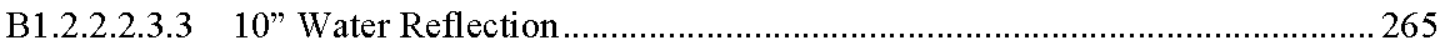

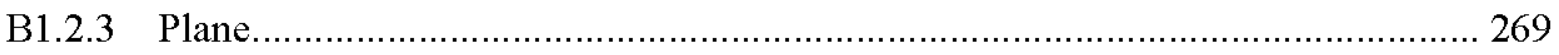

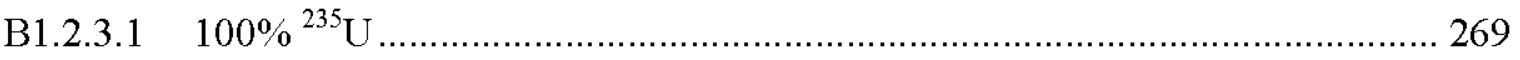

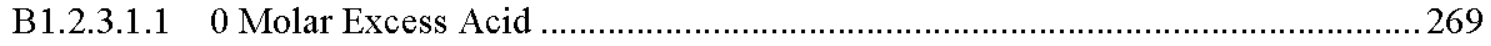

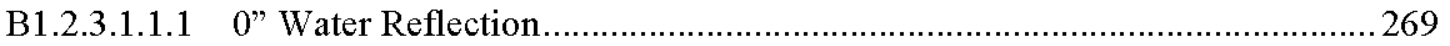

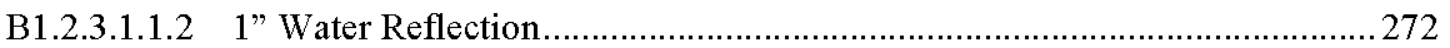

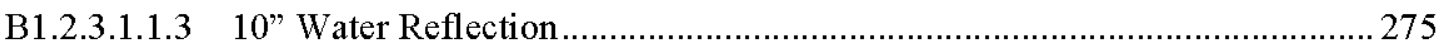

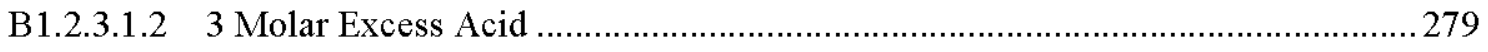

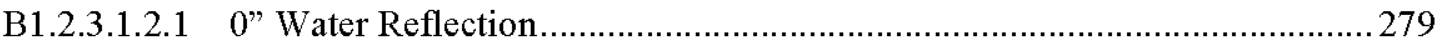

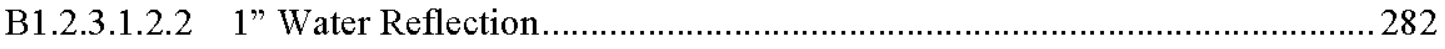

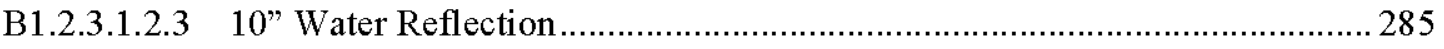

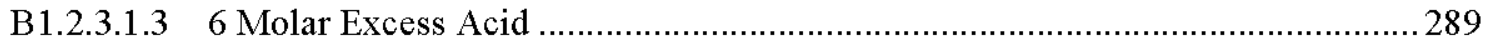

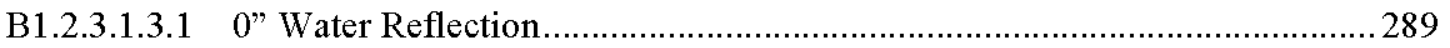

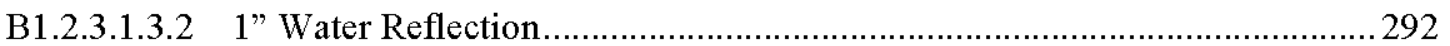

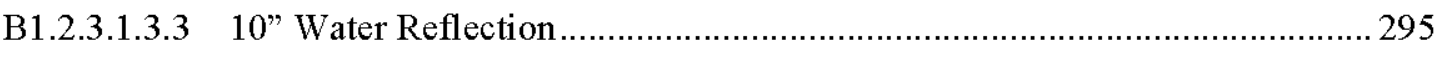

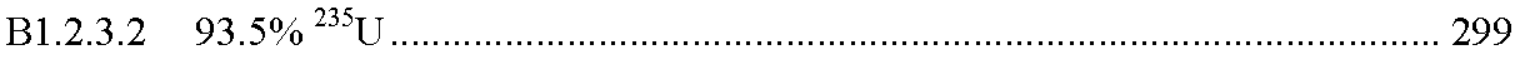

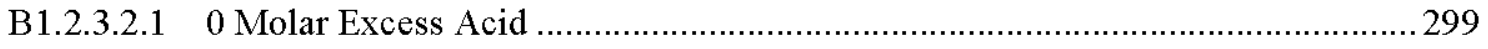

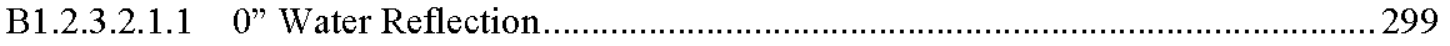

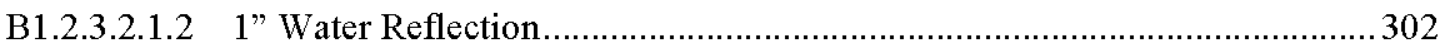

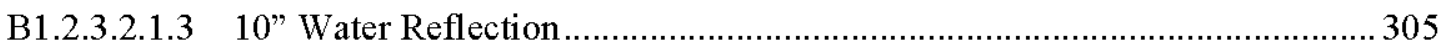




\section{LIST OF FIGURES}

Figure B1.1.1.1.1.1-1. $\mathrm{Pu}\left(\mathrm{NO}_{3}\right) 40 \mathrm{M}$ - Sphere - 100\% ${ }^{239} \mathrm{Pu}-0$ " Water Reflection..................2

Figure B1.1.1.1.1.2-1. Pu(NO 3$) 40 \mathrm{M}-$ Sphere $-100 \%{ }^{239} \mathrm{Pu}$ - 1" Water Reflection...................5

Figure B1.1.1.1.1.3-1. $\mathrm{Pu}\left(\mathrm{NO}_{3}\right) 40 \mathrm{M}-$ Sphere $-100 \%{ }^{239} \mathrm{Pu}-10$ " Water Reflection...............8

Figure B1.1.1.1.2.1-1. $\mathrm{Pu}\left(\mathrm{NO}_{3}\right) 43 \mathrm{M}-$ Sphere $-100 \%{ }^{239} \mathrm{Pu}-0$ " Water Reflection ................11

Figure B1.1.1.1.2.2-1. $\mathrm{Pu}\left(\mathrm{NO}_{3}\right) 43 \mathrm{M}-$ Sphere - $100 \%{ }^{239} \mathrm{Pu}-1$ " Water Reflection................. 14

Figure B1.1.1.1.2.3-1. $\mathrm{Pu}\left(\mathrm{NO}_{3}\right) 43 \mathrm{M}$ - Sphere - $100 \%{ }^{239} \mathrm{Pu}-10$ " Water Reflection..............17

Figure B1.1.1.1.3.1-1. $\mathrm{Pu}\left(\mathrm{NO}_{3}\right) 46 \mathrm{M}-$ Sphere - $100 \%{ }^{239} \mathrm{Pu}-0$ " Water Reflection................20

Figure B1.1.1.1.3.2-1. $\mathrm{Pu}\left(\mathrm{NO}_{3}\right) 46 \mathrm{M}-$ Sphere - $100 \%{ }^{239} \mathrm{Pu}-1$ " Water Reflection................23

Figure B1.1.1.1.3.3-1. $\mathrm{Pu}\left(\mathrm{NO}_{3}\right) 46 \mathrm{M}$ - Sphere - $100 \%{ }^{239} \mathrm{Pu}-10$ " Water Reflection..............26

Figure B1.1.1.2.1.1-1. $\mathrm{Pu}\left(\mathrm{NO}_{3}\right) 40 \mathrm{M}-$ Sphere - 97\% ${ }^{239} \mathrm{Pu}-0$ " Water Reflection...................29

Figure B1.1.1.2.1.2-1. $\mathrm{Pu}\left(\mathrm{NO}_{3}\right) 40 \mathrm{M}-$ Sphere - 97\% ${ }^{239} \mathrm{Pu}-1$ " Water Reflection....................32

Figure B1.1.1.2.1.3-1. $\mathrm{Pu}\left(\mathrm{NO}_{3}\right) 40 \mathrm{M}$ - Sphere - 97\% ${ }^{239} \mathrm{Pu}-10$ " Water Reflection.................35

Figure B1.1.1.2.2.1-1. $\mathrm{Pu}\left(\mathrm{NO}_{3}\right) 43 \mathrm{M}-$ Sphere $-97 \%{ }^{239} \mathrm{Pu}-0$ " Water Reflection ..................38

Figure B1.1.1.2.2.2-1. $\mathrm{Pu}\left(\mathrm{NO}_{3}\right) 43 \mathrm{M}-$ Sphere $-97 \%{ }^{239} \mathrm{Pu}-1$ " Water Reflection .................41

Figure B1.1.1.2.2.3-1. $\mathrm{Pu}\left(\mathrm{NO}_{3}\right) 43 \mathrm{M}-$ Sphere $-97 \%{ }^{239} \mathrm{Pu}-10$ " Water Reflection ...............44

Figure B1.1.1.2.3.1-1. $\mathrm{Pu}\left(\mathrm{NO}_{3}\right) 46 \mathrm{M}-$ Sphere $-97 \%{ }^{239} \mathrm{Pu}-0$ " Water Reflection ................47

Figure B1.1.1.2.3.2-1. $\mathrm{Pu}\left(\mathrm{NO}_{3}\right) 46 \mathrm{M}-$ Sphere $-97 \%{ }^{239} \mathrm{Pu}-1$ " Water Reflection .................50

Figure B1.1.1.2.3.3-1. $\mathrm{Pu}\left(\mathrm{NO}_{3}\right) 46 \mathrm{M}-$ Sphere $-97 \%{ }^{239} \mathrm{Pu}-10$ " Water Reflection ..............53

Figure B1.1.1.3.1.1-1. $\mathrm{Pu}\left(\mathrm{NO}_{3}\right) 40 \mathrm{M}-$ Sphere - 95\% ${ }^{239} \mathrm{Pu}-0$ " Water Reflection..................57

Figure B1.1.1.3.1.2-1. $\mathrm{Pu}\left(\mathrm{NO}_{3}\right) 40 \mathrm{M}-$ Sphere - 95\% ${ }^{239} \mathrm{Pu}-1$ " Water Reflection..................60

Figure B1.1.1.3.1.3-1. $\mathrm{Pu}\left(\mathrm{NO}_{3}\right) 40 \mathrm{M}-$ Sphere - 95\% ${ }^{239} \mathrm{Pu}-10$ " Water Reflection................63

Figure B1.1.2.1.1.1-1. $\mathrm{Pu}\left(\mathrm{NO}_{3}\right) 40 \mathrm{M}-$ Cylinder - $100 \%{ }^{239} \mathrm{Pu}-0$ " Water Reflection .............66

Figure B1.1.2.1.1.2-1. $\mathrm{Pu}\left(\mathrm{NO}_{3}\right) 40 \mathrm{M}-$ Cylinder - $100 \%{ }^{239} \mathrm{Pu}-1$ " Water Reflection .............69

Figure B1.1.2.1.1.3-1. $\mathrm{Pu}\left(\mathrm{NO}_{3}\right) 40 \mathrm{M}-$ Cylinder - $100 \%{ }^{239} \mathrm{Pu}-10$ " Water Reflection............72

Figure B1.1.2.1.2.1-1. $\mathrm{Pu}\left(\mathrm{NO}_{3}\right) 43 \mathrm{M}-$ Cylinder - $100 \%{ }^{239} \mathrm{Pu}-0$ " Water Reflection.............75

Figure B1.1.2.1.2.2-1. $\mathrm{Pu}\left(\mathrm{NO}_{3}\right) 43 \mathrm{M}-$ Cylinder - $100 \%{ }^{239} \mathrm{Pu}-1$ " Water Reflection..............78

Figure B1.1.2.1.2.3-1. $\mathrm{Pu}\left(\mathrm{NO}_{3}\right) 43 \mathrm{M}$ - Cylinder - $100 \%{ }^{239} \mathrm{Pu}-10$ " Water Reflection............81

Figure B1.1.2.1.3.1-1. $\mathrm{Pu}\left(\mathrm{NO}_{3}\right) 46 \mathrm{M}$ - Cylinder - $100 \%{ }^{239} \mathrm{Pu}-0$ " Water Reflection .............84

Figure B1.1.2.1.3.2-1. $\mathrm{Pu}\left(\mathrm{NO}_{3}\right) 46 \mathrm{M}$ - Cylinder - $100 \%{ }^{239} \mathrm{Pu}-1$ " Water Reflection.............87

Figure B1.1.2.1.3.3-1. $\mathrm{Pu}\left(\mathrm{NO}_{3}\right) 46 \mathrm{M}$ - Cylinder - $100 \%{ }^{239} \mathrm{Pu}-10$ " Water Reflection ...........90

Figure B1.1.2.2.1.1-1. $\mathrm{Pu}\left(\mathrm{NO}_{3}\right) 40 \mathrm{M}-$ Cylinder - 97\% ${ }^{239} \mathrm{Pu}-0$ " Water Reflection...............94

Figure B1.1.2.2.1.1-1. $\mathrm{Pu}\left(\mathrm{NO}_{3}\right) 40 \mathrm{M}-$ Cylinder - 97\% ${ }^{239} \mathrm{Pu}-1$ " Water Reflection ...............97

Figure B1.1.2.2.1.3-1. $\mathrm{Pu}\left(\mathrm{NO}_{3}\right) 40 \mathrm{M}-$ Cylinder $-97 \%{ }^{239} \mathrm{Pu}-10$ " Water Reflection ............100 
Figure B1.1.2.2.2.1-1. $\mathrm{Pu}\left(\mathrm{NO}_{3}\right) 43 \mathrm{M}$ - Cylinder - 97\% ${ }^{239} \mathrm{Pu}-0$ " Water Reflection.............103 Figure B1.1.2.2.2.2-1. $\mathrm{Pu}\left(\mathrm{NO}_{3}\right) 43 \mathrm{M}$ - Cylinder - 97\% ${ }^{239} \mathrm{Pu}-1$ " Water Reflection ..............106 Figure B1.1.2.2.2.3-1. $\mathrm{Pu}\left(\mathrm{NO}_{3}\right) 43 \mathrm{M}$ - Cylinder - 97\% ${ }^{239} \mathrm{Pu}-10$ " Water Reflection............109 Figure B1.1.2.2.3.1-1. $\mathrm{Pu}\left(\mathrm{NO}_{3}\right) 46 \mathrm{M}$ - Cylinder - 97\% ${ }^{239} \mathrm{Pu}-0$ " Water Reflection ..............112 Figure B1.1.2.2.3.2-1. $\mathrm{Pu}\left(\mathrm{NO}_{3}\right) 46 \mathrm{M}$ - Cylinder - 97\% ${ }^{239} \mathrm{Pu}-1$ " Water Reflection ..............115 Figure B1.1.2.2.3.3-1. $\mathrm{Pu}\left(\mathrm{NO}_{3}\right) 46 \mathrm{M}$ - Cylinder - 97\% ${ }^{239} \mathrm{Pu}-10$ " Water Reflection............118 Figure B1.1.3.1.1.1-1. $\mathrm{Pu}\left(\mathrm{NO}_{3}\right) 40 \mathrm{M}$ - Plane - $100 \%{ }^{239} \mathrm{Pu}-0$ " Water Reflection................121 Figure B1.1.3.1.1.2-1. $\mathrm{Pu}\left(\mathrm{NO}_{3}\right) 40 \mathrm{M}$ - Plane - $100 \%{ }^{239} \mathrm{Pu}-1$ "Water Reflection.................124 Figure B1.1.3.1.1.3-1. $\mathrm{Pu}\left(\mathrm{NO}_{3}\right) 40 \mathrm{M}$ - Plane - $100 \%{ }^{239} \mathrm{Pu}-10$ " Water Reflection ...............127 Figure B1.1.3.1.2.1-1. $\mathrm{Pu}\left(\mathrm{NO}_{3}\right) 43 \mathrm{M}-\mathrm{Plane}-100 \%{ }^{239} \mathrm{Pu}-0$ " Water Reflection.................131 Figure B1.1.3.1.2.2-1. $\mathrm{Pu}\left(\mathrm{NO}_{3}\right) 43 \mathrm{M}-$ Plane - $100 \%{ }^{239} \mathrm{Pu}-1$ " Water Reflection ..................134 Figure B1.1.3.1.2.3-1. $\mathrm{Pu}\left(\mathrm{NO}_{3}\right) 43 \mathrm{M}-$ Plane - $100 \%{ }^{239} \mathrm{Pu}-10 \%$ Water Reflection ...............137 Figure B1.1.3.1.3.1-1. $\mathrm{Pu}\left(\mathrm{NO}_{3}\right) 46 \mathrm{M}$ - Plane - $100 \%{ }^{239} \mathrm{Pu}-0$ " Water Reflection.................140 Figure B1.1.3.1.3.2-1. $\mathrm{Pu}\left(\mathrm{NO}_{3}\right) 46 \mathrm{M}$ - Plane - $100 \%{ }^{239} \mathrm{Pu}-1$ " Water Reflection ..................143 Figure B1.1.3.1.3.3-1. $\mathrm{Pu}\left(\mathrm{NO}_{3}\right) 46 \mathrm{M}$ - Plane - $100 \%{ }^{239} \mathrm{Pu}-10 \%$ Water Reflection ...............146 Figure B1.1.3.2.1.1-1. $\mathrm{Pu}\left(\mathrm{NO}_{3}\right) 40 \mathrm{M}-\mathrm{Plane}-97 \%{ }^{239} \mathrm{Pu}-0$ " Water Reflection ..................150 Figure B1.1.3.2.1.2-1. $\mathrm{Pu}\left(\mathrm{NO}_{3}\right) 40 \mathrm{M}-\mathrm{Plane}-97 \%{ }^{239} \mathrm{Pu}-1$ "Water Reflection ..................153 Figure B1.1.3.2.1.3-1. $\mathrm{Pu}\left(\mathrm{NO}_{3}\right) 40 \mathrm{M}-\mathrm{Plane}-97 \%{ }^{239} \mathrm{Pu}-10 \%$ Water Reflection ...............156 Figure B1.2.1.1.1.1-1. $\mathrm{UO}_{2}\left(\mathrm{NO}_{3}\right) 20 \mathrm{M}$ - Sphere - 100\% ${ }^{235} \mathrm{U}-0$ " Water Reflection .............159 Figure B1.2.1.1.1.2-1. $\mathrm{UO}_{2}\left(\mathrm{NO}_{3}\right) 20 \mathrm{M}$ - Sphere - $100 \%{ }^{235} \mathrm{U}-1$ " Water Reflection .............162 Figure B1.2.1.1.1.3-1. $\mathrm{UO}_{2}\left(\mathrm{NO}_{3}\right) 20 \mathrm{M}$ - Sphere - $100 \%{ }^{235} \mathrm{U}-10$ " Water Reflection ...........165 Figure B1.2.1.1.2.1-1. $\mathrm{UO}_{2}\left(\mathrm{NO}_{3}\right) 23 \mathrm{M}$ - Sphere - 100\% ${ }^{235} \mathrm{U}-0$ " Water Reflection .............168 Figure B1.2.1.1.2.2-1. $\mathrm{UO}_{2}\left(\mathrm{NO}_{3}\right) 23 \mathrm{M}$ - Sphere - $100 \%{ }^{235} \mathrm{U}-1$ "Water Reflection .............171 Figure B1.2.1.1.2.3-1. $\mathrm{UO}_{2}\left(\mathrm{NO}_{3}\right) 23 \mathrm{M}$ - Sphere - 100\% ${ }^{235} \mathrm{U}-10$ " Water Reflection ...........174 Figure B1.2.1.1.3.1-1. $\mathrm{UO}_{2}\left(\mathrm{NO}_{3}\right) 26 \mathrm{M}$ - Sphere - 100\% ${ }^{235} \mathrm{U}-0$ " Water Reflection .............177 Figure B1.2.1.1.3.2-1. $\mathrm{UO}_{2}\left(\mathrm{NO}_{3}\right) 26 \mathrm{M}$ - Sphere - $100 \%{ }^{235} \mathrm{U}-1$ " Water Reflection .............180 Figure B1.2.1.1.3.3-1. $\mathrm{UO}_{2}\left(\mathrm{NO}_{3}\right) 26 \mathrm{M}$ - Sphere - $100 \%{ }^{235} \mathrm{U}-10$ " Water Reflection ...........183 Figure B1.2.1.2.1.1-1. $\mathrm{UO}_{2}\left(\mathrm{NO}_{3}\right) 20 \mathrm{M}$ - Sphere - 93.5\% ${ }^{235} \mathrm{U}-0$ " Water Reflection............186 Figure B1.2.1.2.1.2-1. $\mathrm{UO}_{2}\left(\mathrm{NO}_{3}\right) 20 \mathrm{M}$ - Sphere - 93.5\% ${ }^{235} \mathrm{U}-1$ " Water Reflection............189 Figure B1.2.1.2.1.3-1. $\mathrm{UO}_{2}\left(\mathrm{NO}_{3}\right) 20 \mathrm{M}$ - Sphere - 93.5\% ${ }^{235} \mathrm{U}-10$ " Water Reflection..........192 Figure B1.2.1.2.2.1-1. $\mathrm{UO}_{2}\left(\mathrm{NO}_{3}\right) 23 \mathrm{M}$ - Sphere - 93.5\% ${ }^{235} \mathrm{U}-0$ " Water Reflection............195 Figure B1.2.1.2.2.2-1. $\mathrm{UO}_{2}\left(\mathrm{NO}_{3}\right) 23 \mathrm{M}$ - Sphere - 93.5\% ${ }^{235} \mathrm{U}-1$ " Water Reflection............198 Figure B1.2.1.2.2.3-1. $\mathrm{UO}_{2}\left(\mathrm{NO}_{3}\right) 23 \mathrm{M}$ - Sphere - 93.5\% ${ }^{235} \mathrm{U}-10$ " Water Reflection..........201 Figure B1.2.1.2.3.1-1. $\mathrm{UO}_{2}\left(\mathrm{NO}_{3}\right) 26 \mathrm{M}$ - Sphere - 93.5\% ${ }^{235} \mathrm{U}-0$ " Water Reflection...........204 
Figure B1.2.1.2.3.2-1. $\mathrm{UO}_{2}\left(\mathrm{NO}_{3}\right) 26 \mathrm{M}$ - Sphere - 93.5\% ${ }^{235} \mathrm{U}-1$ " Water Reflection...........207

Figure B1.2.1.2.3.3-1. $\mathrm{UO}_{2}\left(\mathrm{NO}_{3}\right) 26 \mathrm{M}-$ Sphere - 93.5\% ${ }^{235} \mathrm{U}-10$ " Water Reflection.........210

Figure B1.2.2.1.1.1-1. $\mathrm{UO}_{2}\left(\mathrm{NO}_{3}\right) 20 \mathrm{M}$ - Cylinder - $100 \%{ }^{235} \mathrm{U}-0$ " Water Reflection ..........214

Figure B1.2.2.1.1.2-1. $\mathrm{UO}_{2}\left(\mathrm{NO}_{3}\right) 20 \mathrm{M}-$ Cylinder - $100 \%{ }^{235} \mathrm{U}-1$ "Water Reflection ..........217

Figure B1.2.2.1.1.3-1. $\mathrm{UO}_{2}\left(\mathrm{NO}_{3}\right) 20 \mathrm{M}-$ Cylinder - $100 \%{ }^{235} \mathrm{U}-10$ " Water Reflection ........220

Figure B1.2.2.1.2.1-1. $\mathrm{UO}_{2}\left(\mathrm{NO}_{3}\right) 23 \mathrm{M}-$ Cylinder - $100 \%{ }^{235} \mathrm{U}-0$ " Water Reflection ..........223

Figure B1.2.2.1.2.2-1. $\mathrm{UO}_{2}\left(\mathrm{NO}_{3}\right) 23 \mathrm{M}-$ Cylinder - $100 \%{ }^{235} \mathrm{U}-1$ "Water Reflection ..........226

Figure B1.2.2.1.2.3-1. $\mathrm{UO}_{2}\left(\mathrm{NO}_{3}\right) 23 \mathrm{M}-$ Cylinder - $100 \%{ }^{235} \mathrm{U}-10$ " Water Reflection ........229

Figure B1.2.2.1.3.1-1. $\mathrm{UO}_{2}\left(\mathrm{NO}_{3}\right) 26 \mathrm{M}-$ Cylinder - $100 \%{ }^{235} \mathrm{U}-0$ " Water Reflection ..........232

Figure B1.2.2.1.3.2-1. $\mathrm{UO}_{2}\left(\mathrm{NO}_{3}\right) 26 \mathrm{M}$ - Cylinder - $100 \%{ }^{235} \mathrm{U}-1$ " Water Reflection ..........235

Figure B1.2.2.1.3.3-1. $\mathrm{UO}_{2}\left(\mathrm{NO}_{3}\right) 26 \mathrm{M}-$ Cylinder - $100 \%{ }^{235} \mathrm{U}-10$ " Water Reflection ........238

Figure B1.2.2.2.1.1-1. $\mathrm{UO}_{2}\left(\mathrm{NO}_{3}\right) 20 \mathrm{M}-$ Cylinder $-93.5 \%{ }^{235} \mathrm{U}-0$ " Water Reflection.........242

Figure B1.2.2.2.1.2-1. $\mathrm{UO}_{2}\left(\mathrm{NO}_{3}\right) 20 \mathrm{M}$ - Cylinder - 93.5\% ${ }^{235} \mathrm{U}-1$ " Water Reflection.........245

Figure B1.2.2.2.1.3-1. $\mathrm{UO}_{2}\left(\mathrm{NO}_{3}\right) 20 \mathrm{M}-$ Cylinder $-93.5 \%{ }^{235} \mathrm{U}-10$ " Water

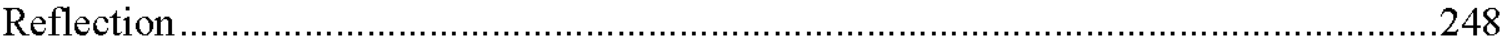

Figure B1.2.2.2.2.1-1. $\mathrm{UO}_{2}\left(\mathrm{NO}_{3}\right) 23 \mathrm{M}-$ Cylinder $-93.5 \%{ }^{235} \mathrm{U}-0$ " Water Reflection........251

Figure B1.2.2.2.2.2-1. $\mathrm{UO}_{2}\left(\mathrm{NO}_{3}\right) 23 \mathrm{M}-$ Cylinder $-93.5 \%{ }^{235} \mathrm{U}-1$ " Water Reflection........254

Figure B1.2.2.2.2.3-1. $\mathrm{UO}_{2}\left(\mathrm{NO}_{3}\right) 23 \mathrm{M}-$ Cylinder $-93.5 \%{ }^{235} \mathrm{U}-10$ " Water

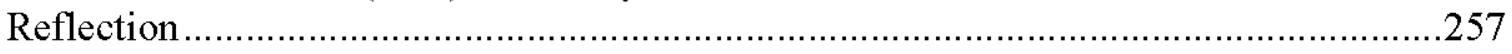

Figure B1.2.2.2.3.1-1. $\mathrm{UO}_{2}\left(\mathrm{NO}_{3}\right) 26 \mathrm{M}-$ Cylinder - 93.5\% ${ }^{235} \mathrm{U}-0$ " Water Reflection........260

Figure B1.2.2.2.3.2-1. $\mathrm{UO}_{2}\left(\mathrm{NO}_{3}\right) 26 \mathrm{M}-$ Cylinder $-93.5 \%{ }^{235} \mathrm{U}-1$ " Water Reflection........263

Figure B1.2.2.2.3.3-1. $\mathrm{UO}_{2}\left(\mathrm{NO}_{3}\right) 26 \mathrm{M}-$ Cylinder $-93.5 \%{ }^{235} \mathrm{U}-10$ " Water

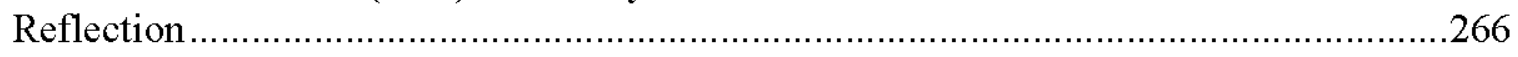

Figure B1.2.3.1.1.1-1. $\mathrm{UO}_{2}\left(\mathrm{NO}_{3}\right) 20 \mathrm{M}$ - Plane - 100\% ${ }^{235} \mathrm{U}-0$ " Water Reflection ..............270

Figure B1.2.3.1.1.2-1. $\mathrm{UO}_{2}\left(\mathrm{NO}_{3}\right) 20 \mathrm{M}-$ Plane - $100 \%{ }^{235} \mathrm{U}-1$ "Water Reflection ..............273

Figure B1.2.3.1.1.3-1. $\mathrm{UO}_{2}\left(\mathrm{NO}_{3}\right) 20 \mathrm{M}-$ Plane - $100 \%{ }^{235} \mathrm{U}-10$ " Water Reflection ............2276

Figure B1.2.3.1.2.1-1. $\mathrm{UO}_{2}\left(\mathrm{NO}_{3}\right) 23 \mathrm{M}-$ Plane - $100 \%{ }^{235} \mathrm{U}-0$ " Water Reflection ..............280

Figure B1.2.3.1.2.2-1. $\mathrm{UO}_{2}\left(\mathrm{NO}_{3}\right) 23 \mathrm{M}-$ Plane - $100 \%{ }^{235} \mathrm{U}-1$ " Water Reflection ..............283

Figure B1.2.3.1.2.3-1. $\mathrm{UO}_{2}\left(\mathrm{NO}_{3}\right) 23 \mathrm{M}-$ Plane - $100 \%{ }^{235} \mathrm{U}-10$ " Water Reflection .............286

Figure B1.2.3.1.3.1-1. $\mathrm{UO}_{2}\left(\mathrm{NO}_{3}\right) 26 \mathrm{M}$ - Plane - $100 \%{ }^{235} \mathrm{U}-0$ " Water Reflection ...............290

Figure B1.2.3.1.3.2-1. $\mathrm{UO}_{2}\left(\mathrm{NO}_{3}\right) 26 \mathrm{M}-$ Plane - $100 \%{ }^{235} \mathrm{U}-1$ " Water Reflection ...............293

Figure B1.2.3.1.3.3-1. $\mathrm{UO}_{2}\left(\mathrm{NO}_{3}\right) 26 \mathrm{M}$ - Plane - 100\% ${ }^{235} \mathrm{U}-10$ " Water Reflection .............296

Figure B1.2.3.2.1.1-1. $\mathrm{UO}_{2}\left(\mathrm{NO}_{3}\right) 20 \mathrm{M}-$ Plane $-93.5 \%{ }^{235} \mathrm{U}-0$ " Water Reflection..............300

Figure B1.2.3.2.1.2-1. $\mathrm{UO}_{2}\left(\mathrm{NO}_{3}\right) 20 \mathrm{M}-$ Plane $-93.5 \%{ }^{235} \mathrm{U}-1$ " Water Reflection..............303

Figure B1.2.3.2.1.3-1. $\mathrm{UO}_{2}\left(\mathrm{NO}_{3}\right) 20 \mathrm{M}$ - Plane - 93.5\% ${ }^{235} \mathrm{U}-10$ " Water Reflection............306 


\section{LIST OF TABLES}

Table B1.1.1.1.1.1-1. Critical Dimensions for $\mathrm{Pu}\left(\mathrm{NO}_{3}\right) 40 \mathrm{M}-\mathrm{Sphere}-100 \%{ }^{239} \mathrm{Pu}-$ 0" Water Reflection

Table B1.1.1.1.1.2-1. Critical Dimensions for $\mathrm{Pu}\left(\mathrm{NO}_{3}\right) 40 \mathrm{M}-\mathrm{Sphere}-100 \%{ }^{239} \mathrm{Pu}-$ 1 "Water Reflection

Table B1.1.1.1.1.3-1. Critical Dimensions for $\mathrm{Pu}\left(\mathrm{NO}_{3}\right) 40 \mathrm{M}-\mathrm{Sphere}-100 \%{ }^{239} \mathrm{Pu}-$ 10" Water Reflection

Table B1.1.1.1.2.1-1. Critical Dimensions for Pu(NO3)4 3M - Sphere - 100\% 239Pu 0" Water Reflections.

Table B1.1.1.1.2.2-1. Critical Dimensions for $\mathrm{Pu}\left(\mathrm{NO}_{3}\right) 43 \mathrm{M}-$ Sphere $-100 \%{ }^{239} \mathrm{Pu}-$ 1 "Water Reflection

Table B1.1.1.1.2.3-1. Critical Dimensions for $\mathrm{Pu}\left(\mathrm{NO}_{3}\right) 43 \mathrm{M}-$ Sphere $-100 \%{ }^{239} \mathrm{Pu}-$ 10" Water Reflection

Table B1.1.1.1.3.1-1. Critical Dimensions for $\mathrm{Pu}\left(\mathrm{NO}_{3}\right) 46 \mathrm{M}-$ Sphere $-100 \%{ }^{239} \mathrm{Pu}-$ 0" Water Reflection

Table B1.1.1.1.3.2-1. Critical Dimensions for $\mathrm{Pu}\left(\mathrm{NO}_{3}\right) 46 \mathrm{M}-$ Sphere $-100 \%{ }^{239} \mathrm{Pu}-$ 1 "Water Reflection

Table B1.1.1.1.3.3-1. Critical Dimensions for $\mathrm{Pu}\left(\mathrm{NO}_{3}\right) 46 \mathrm{M}-$ Sphere $-100 \%{ }^{239} \mathrm{Pu}-$ 10" Water Reflection

Table B1.1.1.2.1.1-1. Critical Dimensions for $\mathrm{Pu}\left(\mathrm{NO}_{3}\right) 40 \mathrm{M}-$ Sphere $-97 \%{ }^{239} \mathrm{Pu}-0$ " Water Reflection

Table B1.1.1.2.1.2-1. Critical Dimensions for $\mathrm{Pu}\left(\mathrm{NO}_{3}\right) 40 \mathrm{M}-$ Sphere $-97 \%{ }^{239} \mathrm{Pu}-1$ " Water Reflection

Table B1.1.1.2.1.3-1. Critical Dimensions for $\mathrm{Pu}\left(\mathrm{NO}_{3}\right) 40 \mathrm{M}-$ Sphere $-97 \%{ }^{239} \mathrm{Pu}-$ 10" Water Reflection

Table B1.1.1.2.2.1-1. Critical Dimensions for $\mathrm{Pu}\left(\mathrm{NO}_{3}\right) 43 \mathrm{M}-$ Sphere $-97 \%{ }^{239} \mathrm{Pu}-0$ " Water Reflection

Table B1.1.1.2.2.2-1. Critical Dimensions for $\mathrm{Pu}\left(\mathrm{NO}_{3}\right) 43 \mathrm{M}-$ Sphere $-97 \%{ }^{239} \mathrm{Pu}-1$ " Water Reflection

Table B1.1.1.2.2.3-1. Critical Dimensions for $\mathrm{Pu}\left(\mathrm{NO}_{3}\right) 43 \mathrm{M}-$ Sphere $-97 \%{ }^{239} \mathrm{Pu}-$ 10" Water Reflection

Table B1.1.1.2.3.1-1. Critical Dimensions for $\mathrm{Pu}\left(\mathrm{NO}_{3}\right) 46 \mathrm{M}-$ Sphere $-97 \%{ }^{239} \mathrm{Pu}-0$ " Water Reflection.

Table B1.1.1.2.3.2-1. Critical Dimensions for $\mathrm{Pu}\left(\mathrm{NO}_{3}\right) 46 \mathrm{M}-$ Sphere $-97 \%{ }^{239} \mathrm{Pu}-1$ " Water Reflection.

Table B1.1.1.2.3.3-1. Critical Dimensions for $\mathrm{Pu}\left(\mathrm{NO}_{3}\right) 46 \mathrm{M}-$ Sphere $-97 \%{ }^{239} \mathrm{Pu}-$ 10" Water Reflection 
Table B1.1.1.3.1.1-1. Critical Dimensions for $\mathrm{Pu}\left(\mathrm{NO}_{3}\right) 40 \mathrm{M}-$ Sphere $-95 \%{ }^{239} \mathrm{Pu}-0$ " Water Reflection

Table B1.1.1.3.1.2-1. Critical Dimensions for $\mathrm{Pu}\left(\mathrm{NO}_{3}\right) 40 \mathrm{M}-$ Sphere $-95 \%{ }^{239} \mathrm{Pu}-1$ " Water Reflection

Table B1.1.1.3.1.3-1. Critical Dimensions for $\mathrm{Pu}\left(\mathrm{NO}_{3}\right) 40 \mathrm{M}-$ Sphere $-95 \%{ }^{239} \mathrm{Pu}-$ 10" Water Reflection

Table B1.1.2.1.1.1-1. Critical Dimensions for $\mathrm{Pu}\left(\mathrm{NO}_{3}\right) 40 \mathrm{M}-$ Cylinder $-100 \%{ }^{239} \mathrm{Pu}-$ 0"Water Reflection

Table B1.1.2.1.1.2-1. Critical Dimensions for $\mathrm{Pu}\left(\mathrm{NO}_{3}\right) 40 \mathrm{M}-$ Cylinder - $100 \%{ }^{239} \mathrm{Pu}-$ 1 "Water Reflection

Table B1.1.2.1.1.3-1. Critical Dimensions for $\mathrm{Pu}\left(\mathrm{NO}_{3}\right) 40 \mathrm{M}-\mathrm{Cylinder}-100 \%{ }^{239} \mathrm{Pu}-$ 10" Water Reflection

Table B1.1.2.1.2.1-1. Critical Dimensions for $\mathrm{Pu}\left(\mathrm{NO}_{3}\right) 43 \mathrm{M}-\mathrm{Cylinder}-100 \%{ }^{239} \mathrm{Pu}-$ 0 "Water Reflection

Table B1.1.2.1.2.2-1. Critical Dimensions for $\mathrm{Pu}\left(\mathrm{NO}_{3}\right) 43 \mathrm{M}-$ Cylinder $-100 \%{ }^{239} \mathrm{Pu}-$ 1 "Water Reflection

Table B1.1.2.1.2.3-1. Critical Dimensions for $\mathrm{Pu}\left(\mathrm{NO}_{3}\right) 43 \mathrm{M}-$ Cylinder $-100 \%{ }^{239} \mathrm{Pu}-$ 10" Water Reflection

Table B1.1.2.1.3.1-1. Critical Dimensions for $\mathrm{Pu}\left(\mathrm{NO}_{3}\right) 46 \mathrm{M}-\mathrm{Cylinder}-100 \%{ }^{239} \mathrm{Pu}-$ 0 " Water Reflection

Table B1.1.2.1.3.2-1. Critical Dimensions for $\mathrm{Pu}\left(\mathrm{NO}_{3}\right) 46 \mathrm{M}-\mathrm{Cylinder}-100 \%{ }^{239} \mathrm{Pu}-$ 1 "Water Reflection

Table B1.1.2.1.3.3-1. Critical Dimensions for $\mathrm{Pu}\left(\mathrm{NO}_{3}\right) 46 \mathrm{M}-$ Cylinder $-100 \%{ }^{239} \mathrm{Pu}-$ 10" Water Reflection

Table B1.1.2.2.1.1-1. Critical Dimensions for $\mathrm{Pu}\left(\mathrm{NO}_{3}\right) 40 \mathrm{M}-$ Cylinder - $97 \%{ }^{239} \mathrm{Pu}-$ 0"Water Reflection

Table B1.1.2.2.1.2-1. Critical Dimensions for $\mathrm{Pu}\left(\mathrm{NO}_{3}\right) 40 \mathrm{M}-$ Cylinder - $97 \%{ }^{239} \mathrm{Pu}-$ 1 "Water Reflection

Table B1.1.2.2.1.3-1. Critical Dimensions for $\mathrm{Pu}\left(\mathrm{NO}_{3}\right) 40 \mathrm{M}-$ Cylinder - $97 \%{ }^{239} \mathrm{Pu}-$ 10" Water Reflection

Table B1.1.2.2.2.1-1. Critical Dimensions for $\mathrm{Pu}\left(\mathrm{NO}_{3}\right) 43 \mathrm{M}-$ Cylinder - 97\% ${ }^{239} \mathrm{Pu}-$ 0 " Water Reflection

Table B1.1.2.2.2.2-1. Critical Dimensions for $\mathrm{Pu}\left(\mathrm{NO}_{3}\right) 43 \mathrm{M}-$ Cylinder $-97 \%{ }^{239} \mathrm{Pu}-$ 1 "Water Reflection

Table B1.1.2.2.2.3-1. Critical Dimensions for $\mathrm{Pu}\left(\mathrm{NO}_{3}\right) 43 \mathrm{M}-$ Cylinder - $97 \%{ }^{239} \mathrm{Pu}-$ 10" Water Reflection

Table B1.1.2.2.3.1-1. Critical Dimensions for $\mathrm{Pu}\left(\mathrm{NO}_{3}\right) 46 \mathrm{M}-$ Cylinder $-97 \%{ }^{239} \mathrm{Pu}-$ 0" Water Reflection

Table B1.1.2.2.3.2-1. Critical Dimensions for $\mathrm{Pu}\left(\mathrm{NO}_{3}\right) 46 \mathrm{M}-\mathrm{Cylinder}-97 \%{ }^{239} \mathrm{Pu}-$ 1 "Water Reflection 
Table B1.1.2.2.3.3-1. Critical Dimensions for $\mathrm{Pu}\left(\mathrm{NO}_{3}\right) 46 \mathrm{M}-$ Cylinder - $97 \%{ }^{239} \mathrm{Pu}-$ 10" Water Reflection

Table B1.1.3.1.1.1-1. Critical Dimensions for $\mathrm{Pu}\left(\mathrm{NO}_{3}\right) 40 \mathrm{M}-\mathrm{Plane}-100 \%{ }^{239} \mathrm{Pu}-0$ " Water Reflection.

Table B1.1.3.1.1.2-1. Critical Dimensions for $\mathrm{Pu}\left(\mathrm{NO}_{3}\right) 40 \mathrm{M}-\mathrm{Plane}-100 \%{ }^{239} \mathrm{Pu}-1$ " Water Reflection

Table B1.1.3.1.1.3-1. Critical Dimensions for $\mathrm{Pu}\left(\mathrm{NO}_{3}\right) 40 \mathrm{M}-\mathrm{Plane}-100 \%{ }^{239} \mathrm{Pu}-$ 10" Water Reflection

Table B1.1.3.1.2.1-1. Critical Dimensions for $\mathrm{Pu}\left(\mathrm{NO}_{3}\right) 43 \mathrm{M}-\mathrm{Plane}-100 \%{ }^{239} \mathrm{Pu}-0$ " Water Reflection

Table B1.1.3.1.2.2-1. Critical Dimensions for $\mathrm{Pu}\left(\mathrm{NO}_{3}\right) 43 \mathrm{M}-\mathrm{Plane}-100 \%{ }^{239} \mathrm{Pu}-1$ " Water Reflection

Table B1.1.3.1.2.3-1. Critical Dimensions for $\mathrm{Pu}\left(\mathrm{NO}_{3}\right) 43 \mathrm{M}-\mathrm{Plane}-100 \%{ }^{239} \mathrm{Pu}-$ 10" Water Reflection

Table B.1.1.3.1.3.1-1. Critical Dimensions for $\mathrm{Pu}\left(\mathrm{NO}_{3}\right) 46 \mathrm{M}-\mathrm{Plane}-100 \%{ }^{239} \mathrm{Pu}-0$ " Water Reflection

Table B1.1.3.1.3.2-1. Critical Dimensions for $\mathrm{Pu}\left(\mathrm{NO}_{3}\right) 46 \mathrm{M}-\mathrm{Plane}-100 \%{ }^{239} \mathrm{Pu}-1$ " Water Reflection

Table B1.1.3.1.3.3-1. Critical Dimensions for $\mathrm{Pu}\left(\mathrm{NO}_{3}\right) 46 \mathrm{M}-\mathrm{Plane}-100 \%{ }^{239} \mathrm{Pu}-$ 10" Water Reflection

Table B1.1.3.2.1.1-1. Critical Dimensions for $\mathrm{Pu}\left(\mathrm{NO}_{3}\right) 40 \mathrm{M}-\mathrm{Plane}-97 \%{ }^{239} \mathrm{Pu}-0$ " Water Reflection

Table B1.1.3.2.1.2-1. Critical Dimensions for $\mathrm{Pu}\left(\mathrm{NO}_{3}\right) 40 \mathrm{M}-\mathrm{Plane}-97 \%{ }^{239} \mathrm{Pu}-1$ " Water Reflection

Table B1.1.3.2.1.3-1. Critical Dimensions for $\mathrm{Pu}\left(\mathrm{NO}_{3}\right) 40 \mathrm{M}-\mathrm{Plane}-97 \%{ }^{239} \mathrm{Pu}-10 "$ Water Reflection

Table B1.2.1.1.1.1-1. Critical Dimensions for $\mathrm{UO}_{2}\left(\mathrm{NO}_{3}\right) 20 \mathrm{M}-$ Sphere $-100 \%{ }^{235} \mathrm{U}-$ 0" Water Reflection

Table B1.2.1.1.1.2-1. Critical Dimensions for $\mathrm{UO}_{2}\left(\mathrm{NO}_{3}\right) 20 \mathrm{M}-$ Sphere $-100 \%{ }^{235} \mathrm{U}-$ 1 "Water Reflection

Table B1.2.1.1.1.3-1. Critical Dimensions for $\mathrm{UO}_{2}\left(\mathrm{NO}_{3}\right) 20 \mathrm{M}-$ Sphere $-100 \%{ }^{235} \mathrm{U}-$ 10" Water Reflection

Table B1.2.1.1.2.1-1. Critical Dimensions for $\mathrm{UO}_{2}\left(\mathrm{NO}_{3}\right) 23 \mathrm{M}-$ Sphere $-100 \%{ }^{235} \mathrm{U}-$ 0"Water Reflection

Table B1.2.1.1.2.2-1. Critical Dimensions for $\mathrm{UO}_{2}\left(\mathrm{NO}_{3}\right) 23 \mathrm{M}-$ Sphere $-100 \%{ }^{235} \mathrm{U}-$ 1 "Water Reflection

Table B1.2.1.1.2.3-1. Critical Dimensions for $\mathrm{UO}_{2}\left(\mathrm{NO}_{3}\right) 23 \mathrm{M}-$ Sphere $-100 \%{ }^{235} \mathrm{U}-$ 10" Water Reflection

Table B1.2.1.1.3.1-1. Critical Dimensions for $\mathrm{UO}_{2}\left(\mathrm{NO}_{3}\right) 26 \mathrm{M}-$ Sphere $-100 \%{ }^{235} \mathrm{U}-$ 0 "Water Reflection 
Table B1.2.1.1.3.2-1. Critical Dimensions for $\mathrm{UO}_{2}\left(\mathrm{NO}_{3}\right) 26 \mathrm{M}-$ Sphere $-100 \%{ }^{235} \mathrm{U}-$ 1 "Water Reflection

Table B1.2.1.1.3.3-1. Critical Dimensions for $\mathrm{UO}_{2}\left(\mathrm{NO}_{3}\right) 26 \mathrm{M}-$ Sphere $-100 \%{ }^{235} \mathrm{U}-$ 10" Water Reflection

Table B1.2.1.2.1.1-1. Critical Dimensions for $\mathrm{UO}_{2}\left(\mathrm{NO}_{3}\right) 20 \mathrm{M}-$ Sphere $-93.5 \%{ }^{235} \mathrm{U}-$ 0"Water Reflection

Table B1.2.1.2.1.2-1. Critical Dimensions for $\mathrm{UO}_{2}\left(\mathrm{NO}_{3}\right) 20 \mathrm{M}-$ Sphere $-93.5 \%{ }^{235} \mathrm{U}-$ 1 "Water Reflection

Table B1.2.1.2.1.3-1. Critical Dimensions for $\mathrm{UO}_{2}\left(\mathrm{NO}_{3}\right) 20 \mathrm{M}-$ Sphere $-93.5 \%{ }^{235} \mathrm{U}-$ 10 "Water Reflection

Table B1.2.1.2.2.1-1. Critical Dimensions for $\mathrm{UO}_{2}\left(\mathrm{NO}_{3}\right) 23 \mathrm{M}-$ Sphere $-93.5 \%{ }^{235} \mathrm{U}-$ 0 " Water Reflection

Table B1.2.1.2.2.2-1. Critical Dimensions for $\mathrm{UO}_{2}\left(\mathrm{NO}_{3}\right) 23 \mathrm{M}-$ Sphere $-93.5 \%{ }^{235} \mathrm{U}-$ 1 "Water Reflection

Table B1.2.1.2.2.3-1. Critical Dimensions for $\mathrm{UO}_{2}\left(\mathrm{NO}_{3}\right) 23 \mathrm{M}-$ Sphere $-93.5 \%{ }^{235} \mathrm{U}-$ 10" Water Reflection

Table B1.2.1.2.3.1-1. Critical Dimensions for $\mathrm{UO}_{2}\left(\mathrm{NO}_{3}\right) 26 \mathrm{M}-$ Sphere $-93.5 \%{ }^{235} \mathrm{U}-$ 0 "Water Reflection .205

Table B1.2.1.2.3.2-1. Critical Dimensions for $\mathrm{UO}_{2}\left(\mathrm{NO}_{3}\right) 26 \mathrm{M}-$ Sphere $-93.5 \%{ }^{235} \mathrm{U}-$ 1 "Water Reflection .208

Table B1.2.1.2.3.3-1. Critical Dimensions for $\mathrm{UO}_{2}\left(\mathrm{NO}_{3}\right) 26 \mathrm{M}-$ Sphere $-93.5 \%{ }^{235} \mathrm{U}-$ 10" Water Reflection

Table B1.2.2.1.1.1-1. Critical Dimensions for $\mathrm{UO}_{2}\left(\mathrm{NO}_{3}\right) 20 \mathrm{M}-$ Cylinder $-100 \%{ }^{235} \mathrm{U}$ - 0" Water Reflection

Table B1.2.2.1.1.2-1. Critical Dimensions for $\mathrm{UO}_{2}\left(\mathrm{NO}_{3}\right) 20 \mathrm{M}-$ Cylinder $-100 \%{ }^{235} \mathrm{U}$ -1 "Water Reflection 218

Table B1.2.2.1.1.3-1. Critical Dimensions for $\mathrm{UO}_{2}\left(\mathrm{NO}_{3}\right) 20 \mathrm{M}-$ Cylinder $-100 \%{ }^{235} \mathrm{U}$ -10 " Water Reflection

Table B1.2.2.1.2.1-1. Critical Dimensions for $\mathrm{UO}_{2}\left(\mathrm{NO}_{3}\right) 23 \mathrm{M}-$ Cylinder $-100 \%{ }^{235} \mathrm{U}$ - 0" Water Reflection .224

Table B1.2.2.1.2.2-1. Critical Dimensions for $\mathrm{UO}_{2}\left(\mathrm{NO}_{3}\right) 23 \mathrm{M}-$ Cylinder $-100 \%{ }^{235} \mathrm{U}$ -1 "Water Reflection

Table B1.2.2.1.2.3-1. Critical Dimensions for $\mathrm{UO}_{2}\left(\mathrm{NO}_{3}\right) 23 \mathrm{M}-$ Cylinder $-100 \%{ }^{235} \mathrm{U}$ - 10" Water Reflection

Table B1.2.2.1.3.1-1. Critical Dimensions for $\mathrm{UO}_{2}\left(\mathrm{NO}_{3}\right) 26 \mathrm{M}-$ Cylinder $-100 \%{ }^{235} \mathrm{U}$ - 0" Water Reflection

Table B1.2.2.1.3.2-1. Critical Dimensions for $\mathrm{UO}_{2}\left(\mathrm{NO}_{3}\right) 26 \mathrm{M}-$ Cylinder $-100 \%{ }^{235} \mathrm{U}$ -1 "Water Reflection .236

Table B1.2.2.1.3.3-1. Critical Dimensions for $\mathrm{UO}_{2}\left(\mathrm{NO}_{3}\right) 26 \mathrm{M}-$ Cylinder $-100 \%{ }^{235} \mathrm{U}$ -10 "Water Reflection 
Table B1.2.2.2.1.1-1. Critical Dimensions for $\mathrm{UO}_{2}\left(\mathrm{NO}_{3}\right) 20 \mathrm{M}-$ Cylinder $-93.5 \%{ }^{235} \mathrm{U}$ - 0" Water Reflection

Table B1.2.2.2.1.2-1. Critical Dimensions for $\mathrm{UO}_{2}\left(\mathrm{NO}_{3}\right) 20 \mathrm{M}-$ Cylinder $-93.5 \%{ }^{235} \mathrm{U}$

- 1" Water Reflection

Table B1.2.2.2.1.3-1. Critical Dimensions for $\mathrm{UO}_{2}\left(\mathrm{NO}_{3}\right) 20 \mathrm{M}-$ Cylinder $-93.5 \%{ }^{235} \mathrm{U}$

- 10" Water Reflection

Table B1.2.2.2.2.1-1. Critical Dimensions for $\mathrm{UO}_{2}\left(\mathrm{NO}_{3}\right) 23 \mathrm{M}-$ Cylinder $-93.5 \%{ }^{235} \mathrm{U}$

- 0" Water Reflection

Table B1.2.2.2.2.2-1. Critical Dimensions for $\mathrm{UO}_{2}\left(\mathrm{NO}_{3}\right) 23 \mathrm{M}-$ Cylinder $-93.5 \%{ }^{235} \mathrm{U}$ -1 "Water Reflection .255

Table B1.2.2.2.2.3-1. Critical Dimensions for $\mathrm{UO}_{2}\left(\mathrm{NO}_{3}\right) 23 \mathrm{M}-$ Cylinder $-93.5 \%{ }^{235} \mathrm{U}$ - 10" Water Reflection .258

Table B1.2.2.2.3.1-1. Critical Dimensions for $\mathrm{UO}_{2}\left(\mathrm{NO}_{3}\right) 26 \mathrm{M}-$ Cylinder $-93.5 \%{ }^{235} \mathrm{U}$ -0" Water Reflection

Table B1.2.2.2.3.2-1. Critical Dimensions for $\mathrm{UO}_{2}\left(\mathrm{NO}_{3}\right) 26 \mathrm{M}-$ Cylinder $-93.5 \%{ }^{235} \mathrm{U}$ -1 " Water Reflection

Table B1.2.2.2.3.3-1. Critical Dimensions for $\mathrm{UO}_{2}\left(\mathrm{NO}_{3}\right) 26 \mathrm{M}-$ Cylinder $-93.5 \%{ }^{235} \mathrm{U}$ -10 " Water Reflection .267

Table B1.2.3.1.1.1-1. Critical Dimensions for $\mathrm{UO}_{2}\left(\mathrm{NO}_{3}\right) 20 \mathrm{M}-$ Plane - $100 \%{ }^{235} \mathrm{U}-$ 0 " Water Reflection

Table B1.2.3.1.1.2-1. Critical Dimensions for $\mathrm{UO}_{2}\left(\mathrm{NO}_{3}\right) 20 \mathrm{M}-$ Plane - $100 \%{ }^{235} \mathrm{U}-$ 1 "Water Reflection .274

Table B1.2.3.1.1.3-1. Critical Dimensions for $\mathrm{UO}_{2}\left(\mathrm{NO}_{3}\right) 20 \mathrm{M}-$ Plane - $100 \%{ }^{235} \mathrm{U}-$ 10" Water Reflection

Table B1.2.3.1.2.1-1. Critical Dimensions for $\mathrm{UO}_{2}\left(\mathrm{NO}_{3}\right) 23 \mathrm{M}-\mathrm{Plane}-100 \%{ }^{235} \mathrm{U}-$ 0 "Water Reflection .281

Table B1.2.3.1.2.2-1. Critical Dimensions for $\mathrm{UO}_{2}\left(\mathrm{NO}_{3}\right) 23 \mathrm{M}-$ Plane - $100 \%{ }^{235} \mathrm{U}-$ 1 "Water Reflection

Table B1.2.3.1.2.3-1 Critical Dimensions for $\mathrm{UO}_{2}\left(\mathrm{NO}_{3}\right) 23 \mathrm{M}-$ Plane - $100 \%{ }^{235} \mathrm{U}-$ 10" Water Reflection

Table B1.2.3.1.3.1-1. Critical Dimensions for $\mathrm{UO}_{2}\left(\mathrm{NO}_{3}\right) 26 \mathrm{M}-$ Plane $-100 \%{ }^{235} \mathrm{U}-$ 0 "Water Reflection

Table B1.2.3.1.3.2-1. Critical Dimensions for $\mathrm{UO}_{2}\left(\mathrm{NO}_{3}\right) 26 \mathrm{M}-$ Plane - $100 \%{ }^{235} \mathrm{U}-$ 1 "Water Reflection

Table B1.2.3.1.3.3-1. Critical Dimensions for $\mathrm{UO}_{2}\left(\mathrm{NO}_{3}\right) 26 \mathrm{M}-$ Plane - $100 \%{ }^{235} \mathrm{U}-$ 10" Water Reflection

Table B1.2.3.2.1.1-1. Critical Dimensions for $\mathrm{UO}_{2}\left(\mathrm{NO}_{3}\right) 20 \mathrm{M}-\mathrm{Plane}-93.5 \%{ }^{235} \mathrm{U}-$ 0 "Water Reflection 301

Table B1.2.3.2.1.2-1. Critical Dimensions for $\mathrm{UO}_{2}\left(\mathrm{NO}_{3}\right) 20 \mathrm{M}-\mathrm{Plane}-93.5 \%{ }^{235} \mathrm{U}-$ 1 "Water Reflection. 
CHPRC-01552, Revision 0

Table B1.2.3.2.1.3-1. Critical Dimensions for $\mathrm{UO}_{2}\left(\mathrm{NO}_{3}\right) 20 \mathrm{M}-\mathrm{Plane}-93.5 \%{ }^{235} \mathrm{U}-$ 10 " Water Reflection ... 
CHPRC-01552, Revision 0

This page is intentionally left blank.

B-xvii 


\section{B1.0 INTRODUCTION}

This Appendix contains a table of the critical dimensions for every case analyzed. The table also includes the first and last dimension, and the step size, for all of the dimensions that were evaluated in arriving at the critical value. The nitrate solution density is also presented in the table. In addition to the table, the critical values are plotted on a graph along with the corresponding ARH600 graph (where applicable).

\section{B1.1 $\mathrm{Pu}\left(\mathrm{NO}_{3}\right)_{4}$ SOLUTION}

\section{B1.1.1 Sphere}

B1.1.1.1 $\quad 100 \%{ }^{239} \mathrm{Pu}$

B1.1.1.1.1 0 Molar Excess Acid

B1.1.1.1.1.1 0" Water Reflector

Case ID: pun40-sp-1000-00

Material: $\mathrm{Pu}\left(\mathrm{NO}_{3}\right)_{4}$ 0 Molar Excess Acid

Geometry: Sphere

Isotopics: $100 \%{ }^{239} \mathrm{Pu}$

Reflector: 0" Water Reflector

Code: MCNP5 v1.40

Analyst: Mark Murphy

Date: $8 / 2011$ 
Figure B1.1.1.1.1.1-1. Pu $\left(\mathrm{NO}_{3}\right) 4$ 0M - Sphere $-100 \%{ }^{239} \mathrm{Pu}-0$ " Water Reflection

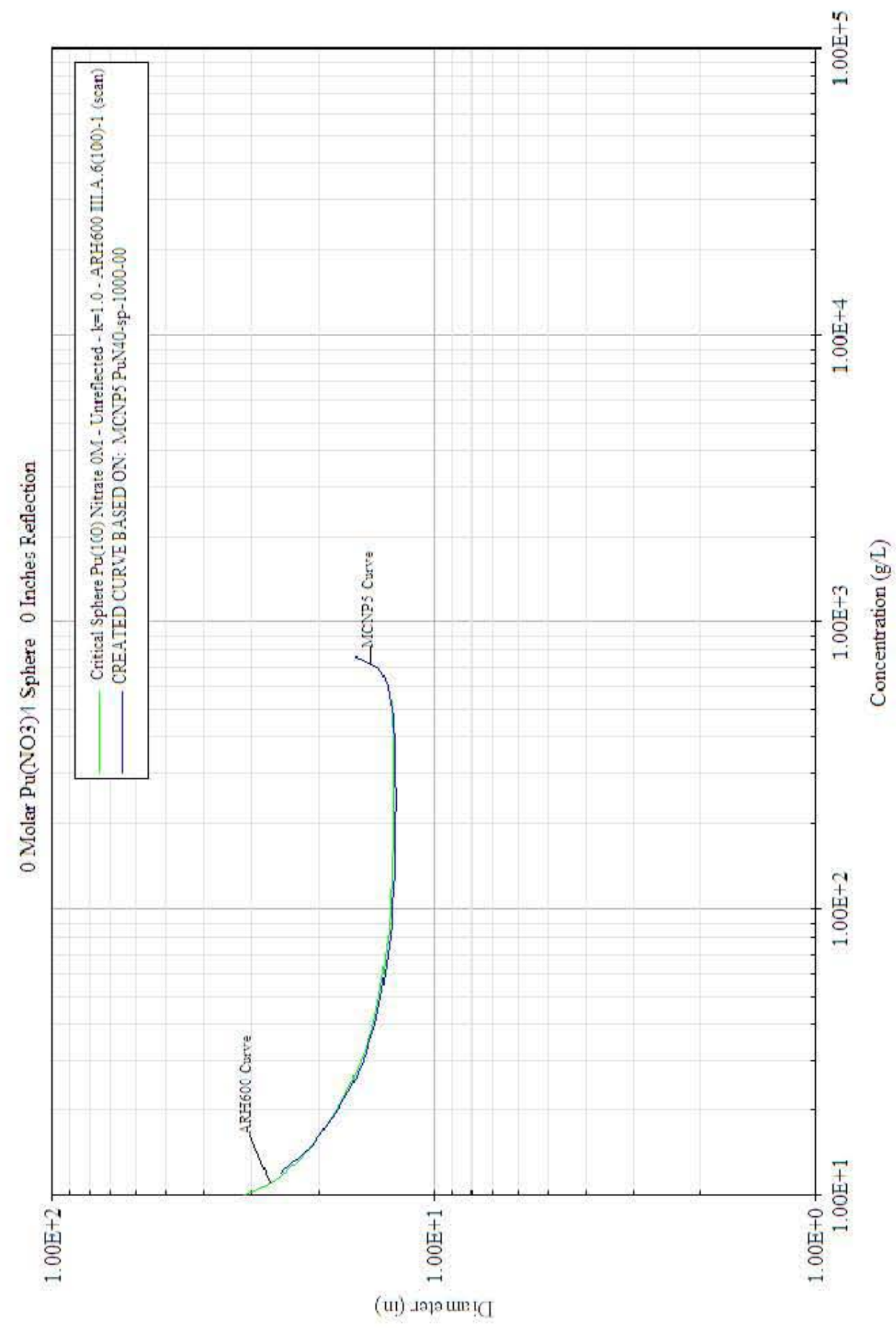


Table B1.1.1.1.1.1. Critical Dimensions for Pu $\left(\mathrm{NO}_{3}\right) 40 \mathrm{M}-$ Sphere $-100 \%{ }^{239} \mathrm{Pu}-0$ " Water Reflection

\begin{tabular}{|c|c|c|c|c|c|}
\hline \multicolumn{6}{|c|}{$100 \% \mathrm{Pu} 239 \mathrm{Pu}(\mathrm{NO} 3) 4$ 0M Sphere - 0 Inches of Reflection } \\
\hline Concentration $(\mathrm{g} / \mathrm{cc})$ & $\begin{array}{l}\text { Density } \\
(\mathrm{g} / \mathrm{cc})\end{array}$ & $\begin{array}{l}\text { First Diameter } \\
\text { (Inches) }\end{array}$ & $\begin{array}{l}\text { Last Diameter } \\
\text { (Inches) }\end{array}$ & $\begin{array}{l}\text { Delta } \\
\text { (Inches) }\end{array}$ & $\begin{array}{l}\text { Critical Diameter } \\
\text { (Inches) }\end{array}$ \\
\hline 0.012 & 1.016 & 21.1 & 26.2 & 0.2 & 25.1951 \\
\hline 0.015 & 1.021 & 18.1 & 21.6 & 0.1 & 20.8696 \\
\hline 0.02 & 1.029 & 15.7 & 18.3 & 0.1 & 17.7486 \\
\hline 0.025 & 1.037 & 14.6 & 16.7 & 0.1 & 16.2314 \\
\hline 0.03 & 1.046 & 13.8 & 15.8 & 0.1 & 15.3321 \\
\hline 0.032 & 1.049 & 13.6 & 15.4 & 0.1 & 15.0608 \\
\hline 0.035 & 1.054 & 13.3 & 15.1 & 0.1 & 14.7224 \\
\hline 0.04 & 1.063 & 13 & 14.7 & 0.05 & 14.2986 \\
\hline 0.05 & 1.08 & 12.5 & 14.2 & 0.05 & 13.7561 \\
\hline 0.06 & 1.097 & 12.2 & 13.8 & 0.05 & 13.4131 \\
\hline 0.07 & 1.113 & 12 & 13.5 & 0.05 & 13.2066 \\
\hline 0.08 & 1.13 & 11.9 & 13.4 & 0.05 & 13.0285 \\
\hline 0.09 & 1.147 & 11.8 & 13.3 & 0.05 & 12.9242 \\
\hline 0.1 & 1.164 & 11.7 & 13.2 & 0.05 & 12.8444 \\
\hline 0.12 & 1.197 & 11.6 & 13.1 & 0.05 & 12.7379 \\
\hline 0.13 & 1.215 & 11.6 & 13 & 0.05 & 12.6975 \\
\hline 0.15 & 1.248 & 11.5 & 13 & 0.05 & 12.6422 \\
\hline 0.2 & 1.332 & 11.4 & 12.9 & 0.05 & 12.6045 \\
\hline 0.239 & 1.397 & 11.5 & 12.9 & 0.05 & 12.5694 \\
\hline 0.25 & 1.415 & 11.4 & 12.9 & 0.05 & 12.5847 \\
\hline 0.359 & 1.594 & 11.5 & 13 & 0.05 & 12.6452 \\
\hline 0.478 & 1.784 & 11.7 & 13.1 & 0.05 & 12.801 \\
\hline 0.5 & 1.818 & 11.7 & 13.2 & 0.05 & 12.8299 \\
\hline 0.6 & 1.962 & 12 & 13.6 & 0.05 & 13.191 \\
\hline 0.65 & 2.026 & 12.3 & 13.9 & 0.05 & 13.505 \\
\hline 0.7 & 2.076 & 12.8 & 14.4 & 0.05 & 14.0332 \\
\hline 0.75 & 2.079 & 14.1 & 15.9 & 0.1 & 15.4784 \\
\hline 0.752 & 2.073 & 14.3 & 16.1 & 0.1 & 15.6692 \\
\hline 0.754 & 2.064 & 14.5 & 16.3 & 0.1 & 15.9074 \\
\hline 0.755 & 2.052 & 14.8 & 16.6 & 0.1 & 16.2095 \\
\hline
\end{tabular}




\section{B1.1.1.1.1.2 1" Water Reflector}

Case ID: PuN40-sp-1000-01

Material: $\mathrm{Pu}\left(\mathrm{NO}_{3}\right)_{4} 0$ Molar Excess Acid

Geometry: Sphere

Isotopics: $100 \%{ }^{239} \mathrm{Pu}$

Reflector: 1"Water Reflector

Code: MCNP5 v1.40

Analyst: Mark Murphy

Date: $8 / 2011$ 
Figure B1.1.1.1.1.2-1. $\mathrm{Pu}\left(\mathrm{NO}_{3}\right) 40 \mathrm{M}-\mathrm{Sphere}-100 \%{ }^{239} \mathrm{Pu}-1$ " Water Reflection

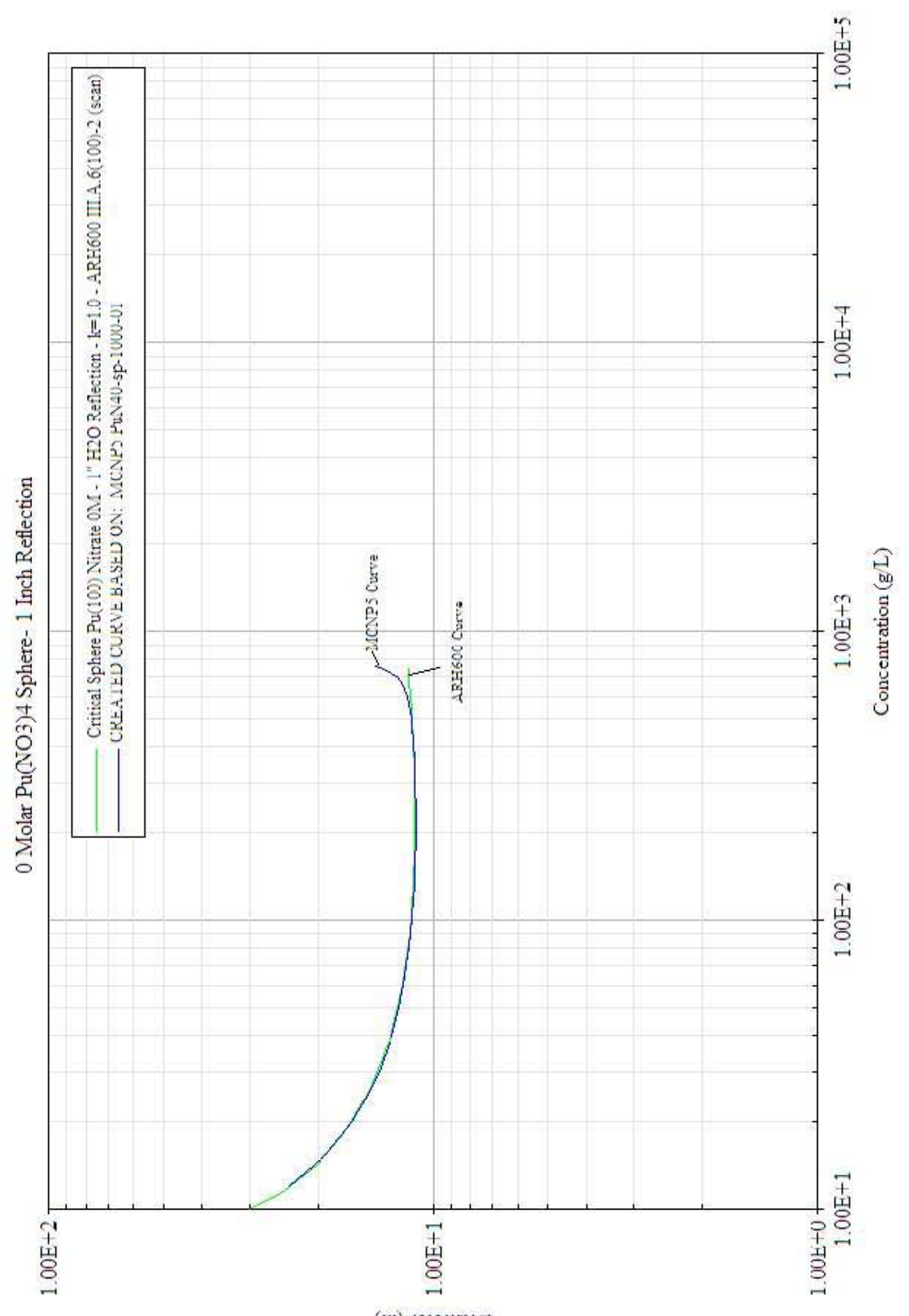

(Ui) .әэวนщ!! 
Table B1.1.1.1.1.2-1. Critical Dimensions for $\mathrm{Pu}\left(\mathrm{NO}_{3}\right) 40 \mathrm{M}-\mathrm{Sphere}-100 \%{ }^{239} \mathrm{Pu}-1$ " Water Reflection

\begin{tabular}{|c|c|c|c|c|c|}
\hline \multicolumn{6}{|c|}{$100 \% \mathrm{Pu} 239 \mathrm{Pu}(\mathrm{NO} 3) 4$ 0M Sphere - 1 Inch of Reflection } \\
\hline Concentration $(\mathrm{g} / \mathrm{cc})$ & \begin{tabular}{|l|} 
Density \\
$(\mathrm{g} / \mathrm{cc})$
\end{tabular} & $\begin{array}{l}\text { First Diameter } \\
\text { (Inches) }\end{array}$ & $\begin{array}{l}\text { Last Diameter } \\
\text { (Inches) }\end{array}$ & \begin{tabular}{|l} 
Delta \\
(Inches)
\end{tabular} & $\begin{array}{l}\text { Critical Diameter } \\
\text { (Inches) } \\
\end{array}$ \\
\hline 0.012 & 1.016 & 19.6 & 24.7 & 0.2 & 23.6804 \\
\hline 0.015 & 1.021 & 16.6 & 20.2 & 0.1 & 19.3958 \\
\hline 0.02 & 1.029 & 14.2 & 16.7 & 0.1 & 16.244 \\
\hline 0.025 & 1.037 & 13.1 & 15.2 & 0.1 & 14.7191 \\
\hline 0.03 & 1.046 & 12.3 & 14.2 & 0.1 & 13.7966 \\
\hline 0.032 & 1.049 & 12.1 & 14 & 0.1 & 13.5365 \\
\hline 0.035 & 1.054 & \begin{tabular}{|l}
1.8 \\
\end{tabular} & 13.6 & 0.1 & 13.2087 \\
\hline 0.04 & 1.063 & 11.4 & 13.2 & 0.1 & 12.7979 \\
\hline 0.05 & 1.08 & 11 & 12.6 & 0.05 & 12.2362 \\
\hline 0.06 & 1.097 & \begin{tabular}{|l|}
10.7 \\
\end{tabular} & 12.2 & \begin{tabular}{|l|}
0.05 \\
\end{tabular} & \begin{tabular}{|l|}
11.883 \\
\end{tabular} \\
\hline 0.07 & 1.113 & 10.5 & 12 & 0.05 & 11.683 \\
\hline 0.08 & 1.13 & 10.4 & 11.8 & 0.05 & 11.5149 \\
\hline 0.09 & 1.147 & 10.3 & 11.7 & 0.05 & 11.399 \\
\hline 0.1 & 1.164 & 10.2 & 11.6 & 0.05 & 11.3167 \\
\hline 0.12 & 1.197 & \begin{tabular}{|l}
10.1 \\
\end{tabular} & 11.5 & 0.05 & 11.2024 \\
\hline 0.13 & 1.215 & 10.1 & 11.5 & 0.05 & 11.1421 \\
\hline 0.15 & 1.248 & 10 & 11.4 & 0.05 & 11.1162 \\
\hline 0.2 & 1.332 & 10 & 11.4 & \begin{tabular}{|l|}
0.05 \\
\end{tabular} & 11.0542 \\
\hline 0.239 & 1.397 & 10 & 11.4 & 0.05 & 11.0426 \\
\hline 0.25 & 1.415 & 10 & 11.4 & 0.05 & 11.0596 \\
\hline 0.359 & 1.594 & 10 & 11.5 & 0.05 & 11.1237 \\
\hline 0.478 & 1.784 & 10.2 & 11.6 & 0.05 & 11.2744 \\
\hline 0.5 & 1.818 & 10.2 & 11.7 & 0.05 & 11.3077 \\
\hline 0.6 & 1.962 & 10.5 & 12 & 0.05 & 11.6531 \\
\hline 0.65 & 2.026 & 10.7 & 12.3 & 0.05 & 11.8933 \\
\hline 0.7 & 2.076 & 11.2 & 12.7 & 0.05 & 12.3762 \\
\hline 0.75 & 2.079 & 12.3 & 14 & 0.05 & 13.6454 \\
\hline 0.752 & 2.073 & 12.4 & 14.2 & 0.1 & 13.803 \\
\hline 0.754 & 2.064 & 12.7 & 14.4 & 0.05 & 14.023 \\
\hline 0.755 & 2.052 & 12.9 & 14.7 & 0.1 & 14.2662 \\
\hline
\end{tabular}




\section{B1.1.1.1.1.3 10" Water Reflector}

Case ID: PuN40-sp-1000-10

Material: $\mathrm{Pu}\left(\mathrm{NO}_{3}\right)_{4} 0$ Molar Excess Acid

Geometry: Sphere

Isotopics: $100 \%{ }^{239} \mathrm{Pu}$

Reflector: 10" Water Reflector

Code: MCNP5 v1.40

Analyst: Mark Murphy

Date: $8 / 2011$ 
Figure B1.1.1.1.1.3-1. $\mathrm{Pu}\left(\mathrm{NO}_{3}\right) 4$ 0M - Sphere $-100 \%{ }^{239} \mathrm{Pu}$ - 10" Water Reflection

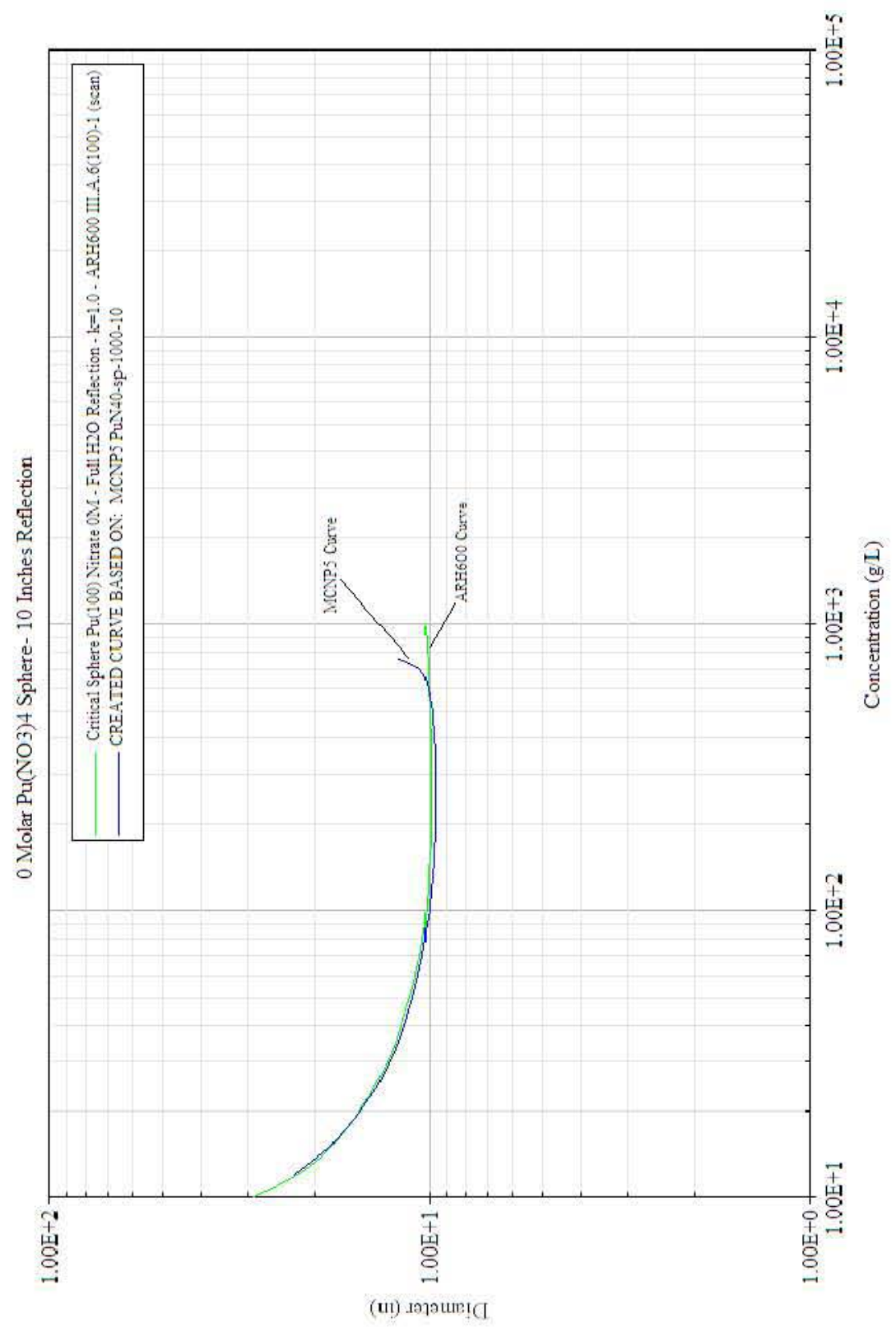


Table B1.1.1.1.1.3-1. Critical Dimensions for $\mathrm{Pu}\left(\mathrm{NO}_{3}\right) 4 \mathrm{OM}-\mathrm{Sphere}-100 \%{ }^{239} \mathrm{Pu}-10 "$ Water Reflection

\begin{tabular}{|c|c|c|c|c|c|}
\hline \multicolumn{6}{|c|}{$100 \% \mathrm{Pu} 239 \mathrm{Pu}(\mathrm{NO} 3) 4$ 0M Sphere - 10 Inches of Reflection } \\
\hline Concentration $(\mathrm{g} / \mathrm{cc})$ & $\begin{array}{l}\text { Density } \\
(\mathrm{g} / \mathrm{cc})\end{array}$ & $\begin{array}{l}\text { First Diameter } \\
\text { (Inches) }\end{array}$ & $\begin{array}{l}\text { Last Diameter } \\
\text { (Inches) }\end{array}$ & $\begin{array}{l}\text { Delta } \\
\text { (Inches) }\end{array}$ & $\begin{array}{l}\text { Critical Diameter } \\
\text { (Inches) } \\
\end{array}$ \\
\hline 0.012 & 1.016 & 18.5 & 23.5 & 0.2 & 22.5941 \\
\hline 0.015 & 1.021 & 15.5 & 18.9 & 0.1 & 18.278 \\
\hline 0.02 & 1.029 & 13.2 & 15.6 & 0.1 & 15.1224 \\
\hline 0.025 & 1.037 & 11.9 & 14 & 0.1 & 13.5784 \\
\hline 0.03 & 1.046 & 11.2 & 13.1 & 0.1 & 12.667 \\
\hline 0.032 & 1.049 & 11 & 12.8 & 0.1 & 12.3805 \\
\hline 0.035 & 1.054 & 10.7 & 12.4 & 0.05 & 12.0395 \\
\hline 0.04 & 1.063 & 10.3 & 12 & 0.05 & 11.6072 \\
\hline 0.05 & 1.08 & 9.8 & 11.4 & 0.05 & 11.0206 \\
\hline 0.06 & 1.097 & 9.5 & 11 & 0.05 & 10.6441 \\
\hline 0.07 & 1.113 & 9.3 & 10.7 & 0.05 & 10.4159 \\
\hline 0.08 & 1.13 & 9.1 & 10.6 & 0.05 & 10.2329 \\
\hline 0.09 & 1.147 & 9 & 10.4 & 0.05 & 10.1082 \\
\hline 0.1 & 1.164 & 8.9 & 10.3 & 0.05 & 9.9986 \\
\hline 0.12 & 1.197 & 8.8 & 10.2 & 0.05 & 9.8696 \\
\hline 0.13 & 1.215 & 8.8 & 10.1 & 0.05 & 9.8238 \\
\hline 0.15 & 1.248 & 8.7 & 10.1 & 0.05 & 9.7448 \\
\hline 0.2 & 1.332 & 8.6 & 10 & 0.05 & 9.6627 \\
\hline 0.239 & 1.397 & 8.6 & 9.9 & 0.05 & 9.6339 \\
\hline 0.25 & 1.415 & 8.6 & 9.9 & 0.05 & 9.6243 \\
\hline 0.359 & 1.594 & 8.6 & 9.9 & 0.05 & 9.6417 \\
\hline 0.478 & 1.784 & 8.7 & 10.1 & 0.05 & 9.7568 \\
\hline 0.5 & 1.818 & 8.7 & 10.1 & 0.05 & 9.7776 \\
\hline 0.6 & 1.962 & 8.9 & 10.4 & 0.05 & 10.0324 \\
\hline 0.65 & 2.026 & 9.1 & 10.6 & 0.05 & 10.2546 \\
\hline 0.7 & 2.076 & 9.4 & 11 & 0.05 & 10.6141 \\
\hline 0.75 & 2.079 & 10.3 & 12 & 0.05 & 11.6064 \\
\hline 0.752 & 2.073 & 10.4 & 12.1 & 0.05 & 11.7187 \\
\hline 0.754 & 2.064 & 10.5 & 12.3 & 0.1 & 11.8904 \\
\hline 0.755 & 2.052 & 10.7 & 12.5 & 0.1 & 12.0879 \\
\hline
\end{tabular}


B1.1.1.1.2 3 Molar Excess Acid

B1.1.1.1.2.1 0" Water Reflector

Case ID: PuN43-sp-1000-00

Material: $\mathrm{Pu}\left(\mathrm{NO}_{3}\right)_{4} 3$ Molar Excess Acid

Geometry: Sphere

Isotopics: $100 \%{ }^{239} \mathrm{Pu}$

Reflector: 0 " water reflected

Code: MCNP5 v1.40

Analyst: Mark Murphy

Date: $8 / 2011$ 
Figure B1.1.1.1.2.1-1. $\mathrm{Pu}\left(\mathrm{NO}_{3}\right) 4$ 3M-Sphere $-100 \%{ }^{239} \mathrm{Pu}-0$ " Water Reflection

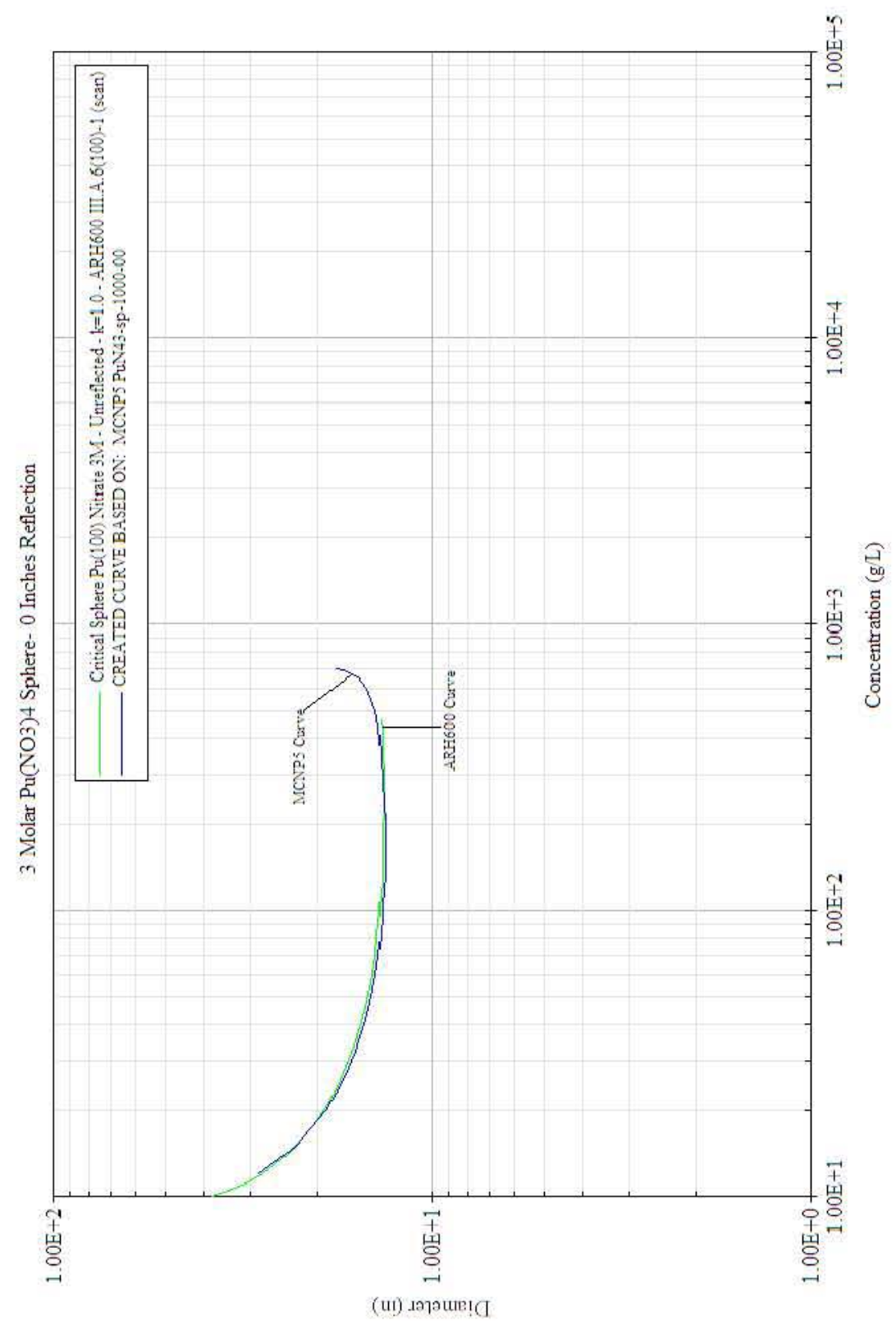


Table B1.1.1.1.2.1-1. Critical Dimensions for Pu(NO3)4 3M - Sphere - 100\% 239Pu - 0" Water Reflections

\begin{tabular}{|c|c|c|c|c|c|}
\hline \multicolumn{6}{|c|}{$100 \% \mathrm{Pu} 239 \mathrm{Pu}(\mathrm{NO} 3) 43 \mathrm{M}$ Sphere - 0 Inches of Reflection } \\
\hline Concentration $(\mathrm{g} / \mathrm{cc})$ & $\begin{array}{l}\text { Density } \\
(\mathrm{g} / \mathrm{cc})\end{array}$ & $\begin{array}{l}\text { First Diameter } \\
\text { (Inches) }\end{array}$ & $\begin{array}{l}\text { Last Diameter } \\
\text { (Inches) }\end{array}$ & $\begin{array}{l}\text { Delta } \\
\text { (Inches) }\end{array}$ & $\begin{array}{l}\text { Critical Diameter } \\
\text { (Inches) }\end{array}$ \\
\hline 0.012 & 1.118 & 23.3 & 29.7 & 0.3 & 28.4707 \\
\hline 0.015 & 1.123 & 19.5 & 23.6 & 0.2 & 22.8116 \\
\hline 0.02 & 1.132 & 16.7 & 19.6 & 0.1 & 18.9681 \\
\hline 0.025 & 1.14 & 15.3 & 17.7 & 0.1 & 17.2009 \\
\hline 0.03 & 1.149 & 14.5 & 16.6 & 0.1 & 16.151 \\
\hline 0.032 & 1.152 & 14.3 & 16.3 & 0.1 & 15.8636 \\
\hline 0.035 & 1.157 & 13.9 & 15.9 & 0.1 & 15.4806 \\
\hline 0.04 & 1.165 & 13.5 & 15.4 & 0.1 & 15.0094 \\
\hline 0.05 & 1.182 & 13 & 14.7 & 0.05 & 14.371 \\
\hline 0.06 & 1.198 & 12.7 & 14.4 & 0.05 & 14.0023 \\
\hline 0.07 & 1.215 & 12.5 & 14.1 & 0.05 & 13.7714 \\
\hline 0.08 & 1.231 & 12.4 & 14 & 0.05 & 13.6007 \\
\hline 0.09 & 1.247 & 12.3 & 13.8 & 0.05 & 13.4759 \\
\hline 0.1 & 1.263 & 12.2 & 13.8 & 0.05 & 13.4063 \\
\hline 0.12 & 1.294 & 12.1 & 13.7 & 0.05 & 13.3144 \\
\hline 0.13 & 1.311 & 12.1 & 13.6 & 0.05 & 13.2571 \\
\hline 0.15 & 1.342 & 12.1 & 13.6 & 0.05 & 13.2353 \\
\hline 0.2 & 1.421 & 12.1 & 13.6 & 0.05 & 13.2372 \\
\hline 0.239 & 1.481 & 12.1 & 13.7 & 0.05 & 13.3043 \\
\hline 0.25 & 1.498 & 12.1 & 13.7 & 0.05 & 13.3231 \\
\hline 0.359 & 1.665 & 12.4 & 14 & 0.05 & 13.5618 \\
\hline 0.478 & 1.841 & 12.7 & 14.3 & 0.05 & 13.9505 \\
\hline 0.5 & 1.871 & 12.8 & 14.4 & 0.05 & 14.07 \\
\hline 0.6 & 1.998 & 13.5 & 15.2 & 0.05 & 14.7937 \\
\hline 0.65 & 2.043 & 14.2 & 15.9 & 0.05 & 15.5452 \\
\hline 0.66 & 2.049 & 14.4 & 16.05 & 0.05 & 15.7538 \\
\hline 0.67 & 2.053 & 14.8 & 16.4 & 0.1 & 16.0442 \\
\hline 0.68 & 2.053 & 15 & 16.8 & 0.1 & 16.4068 \\
\hline 0.69 & 2.045 & 15.5 & 17.4 & 0.1 & 16.9692 \\
\hline 0.698 & 2.017 & 16.5 & 18.5 & 0.1 & 18.0199 \\
\hline
\end{tabular}




\section{B1.1.1.1.2.2 1"Water Reflector}

Case ID: PuN43-sp-1000-01

Material: $\mathrm{Pu}\left(\mathrm{NO}_{3}\right)_{4} 3$ Molar Excess Acid

Geometry: Sphere

Isotopics: $100 \%{ }^{239} \mathrm{Pu}$

Reflector: 1" water reflected

Code: MCNP5 v1.40

Analyst: Mark Murphy

Date: $8 / 2011$ 
Figure B1.1.1.1.2.2-1. Pu(NO $\left.{ }_{3}\right) 43 \mathrm{M}-$ Sphere $-100 \%{ }^{239} \mathrm{Pu}-1$ " Water Reflection

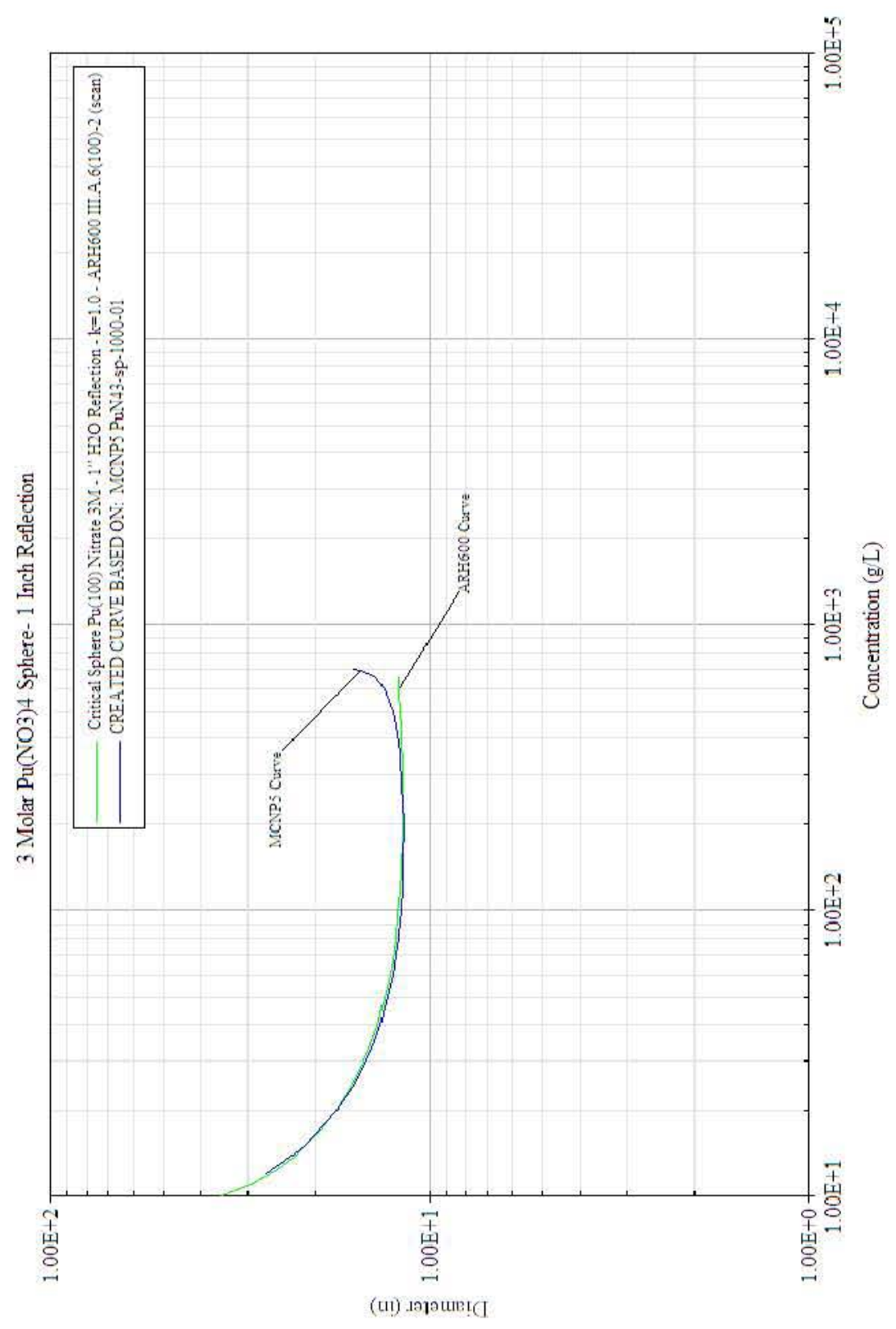


Table B1.1.1.1.2.2-1. Critical Dimensions for $\mathrm{Pu}\left(\mathrm{NO}_{3}\right) 43 \mathrm{M}-\mathrm{Sphere}-100 \%{ }^{239} \mathrm{Pu}-1$ " Water Reflection

\begin{tabular}{|c|c|c|c|c|c|}
\hline \multicolumn{6}{|c|}{$100 \% \mathrm{Pu} 239 \mathrm{Pu}(\mathrm{NO} 3) 43 \mathrm{M}$ Sphere - 1 Inch of Reflection } \\
\hline Concentration $(\mathrm{g} / \mathrm{cc})$ & $\begin{array}{l}\text { Density } \\
(\mathrm{g} / \mathrm{cc})\end{array}$ & $\begin{array}{l}\text { First Diameter } \\
\text { (Inches) }\end{array}$ & $\begin{array}{l}\text { Last Diameter } \\
\text { (Inches) }\end{array}$ & $\begin{array}{l}\text { Delta } \\
\text { (Inches) }\end{array}$ & $\begin{array}{l}\text { Critical Diameter } \\
\text { (Inches) } \\
\end{array}$ \\
\hline 0.012 & 1.118 & 21.8 & 28.2 & 0.3 & 26.9022 \\
\hline 0.015 & 1.123 & 18 & 22.1 & 0.2 & 21.3045 \\
\hline 0.02 & 1.132 & 15.2 & 18 & 0.1 & 17.4545 \\
\hline 0.025 & 1.14 & 13.9 & 16.2 & 0.1 & 15.69 \\
\hline 0.03 & 1.149 & 13 & 15.1 & 0.1 & 14.6221 \\
\hline 0.032 & 1.152 & 12.7 & 14.7 & 0.1 & 14.3224 \\
\hline 0.035 & 1.157 & 12.4 & 14.3 & 0.1 & 13.9325 \\
\hline 0.04 & 1.165 & 12 & 13.9 & 0.1 & 13.4673 \\
\hline 0.05 & 1.182 & 11.5 & 13.2 & 0.05 & 12.8337 \\
\hline 0.06 & 1.198 & 11.2 & 12.8 & 0.05 & 12.467 \\
\hline 0.07 & 1.215 & 11 & 12.6 & 0.05 & 12.2277 \\
\hline 0.08 & 1.231 & 10.8 & 12.4 & 0.05 & 12.0556 \\
\hline 0.09 & 1.247 & 10.7 & 12.3 & 0.05 & 11.9274 \\
\hline 0.1 & 1.263 & 10.7 & 12.2 & 0.05 & 11.8522 \\
\hline 0.12 & 1.294 & 10.6 & 12.1 & 0.05 & 11.7486 \\
\hline 0.13 & 1.311 & 10.6 & 12.1 & 0.05 & 11.6998 \\
\hline 0.15 & 1.342 & 10.5 & 12 & 0.05 & 11.6698 \\
\hline 0.2 & 1.421 & 10.5 & 12 & 0.05 & 11.6634 \\
\hline 0.239 & 1.481 & 10.6 & 12.1 & 0.05 & 11.7204 \\
\hline 0.25 & 1.498 & 10.6 & 12.1 & 0.05 & 11.7404 \\
\hline 0.359 & 1.665 & 10.8 & 12.3 & 0.05 & 11.9651 \\
\hline 0.478 & 1.841 & 11.1 & 12.7 & 0.05 & 12.3227 \\
\hline 0.5 & 1.871 & 11.2 & 12.8 & 0.05 & 12.4224 \\
\hline 0.6 & 1.998 & 11.8 & 13.4 & 0.05 & 13.0912 \\
\hline 0.65 & 2.043 & 12.4 & 14.1 & 0.05 & 13.7548 \\
\hline 0.66 & 2.049 & 12.6 & 14.3 & 0.05 & 13.9309 \\
\hline 0.67 & 2.053 & 12.8 & 14.5 & 0.05 & 14.1749 \\
\hline 0.68 & 2.053 & 13.1 & 14.9 & 0.1 & 14.4869 \\
\hline 0.69 & 2.045 & 13.5 & 15.4 & 0.1 & 14.9934 \\
\hline 0.698 & 2.017 & 14.3 & 16.3 & 0.1 & 15.9224 \\
\hline
\end{tabular}




\section{B1.1.1.1.2.3 10" Water Reflector}

Case ID: PuN43-sp-1000-10

Material: $\mathrm{Pu}\left(\mathrm{NO}_{3}\right)_{4} 3$ Molar Excess Acid

Geometry: Sphere

Isotopics: $100 \%{ }^{239} \mathrm{Pu}$

Reflector: 10 " water reflected

Code: MCNP5 v1.40

Analyst: Mark Murphy

Date: $8 / 2011$ 
Figure B1.1.1.1.2.3-1. $\mathrm{Pu}\left(\mathrm{NO}_{3}\right) 4$ 3M - Sphere - $100 \%{ }^{239} \mathrm{Pu}-10$ " Water Reflection

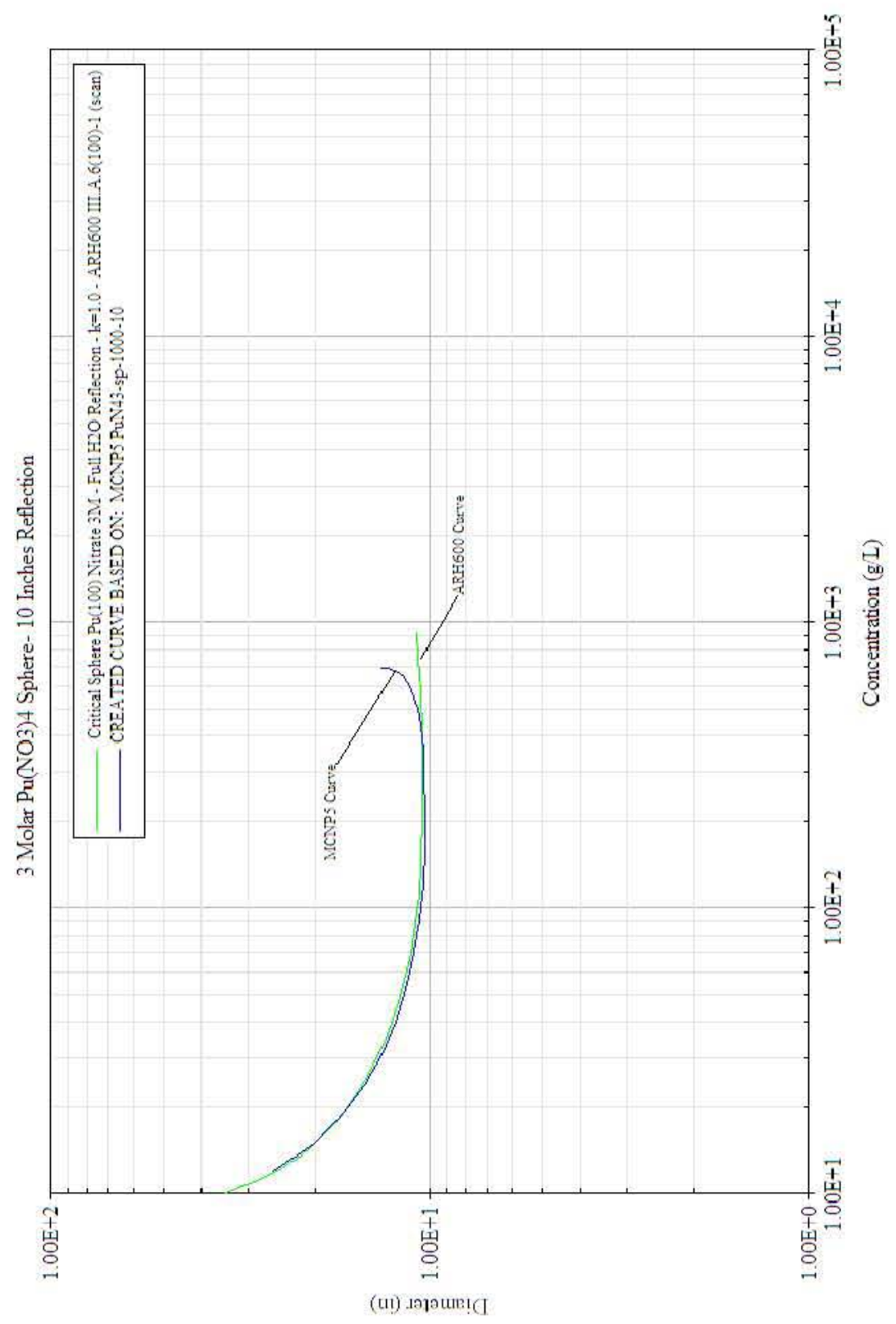


Table B1.1.1.1.2.3-1. Critical Dimensions for $\mathrm{Pu}\left(\mathrm{NO}_{3}\right) 43 \mathrm{M}-$ Sphere $-100 \%{ }^{239} \mathrm{Pu}-10$ " Water Reflection

\begin{tabular}{|c|c|c|c|c|c|}
\hline \multicolumn{6}{|c|}{$100 \% \mathrm{Pu} 239 \mathrm{Pu}(\mathrm{NO} 3) 43 \mathrm{M}$ Sphere - 10 Inches of Reflection } \\
\hline Concentration $(\mathrm{g} / \mathrm{cc})$ & $\begin{array}{l}\text { Density } \\
(\mathrm{g} / \mathrm{cc})\end{array}$ & $\begin{array}{l}\text { First Diameter } \\
\text { (Inches) }\end{array}$ & $\begin{array}{l}\text { Last Diameter } \\
\text { (Inches) }\end{array}$ & $\begin{array}{l}\text { Delta } \\
\text { (Inches) }\end{array}$ & $\begin{array}{l}\text { Critical Diameter } \\
\text { (Inches) } \\
\end{array}$ \\
\hline 0.012 & 1.118 & 20.7 & 26.9 & 0.3 & 25.8375 \\
\hline 0.015 & 1.123 & 16.9 & 20.9 & 0.2 & 20.147 \\
\hline 0.02 & 1.132 & 14.1 & 16.8 & 0.1 & 16.3107 \\
\hline 0.025 & 1.14 & 12.7 & 15 & 0.1 & 14.5013 \\
\hline 0.03 & 1.149 & 11.8 & 13.9 & 0.1 & 13.4248 \\
\hline 0.032 & 1.152 & 11.6 & 13.6 & 0.1 & 13.1199 \\
\hline 0.035 & 1.157 & 11.3 & 13.1 & 0.1 & 12.7283 \\
\hline 0.04 & 1.165 & 10.9 & 12.6 & 0.05 & 12.2358 \\
\hline 0.05 & 1.182 & 10.3 & 11.9 & 0.05 & 11.577 \\
\hline 0.06 & 1.198 & 10 & 11.5 & 0.05 & 11.1796 \\
\hline 0.07 & 1.215 & 9.7 & 11.2 & 0.05 & 10.898 \\
\hline 0.08 & 1.231 & 9.5 & 11 & 0.05 & 10.7152 \\
\hline 0.09 & 1.247 & 9.4 & 10.9 & 0.05 & 10.5771 \\
\hline 0.1 & 1.263 & 9.3 & 10.8 & 0.05 & 10.481 \\
\hline 0.12 & 1.294 & 9.2 & 10.7 & 0.05 & 10.3447 \\
\hline 0.13 & 1.311 & 9.2 & 10.6 & 0.05 & 10.2927 \\
\hline 0.15 & 1.342 & 9.1 & 10.5 & 0.05 & 10.2474 \\
\hline 0.2 & 1.421 & 9.1 & 10.5 & 0.05 & 10.1829 \\
\hline 0.239 & 1.481 & 9.1 & 10.5 & 0.05 & 10.1924 \\
\hline 0.25 & 1.498 & 9.1 & 10.5 & 0.05 & 10.199 \\
\hline 0.359 & 1.665 & 9.2 & 10.7 & 0.05 & 10.3483 \\
\hline 0.478 & 1.841 & 9.4 & 10.9 & 0.05 & 10.611 \\
\hline 0.5 & 1.871 & 9.5 & 11.05 & 0.05 & 10.6835 \\
\hline 0.6 & 1.998 & 10 & 11.5 & 0.05 & 11.2222 \\
\hline 0.65 & 2.043 & 10.4 & 12.1 & 0.05 & 11.7268 \\
\hline 0.66 & 2.049 & 10.6 & 12.3 & 0.05 & 11.8749 \\
\hline 0.67 & 2.053 & 10.7 & 12.4 & 0.05 & 12.0672 \\
\hline 0.68 & 2.053 & 10.9 & 12.7 & 0.1 & 12.3104 \\
\hline 0.69 & 2.045 & 11.2 & 13.1 & 0.1 & 12.7144 \\
\hline 0.698 & 2.017 & 11.9 & 13.8 & 0.1 & 13.405 \\
\hline
\end{tabular}


B1.1.1.1.3 6 Molar Excess Acid

B1.1.1.1.3.1 0"Water Reflector

Case ID: PuN46-sp-1000-00

Material: $\mathrm{Pu}\left(\mathrm{NO}_{3}\right)_{4} 6$ Molar Excess Acid

Geometry: Sphere

Isotopics: $100 \%{ }^{239} \mathrm{Pu}$

Reflector: 0" water reflected

Code: MCNP5 v1.40

Analyst: Mark Murphy

Date: $8 / 2011$ 
Figure B1.1.1.1.3.1-1. $\mathrm{Pu}\left(\mathrm{NO}_{3}\right) 4$ 6M-Sphere - $100 \%{ }^{239} \mathrm{Pu}-0$ " Water Reflection

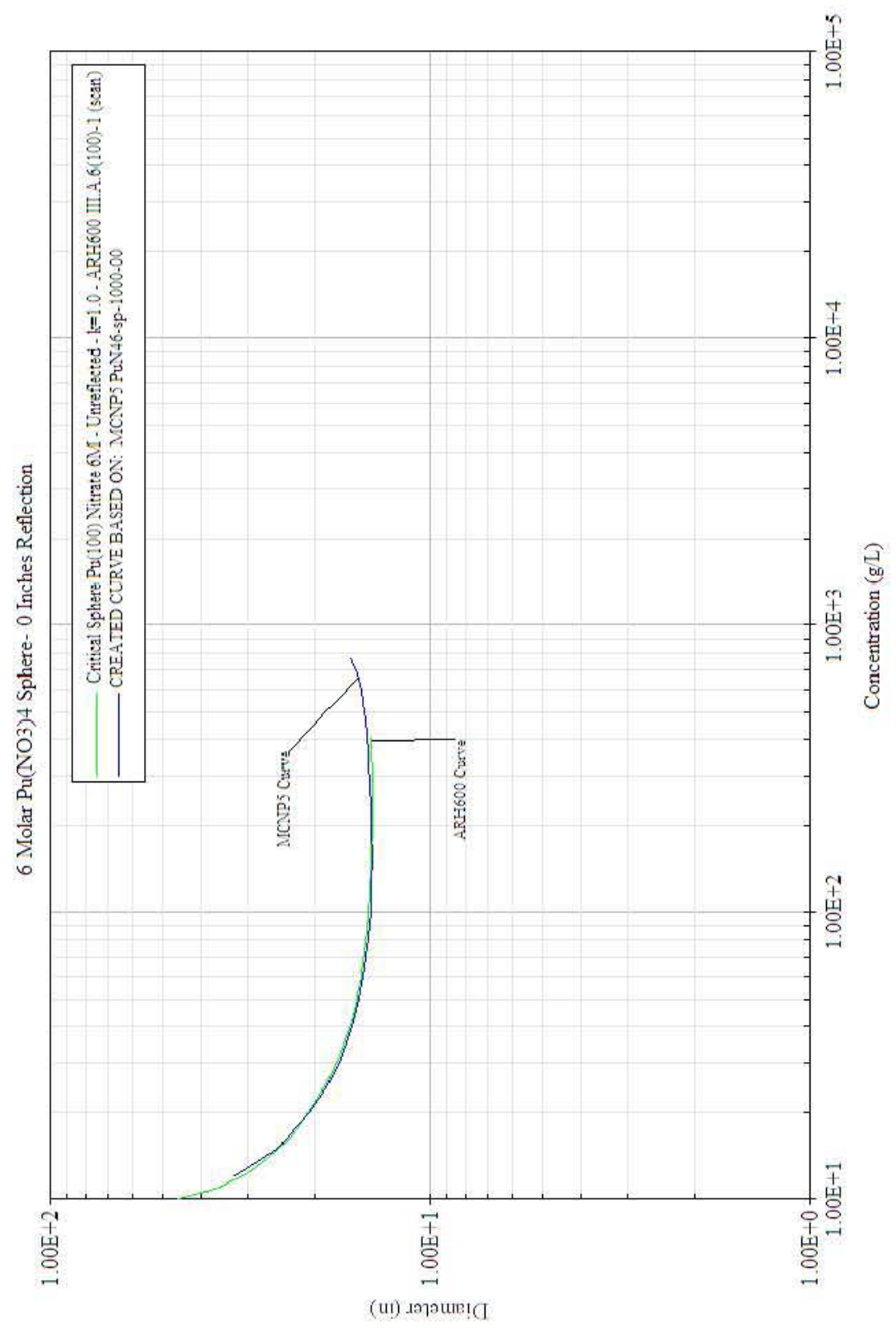


Table B1.1.1.1.3.1-1. Critical Dimensions for $\mathrm{Pu}\left(\mathrm{NO}_{3}\right) 46 \mathrm{M}-\mathrm{Sphere}-100 \%{ }^{239} \mathrm{Pu}-0$ " Water Reflection

\begin{tabular}{|c|c|c|c|c|c|}
\hline \multicolumn{6}{|c|}{$100 \% \mathrm{Pu} 239 \mathrm{Pu}(\mathrm{NO} 3) 46 \mathrm{M}$ Sphere - 0 Inches of Reflection } \\
\hline Concentration $(\mathrm{g} / \mathrm{cc})$ & \begin{tabular}{|l|} 
Density \\
$(\mathrm{g} / \mathrm{cc})$ \\
\end{tabular} & $\begin{array}{l}\text { First Diameter } \\
\text { (Inches) }\end{array}$ & $\begin{array}{l}\text { Last Diameter } \\
\text { (Inches) }\end{array}$ & $\begin{array}{l}\text { Delta } \\
\text { (Inches) }\end{array}$ & $\begin{array}{l}\text { Critical Diameter } \\
\text { (Inches) }\end{array}$ \\
\hline 0.012 & 1.207 & 26.1 & 34 & 0.4 & 32.5438 \\
\hline 0.015 & 1.212 & 21.3 & 26.1 & 0.2 & 25.1795 \\
\hline 0.02 & 1.22 & 18 & 21.2 & 0.1 & 20.5363 \\
\hline 0.025 & 1.229 & 16.4 & 19 & 0.1 & 18.4537 \\
\hline 0.03 & 1.237 & 15.5 & 17.7 & 0.1 & 17.2569 \\
\hline 0.032 & 1.24 & 15.2 & 17.4 & 0.1 & 16.9092 \\
\hline 0.035 & 1.245 & 14.8 & 16.9 & 0.1 & 16.498 \\
\hline 0.04 & 1.253 & 14.4 & 16.4 & 0.1 & 15.9575 \\
\hline 0.05 & 1.27 & 13.8 & 15.7 & 0.1 & 15.2671 \\
\hline 0.06 & 1.286 & 13.5 & 15.2 & 0.05 & 14.8545 \\
\hline 0.07 & 1.301 & 13.3 & 15 & 0.05 & 14.5993 \\
\hline 0.08 & 1.317 & 13.1 & 14.8 & 0.05 & 14.4186 \\
\hline 0.09 & 1.333 & 13 & 14.7 & 0.05 & 14.294 \\
\hline 0.1 & 1.349 & 12.9 & 14.6 & 0.05 & 14.1966 \\
\hline 0.12 & 1.379 & 12.9 & 14.5 & 0.05 & 14.1246 \\
\hline 0.13 & 1.395 & 12.8 & 14.4 & 0.05 & 14.0877 \\
\hline 0.15 & 1.426 & 12.8 & 14.4 & 0.05 & 14.0575 \\
\hline 0.2 & 1.503 & 12.8 & 14.5 & 0.05 & 14.1001 \\
\hline 0.239 & 1.563 & 12.9 & 14.6 & 0.1 & 14.1691 \\
\hline 0.25 & 1.58 & 12.9 & 14.5 & 0.05 & 14.188 \\
\hline 0.359 & 1.748 & 13.2 & 14.8 & 0.05 & 14.4352 \\
\hline 0.478 & 1.935 & 13.4 & 15.1 & 0.05 & 14.6908 \\
\hline 0.5 & 1.969 & 13.5 & 15.1 & 0.05 & 14.7547 \\
\hline 0.6 & 2.122 & 13.7 & 15.5 & 0.1 & 15.0641 \\
\hline 0.65 & 2.197 & 13.9 & 15.7 & 0.1 & 15.2593 \\
\hline 0.7 & 2.268 & 14.2 & 15.9 & 0.05 & 15.5397 \\
\hline 0.75 & 2.335 & 14.5 & 16.3 & 0.1 & 15.9348 \\
\hline 0.76 & 2.348 & 14.6 & 16.4 & 0.1 & 16.0107 \\
\hline 0.765 & 2.354 & 14.6 & 16.4 & 0.1 & 16.0437 \\
\hline 0.77 & 2.36 & 14.7 & 16.5 & 0.1 & 16.1056 \\
\hline 0.775 & 2.367 & 14.7 & 16.6 & 0.1 & 16.1453 \\
\hline
\end{tabular}




\section{B1.1.1.1.3.2 1" Water Reflector}

Case ID: PuN46-sp-1000-01

Material: $\mathrm{Pu}\left(\mathrm{NO}_{3}\right)_{4} 6$ Molar Excess Acid

Geometry: Sphere

Isotopics: $100 \%{ }^{239} \mathrm{Pu}$

Reflector: 1" water reflected

Code: MCNP5 v1.40

Analyst: Mark Murphy

Date: $8 / 2011$ 
Figure B1.1.1.1.3.2-1. $\mathrm{Pu}\left(\mathrm{NO}_{3}\right) 4$ 6M-Sphere - $100 \%{ }^{239} \mathrm{Pu}-1$ " Water Reflection

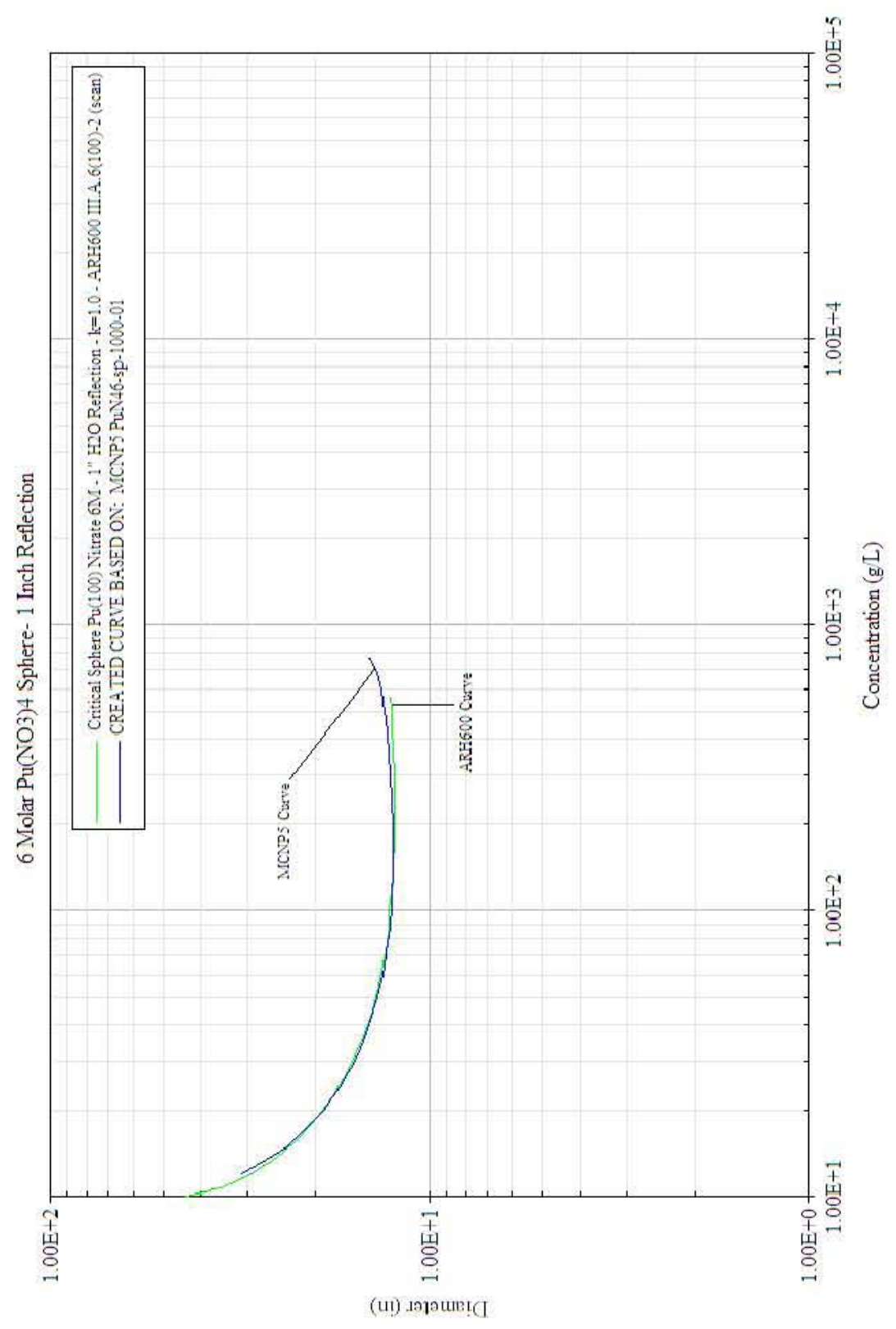


Table B1.1.1.1.3.2-1. Critical Dimensions for $\mathrm{Pu}\left(\mathrm{NO}_{3}\right) 46 \mathrm{M}-\mathrm{Sphere}-100 \%{ }^{239} \mathrm{Pu}-1$ " Water Reflection

\begin{tabular}{|c|c|c|c|c|c|}
\hline \multicolumn{6}{|c|}{$100 \% \mathrm{Pu} 239 \mathrm{Pu}(\mathrm{NO} 3) 46 \mathrm{M}$ Sphere - 1 Inch of Reflection } \\
\hline Concentration $(\mathrm{g} / \mathrm{cc})$ & $\begin{array}{l}\text { Density } \\
(\mathrm{g} / \mathrm{cc})\end{array}$ & $\begin{array}{l}\text { First Diameter } \\
\text { (Inches) }\end{array}$ & $\begin{array}{l}\text { Last Diameter } \\
\text { (Inches) }\end{array}$ & $\begin{array}{l}\text { Delta } \\
\text { (Inches) }\end{array}$ & $\begin{array}{l}\text { Critical Diameter } \\
\text { (Inches) } \\
\end{array}$ \\
\hline 0.012 & 1.207 & 24.4 & 32.6 & 0.4 & 31.0286 \\
\hline 0.015 & 1.212 & 19.8 & 24.5 & 0.2 & 23.6238 \\
\hline 0.02 & 1.22 & 16.4 & 19.6 & 0.1 & 18.9541 \\
\hline 0.025 & 1.229 & 14.9 & 17.3 & 0.1 & 16.8655 \\
\hline 0.03 & 1.237 & 13.9 & 16.2 & 0.1 & 15.6658 \\
\hline 0.032 & 1.24 & 13.6 & 15.8 & 0.1 & 15.3319 \\
\hline 0.035 & 1.245 & 13.3 & 15.3 & 0.1 & 14.9 \\
\hline 0.04 & 1.253 & 12.8 & 14.8 & 0.1 & 14.3639 \\
\hline 0.05 & 1.27 & 12.2 & 14.1 & 0.1 & 13.6668 \\
\hline 0.06 & 1.286 & 11.9 & 13.6 & 0.05 & 13.2492 \\
\hline 0.07 & 1.301 & 11.6 & 13.4 & 0.1 & \begin{tabular}{|l|}
13.0137 \\
\end{tabular} \\
\hline 0.08 & 1.317 & 11.5 & 13.2 & 0.05 & 12.8111 \\
\hline 0.09 & 1.333 & 11.4 & 13.1 & 0.05 & 12.6835 \\
\hline 0.1 & 1.349 & 11.3 & 13 & 0.05 & 12.5983 \\
\hline 0.12 & 1.379 & 11.35 & 12.9 & 0.05 & 12.5064 \\
\hline 0.13 & 1.395 & 11.2 & 12.8 & 0.05 & 12.4635 \\
\hline 0.15 & 1.426 & 11.2 & 12.8 & 0.05 & 12.4378 \\
\hline 0.2 & 1.503 & 11.2 & 12.8 & 0.05 & 12.4367 \\
\hline 0.239 & 1.563 & 11.3 & 12.9 & 0.05 & 12.5225 \\
\hline 0.25 & 1.58 & 11.3 & 12.9 & 0.05 & 12.5355 \\
\hline 0.359 & 1.748 & 11.5 & 13.1 & 0.05 & 12.7834 \\
\hline 0.478 & 1.935 & 11.7 & 13.4 & 0.05 & 13.023 \\
\hline 0.5 & 1.969 & 11.8 & 13.5 & 0.05 & 13.0763 \\
\hline 0.6 & 2.122 & 12.1 & 13.8 & 0.05 & 13.3679 \\
\hline 0.65 & 2.197 & 12.2 & 14 & 0.1 & 13.5487 \\
\hline 0.7 & 2.268 & 12.5 & 14.2 & 0.05 & \begin{tabular}{|l|}
13.811 \\
\end{tabular} \\
\hline 0.75 & 2.335 & \begin{tabular}{|l|}
12.8 \\
\end{tabular} & 14.6 & 0.1 & 14.1695 \\
\hline 0.76 & 2.348 & 12.9 & 14.6 & 0.05 & 14.2362 \\
\hline 0.765 & 2.354 & 12.9 & 14.7 & 0.1 & 14.3011 \\
\hline 0.77 & 2.36 & 12.9 & 14.7 & 0.1 & 14.3402 \\
\hline 0.775 & 2.367 & 13 & 14.8 & 0.1 & 14.3788 \\
\hline
\end{tabular}




\section{B1.1.1.1.3.3 10" Water Reflector}

Case ID: PuN46-sp-1000-10

Material: $\mathrm{Pu}\left(\mathrm{NO}_{3}\right)_{4} 6$ Molar Excess Acid

Geometry: Sphere

Isotopics: $100 \%{ }^{239} \mathrm{Pu}$

Reflector: 10 " water reflected

Code: MCNP5 v1.40

Analyst: Mark Murphy

Date: $8 / 2011$ 
Figure B1.1.1.1.3.3-1. $\mathrm{Pu}\left(\mathrm{NO}_{3}\right) 4$ 6M - Sphere - $100 \%{ }^{239} \mathrm{Pu}-10$ " Water Reflection

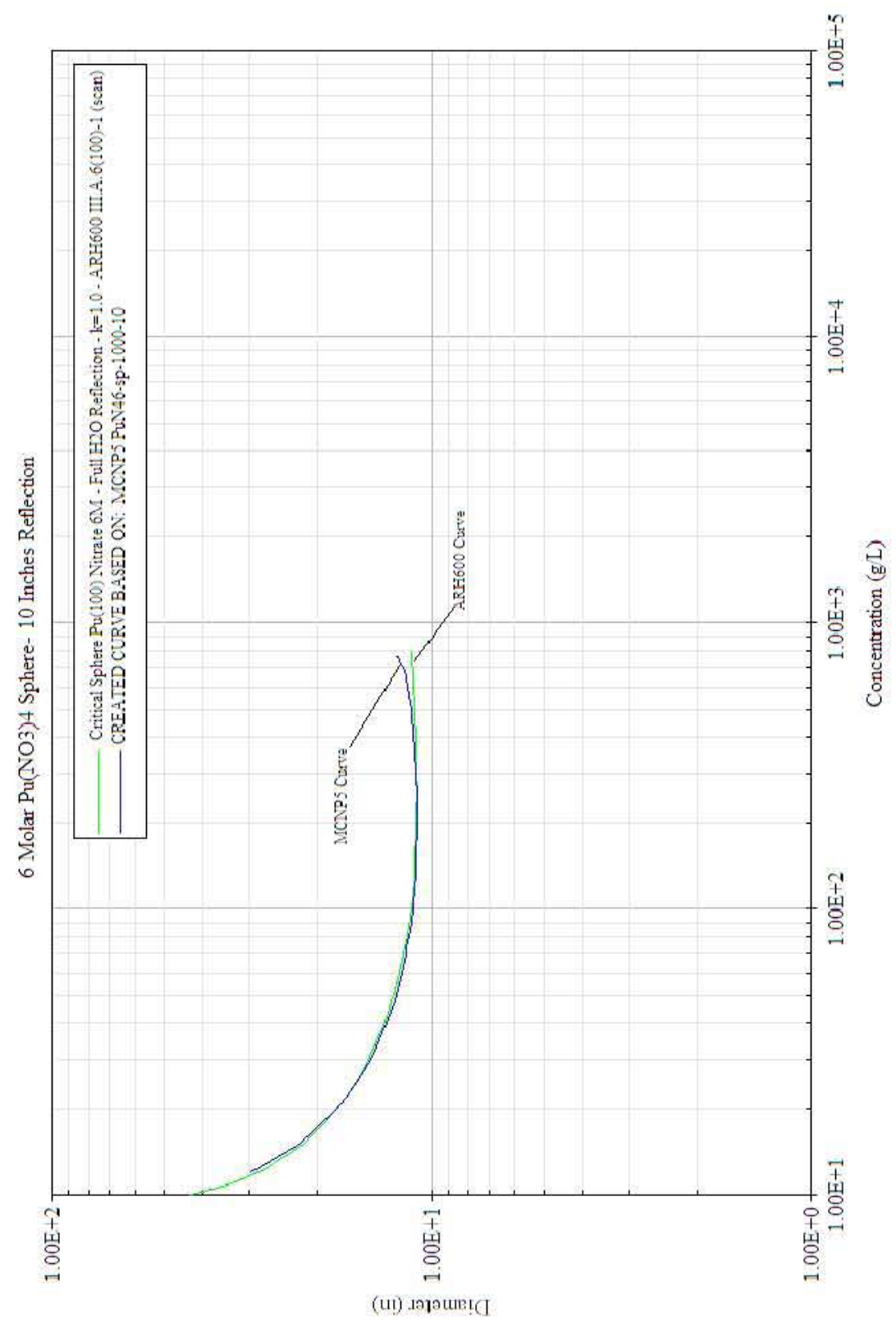


Table B1.1.1.1.3.3-1. Critical Dimensions for $\mathrm{Pu}\left(\mathrm{NO}_{3}\right) 46 \mathrm{M}-$ Sphere $-100 \%{ }^{239} \mathrm{Pu}-10$ " Water Reflection

\begin{tabular}{|c|c|c|c|c|c|}
\hline \multicolumn{6}{|c|}{$100 \% \mathrm{Pu} 239 \mathrm{Pu}(\mathrm{NO} 3) 46 \mathrm{M}$ Sphere - 10 Inches of Reflection } \\
\hline Concentration $(\mathrm{g} / \mathrm{cc})$ & $\begin{array}{l}\text { Density } \\
(\mathrm{g} / \mathrm{cc})\end{array}$ & $\begin{array}{l}\text { First Diameter } \\
\text { (Inches) }\end{array}$ & $\begin{array}{l}\text { Last Diameter } \\
\text { (Inches) }\end{array}$ & $\begin{array}{l}\text { Delta } \\
\text { (Inches) }\end{array}$ & $\begin{array}{l}\text { Critical Diameter } \\
\text { (Inches) }\end{array}$ \\
\hline 0.012 & 1.207 & 23.4 & 31.6 & 0.4 & 29.8142 \\
\hline 0.015 & 1.212 & 18.6 & 23.3 & 0.2 & 22.399 \\
\hline 0.02 & 1.22 & 15.3 & 18.4 & 0.1 & 17.7215 \\
\hline 0.025 & 1.229 & 13.6 & 16.1 & 0.1 & 15.6154 \\
\hline 0.03 & 1.237 & 12.7 & 14.9 & 0.1 & 14.3922 \\
\hline 0.032 & 1.24 & 12.4 & 14.5 & 0.1 & 14.0473 \\
\hline 0.035 & 1.245 & 12 & 14 & 0.1 & 13.6147 \\
\hline 0.04 & 1.253 & 11.6 & 13.5 & 0.1 & 13.0585 \\
\hline 0.05 & 1.27 & 10.9 & 12.7 & 0.1 & 12.3242 \\
\hline 0.06 & 1.286 & 10.6 & 12.2 & 0.05 & 11.8955 \\
\hline 0.07 & 1.301 & 10.3 & 12 & 0.05 & 11.6045 \\
\hline 0.08 & 1.317 & 10.2 & 11.7 & 0.05 & \begin{tabular}{|l}
1.397 \\
\end{tabular} \\
\hline 0.09 & 1.333 & 10 & 11.6 & 0.05 & 11.2398 \\
\hline 0.1 & 1.349 & 9.9 & 11.5 & 0.05 & 11.1352 \\
\hline 0.12 & 1.379 & 9.8 & 11.3 & 0.05 & 11.0109 \\
\hline 0.13 & 1.395 & 9.8 & 11.3 & 0.05 & 10.9592 \\
\hline 0.15 & 1.426 & 9.7 & 11.2 & 0.05 & 10.8943 \\
\hline 0.2 & 1.503 & 9.6 & 11.2 & 0.05 & 10.8481 \\
\hline 0.239 & 1.563 & 9.7 & 11.2 & 0.05 & 10.8672 \\
\hline 0.25 & 1.58 & 9.7 & 11.2 & 0.05 & 10.8865 \\
\hline 0.359 & 1.748 & 9.8 & 11.4 & 0.05 & 11.0397 \\
\hline 0.478 & 1.935 & 10 & 11.6 & 0.05 & 11.2146 \\
\hline 0.5 & 1.969 & 10 & 11.6 & 0.05 & 11.249 \\
\hline 0.6 & 2.122 & 10.2 & 11.8 & 0.05 & 11.4863 \\
\hline 0.65 & 2.197 & 10.3 & 12 & 0.05 & 11.6409 \\
\hline 0.7 & 2.268 & 10.5 & 12.2 & 0.05 & 11.8434 \\
\hline 0.75 & 2.335 & 10.7 & 12.5 & 0.1 & 12.1285 \\
\hline 0.76 & 2.348 & 10.8 & 12.6 & 0.1 & 12.1982 \\
\hline 0.765 & 2.354 & 10.9 & 12.6 & 0.05 & 12.2401 \\
\hline 0.77 & 2.36 & 10.9 & 12.6 & 0.05 & 12.2901 \\
\hline 0.775 & 2.367 & 10.9 & 12.7 & 0.1 & 12.2976 \\
\hline
\end{tabular}


B1.1.1.2 $97 \%{ }^{239} \mathrm{Pu}$

B1.1.1.2.1 0 Molar Excess Acid

B1.1.1.2.1.1 0" Water Reflection

Case ID: PuN40-sp-0970-00

Material: $\mathrm{Pu}\left(\mathrm{NO}_{3}\right)_{4} \quad 0$ Molar Excess Acid

Geometry: Sphere

Isotopics: $97 \%{ }^{239} \mathrm{Pu}$

Reflector: 0" water reflected

Code: MCNP5 v1.40

Analyst: Mark Murphy

Date: $8 / 2011$ 


\section{CHPRC-01552, Revision 0}

Figure B1.1.1.2.1.1-1. Pu(NO $\mathrm{NO}_{3} 40 \mathrm{M}$ - Sphere - 97\% ${ }^{239} \mathrm{Pu}$ - 0" Water Reflection

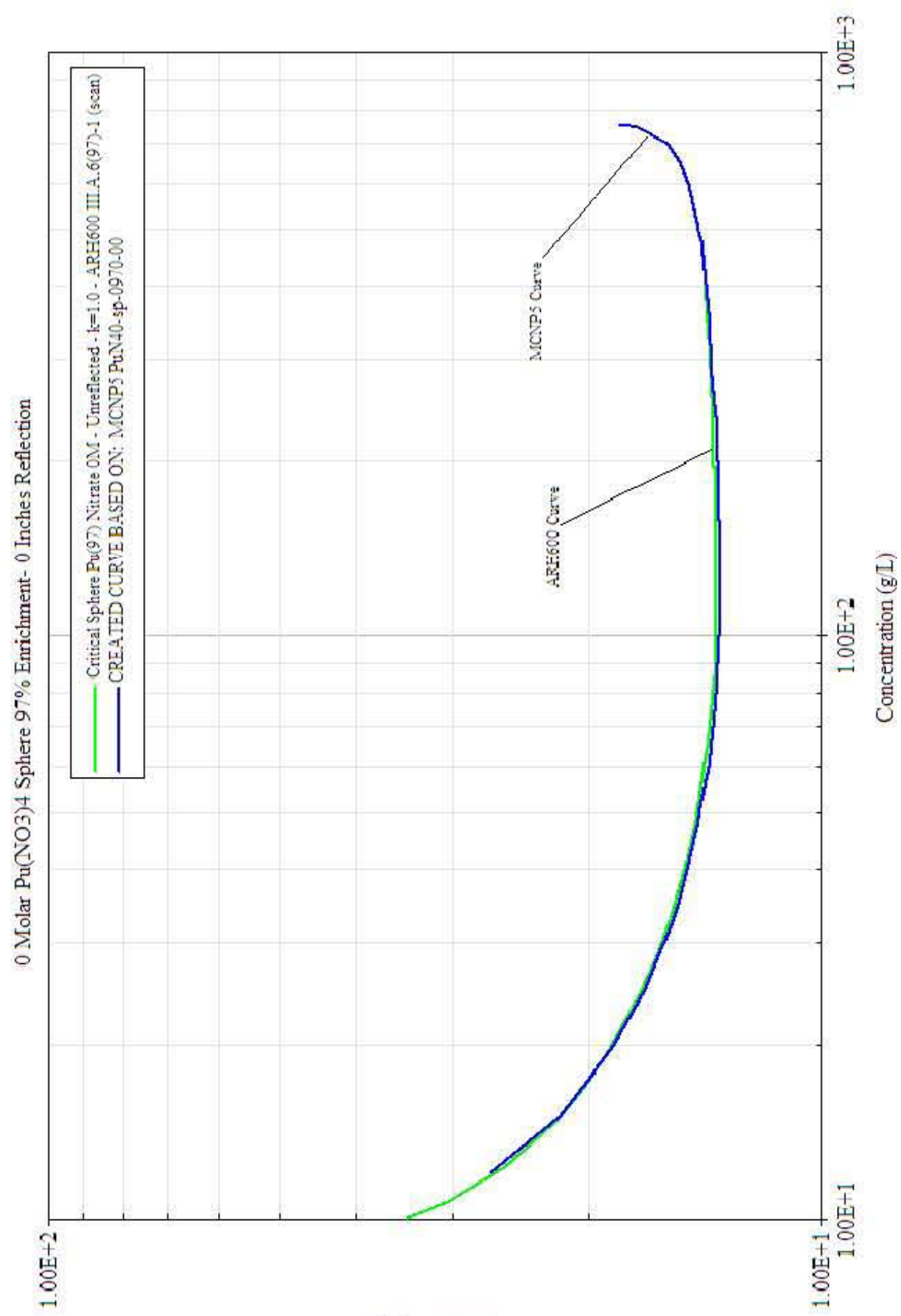

(ui) .эрэщес 
Table B1.1.1.2.1.1-1. Critical Dimensions for $\mathrm{Pu}\left(\mathrm{NO}_{3}\right) 4 \mathrm{OM}-\mathrm{Sphere}-97 \%{ }^{239} \mathrm{Pu}-0$ " Water Reflection

\begin{tabular}{|c|c|c|c|c|c|}
\hline \multicolumn{6}{|c|}{ 97\% Pu239 Pu(NO3)4 0M Sphere- 0 Inches Reflection } \\
\hline Concentration $(\mathrm{g} / \mathrm{cc})$ & $\begin{array}{l}\text { Density } \\
(\mathrm{g} / \mathrm{cc})\end{array}$ & $\begin{array}{l}\text { First Diameter } \\
\text { (Inches) }\end{array}$ & $\begin{array}{l}\text { Last Diameter } \\
\text { (Inches) }\end{array}$ & $\begin{array}{l}\text { Delta } \\
\text { (Inches) }\end{array}$ & $\begin{array}{l}\text { Critical Diameter } \\
\text { (Inches) }\end{array}$ \\
\hline 0.012 & 1.016 & 22.1 & 27.7 & 0.2 & 26.7988 \\
\hline 0.015 & 1.021 & 18.8 & 22.8 & 0.2 & 21.8715 \\
\hline 0.02 & 1.029 & 16.3 & 19 & 0.1 & 18.4693 \\
\hline 0.025 & 1.037 & 15 & 17.3 & 0.1 & 16.8214 \\
\hline 0.03 & 1.046 & 14.2 & 16.3 & 0.1 & 15.8962 \\
\hline 0.032 & 1.049 & 14 & 16 & 0.1 & 15.5977 \\
\hline 0.035 & 1.054 & 13.7 & 15.6 & 0.1 & 15.2625 \\
\hline 0.04 & 1.063 & 13.4 & 15.3 & 0.1 & 14.8278 \\
\hline 0.05 & 1.08 & \begin{tabular}{|l}
12.9 \\
\end{tabular} & 14.7 & 0.1 & 14.2829 \\
\hline 0.06 & 1.097 & 12.6 & 14.3 & 0.05 & 13.9626 \\
\hline 0.07 & 1.113 & 12.5 & 14.1 & 0.05 & 13.7742 \\
\hline 0.08 & 1.13 & 12.4 & 14 & 0.05 & 13.649 \\
\hline 0.09 & 1.147 & 12.3 & 13.9 & 0.05 & 13.5652 \\
\hline 0.1 & 1.164 & 12.3 & 13.9 & 0.05 & 13.5165 \\
\hline 0.12 & 1.197 & 12.2 & 13.9 & 0.05 & 13.4851 \\
\hline 0.13 & 1.215 & 12.2 & 13.8 & 0.05 & 13.4525 \\
\hline 0.15 & 1.248 & 12.2 & 13.8 & 0.05 & 13.4791 \\
\hline 0.2 & 1.332 & 12.3 & 13.9 & 0.05 & 13.5525 \\
\hline 0.239 & 1.397 & 12.4 & 14.1 & 0.05 & 13.6584 \\
\hline 0.25 & 1.415 & 12.4 & 14 & 0.1 & 13.6721 \\
\hline 0.359 & 1.594 & 12.6 & 14.3 & 0.05 & 13.9422 \\
\hline 0.478 & 1.784 & 12.9 & 14.6 & 0.05 & 14.25 \\
\hline 0.5 & 1.818 & 12.9 & 14.7 & 0.1 & 14.346 \\
\hline 0.6 & 1.962 & 13.3 & 15.2 & 0.1 & 14.8084 \\
\hline 0.65 & 2.026 & 13.8 & 15.6 & 0.1 & 15.1717 \\
\hline 0.7 & 2.076 & 14.2 & 16.2 & 0.1 & 15.7801 \\
\hline 0.75 & 2.08 & 15.6 & 17.8 & 0.1 & 17.3634 \\
\hline 0.752 & 2.074 & 15.8 & 18 & 0.1 & 17.5542 \\
\hline 0.754 & 2.064 & 16.1 & 18.3 & 0.1 & 17.8576 \\
\hline 0.755 & 2.054 & 16.3 & 18.6 & 0.1 & 18.131 \\
\hline
\end{tabular}




\section{B1.1.1.2.1.2 1"Water Reflection}

Case ID: PuN40-sp-0970-01

Material: $\mathrm{Pu}\left(\mathrm{NO}_{3}\right)_{4} 0$ Molar Excess Acid

Geometry: Sphere

Isotopics: $97 \%{ }^{239} \mathrm{Pu}$

Reflector: 1" water reflected

Code: MCNP5 v1.40

Analyst: Mark Murphy

Date: $8 / 2011$ 


\section{CHPRC-01552, Revision 0}

Figure B1.1.1.2.1.2-1. $\mathrm{Pu}\left(\mathrm{NO}_{3}\right) 40 \mathrm{M}$ - Sphere - 97\% ${ }^{239} \mathrm{Pu}$ - 1" Water Reflection

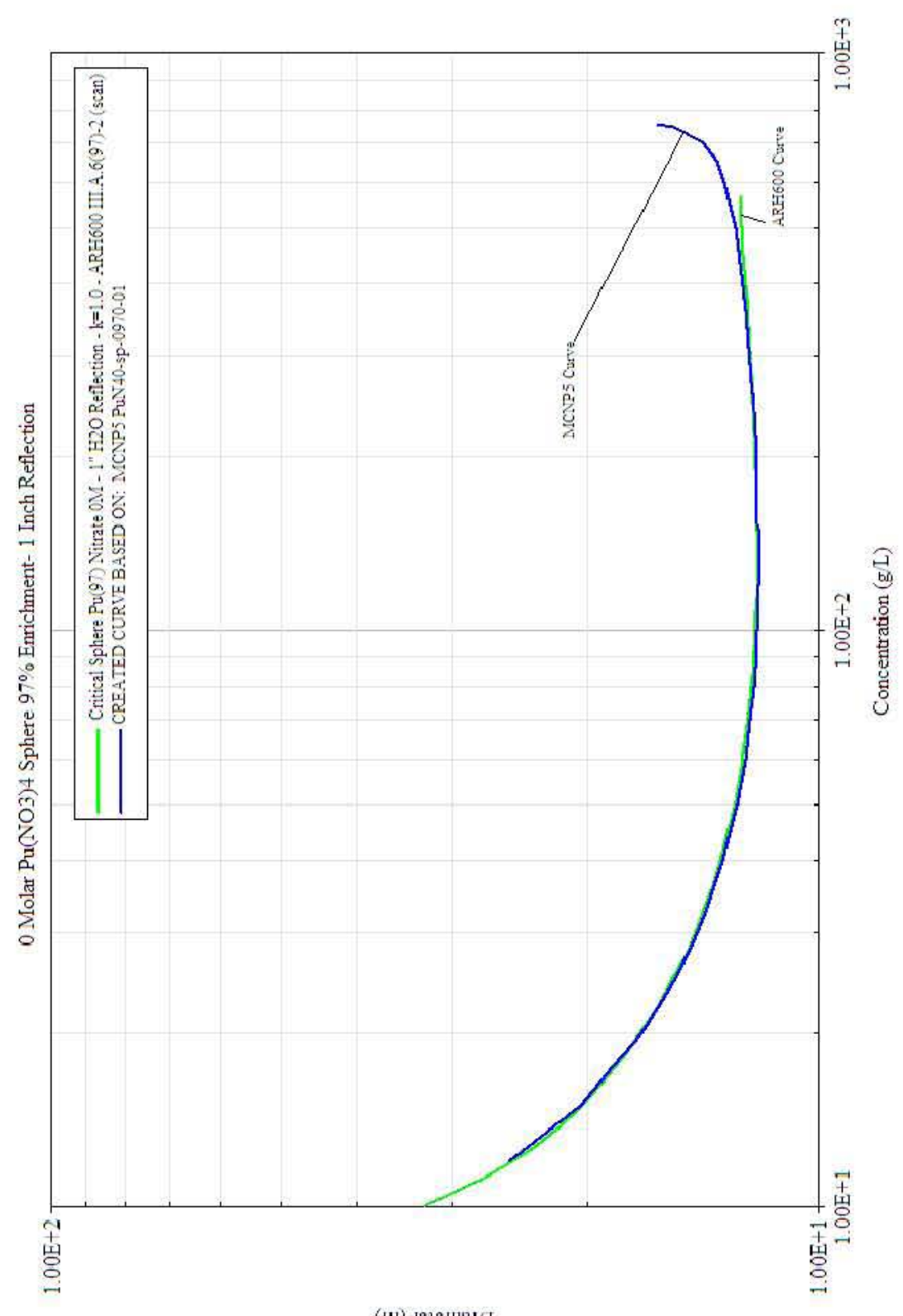

(ui) .17วนแอ!( 
Table B1.1.1.2.1.2-1. Critical Dimensions for $\mathrm{Pu}\left(\mathrm{NO}_{3}\right) 4 \mathrm{OM}-\mathrm{Sphere}-97 \%{ }^{239} \mathrm{Pu}-1$ " Water Reflection

\begin{tabular}{|c|c|c|c|c|c|}
\hline \multicolumn{6}{|c|}{ 97\% Pu239 Pu(NO3)4 0M Sphere- 1 Inch Reflection } \\
\hline Concentration $(\mathrm{g} / \mathrm{cc})$ & $\begin{array}{l}\text { Density } \\
(\mathrm{g} / \mathrm{cc})\end{array}$ & $\begin{array}{l}\text { First Diameter } \\
\text { (Inches) }\end{array}$ & $\begin{array}{l}\text { Last Diameter } \\
\text { (Inches) }\end{array}$ & $\begin{array}{l}\text { Delta } \\
\text { (Inches) }\end{array}$ & $\begin{array}{l}\text { Critical Diameter } \\
\text { (Inches) }\end{array}$ \\
\hline 0.012 & 1.016 & 20.7 & 26.3 & 0.2 & 25.2939 \\
\hline 0.015 & 1.021 & 17.3 & 21.1 & 0.2 & 20.3504 \\
\hline 0.02 & 1.029 & 14.8 & 17.5 & 0.1 & 16.9398 \\
\hline 0.025 & 1.037 & 13.5 & 15.8 & 0.1 & 15.3167 \\
\hline 0.03 & 1.046 & 12.7 & 14.8 & 0.1 & 14.372 \\
\hline 0.032 & 1.049 & 12.5 & 14.5 & 0.1 & 14.0753 \\
\hline 0.035 & 1.054 & 12.3 & 14.2 & 0.1 & 13.7347 \\
\hline 0.04 & 1.063 & 11.9 & 13.7 & 0.1 & 13.3045 \\
\hline 0.05 & 1.08 & 11.4 & 13.1 & 0.05 & 12.7423 \\
\hline 0.06 & 1.097 & 11.1 & 12.8 & 0.05 & 12.4042 \\
\hline 0.07 & 1.113 & 11 & 12.6 & 0.05 & 12.2337 \\
\hline 0.08 & 1.13 & 10.8 & 12.4 & 0.05 & 12.0974 \\
\hline 0.09 & 1.147 & 10.8 & 12.4 & 0.05 & 12.0082 \\
\hline 0.1 & 1.164 & 10.7 & 12.3 & 0.05 & 11.9784 \\
\hline 0.12 & 1.197 & 10.7 & 12.3 & 0.05 & 11.9289 \\
\hline 0.13 & 1.215 & 10.7 & 12.2 & 0.05 & 11.8997 \\
\hline 0.15 & 1.248 & 10.7 & 12.3 & 0.05 & 11.9194 \\
\hline 0.2 & 1.332 & 10.7 & 12.4 & 0.05 & 12.0055 \\
\hline 0.239 & 1.397 & 10.8 & 12.5 & 0.05 & 12.0921 \\
\hline 0.25 & 1.415 & 10.8 & 12.5 & 0.05 & 12.1054 \\
\hline 0.359 & 1.594 & 11 & 12.8 & 0.1 & 12.3994 \\
\hline 0.478 & 1.784 & 11.3 & 13.1 & 0.1 & 12.7192 \\
\hline 0.5 & 1.818 & 11.4 & 13.1 & 0.05 & 12.7904 \\
\hline 0.6 & 1.962 & 11.8 & 13.6 & 0.1 & 13.2142 \\
\hline 0.65 & 2.026 & 12 & 13.9 & 0.1 & 13.5482 \\
\hline 0.7 & 2.076 & 12.5 & 14.5 & 0.1 & 14.0995 \\
\hline 0.75 & 2.08 & 13.8 & 16 & 0.1 & 15.4952 \\
\hline 0.752 & 2.074 & 14 & 16.1 & 0.1 & 15.6722 \\
\hline 0.754 & 2.064 & 14.2 & 16.4 & 0.1 & 15.9412 \\
\hline 0.755 & 2.054 & 14.2 & 16.7 & 0.1 & 16.184 \\
\hline
\end{tabular}




\section{B1.1.1.2.1.3 10" Water Reflection}

Case ID: PuN40-sp-0970-10

Material: $\mathrm{Pu}\left(\mathrm{NO}_{3}\right)_{4} 0$ Molar Excess Acid

Geometry: Sphere

Isotopics: $97 \%{ }^{239} \mathrm{Pu}$

Reflector: 10 " water reflected

Code: MCNP5 v1.40

Analyst: Mark Murphy

Date: $8 / 2011$ 


\section{CHPRC-01552, Revision 0}

Figure B1.1.1.2.1.3-1. Pu(NO $\left.\mathrm{NO}_{3}\right) 4$ 0M - Sphere - 97\% ${ }^{239} \mathrm{Pu}-10$ " Water Reflection

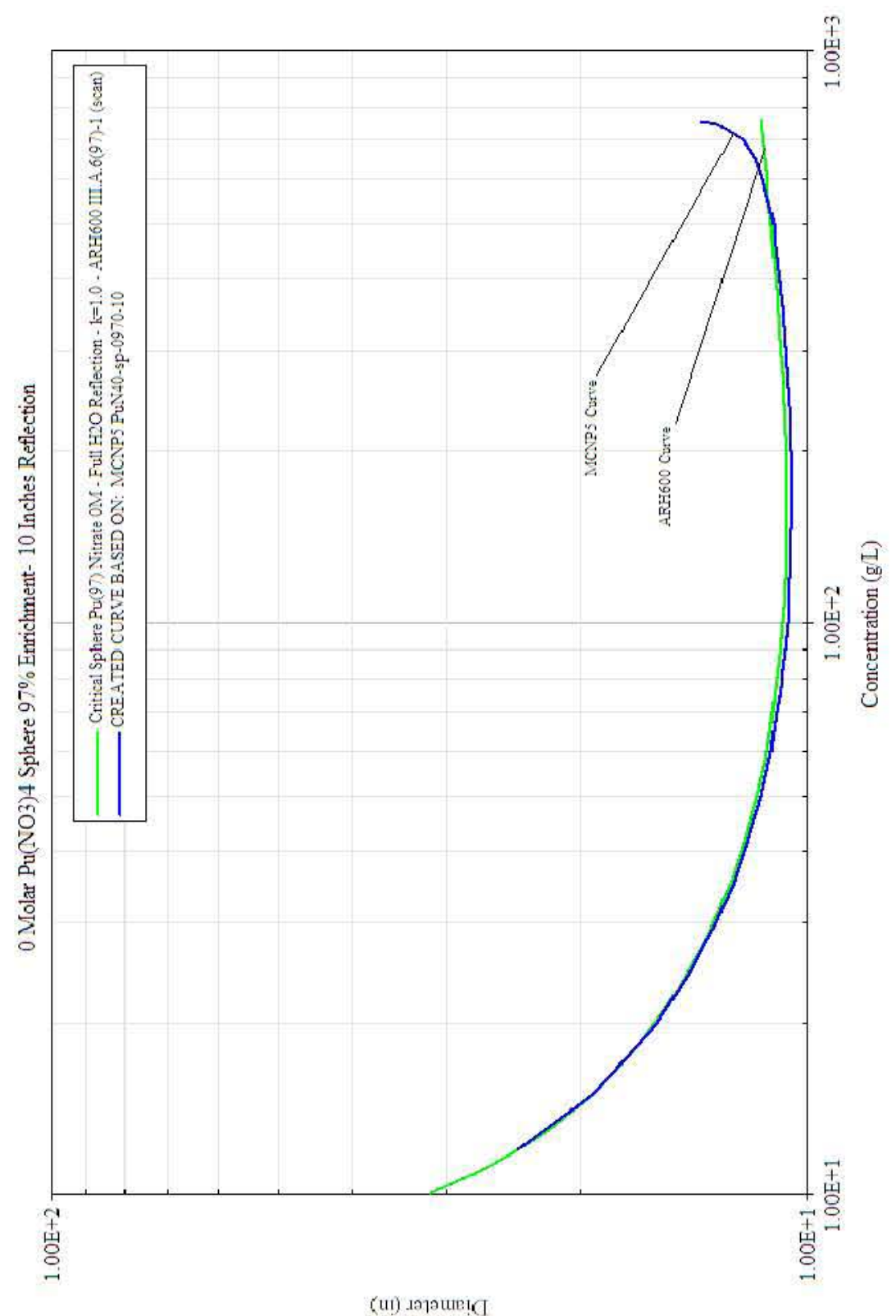

(ui) เәраше! 
Table B1.1.1.2.1.3-1. Critical Dimensions for $\mathrm{Pu}\left(\mathrm{NO}_{3}\right) 40 \mathrm{M}-$ Sphere $-97 \%{ }^{239} \mathrm{Pu}-10$ " Water Reflection

\begin{tabular}{|c|c|c|c|c|c|}
\hline \multicolumn{6}{|c|}{ 97\% Pu239 Pu(NO3)4 0M Sphere- 10 Inches Reflection } \\
\hline Concentration $(\mathrm{g} / \mathrm{cc})$ & $\begin{array}{l}\text { Density } \\
(\mathrm{g} / \mathrm{cc})\end{array}$ & $\begin{array}{l}\text { First Diameter } \\
\text { (Inches) }\end{array}$ & $\begin{array}{l}\text { Last Diameter } \\
\text { (Inches) }\end{array}$ & $\begin{array}{l}\text { Delta } \\
\text { (Inches) }\end{array}$ & $\begin{array}{l}\text { Critical Diameter } \\
\text { (Inches) }\end{array}$ \\
\hline 0.012 & 1.016 & 19.7 & 25.1 & 0.2 & 24.1426 \\
\hline 0.015 & 1.021 & 16.3 & 20 & 0.1 & 19.2421 \\
\hline 0.02 & 1.029 & 13.6 & 16.3 & 0.1 & 15.7884 \\
\hline 0.025 & 1.037 & 12.4 & 14.6 & 0.1 & 14.1646 \\
\hline 0.03 & 1.046 & 11.6 & 13.6 & 0.1 & 13.1872 \\
\hline 0.032 & 1.049 & 11.4 & 13.3 & 0.1 & 12.9124 \\
\hline 0.035 & 1.054 & 11.1 & 13 & 0.1 & 12.5403 \\
\hline 0.04 & 1.063 & 10.7 & 12.5 & 0.1 & 12.1055 \\
\hline 0.05 & 1.08 & 10.3 & 11.9 & 0.05 & 11.5089 \\
\hline 0.06 & 1.097 & 9.9 & 11.5 & 0.05 & 11.1601 \\
\hline 0.07 & 1.113 & 9.7 & 11.3 & 0.05 & 10.9474 \\
\hline 0.08 & 1.13 & 9.5 & 11.1 & 0.05 & 10.7807 \\
\hline 0.09 & 1.147 & 9.5 & 11 & 0.05 & 10.6725 \\
\hline 0.1 & 1.164 & 9.4 & 10.9 & 0.05 & 10.6106 \\
\hline 0.12 & 1.197 & 9.3 & 10.9 & 0.05 & 10.5341 \\
\hline 0.13 & 1.215 & 9.3 & 10.8 & 0.05 & 10.484 \\
\hline 0.15 & 1.248 & 9.2 & 10.8 & 0.05 & 10.4706 \\
\hline 0.2 & 1.332 & 9.3 & 10.8 & 0.05 & 10.5005 \\
\hline 0.239 & 1.397 & 9.3 & 10.9 & 0.05 & 10.5401 \\
\hline 0.25 & 1.415 & 9.3 & 10.9 & 0.05 & 10.5657 \\
\hline 0.359 & 1.594 & 9.5 & 11.1 & 0.05 & 10.7667 \\
\hline 0.478 & 1.784 & 9.6 & 11.4 & 0.1 & 11.0254 \\
\hline 0.5 & 1.818 & 9.7 & 11.4 & 0.05 & 11.0753 \\
\hline 0.6 & 1.962 & 10 & 11.8 & 0.1 & 11.4276 \\
\hline 0.65 & 2.026 & 10.3 & 12 & 0.1 & 11.7138 \\
\hline 0.7 & 2.076 & 10.7 & 12.6 & 0.1 & 12.1355 \\
\hline 0.75 & 2.08 & 11.6 & 13.7 & 0.1 & 13.2636 \\
\hline 0.752 & 2.074 & 11.7 & 13.8 & 0.1 & 13.4101 \\
\hline 0.754 & 2.064 & 11.9 & 14.1 & 0.1 & 13.5844 \\
\hline 0.755 & 2.054 & 12.1 & 14.2 & 0.1 & 13.7982 \\
\hline
\end{tabular}


B1.1.1.2.2 3 Molar Excess Acid

B1.1.1.2.2.1 0"Water Reflection

Case ID: PuN43-sp-0970-00

Material: $\mathrm{Pu}\left(\mathrm{NO}_{3}\right)_{4} 3$ Molar Excess Acid

Geometry: Sphere

Isotopics: $97 \%{ }^{239} \mathrm{Pu}$

Reflector: 0 " water reflected

Code: MCNP5 v1.40

Analyst: Mark Murphy

Date: $8 / 2011$ 
Figure B1.1.1.2.2.1-1. $\mathrm{Pu}\left(\mathrm{NO}_{3}\right) 4$ 3M - Sphere $-97 \%{ }^{239} \mathrm{Pu}-0$ " Water Reflection

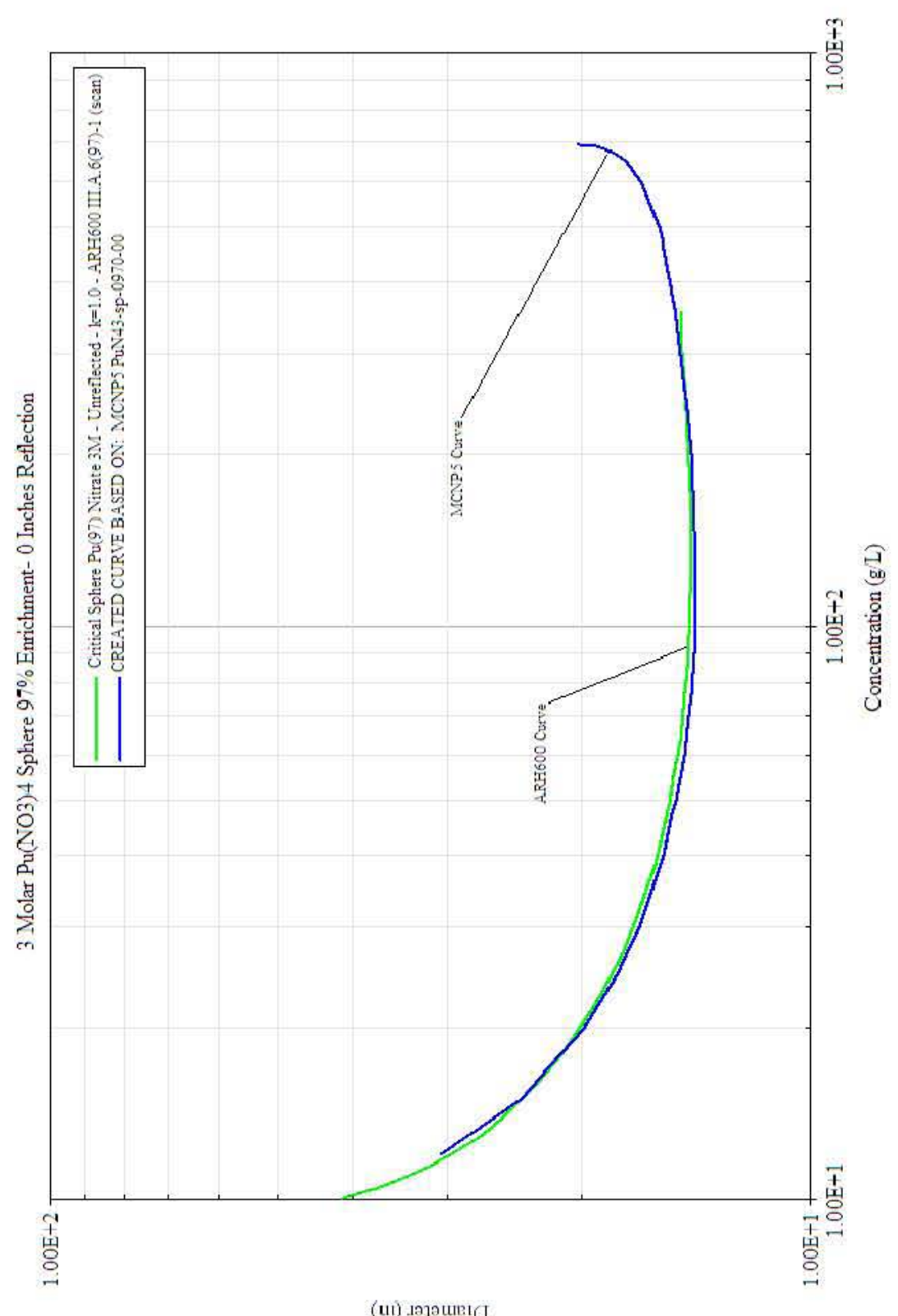

(ui) เәрачนе! (1 
Table B1.1.1.2.2.1-1. Critical Dimensions for $\mathrm{Pu}\left(\mathrm{NO}_{3}\right) 43 \mathrm{M}-$ Sphere $-97 \%{ }^{239} \mathrm{Pu}-0$ " Water Reflection

\begin{tabular}{|c|c|c|c|c|c|}
\hline \multicolumn{6}{|c|}{ 97\% Pu239 Pu(NO3)4 3M Sphere- 0 Inches Reflection } \\
\hline Concentration $(\mathrm{g} / \mathrm{cc})$ & $\begin{array}{l}\text { Density } \\
(\mathrm{g} / \mathrm{cc})\end{array}$ & $\begin{array}{l}\text { First Diameter } \\
\text { (Inches) }\end{array}$ & $\begin{array}{l}\text { Last Diameter } \\
\text { (Inches) }\end{array}$ & $\begin{array}{l}\text { Delta } \\
\text { (Inches) }\end{array}$ & $\begin{array}{l}\text { Critical Diameter } \\
\text { (Inches) }\end{array}$ \\
\hline 0.012 & 1.118 & 24.6 & 32.2 & 0.3 & 30.683 \\
\hline 0.015 & 1.123 & 20.4 & 25 & 0.2 & 24.0455 \\
\hline 0.02 & 1.132 & 17.3 & 20.5 & 0.1 & 19.7943 \\
\hline 0.025 & 1.14 & 16 & 18.4 & 0.1 & 17.8615 \\
\hline 0.03 & 1.149 & 15 & 17.3 & 0.1 & 16.7663 \\
\hline 0.032 & 1.152 & 14.7 & 16.9 & 0.1 & 16.4514 \\
\hline 0.035 & 1.157 & 14.4 & 16.5 & 0.1 & 16.1033 \\
\hline 0.04 & 1.165 & 14 & 16 & 0.1 & 15.5734 \\
\hline 0.05 & 1.182 & 13.5 & 15.4 & 0.1 & 14.9613 \\
\hline 0.06 & 1.198 & 13.2 & 15 & 0.1 & 14.6178 \\
\hline 0.07 & 1.215 & 13 & 14.8 & 0.1 & 14.3966 \\
\hline 0.08 & 1.231 & 12.9 & 14.6 & 0.05 & 14.2625 \\
\hline 0.09 & 1.247 & 12.9 & 14.6 & 0.05 & 14.1859 \\
\hline 0.1 & 1.263 & 12.9 & 14.5 & 0.05 & 14.1366 \\
\hline 0.12 & 1.294 & 12.8 & 14.5 & 0.05 & 14.1291 \\
\hline 0.13 & 1.311 & 12.85 & 14.5 & 0.05 & 14.1062 \\
\hline 0.15 & 1.342 & 12.8 & 14.5 & 0.05 & 14.1593 \\
\hline 0.2 & 1.421 & 12.9 & 14.7 & 0.1 & 14.32 \\
\hline 0.239 & 1.481 & 13.1 & 15 & 0.1 & 14.4825 \\
\hline 0.25 & 1.498 & 13.1 & 15 & 0.1 & 14.533 \\
\hline 0.359 & 1.665 & 13.6 & 15.4 & 0.1 & 15.0366 \\
\hline 0.478 & 1.841 & 14.1 & 16.1 & 0.1 & 15.6306 \\
\hline 0.5 & 1.871 & 14.2 & 16.2 & 0.1 & 15.7755 \\
\hline 0.6 & 1.998 & 15 & 17.1 & 0.1 & 16.6638 \\
\hline 0.65 & 2.043 & 15.8 & 18 & 0.1 & 17.504 \\
\hline 0.66 & 2.049 & 16 & 18.3 & 0.1 & 17.7341 \\
\hline 0.67 & 2.053 & 16.3 & 18.6 & 0.1 & 18.031 \\
\hline 0.68 & 2.053 & 16.7 & 19 & 0.1 & 18.4405 \\
\hline 0.69 & 2.046 & 17.1 & 19.6 & 0.1 & 19.0554 \\
\hline 0.698 & 2.018 & 18.2 & 20.7 & 0.1 & 20.1861 \\
\hline
\end{tabular}




\section{B1.1.1.2.2.2 1"Water Reflection}

Case ID: PuN43-sp-0970-01

Material: $\mathrm{Pu}\left(\mathrm{NO}_{3}\right)_{4} 3$ Molar Excess Acid

Geometry: Sphere

Isotopics: $97 \%{ }^{239} \mathrm{Pu}$

Reflector: 1" water reflected

Code: MCNP5 v1.40

Analyst: Mark Murphy

Date: $8 / 2011$ 
Figure B1.1.1.2.2.2-1. $\mathrm{Pu}\left(\mathrm{NO}_{3}\right) 4$ 3M - Sphere $-97 \%{ }^{239} \mathrm{Pu}-1$ " Water Reflection

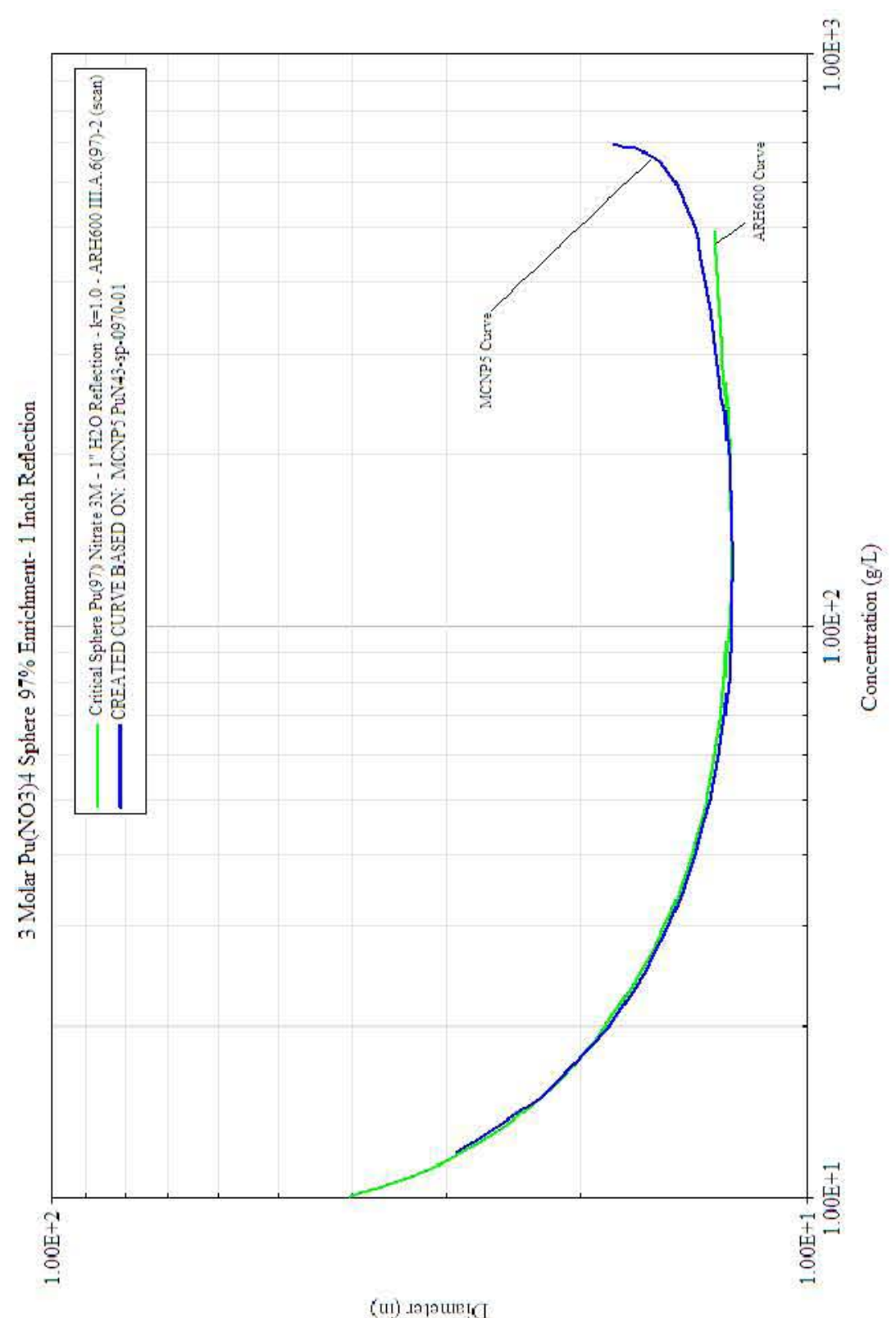

(ui) เәраитея 
Table B1.1.1.2.2.2-1. Critical Dimensions for $\mathrm{Pu}\left(\mathrm{NO}_{3}\right) 43 \mathrm{M}-$ Sphere $-97 \%{ }^{239} \mathrm{Pu}-1$ " Water Reflection

\begin{tabular}{|c|c|c|c|c|c|}
\hline \multicolumn{6}{|c|}{ 97\% Pu239 Pu(NO3)4 3M Sphere- 1 Inch Reflection } \\
\hline Concentration $(\mathrm{g} / \mathrm{cc})$ & $\begin{array}{l}\text { Density } \\
(\mathrm{g} / \mathrm{cc})\end{array}$ & $\begin{array}{l}\text { First Diameter } \\
\text { (Inches) }\end{array}$ & $\begin{array}{l}\text { Last Diameter } \\
\text { (Inches) }\end{array}$ & $\begin{array}{l}\text { Delta } \\
\text { (Inches) }\end{array}$ & $\begin{array}{l}\text { Critical Diameter } \\
\text { (Inches) }\end{array}$ \\
\hline 0.012 & 1.118 & 23 & 30.6 & 0.3 & 29.1391 \\
\hline 0.015 & 1.123 & 18.9 & 23.4 & 0.2 & 22.5105 \\
\hline 0.02 & 1.132 & 15.9 & 18.9 & 0.1 & 18.2778 \\
\hline 0.025 & 1.14 & 14.3 & 16.9 & 0.1 & 16.3283 \\
\hline 0.03 & 1.149 & 13.5 & 15.7 & 0.1 & 15.2456 \\
\hline 0.032 & 1.152 & 13.2 & 15.4 & 0.1 & 14.9106 \\
\hline 0.035 & 1.157 & 12.9 & 15 & 0.1 & 14.5226 \\
\hline 0.04 & 1.165 & 12.5 & 14.4 & 0.1 & 14.0377 \\
\hline 0.05 & 1.182 & 12 & 13.8 & 0.1 & 13.4127 \\
\hline 0.06 & 1.198 & 11.7 & 13.4 & 0.05 & 13.0459 \\
\hline 0.07 & 1.215 & 11.5 & 13.2 & 0.05 & 12.8197 \\
\hline 0.08 & 1.231 & 11.4 & 13 & 0.05 & 12.6972 \\
\hline 0.09 & 1.247 & 11.3 & 13 & 0.05 & 12.6123 \\
\hline 0.1 & 1.263 & 11.2 & 12.9 & 0.05 & 12.5623 \\
\hline 0.12 & 1.294 & 11.2 & 12.9 & 0.05 & 12.5578 \\
\hline 0.13 & 1.311 & 11.2 & 12.9 & 0.05 & 12.5233 \\
\hline 0.15 & 1.342 & 11.2 & 12.9 & 0.05 & 12.5499 \\
\hline 0.2 & 1.421 & 11.4 & 13.1 & 0.05 & 12.6987 \\
\hline 0.239 & 1.481 & 11.5 & 13.2 & 0.05 & 12.8714 \\
\hline 0.25 & 1.498 & 11.6 & 13.2 & 0.05 & \begin{tabular}{|l|}
12.9295 \\
\end{tabular} \\
\hline 0.359 & 1.665 & 12 & 13.8 & 0.1 & 13.3847 \\
\hline 0.478 & 1.841 & 12.4 & 14.4 & 0.1 & 13.949 \\
\hline 0.5 & 1.871 & 12.6 & 14.5 & 0.1 & 14.0776 \\
\hline 0.6 & 1.998 & 13.2 & 15.5 & 0.1 & 14.9093 \\
\hline 0.65 & 2.043 & 14 & 16.1 & 0.1 & 15.6647 \\
\hline 0.66 & 2.049 & 14.1 & 16.4 & 0.1 & 15.8656 \\
\hline 0.67 & 2.053 & 14.4 & 16.6 & 0.1 & 16.1582 \\
\hline 0.68 & 2.053 & 14.7 & 17.6 & 0.1 & 16.503 \\
\hline 0.69 & 2.046 & 15.2 & 17.6 & 0.1 & 17.034 \\
\hline 0.698 & 2.018 & 16.1 & 18.5 & 0.1 & 18.0508 \\
\hline
\end{tabular}




\section{B1.1.1.2.2.3 10" Water Reflection}

Case ID: PuN43-sp-0970-10

Material: $\mathrm{Pu}\left(\mathrm{NO}_{3}\right)_{4} 3$ Molar Excess Acid

Geometry: Sphere

Isotopics: $97 \%{ }^{239} \mathrm{Pu}$

Reflector: 10 " water reflected

Code: MCNP5 v1.40

Analyst: Mark Murphy

Date: $8 / 2011$ 
Figure B1.1.1.2.2.3-1. Pu(NO 3$) 43 \mathrm{M}-$ Sphere $-97 \%{ }^{239} \mathrm{Pu}-10$ " Water Reflection

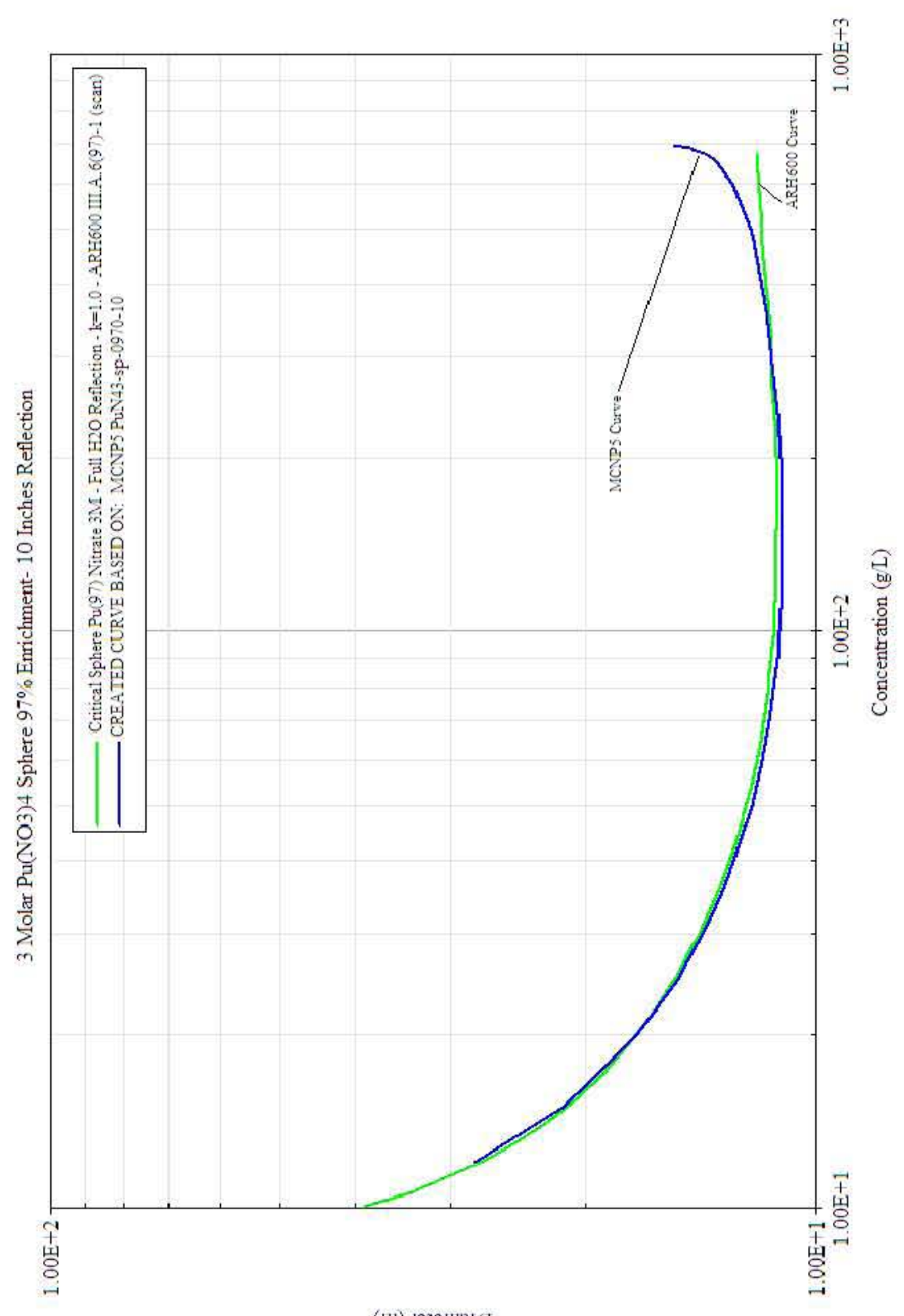

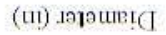


Table B1.1.1.2.2.3-1. Critical Dimensions for $\mathrm{Pu}\left(\mathrm{NO}_{3}\right) 43 \mathrm{M}-\mathrm{Sphere}-97 \%{ }^{239} \mathrm{Pu}-10$ " Water Reflection

\begin{tabular}{|c|c|c|c|c|c|}
\hline \multicolumn{6}{|c|}{ 97\% Pu239 Pu(NO3)4 3M Sphere- 10 Inches Reflection } \\
\hline Concentration $(\mathrm{g} / \mathrm{cc})$ & $\begin{array}{l}\text { Density } \\
(\mathrm{g} / \mathrm{cc})\end{array}$ & $\begin{array}{l}\text { First Diameter } \\
\text { (Inches) }\end{array}$ & $\begin{array}{l}\text { Last Diameter } \\
\text { (Inches) }\end{array}$ & $\begin{array}{l}\text { Delta } \\
\text { (Inches) }\end{array}$ & $\begin{array}{l}\text { Critical Diameter } \\
\text { (Inches) }\end{array}$ \\
\hline 0.012 & 1.118 & 22.1 & 29.4 & 0.3 & 27.9227 \\
\hline 0.015 & 1.123 & 17.8 & 22.2 & 0.2 & 21.3522 \\
\hline 0.02 & 1.132 & 14.7 & 17.6 & 0.1 & 17.0879 \\
\hline 0.025 & 1.14 & 13.2 & 15.6 & 0.1 & 15.1336 \\
\hline 0.03 & 1.149 & 12.3 & 14.5 & 0.1 & 14.0287 \\
\hline 0.032 & 1.152 & 12.1 & 14.2 & 0.1 & 13.6911 \\
\hline 0.035 & 1.157 & 11.7 & 13.7 & 0.1 & 13.2861 \\
\hline 0.04 & 1.165 & 11.3 & 13.2 & 0.1 & 12.7814 \\
\hline 0.05 & 1.182 & 10.7 & 12.5 & 0.1 & 12.1219 \\
\hline 0.06 & 1.198 & 10.4 & 12.1 & 0.05 & 11.732 \\
\hline 0.07 & 1.215 & 10.2 & 11.8 & 0.05 & 11.4786 \\
\hline 0.08 & 1.231 & 10.1 & 11.7 & 0.05 & 11.3267 \\
\hline 0.09 & 1.247 & 10 & 11.55 & 0.05 & 11.2128 \\
\hline 0.1 & 1.263 & 9.9 & 11.5 & 0.05 & 11.1307 \\
\hline 0.12 & 1.294 & 9.8 & 11.4 & 0.05 & 11.0662 \\
\hline 0.13 & 1.311 & 9.7 & 11.4 & 0.05 & 11.0353 \\
\hline 0.15 & 1.342 & 9.8 & 11.4 & 0.05 & 11.0477 \\
\hline 0.2 & 1.421 & 9.8 & 11.5 & 0.05 & 11.0978 \\
\hline 0.239 & 1.481 & 9.9 & 11.6 & 0.05 & 11.2215 \\
\hline 0.25 & 1.498 & 10.05 & 11.55 & 0.05 & 11.2413 \\
\hline 0.359 & 1.665 & 10.3 & 12 & 0.1 & 11.6142 \\
\hline 0.478 & 1.841 & 10.6 & 12.5 & 0.1 & 12.0633 \\
\hline 0.5 & 1.871 & 10.7 & 12.6 & 0.1 & 12.1659 \\
\hline 0.6 & 1.998 & 11.2 & 13.3 & 0.1 & 12.8357 \\
\hline 0.65 & 2.043 & 11.8 & 13.9 & 0.1 & 13.4404 \\
\hline 0.66 & 2.049 & 11.9 & 14 & 0.1 & 13.6075 \\
\hline 0.67 & 2.053 & 12.1 & 14.3 & 0.1 & 13.8291 \\
\hline 0.68 & 2.053 & 12.3 & 14.6 & 0.1 & 14.1053 \\
\hline 0.69 & 2.046 & 12.7 & 14.9 & 0.1 & 14.5477 \\
\hline 0.698 & 2.018 & 13.4 & 15.8 & 0.1 & 15.3337 \\
\hline
\end{tabular}


B1.1.1.2.3 6 Molar Excess Acid

B1.1.1.2.3.1 0"Water Reflection

Case ID: PuN46-sp-0970-00

Material: $\mathrm{Pu}\left(\mathrm{NO}_{3}\right)_{4} 6$ Molar Excess Acid

Geometry: Sphere

Isotopics: $97 \%{ }^{239} \mathrm{Pu}$

Reflector: 0 " water reflected

Code: MCNP5 v1.40

Analyst: Mark Murphy

Date: $8 / 2011$ 
Figure B1.1.1.2.3.1-1. $\mathrm{Pu}\left(\mathrm{NO}_{3}\right) 4$ 6M - Sphere - 97\% ${ }^{239} \mathrm{Pu}-0$ " Water Reflection

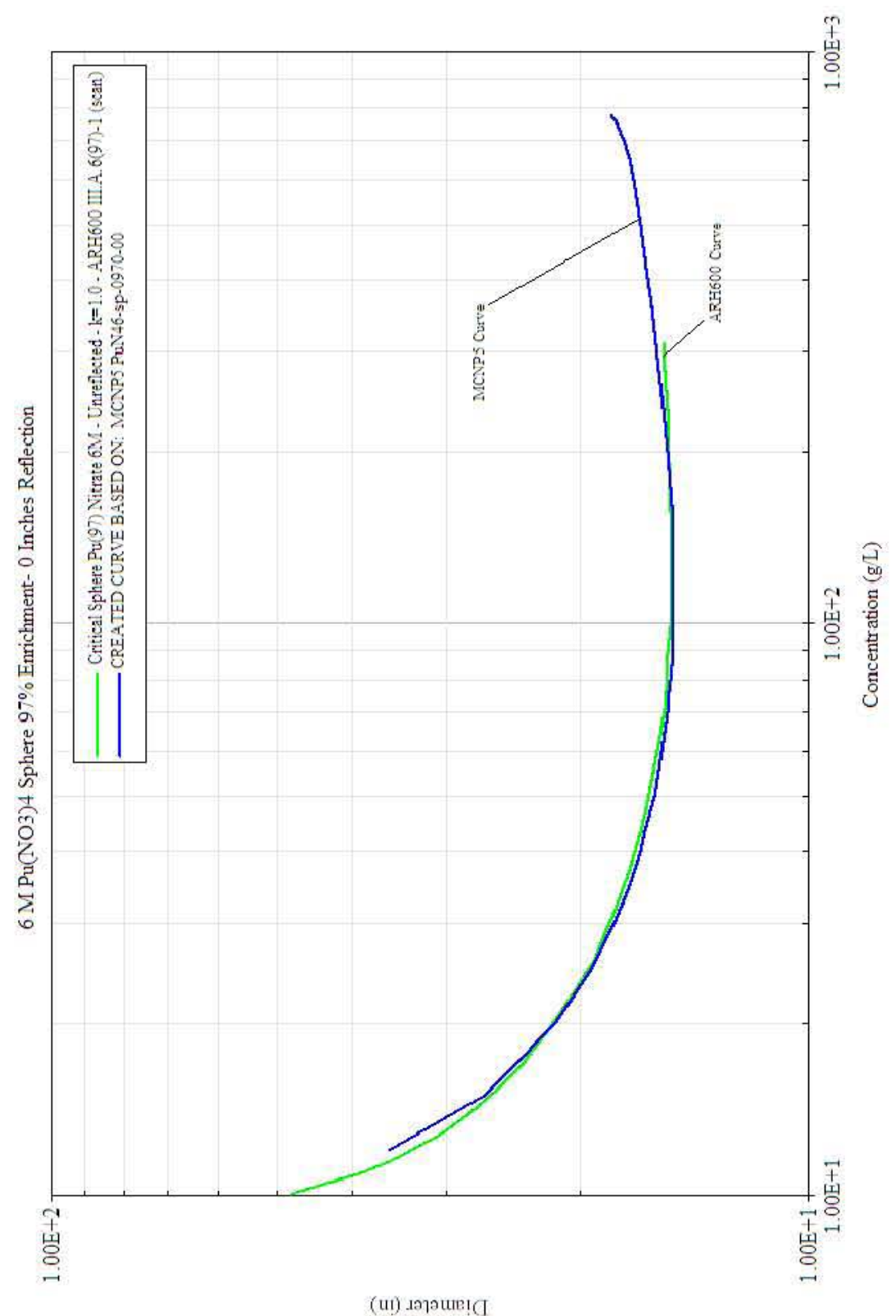

(ui) เәาวแе! ( 
Table B1.1.1.2.3.1-1. Critical Dimensions for $\mathrm{Pu}\left(\mathrm{NO}_{3}\right) 46 \mathrm{M}-$ Sphere $-97 \%{ }^{239} \mathrm{Pu}-0$ " Water Reflection

\begin{tabular}{|c|c|c|c|c|c|}
\hline \multicolumn{6}{|c|}{ 97\% Pu239 Pu(NO3)4 6M Sphere- 0 Inches Reflection } \\
\hline Concentration $(\mathrm{g} / \mathrm{cc})$ & $\begin{array}{l}\text { Density } \\
(\mathrm{g} / \mathrm{cc})\end{array}$ & $\begin{array}{l}\text { First Diameter } \\
\text { (Inches) }\end{array}$ & $\begin{array}{l}\text { Last Diameter } \\
\text { (Inches) }\end{array}$ & $\begin{array}{l}\text { Delta } \\
\text { (Inches) }\end{array}$ & $\begin{array}{l}\text { Critical Diameter } \\
\text { (Inches) }\end{array}$ \\
\hline 0.012 & 1.207 & 28.7 & 37.7 & 0.5 & 35.7046 \\
\hline 0.015 & 1.212 & 22.4 & 27.7 & 0.2 & 26.6893 \\
\hline 0.02 & 1.22 & 18.8 & 22.3 & 0.1 & 21.521 \\
\hline 0.025 & 1.229 & 17 & 19.8 & 0.1 & 19.2538 \\
\hline 0.03 & 1.237 & 16 & 18.5 & 0.1 & 17.9758 \\
\hline 0.032 & 1.24 & 15.8 & 18.1 & 0.1 & 17.6044 \\
\hline 0.035 & 1.245 & 15.4 & 17.7 & 0.1 & 17.1693 \\
\hline 0.04 & 1.253 & 14.9 & 17.1 & 0.1 & 16.6234 \\
\hline 0.05 & 1.27 & 14.4 & 16.4 & 0.1 & 15.91 \\
\hline 0.06 & 1.286 & 14 & 15.9 & 0.1 & 15.5463 \\
\hline 0.07 & 1.301 & 13.9 & 15.7 & 0.1 & 15.3163 \\
\hline 0.08 & 1.317 & 13.8 & 15.6 & 0.1 & 15.1741 \\
\hline 0.09 & 1.333 & 13.7 & 15.5 & 0.1 & 15.0784 \\
\hline 0.1 & 1.349 & 13.6 & 15.3 & 0.1 & 15.0378 \\
\hline 0.12 & 1.379 & 13.6 & 15.3 & 0.1 & 15.0462 \\
\hline 0.13 & 1.395 & 13.6 & 16.1 & 0.1 & 15.0369 \\
\hline 0.15 & 1.426 & 13.6 & 15.5 & 0.1 & 15.0732 \\
\hline 0.2 & 1.503 & 13.8 & 16 & 0.1 & 15.3279 \\
\hline 0.239 & 1.563 & 14 & 15.7 & 0.05 & 15.5214 \\
\hline 0.25 & 1.58 & 14 & 16.8 & 0.1 & 15.5599 \\
\hline 0.359 & 1.748 & 14.5 & 16.3 & 0.1 & 16.0749 \\
\hline 0.478 & 1.935 & 14.9 & 17 & 0.1 & 16.515 \\
\hline 0.5 & 1.969 & 15 & 18.2 & 0.1 & 16.5984 \\
\hline 0.6 & 2.122 & 15.3 & 17.5 & 0.1 & 16.9939 \\
\hline 0.65 & 2.197 & 15.5 & 17.7 & 0.1 & 17.2119 \\
\hline 0.7 & 2.268 & 15.8 & 18 & 0.1 & 17.4874 \\
\hline 0.75 & 2.335 & 16.1 & 19.5 & 0.1 & 17.897 \\
\hline 0.76 & 2.348 & 16.2 & 18.5 & 0.1 & 17.967 \\
\hline 0.765 & 2.354 & 16.3 & 18.6 & 0.1 & 18.0355 \\
\hline 0.77 & 2.36 & 16.3 & 18.6 & 0.1 & 18.0924 \\
\hline 0.775 & 2.367 & 16.3 & 18.5 & 0.1 & 18.13 \\
\hline
\end{tabular}




\section{B1.1.1.2.3.2 1"Water Reflection}

Case ID: PuN46-sp-0970-01

Material: $\mathrm{Pu}\left(\mathrm{NO}_{3}\right)_{4} 6$ Molar Excess Acid

Geometry: Sphere

Isotopics: $97 \%{ }^{239} \mathrm{Pu}$

Reflector: 1" water reflected

Code: MCNP5 v1.40

Analyst: Mark Murphy

Date: $8 / 2011$ 
Figure B1.1.1.2.3.2-1. $\mathrm{Pu}\left(\mathrm{NO}_{3}\right) 4$ 6M - Sphere $-97 \%{ }^{239} \mathrm{Pu}-1$ " Water Reflection

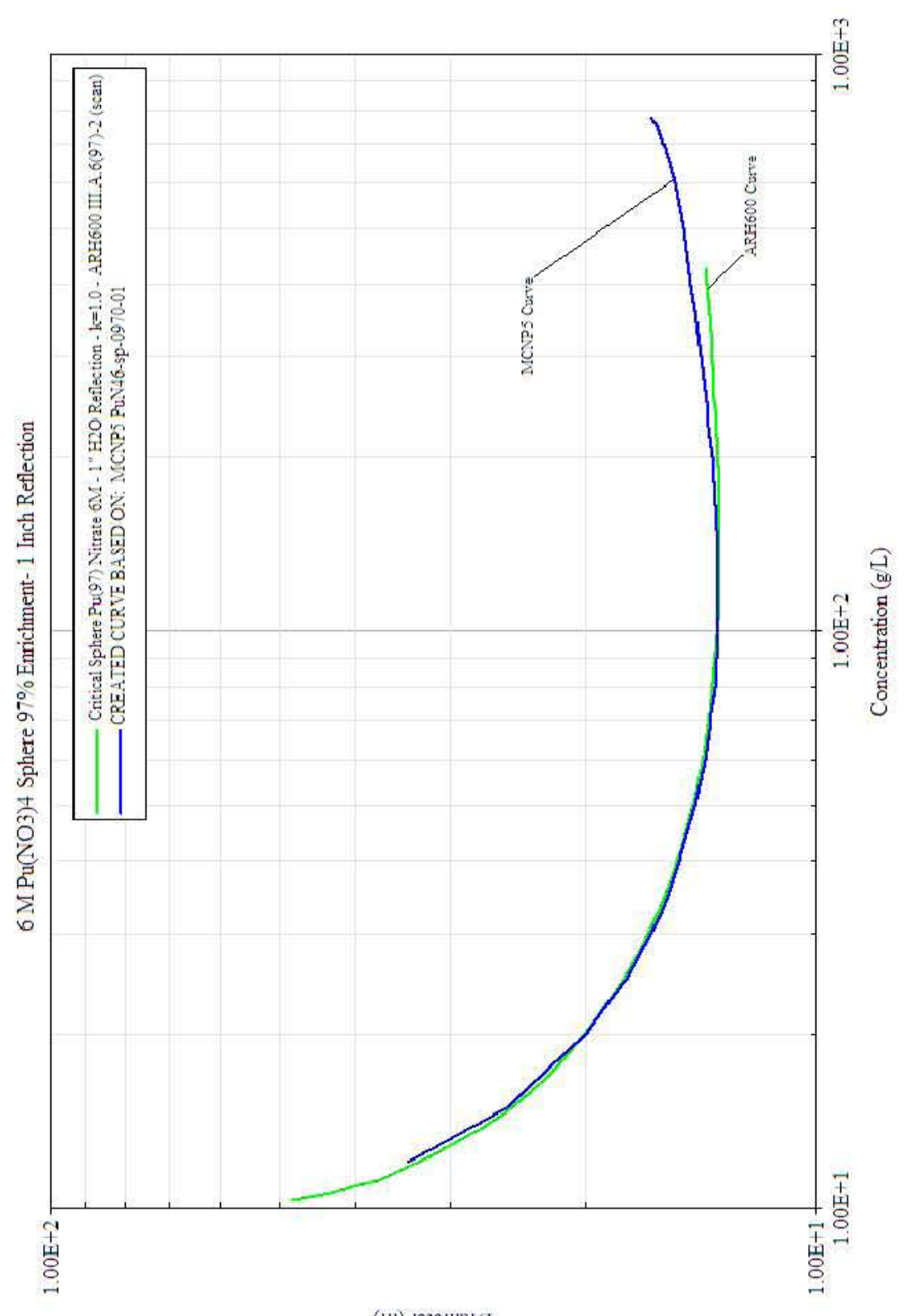

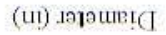


Table B1.1.1.2.3.2-1. Critical Dimensions for $\mathrm{Pu}\left(\mathrm{NO}_{3}\right) 46 \mathrm{M}-$ Sphere $-97 \%{ }^{239} \mathrm{Pu}-1$ " Water Reflection

\begin{tabular}{|c|c|c|c|c|c|}
\hline \multicolumn{6}{|c|}{ 97\% Pu239 Pu(NO3)4 6M Sphere- 1 Inch Reflection } \\
\hline Concentration $(\mathrm{g} / \mathrm{cc})$ & $\begin{array}{l}\text { Density } \\
(\mathrm{g} / \mathrm{cc})\end{array}$ & $\begin{array}{l}\text { First Diameter } \\
\text { (Inches) }\end{array}$ & $\begin{array}{l}\text { Last Diameter } \\
\text { (Inches) }\end{array}$ & $\begin{array}{l}\text { Delta } \\
\text { (Inches) }\end{array}$ & $\begin{array}{l}\text { Critical Diameter } \\
\text { (Inches) }\end{array}$ \\
\hline 0.012 & 1.207 & 27.2 & 36.2 & 0.5 & 34.0721 \\
\hline 0.015 & 1.212 & 20.9 & 26.2 & 0.2 & 25.1257 \\
\hline 0.02 & 1.22 & 17.2 & 20.7 & 0.1 & 19.9429 \\
\hline 0.025 & 1.229 & 15.5 & 18.2 & 0.1 & 17.6589 \\
\hline 0.03 & 1.237 & 14.5 & 16.9 & 0.1 & 16.3864 \\
\hline 0.032 & 1.24 & 14.2 & 16.4 & 0.1 & 16.0123 \\
\hline 0.035 & 1.245 & 14.1 & 16.1 & 0.1 & 15.5826 \\
\hline 0.04 & 1.253 & 13.3 & 15.5 & 0.1 & 15.0204 \\
\hline 0.05 & 1.27 & 12.8 & 14.7 & 0.1 & 14.3314 \\
\hline 0.06 & 1.286 & 12.5 & 14.3 & 0.1 & 13.9198 \\
\hline 0.07 & 1.301 & 12.3 & 14.1 & 0.1 & 13.6882 \\
\hline 0.08 & 1.317 & 12.1 & 13.9 & 0.1 & 13.534 \\
\hline 0.09 & 1.333 & 12.1 & 13.8 & 0.1 & 13.4607 \\
\hline 0.1 & 1.349 & 12 & 13.8 & 0.1 & 13.4074 \\
\hline 0.12 & 1.379 & 12 & 13.8 & 0.1 & 13.4079 \\
\hline 0.13 & 1.395 & 12 & 13.8 & 0.1 & 13.3809 \\
\hline 0.15 & 1.426 & 12 & 13.7 & 0.05 & 13.444 \\
\hline 0.2 & 1.503 & 12.2 & 14 & 0.1 & 13.6385 \\
\hline 0.239 & 1.563 & 12.3 & 15.3 & 0.1 & 13.8158 \\
\hline 0.25 & 1.58 & 12.4 & 14.2 & 0.1 & 13.8754 \\
\hline 0.359 & 1.748 & 12.8 & 14.8 & 0.1 & 14.3642 \\
\hline 0.478 & 1.935 & 13.1 & 15.2 & 0.2 & 14.7812 \\
\hline 0.5 & 1.969 & 13.2 & 15.3 & 0.1 & 14.8685 \\
\hline 0.6 & 2.122 & 13.4 & 15.7 & 0.1 & 15.25 \\
\hline 0.65 & 2.197 & 13.8 & 15.8 & 0.1 & 15.4812 \\
\hline 0.7 & 2.268 & 14 & 16.2 & 0.1 & 15.7541 \\
\hline 0.75 & 2.335 & 14.3 & 16.5 & 0.1 & 16.1141 \\
\hline 0.76 & 2.348 & 14.4 & 16.7 & 0.1 & 16.1926 \\
\hline 0.765 & 2.354 & 14.5 & 16.7 & 0.1 & 16.2489 \\
\hline 0.77 & 2.36 & 14.5 & 17.1 & 0.1 & 16.3036 \\
\hline 0.775 & 2.367 & 14.5 & 16.7 & 0.1 & 16.331 \\
\hline
\end{tabular}




\section{B1.1.1.2.3.3 10" Water Reflection}

Case ID: PuN46-sp-0970-10

Material: $\mathrm{Pu}\left(\mathrm{NO}_{3}\right)_{4} 6$ Molar Excess Acid

Geometry: Sphere

Isotopics: $97 \%{ }^{239} \mathrm{Pu}$

Reflector: 10 " water reflected

Code: MCNP5 v1.40

Analyst: Mark Murphy

Date: $8 / 2011$ 
Figure B1.1.1.2.3.3-1. Pu(NO 3$) 46 \mathrm{M}-$ Sphere $-97 \%{ }^{239} \mathrm{Pu}-10$ " Water Reflection

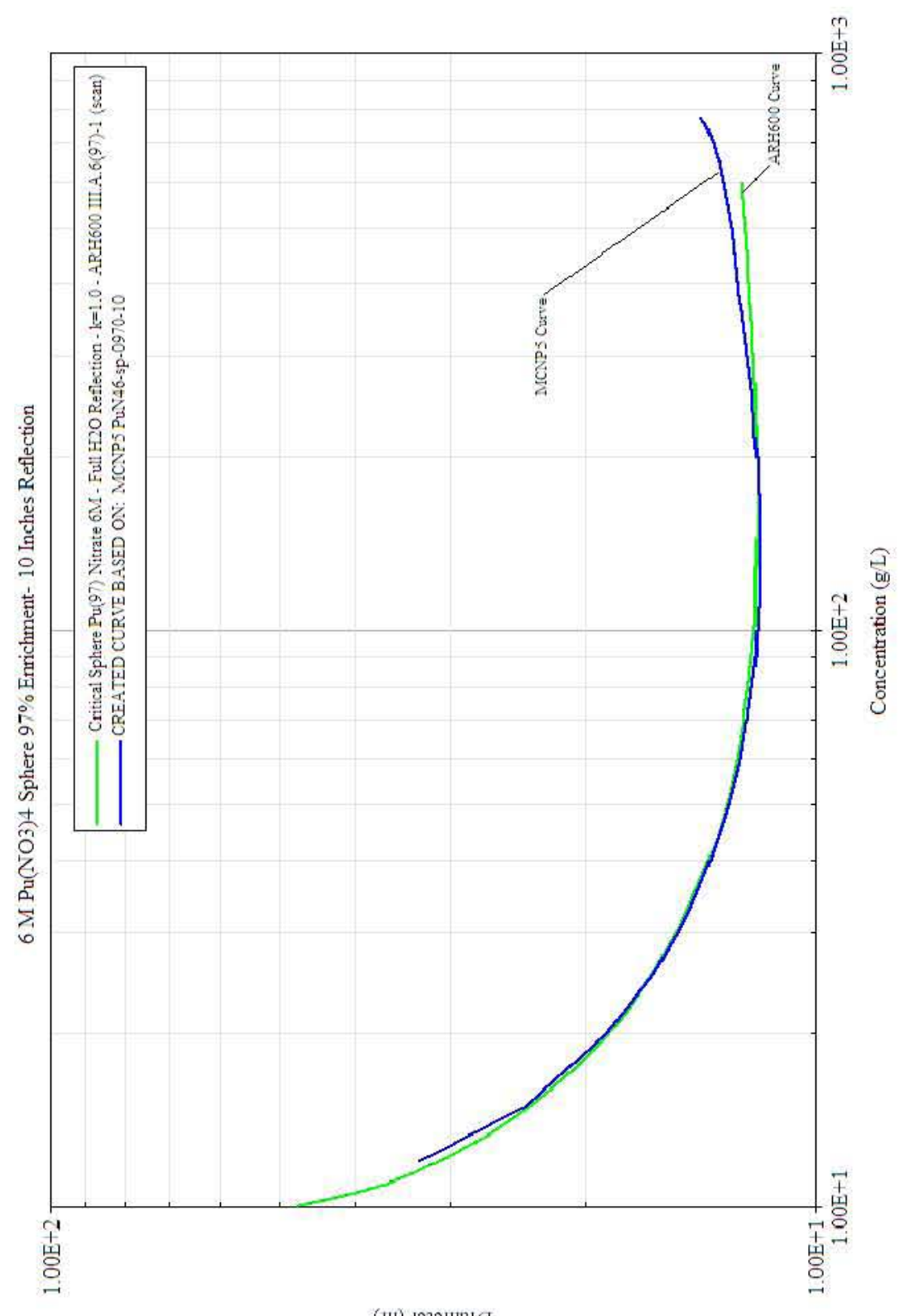

(4i) เฉาวแย!৫ 
Table B1.1.1.2.3.3-1. Critical Dimensions for $\mathrm{Pu}\left(\mathrm{NO}_{3}\right) 46 \mathrm{M}-$ Sphere $-97 \%{ }^{239} \mathrm{Pu}-10 "$ Water Reflection

\begin{tabular}{|c|c|c|c|c|c|}
\hline \multicolumn{6}{|c|}{ 97\% Pu239 Pu(NO3)4 6M Sphere- 10 Inches Reflection } \\
\hline Concentration $(\mathrm{g} / \mathrm{cc})$ & $\begin{array}{l}\text { Density } \\
(\mathrm{g} / \mathrm{cc})\end{array}$ & $\begin{array}{l}\text { First Diameter } \\
\text { (Inches) }\end{array}$ & $\begin{array}{l}\text { Last Diameter } \\
\text { (Inches) }\end{array}$ & $\begin{array}{l}\text { Delta } \\
\text { (Inches) }\end{array}$ & $\begin{array}{l}\text { Critical Diameter } \\
\text { (Inches) }\end{array}$ \\
\hline 0.012 & 1.207 & 26 & 35 & 0.5 & 32.9 \\
\hline 0.015 & 1.212 & 19.6 & 24.9 & 0.2 & 23.9407 \\
\hline 0.02 & 1.22 & 16 & 19.3 & 0.1 & 18.6801 \\
\hline 0.025 & 1.229 & 14.2 & 16.9 & 0.1 & 16.3808 \\
\hline 0.03 & 1.237 & 13.2 & 15.6 & 0.1 & 15.0936 \\
\hline 0.032 & 1.24 & 12.9 & 15.2 & 0.1 & 14.7303 \\
\hline 0.035 & 1.245 & 12.5 & 14.7 & 0.5 & 14.2725 \\
\hline 0.04 & 1.253 & 12.1 & 14.1 & 0.1 & 13.6916 \\
\hline 0.05 & 1.27 & 11.5 & 13.3 & 0.1 & 12.9586 \\
\hline 0.06 & 1.286 & 11.1 & 12.9 & 0.1 & 12.5278 \\
\hline 0.07 & 1.301 & 10.8 & 12.7 & 0.1 & 12.2619 \\
\hline 0.08 & 1.317 & 10.7 & 12.5 & 0.1 & 12.0805 \\
\hline 0.09 & 1.333 & 10.5 & 12.4 & 0.1 & 11.9652 \\
\hline 0.1 & 1.349 & 10.5 & 12.2 & 0.05 & 11.8702 \\
\hline 0.12 & 1.379 & 10.4 & 12.1 & 0.05 & 11.8078 \\
\hline 0.13 & 1.395 & 10.4 & 12.2 & 0.1 & 11.7854 \\
\hline 0.15 & 1.426 & 10.5 & 12.5 & 0.1 & 11.8007 \\
\hline 0.2 & 1.503 & 10.4 & 12.3 & 0.1 & 11.8965 \\
\hline 0.239 & 1.563 & 10.6 & 12.4 & 0.1 & 12.0256 \\
\hline 0.25 & 1.58 & 10.6 & 12.5 & 0.1 & 12.0685 \\
\hline 0.359 & 1.748 & 10.9 & 12.7 & 0.1 & 12.4494 \\
\hline 0.478 & 1.935 & 11.2 & 13 & 0.2 & 12.7739 \\
\hline 0.5 & 1.969 & 11.2 & 13.3 & 0.1 & 12.8627 \\
\hline 0.6 & 2.122 & 11.5 & 13.5 & 0.1 & 13.1733 \\
\hline 0.65 & 2.197 & 11.7 & 13.8 & 0.1 & 13.3626 \\
\hline 0.7 & 2.268 & 11.9 & 14.1 & 0.1 & 13.5964 \\
\hline 0.75 & 2.335 & 12.1 & 14.3 & 0.1 & 13.8787 \\
\hline 0.76 & 2.348 & 12.2 & 14.4 & 0.1 & 13.9575 \\
\hline 0.765 & 2.354 & 12.2 & 14.4 & 0.1 & 14.0037 \\
\hline 0.77 & 2.36 & 12.3 & 14.4 & 0.1 & 14.0274 \\
\hline 0.775 & 2.367 & 12.3 & 14.5 & 0.1 & 14.0692 \\
\hline
\end{tabular}


CHPRC-01552, Revision 0 
B1.1.1.3 $95 \%{ }^{239} \mathrm{Pu}$

B1.1.1.3.1 0 Molar Excess Acid

B1.1.1.3.1.1 0" Water Reflection

Case ID: PuN40-sp-0950-00

Material: $\mathrm{Pu}\left(\mathrm{NO}_{3}\right)_{4} \quad 0$ Molar Excess Acid

Geometry: Sphere

Isotopics: $95 \%{ }^{239} \mathrm{Pu}$

Reflector: 0" water reflected

Code: MCNP5 v1.40

Analyst: Mark Murphy

Date: $8 / 2011$ 


\section{CHPRC-01552, Revision 0}

Figure B1.1.1.3.1.1-1. $\mathrm{Pu}\left(\mathrm{NO}_{3}\right) 40 \mathrm{M}$ - Sphere - 95\% ${ }^{239} \mathrm{Pu}-0$ " Water Reflection

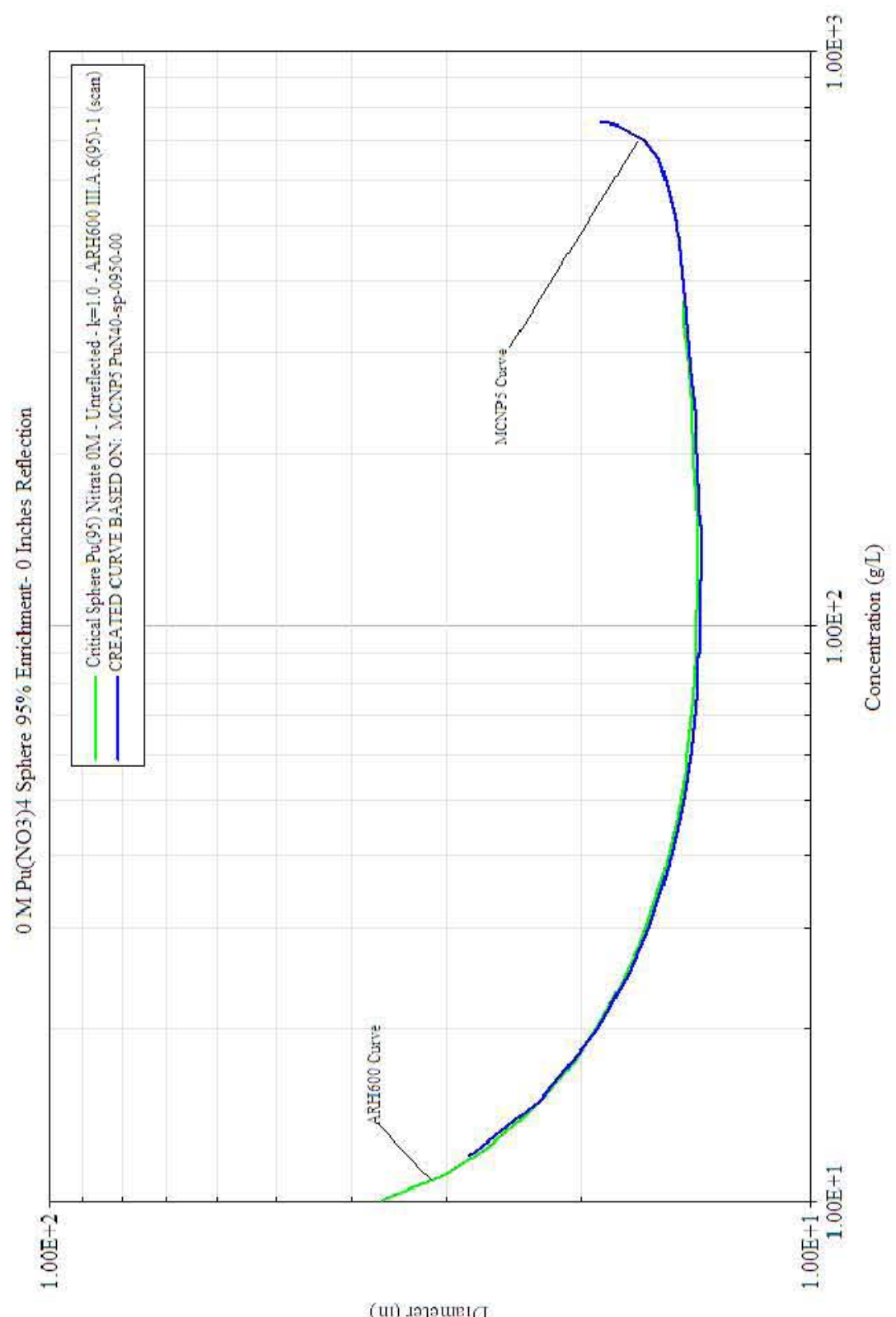

(ui) เәาวแะ!ด 
Table B1.1.1.3.1.1-1. Critical Dimensions for $\mathrm{Pu}\left(\mathrm{NO}_{3}\right) 4 \mathrm{OM}-\mathrm{Sphere}-95 \%{ }^{239} \mathrm{Pu}-0$ " Water Reflection

\begin{tabular}{|c|c|c|c|c|c|}
\hline \multicolumn{6}{|c|}{ 95\% Pu239 Pu(NO3)4 0M Sphere- 0 Inches Reflection } \\
\hline Concentration $(\mathrm{g} / \mathrm{cc})$ & $\begin{array}{l}\text { Density } \\
(\mathrm{g} / \mathrm{cc})\end{array}$ & $\begin{array}{l}\text { First Diameter } \\
\text { (Inches) }\end{array}$ & $\begin{array}{l}\text { Last Diameter } \\
\text { (Inches) }\end{array}$ & $\begin{array}{l}\text { Delta } \\
\text { (Inches) }\end{array}$ & $\begin{array}{l}\text { Critical Diameter } \\
\text { (Inches) }\end{array}$ \\
\hline 0.012 & 1.016 & 22.9 & 29.2 & 0.3 & 27.9794 \\
\hline 0.015 & 1.021 & 19.3 & 23.4 & 0.2 & 22.596 \\
\hline 0.02 & 1.029 & 16.7 & 19.5 & 0.1 & 18.9425 \\
\hline 0.025 & 1.037 & 15.3 & 17.7 & 0.1 & 17.2251 \\
\hline 0.03 & 1.046 & 14.5 & 16.7 & 0.1 & 16.2418 \\
\hline 0.032 & 1.049 & 14.3 & 16.5 & 0.1 & 15.9699 \\
\hline 0.035 & 1.054 & 14 & 16 & 0.1 & 15.6204 \\
\hline 0.04 & 1.063 & 13.6 & 15.6 & 0.1 & 15.1568 \\
\hline 0.05 & 1.08 & 13.2 & 15 & 0.1 & 14.6137 \\
\hline 0.06 & 1.097 & 12.9 & 14.7 & 0.1 & 14.2833 \\
\hline 0.07 & 1.113 & 12.8 & 14.5 & 0.05 & 14.0967 \\
\hline 0.08 & 1.13 & 12.6 & 14.4 & 0.1 & 13.9957 \\
\hline 0.09 & 1.147 & 12.6 & 14.3 & 0.05 & 13.9371 \\
\hline 0.1 & 1.164 & 12.6 & 14.3 & 0.05 & 13.8904 \\
\hline 0.12 & 1.197 & 12.5 & 14.2 & 0.05 & 13.8714 \\
\hline 0.13 & 1.215 & 12.5 & 14.2 & 0.05 & 13.8419 \\
\hline 0.15 & 1.248 & 12.5 & 14.3 & 0.1 & 13.8908 \\
\hline 0.2 & 1.332 & 12.6 & 14.4 & 0.1 & 14.0251 \\
\hline 0.239 & 1.397 & 12.7 & 14.5 & 0.1 & 14.1418 \\
\hline 0.25 & 1.415 & 12.8 & 14.6 & 0.1 & 14.2003 \\
\hline 0.359 & 1.594 & 13.2 & 14.9 & 0.1 & 14.5168 \\
\hline 0.478 & 1.784 & 13.3 & 15.3 & 0.1 & 14.8756 \\
\hline 0.5 & 1.818 & 13.4 & 15.4 & 0.1 & 14.9438 \\
\hline 0.6 & 1.962 & 13.9 & 15.9 & 0.1 & 15.441 \\
\hline 0.65 & 2.026 & 14.2 & 16.2 & 0.1 & 15.805 \\
\hline 0.7 & 2.076 & 14.8 & 16.9 & 0.1 & 16.439 \\
\hline 0.75 & 2.08 & 16.3 & 18.6 & 0.1 & 18.0811 \\
\hline 0.752 & 2.074 & 16.4 & 18.8 & 0.1 & 18.2816 \\
\hline 0.754 & 2.065 & 16.8 & 19.1 & 0.1 & 18.5576 \\
\hline 0.755 & 2.055 & 16.9 & 19.3 & 0.1 & 18.8395 \\
\hline
\end{tabular}




\section{B1.1.1.3.1.2 1"Water Reflection}

Case ID: PuN40-sp-0950-01

Material: $\mathrm{Pu}\left(\mathrm{NO}_{3}\right)_{4} 0$ Molar Excess Acid

Geometry: Sphere

Isotopics: $95 \%{ }^{239} \mathrm{Pu}$

Reflector: 1" water reflected

Code: MCNP5 v1.40

Analyst: Mark Murphy

Date: $8 / 2011$ 


\section{CHPRC-01552, Revision 0}

Figure B1.1.1.3.1.2-1. $\mathrm{Pu}\left(\mathrm{NO}_{3}\right) 40 \mathrm{M}$ - Sphere - 95\% ${ }^{239} \mathrm{Pu}$ - 1" Water Reflection

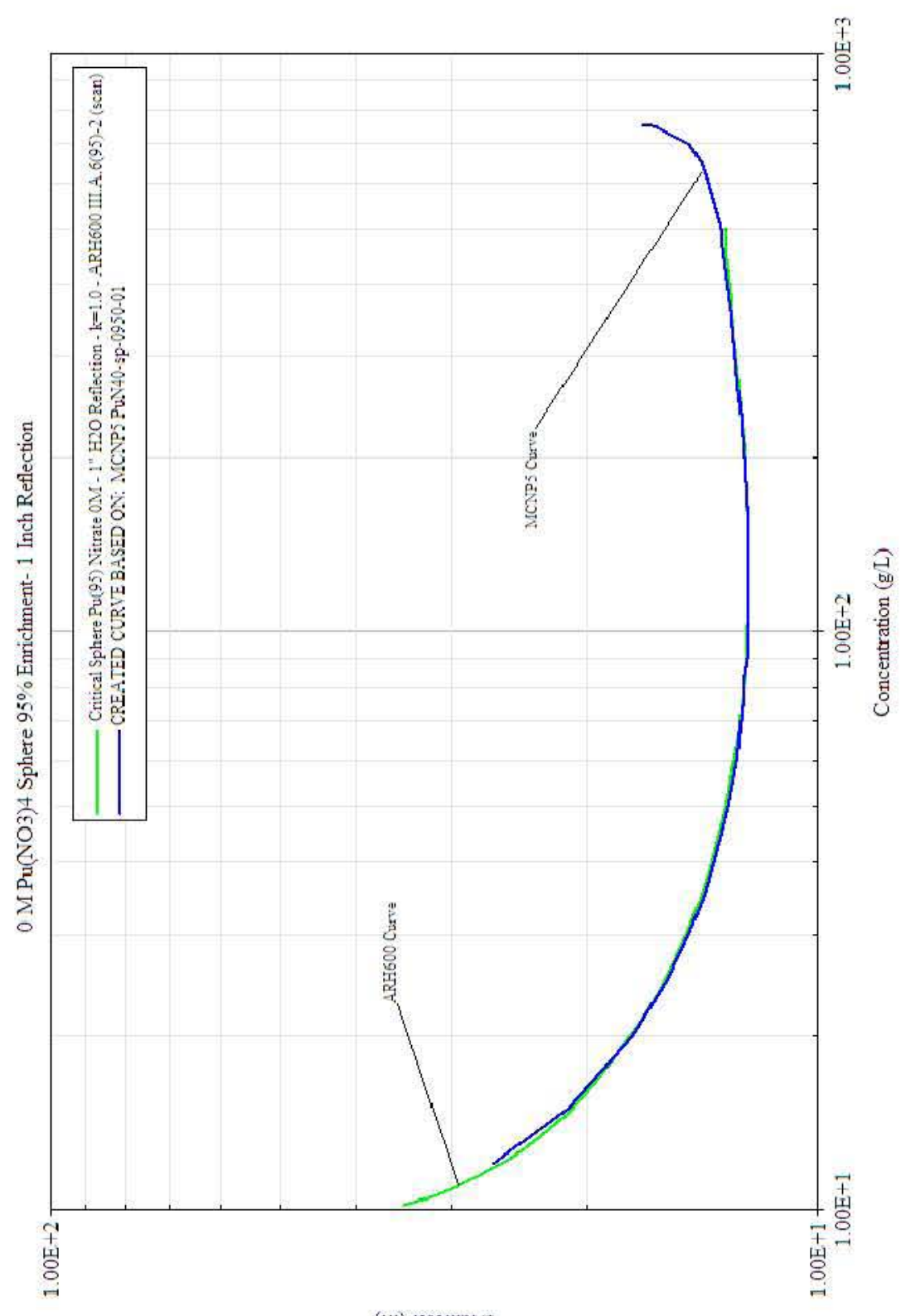

(ui) .әраивเ (1 
Table B1.1.1.3.1.2-1. Critical Dimensions for $\mathrm{Pu}\left(\mathrm{NO}_{3}\right) 4 \mathrm{OM}-\mathrm{Sphere}-95 \%{ }^{239} \mathrm{Pu}-1$ " Water Reflection

\begin{tabular}{|c|c|c|c|c|c|}
\hline \multicolumn{6}{|c|}{ 95\% Pu239 Pu(NO3)4 0M Sphere- 1 Inch Reflection } \\
\hline Concentration $(\mathrm{g} / \mathrm{cc})$ & $\begin{array}{l}\text { Density } \\
(\mathrm{g} / \mathrm{cc})\end{array}$ & $\begin{array}{l}\text { First Diameter } \\
\text { (Inches) }\end{array}$ & $\begin{array}{l}\text { Last Diameter } \\
\text { (Inches) }\end{array}$ & $\begin{array}{l}\text { Delta } \\
\text { (Inches) }\end{array}$ & $\begin{array}{l}\text { Critical Diameter } \\
\text { (Inches) }\end{array}$ \\
\hline 0.012 & 1.016 & 21.4 & 27.8 & 0.3 & 26.4924 \\
\hline 0.015 & 1.021 & 17.8 & 21.8 & 0.2 & 21.0891 \\
\hline 0.02 & 1.029 & 15.1 & 18 & 0.1 & 17.4364 \\
\hline 0.025 & 1.037 & 13.8 & 16.2 & 0.1 & 15.7054 \\
\hline 0.03 & 1.046 & 13 & 15.2 & 0.1 & 14.7142 \\
\hline 0.032 & 1.049 & 12.8 & 14.9 & 0.1 & 14.4369 \\
\hline 0.035 & 1.054 & 12.5 & 14.5 & 0.1 & 14.0593 \\
\hline 0.04 & 1.063 & 12.1 & 14 & 0.1 & 13.635 \\
\hline 0.05 & 1.08 & 11.7 & 13.5 & 0.1 & 13.0681 \\
\hline 0.06 & 1.097 & 11.4 & 13.1 & 0.05 & 12.7511 \\
\hline 0.07 & 1.113 & 11.2 & 12.9 & 0.05 & 12.5783 \\
\hline 0.08 & 1.13 & 11.1 & 12.8 & 0.05 & 12.4427 \\
\hline 0.09 & 1.147 & 11.1 & 12.7 & 0.05 & 12.3748 \\
\hline 0.1 & 1.164 & 11 & 12.7 & 0.05 & 12.3164 \\
\hline 0.12 & 1.197 & 11 & 12.7 & 0.05 & 12.297 \\
\hline 0.13 & 1.215 & 11 & 12.7 & 0.05 & 12.3064 \\
\hline 0.15 & 1.248 & 11 & 12.7 & 0.05 & 12.3275 \\
\hline 0.2 & 1.332 & 11.1 & 12.8 & 0.05 & 12.4449 \\
\hline 0.239 & 1.397 & 11.2 & 12.9 & 0.05 & 12.5545 \\
\hline 0.25 & 1.415 & 11.3 & 12.9 & 0.1 & 12.6066 \\
\hline 0.359 & 1.594 & 11.5 & 13.3 & 0.1 & 12.9504 \\
\hline 0.478 & 1.784 & 11.8 & 13.6 & 0.1 & 13.2904 \\
\hline 0.5 & 1.818 & 11.9 & 13.8 & 0.1 & 13.3705 \\
\hline 0.6 & 1.962 & 12.3 & 14.3 & 0.1 & 13.8482 \\
\hline 0.65 & 2.026 & 12.6 & 14.6 & 0.1 & 14.1638 \\
\hline 0.7 & 2.076 & 13.1 & 15.2 & 0.2 & 14.7443 \\
\hline 0.75 & 2.08 & 14.4 & 16.6 & 0.1 & 16.201 \\
\hline 0.752 & 2.074 & 14.5 & 16.9 & 0.1 & 16.4027 \\
\hline 0.754 & 2.065 & 14.7 & 17.1 & 0.1 & 16.6323 \\
\hline 0.755 & 2.055 & 15.1 & 17.4 & 0.1 & 16.8892 \\
\hline
\end{tabular}




\section{B1.1.1.3.1.3 10" Water Reflection}

Case ID: PuN40-sp-0950-10

Material: $\mathrm{Pu}\left(\mathrm{NO}_{3}\right)_{4} 0$ Molar Excess Acid

Geometry: Sphere

Isotopics: $95 \%{ }^{239} \mathrm{Pu}$

Reflector: 10 " water reflected

Code: MCNP5 v1.40

Analyst: Mark Murphy

Date: $8 / 2011$ 
Figure B1.1.1.3.1.3-1. $\mathrm{Pu}\left(\mathrm{NO}_{3}\right) 40 \mathrm{M}-$ Sphere $-95 \%{ }^{239} \mathrm{Pu}-10$ " Water Reflection

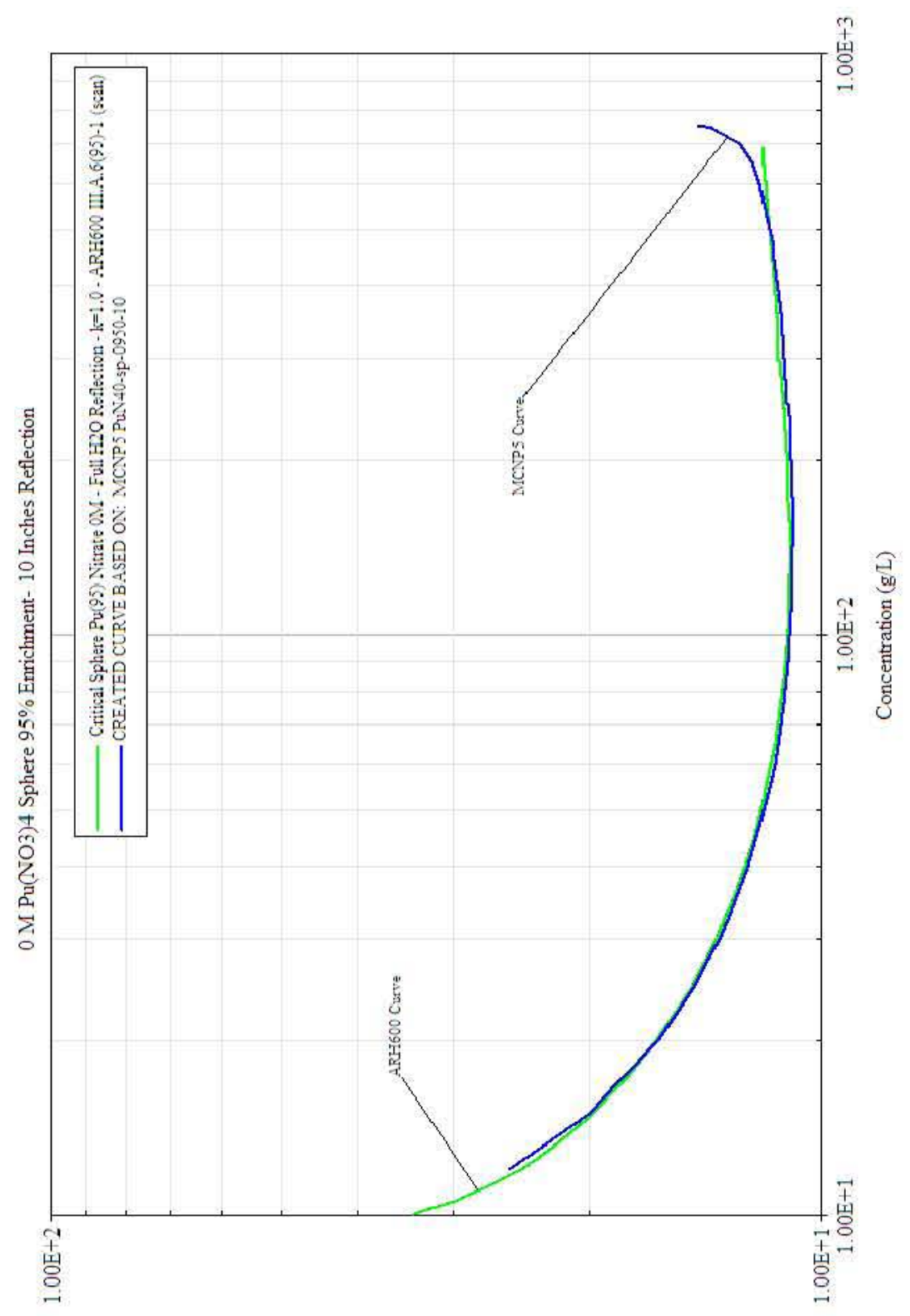

(แ1) .эาวแะ!Ф 
Table B1.1.1.3.1.3-1. Critical Dimensions for $\mathrm{Pu}\left(\mathrm{NO}_{3}\right) 40 \mathrm{M}-$ Sphere $-95 \%{ }^{239} \mathrm{Pu}-10 "$ Water Reflection

\begin{tabular}{|c|c|c|c|c|c|}
\hline \multicolumn{6}{|c|}{ 95\% Pu239 Pu(NO3)4 0M Sphere- 10 Inches Reflection } \\
\hline Concentration $(\mathrm{g} / \mathrm{cc})$ & $\begin{array}{l}\text { Density } \\
(\mathrm{g} / \mathrm{cc})\end{array}$ & $\begin{array}{l}\text { First Diameter } \\
\text { (Inches) }\end{array}$ & $\begin{array}{l}\text { Last Diameter } \\
\text { (Inches) }\end{array}$ & \begin{tabular}{|l|l|} 
Delta \\
(Inches)
\end{tabular} & $\begin{array}{l}\text { Critical Diameter } \\
\text { (Inches) } \\
\end{array}$ \\
\hline 0.012 & 1.016 & 20.3 & 26.5 & 0.3 & 25.3989 \\
\hline 0.015 & 1.021 & 16.8 & 20.7 & 0.2 & 19.9554 \\
\hline 0.02 & 1.029 & 14 & 16.8 & 0.1 & 16.2795 \\
\hline 0.025 & 1.037 & 12.7 & 15 & 0.1 & 14.5428 \\
\hline 0.03 & 1.046 & 11.9 & 14 & 0.1 & 13.5365 \\
\hline 0.032 & 1.049 & 11.6 & 13.7 & 0.1 & 13.2398 \\
\hline 0.035 & 1.054 & 11.3 & 13.3 & 0.1 & 12.8763 \\
\hline 0.04 & 1.063 & 10.9 & 12.8 & 0.1 & 12.4073 \\
\hline 0.05 & 1.08 & 10.5 & 12.2 & 0.05 & 11.8271 \\
\hline 0.06 & 1.097 & 10.1 & 11.8 & 0.05 & 11.4578 \\
\hline 0.07 & 1.113 & 9.9 & 11.6 & 0.05 & 11.2534 \\
\hline 0.08 & 1.13 & 9.8 & 11.4 & 0.05 & \begin{tabular}{|l|l|}
11.109 \\
\end{tabular} \\
\hline 0.09 & 1.147 & 9.7 & 11.3 & 0.05 & 10.9981 \\
\hline 0.1 & 1.164 & 9.7 & 11.3 & 0.05 & 10.9547 \\
\hline 0.12 & 1.197 & 9.65 & 11.2 & 0.05 & 10.87 \\
\hline 0.13 & 1.215 & 9.6 & 11.2 & 0.05 & 10.8515 \\
\hline 0.15 & 1.248 & 9.6 & 11.2 & 0.05 & 10.8382 \\
\hline 0.2 & 1.332 & 9.6 & 11.3 & \begin{tabular}{|l|}
0.05 \\
\end{tabular} & 10.9012 \\
\hline 0.239 & 1.397 & 9.7 & 11.3 & 0.05 & 10.9827 \\
\hline 0.25 & 1.415 & 9.6 & 11.4 & 0.1 & 10.9909 \\
\hline 0.359 & 1.594 & 9.9 & 11.7 & 0.1 & 11.2579 \\
\hline 0.478 & 1.784 & 10.1 & 11.9 & 0.1 & 11.546 \\
\hline 0.5 & 1.818 & 10.1 & 12 & 0.1 & \begin{tabular}{|l|}
11.6191 \\
\end{tabular} \\
\hline 0.6 & 1.962 & 10.5 & 12.4 & 0.1 & 11.9991 \\
\hline 0.65 & 2.026 & 10.7 & 12.7 & 0.1 & 12.2765 \\
\hline 0.7 & 2.076 & 11.1 & 13.1 & 0.1 & 12.7425 \\
\hline 0.75 & 2.08 & 12.1 & 14.3 & 0.1 & \begin{tabular}{|l}
13.8909 \\
\end{tabular} \\
\hline 0.752 & 2.074 & 12.2 & 14.5 & 0.1 & 14.0378 \\
\hline 0.754 & 2.065 & 12.4 & 14.7 & 0.1 & 14.2439 \\
\hline 0.755 & 2.055 & 12.6 & 14.9 & 0.1 & 14.4358 \\
\hline
\end{tabular}


B1.1.2 Cylinder

B1.1.2.1 $\quad 100 \%{ }^{239} \mathrm{Pu}$

B1.1.2.1.1 0 Molar Excess Acid

B1.1.2.1.1.1 0"Water Reflection

Case ID: PuN40-icyl-1000-00

Material: $\mathrm{Pu}\left(\mathrm{NO}_{3}\right)_{4} 0$ Molar Excess Acid

Geometry: Cylinder

Isotopics: $100 \%{ }^{239} \mathrm{Pu}$

Reflector: 0" water reflected

Code: MCNP5 v1.40

Analyst: Mark Murphy

Date: $8 / 2011$ 
Figure B1.1.2.1.1.1-1. $\mathrm{Pu}\left(\mathrm{NO}_{3}\right) 4$ 0M - Cylinder - $100 \%{ }^{239} \mathrm{Pu}-0$ " Water Reflection

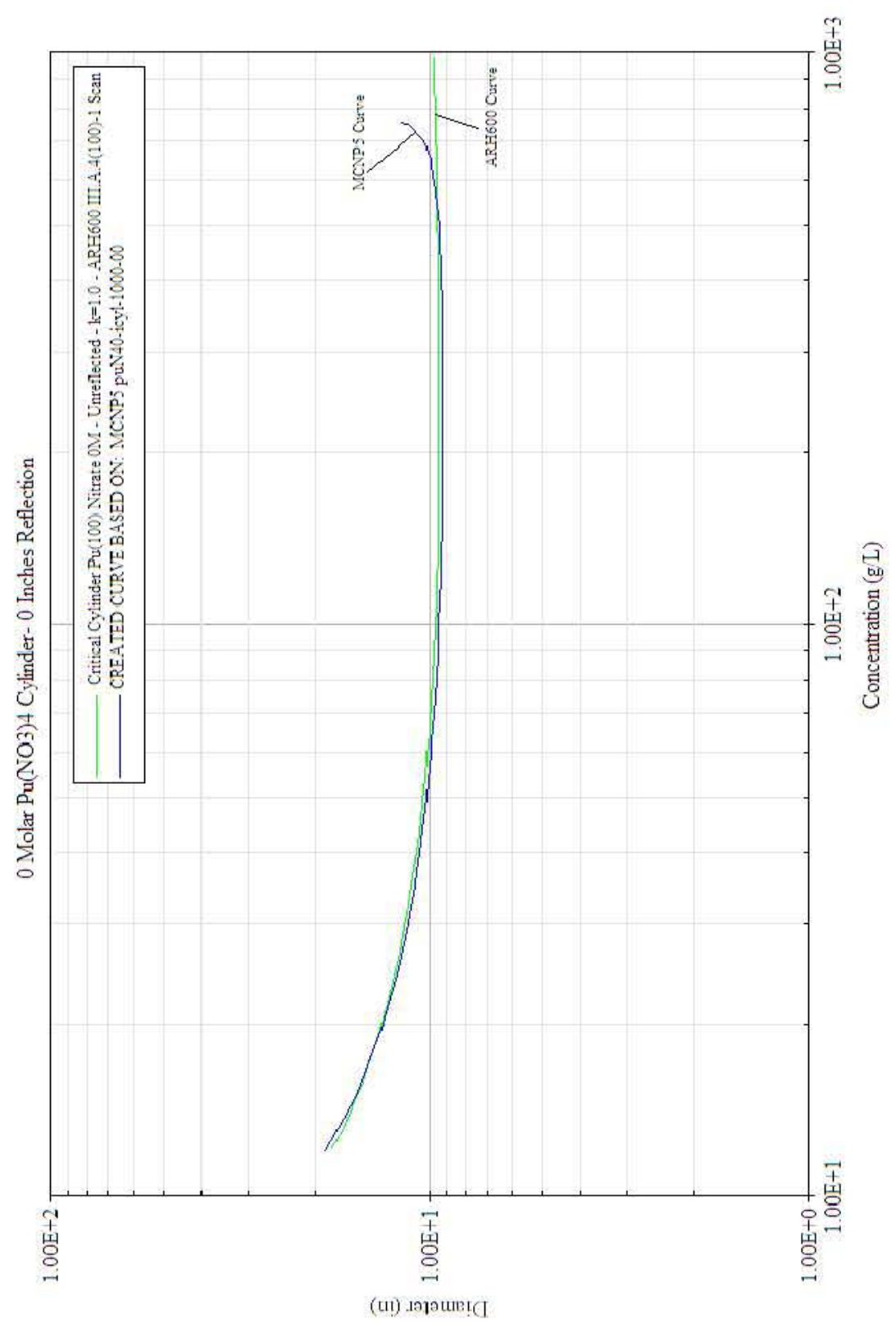


Table B1.1.2.1.1.1-1. Critical Dimensions for $\mathrm{Pu}\left(\mathrm{NO}_{3}\right) 40 \mathrm{M}-\mathrm{Cylinder}-100 \%{ }^{239} \mathrm{Pu}-0$ " Water Reflection

\begin{tabular}{|c|c|c|c|c|c|}
\hline \multicolumn{6}{|c|}{$100 \% \mathrm{Pu} 239 \mathrm{Pu}(\mathrm{NO} 3) 4 \mathrm{0M}$ Cylinder - 0 Inches of Reflection } \\
\hline Concentration $(\mathrm{g} / \mathrm{cc})$ & $\begin{array}{l}\text { Density } \\
(\mathrm{g} / \mathrm{cc})\end{array}$ & $\begin{array}{l}\text { First Diameter } \\
\text { (Inches) }\end{array}$ & $\begin{array}{l}\text { Last Diameter } \\
\text { (Inches) }\end{array}$ & $\begin{array}{l}\text { Delta } \\
\text { (Inches) }\end{array}$ & $\begin{array}{l}\text { Critical Diameter } \\
\text { (Inches) }\end{array}$ \\
\hline 0.012 & 1.016 & 15.7 & 19.6 & 0.2 & 18.7761 \\
\hline 0.015 & 1.021 & 13.5 & 16.1 & 0.1 & 15.5795 \\
\hline 0.02 & 1.029 & 11.6 & 13.6 & 0.1 & 13.1729 \\
\hline 0.025 & 1.037 & 10.7 & 12.4 & 0.05 & 12.0253 \\
\hline 0.03 & 1.046 & 10.2 & 11.7 & 0.05 & 11.3261 \\
\hline 0.032 & 1.049 & 10 & 11.4 & 0.05 & 11.0955 \\
\hline 0.035 & 1.054 & 9.8 & 11.2 & 0.05 & 10.8763 \\
\hline 0.04 & 1.063 & 9.5 & 10.8 & 0.05 & 10.5513 \\
\hline 0.05 & 1.08 & 9.2 & 10.4 & 0.05 & 10.1115 \\
\hline 0.06 & 1.097 & 8.9 & 10.1 & 0.05 & 9.8475 \\
\hline 0.07 & 1.113 & 8.8 & 10 & 0.05 & 9.6909 \\
\hline 0.08 & 1.13 & 8.7 & 9.9 & 0.05 & 9.5692 \\
\hline 0.09 & 1.147 & 8.6 & 9.8 & 0.05 & 9.4825 \\
\hline 0.1 & 1.164 & 8.6 & 9.7 & 0.05 & 9.4255 \\
\hline 0.12 & 1.197 & 8.5 & 9.6 & 0.05 & 9.3308 \\
\hline 0.13 & 1.215 & 8.5 & 9.6 & 0.05 & 9.3016 \\
\hline 0.15 & 1.248 & 8.4 & 9.6 & 0.05 & 9.2506 \\
\hline 0.2 & 1.332 & 8.4 & 9.5 & 0.05 & 9.2135 \\
\hline 0.239 & 1.397 & 8.4 & 9.5 & 0.05 & 9.1932 \\
\hline 0.25 & 1.415 & 8.4 & 9.5 & 0.05 & 9.2177 \\
\hline 0.359 & 1.594 & 8.4 & 9.5 & 0.05 & 9.253 \\
\hline 0.478 & 1.784 & 8.5 & 9.7 & 0.05 & 9.3648 \\
\hline 0.5 & 1.818 & 8.6 & 9.7 & 0.05 & 9.4094 \\
\hline 0.6 & 1.962 & 8.8 & 10 & 0.05 & 9.6687 \\
\hline 0.65 & 2.026 & 9 & 10.2 & 0.05 & 9.8978 \\
\hline 0.7 & 2.076 & 9.4 & 10.6 & 0.05 & 10.2797 \\
\hline 0.75 & 2.079 & 10.3 & 11.7 & 0.05 & 11.3628 \\
\hline 0.752 & 2.073 & 10.5 & 11.8 & 0.05 & 11.5119 \\
\hline 0.754 & 2.064 & 10.6 & 12 & 0.05 & 11.6832 \\
\hline 0.755 & 2.052 & 10.8 & 12.2 & 0.05 & 11.8834 \\
\hline
\end{tabular}


B1.1.2.1.1.2 1"Water Reflection

Case ID: PuN40-icyl-1000-01

Material: $\mathrm{Pu}\left(\mathrm{NO}_{3}\right)_{4} 0$ Molar Excess Acid

Geometry: Cylinder

Isotopics: $100 \%{ }^{239} \mathrm{Pu}$

Reflector: 1" water reflected

Code: MCNP5 v1.40

Analyst: Mark Murphy

Date: $8 / 2011$ 
Figure B1.1.2.1.1.2-1. $\mathrm{Pu}\left(\mathrm{NO}_{3}\right) 40 \mathrm{M}-\mathrm{Cylinder}-100 \%{ }^{239} \mathrm{Pu}-1$ " Water Reflection

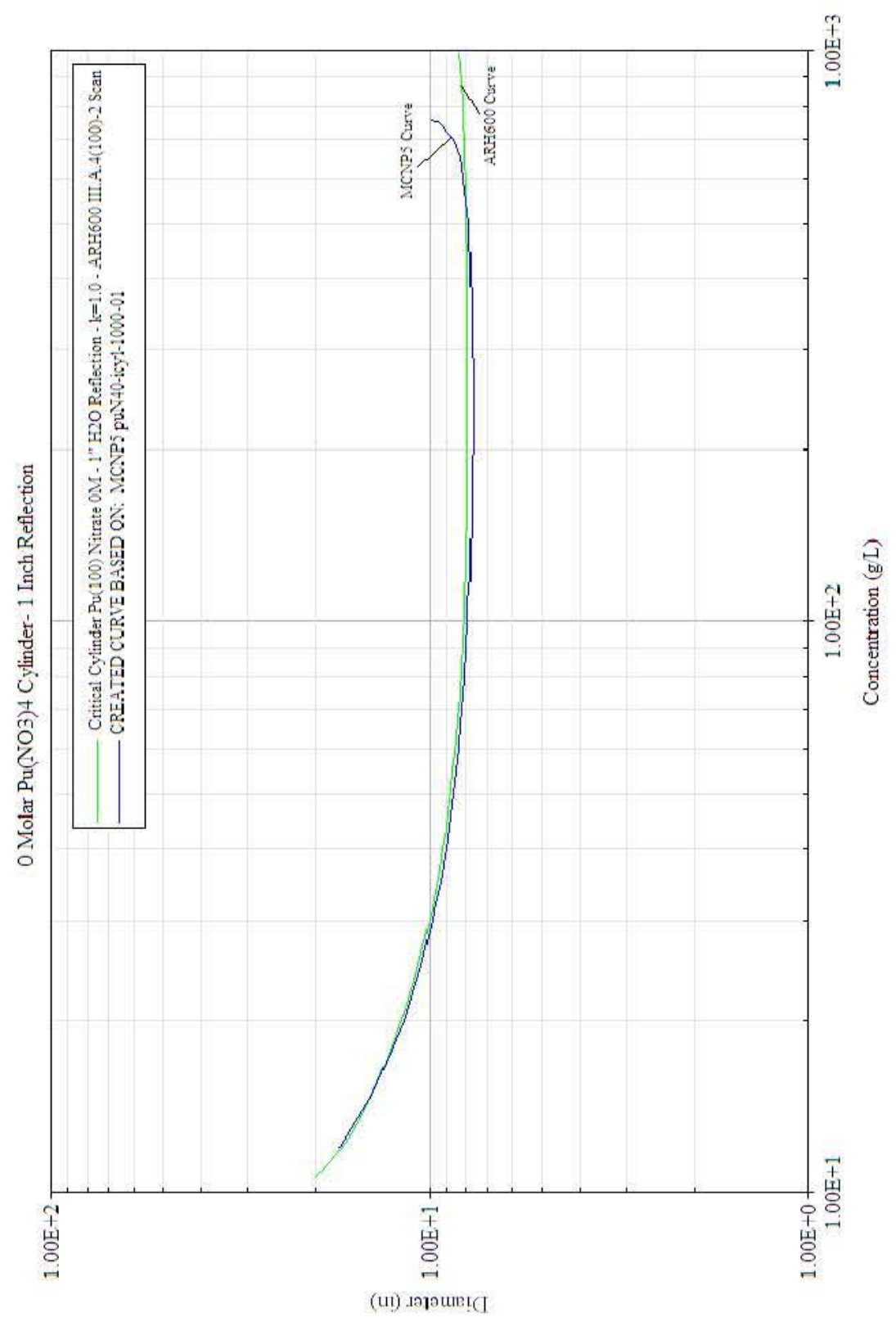


Table B1.1.2.1.1.2-1. Critical Dimensions for $\mathrm{Pu}\left(\mathrm{NO}_{3}\right) 40 \mathrm{M}-\mathrm{Cylinder}-100 \%{ }^{239} \mathrm{Pu}-1$ " Water Reflection

\begin{tabular}{|c|c|c|c|c|c|}
\hline \multicolumn{6}{|c|}{$100 \% \mathrm{Pu} 239 \mathrm{Pu}(\mathrm{NO} 3) 40 \mathrm{M}$ Cylinder- 1 Inch of Reflection } \\
\hline Concentration $(\mathrm{g} / \mathrm{cc})$ & $\begin{array}{l}\text { Density } \\
(\mathrm{g} / \mathrm{cc})\end{array}$ & $\begin{array}{l}\text { First Diameter } \\
\text { (Inches) }\end{array}$ & $\begin{array}{l}\text { Last Diameter } \\
\text { (Inches) }\end{array}$ & $\begin{array}{l}\text { Delta } \\
\text { (Inches) }\end{array}$ & $\begin{array}{l}\text { Critical Diameter } \\
\text { (Inches) }\end{array}$ \\
\hline 0.012 & 1.016 & 14.2 & 18.1 & 0.2 & 17.2 \\
\hline 0.015 & 1.021 & 11.9 & 14.6 & 0.1 & 14.0524 \\
\hline 0.02 & 1.029 & 10.1 & 12.1 & 0.1 & 11.656 \\
\hline 0.025 & 1.037 & 9.2 & 10.8 & 0.05 & 10.4959 \\
\hline 0.03 & 1.046 & 8.7 & 10.1 & 0.05 & 9.8103 \\
\hline 0.032 & 1.049 & 8.5 & 9.9 & 0.05 & 9.5884 \\
\hline 0.035 & 1.054 & 8.3 & 9.6 & 0.05 & 9.3311 \\
\hline 0.04 & 1.063 & 8 & 9.3 & 0.05 & 9.0184 \\
\hline 0.05 & 1.08 & 7.6 & 8.9 & 0.05 & 8.5836 \\
\hline 0.06 & 1.097 & 7.4 & 8.6 & 0.05 & 8.3004 \\
\hline 0.07 & 1.113 & 7.3 & 8.4 & 0.05 & 8.1548 \\
\hline 0.08 & 1.13 & 7.2 & 8.3 & 0.05 & 8.0176 \\
\hline 0.09 & 1.147 & 7.1 & 8.2 & 0.05 & 7.9371 \\
\hline 0.1 & 1.164 & 7 & 8.1 & 0.05 & 7.8817 \\
\hline 0.12 & 1.197 & 7 & 8.1 & 0.05 & 7.7775 \\
\hline 0.13 & 1.215 & 6.9 & 8 & 0.05 & 7.7406 \\
\hline 0.15 & 1.248 & 6.9 & 8 & 0.05 & 7.7079 \\
\hline 0.2 & 1.332 & 6.8 & 7.9 & 0.05 & 7.6509 \\
\hline 0.239 & 1.397 & 6.8 & 7.9 & 0.05 & 7.666 \\
\hline 0.25 & 1.415 & 6.8 & 7.9 & 0.05 & 7.661 \\
\hline 0.359 & 1.594 & 6.9 & 8 & 0.05 & 7.6937 \\
\hline 0.478 & 1.784 & 7 & 8.1 & 0.05 & 7.828 \\
\hline 0.5 & 1.818 & 7 & 8.1 & 0.05 & 7.8591 \\
\hline 0.6 & 1.962 & 7.2 & 8.3 & 0.05 & 8.1035 \\
\hline 0.65 & 2.026 & 7.4 & 8.6 & 0.05 & 8.2772 \\
\hline 0.7 & 2.076 & 7.7 & 8.9 & 0.05 & 8.6195 \\
\hline 0.75 & 2.079 & 8.5 & 9.8 & 0.05 & 9.4956 \\
\hline 0.752 & 2.073 & 8.6 & 9.9 & 0.05 & 9.6257 \\
\hline 0.754 & 2.064 & 8.7 & 10.1 & 0.05 & 9.7716 \\
\hline 0.755 & 2.052 & 8.9 & 10.2 & 0.05 & 9.9276 \\
\hline
\end{tabular}




\section{B1.1.2.1.1.3 10" Water Reflection}

Case ID: PuN40-icyl-1000-10

Material: $\mathrm{Pu}\left(\mathrm{NO}_{3}\right)_{4} 0$ Molar Excess Acid

Geometry: Cylinder

Isotopics: $100 \%{ }^{239} \mathrm{Pu}$

Reflector: 10 " water reflected

Code: MCNP5 v1.40

Analyst: Mark Murphy

Date: $8 / 2011$ 
Figure B1.1.2.1.1.3-1. $\mathrm{Pu}\left(\mathrm{NO}_{3}\right) 40 \mathrm{M}-\mathrm{Cylinder}-100 \%{ }^{239} \mathrm{Pu}-10$ " Water Reflection

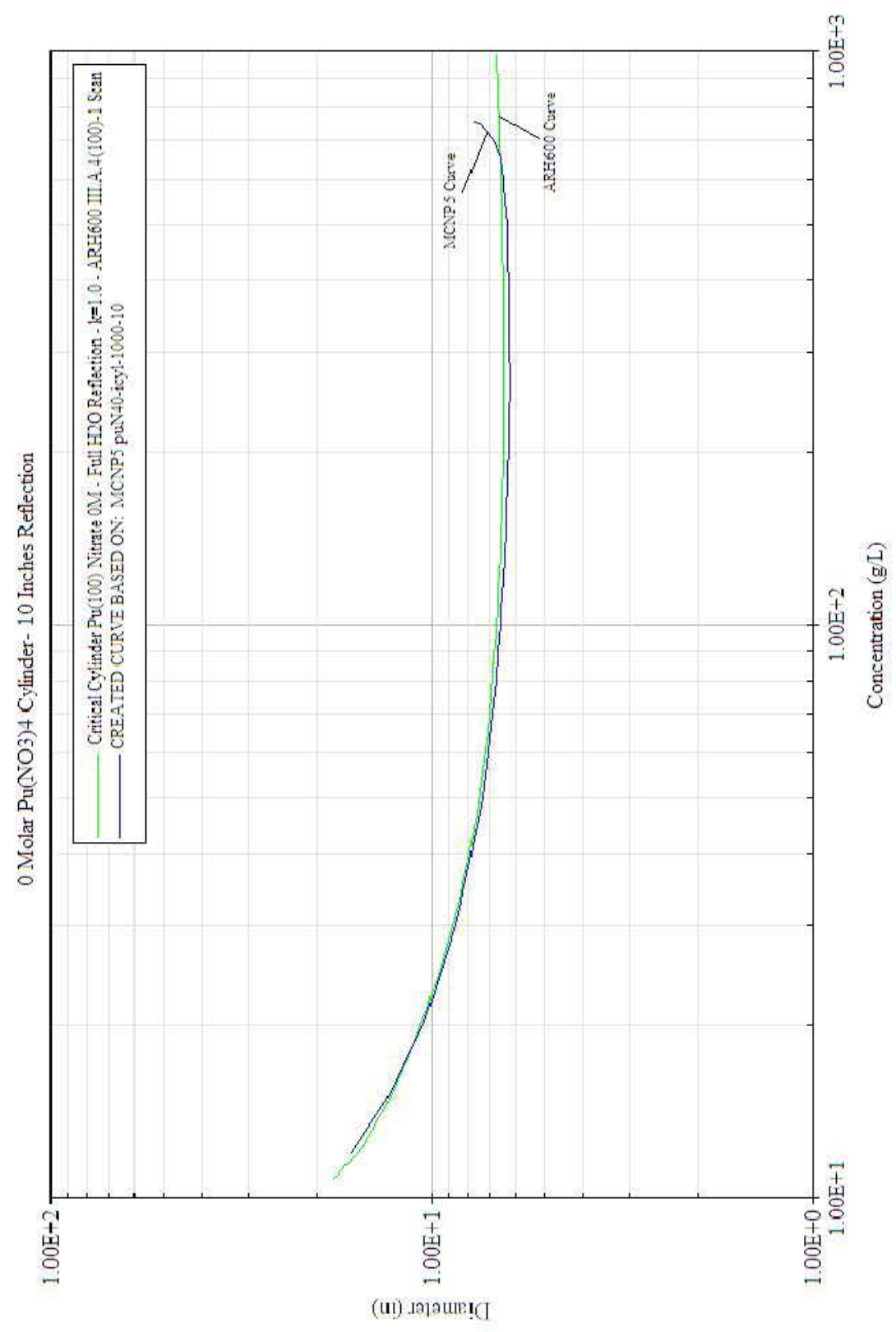


Table B1.1.2.1.1.3-1. Critical Dimensions for $\mathrm{Pu}\left(\mathrm{NO}_{3}\right) 4 \mathrm{OM}-\mathrm{Cylinder}-100 \%{ }^{239} \mathrm{Pu}-10 "$ Water Reflection

\begin{tabular}{|c|c|c|c|c|c|}
\hline \multicolumn{6}{|c|}{$100 \% \mathrm{Pu} 239 \mathrm{Pu}(\mathrm{NO} 3) 40 \mathrm{M}$ Cylinder - 10 Inches of Reflection } \\
\hline Concentration $(\mathrm{g} / \mathrm{cc})$ & $\begin{array}{l}\text { Density } \\
(\mathrm{g} / \mathrm{cc})\end{array}$ & $\begin{array}{l}\text { First Diameter } \\
\text { (Inches) }\end{array}$ & \begin{tabular}{|l}
$\begin{array}{l}\text { Last Diameter } \\
\text { (Inches) }\end{array}$ \\
\end{tabular} & $\begin{array}{l}\text { Delta } \\
\text { (Inches) }\end{array}$ & $\begin{array}{l}\text { Critical Diameter } \\
\text { (Inches) }\end{array}$ \\
\hline 0.012 & 1.016 & 13.1 & 17 & 0.2 & 16.1926 \\
\hline 0.015 & 1.021 & 10.9 & 13.4 & 0.1 & 12.9348 \\
\hline 0.02 & 1.029 & 9 & 10.9 & 0.1 & 10.5112 \\
\hline 0.025 & 1.037 & 8.1 & 9.7 & 0.05 & 9.35 \\
\hline 0.03 & 1.046 & 7.5 & 9 & 0.05 & 8.6283 \\
\hline 0.032 & 1.049 & 7.3 & 8.7 & 0.05 & 8.4145 \\
\hline 0.035 & 1.054 & 7.1 & 8.4 & 0.05 & 8.1592 \\
\hline 0.04 & 1.063 & 6.8 & 8.1 & 0.05 & 7.8122 \\
\hline 0.05 & 1.08 & 6.5 & 7.6 & 0.05 & 7.3452 \\
\hline 0.06 & 1.097 & 6.2 & 7.3 & 0.05 & 7.0539 \\
\hline 0.07 & 1.113 & 6 & 7.1 & 0.05 & 6.8709 \\
\hline 0.08 & 1.13 & 5.9 & 7 & 0.05 & 6.7266 \\
\hline 0.09 & 1.147 & 5.8 & 6.9 & 0.05 & 6.6395 \\
\hline 0.1 & 1.164 & 5.7 & 6.8 & 0.05 & 6.5323 \\
\hline 0.12 & 1.197 & 5.6 & 6.7 & 0.05 & 6.437 \\
\hline 0.13 & 1.215 & 5.5 & 6.6 & 0.05 & 6.3846 \\
\hline 0.15 & 1.248 & 5.5 & 6.6 & 0.05 & 6.3201 \\
\hline 0.2 & 1.332 & 5.4 & 6.5 & 0.05 & 6.2359 \\
\hline 0.239 & 1.397 & \begin{tabular}{|l|}
5.3 \\
\end{tabular} & 6.5 & 0.05 & 6.2051 \\
\hline 0.25 & 1.415 & 5.3 & 6.4 & 0.05 & 6.1832 \\
\hline 0.359 & 1.594 & 5.3 & 6.4 & 0.05 & 6.1997 \\
\hline 0.478 & 1.784 & 5.4 & 6.5 & 0.05 & 6.2572 \\
\hline 0.5 & 1.818 & 5.4 & 6.5 & 0.05 & 6.2785 \\
\hline 0.6 & 1.962 & 5.5 & 6.7 & 0.05 & 6.4355 \\
\hline 0.65 & 2.026 & 5.7 & 6.8 & 0.05 & 6.5714 \\
\hline 0.7 & 2.076 & 5.9 & 7.1 & 0.05 & 6.8096 \\
\hline 0.75 & 2.079 & 6.5 & \begin{tabular}{|l|}
7.7 \\
\end{tabular} & 0.05 & 7.4332 \\
\hline 0.752 & 2.073 & 6.6 & 7.8 & 0.05 & 7.5517 \\
\hline 0.754 & 2.064 & 6.6 & 7.9 & 0.05 & 7.6435 \\
\hline 0.755 & 2.052 & 6.7 & 8.1 & 0.05 & 7.7605 \\
\hline
\end{tabular}


B1.1.2.1.2 3 Molar Excess Acid

B1.1.2.1.2.1 0"Water Reflection

Case ID: PuN43-icyl-1000-00

Material: $\mathrm{Pu}\left(\mathrm{NO}_{3}\right)_{4} 3$ Molar Excess Acid

Geometry: Cylinder

Isotopics: $100 \%{ }^{239} \mathrm{Pu}$

Reflector: 0" water reflected

Code: MCNP5 v1.40

Analyst: Mark Murphy

Date: $8 / 2011$ 
Figure B1.1.2.1.2.1-1. $\mathrm{Pu}\left(\mathrm{NO}_{3}\right) 43 \mathrm{M}-\mathrm{Cylinder}-100 \%{ }^{239} \mathrm{Pu}-0$ " Water Reflection

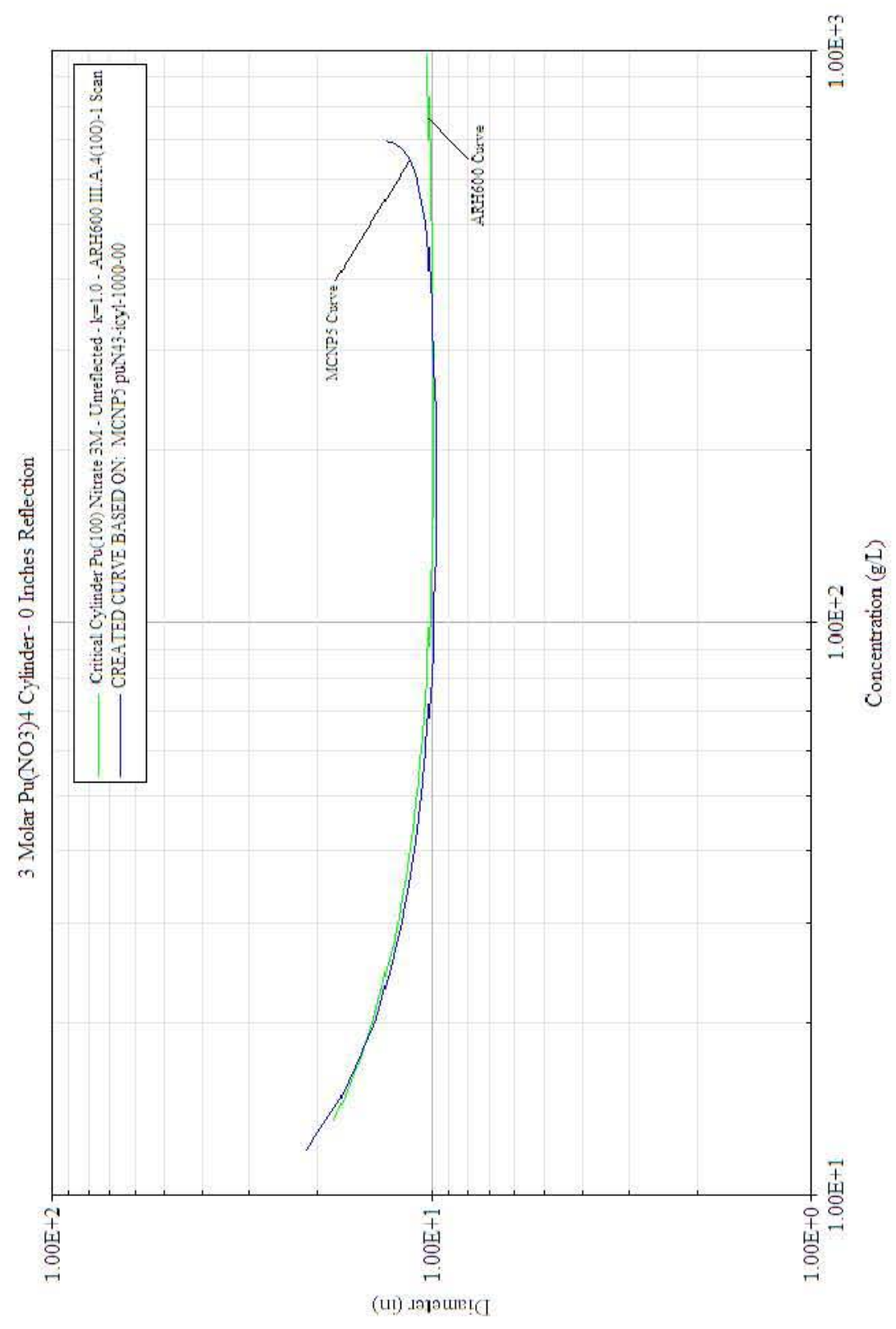


Table B1.1.2.1.2.1-1. Critical Dimensions for $\mathrm{Pu}\left(\mathrm{NO}_{3}\right) 43 \mathrm{M}-\mathrm{Cylinder}-100 \%{ }^{239} \mathrm{Pu}-0$ " Water Reflection

\begin{tabular}{|c|c|c|c|c|c|}
\hline \multicolumn{6}{|c|}{$100 \% \mathrm{Pu} 239 \mathrm{Pu}(\mathrm{NO} 3) 43 \mathrm{M}$ Cylinder - 0 Inches of Reflection } \\
\hline Concentration $(\mathrm{g} / \mathrm{cc})$ & \begin{tabular}{|l|} 
Density \\
$(\mathrm{g} / \mathrm{cc})$ \\
\end{tabular} & $\begin{array}{l}\text { First Diameter } \\
\text { (Inches) }\end{array}$ & $\begin{array}{l}\text { Last Diameter } \\
\text { (Inches) }\end{array}$ & $\begin{array}{l}\text { Delta } \\
\text { (Inches) }\end{array}$ & $\begin{array}{l}\text { Critical Diameter } \\
\text { (Inches) }\end{array}$ \\
\hline 0.012 & 1.118 & 17.4 & 22.3 & 0.2 & 21.3173 \\
\hline 0.015 & 1.123 & 14.5 & 17.7 & 0.1 & 17.0363 \\
\hline 0.02 & 1.132 & 12.4 & 14.6 & 0.1 & 14.0673 \\
\hline 0.025 & 1.14 & 11.4 & 13.1 & 0.05 & 12.7364 \\
\hline 0.03 & 1.149 & 10.7 & 12.3 & 0.05 & 11.9349 \\
\hline 0.032 & 1.152 & 10.5 & 12.1 & 0.05 & 11.7076 \\
\hline 0.035 & 1.157 & 10.3 & 11.8 & 0.05 & 11.4352 \\
\hline 0.04 & 1.165 & 10 & 11.4 & 0.05 & 11.0646 \\
\hline 0.05 & 1.182 & 9.6 & 10.9 & 0.05 & 10.576 \\
\hline 0.06 & 1.198 & 9.3 & 10.6 & 0.05 & 10.3067 \\
\hline 0.07 & 1.215 & 9.2 & 10.4 & 0.05 & 10.1186 \\
\hline 0.08 & 1.231 & 9.1 & 10.3 & 0.05 & 9.9862 \\
\hline 0.09 & 1.247 & 9 & 10.2 & 0.05 & 9.9066 \\
\hline 0.1 & 1.263 & 8.9 & 10.1 & 0.05 & 9.8483 \\
\hline 0.12 & 1.294 & 8.9 & 10 & 0.05 & 9.761 \\
\hline 0.13 & 1.311 & 8.8 & 10 & 0.05 & 9.7422 \\
\hline 0.15 & 1.342 & 8.8 & 10.1 & 0.05 & 9.7084 \\
\hline 0.2 & 1.421 & 8.8 & 10 & 0.05 & 9.7128 \\
\hline 0.239 & 1.481 & 8.8 & 10.1 & 0.05 & 9.7435 \\
\hline 0.25 & 1.498 & 8.9 & 10 & 0.05 & 9.7825 \\
\hline 0.359 & 1.665 & 9 & 10.2 & 0.05 & 9.9383 \\
\hline 0.478 & 1.841 & 9.3 & 10.5 & 0.05 & 10.2316 \\
\hline 0.5 & 1.871 & 9.4 & 10.6 & 0.05 & 10.3235 \\
\hline 0.6 & 1.998 & 9.9 & 11.2 & 0.05 & 10.8459 \\
\hline 0.65 & 2.043 & 10.4 & 11.7 & 0.05 & 11.425 \\
\hline 0.66 & 2.049 & 10.5 & 11.9 & 0.05 & 11.58 \\
\hline 0.67 & 2.053 & 10.7 & 12.1 & 0.05 & 11.7806 \\
\hline 0.68 & 2.053 & 10.9 & 12.4 & 0.05 & 12.0568 \\
\hline 0.69 & 2.045 & 11.3 & 12.8 & 0.05 & 12.466 \\
\hline 0.698 & 2.017 & 12.1 & 13.6 & 0.05 & 13.2338 \\
\hline
\end{tabular}


B1.1.2.1.2.2 1"Water Reflection

Case ID: PuN43-icyl-1000-01

Material: $\mathrm{Pu}\left(\mathrm{NO}_{3}\right)_{4} 3$ Molar Excess Acid

Geometry: Cylinder

Isotopics: $100 \%{ }^{239} \mathrm{Pu}$

Reflector: 1" water reflected

Code: MCNP5 v1.40

Analyst: Mark Murphy

Date: $8 / 2011$ 
Figure B1.1.2.1.2.2-1. $\mathrm{Pu}\left(\mathrm{NO}_{3}\right) 43 \mathrm{M}-\mathrm{Cylinder}-100 \%{ }^{239} \mathrm{Pu}-1$ " Water Reflection

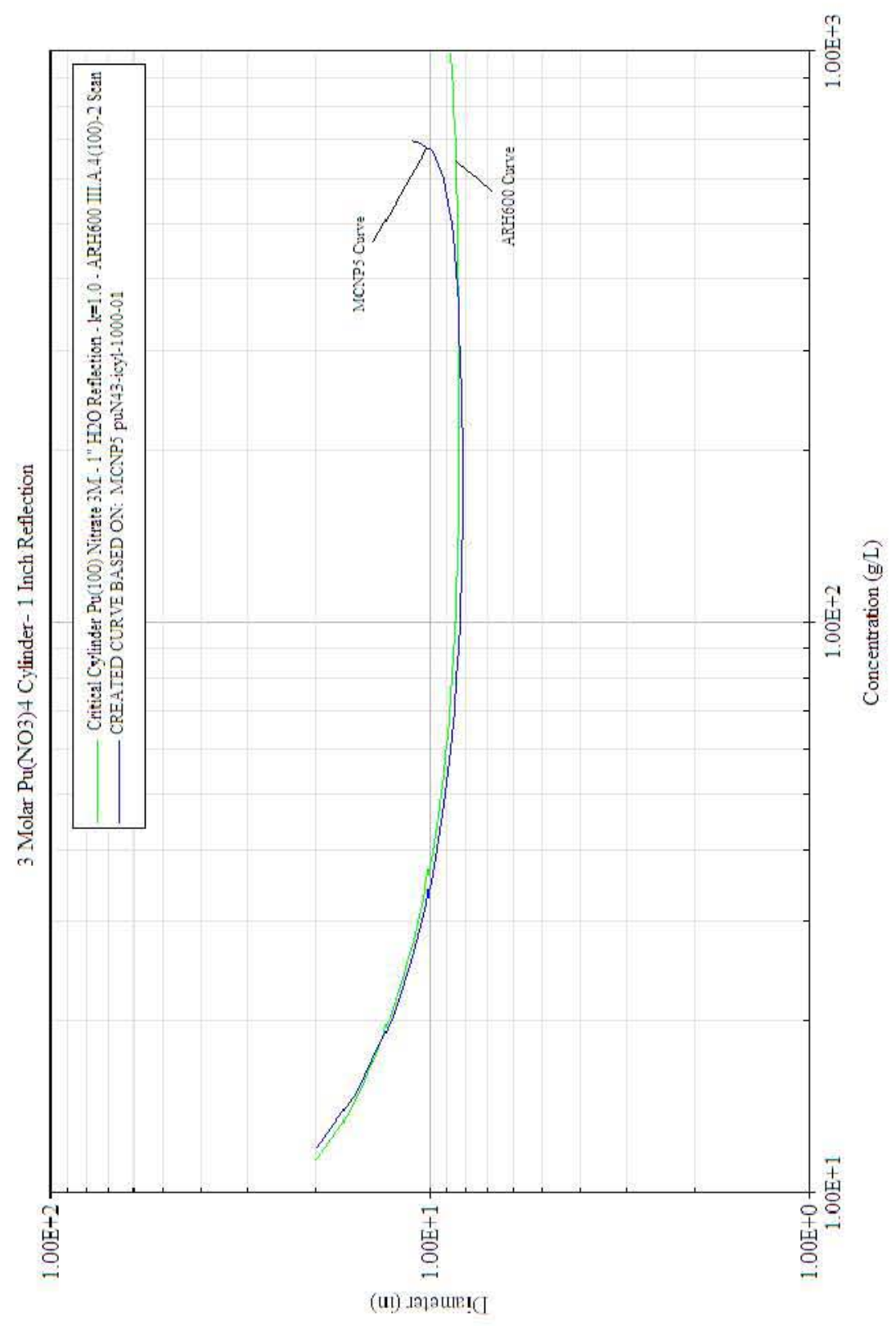


Table B1.1.2.1.2.2-1. Critical Dimensions for $\mathrm{Pu}\left(\mathrm{NO}_{3}\right) 43 \mathrm{M}-\mathrm{Cylinder}-100 \%{ }^{239} \mathrm{Pu}-1$ " Water Reflection

\begin{tabular}{|c|c|c|c|c|c|}
\hline \multicolumn{6}{|c|}{$100 \% \mathrm{Pu} 239 \mathrm{Pu}(\mathrm{NO} 3) 43 \mathrm{M}$ Cylinder - 1 Inch of Reflection } \\
\hline Concentration $(\mathrm{g} / \mathrm{cc})$ & \begin{tabular}{|l|} 
Density \\
$(\mathrm{g} / \mathrm{cc})$ \\
\end{tabular} & $\begin{array}{l}\text { First Diameter } \\
\text { (Inches) }\end{array}$ & $\begin{array}{l}\text { Last Diameter } \\
\text { (Inches) }\end{array}$ & $\begin{array}{l}\text { Delta } \\
\text { (Inches) }\end{array}$ & $\begin{array}{l}\text { Critical Diameter } \\
\text { (Inches) }\end{array}$ \\
\hline 0.012 & 1.118 & 15.8 & 20.7 & 0.2 & 19.7474 \\
\hline 0.015 & 1.123 & 13 & 16.1 & 0.1 & 15.5025 \\
\hline 0.02 & 1.132 & 10.9 & 13 & 0.1 & 12.5503 \\
\hline 0.025 & 1.14 & 9.8 & 11.6 & 0.1 & 11.1838 \\
\hline 0.03 & 1.149 & 9.2 & 10.7 & 0.05 & 10.389 \\
\hline 0.032 & 1.152 & 9 & 10.5 & 0.05 & 10.1707 \\
\hline 0.035 & 1.157 & 8.7 & 10.2 & 0.05 & 9.877 \\
\hline 0.04 & 1.165 & 8.4 & 9.8 & 0.05 & 9.517 \\
\hline 0.05 & 1.182 & 8 & 9.3 & 0.05 & 9.032 \\
\hline 0.06 & 1.198 & 7.8 & 9 & 0.05 & 8.7591 \\
\hline 0.07 & 1.215 & 7.6 & 8.8 & 0.05 & 8.5476 \\
\hline 0.08 & 1.231 & 7.5 & 8.7 & 0.05 & 8.4508 \\
\hline 0.09 & 1.247 & 7.4 & 8.6 & 0.05 & 8.3264 \\
\hline 0.1 & 1.263 & 7.4 & 8.5 & 0.05 & 8.2554 \\
\hline 0.12 & 1.294 & 7.3 & 8.5 & 0.05 & 8.2023 \\
\hline 0.13 & 1.311 & 7.3 & 8.4 & 0.05 & 8.154 \\
\hline 0.15 & 1.342 & 7.3 & 8.4 & 0.05 & 8.127 \\
\hline 0.2 & 1.421 & 7.3 & 8.4 & 0.05 & 8.1185 \\
\hline 0.239 & 1.481 & 7.3 & 8.4 & 0.05 & 8.1602 \\
\hline 0.25 & 1.498 & 7.3 & 8.4 & 0.05 & 8.1551 \\
\hline 0.359 & 1.665 & 7.4 & 8.6 & 0.05 & 8.3307 \\
\hline 0.478 & 1.841 & 7.7 & 8.9 & 0.05 & 8.5847 \\
\hline 0.5 & 1.871 & 7.7 & 8.9 & 0.05 & 8.6616 \\
\hline 0.6 & 1.998 & 8.1 & 9.4 & 0.05 & 9.1176 \\
\hline 0.65 & 2.043 & 8.6 & 9.9 & 0.05 & 9.5847 \\
\hline 0.66 & 2.049 & 8.7 & 10 & 0.05 & 9.7314 \\
\hline 0.67 & 2.053 & 8.8 & 10.2 & 0.05 & 9.898 \\
\hline 0.68 & 2.053 & 9.1 & 10.5 & 0.05 & 10.1248 \\
\hline 0.69 & 2.045 & 9.4 & 10.8 & 0.05 & 10.4858 \\
\hline 0.698 & 2.017 & 10 & 11.4 & 0.05 & 11.1261 \\
\hline
\end{tabular}




\section{B1.1.2.1.2.3 10" Water Reflection}

Case ID: PuN43-icyl-1000-10

Material: $\mathrm{Pu}\left(\mathrm{NO}_{3}\right)_{4} 3$ Molar Excess Acid

Geometry: Cylinder

Isotopics: $100 \%{ }^{239} \mathrm{Pu}$

Reflector: 10 " water reflected

Code: MCNP5 v1.40

Analyst: Mark Murphy

Date: $8 / 2011$ 
Figure B1.1.2.1.2.3-1. $\mathrm{Pu}\left(\mathrm{NO}_{3}\right) 43 \mathrm{M}-\mathrm{Cylinder}-100 \%{ }^{239} \mathrm{Pu}-10$ " Water Reflection

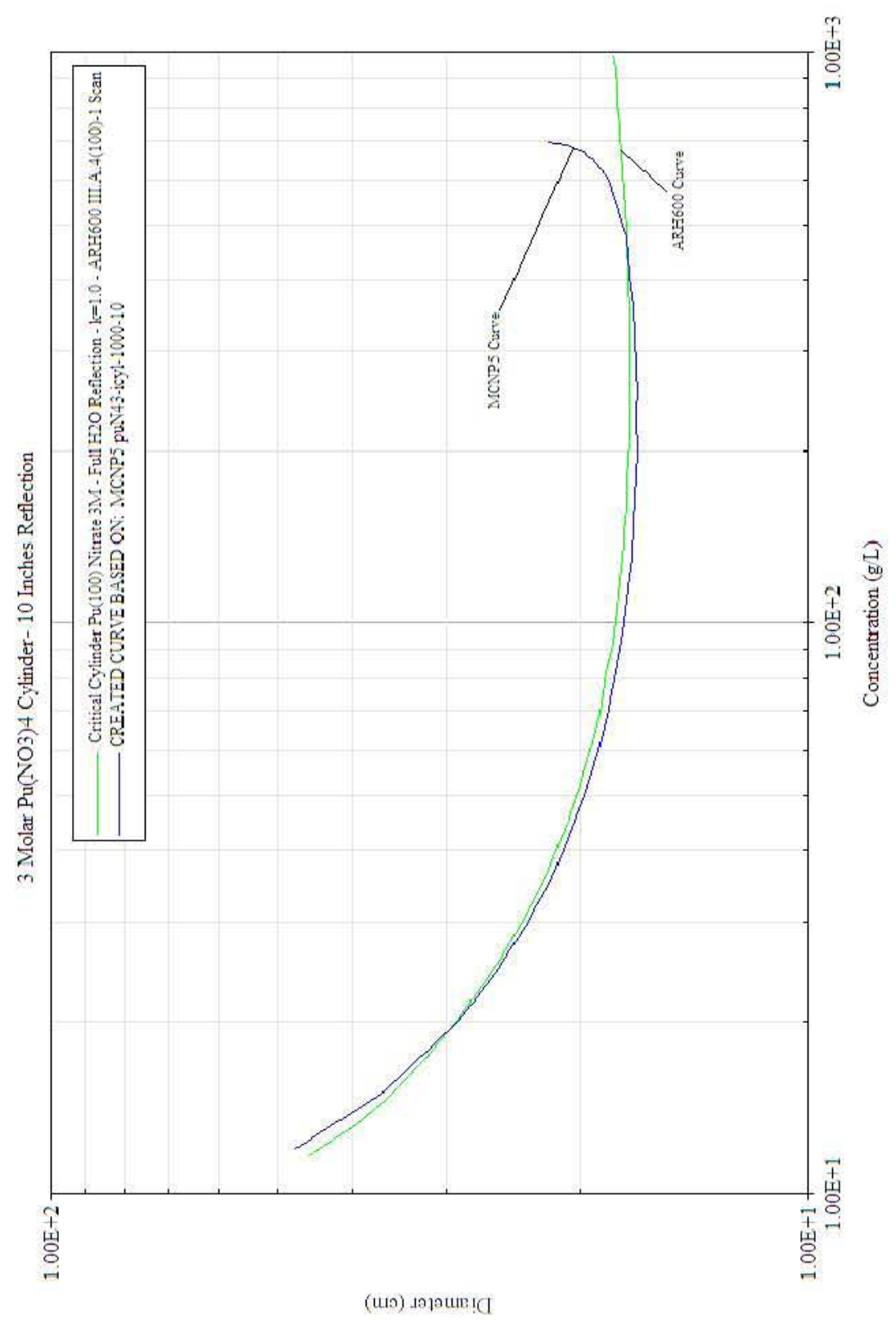


Table B1.1.2.1.2.3-1. Critical Dimensions for $\mathrm{Pu}\left(\mathrm{NO}_{3}\right) 43 \mathrm{M}-\mathrm{Cylinder}-100 \%{ }^{239} \mathrm{Pu}-10 "$ Water Reflection

\begin{tabular}{|c|c|c|c|c|c|}
\hline \multicolumn{6}{|c|}{$100 \% \mathrm{Pu} 239 \mathrm{Pu}(\mathrm{NO} 3) 43 \mathrm{M}$ Cylinder- 10 Inches of Reflection } \\
\hline Concentration $(\mathrm{g} / \mathrm{cc})$ & $\begin{array}{l}\text { Density } \\
(\mathrm{g} / \mathrm{cc})\end{array}$ & $\begin{array}{l}\text { First Diameter } \\
\text { (Inches) }\end{array}$ & $\begin{array}{l}\text { Last Diameter } \\
\text { (Inches) }\end{array}$ & $\begin{array}{l}\text { Delta } \\
\text { (Inches) }\end{array}$ & $\begin{array}{l}\text { Critical Diameter } \\
\text { (Inches) }\end{array}$ \\
\hline 0.012 & 1.118 & 14.7 & 19.6 & 0.2 & 18.6193 \\
\hline 0.015 & 1.123 & 11.9 & 14.9 & 0.1 & 14.3553 \\
\hline 0.02 & 1.132 & 9.7 & 11.8 & 0.1 & 11.401 \\
\hline 0.025 & 1.14 & 8.7 & 10.4 & 0.05 & 10.0215 \\
\hline 0.03 & 1.149 & 8 & 9.5 & 0.05 & 9.1879 \\
\hline 0.032 & 1.152 & 7.8 & 9.3 & 0.05 & 8.9546 \\
\hline 0.035 & 1.157 & 7.6 & 9 & 0.05 & 8.642 \\
\hline 0.04 & 1.165 & 7.2 & 8.6 & 0.05 & 8.2573 \\
\hline 0.05 & 1.182 & 6.8 & 8 & 0.05 & 7.7602 \\
\hline 0.06 & 1.198 & 6.5 & 7.7 & 0.05 & 7.4304 \\
\hline 0.07 & 1.215 & 6.4 & 7.5 & 0.05 & 7.2066 \\
\hline 0.08 & 1.231 & 6.2 & 7.3 & 0.05 & 7.0788 \\
\hline 0.09 & 1.247 & 6.1 & 7.2 & 0.05 & 6.9636 \\
\hline 0.1 & 1.263 & 6 & 7.1 & 0.05 & 6.8843 \\
\hline 0.12 & 1.294 & 5.95 & 7 & 0.05 & 6.7601 \\
\hline 0.13 & 1.311 & 5.9 & 7 & 0.05 & 6.6984 \\
\hline 0.15 & 1.342 & 5.8 & 6.9 & 0.05 & 6.6651 \\
\hline 0.2 & 1.421 & 5.7 & 6.8 & 0.05 & 6.6052 \\
\hline 0.239 & 1.481 & 5.7 & 6.8 & 0.05 & 6.6114 \\
\hline 0.25 & 1.498 & 5.7 & 6.8 & 0.05 & 6.5884 \\
\hline 0.359 & 1.665 & 5.8 & 6.9 & 0.05 & 6.6658 \\
\hline 0.478 & 1.841 & 5.9 & 7.1 & 0.05 & 6.8372 \\
\hline 0.5 & 1.871 & 6 & 7.1 & 0.05 & 6.8977 \\
\hline 0.6 & 1.998 & 6.3 & 7.5 & 0.05 & 7.222 \\
\hline 0.65 & 2.043 & 6.6 & 7.9 & 0.05 & 7.5676 \\
\hline 0.66 & 2.049 & 6.6 & 7.9 & 0.05 & 7.6824 \\
\hline 0.67 & 2.053 & 6.8 & 8.1 & 0.05 & 7.7564 \\
\hline 0.68 & 2.053 & 6.9 & 8.2 & 0.05 & 7.9737 \\
\hline 0.69 & 2.045 & 7.15 & 8.5 & 0.05 & 8.2184 \\
\hline 0.698 & 2.017 & 7.5 & 9 & 0.05 & 8.6798 \\
\hline
\end{tabular}


B1.1.2.1.3 6 Molar Excess Acid

B1.1.2.1.3.1 0"Water Reflection

Case ID: PuN46-icyl-1000-00

Material: $\mathrm{Pu}\left(\mathrm{NO}_{3}\right)_{4} 6$ Molar Excess Acid

Geometry: Cylinder

Isotopics: $100 \%{ }^{239} \mathrm{Pu}$

Reflector: 0 " water reflected

Code: MCNP5 v1.40

Analyst: Mark Murphy

Date: $8 / 2011$ 
Figure B1.1.2.1.3.1-1. $\mathrm{Pu}\left(\mathrm{NO}_{3}\right) 4$ 6M - Cylinder - $100 \%{ }^{239} \mathrm{Pu}-0$ " Water Reflection

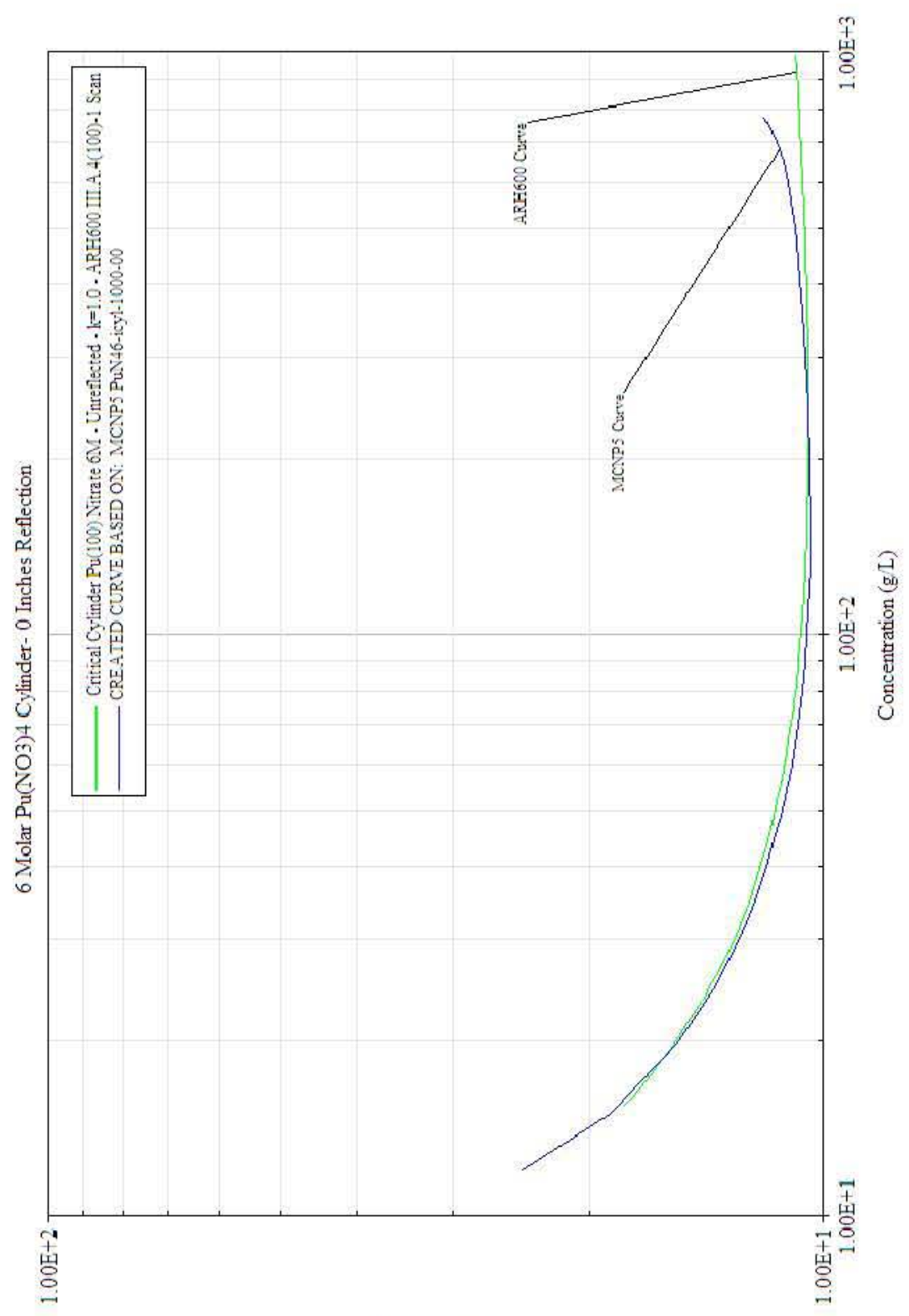

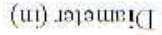


Table B1.1.2.1.3.1-1. Critical Dimensions for $\mathrm{Pu}\left(\mathrm{NO}_{3}\right) 46 \mathrm{M}-\mathrm{Cylinder}-100 \%{ }^{239} \mathrm{Pu}-0$ " Water Reflection

\begin{tabular}{|c|c|c|c|c|c|}
\hline \multicolumn{6}{|c|}{$100 \% \mathrm{Pu} 239 \mathrm{Pu}(\mathrm{NO} 3) 46 \mathrm{M}$ Cylinder - 0 Inches of Reflection } \\
\hline Concentration $(\mathrm{g} / \mathrm{cc})$ & $\begin{array}{l}\text { Density } \\
(\mathrm{g} / \mathrm{cc})\end{array}$ & $\begin{array}{l}\text { First Diameter } \\
\text { (Inches) }\end{array}$ & $\begin{array}{l}\text { Last Diameter } \\
\text { (Inches) }\end{array}$ & $\begin{array}{l}\text { Delta } \\
\text { (Inches) }\end{array}$ & $\begin{array}{l}\text { Critical Diameter } \\
\text { (Inches) }\end{array}$ \\
\hline 0.012 & 1.207 & 19.4 & 25.8 & 0.3 & 24.4075 \\
\hline 0.015 & 1.212 & 15.9 & 19.5 & 0.1 & 18.8338 \\
\hline 0.02 & 1.22 & 13.4 & 15.8 & 0.1 & 15.2923 \\
\hline 0.025 & 1.229 & 12.1 & 14.1 & 0.1 & 13.6885 \\
\hline 0.03 & 1.237 & 11.4 & 13.1 & 0.05 & 12.7775 \\
\hline 0.032 & 1.24 & 11.2 & 12.9 & 0.05 & 12.5027 \\
\hline 0.035 & 1.245 & 10.9 & 12.5 & 0.05 & 12.2089 \\
\hline 0.04 & 1.253 & 10.6 & 12.1 & 0.05 & 11.8001 \\
\hline 0.05 & 1.27 & 10.2 & 11.6 & 0.05 & 11.2439 \\
\hline 0.06 & 1.286 & 9.9 & 11.2 & 0.05 & 10.9336 \\
\hline 0.07 & 1.301 & 9.8 & 11 & 0.05 & 10.7413 \\
\hline 0.08 & 1.317 & 9.6 & 10.9 & 0.05 & 10.6166 \\
\hline 0.09 & 1.333 & 9.5 & 10.8 & 0.05 & 10.515 \\
\hline 0.1 & 1.349 & 9.5 & 10.8 & 0.05 & 10.4594 \\
\hline 0.12 & 1.379 & 9.4 & 10.7 & 0.05 & 10.3749 \\
\hline 0.13 & 1.395 & 9.4 & 10.7 & 0.05 & 10.3375 \\
\hline 0.15 & 1.426 & 9.4 & 10.6 & 0.05 & 10.3303 \\
\hline 0.2 & 1.503 & 9.4 & 10.7 & 0.05 & 10.3564 \\
\hline 0.239 & 1.563 & 9.5 & 10.7 & 0.05 & 10.3958 \\
\hline 0.25 & 1.58 & 9.5 & 10.7 & 0.05 & 10.4222 \\
\hline 0.359 & 1.748 & 9.6 & 10.9 & 0.05 & 10.6134 \\
\hline 0.478 & 1.935 & 9.8 & 11.1 & 0.05 & 10.7839 \\
\hline 0.5 & 1.969 & 9.9 & 11.1 & 0.05 & 10.835 \\
\hline 0.6 & 2.122 & 10.1 & 11.4 & 0.05 & 11.0809 \\
\hline 0.65 & 2.197 & 10.2 & 11.5 & 0.05 & 11.2237 \\
\hline 0.7 & 2.268 & 10.4 & 11.7 & 0.05 & 11.4224 \\
\hline 0.75 & 2.335 & 10.7 & 12 & 0.05 & 11.6914 \\
\hline 0.76 & 2.348 & 10.7 & 12.1 & 0.05 & 11.76 \\
\hline 0.765 & 2.354 & 10.8 & 12.1 & 0.05 & 11.8168 \\
\hline 0.77 & 2.36 & 10.8 & 12.2 & 0.05 & 11.8463 \\
\hline 0.775 & 2.367 & 10.8 & 12.2 & 0.05 & 11.8789 \\
\hline
\end{tabular}




\section{B1.1.2.1.3.2 1"Water Reflection}

Case ID: PuN46-icyl-1000-01

Material: $\mathrm{Pu}\left(\mathrm{NO}_{3}\right)_{4} 6$ Molar Excess Acid

Geometry: Cylinder

Isotopics: $100 \%{ }^{239} \mathrm{Pu}$

Reflector: 1" water reflected

Code: MCNP5 v1.40

Analyst: Mark Murphy

Date: $8 / 2011$ 
Figure B1.1.2.1.3.2-1. $\mathrm{Pu}\left(\mathrm{NO}_{3}\right) 4$ 6M - Cylinder - $100 \%{ }^{239} \mathrm{Pu}-1$ " Water Reflection

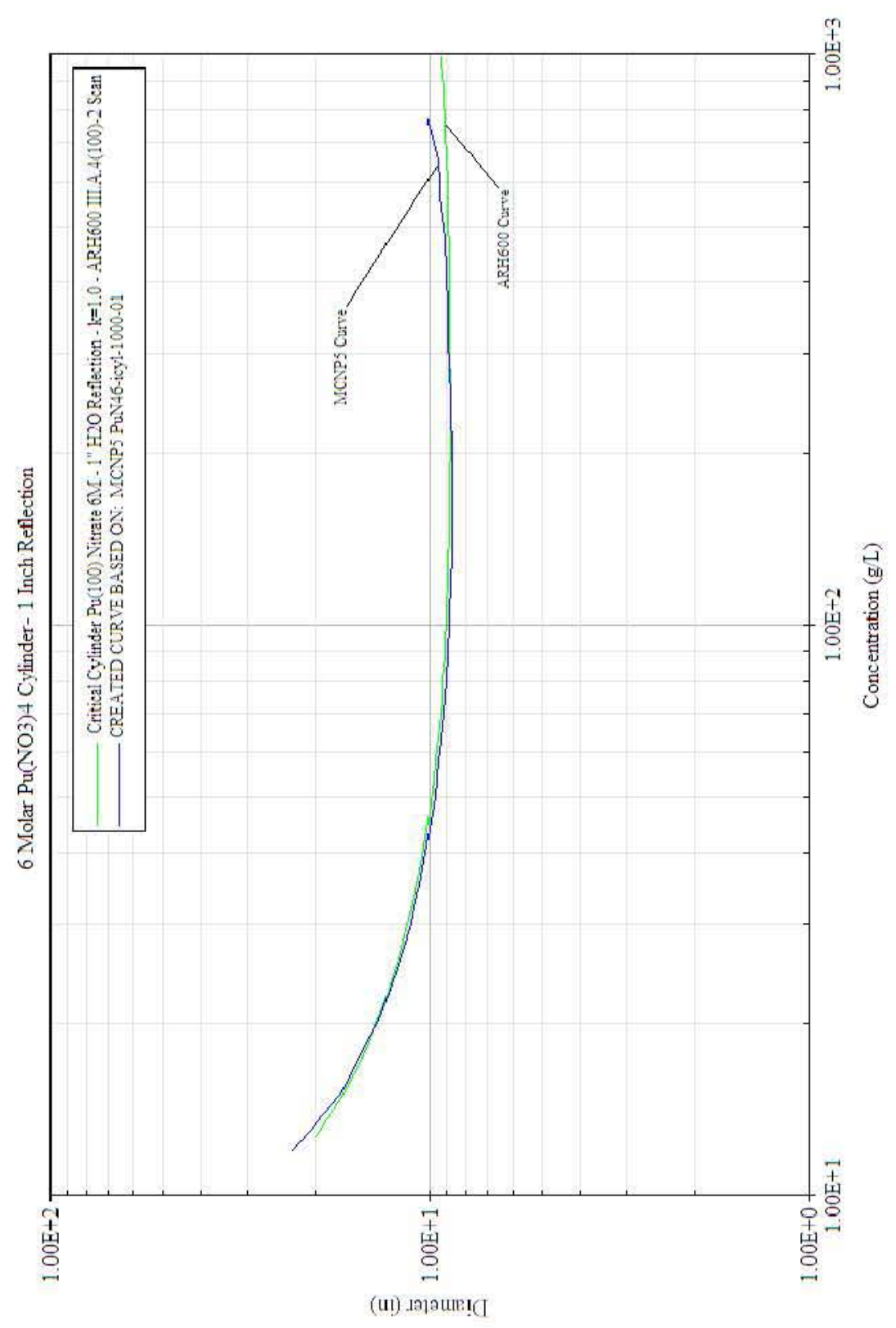


Table B1.1.2.1.3.2-1. Critical Dimensions for $\mathrm{Pu}\left(\mathrm{NO}_{3}\right) 46 \mathrm{M}-\mathrm{Cylinder}-100 \%{ }^{239} \mathrm{Pu}-1$ " Water Reflection

\begin{tabular}{|c|c|c|c|c|c|}
\hline \multicolumn{6}{|c|}{$100 \% \mathrm{Pu} 239 \mathrm{Pu}(\mathrm{NO} 3) 46 \mathrm{M}$ Cylinder - 1 Inch of Reflection } \\
\hline Concentration $(\mathrm{g} / \mathrm{cc})$ & $\begin{array}{l}\text { Density } \\
(\mathrm{g} / \mathrm{cc})\end{array}$ & $\begin{array}{l}\text { First Diameter } \\
\text { (Inches) }\end{array}$ & $\begin{array}{l}\text { Last Diameter } \\
\text { (Inches) }\end{array}$ & $\begin{array}{l}\text { Delta } \\
\text { (Inches) }\end{array}$ & $\begin{array}{l}\text { Critical Diameter } \\
\text { (Inches) }\end{array}$ \\
\hline 0.012 & 1.207 & 17.8 & 24.1 & 0.3 & 22.8535 \\
\hline 0.015 & 1.212 & 14.3 & 17.9 & 0.1 & 17.2257 \\
\hline 0.02 & 1.22 & 11.8 & 14.2 & 0.1 & 13.6965 \\
\hline 0.025 & 1.229 & 10.6 & 12.5 & 0.1 & 12.1036 \\
\hline 0.03 & 1.237 & 9.8 & 11.5 & 0.05 & 11.1767 \\
\hline 0.032 & 1.24 & 9.6 & 11.3 & 0.05 & 10.9263 \\
\hline 0.035 & 1.245 & 9.4 & 11 & 0.05 & 10.6091 \\
\hline 0.04 & 1.253 & 9 & 10.5 & 0.05 & 10.1846 \\
\hline 0.05 & 1.27 & 8.6 & 10 & 0.05 & 9.6547 \\
\hline 0.06 & 1.286 & 8.3 & 9.6 & 0.05 & 9.3416 \\
\hline 0.07 & 1.301 & 8.1 & 9.4 & 0.05 & 9.13 \\
\hline 0.08 & 1.317 & 8 & 9.3 & 0.05 & 9.0033 \\
\hline 0.09 & 1.333 & 7.9 & 9.2 & 0.05 & 8.8851 \\
\hline 0.1 & 1.349 & 7.9 & 9.1 & 0.05 & 8.8226 \\
\hline 0.12 & 1.379 & 7.8 & 9.1 & 0.05 & 8.7419 \\
\hline 0.13 & 1.395 & 7.8 & 9 & 0.05 & 8.7145 \\
\hline 0.15 & 1.426 & 7.7 & 9 & 0.05 & 8.6938 \\
\hline 0.2 & 1.503 & 7.7 & 9 & 0.05 & 8.6941 \\
\hline 0.239 & 1.563 & 7.8 & 9 & 0.05 & 8.7484 \\
\hline 0.25 & 1.58 & 7.8 & 9 & 0.05 & 8.7541 \\
\hline 0.359 & 1.748 & 8 & 9.2 & 0.1 & 8.9245 \\
\hline 0.478 & 1.935 & 8.1 & 9.4 & 0.05 & 9.0965 \\
\hline 0.5 & 1.969 & 8.2 & 9.4 & 0.05 & 9.1457 \\
\hline 0.6 & 2.122 & 8.4 & 9.7 & 0.05 & 9.349 \\
\hline 0.65 & 2.197 & 8.5 & 9.8 & 0.05 & 9.487 \\
\hline 0.7 & 2.268 & 8.7 & 10 & 0.05 & 9.6788 \\
\hline 0.75 & 2.335 & 8.9 & 10.2 & 0.05 & 9.922 \\
\hline 0.76 & 2.348 & 8.9 & 10.3 & 0.05 & 10.0088 \\
\hline 0.765 & 2.354 & 9 & 10.3 & 0.05 & 10.0283 \\
\hline 0.77 & 2.36 & 9 & 10.4 & 0.05 & 10.0809 \\
\hline 0.775 & 2.367 & 9 & 10.4 & 0.05 & 10.0745 \\
\hline
\end{tabular}




\section{B1.1.2.1.3.3 10" Water Reflection}

Case ID: PuN46-icyl-1000-10

Material: $\mathrm{Pu}\left(\mathrm{NO}_{3}\right)_{4} 6$ Molar Excess Acid

Geometry: Cylinder

Isotopics: $100 \%{ }^{239} \mathrm{Pu}$

Reflector: 10 " water reflected

Code: MCNP5 v1.40

Analyst: Mark Murphy

Date: $8 / 2011$ 
Figure B1.1.2.1.3.3-1. $\mathrm{Pu}\left(\mathrm{NO}_{3}\right) 46 \mathrm{M}-\mathrm{Cylinder}-100 \%{ }^{239} \mathrm{Pu}-10$ " Water Reflection

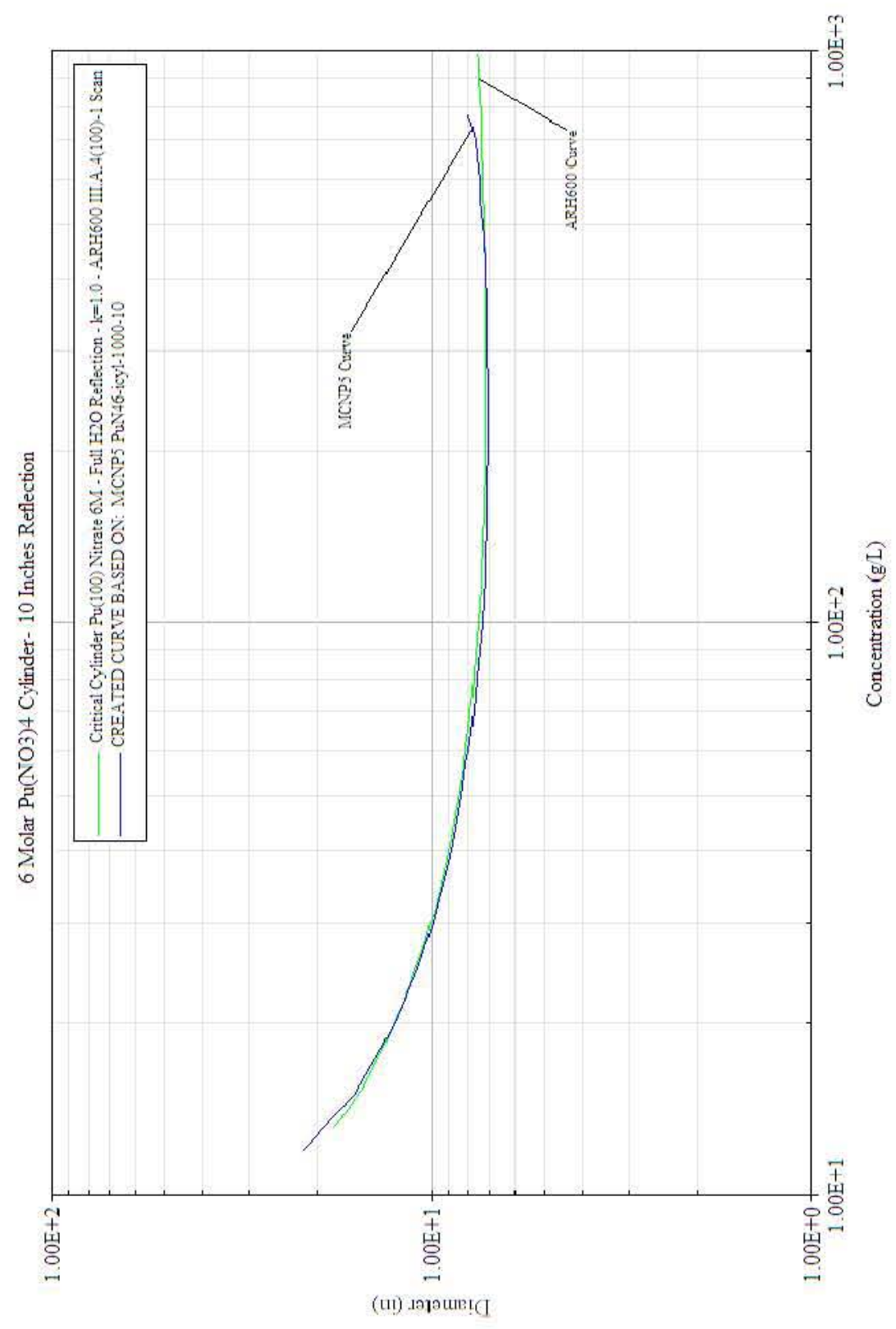


Table B1.1.2.1.3.3-1. Critical Dimensions for $\mathrm{Pu}\left(\mathrm{NO}_{3}\right) 46 \mathrm{M}-\mathrm{Cylinder}-100 \%{ }^{239} \mathrm{Pu}-10 "$ Water Reflection

\begin{tabular}{|c|c|c|c|c|c|}
\hline \multicolumn{6}{|c|}{$100 \% \mathrm{Pu} 239 \mathrm{Pu}(\mathrm{NO} 3) 46 \mathrm{M}$ Cylinder - 10 Inches of Reflection } \\
\hline Concentration $(\mathrm{g} / \mathrm{cc})$ & $\begin{array}{l}\text { Density } \\
(\mathrm{g} / \mathrm{cc})\end{array}$ & $\begin{array}{l}\text { First Diameter } \\
\text { (Inches) }\end{array}$ & $\begin{array}{l}\text { Last Diameter } \\
\text { (Inches) }\end{array}$ & $\begin{array}{l}\text { Delta } \\
\text { (Inches) }\end{array}$ & $\begin{array}{l}\text { Critical Diameter } \\
\text { (Inches) }\end{array}$ \\
\hline 0.012 & 1.207 & 16.7 & 22.9 & 0.3 & 21.6275 \\
\hline 0.015 & 1.212 & 13.1 & 16.7 & 0.1 & 15.989 \\
\hline 0.02 & 1.22 & 10.6 & 12.9 & 0.1 & 12.4653 \\
\hline 0.025 & 1.229 & 9.3 & 11 & 0.1 & 10.8174 \\
\hline 0.03 & 1.237 & 8.6 & 10.3 & 0.05 & 9.8863 \\
\hline 0.032 & 1.24 & 8.4 & 10 & 0.05 & 9.6273 \\
\hline 0.035 & 1.245 & 8.15 & 9.6 & 0.05 & 9.2902 \\
\hline 0.04 & 1.253 & 7.7 & 9.2 & 0.05 & 8.8573 \\
\hline 0.05 & 1.27 & 7.3 & 8.6 & 0.05 & 8.2984 \\
\hline 0.06 & 1.286 & 7 & 8.2 & 0.05 & 7.9429 \\
\hline 0.07 & 1.301 & 6.8 & 8 & 0.05 & 7.7076 \\
\hline 0.08 & 1.317 & 6.6 & 7.8 & 0.05 & 7.5515 \\
\hline 0.09 & 1.333 & 6.5 & 7.7 & 0.05 & 7.4187 \\
\hline 0.1 & 1.349 & 6.4 & 7.6 & 0.05 & 7.3434 \\
\hline 0.12 & 1.379 & 6.3 & 7.5 & 0.05 & 7.2082 \\
\hline 0.13 & 1.395 & 6.3 & 7.4 & 0.05 & 7.178 \\
\hline 0.15 & 1.426 & 6.2 & 7.4 & 0.05 & 7.1303 \\
\hline 0.2 & 1.503 & 6.3 & 7.3 & 0.05 & 7.0706 \\
\hline 0.239 & 1.563 & 6.2 & 7.3 & 0.05 & 7.0639 \\
\hline 0.25 & 1.58 & 6.2 & 7.3 & 0.05 & 7.076 \\
\hline 0.359 & 1.748 & 6.2 & 7.4 & 0.05 & 7.1505 \\
\hline 0.478 & 1.935 & 6.3 & 7.5 & 0.05 & 7.2583 \\
\hline 0.5 & 1.969 & 6.3 & 7.6 & 0.05 & 7.2854 \\
\hline 0.6 & 2.122 & 6.5 & 7.7 & 0.05 & 7.4409 \\
\hline 0.65 & 2.197 & 6.6 & 7.8 & 0.05 & 7.5481 \\
\hline 0.7 & 2.268 & 6.7 & 8 & 0.05 & 7.6681 \\
\hline 0.75 & 2.335 & 6.8 & 8.1 & 0.05 & 7.8659 \\
\hline 0.76 & 2.348 & 6.9 & 8.2 & 0.05 & 7.8994 \\
\hline 0.765 & 2.354 & 6.9 & 8.2 & 0.05 & 7.9464 \\
\hline 0.77 & 2.36 & 6.9 & 8.3 & 0.05 & 7.9485 \\
\hline 0.775 & 2.367 & 6.9 & 8.3 & 0.05 & 7.9778 \\
\hline
\end{tabular}


CHPRC-01552, Revision 0 
B1.1.2.2 $\quad 97 \%{ }^{239} \mathrm{Pu}$

B1.1.2.2.1 0 Molar Excess Acid

B1.1.2.2.1.1 0"Water Reflection

Case ID: PuN40-icyl-0970-00

Material: $\mathrm{Pu}\left(\mathrm{NO}_{3}\right)_{4} 0$ Molar Excess Acid

Geometry: Cylinder

Isotopics: $97 \%{ }^{239} \mathrm{Pu}$

Reflector: 0" water reflected

Code: MCNP5 v1.40

Analyst: Mark Murphy

Date: $8 / 2011$ 
Figure B1.1.2.2.1.1-1. $\mathrm{Pu}\left(\mathrm{NO}_{3}\right) 4 \mathrm{OM}$ - Cylinder - 97\% ${ }^{239} \mathrm{Pu}-0$ " Water Reflection

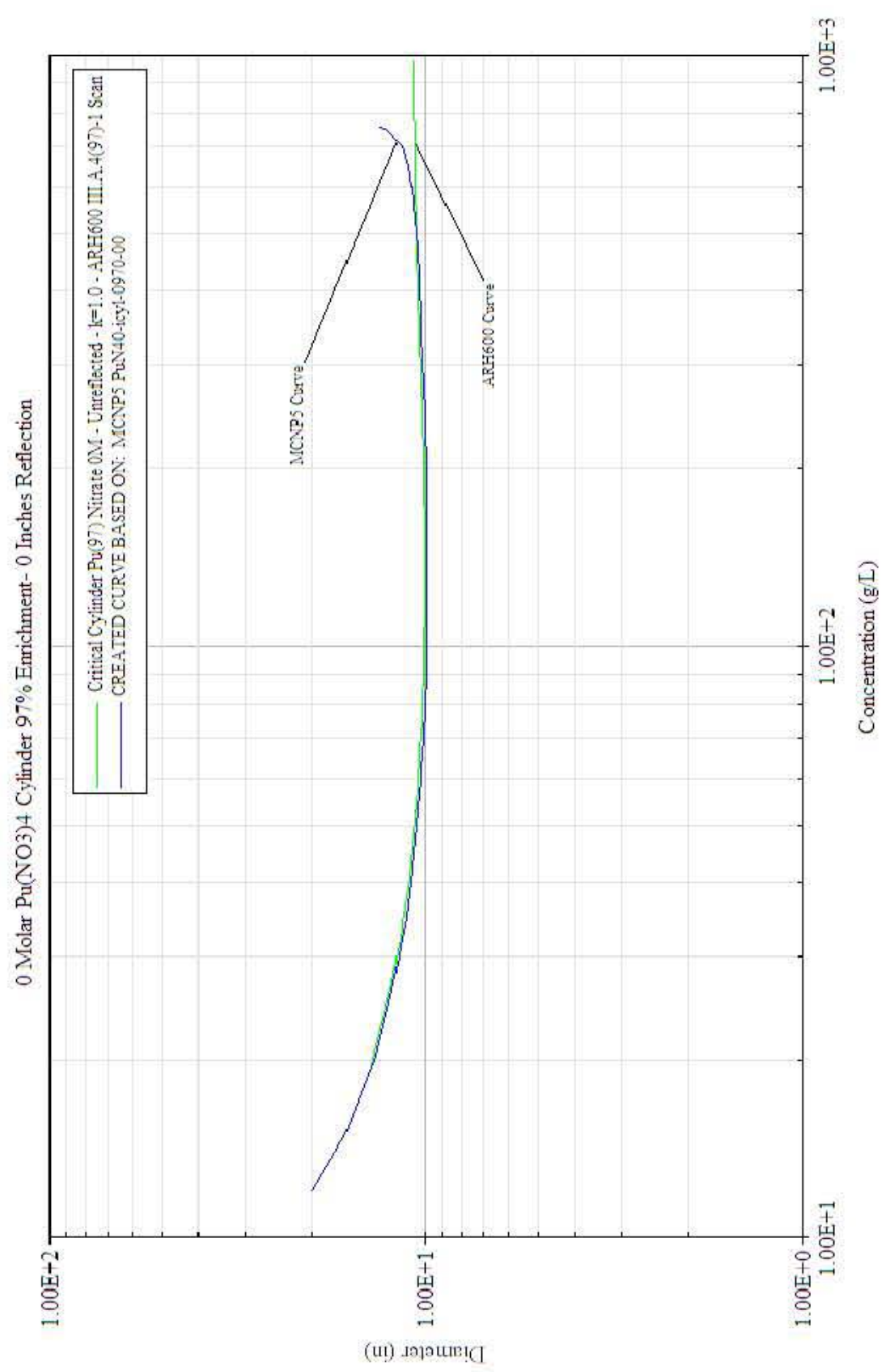


Table B1.1.2.2.1.1-1. Critical Dimensions for $\mathrm{Pu}\left(\mathrm{NO}_{3}\right) 4 \mathrm{OM}-\mathrm{Cylinder}-97 \%{ }^{239} \mathrm{Pu}-0$ " Water Reflection

\begin{tabular}{|c|c|c|c|c|c|}
\hline \multicolumn{6}{|c|}{$97 \% \mathrm{Pu} 239 \mathrm{Pu}(\mathrm{NO} 3) 40 \mathrm{M}$ Cylinder- 0 Inches of Reflection } \\
\hline Concentration $(\mathrm{g} / \mathrm{cc})$ & \begin{tabular}{|l|} 
Density \\
$(\mathrm{g} / \mathrm{cc})$ \\
\end{tabular} & $\begin{array}{l}\text { First Diameter } \\
\text { (Inches) }\end{array}$ & $\begin{array}{l}\text { Last Diameter } \\
\text { (Inches) }\end{array}$ & $\begin{array}{l}\text { Delta } \\
\text { (Inches) }\end{array}$ & $\begin{array}{l}\text { Critical Diameter } \\
\text { (Inches) }\end{array}$ \\
\hline 0.012 & 1.016 & 16.4 & 20.8 & 0.2 & 20.0419 \\
\hline 0.015 & 1.021 & 13.9 & 16.9 & 0.1 & 16.2832 \\
\hline 0.02 & 1.029 & 12 & 14.1 & 0.1 & 13.6883 \\
\hline 0.025 & 1.037 & 11.1 & 12.8 & 0.05 & 12.4409 \\
\hline 0.03 & 1.046 & 10.5 & 12.1 & 0.05 & 11.7233 \\
\hline 0.032 & 1.049 & 10.3 & 11.8 & 0.05 & 11.5081 \\
\hline 0.035 & 1.054 & 10.1 & 11.6 & 0.05 & 11.2518 \\
\hline 0.04 & 1.063 & 9.8 & 11.2 & 0.05 & 10.9168 \\
\hline 0.05 & 1.08 & 9.5 & 10.8 & 0.05 & 10.5076 \\
\hline 0.06 & 1.097 & 9.3 & 10.6 & 0.05 & 10.2621 \\
\hline 0.07 & 1.113 & 9.1 & 10.4 & 0.05 & 10.1253 \\
\hline 0.08 & 1.13 & 9 & 10.3 & 0.05 & 10.0185 \\
\hline 0.09 & 1.147 & 9 & 10.3 & 0.05 & 9.9538 \\
\hline 0.1 & 1.164 & 9 & 10.2 & 0.05 & 9.9223 \\
\hline 0.12 & 1.197 & 9 & 10.2 & 0.05 & 9.8906 \\
\hline 0.13 & 1.215 & 8.9 & 10.1 & 0.05 & 9.8694 \\
\hline 0.15 & 1.248 & 8.9 & 10.2 & 0.05 & 9.8877 \\
\hline 0.2 & \begin{tabular}{|l|}
1.332 \\
\end{tabular} & 9 & 10.2 & 0.05 & 9.9462 \\
\hline 0.239 & 1.397 & 9 & 10.3 & 0.05 & 10.0085 \\
\hline 0.25 & 1.415 & 9 & 10.4 & 0.05 & 10.0319 \\
\hline 0.359 & 1.594 & 9.2 & 10.5 & 0.05 & 10.2398 \\
\hline 0.478 & 1.784 & 9.4 & 10.8 & 0.05 & 10.4771 \\
\hline 0.5 & 1.818 & 9.5 & 10.9 & 0.05 & 10.5291 \\
\hline 0.6 & 1.962 & 9.8 & 11.2 & 0.05 & 10.8705 \\
\hline 0.65 & 2.026 & 10 & 11.5 & 0.1 & 11.1356 \\
\hline 0.7 & 2.076 & 10.4 & 11.9 & 0.05 & 11.5825 \\
\hline 0.75 & 2.08 & 11.4 & 13.1 & 0.05 & 12.7566 \\
\hline 0.752 & 2.074 & 11.6 & 13.3 & 0.05 & 12.9027 \\
\hline 0.754 & 2.064 & 11.8 & 13.5 & 0.05 & 13.1201 \\
\hline 0.755 & 2.054 & 12 & 13.7 & 0.05 & 13.3177 \\
\hline
\end{tabular}


B1.1.2.2.1.2 1"Water Reflection

Case ID: PuN40-icyl-0970-01

Material: $\mathrm{Pu}\left(\mathrm{NO}_{3}\right)_{4} 0$ Molar Excess Acid

Geometry: Cylinder

Isotopics: $97 \%{ }^{239} \mathrm{Pu}$

Reflector: 1" water reflected

Code: MCNP5 v1.40

Analyst: Mark Murphy

Date: $8 / 2011$ 
Figure B1.1.2.2.1.2-1. $\mathrm{Pu}\left(\mathrm{NO}_{3}\right) 4 \mathrm{OM}$ - Cylinder - 97\% ${ }^{239} \mathrm{Pu}-1$ " Water Reflection

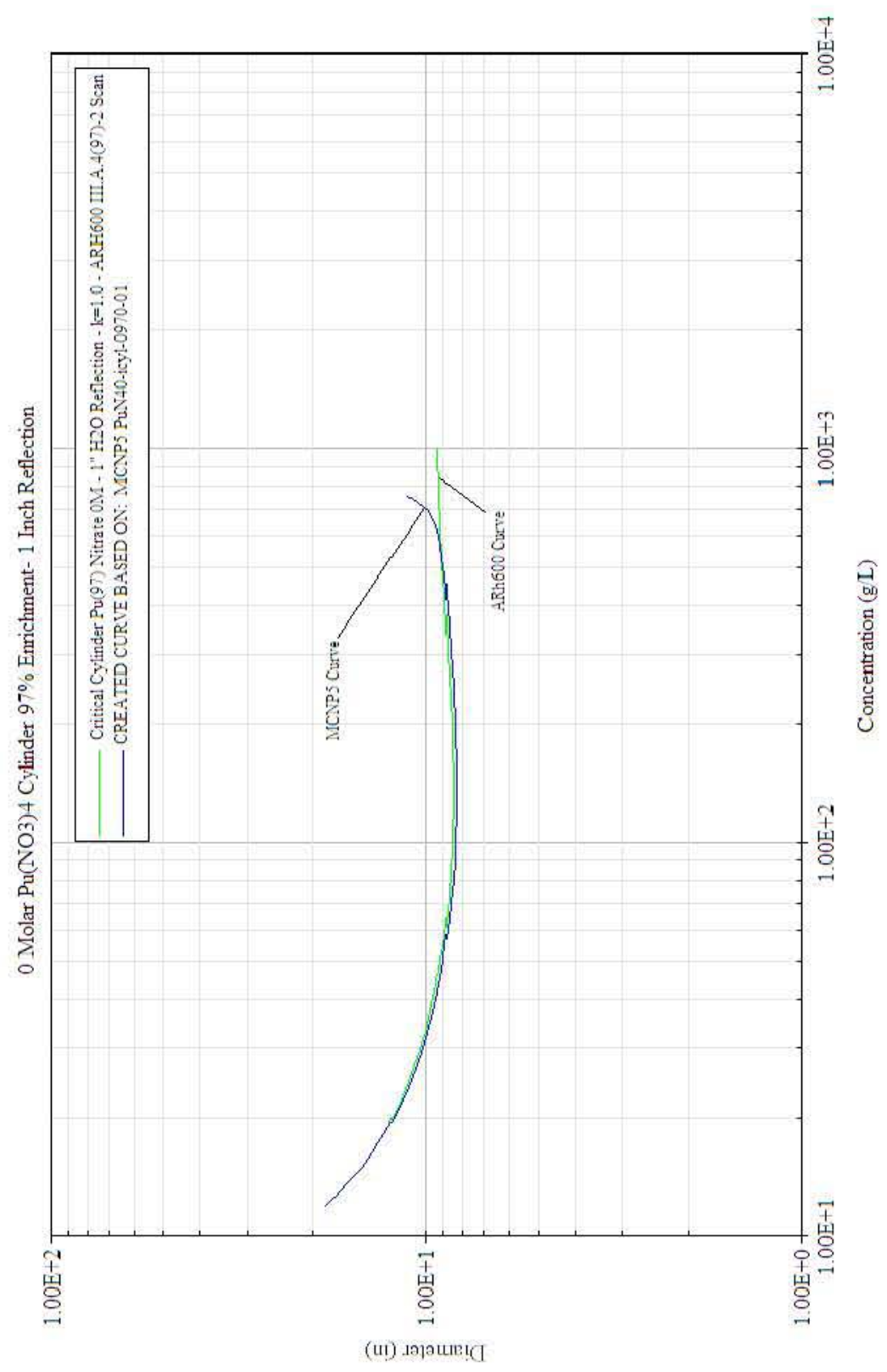


Table B1.1.2.2.1.2-1. Critical Dimensions for $\mathrm{Pu}\left(\mathrm{NO}_{3}\right) 40 \mathrm{M}-\mathrm{Cylinder}-97 \%{ }^{239} \mathrm{Pu}-1$ " Water Reflection

\begin{tabular}{|c|c|c|c|c|c|}
\hline \multicolumn{6}{|c|}{$97 \% \mathrm{Pu} 239 \mathrm{Pu}(\mathrm{NO} 3) 40 \mathrm{M}$ Cylinder- 1 Inch of Reflection } \\
\hline Concentration $(\mathrm{g} / \mathrm{cc})$ & $\begin{array}{l}\text { Density } \\
(\mathrm{g} / \mathrm{cc}) \\
\end{array}$ & $\begin{array}{l}\text { First Diameter } \\
\text { (Inches) }\end{array}$ & $\begin{array}{l}\text { Last Diameter } \\
\text { (Inches) }\end{array}$ & $\begin{array}{l}\text { Delta } \\
\text { (Inches) }\end{array}$ & $\begin{array}{l}\text { Critical Diameter } \\
\text { (Inches) }\end{array}$ \\
\hline 0.012 & 1.016 & 15 & 19.4 & 0.2 & 18.5111 \\
\hline 0.015 & 1.021 & 12.5 & 15.3 & 0.1 & 14.7652 \\
\hline 0.02 & 1.029 & 10.5 & 12.6 & 0.1 & 12.1667 \\
\hline 0.025 & 1.037 & 9.6 & 11.3 & 0.05 & 10.9244 \\
\hline 0.03 & 1.046 & 9 & 10.6 & 0.05 & 10.194 \\
\hline 0.032 & 1.049 & 8.8 & 10.3 & 0.05 & 9.9861 \\
\hline 0.035 & 1.054 & 8.6 & 10.1 & 0.05 & 9.7162 \\
\hline 0.04 & 1.063 & 8.3 & 9.7 & 0.05 & 9.3854 \\
\hline 0.05 & 1.08 & 7.9 & 9.3 & 0.05 & 8.9635 \\
\hline 0.06 & 1.097 & 7.7 & 9 & 0.05 & 8.7116 \\
\hline 0.07 & 1.113 & 7.6 & 8.8 & 0.05 & 8.5725 \\
\hline 0.08 & 1.13 & 7.5 & 8.8 & 0.05 & 8.4603 \\
\hline 0.09 & 1.147 & 7.5 & 8.7 & 0.05 & 8.3962 \\
\hline 0.1 & 1.164 & 7.4 & 8.6 & 0.05 & 8.3554 \\
\hline 0.12 & 1.197 & 7.4 & 8.6 & 0.05 & 8.3185 \\
\hline 0.13 & 1.215 & 7.4 & 8.6 & 0.05 & 8.3012 \\
\hline 0.15 & 1.248 & 7.4 & 8.6 & 0.05 & 8.3076 \\
\hline 0.2 & 1.332 & 7.4 & 8.55 & 0.05 & 8.3627 \\
\hline 0.239 & 1.397 & 7.5 & 8.7 & 0.05 & 8.4323 \\
\hline 0.25 & 1.415 & 7.5 & 8.7 & 0.05 & 8.4505 \\
\hline 0.359 & 1.594 & 7.6 & 8.9 & 0.05 & 8.6454 \\
\hline 0.478 & 1.784 & 7.8 & 9.1 & 0.05 & 8.8849 \\
\hline 0.5 & 1.818 & 7.9 & 9.3 & 0.05 & 8.9487 \\
\hline 0.6 & 1.962 & 8.2 & 9.6 & 0.05 & 9.2585 \\
\hline 0.65 & 2.026 & 8.4 & 9.8 & 0.05 & 9.4952 \\
\hline 0.7 & 2.076 & 8.7 & 10.2 & 0.05 & 9.8849 \\
\hline 0.75 & 2.08 & 9.6 & 11.3 & 0.05 & 10.884 \\
\hline 0.752 & 2.074 & 9.7 & 11.4 & 0.05 & 11.0095 \\
\hline 0.754 & 2.064 & 9.9 & 11.6 & 0.05 & 11.1936 \\
\hline 0.755 & 2.054 & 10 & 11.7 & 0.05 & 11.3479 \\
\hline
\end{tabular}




\section{B1.1.2.2.1.3 10" Water Reflection}

Case ID: PuN40-icyl-0970-10

Material: $\mathrm{Pu}\left(\mathrm{NO}_{3}\right)_{4} 0$ Molar Excess Acid

Geometry: Cylinder

Isotopics: $97 \%{ }^{239} \mathrm{Pu}$

Reflector: 10 " water reflected

Code: MCNP5 v1.40

Analyst: Mark Murphy

Date: $8 / 2011$ 
Figure B1.1.2.2.1.3-1. $\mathrm{Pu}\left(\mathrm{NO}_{3}\right) 4$ 0M - Cylinder - 97\% ${ }^{239} \mathrm{Pu}-10$ " Water Reflection

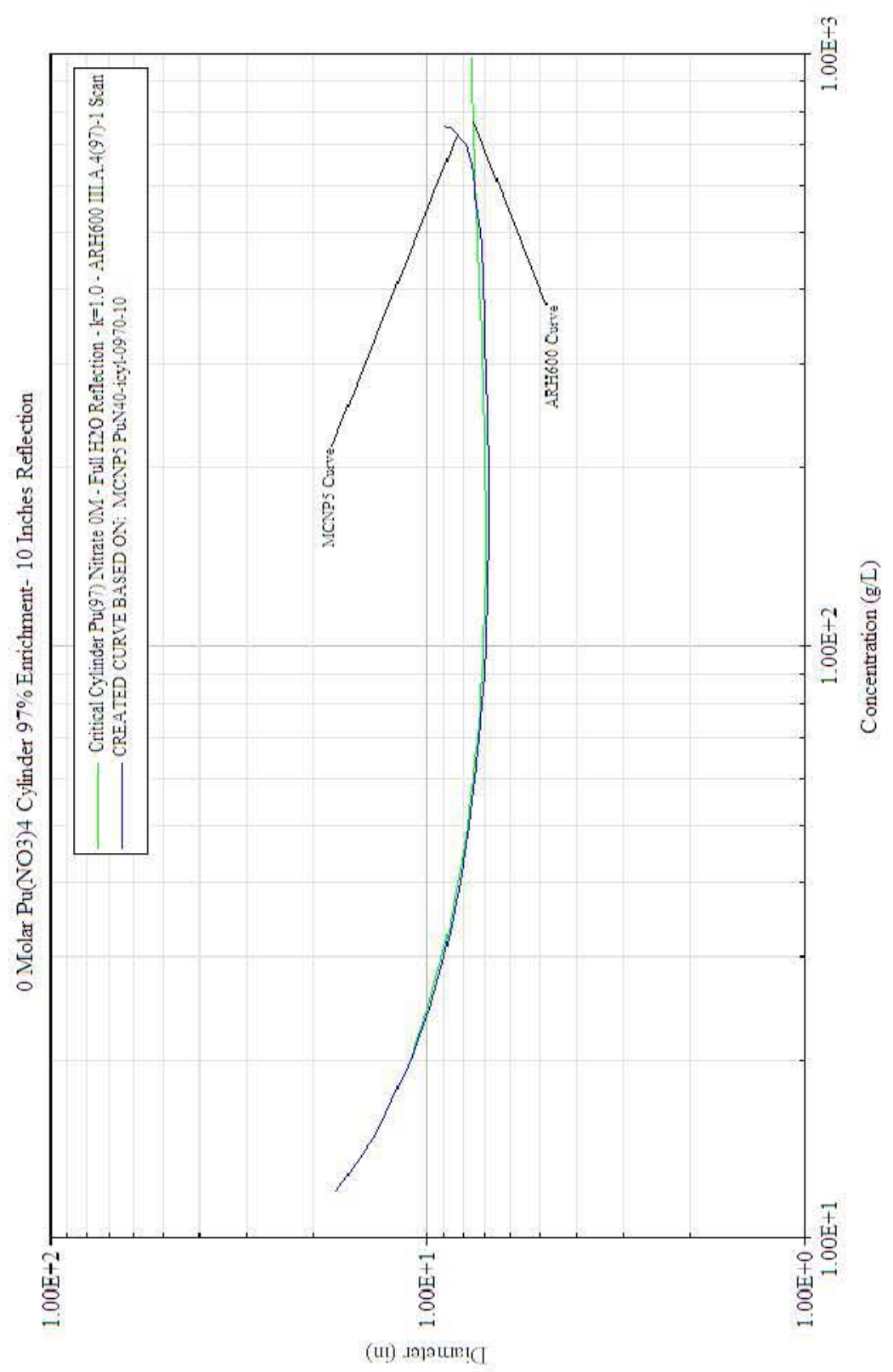


Table B1.1.2.2.1.3-1. Critical Dimensions for $\mathrm{Pu}\left(\mathrm{NO}_{3}\right) 40 \mathrm{M}-\mathrm{Cylinder}-97 \%{ }^{239} \mathrm{Pu}-10 "$ Water Reflection

\begin{tabular}{|c|c|c|c|c|c|}
\hline \multicolumn{6}{|c|}{$97 \% \mathrm{Pu} 239 \mathrm{Pu}(\mathrm{NO} 3) 40 \mathrm{M}$ Cylinder- 10 Inches of Reflection } \\
\hline Concentration $(\mathrm{g} / \mathrm{cc})$ & $\begin{array}{l}\text { Density } \\
(\mathrm{g} / \mathrm{cc}) \\
\end{array}$ & $\begin{array}{l}\text { First Diameter } \\
\text { (Inches) }\end{array}$ & $\begin{array}{l}\text { Last Diameter } \\
\text { (Inches) }\end{array}$ & $\begin{array}{l}\text { Delta } \\
\text { (Inches) }\end{array}$ & \begin{tabular}{|l}
$\begin{array}{l}\text { Critical Diameter } \\
\text { (Inches) }\end{array}$ \\
\end{tabular} \\
\hline 0.012 & 1.016 & 13.9 & 18.2 & 0.2 & 17.3852 \\
\hline 0.015 & 1.021 & 11.4 & 14.2 & 0.1 & 13.6303 \\
\hline 0.02 & 1.029 & 9.4 & 11.4 & 0.1 & 11.0214 \\
\hline 0.025 & 1.037 & 8.4 & 10.1 & 0.05 & 9.7576 \\
\hline 0.03 & 1.046 & 7.8 & 9.3 & 0.05 & 8.9999 \\
\hline 0.032 & 1.049 & 7.6 & 9.1 & 0.05 & 8.7866 \\
\hline 0.035 & 1.054 & 7.4 & 8.8 & 0.05 & 8.5131 \\
\hline 0.04 & 1.063 & 7.1 & 8.5 & 0.05 & 8.1566 \\
\hline 0.05 & 1.08 & 6.7 & 8 & 0.05 & 7.7002 \\
\hline 0.06 & 1.097 & 6.5 & 7.7 & 0.05 & 7.4258 \\
\hline 0.07 & 1.113 & 6.3 & 7.5 & 0.05 & 7.2527 \\
\hline 0.08 & 1.13 & 6.2 & 7.4 & 0.05 & 7.1158 \\
\hline 0.09 & 1.147 & 6.1 & 7.3 & 0.05 & 7.0336 \\
\hline 0.1 & 1.164 & 6.2 & 7.1 & 0.05 & 6.9558 \\
\hline 0.12 & 1.197 & 6 & 6.9 & 0.05 & 6.8877 \\
\hline 0.13 & 1.215 & 6.1 & 6.9 & 0.05 & 6.8571 \\
\hline 0.15 & 1.248 & 5.9 & 7 & 0.05 & 6.8338 \\
\hline 0.2 & 1.332 & 5.9 & 7.1 & 0.05 & 6.8254 \\
\hline 0.239 & 1.397 & 5.9 & 7.1 & 0.05 & 6.8599 \\
\hline 0.25 & 1.415 & 5.9 & 7.1 & 0.05 & 6.8678 \\
\hline 0.359 & 1.594 & 6 & 7.2 & 0.05 & 6.9825 \\
\hline 0.478 & 1.784 & 6.1 & 7.3 & 0.05 & 7.1528 \\
\hline 0.5 & 1.818 & 6.2 & 7.5 & 0.05 & 7.1909 \\
\hline 0.6 & 1.962 & 7.2 & 7.5 & 0.02 & 7.436 \\
\hline 0.65 & 2.026 & 6.5 & 7.7 & 0.05 & 7.6108 \\
\hline 0.7 & 2.076 & 6.8 & 8.2 & 0.05 & 7.8932 \\
\hline 0.75 & 2.08 & 7.4 & 9 & 0.05 & 8.6343 \\
\hline 0.752 & 2.074 & 7.5 & 9 & 0.05 & 8.7289 \\
\hline 0.754 & 2.064 & 7.6 & 9.2 & 0.05 & 8.874 \\
\hline 0.755 & 2.054 & 7.7 & 9.9 & 0.1 & 8.9866 \\
\hline
\end{tabular}


B1.1.2.2.2 3 Molar Excess Acid

B1.1.2.2.2.1 0"Water Reflection

Case ID: PuN43-icyl-0970-00

Material: $\mathrm{Pu}\left(\mathrm{NO}_{3}\right)_{4} 3$ Molar Excess Acid

Geometry: Cylinder

Isotopics: $97 \%{ }^{239} \mathrm{Pu}$

Reflector: 0 " water reflected

Code: MCNP5 v1.40

Analyst: Mark Murphy

Date: $8 / 2011$ 
Figure B1.1.2.2.2.1-1. $\mathrm{Pu}\left(\mathrm{NO}_{3}\right) 43 \mathrm{M}$ - Cylinder - 97\% ${ }^{239} \mathrm{Pu}-0$ " Water Reflection

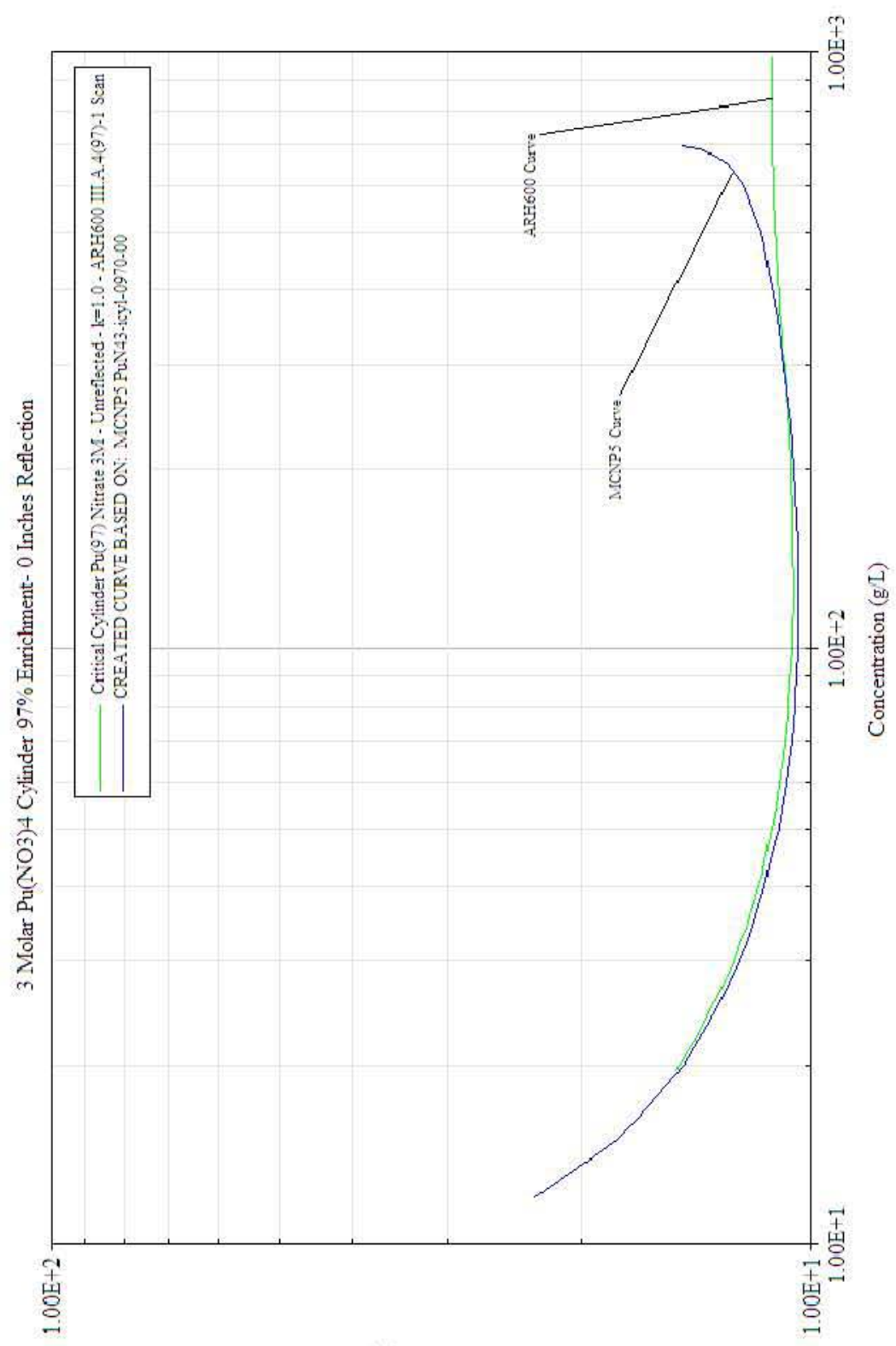

(ui) мәраще! Ф 
Table B1.1.2.2.2.1-1. Critical Dimensions for $\mathrm{Pu}\left(\mathrm{NO}_{3}\right) 43 \mathrm{M}-\mathrm{Cylinder}-97 \%{ }^{239} \mathrm{Pu}-0$ " Water Reflection

\begin{tabular}{|c|c|c|c|c|c|}
\hline \multicolumn{6}{|c|}{$97 \% \mathrm{Pu} 239 \mathrm{Pu}(\mathrm{NO} 3) 43 \mathrm{M}$ Cylinder- 0 Inches of Reflection } \\
\hline Concentration $(\mathrm{g} / \mathrm{cc})$ & $\begin{array}{l}\text { Density } \\
(\mathrm{g} / \mathrm{cc})\end{array}$ & $\begin{array}{l}\text { First Diameter } \\
\text { (Inches) }\end{array}$ & $\begin{array}{l}\text { Last Diameter } \\
\text { (Inches) }\end{array}$ & $\begin{array}{l}\text { Delta } \\
\text { (Inches) }\end{array}$ & $\begin{array}{l}\text { Critical Diameter } \\
\text { (Inches) }\end{array}$ \\
\hline 0.012 & 1.118 & 18.4 & 24.1 & 0.2 & 23.0228 \\
\hline 0.015 & 1.123 & 15.2 & 18.6 & 0.1 & 17.946 \\
\hline 0.02 & 1.132 & 12.8 & 15.2 & 0.1 & 14.718 \\
\hline 0.025 & 1.14 & 11.7 & 13.7 & 0.1 & 13.2455 \\
\hline 0.03 & 1.149 & 11 & 12.8 & 0.1 & 12.401 \\
\hline 0.032 & 1.152 & 10.8 & 12.5 & 0.05 & 12.1607 \\
\hline 0.035 & 1.157 & 10.6 & 12.2 & 0.05 & 11.8652 \\
\hline 0.04 & 1.165 & 10.3 & 11.8 & 0.05 & 11.5023 \\
\hline 0.05 & 1.182 & 10 & 11.3 & 0.05 & 11.0282 \\
\hline 0.06 & 1.198 & 9.7 & 11.1 & 0.05 & 10.7451 \\
\hline 0.07 & 1.215 & 9.5 & 10.9 & 0.05 & 10.5938 \\
\hline 0.08 & 1.231 & 9.5 & 10.8 & 0.05 & 10.496 \\
\hline 0.09 & 1.247 & 9.4 & 10.7 & 0.05 & 10.4254 \\
\hline 0.1 & 1.263 & 9.4 & 10.7 & 0.05 & 10.3895 \\
\hline 0.12 & 1.294 & 9.4 & 10.7 & 0.05 & 10.3774 \\
\hline 0.13 & 1.311 & 9.4 & 10.7 & 0.05 & 10.3688 \\
\hline 0.15 & 1.342 & 9.45 & 10.7 & 0.05 & 10.3933 \\
\hline 0.2 & 1.421 & 9.5 & 10.75 & 0.05 & 10.5191 \\
\hline 0.239 & 1.481 & 9.6 & 11 & 0.05 & 10.641 \\
\hline 0.25 & 1.498 & 9.6 & 11 & 0.05 & 10.6802 \\
\hline 0.359 & 1.665 & 9.9 & 11.4 & 0.05 & 11.0399 \\
\hline 0.478 & 1.841 & 10.3 & 11.8 & 0.05 & 11.4874 \\
\hline 0.5 & 1.871 & 10.4 & 12 & 0.05 & 11.614 \\
\hline 0.6 & 1.998 & 11 & 12.6 & 0.05 & 12.2412 \\
\hline 0.65 & 2.043 & 11.65 & 13.2 & 0.05 & 12.87 \\
\hline 0.66 & 2.049 & 11.7 & 13.4 & 0.05 & 13.0496 \\
\hline 0.67 & 2.053 & 12 & 13.6 & 0.05 & 13.2741 \\
\hline 0.68 & 2.053 & 12.2 & 14 & 0.1 & 13.5914 \\
\hline 0.69 & 2.046 & 12.6 & 14.4 & 0.1 & 14.0211 \\
\hline 0.698 & 2.018 & 13.4 & 15.3 & 0.1 & 14.8525 \\
\hline
\end{tabular}




\section{B1.1.2.2.2.2 1"Water Reflection}

Case ID: PuN43-icyl-0970-01

Material: $\mathrm{Pu}\left(\mathrm{NO}_{3}\right)_{4} 3$ Molar Excess Acid

Geometry: Cylinder

Isotopics: $97 \%{ }^{239} \mathrm{Pu}$

Reflector: 1" water reflected

Code: MCNP5 v1.40

Analyst: Mark Murphy

Date: $8 / 2011$ 
Figure B1.1.2.2.2.2-1. $\mathrm{Pu}\left(\mathrm{NO}_{3}\right) 43 \mathrm{M}$ - Cylinder - 97\% ${ }^{239} \mathrm{Pu}-1$ " Water Reflection

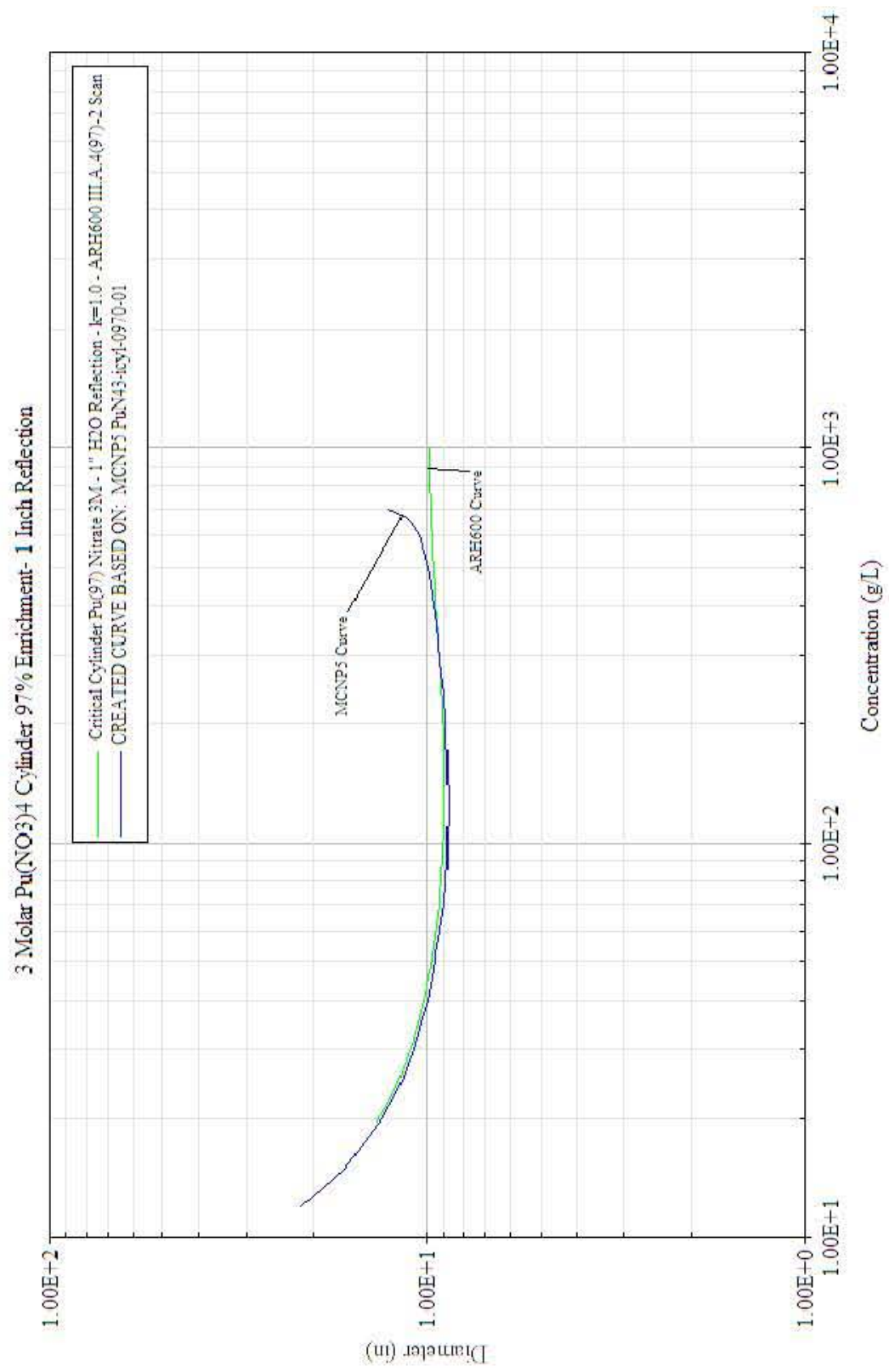


Table B1.1.2.2.2.2-1. Critical Dimensions for $\mathrm{Pu}\left(\mathrm{NO}_{3}\right) 43 \mathrm{M}-\mathrm{Cylinder}-97 \%{ }^{239} \mathrm{Pu}-1$ " Water Reflection

\begin{tabular}{|c|c|c|c|c|c|}
\hline \multicolumn{6}{|c|}{$97 \% \mathrm{Pu} 239 \mathrm{Pu}(\mathrm{NO} 3) 4$ 3M Cylinder- 1 Inch of Reflection } \\
\hline Concentration $(\mathrm{g} / \mathrm{cc})$ & $\begin{array}{l}\text { Density } \\
(\mathrm{g} / \mathrm{cc})\end{array}$ & $\begin{array}{l}\text { First Diameter } \\
\text { (Inches) }\end{array}$ & $\begin{array}{l}\text { Last Diameter } \\
\text { (Inches) }\end{array}$ & $\begin{array}{l}\text { Delta } \\
\text { (Inches) }\end{array}$ & $\begin{array}{l}\text { Critical Diameter } \\
\text { (Inches) }\end{array}$ \\
\hline 0.012 & 1.118 & 16.9 & 22.5 & 0.2 & 21.4679 \\
\hline 0.015 & 1.123 & 13.6 & 17.1 & 0.1 & 16.4142 \\
\hline 0.02 & 1.132 & 11.3 & 13.7 & 0.1 & 13.1751 \\
\hline 0.025 & 1.14 & 10.2 & 12.1 & 0.1 & 11.6868 \\
\hline 0.03 & 1.149 & 9.5 & 11.2 & 0.05 & 10.8662 \\
\hline 0.032 & 1.152 & 9.3 & 11 & 0.05 & 10.616 \\
\hline 0.035 & 1.157 & 9.1 & 10.6 & 0.05 & 10.3254 \\
\hline 0.04 & 1.165 & 8.8 & 10.3 & 0.05 & 9.9346 \\
\hline 0.05 & 1.182 & 8.4 & 9.8 & 0.05 & 9.4676 \\
\hline 0.06 & 1.198 & 8.1 & 9.5 & 0.05 & 9.1904 \\
\hline 0.07 & 1.215 & 8 & 9.3 & 0.05 & 9.0115 \\
\hline 0.08 & 1.231 & 7.9 & 9.2 & 0.05 & 8.9094 \\
\hline 0.09 & 1.247 & 7.8 & 9.1 & 0.05 & 8.8372 \\
\hline 0.1 & 1.263 & 7.8 & 9.1 & 0.05 & 8.7927 \\
\hline 0.12 & 1.294 & 7.8 & 9.1 & 0.05 & 8.7759 \\
\hline 0.13 & 1.311 & 7.8 & 8.95 & 0.05 & 8.76 \\
\hline 0.15 & 1.342 & 7.8 & 9.1 & 0.05 & 8.7907 \\
\hline 0.2 & 1.421 & 7.9 & 9.2 & 0.05 & 8.8963 \\
\hline 0.239 & 1.481 & 8 & 9.3 & 0.05 & 9.0078 \\
\hline 0.25 & 1.498 & 8 & 9.3 & 0.05 & 9.0445 \\
\hline 0.359 & 1.665 & 8.3 & 9.7 & 0.05 & 9.3835 \\
\hline 0.478 & 1.841 & 8.6 & 10.1 & 0.05 & 9.7935 \\
\hline 0.5 & 1.871 & 8.7 & 10.2 & 0.05 & 9.8915 \\
\hline 0.6 & 1.998 & 9.3 & 10.8 & 0.05 & 10.4822 \\
\hline 0.65 & 2.043 & 9.7 & 11.4 & 0.05 & 11.0333 \\
\hline 0.66 & 2.049 & 9.9 & 11.5 & 0.05 & 11.1822 \\
\hline 0.67 & 2.053 & 10 & 11.7 & 0.05 & 11.3837 \\
\hline 0.68 & 2.053 & 10.3 & 12 & 0.05 & 11.6378 \\
\hline 0.69 & 2.046 & 10.6 & 12.4 & 0.1 & 12.0144 \\
\hline 0.698 & 2.018 & 11.3 & 13.1 & 0.1 & 12.7574 \\
\hline
\end{tabular}




\section{B1.1.2.2.2.3 10" Water Reflection}

Case ID: PuN43-icyl-0970-10

Material: $\mathrm{Pu}\left(\mathrm{NO}_{3}\right)_{4} 3$ Molar Excess Acid

Geometry: Cylinder

Isotopics: $97 \%{ }^{239} \mathrm{Pu}$

Reflector: 10 " water reflected

Code: MCNP5 v1.40

Analyst: Mark Murphy

Date: $8 / 2011$ 
Figure B1.1.2.2.2.3-1. $\mathrm{Pu}\left(\mathrm{NO}_{3}\right) 43 \mathrm{M}-\mathrm{Cylinder}-97 \%{ }^{239} \mathrm{Pu}-10$ " Water Reflection

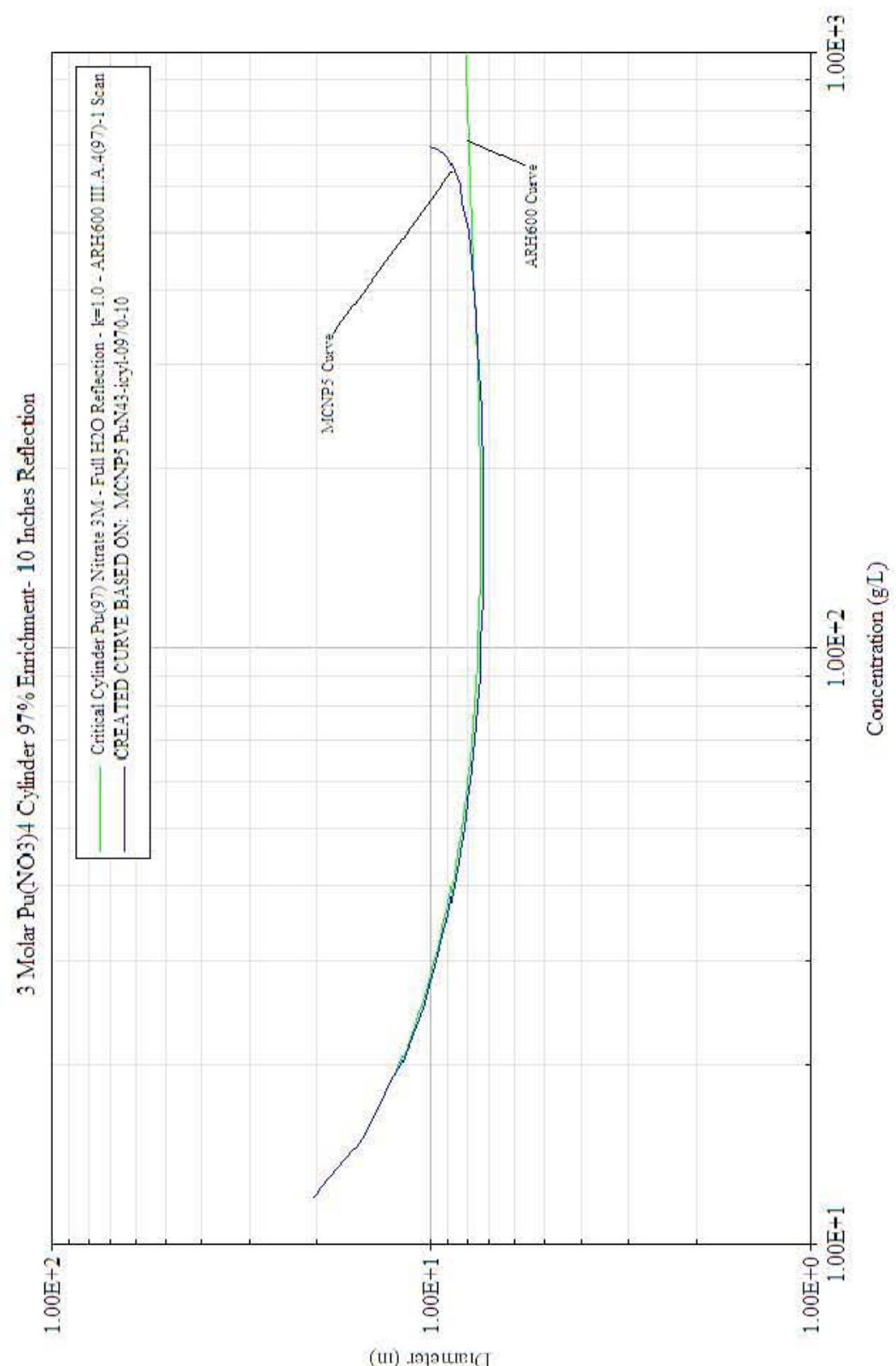

(ui) เэฺวนเ!! 
Table B1.1.2.2.2.3-1. Critical Dimensions for $\mathrm{Pu}\left(\mathrm{NO}_{3}\right) 43 \mathrm{M}-\mathrm{Cylinder}-97 \%{ }^{239} \mathrm{Pu}-10 "$ Water Reflection

\begin{tabular}{|c|c|c|c|c|c|}
\hline \multicolumn{6}{|c|}{$97 \% \mathrm{Pu} 239 \mathrm{Pu}(\mathrm{NO} 3) 43 \mathrm{M}$ Cylinder- 10 Inches of Reflection } \\
\hline Concentration $(\mathrm{g} / \mathrm{cc})$ & $\begin{array}{l}\text { Density } \\
(\mathrm{g} / \mathrm{cc})\end{array}$ & $\begin{array}{l}\text { First Diameter } \\
\text { (Inches) }\end{array}$ & $\begin{array}{l}\text { Last Diameter } \\
\text { (Inches) }\end{array}$ & $\begin{array}{l}\text { Delta } \\
\text { (Inches) }\end{array}$ & $\begin{array}{l}\text { Critical Diameter } \\
\text { (Inches) }\end{array}$ \\
\hline 0.012 & 1.118 & 15.7 & 21.4 & 0.2 & 20.3307 \\
\hline 0.015 & 1.123 & 12.6 & 15.9 & 0.1 & 15.2232 \\
\hline 0.02 & 1.132 & 10.1 & 12.5 & 0.1 & 11.9896 \\
\hline 0.025 & 1.14 & 9 & 10.9 & 0.1 & 10.495 \\
\hline 0.03 & 1.149 & 8.3 & 10 & 0.05 & 9.6291 \\
\hline 0.032 & 1.152 & 8.1 & 9.7 & 0.05 & 9.3777 \\
\hline 0.035 & 1.157 & 7.9 & 9.4 & 0.05 & 9.0594 \\
\hline 0.04 & 1.165 & 7.5 & 9 & 0.05 & 8.6634 \\
\hline 0.05 & 1.182 & 7.1 & 8.5 & 0.05 & 8.1548 \\
\hline 0.06 & 1.198 & 6.8 & 8.1 & 0.05 & 7.8423 \\
\hline 0.07 & 1.215 & 6.7 & 7.9 & 0.05 & 7.6431 \\
\hline 0.08 & 1.231 & 6.5 & 7.8 & 0.05 & 7.5 \\
\hline 0.09 & 1.247 & 6.4 & 7.7 & 0.05 & 7.4144 \\
\hline 0.1 & 1.263 & 6.4 & 7.6 & 0.05 & 7.3543 \\
\hline 0.12 & 1.294 & 6.3 & 7.5 & 0.05 & 7.2738 \\
\hline 0.13 & 1.311 & 6.3 & 7.45 & 0.05 & 7.2543 \\
\hline 0.15 & 1.342 & 6.3 & 7.5 & 0.05 & 7.2403 \\
\hline 0.2 & 1.421 & 6.3 & 7.5 & 0.05 & 7.2542 \\
\hline 0.239 & 1.481 & 6.35 & 7.6 & 0.05 & 7.3323 \\
\hline 0.25 & 1.498 & 6.4 & 7.6 & 0.05 & 7.3399 \\
\hline 0.359 & 1.665 & 6.5 & 7.9 & 0.05 & 7.5781 \\
\hline 0.478 & 1.841 & 6.8 & 8.2 & 0.05 & 7.8817 \\
\hline 0.5 & 1.871 & 6.8 & 8.3 & 0.05 & 7.9559 \\
\hline 0.6 & 1.998 & 7.2 & 8.7 & 0.05 & 8.3905 \\
\hline 0.65 & 2.043 & 7.5 & 9.2 & 0.05 & 8.8014 \\
\hline 0.66 & 2.049 & 7.65 & 9.3 & 0.05 & 8.9137 \\
\hline 0.67 & 2.053 & 7.8 & 9.4 & 0.05 & 9.047 \\
\hline 0.68 & 2.053 & 7.9 & 9.6 & 0.25 & 9.2442 \\
\hline 0.69 & 2.046 & 8.2 & 9.9 & 0.05 & 9.5317 \\
\hline 0.698 & 2.018 & 8.6 & 10.4 & 0.1 & 10.0699 \\
\hline
\end{tabular}


B1.1.2.2.3 6 Molar Excess Acid

B1.1.2.2.3.1 0"Water Reflection

Case ID: PuN46-icyl-0970-00

Material: $\mathrm{Pu}\left(\mathrm{NO}_{3}\right)_{4} 6$ Molar Excess Acid

Geometry: Cylinder

Isotopics: $97 \%{ }^{239} \mathrm{Pu}$

Reflector: 0 " water reflected

Code: MCNP5 v1.40

Analyst: Mark Murphy

Date: $8 / 2011$ 
Figure B1.1.2.2.3.1-1. $\mathrm{Pu}\left(\mathrm{NO}_{3}\right) 46 \mathrm{M}-\mathrm{Cylinder}-97 \%{ }^{239} \mathrm{Pu}-0$ " Water Reflection

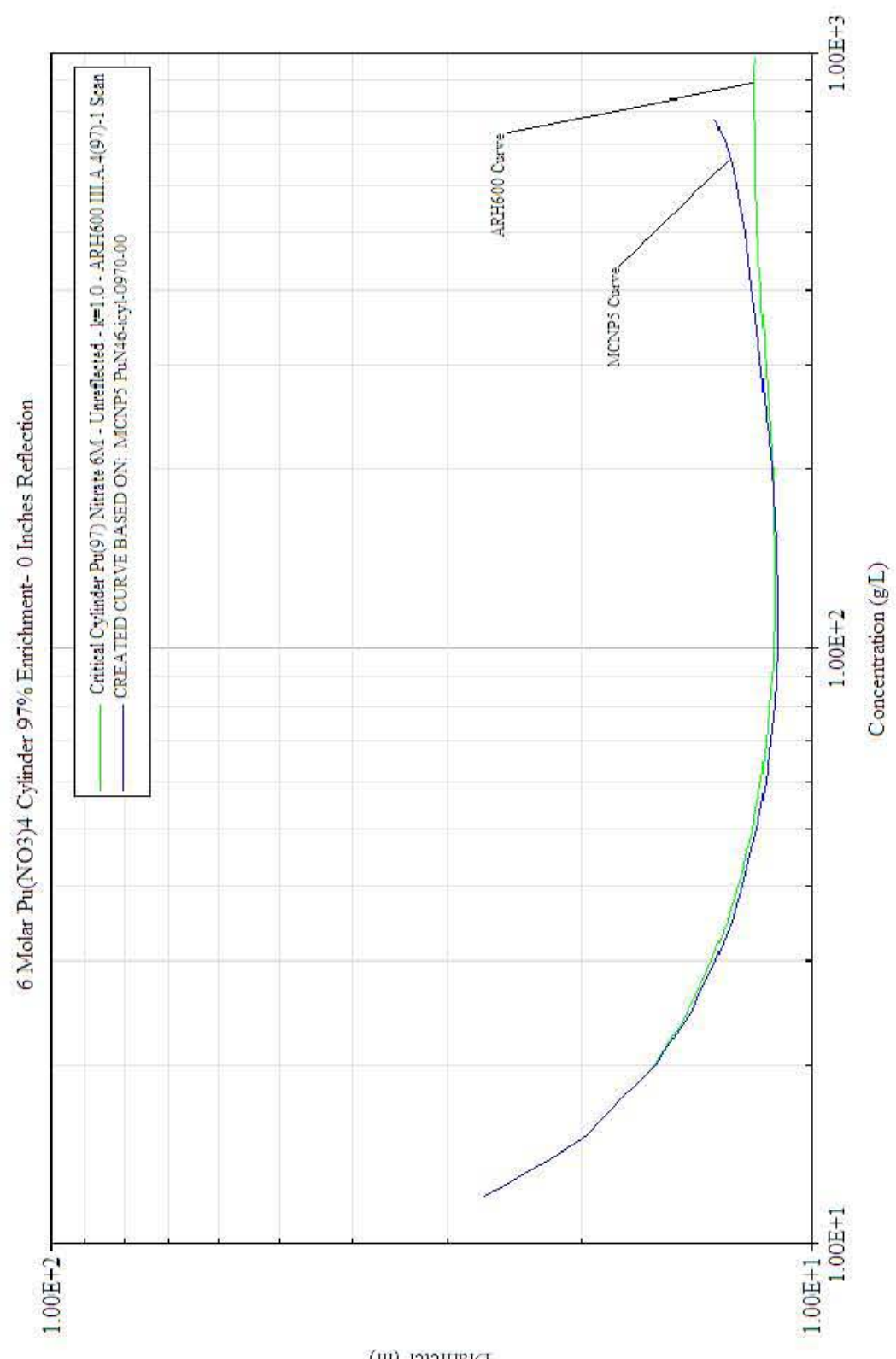

(ui) -әวаแе! 
Table B1.1.2.2.3.1-1. Critical Dimensions for $\mathrm{Pu}\left(\mathrm{NO}_{3}\right) 46 \mathrm{M}-\mathrm{Cylinder}-97 \%{ }^{239} \mathrm{Pu}-0$ " Water Reflection

\begin{tabular}{|c|c|c|c|c|c|}
\hline \multicolumn{6}{|c|}{$97 \% \mathrm{Pu} 239 \mathrm{Pu}(\mathrm{NO} 3) 46 \mathrm{M}$ Cylinder- 0 Inches of Reflection } \\
\hline Concentration $(\mathrm{g} / \mathrm{cc})$ & $\begin{array}{l}\text { Density } \\
(\mathrm{g} / \mathrm{cc})\end{array}$ & $\begin{array}{l}\text { First Diameter } \\
\text { (Inches) }\end{array}$ & $\begin{array}{l}\text { Last Diameter } \\
\text { (Inches) }\end{array}$ & $\begin{array}{l}\text { Delta } \\
\text { (Inches) }\end{array}$ & $\begin{array}{l}\text { Critical Diameter } \\
\text { (Inches) }\end{array}$ \\
\hline 0.012 & 1.207 & 20.7 & 28.4 & 0.3 & 26.8366 \\
\hline 0.015 & 1.212 & 16.6 & 20.8 & 0.2 & 19.9948 \\
\hline 0.02 & 1.22 & 13.9 & 16.6 & 0.1 & 16.0108 \\
\hline 0.025 & 1.229 & 12.6 & 14.7 & 0.1 & 14.2684 \\
\hline 0.03 & 1.237 & 11.8 & 13.7 & 0.1 & 13.3245 \\
\hline 0.032 & 1.24 & 11.6 & 13.4 & 0.1 & 13.0233 \\
\hline 0.035 & 1.245 & 11.4 & 13.1 & 0.05 & 12.7048 \\
\hline 0.04 & 1.253 & 11 & 12.7 & 0.05 & 12.2825 \\
\hline 0.05 & 1.27 & 10.6 & 12.1 & 0.05 & 11.7565 \\
\hline 0.06 & 1.286 & 10.3 & 11.8 & 0.05 & 11.4484 \\
\hline 0.07 & 1.301 & 10.2 & 11.6 & 0.05 & 11.2841 \\
\hline 0.08 & 1.317 & 10.1 & 11.5 & 0.05 & 11.1708 \\
\hline 0.09 & 1.333 & 10 & 11.4 & 0.05 & 11.1008 \\
\hline 0.1 & 1.349 & 9.9 & 11.4 & 0.05 & 11.0623 \\
\hline 0.12 & 1.379 & 10 & 11.4 & 0.05 & 11.0539 \\
\hline 0.13 & 1.395 & 10 & 11.4 & 0.05 & 11.0483 \\
\hline 0.15 & 1.426 & 10 & 11.4 & 0.05 & 11.0981 \\
\hline 0.2 & 1.503 & 10.1 & 11.6 & 0.05 & 11.2536 \\
\hline 0.239 & 1.563 & 10.3 & 11.7 & 0.05 & 11.4031 \\
\hline 0.25 & 1.58 & 10.3 & 11.8 & 0.05 & 11.4474 \\
\hline 0.359 & 1.748 & 10.6 & 12.2 & 0.05 & 11.8328 \\
\hline 0.478 & 1.935 & 10.9 & 12.5 & 0.05 & 12.1613 \\
\hline 0.5 & 1.969 & 11 & 12.6 & 0.05 & 12.2139 \\
\hline 0.6 & 2.122 & 11.2 & 12.9 & 0.05 & 12.5106 \\
\hline 0.65 & 2.197 & 11.4 & 13 & 0.05 & 12.6713 \\
\hline 0.7 & 2.268 & 11.6 & 13.3 & 0.05 & 12.8927 \\
\hline 0.75 & 2.335 & 11.8 & 13.5 & 0.05 & 13.1854 \\
\hline 0.76 & 2.348 & 11.9 & 13.6 & 0.05 & 13.2411 \\
\hline 0.765 & 2.354 & 11.9 & 13.7 & 0.1 & 13.2868 \\
\hline 0.77 & 2.36 & 12 & 13.7 & 0.05 & 13.3394 \\
\hline 0.775 & 2.367 & 12 & 13.7 & 0.05 & 13.3687 \\
\hline
\end{tabular}




\section{B1.1.2.2.3.2 1"Water Reflection}

Case ID: PuN46-icyl-0970-01

Material: $\mathrm{Pu}\left(\mathrm{NO}_{3}\right)_{4} 6$ Molar Excess Acid

Geometry: Cylinder

Isotopics: $97 \%{ }^{239} \mathrm{Pu}$

Reflector: 1" water reflected

Code: MCNP5 v1.40

Analyst: Mark Murphy

Date: $8 / 2011$ 
Figure B1.1.2.2.3.2-1. $\mathrm{Pu}\left(\mathrm{NO}_{3}\right) 46 \mathrm{M}$ - Cylinder - 97\% ${ }^{239} \mathrm{Pu}-1$ " Water Reflection

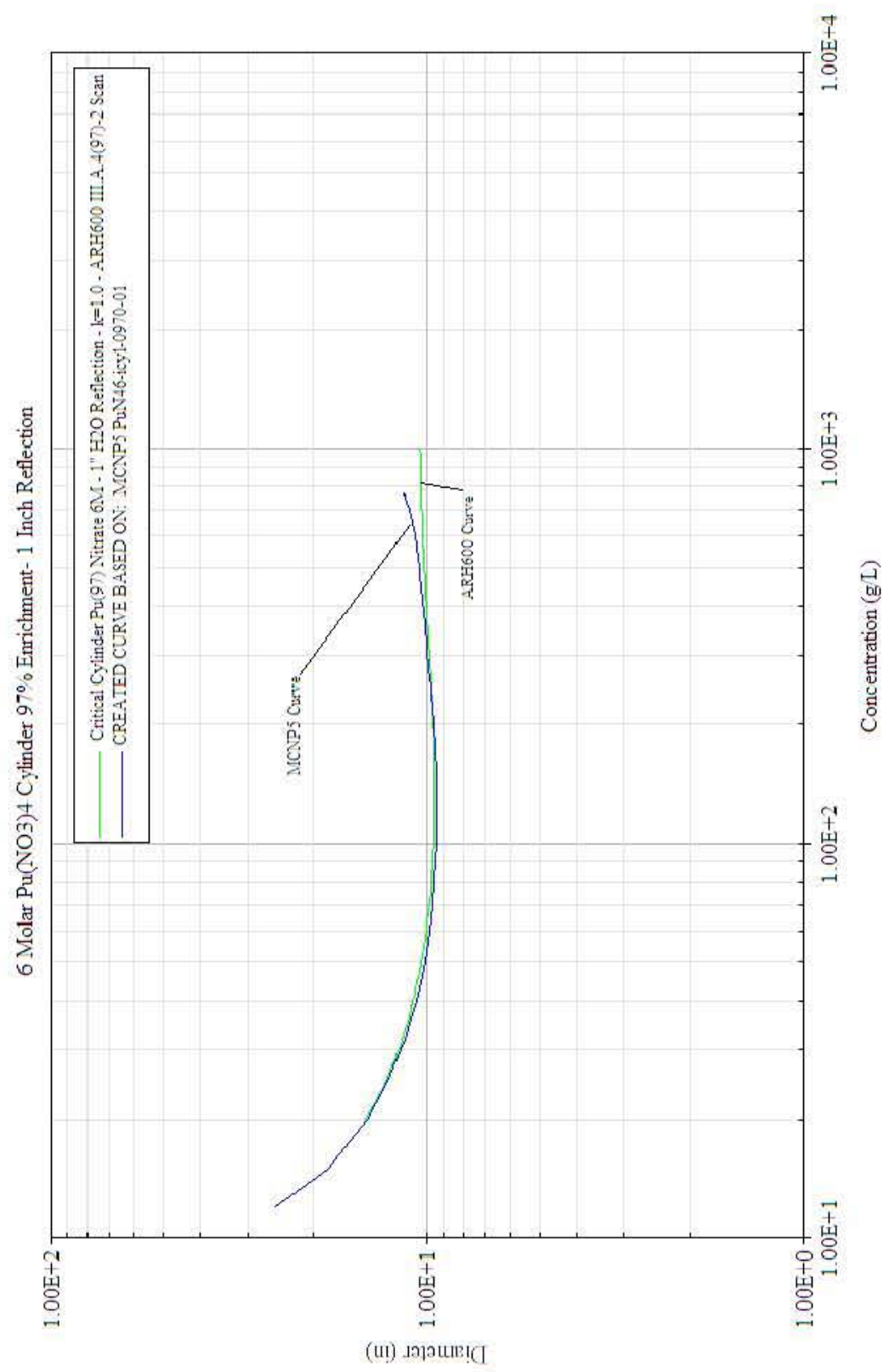


Table B1.1.2.2.3.2-1. Critical Dimensions for $\mathrm{Pu}\left(\mathrm{NO}_{3}\right) 46 \mathrm{M}-\mathrm{Cylinder}-97 \%{ }^{239} \mathrm{Pu}-1$ " Water Reflection

\begin{tabular}{|c|c|c|c|c|c|}
\hline \multicolumn{6}{|c|}{$97 \% \mathrm{Pu} 239 \mathrm{Pu}(\mathrm{NO} 3) 46 \mathrm{M}$ Cylinder- 1 Inch of Reflection } \\
\hline Concentration $(\mathrm{g} / \mathrm{cc})$ & $\begin{array}{l}\text { Density } \\
(\mathrm{g} / \mathrm{cc}) \\
\end{array}$ & $\begin{array}{l}\text { First Diameter } \\
\text { (Inches) }\end{array}$ & $\begin{array}{l}\text { Last Diameter } \\
\text { (Inches) }\end{array}$ & $\begin{array}{l}\text { Delta } \\
\text { (Inches) }\end{array}$ & $\begin{array}{l}\text { Critical Diameter } \\
\text { (Inches) }\end{array}$ \\
\hline 0.012 & 1.207 & 19.2 & 26.8 & 0.3 & 25.198 \\
\hline 0.015 & 1.212 & 15.1 & 19.2 & 0.2 & 18.3879 \\
\hline 0.02 & 1.22 & 12.3 & 14.9 & 0.1 & 14.4374 \\
\hline 0.025 & 1.229 & 11 & 13.1 & 0.1 & 12.6771 \\
\hline 0.03 & 1.237 & 10.3 & 12.1 & 0.1 & 11.7262 \\
\hline 0.032 & 1.24 & 10 & 11.8 & 0.1 & 11.4404 \\
\hline 0.035 & 1.245 & 9.8 & 11.5 & 0.05 & 11.0938 \\
\hline 0.04 & 1.253 & 9.4 & 11 & 0.05 & 10.6735 \\
\hline 0.05 & 1.27 & 9 & 10.5 & 0.05 & 10.126 \\
\hline 0.06 & 1.286 & 8.7 & 10.2 & 0.05 & 9.8299 \\
\hline 0.07 & 1.301 & 8.5 & 10 & 0.05 & 9.6635 \\
\hline 0.08 & 1.317 & 8.4 & 9.8 & 0.05 & 9.538 \\
\hline 0.09 & 1.333 & 8.4 & 9.8 & 0.05 & 9.4737 \\
\hline 0.1 & 1.349 & 8.4 & 9.7 & 0.05 & 9.4155 \\
\hline 0.12 & 1.379 & 8.3 & 9.7 & 0.05 & 9.4093 \\
\hline 0.13 & 1.395 & 8.4 & 9.7 & 0.05 & 9.3951 \\
\hline 0.15 & 1.426 & 8.3 & 9.7 & 0.05 & 9.4317 \\
\hline 0.2 & \begin{tabular}{|l|}
1.503 \\
\end{tabular} & 8.5 & 9.9 & 0.05 & 9.5717 \\
\hline 0.239 & 1.563 & 8.6 & 10 & 0.05 & 9.7071 \\
\hline 0.25 & 1.58 & 8.6 & 10.1 & 0.05 & 9.7379 \\
\hline 0.359 & 1.748 & 8.9 & 10.4 & 0.05 & 10.1035 \\
\hline 0.478 & 1.935 & 9.2 & 10.8 & 0.05 & 10.4174 \\
\hline 0.5 & 1.969 & 9.3 & 10.8 & 0.05 & 10.4822 \\
\hline 0.6 & 2.122 & 9.5 & 11.1 & 0.05 & 10.7574 \\
\hline 0.65 & 2.197 & 9.6 & 11.2 & 0.05 & 10.9275 \\
\hline 0.7 & 2.268 & 9.8 & 11.5 & 0.05 & 11.1276 \\
\hline 0.75 & 2.335 & 10.1 & 11.8 & 0.05 & 11.3892 \\
\hline 0.76 & 2.348 & 10.1 & 11.8 & 0.05 & 11.4472 \\
\hline 0.765 & 2.354 & 10.2 & 11.9 & 0.05 & 11.4993 \\
\hline 0.77 & 2.36 & 10.2 & 11.9 & 0.05 & 11.5302 \\
\hline 0.775 & 2.367 & 10.2 & 11.9 & 0.05 & 11.5585 \\
\hline
\end{tabular}




\section{B1.1.2.2.3.3 10" Water Reflection}

Case ID: PuN46-icyl-0970-10

Material: $\mathrm{Pu}\left(\mathrm{NO}_{3}\right)_{4} 6$ Molar Excess Acid

Geometry: Cylinder

Isotopics: $97 \%{ }^{239} \mathrm{Pu}$

Reflector: 10 " water reflected

Code: MCNP5 v1.40

Analyst: Mark Murphy

Date: $8 / 2011$ 
Figure B1.1.2.2.3.3-1. $\mathrm{Pu}\left(\mathrm{NO}_{3}\right) 46 \mathrm{M}-\mathrm{Cylinder}-97 \%{ }^{239} \mathrm{Pu}-10$ " Water Reflection

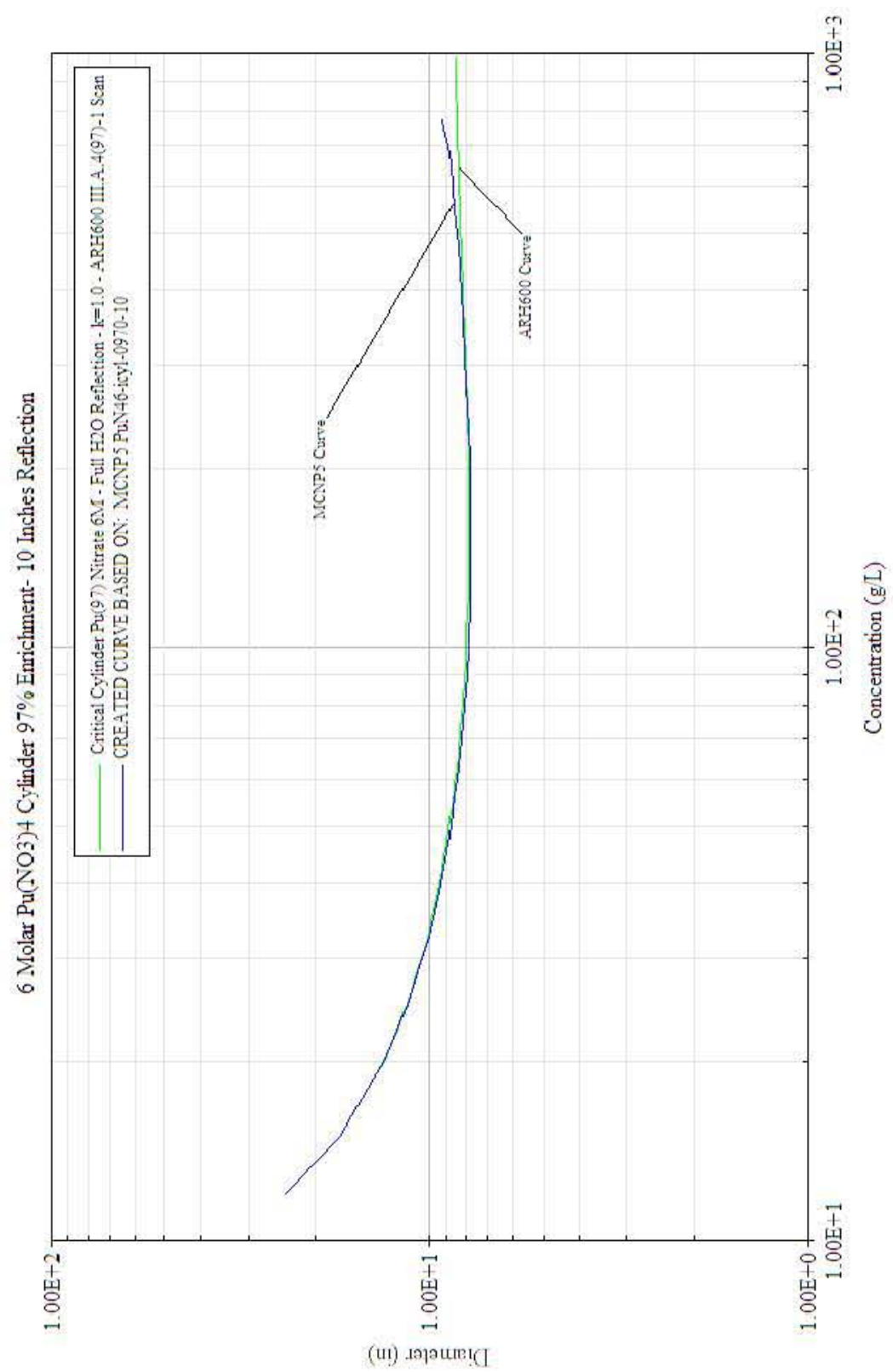


Table B1.1.2.2.3.3-1. Critical Dimensions for $\mathrm{Pu}\left(\mathrm{NO}_{3}\right) 46 \mathrm{M}-\mathrm{Cylinder}-97 \%{ }^{239} \mathrm{Pu}-10 "$ Water Reflection

\begin{tabular}{|c|c|c|c|c|c|}
\hline \multicolumn{6}{|c|}{$97 \% \mathrm{Pu} 239 \mathrm{Pu}(\mathrm{NO} 3) 46 \mathrm{M}$ Cylinder- 10 Inches of Reflection } \\
\hline Concentration $(\mathrm{g} / \mathrm{cc})$ & $\begin{array}{l}\text { Density } \\
(\mathrm{g} / \mathrm{cc})\end{array}$ & $\begin{array}{l}\text { First Diameter } \\
\text { (Inches) }\end{array}$ & $\begin{array}{l}\text { Last Diameter } \\
\text { (Inches) }\end{array}$ & $\begin{array}{l}\text { Delta } \\
\text { (Inches) }\end{array}$ & $\begin{array}{l}\text { Critical Diameter } \\
\text { (Inches) }\end{array}$ \\
\hline 0.012 & 1.207 & 17.9 & 25.6 & 0.3 & 23.9584 \\
\hline 0.015 & 1.212 & 13.9 & 18 & 0.2 & 17.1667 \\
\hline 0.02 & 1.22 & 11.1 & 13.7 & 0.1 & 13.175 \\
\hline 0.025 & 1.229 & 9.7 & 11.8 & 0.1 & 11.4159 \\
\hline 0.03 & 1.237 & 9 & 10.8 & 0.1 & 10.4219 \\
\hline 0.032 & 1.24 & 8.7 & 10.5 & 0.1 & 10.1284 \\
\hline 0.035 & 1.245 & 8.5 & 10.1 & 0.05 & 9.7647 \\
\hline 0.04 & 1.253 & 8.1 & 9.7 & 0.05 & 9.3281 \\
\hline 0.05 & 1.27 & 7.6 & 9 & 0.05 & 8.76 \\
\hline 0.06 & 1.286 & 7.3 & 8.7 & 0.05 & 8.4147 \\
\hline 0.07 & 1.301 & 7.1 & 8.5 & 0.05 & 8.2105 \\
\hline 0.08 & 1.317 & 7 & 8.3 & 0.05 & 8.0517 \\
\hline 0.09 & 1.333 & 6.9 & 8.2 & 0.05 & 7.9426 \\
\hline 0.1 & 1.349 & 6.8 & 8.2 & 0.05 & 7.8749 \\
\hline 0.12 & 1.379 & 6.8 & 8.1 & 0.05 & 7.7985 \\
\hline 0.13 & 1.395 & 6.8 & 8 & 0.05 & 7.7859 \\
\hline 0.15 & 1.426 & 6.7 & 8.1 & 0.05 & 7.7721 \\
\hline 0.2 & 1.503 & 6.8 & 8.1 & 0.05 & 7.8235 \\
\hline 0.239 & 1.563 & 6.8 & 8.2 & 0.05 & 7.8897 \\
\hline 0.25 & 1.58 & 6.8 & 8.2 & 0.05 & 7.9241 \\
\hline 0.359 & 1.748 & 7 & 8.5 & 0.05 & 8.1655 \\
\hline 0.478 & 1.935 & 7.2 & 8.7 & 0.05 & 8.3885 \\
\hline 0.5 & 1.969 & 7.2 & 8.8 & 0.05 & 8.443 \\
\hline 0.6 & 2.122 & 7.4 & 9 & 0.05 & 8.6508 \\
\hline 0.65 & 2.197 & 7.5 & 9.1 & 0.05 & 8.7729 \\
\hline 0.7 & 2.268 & 7.6 & 9.3 & 0.05 & 8.9266 \\
\hline 0.75 & 2.335 & 7.8 & 9.5 & 0.05 & 9.1516 \\
\hline 0.76 & 2.348 & 7.8 & 9.5 & 0.05 & 9.1846 \\
\hline 0.765 & 2.354 & 7.9 & 9.5 & 0.05 & 9.2288 \\
\hline 0.77 & 2.36 & 7.9 & 9.6 & 0.05 & 9.2516 \\
\hline 0.775 & 2.367 & 7.9 & 9.6 & 0.05 & 9.2649 \\
\hline
\end{tabular}


B1.1.3 Plane

B1.1.3.1 $\quad 100 \%{ }^{239} \mathrm{Pu}$

B1.1.3.1.1 0 Molar Excess Acid

B1.1.3.1.1.1 0" Water Reflection

Case ID: PuN40-pl-1000-00

Material: $\mathrm{Pu}\left(\mathrm{NO}_{3}\right)_{4} \quad 0$ Molar Excess Acid

Geometry: Plane

Isotopics: $100 \%{ }^{239} \mathrm{Pu}$

Reflector: 0" water reflected

Code: MCNP5 v1.40

Analyst: Mark Murphy

Date: $8 / 2011$ 
Figure B1.1.3.1.1.1-1. $\mathrm{Pu}\left(\mathrm{NO}_{3}\right) 4$ 0M - Plane - $100 \%{ }^{239} \mathrm{Pu}-0$ " Water Reflection

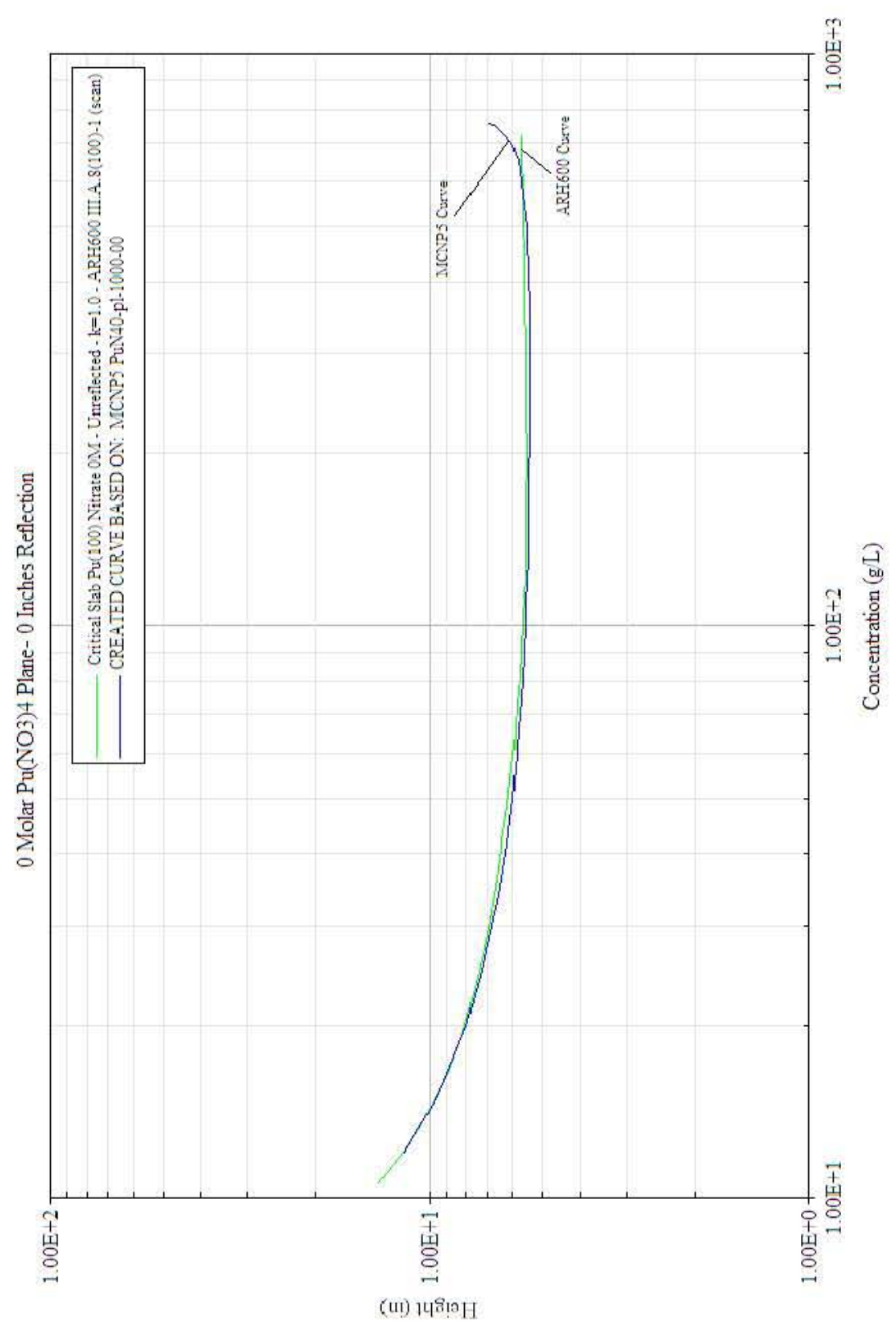


Table B1.1.3.1.1.1-1. Critical Dimensions for $\mathrm{Pu}\left(\mathrm{NO}_{3}\right) 40 \mathrm{M}-\mathrm{Plane}-100 \%{ }^{239} \mathrm{Pu}-0$ " Water Reflection

\begin{tabular}{|c|c|c|c|c|c|}
\hline \multicolumn{6}{|c|}{$100 \% \mathrm{Pu} 239 \mathrm{Pu}(\mathrm{NO} 3) 4$ 0M Plane - 0 Inches of Reflection } \\
\hline Concentration $(\mathrm{g} / \mathrm{cc})$ & $\begin{array}{l}\text { Density } \\
(\mathrm{g} / \mathrm{cc})\end{array}$ & $\begin{array}{l}\text { First Diameter } \\
\text { (Inches) }\end{array}$ & \begin{tabular}{|l|}
$\begin{array}{l}\text { Last Diameter } \\
\text { (Inches) }\end{array}$ \\
\end{tabular} & $\begin{array}{l}\text { Delta } \\
\text { (Inches) }\end{array}$ & \begin{tabular}{|l} 
Critical Height \\
(Inches)
\end{tabular} \\
\hline 0.012 & 1.016 & 9.7 & 12.1 & 0.1 & 11.6392 \\
\hline 0.015 & 1.021 & 8.2 & 9.9 & 0.05 & 9.5107 \\
\hline 0.02 & 1.029 & 7 & 8.3 & 0.05 & 7.976 \\
\hline 0.025 & 1.037 & 6.4 & 7.5 & 0.05 & 7.2443 \\
\hline 0.03 & 1.046 & 6.1 & 7 & 0.05 & 6.8072 \\
\hline 0.032 & 1.049 & 5.9 & 6.9 & 0.05 & 6.6524 \\
\hline 0.035 & 1.054 & 5.8 & 6.7 & 0.05 & 6.5136 \\
\hline 0.04 & 1.063 & 5.6 & 6.5 & 0.05 & 6.2929 \\
\hline 0.05 & 1.08 & 5.4 & 6.2 & 0.05 & 6.0227 \\
\hline 0.06 & 1.097 & 5.3 & 6 & 0.05 & 5.8492 \\
\hline 0.07 & 1.113 & 5.2 & 5.9 & 0.05 & 5.7401 \\
\hline 0.08 & 1.13 & 5.1 & 5.9 & 0.05 & 5.6561 \\
\hline 0.09 & 1.147 & 5 & 5.8 & 0.05 & 5.6029 \\
\hline 0.1 & 1.164 & 5 & 5.8 & 0.05 & 5.5629 \\
\hline 0.12 & 1.197 & 4.9 & 5.7 & 0.05 & 5.5075 \\
\hline 0.13 & 1.215 & 4.9 & 5.7 & 0.05 & 5.4748 \\
\hline 0.15 & 1.248 & 4.9 & 5.7 & 0.05 & 5.4524 \\
\hline 0.2 & 1.332 & 4.9 & 5.6 & 0.05 & 5.4148 \\
\hline 0.239 & 1.397 & 4.9 & 5.6 & 0.05 & 5.4094 \\
\hline 0.25 & 1.415 & 4.9 & 5.6 & 0.05 & 5.4144 \\
\hline 0.359 & 1.594 & 4.9 & 5.7 & 0.05 & 5.4385 \\
\hline 0.478 & 1.784 & 5 & 5.7 & 0.05 & 5.513 \\
\hline 0.5 & 1.818 & 5 & 5.7 & 0.05 & 5.5249 \\
\hline 0.6 & 1.962 & 5.1 & 5.9 & 0.05 & 5.689 \\
\hline 0.65 & 2.026 & 5.3 & 6 & 0.05 & 5.8223 \\
\hline 0.7 & 2.076 & 5.5 & 6.3 & 0.05 & 6.0588 \\
\hline 0.75 & 2.079 & 6 & 6.9 & 0.05 & 6.694 \\
\hline 0.752 & 2.073 & 6.1 & 7 & 0.05 & 6.7666 \\
\hline 0.754 & 2.064 & 6.2 & 7.1 & 0.05 & 6.8822 \\
\hline 0.755 & 2.052 & 6.3 & 7.2 & 0.05 & 6.9957 \\
\hline
\end{tabular}


B1.1.3.1.1.2 1"Water Reflection

Case ID: PuN40-pl-1000-01

Material: $\mathrm{Pu}\left(\mathrm{NO}_{3}\right)_{4} 0$ Molar Excess Acid

Geometry: Plane

Isotopics: $100 \%{ }^{239} \mathrm{Pu}$

Reflector: 1" water reflected

Code: MCNP5 v1.40

Analyst: Mark Murphy

Date: $8 / 2011$ 


\section{CHPRC-01552, Revision 0}

Figure B1.1.3.1.1.2-1. Pu(NO $\left.\mathrm{NO}_{3}\right) 4$ 0M - Plane - 100\% ${ }^{239} \mathrm{Pu}-1$ " Water Reflection

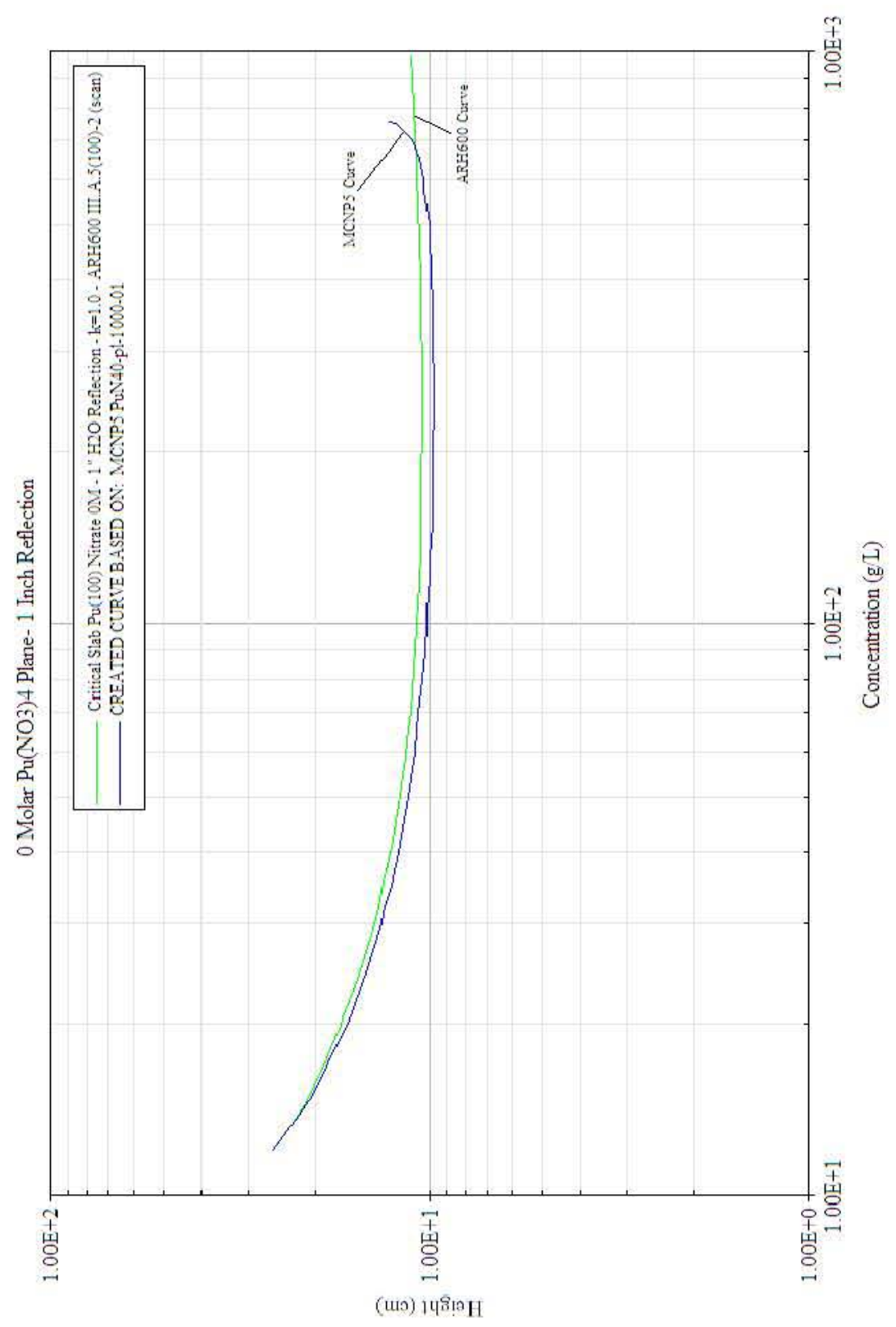


Table B1.1.3.1.1.2-1. Critical Dimensions for $\mathrm{Pu}\left(\mathrm{NO}_{3}\right) 4 \mathrm{MM}-\mathrm{Plane}-100 \%{ }^{239} \mathrm{Pu}-1$ " Water Reflection

\begin{tabular}{|c|c|c|c|c|c|}
\hline \multicolumn{6}{|c|}{$100 \% \mathrm{Pu} 239 \mathrm{Pu}(\mathrm{NO} 3) 4$ 0M Plane- 1 Inch of Reflection } \\
\hline Concentration $(\mathrm{g} / \mathrm{cc})$ & $\begin{array}{l}\text { Density } \\
(\mathrm{g} / \mathrm{cc})\end{array}$ & $\begin{array}{l}\text { First Diameter } \\
\text { (Inches) }\end{array}$ & $\begin{array}{l}\text { Last Diameter } \\
\text { (Inches) }\end{array}$ & $\begin{array}{l}\text { Delta } \\
\text { (Inches) }\end{array}$ & \begin{tabular}{|l} 
Critical Height \\
(Inches)
\end{tabular} \\
\hline 0.012 & 1.016 & 8.1 & 10.6 & 0.1 & 10.1075 \\
\hline 0.015 & 1.021 & 6.7 & 8.4 & 0.05 & 8.0112 \\
\hline 0.02 & 1.029 & 5.5 & 6.7 & 0.05 & 6.4604 \\
\hline 0.025 & 1.037 & 4.9 & 6 & 0.05 & 5.7064 \\
\hline 0.03 & 1.046 & 4.6 & 5.5 & 0.05 & 5.2449 \\
\hline 0.032 & 1.049 & 4.5 & 5.4 & 0.05 & 5.1355 \\
\hline 0.035 & 1.054 & 4.3 & 5.2 & 0.05 & 4.9572 \\
\hline 0.04 & 1.063 & 4.1 & 5 & 0.05 & 4.7477 \\
\hline 0.05 & 1.08 & 3.9 & 4.7 & 0.05 & 4.4618 \\
\hline 0.06 & 1.097 & 3.7 & 4.5 & 0.05 & 4.2864 \\
\hline 0.07 & 1.113 & 3.6 & 4.4 & 0.05 & 4.1814 \\
\hline 0.08 & 1.13 & 3.6 & 4.3 & 0.05 & 4.1006 \\
\hline 0.09 & 1.147 & 3.5 & 4.2 & 0.05 & 4.0338 \\
\hline 0.1 & 1.164 & 3.5 & 4.2 & 0.05 & 3.9873 \\
\hline 0.12 & 1.197 & 3.4 & 4.1 & 0.05 & 3.9202 \\
\hline 0.13 & 1.215 & 3.4 & 4.1 & 0.05 & 3.9091 \\
\hline 0.15 & 1.248 & 3.4 & 4.1 & 0.05 & 3.8667 \\
\hline 0.2 & 1.332 & 3.3 & 4 & 0.05 & 3.839 \\
\hline 0.239 & 1.397 & 3.3 & 4 & 0.05 & 3.8335 \\
\hline 0.25 & 1.415 & 3.3 & 4 & 0.05 & 3.8365 \\
\hline 0.359 & 1.594 & 3.4 & 4 & 0.05 & 3.8485 \\
\hline 0.478 & 1.784 & 3.4 & 4.1 & 0.05 & 3.9149 \\
\hline 0.5 & 1.818 & 3.4 & 4.1 & 0.05 & 3.9339 \\
\hline 0.6 & 1.962 & 3.5 & 4.2 & 0.05 & 4.0598 \\
\hline 0.65 & 2.026 & 3.6 & 4.4 & 0.05 & 4.1688 \\
\hline 0.7 & 2.076 & 3.8 & 4.6 & 0.05 & 4.348 \\
\hline 0.75 & 2.079 & 4.2 & 5 & 0.05 & 4.8082 \\
\hline 0.752 & 2.073 & 4.2 & 5.1 & 0.05 & 4.8791 \\
\hline 0.754 & 2.064 & 4.3 & 5.2 & 0.05 & 4.9594 \\
\hline 0.755 & 2.052 & 4.4 & 5.3 & 0.05 & 5.0499 \\
\hline
\end{tabular}




\section{B1.1.3.1.1.3 10" Water Reflection}

Case ID: PuN40-pl-1000-10

Material: $\mathrm{Pu}\left(\mathrm{NO}_{3}\right)_{4} 0$ Molar Excess Acid

Geometry: Plane

Isotopics: $100 \%{ }^{239} \mathrm{Pu}$

Reflector: 10 " water reflected

Code: MCNP5 v1.40

Analyst: Mark Murphy

Date: $8 / 2011$ 
Figure B1.1.3.1.1.3-1. $\mathrm{Pu}\left(\mathrm{NO}_{3}\right) 40 \mathrm{M}-\mathrm{Plane}-100 \%{ }^{239} \mathrm{Pu}-10$ " Water Reflection

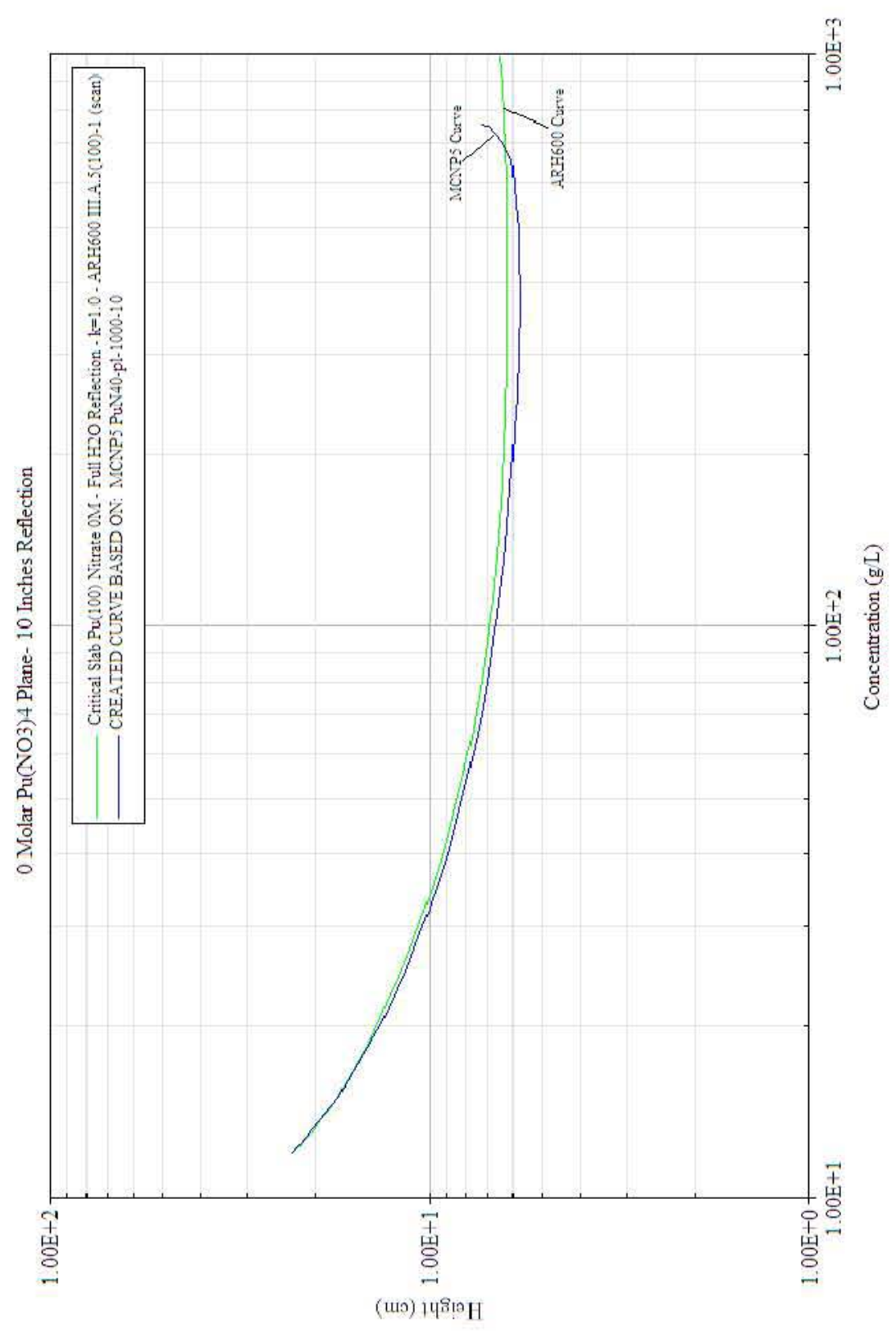


Table B1.1.3.1.1.3-1. Critical Dimensions for $\mathrm{Pu}\left(\mathrm{NO}_{3}\right) 40 \mathrm{M}-\mathrm{Plane}-100 \%{ }^{239} \mathrm{Pu}-10 "$ Water Reflection

\begin{tabular}{|c|c|c|c|c|c|}
\hline \multicolumn{6}{|c|}{$100 \% \mathrm{Pu} 239 \mathrm{Pu}(\mathrm{NO} 3) 40 \mathrm{M}$ Plane - 10 Inches of Reflection } \\
\hline Concentration $(\mathrm{g} / \mathrm{cc})$ & $\begin{array}{l}\text { Density } \\
(\mathrm{g} / \mathrm{cc})\end{array}$ & $\begin{array}{l}\text { First Diameter } \\
\text { (Inches) }\end{array}$ & $\begin{array}{l}\text { Last Diameter } \\
\text { (Inches) }\end{array}$ & $\begin{array}{l}\text { Delta } \\
\text { (Inches) }\end{array}$ & \begin{tabular}{|l} 
Critical Height \\
(Inches)
\end{tabular} \\
\hline 0.012 & 1.016 & 7 & 9.5 & 0.1 & 9.0109 \\
\hline 0.015 & 1.021 & 5.6 & 7.2 & 0.05 & 6.8573 \\
\hline 0.02 & 1.029 & 4.4 & 5.6 & 0.05 & 5.305 \\
\hline 0.025 & 1.037 & 3.8 & 4.8 & 0.05 & 4.5386 \\
\hline 0.03 & 1.046 & 3.4 & 4.3 & 0.05 & 4.0806 \\
\hline 0.032 & 1.049 & 3.3 & 4.1 & 0.05 & 3.9273 \\
\hline 0.035 & 1.054 & 3.1 & 3.9 & 0.05 & 3.7583 \\
\hline 0.04 & 1.063 & 2.9 & 3.7 & 0.05 & 3.5206 \\
\hline 0.05 & 1.08 & 2.7 & 3.4 & 0.05 & 3.2126 \\
\hline 0.06 & 1.097 & 2.5 & 3.2 & 0.05 & 3.0168 \\
\hline 0.07 & 1.113 & 2.4 & 3 & 0.05 & 2.8657 \\
\hline 0.08 & 1.13 & 2.3 & 2.9 & 0.05 & 2.7678 \\
\hline 0.09 & 1.147 & 2.2 & 2.9 & 0.05 & 2.6869 \\
\hline 0.1 & 1.164 & 2.1 & 2.8 & 0.05 & 2.6259 \\
\hline 0.12 & 1.197 & 2 & 2.7 & 0.05 & 2.5404 \\
\hline 0.13 & 1.215 & 2 & 2.7 & 0.05 & 2.4932 \\
\hline 0.15 & 1.248 & 1.9 & 2.6 & 0.05 & 2.4416 \\
\hline 0.2 & 1.332 & 1.9 & 2.5 & 0.05 & 2.3618 \\
\hline 0.239 & 1.397 & 1.8 & 2.5 & 0.05 & 2.3224 \\
\hline 0.25 & 1.415 & 1.8 & 2.5 & 0.05 & 2.308 \\
\hline 0.359 & 1.594 & 1.8 & 2.5 & 0.05 & 2.2759 \\
\hline 0.478 & 1.784 & 1.8 & 2.5 & 0.05 & 2.2822 \\
\hline 0.5 & 1.818 & 1.8 & 2.5 & 0.05 & 2.2923 \\
\hline 0.6 & 1.962 & 1.8 & 2.5 & 0.05 & 2.3428 \\
\hline 0.65 & 2.026 & 1.9 & 2.6 & 0.05 & 2.3908 \\
\hline 0.7 & 2.076 & 1.9 & 2.7 & 0.05 & 2.5072 \\
\hline 0.75 & 2.079 & 2.1 & 2.9 & 0.05 & 2.7435 \\
\hline 0.752 & 2.073 & 2.2 & 3 & 0.05 & 2.8014 \\
\hline 0.754 & 2.064 & 2.2 & 3 & 0.05 & 2.8271 \\
\hline 0.755 & 2.052 & 2.3 & 3.1 & 0.05 & 2.8833 \\
\hline
\end{tabular}


CHPRC-01552, Revision 0 
B1.1.3.1.2 3 Molar Excess Acid

B1.1.3.1.2.1 0"Water Reflection

Case ID: PuN43-pl-1000-00

Material: $\mathrm{Pu}\left(\mathrm{NO}_{3}\right)_{4} 3$ Molar Excess Acid

Geometry: Plane

Isotopics: $100 \%{ }^{239} \mathrm{Pu}$

Reflector: 0 " water reflected

Code: MCNP5 v1.40

Analyst: Mark Murphy

Date: $8 / 2011$ 
Figure B1.1.3.1.2.1-1. $\mathrm{Pu}\left(\mathrm{NO}_{3}\right) 43 \mathrm{M}-\mathrm{Plane}-100 \%{ }^{239} \mathrm{Pu}-0$ " Water Reflection

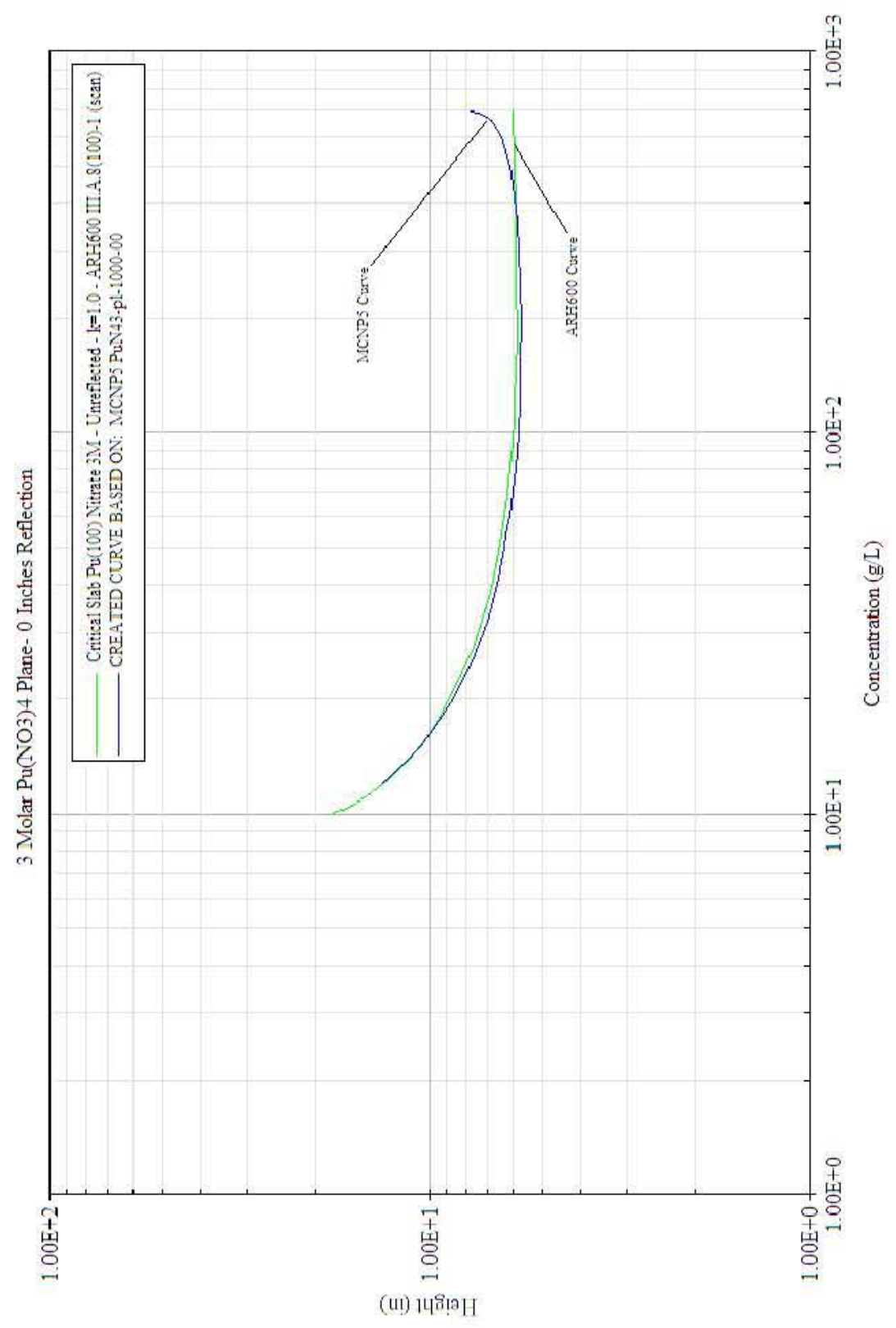


Table B1.1.3.1.2.1-1. Critical Dimensions for $\mathrm{Pu}\left(\mathrm{NO}_{3}\right) 43 \mathrm{M}-\mathrm{Plane}-100 \%{ }^{239} \mathrm{Pu}-0$ " Water Reflection

\begin{tabular}{|c|c|c|c|c|c|}
\hline \multicolumn{6}{|c|}{$100 \% \mathrm{Pu} 239 \mathrm{Pu}(\mathrm{NO} 3) 43 \mathrm{M}$ Plane - 0 Inches of Reflection } \\
\hline Concentration $(\mathrm{g} / \mathrm{cc})$ & $\begin{array}{l}\text { Density } \\
(\mathrm{g} / \mathrm{cc})\end{array}$ & $\begin{array}{l}\text { First Diameter } \\
\text { (Inches) }\end{array}$ & $\begin{array}{l}\text { Last Diameter } \\
\text { (Inches) }\end{array}$ & $\begin{array}{l}\text { Delta } \\
\text { (Inches) }\end{array}$ & \begin{tabular}{|l} 
Critical Height \\
(Inches)
\end{tabular} \\
\hline 0.012 & 1.118 & 10.7 & 13.9 & 0.1 & 13.2494 \\
\hline 0.015 & 1.123 & 8.8 & 10.9 & 0.1 & 10.4597 \\
\hline 0.02 & 1.132 & 7.5 & 8.9 & 0.05 & 8.5809 \\
\hline 0.025 & 1.14 & 6.8 & 8 & 0.05 & 7.7138 \\
\hline 0.03 & 1.149 & 6.4 & 7.4 & 0.05 & 7.1876 \\
\hline 0.032 & 1.152 & 6.3 & 7.3 & 0.05 & 7.0331 \\
\hline 0.035 & 1.157 & 6.1 & 7.1 & 0.05 & 6.8541 \\
\hline 0.04 & 1.165 & 5.9 & 6.8 & 0.05 & 6.6159 \\
\hline 0.05 & 1.182 & 5.7 & 6.5 & 0.05 & 6.315 \\
\hline 0.06 & 1.198 & 5.5 & 6.3 & 0.05 & 6.1357 \\
\hline 0.07 & 1.215 & 5.4 & 6.2 & 0.05 & 5.9998 \\
\hline 0.08 & 1.231 & 5.3 & 6.1 & 0.05 & 5.9261 \\
\hline 0.09 & 1.247 & 5.3 & 6.1 & 0.05 & 5.8743 \\
\hline 0.1 & 1.263 & 5.2 & 6 & 0.05 & 5.8241 \\
\hline 0.12 & 1.294 & 5.2 & 6 & 0.05 & 5.7656 \\
\hline 0.13 & 1.311 & 5.2 & 5.9 & 0.05 & 5.7447 \\
\hline 0.15 & 1.342 & 5.2 & 5.9 & 0.05 & 5.738 \\
\hline 0.2 & 1.421 & 5.2 & 5.9 & 0.05 & 5.7308 \\
\hline 0.239 & 1.481 & 5.2 & 5.9 & 0.05 & 5.7593 \\
\hline 0.25 & 1.498 & 5.2 & 6 & 0.05 & 5.7659 \\
\hline 0.359 & 1.665 & 5.3 & 6.1 & 0.05 & 5.8636 \\
\hline 0.478 & 1.841 & 5.4 & 6.2 & 0.05 & 6.0428 \\
\hline 0.5 & 1.871 & 5.5 & 6.3 & 0.05 & 6.0901 \\
\hline 0.6 & 1.998 & 5.8 & 6.6 & 0.05 & 6.4127 \\
\hline 0.65 & 2.043 & 6.1 & 7 & 0.05 & 6.7495 \\
\hline 0.66 & 2.049 & 6.2 & 7.1 & 0.05 & 6.8476 \\
\hline 0.67 & 2.053 & 6.3 & 7.2 & 0.05 & 6.9698 \\
\hline 0.68 & 2.053 & 6.4 & 7.3 & 0.05 & 7.1268 \\
\hline 0.69 & 2.045 & 6.7 & 7.6 & 0.05 & 7.3782 \\
\hline 0.698 & 2.017 & 7.1 & 8.1 & 0.05 & 7.8535 \\
\hline
\end{tabular}




\section{B1.1.3.1.2.2 1"Water Reflection}

Case ID: PuN43-pl-1000-01

Material: $\mathrm{Pu}\left(\mathrm{NO}_{3}\right)_{4} 3$ Molar Excess Acid

Geometry: Plane

Isotopics: $100 \%{ }^{239} \mathrm{Pu}$

Reflector: 1" water reflected

Code: MCNP5 v1.40

Analyst: Mark Murphy

Date: $8 / 2011$ 


\section{CHPRC-01552, Revision 0}

Figure B1.1.3.1.2.2-1. Pu(NO $\left.\mathrm{NO}_{3}\right) 4 \mathrm{M}-$ Plane - $100 \%{ }^{239} \mathrm{Pu}-1$ " Water Reflection

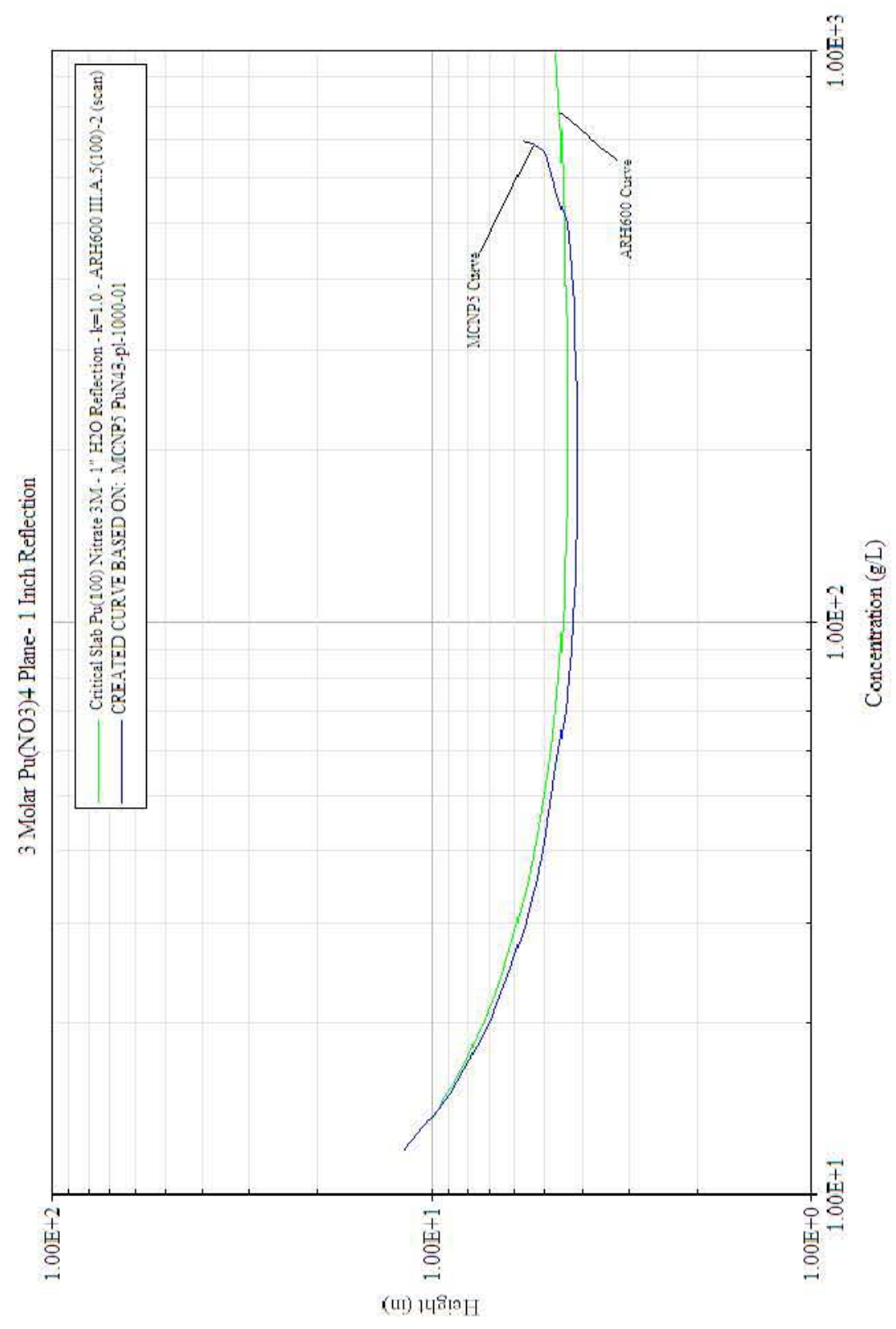


Table B1.1.3.1.2.2-1. Critical Dimensions for $\mathrm{Pu}\left(\mathrm{NO}_{3}\right) 43 \mathrm{M}-\mathrm{Plane}-100 \%{ }^{239} \mathrm{Pu}-1$ " Water Reflection

\begin{tabular}{|c|c|c|c|c|c|}
\hline \multicolumn{6}{|c|}{$100 \% \mathrm{Pu} 239 \mathrm{Pu}(\mathrm{NO} 3) 43 \mathrm{M}$ Plane - 1 Inch of Reflection } \\
\hline Concentration $(\mathrm{g} / \mathrm{cc})$ & $\begin{array}{l}\text { Density } \\
(\mathrm{g} / \mathrm{cc})\end{array}$ & $\begin{array}{l}\text { First Diameter } \\
\text { (Inches) }\end{array}$ & $\begin{array}{l}\text { Last Diameter } \\
\text { (Inches) }\end{array}$ & $\begin{array}{l}\text { Delta } \\
\text { (Inches) }\end{array}$ & $\begin{array}{l}\text { Critical Height } \\
\text { (Inches) }\end{array}$ \\
\hline 0.012 & 1.118 & 9.2 & 12.3 & 0.1 & 11.7075 \\
\hline 0.015 & 1.123 & 7.3 & 9.3 & 0.1 & 8.9095 \\
\hline 0.02 & 1.132 & 5.9 & 7.3 & 0.05 & 7.028 \\
\hline 0.025 & 1.14 & 5.3 & 6.4 & 0.05 & 6.1512 \\
\hline 0.03 & 1.149 & 4.8 & 5.9 & 0.05 & 5.6232 \\
\hline 0.032 & 1.152 & 4.7 & 5.7 & 0.05 & 5.4823 \\
\hline 0.035 & 1.157 & 4.6 & 5.5 & 0.05 & 5.2913 \\
\hline 0.04 & 1.165 & 4.4 & 5.3 & 0.05 & 5.0704 \\
\hline 0.07 & 1.215 & 3.8 & 4.6 & 0.05 & 4.4313 \\
\hline 0.08 & 1.231 & 3.8 & 4.5 & 0.05 & 4.3311 \\
\hline 0.09 & 1.247 & 3.7 & 4.4 & 0.05 & 4.2686 \\
\hline 0.1 & 1.263 & 3.7 & 4.4 & 0.05 & 4.2163 \\
\hline 0.12 & 1.294 & 3.6 & 4.3 & 0.05 & 4.1662 \\
\hline 0.13 & 1.311 & 3.6 & 4.3 & 0.05 & 4.1563 \\
\hline 0.15 & 1.342 & 3.6 & 4.3 & 0.05 & 4.1304 \\
\hline 0.2 & 1.421 & 3.5 & 4.3 & 0.05 & 4.1087 \\
\hline 0.239 & 1.481 & 3.6 & 4.3 & 0.05 & 4.1177 \\
\hline 0.25 & 1.498 & 3.6 & 4.3 & 0.05 & 4.129 \\
\hline 0.359 & 1.665 & 3.6 & 4.4 & 0.05 & 4.204 \\
\hline 0.478 & 1.841 & 3.8 & 4.5 & 0.05 & 4.3407 \\
\hline 0.5 & 1.871 & 3.8 & 4.6 & 0.05 & 4.3962 \\
\hline 0.66 & 2.049 & 4.3 & 5.2 & 0.05 & 4.9835 \\
\hline 0.67 & 2.053 & 4.4 & 5.3 & 0.05 & 5.0503 \\
\hline 0.68 & 2.053 & 4.5 & 5.4 & 0.05 & 5.1906 \\
\hline 0.69 & 2.045 & 4.7 & 5.6 & 0.05 & 5.3809 \\
\hline 0.698 & 2.017 & 5 & 6 & 0.05 & 5.755 \\
\hline
\end{tabular}




\section{B1.1.3.1.2.3 10" Water Reflection}

Case ID: PuN43-pl-1000-10

Material: $\mathrm{Pu}\left(\mathrm{NO}_{3}\right)_{4} 3$ Molar Excess Acid

Geometry: Plane

Isotopics: $100 \%{ }^{239} \mathrm{Pu}$

Reflector: 10 " water reflected

Code: MCNP5 v1.40

Analyst: Mark Murphy

Date: $8 / 2011$ 
Figure B1.1.3.1.2.3-1. $\mathrm{Pu}\left(\mathrm{NO}_{3}\right) 4$ 3M - Plane - 100\% ${ }^{239} \mathrm{Pu}-10$ " Water Reflection

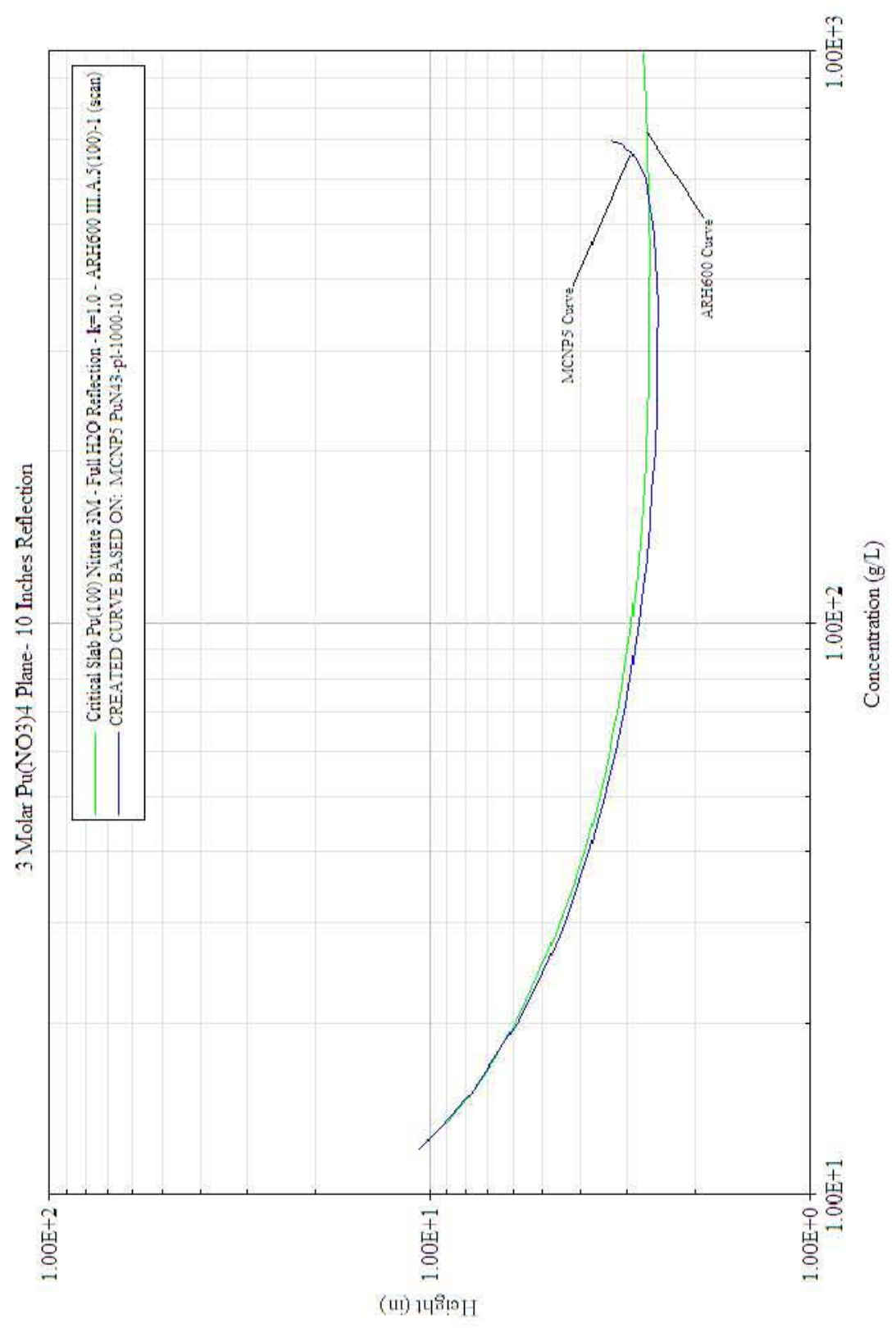


Table B1.1.3.1.2.3-1. Critical Dimensions for $\mathrm{Pu}\left(\mathrm{NO}_{3}\right) 43 \mathrm{M}-\mathrm{Plane}-100 \%{ }^{239} \mathrm{Pu}-10 "$ Water Reflection

\begin{tabular}{|c|c|c|c|c|c|}
\hline \multicolumn{6}{|c|}{$100 \%$ Pu239 Pu(NO3)4 3M Plane - 10 Inches of Reflection } \\
\hline Concentration $(\mathrm{g} / \mathrm{cc})$ & $\begin{array}{l}\text { Density } \\
(\mathrm{g} / \mathrm{cc})\end{array}$ & $\begin{array}{l}\text { First Diameter } \\
\text { (Inches) }\end{array}$ & $\begin{array}{l}\text { Last Diameter } \\
\text { (Inches) }\end{array}$ & $\begin{array}{l}\text { Delta } \\
\text { (Inches) }\end{array}$ & $\begin{array}{l}\text { Critical Height } \\
\text { (Inches) }\end{array}$ \\
\hline 0.012 & 1.118 & 8 & 11.2 & 0.1 & 10.5566 \\
\hline 0.015 & 1.123 & 6.2 & 8.1 & 0.1 & 7.7471 \\
\hline 0.02 & 1.132 & 4.8 & 6.1 & 0.05 & 5.854 \\
\hline 0.025 & 1.14 & 4.1 & 5.2 & 0.05 & 4.9351 \\
\hline 0.03 & 1.149 & 3.6 & 4.6 & 0.05 & 4.386 \\
\hline 0.032 & 1.152 & 3.5 & 4.5 & 0.05 & 4.2385 \\
\hline 0.035 & 1.157 & 3.3 & 4.2 & 0.05 & 4.0406 \\
\hline 0.04 & 1.165 & 3.1 & 4 & 0.05 & 3.7876 \\
\hline 0.05 & 1.182 & 2.8 & 3.6 & 0.05 & 3.4362 \\
\hline 0.06 & 1.198 & 2.6 & 3.4 & 0.05 & 3.2238 \\
\hline 0.07 & 1.215 & 2.5 & 3.2 & 0.05 & 3.0679 \\
\hline 0.08 & 1.231 & 2.4 & 3.1 & 0.05 & 2.9536 \\
\hline 0.09 & 1.247 & 2.3 & 3 & 0.05 & 2.8716 \\
\hline 0.1 & 1.263 & 2.3 & 3 & 0.05 & 2.8076 \\
\hline 0.12 & 1.294 & 2.2 & 2.9 & 0.05 & 2.7075 \\
\hline 0.13 & 1.311 & 2.1 & 2.8 & 0.05 & 2.6733 \\
\hline 0.15 & 1.342 & 2.1 & 2.8 & 0.05 & 2.6176 \\
\hline 0.2 & 1.421 & 2 & 2.7 & 0.05 & 2.541 \\
\hline 0.239 & 1.481 & 2 & 2.7 & 0.05 & 2.5157 \\
\hline 0.25 & 1.498 & 2 & 2.7 & 0.05 & 2.5143 \\
\hline 0.359 & 1.665 & 2 & 2.7 & 0.05 & 2.496 \\
\hline 0.478 & 1.841 & 2 & 2.7 & 0.05 & 2.5598 \\
\hline 0.5 & 1.871 & 2 & 2.7 & 0.05 & 2.5785 \\
\hline 0.6 & 1.998 & 2.1 & 2.9 & 0.05 & 2.6991 \\
\hline 0.65 & 2.043 & 2.2 & 3 & 0.05 & 2.8613 \\
\hline 0.66 & 2.049 & 2.2 & 3.1 & 0.05 & 2.8985 \\
\hline 0.67 & 2.053 & 2.3 & 3.1 & 0.05 & 2.9363 \\
\hline 0.68 & 2.053 & 2.3 & 3.2 & 0.05 & 3.0257 \\
\hline 0.69 & 2.045 & 2.4 & 3.3 & 0.05 & 3.1194 \\
\hline 0.698 & 2.017 & 2.6 & 3.5 & 0.05 & 3.3406 \\
\hline
\end{tabular}


B1.1.3.1.3 6 Molar Excess Acid

B1.1.3.1.3.1 0"Water Reflection

Case ID: PuN46-pl-1000-00

Material: $\mathrm{Pu}\left(\mathrm{NO}_{3}\right)_{4} 6$ Molar Excess Acid

Geometry: Plane

Isotopics: $100 \%{ }^{239} \mathrm{Pu}$

Reflector: 0" water reflected

Code: MCNP5 v1.40

Analyst: Mark Murphy

Date: $8 / 2011$ 


\section{CHPRC-01552, Revision 0}

Figure B1.1.3.1.3.1-1. Pu(NO $\left.\mathrm{NO}_{3}\right) 4$ 6M - Plane - 100\% ${ }^{239} \mathrm{Pu}-0$ " Water Reflection

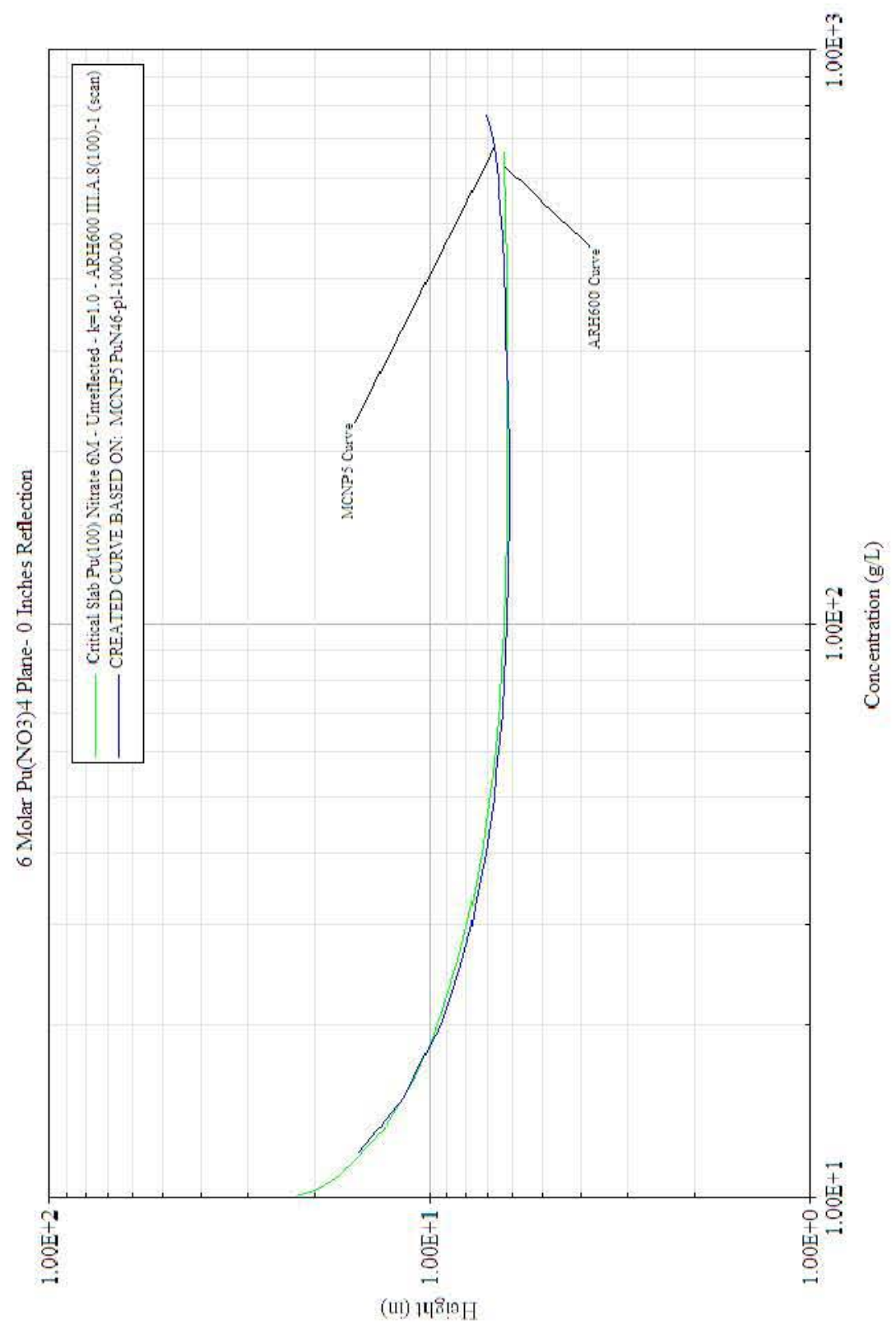


Table B.1.1.3.1.3.1-1. Critical Dimensions for $\mathrm{Pu}\left(\mathrm{NO}_{3}\right) 46 \mathrm{M}-\mathrm{Plane}-100 \%{ }^{239} \mathrm{Pu}-0$ " Water Reflection

\begin{tabular}{|c|c|c|c|c|c|}
\hline \multicolumn{6}{|c|}{$100 \% \mathrm{Pu} 239 \mathrm{Pu}(\mathrm{NO} 3) 46 \mathrm{M}$ Plane - 0 Inches of Reflection } \\
\hline Concentration $(\mathrm{g} / \mathrm{cc})$ & $\begin{array}{l}\text { Density } \\
(\mathrm{g} / \mathrm{cc})\end{array}$ & $\begin{array}{l}\text { First Diameter } \\
\text { (Inches) }\end{array}$ & $\begin{array}{l}\text { Last Diameter } \\
\text { (Inches) }\end{array}$ & $\begin{array}{l}\text { Delta } \\
\text { (Inches) }\end{array}$ & $\begin{array}{l}\text { Critical Height } \\
\text { (Inches) }\end{array}$ \\
\hline 0.012 & 1.207 & 12 & 16.1 & 0.2 & 15.3038 \\
\hline 0.015 & 1.212 & 9.7 & 12.1 & 0.1 & 11.5912 \\
\hline 0.02 & 1.22 & 8.1 & 9.7 & 0.05 & 9.3323 \\
\hline 0.025 & 1.229 & 7.3 & 8.6 & 0.05 & 8.2911 \\
\hline 0.03 & 1.237 & 6.8 & 8 & 0.05 & 7.7143 \\
\hline 0.032 & 1.24 & 6.7 & 7.8 & 0.05 & 7.5414 \\
\hline 0.035 & 1.245 & 6.5 & 7.6 & 0.05 & 7.3602 \\
\hline 0.04 & 1.253 & 6.3 & 7.3 & 0.05 & 7.0857 \\
\hline 0.05 & 1.27 & 6 & 7 & 0.05 & 6.7322 \\
\hline 0.06 & 1.286 & 5.9 & 6.8 & 0.05 & 6.5226 \\
\hline 0.07 & 1.301 & 5.7 & 6.6 & 0.05 & 6.391 \\
\hline 0.08 & 1.317 & 5.7 & 6.6 & 0.05 & 6.3096 \\
\hline 0.09 & 1.333 & 5.6 & 6.5 & 0.05 & 6.2481 \\
\hline 0.1 & 1.349 & 5.5 & 6.4 & 0.05 & 6.2063 \\
\hline 0.12 & 1.379 & 5.5 & 6.4 & 0.05 & 6.1712 \\
\hline 0.13 & 1.395 & 5.5 & 6.4 & 0.05 & 6.1392 \\
\hline 0.15 & 1.426 & 5.4 & 6.3 & 0.05 & 6.1146 \\
\hline 0.2 & 1.503 & 5.5 & 6.4 & 0.05 & 6.1312 \\
\hline 0.239 & 1.563 & 5.5 & 6.4 & 0.05 & 6.1634 \\
\hline 0.25 & 1.58 & 5.5 & 6.4 & 0.05 & 6.172 \\
\hline 0.359 & 1.748 & 5.6 & 6.5 & 0.05 & 6.2801 \\
\hline 0.478 & 1.935 & 5.7 & 6.6 & 0.05 & 6.4006 \\
\hline 0.5 & 1.969 & 5.8 & 6.7 & 0.05 & 6.4188 \\
\hline 0.6 & 2.122 & 5.9 & 6.8 & 0.05 & 6.5479 \\
\hline 0.65 & 2.197 & 6 & 6.9 & 0.05 & 6.6518 \\
\hline 0.7 & 2.268 & 6.1 & 7 & 0.05 & 6.785 \\
\hline 0.75 & 2.335 & 6.3 & 7.2 & 0.05 & 6.964 \\
\hline 0.76 & 2.348 & 6.3 & 7.2 & 0.05 & 6.9967 \\
\hline 0.765 & 2.354 & 6.4 & 7.2 & 0.05 & 7.0219 \\
\hline 0.77 & 2.36 & 6.4 & 7.3 & 0.05 & 7.0406 \\
\hline 0.775 & 2.367 & 6.4 & 7.3 & 0.05 & 7.0672 \\
\hline
\end{tabular}




\section{B1.1.3.1.3.2 1"Water Reflection}

Case ID: PuN46-pl-1000-01

Material: $\mathrm{Pu}\left(\mathrm{NO}_{3}\right)_{4} 6$ Molar Excess Acid

Geometry: Plane

Isotopics: $100 \%{ }^{239} \mathrm{Pu}$

Reflector: 1" water reflected

Code: MCNP5 v1.40

Analyst: Mark Murphy

Date: $8 / 2011$ 
Figure B1.1.3.1.3.2-1. $\mathrm{Pu}\left(\mathrm{NO}_{3}\right) 46 \mathrm{M}-\mathrm{Plane}-100 \%{ }^{239} \mathrm{Pu}-1$ " Water Reflection

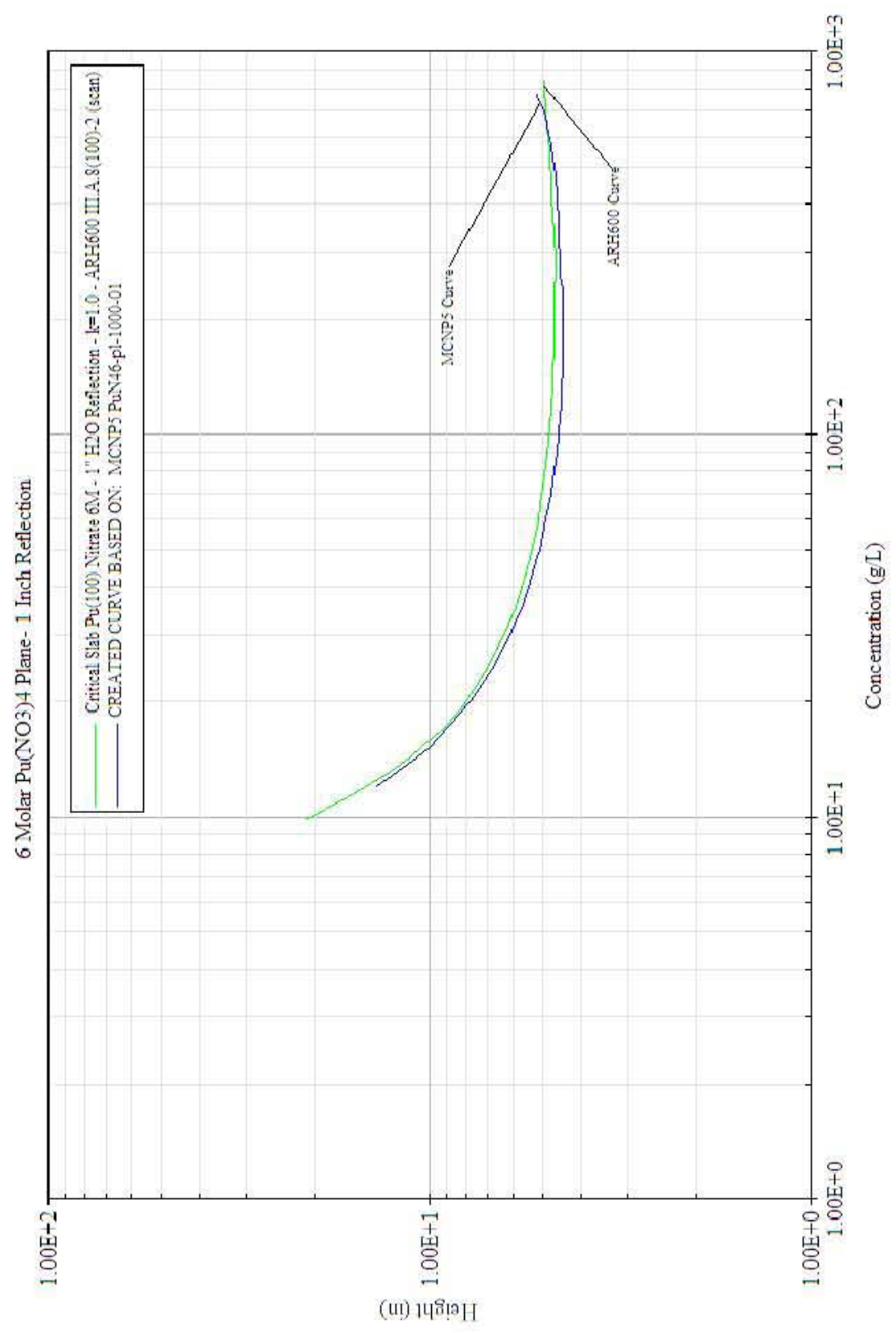


Table B1.1.3.1.3.2-1. Critical Dimensions for $\mathrm{Pu}\left(\mathrm{NO}_{3}\right) 46 \mathrm{M}-\mathrm{Plane}-100 \%{ }^{239} \mathrm{Pu}-1$ " Water Reflection

\begin{tabular}{|c|c|c|c|c|c|}
\hline \multicolumn{6}{|c|}{$100 \%$ Pu239 Pu(NO3)4 6M Plane - 1 Inch of Reflection } \\
\hline Concentration $(\mathrm{g} / \mathrm{cc})$ & $\begin{array}{l}\text { Density } \\
(\mathrm{g} / \mathrm{cc})\end{array}$ & $\begin{array}{l}\text { First Diameter } \\
\text { (Inches) }\end{array}$ & $\begin{array}{l}\text { Last Diameter } \\
\text { (Inches) }\end{array}$ & $\begin{array}{l}\text { Delta } \\
\text { (Inches) }\end{array}$ & \begin{tabular}{|l} 
Critical Height \\
(Inches)
\end{tabular} \\
\hline 0.012 & 1.207 & 10.4 & 14.5 & 0.2 & 13.6948 \\
\hline 0.015 & 1.212 & 8.2 & 10.5 & 0.1 & 10.0379 \\
\hline 0.02 & 1.22 & 6.5 & 8 & 0.05 & 7.7531 \\
\hline 0.025 & 1.229 & 5.7 & 7 & 0.05 & 6.7 \\
\hline 0.03 & 1.237 & 5.3 & 6.4 & 0.05 & 6.1118 \\
\hline 0.032 & 1.24 & 5.1 & 6.2 & 0.05 & 5.9415 \\
\hline 0.035 & 1.245 & 4.9 & 6 & 0.05 & 5.721 \\
\hline 0.04 & 1.253 & 4.7 & 5.7 & 0.05 & 5.475 \\
\hline 0.05 & 1.27 & 4.5 & 5.3 & 0.05 & 5.1219 \\
\hline 0.06 & 1.286 & 4.3 & 5.1 & 0.05 & 4.915 \\
\hline 0.07 & 1.301 & 4.2 & 5 & 0.05 & 4.7794 \\
\hline 0.08 & 1.317 & 4.1 & 4.9 & 0.05 & 4.6688 \\
\hline 0.09 & 1.333 & 4 & 4.8 & 0.05 & 4.608 \\
\hline 0.1 & 1.349 & 3.9 & 4.8 & 0.05 & 4.5683 \\
\hline 0.12 & 1.379 & 3.9 & 4.7 & 0.05 & 4.509 \\
\hline 0.13 & 1.395 & 3.9 & 4.7 & 0.05 & 4.4896 \\
\hline 0.15 & 1.426 & 3.9 & 4.7 & 0.05 & 4.4597 \\
\hline 0.2 & 1.503 & 3.8 & 4.6 & 0.05 & 4.4421 \\
\hline 0.239 & 1.563 & 3.9 & 4.7 & 0.05 & 4.4625 \\
\hline 0.25 & 1.58 & 3.9 & 4.7 & 0.05 & 4.473 \\
\hline 0.359 & 1.748 & 3.9 & 4.8 & 0.05 & 4.5537 \\
\hline 0.478 & 1.935 & 4 & 4.9 & 0.05 & 4.6468 \\
\hline 0.5 & 1.969 & 4.1 & 4.9 & 0.05 & 4.6819 \\
\hline 0.6 & 2.122 & 4.2 & 5 & 0.05 & 4.8074 \\
\hline 0.65 & 2.197 & 4.3 & 5.1 & 0.05 & 4.8826 \\
\hline 0.7 & 2.268 & 4.3 & 5.2 & 0.05 & 4.9831 \\
\hline 0.75 & 2.335 & 4.5 & 5.3 & 0.05 & 5.1336 \\
\hline 0.76 & 2.348 & 4.5 & 5.4 & 0.05 & 5.1823 \\
\hline 0.765 & 2.354 & 4.5 & 5.4 & 0.05 & 5.1831 \\
\hline 0.77 & 2.36 & 4.5 & 5.5 & 0.05 & 5.2141 \\
\hline 0.775 & 2.367 & 4.6 & 5.4 & 0.05 & 5.234 \\
\hline
\end{tabular}




\section{B1.1.3.1.3.3 10" Water Reflection}

Case ID: PuN46-pl-1000-10

Material: $\mathrm{Pu}\left(\mathrm{NO}_{3}\right)_{4} 6$ Molar Excess Acid

Geometry: Plane

Isotopics: $100 \%{ }^{239} \mathrm{Pu}$

Reflector: 10 " water reflected

Code: MCNP5 v1.40

Analyst: Mark Murphy

Date: $8 / 2011$ 
Figure B1.1.3.1.3.3-1. $\mathrm{Pu}\left(\mathrm{NO}_{3}\right) 46 \mathrm{M}-\mathrm{Plane}-100 \%{ }^{239} \mathrm{Pu}-10$ " Water Reflection

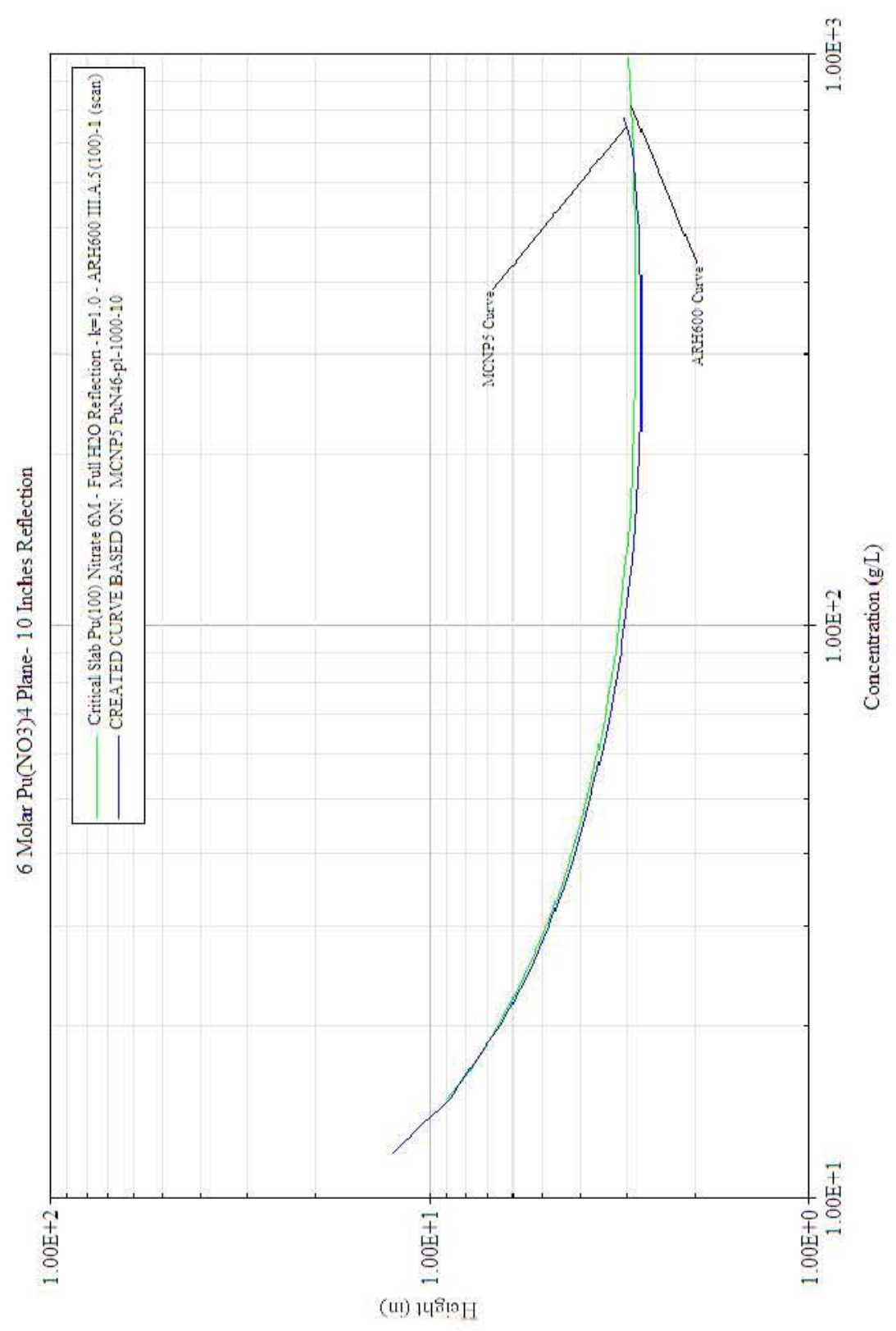


Table B1.1.3.1.3.3-1. Critical Dimensions for $\mathrm{Pu}\left(\mathrm{NO}_{3}\right) 46 \mathrm{M}-\mathrm{Plane}-100 \%{ }^{239} \mathrm{Pu}-10 "$ Water Reflection

\begin{tabular}{|c|c|c|c|c|c|}
\hline \multicolumn{6}{|c|}{$100 \% \mathrm{Pu} 239 \mathrm{Pu}(\mathrm{NO} 3) 46 \mathrm{M}$ Plane - 10 Inches of Reflection } \\
\hline Concentration $(\mathrm{g} / \mathrm{cc})$ & $\begin{array}{l}\text { Density } \\
(\mathrm{g} / \mathrm{cc})\end{array}$ & $\begin{array}{l}\text { First Diameter } \\
\text { (Inches) }\end{array}$ & \begin{tabular}{|l|}
$\begin{array}{l}\text { Last Diameter } \\
\text { (Inches) }\end{array}$ \\
\end{tabular} & $\begin{array}{l}\text { Delta } \\
\text { (Inches) }\end{array}$ & \begin{tabular}{|l} 
Critical Height \\
(Inches)
\end{tabular} \\
\hline 0.012 & 1.207 & 9.3 & 13.2 & 0.2 & 12.4481 \\
\hline 0.015 & 1.212 & 6.9 & 9.3 & 0.1 & 8.7942 \\
\hline 0.02 & 1.22 & 5.3 & 6.8 & 0.05 & 6.4719 \\
\hline 0.025 & 1.229 & 4.5 & 5.7 & 0.05 & 5.4305 \\
\hline 0.03 & 1.237 & 4 & 5 & 0.05 & 4.8115 \\
\hline 0.032 & 1.24 & 3.8 & 4.9 & 0.05 & 4.6369 \\
\hline 0.035 & 1.245 & 3.7 & 4.6 & 0.05 & 4.4169 \\
\hline 0.04 & 1.253 & 3.4 & 4.4 & 0.05 & 4.137 \\
\hline 0.05 & 1.27 & 3.1 & 3.9 & 0.05 & 3.7432 \\
\hline 0.06 & 1.286 & 2.9 & 3.7 & 0.05 & 3.4913 \\
\hline 0.07 & 1.301 & 2.7 & 3.5 & 0.05 & 3.3392 \\
\hline 0.08 & 1.317 & 2.6 & 3.4 & 0.05 & 3.207 \\
\hline 0.09 & 1.333 & 2.6 & 3.3 & 0.05 & 3.1166 \\
\hline 0.1 & 1.349 & 2.5 & 3.2 & 0.05 & 3.0521 \\
\hline 0.12 & 1.379 & 2.4 & 3.1 & 0.05 & 2.9542 \\
\hline 0.13 & 1.395 & 2.4 & 3.1 & 0.05 & 2.9117 \\
\hline 0.15 & 1.426 & 2.3 & 3 & 0.05 & 2.8543 \\
\hline 0.2 & 1.503 & 2.2 & 2.9 & 0.05 & 2.7775 \\
\hline 0.239 & 1.563 & 2.2 & 2.9 & 0.05 & 2.7538 \\
\hline 0.25 & 1.58 & 2.2 & 2.9 & 0.05 & 2.7463 \\
\hline 0.359 & 1.748 & 2.2 & 2.9 & 0.05 & 2.7526 \\
\hline 0.478 & 1.935 & 2.2 & 3 & 0.05 & 2.7759 \\
\hline 0.5 & 1.969 & 2.2 & 3 & 0.05 & 2.775 \\
\hline 0.6 & 2.122 & 2.2 & 3 & 0.05 & 2.8474 \\
\hline 0.65 & 2.197 & 2.3 & 3.1 & 0.05 & 2.8765 \\
\hline 0.7 & 2.268 & 2.3 & 3.1 & 0.05 & 2.9402 \\
\hline 0.75 & 2.335 & 2.4 & 3.2 & 0.05 & \begin{tabular}{|l|}
3.0123 \\
\end{tabular} \\
\hline 0.76 & 2.348 & 2.4 & 3.2 & 0.05 & 3.031 \\
\hline 0.765 & 2.354 & 2.4 & 3.2 & 0.05 & 3.0489 \\
\hline 0.77 & 2.36 & 2.4 & 3.3 & 0.05 & 3.0632 \\
\hline 0.775 & 2.367 & 2.4 & 3.3 & 0.05 & 3.0546 \\
\hline
\end{tabular}


CHPRC-01552, Revision 0

B-148 
B1.1.3.2 $97 \%{ }^{239} \mathrm{Pu}$

B1.1.3.2.1 0 Molar Excess Acid

B1.1.3.2.1.1 0" Water Reflection

Case ID: PuN40-pl-0970-00

Material: $\mathrm{Pu}\left(\mathrm{NO}_{3}\right)_{4} 0$ Molar Excess Acid

Geometry: Plane

Isotopics: $97 \%{ }^{239} \mathrm{Pu}$

Reflector: 0" water reflected

Code: MCNP5 v1.40

Analyst: Mark Murphy

Date: $8 / 2011$ 
CHPRC-01552, Revision 0

Figure B1.1.3.2.1.1-1. $\mathrm{Pu}\left(\mathrm{NO}_{3}\right) 4$ 0M - Plane - 97\% ${ }^{239} \mathrm{Pu}-0$ " Water Reflection

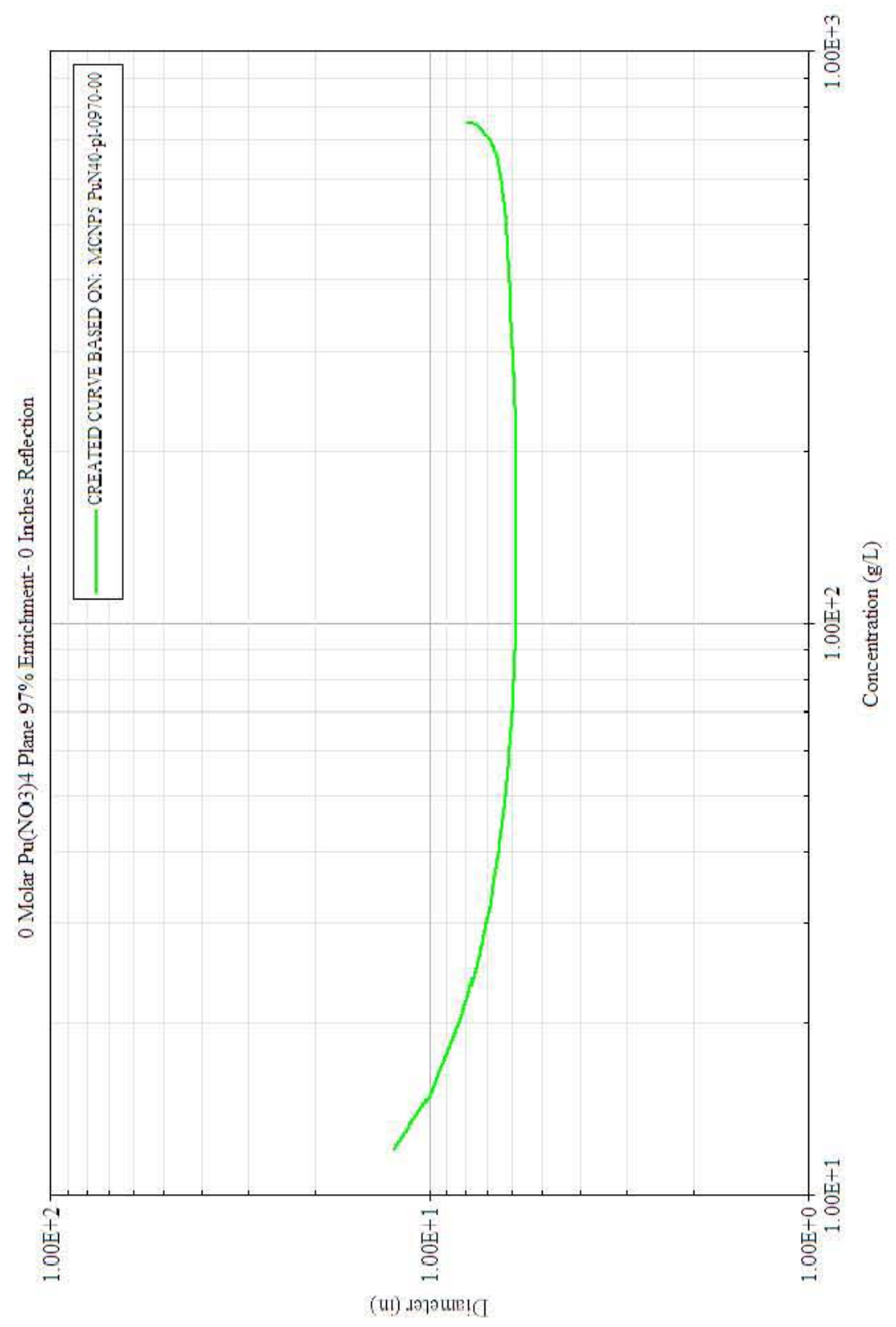


Table B1.1.3.2.1.1-1. Critical Dimensions for $\mathrm{Pu}\left(\mathrm{NO}_{3}\right) 40 \mathrm{M}-\mathrm{Plane}-97 \%{ }^{239} \mathrm{Pu}-0$ " Water Reflection

\begin{tabular}{|c|c|c|c|c|c|}
\hline \multicolumn{6}{|c|}{ 97\% Pu239 Pu(NO3)4 0M Plane- 0 Inches Reflection } \\
\hline Concentration $(\mathrm{g} / \mathrm{cc})$ & $\begin{array}{l}\text { Density } \\
(\mathrm{g} / \mathrm{cc})\end{array}$ & $\begin{array}{l}\text { First Diameter } \\
\text { (Inches) }\end{array}$ & \begin{tabular}{|l|}
$\begin{array}{l}\text { Last Diameter } \\
\text { (Inches) }\end{array}$ \\
\end{tabular} & \begin{tabular}{|l|}
$\begin{array}{l}\text { Delta } \\
\text { (Inches) }\end{array}$ \\
\end{tabular} & 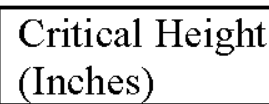 \\
\hline 0.012 & 1.016 & 10.1 & 13 & 0.1 & 12.4087 \\
\hline 0.015 & 1.021 & 8.5 & 10.4 & 0.1 & 9.9922 \\
\hline 0.02 & 1.029 & 7.2 & 8.6 & 0.05 & 8.3229 \\
\hline 0.025 & 1.037 & 6.6 & 7.8 & 0.05 & 7.5272 \\
\hline 0.03 & 1.046 & 6.3 & 7.3 & 0.05 & 7.0496 \\
\hline 0.032 & 1.049 & 6.1 & 7.1 & 0.05 & 6.912 \\
\hline 0.035 & 1.054 & 6 & 7 & 0.05 & 6.7538 \\
\hline 0.04 & 1.063 & 5.8 & 6.8 & 0.05 & 6.5328 \\
\hline 0.05 & 1.08 & 5.6 & 6.5 & 0.05 & 6.2643 \\
\hline 0.06 & 1.097 & 5.5 & 6.3 & 0.05 & 6.1007 \\
\hline 0.07 & 1.113 & 5.4 & 6.2 & 0.05 & 6.0072 \\
\hline 0.08 & 1.13 & 5.3 & 6.1 & 0.05 & 5.9341 \\
\hline 0.09 & 1.147 & 5.3 & 6.1 & 0.05 & 5.8966 \\
\hline 0.1 & 1.164 & 5.2 & 6.1 & 0.05 & 5.8638 \\
\hline 0.12 & 1.197 & 5.2 & 6 & 0.05 & 5.8448 \\
\hline 0.13 & 1.215 & 5.2 & 6 & 0.05 & 5.8341 \\
\hline 0.15 & 1.248 & 5.2 & 6 & 0.05 & 5.8397 \\
\hline 0.2 & 1.332 & 5.2 & 6.1 & 0.05 & 5.8749 \\
\hline 0.239 & 1.397 & 5.3 & 6.1 & 0.05 & 5.9108 \\
\hline 0.25 & 1.415 & 5.3 & 6.1 & 0.05 & 5.926 \\
\hline 0.359 & 1.594 & 5.4 & 6.3 & 0.05 & 6.0512 \\
\hline 0.478 & 1.784 & 5.5 & 6.4 & 0.05 & 6.1978 \\
\hline 0.5 & 1.818 & 5.5 & 6.4 & 0.05 & 6.2307 \\
\hline 0.6 & 1.962 & 5.7 & 6.7 & 0.05 & 6.4404 \\
\hline 0.65 & 2.026 & 5.9 & 6.8 & 0.05 & 6.5999 \\
\hline 0.7 & 2.076 & 6.1 & 7.1 & 0.05 & 6.8758 \\
\hline 0.75 & 2.08 & 6.7 & 7.8 & 0.05 & 7.5745 \\
\hline 0.752 & 2.074 & 6.8 & 7.9 & 0.05 & 7.6569 \\
\hline 0.754 & 2.064 & 6.9 & 8 & 0.05 & 7.7924 \\
\hline 0.755 & 2.054 & 7.1 & 8.2 & 0.05 & 7.9114 \\
\hline
\end{tabular}




\section{B1.1.3.2.1.2 1"Water Reflection}

Case ID: PuN40-pl-0970-01

Material: $\mathrm{Pu}\left(\mathrm{NO}_{3}\right)_{4} \quad 0$ Molar Excess Acid

Geometry: Plane

Isotopics: $97 \%{ }^{239} \mathrm{Pu}$

Reflector: 1" water reflected

Code: MCNP5 v1.40

Analyst: Mark Murphy

Date: $8 / 2011$ 
CHPRC-01552, Revision 0

Figure B1.1.3.2.1.2-1. $\mathrm{Pu}\left(\mathrm{NO}_{3}\right) 4$ 0M-Plane - 97\% ${ }^{239} \mathrm{Pu}-1$ " Water Reflection

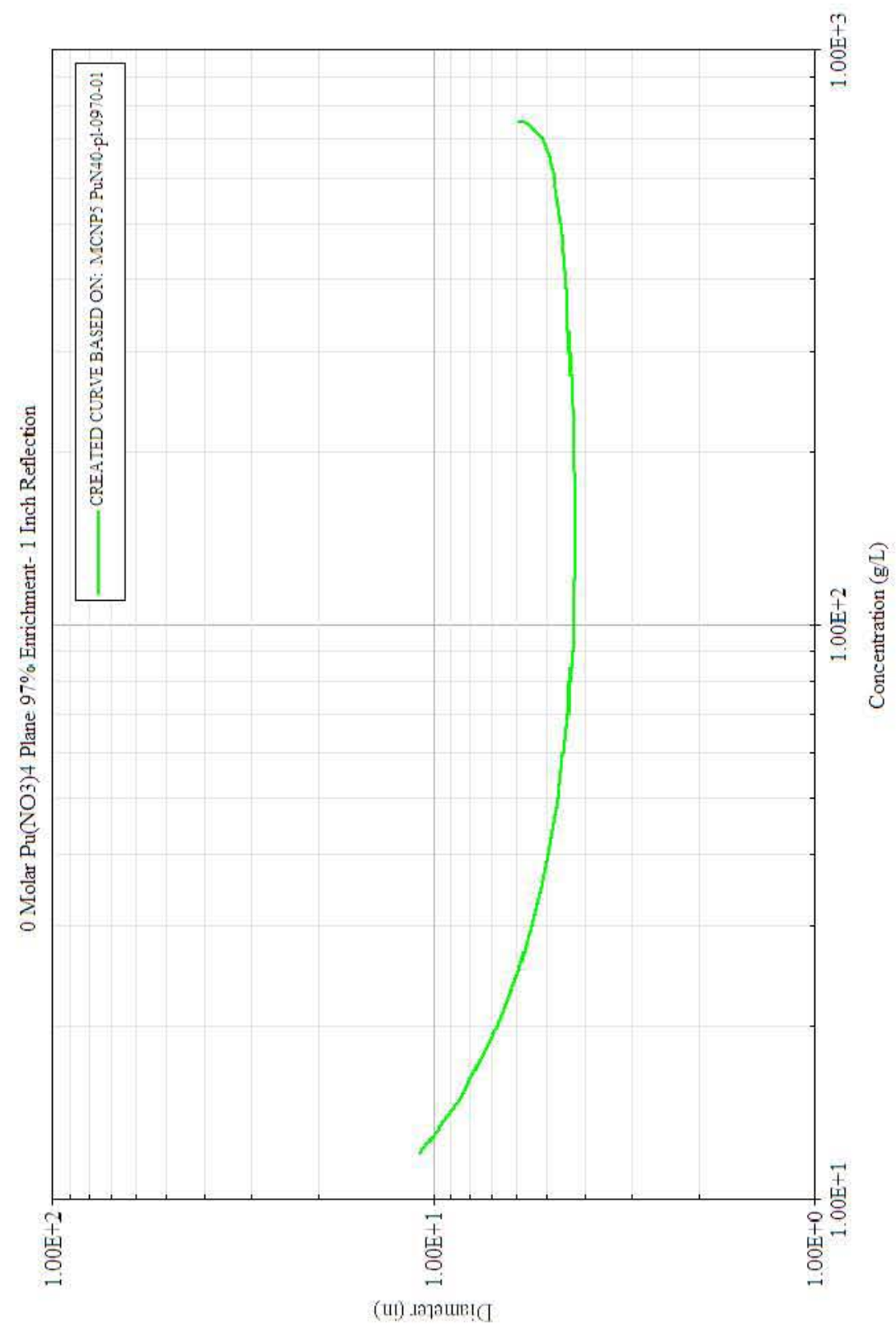


Table B1.1.3.2.1.2-1. Critical Dimensions for $\mathrm{Pu}\left(\mathrm{NO}_{3}\right) 40 \mathrm{M}-\mathrm{Plane}-97 \%{ }^{239} \mathrm{Pu}-1$ " Water Reflection

\begin{tabular}{|c|c|c|c|c|c|}
\hline \multicolumn{6}{|c|}{ 97\% Pu239 Pu(NO3)4 0M Plane- 1 Inch Reflection } \\
\hline Concentration $(\mathrm{g} / \mathrm{cc})$ & $\begin{array}{l}\text { Density } \\
(\mathrm{g} / \mathrm{cc})\end{array}$ & $\begin{array}{l}\text { First Diameter } \\
\text { (Inches) }\end{array}$ & $\begin{array}{l}\text { Last Diameter } \\
\text { (Inches) }\end{array}$ & $\begin{array}{l}\text { Delta } \\
\text { (Inches) }\end{array}$ & $\begin{array}{l}\text { Critical Height } \\
\text { (Inches) }\end{array}$ \\
\hline 0.012 & 1.016 & 8.6 & 11.5 & 0.1 & 10.8698 \\
\hline 0.015 & 1.021 & 7 & 8.8 & 0.1 & 8.4684 \\
\hline 0.02 & 1.029 & 5.7 & 7.1 & 0.05 & 6.7909 \\
\hline 0.025 & 1.037 & 5.1 & 6.2 & 0.05 & 5.983 \\
\hline 0.03 & 1.046 & 4.7 & 5.7 & 0.05 & 5.5103 \\
\hline 0.032 & 1.049 & 4.6 & 5.6 & 0.05 & 5.3734 \\
\hline 0.035 & 1.054 & 4.5 & 5.4 & 0.05 & 5.2048 \\
\hline 0.04 & 1.063 & 4.3 & 5.2 & 0.05 & 4.984 \\
\hline 0.05 & 1.08 & 4.1 & 4.9 & 0.05 & 4.7055 \\
\hline 0.06 & 1.097 & 3.9 & 4.7 & 0.05 & 4.5328 \\
\hline 0.07 & 1.113 & 3.8 & 4.6 & 0.05 & 4.4342 \\
\hline 0.08 & 1.13 & 3.8 & 4.5 & 0.05 & 4.362 \\
\hline 0.09 & 1.147 & 3.7 & 4.5 & 0.05 & 4.316 \\
\hline 0.1 & 1.164 & 3.7 & 4.5 & 0.05 & 4.2815 \\
\hline 0.12 & 1.197 & 3.7 & 4.4 & 0.05 & 4.2511 \\
\hline 0.13 & 1.215 & 3.6 & 4.4 & 0.05 & 4.2405 \\
\hline 0.15 & 1.248 & 3.6 & 4.4 & 0.05 & 4.2371 \\
\hline 0.2 & 1.332 & 3.6 & 4.4 & 0.05 & 4.2679 \\
\hline 0.239 & 1.397 & 3.7 & 4.5 & 0.05 & 4.2988 \\
\hline 0.25 & 1.415 & 3.7 & 4.5 & 0.05 & 4.3141 \\
\hline 0.359 & 1.594 & 3.8 & 4.6 & 0.05 & 4.4292 \\
\hline 0.478 & 1.784 & 3.9 & 4.8 & 0.05 & 4.5766 \\
\hline 0.5 & 1.818 & 3.9 & 4.8 & 0.05 & 4.6054 \\
\hline 0.6 & 1.962 & 4.1 & 5 & 0.2 & 4.7818 \\
\hline 0.65 & 2.026 & 4.2 & 5.1 & 0.05 & 4.9203 \\
\hline 0.7 & 2.076 & 4.4 & 5.4 & 0.05 & 5.1436 \\
\hline 0.75 & 2.08 & 4.9 & 5.9 & 0.05 & 5.6932 \\
\hline 0.752 & 2.074 & 4.9 & 6 & 0.05 & 5.7637 \\
\hline 0.754 & 2.064 & 5 & 6.1 & 0.05 & 5.8631 \\
\hline 0.755 & 2.054 & 5.1 & 6.2 & 0.05 & 5.955 \\
\hline
\end{tabular}




\section{B1.1.3.2.1.3 10" Water Reflection}

Case ID: PuN40-pl-0970-10

Material: $\mathrm{Pu}\left(\mathrm{NO}_{3}\right)_{4} \quad 0$ Molar Excess Acid

Geometry: Plane

Isotopics: $97 \%{ }^{239} \mathrm{Pu}$

Reflector: 10 " water reflected

Code: MCNP5 v1.40

Analyst: Mark Murphy

Date: $8 / 2011$ 


\section{CHPRC-01552, Revision 0}

Figure B1.1.3.2.1.3-1. Pu(NO $\left.\mathrm{NO}_{3}\right) 40 \mathrm{M}$ - Plane - 97\% ${ }^{239} \mathrm{Pu}$ - 10" Water Reflection

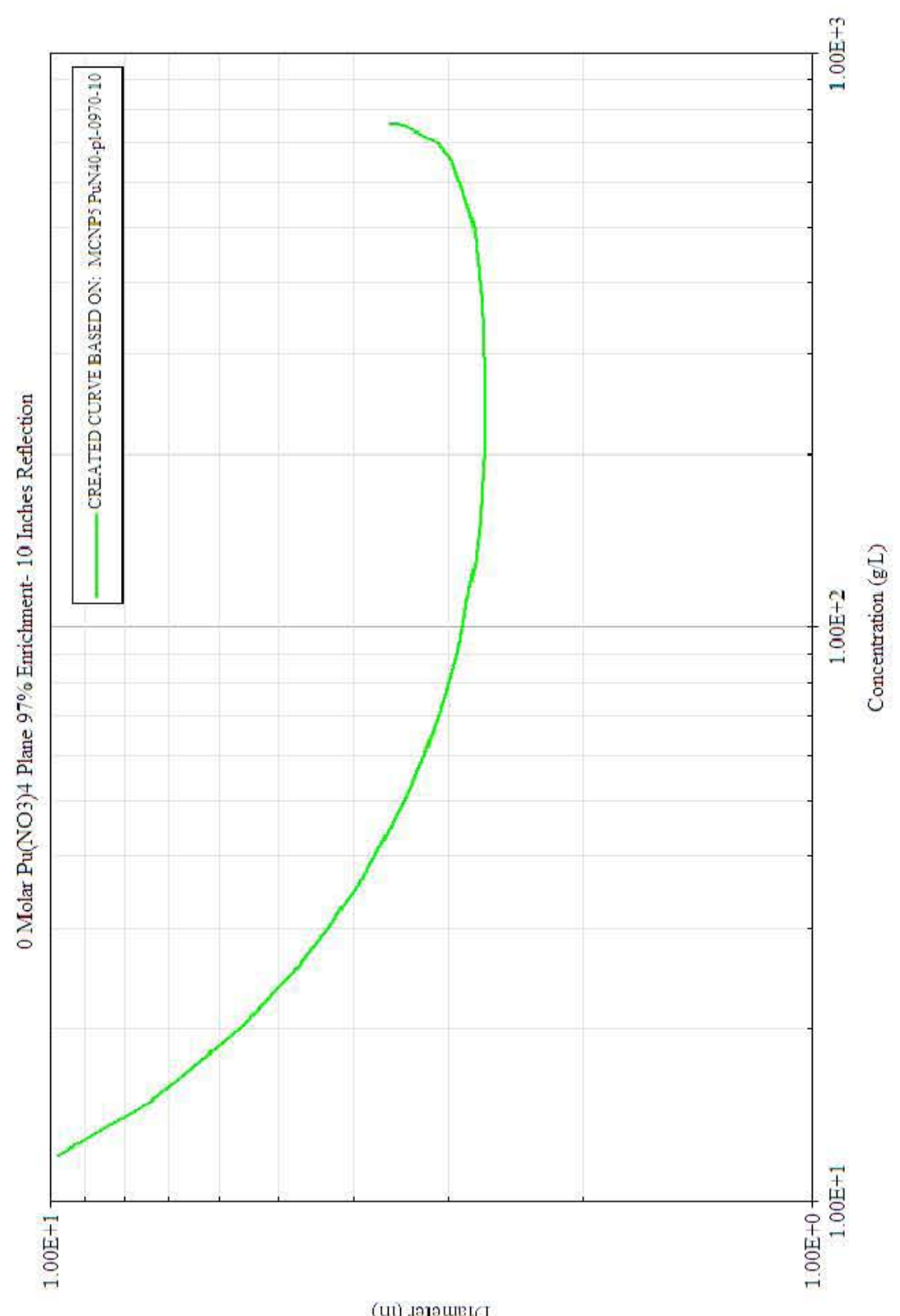

(ui) .әองแะเ(। 
Table B1.1.3.2.1.3-1. Critical Dimensions for $\mathrm{Pu}\left(\mathrm{NO}_{3}\right) 40 \mathrm{M}-\mathrm{Plane}-97 \%{ }^{239} \mathrm{Pu}-10$ " Water Reflection

\begin{tabular}{|c|c|c|c|c|c|}
\hline \multicolumn{6}{|c|}{ 97\% Pu239 Pu(NO3)4 0M Plane- 10 Inches Reflection } \\
\hline Concentration $(\mathrm{g} / \mathrm{cc})$ & $\begin{array}{l}\text { Density } \\
(\mathrm{g} / \mathrm{cc})\end{array}$ & $\begin{array}{l}\text { First Diameter } \\
\text { (Inches) }\end{array}$ & $\begin{array}{l}\text { Last Diameter } \\
\text { (Inches) }\end{array}$ & $\begin{array}{l}\text { Delta } \\
\text { (Inches) }\end{array}$ & $\begin{array}{l}\text { Critical Height } \\
\text { (Inches) }\end{array}$ \\
\hline 0.012 & 1.016 & 7.5 & 10.3 & 0.1 & 9.7609 \\
\hline 0.015 & 1.021 & 5.9 & 7.7 & 0.1 & 7.334 \\
\hline 0.02 & 1.029 & 4.6 & 5.9 & 0.05 & 5.6323 \\
\hline 0.025 & 1.037 & 4 & 5 & 0.05 & 4.8 \\
\hline 0.03 & 1.046 & 3.6 & 4.5 & 0.05 & 4.3142 \\
\hline 0.032 & 1.049 & 3.4 & 4.4 & 0.05 & 4.1642 \\
\hline 0.035 & 1.054 & 3.3 & 4.2 & 0.05 & 3.9806 \\
\hline 0.04 & 1.063 & 3.1 & 3.9 & 0.05 & 3.7381 \\
\hline 0.05 & 1.08 & 2.8 & 3.6 & 0.05 & 3.4267 \\
\hline 0.06 & 1.097 & 2.6 & 3.4 & 0.05 & 3.2238 \\
\hline 0.07 & 1.113 & 2.5 & 3.3 & 0.05 & 3.0936 \\
\hline 0.08 & 1.13 & 2.4 & 3.2 & 0.05 & 2.9937 \\
\hline 0.09 & 1.147 & 2.4 & 3.1 & 0.05 & 2.9223 \\
\hline 0.1 & 1.164 & 2.3 & 3 & 0.05 & 2.8658 \\
\hline 0.12 & 1.197 & 2.2 & 3 & 0.05 & 2.7946 \\
\hline 0.13 & 1.215 & 2.2 & 2.9 & 0.05 & 2.7612 \\
\hline 0.15 & 1.248 & 2.2 & 2.9 & 0.05 & 2.7259 \\
\hline 0.2 & 1.332 & 2.1 & 2.8 & 0.05 & 2.6808 \\
\hline 0.239 & 1.397 & 2.1 & 2.8 & 0.05 & 2.6812 \\
\hline 0.25 & 1.415 & 2.1 & 2.8 & 0.05 & 2.6784 \\
\hline 0.359 & 1.594 & 2.1 & 2.9 & 0.05 & 2.709 \\
\hline 0.478 & 1.784 & 2.1 & 2.9 & 0.05 & 2.7661 \\
\hline 0.5 & 1.818 & 2.1 & 3 & 0.05 & 2.7809 \\
\hline 0.6 & 1.962 & 2.2 & 3.1 & 0.05 & 2.8955 \\
\hline 0.65 & 2.026 & 2.3 & 3.2 & 0.05 & 2.9664 \\
\hline 0.7 & 2.076 & 2.4 & 3.3 & 0.05 & 3.0989 \\
\hline 0.75 & 2.08 & 2.6 & 3.6 & 0.05 & 3.4331 \\
\hline 0.752 & 2.074 & 2.7 & 3.7 & 0.05 & 3.4648 \\
\hline 0.754 & 2.064 & 2.7 & 3.7 & 0.05 & 3.5241 \\
\hline 0.755 & 2.054 & 2.7 & 3.8 & 0.05 & 3.5887 \\
\hline
\end{tabular}




\section{$\mathrm{B} 1.2 \quad \mathrm{UO}_{2}\left(\mathrm{NO}_{3}\right)_{2}$}

\section{B1.2.1 Sphere}

B1.2.1.1 $\quad 100 \%{ }^{235} \mathrm{U}$

B1.2.1.1.1 0 Molar Excess Acid

B1.2.1.1.1.1 0"Water Reflection

Case ID: u5o2N20-sp-1000-00

Material: $\mathrm{UO}_{2}\left(\mathrm{NO}_{3}\right)_{2} \quad 0$ Molar Excess Acid

Geometry: Sphere

Isotopics: $100 \%{ }^{235} \mathrm{U}$

Reflector: 0" water reflected

Code: MCNP5 v1.40

Analyst: Mark Murphy

Date: $8 / 2011$ 
Figure B1.2.1.1.1.1-1. $\mathrm{UO}_{2}\left(\mathrm{NO}_{3}\right) 2 \mathrm{0M}-$ Sphere - 100\% ${ }^{235} \mathrm{U}-0$ " Water Reflection

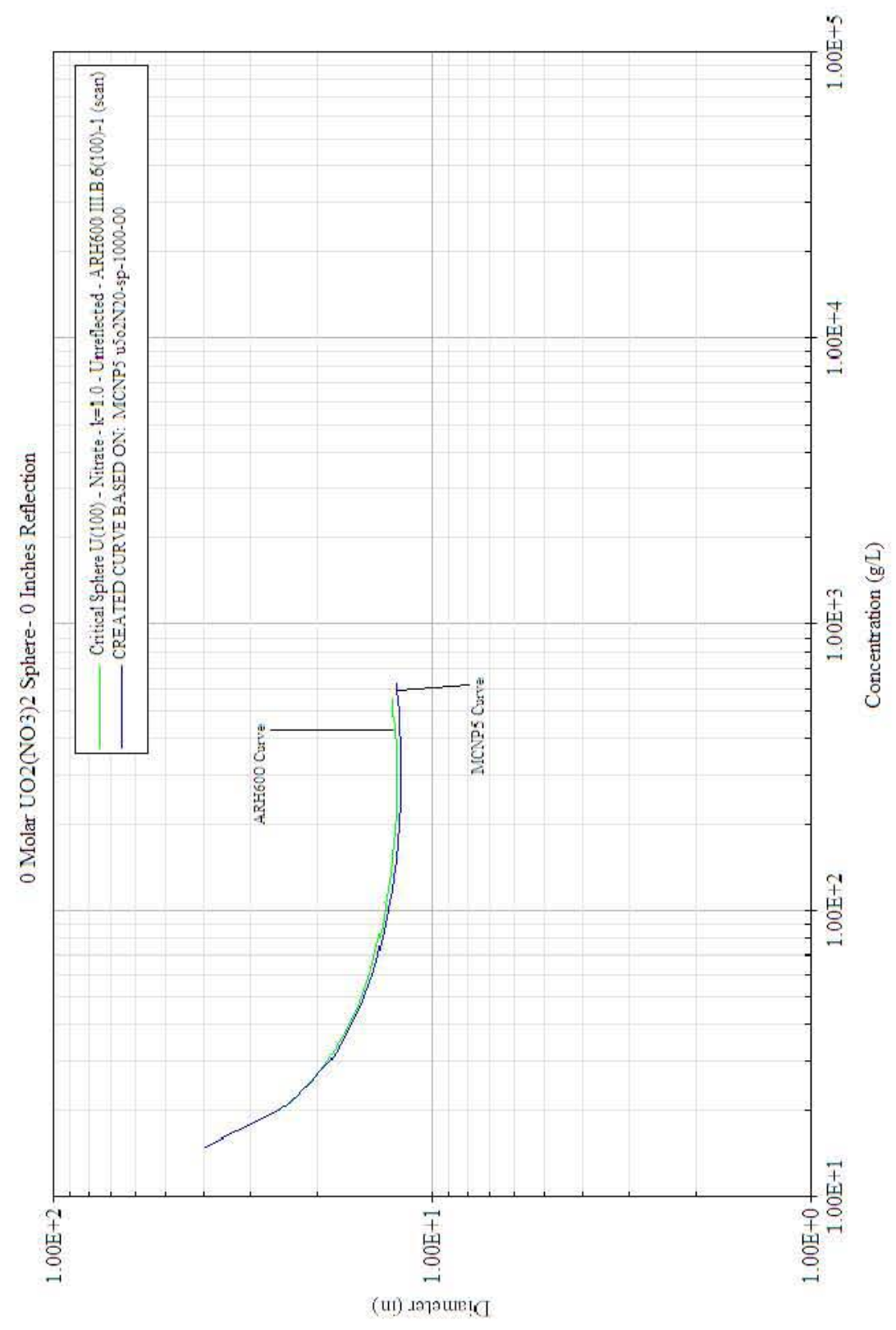


Table B1.2.1.1.1.1- Critical Dimensions for $\mathrm{UO}_{2}\left(\mathrm{NO}_{3}\right) 20 \mathrm{M}-$ Sphere $-100 \%{ }^{235} \mathrm{U}-0$ " Water Reflection

\begin{tabular}{|c|c|c|c|c|c|}
\hline \multicolumn{6}{|c|}{$100 \%$ U235 UO2(NO3)2 0M Sphere - 0 Inches of Reflection } \\
\hline Concentration $(\mathrm{g} / \mathrm{cc})$ & \begin{tabular}{|l} 
Density \\
$(\mathrm{g} / \mathrm{cc})$ \\
\end{tabular} & $\begin{array}{l}\text { First Diameter } \\
\text { (Inches) }\end{array}$ & $\begin{array}{l}\text { Last Diameter } \\
\text { (Inches) }\end{array}$ & $\begin{array}{l}\text { Delta } \\
\text { (Inches) }\end{array}$ & \begin{tabular}{|l} 
Critical Diameter \\
(Inches)
\end{tabular} \\
\hline 0.012 & 1.013 & 24.2 & 57.4 & 1.6 & -- \\
\hline 0.015 & 1.017 & 29.1 & 41.3 & 0.6 & 39.5184 \\
\hline 0.02 & 1.024 & 21 & 26 & 0.2 & 25.0712 \\
\hline 0.025 & 1.031 & 18 & 21.4 & 0.1 & 20.7082 \\
\hline 0.03 & 1.037 & 16.3 & 19.1 & 0.1 & 18.4868 \\
\hline 0.032 & 1.04 & 15.8 & 18.4 & 0.1 & 17.8918 \\
\hline 0.035 & 1.044 & 15.3 & 17.6 & 0.1 & 17.1361 \\
\hline 0.04 & 1.051 & 14.5 & 16.7 & 0.1 & 16.2045 \\
\hline 0.05 & 1.064 & 13.6 & 15.4 & 0.1 & 15.0402 \\
\hline 0.06 & 1.078 & 12.9 & 14.7 & 0.1 & 14.305 \\
\hline 0.07 & 1.091 & 12.5 & 14.2 & 0.05 & 13.8117 \\
\hline 0.08 & 1.105 & 12.2 & 13.8 & 0.05 & 13.4502 \\
\hline 0.09 & 1.118 & 12 & 13.5 & 0.05 & 13.184 \\
\hline 0.1 & 1.131 & 11.8 & 13.3 & 0.05 & 12.9674 \\
\hline 0.118 & 1.155 & 11.6 & 13 & 0.05 & 12.7056 \\
\hline 0.13 & 1.172 & 11.5 & 12.9 & 0.05 & 12.5406 \\
\hline 0.15 & 1.199 & 11.3 & 12.7 & 0.05 & 12.3789 \\
\hline 0.2 & 1.266 & 11.1 & 12.5 & 0.05 & 12.1412 \\
\hline 0.235 & 1.313 & 11 & 12.4 & 0.05 & 12.0469 \\
\hline 0.25 & 1.333 & 11 & 12.3 & 0.05 & 12.0288 \\
\hline 0.353 & 1.471 & 11 & 12.3 & 0.05 & 12.0088 \\
\hline 0.47 & 1.63 & 11 & 12.4 & 0.05 & 12.0901 \\
\hline 0.5 & 1.671 & 11.1 & 12.4 & 0.05 & 12.1107 \\
\hline 0.6 & 1.805 & 11.2 & 12.6 & 0.05 & 12.286 \\
\hline 0.605 & 1.812 & 11.2 & 12.6 & 0.05 & 12.3024 \\
\hline 0.61 & 1.819 & 11.2 & 12.6 & 0.05 & 12.302 \\
\hline 0.615 & 1.826 & 11.2 & 12.6 & 0.05 & 12.2906 \\
\hline 0.62 & 1.832 & 11.2 & 12.6 & 0.05 & 12.3133 \\
\hline 0.625 & 1.839 & 11.2 & 12.6 & 0.05 & 12.3286 \\
\hline 0.629 & 1.844 & 11.3 & 12.6 & 0.05 & 12.3273 \\
\hline
\end{tabular}




\section{B1.2.1.1.1.2 1"Water Reflection}

Case ID: u5o2N20-sp-1000-01

Material: $\mathrm{UO}_{2}\left(\mathrm{NO}_{3}\right)_{2} 0$ Molar Excess Acid

Geometry: Sphere

Isotopics: $100 \%{ }^{235} \mathrm{U}$

Reflector: 1" water reflected

Code: MCNP5 v1.40

Analyst: Mark Murphy

Date: $8 / 2011$ 
Figure B1.2.1.1.1.2-1. $\mathrm{UO}_{2}\left(\mathrm{NO}_{3}\right) 2 \mathrm{OM}-$ Sphere - 100\% ${ }^{235} \mathrm{U}-1$ " Water Reflection

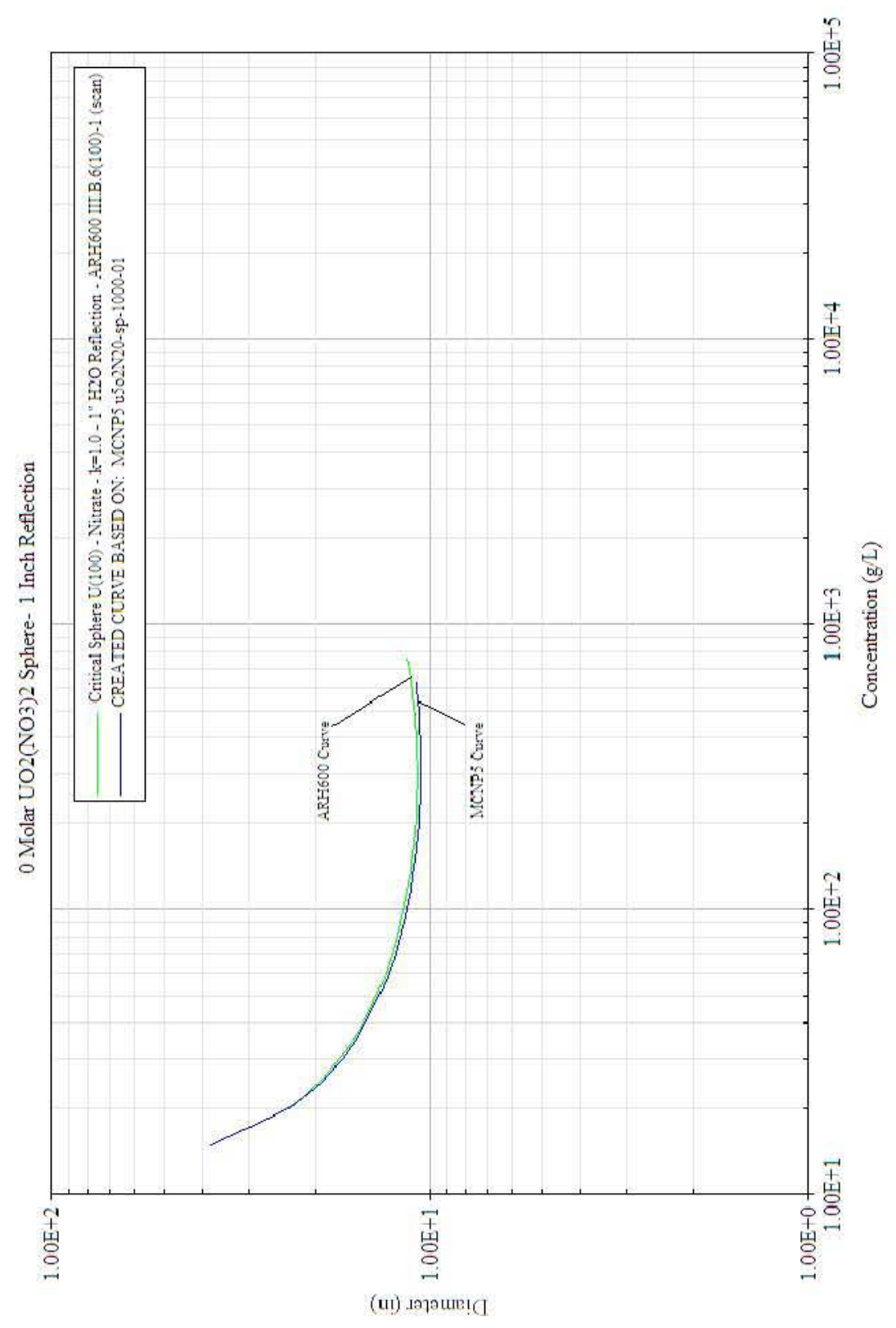


Table B1.2.1.1.1.2-1. Critical Dimensions for $\mathrm{UO}_{2}\left(\mathrm{NO}_{3}\right) 20 \mathrm{M}-$ Sphere $-100 \%{ }^{235} \mathrm{U}-1$ " Water Reflection

\begin{tabular}{|c|c|c|c|c|c|}
\hline \multicolumn{6}{|c|}{$100 \%$ U235 UO2(NO3)2 0M Sphere - 1 Inch of Reflection } \\
\hline Concentration $(\mathrm{g} / \mathrm{cc})$ & $\begin{array}{l}\text { Density } \\
(\mathrm{g} / \mathrm{cc})\end{array}$ & $\begin{array}{l}\text { First Diameter } \\
\text { (Inches) }\end{array}$ & $\begin{array}{l}\text { Last Diameter } \\
\text { (Inches) }\end{array}$ & $\begin{array}{l}\text { Delta } \\
\text { (Inches) }\end{array}$ & $\begin{array}{l}\text { Critical Diameter } \\
\text { (Inches) }\end{array}$ \\
\hline 0.012 & 1.013 & 38.8 & 57 & 0.9 & -- \\
\hline 0.015 & 1.017 & 27.5 & 39.4 & 0.6 & 38.0011 \\
\hline 0.02 & 1.024 & 19.5 & 24.6 & 0.2 & 23.5307 \\
\hline 0.025 & 1.031 & 16.4 & 19.8 & 0.1 & 19.1594 \\
\hline 0.03 & 1.037 & 14.8 & 17.5 & 0.1 & 16.9769 \\
\hline 0.032 & 1.04 & 14.3 & 16.9 & 0.1 & 16.3652 \\
\hline 0.035 & 1.044 & 13.7 & 16.1 & 0.1 & 15.6048 \\
\hline 0.04 & 1.051 & 13 & 15.1 & 0.1 & 14.7039 \\
\hline 0.05 & 1.064 & 12 & 13.9 & 0.1 & 13.5084 \\
\hline 0.06 & 1.078 & 11.4 & 13.2 & 0.1 & 12.783 \\
\hline 0.07 & 1.091 & 11 & 12.6 & 0.05 & 12.2793 \\
\hline 0.08 & 1.105 & 10.7 & 12.3 & 0.05 & 11.9246 \\
\hline 0.09 & 1.118 & 10.5 & 12 & 0.05 & 11.6515 \\
\hline 0.1 & 1.131 & 10.3 & 11.8 & 0.05 & 11.4516 \\
\hline 0.118 & 1.155 & 10.1 & 11.5 & 0.05 & 11.1625 \\
\hline 0.13 & 1.172 & 10 & 11.3 & 0.05 & 11.0426 \\
\hline 0.15 & 1.199 & 9.8 & 11.2 & 0.05 & 10.8522 \\
\hline 0.2 & 1.266 & 9.6 & 10.9 & 0.05 & 10.6136 \\
\hline 0.235 & 1.313 & 9.5 & 10.8 & 0.05 & 10.5214 \\
\hline 0.25 & 1.333 & 9.5 & 10.8 & 0.05 & 10.4977 \\
\hline 0.353 & 1.471 & 9.5 & 10.8 & 0.05 & 10.4607 \\
\hline 0.47 & 1.63 & 9.5 & 10.9 & 0.05 & 10.5302 \\
\hline 0.5 & 1.671 & 9.5 & 10.9 & 0.05 & 10.576 \\
\hline 0.6 & 1.805 & 9.7 & 11 & 0.05 & 10.7287 \\
\hline 0.605 & 1.812 & 9.7 & 11 & 0.05 & 10.7226 \\
\hline 0.61 & 1.819 & 9.7 & 11.1 & 0.05 & 10.7376 \\
\hline 0.615 & 1.826 & 9.7 & 11 & 0.05 & 10.7481 \\
\hline 0.62 & 1.832 & 9.7 & 11 & 0.05 & 10.7549 \\
\hline 0.625 & 1.839 & 9.7 & 11.1 & 0.05 & 10.7562 \\
\hline 0.629 & 1.844 & 9.7 & 11.1 & 0.05 & 10.7747 \\
\hline
\end{tabular}




\section{B1.2.1.1.1.3 10" Water Reflection}

Case ID: u5o2N20-sp-1000-10

Material: $\mathrm{UO}_{2}\left(\mathrm{NO}_{3}\right)_{2} 0$ Molar Excess Acid

Geometry: Sphere

Isotopics: $100 \%{ }^{235} \mathrm{U}$

Reflector: 10 " water reflected

Code: MCNP5 v1.40

Analyst: Mark Murphy

Date: $8 / 2011$ 
CHPRC-01552, Revision 0

Figure B1.2.1.1.1.3-1. $\mathrm{UO}_{2}\left(\mathrm{NO}_{3}\right) 2 \mathrm{OM}-$ Sphere - $100 \%{ }^{235} \mathrm{U}-10$ " Water Reflection

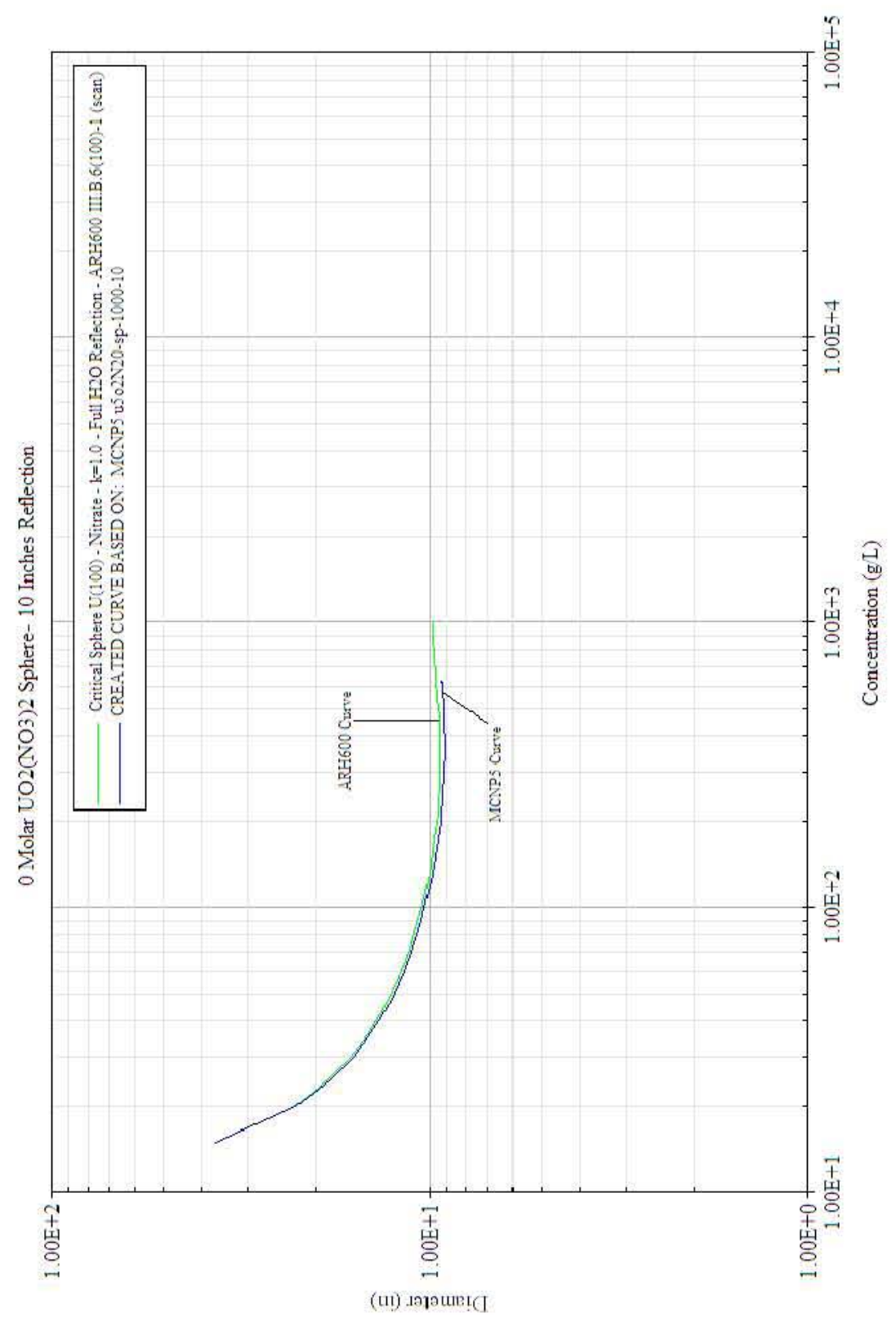


Table B1.2.1.1.1.3-1. Critical Dimensions for $\mathrm{UO}_{2}\left(\mathrm{NO}_{3}\right) 20 \mathrm{M}-$ Sphere $-100 \%{ }^{235} \mathrm{U}-10$ " Water Reflection

\begin{tabular}{|c|c|c|c|c|c|}
\hline \multicolumn{6}{|c|}{ 100\% U235 UO2(NO3)2 0M Sphere - 10 Inches of Reflection } \\
\hline Concentration $(\mathrm{g} / \mathrm{cc})$ & $\begin{array}{l}\text { Density } \\
(\mathrm{g} / \mathrm{cc})\end{array}$ & $\begin{array}{l}\text { First Diameter } \\
\text { (Inches) }\end{array}$ & $\begin{array}{l}\text { Last Diameter } \\
\text { (Inches) }\end{array}$ & $\begin{array}{l}\text { Delta } \\
\text { (Inches) }\end{array}$ & \begin{tabular}{|l} 
Critical Diameter \\
(Inches)
\end{tabular} \\
\hline 0.012 & 1.013 & 41 & 56 & 0.7 & -- \\
\hline 0.015 & 1.017 & 26.3 & 38.7 & 0.6 & 36.901 \\
\hline 0.02 & 1.024 & 18.4 & 23.4 & 0.2 & 22.4478 \\
\hline 0.025 & 1.031 & 15.4 & 18.7 & 0.1 & 18.0664 \\
\hline 0.03 & 1.037 & 13.8 & 16.4 & 0.1 & 15.8688 \\
\hline 0.032 & 1.04 & 13.3 & 15.8 & 0.1 & 15.2536 \\
\hline 0.035 & 1.044 & 12.7 & 15 & 0.1 & 14.4872 \\
\hline 0.04 & 1.051 & 11.9 & 14 & 0.1 & 13.5737 \\
\hline 0.05 & 1.064 & 11 & 12.8 & 0.1 & 12.3637 \\
\hline 0.06 & 1.078 & 10.3 & 12 & 0.05 & 11.6208 \\
\hline 0.07 & 1.091 & 9.9 & 11.5 & 0.05 & 11.1255 \\
\hline 0.08 & 1.105 & 9.6 & 11.1 & 0.05 & 10.7417 \\
\hline 0.09 & 1.118 & 9.4 & 10.8 & 0.05 & 10.465 \\
\hline 0.1 & 1.131 & 9.2 & 10.6 & 0.05 & 10.2606 \\
\hline 0.118 & 1.155 & 8.9 & 10.3 & 0.05 & 9.9594 \\
\hline 0.13 & 1.172 & 8.8 & 10.1 & 0.05 & 9.7964 \\
\hline 0.15 & 1.199 & 8.6 & 9.9 & 0.05 & 9.614 \\
\hline 0.2 & 1.266 & 8.4 & 9.6 & 0.05 & 9.3316 \\
\hline 0.235 & 1.313 & 8.3 & 9.5 & 0.05 & 9.2232 \\
\hline 0.25 & 1.333 & 8.2 & 9.5 & 0.05 & 9.1874 \\
\hline 0.353 & 1.471 & 8.1 & 9.4 & 0.05 & 9.0955 \\
\hline 0.47 & 1.63 & 8.1 & 9.4 & 0.05 & 9.103 \\
\hline 0.5 & 1.671 & 8.1 & 9.4 & 0.05 & 9.1315 \\
\hline 0.6 & 1.805 & 8.2 & 9.5 & 0.05 & 9.2326 \\
\hline 0.605 & 1.812 & 8.2 & 9.6 & 0.05 & 9.2435 \\
\hline 0.61 & 1.819 & 8.2 & 9.5 & 0.05 & 9.2452 \\
\hline 0.615 & 1.826 & 8.2 & 9.5 & 0.05 & 9.2371 \\
\hline 0.62 & 1.832 & 8.2 & 9.6 & 0.05 & 9.2588 \\
\hline 0.625 & 1.839 & 8.2 & 9.6 & 0.05 & 9.2713 \\
\hline 0.629 & 1.844 & 8.3 & 9.6 & 0.05 & 9.2643 \\
\hline
\end{tabular}


B1.2.1.1.2 3 Molar Excess Acid

B1.2.1.1.2.1 0"Water Reflection

Case ID: u5o2N23-sp-1000-00

Material: $\mathrm{UO}_{2}\left(\mathrm{NO}_{3}\right)_{2} 3$ Molar Excess Acid

Geometry: Sphere

Isotopics: $100 \%{ }^{235} \mathrm{U}$

Reflector: 0 " water reflected

Code: MCNP5 v1.40

Analyst: Mark Murphy

Date: $8 / 2011$ 
Figure B1.2.1.1.2.1-1. $\mathrm{UO}_{2}\left(\mathrm{NO}_{3}\right) 23 \mathrm{M}-\mathrm{Sphere}-100 \%{ }^{235} \mathrm{U}-0$ " Water Reflection

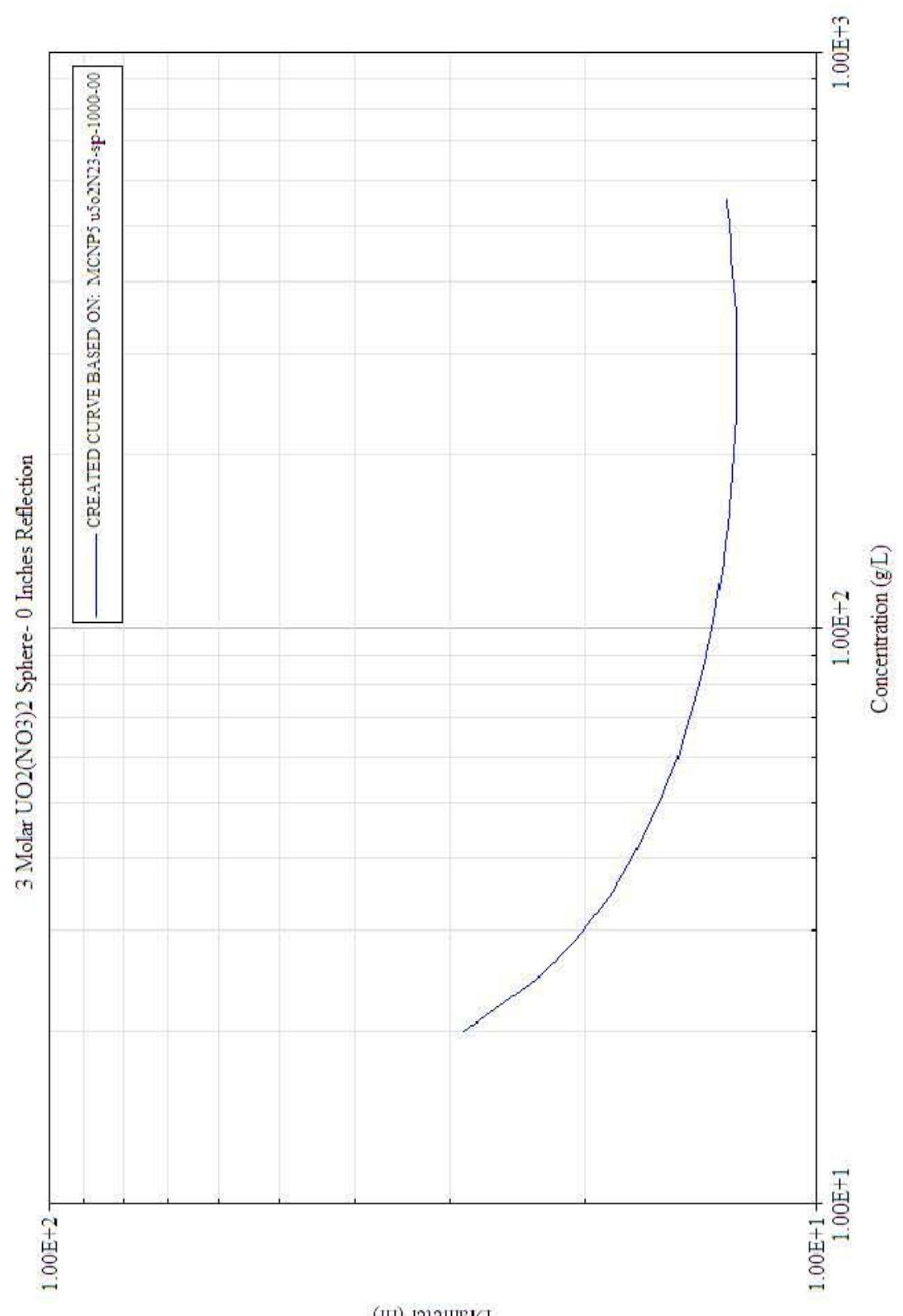

(แ1) เэฺวแย!(1 
Table B1.2.1.1.2.1-1. Critical Dimensions for $\mathrm{UO}_{2}\left(\mathrm{NO}_{3}\right) 23 \mathrm{M}-$ Sphere $-100 \%{ }^{235} \mathrm{U}-0$ " Water Reflection

\begin{tabular}{|c|c|c|c|c|c|}
\hline \multicolumn{6}{|c|}{$100 \%$ U235 UO2(NO3)2 3M Sphere - 0 Inches of Reflection } \\
\hline Concentration $(\mathrm{g} / \mathrm{cc})$ & $\begin{array}{l}\text { Density } \\
(\mathrm{g} / \mathrm{cc})\end{array}$ & $\begin{array}{l}\text { First Diameter } \\
\text { (Inches) }\end{array}$ & $\begin{array}{l}\text { Last Diameter } \\
\text { (Inches) }\end{array}$ & $\begin{array}{l}\text { Delta } \\
\text { (Inches) }\end{array}$ & \begin{tabular}{|l} 
Critical Diameter \\
(Inches)
\end{tabular} \\
\hline 0.012 & 1.112 & 47.4 & 66.5 & 0.9 & -- \\
\hline 0.015 & 1.116 & 35.8 & 52.2 & 0.8 & -- \\
\hline 0.02 & 1.123 & 23.5 & 30 & 0.3 & 28.6621 \\
\hline 0.025 & 1.129 & 19.6 & 23.7 & 0.2 & 22.807 \\
\hline 0.03 & 1.136 & 17.6 & 20.7 & 0.1 & 20.0636 \\
\hline 0.032 & 1.138 & 17 & 19.9 & 0.1 & 19.3174 \\
\hline 0.035 & 1.142 & 16.3 & 19 & 0.1 & 18.4352 \\
\hline 0.04 & 1.149 & 15.5 & 17.9 & 0.1 & 17.3443 \\
\hline 0.05 & 1.162 & 14.4 & 16.4 & 0.1 & 15.9781 \\
\hline 0.06 & 1.175 & 13.7 & 15.5 & 0.1 & 15.1311 \\
\hline 0.07 & 1.188 & 13.2 & 15 & 0.1 & 14.5787 \\
\hline 0.08 & 1.201 & 12.9 & \begin{tabular}{|l|}
14.6 \\
\end{tabular} & 0.05 & 14.1901 \\
\hline 0.09 & 1.215 & 12.6 & 14.2 & 0.05 & 13.8767 \\
\hline 0.1 & 1.228 & 12.5 & 14 & 0.05 & 13.652 \\
\hline 0.118 & 1.251 & 12.2 & 13.7 & 0.05 & 13.3621 \\
\hline 0.13 & 1.267 & 12 & 13.5 & 0.05 & 13.2055 \\
\hline 0.15 & 1.293 & 11.9 & 13.4 & 0.05 & 13.026 \\
\hline 0.2 & 1.359 & 11.7 & 13.1 & 0.05 & 12.7772 \\
\hline 0.235 & 1.405 & 11.6 & 13.1 & 0.05 & 12.6967 \\
\hline 0.25 & 1.425 & 11.6 & 13 & 0.05 & 12.6758 \\
\hline 0.353 & 1.56 & 11.6 & 13 & 0.05 & 12.6944 \\
\hline 0.47 & 1.714 & 11.8 & 13.2 & 0.05 & 12.8756 \\
\hline 0.5 & 1.753 & 11.8 & 13.3 & 0.05 & 12.9574 \\
\hline 0.51 & 1.766 & 11.9 & 13.3 & 0.05 & 12.9808 \\
\hline 0.52 & 1.779 & 11.9 & 13.4 & 0.05 & 12.9966 \\
\hline 0.53 & 1.792 & 11.9 & 13.4 & 0.05 & 13.0261 \\
\hline 0.54 & 1.805 & 11.9 & 13.4 & 0.05 & 13.0478 \\
\hline 0.55 & 1.818 & 11.9 & \begin{tabular}{|l|}
13.4 \\
\end{tabular} & 0.05 & 13.0815 \\
\hline 0.56 & 1.831 & 12 & 13.5 & 0.05 & 13.0919 \\
\hline
\end{tabular}




\section{B1.2.1.1.2.2 1"Water Reflection}

Case ID: u5o2N23-sp-1000-01

Material: $\mathrm{UO}_{2}\left(\mathrm{NO}_{3}\right)_{2} 3$ Molar Excess Acid

Geometry: Sphere

Isotopics: $100 \%{ }^{235} \mathrm{U}$

Reflector: 1" water reflected

Code: MCNP5 v1.40

Analyst: Mark Murphy

Date: $8 / 2011$ 
Figure B1.2.1.1.2.2-1. $\mathrm{UO}_{2}\left(\mathrm{NO}_{3}\right) 2$ 3M-Sphere - 100\% ${ }^{235} \mathrm{U}-1$ " Water Reflection

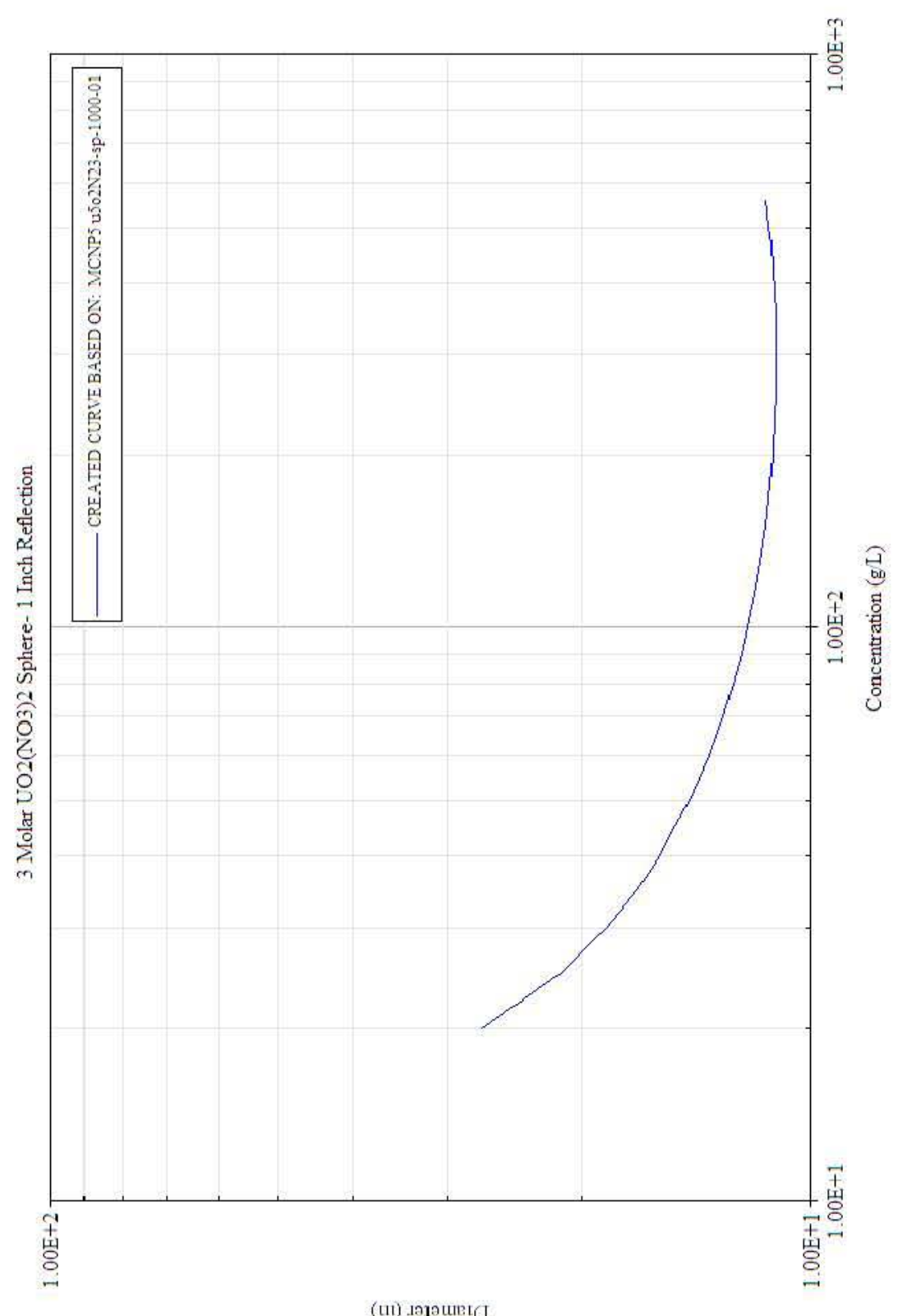

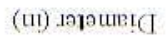


Table B1.2.1.1.2.2-1. Critical Dimensions for $\mathrm{UO}_{2}\left(\mathrm{NO}_{3}\right) 23 \mathrm{M}-$ Sphere $-100 \%{ }^{235} \mathrm{U}-1$ " Water Reflection

\begin{tabular}{|c|c|c|c|c|c|}
\hline \multicolumn{6}{|c|}{$100 \%$ U235 UO2(NO3)2 3M Sphere - 1 Inch of Reflection } \\
\hline Concentration $(\mathrm{g} / \mathrm{cc})$ & $\begin{array}{l}\text { Density } \\
(\mathrm{g} / \mathrm{cc})\end{array}$ & $\begin{array}{l}\text { First Diameter } \\
\text { (Inches) }\end{array}$ & $\begin{array}{l}\text { Last Diameter } \\
\text { (Inches) }\end{array}$ & $\begin{array}{l}\text { Delta } \\
\text { (Inches) }\end{array}$ & \begin{tabular}{|l} 
Critical Diameter \\
(Inches)
\end{tabular} \\
\hline 0.012 & 1.112 & 33.4 & 37.5 & 0.2 & -- \\
\hline 0.015 & 1.116 & 31.6 & 33 & 0.05 & -- \\
\hline 0.02 & 1.123 & 21.8 & 28.3 & 0.3 & 27.099 \\
\hline 0.025 & 1.129 & 18 & 22.1 & 0.2 & 21.2679 \\
\hline 0.03 & 1.136 & 16 & 19.1 & 0.1 & 18.5248 \\
\hline 0.032 & 1.138 & 15.5 & 18.4 & 0.1 & 17.7878 \\
\hline 0.035 & 1.142 & 14.8 & 17.4 & 0.1 & 16.8883 \\
\hline 0.04 & 1.149 & 13.9 & 16.3 & 0.1 & 15.7723 \\
\hline 0.05 & 1.162 & 12.8 & 14.9 & 0.1 & 14.399 \\
\hline 0.06 & 1.175 & 12.2 & 14 & 0.1 & 13.5861 \\
\hline 0.07 & 1.188 & 11.7 & 13.4 & 0.05 & 13.0237 \\
\hline 0.08 & 1.201 & 11.4 & 13 & 0.05 & 12.6116 \\
\hline 0.09 & 1.215 & 11.1 & 12.7 & 0.05 & 12.3126 \\
\hline 0.1 & 1.228 & 10.9 & 12.4 & 0.05 & 12.0864 \\
\hline 0.118 & 1.251 & 10.7 & 12.1 & 0.05 & 11.7848 \\
\hline 0.13 & 1.267 & 10.5 & 12 & 0.05 & 11.6388 \\
\hline 0.15 & 1.293 & 10.4 & 11.8 & 0.05 & 11.4499 \\
\hline 0.2 & 1.359 & 10.2 & 11.5 & 0.05 & 11.2027 \\
\hline 0.235 & 1.405 & 10.1 & 11.4 & 0.05 & 11.1294 \\
\hline 0.25 & 1.425 & 10.1 & 11.4 & 0.05 & \begin{tabular}{|l|}
11.0937 \\
\end{tabular} \\
\hline 0.353 & 1.56 & 10 & 11.4 & 0.05 & 11.1006 \\
\hline 0.47 & 1.714 & 10.2 & 11.6 & 0.05 & 11.2654 \\
\hline 0.5 & 1.753 & 10.2 & 11.7 & 0.05 & 11.3292 \\
\hline 0.51 & 1.766 & 10.3 & 11.7 & 0.05 & 11.3574 \\
\hline 0.52 & 1.779 & 10.3 & 11.7 & 0.05 & 11.3743 \\
\hline 0.53 & 1.792 & 10.3 & 11.7 & 0.05 & 11.3923 \\
\hline 0.54 & 1.805 & 10.3 & 11.8 & 0.05 & 11.4183 \\
\hline 0.55 & 1.818 & 10.3 & \begin{tabular}{|l|}
11.8 \\
\end{tabular} & 0.05 & 11.4375 \\
\hline 0.56 & 1.831 & 10.4 & 11.8 & 0.05 & 11.4624 \\
\hline
\end{tabular}




\section{B1.2.1.1.2.3 10" Water Reflection}

Case ID: u5o2N23-sp-1000-10

Material: $\mathrm{UO}_{2}\left(\mathrm{NO}_{3}\right)_{2} 3$ Molar Excess Acid

Geometry: Sphere

Isotopics: $100 \%{ }^{235} \mathrm{U}$

Reflector: 10 " water reflected

Code: MCNP5 v1.40

Analyst: Mark Murphy

Date: $8 / 2011$ 


\section{CHPRC-01552, Revision 0}

Figure B1.2.1.1.2.3-1. $\mathrm{UO}_{2}\left(\mathrm{NO}_{3}\right) 2 \mathrm{3M}-$ Sphere - $100 \%{ }^{235} \mathrm{U}-10$ " Water Reflection

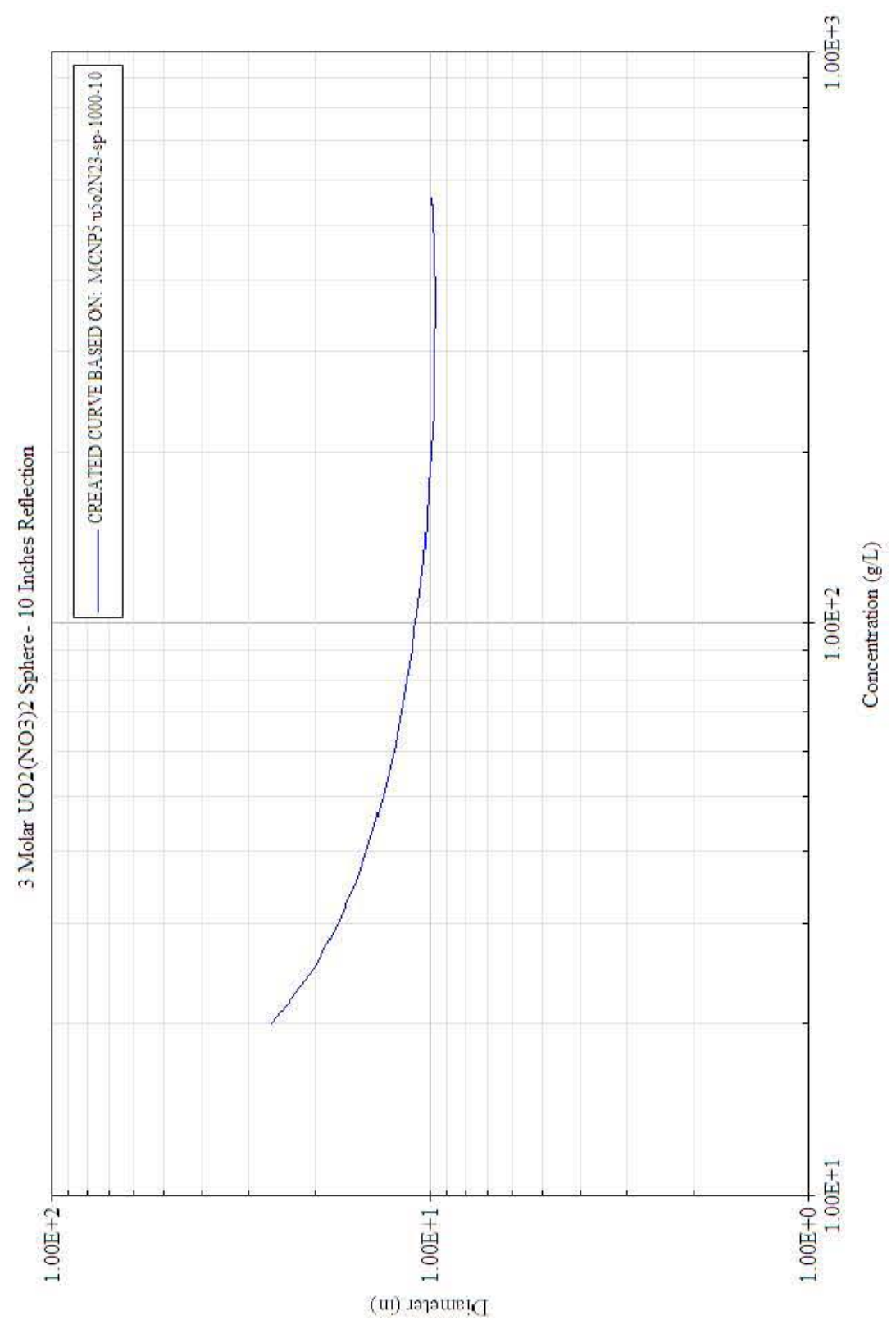


Table B1.2.1.1.2.3-1. Critical Dimensions for $\mathrm{UO}_{2}\left(\mathrm{NO}_{3}\right) 23 \mathrm{M}-$ Sphere $-100 \%{ }^{235} \mathrm{U}-10$ " Water Reflection

\begin{tabular}{|c|c|c|c|c|c|}
\hline \multicolumn{6}{|c|}{$100 \%$ U235 UO2(NO3)2 3M Sphere - 10 Inches of Reflection } \\
\hline Concentration $(\mathrm{g} / \mathrm{cc})$ & $\begin{array}{l}\text { Density } \\
(\mathrm{g} / \mathrm{cc})\end{array}$ & $\begin{array}{l}\text { First Diameter } \\
\text { (Inches) }\end{array}$ & $\begin{array}{l}\text { Last Diameter } \\
\text { (Inches) }\end{array}$ & $\begin{array}{l}\text { Delta } \\
\text { (Inches) }\end{array}$ & \begin{tabular}{|l} 
Critical Diameter \\
(Inches)
\end{tabular} \\
\hline 0.012 & 1.112 & 33.5 & 37.9 & 0.2 & -- \\
\hline 0.015 & 1.116 & 31.8 & 33 & 0.05 & -- \\
\hline 0.02 & 1.123 & 20.7 & 27.2 & 0.3 & 25.9985 \\
\hline 0.025 & 1.129 & 16.9 & 20.9 & 0.2 & 20.0919 \\
\hline 0.03 & 1.136 & 14.9 & 18 & 0.1 & 17.3527 \\
\hline 0.032 & 1.138 & 14.4 & 17.2 & 0.1 & 16.6186 \\
\hline 0.035 & 1.142 & 13.6 & 16.2 & 0.1 & 15.7077 \\
\hline 0.04 & 1.149 & 12.8 & 15.1 & 0.1 & 14.609 \\
\hline 0.05 & 1.162 & 11.7 & 13.6 & 0.1 & 13.2084 \\
\hline 0.06 & 1.175 & 11 & 12.8 & 0.1 & 12.3787 \\
\hline 0.07 & 1.188 & 10.5 & 12.2 & 0.05 & 11.8031 \\
\hline 0.08 & 1.201 & 10.2 & \begin{tabular}{|l|}
11.7 \\
\end{tabular} & 0.05 & 11.3823 \\
\hline 0.09 & 1.215 & 9.9 & 11.4 & 0.05 & 11.066 \\
\hline 0.1 & 1.228 & 9.7 & 11.1 & 0.05 & 10.8194 \\
\hline 0.118 & 1.251 & 9.4 & 10.8 & 0.05 & 10.5057 \\
\hline 0.13 & 1.267 & 9.3 & 10.7 & 0.05 & 10.3447 \\
\hline 0.15 & 1.293 & 9.1 & 10.5 & 0.05 & 10.1372 \\
\hline 0.2 & 1.359 & 8.8 & 10.1 & 0.05 & 9.8377 \\
\hline 0.235 & 1.405 & 8.7 & 10 & 0.05 & 9.731 \\
\hline 0.25 & 1.425 & 8.7 & 10 & 0.05 & 9.7039 \\
\hline 0.353 & 1.56 & 8.6 & 10 & 0.05 & 9.6334 \\
\hline 0.47 & 1.714 & 8.7 & 10 & 0.05 & 9.7135 \\
\hline 0.5 & 1.753 & 8.7 & 10.1 & 0.05 & 9.7547 \\
\hline 0.51 & 1.766 & 8.7 & 10.1 & 0.05 & 9.78 \\
\hline 0.52 & 1.779 & 8.7 & 10.1 & 0.05 & 9.7784 \\
\hline 0.53 & 1.792 & 8.7 & 10.1 & 0.05 & 9.8022 \\
\hline 0.54 & 1.805 & 8.7 & 10.1 & 0.05 & 9.8267 \\
\hline 0.55 & 1.818 & 8.8 & \begin{tabular}{|l|}
10.1 \\
\end{tabular} & 0.05 & 9.833 \\
\hline 0.56 & 1.831 & 8.8 & 10.2 & 0.05 & 9.8543 \\
\hline
\end{tabular}


B1.2.1.1.3 6 Molar Excess Acid

B1.2.1.1.3.1 0"Water Reflection

Case ID: u5o2N26-sp-1000-00

Material: $\mathrm{UO}_{2}\left(\mathrm{NO}_{3}\right)_{2} 6$ Molar Excess Acid

Geometry: Sphere

Isotopics: $100 \%{ }^{235} \mathrm{U}$

Reflector: 0 " water reflected

Code: MCNP5 v1.40

Analyst: Mark Murphy

Date: $8 / 2011$ 
Figure B1.2.1.1.3.1-1. $\mathrm{UO}_{2}\left(\mathrm{NO}_{3}\right) 26 \mathrm{M}-$ Sphere - $100 \%{ }^{235} \mathrm{U}-0$ " Water Reflection

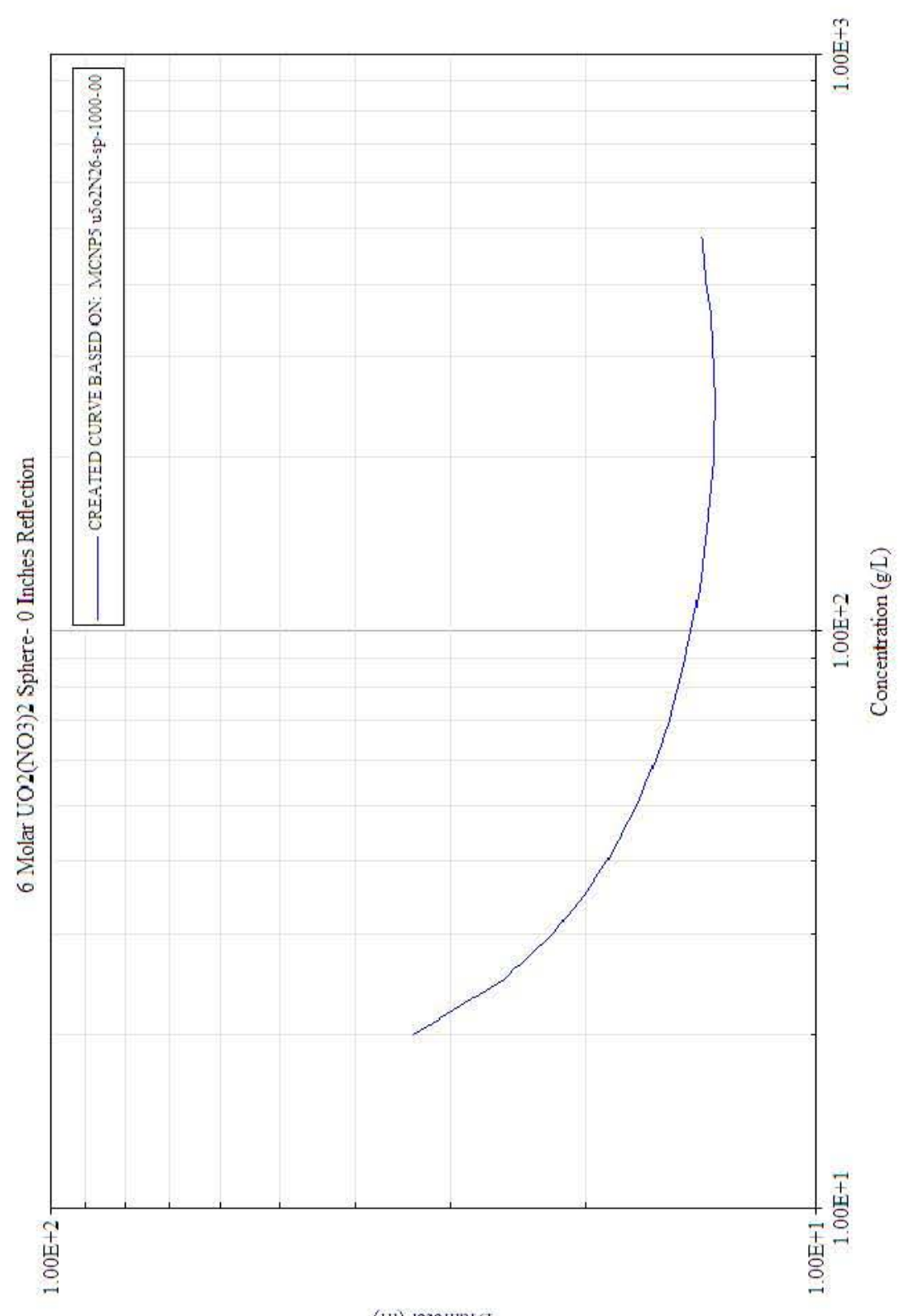

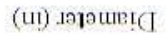


Table B1.2.1.1.3.1-1. Critical Dimensions for $\mathrm{UO}_{2}\left(\mathrm{NO}_{3}\right) 26 \mathrm{M}-$ Sphere $-100 \%{ }^{235} \mathrm{U}-0$ " Water Reflection

\begin{tabular}{|c|c|c|c|c|c|}
\hline \multicolumn{6}{|c|}{$100 \%$ U235 UO2(NO3)2 6M Sphere - 0 Inches of Reflection } \\
\hline Concentration $(\mathrm{g} / \mathrm{cc})$ & $\begin{array}{l}\text { Density } \\
(\mathrm{g} / \mathrm{cc})\end{array}$ & $\begin{array}{l}\text { First Diameter } \\
\text { (Inches) }\end{array}$ & $\begin{array}{l}\text { Last Diameter } \\
\text { (Inches) }\end{array}$ & $\begin{array}{l}\text { Delta } \\
\text { (Inches) }\end{array}$ & $\begin{array}{l}\text { Critical Diameter } \\
\text { (Inches) }\end{array}$ \\
\hline 0.012 & 1.202 & 64.3 & 82.4 & 0.9 & -- \\
\hline 0.015 & 1.206 & 37.7 & 52.5 & 0.7 & -- \\
\hline 0.02 & 1.212 & 26.5 & 36.5 & 0.5 & 33.5161 \\
\hline 0.025 & 1.219 & 21.5 & 26.4 & 0.2 & 25.453 \\
\hline 0.03 & 1.225 & 19.1 & 22.8 & 0.1 & 21.9932 \\
\hline 0.032 & 1.228 & 18.4 & 21.7 & 0.1 & 21.0746 \\
\hline 0.035 & 1.231 & 17.6 & 20.6 & 0.1 & 20.012 \\
\hline 0.04 & 1.238 & 16.6 & 19.3 & 0.1 & 18.7132 \\
\hline 0.05 & 1.251 & 15.4 & 17.6 & 0.1 & 17.1319 \\
\hline 0.06 & 1.264 & 14.6 & 16.6 & 0.1 & 16.1773 \\
\hline 0.07 & 1.277 & 14.1 & 15.9 & 0.1 & 15.5387 \\
\hline 0.08 & 1.289 & 13.7 & 15.5 & 0.1 & 15.1084 \\
\hline 0.09 & 1.302 & 13.4 & 15.1 & 0.05 & 14.7856 \\
\hline 0.1 & 1.315 & 13.3 & 14.9 & 0.05 & 14.5276 \\
\hline 0.118 & 1.338 & 13 & 14.6 & 0.05 & 14.2041 \\
\hline 0.13 & 1.354 & 12.8 & 14.4 & 0.05 & 14.0517 \\
\hline 0.15 & 1.38 & 12.7 & 14.2 & 0.05 & 13.8458 \\
\hline 0.2 & 1.444 & 12.4 & 14 & 0.05 & 13.6021 \\
\hline 0.235 & 1.489 & 12.4 & \begin{tabular}{|l|}
13.9 \\
\end{tabular} & 0.05 & 13.5484 \\
\hline 0.25 & 1.508 & 12.4 & 13.9 & 0.05 & 13.543 \\
\hline 0.353 & 1.638 & 12.5 & 14 & 0.05 & 13.68 \\
\hline 0.47 & 1.784 & 12.8 & 14.4 & 0.05 & 14.0365 \\
\hline 0.475 & 1.79 & 12.8 & 14.5 & 0.05 & 14.0682 \\
\hline 0.48 & 1.796 & 12.9 & 14.4 & 0.05 & 14.0775 \\
\hline 0.486 & 1.804 & 12.9 & 14.5 & 0.05 & 14.1078 \\
\hline
\end{tabular}




\section{B1.2.1.1.3.2 1"Water Reflection}

Case ID: u5o2N26-sp-1000-01

Material: $\mathrm{UO}_{2}\left(\mathrm{NO}_{3}\right)_{2} 6$ Molar Excess Acid

Geometry: Sphere

Isotopics: $100 \%{ }^{235} \mathrm{U}$

Reflector: 1" water reflected

Code: MCNP5 v1.40

Analyst: Mark Murphy

Date: $8 / 2011$ 
Figure B1.2.1.1.3.2-1. $\mathrm{UO}_{2}\left(\mathrm{NO}_{3}\right) 26 \mathrm{M}-$ Sphere - $100 \%{ }^{235} \mathrm{U}-1$ " Water Reflection

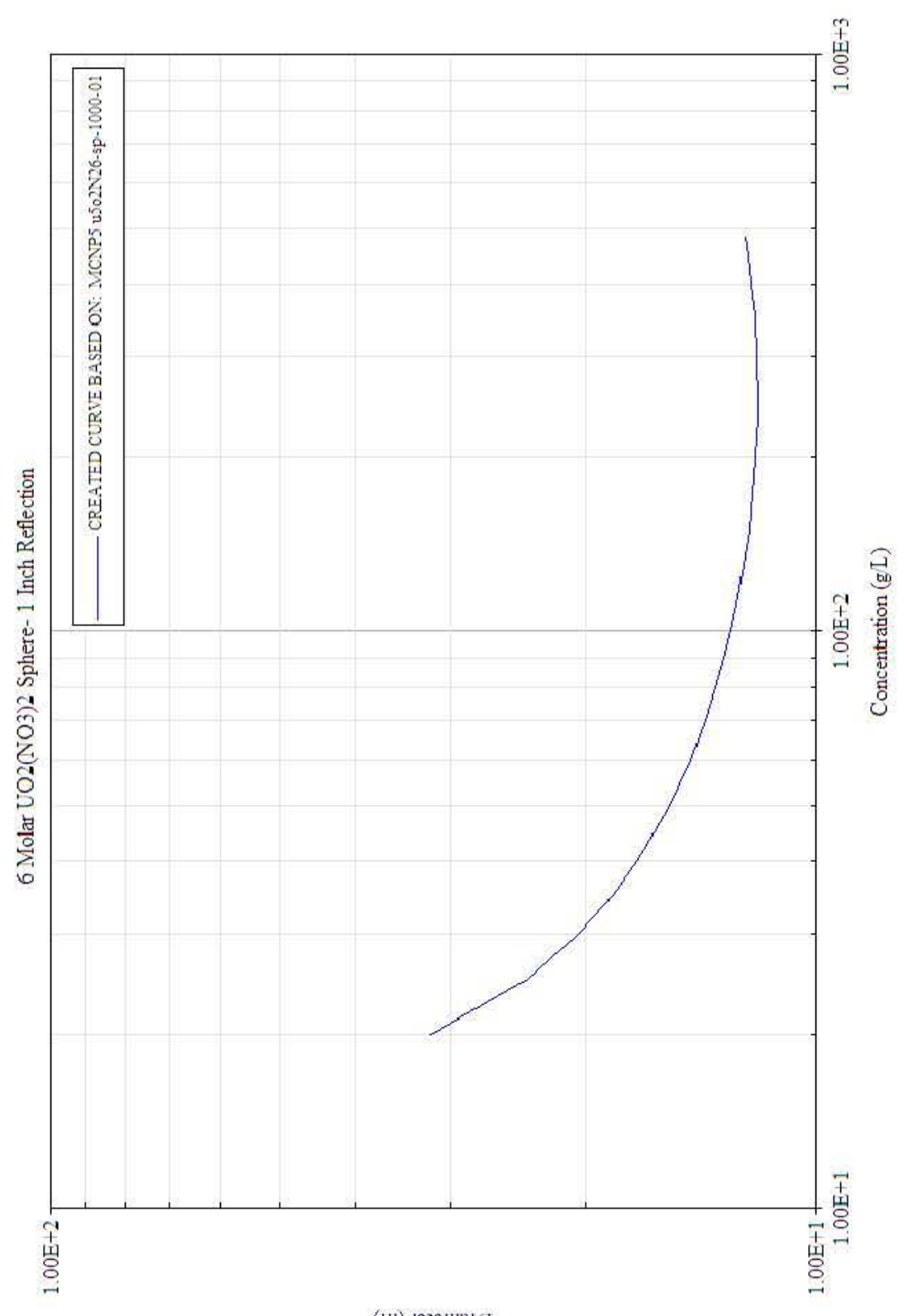

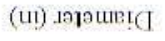


Table B1.2.1.1.3.2-1. Critical Dimensions for $\mathrm{UO}_{2}\left(\mathrm{NO}_{3}\right) 26 \mathrm{M}-$ Sphere $-100 \%{ }^{235} \mathrm{U}-1$ " Water Reflection

\begin{tabular}{|c|c|c|c|c|c|}
\hline \multicolumn{6}{|c|}{$100 \%$ U235 UO2(NO3)2 6M Sphere - 1 Inch of Reflection } \\
\hline Concentration $(\mathrm{g} / \mathrm{cc})$ & $\begin{array}{l}\text { Density } \\
(\mathrm{g} / \mathrm{cc})\end{array}$ & $\begin{array}{l}\text { First Diameter } \\
\text { (Inches) }\end{array}$ & $\begin{array}{l}\text { Last Diameter } \\
\text { (Inches) }\end{array}$ & $\begin{array}{l}\text { Delta } \\
\text { (Inches) }\end{array}$ & $\begin{array}{l}\text { Critical Diameter } \\
\text { (Inches) }\end{array}$ \\
\hline 0.012 & 1.202 & 66.1 & 86.2 & 1 & -- \\
\hline 0.015 & 1.206 & 41 & 51.4 & 0.5 & -- \\
\hline 0.02 & 1.212 & 24.8 & 34.4 & 0.4 & 31.8778 \\
\hline 0.025 & 1.219 & 19.9 & 24.8 & 0.2 & 23.8272 \\
\hline 0.03 & 1.225 & 17.5 & 21 & 0.1 & 20.3917 \\
\hline 0.032 & 1.228 & 16.8 & 20.1 & 0.1 & 19.4818 \\
\hline 0.035 & 1.231 & 16 & 19 & 0.1 & 18.4244 \\
\hline 0.04 & 1.238 & 15.1 & 17.6 & 0.1 & 17.112 \\
\hline 0.05 & 1.251 & 13.8 & 16 & 0.1 & 15.5231 \\
\hline 0.06 & 1.264 & 13 & 15 & 0.1 & 14.5635 \\
\hline 0.07 & 1.277 & 12.5 & 14.3 & 0.1 & 13.9334 \\
\hline 0.08 & 1.289 & 12.1 & 13.9 & 0.1 & 13.4964 \\
\hline 0.09 & 1.302 & 11.8 & 13.5 & 0.05 & 13.1587 \\
\hline 0.1 & 1.315 & 11.6 & 13.3 & 0.05 & 12.8969 \\
\hline 0.118 & 1.338 & 11.3 & 12.9 & 0.05 & 12.5772 \\
\hline 0.13 & 1.354 & 11.2 & 12.7 & 0.05 & 12.407 \\
\hline 0.15 & 1.38 & 11 & 12.5 & 0.05 & 12.211 \\
\hline 0.2 & 1.444 & 10.8 & 12.3 & 0.05 & 11.9716 \\
\hline 0.235 & 1.489 & 10.8 & 12.2 & 0.05 & 11.8966 \\
\hline 0.25 & 1.508 & 10.7 & 12.2 & 0.05 & 11.8832 \\
\hline 0.353 & 1.638 & 10.8 & 12.3 & 0.05 & 11.986 \\
\hline 0.47 & 1.784 & 11.1 & 12.7 & 0.05 & 12.3203 \\
\hline 0.475 & 1.79 & 11.1 & 12.7 & 0.05 & 12.3209 \\
\hline 0.48 & 1.796 & 11.1 & 12.7 & 0.05 & 12.3504 \\
\hline 0.486 & 1.804 & 11.1 & 12.7 & 0.05 & 12.3669 \\
\hline
\end{tabular}




\section{B1.2.1.1.3.3 10" Water Reflection}

Case ID: u5o2N26-sp-1000-10

Material: $\mathrm{UO}_{2}\left(\mathrm{NO}_{3}\right)_{2} 6$ Molar Excess Acid

Geometry: Sphere

Isotopics: $100 \%{ }^{235} \mathrm{U}$

Reflector: 10 " water reflected

Code: MCNP5 v1.40

Analyst: Mark Murphy

Date: $8 / 2011$ 
Figure B1.2.1.1.3.3-1. $\mathrm{UO}_{2}\left(\mathrm{NO}_{3}\right) 2 \mathrm{6M}-$ Sphere - $100 \%{ }^{235} \mathrm{U}-10$ " Water Reflection

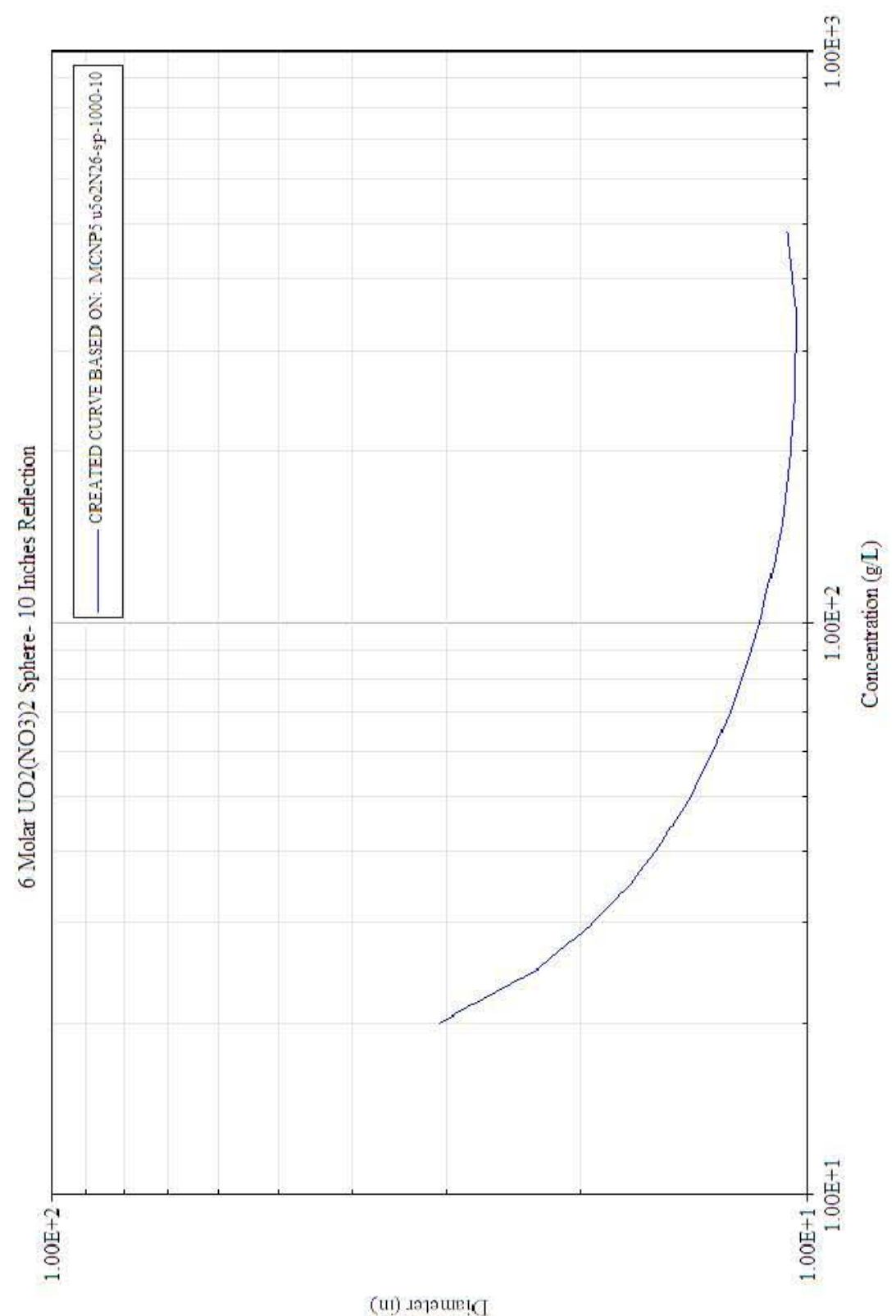

(ui) เәрачеเ 
Table B1.2.1.1.3.3-1. Critical Dimensions for $\mathrm{UO}_{2}\left(\mathrm{NO}_{3}\right) 26 \mathrm{M}-$ Sphere $-100 \%{ }^{235} \mathrm{U}-10$ " Water Reflection

\begin{tabular}{|c|c|c|c|c|c|}
\hline \multicolumn{6}{|c|}{$100 \%$ U235 UO2(NO3)2 6M Sphere - 10 Inches of Reflection } \\
\hline Concentration $(\mathrm{g} / \mathrm{cc})$ & $\begin{array}{l}\text { Density } \\
(\mathrm{g} / \mathrm{cc}) \\
\end{array}$ & $\begin{array}{l}\text { First Diameter } \\
\text { (Inches) }\end{array}$ & $\begin{array}{l}\text { Last Diameter } \\
\text { (Inches) }\end{array}$ & $\begin{array}{l}\text { Delta } \\
\text { (Inches) }\end{array}$ & \begin{tabular}{|l} 
Critical Diameter \\
(Inches)
\end{tabular} \\
\hline 0.012 & 1.202 & 65.3 & 85.7 & 1 & -- \\
\hline 0.015 & 1.206 & 35 & 51.9 & 0.8 & -- \\
\hline 0.02 & 1.212 & 23.6 & 32.5 & 0.4 & 30.6256 \\
\hline 0.025 & 1.219 & 18.7 & 23.5 & 0.2 & 22.6191 \\
\hline 0.03 & 1.225 & 16.3 & 19.9 & 0.1 & 19.1805 \\
\hline 0.032 & 1.228 & 15.6 & 18.9 & 0.1 & 18.2452 \\
\hline 0.035 & 1.231 & 14.8 & 17.7 & 0.1 & 17.1963 \\
\hline 0.04 & 1.238 & 13.8 & 16.4 & 0.1 & 15.8655 \\
\hline 0.05 & 1.251 & 12.5 & 14.7 & 0.1 & 14.2556 \\
\hline 0.06 & 1.264 & 11.7 & 13.7 & 0.1 & 13.2682 \\
\hline 0.07 & 1.277 & 11.2 & 13 & 0.1 & 12.6363 \\
\hline 0.08 & 1.289 & 10.8 & 12.5 & 0.05 & 12.1771 \\
\hline 0.09 & 1.302 & 10.5 & 12.2 & 0.05 & 11.8525 \\
\hline 0.1 & 1.315 & 10.3 & 11.9 & 0.05 & 11.5198 \\
\hline 0.118 & 1.338 & 10 & 11.5 & 0.05 & 11.2071 \\
\hline 0.13 & 1.354 & 9.8 & 11.3 & 0.05 & 11.0317 \\
\hline 0.15 & 1.38 & 9.6 & 11.1 & 0.05 & 10.7869 \\
\hline 0.2 & 1.444 & 9.4 & 10.8 & 0.05 & 10.5099 \\
\hline 0.235 & \begin{tabular}{|l|}
1.489 \\
\end{tabular} & 9.3 & 10.7 & 0.05 & 10.394 \\
\hline 0.25 & 1.508 & 9.3 & 10.7 & 0.05 & 10.3814 \\
\hline 0.353 & 1.638 & 9.3 & 10.7 & 0.05 & 10.3481 \\
\hline 0.47 & 1.784 & 9.4 & 10.9 & 0.05 & 10.5856 \\
\hline 0.475 & 1.79 & 9.4 & 10.9 & 0.05 & 10.5938 \\
\hline 0.48 & 1.796 & 9.4 & 11 & 0.05 & 10.6025 \\
\hline 0.486 & 1.804 & 9.5 & 11 & 0.05 & 10.6232 \\
\hline
\end{tabular}


B1.2.1.2 $\quad 93.5 \%{ }^{235} \mathrm{U}$

B1.2.1.2.1 0 Molar Excess Acid

B1.2.1.2.1.1 0"Water Reflection

Case ID: u5o2N20-sp-0935-00

Material: $\mathrm{UO}_{2}\left(\mathrm{NO}_{3}\right)_{2} 0$ Molar Excess Acid

Geometry: Sphere

Isotopics: $93.5 \%{ }^{235} \mathrm{U}$

Reflector: 0" water reflected

Code: MCNP5 v1.40

Analyst: Mark Murphy

Date: $8 / 2011$ 
Figure B1.2.1.2.1.1-1. $\mathrm{UO}_{2}\left(\mathrm{NO}_{3}\right) 2 \mathrm{OM}-$ Sphere $-93.5 \%{ }^{235} \mathrm{U}-0$ " Water Reflection

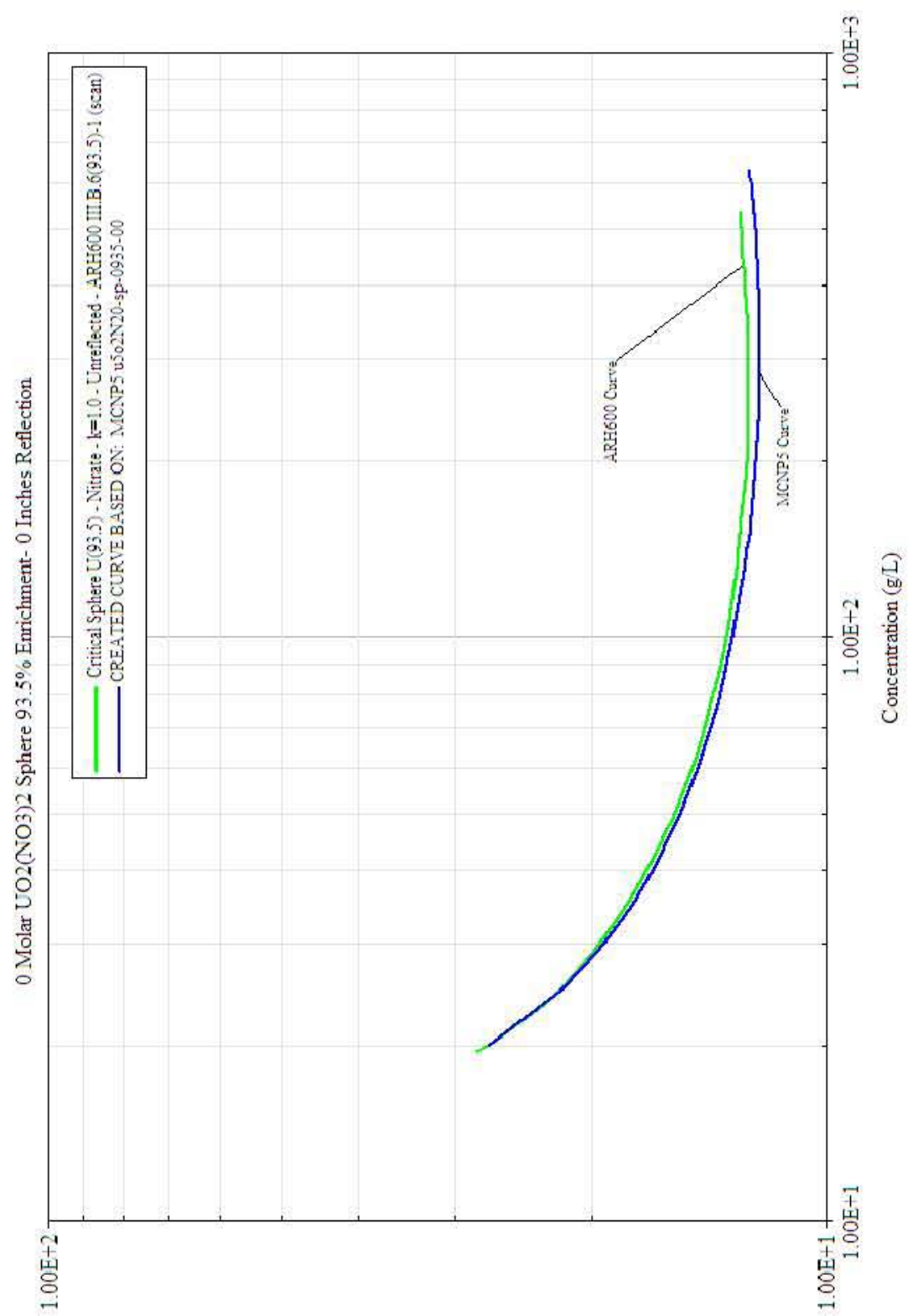

(ui) เวุวuอ ! ( 
Table B1.2.1.2.1.1-1. Critical Dimensions for $\mathrm{UO}_{2}\left(\mathrm{NO}_{3}\right) 2 \mathrm{M}-$ Sphere $-93.5 \%{ }^{235} \mathrm{U}-0$ " Water Reflection

\begin{tabular}{|c|c|c|c|c|c|}
\hline \multicolumn{6}{|c|}{ 93.5\% U235 UO2(NO3)2 0M Sphere- 0 Inches Reflection } \\
\hline Concentration $(\mathrm{g} / \mathrm{cc})$ & $\begin{array}{l}\text { Density } \\
(\mathrm{g} / \mathrm{cc})\end{array}$ & $\begin{array}{l}\text { First Diameter } \\
\text { (Inches) }\end{array}$ & $\begin{array}{l}\text { Last Diameter } \\
\text { (Inches) }\end{array}$ & $\begin{array}{l}\text { Delta } \\
\text { (Inches) }\end{array}$ & $\begin{array}{l}\text { Critical Diameter } \\
\text { (Inches) }\end{array}$ \\
\hline 0.012 & 1.013 & 69 & 115.7 & 2.3 & -- \\
\hline 0.015 & 1.017 & 31.6 & 41.4 & 0.5 & -- \\
\hline 0.02 & 1.024 & 22.4 & 28.3 & 0.3 & 27.1264 \\
\hline 0.025 & 1.031 & 18.8 & 22.5 & 0.1 & 21.8338 \\
\hline 0.03 & 1.037 & 16.9 & 19.8 & 0.1 & 19.2685 \\
\hline 0.032 & 1.04 & 16.4 & 19.2 & 0.1 & 18.5548 \\
\hline 0.035 & 1.044 & 15.7 & 18.3 & 0.1 & 17.7296 \\
\hline 0.04 & 1.051 & 14.9 & 17.2 & 0.1 & 16.7057 \\
\hline 0.05 & 1.064 & 13.9 & 15.8 & 0.1 & 15.395 \\
\hline 0.06 & 1.078 & 13.2 & 15 & 0.1 & 14.5899 \\
\hline 0.07 & 1.091 & 12.8 & 14.4 & 0.05 & 14.0595 \\
\hline 0.08 & 1.105 & 12.4 & 14.1 & 0.05 & 13.6558 \\
\hline 0.09 & 1.118 & 12.2 & 13.7 & 0.05 & 13.3834 \\
\hline 0.1 & 1.131 & 12 & 13.5 & 0.05 & 13.1624 \\
\hline 0.118 & 1.155 & 11.8 & 13.2 & 0.05 & 12.8762 \\
\hline 0.13 & 1.172 & 11.6 & 13 & 0.05 & 12.712 \\
\hline 0.15 & 1.198 & 11.5 & 12.7 & 0.05 & 12.5321 \\
\hline 0.2 & 1.266 & 11.2 & 12.6 & 0.05 & 12.2862 \\
\hline 0.235 & 1.313 & 11.2 & 12.5 & 0.05 & 12.1867 \\
\hline 0.25 & 1.333 & 11.3 & 12.5 & 0.05 & 12.1728 \\
\hline 0.353 & 1.472 & 11.1 & 12.5 & 0.05 & 12.155 \\
\hline 0.47 & 1.631 & 11.2 & 12.6 & 0.05 & 12.237 \\
\hline 0.5 & 1.67 & 11.2 & 12.7 & 0.05 & 12.3 \\
\hline 0.6 & 1.805 & 11.4 & 12.8 & 0.05 & 12.4818 \\
\hline 0.605 & 1.812 & 11.4 & 12.8 & 0.05 & 12.4856 \\
\hline 0.61 & 1.819 & 11.4 & 12.8 & 0.05 & 12.4989 \\
\hline 0.615 & 1.825 & 11.4 & 12.9 & 0.05 & 12.5147 \\
\hline 0.62 & 1.832 & 11.4 & 12.8 & 0.05 & 12.5167 \\
\hline 0.625 & 1.839 & 11.4 & 12.8 & 0.05 & 12.5297 \\
\hline 0.629 & 1.844 & 11.4 & 12.9 & 0.05 & 12.5369 \\
\hline
\end{tabular}


B1.2.1.2.1.2 1"Water Reflection

Case ID: u5o2N20-sp-0935-01

Material: $\mathrm{UO}_{2}\left(\mathrm{NO}_{3}\right)_{2} 0$ Molar Excess Acid

Geometry: Sphere

Isotopics: $93.5 \%{ }^{235} \mathrm{U}$

Reflector: 1" water reflected

Code: MCNP5 v1.40

Analyst: Mark Murphy

Date: $8 / 2011$ 


\section{CHPRC-01552, Revision 0}

Figure B1.2.1.2.1.2-1. $\mathrm{UO}_{2}\left(\mathrm{NO}_{3}\right) 2 \mathrm{0M}-$ Sphere - 93.5\% ${ }^{235} \mathrm{U}-1$ " Water Reflection

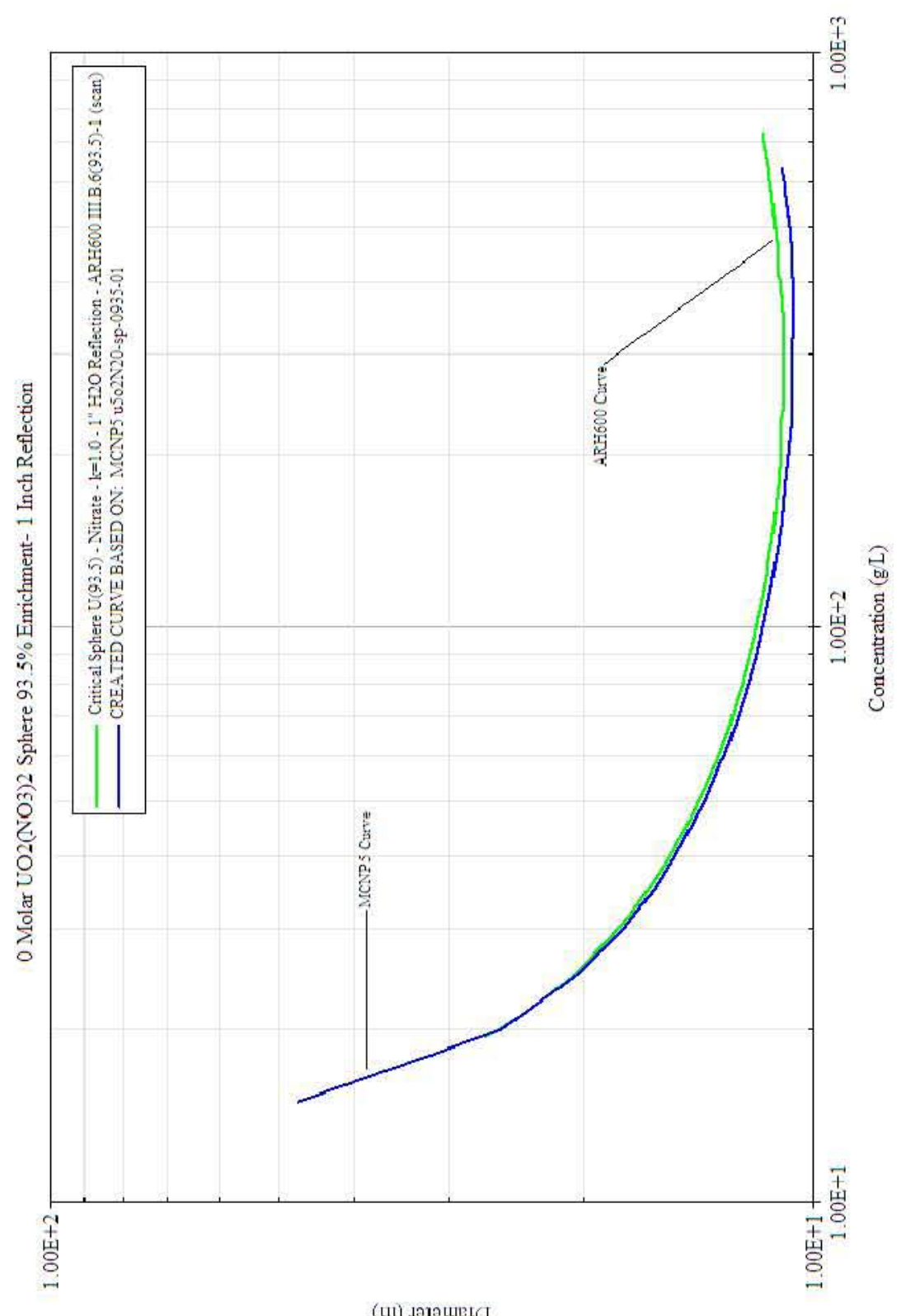

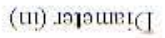


Table B1.2.1.2.1.2-1. Critical Dimensions for $\mathrm{UO}_{2}\left(\mathrm{NO}_{3}\right) 2 \mathrm{OM}-$ Sphere $-93.5 \%{ }^{235} \mathrm{U}-1$ " Water Reflection

\begin{tabular}{|c|c|c|c|c|c|}
\hline \multicolumn{6}{|c|}{ 93.5\% U235 UO2(NO3)2 0M Sphere- 1 Inch Reflection } \\
\hline Concentration $(\mathrm{g} / \mathrm{cc})$ & $\begin{array}{l}\text { Density } \\
(\mathrm{g} / \mathrm{cc})\end{array}$ & $\begin{array}{l}\text { First Diameter } \\
\text { (Inches) }\end{array}$ & $\begin{array}{l}\text { Last Diameter } \\
\text { (Inches) }\end{array}$ & $\begin{array}{l}\text { Delta } \\
\text { (Inches) }\end{array}$ & $\begin{array}{l}\text { Critical Diameter } \\
\text { (Inches) }\end{array}$ \\
\hline 0.012 & 1.013 & 74.7 & 166.1 & 4.5 & -- \\
\hline 0.015 & 1.017 & 30.9 & 52.4 & 1 & 47.2572 \\
\hline 0.02 & 1.024 & 20.8 & 26.5 & 0.2 & 25.6358 \\
\hline 0.025 & 1.031 & 17.2 & 21 & 0.2 & 20.2904 \\
\hline 0.03 & 1.037 & 15.4 & 18.3 & 0.1 & 17.7398 \\
\hline 0.032 & 1.04 & 14.9 & 17.6 & 0.1 & 17.056 \\
\hline 0.035 & 1.044 & 14.2 & 16.7 & 0.1 & 16.216 \\
\hline 0.04 & 1.051 & 13.4 & 15.7 & 0.1 & 15.1769 \\
\hline 0.05 & 1.064 & 12.4 & 14.3 & 0.1 & 13.8842 \\
\hline 0.06 & 1.078 & 11.7 & 13.5 & 0.1 & 13.0848 \\
\hline 0.07 & 1.091 & 11.3 & 12.9 & 0.05 & 12.5335 \\
\hline 0.08 & 1.105 & 10.9 & 12.5 & 0.05 & 12.1382 \\
\hline 0.09 & 1.118 & 10.7 & 12.2 & 0.05 & 11.851 \\
\hline 0.1 & 1.131 & 10.5 & 12 & 0.05 & 11.635 \\
\hline 0.118 & 1.155 & 10.2 & 11.7 & 0.05 & 11.3417 \\
\hline 0.13 & 1.172 & 10.1 & 11.5 & 0.05 & 11.1925 \\
\hline 0.15 & 1.198 & 10 & 11.3 & 0.05 & 11.0091 \\
\hline 0.2 & 1.266 & 9.7 & 11 & 0.05 & 10.759 \\
\hline 0.235 & 1.313 & 9.7 & 11 & 0.05 & 10.6564 \\
\hline 0.25 & 1.333 & 9.6 & 10.9 & 0.05 & 10.6374 \\
\hline 0.353 & 1.472 & 9.6 & 10.9 & 0.05 & 10.582 \\
\hline 0.47 & 1.631 & 9.7 & 11 & 0.05 & 10.6872 \\
\hline 0.5 & 1.67 & 9.8 & 11.1 & 0.05 & 10.7477 \\
\hline 0.6 & 1.805 & 9.8 & 11.2 & 0.05 & 10.904 \\
\hline 0.605 & 1.812 & 9.8 & 11.2 & 0.05 & 10.9185 \\
\hline 0.61 & 1.819 & 9.8 & 11.3 & 0.05 & 10.9259 \\
\hline 0.615 & 1.825 & 9.9 & 11.3 & 0.05 & 10.9495 \\
\hline 0.62 & 1.832 & 9.9 & 11.3 & 0.05 & 10.9485 \\
\hline 0.625 & 1.839 & 9.9 & 11.3 & 0.05 & 10.9633 \\
\hline 0.629 & 1.844 & 9.9 & 11.3 & 0.05 & 10.9727 \\
\hline
\end{tabular}




\section{B1.2.1.2.1.3 10" Water Reflection}

Case ID: u5o2N20-sp-0935-10

Material: $\mathrm{UO}_{2}\left(\mathrm{NO}_{3}\right)_{2} 0$ Molar Excess Acid

Geometry: Sphere

Isotopics: $93.5 \%{ }^{235} \mathrm{U}$

Reflector: 10 " water reflected

Code: MCNP5 v1.40

Analyst: Mark Murphy

Date: $8 / 2011$ 
Figure B1.2.1.2.1.3-1. $\mathrm{UO}_{2}\left(\mathrm{NO}_{3}\right) 2 \mathrm{OM}-$ Sphere $-93.5 \%{ }^{235} \mathrm{U}-10$ " Water Reflection

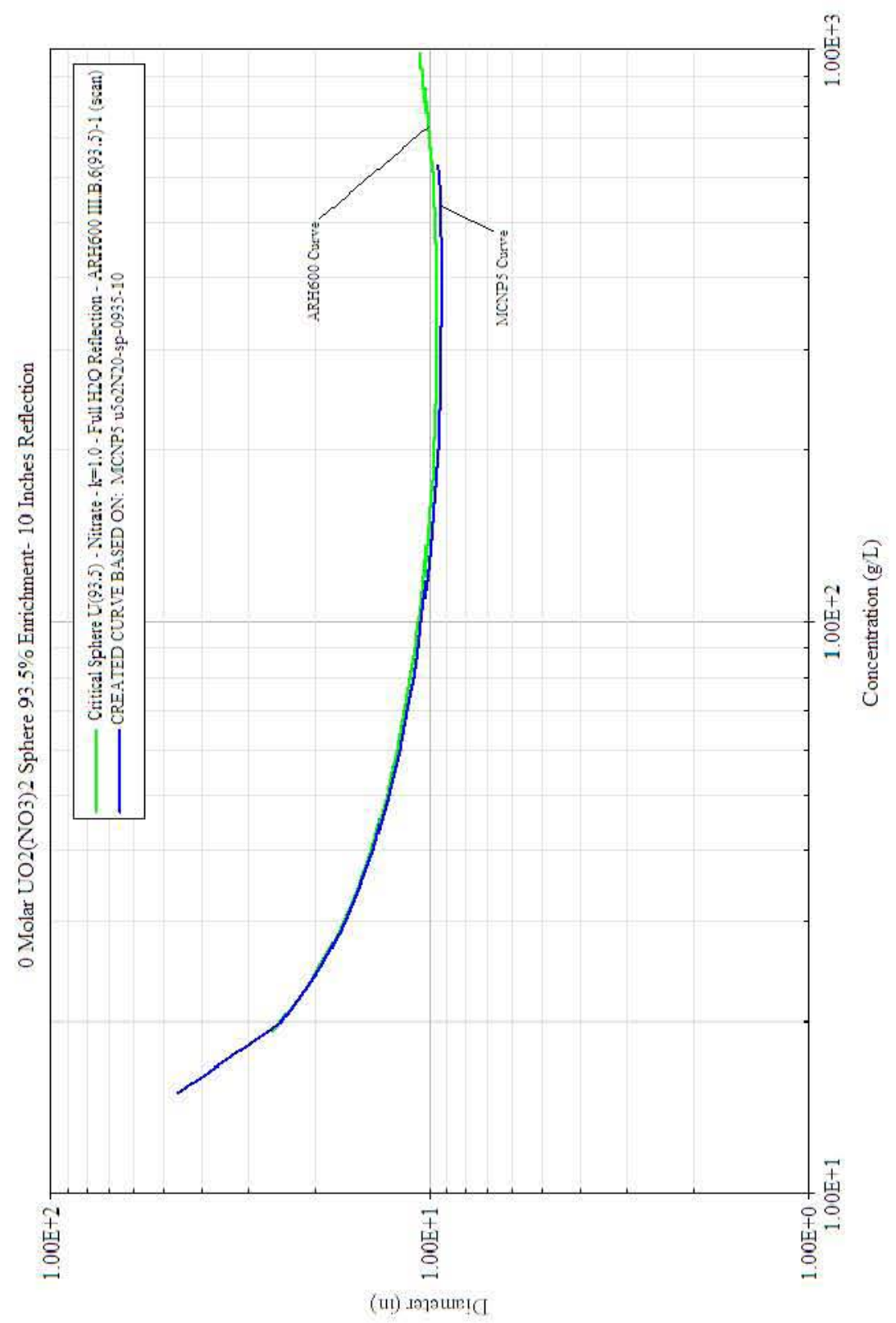


Table B1.2.1.2.1.3-1. Critical Dimensions for $\mathrm{UO}_{2}\left(\mathrm{NO}_{3}\right) 2 \mathrm{OM}-$ Sphere $-93.5 \%{ }^{235} \mathrm{U}-10$ " Water Reflection

\begin{tabular}{|c|c|c|c|c|c|}
\hline \multicolumn{6}{|c|}{ 93.5\% U235 UO2(NO3)2 0M Sphere- 10 Inches Reflection } \\
\hline Concentration $(\mathrm{g} / \mathrm{cc})$ & $\begin{array}{l}\text { Density } \\
(\mathrm{g} / \mathrm{cc})\end{array}$ & $\begin{array}{l}\text { First Diameter } \\
\text { (Inches) }\end{array}$ & $\begin{array}{l}\text { Last Diameter } \\
\text { (Inches) }\end{array}$ & $\begin{array}{l}\text { Delta } \\
\text { (Inches) }\end{array}$ & $\begin{array}{l}\text { Critical Diameter } \\
\text { (Inches) }\end{array}$ \\
\hline 0.012 & 1.013 & 69.9 & 119.9 & 2.5 & -- \\
\hline 0.015 & 1.017 & 30.1 & 50.9 & 1 & 45.8947 \\
\hline 0.02 & 1.024 & 19.7 & 25.4 & 0.3 & 24.4938 \\
\hline 0.025 & 1.031 & 16.2 & 19.9 & 0.1 & 19.1883 \\
\hline 0.03 & 1.037 & 14.3 & 17.2 & 0.1 & 16.6519 \\
\hline 0.032 & 1.04 & 13.8 & 16.5 & 0.1 & 15.9309 \\
\hline 0.035 & 1.044 & 13.1 & 15.6 & 0.1 & 15.114 \\
\hline 0.04 & 1.051 & 12.3 & 14.5 & 0.1 & 14.0597 \\
\hline 0.05 & 1.064 & 11.3 & 13.1 & 0.1 & 12.7401 \\
\hline 0.06 & 1.078 & 10.6 & 12.3 & 0.05 & 11.9283 \\
\hline 0.07 & 1.091 & 10.1 & 11.7 & 0.05 & 11.3748 \\
\hline 0.08 & 1.105 & 9.8 & 11.3 & 0.05 & 10.9668 \\
\hline 0.09 & 1.118 & 9.5 & 11 & 0.05 & 10.6761 \\
\hline 0.1 & 1.131 & 9.3 & 10.7 & 0.05 & 10.4408 \\
\hline 0.118 & 1.155 & 9 & 10.4 & 0.05 & 10.1307 \\
\hline 0.13 & 1.172 & 8.9 & 10.3 & 0.05 & 9.9729 \\
\hline 0.15 & 1.198 & 8.7 & 10.1 & 0.05 & 9.7631 \\
\hline 0.2 & 1.266 & 8.5 & 9.8 & 0.05 & 9.4729 \\
\hline 0.235 & 1.313 & 8.4 & 9.7 & 0.05 & 9.3533 \\
\hline 0.25 & 1.333 & 8.3 & 9.6 & 0.05 & 9.3226 \\
\hline 0.353 & 1.472 & 8.2 & 9.5 & 0.05 & 9.2283 \\
\hline 0.47 & 1.631 & 8.3 & 9.5 & 0.05 & 9.2589 \\
\hline 0.5 & 1.67 & 8.3 & 9.6 & 0.05 & 9.2905 \\
\hline 0.6 & 1.805 & 8.4 & 9.7 & 0.05 & 9.418 \\
\hline 0.605 & 1.812 & 8.4 & 9.7 & 0.05 & 9.4002 \\
\hline 0.61 & 1.819 & 8.3 & 9.7 & 0.05 & 9.4157 \\
\hline 0.615 & 1.825 & 8.4 & 9.7 & 0.05 & 9.4345 \\
\hline 0.62 & 1.832 & 8.4 & 9.7 & 0.05 & 9.4288 \\
\hline 0.625 & 1.839 & 8.4 & 9.7 & 0.05 & 9.4284 \\
\hline 0.629 & 1.844 & 8.4 & 9.7 & 0.05 & 9.4499 \\
\hline
\end{tabular}


B1.2.1.2.2 3 Molar Excess Acid

B1.2.1.2.2.1 0"Water Reflection

Case ID: u5o2N23-sp-0935-00

Material: $\mathrm{UO}_{2}\left(\mathrm{NO}_{3}\right)_{2} 3$ Molar Excess Acid

Geometry: Sphere

Isotopics: $93.5 \%{ }^{235} \mathrm{U}$

Reflector: 0 " water reflected

Code: MCNP5 v1.40

Analyst: Mark Murphy

Date: $8 / 2011$ 
Figure B1.2.1.2.2.1-1. $\mathrm{UO}_{2}\left(\mathrm{NO}_{3}\right) 23 \mathrm{M}-$ Sphere $-93.5 \%{ }^{235} \mathrm{U}-0$ " Water Reflection

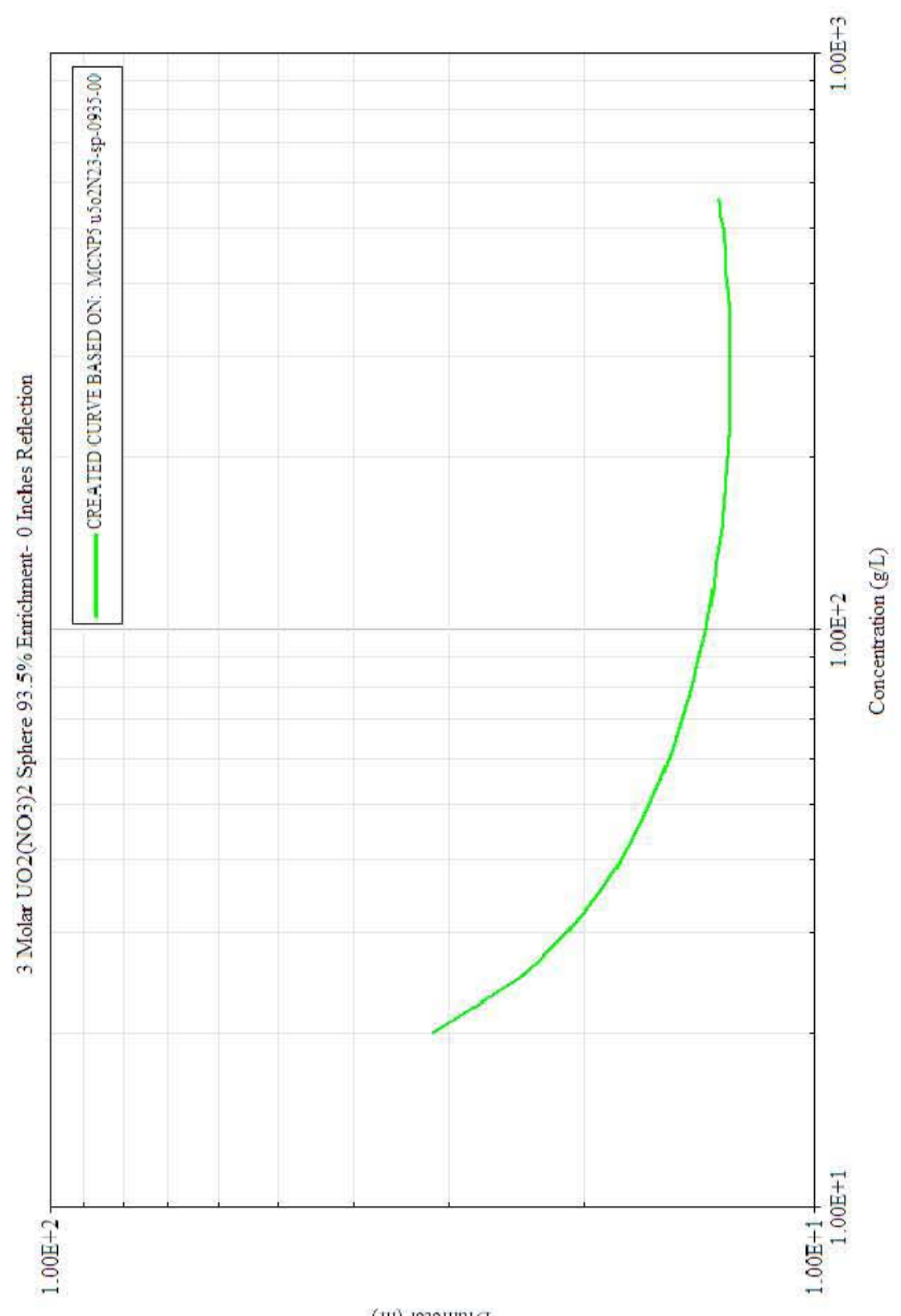

(ui) เวาวแย!( 
Table B1.2.1.2.2.1-1. Critical Dimensions for $\mathrm{UO}_{2}\left(\mathrm{NO}_{3}\right) 23 \mathrm{M}-$ Sphere $-93.5 \%{ }^{235} \mathrm{U}-0$ " Water Reflection

\begin{tabular}{|c|c|c|c|c|c|}
\hline \multicolumn{6}{|c|}{ 93.5\% U235 UO2(NO3)2 3M Sphere- 0 Inches Reflection } \\
\hline Concentration $(\mathrm{g} / \mathrm{cc})$ & \begin{tabular}{|l|} 
Density \\
$(\mathrm{g} / \mathrm{cc})$
\end{tabular} & $\begin{array}{l}\text { First Diameter } \\
\text { (Inches) }\end{array}$ & $\begin{array}{l}\text { Last Diameter } \\
\text { (Inches) }\end{array}$ & $\begin{array}{l}\text { Delta } \\
\text { (Inches) }\end{array}$ & $\begin{array}{l}\text { Critical Diameter } \\
\text { (Inches) } \\
\end{array}$ \\
\hline 0.012 & 1.112 & 107.1 & 207.6 & 5 & -- \\
\hline 0.015 & 1.116 & 41.8 & 62.9 & 1 & -- \\
\hline 0.02 & 1.123 & 25.2 & 33.1 & 0.4 & 31.6145 \\
\hline 0.025 & 1.129 & 20.5 & 25.2 & 0.2 & 24.2514 \\
\hline 0.03 & 1.136 & 18.3 & 21.8 & 0.1 & 21.0167 \\
\hline 0.032 & 1.138 & 17.6 & 20.8 & 0.1 & 20.1656 \\
\hline 0.035 & 1.142 & 16.9 & 19.7 & 0.1 & 19.1454 \\
\hline 0.04 & 1.149 & 15.9 & 18.5 & 0.1 & 17.8941 \\
\hline 0.05 & 1.162 & 14.8 & 16.8 & 0.1 & 16.384 \\
\hline 0.06 & 1.175 & 13.9 & 15.9 & 0.1 & 15.4607 \\
\hline 0.07 & 1.188 & 13.5 & 15.3 & 0.1 & 14.8692 \\
\hline 0.08 & 1.201 & 13.1 & 14.8 & 0.05 & 14.4238 \\
\hline 0.09 & 1.215 & 12.8 & 14.5 & 0.05 & 14.0913 \\
\hline 0.1 & 1.228 & 12.6 & 14.3 & 0.05 & 13.8425 \\
\hline 0.118 & 1.251 & 12.3 & 13.9 & 0.05 & 13.5288 \\
\hline 0.13 & 1.267 & 12.2 & 13.7 & 0.05 & \begin{tabular}{|l|}
13.3819 \\
\end{tabular} \\
\hline 0.15 & 1.293 & 12 & 13.5 & 0.05 & 13.2018 \\
\hline 0.2 & 1.359 & 11.8 & 13.3 & 0.05 & 12.9385 \\
\hline 0.235 & 1.406 & 11.7 & 13.2 & 0.05 & \begin{tabular}{|l|}
12.8424 \\
\end{tabular} \\
\hline 0.25 & 1.425 & 11.7 & 13.2 & 0.05 & 12.8391 \\
\hline 0.353 & 1.561 & 11.8 & 13.2 & 0.05 & 12.8585 \\
\hline 0.47 & 1.715 & 11.9 & 13.4 & 0.05 & 13.0649 \\
\hline 0.5 & 1.753 & 12 & 13.5 & 0.05 & 13.1463 \\
\hline 0.51 & 1.766 & 12 & 13.5 & 0.05 & 13.1835 \\
\hline 0.52 & 1.779 & 12 & 13.6 & 0.1 & \begin{tabular}{|l}
13.2103 \\
\end{tabular} \\
\hline 0.53 & 1.792 & 12.1 & 13.6 & 0.05 & 13.2356 \\
\hline 0.54 & 1.805 & 12.1 & 13.7 & 0.05 & 13.2614 \\
\hline 0.55 & 1.818 & 12.1 & 13.7 & 0.05 & 13.2899 \\
\hline 0.56 & 1.831 & 12.2 & 13.6 & 0.05 & 13.3136 \\
\hline
\end{tabular}


B1.2.1.2.2.2 1"Water Reflection

Case ID: u5o2N23-sp-0935-01

Material: $\mathrm{UO}_{2}\left(\mathrm{NO}_{3}\right)_{2} 3$ Molar Excess Acid

Geometry: Sphere

Isotopics: $93.5 \%{ }^{235} \mathrm{U}$

Reflector: 1" water reflected

Code: MCNP5 v1.40

Analyst: Mark Murphy

Date: $8 / 2011$ 


\section{CHPRC-01552, Revision 0}

Figure B1.2.1.2.2.2-1. $\mathrm{UO}_{2}\left(\mathrm{NO}_{3}\right) 23 \mathrm{M}-$ Sphere $-93.5 \%{ }^{235} \mathrm{U}-1$ " Water Reflection

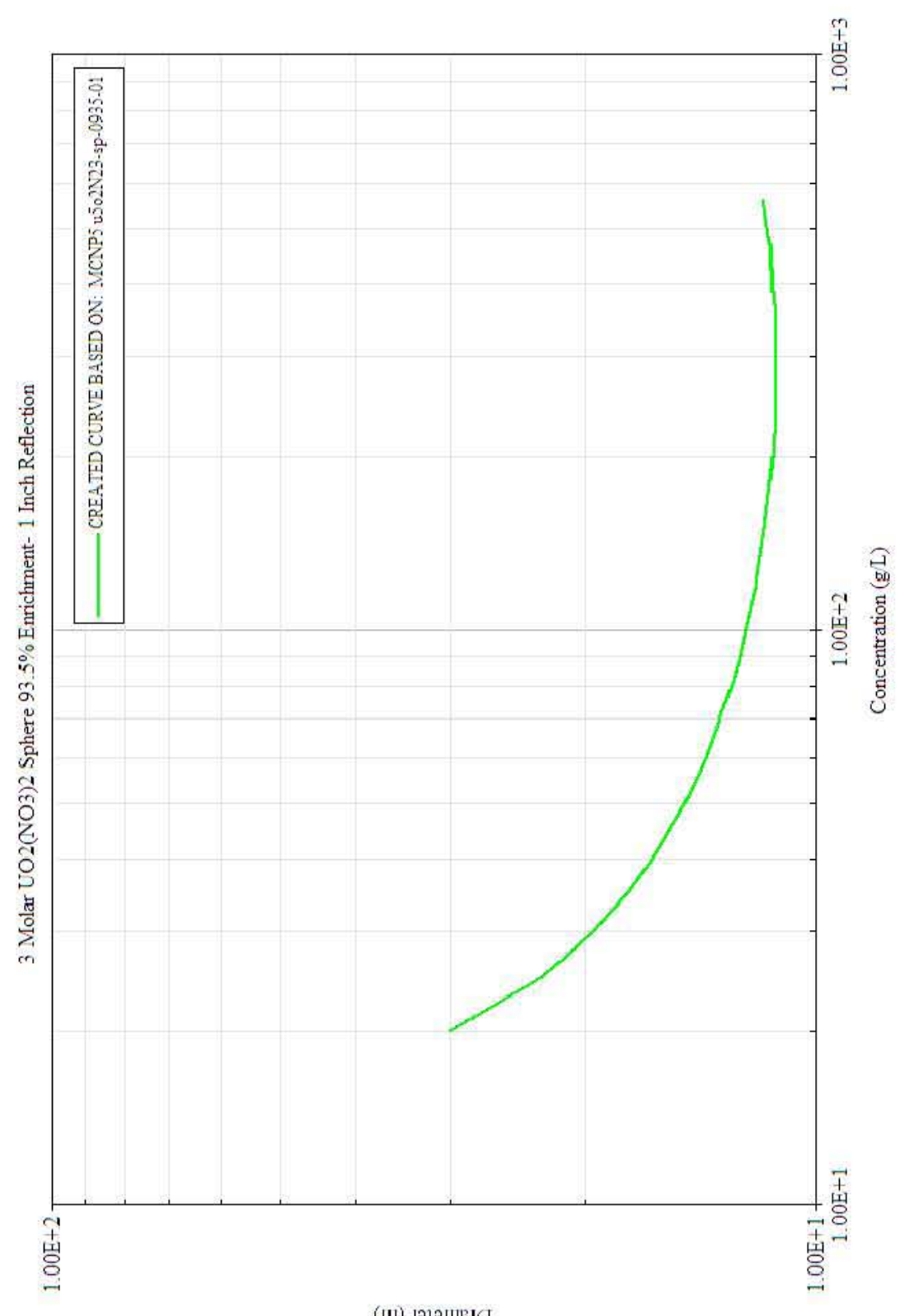

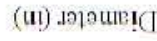


Table B1.2.1.2.2.2-1. Critical Dimensions for $\mathrm{UO}_{2}\left(\mathrm{NO}_{3}\right) 23 \mathrm{M}-$ Sphere $-93.5 \%{ }^{235} \mathrm{U}-1$ " Water Reflection

\begin{tabular}{|c|c|c|c|c|c|}
\hline \multicolumn{6}{|c|}{ 93.5\% U235 UO2(NO3)2 3M Sphere- 1 Inch Reflection } \\
\hline Concentration $(\mathrm{g} / \mathrm{cc})$ & $\begin{array}{l}\text { Density } \\
(\mathrm{g} / \mathrm{cc})\end{array}$ & $\begin{array}{l}\text { First Diameter } \\
\text { (Inches) }\end{array}$ & $\begin{array}{l}\text { Last Diameter } \\
\text { (Inches) }\end{array}$ & $\begin{array}{l}\text { Delta } \\
\text { (Inches) }\end{array}$ & $\begin{array}{l}\text { Critical Diameter } \\
\text { (Inches) }\end{array}$ \\
\hline 0.012 & 1.112 & 144.3 & 234.6 & 4.5 & -- \\
\hline 0.015 & 1.116 & 39.6 & 70 & 1.5 & -- \\
\hline 0.02 & 1.123 & 23.7 & 31.5 & 0.4 & 30.1553 \\
\hline 0.025 & 1.129 & 19 & 23.6 & 0.2 & 22.6883 \\
\hline 0.03 & 1.136 & 16.7 & 20.2 & 0.1 & 19.46 \\
\hline 0.032 & 1.138 & 16.1 & 19.3 & 0.1 & 18.6105 \\
\hline 0.035 & 1.142 & 15.4 & 18.2 & 0.1 & 17.5836 \\
\hline 0.04 & 1.149 & 14.4 & 16.9 & 0.1 & 16.3432 \\
\hline 0.05 & 1.162 & 13.2 & 15.3 & 0.1 & 14.8196 \\
\hline 0.06 & 1.175 & 12.4 & 14.3 & 0.1 & 13.8976 \\
\hline 0.07 & 1.188 & 11.9 & 13.7 & 0.1 & 13.3076 \\
\hline 0.08 & 1.201 & 11.6 & 13.3 & 0.05 & 12.8714 \\
\hline 0.09 & 1.215 & 11.3 & 12.9 & 0.05 & 12.5358 \\
\hline 0.1 & 1.228 & 11.1 & 12.7 & 0.05 & 12.3025 \\
\hline 0.118 & 1.251 & 10.8 & 12.3 & 0.05 & 11.9687 \\
\hline 0.13 & 1.267 & 10.7 & 12.2 & 0.05 & 11.8215 \\
\hline 0.15 & 1.293 & 10.5 & 12 & 0.05 & 11.6231 \\
\hline 0.2 & 1.359 & 10.2 & 11.7 & 0.05 & 11.3577 \\
\hline 0.235 & 1.406 & 10.2 & 11.6 & 0.05 & 11.2597 \\
\hline 0.25 & 1.425 & 10.2 & 11.6 & 0.05 & 11.2673 \\
\hline 0.353 & 1.561 & 10.25 & 11.6 & 0.05 & 11.2539 \\
\hline 0.47 & 1.715 & 10.3 & 11.8 & 0.05 & 11.4515 \\
\hline 0.5 & 1.753 & 10.4 & 11.9 & 0.05 & 11.5241 \\
\hline 0.51 & 1.766 & 10.4 & 11.9 & 0.05 & 11.5533 \\
\hline 0.52 & 1.779 & 10.5 & 11.9 & 0.05 & 11.5727 \\
\hline 0.53 & 1.792 & 10.5 & 11.9 & 0.05 & 11.5937 \\
\hline 0.54 & 1.805 & 10.5 & 11.9 & 0.05 & 11.6292 \\
\hline 0.55 & 1.818 & 10.5 & 12 & 0.05 & 11.6521 \\
\hline 0.56 & 1.831 & 10.5 & 12 & 0.05 & 11.6885 \\
\hline
\end{tabular}




\section{B1.2.1.2.2.3 10" Water Reflection}

Case ID: u5o2N23-sp-0935-10

Material: $\mathrm{UO}_{2}\left(\mathrm{NO}_{3}\right)_{2} 3$ Molar Excess Acid

Geometry: Sphere

Isotopics: $93.5 \%{ }^{235} \mathrm{U}$

Reflector: 10 " water reflected

Code: MCNP5 v1.40

Analyst: Mark Murphy

Date: $8 / 2011$ 
CHPRC-01552, Revision 0

Figure B1.2.1.2.2.3-1. $\mathrm{UO}_{2}\left(\mathrm{NO}_{3}\right) 2$ 3M - Sphere - 93.5\% ${ }^{235} \mathrm{U}-10$ " Water Reflection

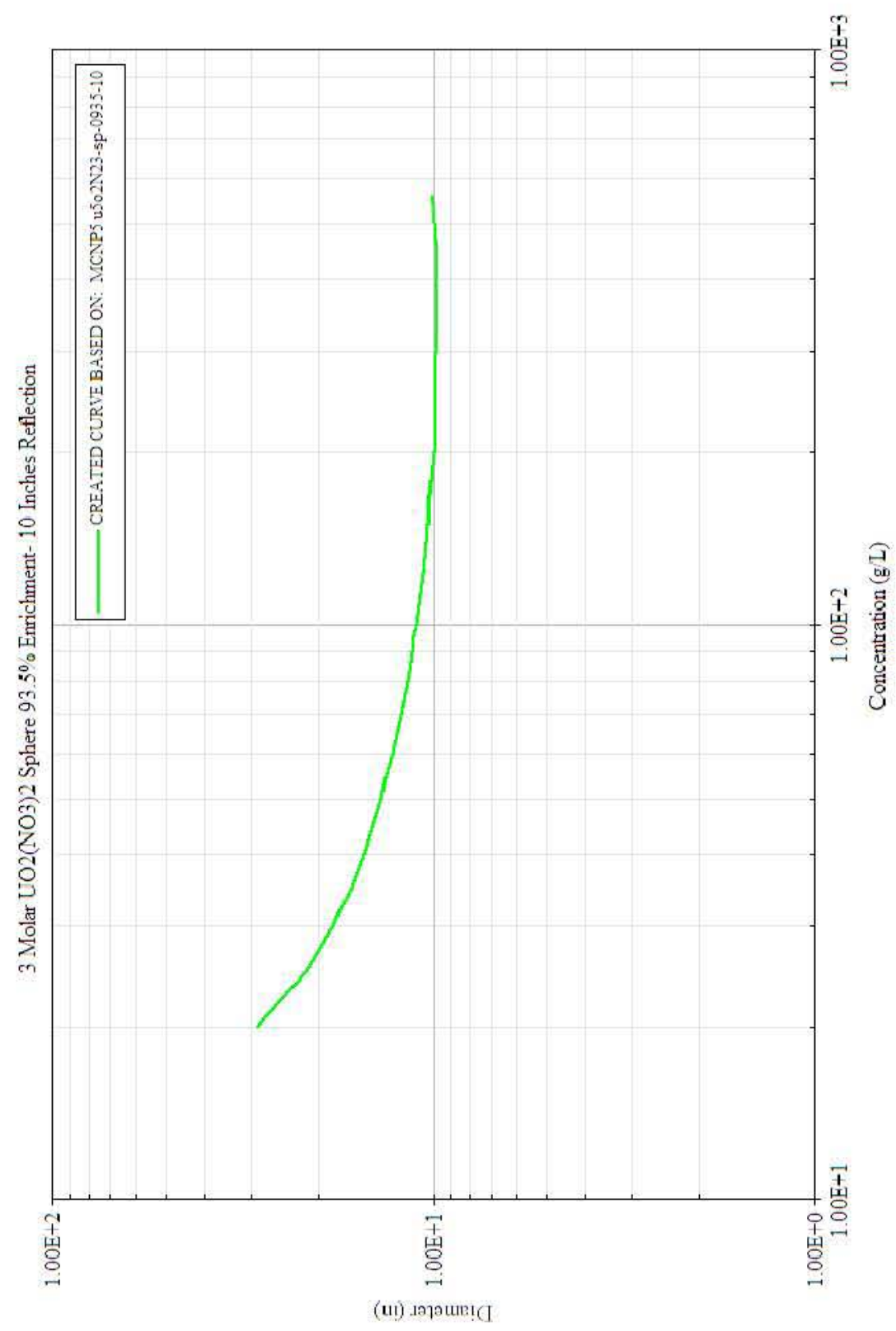


Table B1.2.1.2.2.3-1. Critical Dimensions for $\mathrm{UO}_{2}\left(\mathrm{NO}_{3}\right) 23 \mathrm{M}-$ Sphere $-93.5 \%{ }^{235} \mathrm{U}-10$ " Water Reflection

\begin{tabular}{|c|c|c|c|c|c|}
\hline \multicolumn{6}{|c|}{ 93.5\% U235 UO2(NO3)2 3M Sphere- 10 Inches Reflection } \\
\hline Concentration $(\mathrm{g} / \mathrm{cc})$ & \begin{tabular}{|l|} 
Density \\
$(\mathrm{g} / \mathrm{cc})$
\end{tabular} & $\begin{array}{l}\text { First Diameter } \\
\text { (Inches) }\end{array}$ & $\begin{array}{l}\text { Last Diameter } \\
\text { (Inches) }\end{array}$ & $\begin{array}{l}\text { Delta } \\
\text { (Inches) }\end{array}$ & $\begin{array}{l}\text { Critical Diameter } \\
\text { (Inches) }\end{array}$ \\
\hline 0.012 & 1.112 & 105.5 & 254.3 & 7.4 & -- \\
\hline 0.015 & 1.116 & 39 & 74.1 & 1.7 & -- \\
\hline 0.02 & 1.123 & 22.5 & 30.4 & 0.4 & 28.9724 \\
\hline 0.025 & 1.129 & 17.9 & 22.4 & 0.2 & 21.5734 \\
\hline 0.03 & 1.136 & 15.6 & 19 & 0.1 & 18.304 \\
\hline 0.032 & 1.138 & 15 & 18 & 0.1 & 17.4458 \\
\hline 0.035 & 1.142 & 14.2 & 17 & 0.1 & 16.4132 \\
\hline 0.04 & 1.149 & 13.2 & 15.7 & 0.1 & 15.1759 \\
\hline 0.05 & 1.162 & 12 & 14.1 & 0.1 & 13.6463 \\
\hline 0.06 & 1.175 & 11.3 & 13.1 & 0.1 & 12.7095 \\
\hline 0.07 & 1.188 & 10.8 & 12.35 & 0.05 & 12.0848 \\
\hline 0.08 & 1.201 & 10.4 & 12 & 0.05 & 11.6472 \\
\hline 0.09 & 1.215 & 10 & 11.6 & 0.05 & 11.2911 \\
\hline 0.1 & 1.228 & 9.9 & 11.4 & 0.05 & 11.0278 \\
\hline 0.118 & 1.251 & 9.5 & 11 & 0.05 & 10.7001 \\
\hline 0.13 & 1.267 & 9.4 & 10.8 & 0.05 & 10.5147 \\
\hline 0.15 & 1.293 & 9.2 & 10.6 & 0.05 & 10.3061 \\
\hline 0.2 & 1.359 & 8.9 & 10.3 & 0.05 & 9.9991 \\
\hline 0.235 & 1.406 & 8.8 & 10.2 & 0.05 & 9.8754 \\
\hline 0.25 & 1.425 & 8.8 & 10.2 & 0.1 & 9.8462 \\
\hline 0.353 & 1.561 & 8.7 & 10.1 & 0.05 & 9.7874 \\
\hline 0.47 & 1.715 & 8.8 & 10.2 & 0.05 & 9.8801 \\
\hline 0.5 & 1.753 & 8.8 & 10.3 & 0.05 & 9.9465 \\
\hline 0.51 & 1.766 & 8.8 & 10.3 & 0.05 & 9.9582 \\
\hline 0.52 & 1.779 & 8.9 & 10.3 & 0.05 & 9.9677 \\
\hline 0.53 & 1.792 & 8.9 & 10.3 & 0.05 & 9.997 \\
\hline 0.54 & 1.805 & 8.9 & 10.3 & 0.05 & 10.0132 \\
\hline 0.55 & 1.818 & 8.9 & 10.4 & 0.05 & 10.0246 \\
\hline 0.56 & 1.831 & 8.9 & 10.4 & 0.05 & 10.0515 \\
\hline
\end{tabular}


B1.2.1.2.3 6 Molar Excess Acid

B1.2.1.2.3.1 0"Water Reflection

Case ID: u5o2N26-sp-0935-00

Material: $\mathrm{UO}_{2}\left(\mathrm{NO}_{3}\right)_{2} 6$ Molar Excess Acid

Geometry: Sphere

Isotopics: $93.5 \%{ }^{235} \mathrm{U}$

Reflector: 0 " water reflected

Code: MCNP5 v1.40

Analyst: Mark Murphy

Date: $8 / 2011$ 
Figure B1.2.1.2.3.1-1. $\mathrm{UO}_{2}\left(\mathrm{NO}_{3}\right) 26 \mathrm{M}-$ Sphere $-93.5 \%{ }^{235} \mathrm{U}-0$ " Water Reflection

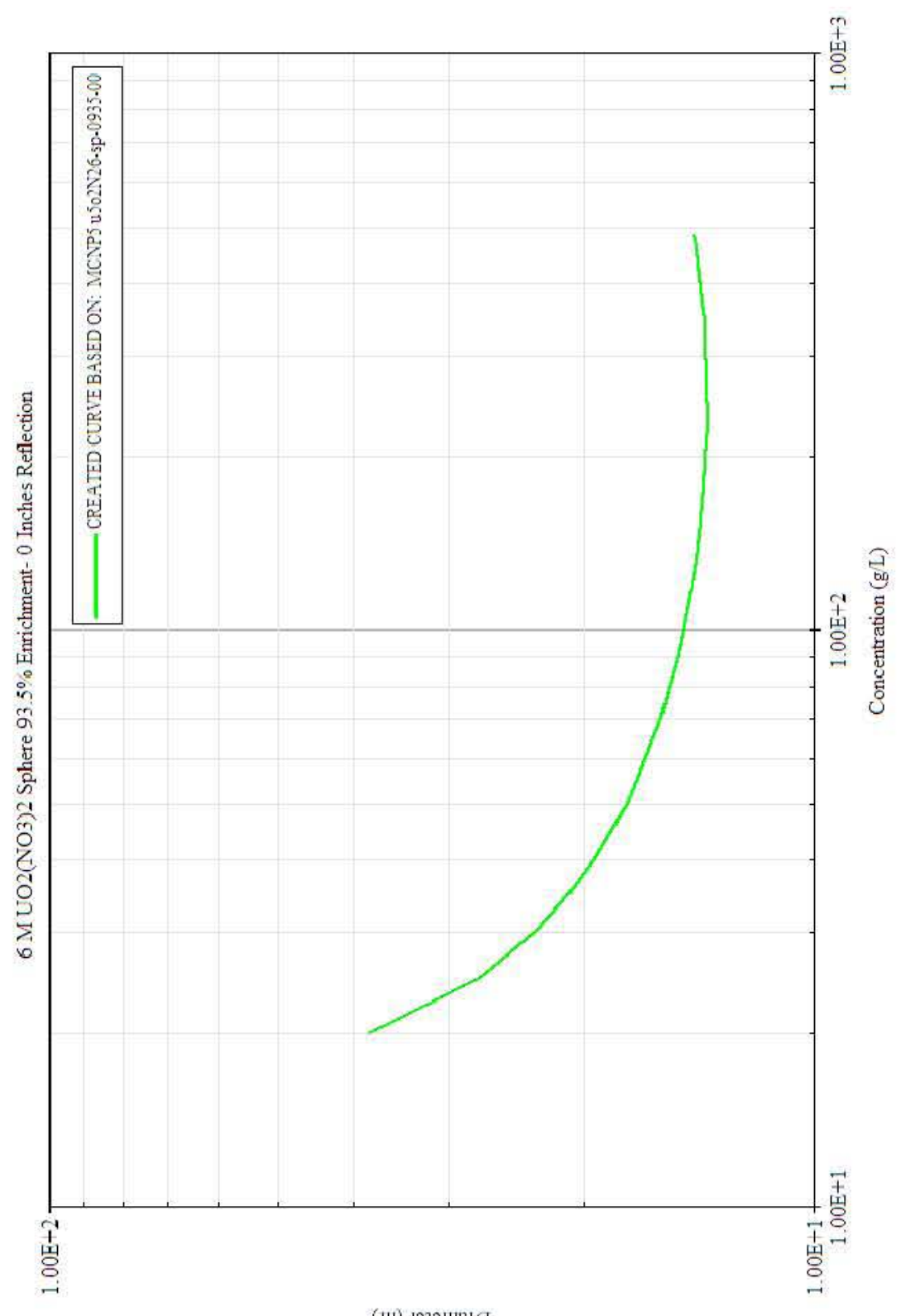

(ui) เәраче! ! 
Table B1.2.1.2.3.1-1. Critical Dimensions for $\mathrm{UO}_{2}\left(\mathrm{NO}_{3}\right) 26 \mathrm{M}-$ Sphere $-93.5 \%{ }^{235} \mathrm{U}-0$ " Water Reflection

\begin{tabular}{|c|c|c|c|c|c|}
\hline \multicolumn{6}{|c|}{ 93.5\% U235 UO2(NO3)2 6M Sphere- 0 Inches Reflection } \\
\hline Concentration $(\mathrm{g} / \mathrm{cc})$ & $\begin{array}{l}\text { Density } \\
(\mathrm{g} / \mathrm{cc})\end{array}$ & $\begin{array}{l}\text { First Diameter } \\
\text { (Inches) }\end{array}$ & $\begin{array}{l}\text { Last Diameter } \\
\text { (Inches) }\end{array}$ & $\begin{array}{l}\text { Delta } \\
\text { (Inches) }\end{array}$ & $\begin{array}{l}\text { Critical Diameter } \\
\text { (Inches) }\end{array}$ \\
\hline 0.012 & 1.202 & 147.8 & 414.6 & 13.3 & -- \\
\hline 0.015 & 1.206 & 58.4 & 133.4 & 3.7 & -- \\
\hline 0.02 & 1.212 & 28.8 & 40.4 & 0.5 & 38.0329 \\
\hline 0.025 & 1.219 & 22.7 & 28.5 & 0.3 & 27.3469 \\
\hline 0.03 & 1.225 & 19.9 & 24 & 0.2 & 23.1782 \\
\hline 0.032 & 1.228 & 19.1 & 22.8 & 0.1 & 22.1309 \\
\hline 0.035 & 1.231 & 18.3 & 21.5 & 0.1 & 20.9007 \\
\hline 0.04 & 1.238 & 17.1 & 20 & 0.1 & 19.4074 \\
\hline 0.05 & 1.251 & 15.7 & 18.1 & 0.1 & 17.5889 \\
\hline 0.06 & 1.264 & 14.9 & 17.1 & 0.1 & 16.5804 \\
\hline 0.07 & 1.277 & 14.3 & 16.3 & 0.1 & 15.8664 \\
\hline 0.08 & 1.289 & 14.2 & 15.8 & 0.1 & 15.4198 \\
\hline 0.09 & 1.302 & 13.8 & 15.5 & 0.1 & 15.0451 \\
\hline 0.1 & 1.315 & 13.5 & 15.2 & 0.05 & 14.7855 \\
\hline 0.118 & 1.338 & 13.1 & 14.8 & 0.05 & 14.437 \\
\hline 0.13 & 1.354 & 13 & 14.6 & 0.05 & 14.2312 \\
\hline 0.15 & 1.38 & 12.95 & 14.35 & 0.05 & 14.0492 \\
\hline 0.2 & 1.444 & 12.6 & 14.1 & 0.05 & 13.7876 \\
\hline 0.235 & 1.489 & 12.5 & 14.1 & 0.05 & 13.7217 \\
\hline 0.25 & 1.508 & 12.5 & 14.1 & 0.05 & 13.7265 \\
\hline 0.353 & 1.639 & 12.7 & 14.2 & 0.05 & 13.855 \\
\hline 0.47 & 1.785 & 13 & 14.6 & 0.05 & 14.25 \\
\hline 0.475 & 1.791 & 13.1 & 14.7 & 0.05 & 14.2626 \\
\hline 0.48 & 1.797 & 13.15 & 14.7 & 0.05 & 14.2869 \\
\hline 0.486 & 1.804 & 13.1 & 14.8 & 0.05 & 14.324 \\
\hline
\end{tabular}




\section{B1.2.1.2.3.2 1"Water Reflection}

Case ID: u5o2N26-sp-0935-01

Material: $\mathrm{UO}_{2}\left(\mathrm{NO}_{3}\right)_{2} 6$ Molar Excess Acid

Geometry: Sphere

Isotopics: $93.5 \%{ }^{235} \mathrm{U}$

Reflector: 1" water reflected

Code: MCNP5 v1.40

Analyst: Mark Murphy

Date: $8 / 2011$ 
Figure B1.2.1.2.3.2-1. $\mathrm{UO}_{2}\left(\mathrm{NO}_{3}\right) 26 \mathrm{M}-$ Sphere $-93.5 \%{ }^{235} \mathrm{U}-1$ " Water Reflection

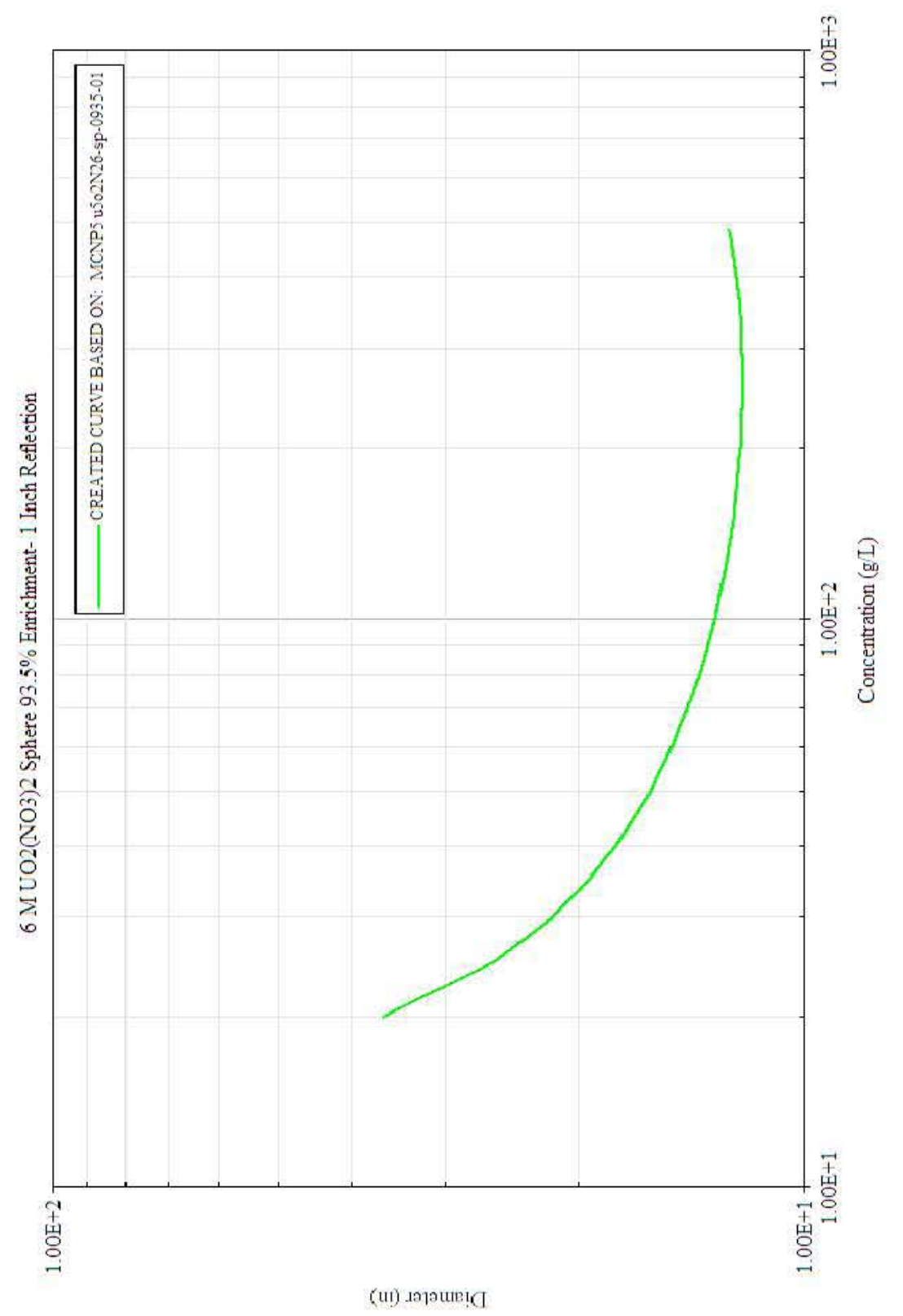


Table B1.2.1.2.3.2-1. Critical Dimensions for $\mathrm{UO}_{2}\left(\mathrm{NO}_{3}\right) 26 \mathrm{M}-$ Sphere $-93.5 \%{ }^{235} \mathrm{U}-1$ " Water Reflection

\begin{tabular}{|c|c|c|c|c|c|}
\hline \multicolumn{6}{|c|}{ 93.5\% U235 UO2(NO3)2 6M Sphere- 1 Inch Reflection } \\
\hline Concentration $(\mathrm{g} / \mathrm{cc})$ & $\begin{array}{l}\text { Density } \\
(\mathrm{g} / \mathrm{cc})\end{array}$ & $\begin{array}{l}\text { First Diameter } \\
\text { (Inches) }\end{array}$ & $\begin{array}{l}\text { Last Diameter } \\
\text { (Inches) }\end{array}$ & $\begin{array}{l}\text { Delta } \\
\text { (Inches) }\end{array}$ & $\begin{array}{l}\text { Critical Diameter } \\
\text { (Inches) }\end{array}$ \\
\hline 0.012 & 1.202 & 189.9 & 438.9 & 12.4 & -- \\
\hline 0.015 & 1.206 & 56.5 & 95 & 1.9 & -- \\
\hline 0.02 & 1.212 & 27.2 & 38.9 & 0.5 & 36.3891 \\
\hline 0.025 & 1.219 & 21.1 & 26.9 & 0.3 & 25.7623 \\
\hline 0.03 & 1.225 & 18.3 & 22.4 & 0.2 & 21.5587 \\
\hline 0.032 & 1.228 & 17.6 & 21.2 & 0.1 & 20.5309 \\
\hline 0.035 & 1.231 & 16.7 & 20 & 0.1 & 19.2801 \\
\hline 0.04 & 1.238 & 15.6 & 18.3 & 0.1 & 17.7833 \\
\hline 0.05 & 1.251 & 14.2 & 16.5 & 0.2 & 16.0069 \\
\hline 0.06 & 1.264 & 13.3 & 15.3 & 0.1 & 14.9559 \\
\hline 0.07 & 1.277 & 12.7 & 14.7 & 0.1 & 14.266 \\
\hline 0.08 & 1.289 & 12.3 & 14.2 & 0.1 & 13.7812 \\
\hline 0.09 & 1.302 & 12.1 & 13.8 & 0.05 & 13.4334 \\
\hline 0.1 & 1.315 & 11.8 & 13.5 & 0.05 & 13.1396 \\
\hline 0.118 & 1.338 & 11.7 & 13.1 & 0.05 & 12.8022 \\
\hline 0.13 & 1.354 & 11.4 & 13 & 0.05 & 12.6073 \\
\hline 0.15 & 1.38 & 11.2 & 12.8 & 0.05 & 12.4026 \\
\hline 0.2 & 1.444 & 11 & 12.5 & 0.05 & 12.1449 \\
\hline 0.235 & \begin{tabular}{|l|}
1.489 \\
\end{tabular} & 10.9 & 12.4 & 0.05 & 12.0795 \\
\hline 0.25 & 1.508 & 10.9 & 12.4 & 0.05 & 12.0409 \\
\hline 0.353 & 1.639 & 11 & 12.5 & 0.05 & 12.1632 \\
\hline 0.47 & 1.785 & 11.3 & 12.9 & 0.05 & 12.5159 \\
\hline 0.475 & 1.791 & 11.3 & 12.9 & 0.05 & 12.5369 \\
\hline 0.48 & 1.797 & 11.3 & 12.9 & 0.05 & 12.5628 \\
\hline 0.486 & 1.804 & 11.4 & 13 & 0.1 & 12.5875 \\
\hline
\end{tabular}




\section{B1.2.1.2.3.3 10" Water Reflection}

Case ID: u5o2N26-sp-0935-10

Material: $\mathrm{UO}_{2}\left(\mathrm{NO}_{3}\right)_{2} 6$ Molar Excess Acid

Geometry: Sphere

Isotopics: $93.5 \%{ }^{235} \mathrm{U}$

Reflector: 10 " water reflected

Code: MCNP5 v1.40

Analyst: Mark Murphy

Date: $8 / 2011$ 
Figure B1.2.1.2.3.3-1. $\mathrm{UO}_{2}\left(\mathrm{NO}_{3}\right) 2$ 6M-Sphere - 93.5\% ${ }^{235} \mathrm{U}-10$ " Water Reflection

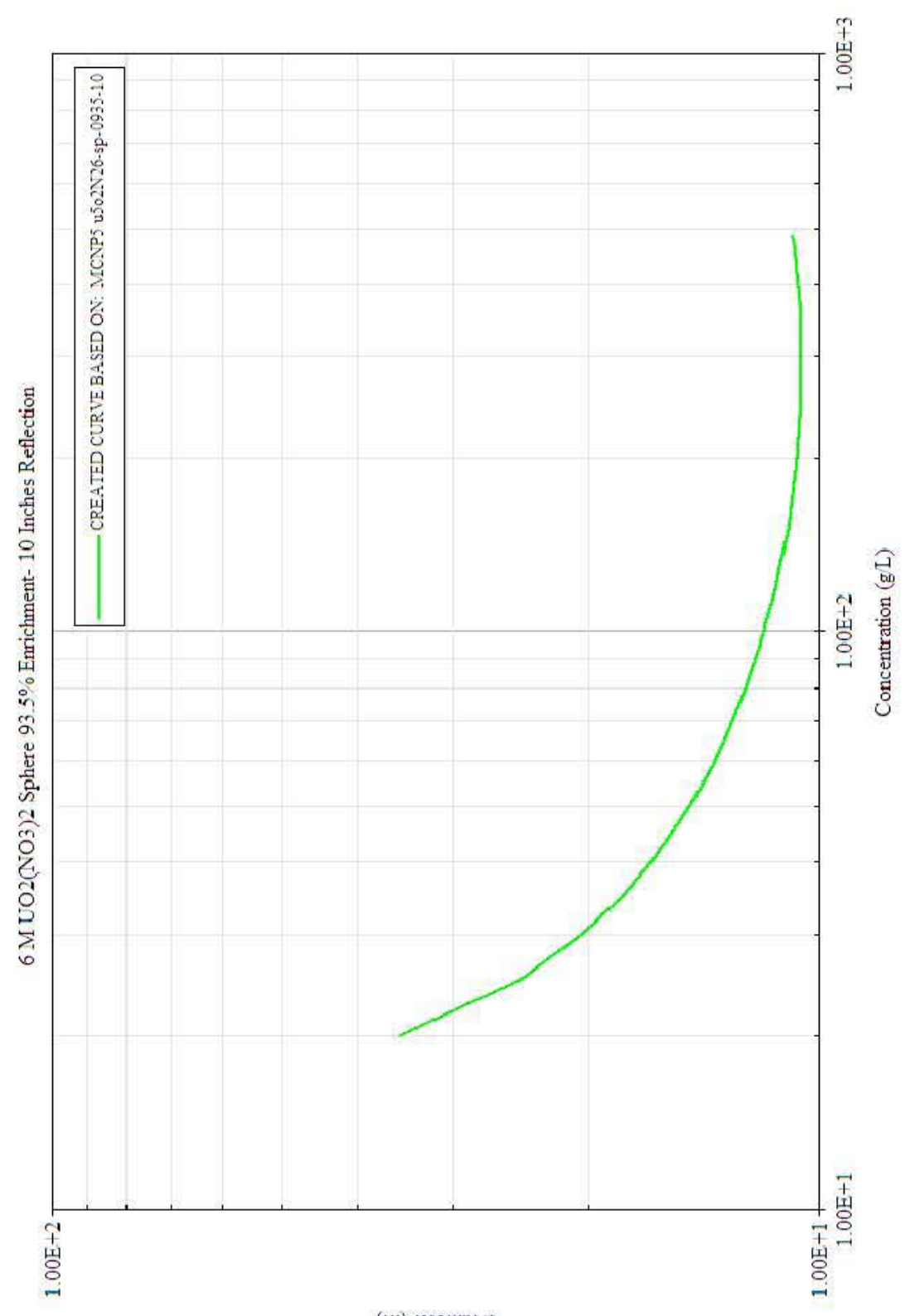

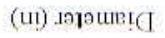


Table B1.2.1.2.3.3-1. Critical Dimensions for $\mathrm{UO}_{2}\left(\mathrm{NO}_{3}\right) 26 \mathrm{M}-$ Sphere $-93.5 \%{ }^{235} \mathrm{U}-10$ " Water Reflection

\begin{tabular}{|c|c|c|c|c|c|}
\hline \multicolumn{6}{|c|}{ 93.5\% U235 UO2(NO3)2 6M Sphere- 10 Inches Reflection } \\
\hline Concentration $(\mathrm{g} / \mathrm{cc})$ & $\begin{array}{l}\text { Density } \\
(\mathrm{g} / \mathrm{cc}) \\
\end{array}$ & $\begin{array}{l}\text { First Diameter } \\
\text { (Inches) }\end{array}$ & $\begin{array}{l}\text { Last Diameter } \\
\text { (Inches) }\end{array}$ & $\begin{array}{l}\text { Delta } \\
\text { (Inches) }\end{array}$ & \begin{tabular}{|l}
$\begin{array}{l}\text { Critical Diameter } \\
\text { (Inches) }\end{array}$ \\
\end{tabular} \\
\hline 0.012 & 1.202 & 134.3 & 430.9 & 14.8 & -- \\
\hline 0.015 & 1.206 & 55.5 & 83.8 & 1.4 & -- \\
\hline 0.02 & 1.212 & 25.7 & 37.7 & 0.6 & 35.2807 \\
\hline 0.025 & 1.219 & 20 & 25.6 & 0.2 & 24.5053 \\
\hline 0.03 & 1.225 & 17.1 & 21.2 & 0.2 & 20.3687 \\
\hline 0.032 & 1.228 & 16.4 & 20 & 0.2 & 19.2771 \\
\hline 0.035 & 1.231 & 15.5 & 18.7 & 0.1 & 18.0048 \\
\hline 0.04 & 1.238 & 14.4 & 17.1 & 0.1 & 16.5359 \\
\hline 0.05 & 1.251 & 12.9 & 15.2 & 0.1 & 14.733 \\
\hline 0.06 & 1.264 & 12.1 & 14.1 & 0.1 & 13.6669 \\
\hline 0.07 & 1.277 & 11.6 & 13.3 & 0.1 & 12.9539 \\
\hline 0.08 & 1.289 & 11.2 & 12.9 & 0.1 & 12.4498 \\
\hline 0.09 & 1.302 & 10.7 & 12.5 & 0.1 & 12.0854 \\
\hline 0.1 & 1.315 & 10.5 & 12.1 & 0.05 & 11.788 \\
\hline 0.118 & 1.338 & 10.2 & 11.8 & 0.05 & 11.4171 \\
\hline 0.13 & 1.354 & 10 & 11.6 & 0.05 & 11.2265 \\
\hline 0.15 & 1.38 & 9.8 & 11.3 & 0.05 & 10.9859 \\
\hline 0.2 & 1.444 & 9.5 & 11 & 0.05 & 10.6787 \\
\hline 0.235 & \begin{tabular}{|l|}
1.489 \\
\end{tabular} & 9.4 & 10.9 & 0.05 & 10.5746 \\
\hline 0.25 & 1.508 & 9.4 & 10.9 & 0.05 & 10.5494 \\
\hline 0.353 & 1.639 & 9.4 & 10.9 & 0.05 & 10.5486 \\
\hline 0.47 & 1.785 & 9.6 & 11.1 & 0.15 & 10.7724 \\
\hline 0.475 & 1.791 & 9.65 & 11.1 & 0.05 & 10.7823 \\
\hline 0.48 & 1.797 & 9.6 & 11.1 & 0.05 & 10.8039 \\
\hline 0.486 & 1.804 & 9.6 & 11.2 & 0.05 & 10.8208 \\
\hline
\end{tabular}


CHPRC-01552, Revision 0 
B1.2.2 Cylinder

B1.2.2.1 $\quad 100 \%{ }^{235} \mathrm{U}$

B1.2.2.1.1 0 Molar Excess Acid

B1.2.2.1.1.1 0"Water Reflection

Case ID: u5o2N20-icyl-1000-00

Material: $\mathrm{UO}_{2}\left(\mathrm{NO}_{3}\right)_{2} 0$ Molar Excess Acid

Geometry: Cylinder

Isotopics: $100 \%{ }^{235} \mathrm{U}$

Reflector: 0" water reflected

Code: MCNP5 v1.40

Analyst: Mark Murphy

Date: $8 / 2011$ 
Figure B1.2.2.1.1.1-1. $\mathrm{UO}_{2}\left(\mathrm{NO}_{3}\right) 2 \mathrm{OM}-\mathrm{Cylinder}-100 \%{ }^{235} \mathrm{U}-0$ " Water Reflection

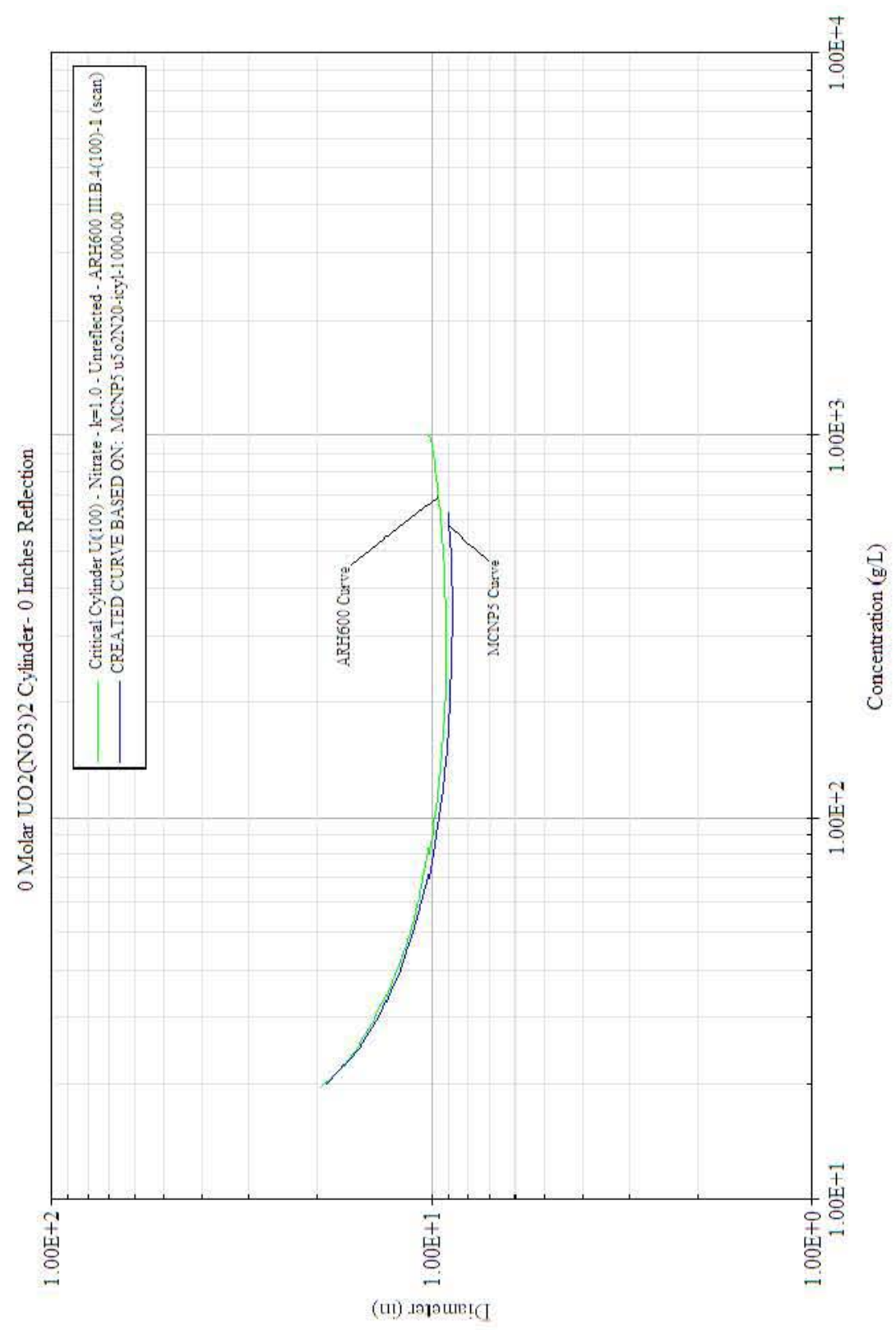


Table B1.2.2.1.1.1- Critical Dimensions for $\mathrm{UO}_{2}\left(\mathrm{NO}_{3}\right) 2 \mathrm{OM}-\mathrm{Cylinder}-100 \%{ }^{235} \mathrm{U}-0$ " Water Reflection

\begin{tabular}{|c|c|c|c|c|c|}
\hline \multicolumn{6}{|c|}{$100 \%$ U235 UO2(NO3) 2 oM Cylinder - 0 Inches of Reflection } \\
\hline Concentration $(\mathrm{g} / \mathrm{cc})$ & $\begin{array}{l}\text { Density } \\
(\mathrm{g} / \mathrm{cc})\end{array}$ & $\begin{array}{l}\text { First Diameter } \\
\text { (Inches) }\end{array}$ & \begin{tabular}{|l}
$\begin{array}{l}\text { Last Diameter } \\
\text { (Inches) }\end{array}$ \\
\end{tabular} & $\begin{array}{l}\text { Delta } \\
\text { (Inches) }\end{array}$ & $\begin{array}{l}\text { Critical Diameter } \\
\text { (Inches) } \\
\end{array}$ \\
\hline 0.012 & 1.013 & 25 & 29.6 & 0.2 & -- \\
\hline 0.015 & 1.017 & 21.7 & 29.6 & 0.4 & -- \\
\hline 0.02 & 1.024 & 15.6 & 19.5 & 0.2 & 18.7408 \\
\hline 0.025 & 1.031 & 13.3 & 16 & 0.1 & 15.3915 \\
\hline 0.03 & 1.037 & 12.1 & 14.2 & 0.1 & 13.6972 \\
\hline 0.032 & 1.04 & 11.7 & 13.7 & 0.1 & 13.2851 \\
\hline 0.035 & 1.044 & 11.3 & 13.1 & 0.1 & 12.7345 \\
\hline 0.04 & 1.051 & 10.7 & 12.4 & 0.05 & 12.0176 \\
\hline 0.05 & 1.064 & 10 & 11.4 & 0.05 & 11.1168 \\
\hline 0.06 & 1.078 & 9.6 & 10.9 & 0.05 & 10.5306 \\
\hline 0.07 & 1.091 & 9.2 & 10.5 & 0.05 & 10.172 \\
\hline 0.08 & 1.105 & 9 & 10.2 & 0.05 & 9.9022 \\
\hline 0.09 & 1.118 & 8.9 & 10 & 0.05 & 9.6949 \\
\hline 0.1 & 1.131 & 8.7 & 9.8 & 0.05 & 9.5471 \\
\hline 0.118 & 1.155 & 8.5 & 9.6 & 0.05 & 9.3249 \\
\hline 0.13 & 1.172 & 8.4 & 9.5 & 0.05 & 9.2254 \\
\hline 0.15 & 1.199 & 8.3 & 9.4 & 0.05 & 9.0685 \\
\hline 0.2 & 1.266 & 8.1 & 9.1 & 0.05 & 8.9077 \\
\hline 0.235 & 1.313 & 8.1 & 9.1 & 0.05 & 8.8109 \\
\hline 0.25 & 1.333 & 8 & 9.1 & 0.05 & 8.8122 \\
\hline 0.353 & 1.471 & 8 & 9.1 & 0.05 & 8.7903 \\
\hline 0.47 & 1.63 & 8.1 & 9.1 & 0.05 & 8.8553 \\
\hline 0.5 & 1.671 & 8.1 & 9.1 & 0.05 & 8.8677 \\
\hline 0.6 & 1.805 & 8.2 & 9.2 & 0.05 & 8.9794 \\
\hline 0.605 & 1.812 & 8.2 & 9.3 & 0.05 & 8.9903 \\
\hline 0.61 & 1.819 & 8.2 & 9.3 & 0.05 & 9.0021 \\
\hline 0.615 & 1.826 & 8.3 & 9.4 & 0.05 & 9.007 \\
\hline 0.62 & 1.832 & 8.2 & 9.3 & 0.05 & 9.0232 \\
\hline 0.625 & 1.839 & 8.2 & 9.3 & 0.05 & 9.0162 \\
\hline 0.629 & 1.844 & 8.2 & 9.3 & 0.05 & 9.0393 \\
\hline
\end{tabular}




\section{B1.2.2.1.1.2 1"Water Reflection}

Case ID: u5o2N20-icyl-1000-01

Material: $\mathrm{UO}_{2}\left(\mathrm{NO}_{3}\right)_{2} 0$ Molar Excess Acid

Geometry: Cylinder

Isotopics: $100 \%{ }^{235} \mathrm{U}$

Reflector: 1" water reflected

Code: MCNP5 v1.40

Analyst: Mark Murphy

Date: $8 / 2011$ 
Figure B1.2.2.1.1.2-1. $\mathrm{UO}_{2}\left(\mathrm{NO}_{3}\right) 2 \mathrm{OM}-\mathrm{Cylinder}-100 \%{ }^{235} \mathrm{U}-1$ " Water Reflection

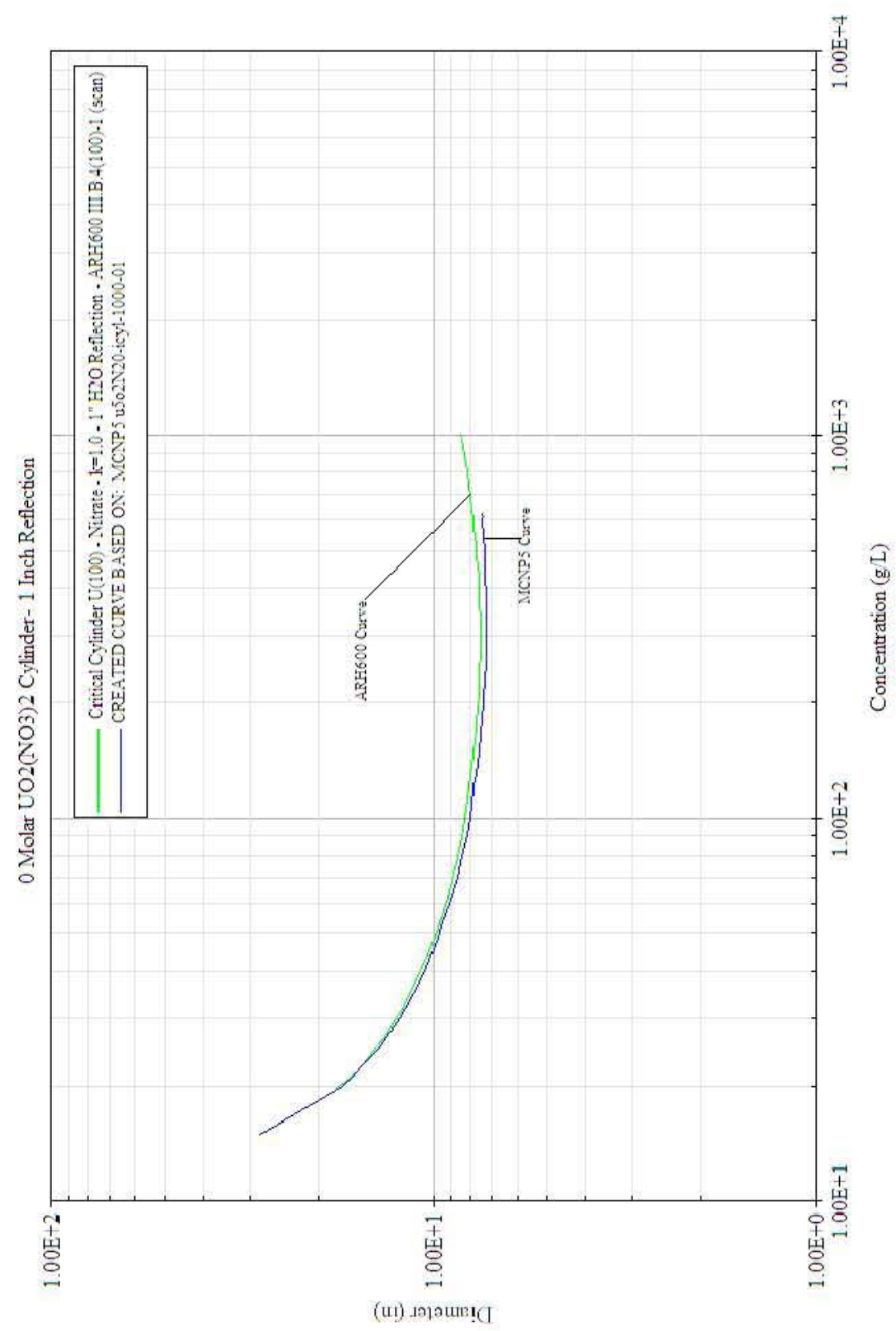


Table B1.2.2.1.1.2-1. Critical Dimensions for $\mathrm{UO}_{2}\left(\mathrm{NO}_{3}\right) 2 \mathrm{OM}-\mathrm{Cylinder}-100 \%{ }^{235} \mathrm{U}-1$ " Water Reflection

\begin{tabular}{|c|c|c|c|c|c|}
\hline \multicolumn{6}{|c|}{$100 \%$ U235 UO2(NO3)2 0M Cylinder - 1 Inch of Reflection } \\
\hline Concentration $(\mathrm{g} / \mathrm{cc})$ & $\begin{array}{l}\text { Density } \\
(\mathrm{g} / \mathrm{cc})\end{array}$ & $\begin{array}{l}\text { First Diameter } \\
\text { (Inches) }\end{array}$ & $\begin{array}{l}\text { Last Diameter } \\
\text { (Inches) }\end{array}$ & $\begin{array}{l}\text { Delta } \\
\text { (Inches) }\end{array}$ & $\begin{array}{l}\text { Critical Diameter } \\
\text { (Inches) } \\
\end{array}$ \\
\hline 0.012 & 1.013 & 25 & 30 & 0.2 & -- \\
\hline 0.015 & 1.017 & 20.2 & 30.1 & 0.5 & 28.1836 \\
\hline 0.02 & 1.024 & 14.2 & 18 & 0.2 & 17.2166 \\
\hline 0.025 & 1.031 & 11.8 & 14.4 & 0.1 & 13.875 \\
\hline 0.03 & 1.037 & 10.6 & 12.7 & 0.1 & 12.2168 \\
\hline 0.032 & 1.04 & 10.2 & 12.2 & 0.1 & 11.7714 \\
\hline 0.035 & 1.044 & 9.8 & 11.6 & 0.1 & 11.1998 \\
\hline 0.04 & 1.051 & 9.2 & 10.8 & 0.05 & 10.4825 \\
\hline 0.05 & 1.064 & 8.5 & 9.9 & 0.05 & 9.5846 \\
\hline 0.06 & 1.078 & 8 & 9.3 & 0.05 & \begin{tabular}{|l|}
9.0271 \\
\end{tabular} \\
\hline 0.07 & 1.091 & 7.7 & 8.9 & 0.05 & 8.635 \\
\hline 0.08 & 1.105 & 7.5 & 8.7 & 0.05 & 8.3673 \\
\hline 0.09 & 1.118 & 7.3 & 8.4 & 0.05 & 8.1411 \\
\hline 0.1 & 1.131 & 7.2 & 8.3 & 0.05 & 8.0036 \\
\hline 0.118 & 1.155 & 7 & 8 & 0.05 & 7.8036 \\
\hline 0.13 & 1.172 & 6.9 & 7.9 & 0.05 & \begin{tabular}{|l}
7.6788 \\
\end{tabular} \\
\hline 0.15 & 1.199 & 6.8 & 7.8 & 0.05 & 7.5422 \\
\hline 0.2 & 1.266 & 6.6 & 7.6 & 0.05 & \begin{tabular}{|l|}
7.356 \\
\end{tabular} \\
\hline 0.235 & 1.313 & 6.5 & 7.5 & 0.05 & 7.2863 \\
\hline 0.25 & 1.333 & 6.5 & 7.5 & 0.05 & 7.2803 \\
\hline 0.353 & 1.471 & 6.5 & 7.5 & 0.05 & 7.2369 \\
\hline 0.47 & 1.63 & 6.5 & 7.5 & 0.05 & 7.2864 \\
\hline 0.5 & 1.671 & 6.5 & 7.5 & 0.05 & 7.3138 \\
\hline 0.6 & 1.805 & 6.6 & 7.7 & 0.05 & 7.4176 \\
\hline 0.605 & 1.812 & 6.6 & 7.7 & 0.05 & 7.4126 \\
\hline 0.61 & 1.819 & 6.6 & 7.7 & 0.05 & 7.4056 \\
\hline 0.615 & 1.826 & 6.6 & 7.7 & 0.05 & 7.431 \\
\hline 0.62 & 1.832 & 6.6 & 7.7 & 0.05 & 7.4379 \\
\hline 0.625 & 1.839 & 6.7 & 7.7 & 0.05 & 7.4234 \\
\hline 0.629 & 1.844 & 6.7 & 7.7 & 0.05 & 7.448 \\
\hline
\end{tabular}




\section{B1.2.2.1.1.3 10" Water Reflection}

Case ID: u5o2N20-icyl-1000-10

Material: $\mathrm{UO}_{2}\left(\mathrm{NO}_{3}\right)_{2} 0$ Molar Excess Acid

Geometry: Cylinder

Isotopics: $100 \%{ }^{235} \mathrm{U}$

Reflector: 10 " water reflected

Code: MCNP5 v1.40

Analyst: Mark Murphy

Date: $8 / 2011$ 
Figure B1.2.2.1.1.3-1. $\mathrm{UO}_{2}\left(\mathrm{NO}_{3}\right) 2 \mathrm{0M}-$ Cylinder - $100 \%{ }^{235} \mathrm{U}-10$ " Water Reflection

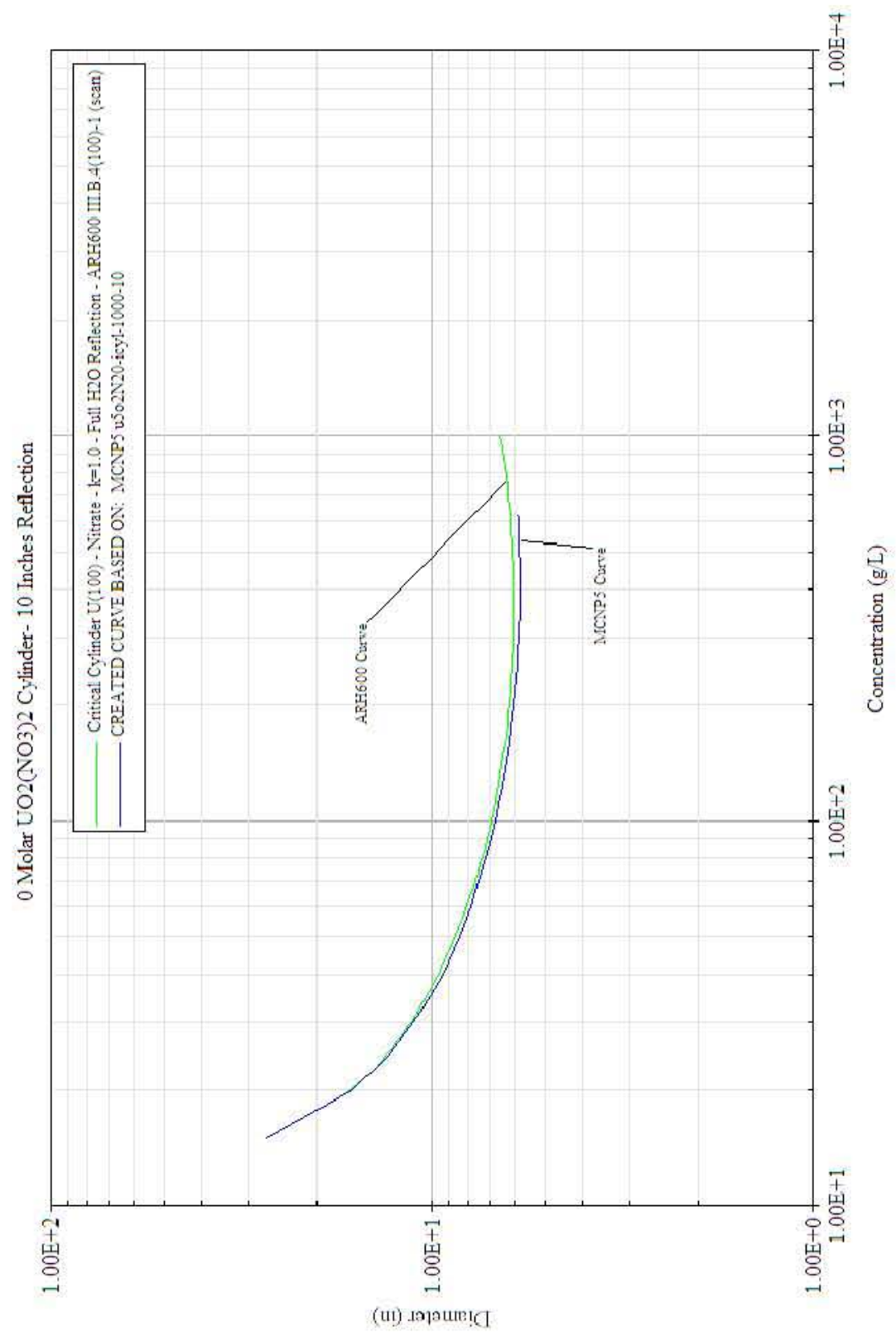


Table B1.2.2.1.1.3-1. Critical Dimensions for $\mathrm{UO}_{2}\left(\mathrm{NO}_{3}\right) 2 \mathrm{OM}-\mathrm{Cylinder}-100 \%{ }^{235} \mathrm{U}-10$ " Water Reflection

\begin{tabular}{|c|c|c|c|c|c|}
\hline \multicolumn{6}{|c|}{$100 \%$ U235 UO2(NO3)2 0M Cylinder - 10 Inches of Reflection } \\
\hline Concentration $(\mathrm{g} / \mathrm{cc})$ & $\begin{array}{l}\text { Density } \\
(\mathrm{g} / \mathrm{cc})\end{array}$ & $\begin{array}{l}\text { First Diameter } \\
\text { (Inches) }\end{array}$ & $\begin{array}{l}\text { Last Diameter } \\
\text { (Inches) }\end{array}$ & $\begin{array}{l}\text { Delta } \\
\text { (Inches) }\end{array}$ & $\begin{array}{l}\text { Critical Diameter } \\
\text { (Inches) } \\
\end{array}$ \\
\hline 0.012 & 1.013 & 25 & 30.2 & 0.2 & -- \\
\hline 0.015 & 1.017 & 19.1 & 28.3 & 0.4 & 27.0299 \\
\hline 0.02 & 1.024 & 13.1 & 16.9 & 0.2 & 16.1253 \\
\hline 0.025 & 1.031 & 10.8 & 13.3 & 0.1 & 12.7855 \\
\hline 0.03 & 1.037 & 9.5 & 11.5 & 0.1 & 11.1028 \\
\hline 0.032 & 1.04 & 9.1 & 11 & 0.1 & 10.6532 \\
\hline 0.035 & 1.044 & 8.7 & 10.4 & 0.05 & 10.057 \\
\hline 0.04 & 1.051 & 8.1 & 9.7 & 0.05 & 9.3302 \\
\hline 0.05 & 1.064 & 7.4 & 8.8 & 0.05 & 8.4217 \\
\hline 0.06 & 1.078 & 6.9 & 8.1 & 0.05 & 7.8489 \\
\hline 0.07 & 1.091 & 6.6 & 7.7 & 0.05 & 7.4828 \\
\hline 0.08 & 1.105 & 6.3 & 7.4 & 0.05 & 7.1655 \\
\hline 0.09 & 1.118 & 6.1 & 7.2 & 0.05 & 6.9537 \\
\hline 0.1 & 1.131 & 6 & 7 & 0.05 & 6.7857 \\
\hline 0.118 & 1.155 & 5.8 & 6.8 & 0.05 & 6.5597 \\
\hline 0.13 & 1.172 & 5.7 & 6.7 & 0.05 & 6.4315 \\
\hline 0.15 & 1.199 & 5.5 & 6.5 & 0.05 & 6.2743 \\
\hline 0.2 & 1.266 & 5.3 & 6.3 & 0.05 & 6.0491 \\
\hline 0.235 & 1.313 & 5.2 & 6.2 & 0.05 & 5.9599 \\
\hline 0.25 & 1.333 & 5.2 & 6.1 & 0.05 & 5.9276 \\
\hline 0.353 & 1.471 & 5.1 & 6 & 0.05 & 5.822 \\
\hline 0.47 & 1.63 & 5.1 & 6 & 0.05 & 5.8133 \\
\hline 0.5 & 1.671 & 5.1 & 6 & 0.05 & 5.8369 \\
\hline 0.6 & 1.805 & 5.1 & 6.1 & 0.05 & 5.8812 \\
\hline 0.605 & 1.812 & 5.1 & 6.1 & 0.05 & 5.8708 \\
\hline 0.61 & 1.819 & 5.1 & 6.1 & 0.05 & 5.8759 \\
\hline 0.615 & 1.826 & 5.2 & 6.1 & 0.05 & 5.8674 \\
\hline 0.62 & 1.832 & 5.1 & 6.1 & 0.05 & 5.8998 \\
\hline 0.625 & 1.839 & 5.1 & 6.1 & 0.05 & 5.9064 \\
\hline 0.629 & 1.844 & 5.1 & 6.1 & 0.05 & 5.9082 \\
\hline
\end{tabular}


B1.2.2.1.2 3 Molar Excess Acid

B1.2.2.1.2.1 0"Water Reflection

Case ID: u5o2N23-icyl-1000-00

Material: $\mathrm{UO}_{2}\left(\mathrm{NO}_{3}\right)_{2} 3$ Molar Excess Acid

Geometry: Cylinder

Isotopics: $100 \%{ }^{235} \mathrm{U}$

Reflector: 0 " water reflected

Code: MCNP5 v1.40

Analyst: Mark Murphy

Date: $8 / 2011$ 
Figure B1.2.2.1.2.1-1. $\mathrm{UO}_{2}\left(\mathrm{NO}_{3}\right) 23 \mathrm{M}-\mathrm{Cylinder}-100 \%{ }^{235} \mathrm{U}-0$ " Water Reflection

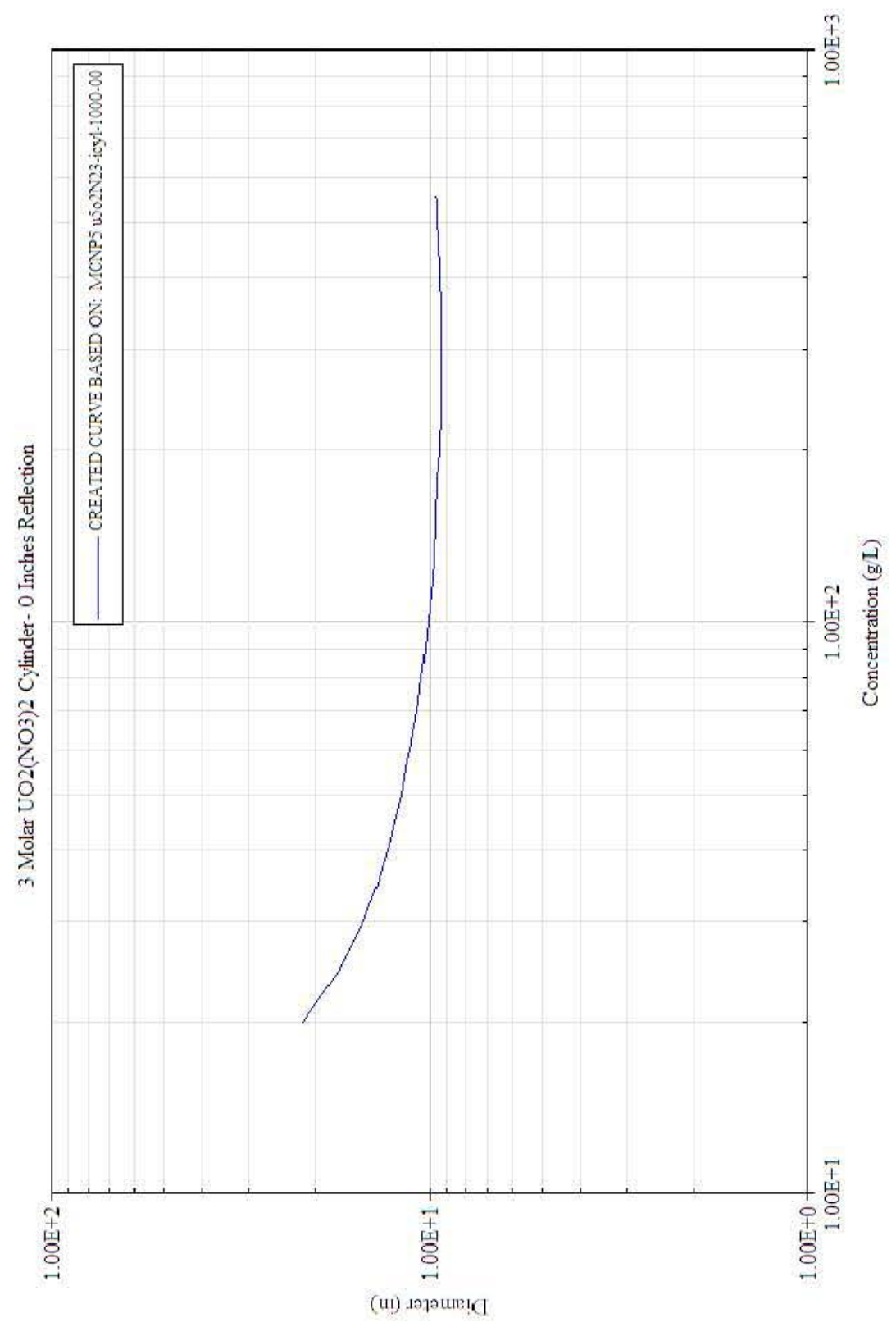


Table B1.2.2.1.2.1-1. Critical Dimensions for $\mathrm{UO}_{2}\left(\mathrm{NO}_{3}\right) 23 \mathrm{M}-\mathrm{Cylinder}-100 \%{ }^{235} \mathrm{U}-0$ " Water Reflection

\begin{tabular}{|c|c|c|c|c|c|}
\hline \multicolumn{6}{|c|}{$100 \%$ U235 UO2(NO3)2 3M Cylinder - 0 Inches of Reflection } \\
\hline Concentration $(\mathrm{g} / \mathrm{cc})$ & \begin{tabular}{|l|} 
Density \\
$(\mathrm{g} / \mathrm{cc})$
\end{tabular} & $\begin{array}{l}\text { First Diameter } \\
\text { (Inches) }\end{array}$ & $\begin{array}{l}\text { Last Diameter } \\
\text { (Inches) }\end{array}$ & $\begin{array}{l}\text { Delta } \\
\text { (Inches) }\end{array}$ & $\begin{array}{l}\text { Critical Diameter } \\
\text { (Inches) }\end{array}$ \\
\hline 0.012 & 1.112 & 28.1 & 31.2 & 0.1 & -- \\
\hline 0.015 & 1.116 & 26.4 & 36.2 & 0.5 & -- \\
\hline 0.02 & 1.123 & 17.5 & 22.4 & 0.2 & 21.4812 \\
\hline 0.025 & 1.129 & 14.6 & 17.7 & 0.1 & 17.0257 \\
\hline 0.03 & 1.136 & 13 & 15.4 & 0.1 & 14.9447 \\
\hline 0.032 & 1.138 & 12.6 & 14.8 & 0.1 & 14.3776 \\
\hline 0.035 & 1.142 & 12.1 & 14.1 & 0.1 & 13.6965 \\
\hline 0.04 & 1.149 & 11.4 & 13.3 & 0.1 & 12.8543 \\
\hline 0.05 & 1.162 & 10.6 & 12.1 & 0.05 & 11.815 \\
\hline 0.06 & 1.175 & 10.1 & 11.5 & 0.05 & 11.1949 \\
\hline 0.07 & 1.188 & 9.7 & 11.1 & 0.05 & 10.7556 \\
\hline 0.08 & 1.201 & 9.5 & 10.8 & 0.05 & 10.4504 \\
\hline 0.09 & 1.215 & 9.3 & 10.5 & 0.05 & 10.201 \\
\hline 0.1 & 1.228 & 9.1 & 10.3 & 0.05 & 10.0615 \\
\hline 0.118 & 1.251 & 8.9 & 10.1 & 0.05 & 9.8218 \\
\hline 0.13 & 1.267 & 8.8 & 10 & 0.05 & 9.7127 \\
\hline 0.15 & 1.293 & 8.7 & 9.8 & 0.05 & 9.5996 \\
\hline 0.2 & 1.359 & 8.6 & 9.7 & 0.05 & 9.3776 \\
\hline 0.235 & 1.405 & 8.5 & 9.6 & 0.05 & 9.3239 \\
\hline 0.25 & 1.425 & 8.5 & 9.6 & 0.05 & 9.3106 \\
\hline 0.353 & 1.56 & 8.5 & 9.6 & 0.05 & 9.3041 \\
\hline 0.47 & 1.714 & 8.6 & 9.7 & 0.05 & 9.4395 \\
\hline 0.5 & 1.753 & 8.7 & 9.8 & 0.05 & 9.5014 \\
\hline 0.51 & 1.766 & 8.7 & 9.8 & 0.05 & 9.5024 \\
\hline 0.52 & 1.779 & 8.75 & 9.8 & 0.05 & 9.5289 \\
\hline 0.53 & 1.792 & 8.7 & 9.8 & 0.05 & 9.5459 \\
\hline 0.54 & 1.805 & 8.7 & 9.8 & 0.05 & 9.5614 \\
\hline 0.55 & 1.818 & 8.7 & 9.9 & 0.05 & 9.5748 \\
\hline 0.56 & 1.831 & 8.7 & 9.9 & 0.05 & 9.6162 \\
\hline
\end{tabular}




\section{B1.2.2.1.2.2 1"Water Reflection}

Case ID: u5o2N23-icyl-1000-01

Material: $\mathrm{UO}_{2}\left(\mathrm{NO}_{3}\right)_{2} 3$ Molar Excess Acid

Geometry: Cylinder

Isotopics: $100 \%{ }^{235} \mathrm{U}$

Reflector: 1" water reflected

Code: MCNP5 v1.40

Analyst: Mark Murphy

Date: $8 / 2011$ 
CHPRC-01552, Revision 0

Figure B1.2.2.1.2.2-1. $\mathrm{UO}_{2}\left(\mathrm{NO}_{3}\right) 23 \mathrm{M}-\mathrm{Cylinder}-100 \%{ }^{235} \mathrm{U}-1$ " Water Reflection

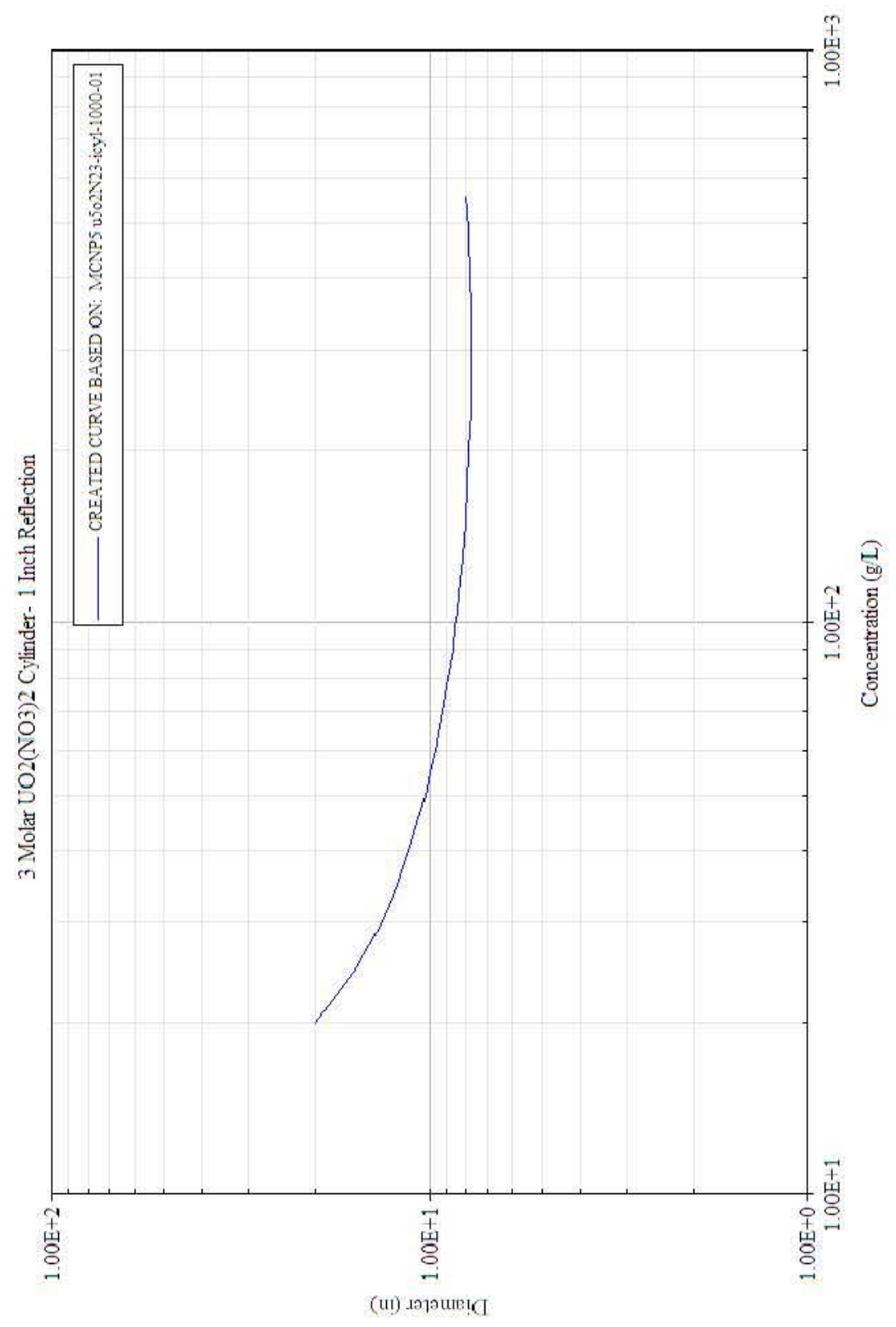


Table B1.2.2.1.2.2-1. Critical Dimensions for $\mathrm{UO}_{2}\left(\mathrm{NO}_{3}\right) 23 \mathrm{M}-\mathrm{Cylinder}-100 \%{ }^{235} \mathrm{U}-1$ " Water Reflection

\begin{tabular}{|c|c|c|c|c|c|}
\hline \multicolumn{6}{|c|}{$100 \%$ U235 UO2(NO3)2 3M Cylinder - 1 Inch of Reflection } \\
\hline Concentration $(\mathrm{g} / \mathrm{cc})$ & $\begin{array}{l}\text { Density } \\
(\mathrm{g} / \mathrm{cc})\end{array}$ & $\begin{array}{l}\text { First Diameter } \\
\text { (Inches) }\end{array}$ & $\begin{array}{l}\text { Last Diameter } \\
\text { (Inches) }\end{array}$ & $\begin{array}{l}\text { Delta } \\
\text { (Inches) }\end{array}$ & $\begin{array}{l}\text { Critical Diameter } \\
\text { (Inches) }\end{array}$ \\
\hline 0.012 & 1.112 & 28.3 & 31.8 & 0.1 & -- \\
\hline 0.015 & 1.116 & 24.9 & 36 & 0.5 & -- \\
\hline 0.02 & 1.123 & 15.9 & 20.9 & 0.2 & 19.9104 \\
\hline 0.025 & 1.129 & 13 & 16.1 & 0.1 & 15.537 \\
\hline 0.03 & 1.136 & 11.5 & 13.9 & 0.1 & 13.3692 \\
\hline 0.032 & 1.138 & 11.1 & 13.3 & 0.1 & 12.8132 \\
\hline 0.035 & 1.142 & 10.6 & 12.5 & 0.1 & 12.1427 \\
\hline 0.04 & 1.149 & 9.9 & 11.7 & 0.1 & 11.2976 \\
\hline 0.05 & 1.162 & 9.1 & 10.6 & 0.05 & 10.2479 \\
\hline 0.06 & 1.175 & 8.5 & 9.9 & 0.05 & 9.6407 \\
\hline 0.07 & 1.188 & 8.2 & 9.5 & 0.05 & 9.2014 \\
\hline 0.08 & 1.201 & 7.9 & 9.2 & 0.05 & 8.8849 \\
\hline 0.09 & 1.215 & 7.7 & 9 & 0.05 & 8.643 \\
\hline 0.1 & 1.228 & 7.6 & 8.8 & 0.05 & 8.4639 \\
\hline 0.118 & 1.251 & 7.4 & 8.5 & 0.05 & 8.2402 \\
\hline 0.13 & 1.267 & 7.3 & 8.4 & 0.05 & 8.0995 \\
\hline 0.15 & 1.293 & 7.2 & 8.3 & 0.05 & 7.9907 \\
\hline 0.2 & 1.359 & 7 & 8 & 0.05 & 7.8087 \\
\hline 0.235 & 1.405 & 6.9 & 8 & 0.05 & 7.7248 \\
\hline 0.25 & 1.425 & 6.9 & 7.9 & 0.05 & 7.7104 \\
\hline 0.353 & 1.56 & 6.9 & 7.9 & 0.05 & 7.7056 \\
\hline 0.47 & 1.714 & 7 & 8.1 & 0.05 & 7.8091 \\
\hline 0.5 & 1.753 & 7 & 8.1 & 0.05 & 7.8437 \\
\hline 0.51 & 1.766 & 7 & 8.1 & 0.05 & 7.8701 \\
\hline 0.52 & 1.779 & 7.1 & 8.1 & 0.05 & 7.9006 \\
\hline 0.53 & 1.792 & 7.1 & 8.1 & 0.05 & 7.9033 \\
\hline 0.54 & 1.805 & 7.1 & 8.2 & 0.05 & 7.9 \\
\hline 0.55 & 1.818 & 7.1 & 8.2 & 0.05 & 7.9482 \\
\hline 0.56 & 1.831 & 7.1 & 8.2 & 0.05 & 7.9489 \\
\hline
\end{tabular}




\section{B1.2.2.1.2.3 10" Water Reflection}

Case ID: u5o2N23-icyl-1000-10

Material: $\mathrm{UO}_{2}\left(\mathrm{NO}_{3}\right)_{2} 3$ Molar Excess Acid

Geometry: Cylinder

Isotopics: $100 \%{ }^{235} \mathrm{U}$

Reflector: 10 " water reflected

Code: MCNP5 v1.40

Analyst: Mark Murphy

Date: $8 / 2011$ 
Figure B1.2.2.1.2.3-1. $\mathrm{UO}_{2}\left(\mathrm{NO}_{3}\right) 23 \mathrm{M}-$ Cylinder - $100 \%{ }^{235} \mathrm{U}-10$ " Water Reflection

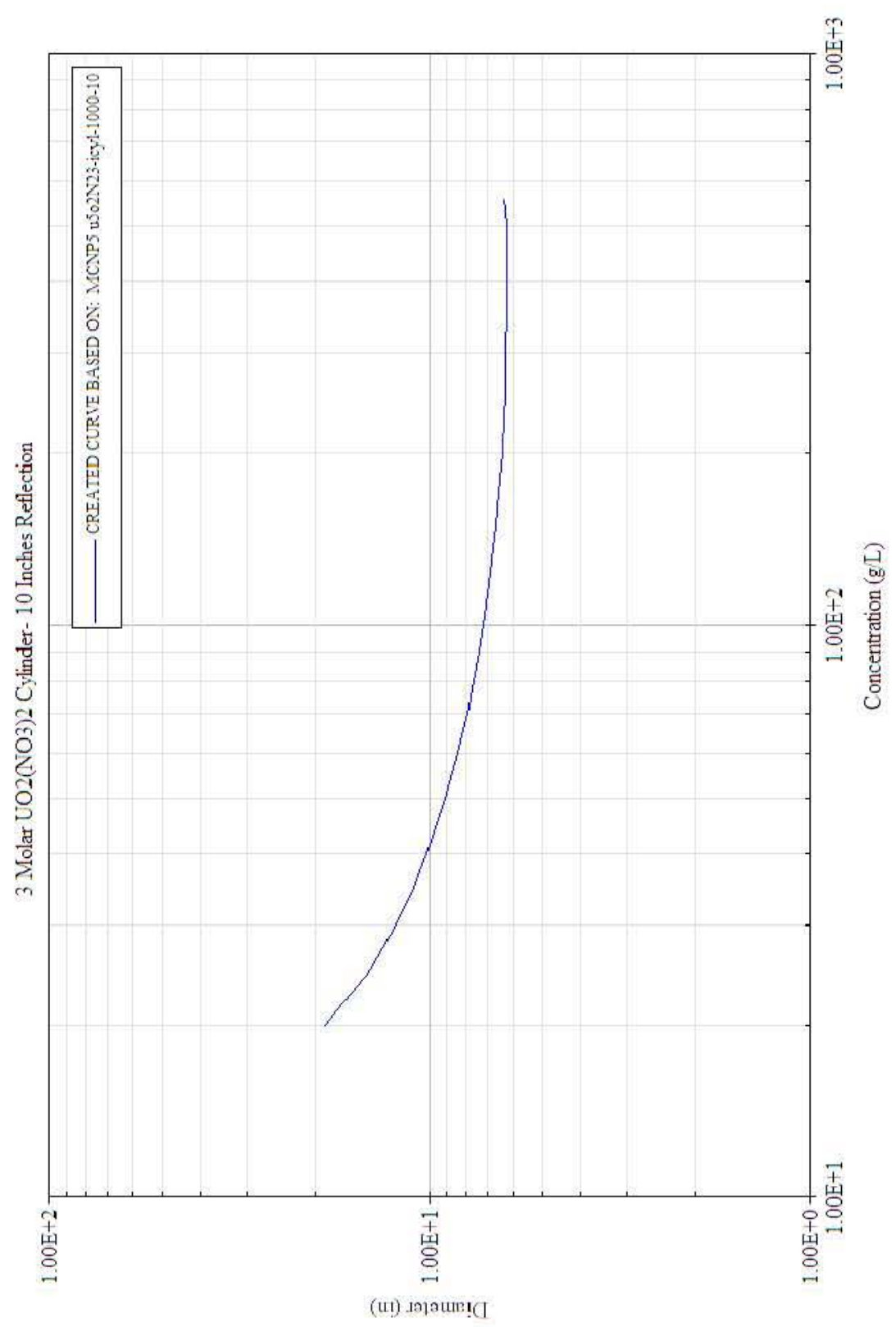


Table B1.2.2.1.2.3-1. Critical Dimensions for $\mathrm{UO}_{2}\left(\mathrm{NO}_{3}\right) 23 \mathrm{M}-\mathrm{Cylinder}-100 \%{ }^{235} \mathrm{U}-10$ " Water Reflection

\begin{tabular}{|c|c|c|c|c|c|}
\hline \multicolumn{6}{|c|}{$100 \%$ U235 UO2(NO3)2 3M Cylinder - 10 Inches of Reflection } \\
\hline Concentration $(\mathrm{g} / \mathrm{cc})$ & $\begin{array}{l}\text { Density } \\
(\mathrm{g} / \mathrm{cc})\end{array}$ & $\begin{array}{l}\text { First Diameter } \\
\text { (Inches) }\end{array}$ & $\begin{array}{l}\text { Last Diameter } \\
\text { (Inches) }\end{array}$ & $\begin{array}{l}\text { Delta } \\
\text { (Inches) }\end{array}$ & $\begin{array}{l}\text { Critical Diameter } \\
\text { (Inches) }\end{array}$ \\
\hline 0.012 & 1.112 & 28.4 & 32.3 & 0.2 & -- \\
\hline 0.015 & 1.116 & 23.7 & 36.1 & 0.6 & -- \\
\hline 0.02 & 1.123 & 14.8 & 19.8 & 0.2 & 18.8404 \\
\hline 0.025 & 1.129 & 11.9 & 14.9 & 0.1 & 14.2793 \\
\hline 0.03 & 1.136 & 10.4 & 12.7 & 0.1 & 12.1867 \\
\hline 0.032 & 1.138 & 9.9 & 12.1 & 0.1 & 11.6619 \\
\hline 0.035 & 1.142 & 9.4 & 11.4 & 0.1 & 10.9736 \\
\hline 0.04 & 1.149 & 8.7 & 10.5 & 0.1 & 10.0943 \\
\hline 0.05 & 1.162 & 7.9 & 9.4 & 0.05 & 9.039 \\
\hline 0.06 & 1.175 & 7.4 & 8.7 & 0.05 & 8.3847 \\
\hline 0.07 & 1.188 & 7 & 8.2 & 0.05 & 7.936 \\
\hline 0.08 & 1.201 & 6.7 & 7.9 & 0.05 & 7.6403 \\
\hline 0.09 & 1.215 & 6.5 & 7.7 & 0.05 & 7.3776 \\
\hline 0.1 & 1.228 & 6.3 & 7.5 & 0.05 & 7.1887 \\
\hline 0.118 & 1.251 & 6.1 & 7.2 & 0.05 & 6.9294 \\
\hline 0.13 & 1.267 & 6 & 7.1 & 0.05 & 6.808 \\
\hline 0.15 & 1.293 & 5.8 & 6.9 & 0.05 & 6.6401 \\
\hline 0.2 & 1.359 & 5.6 & 6.6 & 0.05 & 6.4018 \\
\hline 0.235 & 1.405 & 5.6 & 6.6 & 0.05 & 6.3086 \\
\hline 0.25 & 1.425 & 5.5 & 6.5 & 0.05 & 6.2797 \\
\hline 0.353 & 1.56 & 5.4 & 6.5 & 0.05 & 6.1922 \\
\hline 0.47 & 1.714 & 5.5 & 6.5 & 0.05 & 6.2272 \\
\hline 0.5 & 1.753 & 5.5 & 6.5 & 0.05 & 6.236 \\
\hline 0.51 & 1.766 & 5.5 & 6.5 & 0.05 & 6.2444 \\
\hline 0.52 & 1.779 & 5.5 & 6.5 & 0.05 & 6.2764 \\
\hline 0.53 & 1.792 & 5.5 & 6.5 & 0.05 & 6.2729 \\
\hline 0.54 & 1.805 & 5.5 & 6.5 & 0.05 & 6.2769 \\
\hline 0.55 & 1.818 & 5.5 & 6.6 & 0.05 & 6.303 \\
\hline 0.56 & 1.831 & 5.5 & 6.6 & 0.05 & 6.3007 \\
\hline
\end{tabular}


B1.2.2.1.3 6 Molar Excess Acid

B1.2.2.1.3.1 0"Water Reflection

Case ID: u5o2N26-icyl-1000-00

Material: $\mathrm{UO}_{2}\left(\mathrm{NO}_{3}\right)_{2} 6$ Molar Excess Acid

Geometry: Cylinder

Isotopics: $100 \%{ }^{235} \mathrm{U}$

Reflector: 0 " water reflected

Code: MCNP5 v1.40

Analyst: Mark Murphy

Date: $8 / 2011$ 
CHPRC-01552, Revision 0

Figure B1.2.2.1.3.1-1. $\mathrm{UO}_{2}\left(\mathrm{NO}_{3}\right) 26 \mathrm{M}-\mathrm{Cylinder}-100 \%{ }^{235} \mathrm{U}-0$ " Water Reflection

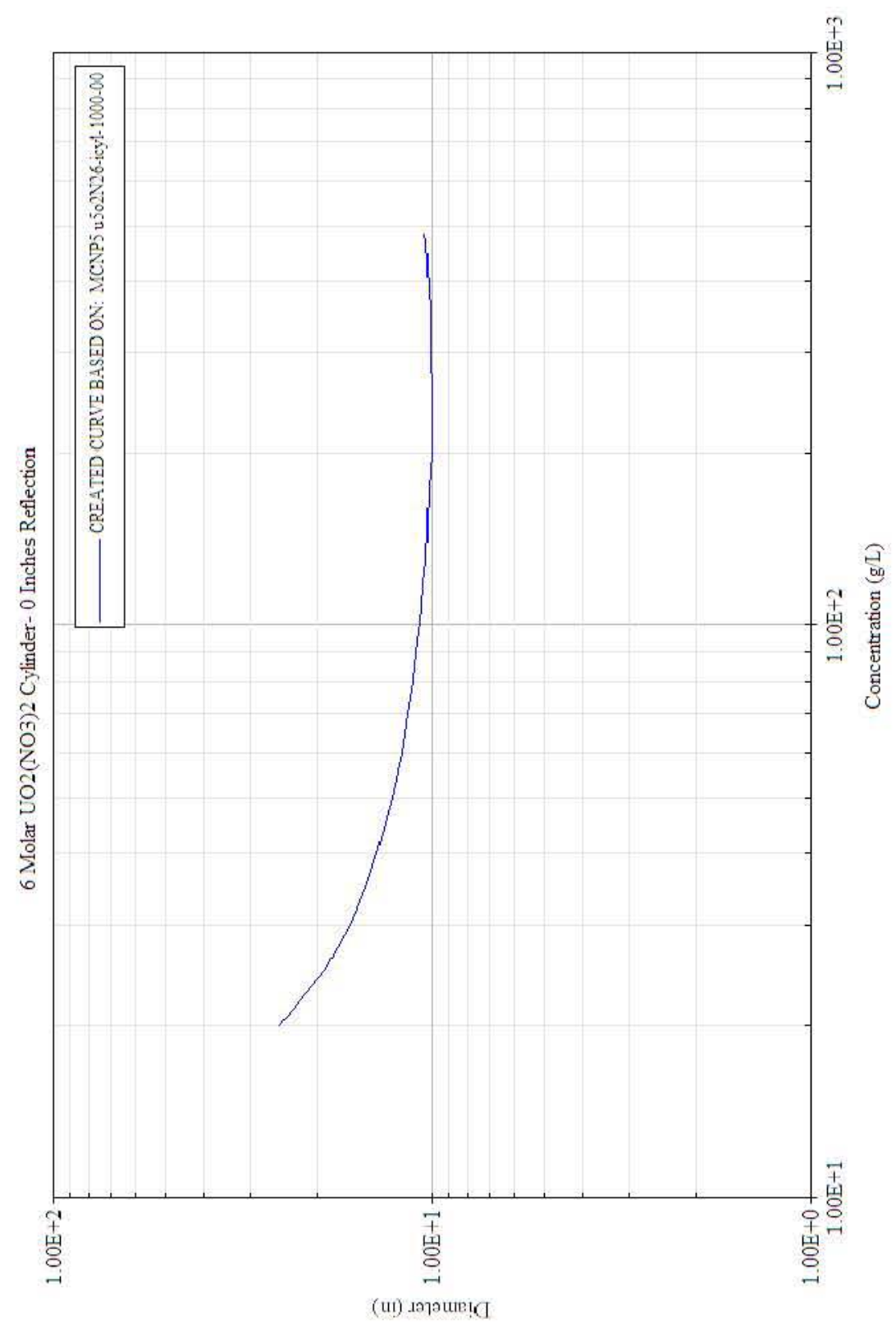


Table B1.2.2.1.3.1-1. Critical Dimensions for $\mathrm{UO}_{2}\left(\mathrm{NO}_{3}\right) 26 \mathrm{M}-\mathrm{Cylinder}-100 \%{ }^{235} \mathrm{U}-0$ " Water Reflection

\begin{tabular}{|c|c|c|c|c|c|}
\hline \multicolumn{6}{|c|}{$100 \%$ U235 UO2(NO3)2 6M Cylinder - 0 Inches of Reflection } \\
\hline Concentration $(\mathrm{g} / \mathrm{cc})$ & $\begin{array}{l}\text { Density } \\
(\mathrm{g} / \mathrm{cc})\end{array}$ & $\begin{array}{l}\text { First Diameter } \\
\text { (Inches) }\end{array}$ & $\begin{array}{l}\text { Last Diameter } \\
\text { (Inches) }\end{array}$ & $\begin{array}{l}\text { Delta } \\
\text { (Inches) }\end{array}$ & \begin{tabular}{|l} 
Critical Diameter \\
(Inches)
\end{tabular} \\
\hline 0.012 & 1.202 & 29.6 & 32.8 & 0.1 & -- \\
\hline 0.015 & 1.206 & 25 & 29.3 & 0.2 & -- \\
\hline 0.02 & 1.212 & 19.7 & 26.4 & 0.3 & 25.198 \\
\hline 0.025 & 1.219 & 16.1 & 19.8 & 0.1 & 19.0428 \\
\hline 0.03 & 1.225 & 14.2 & 17 & 0.1 & 16.3636 \\
\hline 0.032 & 1.228 & 13.7 & 16.2 & 0.1 & 15.6767 \\
\hline 0.035 & 1.231 & 13.1 & 15.4 & 0.1 & 14.8686 \\
\hline 0.04 & 1.238 & 12.3 & 14.3 & 0.1 & 13.885 \\
\hline 0.05 & 1.251 & 11.4 & 13.1 & 0.05 & 12.7008 \\
\hline 0.06 & 1.264 & 10.8 & 12.3 & 0.05 & 11.9626 \\
\hline 0.07 & 1.277 & 10.4 & \begin{tabular}{|l|}
11.8 \\
\end{tabular} & 0.05 & 11.4824 \\
\hline 0.08 & 1.289 & 10.1 & 11.5 & 0.05 & 11.1457 \\
\hline 0.09 & 1.302 & 9.9 & 11.2 & 0.05 & 10.9174 \\
\hline 0.1 & 1.315 & 9.7 & 11 & 0.05 & 10.6998 \\
\hline 0.118 & 1.338 & 9.5 & 10.8 & 0.05 & 10.4669 \\
\hline 0.13 & 1.354 & 9.4 & 10.6 & 0.05 & 10.3325 \\
\hline 0.15 & 1.38 & 9.3 & 10.5 & 0.05 & 10.1846 \\
\hline 0.2 & 1.444 & 9.1 & 10.3 & 0.05 & 9.9921 \\
\hline 0.235 & 1.489 & 9.1 & 10.2 & 0.05 & 9.9631 \\
\hline 0.25 & 1.508 & 9 & 10.2 & 0.05 & 9.9423 \\
\hline 0.353 & 1.638 & 9.1 & 10.3 & 0.05 & 10.043 \\
\hline 0.47 & 1.784 & 9.4 & 10.6 & 0.05 & 10.3112 \\
\hline 0.475 & 1.79 & 9.4 & 10.6 & 0.05 & 10.3209 \\
\hline 0.48 & 1.796 & 9.4 & 10.7 & 0.05 & 10.352 \\
\hline 0.486 & 1.804 & 9.4 & 10.6 & 0.05 & 10.3525 \\
\hline
\end{tabular}




\section{B1.2.2.1.3.2 1"Water Reflection}

Case ID: u5o2N26-icyl-1000-01

Material: $\mathrm{UO}_{2}\left(\mathrm{NO}_{3}\right)_{2} 6$ Molar Excess Acid

Geometry: Cylinder

Isotopics: $100 \%{ }^{235} \mathrm{U}$

Reflector: 1" water reflected

Code: MCNP5 v1.40

Analyst: Mark Murphy

Date: $8 / 2011$ 
CHPRC-01552, Revision 0

Figure B1.2.2.1.3.2-1. $\mathrm{UO}_{2}\left(\mathrm{NO}_{3}\right) 26 \mathrm{M}-\mathrm{Cylinder}-100 \%{ }^{235} \mathrm{U}-1$ " Water Reflection

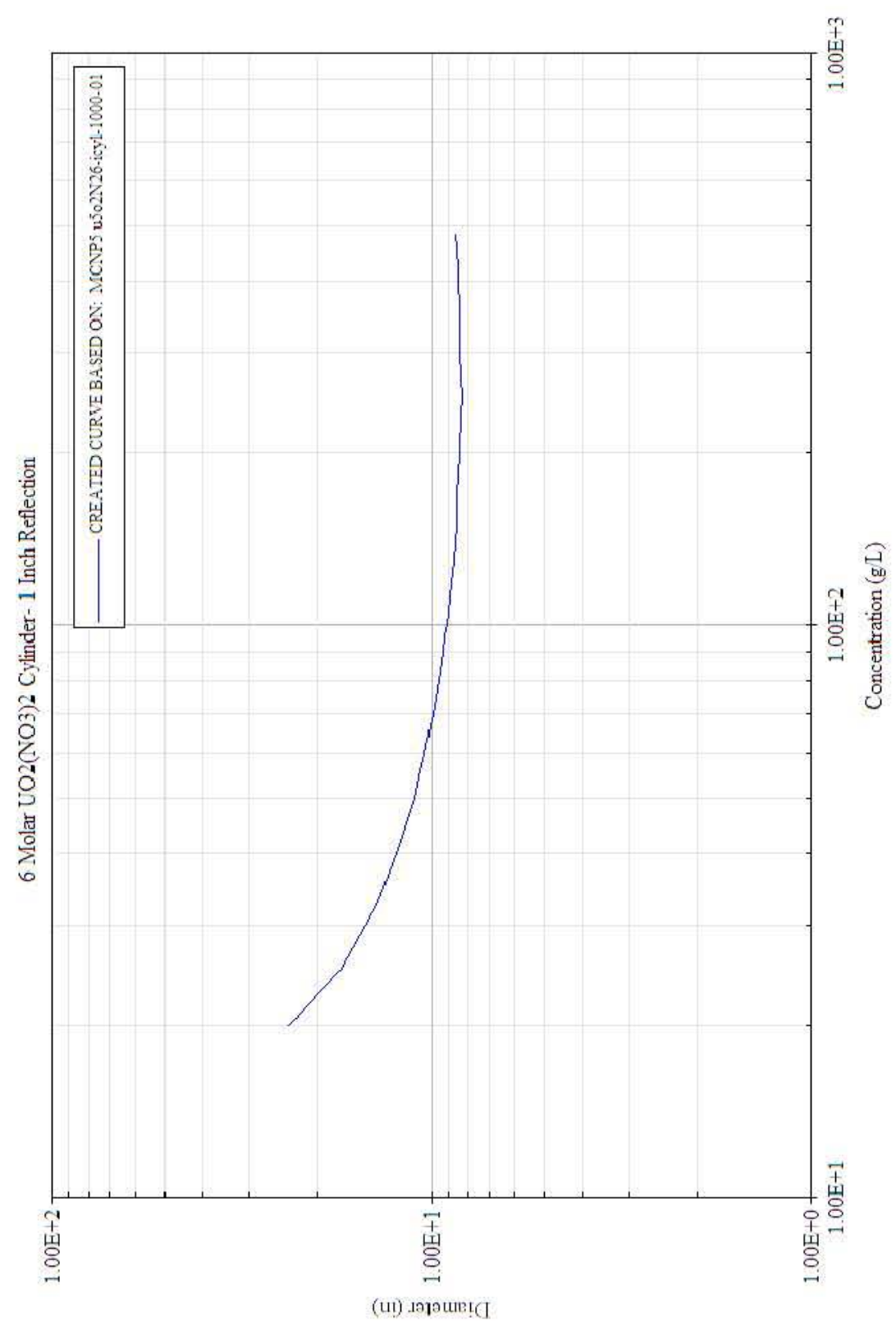


Table B1.2.2.1.3.2-1. Critical Dimensions for $\mathrm{UO}_{2}\left(\mathrm{NO}_{3}\right) 26 \mathrm{M}-\mathrm{Cylinder}-100 \%{ }^{235} \mathrm{U}-1$ " Water Reflection

\begin{tabular}{|c|c|c|c|c|c|}
\hline \multicolumn{6}{|c|}{$100 \%$ U235 UO2(NO3)2 6M Cylinder - 1 Inch of Reflection } \\
\hline Concentration $(\mathrm{g} / \mathrm{cc})$ & \begin{tabular}{|l|} 
Density \\
$(\mathrm{g} / \mathrm{cc})$
\end{tabular} & $\begin{array}{l}\text { First Diameter } \\
\text { (Inches) }\end{array}$ & $\begin{array}{l}\text { Last Diameter } \\
\text { (Inches) }\end{array}$ & $\begin{array}{l}\text { Delta } \\
\text { (Inches) }\end{array}$ & $\begin{array}{l}\text { Critical Diameter } \\
\text { (Inches) }\end{array}$ \\
\hline 0.012 & 1.202 & 29.9 & 33.6 & 0.1 & -- \\
\hline 0.015 & 1.206 & 25 & 29.5 & 0.2 & -- \\
\hline 0.02 & 1.212 & 18.2 & 24.8 & 0.3 & 23.5805 \\
\hline 0.025 & 1.219 & 14.5 & 18.2 & 0.1 & 17.4369 \\
\hline 0.03 & 1.225 & 12.6 & 15.3 & 0.1 & 14.8413 \\
\hline 0.032 & 1.228 & 12.1 & 14.6 & 0.1 & 14.0663 \\
\hline 0.035 & 1.231 & 11.5 & 13.8 & 0.1 & 13.3011 \\
\hline 0.04 & 1.238 & 10.7 & 12.7 & 0.1 & 12.3215 \\
\hline 0.05 & 1.251 & 9.8 & 11.5 & 0.05 & 11.0727 \\
\hline 0.06 & 1.264 & 9.3 & 10.7 & 0.05 & 10.3582 \\
\hline 0.07 & 1.277 & 8.8 & 10.2 & 0.05 & 9.8681 \\
\hline 0.08 & 1.289 & 8.5 & 9.9 & 0.05 & 9.5556 \\
\hline 0.09 & 1.302 & 8.3 & 9.6 & 0.05 & 9.2776 \\
\hline 0.1 & 1.315 & 8.1 & 9.4 & 0.05 & 9.0777 \\
\hline 0.118 & 1.338 & 7.9 & 9.1 & 0.05 & 8.8247 \\
\hline 0.13 & 1.354 & 7.8 & 9 & 0.05 & 8.7188 \\
\hline 0.15 & 1.38 & 7.7 & 8.8 & 0.05 & 8.5401 \\
\hline 0.2 & 1.444 & 7.5 & 8.6 & 0.05 & 8.3653 \\
\hline 0.235 & 1.489 & 7.5 & 8.6 & 0.05 & 8.2988 \\
\hline 0.25 & 1.508 & 7.4 & 8.6 & 0.05 & 8.2803 \\
\hline 0.353 & 1.638 & 7.5 & 8.6 & 0.05 & 8.3579 \\
\hline 0.47 & 1.784 & 7.7 & 8.9 & 0.05 & 8.5667 \\
\hline 0.475 & 1.79 & 7.7 & 8.9 & 0.05 & 8.5726 \\
\hline 0.48 & 1.796 & 7.7 & 8.9 & 0.05 & 8.6123 \\
\hline 0.486 & 1.804 & 7.7 & 8.9 & 0.05 & 8.6067 \\
\hline
\end{tabular}




\section{B1.2.2.1.3.3 10" Water Reflection}

Case ID: u5o2N26-icyl-1000-10

Material: $\mathrm{UO}_{2}\left(\mathrm{NO}_{3}\right)_{2} 6$ Molar Excess Acid

Geometry: Cylinder

Isotopics: $100 \%{ }^{235} \mathrm{U}$

Reflector: 10 " water reflected

Code: MCNP5 v1.40

Analyst: Mark Murphy

Date: $8 / 2011$ 
Figure B1.2.2.1.3.3-1. $\mathrm{UO}_{2}\left(\mathrm{NO}_{3}\right) 26 \mathrm{M}-$ Cylinder - $100 \%{ }^{235} \mathrm{U}-10$ " Water Reflection

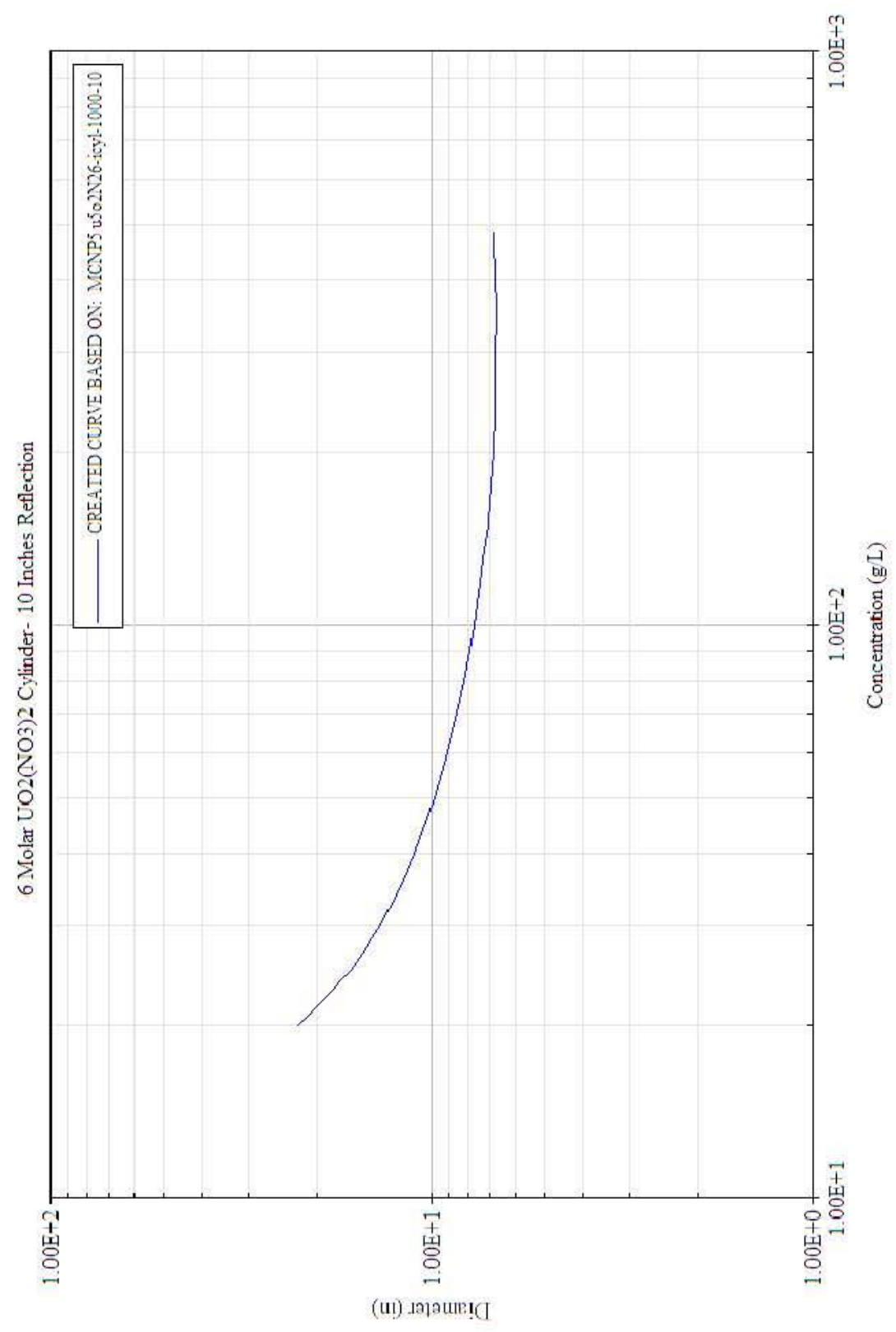


Table B1.2.2.1.3.3-1. Critical Dimensions for $\mathrm{UO}_{2}\left(\mathrm{NO}_{3}\right) 26 \mathrm{M}-\mathrm{Cylinder}-100 \%{ }^{235} \mathrm{U}-10$ " Water Reflection

\begin{tabular}{|c|c|c|c|c|c|}
\hline \multicolumn{6}{|c|}{$100 \%$ U235 UO2(NO3)2 6M Cylinder - 10 Inches of Reflection } \\
\hline Concentration $(\mathrm{g} / \mathrm{cc})$ & $\begin{array}{l}\text { Density } \\
(\mathrm{g} / \mathrm{cc})\end{array}$ & $\begin{array}{l}\text { First Diameter } \\
\text { (Inches) }\end{array}$ & $\begin{array}{l}\text { Last Diameter } \\
\text { (Inches) }\end{array}$ & $\begin{array}{l}\text { Delta } \\
\text { (Inches) }\end{array}$ & $\begin{array}{l}\text { Critical Diameter } \\
\text { (Inches) }\end{array}$ \\
\hline 0.012 & 1.202 & 30.3 & 34.4 & 0.2 & -- \\
\hline 0.015 & 1.206 & 29.5 & 29.7 & 0.02 & -- \\
\hline 0.02 & 1.212 & 17 & 23.7 & 0.3 & 22.3491 \\
\hline 0.025 & 1.219 & 13.2 & 16.9 & 0.1 & 16.2046 \\
\hline 0.03 & 1.225 & 11.4 & 14.1 & 0.1 & 13.5549 \\
\hline 0.032 & 1.228 & 10.9 & 13.4 & 0.1 & 12.8412 \\
\hline 0.035 & 1.231 & 10.3 & 12.5 & 0.1 & 12.031 \\
\hline 0.04 & 1.238 & 9.5 & 11.5 & 0.1 & 11.0481 \\
\hline 0.05 & 1.251 & 8.5 & 10.1 & 0.05 & 9.8176 \\
\hline 0.06 & 1.264 & 7.9 & 9.4 & 0.05 & 9.0473 \\
\hline 0.07 & 1.277 & 7.5 & 8.9 & 0.05 & 8.5436 \\
\hline 0.08 & 1.289 & 7.2 & 8.5 & 0.05 & 8.1829 \\
\hline 0.09 & 1.302 & 7 & 8.2 & 0.05 & 7.9204 \\
\hline 0.1 & 1.315 & 6.8 & 8 & 0.05 & 7.7163 \\
\hline 0.118 & 1.338 & 6.5 & 7.7 & 0.05 & 7.4173 \\
\hline 0.13 & 1.354 & 6.4 & 7.6 & 0.05 & 7.3105 \\
\hline 0.15 & 1.38 & 6.3 & 7.4 & 0.05 & 7.0952 \\
\hline 0.2 & 1.444 & 6 & 7.1 & 0.05 & 6.8495 \\
\hline 0.235 & 1.489 & 5.9 & 7 & 0.05 & 6.7648 \\
\hline 0.25 & 1.508 & 5.9 & 7 & 0.05 & 6.7615 \\
\hline 0.353 & 1.638 & 5.8 & 7 & 0.05 & 6.7103 \\
\hline 0.47 & 1.784 & 5.9 & 7.1 & 0.05 & 6.8065 \\
\hline 0.475 & 1.79 & 6 & 7.1 & 0.05 & 6.8366 \\
\hline 0.48 & 1.796 & 6 & 7.1 & 0.05 & 6.8398 \\
\hline 0.486 & 1.804 & 6 & 7.1 & 0.05 & 6.8354 \\
\hline
\end{tabular}


CHPRC-01552, Revision 0 
B1.2.2.2 $\quad 93.5 \%{ }^{235} \mathrm{U}$

B1.2.2.2.1 0 Molar Excess Acid

B1.2.2.2.1.1 0"Water Reflection

Case ID: u5o2N20-icyl-0935-00

Material: $\mathrm{UO}_{2}\left(\mathrm{NO}_{3}\right)_{2} \quad 0$ Molar Excess Acid

Geometry: Cylinder

Isotopics: $93.5 \%{ }^{235} \mathrm{U}$

Reflector: 0" water reflected

Code: MCNP5 v1.40

Analyst: Mark Murphy

Date: 8/2011 
Figure B1.2.2.2.1.1-1. $\mathrm{UO}_{2}\left(\mathrm{NO}_{3}\right) 2 \mathrm{M}$ - Cylinder - 93.5\% ${ }^{235} \mathrm{U}-0$ " Water Reflection

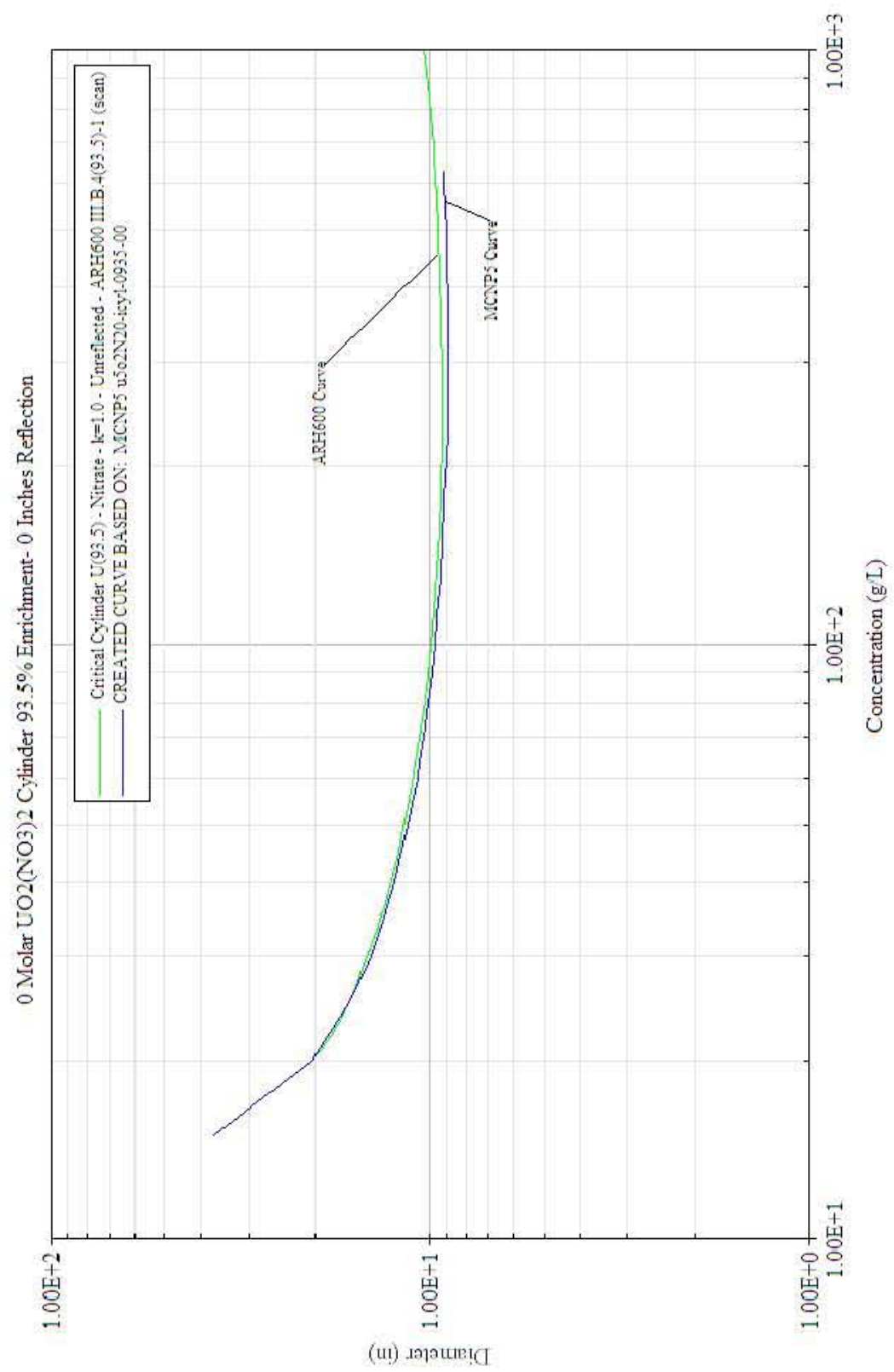


Table B1.2.2.2.1.1-1. Critical Dimensions for $\mathrm{UO}_{2}\left(\mathrm{NO}_{3}\right) 2 \mathrm{M}-\mathrm{Cylinder}-93.5 \%{ }^{235} \mathrm{U}-0$ " Water Reflection

\begin{tabular}{|c|c|c|c|c|c|}
\hline \multicolumn{6}{|c|}{$93.5 \%$ U235 UO2(NO3)2 0M Cylinder- 0 Inches of Reflection } \\
\hline Concentration $(\mathrm{g} / \mathrm{cc})$ & $\begin{array}{l}\text { Density } \\
(\mathrm{g} / \mathrm{cc})\end{array}$ & $\begin{array}{l}\text { First Diameter } \\
\text { (Inches) }\end{array}$ & \begin{tabular}{|l}
$\begin{array}{l}\text { Last Diameter } \\
\text { (Inches) }\end{array}$ \\
\end{tabular} & $\begin{array}{l}\text { Delta } \\
\text { (Inches) }\end{array}$ & $\begin{array}{l}\text { Critical Diameter } \\
\text { (Inches) }\end{array}$ \\
\hline 0.012 & 1.013 & 23.5 & 55.7 & 1.6 & -- \\
\hline 0.015 & 1.017 & 24.5 & 48.4 & 1.2 & 36.9342 \\
\hline 0.02 & 1.024 & 16.6 & 21.2 & 0.2 & 20.3329 \\
\hline 0.025 & 1.031 & 13.9 & 16.8 & 0.1 & 16.2718 \\
\hline 0.03 & 1.037 & 12.6 & 14.8 & 0.1 & 14.2971 \\
\hline 0.032 & 1.04 & 12.1 & 14.3 & 0.1 & 13.8326 \\
\hline 0.035 & 1.044 & 11.6 & 13.6 & 0.1 & 13.1772 \\
\hline 0.04 & 1.051 & 11 & 12.8 & 0.1 & 12.3845 \\
\hline 0.05 & 1.064 & 10.2 & \begin{tabular}{|l|}
11.7 \\
\end{tabular} & 0.05 & 11.4012 \\
\hline 0.06 & 1.078 & 9.7 & 11.1 & 0.05 & 10.7831 \\
\hline 0.07 & 1.091 & 9.4 & 10.7 & 0.05 & 10.3663 \\
\hline 0.08 & 1.105 & 9.1 & 10.4 & 0.05 & 10.0895 \\
\hline 0.09 & 1.118 & 8.9 & 10.1 & 0.05 & 9.8407 \\
\hline 0.1 & 1.131 & 8.8 & 10 & 0.05 & 9.6926 \\
\hline 0.118 & 1.155 & 8.6 & 9.7 & 0.05 & 9.4424 \\
\hline 0.13 & 1.172 & 8.5 & 9.6 & 0.05 & 9.3349 \\
\hline 0.15 & 1.198 & 8.4 & 9.5 & 0.05 & 9.2086 \\
\hline 0.2 & 1.266 & 8.2 & 9.3 & 0.05 & 8.9998 \\
\hline 0.235 & 1.313 & 8.2 & 9.2 & 0.05 & 8.9433 \\
\hline 0.25 & 1.333 & 8.1 & 9.2 & 0.05 & 8.9184 \\
\hline 0.353 & 1.472 & 8.1 & 9.1 & 0.05 & 8.8923 \\
\hline 0.47 & 1.631 & 8.2 & 9.2 & 0.05 & 8.97 \\
\hline 0.5 & 1.67 & 8.2 & 9.3 & 0.05 & 8.9992 \\
\hline 0.6 & 1.805 & 8.5 & 9.5 & 0.05 & 9.1469 \\
\hline 0.605 & 1.812 & 8.3 & 9.4 & 0.05 & 9.1542 \\
\hline 0.61 & 1.819 & 8.3 & 9.4 & 0.05 & 9.1396 \\
\hline 0.615 & 1.825 & 8.3 & 9.4 & 0.05 & 9.1543 \\
\hline 0.62 & 1.832 & 8.3 & 9.4 & 0.05 & 9.1682 \\
\hline 0.625 & 1.839 & 8.3 & 9.4 & 0.05 & 9.1664 \\
\hline 0.629 & 1.844 & 8.3 & 9.5 & 0.05 & 9.1861 \\
\hline
\end{tabular}




\section{B1.2.2.2.1.2 1"Water Reflection}

Case ID: u5o2N20-icyl-0935-01

Material: $\mathrm{UO}_{2}\left(\mathrm{NO}_{3}\right)_{2} 0$ Molar Excess Acid

Geometry: Cylinder

Isotopics: $93.5 \%{ }^{235} \mathrm{U}$

Reflector: 1" water reflected

Code: MCNP5 v1.40

Analyst: Mark Murphy

Date: $8 / 2011$ 
Figure B1.2.2.2.1.2-1. $\mathrm{UO}_{2}\left(\mathrm{NO}_{3}\right) 2 \mathrm{OM}-\mathrm{Cylinder}-93.5 \%{ }^{235} \mathrm{U}-1$ " Water Reflection

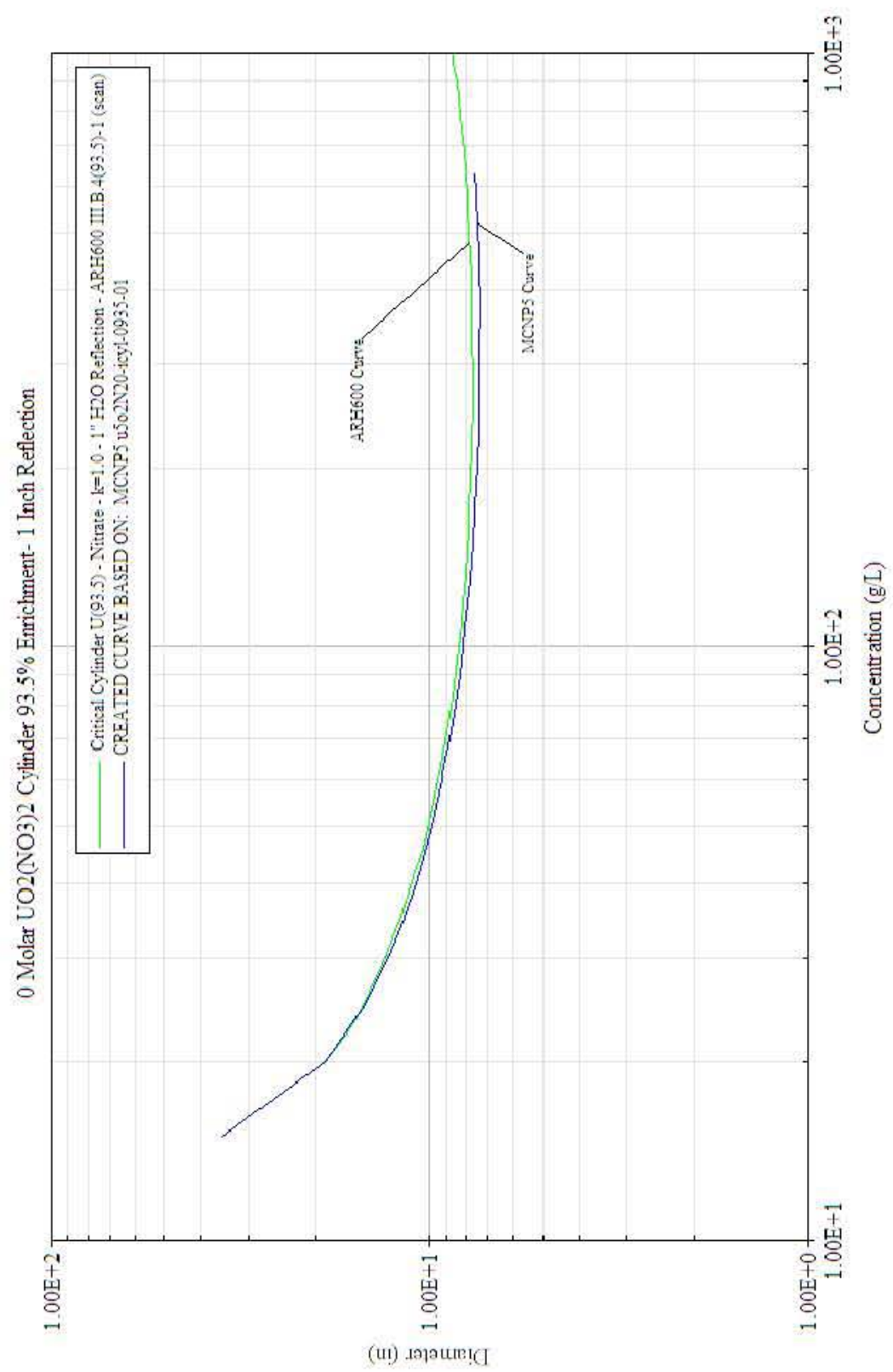


Table B1.2.2.2.1.2-1. Critical Dimensions for $\mathrm{UO}_{2}\left(\mathrm{NO}_{3}\right) 20 \mathrm{M}-\mathrm{Cylinder}-93.5 \%{ }^{235} \mathrm{U}-1$ " Water Reflection

\begin{tabular}{|c|c|c|c|c|c|}
\hline \multicolumn{6}{|c|}{$93.5 \%$ U235 UO2(NO3)2 0M Cylinder- 1 Inch of Reflection } \\
\hline Concentration $(\mathrm{g} / \mathrm{cc})$ & $\begin{array}{l}\text { Density } \\
(\mathrm{g} / \mathrm{cc})\end{array}$ & $\begin{array}{l}\text { First Diameter } \\
\text { (Inches) }\end{array}$ & $\begin{array}{l}\text { Last Diameter } \\
\text { (Inches) }\end{array}$ & $\begin{array}{l}\text { Delta } \\
\text { (Inches) }\end{array}$ & \begin{tabular}{|l}
$\begin{array}{l}\text { Critical Diameter } \\
\text { (Inches) }\end{array}$ \\
\end{tabular} \\
\hline 0.012 & 1.013 & 25 & 31.3 & 0.3 & -- \\
\hline 0.015 & 1.017 & 22.9 & 36.8 & 0.7 & 35.3846 \\
\hline 0.02 & 1.024 & 15.1 & 19.7 & 0.2 & 18.7982 \\
\hline 0.025 & 1.031 & 12.4 & 15.4 & 0.1 & 14.7505 \\
\hline 0.03 & 1.037 & 11 & 13.3 & 0.1 & 12.829 \\
\hline 0.032 & 1.04 & 10.6 & 12.8 & 0.1 & 12.3012 \\
\hline 0.035 & 1.044 & 10.1 & 12 & 0.1 & 11.6228 \\
\hline 0.04 & 1.051 & 9.5 & 11.2 & 0.05 & 10.8707 \\
\hline 0.05 & 1.064 & 8.7 & 10.2 & 0.05 & 9.8652 \\
\hline 0.06 & 1.078 & 8.2 & 9.6 & 0.05 & 9.2377 \\
\hline 0.07 & 1.091 & 7.9 & 9.1 & 0.05 & 8.83 \\
\hline 0.08 & 1.105 & 7.7 & 8.8 & 0.05 & 8.5537 \\
\hline 0.09 & 1.118 & 7.5 & 8.6 & 0.05 & 8.3044 \\
\hline 0.1 & 1.131 & 7.3 & 8.4 & 0.05 & 8.1393 \\
\hline 0.118 & 1.155 & 7.1 & 8.2 & 0.05 & 7.9136 \\
\hline 0.13 & 1.172 & 7 & 8 & 0.05 & 7.8007 \\
\hline 0.15 & 1.198 & 6.9 & 7.9 & 0.05 & 7.6551 \\
\hline 0.2 & 1.266 & 6.7 & 7.7 & 0.05 & 7.4578 \\
\hline 0.235 & 1.313 & 6.6 & 7.6 & 0.05 & 7.388 \\
\hline 0.25 & 1.333 & 6.6 & 7.6 & 0.05 & \begin{tabular}{|l}
7.3757 \\
\end{tabular} \\
\hline 0.353 & 1.472 & 6.6 & 7.6 & 0.05 & 7.3213 \\
\hline 0.47 & 1.631 & 6.6 & 7.7 & 0.05 & 7.4001 \\
\hline 0.5 & 1.67 & 6.6 & 7.7 & 0.05 & 7.4271 \\
\hline 0.6 & 1.805 & 6.7 & 7.8 & 0.05 & 7.543 \\
\hline 0.605 & 1.812 & 6.7 & 7.8 & 0.05 & 7.5477 \\
\hline 0.61 & 1.819 & 6.7 & 7.8 & 0.05 & 7.5691 \\
\hline 0.615 & 1.825 & 6.8 & 7.8 & 0.05 & 7.5792 \\
\hline 0.62 & 1.832 & 6.8 & 7.8 & 0.05 & 7.5795 \\
\hline 0.625 & 1.839 & 6.8 & 7.8 & 0.05 & 7.5809 \\
\hline 0.629 & 1.844 & 6.8 & 7.9 & 0.05 & 7.5846 \\
\hline
\end{tabular}




\section{B1.2.2.2.1.3 10" Water Reflection}

Case ID: u5o2N20-icyl-0935-10

Material: $\mathrm{UO}_{2}\left(\mathrm{NO}_{3}\right)_{2} 0$ Molar Excess Acid

Geometry: Cylinder

Isotopics: $93.5 \%{ }^{235} \mathrm{U}$

Reflector: 10 " water reflected

Code: MCNP5 v1.40

Analyst: Mark Murphy

Date: $8 / 2011$ 
Figure B1.2.2.2.1.3-1. $\mathrm{UO}_{2}\left(\mathrm{NO}_{3}\right) 2 \mathrm{OM}-\mathrm{Cylinder}-93.5 \%{ }^{235} \mathrm{U}-10$ " Water Reflection

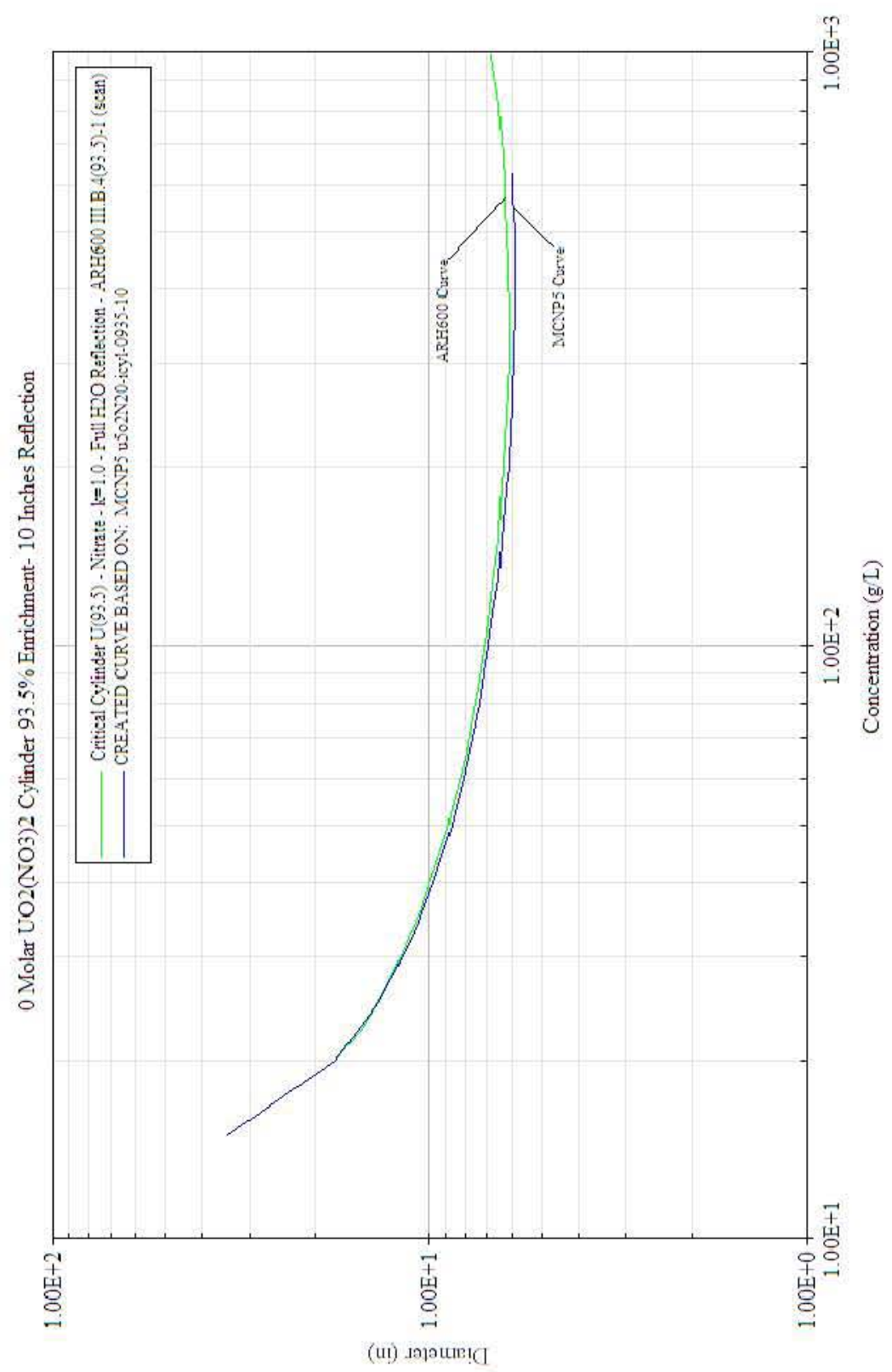


Table B1.2.2.2.1.3-1. Critical Dimensions for $\mathrm{UO}_{2}\left(\mathrm{NO}_{3}\right) 20 \mathrm{M}-\mathrm{Cylinder}-93.5 \%{ }^{235} \mathrm{U}-10$ " Water Reflection

\begin{tabular}{|c|c|c|c|c|c|}
\hline \multicolumn{6}{|c|}{ 93.5\% U235 UO2(NO3)2 0M Cylinder- 10 Inches of Reflection } \\
\hline Concentration $(\mathrm{g} / \mathrm{cc})$ & $\begin{array}{l}\text { Density } \\
(\mathrm{g} / \mathrm{cc})\end{array}$ & $\begin{array}{l}\text { First Diameter } \\
\text { (Inches) }\end{array}$ & $\begin{array}{l}\text { Last Diameter } \\
\text { (Inches) }\end{array}$ & $\begin{array}{l}\text { Delta } \\
\text { (Inches) }\end{array}$ & $\begin{array}{l}\text { Critical Diameter } \\
\text { (Inches) }\end{array}$ \\
\hline 0.012 & 1.013 & 31.7 & 57.1 & 1.2 & -- \\
\hline 0.015 & 1.017 & 21.8 & 36.7 & 0.7 & 34.235 \\
\hline 0.02 & 1.024 & 14.3 & 18.6 & 0.2 & 17.6939 \\
\hline 0.025 & 1.031 & 11.3 & 14 & 0.1 & 13.6407 \\
\hline 0.03 & 1.037 & 9.9 & 12.1 & 0.1 & 11.6926 \\
\hline 0.032 & 1.04 & 9.6 & 11.6 & 0.1 & 11.1437 \\
\hline 0.035 & 1.044 & 9 & 10.9 & 0.1 & 10.5238 \\
\hline 0.04 & 1.051 & 8.4 & 10.1 & 0.05 & 9.7078 \\
\hline 0.05 & 1.064 & 7.6 & 9 & 0.05 & 8.6956 \\
\hline 0.06 & 1.078 & 7.1 & 8.4 & 0.05 & 8.0734 \\
\hline 0.07 & 1.091 & 6.7 & 7.9 & 0.05 & 7.6536 \\
\hline 0.08 & 1.105 & 6.4 & 7.6 & 0.05 & 7.334 \\
\hline 0.09 & 1.118 & 6.2 & 7.4 & 0.05 & 7.1095 \\
\hline 0.1 & 1.131 & 6.1 & 7.2 & 0.05 & 6.9278 \\
\hline 0.118 & 1.155 & 5.9 & 6.9 & 0.05 & 6.6703 \\
\hline 0.13 & 1.172 & 5.7 & 6.8 & 0.05 & 6.537 \\
\hline 0.15 & 1.198 & 5.6 & 6.6 & 0.05 & 6.3902 \\
\hline 0.2 & 1.266 & 5.4 & 6.4 & 0.05 & 6.1411 \\
\hline 0.235 & 1.313 & 5.3 & 6.3 & 0.05 & 6.0496 \\
\hline 0.25 & 1.333 & 5.3 & 6.2 & 0.05 & 6.0198 \\
\hline 0.353 & 1.472 & 5.2 & 6.1 & 0.05 & 5.9178 \\
\hline 0.47 & 1.631 & 5.2 & 6.1 & 0.05 & 5.9202 \\
\hline 0.5 & 1.67 & 5.2 & 6.2 & 0.05 & 5.9369 \\
\hline 0.6 & 1.805 & 5.2 & 6.2 & 0.05 & 6.0058 \\
\hline 0.605 & 1.812 & 5.2 & 6.2 & 0.05 & 6.0017 \\
\hline 0.61 & 1.819 & 5.2 & 6.2 & 0.05 & 6.0103 \\
\hline 0.615 & 1.825 & 5.2 & 6.2 & 0.05 & 6.0165 \\
\hline 0.62 & 1.832 & 5.2 & 6.2 & 0.05 & 6.0144 \\
\hline 0.625 & 1.839 & 5.2 & 6.2 & 0.05 & 6.0238 \\
\hline 0.629 & 1.844 & 5.2 & 6.3 & 0.05 & 6.0211 \\
\hline
\end{tabular}


B1.2.2.2.2 3 Molar Excess Acid

B1.2.2.2.2.1 0"Water Reflection

Case ID: u5o2N23-icyl-0935-00

Material: $\mathrm{UO}_{2}\left(\mathrm{NO}_{3}\right)_{2} 3$ Molar Excess Acid

Geometry: Cylinder

Isotopics: $93.5 \%{ }^{235} \mathrm{U}$

Reflector: 0 " water reflected

Code: MCNP5 v1.40

Analyst: Mark Murphy

Date: $8 / 2011$ 
Figure B1.2.2.2.2.1-1. $\mathrm{UO}_{2}\left(\mathrm{NO}_{3}\right) 23 \mathrm{M}-\mathrm{Cylinder}-93.5 \%{ }^{235} \mathrm{U}-0$ " Water Reflection

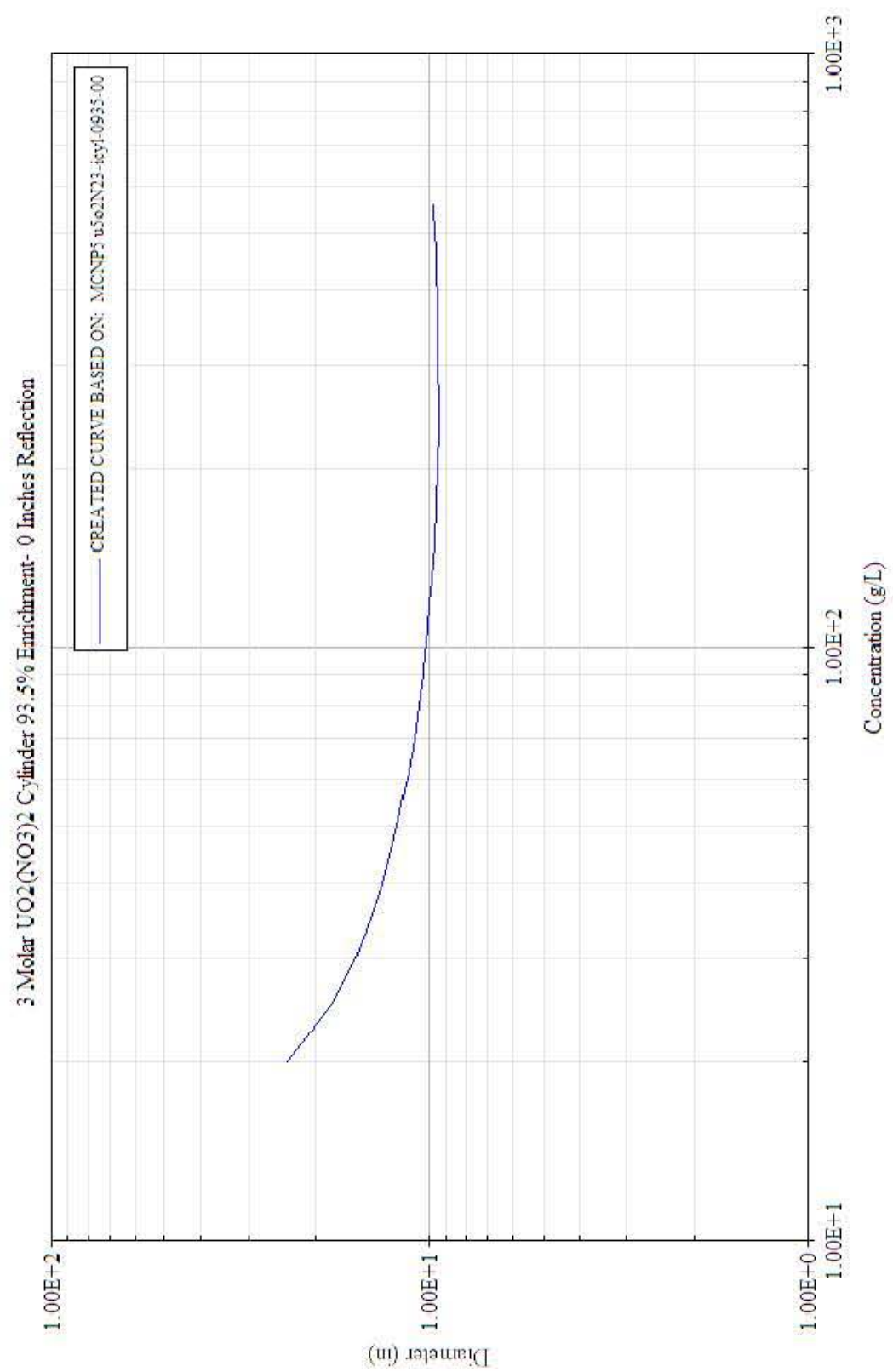


Table B1.2.2.2.2.1-1. Critical Dimensions for $\mathrm{UO}_{2}\left(\mathrm{NO}_{3}\right) 23 \mathrm{M}-\mathrm{Cylinder}-93.5 \%{ }^{235} \mathrm{U}-0$ " Water Reflection

\begin{tabular}{|c|c|c|c|c|c|}
\hline \multicolumn{6}{|c|}{ 93.5\% U235 UO2(NO3)2 3M Cylinder- 0 Inches of Reflection } \\
\hline Concentration $(\mathrm{g} / \mathrm{cc})$ & $\begin{array}{l}\text { Density } \\
(\mathrm{g} / \mathrm{cc})\end{array}$ & $\begin{array}{l}\text { First Diameter } \\
\text { (Inches) }\end{array}$ & $\begin{array}{l}\text { Last Diameter } \\
\text { (Inches) }\end{array}$ & $\begin{array}{l}\text { Delta } \\
\text { (Inches) }\end{array}$ & $\begin{array}{l}\text { Critical Diameter } \\
\text { (Inches) }\end{array}$ \\
\hline 0.012 & 1.112 & 52.1 & 69.5 & 0.8 & -- \\
\hline 0.015 & 1.116 & 29.9 & 38 & 0.4 & -- \\
\hline 0.02 & 1.123 & 18.8 & 25 & 0.3 & 23.8013 \\
\hline 0.025 & 1.129 & 15.3 & 18.8 & 0.1 & 18.1389 \\
\hline 0.03 & 1.136 & 13.6 & 16.2 & 0.1 & 15.6523 \\
\hline 0.032 & 1.138 & 13.1 & 15.5 & 0.1 & 15.0166 \\
\hline 0.035 & 1.142 & 12.5 & 14.7 & 0.1 & 14.2307 \\
\hline 0.04 & 1.149 & 11.8 & 13.7 & 0.1 & 13.2932 \\
\hline 0.05 & 1.162 & 10.9 & 12.5 & 0.05 & 12.121 \\
\hline 0.06 & 1.175 & 10.3 & 11.7 & 0.05 & 11.4348 \\
\hline 0.07 & 1.188 & 9.9 & 11.3 & 0.05 & 10.9784 \\
\hline 0.08 & 1.201 & 9.6 & 10.9 & 0.05 & 10.6466 \\
\hline 0.09 & 1.215 & 9.4 & 10.7 & 0.05 & 10.3858 \\
\hline 0.1 & 1.228 & 9.3 & 10.5 & 0.05 & 10.1994 \\
\hline 0.118 & 1.251 & 9.1 & 10.2 & 0.05 & 9.9617 \\
\hline 0.13 & 1.267 & 8.9 & 10.1 & 0.05 & 9.842 \\
\hline 0.15 & 1.293 & 8.8 & 10 & 0.05 & 9.6929 \\
\hline 0.2 & 1.359 & 8.7 & 9.8 & 0.05 & 9.4909 \\
\hline 0.235 & 1.406 & 8.6 & 9.7 & 0.05 & 9.4258 \\
\hline 0.25 & 1.425 & 8.6 & 9.7 & 0.05 & 9.4181 \\
\hline 0.353 & 1.561 & 8.6 & 9.7 & 0.05 & 9.4388 \\
\hline 0.47 & 1.715 & 8.7 & 9.8 & 0.05 & 9.5727 \\
\hline 0.5 & 1.753 & 8.8 & 9.9 & 0.05 & 9.6429 \\
\hline 0.51 & 1.766 & 8.8 & 10 & 0.05 & 9.6622 \\
\hline 0.52 & 1.779 & 8.8 & 10 & 0.05 & 9.6867 \\
\hline 0.53 & 1.792 & 8.8 & 10 & 0.05 & 9.6972 \\
\hline 0.54 & 1.805 & 8.8 & 10 & 0.05 & 9.7233 \\
\hline 0.55 & 1.818 & 8.9 & 10 & 0.05 & 9.7419 \\
\hline 0.56 & 1.831 & 8.9 & 10 & 0.05 & 9.7641 \\
\hline
\end{tabular}




\section{B1.2.2.2.2.2 1" Water Reflection}

Case ID: u5o2N23-icyl-0935-01

Material: $\mathrm{UO}_{2}\left(\mathrm{NO}_{3}\right)_{2} 3$ Molar Excess Acid

Geometry: Cylinder

Isotopics: $93.5 \%{ }^{235} \mathrm{U}$

Reflector: 1" water reflected

Code: MCNP5 v1.40

Analyst: Mark Murphy

Date: $8 / 2011$ 
Figure B1.2.2.2.2.2-1. $\mathrm{UO}_{2}\left(\mathrm{NO}_{3}\right) 23 \mathrm{M}-\mathrm{Cylinder}-93.5 \%{ }^{235} \mathrm{U}-1$ " Water Reflection

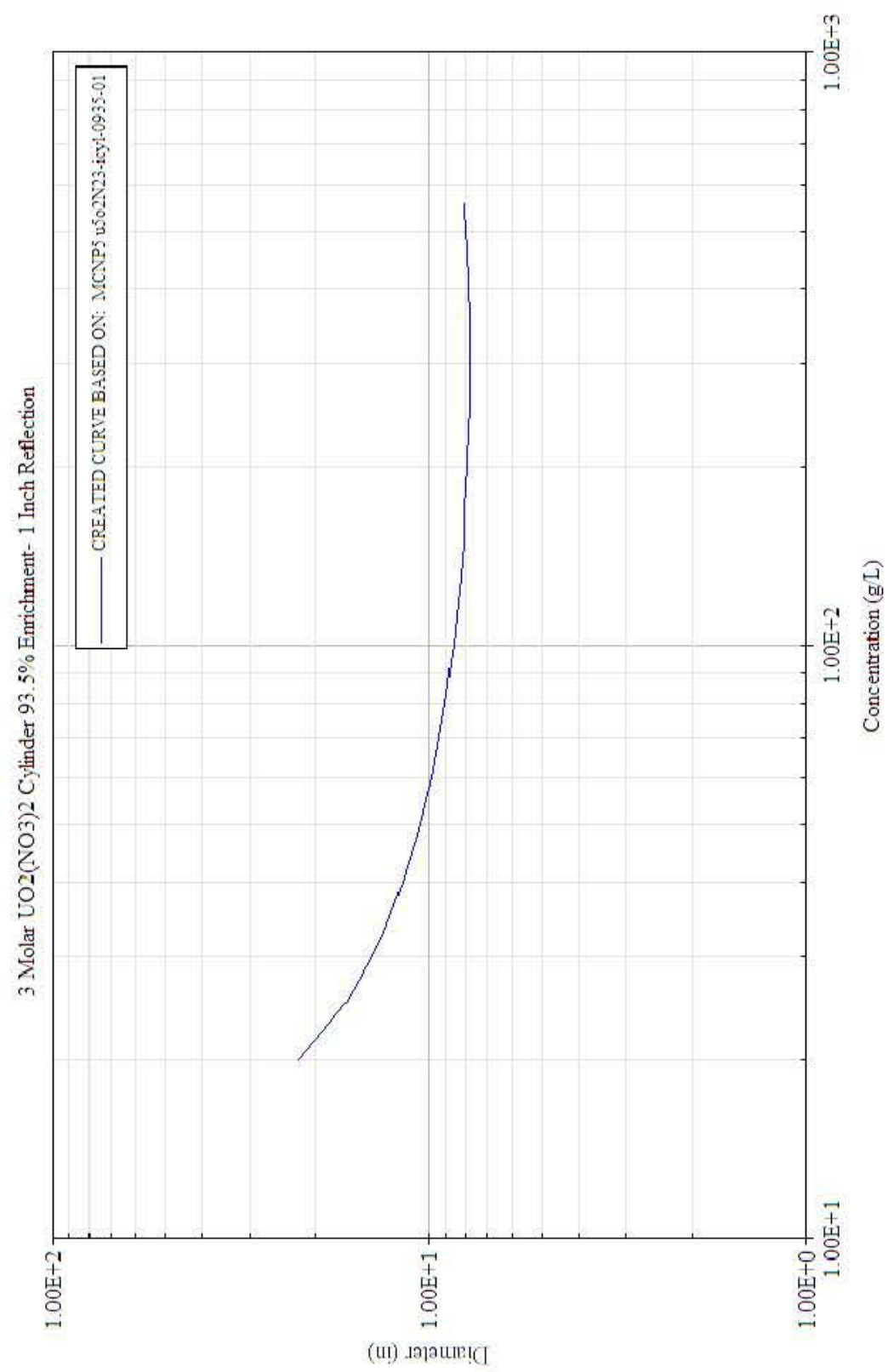


Table B1.2.2.2.2.2-1. Critical Dimensions for $\mathrm{UO}_{2}\left(\mathrm{NO}_{3}\right) 23 \mathrm{M}-\mathrm{Cylinder}-93.5 \%{ }^{235} \mathrm{U}-1$ " Water Reflection

\begin{tabular}{|c|c|c|c|c|c|}
\hline \multicolumn{6}{|c|}{$93.5 \%$ U235 UO2(NO3)2 3M Cylinder- 1 Inch of Reflection } \\
\hline Concentration $(\mathrm{g} / \mathrm{cc})$ & $\begin{array}{l}\text { Density } \\
(\mathrm{g} / \mathrm{cc})\end{array}$ & $\begin{array}{l}\text { First Diameter } \\
\text { (Inches) }\end{array}$ & $\begin{array}{l}\text { Last Diameter } \\
\text { (Inches) }\end{array}$ & $\begin{array}{l}\text { Delta } \\
\text { (Inches) }\end{array}$ & $\begin{array}{l}\text { Critical Diameter } \\
\text { (Inches) }\end{array}$ \\
\hline 0.012 & 1.112 & 38.5 & 73.1 & 1.7 & -- \\
\hline 0.015 & 1.116 & 28.9 & 32.1 & 0.1 & -- \\
\hline 0.02 & 1.123 & 17.2 & 23.5 & 0.3 & 22.2583 \\
\hline 0.025 & 1.129 & 13.8 & 17.2 & 0.1 & 16.5734 \\
\hline 0.03 & 1.136 & 12 & 14.6 & 0.1 & 14.1041 \\
\hline 0.032 & 1.138 & 11.5 & 14 & 0.1 & 13.4448 \\
\hline 0.035 & 1.142 & 10.9 & 13.1 & 0.1 & 12.6809 \\
\hline 0.04 & 1.149 & 10.3 & 12.1 & 0.1 & 11.7429 \\
\hline 0.05 & 1.162 & 9.3 & 10.9 & 0.05 & 10.5669 \\
\hline 0.06 & 1.175 & 8.7 & 10.2 & 0.05 & 9.8635 \\
\hline 0.07 & 1.188 & 8.4 & 9.7 & 0.05 & 9.4103 \\
\hline 0.08 & 1.201 & 8.1 & 9.4 & 0.05 & 9.0703 \\
\hline 0.09 & 1.215 & 7.9 & 9.1 & 0.05 & 8.8214 \\
\hline 0.1 & 1.228 & 7.7 & 8.9 & 0.05 & 8.6209 \\
\hline 0.118 & 1.251 & 7.5 & 8.7 & 0.05 & 8.3757 \\
\hline 0.13 & 1.267 & 7.4 & 8.5 & 0.05 & 8.2545 \\
\hline 0.15 & 1.293 & 7.3 & 8.4 & 0.05 & 8.1059 \\
\hline 0.2 & 1.359 & 7.1 & 8.1 & 0.05 & 7.9002 \\
\hline 0.235 & 1.406 & 7 & 8.1 & 0.05 & 7.8288 \\
\hline 0.25 & 1.425 & 7 & 8.1 & 0.05 & 7.8146 \\
\hline 0.353 & 1.561 & 7 & 8.1 & 0.05 & 7.8077 \\
\hline 0.47 & 1.715 & 7.1 & 8.2 & 0.05 & 7.9427 \\
\hline 0.5 & 1.753 & 7.1 & 8.2 & 0.05 & 7.9914 \\
\hline 0.51 & 1.766 & 7.1 & 8.3 & 0.05 & 8.0166 \\
\hline 0.52 & 1.779 & 7.2 & 8.3 & 0.05 & 8.0301 \\
\hline 0.53 & 1.792 & 7.2 & 8.3 & 0.35 & 8.0487 \\
\hline 0.54 & 1.805 & 7.2 & 8.3 & 0.05 & 8.0631 \\
\hline 0.55 & 1.818 & 7.2 & 8.3 & 0.05 & 8.076 \\
\hline 0.56 & 1.831 & 7.2 & 8.4 & 0.05 & 8.0998 \\
\hline
\end{tabular}




\section{B1.2.2.2.2.3 10" Water Reflection}

Case ID: u5o2N23-icyl-0935-10

Material: $\mathrm{UO}_{2}\left(\mathrm{NO}_{3}\right)_{2} 3$ Molar Excess Acid

Geometry: Cylinder

Isotopics: $93.5 \%{ }^{235} \mathrm{U}$

Reflector: 10 " water reflected

Code: MCNP5 v1.40

Analyst: Mark Murphy

Date: $8 / 2011$ 
Figure B1.2.2.2.2.3-1. $\mathrm{UO}_{2}\left(\mathrm{NO}_{3}\right) 23 \mathrm{M}-\mathrm{Cylinder}-93.5 \%{ }^{235} \mathrm{U}-10$ " Water Reflection

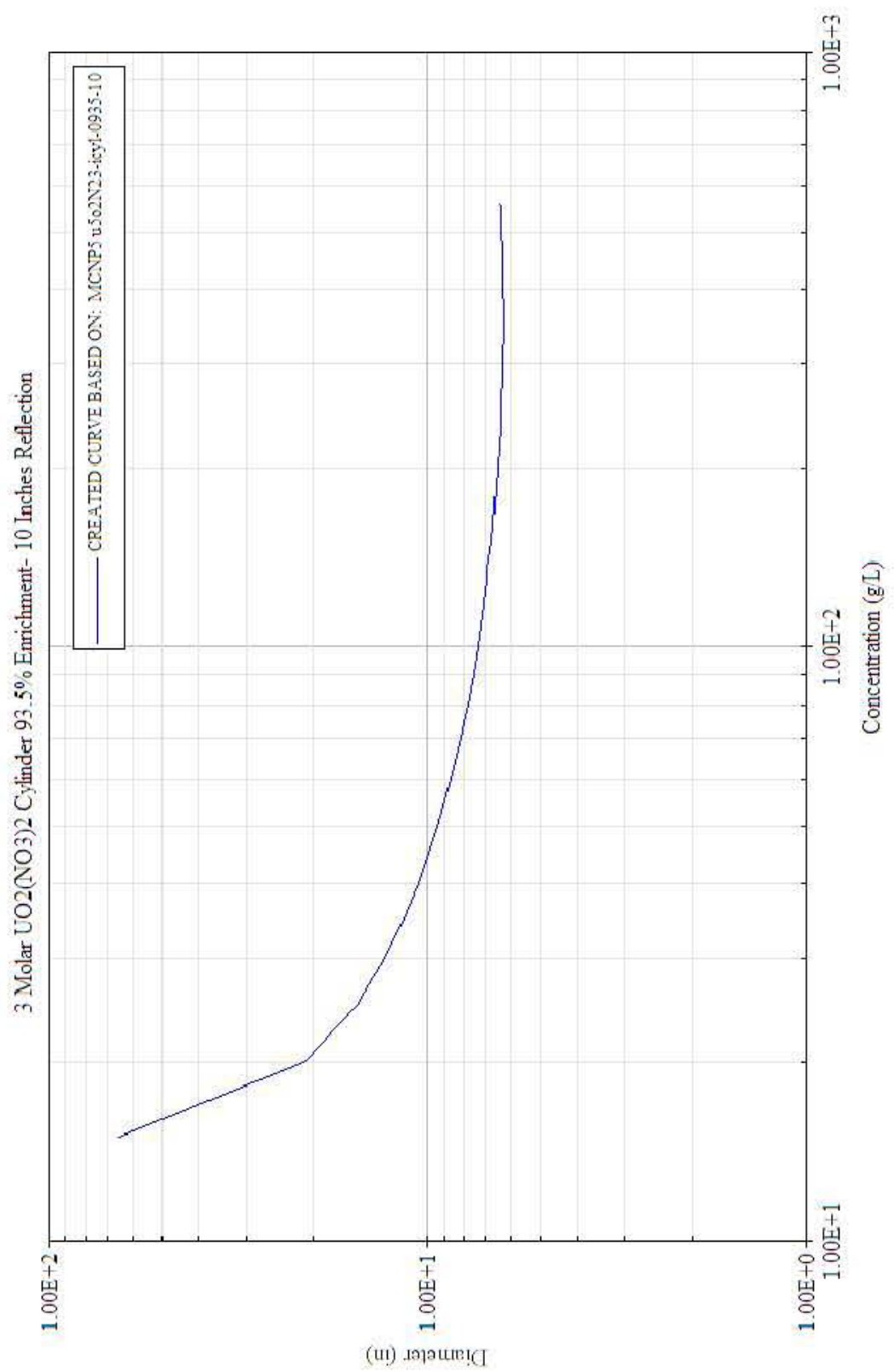


Table B1.2.2.2.2.3-1. Critical Dimensions for $\mathrm{UO}_{2}\left(\mathrm{NO}_{3}\right) 23 \mathrm{M}-\mathrm{Cylinder}-93.5 \%{ }^{235} \mathrm{U}-10$ " Water Reflection

\begin{tabular}{|c|c|c|c|c|c|}
\hline \multicolumn{6}{|c|}{$93.5 \%$ U235 UO2(NO3)2 3M Cylinder- 10 Inches of Reflection } \\
\hline Concentration $(\mathrm{g} / \mathrm{cc})$ & $\begin{array}{l}\text { Density } \\
(\mathrm{g} / \mathrm{cc})\end{array}$ & $\begin{array}{l}\text { First Diameter } \\
\text { (Inches) }\end{array}$ & $\begin{array}{l}\text { Last Diameter } \\
\text { (Inches) }\end{array}$ & $\begin{array}{l}\text { Delta } \\
\text { (Inches) }\end{array}$ & $\begin{array}{l}\text { Critical Diameter } \\
\text { (Inches) }\end{array}$ \\
\hline 0.012 & 1.112 & 42.8 & 79 & 1.8 & -- \\
\hline 0.015 & 1.116 & 28.7 & 74.2 & 2.2 & 65.6534 \\
\hline 0.02 & 1.123 & 16.2 & 22.2 & 0.3 & 21.1077 \\
\hline 0.025 & 1.129 & 12.6 & 16.1 & 0.1 & 15.3782 \\
\hline 0.03 & 1.136 & 10.9 & 13.4 & 0.1 & 12.9314 \\
\hline 0.032 & 1.138 & 10.4 & 12.7 & 0.1 & 12.2641 \\
\hline 0.035 & 1.142 & 9.8 & 11.9 & 0.1 & 11.4902 \\
\hline 0.04 & 1.149 & 9.1 & 10.9 & 0.1 & 10.5426 \\
\hline 0.05 & 1.162 & 8.1 & 9.7 & 0.05 & 9.3699 \\
\hline 0.06 & 1.175 & 7.5 & 9 & 0.05 & 8.6405 \\
\hline 0.07 & 1.188 & 7.1 & 8.4 & 0.05 & 8.1645 \\
\hline 0.08 & 1.201 & 6.9 & 8.1 & 0.05 & \begin{tabular}{|l}
7.8129 \\
\end{tabular} \\
\hline 0.09 & 1.215 & 6.6 & 7.8 & 0.05 & \begin{tabular}{|l}
7.5509 \\
\end{tabular} \\
\hline 0.1 & 1.228 & 6.4 & 7.6 & 0.05 & 7.3434 \\
\hline 0.118 & 1.251 & 6.2 & 7.3 & 0.05 & 7.08 \\
\hline 0.13 & 1.267 & 6.1 & 7.2 & 0.05 & 6.9407 \\
\hline 0.15 & 1.293 & 5.9 & 7 & 0.05 & 6.7659 \\
\hline 0.2 & 1.359 & 5.7 & 6.7 & 0.05 & 6.5204 \\
\hline 0.235 & 1.406 & 5.6 & 6.6 & 0.05 & 6.4152 \\
\hline 0.25 & 1.425 & 5.6 & 6.6 & 0.05 & 6.3878 \\
\hline 0.353 & 1.561 & 5.5 & 6.5 & 0.05 & 6.3021 \\
\hline 0.47 & 1.715 & 5.5 & 6.6 & 0.05 & 6.3398 \\
\hline 0.5 & 1.753 & 5.5 & 6.6 & 0.05 & 6.3883 \\
\hline 0.51 & 1.766 & 5.6 & 6.6 & 0.05 & 6.3903 \\
\hline 0.52 & 1.779 & 5.6 & 6.6 & 0.05 & 6.3992 \\
\hline 0.53 & 1.792 & 5.6 & 6.7 & 0.05 & 6.4147 \\
\hline 0.54 & 1.805 & 5.6 & 6.7 & 0.05 & 6.421 \\
\hline 0.55 & 1.818 & 5.6 & 6.7 & 0.05 & 6.4301 \\
\hline 0.56 & 1.831 & 5.6 & 6.7 & 0.05 & 6.4405 \\
\hline
\end{tabular}


B1.2.2.2.3 6 Molar Excess Acid

B1.2.2.2.3.1 0"Water Reflection

Case ID: u5o2N26-icyl-0935-00

Material: $\mathrm{UO}_{2}\left(\mathrm{NO}_{3}\right)_{2} 6$ Molar Excess Acid

Geometry: Cylinder

Isotopics: $93.5 \%{ }^{235} \mathrm{U}$

Reflector: 0 " water reflected

Code: MCNP5 v1.40

Analyst: Mark Murphy

Date: $8 / 2011$ 
Figure B1.2.2.2.3.1-1. $\mathrm{UO}_{2}\left(\mathrm{NO}_{3}\right) 26 \mathrm{M}-\mathrm{Cylinder}-93.5 \%{ }^{235} \mathrm{U}-0$ " Water Reflection

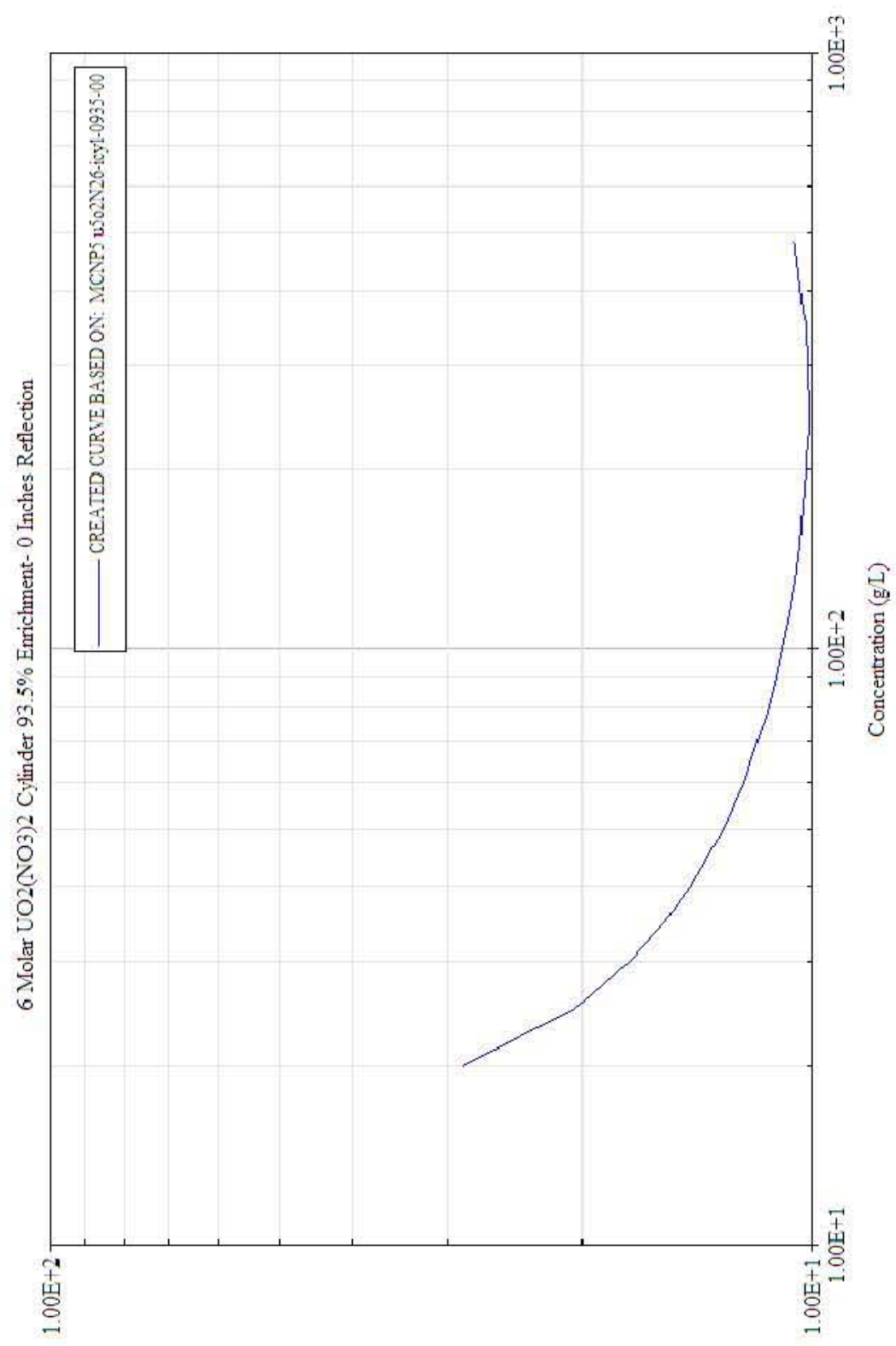

(ui) -әращт!( 
Table B1.2.2.2.3.1-1. Critical Dimensions for $\mathrm{UO}_{2}\left(\mathrm{NO}_{3}\right) 26 \mathrm{M}-\mathrm{Cylinder}-93.5 \%{ }^{235} \mathrm{U}-0$ " Water Reflection

\begin{tabular}{|c|c|c|c|c|c|}
\hline \multicolumn{6}{|c|}{$93.5 \%$ U235 UO2(NO3)2 6M Cylinder- 0 Inches of Reflection } \\
\hline Concentration $(\mathrm{g} / \mathrm{cc})$ & $\begin{array}{l}\text { Density } \\
(\mathrm{g} / \mathrm{cc})\end{array}$ & $\begin{array}{l}\text { First Diameter } \\
\text { (Inches) }\end{array}$ & $\begin{array}{l}\text { Last Diameter } \\
\text { (Inches) }\end{array}$ & $\begin{array}{l}\text { Delta } \\
\text { (Inches) }\end{array}$ & \begin{tabular}{|l}
$\begin{array}{l}\text { Critical Diameter } \\
\text { (Inches) }\end{array}$ \\
\end{tabular} \\
\hline 0.012 & 1.202 & 63.8 & 83.4 & 0.9 & -- \\
\hline 0.015 & 1.206 & 26.5 & 50.1 & 1.1 & -- \\
\hline 0.02 & 1.212 & 21.6 & 30.6 & 0.4 & 28.6531 \\
\hline 0.025 & 1.219 & 17 & 21.3 & 0.2 & 20.4587 \\
\hline 0.03 & 1.225 & 14.8 & 17.9 & 0.1 & 17.299 \\
\hline 0.032 & 1.228 & 14.2 & 17 & 0.1 & 16.4811 \\
\hline 0.035 & 1.231 & 13.6 & 16 & 0.1 & 15.5703 \\
\hline 0.04 & 1.238 & 12.7 & 14.8 & 0.1 & 14.3951 \\
\hline 0.05 & 1.251 & 11.8 & 13.4 & 0.1 & 13.0453 \\
\hline 0.06 & 1.264 & 11.3 & 12.6 & 0.05 & 12.2559 \\
\hline 0.07 & 1.277 & 10.6 & 12.1 & 0.05 & 11.7422 \\
\hline 0.08 & 1.289 & 10.3 & 11.7 & 0.05 & 11.3681 \\
\hline 0.09 & 1.302 & 10.1 & 11.4 & 0.05 & 11.0901 \\
\hline 0.1 & 1.315 & 9.9 & 11.2 & 0.05 & 10.8855 \\
\hline 0.118 & 1.338 & 9.7 & 10.85 & 0.05 & 10.6128 \\
\hline 0.13 & 1.354 & 9.5 & 10.8 & 0.05 & 10.4833 \\
\hline 0.15 & 1.38 & 9.4 & 10.55 & 0.05 & 10.3414 \\
\hline 0.2 & 1.444 & 9.25 & 10.4 & 0.05 & 10.1356 \\
\hline 0.235 & 1.489 & 9.25 & 10.4 & 0.05 & 10.0841 \\
\hline 0.25 & 1.508 & 9.2 & 10.4 & 0.1 & 10.0857 \\
\hline 0.353 & 1.639 & 9.3 & 10.5 & 0.05 & 10.1661 \\
\hline 0.47 & 1.785 & 9.55 & 10.8 & 0.05 & 10.4595 \\
\hline 0.475 & 1.791 & 9.5 & 10.8 & 0.05 & 10.4834 \\
\hline 0.48 & 1.797 & 9.5 & 10.7 & 0.1 & 10.5006 \\
\hline 0.486 & 1.804 & 9.6 & 10.8 & 0.05 & 10.5272 \\
\hline
\end{tabular}




\section{B1.2.2.2.3.2 1" Water Reflection}

Case ID: u5o2N26-icyl-0935-01

Material: $\mathrm{UO}_{2}\left(\mathrm{NO}_{3}\right)_{2} 6$ Molar Excess Acid

Geometry: Cylinder

Isotopics: $93.5 \%{ }^{235} \mathrm{U}$

Reflector: 1" water reflected

Code: MCNP5 v1.40

Analyst: Mark Murphy

Date: $8 / 2011$ 
Figure B1.2.2.2.3.2-1. $\mathrm{UO}_{2}\left(\mathrm{NO}_{3}\right) 26 \mathrm{M}-\mathrm{Cylinder}-93.5 \%{ }^{235} \mathrm{U}-1$ " Water Reflection

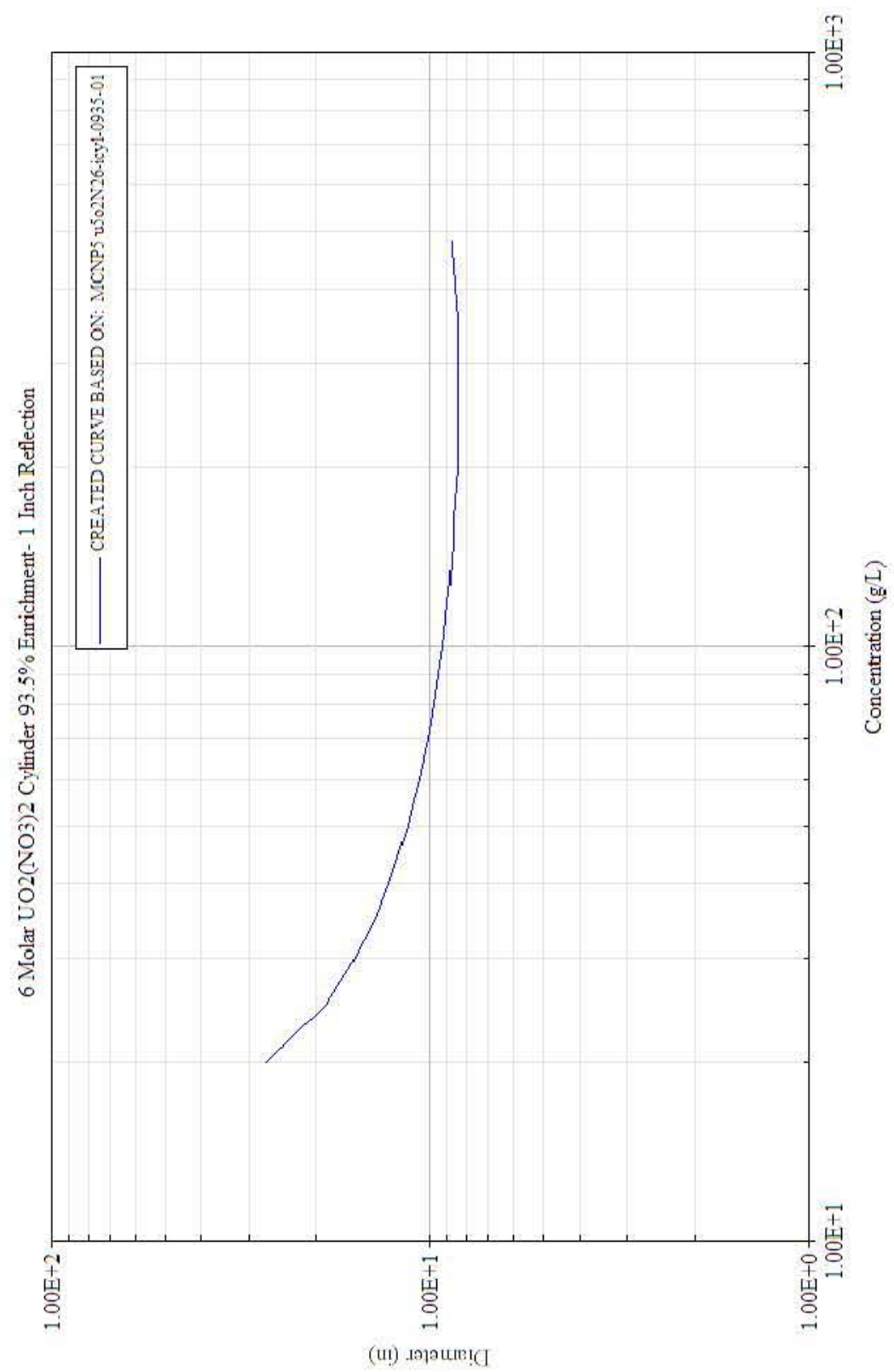


Table B1.2.2.2.3.2-1. Critical Dimensions for $\mathrm{UO}_{2}\left(\mathrm{NO}_{3}\right) 26 \mathrm{M}-\mathrm{Cylinder}-93.5 \%{ }^{235} \mathrm{U}-1$ " Water Reflection

\begin{tabular}{|c|c|c|c|c|c|}
\hline \multicolumn{6}{|c|}{$93.5 \%$ U235 UO2(NO3)2 6M Cylinder- 1 Inch of Reflection } \\
\hline Concentration $(\mathrm{g} / \mathrm{cc})$ & $\begin{array}{l}\text { Density } \\
(\mathrm{g} / \mathrm{cc}) \\
\end{array}$ & $\begin{array}{l}\text { First Diameter } \\
\text { (Inches) }\end{array}$ & $\begin{array}{l}\text { Last Diameter } \\
\text { (Inches) }\end{array}$ & $\begin{array}{l}\text { Delta } \\
\text { (Inches) }\end{array}$ & \begin{tabular}{|l}
$\begin{array}{l}\text { Critical Diameter } \\
\text { (Inches) }\end{array}$ \\
\end{tabular} \\
\hline 0.012 & 1.202 & 68.8 & 92.3 & 1.1 & -- \\
\hline 0.015 & 1.206 & 50.4 & 51.2 & 0.1 & -- \\
\hline 0.02 & 1.212 & 20.1 & 28.7 & 0.4 & 27.0445 \\
\hline 0.025 & 1.219 & 15.4 & 19.7 & 0.4 & 18.8738 \\
\hline 0.03 & 1.225 & 13.2 & 16.3 & 0.1 & 15.6895 \\
\hline 0.032 & 1.228 & 12.7 & 15.5 & 0.1 & 14.8745 \\
\hline 0.035 & 1.231 & 12 & 14.4 & 0.1 & 13.9444 \\
\hline 0.04 & 1.238 & 11.1 & 13.2 & 0.1 & 12.8109 \\
\hline 0.05 & 1.251 & 10 & 11.8 & 0.1 & 11.4625 \\
\hline 0.06 & 1.264 & 9.4 & 11 & 0.1 & 10.6508 \\
\hline 0.07 & 1.277 & 9.05 & 10.35 & 0.1 & 10.1279 \\
\hline 0.08 & 1.289 & 8.7 & 10.1 & 0.05 & 9.7401 \\
\hline 0.09 & 1.302 & 8.4 & 9.8 & 0.05 & 9.4635 \\
\hline 0.1 & 1.315 & 8.3 & 9.5 & 0.05 & 9.2599 \\
\hline 0.118 & 1.338 & 8 & 9.3 & 0.1 & 8.978 \\
\hline 0.13 & 1.354 & 7.9 & 9.1 & 0.05 & 8.8415 \\
\hline 0.15 & 1.38 & 7.85 & 8.9 & 0.05 & 8.6794 \\
\hline 0.2 & 1.444 & 7.6 & 8.7 & 0.05 & 8.4746 \\
\hline 0.235 & \begin{tabular}{|l|}
1.489 \\
\end{tabular} & 7.5 & 8.7 & 0.05 & 8.4154 \\
\hline 0.25 & 1.508 & 7.5 & 8.7 & 0.05 & 8.4064 \\
\hline 0.353 & 1.639 & 7.6 & 8.7 & 0.05 & 8.4687 \\
\hline 0.47 & 1.785 & 7.8 & 9 & 0.05 & 8.7242 \\
\hline 0.475 & 1.791 & 7.8 & 9 & 0.1 & 8.7445 \\
\hline 0.48 & 1.797 & 7.8 & 9 & 0.1 & 8.7484 \\
\hline 0.486 & 1.804 & 7.95 & 9.1 & 0.05 & 8.7734 \\
\hline
\end{tabular}




\section{B1.2.2.2.3.3 10" Water Reflection}

Case ID: u5o2N26-icyl-0935-10

Material: $\mathrm{UO}_{2}\left(\mathrm{NO}_{3}\right)_{2} 6$ Molar Excess Acid

Geometry: Cylinder

Isotopics: $93.5 \%{ }^{235} \mathrm{U}$

Reflector: 10 " water reflected

Code: MCNP5 v1.40

Analyst: Mark Murphy

Date: $8 / 2011$ 
Figure B1.2.2.2.3.3-1. $\mathrm{UO}_{2}\left(\mathrm{NO}_{3}\right) 2$ 6M-Cylinder - 93.5\% ${ }^{235} \mathrm{U}-10$ " Water Reflection

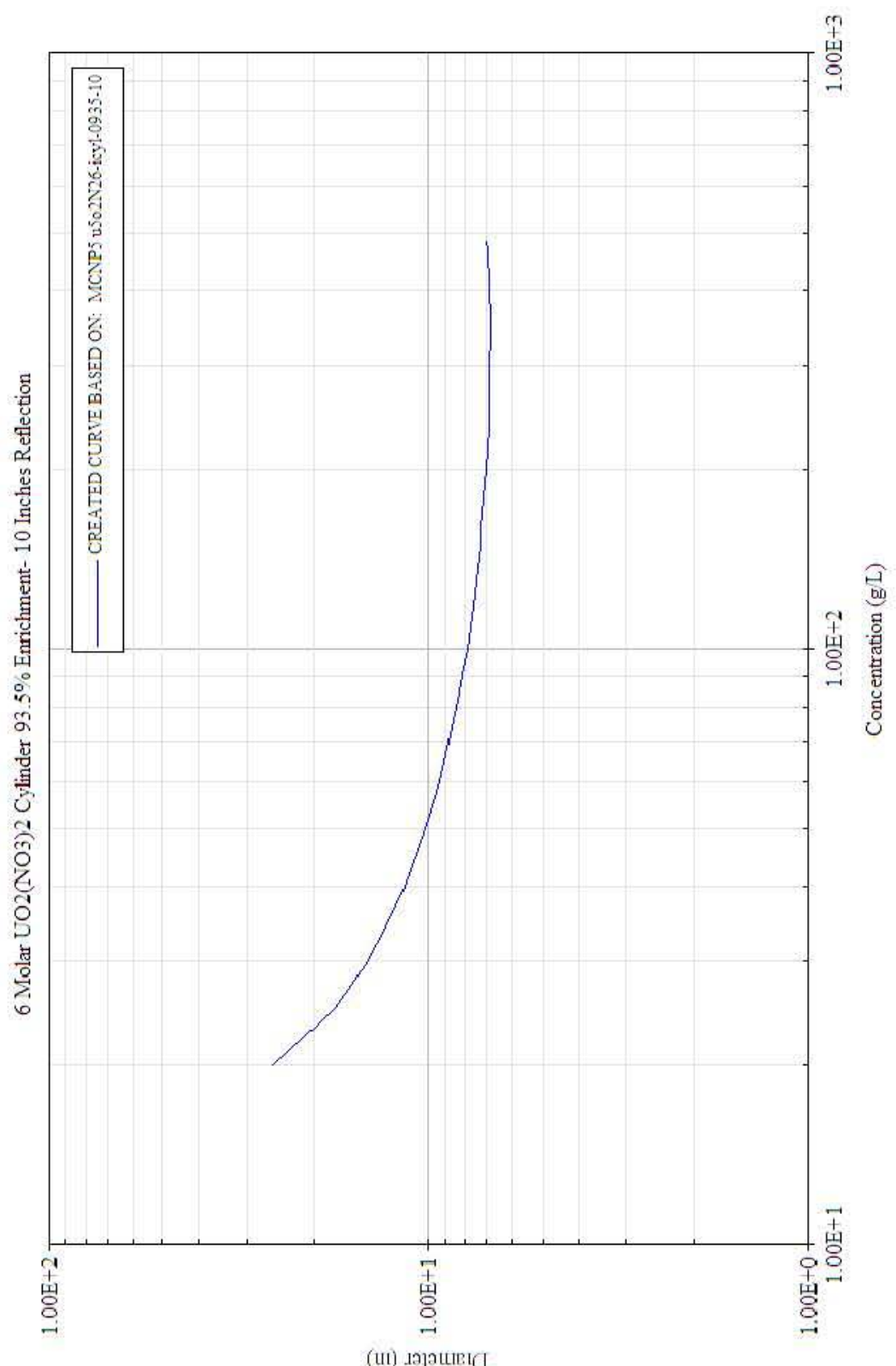

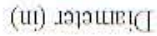


Table B1.2.2.2.3.3-1. Critical Dimensions for $\mathrm{UO}_{2}\left(\mathrm{NO}_{3}\right) 26 \mathrm{M}-\mathrm{Cylinder}-93.5 \%{ }^{235} \mathrm{U}-10$ " Water Reflection

\begin{tabular}{|c|c|c|c|c|c|}
\hline \multicolumn{6}{|c|}{ 93.5\% U235 UO2(NO3)2 6M Cylinder- 10 Inches of Reflection } \\
\hline Concentration $(\mathrm{g} / \mathrm{cc})$ & $\begin{array}{l}\text { Density } \\
(\mathrm{g} / \mathrm{cc})\end{array}$ & $\begin{array}{l}\text { First Diameter } \\
\text { (Inches) }\end{array}$ & $\begin{array}{l}\text { Last Diameter } \\
\text { (Inches) }\end{array}$ & $\begin{array}{l}\text { Delta } \\
\text { (Inches) }\end{array}$ & $\begin{array}{l}\text { Critical Diameter } \\
\text { (Inches) }\end{array}$ \\
\hline 0.012 & 1.202 & 73.7 & 101 & 1.3 & -- \\
\hline 0.015 & 1.206 & 2.61 & 52.6 & 5.2 & -- \\
\hline 0.02 & 1.212 & 18.8 & 27.2 & 0.4 & 25.7407 \\
\hline 0.025 & 1.219 & 14.2 & 18.5 & 0.4 & 17.5967 \\
\hline 0.03 & 1.225 & 12 & 15.1 & 0.1 & 14.4659 \\
\hline 0.032 & 1.228 & 11.4 & 14.2 & 0.1 & 13.6342 \\
\hline 0.035 & 1.231 & 10.7 & 13.2 & 0.1 & 12.6733 \\
\hline 0.04 & 1.238 & 9.9 & 12 & 0.1 & 11.549 \\
\hline 0.05 & 1.251 & 8.85 & 10.45 & 0.05 & 10.1539 \\
\hline 0.06 & 1.264 & 8.5 & 9.65 & 0.05 & 9.3417 \\
\hline 0.07 & 1.277 & 7.7 & 9.1 & 0.05 & 8.7915 \\
\hline 0.08 & 1.289 & 7.4 & 8.7 & 0.05 & 8.4064 \\
\hline 0.09 & 1.302 & 7.25 & 8.4 & 0.05 & 8.117 \\
\hline 0.1 & 1.315 & 6.95 & 8.2 & 0.05 & 7.8816 \\
\hline 0.118 & 1.338 & 6.7 & 7.8 & 0.05 & 7.5875 \\
\hline 0.13 & 1.354 & 6.5 & 7.7 & 0.05 & \begin{tabular}{|l}
7.4378 \\
\end{tabular} \\
\hline 0.15 & 1.38 & 6.45 & 7.5 & 0.05 & 7.2554 \\
\hline 0.2 & 1.444 & 6.1 & 7.2 & 0.05 & 6.9888 \\
\hline 0.235 & 1.489 & 6 & 7.1 & 0.1 & 6.8894 \\
\hline 0.25 & 1.508 & 6 & 7.1 & 0.05 & 6.8653 \\
\hline 0.353 & 1.639 & 6 & 7.1 & 0.05 & 6.8294 \\
\hline 0.47 & 1.785 & 6 & 7.15 & 0.05 & 6.9498 \\
\hline 0.475 & 1.791 & 6.1 & 7.2 & 0.05 & 6.9626 \\
\hline 0.48 & 1.797 & 6.15 & 7.2 & 0.05 & 6.9803 \\
\hline 0.486 & 1.804 & 6.1 & 7.2 & 0.05 & 6.9863 \\
\hline
\end{tabular}


CHPRC-01552, Revision 0 
B1.2.3 Plane

B1.2.3.1 $\quad 100 \%{ }^{235} \mathrm{U}$

B1.2.3.1.1 0 Molar Excess Acid

B1.2.3.1.1.1 0"Water Reflection

Case ID: u5o2N20-pl-1000-00

Material: $\mathrm{UO}_{2}\left(\mathrm{NO}_{3}\right)_{2} \quad 0$ Molar Excess Acid

Geometry: Plane

Isotopics: $100 \%{ }^{235} \mathrm{U}$

Reflector: 0" water reflected

Code: MCNP5 v1.40

Analyst: Mark Murphy

Date: $8 / 2011$ 
Figure B1.2.3.1.1.1-1. $\mathrm{UO}_{2}\left(\mathrm{NO}_{3}\right) 2 \mathrm{OM}-$ Plane - $100 \%{ }^{235} \mathrm{U}-0$ " Water Reflection

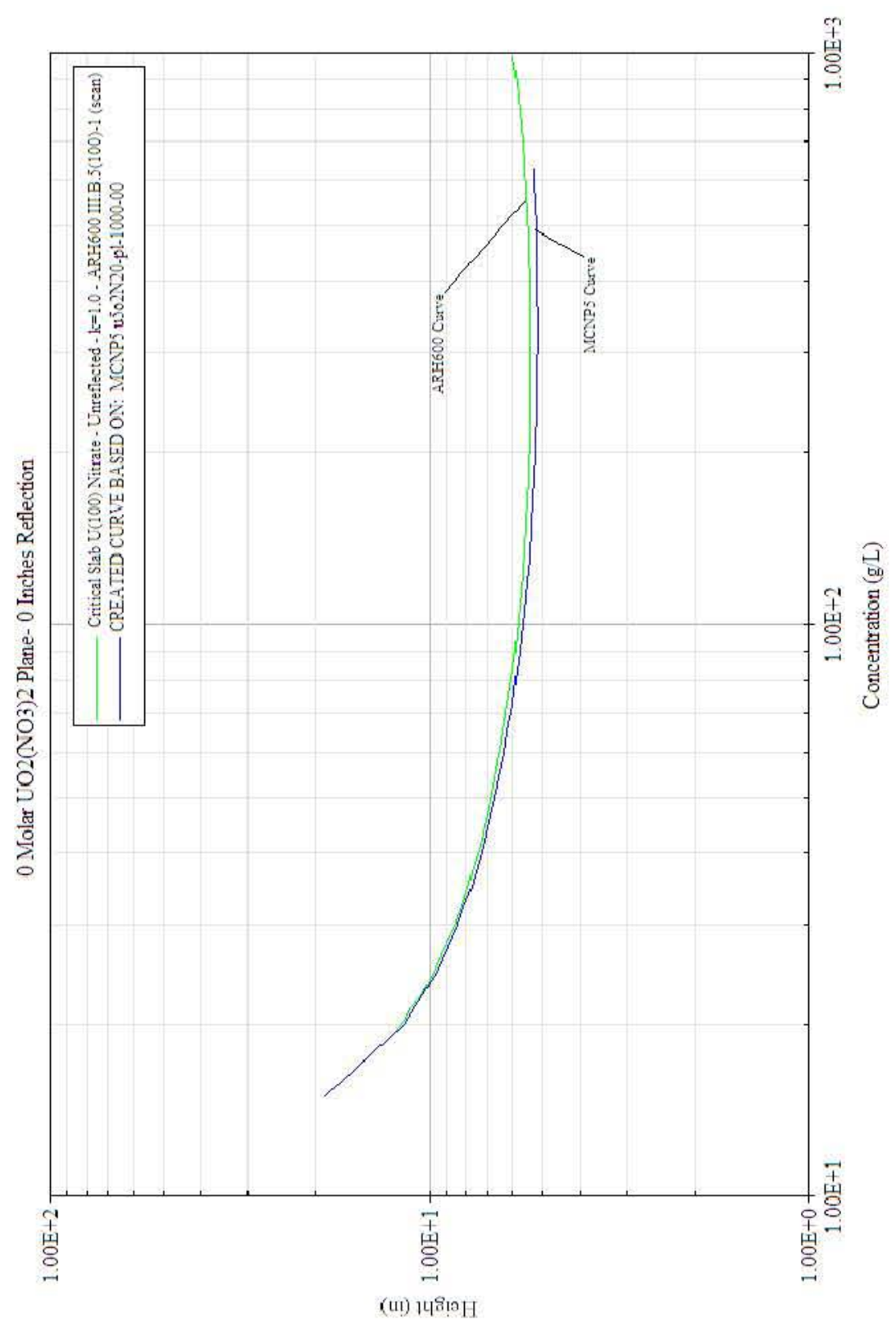


Table B1.2.3.1.1.1-1. Critical Dimensions for $\mathrm{UO}_{2}\left(\mathrm{NO}_{3}\right) 20 \mathrm{M}-$ Plane $-100 \%{ }^{235} \mathrm{U}-0$ " Water Reflection

\begin{tabular}{|c|c|c|c|c|c|}
\hline \multicolumn{6}{|c|}{$100 \%$ U235 UO2(NO3)2 0M Plane - 0 Inches of Reflection } \\
\hline Concentration $(\mathrm{g} / \mathrm{cc})$ & $\begin{array}{l}\text { Density } \\
(\mathrm{g} / \mathrm{cc})\end{array}$ & $\begin{array}{l}\text { First Diameter } \\
\text { (Inches) }\end{array}$ & $\begin{array}{l}\text { Last Diameter } \\
\text { (Inches) }\end{array}$ & $\begin{array}{l}\text { Delta } \\
\text { (Inches) }\end{array}$ & $\begin{array}{l}\text { Critical Height } \\
\text { (Inches) }\end{array}$ \\
\hline 0.012 & 1.013 & 23.8 & 46 & 1.1 & -- \\
\hline 0.015 & 1.017 & 13.6 & 20.1 & 0.3 & 18.7979 \\
\hline 0.02 & 1.024 & 9.6 & 12.1 & 0.1 & 11.6188 \\
\hline 0.025 & 1.031 & 8.1 & 9.8 & 0.05 & 9.4489 \\
\hline 0.03 & 1.037 & 7.3 & 8.7 & 0.05 & 8.3742 \\
\hline 0.032 & 1.04 & 7.1 & 8.4 & 0.05 & 8.0785 \\
\hline 0.035 & 1.044 & 6.8 & 8 & 0.05 & 7.7163 \\
\hline 0.04 & 1.051 & 6.5 & 7.5 & 0.05 & 7.262 \\
\hline 0.05 & 1.064 & 6 & 6.9 & 0.05 & 6.6889 \\
\hline 0.06 & 1.078 & 5.7 & 6.6 & 0.05 & 6.3236 \\
\hline 0.07 & 1.091 & 5.5 & 6.3 & 0.05 & 6.08 \\
\hline 0.08 & 1.105 & 5.3 & 6.1 & 0.05 & 5.8956 \\
\hline 0.09 & 1.118 & 5.2 & 6 & 0.05 & 5.7651 \\
\hline 0.1 & 1.131 & 5.1 & 5.9 & 0.05 & 5.6603 \\
\hline 0.118 & 1.155 & 5 & 5.7 & 0.05 & 5.5283 \\
\hline 0.13 & 1.172 & 4.9 & 5.7 & 0.05 & 5.4447 \\
\hline 0.15 & 1.199 & 4.8 & 5.6 & 0.05 & 5.3663 \\
\hline 0.2 & 1.266 & 4.7 & 5.5 & 0.05 & 5.2467 \\
\hline 0.235 & 1.313 & 4.7 & 5.4 & 0.05 & 5.2087 \\
\hline 0.25 & 1.333 & 4.6 & 5.4 & 0.05 & 5.1877 \\
\hline 0.353 & 1.471 & 4.6 & 5.4 & 0.05 & 5.1583 \\
\hline 0.47 & 1.63 & 4.7 & 5.4 & 0.05 & 5.2036 \\
\hline 0.5 & 1.671 & 4.7 & 5.4 & 0.05 & 5.2095 \\
\hline 0.6 & 1.805 & 4.7 & 5.5 & 0.05 & 5.2753 \\
\hline 0.605 & 1.812 & 4.7 & 5.5 & 0.05 & 5.2729 \\
\hline 0.61 & 1.819 & 4.7 & 5.5 & 0.05 & 5.2742 \\
\hline 0.615 & 1.826 & 4.7 & 5.5 & 0.05 & 5.2903 \\
\hline 0.62 & 1.832 & 4.7 & 5.5 & 0.05 & 5.2875 \\
\hline 0.625 & 1.839 & 4.8 & 5.5 & 0.05 & 5.3071 \\
\hline 0.629 & 1.844 & 4.8 & 5.5 & 0.05 & 5.3091 \\
\hline
\end{tabular}




\section{B1.2.3.1.1.2 1"Water Reflection}

Case ID: u5o2N20-pl-1000-01

Material: $\mathrm{UO}_{2}\left(\mathrm{NO}_{3}\right)_{2} 0$ Molar Excess Acid

Geometry: Plane

Isotopics: $100 \%{ }^{235} \mathrm{U}$

Reflector: 1" water reflected

Code: MCNP5 v1.40

Analyst: Mark Murphy

Date: $8 / 2011$ 
Figure B1.2.3.1.1.2-1. $\mathrm{UO}_{2}\left(\mathrm{NO}_{3}\right) 2 \mathrm{OM}-$ Plane $-100 \%{ }^{235} \mathrm{U}-1$ " Water Reflection

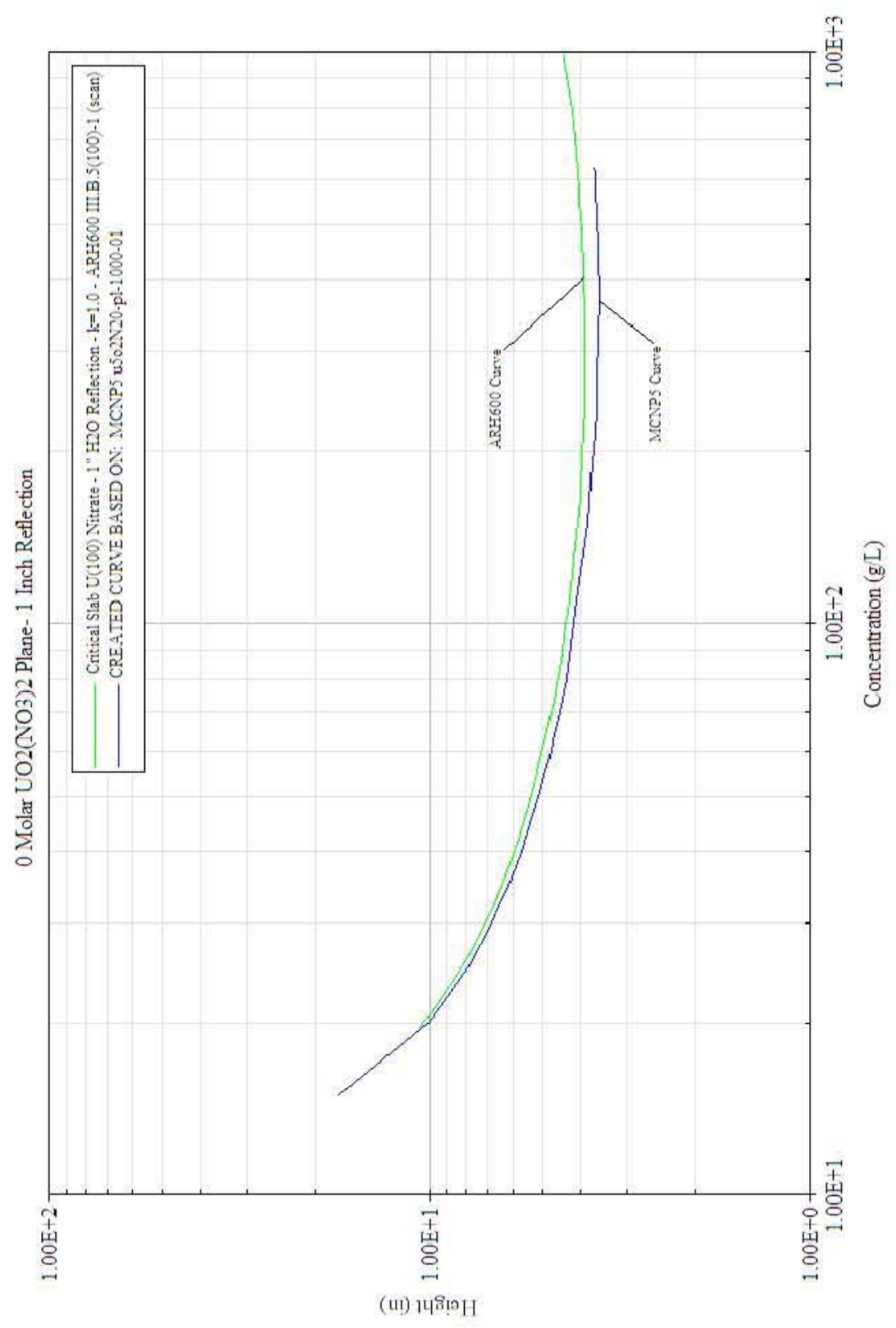


Table B1.2.3.1.1.2-1. Critical Dimensions for $\mathrm{UO}_{2}\left(\mathrm{NO}_{3}\right) 20 \mathrm{M}-$ Plane $-100 \%{ }^{235} \mathrm{U}-1$ " Water Reflection

\begin{tabular}{|c|c|c|c|c|c|}
\hline \multicolumn{6}{|c|}{$100 \%$ U235 UO2(NO3)2 0M Plane - 1 Inch of Reflection } \\
\hline Concentration $(\mathrm{g} / \mathrm{cc})$ & $\begin{array}{l}\text { Density } \\
(\mathrm{g} / \mathrm{cc})\end{array}$ & $\begin{array}{l}\text { First Diameter } \\
\text { (Inches) }\end{array}$ & $\begin{array}{l}\text { Last Diameter } \\
\text { (Inches) }\end{array}$ & $\begin{array}{l}\text { Delta } \\
\text { (Inches) }\end{array}$ & \begin{tabular}{|l} 
Critical Height \\
(Inches)
\end{tabular} \\
\hline 0.012 & 1.013 & 22.3 & 47.1 & 1.2 & -- \\
\hline 0.015 & 1.017 & 12 & 18.6 & 0.3 & 17.2024 \\
\hline 0.02 & 1.024 & 8.1 & 10.6 & 0.1 & 10.071 \\
\hline 0.025 & 1.031 & 6.6 & 8.3 & 0.05 & 7.8998 \\
\hline 0.03 & 1.037 & 5.8 & 7.1 & 0.05 & 6.8476 \\
\hline 0.032 & 1.04 & 5.6 & 6.8 & 0.05 & 6.5438 \\
\hline 0.035 & 1.044 & 5.3 & 6.4 & 0.05 & 6.1839 \\
\hline 0.04 & 1.051 & 4.9 & 6 & 0.05 & 5.7283 \\
\hline 0.05 & 1.064 & 4.3 & 5.4 & 0.05 & 5.1467 \\
\hline 0.06 & 1.078 & 1.6 & 5 & 0.1 & 4.7775 \\
\hline 0.07 & 1.091 & 0.6 & 4.6 & 0.2 & 4.5297 \\
\hline 0.08 & 1.105 & 0.21 & 4.4 & 0.2 & 4.3488 \\
\hline 0.09 & 1.118 & 0.21 & 4.2 & 0.2 & 4.2184 \\
\hline 0.15 & 1.199 & 0.21 & 5 & 0.2 & 3.8126 \\
\hline 0.2 & 1.266 & 0.01 & 5 & 0.2 & 3.6837 \\
\hline 0.235 & 1.313 & 0.01 & 5 & 0.2 & 3.6251 \\
\hline 0.25 & 1.333 & 0.01 & 5 & 0.2 & 3.6139 \\
\hline 0.353 & 1.471 & 0.01 & 5 & 0.2 & 3.572 \\
\hline 0.47 & 1.63 & 0.21 & 5 & 0.2 & 3.6058 \\
\hline 0.5 & 1.671 & 0.01 & 5 & 0.2 & 3.6141 \\
\hline 0.6 & 1.805 & 0.01 & 5 & 0.2 & 3.6579 \\
\hline 0.605 & 1.812 & 0.01 & 5 & 0.2 & 3.6647 \\
\hline 0.61 & 1.819 & 0.01 & 5 & 0.2 & 3.6656 \\
\hline 0.615 & 1.826 & 0.01 & 5 & 0.2 & 3.6569 \\
\hline 0.62 & 1.832 & 0.01 & 5 & 0.2 & 3.6668 \\
\hline 0.625 & 1.839 & 0.01 & 5 & 0.2 & 3.6867 \\
\hline 0.629 & 1.844 & 0.01 & 5 & 0.2 & 3.6831 \\
\hline
\end{tabular}




\section{B1.2.3.1.1.3 10" Water Reflection}

Case ID: u5o2N20-pl-1000-10

Material: $\mathrm{UO}_{2}\left(\mathrm{NO}_{3}\right)_{2} 0$ Molar Excess Acid

Geometry: Plane

Isotopics: $100 \%{ }^{235} \mathrm{U}$

Reflector: 10 " water reflected

Code: MCNP5 v1.40

Analyst: Mark Murphy

Date: $8 / 2011$ 
Figure B1.2.3.1.1.3-1. $\mathrm{UO}_{2}\left(\mathrm{NO}_{3}\right) 2 \mathrm{OM}-$ Plane - $100 \%{ }^{235} \mathrm{U}-10$ " Water Reflection

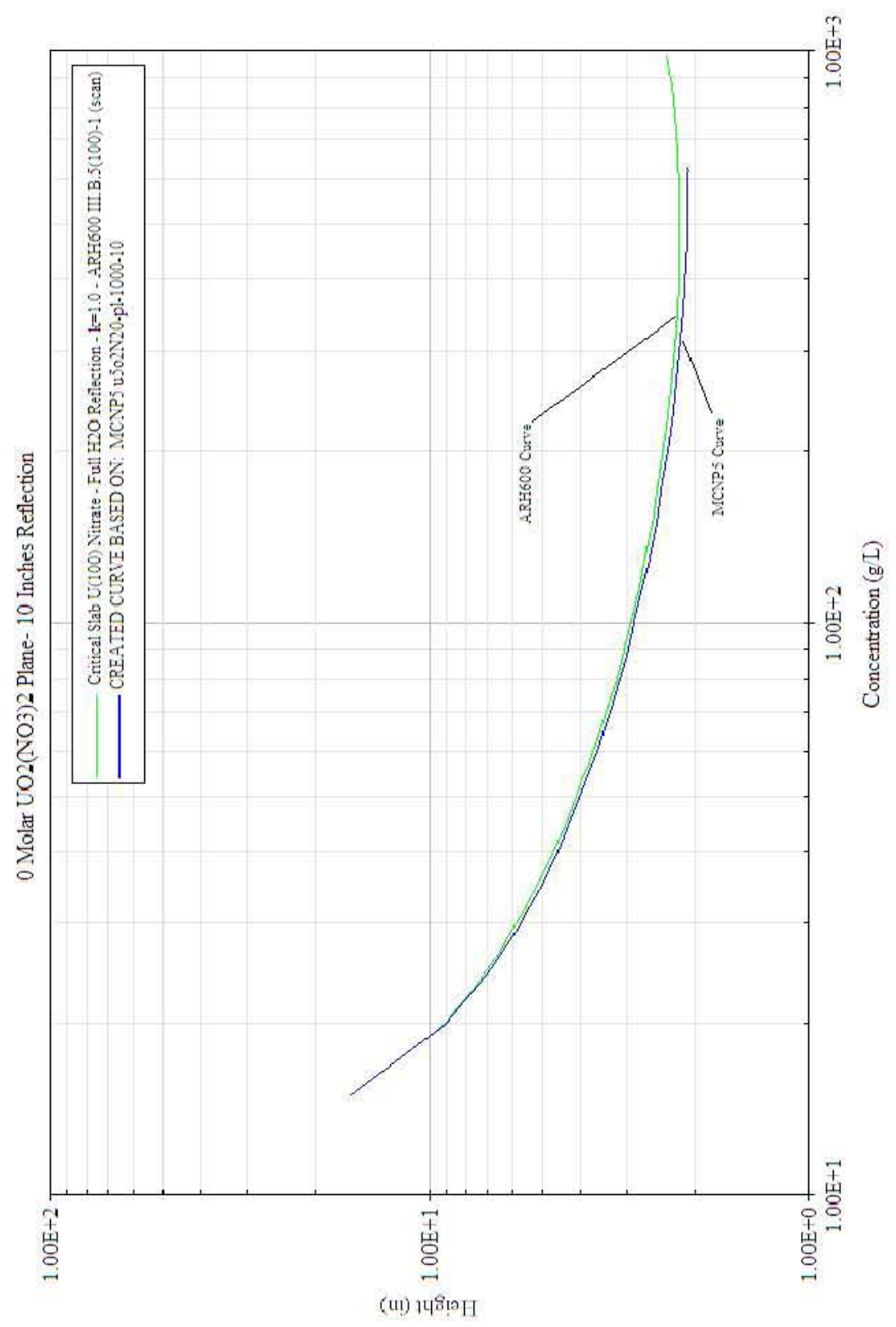


Table B1.2.3.1.1.3-1. Critical Dimensions for $\mathrm{UO}_{2}\left(\mathrm{NO}_{3}\right) 2 \mathrm{OM}-\mathrm{Plane}-100 \%{ }^{235} \mathrm{U}-10$ " Water Reflection

\begin{tabular}{|c|c|c|c|c|c|}
\hline \multicolumn{6}{|c|}{$100 \%$ U235 UO2(NO3)2 0M Plane - 10 Inches of Reflection } \\
\hline Concentration $(\mathrm{g} / \mathrm{cc})$ & \begin{tabular}{|l} 
Density \\
$(\mathrm{g} / \mathrm{cc})$
\end{tabular} & $\begin{array}{l}\text { First Diameter } \\
\text { (Inches) }\end{array}$ & $\begin{array}{l}\text { Last Diameter } \\
\text { (Inches) }\end{array}$ & $\begin{array}{l}\text { Delta } \\
\text { (Inches) }\end{array}$ & \begin{tabular}{|l|}
$\begin{array}{l}\text { Critical Height } \\
\text { (Inches) }\end{array}$ \\
\end{tabular} \\
\hline 0.012 & 1.013 & 21.3 & 42.5 & 1 & -- \\
\hline 0.015 & 1.017 & 10.9 & 17.4 & 0.3 & 16.0469 \\
\hline 0.02 & 1.024 & 7 & 9.4 & 0.1 & 8.9471 \\
\hline 0.025 & 1.031 & 5.5 & 7.2 & 0.05 & 6.8052 \\
\hline 0.03 & 1.037 & 4.7 & 6 & 0.05 & 5.7151 \\
\hline 0.032 & 1.04 & 4.4 & 5.7 & 0.05 & 5.4136 \\
\hline 0.035 & 1.044 & 4 & 5.3 & 0.05 & 5.0405 \\
\hline 0.04 & 1.051 & 0.2 & 4.8 & 0.2 & 4.5852 \\
\hline 0.05 & 1.064 & 0.01 & 3.9 & 0.2 & 3.9763 \\
\hline 0.06 & 1.078 & 0.01 & 5 & 0.2 & 3.6003 \\
\hline 0.07 & 1.091 & 0.01 & 5 & 0.2 & 3.3383 \\
\hline 0.08 & 1.105 & 0.01 & 5 & 0.2 & 3.1373 \\
\hline 0.09 & 1.118 & 0.01 & 5 & 0.2 & 2.9852 \\
\hline 0.1 & 1.131 & 0.01 & 5 & 0.2 & 2.8682 \\
\hline 0.118 & 1.155 & 0.01 & 5 & 0.2 & 2.7081 \\
\hline 0.13 & 1.172 & 0.01 & 5 & 0.2 & 2.6279 \\
\hline 0.15 & 1.199 & 0.01 & 5 & 0.2 & 2.5154 \\
\hline 0.2 & 1.266 & 0.01 & 5 & 0.2 & \begin{tabular}{|l|}
2.3447 \\
\end{tabular} \\
\hline 0.235 & 1.313 & 0.01 & 5 & 0.2 & 2.2615 \\
\hline 0.25 & 1.333 & 0.01 & 5 & 0.2 & 2.2399 \\
\hline 0.353 & 1.471 & 0.01 & 5 & 0.2 & 2.1302 \\
\hline 0.47 & 1.63 & 0.01 & 5 & 0.2 & 2.0839 \\
\hline 0.5 & 1.671 & 0.01 & 5 & 0.2 & 2.0812 \\
\hline 0.6 & 1.805 & 0.01 & 5 & 0.2 & 2.0825 \\
\hline 0.605 & 1.812 & 0.01 & 5 & 0.2 & 2.0878 \\
\hline 0.61 & \begin{tabular}{|l|}
1.819 \\
\end{tabular} & 0.01 & 5 & 0.2 & 2.0766 \\
\hline 0.615 & 1.826 & 0.01 & 5 & 0.2 & 2.0729 \\
\hline 0.62 & 1.832 & 0.01 & 5 & 0.2 & 2.0693 \\
\hline 0.625 & 1.839 & 0.01 & 5 & 0.2 & 2.0857 \\
\hline 0.629 & 1.844 & 0.01 & 5 & 0.2 & 2.0811 \\
\hline
\end{tabular}


CHPRC-01552, Revision 0 
B1.2.3.1.2 3 Molar Excess Acid

B1.2.3.1.2.1 0"Water Reflection

Case ID: u5o2N23-pl-1000-00

Material: $\mathrm{UO}_{2}\left(\mathrm{NO}_{3}\right)_{2} 3$ Molar Excess Acid

Geometry: Plane

Isotopics: $100 \%{ }^{235} \mathrm{U}$

Reflector: 0 " water reflected

Code: MCNP5 v1.40

Analyst: Mark Murphy

Date: $8 / 2011$ 
Figure B1.2.3.1.2.1-1. $\mathrm{UO}_{2}\left(\mathrm{NO}_{3}\right) 2$ 3M - Plane - $100 \%{ }^{235} \mathrm{U}-0$ " Water Reflection

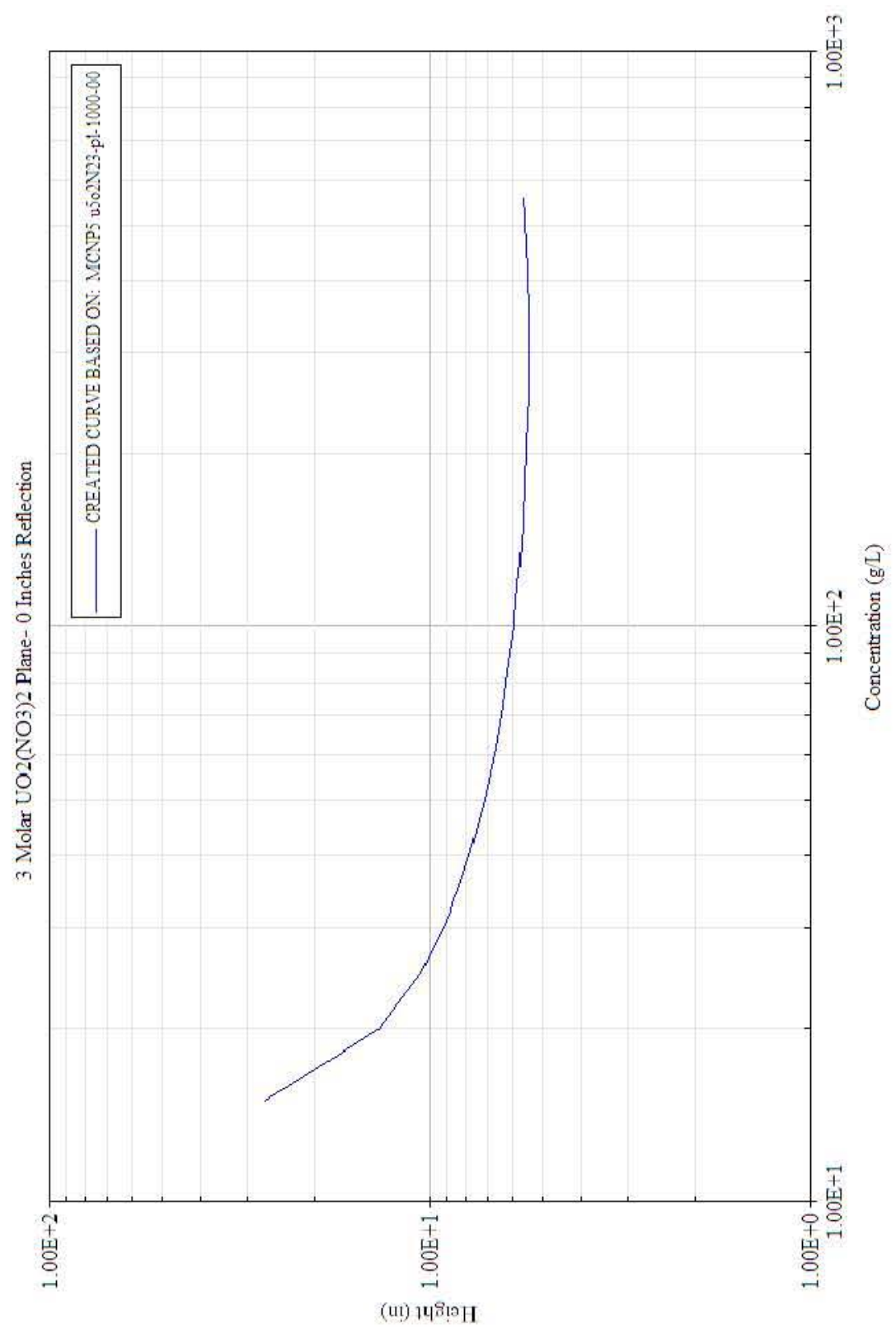


Table B1.2.3.1.2.1-1. Critical Dimensions for $\mathrm{UO}_{2}\left(\mathrm{NO}_{3}\right) 23 \mathrm{M}-$ Plane $-100 \%{ }^{235} \mathrm{U}-0$ " Water Reflection

\begin{tabular}{|c|c|c|c|c|c|}
\hline \multicolumn{6}{|c|}{$100 \%$ U235 UO2(NO3)2 3M Plane - 0 Inches of Reflection } \\
\hline Concentration $(\mathrm{g} / \mathrm{cc})$ & $\begin{array}{l}\text { Density } \\
(\mathrm{g} / \mathrm{cc})\end{array}$ & $\begin{array}{l}\text { First Diameter } \\
\text { (Inches) }\end{array}$ & $\begin{array}{l}\text { Last Diameter } \\
\text { (Inches) }\end{array}$ & $\begin{array}{l}\text { Delta } \\
\text { (Inches) }\end{array}$ & $\begin{array}{l}\text { Critical Height } \\
\text { (Inches) }\end{array}$ \\
\hline 0.012 & 1.112 & 25 & 30.3 & 0.2 & -- \\
\hline 0.015 & 1.116 & 16.5 & 27.9 & 0.5 & 26.969 \\
\hline 0.02 & 1.123 & 10.8 & 14.1 & 0.1 & 13.4134 \\
\hline 0.025 & 1.129 & 8.9 & 10.9 & 0.1 & 10.4911 \\
\hline 0.03 & 1.136 & 7.9 & 9.5 & 0.05 & 9.1544 \\
\hline 0.032 & 1.138 & 7.7 & 9.1 & 0.05 & 8.7747 \\
\hline 0.035 & 1.142 & 7.3 & 8.7 & 0.05 & 8.3651 \\
\hline 0.04 & 1.149 & 6.9 & 8.1 & 0.05 & 7.8103 \\
\hline 0.05 & 1.162 & 6.4 & 7.4 & 0.05 & 7.1352 \\
\hline 0.06 & 1.175 & 6 & 7 & 0.05 & 6.7138 \\
\hline 0.07 & 1.188 & 5.8 & 6.7 & 0.05 & 6.442 \\
\hline 0.08 & 1.201 & 5.6 & 6.5 & 0.05 & 6.2582 \\
\hline 0.09 & 1.215 & 5.5 & 6.3 & 0.05 & 6.0991 \\
\hline 0.1 & 1.228 & 5.4 & 6.2 & 0.05 & 5.9794 \\
\hline 0.118 & 1.251 & 5.3 & 6 & 0.05 & 5.8321 \\
\hline 0.13 & 1.267 & 5.2 & 6 & 0.05 & 5.7633 \\
\hline 0.15 & 1.293 & 5.1 & 5.9 & 0.05 & 5.6807 \\
\hline 0.2 & 1.359 & 5 & 5.8 & 0.05 & 5.5438 \\
\hline 0.235 & 1.405 & 5 & 5.7 & 0.05 & 5.5082 \\
\hline 0.25 & 1.425 & 5 & 5.7 & 0.05 & 5.4896 \\
\hline 0.353 & 1.56 & 5 & 5.7 & 0.05 & 5.4868 \\
\hline 0.47 & 1.714 & 5 & 5.8 & 0.05 & 5.5691 \\
\hline 0.5 & 1.753 & 5.1 & 5.8 & 0.05 & 5.6031 \\
\hline 0.51 & 1.766 & 5.1 & 5.8 & 0.05 & 5.5905 \\
\hline 0.52 & 1.779 & 5.1 & 5.8 & 0.05 & 5.6176 \\
\hline 0.53 & 1.792 & 5.1 & 5.8 & 0.05 & 5.6273 \\
\hline 0.54 & 1.805 & 5.1 & 5.9 & 0.05 & 5.6393 \\
\hline 0.55 & 1.818 & 5.1 & 5.9 & 0.05 & 5.6617 \\
\hline 0.56 & 1.831 & 5.1 & 5.9 & 0.05 & 5.6751 \\
\hline
\end{tabular}




\section{B1.2.3.1.2.2 1"Water Reflection}

Case ID: u5o2N23-pl-1000-01

Material: $\mathrm{UO}_{2}\left(\mathrm{NO}_{3}\right)_{2} 3$ Molar Excess Acid

Geometry: Plane

Isotopics: $100 \%{ }^{235} \mathrm{U}$

Reflector: 1" water reflected

Code: MCNP5 v1.40

Analyst: Mark Murphy

Date: $8 / 2011$ 
Figure B1.2.3.1.2.2-1. $\mathrm{UO}_{2}\left(\mathrm{NO}_{3}\right) 2$ 3M - Plane - $100 \%{ }^{235} \mathrm{U}-1$ " Water Reflection

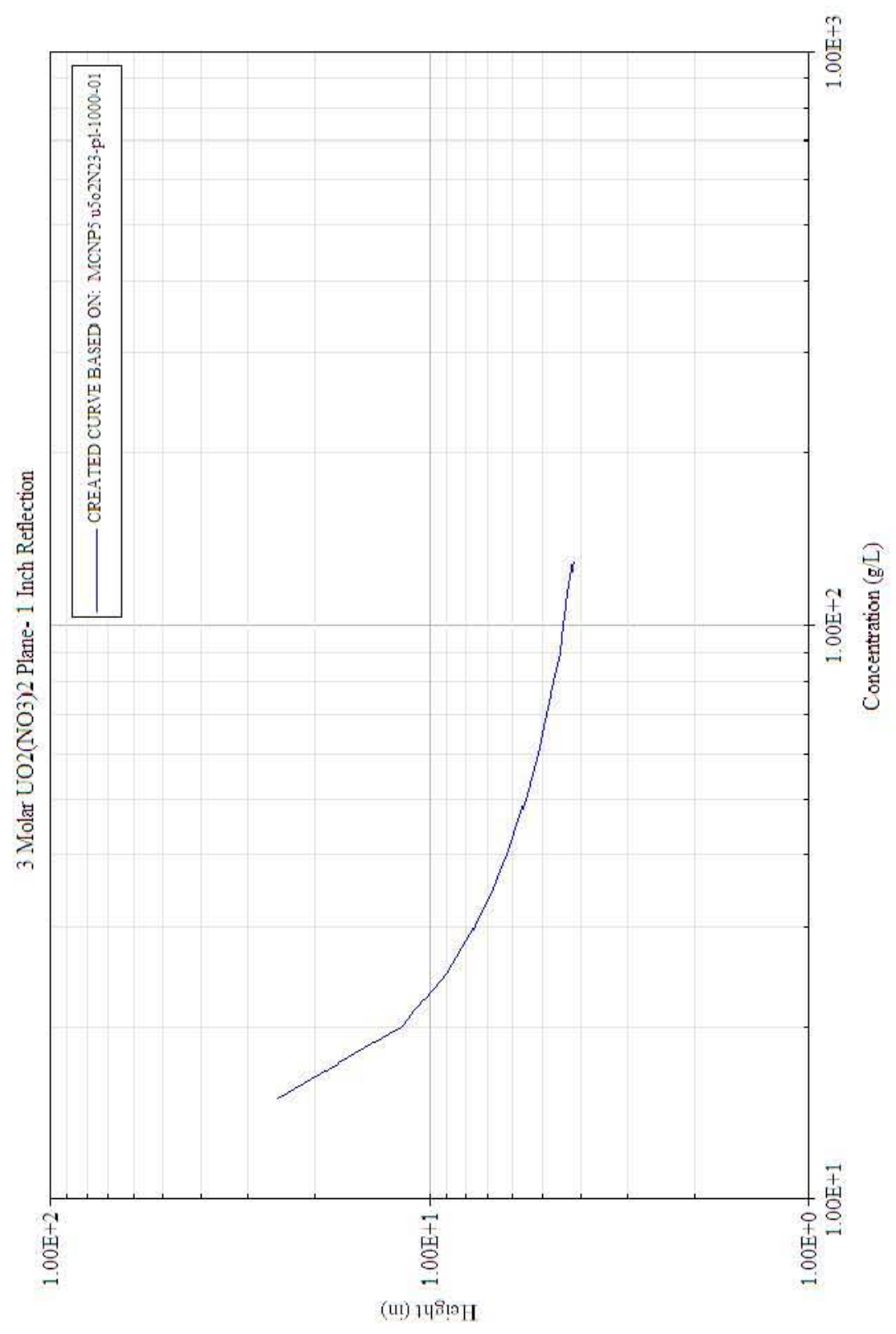


Table B1.2.3.1.2.2-1. Critical Dimensions for $\mathrm{UO}_{2}\left(\mathrm{NO}_{3}\right) 23 \mathrm{M}-\mathrm{Plane}-100 \%{ }^{235} \mathrm{U}-1$ " Water Reflection

\begin{tabular}{|l|l|l|l|l|l|}
\hline \multicolumn{7}{|c|}{$\begin{array}{l}\text { 100\% U235 UO2(NO3)2 3M Plane - 1 Inch of Reflection } \\
\text { Concentration(g/cc) }\end{array}$} & $\begin{array}{l}\text { Density } \\
(\mathrm{g} / \mathrm{cc})\end{array}$ & $\begin{array}{l}\text { First Diameter } \\
\text { (Inches) }\end{array}$ & $\begin{array}{l}\text { Last Diameter } \\
\text { (Inches) }\end{array}$ & $\begin{array}{l}\text { Delta } \\
\text { (nches) }\end{array}$ & $\begin{array}{l}\text { Critical Height } \\
\text { (Inches) }\end{array}$ \\
\hline 0.012 & 1.112 & 25 & 31.7 & 0.3 & -- \\
\hline 0.015 & 1.116 & 15 & 28 & 0.6 & 25.0671 \\
\hline 0.02 & 1.123 & 9.2 & 12.5 & 0.1 & 11.7976 \\
\hline 0.025 & 1.129 & 7.4 & 9.3 & 0.1 & 8.9077 \\
\hline 0.03 & 1.136 & 6.4 & 7.9 & 0.05 & 7.5718 \\
\hline 0.032 & 1.138 & 6.1 & 7.5 & 0.05 & 7.1945 \\
\hline 0.035 & 1.142 & 5.8 & 7 & 0.05 & 6.7635 \\
\hline 0.04 & 1.149 & 5.4 & 6.5 & 0.05 & 6.2289 \\
\hline 0.05 & 1.162 & 4.8 & 5.8 & 0.05 & 5.5532 \\
\hline 0.06 & 1.175 & 4.4 & 5.4 & 0.05 & 5.1484 \\
\hline 0.07 & 1.188 & 4.1 & 5 & 0.05 & 4.8725 \\
\hline 0.08 & 1.201 & 1.3 & 4.8 & 0.1 & 4.6702 \\
\hline 0.09 & 1.215 & 0.6 & 4.6 & 0.2 & 4.5062 \\
\hline 0.1 & 1.228 & 0.1 & 4.5 & 0.2 & 4.3983 \\
\hline 0.118 & 1.251 & 0.21 & 4.3 & 0.2 & 4.2517 \\
\hline 0.13 & 1.267 & 0.21 & 4.2 & 0.2 & 4.1662 \\
\hline & & & & & \\
\hline
\end{tabular}




\section{B1.2.3.1.2.3 10" Water Reflection}

Case ID: u5o2N23-pl-1000-10

Material: $\mathrm{UO}_{2}\left(\mathrm{NO}_{3}\right)_{2} 3$ Molar Excess Acid

Geometry: Plane

Isotopics: $100 \%{ }^{235} \mathrm{U}$

Reflector: 10 " water reflected

Code: MCNP5 v1.40

Analyst: Mark Murphy

Date: $8 / 2011$ 


\section{CHPRC-01552, Revision 0}

Figure B1.2.3.1.2.3-1. $\mathrm{UO}_{2}\left(\mathrm{NO}_{3}\right) 2$ 3M - Plane - 100\% ${ }^{235} \mathrm{U}-10$ " Water Reflection

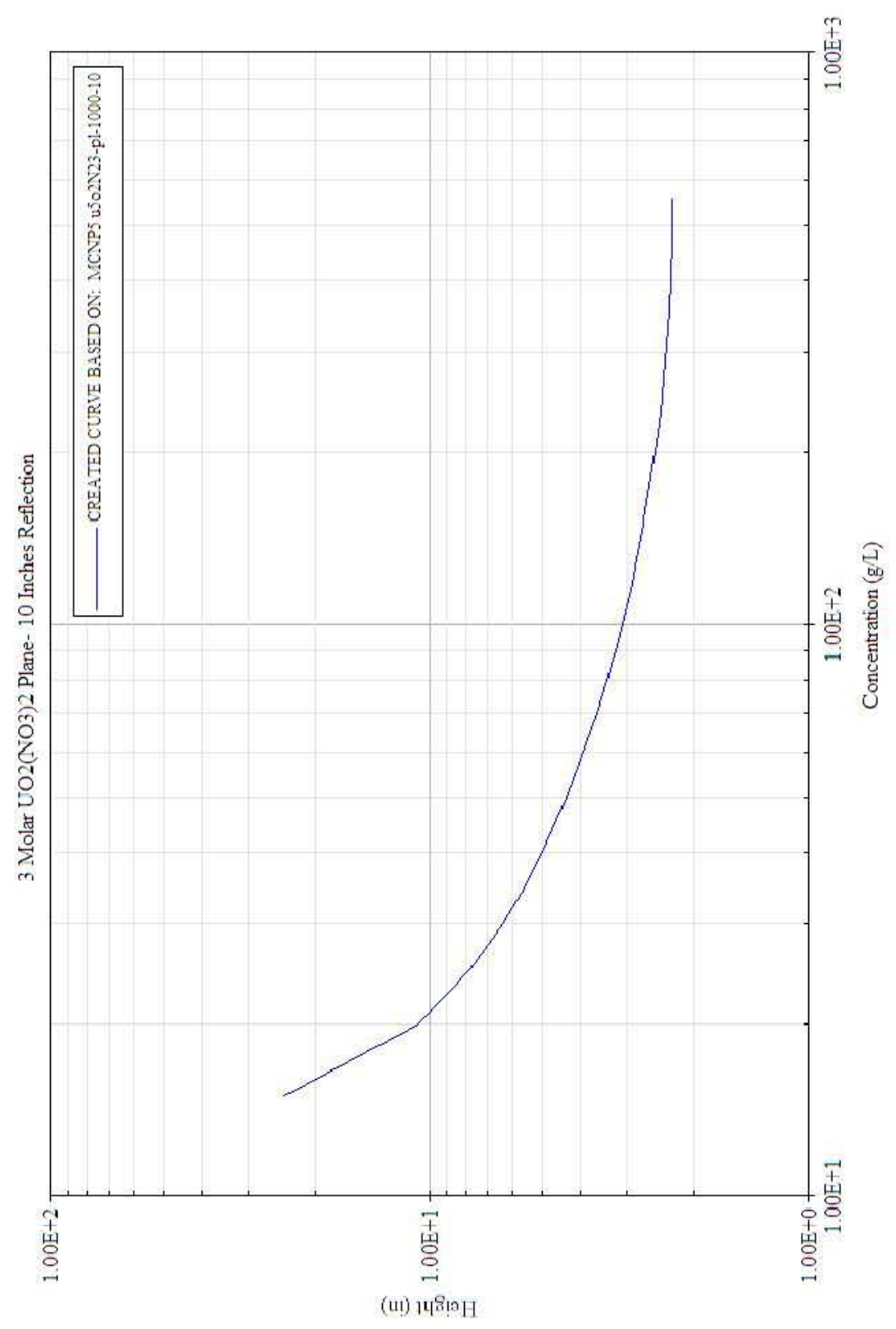


Table B1.2.3.1.2.3-1 Critical Dimensions for $\mathrm{UO}_{2}\left(\mathrm{NO}_{3}\right) 23 \mathrm{M}-\mathrm{Plane}-100 \%{ }^{235} \mathrm{U}-10$ " Water Reflection

\begin{tabular}{|c|c|c|c|c|c|}
\hline \multicolumn{6}{|c|}{$100 \%$ U235 UO2(NO3)2 3M Plane - 10 Inches of Reflection } \\
\hline Concentration $(\mathrm{g} / \mathrm{cc})$ & $\begin{array}{l}\text { Density } \\
(\mathrm{g} / \mathrm{cc})\end{array}$ & $\begin{array}{l}\text { First Diameter } \\
\text { (Inches) }\end{array}$ & $\begin{array}{l}\text { Last Diameter } \\
\text { (Inches) }\end{array}$ & $\begin{array}{l}\text { Delta } \\
\text { (Inches) }\end{array}$ & $\begin{array}{l}\text { Critical Height } \\
\text { (Inches) }\end{array}$ \\
\hline 0.012 & 1.112 & 25 & 32.8 & 0.4 & -- \\
\hline 0.015 & 1.116 & 13.8 & 26.5 & 0.6 & 24.0236 \\
\hline 0.02 & 1.123 & 8.1 & 11.3 & 0.1 & 10.6632 \\
\hline 0.025 & 1.129 & 6.2 & 8.2 & 0.1 & 7.7524 \\
\hline 0.03 & 1.136 & 5.2 & 6.7 & 0.05 & 6.4056 \\
\hline 0.032 & 1.138 & 4.9 & 6.3 & 0.05 & 6.0319 \\
\hline 0.035 & 1.142 & 4.5 & 5.9 & 0.05 & 5.5857 \\
\hline 0.04 & 1.149 & 4 & 5.3 & 0.05 & 5.0274 \\
\hline 0.05 & 1.162 & 0.01 & 4.4 & 0.2 & 4.3289 \\
\hline 0.06 & 1.175 & 0.01 & 3.8 & 0.1 & 3.9021 \\
\hline 0.07 & 1.188 & 0.01 & 5 & 0.2 & 3.6086 \\
\hline 0.08 & 1.201 & 0.01 & 5 & 0.2 & 3.398 \\
\hline 0.09 & 1.215 & 0.01 & 5 & 0.2 & 3.2302 \\
\hline 0.1 & 1.228 & 0.01 & 5 & 0.2 & 3.0937 \\
\hline 0.118 & 1.251 & 0.01 & 5 & 0.2 & 2.9129 \\
\hline 0.13 & 1.267 & 0.01 & 5 & 0.2 & 2.8265 \\
\hline 0.15 & 1.293 & 0.01 & 5 & 0.2 & 2.7133 \\
\hline 0.2 & 1.359 & 0.01 & 5 & 0.2 & 2.5223 \\
\hline 0.235 & 1.405 & 0.01 & 5 & 0.2 & 2.438 \\
\hline 0.25 & 1.425 & 0.01 & 5 & 0.2 & 2.4251 \\
\hline 0.353 & 1.56 & 0.01 & 5 & 0.2 & 2.3143 \\
\hline 0.47 & 1.714 & 0.01 & 5 & 0.2 & 2.2766 \\
\hline 0.5 & 1.753 & 0.01 & 5 & 0.2 & 2.2759 \\
\hline 0.51 & 1.766 & 0.01 & 5 & 0.2 & 2.2853 \\
\hline 0.52 & 1.779 & 0.01 & 5 & 0.2 & 2.285 \\
\hline 0.53 & 1.792 & 0.01 & 5 & 0.2 & 2.2778 \\
\hline 0.54 & 1.805 & 0.01 & 5 & 0.2 & 2.287 \\
\hline 0.55 & 1.818 & 0.01 & 5 & 0.2 & 2.2901 \\
\hline 0.56 & 1.831 & 0.01 & 5 & 0.2 & 2.2884 \\
\hline
\end{tabular}


CHPRC-01552, Revision 0 
B1.2.3.1.3 6 Molar Excess Acid

B1.2.3.1.3.1 0"Water Reflection

Case ID: u5o2N26-pl-1000-00

Material: $\mathrm{UO}_{2}\left(\mathrm{NO}_{3}\right)_{2} 6$ Molar Excess Acid

Geometry: Plane

Isotopics: $100 \%{ }^{235} \mathrm{U}$

Reflector: 0" water reflected

Code: MCNP5 v1.40

Analyst: Mark Murphy

Date: $8 / 2011$ 
Figure B1.2.3.1.3.1-1. $\mathrm{UO}_{2}\left(\mathrm{NO}_{3}\right) 2$ 6M - Plane - 100\% ${ }^{235} \mathrm{U}-0$ " Water Reflection

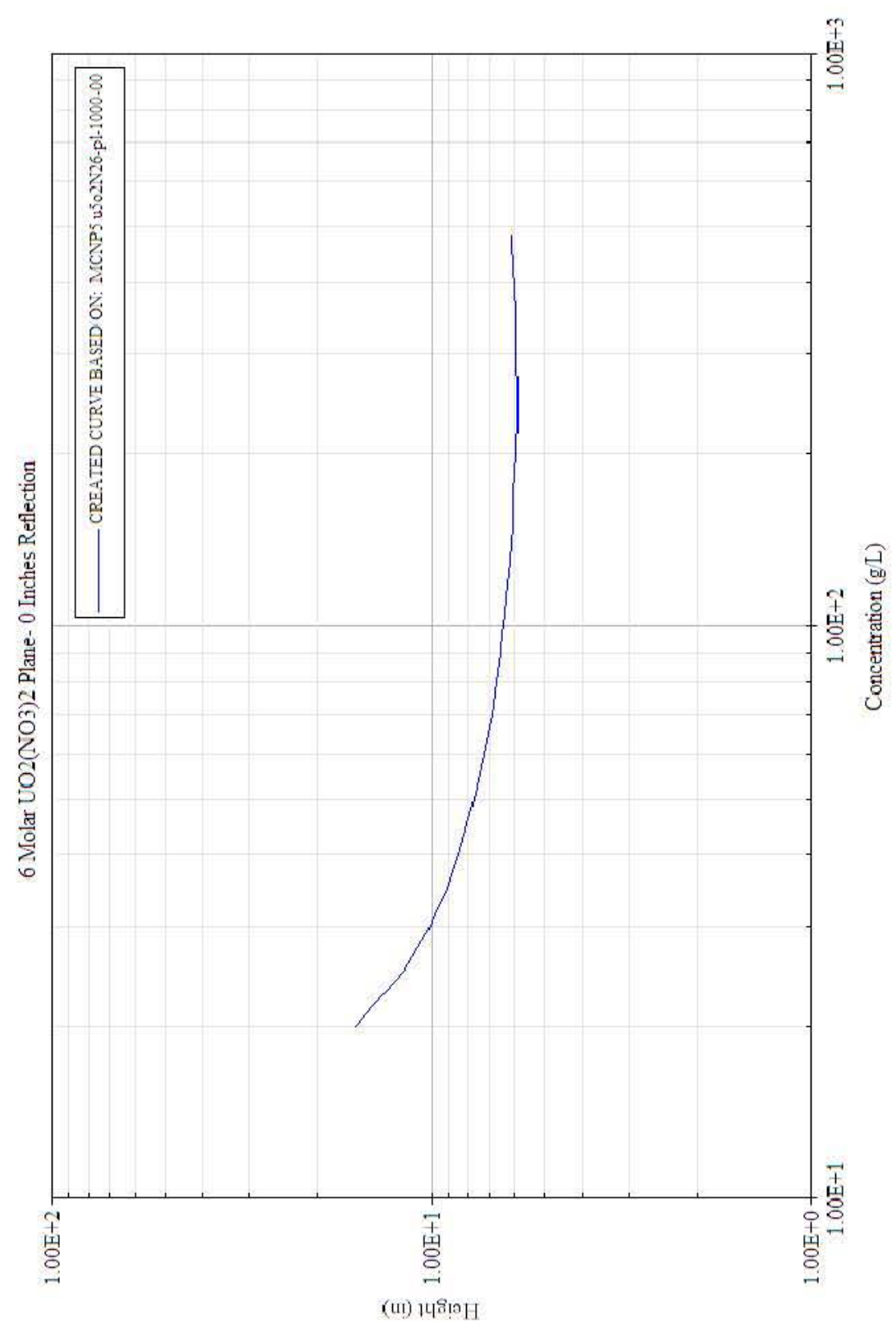


Table B1.2.3.1.3.1-1. Critical Dimensions for $\mathrm{UO}_{2}\left(\mathrm{NO}_{3}\right) 26 \mathrm{M}-$ Plane $-100 \%{ }^{235} \mathrm{U}-0$ " Water Reflection

\begin{tabular}{|c|c|c|c|c|c|}
\hline \multicolumn{6}{|c|}{$100 \%$ U235 UO2(NO3)2 6M Plane - 0 Inches of Reflection } \\
\hline Concentration $(\mathrm{g} / \mathrm{cc})$ & $\begin{array}{l}\text { Density } \\
(\mathrm{g} / \mathrm{cc})\end{array}$ & $\begin{array}{l}\text { First Diameter } \\
\text { (Inches) }\end{array}$ & $\begin{array}{l}\text { Last Diameter } \\
\text { (Inches) }\end{array}$ & \begin{tabular}{|l|} 
Delta \\
(Inches)
\end{tabular} & $\begin{array}{l}\text { Critical Height } \\
\text { (Inches) }\end{array}$ \\
\hline 0.012 & 1.202 & 25 & 32.2 & 0.3 & -- \\
\hline 0.015 & 1.206 & 21.2 & 37.6 & 0.8 & -- \\
\hline 0.02 & 1.212 & 12.3 & 16.6 & 0.2 & 15.7409 \\
\hline 0.025 & 1.219 & 9.8 & 12.2 & 0.1 & 11.7665 \\
\hline 0.03 & 1.225 & 8.7 & 10.5 & 0.1 & 10.0652 \\
\hline 0.032 & 1.228 & 8.3 & 10 & 0.05 & 9.6374 \\
\hline 0.035 & 1.231 & 7.9 & 9.4 & 0.05 & 9.0939 \\
\hline 0.04 & 1.238 & 7.5 & 8.8 & 0.05 & 8.4707 \\
\hline 0.05 & 1.251 & 6.8 & 8 & 0.05 & 7.6849 \\
\hline 0.06 & 1.264 & 6.5 & 7.5 & 0.05 & 7.2256 \\
\hline 0.07 & 1.277 & 6.2 & 7.1 & 0.05 & 6.9088 \\
\hline 0.08 & 1.289 & 6 & 6.9 & 0.05 & 6.7009 \\
\hline 0.09 & 1.302 & 5.9 & 6.8 & 0.05 & 6.535 \\
\hline 0.1 & 1.315 & \begin{tabular}{|l|}
5.8 \\
\end{tabular} & 6.6 & 0.05 & 6.4094 \\
\hline 0.118 & 1.338 & 5.6 & 6.5 & 0.05 & 6.2484 \\
\hline 0.13 & 1.354 & 5.6 & 6.4 & 0.05 & 6.1646 \\
\hline 0.15 & 1.38 & 5.5 & 6.3 & 0.05 & 6.0589 \\
\hline 0.2 & 1.444 & 5.4 & 6.1 & 0.05 & 5.9434 \\
\hline 0.235 & 1.489 & \begin{tabular}{|l}
5.4 \\
\end{tabular} & 6.1 & 0.05 & 5.9068 \\
\hline 0.25 & 1.508 & 5.4 & 6.1 & 0.05 & 5.8925 \\
\hline 0.353 & 1.638 & 5.4 & 6.1 & 0.05 & 5.9451 \\
\hline 0.47 & 1.784 & 5.5 & 6.3 & 0.05 & 6.1101 \\
\hline 0.475 & 1.79 & 5.5 & 6.3 & 0.05 & 6.1112 \\
\hline 0.48 & 1.796 & 5.5 & 6.3 & 0.05 & 6.1159 \\
\hline 0.486 & 1.804 & 5.5 & 6.3 & 0.05 & 6.1105 \\
\hline
\end{tabular}




\section{B1.2.3.1.3.2 1"Water Reflection}

Case ID: u5o2N26-pl-1000-01

Material: $\mathrm{UO}_{2}\left(\mathrm{NO}_{3}\right)_{2} 6$ Molar Excess Acid

Geometry: Plane

Isotopics: $100 \%{ }^{235} \mathrm{U}$

Reflector: 1" water reflected

Code: MCNP5 v1.40

Analyst: Mark Murphy

Date: 8/2011 
Figure B1.2.3.1.3.2-1. $\mathrm{UO}_{2}\left(\mathrm{NO}_{3}\right) 2$ 6M - Plane - $100 \%{ }^{235} \mathrm{U}-1$ " Water Reflection

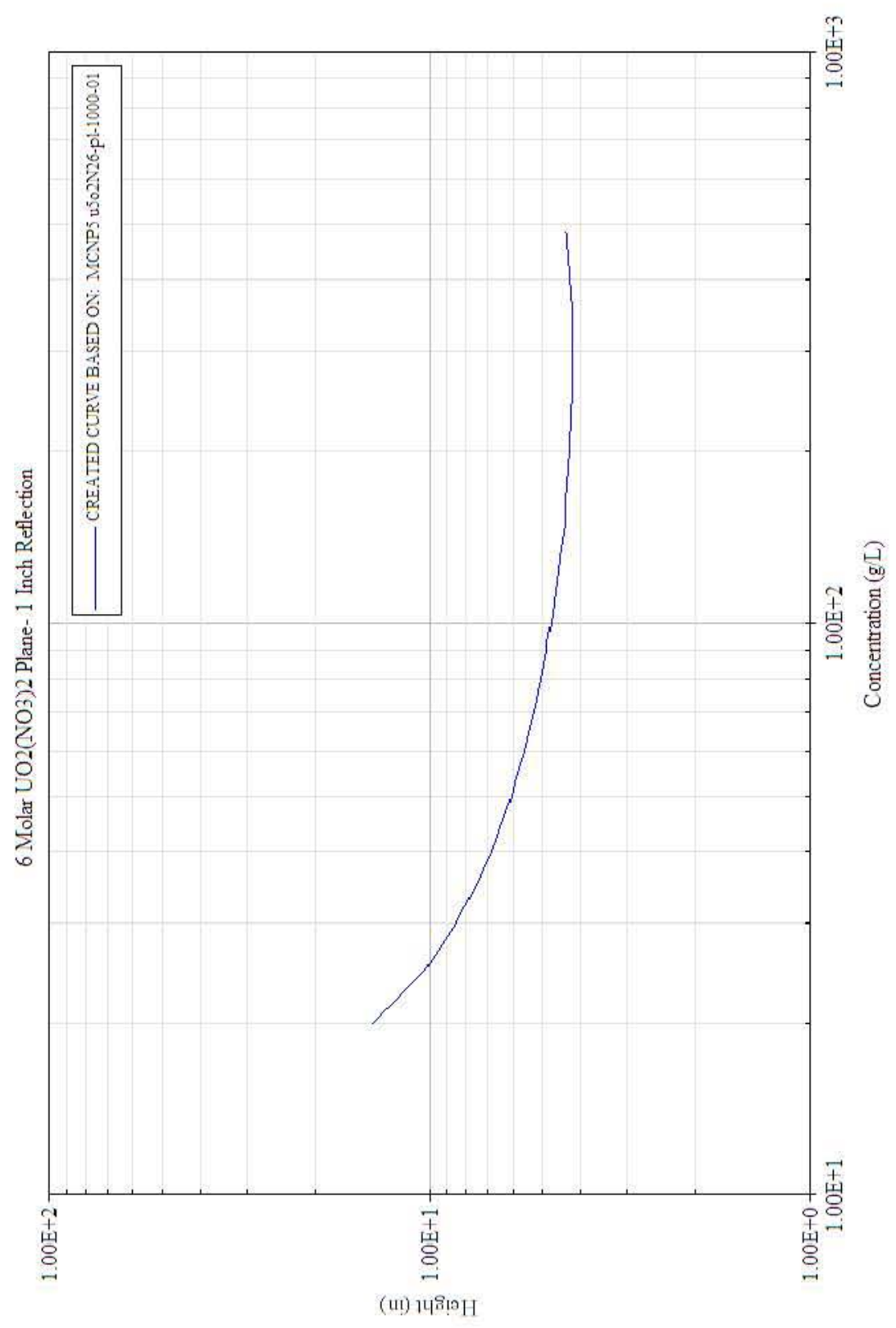


Table B1.2.3.1.3.2-1. Critical Dimensions for $\mathrm{UO}_{2}\left(\mathrm{NO}_{3}\right) 26 \mathrm{M}-$ Plane - $100 \%{ }^{235} \mathrm{U}-1$ " Water Reflection

\begin{tabular}{|c|c|c|c|c|c|}
\hline \multicolumn{6}{|c|}{$100 \%$ U235 UO2(NO3)2 6M Plane - 1 Inch of Reflection } \\
\hline Concentration $(\mathrm{g} / \mathrm{cc})$ & $\begin{array}{l}\text { Density } \\
(\mathrm{g} / \mathrm{cc})\end{array}$ & $\begin{array}{l}\text { First Diameter } \\
\text { (Inches) }\end{array}$ & \begin{tabular}{|l|}
$\begin{array}{l}\text { Last Diameter } \\
\text { (Inches) }\end{array}$ \\
\end{tabular} & \begin{tabular}{|l|}
$\begin{array}{l}\text { Delta } \\
\text { (Inches) }\end{array}$ \\
\end{tabular} & \begin{tabular}{|l}
$\begin{array}{l}\text { Critical Height } \\
\text { (Inches) }\end{array}$ \\
\end{tabular} \\
\hline 0.012 & 1.202 & 25 & 31.5 & 0.3 & -- \\
\hline 0.015 & 1.206 & 19.6 & 35.3 & 0.7 & -- \\
\hline 0.02 & 1.212 & 10.7 & 15 & 0.2 & 14.08 \\
\hline 0.025 & 1.219 & 8.3 & 10.6 & 0.1 & 10.1575 \\
\hline 0.03 & 1.225 & 7.1 & 8.8 & 0.05 & 8.4583 \\
\hline 0.032 & 1.228 & 6.7 & 8.4 & 0.05 & 8.0154 \\
\hline 0.035 & 1.231 & 6.3 & 7.8 & 0.05 & 7.5094 \\
\hline 0.04 & 1.238 & 5.9 & 7.2 & 0.05 & 6.8373 \\
\hline 0.05 & 1.251 & 5.2 & 6.3 & 0.05 & 6.0758 \\
\hline 0.06 & 1.264 & 4.9 & 5.8 & 0.05 & 5.5914 \\
\hline 0.07 & 1.277 & 4.6 & 5.5 & 0.05 & 5.2733 \\
\hline 0.08 & 1.289 & 4.4 & 5.3 & 0.05 & 5.0641 \\
\hline 0.09 & 1.302 & 4.3 & 5.1 & 0.05 & 4.8882 \\
\hline 0.1 & 1.315 & 4.2 & 5 & 0.05 & 4.7578 \\
\hline 0.118 & 1.338 & 4 & 4.8 & 0.05 & 4.6024 \\
\hline 0.13 & 1.354 & 3.9 & 4.7 & 0.05 & 4.5186 \\
\hline 0.15 & 1.38 & 3.9 & 4.6 & 0.05 & 4.393 \\
\hline 0.2 & 1.444 & 3.7 & 4.4 & 0.05 & 4.2691 \\
\hline 0.235 & 1.489 & 3.7 & 4.4 & 0.05 & 4.216 \\
\hline 0.25 & 1.508 & 3.7 & 4.4 & 0.05 & 4.2044 \\
\hline 0.353 & 1.638 & 3.7 & 4.4 & 0.05 & 4.2055 \\
\hline 0.47 & 1.784 & 3.8 & 4.5 & 0.05 & 4.3502 \\
\hline 0.475 & 1.79 & 3.8 & 4.5 & 0.05 & 4.3536 \\
\hline 0.48 & 1.796 & 3.8 & 4.5 & 0.05 & 4.3485 \\
\hline 0.486 & 1.804 & 3.8 & 4.5 & 0.05 & 4.3659 \\
\hline
\end{tabular}




\section{B1.2.3.1.3.3 10" Water Reflection}

Case ID: u5o2N26-pl-1000-10

Material: $\mathrm{UO}_{2}\left(\mathrm{NO}_{3}\right)_{2} 6$ Molar Excess Acid

Geometry: Plane

Isotopics: $100 \%{ }^{235} \mathrm{U}$

Reflector: 10 " water reflected

Code: MCNP5 v1.40

Analyst: Mark Murphy

Date: $8 / 2011$ 


\section{CHPRC-01552, Revision 0}

Figure B1.2.3.1.3.3-1. $\mathrm{UO}_{2}\left(\mathrm{NO}_{3}\right) 26 \mathrm{M}-$ Plane - $100 \%{ }^{235} \mathrm{U}-10$ " Water Reflection

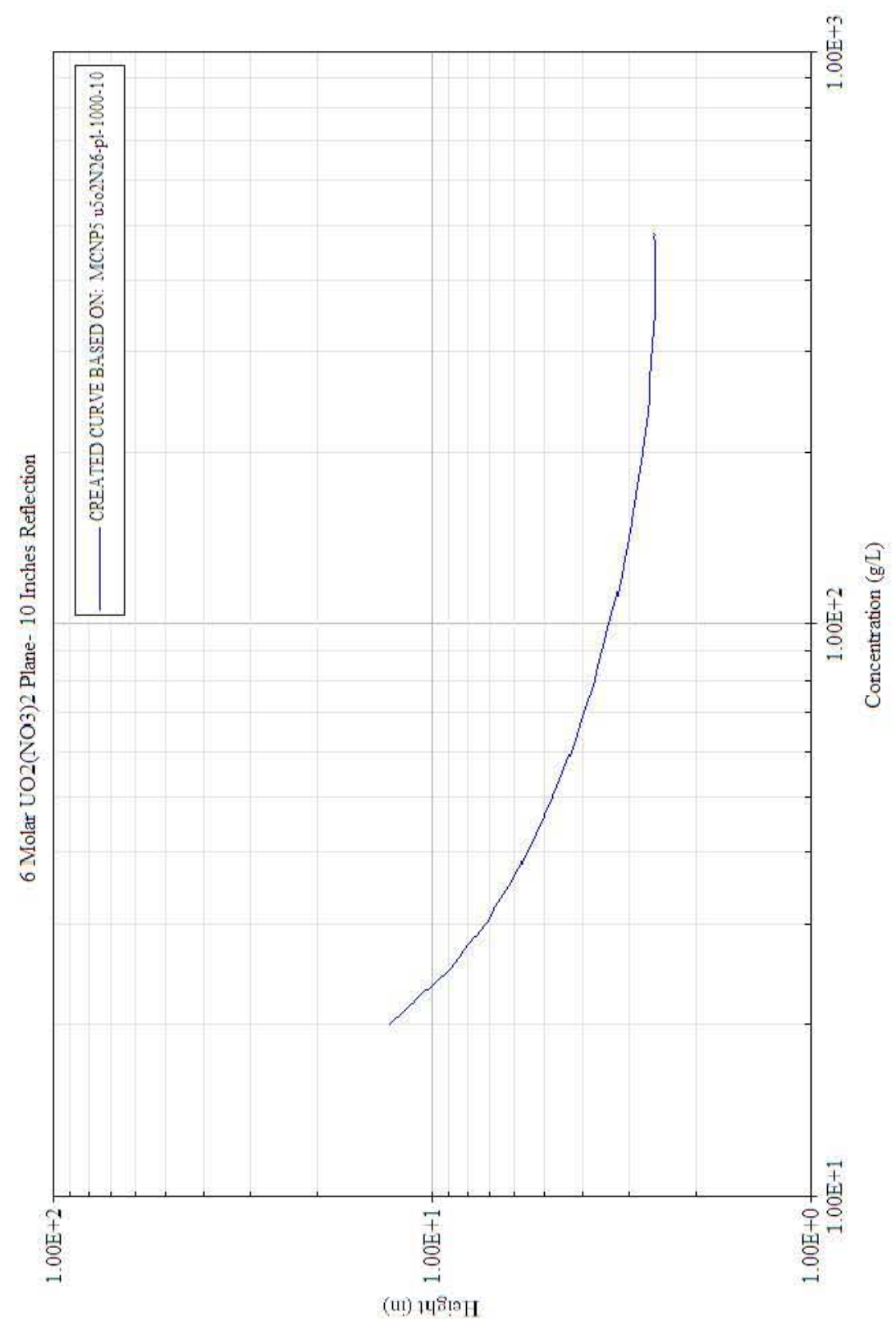


Table B1.2.3.1.3.3-1. Critical Dimensions for $\mathrm{UO}_{2}\left(\mathrm{NO}_{3}\right) 26 \mathrm{M}-\mathrm{Plane}-100 \%{ }^{235} \mathrm{U}-10$ " Water Reflection

\begin{tabular}{|c|c|c|c|c|c|}
\hline \multicolumn{6}{|c|}{$100 \%$ U235 UO2(NO3)2 6M Plane - 10 Inches of Reflection } \\
\hline Concentration $(\mathrm{g} / \mathrm{cc})$ & \begin{tabular}{|l|} 
Density \\
$(\mathrm{g} / \mathrm{cc})$
\end{tabular} & $\begin{array}{l}\text { First Diameter } \\
\text { (Inches) }\end{array}$ & $\begin{array}{l}\text { Last Diameter } \\
\text { (Inches) }\end{array}$ & $\begin{array}{l}\text { Delta } \\
\text { (Inches) }\end{array}$ & \begin{tabular}{|l}
$\begin{array}{l}\text { Critical Height } \\
\text { (Inches) }\end{array}$ \\
\end{tabular} \\
\hline 0.012 & 1.202 & 25 & 31 & 0.3 & -- \\
\hline 0.015 & 1.206 & 18.4 & 34.5 & 0.8 & -- \\
\hline 0.02 & 1.212 & 9.5 & 13.7 & 0.2 & 12.8796 \\
\hline 0.025 & 1.219 & 7 & 9.4 & 0.1 & 8.8902 \\
\hline 0.03 & 1.225 & 5.8 & 7.6 & 0.1 & 7.2192 \\
\hline 0.032 & 1.228 & 5.5 & 7.1 & 0.05 & 6.767 \\
\hline 0.035 & 1.231 & 5.1 & 6.5 & 0.05 & 6.218 \\
\hline 0.04 & 1.238 & 4.6 & 5.8 & 0.05 & 5.5751 \\
\hline 0.05 & 1.251 & 4 & 5 & 0.05 & 4.7624 \\
\hline 0.06 & 1.264 & 3.6 & 4.5 & 0.05 & 4.2772 \\
\hline 0.07 & 1.277 & 3.3 & 4.1 & 0.05 & 3.944 \\
\hline 0.08 & 1.289 & 3.1 & 3.9 & 0.05 & 3.7 \\
\hline 0.09 & 1.302 & 2.9 & 3.7 & 0.05 & 3.5213 \\
\hline 0.1 & 1.315 & 2.8 & 3.6 & 0.05 & 3.3765 \\
\hline 0.118 & 1.338 & 2.6 & 3.4 & 0.05 & 3.1753 \\
\hline 0.13 & 1.354 & 2.5 & 3.3 & 0.05 & 3.0776 \\
\hline 0.15 & 1.38 & 2.4 & 3.1 & 0.05 & 2.9572 \\
\hline 0.2 & 1.444 & 2.2 & 2.9 & 0.05 & 2.7554 \\
\hline 0.235 & 1.489 & 2.2 & 2.9 & 0.05 & 2.6664 \\
\hline 0.25 & 1.508 & 2.1 & 2.8 & 0.05 & 2.6378 \\
\hline 0.353 & 1.638 & 2 & 2.7 & 0.05 & 2.5478 \\
\hline 0.47 & 1.784 & 2 & 2.7 & 0.05 & 2.5488 \\
\hline 0.475 & 1.79 & 2 & 2.7 & 0.05 & 2.5663 \\
\hline 0.48 & 1.796 & 2 & 2.8 & 0.05 & 2.56 \\
\hline 0.486 & 1.804 & 2 & 2.7 & 0.05 & 2.5743 \\
\hline
\end{tabular}


CHPRC-01552, Revision 0 
B1.2.3.2 $\quad 93.5 \%{ }^{235} \mathrm{U}$

B1.2.3.2.1 0 Molar Excess Acid

B1.2.3.2.1.1 0"Water Reflection

Case ID: u5o2N20-pl-0935-00

Material: $\mathrm{UO}_{2}\left(\mathrm{NO}_{3}\right)_{2} 0$ Molar Excess Acid

Geometry: Plane

Isotopics: $93.5 \%{ }^{235} \mathrm{U}$

Reflector: 0" water reflected

Code: MCNP5 v1.40

Analyst: Mark Murphy

Date: $8 / 2011$ 
Figure B1.2.3.2.1.1-1. $\mathrm{UO}_{2}\left(\mathrm{NO}_{3}\right) 2 \mathrm{OM}-$ Plane $-93.5 \%{ }^{235} \mathrm{U}-0$ " Water Reflection

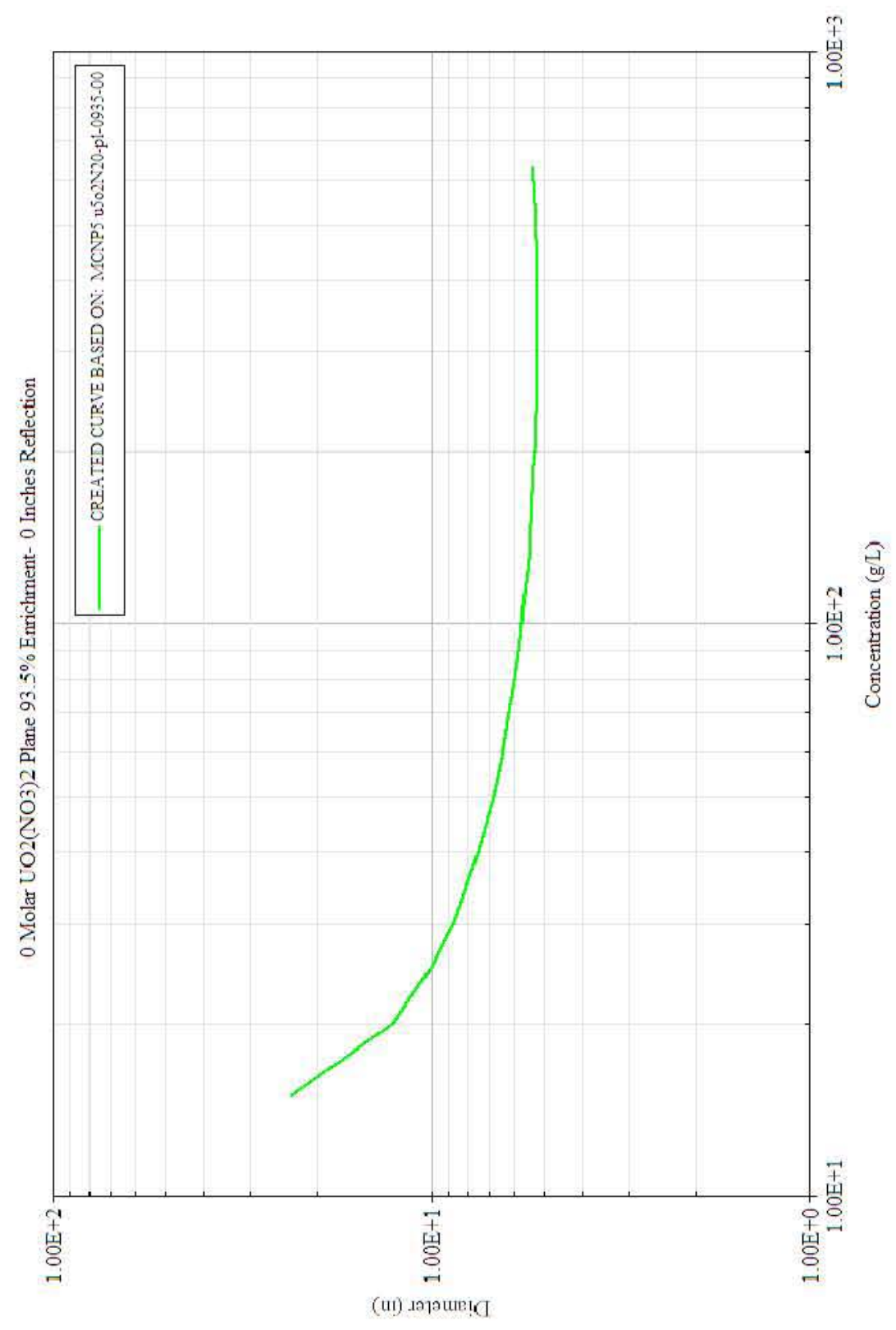


Table B1.2.3.2.1.1-1. Critical Dimensions for $\mathrm{UO}_{2}\left(\mathrm{NO}_{3}\right) 2 \mathrm{OM}-$ Plane $-93.5 \%{ }^{235} \mathrm{U}-0$ " Water Reflection

\begin{tabular}{|c|c|c|c|c|c|}
\hline \multicolumn{6}{|c|}{ 93.5\% U235 UO2(NO3)2 0M Plane- 0 Inches Reflection } \\
\hline Concentration $(\mathrm{g} / \mathrm{cc})$ & $\begin{array}{l}\text { Density } \\
(\mathrm{g} / \mathrm{cc})\end{array}$ & $\begin{array}{l}\text { First Diameter } \\
\text { (Inches) }\end{array}$ & $\begin{array}{l}\text { Last Diameter } \\
\text { (Inches) }\end{array}$ & $\begin{array}{l}\text { Delta } \\
\text { (Inches) }\end{array}$ & \begin{tabular}{|l} 
Critical Height \\
(Inches)
\end{tabular} \\
\hline 0.012 & 1.013 & 32.5 & 50.6 & 0.9 & -- \\
\hline 0.015 & 1.017 & 15.3 & 25.8 & 0.5 & 23.4106 \\
\hline 0.02 & 1.024 & 10.3 & 13.2 & 0.1 & 12.6306 \\
\hline 0.025 & 1.031 & 8.5 & 10.4 & 0.1 & 10.0112 \\
\hline 0.03 & 1.037 & 7.6 & 9.1 & 0.05 & 8.7599 \\
\hline 0.032 & 1.04 & 7.3 & 8.7 & 0.05 & 8.4125 \\
\hline 0.035 & 1.044 & 7 & 8.3 & 0.05 & 8.0107 \\
\hline 0.04 & 1.051 & 6.6 & 7.8 & 0.05 & 7.4985 \\
\hline 0.05 & 1.064 & 6.1 & 7.1 & 0.05 & 6.8594 \\
\hline 0.06 & 1.078 & 5.8 & 6.7 & 0.05 & 6.4623 \\
\hline 0.07 & 1.091 & 5.6 & 6.4 & 0.05 & 6.2018 \\
\hline 0.08 & 1.105 & 5.4 & 6.2 & 0.05 & 6.0051 \\
\hline 0.09 & 1.118 & 5.3 & 6.1 & 0.05 & 5.8669 \\
\hline 0.1 & 1.131 & 5.2 & 5.9 & 0.05 & 5.7563 \\
\hline 0.118 & 1.155 & 5.1 & 5.8 & 0.05 & 5.6115 \\
\hline 0.13 & 1.172 & 5 & 5.7 & 0.05 & 5.5348 \\
\hline 0.15 & 1.198 & 4.9 & 5.6 & 0.05 & 5.4505 \\
\hline 0.2 & 1.266 & 4.8 & 5.5 & 0.05 & 5.3108 \\
\hline 0.235 & 1.313 & 4.8 & 5.4 & 0.05 & 5.2683 \\
\hline 0.25 & 1.333 & 4.7 & 5.4 & 0.05 & 5.2526 \\
\hline 0.353 & 1.472 & 4.7 & 5.4 & 0.05 & 5.2284 \\
\hline 0.47 & 1.631 & 4.8 & 5.5 & 0.05 & 5.2675 \\
\hline 0.5 & 1.67 & 4.8 & 5.5 & 0.05 & 5.2913 \\
\hline 0.6 & 1.805 & 4.8 & 5.6 & 0.05 & 5.3721 \\
\hline 0.605 & 1.812 & 4.8 & 5.6 & 0.05 & 5.3737 \\
\hline 0.61 & 1.819 & 4.8 & 5.6 & 0.05 & 5.3751 \\
\hline 0.615 & 1.825 & 4.8 & 5.6 & 0.05 & 5.3873 \\
\hline 0.62 & 1.832 & 4.8 & 5.6 & 0.05 & 5.3897 \\
\hline 0.625 & 1.839 & 4.8 & 5.6 & 0.05 & 5.3968 \\
\hline 0.629 & 1.844 & 4.9 & 5.6 & 0.05 & 5.4023 \\
\hline
\end{tabular}




\section{B1.2.3.2.1.2 1" Water Reflection}

Case ID: u5o2N20-p1-0935-01

Material: $\mathrm{UO}_{2}\left(\mathrm{NO}_{3}\right)_{2} \quad 0$ Molar Excess Acid

Geometry: Plane

Isotopics: $93.5 \%{ }^{235} \mathrm{U}$

Reflector: 1" water reflected

Code: MCNP5 v1.40

Analyst: Mark Murphy

Date: $8 / 2011$ 
Figure B1.2.3.2.1.2-1. $\mathrm{UO}_{2}\left(\mathrm{NO}_{3}\right) 2 \mathrm{OM}-$ Plane $-93.5 \%{ }^{235} \mathrm{U}-1$ " Water Reflection

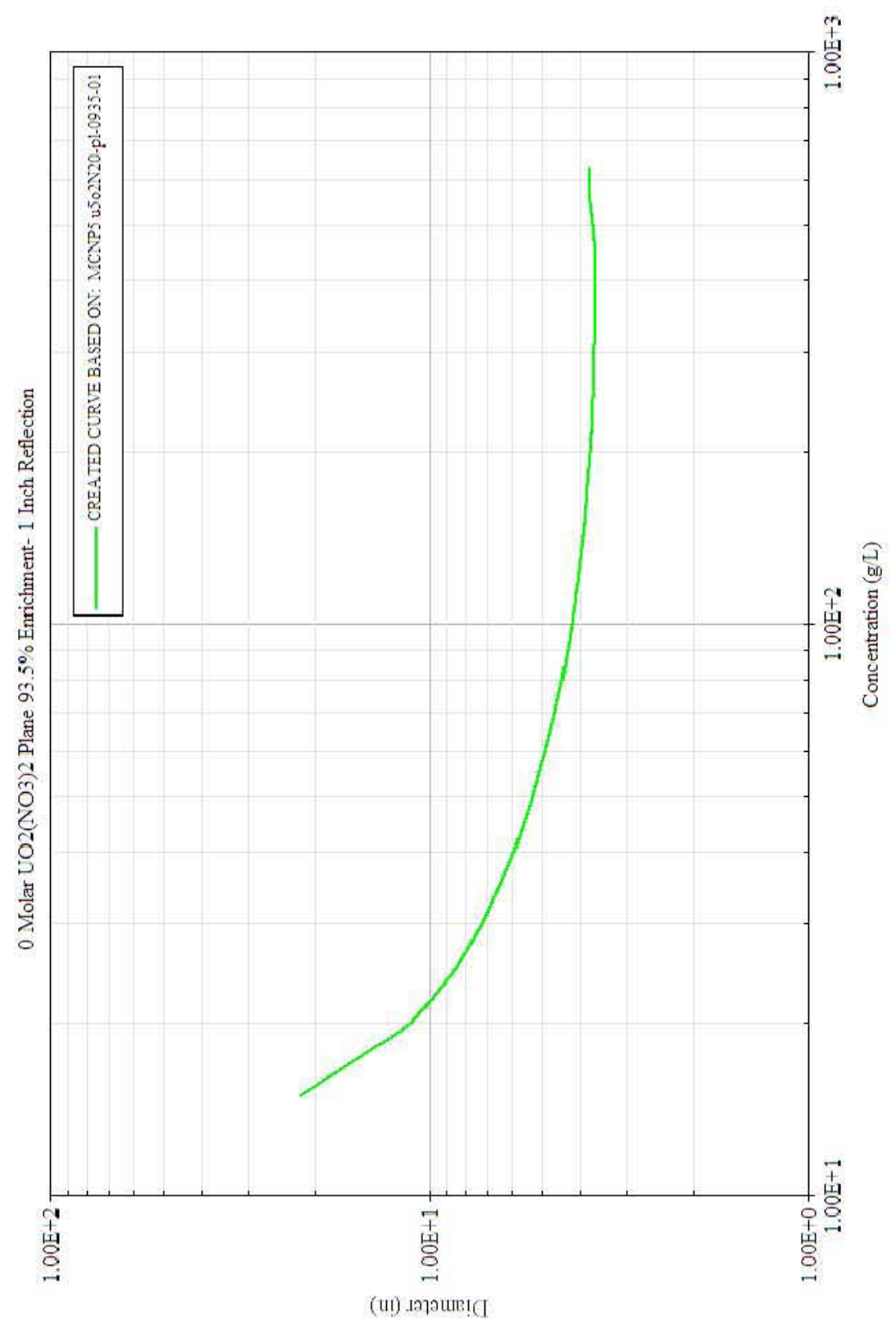


Table B1.2.3.2.1.2-1. Critical Dimensions for $\mathrm{UO}_{2}\left(\mathrm{NO}_{3}\right) 2 \mathrm{OM}-\mathrm{Plane}-93.5 \%{ }^{235} \mathrm{U}-1$ " Water Reflection

\begin{tabular}{|c|c|c|c|c|c|}
\hline \multicolumn{6}{|c|}{ 93.5\% U235 UO2(NO3)2 0M Plane- 1 Inch Reflection } \\
\hline Concentration $(\mathrm{g} / \mathrm{cc})$ & $\begin{array}{l}\text { Density } \\
(\mathrm{g} / \mathrm{cc})\end{array}$ & $\begin{array}{l}\text { First Diameter } \\
\text { (Inches) }\end{array}$ & $\begin{array}{l}\text { Last Diameter } \\
\text { (Inches) }\end{array}$ & $\begin{array}{l}\text { Delta } \\
\text { (Inches) }\end{array}$ & $\begin{array}{l}\text { Critical Height } \\
\text { (Inches) }\end{array}$ \\
\hline 0.012 & 1.013 & 39.2 & 120.9 & 4 & -- \\
\hline 0.015 & 1.017 & 13.8 & 24.3 & 0.5 & 21.8698 \\
\hline 0.02 & 1.024 & 8.7 & 11.7 & 0.1 & 11.098 \\
\hline 0.025 & 1.031 & 7 & 8.9 & 0.1 & 8.4795 \\
\hline 0.03 & 1.037 & 6.1 & 7.5 & 0.05 & 7.226 \\
\hline 0.032 & 1.04 & 5.8 & 7.2 & 0.05 & 6.879 \\
\hline 0.035 & 1.044 & 5.5 & 6.7 & 0.05 & 6.4705 \\
\hline 0.04 & 1.051 & 5.1 & 6.2 & 0.05 & 5.9665 \\
\hline 0.05 & 1.064 & 4.6 & 5.5 & 0.05 & 5.32 \\
\hline 0.06 & 1.078 & 4.3 & 5.1 & 0.05 & 4.9234 \\
\hline 0.07 & 1.091 & 4 & 4.9 & 0.05 & 4.6545 \\
\hline 0.08 & 1.105 & 3.9 & 4.7 & 0.05 & 4.4608 \\
\hline 0.09 & 1.118 & 3.8 & 4.5 & 0.05 & 4.3172 \\
\hline 0.1 & 1.131 & 3.6 & 4.4 & 0.05 & 4.206 \\
\hline 0.118 & 1.155 & 3.5 & 4.2 & 0.05 & 4.0484 \\
\hline 0.13 & 1.172 & 3.4 & 4.1 & 0.05 & 3.9733 \\
\hline 0.15 & 1.198 & 3.4 & 4 & 0.05 & 3.8777 \\
\hline 0.2 & 1.266 & 3.2 & 3.9 & 0.05 & 3.7389 \\
\hline 0.235 & 1.313 & 3.2 & 3.9 & 0.05 & 3.6917 \\
\hline 0.25 & 1.333 & 3.2 & 3.8 & 0.05 & 3.6764 \\
\hline 0.353 & 1.472 & 3.1 & 3.8 & 0.05 & 3.6334 \\
\hline 0.47 & 1.631 & 3.2 & 3.8 & 0.05 & 3.6629 \\
\hline 0.5 & 1.67 & 3.2 & 3.9 & 0.05 & 3.6829 \\
\hline 0.6 & 1.805 & 3.2 & 3.9 & 0.05 & 3.7524 \\
\hline 0.605 & 1.812 & 3.2 & 3.9 & 0.05 & 3.7488 \\
\hline 0.61 & 1.819 & 3.2 & 3.9 & 0.05 & 3.7504 \\
\hline 0.615 & 1.825 & 3.2 & 3.9 & 0.05 & 3.7591 \\
\hline 0.62 & 1.832 & 3.2 & 3.9 & 0.05 & 3.764 \\
\hline 0.625 & 1.839 & 3.2 & 3.9 & 0.05 & 3.7645 \\
\hline 0.629 & 1.844 & 3.2 & 3.9 & 0.05 & 3.7671 \\
\hline
\end{tabular}




\section{B1.2.3.2.1.3 10" Water Reflection}

Case ID: u5o2N20-pl-0935-10

Material: $\mathrm{UO}_{2}\left(\mathrm{NO}_{3}\right)_{2} 0$ Molar Excess Acid

Geometry: Plane

Isotopics: $93.5 \%{ }^{235} \mathrm{U}$

Reflector: 10 " water reflected

Code: MCNP5 v1.40

Analyst: Mark Murphy

Date: $8 / 2011$ 
Figure B1.2.3.2.1.3-1. $\mathrm{UO}_{2}\left(\mathrm{NO}_{3}\right) 2 \mathrm{OM}-$ Plane $-93.5 \%{ }^{235} \mathrm{U}-10$ " Water Reflection

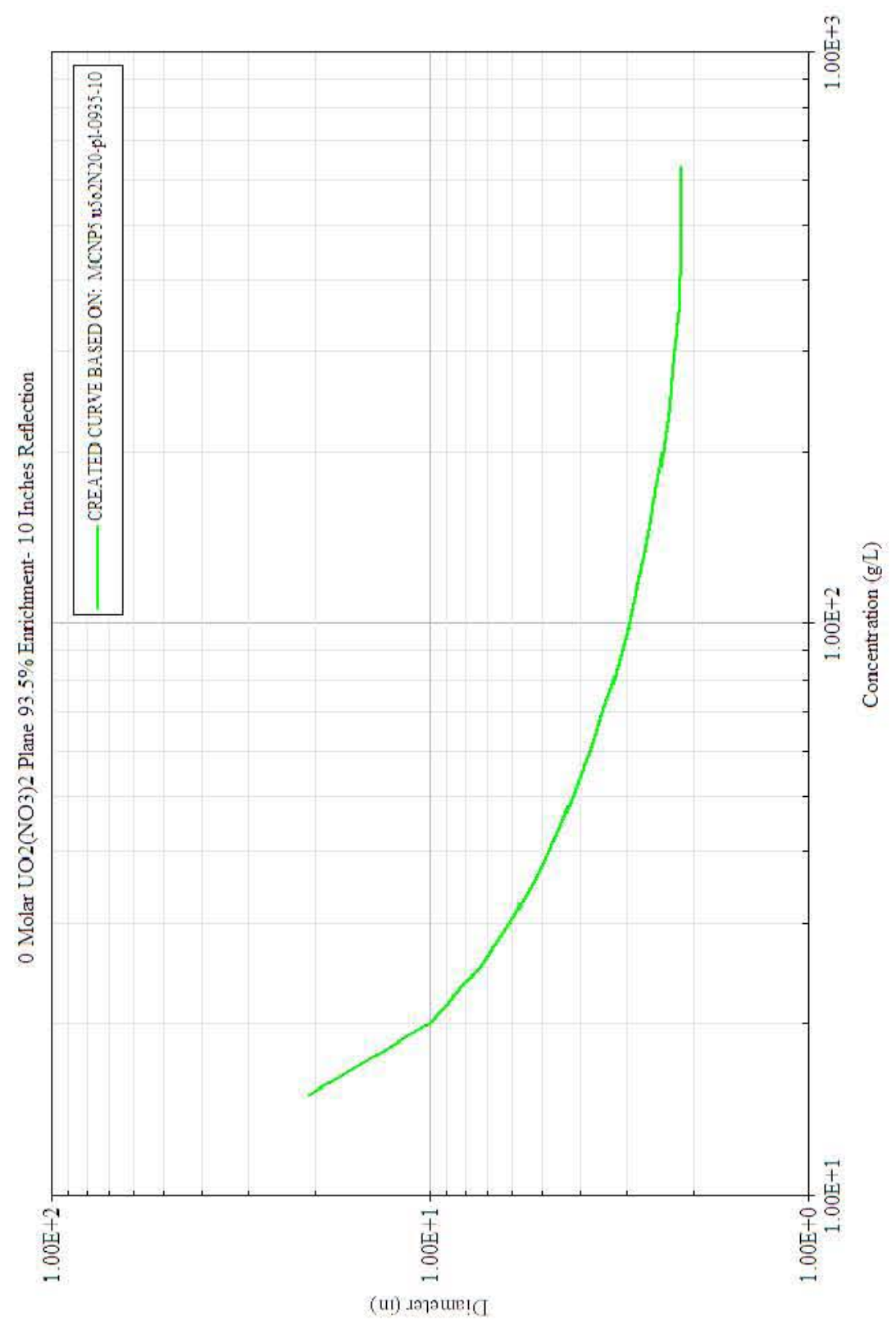


Table B1.2.3.2.1.3-1. Critical Dimensions for $\mathrm{UO}_{2}\left(\mathrm{NO}_{3}\right) 20 \mathrm{M}-$ Plane $-93.5 \%{ }^{235} \mathrm{U}-10$ " Water Reflection

\begin{tabular}{|c|c|c|c|c|c|}
\hline \multicolumn{6}{|c|}{ 93.5\% U235 UO2(NO3)2 0M Plane- 10 Inches Reflection } \\
\hline Concentration $(\mathrm{g} / \mathrm{cc})$ & $\begin{array}{l}\text { Density } \\
(\mathrm{g} / \mathrm{cc})\end{array}$ & $\begin{array}{l}\text { First Diameter } \\
\text { (Inches) }\end{array}$ & $\begin{array}{l}\text { Last Diameter } \\
\text { (Inches) }\end{array}$ & $\begin{array}{l}\text { Delta } \\
\text { (Inches) }\end{array}$ & $\begin{array}{l}\text { Critical Height } \\
\text { (Inches) }\end{array}$ \\
\hline 0.012 & 1.013 & 34.5 & 129.5 & 4.7 & -- \\
\hline 0.015 & 1.017 & 12.7 & 23.2 & 0.5 & 20.7472 \\
\hline 0.02 & 1.024 & 7.7 & 10.5 & 0.1 & 9.994 \\
\hline 0.025 & 1.031 & 5.9 & 7.7 & 0.1 & 7.3539 \\
\hline 0.03 & 1.037 & 5 & 6.4 & 0.05 & 6.107 \\
\hline 0.032 & 1.04 & 4.7 & 6 & 0.05 & 5.7456 \\
\hline 0.035 & 1.044 & 4.4 & 5.6 & 0.05 & 5.3356 \\
\hline 0.04 & 1.051 & 4 & 5 & 0.05 & 4.8175 \\
\hline 0.05 & 1.064 & 3.5 & 4.4 & 0.05 & 4.1583 \\
\hline 0.06 & 1.078 & 3.1 & 3.9 & 0.05 & 3.7434 \\
\hline 0.07 & 1.091 & 2.9 & 3.6 & 0.05 & 3.4616 \\
\hline 0.08 & 1.105 & 2.7 & 3.4 & 0.05 & 3.2491 \\
\hline 0.09 & 1.118 & 2.5 & 3.3 & 0.05 & 3.0931 \\
\hline 0.1 & 1.131 & 2.4 & 3.1 & 0.05 & 2.9638 \\
\hline 0.118 & 1.155 & 2.3 & 3 & 0.05 & 2.7938 \\
\hline 0.13 & 1.172 & 2.2 & 2.9 & 0.05 & 2.7055 \\
\hline 0.15 & 1.198 & 2.25 & 2.7 & 0.05 & 2.592 \\
\hline 0.2 & 1.266 & 1.9 & 2.5 & 0.05 & 2.4008 \\
\hline 0.235 & 1.313 & 1.9 & 2.5 & 0.05 & 2.327 \\
\hline 0.25 & 1.333 & 1.8 & 2.4 & 0.05 & 2.2988 \\
\hline 0.353 & 1.472 & 1.7 & 2.3 & 0.05 & 2.1902 \\
\hline 0.47 & 1.631 & 1.7 & 2.3 & 0.05 & 2.145 \\
\hline 0.5 & 1.67 & 1.7 & 2.3 & 0.05 & 2.1481 \\
\hline 0.6 & 1.805 & 1.7 & 2.3 & 0.05 & 2.1516 \\
\hline 0.605 & 1.812 & 1.7 & 2.3 & 0.05 & 2.1529 \\
\hline 0.61 & 1.819 & 1.7 & 2.3 & 0.05 & 2.1459 \\
\hline 0.615 & 1.825 & 1.7 & 2.3 & 0.05 & 2.1514 \\
\hline 0.62 & 1.832 & 1.7 & 2.3 & 0.05 & 2.1546 \\
\hline 0.625 & 1.839 & 1.7 & 2.3 & 0.05 & 2.153 \\
\hline 0.629 & 1.844 & 1.7 & 2.3 & 0.05 & 2.1539 \\
\hline
\end{tabular}


CHPRC-01552, Revision 0

This page is intentionally left blank. 
CHPRC-01552, Revision 0

\section{APPENDIX C - CRITVIEW COMPATIBLE DATA FILES}


CHPRC-01552, Revision 0

This page is intentionally left blank. 


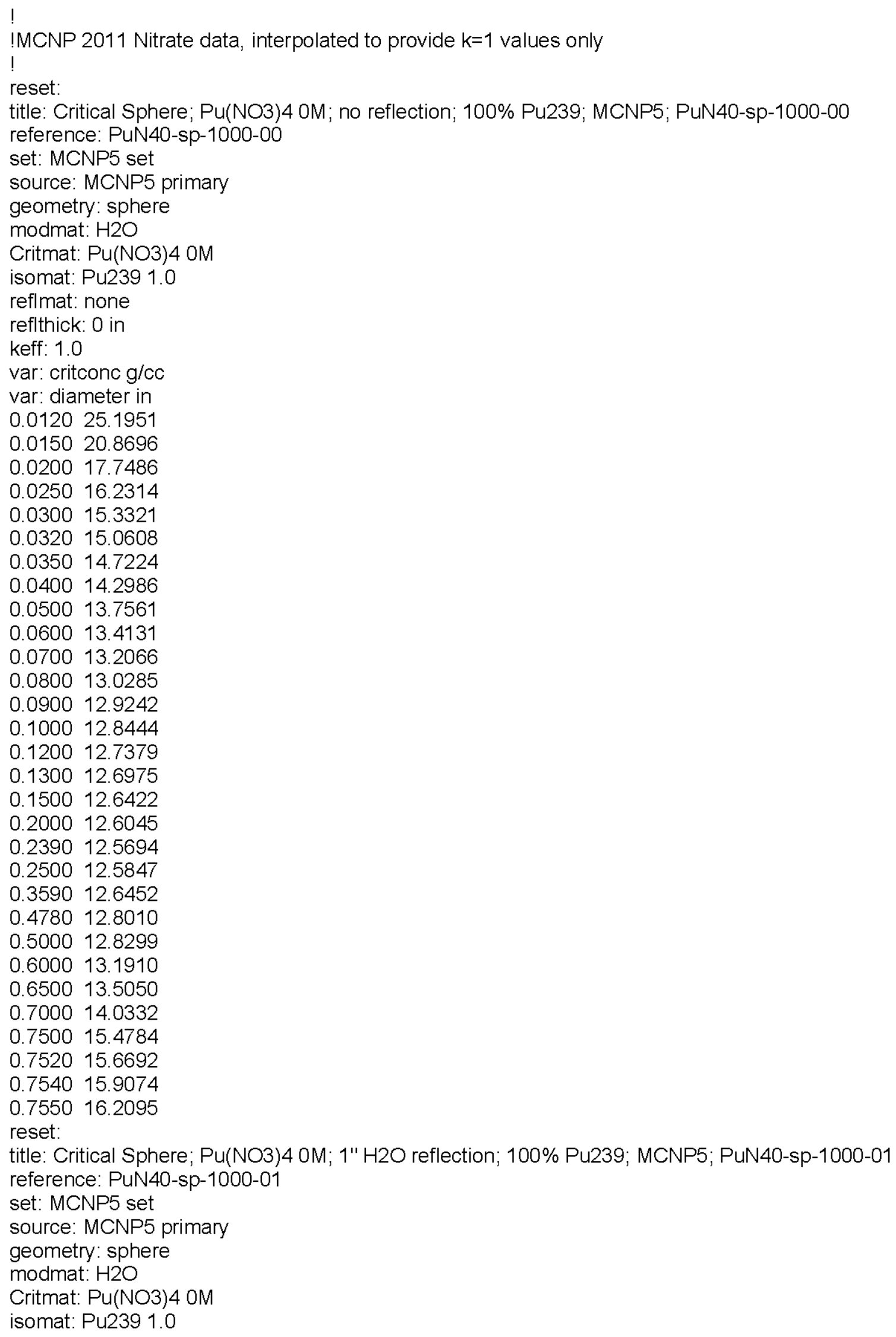




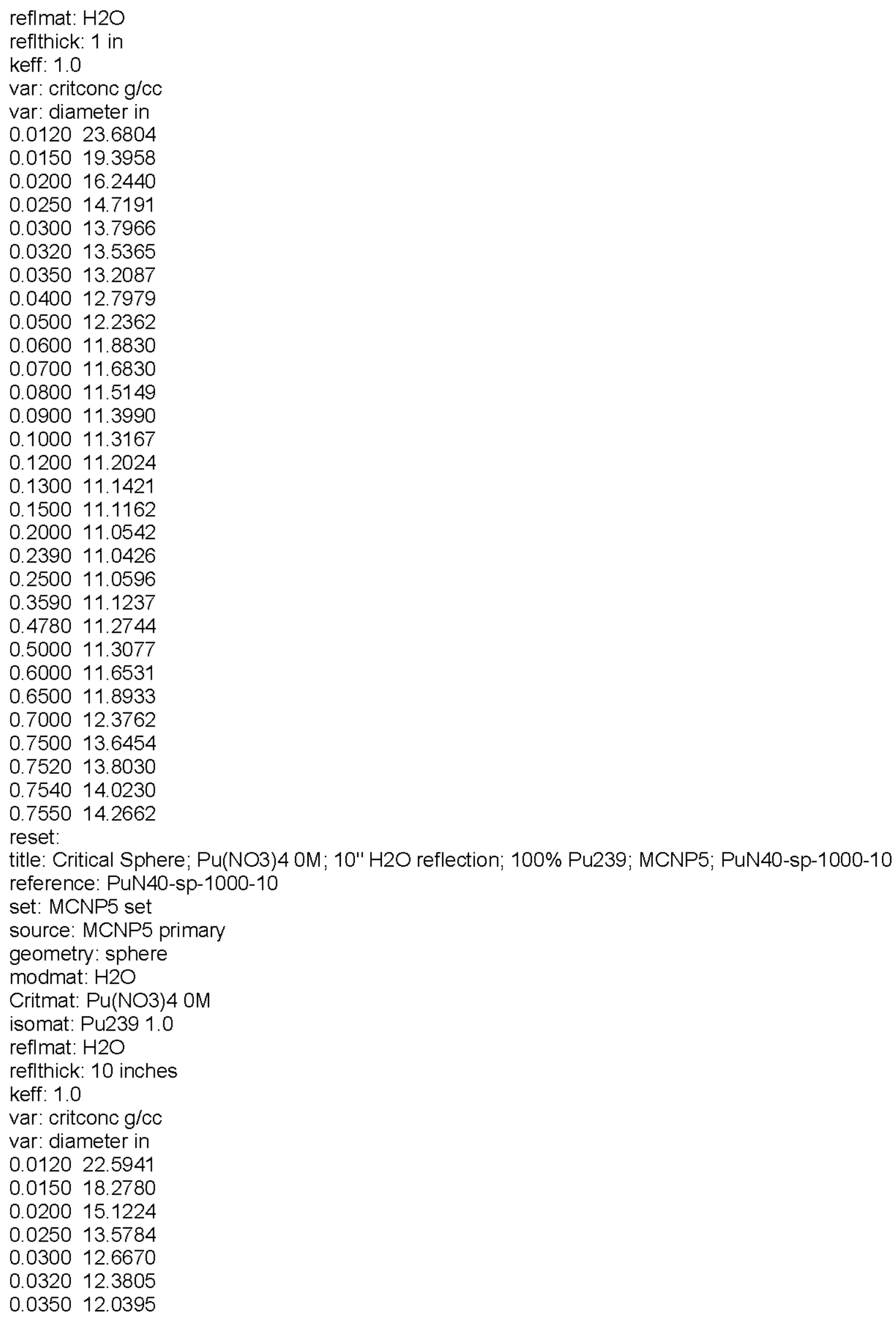




\section{CHPRC-01552, Revision 0}

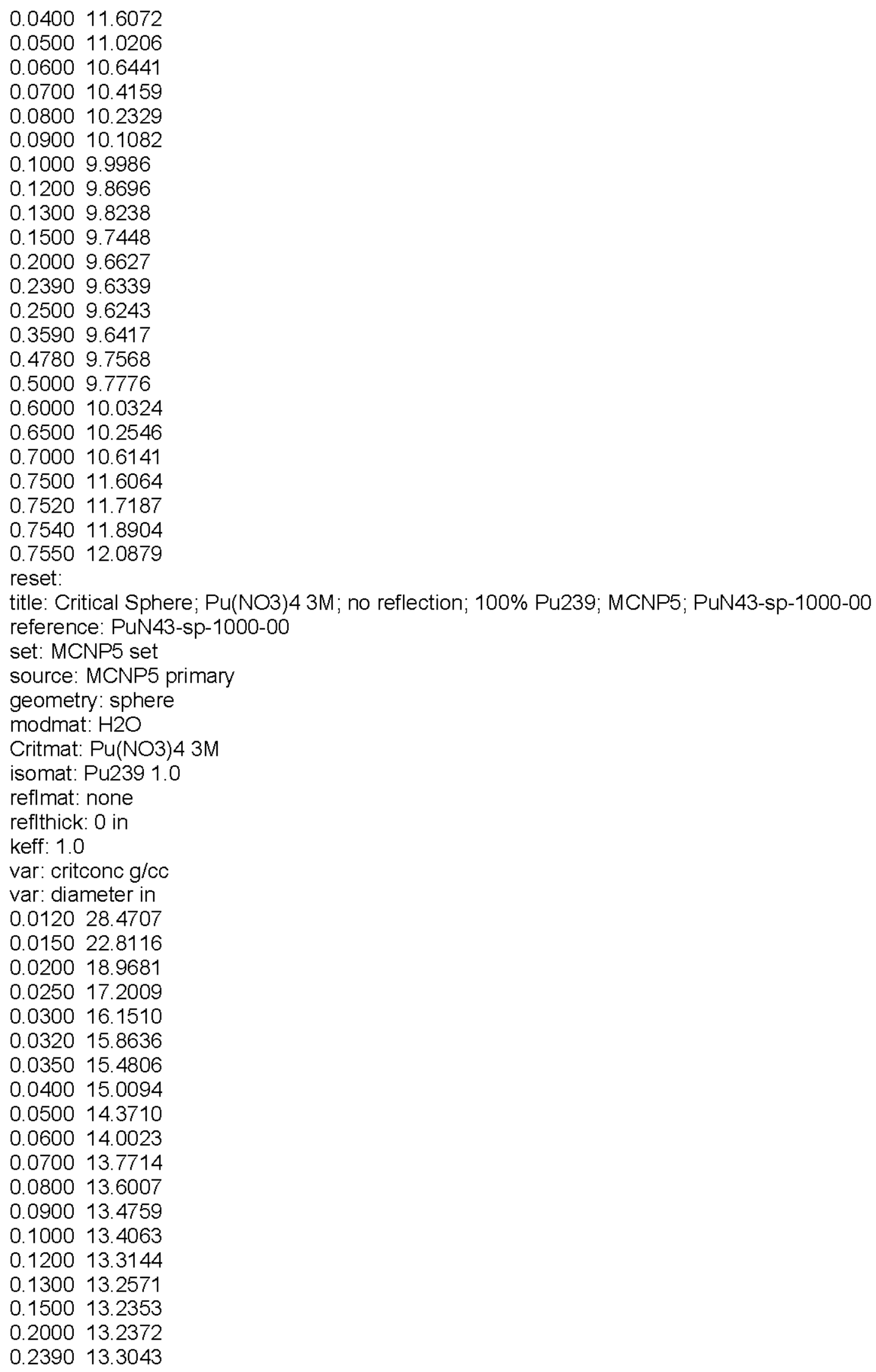




\section{CHPRC-01552, Revision 0}

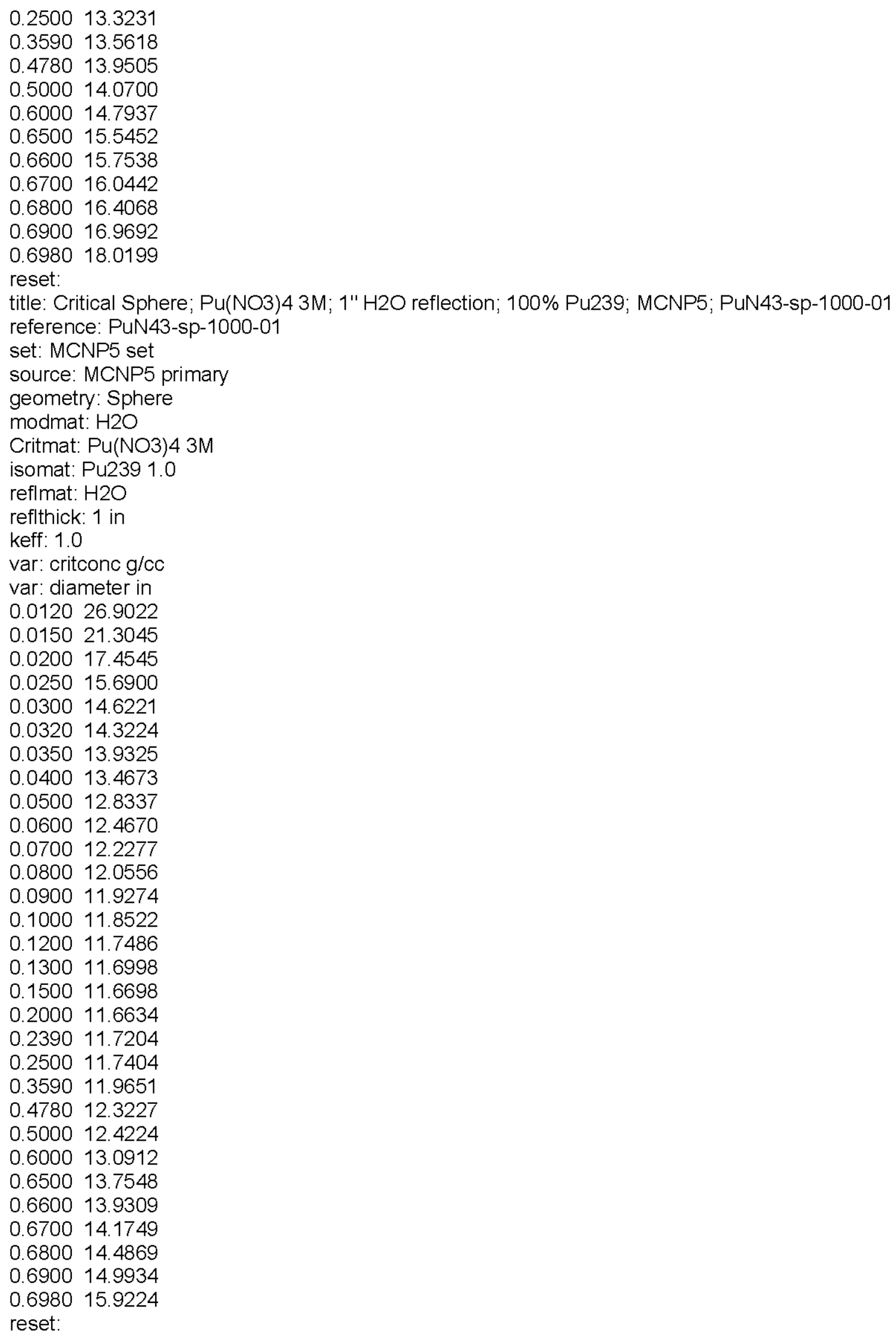


title: Critical Sphere; Pu(NO3)4 3M; 10" H2O reflection; 100\% Pu239; MCNP5; PuN43-sp-1000-10 reference: PuN43-sp-1000-10

set: MCNP5 set

source: MCNP5 primary

geometry: sphere

modmat: $\mathrm{H} 2 \mathrm{O}$

Critmat: $\mathrm{Pu}(\mathrm{NO} 3) 43 \mathrm{M}$

isomat: Pu239 1.0

reflmat: $\mathrm{H} 2 \mathrm{O}$

reflthick: 10 in

keff: 1.0

var: critconc g/cc

var: diameter in

0.012025 .8375

0.015020 .1470

0.020016 .3107

0.025014 .5013

0.030013 .4248

0.032013 .1199

0.035012 .7283

0.040012 .2358

0.050011 .5770

0.060011 .1796

0.070010 .8980

0.080010 .7152

0.090010 .5771

0.100010 .4810

0.120010 .3447

$0.1300 \quad 10.2927$

$0.1500 \quad 10.2474$

0.200010 .1829

0.239010 .1924

0.250010 .1990

$0.3590 \quad 10.3483$

0.478010 .6110

0.500010 .6835

0.600011 .2222

0.650011 .7268

0.660011 .8749

0.670012 .0672

$0.6800 \quad 12.3104$

0.690012 .7144

0.698013 .4050

reset:

title: Critical Sphere; Pu(NO3)4 6M; no reflection; 100\% Pu239; MCNP5; PuN46-sp-1000-00

reference: PuN46-sp-1000-00

set: MCNP5 set

source: MCNP5 primary

geometry: sphere

isomat: Pu239 1.0

modmat: $\mathrm{H} 2 \mathrm{O}$

Critmat: $\mathrm{Pu}(\mathrm{NO} 3) 46 \mathrm{M}$

reflmat: none

reflthick: 0 in

keff: 1.0

var: critconc $\mathrm{g} / \mathrm{cc}$ 
CHPRC-01552, Revision 0

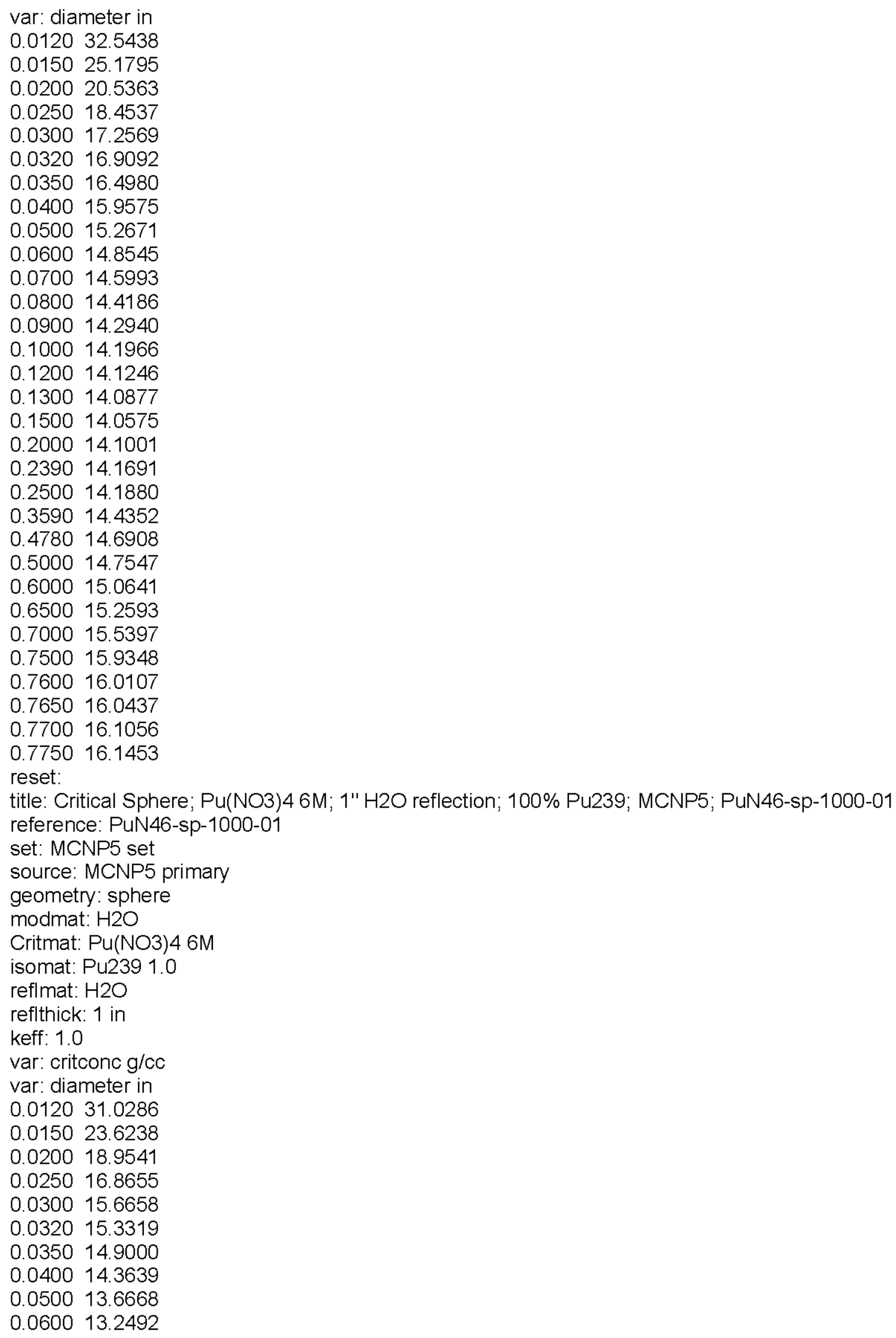




\section{CHPRC-01552, Revision 0}

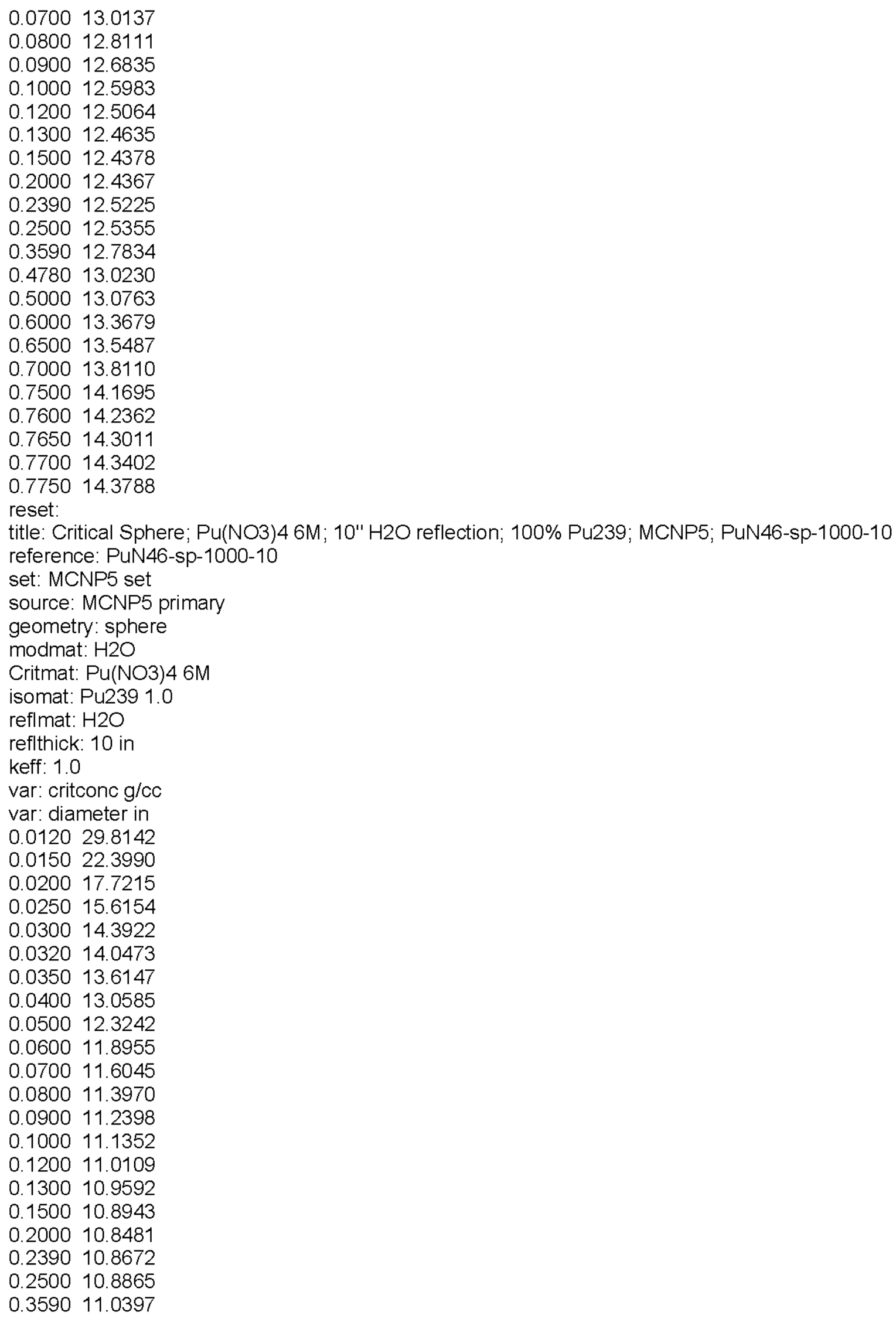




\section{CHPRC-01552, Revision 0}

$\begin{array}{ll}0.4780 & 11.2146 \\ 0.5000 & 11.2490 \\ 0.6000 & 11.4863 \\ 0.6500 & 11.6409 \\ 0.7000 & 11.8434 \\ 0.7500 & 12.1285 \\ 0.7600 & 12.1982 \\ 0.7650 & 12.2401 \\ 0.7700 & 12.2901 \\ 0.7750 & 12.2976 \\ \text { reset: }\end{array}$

title: Critical Sphere; Pu(NO3)4 OM; no reflection; 97\% Pu239; MCNP5; PuN40-sp-0970-00 reference: PuN40-sp-0970-00

set: MCNP5 set

source: MCNP5 primary

geometry: sphere

modmat: $\mathrm{H} 2 \mathrm{O}$

critmat: $\mathrm{Pu}(\mathrm{NO} 3) 40 \mathrm{M}$

isomat: Pu239 0.970 Pu240 0.030

reflmat: none

reflthick: 0 in

keff: 1.0

var: critconc g/cc

var: diameter in

0.012026 .7988

0.015021 .8715

0.020018 .4693

0.025016 .8214

0.030015 .8962

0.032015 .5977

0.035015 .2625

0.040014 .8278

0.050014 .2829

0.060013 .9626

0.070013 .7742

0.080013 .6490

0.090013 .5652

0.100013 .5165

0.120013 .4851

0.130013 .4525

0.150013 .4791

0.200013 .5525

0.239013 .6584

0.250013 .6721

0.359013 .9422

0.478014 .2500

0.500014 .3460

0.600014 .8084

0.650015 .1717

0.700015 .7801

0.750017 .3634

0.752017 .5542

$0.7540 \quad 17.8576$

0.755018 .1310

reset:

title: Critical Sphere; Pu(NO3)4 0M; 1" H2O reflection; 97\% Pu239; MCNP5; PuN40-sp-0970-01 
CHPRC-01552, Revision 0

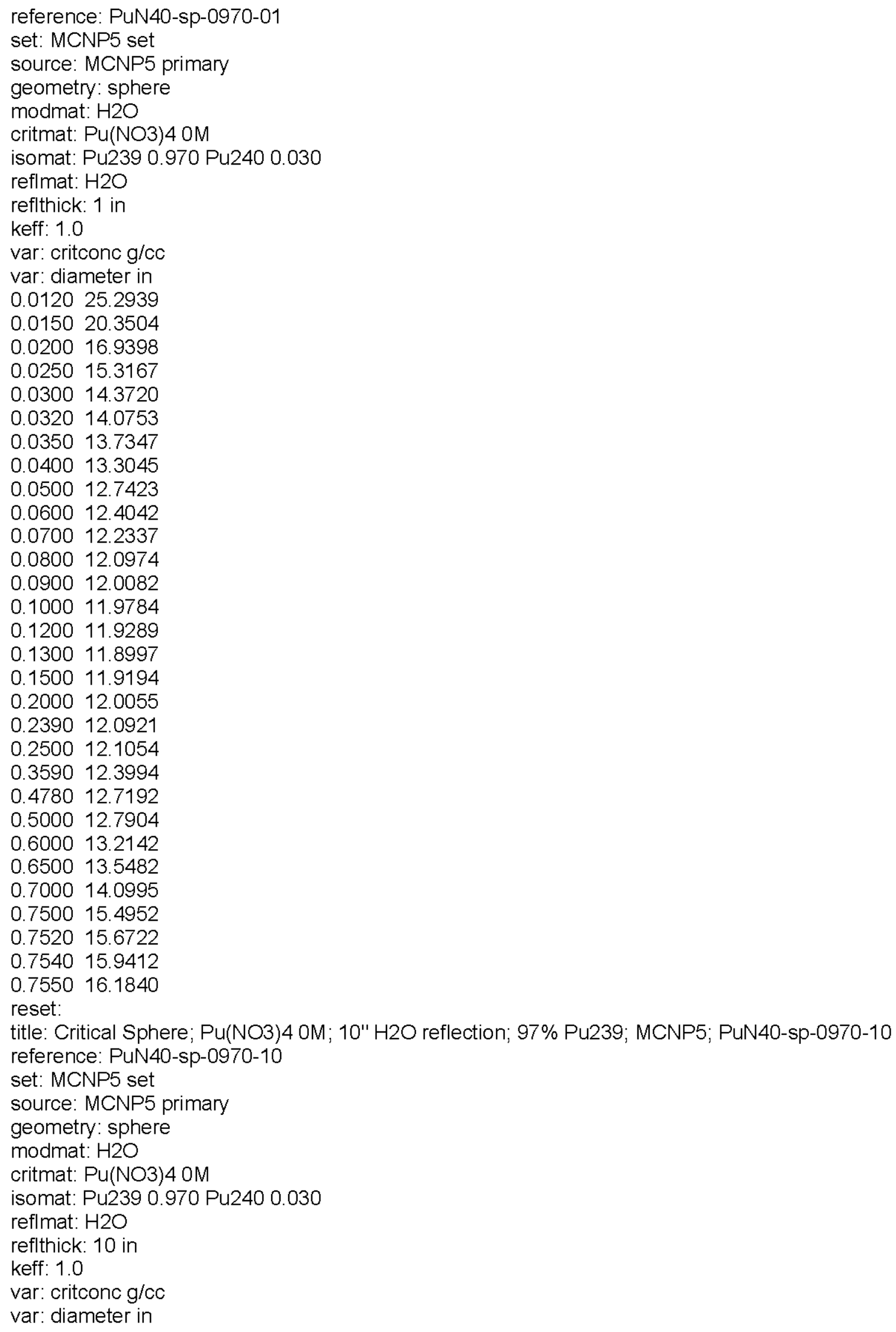


CHPRC-01552, Revision 0

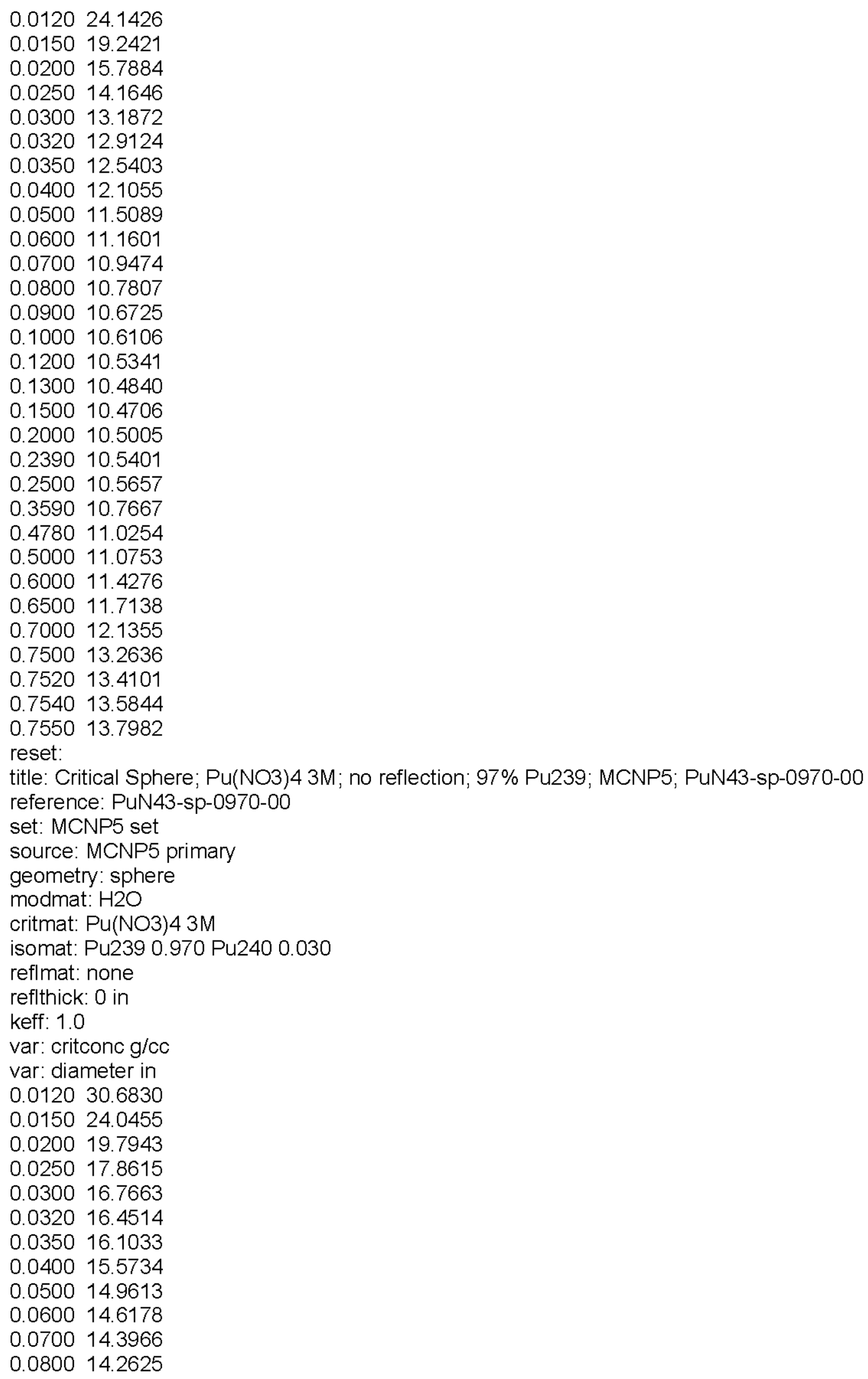


CHPRC-01552, Revision 0

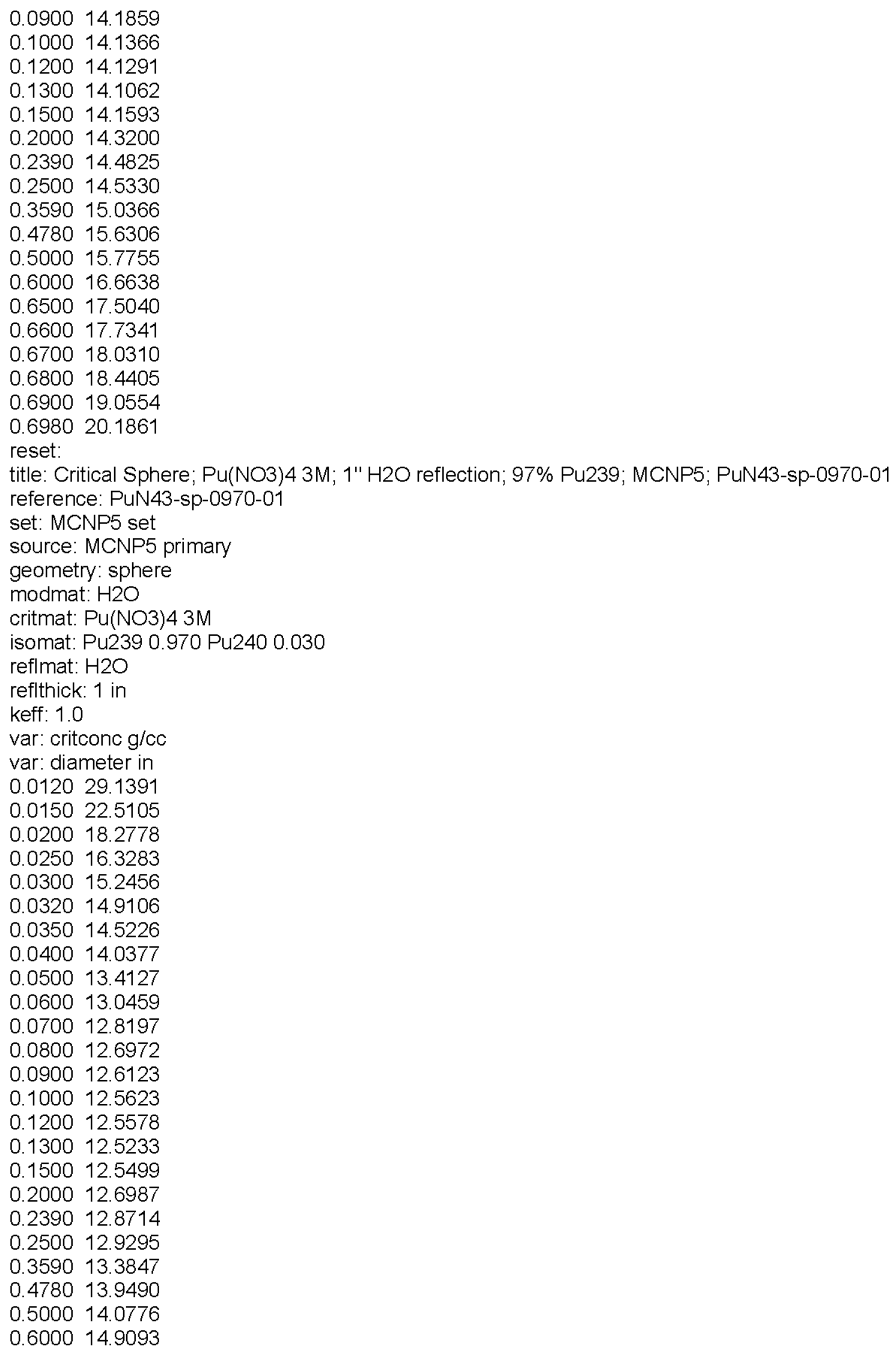




\section{CHPRC-01552, Revision 0}

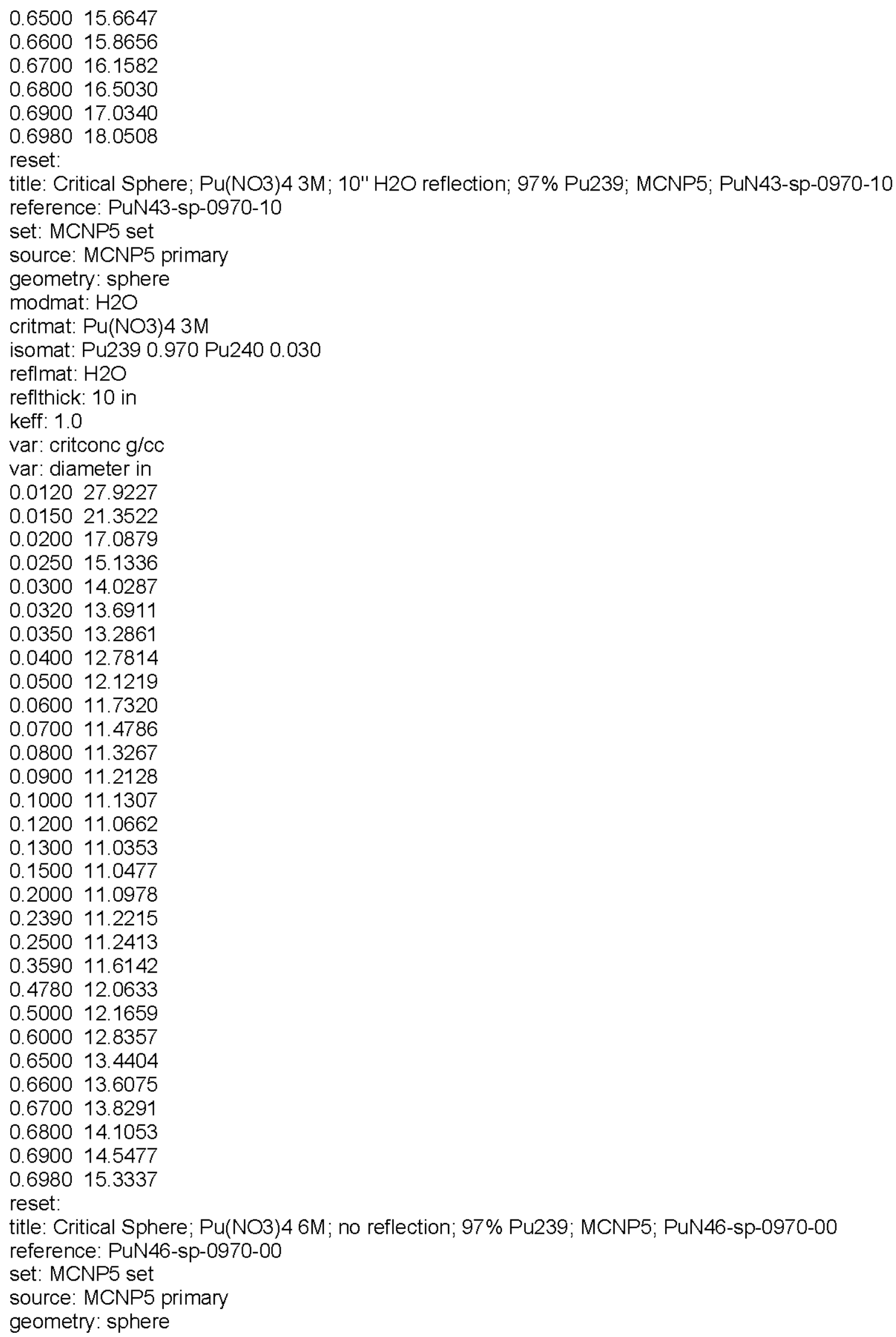


CHPRC-01552, Revision 0

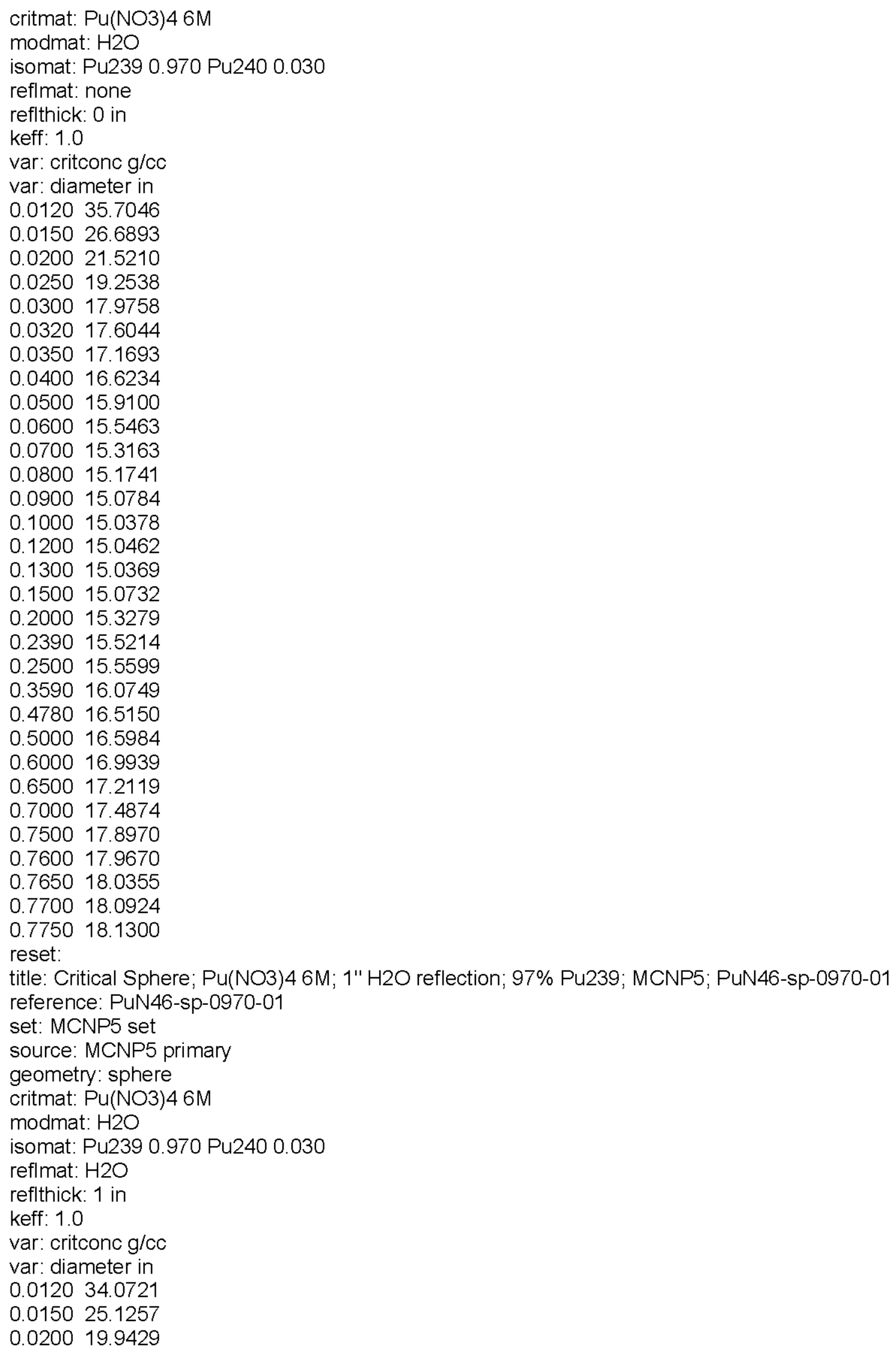


CHPRC-01552, Revision 0

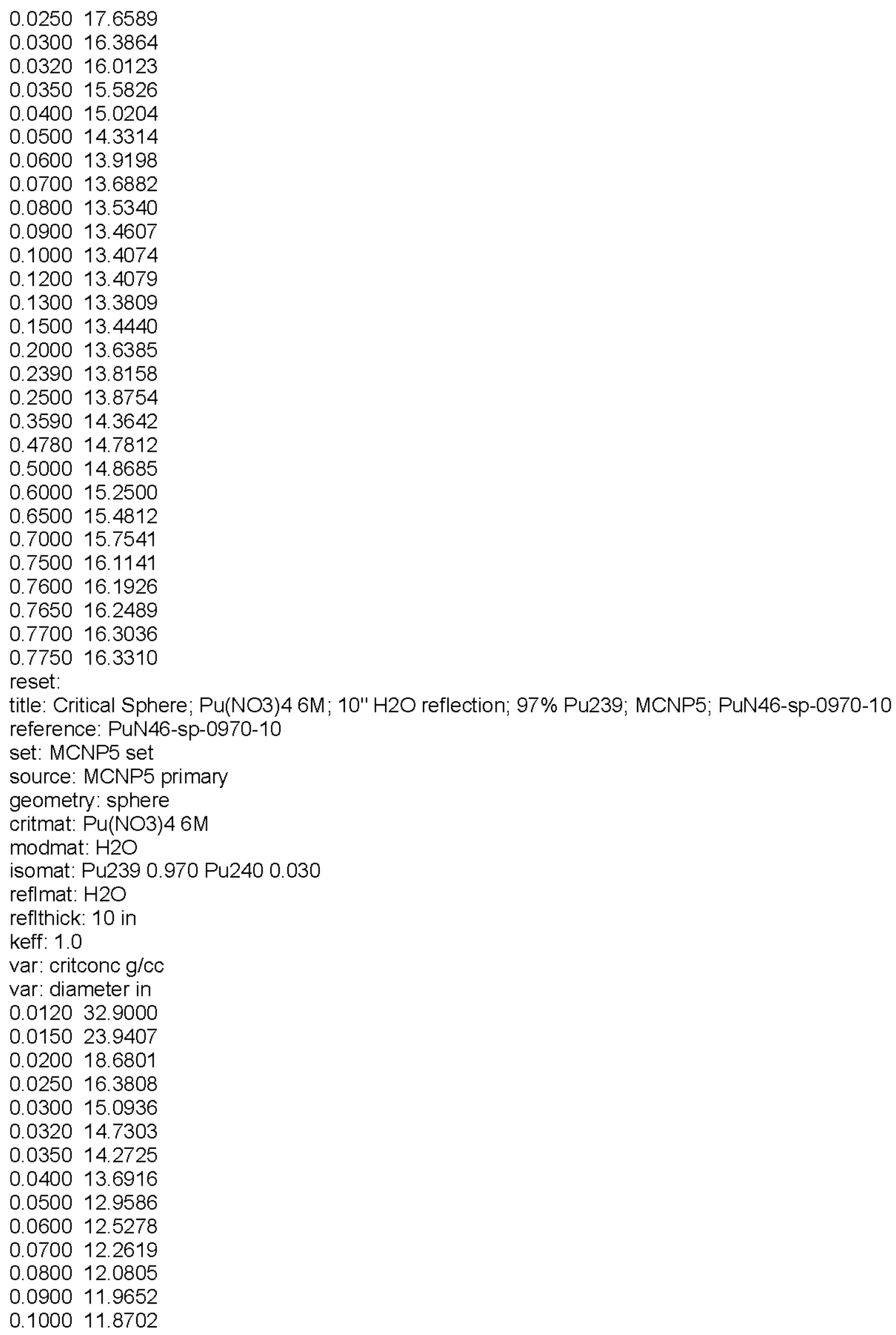




\section{CHPRC-01552, Revision 0}

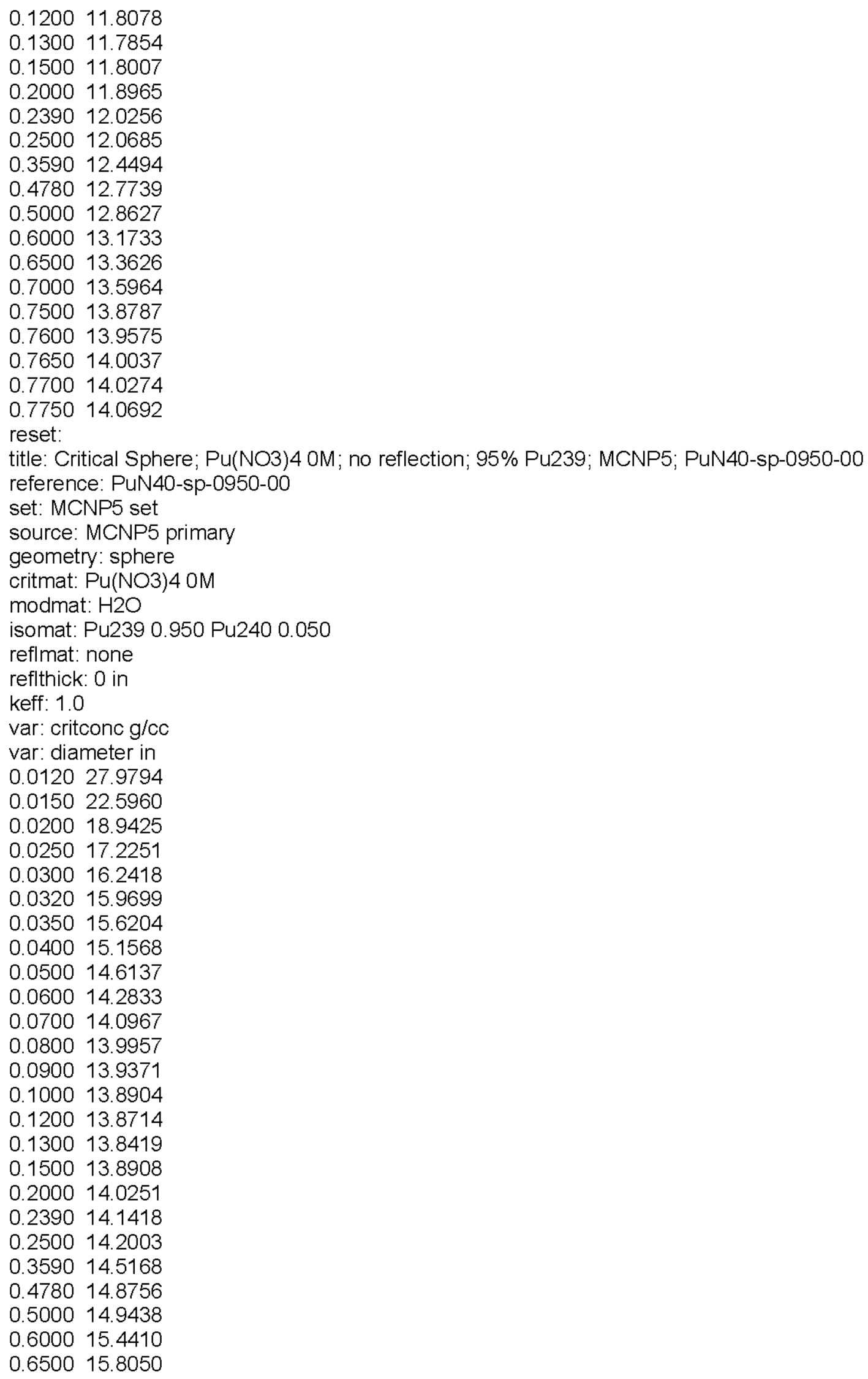


CHPRC-01552, Revision 0

0.700016 .4390

0.750018 .0811

0.752018 .2816

0.754018 .5576

0.755018 .8395

reset:

title: Critical Sphere; Pu(NO3)4 OM; 1" H2O reflection; 95\% Pu239; MCNP5; PuN40-sp-0950-01

reference: PuN40-sp-0950-01

set: MCNP5 set

source: MCNP5 primary

geometry: sphere

critmat: $\mathrm{Pu}(\mathrm{NO} 3) 4 \mathrm{OM}$

modmat: $\mathrm{H} 2 \mathrm{O}$

isomat: Pu239 0.950 Pu240 0.050

reflmat: $\mathrm{H} 2 \mathrm{O}$

reflthick: 1 in

keff: 1.0

var: critconc $\mathrm{g} / \mathrm{cc}$

var: diameter in

0.012026 .4924

0.015021 .0891

0.020017 .4364

0.025015 .7054

0.030014 .7142

0.032014 .4369

0.035014 .0593

0.040013 .6350

0.050013 .0681

0.060012 .7511

0.070012 .5783

0.080012 .4427

0.090012 .3748

0.100012 .3164

0.120012 .2970

0.130012 .3064

$0.1500 \quad 12.3275$

0.200012 .4449

0.239012 .5545

0.250012 .6066

0.359012 .9504

$0.4780 \quad 13.2904$

0.500013 .3705

0.600013 .8482

0.650014 .1638

0.700014 .7443

$0.7500 \quad 16.2010$

$0.7520 \quad 16.4027$

$0.7540 \quad 16.6323$

0.755016 .8892

reset:

title: Critical Sphere; Pu(NO3)4 OM; 10" H2O reflection; 95\% Pu239; MCNP5; PuN40-sp-0950-10

reference: PuN40-sp-0950-10

set: MCNP5 set

source: MCNP5 primary

geometry: sphere

critmat: $\mathrm{Pu}(\mathrm{NO} 3) 40 \mathrm{M}$ 
CHPRC-01552, Revision 0

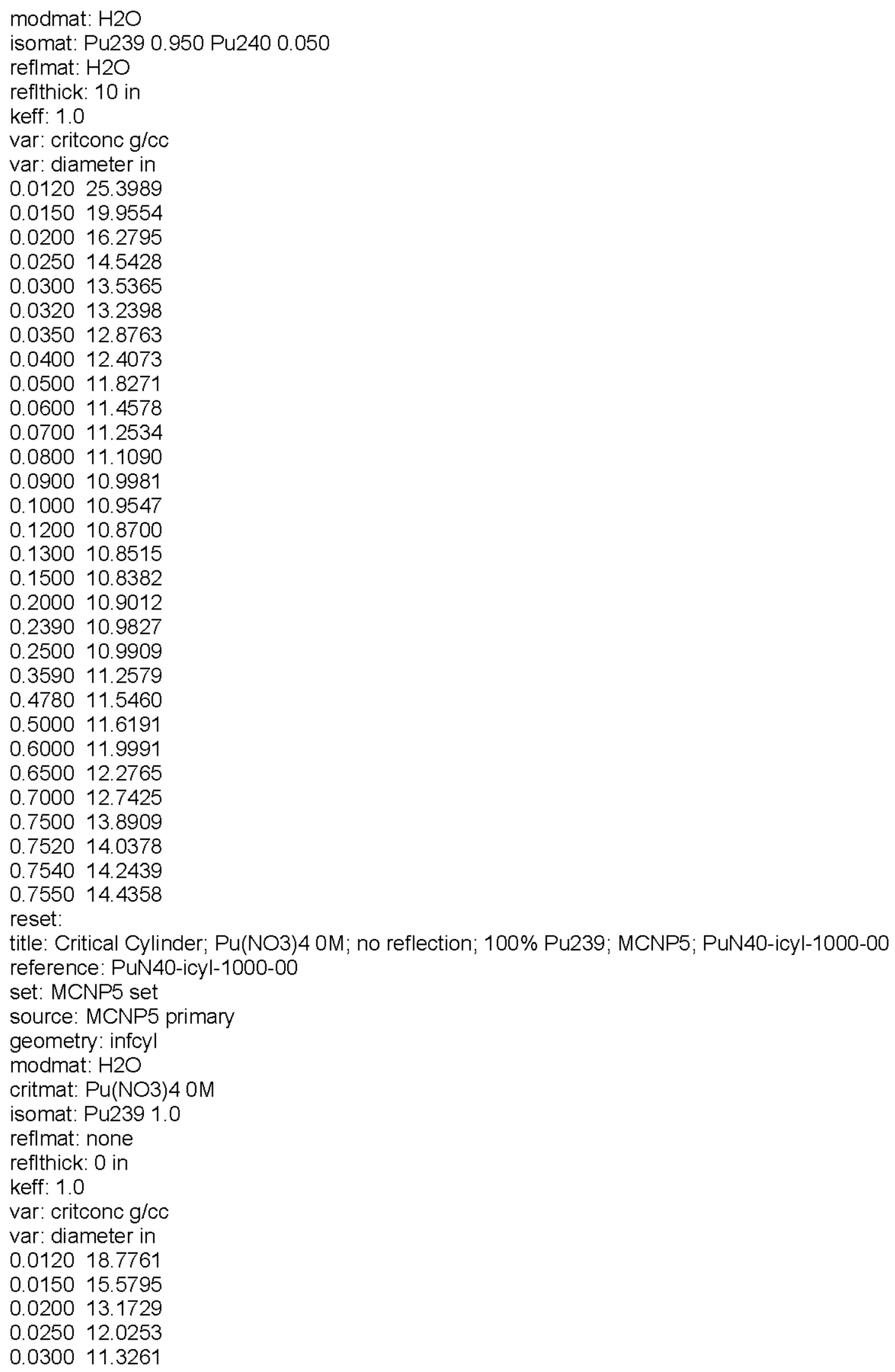


CHPRC-01552, Revision 0

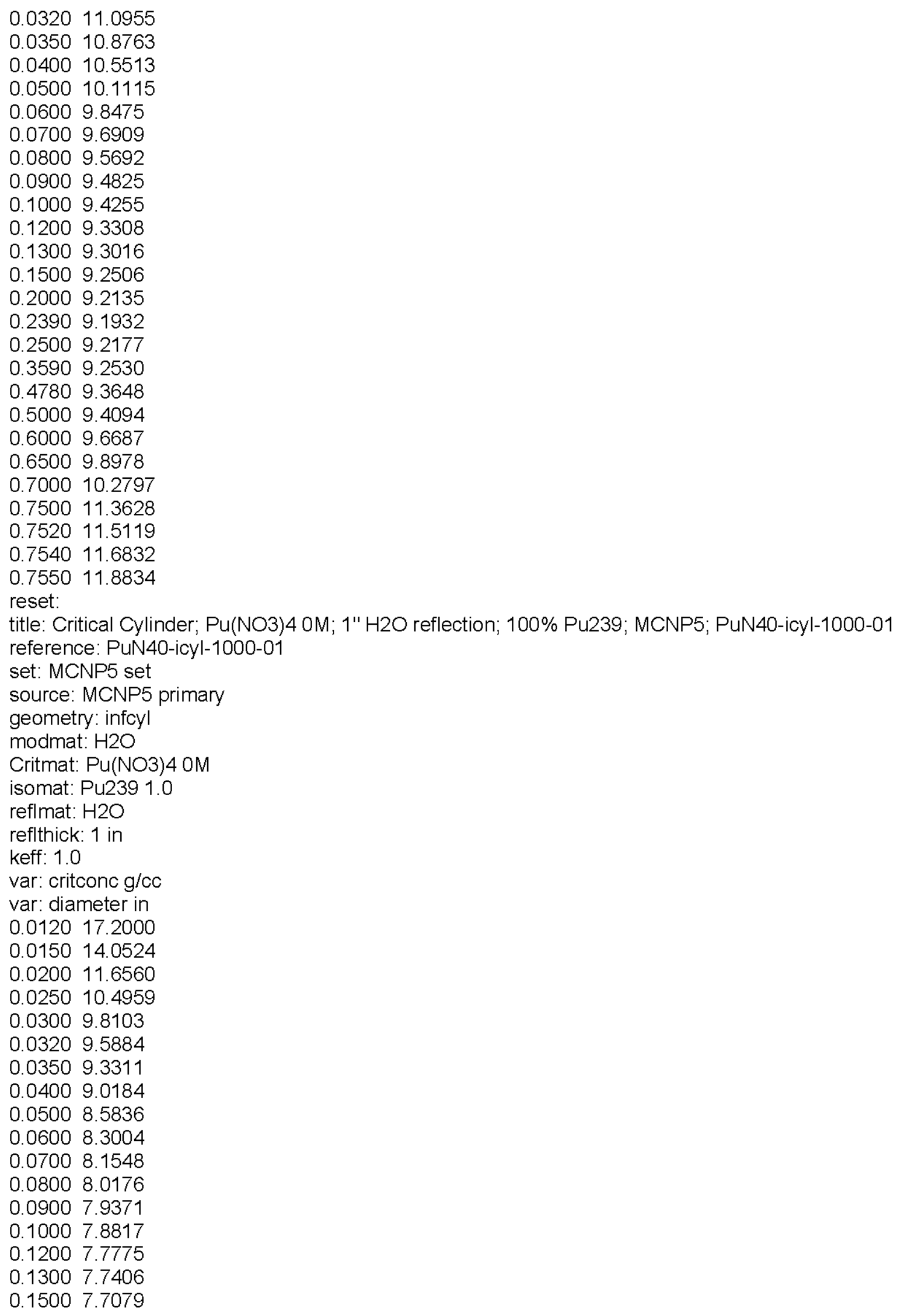




\section{CHPRC-01552, Revision 0}

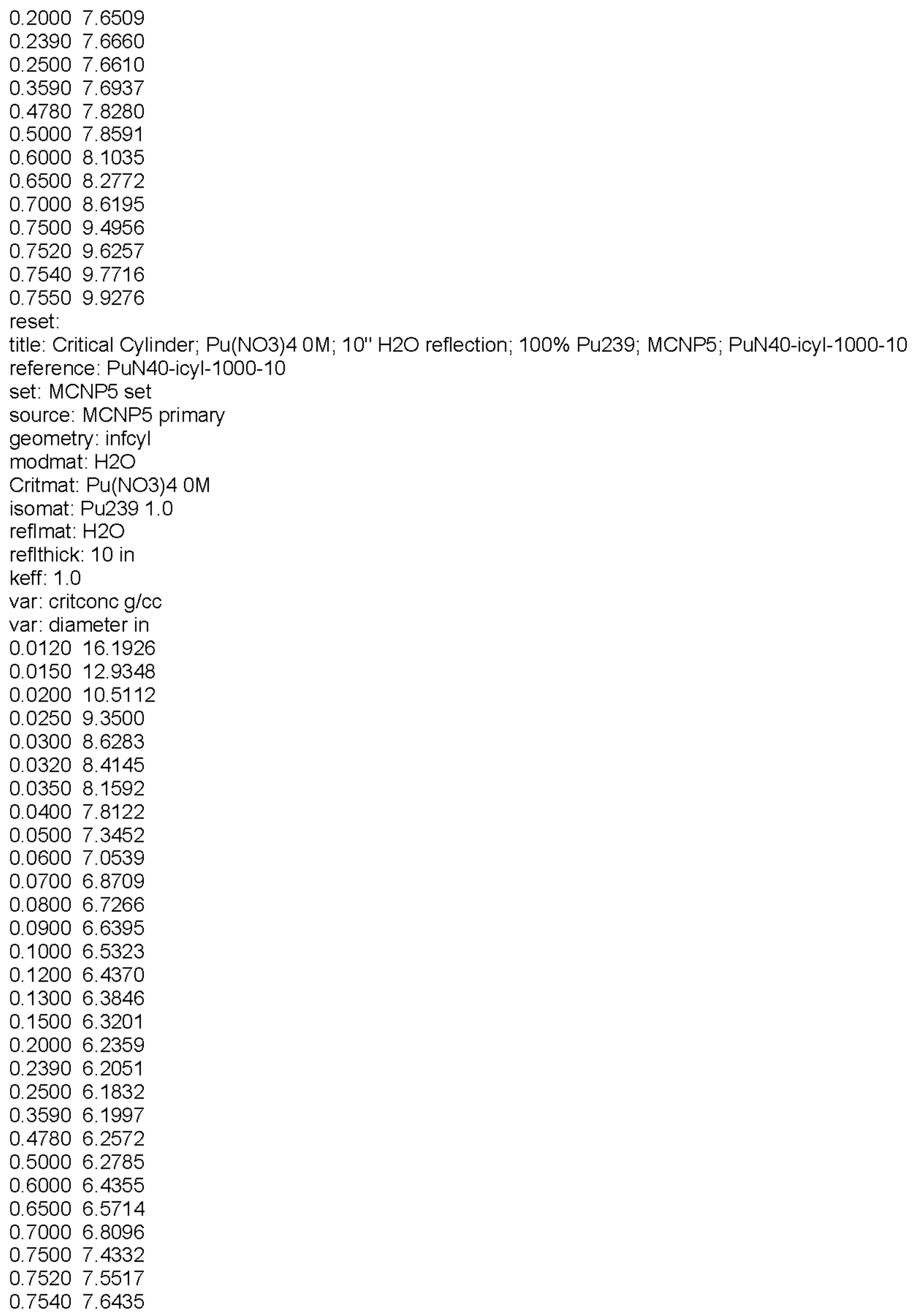




\section{CHPRC-01552, Revision 0}

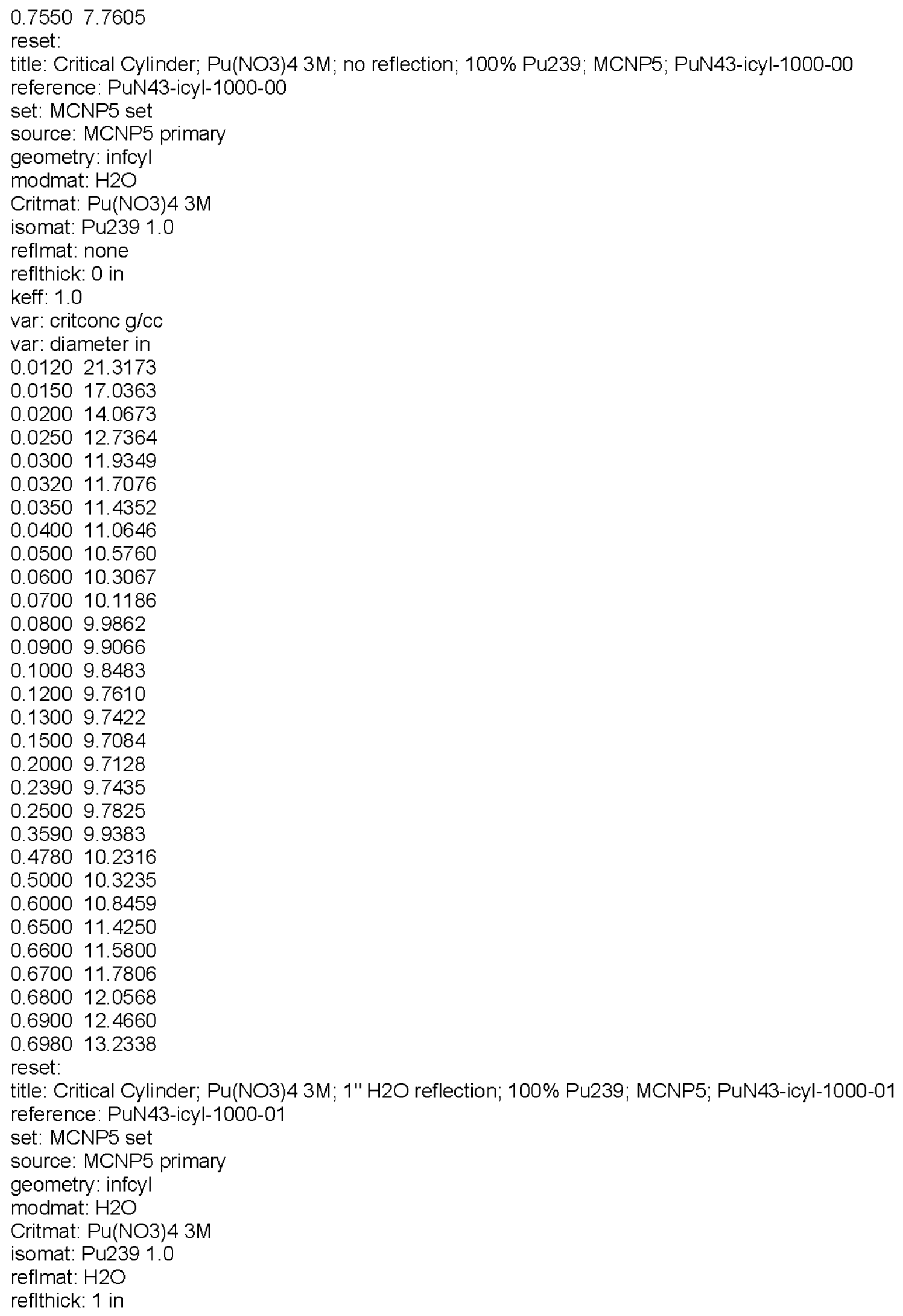


CHPRC-01552, Revision 0

keff: 1.0

var: critconc g/cc

var: diameter in

0.012019 .7474

0.015015 .5025

0.020012 .5503

0.025011 .1838

0.030010 .3890

$\begin{array}{lll}0.0320 & 10.1707\end{array}$

0.03509 .8770

0.04009 .5170

0.05009 .0320

0.06008 .7591

0.07008 .5476

0.08008 .4508

0.09008 .3264

0.10008 .2554

0.12008 .2023

0.13008 .1540

0.15008 .1270

0.20008 .1185

0.23908 .1602

0.25008 .1551

0.35908 .3307

0.47808 .5847

0.50008 .6616

0.60009 .1176

0.65009 .5847

0.66009 .7314

0.67009 .8980

$0.6800 \quad 10.1248$

0.690010 .4858

0.698011 .1261

reset:

title: Critical Cylinder; Pu(NO3)4 3M; 10" H2O reflection; 100\% Pu239; MCNP5; PuN43-icyl-1000-10 reference: PuN43-icyl-1000-10

set: MCNP5 set

source: MCNP5 primary

geometry: infcyl

modmat: $\mathrm{H} 2 \mathrm{O}$

Critmat: $\mathrm{Pu}(\mathrm{NO} 3) 43 \mathrm{M}$

isomat: Pu239 1.0

reflmat: $\mathrm{H} 2 \mathrm{O}$

reflthick: 10 in

keff: 1.0

var: critconc g/cc

var: diameter in

0.012018 .6193

0.015014 .3553

0.020011 .4010

0.025010 .0215

0.03009 .1879

0.03208 .9546

0.03508 .6420

0.04008 .2573

0.05007 .7602 
CHPRC-01552, Revision 0

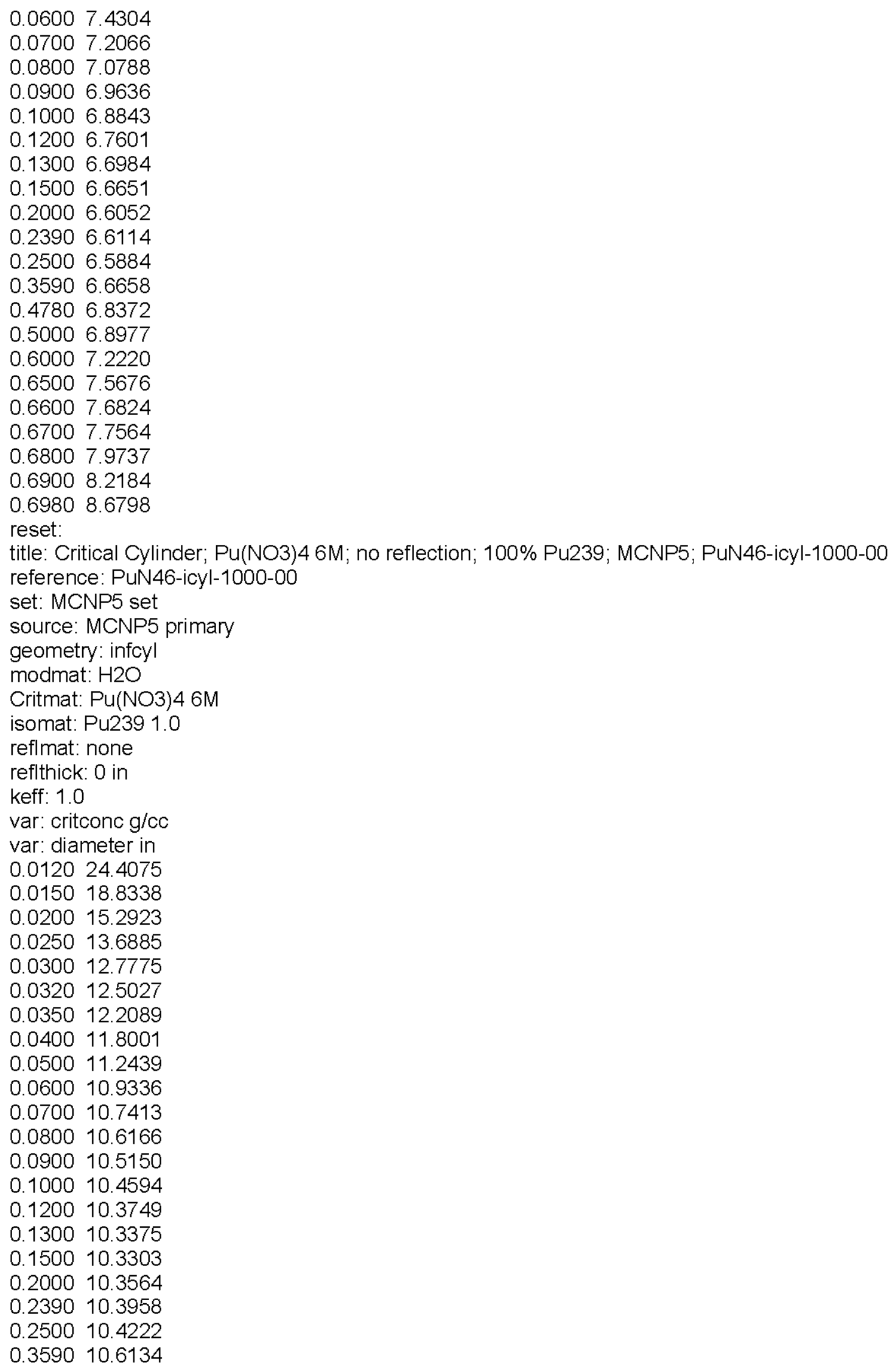




\title{
CHPRC-01552, Revision 0
}

\begin{abstract}
0.478010 .7839
0.500010 .8350

0.600011 .0809

0.650011 .2237

0.700011 .4224

0.750011 .6914

0.760011 .7600

0.765011 .8168

0.770011 .8463

0.775011 .8789

reset:
\end{abstract}

title: Critical Cylinder; Pu(NO3)4 6M; 1" H2O reflection; 100\% Pu239; MCNP5; PuN46-icyl-1000-01 reference: PuN46-icyl-1000-01

set: MCNP5 set

source: MCNP5 primary

geometry: infcyl

modmat: $\mathrm{H} 2 \mathrm{O}$

Critmat: $\mathrm{Pu}(\mathrm{NO} 3) 46 \mathrm{M}$

isomat: Pu239 1.0

reflmat: $\mathrm{H} 2 \mathrm{O}$

reflthick: 1 in

keff: 1.0

var: critconc $\mathrm{g} / \mathrm{cc}$

var: diameter in

0.012022 .8535

0.015017 .2257

0.020013 .6965

0.025012 .1036

0.030011 .1767

0.032010 .9263

0.035010 .6091

0.040010 .1846

$0.0500 \quad 9.6547$

0.06009 .3416

0.07009 .1300

0.08009 .0033

0.09008 .8851

0.10008 .8226

0.12008 .7419

0.13008 .7145

0.15008 .6938

0.20008 .6941

0.23908 .7484

0.25008 .7541

0.35908 .9245

$0.4780 \quad 9.0965$

0.50009 .1457

0.60009 .3490

0.65009 .4870

0.70009 .6788

0.75009 .9220

0.760010 .0088

$0.7650 \quad 10.0283$

$0.7700 \quad 10.0809$

$0.7750 \quad 10.0745$

reset: 
title: Critical Cylinder; Pu(NO3)4 6M; 10" H2O reflection; 100\% Pu239; MCNP5; PuN46-icyl-1000-10 reference: PuN46-icyl-1000-10

set: MCNP5 set

source: MCNP5 primary

geometry: infcyl

modmat: $\mathrm{H} 2 \mathrm{O}$

Critmat: $\mathrm{Pu}(\mathrm{NO} 3) 46 \mathrm{M}$

isomat: Pu239 1.0

reflmat: $\mathrm{H} 2 \mathrm{O}$

reflthick: 10 in

keff: 1.0

var: critconc g/cc

var: diameter in

0.012021 .6275

0.015015 .9890

0.020012 .4653

0.025010 .8174

0.03009 .8863

$0.0320 \quad 9.6273$

$0.0350 \quad 9.2902$

0.04008 .8573

0.05008 .2984

0.06007 .9429

0.07007 .7076

0.08007 .5515

0.09007 .4187

0.10007 .3434

0.12007 .2082

0.13007 .1780

0.15007 .1303

0.20007 .0706

0.23907 .0639

0.25007 .0760

0.35907 .1505

0.47807 .2583

0.50007 .2854

0.60007 .4409

0.65007 .5481

0.70007 .6681

0.75007 .8659

0.76007 .8994

0.76507 .9464

0.77007 .9485

0.77507 .9778

reset:

title: Critical Cylinder; Pu(NO3)4 OM; no reflection; 97\% Pu239; MCNP5; PuN40-icyl-0970-00

reference: PuN40-icyl-0970-00

set: MCNP5 set

source: MCNP5 primary

geometry: infcyl

modmat: $\mathrm{H} 2 \mathrm{O}$

critmat: $\mathrm{Pu}(\mathrm{NO} 3) 40 \mathrm{M}$

isomat: Pu239 0.970 Pu240 0.030

reflmat: none

reflthick: 0 in

keff: 1.0 
CHPRC-01552, Revision 0

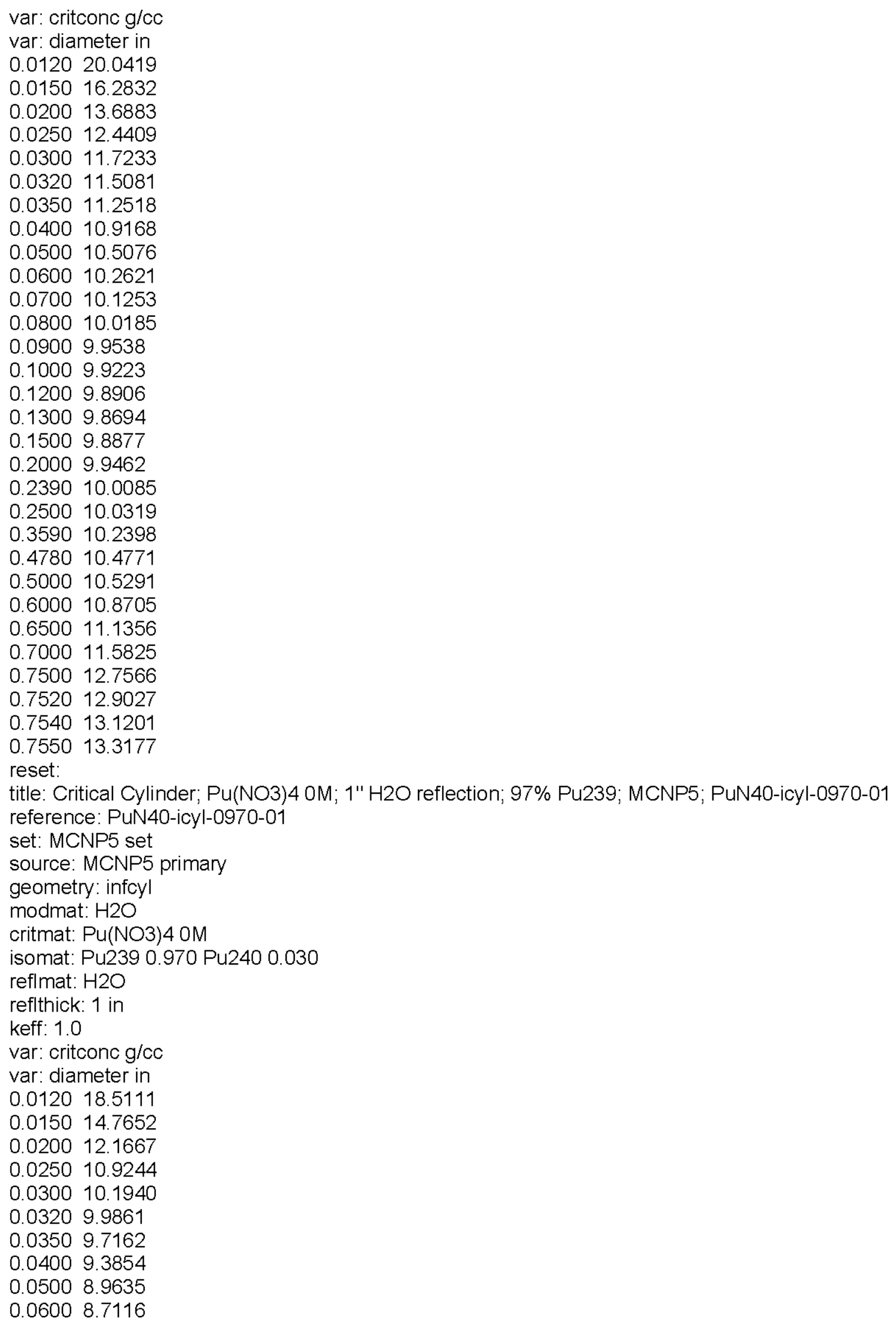


CHPRC-01552, Revision 0

0.07008 .5725

0.08008 .4603

0.09008 .3962

0.10008 .3554

0.12008 .3185

0.13008 .3012

0.15008 .3076

0.20008 .3627

0.23908 .4323

0.25008 .4505

0.35908 .6454

0.47808 .8849

0.50008 .9487

0.60009 .2585

0.65009 .4952

0.70009 .8849

$0.7500 \quad 10.8840$

0.752011 .0095

0.754011 .1936

0.755011 .3479

reset:

title: Critical Cylinder; Pu(NO3)4 0M; 10" H2O reflection; 97\% Pu239; MCNP5; PuN40-icyl-0970-10 reference: PuN40-icyl-0970-10

set: MCNP5 set

source: MCNP5 primary

geometry: infcyl

modmat: $\mathrm{H} 2 \mathrm{O}$

critmat: $\mathrm{Pu}(\mathrm{NO} 3) 4 \mathrm{OM}$

isomat: Pu239 0.970 Pu240 0.030

reflmat: $\mathrm{H} 2 \mathrm{O}$

reflthick: 10 in

keff: 1.0

var: critconc g/cc

var: diameter in

0.012017 .3852

0.015013 .6303

0.020011 .0214

0.02509 .7576

0.03008 .9999

0.03208 .7866

0.03508 .5131

0.04008 .1566

0.05007 .7002

0.06007 .4258

0.07007 .2527

0.08007 .1158

0.09007 .0336

0.10006 .9558

$0.1200 \quad 6.8877$

0.13006 .8571

0.15006 .8338

0.20006 .8254

0.23906 .8599

0.25006 .8678

0.35906 .9825

0.47807 .1528 


\section{CHPRC-01552, Revision 0}

0.50007 .1909

0.60007 .4360

0.65007 .6108

0.70007 .8932

0.75008 .6343

0.75208 .7289

0.75408 .8740

0.75508 .9866

reset:

title: Critical Cylinder; Pu(NO3)4 3M; no reflection; 97\% Pu239; MCNP5; PuN43-icyl-0970-00

reference: PuN43-icyl-0970-00

set: MCNP5 set

source: MCNP5 primary

geometry: infcyl

modmat: $\mathrm{H} 2 \mathrm{O}$

critmat: $\mathrm{Pu}(\mathrm{NO} 3) 43 \mathrm{M}$

isomat: Pu239 0.970 Pu240 0.030

reflmat: none

reflthick: 0 in

keff: 1.0

var: critconc $\mathrm{g} / \mathrm{cc}$

var: diameter in

0.012023 .0228

0.015017 .9460

0.020014 .7180

0.025013 .2455

0.030012 .4010

0.032012 .1607

0.035011 .8652

0.040011 .5023

0.050011 .0282

0.060010 .7451

0.070010 .5938

0.080010 .4960

0.090010 .4254

0.100010 .3895

0.120010 .3774

0.130010 .3688

$0.1500 \quad 10.3933$

0.200010 .5191

0.239010 .6410

$0.2500 \quad 10.6802$

0.359011 .0399

0.478011 .4874

0.500011 .6140

0.600012 .2412

0.650012 .8700

0.660013 .0496

0.670013 .2741

0.680013 .5914

0.690014 .0211

0.698014 .8525

reset:

title: Critical Cylinder; Pu(NO3)4 3M; 1" H2O reflection; 97\% Pu239; MCNP5; PuN43-icyl-0970-01 reference: PuN43-icyl-0970-01

set: MCNP5 set 
CHPRC-01552, Revision 0

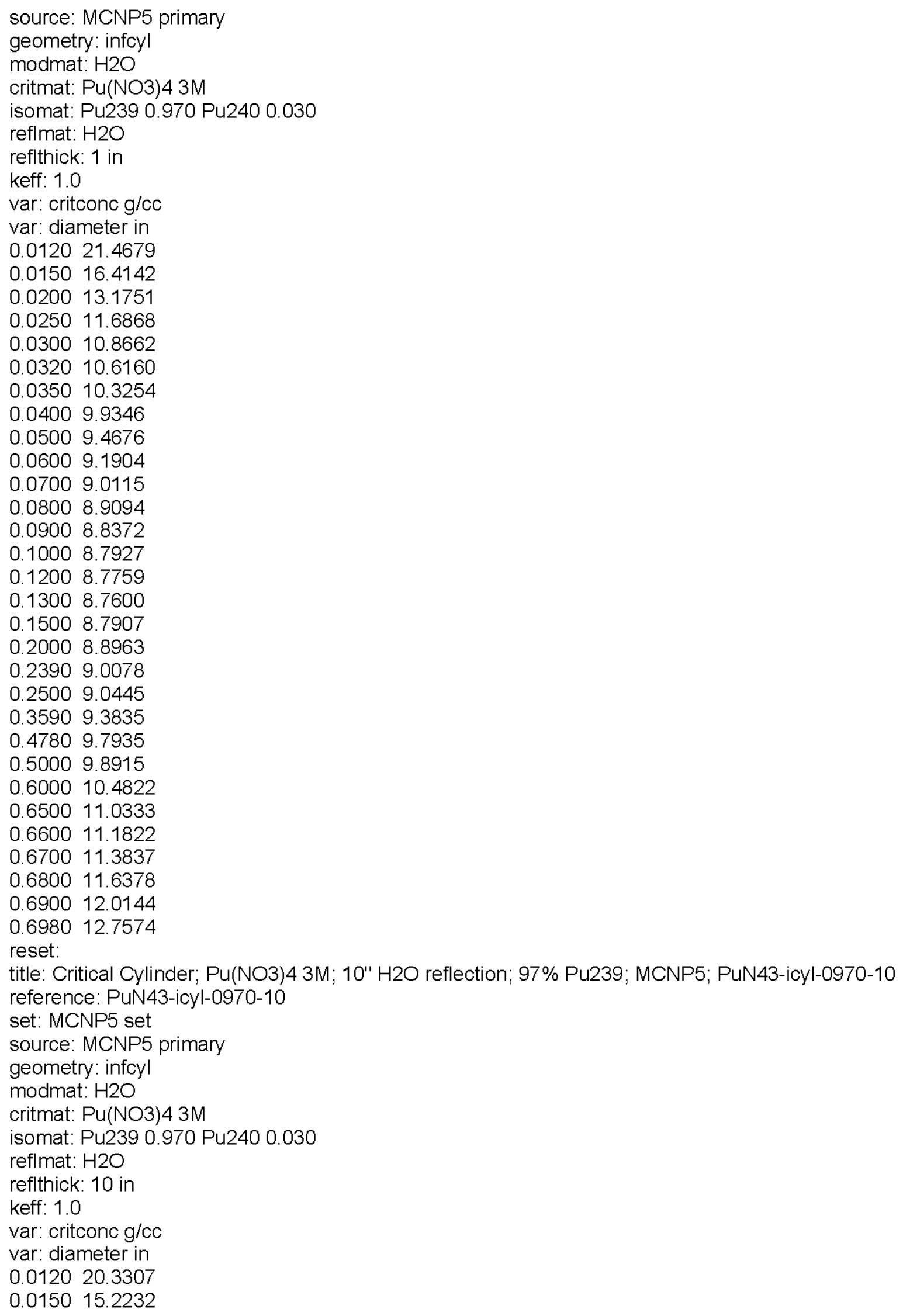


CHPRC-01552, Revision 0

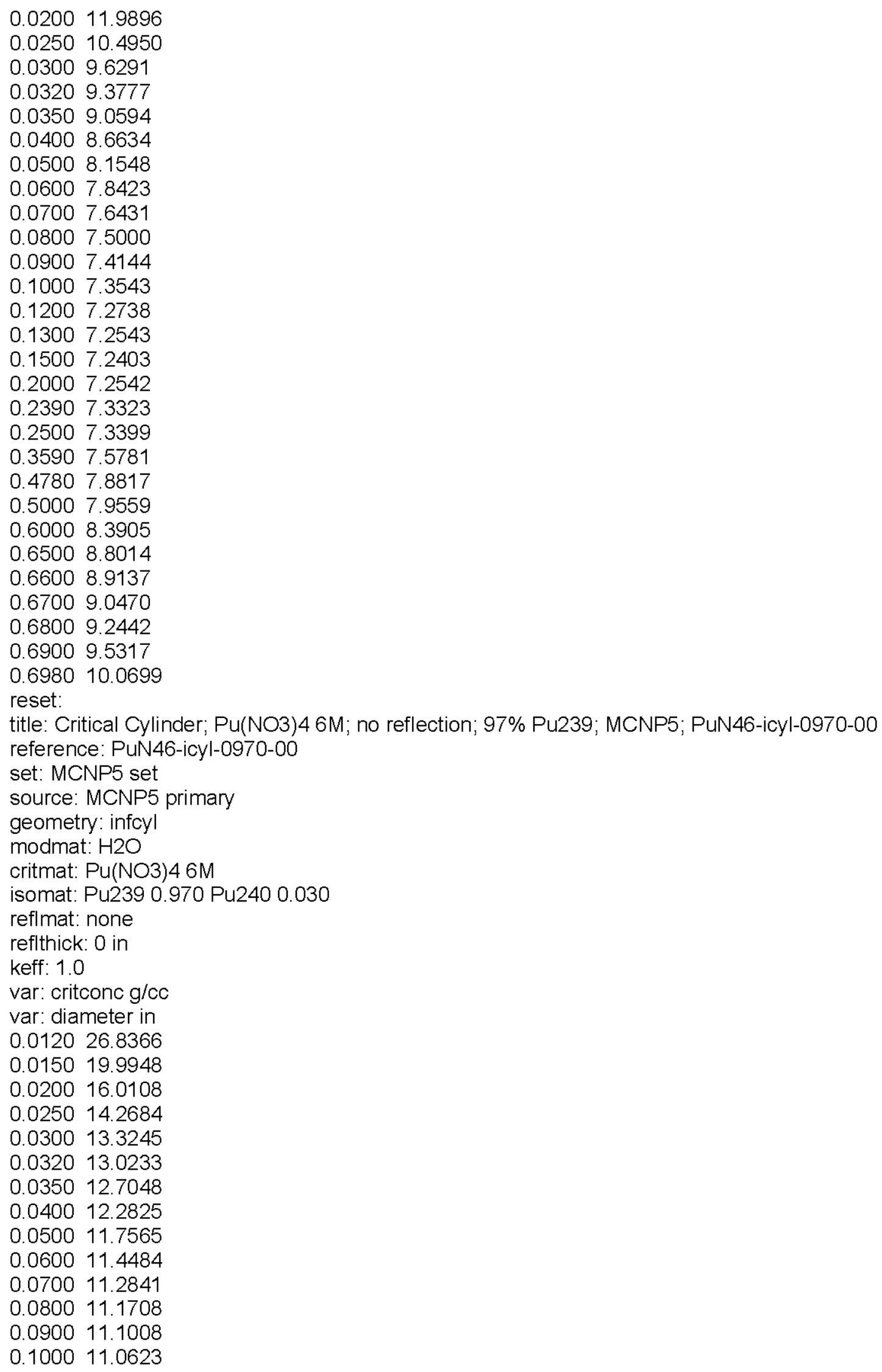


CHPRC-01552, Revision 0

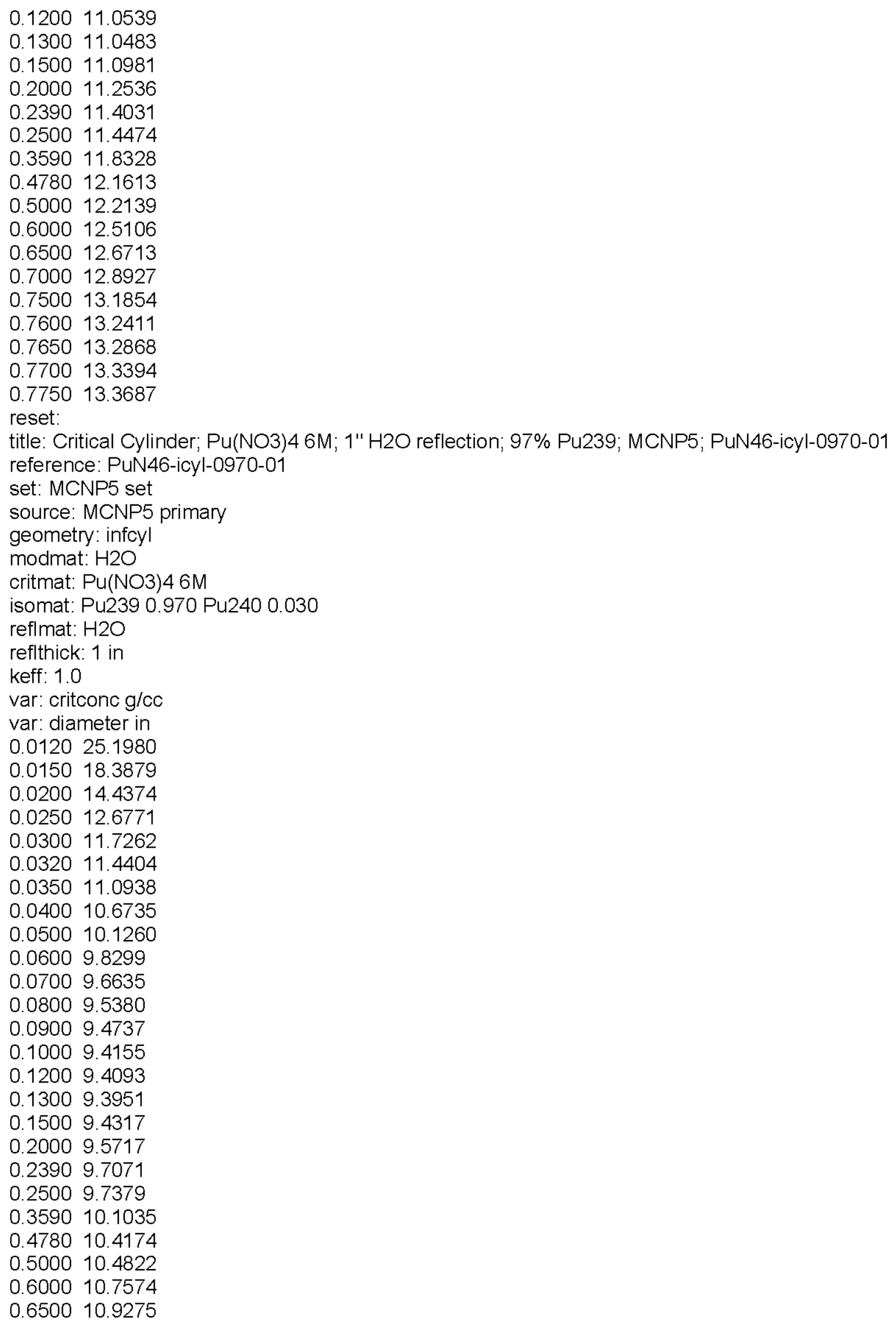




\section{CHPRC-01552, Revision 0}

0.700011 .1276

0.750011 .3892

0.760011 .4472

0.765011 .4993

0.770011 .5302

0.775011 .5585

reset:

title: Critical Cylinder; Pu(NO3)4 6M; 10" H2O reflection; 97\% Pu239; MCNP5; PuN46-icyl-0970-10

reference: PuN46-icyl-0970-10

set: MCNP5 set

source: MCNP5 primary

geometry: infcyl

modmat: $\mathrm{H} 2 \mathrm{O}$

critmat: $\mathrm{Pu}(\mathrm{NO} 3) 46 \mathrm{M}$

isomat: Pu239 0.970 Pu240 0.030

reflmat: $\mathrm{H} 2 \mathrm{O}$

reflthick: 10 in

keff: 1.0

var: critconc g/cc

var: diameter in

0.012023 .9584

0.015017 .1667

0.020013 .1750

0.025011 .4159

0.030010 .4219

0.032010 .1284

0.03509 .7647

0.04009 .3281

0.05008 .7600

0.06008 .4147

0.07008 .2105

0.08008 .0517

0.09007 .9426

0.10007 .8749

0.12007 .7985

0.13007 .7859

0.15007 .7721

0.20007 .8235

0.23907 .8897

0.25007 .9241

0.35908 .1655

0.47808 .3885

0.50008 .4430

0.60008 .6508

0.65008 .7729

0.70008 .9266

0.75009 .1516

0.76009 .1846

$0.7650 \quad 9.2288$

0.77009 .2516

$0.7750 \quad 9.2649$

reset:

title: Critical Slab; Pu(NO3)4 OM; no reflection; 100\% Pu239; MCNP5; PuN40-pl-1000-00

reference: PuN40-pl-1000-00

set: MCNP5 set

source: MCNP5 primary 


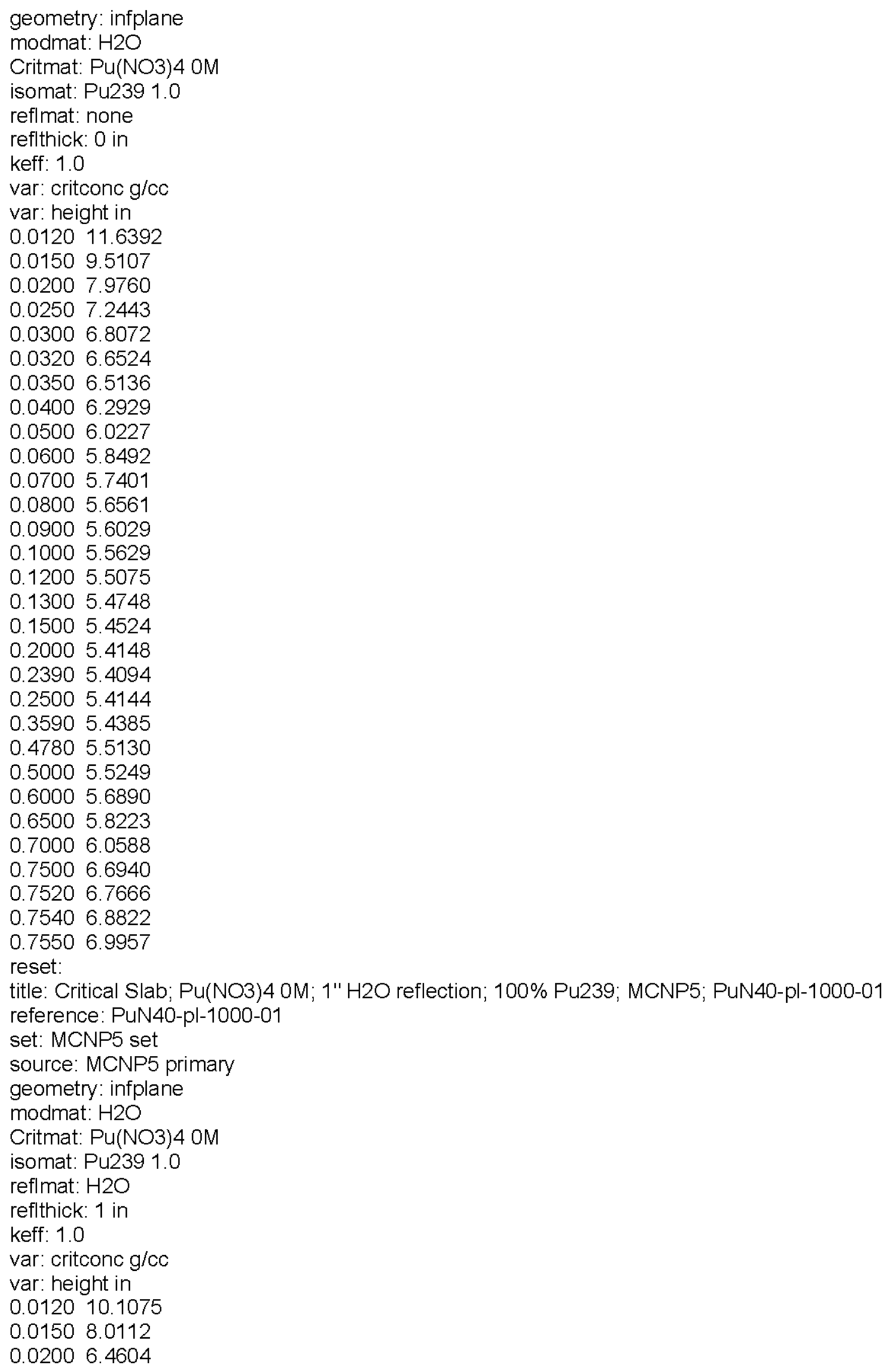


CHPRC-01552, Revision 0

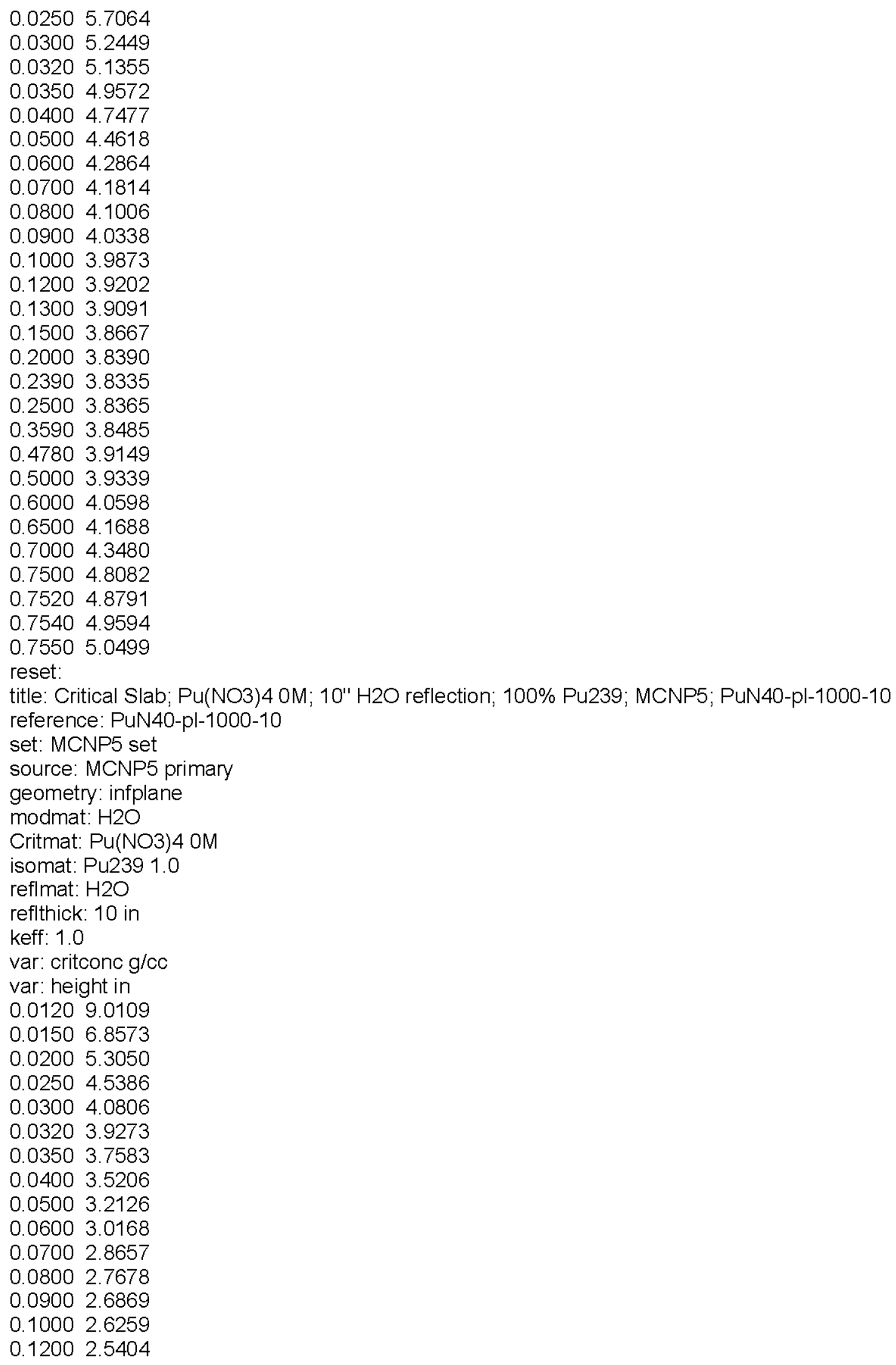




\section{CHPRC-01552, Revision 0}

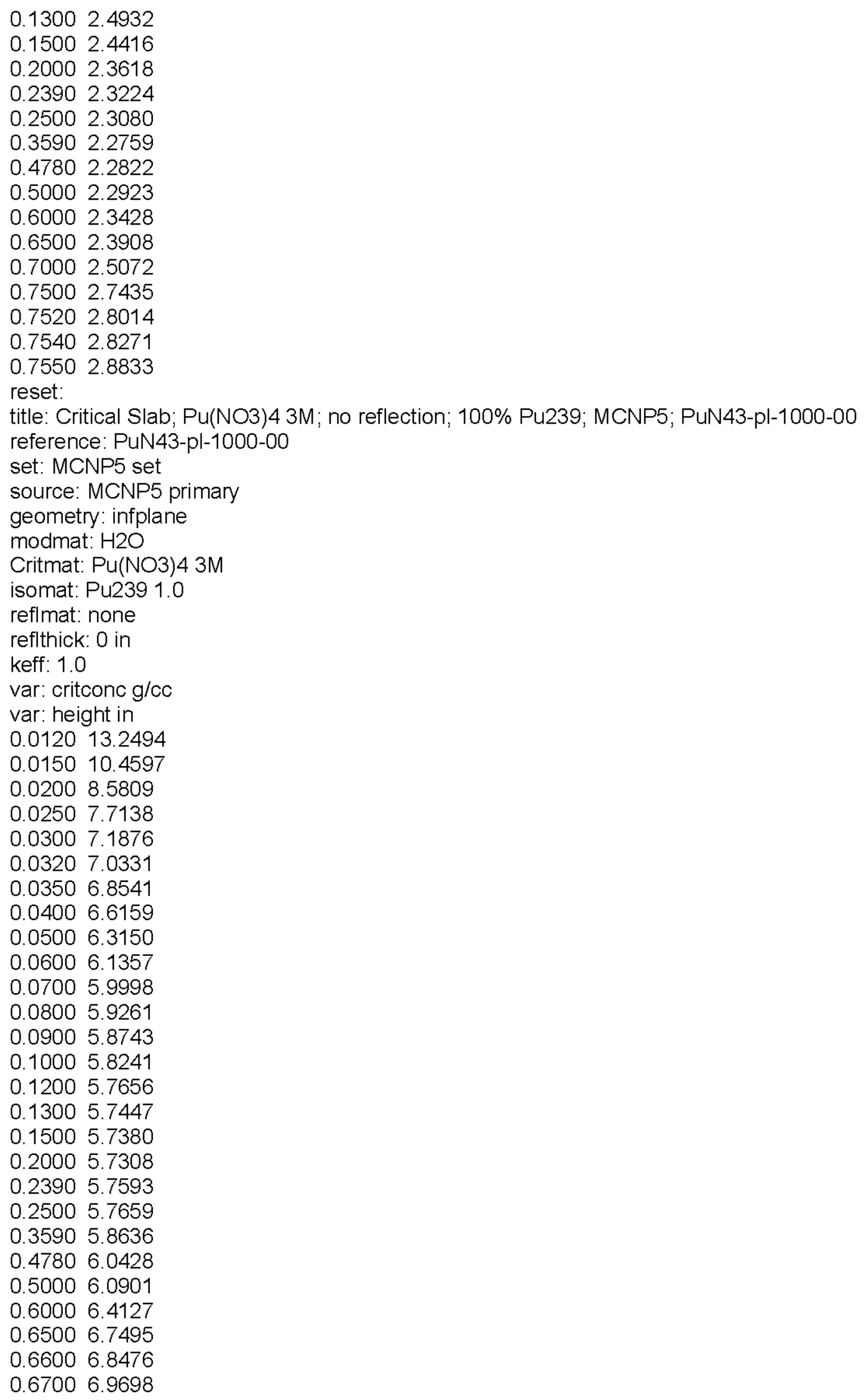


CHPRC-01552, Revision 0

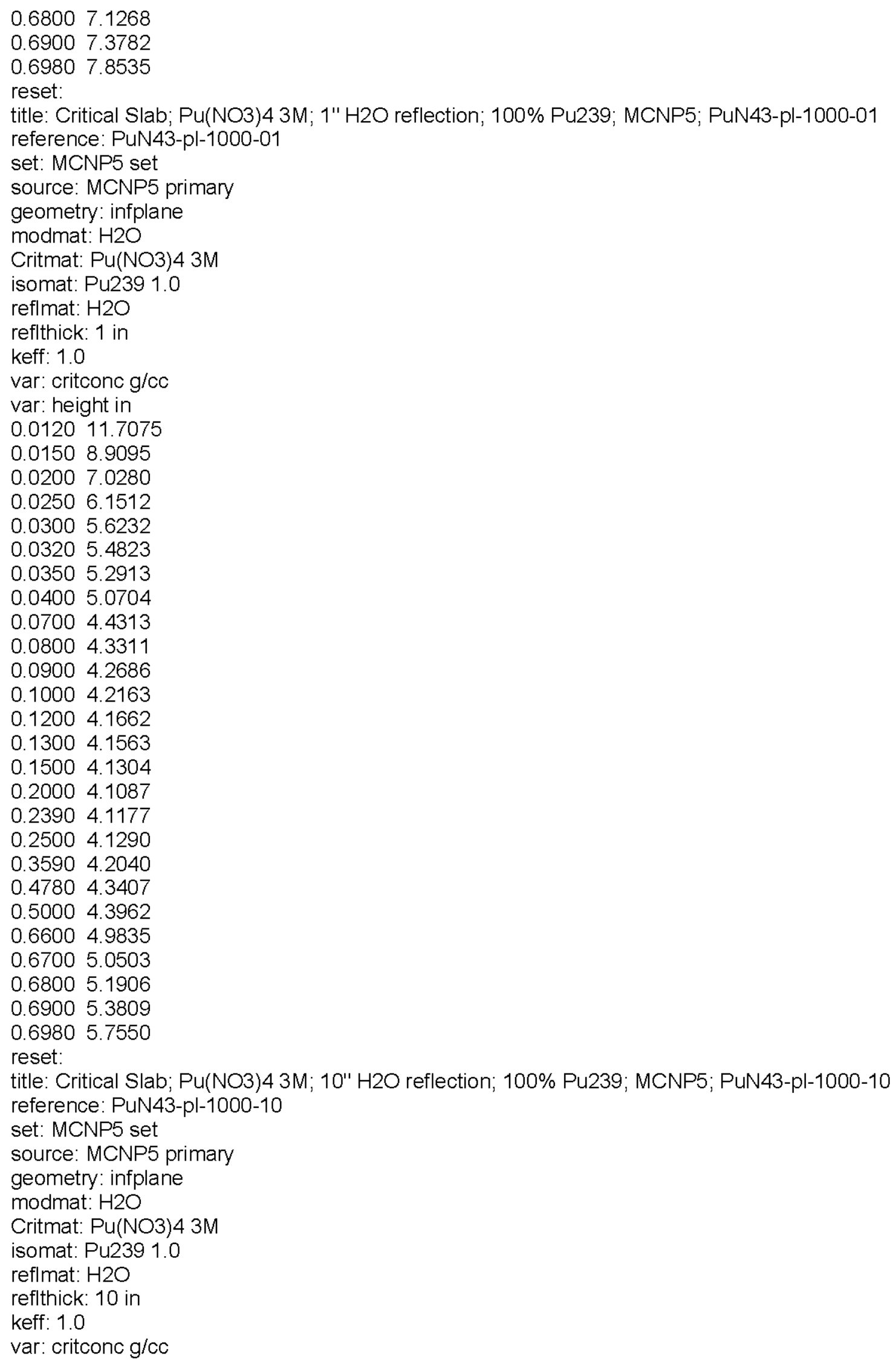




\section{CHPRC-01552, Revision 0}

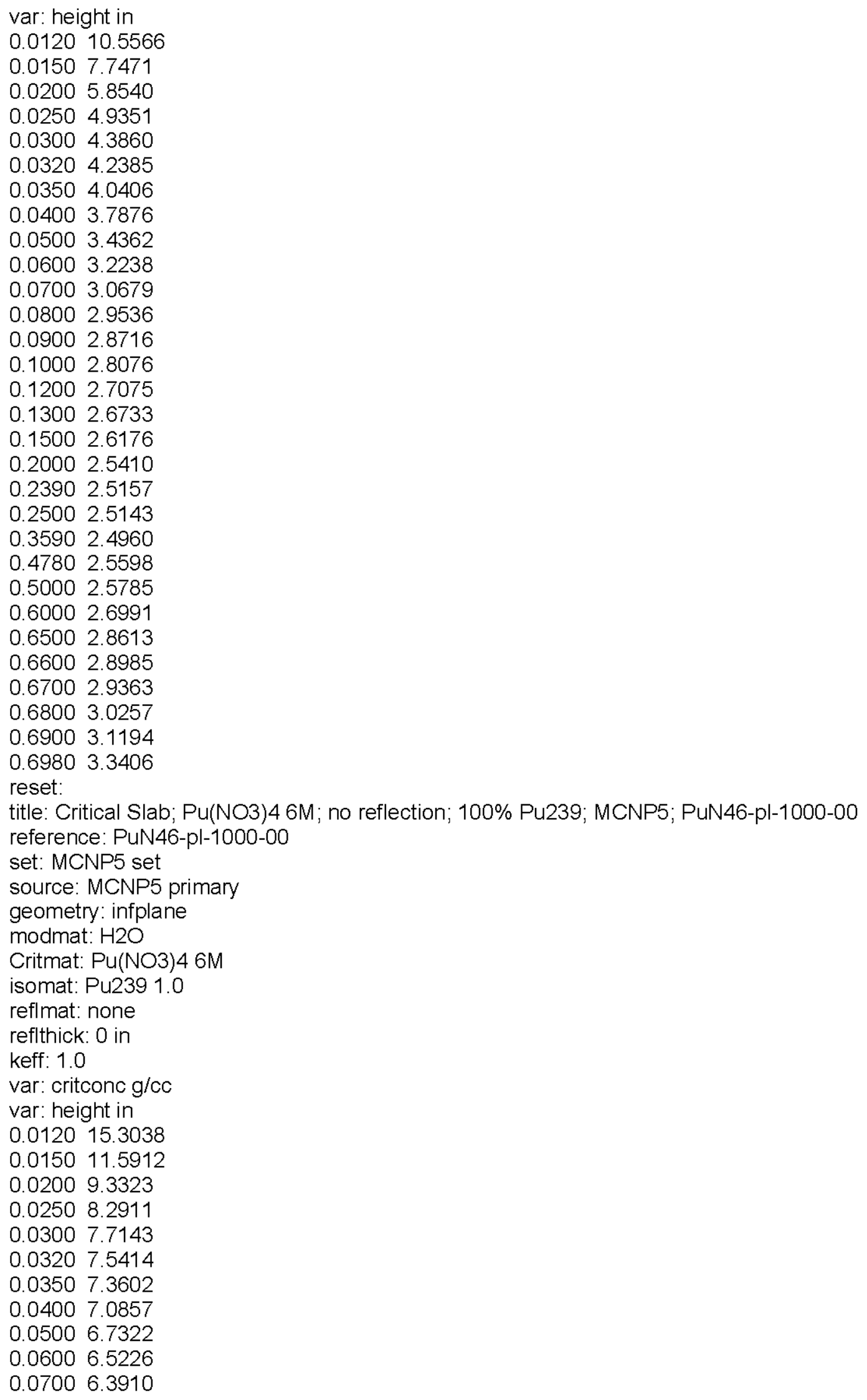


CHPRC-01552, Revision 0

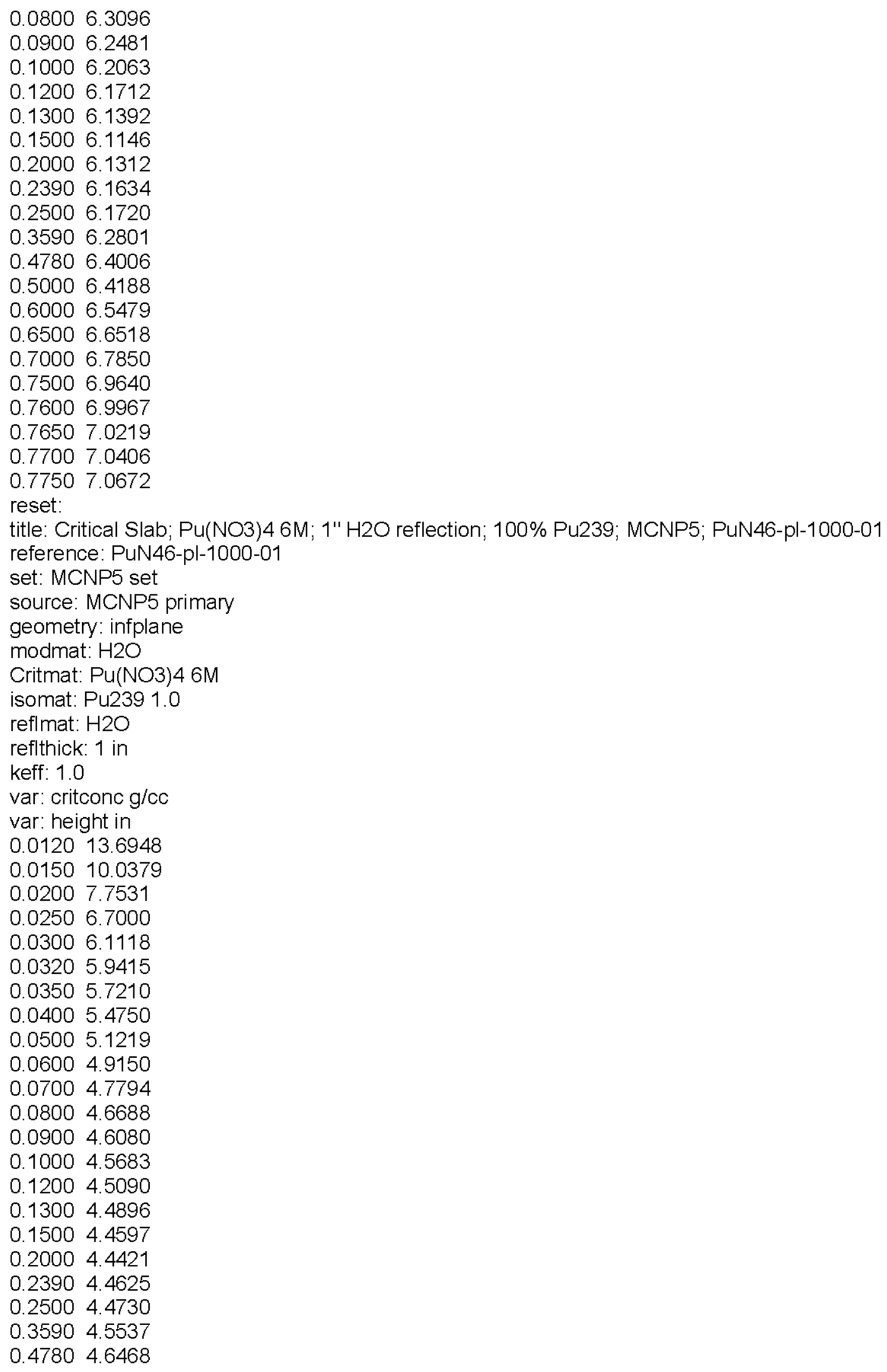




\section{CHPRC-01552, Revision 0}

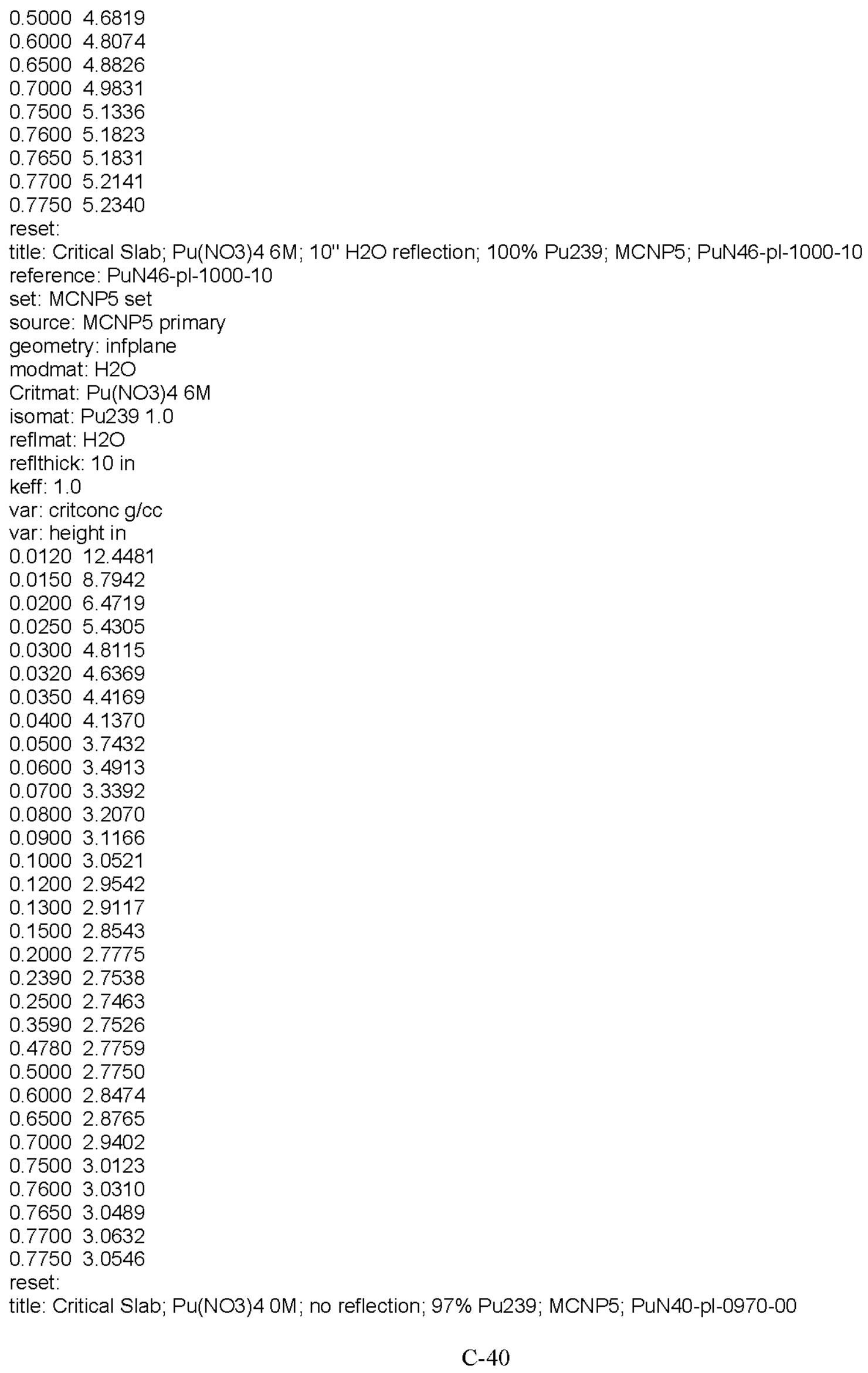


CHPRC-01552, Revision 0

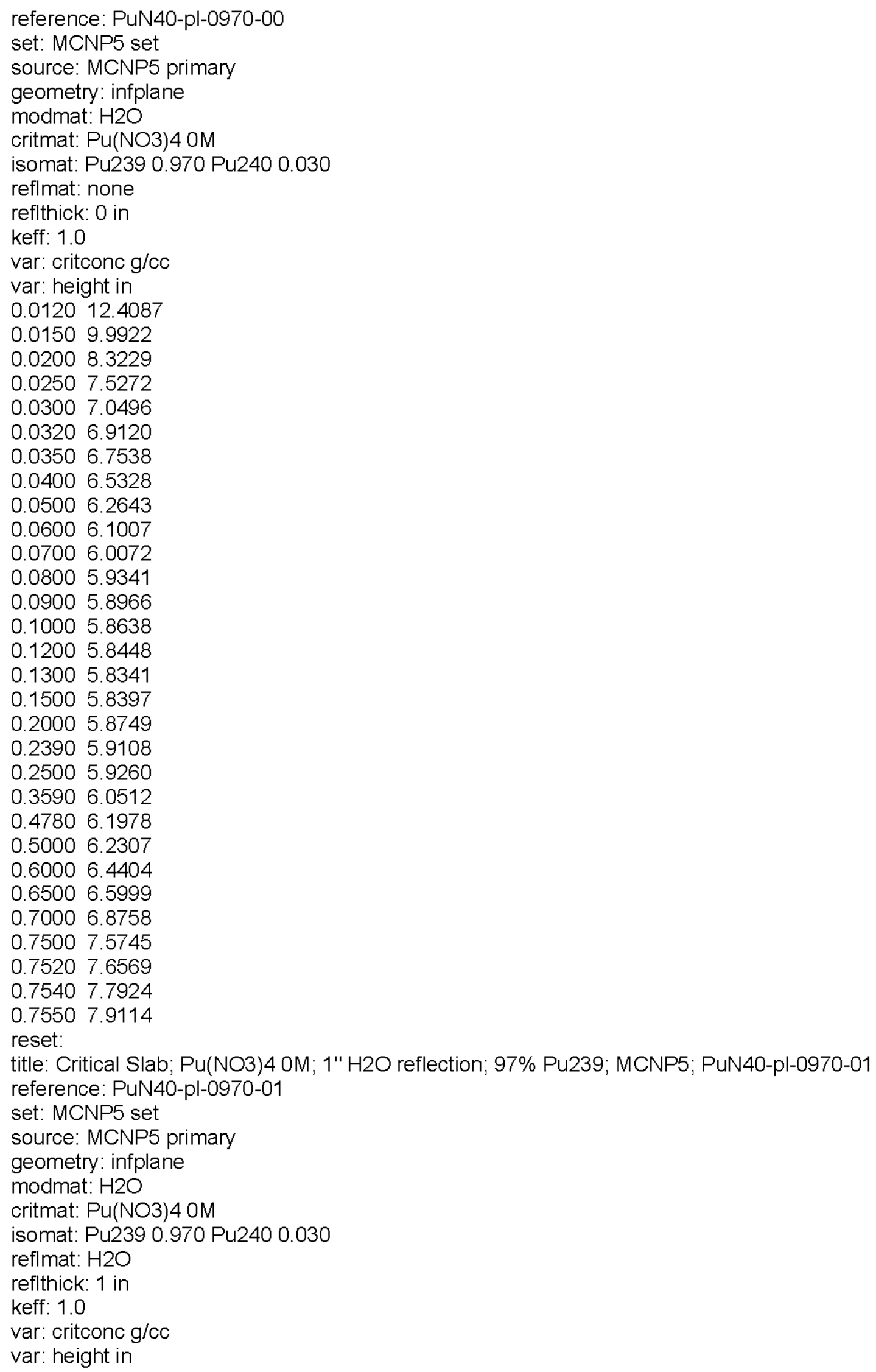


CHPRC-01552, Revision 0

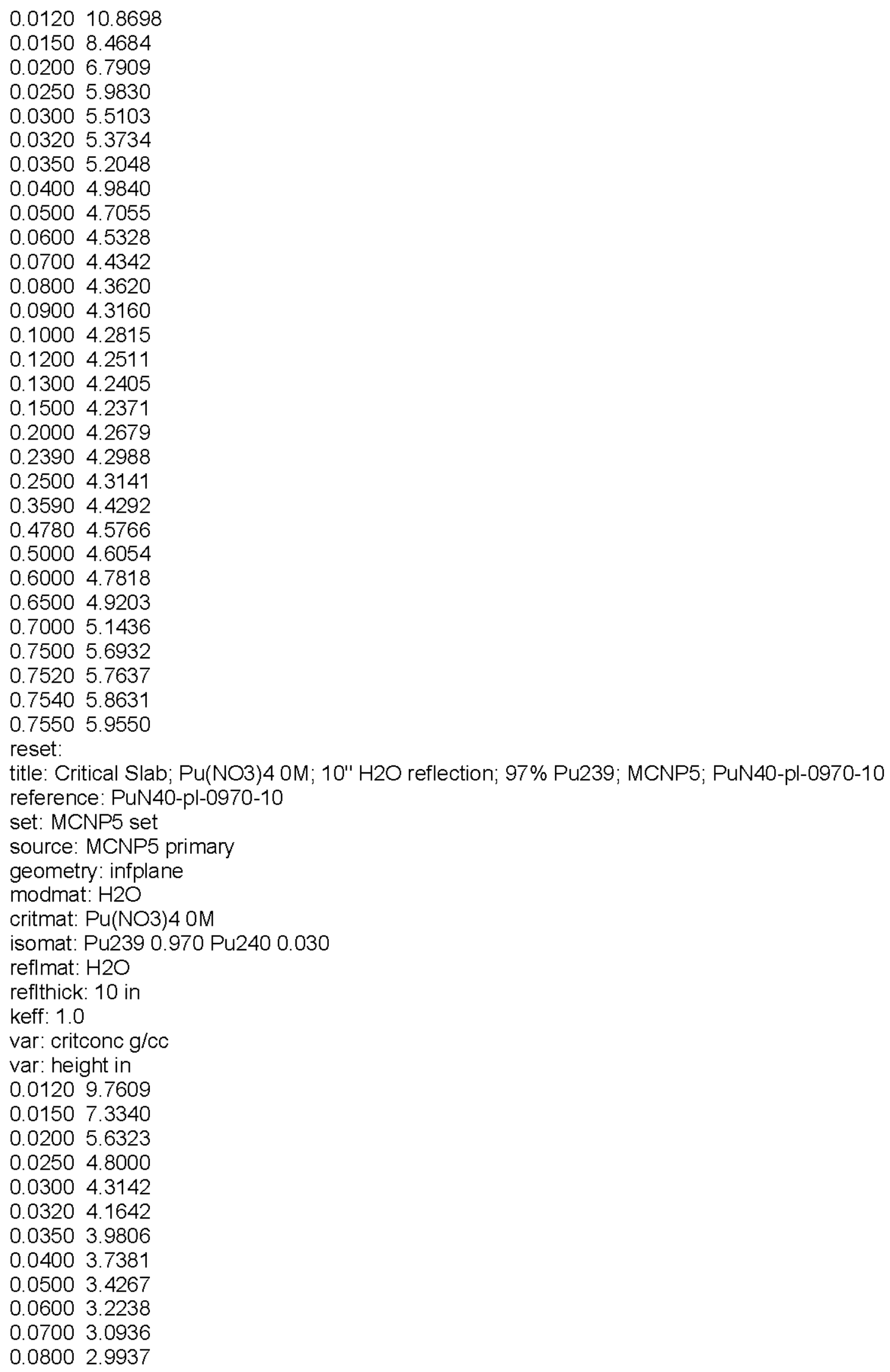




\section{CHPRC-01552, Revision 0}

0.09002 .9223

0.10002 .8658

0.12002 .7946

0.13002 .7612

$0.1500 \quad 2.7259$

0.20002 .6808

0.23902 .6812

0.25002 .6784

0.35902 .7090

0.47802 .7661

0.50002 .7809

0.60002 .8955

0.65002 .9664

0.70003 .0989

0.75003 .4331

0.75203 .4648

$0.7540 \quad 3.5241$

0.75503 .5887

!

!Uranyl Nitrate

I

reset:

title: Critical Sphere; UO2(NO3)2 OM; no reflection; 100\% U235; MCNP5; u5o2N20-sp-1000-00

reference: u5o2N20-sp-1000-00

title: MCNP5 u5o2N20-sp-1000-00

reference: u5o2N20-sp-1000-00

set: MCNP5 set

source: MCNP5 primary

geometry: Sphere

modmat: $\mathrm{H} 2 \mathrm{O}$

Critmat: UO2(NO3)2 OM

isomat: U235 1.0

reflmat: none

reflthick: 0 in

keff: 1.0

var: critconc $\mathrm{g} / \mathrm{cc}$

var: diameter in

0.015039 .5184

0.020025 .0712

0.025020 .7082

0.030018 .4868

0.032017 .8918

0.035017 .1361

0.040016 .2045

0.050015 .0402

0.060014 .3050

0.070013 .8117

0.080013 .4502

0.090013 .1840

0.100012 .9674

0.118012 .7056

0.130012 .5406

$0.1500 \quad 12.3789$

0.200012 .1412

0.235012 .0469

0.250012 .0288 


\section{CHPRC-01552, Revision 0}

$$
\begin{array}{ll}
0.3530 & 12.0088 \\
0.4700 & 12.0901 \\
0.5000 & 12.1107 \\
0.6000 & 12.2860 \\
0.6050 & 12.3024 \\
0.6100 & 12.3020 \\
0.6150 & 12.2906 \\
0.6200 & 12.3133 \\
0.6250 & 12.3286 \\
0.6290 & 12.3273 \\
\text { reset: } &
\end{array}
$$

title: Critical Sphere; UO2(NO3)2 OM; 1" H2O reflection; 100\% U235; MCNP5; u5o2N2O-sp-1000-01 reference: u5o2N20-sp-1000-01

set: MCNP5 set

source: MCNP5 primary

geometry: sphere

modmat: $\mathrm{H} 2 \mathrm{O}$

Critmat: UO2(NO3)2 OM

isomat: U235 1.0

reflmat: $\mathrm{H} 2 \mathrm{O}$

reflthick: 1 in

keff: 1.0

var: critconc g/cc

var: diameter in

0.015038 .0011

0.020023 .5307

$0.0250 \quad 19.1594$

0.030016 .9769

$0.0320 \quad 16.3652$

0.035015 .6048

0.040014 .7039

0.050013 .5084

0.060012 .7830

$0.0700 \quad 12.2793$

0.080011 .9246

0.090011 .6515

0.100011 .4516

0.118011 .1625

0.130011 .0426

0.150010 .8522

0.200010 .6136

0.235010 .5214

0.250010 .4977

$\begin{array}{lll}0.3530 & 10.4607\end{array}$

0.470010 .5302

0.500010 .5760

0.600010 .7287

0.605010 .7226

0.610010 .7376

0.615010 .7481

0.620010 .7549

0.625010 .7562

$0.6290 \quad 10.7747$

reset:

title: Critical Sphere; UO2(NO3)2 OM; 10" H2O reflection; 100\% U235; MCNP5; u5o2N2O-sp-1000-10 reference: u5o2N20-sp-1000-10 
set: MCNP5 set

source: MCNP5 primary

geometry: sphere

modmat: $\mathrm{H} 2 \mathrm{O}$

Critmat: UO2(NO3)2 OM

isomat: U235 1.0

reflmat: $\mathrm{H} 2 \mathrm{O}$

reflthick: 10 in

keff: 1.0

var: critconc g/cc

var: diameter in

0.015036 .9010

0.020022 .4478

0.025018 .0664

0.030015 .8688

0.032015 .2536

0.035014 .4872

0.040013 .5737

0.050012 .3637

0.060011 .6208

0.070011 .1255

0.080010 .7417

0.090010 .4650

0.100010 .2606

0.11809 .9594

0.13009 .7964

0.15009 .6140

0.20009 .3316

$0.2350 \quad 9.2232$

$0.2500 \quad 9.1874$

0.35309 .0955

0.47009 .1030

0.50009 .1315

0.60009 .2326

0.60509 .2435

0.61009 .2452

0.61509 .2371

0.62009 .2588

0.62509 .2713

0.62909 .2643

reset:

title: Critical Sphere; UO2(NO3)2 3M; no reflection; 100\% U235; MCNP5; u5o2N23-sp-1000-00

reference: u5o2N23-sp-1000-00

set: MCNP5 set

source: MCNP5 primary

geometry: sphere

modmat: $\mathrm{H} 2 \mathrm{O}$

Critmat: UO2(NO3)2 3M

isomat: U235 1.0

reflmat: none

reflthick: 0 in

keff: 1.0

var: critconc g/cc

var: diameter in

0.020028 .6621

0.025022 .8070 
CHPRC-01552, Revision 0

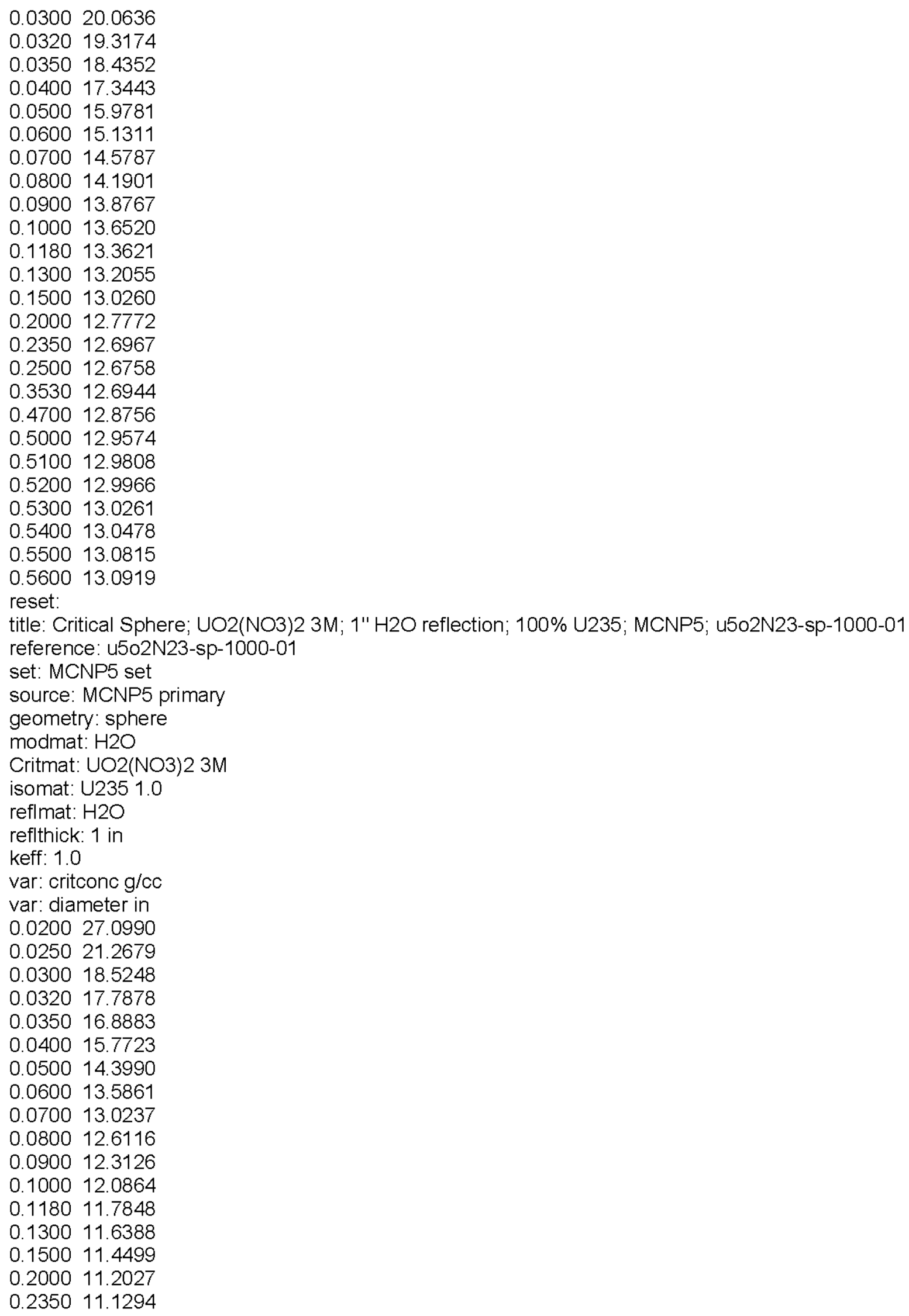




\section{CHPRC-01552, Revision 0}

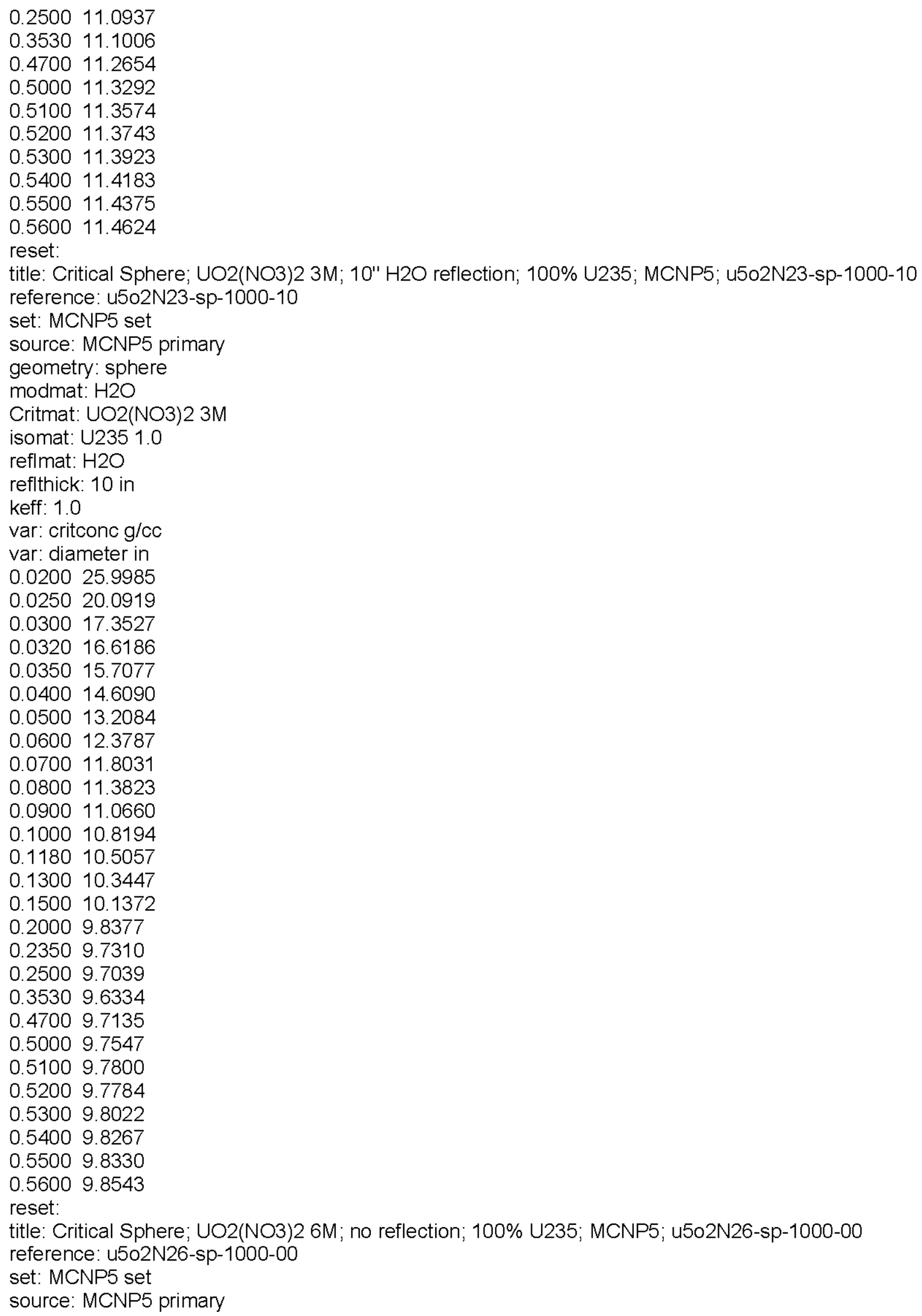


CHPRC-01552, Revision 0

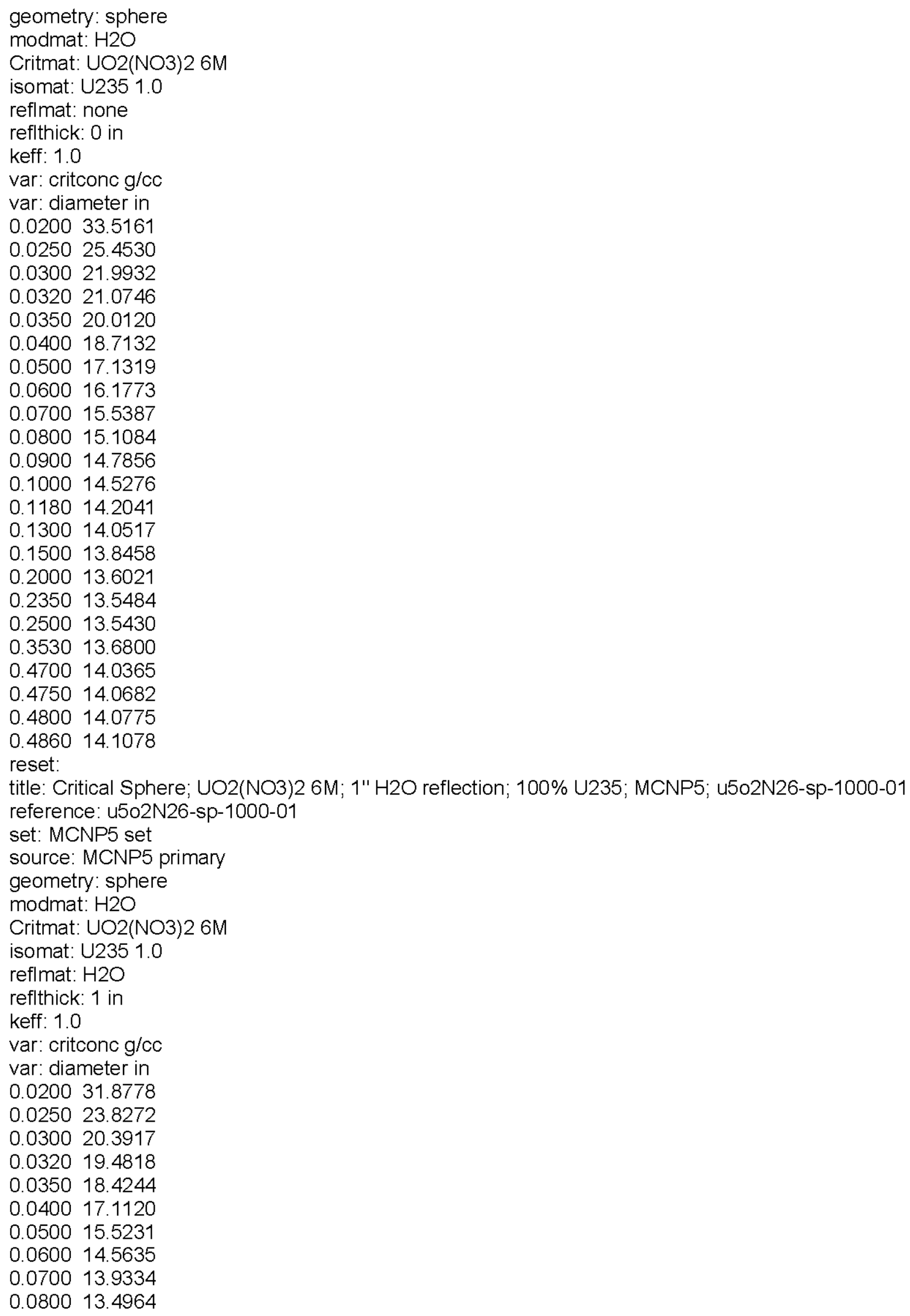




\section{CHPRC-01552, Revision 0}

0.090013 .1587

0.100012 .8969

0.118012 .5772

0.130012 .4070

0.150012 .2110

0.200011 .9716

0.235011 .8966

0.250011 .8832

0.353011 .9860

0.470012 .3203

$0.4750 \quad 12.3209$

0.480012 .3504

0.486012 .3669

reset:

title: Critical Sphere; UO2(NO3)2 6M; 10 " H2O reflection; 100\% U235; MCNP5; u5o2N26-sp-1000-10 reference: u5o2N26-sp-1000-10

set: MCNP5 set

source: MCNP5 primary

geometry: sphere

modmat: $\mathrm{H} 2 \mathrm{O}$

Critmat: UO2(NO3)2 $6 \mathrm{M}$

isomat: U235 1.0

reflmat: $\mathrm{H} 2 \mathrm{O}$

reflthick: 10 in

keff: 1.0

var: critconc $\mathrm{g} / \mathrm{cc}$

var: diameter in

0.020030 .6256

0.025022 .6191

0.030019 .1805

0.032018 .2452

0.035017 .1963

0.040015 .8655

0.050014 .2556

0.060013 .2682

0.070012 .6363

0.080012 .1771

0.090011 .8525

0.100011 .5198

0.118011 .2071

0.130011 .0317

0.150010 .7869

0.200010 .5099

0.235010 .3940

0.250010 .3814

0.353010 .3481

0.470010 .5856

0.475010 .5938

0.480010 .6025

0.486010 .6232

reset:

title: Critical Sphere; UO2(NO3)2 OM; no reflection; 93.5\% U235; MCNP5; u5o2N20-sp-0935-00

reference: u5o2N20-sp-0935-00

set: MCNP5 set

source: MCNP5 primary

geometry: sphere 
CHPRC-01552, Revision 0

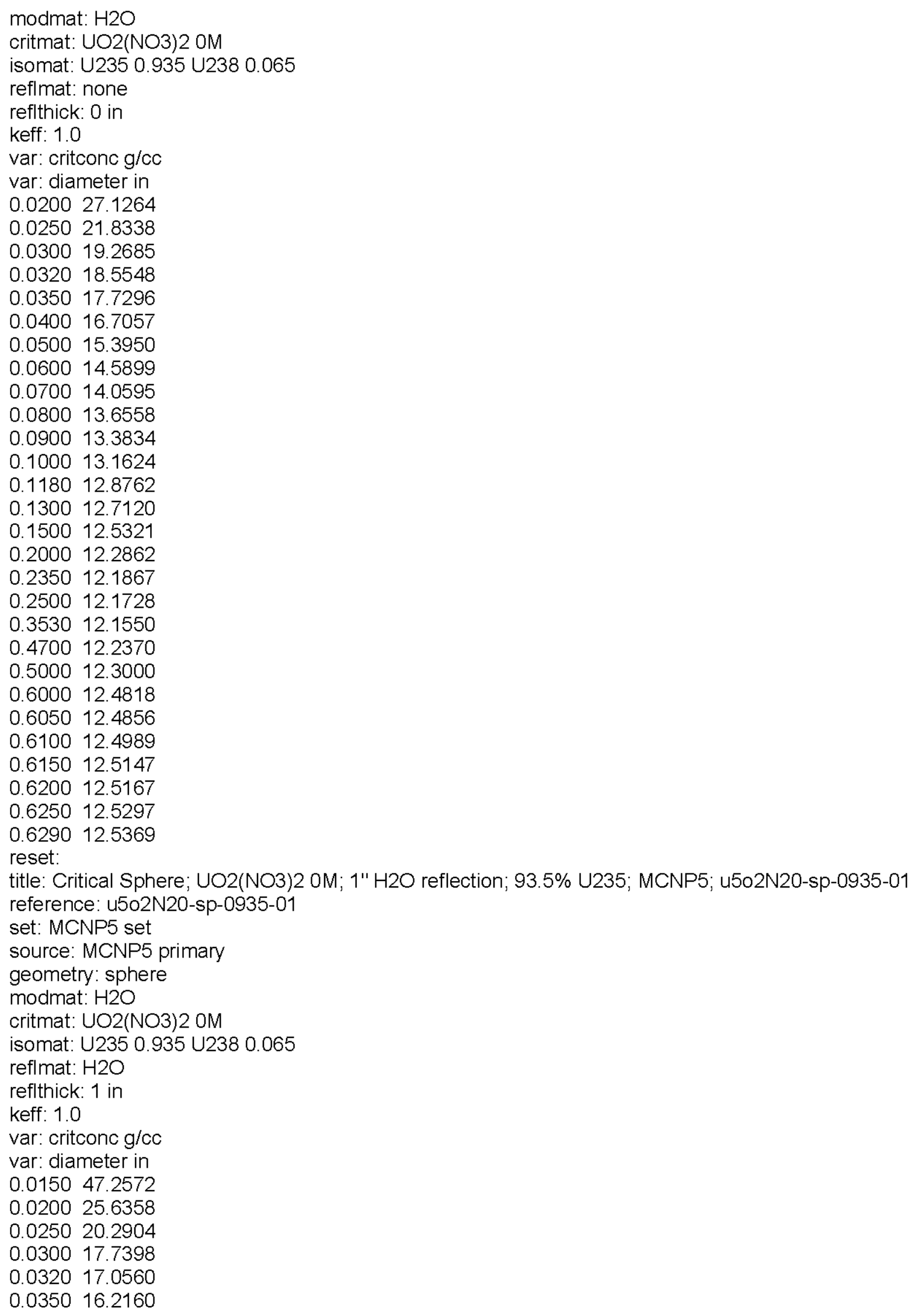


CHPRC-01552, Revision 0

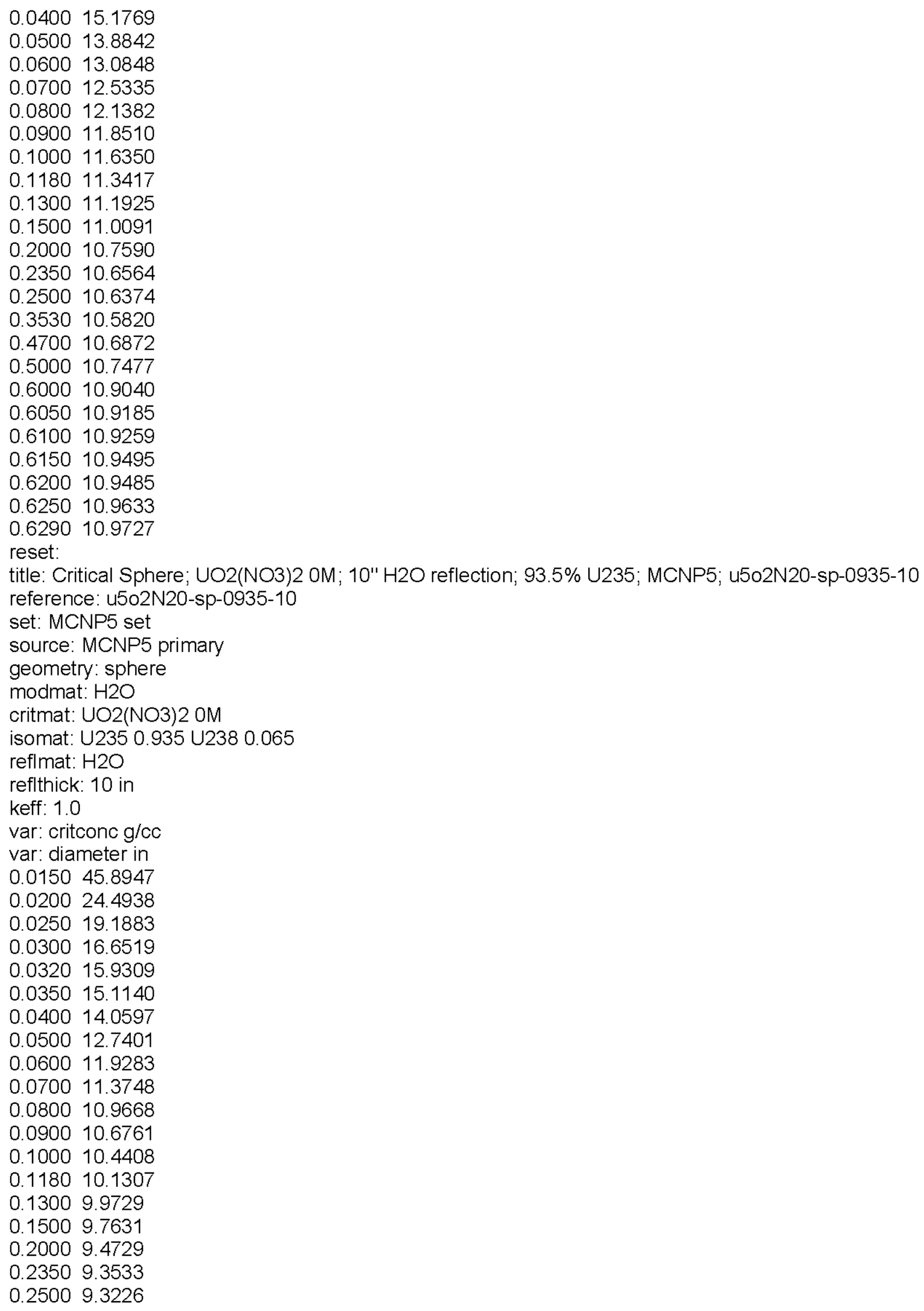




\title{
CHPRC-01552, Revision 0
}

\begin{abstract}
0.35309 .2283
0.47009 .2589

0.50009 .2905

0.60009 .4180

0.60509 .4002

$0.6100 \quad 9.4157$

$0.6150 \quad 9.4345$

0.62009 .4288

0.62509 .4284

$0.6290 \quad 9.4499$

reset:
\end{abstract}

title: Critical Sphere; UO2(NO3)2 3M; no reflection; 93.5\% U235; MCNP5; u5o2N23-sp-0935-00

reference: u5o2N23-sp-0935-00

set: MCNP5 set

source: MCNP5 primary

geometry: sphere

modmat: $\mathrm{H} 2 \mathrm{O}$

critmat: UO2(NO3)2 3M

isomat: U235 $0.935 \mathrm{U} 2380.065$

reflmat: none

reflthick: 0 in

keff: 1.0

var: critconc $\mathrm{g} / \mathrm{cc}$

var: diameter in

0.020031 .6145

0.025024 .2514

0.030021 .0167

0.032020 .1656

0.035019 .1454

0.040017 .8941

0.050016 .3840

0.060015 .4607

0.070014 .8692

0.080014 .4238

0.090014 .0913

0.100013 .8425

0.118013 .5288

0.130013 .3819

0.150013 .2018

0.200012 .9385

0.235012 .8424

0.250012 .8391

0.353012 .8585

0.470013 .0649

0.500013 .1463

0.510013 .1835

0.520013 .2103

0.530013 .2356

0.540013 .2614

0.550013 .2899

0.560013 .3136

reset:

title: Critical Sphere; UO2(NO3)2 3M; 1" H2O reflection; 93.5\% U235; MCNP5; u5o2N23-sp-0935-01

reference: u5o2N23-sp-0935-01

set: MCNP5 set

source: MCNP5 primary 


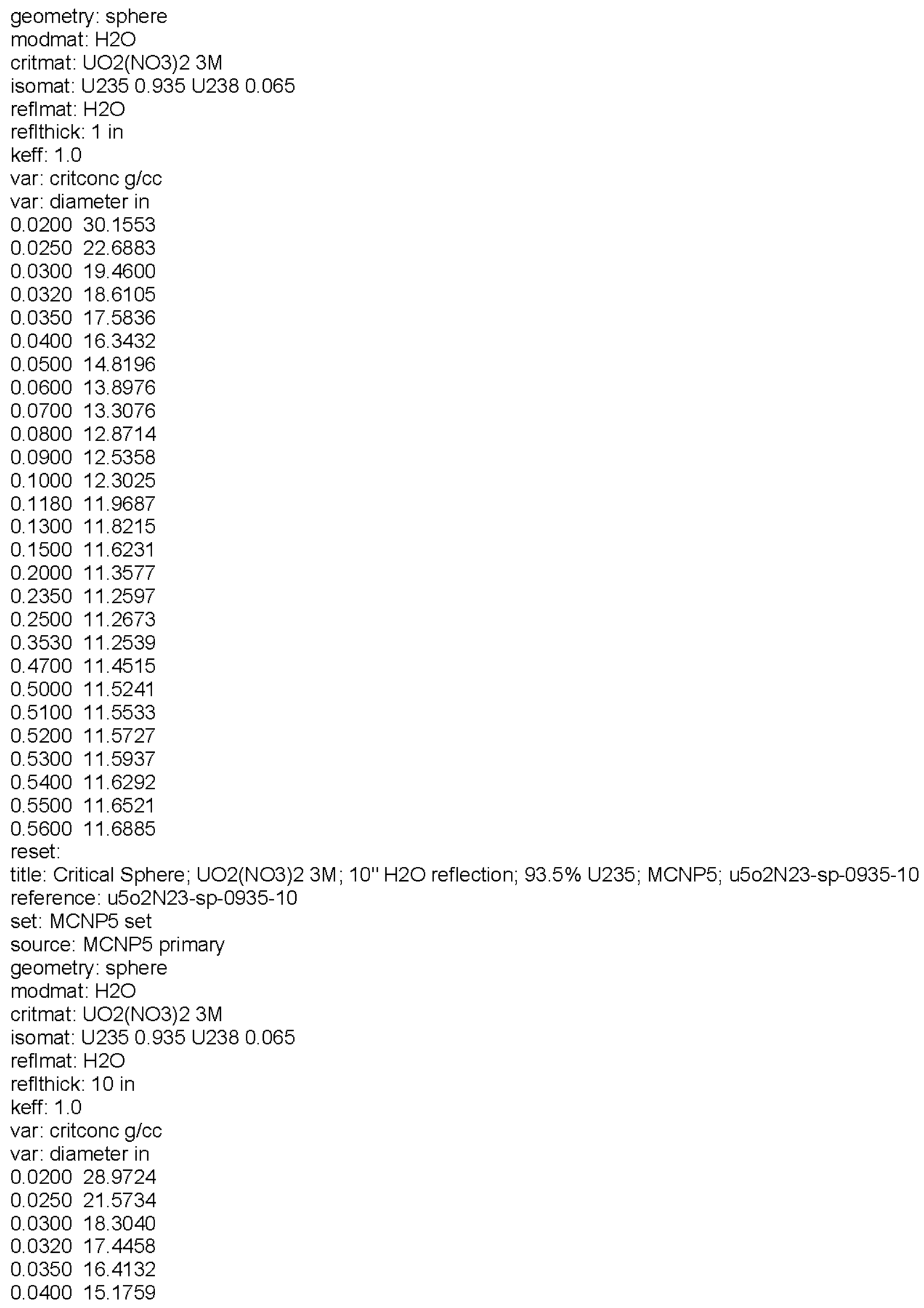


CHPRC-01552, Revision 0

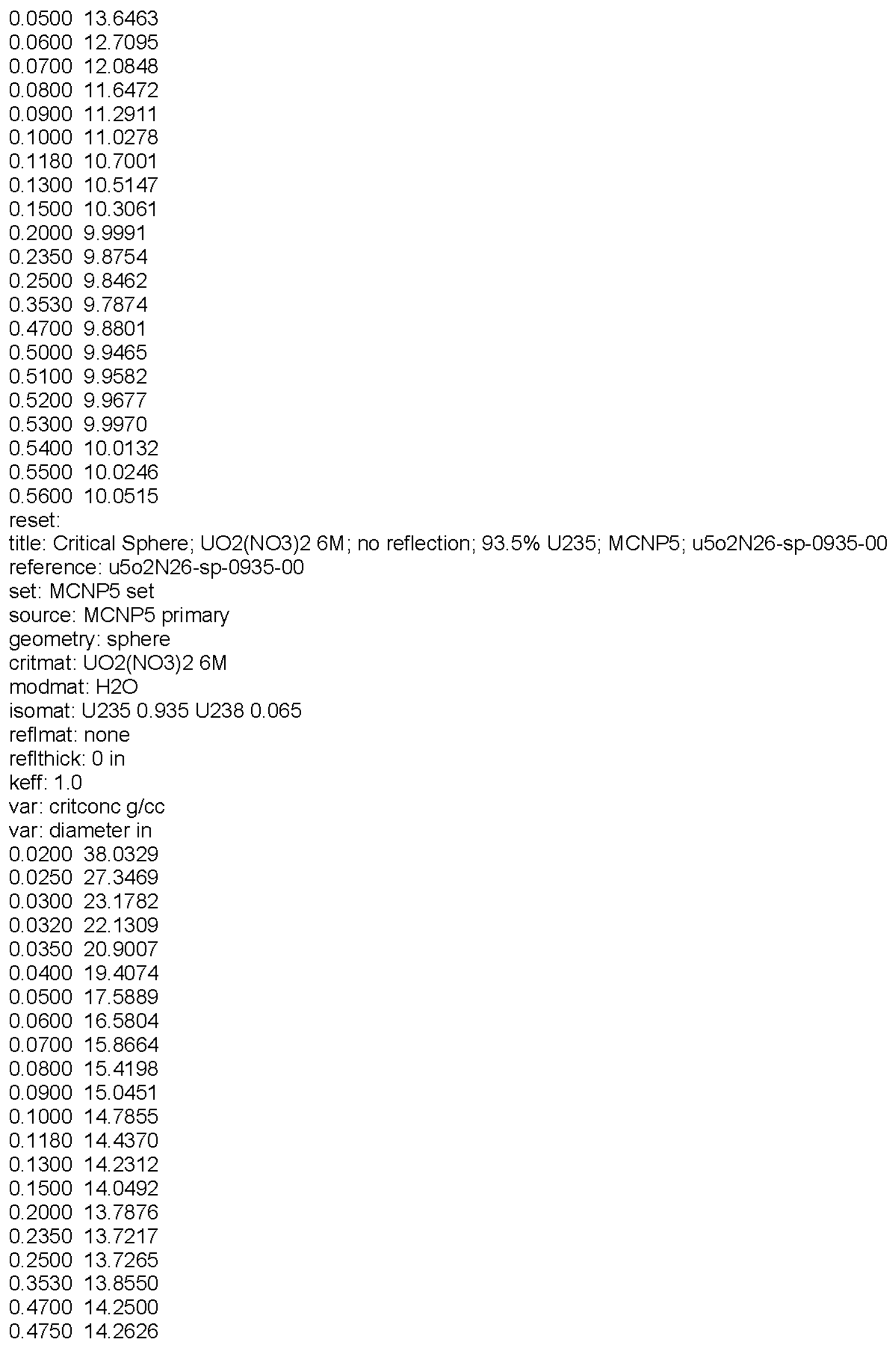




\section{CHPRC-01552, Revision 0}

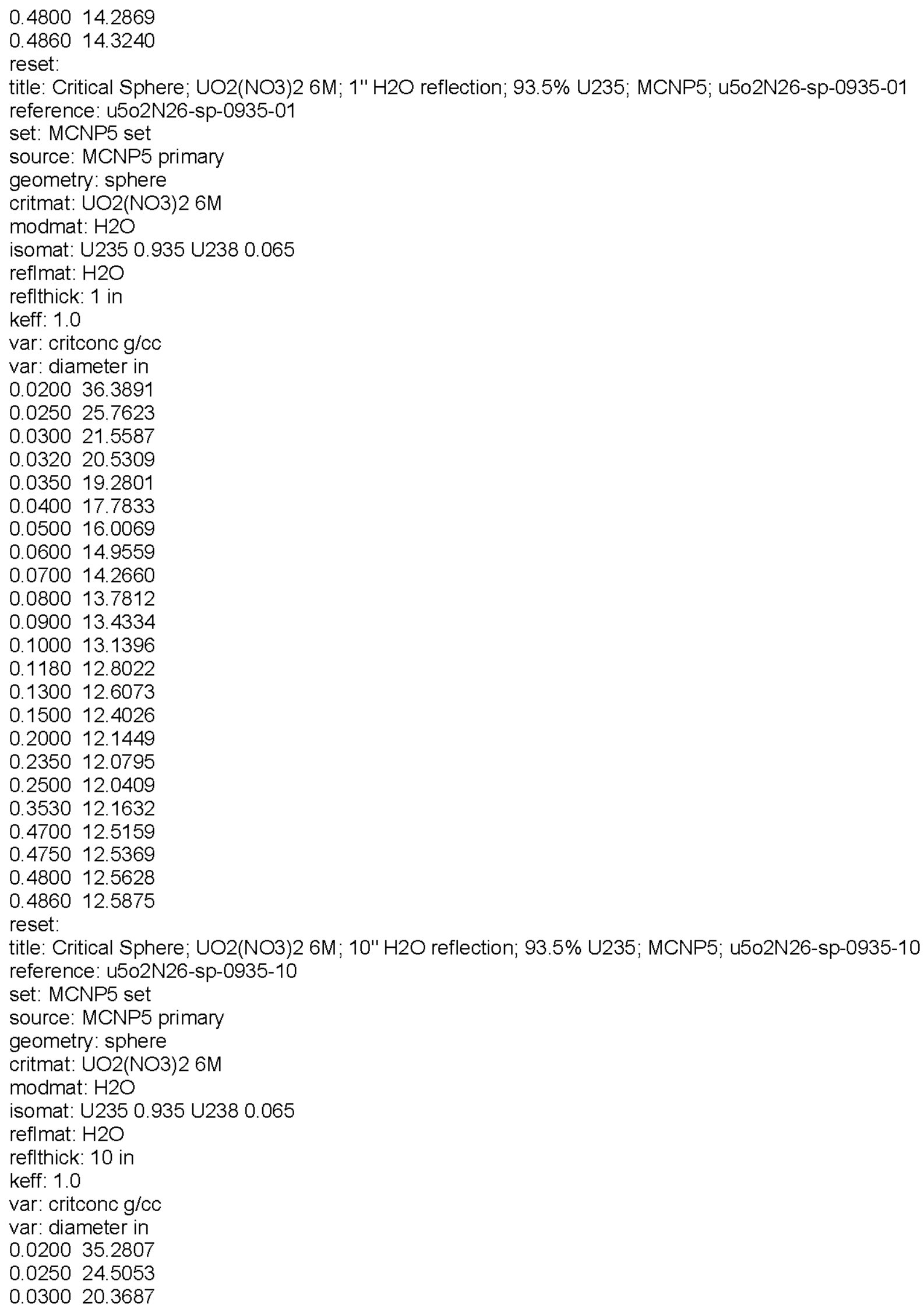


CHPRC-01552, Revision 0

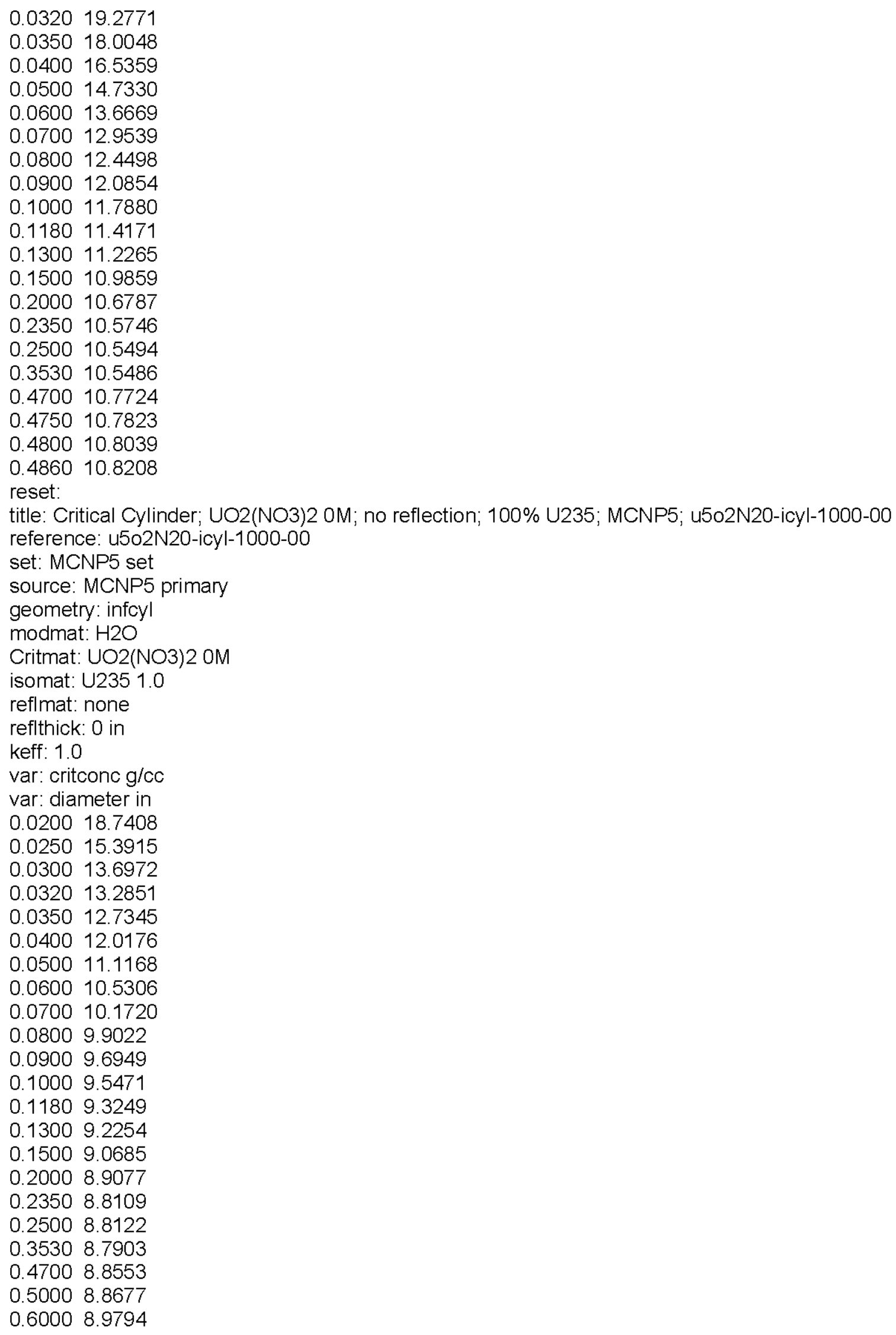




\section{CHPRC-01552, Revision 0}

0.60508 .9903

0.61009 .0021

0.61509 .0070

0.62009 .0232

0.62509 .0162

0.62909 .0393

reset:

title: Critical Cylinder; UO2(NO3)2 0M; 1" H2O reflection; 100\% U235; MCNP5; u5o2N2O-icyl-1000-01

reference: u5o2N20-icyl-1000-01

set: MCNP5 set

source: MCNP5 primary

geometry: infcyl

modmat: $\mathrm{H} 2 \mathrm{O}$

Critmat: UO2(NO3)2 OM

isomat: U235 1.0

reflmat: $\mathrm{H} 2 \mathrm{O}$

reflthick: 1 in

keff: 1.0

var: critconc $\mathrm{g} / \mathrm{cc}$

var: diameter in

0.015028 .1836

0.020017 .2166

0.025013 .8750

0.030012 .2168

0.032011 .7714

0.035011 .1998

0.040010 .4825

0.05009 .5846

0.06009 .0271

0.07008 .6350

0.08008 .3673

0.09008 .1411

0.10008 .0036

0.11807 .8036

0.13007 .6788

0.15007 .5422

0.20007 .3560

0.23507 .2863

0.25007 .2803

0.35307 .2369

0.47007 .2864

0.50007 .3138

0.60007 .4176

0.60507 .4126

0.61007 .4056

0.61507 .4310

0.62007 .4379

0.62507 .4234

0.62907 .4480

reset:

title: Critical Cylinder; UO2(NO3)2 OM; 10" H2O reflection; 100\% U235; MCNP5; u5o2N2O-icyl-1000-10 reference: u5o2N20-icyl-1000-10

set: MCNP5 set

source: MCNP5 primary

geometry: infcyl

isomat: U235 1.0 


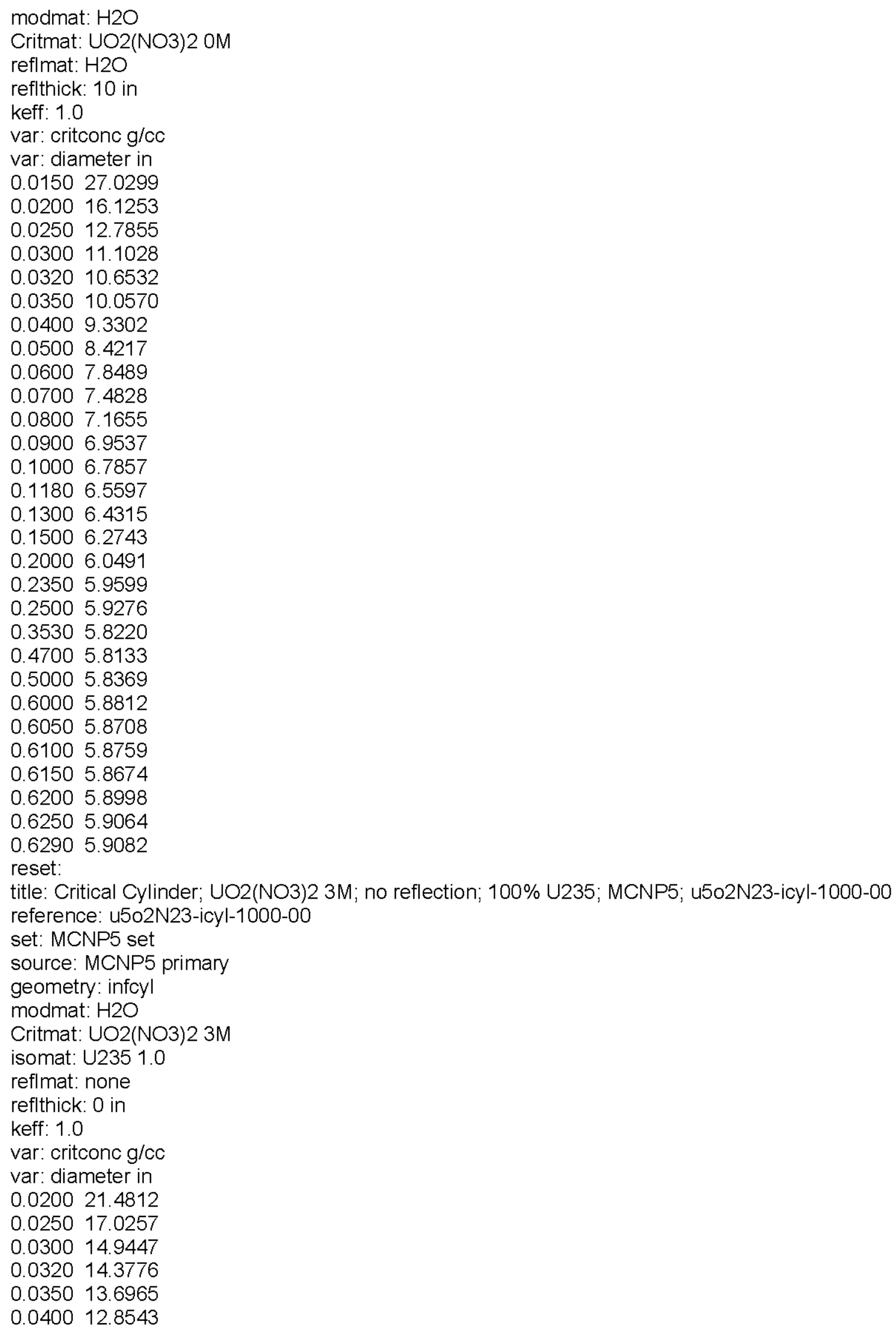


CHPRC-01552, Revision 0

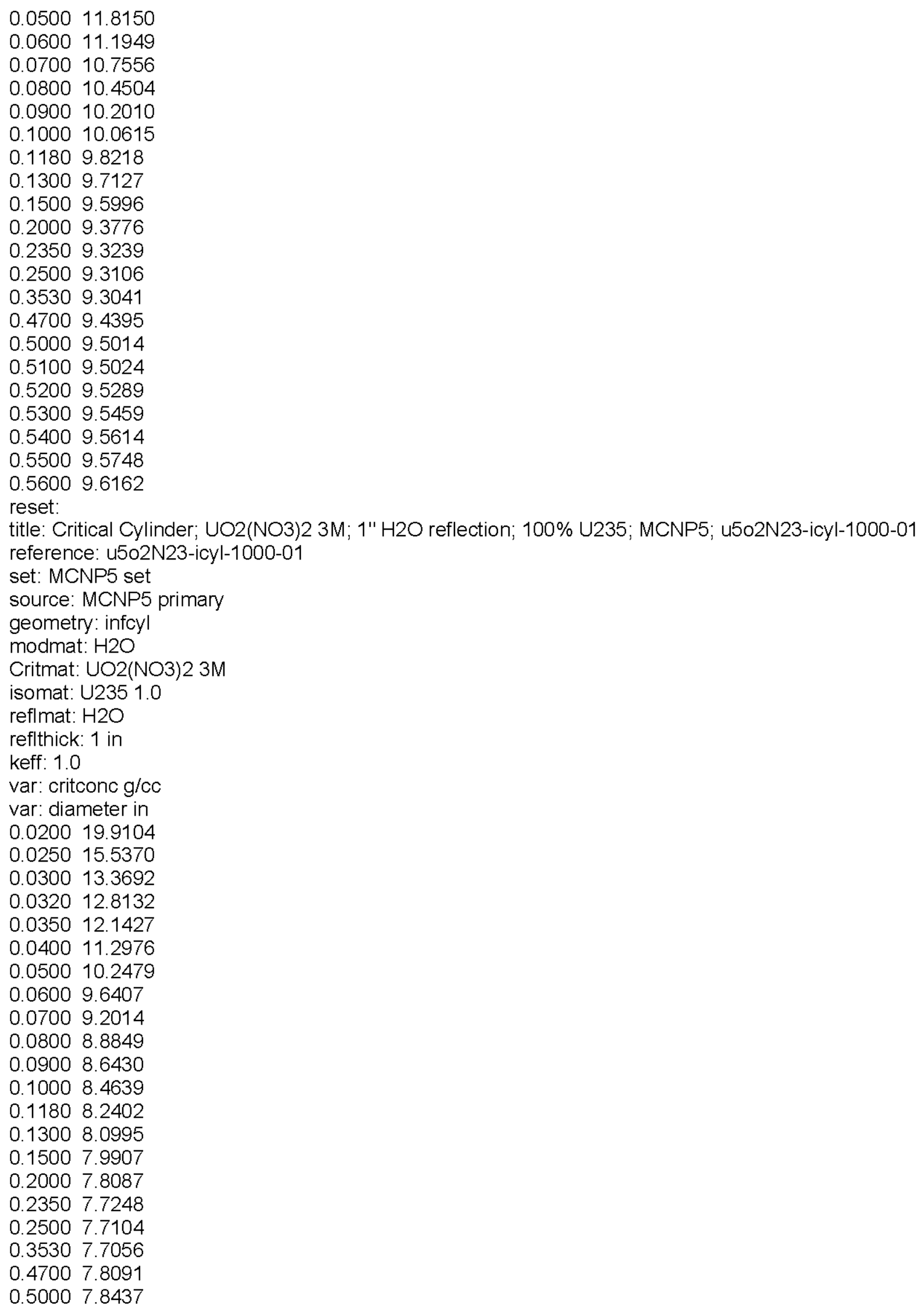




\section{CHPRC-01552, Revision 0}

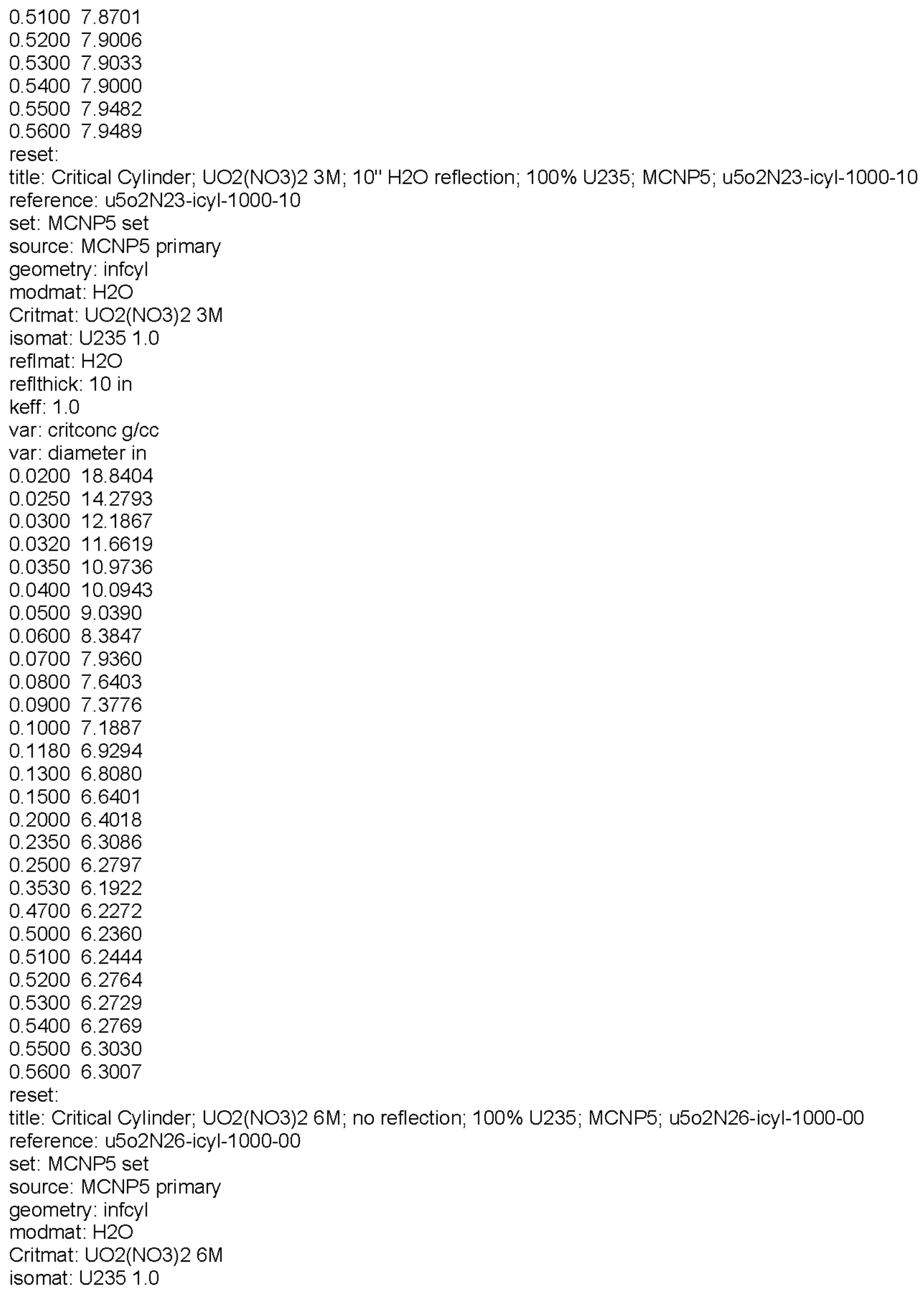




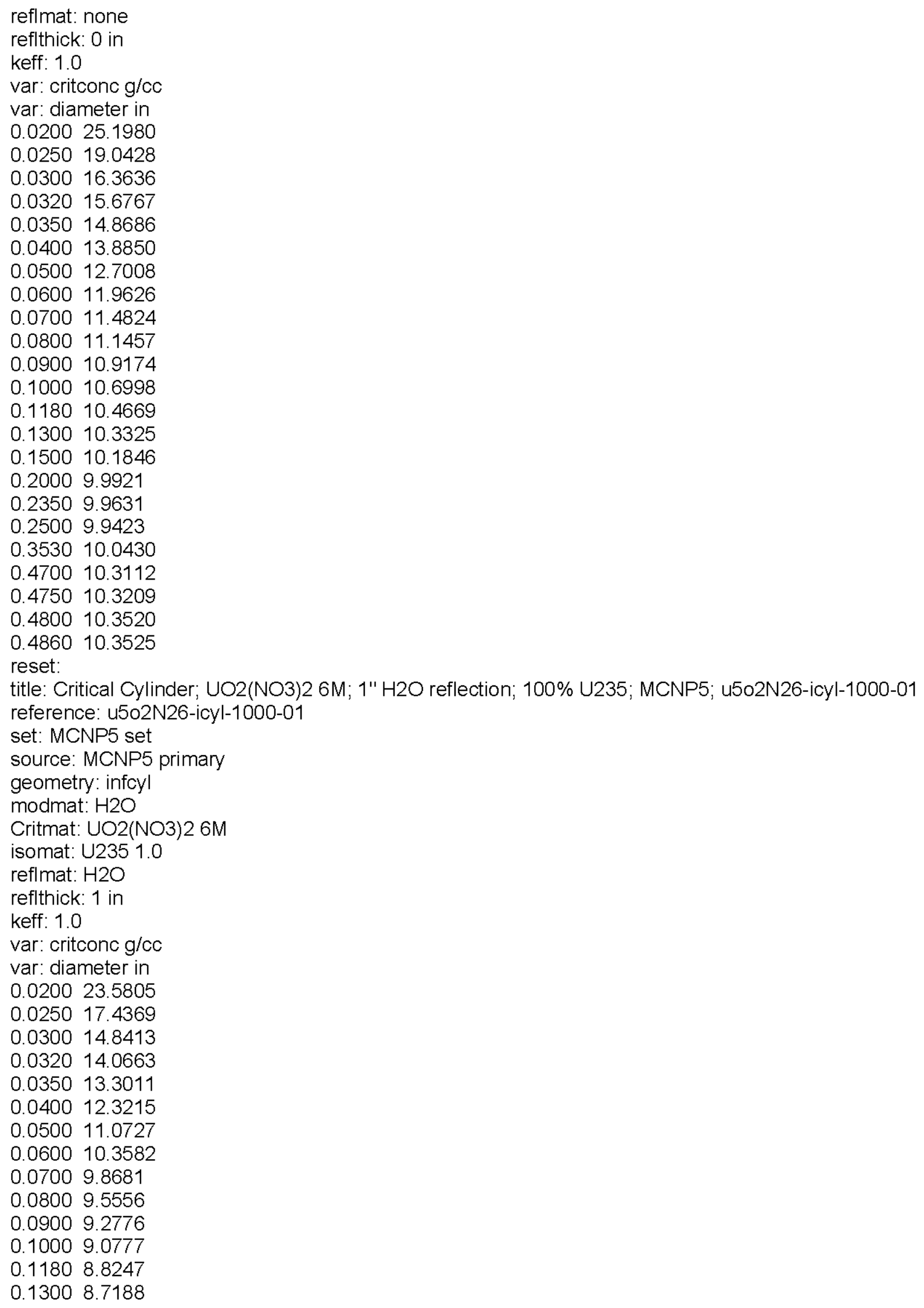




\section{CHPRC-01552, Revision 0}

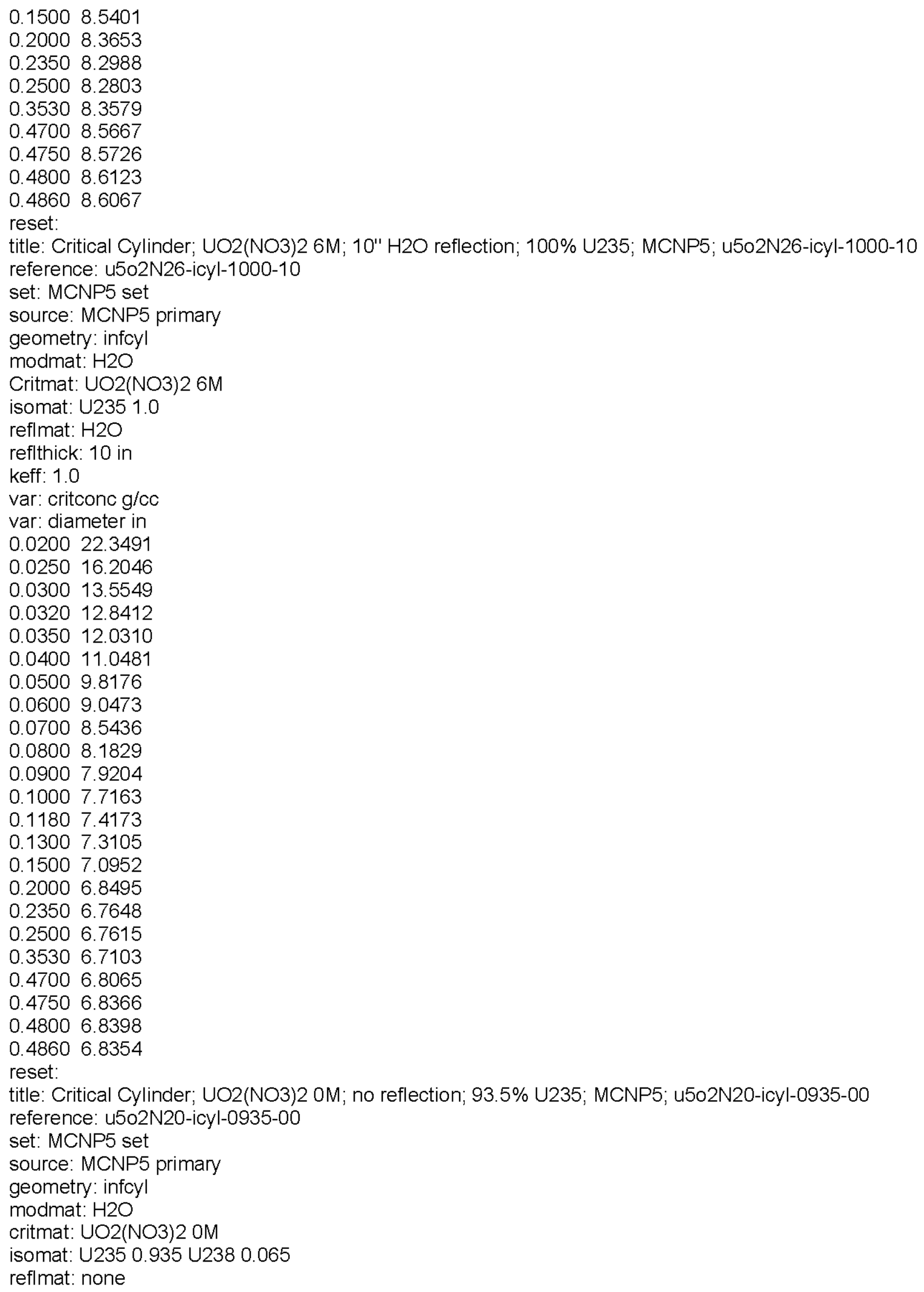


CHPRC-01552, Revision 0

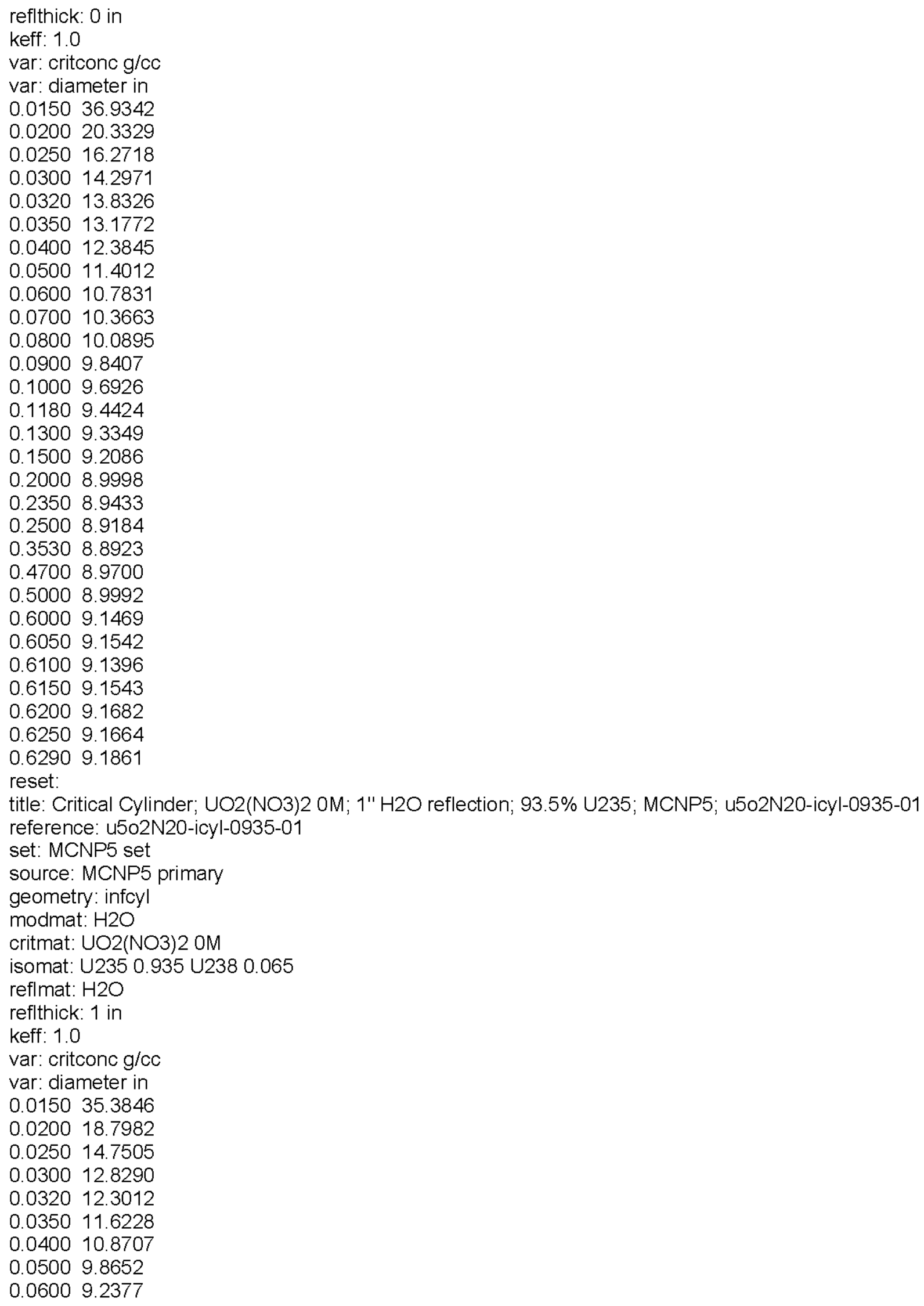




\section{CHPRC-01552, Revision 0}

\subsection{8 .8300 \\ 0.08008 .5537 \\ 0.09008 .3044 \\ 0.10008 .1393 \\ 0.11807 .9136 \\ 0.13007 .8007 \\ 0.15007 .6551 \\ 0.20007 .4578 \\ 0.23507 .3880 \\ 0.25007 .3757 \\ 0.35307 .3213 \\ 0.47007 .4001 \\ 0.50007 .4271 \\ 0.60007 .5430 \\ 0.60507 .5477 \\ 0.61007 .5691 \\ 0.61507 .5792 \\ 0.62007 .5795 \\ 0.62507 .5809 \\ 0.62907 .5846 \\ reset:}

title: Critical Cylinder; UO2(NO3)2 OM; 10" H2O reflection; 93.5\% U235; MCNP5; u5o2N20-icyl-0935-10 reference: u5o2N20-icyl-0935-10

set: MCNP5 set

source: MCNP5 primary

geometry: infcyl

modmat: $\mathrm{H} 2 \mathrm{O}$

critmat: $\mathrm{UO} 2(\mathrm{NO} 3) 2 \mathrm{OM}$

isomat: $\mathrm{U} 2350.935 \mathrm{U} 2380.065$

reflmat: $\mathrm{H} 2 \mathrm{O}$

reflthick: 10 in

keff: 1.0

var: critconc g/cc

var: diameter in

0.015034 .2350

0.020017 .6939

0.025013 .6407

0.030011 .6926

0.032011 .1437

0.035010 .5238

0.04009 .7078

0.05008 .6956

0.06008 .0734

0.07007 .6536

0.08007 .3340

0.09007 .1095

0.10006 .9278

0.11806 .6703

0.13006 .5370

0.15006 .3902

0.20006 .1411

0.23506 .0496

0.25006 .0198

0.35305 .9178

0.47005 .9202

0.50005 .9369 


\section{CHPRC-01552, Revision 0}

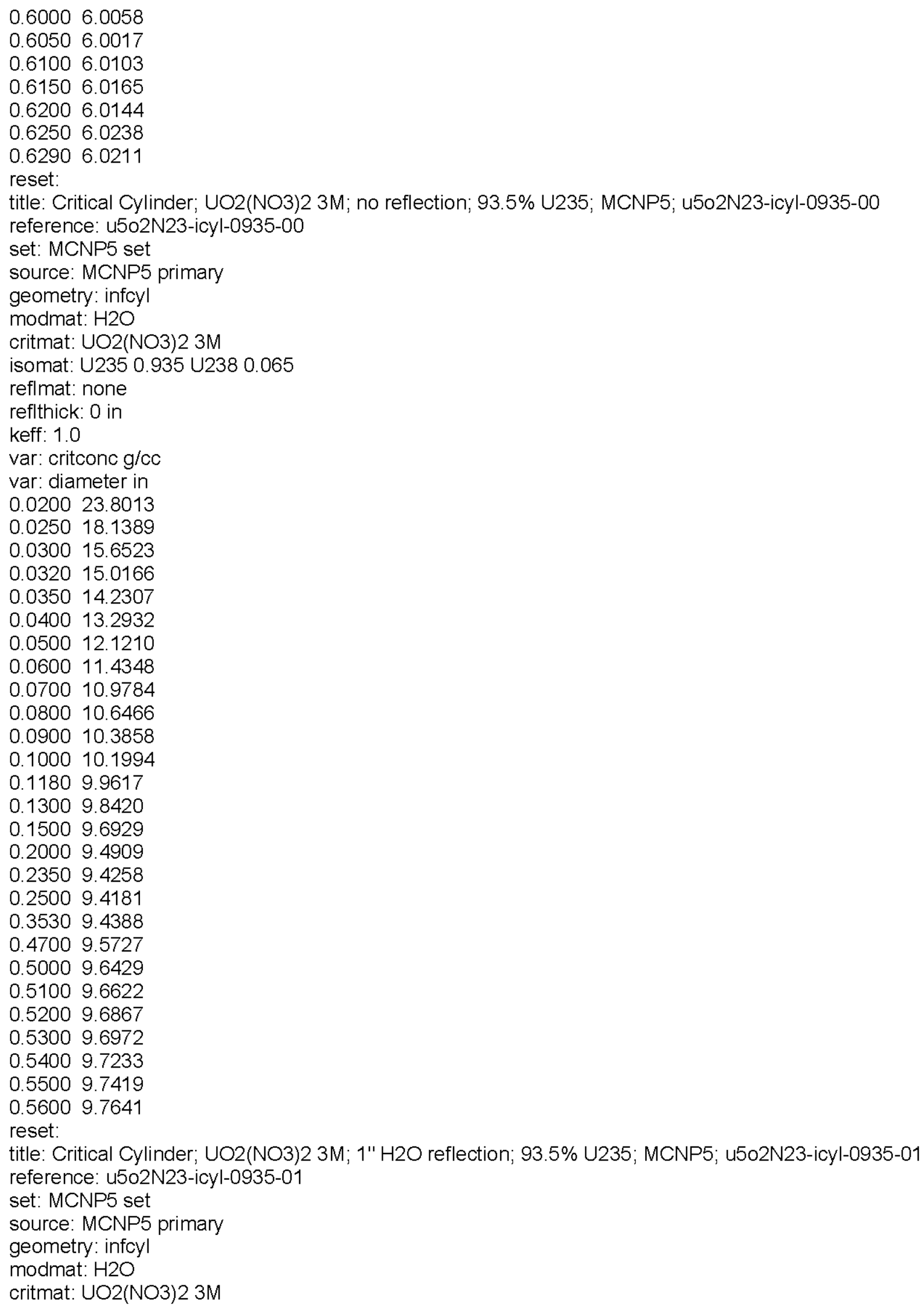




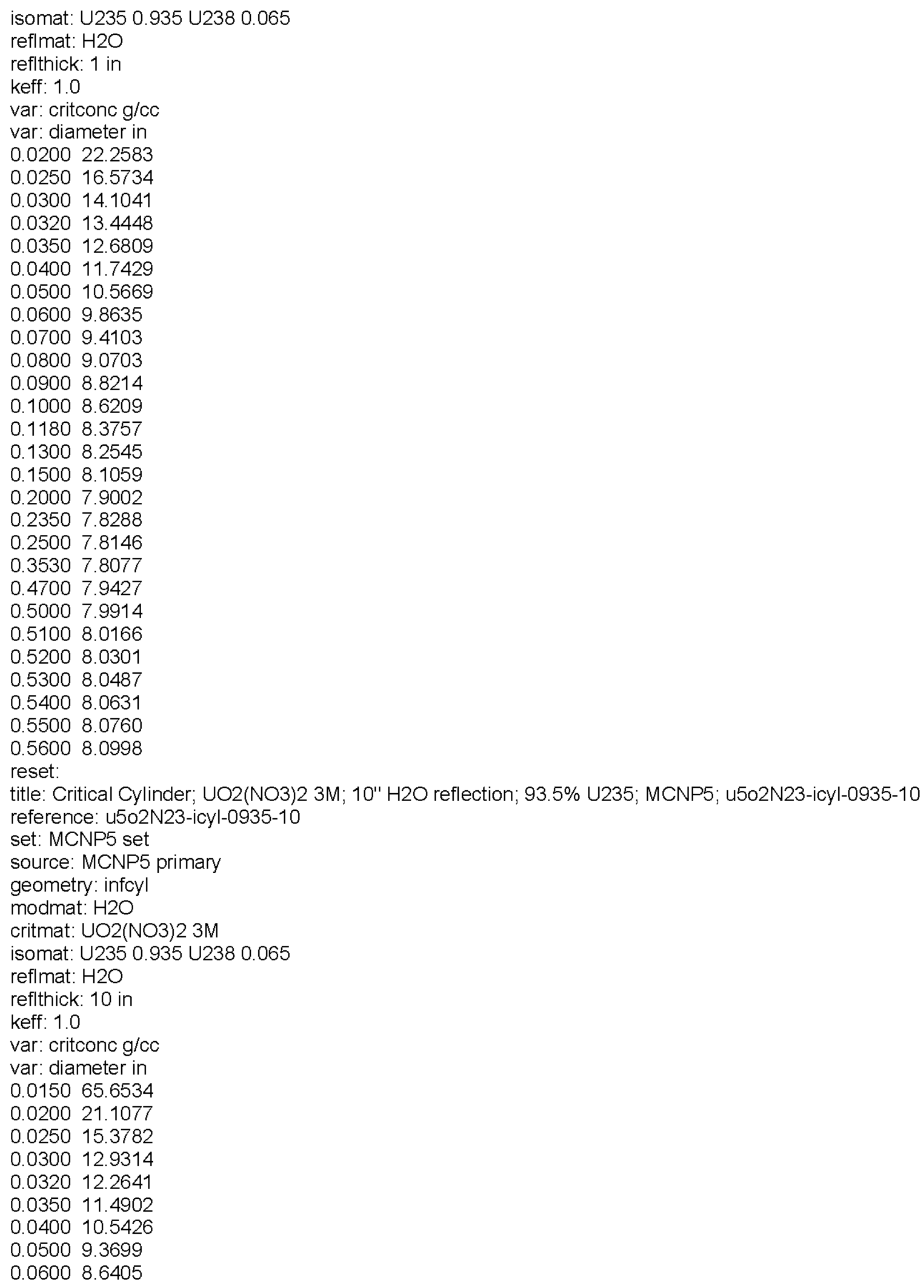




\section{CHPRC-01552, Revision 0}

\subsection{8 .1645 \\ 0.08007 .8129 \\ 0.09007 .5509 \\ 0.10007 .3434 \\ 0.11807 .0800 \\ 0.13006 .9407 \\ 0.15006 .7659 \\ 0.20006 .5204 \\ 0.23506 .4152 \\ 0.25006 .3878 \\ $0.3530 \quad 6.3021$ \\ 0.47006 .3398 \\ 0.50006 .3883 \\ 0.51006 .3903 \\ $0.5200 \quad 6.3992$ \\ 0.53006 .4147 \\ 0.54006 .4210 \\ $0.5500 \quad 6.4301$ \\ 0.56006 .4405 \\ reset:}

title: Critical Cylinder; UO2(NO3)2 6M; no reflection; 93.5\% U235; MCNP5; u5o2N26-icyl-0935-00

reference: u5o2N26-icyl-0935-00

set: MCNP5 set

source: MCNP5 primary

geometry: infcyl

modmat: $\mathrm{H} 2 \mathrm{O}$

critmat: UO2(NO3) $26 \mathrm{M}$

isomat: U235 $0.935 \mathrm{U} 2380.065$

reflmat: none

reflthick: 0 in

keff: 1.0

var: critconc g/cc

var: diameter in

0.020028 .6531

0.025020 .4587

$0.0300 \quad 17.2990$

0.032016 .4811

0.035015 .5703

0.040014 .3951

0.050013 .0453

$0.0600 \quad 12.2559$

0.070011 .7422

0.080011 .3681

0.090011 .0901

0.100010 .8855

0.118010 .6128

0.130010 .4833

0.150010 .3414

0.200010 .1356

0.235010 .0841

0.250010 .0857

0.353010 .1661

$0.4700 \quad 10.4595$

0.475010 .4834

0.480010 .5006

0.486010 .5272 


\section{CHPRC-01552, Revision 0}

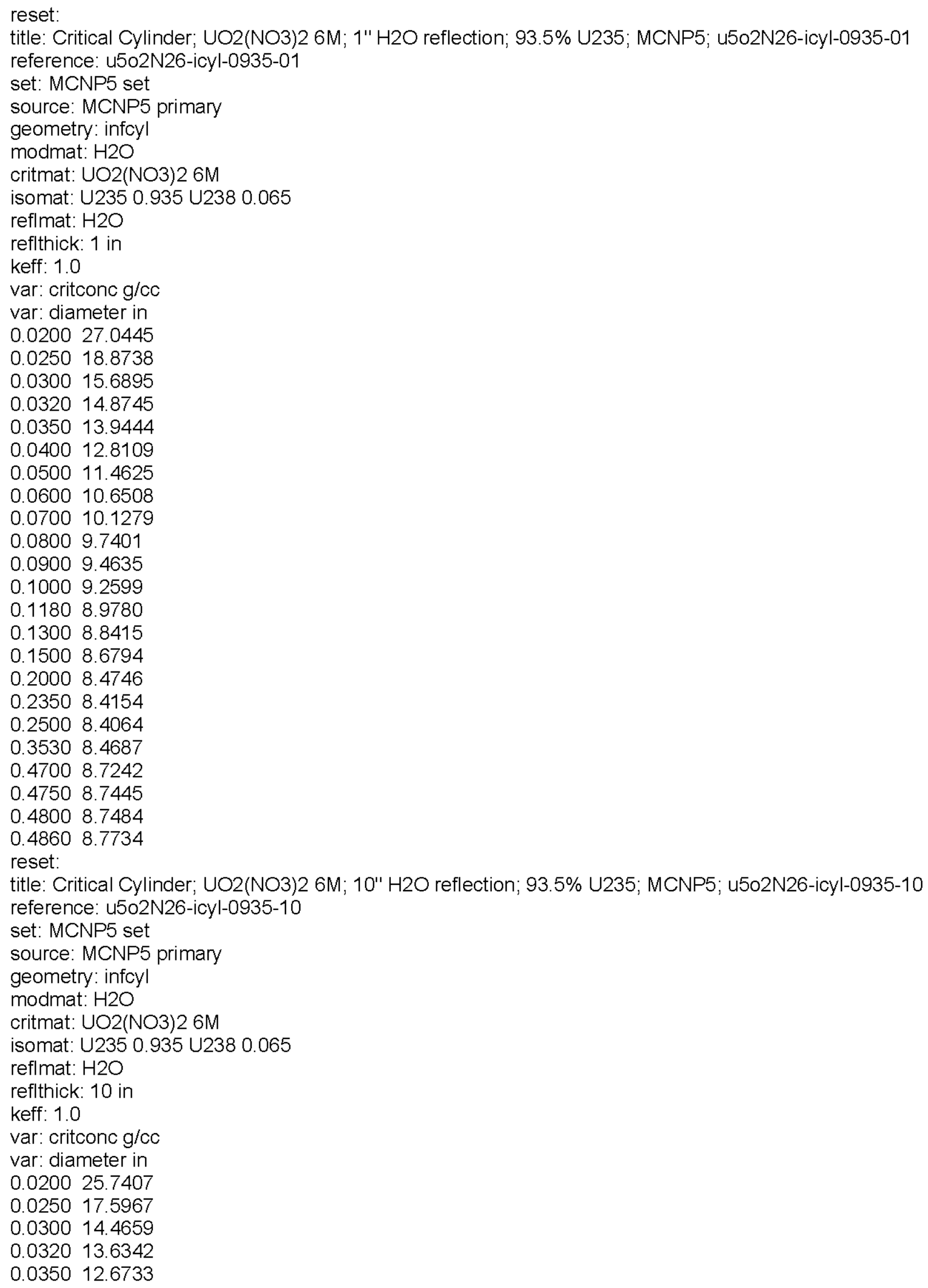




\section{CHPRC-01552, Revision 0}

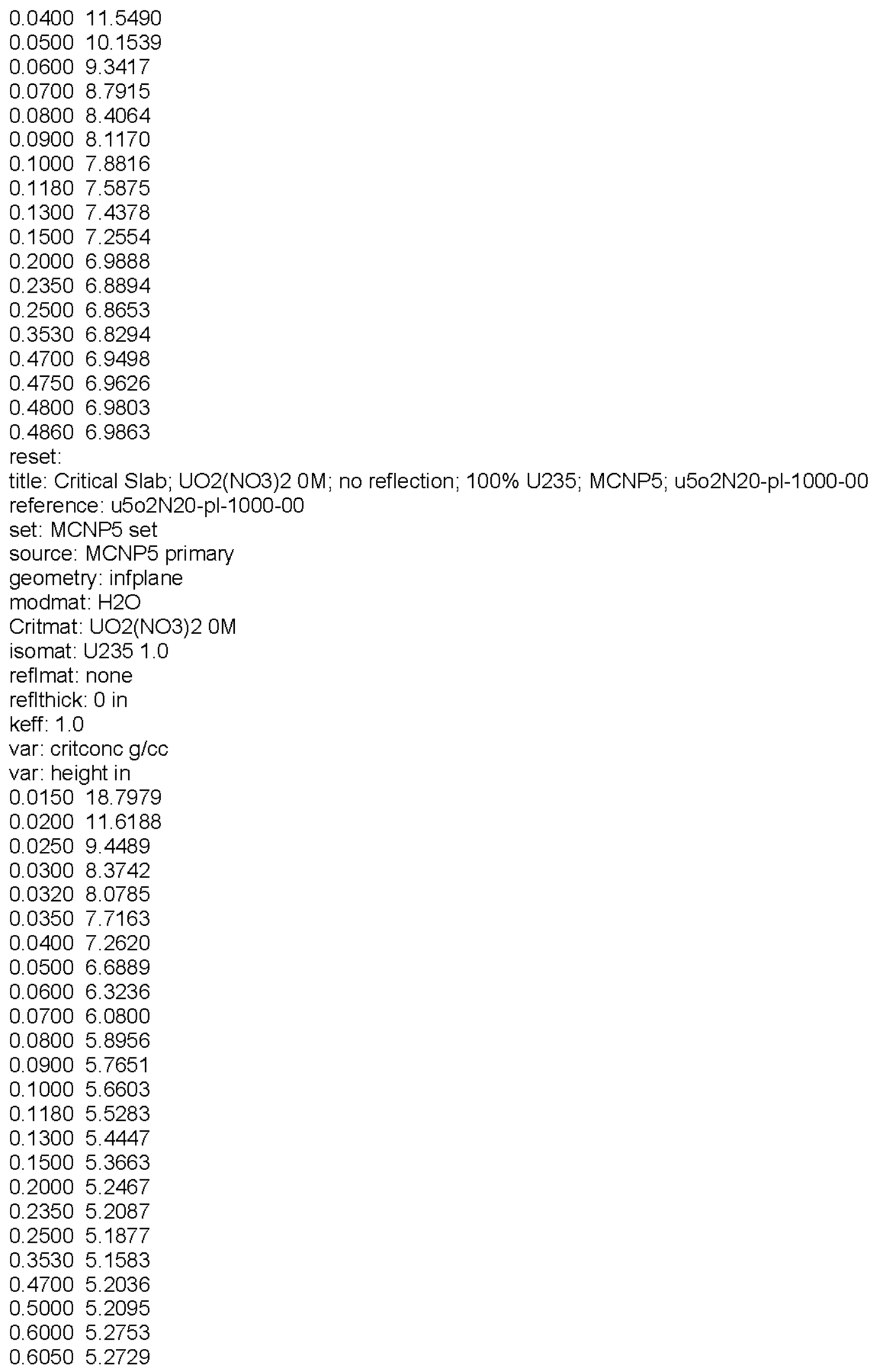




\section{CHPRC-01552, Revision 0}

0.61005 .2742

0.61505 .2903

0.62005 .2875

0.62505 .3071

0.62905 .3091

reset:

title: Critical Slab; UO2(NO3)2 OM; 1" H2O reflection; 100\% U235; MCNP5; u5o2N2O-pl-1000-01

reference: u5o2N20-pl-1000-01

set: MCNP5 set

source: MCNP5 primary

geometry: infplane

modmat: $\mathrm{H} 2 \mathrm{O}$

Critmat: UO2(NO3)2 OM

isomat: U235 1.0

reflmat: $\mathrm{H} 2 \mathrm{O}$

reflthick: 1 in

keff: 1.0

var: critconc $\mathrm{g} / \mathrm{cc}$

var: height in

$0.0150 \quad 17.2024$

0.020010 .0710

0.02507 .8998

0.03006 .8476

0.03206 .5438

0.03506 .1839

0.04005 .7283

0.05005 .1467

0.06004 .7775

0.07004 .5297

0.08004 .3488

$0.0900 \quad 4.2184$

0.15003 .8126

0.20003 .6837

0.23503 .6251

0.25003 .6139

0.35303 .5720

0.47003 .6058

0.50003 .6141

0.60003 .6579

0.60503 .6647

0.61003 .6656

0.61503 .6569

0.62003 .6668

0.62503 .6867

0.62903 .6831

reset:

title: Critical Slab; UO2(NO3)2 OM; 10 " H2O reflection; 100\% U235; MCNP5; u5o2N2O-pl-1000-10 reference: u5o2N20-pl-1000-10

set: MCNP5 set

source: MCNP5 primary

geometry: infplane

isomat: U235 1.0

modmat: $\mathrm{H} 2 \mathrm{O}$

Critmat: UO2(NO3)2 OM

reflmat: $\mathrm{H} 2 \mathrm{O}$

reflthick: 10 in 
CHPRC-01552, Revision 0

keff: 1.0

var: critconc g/cc

var: height in

0.015016 .0469

0.02008 .9471

0.02506 .8052

0.03005 .7151

0.03205 .4136

0.03505 .0405

0.04004 .5852

0.05003 .9763

0.06003 .6003

0.07003 .3383

0.08003 .1373

0.09002 .9852

0.10002 .8682

0.11802 .7081

0.13002 .6279

0.15002 .5154

$0.2000 \quad 2.3447$

$0.2350 \quad 2.2615$

$0.2500 \quad 2.2399$

0.35302 .1302

0.47002 .0839

0.50002 .0812

0.60002 .0825

0.60502 .0878

0.61002 .0766

0.61502 .0729

0.62002 .0693

0.62502 .0857

0.62902 .0811

reset:

title: Critical Slab; UO2(NO3)2 3M; no reflection; 100\% U235; MCNP5; u5o2N23-pl-1000-00

reference: $u 502 \mathrm{~N} 23-\mathrm{pl}-1000-00$

set: MCNP5 set

source: MCNP5 primary

geometry: infplane

modmat: $\mathrm{H} 2 \mathrm{O}$

Critmat: UO2(NO3)2 3M

isomat: U235 1.0

reflmat: none

reflthick: 0 in

keff: 1.0

var: critconc g/cc

var: height in

0.015026 .9690

0.020013 .4134

0.025010 .4911

0.03009 .1544

0.03208 .7747

0.03508 .3651

0.04007 .8103

0.05007 .1352

0.06006 .7138

0.07006 .4420 


\section{CHPRC-01552, Revision 0}

$0.0800 \quad 6.2582$

0.09006 .0991

0.10005 .9794

0.11805 .8321

0.13005 .7633

0.15005 .6807

0.20005 .5438

0.23505 .5082

0.25005 .4896

0.35305 .4868

0.47005 .5691

0.50005 .6031

0.51005 .5905

0.52005 .6176

0.53005 .6273

0.54005 .6393

0.55005 .6617

0.56005 .6751

reset:

title: Critical Slab; UO2(NO3)2 3M; 1" H2O reflection; 100\% U235; MCNP5; u5o2N23-pl-1000-01

reference: u5o2N23-pl-1000-01

set: MCNP5 set

source: MCNP5 primary

geometry: infplane

modmat: $\mathrm{H} 2 \mathrm{O}$

Critmat: UO2(NO3)2 $3 \mathrm{M}$

isomat: $\mathrm{U} 2351.0$

reflmat: $\mathrm{H} 2 \mathrm{O}$

reflthick: 1 in

keff: 1.0

var: critconc $\mathrm{g} / \mathrm{cc}$

var: height in

0.015025 .0671

0.020011 .7976

0.02508 .9077

0.03007 .5718

0.03207 .1945

0.03506 .7635

0.04006 .2289

0.05005 .5532

0.06005 .1484

0.07004 .8725

0.08004 .6702

0.09004 .5062

0.10004 .3983

$0.1180 \quad 4.2517$

0.13004 .1662

reset:

title: Critical Slab; UO2(NO3)2 3M; 10" H2O reflection; 100\% U235; MCNP5; u5o2N23-pl-1000-10 reference: u5o2N23-pl-1000-10

set: MCNP5 set

source: MCNP5 primary

geometry: infplane

modmat: $\mathrm{H} 2 \mathrm{O}$

Critmat: UO2(NO3)2 3M

isomat: U235 1.0 


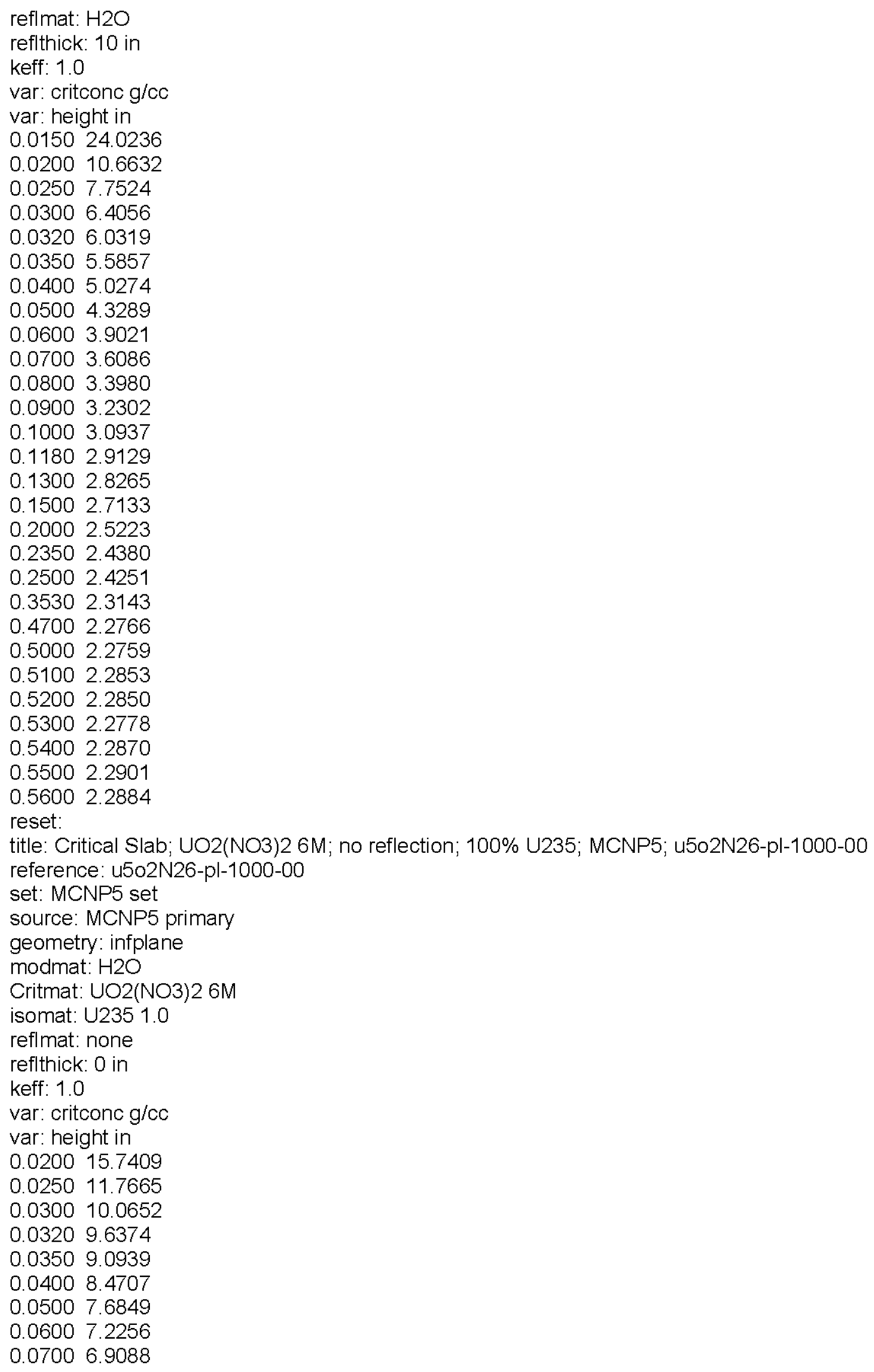




\section{CHPRC-01552, Revision 0}

0.08006 .7009

0.09006 .5350

0.10006 .4094

$0.1180 \quad 6.2484$

0.13006 .1646

0.15006 .0589

0.20005 .9434

0.23505 .9068

0.25005 .8925

0.35305 .9451

0.47006 .1101

0.47506 .1112

0.48006 .1159

0.48606 .1105

reset:

title: Critical Slab; UO2(NO3)2 6M; 1" H2O reflection; 100\% U235; MCNP5; u5o2N26-pl-1000-01 reference: u5o2N26-pl-1000-01

set: MCNP5 set

source: MCNP5 primary

geometry: infplane

modmat: $\mathrm{H} 2 \mathrm{O}$

Critmat: UO2(NO3)2 $6 \mathrm{M}$

isomat: U235 1.0

reflmat: $\mathrm{H} 2 \mathrm{O}$

reflthick: 1 in

keff: 1.0

var: critconc g/cc

var: height in

0.020014 .0800

0.025010 .1575

0.03008 .4583

0.03208 .0154

0.03507 .5094

0.04006 .8373

0.05006 .0758

0.06005 .5914

0.07005 .2733

0.08005 .0641

0.09004 .8882

0.10004 .7578

0.11804 .6024

0.13004 .5186

0.15004 .3930

0.20004 .2691

0.23504 .2160

0.25004 .2044

$0.3530 \quad 4.2055$

0.47004 .3502

0.47504 .3536

$0.4800 \quad 4.3485$

0.48604 .3659

reset:

title: Critical Slab; UO2(NO3)2 6M; 10" H2O reflection; 100\% U235; MCNP5; u5o2N26-pl-1000-10 reference: $\mathrm{u} 5 \mathrm{o} 2 \mathrm{~N} 26-\mathrm{pl}-1000-10$

set: MCNP5 set

source: MCNP5 primary 
CHPRC-01552, Revision 0

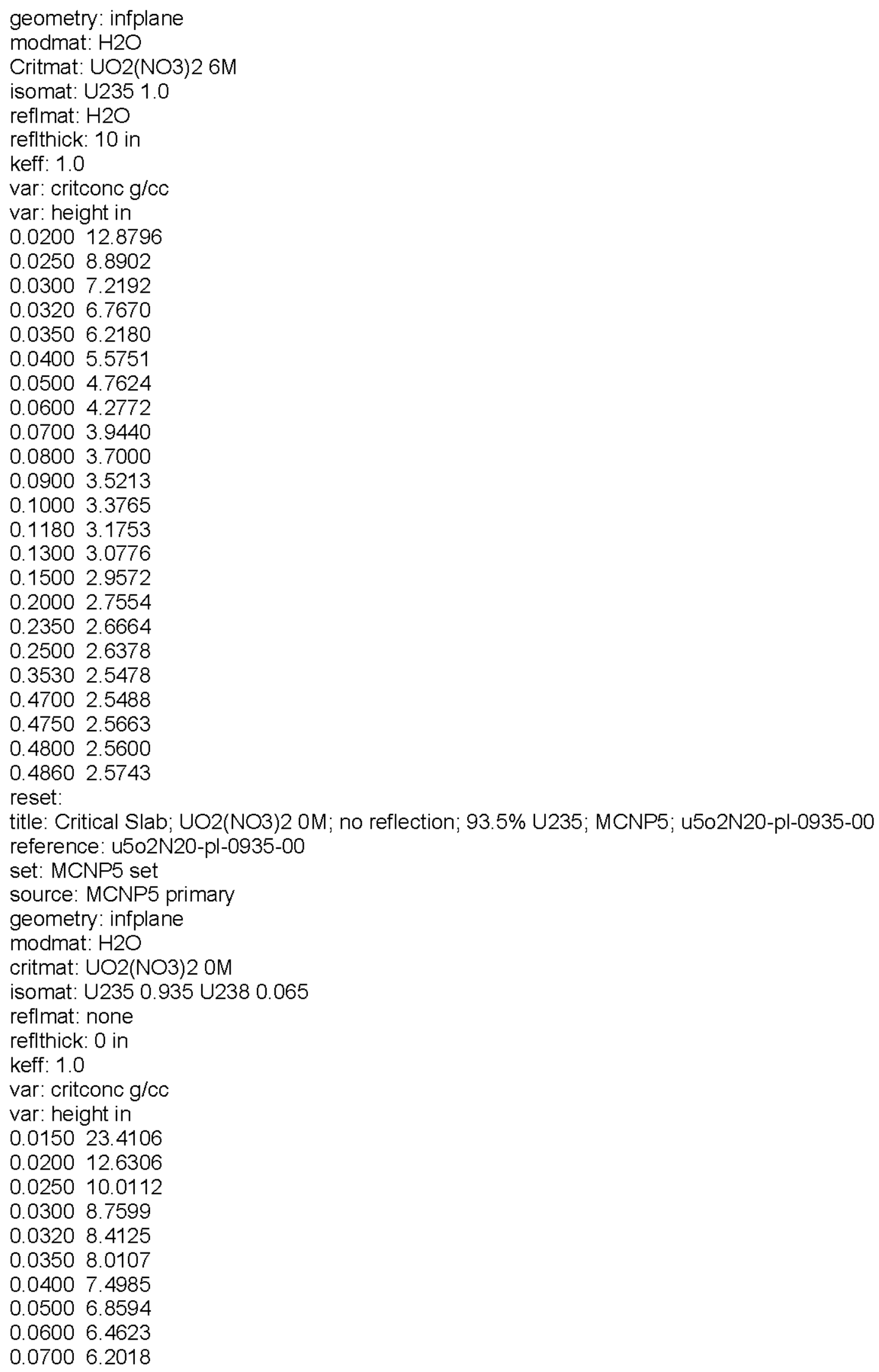


CHPRC-01552, Revision 0

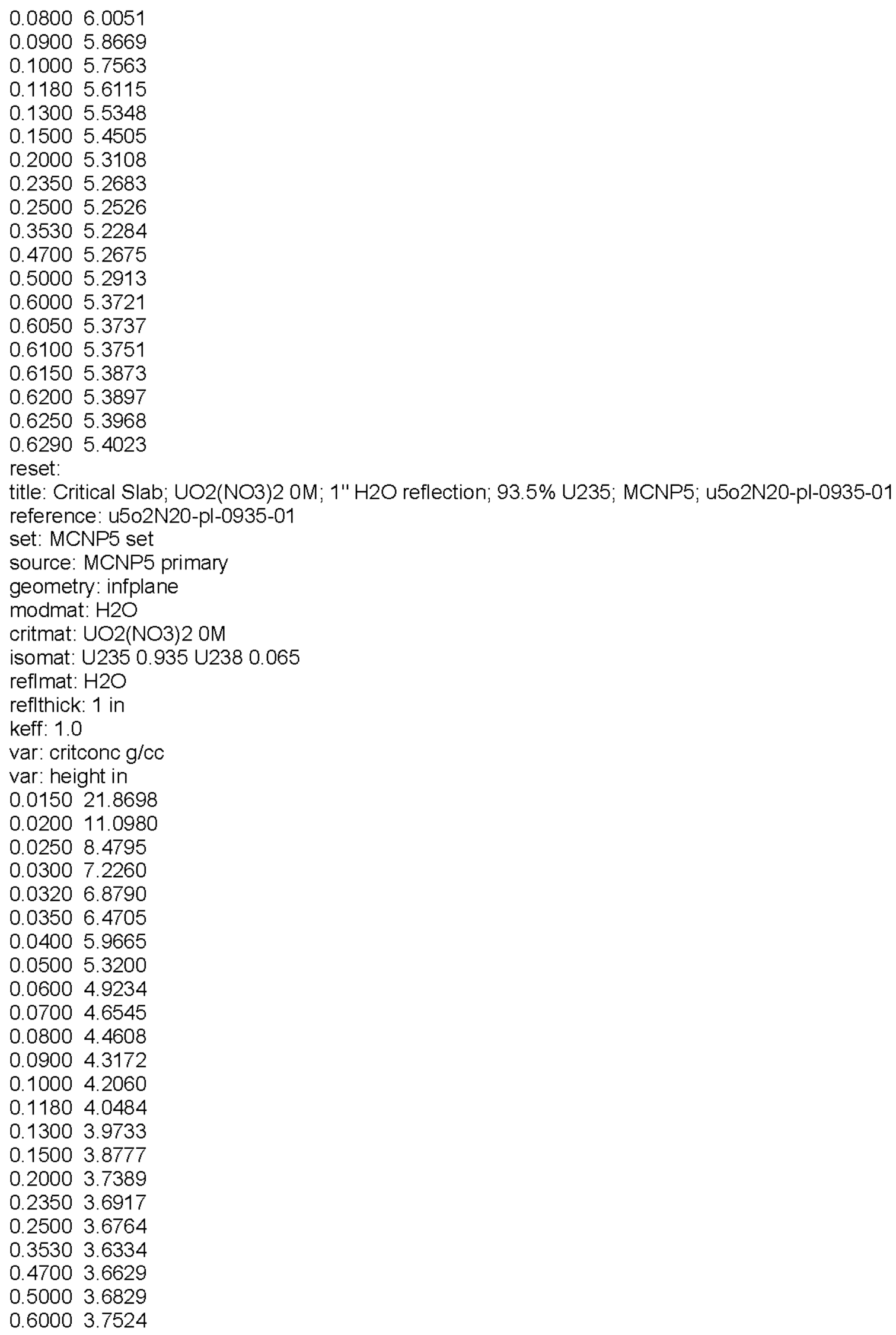




\section{CHPRC-01552, Revision 0}

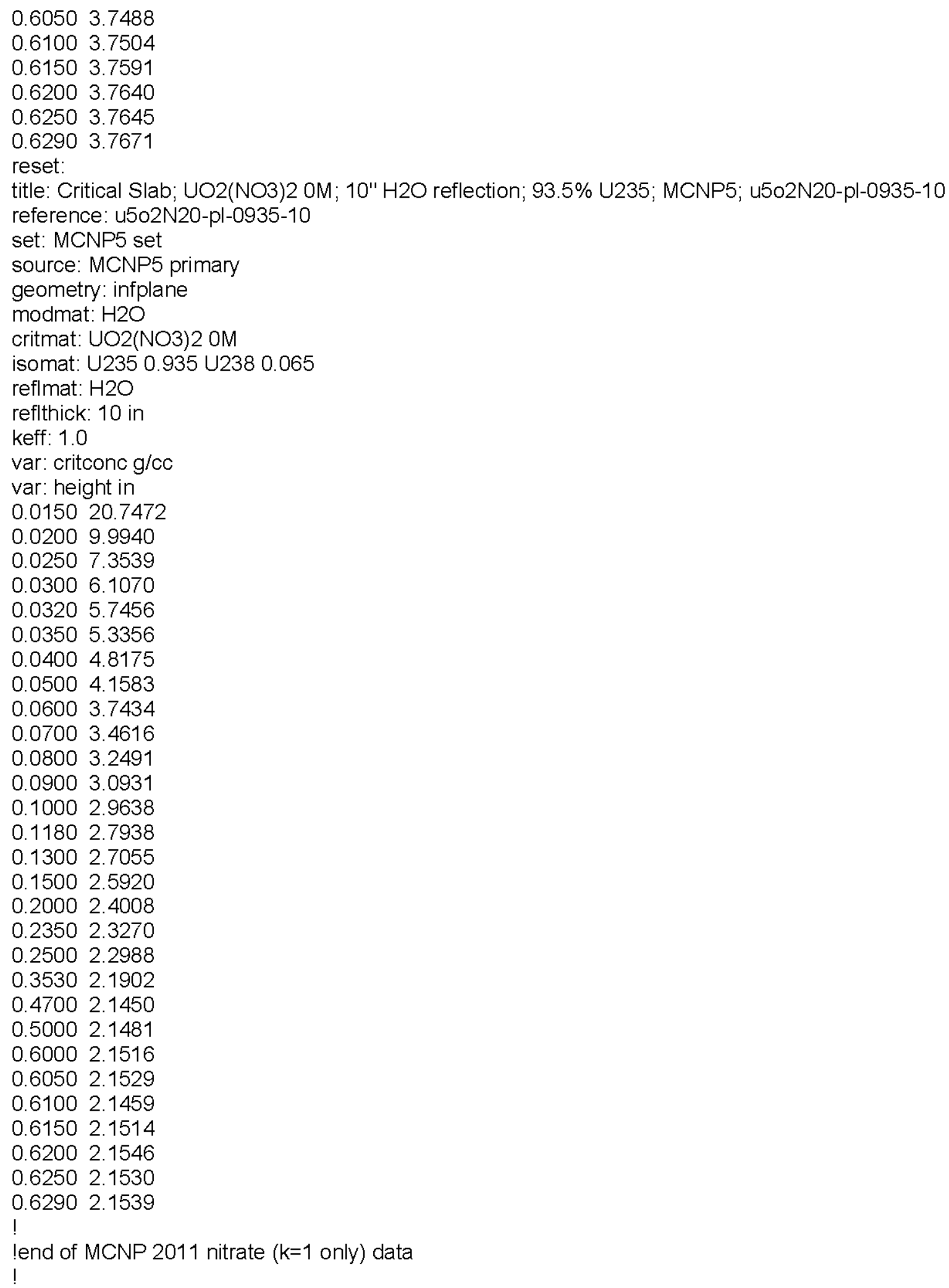


CHPRC-01552, Revision 0

This page is intentionally left blank. 
CHPRC-01552, Revision 0

\section{APPENDIX D - TEMPLATE FILES FOR CASES}


CHPRC-01552, Revision 0

This page is intentionally left blank.

D-2 


\section{Template File for Case: pun40-sp-1000-00}

sp, 100.0 wt\% Pu239, ZZZ1 milli-g/cc, in Pu(NO3)4, with 0 Molar HNO3

$\mathrm{c}$

c worm notes: format of the form |n.m for floating point, $\mid \mathrm{n}$ for integers

c $\quad$ zzz0 (in upper case) is replaced with the number of cyles

c $\quad$ zzz1 $=$ the actual concentration in units of milli-g/cc

c $\quad$ zzz2 $=$ solution density in $\mathrm{g} / \mathrm{cc}$

c $\quad \mathrm{qqq} 1=$ the minimum dimensions (in hundreths of inches)

c $\quad \mathrm{qqq} 2=$ the maximum dimensions (in hundreths of inches)

c $\quad \mathrm{qqq} 3=$ the delta (in hundreths of inches)

c

c Diameter $=<\mathrm{d} 1=$ QQQ1:QQQ2:QQQ3 $\mid 5>$ hundreths of inches - must be integer

$\mathrm{c}$ radius $=<\mathrm{rd}=\mathrm{d} 1 * 2.54 / 100 / 2 \mid 5.4>\mathrm{cm}$

c note for perl, numbers with leading zeros must be enclosed in single quotes or else

c they will be interpretted as octal

$\mathrm{c}$ Conc $=<\mathrm{c}={ }^{\prime} \mathrm{ZZZ1} 1 \mid 5>$ milli-g Pu/cc

c Reflector width $=<\mathrm{rw}=0.0 \mid 2.1>$ inches

c Enrichment $=<$ ena $=1.0 \mid 1.2>$ atom fraction Pu239

c Mols of Acid per liter $<$ molAcid $=0.0 \mid 1.2>$

$\mathrm{c}$

$\mathrm{c}$

c Density of solution $<$ denSol $=Z Z Z 2 / 1 \mathrm{e} 5 \mid 1.5>$

c

c

c Atomic Weights

c $\mathrm{H} 2 \mathrm{O}=\langle$ atwH2O $=18.0152| 3.4>$

$\mathrm{c} \mathrm{NO3}=\langle$ atwNO3 $=62.0049| 3.4>$

c HNO3 $=<$ atwHNO3 $=63.0128 \mid 3.4>$

c Pu239 $=<$ atwPu239 $=239.0522 \mid 3.4>$

c Pu240 $=<$ atwPu240 $=240.0538 \mid 3.4>$

$\mathrm{c} \mathrm{Pu}=<\mathrm{atwPu}=\mathrm{ena}^{*}$ atwPu239+(1-ena)*atwPu240|3.4>

$\mathrm{c}$

c Molarity of solution components

c Mols of $\mathrm{Pu}$ per liter $<\mathrm{molPu}=\mathrm{c} / \mathrm{atwPu} \mid 3.4>$

c Mols of $\mathrm{H} 2 \mathrm{O}$ per liter $<$ molH2O $=(1000 *$ denSol-molPu* $(a t w P u+4 * a t w N O 3)$ molAcid*atwHNO3)/atwH2O $3.4>$

$\mathrm{c}$

$\mathrm{c}$

$<$ perl $>$

sub IMP

\{

return 1 if $(\$[0]>0)$;

return 0 ;

\}

$</$ perl $>$

c sphere

$2011<$-denSol|3.6> $-1 \quad$ imp:n=1

$212-1.000-21 \quad$ imp:n=<\&IMP(rw) $\mid 1>$ \$zero importance if no reflector

$220 \quad 2$ imp:n=0 


$$
\begin{array}{lll}
1 & \text { so }<\text { rd } \mid 4.4> & \text { \$ Sphere } \\
2 & \text { so }<\text { rd }+ \text { rw*in|4.4> } & \text { \$Reflection }
\end{array}
$$

mode $\mathrm{n}$

kcode 20001.0100 ZZZ0

$\begin{array}{llll}\text { ksrc } & 0.00 & 0.00 & 0.00\end{array}$

$\begin{array}{llll}0.00 & 0.00 & 0.10\end{array}$

$\begin{array}{lll}0.00 & 0.00 & 0.50\end{array}$

$\begin{array}{lll}0.00 & 0.00 & 1.00\end{array}$

$\begin{array}{lll}0.00 & 0.00 & 2.00\end{array}$

$\begin{array}{lll}0.00 & 0.00 & 3.00\end{array}$

$\begin{array}{lll}1.00 & 1.00 & 1.00\end{array}$

$\begin{array}{lll}1.00 & 1.00 & 2.00\end{array}$

$\begin{array}{lll}1.00 & 1.00 & 3.00\end{array}$

$\begin{array}{lll}2.00 & 2.00 & 1.00\end{array}$

$\begin{array}{lll}2.00 & 2.00 & 2.00\end{array}$

$\begin{array}{lll}2.00 & 2.00 & 3.00\end{array}$

$\begin{array}{lll}-1.00 & -1.00 & -1.00\end{array}$

$-10.00 \quad-10.00 \quad-20.00$

$\begin{array}{llll}-10.00 & -10.00 & -30.00\end{array}$

c

m2 \$water

$1001.66 \mathrm{c} \quad 2$

$8016.66 \mathrm{c} \quad 1$

$\mathrm{mt} 2$ 1wtr.60t

c $\mathrm{pu} /$ water mixture

m11 \$ Pu(NO3)4 - NO3 - H2O, 100.0 wt\% Pu239, ZZZ1 milli-g Pu/cc, $<$ denSol|3.4>

c molarity $\mathrm{pu}=\langle\operatorname{molPu}| 3.4>$, acid $=\langle\operatorname{mol} A \mathrm{cid}| 3.4>, \mathrm{H} 2 \mathrm{O}=\langle\operatorname{molH} 2 \mathrm{O}| 3.4>$

c Pu in salt

$94239.66 \mathrm{c}<\mathrm{molPu} * \mathrm{ena} \mid 3.4>$

c $94240.66 \mathrm{c}<\mathrm{molPu} *(1$-ena) $\mid 3.4>$

c NO3 in salt plus acid

$7014.66 \mathrm{c}<4 * \mathrm{molPu}+$ molAcid $\mid 3.4>$

$8016.66 \mathrm{c}<12 * \mathrm{molPu}+3 * \operatorname{mol} \mathrm{Acid} \mid 3.4>$

c water

$1001.66 \mathrm{c}<2 * \mathrm{molH} 2 \mathrm{O}+\operatorname{molAcid} \mid 3.4>$ \$Includes Free Hydrogen Ion

$8016.66 \mathrm{c}<\mathrm{molH} 2 \mathrm{O} \mid 3.4>$

mt11 1wtr.60t

c

c 


\section{Template File for Case: pun40-sp-1000-01}

sp, 100.0 wt\% Pu239, ZZZ1 milli-g/cc, in Pu(NO3)4, with 0 Molar HNO3

$\mathrm{c}$

c worm notes: format of the form |n.m for floating point, $\mid \mathrm{n}$ for integers

c $\quad$ zzz0 (in upper case) is replaced with the number of cyles

c $\quad$ zzz1 $=$ the actual concentration in units of milli-g/cc

c $\quad$ zzz2 $=$ solution density in $\mathrm{g} / \mathrm{cc}$

c $\quad \mathrm{qqq} 1=$ the minimum dimensions (in hundreths of inches)

c $\quad \mathrm{qqq} 2=$ the maximum dimensions (in hundreths of inches)

c $\quad \mathrm{qqq} 3=$ the delta (in hundreths of inches)

c

c Diameter $=<\mathrm{d} 1=$ QQQ1:QQQ2:QQQ3 $\mid 5>$ hundreths of inches - must be integer

$\mathrm{c}$ radius $=<\mathrm{rd}=\mathrm{d} 1 * 2.54 / 100 / 2 \mid 5.4>\mathrm{cm}$

c note for perl, numbers with leading zeros must be enclosed in single quotes or else

c they will be interpretted as octal

$\mathrm{c}$ Conc $=<\mathrm{c}={ }^{\prime} \mathrm{ZZZ1} 1 \mid 5>$ milli-g Pu/cc

c Reflector width $=<\mathrm{rw}=1.0 \mid 2.1>$ inches

c Enrichment $=<$ ena $=1.0 \mid 1.2>$ atom fraction Pu239

c Mols of Acid per liter $<$ molAcid $=0.0 \mid 1.2>$

$\mathrm{c}$

$\mathrm{c}$

c Density of solution $<$ denSol $=Z Z Z 2 / 1 \mathrm{e} 5 \mid 1.5>$

c

c

c Atomic Weights

c $\mathrm{H} 2 \mathrm{O}=\langle$ atwH2O $=18.0152| 3.4>$

$\mathrm{c} \mathrm{NO3}=\langle$ atwNO3 $=62.0049| 3.4>$

c HNO3 $=<$ atwHNO3 $=63.0128 \mid 3.4>$

c Pu239 $=<$ atwPu239 $=239.0522 \mid 3.4>$

c Pu240 $=<$ atwPu240 $=240.0538 \mid 3.4>$

$\mathrm{c} \mathrm{Pu}=<\mathrm{atwPu}=\mathrm{ena}^{*}$ atwPu239+(1-ena)*atwPu240|3.4>

$\mathrm{c}$

c Molarity of solution components

c Mols of $\mathrm{Pu}$ per liter $<\mathrm{molPu}=\mathrm{c} / \mathrm{atwPu} \mid 3.4>$

c Mols of $\mathrm{H} 2 \mathrm{O}$ per liter $<$ molH2O $=(1000 *$ denSol-molPu* $(a t w P u+4 * a t w N O 3)$ molAcid*atwHNO3)/atwH2O $3.4>$

$\mathrm{c}$

$\mathrm{c}$

$<$ perl $>$

sub IMP

\{

return 1 if $(\$[0]>0)$;

return 0 ;

\}

$</$ perl $>$

c sphere

$2011<$-denSol|3.6> $-1 \quad$ imp:n=1

$212-1.000-21 \quad$ imp:n=<\&IMP(rw) $\mid 1>$ \$zero importance if no reflector

$220 \quad 2$ imp:n=0 


$$
\begin{array}{lll}
1 & \text { so }<\text { rd } \mid 4.4> & \text { \$ Sphere } \\
2 & \text { so }<\text { rd }+ \text { rw*in|4.4> } & \text { \$Reflection }
\end{array}
$$

mode $\mathrm{n}$

kcode 20001.0100 ZZZ0

$\begin{array}{llll}\text { ksrc } & 0.00 & 0.00 & 0.00\end{array}$

$\begin{array}{llll}0.00 & 0.00 & 0.10\end{array}$

$\begin{array}{lll}0.00 & 0.00 & 0.50\end{array}$

$\begin{array}{lll}0.00 & 0.00 & 1.00\end{array}$

$\begin{array}{lll}0.00 & 0.00 & 2.00\end{array}$

$\begin{array}{lll}0.00 & 0.00 & 3.00\end{array}$

$\begin{array}{lll}1.00 & 1.00 & 1.00\end{array}$

$\begin{array}{lll}1.00 & 1.00 & 2.00\end{array}$

$\begin{array}{lll}1.00 & 1.00 & 3.00\end{array}$

$\begin{array}{lll}2.00 & 2.00 & 1.00\end{array}$

$\begin{array}{lll}2.00 & 2.00 & 2.00\end{array}$

$\begin{array}{lll}2.00 & 2.00 & 3.00\end{array}$

$\begin{array}{lll}-1.00 & -1.00 & -1.00\end{array}$

$-10.00 \quad-10.00 \quad-20.00$

$\begin{array}{llll}-10.00 & -10.00 & -30.00\end{array}$

c

m2 \$water

$1001.66 \mathrm{c} \quad 2$

$8016.66 \mathrm{c} \quad 1$

$\mathrm{mt} 2$ 1wtr.60t

c $\mathrm{pu} /$ water mixture

m11 \$ Pu(NO3)4 - NO3 - H2O, 100.0 wt\% Pu239, ZZZ1 milli-g Pu/cc, $<$ denSol|3.4>

c molarity $\mathrm{pu}=<\operatorname{molPu} \mid 3.4>$, acid $=<\operatorname{molAcid} \mid 3.4>, \mathrm{H} 2 \mathrm{O}=\langle\operatorname{molH} 2 \mathrm{O}| 3.4>$

c Pu in salt

$94239.66 \mathrm{c}<\mathrm{molPu} * \mathrm{ena} \mid 3.4>$

c $94240.66 \mathrm{c}<\mathrm{molPu} *(1$-ena) $\mid 3.4>$

c NO3 in salt plus acid

$7014.66 \mathrm{c}<4 * \mathrm{molPu}+$ molAcid $\mid 3.4>$

$8016.66 \mathrm{c}<12 * \mathrm{molPu}+3 * \operatorname{mol} \mathrm{Acid} \mid 3.4>$

c water

$1001.66 \mathrm{c}<2 * \mathrm{molH} 2 \mathrm{O}+\operatorname{molAcid} \mid 3.4>$ \$Includes Free Hydrogen Ion

$8016.66 \mathrm{c}<\mathrm{molH} 2 \mathrm{O} \mid 3.4>$

mt11 1wtr.60t

c

c 


\section{Template File for Case: pun40-sp-1000-10}

sp, 100.0 wt\% Pu239, ZZZ1 milli-g/cc, in Pu(NO3)4, with 0 Molar HNO3

$\mathrm{c}$

c worm notes: format of the form |n.m for floating point, $\mid \mathrm{n}$ for integers

c $\quad$ zzz0 (in upper case) is replaced with the number of cyles

c $\quad$ zzz1 $=$ the actual concentration in units of milli-g/cc

c $\quad$ zzz2 $=$ solution density in $\mathrm{g} / \mathrm{cc}$

c $\quad \mathrm{qqq} 1=$ the minimum dimensions (in hundreths of inches)

c $\quad \mathrm{qqq} 2=$ the maximum dimensions (in hundreths of inches)

c $\quad \mathrm{qqq} 3=$ the delta (in hundreths of inches)

c

c Diameter $=<\mathrm{d} 1=$ QQQ1:QQQ2:QQQ3 $\mid 5>$ hundreths of inches - must be integer

$\mathrm{c}$ radius $=<\mathrm{rd}=\mathrm{d} 1 * 2.54 / 100 / 2 \mid 5.4>\mathrm{cm}$

c note for perl, numbers with leading zeros must be enclosed in single quotes or else

c they will be interpretted as octal

$\mathrm{c}$ Conc $=<\mathrm{c}={ }^{\prime} \mathrm{ZZZ1} 1 \mid 5>$ milli-g Pu/cc

c Reflector width $=<\mathrm{rw}=10.0 \mid 2.1>$ inches

c Enrichment $=<$ ena $=1.0 \mid 1.2>$ atom fraction Pu239

c Mols of Acid per liter $<$ molAcid $=0.0 \mid 1.2>$

$\mathrm{c}$

$\mathrm{c}$

c Density of solution $<$ denSol $=Z Z Z 2 / 1 \mathrm{e} 5 \mid 1.5>$

c

c

c Atomic Weights

c $\mathrm{H} 2 \mathrm{O}=\langle$ atwH2O $=18.0152| 3.4>$

$\mathrm{c} \mathrm{NO3}=\langle$ atwNO3 $=62.0049| 3.4>$

c HNO3 $=<$ atwHNO3 $=63.0128 \mid 3.4>$

c Pu239 $=<$ atwPu239 $=239.0522 \mid 3.4>$

c Pu240 $=<$ atwPu240 $=240.0538 \mid 3.4>$

$\mathrm{c} \mathrm{Pu}=<\mathrm{atwPu}=\mathrm{ena}^{*}$ atwPu239+(1-ena)*atwPu240|3.4>

$\mathrm{c}$

c Molarity of solution components

c Mols of $\mathrm{Pu}$ per liter $<\mathrm{molPu}=\mathrm{c} / \mathrm{atwPu} \mid 3.4>$

c Mols of $\mathrm{H} 2 \mathrm{O}$ per liter $<$ molH2O $=(1000 *$ denSol-molPu* $(\operatorname{atwPu}+4 *$ atwNO3)molAcid*atwHNO3)/atwH2O $3.4>$

$\mathrm{c}$

$\mathrm{c}$

$<$ perl $>$

sub IMP

\{

return 1 if $(\$[0]>0)$;

return 0 ;

\}

$</$ perl $>$

c sphere

$2011<$-denSol|3.6> $-1 \quad$ imp:n=1

$212-1.000-21 \quad$ imp:n=<\&IMP(rw) $\mid 1>$ \$zero importance if no reflector

$220 \quad 2$ imp:n=0 


$$
\begin{array}{lll}
1 & \text { so }<\text { rd } \mid 4.4> & \text { \$ Sphere } \\
2 & \text { so }<\text { rd }+ \text { rw*in|4.4> } & \text { \$Reflection }
\end{array}
$$

mode $\mathrm{n}$

kcode 20001.0100 ZZZ0

$\begin{array}{llll}\text { ksrc } & 0.00 & 0.00 & 0.00\end{array}$

$\begin{array}{llll}0.00 & 0.00 & 0.10\end{array}$

$\begin{array}{lll}0.00 & 0.00 & 0.50\end{array}$

$\begin{array}{lll}0.00 & 0.00 & 1.00\end{array}$

$\begin{array}{lll}0.00 & 0.00 & 2.00\end{array}$

$\begin{array}{lll}0.00 & 0.00 & 3.00\end{array}$

$\begin{array}{lll}1.00 & 1.00 & 1.00\end{array}$

$\begin{array}{lll}1.00 & 1.00 & 2.00\end{array}$

$\begin{array}{lll}1.00 & 1.00 & 3.00\end{array}$

$\begin{array}{lll}2.00 & 2.00 & 1.00\end{array}$

$\begin{array}{lll}2.00 & 2.00 & 2.00\end{array}$

$\begin{array}{lll}2.00 & 2.00 & 3.00\end{array}$

$\begin{array}{lll}-1.00 & -1.00 & -1.00\end{array}$

$-10.00 \quad-10.00 \quad-20.00$

$\begin{array}{llll}-10.00 & -10.00 & -30.00\end{array}$

c

m2 \$water

$1001.66 \mathrm{c} \quad 2$

$8016.66 \mathrm{c} \quad 1$

$\mathrm{mt} 2$ 1wtr.60t

c $\mathrm{pu} /$ water mixture

m11 \$ Pu(NO3)4 - NO3 - H2O, 100.0 wt\% Pu239, ZZZ1 milli-g Pu/cc, $<$ denSol|3.4>

c molarity $\mathrm{pu}=<\operatorname{molPu} \mid 3.4>$, acid $=<\operatorname{molAcid} \mid 3.4>, \mathrm{H} 2 \mathrm{O}=\langle\operatorname{molH} 2 \mathrm{O}| 3.4>$

c Pu in salt

$94239.66 \mathrm{c}<\mathrm{molPu} * \mathrm{ena} \mid 3.4>$

c $94240.66 \mathrm{c}<\mathrm{molPu} *(1$-ena) $\mid 3.4>$

c NO3 in salt plus acid

$7014.66 \mathrm{c}<4 * \mathrm{molPu}+$ molAcid $\mid 3.4>$

$8016.66 \mathrm{c}<12 * \mathrm{molPu}+3 * \operatorname{mol} \mathrm{Acid} \mid 3.4>$

c water

$1001.66 \mathrm{c}<2 * \mathrm{molH} 2 \mathrm{O}+\operatorname{molAcid} \mid 3.4>$ \$Includes Free Hydrogen Ion

$8016.66 \mathrm{c}<\mathrm{molH} 2 \mathrm{O} \mid 3.4>$

mt11 1wtr.60t

c

c 


\section{Template File for Case: PuN43-sp-1000-00}

sp, 100.0 wt\% Pu239, ZZZ1 milli-g/cc, in Pu(NO3)4, with 3 Molar HNO3

$\mathrm{c}$

c worm notes: format of the form $\mid n . m$ for floating point, $\mid \mathrm{n}$ for integers

c $\quad$ zzz0 (in upper case) is replaced with the number of cyles

c $\quad$ zzz1 $=$ the actual concentration in units of milli-g/cc

c $\quad$ zzz2 $=$ solution density in $\mathrm{g} / \mathrm{cc}$

c $\quad \mathrm{qqq} 1=$ the minimum dimensions (in hundreths of inches)

c $\quad$ qqq $2=$ the maximum dimensions (in hundreths of inches)

c $\quad \mathrm{qqq} 3=$ the delta (in hundreths of inches)

$\mathrm{c}$

$\mathrm{c}$ Diameter $=<\mathrm{d} 1=\mathrm{QQQ1}: \mathrm{QQQ} 2: \mathrm{QQQ} 3 \mid 5>$ hundreths of inches - must be integer

$\mathrm{c}$ radius $=<\mathrm{rd}=\mathrm{d} 1 * 2.54 / 100 / 2 \mid 5.4>\mathrm{cm}$

c note for perl, numbers with leading zeros must be enclosed in single quotes or else

$c$ they will be interpretted as octal

$\mathrm{c}$ Conc $=<\mathrm{c}={ }^{\prime} \mathrm{ZZZ1}^{\prime} \mid 5>$ milli-g Pu/cc

c Reflector width $=<\mathrm{rw}=0.0 \mid 2.1>$ inches

c Enrichment $=<$ ena $=1.0 \mid 1.2>$ atom fraction $\mathrm{Pu} 239$

c Mols of Acid per liter $<$ molAcid $=3.0 \mid 1.2>$

$\mathrm{c}$

$\mathrm{c}$

c Density of solution $<$ denSol=ZZZ2/1e $5 \mid 1.5>$

$\mathrm{c}$

$\mathrm{c}$

c Atomic Weights

$\mathrm{c} \mathrm{H} 2 \mathrm{O}=\langle\mathrm{atwH} 2 \mathrm{O}=18.0152| 3.4>$

c NO3 $=<$ atwNO3 $=62.0049 \mid 3.4>$

c HNO3 $=<$ atwHNO3 $=63.0128 \mid 3.4>$

c Pu239 $=<$ atwPu239 $=239.0522 \mid 3.4>$

c Pu240 $=<$ atwPu240 $=240.0538 \mid 3.4>$

$\mathrm{c} \mathrm{Pu}=<\mathrm{atwPu}=$ ena*atwPu239+(1-ena)*atwPu240|3.4 $>$

$\mathrm{c}$

c Molarity of solution components

c Mols of $\mathrm{Pu}$ per liter $<\mathrm{molPu}=\mathrm{c} / \mathrm{atwPu} \mid 3.4>$

c Mols of $\mathrm{H} 2 \mathrm{O}$ per liter $<\mathrm{molH} 2 \mathrm{O}=(1000 *$ denSol-molPu* $(\mathrm{atwPu}+4 *$ atwNO3)

molAcid*atwHNO3)/atwH2O|3.4>

$\mathrm{c}$

$\mathrm{c}$

$<$ perl $>$

sub IMP

\{

return 1 if $\left(\$ \_[0]>0\right)$;

return 0 ;

\}

$</$ perl $>$

c sphere

$2011<$-denSol|3.6> $-1 \quad$ imp:n=1

$212-1.000-21 \quad \mathrm{imp}: \mathrm{n}=<\& \operatorname{IMP}(\mathrm{rw}) \mid 1>$ \$zero importance if no reflector

$220 \quad 2 \quad$ imp:n $=0$ 


$$
\begin{array}{lll}
1 & \text { so }<\text { rd } \mid 4.4> & \text { \$ Sphere } \\
2 & \text { so }<\text { rd }+ \text { rw*in|4.4> } & \text { \$Reflection }
\end{array}
$$

mode $\mathrm{n}$

kcode 20001.0100 ZZZ0

$\begin{array}{llll}\text { ksrc } & 0.00 & 0.00 & 0.00\end{array}$

$\begin{array}{llll}0.00 & 0.00 & 0.10\end{array}$

$\begin{array}{lll}0.00 & 0.00 & 0.50\end{array}$

$\begin{array}{lll}0.00 & 0.00 & 1.00\end{array}$

$\begin{array}{lll}0.00 & 0.00 & 2.00\end{array}$

$\begin{array}{lll}0.00 & 0.00 & 3.00\end{array}$

$\begin{array}{lll}1.00 & 1.00 & 1.00\end{array}$

$\begin{array}{lll}1.00 & 1.00 & 2.00\end{array}$

$\begin{array}{lll}1.00 & 1.00 & 3.00\end{array}$

$\begin{array}{lll}2.00 & 2.00 & 1.00\end{array}$

$\begin{array}{lll}2.00 & 2.00 & 2.00\end{array}$

$\begin{array}{lll}2.00 & 2.00 & 3.00\end{array}$

$\begin{array}{lll}-1.00 & -1.00 & -1.00\end{array}$

$-10.00 \quad-10.00 \quad-20.00$

$\begin{array}{llll}-10.00 & -10.00 & -30.00\end{array}$

c

m2 \$water

$1001.66 \mathrm{c} \quad 2$

$8016.66 \mathrm{c} \quad 1$

$\mathrm{mt} 2$ 1wtr.60t

c $\mathrm{pu} /$ water mixture

m11 \$ Pu(NO3)4 - NO3 - H2O, 100.0 wt\% Pu239, ZZZ1 milli-g Pu/cc, $<$ denSol|3.4>

c molarity $\mathrm{pu}=<\operatorname{molPu} \mid 3.4>$, acid $=<\operatorname{molAcid} \mid 3.4>, \mathrm{H} 2 \mathrm{O}=\langle\operatorname{molH} 2 \mathrm{O}| 3.4>$

c Pu in salt

$94239.66 \mathrm{c}<\mathrm{molPu} * \mathrm{ena} \mid 3.4>$

c $94240.66 \mathrm{c}<\mathrm{molPu} *(1$-ena) $\mid 3.4>$

c NO3 in salt plus acid

$7014.66 \mathrm{c}<4 * \mathrm{molPu}+$ molAcid $\mid 3.4>$

$8016.66 \mathrm{c}<12 * \mathrm{molPu}+3 * \operatorname{mol} \mathrm{Acid} \mid 3.4>$

c water

$1001.66 \mathrm{c}<2 * \mathrm{molH} 2 \mathrm{O}+\operatorname{molAcid} \mid 3.4>$ \$Includes Free Hydrogen Ion

$8016.66 \mathrm{c}<\mathrm{molH} 2 \mathrm{O} \mid 3.4>$

mt11 1wtr.60t

c

c 
Template File for Case: PuN43-sp-1000-01

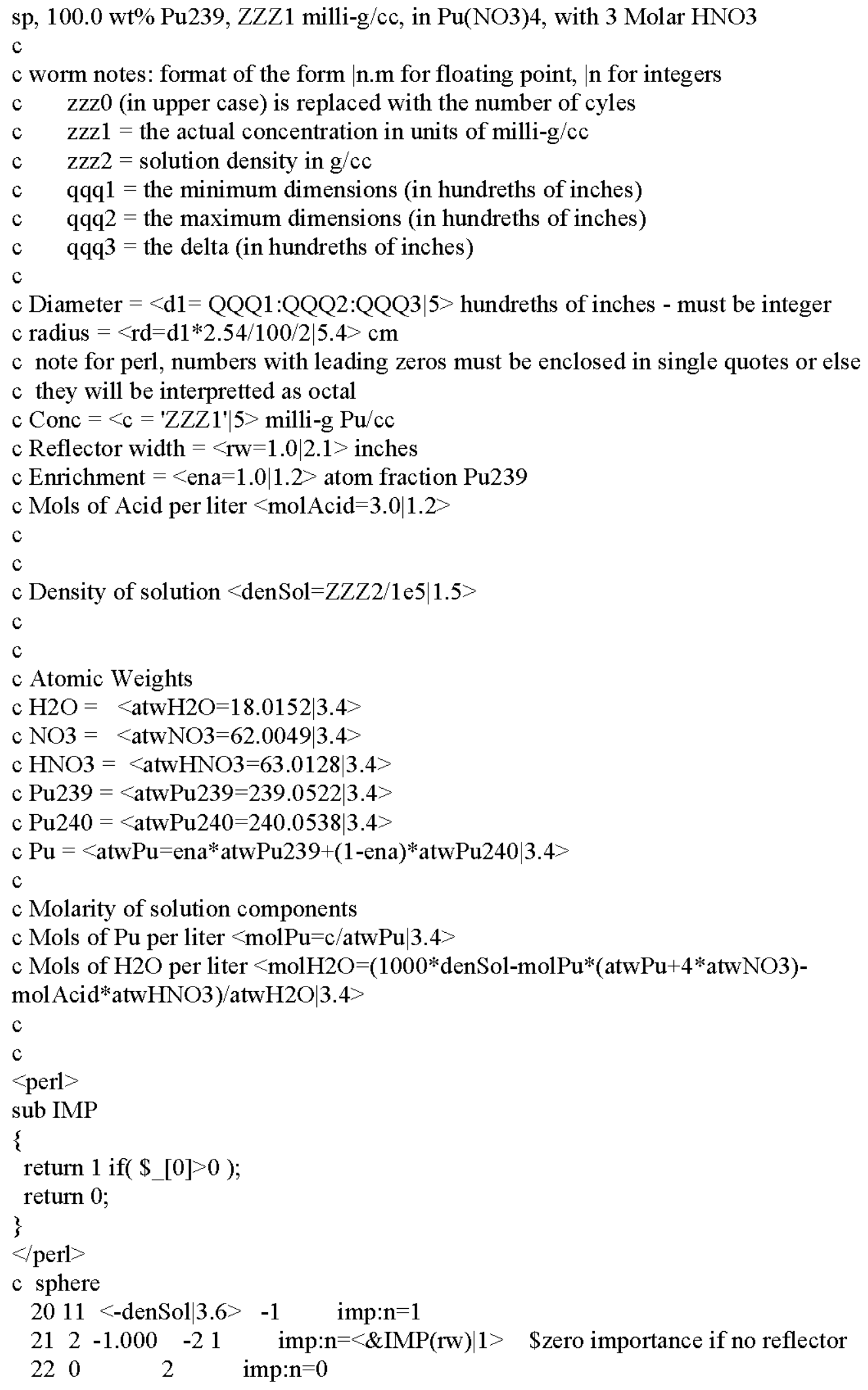




$$
\begin{array}{lll}
1 & \text { so }<\text { rd } \mid 4.4> & \text { \$ Sphere } \\
2 & \text { so }<\text { rd }+ \text { rw*in|4.4> } & \text { \$Reflection }
\end{array}
$$

mode $\mathrm{n}$

kcode 20001.0100 ZZZ0

$\begin{array}{llll}\text { ksrc } & 0.00 & 0.00 & 0.00\end{array}$

$\begin{array}{llll}0.00 & 0.00 & 0.10\end{array}$

$\begin{array}{lll}0.00 & 0.00 & 0.50\end{array}$

$\begin{array}{lll}0.00 & 0.00 & 1.00\end{array}$

$\begin{array}{lll}0.00 & 0.00 & 2.00\end{array}$

$\begin{array}{lll}0.00 & 0.00 & 3.00\end{array}$

$\begin{array}{lll}1.00 & 1.00 & 1.00\end{array}$

$\begin{array}{lll}1.00 & 1.00 & 2.00\end{array}$

$\begin{array}{lll}1.00 & 1.00 & 3.00\end{array}$

$\begin{array}{lll}2.00 & 2.00 & 1.00\end{array}$

$\begin{array}{lll}2.00 & 2.00 & 2.00\end{array}$

$\begin{array}{lll}2.00 & 2.00 & 3.00\end{array}$

$\begin{array}{lll}-1.00 & -1.00 & -1.00\end{array}$

$-10.00 \quad-10.00 \quad-20.00$

$\begin{array}{llll}-10.00 & -10.00 & -30.00\end{array}$

c

m2 \$water

$1001.66 \mathrm{c} \quad 2$

$8016.66 \mathrm{c} \quad 1$

$\mathrm{mt} 2$ 1wtr.60t

c $\mathrm{pu} /$ water mixture

m11 \$ Pu(NO3)4 - NO3 - H2O, 100.0 wt\% Pu239, ZZZ1 milli-g Pu/cc, $<$ denSol|3.4>

c molarity $\mathrm{pu}=<\operatorname{molPu} \mid 3.4>$, acid $=<\operatorname{molAcid} \mid 3.4>, \mathrm{H} 2 \mathrm{O}=\langle\operatorname{molH} 2 \mathrm{O}| 3.4>$

c Pu in salt

$94239.66 \mathrm{c}<\mathrm{molPu} * \mathrm{ena} \mid 3.4>$

c $94240.66 \mathrm{c}<\mathrm{molPu} *(1$-ena) $\mid 3.4>$

c NO3 in salt plus acid

$7014.66 \mathrm{c}<4 * \mathrm{molPu}+$ molAcid $\mid 3.4>$

$8016.66 \mathrm{c}<12 * \mathrm{molPu}+3 * \operatorname{mol} \mathrm{Acid} \mid 3.4>$

c water

$1001.66 \mathrm{c}<2 * \mathrm{molH} 2 \mathrm{O}+\operatorname{molAcid} \mid 3.4>$ \$Includes Free Hydrogen Ion

$8016.66 \mathrm{c}<\mathrm{molH} 2 \mathrm{O} \mid 3.4>$

mt11 1wtr.60t

c

c 
Template File for Case: PuN43-sp-1000-10

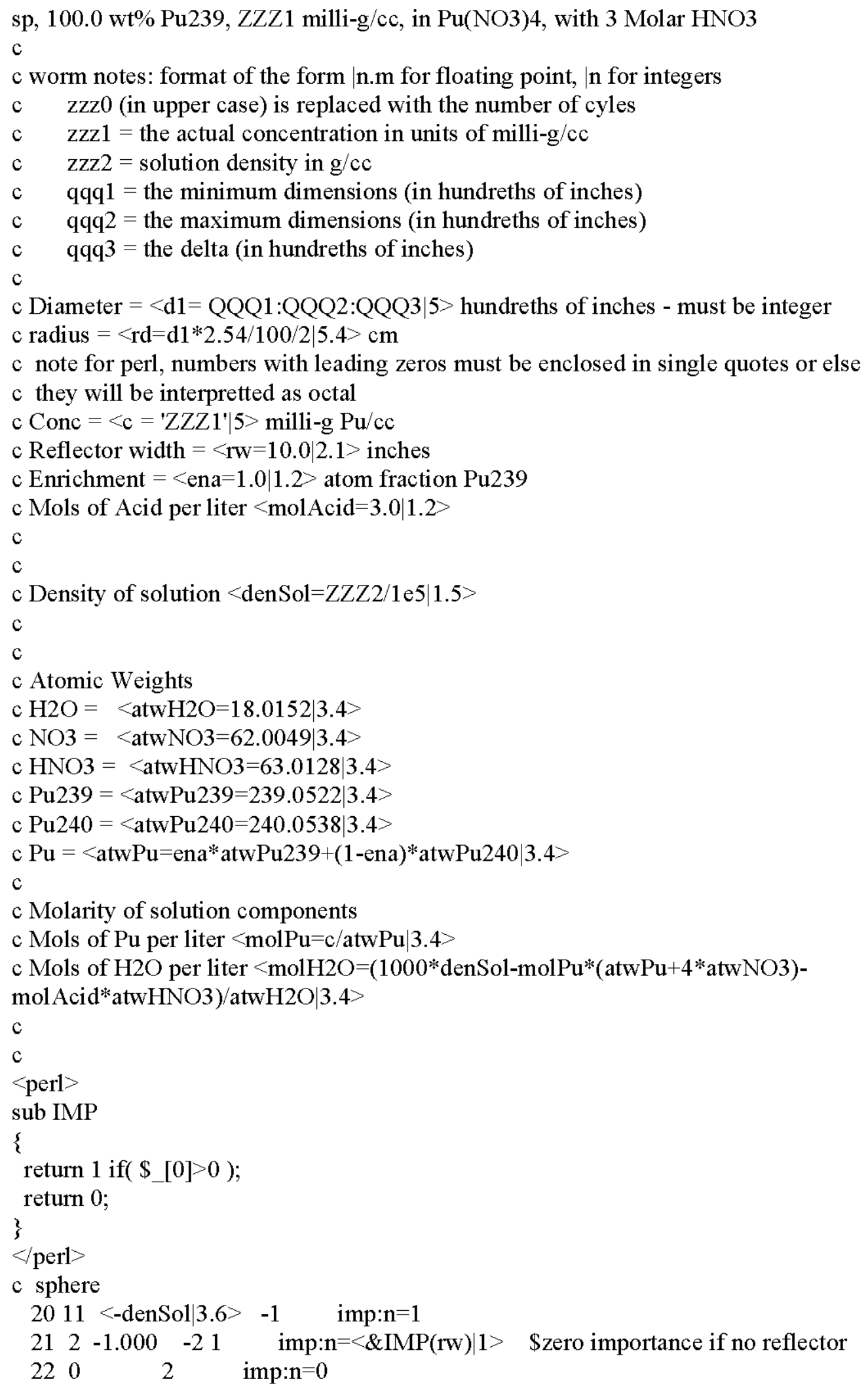




$$
\begin{array}{lll}
1 & \text { so }<\text { rd } \mid 4.4> & \text { \$ Sphere } \\
2 & \text { so }<\text { rd }+ \text { rw*in|4.4> } & \text { \$Reflection }
\end{array}
$$

mode $\mathrm{n}$

kcode 20001.0100 ZZZ0

$\begin{array}{llll}\text { ksrc } & 0.00 & 0.00 & 0.00\end{array}$

$\begin{array}{llll}0.00 & 0.00 & 0.10\end{array}$

$\begin{array}{lll}0.00 & 0.00 & 0.50\end{array}$

$\begin{array}{lll}0.00 & 0.00 & 1.00\end{array}$

$\begin{array}{lll}0.00 & 0.00 & 2.00\end{array}$

$\begin{array}{lll}0.00 & 0.00 & 3.00\end{array}$

$\begin{array}{lll}1.00 & 1.00 & 1.00\end{array}$

$\begin{array}{lll}1.00 & 1.00 & 2.00\end{array}$

$\begin{array}{lll}1.00 & 1.00 & 3.00\end{array}$

$\begin{array}{lll}2.00 & 2.00 & 1.00\end{array}$

$\begin{array}{lll}2.00 & 2.00 & 2.00\end{array}$

$\begin{array}{lll}2.00 & 2.00 & 3.00\end{array}$

$\begin{array}{lll}-1.00 & -1.00 & -1.00\end{array}$

$-10.00 \quad-10.00 \quad-20.00$

$\begin{array}{llll}-10.00 & -10.00 & -30.00\end{array}$

c

m2 \$water

$1001.66 \mathrm{c} \quad 2$

$8016.66 \mathrm{c} \quad 1$

$\mathrm{mt} 2$ 1wtr.60t

c $\mathrm{pu} /$ water mixture

m11 \$ Pu(NO3)4 - NO3 - H2O, 100.0 wt\% Pu239, ZZZ1 milli-g Pu/cc, $<$ denSol|3.4>

c molarity $\mathrm{pu}=\langle\operatorname{molPu}| 3.4>$, acid $=\langle\operatorname{mol} A \mathrm{cid}| 3.4>, \mathrm{H} 2 \mathrm{O}=\langle\operatorname{molH} 2 \mathrm{O}| 3.4>$

c Pu in salt

$94239.66 \mathrm{c}<\mathrm{molPu} * \mathrm{ena} \mid 3.4>$

c $94240.66 \mathrm{c}<\mathrm{molPu} *(1$-ena) $\mid 3.4>$

c NO3 in salt plus acid

$7014.66 \mathrm{c}<4 * \mathrm{molPu}+$ molAcid $\mid 3.4>$

$8016.66 \mathrm{c}<12 * \mathrm{molPu}+3 * \operatorname{mol} \mathrm{Acid} \mid 3.4>$

c water

$1001.66 \mathrm{c}<2 * \mathrm{molH} 2 \mathrm{O}+\operatorname{molAcid} \mid 3.4>$ \$Includes Free Hydrogen Ion

$8016.66 \mathrm{c}<\mathrm{molH} 2 \mathrm{O} \mid 3.4>$

mt11 1wtr.60t

c

c 
Template File for Case: PuN46-sp-1000-00

sp, 100.0 wt\% Pu239, ZZZ1 milli-g/cc, in Pu(NO3)4, with 6 Molar HNO3

$\mathrm{c}$

c worm notes: format of the form $\mid n . m$ for floating point, $\mid \mathrm{n}$ for integers

c zzz0 (in upper case) is replaced with the number of cyles

c $\quad$ zzz1 $=$ the actual concentration in units of milli-g/cc

c $\quad$ zzz2 $=$ solution density in $\mathrm{g} / \mathrm{cc}$

c $\quad \mathrm{qqq1}=$ the minimum dimensions (in hundreths of inches)

c $\quad \mathrm{qqq} 2=$ the maximum dimensions (in hundreths of inches)

c $\quad \mathrm{qqq} 3=$ the delta (in hundreths of inches)

c

c Diameter $=<\mathrm{d} 1=$ QQQ1:QQQ2:QQQ3 $\mid 5>$ hundreths of inches - must be integer

$\mathrm{c}$ radius $=<\mathrm{rd}=\mathrm{d} 1 * 2.54 / 100 / 2 \mid 5.4>\mathrm{cm}$

c note for perl, numbers with leading zeros must be enclosed in single quotes or else

c they will be interpretted as octal

$\mathrm{c}$ Conc $=<\mathrm{c}={ }^{\prime} \mathrm{ZZZ1} 1 \mid 5>$ milli-g Pu/cc

c Reflector width $=<\mathrm{rw}=0.0 \mid 2.1>$ inches

c Enrichment $=<$ ena $=1.0 \mid 1.2>$ atom fraction Pu239

c Mols of Acid per liter $<$ molAcid $=6.0 \mid 1.2>$

c

$\mathrm{c}$

c Density of solution $<$ denSol=ZZZ2/1e5 $\mid 1.5>$

c

C

c Atomic Weights

c $\mathrm{H} 2 \mathrm{O}=\langle$ atwH2O $=18.0152| 3.4>$

$\mathrm{c} \mathrm{NO3}=\langle$ atwNO3 $=62.0049| 3.4>$

c $\mathrm{HNO} 3=<\mathrm{atwHNO} 3=63.0128 \mid 3.4>$

c Pu239 $=<$ atwPu239 $=239.0522 \mid 3.4>$

c Pu240 $=<$ atwPu240 $=240.0538 \mid 3.4>$

c $\mathrm{Pu}=<\mathrm{atwPu}=\mathrm{ena}^{*}$ atwPu239+(1-ena)*atwPu240|3.4>

c

c Molarity of solution components

c Mols of $\mathrm{Pu}$ per liter $<\mathrm{molPu}=\mathrm{c} / \mathrm{atwPu} \mid 3.4>$

c Mols of $\mathrm{H} 2 \mathrm{O}$ per liter $<$ molH2O $=(1000 *$ denSol-molPu* $(a+w P u+4 * a t w N O 3)$ -

molAcid*atwHNO3)/atwH2O $3.4>$

$\mathrm{c}$

$\mathrm{c}$

$<$ perl $>$

sub IMP

\{

return 1 if $\left(\$ \_[0]>0\right)$;

return 0 ;

\}

$<$ perl $>$

c sphere

$2011<-$ denSol|3.6> $-1 \quad$ imp:n=1

$21 \quad 2-1.000-21 \quad$ imp:n=<\&IMP(rw) $\mid 1>$ \$zero importance if no reflector

$220 \quad 2 \quad$ imp:n $=0$ 


$$
\begin{array}{lll}
1 & \text { so }<\text { rd } \mid 4.4> & \text { \$ Sphere } \\
2 & \text { so }<\text { rd }+ \text { rw*in|4.4> } & \text { \$Reflection }
\end{array}
$$

mode $\mathrm{n}$

kcode 20001.0100 ZZZ0

$\begin{array}{llll}\text { ksrc } & 0.00 & 0.00 & 0.00\end{array}$

$\begin{array}{llll}0.00 & 0.00 & 0.10\end{array}$

$\begin{array}{lll}0.00 & 0.00 & 0.50\end{array}$

$\begin{array}{lll}0.00 & 0.00 & 1.00\end{array}$

$\begin{array}{lll}0.00 & 0.00 & 2.00\end{array}$

$\begin{array}{lll}0.00 & 0.00 & 3.00\end{array}$

$\begin{array}{lll}1.00 & 1.00 & 1.00\end{array}$

$\begin{array}{lll}1.00 & 1.00 & 2.00\end{array}$

$\begin{array}{lll}1.00 & 1.00 & 3.00\end{array}$

$\begin{array}{lll}2.00 & 2.00 & 1.00\end{array}$

$\begin{array}{lll}2.00 & 2.00 & 2.00\end{array}$

$\begin{array}{lll}2.00 & 2.00 & 3.00\end{array}$

$\begin{array}{lll}-1.00 & -1.00 & -1.00\end{array}$

$-10.00 \quad-10.00 \quad-20.00$

$\begin{array}{llll}-10.00 & -10.00 & -30.00\end{array}$

c

m2 \$water

$1001.66 \mathrm{c} \quad 2$

$8016.66 \mathrm{c} \quad 1$

$\mathrm{mt} 2$ 1wtr.60t

c $\mathrm{pu} /$ water mixture

m11 \$ Pu(NO3)4 - NO3 - H2O, 100.0 wt\% Pu239, ZZZ1 milli-g Pu/cc, $<$ denSol|3.4>

c molarity $\mathrm{pu}=<\operatorname{molPu} \mid 3.4>$, acid $=<\operatorname{molAcid} \mid 3.4>, \mathrm{H} 2 \mathrm{O}=\langle\operatorname{molH} 2 \mathrm{O}| 3.4>$

c Pu in salt

$94239.66 \mathrm{c}<\mathrm{molPu} * \mathrm{ena} \mid 3.4>$

c $94240.66 \mathrm{c}<\mathrm{molPu} *(1$-ena) $\mid 3.4>$

c NO3 in salt plus acid

$7014.66 \mathrm{c}<4 * \mathrm{molPu}+$ molAcid $\mid 3.4>$

$8016.66 \mathrm{c}<12 * \mathrm{molPu}+3 * \operatorname{mol} \mathrm{Acid} \mid 3.4>$

c water

$1001.66 \mathrm{c}<2 * \mathrm{molH} 2 \mathrm{O}+\operatorname{molAcid} \mid 3.4>$ \$Includes Free Hydrogen Ion

$8016.66 \mathrm{c}<\mathrm{molH} 2 \mathrm{O} \mid 3.4>$

mt11 1wtr.60t

c

c 
Template File for Case: PuN46-sp-1000-01

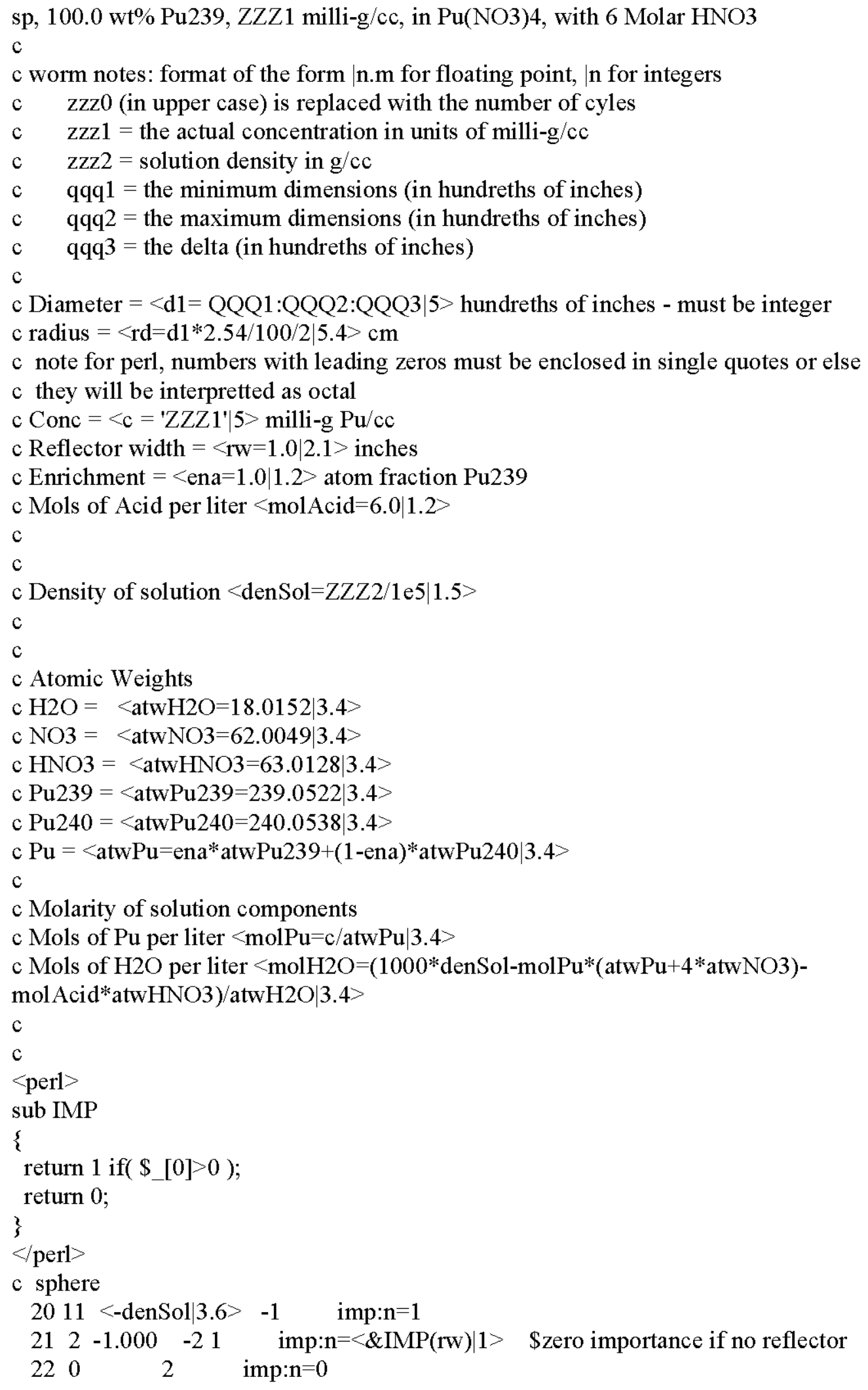




$$
\begin{array}{lll}
1 & \text { so }<\text { rd } \mid 4.4> & \text { \$ Sphere } \\
2 & \text { so }<\text { rd }+ \text { rw*in|4.4> } & \text { \$Reflection }
\end{array}
$$

mode $\mathrm{n}$

kcode 20001.0100 ZZZ0

$\begin{array}{llll}\text { ksrc } & 0.00 & 0.00 & 0.00\end{array}$

$\begin{array}{llll}0.00 & 0.00 & 0.10\end{array}$

$\begin{array}{lll}0.00 & 0.00 & 0.50\end{array}$

$\begin{array}{lll}0.00 & 0.00 & 1.00\end{array}$

$\begin{array}{lll}0.00 & 0.00 & 2.00\end{array}$

$\begin{array}{lll}0.00 & 0.00 & 3.00\end{array}$

$\begin{array}{lll}1.00 & 1.00 & 1.00\end{array}$

$\begin{array}{lll}1.00 & 1.00 & 2.00\end{array}$

$\begin{array}{lll}1.00 & 1.00 & 3.00\end{array}$

$\begin{array}{lll}2.00 & 2.00 & 1.00\end{array}$

$\begin{array}{lll}2.00 & 2.00 & 2.00\end{array}$

$\begin{array}{lll}2.00 & 2.00 & 3.00\end{array}$

$\begin{array}{lll}-1.00 & -1.00 & -1.00\end{array}$

$-10.00 \quad-10.00 \quad-20.00$

$\begin{array}{llll}-10.00 & -10.00 & -30.00\end{array}$

c

m2 \$water

$1001.66 \mathrm{c} \quad 2$

$8016.66 \mathrm{c} \quad 1$

$\mathrm{mt} 2$ 1wtr.60t

c $\mathrm{pu} /$ water mixture

m11 \$ Pu(NO3)4 - NO3 - H2O, 100.0 wt\% Pu239, ZZZ1 milli-g Pu/cc, $<$ denSol|3.4>

c molarity $\mathrm{pu}=<\operatorname{molPu} \mid 3.4>$, acid $=<\operatorname{molAcid} \mid 3.4>, \mathrm{H} 2 \mathrm{O}=\langle\operatorname{molH} 2 \mathrm{O}| 3.4>$

c Pu in salt

$94239.66 \mathrm{c}<\mathrm{molPu} * \mathrm{ena} \mid 3.4>$

c $94240.66 \mathrm{c}<\mathrm{molPu} *(1$-ena) $\mid 3.4>$

c NO3 in salt plus acid

$7014.66 \mathrm{c}<4 * \mathrm{molPu}+$ molAcid $\mid 3.4>$

$8016.66 \mathrm{c}<12 * \mathrm{molPu}+3 * \operatorname{mol} \mathrm{Acid} \mid 3.4>$

c water

$1001.66 \mathrm{c}<2 * \mathrm{molH} 2 \mathrm{O}+\operatorname{molAcid} \mid 3.4>$ \$Includes Free Hydrogen Ion

$8016.66 \mathrm{c}<\mathrm{molH} 2 \mathrm{O} \mid 3.4>$

mt11 1wtr.60t

c

c 


\section{Template File for Case: PuN46-sp-1000-10}

sp, 100.0 wt\% Pu239, ZZZ1 milli-g/cc, in Pu(NO3)4, with 6 Molar HNO3

$\mathrm{c}$

c worm notes: format of the form $\mid n . m$ for floating point, $\mid \mathrm{n}$ for integers

c $\quad$ zzz0 (in upper case) is replaced with the number of cyles

c $\quad$ zzz1 $=$ the actual concentration in units of milli-g/cc

c $\quad$ zzz2 $=$ solution density in $\mathrm{g} / \mathrm{cc}$

c $\quad \mathrm{qqq} 1=$ the minimum dimensions (in hundreths of inches)

c $\quad \mathrm{qqq} 2=$ the maximum dimensions (in hundreths of inches)

c $\quad \mathrm{qqq} 3=$ the delta (in hundreths of inches)

c

c Diameter $=<\mathrm{d} 1=$ QQQ1:QQQ2:QQQ3 $\mid 5>$ hundreths of inches - must be integer

$\mathrm{c}$ radius $=<\mathrm{rd}=\mathrm{d} 1 * 2.54 / 100 / 2 \mid 5.4>\mathrm{cm}$

c note for perl, numbers with leading zeros must be enclosed in single quotes or else

c they will be interpretted as octal

$\mathrm{c}$ Conc $=<\mathrm{c}={ }^{\prime} \mathrm{ZZZ1} 1 \mid 5>$ milli-g Pu/cc

c Reflector width $=<\mathrm{rw}=10.0 \mid 2.1>$ inches

c Enrichment $=<$ ena $=1.0 \mid 1.2>$ atom fraction Pu239

c Mols of Acid per liter $<$ molAcid $=6.0 \mid 1.2>$

$\mathrm{c}$

$\mathrm{c}$

c Density of solution $<$ denSol $=Z Z Z 2 / 1 \mathrm{e} 5 \mid 1.5>$

c

c

c Atomic Weights

c $\mathrm{H} 2 \mathrm{O}=\langle$ atwH2O $=18.0152| 3.4>$

$\mathrm{c} \mathrm{NO3}=\langle$ atwNO3 $=62.0049| 3.4>$

c HNO3 $=<$ atwHNO3 $=63.0128 \mid 3.4>$

c Pu239 $=<$ atwPu239 $=239.0522 \mid 3.4>$

c Pu240 $=<$ atwPu240 $=240.0538 \mid 3.4>$

$\mathrm{c} \mathrm{Pu}=<\mathrm{atwPu}=\mathrm{ena}^{*}$ atwPu239+(1-ena)*atwPu240|3.4>

$\mathrm{c}$

c Molarity of solution components

c Mols of $\mathrm{Pu}$ per liter $<\mathrm{molPu}=\mathrm{c} / \mathrm{atwPu} \mid 3.4>$

c Mols of $\mathrm{H} 2 \mathrm{O}$ per liter $<$ molH2O $=(1000 *$ denSol-molPu* $(a t w P u+4 * a t w N O 3)$ molAcid*atwHNO3)/atwH2O $3.4>$

$\mathrm{c}$

$\mathrm{c}$

$<$ perl $>$

sub IMP

\{

return 1 if $(\$[0]>0)$;

return 0 ;

\}

$</$ perl $>$

c sphere

$2011<$-denSol|3.6> $-1 \quad$ imp:n=1

$212-1.000-21 \quad$ imp:n=<\&IMP(rw) $\mid 1>$ \$zero importance if no reflector

$220 \quad 2 \quad$ imp:n $=0$ 


$$
\begin{array}{lll}
1 & \text { so }<\text { rd } \mid 4.4> & \text { \$ Sphere } \\
2 & \text { so }<\text { rd }+ \text { rw*in|4.4> } & \text { \$Reflection }
\end{array}
$$

mode $\mathrm{n}$

kcode 20001.0100 ZZZ0

$\begin{array}{llll}\text { ksrc } & 0.00 & 0.00 & 0.00\end{array}$

$\begin{array}{llll}0.00 & 0.00 & 0.10\end{array}$

$\begin{array}{lll}0.00 & 0.00 & 0.50\end{array}$

$\begin{array}{lll}0.00 & 0.00 & 1.00\end{array}$

$\begin{array}{lll}0.00 & 0.00 & 2.00\end{array}$

$\begin{array}{lll}0.00 & 0.00 & 3.00\end{array}$

$\begin{array}{lll}1.00 & 1.00 & 1.00\end{array}$

$\begin{array}{lll}1.00 & 1.00 & 2.00\end{array}$

$\begin{array}{lll}1.00 & 1.00 & 3.00\end{array}$

$\begin{array}{lll}2.00 & 2.00 & 1.00\end{array}$

$\begin{array}{lll}2.00 & 2.00 & 2.00\end{array}$

$\begin{array}{lll}2.00 & 2.00 & 3.00\end{array}$

$\begin{array}{lll}-1.00 & -1.00 & -1.00\end{array}$

$-10.00 \quad-10.00 \quad-20.00$

$\begin{array}{llll}-10.00 & -10.00 & -30.00\end{array}$

c

m2 \$water

$1001.66 \mathrm{c} \quad 2$

$8016.66 \mathrm{c} \quad 1$

$\mathrm{mt} 2$ 1wtr.60t

c $\mathrm{pu} /$ water mixture

m11 \$ Pu(NO3)4 - NO3 - H2O, 100.0 wt\% Pu239, ZZZ1 milli-g Pu/cc, $<$ denSol|3.4>

c molarity $\mathrm{pu}=<\operatorname{molPu} \mid 3.4>$, acid $=<\operatorname{molAcid} \mid 3.4>, \mathrm{H} 2 \mathrm{O}=\langle\operatorname{molH} 2 \mathrm{O}| 3.4>$

c Pu in salt

$94239.66 \mathrm{c}<\mathrm{molPu} * \mathrm{ena} \mid 3.4>$

c $94240.66 \mathrm{c}<\mathrm{molPu} *(1$-ena) $\mid 3.4>$

c NO3 in salt plus acid

$7014.66 \mathrm{c}<4 * \mathrm{molPu}+$ molAcid $\mid 3.4>$

$8016.66 \mathrm{c}<12 * \mathrm{molPu}+3 * \operatorname{mol} \mathrm{Acid} \mid 3.4>$

c water

$1001.66 \mathrm{c}<2 * \mathrm{molH} 2 \mathrm{O}+\operatorname{molAcid} \mid 3.4>$ \$Includes Free Hydrogen Ion

$8016.66 \mathrm{c}<\mathrm{molH} 2 \mathrm{O} \mid 3.4>$

mt11 1wtr.60t

c

c 


\section{Template File for Case: PuN40-sp-0970-00}

sp, 97.0 wt\% Pu239, ZZZ1 milli-g/cc, in Pu(NO3)4, with 0 Molar HNO3

c

$\mathrm{c}$ worm notes: format of the form $\mid \mathrm{n} . \mathrm{m}$ for floating point, $\mid \mathrm{n}$ for integers

c zzz0 (in upper case) is replaced with the number of cyles

c $\quad$ zzz1 $=$ the actual concentration in units of milli-g/cc

c $\quad$ zzz2 $=$ solution density in $\mathrm{g} / \mathrm{cc}$

c $\quad \mathrm{qqq} 1=$ the minimum dimensions (in hundreths of inches)

c $\quad \mathrm{qqq} 2=$ the maximum dimensions (in hundreths of inches)

c $\quad \mathrm{qqq} 3=$ the delta (in hundreths of inches)

c

c Diameter $=<\mathrm{d} 1=$ QQQ1:QQQ2:QQQ3 $\mid 5>$ hundreths of inches - must be integer

$\mathrm{c}$ radius $=<\mathrm{rd}=\mathrm{d} 1 * 2.54 / 100 / 2 \mid 5.4>\mathrm{cm}$

c note for perl, numbers with leading zeros must be enclosed in single quotes or else

c they will be interpretted as octal

$\mathrm{c}$ Conc $=<\mathrm{c}={ }^{\prime} \mathrm{ZZZ1} 1 \mid 5>$ milli-g Pu/cc

c Reflector width $=<\mathrm{rw}=0.0 \mid 2.1>$ inches

c Enrichment $=<$ ena $=0.97 \mid 1.2>$ atom fraction Pu239

c Mols of Acid per liter $<$ molAcid $=0.0 \mid 1.2>$

$\mathrm{c}$

$\mathrm{c}$

c Density of solution $<$ denSol $=Z Z Z 2 / 1 \mathrm{e} 5 \mid 1.5>$

c

c

c Atomic Weights

c $\mathrm{H} 2 \mathrm{O}=\langle$ atwH2O $=18.0152| 3.4>$

$\mathrm{c} \mathrm{NO3}=\langle$ atwNO3 $=62.0049| 3.4>$

c HNO3 $=<$ atwHNO3 $=63.0128 \mid 3.4>$

c Pu239 $=<$ atwPu239 $=239.0522 \mid 3.4>$

c Pu240 $=<$ atwPu240 $=240.0538 \mid 3.4>$

$\mathrm{c} \mathrm{Pu}=<\mathrm{atwPu}=\mathrm{ena}^{*}$ atwPu239+(1-ena)*atwPu240|3.4>

$\mathrm{c}$

c Molarity of solution components

c Mols of $\mathrm{Pu}$ per liter $<\mathrm{molPu}=\mathrm{c} / \mathrm{atwPu} \mid 3.4>$

c Mols of $\mathrm{H} 2 \mathrm{O}$ per liter $<$ molH2O $=(1000 *$ denSol-molPu* $(\operatorname{atwPu}+4 *$ atwNO3)molAcid*atwHNO3)/atwH2O|3.4>

$\mathrm{c}$

$\mathrm{c}$

$<$ perl $>$

sub IMP

\{

return 1 if $(\$[0]>0)$;

return 0 ;

\}

$</$ perl $>$

c sphere

$2011<$-denSol|3.6> $-1 \quad$ imp:n=1

$212-1.000-21 \quad$ imp:n=<\&IMP(rw) $\mid 1>$ \$zero importance if no reflector

$220 \quad 2$ imp:n=0 


$$
\begin{array}{lll}
1 & \text { so }<\mathrm{rd} \mid 4.4> & \text { \$ Sphere } \\
2 & \text { so }<\mathrm{rd}+\mathrm{rw}^{*} \text { in } \mid 4.4> & \text { \$Reflection }
\end{array}
$$

mode $\mathrm{n}$

kcode $20001.0100 \mathrm{ZZZ0}$

$\begin{array}{llll}\text { ksrc } & 0.00 & 0.00 & 0.00\end{array}$

$\begin{array}{llll}0.00 & 0.00 & 0.10\end{array}$

$\begin{array}{lll}0.00 & 0.00 & 0.50\end{array}$

$\begin{array}{lll}0.00 & 0.00 & 1.00\end{array}$

$\begin{array}{lll}0.00 & 0.00 & 2.00\end{array}$

$\begin{array}{lll}0.00 & 0.00 & 3.00\end{array}$

$\begin{array}{lll}1.00 & 1.00 & 1.00\end{array}$

$\begin{array}{lll}1.00 & 1.00 & 2.00\end{array}$

$\begin{array}{lll}1.00 & 1.00 & 3.00\end{array}$

$\begin{array}{lll}2.00 & 2.00 & 1.00\end{array}$

$\begin{array}{lll}2.00 & 2.00 & 2.00\end{array}$

$\begin{array}{lll}2.00 & 2.00 & 3.00\end{array}$

$\begin{array}{lll}-1.00 & -1.00 & -1.00\end{array}$

$\begin{array}{lll}-10.00 & -10.00 & -20.00\end{array}$

$\begin{array}{lll}-10.00 & -10.00 & -30.00\end{array}$

c

m2 \$water

$1001.66 \mathrm{c} \quad 2$

$8016.66 \mathrm{c} \quad 1$

$\mathrm{mt} 2$ 1wtr.60t

c $\mathrm{pu} /$ water mixture

m11 \$ Pu(NO3)4 - NO3 - H2O, 100.0 wt\% Pu239, ZZZ1 milli-g Pu/cc, $<$ denSol|3.4 $>$

c molarity $\mathrm{pu}=<\operatorname{molPu} \mid 3.4>$, acid $=<\operatorname{molAcid} \mid 3.4>, \mathrm{H} 2 \mathrm{O}=\langle\operatorname{molH} 2 \mathrm{O}| 3.4>$

c Pu in salt

$94239.66 \mathrm{c}<\mathrm{molPu}$ *ena $\mid 3.4>$

$94240.66 \mathrm{c}<\mathrm{molPu}^{*}(1$-ena $) \mid 3.4>$

c NO3 in salt plus acid

$7014.66 \mathrm{c}<4 * \mathrm{molPu}+$ molAcid $\mid 3.4>$

$8016.66 \mathrm{c}<12 * \mathrm{molPu}+3 * \operatorname{molAcid} \mid 3.4>$

c water

$1001.66 \mathrm{c}<2 *$ molH2O + molAcid $\mid 3.4>$ \$Includes Free Hydrogen Ion

$8016.66 \mathrm{c}<\mathrm{molH} 2 \mathrm{O} \mid 3.4>$

mt11 1wtr.60t

$\mathrm{c}$

c 
Template File for Case: PuN40-sp-0970-01

sp, 97.0 wt\% Pu239, ZZZ1 milli-g/cc, in Pu(NO3)4, with 0 Molar HNO3

$\mathrm{c}$

c worm notes: format of the form $\mid$ n.m for floating point, $\mid \mathrm{n}$ for integers

c zzz0 (in upper case) is replaced with the number of cyles

c $\quad$ zzz1 $=$ the actual concentration in units of milli-g/cc

c $\quad$ zzz2 $=$ solution density in $\mathrm{g} / \mathrm{cc}$

c $\quad \mathrm{qqq1}=$ the minimum dimensions (in hundreths of inches)

c $\quad \mathrm{qqq} 2=$ the maximum dimensions (in hundreths of inches)

c $\quad \mathrm{qqq} 3=$ the delta (in hundreths of inches)

c

c Diameter $=<\mathrm{d} 1=$ QQQ1:QQQ2:QQQ3 $\mid 5>$ hundreths of inches - must be integer

$\mathrm{c}$ radius $=<\mathrm{rd}=\mathrm{d} 1 * 2.54 / 100 / 2 \mid 5.4>\mathrm{cm}$

c note for perl, numbers with leading zeros must be enclosed in single quotes or else

c they will be interpretted as octal

$\mathrm{c}$ Conc $=<\mathrm{c}={ }^{\prime} \mathrm{ZZZ1} 1 \mid 5>$ milli-g Pu/cc

c Reflector width $=<\mathrm{rw}=1.0 \mid 2.1>$ inches

c Enrichment $=<$ ena $=0.97 \mid 1.2>$ atom fraction Pu239

c Mols of Acid per liter $<$ molAcid $=0.0 \mid 1.2>$

$\mathrm{c}$

$\mathrm{c}$

c Density of solution $<$ denSol $=Z Z Z 2 / 1$ e $5 \mid 1.5>$

c

C

c Atomic Weights

c $\mathrm{H} 2 \mathrm{O}=\langle$ atwH2O $=18.0152| 3.4>$

$\mathrm{c} \mathrm{NO3}=\langle$ atwNO3 $=62.0049| 3.4>$

c $\mathrm{HNO} 3=<\mathrm{atwHNO} 3=63.0128 \mid 3.4>$

c Pu239 $=<$ atwPu239 $=239.0522 \mid 3.4>$

c Pu240 $=<$ atwPu240 $=240.0538 \mid 3.4>$

c $\mathrm{Pu}=<\mathrm{atwPu}=\mathrm{ena}^{*}$ atwPu239+(1-ena)*atwPu240|3.4>

c

c Molarity of solution components

c Mols of $\mathrm{Pu}$ per liter $<\mathrm{molPu}=\mathrm{c} / \mathrm{atwPu} \mid 3.4>$

c Mols of $\mathrm{H} 2 \mathrm{O}$ per liter $<$ molH2O $=(1000 *$ denSol-molPu* $(a+w P u+4 * a t w N O 3)$ -

molAcid*atwHNO3)/atwH2O $3.4>$

$\mathrm{c}$

c

$<$ perl $>$

sub IMP

\{

return 1 if $\left(\$ \_[0]>0\right)$;

return 0 ;

\}

$</$ perl $>$

c sphere

$2011<$-denSol|3.6> $-1 \quad$ imp:n=1

$21 \quad 2-1.000-21 \quad$ imp:n=<\&IMP(rw) $\mid 1>$ \$zero importance if no reflector

$220 \quad 2 \quad$ imp:n $=0$ 


$$
\begin{array}{lll}
1 & \text { so }<\mathrm{rd} \mid 4.4> & \text { \$ Sphere } \\
2 & \text { so }<\mathrm{rd}+\mathrm{rw}^{*} \text { in } \mid 4.4> & \text { \$Reflection }
\end{array}
$$

mode $\mathrm{n}$

kcode $20001.0100 \mathrm{ZZZ0}$

$\begin{array}{llll}\text { ksrc } & 0.00 & 0.00 & 0.00\end{array}$

$\begin{array}{llll}0.00 & 0.00 & 0.10\end{array}$

$\begin{array}{lll}0.00 & 0.00 & 0.50\end{array}$

$\begin{array}{lll}0.00 & 0.00 & 1.00\end{array}$

$\begin{array}{lll}0.00 & 0.00 & 2.00\end{array}$

$\begin{array}{lll}0.00 & 0.00 & 3.00\end{array}$

$\begin{array}{lll}1.00 & 1.00 & 1.00\end{array}$

$\begin{array}{lll}1.00 & 1.00 & 2.00\end{array}$

$\begin{array}{lll}1.00 & 1.00 & 3.00\end{array}$

$\begin{array}{lll}2.00 & 2.00 & 1.00\end{array}$

$\begin{array}{lll}2.00 & 2.00 & 2.00\end{array}$

$\begin{array}{lll}2.00 & 2.00 & 3.00\end{array}$

$\begin{array}{lll}-1.00 & -1.00 & -1.00\end{array}$

$\begin{array}{lll}-10.00 & -10.00 & -20.00\end{array}$

$\begin{array}{lll}-10.00 & -10.00 & -30.00\end{array}$

c

m2 \$water

$1001.66 \mathrm{c} \quad 2$

$8016.66 \mathrm{c} \quad 1$

$\mathrm{mt} 2$ 1wtr.60t

c $\mathrm{pu} /$ water mixture

m11 \$ Pu(NO3)4 - NO3 - H2O, 100.0 wt\% Pu239, ZZZ1 milli-g Pu/cc, $<$ denSol|3.4 $>$

c molarity $\mathrm{pu}=<\operatorname{molPu} \mid 3.4>$, acid $=<\operatorname{molAcid} \mid 3.4>, \mathrm{H} 2 \mathrm{O}=\langle\operatorname{molH} 2 \mathrm{O}| 3.4>$

c Pu in salt

$94239.66 \mathrm{c}<\mathrm{molPu}$ *ena $\mid 3.4>$

$94240.66 \mathrm{c}<\mathrm{molPu}^{*}(1$-ena $) \mid 3.4>$

c NO3 in salt plus acid

$7014.66 \mathrm{c}<4 * \mathrm{molPu}+$ molAcid $\mid 3.4>$

$8016.66 \mathrm{c}<12 * \mathrm{molPu}+3 * \operatorname{molAcid} \mid 3.4>$

c water

$1001.66 \mathrm{c}<2 *$ molH2O + molAcid $\mid 3.4>$ \$Includes Free Hydrogen Ion

$8016.66 \mathrm{c}<\mathrm{molH} 2 \mathrm{O} \mid 3.4>$

mt11 1wtr.60t

$\mathrm{c}$

c 
Template File for Case: PuN40-sp-0970-10

sp, 97.0 wt\% Pu239, ZZZ1 milli-g/cc, in Pu(NO3)4, with 0 Molar HNO3

$\mathrm{c}$

c worm notes: format of the form $\mid$ n.m for floating point, $\mid \mathrm{n}$ for integers

c zzz0 (in upper case) is replaced with the number of cyles

c $\quad$ zzz1 $=$ the actual concentration in units of milli-g/cc

c $\quad$ zzz2 $=$ solution density in $\mathrm{g} / \mathrm{cc}$

c $\quad \mathrm{qqq1}=$ the minimum dimensions (in hundreths of inches)

c $\quad \mathrm{qqq} 2=$ the maximum dimensions (in hundreths of inches)

c $\quad \mathrm{qqq} 3=$ the delta (in hundreths of inches)

c

c Diameter $=<\mathrm{d} 1=$ QQQ1:QQQ2:QQQ3 $\mid 5>$ hundreths of inches - must be integer

$\mathrm{c}$ radius $=<\mathrm{rd}=\mathrm{d} 1 * 2.54 / 100 / 2 \mid 5.4>\mathrm{cm}$

c note for perl, numbers with leading zeros must be enclosed in single quotes or else

c they will be interpretted as octal

$\mathrm{c}$ Conc $=<\mathrm{c}={ }^{\prime} \mathrm{ZZZ1} 1 \mid 5>$ milli-g Pu/cc

c Reflector width $=\langle\mathrm{rw}=10.0| 2.1>$ inches

c Enrichment $=<$ ena $=0.97 \mid 1.2>$ atom fraction $\mathrm{Pu} 239$

c Mols of Acid per liter $<$ molAcid $=0.0 \mid 1.2>$

c

$\mathrm{c}$

c Density of solution $<$ denSol $=Z Z Z 2 / 1$ e $5 \mid 1.5>$

c

C

c Atomic Weights

c $\mathrm{H} 2 \mathrm{O}=\langle$ atwH2O $=18.0152| 3.4>$

$\mathrm{c} \mathrm{NO3}=\langle$ atwNO3 $=62.0049| 3.4>$

c $\mathrm{HNO} 3=<\mathrm{atwHNO} 3=63.0128 \mid 3.4>$

c Pu239 $=<$ atwPu239 $=239.0522 \mid 3.4>$

c Pu240 $=<$ atwPu240 $=240.0538 \mid 3.4>$

c $\mathrm{Pu}=<\mathrm{atwPu}=\mathrm{ena}^{*}$ atwPu239+(1-ena)*atwPu240|3.4>

c

c Molarity of solution components

c Mols of $\mathrm{Pu}$ per liter $<\mathrm{molPu}=\mathrm{c} / \mathrm{atwPu} \mid 3.4>$

c Mols of $\mathrm{H} 2 \mathrm{O}$ per liter $<$ molH2O $=(1000 *$ denSol-molPu* $(a+w P u+4 * a t w N O 3)$ -

molAcid*atwHNO3)/atwH2O $3.4>$

$\mathrm{c}$

c

$<$ perl $>$

sub IMP

\{

return 1 if $\left(\$ \_[0]>0\right)$;

return 0 ;

\}

$</$ perl $>$

c sphere

$2011<$-denSol|3.6> $-1 \quad$ imp:n=1

$21 \quad 2-1.000-21 \quad$ imp:n=<\&IMP(rw) $\mid 1>$ \$zero importance if no reflector

$220 \quad 2 \quad$ imp:n $=0$ 


$$
\begin{array}{lll}
1 & \text { so }<\mathrm{rd} \mid 4.4> & \text { \$ Sphere } \\
2 & \text { so }<\mathrm{rd}+\mathrm{rw}^{*} \text { in } \mid 4.4> & \text { \$Reflection }
\end{array}
$$

mode $\mathrm{n}$

kcode $20001.0100 \mathrm{ZZZ0}$

$\begin{array}{llll}\text { ksrc } & 0.00 & 0.00 & 0.00\end{array}$

$\begin{array}{llll}0.00 & 0.00 & 0.10\end{array}$

$\begin{array}{lll}0.00 & 0.00 & 0.50\end{array}$

$\begin{array}{lll}0.00 & 0.00 & 1.00\end{array}$

$\begin{array}{lll}0.00 & 0.00 & 2.00\end{array}$

$\begin{array}{lll}0.00 & 0.00 & 3.00\end{array}$

$\begin{array}{lll}1.00 & 1.00 & 1.00\end{array}$

$\begin{array}{lll}1.00 & 1.00 & 2.00\end{array}$

$\begin{array}{lll}1.00 & 1.00 & 3.00\end{array}$

$\begin{array}{lll}2.00 & 2.00 & 1.00\end{array}$

$\begin{array}{lll}2.00 & 2.00 & 2.00\end{array}$

$\begin{array}{lll}2.00 & 2.00 & 3.00\end{array}$

$\begin{array}{lll}-1.00 & -1.00 & -1.00\end{array}$

$\begin{array}{lll}-10.00 & -10.00 & -20.00\end{array}$

$\begin{array}{lll}-10.00 & -10.00 & -30.00\end{array}$

c

m2 \$water

$1001.66 \mathrm{c} \quad 2$

$8016.66 \mathrm{c} \quad 1$

$\mathrm{mt} 2$ 1wtr.60t

c $\mathrm{pu} /$ water mixture

m11 \$ Pu(NO3)4 - NO3 - H2O, 100.0 wt\% Pu239, ZZZ1 milli-g Pu/cc, $<$ denSol|3.4 $>$

c molarity $\mathrm{pu}=<\operatorname{molPu} \mid 3.4>$, acid $=<\operatorname{molAcid} \mid 3.4>, \mathrm{H} 2 \mathrm{O}=\langle\operatorname{molH} 2 \mathrm{O}| 3.4>$

c Pu in salt

$94239.66 \mathrm{c}<\mathrm{molPu}$ *ena $\mid 3.4>$

$94240.66 \mathrm{c}<\mathrm{molPu}^{*}(1$-ena $) \mid 3.4>$

c NO3 in salt plus acid

$7014.66 \mathrm{c}<4 * \mathrm{molPu}+$ molAcid $\mid 3.4>$

$8016.66 \mathrm{c}<12 * \mathrm{molPu}+3 * \operatorname{molAcid} \mid 3.4>$

c water

$1001.66 \mathrm{c}<2 *$ molH2O + molAcid $\mid 3.4>$ \$Includes Free Hydrogen Ion

$8016.66 \mathrm{c}<\mathrm{molH} 2 \mathrm{O} \mid 3.4>$

mt11 1wtr.60t

$\mathrm{c}$

c 


\section{Template File for Case: PuN43-sp-0970-00}

sp, 97.0 wt\% Pu239, ZZZ1 milli-g/cc, in Pu(NO3)4, with 3 Molar HNO3

c

c worm notes: format of the form $\mid n . m$ for floating point, $\mid \mathrm{n}$ for integers

c zzz0 (in upper case) is replaced with the number of cyles

c $\quad$ zzz1 $=$ the actual concentration in units of milli-g/cc

c $\quad$ zzz2 $=$ solution density in $\mathrm{g} / \mathrm{cc}$

c $\quad \mathrm{qqq} 1=$ the minimum dimensions (in hundreths of inches)

c $\quad \mathrm{qqq} 2=$ the maximum dimensions (in hundreths of inches)

c $\quad \mathrm{qqq} 3=$ the delta (in hundreths of inches)

c

c Diameter $=<\mathrm{d} 1=$ QQQ1:QQQ2:QQQ3 $\mid 5>$ hundreths of inches - must be integer

$\mathrm{c}$ radius $=<\mathrm{rd}=\mathrm{d} 1 * 2.54 / 100 / 2 \mid 5.4>\mathrm{cm}$

c note for perl, numbers with leading zeros must be enclosed in single quotes or else

c they will be interpretted as octal

$\mathrm{c}$ Conc $=<\mathrm{c}={ }^{\prime} \mathrm{ZZZ1} 1 \mid 5>$ milli-g Pu/cc

c Reflector width $=<\mathrm{rw}=0.0 \mid 2.1>$ inches

c Enrichment $=<$ ena $=0.97 \mid 1.2>$ atom fraction Pu239

c Mols of Acid per liter $<$ molAcid $=3.0 \mid 1.2>$

$\mathrm{c}$

$\mathrm{c}$

c Density of solution $<$ denSol $=Z Z Z 2 / 1 \mathrm{e} 5 \mid 1.5>$

c

c

c Atomic Weights

c $\mathrm{H} 2 \mathrm{O}=\langle$ atwH2O $=18.0152| 3.4>$

$\mathrm{c} \mathrm{NO3}=\langle$ atwNO3 $=62.0049| 3.4>$

c HNO3 $=<$ atwHNO3 $=63.0128 \mid 3.4>$

c Pu239 $=<$ atwPu239 $=239.0522 \mid 3.4>$

c Pu240 $=<$ atwPu240 $=240.0538 \mid 3.4>$

$\mathrm{c} \mathrm{Pu}=<\mathrm{atwPu}=\mathrm{ena}^{*}$ atwPu239+(1-ena)*atwPu240|3.4>

$\mathrm{c}$

c Molarity of solution components

c Mols of $\mathrm{Pu}$ per liter $<\mathrm{molPu}=\mathrm{c} / \mathrm{atwPu} \mid 3.4>$

c Mols of $\mathrm{H} 2 \mathrm{O}$ per liter $<$ molH2O $=(1000 *$ denSol-molPu* $(\operatorname{atwPu}+4 *$ atwNO3)molAcid*atwHNO3)/atwH2O|3.4>

$\mathrm{c}$

$\mathrm{c}$

$<$ perl $>$

sub IMP

\{

return 1 if $(\$[0]>0)$;

return 0 ;

\}

$</$ perl $>$

c sphere

$2011<$-denSol|3.6> $-1 \quad$ imp:n=1

$212-1.000-21 \quad$ imp:n=<\&IMP(rw) $\mid 1>$ \$zero importance if no reflector

$220 \quad 2$ imp:n=0 


$$
\begin{array}{lll}
1 & \text { so }<\mathrm{rd} \mid 4.4> & \text { \$ Sphere } \\
2 & \text { so }<\mathrm{rd}+\mathrm{rw}^{*} \text { in } \mid 4.4> & \text { \$Reflection }
\end{array}
$$

mode $\mathrm{n}$

kcode $20001.0100 \mathrm{ZZZ0}$

$\begin{array}{llll}\text { ksrc } & 0.00 & 0.00 & 0.00\end{array}$

$\begin{array}{llll}0.00 & 0.00 & 0.10\end{array}$

$\begin{array}{lll}0.00 & 0.00 & 0.50\end{array}$

$\begin{array}{lll}0.00 & 0.00 & 1.00\end{array}$

$\begin{array}{lll}0.00 & 0.00 & 2.00\end{array}$

$\begin{array}{lll}0.00 & 0.00 & 3.00\end{array}$

$\begin{array}{lll}1.00 & 1.00 & 1.00\end{array}$

$\begin{array}{lll}1.00 & 1.00 & 2.00\end{array}$

$\begin{array}{lll}1.00 & 1.00 & 3.00\end{array}$

$\begin{array}{lll}2.00 & 2.00 & 1.00\end{array}$

$\begin{array}{lll}2.00 & 2.00 & 2.00\end{array}$

$\begin{array}{lll}2.00 & 2.00 & 3.00\end{array}$

$\begin{array}{lll}-1.00 & -1.00 & -1.00\end{array}$

$\begin{array}{lll}-10.00 & -10.00 & -20.00\end{array}$

$\begin{array}{lll}-10.00 & -10.00 & -30.00\end{array}$

c

m2 \$water

$1001.66 \mathrm{c} \quad 2$

$8016.66 \mathrm{c} \quad 1$

$\mathrm{mt} 2$ 1wtr.60t

c $\mathrm{pu} /$ water mixture

m11 \$ Pu(NO3)4 - NO3 - H2O, 100.0 wt\% Pu239, ZZZ1 milli-g Pu/cc, $<$ denSol|3.4 $>$

c molarity $\mathrm{pu}=<\operatorname{molPu} \mid 3.4>$, acid $=<\operatorname{molAcid} \mid 3.4>, \mathrm{H} 2 \mathrm{O}=\langle\operatorname{molH} 2 \mathrm{O}| 3.4>$

c Pu in salt

$94239.66 \mathrm{c}<\mathrm{molPu}$ *ena $\mid 3.4>$

$94240.66 \mathrm{c}<\mathrm{molPu}^{*}(1$-ena $) \mid 3.4>$

c NO3 in salt plus acid

$7014.66 \mathrm{c}<4 * \mathrm{molPu}+$ molAcid $\mid 3.4>$

$8016.66 \mathrm{c}<12 * \mathrm{molPu}+3 * \operatorname{molAcid} \mid 3.4>$

c water

$1001.66 \mathrm{c}<2 *$ molH2O + molAcid $\mid 3.4>$ \$Includes Free Hydrogen Ion

$8016.66 \mathrm{c}<\mathrm{molH} 2 \mathrm{O} \mid 3.4>$

mt11 1wtr.60t

$\mathrm{c}$

c 
Template File for Case: PuN43-sp-0970-01

sp, 97.0 wt\% Pu239, ZZZ1 milli-g/cc, in Pu(NO3)4, with 3 Molar HNO3

$\mathrm{c}$

c worm notes: format of the form |n.m for floating point, $\mid \mathrm{n}$ for integers

c zzz0 (in upper case) is replaced with the number of cyles

c $\quad$ zzz1 $=$ the actual concentration in units of milli-g/cc

c $\quad$ zzz2 $=$ solution density in $\mathrm{g} / \mathrm{cc}$

c $\quad \mathrm{qqq1}=$ the minimum dimensions (in hundreths of inches)

c $\quad \mathrm{qqq} 2=$ the maximum dimensions (in hundreths of inches)

c $\quad \mathrm{qqq} 3=$ the delta (in hundreths of inches)

c

c Diameter $=<\mathrm{d} 1=$ QQQ1:QQQ2:QQQ3 $\mid 5>$ hundreths of inches - must be integer

$\mathrm{c}$ radius $=<\mathrm{rd}=\mathrm{d} 1 * 2.54 / 100 / 2 \mid 5.4>\mathrm{cm}$

c note for perl, numbers with leading zeros must be enclosed in single quotes or else

c they will be interpretted as octal

$\mathrm{c}$ Conc $=<\mathrm{c}={ }^{\prime} \mathrm{ZZZ1} 1 \mid 5>$ milli-g Pu/cc

c Reflector width $=<\mathrm{rw}=1.0 \mid 2.1>$ inches

c Enrichment $=<$ ena $=0.97 \mid 1.2>$ atom fraction Pu239

c Mols of Acid per liter $<$ molAcid $=3.0 \mid 1.2>$

$\mathrm{c}$

$\mathrm{c}$

c Density of solution $<$ denSol $=Z Z Z 2 / 1 \mathrm{e} 5 \mid 1.5>$

c

C

c Atomic Weights

c $\mathrm{H} 2 \mathrm{O}=\langle$ atwH2O $=18.0152| 3.4>$

$\mathrm{c} \mathrm{NO3}=\langle$ atwNO3 $=62.0049| 3.4>$

c $\mathrm{HNO} 3=<\mathrm{atwHNO} 3=63.0128 \mid 3.4>$

c Pu239 $=<$ atwPu239 $=239.0522 \mid 3.4>$

c Pu240 $=<$ atwPu240 $=240.0538 \mid 3.4>$

$\mathrm{c} \mathrm{Pu}=<\mathrm{atwPu}=\mathrm{ena}^{*}$ atwPu239+(1-ena)*atwPu240|3.4>

c

c Molarity of solution components

c Mols of $\mathrm{Pu}$ per liter $<\mathrm{molPu}=\mathrm{c} / \mathrm{atwPu} \mid 3.4>$

c Mols of $\mathrm{H} 2 \mathrm{O}$ per liter $<$ molH2O $=(1000 *$ denSol-molPu* $(a+w P u+4 * a t w N O 3)$ -

molAcid*atwHNO3)/atwH2O $3.4>$

$\mathrm{c}$

c

$<$ perl $>$

sub IMP

\{

return 1 if $(\$[0]>0)$;

return 0 ;

\}

$</$ perl $>$

c sphere

$2011<$-denSol|3.6> $-1 \quad$ imp:n=1

$21 \quad 2-1.000-21 \quad$ imp:n=<\&IMP(rw) $\mid 1>$ \$zero importance if no reflector

$220 \quad 2 \quad$ imp:n $=0$ 


$$
\begin{array}{lll}
1 & \text { so }<\mathrm{rd} \mid 4.4> & \text { \$ Sphere } \\
2 & \text { so }<\mathrm{rd}+\mathrm{rw}^{*} \text { in } \mid 4.4> & \text { \$Reflection }
\end{array}
$$

mode $\mathrm{n}$

kcode $20001.0100 \mathrm{ZZZ0}$

$\begin{array}{llll}\text { ksrc } & 0.00 & 0.00 & 0.00\end{array}$

$\begin{array}{llll}0.00 & 0.00 & 0.10\end{array}$

$\begin{array}{lll}0.00 & 0.00 & 0.50\end{array}$

$\begin{array}{lll}0.00 & 0.00 & 1.00\end{array}$

$\begin{array}{lll}0.00 & 0.00 & 2.00\end{array}$

$\begin{array}{lll}0.00 & 0.00 & 3.00\end{array}$

$\begin{array}{lll}1.00 & 1.00 & 1.00\end{array}$

$\begin{array}{lll}1.00 & 1.00 & 2.00\end{array}$

$\begin{array}{lll}1.00 & 1.00 & 3.00\end{array}$

$\begin{array}{lll}2.00 & 2.00 & 1.00\end{array}$

$\begin{array}{lll}2.00 & 2.00 & 2.00\end{array}$

$\begin{array}{lll}2.00 & 2.00 & 3.00\end{array}$

$\begin{array}{lll}-1.00 & -1.00 & -1.00\end{array}$

$\begin{array}{lll}-10.00 & -10.00 & -20.00\end{array}$

$\begin{array}{lll}-10.00 & -10.00 & -30.00\end{array}$

c

m2 \$water

$1001.66 \mathrm{c} \quad 2$

$8016.66 \mathrm{c} \quad 1$

$\mathrm{mt} 2$ 1wtr.60t

c $\mathrm{pu} /$ water mixture

m11 \$ Pu(NO3)4 - NO3 - H2O, 100.0 wt\% Pu239, ZZZ1 milli-g Pu/cc, $<$ denSol|3.4 $>$

c molarity $\mathrm{pu}=<\operatorname{molPu} \mid 3.4>$, acid $=<\operatorname{molAcid} \mid 3.4>, \mathrm{H} 2 \mathrm{O}=\langle\operatorname{molH} 2 \mathrm{O}| 3.4>$

c Pu in salt

$94239.66 \mathrm{c}<\mathrm{molPu}$ *ena $\mid 3.4>$

$94240.66 \mathrm{c}<\mathrm{molPu}^{*}(1$-ena $) \mid 3.4>$

c NO3 in salt plus acid

$7014.66 \mathrm{c}<4 * \mathrm{molPu}+$ molAcid $\mid 3.4>$

$8016.66 \mathrm{c}<12 * \mathrm{molPu}+3 * \operatorname{molAcid} \mid 3.4>$

c water

$1001.66 \mathrm{c}<2 *$ molH2O + molAcid $\mid 3.4>$ \$Includes Free Hydrogen Ion

$8016.66 \mathrm{c}<\mathrm{molH} 2 \mathrm{O} \mid 3.4>$

mt11 1wtr.60t

$\mathrm{c}$

c 
Template File for Case: PuN43-sp-0970-10

sp, 97.0 wt\% Pu239, ZZZ1 milli-g/cc, in Pu(NO3)4, with 3 Molar HNO3

$\mathrm{c}$

c worm notes: format of the form $\mid$ n.m for floating point, $\mid \mathrm{n}$ for integers

c zzz0 (in upper case) is replaced with the number of cyles

c $\quad$ zzz1 $=$ the actual concentration in units of milli-g/cc

c $\quad$ zzz2 $=$ solution density in $\mathrm{g} / \mathrm{cc}$

c $\quad \mathrm{qqq1}=$ the minimum dimensions (in hundreths of inches)

c $\quad \mathrm{qqq} 2=$ the maximum dimensions (in hundreths of inches)

c $\quad \mathrm{qqq} 3=$ the delta (in hundreths of inches)

c

c Diameter $=<\mathrm{d} 1=$ QQQ1:QQQ2:QQQ3 $\mid 5>$ hundreths of inches - must be integer

$\mathrm{c}$ radius $=<\mathrm{rd}=\mathrm{d} 1 * 2.54 / 100 / 2 \mid 5.4>\mathrm{cm}$

c note for perl, numbers with leading zeros must be enclosed in single quotes or else

c they will be interpretted as octal

$\mathrm{c}$ Conc $=<\mathrm{c}={ }^{\prime} \mathrm{ZZZ1} 1 \mid 5>$ milli-g Pu/cc

c Reflector width $=\langle\mathrm{rw}=10.0| 2.1>$ inches

c Enrichment $=<$ ena $=0.97 \mid 1.2>$ atom fraction Pu239

c Mols of Acid per liter $<$ molAcid $=3.0 \mid 1.2>$

c

$\mathrm{c}$

c Density of solution $<$ denSol $=Z Z Z 2 / 1$ e $5 \mid 1.5>$

c

C

c Atomic Weights

c $\mathrm{H} 2 \mathrm{O}=\langle$ atwH2O $=18.0152| 3.4>$

$\mathrm{c} \mathrm{NO3}=\langle$ atwNO3 $=62.0049| 3.4>$

c $\mathrm{HNO} 3=<\mathrm{atwHNO} 3=63.0128 \mid 3.4>$

c Pu239 $=<$ atwPu239 $=239.0522 \mid 3.4>$

c Pu240 $=<$ atwPu240 $=240.0538 \mid 3.4>$

c $\mathrm{Pu}=<\mathrm{atwPu}=\mathrm{ena}^{*}$ atwPu239+(1-ena)*atwPu240|3.4>

c

c Molarity of solution components

c Mols of $\mathrm{Pu}$ per liter $<\mathrm{molPu}=\mathrm{c} / \mathrm{atwPu} \mid 3.4>$

c Mols of $\mathrm{H} 2 \mathrm{O}$ per liter $<$ molH2O $=(1000 *$ denSol-molPu* $(a+w P u+4 * a t w N O 3)$ -

molAcid*atwHNO3)/atwH2O $3.4>$

$\mathrm{c}$

c

$<$ perl $>$

sub IMP

\{

return 1 if $\left(\$ \_[0]>0\right)$;

return 0 ;

\}

$</$ perl $>$

c sphere

$2011<$-denSol|3.6> $-1 \quad$ imp:n=1

$21 \quad 2-1.000-21 \quad$ imp:n=<\&IMP(rw) $\mid 1>$ \$zero importance if no reflector

$220 \quad 2 \quad$ imp:n $=0$ 


$$
\begin{array}{lll}
1 & \text { so }<\mathrm{rd} \mid 4.4> & \text { \$ Sphere } \\
2 & \text { so }<\mathrm{rd}+\mathrm{rw}^{*} \text { in } \mid 4.4> & \text { \$Reflection }
\end{array}
$$

mode $\mathrm{n}$

kcode $20001.0100 \mathrm{ZZZ0}$

$\begin{array}{llll}\text { ksrc } & 0.00 & 0.00 & 0.00\end{array}$

$\begin{array}{llll}0.00 & 0.00 & 0.10\end{array}$

$\begin{array}{lll}0.00 & 0.00 & 0.50\end{array}$

$\begin{array}{lll}0.00 & 0.00 & 1.00\end{array}$

$\begin{array}{lll}0.00 & 0.00 & 2.00\end{array}$

$\begin{array}{lll}0.00 & 0.00 & 3.00\end{array}$

$\begin{array}{lll}1.00 & 1.00 & 1.00\end{array}$

$\begin{array}{lll}1.00 & 1.00 & 2.00\end{array}$

$\begin{array}{lll}1.00 & 1.00 & 3.00\end{array}$

$\begin{array}{lll}2.00 & 2.00 & 1.00\end{array}$

$\begin{array}{lll}2.00 & 2.00 & 2.00\end{array}$

$\begin{array}{lll}2.00 & 2.00 & 3.00\end{array}$

$\begin{array}{lll}-1.00 & -1.00 & -1.00\end{array}$

$\begin{array}{lll}-10.00 & -10.00 & -20.00\end{array}$

$\begin{array}{lll}-10.00 & -10.00 & -30.00\end{array}$

c

m2 \$water

$1001.66 \mathrm{c} \quad 2$

$8016.66 \mathrm{c} \quad 1$

$\mathrm{mt} 2$ 1wtr.60t

c $\mathrm{pu} /$ water mixture

m11 \$ Pu(NO3)4 - NO3 - H2O, 100.0 wt\% Pu239, ZZZ1 milli-g Pu/cc, $<$ denSol|3.4 $>$

c molarity $\mathrm{pu}=<\operatorname{molPu} \mid 3.4>$, acid $=<\operatorname{molAcid} \mid 3.4>, \mathrm{H} 2 \mathrm{O}=\langle\operatorname{molH} 2 \mathrm{O}| 3.4>$

c Pu in salt

$94239.66 \mathrm{c}<\mathrm{molPu}$ *ena $\mid 3.4>$

$94240.66 \mathrm{c}<\mathrm{molPu}^{*}(1$-ena $) \mid 3.4>$

c NO3 in salt plus acid

$7014.66 \mathrm{c}<4 * \mathrm{molPu}+$ molAcid $\mid 3.4>$

$8016.66 \mathrm{c}<12 * \mathrm{molPu}+3 * \operatorname{molAcid} \mid 3.4>$

c water

$1001.66 \mathrm{c}<2 *$ molH2O + molAcid $\mid 3.4>$ \$Includes Free Hydrogen Ion

$8016.66 \mathrm{c}<\mathrm{molH} 2 \mathrm{O} \mid 3.4>$

mt11 1wtr.60t

$\mathrm{c}$

c 


\section{Template File for Case: PuN46-sp-0970-00}

sp, 97.0 wt\% Pu239, ZZZ1 milli-g/cc, in Pu(NO3)4, with 6 Molar HNO3

c

c worm notes: format of the form |n.m for floating point, $\mid \mathrm{n}$ for integers

c $\quad$ zzz0 (in upper case) is replaced with the number of cyles

c $\quad$ zzz1 $=$ the actual concentration in units of milli-g/cc

c $\quad$ zzz2 $=$ solution density in $\mathrm{g} / \mathrm{cc}$

c $\quad \mathrm{qqq} 1=$ the minimum dimensions (in hundreths of inches)

c $\quad \mathrm{qqq} 2=$ the maximum dimensions (in hundreths of inches)

c $\quad \mathrm{qqq} 3=$ the delta (in hundreths of inches)

c

c Diameter $=<\mathrm{d} 1=$ QQQ1:QQQ2:QQQ3 $\mid 5>$ hundreths of inches - must be integer

$\mathrm{c}$ radius $=<\mathrm{rd}=\mathrm{d} 1 * 2.54 / 100 / 2 \mid 5.4>\mathrm{cm}$

c note for perl, numbers with leading zeros must be enclosed in single quotes or else

c they will be interpretted as octal

$\mathrm{c}$ Conc $=<\mathrm{c}={ }^{\prime} \mathrm{ZZZ1} 1 \mid 5>$ milli-g Pu/cc

c Reflector width $=<\mathrm{rw}=0.0 \mid 2.1>$ inches

c Enrichment $=<$ ena $=0.97 \mid 1.2>$ atom fraction Pu239

c Mols of Acid per liter $<$ molAcid $=6.0 \mid 1.2>$

$\mathrm{c}$

$\mathrm{c}$

c Density of solution $<$ denSol $=Z Z Z 2 / 1 \mathrm{e} 5 \mid 1.5>$

c

c

c Atomic Weights

c $\mathrm{H} 2 \mathrm{O}=\langle$ atwH2O $=18.0152| 3.4>$

$\mathrm{c} \mathrm{NO3}=\langle$ atwNO3 $=62.0049| 3.4>$

c HNO3 $=<$ atwHNO3 $=63.0128 \mid 3.4>$

c Pu239 $=<$ atwPu239 $=239.0522 \mid 3.4>$

c Pu240 $=<$ atwPu240 $=240.0538 \mid 3.4>$

$\mathrm{c} \mathrm{Pu}=<\mathrm{atwPu}=\mathrm{ena}^{*}$ atwPu239+(1-ena)*atwPu240|3.4>

$\mathrm{c}$

c Molarity of solution components

c Mols of $\mathrm{Pu}$ per liter $<\mathrm{molPu}=\mathrm{c} / \mathrm{atwPu} \mid 3.4>$

c Mols of $\mathrm{H} 2 \mathrm{O}$ per liter $<$ molH2O $=(1000 *$ denSol-molPu* $(a t w P u+4 * a t w N O 3)$ molAcid*atwHNO3)/atwH2O|3.4>

$\mathrm{c}$

$\mathrm{c}$

$<$ perl $>$

sub IMP

\{

return 1 if $(\$[0]>0)$;

return 0 ;

\}

$</$ perl $>$

c sphere

$2011<$-denSol|3.6> $-1 \quad$ imp:n=1

$212-1.000-21 \quad$ imp:n=<\&IMP(rw) $\mid 1>$ \$zero importance if no reflector

$220 \quad 2$ imp:n=0 


$$
\begin{array}{lll}
1 & \text { so }<\mathrm{rd} \mid 4.4> & \text { \$ Sphere } \\
2 & \text { so }<\mathrm{rd}+\mathrm{rw}^{*} \text { in } \mid 4.4> & \text { \$Reflection }
\end{array}
$$

mode $\mathrm{n}$

kcode $20001.0100 \mathrm{ZZZ0}$

$\begin{array}{llll}\text { ksrc } & 0.00 & 0.00 & 0.00\end{array}$

$\begin{array}{llll}0.00 & 0.00 & 0.10\end{array}$

$\begin{array}{lll}0.00 & 0.00 & 0.50\end{array}$

$\begin{array}{lll}0.00 & 0.00 & 1.00\end{array}$

$\begin{array}{lll}0.00 & 0.00 & 2.00\end{array}$

$\begin{array}{lll}0.00 & 0.00 & 3.00\end{array}$

$\begin{array}{lll}1.00 & 1.00 & 1.00\end{array}$

$\begin{array}{lll}1.00 & 1.00 & 2.00\end{array}$

$\begin{array}{lll}1.00 & 1.00 & 3.00\end{array}$

$\begin{array}{lll}2.00 & 2.00 & 1.00\end{array}$

$\begin{array}{lll}2.00 & 2.00 & 2.00\end{array}$

$\begin{array}{lll}2.00 & 2.00 & 3.00\end{array}$

$\begin{array}{lll}-1.00 & -1.00 & -1.00\end{array}$

$\begin{array}{lll}-10.00 & -10.00 & -20.00\end{array}$

$\begin{array}{lll}-10.00 & -10.00 & -30.00\end{array}$

c

m2 \$water

$1001.66 \mathrm{c} \quad 2$

$8016.66 \mathrm{c} \quad 1$

$\mathrm{mt} 2$ 1wtr.60t

c $\mathrm{pu} /$ water mixture

m11 \$ Pu(NO3)4 - NO3 - H2O, 100.0 wt\% Pu239, ZZZ1 milli-g Pu/cc, $<$ denSol|3.4 $>$

c molarity $\mathrm{pu}=<\operatorname{molPu} \mid 3.4>$, acid $=<\operatorname{molAcid} \mid 3.4>, \mathrm{H} 2 \mathrm{O}=\langle\operatorname{molH} 2 \mathrm{O}| 3.4>$

c Pu in salt

$94239.66 \mathrm{c}<\mathrm{molPu}$ *ena $\mid 3.4>$

$94240.66 \mathrm{c}<\mathrm{molPu}^{*}(1$-ena $) \mid 3.4>$

c NO3 in salt plus acid

$7014.66 \mathrm{c}<4 * \mathrm{molPu}+$ molAcid $\mid 3.4>$

$8016.66 \mathrm{c}<12 * \mathrm{molPu}+3 * \operatorname{molAcid} \mid 3.4>$

c water

$1001.66 \mathrm{c}<2 *$ molH2O + molAcid $\mid 3.4>$ \$Includes Free Hydrogen Ion

$8016.66 \mathrm{c}<\mathrm{molH} 2 \mathrm{O} \mid 3.4>$

mt11 1wtr.60t

$\mathrm{c}$

c 
Template File for Case: PuN46-sp-0970-01

sp, 97.0 wt\% Pu239, ZZZ1 milli-g/cc, in Pu(NO3)4, with 6 Molar HNO3

$\mathrm{c}$

c worm notes: format of the form $\mid$ n.m for floating point, $\mid \mathrm{n}$ for integers

c zzz0 (in upper case) is replaced with the number of cyles

c $\quad$ zzz1 $=$ the actual concentration in units of milli-g/cc

c $\quad$ zzz2 $=$ solution density in $\mathrm{g} / \mathrm{cc}$

c $\quad \mathrm{qqq1}=$ the minimum dimensions (in hundreths of inches)

c $\quad \mathrm{qqq} 2=$ the maximum dimensions (in hundreths of inches)

c $\quad \mathrm{qqq} 3=$ the delta (in hundreths of inches)

c

c Diameter $=<\mathrm{d} 1=$ QQQ1:QQQ2:QQQ3 $\mid 5>$ hundreths of inches - must be integer

$\mathrm{c}$ radius $=<\mathrm{rd}=\mathrm{d} 1 * 2.54 / 100 / 2 \mid 5.4>\mathrm{cm}$

c note for perl, numbers with leading zeros must be enclosed in single quotes or else

c they will be interpretted as octal

$\mathrm{c}$ Conc $=<\mathrm{c}={ }^{\prime} \mathrm{ZZZ1} 1 \mid 5>$ milli-g Pu/cc

c Reflector width $=<\mathrm{rw}=1.0 \mid 2.1>$ inches

c Enrichment $=<$ ena $=0.97 \mid 1.2>$ atom fraction Pu239

c Mols of Acid per liter $<$ molAcid $=6.0 \mid 1.2>$

$\mathrm{c}$

$\mathrm{c}$

c Density of solution $<$ denSol $=Z Z Z 2 / 1$ e $5 \mid 1.5>$

c

C

c Atomic Weights

c $\mathrm{H} 2 \mathrm{O}=\langle$ atwH2O $=18.0152 \mid 3.4\rangle$

c NO3 $=\langle$ atwNO3 $=62.0049| 3.4>$

c $\mathrm{HNO} 3=<\mathrm{atwHNO} 3=63.0128 \mid 3.4>$

c Pu239 $=<$ atwPu239 $=239.0522 \mid 3.4>$

c Pu240 $=\langle$ atwPu240 $=240.0538| 3.4>$

c $\mathrm{Pu}=<\mathrm{atwPu}=\mathrm{ena}^{*}$ atwPu239+(1-ena)*atwPu240|3.4>

c

c Molarity of solution components

c Mols of $\mathrm{Pu}$ per liter $<\mathrm{molPu}=\mathrm{c} / \mathrm{atwPu} \mid 3.4>$

c Mols of $\mathrm{H} 2 \mathrm{O}$ per liter $<$ molH2O $=(1000 *$ denSol-molPu* $(\operatorname{atwPu}+4 * a+w N O 3)$ -

molAcid*atwHNO3)/atwH2O $3.4>$

$\mathrm{c}$

c

$<$ perl $>$

sub IMP

\{

return 1 if $\left(\$ \_[0]>0\right)$;

return 0 ;

\}

$</$ perl $>$

c sphere

$2011<$-denSol|3.6> -1 imp:n=1

$21 \quad 2-1.000-21 \quad$ imp:n=<\&IMP(rw) $\mid 1>$ \$zero importance if no reflector

$220 \quad 2$ imp:n=0 


$$
\begin{array}{lll}
1 & \text { so }<\mathrm{rd} \mid 4.4> & \text { \$ Sphere } \\
2 & \text { so }<\mathrm{rd}+\mathrm{rw}^{*} \text { in } \mid 4.4> & \text { \$Reflection }
\end{array}
$$

mode $\mathrm{n}$

kcode $20001.0100 \mathrm{ZZZ0}$

$\begin{array}{llll}\text { ksrc } & 0.00 & 0.00 & 0.00\end{array}$

$\begin{array}{llll}0.00 & 0.00 & 0.10\end{array}$

$\begin{array}{lll}0.00 & 0.00 & 0.50\end{array}$

$\begin{array}{lll}0.00 & 0.00 & 1.00\end{array}$

$\begin{array}{lll}0.00 & 0.00 & 2.00\end{array}$

$\begin{array}{lll}0.00 & 0.00 & 3.00\end{array}$

$\begin{array}{lll}1.00 & 1.00 & 1.00\end{array}$

$\begin{array}{lll}1.00 & 1.00 & 2.00\end{array}$

$\begin{array}{lll}1.00 & 1.00 & 3.00\end{array}$

$\begin{array}{lll}2.00 & 2.00 & 1.00\end{array}$

$\begin{array}{lll}2.00 & 2.00 & 2.00\end{array}$

$\begin{array}{lll}2.00 & 2.00 & 3.00\end{array}$

$\begin{array}{lll}-1.00 & -1.00 & -1.00\end{array}$

$\begin{array}{lll}-10.00 & -10.00 & -20.00\end{array}$

$\begin{array}{lll}-10.00 & -10.00 & -30.00\end{array}$

c

m2 \$water

$1001.66 \mathrm{c} \quad 2$

$8016.66 \mathrm{c} \quad 1$

$\mathrm{mt} 2$ 1wtr.60t

c $\mathrm{pu} /$ water mixture

m11 \$ Pu(NO3)4 - NO3 - H2O, 100.0 wt\% Pu239, ZZZ1 milli-g Pu/cc, $<$ denSol|3.4 $>$

c molarity $\mathrm{pu}=<\operatorname{molPu} \mid 3.4>$, acid $=<\operatorname{molAcid} \mid 3.4>, \mathrm{H} 2 \mathrm{O}=\langle\operatorname{molH} 2 \mathrm{O}| 3.4>$

c Pu in salt

$94239.66 \mathrm{c}<\mathrm{molPu}$ *ena $\mid 3.4>$

$94240.66 \mathrm{c}<\mathrm{molPu}^{*}(1$-ena $) \mid 3.4>$

c NO3 in salt plus acid

$7014.66 \mathrm{c}<4 * \mathrm{molPu}+$ molAcid $\mid 3.4>$

$8016.66 \mathrm{c}<12 * \mathrm{molPu}+3 * \operatorname{molAcid} \mid 3.4>$

c water

$1001.66 \mathrm{c}<2 *$ molH2O + molAcid $\mid 3.4>$ \$Includes Free Hydrogen Ion

$8016.66 \mathrm{c}<\mathrm{molH} 2 \mathrm{O} \mid 3.4>$

mt11 1wtr.60t

$\mathrm{c}$

c 
Template File for Case: PuN46-sp-0970-10

sp, 97.0 wt\% Pu239, ZZZ1 milli-g/cc, in Pu(NO3)4, with 6 Molar HNO3

$\mathrm{c}$

c worm notes: format of the form $\mid$ n.m for floating point, $\mid \mathrm{n}$ for integers

c zzz0 (in upper case) is replaced with the number of cyles

c $\quad$ zzz1 $=$ the actual concentration in units of milli-g/cc

c $\quad$ zzz2 $=$ solution density in $\mathrm{g} / \mathrm{cc}$

c $\quad \mathrm{qqq1}=$ the minimum dimensions (in hundreths of inches)

c $\quad \mathrm{qqq} 2=$ the maximum dimensions (in hundreths of inches)

c $\quad \mathrm{qqq} 3=$ the delta (in hundreths of inches)

c

c Diameter $=<\mathrm{d} 1=$ QQQ1:QQQ2:QQQ3 $\mid 5>$ hundreths of inches - must be integer

$\mathrm{c}$ radius $=<\mathrm{rd}=\mathrm{d} 1 * 2.54 / 100 / 2 \mid 5.4>\mathrm{cm}$

c note for perl, numbers with leading zeros must be enclosed in single quotes or else

c they will be interpretted as octal

$\mathrm{c}$ Conc $=<\mathrm{c}={ }^{\prime} \mathrm{ZZZ1} 1 \mid 5>$ milli-g Pu/cc

c Reflector width $=\langle\mathrm{rw}=10.0| 2.1>$ inches

c Enrichment $=<$ ena $=0.97 \mid 1.2>$ atom fraction Pu239

c Mols of Acid per liter $<$ molAcid $=6.0 \mid 1.2>$

c

$\mathrm{c}$

c Density of solution $<$ denSol $=Z Z Z 2 / 1$ e $5 \mid 1.5>$

c

C

c Atomic Weights

c $\mathrm{H} 2 \mathrm{O}=\langle$ atwH2O $=18.0152| 3.4>$

$\mathrm{c} \mathrm{NO3}=\langle$ atwNO3 $=62.0049| 3.4>$

c $\mathrm{HNO} 3=<\mathrm{atwHNO} 3=63.0128 \mid 3.4>$

c Pu239 $=<$ atwPu239 $=239.0522 \mid 3.4>$

c Pu240 $=<$ atwPu240 $=240.0538 \mid 3.4>$

c $\mathrm{Pu}=<\mathrm{atwPu}=\mathrm{ena}^{*}$ atwPu239+(1-ena)*atwPu240|3.4>

c

c Molarity of solution components

c Mols of $\mathrm{Pu}$ per liter $<\mathrm{molPu}=\mathrm{c} / \mathrm{atwPu} \mid 3.4>$

c Mols of $\mathrm{H} 2 \mathrm{O}$ per liter $<$ molH2O $=(1000 *$ denSol-molPu* $(a+w P u+4 * a t w N O 3)$ -

molAcid*atwHNO3)/atwH2O $3.4>$

$\mathrm{c}$

c

$<$ perl $>$

sub IMP

\{

return 1 if $\left(\$ \_[0]>0\right)$;

return 0 ;

\}

$</$ perl $>$

c sphere

$2011<$-denSol|3.6> $-1 \quad$ imp:n=1

$21 \quad 2-1.000-21 \quad$ imp:n=<\&IMP(rw) $\mid 1>$ \$zero importance if no reflector

$220 \quad 2$ imp:n=0 


$$
\begin{array}{lll}
1 & \text { so }<\mathrm{rd} \mid 4.4> & \text { \$ Sphere } \\
2 & \text { so }<\mathrm{rd}+\mathrm{rw}^{*} \text { in } \mid 4.4> & \text { \$Reflection }
\end{array}
$$

mode $\mathrm{n}$

kcode $20001.0100 \mathrm{ZZZ0}$

$\begin{array}{llll}\text { ksrc } & 0.00 & 0.00 & 0.00\end{array}$

$\begin{array}{llll}0.00 & 0.00 & 0.10\end{array}$

$\begin{array}{lll}0.00 & 0.00 & 0.50\end{array}$

$\begin{array}{lll}0.00 & 0.00 & 1.00\end{array}$

$\begin{array}{lll}0.00 & 0.00 & 2.00\end{array}$

$\begin{array}{lll}0.00 & 0.00 & 3.00\end{array}$

$\begin{array}{lll}1.00 & 1.00 & 1.00\end{array}$

$\begin{array}{lll}1.00 & 1.00 & 2.00\end{array}$

$\begin{array}{lll}1.00 & 1.00 & 3.00\end{array}$

$\begin{array}{lll}2.00 & 2.00 & 1.00\end{array}$

$\begin{array}{lll}2.00 & 2.00 & 2.00\end{array}$

$\begin{array}{lll}2.00 & 2.00 & 3.00\end{array}$

$\begin{array}{lll}-1.00 & -1.00 & -1.00\end{array}$

$\begin{array}{lll}-10.00 & -10.00 & -20.00\end{array}$

$\begin{array}{lll}-10.00 & -10.00 & -30.00\end{array}$

c

m2 \$water

$1001.66 \mathrm{c} \quad 2$

$8016.66 \mathrm{c} \quad 1$

$\mathrm{mt} 2$ 1wtr.60t

c $\mathrm{pu} /$ water mixture

m11 \$ Pu(NO3)4 - NO3 - H2O, 100.0 wt\% Pu239, ZZZ1 milli-g Pu/cc, $<$ denSol|3.4 $>$

c molarity $\mathrm{pu}=<\operatorname{molPu} \mid 3.4>$, acid $=<\operatorname{molAcid} \mid 3.4>, \mathrm{H} 2 \mathrm{O}=\langle\operatorname{molH} 2 \mathrm{O}| 3.4>$

c Pu in salt

$94239.66 \mathrm{c}<\mathrm{molPu}$ *ena $\mid 3.4>$

$94240.66 \mathrm{c}<\mathrm{molPu}^{*}(1$-ena $) \mid 3.4>$

c NO3 in salt plus acid

$7014.66 \mathrm{c}<4 * \mathrm{molPu}+$ molAcid $\mid 3.4>$

$8016.66 \mathrm{c}<12 * \mathrm{molPu}+3 * \operatorname{molAcid} \mid 3.4>$

c water

$1001.66 \mathrm{c}<2 *$ molH2O + molAcid $\mid 3.4>$ \$Includes Free Hydrogen Ion

$8016.66 \mathrm{c}<\mathrm{molH} 2 \mathrm{O} \mid 3.4>$

mt11 1wtr.60t

$\mathrm{c}$

c 


\section{Template File for Case: PuN40-sp-0950-00}

sp, 95.0 wt\% Pu239, ZZZ1 milli-g/cc, in Pu(NO3)4, with 0 Molar HNO3

c

c worm notes: format of the form |n.m for floating point, $\mid \mathrm{n}$ for integers

c $\quad$ zzz0 (in upper case) is replaced with the number of cyles

c $\quad$ zzz1 $=$ the actual concentration in units of milli-g/cc

c $\quad$ zzz2 $=$ solution density in $\mathrm{g} / \mathrm{cc}$

c $\quad \mathrm{qqq} 1=$ the minimum dimensions (in hundreths of inches)

c $\quad \mathrm{qqq} 2=$ the maximum dimensions (in hundreths of inches)

c $\quad \mathrm{qqq} 3=$ the delta (in hundreths of inches)

c

c Diameter $=<\mathrm{d} 1=$ QQQ1:QQQ2:QQQ3 $\mid 5>$ hundreths of inches - must be integer

$\mathrm{c}$ radius $=<\mathrm{rd}=\mathrm{d} 1 * 2.54 / 100 / 2 \mid 5.4>\mathrm{cm}$

c note for perl, numbers with leading zeros must be enclosed in single quotes or else

c they will be interpretted as octal

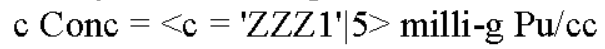

c Reflector width $=<\mathrm{rw}=0.0 \mid 2.1>$ inches

c Enrichment $=<$ ena $=0.95 \mid 1.2>$ atom fraction Pu239

c Mols of Acid per liter $<$ molAcid $=0.0 \mid 1.2>$

$\mathrm{c}$

$\mathrm{c}$

c Density of solution $<$ denSol $=Z Z Z 2 / 1 \mathrm{e} 5 \mid 1.5>$

c

c

c Atomic Weights

c $\mathrm{H} 2 \mathrm{O}=\langle$ atwH2O $=18.0152| 3.4>$

$\mathrm{c} \mathrm{NO3}=\langle$ atwNO3 $=62.0049| 3.4>$

c HNO3 $=<$ atwHNO3 $=63.0128 \mid 3.4>$

c Pu239 $=<$ atwPu239 $=239.0522 \mid 3.4>$

c Pu240 $=<$ atwPu240 $=240.0538 \mid 3.4>$

$\mathrm{c} \mathrm{Pu}=<\mathrm{atwPu}=\mathrm{ena}^{*}$ atwPu239+(1-ena)*atwPu240|3.4>

$\mathrm{c}$

c Molarity of solution components

c Mols of $\mathrm{Pu}$ per liter $<\mathrm{molPu}=\mathrm{c} / \mathrm{atwPu} \mid 3.4>$

c Mols of $\mathrm{H} 2 \mathrm{O}$ per liter $<$ molH2O $=(1000 *$ denSol-molPu* $(\operatorname{atwPu}+4 *$ atwNO3)molAcid*atwHNO3)/atwH2O|3.4>

$\mathrm{c}$

$\mathrm{c}$

$<$ perl $>$

sub IMP

\{

return 1 if $(\$[0]>0)$;

return 0 ;

\}

$</$ perl $>$

c sphere

$2011<$-denSol|3.6> $-1 \quad$ imp:n=1

$212-1.000-21 \quad$ imp:n=<\&IMP(rw) $\mid 1>$ \$zero importance if no reflector

$220 \quad 2 \quad$ imp:n $=0$ 


$$
\begin{array}{lll}
1 & \text { so }<\mathrm{rd} \mid 4.4> & \text { \$ Sphere } \\
2 & \text { so }<\mathrm{rd}+\mathrm{rw}^{*} \text { in } \mid 4.4> & \text { \$Reflection }
\end{array}
$$

mode $\mathrm{n}$

kcode $20001.0100 \mathrm{ZZZ0}$

$\begin{array}{llll}\text { ksrc } & 0.00 & 0.00 & 0.00\end{array}$

$\begin{array}{llll}0.00 & 0.00 & 0.10\end{array}$

$\begin{array}{lll}0.00 & 0.00 & 0.50\end{array}$

$\begin{array}{lll}0.00 & 0.00 & 1.00\end{array}$

$\begin{array}{lll}0.00 & 0.00 & 2.00\end{array}$

$\begin{array}{lll}0.00 & 0.00 & 3.00\end{array}$

$\begin{array}{lll}1.00 & 1.00 & 1.00\end{array}$

$\begin{array}{lll}1.00 & 1.00 & 2.00\end{array}$

$\begin{array}{lll}1.00 & 1.00 & 3.00\end{array}$

$\begin{array}{lll}2.00 & 2.00 & 1.00\end{array}$

$\begin{array}{lll}2.00 & 2.00 & 2.00\end{array}$

$\begin{array}{lll}2.00 & 2.00 & 3.00\end{array}$

$\begin{array}{lll}-1.00 & -1.00 & -1.00\end{array}$

$\begin{array}{lll}-10.00 & -10.00 & -20.00\end{array}$

$\begin{array}{lll}-10.00 & -10.00 & -30.00\end{array}$

c

m2 \$water

$1001.66 \mathrm{c} \quad 2$

$8016.66 \mathrm{c} \quad 1$

$\mathrm{mt} 2$ 1wtr.60t

c $\mathrm{pu} /$ water mixture

m11 \$ Pu(NO3)4 - NO3 - H2O, 100.0 wt\% Pu239, ZZZ1 milli-g Pu/cc, $<$ denSol|3.4 $>$

c molarity $\mathrm{pu}=<\operatorname{molPu} \mid 3.4>$, acid $=<\operatorname{molAcid} \mid 3.4>, \mathrm{H} 2 \mathrm{O}=\langle\operatorname{molH} 2 \mathrm{O}| 3.4>$

c Pu in salt

$94239.66 \mathrm{c}<\mathrm{molPu}$ *ena $\mid 3.4>$

$94240.66 \mathrm{c}<\mathrm{molPu}^{*}(1$-ena $) \mid 3.4>$

c NO3 in salt plus acid

$7014.66 \mathrm{c}<4 * \mathrm{molPu}+$ molAcid $\mid 3.4>$

$8016.66 \mathrm{c}<12 * \mathrm{molPu}+3 * \operatorname{molAcid} \mid 3.4>$

c water

$1001.66 \mathrm{c}<2 *$ molH2O + molAcid $\mid 3.4>$ \$Includes Free Hydrogen Ion

$8016.66 \mathrm{c}<\mathrm{molH} 2 \mathrm{O} \mid 3.4>$

mt11 1wtr.60t

$\mathrm{c}$

c 
Template File for Case: PuN40-sp-0950-01

sp, 95.0 wt\% Pu239, ZZZ1 milli-g/cc, in Pu(NO3)4, with 0 Molar HNO3

$\mathrm{c}$

c worm notes: format of the form |n.m for floating point, $\mid \mathrm{n}$ for integers

c zzz0 (in upper case) is replaced with the number of cyles

c $\quad$ zzz1 $=$ the actual concentration in units of milli-g/cc

c $\quad$ zzz2 $=$ solution density in $\mathrm{g} / \mathrm{cc}$

c $\quad \mathrm{qqq1}=$ the minimum dimensions (in hundreths of inches)

c $\quad \mathrm{qqq} 2=$ the maximum dimensions (in hundreths of inches)

c $\quad \mathrm{qqq} 3=$ the delta (in hundreths of inches)

c

c Diameter $=<\mathrm{d} 1=$ QQQ1:QQQ2:QQQ3 $\mid 5>$ hundreths of inches - must be integer

$\mathrm{c}$ radius $=<\mathrm{rd}=\mathrm{d} 1 * 2.54 / 100 / 2 \mid 5.4>\mathrm{cm}$

c note for perl, numbers with leading zeros must be enclosed in single quotes or else

c they will be interpretted as octal

$\mathrm{c}$ Conc $=<\mathrm{c}={ }^{\prime} \mathrm{ZZZ1} 1 \mid 5>$ milli-g Pu/cc

c Reflector width $=<\mathrm{rw}=1.0 \mid 2.1>$ inches

c Enrichment $=<$ ena $=0.95 \mid 1.2>$ atom fraction Pu239

c Mols of Acid per liter $<$ molAcid $=0.0 \mid 1.2>$

$\mathrm{c}$

$\mathrm{c}$

c Density of solution $<$ denSol $=Z Z Z 2 / 1$ e $5 \mid 1.5>$

c

C

c Atomic Weights

c $\mathrm{H} 2 \mathrm{O}=\langle$ atwH2O $=18.0152| 3.4>$

$\mathrm{c} \mathrm{NO3}=\langle$ atwNO3 $=62.0049| 3.4>$

c $\mathrm{HNO} 3=<\mathrm{atwHNO} 3=63.0128 \mid 3.4>$

c Pu239 $=<$ atwPu239 $=239.0522 \mid 3.4>$

c Pu240 $=<$ atwPu240 $=240.0538 \mid 3.4>$

c $\mathrm{Pu}=<\mathrm{atwPu}=\mathrm{ena}^{*}$ atwPu239+(1-ena)*atwPu240|3.4>

c

c Molarity of solution components

c Mols of $\mathrm{Pu}$ per liter $<\mathrm{molPu}=\mathrm{c} / \mathrm{atwPu} \mid 3.4>$

c Mols of $\mathrm{H} 2 \mathrm{O}$ per liter $<$ molH2O $=(1000 *$ denSol-molPu* $(a+w P u+4 * a t w N O 3)$ -

molAcid*atwHNO3)/atwH2O $3.4>$

$\mathrm{c}$

c

$<$ perl $>$

sub IMP

\{

return 1 if $\left(\$ \_[0]>0\right)$;

return 0 ;

\}

$</$ perl $>$

c sphere

$2011<$-denSol|3.6> $-1 \quad$ imp:n=1

$21 \quad 2-1.000-21 \quad$ imp:n=<\&IMP(rw) $\mid 1>$ \$zero importance if no reflector

$220 \quad 2 \quad$ imp:n $=0$ 


$$
\begin{array}{lll}
1 & \text { so }<\mathrm{rd} \mid 4.4> & \text { \$ Sphere } \\
2 & \text { so }<\mathrm{rd}+\mathrm{rw}^{*} \text { in } \mid 4.4> & \text { \$Reflection }
\end{array}
$$

mode $\mathrm{n}$

kcode $20001.0100 \mathrm{ZZZ0}$

$\begin{array}{llll}\text { ksrc } & 0.00 & 0.00 & 0.00\end{array}$

$\begin{array}{llll}0.00 & 0.00 & 0.10\end{array}$

$\begin{array}{lll}0.00 & 0.00 & 0.50\end{array}$

$\begin{array}{lll}0.00 & 0.00 & 1.00\end{array}$

$\begin{array}{lll}0.00 & 0.00 & 2.00\end{array}$

$\begin{array}{lll}0.00 & 0.00 & 3.00\end{array}$

$\begin{array}{lll}1.00 & 1.00 & 1.00\end{array}$

$\begin{array}{lll}1.00 & 1.00 & 2.00\end{array}$

$\begin{array}{lll}1.00 & 1.00 & 3.00\end{array}$

$\begin{array}{lll}2.00 & 2.00 & 1.00\end{array}$

$\begin{array}{lll}2.00 & 2.00 & 2.00\end{array}$

$\begin{array}{lll}2.00 & 2.00 & 3.00\end{array}$

$\begin{array}{lll}-1.00 & -1.00 & -1.00\end{array}$

$\begin{array}{lll}-10.00 & -10.00 & -20.00\end{array}$

$\begin{array}{lll}-10.00 & -10.00 & -30.00\end{array}$

c

m2 \$water

$1001.66 \mathrm{c} \quad 2$

$8016.66 \mathrm{c} \quad 1$

$\mathrm{mt} 2$ 1wtr.60t

c $\mathrm{pu} /$ water mixture

m11 \$ Pu(NO3)4 - NO3 - H2O, 100.0 wt\% Pu239, ZZZ1 milli-g Pu/cc, $<$ denSol|3.4 $>$

c molarity $\mathrm{pu}=<\operatorname{molPu} \mid 3.4>$, acid $=<\operatorname{molAcid} \mid 3.4>, \mathrm{H} 2 \mathrm{O}=\langle\operatorname{molH} 2 \mathrm{O}| 3.4>$

c Pu in salt

$94239.66 \mathrm{c}<\mathrm{molPu}$ *ena $\mid 3.4>$

$94240.66 \mathrm{c}<\mathrm{molPu}^{*}(1$-ena $) \mid 3.4>$

c NO3 in salt plus acid

$7014.66 \mathrm{c}<4 * \mathrm{molPu}+$ molAcid $\mid 3.4>$

$8016.66 \mathrm{c}<12 * \mathrm{molPu}+3 * \operatorname{molAcid} \mid 3.4>$

c water

$1001.66 \mathrm{c}<2 *$ molH2O + molAcid $\mid 3.4>$ \$Includes Free Hydrogen Ion

$8016.66 \mathrm{c}<\mathrm{molH} 2 \mathrm{O} \mid 3.4>$

mt11 1wtr.60t

$\mathrm{c}$

c 
Template File for Case: PuN40-sp-0950-10

sp, 95.0 wt\% Pu239, ZZZ1 milli-g/cc, in Pu(NO3)4, with 0 Molar HNO3

$\mathrm{c}$

c worm notes: format of the form $\mid$ n.m for floating point, $\mid \mathrm{n}$ for integers

c zzz0 (in upper case) is replaced with the number of cyles

c $\quad$ zzz1 $=$ the actual concentration in units of milli-g/cc

c $\quad$ zzz2 $=$ solution density in $\mathrm{g} / \mathrm{cc}$

c $\quad \mathrm{qqq1}=$ the minimum dimensions (in hundreths of inches)

c $\quad \mathrm{qqq} 2=$ the maximum dimensions (in hundreths of inches)

c $\quad \mathrm{qqq} 3=$ the delta (in hundreths of inches)

c

c Diameter $=<\mathrm{d} 1=$ QQQ1:QQQ2:QQQ3 $\mid 5>$ hundreths of inches - must be integer

$\mathrm{c}$ radius $=<\mathrm{rd}=\mathrm{d} 1 * 2.54 / 100 / 2 \mid 5.4>\mathrm{cm}$

c note for perl, numbers with leading zeros must be enclosed in single quotes or else

c they will be interpretted as octal

$\mathrm{c}$ Conc $=<\mathrm{c}={ }^{\prime} \mathrm{ZZZ1} 1 \mid 5>$ milli-g Pu/cc

c Reflector width $=<\mathrm{rw}=10.0 \mid 2.1>$ inches

c Enrichment $=<$ ena $=0.95 \mid 1.2>$ atom fraction Pu239

c Mols of Acid per liter $<$ molAcid $=0.0 \mid 1.2>$

$\mathrm{c}$

$\mathrm{c}$

c Density of solution $<$ denSol $=Z Z Z 2 / 1$ e $5 \mid 1.5>$

c

C

c Atomic Weights

c $\mathrm{H} 2 \mathrm{O}=\langle$ atwH2O $=18.0152| 3.4>$

$\mathrm{c} \mathrm{NO3}=\langle$ atwNO3 $=62.0049| 3.4>$

c $\mathrm{HNO} 3=<\mathrm{atwHNO} 3=63.0128 \mid 3.4>$

c Pu239 $=<$ atwPu239 $=239.0522 \mid 3.4>$

c Pu240 $=<$ atwPu240 $=240.0538 \mid 3.4>$

c $\mathrm{Pu}=<\mathrm{atwPu}=\mathrm{ena}^{*}$ atwPu239+(1-ena)*atwPu240|3.4>

c

c Molarity of solution components

c Mols of $\mathrm{Pu}$ per liter $<\mathrm{molPu}=\mathrm{c} / \mathrm{atwPu} \mid 3.4>$

c Mols of $\mathrm{H} 2 \mathrm{O}$ per liter $<$ molH2O $=(1000 *$ denSol-molPu* $(a+w P u+4 * a t w N O 3)$ -

molAcid*atwHNO3)/atwH2O $3.4>$

$\mathrm{c}$

c

$<$ perl $>$

sub IMP

\{

return 1 if $\left(\$ \_[0]>0\right)$;

return 0 ;

\}

$</$ perl $>$

c sphere

$2011<$-denSol|3.6> $-1 \quad$ imp:n=1

$21 \quad 2-1.000-21 \quad$ imp:n=<\&IMP(rw) $\mid 1>$ \$zero importance if no reflector

$220 \quad 2$ imp:n=0 


$$
\begin{array}{lll}
1 & \text { so }<\mathrm{rd} \mid 4.4> & \text { \$ Sphere } \\
2 & \text { so }<\mathrm{rd}+\mathrm{rw}^{*} \text { in } \mid 4.4> & \text { \$Reflection }
\end{array}
$$

mode $\mathrm{n}$

kcode $20001.0100 \mathrm{ZZZ0}$

$\begin{array}{llll}\text { ksrc } & 0.00 & 0.00 & 0.00\end{array}$

$\begin{array}{llll}0.00 & 0.00 & 0.10\end{array}$

$\begin{array}{lll}0.00 & 0.00 & 0.50\end{array}$

$\begin{array}{lll}0.00 & 0.00 & 1.00\end{array}$

$\begin{array}{lll}0.00 & 0.00 & 2.00\end{array}$

$\begin{array}{lll}0.00 & 0.00 & 3.00\end{array}$

$\begin{array}{lll}1.00 & 1.00 & 1.00\end{array}$

$\begin{array}{lll}1.00 & 1.00 & 2.00\end{array}$

$\begin{array}{lll}1.00 & 1.00 & 3.00\end{array}$

$\begin{array}{lll}2.00 & 2.00 & 1.00\end{array}$

$\begin{array}{lll}2.00 & 2.00 & 2.00\end{array}$

$\begin{array}{lll}2.00 & 2.00 & 3.00\end{array}$

$\begin{array}{lll}-1.00 & -1.00 & -1.00\end{array}$

$\begin{array}{lll}-10.00 & -10.00 & -20.00\end{array}$

$\begin{array}{lll}-10.00 & -10.00 & -30.00\end{array}$

c

m2 \$water

$1001.66 \mathrm{c} \quad 2$

$8016.66 \mathrm{c} \quad 1$

$\mathrm{mt} 2$ 1wtr.60t

c $\mathrm{pu} /$ water mixture

m11 \$ Pu(NO3)4 - NO3 - H2O, 100.0 wt\% Pu239, ZZZ1 milli-g Pu/cc, $<$ denSol|3.4 $>$

c molarity $\mathrm{pu}=<\operatorname{molPu} \mid 3.4>$, acid $=<\operatorname{molAcid} \mid 3.4>, \mathrm{H} 2 \mathrm{O}=\langle\operatorname{molH} 2 \mathrm{O}| 3.4>$

c Pu in salt

$94239.66 \mathrm{c}<\mathrm{molPu}$ *ena $\mid 3.4>$

$94240.66 \mathrm{c}<\mathrm{molPu}^{*}(1$-ena $) \mid 3.4>$

c NO3 in salt plus acid

$7014.66 \mathrm{c}<4 * \mathrm{molPu}+$ molAcid $\mid 3.4>$

$8016.66 \mathrm{c}<12 * \mathrm{molPu}+3 * \operatorname{molAcid} \mid 3.4>$

c water

$1001.66 \mathrm{c}<2 *$ molH2O + molAcid $\mid 3.4>$ \$Includes Free Hydrogen Ion

$8016.66 \mathrm{c}<\mathrm{molH} 2 \mathrm{O} \mid 3.4>$

mt11 1wtr.60t

$\mathrm{c}$

c 


\section{Template File for Case: PuN40-icyl-1000-00}

icyl, 100.0 wt\% Pu239, ZZZ1 milli-g/cc, in Pu(NO3)4, with 0 Molar HNO3

$\mathrm{c}$

c worm notes: format of the form $\mid$ n.m for floating point, $\mid \mathrm{n}$ for integers

c $\quad$ zzz0 (in upper case) is replaced with the number of cyles

c $\quad$ zzz1 $=$ the actual concentration in units of milli-g/cc

c $\quad$ zzz2 $=$ solution density in $\mathrm{g} / \mathrm{cc}$

c $\quad \mathrm{qqq1}=$ the minimum dimensions (in hundreths of inches)

c $\quad \mathrm{qqq} 2=$ the maximum dimensions (in hundreths of inches)

c $\quad \mathrm{qqq} 3=$ the delta (in hundreths of inches)

C

c Diameter $=<\mathrm{d} 1=$ QQQ1:QQQ2:QQQ3 $\mid 5>$ hundreths of inches - must be integer

$\mathrm{c}$ radius $=\langle\mathrm{rd}=\mathrm{d} 1 * 2.54 / 100 / 2| 5.4>\mathrm{cm}$

c note for perl, numbers with leading zeros must be enclosed in single quotes or else

$c$ they will be interpretted as octal

$\mathrm{c}$ Conc $=<\mathrm{c}={ }^{\prime} \mathrm{ZZZ1} \mid 5>$ milli-g Pu/cc

c Reflector width $=<\mathrm{rw}=0.0 \mid 2.1>$ inches

c Enrichment $=<$ ena $=1.0 \mid 1.2>$ atom fraction Pu239

c Mols of Acid per liter $<$ molAcid $=0.0 \mid 1.2>$

$\mathrm{c}$

$\mathrm{c}$

c Density of solution $<$ denSol $=$ ZZZ2/1e $5 \mid 1.5>$

c

$\mathrm{c}$

c Atomic Weights

c $\mathrm{H} 2 \mathrm{O}=\langle$ atwH2O $=18.0152| 3.4>$

c NO3 $=\langle$ atwNO3 $=62.0049| 3.4>$

c HNO3 $=\langle$ atwHNO3 $=63.0128| 3.4>$

c Pu239 $=\langle$ atwPu239 $=239.0522 \mid 3.4\rangle$

c Pu240 $=\langle$ atwPu240 $=240.0538| 3.4>$

c $\mathrm{Pu}=<$ atwPu=ena*atwPu239+(1-ena)*atwPu240|3.4>

$\mathrm{c}$

c Molarity of solution components

c Mols of $\mathrm{Pu}$ per liter $<\mathrm{molPu}=\mathrm{c} / \mathrm{atwPu} \mid 3.4>$

c Mols of $\mathrm{H} 2 \mathrm{O}$ per liter $<$ molH2O $=(1000 *$ denSol-molPu* $(\operatorname{atwPu}+4 * a+w N O 3)$ -

molAcid*atwHNO3)/atwH2O|3.4>

$\mathrm{c}$

$\mathrm{c}$

$<$ perl $>$

sub IMP

\{

return 1 if $(\$[0]>0)$;

return 0 ;

\}

$</$ perl $>$

c sphere

$2011<-$ denSol|3.6> $-1 \quad$ imp:n=1

$212-1.000 \quad-21 \quad$ imp:n=<\&IMP(rw) $\mid 1>$ \$zero importance if no reflector

$220 \quad 2$ imp:n=0 


$$
\begin{aligned}
& 1 \quad \mathrm{cz}<\mathrm{rd} \mid 4.4>\quad \text { \$ Cylinder } \\
& 2 \mathrm{cz}<\mathrm{rd}+\mathrm{rw}^{*} \text { in } \mid 4.4>\quad \text { \$ Reflection }
\end{aligned}
$$

mode $\mathrm{n}$

kcode 20001.0100 ZZZ0

$\begin{array}{llll}\text { ksrc } & 0.00 & 0.00 & 0.00\end{array}$

$\begin{array}{llll}0.00 & 0.00 & 0.10\end{array}$

$\begin{array}{lll}0.00 & 0.00 & 0.50\end{array}$

$\begin{array}{lll}0.00 & 0.00 & 1.00\end{array}$

$\begin{array}{lll}0.00 & 0.00 & 2.00\end{array}$

$\begin{array}{lll}0.00 & 0.00 & 3.00\end{array}$

$\begin{array}{lll}1.00 & 1.00 & 1.00\end{array}$

$\begin{array}{lll}1.00 & 1.00 & 2.00\end{array}$

$\begin{array}{lll}1.00 & 1.00 & 3.00\end{array}$

$\begin{array}{lll}2.00 & 2.00 & 1.00\end{array}$

$\begin{array}{lll}2.00 & 2.00 & 2.00\end{array}$

$\begin{array}{lll}2.00 & 2.00 & 3.00\end{array}$

$\begin{array}{lll}-1.00 & -1.00 & -1.00\end{array}$

$-10.00 \quad-10.00 \quad-20.00$

$\begin{array}{llll}-10.00 & -10.00 & -30.00\end{array}$

c

m2 \$water

$1001.66 \mathrm{c} \quad 2$

$8016.66 \mathrm{c} \quad 1$

$\mathrm{mt} 2$ 1wtr.60t

c $\mathrm{pu} /$ water mixture

m11 \$ Pu(NO3)4 - NO3 - H2O, 100.0 wt\% Pu239, ZZZ1 milli-g Pu/cc, $<$ denSol|3.4>

c molarity $\mathrm{pu}=<\operatorname{molPu} \mid 3.4>$, acid $=<\operatorname{molAcid} \mid 3.4>, \mathrm{H} 2 \mathrm{O}=\langle\operatorname{molH} 2 \mathrm{O}| 3.4>$

c Pu in salt

$94239.66 \mathrm{c}<\mathrm{molPu} * \mathrm{ena} \mid 3.4>$

c $94240.66 \mathrm{c}<\mathrm{molPu} *(1$-ena) $\mid 3.4>$

c NO3 in salt plus acid

$7014.66 \mathrm{c}<4 * \mathrm{molPu}+$ molAcid $\mid 3.4>$

$8016.66 \mathrm{c}<12 * \mathrm{molPu}+3 * \operatorname{mol} \mathrm{Acid} \mid 3.4>$

c water

$1001.66 \mathrm{c}<2 * \mathrm{molH} 2 \mathrm{O}+\operatorname{molAcid} \mid 3.4>$ \$Includes Free Hydrogen Ion

$8016.66 \mathrm{c}<\mathrm{molH} 2 \mathrm{O} \mid 3.4>$

mt11 1wtr.60t

c

c 


\section{Template File for Case: PuN40-icyl-1000-01}

icyl, 100.0 wt\% Pu239, ZZZ1 milli-g/cc, in Pu(NO3)4, with 0 Molar HNO3

c

c worm notes: format of the form |n.m for floating point, $\mid \mathrm{n}$ for integers

c $\quad$ zzz0 (in upper case) is replaced with the number of cyles

c $\quad$ zzz1 $=$ the actual concentration in units of milli-g/cc

c $\quad$ zzz2 $=$ solution density in $\mathrm{g} / \mathrm{cc}$

c $\quad \mathrm{qqq} 1=$ the minimum dimensions (in hundreths of inches)

c $\quad \mathrm{qqq} 2$ = the maximum dimensions (in hundreths of inches)

c $\quad \mathrm{qqq} 3=$ the delta (in hundreths of inches)

$\mathrm{c}$

c Diameter $=<\mathrm{d} 1=$ QQQ1:QQQ2:QQQ3 $\mid 5>$ hundreths of inches - must be integer

$\mathrm{c}$ radius $=<\mathrm{rd}=\mathrm{d} 1 * 2.54 / 100 / 2 \mid 5.4>\mathrm{cm}$

c note for perl, numbers with leading zeros must be enclosed in single quotes or else

c they will be interpretted as octal

$\mathrm{c}$ Conc $=<\mathrm{c}={ }^{\prime} \mathrm{ZZZ1} 1 \mid 5>$ milli-g Pu/cc

c Reflector width $=<\mathrm{rw}=1.0 \mid 2.1>$ inches

c Enrichment $=<$ ena $=1.0 \mid 1.2>$ atom fraction Pu239

c Mols of Acid per liter $<$ molAcid $=0.0 \mid 1.2>$

$\mathrm{c}$

$\mathrm{c}$

c Density of solution $<$ denSol $=Z Z Z 2 / 1 \mathrm{e} 5 \mid 1.5>$

c

c

c Atomic Weights

c $\mathrm{H} 2 \mathrm{O}=\langle$ atwH2O $=18.0152| 3.4>$

$\mathrm{c} \mathrm{NO3}=\langle$ atwNO3 $=62.0049| 3.4>$

c HNO3 $=<$ atwHNO3 $=63.0128 \mid 3.4>$

c Pu239 $=<$ atwPu239 $=239.0522 \mid 3.4>$

c Pu240 $=<$ atwPu240 $=240.0538 \mid 3.4>$

$\mathrm{c} \mathrm{Pu}=<\mathrm{atwPu}=\mathrm{ena}^{*}$ atwPu239+(1-ena)*atwPu240|3.4>

$\mathrm{c}$

c Molarity of solution components

c Mols of $\mathrm{Pu}$ per liter $<\mathrm{molPu}=\mathrm{c} / \mathrm{atwPu} \mid 3.4>$

c Mols of $\mathrm{H} 2 \mathrm{O}$ per liter $<$ molH2O $=(1000 *$ denSol-molPu* $(a t w P u+4 * a t w N O 3)$ molAcid*atwHNO3)/atwH2O $3.4>$

$\mathrm{c}$

$\mathrm{c}$

$<$ perl $>$

sub IMP

\{

return 1 if $(\$[0]>0)$;

return 0 ;

\}

$</$ perl $>$

c sphere

$2011<$-denSol|3.6> $-1 \quad$ imp:n=1

$212-1.000-21 \quad$ imp:n=<\&IMP(rw) $\mid 1>$ \$zero importance if no reflector

$220 \quad 2 \quad$ imp:n $=0$ 


$$
\begin{aligned}
& 1 \quad \mathrm{cz}<\mathrm{rd} \mid 4.4>\quad \text { \$ Cylinder } \\
& 2 \mathrm{cz}<\mathrm{rd}+\mathrm{rw}^{*} \text { in } \mid 4.4>\quad \text { \$ Reflection }
\end{aligned}
$$

mode $\mathrm{n}$

kcode 20001.0100 ZZZ0

$\begin{array}{llll}\text { ksrc } & 0.00 & 0.00 & 0.00\end{array}$

$\begin{array}{llll}0.00 & 0.00 & 0.10\end{array}$

$\begin{array}{lll}0.00 & 0.00 & 0.50\end{array}$

$\begin{array}{lll}0.00 & 0.00 & 1.00\end{array}$

$\begin{array}{lll}0.00 & 0.00 & 2.00\end{array}$

$\begin{array}{lll}0.00 & 0.00 & 3.00\end{array}$

$\begin{array}{lll}1.00 & 1.00 & 1.00\end{array}$

$\begin{array}{lll}1.00 & 1.00 & 2.00\end{array}$

$\begin{array}{lll}1.00 & 1.00 & 3.00\end{array}$

$\begin{array}{lll}2.00 & 2.00 & 1.00\end{array}$

$\begin{array}{lll}2.00 & 2.00 & 2.00\end{array}$

$\begin{array}{lll}2.00 & 2.00 & 3.00\end{array}$

$\begin{array}{lll}-1.00 & -1.00 & -1.00\end{array}$

$-10.00 \quad-10.00 \quad-20.00$

$\begin{array}{llll}-10.00 & -10.00 & -30.00\end{array}$

c

m2 \$water

$1001.66 \mathrm{c} \quad 2$

$8016.66 \mathrm{c} \quad 1$

$\mathrm{mt} 2$ 1wtr.60t

c $\mathrm{pu} /$ water mixture

m11 \$ Pu(NO3)4 - NO3 - H2O, 100.0 wt\% Pu239, ZZZ1 milli-g Pu/cc, $<$ denSol|3.4>

c molarity $\mathrm{pu}=<\operatorname{molPu} \mid 3.4>$, acid $=<\operatorname{molAcid} \mid 3.4>, \mathrm{H} 2 \mathrm{O}=\langle\operatorname{molH} 2 \mathrm{O}| 3.4>$

c Pu in salt

$94239.66 \mathrm{c}<\mathrm{molPu} * \mathrm{ena} \mid 3.4>$

c $94240.66 \mathrm{c}<\mathrm{molPu} *(1$-ena) $\mid 3.4>$

c NO3 in salt plus acid

$7014.66 \mathrm{c}<4 * \mathrm{molPu}+$ molAcid $\mid 3.4>$

$8016.66 \mathrm{c}<12 * \mathrm{molPu}+3 * \operatorname{mol} \mathrm{Acid} \mid 3.4>$

c water

$1001.66 \mathrm{c}<2 * \mathrm{molH} 2 \mathrm{O}+\operatorname{molAcid} \mid 3.4>$ \$Includes Free Hydrogen Ion

$8016.66 \mathrm{c}<\mathrm{molH} 2 \mathrm{O} \mid 3.4>$

mt11 1wtr.60t

c

c 


\section{Template File for Case: PuN40-icyl-1000-10}

icyl, 100.0 wt\% Pu239, ZZZ1 milli-g/cc, in Pu(NO3)4, with 0 Molar HNO3

c

c worm notes: format of the form |n.m for floating point, $\mid \mathrm{n}$ for integers

c $\quad$ zzz0 (in upper case) is replaced with the number of cyles

c $\quad$ zzz1 $=$ the actual concentration in units of milli-g/cc

c $\quad$ zzz2 $=$ solution density in $\mathrm{g} / \mathrm{cc}$

c $\quad \mathrm{qqq} 1=$ the minimum dimensions (in hundreths of inches)

c $\quad \mathrm{qqq} 2$ = the maximum dimensions (in hundreths of inches)

c $\quad \mathrm{qqq} 3=$ the delta (in hundreths of inches)

$\mathrm{c}$

c Diameter $=<\mathrm{d} 1=$ QQQ1:QQQ2:QQQ3 $\mid 5>$ hundreths of inches - must be integer

$\mathrm{c}$ radius $=<\mathrm{rd}=\mathrm{d} 1 * 2.54 / 100 / 2 \mid 5.4>\mathrm{cm}$

c note for perl, numbers with leading zeros must be enclosed in single quotes or else

c they will be interpretted as octal

$\mathrm{c}$ Conc $=<\mathrm{c}={ }^{\prime} \mathrm{ZZZ1} 1 \mid 5>$ milli-g Pu/cc

c Reflector width $=<\mathrm{rw}=10.0 \mid 2.1>$ inches

c Enrichment $=<$ ena $=1.0 \mid 1.2>$ atom fraction Pu239

c Mols of Acid per liter $<$ molAcid $=0.0 \mid 1.2>$

$\mathrm{c}$

$\mathrm{c}$

c Density of solution $<$ denSol $=Z Z Z 2 / 1 \mathrm{e} 5 \mid 1.5>$

c

c

c Atomic Weights

c $\mathrm{H} 2 \mathrm{O}=\langle$ atwH2O $=18.0152| 3.4>$

$\mathrm{c} \mathrm{NO3}=\langle$ atwNO3 $=62.0049| 3.4>$

c HNO3 $=<$ atwHNO3 $=63.0128 \mid 3.4>$

c $\mathrm{Pu} 239=<\mathrm{atwPu} 239=239.0522 \mid 3.4>$

c Pu240 $=<$ atwPu240 $=240.0538 \mid 3.4>$

$\mathrm{c} \mathrm{Pu}=<\mathrm{atwPu}=\mathrm{ena}^{*}$ atwPu239+(1-ena)*atwPu240|3.4>

$\mathrm{c}$

c Molarity of solution components

c Mols of $\mathrm{Pu}$ per liter $<\mathrm{molPu}=\mathrm{c} / \mathrm{atwPu} \mid 3.4>$

c Mols of $\mathrm{H} 2 \mathrm{O}$ per liter $<$ molH2O $=(1000 *$ denSol-molPu* $(\operatorname{atwPu}+4 *$ atwNO3)molAcid*atwHNO3)/atwH2O $3.4>$

$\mathrm{c}$

$\mathrm{c}$

$<$ perl $>$

sub IMP

\{

return 1 if $\left(\$ \_[0]>0\right)$;

return 0 ;

\}

$</$ perl $>$

c sphere

$2011<$-denSol|3.6> $-1 \quad$ imp:n=1

$212-1.000-21 \quad$ imp:n=<\&IMP(rw) $\mid 1>$ \$zero importance if no reflector

$220 \quad 2 \quad$ imp:n $=0$ 


$$
\begin{aligned}
& 1 \quad \mathrm{cz}<\mathrm{rd} \mid 4.4>\quad \text { \$ Cylinder } \\
& 2 \mathrm{cz}<\mathrm{rd}+\mathrm{rw}^{*} \text { in } \mid 4.4>\quad \text { \$ Reflection }
\end{aligned}
$$

mode $\mathrm{n}$

kcode 20001.0100 ZZZ0

$\begin{array}{llll}\text { ksrc } & 0.00 & 0.00 & 0.00\end{array}$

$\begin{array}{llll}0.00 & 0.00 & 0.10\end{array}$

$\begin{array}{lll}0.00 & 0.00 & 0.50\end{array}$

$\begin{array}{lll}0.00 & 0.00 & 1.00\end{array}$

$\begin{array}{lll}0.00 & 0.00 & 2.00\end{array}$

$\begin{array}{lll}0.00 & 0.00 & 3.00\end{array}$

$\begin{array}{lll}1.00 & 1.00 & 1.00\end{array}$

$\begin{array}{lll}1.00 & 1.00 & 2.00\end{array}$

$\begin{array}{lll}1.00 & 1.00 & 3.00\end{array}$

$\begin{array}{lll}2.00 & 2.00 & 1.00\end{array}$

$\begin{array}{lll}2.00 & 2.00 & 2.00\end{array}$

$\begin{array}{lll}2.00 & 2.00 & 3.00\end{array}$

$\begin{array}{lll}-1.00 & -1.00 & -1.00\end{array}$

$-10.00 \quad-10.00 \quad-20.00$

$\begin{array}{llll}-10.00 & -10.00 & -30.00\end{array}$

c

m2 \$water

$1001.66 \mathrm{c} \quad 2$

$8016.66 \mathrm{c} \quad 1$

$\mathrm{mt} 2$ 1wtr.60t

c $\mathrm{pu} /$ water mixture

m11 \$ Pu(NO3)4 - NO3 - H2O, 100.0 wt\% Pu239, ZZZ1 milli-g Pu/cc, $<$ denSol|3.4>

c molarity $\mathrm{pu}=<\operatorname{molPu} \mid 3.4>$, acid $=<\operatorname{molAcid} \mid 3.4>, \mathrm{H} 2 \mathrm{O}=\langle\operatorname{molH} 2 \mathrm{O}| 3.4>$

c Pu in salt

$94239.66 \mathrm{c}<\mathrm{molPu} * \mathrm{ena} \mid 3.4>$

c $94240.66 \mathrm{c}<\mathrm{molPu} *(1$-ena) $\mid 3.4>$

c NO3 in salt plus acid

$7014.66 \mathrm{c}<4 * \mathrm{molPu}+$ molAcid $\mid 3.4>$

$8016.66 \mathrm{c}<12 * \mathrm{molPu}+3 * \operatorname{mol} \mathrm{Acid} \mid 3.4>$

c water

$1001.66 \mathrm{c}<2 * \mathrm{molH} 2 \mathrm{O}+\operatorname{molAcid} \mid 3.4>$ \$Includes Free Hydrogen Ion

$8016.66 \mathrm{c}<\mathrm{molH} 2 \mathrm{O} \mid 3.4>$

mt11 1wtr.60t

c

c 


\section{Template File for Case: PuN43-icyl-1000-00}

icyl, 100.0 wt\% Pu239, ZZZ1 milli-g/cc, in Pu(NO3)4, with 3 Molar HNO3

c

c worm notes: format of the form |n.m for floating point, $\mid \mathrm{n}$ for integers

c $\quad$ zzz0 (in upper case) is replaced with the number of cyles

c $\quad$ zzz1 $=$ the actual concentration in units of milli-g/cc

c $\quad$ zzz2 $=$ solution density in $\mathrm{g} / \mathrm{cc}$

c $\quad \mathrm{qqq} 1=$ the minimum dimensions (in hundreths of inches)

c $\quad \mathrm{qqq} 2$ = the maximum dimensions (in hundreths of inches)

c $\quad \mathrm{qqq} 3=$ the delta (in hundreths of inches)

$\mathrm{c}$

c Diameter $=<\mathrm{d} 1=$ QQQ1:QQQ2:QQQ3 $\mid 5>$ hundreths of inches - must be integer

$\mathrm{c}$ radius $=<\mathrm{rd}=\mathrm{d} 1 * 2.54 / 100 / 2 \mid 5.4>\mathrm{cm}$

c note for perl, numbers with leading zeros must be enclosed in single quotes or else

c they will be interpretted as octal

$\mathrm{c}$ Conc $=<\mathrm{c}={ }^{\prime} \mathrm{ZZZ1} 1 \mid 5>$ milli-g Pu/cc

c Reflector width $=<\mathrm{rw}=0.0 \mid 2.1>$ inches

c Enrichment $=<$ ena $=1.0 \mid 1.2>$ atom fraction Pu239

c Mols of Acid per liter $<$ molAcid $=3.0 \mid 1.2>$

$\mathrm{c}$

$\mathrm{c}$

c Density of solution $<$ denSol $=Z Z Z 2 / 1 \mathrm{e} 5 \mid 1.5>$

c

c

c Atomic Weights

c $\mathrm{H} 2 \mathrm{O}=\langle$ atwH2O $=18.0152| 3.4>$

$\mathrm{c} \mathrm{NO3}=\langle$ atwNO3 $=62.0049| 3.4>$

c HNO3 $=<$ atwHNO3 $=63.0128 \mid 3.4>$

c Pu239 $=<$ atwPu239 $=239.0522 \mid 3.4>$

c Pu240 $=<$ atwPu240 $=240.0538 \mid 3.4>$

$\mathrm{c} \mathrm{Pu}=<\mathrm{atwPu}=\mathrm{ena}^{*}$ atwPu239+(1-ena)*atwPu240|3.4>

$\mathrm{c}$

c Molarity of solution components

c Mols of $\mathrm{Pu}$ per liter $<\mathrm{molPu}=\mathrm{c} / \mathrm{atwPu} \mid 3.4>$

c Mols of $\mathrm{H} 2 \mathrm{O}$ per liter $<$ molH2O $=(1000 *$ denSol-molPu* $(\operatorname{atwPu}+4 *$ atwNO3)molAcid*atwHNO3)/atwH2O $3.4>$

$\mathrm{c}$

$\mathrm{c}$

$<$ perl $>$

sub IMP

\{

return 1 if $(\$[0]>0)$;

return 0 ;

\}

$</$ perl $>$

c sphere

$2011<$-denSol|3.6> $-1 \quad$ imp:n=1

$212-1.000-21 \quad$ imp:n=<\&IMP(rw) $\mid 1>$ \$zero importance if no reflector

$220 \quad 2$ imp:n=0 


$$
\begin{aligned}
& 1 \quad \mathrm{cz}<\mathrm{rd} \mid 4.4>\quad \text { \$ Cylinder } \\
& 2 \mathrm{cz}<\mathrm{rd}+\mathrm{rw}^{*} \text { in } \mid 4.4>\quad \text { \$ Reflection }
\end{aligned}
$$

mode $\mathrm{n}$

kcode 20001.0100 ZZZ0

$\begin{array}{llll}\text { ksrc } & 0.00 & 0.00 & 0.00\end{array}$

$\begin{array}{llll}0.00 & 0.00 & 0.10\end{array}$

$\begin{array}{lll}0.00 & 0.00 & 0.50\end{array}$

$\begin{array}{lll}0.00 & 0.00 & 1.00\end{array}$

$\begin{array}{lll}0.00 & 0.00 & 2.00\end{array}$

$\begin{array}{lll}0.00 & 0.00 & 3.00\end{array}$

$\begin{array}{lll}1.00 & 1.00 & 1.00\end{array}$

$\begin{array}{lll}1.00 & 1.00 & 2.00\end{array}$

$\begin{array}{lll}1.00 & 1.00 & 3.00\end{array}$

$\begin{array}{lll}2.00 & 2.00 & 1.00\end{array}$

$\begin{array}{lll}2.00 & 2.00 & 2.00\end{array}$

$\begin{array}{lll}2.00 & 2.00 & 3.00\end{array}$

$\begin{array}{lll}-1.00 & -1.00 & -1.00\end{array}$

$-10.00 \quad-10.00 \quad-20.00$

$\begin{array}{llll}-10.00 & -10.00 & -30.00\end{array}$

c

m2 \$water

$1001.66 \mathrm{c} \quad 2$

$8016.66 \mathrm{c} \quad 1$

$\mathrm{mt} 2$ 1wtr.60t

c $\mathrm{pu} /$ water mixture

m11 \$ Pu(NO3)4 - NO3 - H2O, 100.0 wt\% Pu239, ZZZ1 milli-g Pu/cc, $<$ denSol|3.4>

c molarity $\mathrm{pu}=<\operatorname{molPu} \mid 3.4>$, acid $=<\operatorname{molAcid} \mid 3.4>, \mathrm{H} 2 \mathrm{O}=\langle\operatorname{molH} 2 \mathrm{O}| 3.4>$

c Pu in salt

$94239.66 \mathrm{c}<\mathrm{molPu} * \mathrm{ena} \mid 3.4>$

c $94240.66 \mathrm{c}<\mathrm{molPu} *(1$-ena) $\mid 3.4>$

c NO3 in salt plus acid

$7014.66 \mathrm{c}<4 * \mathrm{molPu}+$ molAcid $\mid 3.4>$

$8016.66 \mathrm{c}<12 * \mathrm{molPu}+3 * \operatorname{mol} \mathrm{Acid} \mid 3.4>$

c water

$1001.66 \mathrm{c}<2 * \mathrm{molH} 2 \mathrm{O}+\operatorname{molAcid} \mid 3.4>$ \$Includes Free Hydrogen Ion

$8016.66 \mathrm{c}<\mathrm{molH} 2 \mathrm{O} \mid 3.4>$

mt11 1wtr.60t

c

c 


\section{Template File for Case: PuN43-icyl-1000-01}

icyl, 100.0 wt\% Pu239, ZZZ1 milli-g/cc, in Pu(NO3)4, with 3 Molar HNO3

c

c worm notes: format of the form |n.m for floating point, $\mid \mathrm{n}$ for integers

c $\quad$ zzz0 (in upper case) is replaced with the number of cyles

c $\quad$ zzz1 $=$ the actual concentration in units of milli-g/cc

c $\quad$ zzz2 $=$ solution density in $\mathrm{g} / \mathrm{cc}$

c $\quad \mathrm{qqq} 1=$ the minimum dimensions (in hundreths of inches)

c $\quad \mathrm{qqq} 2$ = the maximum dimensions (in hundreths of inches)

c $\quad \mathrm{qqq} 3=$ the delta (in hundreths of inches)

$\mathrm{c}$

c Diameter $=<\mathrm{d} 1=$ QQQ1:QQQ2:QQQ3 $\mid 5>$ hundreths of inches - must be integer

$\mathrm{c}$ radius $=<\mathrm{rd}=\mathrm{d} 1 * 2.54 / 100 / 2 \mid 5.4>\mathrm{cm}$

c note for perl, numbers with leading zeros must be enclosed in single quotes or else

c they will be interpretted as octal

$\mathrm{c}$ Conc $=<\mathrm{c}={ }^{\prime} \mathrm{ZZZ1} 1 \mid 5>$ milli-g Pu/cc

c Reflector width $=<\mathrm{rw}=1.0 \mid 2.1>$ inches

c Enrichment $=<$ ena $=1.0 \mid 1.2>$ atom fraction Pu239

c Mols of Acid per liter $<$ molAcid $=3.0 \mid 1.2>$

$\mathrm{c}$

$\mathrm{c}$

c Density of solution $<$ denSol $=Z Z Z 2 / 1 \mathrm{e} 5 \mid 1.5>$

c

c

c Atomic Weights

c $\mathrm{H} 2 \mathrm{O}=\langle$ atwH2O $=18.0152| 3.4>$

$\mathrm{c} \mathrm{NO3}=\langle$ atwNO3 $=62.0049| 3.4>$

c HNO3 $=<$ atwHNO3 $=63.0128 \mid 3.4>$

c Pu239 $=<$ atwPu239 $=239.0522 \mid 3.4>$

c Pu240 $=<$ atwPu240 $=240.0538 \mid 3.4>$

$\mathrm{c} \mathrm{Pu}=<$ atwPu$=$ ena*atwPu239+(1-ena)*atwPu240|3.4 $>$

$\mathrm{c}$

c Molarity of solution components

c Mols of $\mathrm{Pu}$ per liter $<\mathrm{molPu}=\mathrm{c} / \mathrm{atwPu} \mid 3.4>$

c Mols of $\mathrm{H} 2 \mathrm{O}$ per liter $<$ molH2O $=(1000 *$ denSol-molPu* $(a t w P u+4 * a t w N O 3)$ molAcid*atwHNO3)/atwH2O|3.4>

$\mathrm{c}$

$\mathrm{c}$

$<$ perl $>$

sub IMP

\{

return 1 if $(\$[0]>0)$;

return 0 ;

\}

$</$ perl $>$

c sphere

$2011<$-denSol|3.6> $-1 \quad$ imp:n=1

$212-1.000-21 \quad$ imp:n=<\&IMP(rw) $\mid 1>$ \$zero importance if no reflector

$220 \quad 2 \quad$ imp:n $=0$ 


$$
\begin{aligned}
& 1 \quad \mathrm{cz}<\mathrm{rd} \mid 4.4>\quad \text { \$ Cylinder } \\
& 2 \mathrm{cz}<\mathrm{rd}+\mathrm{rw}^{*} \text { in } \mid 4.4>\quad \text { \$ Reflection }
\end{aligned}
$$

mode $\mathrm{n}$

kcode 20001.0100 ZZZ0

$\begin{array}{llll}\text { ksrc } & 0.00 & 0.00 & 0.00\end{array}$

$\begin{array}{llll}0.00 & 0.00 & 0.10\end{array}$

$\begin{array}{lll}0.00 & 0.00 & 0.50\end{array}$

$\begin{array}{lll}0.00 & 0.00 & 1.00\end{array}$

$\begin{array}{lll}0.00 & 0.00 & 2.00\end{array}$

$\begin{array}{lll}0.00 & 0.00 & 3.00\end{array}$

$\begin{array}{lll}1.00 & 1.00 & 1.00\end{array}$

$\begin{array}{lll}1.00 & 1.00 & 2.00\end{array}$

$\begin{array}{lll}1.00 & 1.00 & 3.00\end{array}$

$\begin{array}{lll}2.00 & 2.00 & 1.00\end{array}$

$\begin{array}{lll}2.00 & 2.00 & 2.00\end{array}$

$\begin{array}{lll}2.00 & 2.00 & 3.00\end{array}$

$\begin{array}{lll}-1.00 & -1.00 & -1.00\end{array}$

$-10.00 \quad-10.00 \quad-20.00$

$\begin{array}{llll}-10.00 & -10.00 & -30.00\end{array}$

c

m2 \$water

$1001.66 \mathrm{c} \quad 2$

$8016.66 \mathrm{c} \quad 1$

$\mathrm{mt} 2$ 1wtr.60t

c $\mathrm{pu} /$ water mixture

m11 \$ Pu(NO3)4 - NO3 - H2O, 100.0 wt\% Pu239, ZZZ1 milli-g Pu/cc, $<$ denSol|3.4>

c molarity $\mathrm{pu}=<\operatorname{molPu} \mid 3.4>$, acid $=<\operatorname{molAcid} \mid 3.4>, \mathrm{H} 2 \mathrm{O}=\langle\operatorname{molH} 2 \mathrm{O}| 3.4>$

c Pu in salt

$94239.66 \mathrm{c}<\mathrm{molPu} * \mathrm{ena} \mid 3.4>$

c $94240.66 \mathrm{c}<\mathrm{molPu} *(1$-ena) $\mid 3.4>$

c NO3 in salt plus acid

$7014.66 \mathrm{c}<4 * \mathrm{molPu}+$ molAcid $\mid 3.4>$

$8016.66 \mathrm{c}<12 * \mathrm{molPu}+3 * \operatorname{mol} \mathrm{Acid} \mid 3.4>$

c water

$1001.66 \mathrm{c}<2 * \mathrm{molH} 2 \mathrm{O}+\operatorname{molAcid} \mid 3.4>$ \$Includes Free Hydrogen Ion

$8016.66 \mathrm{c}<\mathrm{molH} 2 \mathrm{O} \mid 3.4>$

mt11 1wtr.60t

c

c 


\section{Template File for Case: PuN43-icyl-1000-10}

icyl, 100.0 wt\% Pu239, ZZZ1 milli-g/cc, in Pu(NO3)4, with 3 Molar HNO3

c

c worm notes: format of the form |n.m for floating point, $\mid \mathrm{n}$ for integers

c $\quad$ zzz0 (in upper case) is replaced with the number of cyles

c $\quad$ zzz1 $=$ the actual concentration in units of milli-g/cc

c $\quad$ zzz2 $=$ solution density in $\mathrm{g} / \mathrm{cc}$

c $\quad \mathrm{qqq} 1=$ the minimum dimensions (in hundreths of inches)

c $\quad \mathrm{qqq} 2$ = the maximum dimensions (in hundreths of inches)

c $\quad \mathrm{qqq} 3=$ the delta (in hundreths of inches)

$\mathrm{c}$

c Diameter $=<\mathrm{d} 1=$ QQQ1:QQQ2:QQQ3 $\mid 5>$ hundreths of inches - must be integer

$\mathrm{c}$ radius $=<\mathrm{rd}=\mathrm{d} 1 * 2.54 / 100 / 2 \mid 5.4>\mathrm{cm}$

c note for perl, numbers with leading zeros must be enclosed in single quotes or else

c they will be interpretted as octal

$\mathrm{c}$ Conc $=<\mathrm{c}={ }^{\prime} \mathrm{ZZZ1} 1 \mid 5>$ milli-g Pu/cc

c Reflector width $=<\mathrm{rw}=10.0 \mid 2.1>$ inches

c Enrichment $=<$ ena $=1.0 \mid 1.2>$ atom fraction Pu239

c Mols of Acid per liter $<$ molAcid $=3.0 \mid 1.2>$

$\mathrm{c}$

$\mathrm{c}$

c Density of solution $<$ denSol $=Z Z Z 2 / 1 \mathrm{e} 5 \mid 1.5>$

c

c

c Atomic Weights

c $\mathrm{H} 2 \mathrm{O}=\langle$ atwH2O $=18.0152| 3.4>$

$\mathrm{c} \mathrm{NO3}=\langle$ atwNO3 $=62.0049| 3.4>$

c HNO3 $=<$ atwHNO3 $=63.0128 \mid 3.4>$

c Pu239 $=<$ atwPu239 $=239.0522 \mid 3.4>$

c Pu240 $=<$ atwPu240 $=240.0538 \mid 3.4>$

$\mathrm{c} \mathrm{Pu}=<\mathrm{atwPu}=\mathrm{ena}^{*}$ atwPu239+(1-ena)*atwPu240|3.4>

$\mathrm{c}$

c Molarity of solution components

c Mols of $\mathrm{Pu}$ per liter $<\mathrm{molPu}=\mathrm{c} / \mathrm{atwPu} \mid 3.4>$

c Mols of $\mathrm{H} 2 \mathrm{O}$ per liter $<$ molH2O $=(1000 *$ denSol-molPu* $(\operatorname{atwPu}+4 *$ atwNO3)molAcid*atwHNO3)/atwH2O|3.4>

$\mathrm{c}$

$\mathrm{c}$

$<$ perl $>$

sub IMP

\{

return 1 if $(\$[0]>0)$;

return 0 ;

\}

$</$ perl $>$

c sphere

$2011<$-denSol|3.6> $-1 \quad$ imp:n=1

$212-1.000-21 \quad$ imp:n=<\&IMP(rw) $\mid 1>$ \$zero importance if no reflector

$220 \quad 2$ imp:n=0 


$$
\begin{aligned}
& 1 \quad \mathrm{cz}<\mathrm{rd} \mid 4.4>\quad \text { \$ Cylinder } \\
& 2 \mathrm{cz}<\mathrm{rd}+\mathrm{rw}^{*} \text { in } \mid 4.4>\quad \text { \$ Reflection }
\end{aligned}
$$

mode $\mathrm{n}$

kcode 20001.0100 ZZZ0

$\begin{array}{llll}\text { ksrc } & 0.00 & 0.00 & 0.00\end{array}$

$\begin{array}{llll}0.00 & 0.00 & 0.10\end{array}$

$\begin{array}{lll}0.00 & 0.00 & 0.50\end{array}$

$\begin{array}{lll}0.00 & 0.00 & 1.00\end{array}$

$\begin{array}{lll}0.00 & 0.00 & 2.00\end{array}$

$\begin{array}{lll}0.00 & 0.00 & 3.00\end{array}$

$\begin{array}{lll}1.00 & 1.00 & 1.00\end{array}$

$\begin{array}{lll}1.00 & 1.00 & 2.00\end{array}$

$\begin{array}{lll}1.00 & 1.00 & 3.00\end{array}$

$\begin{array}{lll}2.00 & 2.00 & 1.00\end{array}$

$\begin{array}{lll}2.00 & 2.00 & 2.00\end{array}$

$\begin{array}{lll}2.00 & 2.00 & 3.00\end{array}$

$\begin{array}{lll}-1.00 & -1.00 & -1.00\end{array}$

$-10.00-10.00 \quad-20.00$

$\begin{array}{llll}-10.00 & -10.00 & -30.00\end{array}$

c

m2 \$water

$1001.66 \mathrm{c} \quad 2$

$8016.66 \mathrm{c} \quad 1$

$\mathrm{mt} 2$ 1wtr.60t

c $\mathrm{pu} /$ water mixture

m11 \$ Pu(NO3)4 - NO3 - H2O, 100.0 wt\% Pu239, ZZZ1 milli-g Pu/cc, $<$ denSol|3.4>

c molarity $\mathrm{pu}=<\operatorname{molPu} \mid 3.4>$, acid $=<\operatorname{molAcid} \mid 3.4>, \mathrm{H} 2 \mathrm{O}=\langle\operatorname{molH} 2 \mathrm{O}| 3.4>$

c Pu in salt

$94239.66 \mathrm{c}<\mathrm{molPu} * \mathrm{ena} \mid 3.4>$

c $94240.66 \mathrm{c}<\mathrm{molPu} *(1$-ena) $\mid 3.4>$

c NO3 in salt plus acid

$7014.66 \mathrm{c}<4 * \mathrm{molPu}+$ molAcid $\mid 3.4>$

$8016.66 \mathrm{c}<12 * \mathrm{molPu}+3 * \operatorname{mol} \mathrm{Acid} \mid 3.4>$

c water

$1001.66 \mathrm{c}<2 * \mathrm{molH} 2 \mathrm{O}+\operatorname{molAcid} \mid 3.4>$ \$Includes Free Hydrogen Ion

$8016.66 \mathrm{c}<\mathrm{molH} 2 \mathrm{O} \mid 3.4>$

mt11 1wtr.60t

c

c 


\section{Template File for Case: PuN46-icyl-1000-00}

icyl, 100.0 wt\% Pu239, ZZZ1 milli-g/cc, in Pu(NO3)4, with 6 Molar HNO3

c

c worm notes: format of the form |n.m for floating point, $\mid \mathrm{n}$ for integers

c $\quad$ zzz0 (in upper case) is replaced with the number of cyles

c $\quad$ zzz1 $=$ the actual concentration in units of milli-g/cc

c $\quad$ zzz2 $=$ solution density in $\mathrm{g} / \mathrm{cc}$

c $\quad \mathrm{qqq} 1=$ the minimum dimensions (in hundreths of inches)

c $\quad \mathrm{qqq} 2$ = the maximum dimensions (in hundreths of inches)

c $\quad \mathrm{qqq} 3=$ the delta (in hundreths of inches)

$\mathrm{c}$

c Diameter $=<\mathrm{d} 1=$ QQQ1:QQQ2:QQQ3 $\mid 5>$ hundreths of inches - must be integer

$\mathrm{c}$ radius $=<\mathrm{rd}=\mathrm{d} 1 * 2.54 / 100 / 2 \mid 5.4>\mathrm{cm}$

c note for perl, numbers with leading zeros must be enclosed in single quotes or else

c they will be interpretted as octal

$\mathrm{c}$ Conc $=<\mathrm{c}={ }^{\prime} \mathrm{ZZZ1} 1 \mid 5>$ milli-g Pu/cc

c Reflector width $=<\mathrm{rw}=0.0 \mid 2.1>$ inches

c Enrichment $=<$ ena $=1.0 \mid 1.2>$ atom fraction Pu239

c Mols of Acid per liter $<$ molAcid $=6.0 \mid 1.2>$

$\mathrm{c}$

$\mathrm{c}$

c Density of solution $<$ denSol $=Z Z Z 2 / 1 \mathrm{e} 5 \mid 1.5>$

c

c

c Atomic Weights

c $\mathrm{H} 2 \mathrm{O}=\langle$ atwH2O $=18.0152| 3.4>$

$\mathrm{c} \mathrm{NO3}=\langle$ atwNO3 $=62.0049| 3.4>$

c HNO3 $=<$ atwHNO3 $=63.0128 \mid 3.4>$

c Pu239 $=<$ atwPu239 $=239.0522 \mid 3.4>$

c Pu240 $=<$ atwPu240 $=240.0538 \mid 3.4>$

$\mathrm{c} \mathrm{Pu}=<\mathrm{atwPu}=\mathrm{ena}^{*}$ atwPu239+(1-ena)*atwPu240|3.4>

$\mathrm{c}$

c Molarity of solution components

c Mols of $\mathrm{Pu}$ per liter $<\mathrm{molPu}=\mathrm{c} / \mathrm{atwPu} \mid 3.4>$

c Mols of $\mathrm{H} 2 \mathrm{O}$ per liter $<$ molH2O $=(1000 *$ denSol-molPu* $(\operatorname{atwPu}+4 *$ atwNO3)molAcid*atwHNO3)/atwH2O $3.4>$

$\mathrm{c}$

$\mathrm{c}$

$<$ perl $>$

sub IMP

\{

return 1 if $(\$[0]>0)$;

return 0 ;

\}

$</$ perl $>$

c sphere

$2011<$-denSol|3.6> $-1 \quad$ imp:n=1

$212-1.000-21 \quad$ imp:n=<\&IMP(rw) $\mid 1>$ \$zero importance if no reflector

$220 \quad 2$ imp:n=0 


$$
\begin{aligned}
& 1 \quad \mathrm{cz}<\mathrm{rd} \mid 4.4>\quad \text { \$ Cylinder } \\
& 2 \mathrm{cz}<\mathrm{rd}+\mathrm{rw}^{*} \text { in } \mid 4.4>\quad \text { \$ Reflection }
\end{aligned}
$$

mode $\mathrm{n}$

kcode 20001.0100 ZZZ0

$\begin{array}{llll}\text { ksrc } & 0.00 & 0.00 & 0.00\end{array}$

$\begin{array}{llll}0.00 & 0.00 & 0.10\end{array}$

$\begin{array}{lll}0.00 & 0.00 & 0.50\end{array}$

$\begin{array}{lll}0.00 & 0.00 & 1.00\end{array}$

$\begin{array}{lll}0.00 & 0.00 & 2.00\end{array}$

$\begin{array}{lll}0.00 & 0.00 & 3.00\end{array}$

$\begin{array}{lll}1.00 & 1.00 & 1.00\end{array}$

$\begin{array}{lll}1.00 & 1.00 & 2.00\end{array}$

$\begin{array}{lll}1.00 & 1.00 & 3.00\end{array}$

$\begin{array}{lll}2.00 & 2.00 & 1.00\end{array}$

$\begin{array}{lll}2.00 & 2.00 & 2.00\end{array}$

$\begin{array}{lll}2.00 & 2.00 & 3.00\end{array}$

$\begin{array}{lll}-1.00 & -1.00 & -1.00\end{array}$

$-10.00 \quad-10.00 \quad-20.00$

$\begin{array}{llll}-10.00 & -10.00 & -30.00\end{array}$

c

m2 \$water

$1001.66 \mathrm{c} \quad 2$

$8016.66 \mathrm{c} \quad 1$

$\mathrm{mt} 2$ 1wtr.60t

c $\mathrm{pu} /$ water mixture

m11 \$ Pu(NO3)4 - NO3 - H2O, 100.0 wt\% Pu239, ZZZ1 milli-g Pu/cc, $<$ denSol|3.4>

c molarity $\mathrm{pu}=<\operatorname{molPu} \mid 3.4>$, acid $=<\operatorname{molAcid} \mid 3.4>, \mathrm{H} 2 \mathrm{O}=\langle\operatorname{molH} 2 \mathrm{O}| 3.4>$

c Pu in salt

$94239.66 \mathrm{c}<\mathrm{molPu} * \mathrm{ena} \mid 3.4>$

c $94240.66 \mathrm{c}<\mathrm{molPu} *(1$-ena) $\mid 3.4>$

c NO3 in salt plus acid

$7014.66 \mathrm{c}<4 * \mathrm{molPu}+$ molAcid $\mid 3.4>$

$8016.66 \mathrm{c}<12 * \mathrm{molPu}+3 * \operatorname{mol} \mathrm{Acid} \mid 3.4>$

c water

$1001.66 \mathrm{c}<2 * \mathrm{molH} 2 \mathrm{O}+\operatorname{molAcid} \mid 3.4>$ \$Includes Free Hydrogen Ion

$8016.66 \mathrm{c}<\mathrm{molH} 2 \mathrm{O} \mid 3.4>$

mt11 1wtr.60t

c

c 
Template File for Case: PuN46-icyl-1000-01

icyl, 100.0 wt\% Pu239, ZZZ1 milli-g/cc, in Pu(NO3)4, with 6 Molar HNO3

$\mathrm{c}$

c worm notes: format of the form $\mid n . m$ for floating point, $\mid n$ for integers

c $\quad$ zzz0 (in upper case) is replaced with the number of cyles

c $\quad$ zzz1 $=$ the actual concentration in units of milli-g/cc

c $\quad$ zzz2 $=$ solution density in $\mathrm{g} / \mathrm{cc}$

c $\quad \mathrm{qqq} 1=$ the minimum dimensions (in hundreths of inches)

c $\quad$ qqq 2 the maximum dimensions (in hundreths of inches)

c $\quad \mathrm{qqq} 3=$ the delta (in hundreths of inches)

$\mathrm{c}$

c Diameter $=<\mathrm{d} 1=$ QQQ1:QQQ2:QQQ3 $\mid 5>$ hundreths of inches - must be integer

$\mathrm{c}$ radius $=\langle\mathrm{rd}=\mathrm{d} 1 * 2.54 / 100 / 2| 5.4>\mathrm{cm}$

c note for perl, numbers with leading zeros must be enclosed in single quotes or else

c they will be interpretted as octal

$\mathrm{c}$ Conc $=<\mathrm{c}={ }^{\prime} \mathrm{ZZZ1}{ }^{\prime} \mid 5>$ milli-g Pu/cc

c Reflector width $=<\mathrm{rw}=1.0 \mid 2.1>$ inches

c Enrichment $=<$ ena $=1.0 \mid 1.2>$ atom fraction $\mathrm{Pu} 239$

c Mols of Acid per liter $<$ molAcid $=6.0 \mid 1.2>$

$\mathrm{c}$

$\mathrm{c}$

c Density of solution $<$ denSol $=Z Z Z 2 / 1 \mathrm{e} 5 \mid 1.5>$

c

C

c Atomic Weights

$\mathrm{c} \mathrm{H} 2 \mathrm{O}=\langle\mathrm{atwH} 2 \mathrm{O}=18.0152| 3.4>$

$\mathrm{c} \mathrm{NO3}=\langle$ atwNO3 $=62.0049| 3.4>$

c HNO3 $=<$ atwHNO3 $=63.0128 \mid 3.4>$

c Pu239 $=<$ atwPu239 $=239.0522 \mid 3.4>$

c $\mathrm{Pu} 240=<\mathrm{atwPu} 240=240.0538 \mid 3.4>$

$\mathrm{c} \mathrm{Pu}=<$ atwPu$=$ ena*atwPu239+(1-ena)*atwPu240|3.4 $>$

$\mathrm{c}$

c Molarity of solution components

c Mols of $\mathrm{Pu}$ per liter $<\mathrm{molPu}=\mathrm{c} / \mathrm{atwPu} \mid 3.4>$

c Mols of $\mathrm{H} 2 \mathrm{O}$ per liter $<$ molH $2 \mathrm{O}=(1000 *$ denSol-molPu* $(\mathrm{atwPu}+4 * \mathrm{atwNO})$ -

molAcid*atwHNO3)/atwH2O|3.4>

$\mathrm{c}$

$\mathrm{c}$

$<$ perl $>$

sub IMP

\{

return 1 if $\left(\$ \_[0]>0\right)$;

return 0 ;

\}

$</$ perl $>$

c sphere

$2011<$-denSol|3.6> $-1 \quad$ imp:n=1

$212-1.000-21 \quad$ imp:n=<\&IMP(rw) $\mid 1>$ \$zero importance if no reflector

$220 \quad 2$ imp:n=0 


$$
\begin{array}{lll}
1 & \mathrm{cz}<\mathrm{rd} \mid 4.4> & \text { \$Cylinder } \\
2 & \mathrm{cz}<\mathrm{rd}+\mathrm{rw} * \mathrm{in} \mid 4.4> & \text { \$Reflection }
\end{array}
$$

mode $\mathrm{n}$

kcode 20001.0100 ZZZ0

$\begin{array}{llll}\text { ksrc } & 0.00 & 0.00 & 0.00\end{array}$

$\begin{array}{lll}0.00 & 0.00 & 0.10\end{array}$

$\begin{array}{lll}0.00 & 0.00 & 0.50\end{array}$

$\begin{array}{lll}0.00 & 0.00 & 1.00\end{array}$

$\begin{array}{lll}0.00 & 0.00 & 2.00\end{array}$

$\begin{array}{lll}0.00 & 0.00 & 3.00\end{array}$

$\begin{array}{lll}1.00 & 1.00 & 1.00\end{array}$

$\begin{array}{lll}1.00 & 1.00 & 2.00\end{array}$

$\begin{array}{lll}1.00 & 1.00 & 3.00\end{array}$

$\begin{array}{lll}2.00 & 2.00 & 1.00\end{array}$

$\begin{array}{lll}2.00 & 2.00 & 2.00\end{array}$

$\begin{array}{lll}2.00 & 2.00 & 3.00\end{array}$

$\begin{array}{lll}-1.00 & -1.00 & -1.00\end{array}$

$-10.00 \quad-10.00 \quad-20.00$

$\begin{array}{lll}-10.00 & -10.00 & -30.00\end{array}$

c

m2 \$water

$1001.66 \mathrm{c} \quad 2$

$8016.66 \mathrm{c} \quad 1$

$\mathrm{mt} 2$ 1wtr.60t

c pu/water mixture

m11 \$ Pu(NO3)4 - NO3 - H2O, 100.0 wt\% Pu239, ZZZ1 milli-g Pu/cc, $<$ denSol|3.4 $>$

c molarity $\mathrm{pu}=<\operatorname{molPu} \mid 3.4>$, acid $=<\operatorname{molAcid} \mid 3.4>, \mathrm{H} 2 \mathrm{O}=\langle\operatorname{molH} 2 \mathrm{O}| 3.4>$

c Pu in salt

94239.66 $<$ molPu*ena|3.4>

c $94240.66 \mathrm{c}<\mathrm{molPu} *(1$-ena) $\mid 3.4>$

c NO3 in salt plus acid

$7014.66 \mathrm{c}<4 * \mathrm{molPu}+\operatorname{molAcid} \mid 3.4>$

$8016.66 \mathrm{c}<12 * \mathrm{molPu}+3 * \operatorname{molAcid} \mid 3.4>$

c water

$1001.66 \mathrm{c}<2 *$ molH2O + molAcid $\mid 3.4>$ \$Includes Free Hydrogen Ion

$8016.66 \mathrm{c}<\mathrm{molH} 2 \mathrm{O} \mid 3.4>$

mt11 1wtr.60t

c

c 


\section{Template File for Case: PuN46-icyl-1000-10}

icyl, 100.0 wt\% Pu239, ZZZ1 milli-g/cc, in Pu(NO3)4, with 6 Molar HNO3

c

c worm notes: format of the form |n.m for floating point, $\mid \mathrm{n}$ for integers

c $\quad$ zzz0 (in upper case) is replaced with the number of cyles

c $\quad$ zzz1 $=$ the actual concentration in units of milli-g/cc

c $\quad$ zzz2 $=$ solution density in $\mathrm{g} / \mathrm{cc}$

c $\quad \mathrm{qqq} 1=$ the minimum dimensions (in hundreths of inches)

c $\quad \mathrm{qqq} 2$ = the maximum dimensions (in hundreths of inches)

c $\quad \mathrm{qqq} 3=$ the delta (in hundreths of inches)

$\mathrm{c}$

c Diameter $=<\mathrm{d} 1=$ QQQ1:QQQ2:QQQ3 $\mid 5>$ hundreths of inches - must be integer

$\mathrm{c}$ radius $=<\mathrm{rd}=\mathrm{d} 1 * 2.54 / 100 / 2 \mid 5.4>\mathrm{cm}$

c note for perl, numbers with leading zeros must be enclosed in single quotes or else

c they will be interpretted as octal

$\mathrm{c}$ Conc $=<\mathrm{c}={ }^{\prime} \mathrm{ZZZ1} 1 \mid 5>$ milli-g Pu/cc

c Reflector width $=<\mathrm{rw}=10.0 \mid 2.1>$ inches

c Enrichment $=<$ ena $=1.0 \mid 1.2>$ atom fraction Pu239

c Mols of Acid per liter $<$ molAcid $=6.0 \mid 1.2>$

$\mathrm{c}$

$\mathrm{c}$

c Density of solution $<$ denSol $=Z Z Z 2 / 1 \mathrm{e} 5 \mid 1.5>$

c

c

c Atomic Weights

c $\mathrm{H} 2 \mathrm{O}=\langle$ atwH2O $=18.0152| 3.4>$

$\mathrm{c} \mathrm{NO3}=\langle$ atwNO3 $=62.0049| 3.4>$

c HNO3 $=<$ atwHNO3 $=63.0128 \mid 3.4>$

c Pu239 $=<$ atwPu239 $=239.0522 \mid 3.4>$

c Pu240 $=<$ atwPu240 $=240.0538 \mid 3.4>$

$\mathrm{c} \mathrm{Pu}=<\mathrm{atwPu}=\mathrm{ena}^{*}$ atwPu239+(1-ena)*atwPu240|3.4>

$\mathrm{c}$

c Molarity of solution components

c Mols of $\mathrm{Pu}$ per liter $<\mathrm{molPu}=\mathrm{c} / \mathrm{atwPu} \mid 3.4>$

c Mols of $\mathrm{H} 2 \mathrm{O}$ per liter $<$ molH2O $=(1000 *$ denSol-molPu* $(\operatorname{atwPu}+4 *$ atwNO3)molAcid*atwHNO3)/atwH2O|3.4>

$\mathrm{c}$

$\mathrm{c}$

$<$ perl $>$

sub IMP

\{

return 1 if $(\$[0]>0)$;

return 0 ;

\}

$</$ perl $>$

c sphere

$2011<$-denSol|3.6> $-1 \quad$ imp:n=1

$212-1.000-21 \quad$ imp:n=<\&IMP(rw) $\mid 1>$ \$zero importance if no reflector

$220 \quad 2$ imp:n=0 


$$
\begin{aligned}
& 1 \quad \mathrm{cz}<\mathrm{rd} \mid 4.4>\quad \text { \$ Cylinder } \\
& 2 \mathrm{cz}<\mathrm{rd}+\mathrm{rw}^{*} \text { in } \mid 4.4>\quad \text { \$ Reflection }
\end{aligned}
$$

mode $\mathrm{n}$

kcode 20001.0100 ZZZ0

$\begin{array}{llll}\text { ksrc } & 0.00 & 0.00 & 0.00\end{array}$

$\begin{array}{llll}0.00 & 0.00 & 0.10\end{array}$

$\begin{array}{lll}0.00 & 0.00 & 0.50\end{array}$

$\begin{array}{lll}0.00 & 0.00 & 1.00\end{array}$

$\begin{array}{lll}0.00 & 0.00 & 2.00\end{array}$

$\begin{array}{lll}0.00 & 0.00 & 3.00\end{array}$

$\begin{array}{lll}1.00 & 1.00 & 1.00\end{array}$

$\begin{array}{lll}1.00 & 1.00 & 2.00\end{array}$

$\begin{array}{lll}1.00 & 1.00 & 3.00\end{array}$

$\begin{array}{lll}2.00 & 2.00 & 1.00\end{array}$

$\begin{array}{lll}2.00 & 2.00 & 2.00\end{array}$

$\begin{array}{lll}2.00 & 2.00 & 3.00\end{array}$

$\begin{array}{lll}-1.00 & -1.00 & -1.00\end{array}$

$-10.00 \quad-10.00 \quad-20.00$

$\begin{array}{llll}-10.00 & -10.00 & -30.00\end{array}$

c

m2 \$water

$1001.66 \mathrm{c} \quad 2$

$8016.66 \mathrm{c} \quad 1$

$\mathrm{mt} 2$ 1wtr.60t

c $\mathrm{pu} /$ water mixture

m11 \$ Pu(NO3)4 - NO3 - H2O, 100.0 wt\% Pu239, ZZZ1 milli-g Pu/cc, $<$ denSol|3.4>

c molarity $\mathrm{pu}=<\operatorname{molPu} \mid 3.4>$, acid $=<\operatorname{molAcid} \mid 3.4>, \mathrm{H} 2 \mathrm{O}=\langle\operatorname{molH} 2 \mathrm{O}| 3.4>$

c Pu in salt

$94239.66 \mathrm{c}<\mathrm{molPu} * \mathrm{ena} \mid 3.4>$

c $94240.66 \mathrm{c}<\mathrm{molPu} *(1$-ena) $\mid 3.4>$

c NO3 in salt plus acid

$7014.66 \mathrm{c}<4 * \mathrm{molPu}+$ molAcid $\mid 3.4>$

$8016.66 \mathrm{c}<12 * \mathrm{molPu}+3 * \operatorname{mol} \mathrm{Acid} \mid 3.4>$

c water

$1001.66 \mathrm{c}<2 * \mathrm{molH} 2 \mathrm{O}+\operatorname{molAcid} \mid 3.4>$ \$Includes Free Hydrogen Ion

$8016.66 \mathrm{c}<\mathrm{molH} 2 \mathrm{O} \mid 3.4>$

mt11 1wtr.60t

c

c 


\section{Template File for Case: PuN40-icyl-0970-00}

icyl, 97.0 wt\% Pu239, ZZZ1 milli-g/cc, in Pu(NO3)4, with 0 Molar HNO3

c

c worm notes: format of the form |n.m for floating point, $\mid \mathrm{n}$ for integers

c $\quad$ zzz0 (in upper case) is replaced with the number of cyles

c $\quad$ zzz1 $=$ the actual concentration in units of milli-g/cc

c $\quad$ zzz2 $=$ solution density in $\mathrm{g} / \mathrm{cc}$

c $\quad \mathrm{qqq} 1=$ the minimum dimensions (in hundreths of inches)

c $\quad \mathrm{qqq} 2$ = the maximum dimensions (in hundreths of inches)

c $\quad \mathrm{qqq} 3=$ the delta (in hundreths of inches)

$\mathrm{c}$

c Diameter $=<\mathrm{d} 1=$ QQQ1:QQQ2:QQQ3 $\mid 5>$ hundreths of inches - must be integer

$\mathrm{c}$ radius $=<\mathrm{rd}=\mathrm{d} 1 * 2.54 / 100 / 2 \mid 5.4>\mathrm{cm}$

c note for perl, numbers with leading zeros must be enclosed in single quotes or else

c they will be interpretted as octal

$\mathrm{c}$ Conc $=<\mathrm{c}={ }^{\prime} \mathrm{ZZZ1} 1 \mid 5>$ milli-g Pu/cc

c Reflector width $=<\mathrm{rw}=0.0 \mid 2.1>$ inches

c Enrichment $=<$ ena $=0.97 \mid 1.2>$ atom fraction Pu239

c Mols of Acid per liter $<$ molAcid $=0.0 \mid 1.2>$

$\mathrm{c}$

$\mathrm{c}$

c Density of solution $<$ denSol $=Z Z Z 2 / 1 \mathrm{e} 5 \mid 1.5>$

c

c

c Atomic Weights

c $\mathrm{H} 2 \mathrm{O}=\langle$ atwH2O $=18.0152| 3.4>$

$\mathrm{c} \mathrm{NO3}=\langle$ atwNO3 $=62.0049| 3.4>$

c HNO3 $=<$ atwHNO3 $=63.0128 \mid 3.4>$

c Pu239 $=<$ atwPu239 $=239.0522 \mid 3.4>$

c Pu240 $=<$ atwPu240 $=240.0538 \mid 3.4>$

$\mathrm{c} \mathrm{Pu}=<$ atwPu$=$ ena*atwPu239+(1-ena)*atwPu240|3.4 $>$

$\mathrm{c}$

c Molarity of solution components

c Mols of $\mathrm{Pu}$ per liter $<\mathrm{molPu}=\mathrm{c} / \mathrm{atwPu} \mid 3.4>$

c Mols of $\mathrm{H} 2 \mathrm{O}$ per liter $<$ molH2O $=(1000 *$ denSol-molPu* $(a t w P u+4 * a t w N O 3)$ molAcid*atwHNO3)/atwH2O|3.4>

$\mathrm{c}$

$\mathrm{c}$

$<$ perl $>$

sub IMP

\{

return 1 if $(\$[0]>0)$;

return 0 ;

\}

$</$ perl $>$

c sphere

$2011<$-denSol|3.6> $-1 \quad$ imp:n=1

$212-1.000-21 \quad$ imp:n=<\&IMP(rw) $\mid 1>$ \$zero importance if no reflector

$220 \quad 2$ imp:n=0 


$$
\begin{aligned}
& 1 \quad \mathrm{cz}<\mathrm{rd} \mid 4.4>\quad \text { \$ Cylinder } \\
& 2 \mathrm{cz}<\mathrm{rd}+\mathrm{rw}^{*} \text { in } \mid 4.4>\quad \text { \$ Reflection }
\end{aligned}
$$

mode $\mathrm{n}$

kcode 20001.0100 ZZZ0

$\begin{array}{llll}\text { ksrc } & 0.00 & 0.00 & 0.00\end{array}$

$\begin{array}{llll}0.00 & 0.00 & 0.10\end{array}$

$\begin{array}{lll}0.00 & 0.00 & 0.50\end{array}$

$\begin{array}{lll}0.00 & 0.00 & 1.00\end{array}$

$\begin{array}{lll}0.00 & 0.00 & 2.00\end{array}$

$\begin{array}{lll}0.00 & 0.00 & 3.00\end{array}$

$\begin{array}{lll}1.00 & 1.00 & 1.00\end{array}$

$\begin{array}{lll}1.00 & 1.00 & 2.00\end{array}$

$\begin{array}{lll}1.00 & 1.00 & 3.00\end{array}$

$\begin{array}{lll}2.00 & 2.00 & 1.00\end{array}$

$\begin{array}{lll}2.00 & 2.00 & 2.00\end{array}$

$\begin{array}{lll}2.00 & 2.00 & 3.00\end{array}$

$\begin{array}{lll}-1.00 & -1.00 & -1.00\end{array}$

$-10.00 \quad-10.00 \quad-20.00$

$\begin{array}{llll}-10.00 & -10.00 & -30.00\end{array}$

c

m2 \$water

$1001.66 \mathrm{c} \quad 2$

$8016.66 \mathrm{c} \quad 1$

$\mathrm{mt} 2$ 1wtr.60t

c $\mathrm{pu} /$ water mixture

m11 \$ Pu(NO3)4 - NO3 - H2O, 100.0 wt\% Pu239, ZZZ1 milli-g Pu/cc, $<$ denSol|3.4>

c molarity $\mathrm{pu}=<\operatorname{molPu} \mid 3.4>$, acid $=<\operatorname{molAcid} \mid 3.4>, \mathrm{H} 2 \mathrm{O}=\langle\operatorname{molH} 2 \mathrm{O}| 3.4>$

c Pu in salt

$94239.66 \mathrm{c}<\mathrm{molPu} *$ ena $\mid 3.4>$

$94240.66 \mathrm{c}<\mathrm{molPu}^{*}(1-\mathrm{ena}) \mid 3.4>$

c NO3 in salt plus acid

$7014.66 \mathrm{c}<4 * \mathrm{molPu}+$ molAcid $\mid 3.4>$

$8016.66 \mathrm{c}<12 * \mathrm{molPu}+3 * \operatorname{mol} \mathrm{Acid} \mid 3.4>$

c water

$1001.66 \mathrm{c}<2 * \mathrm{molH} 2 \mathrm{O}+\operatorname{molAcid} \mid 3.4>$ \$Includes Free Hydrogen Ion

$8016.66 \mathrm{c}<\mathrm{molH} 2 \mathrm{O} \mid 3.4>$

mt11 1wtr.60t

c

c 


\section{Template File for Case: PuN40-icyl-0970-01}

icyl, 97.0 wt\% Pu239, ZZZ1 milli-g/cc, in Pu(NO3)4, with 0 Molar HNO3

c

c worm notes: format of the form |n.m for floating point, $\mid \mathrm{n}$ for integers

c $\quad$ zzz0 (in upper case) is replaced with the number of cyles

c $\quad$ zzz1 $=$ the actual concentration in units of milli-g/cc

c $\quad$ zzz2 $=$ solution density in $\mathrm{g} / \mathrm{cc}$

c $\quad \mathrm{qqq} 1=$ the minimum dimensions (in hundreths of inches)

c $\quad \mathrm{qqq} 2$ = the maximum dimensions (in hundreths of inches)

c $\quad \mathrm{qqq} 3=$ the delta (in hundreths of inches)

$\mathrm{c}$

c Diameter $=<\mathrm{d} 1=$ QQQ1:QQQ2:QQQ3 $\mid 5>$ hundreths of inches - must be integer

$\mathrm{c}$ radius $=<\mathrm{rd}=\mathrm{d} 1 * 2.54 / 100 / 2 \mid 5.4>\mathrm{cm}$

c note for perl, numbers with leading zeros must be enclosed in single quotes or else

c they will be interpretted as octal

$\mathrm{c}$ Conc $=<\mathrm{c}={ }^{\prime} \mathrm{ZZZ1} 1 \mid 5>$ milli-g Pu/cc

c Reflector width $=<\mathrm{rw}=1.0 \mid 2.1>$ inches

c Enrichment $=<$ ena $=0.97 \mid 1.2>$ atom fraction Pu239

c Mols of Acid per liter $<$ molAcid $=0.0 \mid 1.2>$

$\mathrm{c}$

$\mathrm{c}$

c Density of solution $<$ denSol $=Z Z Z 2 / 1 \mathrm{e} 5 \mid 1.5>$

c

c

c Atomic Weights

c $\mathrm{H} 2 \mathrm{O}=\langle$ atwH2O $=18.0152| 3.4>$

$\mathrm{c} \mathrm{NO3}=\langle$ atwNO3 $=62.0049| 3.4>$

c HNO3 $=<$ atwHNO3 $=63.0128 \mid 3.4>$

c Pu239 $=<$ atwPu239 $=239.0522 \mid 3.4>$

c Pu240 $=<$ atwPu240 $=240.0538 \mid 3.4>$

$\mathrm{c} \mathrm{Pu}=<$ atwPu$=$ ena*atwPu239+(1-ena)*atwPu240|3.4 $>$

$\mathrm{c}$

c Molarity of solution components

c Mols of $\mathrm{Pu}$ per liter $<\mathrm{molPu}=\mathrm{c} / \mathrm{atwPu} \mid 3.4>$

c Mols of $\mathrm{H} 2 \mathrm{O}$ per liter $<$ molH2O $=(1000 *$ denSol-molPu* $(a t w P u+4 * a t w N O 3)$ molAcid*atwHNO3)/atwH2O|3.4>

$\mathrm{c}$

$\mathrm{c}$

$<$ perl $>$

sub IMP

\{

return 1 if $(\$[0]>0)$;

return 0 ;

\}

$</$ perl $>$

c sphere

$2011<$-denSol|3.6> $-1 \quad$ imp:n=1

$212-1.000-21 \quad$ imp:n=<\&IMP(rw) $\mid 1>$ \$zero importance if no reflector

$220 \quad 2 \quad$ imp:n $=0$ 


$$
\begin{aligned}
& 1 \quad \mathrm{cz}<\mathrm{rd} \mid 4.4>\quad \text { \$ Cylinder } \\
& 2 \mathrm{cz}<\mathrm{rd}+\mathrm{rw}^{*} \text { in } \mid 4.4>\quad \text { \$ Reflection }
\end{aligned}
$$

mode $\mathrm{n}$

kcode 20001.0100 ZZZ0

$\begin{array}{llll}\text { ksrc } & 0.00 & 0.00 & 0.00\end{array}$

$\begin{array}{llll}0.00 & 0.00 & 0.10\end{array}$

$\begin{array}{lll}0.00 & 0.00 & 0.50\end{array}$

$\begin{array}{lll}0.00 & 0.00 & 1.00\end{array}$

$\begin{array}{lll}0.00 & 0.00 & 2.00\end{array}$

$\begin{array}{lll}0.00 & 0.00 & 3.00\end{array}$

$\begin{array}{lll}1.00 & 1.00 & 1.00\end{array}$

$\begin{array}{lll}1.00 & 1.00 & 2.00\end{array}$

$\begin{array}{lll}1.00 & 1.00 & 3.00\end{array}$

$\begin{array}{lll}2.00 & 2.00 & 1.00\end{array}$

$\begin{array}{lll}2.00 & 2.00 & 2.00\end{array}$

$\begin{array}{lll}2.00 & 2.00 & 3.00\end{array}$

$\begin{array}{lll}-1.00 & -1.00 & -1.00\end{array}$

$-10.00 \quad-10.00 \quad-20.00$

$\begin{array}{llll}-10.00 & -10.00 & -30.00\end{array}$

c

m2 \$water

$1001.66 \mathrm{c} \quad 2$

$8016.66 \mathrm{c} \quad 1$

$\mathrm{mt} 2$ 1wtr.60t

c $\mathrm{pu} /$ water mixture

m11 \$ Pu(NO3)4 - NO3 - H2O, 100.0 wt\% Pu239, ZZZ1 milli-g Pu/cc, $<$ denSol|3.4>

c molarity $\mathrm{pu}=<\operatorname{molPu} \mid 3.4>$, acid $=<\operatorname{molAcid} \mid 3.4>, \mathrm{H} 2 \mathrm{O}=\langle\operatorname{molH} 2 \mathrm{O}| 3.4>$

c Pu in salt

$94239.66 \mathrm{c}<\mathrm{molPu} *$ ena $\mid 3.4>$

$94240.66 \mathrm{c}<\mathrm{molPu}^{*}(1-\mathrm{ena}) \mid 3.4>$

c NO3 in salt plus acid

$7014.66 \mathrm{c}<4 * \mathrm{molPu}+$ molAcid $\mid 3.4>$

$8016.66 \mathrm{c}<12 * \mathrm{molPu}+3 * \operatorname{mol} \mathrm{Acid} \mid 3.4>$

c water

$1001.66 \mathrm{c}<2 * \mathrm{molH} 2 \mathrm{O}+\operatorname{molAcid} \mid 3.4>$ \$Includes Free Hydrogen Ion

$8016.66 \mathrm{c}<\mathrm{molH} 2 \mathrm{O} \mid 3.4>$

mt11 1wtr.60t

c

c 


\section{Template File for Case: PuN40-icyl-0970-10}

icyl, 97.0 wt\% Pu239, ZZZ1 milli-g/cc, in Pu(NO3)4, with 0 Molar HNO3

c

c worm notes: format of the form |n.m for floating point, $\mid \mathrm{n}$ for integers

c $\quad$ zzz0 (in upper case) is replaced with the number of cyles

c $\quad$ zzz1 $=$ the actual concentration in units of milli-g/cc

c $\quad$ zzz2 $=$ solution density in $\mathrm{g} / \mathrm{cc}$

c $\quad \mathrm{qqq} 1=$ the minimum dimensions (in hundreths of inches)

c $\quad \mathrm{qqq} 2$ = the maximum dimensions (in hundreths of inches)

c $\quad \mathrm{qqq} 3=$ the delta (in hundreths of inches)

$\mathrm{c}$

c Diameter $=<\mathrm{d} 1=$ QQQ1:QQQ2:QQQ3 $\mid 5>$ hundreths of inches - must be integer

$\mathrm{c}$ radius $=<\mathrm{rd}=\mathrm{d} 1 * 2.54 / 100 / 2 \mid 5.4>\mathrm{cm}$

c note for perl, numbers with leading zeros must be enclosed in single quotes or else

c they will be interpretted as octal

$\mathrm{c}$ Conc $=<\mathrm{c}={ }^{\prime} \mathrm{ZZZ1} 1 \mid 5>$ milli-g Pu/cc

c Reflector width $=<\mathrm{rw}=10.0 \mid 2.1>$ inches

c Enrichment $=<$ ena $=0.97 \mid 1.2>$ atom fraction Pu239

c Mols of Acid per liter $<$ molAcid $=0.0 \mid 1.2>$

$\mathrm{c}$

$\mathrm{c}$

c Density of solution $<$ denSol $=Z Z Z 2 / 1 \mathrm{e} 5 \mid 1.5>$

c

c

c Atomic Weights

c $\mathrm{H} 2 \mathrm{O}=\langle$ atwH2O $=18.0152| 3.4>$

$\mathrm{c} \mathrm{NO3}=\langle$ atwNO3 $=62.0049| 3.4>$

c HNO3 $=<$ atwHNO3 $=63.0128 \mid 3.4>$

c Pu239 $=<$ atwPu239 $=239.0522 \mid 3.4>$

c Pu240 $=<$ atwPu240 $=240.0538 \mid 3.4>$

$\mathrm{c} \mathrm{Pu}=<\mathrm{atwPu}=\mathrm{ena}^{*}$ atwPu239+(1-ena)*atwPu240|3.4>

$\mathrm{c}$

c Molarity of solution components

c Mols of $\mathrm{Pu}$ per liter $<\mathrm{molPu}=\mathrm{c} / \mathrm{atwPu} \mid 3.4>$

c Mols of $\mathrm{H} 2 \mathrm{O}$ per liter $<$ molH2O $=(1000 *$ denSol-molPu* $(\operatorname{atwPu}+4 *$ atwNO3)molAcid*atwHNO3)/atwH2O|3.4>

$\mathrm{c}$

$\mathrm{c}$

$<$ perl $>$

sub IMP

\{

return 1 if $(\$[0]>0)$;

return 0 ;

\}

$</$ perl $>$

c sphere

$2011<$-denSol|3.6> $-1 \quad$ imp:n=1

$212-1.000-21 \quad$ imp:n=<\&IMP(rw) $\mid 1>$ \$zero importance if no reflector

$220 \quad 2$ imp:n=0 
$\begin{array}{lll}1 & \mathrm{cz}<\mathrm{rd} \mid 4.4> & \text { \$Sphere } \\ 2 & \mathrm{cz}<\mathrm{rd}+\mathrm{rw} * \mathrm{in} \mid 4.4> & \text { \$Reflection }\end{array}$

mode $\mathrm{n}$

kcode $20001.0100 \mathrm{ZZZ0}$

$\begin{array}{llll}\text { ksrc } & 0.00 & 0.00 & 0.00\end{array}$

$\begin{array}{llll}0.00 & 0.00 & 0.10\end{array}$

$\begin{array}{lll}0.00 & 0.00 & 0.50\end{array}$

$\begin{array}{lll}0.00 & 0.00 & 1.00\end{array}$

$\begin{array}{lll}0.00 & 0.00 & 2.00\end{array}$

$\begin{array}{lll}0.00 & 0.00 & 3.00\end{array}$

$\begin{array}{lll}1.00 & 1.00 & 1.00\end{array}$

$\begin{array}{lll}1.00 & 1.00 & 2.00\end{array}$

$\begin{array}{lll}1.00 & 1.00 & 3.00\end{array}$

$\begin{array}{lll}2.00 & 2.00 & 1.00\end{array}$

$\begin{array}{lll}2.00 & 2.00 & 2.00\end{array}$

$\begin{array}{lll}2.00 & 2.00 & 3.00\end{array}$

$\begin{array}{lll}-1.00 & -1.00 & -1.00\end{array}$

$\begin{array}{lll}-10.00 & -10.00 & -20.00\end{array}$

$\begin{array}{lll}-10.00 & -10.00 & -30.00\end{array}$

c

m2 \$water

$1001.66 \mathrm{c} \quad 2$

$8016.66 \mathrm{c} \quad 1$

$\mathrm{mt} 2$ 1wtr.60t

c $\mathrm{pu} /$ water mixture

m11 \$ Pu(NO3)4 - NO3 - H2O, 100.0 wt\% Pu239, ZZZ1 milli-g Pu/cc, $<$ denSol|3.4 $>$

c molarity $\mathrm{pu}=<\operatorname{molPu} \mid 3.4>$, acid $=<\operatorname{molAcid} \mid 3.4>, \mathrm{H} 2 \mathrm{O}=\langle\operatorname{molH} 2 \mathrm{O}| 3.4>$

c Pu in salt

$94239.66 \mathrm{c}<\mathrm{molPu}$ *ena $\mid 3.4>$

$94240.66 \mathrm{c}<\mathrm{molPu}^{*}(1$-ena $) \mid 3.4>$

c NO3 in salt plus acid

$7014.66 \mathrm{c}<4 * \mathrm{molPu}+$ molAcid $\mid 3.4>$

$8016.66 \mathrm{c}<12 * \mathrm{molPu}+3 * \operatorname{molAcid} \mid 3.4>$

c water

$1001.66 \mathrm{c}<2 * \mathrm{molH} 2 \mathrm{O}+\operatorname{molAcid} \mid 3.4>$ \$ncludes Free Hydrogen Ion

$8016.66 \mathrm{c}<\mathrm{molH} 2 \mathrm{O} \mid 3.4>$

mt11 1wtr.60t

c

c 


\section{Template File for Case: PuN43-icyl-0970-00}

icyl, 97.0 wt\% Pu239, ZZZ1 milli-g/cc, in Pu(NO3)4, with 3 Molar HNO3

c

c worm notes: format of the form |n.m for floating point, $\mid \mathrm{n}$ for integers

c $\quad$ zzz0 (in upper case) is replaced with the number of cyles

c $\quad$ zzz1 $=$ the actual concentration in units of milli-g/cc

c $\quad$ zzz2 $=$ solution density in $\mathrm{g} / \mathrm{cc}$

c $\quad \mathrm{qqq} 1=$ the minimum dimensions (in hundreths of inches)

c $\quad \mathrm{qqq} 2$ = the maximum dimensions (in hundreths of inches)

c $\quad \mathrm{qqq} 3=$ the delta (in hundreths of inches)

$\mathrm{c}$

c Diameter $=<\mathrm{d} 1=$ QQQ1:QQQ2:QQQ3 $\mid 5>$ hundreths of inches - must be integer

$\mathrm{c}$ radius $=<\mathrm{rd}=\mathrm{d} 1 * 2.54 / 100 / 2 \mid 5.4>\mathrm{cm}$

c note for perl, numbers with leading zeros must be enclosed in single quotes or else

c they will be interpretted as octal

$\mathrm{c}$ Conc $=<\mathrm{c}={ }^{\prime} \mathrm{ZZZ1} 1 \mid 5>$ milli-g Pu/cc

c Reflector width $=<\mathrm{rw}=0.0 \mid 2.1>$ inches

c Enrichment $=<$ ena $=0.97 \mid 1.2>$ atom fraction $\mathrm{Pu} 239$

c Mols of Acid per liter $<$ molAcid $=3.0 \mid 1.2>$

$\mathrm{c}$

$\mathrm{c}$

c Density of solution $<$ denSol $=Z Z Z 2 / 1 \mathrm{e} 5 \mid 1.5>$

c

c

c Atomic Weights

c $\mathrm{H} 2 \mathrm{O}=\langle$ atwH2O $=18.0152| 3.4>$

$\mathrm{c} \mathrm{NO3}=\langle$ atwNO3 $=62.0049| 3.4>$

c HNO3 $=<$ atwHNO3 $=63.0128 \mid 3.4>$

c Pu239 $=<$ atwPu239 $=239.0522 \mid 3.4>$

c Pu240 $=<$ atwPu240 $=240.0538 \mid 3.4>$

$\mathrm{c} \mathrm{Pu}=<$ atwPu$=$ ena*atwPu239+(1-ena)*atwPu240|3.4 $>$

$\mathrm{c}$

c Molarity of solution components

c Mols of $\mathrm{Pu}$ per liter $<\mathrm{molPu}=\mathrm{c} / \mathrm{atwPu} \mid 3.4>$

c Mols of $\mathrm{H} 2 \mathrm{O}$ per liter $<$ molH2O $=(1000 *$ denSol-molPu* $(a t w P u+4 * a t w N O 3)$ molAcid*atwHNO3)/atwH2O|3.4>

$\mathrm{c}$

$\mathrm{c}$

$<$ perl $>$

sub IMP

\{

return 1 if $(\$[0]>0)$;

return 0 ;

\}

$</$ perl $>$

c sphere

$2011<$-denSol|3.6> $-1 \quad$ imp:n=1

$212-1.000-21 \quad$ imp:n=<\&IMP(rw) $\mid 1>$ \$zero importance if no reflector

$220 \quad 2$ imp:n=0 


$$
\begin{aligned}
& 1 \quad \mathrm{cz}<\mathrm{rd} \mid 4.4>\quad \text { \$ Cylinder } \\
& 2 \mathrm{cz}<\mathrm{rd}+\mathrm{rw}^{*} \text { in } \mid 4.4>\quad \text { \$ Reflection }
\end{aligned}
$$

mode $\mathrm{n}$

kcode 20001.0100 ZZZ0

$\begin{array}{llll}\text { ksrc } & 0.00 & 0.00 & 0.00\end{array}$

$\begin{array}{llll}0.00 & 0.00 & 0.10\end{array}$

$\begin{array}{lll}0.00 & 0.00 & 0.50\end{array}$

$\begin{array}{lll}0.00 & 0.00 & 1.00\end{array}$

$\begin{array}{lll}0.00 & 0.00 & 2.00\end{array}$

$\begin{array}{lll}0.00 & 0.00 & 3.00\end{array}$

$\begin{array}{lll}1.00 & 1.00 & 1.00\end{array}$

$\begin{array}{lll}1.00 & 1.00 & 2.00\end{array}$

$\begin{array}{lll}1.00 & 1.00 & 3.00\end{array}$

$\begin{array}{lll}2.00 & 2.00 & 1.00\end{array}$

$\begin{array}{lll}2.00 & 2.00 & 2.00\end{array}$

$\begin{array}{lll}2.00 & 2.00 & 3.00\end{array}$

$\begin{array}{lll}-1.00 & -1.00 & -1.00\end{array}$

$-10.00 \quad-10.00 \quad-20.00$

$\begin{array}{llll}-10.00 & -10.00 & -30.00\end{array}$

c

m2 \$water

$1001.66 \mathrm{c} \quad 2$

$8016.66 \mathrm{c} \quad 1$

$\mathrm{mt} 2$ 1wtr.60t

c $\mathrm{pu} /$ water mixture

m11 \$ Pu(NO3)4 - NO3 - H2O, 100.0 wt\% Pu239, ZZZ1 milli-g Pu/cc, $<$ denSol|3.4>

c molarity $\mathrm{pu}=<\operatorname{molPu} \mid 3.4>$, acid $=<\operatorname{molAcid} \mid 3.4>, \mathrm{H} 2 \mathrm{O}=\langle\operatorname{molH} 2 \mathrm{O}| 3.4>$

c Pu in salt

$94239.66 \mathrm{c}<\mathrm{molPu} *$ ena $\mid 3.4>$

$94240.66 \mathrm{c}<\mathrm{molPu}^{*}(1-\mathrm{ena}) \mid 3.4>$

c NO3 in salt plus acid

$7014.66 \mathrm{c}<4 * \mathrm{molPu}+$ molAcid $\mid 3.4>$

$8016.66 \mathrm{c}<12 * \mathrm{molPu}+3 * \operatorname{mol} \mathrm{Acid} \mid 3.4>$

c water

$1001.66 \mathrm{c}<2 * \mathrm{molH} 2 \mathrm{O}+\operatorname{molAcid} \mid 3.4>$ \$Includes Free Hydrogen Ion

$8016.66 \mathrm{c}<\mathrm{molH} 2 \mathrm{O} \mid 3.4>$

mt11 1wtr.60t

c

c 


\section{Template File for Case: PuN43-icyl-0970-01}

icyl, 97.0 wt\% Pu239, ZZZ1 milli-g/cc, in Pu(NO3)4, with 3 Molar HNO3

c

c worm notes: format of the form |n.m for floating point, $\mid \mathrm{n}$ for integers

c $\quad$ zzz0 (in upper case) is replaced with the number of cyles

c $\quad$ zzz1 $=$ the actual concentration in units of milli-g/cc

c $\quad$ zzz2 $=$ solution density in $\mathrm{g} / \mathrm{cc}$

c $\quad \mathrm{qqq} 1=$ the minimum dimensions (in hundreths of inches)

c $\quad \mathrm{qqq} 2$ = the maximum dimensions (in hundreths of inches)

c $\quad \mathrm{qqq} 3=$ the delta (in hundreths of inches)

$\mathrm{c}$

c Diameter $=<\mathrm{d} 1=$ QQQ1:QQQ2:QQQ3 $\mid 5>$ hundreths of inches - must be integer

$\mathrm{c}$ radius $=<\mathrm{rd}=\mathrm{d} 1 * 2.54 / 100 / 2 \mid 5.4>\mathrm{cm}$

c note for perl, numbers with leading zeros must be enclosed in single quotes or else

c they will be interpretted as octal

$\mathrm{c}$ Conc $=<\mathrm{c}={ }^{\prime} \mathrm{ZZZ1} 1 \mid 5>$ milli-g Pu/cc

c Reflector width $=<\mathrm{rw}=1.0 \mid 2.1>$ inches

c Enrichment $=<$ ena $=0.97 \mid 1.2>$ atom fraction Pu239

c Mols of Acid per liter $<$ molAcid $=3.0 \mid 1.2>$

$\mathrm{c}$

$\mathrm{c}$

c Density of solution $<$ denSol $=Z Z Z 2 / 1 \mathrm{e} 5 \mid 1.5>$

c

c

c Atomic Weights

c $\mathrm{H} 2 \mathrm{O}=\langle$ atwH2O $=18.0152| 3.4>$

$\mathrm{c} \mathrm{NO3}=\langle$ atwNO3 $=62.0049| 3.4>$

c HNO3 $=<$ atwHNO3 $=63.0128 \mid 3.4>$

c Pu239 $=<$ atwPu239 $=239.0522 \mid 3.4>$

c Pu240 $=<$ atwPu240 $=240.0538 \mid 3.4>$

$\mathrm{c} \mathrm{Pu}=<$ atwPu$=$ ena*atwPu239+(1-ena)*atwPu240|3.4 $>$

$\mathrm{c}$

c Molarity of solution components

c Mols of $\mathrm{Pu}$ per liter $<\mathrm{molPu}=\mathrm{c} / \mathrm{atwPu} \mid 3.4>$

c Mols of $\mathrm{H} 2 \mathrm{O}$ per liter $<$ molH2O $=(1000 *$ denSol-molPu* $(a t w P u+4 * a t w N O 3)$ molAcid*atwHNO3)/atwH2O|3.4>

$\mathrm{c}$

$\mathrm{c}$

$<$ perl $>$

sub IMP

\{

return 1 if $(\$[0]>0)$;

return 0 ;

\}

$</$ perl $>$

c sphere

$2011<$-denSol|3.6> $-1 \quad$ imp:n=1

$212-1.000-21 \quad$ imp:n=<\&IMP(rw) $\mid 1>$ \$zero importance if no reflector

$220 \quad 2$ imp:n=0 


$$
\begin{aligned}
& 1 \quad \mathrm{cz}<\mathrm{rd} \mid 4.4>\quad \text { \$ Cylinder } \\
& 2 \mathrm{cz}<\mathrm{rd}+\mathrm{rw}^{*} \text { in } \mid 4.4>\quad \text { \$ Reflection }
\end{aligned}
$$

mode $\mathrm{n}$

kcode 20001.0100 ZZZ0

$\begin{array}{llll}\text { ksrc } & 0.00 & 0.00 & 0.00\end{array}$

$\begin{array}{llll}0.00 & 0.00 & 0.10\end{array}$

$\begin{array}{lll}0.00 & 0.00 & 0.50\end{array}$

$\begin{array}{lll}0.00 & 0.00 & 1.00\end{array}$

$\begin{array}{lll}0.00 & 0.00 & 2.00\end{array}$

$\begin{array}{lll}0.00 & 0.00 & 3.00\end{array}$

$\begin{array}{lll}1.00 & 1.00 & 1.00\end{array}$

$\begin{array}{lll}1.00 & 1.00 & 2.00\end{array}$

$\begin{array}{lll}1.00 & 1.00 & 3.00\end{array}$

$\begin{array}{lll}2.00 & 2.00 & 1.00\end{array}$

$\begin{array}{lll}2.00 & 2.00 & 2.00\end{array}$

$\begin{array}{lll}2.00 & 2.00 & 3.00\end{array}$

$\begin{array}{lll}-1.00 & -1.00 & -1.00\end{array}$

$-10.00 \quad-10.00 \quad-20.00$

$\begin{array}{llll}-10.00 & -10.00 & -30.00\end{array}$

c

m2 \$water

$1001.66 \mathrm{c} \quad 2$

$8016.66 \mathrm{c} \quad 1$

$\mathrm{mt} 2$ 1wtr.60t

c $\mathrm{pu} /$ water mixture

m11 \$ Pu(NO3)4 - NO3 - H2O, 100.0 wt\% Pu239, ZZZ1 milli-g Pu/cc, $<$ denSol|3.4>

c molarity $\mathrm{pu}=\langle\operatorname{molPu}| 3.4>$, acid $=\langle\operatorname{mol} A \mathrm{cid}| 3.4>, \mathrm{H} 2 \mathrm{O}=\langle\operatorname{molH} 2 \mathrm{O}| 3.4>$

c Pu in salt

$94239.66 \mathrm{c}<\mathrm{molPu}$ *ena $\mid 3.4>$

$94240.66 \mathrm{c}<\mathrm{molPu}^{*}(1-\mathrm{ena}) \mid 3.4>$

c $\quad \mathrm{NO} 3$ in salt plus acid

$7014.66 \mathrm{c}<4 * \mathrm{molPu}+$ molAcid $\mid 3.4>$

$8016.66 \mathrm{c}<12 * \mathrm{molPu}+3 * \operatorname{molAcid} \mid 3.4>$

c water

$1001.66 \mathrm{c}<2 *$ molH2O + molAcid $\mid 3.4>$ \$Includes Free Hydrogen Ion

$8016.66 \mathrm{c}<\mathrm{molH} 2 \mathrm{O} \mid 3.4>$

mt11 1wtr.60t

c

c 


\section{Template File for Case: PuN43-icyl-0970-10}

icyl, 97.0 wt\% Pu239, ZZZ1 milli-g/cc, in Pu(NO3)4, with 3 Molar HNO3

c

c worm notes: format of the form |n.m for floating point, $\mid \mathrm{n}$ for integers

c $\quad$ zzz0 (in upper case) is replaced with the number of cyles

c $\quad$ zzz1 $=$ the actual concentration in units of milli-g/cc

c $\quad$ zzz2 $=$ solution density in $\mathrm{g} / \mathrm{cc}$

c $\quad \mathrm{qqq} 1=$ the minimum dimensions (in hundreths of inches)

c $\quad \mathrm{qqq} 2$ = the maximum dimensions (in hundreths of inches)

c $\quad \mathrm{qqq} 3=$ the delta (in hundreths of inches)

$\mathrm{c}$

c Diameter $=<\mathrm{d} 1=$ QQQ1:QQQ2:QQQ3 $\mid 5>$ hundreths of inches - must be integer

$\mathrm{c}$ radius $=<\mathrm{rd}=\mathrm{d} 1 * 2.54 / 100 / 2 \mid 5.4>\mathrm{cm}$

c note for perl, numbers with leading zeros must be enclosed in single quotes or else

c they will be interpretted as octal

$\mathrm{c}$ Conc $=<\mathrm{c}={ }^{\prime} \mathrm{ZZZ1} 1 \mid 5>$ milli-g Pu/cc

c Reflector width $=<\mathrm{rw}=10.0 \mid 2.1>$ inches

c Enrichment $=<$ ena $=0.97 \mid 1.2>$ atom fraction Pu239

c Mols of Acid per liter $<$ molAcid $=3.0 \mid 1.2>$

$\mathrm{c}$

$\mathrm{c}$

c Density of solution $<$ denSol $=Z Z Z 2 / 1 \mathrm{e} 5 \mid 1.5>$

c

c

c Atomic Weights

c $\mathrm{H} 2 \mathrm{O}=\langle$ atwH2O $=18.0152| 3.4>$

$\mathrm{c} \mathrm{NO3}=\langle$ atwNO3 $=62.0049| 3.4>$

c HNO3 $=<$ atwHNO3 $=63.0128 \mid 3.4>$

c Pu239 $=<$ atwPu239 $=239.0522 \mid 3.4>$

c Pu240 $=<$ atwPu240 $=240.0538 \mid 3.4>$

$\mathrm{c} \mathrm{Pu}=<\mathrm{atwPu}=\mathrm{ena}^{*}$ atwPu239+(1-ena)*atwPu240|3.4>

$\mathrm{c}$

c Molarity of solution components

c Mols of $\mathrm{Pu}$ per liter $<\mathrm{molPu}=\mathrm{c} / \mathrm{atwPu} \mid 3.4>$

c Mols of $\mathrm{H} 2 \mathrm{O}$ per liter $<$ molH2O $=(1000 *$ denSol-molPu* $(\operatorname{atwPu}+4 *$ atwNO3)molAcid*atwHNO3)/atwH2O|3.4>

$\mathrm{c}$

$\mathrm{c}$

$<$ perl $>$

sub IMP

\{

return 1 if $(\$[0]>0)$;

return 0 ;

\}

$</$ perl $>$

c sphere

$2011<$-denSol|3.6> $-1 \quad$ imp:n=1

$212-1.000-21 \quad$ imp:n=<\&IMP(rw) $\mid 1>$ \$zero importance if no reflector

$220 \quad 2$ imp:n=0 


$$
\begin{aligned}
& 1 \quad \mathrm{cz}<\mathrm{rd} \mid 4.4>\quad \text { \$ Cylinder } \\
& 2 \mathrm{cz}<\mathrm{rd}+\mathrm{rw}^{*} \text { in } \mid 4.4>\quad \text { \$ Reflection }
\end{aligned}
$$

mode $\mathrm{n}$

kcode 20001.0100 ZZZ0

$\begin{array}{llll}\text { ksrc } & 0.00 & 0.00 & 0.00\end{array}$

$\begin{array}{llll}0.00 & 0.00 & 0.10\end{array}$

$\begin{array}{lll}0.00 & 0.00 & 0.50\end{array}$

$\begin{array}{lll}0.00 & 0.00 & 1.00\end{array}$

$\begin{array}{lll}0.00 & 0.00 & 2.00\end{array}$

$\begin{array}{lll}0.00 & 0.00 & 3.00\end{array}$

$\begin{array}{lll}1.00 & 1.00 & 1.00\end{array}$

$\begin{array}{lll}1.00 & 1.00 & 2.00\end{array}$

$\begin{array}{lll}1.00 & 1.00 & 3.00\end{array}$

$\begin{array}{lll}2.00 & 2.00 & 1.00\end{array}$

$\begin{array}{lll}2.00 & 2.00 & 2.00\end{array}$

$\begin{array}{lll}2.00 & 2.00 & 3.00\end{array}$

$\begin{array}{lll}-1.00 & -1.00 & -1.00\end{array}$

$-10.00 \quad-10.00 \quad-20.00$

$\begin{array}{llll}-10.00 & -10.00 & -30.00\end{array}$

c

m2 \$water

$1001.66 \mathrm{c} \quad 2$

$8016.66 \mathrm{c} \quad 1$

$\mathrm{mt} 2$ 1wtr.60t

c $\mathrm{pu} /$ water mixture

m11 \$ Pu(NO3)4 - NO3 - H2O, 100.0 wt\% Pu239, ZZZ1 milli-g Pu/cc, $<$ denSol|3.4>

c molarity $\mathrm{pu}=\langle\operatorname{molPu}| 3.4>$, acid $=\langle\operatorname{mol} A \mathrm{cid}| 3.4>, \mathrm{H} 2 \mathrm{O}=\langle\operatorname{molH} 2 \mathrm{O}| 3.4>$

c Pu in salt

$94239.66 \mathrm{c}<\mathrm{molPu}$ *ena $\mid 3.4>$

$94240.66 \mathrm{c}<\mathrm{molPu}^{*}(1-\mathrm{ena}) \mid 3.4>$

c $\quad \mathrm{NO} 3$ in salt plus acid

$7014.66 \mathrm{c}<4 * \mathrm{molPu}+$ molAcid $\mid 3.4>$

$8016.66 \mathrm{c}<12 * \mathrm{molPu}+3 * \operatorname{molAcid} \mid 3.4>$

c water

$1001.66 \mathrm{c}<2 *$ molH2O + molAcid $\mid 3.4>$ \$Includes Free Hydrogen Ion

$8016.66 \mathrm{c}<\mathrm{molH} 2 \mathrm{O} \mid 3.4>$

mt11 1wtr.60t

c

c 


\section{Template File for Case: PuN46-icyl-0970-00}

icyl, 97.0 wt\% Pu239, ZZZ1 milli-g/cc, in Pu(NO3)4, with 6 Molar HNO3

c

c worm notes: format of the form |n.m for floating point, $\mid \mathrm{n}$ for integers

c $\quad$ zzz0 (in upper case) is replaced with the number of cyles

c $\quad$ zzz1 $=$ the actual concentration in units of milli-g/cc

c $\quad$ zzz2 $=$ solution density in $\mathrm{g} / \mathrm{cc}$

c $\quad \mathrm{qqq} 1=$ the minimum dimensions (in hundreths of inches)

c $\quad \mathrm{qqq} 2$ = the maximum dimensions (in hundreths of inches)

c $\quad \mathrm{qqq} 3=$ the delta (in hundreths of inches)

$\mathrm{c}$

c Diameter $=<\mathrm{d} 1=$ QQQ1:QQQ2:QQQ3 $\mid 5>$ hundreths of inches - must be integer

$\mathrm{c}$ radius $=<\mathrm{rd}=\mathrm{d} 1 * 2.54 / 100 / 2 \mid 5.4>\mathrm{cm}$

c note for perl, numbers with leading zeros must be enclosed in single quotes or else

c they will be interpretted as octal

$\mathrm{c}$ Conc $=<\mathrm{c}={ }^{\prime} \mathrm{ZZZ1} 1 \mid 5>$ milli-g Pu/cc

c Reflector width $=<\mathrm{rw}=0.0 \mid 2.1>$ inches

c Enrichment $=<$ ena $=0.97 \mid 1.2>$ atom fraction Pu239

c Mols of Acid per liter $<$ molAcid $=6.0 \mid 1.2>$

$\mathrm{c}$

$\mathrm{c}$

c Density of solution $<$ denSol $=Z Z Z 2 / 1 \mathrm{e} 5 \mid 1.5>$

c

c

c Atomic Weights

c $\mathrm{H} 2 \mathrm{O}=\langle$ atwH2O $=18.0152| 3.4>$

$\mathrm{c} \mathrm{NO3}=\langle$ atwNO3 $=62.0049| 3.4>$

c HNO3 $=<$ atwHNO3 $=63.0128 \mid 3.4>$

c Pu239 $=<$ atwPu239 $=239.0522 \mid 3.4>$

c Pu240 $=<$ atwPu240 $=240.0538 \mid 3.4>$

$\mathrm{c} \mathrm{Pu}=<\mathrm{atwPu}=\mathrm{ena}^{*}$ atwPu239+(1-ena)*atwPu240|3.4>

$\mathrm{c}$

c Molarity of solution components

c Mols of $\mathrm{Pu}$ per liter $<\mathrm{molPu}=\mathrm{c} / \mathrm{atwPu} \mid 3.4>$

c Mols of $\mathrm{H} 2 \mathrm{O}$ per liter $<$ molH2O $=(1000 *$ denSol-molPu* $(a t w P u+4 * a t w N O 3)$ molAcid*atwHNO3)/atwH2O $3.4>$

$\mathrm{c}$

$\mathrm{c}$

$<$ perl $>$

sub IMP

\{

return 1 if $(\$[0]>0)$;

return 0 ;

\}

$</$ perl $>$

c sphere

$2011<$-denSol|3.6> $-1 \quad$ imp:n=1

$212-1.000-21 \quad$ imp:n=<\&IMP(rw) $\mid 1>$ \$zero importance if no reflector

$220 \quad 2$ imp:n=0 


$$
\begin{aligned}
& 1 \quad \mathrm{cz}<\mathrm{rd} \mid 4.4>\quad \text { \$ Cylinder } \\
& 2 \mathrm{cz}<\mathrm{rd}+\mathrm{rw}^{*} \text { in } \mid 4.4>\quad \text { \$ Reflection }
\end{aligned}
$$

mode $\mathrm{n}$

kcode 20001.0100 ZZZ0

$\begin{array}{llll}\text { ksrc } & 0.00 & 0.00 & 0.00\end{array}$

$\begin{array}{llll}0.00 & 0.00 & 0.10\end{array}$

$\begin{array}{lll}0.00 & 0.00 & 0.50\end{array}$

$\begin{array}{lll}0.00 & 0.00 & 1.00\end{array}$

$\begin{array}{lll}0.00 & 0.00 & 2.00\end{array}$

$\begin{array}{lll}0.00 & 0.00 & 3.00\end{array}$

$\begin{array}{lll}1.00 & 1.00 & 1.00\end{array}$

$\begin{array}{lll}1.00 & 1.00 & 2.00\end{array}$

$\begin{array}{lll}1.00 & 1.00 & 3.00\end{array}$

$\begin{array}{lll}2.00 & 2.00 & 1.00\end{array}$

$\begin{array}{lll}2.00 & 2.00 & 2.00\end{array}$

$\begin{array}{lll}2.00 & 2.00 & 3.00\end{array}$

$\begin{array}{lll}-1.00 & -1.00 & -1.00\end{array}$

$-10.00 \quad-10.00 \quad-20.00$

$\begin{array}{llll}-10.00 & -10.00 & -30.00\end{array}$

c

m2 \$water

$1001.66 \mathrm{c} \quad 2$

$8016.66 \mathrm{c} \quad 1$

$\mathrm{mt} 2$ 1wtr.60t

c $\mathrm{pu} /$ water mixture

m11 \$ Pu(NO3)4 - NO3 - H2O, 100.0 wt\% Pu239, ZZZ1 milli-g Pu/cc, $<$ denSol|3.4>

c molarity $\mathrm{pu}=<\operatorname{molPu} \mid 3.4>$, acid $=<\operatorname{molAcid} \mid 3.4>, \mathrm{H} 2 \mathrm{O}=\langle\operatorname{molH} 2 \mathrm{O}| 3.4>$

c Pu in salt

$94239.66 \mathrm{c}<\mathrm{molPu}$ *ena $\mid 3.4>$

$94240.66 \mathrm{c}<\mathrm{molPu}^{*}(1-\mathrm{ena}) \mid 3.4>$

c $\quad \mathrm{NO} 3$ in salt plus acid

$7014.66 \mathrm{c}<4 * \mathrm{molPu}+$ molAcid $\mid 3.4>$

$8016.66 \mathrm{c}<12 * \mathrm{molPu}+3 * \operatorname{molAcid} \mid 3.4>$

c water

$1001.66 \mathrm{c}<2 *$ molH2O + molAcid $\mid 3.4>$ \$Includes Free Hydrogen Ion

$8016.66 \mathrm{c}<\mathrm{molH} 2 \mathrm{O} \mid 3.4>$

mt11 1wtr.60t

c

c 


\section{Template File for Case: PuN46-icyl-0970-01}

icyl, 97.0 wt\% Pu239, ZZZ1 milli-g/cc, in Pu(NO3)4, with 6 Molar HNO3

c

c worm notes: format of the form |n.m for floating point, $\mid \mathrm{n}$ for integers

c $\quad$ zzz0 (in upper case) is replaced with the number of cyles

c $\quad$ zzz1 $=$ the actual concentration in units of milli-g/cc

c $\quad$ zzz2 $=$ solution density in $\mathrm{g} / \mathrm{cc}$

c $\quad \mathrm{qqq} 1=$ the minimum dimensions (in hundreths of inches)

c $\quad \mathrm{qqq} 2$ = the maximum dimensions (in hundreths of inches)

c $\quad \mathrm{qqq} 3=$ the delta (in hundreths of inches)

$\mathrm{c}$

c Diameter $=<\mathrm{d} 1=$ QQQ1:QQQ2:QQQ3 $\mid 5>$ hundreths of inches - must be integer

$\mathrm{c}$ radius $=<\mathrm{rd}=\mathrm{d} 1 * 2.54 / 100 / 2 \mid 5.4>\mathrm{cm}$

c note for perl, numbers with leading zeros must be enclosed in single quotes or else

c they will be interpretted as octal

$\mathrm{c}$ Conc $=<\mathrm{c}={ }^{\prime} \mathrm{ZZZ1} 1 \mid 5>$ milli-g Pu/cc

c Reflector width $=<\mathrm{rw}=1.0 \mid 2.1>$ inches

c Enrichment $=<$ ena $=0.97 \mid 1.2>$ atom fraction Pu239

c Mols of Acid per liter $<$ molAcid $=6.0 \mid 1.2>$

$\mathrm{c}$

$\mathrm{c}$

c Density of solution $<$ denSol $=Z Z Z 2 / 1 \mathrm{e} 5 \mid 1.5>$

c

c

c Atomic Weights

c $\mathrm{H} 2 \mathrm{O}=\langle$ atwH2O $=18.0152| 3.4>$

$\mathrm{c} \mathrm{NO3}=\langle$ atwNO3 $=62.0049| 3.4>$

c HNO3 $=<$ atwHNO3 $=63.0128 \mid 3.4>$

c Pu239 $=<$ atwPu239 $=239.0522 \mid 3.4>$

c Pu240 $=<$ atwPu240 $=240.0538 \mid 3.4>$

$\mathrm{c} \mathrm{Pu}=<$ atwPu$=$ ena*atwPu239+(1-ena)*atwPu240|3.4 $>$

$\mathrm{c}$

c Molarity of solution components

c Mols of $\mathrm{Pu}$ per liter $<\mathrm{molPu}=\mathrm{c} / \mathrm{atwPu} \mid 3.4>$

c Mols of $\mathrm{H} 2 \mathrm{O}$ per liter $<$ molH2O $=(1000 *$ denSol-molPu* $(a t w P u+4 * a t w N O 3)$ molAcid*atwHNO3)/atwH2O|3.4>

$\mathrm{c}$

$\mathrm{c}$

$<$ perl $>$

sub IMP

\{

return 1 if $(\$[0]>0)$;

return 0 ;

\}

$</$ perl $>$

c sphere

$2011<$-denSol|3.6> $-1 \quad$ imp:n=1

$212-1.000-21 \quad$ imp:n=<\&IMP(rw) $\mid 1>$ \$zero importance if no reflector

$220 \quad 2 \quad$ imp:n $=0$ 


$$
\begin{aligned}
& 1 \quad \mathrm{cz}<\mathrm{rd} \mid 4.4>\quad \text { \$ Cylinder } \\
& 2 \mathrm{cz}<\mathrm{rd}+\mathrm{rw}^{*} \text { in } \mid 4.4>\quad \text { \$ Reflection }
\end{aligned}
$$

mode $\mathrm{n}$

kcode 20001.0100 ZZZ0

$\begin{array}{llll}\text { ksrc } & 0.00 & 0.00 & 0.00\end{array}$

$\begin{array}{llll}0.00 & 0.00 & 0.10\end{array}$

$\begin{array}{lll}0.00 & 0.00 & 0.50\end{array}$

$\begin{array}{lll}0.00 & 0.00 & 1.00\end{array}$

$\begin{array}{lll}0.00 & 0.00 & 2.00\end{array}$

$\begin{array}{lll}0.00 & 0.00 & 3.00\end{array}$

$\begin{array}{lll}1.00 & 1.00 & 1.00\end{array}$

$\begin{array}{lll}1.00 & 1.00 & 2.00\end{array}$

$\begin{array}{lll}1.00 & 1.00 & 3.00\end{array}$

$\begin{array}{lll}2.00 & 2.00 & 1.00\end{array}$

$\begin{array}{lll}2.00 & 2.00 & 2.00\end{array}$

$\begin{array}{lll}2.00 & 2.00 & 3.00\end{array}$

$\begin{array}{lll}-1.00 & -1.00 & -1.00\end{array}$

$-10.00 \quad-10.00 \quad-20.00$

$\begin{array}{llll}-10.00 & -10.00 & -30.00\end{array}$

c

m2 \$water

$1001.66 \mathrm{c} \quad 2$

$8016.66 \mathrm{c} \quad 1$

$\mathrm{mt} 2$ 1wtr.60t

c $\mathrm{pu} /$ water mixture

m11 \$ Pu(NO3)4 - NO3 - H2O, 100.0 wt\% Pu239, ZZZ1 milli-g Pu/cc, $<$ denSol|3.4>

c molarity $\mathrm{pu}=<\operatorname{molPu} \mid 3.4>$, acid $=<\operatorname{molAcid} \mid 3.4>, \mathrm{H} 2 \mathrm{O}=\langle\operatorname{molH} 2 \mathrm{O}| 3.4>$

c Pu in salt

$94239.66 \mathrm{c}<\mathrm{molPu}$ *ena $\mid 3.4>$

$94240.66 \mathrm{c}<\mathrm{molPu}^{*}(1-\mathrm{ena}) \mid 3.4>$

c $\quad \mathrm{NO} 3$ in salt plus acid

$7014.66 \mathrm{c}<4 * \mathrm{molPu}+$ molAcid $\mid 3.4>$

$8016.66 \mathrm{c}<12 * \mathrm{molPu}+3 * \operatorname{molAcid} \mid 3.4>$

c water

$1001.66 \mathrm{c}<2 *$ molH2O + molAcid $\mid 3.4>$ \$Includes Free Hydrogen Ion

$8016.66 \mathrm{c}<\mathrm{molH} 2 \mathrm{O} \mid 3.4>$

mt11 1wtr.60t

c

c 


\section{Template File for Case: PuN46-icyl-0970-10}

icyl, 97.0 wt\% Pu239, ZZZ1 milli-g/cc, in Pu(NO3)4, with 6 Molar HNO3

c

c worm notes: format of the form |n.m for floating point, $\mid \mathrm{n}$ for integers

c $\quad$ zzz0 (in upper case) is replaced with the number of cyles

c $\quad$ zzz1 $=$ the actual concentration in units of milli-g/cc

c $\quad$ zzz2 $=$ solution density in $\mathrm{g} / \mathrm{cc}$

c $\quad \mathrm{qqq} 1=$ the minimum dimensions (in hundreths of inches)

c $\quad \mathrm{qqq} 2$ = the maximum dimensions (in hundreths of inches)

c $\quad \mathrm{qqq} 3=$ the delta (in hundreths of inches)

$\mathrm{c}$

c Diameter $=<\mathrm{d} 1=$ QQQ1:QQQ2:QQQ3 $\mid 5>$ hundreths of inches - must be integer

$\mathrm{c}$ radius $=<\mathrm{rd}=\mathrm{d} 1 * 2.54 / 100 / 2 \mid 5.4>\mathrm{cm}$

c note for perl, numbers with leading zeros must be enclosed in single quotes or else

c they will be interpretted as octal

$\mathrm{c}$ Conc $=<\mathrm{c}={ }^{\prime} \mathrm{ZZZ1} 1 \mid 5>$ milli-g Pu/cc

c Reflector width $=<\mathrm{rw}=10.0 \mid 2.1>$ inches

c Enrichment $=<$ ena $=0.97 \mid 1.2>$ atom fraction Pu239

c Mols of Acid per liter $<$ molAcid $=6.0 \mid 1.2>$

$\mathrm{c}$

$\mathrm{c}$

c Density of solution $<$ denSol $=Z Z Z 2 / 1 \mathrm{e} 5 \mid 1.5>$

c

c

c Atomic Weights

c $\mathrm{H} 2 \mathrm{O}=\langle$ atwH2O $=18.0152| 3.4>$

$\mathrm{c} \mathrm{NO3}=\langle$ atwNO3 $=62.0049| 3.4>$

c HNO3 $=<$ atwHNO3 $=63.0128 \mid 3.4>$

$\mathrm{c} \mathrm{Pu} 239=<\mathrm{atwPu} 239=239.0522 \mid 3.4>$

c Pu240 $=<$ atwPu240 $=240.0538 \mid 3.4>$

$\mathrm{c} \mathrm{Pu}=<\mathrm{atwPu}=\mathrm{ena}^{*}$ atwPu239+(1-ena)*atwPu240|3.4>

$\mathrm{c}$

c Molarity of solution components

c Mols of $\mathrm{Pu}$ per liter $<\mathrm{molPu}=\mathrm{c} / \mathrm{atwPu} \mid 3.4>$

c Mols of $\mathrm{H} 2 \mathrm{O}$ per liter $<$ molH2O $=(1000 *$ denSol-molPu* $(\operatorname{atwPu}+4 *$ atwNO3)molAcid*atwHNO3)/atwH2O|3.4>

$\mathrm{c}$

$\mathrm{c}$

$<$ perl $>$

sub IMP

\{

return 1 if $(\$[0]>0)$;

return 0 ;

\}

$</$ perl $>$

c sphere

$2011<$-denSol|3.6> $-1 \quad$ imp:n=1

$212-1.000-21 \quad$ imp:n=<\&IMP(rw) $\mid 1>$ \$zero importance if no reflector

$220 \quad 2$ imp:n=0 


$$
\begin{aligned}
& 1 \quad \mathrm{cz}<\mathrm{rd} \mid 4.4>\quad \text { \$ Cylinder } \\
& 2 \mathrm{cz}<\mathrm{rd}+\mathrm{rw}^{*} \text { in } \mid 4.4>\quad \text { \$ Reflection }
\end{aligned}
$$

mode $\mathrm{n}$

kcode 20001.0100 ZZZ0

$\begin{array}{llll}\text { ksrc } & 0.00 & 0.00 & 0.00\end{array}$

$\begin{array}{llll}0.00 & 0.00 & 0.10\end{array}$

$\begin{array}{lll}0.00 & 0.00 & 0.50\end{array}$

$\begin{array}{lll}0.00 & 0.00 & 1.00\end{array}$

$\begin{array}{lll}0.00 & 0.00 & 2.00\end{array}$

$\begin{array}{lll}0.00 & 0.00 & 3.00\end{array}$

$\begin{array}{lll}1.00 & 1.00 & 1.00\end{array}$

$\begin{array}{lll}1.00 & 1.00 & 2.00\end{array}$

$\begin{array}{lll}1.00 & 1.00 & 3.00\end{array}$

$\begin{array}{lll}2.00 & 2.00 & 1.00\end{array}$

$\begin{array}{lll}2.00 & 2.00 & 2.00\end{array}$

$\begin{array}{lll}2.00 & 2.00 & 3.00\end{array}$

$\begin{array}{lll}-1.00 & -1.00 & -1.00\end{array}$

$-10.00 \quad-10.00 \quad-20.00$

$\begin{array}{llll}-10.00 & -10.00 & -30.00\end{array}$

c

m2 \$water

$1001.66 \mathrm{c} \quad 2$

$8016.66 \mathrm{c} \quad 1$

$\mathrm{mt} 2$ 1wtr.60t

c $\mathrm{pu} /$ water mixture

m11 \$ Pu(NO3)4 - NO3 - H2O, 100.0 wt\% Pu239, ZZZ1 milli-g Pu/cc, $<$ denSol|3.4>

c molarity $\mathrm{pu}=<\operatorname{molPu} \mid 3.4>$, acid $=<\operatorname{molAcid} \mid 3.4>, \mathrm{H} 2 \mathrm{O}=\langle\operatorname{molH} 2 \mathrm{O}| 3.4>$

c Pu in salt

$94239.66 \mathrm{c}<\mathrm{molPu}$ *ena $\mid 3.4>$

$94240.66 \mathrm{c}<\mathrm{molPu}^{*}(1-\mathrm{ena}) \mid 3.4>$

c $\quad \mathrm{NO} 3$ in salt plus acid

$7014.66 \mathrm{c}<4 * \mathrm{molPu}+$ molAcid $\mid 3.4>$

$8016.66 \mathrm{c}<12 * \mathrm{molPu}+3 * \operatorname{molAcid} \mid 3.4>$

c water

$1001.66 \mathrm{c}<2 *$ molH2O + molAcid $\mid 3.4>$ \$Includes Free Hydrogen Ion

$8016.66 \mathrm{c}<\mathrm{molH} 2 \mathrm{O} \mid 3.4>$

mt11 1wtr.60t

c

c 


\section{Template File for Case: PuN40-pl-1000-00}

pl, 100.0 wt\% Pu239, ZZZ1 milli-g/cc, in Pu(NO3)4, with 0 Molar HNO3

c

c worm notes: format of the form |n.m for floating point, $\mid \mathrm{n}$ for integers

c $\quad$ zzz0 (in upper case) is replaced with the number of cyles

c $\quad$ zzz1 $=$ the actual concentration in units of milli-g/cc

c $\quad$ zzz2 $=$ solution density in $\mathrm{g} / \mathrm{cc}$

c $\quad \mathrm{qqq} 1=$ the minimum dimensions (in hundreths of inches)

c $\quad \mathrm{qqq} 2$ = the maximum dimensions (in hundreths of inches)

c $\quad \mathrm{qqq} 3=$ the delta (in hundreths of inches)

$\mathrm{c}$

c Diameter $=<\mathrm{d} 1=$ QQQ1:QQQ2:QQQ3 $\mid 5>$ hundreths of inches - must be integer

$\mathrm{c}$ radius $=<\mathrm{rd}=\mathrm{d} 1 * 2.54 / 100 / 2 \mid 5.4>\mathrm{cm}$

c note for perl, numbers with leading zeros must be enclosed in single quotes or else

c they will be interpretted as octal

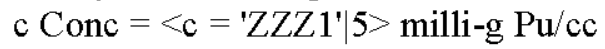

c Reflector width $=<\mathrm{rw}=0.0 \mid 2.1>$ inches

c Enrichment $=<$ ena $=1.0 \mid 1.2>$ atom fraction Pu239

c Mols of Acid per liter $<$ molAcid $=0.0 \mid 1.2>$

c

$\mathrm{c}$

c Density of solution $<$ denSol $=Z Z Z 2 / 1 \mathrm{e} 5 \mid 1.5>$

c

c

c Atomic Weights

c $\mathrm{H} 2 \mathrm{O}=\langle$ atwH2O $=18.0152| 3.4>$

$\mathrm{c} \mathrm{NO3}=\langle$ atwNO3 $=62.0049| 3.4>$

c HNO3 $=<$ atwHNO3 $=63.0128 \mid 3.4>$

c $\mathrm{Pu} 239=<\mathrm{atwPu} 239=239.0522 \mid 3.4>$

c Pu240 $=<$ atwPu240 $=240.0538 \mid 3.4>$

$\mathrm{c} \mathrm{Pu}=<\mathrm{atwPu}=\mathrm{ena}^{*}$ atwPu239+(1-ena)*atwPu240|3.4>

$\mathrm{c}$

c Molarity of solution components

c Mols of $\mathrm{Pu}$ per liter $<\mathrm{molPu}=\mathrm{c} / \mathrm{atwPu} \mid 3.4>$

c Mols of $\mathrm{H} 2 \mathrm{O}$ per liter $<$ molH2O $=(1000 *$ denSol-molPu* $(\operatorname{atwPu}+4 *$ atwNO3)-

molAcid*atwHNO3)/atwH2O $3.4>$

c

$\mathrm{c}$

$<$ perl $>$

sub IMP

\{

return 1 if $(\$[0]>0)$;

return 0

\}

$</$ perl $>$

c sphere

$2011<$-denSol|3.6> $-12-7-586 \quad$ imp:n=1

$2120-1.000 \quad-3468-7-5((1-3):(-24)) \quad$ imp:n=<\&IMP(rw) $\mid 1>$ \$zero importance if no reflector

$220 \quad 3:-4: 7: 5:-8:-6 \quad$ imp:n=0 


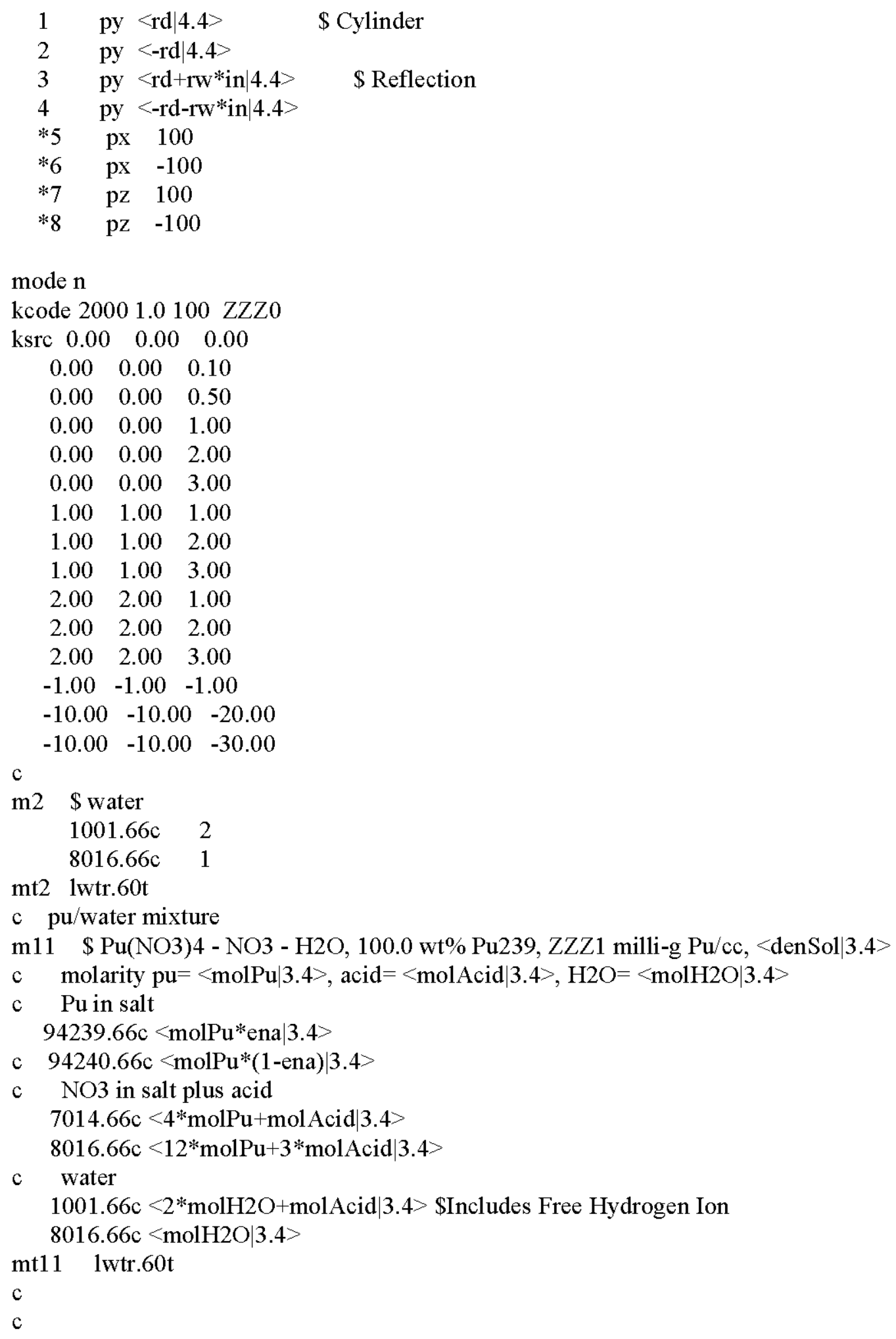




\section{Template File for Case: PuN40-pl-1000-01}

pl, 100.0 wt\% Pu239, ZZZ1 milli-g/cc, in Pu(NO3)4, with 0 Molar HNO3

$\mathrm{c}$

c worm notes: format of the form |n.m for floating point, $\mid \mathrm{n}$ for integers

c $\quad$ zzz0 (in upper case) is replaced with the number of cyles

c $\quad$ zzz1 $=$ the actual concentration in units of milli-g/cc

c $\quad$ zzz2 $=$ solution density in $\mathrm{g} / \mathrm{cc}$

c $\quad \mathrm{qqq} 1=$ the minimum dimensions (in hundreths of inches)

c $\quad \mathrm{qqq} 2$ = the maximum dimensions (in hundreths of inches)

c $\quad \mathrm{qqq} 3=$ the delta (in hundreths of inches)

$\mathrm{c}$

c Diameter $=<\mathrm{d} 1=$ QQQ1:QQQ2:QQQ3 $\mid 5>$ hundreths of inches - must be integer

$\mathrm{c}$ radius $=<\mathrm{rd}=\mathrm{d} 1 * 2.54 / 100 / 2 \mid 5.4>\mathrm{cm}$

c note for perl, numbers with leading zeros must be enclosed in single quotes or else

c they will be interpretted as octal

$\mathrm{c}$ Conc $=<\mathrm{c}={ }^{\prime} \mathrm{ZZZ1} 1 \mid 5>$ milli-g Pu/cc

c Reflector width $=<\mathrm{rw}=1.0 \mid 2.1>$ inches

c Enrichment $=<$ ena $=1.0 \mid 1.2>$ atom fraction Pu239

c Mols of Acid per liter $<$ molAcid $=0.0 \mid 1.2>$

c

$\mathrm{c}$

c Density of solution $<$ denSol $=Z Z Z 2 / 1 \mathrm{e} 5 \mid 1.5>$

c

c

c Atomic Weights

c $\mathrm{H} 2 \mathrm{O}=\langle$ atwH2O $=18.0152| 3.4>$

$\mathrm{c} \mathrm{NO3}=\langle$ atwNO3 $=62.0049| 3.4>$

c HNO3 $=<$ atwHNO3 $=63.0128 \mid 3.4>$

c $\mathrm{Pu} 239=<\mathrm{atwPu} 239=239.0522 \mid 3.4>$

c Pu240 $=<$ atwPu240 $=240.0538 \mid 3.4>$

$\mathrm{c} \mathrm{Pu}=<\mathrm{atwPu}=\mathrm{ena}^{*}$ atwPu239+(1-ena)*atwPu240|3.4>

$\mathrm{c}$

c Molarity of solution components

c Mols of $\mathrm{Pu}$ per liter $<\mathrm{molPu}=\mathrm{c} / \mathrm{atwPu} \mid 3.4>$

c Mols of $\mathrm{H} 2 \mathrm{O}$ per liter $<$ molH2O $=(1000 *$ denSol-molPu* $(\operatorname{atwPu}+4 *$ atwNO3)-

molAcid*atwHNO3)/atwH2O $3.4>$

c

$\mathrm{c}$

$<$ perl $>$

sub IMP

\{

return 1 if $(\$[0]>0)$;

return 0

\}

$</$ perl $>$

c sphere

$2011<$-denSol|3.6> $-12-7-586 \quad$ imp:n=1

$212-1.000 \quad-3468-7-5((1-3):(-24)) \quad$ imp:n=<\&IMP(rw) $\mid 1>\quad$ \$zero importance if no reflector

$220 \quad 3:-4: 7: 5:-8:-6 \quad$ imp:n=0 


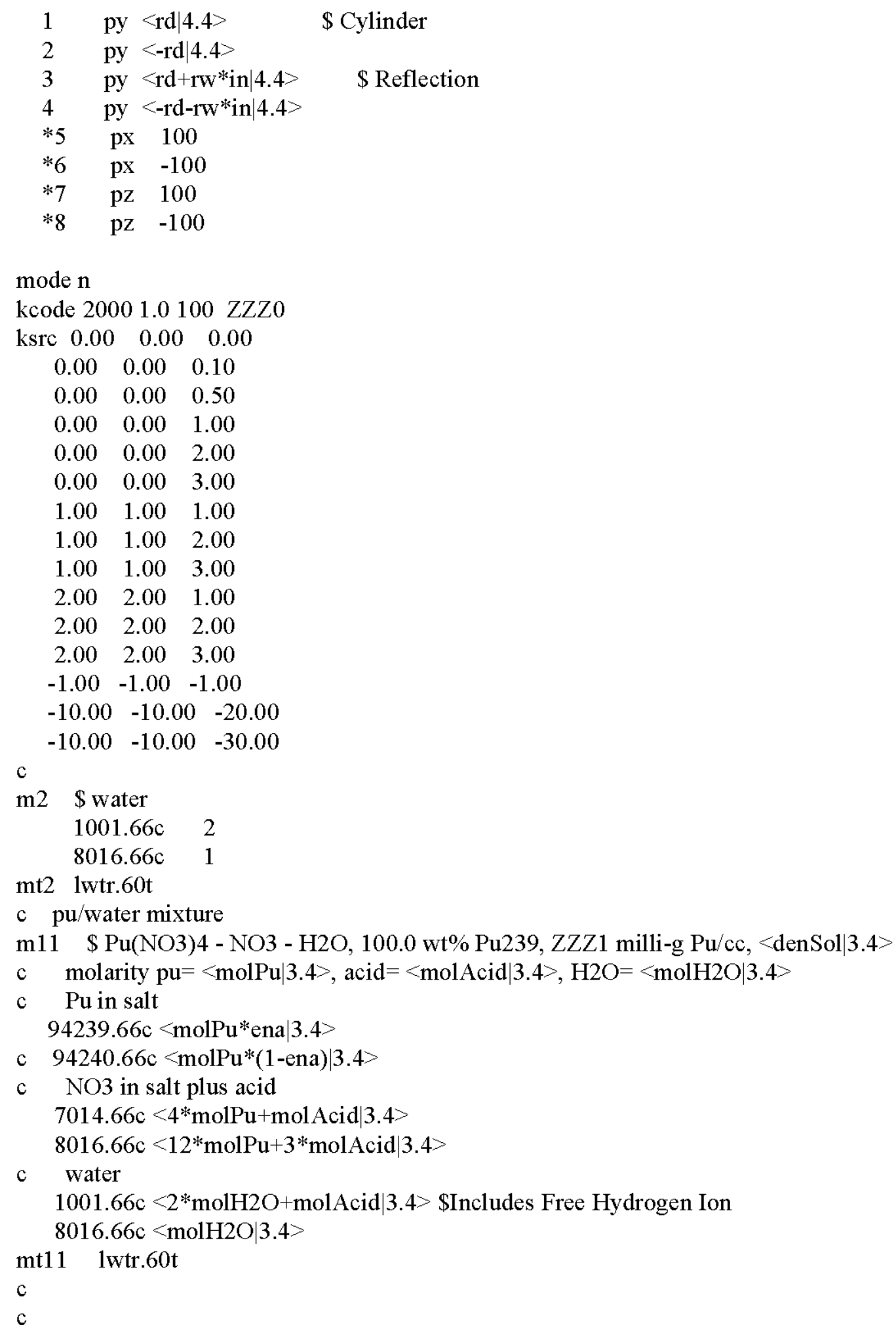




\section{Template File for Case: PuN40-pl-1000-10}

pl, 100.0 wt\% Pu239, ZZZ1 milli-g/cc, in Pu(NO3)4, with 0 Molar HNO3

$\mathrm{c}$

c worm notes: format of the form |n.m for floating point, $\mid \mathrm{n}$ for integers

c $\quad$ zzz0 (in upper case) is replaced with the number of cyles

c $\quad$ zzz1 $=$ the actual concentration in units of milli-g/cc

c $\quad$ zzz2 $=$ solution density in $\mathrm{g} / \mathrm{cc}$

c $\quad \mathrm{qqq} 1=$ the minimum dimensions (in hundreths of inches)

c $\quad \mathrm{qqq} 2$ = the maximum dimensions (in hundreths of inches)

c $\quad \mathrm{qqq} 3=$ the delta (in hundreths of inches)

$\mathrm{c}$

c Diameter $=<\mathrm{d} 1=$ QQQ1:QQQ2:QQQ3 $\mid 5>$ hundreths of inches - must be integer

$\mathrm{c}$ radius $=<\mathrm{rd}=\mathrm{d} 1 * 2.54 / 100 / 2 \mid 5.4>\mathrm{cm}$

c note for perl, numbers with leading zeros must be enclosed in single quotes or else

c they will be interpretted as octal

$\mathrm{c}$ Conc $=<\mathrm{c}={ }^{\prime} \mathrm{ZZZ1} 1 \mid 5>$ milli-g Pu/cc

c Reflector width $=<\mathrm{rw}=10.0 \mid 2.1>$ inches

c Enrichment $=<$ ena $=1.0 \mid 1.2>$ atom fraction Pu239

c Mols of Acid per liter $<$ molAcid $=0.0 \mid 1.2>$

c

$\mathrm{c}$

c Density of solution $<$ denSol $=Z Z Z 2 / 1$ e $5 \mid 1.5>$

c

c

c Atomic Weights

c $\mathrm{H} 2 \mathrm{O}=\langle$ atwH2O $=18.0152| 3.4>$

$\mathrm{c} \mathrm{NO3}=\langle$ atwNO3 $=62.0049| 3.4>$

c $\mathrm{HNO} 3=<\mathrm{atwHNO} 3=63.0128 \mid 3.4>$

c Pu239 $=<$ atwPu239 $=239.0522 \mid 3.4>$

c Pu240 $=<$ atwPu240 $=240.0538 \mid 3.4>$

$\mathrm{c} \mathrm{Pu}=<\mathrm{atwPu}=\mathrm{ena}^{*}$ atwPu239+(1-ena)*atwPu240|3.4>

c

c Molarity of solution components

c Mols of $\mathrm{Pu}$ per liter $<\mathrm{molPu}=\mathrm{c} / \mathrm{atwPu} \mid 3.4>$

c Mols of $\mathrm{H} 2 \mathrm{O}$ per liter $<$ molH2O $=(1000 *$ denSol-molPu* $(a+w P u+4 * a t w N O 3)$ -

molAcid*atwHNO3)/atwH2O $3.4>$

$\mathrm{c}$

$\mathrm{c}$

$<$ perl $>$

sub IMP

\{

return 1 if $(\$[0]>0)$;

return 0 ;

\}

$</$ perl $>$

c sphere

$2011<$-denSol|3.6> $-12-7-586 \quad$ imp:n=1

$212-1.000 \quad-3468-7-5((1-3):(-24)) \quad$ imp:n=<\&IMP(rw) $\mid 1>\quad$ \$zero importance if no reflector

$220 \quad 3:-4: 7: 5:-8:-6 \quad$ imp:n=0 


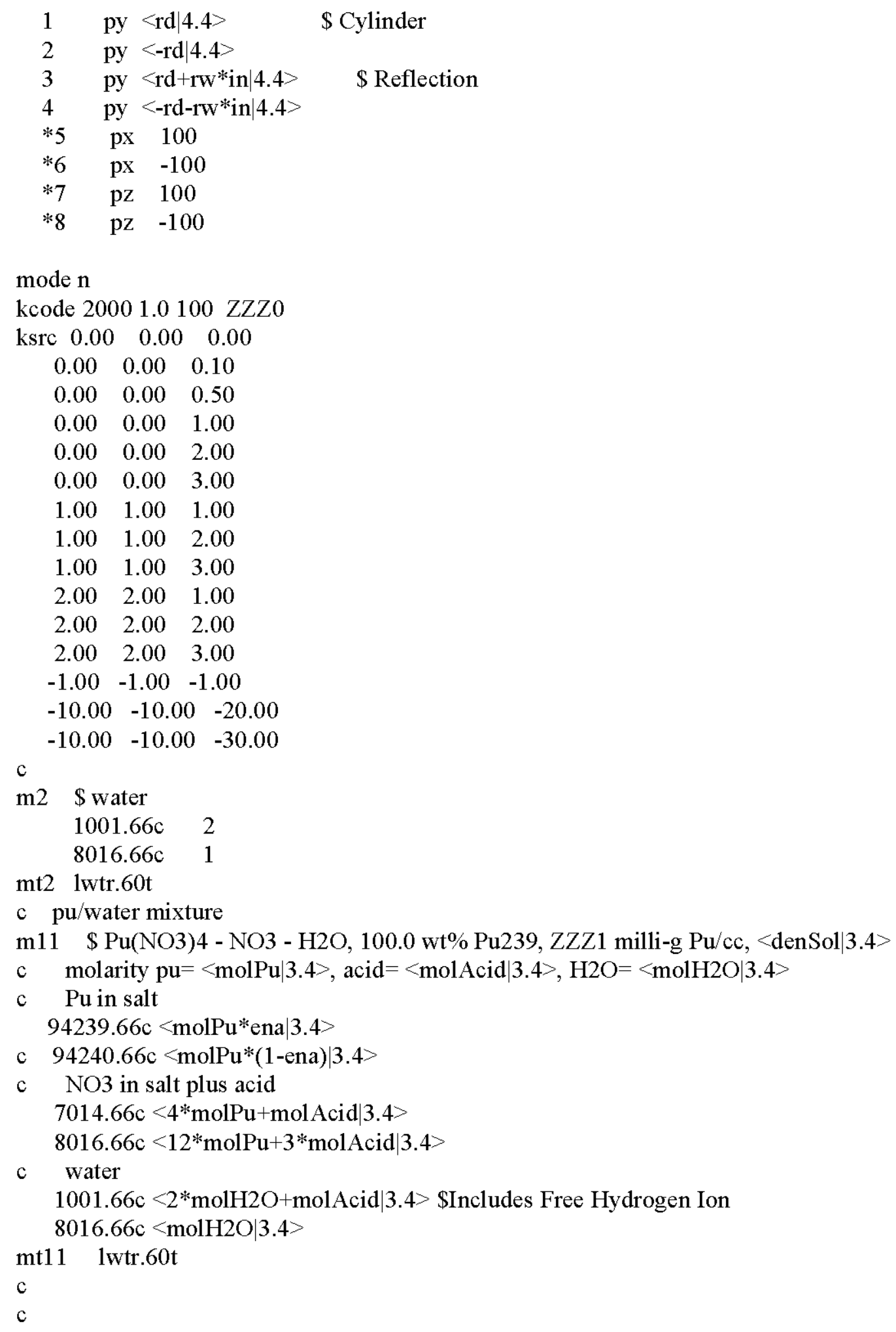


CHPRC-01552, Revision 0 
Template File for Case: PuN43-pl-1000-00

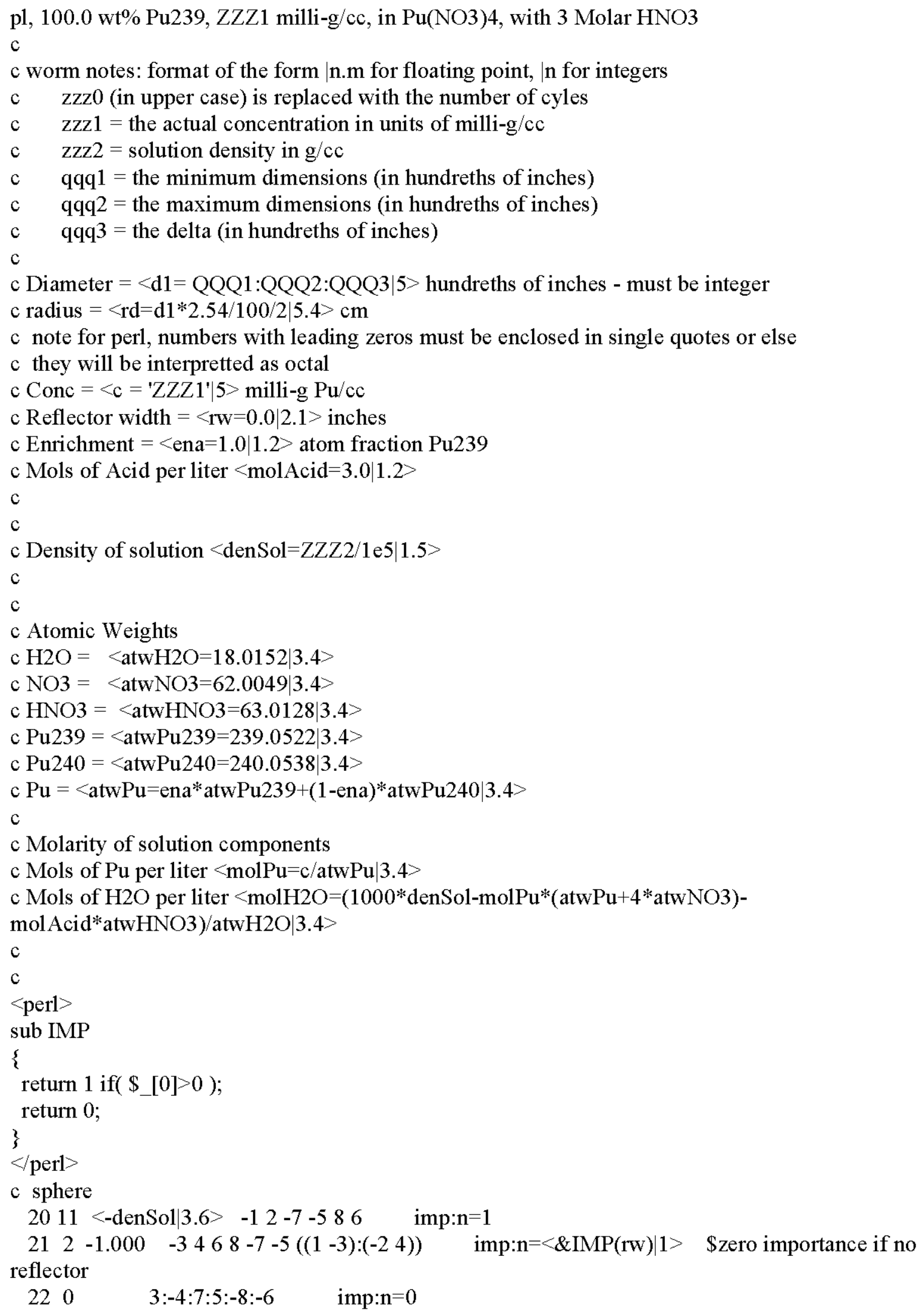




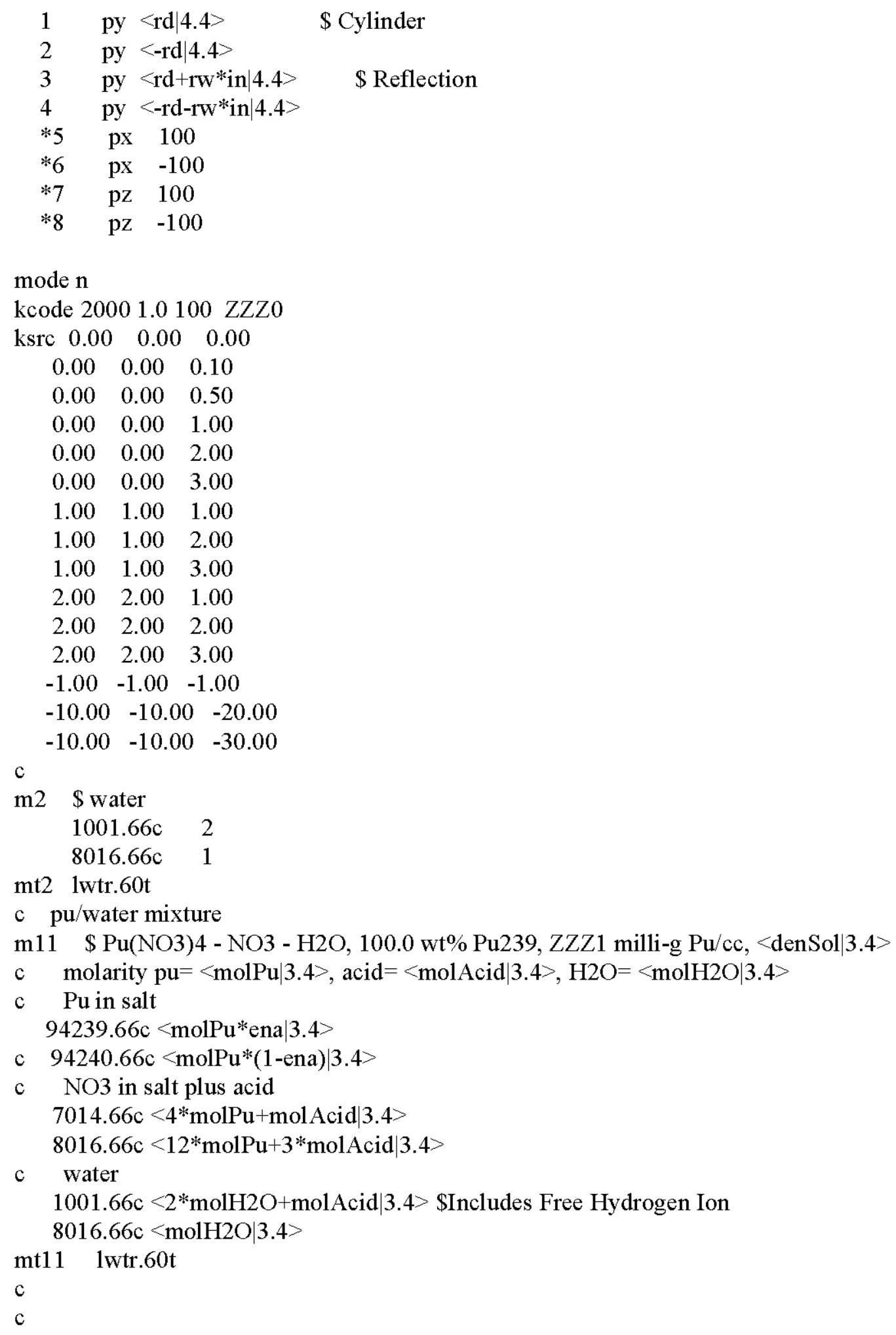


Template File for Case: PuN43-pl-1000-01

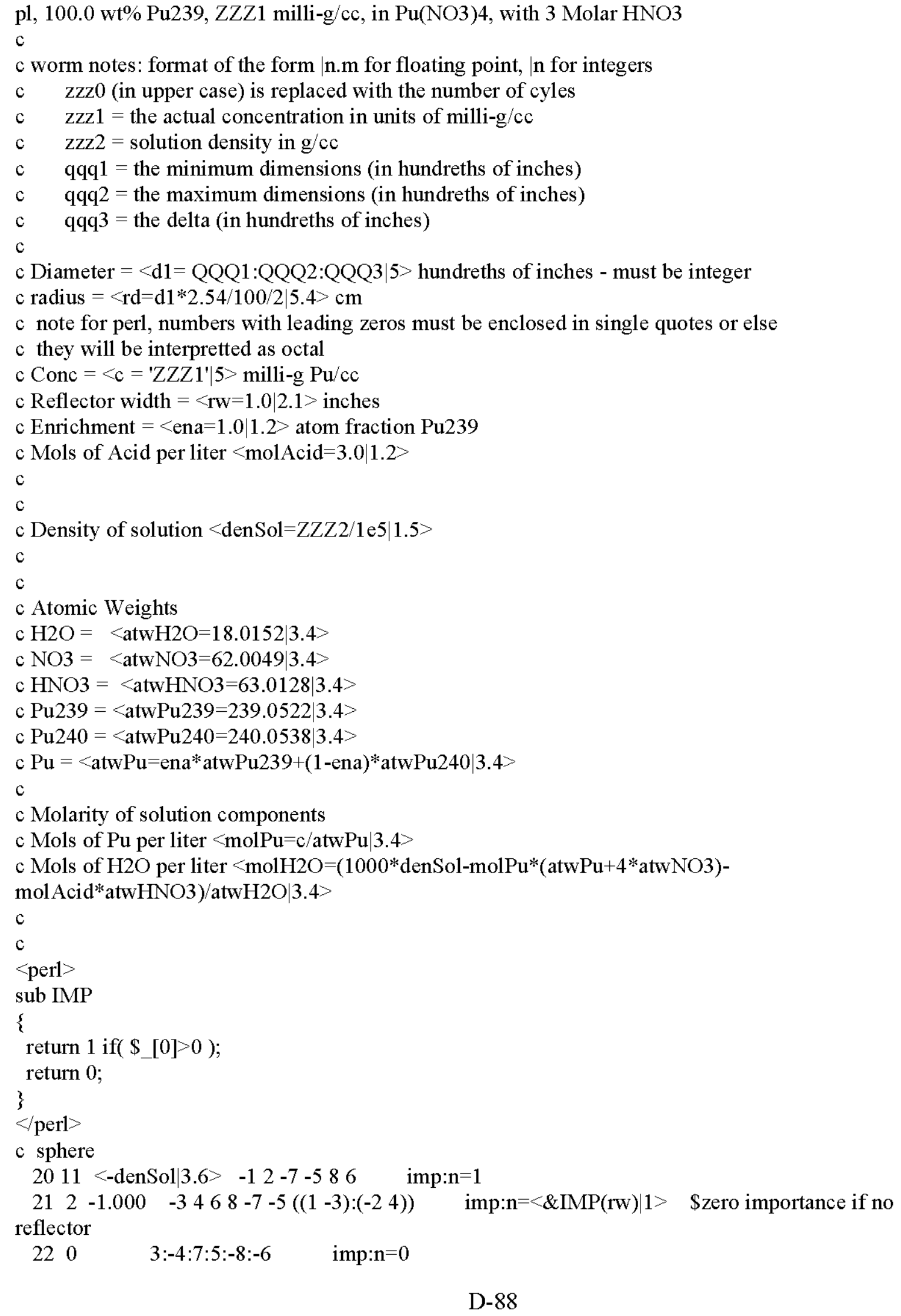




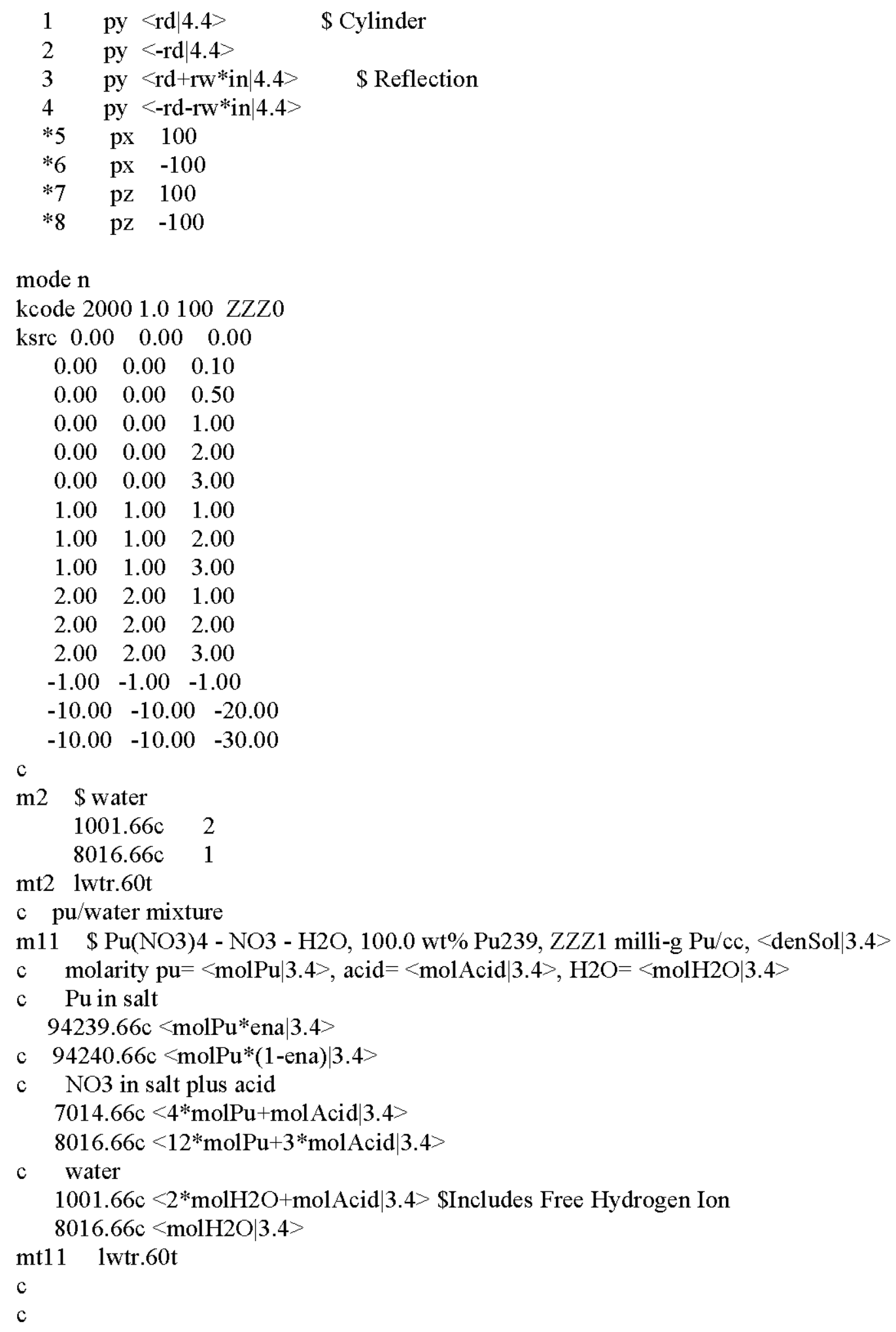


Template File for Case: PuN43-pl-1000-10

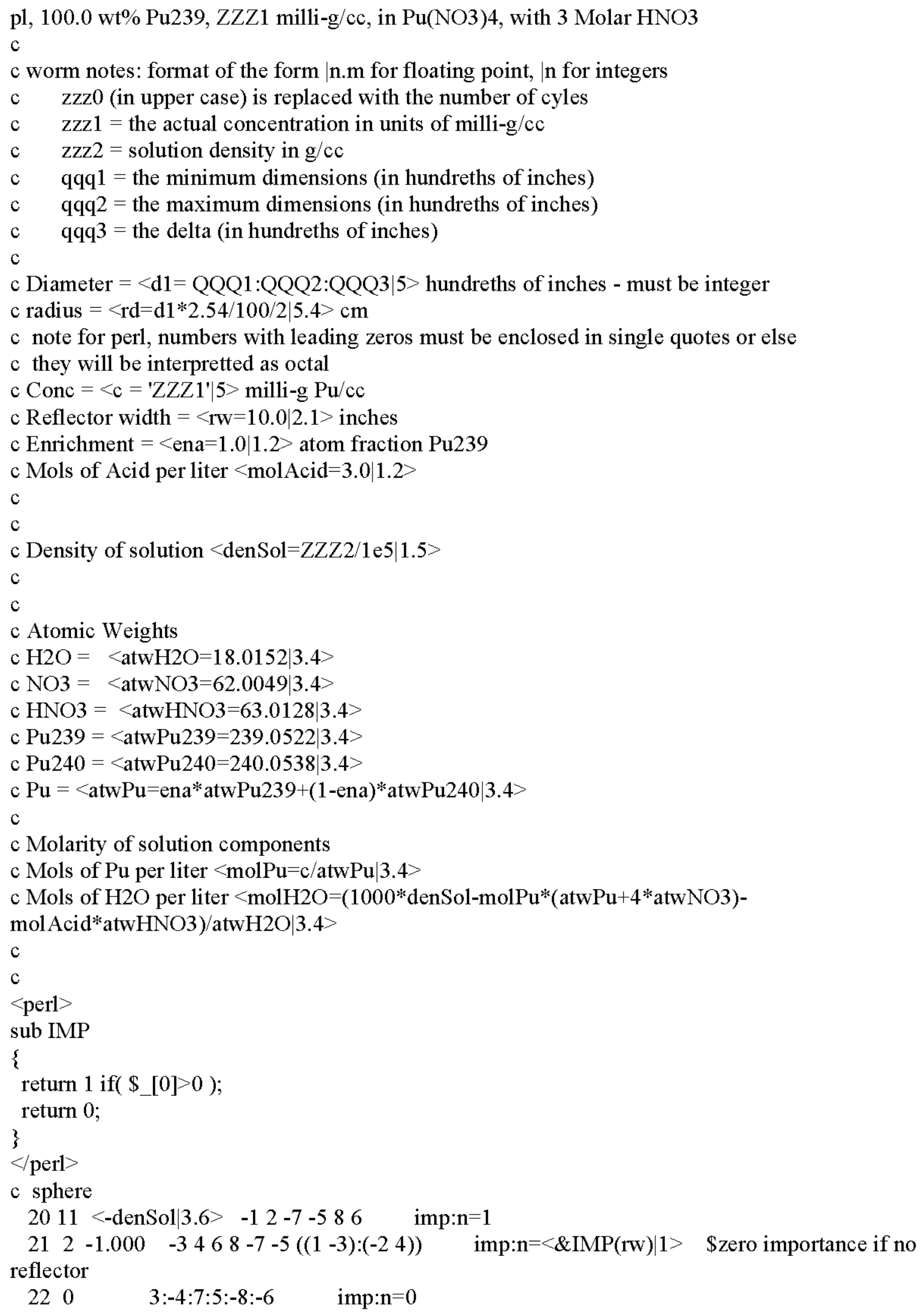




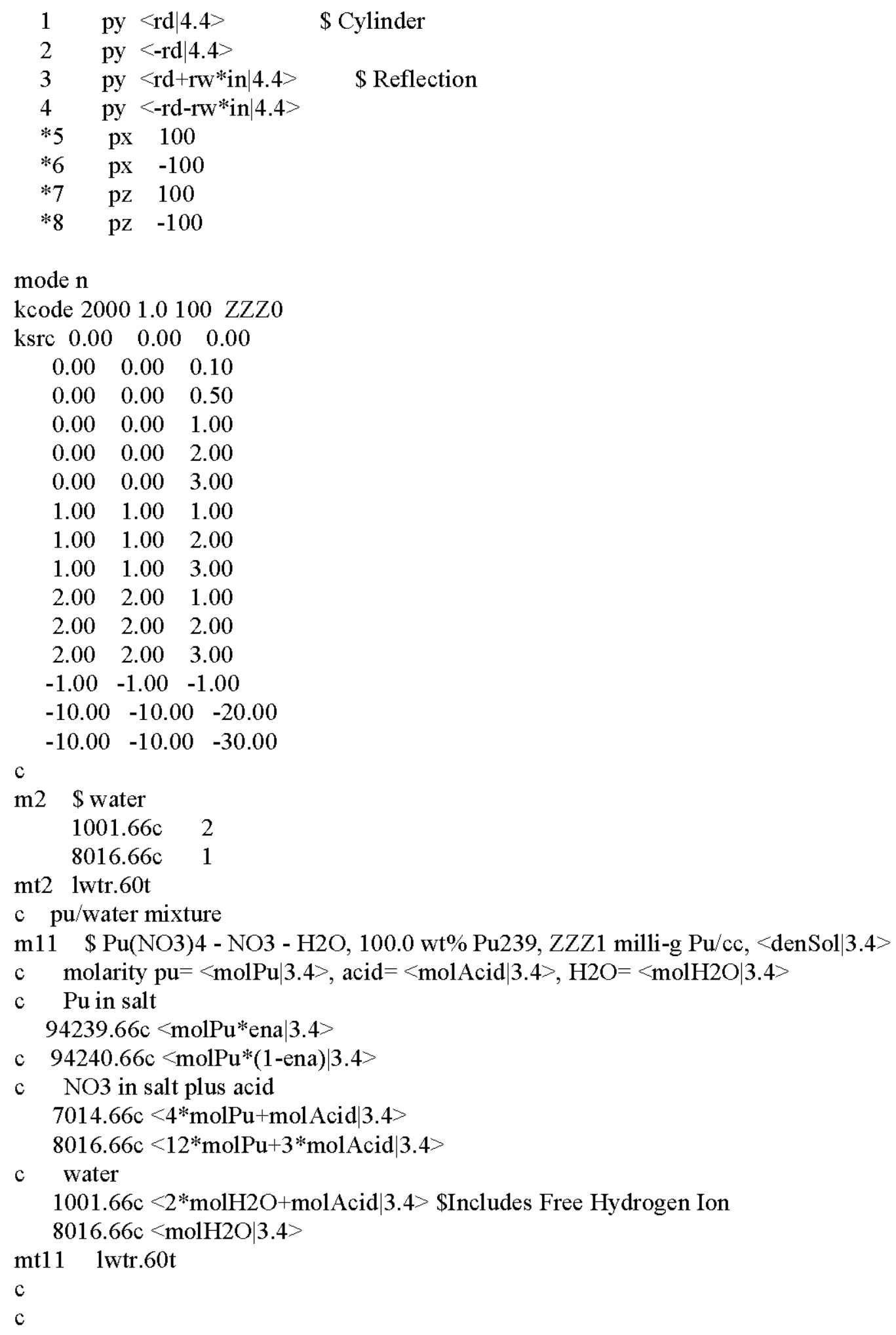


CHPRC-01552, Revision 0 


\section{Template File for Case: PuN46-pl-1000-00}

pl, 100.0 wt\% Pu239, ZZZ1 milli-g/cc, in Pu(NO3)4, with 6 Molar HNO3

c

c worm notes: format of the form |n.m for floating point, $\mid \mathrm{n}$ for integers

c $\quad$ zzz0 (in upper case) is replaced with the number of cyles

c $\quad$ zzz1 $=$ the actual concentration in units of milli-g/cc

c $\quad$ zzz2 $=$ solution density in $\mathrm{g} / \mathrm{cc}$

c $\quad \mathrm{qqq} 1=$ the minimum dimensions (in hundreths of inches)

c $\quad \mathrm{qqq} 2$ = the maximum dimensions (in hundreths of inches)

c $\quad \mathrm{qqq} 3=$ the delta (in hundreths of inches)

$\mathrm{c}$

c Diameter $=<\mathrm{d} 1=$ QQQ1:QQQ2:QQQ3 $\mid 5>$ hundreths of inches - must be integer

$\mathrm{c}$ radius $=<\mathrm{rd}=\mathrm{d} 1 * 2.54 / 100 / 2 \mid 5.4>\mathrm{cm}$

c note for perl, numbers with leading zeros must be enclosed in single quotes or else

c they will be interpretted as octal

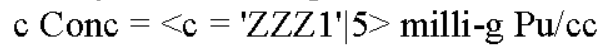

c Reflector width $=<\mathrm{rw}=0.0 \mid 2.1>$ inches

c Enrichment $=<$ ena $=1.0 \mid 1.2>$ atom fraction Pu239

c Mols of Acid per liter $<$ molAcid $=6.0 \mid 1.2>$

c

$\mathrm{c}$

c Density of solution $<$ denSol $=Z Z Z 2 / 1 \mathrm{e} 5 \mid 1.5>$

c

c

c Atomic Weights

c $\mathrm{H} 2 \mathrm{O}=\langle$ atwH2O $=18.0152| 3.4>$

$\mathrm{c} \mathrm{NO3}=\langle$ atwNO3 $=62.0049| 3.4>$

c HNO3 $=<$ atwHNO3 $=63.0128 \mid 3.4>$

c $\mathrm{Pu} 239=<\mathrm{atwPu} 239=239.0522 \mid 3.4>$

c Pu240 $=<$ atwPu240 $=240.0538 \mid 3.4>$

$\mathrm{c} \mathrm{Pu}=<\mathrm{atwPu}=\mathrm{ena}^{*}$ atwPu239+(1-ena)*atwPu240|3.4>

$\mathrm{c}$

c Molarity of solution components

c Mols of $\mathrm{Pu}$ per liter $<\mathrm{molPu}=\mathrm{c} / \mathrm{atwPu} \mid 3.4>$

c Mols of $\mathrm{H} 2 \mathrm{O}$ per liter $<$ molH2O $=(1000 *$ denSol-molPu* $(\operatorname{atwPu}+4 *$ atwNO3)-

molAcid*atwHNO3)/atwH2O $3.4>$

c

$\mathrm{c}$

$<$ perl $>$

sub IMP

\{

return 1 if $(\$[0]>0)$;

return 0

\}

$</$ perl $>$

c sphere

$2011<$-denSol|3.6> $-12-7-586 \quad$ imp:n=1

$212-1.000 \quad-3468-7-5((1-3):(-24)) \quad$ imp:n=<\&IMP(rw) $\mid 1>\quad$ \$zero importance if no reflector

$220 \quad 3:-4: 7: 5:-8:-6 \quad$ imp:n=0 


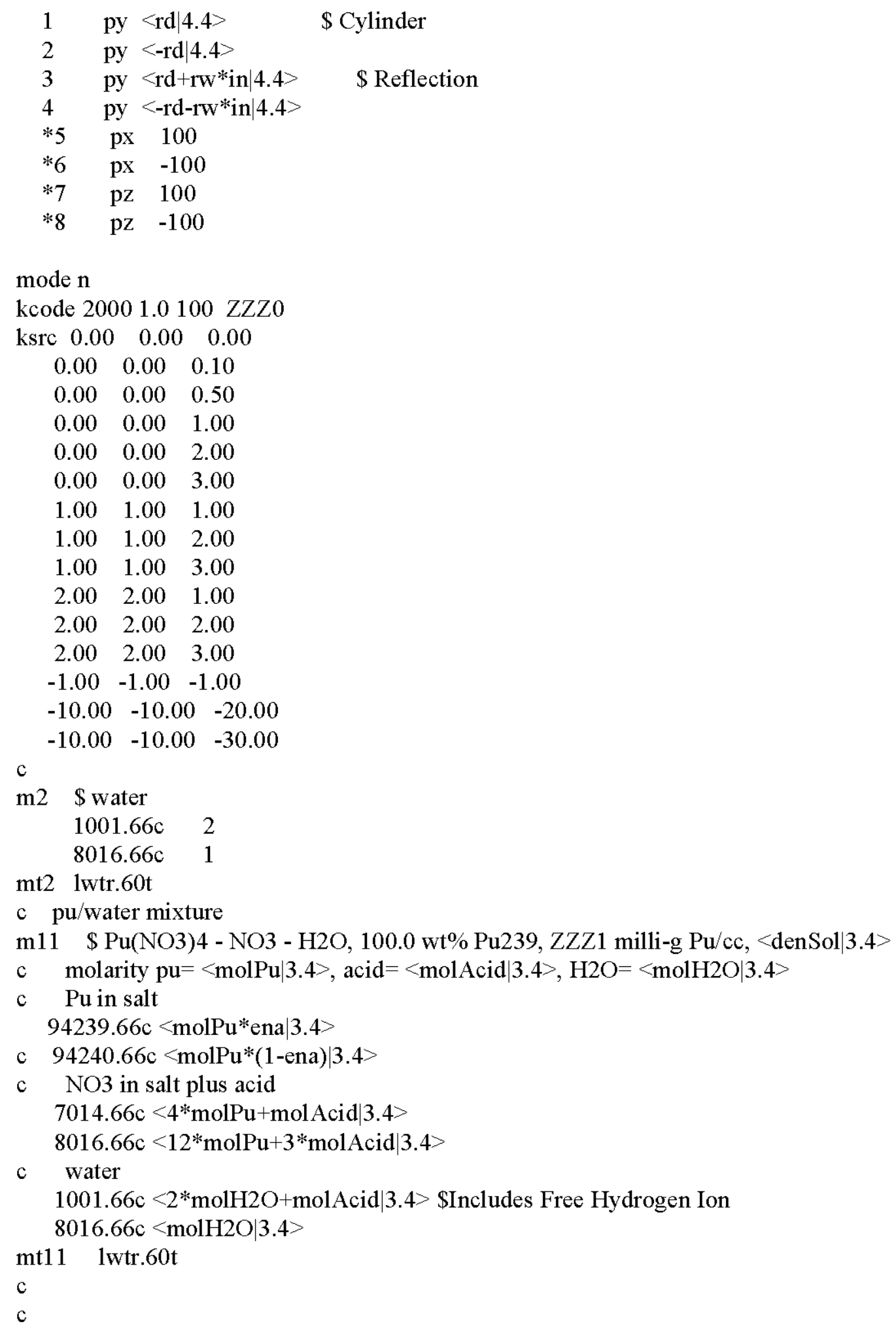




\section{Template File for Case: PuN46-pl-1000-01}

pl, 100.0 wt\% Pu239, ZZZ1 milli-g/cc, in Pu(NO3)4, with 6 Molar HNO3

c

c worm notes: format of the form |n.m for floating point, $\mid \mathrm{n}$ for integers

c $\quad$ zzz0 (in upper case) is replaced with the number of cyles

c $\quad$ zzz1 $=$ the actual concentration in units of milli-g/cc

c $\quad$ zzz2 $=$ solution density in $\mathrm{g} / \mathrm{cc}$

c $\quad \mathrm{qqq} 1=$ the minimum dimensions (in hundreths of inches)

c $\quad \mathrm{qqq} 2$ = the maximum dimensions (in hundreths of inches)

c $\quad \mathrm{qqq} 3=$ the delta (in hundreths of inches)

$\mathrm{c}$

c Diameter $=<\mathrm{d} 1=$ QQQ1:QQQ2:QQQ3 $\mid 5>$ hundreths of inches - must be integer

$\mathrm{c}$ radius $=<\mathrm{rd}=\mathrm{d} 1 * 2.54 / 100 / 2 \mid 5.4>\mathrm{cm}$

c note for perl, numbers with leading zeros must be enclosed in single quotes or else

c they will be interpretted as octal

$\mathrm{c}$ Conc $=<\mathrm{c}={ }^{\prime} \mathrm{ZZZ1} 1 \mid 5>$ milli-g Pu/cc

c Reflector width $=<\mathrm{rw}=1.0 \mid 2.1>$ inches

c Enrichment $=<$ ena $=1.0 \mid 1.2>$ atom fraction Pu239

c Mols of Acid per liter $<$ molAcid $=6.0 \mid 1.2>$

c

$\mathrm{c}$

c Density of solution $<$ denSol $=Z Z Z 2 / 1 \mathrm{e} 5 \mid 1.5>$

c

c

c Atomic Weights

c $\mathrm{H} 2 \mathrm{O}=\langle$ atwH2O $=18.0152| 3.4>$

$\mathrm{c} \mathrm{NO3}=\langle$ atwNO3 $=62.0049| 3.4>$

c HNO3 $=<$ atwHNO3 $=63.0128 \mid 3.4>$

c $\mathrm{Pu} 239=<\mathrm{atwPu} 239=239.0522 \mid 3.4>$

c Pu240 $=<$ atwPu240 $=240.0538 \mid 3.4>$

$\mathrm{c} \mathrm{Pu}=<\mathrm{atwPu}=\mathrm{ena}^{*}$ atwPu239+(1-ena)*atwPu240|3.4>

$\mathrm{c}$

c Molarity of solution components

c Mols of $\mathrm{Pu}$ per liter $<\mathrm{molPu}=\mathrm{c} / \mathrm{atwPu} \mid 3.4>$

c Mols of $\mathrm{H} 2 \mathrm{O}$ per liter $<$ molH2O $=(1000 *$ denSol-molPu* $(\operatorname{atwPu}+4 *$ atwNO3)-

molAcid*atwHNO3)/atwH2O $3.4>$

c

$\mathrm{c}$

$<$ perl $>$

sub IMP

\{

return 1 if $(\$[0]>0)$;

return 0 ;

\}

$</$ perl $>$

c sphere

$2011<-$ denSol|3.6> $-12-7-586 \quad$ imp:n=1

$2120-1.000 \quad-3468-7-5((1-3):(-24)) \quad$ imp:n=<\&IMP(rw) $\mid 1>$ \$zero importance if no reflector

$220 \quad 3:-4: 7: 5:-8:-6 \quad$ imp:n=0 


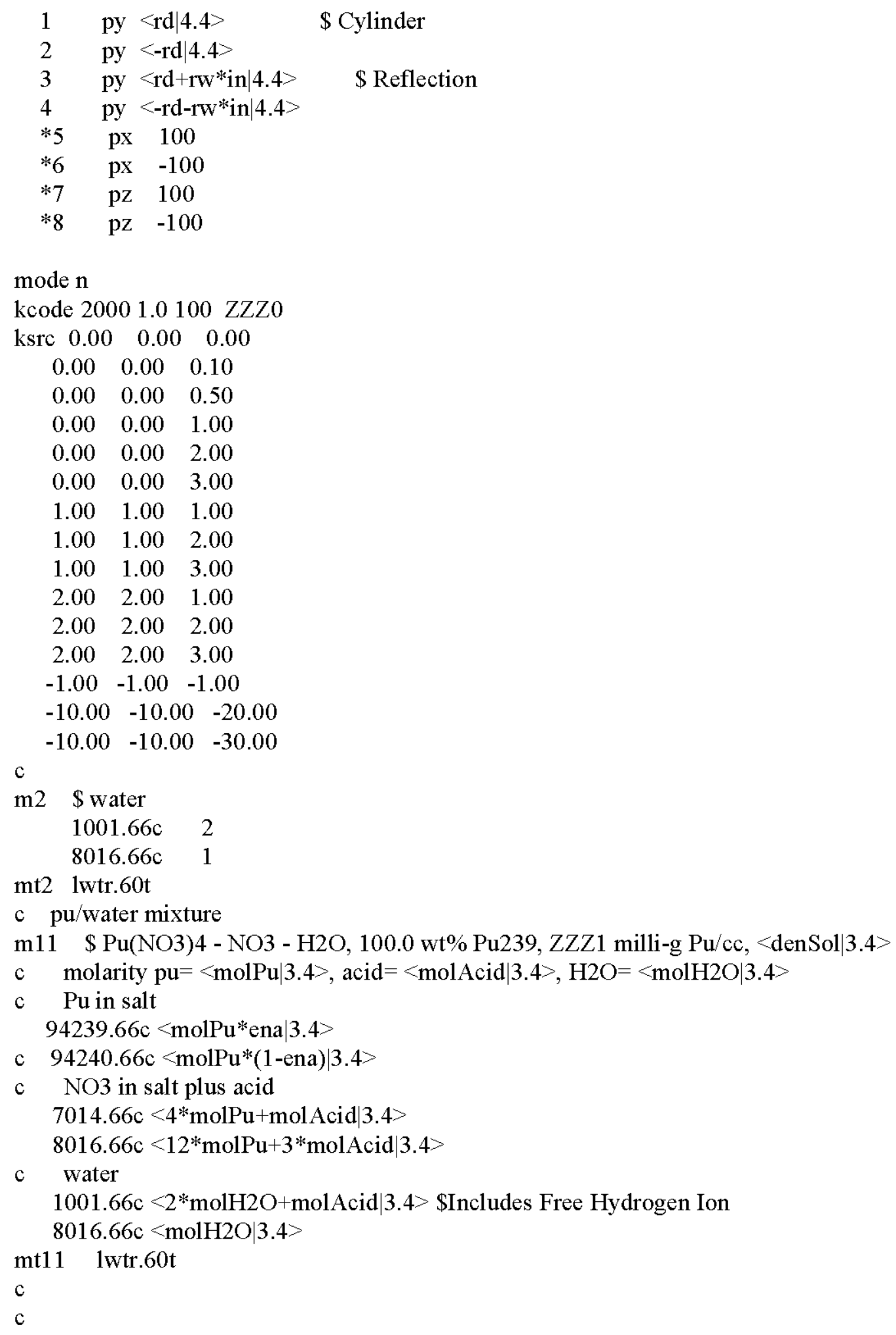




\section{Template File for Case: PuN46-pl-1000-10}

pl, 100.0 wt\% Pu239, ZZZ1 milli-g/cc, in Pu(NO3)4, with 6 Molar HNO3

c

c worm notes: format of the form $\mid n . m$ for floating point, $\mid \mathrm{n}$ for integers

c zzz0 (in upper case) is replaced with the number of cyles

c $\quad$ zzz1 $=$ the actual concentration in units of milli-g/cc

c $\quad$ zzz2 $=$ solution density in $\mathrm{g} / \mathrm{cc}$

c $\quad \mathrm{qqq} 1=$ the minimum dimensions (in hundreths of inches)

c $\quad \mathrm{qqq} 2=$ the maximum dimensions (in hundreths of inches)

c $\quad \mathrm{qqq} 3=$ the delta (in hundreths of inches)

$\mathrm{c}$

c Diameter $=<\mathrm{d} 1=$ QQQ1:QQQ2:QQQ3 $\mid 5>$ hundreths of inches - must be integer

$\mathrm{c}$ radius $=<\mathrm{rd}=\mathrm{d} 1 * 2.54 / 100 / 2 \mid 5.4>\mathrm{cm}$

c note for perl, numbers with leading zeros must be enclosed in single quotes or else

$c$ they will be interpretted as octal

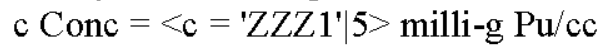

c Reflector width $=<\mathrm{rw}=10.0 \mid 2.1>$ inches

c Enrichment $=<$ ena $=1.0 \mid 1.2>$ atom fraction Pu239

c Mols of Acid per liter $<$ molAcid $=6.0 \mid 1.2>$

$\mathrm{c}$

$\mathrm{c}$

c Density of solution $<$ denSol $=Z Z Z 2 / 1 \mathrm{e} 5 \mid 1.5>$

c

C

c Atomic Weights

c $\mathrm{H} 2 \mathrm{O}=\langle$ atwH2O $=18.0152| 3.4>$

c NO3 $=\langle$ atwNO3 $=62.0049| 3.4>$

c HNO3 $=<$ atwHNO3 $=63.0128 \mid 3.4>$

$\mathrm{c} \mathrm{Pu} 239=<\mathrm{atwPu} 239=239.0522 \mid 3.4>$

c Pu240 $=<$ atwPu240 $=240.0538 \mid 3.4>$

$\mathrm{c} \mathrm{Pu}=<\mathrm{atwPu}=\mathrm{ena}^{*}$ atwPu239+(1-ena)*atwPu240|3.4>

$\mathrm{c}$

c Molarity of solution components

c Mols of $\mathrm{Pu}$ per liter $<\mathrm{molPu}=\mathrm{c} / \mathrm{atwPu} \mid 3.4>$

c Mols of $\mathrm{H} 2 \mathrm{O}$ per liter $<$ molH2O $=(1000 *$ denSol-molPu* $(\operatorname{atwPu}+4 *$ atwNO3)-

molAcid*atwHNO3)/atwH2O $3.4>$

$\mathrm{c}$

$\mathrm{c}$

$<$ perl $>$

sub IMP

\{

return 1 if $(\$[0]>0)$;

return 0 ;

\}

$</$ perl $>$

c sphere

$2011<$-denSol|3.6> $-12-7-586 \quad$ imp:n=1

$212-1.000 \quad-3468-7-5((1-3):(-24)) \quad$ imp:n=<\&IMP(rw) $\mid 1>\quad$ \$zero importance if no reflector

$220 \quad 3:-4: 7: 5:-8:-6 \quad$ imp: $n=0$ 


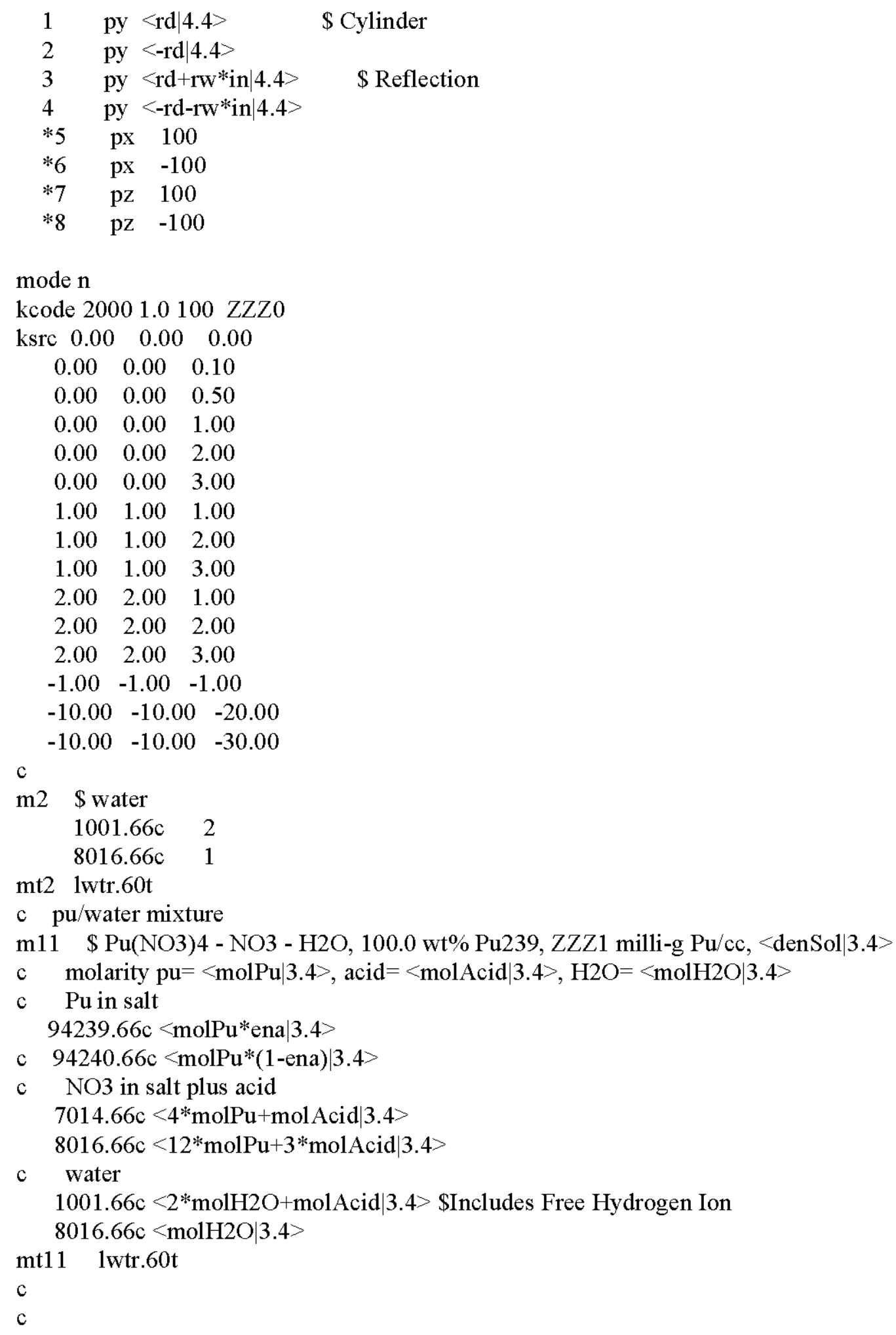


Template File for Case: PuN40-pl-0970-00

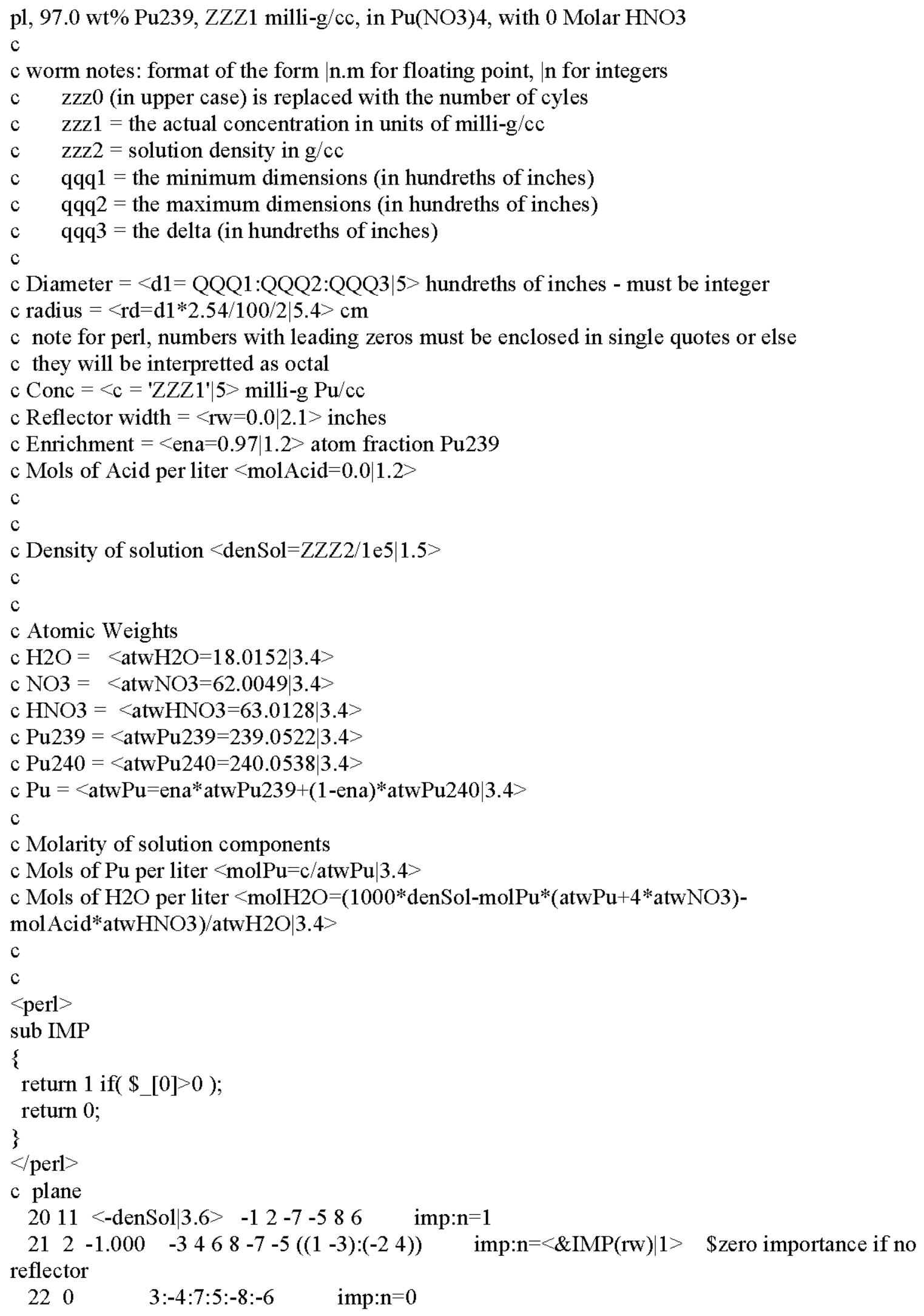




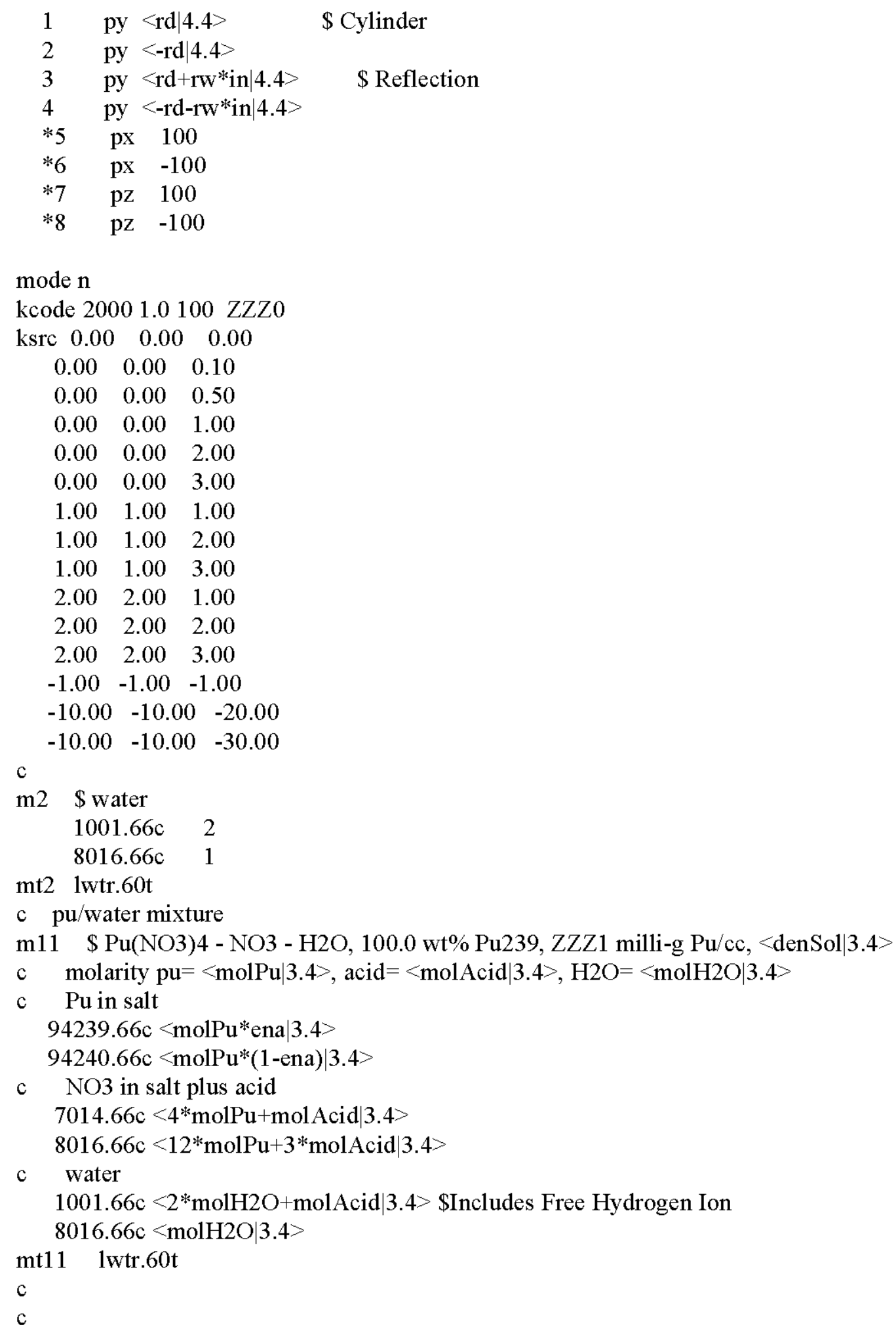


Template File for Case: PuN40-pl-0970-01

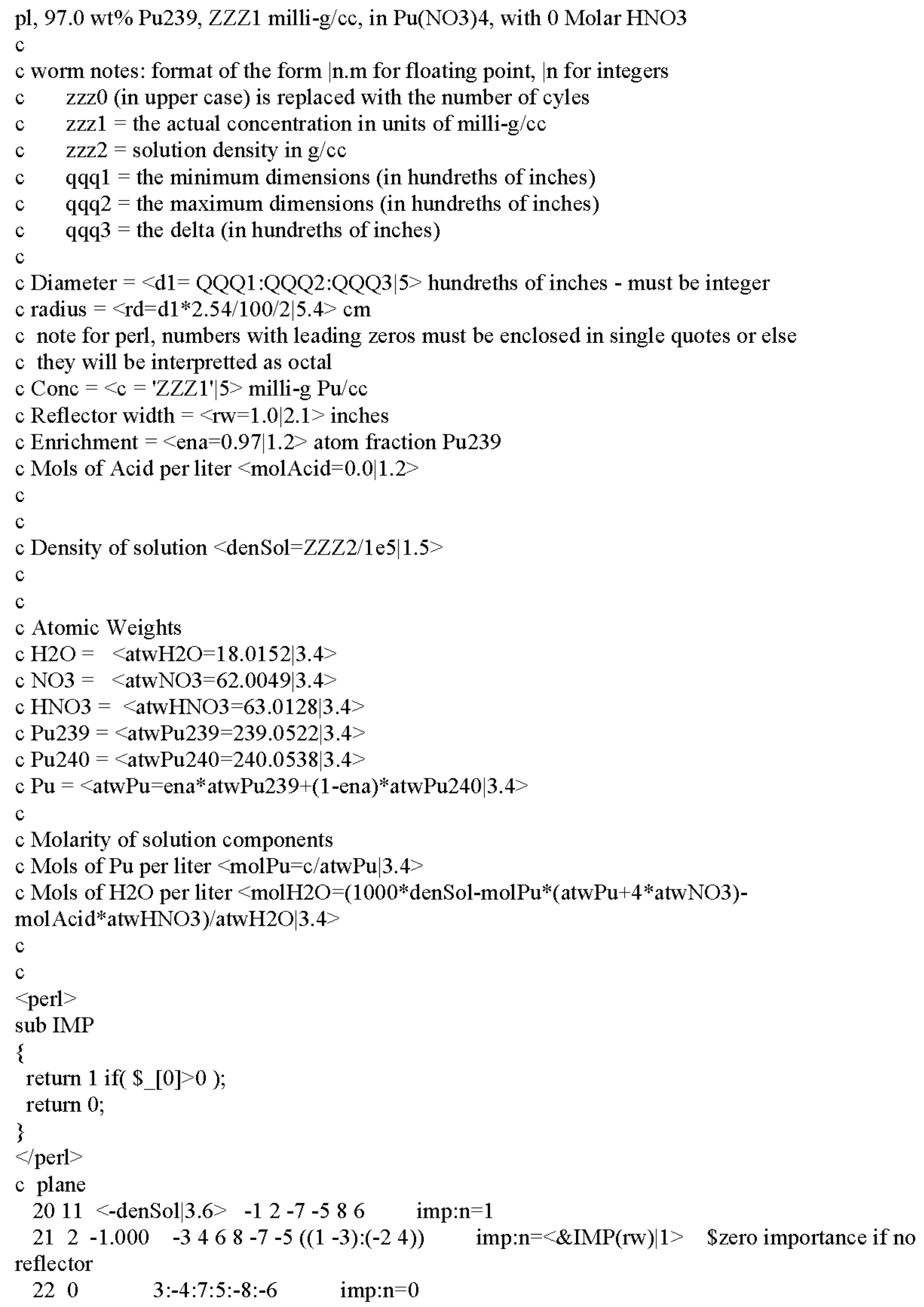




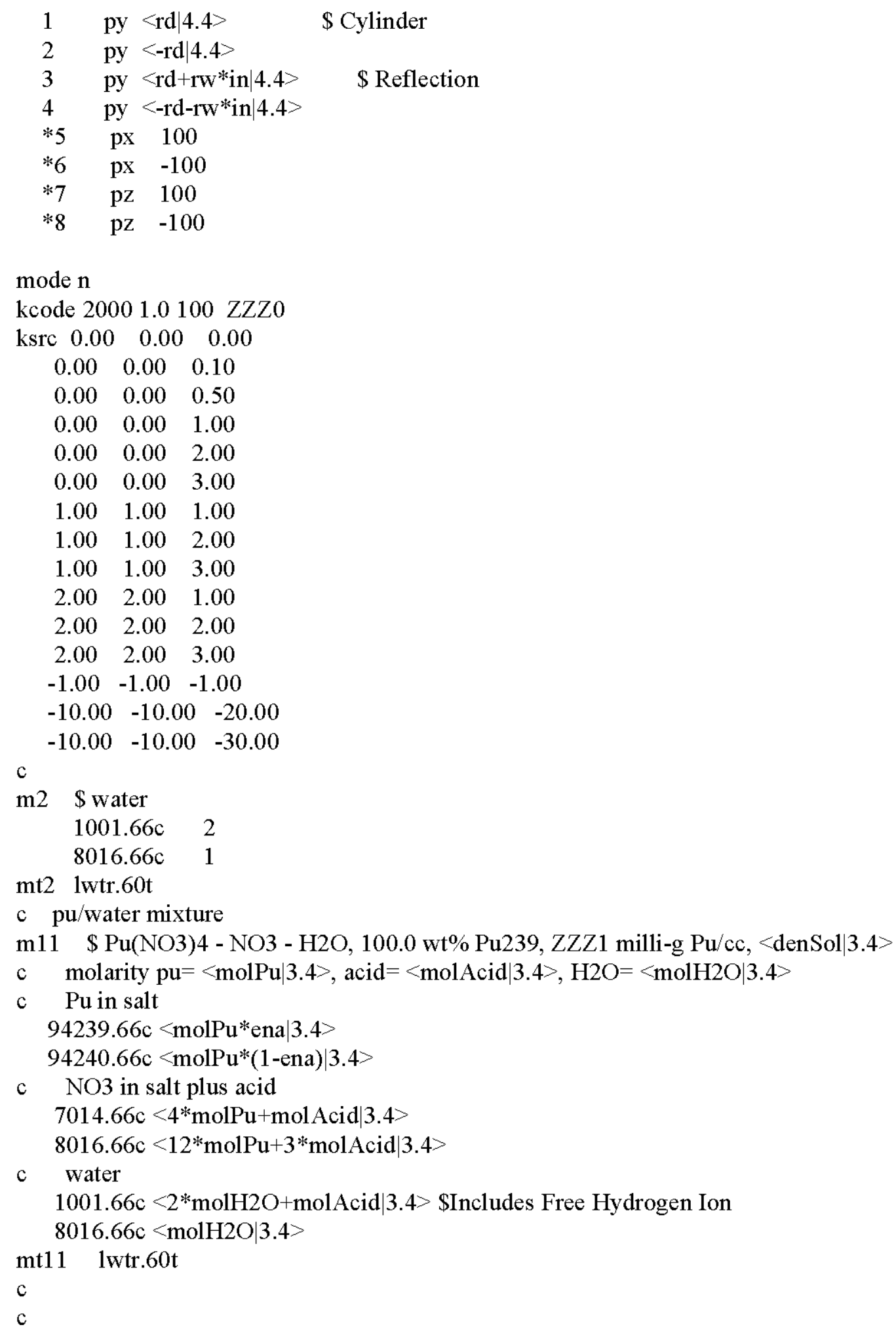


Template File for Case: PuN40-pl-0970-10

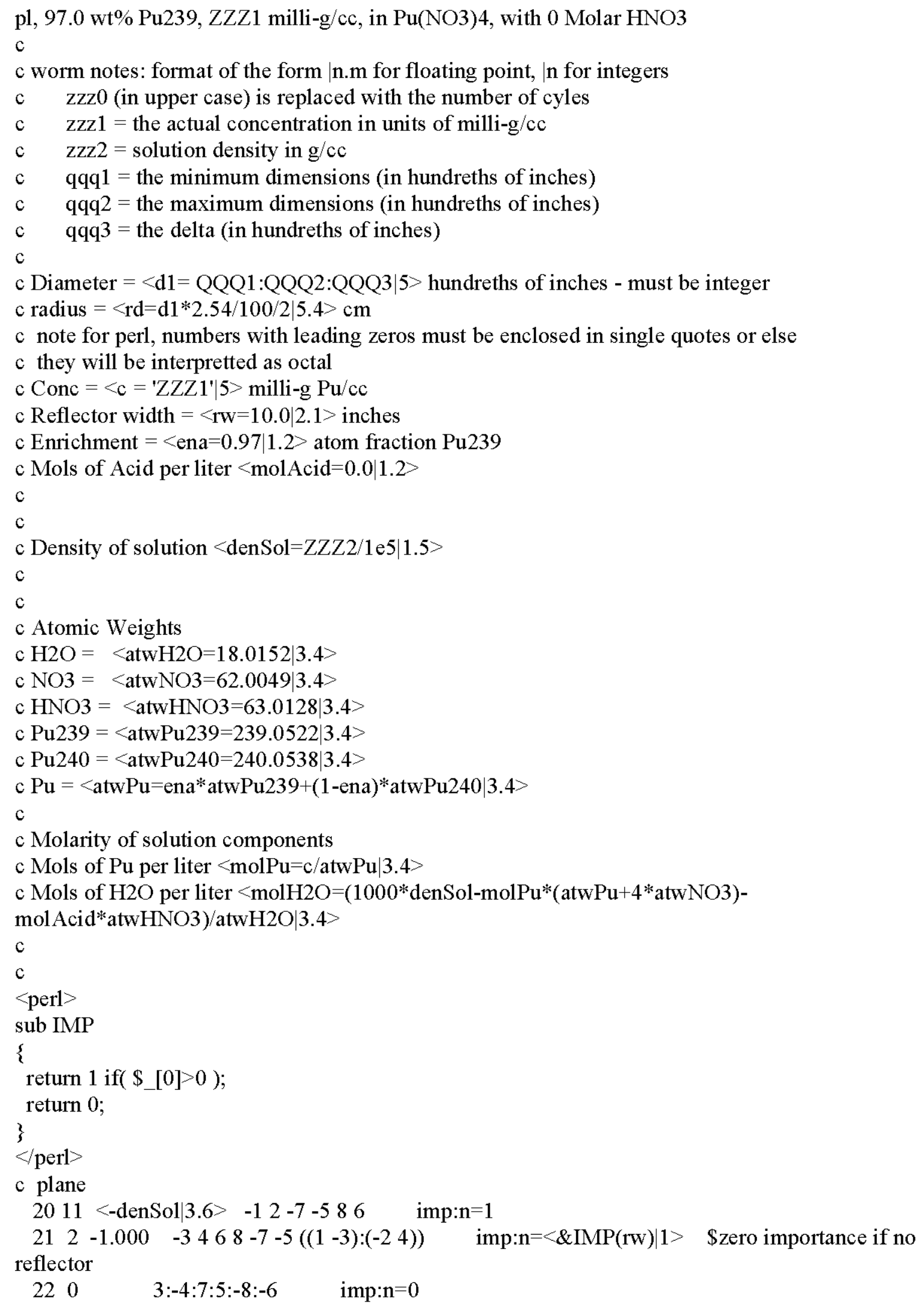




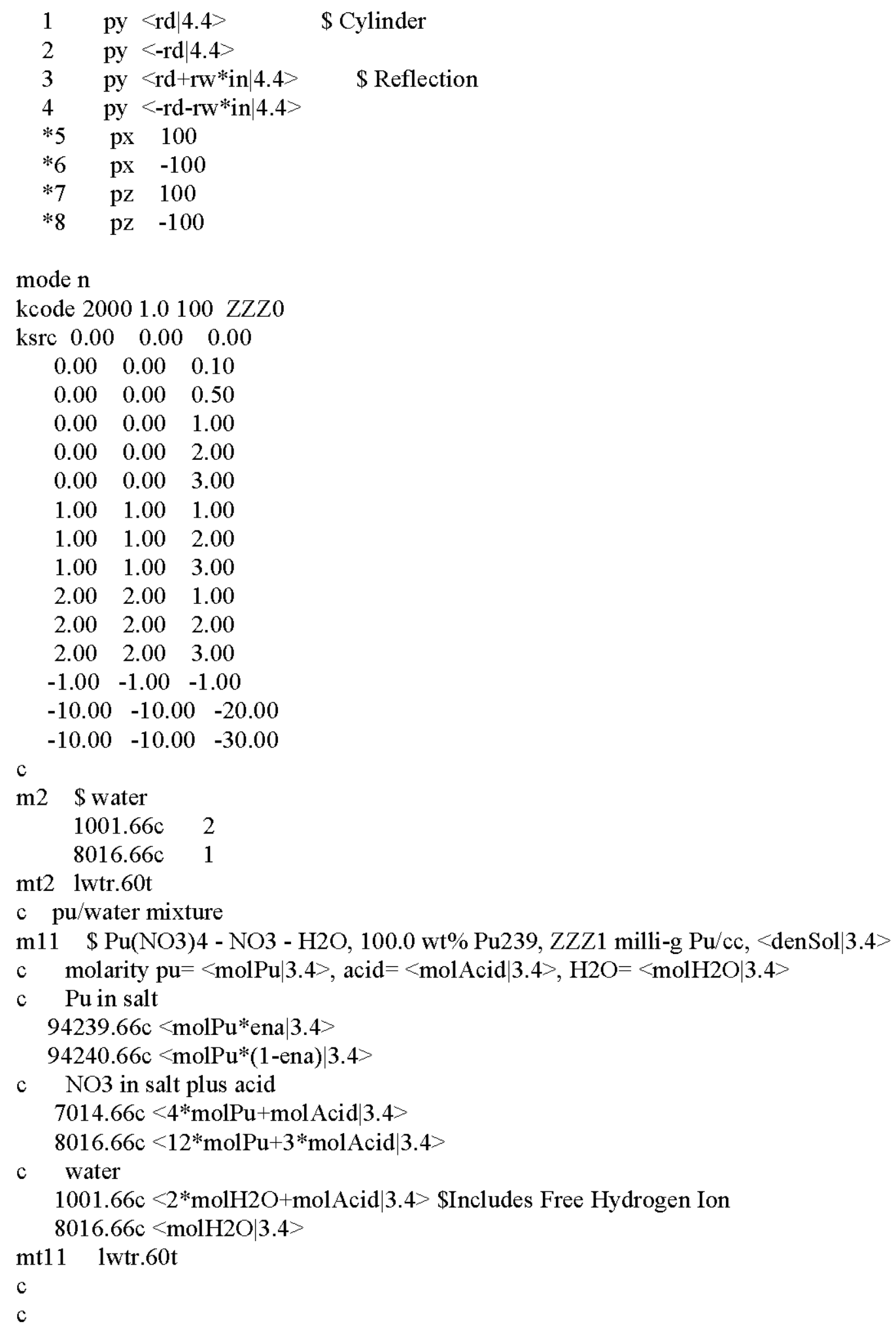


Template File for Case: u5o2N20-sp-1000-00

sp, $100.0 \mathrm{wt} \%$ UO2, ZZZ1 milli-g/cc, in UO2(NO3)2, with 0 Molar HNO3

$\mathrm{c}$

c worm notes: format of the form $\mid n . m$ for floating point, $\mid \mathrm{n}$ for integers

c zzz0 (in upper case) is replaced with the number of cyles

c $\quad$ zzz1 $=$ the actual concentration in units of milli-g/cc

c $\quad$ zzz2 $=$ solution density in $\mathrm{g} / \mathrm{cc}$

c $\quad \mathrm{qqq1}=$ the minimum dimensions (in hundreths of inches)

c $\quad \mathrm{qqq} 2=$ the maximum dimensions (in hundreths of inches)

c $\quad \mathrm{qqq} 3=$ the delta (in hundreths of inches)

c

c Diameter $=<\mathrm{d} 1=$ QQQ1:QQQ2:QQQ3 $\mid 5>$ hundreths of inches - must be integer

$\mathrm{c}$ radius $=<\mathrm{rd}=\mathrm{d} 1 * 2.54 / 100 / 2 \mid 5.4>\mathrm{cm}$

c note for perl, numbers with leading zeros must be enclosed in single quotes or else

c they will be interpretted as octal

$\mathrm{c}$ Conc $=<\mathrm{c}={ }^{\prime} \mathrm{ZZZ1} 1 \mid 5>$ milli-g U/cc

c Reflector width $=<\mathrm{rw}=0.0 \mid 2.1>$ inches

c Enrichment $=<$ ena $=1.0 \mid 1.2>$ atom fraction $\mathrm{U}$

c Mols of Acid per liter $<$ molAcid $=0.0 \mid 1.2>$

$\mathrm{c}$

$\mathrm{c}$

c Density of solution $<$ denSol=ZZZ2/1e5 $\mid 1.5>$

c

C

c Atomic Weights

c $\mathrm{H} 2 \mathrm{O}=\langle$ atwH2O $=18.0152| 3.4>$

$\mathrm{c} \mathrm{NO3}=\langle$ atwNO3 $=62.0049| 3.4>$

c $\mathrm{O}=\langle$ atwO $=15.9994| 3.4>$

$\mathrm{c} \mathrm{HNO} 3=<$ atwHNO3 $=63.0128 \mid 3.4>$

c U235 $=<$ atwU235 $=235.0439 \mid 3.4>$

c U238 $=<$ atwU238 $=238.0508 \mid 3.4>$

$\mathrm{c} \mathrm{U}=<$ atwU $=$ ena*atwU235+(1-ena)*atwU238|3.4>

c

c Molarity of solution components

c Mols of $\mathrm{U}$ per liter $<$ molU $=\mathrm{c} / \mathrm{atwU} \mid 3.4>$

c Mols of $\mathrm{H} 2 \mathrm{O}$ per liter $<\operatorname{molH} 2 \mathrm{O}=(1000 *$ denSol-molU* $*$ atwU $+2 * a+w N O 3+2 * a t w O)$ molAcid*atwHNO3)/atwH2O|3.4>

$\mathrm{c}$

c

$<$ perl $>$

sub IMP

\{

return 1 if $\left(\$ \_[0]>0\right)$;

return 0 ;

\}

$</$ perl $>$

c sphere

$2011<$-denSol|3.6> $-1 \quad$ imp:n=1

$212-1.000-21 \quad$ imp:n=<\&IMP(rw) $\mid 1>$ \$zero importance if no reflector

$220 \quad 2$ imp:n=0 


$$
\begin{array}{lll}
1 & \text { so }<\text { rd } \mid 4.4> & \text { \$ Sphere } \\
2 & \text { so }<\text { rd }+ \text { rw*in|4.4 }> & \text { \$Reflection }
\end{array}
$$

mode $\mathrm{n}$

kcode 20001.0100 ZZZ0

$\begin{array}{llll}\text { ksrc } & 0.00 & 0.00 & 0.00\end{array}$

$\begin{array}{lll}0.00 & 0.00 & 0.10\end{array}$

$\begin{array}{lll}0.00 & 0.00 & 0.50\end{array}$

$\begin{array}{lll}0.00 & 0.00 & 1.00\end{array}$

$\begin{array}{lll}0.00 & 0.00 & 2.00\end{array}$

$\begin{array}{lll}0.00 & 0.00 & 3.00\end{array}$

$\begin{array}{lll}1.00 & 1.00 & 1.00\end{array}$

$\begin{array}{lll}1.00 & 1.00 & 2.00\end{array}$

$\begin{array}{lll}1.00 & 1.00 & 3.00\end{array}$

$\begin{array}{lll}2.00 & 2.00 & 1.00\end{array}$

$\begin{array}{lll}2.00 & 2.00 & 2.00\end{array}$

$\begin{array}{lll}2.00 & 2.00 & 3.00\end{array}$

$\begin{array}{lll}-1.00 & -1.00 & -1.00\end{array}$

$-10.00 \quad-10.00 \quad-20.00$

$\begin{array}{lll}-10.00 & -10.00 & -30.00\end{array}$

c

m2 \$water

$1001.66 \mathrm{c} \quad 2$

$8016.66 \mathrm{c} \quad 1$

$\mathrm{mt} 2$ 1wtr.60t

c UO2/water mixture

m11 \$ UO2(NO3)2 - NO3 - H2O, $100.0 \mathrm{wt} \% \mathrm{U} 235, \mathrm{ZZZ1} \mathrm{milli-g} \mathrm{U/cc,}<\mathrm{denSol} \mid 3.4>$

c molarity $\mathrm{U}=\langle\operatorname{molU}| 3.4>$, acid $=\langle\operatorname{mol} A \operatorname{Acid} \mid 3.4\rangle, \mathrm{H} 2 \mathrm{O}=\langle\operatorname{molH} 2 \mathrm{O} \mid 3.4\rangle$

c U in salt

$92235.66 \mathrm{c}<$ molU*ena $3.4>$

c $92238.66 \mathrm{c}<$ molU* $* 1$-ena $) \mid 3.4>$

c NO3 in salt plus acid

$7014.66 \mathrm{c}<2 * \mathrm{molU}+\operatorname{molAcid} \mid 3.4>$

$8016.66 \mathrm{c}<6 * \mathrm{molU}+3 * \operatorname{mol} \mathrm{Acid} \mid 3.4>$

c water

1001.66c $<2 *$ molH2O + molAcid $\mid 3.4>$ \$ncludes Free Hydrogen Ion

$8016.66 \mathrm{c}<\mathrm{molH} 2 \mathrm{O} \mid 3.4>$

mt11 1wtr.60t

c

c 
Template File for Case: u5o2N20-sp-1000-01

sp, $100.0 \mathrm{wt} \%$ UO2, ZZZ1 milli-g/cc, in UO2(NO3)2, with 0 Molar HNO3

$\mathrm{c}$

c worm notes: format of the form $\mid n . m$ for floating point, $\mid \mathrm{n}$ for integers

c zzz0 (in upper case) is replaced with the number of cyles

c $\quad$ zzz1 $=$ the actual concentration in units of milli-g/cc

c $\quad$ zzz2 $=$ solution density in $\mathrm{g} / \mathrm{cc}$

c $\quad \mathrm{qqq1}=$ the minimum dimensions (in hundreths of inches)

c $\quad \mathrm{qqq} 2=$ the maximum dimensions (in hundreths of inches)

c $\quad \mathrm{qqq} 3=$ the delta (in hundreths of inches)

c

c Diameter $=<\mathrm{d} 1=$ QQQ1:QQQ2:QQQ3 $\mid 5>$ hundreths of inches - must be integer

$\mathrm{c}$ radius $=<\mathrm{rd}=\mathrm{d} 1 * 2.54 / 100 / 2 \mid 5.4>\mathrm{cm}$

c note for perl, numbers with leading zeros must be enclosed in single quotes or else

c they will be interpretted as octal

$\mathrm{c}$ Conc $=<\mathrm{c}={ }^{\prime}$ ZZZ1' $\mid 5>$ milli-g U/cc

c Reflector width $=<\mathrm{rw}=1.0 \mid 2.1>$ inches

c Enrichment $=<$ ena $=1.0 \mid 1.2>$ atom fraction $\mathrm{U}$

c Mols of Acid per liter $<$ molAcid $=0.0 \mid 1.2>$

c

$\mathrm{c}$

c Density of solution $<$ denSol=ZZZ2/1e5 $\mid 1.5>$

c

C

c Atomic Weights

c $\mathrm{H} 2 \mathrm{O}=\langle$ atwH2O $=18.0152| 3.4>$

$\mathrm{c} \mathrm{NO3}=\langle$ atwNO3 $=62.0049| 3.4>$

c $\mathrm{O}=\langle$ atwO $=15.9994| 3.4>$

$\mathrm{c} \mathrm{HNO} 3=<$ atwHNO3 $=63.0128 \mid 3.4>$

c U235 $=<$ atwU235 $=235.0439 \mid 3.4>$

c U238 $=<$ atwU238 $=238.0508 \mid 3.4>$

$\mathrm{c} \mathrm{U}=<$ atwU $=$ ena*atwU235+(1-ena)*atwU238|3.4>

c

c Molarity of solution components

c Mols of $\mathrm{U}$ per liter $<$ molU $=\mathrm{c} / \mathrm{atwU} \mid 3.4>$

c Mols of $\mathrm{H} 2 \mathrm{O}$ per liter $<\operatorname{molH} 2 \mathrm{O}=(1000 *$ denSol-molU* $*$ atwU $+2 * a+w N O 3+2 * a t w O)$ molAcid*atwHNO3)/atwH2O|3.4>

$\mathrm{c}$

c

$<$ perl $>$

sub IMP

\{

return 1 if $\left(\$ \_[0]>0\right)$;

return 0 ;

\}

$</$ perl $>$

c sphere

$2011<$-denSol|3.6> $-1 \quad$ imp:n=1

$212-1.000-21 \quad$ imp:n=<\&IMP(rw) $\mid 1>$ \$zero importance if no reflector

$220 \quad 2 \quad$ imp:n=0 


$$
\begin{array}{lll}
1 & \text { so }<\text { rd } \mid 4.4> & \text { \$ Sphere } \\
2 & \text { so }<\text { rd }+ \text { rw*in|4.4 }> & \text { \$Reflection }
\end{array}
$$

mode $\mathrm{n}$

kcode $20001.0100 \mathrm{ZZZ0}$

$\begin{array}{llll}\text { ksrc } & 0.00 & 0.00 & 0.00\end{array}$

$\begin{array}{lll}0.00 & 0.00 & 0.10\end{array}$

$\begin{array}{lll}0.00 & 0.00 & 0.50\end{array}$

$\begin{array}{lll}0.00 & 0.00 & 1.00\end{array}$

$\begin{array}{lll}0.00 & 0.00 & 2.00\end{array}$

$\begin{array}{lll}0.00 & 0.00 & 3.00\end{array}$

$\begin{array}{lll}1.00 & 1.00 & 1.00\end{array}$

$\begin{array}{lll}1.00 & 1.00 & 2.00\end{array}$

$\begin{array}{lll}1.00 & 1.00 & 3.00\end{array}$

$\begin{array}{lll}2.00 & 2.00 & 1.00\end{array}$

$\begin{array}{lll}2.00 & 2.00 & 2.00\end{array}$

$\begin{array}{lll}2.00 & 2.00 & 3.00\end{array}$

$\begin{array}{lll}-1.00 & -1.00 & -1.00\end{array}$

$-10.00 \quad-10.00 \quad-20.00$

$\begin{array}{lll}-10.00 & -10.00 & -30.00\end{array}$

c

m2 \$water

$1001.66 \mathrm{c} \quad 2$

$8016.66 \mathrm{c} \quad 1$

$\mathrm{mt} 2$ 1wtr.60t

c UO2/water mixture

m11 \$ UO2(NO3)2 - NO3 - H2O, $100.0 \mathrm{wt} \% \mathrm{U} 235, \mathrm{ZZZ1} \mathrm{milli-g} \mathrm{U/cc,}<\mathrm{denSol} \mid 3.4>$

c molarity $\mathrm{U}=\langle\operatorname{molU}| 3.4>$, acid $=\langle\operatorname{mol} A$ cid $| 3.4>, \mathrm{H} 2 \mathrm{O}=\langle\operatorname{molH} 2 \mathrm{O} \mid 3.4\rangle$

c $\mathrm{U}$ in salt

$92235.66 \mathrm{c}<$ molU*ena $3.4>$

c $92238.66 \mathrm{c}<$ molU* $* 1$-ena $) \mid 3.4>$

c NO3 in salt plus acid

$7014.66 \mathrm{c}<2 * \mathrm{molU}+\operatorname{molAcid} \mid 3.4>$

8016.66c $<6 * \mathrm{molU}+3 * \operatorname{molAcid} \mid 3.4>$

c water

1001.66c $<2 *$ molH2O + molAcid $\mid 3.4>$ \$ncludes Free Hydrogen Ion

$8016.66 \mathrm{c}<\mathrm{molH} 2 \mathrm{O} \mid 3.4>$

mt11 1wtr.60t

c

c 
Template File for Case: u5o2N20-sp-1000-10

sp, $100.0 \mathrm{wt} \%$ UO2, ZZZ1 milli-g/cc, in UO2(NO3)2, with 0 Molar HNO3

$\mathrm{c}$

c worm notes: format of the form $\mid n . m$ for floating point, $\mid \mathrm{n}$ for integers

c $\quad$ zzz0 (in upper case) is replaced with the number of cyles

c $\quad$ zzz1 $=$ the actual concentration in units of milli-g/cc

c $\quad \mathrm{zzz} 2=$ solution density in $\mathrm{g} / \mathrm{cc}$

c $\quad \mathrm{qqq1}=$ the minimum dimensions (in hundreths of inches)

c $\quad \mathrm{qqq} 2=$ the maximum dimensions (in hundreths of inches)

c $\quad \mathrm{qqq} 3=$ the delta (in hundreths of inches)

c

c Diameter $=<\mathrm{d} 1=$ QQQ1:QQQ2:QQQ3 $\mid 5>$ hundreths of inches - must be integer

$\mathrm{c}$ radius $=<\mathrm{rd}=\mathrm{d} 1 * 2.54 / 100 / 2 \mid 5.4>\mathrm{cm}$

c note for perl, numbers with leading zeros must be enclosed in single quotes or else

c they will be interpretted as octal

$\mathrm{c}$ Conc $=<\mathrm{c}={ }^{\prime}$ ZZZ1' $\mid 5>$ milli-g U/cc

c Reflector width $=<\mathrm{rw}=10.0 \mid 2.1>$ inches

c Enrichment $=<$ ena $=1.0 \mid 1.2>$ atom fraction $\mathrm{U}$

c Mols of Acid per liter $<$ molAcid $=0.0 \mid 1.2>$

$\mathrm{c}$

$\mathrm{c}$

c Density of solution $<$ denSol $=Z Z Z 2 / 1 \mathrm{e} 5 \mid 1.5>$

c

c

c Atomic Weights

c $\mathrm{H} 2 \mathrm{O}=\langle$ atwH2O $=18.0152| 3.4>$

$\mathrm{c} \mathrm{NO3}=\langle$ atwNO3 $=62.0049| 3.4>$

c $\mathrm{O}=\langle$ atwO $=15.9994| 3.4>$

$\mathrm{c} \mathrm{HNO} 3=<$ atwHNO3 $=63.0128 \mid 3.4>$

c U235 $=<$ atwU235 $=235.0439 \mid 3.4>$

c U238 $=<$ atwU238 $=238.0508 \mid 3.4>$

c U $=<$ atwU $=$ ena*atwU235+(1-ena)*atwU238 $3.4>$

c

c Molarity of solution components

c Mols of $\mathrm{U}$ per liter $<$ molU $=\mathrm{c} / \mathrm{atwU} \mid 3.4>$

c Mols of $\mathrm{H} 2 \mathrm{O}$ per liter $<\operatorname{molH} 2 \mathrm{O}=(1000 *$ denSol-molU* $*$ atwU $+2 * a+w N O 3+2 * a t w O)$ molAcid*atwHNO3)/atwH2O|3.4>

$\mathrm{c}$

$\mathrm{c}$

$<$ perl $>$

sub IMP

\{

return 1 if $\left(\$ \_[0]>0\right)$;

return 0 ;

\}

$</$ perl $>$

c sphere

$2011<$-denSol|3.6> $-1 \quad$ imp:n=1

$212-1.000-21 \quad$ imp:n=<\&IMP(rw) $\mid 1>$ \$zero importance if no reflector

$220 \quad 2 \quad$ imp:n=0 


$$
\begin{array}{lll}
1 & \text { so }<\text { rd } \mid 4.4> & \text { \$ Sphere } \\
2 & \text { so }<\text { rd }+ \text { rw*in|4.4 }> & \text { \$Reflection }
\end{array}
$$

mode $\mathrm{n}$

kcode 20001.0100 ZZZ0

$\begin{array}{llll}\text { ksrc } & 0.00 & 0.00 & 0.00\end{array}$

$\begin{array}{lll}0.00 & 0.00 & 0.10\end{array}$

$\begin{array}{lll}0.00 & 0.00 & 0.50\end{array}$

$\begin{array}{lll}0.00 & 0.00 & 1.00\end{array}$

$\begin{array}{lll}0.00 & 0.00 & 2.00\end{array}$

$\begin{array}{lll}0.00 & 0.00 & 3.00\end{array}$

$\begin{array}{lll}1.00 & 1.00 & 1.00\end{array}$

$\begin{array}{lll}1.00 & 1.00 & 2.00\end{array}$

$\begin{array}{lll}1.00 & 1.00 & 3.00\end{array}$

$\begin{array}{lll}2.00 & 2.00 & 1.00\end{array}$

$\begin{array}{lll}2.00 & 2.00 & 2.00\end{array}$

$\begin{array}{lll}2.00 & 2.00 & 3.00\end{array}$

$\begin{array}{lll}-1.00 & -1.00 & -1.00\end{array}$

$-10.00 \quad-10.00 \quad-20.00$

$\begin{array}{lll}-10.00 & -10.00 & -30.00\end{array}$

c

m2 \$water

$1001.66 \mathrm{c} \quad 2$

$8016.66 \mathrm{c} \quad 1$

$\mathrm{mt} 2$ 1wtr.60t

c UO2/water mixture

m11 \$ UO2(NO3)2 - NO3 - H2O, $100.0 \mathrm{wt} \% \mathrm{U} 235, \mathrm{ZZZ1} \mathrm{milli-g} \mathrm{U/cc,}<\mathrm{denSol} \mid 3.4>$

c molarity $\mathrm{U}=\langle\operatorname{molU}| 3.4>$, acid $=\langle\operatorname{mol} A \operatorname{Acid} \mid 3.4\rangle, \mathrm{H} 2 \mathrm{O}=\langle\operatorname{molH} 2 \mathrm{O} \mid 3.4\rangle$

c U in salt

$92235.66 \mathrm{c}<$ molU*ena $3.4>$

c $92238.66 \mathrm{c}<$ molU* $* 1$-ena $) \mid 3.4>$

c NO3 in salt plus acid

$7014.66 \mathrm{c}<2 * \mathrm{molU}+\operatorname{molAcid} \mid 3.4>$

$8016.66 \mathrm{c}<6 * \mathrm{molU}+3 * \operatorname{mol} \mathrm{Acid} \mid 3.4>$

c water

1001.66c $<2 *$ molH2O + molAcid $\mid 3.4>$ \$ncludes Free Hydrogen Ion

$8016.66 \mathrm{c}<\mathrm{molH} 2 \mathrm{O} \mid 3.4>$

mt11 1wtr.60t

c

c 
Template File for Case: u5o2N23-sp-1000-00

sp, $100.0 \mathrm{wt} \%$ UO2, ZZZ1 milli-g/cc, in UO2(NO3)2, with 3 Molar HNO3

$\mathrm{c}$

c worm notes: format of the form $\mid n . m$ for floating point, $\mid \mathrm{n}$ for integers

c zzz0 (in upper case) is replaced with the number of cyles

c $\quad$ zzz1 $=$ the actual concentration in units of milli-g/cc

c $\quad$ zzz2 $=$ solution density in $\mathrm{g} / \mathrm{cc}$

c $\quad \mathrm{qqq1}=$ the minimum dimensions (in hundreths of inches)

c $\quad \mathrm{qqq} 2=$ the maximum dimensions (in hundreths of inches)

c $\quad \mathrm{qqq} 3=$ the delta (in hundreths of inches)

c

c Diameter $=<\mathrm{d} 1=$ QQQ1:QQQ2:QQQ3 $\mid 5>$ hundreths of inches - must be integer

$\mathrm{c}$ radius $=<\mathrm{rd}=\mathrm{d} 1 * 2.54 / 100 / 2 \mid 5.4>\mathrm{cm}$

c note for perl, numbers with leading zeros must be enclosed in single quotes or else

c they will be interpretted as octal

$\mathrm{c}$ Conc $=<\mathrm{c}={ }^{\prime} \mathrm{ZZZ1} 1 \mid 5>$ milli-g U/cc

c Reflector width $=<\mathrm{rw}=0.0 \mid 2.1>$ inches

c Enrichment $=<$ ena $=1.0 \mid 1.2>$ atom fraction $\mathrm{U}$

c Mols of Acid per liter $<$ molAcid $=3.0 \mid 1.2>$

$\mathrm{c}$

$\mathrm{c}$

c Density of solution $<$ denSol=ZZZ2/1e5 $\mid 1.5>$

c

c

c Atomic Weights

c $\mathrm{H} 2 \mathrm{O}=\langle$ atwH2O $=18.0152| 3.4>$

$\mathrm{c} \mathrm{NO3}=\langle$ atwNO3 $=62.0049| 3.4>$

c $\mathrm{O}=\langle$ atwO $=15.9994| 3.4>$

$\mathrm{c} \mathrm{HNO} 3=<$ atwHNO3 $=63.0128 \mid 3.4>$

c U235 $=<$ atwU235 $=235.0439 \mid 3.4>$

c U238 $=<$ atwU238 $=238.0508 \mid 3.4>$

$\mathrm{c} \mathrm{U}=<$ atwU $=$ ena*atwU235+(1-ena)*atwU238|3.4>

c

c Molarity of solution components

c Mols of $\mathrm{U}$ per liter $<$ molU $=\mathrm{c} / \mathrm{atwU} \mid 3.4>$

c Mols of $\mathrm{H} 2 \mathrm{O}$ per liter $<\operatorname{molH} 2 \mathrm{O}=(1000 *$ denSol-molU* $*$ atwU $+2 * a+w N O 3+2 * a t w O)$ molAcid*atwHNO3)/atwH2O|3.4>

$\mathrm{c}$

c

$<$ perl $>$

sub IMP

\{

return 1 if $\left(\$ \_[0]>0\right)$;

return 0 ;

\}

$</$ perl $>$

c sphere

$2011<$-denSol|3.6> $-1 \quad$ imp:n=1

$212-1.000-21 \quad$ imp:n=<\&IMP(rw) $\mid 1>$ \$zero importance if no reflector

$220 \quad 2 \quad$ imp:n=0 


$$
\begin{array}{lll}
1 & \text { so }<\mathrm{rd} \mid 4.4> & \text { \$ Sphere } \\
2 & \text { so }<\mathrm{rd}+\mathrm{rw}^{*} \text { in } \mid 4.4> & \text { \$Reflection }
\end{array}
$$

mode $\mathrm{n}$

kcode $20001.0100 \mathrm{ZZZ0}$

$\begin{array}{llll}\text { ksrc } & 0.00 & 0.00 & 0.00\end{array}$

$\begin{array}{lll}0.00 & 0.00 & 0.10\end{array}$

$\begin{array}{lll}0.00 & 0.00 & 0.50\end{array}$

$\begin{array}{lll}0.00 & 0.00 & 1.00\end{array}$

$\begin{array}{lll}0.00 & 0.00 & 2.00\end{array}$

$\begin{array}{lll}0.00 & 0.00 & 3.00\end{array}$

$\begin{array}{lll}1.00 & 1.00 & 1.00\end{array}$

$\begin{array}{lll}1.00 & 1.00 & 2.00\end{array}$

$\begin{array}{lll}1.00 & 1.00 & 3.00\end{array}$

$\begin{array}{lll}2.00 & 2.00 & 1.00\end{array}$

$\begin{array}{lll}2.00 & 2.00 & 2.00\end{array}$

$\begin{array}{lll}2.00 & 2.00 & 3.00\end{array}$

$\begin{array}{lll}-1.00 & -1.00 & -1.00\end{array}$

$-10.00 \quad-10.00 \quad-20.00$

$\begin{array}{lll}-10.00 & -10.00 & -30.00\end{array}$

c

m2 \$water

$1001.66 \mathrm{c} \quad 2$

$8016.66 \mathrm{c} \quad 1$

$\mathrm{mt} 2$ 1wtr.60t

c UO2/water mixture

m11 \$ UO2(NO3)2 - NO3 - H2O, $100.0 \mathrm{wt} \% \mathrm{U} 235, \mathrm{ZZZ1} \mathrm{milli-g} \mathrm{U/cc,}<\mathrm{denSol} \mid 3.4>$

c molarity $\mathrm{U}=\langle\operatorname{molU}| 3.4>$, acid $=\langle\operatorname{mol} A$ cid $| 3.4>, \mathrm{H} 2 \mathrm{O}=\langle\operatorname{molH} 2 \mathrm{O} \mid 3.4\rangle$

c $\mathrm{U}$ in salt

$92235.66 \mathrm{c}<$ molU*ena $3.4>$

c $92238.66 \mathrm{c}<$ molU* $* 1$-ena $) \mid 3.4>$

c NO3 in salt plus acid

$7014.66 \mathrm{c}<2 * \mathrm{molU}+\operatorname{molAcid} \mid 3.4>$

8016.66c $<6 * \mathrm{molU}+3 * \operatorname{molAcid} \mid 3.4>$

c water

1001.66c $<2 *$ molH2O + molAcid $\mid 3.4>$ \$ncludes Free Hydrogen Ion

$8016.66 \mathrm{c}<\mathrm{molH} 2 \mathrm{O} \mid 3.4>$

mt11 1wtr.60t

c

$\mathrm{c}$ 
Template File for Case: u5o2N23-sp-1000-01

sp, $100.0 \mathrm{wt} \%$ UO2, ZZZ1 milli-g/cc, in UO2(NO3)2, with 3 Molar HNO3

$\mathrm{c}$

c worm notes: format of the form $\mid n . m$ for floating point, $\mid \mathrm{n}$ for integers

c zzz0 (in upper case) is replaced with the number of cyles

c $\quad$ zzz1 $=$ the actual concentration in units of milli-g/cc

c $\quad$ zzz2 $=$ solution density in $\mathrm{g} / \mathrm{cc}$

c $\quad \mathrm{qqq1}=$ the minimum dimensions (in hundreths of inches)

c $\quad \mathrm{qqq} 2=$ the maximum dimensions (in hundreths of inches)

c $\quad \mathrm{qqq} 3=$ the delta (in hundreths of inches)

c

c Diameter $=<\mathrm{d} 1=$ QQQ1:QQQ2:QQQ3 $\mid 5>$ hundreths of inches - must be integer

$\mathrm{c}$ radius $=<\mathrm{rd}=\mathrm{d} 1 * 2.54 / 100 / 2 \mid 5.4>\mathrm{cm}$

c note for perl, numbers with leading zeros must be enclosed in single quotes or else

c they will be interpretted as octal

$\mathrm{c}$ Conc $=<\mathrm{c}={ }^{\prime}$ ZZZ1' $\mid 5>$ milli-g U/cc

c Reflector width $=<\mathrm{rw}=1.0 \mid 2.1>$ inches

c Enrichment $=<$ ena $=1.0 \mid 1.2>$ atom fraction $\mathrm{U}$

c Mols of Acid per liter $<$ molAcid $=3.0 \mid 1.2>$

$\mathrm{c}$

$\mathrm{c}$

c Density of solution $<$ denSol=ZZZ2/1e5 $\mid 1.5>$

c

C

c Atomic Weights

c $\mathrm{H} 2 \mathrm{O}=\langle$ atwH2O $=18.0152| 3.4>$

$\mathrm{c} \mathrm{NO3}=\langle$ atwNO3 $=62.0049| 3.4>$

c $\mathrm{O}=\langle$ atwO $=15.9994| 3.4>$

$\mathrm{c} \mathrm{HNO} 3=<$ atwHNO3 $=63.0128 \mid 3.4>$

c U235 $=<$ atwU235 $=235.0439 \mid 3.4>$

c U238 $=<$ atwU238 $=238.0508 \mid 3.4>$

$\mathrm{c} \mathrm{U}=<$ atwU $=$ ena*atwU235+(1-ena)*atwU238|3.4>

c

c Molarity of solution components

c Mols of $\mathrm{U}$ per liter $<$ molU $=\mathrm{c} / \mathrm{atwU} \mid 3.4>$

c Mols of $\mathrm{H} 2 \mathrm{O}$ per liter $<\operatorname{molH} 2 \mathrm{O}=(1000 *$ denSol-molU* $*$ atwU $+2 * a+w N O 3+2 * a t w O)$ molAcid*atwHNO3)/atwH2O|3.4>

$\mathrm{c}$

c

$<$ perl $>$

sub IMP

\{

return 1 if $\left(\$ \_[0]>0\right)$;

return 0 ;

\}

$</$ perl $>$

c sphere

$2011<$-denSol|3.6> $-1 \quad$ imp:n=1

$212-1.000-21 \quad$ imp:n=<\&IMP(rw) $\mid 1>$ \$zero importance if no reflector

$220 \quad 2 \quad$ imp:n=0 


$$
\begin{array}{lll}
1 & \text { so }<\text { rd } \mid 4.4> & \text { \$ Sphere } \\
2 & \text { so }<\text { rd }+ \text { rw*in|4.4 }> & \text { \$Reflection }
\end{array}
$$

mode $\mathrm{n}$

kcode $20001.0100 \mathrm{ZZZ0}$

$\begin{array}{llll}\text { ksrc } & 0.00 & 0.00 & 0.00\end{array}$

$\begin{array}{lll}0.00 & 0.00 & 0.10\end{array}$

$\begin{array}{lll}0.00 & 0.00 & 0.50\end{array}$

$\begin{array}{lll}0.00 & 0.00 & 1.00\end{array}$

$\begin{array}{lll}0.00 & 0.00 & 2.00\end{array}$

$\begin{array}{lll}0.00 & 0.00 & 3.00\end{array}$

$\begin{array}{lll}1.00 & 1.00 & 1.00\end{array}$

$\begin{array}{lll}1.00 & 1.00 & 2.00\end{array}$

$\begin{array}{lll}1.00 & 1.00 & 3.00\end{array}$

$\begin{array}{lll}2.00 & 2.00 & 1.00\end{array}$

$\begin{array}{lll}2.00 & 2.00 & 2.00\end{array}$

$\begin{array}{lll}2.00 & 2.00 & 3.00\end{array}$

$\begin{array}{lll}-1.00 & -1.00 & -1.00\end{array}$

$-10.00 \quad-10.00 \quad-20.00$

$\begin{array}{lll}-10.00 & -10.00 & -30.00\end{array}$

c

m2 \$water

$1001.66 \mathrm{c} \quad 2$

$8016.66 \mathrm{c} \quad 1$

$\mathrm{mt} 2$ 1wtr.60t

c UO2/water mixture

m11 \$ UO2(NO3)2 - NO3 - H2O, $100.0 \mathrm{wt} \% \mathrm{U} 235, \mathrm{ZZZ1} \mathrm{milli-g} \mathrm{U/cc,}<\mathrm{denSol} \mid 3.4>$

c molarity $\mathrm{U}=\langle\operatorname{molU}| 3.4>$, acid $=\langle\operatorname{mol} A$ cid $| 3.4>, \mathrm{H} 2 \mathrm{O}=\langle\operatorname{molH} 2 \mathrm{O} \mid 3.4\rangle$

c $\mathrm{U}$ in salt

$92235.66 \mathrm{c}<$ molU*ena $3.4>$

c $92238.66 \mathrm{c}<$ molU* $* 1$-ena $) \mid 3.4>$

c NO3 in salt plus acid

$7014.66 \mathrm{c}<2 * \mathrm{molU}+\operatorname{molAcid} \mid 3.4>$

8016.66c $<6 * \mathrm{molU}+3 * \operatorname{molAcid} \mid 3.4>$

c water

1001.66c $<2 *$ molH2O + molAcid $\mid 3.4>$ \$ncludes Free Hydrogen Ion

$8016.66 \mathrm{c}<\mathrm{molH} 2 \mathrm{O} \mid 3.4>$

mt11 1wtr.60t

c

c 
Template File for Case: u5o2N23-sp-1000-10

sp, $100.0 \mathrm{wt} \%$ UO2, ZZZ1 milli-g/cc, in UO2(NO3)2, with 3 Molar HNO3

$\mathrm{c}$

c worm notes: format of the form |n.m for floating point, $\mid \mathrm{n}$ for integers

c $\quad$ zzz0 (in upper case) is replaced with the number of cyles

c $\quad$ zzz1 $=$ the actual concentration in units of milli-g/cc

c $\quad \mathrm{zzz} 2=$ solution density in $\mathrm{g} / \mathrm{cc}$

c $\quad \mathrm{qqq1}=$ the minimum dimensions (in hundreths of inches)

c $\quad \mathrm{qqq} 2=$ the maximum dimensions (in hundreths of inches)

c $\quad \mathrm{qqq} 3=$ the delta (in hundreths of inches)

c

c Diameter $=<\mathrm{d} 1=$ QQQ1:QQQ2:QQQ3 $\mid 5>$ hundreths of inches - must be integer

$\mathrm{c}$ radius $=<\mathrm{rd}=\mathrm{d} 1 * 2.54 / 100 / 2 \mid 5.4>\mathrm{cm}$

c note for perl, numbers with leading zeros must be enclosed in single quotes or else

c they will be interpretted as octal

$\mathrm{c}$ Conc $=<\mathrm{c}={ }^{\prime}$ ZZZ1' $\mid 5>$ milli-g U/cc

c Reflector width $=<\mathrm{rw}=10.0 \mid 2.1>$ inches

c Enrichment $=<$ ena $=1.0 \mid 1.2>$ atom fraction $\mathrm{U}$

c Mols of Acid per liter $<$ molAcid $=3.0 \mid 1.2>$

$\mathrm{c}$

$\mathrm{c}$

c Density of solution $<$ denSol $=Z Z Z 2 / 1$ e $5 \mid 1.5>$

c

c

c Atomic Weights

c $\mathrm{H} 2 \mathrm{O}=\langle$ atwH2O $=18.0152| 3.4>$

$\mathrm{c} \mathrm{NO3}=\langle$ atwNO3 $=62.0049| 3.4>$

c $\mathrm{O}=\langle$ atwO $=15.9994| 3.4>$

$\mathrm{c} \mathrm{HNO} 3=<$ atwHNO3 $=63.0128 \mid 3.4>$

c U235 $=<$ atwU235 $=235.0439 \mid 3.4>$

c U238 $=<$ atwU238 $=238.0508 \mid 3.4>$

c U $=<$ atwU $=$ ena*atwU235+(1-ena)*atwU238 $3.4>$

c

c Molarity of solution components

c Mols of $\mathrm{U}$ per liter $<$ molU $=\mathrm{c} / \mathrm{atwU} \mid 3.4>$

c Mols of $\mathrm{H} 2 \mathrm{O}$ per liter $<\operatorname{molH} 2 \mathrm{O}=(1000 *$ denSol-molU* $*$ atwU $+2 * a+w N O 3+2 * a t w O)$ molAcid*atwHNO3)/atwH2O|3.4>

$\mathrm{c}$

$\mathrm{c}$

$<$ perl $>$

sub IMP

\{

return 1 if $\left(\$ \_[0]>0\right)$;

return 0 ;

\}

$</$ perl $>$

c sphere

$2011<$-denSol|3.6> $-1 \quad$ imp:n=1

$212-1.000-21 \quad$ imp:n=<\&IMP(rw) $\mid 1>$ \$zero importance if no reflector

$220 \quad 2$ imp:n=0 


$$
\begin{array}{lll}
1 & \text { so }<\text { rd } \mid 4.4> & \text { \$ Sphere } \\
2 & \text { so }<\text { rd }+ \text { rw*in|4.4 }> & \text { \$Reflection }
\end{array}
$$

mode $\mathrm{n}$

kcode 20001.0100 ZZZ0

$\begin{array}{llll}\text { ksrc } & 0.00 & 0.00 & 0.00\end{array}$

$\begin{array}{lll}0.00 & 0.00 & 0.10\end{array}$

$\begin{array}{lll}0.00 & 0.00 & 0.50\end{array}$

$\begin{array}{lll}0.00 & 0.00 & 1.00\end{array}$

$\begin{array}{lll}0.00 & 0.00 & 2.00\end{array}$

$\begin{array}{lll}0.00 & 0.00 & 3.00\end{array}$

$\begin{array}{lll}1.00 & 1.00 & 1.00\end{array}$

$\begin{array}{lll}1.00 & 1.00 & 2.00\end{array}$

$\begin{array}{lll}1.00 & 1.00 & 3.00\end{array}$

$\begin{array}{lll}2.00 & 2.00 & 1.00\end{array}$

$\begin{array}{lll}2.00 & 2.00 & 2.00\end{array}$

$\begin{array}{lll}2.00 & 2.00 & 3.00\end{array}$

$\begin{array}{lll}-1.00 & -1.00 & -1.00\end{array}$

$-10.00 \quad-10.00 \quad-20.00$

$\begin{array}{lll}-10.00 & -10.00 & -30.00\end{array}$

c

m2 \$water

$1001.66 \mathrm{c} \quad 2$

$8016.66 \mathrm{c} \quad 1$

$\mathrm{mt} 2$ 1wtr.60t

c UO2/water mixture

m11 \$ UO2(NO3)2 - NO3 - H2O, $100.0 \mathrm{wt} \% \mathrm{U} 235, \mathrm{ZZZ1} \mathrm{milli-g} \mathrm{U/cc,}<\mathrm{denSol} \mid 3.4>$

c molarity $\mathrm{U}=\langle\operatorname{molU}| 3.4>$, acid $=\langle\operatorname{mol} A \operatorname{Acid} \mid 3.4\rangle, \mathrm{H} 2 \mathrm{O}=\langle\operatorname{molH} 2 \mathrm{O} \mid 3.4\rangle$

c U in salt

$92235.66 \mathrm{c}<$ molU*ena $3.4>$

c $92238.66 \mathrm{c}<$ molU* $* 1$-ena $) \mid 3.4>$

c NO3 in salt plus acid

$7014.66 \mathrm{c}<2 * \mathrm{molU}+\operatorname{molAcid} \mid 3.4>$

$8016.66 \mathrm{c}<6 * \mathrm{molU}+3 * \operatorname{mol} \mathrm{Acid} \mid 3.4>$

c water

1001.66c $<2 *$ molH2O + molAcid $\mid 3.4>$ \$ncludes Free Hydrogen Ion

$8016.66 \mathrm{c}<\mathrm{molH} 2 \mathrm{O} \mid 3.4>$

mt11 1wtr.60t

c

c 
Template File for Case: u5o2N26-sp-1000-00

sp, $100.0 \mathrm{wt} \%$ UO2, ZZZ1 milli-g/cc, in UO2(NO3)2, with 6 Molar HNO3

$\mathrm{c}$

c worm notes: format of the form $\mid n . m$ for floating point, $\mid \mathrm{n}$ for integers

c zzz0 (in upper case) is replaced with the number of cyles

c $\quad$ zzz1 $=$ the actual concentration in units of milli-g/cc

c $\quad$ zzz2 $=$ solution density in $\mathrm{g} / \mathrm{cc}$

c $\quad \mathrm{qqq1}=$ the minimum dimensions (in hundreths of inches)

c $\quad \mathrm{qqq} 2=$ the maximum dimensions (in hundreths of inches)

c $\quad \mathrm{qqq} 3=$ the delta (in hundreths of inches)

c

c Diameter $=<\mathrm{d} 1=$ QQQ1:QQQ2:QQQ3 $\mid 5>$ hundreths of inches - must be integer

$\mathrm{c}$ radius $=<\mathrm{rd}=\mathrm{d} 1 * 2.54 / 100 / 2 \mid 5.4>\mathrm{cm}$

c note for perl, numbers with leading zeros must be enclosed in single quotes or else

c they will be interpretted as octal

$\mathrm{c}$ Conc $=<\mathrm{c}={ }^{\prime} \mathrm{ZZZ1} 1 \mid 5>$ milli-g U/cc

c Reflector width $=<\mathrm{rw}=0.0 \mid 2.1>$ inches

c Enrichment $=<$ ena $=1.0 \mid 1.2>$ atom fraction $\mathrm{U}$

c Mols of Acid per liter $<$ molAcid $=6.0 \mid 1.2>$

$\mathrm{c}$

$\mathrm{c}$

c Density of solution $<$ denSol=ZZZ2/1e5 $\mid 1.5>$

c

C

c Atomic Weights

c $\mathrm{H} 2 \mathrm{O}=\langle$ atwH2O $=18.0152| 3.4>$

$\mathrm{c} \mathrm{NO3}=\langle$ atwNO3 $=62.0049| 3.4>$

c $\mathrm{O}=\langle$ atwO $=15.9994| 3.4>$

$\mathrm{c} \mathrm{HNO} 3=<$ atwHNO3 $=63.0128 \mid 3.4>$

c U235 $=<$ atwU235 $=235.0439 \mid 3.4>$

c U238 $=<$ atwU238 $=238.0508 \mid 3.4>$

$\mathrm{c} \mathrm{U}=<$ atwU $=$ ena*atwU235+(1-ena)*atwU238|3.4>

c

c Molarity of solution components

c Mols of $\mathrm{U}$ per liter $<$ molU $=\mathrm{c} / \mathrm{atwU} \mid 3.4>$

c Mols of $\mathrm{H} 2 \mathrm{O}$ per liter $<\operatorname{molH} 2 \mathrm{O}=(1000 *$ denSol-molU* $*$ atwU $+2 * a+w N O 3+2 * a t w O)$ molAcid*atwHNO3)/atwH2O|3.4>

$\mathrm{c}$

c

$<$ perl $>$

sub IMP

\{

return 1 if $\left(\$ \_[0]>0\right)$;

return 0 ;

\}

$</$ perl $>$

c sphere

$2011<$-denSol|3.6> $-1 \quad$ imp:n=1

$212-1.000-21 \quad$ imp:n=<\&IMP(rw) $\mid 1>$ \$zero importance if no reflector

$220 \quad 2$ imp:n=0 


$$
\begin{array}{lll}
1 & \text { so }<\text { rd } \mid 4.4> & \text { \$ Sphere } \\
2 & \text { so }<\text { rd }+ \text { rw*in|4.4 }> & \text { \$Reflection }
\end{array}
$$

mode $\mathrm{n}$

kcode 20001.0100 ZZZ0

$\begin{array}{llll}\text { ksrc } & 0.00 & 0.00 & 0.00\end{array}$

$\begin{array}{lll}0.00 & 0.00 & 0.10\end{array}$

$\begin{array}{lll}0.00 & 0.00 & 0.50\end{array}$

$\begin{array}{lll}0.00 & 0.00 & 1.00\end{array}$

$\begin{array}{lll}0.00 & 0.00 & 2.00\end{array}$

$\begin{array}{lll}0.00 & 0.00 & 3.00\end{array}$

$\begin{array}{lll}1.00 & 1.00 & 1.00\end{array}$

$\begin{array}{lll}1.00 & 1.00 & 2.00\end{array}$

$\begin{array}{lll}1.00 & 1.00 & 3.00\end{array}$

$\begin{array}{lll}2.00 & 2.00 & 1.00\end{array}$

$\begin{array}{lll}2.00 & 2.00 & 2.00\end{array}$

$\begin{array}{lll}2.00 & 2.00 & 3.00\end{array}$

$\begin{array}{lll}-1.00 & -1.00 & -1.00\end{array}$

$-10.00 \quad-10.00 \quad-20.00$

$\begin{array}{lll}-10.00 & -10.00 & -30.00\end{array}$

c

m2 \$water

$1001.66 \mathrm{c} \quad 2$

$8016.66 \mathrm{c} \quad 1$

$\mathrm{mt} 2$ 1wtr.60t

c UO2/water mixture

m11 \$ UO2(NO3)2 - NO3 - H2O, $100.0 \mathrm{wt} \% \mathrm{U} 235, \mathrm{ZZZ1} \mathrm{milli-g} \mathrm{U/cc,}<\mathrm{denSol} \mid 3.4>$

c molarity $\mathrm{U}=\langle\operatorname{molU}| 3.4>$, acid $=\langle\operatorname{mol} A \operatorname{Acid} \mid 3.4\rangle, \mathrm{H} 2 \mathrm{O}=\langle\operatorname{molH} 2 \mathrm{O} \mid 3.4\rangle$

c U in salt

$92235.66 \mathrm{c}<$ molU*ena $3.4>$

c $92238.66 \mathrm{c}<$ molU* $* 1$-ena $) \mid 3.4>$

c NO3 in salt plus acid

$7014.66 \mathrm{c}<2 * \mathrm{molU}+\operatorname{molAcid} \mid 3.4>$

$8016.66 \mathrm{c}<6 * \mathrm{molU}+3 * \operatorname{mol} \mathrm{Acid} \mid 3.4>$

c water

1001.66c $<2 *$ molH2O + molAcid $\mid 3.4>$ \$ncludes Free Hydrogen Ion

$8016.66 \mathrm{c}<\mathrm{molH} 2 \mathrm{O} \mid 3.4>$

mt11 1wtr.60t

c

c 
Template File for Case: u5o2N26-sp-1000-01

sp, $100.0 \mathrm{wt} \%$ UO2, ZZZ1 milli-g/cc, in UO2(NO3)2, with 6 Molar HNO3

$\mathrm{c}$

c worm notes: format of the form $\mid n . m$ for floating point, $\mid \mathrm{n}$ for integers

c zzz0 (in upper case) is replaced with the number of cyles

c $\quad$ zzz1 $=$ the actual concentration in units of milli-g/cc

c $\quad$ zzz2 $=$ solution density in $\mathrm{g} / \mathrm{cc}$

c $\quad \mathrm{qqq1}=$ the minimum dimensions (in hundreths of inches)

c $\quad \mathrm{qqq} 2=$ the maximum dimensions (in hundreths of inches)

c $\quad \mathrm{qqq} 3=$ the delta (in hundreths of inches)

c

c Diameter $=<\mathrm{d} 1=$ QQQ1:QQQ2:QQQ3 $\mid 5>$ hundreths of inches - must be integer

$\mathrm{c}$ radius $=<\mathrm{rd}=\mathrm{d} 1 * 2.54 / 100 / 2 \mid 5.4>\mathrm{cm}$

c note for perl, numbers with leading zeros must be enclosed in single quotes or else

c they will be interpretted as octal

$\mathrm{c}$ Conc $=<\mathrm{c}={ }^{\prime}$ ZZZ1' $\mid 5>$ milli-g U/cc

c Reflector width $=<\mathrm{rw}=1.0 \mid 2.1>$ inches

c Enrichment $=<$ ena $=1.0 \mid 1.2>$ atom fraction $\mathrm{U}$

c Mols of Acid per liter $<$ molAcid $=6.0 \mid 1.2>$

$\mathrm{c}$

$\mathrm{c}$

c Density of solution $<$ denSol=ZZZ2/1e5 $\mid 1.5>$

c

C

c Atomic Weights

c $\mathrm{H} 2 \mathrm{O}=\langle$ atwH2O $=18.0152| 3.4>$

$\mathrm{c} \mathrm{NO3}=\langle$ atwNO3 $=62.0049| 3.4>$

c $\mathrm{O}=\langle$ atwO $=15.9994| 3.4>$

$\mathrm{c} \mathrm{HNO} 3=<$ atwHNO3 $=63.0128 \mid 3.4>$

c U235 $=<$ atwU235 $=235.0439 \mid 3.4>$

c U238 $=<$ atwU238 $=238.0508 \mid 3.4>$

$\mathrm{c} \mathrm{U}=<$ atwU $=$ ena*atwU235+(1-ena)*atwU238|3.4>

c

c Molarity of solution components

c Mols of $\mathrm{U}$ per liter $<$ molU $=\mathrm{c} / \mathrm{atwU} \mid 3.4>$

c Mols of $\mathrm{H} 2 \mathrm{O}$ per liter $<\operatorname{molH} 2 \mathrm{O}=(1000 *$ denSol-molU* $*$ atwU $+2 * a+w N O 3+2 * a t w O)$ molAcid*atwHNO3)/atwH2O|3.4>

$\mathrm{c}$

c

$<$ perl $>$

sub IMP

\{

return 1 if $\left(\$ \_[0]>0\right)$;

return 0 ;

\}

$</$ perl $>$

c sphere

$2011<$-denSol|3.6> $-1 \quad$ imp:n=1

$212-1.000-21 \quad$ imp:n=<\&IMP(rw) $\mid 1>$ \$zero importance if no reflector

$220 \quad 2 \quad$ imp:n=0 


$$
\begin{array}{lll}
1 & \text { so }<\text { rd } \mid 4.4> & \text { \$ Sphere } \\
2 & \text { so }<\text { rd }+ \text { rw*in|4.4 }> & \text { \$Reflection }
\end{array}
$$

mode $\mathrm{n}$

kcode 20001.0100 ZZZ0

$\begin{array}{llll}\text { ksrc } & 0.00 & 0.00 & 0.00\end{array}$

$\begin{array}{lll}0.00 & 0.00 & 0.10\end{array}$

$\begin{array}{lll}0.00 & 0.00 & 0.50\end{array}$

$\begin{array}{lll}0.00 & 0.00 & 1.00\end{array}$

$\begin{array}{lll}0.00 & 0.00 & 2.00\end{array}$

$\begin{array}{lll}0.00 & 0.00 & 3.00\end{array}$

$\begin{array}{lll}1.00 & 1.00 & 1.00\end{array}$

$\begin{array}{lll}1.00 & 1.00 & 2.00\end{array}$

$\begin{array}{lll}1.00 & 1.00 & 3.00\end{array}$

$\begin{array}{lll}2.00 & 2.00 & 1.00\end{array}$

$\begin{array}{lll}2.00 & 2.00 & 2.00\end{array}$

$\begin{array}{lll}2.00 & 2.00 & 3.00\end{array}$

$\begin{array}{lll}-1.00 & -1.00 & -1.00\end{array}$

$-10.00 \quad-10.00 \quad-20.00$

$\begin{array}{lll}-10.00 & -10.00 & -30.00\end{array}$

c

m2 \$water

$1001.66 \mathrm{c} \quad 2$

$8016.66 \mathrm{c} \quad 1$

$\mathrm{mt} 2$ 1wtr.60t

c UO2/water mixture

m11 \$ UO2(NO3)2 - NO3 - H2O, $100.0 \mathrm{wt} \% \mathrm{U} 235, \mathrm{ZZZ1} \mathrm{milli-g} \mathrm{U/cc,}<\mathrm{denSol} \mid 3.4>$

c molarity $\mathrm{U}=\langle\operatorname{molU}| 3.4>$, acid $=\langle\operatorname{mol} A$ cid $| 3.4>, \mathrm{H} 2 \mathrm{O}=\langle\operatorname{molH} 2 \mathrm{O} \mid 3.4\rangle$

c U in salt

$92235.66 \mathrm{c}<$ molU*ena $3.4>$

c $92238.66 \mathrm{c}<$ molU* $* 1$-ena $) \mid 3.4>$

c NO3 in salt plus acid

$7014.66 \mathrm{c}<2 * \mathrm{molU}+\operatorname{molAcid} \mid 3.4>$

$8016.66 \mathrm{c}<6 * \mathrm{molU}+3 * \operatorname{mol} \mathrm{Acid} \mid 3.4>$

c water

1001.66c $<2 *$ molH2O + molAcid $\mid 3.4>$ \$ncludes Free Hydrogen Ion

$8016.66 \mathrm{c}<\mathrm{molH} 2 \mathrm{O} \mid 3.4>$

mt11 1wtr.60t

c

c 
Template File for Case: u5o2N26-sp-1000-10

sp, $100.0 \mathrm{wt} \%$ UO2, ZZZ1 milli-g/cc, in UO2(NO3)2, with 6 Molar HNO3

$\mathrm{c}$

c worm notes: format of the form |n.m for floating point, $\mid \mathrm{n}$ for integers

c $\quad$ zzz0 (in upper case) is replaced with the number of cyles

c $\quad$ zzz1 $=$ the actual concentration in units of milli-g/cc

c $\quad \mathrm{zzz} 2=$ solution density in $\mathrm{g} / \mathrm{cc}$

c $\quad \mathrm{qqq1}=$ the minimum dimensions (in hundreths of inches)

c $\quad \mathrm{qqq} 2=$ the maximum dimensions (in hundreths of inches)

c $\quad \mathrm{qqq} 3=$ the delta (in hundreths of inches)

c

c Diameter $=<\mathrm{d} 1=$ QQQ1:QQQ2:QQQ3 $\mid 5>$ hundreths of inches - must be integer

$\mathrm{c}$ radius $=<\mathrm{rd}=\mathrm{d} 1 * 2.54 / 100 / 2 \mid 5.4>\mathrm{cm}$

c note for perl, numbers with leading zeros must be enclosed in single quotes or else

c they will be interpretted as octal

$\mathrm{c}$ Conc $=<\mathrm{c}={ }^{\prime}$ ZZZ1' $\mid 5>$ milli-g U/cc

c Reflector width $=<\mathrm{rw}=10.0 \mid 2.1>$ inches

c Enrichment $=<$ ena $=1.0 \mid 1.2>$ atom fraction $\mathrm{U}$

c Mols of Acid per liter $<$ molAcid $=6.0 \mid 1.2>$

$\mathrm{c}$

$\mathrm{c}$

c Density of solution $<$ denSol $=Z Z Z 2 / 1$ e $5 \mid 1.5>$

c

c

c Atomic Weights

c $\mathrm{H} 2 \mathrm{O}=\langle$ atwH2O $=18.0152| 3.4>$

$\mathrm{c} \mathrm{NO3}=\langle$ atwNO3 $=62.0049| 3.4>$

c $\mathrm{O}=\langle$ atwO $=15.9994| 3.4>$

$\mathrm{c} \mathrm{HNO} 3=<$ atwHNO3 $=63.0128 \mid 3.4>$

c U235 $=<$ atwU235 $=235.0439 \mid 3.4>$

c U238 $=<$ atwU238 $=238.0508 \mid 3.4>$

c U $=<$ atwU $=$ ena*atwU235+(1-ena)*atwU238 $3.4>$

c

c Molarity of solution components

c Mols of $\mathrm{U}$ per liter $<$ molU $=\mathrm{c} / \mathrm{atwU} \mid 3.4>$

c Mols of $\mathrm{H} 2 \mathrm{O}$ per liter $<\operatorname{molH} 2 \mathrm{O}=(1000 *$ denSol-molU* $*$ atwU $+2 * a+w N O 3+2 * a t w O)$ molAcid*atwHNO3)/atwH2O|3.4>

$\mathrm{c}$

$\mathrm{c}$

$<$ perl $>$

sub IMP

\{

return 1 if $\left(\$ \_[0]>0\right)$;

return 0 ;

\}

$</$ perl $>$

c sphere

$2011<$-denSol|3.6> $-1 \quad$ imp:n=1

$212-1.000-21 \quad$ imp:n=<\&IMP(rw) $\mid 1>$ \$zero importance if no reflector

$220 \quad 2$ imp:n=0 


$$
\begin{array}{lll}
1 & \text { so }<\text { rd } \mid 4.4> & \text { \$ Sphere } \\
2 & \text { so }<\text { rd }+ \text { rw*in|4.4 }> & \text { \$Reflection }
\end{array}
$$

mode $\mathrm{n}$

kcode 20001.0100 ZZZ0

$\begin{array}{llll}\text { ksrc } & 0.00 & 0.00 & 0.00\end{array}$

$\begin{array}{lll}0.00 & 0.00 & 0.10\end{array}$

$\begin{array}{lll}0.00 & 0.00 & 0.50\end{array}$

$\begin{array}{lll}0.00 & 0.00 & 1.00\end{array}$

$\begin{array}{lll}0.00 & 0.00 & 2.00\end{array}$

$\begin{array}{lll}0.00 & 0.00 & 3.00\end{array}$

$\begin{array}{lll}1.00 & 1.00 & 1.00\end{array}$

$\begin{array}{lll}1.00 & 1.00 & 2.00\end{array}$

$\begin{array}{lll}1.00 & 1.00 & 3.00\end{array}$

$\begin{array}{lll}2.00 & 2.00 & 1.00\end{array}$

$\begin{array}{lll}2.00 & 2.00 & 2.00\end{array}$

$\begin{array}{lll}2.00 & 2.00 & 3.00\end{array}$

$\begin{array}{lll}-1.00 & -1.00 & -1.00\end{array}$

$-10.00 \quad-10.00 \quad-20.00$

$\begin{array}{lll}-10.00 & -10.00 & -30.00\end{array}$

c

m2 \$water

$1001.66 \mathrm{c} \quad 2$

$8016.66 \mathrm{c} \quad 1$

$\mathrm{mt} 2$ 1wtr.60t

c UO2/water mixture

m11 \$ UO2(NO3)2 - NO3 - H2O, $100.0 \mathrm{wt} \% \mathrm{U} 235, \mathrm{ZZZ1} \mathrm{milli-g} \mathrm{U/cc,}<\mathrm{denSol} \mid 3.4>$

c molarity $\mathrm{U}=\langle\operatorname{molU}| 3.4>$, acid $=\langle\operatorname{mol} A$ cid $| 3.4>, \mathrm{H} 2 \mathrm{O}=\langle\operatorname{molH} 2 \mathrm{O} \mid 3.4\rangle$

c U in salt

$92235.66 \mathrm{c}<$ molU*ena $3.4>$

c $92238.66 \mathrm{c}<$ molU* $* 1$-ena $) \mid 3.4>$

c NO3 in salt plus acid

$7014.66 \mathrm{c}<2 * \mathrm{molU}+\operatorname{molAcid} \mid 3.4>$

$8016.66 \mathrm{c}<6 * \mathrm{molU}+3 * \operatorname{mol} \mathrm{Acid} \mid 3.4>$

c water

1001.66c $<2 *$ molH2O + molAcid $\mid 3.4>$ \$ncludes Free Hydrogen Ion

$8016.66 \mathrm{c}<\mathrm{molH} 2 \mathrm{O} \mid 3.4>$

mt11 1wtr.60t

c

c 
Template File for Case: u5o2N20-sp-0935-00

sp, 93.5 wt\% UO2, ZZZ1 milli-g/cc, in UO2(NO3)2, with 0 Molar HNO3

$\mathrm{c}$

c worm notes: format of the form $\mid$ n.m for floating point, $\mid \mathrm{n}$ for integers

c zzz0 (in upper case) is replaced with the number of cyles

c $\quad$ zzz1 $=$ the actual concentration in units of milli-g/cc

c $\quad$ zzz2 $=$ solution density in $\mathrm{g} / \mathrm{cc}$

c $\quad \mathrm{qqq1}=$ the minimum dimensions (in hundreths of inches)

c $\quad \mathrm{qqq} 2=$ the maximum dimensions (in hundreths of inches)

c $\quad \mathrm{qqq} 3=$ the delta (in hundreths of inches)

c

$\mathrm{c}$ Diameter $=<\mathrm{d} 1=$ QQQ1:QQQ2:QQQ3 $\mid 5>$ hundreths of inches - must be integer

$\mathrm{c}$ radius $=<\mathrm{rd}=\mathrm{d} 1 * 2.54 / 100 / 2 \mid 5.4>\mathrm{cm}$

c note for perl, numbers with leading zeros must be enclosed in single quotes or else

c they will be interpretted as octal

$\mathrm{c}$ Conc $=<\mathrm{c}={ }^{\prime} \mathrm{ZZZ1} 1 \mid 5>$ milli-g U/cc

c Reflector width $=<\mathrm{rw}=0.0 \mid 2.1>$ inches

c Enrichment $=<$ ena $=0.935 \mid 1.3>$ atom fraction $\mathrm{U}$

c Mols of Acid per liter $<$ molAcid $=0.0 \mid 1.2>$

$\mathrm{c}$

$\mathrm{c}$

c Density of solution $<$ denSol $=Z Z Z 2 / 1 \mathrm{e} 5 \mid 1.5>$

c

c

c Atomic Weights

c $\mathrm{H} 2 \mathrm{O}=\langle$ atwH2O $=18.0152| 3.4>$

$\mathrm{c} \mathrm{NO3}=\langle$ atwNO3 $=62.0049| 3.4>$

c $\mathrm{O}=\langle$ atwO $=15.9994| 3.4>$

$\mathrm{c} \mathrm{HNO} 3=<$ atwHNO3 $=63.0128 \mid 3.4>$

c U235 $=<$ atwU235 $=235.0439 \mid 3.4>$

c U238 $=<$ atwU238 $=238.0508 \mid 3.4>$

c U $=<$ atwU $=$ ena*atwU235+(1-ena)*atwU238 $3.4>$

c

c Molarity of solution components

c Mols of $\mathrm{U}$ per liter $<$ molU $=\mathrm{c} / \mathrm{atwU} \mid 3.4>$

c Mols of $\mathrm{H} 2 \mathrm{O}$ per liter $<\operatorname{molH} 2 \mathrm{O}=(1000 *$ denSol-molU* $*$ atwU $+2 * a+w N O 3+2 * a t w O)$ molAcid*atwHNO3)/atwH2O|3.4>

$\mathrm{c}$

c

$<$ perl $>$

sub IMP

\{

return 1 if $\left(\$ \_[0]>0\right)$;

return 0 ;

\}

$</$ perl $>$

c sphere

$2011<$-denSol|3.6> $-1 \quad$ imp:n=1

$212-1.000-21 \quad$ imp:n=<\&IMP(rw) $\mid 1>$ \$zero importance if no reflector

$220 \quad 2$ imp:n=0 


$$
\begin{array}{lll}
1 & \text { so }<\mathrm{rd} \mid 4.4> & \text { \$ Sphere } \\
2 & \text { so }<\mathrm{rd}+\mathrm{rw}^{*} \text { in } \mid 4.4> & \text { \$ Reflection }
\end{array}
$$

mode $\mathrm{n}$

kcode $20001.0100 \mathrm{ZZZ0}$

$\begin{array}{llll}\text { ksrc } & 0.00 & 0.00 & 0.00\end{array}$

$\begin{array}{lll}0.00 & 0.00 & 0.10\end{array}$

$\begin{array}{lll}0.00 & 0.00 & 0.50\end{array}$

$\begin{array}{lll}0.00 & 0.00 & 1.00\end{array}$

$\begin{array}{lll}0.00 & 0.00 & 2.00\end{array}$

$\begin{array}{lll}0.00 & 0.00 & 3.00\end{array}$

$\begin{array}{lll}1.00 & 1.00 & 1.00\end{array}$

$\begin{array}{lll}1.00 & 1.00 & 2.00\end{array}$

$\begin{array}{lll}1.00 & 1.00 & 3.00\end{array}$

$\begin{array}{lll}2.00 & 2.00 & 1.00\end{array}$

$\begin{array}{lll}2.00 & 2.00 & 2.00\end{array}$

$\begin{array}{lll}2.00 & 2.00 & 3.00\end{array}$

$\begin{array}{lll}-1.00 & -1.00 & -1.00\end{array}$

$-10.00 \quad-10.00 \quad-20.00$

$\begin{array}{lll}-10.00 & -10.00 & -30.00\end{array}$

c

m2 \$water

$1001.66 \mathrm{c} \quad 2$

$8016.66 \mathrm{c} \quad 1$

$\mathrm{mt} 2$ 1wtr.60t

c UO2/water mixture

m11 \$ UO2(NO3)2 - NO3 - H2O, $100.0 \mathrm{wt} \% \mathrm{U} 235, \mathrm{ZZZ1} \mathrm{milli-g} \mathrm{U/cc,}<\mathrm{denSol} \mid 3.4>$

c molarity $\mathrm{U}=\langle\operatorname{molU}| 3.4>$, acid $=\langle\operatorname{mol} A$ cid $| 3.4>, \mathrm{H} 2 \mathrm{O}=\langle\operatorname{molH} 2 \mathrm{O} \mid 3.4\rangle$

c $\mathrm{U}$ in salt

$92235.66 \mathrm{c}<$ molU*ena $3.4>$

$92238.66 \mathrm{c}<$ molU* $* 1$-ena $) \mid 3.4>$

c NO3 in salt plus acid

$7014.66 \mathrm{c}<2 * \mathrm{molU}+\operatorname{molAcid} \mid 3.4>$

$8016.66 \mathrm{c}<6 * \mathrm{molU}+3 * \operatorname{mol} \mathrm{Acid} \mid 3.4>$

c water

1001.66c $<2 *$ molH2O + molAcid $\mid 3.4>$ \$ncludes Free Hydrogen Ion

$8016.66 \mathrm{c}<\mathrm{molH} 2 \mathrm{O} \mid 3.4>$

mt11 1wtr.60t

c

$\mathrm{c}$ 
Template File for Case: u5o2N20-sp-0935-01

sp, 93.5 wt\% UO2, ZZZ1 milli-g/cc, in UO2(NO3)2, with 0 Molar HNO3

$\mathrm{c}$

c worm notes: format of the form |n.m for floating point, $\mid \mathrm{n}$ for integers

c zzz0 (in upper case) is replaced with the number of cyles

c $\quad$ zzz1 $=$ the actual concentration in units of milli-g/cc

c $\quad$ zzz2 $=$ solution density in $\mathrm{g} / \mathrm{cc}$

c $\quad \mathrm{qqq1}=$ the minimum dimensions (in hundreths of inches)

c $\quad \mathrm{qqq} 2=$ the maximum dimensions (in hundreths of inches)

c $\quad \mathrm{qqq} 3=$ the delta (in hundreths of inches)

c

c Diameter $=<\mathrm{d} 1=$ QQQ1:QQQ2:QQQ3 $\mid 5>$ hundreths of inches - must be integer

$\mathrm{c}$ radius $=<\mathrm{rd}=\mathrm{d} 1 * 2.54 / 100 / 2 \mid 5.4>\mathrm{cm}$

c note for perl, numbers with leading zeros must be enclosed in single quotes or else

c they will be interpretted as octal

$\mathrm{c}$ Conc $=<\mathrm{c}={ }^{\prime}$ ZZZ1' $\mid 5>$ milli-g U/cc

c Reflector width $=<\mathrm{rw}=1.0 \mid 2.1>$ inches

c Enrichment $=<$ ena $=0.935 \mid 1.3>$ atom fraction $\mathrm{U}$

c Mols of Acid per liter $<$ molAcid $=0.0 \mid 1.2>$

c

$\mathrm{c}$

c Density of solution $<$ denSol=ZZZ2/1e5 $\mid 1.5>$

c

c

c Atomic Weights

c $\mathrm{H} 2 \mathrm{O}=\langle$ atwH2O $=18.0152| 3.4>$

c NO3 $=<$ atwNO3 $=62.0049 \mid 3.4>$

c $\mathrm{O}=\langle$ atwO $=15.9994| 3.4>$

$\mathrm{c} \mathrm{HNO} 3=<$ atwHNO3 $=63.0128 \mid 3.4>$

c U235 $=<$ atwU235 $=235.0439 \mid 3.4>$

c U238 $=<$ atwU238 $=238.0508 \mid 3.4>$

$\mathrm{c} \mathrm{U}=<$ atwU $=$ ena*atwU235+(1-ena)*atwU238|3.4>

c

c Molarity of solution components

c Mols of $\mathrm{U}$ per liter $<$ molU $=\mathrm{c} / \mathrm{atwU} \mid 3.4>$

c Mols of $\mathrm{H} 2 \mathrm{O}$ per liter $<\operatorname{molH} 2 \mathrm{O}=(1000 *$ denSol-molU* $(\operatorname{atwU}+2 *$ atwNO3 $+2 *$ atwO $)-$ molAcid*atwHNO3)/atwH2O|3.4>

$\mathrm{c}$

c

$<$ perl $>$

sub IMP

\{

return 1 if $\left(\$ \_[0]>0\right)$;

return 0 ;

\}

$</$ perl $>$

c sphere

$2011<$-denSol|3.6> $-1 \quad$ imp:n=1

$212-1.000-21 \quad$ imp:n=<\&IMP(rw) $\mid 1>$ \$zero importance if no reflector

$220 \quad 2$ imp:n=0 


$$
\begin{array}{lll}
1 & \text { so }<\mathrm{rd} \mid 4.4> & \text { \$ Sphere } \\
2 & \text { so }<\mathrm{rd}+\mathrm{rw}^{*} \text { in } \mid 4.4> & \text { \$ Reflection }
\end{array}
$$

mode $\mathrm{n}$

kcode $20001.0100 \mathrm{ZZZ0}$

$\begin{array}{llll}\text { ksrc } & 0.00 & 0.00 & 0.00\end{array}$

$\begin{array}{lll}0.00 & 0.00 & 0.10\end{array}$

$\begin{array}{lll}0.00 & 0.00 & 0.50\end{array}$

$\begin{array}{lll}0.00 & 0.00 & 1.00\end{array}$

$\begin{array}{lll}0.00 & 0.00 & 2.00\end{array}$

$\begin{array}{lll}0.00 & 0.00 & 3.00\end{array}$

$\begin{array}{lll}1.00 & 1.00 & 1.00\end{array}$

$\begin{array}{lll}1.00 & 1.00 & 2.00\end{array}$

$\begin{array}{lll}1.00 & 1.00 & 3.00\end{array}$

$\begin{array}{lll}2.00 & 2.00 & 1.00\end{array}$

$\begin{array}{lll}2.00 & 2.00 & 2.00\end{array}$

$\begin{array}{lll}2.00 & 2.00 & 3.00\end{array}$

$\begin{array}{lll}-1.00 & -1.00 & -1.00\end{array}$

$-10.00 \quad-10.00 \quad-20.00$

$\begin{array}{lll}-10.00 & -10.00 & -30.00\end{array}$

c

m2 \$water

$1001.66 \mathrm{c} \quad 2$

$8016.66 \mathrm{c} \quad 1$

$\mathrm{mt} 2$ 1wtr.60t

c UO2/water mixture

m11 \$ UO2(NO3)2 - NO3 - H2O, $100.0 \mathrm{wt} \% \mathrm{U} 235, \mathrm{ZZZ1} \mathrm{milli-g} \mathrm{U/cc,}<\mathrm{denSol} \mid 3.4>$

c molarity $\mathrm{U}=\langle\operatorname{molU}| 3.4>$, acid $=\langle\operatorname{mol} A$ cid $| 3.4>, \mathrm{H} 2 \mathrm{O}=\langle\operatorname{molH} 2 \mathrm{O} \mid 3.4\rangle$

c $\mathrm{U}$ in salt

$92235.66 \mathrm{c}<$ molU*ena $3.4>$

$92238.66 \mathrm{c}<$ molU* $* 1$-ena $) \mid 3.4>$

c NO3 in salt plus acid

$7014.66 \mathrm{c}<2 * \mathrm{molU}+\operatorname{molAcid} \mid 3.4>$

$8016.66 \mathrm{c}<6 * \mathrm{molU}+3 * \operatorname{mol} \mathrm{Acid} \mid 3.4>$

c water

1001.66c $<2 *$ molH2O + molAcid $\mid 3.4>$ \$ncludes Free Hydrogen Ion

$8016.66 \mathrm{c}<\mathrm{molH} 2 \mathrm{O} \mid 3.4>$

mt11 1wtr.60t

c

$\mathrm{c}$ 
Template File for Case: u5o2N20-sp-0935-10

sp, 93.5 wt\% UO2, ZZZ1 milli-g/cc, in UO2(NO3)2, with 0 Molar HNO3

$\mathrm{c}$

c worm notes: format of the form $\mid$ n.m for floating point, $\mid \mathrm{n}$ for integers

c zzz0 (in upper case) is replaced with the number of cyles

c $\quad$ zzz1 $=$ the actual concentration in units of milli-g/cc

c $\quad$ zzz2 $=$ solution density in $\mathrm{g} / \mathrm{cc}$

c $\quad \mathrm{qqq1}=$ the minimum dimensions (in hundreths of inches)

c $\quad \mathrm{qqq} 2=$ the maximum dimensions (in hundreths of inches)

c $\quad \mathrm{qqq} 3=$ the delta (in hundreths of inches)

c

$\mathrm{c}$ Diameter $=<\mathrm{d} 1=$ QQQ1:QQQ2:QQQ3 $\mid 5>$ hundreths of inches - must be integer

$\mathrm{c}$ radius $=<\mathrm{rd}=\mathrm{d} 1 * 2.54 / 100 / 2 \mid 5.4>\mathrm{cm}$

c note for perl, numbers with leading zeros must be enclosed in single quotes or else

c they will be interpretted as octal

$\mathrm{c}$ Conc $=<\mathrm{c}={ }^{\prime}$ ZZZ1' $\mid 5>$ milli-g U/cc

c Reflector width $=<\mathrm{rw}=10.0 \mid 2.1>$ inches

c Enrichment $=<$ ena $=0.935 \mid 1.3>$ atom fraction $\mathrm{U}$

c Mols of Acid per liter $<$ molAcid $=0.0 \mid 1.2>$

c

$\mathrm{c}$

c Density of solution $<$ denSol $=Z Z Z 2 / 1$ e $5 \mid 1.5>$

c

c

c Atomic Weights

c $\mathrm{H} 2 \mathrm{O}=\langle$ atwH2O $=18.0152| 3.4>$

$\mathrm{c} \mathrm{NO3}=\langle$ atwNO3 $=62.0049| 3.4>$

c $\mathrm{O}=\langle$ atwO $=15.9994| 3.4>$

$\mathrm{c} \mathrm{HNO} 3=<$ atwHNO3 $=63.0128 \mid 3.4>$

c U235 $=<$ atwU235 $=235.0439 \mid 3.4>$

c U238 $=<$ atwU238 $=238.0508 \mid 3.4>$

c U $=<$ atwU $=$ ena*atwU235+(1-ena)*atwU238 $3.4>$

c

c Molarity of solution components

c Mols of $\mathrm{U}$ per liter $<$ molU $=\mathrm{c} / \mathrm{atwU} \mid 3.4>$

c Mols of $\mathrm{H} 2 \mathrm{O}$ per liter $<\operatorname{molH} 2 \mathrm{O}=(1000 *$ denSol-molU* $*$ atwU $+2 * a+w N O 3+2 * a t w O)$ molAcid*atwHNO3)/atwH2O|3.4>

$\mathrm{c}$

c

$<$ perl $>$

sub IMP

\{

return 1 if $\left(\$ \_[0]>0\right)$;

return 0 ;

\}

$</$ perl $>$

c sphere

$2011<$-denSol|3.6> $-1 \quad$ imp:n=1

$212-1.000-21 \quad$ imp:n=<\&IMP(rw) $\mid 1>$ \$zero importance if no reflector

$220 \quad 2 \quad$ imp:n $=0$ 


$$
\begin{array}{lll}
1 & \text { so }<\mathrm{rd} \mid 4.4> & \text { \$ Sphere } \\
2 & \text { so }<\mathrm{rd}+\mathrm{rw}^{*} \text { in } \mid 4.4> & \text { \$ Reflection }
\end{array}
$$

mode $\mathrm{n}$

kcode $20001.0100 \mathrm{ZZZ0}$

$\begin{array}{llll}\text { ksrc } & 0.00 & 0.00 & 0.00\end{array}$

$\begin{array}{lll}0.00 & 0.00 & 0.10\end{array}$

$\begin{array}{lll}0.00 & 0.00 & 0.50\end{array}$

$\begin{array}{lll}0.00 & 0.00 & 1.00\end{array}$

$\begin{array}{lll}0.00 & 0.00 & 2.00\end{array}$

$\begin{array}{lll}0.00 & 0.00 & 3.00\end{array}$

$\begin{array}{lll}1.00 & 1.00 & 1.00\end{array}$

$\begin{array}{lll}1.00 & 1.00 & 2.00\end{array}$

$\begin{array}{lll}1.00 & 1.00 & 3.00\end{array}$

$\begin{array}{lll}2.00 & 2.00 & 1.00\end{array}$

$\begin{array}{lll}2.00 & 2.00 & 2.00\end{array}$

$\begin{array}{lll}2.00 & 2.00 & 3.00\end{array}$

$\begin{array}{lll}-1.00 & -1.00 & -1.00\end{array}$

$-10.00 \quad-10.00 \quad-20.00$

$\begin{array}{lll}-10.00 & -10.00 & -30.00\end{array}$

c

m2 \$water

$1001.66 \mathrm{c} \quad 2$

$8016.66 \mathrm{c} \quad 1$

$\mathrm{mt} 2$ 1wtr.60t

c UO2/water mixture

m11 \$ UO2(NO3)2 - NO3 - H2O, $100.0 \mathrm{wt} \% \mathrm{U} 235, \mathrm{ZZZ1} \mathrm{milli-g} \mathrm{U/cc,}<\mathrm{denSol} \mid 3.4>$

c molarity $\mathrm{U}=\langle\operatorname{molU}| 3.4>$, acid $=\langle\operatorname{mol} A$ cid $| 3.4>, \mathrm{H} 2 \mathrm{O}=\langle\operatorname{molH} 2 \mathrm{O} \mid 3.4\rangle$

c $\mathrm{U}$ in salt

$92235.66 \mathrm{c}<$ molU*ena $3.4>$

$92238.66 \mathrm{c}<$ molU* $* 1$-ena $) \mid 3.4>$

c NO3 in salt plus acid

$7014.66 \mathrm{c}<2 * \mathrm{molU}+\operatorname{molAcid} \mid 3.4>$

$8016.66 \mathrm{c}<6 * \mathrm{molU}+3 * \operatorname{mol} \mathrm{Acid} \mid 3.4>$

c water

1001.66c $<2 *$ molH2O + molAcid $\mid 3.4>$ \$ncludes Free Hydrogen Ion

$8016.66 \mathrm{c}<\mathrm{molH} 2 \mathrm{O} \mid 3.4>$

mt11 1wtr.60t

c

$\mathrm{c}$ 
Template File for Case: u5o2N23-sp-0935-00

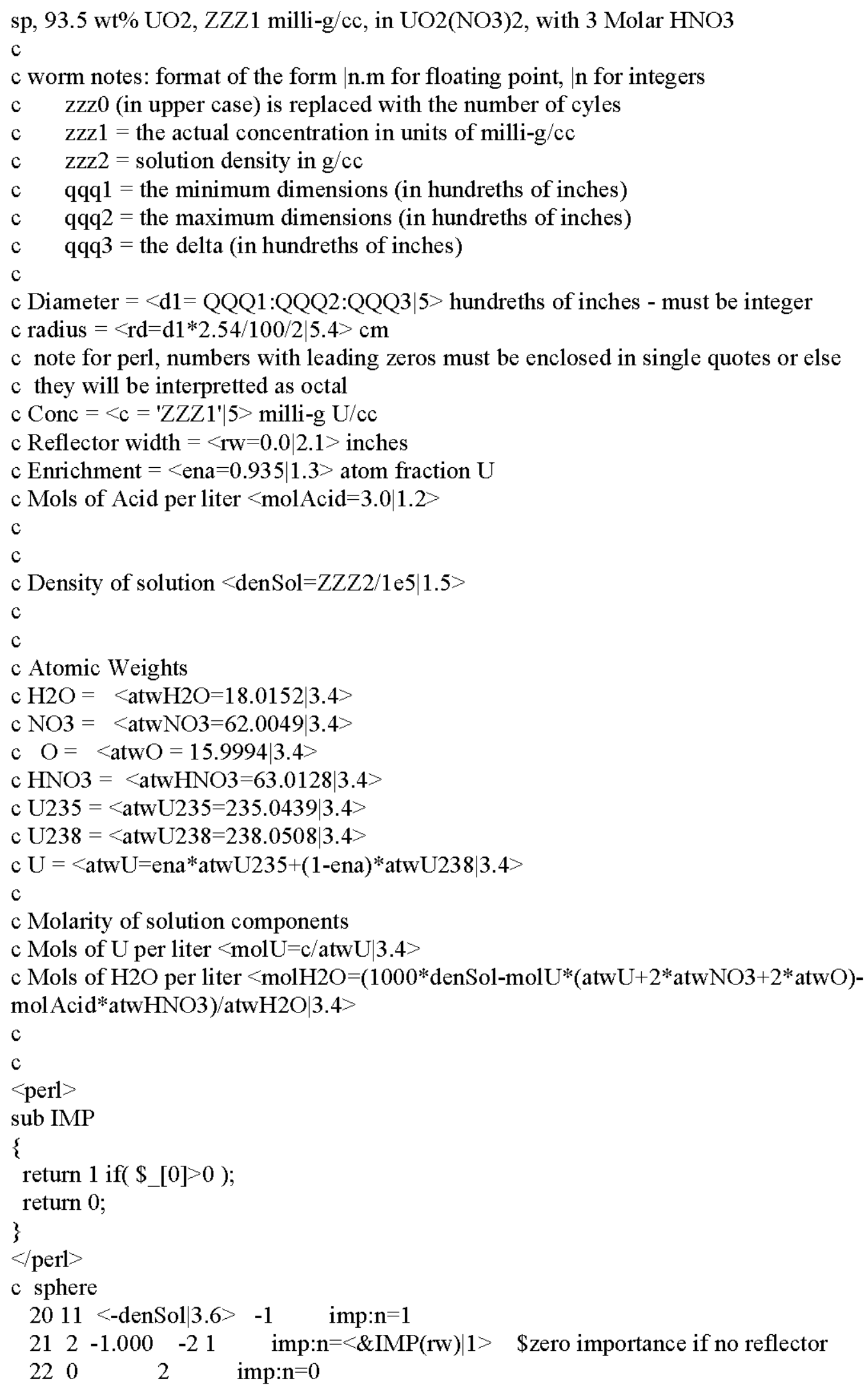




$$
\begin{array}{lll}
1 & \text { so }<\mathrm{rd} \mid 4.4> & \text { \$ Sphere } \\
2 & \text { so }<\mathrm{rd}+\mathrm{rw}^{*} \text { in } \mid 4.4> & \text { \$ Reflection }
\end{array}
$$

mode $\mathrm{n}$

kcode $20001.0100 \mathrm{ZZZ0}$

$\begin{array}{llll}\text { ksrc } & 0.00 & 0.00 & 0.00\end{array}$

$\begin{array}{lll}0.00 & 0.00 & 0.10\end{array}$

$\begin{array}{lll}0.00 & 0.00 & 0.50\end{array}$

$\begin{array}{lll}0.00 & 0.00 & 1.00\end{array}$

$\begin{array}{lll}0.00 & 0.00 & 2.00\end{array}$

$\begin{array}{lll}0.00 & 0.00 & 3.00\end{array}$

$\begin{array}{lll}1.00 & 1.00 & 1.00\end{array}$

$\begin{array}{lll}1.00 & 1.00 & 2.00\end{array}$

$\begin{array}{lll}1.00 & 1.00 & 3.00\end{array}$

$\begin{array}{lll}2.00 & 2.00 & 1.00\end{array}$

$\begin{array}{lll}2.00 & 2.00 & 2.00\end{array}$

$\begin{array}{lll}2.00 & 2.00 & 3.00\end{array}$

$\begin{array}{lll}-1.00 & -1.00 & -1.00\end{array}$

$-10.00 \quad-10.00 \quad-20.00$

$\begin{array}{lll}-10.00 & -10.00 & -30.00\end{array}$

c

m2 \$water

$1001.66 \mathrm{c} \quad 2$

$8016.66 \mathrm{c} \quad 1$

$\mathrm{mt} 2$ 1wtr.60t

c UO2/water mixture

m11 \$ UO2(NO3)2 - NO3 - H2O, $100.0 \mathrm{wt} \% \mathrm{U} 235, \mathrm{ZZZ1} \mathrm{milli-g} \mathrm{U/cc,}<\mathrm{denSol} \mid 3.4>$

c molarity $\mathrm{U}=\langle\operatorname{molU}| 3.4>$, acid $=\langle\operatorname{mol} A$ cid $| 3.4>, \mathrm{H} 2 \mathrm{O}=\langle\operatorname{molH} 2 \mathrm{O} \mid 3.4\rangle$

c $\mathrm{U}$ in salt

$92235.66 \mathrm{c}<$ molU*ena $3.4>$

$92238.66 \mathrm{c}<$ molU* $* 1$-ena $) \mid 3.4>$

c NO3 in salt plus acid

$7014.66 \mathrm{c}<2 * \mathrm{molU}+\operatorname{molAcid} \mid 3.4>$

$8016.66 \mathrm{c}<6 * \mathrm{molU}+3 * \operatorname{mol} \mathrm{Acid} \mid 3.4>$

c water

1001.66c $<2 *$ molH2O + molAcid $\mid 3.4>$ \$ncludes Free Hydrogen Ion

$8016.66 \mathrm{c}<\mathrm{molH} 2 \mathrm{O} \mid 3.4>$

mt11 1wtr.60t

c

$\mathrm{c}$ 
Template File for Case: u5o2N23-sp-0935-01

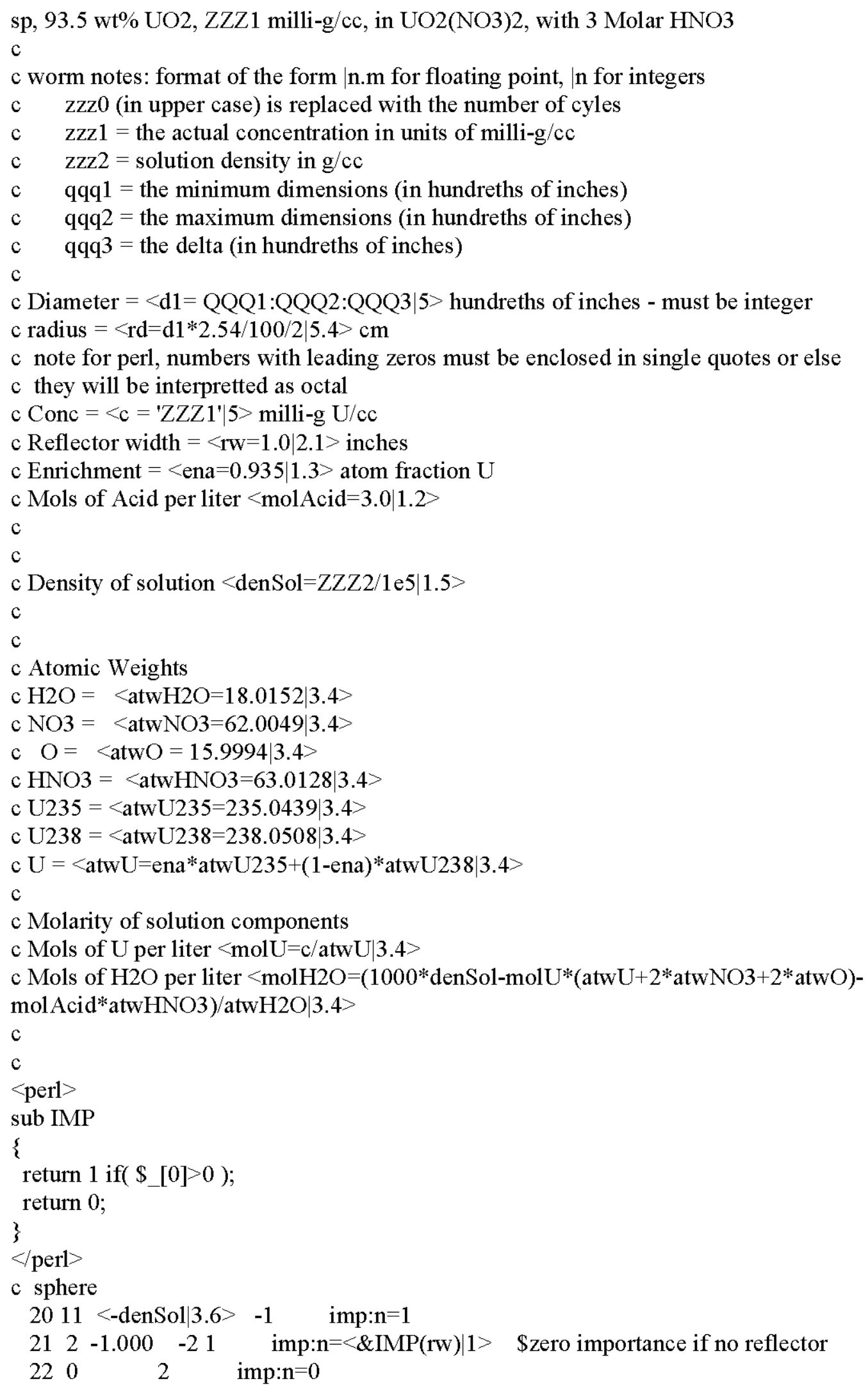




$$
\begin{array}{lll}
1 & \text { so }<\mathrm{rd} \mid 4.4> & \text { \$ Sphere } \\
2 & \text { so }<\mathrm{rd}+\mathrm{rw}^{*} \text { in } \mid 4.4> & \text { \$ Reflection }
\end{array}
$$

mode $\mathrm{n}$

kcode $20001.0100 \mathrm{ZZZ0}$

$\begin{array}{llll}\text { ksrc } & 0.00 & 0.00 & 0.00\end{array}$

$\begin{array}{lll}0.00 & 0.00 & 0.10\end{array}$

$\begin{array}{lll}0.00 & 0.00 & 0.50\end{array}$

$\begin{array}{lll}0.00 & 0.00 & 1.00\end{array}$

$\begin{array}{lll}0.00 & 0.00 & 2.00\end{array}$

$\begin{array}{lll}0.00 & 0.00 & 3.00\end{array}$

$\begin{array}{lll}1.00 & 1.00 & 1.00\end{array}$

$\begin{array}{lll}1.00 & 1.00 & 2.00\end{array}$

$\begin{array}{lll}1.00 & 1.00 & 3.00\end{array}$

$\begin{array}{lll}2.00 & 2.00 & 1.00\end{array}$

$\begin{array}{lll}2.00 & 2.00 & 2.00\end{array}$

$\begin{array}{lll}2.00 & 2.00 & 3.00\end{array}$

$\begin{array}{lll}-1.00 & -1.00 & -1.00\end{array}$

$-10.00 \quad-10.00 \quad-20.00$

$\begin{array}{lll}-10.00 & -10.00 & -30.00\end{array}$

c

m2 \$water

$1001.66 \mathrm{c} \quad 2$

$8016.66 \mathrm{c} \quad 1$

$\mathrm{mt} 2$ 1wtr.60t

c UO2/water mixture

m11 \$ UO2(NO3)2 - NO3 - H2O, $100.0 \mathrm{wt} \% \mathrm{U} 235, \mathrm{ZZZ1} \mathrm{milli-g} \mathrm{U/cc,}<\mathrm{denSol} \mid 3.4>$

c molarity $\mathrm{U}=\langle\operatorname{molU}| 3.4>$, acid $=\langle\operatorname{mol} A$ cid $| 3.4>, \mathrm{H} 2 \mathrm{O}=\langle\operatorname{molH} 2 \mathrm{O} \mid 3.4\rangle$

c $\mathrm{U}$ in salt

$92235.66 \mathrm{c}<$ molU*ena $3.4>$

$92238.66 \mathrm{c}<$ molU* $* 1$-ena $) \mid 3.4>$

c NO3 in salt plus acid

$7014.66 \mathrm{c}<2 * \mathrm{molU}+\operatorname{molAcid} \mid 3.4>$

$8016.66 \mathrm{c}<6 * \mathrm{molU}+3 * \operatorname{mol} \mathrm{Acid} \mid 3.4>$

c water

1001.66c $<2 *$ molH2O + molAcid $\mid 3.4>$ \$ncludes Free Hydrogen Ion

$8016.66 \mathrm{c}<\mathrm{molH} 2 \mathrm{O} \mid 3.4>$

mt11 1wtr.60t

c

$\mathrm{c}$ 
Template File for Case: u5o2N23-sp-0935-10

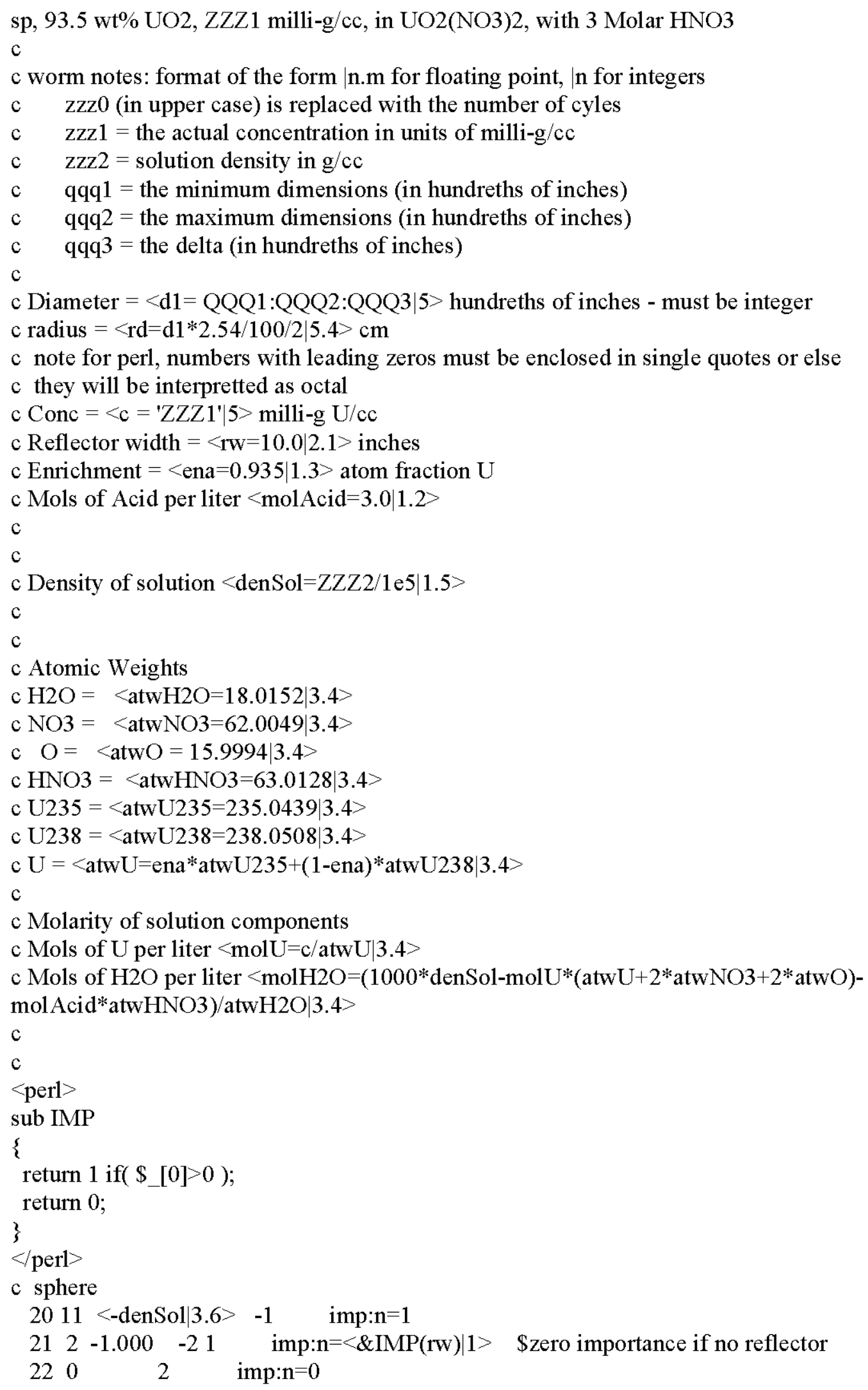




$$
\begin{array}{lll}
1 & \text { so }<\mathrm{rd} \mid 4.4> & \text { \$ Sphere } \\
2 & \text { so }<\mathrm{rd}+\mathrm{rw}^{*} \text { in } \mid 4.4> & \text { \$ Reflection }
\end{array}
$$

mode $\mathrm{n}$

kcode $20001.0100 \mathrm{ZZZ0}$

$\begin{array}{llll}\text { ksrc } & 0.00 & 0.00 & 0.00\end{array}$

$\begin{array}{lll}0.00 & 0.00 & 0.10\end{array}$

$\begin{array}{lll}0.00 & 0.00 & 0.50\end{array}$

$\begin{array}{lll}0.00 & 0.00 & 1.00\end{array}$

$\begin{array}{lll}0.00 & 0.00 & 2.00\end{array}$

$\begin{array}{lll}0.00 & 0.00 & 3.00\end{array}$

$\begin{array}{lll}1.00 & 1.00 & 1.00\end{array}$

$\begin{array}{lll}1.00 & 1.00 & 2.00\end{array}$

$\begin{array}{lll}1.00 & 1.00 & 3.00\end{array}$

$\begin{array}{lll}2.00 & 2.00 & 1.00\end{array}$

$\begin{array}{lll}2.00 & 2.00 & 2.00\end{array}$

$\begin{array}{lll}2.00 & 2.00 & 3.00\end{array}$

$\begin{array}{lll}-1.00 & -1.00 & -1.00\end{array}$

$-10.00 \quad-10.00 \quad-20.00$

$\begin{array}{lll}-10.00 & -10.00 & -30.00\end{array}$

c

m2 \$water

$1001.66 \mathrm{c} \quad 2$

$8016.66 \mathrm{c} \quad 1$

$\mathrm{mt} 2$ 1wtr.60t

c UO2/water mixture

m11 \$ UO2(NO3)2 - NO3 - H2O, $100.0 \mathrm{wt} \% \mathrm{U} 235, \mathrm{ZZZ1} \mathrm{milli-g} \mathrm{U/cc,}<\mathrm{denSol} \mid 3.4>$

c molarity $\mathrm{U}=\langle\operatorname{molU}| 3.4>$, acid $=\langle\operatorname{mol} A$ cid $| 3.4>, \mathrm{H} 2 \mathrm{O}=\langle\operatorname{molH} 2 \mathrm{O} \mid 3.4\rangle$

c $\mathrm{U}$ in salt

$92235.66 \mathrm{c}<$ molU*ena $3.4>$

$92238.66 \mathrm{c}<$ molU* $* 1$-ena $) \mid 3.4>$

c NO3 in salt plus acid

$7014.66 \mathrm{c}<2 * \mathrm{molU}+\operatorname{molAcid} \mid 3.4>$

$8016.66 \mathrm{c}<6 * \mathrm{molU}+3 * \operatorname{mol} \mathrm{Acid} \mid 3.4>$

c water

1001.66c $<2 *$ molH2O + molAcid $\mid 3.4>$ \$ncludes Free Hydrogen Ion

$8016.66 \mathrm{c}<\mathrm{molH} 2 \mathrm{O} \mid 3.4>$

mt11 1wtr.60t

c

$\mathrm{c}$ 
Template File for Case: u5o2N26-sp-0935-00

sp, 93.5 wt\% UO2, ZZZ1 milli-g/cc, in UO2(NO3)2, with 6 Molar HNO3

$\mathrm{c}$

c worm notes: format of the form $\mid$ n.m for floating point, $\mid \mathrm{n}$ for integers

c zzz0 (in upper case) is replaced with the number of cyles

c $\quad$ zzz1 $=$ the actual concentration in units of milli-g/cc

c $\quad$ zzz2 $=$ solution density in $\mathrm{g} / \mathrm{cc}$

c $\quad \mathrm{qqq1}=$ the minimum dimensions (in hundreths of inches)

c $\quad \mathrm{qqq} 2=$ the maximum dimensions (in hundreths of inches)

c $\quad \mathrm{qqq} 3=$ the delta (in hundreths of inches)

c

c Diameter $=<\mathrm{d} 1=$ QQQ1:QQQ2:QQQ3 $\mid 5>$ hundreths of inches - must be integer

$\mathrm{c}$ radius $=<\mathrm{rd}=\mathrm{d} 1 * 2.54 / 100 / 2 \mid 5.4>\mathrm{cm}$

c note for perl, numbers with leading zeros must be enclosed in single quotes or else

c they will be interpretted as octal

$\mathrm{c}$ Conc $=<\mathrm{c}={ }^{\prime} \mathrm{ZZZ1} 1 \mid 5>$ milli-g U/cc

c Reflector width $=<\mathrm{rw}=0.0 \mid 2.1>$ inches

c Enrichment $=<$ ena $=0.935 \mid 1.3>$ atom fraction $\mathrm{U}$

c Mols of Acid per liter $<$ molAcid $=6.0 \mid 1.2>$

$\mathrm{c}$

$\mathrm{c}$

c Density of solution $<$ denSol $=Z Z Z 2 / 1 \mathrm{e} 5 \mid 1.5>$

c

c

c Atomic Weights

c $\mathrm{H} 2 \mathrm{O}=\langle$ atwH2O $=18.0152| 3.4>$

$\mathrm{c} \mathrm{NO3}=\langle$ atwNO3 $=62.0049| 3.4>$

c $\mathrm{O}=\langle$ atwO $=15.9994| 3.4>$

$\mathrm{c} \mathrm{HNO} 3=<$ atwHNO3 $=63.0128 \mid 3.4>$

c U235 $=<$ atwU235 $=235.0439 \mid 3.4>$

c U238 $=<$ atwU238 $=238.0508 \mid 3.4>$

c U $=<$ atwU $=$ ena*atwU235+(1-ena)*atwU238 $3.4>$

c

c Molarity of solution components

c Mols of $\mathrm{U}$ per liter $<$ molU $=\mathrm{c} / \mathrm{atwU} \mid 3.4>$

c Mols of $\mathrm{H} 2 \mathrm{O}$ per liter $<\operatorname{molH} 2 \mathrm{O}=(1000 *$ denSol-molU* $*$ atwU $+2 * a+w N O 3+2 * a t w O)$ molAcid*atwHNO3)/atwH2O|3.4>

$\mathrm{c}$

c

$<$ perl $>$

sub IMP

\{

return 1 if $\left(\$ \_[0]>0\right)$;

return 0 ;

\}

$</$ perl $>$

c sphere

$2011<$-denSol|3.6> $-1 \quad$ imp:n=1

$212-1.000-21 \quad$ imp:n=<\&IMP(rw) $\mid 1>$ \$zero importance if no reflector

$220 \quad 2 \quad$ imp:n=0 


$$
\begin{array}{lll}
1 & \text { so }<\mathrm{rd} \mid 4.4> & \text { \$ Sphere } \\
2 & \text { so }<\mathrm{rd}+\mathrm{rw}^{*} \text { in } \mid 4.4> & \text { \$ Reflection }
\end{array}
$$

mode $\mathrm{n}$

kcode $20001.0100 \mathrm{ZZZ0}$

$\begin{array}{llll}\text { ksrc } & 0.00 & 0.00 & 0.00\end{array}$

$\begin{array}{lll}0.00 & 0.00 & 0.10\end{array}$

$\begin{array}{lll}0.00 & 0.00 & 0.50\end{array}$

$\begin{array}{lll}0.00 & 0.00 & 1.00\end{array}$

$\begin{array}{lll}0.00 & 0.00 & 2.00\end{array}$

$\begin{array}{lll}0.00 & 0.00 & 3.00\end{array}$

$\begin{array}{lll}1.00 & 1.00 & 1.00\end{array}$

$\begin{array}{lll}1.00 & 1.00 & 2.00\end{array}$

$\begin{array}{lll}1.00 & 1.00 & 3.00\end{array}$

$\begin{array}{lll}2.00 & 2.00 & 1.00\end{array}$

$\begin{array}{lll}2.00 & 2.00 & 2.00\end{array}$

$\begin{array}{lll}2.00 & 2.00 & 3.00\end{array}$

$\begin{array}{lll}-1.00 & -1.00 & -1.00\end{array}$

$-10.00 \quad-10.00 \quad-20.00$

$\begin{array}{lll}-10.00 & -10.00 & -30.00\end{array}$

c

m2 \$water

$1001.66 \mathrm{c} \quad 2$

$8016.66 \mathrm{c} \quad 1$

$\mathrm{mt} 2$ 1wtr.60t

c UO2/water mixture

m11 \$ UO2(NO3)2 - NO3 - H2O, $100.0 \mathrm{wt} \% \mathrm{U} 235, \mathrm{ZZZ1} \mathrm{milli-g} \mathrm{U/cc,}<\mathrm{denSol} \mid 3.4>$

c molarity $\mathrm{U}=\langle\operatorname{molU}| 3.4>$, acid $=\langle\operatorname{mol} A$ cid $| 3.4>, \mathrm{H} 2 \mathrm{O}=\langle\operatorname{molH} 2 \mathrm{O} \mid 3.4\rangle$

c $\mathrm{U}$ in salt

$92235.66 \mathrm{c}<$ molU*ena $3.4>$

$92238.66 \mathrm{c}<$ molU* $* 1$-ena $) \mid 3.4>$

c NO3 in salt plus acid

$7014.66 \mathrm{c}<2 * \mathrm{molU}+\operatorname{molAcid} \mid 3.4>$

$8016.66 \mathrm{c}<6 * \mathrm{molU}+3 * \operatorname{mol} \mathrm{Acid} \mid 3.4>$

c water

1001.66c $<2 *$ molH2O + molAcid $\mid 3.4>$ \$ncludes Free Hydrogen Ion

$8016.66 \mathrm{c}<\mathrm{molH} 2 \mathrm{O} \mid 3.4>$

mt11 1wtr.60t

c

$\mathrm{c}$ 
Template File for Case: u5o2N26-sp-0935-01

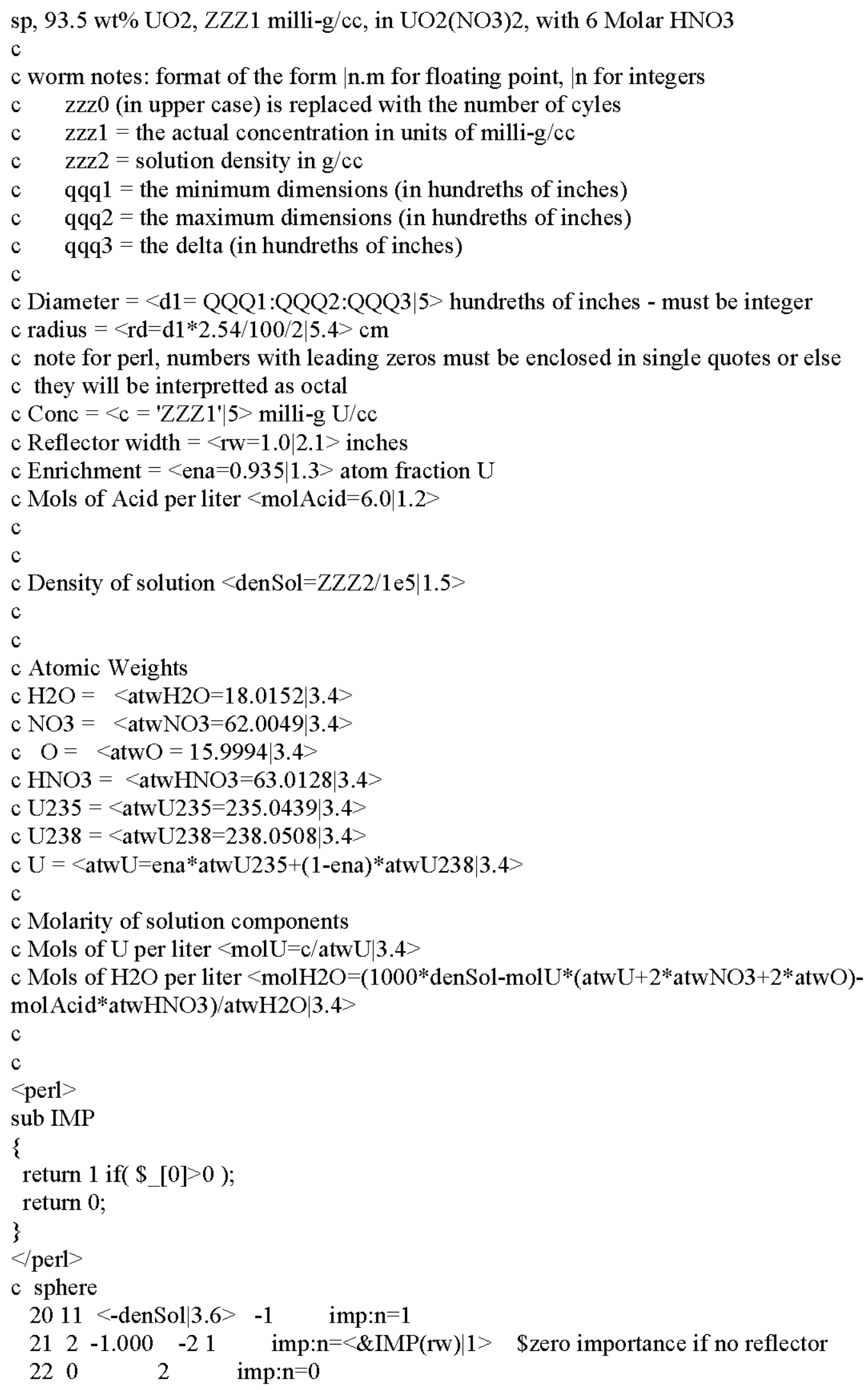




$$
\begin{array}{lll}
1 & \text { so }<\mathrm{rd} \mid 4.4> & \text { \$ Sphere } \\
2 & \text { so }<\mathrm{rd}+\mathrm{rw}^{*} \text { in } \mid 4.4> & \text { \$ Reflection }
\end{array}
$$

mode $\mathrm{n}$

kcode $20001.0100 \mathrm{ZZZ0}$

$\begin{array}{llll}\text { ksrc } & 0.00 & 0.00 & 0.00\end{array}$

$\begin{array}{lll}0.00 & 0.00 & 0.10\end{array}$

$\begin{array}{lll}0.00 & 0.00 & 0.50\end{array}$

$\begin{array}{lll}0.00 & 0.00 & 1.00\end{array}$

$\begin{array}{lll}0.00 & 0.00 & 2.00\end{array}$

$\begin{array}{lll}0.00 & 0.00 & 3.00\end{array}$

$\begin{array}{lll}1.00 & 1.00 & 1.00\end{array}$

$\begin{array}{lll}1.00 & 1.00 & 2.00\end{array}$

$\begin{array}{lll}1.00 & 1.00 & 3.00\end{array}$

$\begin{array}{lll}2.00 & 2.00 & 1.00\end{array}$

$\begin{array}{lll}2.00 & 2.00 & 2.00\end{array}$

$\begin{array}{lll}2.00 & 2.00 & 3.00\end{array}$

$\begin{array}{lll}-1.00 & -1.00 & -1.00\end{array}$

$-10.00 \quad-10.00 \quad-20.00$

$\begin{array}{lll}-10.00 & -10.00 & -30.00\end{array}$

c

m2 \$water

$1001.66 \mathrm{c} \quad 2$

$8016.66 \mathrm{c} \quad 1$

$\mathrm{mt} 2$ 1wtr.60t

c UO2/water mixture

m11 \$ UO2(NO3)2 - NO3 - H2O, $100.0 \mathrm{wt} \% \mathrm{U} 235, \mathrm{ZZZ1} \mathrm{milli-g} \mathrm{U/cc,}<\mathrm{denSol} \mid 3.4>$

c molarity $\mathrm{U}=\langle\operatorname{molU}| 3.4>$, acid $=\langle\operatorname{mol} A$ cid $| 3.4>, \mathrm{H} 2 \mathrm{O}=\langle\operatorname{molH} 2 \mathrm{O} \mid 3.4\rangle$

c $\mathrm{U}$ in salt

$92235.66 \mathrm{c}<$ molU*ena $3.4>$

$92238.66 \mathrm{c}<$ molU* $* 1$-ena $) \mid 3.4>$

c NO3 in salt plus acid

$7014.66 \mathrm{c}<2 * \mathrm{molU}+\operatorname{molAcid} \mid 3.4>$

$8016.66 \mathrm{c}<6 * \mathrm{molU}+3 * \operatorname{mol} \mathrm{Acid} \mid 3.4>$

c water

1001.66c $<2 *$ molH2O + molAcid $\mid 3.4>$ \$ncludes Free Hydrogen Ion

$8016.66 \mathrm{c}<\mathrm{molH} 2 \mathrm{O} \mid 3.4>$

mt11 1wtr.60t

c

$\mathrm{c}$ 
Template File for Case: u5o2N26-sp-0935-10

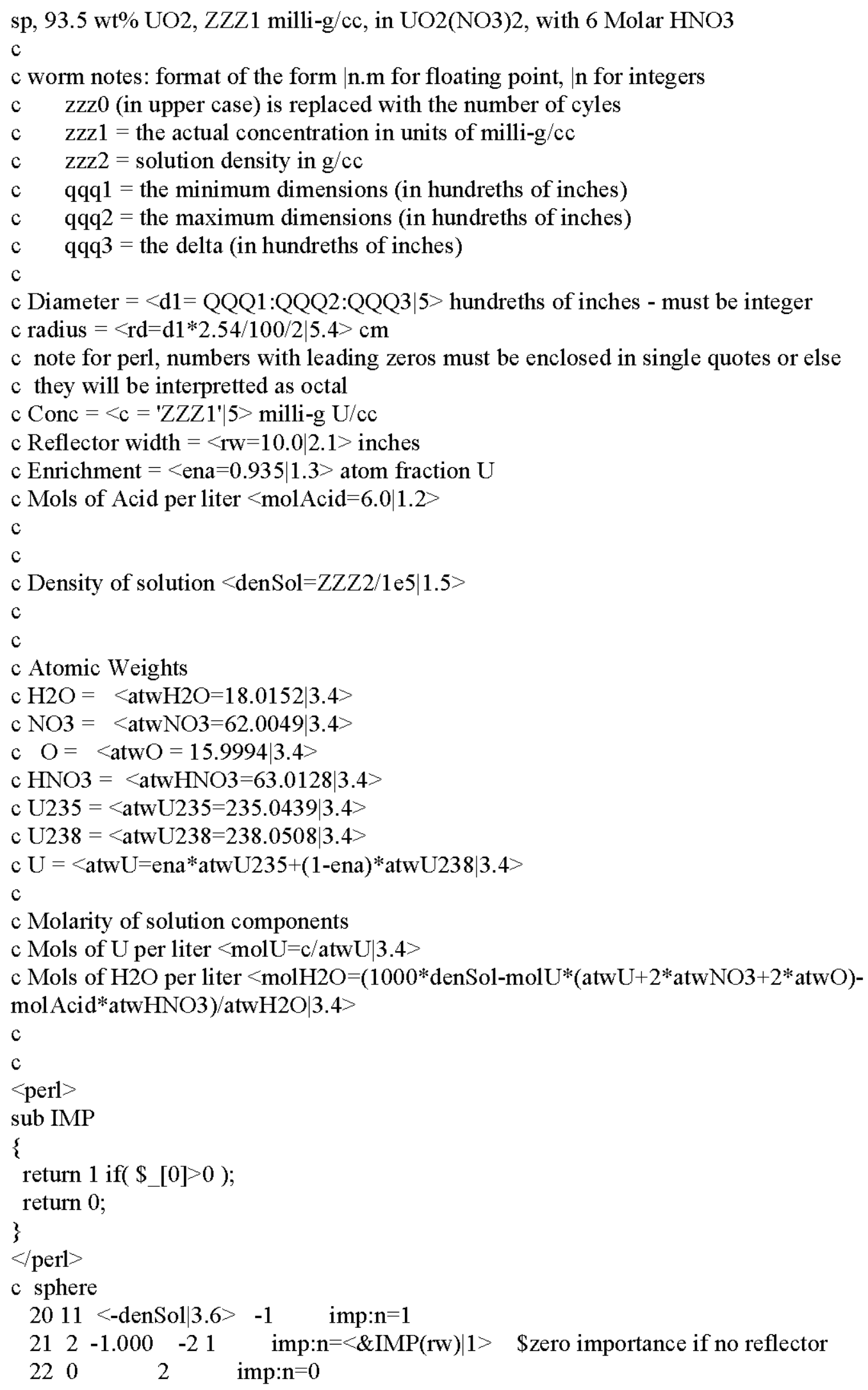




$$
\begin{array}{lll}
1 & \text { so }<\mathrm{rd} \mid 4.4> & \text { \$ Sphere } \\
2 & \text { so }<\mathrm{rd}+\mathrm{rw}^{*} \text { in } \mid 4.4> & \text { \$ Reflection }
\end{array}
$$

mode $\mathrm{n}$

kcode $20001.0100 \mathrm{ZZZ0}$

$\begin{array}{llll}\text { ksrc } & 0.00 & 0.00 & 0.00\end{array}$

$\begin{array}{lll}0.00 & 0.00 & 0.10\end{array}$

$\begin{array}{lll}0.00 & 0.00 & 0.50\end{array}$

$\begin{array}{lll}0.00 & 0.00 & 1.00\end{array}$

$\begin{array}{lll}0.00 & 0.00 & 2.00\end{array}$

$\begin{array}{lll}0.00 & 0.00 & 3.00\end{array}$

$\begin{array}{lll}1.00 & 1.00 & 1.00\end{array}$

$\begin{array}{lll}1.00 & 1.00 & 2.00\end{array}$

$\begin{array}{lll}1.00 & 1.00 & 3.00\end{array}$

$\begin{array}{lll}2.00 & 2.00 & 1.00\end{array}$

$\begin{array}{lll}2.00 & 2.00 & 2.00\end{array}$

$\begin{array}{lll}2.00 & 2.00 & 3.00\end{array}$

$\begin{array}{lll}-1.00 & -1.00 & -1.00\end{array}$

$-10.00 \quad-10.00 \quad-20.00$

$\begin{array}{lll}-10.00 & -10.00 & -30.00\end{array}$

c

m2 \$water

$1001.66 \mathrm{c} \quad 2$

$8016.66 \mathrm{c} \quad 1$

$\mathrm{mt} 2$ 1wtr.60t

c UO2/water mixture

m11 \$ UO2(NO3)2 - NO3 - H2O, $100.0 \mathrm{wt} \% \mathrm{U} 235, \mathrm{ZZZ1} \mathrm{milli-g} \mathrm{U/cc,}<\mathrm{denSol} \mid 3.4>$

c molarity $\mathrm{U}=\langle\operatorname{molU}| 3.4>$, acid $=\langle\operatorname{mol} A$ cid $| 3.4>, \mathrm{H} 2 \mathrm{O}=\langle\operatorname{molH} 2 \mathrm{O} \mid 3.4\rangle$

c $\mathrm{U}$ in salt

$92235.66 \mathrm{c}<$ molU*ena $3.4>$

$92238.66 \mathrm{c}<$ molU* $* 1$-ena $) \mid 3.4>$

c NO3 in salt plus acid

$7014.66 \mathrm{c}<2 * \mathrm{molU}+\operatorname{molAcid} \mid 3.4>$

$8016.66 \mathrm{c}<6 * \mathrm{molU}+3 * \operatorname{mol} \mathrm{Acid} \mid 3.4>$

c water

1001.66c $<2 *$ molH2O + molAcid $\mid 3.4>$ \$ncludes Free Hydrogen Ion

$8016.66 \mathrm{c}<\mathrm{molH} 2 \mathrm{O} \mid 3.4>$

mt11 1wtr.60t

c

$\mathrm{c}$ 
Template File for Case: u5o2N20-icyl-1000-00

icyl, $100.0 \mathrm{wt} \% \mathrm{UO} 2, \mathrm{ZZZ1}$ milli-g/cc, in UO2(NO3)2, with 0 Molar HNO3

c

c worm notes: format of the form |n.m for floating point, $\mid \mathrm{n}$ for integers

c zzz0 (in upper case) is replaced with the number of cyles

c $\quad$ zzz1 $=$ the actual concentration in units of milli-g/cc

c $\quad$ zzz2 $=$ solution density in $\mathrm{g} / \mathrm{cc}$

c $\quad \mathrm{qqq1}=$ the minimum dimensions (in hundreths of inches)

c $\quad \mathrm{qqq} 2=$ the maximum dimensions (in hundreths of inches)

c $\quad \mathrm{qqq} 3=$ the delta (in hundreths of inches)

c

c Diameter $=<\mathrm{d} 1=$ QQQ1:QQQ2:QQQ3 $\mid 5>$ hundreths of inches - must be integer

$\mathrm{c}$ radius $=<\mathrm{rd}=\mathrm{d} 1 * 2.54 / 100 / 2 \mid 5.4>\mathrm{cm}$

c note for perl, numbers with leading zeros must be enclosed in single quotes or else

c they will be interpretted as octal

$\mathrm{c}$ Conc $=<\mathrm{c}={ }^{\prime} \mathrm{ZZZ1} 1 \mid 5>$ milli-g U/cc

c Reflector width $=<\mathrm{rw}=0.0 \mid 2.1>$ inches

c Enrichment $=<$ ena $=1.0 \mid 1.2>$ atom fraction $\mathrm{U}$

c Mols of Acid per liter $<$ molAcid $=0.0 \mid 1.2>$

c

$\mathrm{c}$

c Density of solution $<$ denSol=ZZZ2/1e5 $\mid 1.5>$

c

C

c Atomic Weights

c $\mathrm{H} 2 \mathrm{O}=\langle$ atwH2O $=18.0152| 3.4>$

$\mathrm{c} \mathrm{NO3}=\langle$ atwNO3 $=62.0049| 3.4>$

c $\mathrm{O}=\langle$ atwO $=15.9994| 3.4>$

$\mathrm{c} \mathrm{HNO} 3=<$ atwHNO3 $=63.0128 \mid 3.4>$

c U235 $=<$ atwU235 $=235.0439 \mid 3.4>$

c U238 $=<$ atwU238 $=238.0508 \mid 3.4>$

$\mathrm{c} \mathrm{U}=<$ atwU $=$ ena*atwU235+(1-ena)*atwU238|3.4>

c

c Molarity of solution components

c Mols of $\mathrm{U}$ per liter $<$ molU $=\mathrm{c} / \mathrm{atwU} \mid 3.4>$

c Mols of $\mathrm{H} 2 \mathrm{O}$ per liter $<\operatorname{molH} 2 \mathrm{O}=(1000 *$ denSol-molU* $*$ atwU $+2 * a+w N O 3+2 * a t w O)$ molAcid*atwHNO3)/atwH2O|3.4>

$\mathrm{c}$

c

$<$ perl $>$

sub IMP

\{

return 1 if $\left(\$ \_[0]>0\right)$;

return 0 ;

\}

$</$ perl $>$

c sphere

$2011<$-denSol|3.6> -1 imp:n=1

$212-1.000-21 \quad$ imp:n=<\&IMP(rw) $\mid 1>$ \$zero importance if no reflector

$220 \quad 2$ imp:n=0 


$$
\begin{aligned}
& 1 \quad \mathrm{cz}<\mathrm{rd} \mid 4.4>\quad \text { \$ Sphere } \\
& 2 \mathrm{cz}<\mathrm{rd}+\mathrm{rw} * \mathrm{in} \mid 4.4>\quad \text { \$Reflection }
\end{aligned}
$$

mode $\mathrm{n}$

kcode $20001.0100 \mathrm{ZZZ0}$

$\begin{array}{llll}\text { ksrc } & 0.00 & 0.00 & 0.00\end{array}$

$\begin{array}{lll}0.00 & 0.00 & 0.10\end{array}$

$\begin{array}{lll}0.00 & 0.00 & 0.50\end{array}$

$\begin{array}{lll}0.00 & 0.00 & 1.00\end{array}$

$\begin{array}{lll}0.00 & 0.00 & 2.00\end{array}$

$\begin{array}{lll}0.00 & 0.00 & 3.00\end{array}$

$\begin{array}{lll}1.00 & 1.00 & 1.00\end{array}$

$\begin{array}{lll}1.00 & 1.00 & 2.00\end{array}$

$\begin{array}{lll}1.00 & 1.00 & 3.00\end{array}$

$\begin{array}{lll}2.00 & 2.00 & 1.00\end{array}$

$\begin{array}{lll}2.00 & 2.00 & 2.00\end{array}$

$\begin{array}{lll}2.00 & 2.00 & 3.00\end{array}$

$\begin{array}{lll}-1.00 & -1.00 & -1.00\end{array}$

$-10.00 \quad-10.00 \quad-20.00$

$\begin{array}{lll}-10.00 & -10.00 & -30.00\end{array}$

c

m2 \$water

$1001.66 \mathrm{c} \quad 2$

$8016.66 \mathrm{c} \quad 1$

$\mathrm{mt} 2$ 1wtr.60t

c UO2/water mixture

m11 \$ UO2(NO3)2 - NO3 - H2O, $100.0 \mathrm{wt} \% \mathrm{U} 235, \mathrm{ZZZ1} \mathrm{milli-g} \mathrm{U/cc,}<\mathrm{denSol} \mid 3.4>$

c molarity $\mathrm{U}=\langle\operatorname{molU}| 3.4>$, acid $=\langle\operatorname{mol} A$ cid $| 3.4>, \mathrm{H} 2 \mathrm{O}=\langle\operatorname{molH} 2 \mathrm{O} \mid 3.4\rangle$

c $\mathrm{U}$ in salt

$92235.66 \mathrm{c}<$ molU*ena $3.4>$

c $92238.66 \mathrm{c}<$ molU* $* 1$-ena $) \mid 3.4>$

c NO3 in salt plus acid

$7014.66 \mathrm{c}<2 * \mathrm{molU}+\operatorname{molAcid} \mid 3.4>$

8016.66c $<6 * \mathrm{molU}+3 * \operatorname{molAcid} \mid 3.4>$

c water

1001.66c $<2 *$ molH2O + molAcid $\mid 3.4>$ \$ncludes Free Hydrogen Ion

$8016.66 \mathrm{c}<\mathrm{molH} 2 \mathrm{O} \mid 3.4>$

mt11 1wtr.60t

c

c 
Template File for Case: u5o2N20-icyl-1000-01

icyl, $100.0 \mathrm{wt} \% \mathrm{UO} 2, \mathrm{ZZZ1}$ milli-g/cc, in UO2(NO3)2, with 0 Molar HNO3

c

c worm notes: format of the form |n.m for floating point, $\mid \mathrm{n}$ for integers

c zzz0 (in upper case) is replaced with the number of cyles

c $\quad$ zzz1 $=$ the actual concentration in units of milli-g/cc

c $\quad$ zzz2 $=$ solution density in $\mathrm{g} / \mathrm{cc}$

c $\quad \mathrm{qqq1}=$ the minimum dimensions (in hundreths of inches)

c $\quad \mathrm{qqq} 2=$ the maximum dimensions (in hundreths of inches)

c $\quad \mathrm{qqq} 3=$ the delta (in hundreths of inches)

c

$\mathrm{c}$ Diameter $=<\mathrm{d} 1=$ QQQ1:QQQ2:QQQ3 $\mid 5>$ hundreths of inches - must be integer

$\mathrm{c}$ radius $=<\mathrm{rd}=\mathrm{d} 1 * 2.54 / 100 / 2 \mid 5.4>\mathrm{cm}$

c note for perl, numbers with leading zeros must be enclosed in single quotes or else

c they will be interpretted as octal

$\mathrm{c}$ Conc $=<\mathrm{c}={ }^{\prime}$ ZZZ1' $\mid 5>$ milli-g U/cc

c Reflector width $=<\mathrm{rw}=1.0 \mid 2.1>$ inches

c Enrichment $=<$ ena $=1.0 \mid 1.2>$ atom fraction $\mathrm{U}$

c Mols of Acid per liter $<$ molAcid $=0.0 \mid 1.2>$

c

$\mathrm{c}$

c Density of solution $<$ denSol=ZZZ2/1e5 $\mid 1.5>$

c

C

c Atomic Weights

c $\mathrm{H} 2 \mathrm{O}=\langle$ atwH2O $=18.0152| 3.4>$

$\mathrm{c} \mathrm{NO3}=\langle$ atwNO3 $=62.0049| 3.4>$

c $\mathrm{O}=\langle$ atwO $=15.9994| 3.4>$

$\mathrm{c} \mathrm{HNO} 3=<$ atwHNO3 $=63.0128 \mid 3.4>$

c U235 $=<$ atwU235 $=235.0439 \mid 3.4>$

c U238 $=<$ atwU238 $=238.0508 \mid 3.4>$

$\mathrm{c} \mathrm{U}=<$ atwU $=$ ena*atwU235+(1-ena)*atwU238|3.4>

c

c Molarity of solution components

c Mols of $\mathrm{U}$ per liter $<$ molU $=\mathrm{c} / \mathrm{atwU} \mid 3.4>$

c Mols of $\mathrm{H} 2 \mathrm{O}$ per liter $<\operatorname{molH} 2 \mathrm{O}=(1000 *$ denSol-molU* $*$ atwU $+2 * a+w N O 3+2 * a t w O)$ molAcid*atwHNO3)/atwH2O|3.4>

$\mathrm{c}$

c

$<$ perl $>$

sub IMP

\{

return 1 if $\left(\$ \_[0]>0\right)$;

return 0 ;

\}

$</$ perl $>$

c sphere

$2011<$-denSol|3.6> $-1 \quad$ imp:n=1

$212-1.000-21 \quad$ imp:n=<\&IMP(rw) $\mid 1>$ \$zero importance if no reflector

$220 \quad 2$ imp:n=0 


$$
\begin{aligned}
& 1 \quad \mathrm{cz}<\mathrm{rd} \mid 4.4>\quad \text { \$ Sphere } \\
& 2 \mathrm{cz}<\mathrm{rd}+\mathrm{rw} * \mathrm{in} \mid 4.4>\quad \text { \$Reflection }
\end{aligned}
$$

mode $\mathrm{n}$

kcode $20001.0100 \mathrm{ZZZ} 0$

$\begin{array}{llll}\text { ksrc } & 0.00 & 0.00 & 0.00\end{array}$

$\begin{array}{lll}0.00 & 0.00 & 0.10\end{array}$

$\begin{array}{lll}0.00 & 0.00 & 0.50\end{array}$

$\begin{array}{lll}0.00 & 0.00 & 1.00\end{array}$

$\begin{array}{lll}0.00 & 0.00 & 2.00\end{array}$

$\begin{array}{lll}0.00 & 0.00 & 3.00\end{array}$

$\begin{array}{lll}1.00 & 1.00 & 1.00\end{array}$

$\begin{array}{lll}1.00 & 1.00 & 2.00\end{array}$

$\begin{array}{lll}1.00 & 1.00 & 3.00\end{array}$

$\begin{array}{lll}2.00 & 2.00 & 1.00\end{array}$

$\begin{array}{lll}2.00 & 2.00 & 2.00\end{array}$

$\begin{array}{lll}2.00 & 2.00 & 3.00\end{array}$

$\begin{array}{lll}-1.00 & -1.00 & -1.00\end{array}$

$-10.00 \quad-10.00 \quad-20.00$

$\begin{array}{lll}-10.00 & -10.00 & -30.00\end{array}$

c

m2 \$water

$1001.66 \mathrm{c} \quad 2$

$8016.66 \mathrm{c} \quad 1$

$\mathrm{mt} 2$ 1wtr.60t

c UO2/water mixture

m11 \$ UO2(NO3)2 - NO3 - H2O, $100.0 \mathrm{wt} \% \mathrm{U} 235, \mathrm{ZZZ1} \mathrm{milli-g} \mathrm{U/cc,}<\mathrm{denSol} \mid 3.4>$

c molarity $\mathrm{U}=\langle\operatorname{molU}| 3.4>$, acid $=\langle\operatorname{mol} A$ cid $| 3.4>, \mathrm{H} 2 \mathrm{O}=\langle\operatorname{molH} 2 \mathrm{O} \mid 3.4\rangle$

c $\mathrm{U}$ in salt

$92235.66 \mathrm{c}<$ molU*ena $3.4>$

c $92238.66 \mathrm{c}<$ molU* $(1-$ ena $) \mid 3.4>$

c NO3 in salt plus acid

$7014.66 \mathrm{c}<2 * \mathrm{molU}+\operatorname{molAcid} \mid 3.4>$

8016.66c $<6 * \mathrm{molU}+3 * \operatorname{molAcid} \mid 3.4>$

c water

1001.66c $<2 *$ molH2O + molAcid $\mid 3.4>$ \$ncludes Free Hydrogen Ion

$8016.66 \mathrm{c}<\mathrm{molH} 2 \mathrm{O} \mid 3.4>$

mt11 1wtr.60t

c

c 
Template File for Case: u5o2N20-icyl-1000-10

icyl, $100.0 \mathrm{wt} \% \mathrm{UO} 2, \mathrm{ZZZ1}$ milli-g/cc, in UO2(NO3)2, with 0 Molar HNO3

c

c worm notes: format of the form |n.m for floating point, $\mid \mathrm{n}$ for integers

c zzz0 (in upper case) is replaced with the number of cyles

c $\quad$ zzz1 $=$ the actual concentration in units of milli-g/cc

c $\quad$ zzz2 $=$ solution density in $\mathrm{g} / \mathrm{cc}$

c $\quad \mathrm{qqq1}=$ the minimum dimensions (in hundreths of inches)

c $\quad \mathrm{qqq} 2=$ the maximum dimensions (in hundreths of inches)

c $\quad \mathrm{qqq} 3=$ the delta (in hundreths of inches)

c

$\mathrm{c}$ Diameter $=<\mathrm{d} 1=$ QQQ1:QQQ2:QQQ3 $\mid 5>$ hundreths of inches - must be integer

$\mathrm{c}$ radius $=<\mathrm{rd}=\mathrm{d} 1 * 2.54 / 100 / 2 \mid 5.4>\mathrm{cm}$

c note for perl, numbers with leading zeros must be enclosed in single quotes or else

c they will be interpretted as octal

$\mathrm{c}$ Conc $=<\mathrm{c}={ }^{\prime}$ ZZZ1' $\mid 5>$ milli-g U/cc

c Reflector width $=<\mathrm{rw}=10.0 \mid 2.1>$ inches

c Enrichment $=<$ ena $=1.0 \mid 1.2>$ atom fraction $\mathrm{U}$

c Mols of Acid per liter $<$ molAcid $=0.0 \mid 1.2>$

c

$\mathrm{c}$

c Density of solution $<$ denSol=ZZZ2/1e5 $\mid 1.5>$

c

C

c Atomic Weights

c $\mathrm{H} 2 \mathrm{O}=\langle$ atwH2O $=18.0152| 3.4>$

c NO3 $=<$ atwNO3 $=62.0049 \mid 3.4>$

c $\mathrm{O}=\langle$ atwO $=15.9994| 3.4>$

$\mathrm{c} \mathrm{HNO} 3=<$ atwHNO3 $=63.0128 \mid 3.4>$

c U235 $=<$ atwU235 $=235.0439 \mid 3.4>$

c U238 $=<$ atwU238 $=238.0508 \mid 3.4>$

$\mathrm{c} \mathrm{U}=<$ atwU $=$ ena*atwU235+(1-ena)*atwU238|3.4>

c

c Molarity of solution components

c Mols of $\mathrm{U}$ per liter $<$ molU $=\mathrm{c} / \mathrm{atwU} \mid 3.4>$

c Mols of $\mathrm{H} 2 \mathrm{O}$ per liter $<\operatorname{molH} 2 \mathrm{O}=(1000 *$ denSol-molU* $*$ atwU $+2 * a+w N O 3+2 * a t w O)$ molAcid*atwHNO3)/atwH2O|3.4>

$\mathrm{c}$

c

$<$ perl $>$

sub IMP

\{

return 1 if $\left(\$ \_[0]>0\right)$;

return 0 ;

\}

$</$ perl $>$

c sphere

$2011<$-denSol|3.6> $-1 \quad$ imp:n=1

$212-1.000-21 \quad$ imp:n=<\&IMP(rw) $\mid 1>$ \$zero importance if no reflector

$220 \quad 2$ imp:n=0 


$$
\begin{aligned}
& 1 \quad \mathrm{cz}<\mathrm{rd} \mid 4.4>\quad \text { \$ Sphere } \\
& 2 \mathrm{cz}<\mathrm{rd}+\mathrm{rw} * \mathrm{in} \mid 4.4>\quad \text { \$Reflection }
\end{aligned}
$$

mode $\mathrm{n}$

kcode $20001.0100 \mathrm{ZZZ0}$

$\begin{array}{llll}\text { ksrc } & 0.00 & 0.00 & 0.00\end{array}$

$\begin{array}{lll}0.00 & 0.00 & 0.10\end{array}$

$\begin{array}{lll}0.00 & 0.00 & 0.50\end{array}$

$\begin{array}{lll}0.00 & 0.00 & 1.00\end{array}$

$\begin{array}{lll}0.00 & 0.00 & 2.00\end{array}$

$\begin{array}{lll}0.00 & 0.00 & 3.00\end{array}$

$\begin{array}{lll}1.00 & 1.00 & 1.00\end{array}$

$\begin{array}{lll}1.00 & 1.00 & 2.00\end{array}$

$\begin{array}{lll}1.00 & 1.00 & 3.00\end{array}$

$\begin{array}{lll}2.00 & 2.00 & 1.00\end{array}$

$\begin{array}{lll}2.00 & 2.00 & 2.00\end{array}$

$\begin{array}{lll}2.00 & 2.00 & 3.00\end{array}$

$\begin{array}{lll}-1.00 & -1.00 & -1.00\end{array}$

$-10.00 \quad-10.00 \quad-20.00$

$\begin{array}{lll}-10.00 & -10.00 & -30.00\end{array}$

c

m2 \$water

$1001.66 \mathrm{c} \quad 2$

$8016.66 \mathrm{c} \quad 1$

$\mathrm{mt} 2$ 1wtr.60t

c UO2/water mixture

m11 \$ UO2(NO3)2 - NO3 - H2O, $100.0 \mathrm{wt} \% \mathrm{U} 235, \mathrm{ZZZ1} \mathrm{milli-g} \mathrm{U/cc,}<\mathrm{denSol} \mid 3.4>$

c molarity $\mathrm{U}=\langle\operatorname{molU}| 3.4>$, acid $=\langle\operatorname{mol} A$ cid $| 3.4>, \mathrm{H} 2 \mathrm{O}=\langle\operatorname{molH} 2 \mathrm{O} \mid 3.4\rangle$

c $\mathrm{U}$ in salt

$92235.66 \mathrm{c}<$ molU*ena $3.4>$

c $92238.66 \mathrm{c}<$ molU* $(1-$ ena $) \mid 3.4>$

c NO3 in salt plus acid

$7014.66 \mathrm{c}<2 * \mathrm{molU}+\operatorname{molAcid} \mid 3.4>$

8016.66c $<6 * \mathrm{molU}+3 * \operatorname{molAcid} \mid 3.4>$

c water

1001.66c $<2 *$ molH2O + molAcid $\mid 3.4>$ \$ncludes Free Hydrogen Ion

$8016.66 \mathrm{c}<\mathrm{molH} 2 \mathrm{O} \mid 3.4>$

mt11 1wtr.60t

c

$\mathrm{c}$ 
Template File for Case: u5o2N23-icyl-1000-00

icyl, $100.0 \mathrm{wt} \% \mathrm{UO} 2, \mathrm{ZZZ1}$ milli-g/cc, in UO2(NO3)2, with 3 Molar HNO3

c

c worm notes: format of the form |n.m for floating point, $\mid \mathrm{n}$ for integers

c zzz0 (in upper case) is replaced with the number of cyles

c $\quad$ zzz1 $=$ the actual concentration in units of milli-g/cc

c $\quad$ zzz2 $=$ solution density in $\mathrm{g} / \mathrm{cc}$

c $\quad \mathrm{qqq1}=$ the minimum dimensions (in hundreths of inches)

c $\quad \mathrm{qqq} 2=$ the maximum dimensions (in hundreths of inches)

c $\quad \mathrm{qqq} 3=$ the delta (in hundreths of inches)

c

c Diameter $=<\mathrm{d} 1=$ QQQ1:QQQ2:QQQ3 $\mid 5>$ hundreths of inches - must be integer

$\mathrm{c}$ radius $=<\mathrm{rd}=\mathrm{d} 1 * 2.54 / 100 / 2 \mid 5.4>\mathrm{cm}$

c note for perl, numbers with leading zeros must be enclosed in single quotes or else

c they will be interpretted as octal

$\mathrm{c}$ Conc $=<\mathrm{c}={ }^{\prime} \mathrm{ZZZ1} 1 \mid 5>$ milli-g U/cc

c Reflector width $=<\mathrm{rw}=0.0 \mid 2.1>$ inches

c Enrichment $=<$ ena $=1.0 \mid 1.2>$ atom fraction $\mathrm{U}$

c Mols of Acid per liter $<$ molAcid $=3.0 \mid 1.2>$

c

$\mathrm{c}$

c Density of solution $<$ denSol=ZZZ2/1e5 $\mid 1.5>$

c

C

c Atomic Weights

c $\mathrm{H} 2 \mathrm{O}=\langle$ atwH2O $=18.0152| 3.4>$

$\mathrm{c} \mathrm{NO3}=\langle$ atwNO3 $=62.0049| 3.4>$

c $\mathrm{O}=\langle$ atwO $=15.9994| 3.4>$

$\mathrm{c} \mathrm{HNO} 3=<$ atwHNO3 $=63.0128 \mid 3.4>$

c U235 $=<$ atwU235 $=235.0439 \mid 3.4>$

c U238 $=<$ atwU238 $=238.0508 \mid 3.4>$

$\mathrm{c} \mathrm{U}=<$ atwU $=$ ena*atwU235+(1-ena)*atwU238|3.4>

c

c Molarity of solution components

c Mols of $\mathrm{U}$ per liter $<$ molU $=\mathrm{c} / \mathrm{atwU} \mid 3.4>$

c Mols of $\mathrm{H} 2 \mathrm{O}$ per liter $<\operatorname{molH} 2 \mathrm{O}=(1000 *$ denSol-molU* $*$ atwU $+2 * a+w N O 3+2 * a t w O)$ molAcid*atwHNO3)/atwH2O|3.4>

$\mathrm{c}$

c

$<$ perl $>$

sub IMP

\{

return 1 if $\left(\$ \_[0]>0\right)$;

return 0 ;

\}

$</$ perl $>$

c sphere

$2011<$-denSol|3.6> -1 imp:n=1

$212-1.000-21 \quad$ imp:n=<\&IMP(rw) $\mid 1>$ \$zero importance if no reflector

$220 \quad 2$ imp:n=0 


$$
\begin{aligned}
& 1 \quad \mathrm{cz}<\mathrm{rd} \mid 4.4>\quad \text { \$ Sphere } \\
& 2 \mathrm{cz}<\mathrm{rd}+\mathrm{rw} * \mathrm{in} \mid 4.4>\quad \text { \$Reflection }
\end{aligned}
$$

mode $\mathrm{n}$

kcode $20001.0100 \mathrm{ZZZ} 0$

$\begin{array}{llll}\text { ksrc } & 0.00 & 0.00 & 0.00\end{array}$

$\begin{array}{lll}0.00 & 0.00 & 0.10\end{array}$

$\begin{array}{lll}0.00 & 0.00 & 0.50\end{array}$

$\begin{array}{lll}0.00 & 0.00 & 1.00\end{array}$

$\begin{array}{lll}0.00 & 0.00 & 2.00\end{array}$

$\begin{array}{lll}0.00 & 0.00 & 3.00\end{array}$

$\begin{array}{lll}1.00 & 1.00 & 1.00\end{array}$

$\begin{array}{lll}1.00 & 1.00 & 2.00\end{array}$

$\begin{array}{lll}1.00 & 1.00 & 3.00\end{array}$

$\begin{array}{lll}2.00 & 2.00 & 1.00\end{array}$

$\begin{array}{lll}2.00 & 2.00 & 2.00\end{array}$

$\begin{array}{lll}2.00 & 2.00 & 3.00\end{array}$

$\begin{array}{lll}-1.00 & -1.00 & -1.00\end{array}$

$-10.00 \quad-10.00 \quad-20.00$

$\begin{array}{lll}-10.00 & -10.00 & -30.00\end{array}$

c

m2 \$water

$1001.66 \mathrm{c} \quad 2$

$8016.66 \mathrm{c} \quad 1$

$\mathrm{mt} 2$ 1wtr.60t

c UO2/water mixture

m11 \$ UO2(NO3)2 - NO3 - H2O, $100.0 \mathrm{wt} \% \mathrm{U} 235, \mathrm{ZZZ1} \mathrm{milli-g} \mathrm{U/cc,}<\mathrm{denSol} \mid 3.4>$

c molarity $\mathrm{U}=\langle\operatorname{molU}| 3.4>$, acid $=\langle\operatorname{mol} A$ cid $| 3.4>, \mathrm{H} 2 \mathrm{O}=\langle\operatorname{molH} 2 \mathrm{O} \mid 3.4\rangle$

c $\mathrm{U}$ in salt

$92235.66 \mathrm{c}<$ molU*ena $3.4>$

c $92238.66 \mathrm{c}<$ molU* $(1-$ ena $) \mid 3.4>$

c NO3 in salt plus acid

$7014.66 \mathrm{c}<2 * \mathrm{molU}+\operatorname{molAcid} \mid 3.4>$

8016.66c $<6 * \mathrm{molU}+3 * \operatorname{molAcid} \mid 3.4>$

c water

1001.66c $<2 *$ molH2O + molAcid $\mid 3.4>$ \$ncludes Free Hydrogen Ion

$8016.66 \mathrm{c}<\mathrm{molH} 2 \mathrm{O} \mid 3.4>$

mt11 1wtr.60t

c

c 
Template File for Case: u5o2N23-icyl-1000-01

icyl, $100.0 \mathrm{wt} \% \mathrm{UO} 2, \mathrm{ZZZ1}$ milli-g/cc, in UO2(NO3)2, with 3 Molar HNO3

c

c worm notes: format of the form |n.m for floating point, $\mid \mathrm{n}$ for integers

c zzz0 (in upper case) is replaced with the number of cyles

c $\quad$ zzz1 $=$ the actual concentration in units of milli-g/cc

c $\quad$ zzz2 $=$ solution density in $\mathrm{g} / \mathrm{cc}$

c $\quad \mathrm{qqq1}=$ the minimum dimensions (in hundreths of inches)

c $\quad \mathrm{qqq} 2=$ the maximum dimensions (in hundreths of inches)

c $\quad \mathrm{qqq} 3=$ the delta (in hundreths of inches)

c

c Diameter $=<\mathrm{d} 1=$ QQQ1:QQQ2:QQQ3 $\mid 5>$ hundreths of inches - must be integer

$\mathrm{c}$ radius $=<\mathrm{rd}=\mathrm{d} 1 * 2.54 / 100 / 2 \mid 5.4>\mathrm{cm}$

c note for perl, numbers with leading zeros must be enclosed in single quotes or else

c they will be interpretted as octal

$\mathrm{c}$ Conc $=<\mathrm{c}={ }^{\prime}$ ZZZ1' $\mid 5>$ milli-g U/cc

c Reflector width $=<\mathrm{rw}=1.0 \mid 2.1>$ inches

c Enrichment $=<$ ena $=1.0 \mid 1.2>$ atom fraction $\mathrm{U}$

c Mols of Acid per liter $<$ molAcid=3.0 $\mid 1.2>$

c

$\mathrm{c}$

c Density of solution $<$ denSol=ZZZ2/1e5 $\mid 1.5>$

c

c

c Atomic Weights

c $\mathrm{H} 2 \mathrm{O}=\langle$ atwH2O $=18.0152| 3.4>$

$\mathrm{c} \mathrm{NO3}=\langle$ atwNO3 $=62.0049| 3.4>$

c $\mathrm{O}=\langle$ atwO $=15.9994| 3.4>$

$\mathrm{c} \mathrm{HNO} 3=<$ atwHNO3 $=63.0128 \mid 3.4>$

c U235 $=<$ atwU235 $=235.0439 \mid 3.4>$

c U238 $=<$ atwU238 $=238.0508 \mid 3.4>$

$\mathrm{c} \mathrm{U}=<$ atwU $=$ ena*atwU235+(1-ena)*atwU238|3.4>

c

c Molarity of solution components

c Mols of $\mathrm{U}$ per liter $<$ molU $=\mathrm{c} / \mathrm{atwU} \mid 3.4>$

c Mols of $\mathrm{H} 2 \mathrm{O}$ per liter $<\operatorname{molH} 2 \mathrm{O}=(1000 *$ denSol-molU* $*$ atwU $+2 * a+w N O 3+2 * a t w O)$ molAcid*atwHNO3)/atwH2O|3.4>

$\mathrm{c}$

c

$<$ perl $>$

sub IMP

\{

return 1 if $\left(\$ \_[0]>0\right)$;

return 0 ;

\}

$</$ perl $>$

c sphere

$2011<$-denSol|3.6> -1 imp:n=1

$212-1.000-21 \quad$ imp:n=<\&IMP(rw) $\mid 1>$ \$zero importance if no reflector

$220 \quad 2$ imp:n=0 


$$
\begin{aligned}
& 1 \quad \mathrm{cz}<\mathrm{rd} \mid 4.4>\quad \text { \$ Sphere } \\
& 2 \mathrm{cz}<\mathrm{rd}+\mathrm{rw} * \mathrm{in} \mid 4.4>\quad \text { \$Reflection }
\end{aligned}
$$

mode $\mathrm{n}$

kcode $20001.0100 \mathrm{ZZZ} 0$

$\begin{array}{llll}\text { ksrc } & 0.00 & 0.00 & 0.00\end{array}$

$\begin{array}{lll}0.00 & 0.00 & 0.10\end{array}$

$\begin{array}{lll}0.00 & 0.00 & 0.50\end{array}$

$\begin{array}{lll}0.00 & 0.00 & 1.00\end{array}$

$\begin{array}{lll}0.00 & 0.00 & 2.00\end{array}$

$\begin{array}{lll}0.00 & 0.00 & 3.00\end{array}$

$\begin{array}{lll}1.00 & 1.00 & 1.00\end{array}$

$\begin{array}{lll}1.00 & 1.00 & 2.00\end{array}$

$\begin{array}{lll}1.00 & 1.00 & 3.00\end{array}$

$\begin{array}{lll}2.00 & 2.00 & 1.00\end{array}$

$\begin{array}{lll}2.00 & 2.00 & 2.00\end{array}$

$\begin{array}{lll}2.00 & 2.00 & 3.00\end{array}$

$\begin{array}{lll}-1.00 & -1.00 & -1.00\end{array}$

$-10.00 \quad-10.00 \quad-20.00$

$\begin{array}{lll}-10.00 & -10.00 & -30.00\end{array}$

c

m2 \$water

$1001.66 \mathrm{c} \quad 2$

$8016.66 \mathrm{c} \quad 1$

$\mathrm{mt} 2$ 1wtr.60t

c UO2/water mixture

m11 \$ UO2(NO3)2 - NO3 - H2O, $100.0 \mathrm{wt} \% \mathrm{U} 235, \mathrm{ZZZ1} \mathrm{milli-g} \mathrm{U/cc,}<\mathrm{denSol} \mid 3.4>$

c molarity $\mathrm{U}=\langle\operatorname{molU}| 3.4>$, acid $=\langle\operatorname{mol} A$ cid $| 3.4>, \mathrm{H} 2 \mathrm{O}=\langle\operatorname{molH} 2 \mathrm{O} \mid 3.4\rangle$

c $\mathrm{U}$ in salt

$92235.66 \mathrm{c}<$ molU*ena $3.4>$

c $92238.66 \mathrm{c}<$ molU* $* 1$-ena $) \mid 3.4>$

c NO3 in salt plus acid

$7014.66 \mathrm{c}<2 * \mathrm{molU}+\operatorname{molAcid} \mid 3.4>$

8016.66c $<6 * \mathrm{molU}+3 * \operatorname{molAcid} \mid 3.4>$

c water

1001.66c $<2 *$ molH2O + molAcid $\mid 3.4>$ \$ncludes Free Hydrogen Ion

$8016.66 \mathrm{c}<\mathrm{molH} 2 \mathrm{O} \mid 3.4>$

mt11 1wtr.60t

c

c 
Template File for Case: u5o2N23-icyl-1000-10

icyl, $100.0 \mathrm{wt} \% \mathrm{UO} 2, \mathrm{ZZZ1}$ milli-g/cc, in UO2(NO3)2, with 3 Molar HNO3

c

c worm notes: format of the form |n.m for floating point, $\mid \mathrm{n}$ for integers

c zzz0 (in upper case) is replaced with the number of cyles

c $\quad$ zzz1 $=$ the actual concentration in units of milli-g/cc

c $\quad$ zzz2 $=$ solution density in $\mathrm{g} / \mathrm{cc}$

c $\quad \mathrm{qqq1}=$ the minimum dimensions (in hundreths of inches)

c $\quad \mathrm{qqq} 2=$ the maximum dimensions (in hundreths of inches)

c $\quad \mathrm{qqq} 3=$ the delta (in hundreths of inches)

c

c Diameter $=<\mathrm{d} 1=$ QQQ1:QQQ2:QQQ3 $\mid 5>$ hundreths of inches - must be integer

$\mathrm{c}$ radius $=<\mathrm{rd}=\mathrm{d} 1 * 2.54 / 100 / 2 \mid 5.4>\mathrm{cm}$

c note for perl, numbers with leading zeros must be enclosed in single quotes or else

c they will be interpretted as octal

$\mathrm{c}$ Conc $=<\mathrm{c}={ }^{\prime}$ ZZZ1' $\mid 5>$ milli-g U/cc

c Reflector width $=<\mathrm{rw}=10.0 \mid 2.1>$ inches

c Enrichment $=<$ ena $=1.0 \mid 1.2>$ atom fraction $\mathrm{U}$

c Mols of Acid per liter $<$ molAcid $=3.0 \mid 1.2>$

c

$\mathrm{c}$

c Density of solution $<$ denSol=ZZZ2/1e5 $\mid 1.5>$

c

c

c Atomic Weights

c $\mathrm{H} 2 \mathrm{O}=\langle$ atwH2O $=18.0152| 3.4>$

$\mathrm{c} \mathrm{NO3}=\langle$ atwNO3 $=62.0049| 3.4>$

c $\mathrm{O}=\langle$ atwO $=15.9994| 3.4>$

$\mathrm{c} \mathrm{HNO} 3=<$ atwHNO3 $=63.0128 \mid 3.4>$

c U235 $=<$ atwU235 $=235.0439 \mid 3.4>$

c U238 $=<$ atwU238 $=238.0508 \mid 3.4>$

$\mathrm{c} \mathrm{U}=<$ atwU $=$ ena*atwU235+(1-ena)*atwU238|3.4>

c

c Molarity of solution components

c Mols of $\mathrm{U}$ per liter $<$ molU $=\mathrm{c} / \mathrm{atwU} \mid 3.4>$

c Mols of $\mathrm{H} 2 \mathrm{O}$ per liter $<\operatorname{molH} 2 \mathrm{O}=(1000 *$ denSol-molU* $*$ atwU $+2 * a+w N O 3+2 * a t w O)$ molAcid*atwHNO3)/atwH2O|3.4>

$\mathrm{c}$

c

$<$ perl $>$

sub IMP

\{

return 1 if $\left(\$ \_[0]>0\right)$;

return 0 ;

\}

$</$ perl $>$

c sphere

$2011<$-denSol|3.6> $-1 \quad$ imp:n=1

$212-1.000-21 \quad$ imp:n=<\&IMP(rw) $\mid 1>$ \$zero importance if no reflector

$220 \quad 2$ imp:n=0 


$$
\begin{aligned}
& 1 \quad \mathrm{cz}<\mathrm{rd} \mid 4.4>\quad \text { \$ Sphere } \\
& 2 \mathrm{cz}<\mathrm{rd}+\mathrm{rw} * \mathrm{in} \mid 4.4>\quad \text { \$Reflection }
\end{aligned}
$$

mode $\mathrm{n}$

kcode $20001.0100 \mathrm{ZZZ0}$

$\begin{array}{llll}\text { ksrc } & 0.00 & 0.00 & 0.00\end{array}$

$\begin{array}{lll}0.00 & 0.00 & 0.10\end{array}$

$\begin{array}{lll}0.00 & 0.00 & 0.50\end{array}$

$\begin{array}{lll}0.00 & 0.00 & 1.00\end{array}$

$\begin{array}{lll}0.00 & 0.00 & 2.00\end{array}$

$\begin{array}{lll}0.00 & 0.00 & 3.00\end{array}$

$\begin{array}{lll}1.00 & 1.00 & 1.00\end{array}$

$\begin{array}{lll}1.00 & 1.00 & 2.00\end{array}$

$\begin{array}{lll}1.00 & 1.00 & 3.00\end{array}$

$\begin{array}{lll}2.00 & 2.00 & 1.00\end{array}$

$\begin{array}{lll}2.00 & 2.00 & 2.00\end{array}$

$\begin{array}{lll}2.00 & 2.00 & 3.00\end{array}$

$\begin{array}{lll}-1.00 & -1.00 & -1.00\end{array}$

$-10.00 \quad-10.00 \quad-20.00$

$\begin{array}{lll}-10.00 & -10.00 & -30.00\end{array}$

c

m2 \$water

$1001.66 \mathrm{c} \quad 2$

$8016.66 \mathrm{c} \quad 1$

$\mathrm{mt} 2$ 1wtr.60t

c UO2/water mixture

m11 \$ UO2(NO3)2 - NO3 - H2O, $100.0 \mathrm{wt} \% \mathrm{U} 235, \mathrm{ZZZ1} \mathrm{milli-g} \mathrm{U/cc,}<\mathrm{denSol} \mid 3.4>$

c molarity $\mathrm{U}=\langle\operatorname{molU}| 3.4>$, acid $=\langle\operatorname{mol} A$ cid $| 3.4>, \mathrm{H} 2 \mathrm{O}=\langle\operatorname{molH} 2 \mathrm{O} \mid 3.4\rangle$

c $\mathrm{U}$ in salt

$92235.66 \mathrm{c}<$ molU*ena $3.4>$

c $92238.66 \mathrm{c}<$ molU* $* 1$-ena $) \mid 3.4>$

c NO3 in salt plus acid

$7014.66 \mathrm{c}<2 * \mathrm{molU}+\operatorname{molAcid} \mid 3.4>$

8016.66c $<6 * \mathrm{molU}+3 * \operatorname{molAcid} \mid 3.4>$

c water

1001.66c $<2 *$ molH2O + molAcid $\mid 3.4>$ \$ncludes Free Hydrogen Ion

$8016.66 \mathrm{c}<\mathrm{molH} 2 \mathrm{O} \mid 3.4>$

mt11 1wtr.60t

c

c 


\section{Template File for Case: u5o2N26-icyl-1000-00}

icyl, $100.0 \mathrm{wt} \% \mathrm{UO} 2, \mathrm{ZZZ1}$ milli-g/cc, in UO2(NO3)2, with 6 Molar HNO3

c

c worm notes: format of the form |n.m for floating point, $\mid \mathrm{n}$ for integers

c $\quad$ zzz0 (in upper case) is replaced with the number of cyles

c $\quad$ zzz1 $=$ the actual concentration in units of milli-g/cc

c $\quad$ zzz2 $=$ solution density in $\mathrm{g} / \mathrm{cc}$

c $\quad \mathrm{qqq} 1=$ the minimum dimensions (in hundreths of inches)

c $\quad \mathrm{qqq} 2=$ the maximum dimensions (in hundreths of inches)

c $\quad \mathrm{qqq} 3=$ the delta (in hundreths of inches)

c

c Diameter $=<\mathrm{d} 1=$ QQQ1:QQQ2:QQQ3 $\mid 5>$ hundreths of inches - must be integer

$\mathrm{c}$ radius $=<\mathrm{rd}=\mathrm{d} 1 * 2.54 / 100 / 2 \mid 5.4>\mathrm{cm}$

c note for perl, numbers with leading zeros must be enclosed in single quotes or else

c they will be interpretted as octal

$\mathrm{c}$ Conc $=<\mathrm{c}={ }^{\prime} \mathrm{ZZZ1} 1 \mid 5>$ milli-g U/cc

c Reflector width $=<\mathrm{rw}=0.0 \mid 2.1>$ inches

c Enrichment $=<$ ena $=1.0 \mid 1.2>$ atom fraction $\mathrm{U}$

c Mols of Acid per liter $<$ molAcid $=6.0 \mid 1.2>$

$\mathrm{c}$

$\mathrm{c}$

c Density of solution $<$ denSol $=Z Z Z 2 / 1$ e $5 \mid 1.5>$

c

c

c Atomic Weights

c $\mathrm{H} 2 \mathrm{O}=\langle$ atwH2O $=18.0152| 3.4>$

$\mathrm{c} \mathrm{NO3}=\langle$ atwNO3 $=62.0049| 3.4>$

c $\mathrm{O}=\langle$ atwO $=15.9994| 3.4>$

c HNO3 $=<$ atwHNO3 $=63.0128 \mid 3.4>$

c U235 $=<$ atwU235 $=235.0439 \mid 3.4>$

c U238 $=<$ atwU238 $=238.0508 \mid 3.4>$

c U $=<$ atwU $=$ ena*atwU235+(1-ena)*atwU238 $3.4>$

c

c Molarity of solution components

c Mols of $\mathrm{U}$ per liter $<\mathrm{molU}=\mathrm{c} / \mathrm{atwU} \mid 3.4>$

c Mols of $\mathrm{H} 2 \mathrm{O}$ per liter $<$ molH2O $=(1000 *$ denSol-molU* $(\operatorname{atwU}+2 *$ atwNO3+2*atwO $)-$ molAcid*atwHNO3)/atwH2O|3.4>

$\mathrm{c}$

c

$<$ perl $>$

sub IMP

\{

return 1 if $\left(\$ \_[0]>0\right)$;

return 0 ;

\}

$</$ perl $>$

c sphere

$2011<$-denSol|3.6> $-1 \quad$ imp:n=1

$212-1.000-21 \quad$ imp:n=<\&IMP(rw) $\mid 1>$ \$zero importance if no reflector

$220 \quad 2$ imp:n=0 


$$
\begin{aligned}
& 1 \quad \mathrm{cz}<\mathrm{rd} \mid 4.4>\quad \text { \$ Sphere } \\
& 2 \mathrm{cz}<\mathrm{rd}+\mathrm{rw} * \mathrm{in} \mid 4.4>\quad \text { \$Reflection }
\end{aligned}
$$

mode $\mathrm{n}$

kcode $20001.0100 \mathrm{ZZZ0}$

$\begin{array}{llll}\text { ksrc } & 0.00 & 0.00 & 0.00\end{array}$

$\begin{array}{lll}0.00 & 0.00 & 0.10\end{array}$

$\begin{array}{lll}0.00 & 0.00 & 0.50\end{array}$

$\begin{array}{lll}0.00 & 0.00 & 1.00\end{array}$

$\begin{array}{lll}0.00 & 0.00 & 2.00\end{array}$

$\begin{array}{lll}0.00 & 0.00 & 3.00\end{array}$

$\begin{array}{lll}1.00 & 1.00 & 1.00\end{array}$

$\begin{array}{lll}1.00 & 1.00 & 2.00\end{array}$

$\begin{array}{lll}1.00 & 1.00 & 3.00\end{array}$

$\begin{array}{lll}2.00 & 2.00 & 1.00\end{array}$

$\begin{array}{lll}2.00 & 2.00 & 2.00\end{array}$

$\begin{array}{lll}2.00 & 2.00 & 3.00\end{array}$

$\begin{array}{lll}-1.00 & -1.00 & -1.00\end{array}$

$-10.00 \quad-10.00 \quad-20.00$

$\begin{array}{lll}-10.00 & -10.00 & -30.00\end{array}$

c

m2 \$water

$1001.66 \mathrm{c} \quad 2$

$8016.66 \mathrm{c} \quad 1$

$\mathrm{mt} 2$ 1wtr.60t

c UO2/water mixture

m11 \$ UO2(NO3)2 - NO3 - H2O, $100.0 \mathrm{wt} \% \mathrm{U} 235, \mathrm{ZZZ1} \mathrm{milli-g} \mathrm{U/cc,}<\mathrm{denSol} \mid 3.4>$

c molarity $\mathrm{U}=\langle\operatorname{molU}| 3.4>$, acid $=\langle\operatorname{mol} A$ cid $| 3.4>, \mathrm{H} 2 \mathrm{O}=\langle\operatorname{molH} 2 \mathrm{O} \mid 3.4\rangle$

c $\mathrm{U}$ in salt

$92235.66 \mathrm{c}<$ molU*ena $3.4>$

c $92238.66 \mathrm{c}<$ molU* $(1-$ ena $) \mid 3.4>$

c NO3 in salt plus acid

$7014.66 \mathrm{c}<2 * \mathrm{molU}+\operatorname{molAcid} \mid 3.4>$

8016.66c $<6 * \mathrm{molU}+3 * \operatorname{molAcid} \mid 3.4>$

c water

1001.66c $<2 *$ molH2O + molAcid $\mid 3.4>$ \$ncludes Free Hydrogen Ion

$8016.66 \mathrm{c}<\mathrm{molH} 2 \mathrm{O} \mid 3.4>$

mt11 1wtr.60t

c

c 
Template File for Case: u5o2N26-icyl-1000-01

icyl, $100.0 \mathrm{wt} \% \mathrm{UO} 2, \mathrm{ZZZ1}$ milli-g/cc, in UO2(NO3)2, with 6 Molar HNO3

c

c worm notes: format of the form |n.m for floating point, $\mid \mathrm{n}$ for integers

c zzz0 (in upper case) is replaced with the number of cyles

c $\quad$ zzz1 $=$ the actual concentration in units of milli-g/cc

c $\quad$ zzz2 $=$ solution density in $\mathrm{g} / \mathrm{cc}$

c $\quad \mathrm{qqq1}=$ the minimum dimensions (in hundreths of inches)

c $\quad \mathrm{qqq} 2=$ the maximum dimensions (in hundreths of inches)

c $\quad \mathrm{qqq} 3=$ the delta (in hundreths of inches)

c

$\mathrm{c}$ Diameter $=<\mathrm{d} 1=$ QQQ1:QQQ2:QQQ3 $\mid 5>$ hundreths of inches - must be integer

$\mathrm{c}$ radius $=<\mathrm{rd}=\mathrm{d} 1 * 2.54 / 100 / 2 \mid 5.4>\mathrm{cm}$

c note for perl, numbers with leading zeros must be enclosed in single quotes or else

c they will be interpretted as octal

$\mathrm{c}$ Conc $=<\mathrm{c}={ }^{\prime}$ ZZZ1' $\mid 5>$ milli-g U/cc

c Reflector width $=<\mathrm{rw}=1.0 \mid 2.1>$ inches

c Enrichment $=<$ ena $=1.0 \mid 1.2>$ atom fraction $\mathrm{U}$

c Mols of Acid per liter $<$ molAcid $=6.0 \mid 1.2>$

c

$\mathrm{c}$

c Density of solution $<$ denSol=ZZZ2/1e5 $\mid 1.5>$

c

C

c Atomic Weights

c $\mathrm{H} 2 \mathrm{O}=\langle$ atwH2O $=18.0152| 3.4>$

c NO3 $=<$ atwNO3 $=62.0049 \mid 3.4>$

c $\mathrm{O}=\langle$ atwO $=15.9994| 3.4>$

$\mathrm{c} \mathrm{HNO} 3=<$ atwHNO3 $=63.0128 \mid 3.4>$

c U235 $=<$ atwU235 $=235.0439 \mid 3.4>$

c U238 $=<$ atwU238 $=238.0508 \mid 3.4>$

$\mathrm{c} \mathrm{U}=<$ atwU $=$ ena*atwU235+(1-ena)*atwU238|3.4>

c

c Molarity of solution components

c Mols of $\mathrm{U}$ per liter $<$ molU $=\mathrm{c} / \mathrm{atwU} \mid 3.4>$

c Mols of $\mathrm{H} 2 \mathrm{O}$ per liter $<\operatorname{molH} 2 \mathrm{O}=(1000 *$ denSol-molU* $*$ atwU $+2 * a+w N O 3+2 * a t w O)$ molAcid*atwHNO3)/atwH2O|3.4>

$\mathrm{c}$

c

$<$ perl $>$

sub IMP

\{

return 1 if $\left(\$ \_[0]>0\right)$;

return 0 ;

\}

$</$ perl $>$

c sphere

$2011<$-denSol|3.6> $-1 \quad$ imp:n=1

$212-1.000-21 \quad$ imp:n=<\&IMP(rw) $\mid 1>$ \$zero importance if no reflector

$220 \quad 2$ imp:n=0 


$$
\begin{aligned}
& 1 \quad \mathrm{cz}<\mathrm{rd} \mid 4.4>\quad \text { \$ Sphere } \\
& 2 \mathrm{cz}<\mathrm{rd}+\mathrm{rw} * \mathrm{in} \mid 4.4>\quad \text { \$Reflection }
\end{aligned}
$$

mode $\mathrm{n}$

kcode $20001.0100 \mathrm{ZZZ} 0$

$\begin{array}{llll}\text { ksrc } & 0.00 & 0.00 & 0.00\end{array}$

$\begin{array}{lll}0.00 & 0.00 & 0.10\end{array}$

$\begin{array}{lll}0.00 & 0.00 & 0.50\end{array}$

$\begin{array}{lll}0.00 & 0.00 & 1.00\end{array}$

$\begin{array}{lll}0.00 & 0.00 & 2.00\end{array}$

$\begin{array}{lll}0.00 & 0.00 & 3.00\end{array}$

$\begin{array}{lll}1.00 & 1.00 & 1.00\end{array}$

$\begin{array}{lll}1.00 & 1.00 & 2.00\end{array}$

$\begin{array}{lll}1.00 & 1.00 & 3.00\end{array}$

$\begin{array}{lll}2.00 & 2.00 & 1.00\end{array}$

$\begin{array}{lll}2.00 & 2.00 & 2.00\end{array}$

$\begin{array}{lll}2.00 & 2.00 & 3.00\end{array}$

$\begin{array}{lll}-1.00 & -1.00 & -1.00\end{array}$

$-10.00 \quad-10.00 \quad-20.00$

$\begin{array}{lll}-10.00 & -10.00 & -30.00\end{array}$

c

m2 \$water

$1001.66 \mathrm{c} \quad 2$

$8016.66 \mathrm{c} \quad 1$

$\mathrm{mt} 2$ 1wtr.60t

c UO2/water mixture

m11 \$ UO2(NO3)2 - NO3 - H2O, $100.0 \mathrm{wt} \% \mathrm{U} 235, \mathrm{ZZZ1} \mathrm{milli-g} \mathrm{U/cc,}<\mathrm{denSol} \mid 3.4>$

c molarity $\mathrm{U}=\langle\operatorname{molU}| 3.4>$, acid $=\langle\operatorname{mol} A$ cid $| 3.4>, \mathrm{H} 2 \mathrm{O}=\langle\operatorname{molH} 2 \mathrm{O} \mid 3.4\rangle$

c $\mathrm{U}$ in salt

$92235.66 \mathrm{c}<$ molU*ena $3.4>$

c $92238.66 \mathrm{c}<$ molU* $(1-$ ena $) \mid 3.4>$

c NO3 in salt plus acid

$7014.66 \mathrm{c}<2 * \mathrm{molU}+\operatorname{molAcid} \mid 3.4>$

8016.66c $<6 * \mathrm{molU}+3 * \operatorname{molAcid} \mid 3.4>$

c water

1001.66c $<2 *$ molH2O + molAcid $\mid 3.4>$ \$ncludes Free Hydrogen Ion

$8016.66 \mathrm{c}<\mathrm{molH} 2 \mathrm{O} \mid 3.4>$

mt11 1wtr.60t

c

$\mathrm{c}$ 
Template File for Case: u5o2N26-icyl-1000-10

icyl, $100.0 \mathrm{wt} \% \mathrm{UO} 2, \mathrm{ZZZ1}$ milli-g/cc, in UO2(NO3)2, with 6 Molar HNO3

c

c worm notes: format of the form |n.m for floating point, $\mid \mathrm{n}$ for integers

c zzz0 (in upper case) is replaced with the number of cyles

c $\quad$ zzz1 $=$ the actual concentration in units of milli-g/cc

c $\quad$ zzz2 $=$ solution density in $\mathrm{g} / \mathrm{cc}$

c $\quad \mathrm{qqq1}=$ the minimum dimensions (in hundreths of inches)

c $\quad \mathrm{qqq} 2=$ the maximum dimensions (in hundreths of inches)

c $\quad \mathrm{qqq} 3=$ the delta (in hundreths of inches)

c

c Diameter $=<\mathrm{d} 1=$ QQQ1:QQQ2:QQQ3 $\mid 5>$ hundreths of inches - must be integer

$\mathrm{c}$ radius $=<\mathrm{rd}=\mathrm{d} 1 * 2.54 / 100 / 2 \mid 5.4>\mathrm{cm}$

c note for perl, numbers with leading zeros must be enclosed in single quotes or else

c they will be interpretted as octal

$\mathrm{c}$ Conc $=<\mathrm{c}={ }^{\prime}$ ZZZ1' $\mid 5>$ milli-g U/cc

c Reflector width $=<\mathrm{rw}=10.0 \mid 2.1>$ inches

c Enrichment $=<$ ena $=1.0 \mid 1.2>$ atom fraction $\mathrm{U}$

c Mols of Acid per liter $<$ molAcid $=6.0 \mid 1.2>$

c

$\mathrm{c}$

c Density of solution $<$ denSol=ZZZ2/1e5 $\mid 1.5>$

c

c

c Atomic Weights

c $\mathrm{H} 2 \mathrm{O}=\langle$ atwH2O $=18.0152| 3.4>$

c NO3 $=<$ atwNO3 $=62.0049 \mid 3.4>$

c $\mathrm{O}=\langle$ atwO $=15.9994| 3.4>$

$\mathrm{c} \mathrm{HNO} 3=<$ atwHNO3 $=63.0128 \mid 3.4>$

c U235 $=<$ atwU235 $=235.0439 \mid 3.4>$

c U238 $=<$ atwU238 $=238.0508 \mid 3.4>$

$\mathrm{c} \mathrm{U}=<$ atwU $=$ ena*atwU235+(1-ena)*atwU238|3.4>

c

c Molarity of solution components

c Mols of $\mathrm{U}$ per liter $<$ molU $=\mathrm{c} / \mathrm{atwU} \mid 3.4>$

c Mols of $\mathrm{H} 2 \mathrm{O}$ per liter $<\operatorname{molH} 2 \mathrm{O}=(1000 *$ denSol-molU* $*$ atwU $+2 * a+w N O 3+2 * a t w O)$ molAcid*atwHNO3)/atwH2O|3.4>

$\mathrm{c}$

c

$<$ perl $>$

sub IMP

\{

return 1 if $\left(\$ \_[0]>0\right)$;

return 0 ;

\}

$</$ perl $>$

c sphere

$2011<$-denSol|3.6> -1 imp:n=1

$212-1.000-21 \quad$ imp:n=<\&IMP(rw) $\mid 1>$ \$zero importance if no reflector

$220 \quad 2$ imp:n=0 


$$
\begin{aligned}
& 1 \quad \mathrm{cz}<\mathrm{rd} \mid 4.4>\quad \text { \$ Sphere } \\
& 2 \mathrm{cz}<\mathrm{rd}+\mathrm{rw} * \mathrm{in} \mid 4.4>\quad \text { \$Reflection }
\end{aligned}
$$

mode $\mathrm{n}$

kcode $20001.0100 \mathrm{ZZZ} 0$

$\begin{array}{llll}\text { ksrc } & 0.00 & 0.00 & 0.00\end{array}$

$\begin{array}{lll}0.00 & 0.00 & 0.10\end{array}$

$\begin{array}{lll}0.00 & 0.00 & 0.50\end{array}$

$\begin{array}{lll}0.00 & 0.00 & 1.00\end{array}$

$\begin{array}{lll}0.00 & 0.00 & 2.00\end{array}$

$\begin{array}{lll}0.00 & 0.00 & 3.00\end{array}$

$\begin{array}{lll}1.00 & 1.00 & 1.00\end{array}$

$\begin{array}{lll}1.00 & 1.00 & 2.00\end{array}$

$\begin{array}{lll}1.00 & 1.00 & 3.00\end{array}$

$\begin{array}{lll}2.00 & 2.00 & 1.00\end{array}$

$\begin{array}{lll}2.00 & 2.00 & 2.00\end{array}$

$\begin{array}{lll}2.00 & 2.00 & 3.00\end{array}$

$\begin{array}{lll}-1.00 & -1.00 & -1.00\end{array}$

$-10.00 \quad-10.00 \quad-20.00$

$\begin{array}{lll}-10.00 & -10.00 & -30.00\end{array}$

c

m2 \$water

$1001.66 \mathrm{c} \quad 2$

$8016.66 \mathrm{c} \quad 1$

$\mathrm{mt} 2$ 1wtr.60t

c UO2/water mixture

m11 \$ UO2(NO3)2 - NO3 - H2O, $100.0 \mathrm{wt} \% \mathrm{U} 235, \mathrm{ZZZ1} \mathrm{milli-g} \mathrm{U/cc,}<\mathrm{denSol} \mid 3.4>$

c molarity $\mathrm{U}=\langle\operatorname{molU}| 3.4>$, acid $=\langle\operatorname{mol} A$ cid $| 3.4>, \mathrm{H} 2 \mathrm{O}=\langle\operatorname{molH} 2 \mathrm{O} \mid 3.4\rangle$

c $\mathrm{U}$ in salt

$92235.66 \mathrm{c}<$ molU*ena $3.4>$

c $92238.66 \mathrm{c}<$ molU* $(1-$ ena $) \mid 3.4>$

c NO3 in salt plus acid

$7014.66 \mathrm{c}<2 * \mathrm{molU}+\operatorname{molAcid} \mid 3.4>$

8016.66c $<6 * \mathrm{molU}+3 * \operatorname{molAcid} \mid 3.4>$

c water

1001.66c $<2 *$ molH2O + molAcid $\mid 3.4>$ \$ncludes Free Hydrogen Ion

$8016.66 \mathrm{c}<\mathrm{molH} 2 \mathrm{O} \mid 3.4>$

mt11 1wtr.60t

c

c 
Template File for Case: u5o2N20-icyl-0935-00

icyl, 93.5 wt\% UO2, ZZZ1 milli-g/cc, in UO2(NO3)2, with 0 Molar HNO3

c

c worm notes: format of the form |n.m for floating point, $\mid \mathrm{n}$ for integers

c zzz0 (in upper case) is replaced with the number of cyles

c $\quad$ zzz1 $=$ the actual concentration in units of milli-g/cc

c $\quad$ zzz2 $=$ solution density in $\mathrm{g} / \mathrm{cc}$

c $\quad \mathrm{qqq1}=$ the minimum dimensions (in hundreths of inches)

c $\quad \mathrm{qqq} 2=$ the maximum dimensions (in hundreths of inches)

c $\quad \mathrm{qqq} 3=$ the delta (in hundreths of inches)

c

c Diameter $=<\mathrm{d} 1=$ QQQ1:QQQ2:QQQ3 $\mid 5>$ hundreths of inches - must be integer

$\mathrm{c}$ radius $=<\mathrm{rd}=\mathrm{d} 1 * 2.54 / 100 / 2 \mid 5.4>\mathrm{cm}$

c note for perl, numbers with leading zeros must be enclosed in single quotes or else

c they will be interpretted as octal

$\mathrm{c}$ Conc $=<\mathrm{c}={ }^{\prime} \mathrm{ZZZ1} 1 \mid 5>$ milli-g U/cc

c Reflector width $=<\mathrm{rw}=0.0 \mid 2.1>$ inches

c Enrichment $=<$ ena $=0.935 \mid 1.3>$ atom fraction $\mathrm{U}$

c Mols of Acid per liter $<$ molAcid $=0.0 \mid 1.2>$

$\mathrm{c}$

$\mathrm{c}$

c Density of solution $<$ denSol $=Z Z Z 2 / 1 \mathrm{e} 5 \mid 1.5>$

c

c

c Atomic Weights

c $\mathrm{H} 2 \mathrm{O}=\langle$ atwH2O $=18.0152| 3.4>$

$\mathrm{c} \mathrm{NO3}=\langle$ atwNO3 $=62.0049| 3.4>$

c $\mathrm{O}=\langle$ atwO $=15.9994| 3.4>$

$\mathrm{c} \mathrm{HNO} 3=<$ atwHNO3 $=63.0128 \mid 3.4>$

c U235 $=<$ atwU235 $=235.0439 \mid 3.4>$

c U238 $=<$ atwU238 $=238.0508 \mid 3.4>$

c U $=<$ atwU $=$ ena*atwU235+(1-ena)*atwU238 $3.4>$

c

c Molarity of solution components

c Mols of $\mathrm{U}$ per liter $<$ molU $=\mathrm{c} / \mathrm{atwU} \mid 3.4>$

c Mols of $\mathrm{H} 2 \mathrm{O}$ per liter $<\operatorname{molH} 2 \mathrm{O}=(1000 *$ denSol-molU* $*$ atwU $+2 * a+w N O 3+2 * a t w O)$ molAcid*atwHNO3)/atwH2O|3.4>

$\mathrm{c}$

c

$<$ perl $>$

sub IMP

\{

return 1 if $\left(\$ \_[0]>0\right)$;

return 0 ;

\}

$</$ perl $>$

c Cylinder

$2011<$-denSol|3.6> $-1 \quad$ imp:n=1

$212-1.000-21 \quad$ imp:n=<\&IMP(rw) $\mid 1>$ \$zero importance if no reflector

$220 \quad 2$ imp:n=0 


$$
\begin{aligned}
& 1 \quad \mathrm{cz}<\mathrm{rd} \mid 4.4>\quad \text { \$ Sphere } \\
& 2 \mathrm{cz}<\mathrm{rd}+\mathrm{rw} * \mathrm{in} \mid 4.4>\quad \text { \$Reflection }
\end{aligned}
$$

mode $\mathrm{n}$

kcode $20001.0100 \mathrm{ZZZ0}$

$\begin{array}{llll}\text { ksrc } & 0.00 & 0.00 & 0.00\end{array}$

$\begin{array}{lll}0.00 & 0.00 & 0.10\end{array}$

$\begin{array}{lll}0.00 & 0.00 & 0.50\end{array}$

$\begin{array}{lll}0.00 & 0.00 & 1.00\end{array}$

$\begin{array}{lll}0.00 & 0.00 & 2.00\end{array}$

$\begin{array}{lll}0.00 & 0.00 & 3.00\end{array}$

$\begin{array}{lll}1.00 & 1.00 & 1.00\end{array}$

$\begin{array}{lll}1.00 & 1.00 & 2.00\end{array}$

$\begin{array}{lll}1.00 & 1.00 & 3.00\end{array}$

$\begin{array}{lll}2.00 & 2.00 & 1.00\end{array}$

$\begin{array}{lll}2.00 & 2.00 & 2.00\end{array}$

$\begin{array}{lll}2.00 & 2.00 & 3.00\end{array}$

$\begin{array}{lll}-1.00 & -1.00 & -1.00\end{array}$

$-10.00 \quad-10.00 \quad-20.00$

$\begin{array}{lll}-10.00 & -10.00 & -30.00\end{array}$

c

m2 \$water

$1001.66 \mathrm{c} \quad 2$

$8016.66 \mathrm{c} \quad 1$

$\mathrm{mt} 2$ 1wtr.60t

c UO2/water mixture

m11 \$ UO2(NO3)2 - NO3 - H2O, $100.0 \mathrm{wt} \% \mathrm{U} 235, \mathrm{ZZZ1} \mathrm{milli-g} \mathrm{U/cc,}<\mathrm{denSol} \mid 3.4>$

c molarity $\mathrm{U}=\langle\operatorname{molU}| 3.4>$, acid $=\langle\operatorname{mol} A$ cid $| 3.4>, \mathrm{H} 2 \mathrm{O}=\langle\operatorname{molH} 2 \mathrm{O} \mid 3.4\rangle$

c $\mathrm{U}$ in salt

$92235.66 \mathrm{c}<$ molU*ena $3.4>$

$92238.66 \mathrm{c}<$ molU* $* 1$-ena $) \mid 3.4>$

c NO3 in salt plus acid

$7014.66 \mathrm{c}<2 * \mathrm{molU}+\operatorname{molAcid} \mid 3.4>$

$8016.66 \mathrm{c}<6 * \mathrm{molU}+3 * \operatorname{mol} \mathrm{Acid} \mid 3.4>$

c water

1001.66c $<2 *$ molH2O + molAcid $\mid 3.4>$ \$ncludes Free Hydrogen Ion

$8016.66 \mathrm{c}<\mathrm{molH} 2 \mathrm{O} \mid 3.4>$

mt11 1wtr.60t

c

c 
Template File for Case: u5o2N20-icyl-0935-01

icyl, 93.5 wt\% UO2, ZZZ1 milli-g/cc, in UO2(NO3)2, with 0 Molar HNO3

c

c worm notes: format of the form |n.m for floating point, $\mid \mathrm{n}$ for integers

c zzz0 (in upper case) is replaced with the number of cyles

c $\quad$ zzz1 $=$ the actual concentration in units of milli-g/cc

c $\quad$ zzz2 $=$ solution density in $\mathrm{g} / \mathrm{cc}$

c $\quad \mathrm{qqq1}=$ the minimum dimensions (in hundreths of inches)

c $\quad \mathrm{qqq} 2=$ the maximum dimensions (in hundreths of inches)

c $\quad \mathrm{qqq} 3=$ the delta (in hundreths of inches)

c

c Diameter $=<\mathrm{d} 1=$ QQQ1:QQQ2:QQQ3 $\mid 5>$ hundreths of inches - must be integer

$\mathrm{c}$ radius $=<\mathrm{rd}=\mathrm{d} 1 * 2.54 / 100 / 2 \mid 5.4>\mathrm{cm}$

c note for perl, numbers with leading zeros must be enclosed in single quotes or else

c they will be interpretted as octal

$\mathrm{c}$ Conc $=<\mathrm{c}={ }^{\prime}$ ZZZ1' $\mid 5>$ milli-g U/cc

c Reflector width $=<\mathrm{rw}=1.0 \mid 2.1>$ inches

c Enrichment $=<$ ena $=0.935 \mid 1.3>$ atom fraction $\mathrm{U}$

c Mols of Acid per liter $<$ molAcid $=0.0 \mid 1.2>$

c

$\mathrm{c}$

c Density of solution $<$ denSol=ZZZ2/1e5 $\mid 1.5>$

c

C

c Atomic Weights

c $\mathrm{H} 2 \mathrm{O}=\langle$ atwH2O $=18.0152| 3.4>$

c NO3 $=<$ atwNO3 $=62.0049 \mid 3.4>$

c $\mathrm{O}=\langle$ atwO $=15.9994| 3.4>$

$\mathrm{c} \mathrm{HNO} 3=<$ atwHNO3 $=63.0128 \mid 3.4>$

c U235 $=<$ atwU235 $=235.0439 \mid 3.4>$

c U238 $=<$ atwU238 $=238.0508 \mid 3.4>$

$\mathrm{c} \mathrm{U}=<$ atwU $=$ ena*atwU235+(1-ena)*atwU238|3.4>

c

c Molarity of solution components

c Mols of $\mathrm{U}$ per liter $<$ molU $=\mathrm{c} / \mathrm{atwU} \mid 3.4>$

c Mols of $\mathrm{H} 2 \mathrm{O}$ per liter $<\operatorname{molH} 2 \mathrm{O}=(1000 *$ denSol-molU* $*$ atwU $+2 * a+w N O 3+2 * a t w O)$ molAcid*atwHNO3)/atwH2O|3.4>

$\mathrm{c}$

c

$<$ perl $>$

sub IMP

\{

return 1 if $\left(\$ \_[0]>0\right)$;

return 0 ;

\}

$</$ perl $>$

c Cylinder

$2011<$-denSol|3.6> -1 imp:n=1

$212-1.000-21 \quad$ imp:n=<\&IMP(rw) $\mid 1>$ \$zero importance if no reflector

$220 \quad 2$ imp:n=0 


$$
\begin{aligned}
& 1 \quad \mathrm{cz}<\mathrm{rd} \mid 4.4>\quad \text { \$ Sphere } \\
& 2 \mathrm{cz}<\mathrm{rd}+\mathrm{rw} * \mathrm{in} \mid 4.4>\quad \text { \$Reflection }
\end{aligned}
$$

mode $\mathrm{n}$

kcode $20001.0100 \mathrm{ZZZ0}$

$\begin{array}{llll}\text { ksrc } & 0.00 & 0.00 & 0.00\end{array}$

$\begin{array}{lll}0.00 & 0.00 & 0.10\end{array}$

$\begin{array}{lll}0.00 & 0.00 & 0.50\end{array}$

$\begin{array}{lll}0.00 & 0.00 & 1.00\end{array}$

$\begin{array}{lll}0.00 & 0.00 & 2.00\end{array}$

$\begin{array}{lll}0.00 & 0.00 & 3.00\end{array}$

$\begin{array}{lll}1.00 & 1.00 & 1.00\end{array}$

$\begin{array}{lll}1.00 & 1.00 & 2.00\end{array}$

$\begin{array}{lll}1.00 & 1.00 & 3.00\end{array}$

$\begin{array}{lll}2.00 & 2.00 & 1.00\end{array}$

$\begin{array}{lll}2.00 & 2.00 & 2.00\end{array}$

$\begin{array}{lll}2.00 & 2.00 & 3.00\end{array}$

$\begin{array}{lll}-1.00 & -1.00 & -1.00\end{array}$

$-10.00 \quad-10.00 \quad-20.00$

$\begin{array}{lll}-10.00 & -10.00 & -30.00\end{array}$

c

m2 \$water

$1001.66 \mathrm{c} \quad 2$

$8016.66 \mathrm{c} \quad 1$

$\mathrm{mt} 2$ 1wtr.60t

c UO2/water mixture

m11 \$ UO2(NO3)2 - NO3 - H2O, $100.0 \mathrm{wt} \% \mathrm{U} 235, \mathrm{ZZZ1} \mathrm{milli-g} \mathrm{U/cc,}<\mathrm{denSol} \mid 3.4>$

c molarity $\mathrm{U}=\langle\operatorname{molU}| 3.4>$, acid $=\langle\operatorname{mol} A$ cid $| 3.4>, \mathrm{H} 2 \mathrm{O}=\langle\operatorname{molH} 2 \mathrm{O} \mid 3.4\rangle$

c $\mathrm{U}$ in salt

$92235.66 \mathrm{c}<$ molU*ena $3.4>$

$92238.66 \mathrm{c}<$ molU* $* 1$-ena $) \mid 3.4>$

c NO3 in salt plus acid

$7014.66 \mathrm{c}<2 * \mathrm{molU}+\operatorname{molAcid} \mid 3.4>$

$8016.66 \mathrm{c}<6 * \mathrm{molU}+3 * \operatorname{mol} \mathrm{Acid} \mid 3.4>$

c water

1001.66c $<2 *$ molH2O + molAcid $\mid 3.4>$ \$ncludes Free Hydrogen Ion

$8016.66 \mathrm{c}<\mathrm{molH} 2 \mathrm{O} \mid 3.4>$

mt11 1wtr.60t

c

$\mathrm{c}$ 


\section{Template File for Case: u5o2N20-icyl-0935-10}

icyl, 93.5 wt\% UO2, ZZZ1 milli-g/cc, in UO2(NO3)2, with 0 Molar HNO3

c

c worm notes: format of the form |n.m for floating point, $\mid \mathrm{n}$ for integers

c $\quad$ zzz0 (in upper case) is replaced with the number of cyles

c $\quad$ zzz1 $=$ the actual concentration in units of milli-g/cc

c $\quad$ zzz2 $=$ solution density in $\mathrm{g} / \mathrm{cc}$

c $\quad \mathrm{qqq} 1=$ the minimum dimensions (in hundreths of inches)

c $\quad \mathrm{qqq} 2$ = the maximum dimensions (in hundreths of inches)

c $\quad \mathrm{qqq} 3=$ the delta (in hundreths of inches)

$\mathrm{c}$

c Diameter $=<\mathrm{d} 1=$ QQQ1:QQQ2:QQQ3 $\mid 5>$ hundreths of inches - must be integer

$\mathrm{c}$ radius $=<\mathrm{rd}=\mathrm{d} 1 * 2.54 / 100 / 2 \mid 5.4>\mathrm{cm}$

c note for perl, numbers with leading zeros must be enclosed in single quotes or else

c they will be interpretted as octal

$\mathrm{c}$ Conc $=<\mathrm{c}={ }^{\prime}$ ZZZ1' $\mid 5>$ milli-g U/cc

c Reflector width $=\langle\mathrm{rw}=10.0| 2.1>$ inches

c Enrichment $=<$ ena $=0.935 \mid 1.3>$ atom fraction $\mathrm{U}$

c Mols of Acid per liter $<$ molAcid $=0.0 \mid 1.2>$

$\mathrm{c}$

$\mathrm{c}$

c Density of solution $<$ denSol $=Z Z Z 2 / 1$ e $5 \mid 1.5>$

c

c

c Atomic Weights

c $\mathrm{H} 2 \mathrm{O}=\langle$ atwH2O $=18.0152 \mid 3.4\rangle$

c NO3 $=<$ atwNO3 $=62.0049 \mid 3.4>$

c $\mathrm{O}=\langle$ atwO $=15.9994| 3.4>$

c HNO3 $=<$ atwHNO3 $=63.0128 \mid 3.4>$

c U235 $=<$ atwU235 $=235.0439 \mid 3.4>$

c U238 $=<$ atwU238 $=238.0508 \mid 3.4>$

c U $=<$ atwU $=$ ena*atwU235+(1-ena)*atwU238 $3.4>$

c

c Molarity of solution components

c Mols of $\mathrm{U}$ per liter $<$ molU $=\mathrm{c} / \mathrm{atwU} \mid 3.4>$

c Mols of $\mathrm{H} 2 \mathrm{O}$ per liter $<$ molH2O $=(1000 *$ denSol-molU* $(\operatorname{atwU}+2 *$ atwNO3+2*atwO $)-$ molAcid*atwHNO3)/atwH2O|3.4>

$\mathrm{c}$

c

$<$ perl $>$

sub IMP

\{

return 1 if $\left(\$ \_[0]>0\right)$;

return 0 ;

\}

$</$ perl $>$

c Cylinder

$2011<$-denSol|3.6> $-1 \quad$ imp:n=1

$212-1.000-21 \quad$ imp:n=<\&IMP(rw) $\mid 1>$ \$zero importance if no reflector

$220 \quad 2 \quad$ imp:n=0 


$$
\begin{aligned}
& 1 \quad \mathrm{cz}<\mathrm{rd} \mid 4.4>\quad \text { \$ Sphere } \\
& 2 \mathrm{cz}<\mathrm{rd}+\mathrm{rw} * \mathrm{in} \mid 4.4>\quad \text { \$Reflection }
\end{aligned}
$$

mode $\mathrm{n}$

kcode $20001.0100 \mathrm{ZZZ} 0$

$\begin{array}{llll}\text { ksrc } & 0.00 & 0.00 & 0.00\end{array}$

$\begin{array}{lll}0.00 & 0.00 & 0.10\end{array}$

$\begin{array}{lll}0.00 & 0.00 & 0.50\end{array}$

$\begin{array}{lll}0.00 & 0.00 & 1.00\end{array}$

$\begin{array}{lll}0.00 & 0.00 & 2.00\end{array}$

$\begin{array}{lll}0.00 & 0.00 & 3.00\end{array}$

$\begin{array}{lll}1.00 & 1.00 & 1.00\end{array}$

$\begin{array}{lll}1.00 & 1.00 & 2.00\end{array}$

$\begin{array}{lll}1.00 & 1.00 & 3.00\end{array}$

$\begin{array}{lll}2.00 & 2.00 & 1.00\end{array}$

$\begin{array}{lll}2.00 & 2.00 & 2.00\end{array}$

$\begin{array}{lll}2.00 & 2.00 & 3.00\end{array}$

$\begin{array}{lll}-1.00 & -1.00 & -1.00\end{array}$

$-10.00 \quad-10.00 \quad-20.00$

$\begin{array}{lll}-10.00 & -10.00 & -30.00\end{array}$

c

m2 \$water

$1001.66 \mathrm{c} \quad 2$

$8016.66 \mathrm{c} \quad 1$

$\mathrm{mt} 2$ 1wtr.60t

c UO2/water mixture

m11 \$ UO2(NO3)2 - NO3 - H2O, $100.0 \mathrm{wt} \% \mathrm{U} 235, \mathrm{ZZZ1} \mathrm{milli-g} \mathrm{U/cc,}<\mathrm{denSol} \mid 3.4>$

c molarity $\mathrm{U}=\langle\operatorname{molU}| 3.4>$, acid $=\langle\operatorname{mol} A$ cid $| 3.4>, \mathrm{H} 2 \mathrm{O}=\langle\operatorname{molH} 2 \mathrm{O} \mid 3.4\rangle$

c $\mathrm{U}$ in salt

$92235.66 \mathrm{c}<$ molU*ena $3.4>$

$92238.66 \mathrm{c}<$ molU* $* 1$-ena $) \mid 3.4>$

c NO3 in salt plus acid

$7014.66 \mathrm{c}<2 * \mathrm{molU}+\operatorname{molAcid} \mid 3.4>$

$8016.66 \mathrm{c}<6 * \mathrm{molU}+3 * \operatorname{mol} \mathrm{Acid} \mid 3.4>$

c water

1001.66c $<2 *$ molH2O + molAcid $\mid 3.4>$ \$ncludes Free Hydrogen Ion

$8016.66 \mathrm{c}<\mathrm{molH} 2 \mathrm{O} \mid 3.4>$

mt11 1wtr.60t

c

c 


\section{Template File for Case: u5o2N23-icyl-0935-00}

icyl, 93.5 wt\% UO2, ZZZ1 milli-g/cc, in UO2(NO3)2, with 3 Molar HNO3

c

c worm notes: format of the form |n.m for floating point, $\mid \mathrm{n}$ for integers

c $\quad$ zzz0 (in upper case) is replaced with the number of cyles

c $\quad$ zzz1 $=$ the actual concentration in units of milli-g/cc

c $\quad$ zzz2 $=$ solution density in $\mathrm{g} / \mathrm{cc}$

c $\quad \mathrm{qqq} 1=$ the minimum dimensions (in hundreths of inches)

c $\quad \mathrm{qqq} 2$ = the maximum dimensions (in hundreths of inches)

c $\quad \mathrm{qqq} 3=$ the delta (in hundreths of inches)

$\mathrm{c}$

c Diameter $=<\mathrm{d} 1=$ QQQ1:QQQ2:QQQ3 $\mid 5>$ hundreths of inches - must be integer

$\mathrm{c}$ radius $=<\mathrm{rd}=\mathrm{d} 1 * 2.54 / 100 / 2 \mid 5.4>\mathrm{cm}$

c note for perl, numbers with leading zeros must be enclosed in single quotes or else

c they will be interpretted as octal

$\mathrm{c}$ Conc $=<\mathrm{c}={ }^{\prime} \mathrm{ZZZ1} 1 \mid 5>$ milli-g U/cc

c Reflector width $=<\mathrm{rw}=0.0 \mid 2.1>$ inches

c Enrichment $=<$ ena $=0.935 \mid 1.3>$ atom fraction $\mathrm{U}$

c Mols of Acid per liter $<$ molAcid $=3.0 \mid 1.2>$

$\mathrm{c}$

$\mathrm{c}$

c Density of solution $<$ denSol $=Z Z Z 2 / 1 \mathrm{e} 5 \mid 1.5>$

c

c

c Atomic Weights

c $\mathrm{H} 2 \mathrm{O}=\langle$ atwH2O $=18.0152 \mid 3.4\rangle$

$\mathrm{c} \mathrm{NO3}=\langle$ atwNO3 $=62.0049 \mid 3.4\rangle$

c $\mathrm{O}=\langle$ atwO $=15.9994| 3.4>$

c $\mathrm{HNO} 3=<$ atwHNO3 $=63.0128 \mid 3.4>$

c U235 $=<$ atwU235 $=235.0439 \mid 3.4>$

c U238 $=<$ atwU238 $=238.0508 \mid 3.4>$

c U $=<$ atwU $=$ ena*atwU235+(1-ena)*atwU238 $3.4>$

$\mathrm{c}$

c Molarity of solution components

c Mols of $\mathrm{U}$ per liter $<$ molU $=\mathrm{c} / \mathrm{atwU} \mid 3.4>$

c Mols of $\mathrm{H} 2 \mathrm{O}$ per liter $<$ molH2O $=(1000 *$ denSol-molU* $(\operatorname{atwU}+2 *$ atwNO3+2*atwO $)-$ molAcid*atwHNO3)/atwH2O|3.4>

$\mathrm{c}$

$\mathrm{c}$

$<$ perl $>$

sub IMP

\{

return 1 if $\left(\$ \_[0]>0\right)$;

return 0 ;

\}

$</$ perl $>$

c Cylinder

$2011<$-denSol|3.6> $-1 \quad$ imp:n=1

$212-1.000-21 \quad$ imp:n=<\&IMP(rw) $\mid 1>$ \$zero importance if no reflector

$220 \quad 2$ imp:n=0 


$$
\begin{aligned}
& 1 \quad \mathrm{cz}<\mathrm{rd} \mid 4.4>\quad \text { \$ Cylinder } \\
& 2 \mathrm{cz}<\mathrm{rd}+\mathrm{rw} * \mathrm{in} \mid 4.4>\quad \text { \$ Reflection }
\end{aligned}
$$

mode $\mathrm{n}$

kcode $20001.0100 \mathrm{ZZZ0}$

$\begin{array}{llll}\text { ksrc } & 0.00 & 0.00 & 0.00\end{array}$

$\begin{array}{lll}0.00 & 0.00 & 0.10\end{array}$

$\begin{array}{lll}0.00 & 0.00 & 0.50\end{array}$

$\begin{array}{lll}0.00 & 0.00 & 1.00\end{array}$

$\begin{array}{lll}0.00 & 0.00 & 2.00\end{array}$

$\begin{array}{lll}0.00 & 0.00 & 3.00\end{array}$

$\begin{array}{lll}1.00 & 1.00 & 1.00\end{array}$

$\begin{array}{lll}1.00 & 1.00 & 2.00\end{array}$

$\begin{array}{lll}1.00 & 1.00 & 3.00\end{array}$

$\begin{array}{lll}2.00 & 2.00 & 1.00\end{array}$

$\begin{array}{lll}2.00 & 2.00 & 2.00\end{array}$

$\begin{array}{lll}2.00 & 2.00 & 3.00\end{array}$

$\begin{array}{lll}-1.00 & -1.00 & -1.00\end{array}$

$-10.00 \quad-10.00 \quad-20.00$

$\begin{array}{lll}-10.00 & -10.00 & -30.00\end{array}$

c

m2 \$water

$1001.66 \mathrm{c} \quad 2$

$8016.66 \mathrm{c} \quad 1$

$\mathrm{mt} 2$ 1wtr.60t

c UO2/water mixture

m11 \$ UO2(NO3)2 - NO3 - H2O, $100.0 \mathrm{wt} \% \mathrm{U} 235, \mathrm{ZZZ1} \mathrm{milli-g} \mathrm{U/cc,}<\mathrm{denSol} \mid 3.4>$

c molarity $\mathrm{U}=\langle\operatorname{molU}| 3.4>$, acid $=\langle\operatorname{mol} A$ cid $| 3.4>, \mathrm{H} 2 \mathrm{O}=\langle\operatorname{molH} 2 \mathrm{O} \mid 3.4\rangle$

c $\mathrm{U}$ in salt

$92235.66 \mathrm{c}<$ molU*ena $3.4>$

$92238.66 \mathrm{c}<$ molU* $* 1$-ena $) \mid 3.4>$

c NO3 in salt plus acid

$7014.66 \mathrm{c}<2 * \mathrm{molU}+\operatorname{molAcid} \mid 3.4>$

8016.66c $<6 * \mathrm{molU}+3 * \operatorname{molAcid} \mid 3.4>$

c water

1001.66c $<2 *$ molH2O + molAcid $\mid 3.4>$ \$ncludes Free Hydrogen Ion

$8016.66 \mathrm{c}<\mathrm{molH} 2 \mathrm{O} \mid 3.4>$

mt11 1wtr.60t

$\mathrm{c}$

$\mathrm{c}$ 
Template File for Case: u5o2N23-icyl-0935-01

icyl, 93.5 wt\% UO2, ZZZ1 milli-g/cc, in UO2(NO3)2, with 3 Molar HNO3

c

c worm notes: format of the form |n.m for floating point, $\mid \mathrm{n}$ for integers

c zzz0 (in upper case) is replaced with the number of cyles

c $\quad$ zzz1 $=$ the actual concentration in units of milli-g/cc

c $\quad$ zzz2 $=$ solution density in $\mathrm{g} / \mathrm{cc}$

c $\quad \mathrm{qqq1}=$ the minimum dimensions (in hundreths of inches)

c $\quad \mathrm{qqq} 2=$ the maximum dimensions (in hundreths of inches)

c $\quad \mathrm{qqq} 3=$ the delta (in hundreths of inches)

c

$\mathrm{c}$ Diameter $=<\mathrm{d} 1=$ QQQ1:QQQ2:QQQ3 $\mid 5>$ hundreths of inches - must be integer

$\mathrm{c}$ radius $=<\mathrm{rd}=\mathrm{d} 1 * 2.54 / 100 / 2 \mid 5.4>\mathrm{cm}$

c note for perl, numbers with leading zeros must be enclosed in single quotes or else

c they will be interpretted as octal

$\mathrm{c}$ Conc $=<\mathrm{c}={ }^{\prime}$ ZZZ1' $\mid 5>$ milli-g U/cc

c Reflector width $=<\mathrm{rw}=1.0 \mid 2.1>$ inches

c Enrichment $=<$ ena $=0.935 \mid 1.3>$ atom fraction $\mathrm{U}$

c Mols of Acid per liter $<$ molAcid $=3.0 \mid 1.2>$

$\mathrm{c}$

$\mathrm{c}$

c Density of solution $<$ denSol=ZZZ2/1e5 $\mid 1.5>$

c

c

c Atomic Weights

c $\mathrm{H} 2 \mathrm{O}=\langle$ atwH2O $=18.0152| 3.4>$

$\mathrm{c} \mathrm{NO3}=\langle$ atwNO3 $=62.0049| 3.4>$

c $\mathrm{O}=\langle$ atwO $=15.9994| 3.4>$

$\mathrm{c} \mathrm{HNO} 3=<$ atwHNO3 $=63.0128 \mid 3.4>$

c U235 $=<$ atwU235 $=235.0439 \mid 3.4>$

c U238 $=<$ atwU238 $=238.0508 \mid 3.4>$

$\mathrm{c} \mathrm{U}=<$ atwU $=$ ena*atwU235+(1-ena)*atwU238|3.4>

c

c Molarity of solution components

c Mols of $\mathrm{U}$ per liter $<$ molU $=\mathrm{c} / \mathrm{atwU} \mid 3.4>$

c Mols of $\mathrm{H} 2 \mathrm{O}$ per liter $<\operatorname{molH} 2 \mathrm{O}=(1000 *$ denSol-molU* $*$ atwU $+2 * a+w N O 3+2 * a t w O)$ molAcid*atwHNO3)/atwH2O|3.4>

$\mathrm{c}$

c

$<$ perl $>$

sub IMP

\{

return 1 if $\left(\$ \_[0]>0\right)$;

return 0 ;

\}

$</$ perl $>$

c Cylinder

$2011<$-denSol|3.6> $-1 \quad$ imp:n=1

$212-1.000-21 \quad$ imp:n=<\&IMP(rw) $\mid 1>$ \$zero importance if no reflector

$220 \quad 2$ imp:n=0 


$$
\begin{array}{llll}
1 & \mathrm{cz} & <\mathrm{rd} \mid 4.4> & \text { \$Cylinder } \\
2 & \mathrm{cz} & <\mathrm{rd}+\mathrm{rw} * \mathrm{in} \mid 4.4> & \text { \$ Reflection }
\end{array}
$$

mode $\mathrm{n}$

kcode $20001.0100 \mathrm{ZZZ0}$

$\begin{array}{llll}\text { ksrc } & 0.00 & 0.00 & 0.00\end{array}$

$\begin{array}{lll}0.00 & 0.00 & 0.10\end{array}$

$\begin{array}{lll}0.00 & 0.00 & 0.50\end{array}$

$\begin{array}{lll}0.00 & 0.00 & 1.00\end{array}$

$\begin{array}{lll}0.00 & 0.00 & 2.00\end{array}$

$\begin{array}{lll}0.00 & 0.00 & 3.00\end{array}$

$\begin{array}{lll}1.00 & 1.00 & 1.00\end{array}$

$\begin{array}{lll}1.00 & 1.00 & 2.00\end{array}$

$\begin{array}{lll}1.00 & 1.00 & 3.00\end{array}$

$\begin{array}{lll}2.00 & 2.00 & 1.00\end{array}$

$\begin{array}{lll}2.00 & 2.00 & 2.00\end{array}$

$\begin{array}{lll}2.00 & 2.00 & 3.00\end{array}$

$\begin{array}{lll}-1.00 & -1.00 & -1.00\end{array}$

$-10.00 \quad-10.00 \quad-20.00$

$\begin{array}{lll}-10.00 & -10.00 & -30.00\end{array}$

c

m2 \$water

$1001.66 \mathrm{c} \quad 2$

$8016.66 \mathrm{c} \quad 1$

$\mathrm{mt} 2$ 1wtr.60t

c UO2/water mixture

m11 \$ UO2(NO3)2 - NO3 - H2O, $100.0 \mathrm{wt} \% \mathrm{U} 235, \mathrm{ZZZ1} \mathrm{milli-g} \mathrm{U/cc,}<\mathrm{denSol} \mid 3.4>$

c molarity $\mathrm{U}=\langle\operatorname{molU}| 3.4>$, acid $=\langle\operatorname{mol} A$ cid $| 3.4>, \mathrm{H} 2 \mathrm{O}=\langle\operatorname{molH} 2 \mathrm{O} \mid 3.4\rangle$

c $\mathrm{U}$ in salt

$92235.66 \mathrm{c}<$ molU*ena $3.4>$

$92238.66 \mathrm{c}<$ molU* $* 1$-ena $) \mid 3.4>$

c NO3 in salt plus acid

$7014.66 \mathrm{c}<2 * \mathrm{molU}+\operatorname{molAcid} \mid 3.4>$

8016.66c $<6 * \mathrm{molU}+3 * \operatorname{molAcid} \mid 3.4>$

c water

1001.66c $<2 *$ molH2O + molAcid $\mid 3.4>$ \$ncludes Free Hydrogen Ion

$8016.66 \mathrm{c}<\mathrm{molH} 2 \mathrm{O} \mid 3.4>$

mt11 1wtr.60t

$\mathrm{c}$

$\mathrm{c}$ 


\section{Template File for Case: u502N23-icyl-0935-10}

icyl, 93.5 wt\% UO2, ZZZ1 milli-g/cc, in UO2(NO3)2, with 3 Molar HNO3

c

c worm notes: format of the form |n.m for floating point, $\mid \mathrm{n}$ for integers

c $\quad$ zzz0 (in upper case) is replaced with the number of cyles

c $\quad$ zzz1 $=$ the actual concentration in units of milli-g/cc

c $\quad$ zzz2 $=$ solution density in $\mathrm{g} / \mathrm{cc}$

c $\quad \mathrm{qqq} 1=$ the minimum dimensions (in hundreths of inches)

c $\quad \mathrm{qqq} 2$ = the maximum dimensions (in hundreths of inches)

c $\quad \mathrm{qqq} 3=$ the delta (in hundreths of inches)

$\mathrm{c}$

c Diameter $=<\mathrm{d} 1=$ QQQ1:QQQ2:QQQ3 $\mid 5>$ hundreths of inches - must be integer

$\mathrm{c}$ radius $=<\mathrm{rd}=\mathrm{d} 1 * 2.54 / 100 / 2 \mid 5.4>\mathrm{cm}$

c note for perl, numbers with leading zeros must be enclosed in single quotes or else

c they will be interpretted as octal

$\mathrm{c}$ Conc $=<\mathrm{c}={ }^{\prime}$ ZZZ1' $\mid 5>$ milli-g U/cc

c Reflector width $=\langle\mathrm{rw}=10.0| 2.1>$ inches

c Enrichment $=<$ ena $=0.935 \mid 1.3>$ atom fraction $\mathrm{U}$

c Mols of Acid per liter $<$ molAcid $=3.0 \mid 1.2>$

$\mathrm{c}$

$\mathrm{c}$

c Density of solution $<$ denSol $=Z Z Z 2 / 1 \mathrm{e} 5 \mid 1.5>$

c

c

c Atomic Weights

c $\mathrm{H} 2 \mathrm{O}=\langle$ atwH2O $=18.0152 \mid 3.4\rangle$

$\mathrm{c} \mathrm{NO3}=\langle$ atwNO3 $=62.0049 \mid 3.4\rangle$

c $\mathrm{O}=\langle$ atwO $=15.9994| 3.4>$

c HNO3 $=\langle$ atwHNO3 $=63.0128| 3.4>$

c U235 $=<$ atwU235 $=235.0439 \mid 3.4>$

c U238 $=<$ atwU238 $=238.0508 \mid 3.4>$

c U $=<$ atwU $=$ ena*atwU235+(1-ena)*atwU238 $3.4>$

$\mathrm{c}$

c Molarity of solution components

c Mols of $\mathrm{U}$ per liter $<$ molU $=\mathrm{c} / \mathrm{atwU} \mid 3.4>$

c Mols of $\mathrm{H} 2 \mathrm{O}$ per liter $<$ molH2O $=(1000 *$ denSol-molU* $(\operatorname{atwU}+2 *$ atwNO3+2*atwO $)-$ molAcid*atwHNO3)/atwH2O|3.4>

$\mathrm{c}$

c

$<$ perl $>$

sub IMP

\{

return 1 if $\left(\$ \_[0]>0\right)$;

return 0 ;

\}

$</$ perl $>$

c Cylinder

$2011<$-denSol|3.6> $-1 \quad$ imp:n=1

$212-1.000-21 \quad$ imp:n=<\&IMP(rw) $\mid 1>$ \$zero importance if no reflector

$220 \quad 2$ imp:n=0 


$$
\begin{array}{llll}
1 & \mathrm{cz} & <\mathrm{rd} \mid 4.4> & \text { \$Cylinder } \\
2 & \mathrm{cz} & <\mathrm{rd}+\mathrm{rw} * \mathrm{in} \mid 4.4> & \text { \$ Reflection }
\end{array}
$$

mode $\mathrm{n}$

kcode $20001.0100 \mathrm{ZZZ0}$

$\begin{array}{llll}\text { ksrc } & 0.00 & 0.00 & 0.00\end{array}$

$\begin{array}{lll}0.00 & 0.00 & 0.10\end{array}$

$\begin{array}{lll}0.00 & 0.00 & 0.50\end{array}$

$\begin{array}{lll}0.00 & 0.00 & 1.00\end{array}$

$\begin{array}{lll}0.00 & 0.00 & 2.00\end{array}$

$\begin{array}{lll}0.00 & 0.00 & 3.00\end{array}$

$\begin{array}{lll}1.00 & 1.00 & 1.00\end{array}$

$\begin{array}{lll}1.00 & 1.00 & 2.00\end{array}$

$\begin{array}{lll}1.00 & 1.00 & 3.00\end{array}$

$\begin{array}{lll}2.00 & 2.00 & 1.00\end{array}$

$\begin{array}{lll}2.00 & 2.00 & 2.00\end{array}$

$\begin{array}{lll}2.00 & 2.00 & 3.00\end{array}$

$\begin{array}{lll}-1.00 & -1.00 & -1.00\end{array}$

$-10.00 \quad-10.00 \quad-20.00$

$\begin{array}{lll}-10.00 & -10.00 & -30.00\end{array}$

c

m2 \$water

$1001.66 \mathrm{c} \quad 2$

$8016.66 \mathrm{c} \quad 1$

$\mathrm{mt} 2$ 1wtr.60t

c UO2/water mixture

m11 \$ UO2(NO3)2 - NO3 - H2O, $100.0 \mathrm{wt} \% \mathrm{U} 235, \mathrm{ZZZ1} \mathrm{milli-g} \mathrm{U/cc,}<\mathrm{denSol} \mid 3.4>$

c molarity $\mathrm{U}=\langle\operatorname{molU}| 3.4>$, acid $=\langle\operatorname{mol} A$ cid $| 3.4>, \mathrm{H} 2 \mathrm{O}=\langle\operatorname{molH} 2 \mathrm{O} \mid 3.4\rangle$

c $\mathrm{U}$ in salt

$92235.66 \mathrm{c}<$ molU*ena $3.4>$

$92238.66 \mathrm{c}<$ molU* $* 1$-ena $) \mid 3.4>$

c NO3 in salt plus acid

$7014.66 \mathrm{c}<2 * \mathrm{molU}+\operatorname{molAcid} \mid 3.4>$

8016.66c $<6 * \mathrm{molU}+3 * \operatorname{molAcid} \mid 3.4>$

c water

1001.66c $<2 *$ molH2O + molAcid $\mid 3.4>$ \$ncludes Free Hydrogen Ion

$8016.66 \mathrm{c}<\mathrm{molH} 2 \mathrm{O} \mid 3.4>$

mt11 1wtr.60t

$\mathrm{c}$

$\mathrm{c}$ 


\section{Template File for Case: u5o2N26-icyl-0935-00}

icyl, 93.5 wt\% UO2, ZZZ1 milli-g/cc, in UO2(NO3)2, with 6 Molar HNO3

c

c worm notes: format of the form |n.m for floating point, $\mid \mathrm{n}$ for integers

c $\quad$ zzz0 (in upper case) is replaced with the number of cyles

c $\quad$ zzz1 $=$ the actual concentration in units of milli-g/cc

c $\quad$ zzz2 $=$ solution density in $\mathrm{g} / \mathrm{cc}$

c $\quad \mathrm{qqq} 1=$ the minimum dimensions (in hundreths of inches)

c $\quad \mathrm{qqq} 2$ = the maximum dimensions (in hundreths of inches)

c $\quad \mathrm{qqq} 3=$ the delta (in hundreths of inches)

$\mathrm{c}$

c Diameter $=<\mathrm{d} 1=$ QQQ1:QQQ2:QQQ3 $\mid 5>$ hundreths of inches - must be integer

$\mathrm{c}$ radius $=<\mathrm{rd}=\mathrm{d} 1 * 2.54 / 100 / 2 \mid 5.4>\mathrm{cm}$

c note for perl, numbers with leading zeros must be enclosed in single quotes or else

c they will be interpretted as octal

$\mathrm{c}$ Conc $=<\mathrm{c}={ }^{\prime} \mathrm{ZZZ1} 1 \mid 5>$ milli-g U/cc

c Reflector width $=<\mathrm{rw}=0.0 \mid 2.1>$ inches

c Enrichment $=<$ ena $=0.935 \mid 1.3>$ atom fraction $\mathrm{U}$

c Mols of Acid per liter $<$ molAcid $=6.0 \mid 1.2>$

$\mathrm{c}$

$\mathrm{c}$

c Density of solution $<$ denSol $=Z Z Z 2 / 1 \mathrm{e} 5 \mid 1.5>$

c

c

c Atomic Weights

c $\mathrm{H} 2 \mathrm{O}=\langle$ atwH2O $=18.0152 \mid 3.4\rangle$

$\mathrm{c} \mathrm{NO3}=\langle$ atwNO3 $=62.0049 \mid 3.4\rangle$

c $\mathrm{O}=\langle$ atwO $=15.9994| 3.4>$

c HNO3 $=\langle$ atwHNO3 $=63.0128| 3.4>$

c U235 $=<$ atwU235 $=235.0439 \mid 3.4>$

c U238 $=<$ atwU238 $=238.0508 \mid 3.4>$

c U $=<$ atwU $=$ ena*atwU235+(1-ena)*atwU238 $3.4>$

$\mathrm{c}$

c Molarity of solution components

c Mols of $\mathrm{U}$ per liter $<$ molU $=\mathrm{c} / \mathrm{atwU} \mid 3.4>$

c Mols of $\mathrm{H} 2 \mathrm{O}$ per liter $<$ molH2O $=(1000 *$ denSol-molU* $(\operatorname{atwU}+2 *$ atwNO3+2*atwO $)-$ molAcid*atwHNO3)/atwH2O|3.4>

$\mathrm{c}$

c

$<$ perl $>$

sub IMP

\{

return 1 if $\left(\$ \_[0]>0\right)$;

return 0 ;

\}

$</$ perl $>$

c Cylinder

$2011<$-denSol|3.6> $-1 \quad$ imp:n=1

$212-1.000-21 \quad$ imp:n=<\&IMP(rw) $\mid 1>$ \$zero importance if no reflector

$220 \quad 2$ imp:n=0 


$$
\begin{array}{llll}
1 & \mathrm{cz} & <\mathrm{rd} \mid 4.4> & \text { \$Cylinder } \\
2 & \mathrm{cz} & <\mathrm{rd}+\mathrm{rw} * \mathrm{in} \mid 4.4> & \text { \$ Reflection }
\end{array}
$$

mode $\mathrm{n}$

kcode $20001.0100 \mathrm{ZZZ0}$

$\begin{array}{llll}\text { ksrc } & 0.00 & 0.00 & 0.00\end{array}$

$\begin{array}{lll}0.00 & 0.00 & 0.10\end{array}$

$\begin{array}{lll}0.00 & 0.00 & 0.50\end{array}$

$\begin{array}{lll}0.00 & 0.00 & 1.00\end{array}$

$\begin{array}{lll}0.00 & 0.00 & 2.00\end{array}$

$\begin{array}{lll}0.00 & 0.00 & 3.00\end{array}$

$\begin{array}{lll}1.00 & 1.00 & 1.00\end{array}$

$\begin{array}{lll}1.00 & 1.00 & 2.00\end{array}$

$\begin{array}{lll}1.00 & 1.00 & 3.00\end{array}$

$\begin{array}{lll}2.00 & 2.00 & 1.00\end{array}$

$\begin{array}{lll}2.00 & 2.00 & 2.00\end{array}$

$\begin{array}{lll}2.00 & 2.00 & 3.00\end{array}$

$\begin{array}{lll}-1.00 & -1.00 & -1.00\end{array}$

$-10.00 \quad-10.00 \quad-20.00$

$\begin{array}{lll}-10.00 & -10.00 & -30.00\end{array}$

c

m2 \$water

$1001.66 \mathrm{c} \quad 2$

$8016.66 \mathrm{c} \quad 1$

$\mathrm{mt} 2$ 1wtr.60t

c UO2/water mixture

m11 \$ UO2(NO3)2 - NO3 - H2O, $100.0 \mathrm{wt} \% \mathrm{U} 235, \mathrm{ZZZ1} \mathrm{milli-g} \mathrm{U/cc,}<\mathrm{denSol} \mid 3.4>$

c molarity $\mathrm{U}=\langle\operatorname{molU}| 3.4>$, acid $=\langle\operatorname{mol} A$ cid $| 3.4>, \mathrm{H} 2 \mathrm{O}=\langle\operatorname{molH} 2 \mathrm{O} \mid 3.4\rangle$

c $\mathrm{U}$ in salt

$92235.66 \mathrm{c}<$ molU*ena $3.4>$

$92238.66 \mathrm{c}<$ molU* $* 1$-ena $) \mid 3.4>$

c NO3 in salt plus acid

$7014.66 \mathrm{c}<2 * \mathrm{molU}+\operatorname{molAcid} \mid 3.4>$

8016.66c $<6 * \mathrm{molU}+3 * \operatorname{molAcid} \mid 3.4>$

c water

1001.66c $<2 *$ molH2O + molAcid $\mid 3.4>$ \$ncludes Free Hydrogen Ion

$8016.66 \mathrm{c}<\mathrm{molH} 2 \mathrm{O} \mid 3.4>$

mt11 1wtr.60t

$\mathrm{c}$

$\mathrm{c}$ 
Template File for Case: u5o2N26-icyl-0935-01

icyl, 93.5 wt\% UO2, ZZZ1 milli-g/cc, in UO2(NO3)2, with 6 Molar HNO3

c

c worm notes: format of the form $\mid n . m$ for floating point, $\mid \mathrm{n}$ for integers

c zzz0 (in upper case) is replaced with the number of cyles

c $\quad$ zzz1 $=$ the actual concentration in units of milli-g/cc

c $\quad$ zzz2 $=$ solution density in $\mathrm{g} / \mathrm{cc}$

c $\quad \mathrm{qqq1}=$ the minimum dimensions (in hundreths of inches)

c $\quad \mathrm{qqq} 2=$ the maximum dimensions (in hundreths of inches)

c $\quad \mathrm{qqq} 3=$ the delta (in hundreths of inches)

c

c Diameter $=<\mathrm{d} 1=$ QQQ1:QQQ2:QQQ3 $\mid 5>$ hundreths of inches - must be integer

$\mathrm{c}$ radius $=<\mathrm{rd}=\mathrm{d} 1 * 2.54 / 100 / 2 \mid 5.4>\mathrm{cm}$

c note for perl, numbers with leading zeros must be enclosed in single quotes or else

c they will be interpretted as octal

$\mathrm{c}$ Conc $=<\mathrm{c}={ }^{\prime}$ ZZZ1' $\mid 5>$ milli-g U/cc

c Reflector width $=<\mathrm{rw}=1.0 \mid 2.1>$ inches

c Enrichment $=<$ ena $=0.935 \mid 1.3>$ atom fraction $\mathrm{U}$

c Mols of Acid per liter $<$ molAcid $=6.0 \mid 1.2>$

$\mathrm{c}$

$\mathrm{c}$

c Density of solution $<$ denSol $=Z Z Z 2 / 1 \mathrm{e} 5 \mid 1.5>$

c

c

c Atomic Weights

c $\mathrm{H} 2 \mathrm{O}=\langle$ atwH2O $=18.0152| 3.4>$

$\mathrm{c} \mathrm{NO3}=\langle$ atwNO3 $=62.0049| 3.4>$

c $\mathrm{O}=\langle$ atwO $=15.9994| 3.4>$

$\mathrm{c} \mathrm{HNO} 3=<$ atwHNO3 $=63.0128 \mid 3.4>$

c U235 $=<$ atwU235 $=235.0439 \mid 3.4>$

c U238 $=<$ atwU238 $=238.0508 \mid 3.4>$

c U $=<$ atwU $=$ ena*atwU235+(1-ena)*atwU238 $3.4>$

c

c Molarity of solution components

c Mols of $\mathrm{U}$ per liter $<$ molU $=\mathrm{c} / \mathrm{atwU} \mid 3.4>$

c Mols of $\mathrm{H} 2 \mathrm{O}$ per liter $<\operatorname{molH} 2 \mathrm{O}=(1000 *$ denSol-molU* $*$ atwU $+2 * a+w N O 3+2 * a t w O)$ molAcid*atwHNO3)/atwH2O|3.4>

$\mathrm{c}$

c

$<$ perl $>$

sub IMP

\{

return 1 if $\left(\$ \_[0]>0\right)$;

return 0 ;

\}

$</$ perl $>$

c Cylinder

$2011<$-denSol|3.6> $-1 \quad$ imp:n=1

$212-1.000-21 \quad$ imp:n=<\&IMP(rw) $\mid 1>$ \$zero importance if no reflector

$220 \quad 2$ imp:n=0 


$$
\begin{array}{llll}
1 & \mathrm{cz} & <\mathrm{rd} \mid 4.4> & \text { \$Cylinder } \\
2 & \mathrm{cz} & <\mathrm{rd}+\mathrm{rw} * \mathrm{in} \mid 4.4> & \text { \$ Reflection }
\end{array}
$$

mode $\mathrm{n}$

kcode $20001.0100 \mathrm{ZZZ0}$

$\begin{array}{llll}\text { ksrc } & 0.00 & 0.00 & 0.00\end{array}$

$\begin{array}{lll}0.00 & 0.00 & 0.10\end{array}$

$\begin{array}{lll}0.00 & 0.00 & 0.50\end{array}$

$\begin{array}{lll}0.00 & 0.00 & 1.00\end{array}$

$\begin{array}{lll}0.00 & 0.00 & 2.00\end{array}$

$\begin{array}{lll}0.00 & 0.00 & 3.00\end{array}$

$\begin{array}{lll}1.00 & 1.00 & 1.00\end{array}$

$\begin{array}{lll}1.00 & 1.00 & 2.00\end{array}$

$\begin{array}{lll}1.00 & 1.00 & 3.00\end{array}$

$\begin{array}{lll}2.00 & 2.00 & 1.00\end{array}$

$\begin{array}{lll}2.00 & 2.00 & 2.00\end{array}$

$\begin{array}{lll}2.00 & 2.00 & 3.00\end{array}$

$\begin{array}{lll}-1.00 & -1.00 & -1.00\end{array}$

$-10.00 \quad-10.00 \quad-20.00$

$\begin{array}{lll}-10.00 & -10.00 & -30.00\end{array}$

c

m2 \$water

$1001.66 \mathrm{c} \quad 2$

$8016.66 \mathrm{c} \quad 1$

$\mathrm{mt} 2$ 1wtr.60t

c UO2/water mixture

m11 \$ UO2(NO3)2 - NO3 - H2O, $100.0 \mathrm{wt} \% \mathrm{U} 235, \mathrm{ZZZ1} \mathrm{milli-g} \mathrm{U/cc,}<\mathrm{denSol} \mid 3.4>$

c molarity $\mathrm{U}=\langle\operatorname{molU}| 3.4>$, acid $=\langle\operatorname{mol} A$ cid $| 3.4>, \mathrm{H} 2 \mathrm{O}=\langle\operatorname{molH} 2 \mathrm{O} \mid 3.4\rangle$

c $\mathrm{U}$ in salt

$92235.66 \mathrm{c}<$ molU*ena $3.4>$

$92238.66 \mathrm{c}<$ molU* $* 1$-ena $) \mid 3.4>$

c NO3 in salt plus acid

$7014.66 \mathrm{c}<2 * \mathrm{molU}+\operatorname{molAcid} \mid 3.4>$

8016.66c $<6 * \mathrm{molU}+3 * \operatorname{molAcid} \mid 3.4>$

c water

1001.66c $<2 *$ molH2O + molAcid $\mid 3.4>$ \$ncludes Free Hydrogen Ion

$8016.66 \mathrm{c}<\mathrm{molH} 2 \mathrm{O} \mid 3.4>$

mt11 1wtr.60t

$\mathrm{c}$

$\mathrm{c}$ 


\section{Template File for Case: u5o2N26-icyl-0935-10}

icyl, 93.5 wt\% UO2, ZZZ1 milli-g/cc, in UO2(NO3)2, with 6 Molar HNO3

c

c worm notes: format of the form |n.m for floating point, $\mid \mathrm{n}$ for integers

c $\quad$ zzz0 (in upper case) is replaced with the number of cyles

c $\quad$ zzz1 $=$ the actual concentration in units of milli-g/cc

c $\quad$ zzz2 $=$ solution density in $\mathrm{g} / \mathrm{cc}$

c $\quad \mathrm{qqq} 1=$ the minimum dimensions (in hundreths of inches)

c $\quad \mathrm{qqq} 2$ = the maximum dimensions (in hundreths of inches)

c $\quad \mathrm{qqq} 3=$ the delta (in hundreths of inches)

$\mathrm{c}$

c Diameter $=<\mathrm{d} 1=$ QQQ1:QQQ2:QQQ3 $\mid 5>$ hundreths of inches - must be integer

$\mathrm{c}$ radius $=<\mathrm{rd}=\mathrm{d} 1 * 2.54 / 100 / 2 \mid 5.4>\mathrm{cm}$

c note for perl, numbers with leading zeros must be enclosed in single quotes or else

c they will be interpretted as octal

$\mathrm{c}$ Conc $=<\mathrm{c}={ }^{\prime}$ ZZZ1' $\mid 5>$ milli-g U/cc

c Reflector width $=\langle\mathrm{rw}=10.0| 2.1>$ inches

c Enrichment $=<$ ena $=0.935 \mid 1.3>$ atom fraction $\mathrm{U}$

c Mols of Acid per liter $<$ molAcid $=6.0 \mid 1.2>$

$\mathrm{c}$

$\mathrm{c}$

c Density of solution $<$ denSol $=Z Z Z 2 / 1 \mathrm{e} 5 \mid 1.5>$

c

c

c Atomic Weights

c $\mathrm{H} 2 \mathrm{O}=\langle$ atwH2O $=18.0152 \mid 3.4\rangle$

$\mathrm{c} \mathrm{NO3}=\langle$ atwNO3 $=62.0049 \mid 3.4\rangle$

c $\mathrm{O}=\langle$ atwO $=15.9994| 3.4>$

c HNO3 $=\langle$ atwHNO3 $=63.0128| 3.4>$

c U235 $=<$ atwU235 $=235.0439 \mid 3.4>$

c U238 $=<$ atwU238 $=238.0508 \mid 3.4>$

c U $=<$ atwU $=$ ena*atwU235+(1-ena)*atwU238 $3.4>$

$\mathrm{c}$

c Molarity of solution components

c Mols of $\mathrm{U}$ per liter $<$ molU $=\mathrm{c} / \mathrm{atwU} \mid 3.4>$

c Mols of $\mathrm{H} 2 \mathrm{O}$ per liter $<$ molH2O $=(1000 *$ denSol-molU* $(\operatorname{atwU}+2 *$ atwNO3+2*atwO $)-$ molAcid*atwHNO3)/atwH2O|3.4>

$\mathrm{c}$

c

$<$ perl $>$

sub IMP

\{

return 1 if $\left(\$ \_[0]>0\right)$;

return 0 ;

\}

$</$ perl $>$

c Cylinder

$2011<$-denSol|3.6> $-1 \quad$ imp:n=1

$212-1.000-21 \quad$ imp:n=<\&IMP(rw) $\mid 1>$ \$zero importance if no reflector

$220 \quad 2$ imp:n=0 


$$
\begin{aligned}
& 1 \quad \mathrm{cz}<\mathrm{rd} \mid 4.4>\quad \text { \$ cylinder } \\
& 2 \mathrm{cz}<\mathrm{rd}+\mathrm{rw} * \mathrm{in} \mid 4.4>\quad \text { \$ Reflection }
\end{aligned}
$$

mode $\mathrm{n}$

kcode $20001.0100 \mathrm{ZZZ0}$

$\begin{array}{llll}\text { ksrc } & 0.00 & 0.00 & 0.00\end{array}$

$\begin{array}{lll}0.00 & 0.00 & 0.10\end{array}$

$\begin{array}{lll}0.00 & 0.00 & 0.50\end{array}$

$\begin{array}{lll}0.00 & 0.00 & 1.00\end{array}$

$\begin{array}{lll}0.00 & 0.00 & 2.00\end{array}$

$\begin{array}{lll}0.00 & 0.00 & 3.00\end{array}$

$\begin{array}{lll}1.00 & 1.00 & 1.00\end{array}$

$\begin{array}{lll}1.00 & 1.00 & 2.00\end{array}$

$\begin{array}{lll}1.00 & 1.00 & 3.00\end{array}$

$\begin{array}{lll}2.00 & 2.00 & 1.00\end{array}$

$\begin{array}{lll}2.00 & 2.00 & 2.00\end{array}$

$\begin{array}{lll}2.00 & 2.00 & 3.00\end{array}$

$\begin{array}{lll}-1.00 & -1.00 & -1.00\end{array}$

$\begin{array}{lll}-10.00 & -10.00 & -20.00\end{array}$

$\begin{array}{lll}-10.00 & -10.00 & -30.00\end{array}$

c

m2 \$water

$1001.66 \mathrm{c} \quad 2$

$8016.66 \mathrm{c} \quad 1$

$\mathrm{mt} 2$ 1wtr.60t

c UO2/water mixture

m11 \$ UO2(NO3)2 - NO3 - H2O, $100.0 \mathrm{wt} \% \mathrm{U} 235, \mathrm{ZZZ1} \mathrm{milli-g} \mathrm{U/cc,}<\mathrm{denSol} \mid 3.4>$

c molarity $\mathrm{U}=\langle\operatorname{molU}| 3.4>$, acid $=\langle\operatorname{mol} A$ cid $| 3.4>, \mathrm{H} 2 \mathrm{O}=\langle\operatorname{molH} 2 \mathrm{O} \mid 3.4\rangle$

c $\mathrm{U}$ in salt

$92235.66 \mathrm{c}<$ molU*ena $3.4>$

$92238.66 \mathrm{c}<$ molU* $* 1$-ena $) \mid 3.4>$

c NO3 in salt plus acid

$7014.66 \mathrm{c}<2 * \mathrm{molU}+\operatorname{molAcid} \mid 3.4>$

8016.66c $<6 * \mathrm{molU}+3 * \operatorname{molAcid} \mid 3.4>$

c water

1001.66c $<2 *$ molH2O + molAcid $\mid 3.4>$ \$ncludes Free Hydrogen Ion

$8016.66 \mathrm{c}<\mathrm{molH} 2 \mathrm{O} \mid 3.4>$

mt11 1wtr.60t

$\mathrm{c}$

$\mathrm{c}$ 


\section{Template File for Case: u502N20-pl-1000-00}

$\mathrm{pl}, 100.0 \mathrm{wt} \% \mathrm{UO} 2, \mathrm{ZZZ1}$ milli-g/cc, in UO2(NO3)2, with 0 Molar HNO3

c

c worm notes: format of the form $\mid n . m$ for floating point, $\mid \mathrm{n}$ for integers

c $\quad$ zzz0 (in upper case) is replaced with the number of cyles

c $\quad$ zzz1 $=$ the actual concentration in units of milli-g/cc

c $\quad$ zzz2 $=$ solution density in $\mathrm{g} / \mathrm{cc}$

c $\quad \mathrm{qqq} 1=$ the minimum dimensions (in hundreths of inches)

c $\quad \mathrm{qqq} 2$ = the maximum dimensions (in hundreths of inches)

c $\quad \mathrm{qqq} 3=$ the delta (in hundreths of inches)

$\mathrm{c}$

c Diameter $=<\mathrm{d} 1=$ QQQ1:QQQ2:QQQ3 $\mid 5>$ hundreths of inches - must be integer

$\mathrm{c}$ radius $=<\mathrm{rd}=\mathrm{d} 1 * 2.54 / 100 / 2 \mid 5.4>\mathrm{cm}$

c note for perl, numbers with leading zeros must be enclosed in single quotes or else

c they will be interpretted as octal

$\mathrm{c}$ Conc $=<\mathrm{c}={ }^{\prime} \mathrm{ZZZ1} 1 \mid 5>$ milli-g U/cc

c Reflector width $=<\mathrm{rw}=0.0 \mid 2.1>$ inches

c Enrichment $=<$ ena $=1.0 \mid 1.2>$ atom fraction $\mathrm{U}$

c Mols of Acid per liter $<$ molAcid $=0.0 \mid 1.2>$

$\mathrm{c}$

$\mathrm{c}$

c Density of solution $<$ denSol $=Z Z Z 2 / 1$ e $5 \mid 1.5>$

c

c

c Atomic Weights

c $\mathrm{H} 2 \mathrm{O}=\langle$ atwH2O $=18.0152 \mid 3.4\rangle$

c NO3 $=<$ atwNO3 $=62.0049 \mid 3.4>$

c $\mathrm{O}=\langle$ atwO $=15.9994| 3.4>$

c HNO3 $=<$ atwHNO3 $=63.0128 \mid 3.4>$

c U235 $=<$ atwU235 $=235.0439 \mid 3.4>$

c U238 $=<$ atwU238 $=238.0508 \mid 3.4>$

c U $=<$ atwU $=$ ena*atwU235+(1-ena)*atwU238 $3.4>$

c

c Molarity of solution components

c Mols of $\mathrm{U}$ per liter $<$ molU $=\mathrm{c} / \mathrm{atwU} \mid 3.4>$

c Mols of $\mathrm{H} 2 \mathrm{O}$ per liter $<$ molH2O $=(1000 *$ denSol-molU* $(\operatorname{atwU}+2 *$ atwNO3+2*atwO $)-$ molAcid*atwHNO3)/atwH2O|3.4>

$\mathrm{c}$

c

$<$ perl $>$

sub IMP

\{

return 1 if $\left(\$ \_[0]>0\right)$;

return 0 ;

\}

$</$ perl $>$

c semi inifinite plane

$2011<-$ denSol|3.6> $-12-7-586 \quad$ imp:n=1

$212-1.000 \quad-3468-7-5((1-3):(-24)) \quad$ imp:n=<\&IMP(rw) $\mid 1>$ \$zero importance if no reflector 


\begin{tabular}{|c|c|c|c|}
\hline 220 & & $3:-4: 7: 5:-8:-6$ & imp: $n=0$ \\
\hline 1 & py & $\langle\mathrm{rd}| 4.4>$ & \$ Cylinder \\
\hline 2 & py & $<-\operatorname{rd} \mid 4.4>$ & \\
\hline 3 & py & $<\mathrm{rd}+\mathrm{rw}{ }^{*} \mathrm{in} \mid 4.4>$ & \$ Reflection \\
\hline 4 & py & $<-$ rd-rw*in $\mid 4.4>$ & \\
\hline$* 5$ & px & 100 & \\
\hline$* 6$ & $\mathrm{px}$ & -100 & \\
\hline$* 7$ & $\mathrm{pz}$ & 100 & \\
\hline$* 8$ & $\mathrm{pz}$ & -100 & \\
\hline
\end{tabular}

mode $\mathrm{n}$

kcode 20001.0100 ZZZ0

$\begin{array}{llll}\text { ksrc } & 0.00 & 0.00 & 0.00\end{array}$

$\begin{array}{lll}0.00 & 0.00 & 0.10\end{array}$

$\begin{array}{lll}0.00 & 0.00 & 0.50\end{array}$

$\begin{array}{lll}0.00 & 0.00 & 1.00\end{array}$

$\begin{array}{lll}0.00 & 0.00 & 2.00\end{array}$

$\begin{array}{lll}0.00 & 0.00 & 3.00\end{array}$

$\begin{array}{lll}1.00 & 1.00 & 1.00\end{array}$

$\begin{array}{lll}1.00 & 1.00 & 2.00\end{array}$

$\begin{array}{lll}1.00 & 1.00 & 3.00\end{array}$

$\begin{array}{lll}2.00 & 2.00 & 1.00\end{array}$

$\begin{array}{lll}2.00 & 2.00 & 2.00\end{array}$

$\begin{array}{lll}2.00 & 2.00 & 3.00\end{array}$

$\begin{array}{lll}-1.00 & -1.00 & -1.00\end{array}$

$\begin{array}{lll}-10.00 & -10.00 & -20.00\end{array}$

$\begin{array}{lll}-10.00 & -10.00 & -30.00\end{array}$

c

m2 \$ water

$1001.66 \mathrm{c} \quad 2$

$8016.66 \mathrm{c} \quad 1$

$\mathrm{mt} 2$ 1wtr.60t

c UO2/water mixture

m11 \$ UO2(NO3)2 - NO3 - H2O, $100.0 \mathrm{wt} \% \mathrm{U} 235, \mathrm{ZZZ1} \mathrm{milli-g} \mathrm{U/cc,}<\mathrm{denSol} \mid 3.4>$

c molarity $\mathrm{U}=\langle\operatorname{molU}| 3.4>$, acid $=\langle\operatorname{mol} A c i d| 3.4>, \mathrm{H} 2 \mathrm{O}=\langle\operatorname{molH} 2 \mathrm{O} \mid 3.4\rangle$

c U in salt

$92235.66 \mathrm{c}<$ molU*ena $3.4>$

c $92238.66 \mathrm{c}<$ molU* $(1$-ena $) \mid 3.4>$

c NO3 in salt plus acid

$7014.66 \mathrm{c}<2 * \operatorname{molU}+\operatorname{molAcid} \mid 3.4>$

$8016.66 \mathrm{c}<6 * \operatorname{molU}+3 * \operatorname{mol} \mathrm{Acid} \mid 3.4>$

c water

1001.66c $<2 *$ molH2O + molAcid|3.4 $>$ \$ncludes Free Hydrogen Ion

$8016.66 \mathrm{c}<\mathrm{molH} 2 \mathrm{O} \mid 3.4>$

mt11 1wtr.60t

c

c 


\section{Template File for Case: u502N20-pl-1000-01}

$\mathrm{pl}, 100.0 \mathrm{wt} \% \mathrm{UO} 2, \mathrm{ZZZ1}$ milli-g/cc, in UO2(NO3)2, with 0 Molar HNO3

c

c worm notes: format of the form |n.m for floating point, $\mid \mathrm{n}$ for integers

c $\quad$ zzz0 (in upper case) is replaced with the number of cyles

c $\quad$ zzz1 $=$ the actual concentration in units of milli-g/cc

c $\quad$ zzz2 $=$ solution density in $\mathrm{g} / \mathrm{cc}$

c $\quad \mathrm{qqq} 1=$ the minimum dimensions (in hundreths of inches)

c $\quad \mathrm{qqq} 2$ = the maximum dimensions (in hundreths of inches)

c $\quad \mathrm{qqq} 3=$ the delta (in hundreths of inches)

$\mathrm{c}$

c Diameter $=<\mathrm{d} 1=$ QQQ1:QQQ2:QQQ3 $\mid 5>$ hundreths of inches - must be integer

$\mathrm{c}$ radius $=<\mathrm{rd}=\mathrm{d} 1 * 2.54 / 100 / 2 \mid 5.4>\mathrm{cm}$

c note for perl, numbers with leading zeros must be enclosed in single quotes or else

c they will be interpretted as octal

$\mathrm{c}$ Conc $=<\mathrm{c}={ }^{\prime}$ ZZZ1' $\mid 5>$ milli-g U/cc

c Reflector width $=<\mathrm{rw}=1.0 \mid 2.1>$ inches

c Enrichment $=<$ ena $=1.0 \mid 1.2>$ atom fraction $\mathrm{U}$

c Mols of Acid per liter $<$ molAcid $=0.0 \mid 1.2>$

$\mathrm{c}$

$\mathrm{c}$

c Density of solution $<$ denSol $=Z Z Z 2 / 1$ e $5 \mid 1.5>$

c

c

c Atomic Weights

c $\mathrm{H} 2 \mathrm{O}=\langle$ atwH2O $=18.0152| 3.4>$

$\mathrm{c} \mathrm{NO3}=\langle$ atwNO3 $=62.0049| 3.4>$

c $\mathrm{O}=\langle$ atwO $=15.9994| 3.4>$

$\mathrm{c} \mathrm{HNO} 3=<$ atwHNO3 $=63.0128 \mid 3.4>$

c U235 $=<$ atwU235 $=235.0439 \mid 3.4>$

c U238 $=<$ atwU238 $=238.0508 \mid 3.4>$

c U $=<$ atwU $=$ ena*atwU235+(1-ena)*atwU238 $3.4>$

c

c Molarity of solution components

c Mols of $\mathrm{U}$ per liter $<\mathrm{molU}=\mathrm{c} / \mathrm{atwU} \mid 3.4>$

c Mols of $\mathrm{H} 2 \mathrm{O}$ per liter $<$ molH2O $=(1000 *$ denSol-molU* $(\operatorname{atwU}+2 *$ atwNO3+2*atwO $)-$ molAcid*atwHNO3)/atwH2O|3.4>

c

c

$<$ perl $>$

sub IMP

\{

return 1 if $\left(\$ \_[0]>0\right)$;

return 0 ;

\}

$</$ perl $>$

c semi inifinite plane

$2011<-$ denSol|3.6> $-12-7-586 \quad$ imp:n=1

$212-1.000 \quad-3468-7-5((1-3):(-24)) \quad$ imp:n=<\&IMP(rw) $\mid 1>$ \$zero importance if no reflector 


\begin{tabular}{|c|c|c|c|}
\hline 220 & & $3:-4: 7: 5:-8:-6$ & imp: $n=0$ \\
\hline 1 & py & $<\mathrm{rd} \mid 4.4>$ & \$ Cylinder \\
\hline 2 & py & $<-\operatorname{rd} \mid 4.4>$ & \\
\hline 3 & py & $<\mathrm{rd}+\mathrm{rw}^{*}$ in $\mid 4.4>$ & \$ Reflection \\
\hline 4 & py & $<-$ rd-rw*in $\mid 4.4>$ & \\
\hline$* 5$ & $\mathrm{px}$ & 100 & \\
\hline$* 6$ & $\mathrm{px}$ & -100 & \\
\hline$* 7$ & $\mathrm{pz}$ & 100 & \\
\hline$* 8$ & $\mathrm{pz}$ & -100 & \\
\hline
\end{tabular}

mode $\mathrm{n}$

kcode 20001.0100 ZZZ0

$\begin{array}{llll}\text { ksrc } & 0.00 & 0.00 & 0.00\end{array}$

$\begin{array}{lll}0.00 & 0.00 & 0.10\end{array}$

$\begin{array}{lll}0.00 & 0.00 & 0.50\end{array}$

$\begin{array}{lll}0.00 & 0.00 & 1.00\end{array}$

$\begin{array}{lll}0.00 & 0.00 & 2.00\end{array}$

$\begin{array}{lll}0.00 & 0.00 & 3.00\end{array}$

$\begin{array}{lll}1.00 & 1.00 & 1.00\end{array}$

$\begin{array}{lll}1.00 & 1.00 & 2.00\end{array}$

$\begin{array}{lll}1.00 & 1.00 & 3.00\end{array}$

$\begin{array}{lll}2.00 & 2.00 & 1.00\end{array}$

$\begin{array}{lll}2.00 & 2.00 & 2.00\end{array}$

$\begin{array}{lll}2.00 & 2.00 & 3.00\end{array}$

$\begin{array}{lll}-1.00 & -1.00 & -1.00\end{array}$

$\begin{array}{lll}-10.00 & -10.00 & -20.00\end{array}$

$\begin{array}{lll}-10.00 & -10.00 & -30.00\end{array}$

c

m2 \$ water

$1001.66 \mathrm{c} \quad 2$

$8016.66 \mathrm{c} \quad 1$

$\mathrm{mt} 2$ 1wtr.60t

c UO2/water mixture

m11 \$ UO2(NO3)2 - NO3 - H2O, $100.0 \mathrm{wt} \% \mathrm{U} 235, \mathrm{ZZZ1} \mathrm{milli-g} \mathrm{U/cc,}<\mathrm{denSol} \mid 3.4>$

c molarity $\mathrm{U}=\langle\operatorname{molU}| 3.4>$, acid $=\langle\operatorname{mol} A c i d| 3.4>, \mathrm{H} 2 \mathrm{O}=\langle\operatorname{molH} 2 \mathrm{O} \mid 3.4\rangle$

c U in salt

$92235.66 \mathrm{c}<$ molU*ena $3.4>$

c $92238.66 \mathrm{c}<$ molU* $(1$-ena $) \mid 3.4>$

c NO3 in salt plus acid

$7014.66 \mathrm{c}<2 * \operatorname{molU}+\operatorname{molAcid} \mid 3.4>$

$8016.66 \mathrm{c}<6 * \operatorname{molU}+3 * \operatorname{mol} \mathrm{Acid} \mid 3.4>$

c water

1001.66c $<2 *$ molH2O + molAcid|3.4 $>$ \$ncludes Free Hydrogen Ion

$8016.66 \mathrm{c}<\mathrm{molH} 2 \mathrm{O} \mid 3.4>$

mt11 1wtr.60t

c

c 


\section{Template File for Case: u5o2N20-pl-1000-10}

$\mathrm{pl}, 100.0 \mathrm{wt} \% \mathrm{UO} 2, \mathrm{ZZZ1}$ milli-g/cc, in UO2(NO3)2, with 0 Molar HNO3

c

c worm notes: format of the form $\mid n . m$ for floating point, $\mid \mathrm{n}$ for integers

c $\quad$ zzz0 (in upper case) is replaced with the number of cyles

c $\quad$ zzz1 $=$ the actual concentration in units of milli-g/cc

c $\quad$ zzz2 $=$ solution density in $\mathrm{g} / \mathrm{cc}$

c $\quad \mathrm{qqq} 1=$ the minimum dimensions (in hundreths of inches)

c $\quad \mathrm{qqq} 2$ = the maximum dimensions (in hundreths of inches)

c $\quad \mathrm{qqq} 3=$ the delta (in hundreths of inches)

$\mathrm{c}$

c Diameter $=<\mathrm{d} 1=$ QQQ1:QQQ2:QQQ3 $\mid 5>$ hundreths of inches - must be integer

$\mathrm{c}$ radius $=<\mathrm{rd}=\mathrm{d} 1 * 2.54 / 100 / 2 \mid 5.4>\mathrm{cm}$

c note for perl, numbers with leading zeros must be enclosed in single quotes or else

c they will be interpretted as octal

$\mathrm{c}$ Conc $=<\mathrm{c}={ }^{\prime}$ ZZZ1' $\mid 5>$ milli-g U/cc

c Reflector width $=<\mathrm{rw}=10.0 \mid 2.1>$ inches

c Enrichment $=<$ ena $=1.0 \mid 1.2>$ atom fraction $\mathrm{U}$

c Mols of Acid per liter $<$ molAcid $=0.0 \mid 1.2>$

c

$\mathrm{c}$

c Density of solution $<$ denSol $=Z Z Z 2 / 1$ e $5 \mid 1.5>$

c

c

c Atomic Weights

c $\mathrm{H} 2 \mathrm{O}=\langle$ atwH2O $=18.0152| 3.4>$

$\mathrm{c} \mathrm{NO3}=\langle$ atwNO3 $=62.0049| 3.4>$

c $\mathrm{O}=\langle$ atwO $=15.9994| 3.4>$

$\mathrm{c} \mathrm{HNO} 3=<$ atwHNO3 $=63.0128 \mid 3.4>$

c U235 $=<$ atwU235 $=235.0439 \mid 3.4>$

c U238 $=<$ atwU238 $=238.0508 \mid 3.4>$

c U $=<$ atwU $=$ ena*atwU235+(1-ena)*atwU238 $3.4>$

c

c Molarity of solution components

c Mols of $\mathrm{U}$ per liter $<\mathrm{molU}=\mathrm{c} / \mathrm{atwU} \mid 3.4>$

c Mols of $\mathrm{H} 2 \mathrm{O}$ per liter $<$ molH2O $=(1000 *$ denSol-molU* $(\operatorname{atwU}+2 *$ atwNO3+2*atwO $)-$ molAcid*atwHNO3)/atwH2O|3.4>

c

c

$<$ perl $>$

sub IMP

\{

return 1 if $\left(\$ \_[0]>0\right)$;

return 0 ;

\}

$</$ perl $>$

c semi inifinite plane

$2011<-$ denSol|3.6> $-12-7-586 \quad$ imp:n=1

$212-1.000 \quad-3468-7-5((1-3):(-24)) \quad$ imp:n=<\&IMP(rw) $\mid 1>$ \$zero importance if no reflector 


\begin{tabular}{|c|c|c|c|}
\hline 220 & & $3:-4: 7: 5:-8:-6$ & imp: $n=0$ \\
\hline 1 & py & $\langle\mathrm{rd}| 4.4>$ & \$ Cylinder \\
\hline 2 & py & $<-\operatorname{rd} \mid 4.4>$ & \\
\hline 3 & py & $<\mathrm{rd}+\mathrm{rw}{ }^{*} \mathrm{in} \mid 4.4>$ & \$ Reflection \\
\hline 4 & py & $<-$ rd-rw*in $\mid 4.4>$ & \\
\hline$* 5$ & px & 100 & \\
\hline$* 6$ & $\mathrm{px}$ & -100 & \\
\hline$* 7$ & $\mathrm{pz}$ & 100 & \\
\hline$* 8$ & $\mathrm{pz}$ & -100 & \\
\hline
\end{tabular}

mode $\mathrm{n}$

kcode 20001.0100 ZZZ0

$\begin{array}{llll}\text { ksrc } & 0.00 & 0.00 & 0.00\end{array}$

$\begin{array}{lll}0.00 & 0.00 & 0.10\end{array}$

$\begin{array}{lll}0.00 & 0.00 & 0.50\end{array}$

$\begin{array}{lll}0.00 & 0.00 & 1.00\end{array}$

$\begin{array}{lll}0.00 & 0.00 & 2.00\end{array}$

$\begin{array}{lll}0.00 & 0.00 & 3.00\end{array}$

$\begin{array}{lll}1.00 & 1.00 & 1.00\end{array}$

$\begin{array}{lll}1.00 & 1.00 & 2.00\end{array}$

$\begin{array}{lll}1.00 & 1.00 & 3.00\end{array}$

$\begin{array}{lll}2.00 & 2.00 & 1.00\end{array}$

$\begin{array}{lll}2.00 & 2.00 & 2.00\end{array}$

$\begin{array}{lll}2.00 & 2.00 & 3.00\end{array}$

$\begin{array}{lll}-1.00 & -1.00 & -1.00\end{array}$

$\begin{array}{lll}-10.00 & -10.00 & -20.00\end{array}$

$\begin{array}{lll}-10.00 & -10.00 & -30.00\end{array}$

c

m2 \$ water

$1001.66 \mathrm{c} \quad 2$

$8016.66 \mathrm{c} \quad 1$

$\mathrm{mt} 2$ 1wtr.60t

c UO2/water mixture

m11 \$ UO2(NO3)2 - NO3 - H2O, $100.0 \mathrm{wt} \% \mathrm{U} 235, \mathrm{ZZZ1} \mathrm{milli-g} \mathrm{U/cc,}<\mathrm{denSol} \mid 3.4>$

c molarity $\mathrm{U}=\langle\operatorname{molU}| 3.4>$, acid $=\langle\operatorname{mol} A c i d| 3.4>, \mathrm{H} 2 \mathrm{O}=\langle\operatorname{molH} 2 \mathrm{O} \mid 3.4\rangle$

c U in salt

$92235.66 \mathrm{c}<$ molU*ena $3.4>$

c $92238.66 \mathrm{c}<$ molU* $(1$-ena $) \mid 3.4>$

c NO3 in salt plus acid

$7014.66 \mathrm{c}<2 * \operatorname{molU}+\operatorname{molAcid} \mid 3.4>$

$8016.66 \mathrm{c}<6 * \operatorname{molU}+3 * \operatorname{mol} \mathrm{Acid} \mid 3.4>$

c water

1001.66c $<2 *$ molH2O + molAcid|3.4 $>$ \$ncludes Free Hydrogen Ion

$8016.66 \mathrm{c}<\mathrm{molH} 2 \mathrm{O} \mid 3.4>$

mt11 1wtr.60t

c

c 
CHPRC-01552, Revision 0

D-183 


\section{Template File for Case: u5o2N23-pl-1000-00}

$\mathrm{pl}, 100.0 \mathrm{wt} \% \mathrm{UO} 2, \mathrm{ZZZ1}$ milli-g/cc, in UO2(NO3)2, with 3 Molar HNO3

c

c worm notes: format of the form |n.m for floating point, $\mid \mathrm{n}$ for integers

c $\quad$ zzz0 (in upper case) is replaced with the number of cyles

c $\quad$ zzz1 $=$ the actual concentration in units of milli-g/cc

c $\quad$ zzz2 $=$ solution density in $\mathrm{g} / \mathrm{cc}$

c $\quad \mathrm{qqq} 1=$ the minimum dimensions (in hundreths of inches)

c $\quad \mathrm{qqq} 2$ = the maximum dimensions (in hundreths of inches)

c $\quad \mathrm{qqq} 3=$ the delta (in hundreths of inches)

$\mathrm{c}$

c Diameter $=<\mathrm{d} 1=$ QQQ1:QQQ2:QQQ3 $\mid 5>$ hundreths of inches - must be integer

$\mathrm{c}$ radius $=<\mathrm{rd}=\mathrm{d} 1 * 2.54 / 100 / 2 \mid 5.4>\mathrm{cm}$

c note for perl, numbers with leading zeros must be enclosed in single quotes or else

c they will be interpretted as octal

$\mathrm{c}$ Conc $=<\mathrm{c}={ }^{\prime} \mathrm{ZZZ1} 1 \mid 5>$ milli-g U/cc

c Reflector width $=<\mathrm{rw}=0.0 \mid 2.1>$ inches

c Enrichment $=<$ ena $=1.0 \mid 1.2>$ atom fraction $\mathrm{U}$

c Mols of Acid per liter $<$ molAcid $=3.0 \mid 1.2>$

$\mathrm{c}$

$\mathrm{c}$

c Density of solution $<$ denSol $=Z Z Z 2 / 1$ e $5 \mid 1.5>$

c

c

c Atomic Weights

c $\mathrm{H} 2 \mathrm{O}=\langle$ atwH2O $=18.0152| 3.4>$

$\mathrm{c} \mathrm{NO3}=\langle$ atwNO3 $=62.0049| 3.4>$

c $\mathrm{O}=\langle$ atwO $=15.9994| 3.4>$

$\mathrm{c} \mathrm{HNO} 3=<$ atwHNO3 $=63.0128 \mid 3.4>$

c U235 $=<$ atwU235 $=235.0439 \mid 3.4>$

c U238 $=<$ atwU238 $=238.0508 \mid 3.4>$

c U $=<$ atwU $=$ ena*atwU235+(1-ena)*atwU238 $3.4>$

c

c Molarity of solution components

c Mols of $\mathrm{U}$ per liter $<\mathrm{molU}=\mathrm{c} / \mathrm{atwU} \mid 3.4>$

c Mols of $\mathrm{H} 2 \mathrm{O}$ per liter $<$ molH2O $=(1000 *$ denSol-molU* $(\operatorname{atwU}+2 *$ atwNO3+2*atwO $)-$ molAcid*atwHNO3)/atwH2O|3.4>

$\mathrm{c}$

c

$<$ perl $>$

sub IMP

\{

return 1 if $(\$[0]>0)$;

return 0 ;

\}

$</$ perl $>$

c semi inifinite plane

$2011<-$ denSol|3.6> $-12-7-586 \quad$ imp:n=1

$212-1.000 \quad-3468-7-5((1-3):(-24)) \quad$ imp:n=<\&IMP(rw) $\mid 1>$ \$zero importance if no reflector 


\begin{tabular}{|c|c|c|c|}
\hline 220 & & $3:-4: 7: 5:-8:-6$ & imp: $n=0$ \\
\hline 1 & py & $\langle\mathrm{rd}| 4.4>$ & \$ Cylinder \\
\hline 2 & py & $<-\operatorname{rd} \mid 4.4>$ & \\
\hline 3 & py & $<\mathrm{rd}+\mathrm{rw}{ }^{*} \mathrm{in} \mid 4.4>$ & \$ Reflection \\
\hline 4 & py & $<-$ rd-rw*in $\mid 4.4>$ & \\
\hline$* 5$ & px & 100 & \\
\hline$* 6$ & $\mathrm{px}$ & -100 & \\
\hline$* 7$ & $\mathrm{pz}$ & 100 & \\
\hline$* 8$ & $\mathrm{pz}$ & -100 & \\
\hline
\end{tabular}

mode $\mathrm{n}$

kcode 20001.0100 ZZZ0

$\begin{array}{llll}\text { ksrc } & 0.00 & 0.00 & 0.00\end{array}$

$\begin{array}{lll}0.00 & 0.00 & 0.10\end{array}$

$\begin{array}{lll}0.00 & 0.00 & 0.50\end{array}$

$\begin{array}{lll}0.00 & 0.00 & 1.00\end{array}$

$\begin{array}{lll}0.00 & 0.00 & 2.00\end{array}$

$\begin{array}{lll}0.00 & 0.00 & 3.00\end{array}$

$\begin{array}{lll}1.00 & 1.00 & 1.00\end{array}$

$\begin{array}{lll}1.00 & 1.00 & 2.00\end{array}$

$\begin{array}{lll}1.00 & 1.00 & 3.00\end{array}$

$\begin{array}{lll}2.00 & 2.00 & 1.00\end{array}$

$\begin{array}{lll}2.00 & 2.00 & 2.00\end{array}$

$\begin{array}{lll}2.00 & 2.00 & 3.00\end{array}$

$\begin{array}{lll}-1.00 & -1.00 & -1.00\end{array}$

$\begin{array}{lll}-10.00 & -10.00 & -20.00\end{array}$

$\begin{array}{lll}-10.00 & -10.00 & -30.00\end{array}$

c

m2 \$ water

$1001.66 \mathrm{c} \quad 2$

$8016.66 \mathrm{c} \quad 1$

$\mathrm{mt} 2$ 1wtr.60t

c UO2/water mixture

m11 \$ UO2(NO3)2 - NO3 - H2O, $100.0 \mathrm{wt} \%$ U235, ZZZ1 milli-g U/cc, $<$ denSol|3.4 $>$

c molarity $\mathrm{U}=\langle\operatorname{molU}| 3.4>$, acid $=\langle\operatorname{mol} A c i d| 3.4>, \mathrm{H} 2 \mathrm{O}=\langle\operatorname{molH} 2 \mathrm{O} \mid 3.4\rangle$

c U in salt

$92235.66 \mathrm{c}<$ molU*ena $3.4>$

c $92238.66 \mathrm{c}<$ molU* $(1$-ena $) \mid 3.4>$

c NO3 in salt plus acid

$7014.66 \mathrm{c}<2 * \mathrm{molU}+\operatorname{molAcid} \mid 3.4>$

$8016.66 \mathrm{c}<6 * \operatorname{molU}+3 * \operatorname{mol} \mathrm{Acid} \mid 3.4>$

c water

1001.66c $<2 *$ molH2O + molAcid|3.4 $>$ \$ncludes Free Hydrogen Ion

$8016.66 \mathrm{c}<\mathrm{molH} 2 \mathrm{O} \mid 3.4>$

mt11 1wtr.60t

c

c 


\section{Template File for Case: u5o2N23-pl-1000-01}

$\mathrm{pl}, 100.0 \mathrm{wt} \% \mathrm{UO} 2, \mathrm{ZZZ1}$ milli-g/cc, in UO2(NO3)2, with 3 Molar HNO3

c

c worm notes: format of the form $\mid n . m$ for floating point, $\mid \mathrm{n}$ for integers

c $\quad$ zzz0 (in upper case) is replaced with the number of cyles

c $\quad$ zzz1 $=$ the actual concentration in units of milli-g/cc

c $\quad$ zzz2 $=$ solution density in $\mathrm{g} / \mathrm{cc}$

c $\quad \mathrm{qqq} 1=$ the minimum dimensions (in hundreths of inches)

c $\quad \mathrm{qqq} 2$ = the maximum dimensions (in hundreths of inches)

c $\quad \mathrm{qqq} 3=$ the delta (in hundreths of inches)

$\mathrm{c}$

c Diameter $=<\mathrm{d} 1=$ QQQ1:QQQ2:QQQ3 $\mid 5>$ hundreths of inches - must be integer

$\mathrm{c}$ radius $=<\mathrm{rd}=\mathrm{d} 1 * 2.54 / 100 / 2 \mid 5.4>\mathrm{cm}$

c note for perl, numbers with leading zeros must be enclosed in single quotes or else

c they will be interpretted as octal

$\mathrm{c}$ Conc $=<\mathrm{c}={ }^{\prime}$ ZZZ1' $\mid 5>$ milli-g U/cc

c Reflector width $=<\mathrm{rw}=1.0 \mid 2.1>$ inches

c Enrichment $=<$ ena $=1.0 \mid 1.2>$ atom fraction $\mathrm{U}$

c Mols of Acid per liter $<$ molAcid $=3.0 \mid 1.2>$

$\mathrm{c}$

$\mathrm{c}$

c Density of solution $<$ denSol $=Z Z Z 2 / 1$ e $5 \mid 1.5>$

c

c

c Atomic Weights

c $\mathrm{H} 2 \mathrm{O}=\langle$ atwH2O $=18.0152 \mid 3.4\rangle$

c NO3 $=<$ atwNO3 $=62.0049 \mid 3.4>$

c $\mathrm{O}=\langle$ atwO $=15.9994| 3.4>$

c HNO3 $=<$ atwHNO3 $=63.0128 \mid 3.4>$

c U235 $=<$ atwU235 $=235.0439 \mid 3.4>$

c U238 $=<$ atwU238 $=238.0508 \mid 3.4>$

c U $=<$ atwU $=$ ena*atwU235+(1-ena)*atwU238 $3.4>$

c

c Molarity of solution components

c Mols of $\mathrm{U}$ per liter $<$ molU $=\mathrm{c} / \mathrm{atwU} \mid 3.4>$

c Mols of $\mathrm{H} 2 \mathrm{O}$ per liter $<$ molH2O $=(1000 *$ denSol-molU* $(\operatorname{atwU}+2 *$ atwNO3+2*atwO $)-$ molAcid*atwHNO3)/atwH2O|3.4>

$\mathrm{c}$

c

$<$ perl $>$

sub IMP

\{

return 1 if $\left(\$ \_[0]>0\right)$;

return 0 ;

\}

$</$ perl $>$

c semi inifinite plane

$2011<-$ denSol|3.6> $-12-7-586 \quad$ imp:n=1

$212-1.000 \quad-3468-7-5((1-3):(-24)) \quad$ imp:n=<\&IMP(rw) $\mid 1>$ \$zero importance if no reflector 


\begin{tabular}{|c|c|c|c|}
\hline 220 & & $3:-4: 7: 5:-8:-6$ & imp: $n=0$ \\
\hline 1 & py & $\langle\mathrm{rd}| 4.4>$ & \$ Cylinder \\
\hline 2 & py & $<-\operatorname{rd} \mid 4.4>$ & \\
\hline 3 & py & $<\mathrm{rd}+\mathrm{rw}{ }^{*} \mathrm{in} \mid 4.4>$ & \$ Reflection \\
\hline 4 & py & $<-$ rd-rw*in $\mid 4.4>$ & \\
\hline$* 5$ & px & 100 & \\
\hline$* 6$ & $\mathrm{px}$ & -100 & \\
\hline$* 7$ & $\mathrm{pz}$ & 100 & \\
\hline$* 8$ & $\mathrm{pz}$ & -100 & \\
\hline
\end{tabular}

mode $\mathrm{n}$

kcode 20001.0100 ZZZ0

$\begin{array}{llll}\text { ksrc } & 0.00 & 0.00 & 0.00\end{array}$

$\begin{array}{lll}0.00 & 0.00 & 0.10\end{array}$

$\begin{array}{lll}0.00 & 0.00 & 0.50\end{array}$

$\begin{array}{lll}0.00 & 0.00 & 1.00\end{array}$

$\begin{array}{lll}0.00 & 0.00 & 2.00\end{array}$

$\begin{array}{lll}0.00 & 0.00 & 3.00\end{array}$

$\begin{array}{lll}1.00 & 1.00 & 1.00\end{array}$

$\begin{array}{lll}1.00 & 1.00 & 2.00\end{array}$

$\begin{array}{lll}1.00 & 1.00 & 3.00\end{array}$

$\begin{array}{lll}2.00 & 2.00 & 1.00\end{array}$

$\begin{array}{lll}2.00 & 2.00 & 2.00\end{array}$

$\begin{array}{lll}2.00 & 2.00 & 3.00\end{array}$

$\begin{array}{lll}-1.00 & -1.00 & -1.00\end{array}$

$\begin{array}{lll}-10.00 & -10.00 & -20.00\end{array}$

$\begin{array}{lll}-10.00 & -10.00 & -30.00\end{array}$

c

m2 \$ water

$1001.66 \mathrm{c} \quad 2$

$8016.66 \mathrm{c} \quad 1$

$\mathrm{mt} 2$ 1wtr.60t

c UO2/water mixture

m11 \$ UO2(NO3)2 - NO3 - H2O, $100.0 \mathrm{wt} \% \mathrm{U} 235, \mathrm{ZZZ1} \mathrm{milli-g} \mathrm{U/cc,}<\mathrm{denSol} \mid 3.4>$

c molarity $\mathrm{U}=\langle\operatorname{molU}| 3.4>$, acid $=\langle\operatorname{mol} A c i d| 3.4>, \mathrm{H} 2 \mathrm{O}=\langle\operatorname{molH} 2 \mathrm{O} \mid 3.4\rangle$

c U in salt

$92235.66 \mathrm{c}<$ molU*ena $3.4>$

c $92238.66 \mathrm{c}<$ molU* $(1$-ena $) \mid 3.4>$

c NO3 in salt plus acid

$7014.66 \mathrm{c}<2 * \operatorname{molU}+\operatorname{molAcid} \mid 3.4>$

$8016.66 \mathrm{c}<6 * \operatorname{molU}+3 * \operatorname{mol} \mathrm{Acid} \mid 3.4>$

c water

1001.66c $<2 *$ molH2O + molAcid|3.4 $>$ \$ncludes Free Hydrogen Ion

$8016.66 \mathrm{c}<\mathrm{molH} 2 \mathrm{O} \mid 3.4>$

mt11 1wtr.60t

c

c 


\section{Template File for Case: u5o2N23-pl-1000-10}

$\mathrm{pl}, 100.0 \mathrm{wt} \% \mathrm{UO} 2, \mathrm{ZZZ1}$ milli-g/cc, in UO2(NO3)2, with 3 Molar HNO3

c

c worm notes: format of the form $\mid n . m$ for floating point, $\mid \mathrm{n}$ for integers

c $\quad$ zzz0 (in upper case) is replaced with the number of cyles

c $\quad$ zzz1 $=$ the actual concentration in units of milli-g/cc

c $\quad$ zzz2 $=$ solution density in $\mathrm{g} / \mathrm{cc}$

c $\quad \mathrm{qqq} 1=$ the minimum dimensions (in hundreths of inches)

c $\quad \mathrm{qqq} 2$ = the maximum dimensions (in hundreths of inches)

c $\quad \mathrm{qqq} 3=$ the delta (in hundreths of inches)

$\mathrm{c}$

c Diameter $=<\mathrm{d} 1=$ QQQ1:QQQ2:QQQ3 $\mid 5>$ hundreths of inches - must be integer

$\mathrm{c}$ radius $=<\mathrm{rd}=\mathrm{d} 1 * 2.54 / 100 / 2 \mid 5.4>\mathrm{cm}$

c note for perl, numbers with leading zeros must be enclosed in single quotes or else

c they will be interpretted as octal

$\mathrm{c}$ Conc $=<\mathrm{c}={ }^{\prime}$ ZZZ1' $\mid 5>$ milli-g U/cc

c Reflector width $=<\mathrm{rw}=10.0 \mid 2.1>$ inches

c Enrichment $=<$ ena $=1.0 \mid 1.2>$ atom fraction $\mathrm{U}$

c Mols of Acid per liter $<$ molAcid $=3.0 \mid 1.2>$

$\mathrm{c}$

$\mathrm{c}$

c Density of solution $<$ denSol $=Z Z Z 2 / 1$ e $5 \mid 1.5>$

c

c

c Atomic Weights

c $\mathrm{H} 2 \mathrm{O}=\langle$ atwH2O $=18.0152 \mid 3.4\rangle$

c NO3 $=<$ atwNO3 $=62.0049 \mid 3.4>$

c $\mathrm{O}=\langle$ atwO $=15.9994| 3.4>$

c HNO3 $=<$ atwHNO3 $=63.0128 \mid 3.4>$

c U235 $=<$ atwU235 $=235.0439 \mid 3.4>$

c U238 $=<$ atwU238 $=238.0508 \mid 3.4>$

c U $=<$ atwU $=$ ena*atwU235+(1-ena)*atwU238 $3.4>$

c

c Molarity of solution components

c Mols of $\mathrm{U}$ per liter $<$ molU $=\mathrm{c} / \mathrm{atwU} \mid 3.4>$

c Mols of $\mathrm{H} 2 \mathrm{O}$ per liter $<$ molH2O $=(1000 *$ denSol-molU* $(\operatorname{atwU}+2 *$ atwNO3+2*atwO $)-$ molAcid*atwHNO3)/atwH2O|3.4>

$\mathrm{c}$

c

$<$ perl $>$

sub IMP

\{

return 1 if $\left(\$ \_[0]>0\right)$;

return 0 ;

\}

$</$ perl $>$

c semi inifinite plane

$2011<-$ denSol|3.6> $-12-7-586 \quad$ imp:n=1

$212-1.000 \quad-3468-7-5((1-3):(-24)) \quad$ imp:n=<\&IMP(rw) $\mid 1>$ \$zero importance if no reflector 


\begin{tabular}{|c|c|c|c|}
\hline 220 & & $3:-4: 7: 5:-8:-6$ & imp: $n=0$ \\
\hline 1 & py & $<\mathrm{rd} \mid 4.4>$ & \$ Cylinder \\
\hline 2 & py & $<-\operatorname{rd} \mid 4.4>$ & \\
\hline 3 & py & $<\mathrm{rd}+\mathrm{rw}^{*}$ in $\mid 4.4>$ & \$ Reflection \\
\hline 4 & py & $<-$ rd-rw*in $\mid 4.4>$ & \\
\hline$* 5$ & $\mathrm{px}$ & 100 & \\
\hline$* 6$ & $\mathrm{px}$ & -100 & \\
\hline$* 7$ & $\mathrm{pz}$ & 100 & \\
\hline$* 8$ & $\mathrm{pz}$ & -100 & \\
\hline
\end{tabular}

mode $\mathrm{n}$

kcode 20001.0100 ZZZ0

$\begin{array}{llll}\text { ksrc } & 0.00 & 0.00 & 0.00\end{array}$

$\begin{array}{lll}0.00 & 0.00 & 0.10\end{array}$

$\begin{array}{lll}0.00 & 0.00 & 0.50\end{array}$

$\begin{array}{lll}0.00 & 0.00 & 1.00\end{array}$

$\begin{array}{lll}0.00 & 0.00 & 2.00\end{array}$

$\begin{array}{lll}0.00 & 0.00 & 3.00\end{array}$

$\begin{array}{lll}1.00 & 1.00 & 1.00\end{array}$

$\begin{array}{lll}1.00 & 1.00 & 2.00\end{array}$

$\begin{array}{lll}1.00 & 1.00 & 3.00\end{array}$

$\begin{array}{lll}2.00 & 2.00 & 1.00\end{array}$

$\begin{array}{lll}2.00 & 2.00 & 2.00\end{array}$

$\begin{array}{lll}2.00 & 2.00 & 3.00\end{array}$

$\begin{array}{lll}-1.00 & -1.00 & -1.00\end{array}$

$\begin{array}{lll}-10.00 & -10.00 & -20.00\end{array}$

$\begin{array}{lll}-10.00 & -10.00 & -30.00\end{array}$

c

m2 \$ water

$1001.66 \mathrm{c} \quad 2$

$8016.66 \mathrm{c} \quad 1$

$\mathrm{mt} 2$ 1wtr.60t

c UO2/water mixture

m11 \$ UO2(NO3)2 - NO3 - H2O, $100.0 \mathrm{wt} \% \mathrm{U} 235, \mathrm{ZZZ1} \mathrm{milli-g} \mathrm{U/cc,}<\mathrm{denSol} \mid 3.4>$

c molarity $\mathrm{U}=\langle\operatorname{molU}| 3.4>$, acid $=\langle\operatorname{mol} A c i d| 3.4>, \mathrm{H} 2 \mathrm{O}=\langle\operatorname{molH} 2 \mathrm{O} \mid 3.4\rangle$

c U in salt

$92235.66 \mathrm{c}<$ molU*ena $3.4>$

c $92238.66 \mathrm{c}<$ molU* $(1$-ena $) \mid 3.4>$

c NO3 in salt plus acid

$7014.66 \mathrm{c}<2 * \operatorname{molU}+\operatorname{molAcid} \mid 3.4>$

$8016.66 \mathrm{c}<6 * \operatorname{molU}+3 * \operatorname{mol} \mathrm{Acid} \mid 3.4>$

c water

1001.66c $<2 *$ molH2O + molAcid|3.4 $>$ \$ncludes Free Hydrogen Ion

$8016.66 \mathrm{c}<\mathrm{molH} 2 \mathrm{O} \mid 3.4>$

mt11 1wtr.60t

c

c 
CHPRC-01552, Revision 0

D-190 


\section{Template File for Case: u5o2N26-pl-1000-00}

$\mathrm{pl}, 100.0 \mathrm{wt} \% \mathrm{UO} 2, \mathrm{ZZZ1}$ milli-g/cc, in UO2(NO3)2, with 6 Molar HNO3

c

c worm notes: format of the form $\mid n . m$ for floating point, $\mid \mathrm{n}$ for integers

c $\quad$ zzz0 (in upper case) is replaced with the number of cyles

c $\quad$ zzz1 $=$ the actual concentration in units of milli-g/cc

c $\quad$ zzz2 $=$ solution density in $\mathrm{g} / \mathrm{cc}$

c $\quad \mathrm{qqq} 1=$ the minimum dimensions (in hundreths of inches)

c $\quad \mathrm{qqq} 2$ = the maximum dimensions (in hundreths of inches)

c $\quad \mathrm{qqq} 3=$ the delta (in hundreths of inches)

$\mathrm{c}$

c Diameter $=<\mathrm{d} 1=$ QQQ1:QQQ2:QQQ3 $\mid 5>$ hundreths of inches - must be integer

$\mathrm{c}$ radius $=<\mathrm{rd}=\mathrm{d} 1 * 2.54 / 100 / 2 \mid 5.4>\mathrm{cm}$

c note for perl, numbers with leading zeros must be enclosed in single quotes or else

c they will be interpretted as octal

$\mathrm{c}$ Conc $=<\mathrm{c}={ }^{\prime} \mathrm{ZZZ1} 1 \mid 5>$ milli-g U/cc

c Reflector width $=<\mathrm{rw}=0.0 \mid 2.1>$ inches

c Enrichment $=<$ ena $=1.0 \mid 1.2>$ atom fraction $\mathrm{U}$

c Mols of Acid per liter $<$ molAcid $=6.0 \mid 1.2>$

$\mathrm{c}$

$\mathrm{c}$

c Density of solution $<$ denSol $=Z Z Z 2 / 1$ e $5 \mid 1.5>$

c

c

c Atomic Weights

c $\mathrm{H} 2 \mathrm{O}=\langle$ atwH2O $=18.0152 \mid 3.4\rangle$

c NO3 $=<$ atwNO3 $=62.0049 \mid 3.4>$

c $\mathrm{O}=\langle$ atwO $=15.9994| 3.4>$

c HNO3 $=<$ atwHNO3 $=63.0128 \mid 3.4>$

c U235 $=<$ atwU235 $=235.0439 \mid 3.4>$

c U238 $=<$ atwU238 $=238.0508 \mid 3.4>$

c U $=<$ atwU $=$ ena*atwU235+(1-ena)*atwU238 $3.4>$

c

c Molarity of solution components

c Mols of $\mathrm{U}$ per liter $<$ molU $=\mathrm{c} / \mathrm{atwU} \mid 3.4>$

c Mols of $\mathrm{H} 2 \mathrm{O}$ per liter $<$ molH2O $=(1000 *$ denSol-molU* $(\operatorname{atwU}+2 *$ atwNO3+2*atwO $)-$ molAcid*atwHNO3)/atwH2O|3.4>

$\mathrm{c}$

c

$<$ perl $>$

sub IMP

\{

return 1 if $\left(\$ \_[0]>0\right)$;

return 0 ;

\}

$</$ perl $>$

c semi inifinite plane

$2011<-$ denSol|3.6> $-12-7-586 \quad$ imp:n=1

$212-1.000 \quad-3468-7-5((1-3):(-24)) \quad$ imp:n=<\&IMP(rw) $\mid 1>$ \$zero importance if no reflector 


\begin{tabular}{|c|c|c|c|}
\hline 220 & & $3:-4: 7: 5:-8:-6$ & imp: $n=0$ \\
\hline 1 & py & $\langle\mathrm{rd}| 4.4>$ & \$ Cylinder \\
\hline 2 & py & $<-\operatorname{rd} \mid 4.4>$ & \\
\hline 3 & py & $<\mathrm{rd}+\mathrm{rw}{ }^{*} \mathrm{in} \mid 4.4>$ & \$ Reflection \\
\hline 4 & py & $<-$ rd-rw*in $\mid 4.4>$ & \\
\hline$* 5$ & px & 100 & \\
\hline$* 6$ & $\mathrm{px}$ & -100 & \\
\hline$* 7$ & $\mathrm{pz}$ & 100 & \\
\hline$* 8$ & $\mathrm{pz}$ & -100 & \\
\hline
\end{tabular}

mode $\mathrm{n}$

kcode 20001.0100 ZZZ0

$\begin{array}{llll}\text { ksrc } & 0.00 & 0.00 & 0.00\end{array}$

$\begin{array}{lll}0.00 & 0.00 & 0.10\end{array}$

$\begin{array}{lll}0.00 & 0.00 & 0.50\end{array}$

$\begin{array}{lll}0.00 & 0.00 & 1.00\end{array}$

$\begin{array}{lll}0.00 & 0.00 & 2.00\end{array}$

$\begin{array}{lll}0.00 & 0.00 & 3.00\end{array}$

$\begin{array}{lll}1.00 & 1.00 & 1.00\end{array}$

$\begin{array}{lll}1.00 & 1.00 & 2.00\end{array}$

$\begin{array}{lll}1.00 & 1.00 & 3.00\end{array}$

$\begin{array}{lll}2.00 & 2.00 & 1.00\end{array}$

$\begin{array}{lll}2.00 & 2.00 & 2.00\end{array}$

$\begin{array}{lll}2.00 & 2.00 & 3.00\end{array}$

$\begin{array}{lll}-1.00 & -1.00 & -1.00\end{array}$

$\begin{array}{lll}-10.00 & -10.00 & -20.00\end{array}$

$\begin{array}{lll}-10.00 & -10.00 & -30.00\end{array}$

c

m2 \$ water

$1001.66 \mathrm{c} \quad 2$

$8016.66 \mathrm{c} \quad 1$

$\mathrm{mt} 2$ 1wtr.60t

c UO2/water mixture

m11 \$ UO2(NO3)2 - NO3 - H2O, $100.0 \mathrm{wt} \% \mathrm{U} 235, \mathrm{ZZZ1} \mathrm{milli-g} \mathrm{U/cc,}<\mathrm{denSol} \mid 3.4>$

c molarity $\mathrm{U}=\langle\operatorname{molU}| 3.4>$, acid $=\langle\operatorname{mol} A c i d| 3.4>, \mathrm{H} 2 \mathrm{O}=\langle\operatorname{molH} 2 \mathrm{O} \mid 3.4\rangle$

c U in salt

$92235.66 \mathrm{c}<$ molU*ena $3.4>$

c $92238.66 \mathrm{c}<$ molU* $(1$-ena $) \mid 3.4>$

c NO3 in salt plus acid

$7014.66 \mathrm{c}<2 * \operatorname{molU}+\operatorname{molAcid} \mid 3.4>$

$8016.66 \mathrm{c}<6 * \operatorname{molU}+3 * \operatorname{mol} \mathrm{Acid} \mid 3.4>$

c water

1001.66c $<2 *$ molH2O + molAcid|3.4 $>$ \$ncludes Free Hydrogen Ion

$8016.66 \mathrm{c}<\mathrm{molH} 2 \mathrm{O} \mid 3.4>$

mt11 1wtr.60t

c

c 


\section{Template File for Case: u5o2N26-pl-1000-01}

$\mathrm{pl}, 100.0 \mathrm{wt} \% \mathrm{UO} 2, \mathrm{ZZZ1}$ milli-g/cc, in UO2(NO3)2, with 6 Molar HNO3

c

c worm notes: format of the form |n.m for floating point, $\mid \mathrm{n}$ for integers

c $\quad$ zzz0 (in upper case) is replaced with the number of cyles

c $\quad$ zzz1 $=$ the actual concentration in units of milli-g/cc

c $\quad$ zzz2 $=$ solution density in $\mathrm{g} / \mathrm{cc}$

c $\quad \mathrm{qqq} 1=$ the minimum dimensions (in hundreths of inches)

c $\quad \mathrm{qqq} 2=$ the maximum dimensions (in hundreths of inches)

c $\quad \mathrm{qqq} 3=$ the delta (in hundreths of inches)

$\mathrm{c}$

c Diameter $=<\mathrm{d} 1=$ QQQ1:QQQ2:QQQ3 $\mid 5>$ hundreths of inches - must be integer

$\mathrm{c}$ radius $=<\mathrm{rd}=\mathrm{d} 1 * 2.54 / 100 / 2 \mid 5.4>\mathrm{cm}$

c note for perl, numbers with leading zeros must be enclosed in single quotes or else

c they will be interpretted as octal

$\mathrm{c}$ Conc $=<\mathrm{c}={ }^{\prime}$ ZZZ1' $\mid 5>$ milli-g U/cc

c Reflector width $=<\mathrm{rw}=1.0 \mid 2.1>$ inches

c Enrichment $=<$ ena $=1.0 \mid 1.2>$ atom fraction $\mathrm{U}$

c Mols of Acid per liter $<$ molAcid $=6.0 \mid 1.2>$

$\mathrm{c}$

$\mathrm{c}$

c Density of solution $<$ denSol $=Z Z Z 2 / 1 \mathrm{e} 5 \mid 1.5>$

c

c

c Atomic Weights

c $\mathrm{H} 2 \mathrm{O}=\langle$ atwH2O $=18.0152 \mid 3.4\rangle$

c NO3 $=<$ atwNO3 $=62.0049 \mid 3.4>$

c $\mathrm{O}=\langle$ atwO $=15.9994| 3.4>$

c HNO3 $=<$ atwHNO3 $=63.0128 \mid 3.4>$

c U235 $=<$ atwU235 $=235.0439 \mid 3.4>$

c U238 $=<$ atwU238 $=238.0508 \mid 3.4>$

c U $=<$ atwU $=$ ena*atwU235+(1-ena)*atwU238 $3.4>$

$\mathrm{c}$

c Molarity of solution components

c Mols of $\mathrm{U}$ per liter $<$ molU $=\mathrm{c} / \mathrm{atwU} \mid 3.4>$

c Mols of $\mathrm{H} 2 \mathrm{O}$ per liter $<$ molH2O $=(1000 *$ denSol-molU* $(\operatorname{atwU}+2 *$ atwNO3+2*atwO $)-$ molAcid*atwHNO3)/atwH2O|3.4>

$\mathrm{c}$

c

$<$ perl $>$

sub IMP

\{

return 1 if $\left(\$ \_[0]>0\right)$;

return 0 ;

\}

$</$ perl $>$

c semi inifinite plane

$2011<-$ denSol|3.6> $-12-7-586 \quad$ imp:n=1

$212-1.000 \quad-3468-7-5((1-3):(-24)) \quad$ imp:n=<\&IMP(rw) $\mid 1>$ \$zero importance if no reflector 


\begin{tabular}{|c|c|c|c|}
\hline 220 & & $3:-4: 7: 5:-8:-6$ & imp: $n=0$ \\
\hline 1 & py & $\langle\mathrm{rd}| 4.4>$ & \$ Cylinder \\
\hline 2 & py & $<-\operatorname{rd} \mid 4.4>$ & \\
\hline 3 & py & $<\mathrm{rd}+\mathrm{rw}{ }^{*} \mathrm{in} \mid 4.4>$ & \$ Reflection \\
\hline 4 & py & $<-$ rd-rw*in $\mid 4.4>$ & \\
\hline$* 5$ & px & 100 & \\
\hline$* 6$ & $\mathrm{px}$ & -100 & \\
\hline$* 7$ & $\mathrm{pz}$ & 100 & \\
\hline$* 8$ & $\mathrm{pz}$ & -100 & \\
\hline
\end{tabular}

mode $\mathrm{n}$

kcode 20001.0100 ZZZ0

$\begin{array}{llll}\text { ksrc } & 0.00 & 0.00 & 0.00\end{array}$

$\begin{array}{lll}0.00 & 0.00 & 0.10\end{array}$

$\begin{array}{lll}0.00 & 0.00 & 0.50\end{array}$

$\begin{array}{lll}0.00 & 0.00 & 1.00\end{array}$

$\begin{array}{lll}0.00 & 0.00 & 2.00\end{array}$

$\begin{array}{lll}0.00 & 0.00 & 3.00\end{array}$

$\begin{array}{lll}1.00 & 1.00 & 1.00\end{array}$

$\begin{array}{lll}1.00 & 1.00 & 2.00\end{array}$

$\begin{array}{lll}1.00 & 1.00 & 3.00\end{array}$

$\begin{array}{lll}2.00 & 2.00 & 1.00\end{array}$

$\begin{array}{lll}2.00 & 2.00 & 2.00\end{array}$

$\begin{array}{lll}2.00 & 2.00 & 3.00\end{array}$

$\begin{array}{lll}-1.00 & -1.00 & -1.00\end{array}$

$\begin{array}{lll}-10.00 & -10.00 & -20.00\end{array}$

$\begin{array}{lll}-10.00 & -10.00 & -30.00\end{array}$

c

m2 \$ water

$1001.66 \mathrm{c} \quad 2$

$8016.66 \mathrm{c} \quad 1$

$\mathrm{mt} 2$ 1wtr.60t

c UO2/water mixture

m11 \$ UO2(NO3)2 - NO3 - H2O, $100.0 \mathrm{wt} \%$ U235, ZZZ1 milli-g U/cc, $<$ denSol|3.4 $>$

c molarity $\mathrm{U}=\langle\operatorname{molU}| 3.4>$, acid $=\langle\operatorname{mol} A c i d| 3.4>, \mathrm{H} 2 \mathrm{O}=\langle\operatorname{molH} 2 \mathrm{O} \mid 3.4\rangle$

c U in salt

$92235.66 \mathrm{c}<$ molU*ena $3.4>$

c $92238.66 \mathrm{c}<$ molU* $(1$-ena $) \mid 3.4>$

c NO3 in salt plus acid

$7014.66 \mathrm{c}<2 * \mathrm{molU}+\operatorname{molAcid} \mid 3.4>$

$8016.66 \mathrm{c}<6 * \operatorname{molU}+3 * \operatorname{mol} \mathrm{Acid} \mid 3.4>$

c water

1001.66c $<2 *$ molH2O + molAcid|3.4 $>$ \$ncludes Free Hydrogen Ion

$8016.66 \mathrm{c}<\mathrm{molH} 2 \mathrm{O} \mid 3.4>$

mt11 1wtr.60t

c

c 


\section{Template File for Case: u5o2N26-pl-1000-10}

$\mathrm{pl}, 100.0 \mathrm{wt} \% \mathrm{UO} 2, \mathrm{ZZZ1}$ milli-g/cc, in UO2(NO3)2, with 6 Molar HNO3

c

c worm notes: format of the form $\mid n . m$ for floating point, $\mid \mathrm{n}$ for integers

c $\quad$ zzz0 (in upper case) is replaced with the number of cyles

c $\quad$ zzz1 $=$ the actual concentration in units of milli-g/cc

c $\quad$ zzz2 $=$ solution density in $\mathrm{g} / \mathrm{cc}$

c $\quad \mathrm{qqq} 1=$ the minimum dimensions (in hundreths of inches)

c $\quad \mathrm{qqq} 2$ = the maximum dimensions (in hundreths of inches)

c $\quad \mathrm{qqq} 3=$ the delta (in hundreths of inches)

$\mathrm{c}$

c Diameter $=<\mathrm{d} 1=$ QQQ1:QQQ2:QQQ3 $\mid 5>$ hundreths of inches - must be integer

$\mathrm{c}$ radius $=<\mathrm{rd}=\mathrm{d} 1 * 2.54 / 100 / 2 \mid 5.4>\mathrm{cm}$

c note for perl, numbers with leading zeros must be enclosed in single quotes or else

c they will be interpretted as octal

$\mathrm{c}$ Conc $=<\mathrm{c}={ }^{\prime}$ ZZZ1' $\mid 5>$ milli-g U/cc

c Reflector width $=<\mathrm{rw}=10.0 \mid 2.1>$ inches

c Enrichment $=<$ ena $=1.0 \mid 1.2>$ atom fraction $\mathrm{U}$

c Mols of Acid per liter $<$ molAcid $=6.0 \mid 1.2>$

$\mathrm{c}$

$\mathrm{c}$

c Density of solution $<$ denSol $=Z Z Z 2 / 1$ e $5 \mid 1.5>$

c

c

c Atomic Weights

c $\mathrm{H} 2 \mathrm{O}=\langle$ atwH2O $=18.0152| 3.4>$

$\mathrm{c} \mathrm{NO3}=\langle$ atwNO3 $=62.0049| 3.4>$

c $\mathrm{O}=\langle$ atwO $=15.9994| 3.4>$

$\mathrm{c} \mathrm{HNO} 3=<$ atwHNO3 $=63.0128 \mid 3.4>$

c U235 $=<$ atwU235 $=235.0439 \mid 3.4>$

c U238 $=<$ atwU238 $=238.0508 \mid 3.4>$

c U $=<$ atwU $=$ ena*atwU235+(1-ena)*atwU238 $3.4>$

c

c Molarity of solution components

c Mols of $\mathrm{U}$ per liter $<\mathrm{molU}=\mathrm{c} / \mathrm{atwU} \mid 3.4>$

c Mols of $\mathrm{H} 2 \mathrm{O}$ per liter $<$ molH2O $=(1000 *$ denSol-molU* $(\operatorname{atwU}+2 *$ atwNO3+2*atwO $)-$ molAcid*atwHNO3)/atwH2O|3.4>

c

c

$<$ perl $>$

sub IMP

\{

return 1 if $\left(\$ \_[0]>0\right)$;

return 0 ;

\}

$</$ perl $>$

c semi inifinite plane

$2011<-$ denSol|3.6> $-12-7-586 \quad$ imp:n=1

$212-1.000 \quad-3468-7-5((1-3):(-24)) \quad$ imp:n=<\&IMP(rw) $\mid 1>$ \$zero importance if no reflector 


\begin{tabular}{|c|c|c|c|}
\hline 220 & & $3:-4: 7: 5:-8:-6$ & imp: $n=0$ \\
\hline 1 & py & $\langle\mathrm{rd}| 4.4>$ & \$ Cylinder \\
\hline 2 & py & $<-\operatorname{rd} \mid 4.4>$ & \\
\hline 3 & py & $<\mathrm{rd}+\mathrm{rw}{ }^{*} \mathrm{in} \mid 4.4>$ & \$ Reflection \\
\hline 4 & py & $<-$ rd-rw*in $\mid 4.4>$ & \\
\hline$* 5$ & px & 100 & \\
\hline$* 6$ & $\mathrm{px}$ & -100 & \\
\hline$* 7$ & $\mathrm{pz}$ & 100 & \\
\hline$* 8$ & $\mathrm{pz}$ & -100 & \\
\hline
\end{tabular}

mode $\mathrm{n}$

kcode 20001.0100 ZZZ0

$\begin{array}{llll}\text { ksrc } & 0.00 & 0.00 & 0.00\end{array}$

$\begin{array}{lll}0.00 & 0.00 & 0.10\end{array}$

$\begin{array}{lll}0.00 & 0.00 & 0.50\end{array}$

$\begin{array}{lll}0.00 & 0.00 & 1.00\end{array}$

$\begin{array}{lll}0.00 & 0.00 & 2.00\end{array}$

$\begin{array}{lll}0.00 & 0.00 & 3.00\end{array}$

$\begin{array}{lll}1.00 & 1.00 & 1.00\end{array}$

$\begin{array}{lll}1.00 & 1.00 & 2.00\end{array}$

$\begin{array}{lll}1.00 & 1.00 & 3.00\end{array}$

$\begin{array}{lll}2.00 & 2.00 & 1.00\end{array}$

$\begin{array}{lll}2.00 & 2.00 & 2.00\end{array}$

$\begin{array}{lll}2.00 & 2.00 & 3.00\end{array}$

$\begin{array}{lll}-1.00 & -1.00 & -1.00\end{array}$

$\begin{array}{lll}-10.00 & -10.00 & -20.00\end{array}$

$\begin{array}{lll}-10.00 & -10.00 & -30.00\end{array}$

c

m2 \$ water

$1001.66 \mathrm{c} \quad 2$

$8016.66 \mathrm{c} \quad 1$

$\mathrm{mt} 2$ 1wtr.60t

c UO2/water mixture

m11 \$ UO2(NO3)2 - NO3 - H2O, $100.0 \mathrm{wt} \% \mathrm{U} 235, \mathrm{ZZZ1} \mathrm{milli-g} \mathrm{U/cc,}<\mathrm{denSol} \mid 3.4>$

c molarity $\mathrm{U}=\langle\operatorname{molU}| 3.4>$, acid $=\langle\operatorname{mol} A c i d| 3.4>, \mathrm{H} 2 \mathrm{O}=\langle\operatorname{molH} 2 \mathrm{O} \mid 3.4\rangle$

c U in salt

$92235.66 \mathrm{c}<$ molU*ena $3.4>$

c $92238.66 \mathrm{c}<$ molU* $(1$-ena $) \mid 3.4>$

c NO3 in salt plus acid

$7014.66 \mathrm{c}<2 * \operatorname{molU}+\operatorname{molAcid} \mid 3.4>$

$8016.66 \mathrm{c}<6 * \operatorname{molU}+3 * \operatorname{mol} \mathrm{Acid} \mid 3.4>$

c water

1001.66c $<2 *$ molH2O + molAcid|3.4 $>$ \$ncludes Free Hydrogen Ion

$8016.66 \mathrm{c}<\mathrm{molH} 2 \mathrm{O} \mid 3.4>$

mt11 1wtr.60t

c

c 
CHPRC-01552, Revision 0

D-197 
CHPRC-01552, Revision 0

D-198 


\section{Template File for Case: u5o2N20-pl-0935-00}

$\mathrm{pl}, 93.5 \mathrm{wt} \% \mathrm{UO} 2, \mathrm{ZZZ1}$ milli-g/cc, in UO2(NO3)2, with 0 Molar HNO3

c

c worm notes: format of the form $\mid n . m$ for floating point, $\mid \mathrm{n}$ for integers

c $\quad$ zzz0 (in upper case) is replaced with the number of cyles

c $\quad$ zzz1 $=$ the actual concentration in units of milli-g/cc

c $\quad$ zzz2 $=$ solution density in $\mathrm{g} / \mathrm{cc}$

c $\quad \mathrm{qqq} 1=$ the minimum dimensions (in hundreths of inches)

c $\quad \mathrm{qqq} 2$ = the maximum dimensions (in hundreths of inches)

c $\quad \mathrm{qqq} 3=$ the delta (in hundreths of inches)

$\mathrm{c}$

c Diameter $=<\mathrm{d} 1=$ QQQ1:QQQ2:QQQ3 $\mid 5>$ hundreths of inches - must be integer

$\mathrm{c}$ radius $=<\mathrm{rd}=\mathrm{d} 1 * 2.54 / 100 / 2 \mid 5.4>\mathrm{cm}$

c note for perl, numbers with leading zeros must be enclosed in single quotes or else

c they will be interpretted as octal

$\mathrm{c}$ Conc $=<\mathrm{c}={ }^{\prime} \mathrm{ZZZ1} 1 \mid 5>$ milli-g U/cc

c Reflector width $=<\mathrm{rw}=0.0 \mid 2.1>$ inches

c Enrichment $=<$ ena $=0.935 \mid 1.3>$ atom fraction $\mathrm{U}$

c Mols of Acid per liter $<$ molAcid $=0.0 \mid 1.2>$

$\mathrm{c}$

$\mathrm{c}$

c Density of solution $<$ denSol $=Z Z Z 2 / 1 \mathrm{e} 5 \mid 1.5>$

c

C

c Atomic Weights

c $\mathrm{H} 2 \mathrm{O}=\langle$ atwH2O $=18.0152 \mid 3.4\rangle$

c NO3 $=<$ atwNO3 $=62.0049 \mid 3.4>$

c $\mathrm{O}=\langle$ atwO $=15.9994| 3.4>$

c HNO3 $=<$ atwHNO3 $=63.0128 \mid 3.4>$

c U235 $=<$ atwU235 $=235.0439 \mid 3.4>$

c U238 $=<$ atwU238 $=238.0508 \mid 3.4>$

c U $=<$ atwU $=$ ena*atwU235+(1-ena)*atwU238 $3.4>$

$\mathrm{c}$

c Molarity of solution components

c Mols of $\mathrm{U}$ per liter $<$ molU $=\mathrm{c} / \mathrm{atwU} \mid 3.4>$

c Mols of $\mathrm{H} 2 \mathrm{O}$ per liter $<$ molH2O $=(1000 *$ denSol-molU* $(\operatorname{atwU}+2 *$ atwNO3+2*atwO $)-$ molAcid*atwHNO3)/atwH2O|3.4>

$\mathrm{c}$

c

$<$ perl $>$

sub IMP

\{

return 1 if $\left(\$ \_[0]>0\right)$;

return 0 ;

\}

$</$ perl $>$

c semi inifinite plane

$2011<-$ denSol|3.6> $-12-7-586 \quad$ imp:n=1

$212-1.000 \quad-3468-7-5((1-3):(-24)) \quad$ imp:n=<\&IMP(rw) $\mid 1>\quad$ \$zero importance if no reflector 


\begin{tabular}{|c|c|c|c|}
\hline 220 & & $3:-4: 7: 5:-8:-6$ & imp: $n=0$ \\
\hline 1 & py & $<\mathrm{rd} \mid 4.4>$ & \$ Cylinder \\
\hline 2 & py & $<-\operatorname{rd} \mid 4.4>$ & \\
\hline 3 & py & $<\mathrm{rd}+\mathrm{rw}^{*}$ in $\mid 4.4>$ & \$ Reflection \\
\hline 4 & py & $<-$ rd-rw*in $\mid 4.4>$ & \\
\hline$* 5$ & $\mathrm{px}$ & 100 & \\
\hline$* 6$ & $\mathrm{px}$ & -100 & \\
\hline$* 7$ & $\mathrm{pz}$ & 100 & \\
\hline$* 8$ & $\mathrm{pz}$ & -100 & \\
\hline
\end{tabular}

mode $\mathrm{n}$

kcode 20001.0100 ZZZ0

$\begin{array}{llll}\text { ksrc } & 0.00 & 0.00 & 0.00\end{array}$

$\begin{array}{lll}0.00 & 0.00 & 0.10\end{array}$

$\begin{array}{lll}0.00 & 0.00 & 0.50\end{array}$

$\begin{array}{lll}0.00 & 0.00 & 1.00\end{array}$

$\begin{array}{lll}0.00 & 0.00 & 2.00\end{array}$

$\begin{array}{lll}0.00 & 0.00 & 3.00\end{array}$

$\begin{array}{lll}1.00 & 1.00 & 1.00\end{array}$

$\begin{array}{lll}1.00 & 1.00 & 2.00\end{array}$

$\begin{array}{lll}1.00 & 1.00 & 3.00\end{array}$

$\begin{array}{lll}2.00 & 2.00 & 1.00\end{array}$

$\begin{array}{lll}2.00 & 2.00 & 2.00\end{array}$

$\begin{array}{lll}2.00 & 2.00 & 3.00\end{array}$

$\begin{array}{lll}-1.00 & -1.00 & -1.00\end{array}$

$\begin{array}{lll}-10.00 & -10.00 & -20.00\end{array}$

$\begin{array}{lll}-10.00 & -10.00 & -30.00\end{array}$

c

m2 \$ water

$1001.66 \mathrm{c} \quad 2$

$8016.66 \mathrm{c} \quad 1$

$\mathrm{mt} 2$ 1wtr.60t

c UO2/water mixture

m11 \$ UO2(NO3)2 - NO3 - H2O, $100.0 \mathrm{wt} \%$ U235, ZZZ1 milli-g U/cc, $<$ denSol|3.4 $>$

c molarity $\mathrm{U}=\langle\operatorname{molU}| 3.4>$, acid $=\langle\operatorname{mol} A c i d| 3.4>, \mathrm{H} 2 \mathrm{O}=\langle\operatorname{molH} 2 \mathrm{O} \mid 3.4\rangle$

c U in salt

$92235.66 \mathrm{c}<$ molU*ena $3.4>$

$92238.66 \mathrm{c}<$ molU* $(1$-ena $) \mid 3.4>$

c NO3 in salt plus acid

$7014.66 \mathrm{c}<2 * \mathrm{molU}+\operatorname{molAcid} \mid 3.4>$

$8016.66 \mathrm{c}<6 * \operatorname{molU}+3 * \operatorname{mol} \mathrm{Acid} \mid 3.4>$

c water

$1001.66 \mathrm{c}<2 * \mathrm{molH} 2 \mathrm{O}+$ molAcid|3.4 $>$ \$Includes Free Hydrogen Ion

$8016.66 \mathrm{c}<\mathrm{molH} 2 \mathrm{O} \mid 3.4>$

mt11 1wtr.60t

c

c 


\section{Template File for Case: u5o2N20-pl-0935-01}

$\mathrm{pl}, 93.5 \mathrm{wt} \% \mathrm{UO} 2, \mathrm{ZZZ1}$ milli-g/cc, in UO2(NO3)2, with 0 Molar HNO3

c

c worm notes: format of the form $\mid n . m$ for floating point, $\mid \mathrm{n}$ for integers

c $\quad$ zzz0 (in upper case) is replaced with the number of cyles

c $\quad$ zzz1 $=$ the actual concentration in units of milli-g/cc

c $\quad$ zzz2 $=$ solution density in $\mathrm{g} / \mathrm{cc}$

c $\quad \mathrm{qqq} 1=$ the minimum dimensions (in hundreths of inches)

c $\quad \mathrm{qqq} 2$ = the maximum dimensions (in hundreths of inches)

c $\quad \mathrm{qqq} 3=$ the delta (in hundreths of inches)

$\mathrm{c}$

c Diameter $=<\mathrm{d} 1=$ QQQ1:QQQ2:QQQ3 $\mid 5>$ hundreths of inches - must be integer

$\mathrm{c}$ radius $=<\mathrm{rd}=\mathrm{d} 1 * 2.54 / 100 / 2 \mid 5.4>\mathrm{cm}$

c note for perl, numbers with leading zeros must be enclosed in single quotes or else

c they will be interpretted as octal

$\mathrm{c}$ Conc $=<\mathrm{c}={ }^{\prime}$ ZZZ1' $\mid 5>$ milli-g U/cc

c Reflector width $=<\mathrm{rw}=1.0 \mid 2.1>$ inches

c Enrichment $=<$ ena $=0.935 \mid 1.3>$ atom fraction $\mathrm{U}$

c Mols of Acid per liter $<$ molAcid $=0.0 \mid 1.2>$

$\mathrm{c}$

$\mathrm{c}$

c Density of solution $<$ denSol $=Z Z Z 2 / 1 \mathrm{e} 5 \mid 1.5>$

c

C

c Atomic Weights

c $\mathrm{H} 2 \mathrm{O}=\langle$ atwH2O $=18.0152| 3.4>$

$\mathrm{c} \mathrm{NO3}=\langle$ atwNO3 $=62.0049| 3.4>$

c $\mathrm{O}=\langle$ atwO $=15.9994| 3.4>$

c HNO3 $=<$ atwHNO3 $=63.0128 \mid 3.4>$

c U235 $=<$ atwU235 $=235.0439 \mid 3.4>$

c U238 $=<$ atwU238 $=238.0508 \mid 3.4>$

c U $=<$ atwU $=$ ena*atwU235+(1-ena)*atwU238 $3.4>$

$\mathrm{c}$

c Molarity of solution components

c Mols of $\mathrm{U}$ per liter $<\mathrm{molU}=\mathrm{c} / \mathrm{atwU} \mid 3.4>$

c Mols of $\mathrm{H} 2 \mathrm{O}$ per liter $<$ molH2O $=(1000 *$ denSol-molU* $(\operatorname{atwU}+2 *$ atwNO3+2*atwO $)-$ molAcid*atwHNO3)/atwH2O|3.4>

c

c

$<$ perl $>$

sub IMP

\{

return 1 if $\left(\$ \_[0]>0\right)$;

return 0 ;

\}

$</$ perl $>$

c semi inifinite plane

$2011<-$ denSol|3.6> $-12-7-586 \quad$ imp:n=1

$212-1.000 \quad-3468-7-5((1-3):(-24)) \quad$ imp:n=<\&IMP(rw) $\mid 1>$ \$zero importance if no reflector 


\begin{tabular}{|c|c|c|c|}
\hline 220 & & $3:-4: 7: 5:-8:-6$ & imp: $n=0$ \\
\hline 1 & py & $<\mathrm{rd} \mid 4.4>$ & \$ Cylinder \\
\hline 2 & py & $<-\operatorname{rd} \mid 4.4>$ & \\
\hline 3 & py & $<\mathrm{rd}+\mathrm{rw}^{*}$ in $\mid 4.4>$ & \$ Reflection \\
\hline 4 & py & $<-$ rd-rw*in $\mid 4.4>$ & \\
\hline$* 5$ & $\mathrm{px}$ & 100 & \\
\hline$* 6$ & $\mathrm{px}$ & -100 & \\
\hline$* 7$ & $\mathrm{pz}$ & 100 & \\
\hline$* 8$ & $\mathrm{pz}$ & -100 & \\
\hline
\end{tabular}

mode $\mathrm{n}$

kcode 20001.0100 ZZZ0

$\begin{array}{llll}\text { ksrc } & 0.00 & 0.00 & 0.00\end{array}$

$\begin{array}{lll}0.00 & 0.00 & 0.10\end{array}$

$\begin{array}{lll}0.00 & 0.00 & 0.50\end{array}$

$\begin{array}{lll}0.00 & 0.00 & 1.00\end{array}$

$\begin{array}{lll}0.00 & 0.00 & 2.00\end{array}$

$\begin{array}{lll}0.00 & 0.00 & 3.00\end{array}$

$\begin{array}{lll}1.00 & 1.00 & 1.00\end{array}$

$\begin{array}{lll}1.00 & 1.00 & 2.00\end{array}$

$\begin{array}{lll}1.00 & 1.00 & 3.00\end{array}$

$\begin{array}{lll}2.00 & 2.00 & 1.00\end{array}$

$\begin{array}{lll}2.00 & 2.00 & 2.00\end{array}$

$\begin{array}{lll}2.00 & 2.00 & 3.00\end{array}$

$\begin{array}{lll}-1.00 & -1.00 & -1.00\end{array}$

$\begin{array}{lll}-10.00 & -10.00 & -20.00\end{array}$

$\begin{array}{lll}-10.00 & -10.00 & -30.00\end{array}$

c

m2 \$ water

$1001.66 \mathrm{c} \quad 2$

$8016.66 \mathrm{c} \quad 1$

$\mathrm{mt} 2$ 1wtr.60t

c UO2/water mixture

m11 \$ UO2(NO3)2 - NO3 - H2O, $100.0 \mathrm{wt} \%$ U235, ZZZ1 milli-g U/cc, $<$ denSol|3.4 $>$

c molarity $\mathrm{U}=\langle\operatorname{molU}| 3.4>$, acid $=\langle\operatorname{mol} A c i d| 3.4>, \mathrm{H} 2 \mathrm{O}=\langle\operatorname{molH} 2 \mathrm{O} \mid 3.4\rangle$

c U in salt

$92235.66 \mathrm{c}<$ molU*ena $3.4>$

$92238.66 \mathrm{c}<$ molU* $(1$-ena $) \mid 3.4>$

c NO3 in salt plus acid

$7014.66 \mathrm{c}<2 * \mathrm{molU}+\operatorname{molAcid} \mid 3.4>$

$8016.66 \mathrm{c}<6 * \operatorname{molU}+3 * \operatorname{mol} \mathrm{Acid} \mid 3.4>$

c water

$1001.66 \mathrm{c}<2 * \mathrm{molH} 2 \mathrm{O}+$ molAcid|3.4 $>$ \$Includes Free Hydrogen Ion

$8016.66 \mathrm{c}<\mathrm{molH} 2 \mathrm{O} \mid 3.4>$

mt11 1wtr.60t

c

c 


\section{Template File for Case: u5o2N20-pl-0935-10}

$\mathrm{pl}, 93.5 \mathrm{wt} \% \mathrm{UO} 2, \mathrm{ZZZ1}$ milli-g/cc, in UO2(NO3)2, with 0 Molar HNO3

c

c worm notes: format of the form $\mid n . m$ for floating point, $\mid \mathrm{n}$ for integers

c $\quad$ zzz0 (in upper case) is replaced with the number of cyles

c $\quad$ zzz1 $=$ the actual concentration in units of milli-g/cc

c $\quad$ zzz2 $=$ solution density in $\mathrm{g} / \mathrm{cc}$

c $\quad \mathrm{qqq} 1=$ the minimum dimensions (in hundreths of inches)

c $\quad \mathrm{qqq} 2$ = the maximum dimensions (in hundreths of inches)

c $\quad \mathrm{qqq} 3=$ the delta (in hundreths of inches)

$\mathrm{c}$

c Diameter $=<\mathrm{d} 1=$ QQQ1:QQQ2:QQQ3 $\mid 5>$ hundreths of inches - must be integer

$\mathrm{c}$ radius $=<\mathrm{rd}=\mathrm{d} 1 * 2.54 / 100 / 2 \mid 5.4>\mathrm{cm}$

c note for perl, numbers with leading zeros must be enclosed in single quotes or else

c they will be interpretted as octal

$\mathrm{c}$ Conc $=<\mathrm{c}={ }^{\prime}$ ZZZ1' $\mid 5>$ milli-g U/cc

c Reflector width $=<\mathrm{rw}=10.0 \mid 2.1>$ inches

c Enrichment $=<$ ena $=0.935 \mid 1.2>$ atom fraction $\mathrm{U}$

c Mols of Acid per liter $<$ molAcid $=0.0 \mid 1.2>$

$\mathrm{c}$

$\mathrm{c}$

c Density of solution $<$ denSol $=Z Z Z 2 / 1 \mathrm{e} 5 \mid 1.5>$

c

c

c Atomic Weights

c $\mathrm{H} 2 \mathrm{O}=\langle$ atwH2O $=18.0152| 3.4>$

$\mathrm{c} \mathrm{NO3}=\langle$ atwNO3 $=62.0049| 3.4>$

c $\mathrm{O}=\langle$ atwO $=15.9994| 3.4>$

c HNO3 $=<$ atwHNO3 $=63.0128 \mid 3.4>$

c U235 $=<$ atwU235 $=235.0439 \mid 3.4>$

c U238 $=<$ atwU238 $=238.0508 \mid 3.4>$

c U $=<$ atwU $=$ ena*atwU235+(1-ena)*atwU238 $3.4>$

$\mathrm{c}$

c Molarity of solution components

c Mols of $\mathrm{U}$ per liter $<\mathrm{molU}=\mathrm{c} / \mathrm{atwU} \mid 3.4>$

c Mols of $\mathrm{H} 2 \mathrm{O}$ per liter $<$ molH2O $=(1000 *$ denSol-molU* $(\operatorname{atwU}+2 *$ atwNO3+2*atwO $)-$ molAcid*atwHNO3)/atwH2O|3.4>

c

c

$<$ perl $>$

sub IMP

\{

return 1 if $\left(\$ \_[0]>0\right)$;

return 0 ;

\}

$</$ perl $>$

c semi inifinite plane

$2011<-$ denSol|3.6> $-12-7-586 \quad$ imp:n=1

$212-1.000 \quad-3468-7-5((1-3):(-24)) \quad$ imp:n=<\&IMP(rw) $\mid 1>$ \$zero importance if no reflector 


\begin{tabular}{|c|c|c|c|}
\hline 220 & & $3:-4: 7: 5:-8:-6$ & imp: $n=0$ \\
\hline 1 & py & $<\mathrm{rd} \mid 4.4>$ & \$ Cylinder \\
\hline 2 & py & $<-\operatorname{rd} \mid 4.4>$ & \\
\hline 3 & py & $<\mathrm{rd}+\mathrm{rw}^{*}$ in $\mid 4.4>$ & \$ Reflection \\
\hline 4 & py & $<-$ rd-rw*in $\mid 4.4>$ & \\
\hline$* 5$ & $\mathrm{px}$ & 100 & \\
\hline$* 6$ & $\mathrm{px}$ & -100 & \\
\hline$* 7$ & $\mathrm{pz}$ & 100 & \\
\hline$* 8$ & $\mathrm{pz}$ & -100 & \\
\hline
\end{tabular}

mode $\mathrm{n}$

kcode 20001.0100 ZZZ0

$\begin{array}{llll}\text { ksrc } & 0.00 & 0.00 & 0.00\end{array}$

$\begin{array}{lll}0.00 & 0.00 & 0.10\end{array}$

$\begin{array}{lll}0.00 & 0.00 & 0.50\end{array}$

$\begin{array}{lll}0.00 & 0.00 & 1.00\end{array}$

$\begin{array}{lll}0.00 & 0.00 & 2.00\end{array}$

$\begin{array}{lll}0.00 & 0.00 & 3.00\end{array}$

$\begin{array}{lll}1.00 & 1.00 & 1.00\end{array}$

$\begin{array}{lll}1.00 & 1.00 & 2.00\end{array}$

$\begin{array}{lll}1.00 & 1.00 & 3.00\end{array}$

$\begin{array}{lll}2.00 & 2.00 & 1.00\end{array}$

$\begin{array}{lll}2.00 & 2.00 & 2.00\end{array}$

$\begin{array}{lll}2.00 & 2.00 & 3.00\end{array}$

$\begin{array}{lll}-1.00 & -1.00 & -1.00\end{array}$

$\begin{array}{lll}-10.00 & -10.00 & -20.00\end{array}$

$\begin{array}{lll}-10.00 & -10.00 & -30.00\end{array}$

c

m2 \$ water

$1001.66 \mathrm{c} \quad 2$

$8016.66 \mathrm{c} \quad 1$

$\mathrm{mt} 2$ 1wtr.60t

c UO2/water mixture

m11 \$ UO2(NO3)2 - NO3 - H2O, $100.0 \mathrm{wt} \%$ U235, ZZZ1 milli-g U/cc, $<$ denSol|3.4 $>$

c molarity $\mathrm{U}=\langle\operatorname{molU}| 3.4>$, acid $=\langle\operatorname{mol} A c i d| 3.4>, \mathrm{H} 2 \mathrm{O}=\langle\operatorname{molH} 2 \mathrm{O} \mid 3.4\rangle$

c U in salt

$92235.66 \mathrm{c}<$ molU*ena $3.4>$

$92238.66 \mathrm{c}<$ molU* $(1$-ena $) \mid 3.4>$

c NO3 in salt plus acid

$7014.66 \mathrm{c}<2 * \mathrm{molU}+\operatorname{molAcid} \mid 3.4>$

$8016.66 \mathrm{c}<6 * \operatorname{molU}+3 * \operatorname{mol} \mathrm{Acid} \mid 3.4>$

c water

$1001.66 \mathrm{c}<2 * \mathrm{molH} 2 \mathrm{O}+$ molAcid|3.4 $>$ \$Includes Free Hydrogen Ion

$8016.66 \mathrm{c}<\mathrm{molH} 2 \mathrm{O} \mid 3.4>$

mt11 1wtr.60t

c

c 
CHPRC-01552, Revision 0 
CHPRC-01552, Revision 0

\section{APPENDIX E - EXAMPLE INPUT FILES}

E-1 
CHPRC-01552, Revision 0

This page is intentionally left blank. 
Appendix E Sample Input Files

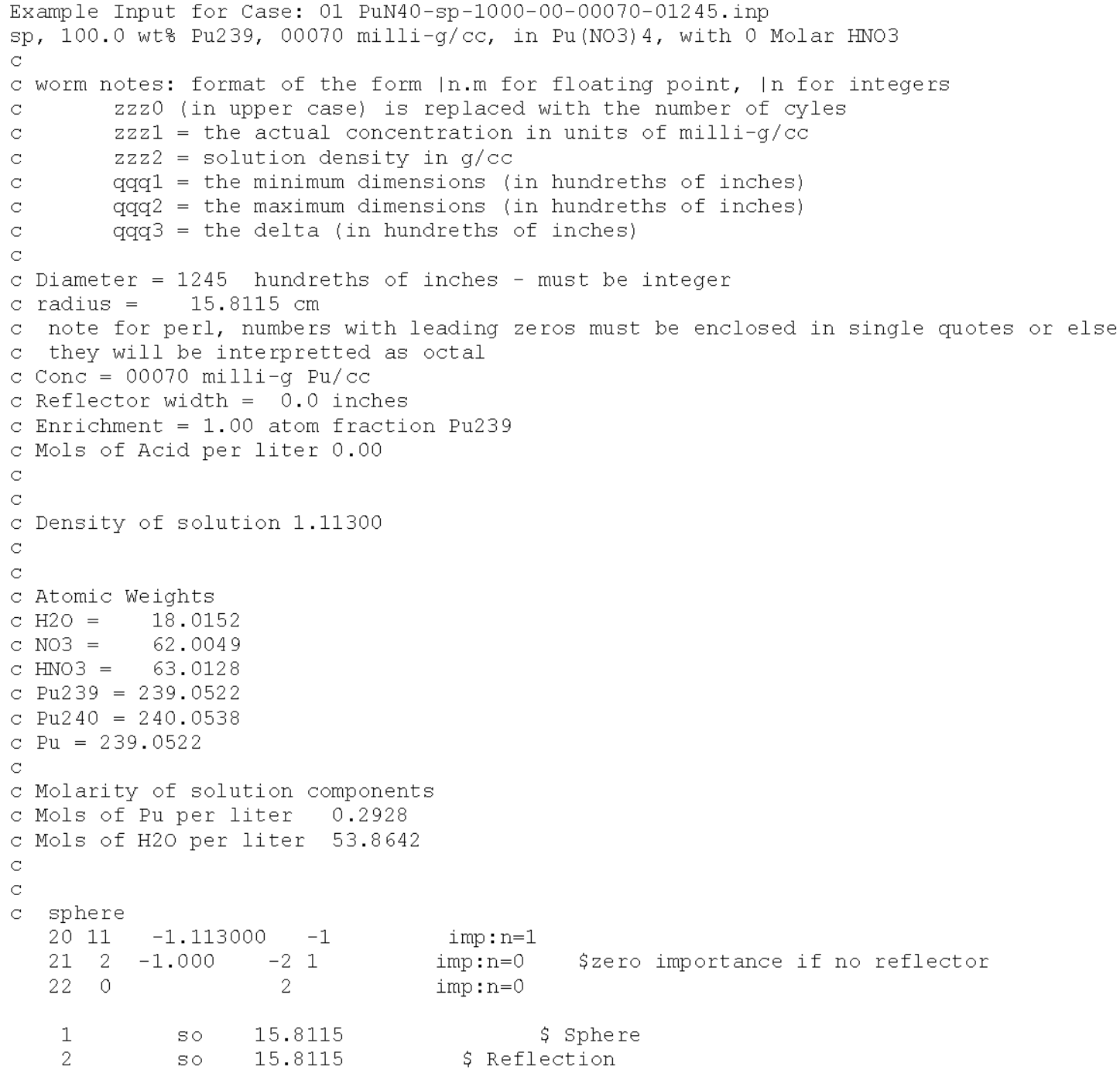




\section{CHPRC-01552, Revision 0}
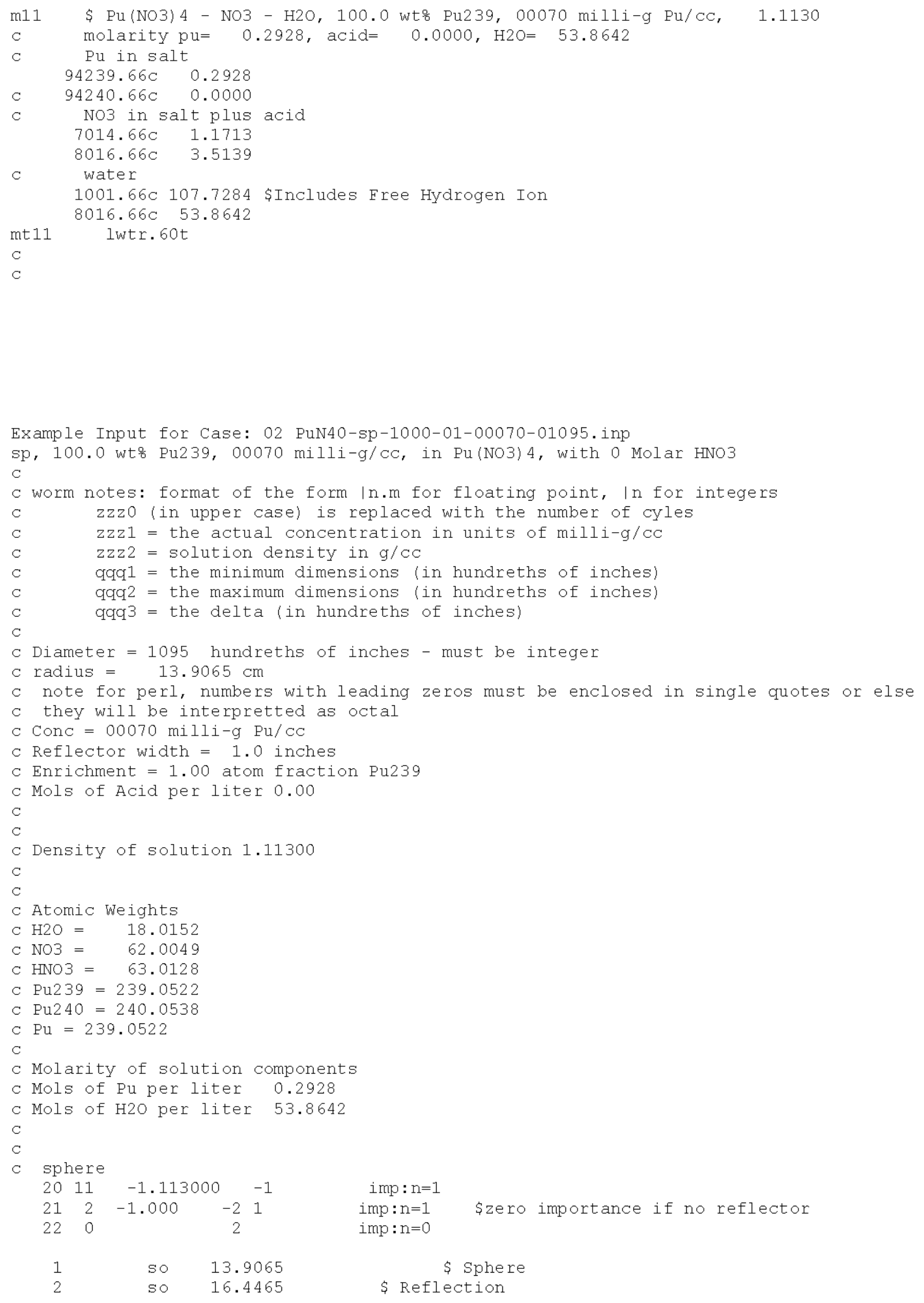

mode $n$

\begin{tabular}{|c|c|c|c|}
\hline ode & 2000 & 1.0100 & 2000 \\
\hline $\mathrm{ksrc}$ & 0.00 & 0.00 & 0.00 \\
\hline & 0.0 & 0.00 & 0.10 \\
\hline
\end{tabular}




\section{CHPRC-01552, Revision 0}

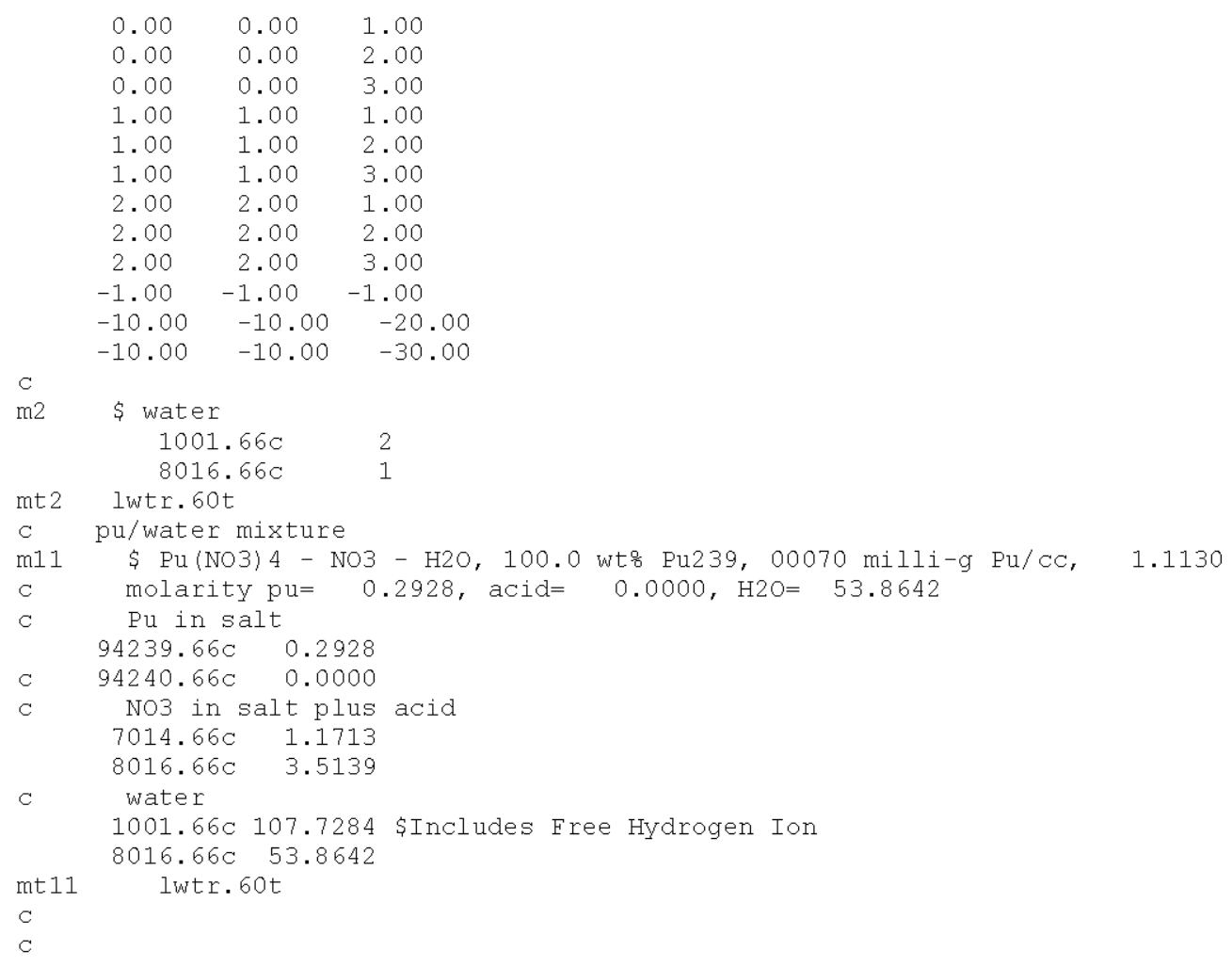




\section{CHPRC-01552, Revision 0}

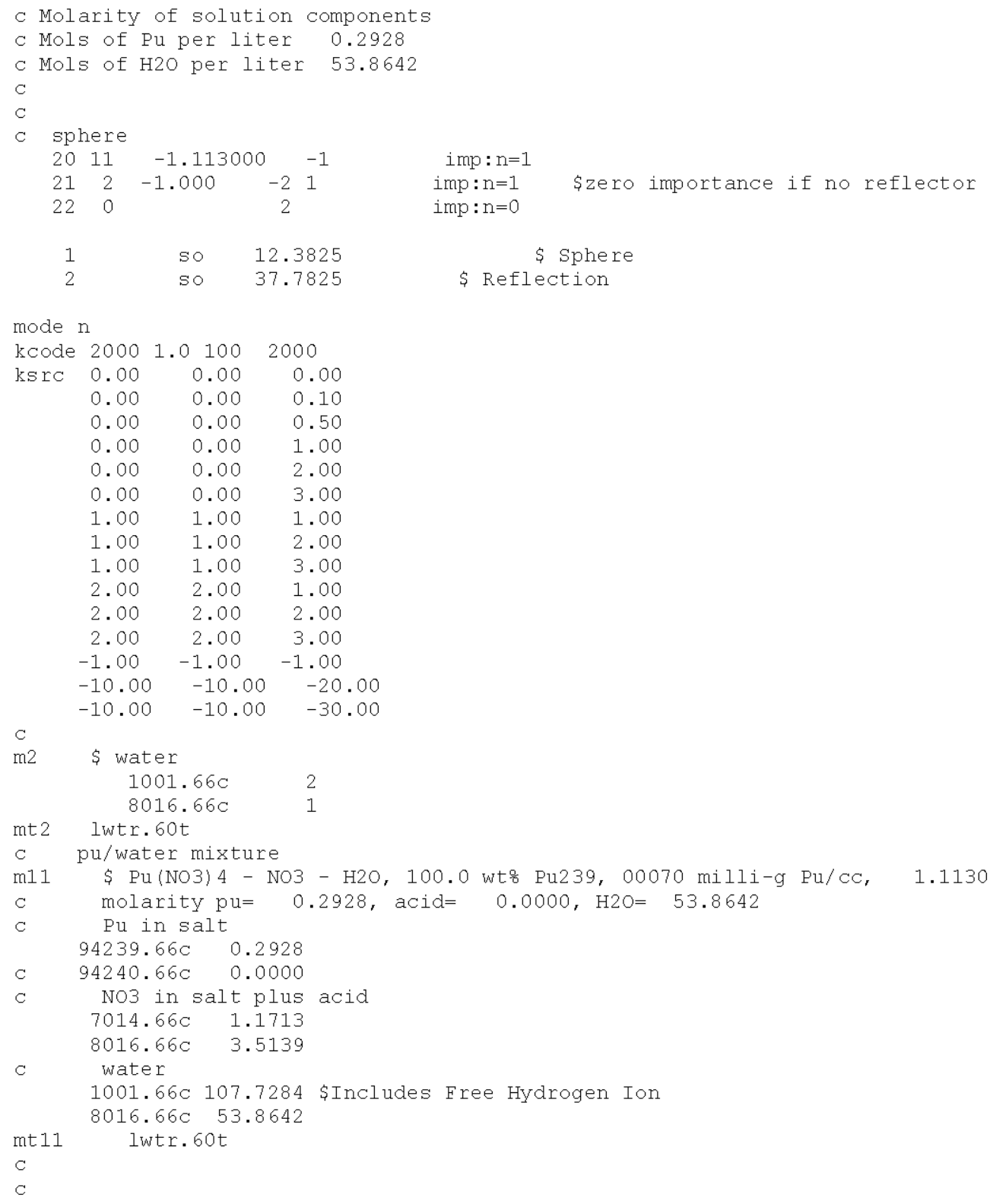




\section{CHPRC-01552, Revision 0}

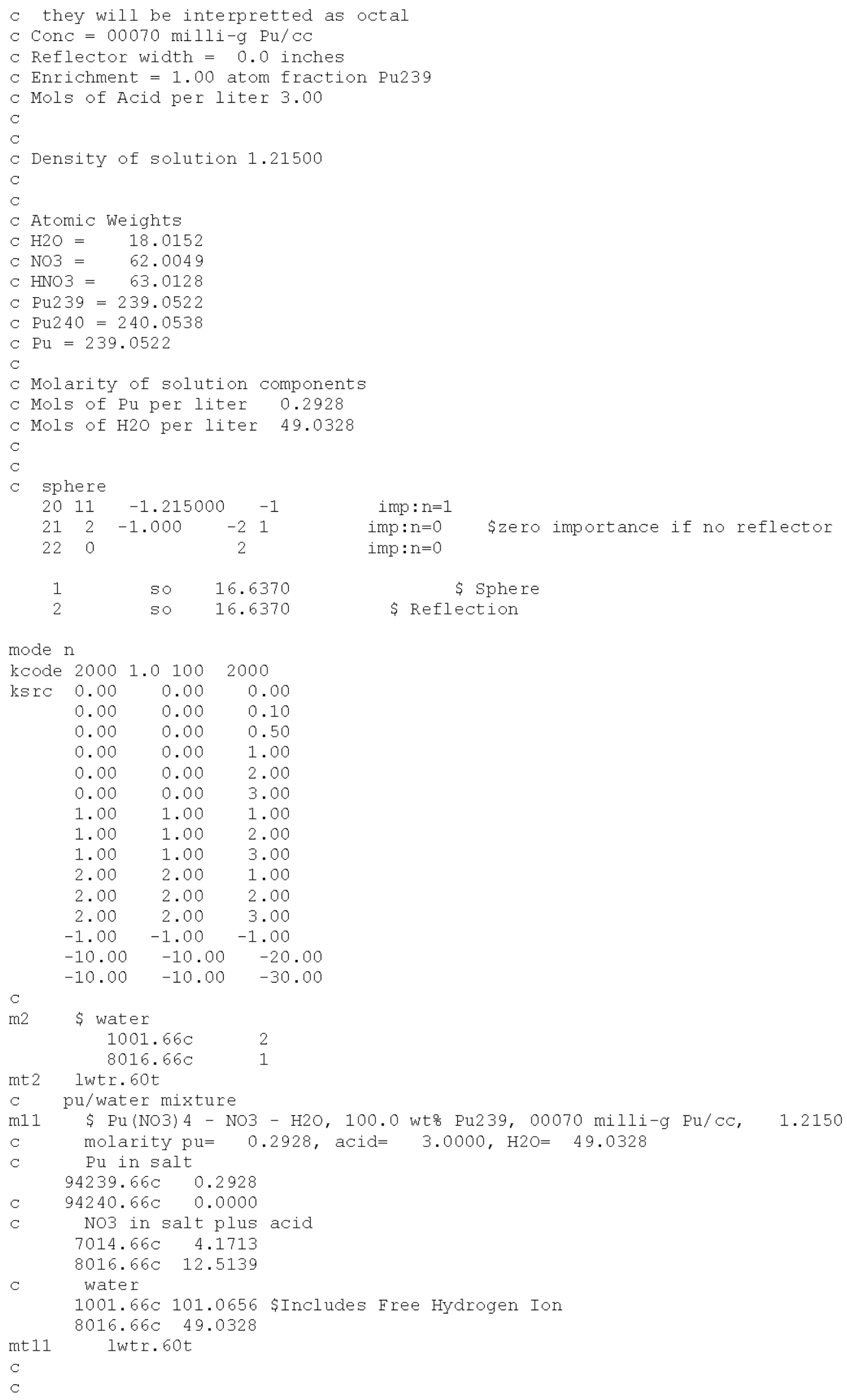




\section{CHPRC-01552, Revision 0}

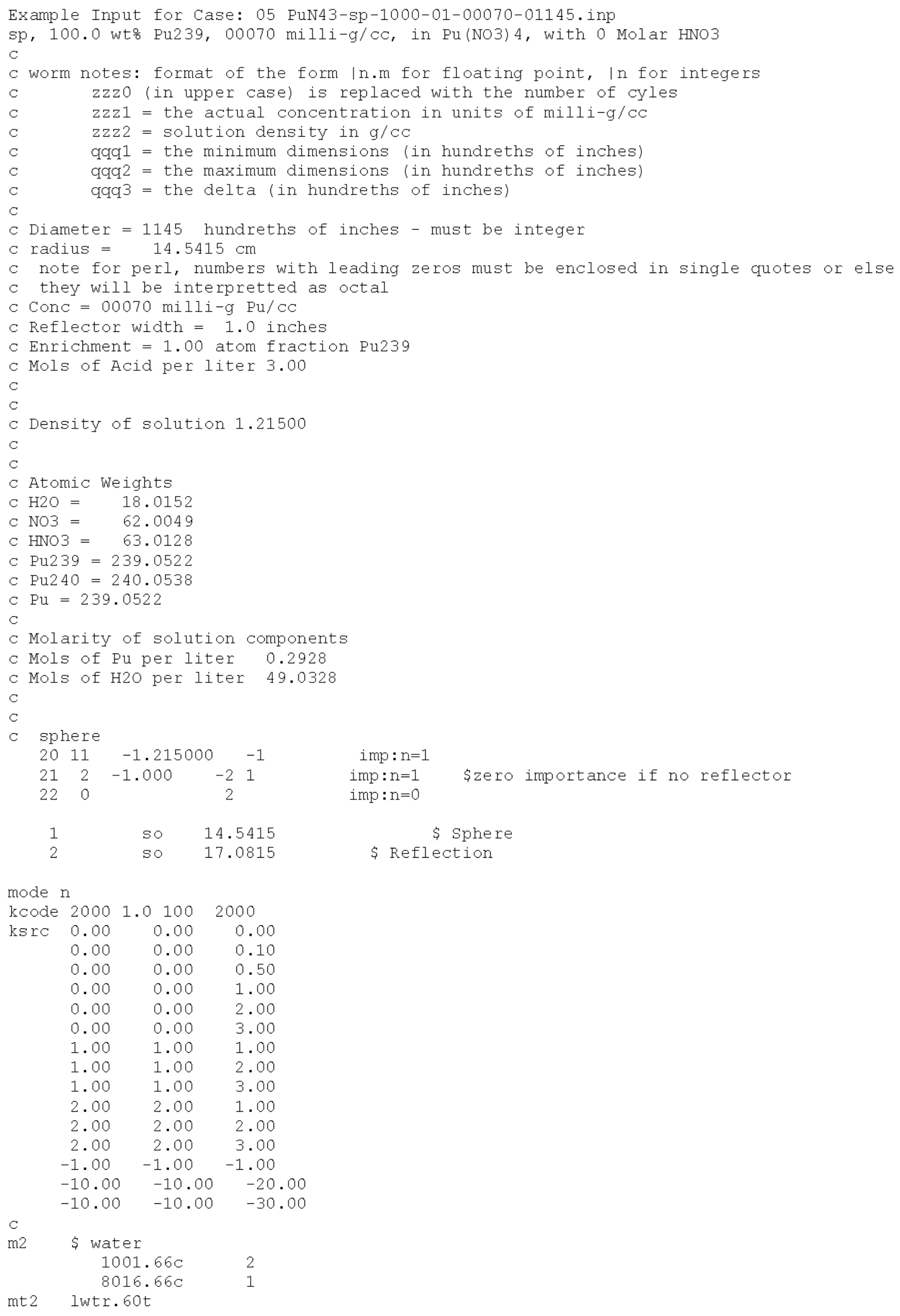




\section{CHPRC-01552, Revision 0}

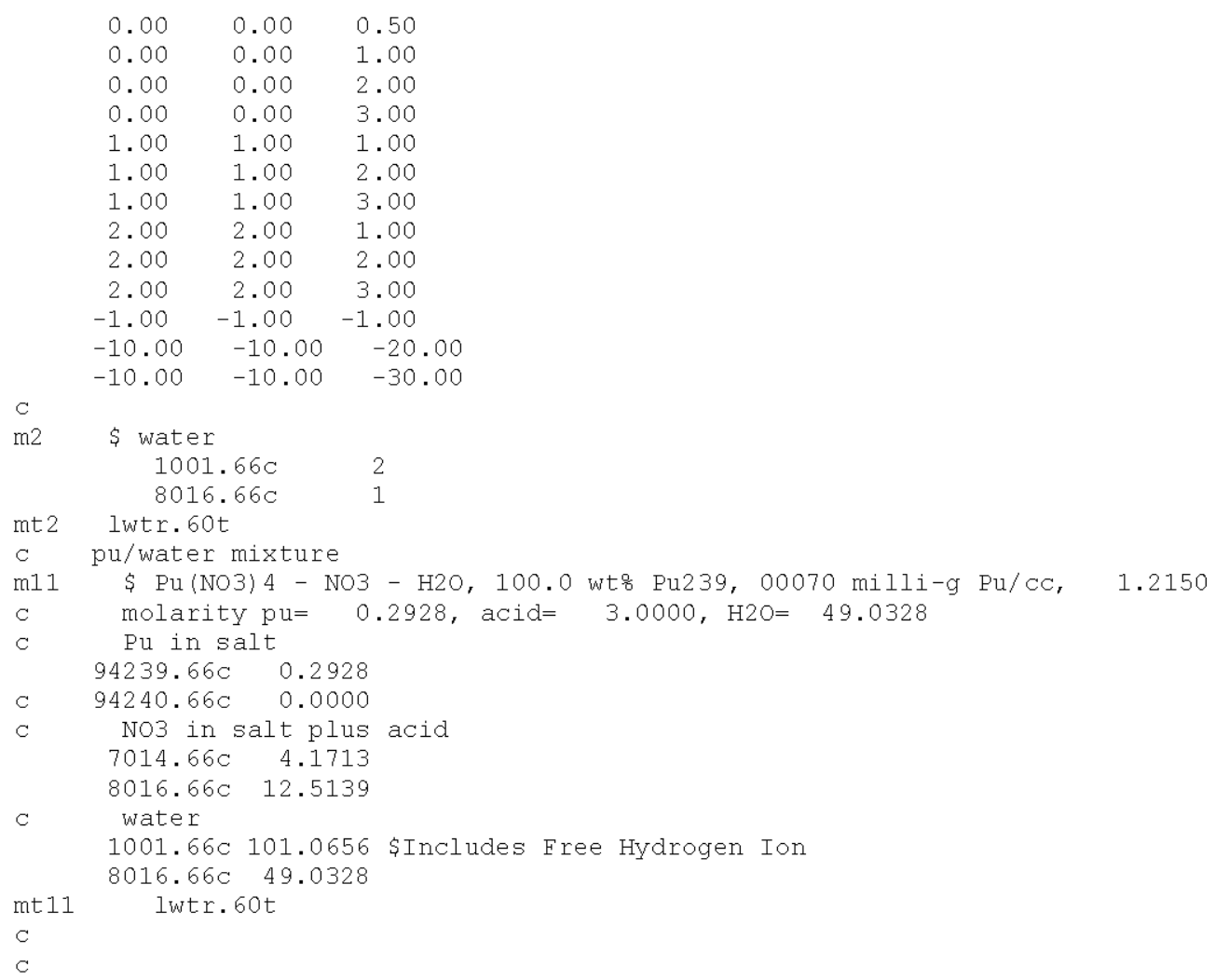




\section{CHPRC-01552, Revision 0}

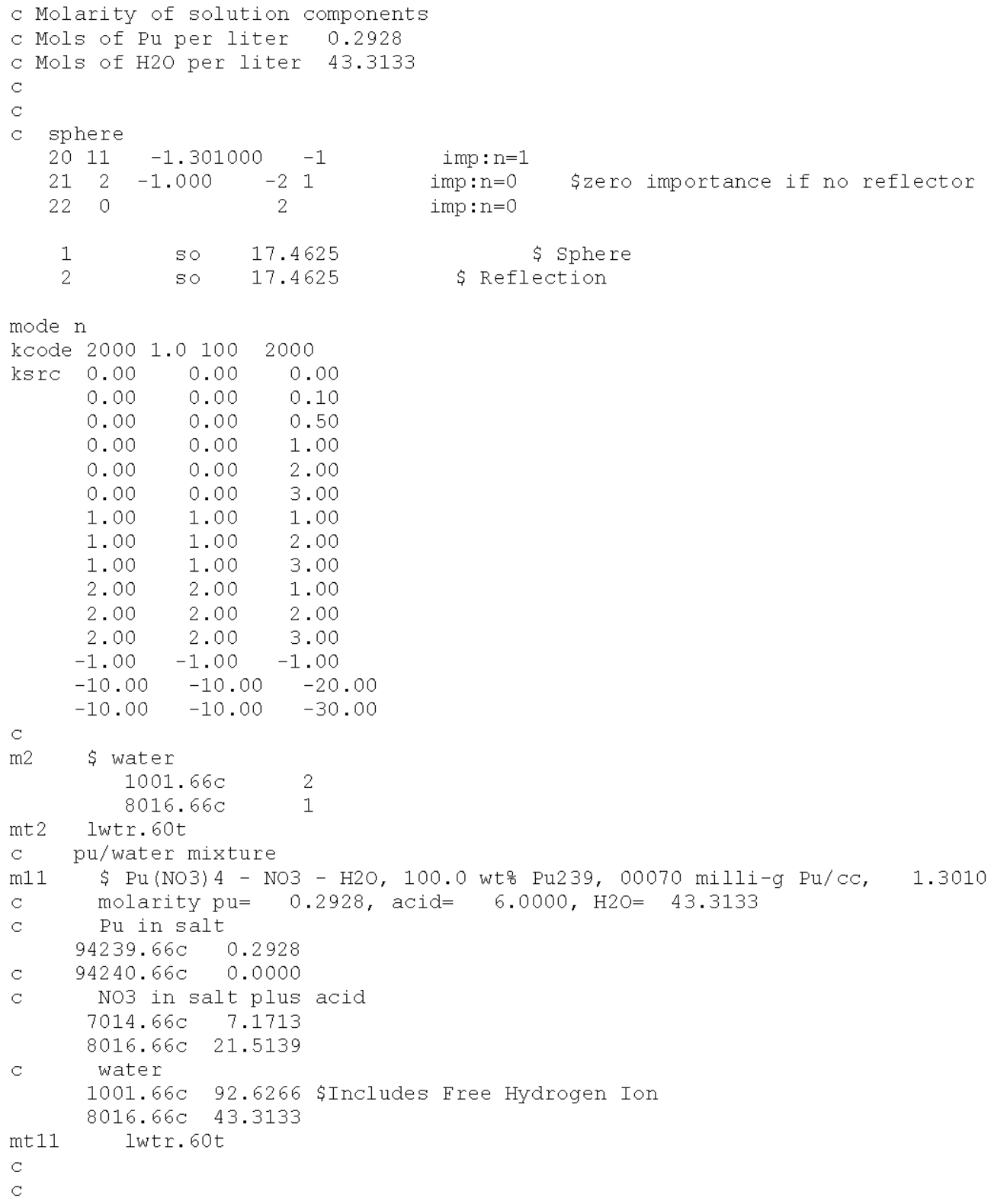




\section{CHPRC-01552, Revision 0}

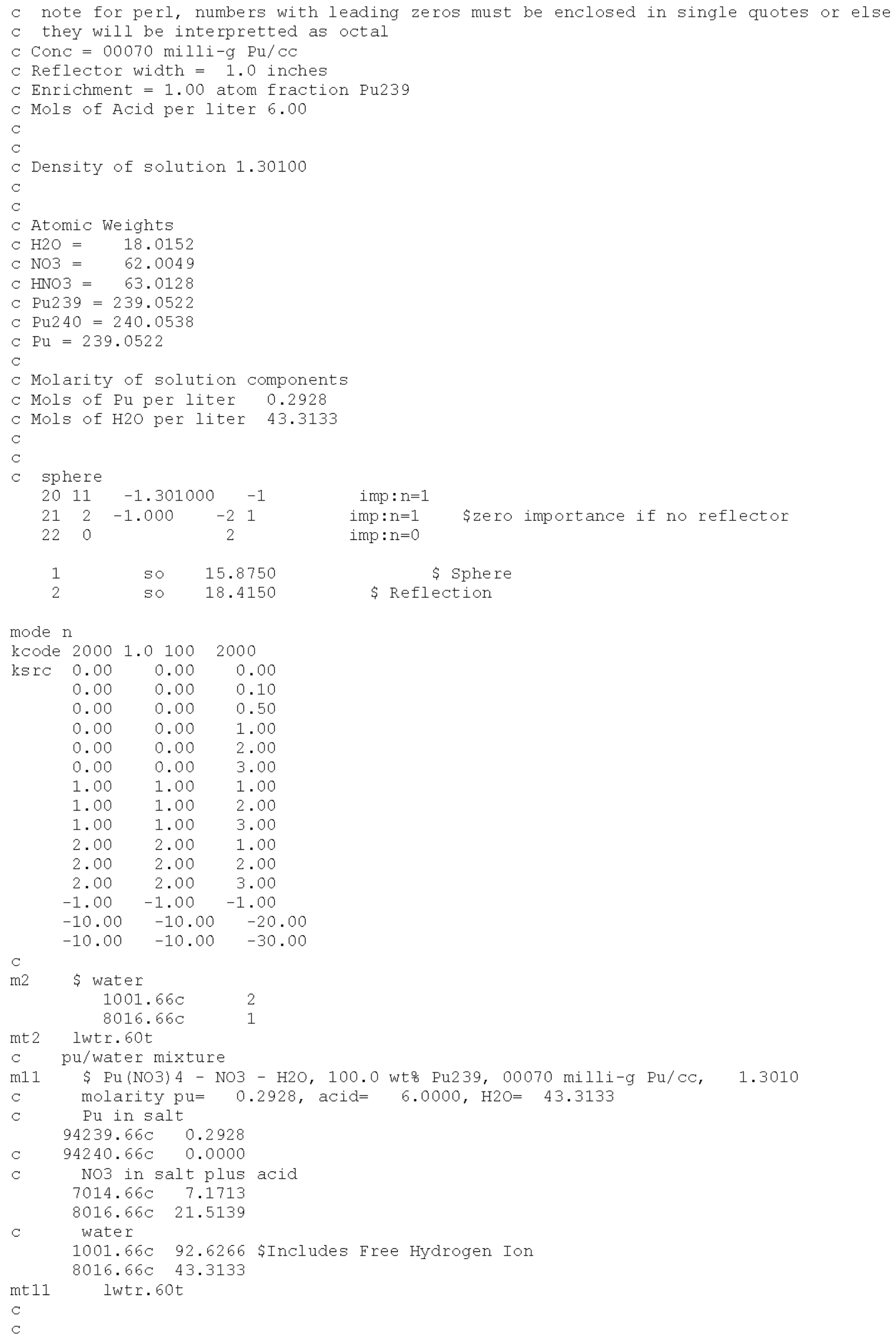




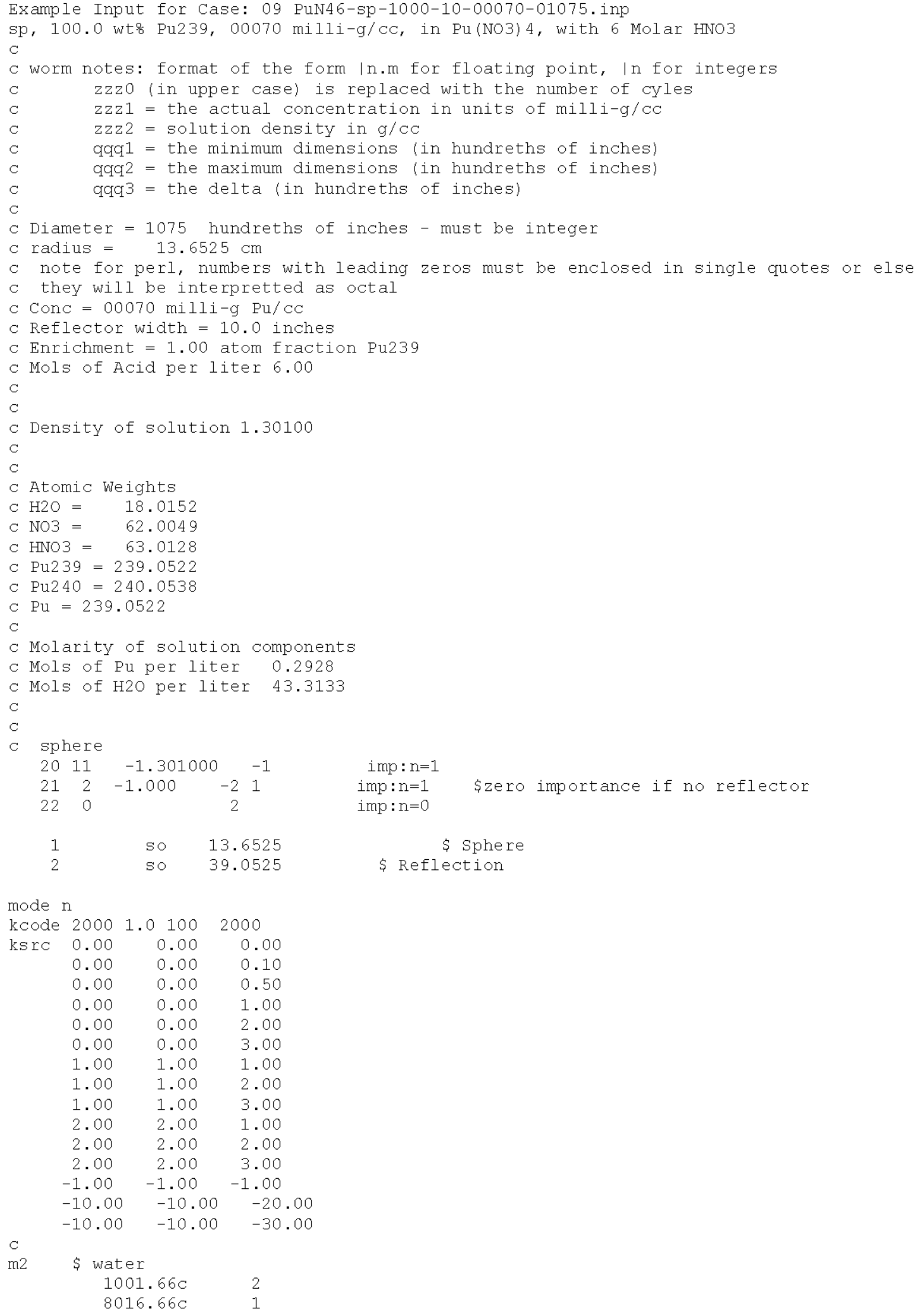




\section{CHPRC-01552, Revision 0}

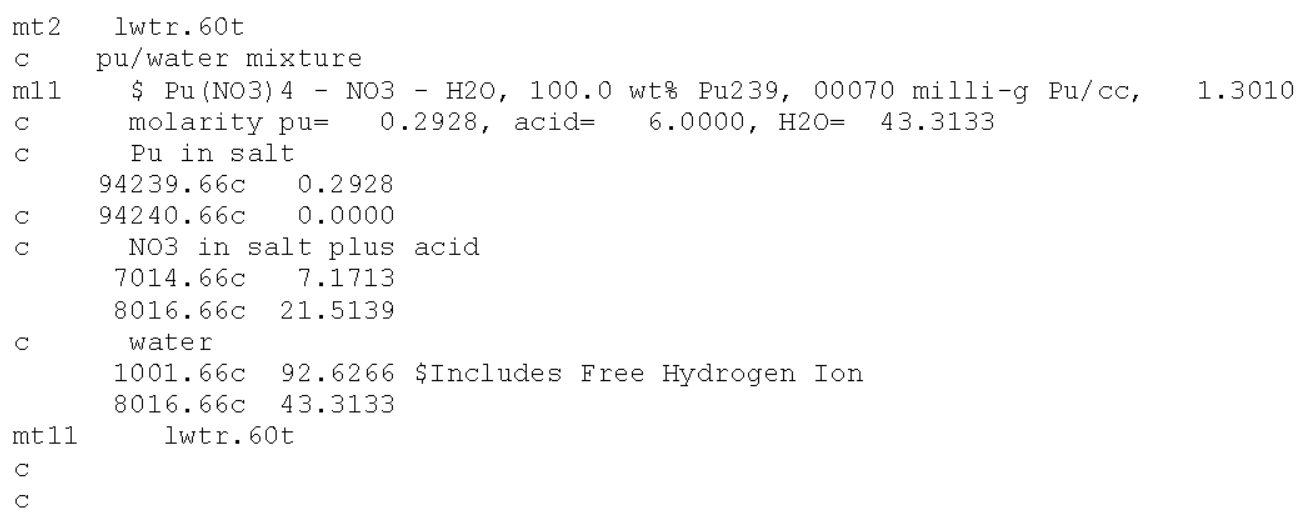




\section{CHPRC-01552, Revision 0}

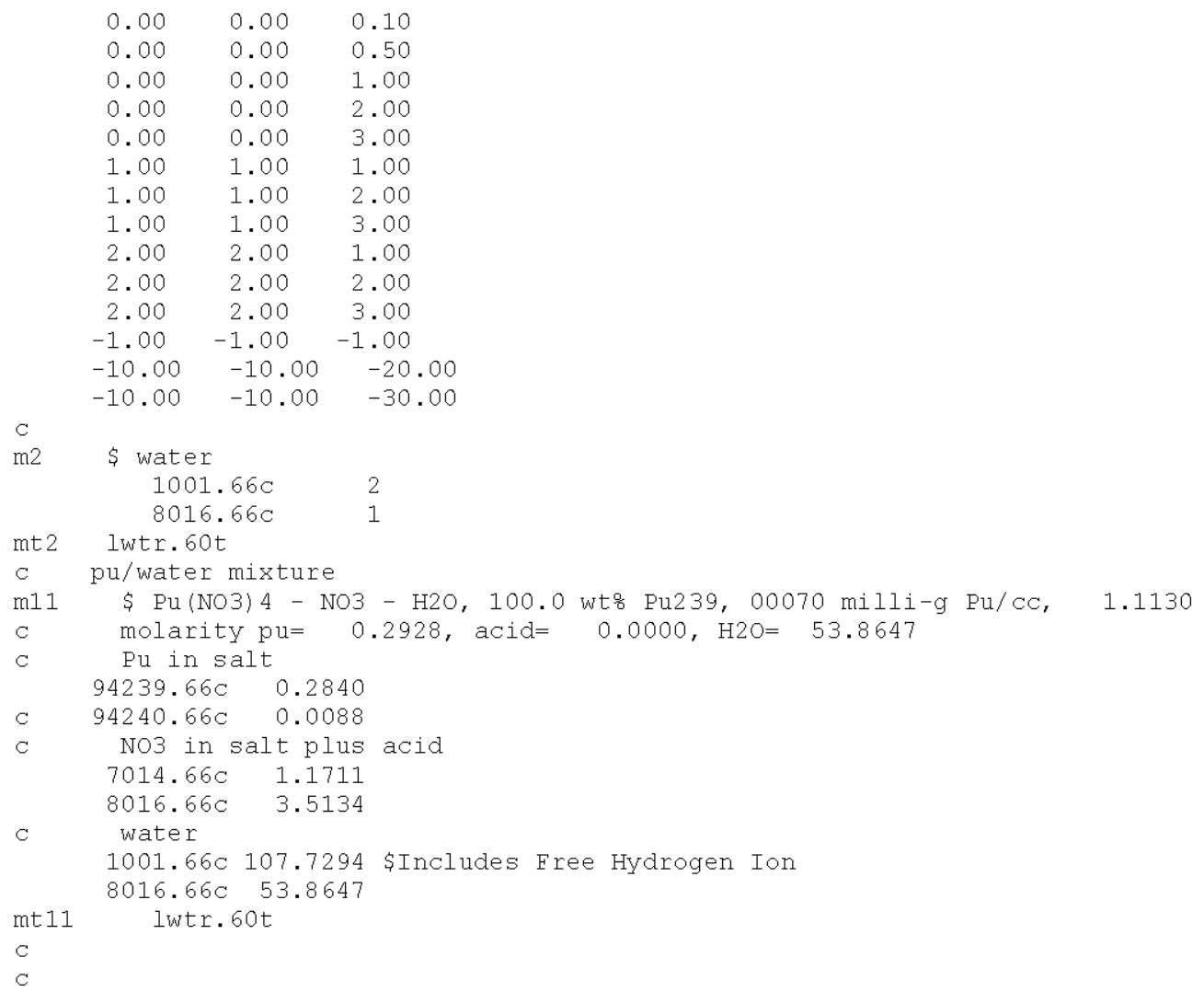




\section{CHPRC-01552, Revision 0}

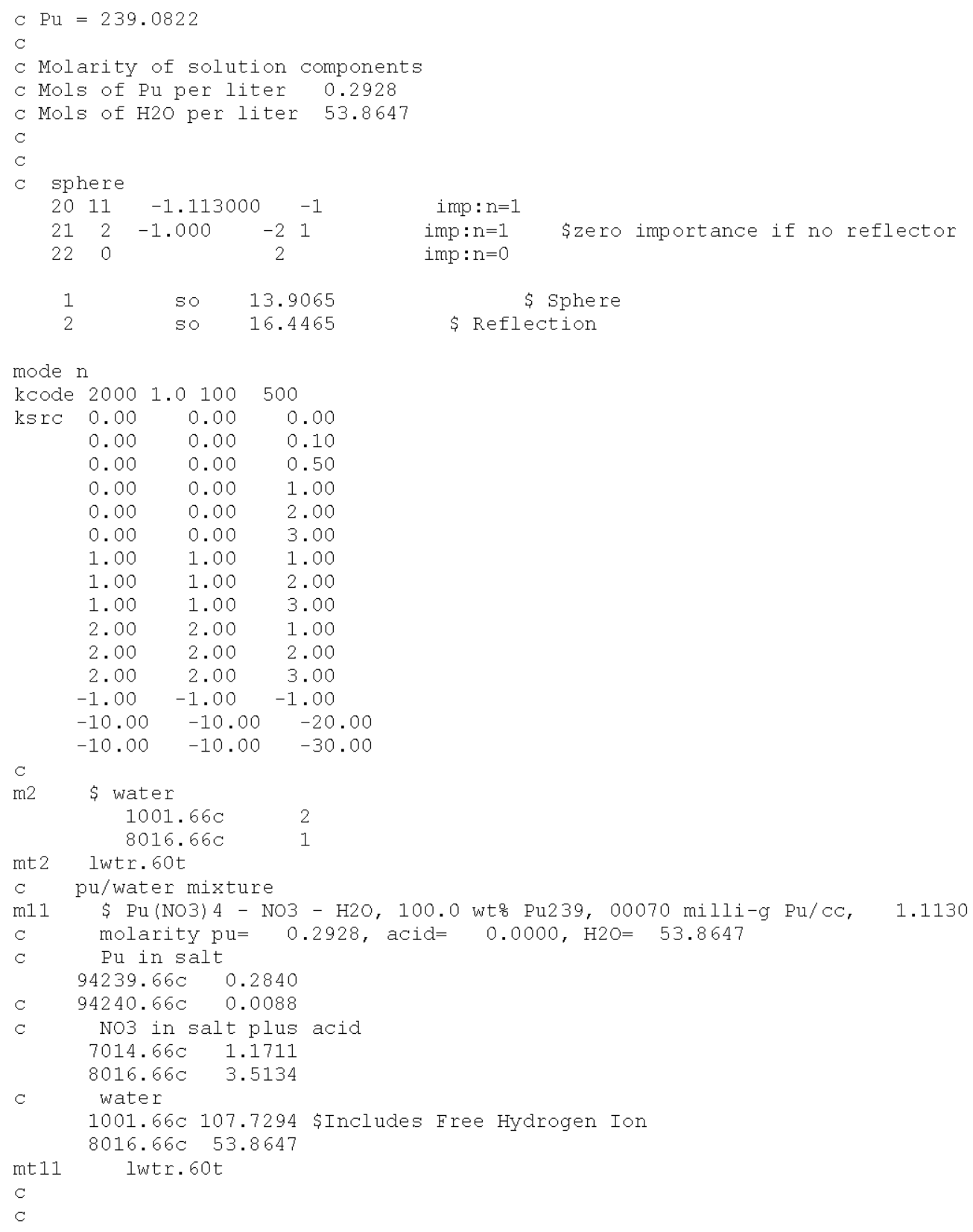




\section{CHPRC-01552, Revision 0}

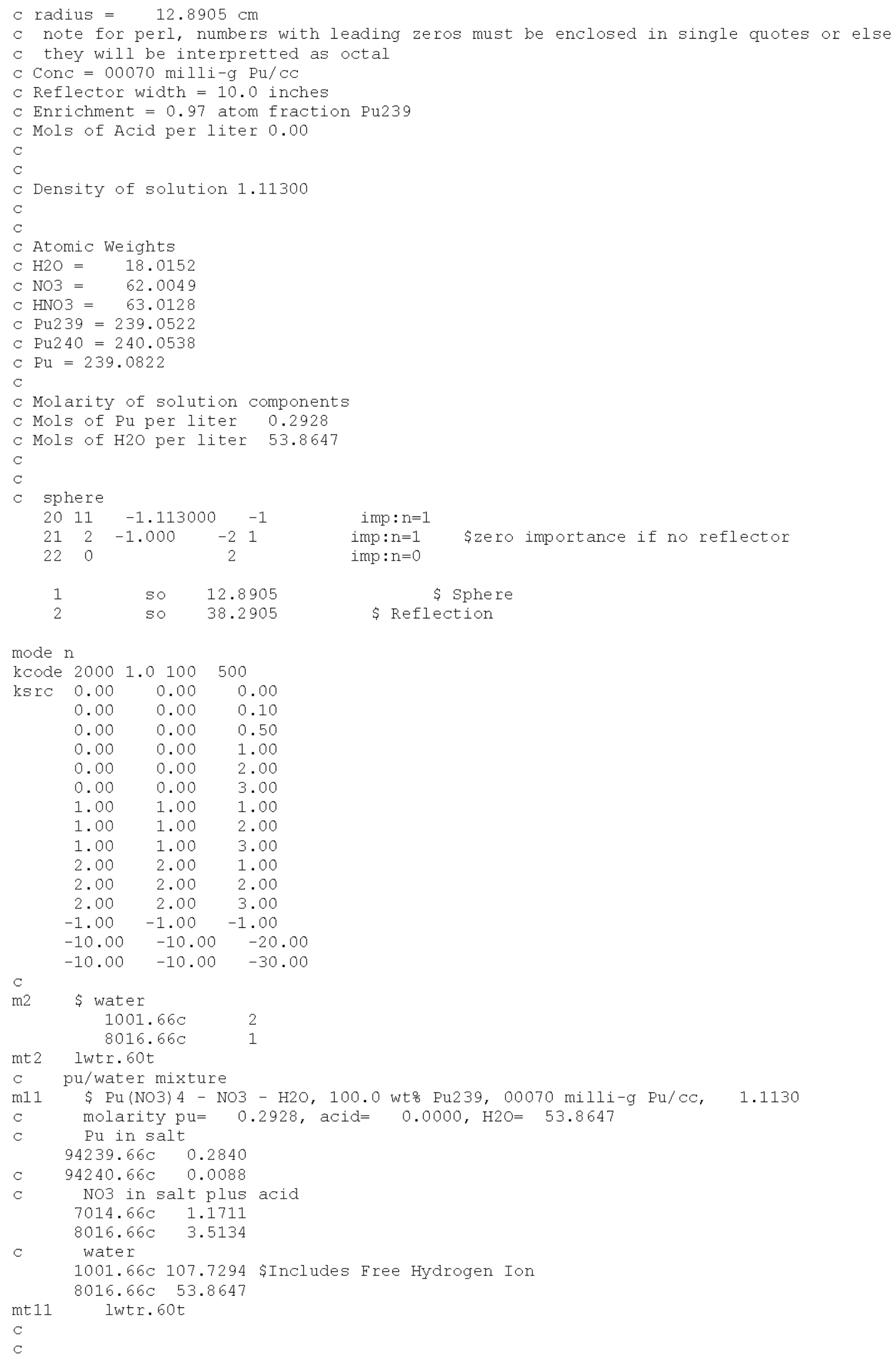




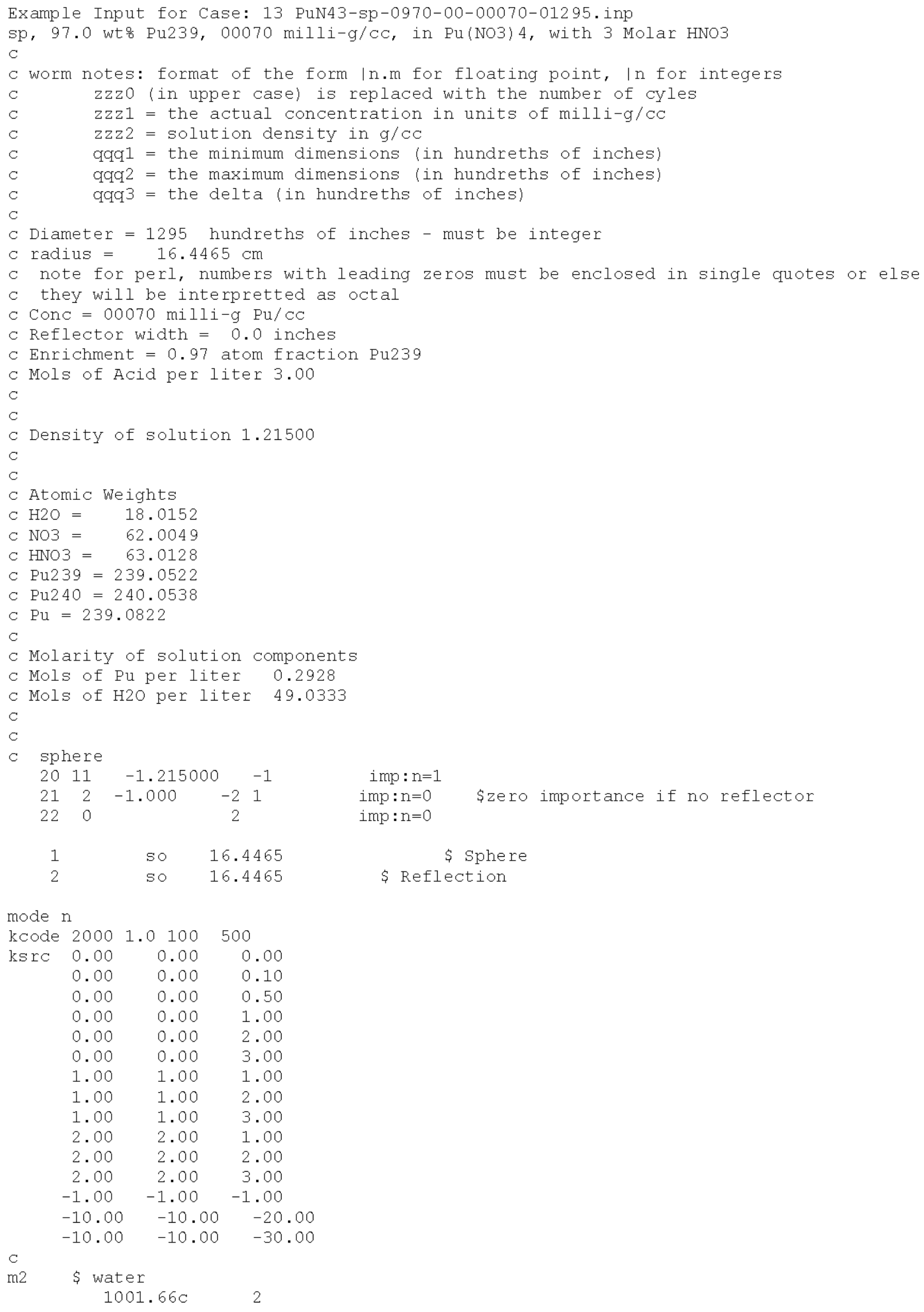




\section{CHPRC-01552, Revision 0}

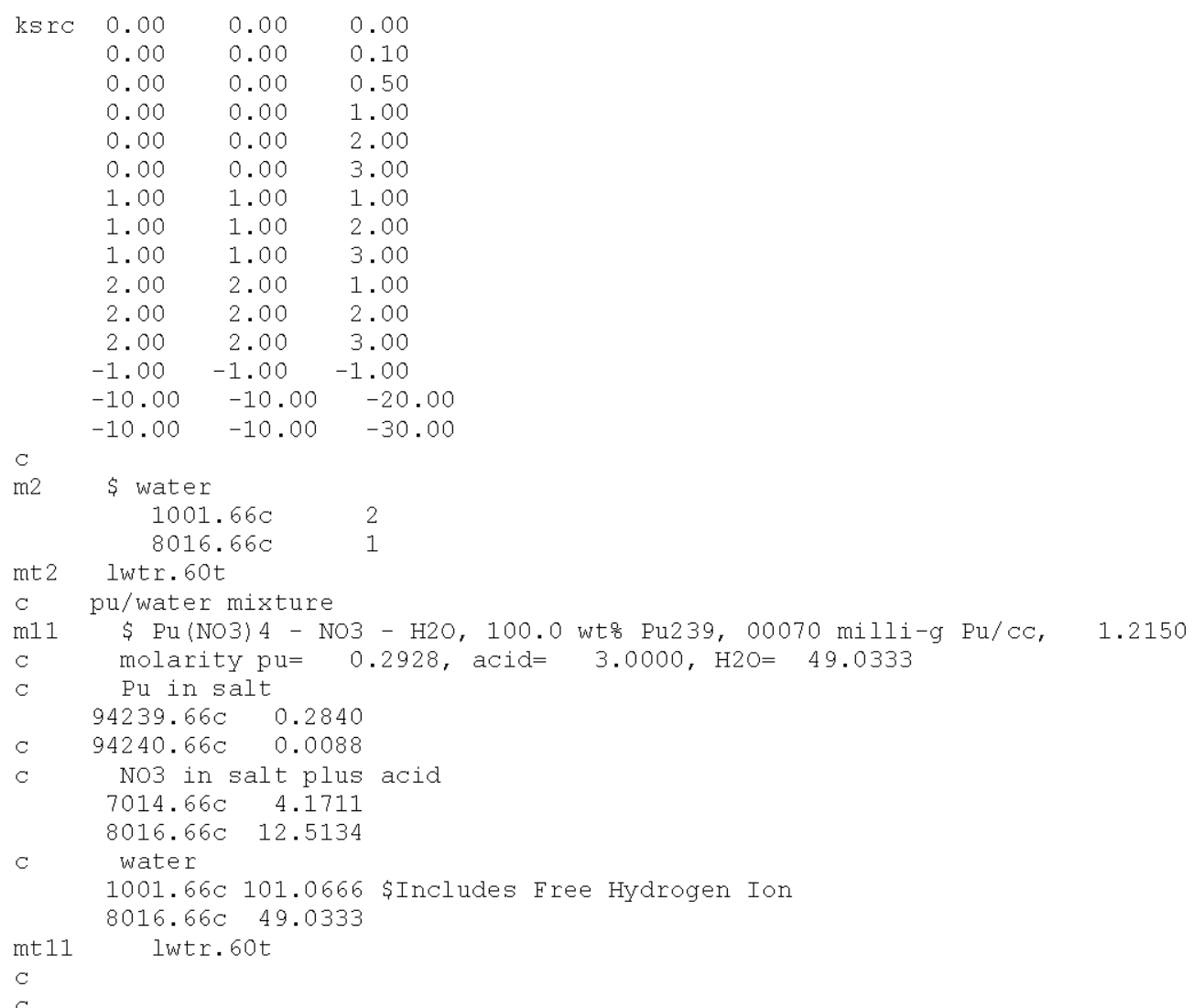




\section{CHPRC-01552, Revision 0}

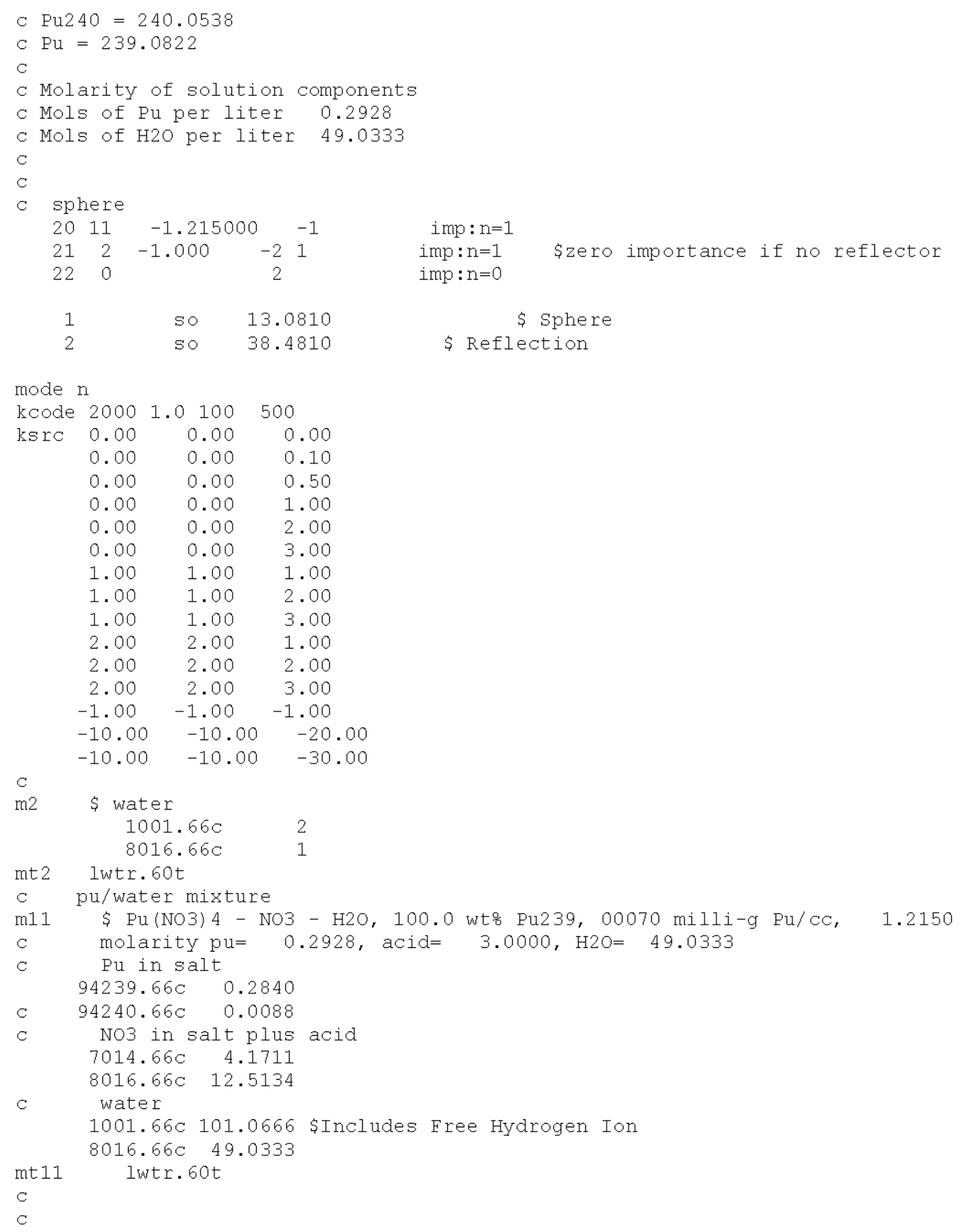




\section{CHPRC-01552, Revision 0}

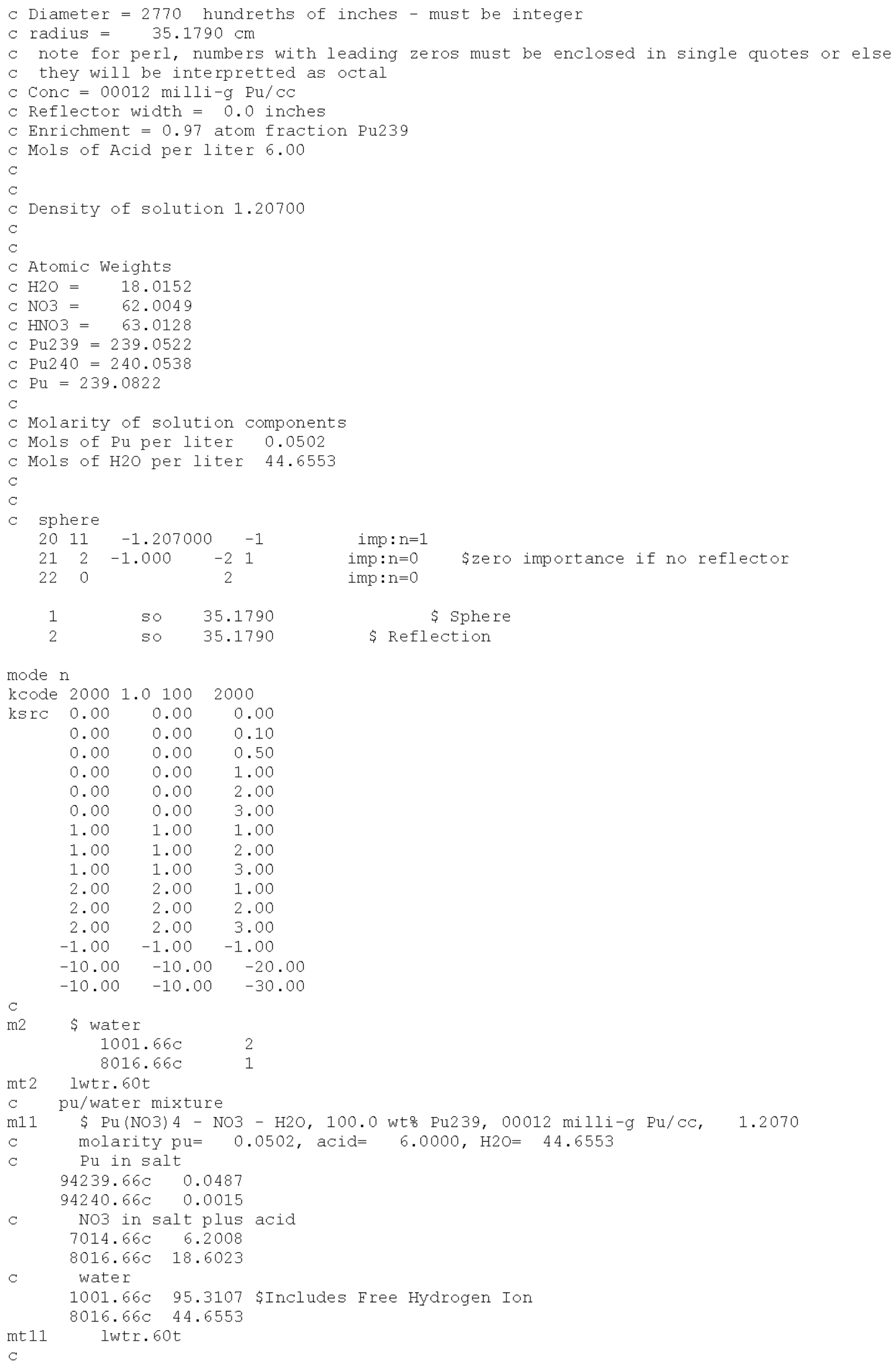




\section{CHPRC-01552, Revision 0}

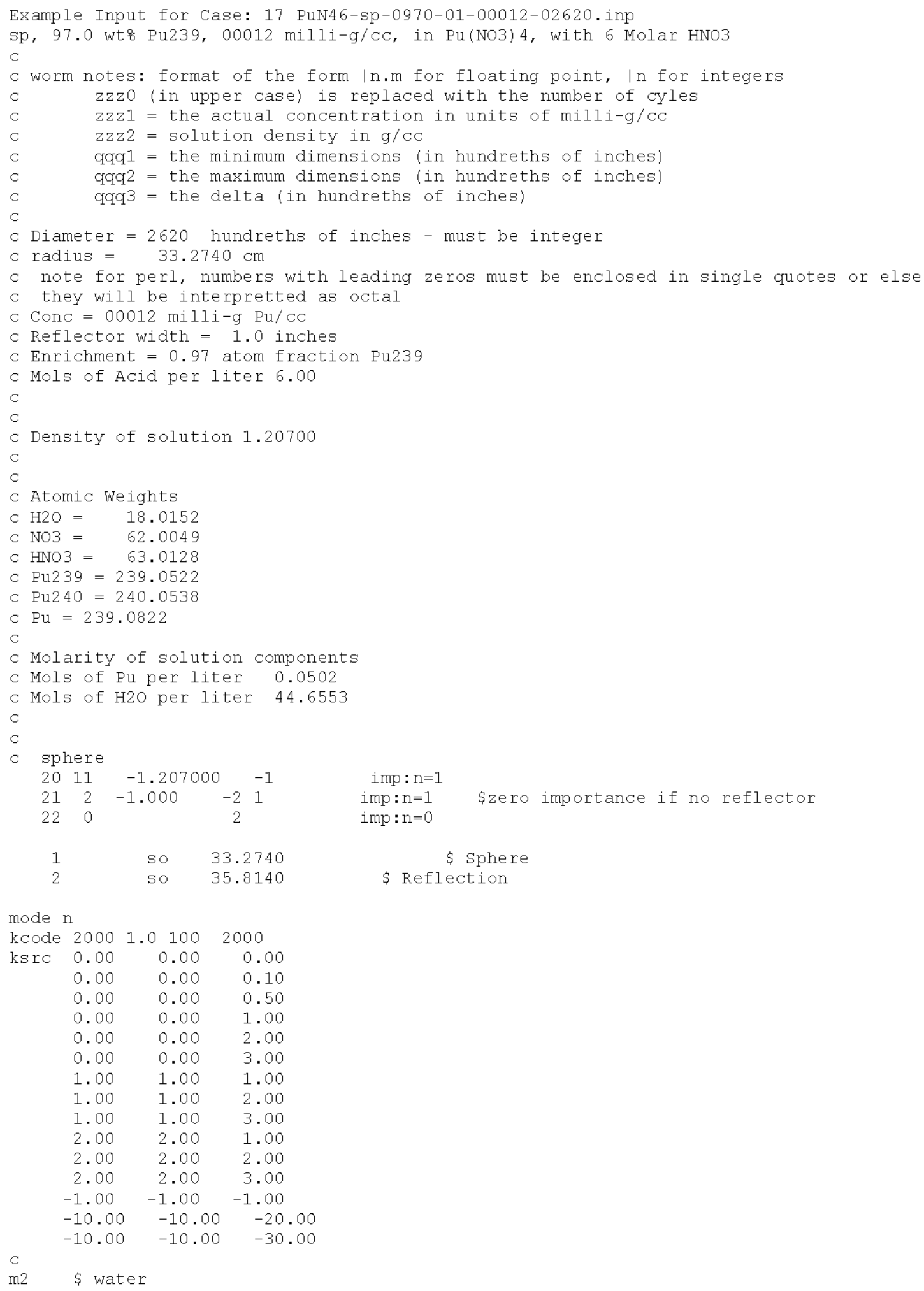




\section{CHPRC-01552, Revision 0}

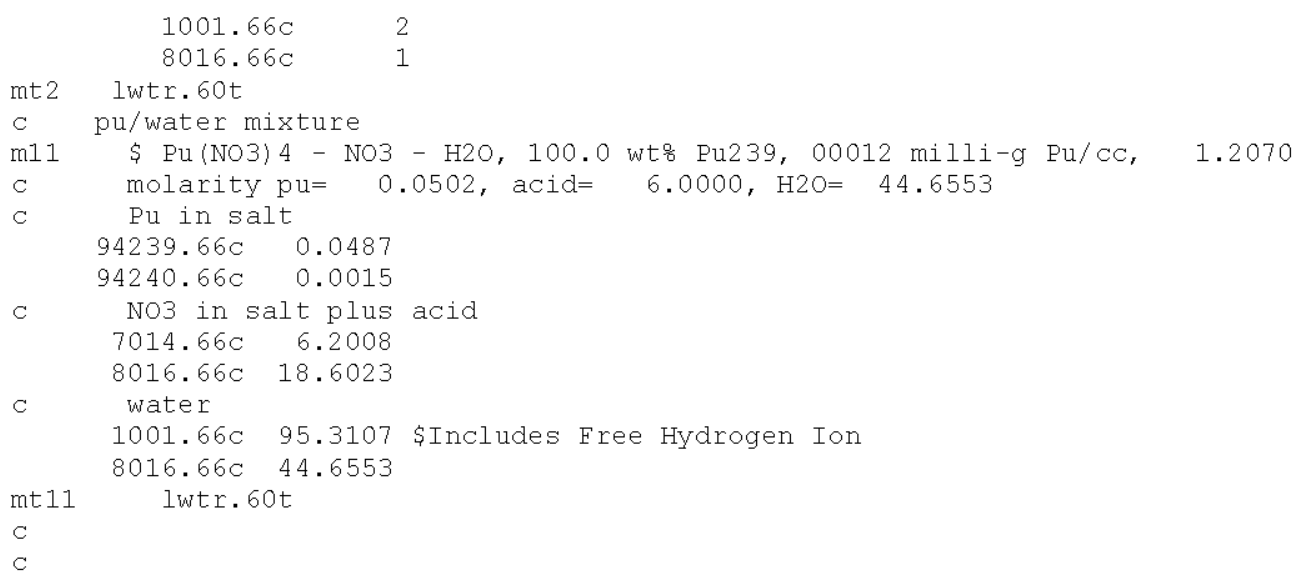

mode $n$ 


\section{CHPRC-01552, Revision 0}

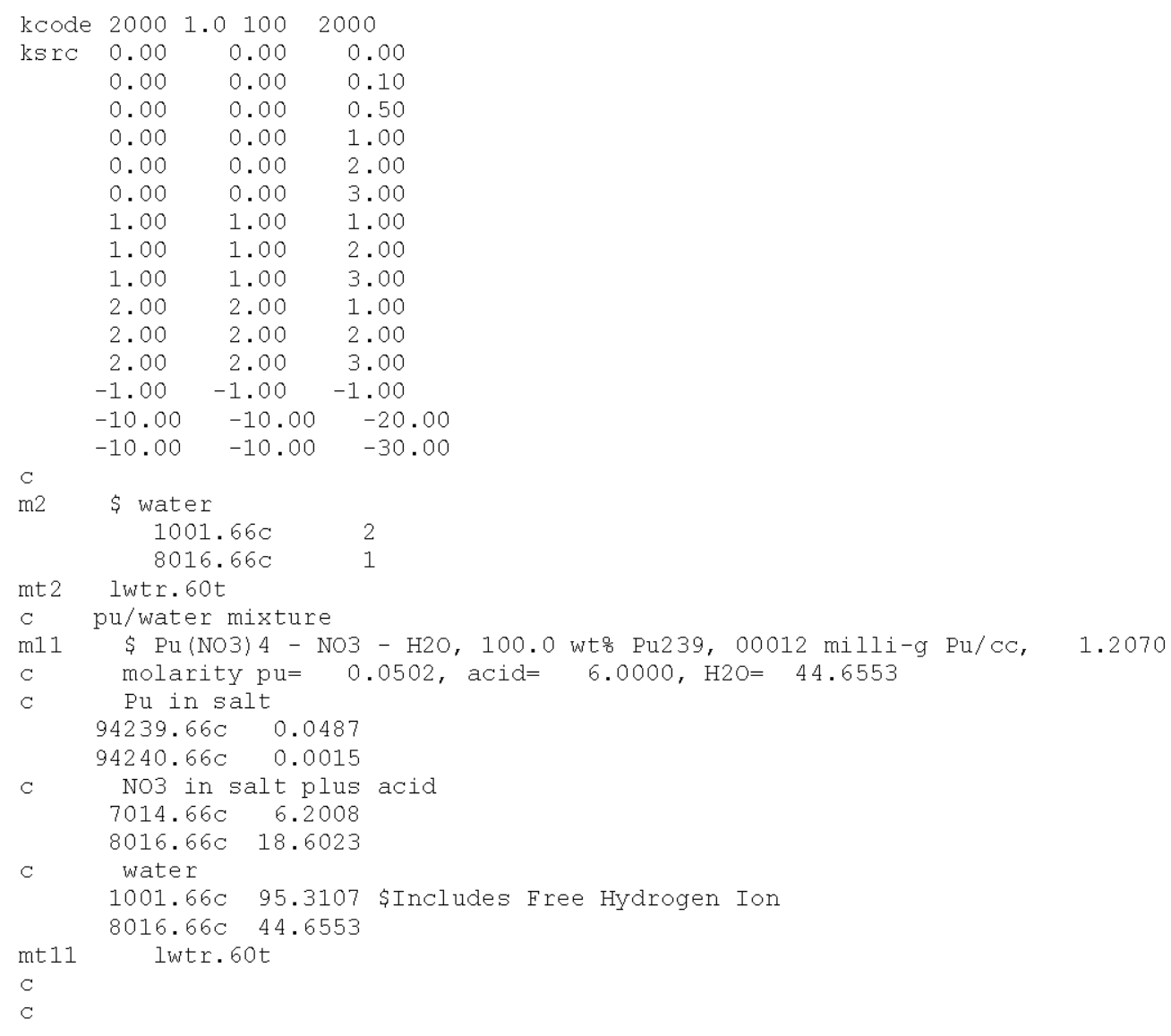




\section{CHPRC-01552, Revision 0}

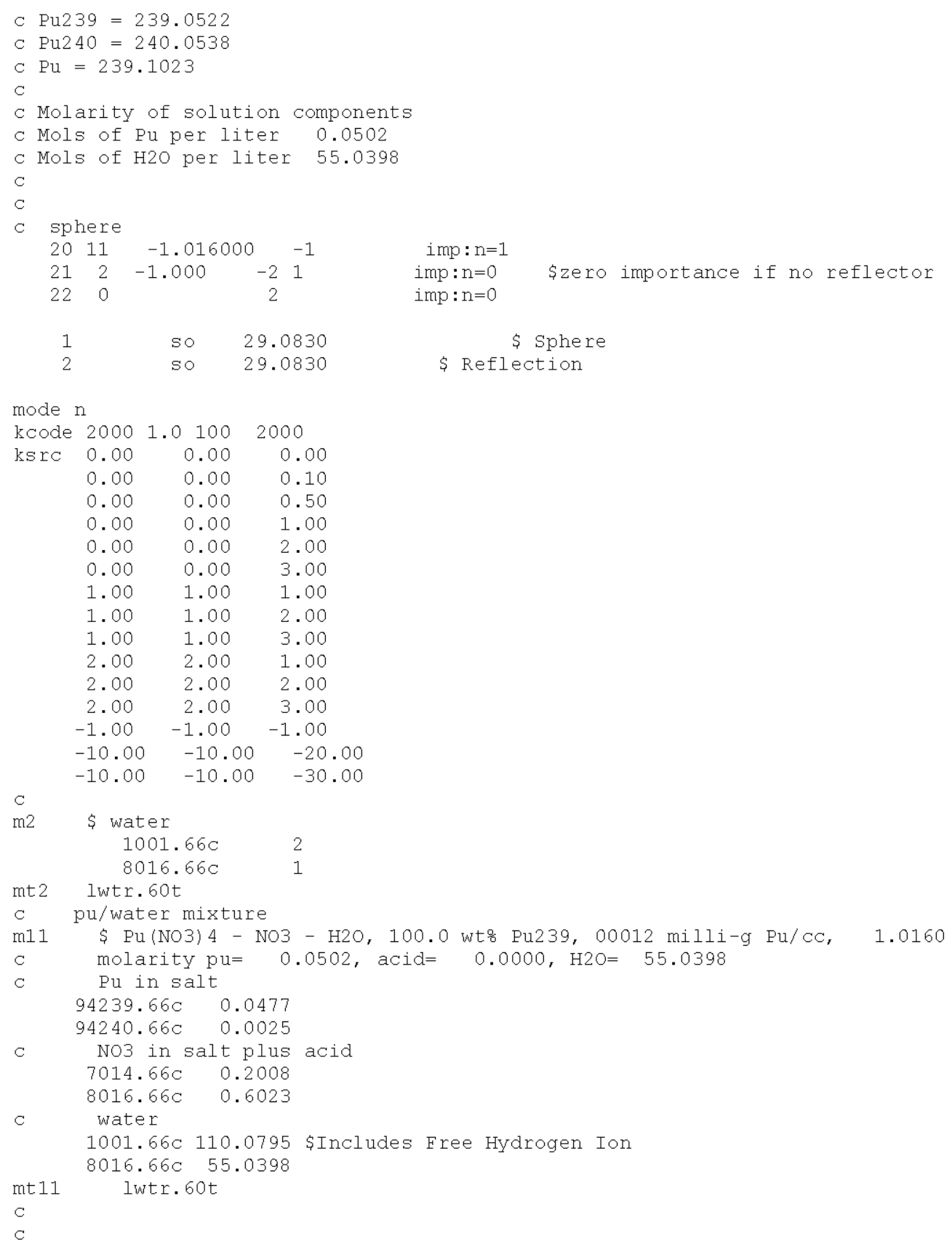




\section{CHPRC-01552, Revision 0}

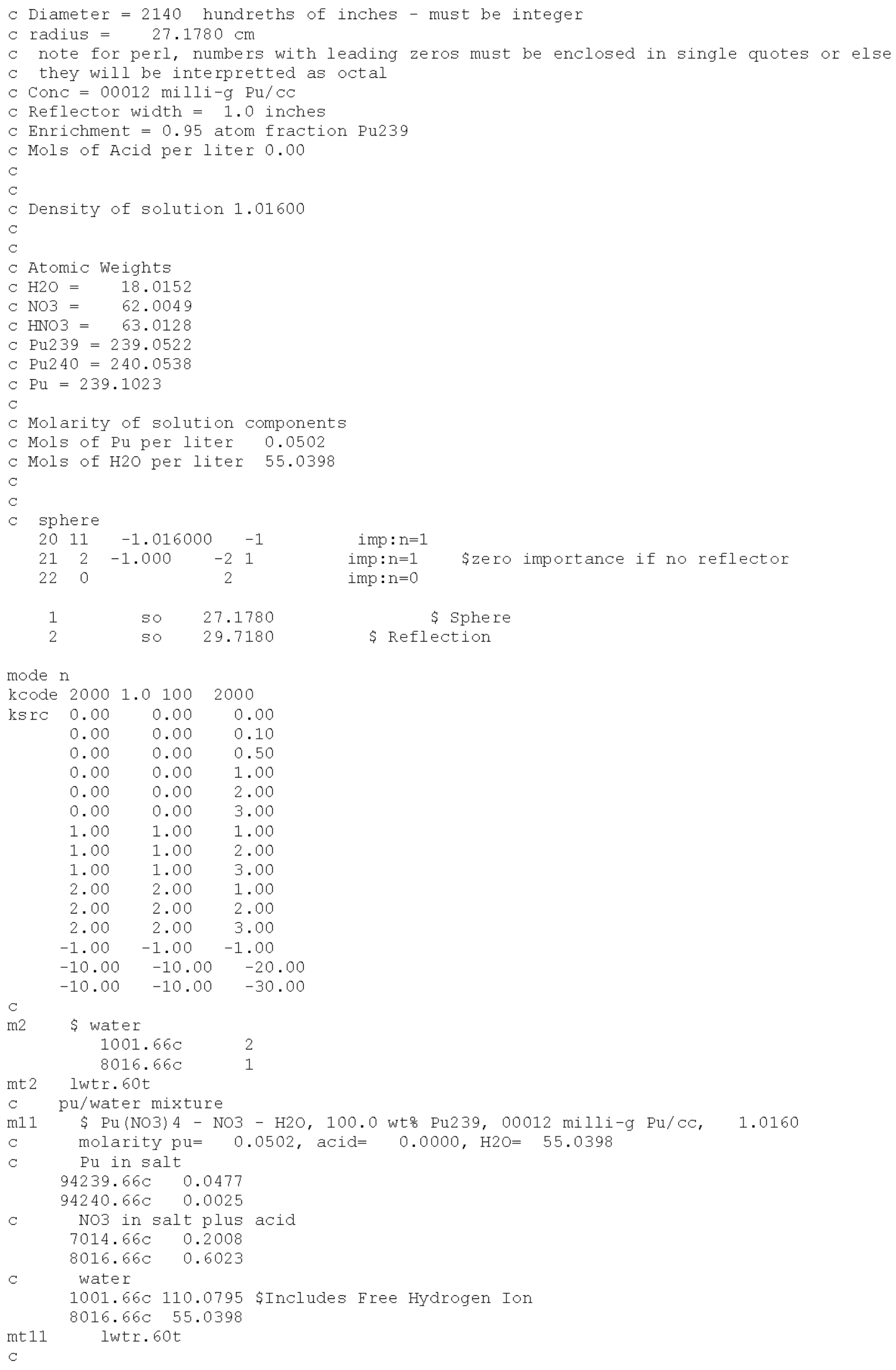




\section{CHPRC-01552, Revision 0}

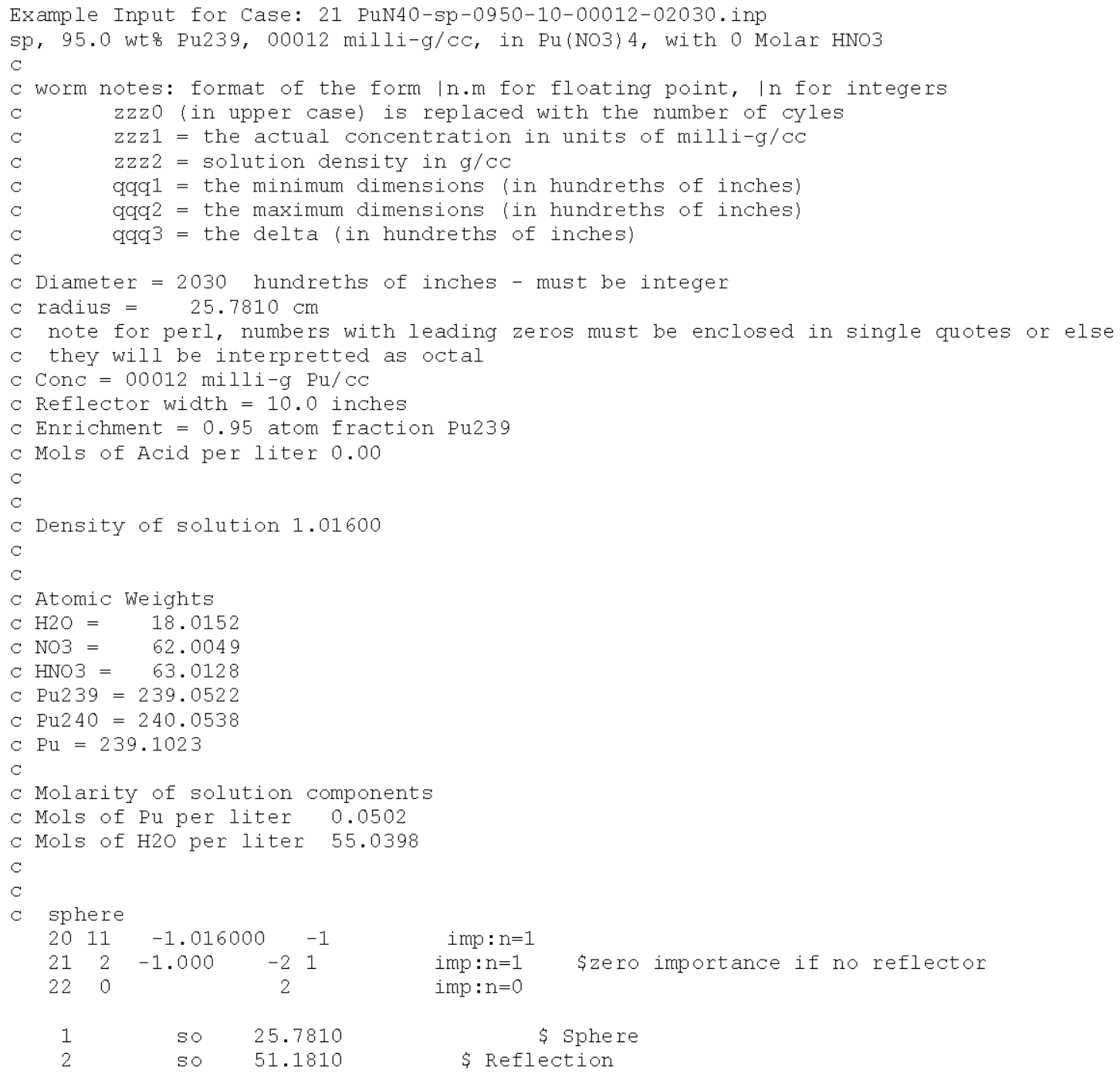




\section{CHPRC-01552, Revision 0}

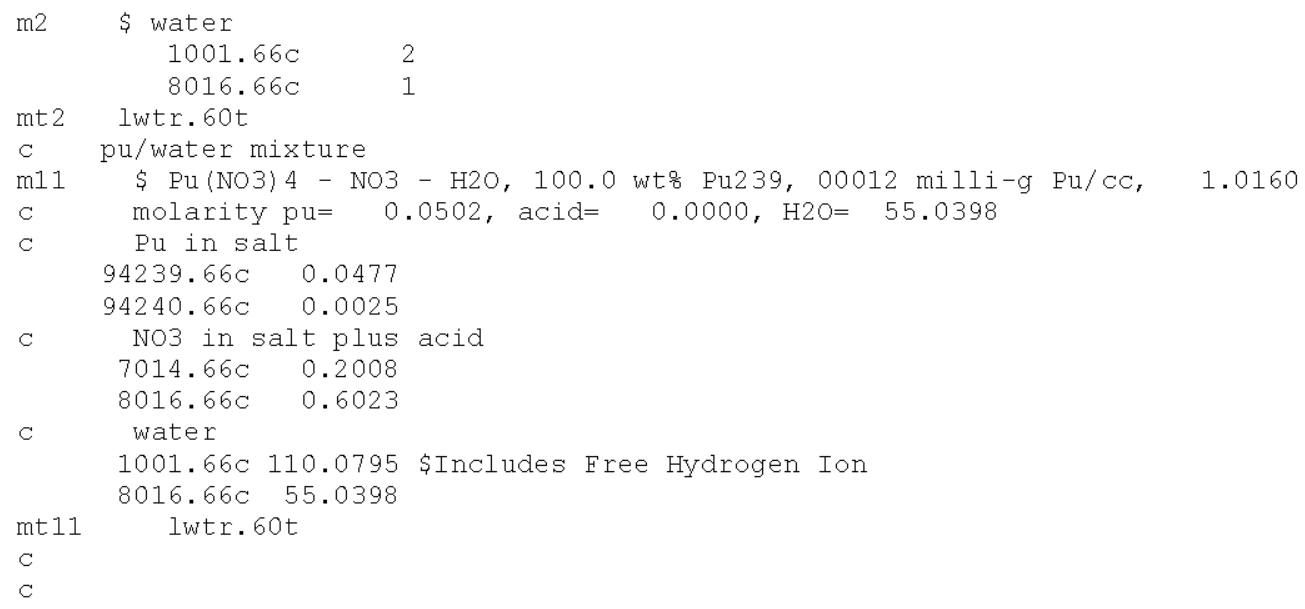




\section{CHPRC-01552, Revision 0}

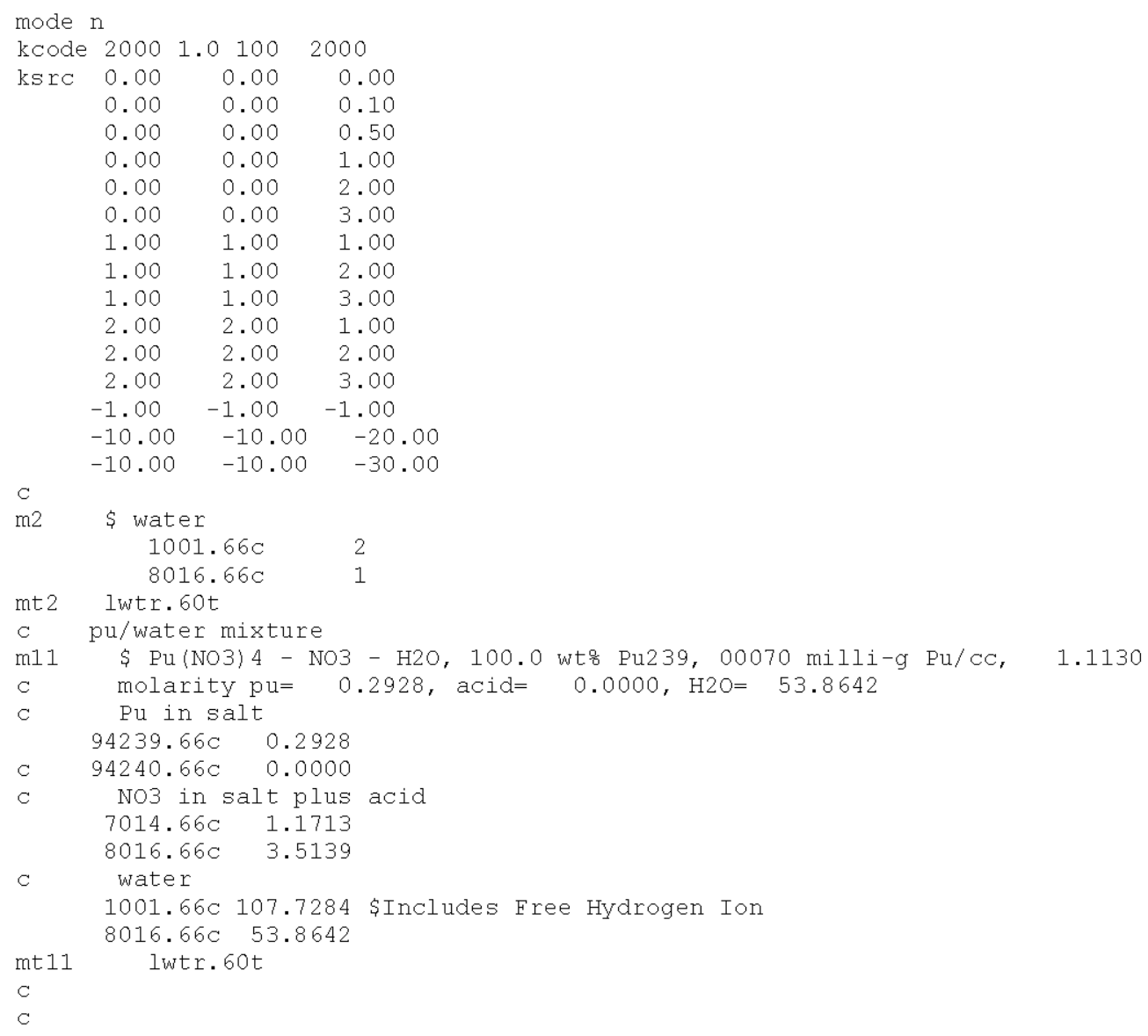




\section{CHPRC-01552, Revision 0}

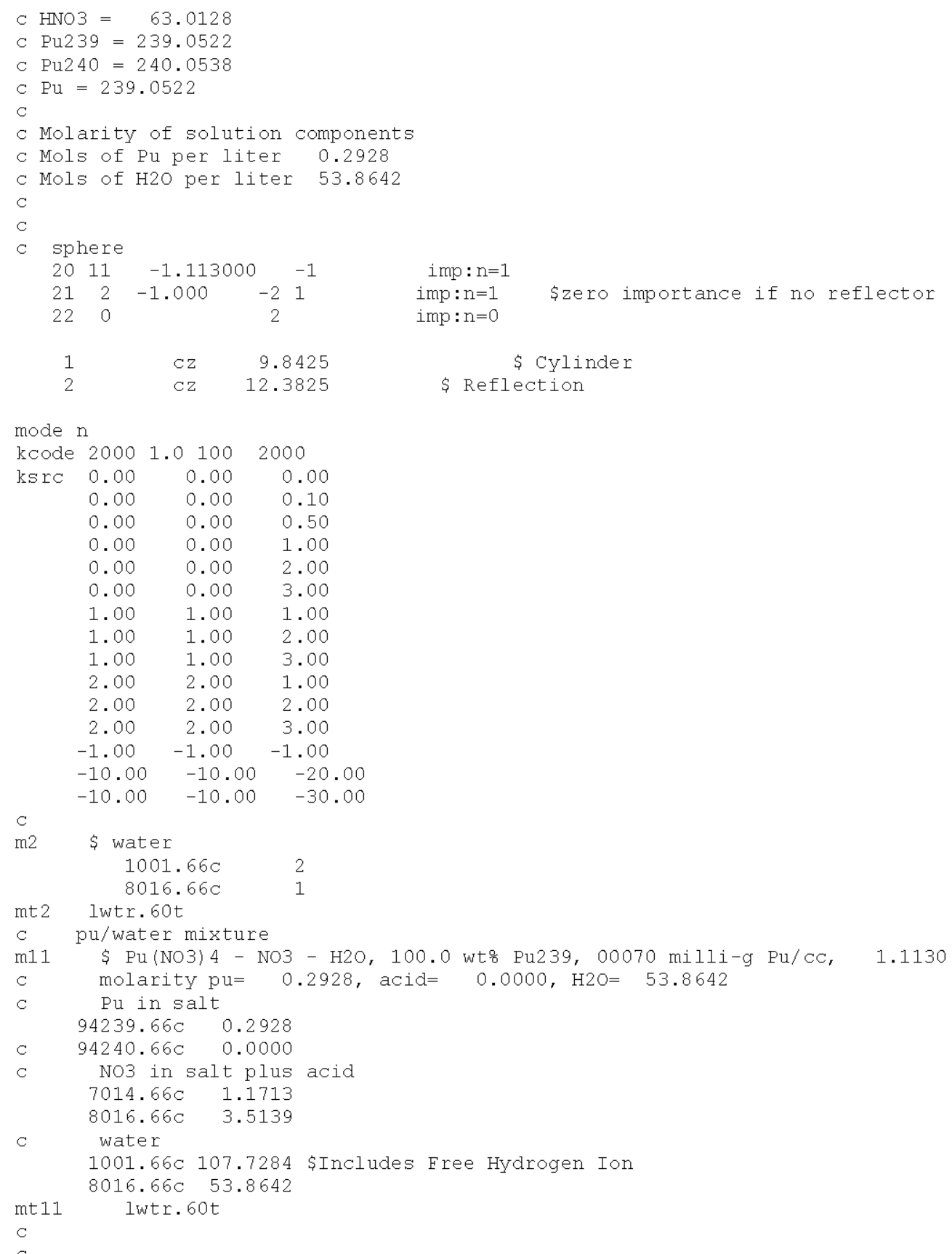




\section{CHPRC-01552, Revision 0}

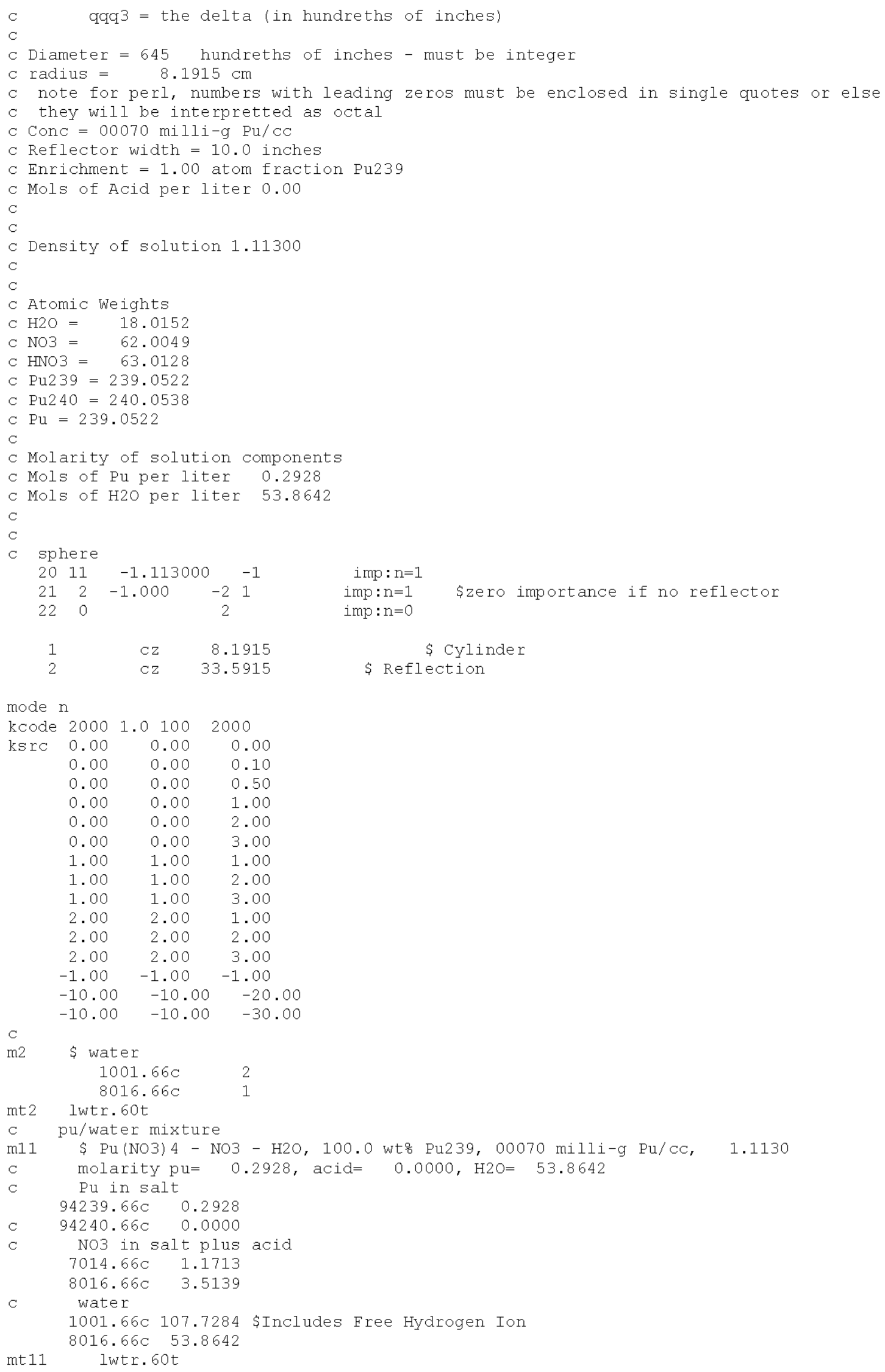




\section{CHPRC-01552, Revision 0}

Example Input for Case: 25 PuN43-icyl-1000-00-00070-00965.inp icyl, 100.0 wto Pu239, $00070 \mathrm{milli-g/CC}$, in Pu(No3) 4, with 3 Molar HNO3 $\mathrm{C}$

C worm notes: format of the form ln.m for floating point, In for integers

c zzzo (in upper case) is replaced with the number of cyles

C $\quad \mathrm{zzzl}=$ the actual concentration in units of $\mathrm{milli-g/cc}$

zzz2 = solution density in $g / \mathrm{CC}$

qqq1 = the minimum dimensions (in hundreths of inches)

qqq2 = the maximum dimensions (in hundreths of inches)

qq93 3 the delta (in hundreths of inches)

Diameter $=965$ hundreths of inches - must be integer

$\mathrm{C}$ radius $=12.2555 \mathrm{~cm}$

c note for perl, numbers with leading zeros must be enclosed in single quotes or else

c they will be interpretted as octal

c Conc $=00070 \mathrm{milli-g} \mathrm{Pu/CC}$

C Reflector width $=0.0$ inches

C Enrichment $=1.00$ atom fraction Pu239

C Mols of Acid per liter 3.00

C

$\mathrm{C}$

C Density of solution 1.21500

$\mathrm{C}$

C

C Atomic Weights

C $\mathrm{H} 2 \mathrm{O}=18.0152$

C $\mathrm{NO}=62.0049$

$\mathrm{C}$ HNO3 $=63.0128$

C $\operatorname{Pu} 239=239.0522$

C Pu240 = 240.0538

$\mathrm{C} \mathrm{Pu}=239.0522$

$\mathrm{C}$

C Molarity of solution components

C Mols of Pu per liter 0.2928

C Mols of H2O per liter 49.0328

C

C

C sphere

$2011-1.215000 \quad-1 \quad$ imp: $n=1$

$212-1.000 \quad-21 \quad$ imp:n=0 \$zero importance if no reflector

220

$1 \quad \mathrm{CZ} \quad 12.2555$

mode $n$

kcode 20001.01002000

$\begin{array}{llll}\text { ksre } & 0.00 \quad 0.00 & 0.00\end{array}$

$0.00 \quad 0.00 \quad 0.10$

$\begin{array}{lll}0.00 & 0.00 & 0.50\end{array}$

$0.00 \quad 0.00 \quad 1.00$

$\begin{array}{lll}0.00 & 0.00 \quad 2.00\end{array}$

$\begin{array}{lll}0.00 & 0.00 \quad 3.00\end{array}$

$1.001 .00 \quad 1.00$

$1.00 \quad 1.00 \quad 2.00$

$1.00 \quad 1.00 \quad 3.00$

$\begin{array}{lll}2.00 & 2.00 \quad 1.00\end{array}$

$\begin{array}{lll}2.00 & 2.00 \quad 2.00\end{array}$

$\begin{array}{lll}2.00 & 2.00 \quad 3.00\end{array}$

$-1.00-1.00 \quad-1.00$

$\begin{array}{lll}-10.00 & -10.00 & -20.00\end{array}$

$-10.00 \quad-10.00 \quad-30.00$ 


\section{CHPRC-01552, Revision 0}

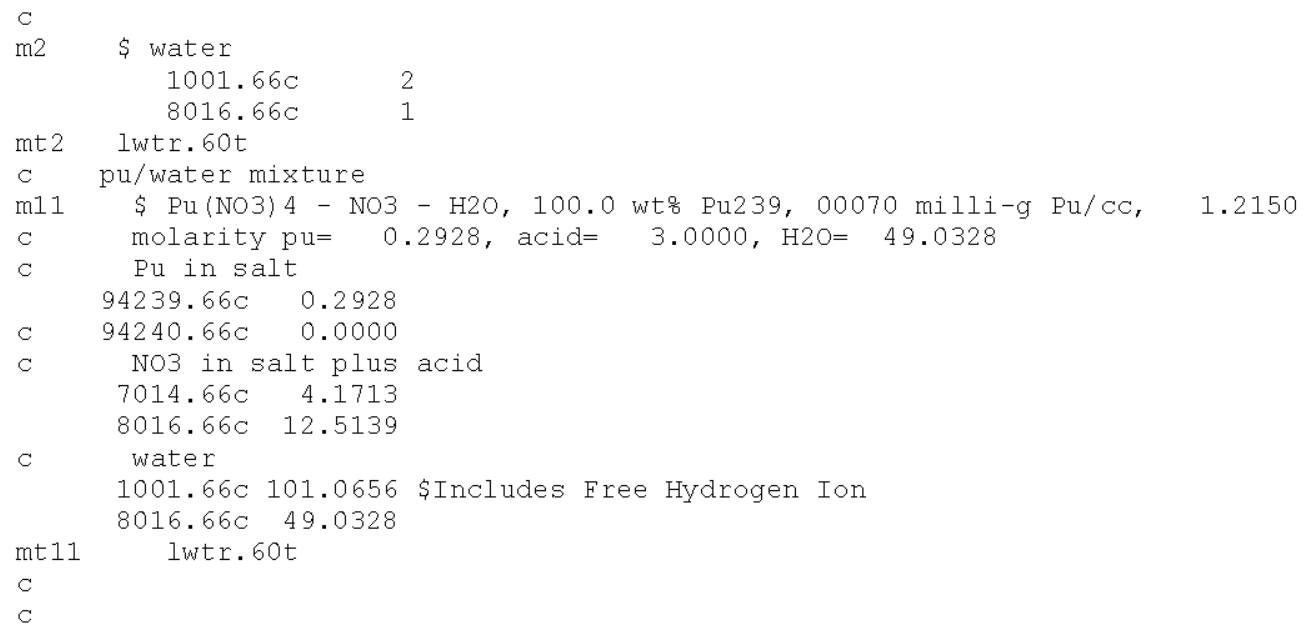

Example Input for Case: 26 PuN43-icyl-1000-01-00070-00805.inp icyl, 100.0 wt: Pu239, $00070 \mathrm{milli-g/CC}$, in Pu(NO3) 4, with 3 Molar HNO3

$c$

$c$ worm notes: format of the form $\mid \mathrm{n} . \mathrm{m}$ for floating point, In for integers

c zzzo (in upper case) is replaced with the number of cyles

c $\quad$ zzzl = the actual concentration in units of milli-g/cc

c $\quad z z z 2$ = solution density in $\mathrm{g} / \mathrm{CC}$

c qqq1 $=$ the minimum dimensions (in hundreths of inches)

c $\quad$ qqq 2 = the maximum dimensions (in hundreths of inches)

qq93 $=$ the delta (in hundreths of inches)

C Diameter $=805$ hundreths of inches - must be integer

$\mathrm{C}$ radius $=10.2235 \mathrm{~cm}$

c note for perl, numbers with leading zeros must be enclosed in single quotes or else

they will be interpretted as octal

Conc $=00070 \mathrm{milli}-\mathrm{g} \mathrm{Pu} / \mathrm{CC}$

C Reflector width $=1.0$ inches

C Enrichment $=1.00$ atom fraction Pu239

c Mols of Acid per liter 3.00

$\mathrm{C}$

C Density of solution 1.21500

C Atomic Weights

$\mathrm{C} \mathrm{H} 2 \mathrm{O}=18.0152$

$\mathrm{C}$ NO3 $=62.0049$

C $\mathrm{HNO} 3=63.0128$

C $\operatorname{Pu} 239=239.0522$

C Pu240 $=240.0538$

$\mathrm{C} \mathrm{Pu}=239.0522$

C Molarity of solution components

C Mols of $\mathrm{Pu}$ per liter 0.2928

c Mols of H2O per liter 49.0328

C

C sphere

$2011-1.215000-1 \quad$ imp: $n=1$

$212-1.000-21 \quad$ imp:n=1 \$zero importance if no reflector

$220 \quad 2 \quad$ imp $: \mathrm{n}=0$

$1 \quad \mathrm{CZ} \quad 10.2235 \quad$ \$ Cylinder

$2 \quad C z \quad 12.7635 \quad$ \$ Reflection 


\section{CHPRC-01552, Revision 0}

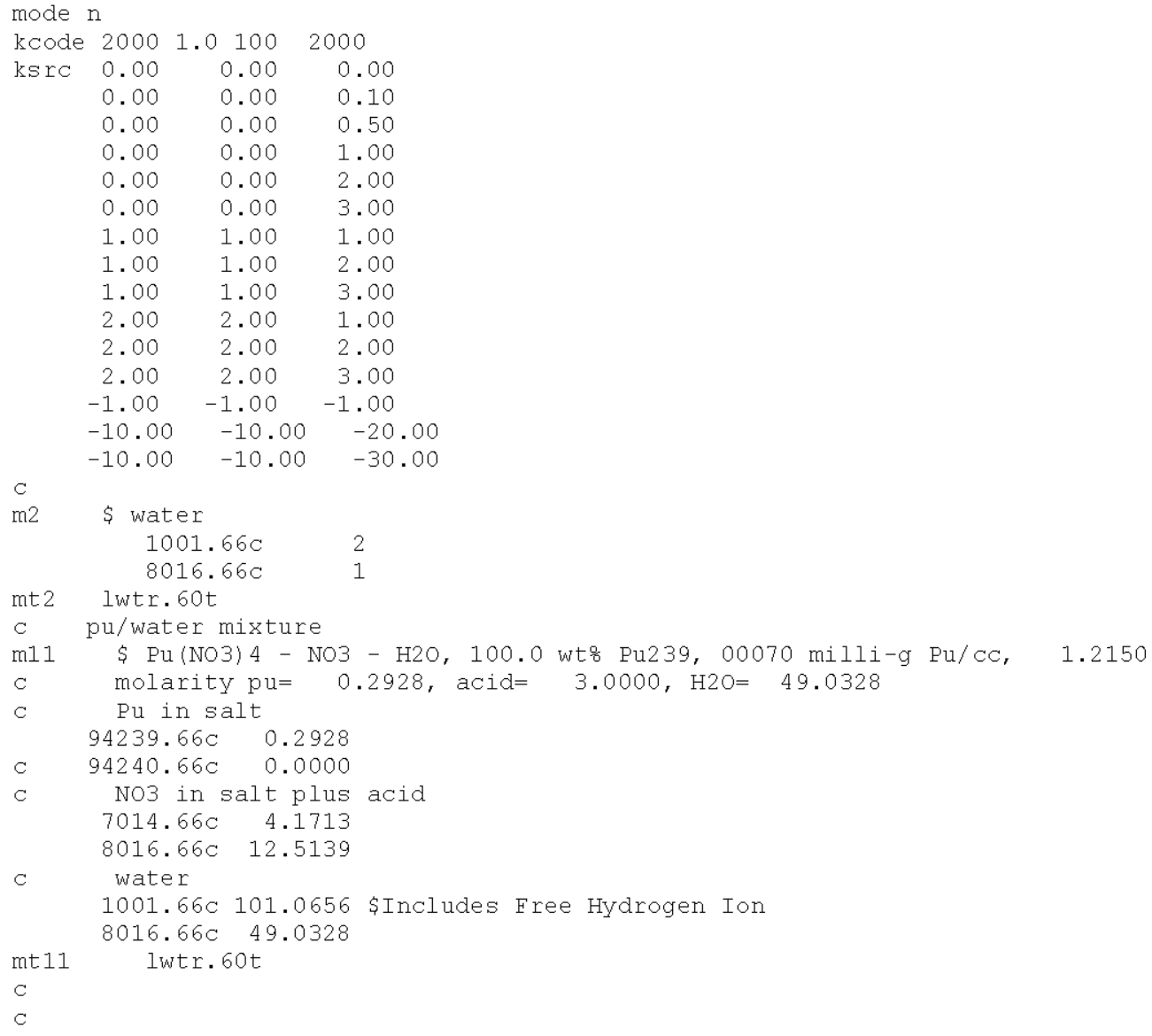




\section{CHPRC-01552, Revision 0}

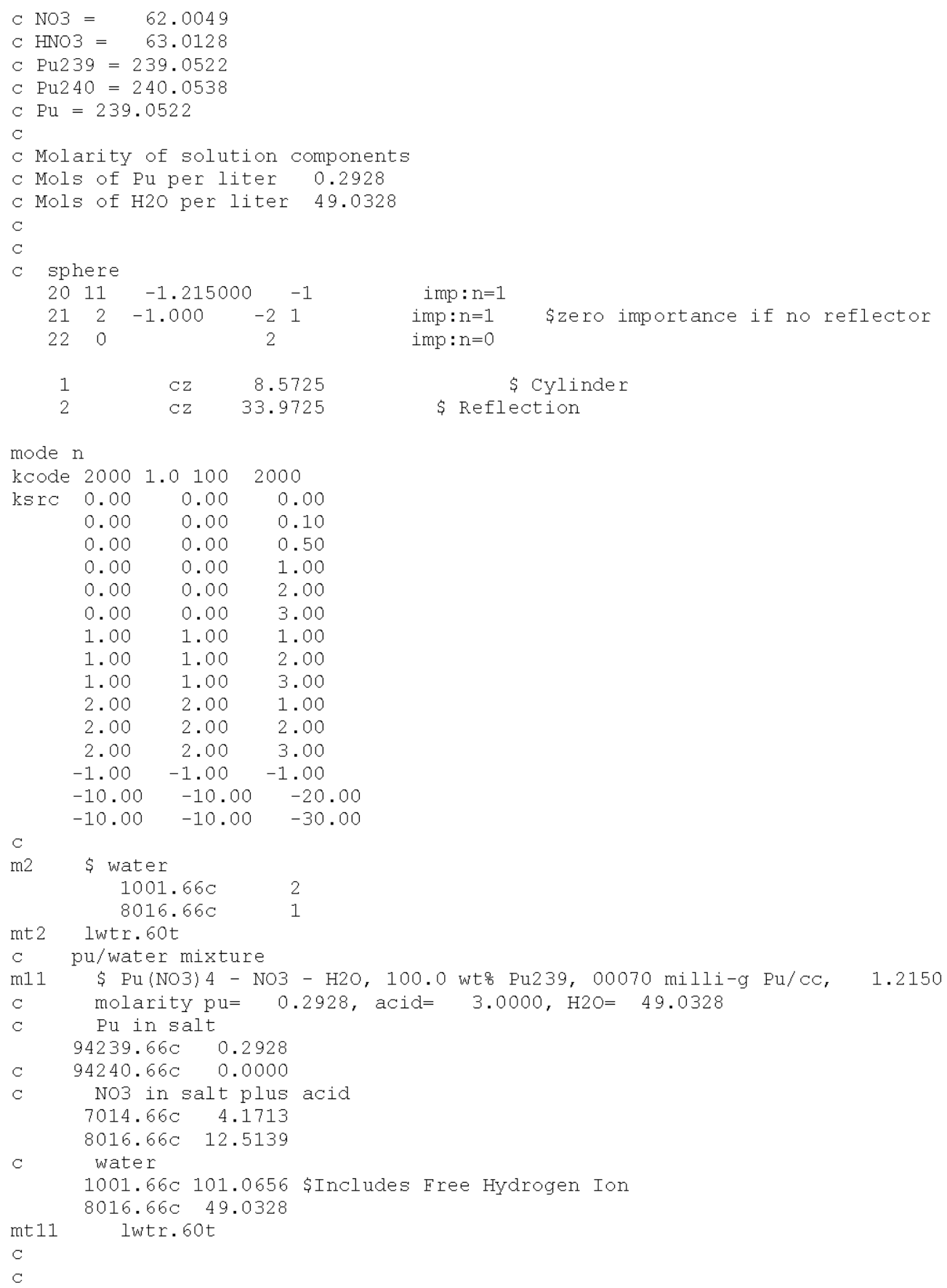




\section{CHPRC-01552, Revision 0}

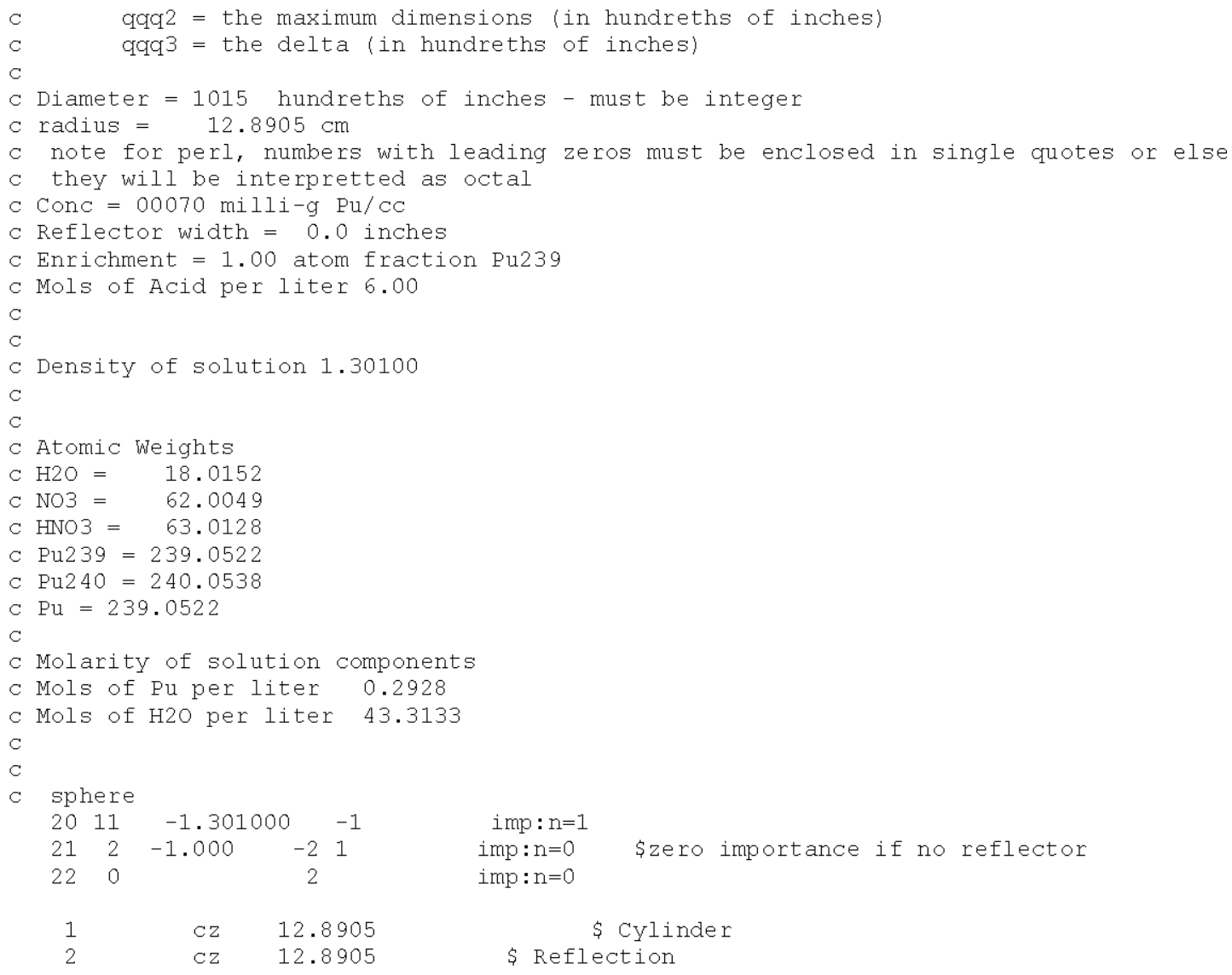




\section{CHPRC-01552, Revision 0}

$\operatorname{mt11}$
$\mathrm{C}$

Example Input for Case: 29 PuN46-icyl-1000-01-00070-00855.inp icyl, 100.0 wt: Pu239, $00070 \mathrm{milli}-\mathrm{g} / \mathrm{CC}$, in Pu(NO3) 4, with 6 Molar HNO3 $\mathrm{C}$

C worm notes: format of the form $\mathrm{n} . \mathrm{m}$ for floating point, In for integers

c zzzo (in upper case) is replaced with the number of cyles

c $\quad$ zzzl = the actual concentration in units of milli-g/cC

C $\quad z z z 2$ = solution density in $\mathrm{g} / \mathrm{CC}$

q9q1 = the minimum dimensions (in hundreths of inches)

qq92 = the maximum dimensions (in hundreths of inches)

qqq3 = the delta (in hundreths of inches)

Diameter $=855$ hundreths of inches - must be integer

$\mathrm{C}$ radius $=10.8585 \mathrm{~cm}$

C note for perl, numbers with leading zeros must be enclosed in single quotes or else

c they will be interpretted as octal

c $\mathrm{Conc}=00070 \mathrm{milli}-\mathrm{g} \mathrm{Pu} / \mathrm{CC}$

C Reflector width $=1.0$ inches

C Enrichment $=1.00$ atom Eraction Pu239

C Mols of Acid per liter 6.00

$\mathrm{C}$

C Density of solution 1.30100

Atomic Weights

$\mathrm{C} \mathrm{H} 2 \mathrm{O}=18.0152$

$\mathrm{C}$ NO3 $=62.0049$

C $\mathrm{HNO}=63.0128$

C Pu239 $=239.0522$

C Pu240 $=240.0538$

$\mathrm{C} \mathrm{Pu}=239.0522$

C Molarity of solution components

C Mols of Pu per liter 0.2928

C Mols of H2O per liter 43.3133

C

C sphere

$2011-1.301000-1 \quad$ imp $: n=1$

$\begin{array}{llllll}21 & 2 & -1.000 & -2 & 1 & \end{array}$

$220 \quad 2 \quad$ imp $: n=0$

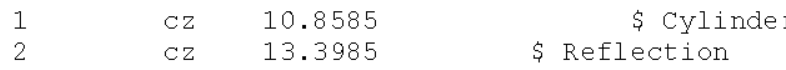

mode $\mathrm{n}$

kcode 20001.01002000

$\begin{array}{llll}\text { ksrc } & 0.00 & 0.00 & 0.00\end{array}$

$0.00 \quad 0.00 \quad 0.10$

$\begin{array}{lll}0.00 & 0.00 \quad 0.50\end{array}$

$0.00 \quad 0.00 \quad 1.00$

$\begin{array}{lll}0.00 & 0.00 \quad 2.00\end{array}$

$\begin{array}{lll}0.00 & 0.00 \quad 3.00\end{array}$

$\begin{array}{lll}1.00 & 1.00 & 1.00\end{array}$

$\begin{array}{lll}1.00 & 1.00 \quad 2.00\end{array}$

$\begin{array}{lll}1.00 & 1.00 \quad 3.00\end{array}$

$\begin{array}{lll}2.00 & 2.00 \quad 1.00\end{array}$

$\begin{array}{lll}2.00 & 2.00 \quad 2.00\end{array}$

$\begin{array}{lll}2.00 & 2.00 \quad 3.00\end{array}$

$\begin{array}{lll}-1.00 & -1.00 & -1.00\end{array}$

$\begin{array}{lll}-10.00 & -10.00 \quad-20.00\end{array}$ 


\section{CHPRC-01552, Revision 0}

$2 \mathrm{Cz} \quad 34.6075 \quad$ \$ Reflection

mode $n$

kcode 20001.01002000

$\begin{array}{llll}\text { ksre } & 0.00 & 0.00 & 0.00\end{array}$

$\begin{array}{lll}0.00 & 0.00 \quad 0.10\end{array}$

$\begin{array}{lll}0.00 & 0.00 \quad 0.50\end{array}$

$0.00 \quad 0.00 \quad 1.00$

$\begin{array}{lll}0.00 & 0.00 \quad 2.00\end{array}$

$\begin{array}{lll}0.00 & 0.00 \quad 3.00\end{array}$

$1.00 \quad 1.00 \quad 1.00$

$\begin{array}{lll}1.00 & 1.00 \quad 2.00\end{array}$

$\begin{array}{lll}1.00 & 1.00 \quad 3.00\end{array}$

$\begin{array}{lll}2.00 & 2.00 \quad 1.00\end{array}$

$\begin{array}{lll}2.00 & 2.00 \quad 2.00\end{array}$

$2.00 \quad 2.00 \quad 3.00$

$\begin{array}{lll}-1.00 & -1.00 & -1.00\end{array}$

$-10.00 \quad-10.00 \quad-20.00$

$\begin{array}{lll}-10.00 & -10.00 & -30.00\end{array}$

m2 \$ water

$1001.66 \mathrm{C} \quad 2$

$8016.66 \mathrm{C} \quad 1$

mt2 lwtr.60t

C pu/water mixture

m11 \$ $\mathrm{Pu}(\mathrm{NO} 3) 4-\mathrm{NO3}-\mathrm{H} 2 \mathrm{O}, 100.0$ wto Pu239, $00070 \mathrm{milli-g} \mathrm{Pu/CC,} 1.3010$

C molarity $\mathrm{pu}=0.2928$, acid= 6.0000, $\mathrm{H} 20=43.3133$

C Pu in salt

$94239.66 \mathrm{C} \quad 0.2928$

$94240.66 \mathrm{C} \quad 0.0000$

No3 in salt plus acid

$7014.66 \mathrm{c} \quad 7.1713$

$8016.66 \mathrm{c} \quad 21.5139$

c water

1001.66c 92.6266 \$Includes Free Hydrogen Ion

$8016.66 \mathrm{C} \quad 43.3133$

mt11 lwtr. $60 t$

C

$\mathrm{C}$

Example Input for Case: 31 Pun40-icyl-0970-00-00012-01640.inp

icyl, 97.0 wto Pu239, $00012 \mathrm{milli-g/CC}$, in Pu(NO3) 4, with 0 Molar HNO3

$\mathrm{C}$

c worm notes: format of the form |n.m for floating point, In for integers

c zzzo (in upper case) is replaced with the number of cyles

c $\quad \mathrm{zzzl}=$ the actual concentration in units of milli-g/cc

C $\quad$ zzz2 = solution density in $\mathrm{g} / \mathrm{CC}$

c qqq1 = the minimum dimensions (in hundreths of inches)

c $\quad$ qq2 2 the maximum dimensions (in hundreths of inches)

c $\quad$ qq93 $=$ the delta (in hundreths of inches)

$\mathrm{C}$ Diameter $=1640$ hundreths of inches - must be integer

$\mathrm{C}$ radius $=20.8280 \mathrm{~cm}$

c note for perl, numbers with leading zeros must be enclosed in single quotes or else

c they will be interpretted as octal

$\mathrm{C}$ Conc $=00012 \mathrm{milli}-\mathrm{g} \mathrm{Pu} / \mathrm{CC}$

C Reflector width $=0.0$ inches

C Enrichment $=0.97$ atom fraction Pu239

C Mols of Acid per liter 0.00

$\mathrm{C}$

$\mathrm{C}$

C Density of solution 1.01600

$\mathrm{C}$

C Atomic Weights 


\section{CHPRC-01552, Revision 0}

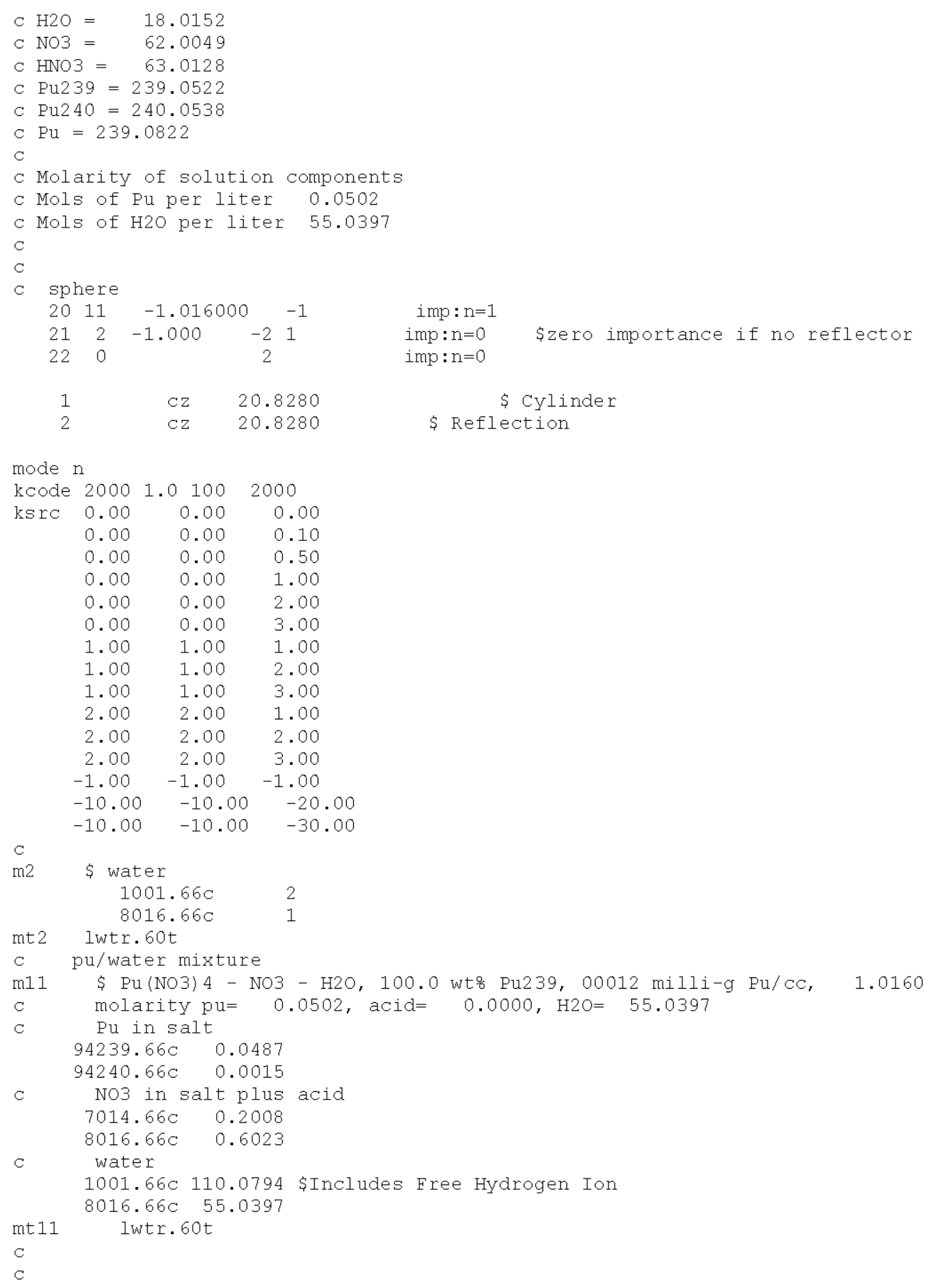




\section{CHPRC-01552, Revision 0}

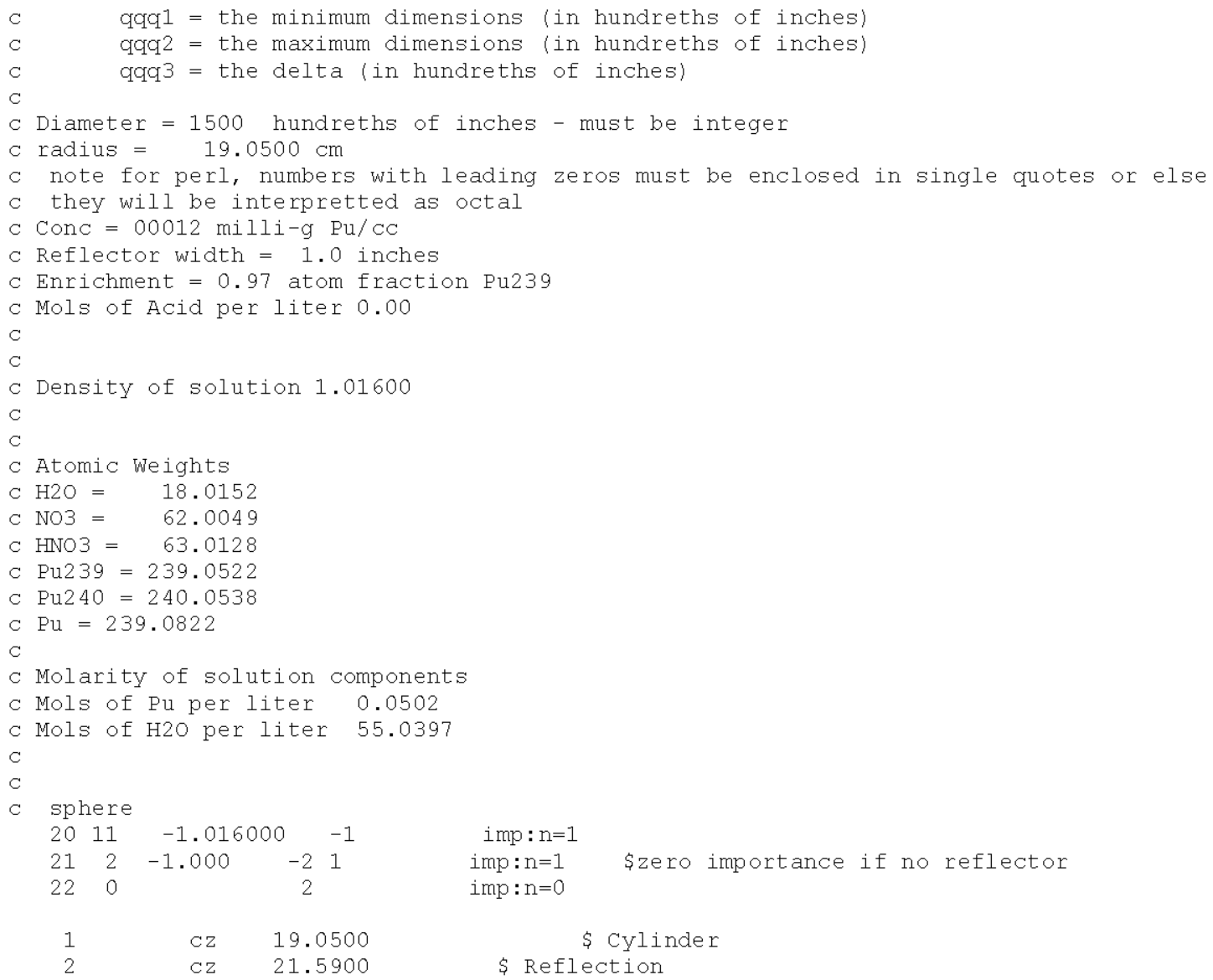




\section{CHPRC-01552, Revision 0}

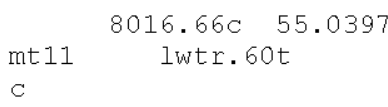




\section{CHPRC-01552, Revision 0}

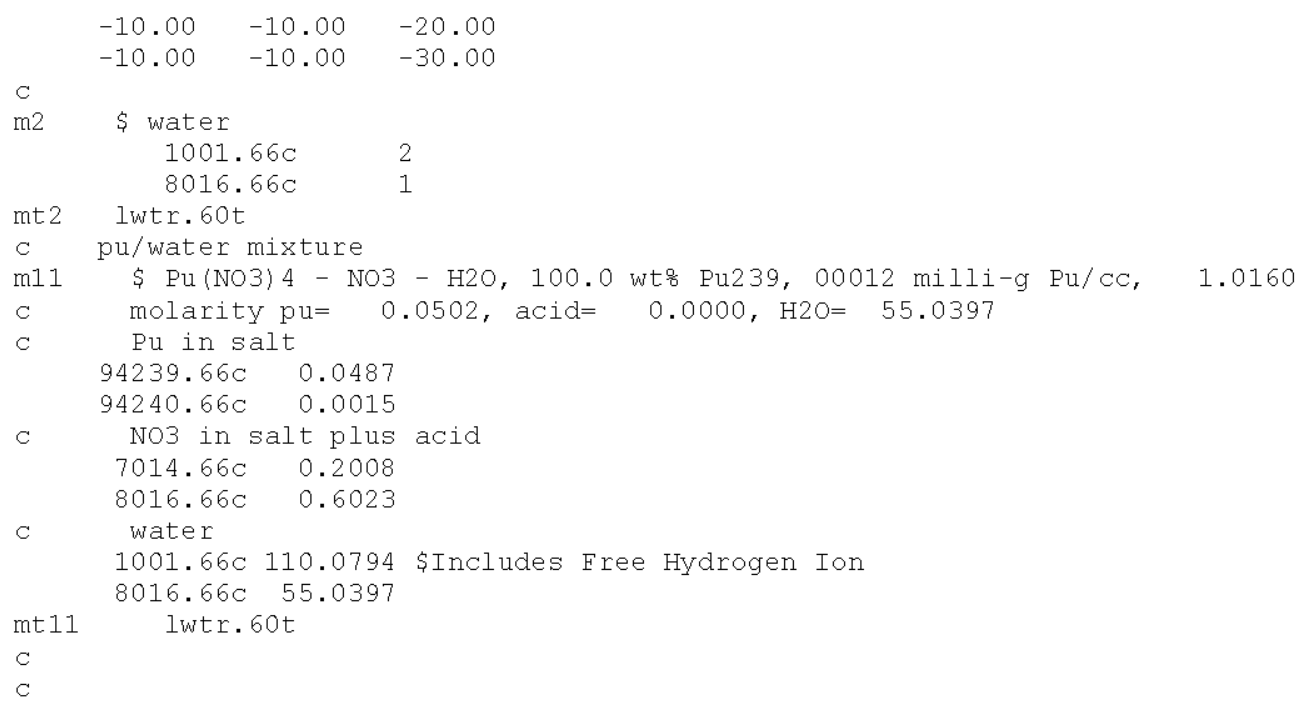




\section{CHPRC-01552, Revision 0}

$\begin{array}{rrrr}1 & C z & 23.3680 & \text { \$Cylinder } \\ 2 & \mathrm{CZ} & 23.3680 & \text { \$ Reflection }\end{array}$

mode $\mathrm{n}$

kcode 20001.01002000

$\begin{array}{llll}\text { ksrc } & 0.00 & 0.00 & 0.00\end{array}$

$0.00 \quad 0.00 \quad 0.10$

$\begin{array}{lll}0.00 & 0.00 \quad 0.50\end{array}$

$\begin{array}{lll}0.00 & 0.00 \quad 1.00\end{array}$

$\begin{array}{lll}0.00 & 0.00 \quad 2.00\end{array}$

$\begin{array}{lll}0.00 & 0.00 \quad 3.00\end{array}$

$\begin{array}{lll}1.00 & 1.00 \quad 1.00\end{array}$

$\begin{array}{lll}1.00 & 1.00 \quad 2.00\end{array}$

$\begin{array}{lll}1.00 & 1.00 \quad 3.00\end{array}$

$\begin{array}{lll}2.00 & 2.00 \quad 1.00\end{array}$

$2.00 \quad 2.00 \quad 2.00$

$\begin{array}{lll}2.00 & 2.00 \quad 3.00\end{array}$

$-1.00 \quad-1.00 \quad-1.00$

$\begin{array}{lll}-10.00 & -10.00 & -20.00\end{array}$

$\begin{array}{lll}-10.00 & -10.00 & -30.00\end{array}$

m2 \$ water

$1001.66 \mathrm{C} \quad 2$

mt2 lwtr.60t

C pu/water mixture

m11 \$ Pu (NO3) 4 - NO3 - H20, 100.0 wto Pu239, 00012 milli-g Pu/CC, 1.1180

c molarity $\mathrm{pu}=0.0502$, acid $=3.0000, \mathrm{H} 2 \mathrm{O}=50.2083$

C Pu in salt

$94239.66 \mathrm{c} \quad 0.0487$

$94240.66 \mathrm{C} \quad 0.0015$

C NO3 in salt plus acid

$7014.66 \mathrm{c} \quad 3.2008$

$8016.66 \mathrm{C} \quad 9.6023$

C water

1001.66 103.4167 \$Includes Free Hydrogen Ion

$8016.66 \mathrm{C} \quad 50.2083$

mt11 lwtr. $60 t$

$\mathrm{C}$

c

Example Input for Case: 35 PuN43-icyl-0970-01-00012-01690.inp

icyl, 97.0 wto Pu239, $00012 \mathrm{milli-g/CC}$, in Pu(NO3) 4, with 3 Molar HNO3

C

C worm notes: format of the form $\mid \mathrm{n} . \mathrm{m}$ for floating point, In for integers

c zzzo (in upper case) is replaced with the number of cyles

c $\quad$ zzzl = the actual concentration in units of milli-g/cc

c $\quad$ zzz2 = solution density in $g / C C$

C $\quad$ qqq1 $=$ the minimum dimensions (in hundreths of inches)

c $\quad$ qवq 2 the maximum dimensions (in hundreths of inches)

c $\quad$ qq9 3 = the delta (in hundreths of inches)

Diameter $=1690$ hundreths of inches - must be integer

$\mathrm{C}$ radius $=21.4630 \mathrm{~cm}$

C note for perl, numbers with leading zeros must be enclosed in single quotes or else

they will be interpretted as octal

Conc $=00012$ milli-g $\mathrm{Pu} / \mathrm{CC}$

c Reflector width $=1.0$ inches

C Enrichment $=0.97$ atom fraction Pu239

C Mols of Acid per liter 3.00

$\mathrm{C}$

$\mathrm{C}$

C Density of solution 1.11800 


\section{CHPRC-01552, Revision 0}

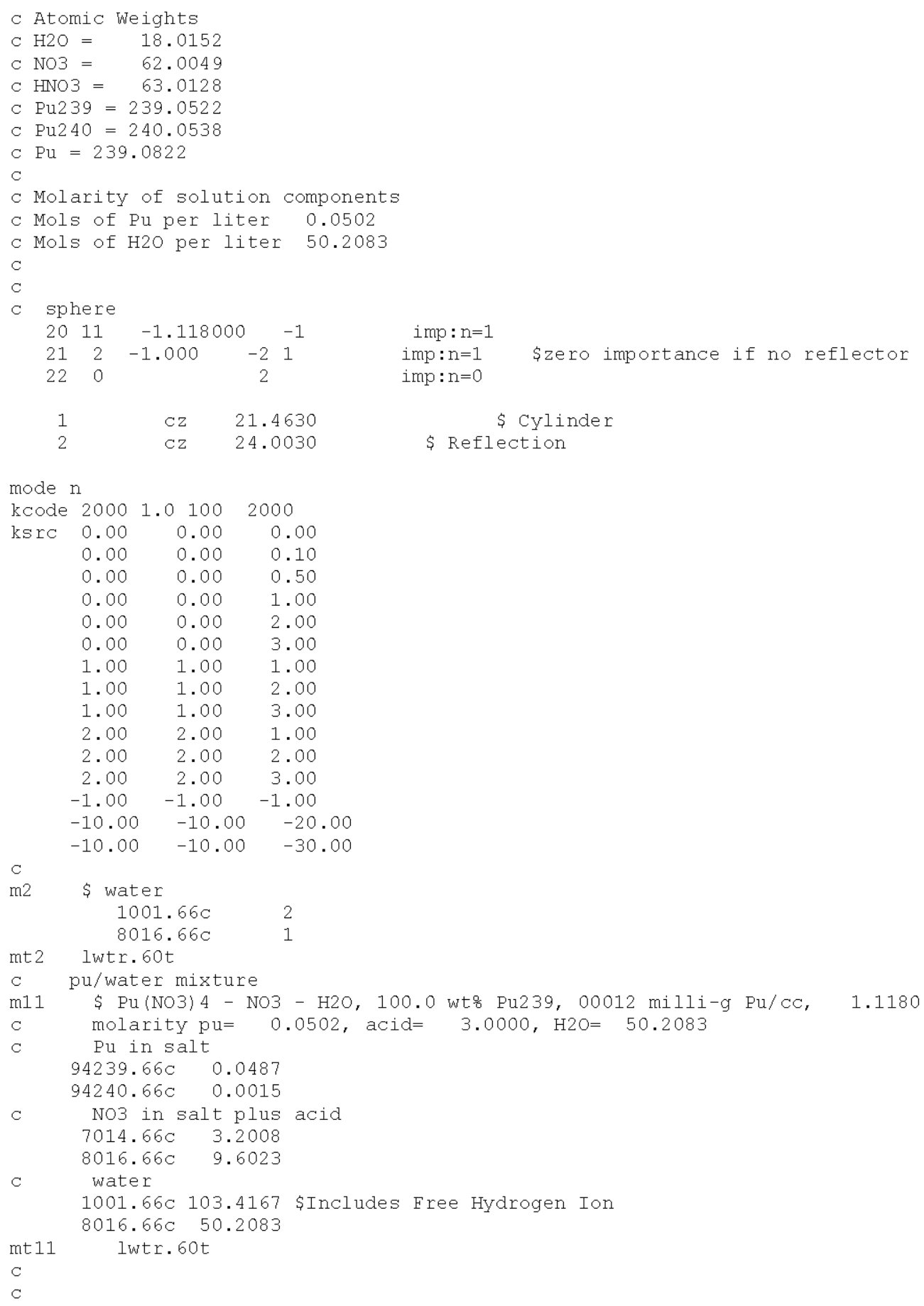




\section{CHPRC-01552, Revision 0}

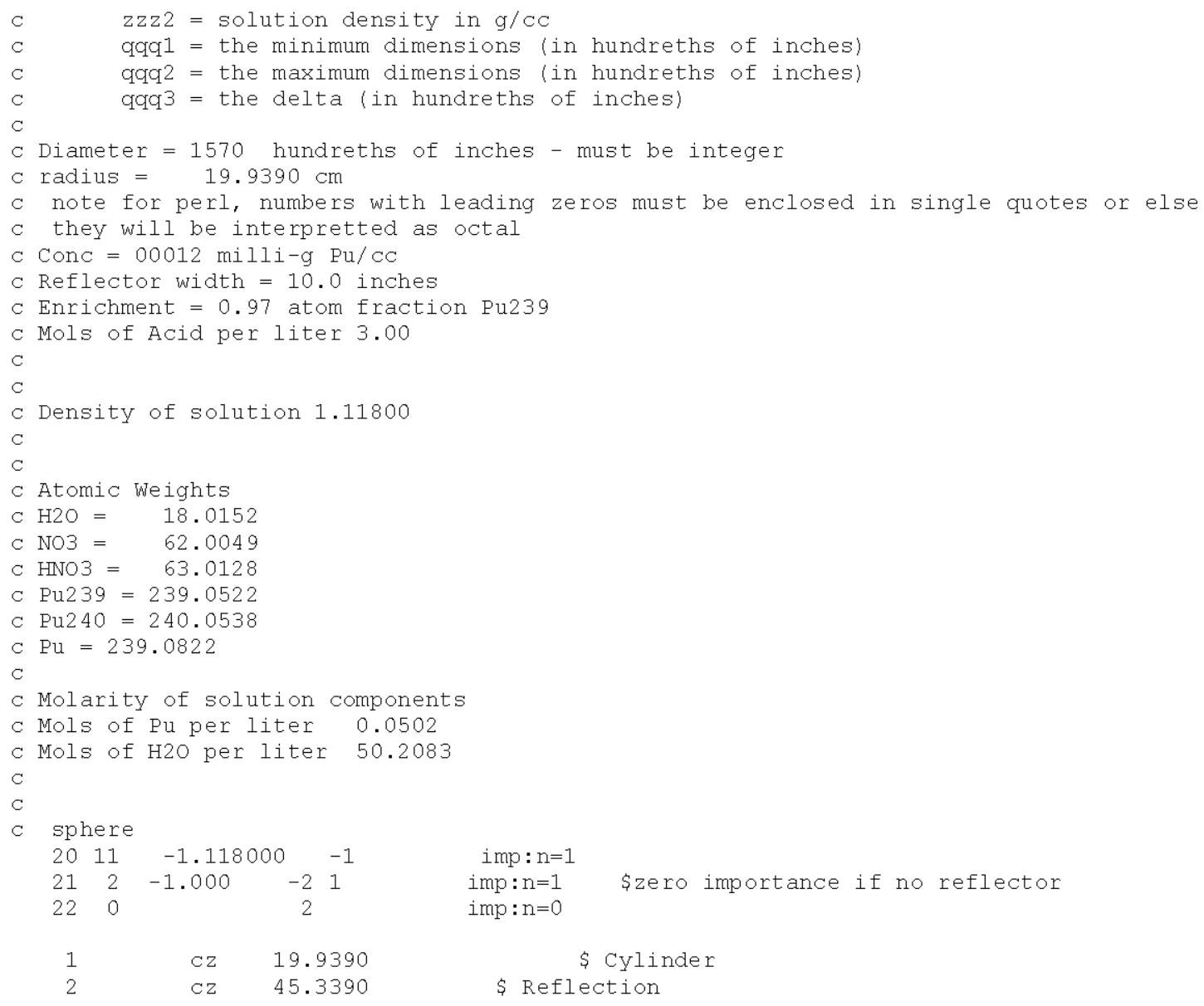




\section{CHPRC-01552, Revision 0}

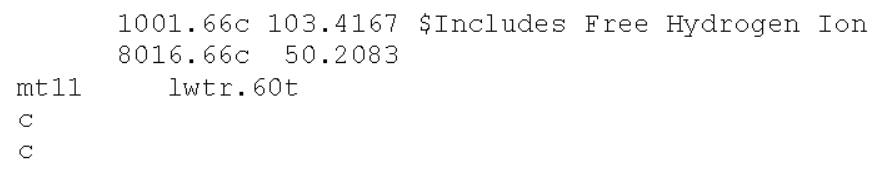




\section{CHPRC-01552, Revision 0}

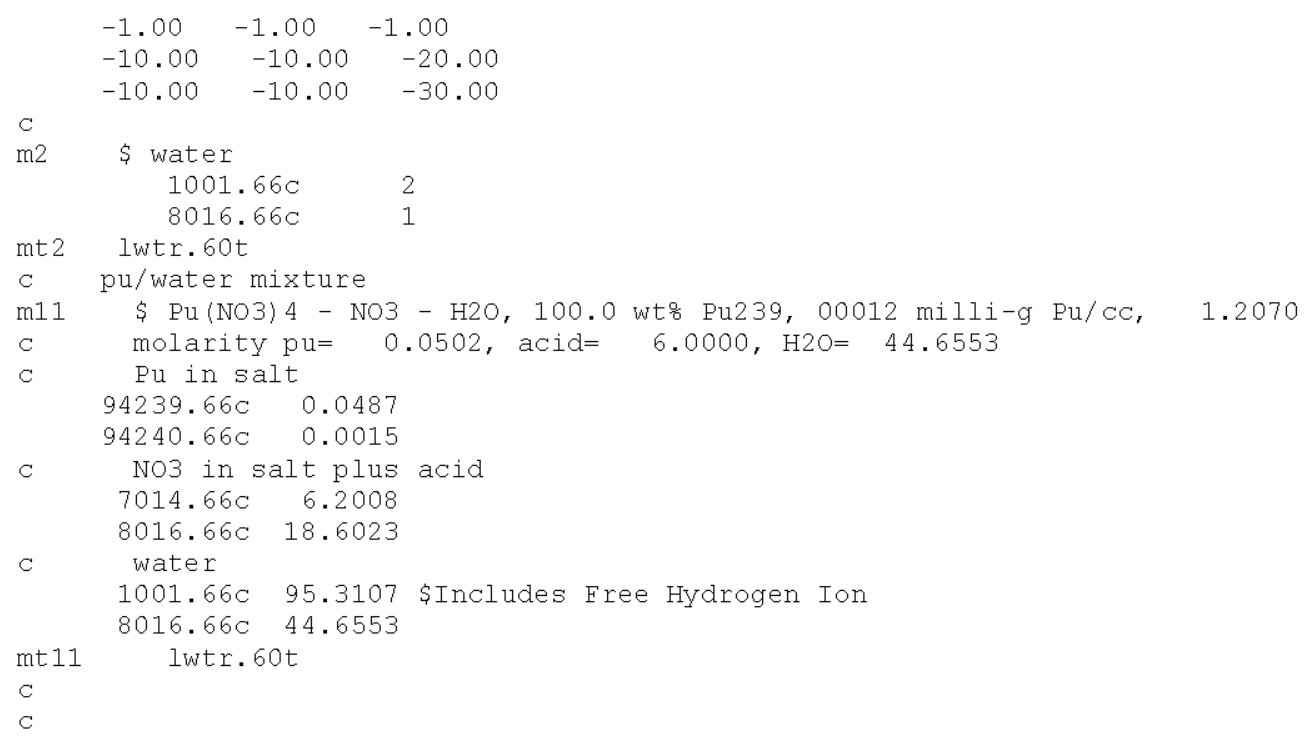




\section{CHPRC-01552, Revision 0}

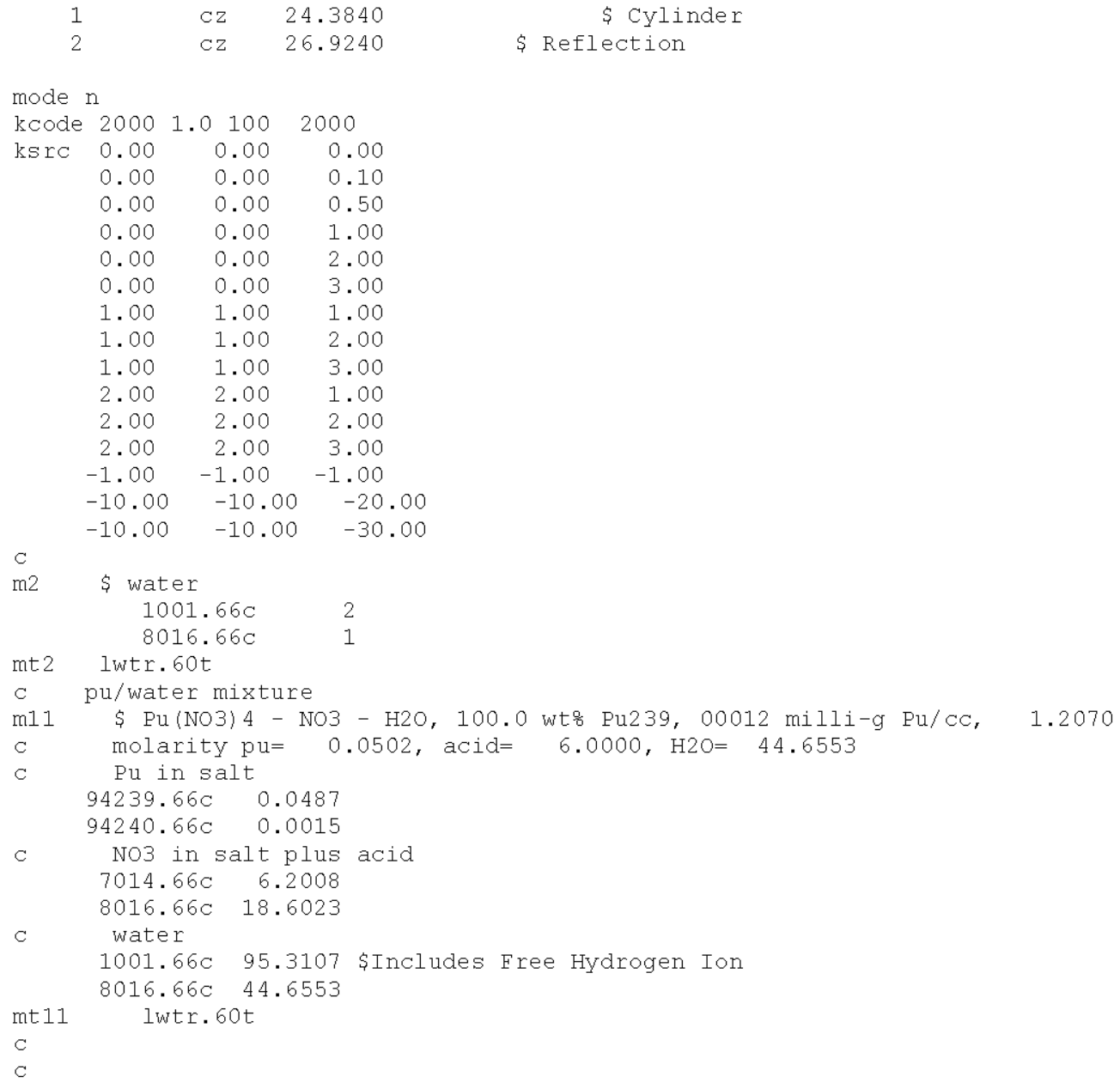




\section{CHPRC-01552, Revision 0}

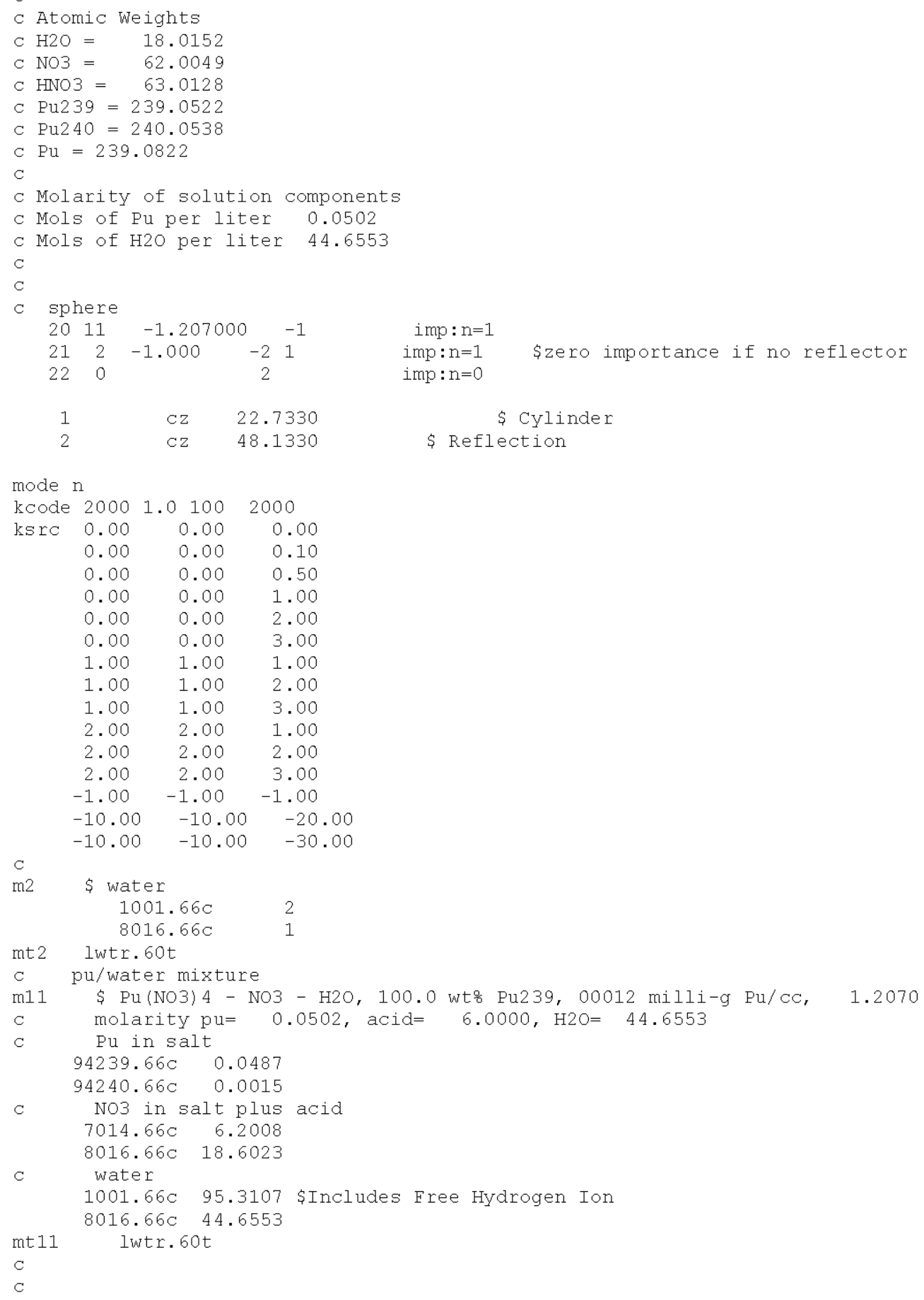




\section{CHPRC-01552, Revision 0}

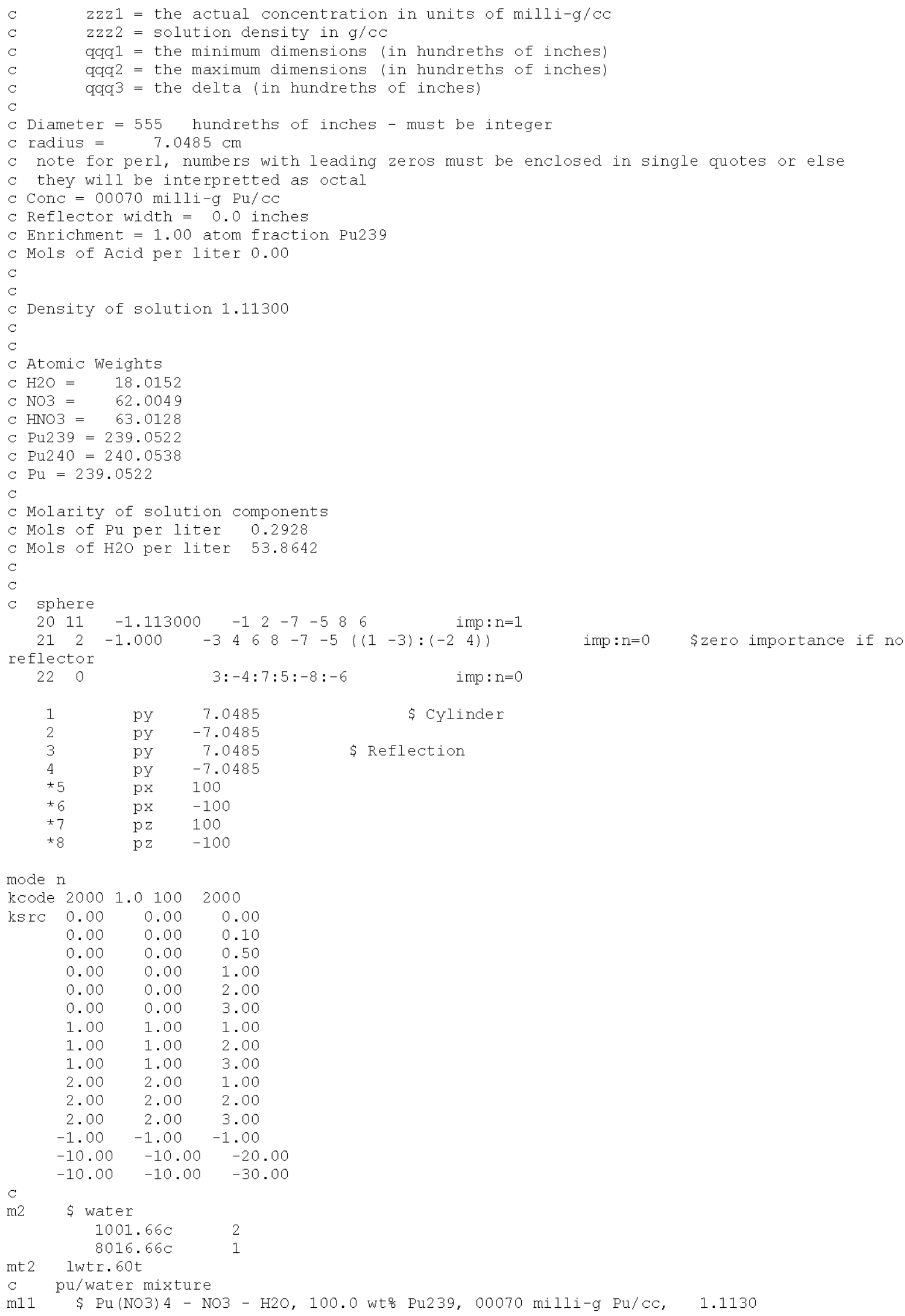




\section{CHPRC-01552, Revision 0}

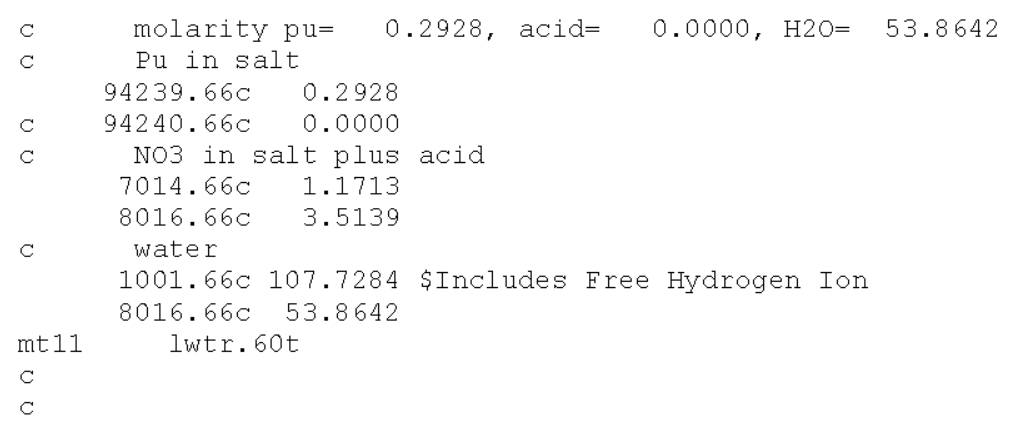

Example Input for Case: 41 PuN40-pl-1000-01-00070-00390.inp

pl, 100.0 wto Pu239, $00070 \mathrm{milli-g/CC}$, in Pu(NO3) 4, with 0 Molar HNO3

$\mathrm{C}$

C worm notes: format of the form |n.m for floating point, In for integers

c zzzo (in upper case) is replaced with the number of cyles

C $\quad \mathrm{zzzl}=$ the actual concentration in units of milli-g/cc

zzz2 = solution density in $g / \mathrm{CC}$

qqa1 = the minimum dimensions (in hundreths of inches)

qq92 = the maximum dimensions (in hundreths of inches)

q993 = the delta (in hundreths of inches)

Diameter $=390$ hundreths of inches - must be integer

C radius $=4.9530 \mathrm{~cm}$

c note for perl, numbers with leading zeros must be enclosed in single quotes or else

c they will be interpretted as octal

C $\mathrm{Conc}=00070 \mathrm{milli-g} \mathrm{Pu} / \mathrm{CC}$

C Reflector width $=1.0$ inches

C Enrichment $=1.00$ atom fraction Pu239

C Mols of Acid per liter 0.00

$\mathrm{C}$

C

C Density of solution 1.11300

C

$\mathrm{C}$

C Atomic Weights

C $\mathrm{H} 2 \mathrm{O}=18.0152$

C $\mathrm{NO} 3=62.0049$

$\mathrm{C}$ HNO3 $=63.0128$

C $\mathrm{Pu} 239=239.0522$

C Pu $240=240.0538$

C $\mathrm{Pu}=239.0522$

C Molarity of solution components

C Mols of Pu per liter 0.2928

C Mols of H2O per liter 53.8642

$\mathrm{C}$

C

C sphere

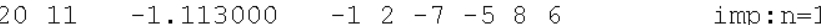

reflector

220

$3:-4: 7: 5:-8:-6 \quad$ imp $: n=0$

$\begin{array}{llll}1 & \text { py } & 4.9530 & \text { \$Cylinder } \\ 2 & \text { py } & -4.9530 & \\ 3 & \text { py } & 7.4930 & \text { \$Reflection } \\ 4 & \text { py } & -7.4930 & \\ * 5 & \text { px } & 100 & \\ * 6 & \text { px } & -100 & \\ \star 7 & \text { pz } & 100 & \\ \star 8 & \text { pz } & -100 & \end{array}$




\section{CHPRC-01552, Revision 0}

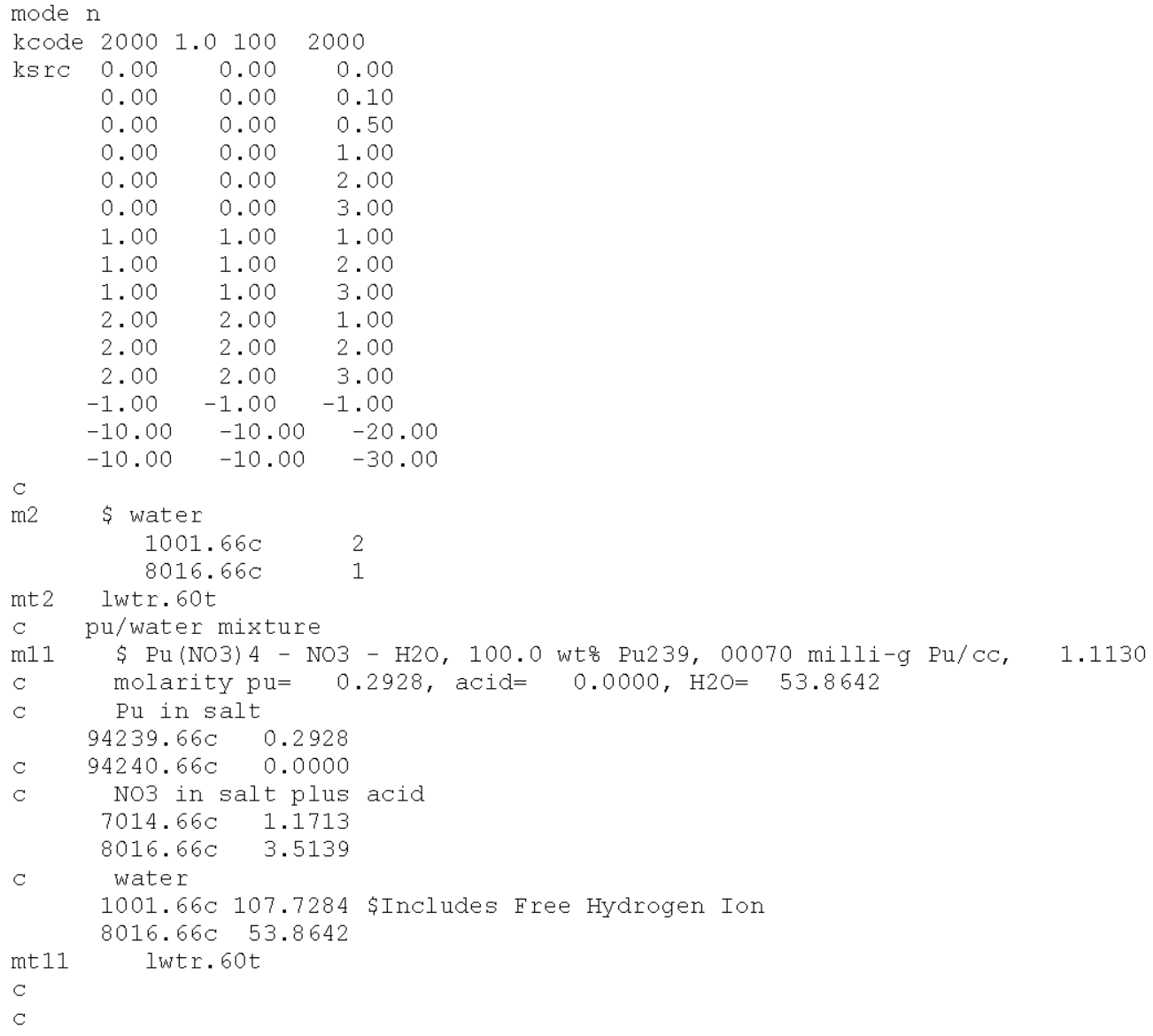




\section{CHPRC-01552, Revision 0}

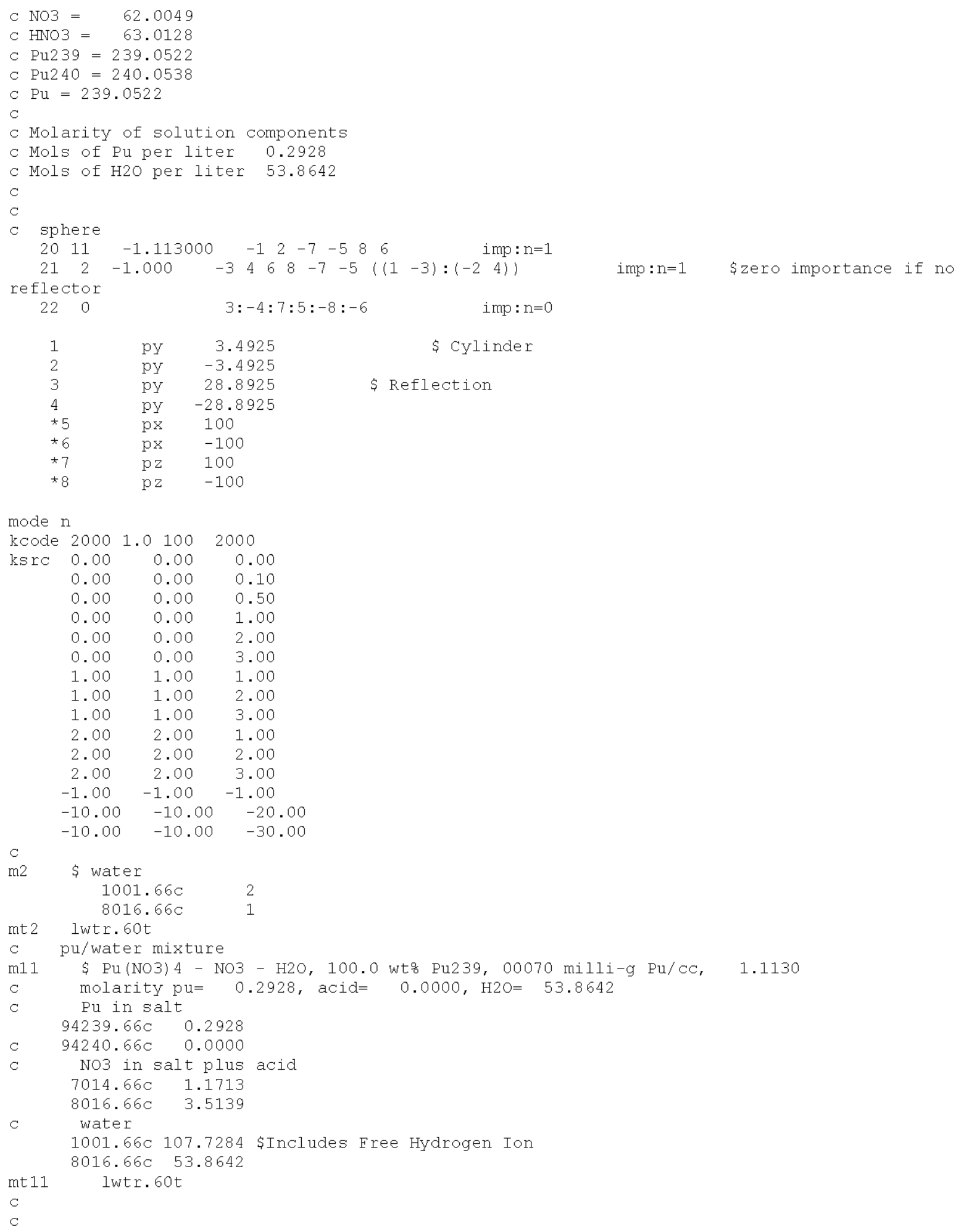




\section{CHPRC-01552, Revision 0}

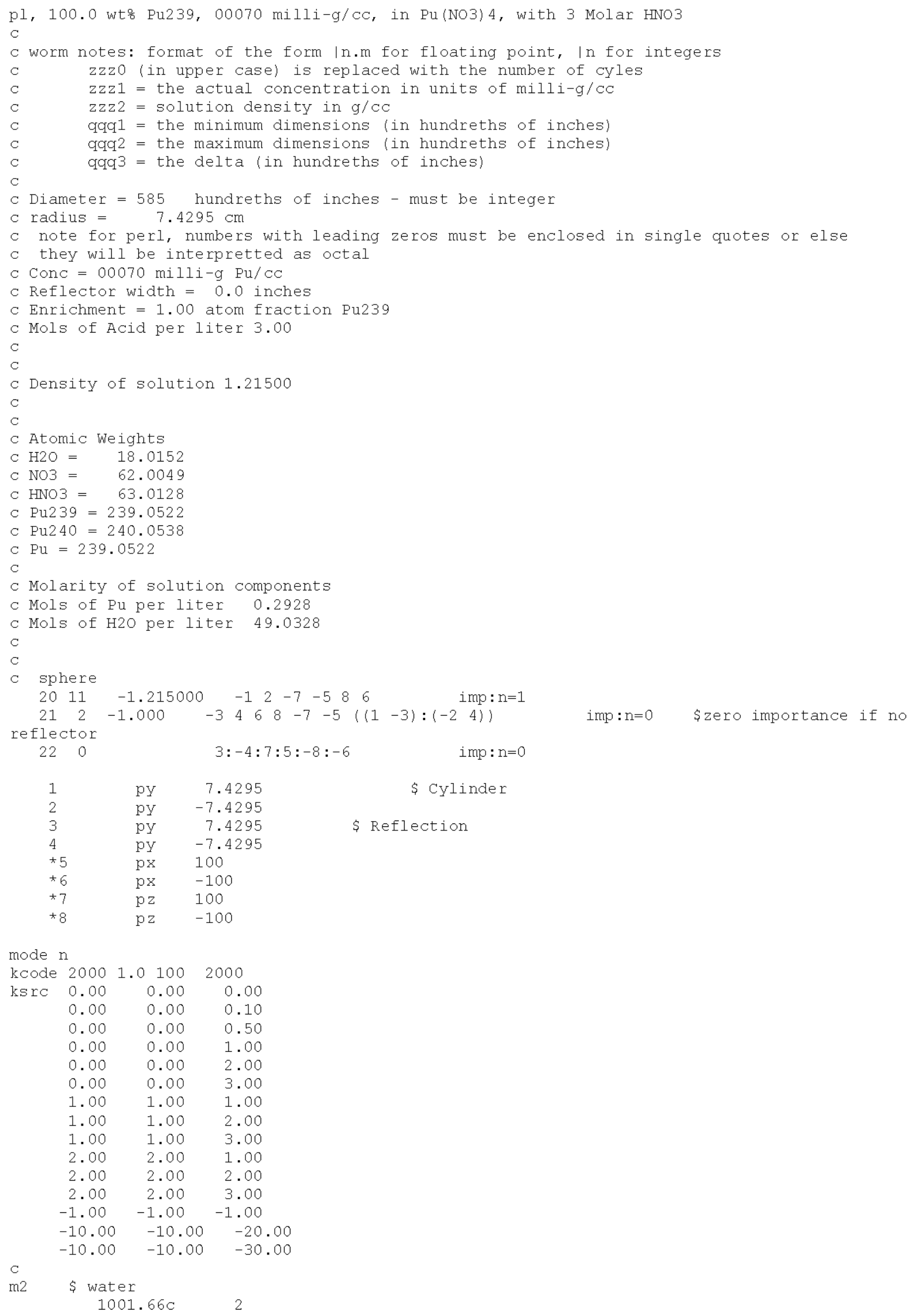




\section{CHPRC-01552, Revision 0}
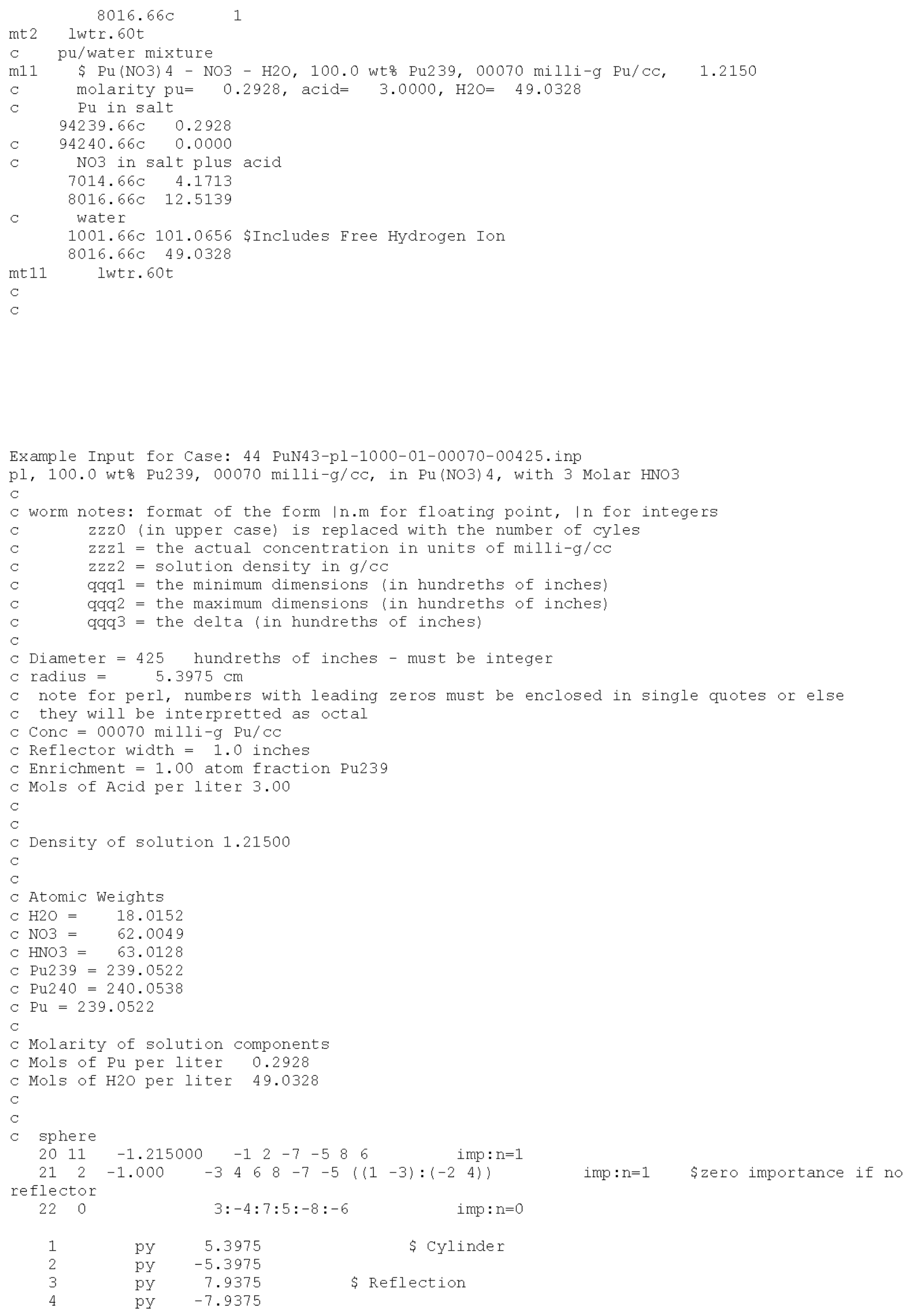


\section{CHPRC-01552, Revision 0}

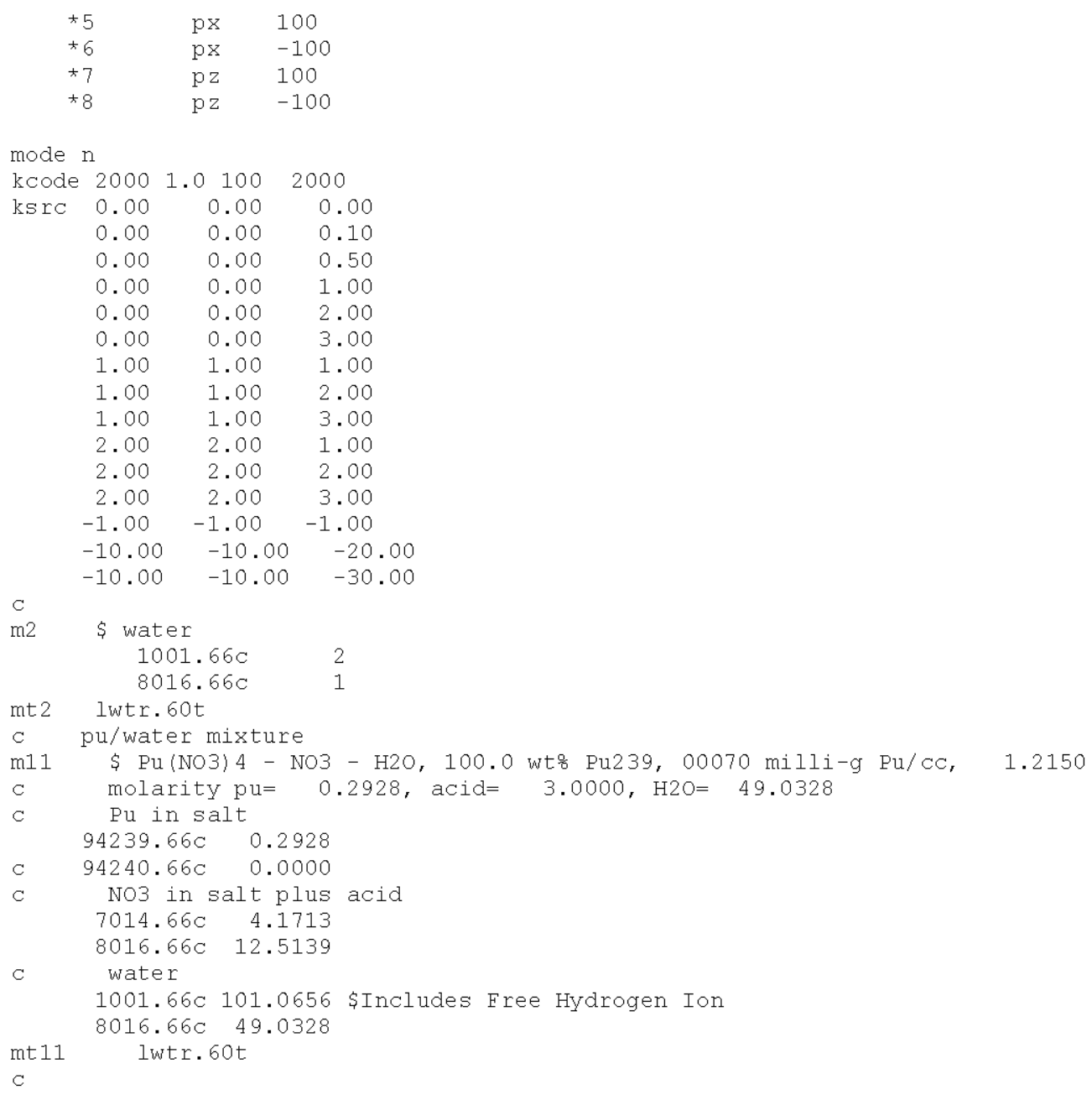




\section{CHPRC-01552, Revision 0}

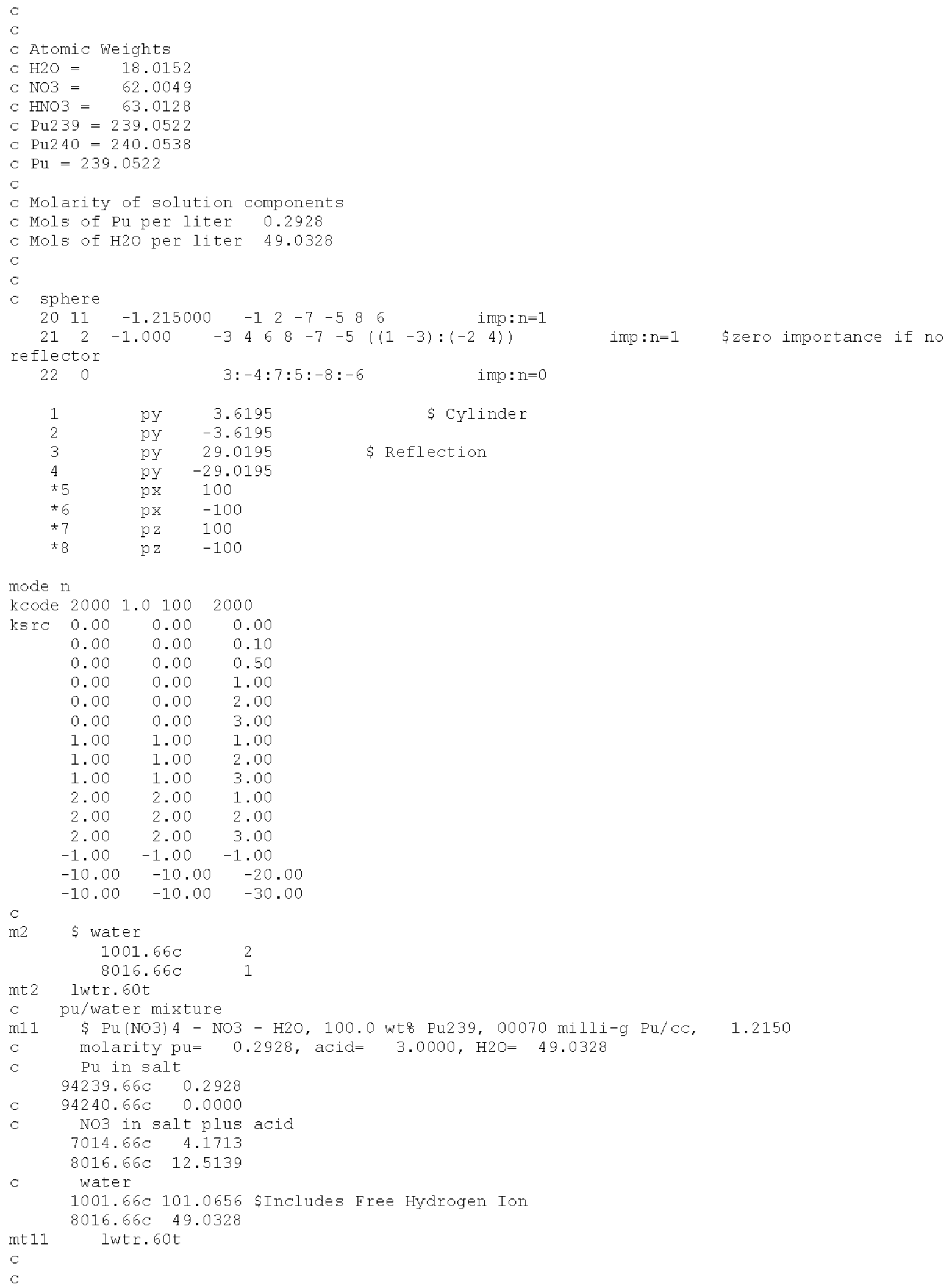




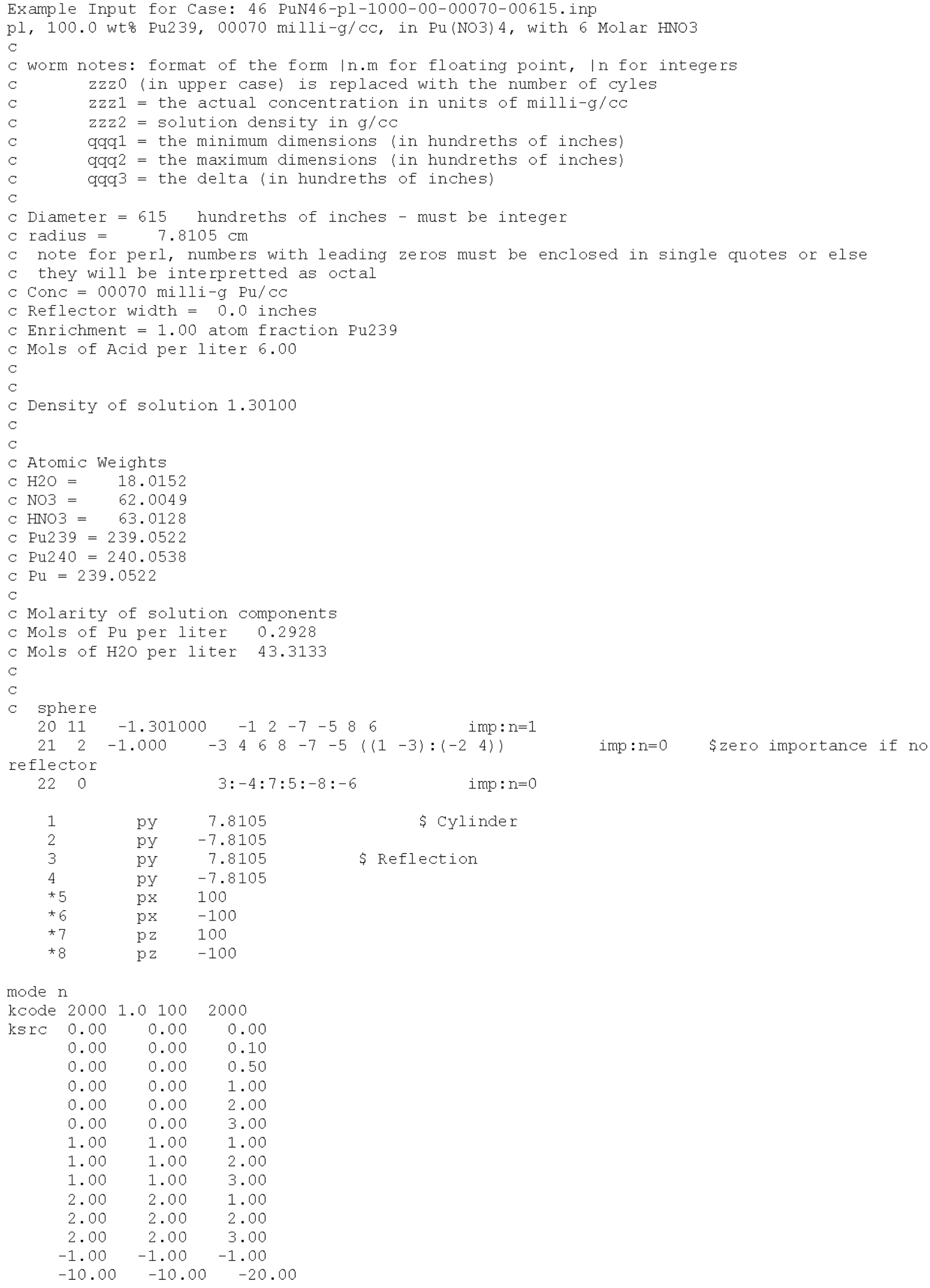




\section{CHPRC-01552, Revision 0}

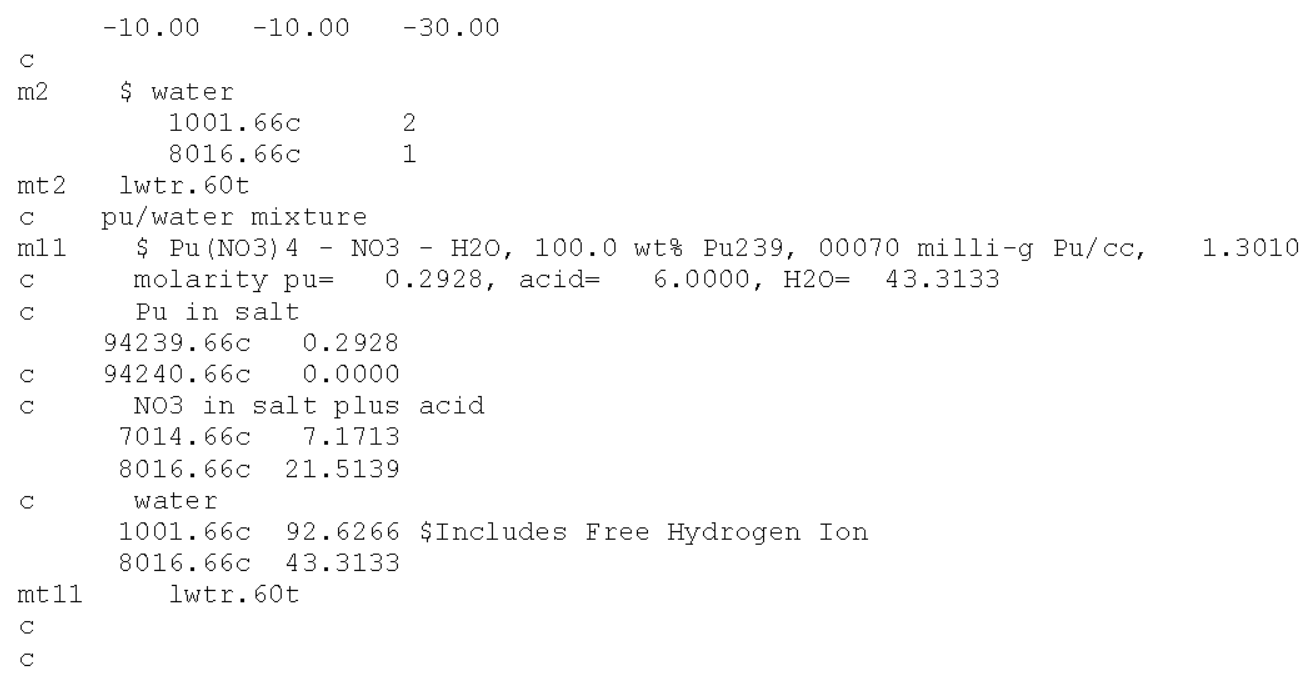

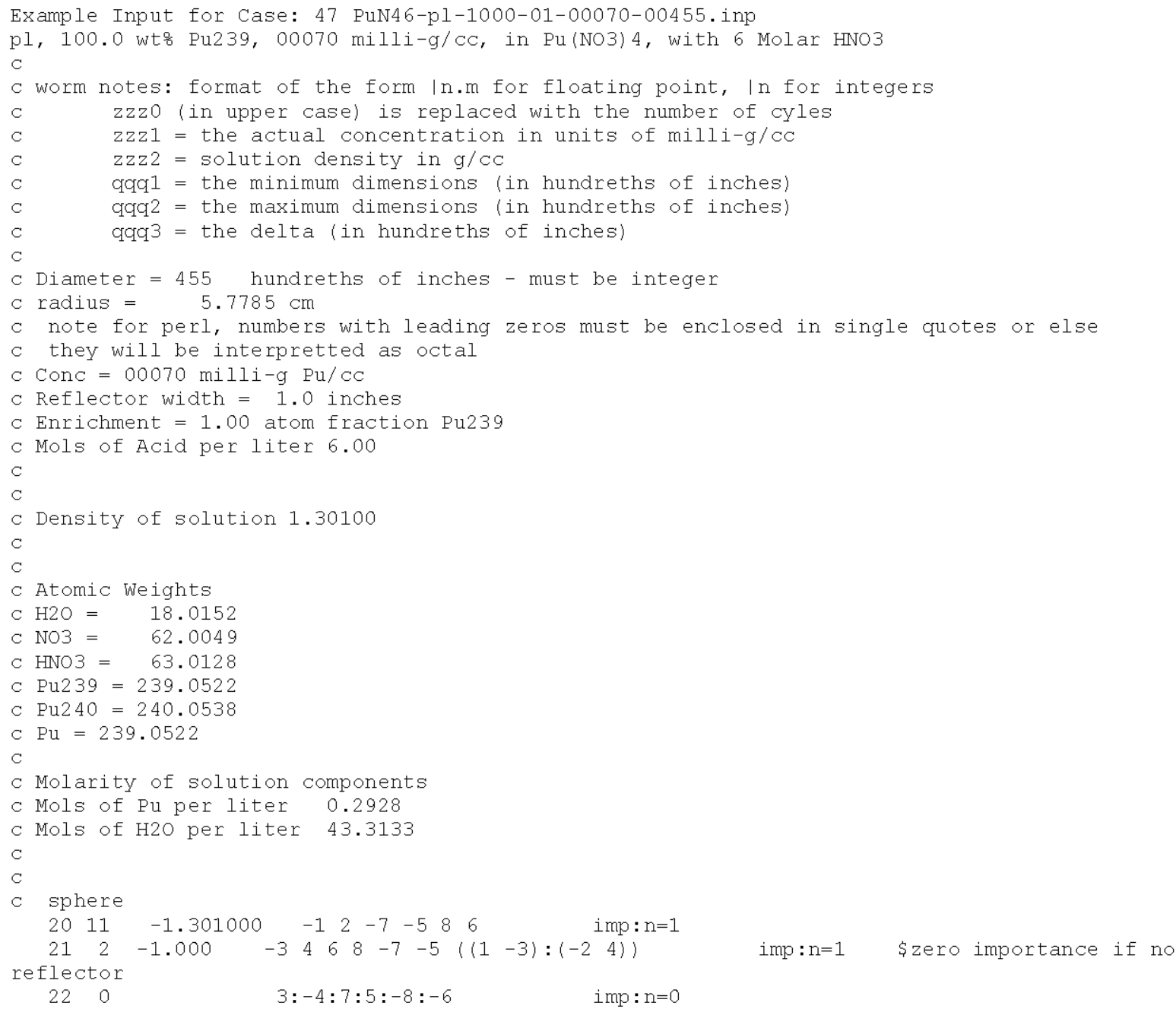




\section{CHPRC-01552, Revision 0}

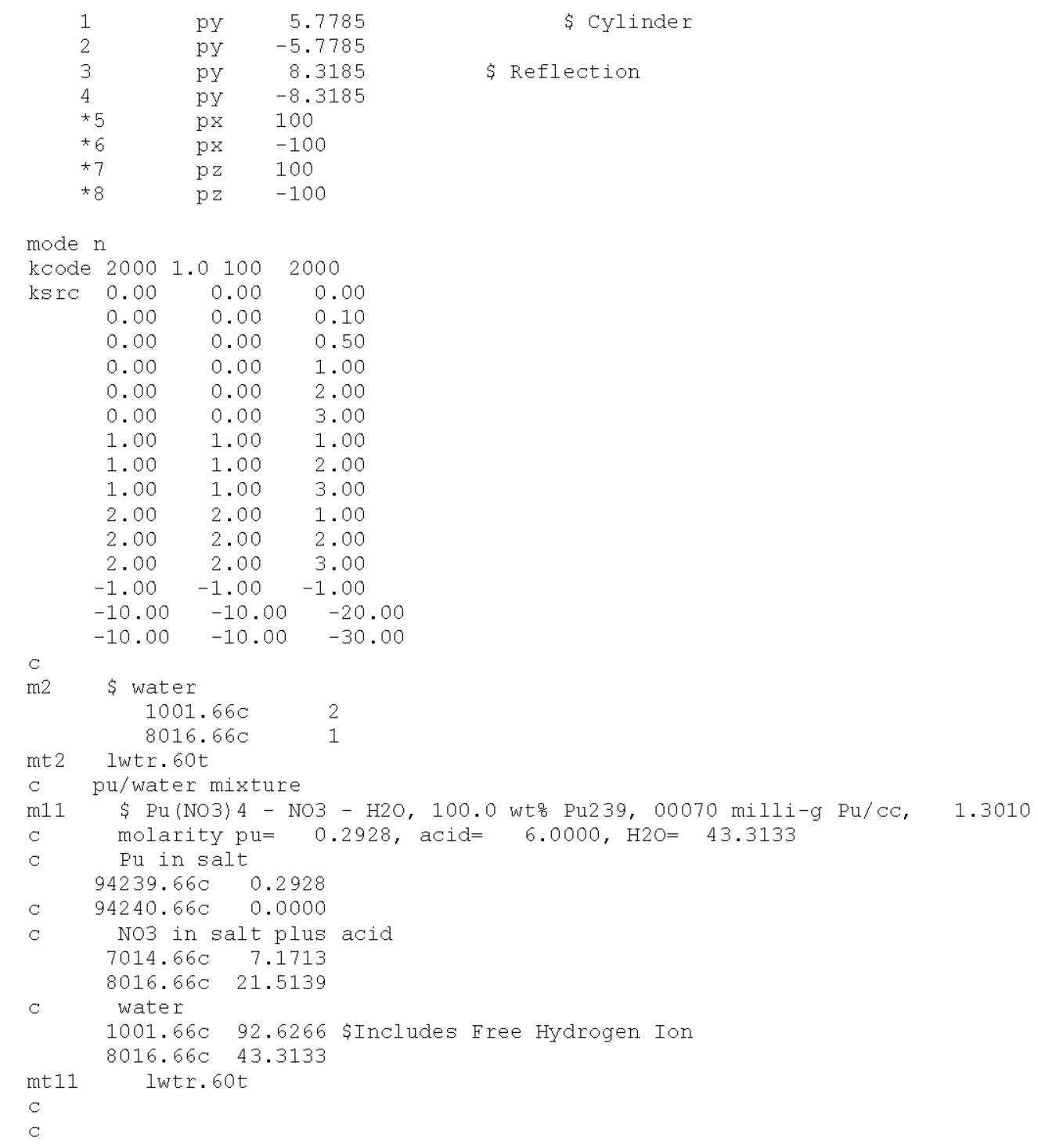

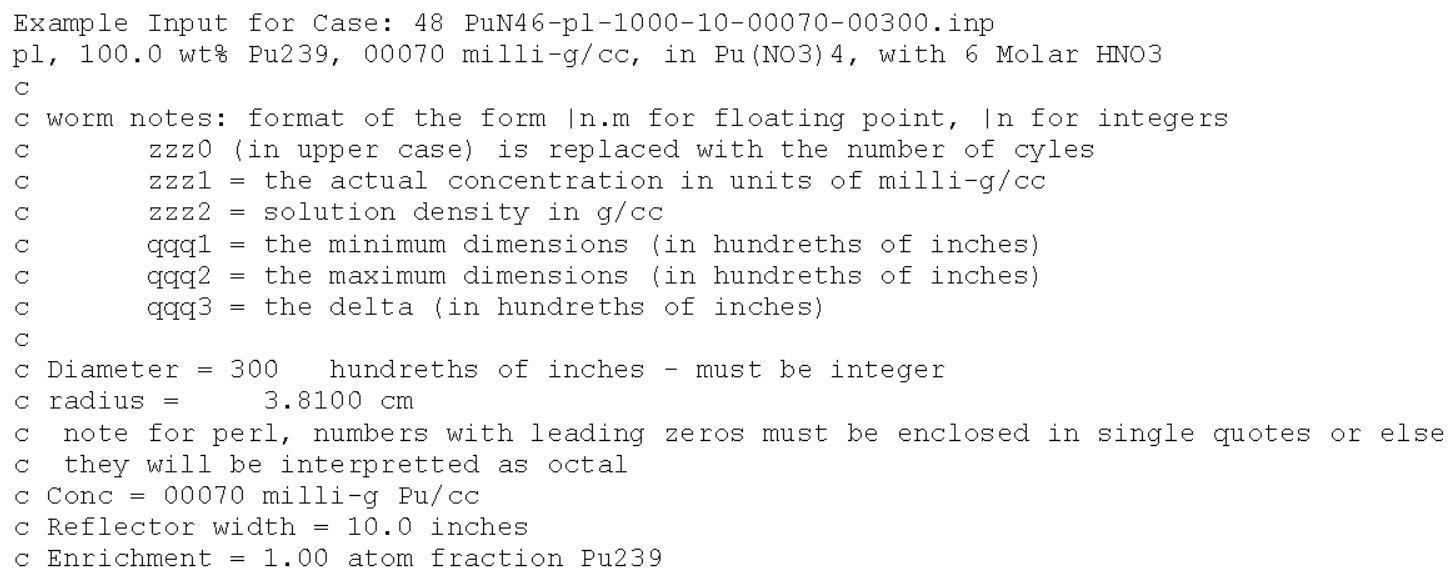




\section{CHPRC-01552, Revision 0}

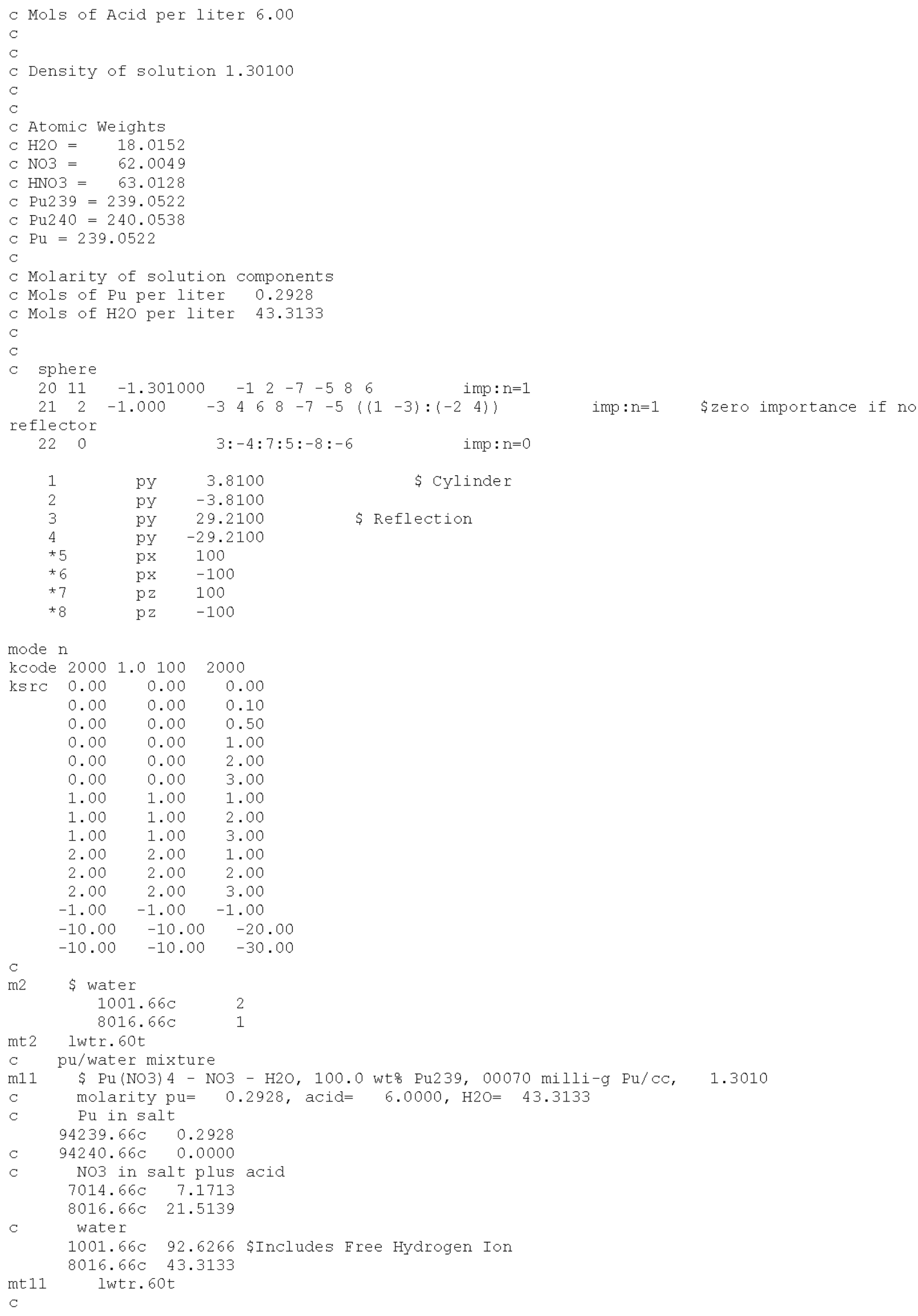




\section{CHPRC-01552, Revision 0}

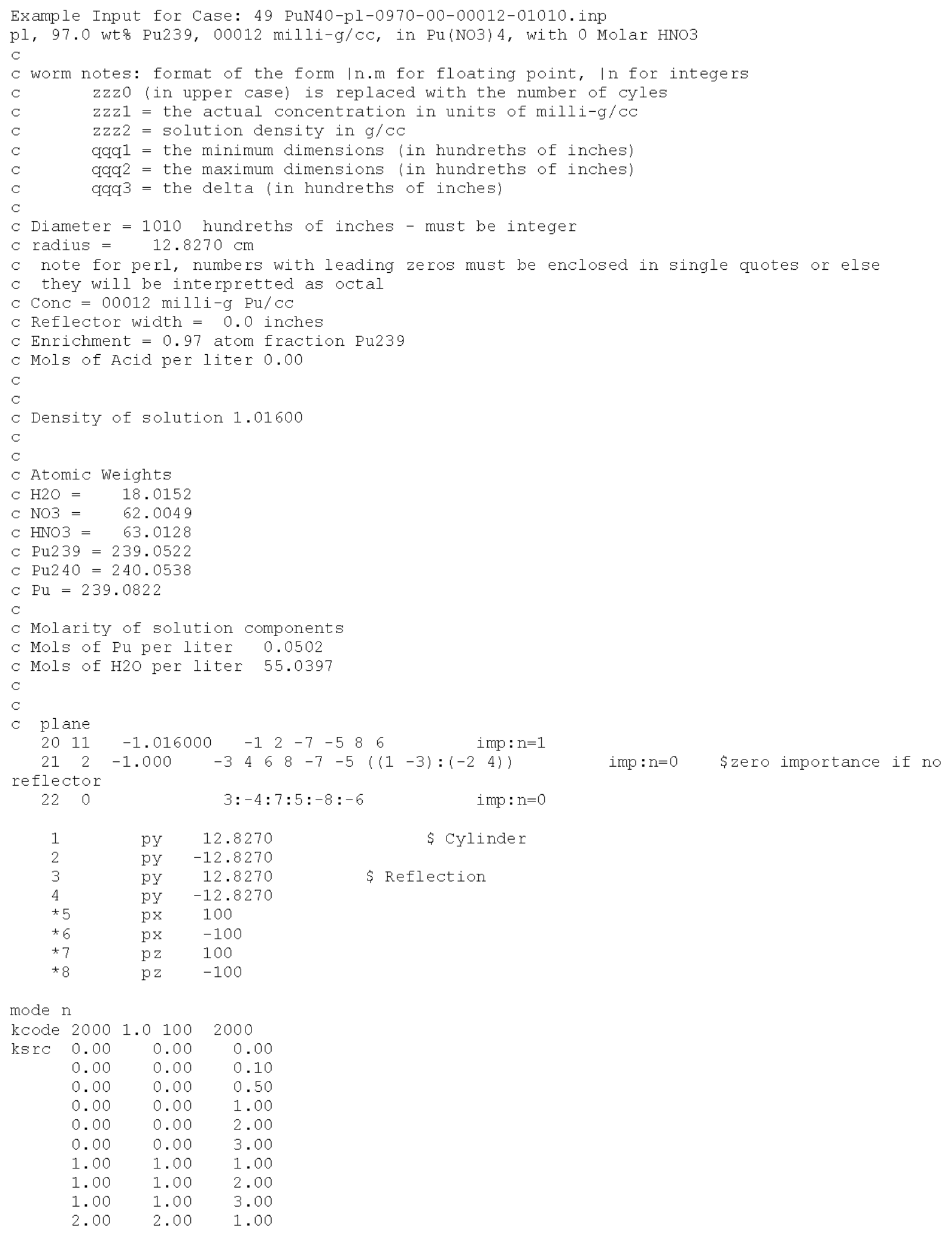




\section{CHPRC-01552, Revision 0}

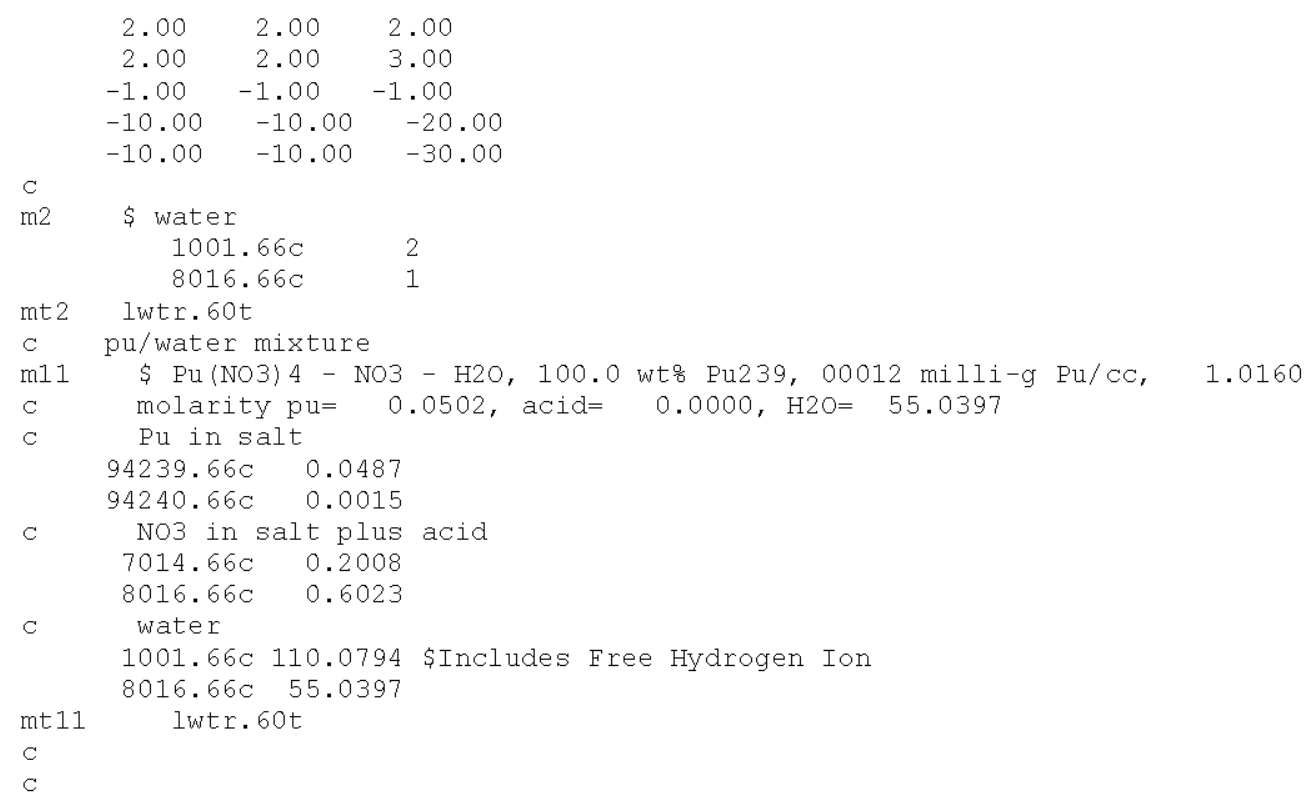




\section{CHPRC-01552, Revision 0}

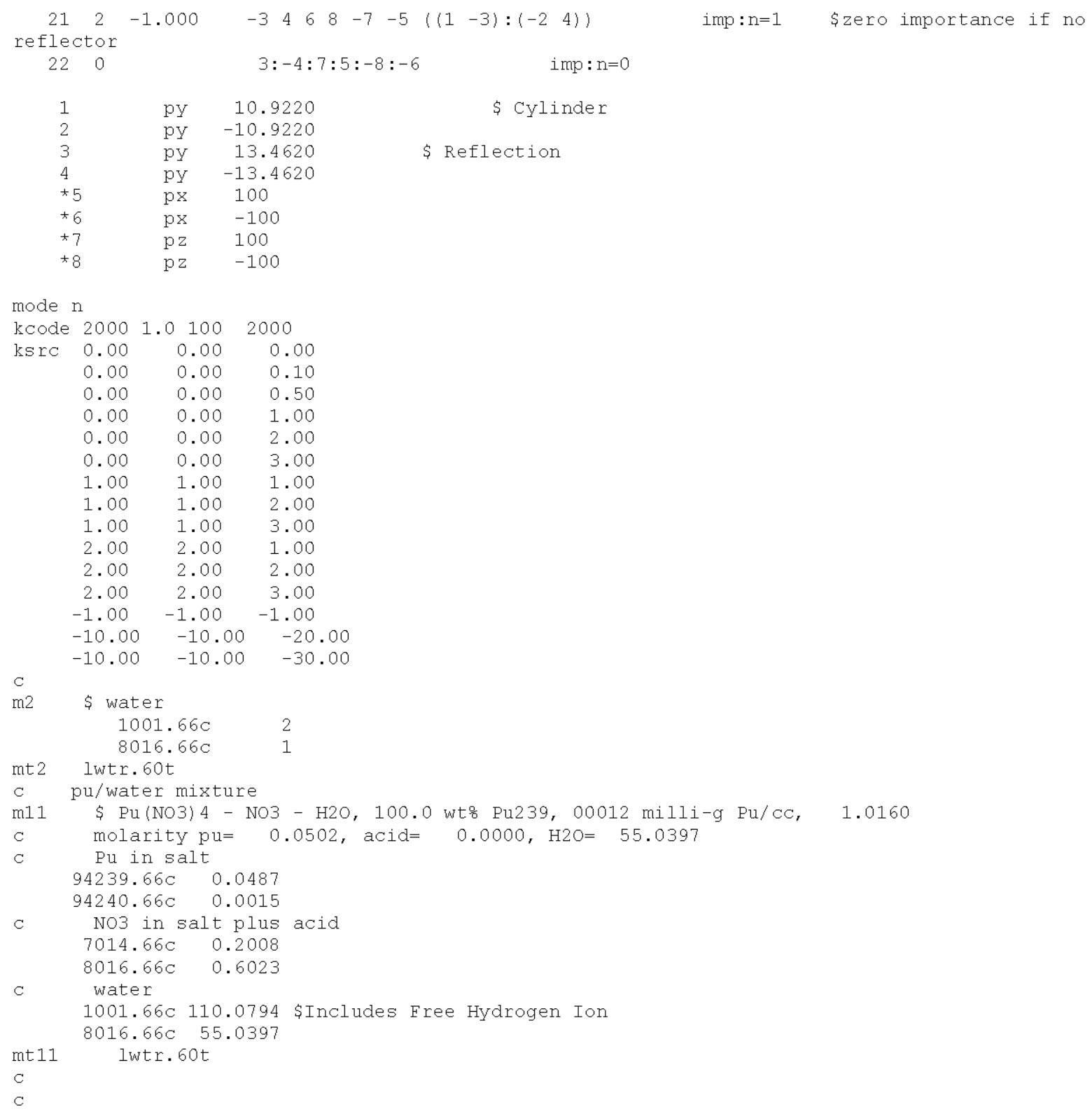




\section{CHPRC-01552, Revision 0}

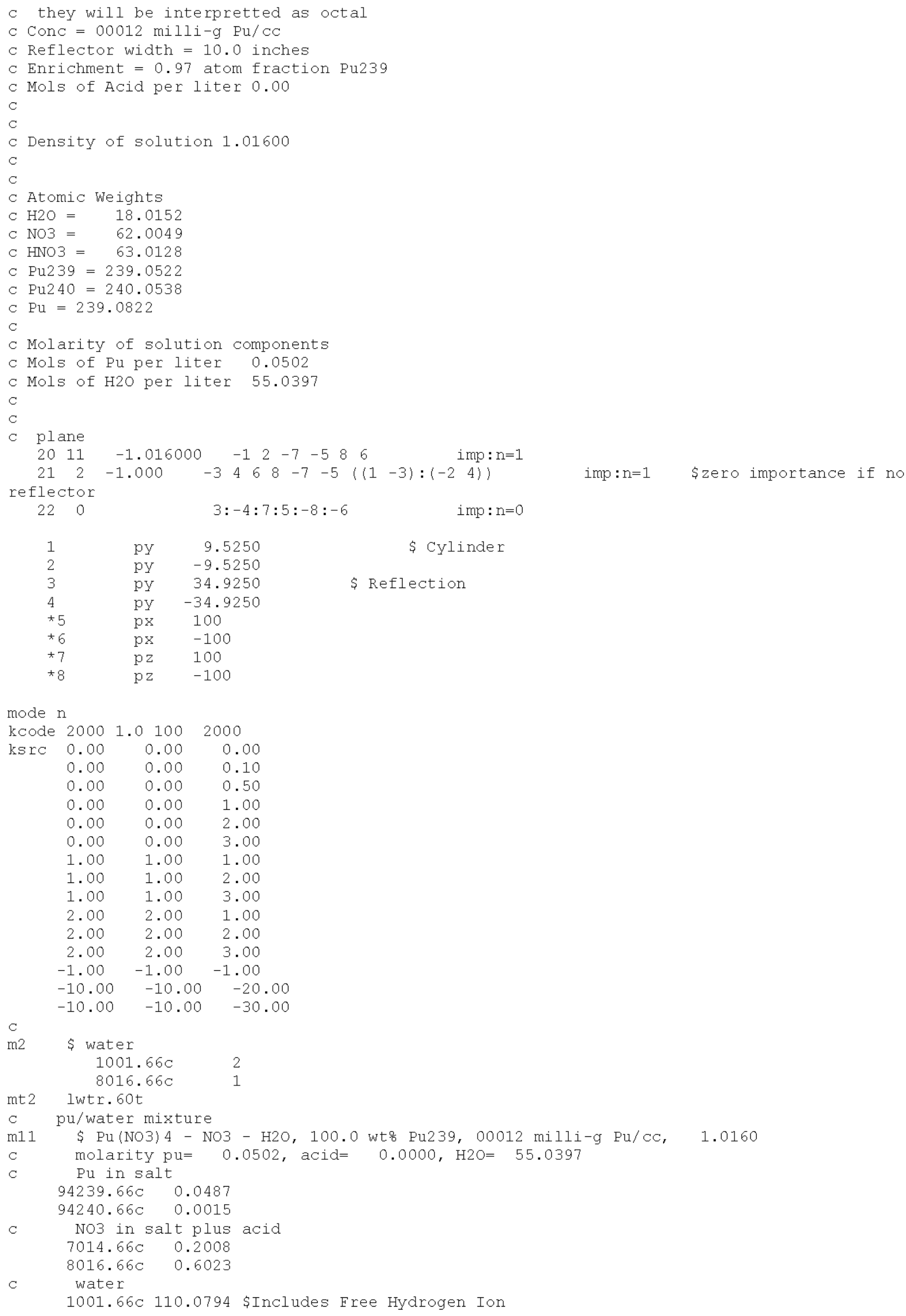




\section{CHPRC-01552, Revision 0}

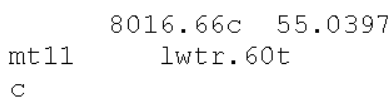




\section{CHPRC-01552, Revision 0}

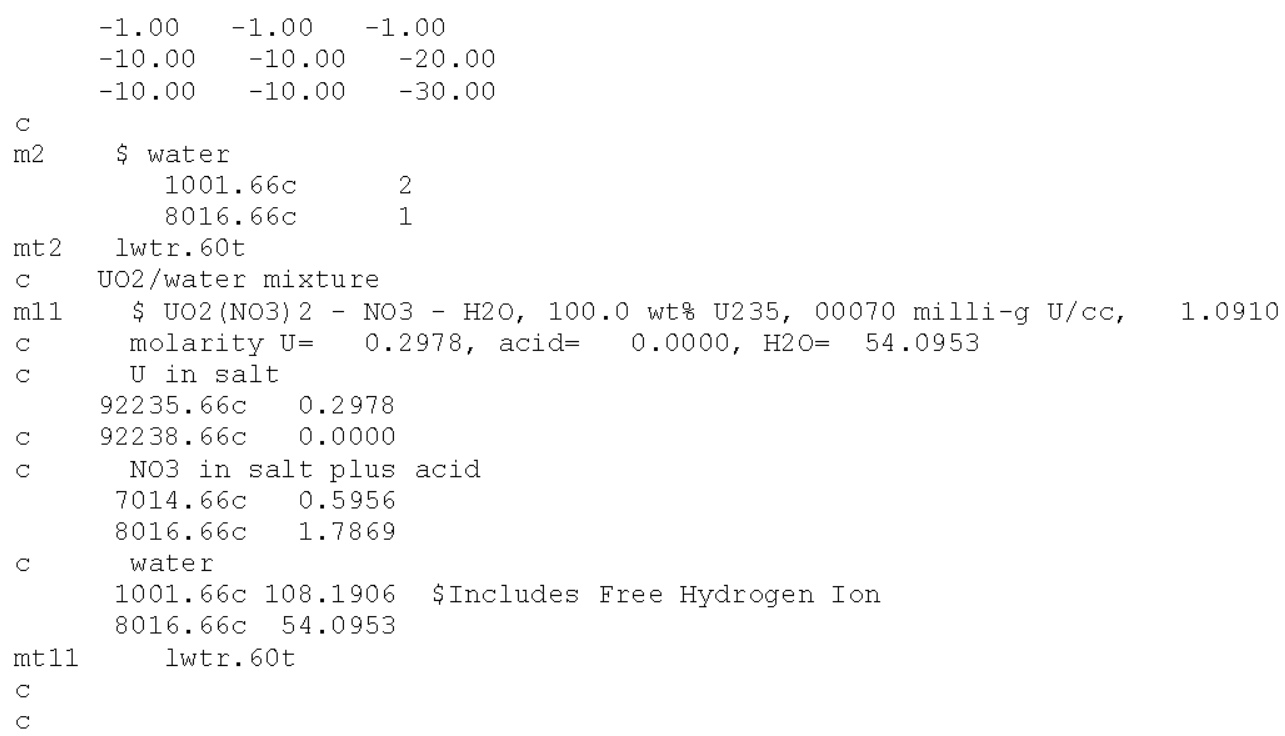




\section{CHPRC-01552, Revision 0}

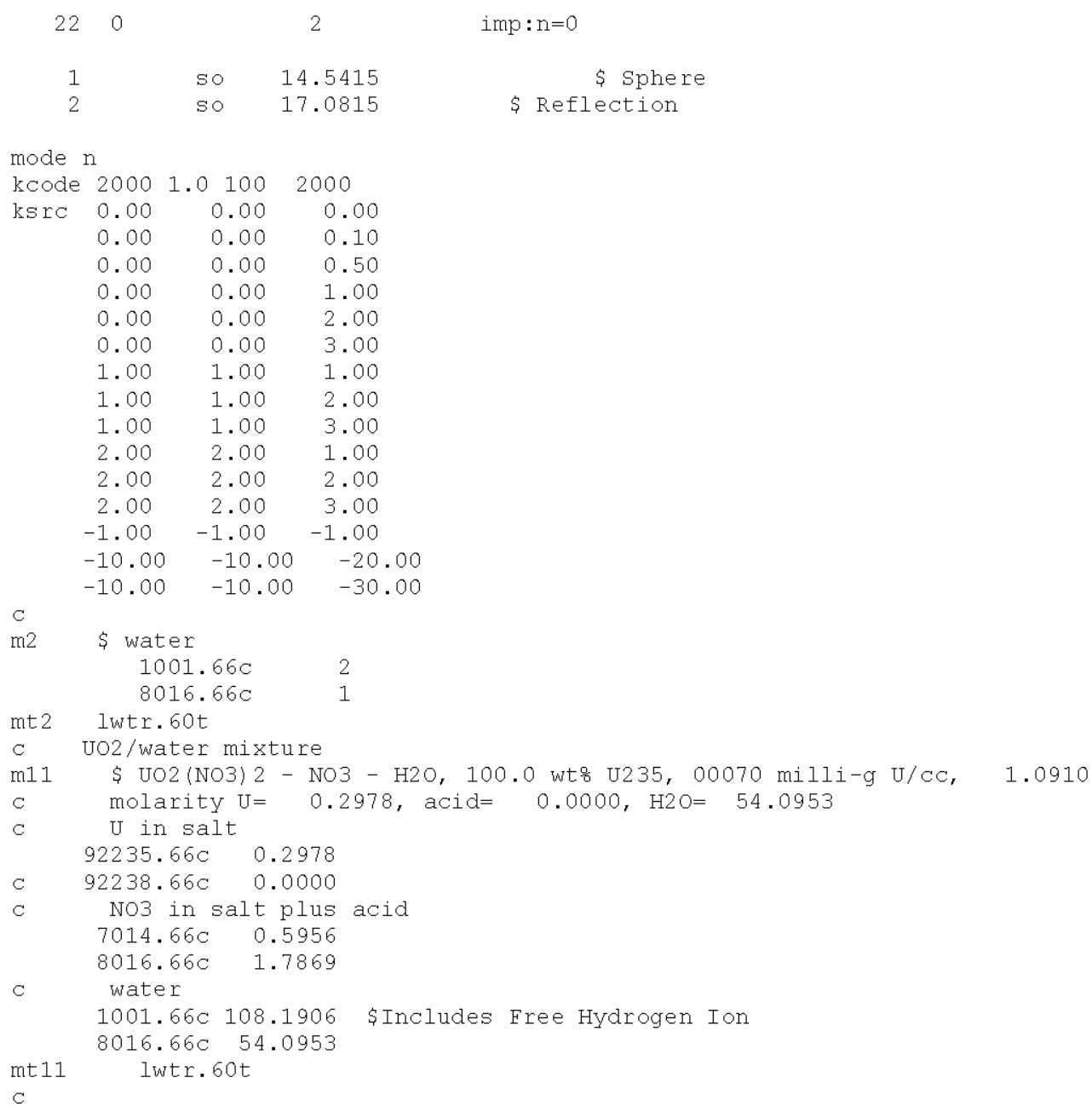




\section{CHPRC-01552, Revision 0}

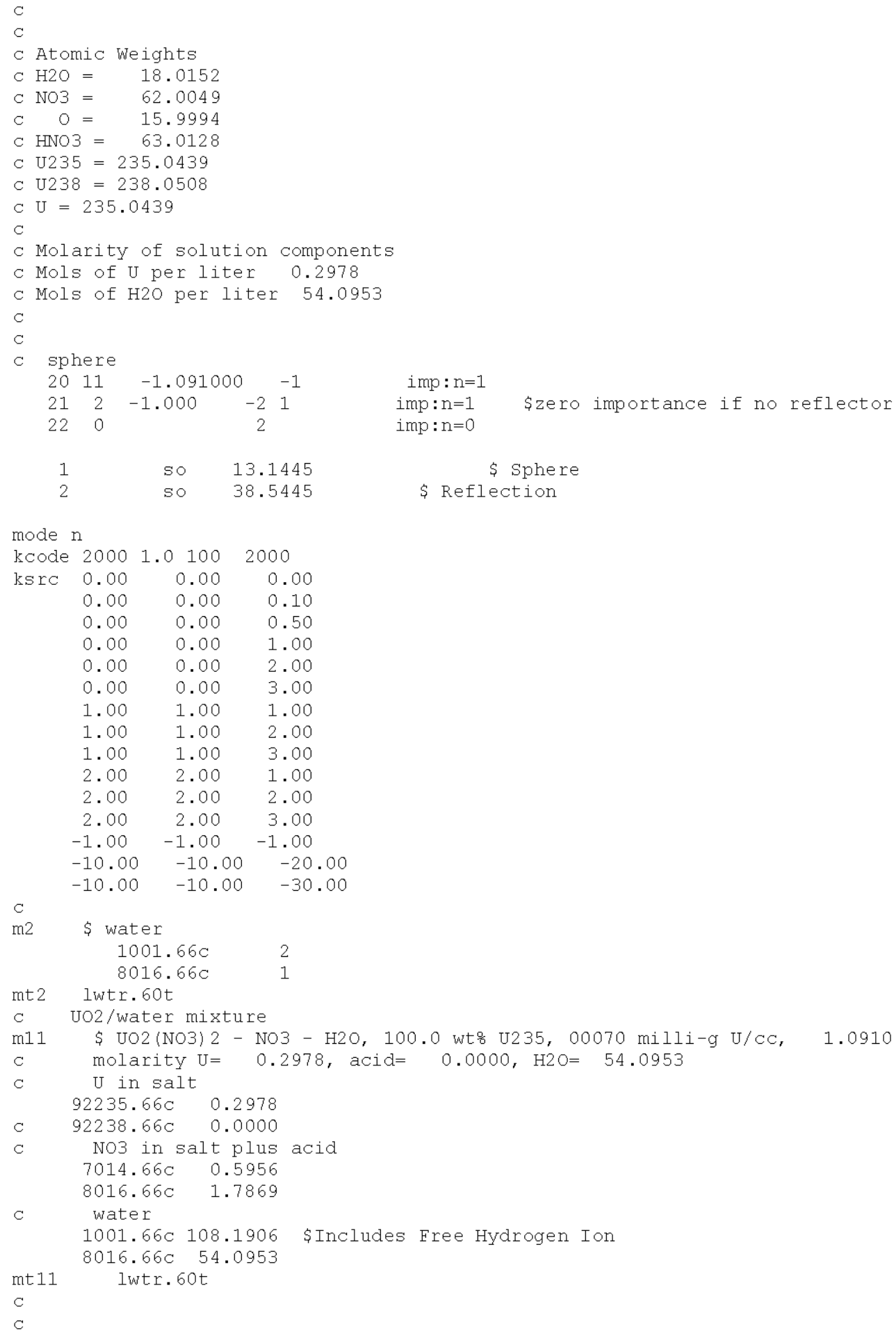




\section{CHPRC-01552, Revision 0}

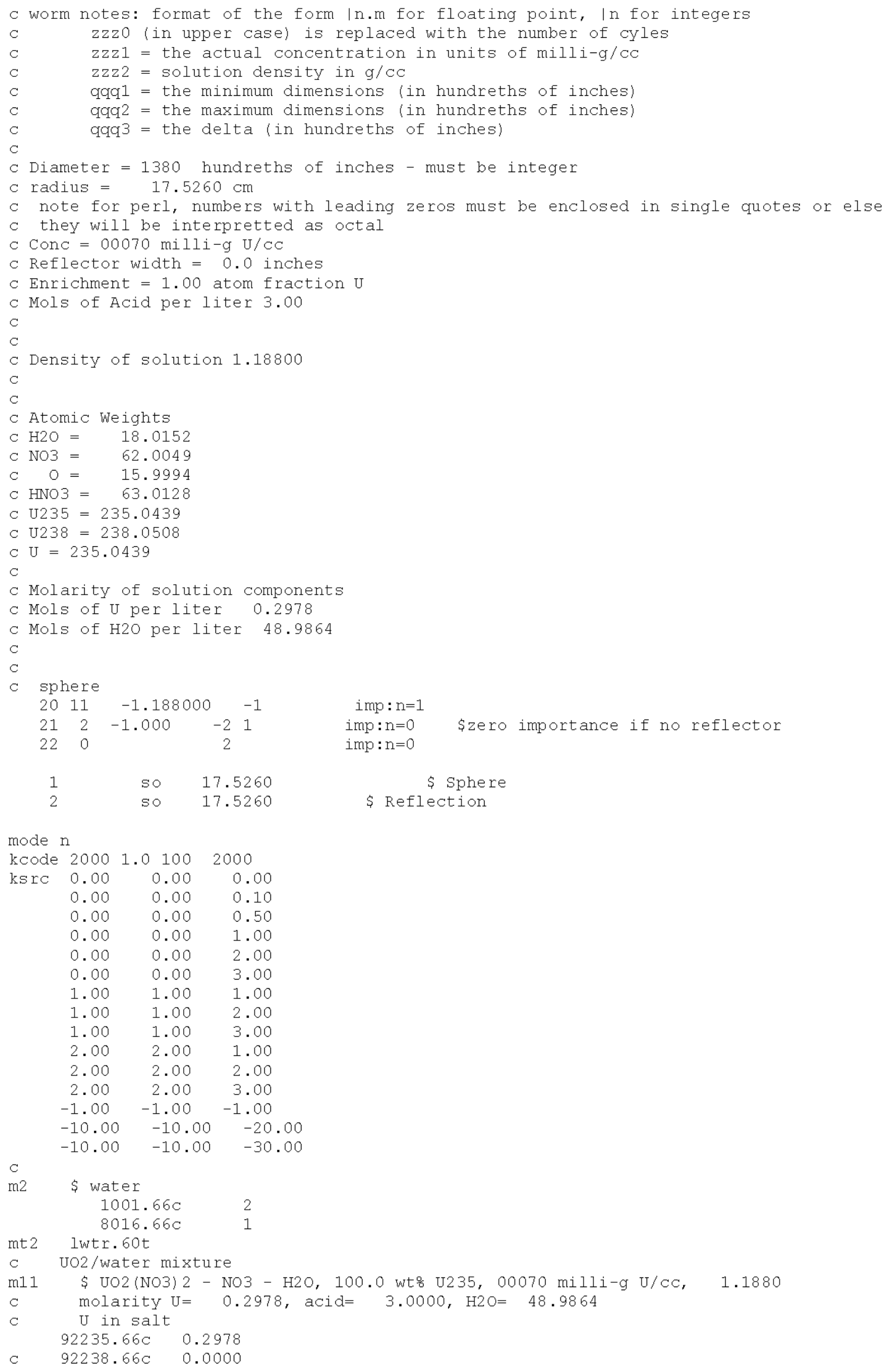




\section{CHPRC-01552, Revision 0}

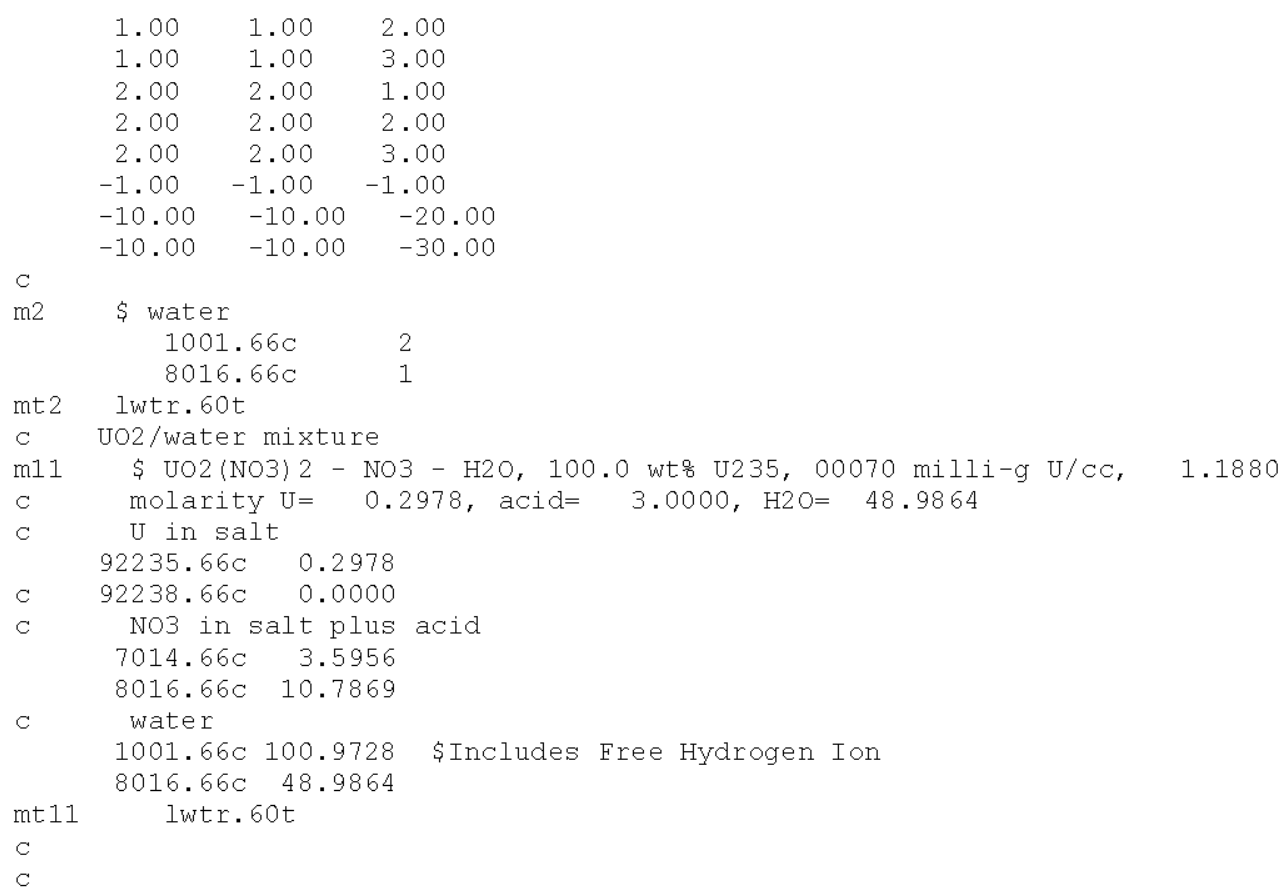




\section{CHPRC-01552, Revision 0}

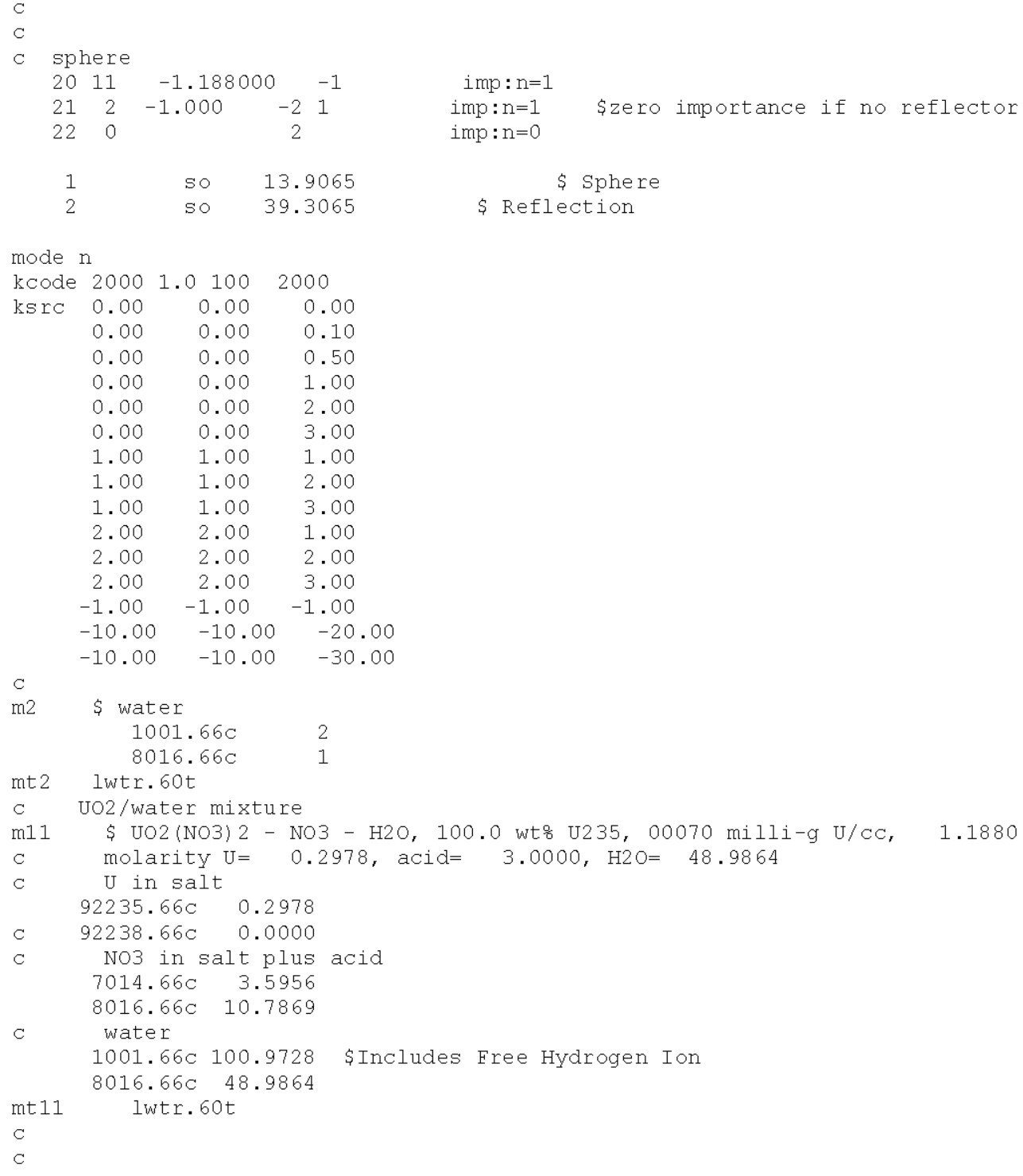




\section{CHPRC-01552, Revision 0}

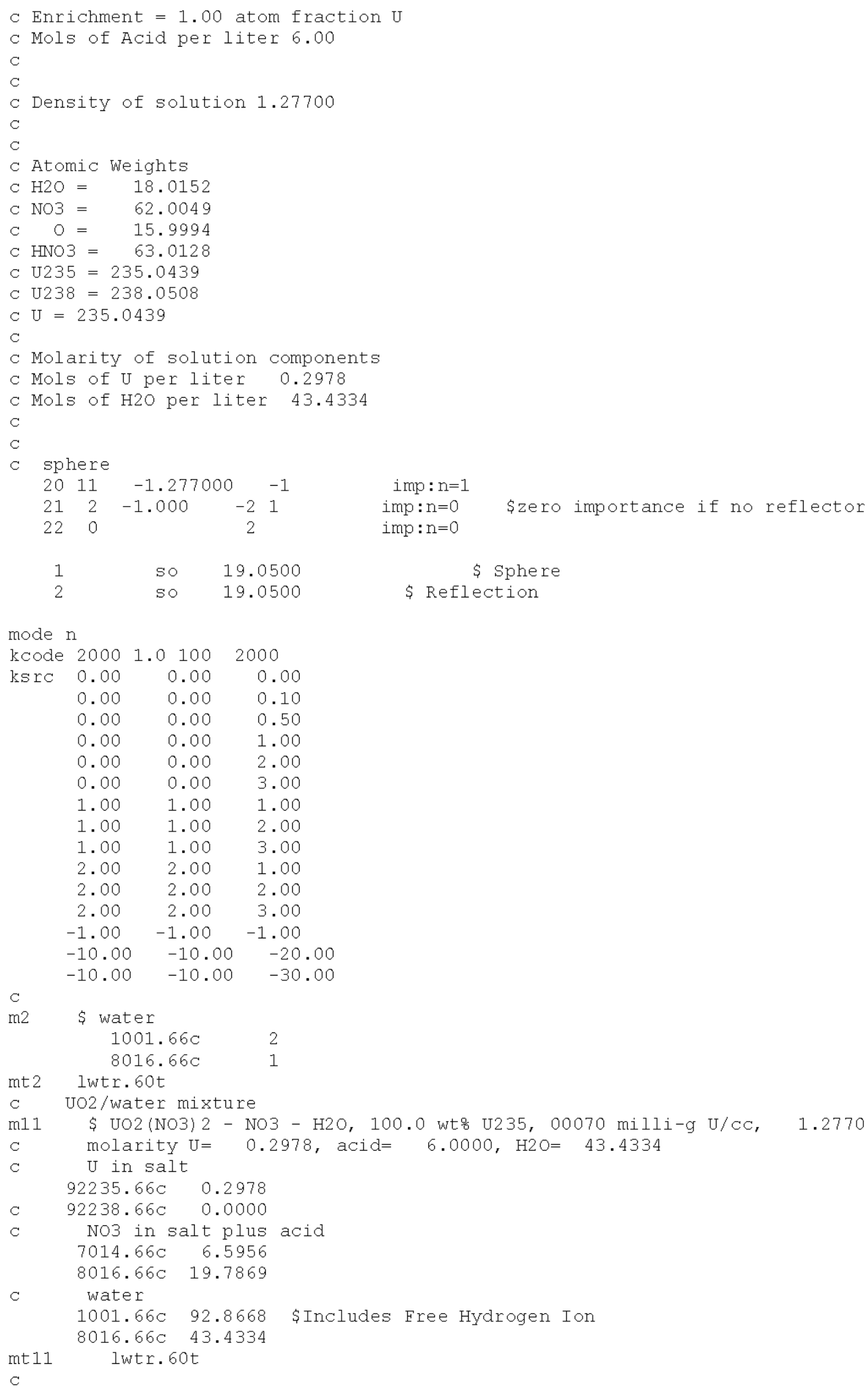




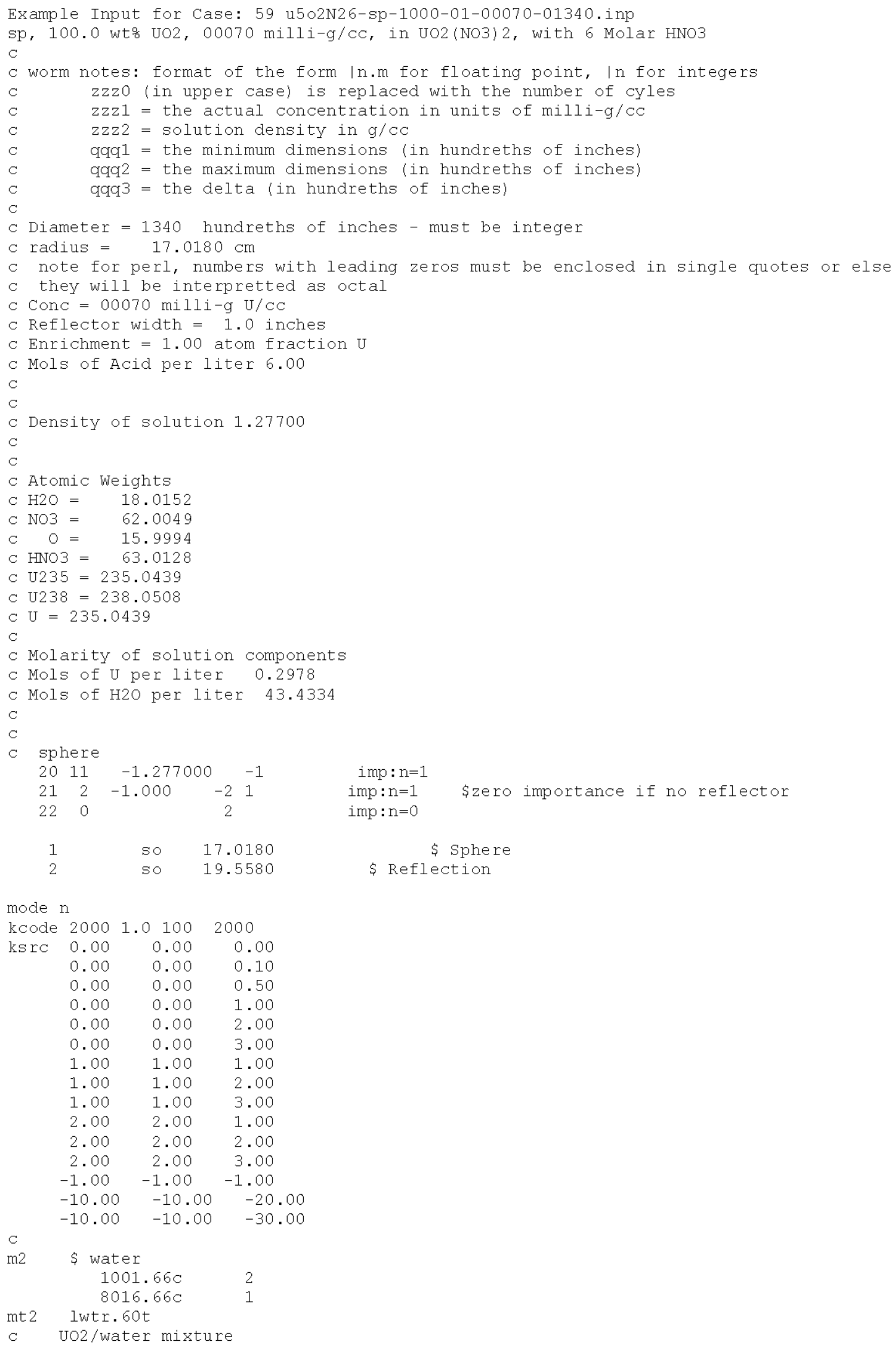




\section{CHPRC-01552, Revision 0}
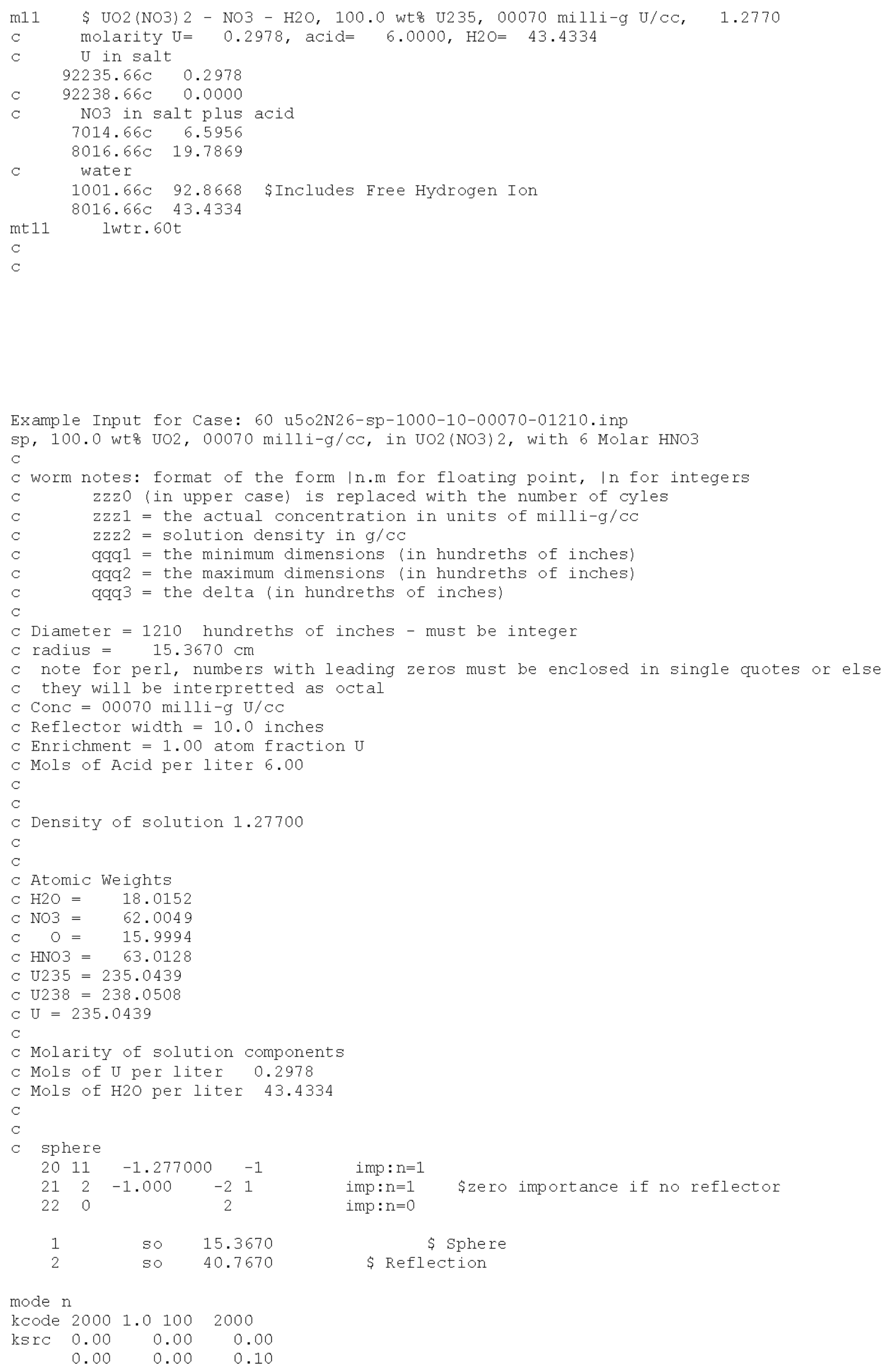


\section{CHPRC-01552, Revision 0}

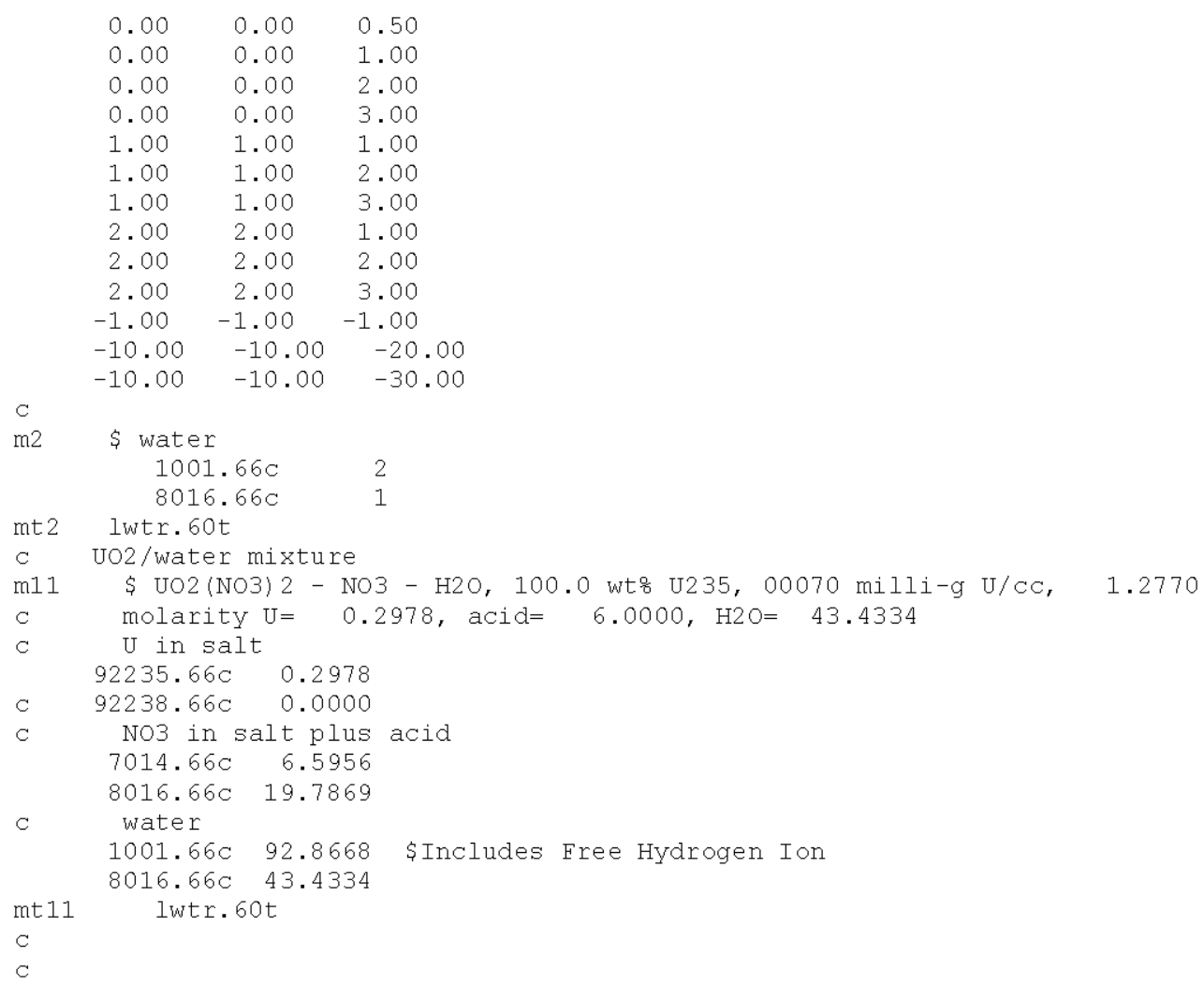




\section{CHPRC-01552, Revision 0}

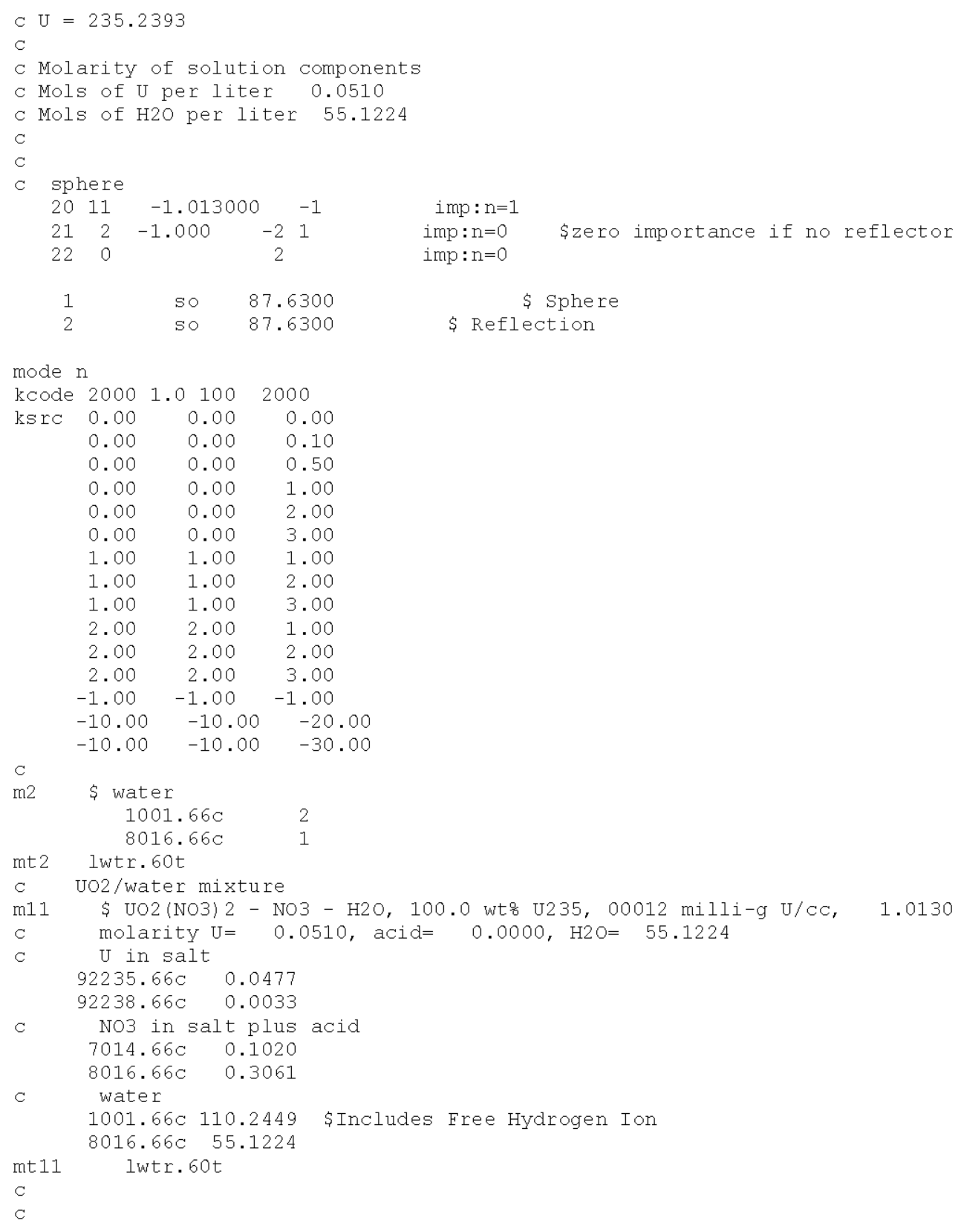




\section{CHPRC-01552, Revision 0}

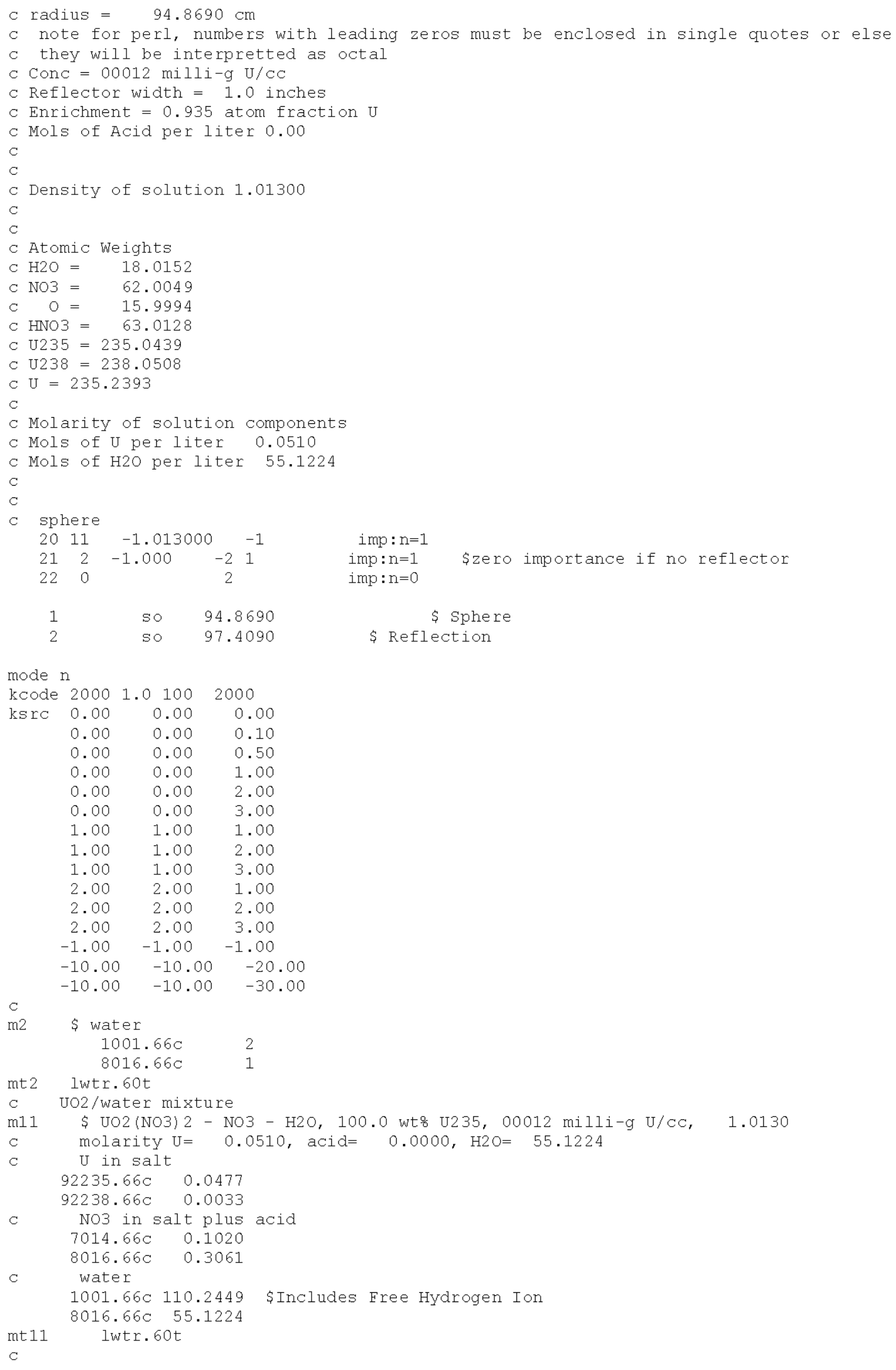




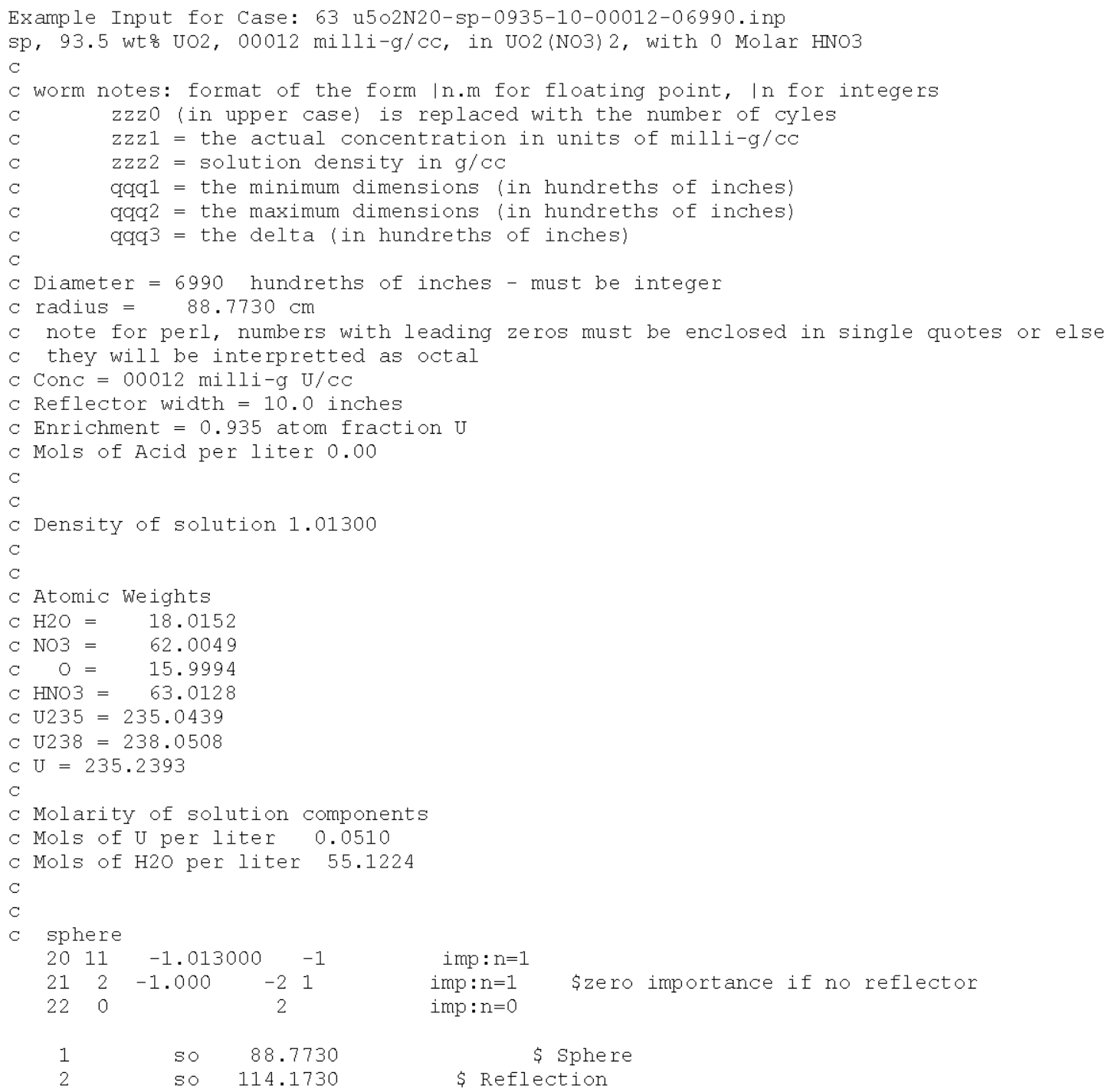




\section{CHPRC-01552, Revision 0}

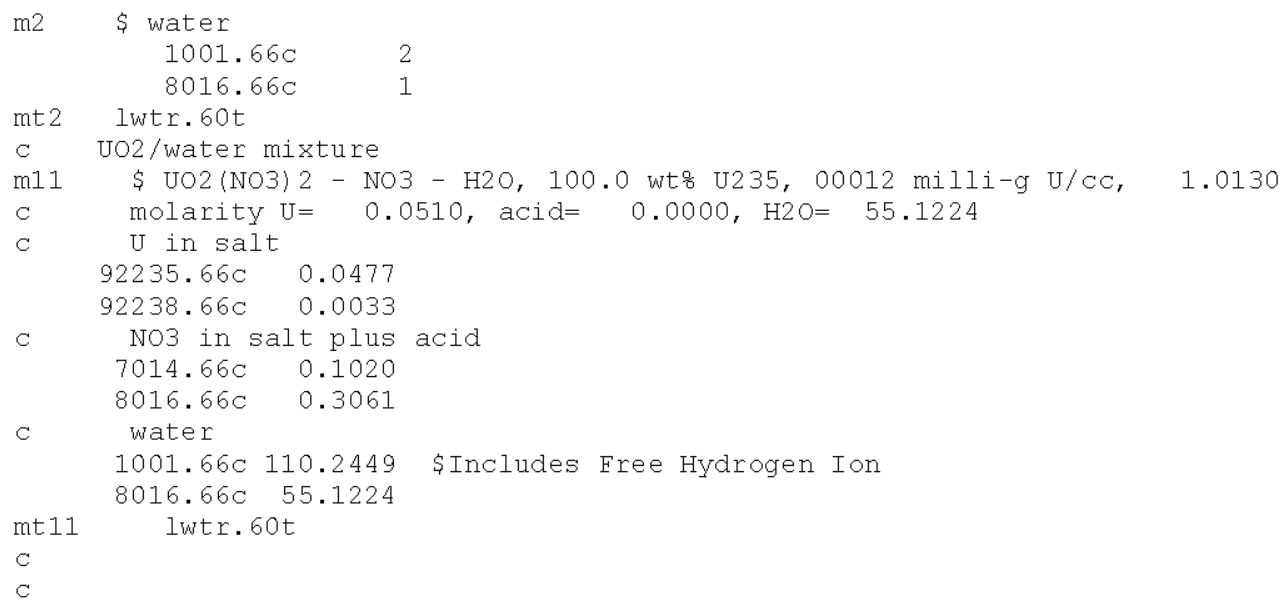




\section{CHPRC-01552, Revision 0}

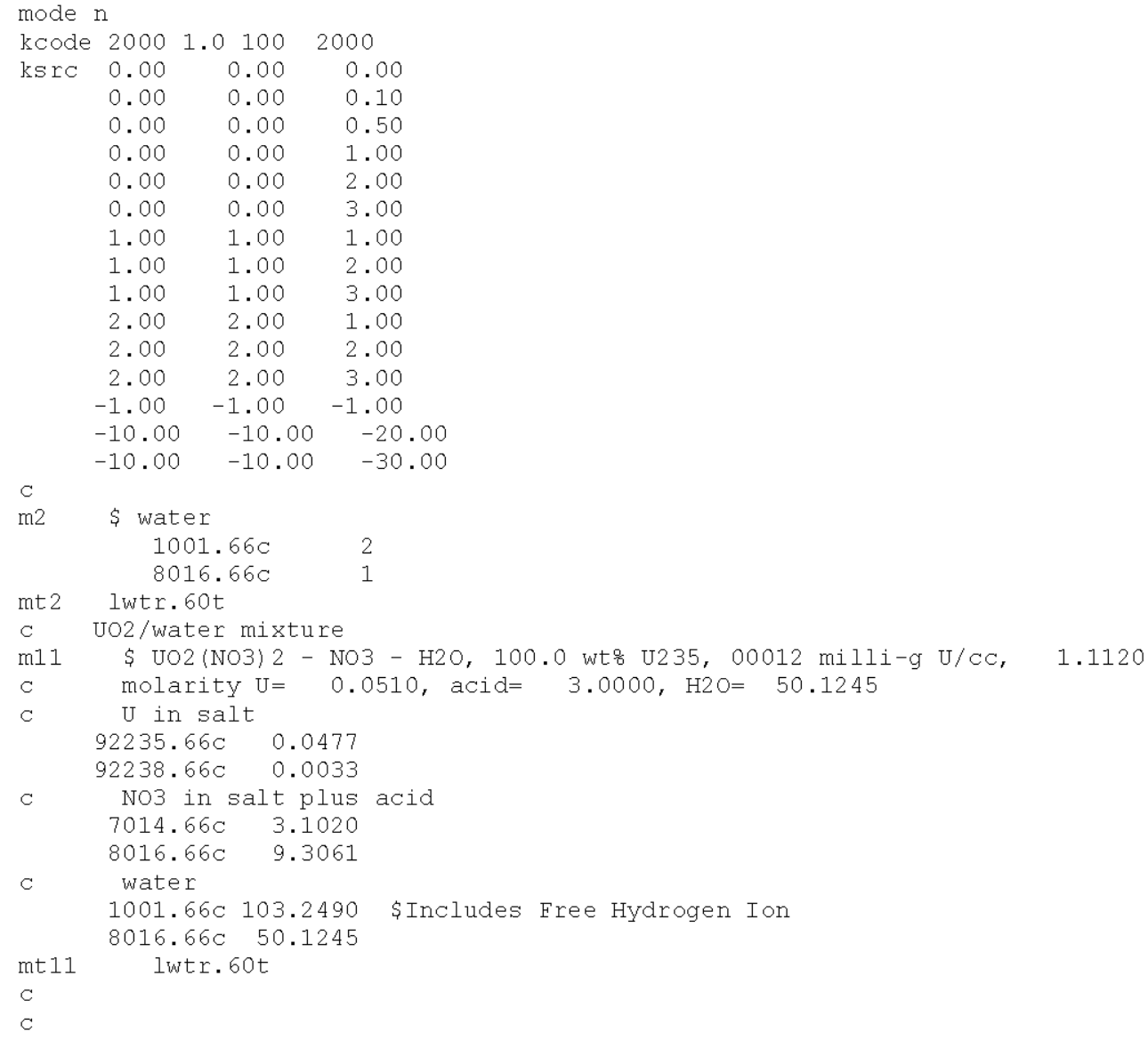




\section{CHPRC-01552, Revision 0}

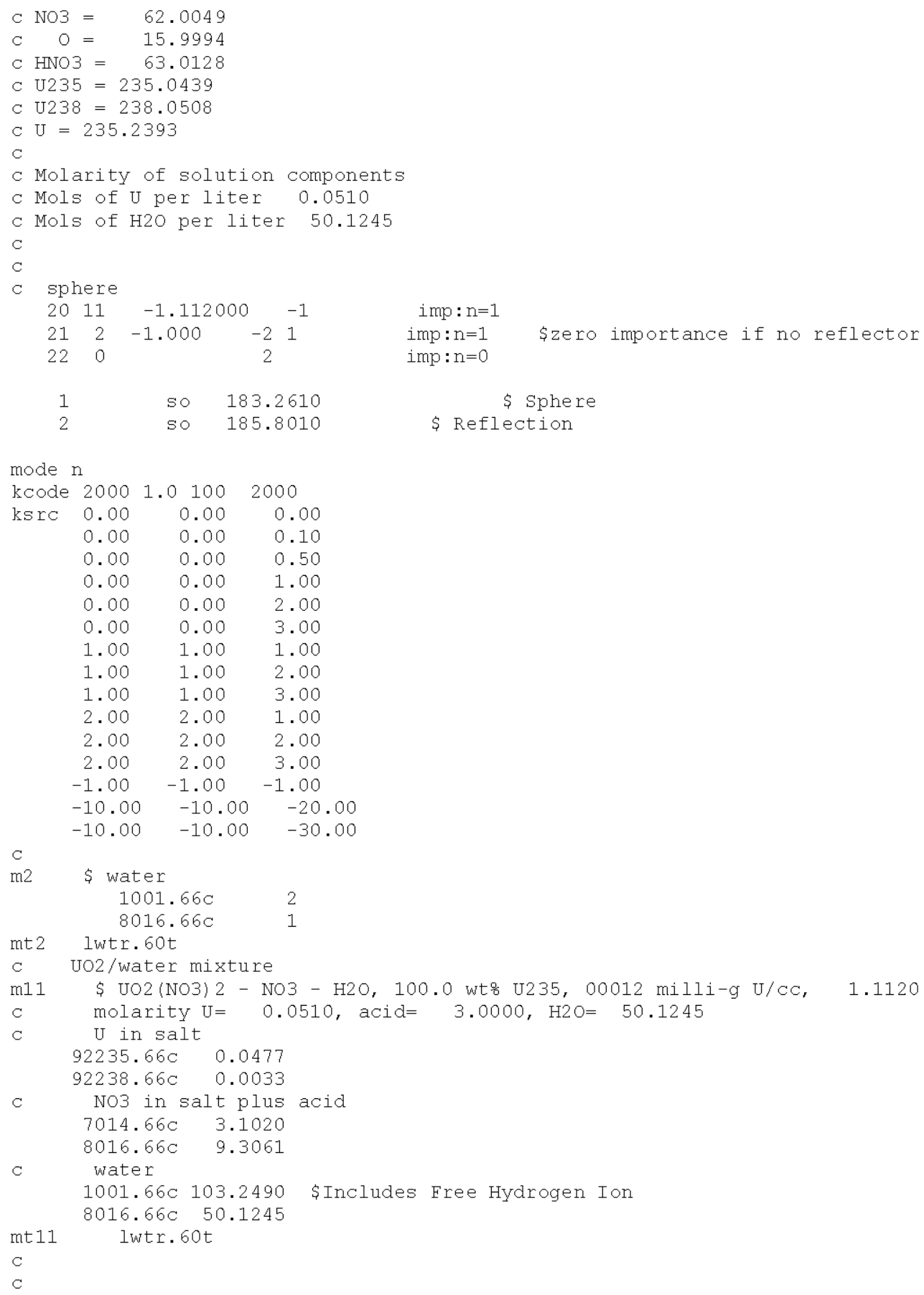




\section{CHPRC-01552, Revision 0}

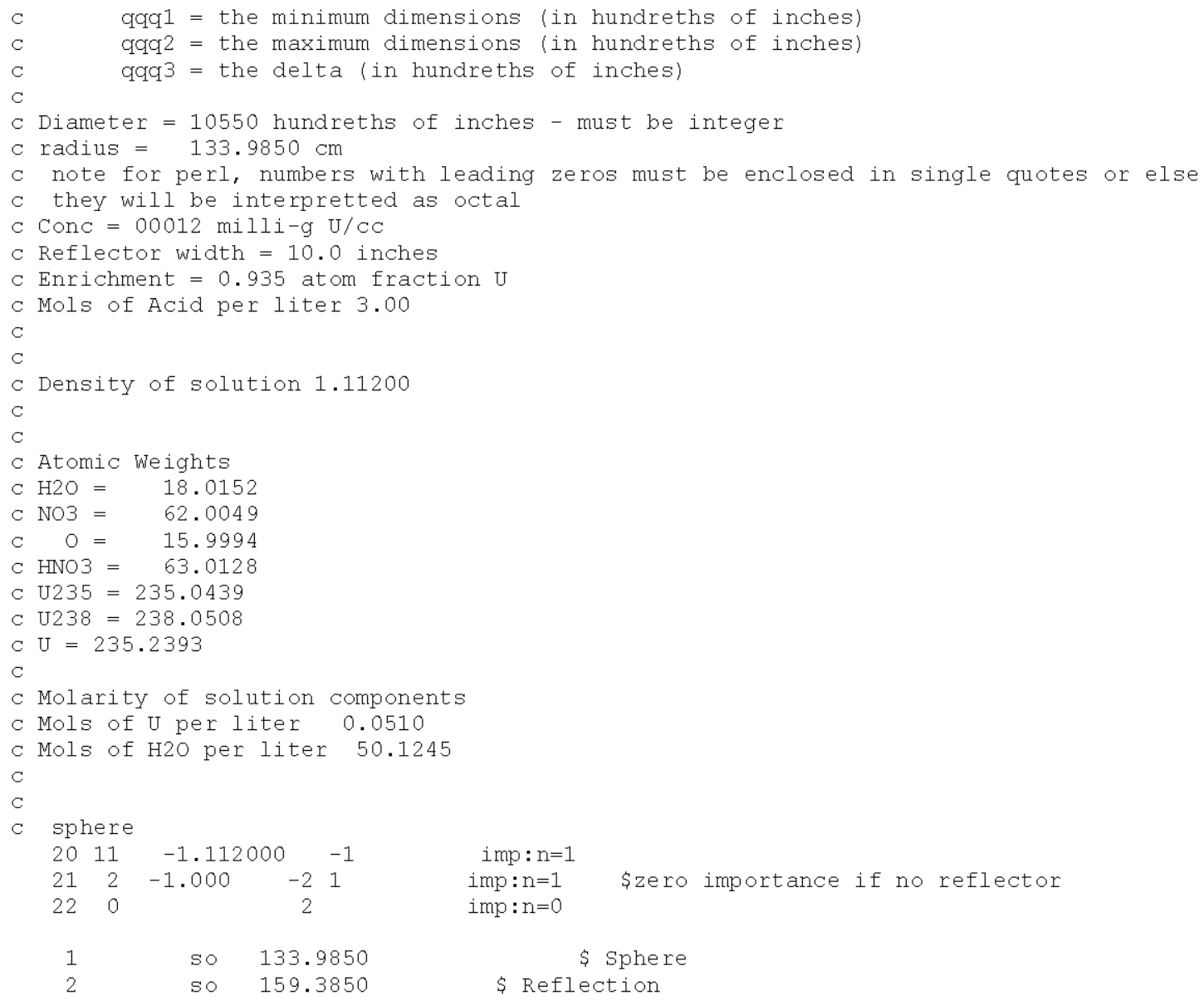




\section{CHPRC-01552, Revision 0}

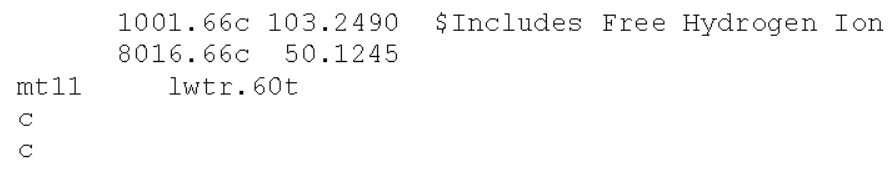




\section{CHPRC-01552, Revision 0}

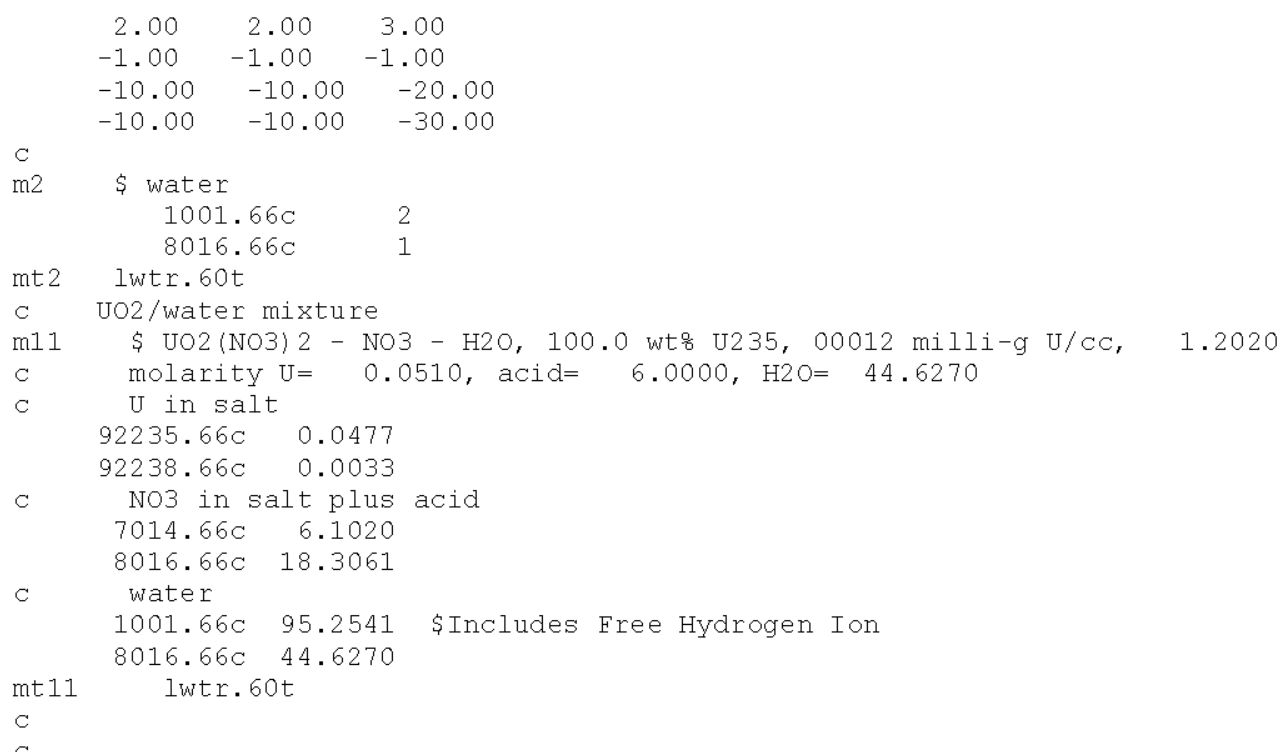




\section{CHPRC-01552, Revision 0}

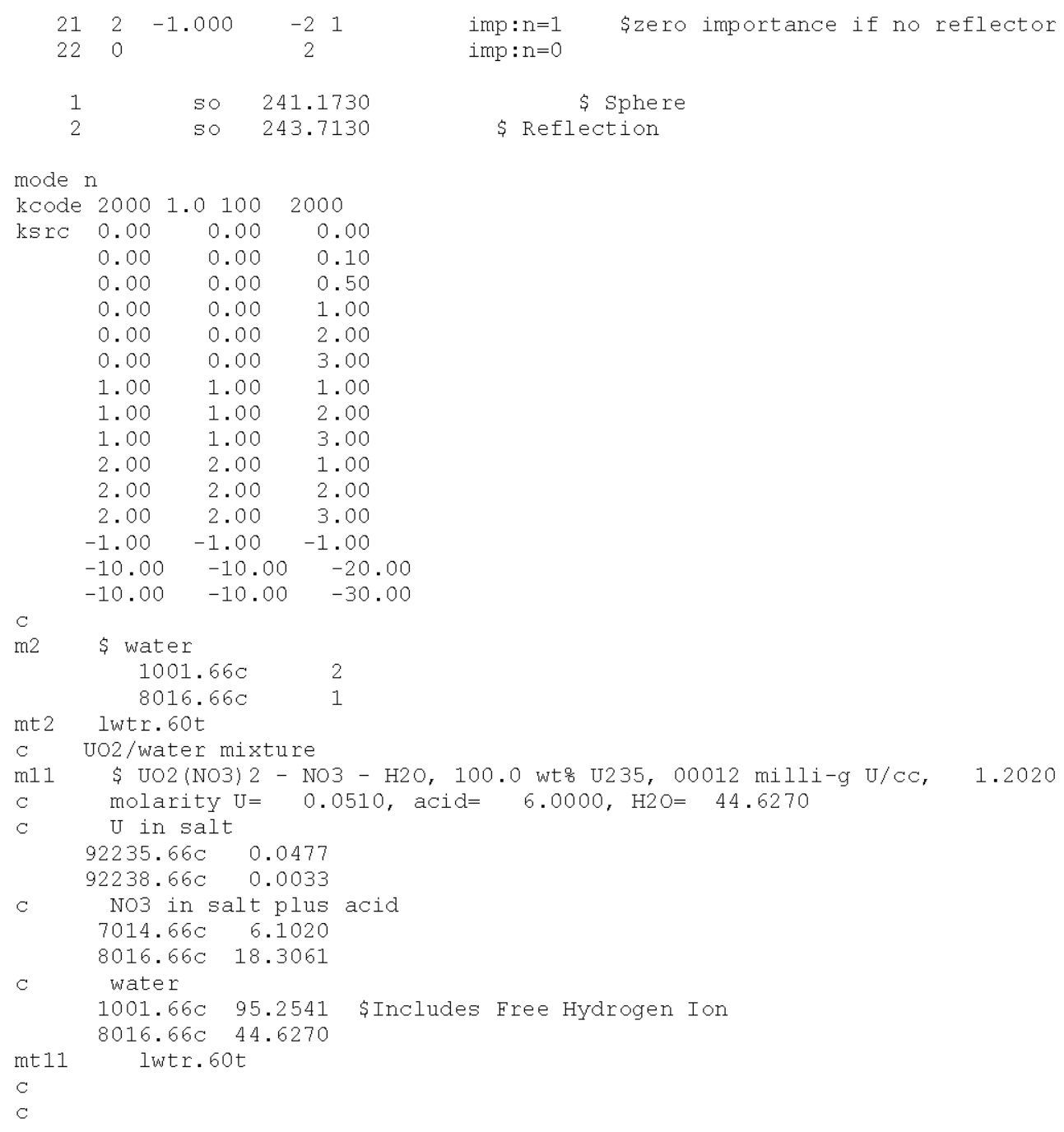




\section{CHPRC-01552, Revision 0}

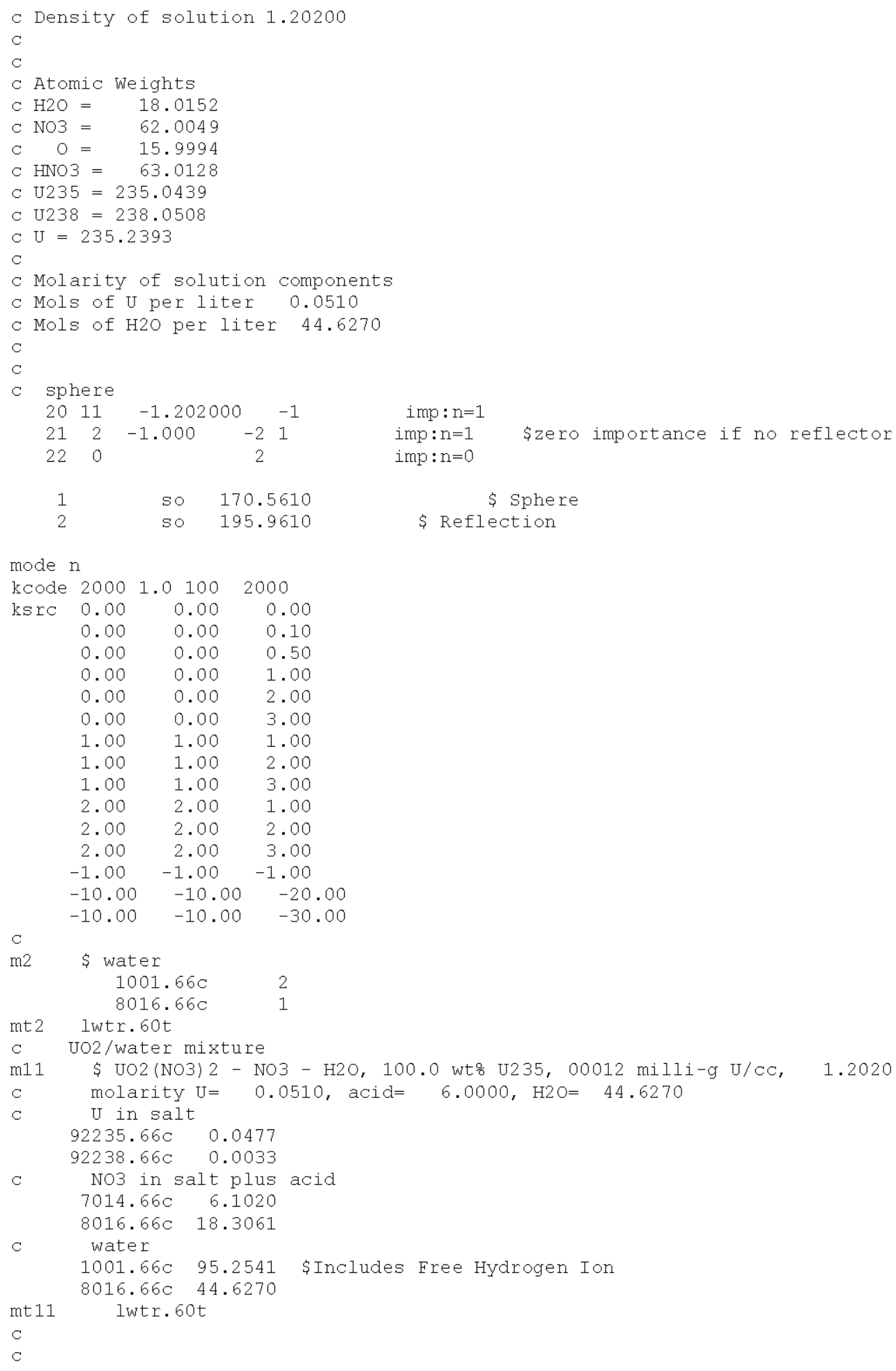

Example Input for Case: 70 u502N20-icyl-1000-00-00070-00950.inp icyl, 100.0 wt: U02, $00070 \mathrm{milli}-\mathrm{g} / \mathrm{CC}$, in UO2(NO3) 2, with 0 Molar HNO3 


\section{CHPRC-01552, Revision 0}

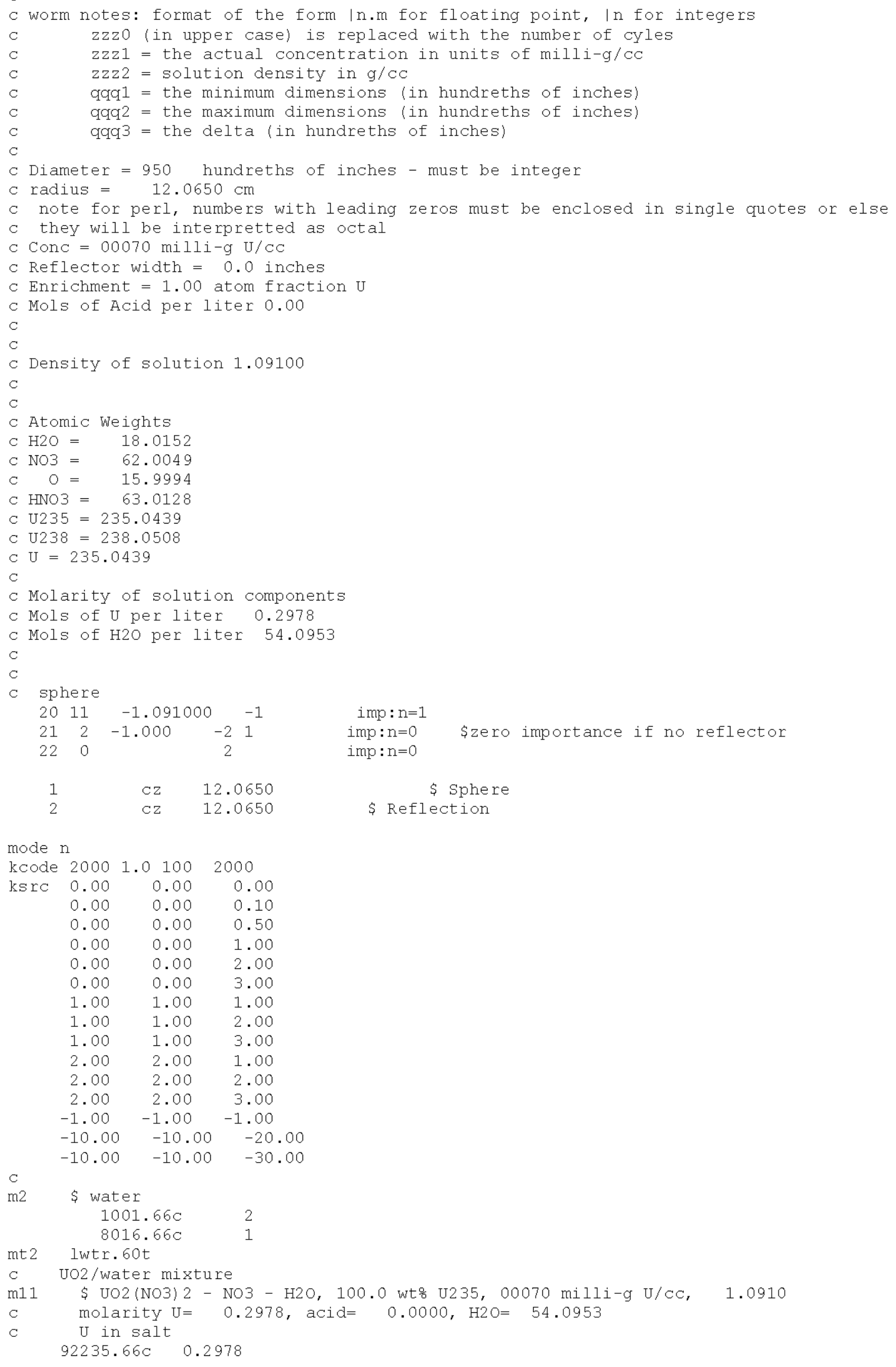




\section{CHPRC-01552, Revision 0}

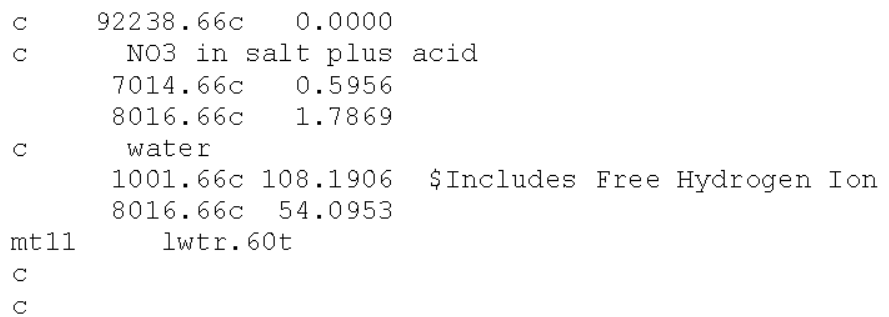

Example Input for Case: 71 u5o2N20-icyl-1000-01-00070-00800.inp icyl, 100.0 wto U02, $00070 \mathrm{milli-g/CC}$, in UO2(NO3) 2, with 0 Molar HNO3

$\mathrm{C}$

c worm notes: format of the form $\mid \mathrm{n} . \mathrm{m}$ for floating point, In for integers

c zzzo (in upper case) is replaced with the number of cyles

c $\quad z z z 1=$ the actual concentration in units of milli-g/cc

C $\quad$ zzz2 = solution density in $\mathrm{g} / \mathrm{CC}$

c qq91 = the minimum dimensions (in hundreths of inches)

c $\quad$ qqq2 = the maximum dimensions (in hundreths of inches)

c $\quad$ q99 3 = the delta (in hundreths of inches)

C Diameter $=800$ hundreths of inches - must be integer

$\mathrm{C}$ radius $=10.1600 \mathrm{~cm}$

c note for perl, numbers with leading zeros must be enclosed in single quotes or else

c they will be interpretted as octal

$\mathrm{C}$ Conc $=00070 \mathrm{milli}-\mathrm{g} \mathrm{U} / \mathrm{CC}$

c Reflector width $=1.0$ inches

C Enrichment $=1.00$ atom fraction U

C Mols of Acid per liter 0.00

$\mathrm{C}$

C Density of solution 1.09100

$\mathrm{C}$

Atomic Weights

$\mathrm{C} \mathrm{H} 2 \mathrm{O}=18.0152$

$\mathrm{C} \mathrm{NO3}=62.0049$

C $\mathrm{O}=15.9994$

C $\mathrm{HNO}=63.0128$

$\mathrm{C} U 235=235.0439$

$\mathrm{C} U 238=238.0508$

C $U=235.0439$

C Molarity of solution components

C Mols of u per liter 0.2978

C Mols of H2O per liter 54.0953

C

C sphere

$212-1.000 \quad-21 \quad$ imp:n=1 \$zero importance if no reflector

220

$1 \quad \mathrm{Cz} \quad 10.1600$

imp $: n=0$

10.1600

mode $n$

kcode 20001.01002000

$\begin{array}{llll}\text { ksrc } & 0.00 & 0.00 & 0.00\end{array}$

$0.00 \quad 0.00 \quad 0.10$

$\begin{array}{lll}0.00 & 0.00 \quad 0.50\end{array}$

$0.00 \quad 0.00 \quad 1.00$

$\begin{array}{lll}0.00 & 0.00 & 2.00\end{array}$

$0.00 \quad 0.00 \quad 3.00$ 


\section{CHPRC-01552, Revision 0}

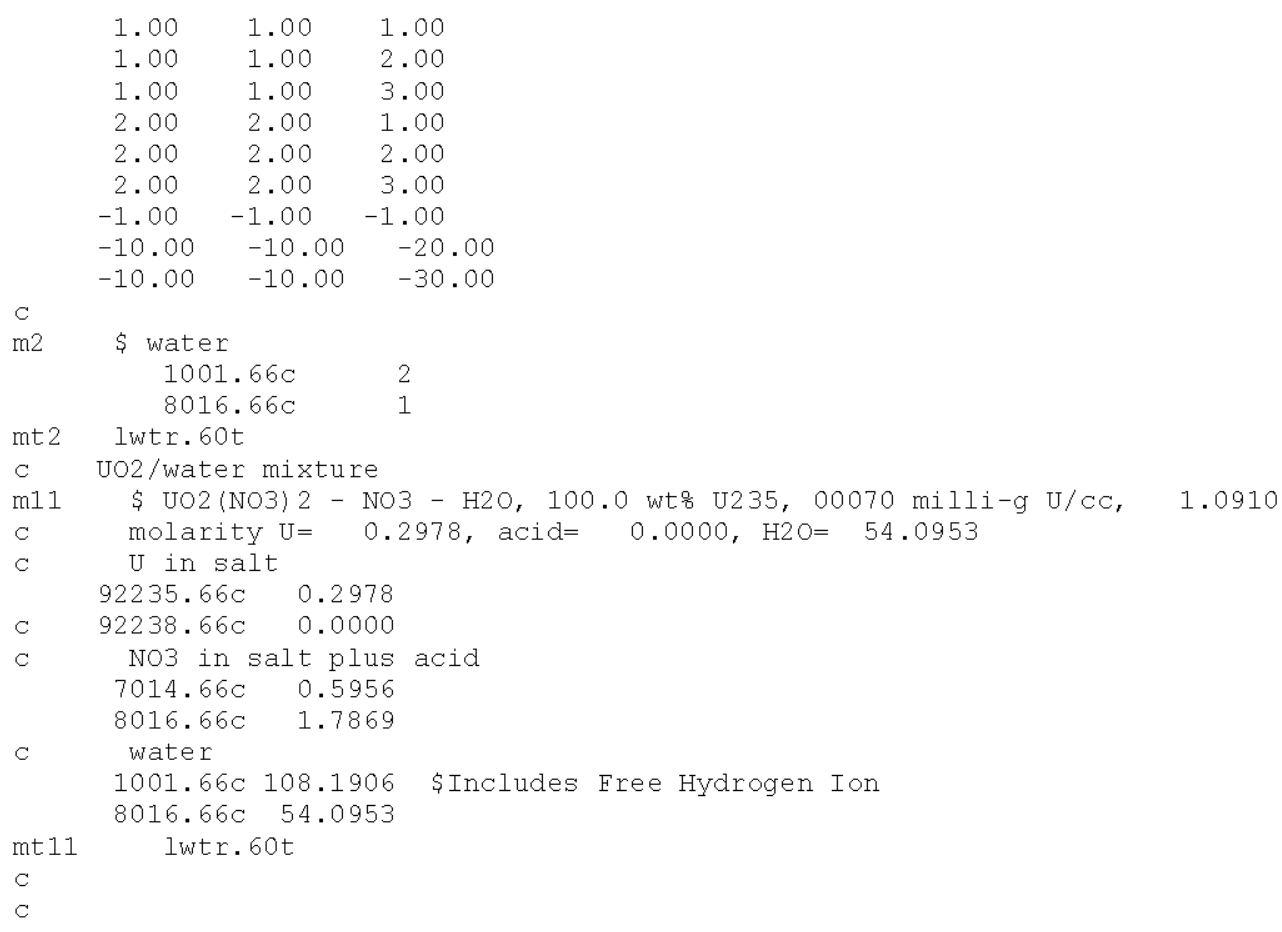




\section{CHPRC-01552, Revision 0}

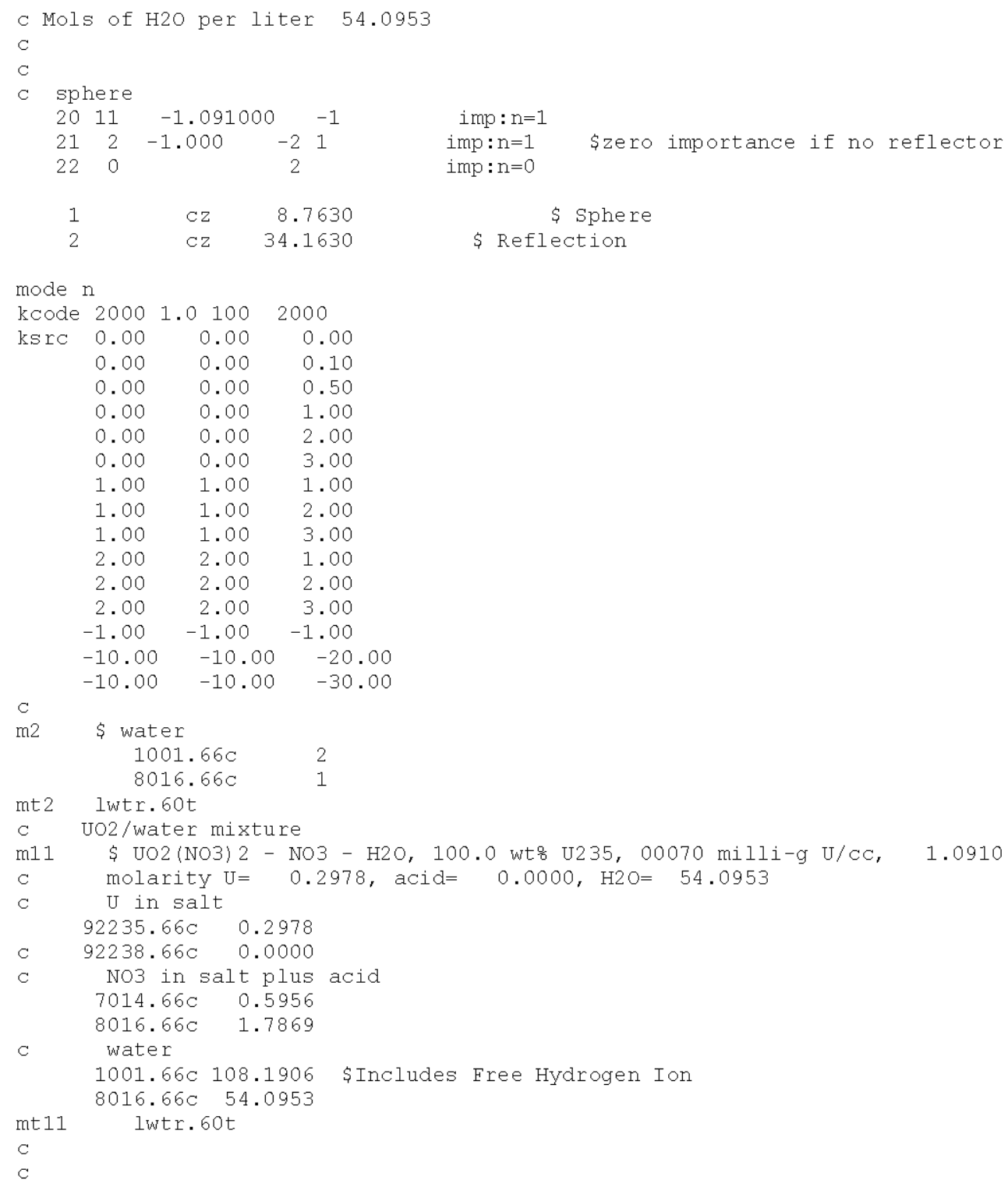




\section{CHPRC-01552, Revision 0}

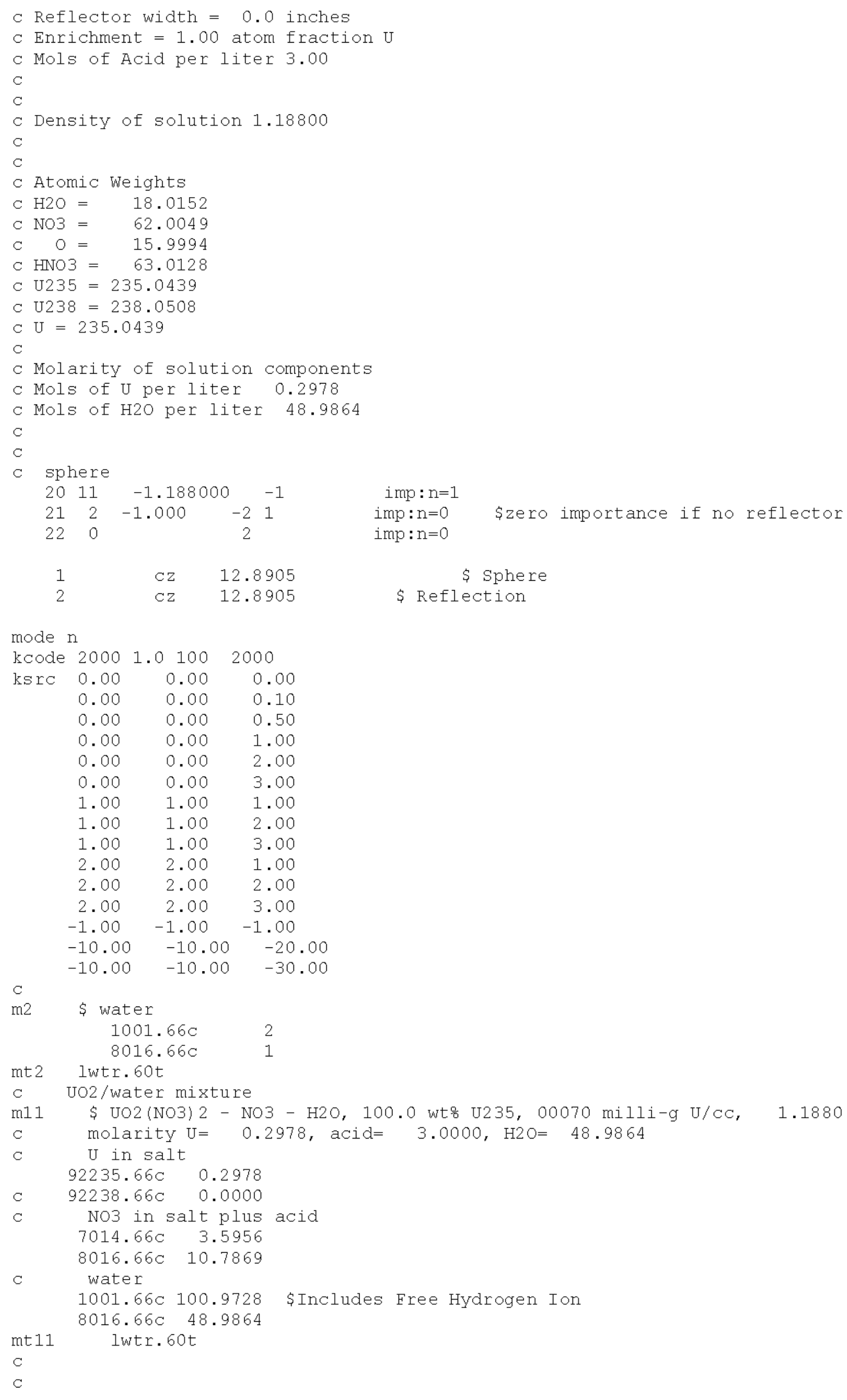




\section{CHPRC-01552, Revision 0}

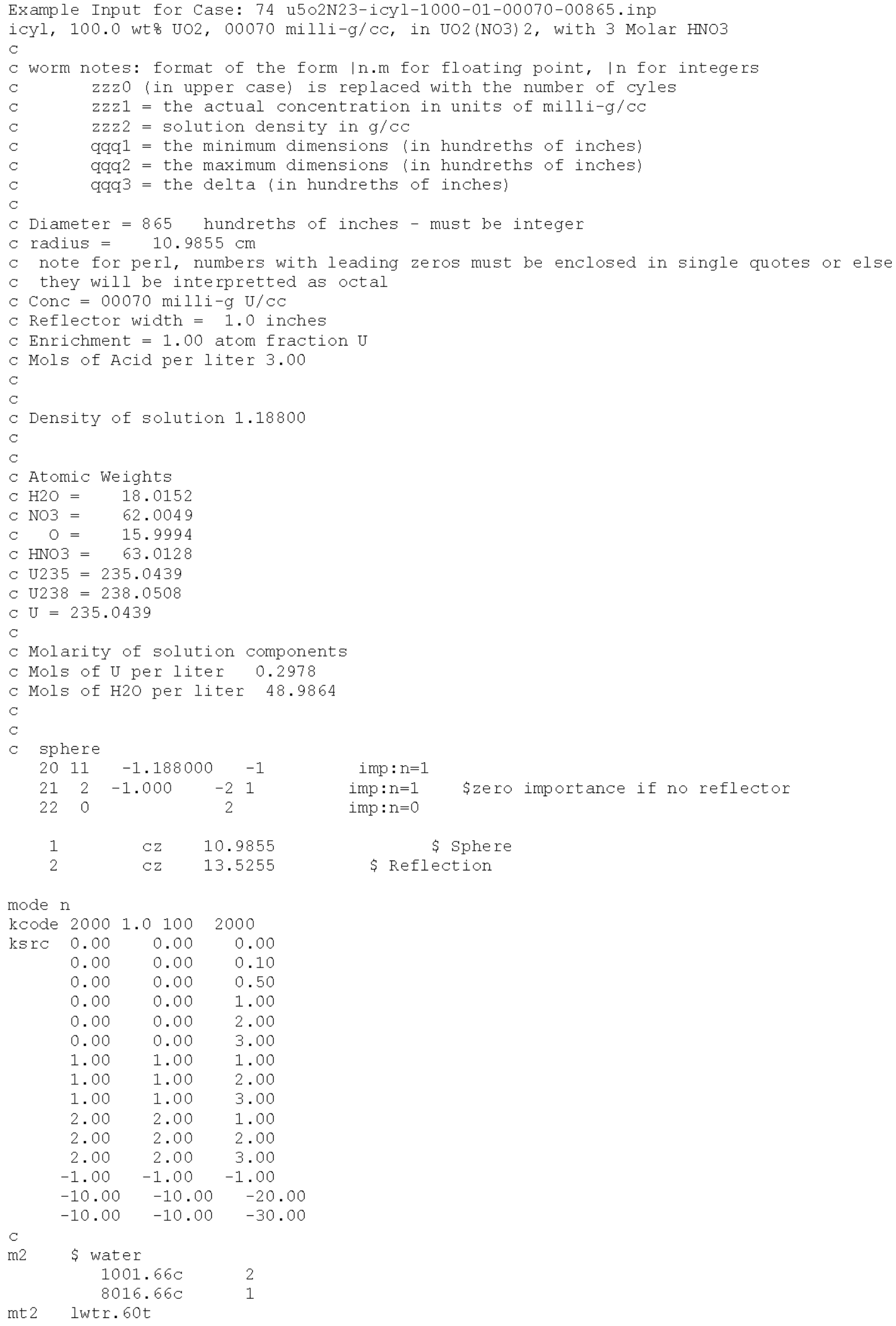




\section{CHPRC-01552, Revision 0}

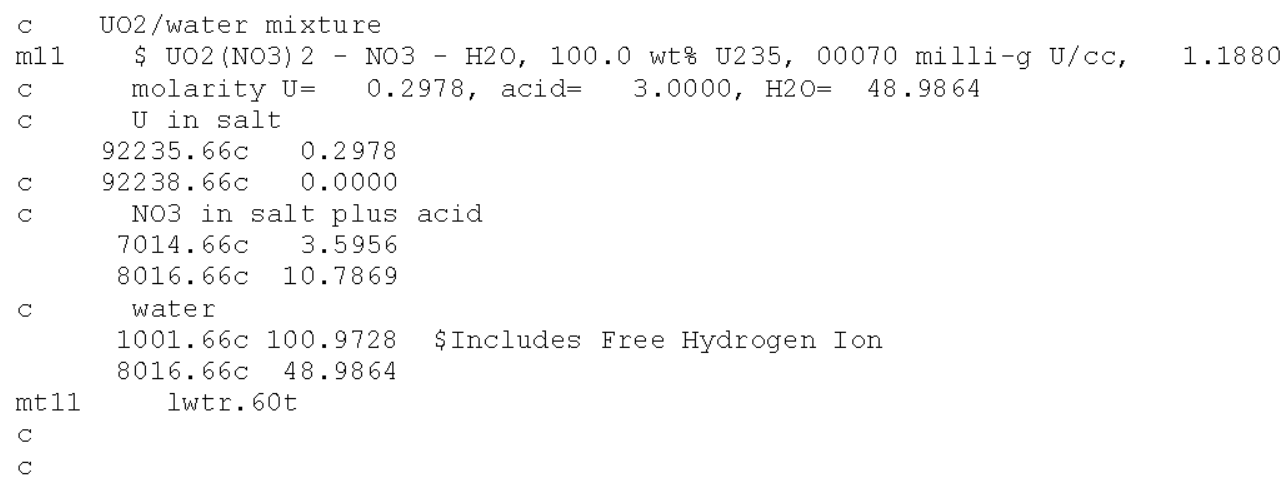

Example Input for Case: 75 u5o2N23-icyl-1000-10-00070-00760.inp icyl, 100.0 wt: U02, $00070 \mathrm{milli-g/CC}$, in UO2(NO3)2, with 3 Molar HNO3 $\mathrm{C}$ 


\section{CHPRC-01552, Revision 0}

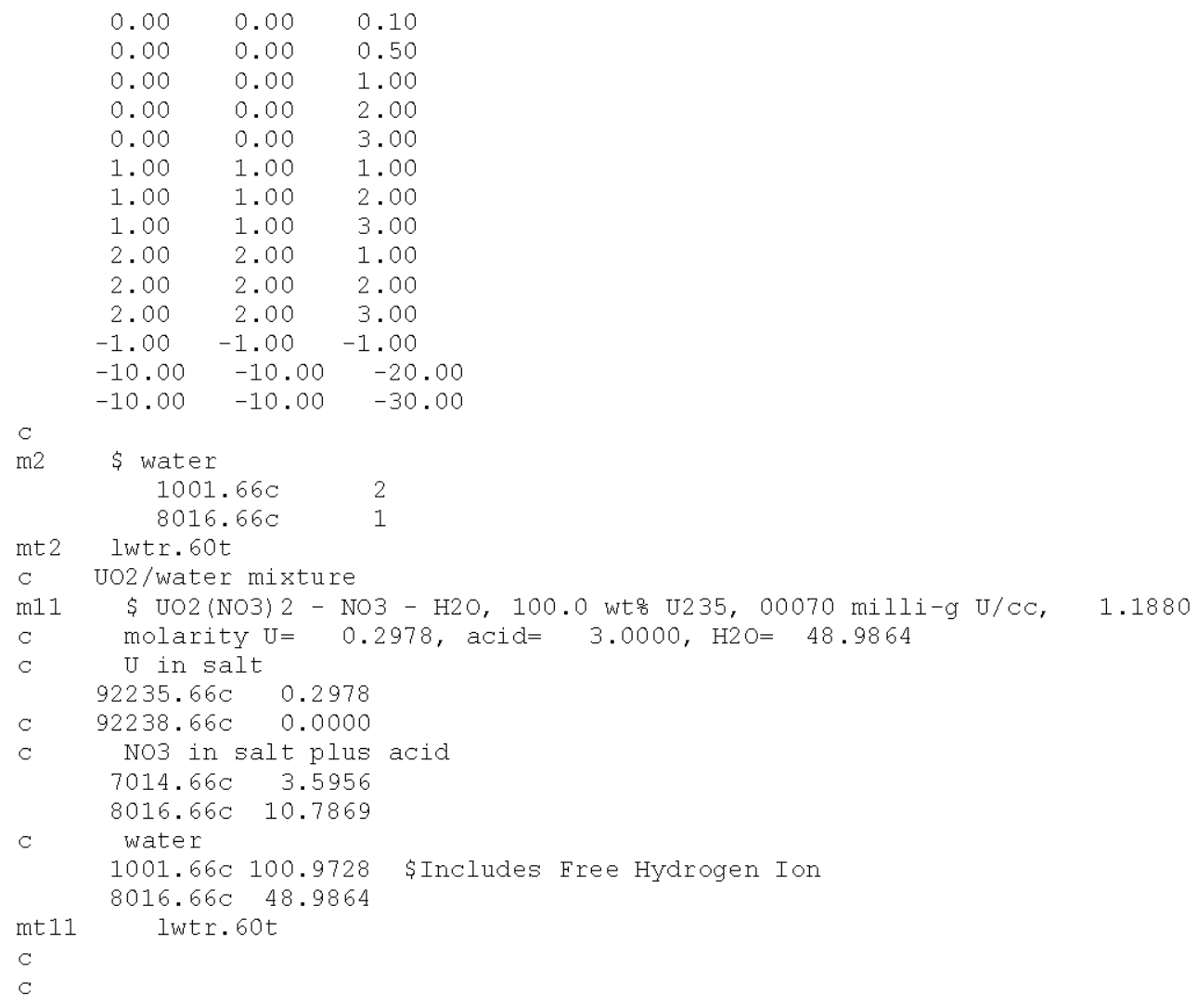




\section{CHPRC-01552, Revision 0}

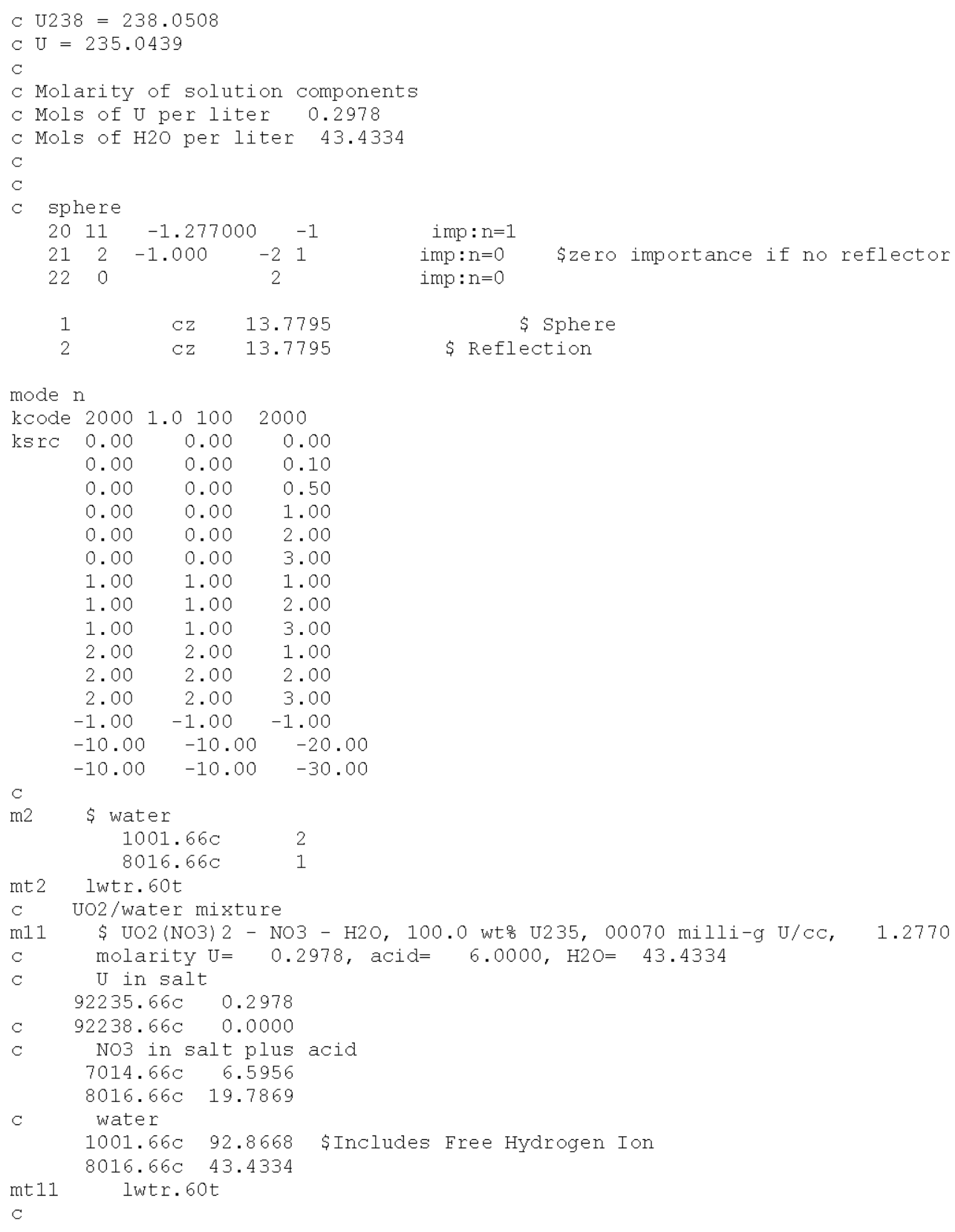




\section{CHPRC-01552, Revision 0}

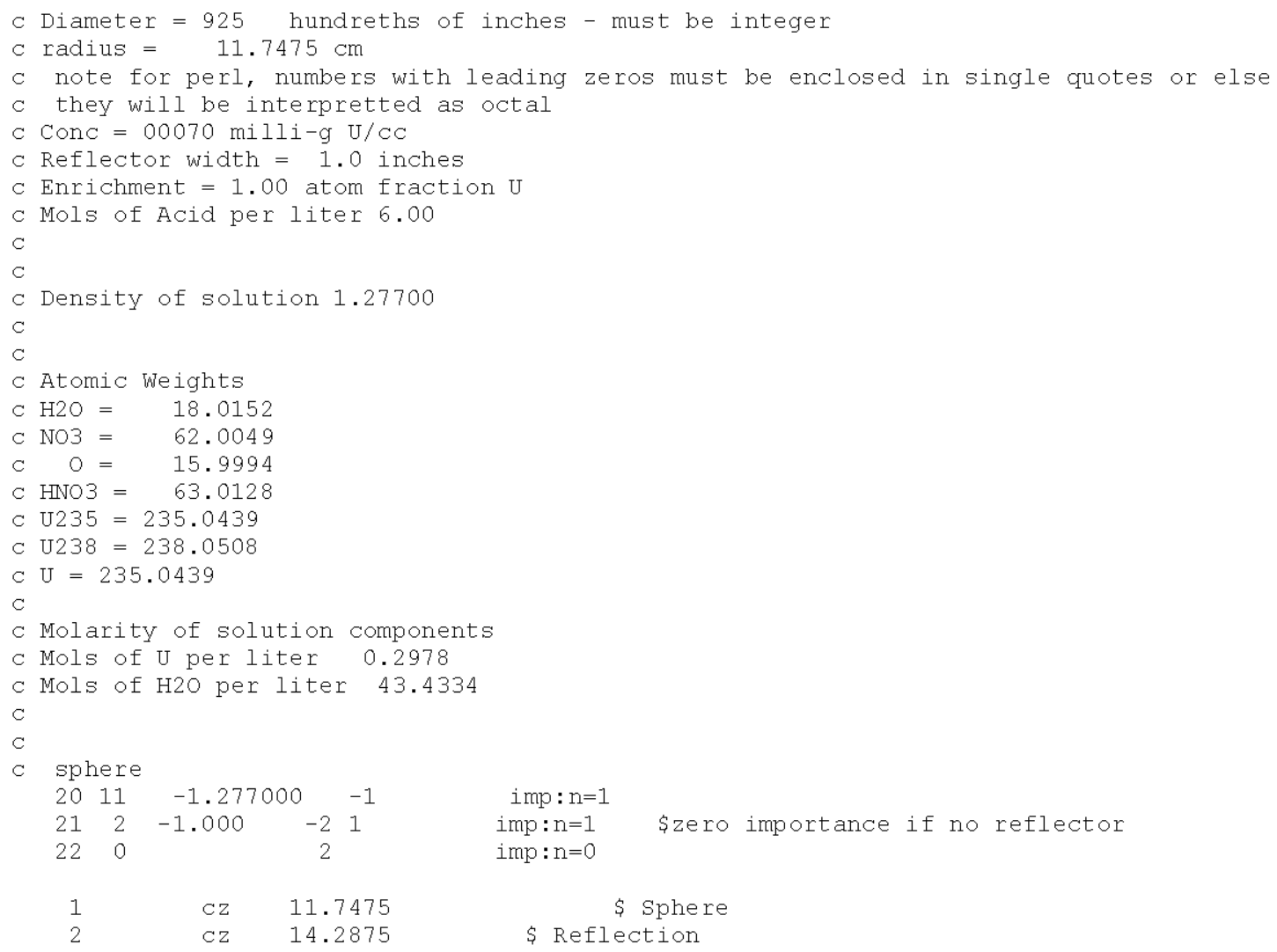




\section{CHPRC-01552, Revision 0}

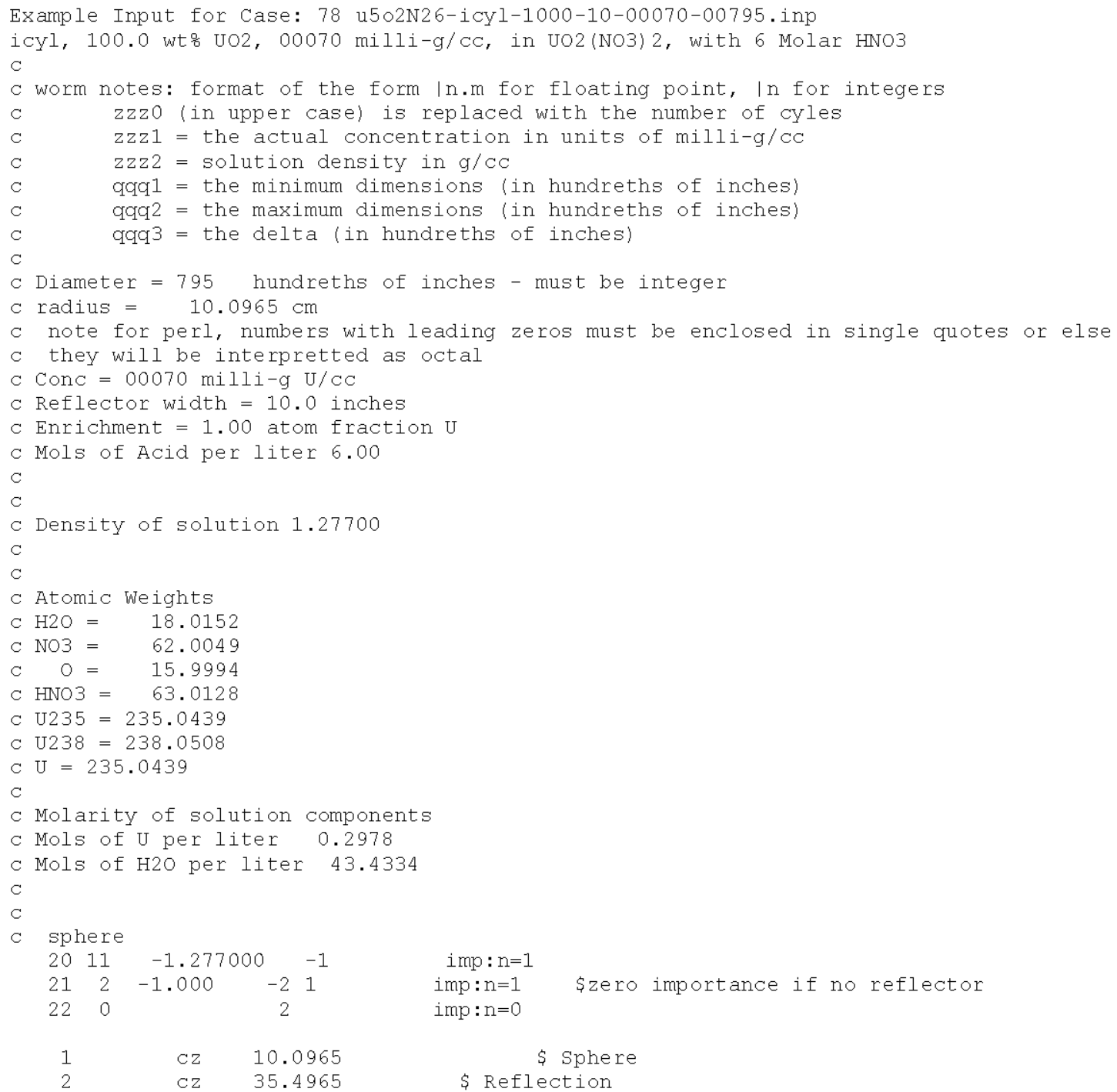




\section{CHPRC-01552, Revision 0}

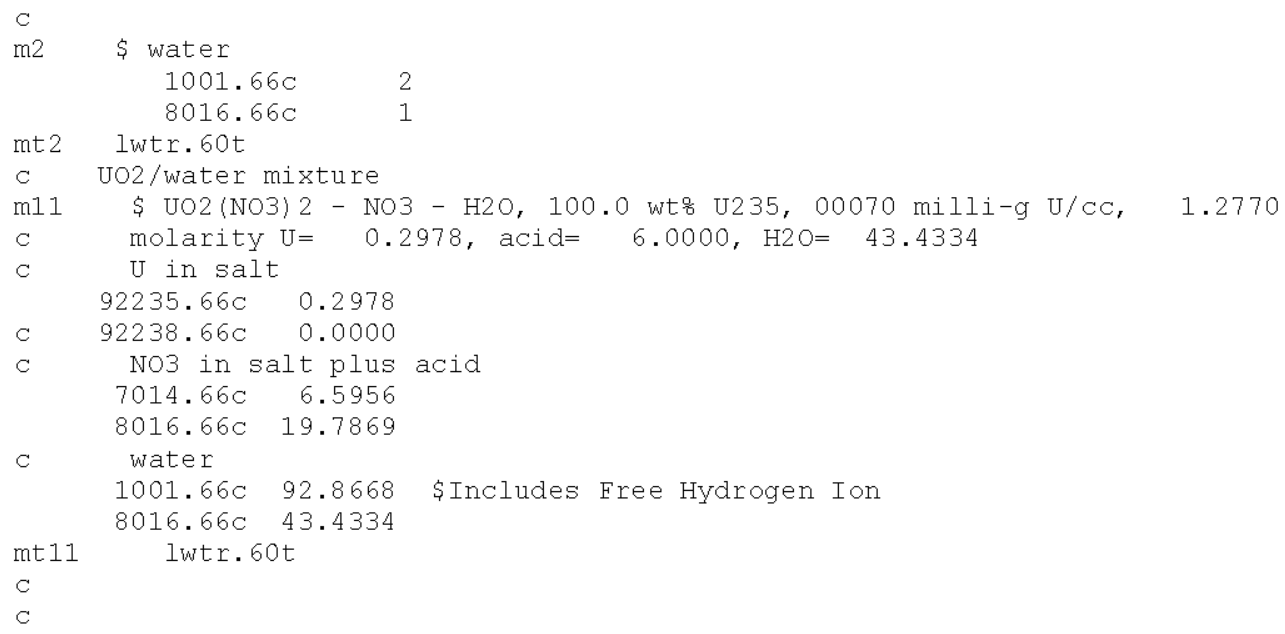

Example Input for Case: 79 u5o2N20-icyl-0935-00-00012-02350.inp icyl, 93.5 wto UO2, $00012 \mathrm{milli-g} / \mathrm{CC}$, in UO2(NO3)2, with 0 Molar HNO3

c worm notes: format of the form $\mid \mathrm{n} . \mathrm{m}$ for floating point, In for integers

c zzzo (in upper case) is replaced with the number of cyles

c $\quad$ zzzl = the actual concentration in units of milli-g/cc

c $\quad z z z 2$ = solution density in $\mathrm{g} / \mathrm{CC}$

c $\quad$ qqq1 $=$ the minimum dimensions (in hundreths of inches)

c qवq 2 the maximum dimensions (in hundreths of inches)

qq93 $=$ the delta (in hundreths of inches)

Diameter $=2350$ hundreths of inches - must be integer

$\mathrm{C}$ radius $=29.8450 \mathrm{~cm}$

c note for perl, numbers with leading zeros must be enclosed in single quotes or else

they will be interpretted as octal

Conc $=00012$ milli-g $\mathrm{U} / \mathrm{CC}$

Reflector width $=0.0$ inches

C Enrichment $=0.935$ atom fraction U

c Mols of Acid per liter 0.00

$\mathrm{C}$

C Density of solution 1.01300

C Atomic Weights

$\mathrm{C} \mathrm{H} 2 \mathrm{O}=18.0152$

C NO3 $=62.0049$

$\mathrm{O}=15.9994$

$\mathrm{C}$ HNO3 $=63.0128$

C U235 $=235.0439$

C $\mathrm{U} 238=238.0508$

$\mathrm{C} U=235.2393$

C Molarity of solution components

C Mols of U per liter 0.0510

C Mols of H2O per liter 55.1224

C

Cylinder

$2011-1.013000-1 \quad$ imp $: n=1$

$212-1.000-21 \quad$ imp $: \mathrm{n}=0$ \$zero importance if no reflector

$1 \quad \mathrm{CZ} \quad 29.8450 \quad$ \$ Sphere 


\section{CHPRC-01552, Revision 0}
2
$C z$
29.8450
\$Reflection

mode $n$

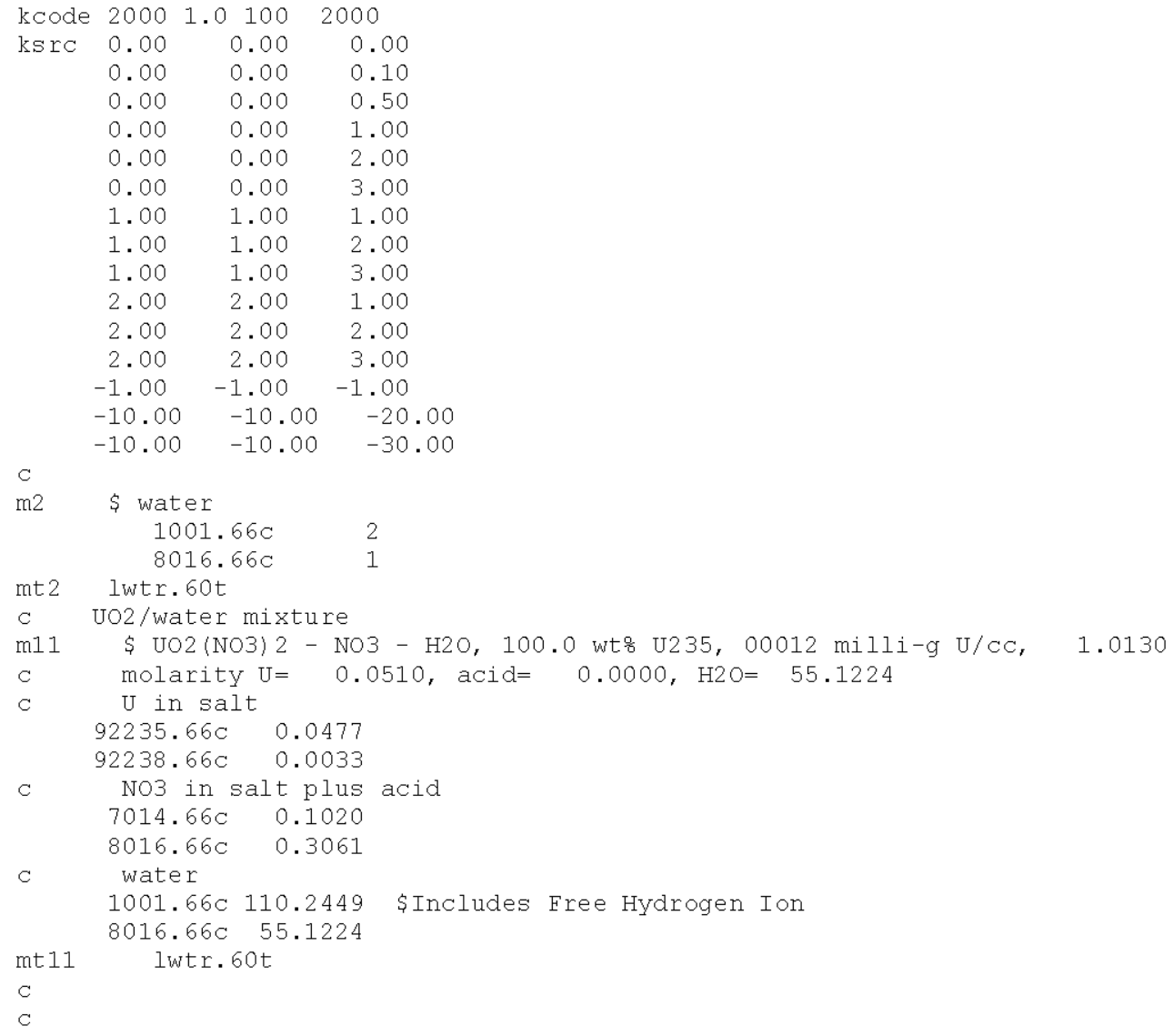




\section{CHPRC-01552, Revision 0}

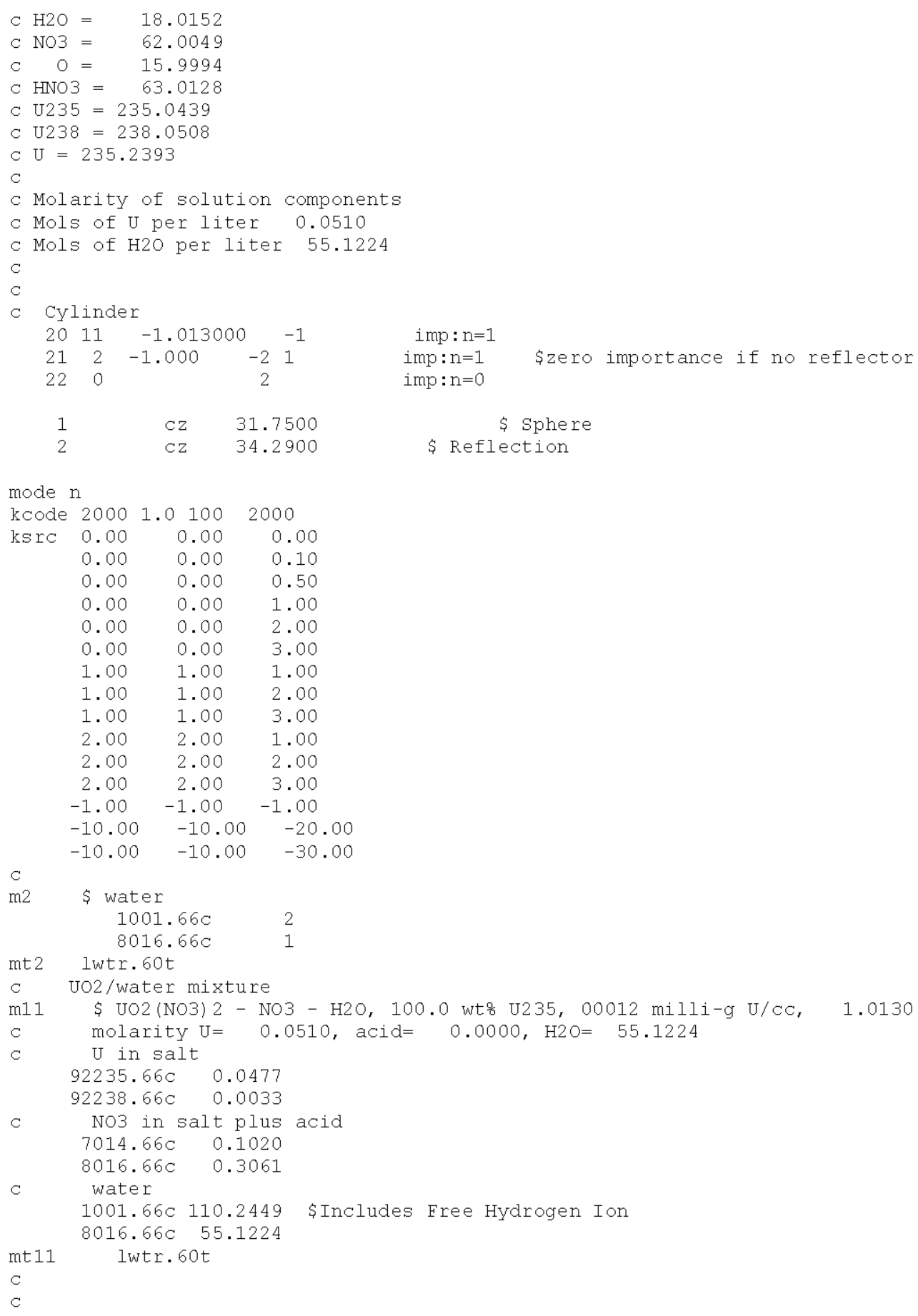




\section{CHPRC-01552, Revision 0}

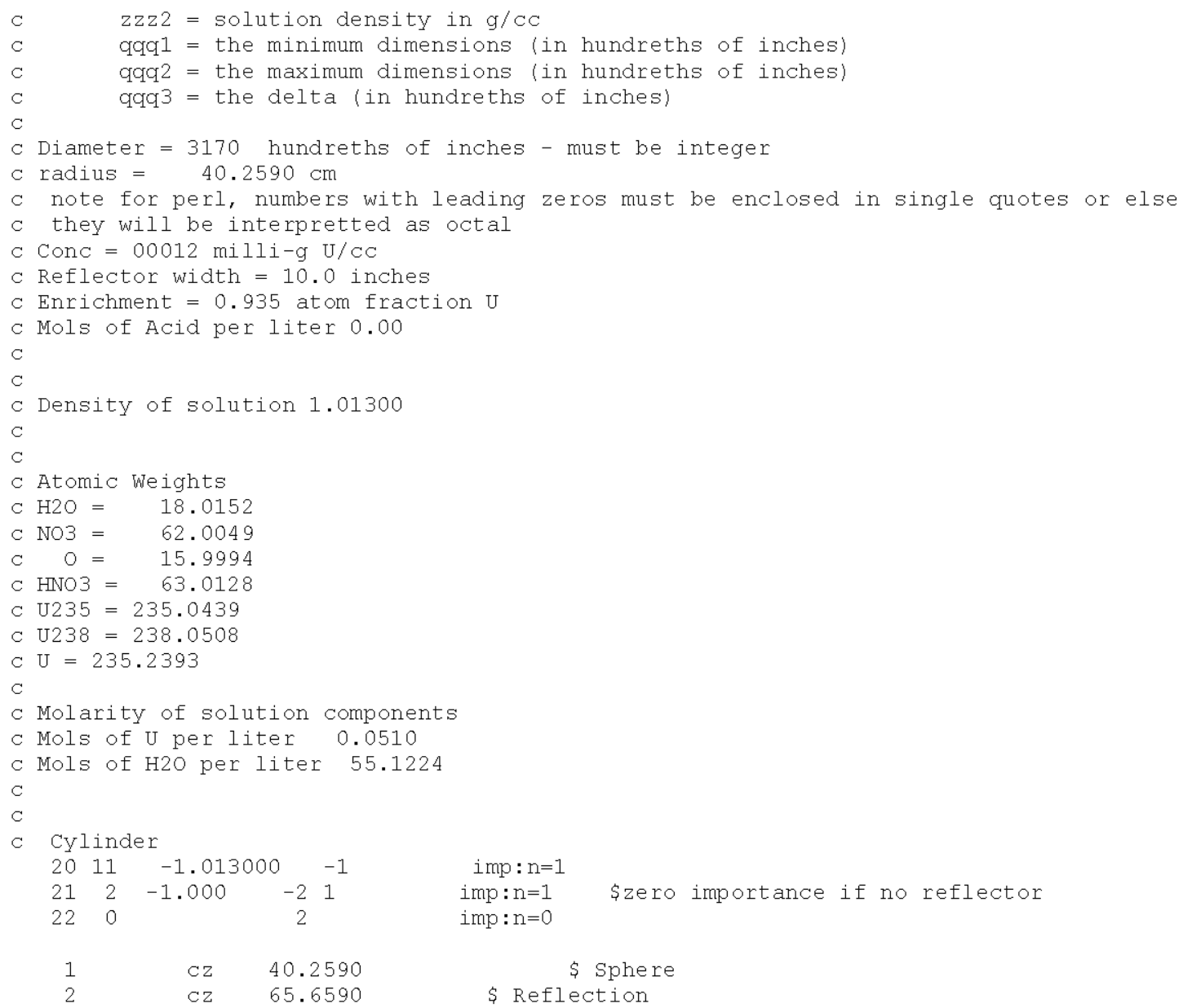




\section{CHPRC-01552, Revision 0}

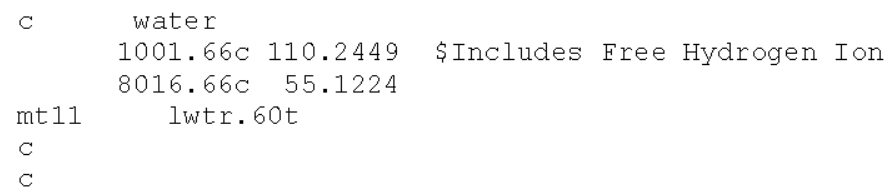




\section{CHPRC-01552, Revision 0}

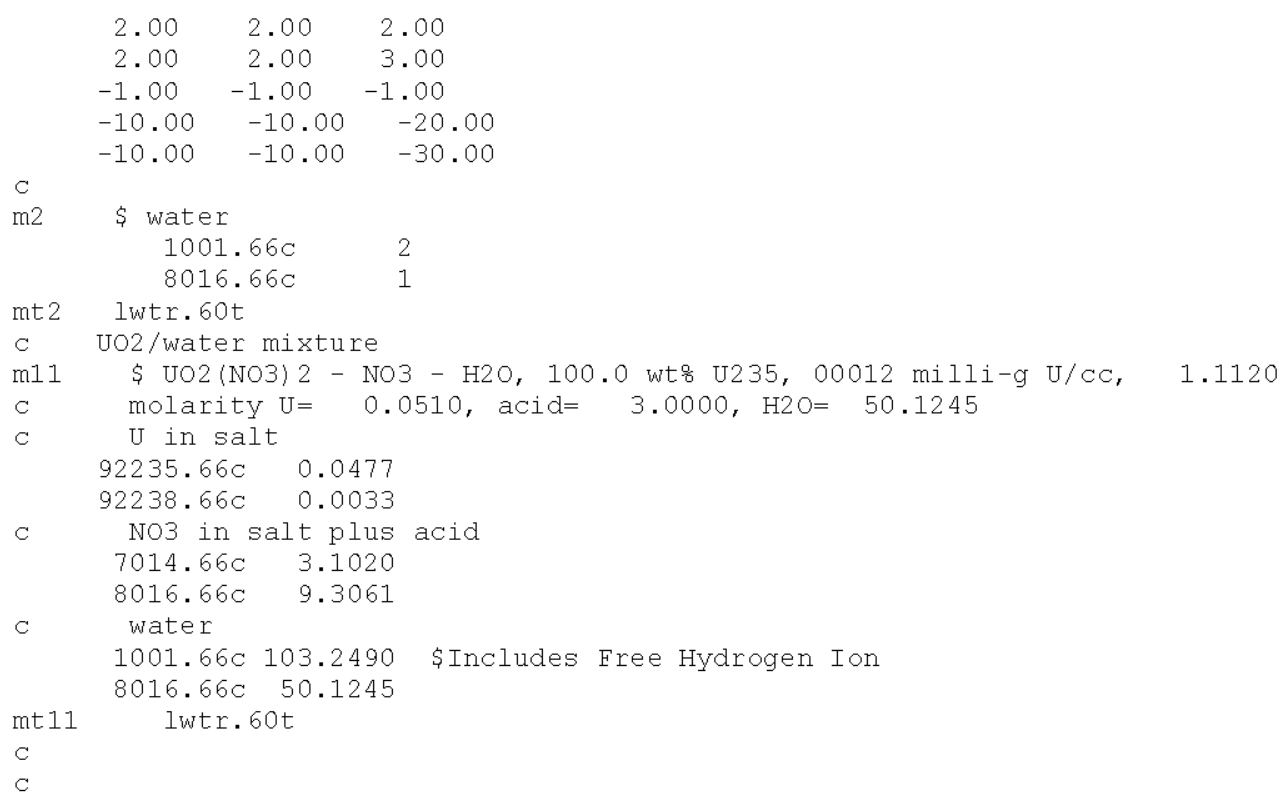




\section{CHPRC-01552, Revision 0}

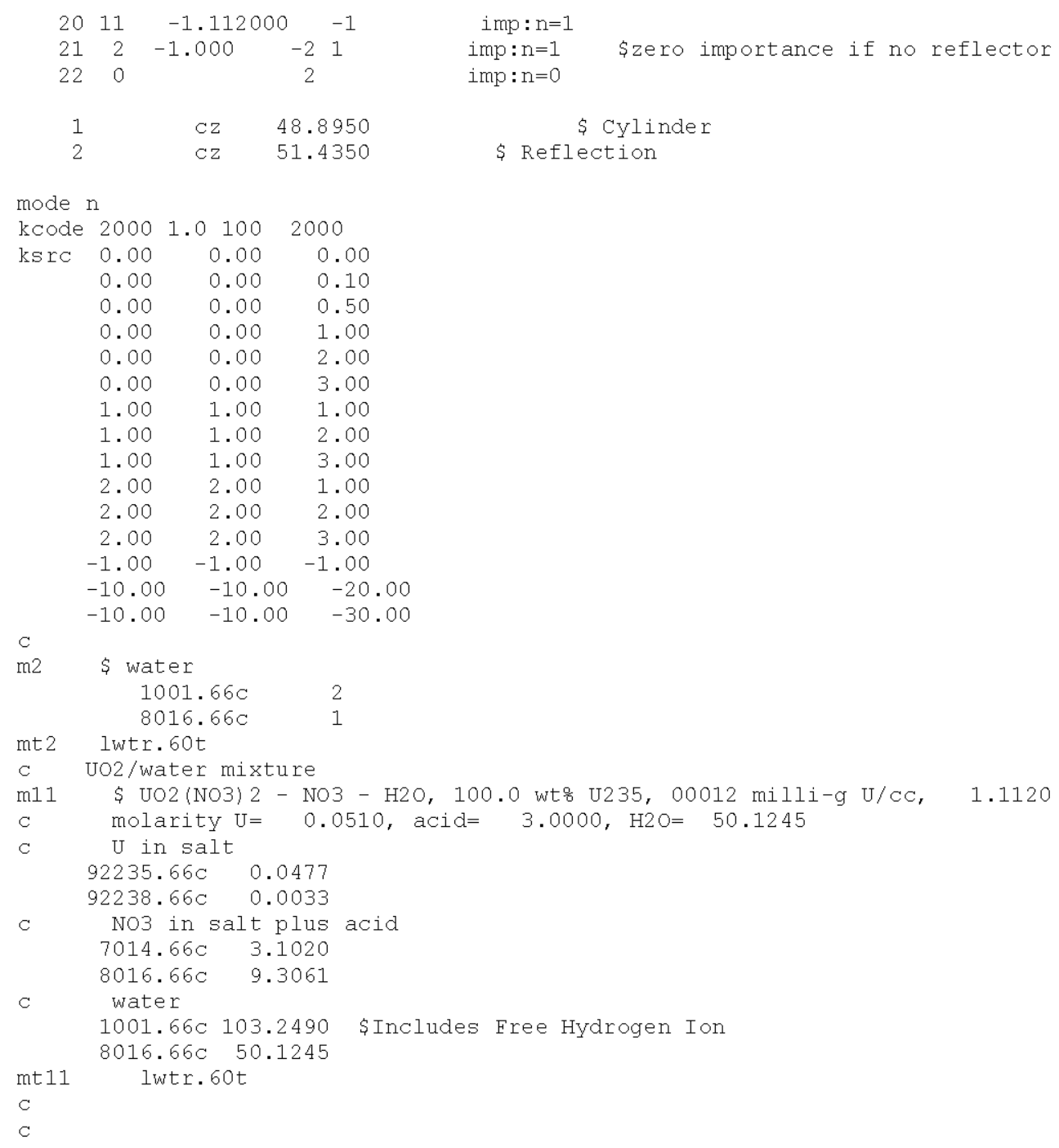




\section{CHPRC-01552, Revision 0}

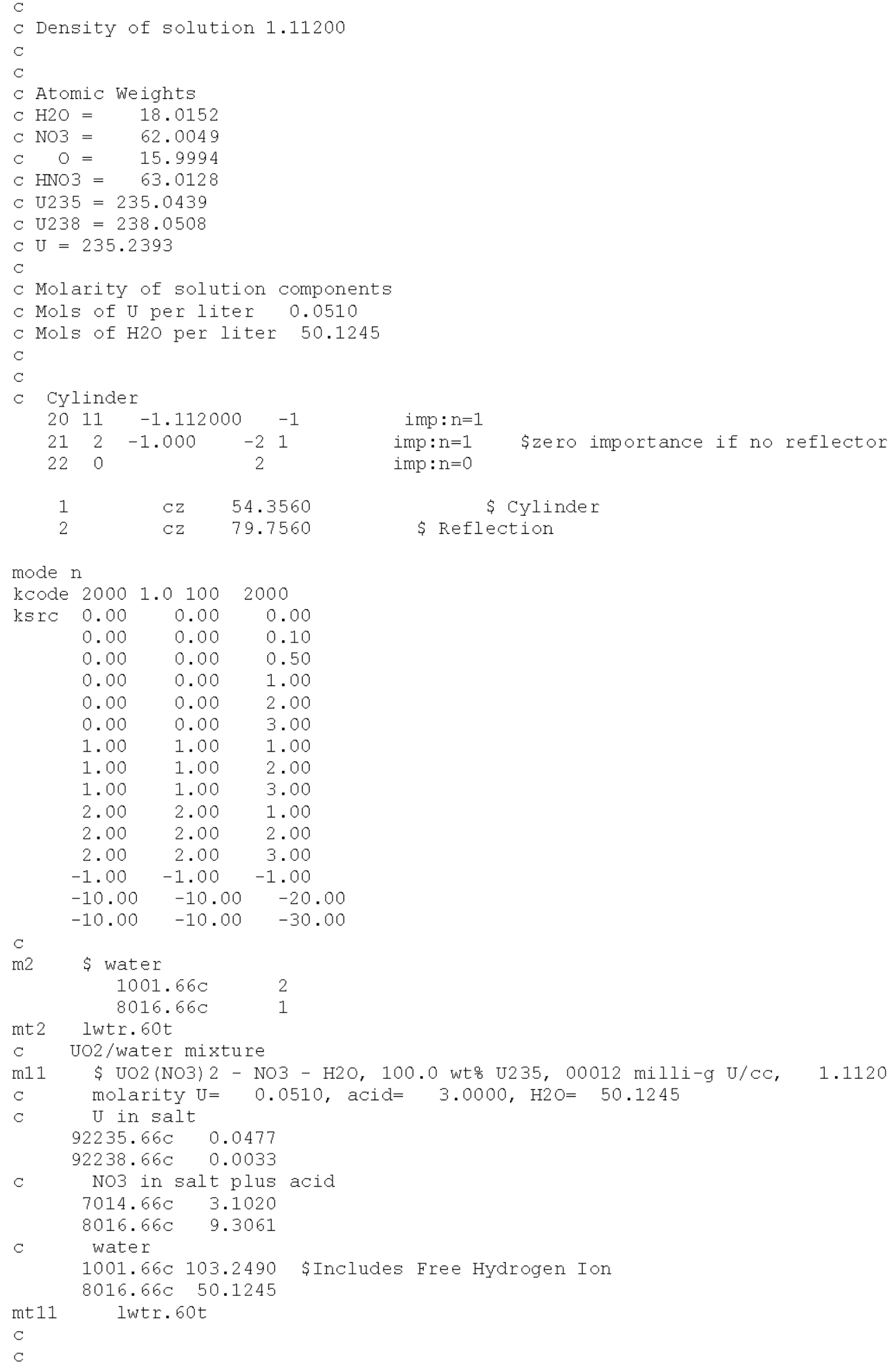




\section{CHPRC-01552, Revision 0}

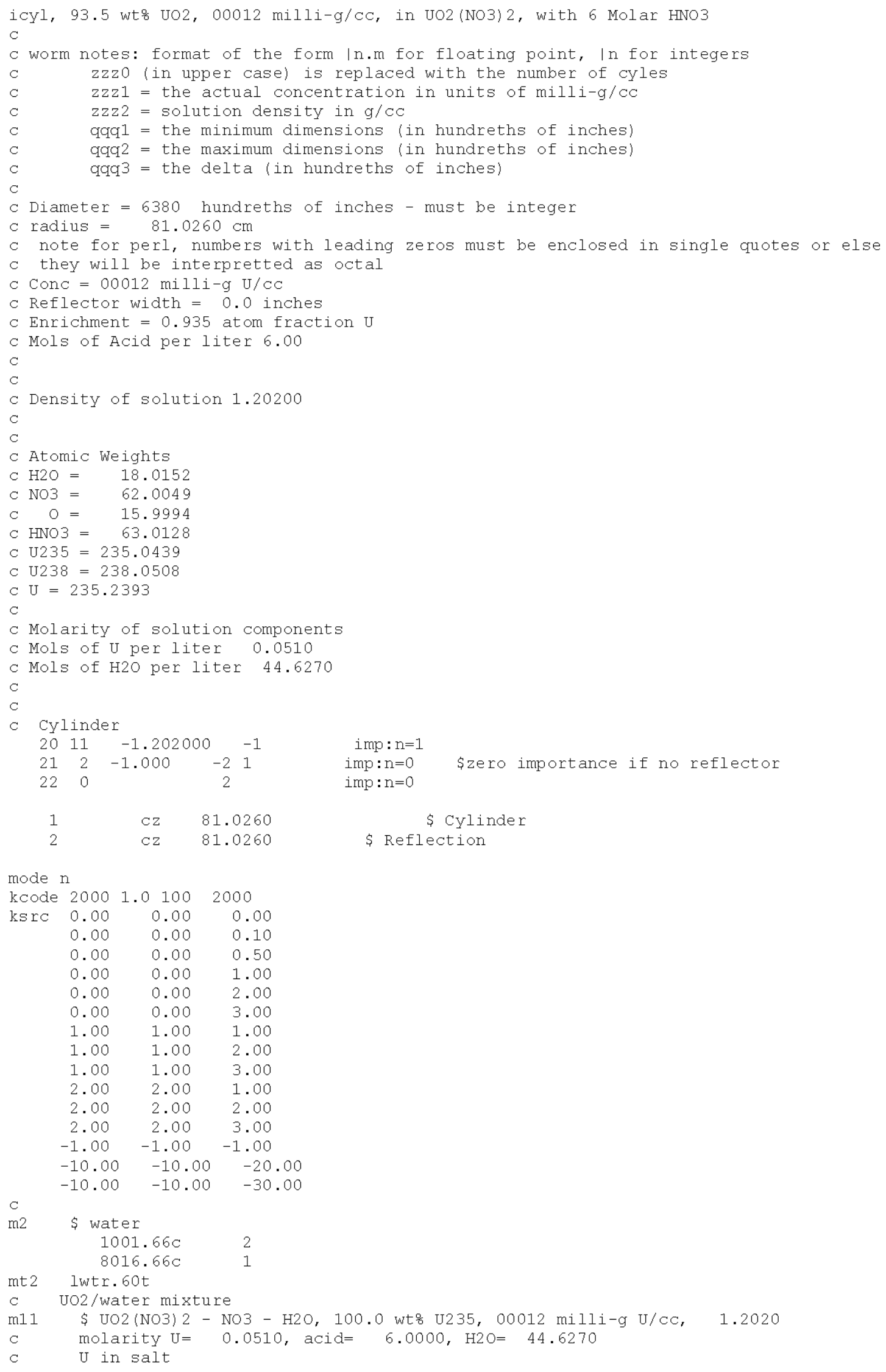




\section{CHPRC-01552, Revision 0}

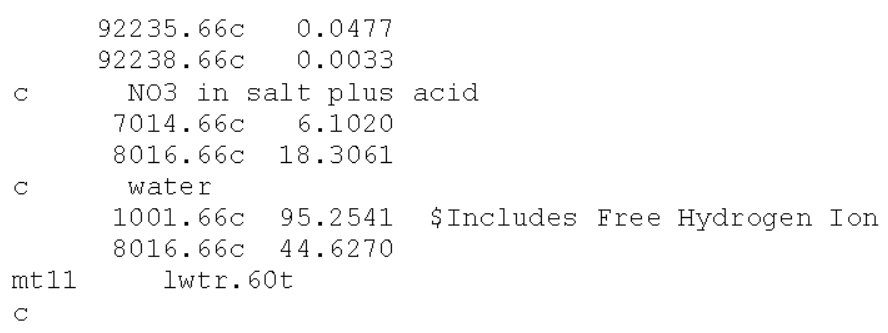




\section{CHPRC-01552, Revision 0}

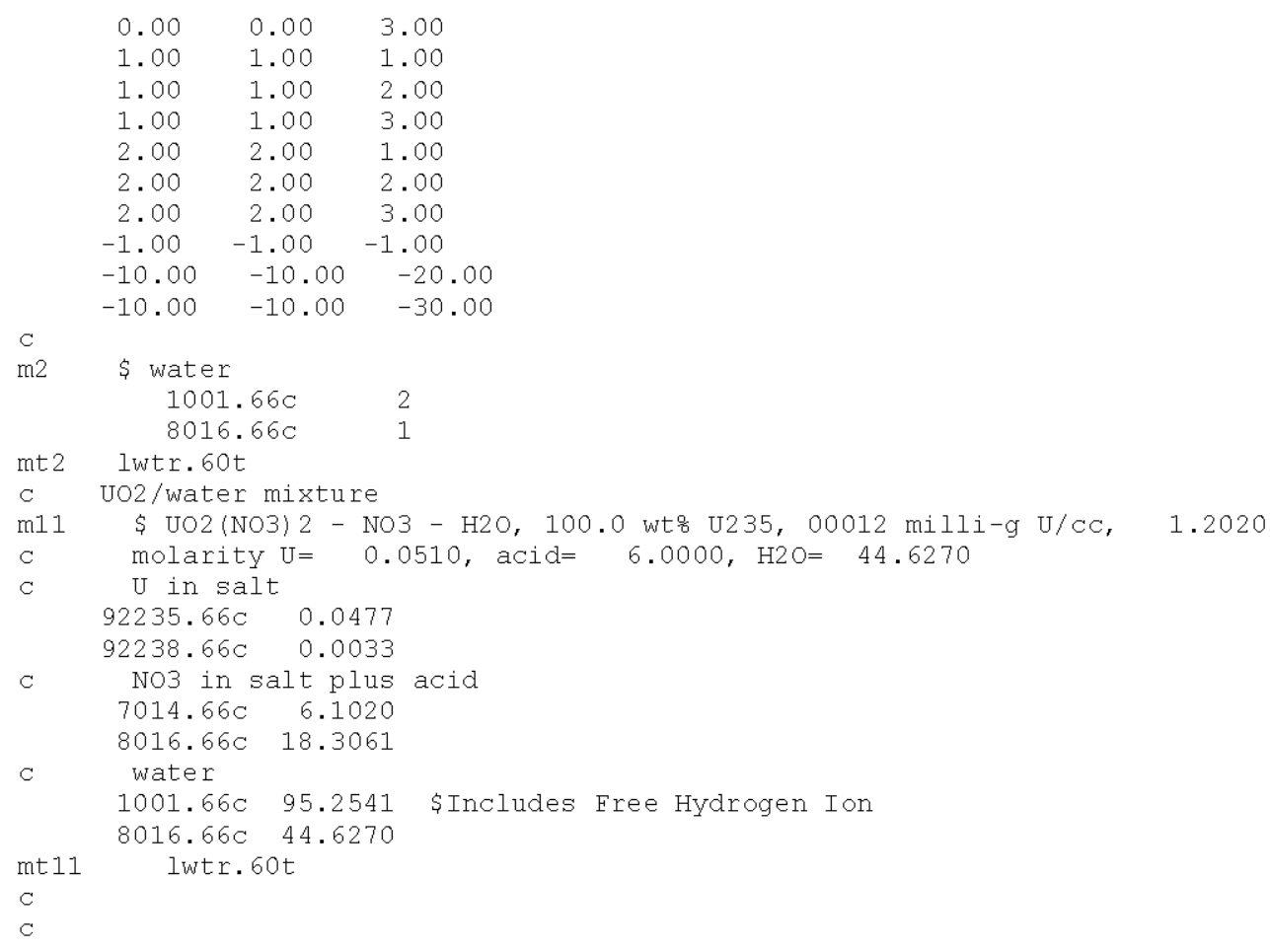




\section{CHPRC-01552, Revision 0}

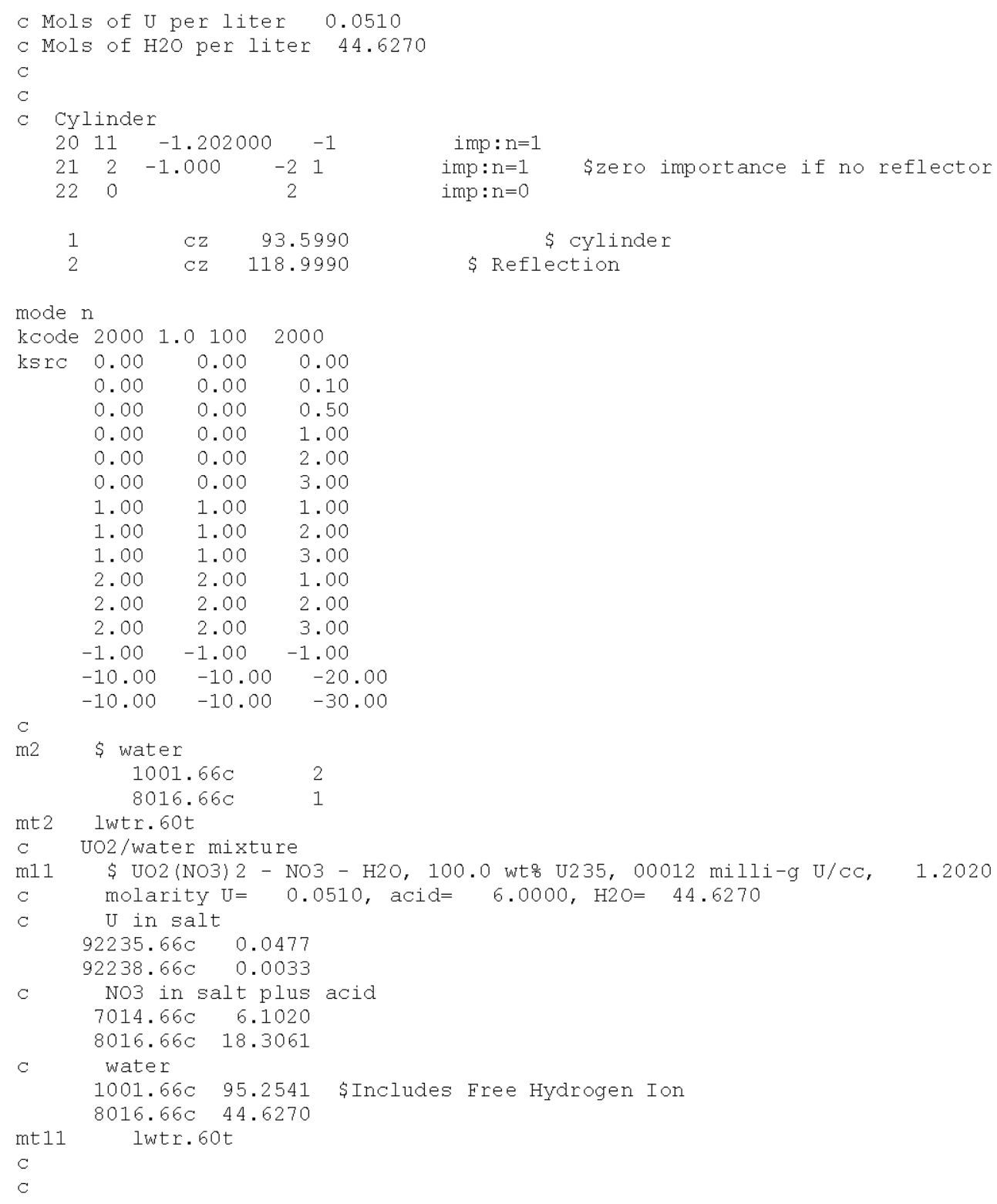




\section{CHPRC-01552, Revision 0}

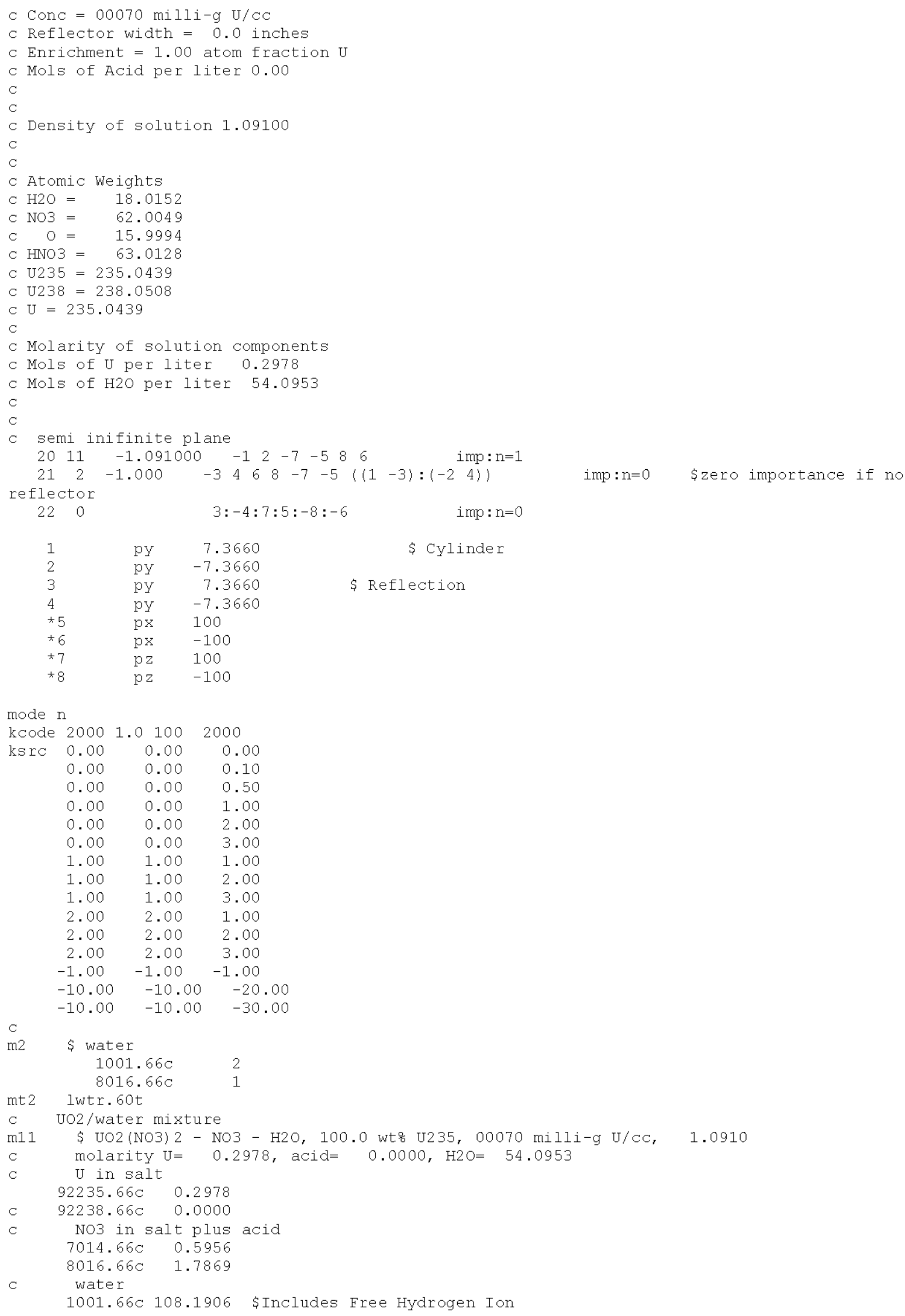




\section{CHPRC-01552, Revision 0}

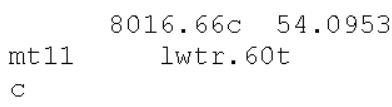




\section{CHPRC-01552, Revision 0}

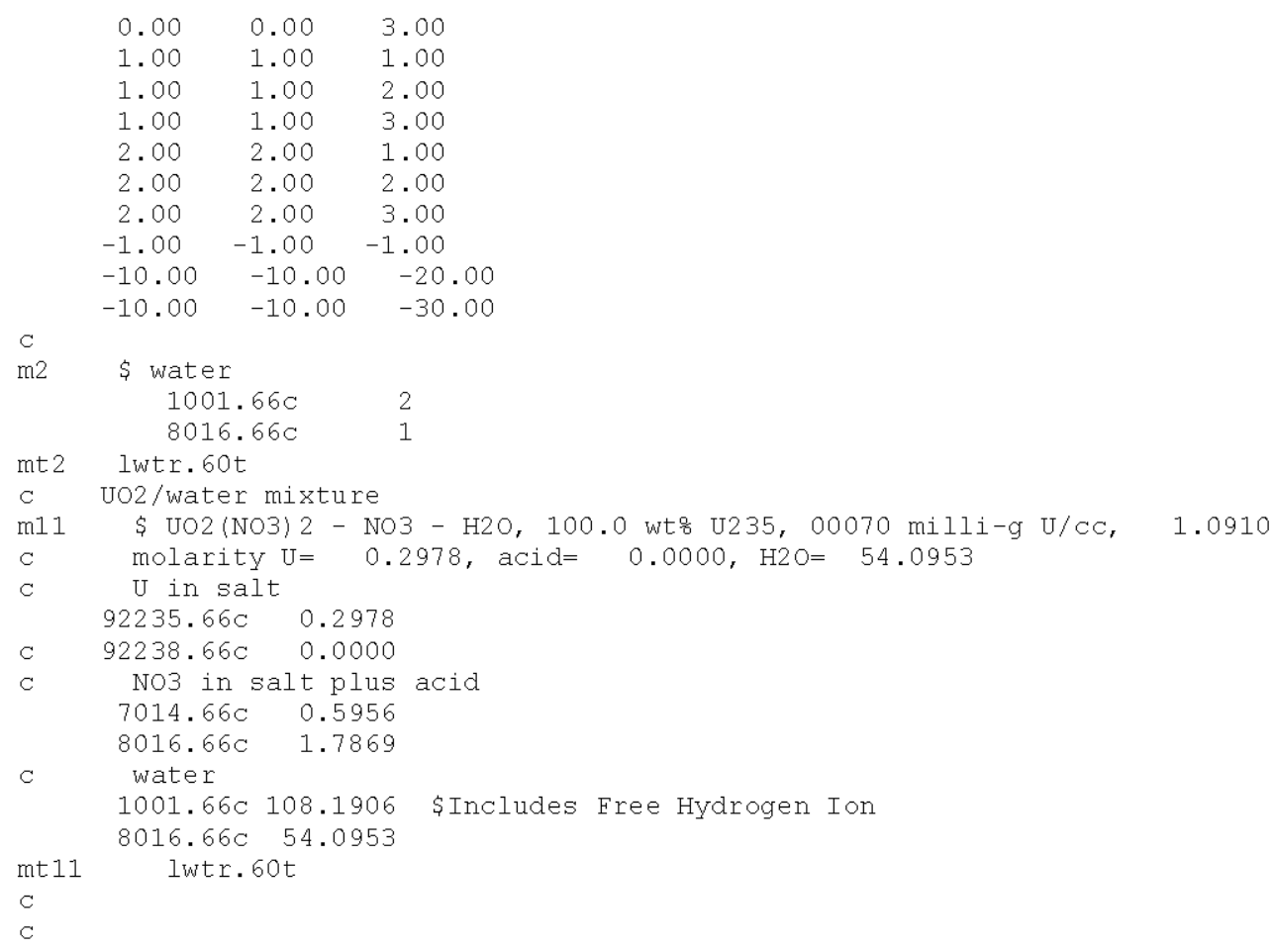




\section{CHPRC-01552, Revision 0}

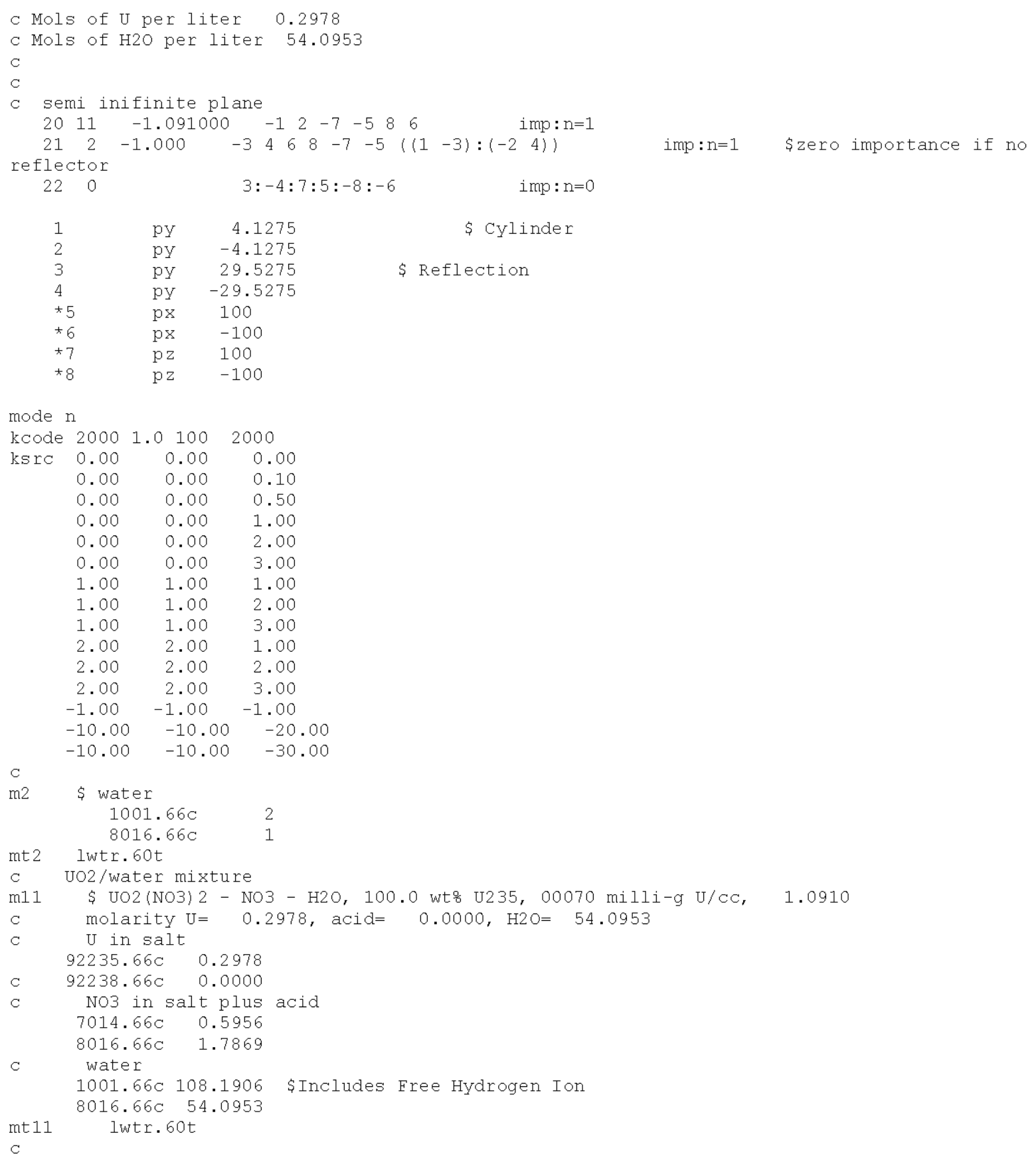




\section{CHPRC-01552, Revision 0}

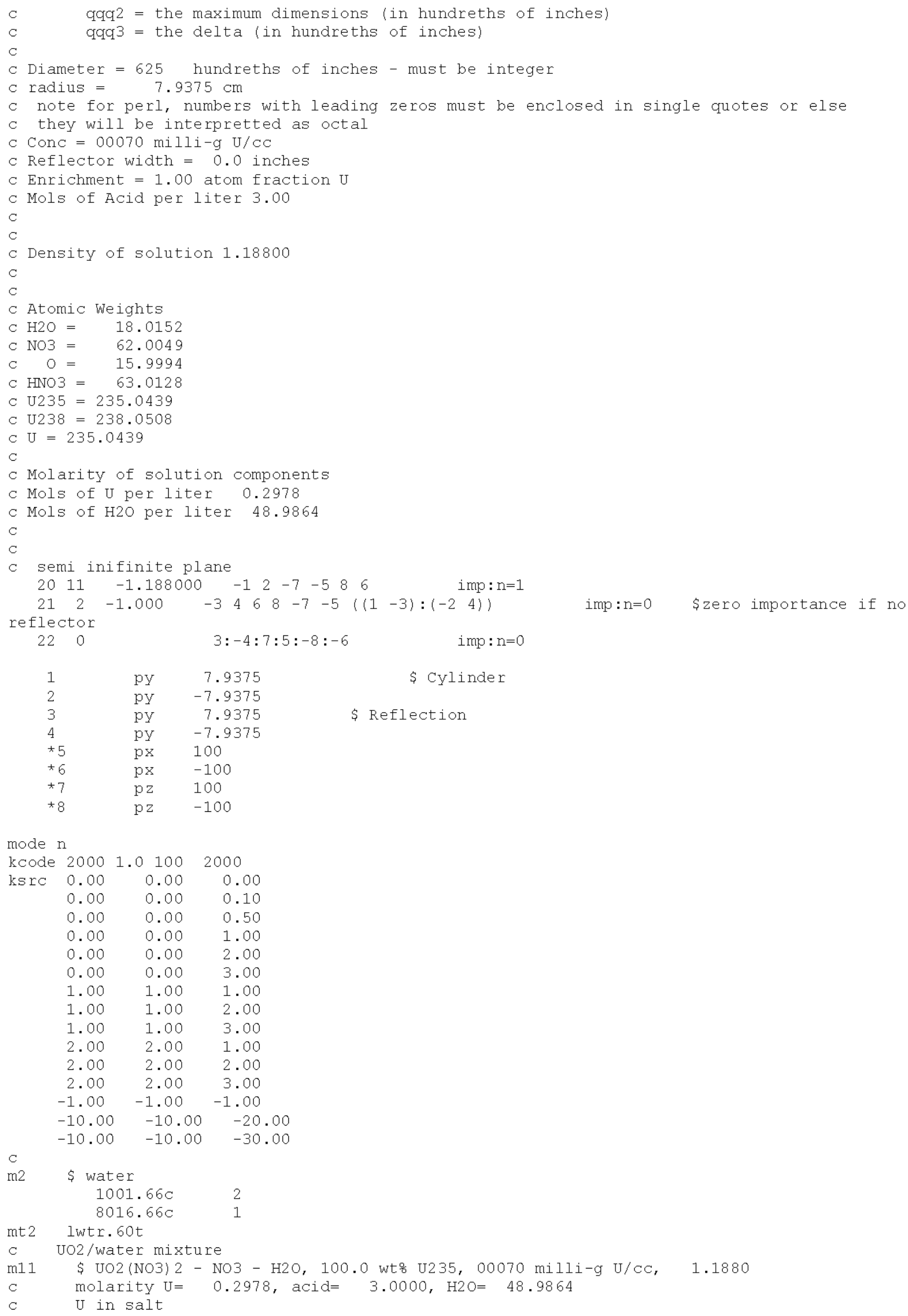




\section{CHPRC-01552, Revision 0}

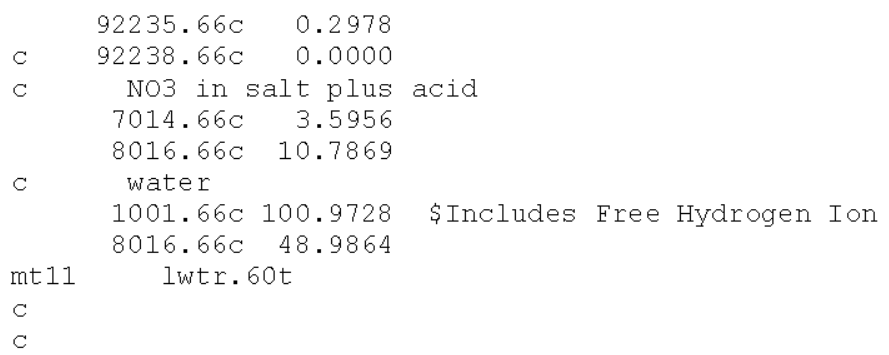

Example Input for Case: 92 u5o2N23-pl-1000-01-00070-00460.inp pl, 100.0 wto U02, $00070 \mathrm{milli-g/cC,}$ in UO2(NO3)2, with 3 Molar HNO3

$\mathrm{C}$

C worm notes: format of the form |n.m for floating point, In for integers

c zzzo (in upper case) is replaced with the number of cyles

$z z z 1=$ the actual concentration in units of milli-g/co

zzz2 = solution density in $\mathrm{g} / \mathrm{CC}$

qqal = the minimum dimensions (in hundreths of inches)

qवq2 = the maximum dimensions (in hundreths of inches)

q993 = the delta (in hundreths of inches)

Diameter $=460$ hundreths of inches - must be integer

C radius $=5.8420 \mathrm{~cm}$

C note for perl, numbers with leading zeros must be enclosed in single quotes or else

c they will be interpretted as octal

$\mathrm{C}$ Conc $=00070 \mathrm{milli-g} \mathrm{U} / \mathrm{CC}$

C Reflector width $=1.0$ inches

$\mathrm{C}$ Enrichment $=1.00$ atom fraction $\mathrm{U}$

C Mols of Acid per liter 3.00

C

C

C Density of solution 1.18800

C Atomic Weights

$\mathrm{C} \mathrm{H} 2 \mathrm{O}=18.0152$

$\mathrm{C}$ NO3 $=62.0049$

$\mathrm{O}=15.9994$

$\mathrm{C}$ HNO3 $=63.0128$

$\mathrm{C} U 235=235.0439$

C $\mathrm{U} 238=238.0508$

$\mathrm{C} U=235.0439$

C Molarity of solution components

C Mols of U per liter 0.2978

C Mols of H2O per liter 48.9864

$\mathrm{C}$

C semi inifinite plane

$\begin{array}{llllllllll}20 & 11 & -1.188000 & -1 & 2 & -7 & -5 & 8 & 6\end{array}$ imp: $\mathrm{n}=1$

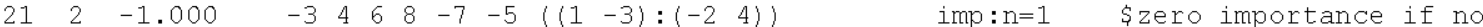

reflector

220

$3:-4: 7: 5:-8:-6 \quad$ imp $: n=0$

$1 \quad$ py $\quad 5.8420$

py $\quad-5.8420$

py $\quad 8.3820$

py $\quad-8.3820$

px 100

px -100

$\begin{array}{lll}* 7 & \mathrm{pz} & 100 \\ \star 8 & \mathrm{pz} & -100\end{array}$ 


\section{CHPRC-01552, Revision 0}

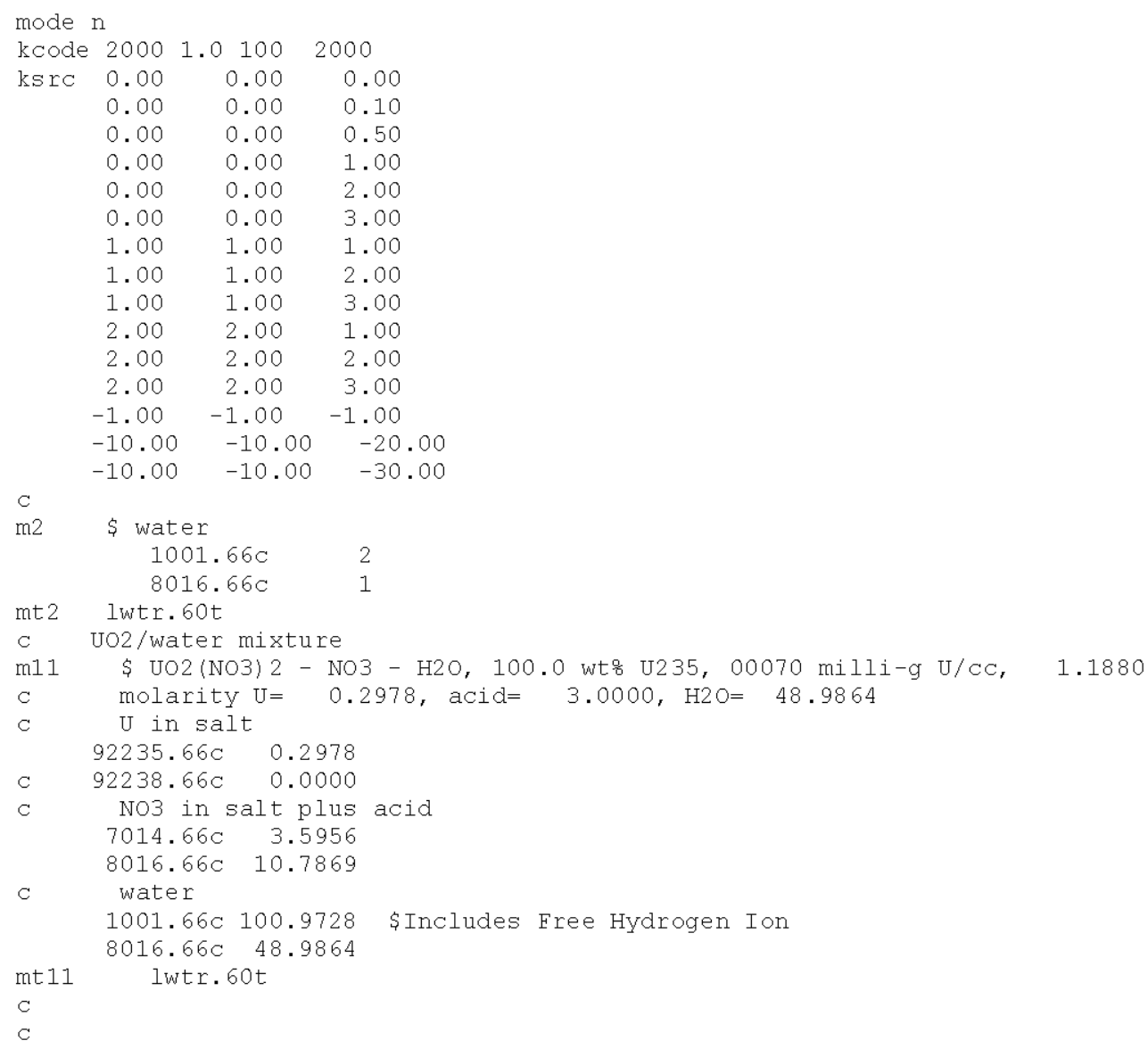




\section{CHPRC-01552, Revision 0}

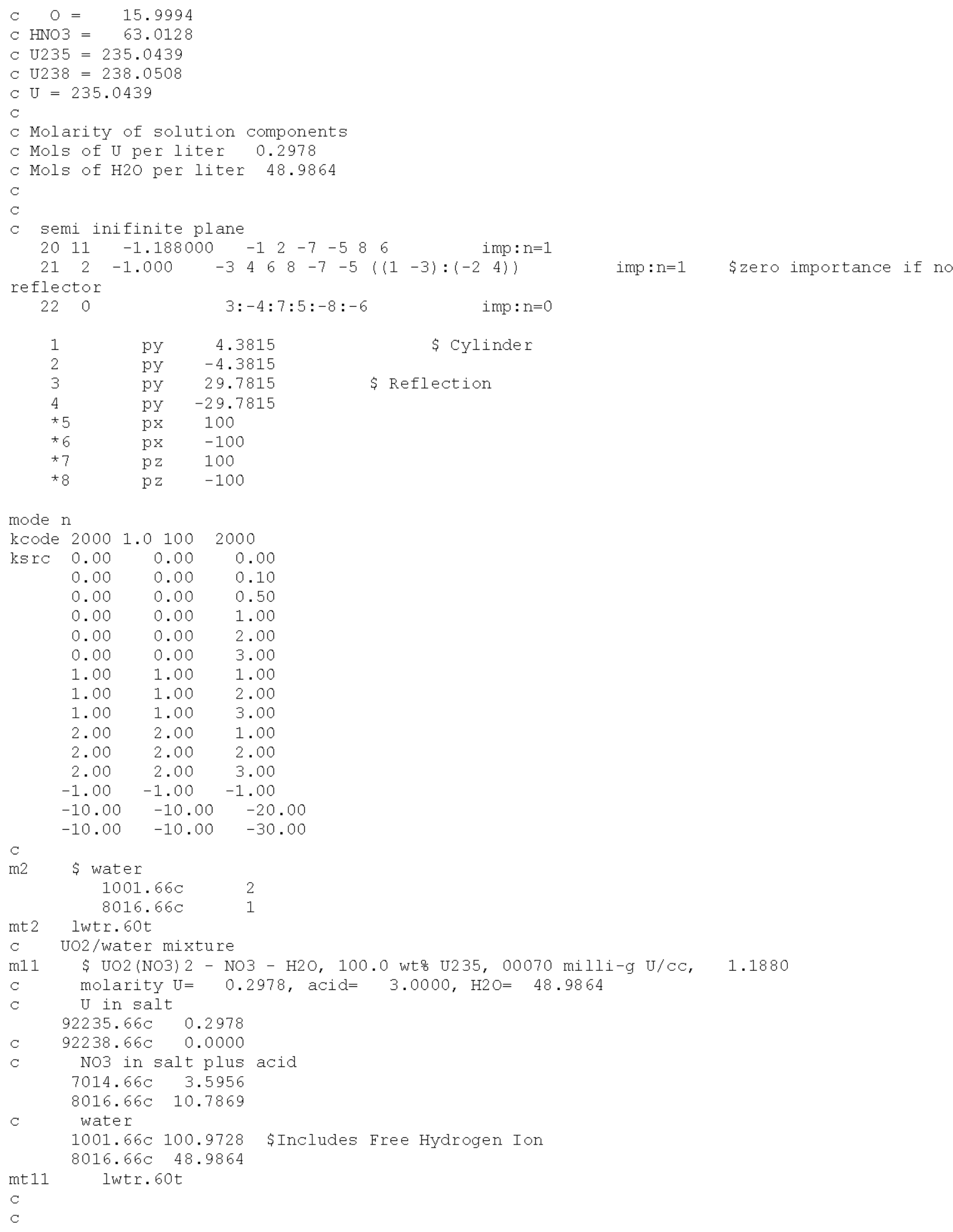




\section{CHPRC-01552, Revision 0}

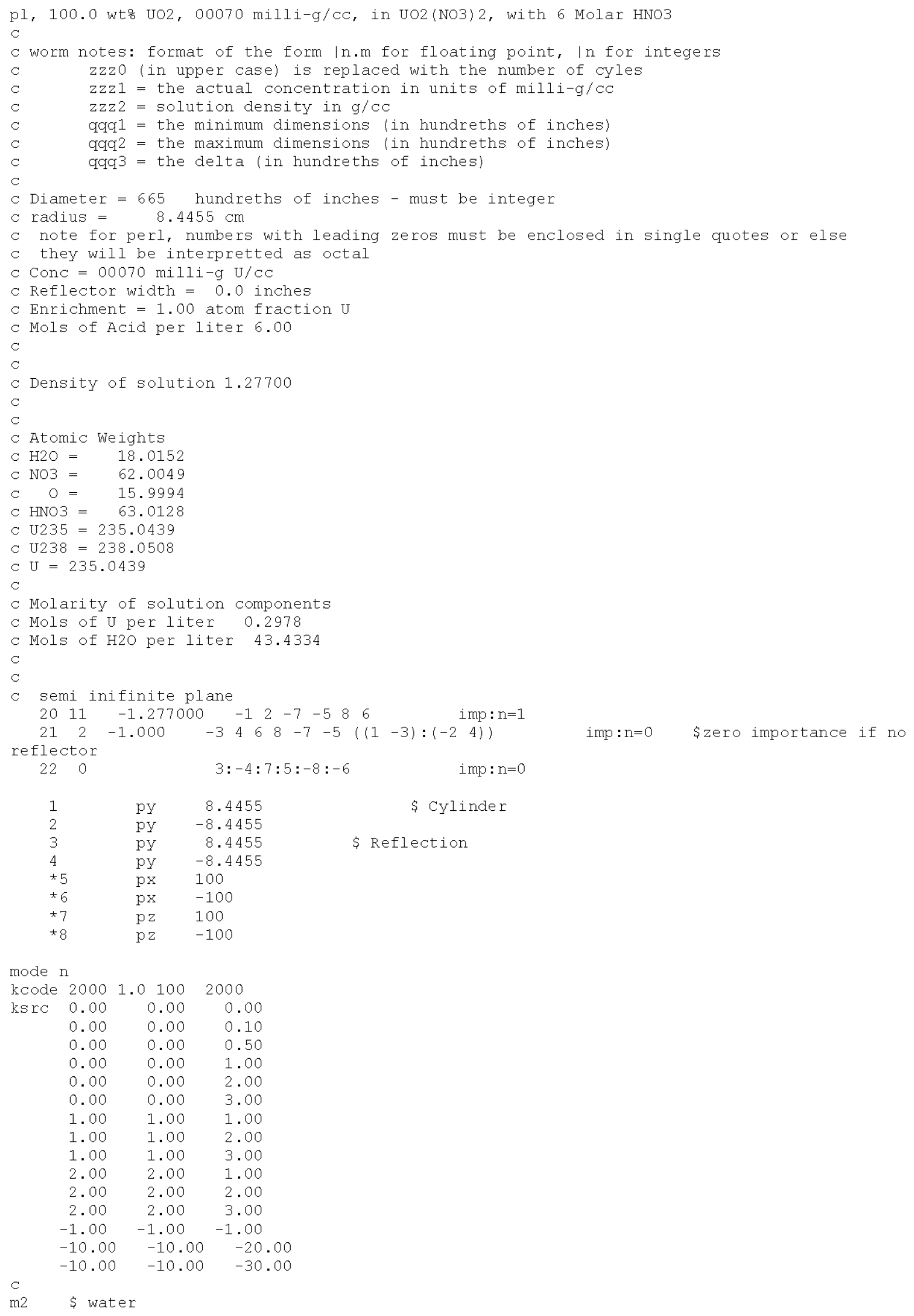




\section{CHPRC-01552, Revision 0}

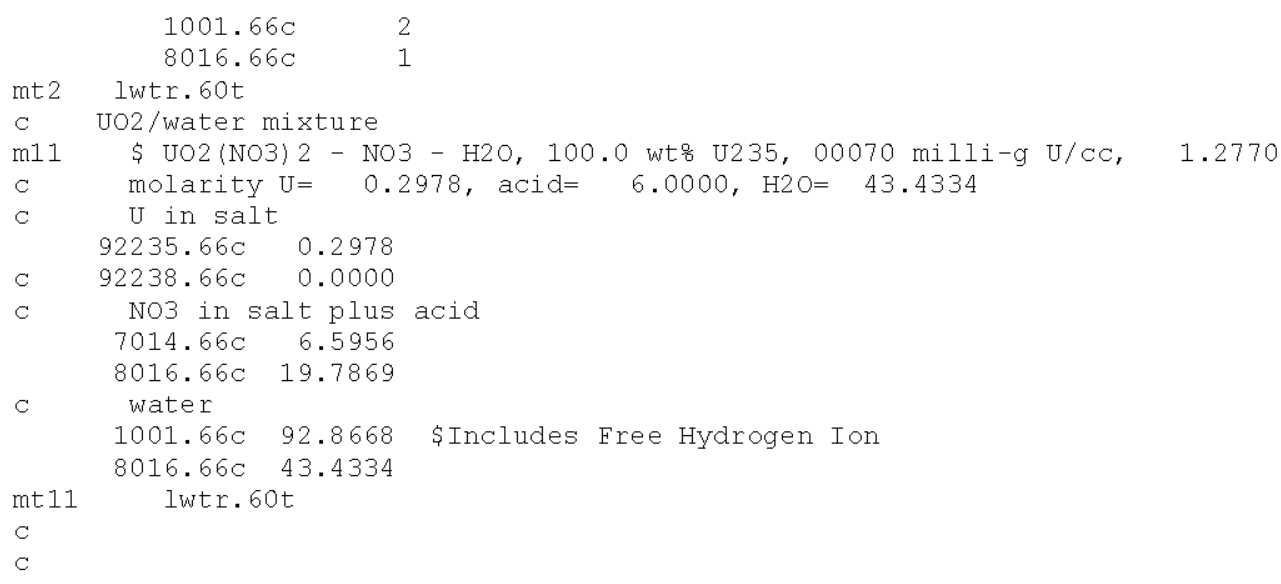




\section{CHPRC-01552, Revision 0}

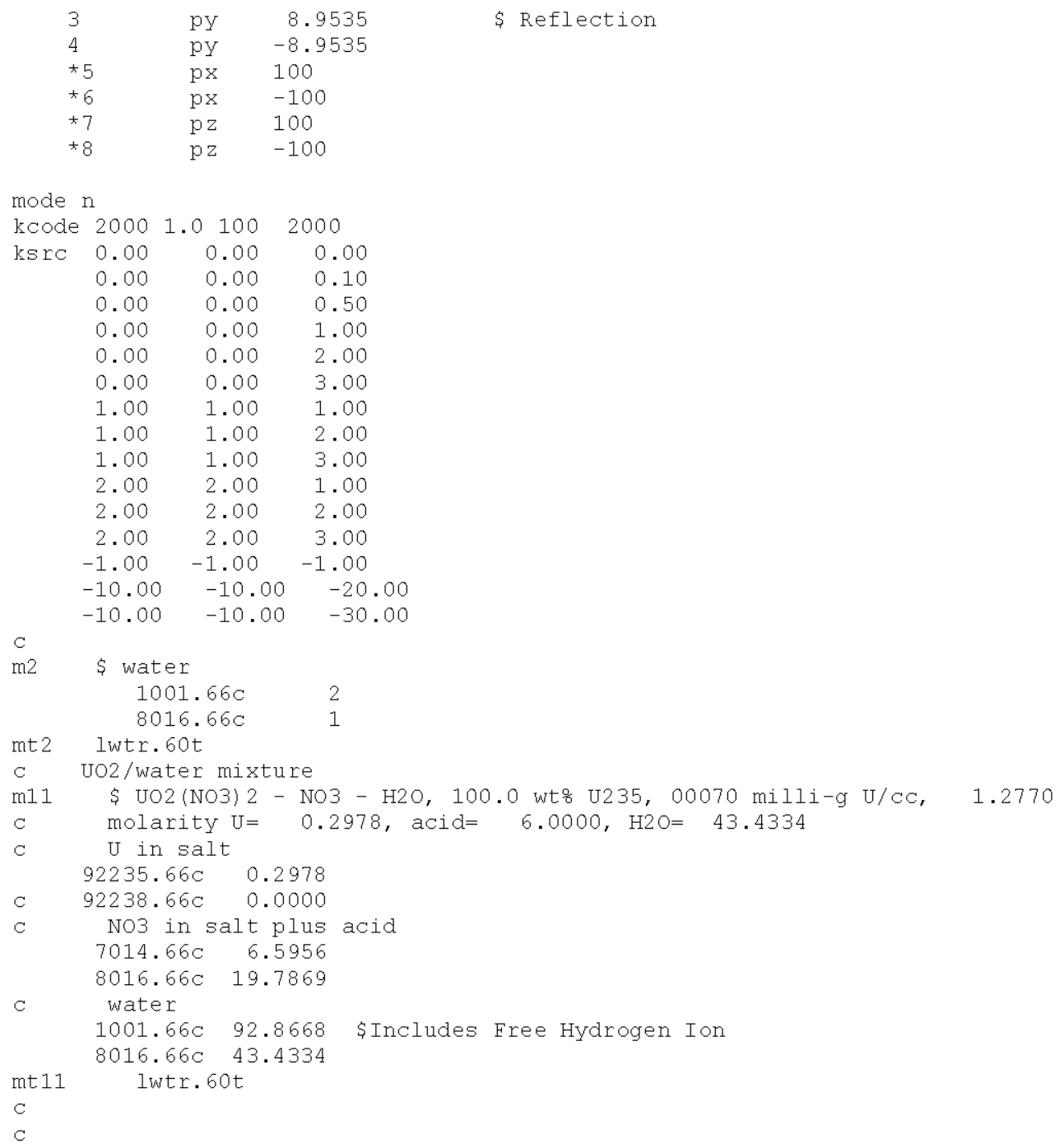




\section{CHPRC-01552, Revision 0}

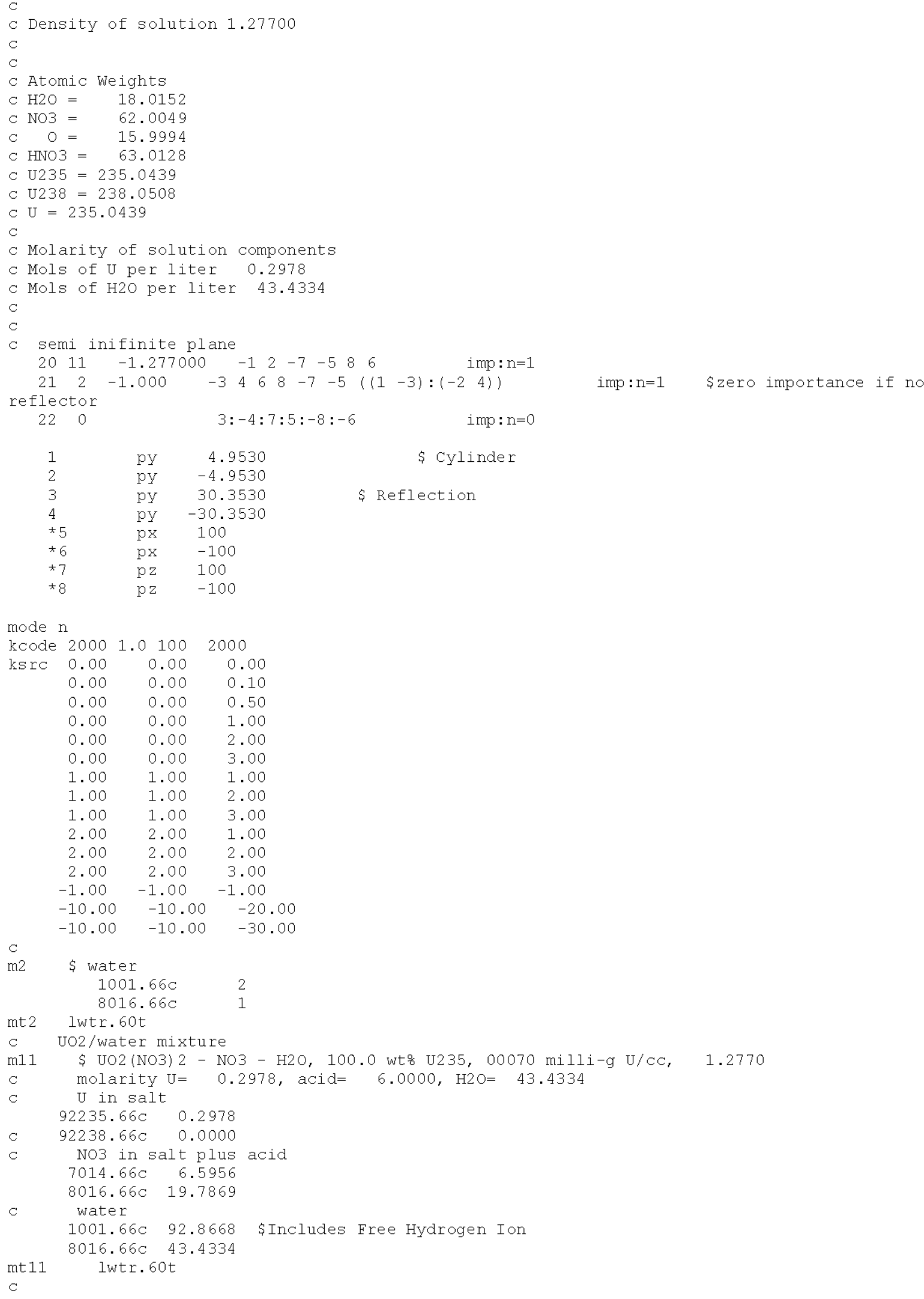




\section{CHPRC-01552, Revision 0}

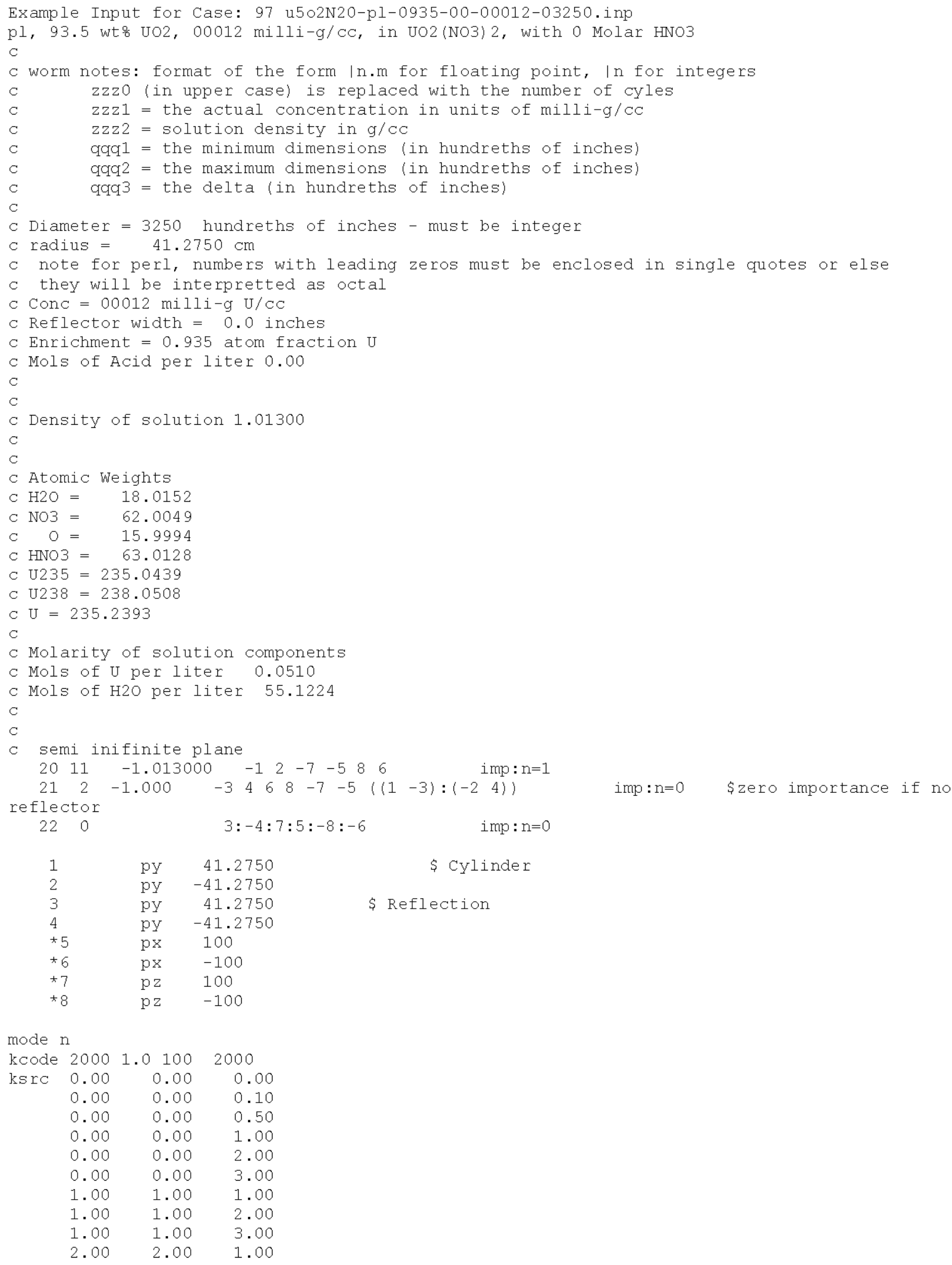




\section{CHPRC-01552, Revision 0}

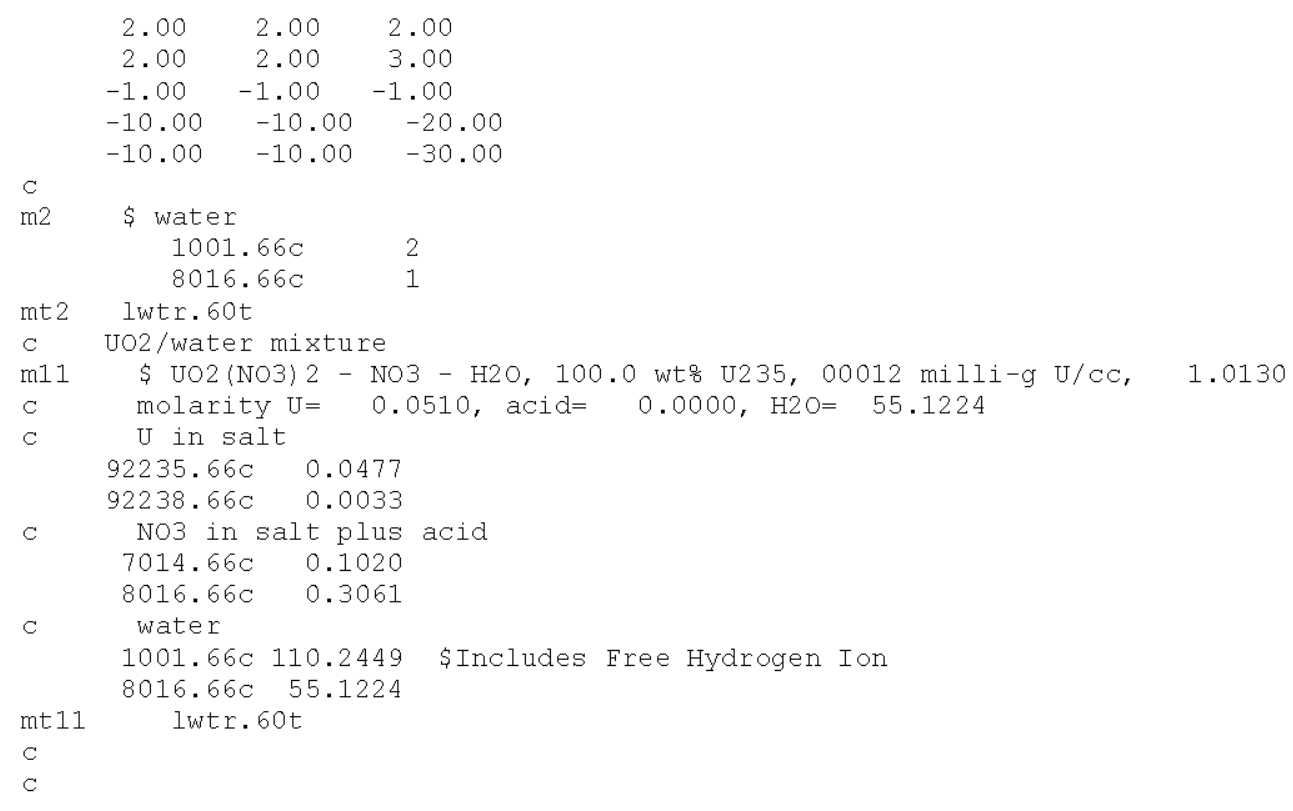




\section{CHPRC-01552, Revision 0}

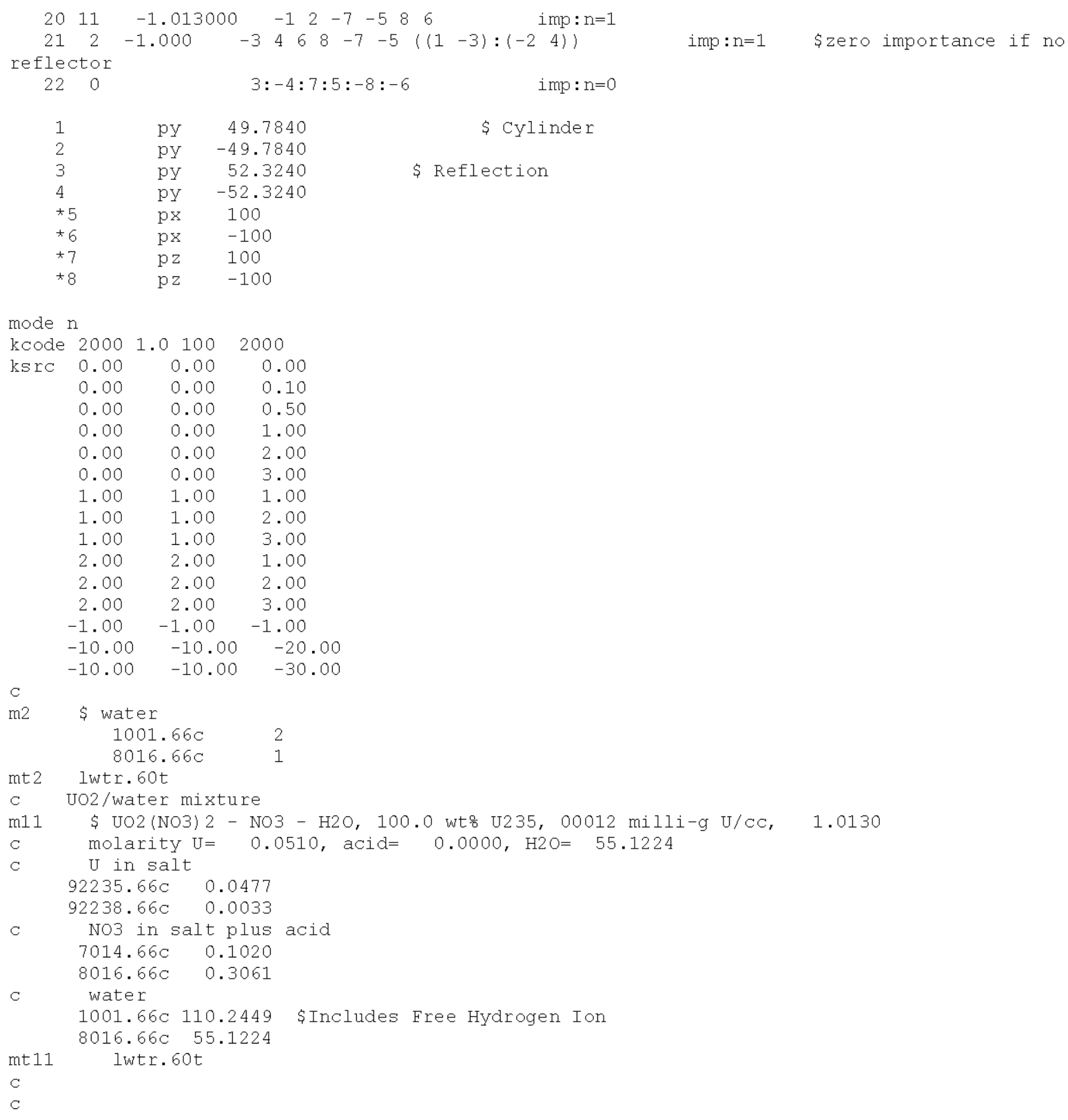




\section{CHPRC-01552, Revision 0}

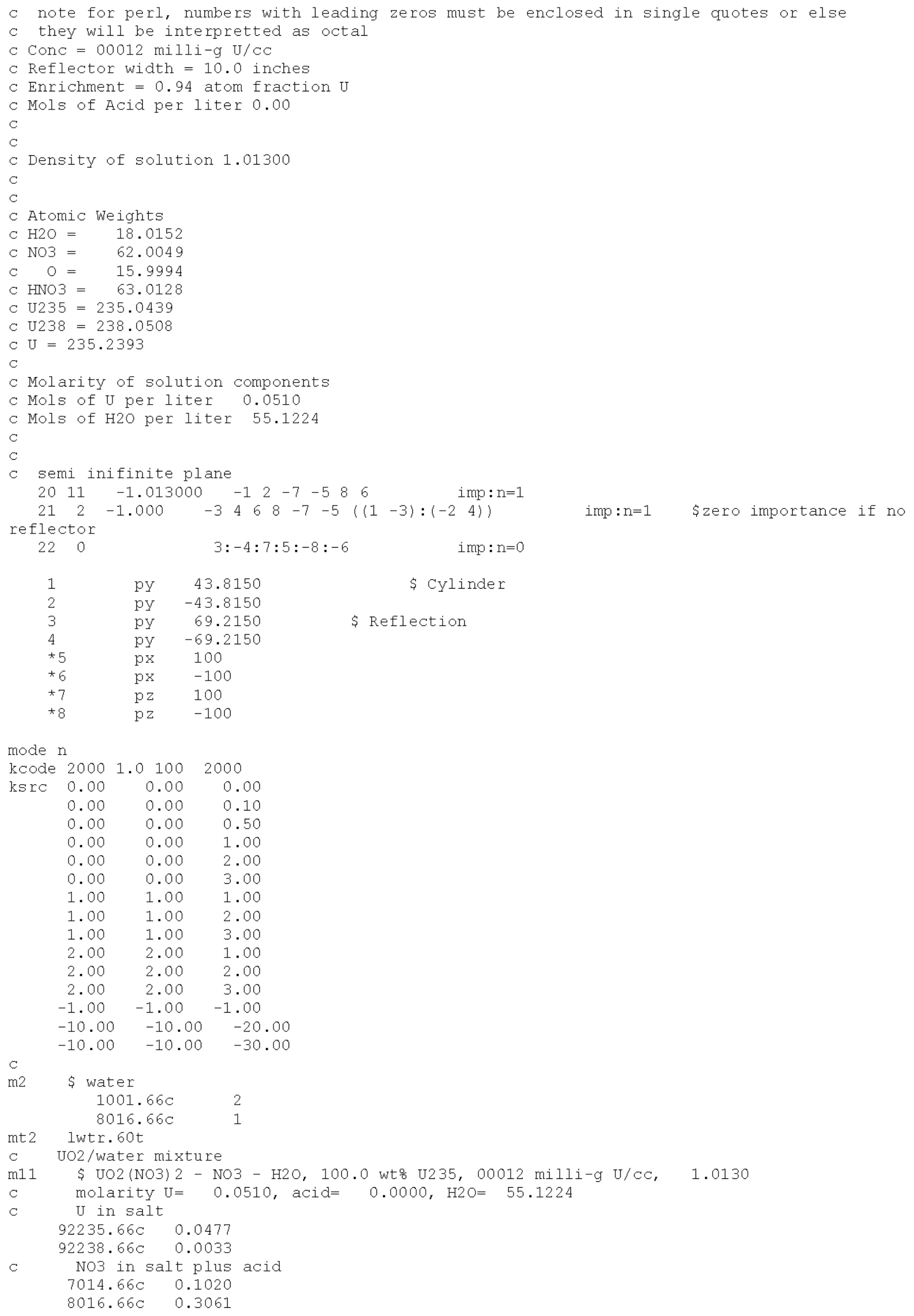


CHPRC-01552, Revision 0

$\begin{array}{lll}\text { C } & \text { water } & \\ & 1001.66 \mathrm{c} & 110.2449 \text { \$Includes Free Hydrogen Ion } \\ & 8016.66 \mathrm{C} \quad 55.1224 & \\ \text { mt11 } & \text { lwtr.60t } \\ \text { C } & & \\ \text { C } & \end{array}$


CHPRC-01552, Revision 0

APPENDIX F - RAW DATA LISTING

F-1 
CHPRC-01552, Revision 0

This page is intentionally left blank. 
Pun40-sp-1000-00

12

21.100 .923100 .00033

21.300 .928580 .00032

21.500 .932530 .00032

21.700 .937200 .00034

21.900 .941430 .00033

22.100 .946030 .00031

22.300 .949410 .00032

22.500 .953290 .00032

22.700 .957810 .00032

22.900 .961640 .00031

23.100 .965050 .00032

23.300 .968210 .00031

23.500 .971140 .00032

23.700 .976680 .00032

23.900 .978970 .00032

24.100 .982460 .00031

24.300 .985900 .00032

24.500 .989330 .00031

24.700 .992250 .00032

24.900 .995780 .00031

25.100 .998240 .00031

25.301 .001940 .00030

25.501 .004830 .00031

25.701 .008180 .00031

25.901 .010950 .00030

26.101 .014270 .00030

26.201 .015370 .00029

25

14.600 .923370 .00044 14.700 .927540 .00043 14.800 .933020 .00043 14.900 .938760 .00045 15.000 .943250 .00044 15.100 .948070 .00044
15

18.100 .922100 .00037

18.200 .925400 .00037

18.300 .928300 .00036

18.400 .931810 .00037

18.500 .935020 .00036

18.600 .938210 .00035

18.700 .941110 .00036

18.800 .944220 .00036

18.900 .946850 .00037

19.000 .949890 .00036

19.100 .952750 .00037

19.200 .954560 .00035

19.300 .958520 .00036

19.400 .961580 .00036

19.500 .964810 .00037

19.600 .966610 .00035

19.700 .969290 .00037

19.800 .972930 .00037

19.900 .975010 .00036

20.000 .977270 .00036

20.100 .979870 .00036

20.200 .982500 .00036

20.300 .985170 .00036

20.400 .986800 .00035

20.500 .990550 .00036

20.600 .992780 .00036

20.700 .995230 .00036

20.800 .998120 .00035

20.901 .000820 .00034

21.001 .002680 .00035

21.101 .005510 .00034

21.201 .007940 .00035

21.301 .009500 .00036

21.401 .012410 .00035

21.501 .013980 .00035

21.601 .016500 .00036

30

13.800 .918950 .00046 13.900 .925630 .00046 14.000 .930140 .00046 14.100 .936190 .00047 14.200 .942420 .00045 14.300 .947700 .00045
20

15.700 .918860 .00041

15.800 .923530 .00041

15.900 .927190 .00042

16.000 .931670 .00041

16.100 .935860 .00042

16.200 .940300 .00042

16.300 .944850 .00040

16.400 .948820 .00042

16.500 .952310 .00041

16.600 .956950 .00041

16.700 .961460 .00041

16.800 .964130 .00041

16.900 .968590 .00041

17.000 .973110 .00041

17.100 .976720 .00041

17.200 .980120 .00040

17.300 .984210 .00040

17.400 .987560 .00040

17.500 .991760 .00040

17.600 .994240 .00041

17.700 .998040 .00040

17.801 .002070 .00041

17.901 .005010 .00041

18.001 .008190 .00041

18.101 .012240 .00039

18.201 .016080 .00042

18.301 .019340 .00041 
15.200 .952530 .00043 15.300 .957460 .00043 15.400 .962230 .00045 15.500 .967580 .00044 15.600 .971670 .00045 15.700 .975510 .00044 15.800 .980930 .00044 15.900 .985040 .00043 16.000 .989030 .00043 16.100 .993910 .00045 16.200 .998460 .00044 16.301 .003360 .00044 16.401 .006670 .00044 16.501 .011500 .00044 16.601 .014790 .00044 16.701 .019430 .00043
14.400 .953060 .00047 14.500 .958510 .00047 14.600 .963780 .00046 14.700 .968510 .00046 14.800 .973100 .00045 14.900 .979170 .00047 15.000 .984400 .00046 15.100 .988700 .00046 15.200 .993620 .00046 15.300 .998500 .00046 15.401 .003180 .00046 15.501 .008600 .00046 15.601 .013090 .00044 15.701 .018420 .00046 15.801 .022770 .00045
14.200 .954350 .00046 14.300 .960050 .00045 14.400 .966260 .00045 14.500 .970820 .00046 14.600 .975330 .00047 14.700 .980710 .00046 14.800 .986280 .00047 14.900 .992440 .00047 15.000 .996550 .00047 15.101 .002220 .00047 15.201 .007510 .00047 15.301 .012630 .00047 15.401 .016870 .00047
35

13.300 .918330 .00050 13.400 .924140 .00048 13.500 .930200 .00047 13.600 .936760 .00047 13.700 .942810 .00048 13.800 .948040 .00047 13.900 .954600 .00050 14.000 .960160 .00048 14.100 .965560 .00048 14.200 .971320 .00049 14.300 .977200 .00048 14.400 .981800 .00047 14.500 .988020 .00047 14.600 .993560 .00047 14.700 .998740 .00048 14.801 .004360 .00047 14.901 .008480 .00048 15.001 .013970 .00049 15.101 .019230 .00047
40

13.000 .921250 .00049

13.050 .924510 .00049

13.100 .927920 .00049

13.150 .931370 .00049

13.200 .934240 .00051

13.250 .937520 .00048

13.300 .941040 .00049

13.350 .942610 .00049

13.400 .946050 .00047

13.450 .949870 .00048

13.500 .953060 .00049

13.550 .956520 .00049

13.600 .958810 .00050

13.650 .961740 .00049

13.700 .965470 .00049

13.750 .968450 .00049

13.800 .970790 .00049

13.850 .973890 .00048

13.900 .977640 .00048

13.950 .979970 .00048

14.000 .982900 .00049

14.050 .986090 .00049

14.100 .988000 .00049

14.150 .991160 .00049

14.200 .993810 .00048

14.250 .996900 .00050

14.301 .000090 .00050

14.351 .001980 .00049

14.401 .006260 .00048

14.451 .008410 .00050 14.501 .010290 .00048
50

12.500 .918930 .00051

12.550 .922070 .00050

12.600 .925500 .00049

12.650 .929690 .00049

12.700 .933070 .00050

12.750 .935860 .00051

12.800 .940070 .00050

12.850 .944030 .00052

12.900 .945910 .00051

12.950 .950390 .00050

13.000 .952760 .00049

13.050 .956470 .00050

13.100 .959100 .00049

13.150 .962560 .00050

13.200 .966120 .00051

13.250 .969260 .00050

13.300 .971890 .00051

13.350 .975620 .00050

13.400 .978800 .00050

13.450 .981620 .00050

13.500 .985490 .00051

13.550 .986640 .00053

13.600 .990570 .00049

13.650 .993720 .00050

13.700 .997150 .00050

13.750 .999630 .00049

13.801 .002660 .00050

13.851 .005760 .00052

13.901 .009140 .00049

13.951 .011760 .00050

14.001 .014750 .00052 
14.551 .012750 .00049 14.601 .016710 .00049 14.651 .019150 .00047 14.701 .022930 .00047

60

12.200 .917720 .00051 12.250 .920980 .00051 12.300 .925490 .00051 12.350 .928020 .00052 12.400 .932020 .00052 12.450 .936040 .00050 12.500 .939910 .00051 12.550 .943110 .00051 12.600 .946850 .00052 12.650 .949830 .00051 12.700 .953300 .00051 12.750 .956550 .00050 12.800 .960980 .00052 12.850 .962740 .00053 12.900 .968400 .00051 12.950 .970450 .00050 13.000 .973850 .00052 13.050 .977200 .00051 13.100 .980970 .00051 13.150 .982870 .00052 13.200 .985860 .00051 13.250 .989530 .00051 13.300 .993510 .00051 13.350 .996220 .00050 13.400 .999310 .00050 13.451 .001950 .00052 13.501 .005710 .00050 13.551 .008520 .00052 13.601 .013140 .00051 13.651 .014860 .00051 13.701 .018730 .00051 13.751 .021410 .00053 13.801 .024730 .00051

90 11.800 .918550 .00053 11.850 .923280 .00052 11.900 .926310 .00052 11.950 .930310 .00053 12.000 .934150 .00052 12.050 .938290 .00053
70

12.000 .916930 .00053 12.050 .920020 .00051

12.100 .925190 .00052

12.150 .927950 .00053

12.200 .931290 .00050

12.250 .935810 .00053

12.300 .937700 .00051

12.350 .942530 .00052

12.400 .945700 .00052

12.450 .950180 .00052

12.500 .952860 .00052

12.550 .955650 .00052

12.600 .960260 .00051

12.650 .962910 .00053

12.700 .966640 .00053

12.750 .970300 .00053

12.800 .972990 .00053

12.850 .977100 .00053

12.900 .980380 .00052

12.950 .984420 .00050

13.000 .987190 .00052

13.050 .990550 .00051

13.100 .992380 .00052

13.150 .996800 .00052

13.200 .999640 .00052

13.251 .002360 .00052

13.301 .006720 .00051

13.351 .009920 .00052

13.401 .013000 .00052

13.451 .015810 .00050

13.501 .019440 .00053
100 11.700 .916390 .00052 11.750 .920170 .00052 11.800 .925510 .00053 11.850 .928410 .00052 11.900 .932220 .00053 11.950 .936070 .00052
14.051 .018280 .00050

14.101 .021290 .00051

14.151 .024150 .00049

14.201 .025920 .00051

80
11.900 .919460 .00052 11.950 .923510 .00052 12.000 .926970 .00051 12.050 .931030 .00052 12.100 .933760 .00052 12.150 .938840 .00054 12.200 .941230 .00053 12.250 .945440 .00052 12.300 .949860 .00051 12.350 .951910 .00052 12.400 .955390 .00051 12.450 .960320 .00053 12.500 .963470 .00053 12.550 .966310 .00052 12.600 .970720 .00051 12.650 .973360 .00053 12.700 .977810 .00052 12.750 .981850 .00052 12.800 .984270 .00053 12.850 .986840 .00052 12.900 .991380 .00054 12.950 .993280 .00052 13.000 .997330 .00052 13.051 .002020 .00050 13.101 .003640 .00052 13.151 .006820 .00053 13.201 .011230 .00052 13.251 .013740 .00052 13.301 .017150 .00053 13.351 .019740 .00053 13.401 .023130 .00052

120

11.600 .916530 .00052 11.650 .921130 .00053 11.700 .924530 .00052 11.750 .927440 .00053 11.800 .931670 .00053 11.850 .936010 .00054 
12.100 .941140 .00053

12.150 .946090 .00052

12.200 .949030 .00051

12.250 .952810 .00054

12.300 .957870 .00051

12.350 .959860 .00054

12.400 .963810 .00052

12.450 .966280 .00054

12.500 .970220 .00052

12.550 .974190 .00052

12.600 .978990 .00051

12.650 .982320 .00053

12.700 .984540 .00052

12.750 .987590 .00053

12.800 .991200 .00052

12.850 .994400 .00053

12.900 .998440 .00053

12.951 .001660 .00053

13.001 .005130 .00052

13.051 .007920 .00052

13.101 .010240 .00053

13.151 .014170 .00052

13.201 .019260 .00054

13.251 .021340 .00055

13.301 .023750 .00053
12.000 .940480 .00053

12.050 .943700 .00052

12.100 .946870 .00053

12.150 .949930 .00051

12.200 .955000 .00051

12.250 .958670 .00053

12.300 .962650 .00053

12.350 .965050 .00054

12.400 .969010 .00053

12.450 .972510 .00053

12.500 .975370 .00054

12.550 .980610 .00053

12.600 .983620 .00053

12.650 .987330 .00053

12.700 .990220 .00054

12.750 .993090 .00053

12.800 .997220 .00053

12.851 .000350 .00052

12.901 .003260 .00053

12.951 .008620 .00053

13.001 .009460 .00053

13.051 .014230 .00054

13.101 .016530 .00052

13.151 .020720 .00053

13.201 .023930 .00052
11.900 .939830 .00052

11.950 .943200 .00053

12.000 .946790 .00054

12.050 .951040 .00054

12.100 .954830 .00053

12.150 .958270 .00052

12.200 .961990 .00053

12.250 .964900 .00053

12.300 .968390 .00053

12.350 .972370 .00053

12.400 .975880 .00052

12.450 .980550 .00052

12.500 .983170 .00053

12.550 .985940 .00052

12.600 .990030 .00052

12.650 .994590 .00053

12.700 .997220 .00053

12.751 .000890 .00053

12.801 .003810 .00053

12.851 .006900 .00053

12.901 .010370 .00053

12.951 .013520 .00055

13.001 .017720 .00053

13.051 .020280 .00053

13.101 .023760 .00053
130

11.600 .919590 .00053

11.650 .924680 .00052

11.700 .927540 .00054

11.750 .931660 .00054

11.800 .935200 .00054

11.850 .939930 .00051

11.900 .942670 .00052

11.950 .946760 .00052

12.000 .950350 .00054

12.050 .954890 .00054

12.100 .958370 .00053

12.150 .961760 .00054

12.200 .965250 .00052

12.250 .968560 .00053

12.300 .972840 .00054

12.350 .976680 .00053

12.400 .979520 .00052

12.450 .983240 .00053

12.500 .987360 .00054

12.550 .990150 .00055

12.600 .992950 .00053

12.650 .996740 .00053
150

11.500 .915520 .00053

11.550 .919890 .00052

11.600 .923580 .00052

11.650 .926870 .00051

11.700 .930360 .00052

11.750 .934210 .00054

11.800 .938320 .00052

11.850 .941400 .00053

11.900 .946740 .00054

11.950 .950360 .00052

12.000 .953990 .00054

12.050 .956240 .00052

12.100 .961050 .00054

12.150 .965050 .00054

12.200 .967840 .00053

12.250 .971300 .00053

12.300 .974170 .00052

12.350 .979640 .00054

12.400 .982380 .00053

12.450 .986940 .00052

12.500 .990020 .00054

12.550 .992800 .00054
200

11.400 .912700 .00053

11.450 .915870 .00053

11.500 .918660 .00053

11.550 .923620 .00053

11.600 .927980 .00055

11.650 .931720 .00054

11.700 .934940 .00053

11.750 .939100 .00052

11.800 .942340 .00054

11.850 .946520 .00053

11.900 .950730 .00053

11.950 .953860 .00052

12.000 .958370 .00053

12.050 .961000 .00053

12.100 .965450 .00054

12.150 .969350 .00053

12.200 .971290 .00052

12.250 .975840 .00053

12.300 .979940 .00053

12.350 .984820 .00052

12.400 .986200 .00054

12.450 .991240 .00052 
12.701 .000170 .00054

12.751 .004160 .00053

12.801 .008020 .00052

12.851 .011580 .00055

12.901 .015170 .00052

12.951 .017230 .00053

13.001 .020980 .00053

239

11.500 .920000 .00054 11.550 .924020 .00053 11.600 .928370 .00053 11.650 .932110 .00053 11.700 .935920 .00053 11.750 .940170 .00054 11.800 .943740 .00053 11.850 .947390 .00052 11.900 .950950 .00052 11.950 .954020 .00052 12.000 .957790 .00052 12.050 .962580 .00053 12.100 .966660 .00052 12.150 .969510 .00055 12.200 .972770 .00054 12.250 .975990 .00054 12.300 .980470 .00055 12.350 .983620 .00053 12.400 .987390 .00053 12.450 .991250 .00054 12.500 .994820 .00053 12.550 .998510 .00053 12.601 .002360 .00053 12.651 .004910 .00053 12.701 .008210 .00052 12.751 .011510 .00054 12.801 .015120 .00053 12.851 .017990 .00053 12.901 .021170 .00053

478

11.700 .920510 .00054 11.750 .923950 .00052 11.800 .928130 .00052
12.600 .996190 .00054

12.651 .000700 .00053

12.701 .003530 .00053

12.751 .006350 .00053

12.801 .011280 .00054

12.851 .013920 .00054

12.901 .016700 .00053

12.951 .020700 .00053

13.001 .024320 .00052

250

11.400 .912300 .00053

11.450 .916190 .00054

11.500 .920140 .00053

11.550 .924730 .00055

11.600 .928130 .00052

11.650 .932030 .00054

11.700 .935320 .00053

11.750 .938770 .00054

11.800 .942830 .00051

11.850 .946870 .00051

11.900 .951870 .00052

11.950 .954140 .00053

12.000 .957650 .00054

12.050 .962130 .00052

12.100 .965750 .00054

12.150 .968810 .00053

12.200 .972940 .00054

12.250 .976440 .00054

12.300 .980200 .00053

12.350 .983420 .00052

12.400 .987900 .00052

12.450 .990070 .00052

12.500 .994220 .00054

12.550 .998180 .00052

12.601 .000800 .00054

12.651 .004800 .00051

12.701 .008010 .00054

12.751 .011330 .00053

12.801 .014660 .00053

12.851 .017510 .00054

12.901 .021510 .00052

12.500 .993640 .00055

12.550 .996960 .00053

12.600 .999570 .00054

12.651 .004400 .00052

12.701 .007340 .00053

12.751 .011250 .00055

12.801 .014800 .00053

12.851 .017640 .00053

12.901 .022050 .00052
11.500 .916480 .00052

11.550 .919830 .00053

11.600 .923800 .00052

11.650 .928810 .00051

11.700 .932980 .00052

11.750 .935510 .00052

11.800 .939330 .00053

11.850 .943200 .00052

11.900 .946440 .00053

11.950 .949140 .00053

12.000 .953360 .00050

12.050 .957870 .00053

12.100 .961440 .00052

12.150 .965440 .00051

12.200 .968870 .00053

12.250 .971960 .00052

12.300 .975790 .00053

12.350 .979210 .00053

12.400 .983000 .00053

12.450 .985930 .00053

12.500 .990030 .00053

12.550 .994170 .00053

12.600 .997180 .00053

12.651 .000300 .00054

12.701 .003400 .00054

12.751 .007030 .00053

12.801 .010410 .00052

12.851 .014060 .00050

12.901 .017700 .00053

12.951 .020210 .00053

13.001 .024090 .00052
359
500

11.700 .917750 .00051

11.750 .920980 .00052

11.800 .925230 .00052
600

12.000 .916200 .00054

12.050 .920480 .00050

12.100 .923430 .00051 
11.850 .932760 .00053 11.900 .935230 .00051 11.950 .940870 .00053 12.000 .942450 .00051 12.050 .947590 .00051 12.100 .950990 .00052 12.150 .954370 .00051 12.200 .957590 .00052 12.250 .962820 .00052 12.300 .965130 .00052 12.350 .968800 .00051 12.400 .972500 .00053 12.450 .976590 .00052 12.500 .979730 .00052 12.550 .982440 .00053 12.600 .986970 .00052 12.650 .990520 .00053 12.700 .992740 .00052 12.750 .996000 .00052 12.800 .999930 .00051 12.851 .003380 .00052 12.901 .006730 .00052 12.951 .010070 .00051 13.001 .013970 .00052 13.051 .017480 .00054 13.101 .020740 .00052 13.151 .020420 .00053 13.201 .023780 .00052

650

12.300 .917820 .00052 12.350 .921480 .00051 12.400 .924290 .00052 12.450 .928650 .00052 12.500 .931610 .00052 12.550 .935580 .00051 12.600 .939130 .00051 12.650 .942600 .00051 12.700 .946610 .00051 12.750 .950000 .00053 12.800 .952540 .00050 12.850 .956790 .00051 12.900 .960350 .00052 12.950 .963500 .00052 13.000 .967290 .00052 13.050 .970130 .00052 13.100 .973510 .00051
11.850 .928680 .00052 11.900 .931780 .00050 11.950 .937030 .00051 12.000 .940220 .00052 12.050 .943410 .00054 12.100 .947770 .00052 12.150 .951660 .00051 12.200 .954960 .00053 12.250 .959010 .00053 12.300 .961940 .00053 12.350 .966750 .00053 12.400 .969880 .00053 12.450 .973000 .00052 12.500 .976430 .00052 12.550 .980180 .00052 12.600 .983200 .00051 12.650 .986790 .00051 12.700 .990460 .00053 12.750 .993310 .00052 12.800 .997700 .00052 12.851 .001550 .00053 12.901 .003410 .00051 12.951 .007790 .00053 13.001 .011440 .00053 13.051 .013700 .00052 13.101 .017150 .00053 13.451 .016640 .00052 13.501 .020000 .00052 13.551 .023580 .00052 13.601 .025510 .00053

700

12.800 .918010 .00052 12.850 .921990 .00051 12.900 .925050 .00051 12.950 .928430 .00052 13.000 .932230 .00050 13.050 .935420 .00051 13.100 .938570 .00050 13.150 .942930 .00053 13.200 .945660 .00052 13.250 .949670 .00052 13.300 .953550 .00052 13.350 .955680 .00051 13.400 .958260 .00052 13.450 .962460 .00052 13.500 .966040 .00052 13.550 .969820 .00053 13.600 .972180 .00050
12.150 .927280 .00051 12.200 .931330 .00052 12.250 .935070 .00052 12.300 .937500 .00052 12.350 .942610 .00051 12.400 .945360 .00052 12.450 .948710 .00051 12.500 .952430 .00053 12.550 .956450 .00052 12.600 .959250 .00051 12.650 .963470 .00053 12.700 .966600 .00053 12.750 .968750 .00053 12.800 .973980 .00052 12.850 .977300 .00053 12.900 .980570 .00052 12.950 .984290 .00051 13.000 .987000 .00053 13.050 .990110 .00051 13.100 .993730 .00052 13.150 .997510 .00050 13.201 .000550 .00053 13.251 .004030 .00052 13.301 .006320 .00053 13.351 .010630 .00052 13.401 .014280 .00052
750
14.100 .916160 .00052 14.200 .923110 .00051 14.300 .928620 .00051 14.400 .935370 .00052 14.500 .940490 .00051 14.600 .947680 .00053 14.700 .953100 .00051 14.800 .960340 .00051 14.900 .965270 .00052 15.000 .972580 .00052 15.100 .977240 .00051 15.200 .983830 .00052 15.300 .989530 .00052 15.400 .995210 .00051 15.501 .001320 .00051 15.601 .007160 .00052 15.701 .011410 .00052 
13.150 .977390 .00051 13.200 .980510 .00053 13.250 .983820 .00053 13.300 .987500 .00051 13.350 .990950 .00053 13.400 .993850 .00051 13.450 .997680 .00051 13.500 .999630 .00052 13.551 .003360 .00051 13.601 .007650 .00052 13.651 .009640 .00052 13.701 .012660 .00051 13.751 .017070 .00052 13.801 .019830 .00052 13.851 .022770 .00051 13.901 .025690 .00052
13.650 .976540 .00052 13.700 .978190 .00052 13.750 .981450 .00051 13.800 .984520 .00053 13.850 .987610 .00052 13.900 .992490 .00052 13.950 .994340 .00052 14.000 .997640 .00051 14.051 .001190 .00052 14.101 .003640 .00051 14.151 .007490 .00052 14.201 .009780 .00050 14.251 .012770 .00050 14.301 .017090 .00051 14.351 .019810 .00052 14.401 .023120 .00053
752

14.300 .917900 .00052 14.400 .923560 .00050 14.500 .930660 .00051 14.600 .937000 .00052 14.700 .943310 .00052 14.800 .949030 .00051 14.900 .955700 .00051 15.000 .961770 .00052 15.100 .966500 .00052 15.200 .973460 .00051 15.300 .978600 .00052 15.400 .984740 .00051 15.500 .991450 .00051 15.600 .995130 .00050 15.701 .002170 .00052 15.801 .006990 .00051 15.901 .013330 .00052 16.001 .019020 .00053 16.101 .024720 .00052
754

14.500 .916140 .00052 14.600 .921940 .00050 14.700 .928760 .00051 14.800 .933640 .00053 14.900 .940350 .00051 15.000 .947050 .00054 15.100 .953190 .00051 15.200 .958400 .00051 15.300 .965070 .00051 15.400 .970440 .00054 15.500 .976760 .00051 15.600 .981790 .00050 15.700 .988110 .00052 15.800 .993770 .00053 15.900 .999680 .00052 16.001 .003980 .00051 16.101 .010370 .00051 16.201 .016810 .00051 16.301 .021190 .00052
15.801 .018230 .00051 15.901 .024100 .00052

$\begin{array}{ccc} & 755 \\ 14.80 & 0.91844 & 0.00052 \\ 14.90 & 0.92467 & 0.00051 \\ 15.00 & 0.93035 & 0.00052 \\ 15.10 & 0.93600 & 0.00051 \\ 15.20 & 0.94324 & 0.00053 \\ 15.30 & 0.94851 & 0.00052 \\ 15.40 & 0.95464 & 0.00051 \\ 15.50 & 0.95974 & 0.00052 \\ 15.60 & 0.96609 & 0.00051 \\ 15.70 & 0.97166 & 0.00052 \\ 15.80 & 0.97798 & 0.00052 \\ 15.90 & 0.98313 & 0.00052 \\ 16.00 & 0.98920 & 0.00051 \\ 16.10 & 0.99408 & 0.00051 \\ 16.20 & 0.99940 & 0.00051 \\ 16.30 & 1.00573 & 0.00052 \\ 16.40 & 1.00970 & 0.00052 \\ 16.50 & 1.01614 & 0.00052 \\ 16.60 & 1.02208 & 0.00052\end{array}$


Pun40-sp-1000-01

12

19.600 .924450 .00032 19.800 .928260 .00032 20.000 .932960 .00032 20.200 .937260 .00032 20.400 .941170 .00030 20.600 .945680 .00031 20.800 .949640 .00031 21.000 .953380 .00031 21.200 .957520 .00031 21.400 .961940 .00031 21.600 .964830 .00031 21.800 .968010 .00030 22.000 .972000 .00030 22.200 .975720 .00031 22.400 .978910 .00030 22.600 .982000 .00030 22.800 .985810 .00029 23.000 .989320 .00030 23.200 .992730 .00029 23.400 .996120 .00029 23.600 .998910 .00029 23.801 .001620 .00030 24.001 .004980 .00030 24.201 .008170 .00030 24.401 .010280 .00030 24.601 .014140 .00029 24.701 .014780 .00029
25

13.100 .923800 .00043 13.200 .928730 .00042 13.300 .933170 .00043 13.400 .938690 .00043 13.500 .943600 .00043 13.600 .947880 .00042 13.700 .953080 .00042
15

16.600 .922590 .00036 16.700 .924880 .00035 16.800 .928200 .00036 16.900 .931250 .00035 17.000 .934890 .00035 17.100 .938390 .00034 17.200 .941260 .00035 17.300 .944210 .00035 17.400 .947030 .00034 17.500 .949830 .00035 17.600 .952430 .00035 17.700 .955770 .00034 17.800 .957890 .00035 17.900 .961100 .00035 18.000 .964510 .00035 18.100 .966550 .00034 18.200 .970220 .00035 18.300 .972420 .00035 18.400 .975320 .00035 18.500 .977880 .00035 18.600 .979940 .00034 18.700 .983130 .00035 18.800 .985320 .00035 18.900 .988970 .00036 19.000 .990480 .00034 19.100 .993250 .00035 19.200 .996450 .00034 19.300 .997970 .00036 19.401 .000090 .00035 19.501 .002820 .00034 19.601 .004650 .00035 19.701 .007760 .00033 19.801 .010270 .00035 19.901 .011690 .00036 20.001 .014920 .00035 20.101 .016230 .00034 20.201 .019720 .00034

30

12.300 .918790 .00044 12.400 .925530 .00044 12.500 .930640 .00044 12.600 .936360 .00044 12.700 .941860 .00044 12.800 .947850 .00044 12.900 .953730 .00047
20

14.200 .919680 .00039 14.300 .924130 .00040 14.400 .928280 .00040 14.500 .932660 .00040 14.600 .936290 .00040 14.700 .940290 .00041 14.800 .944530 .00039 14.900 .949000 .00040 15.000 .952670 .00040 15.100 .957390 .00040 15.200 .961960 .00040 15.300 .965200 .00040 15.400 .968990 .00040 15.500 .972660 .00040 15.600 .976090 .00040 15.700 .979980 .00040 15.800 .984620 .00040 15.900 .987800 .00040 16.000 .991470 .00038 16.100 .994400 .00039 16.200 .998500 .00039 16.301 .001910 .00040 16.401 .005480 .00039 16.501 .008730 .00040 16.601 .012800 .00040 16.701 .016290 .00039 
13.800 .957540 .00043 13.900 .962720 .00042 14.000 .968400 .00043 14.100 .971940 .00042 14.200 .977000 .00042 14.300 .981350 .00043 14.400 .985000 .00042 14.500 .990750 .00043 14.600 .994550 .00043 14.700 .999090 .00042 14.801 .003850 .00043 14.901 .007730 .00043 15.001 .010920 .00042 15.101 .016040 .00043 15.201 .019530 .00043

35

11.800 .919570 .00047 11.900 .925300 .00046 12.000 .931640 .00045 12.100 .936540 .00045 12.200 .943030 .00046 12.300 .948980 .00044 12.400 .955830 .00045 12.500 .961310 .00046 12.600 .966160 .00046 12.700 .972580 .00045 12.800 .977190 .00046 12.900 .982490 .00045 13.000 .987630 .00045 13.100 .994270 .00046 13.200 .999530 .00046 13.301 .004930 .00047 13.401 .009750 .00045 13.501 .015100 .00045 13.601 .020520 .00045
13.000 .958250 .00044 13.100 .963780 .00044 13.200 .969730 .00044 13.300 .975130 .00045 13.400 .979250 .00044 13.500 .985030 .00044 13.600 .988810 .00046 13.700 .994030 .00045 13.801 .000210 .00044 13.901 .005470 .00045 14.001 .008970 .00044 14.101 .014110 .00045 14.201 .018350 .00043
12.800 .959720 .00044 12.900 .966590 .00045 13.000 .971560 .00044 13.100 .976580 .00044 13.200 .982120 .00045 13.300 .987210 .00045 13.400 .992140 .00045 13.500 .997940 .00045 13.601 .003580 .00046 13.701 .007460 .00047 13.801 .012330 .00044 13.901 .017520 .00046 14.001 .021940 .00043
40

11.400 .916250 .00047 11.500 .922300 .00047 11.600 .928450 .00047 11.700 .935040 .00047 11.800 .941570 .00047 11.900 .947490 .00047 12.000 .954160 .00048 12.100 .960170 .00046 12.200 .966070 .00047 12.300 .971850 .00046 12.400 .977590 .00047 12.500 .983670 .00048 12.600 .989550 .00047 12.700 .995380 .00046 12.801 .000100 .00047 12.901 .006490 .00046 13.001 .012140 .00045 13.101 .018980 .00046 13.201 .023150 .00047
50

11.000 .920410 .00048 11.050 .923350 .00049 11.100 .926870 .00048 11.150 .930320 .00048 11.200 .935100 .00048 11.250 .937480 .00048 11.300 .940430 .00047 11.350 .943140 .00048 11.400 .946910 .00048 11.450 .950820 .00049 11.500 .953900 .00047 11.550 .958070 .00048 11.600 .960310 .00049 11.650 .963330 .00047 11.700 .966810 .00048 11.750 .970440 .00048 11.800 .973310 .00048 11.850 .977330 .00047 11.900 .978960 .00048 11.950 .981880 .00048 12.000 .985570 .00048 12.050 .988360 .00047 12.100 .991500 .00048 12.150 .995000 .00048 12.200 .997760 .00047 12.251 .000850 .00048 12.301 .004970 .00048 12.351 .007190 .00050 12.401 .010180 .00047 12.451 .012960 .00048 12.501 .016620 .00048 12.551 .018890 .00048 12.601 .022290 .00047 
60

10.700 .920260 .00048

10.750 .923800 .00047

10.800 .926860 .00048

10.850 .929200 .00049

10.900 .933480 .00049

10.950 .937730 .00049

11.000 .940610 .00049

11.050 .943910 .00049

11.100 .947430 .00050

11.150 .950370 .00049

11.200 .954870 .00049

11.250 .957330 .00050

11.300 .961010 .00047

11.350 .964200 .00050

11.400 .967770 .00049

11.450 .971700 .00050

11.500 .974550 .00051

11.550 .978580 .00049

11.600 .981330 .00050

11.650 .985190 .00050

11.700 .987640 .00049

11.750 .990730 .00050

11.800 .994440 .00049

11.850 .997700 .00050

11.901 .001190 .00049

11.951 .002960 .00050

12.001 .006770 .00050

12.051 .010030 .00048

12.101 .013790 .00048

12.151 .016460 .00049

12.201 .019270 .00047

90

10.300 .921170 .00051

10.350 .924560 .00050

10.400 .929460 .00052

10.450 .932550 .00050

10.500 .935910 .00052

10.550 .939600 .00051

10.600 .942680 .00050

10.650 .946260 .00049

10.700 .952130 .00050

10.750 .953610 .00051

10.800 .958240 .00050

10.850 .960690 .00050

10.900 .965730 .00051
70

10.500 .918780 .00049

10.550 .921900 .00049

10.600 .924980 .00050

10.650 .929840 .00050

10.700 .933240 .00050

10.750 .936010 .00050

10.800 .939230 .00050

10.850 .943630 .00048

10.900 .947020 .00049

10.950 .951410 .00050

11.000 .954460 .00050

11.050 .957720 .00050

11.100 .961190 .00050

11.150 .964370 .00050

11.200 .967600 .00049

11.250 .972390 .00049

11.300 .975210 .00051

11.350 .977540 .00051

11.400 .980910 .00051

11.450 .985330 .00050

11.500 .988050 .00050

11.550 .992110 .00049

11.600 .996040 .00049

11.650 .997880 .00049

11.701 .001090 .00050

11.751 .004580 .00048

11.801 .008730 .00049

11.851 .011600 .00050

11.901 .014550 .00051

11.951 .018450 .00049

12.001 .021530 .00050
80

10.400 .920160 .00049

10.450 .925460 .00052

10.500 .927750 .00049

10.550 .932920 .00050

10.600 .935620 .00049

10.650 .939850 .00049

10.700 .942260 .00051

10.750 .946010 .00050

10.800 .950150 .00050

10.850 .954140 .00051

10.900 .957400 .00051

10.950 .959890 .00050

11.000 .964860 .00051

11.050 .967890 .00050

11.100 .971740 .00050

11.150 .975880 .00050

11.200 .977720 .00051

11.250 .981980 .00050

11.300 .986280 .00049

11.350 .989330 .00051

11.400 .992690 .00049

11.450 .995530 .00050

11.500 .998700 .00051

11.551 .003070 .00051

11.601 .005730 .00049

11.651 .009540 .00051

11.701 .012540 .00051

11.751 .015990 .00052

11.801 .019270 .00051

100

120

10.200 .918440 .00050

10.250 .922670 .00052

10.300 .926280 .00050

10.350 .931100 .00050

10.400 .934730 .00052

10.450 .939150 .00050

10.500 .942030 .00051

10.550 .945750 .00050

10.600 .949810 .00050

10.650 .953250 .00050

10.700 .957200 .00053

10.750 .960310 .00051

10.800 .964100 .00051
10.100 .918930 .00050

10.150 .922830 .00049

10.200 .925940 .00051

10.250 .930090 .00051

10.300 .934070 .00050

10.350 .938530 .00051

10.400 .941760 .00050

10.450 .944220 .00052

10.500 .949630 .00051

10.550 .953020 .00052

10.600 .955800 .00050

10.650 .959320 .00051

10.700 .965100 .00050 
10.950 .969200 .00051 11.000 .972910 .00052 11.050 .975430 .00050 11.100 .979700 .00050 11.150 .983410 .00049 11.200 .985510 .00053 11.250 .989570 .00049 11.300 .994530 .00051 11.350 .996630 .00051 11.401 .000070 .00049 11.451 .004460 .00051 11.501 .006880 .00050 11.551 .009700 .00049 11.601 .014120 .00051 11.651 .017100 .00049 11.701 .020670 .00051
10.850 .968160 .00050 10.900 .971420 .00051 10.950 .974000 .00051 11.000 .978590 .00050 11.050 .982140 .00052 11.100 .985020 .00051 11.150 .989320 .00051 11.200 .991960 .00050 11.250 .994960 .00051 11.300 .999020 .00052 11.351 .001950 .00051 11.401 .005870 .00051 11.451 .010040 .00052 11.501 .013250 .00052 11.551 .015470 .00053 11.601 .019420 .00052
10.750 .967860 .00051 10.800 .970560 .00051 10.850 .975400 .00050 10.900 .977990 .00051 10.950 .980610 .00052 11.000 .984700 .00052 11.050 .988730 .00051 11.100 .992640 .00051 11.150 .995850 .00051 11.200 .999820 .00052 11.251 .003560 .00049 11.301 .006740 .00049 11.351 .009010 .00051 11.401 .012780 .00051 11.451 .016450 .00051 11.501 .019680 .00051
130

10.100 .923020 .00052 10.150 .925760 .00050 10.200 .930080 .00050 10.250 .933940 .00051 10.300 .937790 .00052 10.350 .940860 .00051 10.400 .945500 .00052 10.450 .949260 .00051 10.500 .952300 .00051 10.550 .955720 .00052 10.600 .960500 .00051 10.650 .963910 .00051 10.700 .967790 .00052 10.750 .971400 .00053 10.800 .974460 .00051 10.850 .978200 .00051 10.900 .981760 .00052 10.950 .985410 .00051 11.000 .988040 .00052 11.050 .992200 .00051 11.100 .996560 .00052 11.151 .000650 .00051 11.201 .003180 .00051 11.251 .006340 .00050 11.301 .009550 .00051 11.351 .013270 .00051 11.401 .017150 .00052 11.451 .020250 .00052 11.501 .023520 .00051
150

10.000 .917490 .00051 10.050 .920950 .00050 10.100 .924860 .00049 10.150 .929820 .00051 10.200 .934110 .00051 10.250 .937370 .00051 10.300 .941490 .00052 10.350 .944500 .00052 10.400 .948240 .00050 10.450 .951090 .00051 10.500 .955010 .00052 10.550 .959040 .00052 10.600 .962700 .00051 10.650 .966930 .00052 10.700 .969360 .00052 10.750 .974910 .00052 10.800 .977320 .00051 10.850 .981390 .00051 10.900 .985110 .00051 10.950 .989310 .00050 11.000 .992810 .00051 11.050 .995780 .00052 11.100 .998750 .00052 11.151 .002600 .00051 11.201 .006680 .00051 11.251 .009940 .00051 11.301 .012770 .00051 11.351 .016200 .00053 11.401 .018810 .00051
200

10.000 .922030 .00050 10.050 .925510 .00052 10.100 .928540 .00052 10.150 .934000 .00052 10.200 .937240 .00051 10.250 .941060 .00051 10.300 .944940 .00051 10.350 .949370 .00051 10.400 .952880 .00051 10.450 .956170 .00051 10.500 .959670 .00051 10.550 .963790 .00053 10.600 .967540 .00051 10.650 .971340 .00050 10.700 .974030 .00052 10.750 .978210 .00051 10.800 .981750 .00052 10.850 .985310 .00052 10.900 .987400 .00053 10.950 .992410 .00052 11.000 .996670 .00051 11.050 .999670 .00050 11.101 .003570 .00050 11.151 .006330 .00050 11.201 .008800 .00052 11.251 .013050 .00052 11.301 .016330 .00051 11.351 .020490 .00051 11.401 .022850 .00052 


\begin{abstract}
239
10.000 .922770 .00051 10.050 .926750 .00051 10.100 .930100 .00051 10.150 .933280 .00050 10.200 .938340 .00051 10.250 .940650 .00050 10.300 .945990 .00051 10.350 .948890 .00052 10.400 .953030 .00050 10.450 .956960 .00052 10.500 .959380 .00051 10.550 .964230 .00051 10.600 .967850 .00052 10.650 .971520 .00052 10.700 .974700 .00051 10.750 .979160 .00052 10.800 .982560 .00051 10.850 .985370 .00051 10.900 .988550 .00052 10.950 .993050 .00051 11.000 .996290 .00050 11.051 .000640 .00051 11.101 .003050 .00052 11.151 .007150 .00052 11.201 .009460 .00052 11.251 .012240 .00051 11.301 .016750 .00051 11.351 .020130 .00051 11.401 .024500 .00051
\end{abstract}

250

10.000 .922130 .00052 10.050 .926440 .00051 10.100 .929110 .00050 10.150 .933320 .00051 10.200 .937260 .00053 10.250 .941600 .00052 10.300 .944060 .00051 10.350 .948290 .00054 10.400 .952520 .00052 10.450 .955620 .00051 10.500 .958790 .00052 10.550 .963500 .00051 10.600 .966920 .00052 10.650 .971510 .00052 10.700 .974570 .00049 10.750 .978160 .00050 10.800 .980960 .00052 10.850 .985490 .00053 10.900 .989160 .00051 10.950 .992890 .00052 11.000 .996370 .00051 11.050 .999220 .00051 11.101 .003290 .00051 11.151 .006720 .00052 11.201 .009130 .00051 11.251 .012090 .00052 11.301 .016650 .00052 11.351 .020380 .00050 11.401 .023100 .00051
478

10.200 .922080 .00050 10.250 .925650 .00051 10.300 .930040 .00051 10.350 .934160 .00050 10.400 .936850 .00050 10.450 .941280 .00051 10.500 .944790 .00051 10.550 .948830 .00050 10.600 .952890 .00050 10.650 .954960 .00051 10.700 .959940 .00050 10.750 .962700 .00050 10.800 .967500 .00054 10.850 .970420 .00052
500

10.200 .918810 .00050 10.250 .923490 .00051 10.300 .927640 .00052 10.350 .931610 .00053 10.400 .934040 .00051 10.450 .939290 .00052 10.500 .940930 .00051 10.550 .945570 .00052 10.600 .950170 .00052 10.650 .953450 .00051 10.700 .956470 .00052 10.750 .960910 .00051 10.800 .963920 .00051 10.850 .967070 .00050
359

10.000 .916080 .00050 10.050 .921300 .00051 10.100 .924940 .00051 10.150 .928790 .00051 10.200 .932490 .00050 10.250 .936170 .00052 10.300 .939570 .00049 10.350 .944010 .00052 10.400 .948870 .00051 10.450 .951160 .00051 10.500 .955580 .00049 10.550 .959490 .00052 10.600 .963100 .00051 10.650 .966240 .00049 10.700 .970170 .00050 10.750 .974330 .00052 10.800 .976220 .00053 10.850 .981220 .00051 10.900 .985440 .00051 10.950 .987080 .00051 11.000 .991350 .00052 11.050 .994820 .00050 11.100 .997810 .00050 11.151 .002430 .00052 11.201 .004890 .00053 11.251 .009200 .00050 11.301 .012250 .00051 11.351 .015690 .00052 11.401 .018000 .00050 11.451 .021800 .00051 11.501 .024700 .00050

600 10.500 .919800 .00051 10.550 .923200 .00051 10.600 .927390 .00051 10.650 .930750 .00052 10.700 .933940 .00052 10.750 .939410 .00053 10.800 .943150 .00051 10.850 .944930 .00052 10.900 .948550 .00051 10.950 .953070 .00051 11.000 .956650 .00052 11.050 .959810 .00050 11.100 .963090 .00051 11.150 .966740 .00052 
10.900 .974600 .00051 10.950 .977340 .00051 11.000 .981340 .00051 11.050 .985300 .00051 11.100 .988480 .00050 11.150 .992070 .00052 11.200 .994610 .00052 11.301 .001850 .00051 11.351 .005850 .00051 11.401 .009180 .00052 11.451 .012470 .00050 11.501 .015590 .00051 11.551 .018840 .00051 11.601 .021350 .00050
650

10.700 .916980 .00050 10.750 .920950 .00051 10.800 .923860 .00050 10.850 .928180 .00051 10.900 .931240 .00051 10.950 .934800 .00052 11.000 .938360 .00051 11.050 .942640 .00051 11.100 .945110 .00051 11.150 .948470 .00050 11.200 .952580 .00051 11.250 .956700 .00050 11.300 .959570 .00051 11.350 .963360 .00051 11.400 .967140 .00053 11.450 .970370 .00051 11.500 .972530 .00050 11.550 .976800 .00051 11.600 .980240 .00051 11.650 .984010 .00051 11.700 .985820 .00050 11.750 .989620 .00051 11.800 .993160 .00049 11.850 .996400 .00051 11.901 .000560 .00051 11.951 .003570 .00052 12.001 .006750 .00051 12.051 .009240 .00051 12.101 .011860 .00052 12.151 .016420 .00051
10.900 .969780 .00051 10.950 .973850 .00052 11.000 .977310 .00051 11.050 .981530 .00053 11.100 .985700 .00051 11.150 .988650 .00051 11.200 .992880 .00051 11.250 .995310 .00052 11.300 .999510 .00050 11.351 .002690 .00052 11.401 .004800 .00051 11.451 .008780 .00051 11.501 .012580 .00051 11.551 .015430 .00053 11.601 .018890 .00053 11.651 .022960 .00050 11.701 .024580 .00051
11.250 .973680 .00050 11.300 .977580 .00050 11.350 .980730 .00050 11.400 .983810 .00050 11.450 .987380 .00051 11.500 .990680 .00051 11.550 .994330 .00049 11.600 .997850 .00050 11.650 .999700 .00053 11.701 .004580 .00051 11.751 .006720 .00050 11.801 .011190 .00050 11.851 .013690 .00053 11.901 .017750 .00050 11.951 .019270 .00050 12.001 .022970 .00052
700

11.200 .922530 .00050 11.250 .925090 .00051 11.300 .928250 .00051 11.350 .932490 .00050 11.400 .935800 .00051 11.450 .939820 .00049 11.500 .942990 .00050 11.550 .944800 .00051 11.600 .949540 .00053 11.650 .953520 .00052 11.700 .955990 .00050 11.750 .960970 .00050 11.800 .962480 .00052 11.850 .966290 .00049 11.900 .969000 .00051 11.950 .972680 .00051 12.000 .975490 .00052 12.050 .979420 .00052 12.100 .981650 .00050 12.150 .985630 .00051 12.200 .988290 .00052 12.250 .991960 .00052 12.300 .993760 .00052 12.350 .997860 .00053 12.401 .001950 .00052 12.451 .003940 .00051 12.501 .007440 .00050 12.551 .011250 .00051 12.601 .013310 .00051 12.651 .017100 .00051
750

12.300 .919600 .00051 12.350 .922200 .00052 12.400 .925560 .00050 12.450 .928780 .00051 12.500 .932360 .00050 12.550 .935480 .00049 12.600 .937390 .00050 12.650 .941790 .00052 12.700 .944120 .00049 12.750 .947250 .00050 12.800 .950100 .00048 12.850 .953450 .00052 12.900 .956480 .00050 12.950 .958770 .00050 13.000 .963130 .00051 13.050 .966670 .00051 13.100 .969030 .00051 13.150 .971170 .00051 13.200 .974060 .00051 13.250 .976990 .00051 13.300 .979850 .00050 13.350 .983240 .00050 13.400 .985190 .00052 13.450 .987750 .00050 13.500 .992110 .00051 13.550 .994390 .00052 13.600 .997510 .00050 13.651 .000250 .00051 13.701 .003880 .00051 13.751 .005570 .00051 
CHPRC-01552, Revision 0

12.201 .019150 .00051

12.251 .022150 .00051

12.301 .025060 .00050
12.701 .019770 .00051

752

12.400 .916270 .00050

12.500 .923230 .00049

12.600 .929310 .00051

12.700 .934460 .00051

12.800 .941390 .00050

12.900 .948080 .00051

13.000 .953260 .00049

13.100 .959410 .00051

13.200 .965170 .00050

13.300 .971730 .00050

13.400 .977140 .00050

13.500 .983170 .00051

13.600 .988730 .00052

13.700 .994780 .00051

13.800 .999800 .00051

13.901 .006390 .00050

14.001 .011410 .00051

14.101 .016950 .00052

14.201 .021550 .00051
754

12.700 .922060 .00051

12.750 .926470 .00050

12.800 .928320 .00051

12.850 .932210 .00051

12.900 .934820 .00052

12.950 .938550 .00051

13.000 .942080 .00051

13.050 .943950 .00051

13.100 .947700 .00051

13.150 .950940 .00051

13.200 .952690 .00051

13.250 .955920 .00051

13.300 .958960 .00051

13.350 .961650 .00050

13.400 .964730 .00049

13.450 .967720 .00050

13.500 .970300 .00052

13.550 .973950 .00051

13.600 .976040 .00052

13.650 .978490 .00051

13.700 .981850 .00050

13.750 .985450 .00051

13.800 .988090 .00053

13.850 .990520 .00053

13.900 .993510 .00052

13.950 .996100 .00052

14.000 .999090 .00051

14.051 .001070 .00051

14.101 .004270 .00052

14.151 .007990 .00052

14.201 .008900 .00051

14.251 .012790 .00050

14.301 .015310 .00049

14.351 .017220 .00051

14.401 .021060 .00052
13.801 .009760 .00052

13.851 .012890 .00051

13.901 .014290 .00050

13.951 .016620 .00052

14.001 .020950 .00050

\section{5}

12.900 .921790 .00052

13.000 .927550 .00050

13.100 .932860 .00051

13.200 .939150 .00052

13.300 .945670 .00050

13.400 .951010 .00051

13.500 .955300 .00052

13.600 .961740 .00051

13.700 .967800 .00049

13.800 .973440 .00049

13.900 .978820 .00049

14.000 .985050 .00050

14.100 .990150 .00051

14.200 .995620 .00050

14.301 .002240 .00051

14.401 .006440 .00050

14.501 .012330 .00051

14.601 .018240 .00053

14.701 .022990 .00052 
pun40-sp-1000-10

12

18.500 .923040 .00030

18.700 .928230 .00030

18.900 .932520 .00030

19.100 .936610 .00030

19.300 .940800 .00030

19.500 .944940 .00030

19.700 .949170 .00029

19.900 .952870 .00029

20.100 .956690 .00029

20.300 .961190 .00030

20.500 .964900 .00030

20.700 .967870 .00030

20.900 .972100 .00030

21.100 .975270 .00029

21.300 .979590 .00029

21.500 .982270 .00029

21.700 .986210 .00029

21.900 .988490 .00029

22.100 .992060 .00030

22.300 .995270 .00029

22.500 .998730 .00029

22.701 .001430 .00028

22.901 .004580 .00028

23.101 .007570 .00027

23.301 .011510 .00029

23.501 .013330 .00029
25

11.900 .918690 .00039

12.000 .923600 .00040

12.100 .930280 .00041

12.200 .935380 .00040

12.300 .939230 .00040

12.400 .944380 .00040

12.500 .949550 .00039

12.600 .954370 .00039

12.700 .959970 .00040
15

15.500 .921570 .00034

15.600 .925660 .00034

15.700 .928300 .00033

15.800 .931450 .00034

15.900 .934160 .00033

16.000 .938210 .00034

16.100 .940800 .00034

16.200 .944100 .00034

16.300 .946330 .00034

16.400 .950080 .00033

16.500 .952240 .00033

16.600 .955960 .00034

16.700 .957750 .00033

16.800 .961140 .00032

16.900 .963150 .00034

17.000 .966470 .00034

17.100 .969660 .00034

17.200 .971850 .00033

17.300 .974780 .00033

17.400 .977900 .00034

17.500 .980100 .00033

17.600 .983450 .00032

17.700 .985470 .00032

17.800 .987920 .00034

17.900 .990460 .00033

18.000 .993040 .00033

18.100 .995840 .00033

18.200 .998400 .00032

18.301 .000450 .00032

18.401 .002110 .00033

18.501 .005670 .00033

18.601 .007490 .00033

18.701 .010420 .00032

18.801 .012330 .00032

18.901 .014560 .00032

30

11.200 .921560 .00042

11.300 .927430 .00042

11.400 .932670 .00041

11.500 .938220 .00042

11.600 .944560 .00042

11.700 .949690 .00042

11.800 .954770 .00042

11.900 .960570 .00042

12.000 .965590 .00041
20

13.200 .923040 .00037

13.300 .928440 .00036

13.400 .931670 .00038

13.500 .936520 .00038

13.600 .940860 .00037

13.700 .943980 .00038

13.800 .949760 .00037

13.900 .953570 .00038

14.000 .957640 .00037

14.100 .960570 .00037

14.200 .964840 .00036

14.300 .969240 .00037

14.400 .972650 .00037

14.500 .977810 .00038

14.600 .980470 .00037

$\begin{array}{lll}14.70 & 0.98420 & 0.00037\end{array}$

14.800 .988430 .00037

14.900 .992200 .00036

15.000 .996310 .00038

15.100 .998950 .00038

15.201 .003640 .00037

15.301 .006330 .00036

15.401 .010100 .00037

15.501 .013470 .00037

15.601 .016530 .00037 
12.800 .964410 .00040

12.900 .969980 .00040

13.000 .972990 .00041

13.100 .978020 .00039

13.200 .982460 .00039

13.300 .987600 .00040

13.400 .991290 .00040

13.500 .996870 .00040

13.601 .000860 .00040

13.701 .004310 .00039

13.801 .008520 .00039

13.901 .013550 .00040

14.001 .017260 .00041

35

10.700 .920970 .00044

10.750 .924390 .00042

10.800 .927970 .00041

10.850 .930910 .00042

10.900 .933750 .00042

10.950 .936080 .00043

11.000 .939390 .00043

11.050 .943260 .00043

11.100 .946340 .00043

11.150 .949000 .00043

11.200 .952090 .00042

11.250 .954750 .00042

11.300 .958220 .00043

11.350 .961090 .00043

11.400 .964370 .00042

11.450 .967240 .00043

11.500 .969160 .00043

11.550 .972690 .00042

11.600 .974630 .00044

11.650 .976980 .00042

11.700 .980460 .00044

11.750 .983010 .00041

11.800 .986510 .00043

11.850 .989630 .00042

11.900 .992560 .00042

11.950 .995070 .00042

12.000 .997970 .00041

12.051 .000540 .00042

12.101 .002370 .00042

12.151 .005220 .00042

12.201 .008460 .00044

12.251 .011070 .00043

12.301 .014450 .00043

12.351 .015800 .00043

12.401 .017960 .00042
12.100 .971360 .00041

12.200 .976400 .00042

12.300 .981350 .00042

12.400 .987080 .00040

12.500 .992450 .00040

12.600 .996900 .00040

12.701 .001530 .00041

12.801 .006700 .00041

12.901 .011690 .00041

13.001 .016440 .00042

13.101 .021030 .00041
11.900 .974220 .00042 12.000 .979840 .00041 12.100 .984980 .00041 12.200 .990000 .00042 12.300 .994770 .00042 12.401 .001270 .00044 12.501 .006200 .00042 12.601 .010900 .00041 12.701 .016160 .00042 12.801 .020130 .00041
40
10.300 .919310 .00042

10.350 .922590 .00043

10.400 .926000 .00043

10.450 .928400 .00043

10.500 .932650 .00042

10.550 .935390 .00042

10.600 .939100 .00044

10.650 .941740 .00043

10.700 .944970 .00042

10.750 .948940 .00044

10.800 .950280 .00043

10.850 .955110 .00043

10.900 .957800 .00043

10.950 .961000 .00044

11.000 .965440 .00042

11.050 .967370 .00044

11.100 .970390 .00044

11.150 .973110 .00044

11.200 .976180 .00044

11.250 .978340 .00044

11.300 .982590 .00043

11.350 .986260 .00042

11.400 .988180 .00043

11.450 .990390 .00044

11.500 .993760 .00044

11.550 .996670 .00043

11.600 .999380 .00045

11.651 .003660 .00043

11.701 .005610 .00043

11.751 .008270 .00043

11.801 .011890 .00044

11.851 .013750 .00044

11.901 .016090 .00043

11.951 .019680 .00043

12.001 .022600 .00044
50

9.800 .918240 .00044

9.850 .923420 .00045

9.900 .925010 .00044

9.950 .929290 .00045

10.000 .932750 .00045

10.050 .935940 .00045

10.100 .939940 .00044

10.150 .943150 .00044

10.200 .946350 .00045

10.250 .949530 .00045

10.300 .953070 .00044

10.350 .956960 .00044

10.400 .960710 .00045

10.450 .963190 .00045

10.500 .966360 .00045

10.550 .969850 .00046

10.600 .973920 .00045

10.650 .977110 .00046

10.700 .979490 .00046

10.750 .983460 .00043

10.800 .985480 .00045

10.850 .988420 .00044

10.900 .992130 .00045

10.950 .995670 .00045

11.000 .999070 .00046

11.051 .001330 .00046

11.101 .004000 .00045

11.151 .007260 .00045

11.201 .010510 .00044

11.251 .015190 .00045

11.301 .017220 .00045

11.351 .020240 .00045

11.401 .022740 .00044 
60

9.500 .919210 .00045

9.550 .923780 .00046

9.600 .926710 .00045

9.650 .930480 .00046

9.700 .933890 .00045

9.750 .938720 .00045

9.800 .941410 .00045

9.850 .945170 .00045

9.900 .948430 .00045

9.950 .953450 .00046

10.000 .956380 .00047

10.100 .962760 .00046

10.150 .966760 .00046

10.200 .970010 .00046

10.250 .973640 .00045

10.300 .977410 .00046

10.350 .979250 .00046

10.400 .983460 .00047

10.450 .987040 .00046

10.500 .989540 .00046

10.550 .992520 .00046

10.600 .996490 .00045

10.651 .000470 .00045

10.701 .002840 .00045

10.751 .007540 .00046

10.801 .010340 .00046

10.851 .012220 .00046

10.901 .014800 .00045

10.951 .019640 .00045

11.001 .021590 .00045
70

9.300 .920740 .00046

9.350 .923340 .00045

9.400 .927980 .00045

9.450 .931540 .00047

9.500 .934710 .00047

9.550 .938370 .00047

9.600 .941970 .00047

9.650 .946370 .00047

9.700 .949370 .00046

9.750 .953840 .00046

9.800 .956550 .00046

9.850 .960880 .00046

9.900 .963890 .00046

9.950 .969030 .00045

10.000 .971470 .00047

10.050 .975550 .00046

10.100 .978660 .00046

10.150 .982340 .00046

10.200 .984710 .00047

10.250 .989330 .00044

10.300 .992260 .00046

10.350 .995780 .00047

10.400 .998820 .00047

10.451 .002520 .00048

10.501 .006740 .00047

10.551 .009000 .00045

10.601 .011600 .00047

10.651 .015750 .00046

10.701 .018290 .00046
90

9.000 .917620 .00046

9.050 .922330 .00047

9.100 .925300 .00046

9.150 .930020 .00047

9.200 .933120 .00047

9.250 .936850 .00047

9.300 .940760 .00047

9.350 .945190 .00046

9.400 .948940 .00046

9.450 .952510 .00047

9.500 .956270 .00048

9.550 .960420 .00046

9.600 .963690 .00047
100

8.900 .917070 .00046

8.950 .921570 .00047

9.000 .924780 .00046

9.050 .928780 .00047

9.100 .932630 .00046

9.150 .936000 .00047

9.200 .940600 .00046

9.250 .944670 .00047

9.300 .948660 .00046

9.350 .952620 .00047

9.400 .956530 .00047

9.450 .959290 .00045

9.500 .963210 .00047
80

9.100 .916450 .00046

9.150 .920150 .00045

9.200 .924990 .00047

9.250 .928490 .00047

9.300 .932340 .00047

9.350 .935730 .00047

9.400 .939250 .00046

9.450 .943490 .00046

9.500 .946470 .00048

9.550 .950710 .00046

9.600 .954220 .00046

9.650 .958500 .00046

9.700 .962150 .00046

9.750 .965520 .00046

9.800 .969470 .00047

9.850 .972680 .00046

9.900 .976890 .00046

9.950 .980250 .00046

10.000 .983160 .00047

10.050 .986340 .00047

10.100 .989570 .00047

10.150 .993960 .00046

10.200 .996690 .00046

10.251 .001720 .00048

10.301 .004150 .00048

10.351 .007540 .00047

10.401 .011860 .00047

10.451 .013710 .00046

10.501 .018010 .00047

10.551 .021050 .00045

10.601 .024630 .00047

120

8.800 .918870 .00048

8.850 .922540 .00047

8.900 .927250 .00047

8.950 .930670 .00047

9.000 .934410 .00047

9.050 .937820 .00046

9.100 .942550 .00048

9.150 .946280 .00046

9.200 .950480 .00047

9.250 .953920 .00046

9.300 .957130 .00047

9.350 .961280 .00047

9.450 .969500 .00047 
9.650 .966840 .00047

9.700 .971100 .00046

9.750 .975090 .00046

9.800 .977940 .00046

9.850 .981360 .00046

9.900 .985180 .00048

9.950 .988580 .00046

10.000 .993180 .00048

10.050 .995800 .00048

10.100 .999590 .00046

10.151 .002090 .00047

10.201 .006590 .00048

10.251 .009880 .00046

10.301 .013390 .00047

10.351 .017120 .00045

10.401 .019900 .00047
9.550 .966470 .00048

9.600 .970610 .00046

9.650 .974960 .00047

9.700 .977920 .00047

9.750 .982060 .00049

9.800 .985230 .00047

9.850 .988860 .00047

9.900 .992730 .00047

9.950 .994970 .00046

10.001 .000150 .00047

10.051 .002680 .00046

10.101 .006280 .00048

10.151 .010120 .00046

10.201 .013200 .00049

10.251 .016420 .00047

10.301 .020630 .00046
9.500 .972790 .00047

9.550 .976130 .00048

9.600 .980570 .00048

9.650 .983780 .00046

9.700 .986730 .00047

9.750 .991370 .00047

9.800 .994570 .00047

9.850 .998980 .00046

9.901 .001580 .00047

9.951 .004910 .00048

10.001 .008180 .00047

10.051 .012890 .00047

10.101 .015460 .00046

10.151 .019590 .00046

10.201 .022030 .00048
130

8.800 .923020 .00048

8.850 .927190 .00047

8.900 .930150 .00047

8.950 .935220 .00047

9.000 .938810 .00046

9.050 .943020 .00046

9.100 .946790 .00048

9.150 .949690 .00047

9.200 .954190 .00048

9.250 .958540 .00047

9.300 .961800 .00047

9.350 .965840 .00047

9.400 .969010 .00047

9.450 .973420 .00047

9.500 .976530 .00047

9.550 .980790 .00047

9.600 .984550 .00049

9.650 .987750 .00047

9.700 .992290 .00047

9.750 .995270 .00046

9.800 .998450 .00047

9.851 .001710 .00048

9.901 .006060 .00047

9.951 .009180 .00048 10.001 .012720 .00047 10.051 .016670 .00048 10.101 .019540 .00048
150

8.700 .919230 .00047

8.750 .923990 .00048

8.800 .927900 .00046

8.850 .931770 .00046

8.900 .935320 .00048

8.950 .940260 .00048

9.000 .943950 .00047

9.050 .947750 .00047

9.100 .951530 .00049

9.150 .955490 .00048

9.200 .960200 .00047

9.250 .963620 .00047

9.300 .966530 .00047

9.350 .970920 .00048

9.400 .974140 .00047

9.450 .978070 .00047

9.500 .981800 .00048

9.550 .985640 .00048

9.600 .988880 .00049

9.650 .993490 .00048

9.700 .996220 .00047

9.751 .000440 .00047

9.801 .002680 .00047

9.851 .007530 .00048

9.901 .010070 .00048

9.951 .014800 .00047

10.001 .017540 .00045

10.051 .021460 .00048

10.101 .025390 .00048
200

8.600 .920040 .00047

8.650 .923640 .00047

8.700 .927320 .00047

8.750 .931340 .00047

8.800 .935040 .00047

8.850 .938830 .00048

8.900 .943460 .00048

8.950 .946510 .00048

9.000 .950860 .00047

9.050 .955000 .00047

9.100 .958490 .00047

9.150 .962570 .00048

9.200 .966170 .00047

9.250 .969310 .00048

9.300 .974210 .00048

9.350 .977650 .00048

9.400 .982130 .00047

9.450 .985420 .00048

9.500 .988970 .00045

9.550 .992040 .00047

9.600 .996620 .00048

9.650 .999050 .00047

9.701 .002780 .00046

9.751 .006910 .00047

9.801 .010530 .00047

9.851 .013910 .00048

9.901 .017170 .00047

9.951 .021420 .00047

10.001 .025180 .00049 
239

8.600 .921870 .00047 8.650 .925580 .00047 8.700 .930270 .00047 8.750 .933020 .00049 8.800 .937860 .00047 8.850 .940210 .00047 8.900 .945090 .00048 8.950 .949060 .00048 9.000 .952590 .00046 9.050 .957240 .00046 9.100 .961700 .00046 9.150 .964690 .00047 9.200 .967870 .00048 9.250 .972510 .00049 9.300 .976480 .00048 9.350 .979690 .00047 9.400 .983040 .00048 9.450 .987680 .00048 9.500 .990820 .00046 9.550 .994410 .00047 9.600 .998130 .00048 9.651 .000890 .00047 9.701 .005720 .00047 9.751 .009370 .00048 9.801 .012090 .00048 9.851 .015700 .00047 9.901 .018200 .00048

\section{8}

8.700 .922150 .00047 8.750 .925940 .00048 8.800 .929630 .00047 8.850 .933390 .00047 8.900 .937380 .00046 8.950 .941280 .00047 9.000 .943930 .00048 9.050 .948730 .00048 9.100 .952600 .00047 9.150 .956630 .00047 9.200 .960410 .00048 9.250 .962970 .00049 9.300 .967170 .00047 9.350 .971160 .00049 9.400 .975010 .00048 9.450 .978210 .00047 9.500 .982140 .00047 9.550 .984820 .00047
250

8.600 .922570 .00050

8.650 .926520 .00047

8.700 .929290 .00048

8.750 .933730 .00047

8.800 .937400 .00047

8.850 .941870 .00047

8.900 .945150 .00047

8.950 .950280 .00048

9.000 .953850 .00048

9.050 .957630 .00047

9.100 .961220 .00048

9.150 .965480 .00046

9.200 .968290 .00047

9.250 .972140 .00049

9.300 .976040 .00048

9.350 .980890 .00047

9.400 .983670 .00048

9.450 .986840 .00048

9.500 .990460 .00047

9.550 .994070 .00049

9.600 .998560 .00048

9.651 .001520 .00048

9.701 .005200 .00048

9.751 .009910 .00046

9.801 .012120 .00047

9.851 .015880 .00048

9.901 .018930 .00047
359

8.600 .920630 .00046

8.650 .925810 .00048

8.700 .929400 .00048

8.750 .932450 .00048

8.800 .937040 .00047

8.850 .941150 .00049

8.900 .944100 .00047

8.950 .948190 .00048

9.000 .951900 .00046

9.050 .955970 .00047

9.100 .959920 .00047

9.150 .962630 .00046

9.200 .967030 .00047

9.250 .971320 .00047

9.300 .975050 .00045

9.350 .978440 .00047

9.400 .982630 .00049

9.450 .985280 .00048

9.500 .988670 .00047

9.550 .992810 .00048

9.600 .995900 .00047

9.651 .000820 .00049

9.701 .004330 .00049

9.751 .006820 .00048

9.801 .010710 .00048

9.851 .014330 .00047

9.901 .017030 .00047
500

8.700 .918720 .00046

8.750 .924050 .00047

8.800 .926780 .00048

8.850 .931040 .00048

8.900 .934970 .00047

8.950 .939080 .00049

9.000 .942510 .00047

9.050 .946130 .00046

9.100 .950060 .00048

9.150 .954670 .00047

9.200 .958370 .00048

9.250 .960930 .00048

9.300 .965190 .00048

9.350 .969150 .00047

9.400 .972740 .00048

9.450 .976460 .00047

9.500 .980100 .00048

9.550 .983200 .00048
600

8.900 .919050 .00046

8.950 .921060 .00046

9.000 .926120 .00048

9.050 .929940 .00047

9.100 .933850 .00047

9.150 .936920 .00047

9.200 .941390 .00048

9.250 .944540 .00047

9.300 .948100 .00048

9.350 .952280 .00049

9.400 .954940 .00047

9.450 .959700 .00048

9.500 .962190 .00047

9.550 .966900 .00047

9.600 .970070 .00047

9.650 .973680 .00047

9.700 .976530 .00047

9.750 .979690 .00048 
9.600 .989320 .00047

9.650 .992810 .00047

9.700 .996310 .00047

9.750 .999440 .00050

9.801 .003570 .00049

9.851 .006780 .00048

9.901 .010210 .00049

9.951 .012770 .00048

10.001 .017000 .00048

10.051 .020330 .00049

10.101 .023010 .00049
9.650 .989730 .00048

9.700 .993660 .00048

9.750 .997770 .00048

9.801 .001810 .00047

9.851 .005060 .00047

9.901 .008090 .00046

9.951 .010800 .00048

10.001 .014370 .00048

10.051 .019060 .00047

10.101 .021230 .00048
650

9.100 .919340 .00048

9.150 .922770 .00047

9.200 .927090 .00048

9.250 .931040 .00048

9.300 .934610 .00047

9.350 .938220 .00049

9.400 .941630 .00048

9.450 .945500 .00048

9.500 .949030 .00048

9.550 .951680 .00049

9.600 .955200 .00046

9.650 .959680 .00048

9.700 .962850 .00047

9.750 .966490 .00047

9.800 .969160 .00047

9.850 .973200 .00048

9.900 .975980 .00048

9.950 .979660 .00048

10.000 .984160 .00048

10.050 .985900 .00048

10.100 .990000 .00047

10.150 .992590 .00049

10.200 .996110 .00047

10.250 .999570 .00047

10.301 .004220 .00049

10.351 .006470 .00047

10.401 .010060 .00048

10.451 .013920 .00048

10.501 .015820 .00048

10.551 .019890 .00048

10.601 .023250 .00049
9.800 .983000 .00047

9.850 .987520 .00049

9.900 .990530 .00047

10.051 .001260 .00048

10.101 .004740 .00047

10.151 .007130 .00047

10.201 .011460 .00047

10.251 .014700 .00048

10.301 .017960 .00047

10.351 .020370 .00047

10.401 .024010 .00047
700

9.400 .918900 .00046

9.450 .921690 .00048

9.500 .925340 .00047

9.550 .928550 .00047

9.600 .932130 .00048

9.650 .936560 .00046

9.700 .939780 .00047

9.750 .942940 .00047

9.800 .946550 .00047

9.850 .949500 .00049

9.900 .953090 .00047

9.950 .957370 .00047

10.000 .959120 .00048

10.050 .962810 .00047

10.100 .967190 .00047

10.150 .969640 .00048

10.200 .972520 .00048

10.250 .975560 .00047

10.300 .979600 .00048

10.350 .983040 .00047

10.400 .985830 .00048

10.600 .998970 .00049

10.651 .002630 .00047

10.701 .005090 .00046

10.751 .007190 .00047

10.801 .012180 .00048

10.851 .015290 .00048

10.901 .017940 .00047

10.951 .020060 .00049

11.001 .023300 .00047
750

10.300 .921520 .00046

10.350 .924540 .00046

10.400 .927620 .00046

10.450 .930630 .00047

10.500 .934220 .00047

10.550 .937220 .00047

10.600 .940500 .00047

10.650 .943450 .00048

10.700 .946510 .00047

10.900 .959060 .00048

10.950 .960890 .00048

11.000 .965570 .00047

11.050 .968670 .00047

11.100 .970860 .00047

11.150 .973650 .00046

11.200 .977860 .00048

11.250 .979840 .00047

11.300 .982540 .00047

11.350 .985090 .00047

11.400 .989110 .00047

11.450 .991410 .00049

11.500 .994510 .00048

11.550 .996790 .00046

11.600 .999580 .00049

11.651 .002860 .00049

11.701 .006820 .00047

11.751 .008540 .00046

11.801 .011480 .00048

11.851 .014940 .00046

11.901 .017230 .00046

11.951 .020010 .00049

12.001 .022880 .00047 
752

10.400 .920620 .00047 10.450 .925380 .00046 10.500 .926940 .00048 10.550 .930300 .00047 10.600 .933830 .00048 10.650 .936710 .00047 10.700 .939850 .00049 10.750 .941470 .00048 10.800 .945700 .00047 10.850 .948480 .00047 10.900 .951440 .00047 10.950 .954990 .00047 11.000 .957480 .00048 11.050 .961200 .00047 11.100 .963910 .00047 11.150 .966170 .00047 11.200 .969180 .00047 11.250 .972620 .00048 11.300 .975940 .00048 11.350 .979100 .00046 11.400 .981380 .00047 11.450 .984860 .00048 11.500 .987930 .00048 11.550 .990530 .00047 11.600 .994000 .00047 11.650 .995950 .00047 11.700 .999000 .00047 11.751 .001670 .00048 11.801 .004320 .00046 11.851 .006800 .00047 11.901 .010230 .00048 11.951 .013270 .00048 12.001 .016350 .00048 12.051 .017790 .00048 12.101 .021310 .00046
754

10.500 .918390 .00048 10.600 .925670 .00047 10.700 .929350 .00047 10.800 .936700 .00047 10.900 .941950 .00047 11.000 .949060 .00047 11.100 .954260 .00047 11.200 .961200 .00048 11.300 .965900 .00046 11.400 .972650 .00048 11.500 .978020 .00049 11.600 .983990 .00047 11.700 .989230 .00047 11.800 .994610 .00047 11.901 .000570 .00047 12.001 .006450 .00048 12.101 .011510 .00048 12.201 .016400 .00047 12.301 .022980 .00047
755

10.700 .920920 .00046 10.800 .926890 .00047 10.900 .932300 .00048 11.000 .938540 .00047 11.100 .944780 .00047 11.200 .949850 .00047 11.300 .955510 .00047 11.400 .961480 .00047 11.500 .967850 .00048 11.600 .973590 .00046 11.700 .979540 .00047 11.800 .985190 .00047 11.900 .990770 .00046 12.000 .995210 .00048 12.101 .000660 .00047 12.201 .006490 .00047 12.301 .011620 .00047 12.401 .017770 .00048 12.501 .021770 .00049 
Pun43-sp-1000-00

12

23.300 .924880 .00031

23.600 .929680 .00031

23.900 .935240 .00031

24.200 .939600 .00032

24.500 .944810 .00030

24.800 .949470 .00031

25.100 .954860 .00030

25.400 .960060 .00031

25.700 .964770 .00030

26.000 .967860 .00029

26.300 .972600 .00030

26.600 .976130 .00030

26.900 .980430 .00029

27.200 .984250 .00029

27.500 .988360 .00030

27.800 .991800 .00029

28.100 .996160 .00029

28.400 .999170 .00029

28.701 .002690 .00029

29.001 .005650 .00028

29.301 .009060 .00028

29.601 .011820 .00029

29.701 .013510 .00029
15

19.500 .921840 .00035

19.700 .925980 .00035

19.900 .932840 .00036

20.100 .937260 .00035

20.300 .943500 .00036

20.500 .948290 .00034

20.700 .952630 .00035

20.900 .957330 .00035

21.100 .961930 .00036

21.300 .967210 .00034

21.500 .972050 .00034

21.700 .976610 .00033

21.900 .980650 .00034

22.100 .985300 .00034

22.300 .989350 .00035

22.500 .993700 .00034

22.700 .997400 .00034

22.901 .002060 .00034

23.101 .005430 .00033

23.301 .009400 .00033

23.501 .013440 .00034

23.601 .015300 .00034
25

15.300 .918600 .00043

15.400 .922510 .00043

15.500 .927720 .00043

15.600 .932450 .00044

15.700 .937260 .00043

15.800 .941640 .00044

15.900 .945880 .00043

16.000 .950130 .00043

16.100 .954420 .00044

16.200 .959750 .00041

16.300 .963260 .00043

16.400 .967390 .00043

16.500 .971900 .00044

16.600 .975950 .00043
30
14.500 .919460 .00045

14.600 .925360 .00046

14.700 .929620 .00044

14.800 .934420 .00045

14.900 .941170 .00046

15.000 .945500 .00047

15.100 .951300 .00045

15.200 .955370 .00045

15.300 .960190 .00046

15.400 .965580 .00046

15.500 .970880 .00045

15.600 .975250 .00046

15.700 .978990 .00046

15.800 .983700 .00046
20

16.700 .920450 .00040

16.800 .924400 .00040

16.900 .928830 .00039

17.000 .931510 .00040

17.100 .935360 .00040

17.200 .938650 .00041

17.300 .942610 .00040

17.400 .947020 .00040

17.500 .951440 .00039

17.600 .954370 .00040

17.700 .957200 .00040

17.800 .960500 .00039

17.900 .965220 .00040

18.000 .966770 .00039

18.100 .971950 .00041

18.200 .974840 .00039

18.300 .977180 .00040

18.400 .980900 .00040

18.500 .984810 .00041

18.600 .987490 .00040

18.700 .991500 .00040

18.800 .994910 .00039

18.900 .997780 .00040

19.001 .001040 .00039

19.101 .003670 .00039

19.201 .006790 .00038

19.301 .009120 .00040

19.401 .012670 .00038

19.501 .015870 .00039

19.601 .019430 .00039

32

14.300 .921700 .00047

14.400 .927640 .00045

14.500 .932560 .00046

14.600 .937460 .00047

14.700 .942990 .00047

14.800 .948850 .00047

14.900 .953380 .00046

15.000 .957860 .00045

15.100 .964060 .00046

15.200 .968340 .00046

15.300 .973260 .00046

15.400 .978330 .00045

15.500 .983700 .00046

15.600 .987860 .00047 
16.700 .980150 .00043 16.800 .984620 .00043 16.900 .987380 .00044 17.000 .991640 .00043 17.100 .996420 .00043 17.200 .999970 .00042 17.301 .003160 .00043 17.401 .008990 .00042 17.501 .012160 .00044 17.601 .014390 .00041 17.701 .019390 .00042
15.900 .989010 .00044 16.000 .992540 .00045 16.100 .997480 .00044 16.201 .002420 .00044 16.301 .006340 .00046 16.401 .010900 .00045 16.501 .015400 .00047 16.601 .019780 .00045
15.700 .993230 .00046 15.800 .997710 .00046 15.901 .001310 .00045 16.001 .006890 .00047 16.101 .011430 .00045 16.201 .016660 .00046 16.301 .020210 .00046
35

13.900 .915360 .00047 14.000 .921790 .00045 14.100 .927520 .00047 14.200 .933510 .00047 14.300 .938270 .00048 14.400 .943880 .00047 14.500 .950200 .00047 14.600 .954940 .00045 14.700 .960360 .00047 14.800 .966120 .00047 14.900 .971510 .00046 15.000 .975880 .00046 15.100 .981940 .00046 15.200 .986510 .00047 15.300 .991320 .00046 15.400 .996800 .00047 15.501 .000770 .00048 15.601 .006180 .00046 15.701 .010860 .00046 15.801 .016390 .00046 15.901 .020370 .00046
40

13.500 .914820 .00049 13.600 .920770 .00048 13.700 .927550 .00048 13.800 .933420 .00047 13.900 .940160 .00048 14.000 .944860 .00048 14.100 .951260 .00049 14.200 .956580 .00047 14.300 .962420 .00048 14.400 .968000 .00049 14.500 .973890 .00047 14.600 .978840 .00048 14.700 .983650 .00048 14.800 .989840 .00048 14.900 .994790 .00048 15.000 .999390 .00047 15.101 .005870 .00047 15.201 .010160 .00048 15.301 .014940 .00048 15.401 .021540 .00048
50

13.000 .915820 .00050 13.050 .920430 .00050 13.100 .923130 .00050 13.150 .926360 .00049 13.200 .929890 .00050 13.250 .932690 .00050 13.300 .934610 .00049 13.350 .938330 .00051 13.400 .941530 .00050 13.450 .944990 .00050 13.500 .948320 .00049 13.550 .951890 .00050 13.600 .954380 .00051 13.650 .955660 .00051 13.700 .960790 .00049 13.750 .963330 .00051 13.800 .967020 .00051 13.850 .969660 .00050 13.900 .972540 .00050 13.950 .975400 .00050 14.000 .978800 .00051 14.050 .981060 .00049 14.100 .984560 .00050 14.200 .989630 .00049 14.250 .993820 .00049 14.300 .996350 .00049 14.350 .998900 .00049 14.401 .001520 .00050 14.451 .004720 .00049 14.501 .007060 .00049 14.551 .010100 .00050 14.601 .012610 .00048 14.651 .015350 .00049 14.701 .017890 .00049 
60

12.700 .915360 .00051

12.750 .919740 .00050

12.800 .922350 .00050

12.850 .927580 .00050

12.900 .929890 .00050

12.950 .932750 .00050

13.000 .936710 .00050

13.050 .939890 .00050

13.100 .943170 .00050

13.150 .946490 .00050

13.200 .950140 .00051

13.250 .952430 .00050

13.300 .956210 .00051

13.350 .960440 .00051

13.400 .962490 .00052

13.450 .965980 .00052

13.500 .969230 .00049

13.550 .971540 .00050

13.600 .974390 .00051

13.650 .977950 .00050

13.700 .981660 .00051

13.750 .983920 .00051

13.800 .987780 .00051

13.850 .990610 .00051

13.900 .992900 .00050

13.950 .997700 .00053

14.000 .999870 .00051

14.051 .002730 .00050

14.101 .005230 .00050

14.151 .008160 .00051

14.201 .012060 .00050

14.251 .014560 .00050

14.301 .016450 .00051

14.351 .020340 .00051

14.401 .023510 .00051
70

12.500 .917490 .00050

12.550 .920690 .00051

12.600 .924440 .00050

12.650 .927860 .00051

12.700 .931520 .00051

12.750 .934410 .00051

12.800 .938400 .00050

12.850 .941530 .00051

12.900 .944390 .00052

12.950 .948220 .00052

13.000 .951680 .00053

13.050 .955170 .00051

13.100 .958280 .00051

13.150 .961930 .00051

13.200 .964330 .00049

13.250 .967300 .00050

13.300 .971460 .00051

13.350 .974200 .00051

13.400 .978330 .00052

13.450 .980540 .00052

13.500 .984540 .00052

13.550 .986970 .00050

13.600 .991260 .00051

13.650 .993600 .00051

13.700 .996850 .00051

13.750 .998870 .00052

13.801 .001510 .00053

13.851 .005730 .00051

13.901 .009530 .00051

13.951 .010680 .00052

14.001 .014500 .00052

14.051 .016790 .00050

14.101 .022320 .00051
80

12.400 .919110 .00053

12.450 .923050 .00051

12.500 .927220 .00051

12.550 .930210 .00051

12.600 .934110 .00051

12.650 .937540 .00052

12.700 .941450 .00053

12.750 .945340 .00052

12.800 .948530 .00053

12.850 .950770 .00051

12.900 .954450 .00054

12.950 .957300 .00053

13.000 .961010 .00051

13.050 .964060 .00052

13.100 .967880 .00052

13.150 .970920 .00053

13.200 .974930 .00053

13.250 .977740 .00052

13.300 .981500 .00053

13.350 .984860 .00053

13.400 .988430 .00051

13.450 .991080 .00053

13.500 .993580 .00052

13.600 .999950 .00052

13.651 .003530 .00052

13.701 .006750 .00052

13.751 .009570 .00053

13.801 .012430 .00052

13.851 .015700 .00050

13.901 .018860 .00054

13.951 .022050 .00052

14.001 .024240 .00050
90

12.300 .920400 .00052

12.350 .923170 .00053

12.400 .927790 .00053

12.450 .930890 .00051

12.500 .933820 .00055

12.550 .938530 .00054

12.600 .941690 .00052

12.650 .945100 .00052

12.700 .947650 .00052

12.750 .951740 .00052
100

12.200 .917550 .00052

12.250 .921120 .00052

12.300 .924560 .00052

12.350 .928540 .00053

12.400 .931710 .00054

12.450 .935880 .00053

12.500 .937500 .00050

12.550 .942400 .00053

12.600 .946220 .00052

12.650 .949650 .00051
120

12.100 .916100 .00053

12.150 .920290 .00052

12.200 .922280 .00052

12.250 .926640 .00054

12.300 .930860 .00053

12.350 .933480 .00052

12.400 .936750 .00055

12.450 .940280 .00052

12.500 .944660 .00053

12.550 .948040 .00053 
12.800 .954690 .00051

12.850 .958470 .00052

12.900 .961840 .00053

12.950 .965390 .00052

13.000 .969120 .00053

13.050 .971290 .00052

13.100 .974760 .00052

13.150 .978450 .00050

13.200 .982770 .00052

13.250 .984700 .00054

13.300 .989120 .00053

13.350 .991630 .00053

13.400 .994290 .00053

13.450 .997880 .00052

13.501 .001970 .00053

13.551 .004180 .00053

13.601 .007780 .00053

13.651 .010100 .00053

13.701 .015180 .00051

13.751 .017650 .00052

13.801 .019870 .00053

130

12.100 .919020 .00053

12.150 .922780 .00052

12.200 .926490 .00052

12.250 .929200 .00052

12.300 .931920 .00053

12.350 .936710 .00053

12.400 .939910 .00053

12.450 .944720 .00053

12.500 .948080 .00051

12.550 .951300 .00053

12.600 .955100 .00053

12.650 .957840 .00052

12.700 .960960 .00051

12.750 .964370 .00053

12.800 .967920 .00054

12.850 .972470 .00052

12.900 .974570 .00051

12.950 .979090 .00055

13.000 .982000 .00052

13.050 .985630 .00054

13.100 .990090 .00053

13.150 .993240 .00053

13.200 .995950 .00052

13.250 .999700 .00052
12.700 .953040 .00052

12.750 .956180 .00054

12.800 .960540 .00054

12.850 .964890 .00052

12.900 .967630 .00052

12.950 .969880 .00052

13.000 .973240 .00053

13.050 .976950 .00052

13.100 .981150 .00053

13.150 .983520 .00053

13.200 .986960 .00053

13.250 .991710 .00053

13.300 .993520 .00053

13.350 .997110 .00054

13.400 .999540 .00052

13.451 .003210 .00054

13.501 .005190 .00053

13.551 .009580 .00053

13.601 .013670 .00051

13.651 .016070 .00051

13.701 .019140 .00052

13.751 .021950 .00052

13.801 .025150 .00053
12.600 .952400 .00052

12.650 .955020 .00054

12.700 .958270 .00054

12.750 .962600 .00052

12.800 .965480 .00053

12.850 .968680 .00053

12.900 .972190 .00053

12.950 .975400 .00053

13.000 .977930 .00052

13.050 .982560 .00052

13.100 .985430 .00052

13.150 .988130 .00053

13.200 .992830 .00052

13.250 .996040 .00053

13.300 .999150 .00052

13.351 .002100 .00052

13.401 .004840 .00052

13.451 .008750 .00054

13.501 .013300 .00053

13.551 .014910 .00053

13.601 .018010 .00052

13.651 .021760 .00053

13.701 .024750 .00055
12.100 .919640 .00054

12.150 .924300 .00053

12.200 .927180 .00053

12.250 .930410 .00054

12.300 .934240 .00053

12.350 .938940 .00052

12.400 .942280 .00054

12.450 .945860 .00052

12.500 .948720 .00053

12.550 .952940 .00052

12.600 .956470 .00052

12.650 .958980 .00054

12.750 .967270 .00052

12.800 .970220 .00051

12.850 .972410 .00052

12.900 .976190 .00052

12.950 .980760 .00054

13.000 .983210 .00053

13.050 .986100 .00052

13.100 .990220 .00053

13.150 .993650 .00051

13.200 .996200 .00054

13.251 .001580 .00052

13.301 .003360 .00053
200

12.100 .921520 .00052

12.150 .923670 .00052

12.200 .927470 .00052

12.250 .931600 .00053

12.300 .934570 .00052

12.350 .938910 .00052

12.400 .940850 .00053

12.450 .945380 .00054

12.500 .949000 .00053

12.550 .952490 .00053

12.600 .955810 .00053

12.650 .959800 .00053

12.700 .962840 .00052

12.750 .966630 .00053

12.800 .970110 .00053

12.850 .973750 .00053

12.900 .976780 .00052

12.950 .980060 .00052

13.000 .982690 .00055

13.050 .987130 .00053

13.100 .990960 .00054

13.150 .994520 .00052

13.200 .996660 .00052

13.251 .001150 .00053 
13.301 .001800 .00051 13.351 .005660 .00053 13.401 .008500 .00053 13.451 .011590 .00052 13.501 .015220 .00053 13.551 .017200 .00052 13.601 .021170 .00053
13.351 .007070 .00054 13.401 .010260 .00052 13.451 .013050 .00053 13.501 .017270 .00053 13.551 .020470 .00053 13.601 .022350 .00052
13.301 .003340 .00052 13.351 .007460 .00052 13.401 .010270 .00052 13.451 .013120 .00054 13.501 .016780 .00053 13.551 .019390 .00052 13.601 .022880 .00052
239

12.100 .916730 .00053

12.150 .920430 .00052

12.200 .923290 .00053

12.250 .926270 .00052

12.300 .931660 .00053

12.350 .935420 .00053

12.400 .937270 .00053

12.450 .940680 .00053

12.500 .944040 .00052

12.550 .948400 .00051

12.600 .950790 .00052

12.650 .955430 .00052

12.700 .958760 .00053

12.750 .962600 .00052

12.800 .966620 .00050

12.850 .969250 .00053

12.900 .973150 .00052

12.950 .976630 .00053

13.000 .979490 .00053

13.050 .983950 .00053

13.100 .986880 .00052

13.150 .989640 .00053

13.200 .993250 .00052

13.250 .995740 .00051

13.300 .999640 .00052

13.351 .003790 .00053

13.401 .006530 .00052

13.451 .009800 .00054

13.501 .012660 .00052

13.551 .016070 .00054

13.601 .019520 .00053

13.651 .021730 .00053

13.701 .025390 .00054
250

12.100 .914670 .00051

12.150 .918170 .00050

12.200 .922090 .00052

12.250 .925610 .00055

12.300 .929720 .00052

12.350 .933240 .00052

12.400 .936910 .00051

12.450 .940630 .00052

12.500 .944060 .00053

12.550 .947630 .00054

12.600 .950530 .00051

12.650 .954340 .00053

12.700 .957690 .00052

12.750 .961100 .00053

12.800 .965020 .00053

12.850 .968610 .00053

12.900 .971250 .00051

12.950 .974430 .00054

13.000 .977520 .00053

13.050 .981500 .00052

13.100 .985240 .00052

13.150 .988530 .00051

13.200 .991660 .00053

13.250 .994360 .00052

13.300 .998300 .00052

13.351 .001980 .00052

13.401 .004890 .00054

13.451 .008190 .00053

13.501 .011290 .00052

13.551 .014380 .00053

13.601 .017770 .00054

13.651 .021170 .00052

13.701 .024210 .00053
359

12.400 .919740 .00052

12.450 .924400 .00053

12.500 .928800 .00052

12.550 .931340 .00052

12.600 .935370 .00052

12.650 .938260 .00052

12.700 .942750 .00051

12.750 .945830 .00052

12.800 .949670 .00052

12.850 .952480 .00050

12.900 .956170 .00053

12.950 .960390 .00053

13.000 .963750 .00052

13.050 .965000 .00050

13.100 .969680 .00053

13.150 .973560 .00052

13.200 .976380 .00052

13.250 .980280 .00052

13.300 .981850 .00052

13.350 .985970 .00053

13.400 .988730 .00054

13.450 .992570 .00053

13.500 .995880 .00051

13.550 .999460 .00052

13.601 .001740 .00052

13.651 .005790 .00053

13.701 .008560 .00052

13.751 .011610 .00051

13.801 .015260 .00053

13.851 .017170 .00053

13.901 .021350 .00051

13.951 .024540 .00052

14.001 .027330 .00054
478

12.700 .915720 .00053

12.750 .920130 .00051

12.800 .923130 .00052
500

12.800 .916140 .00052

12.850 .919390 .00050

12.900 .922460 .00050
600

13.500 .917910 .00053

13.550 .920970 .00051

13.600 .924460 .00051 
12.850 .927000 .00051 12.900 .930570 .00052 12.950 .933990 .00052 13.000 .936980 .00051 13.050 .941500 .00052 13.100 .944880 .00051 13.150 .948100 .00051 13.200 .951410 .00052 13.250 .954860 .00053 13.300 .958140 .00052 13.350 .960990 .00052 13.400 .964090 .00051 13.450 .967530 .00052 13.500 .972220 .00052 13.550 .974990 .00052 13.600 .977850 .00050 13.650 .980480 .00052 13.700 .984560 .00050 13.750 .987800 .00051 13.800 .990510 .00052 13.850 .993840 .00052 13.900 .997520 .00052 13.950 .999970 .00051 14.001 .003130 .00053 14.051 .005020 .00053 14.101 .009150 .00051 14.151 .012370 .00052 14.201 .015760 .00053 14.251 .017600 .00053 14.301 .021060 .00051
12.950 .925700 .00052 13.000 .929800 .00051 13.050 .933490 .00052 13.100 .936360 .00050 13.150 .940020 .00054 13.200 .942630 .00051 13.250 .947190 .00052 13.300 .950310 .00051 13.350 .954080 .00052 13.400 .957550 .00053 13.450 .961100 .00052 13.500 .964170 .00051 13.550 .965950 .00054 13.600 .969230 .00052 13.650 .972990 .00051 13.700 .976670 .00051 13.750 .979470 .00054 13.800 .982860 .00052 13.850 .986400 .00051 13.900 .988910 .00051 13.950 .993100 .00053 14.000 .995290 .00053 14.050 .998530 .00051 14.101 .002210 .00051 14.151 .005520 .00053 14.201 .007430 .00053 14.251 .010830 .00051 14.301 .013750 .00052 14.351 .017280 .00052 14.401 .019800 .00052
13.650 .929030 .00051

13.700 .931040 .00052

13.750 .935090 .00052

13.800 .938570 .00052

13.850 .941590 .00052

13.900 .945220 .00052 13.950 .947630 .00054 14.000 .950380 .00053 14.100 .957490 .00051 14.150 .959810 .00052 14.200 .962990 .00052 14.250 .966850 .00052 14.300 .969680 .00052 14.350 .973540 .00052 14.400 .976430 .00053 14.450 .979750 .00052 14.500 .982370 .00052 14.550 .985310 .00053 14.600 .988550 .00051 14.650 .991140 .00053 14.700 .995420 .00053 14.750 .996750 .00051 14.801 .000470 .00051 14.851 .002820 .00051 14.901 .007150 .00052 14.951 .009130 .00051 15.001 .011430 .00051 15.051 .015360 .00052 15.101 .017800 .00051 15.151 .020430 .00050 15.201 .024500 .00051

650

14.200 .918930 .00050 14.250 .922490 .00052 14.300 .926690 .00052 14.350 .928030 .00050 14.400 .932160 .00052 14.450 .935330 .00050 14.500 .938860 .00052 14.550 .940140 .00051 14.700 .950950 .00052 14.750 .953060 .00050 14.800 .956590 .00053 14.850 .959410 .00052 14.900 .962060 .00051 14.950 .965850 .00050 15.000 .967830 .00052 15.050 .971180 .00051
14.400 .918180 .00051

14.450 .921280 .00051

14.500 .925630 .00050

14.550 .928830 .00050

14.600 .931120 .00050 14.650 .935080 .00051 14.700 .937170 .00050 14.750 .940100 .00052 14.800 .943280 .00051 14.850 .946310 .00052 14.900 .949870 .00051 14.950 .952080 .00053 15.000 .955380 .00051 15.100 .961440 .00052 15.150 .964570 .00051 15.050 .958140 .00051
670

14.800 .929380 .00051 14.900 .934460 .00051 15.000 .940400 .00050 15.100 .946060 .00051 15.200 .952080 .00051 15.300 .958330 .00051 15.400 .963800 .00052 15.500 .969660 .00052 15.600 .975160 .00051 15.700 .981490 .00051 15.800 .987120 .00052 15.900 .992030 .00053 16.000 .997660 .00053 16.101 .002950 .00051 16.201 .009190 .00051 16.301 .014540 .00053 
15.100 .974420 .00051 15.150 .976780 .00051 15.200 .979480 .00051 15.250 .982940 .00051 15.300 .986510 .00052 15.350 .988380 .00051 15.400 .990570 .00051 15.450 .995190 .00051 15.500 .997910 .00051 15.551 .000220 .00051 15.601 .001600 .00052 15.651 .006010 .00051 15.701 .008210 .00052 15.751 .011180 .00052 15.801 .013740 .00050 15.851 .017530 .00053 15.901 .020010 .00051

680

15.000 .919490 .00051 15.100 .926090 .00052 15.200 .931030 .00051 15.700 .961260 .00052 15.800 .966790 .00051 15.900 .972410 .00052 16.000 .977440 .00052 16.100 .984520 .00051 16.200 .988400 .00052 16.300 .995040 .00051 16.400 .999530 .00050 16.501 .006420 .00052 16.601 .009530 .00053 16.701 .015790 .00051 16.801 .021530 .00051
15.200 .967190 .00050 15.250 .970200 .00051 15.300 .973230 .00052 15.350 .976540 .00052 15.400 .979510 .00051 15.450 .982000 .00052 15.500 .984940 .00051 15.550 .987730 .00052 15.600 .990340 .00051 15.650 .993870 .00051 15.700 .996050 .00051 15.750 .999810 .00052 15.801 .002310 .00053 15.851 .004060 .00052 15.901 .008420 .00052 15.951 .010500 .00052 16.001 .012580 .00052 16.051 .015900 .00052
16.401 .019410 .00051
690

15.500 .918680 .00051 15.600 .925200 .00051 15.700 .930680 .00051 15.800 .936070 .00051 15.900 .942370 .00052 16.000 .946240 .00051 16.100 .952880 .00051 16.200 .959180 .00051 16.300 .963340 .00052 16.400 .970330 .00052 16.500 .974800 .00051 16.600 .979550 .00051 16.700 .985440 .00050 16.800 .990530 .00052 16.900 .996120 .00050 17.001 .001730 .00051 17.101 .006090 .00051 17.201 .011980 .00050 17.301 .017210 .00052 17.401 .021990 .00051
698

16.500 .920810 .00050 16.600 .926360 .00053 16.700 .931890 .00052 16.800 .937680 .00052 16.900 .943210 .00051 17.000 .947710 .00050 17.100 .953700 .00052 17.200 .958690 .00051 17.300 .963310 .00051 17.400 .969490 .00051 17.500 .974720 .00050 17.600 .979580 .00050 17.700 .984660 .00050 17.800 .988600 .00051 17.900 .993620 .00052 18.000 .998950 .00052 18.101 .004220 .00050 18.201 .009000 .00052 18.301 .014580 .00051 18.401 .018650 .00054 18.501 .023860 .00052 
Pun43-sp-1000-01

12

21.800 .925860 .00030

22.100 .929880 .00029

22.400 .936190 .00031

23.000 .945880 .00030

23.300 .950380 .00029

23.600 .955370 .00030

23.900 .960090 .00030

24.200 .964490 .00029

24.500 .968250 .00029

24.800 .972440 .00029

25.100 .977600 .00029

25.400 .981490 .00028

25.700 .985390 .00029

26.000 .988380 .00029

26.300 .992350 .00028

26.600 .995450 .00029

26.900 .999980 .00028

27.201 .002680 .00028

27.501 .006540 .00027

27.801 .009630 .00028

28.101 .013100 .00028

28.201 .014470 .00027
15

18.000 .922760 .00035

18.200 .927360 .00035

18.400 .933100 .00034

18.600 .938230 .00035

18.800 .942930 .00034

19.000 .948570 .00034

19.200 .953350 .00034

19.400 .957970 .00034

19.600 .963150 .00034

19.800 .967910 .00034

20.000 .973040 .00035

20.200 .977170 .00033

20.400 .981590 .00034

20.600 .985640 .00033

20.800 .990000 .00034

21.000 .993640 .00033

21.200 .997780 .00033

21.401 .002030 .00033

21.601 .006380 .00032

21.801 .009900 .00033

22.001 .012580 .00033

22.101 .015690 .00032
25

13.900 .924530 .00042

14.000 .928870 .00042

14.100 .933460 .00041

14.200 .938310 .00041

14.300 .942630 .00041

14.400 .947150 .00040

14.500 .951330 .00042

14.600 .956080 .00042

14.700 .960770 .00041

14.800 .964770 .00042

14.900 .968810 .00041

15.000 .972750 .00041

15.100 .978570 .00042

15.200 .980530 .00042

15.300 .985310 .00041
30
13.000 .921760 .00043

13.100 .927040 .00044

13.200 .931900 .00043

13.300 .936250 .00044

13.400 .941620 .00044

13.500 .946990 .00043

13.600 .952550 .00042

13.700 .956290 .00044

13.900 .968170 .00043

14.000 .971870 .00043

14.100 .976510 .00043

14.200 .979810 .00044

14.300 .985570 .00042

14.400 .990750 .00044

14.500 .994730 .00044
20

15.200 .920610 .00039

15.300 .924640 .00039

15.400 .928570 .00039

15.500 .932830 .00039

15.600 .936930 .00039

15.700 .940600 .00039

15.800 .943650 .00040

15.900 .947270 .00038

16.000 .951240 .00038

16.100 .955420 .00039

16.200 .958070 .00038

16.300 .963330 .00039

16.400 .965660 .00040

16.500 .968610 .00040

16.600 .972240 .00039

16.700 .975390 .00039

16.800 .978810 .00039

16.900 .982370 .00038

17.000 .985940 .00039

17.100 .989290 .00038

17.200 .992560 .00038

17.300 .994750 .00039

17.400 .997630 .00038

17.501 .001980 .00039

17.601 .005120 .00037

17.701 .007500 .00038

17.801 .011080 .00038

17.901 .013830 .00038

18.001 .016380 .00038

32

12.700 .918020 .00044

12.800 .924290 .00044

12.900 .929160 .00044

13.000 .934000 .00044

13.100 .939750 .00044

13.200 .944820 .00045

13.300 .950680 .00043

13.400 .954480 .00044

13.500 .961460 .00044

13.600 .965080 .00042

13.700 .970470 .00044

13.800 .975800 .00045

13.900 .979880 .00043

14.000 .985310 .00043

14.100 .990690 .00044 
15.400 .989760 .00042 15.500 .993120 .00041 15.600 .997580 .00042 15.701 .000270 .00041 15.801 .005520 .00042 15.901 .008330 .00042 16.001 .012540 .00042 16.101 .015870 .00041 16.201 .020330 .00041

35

12.400 .918580 .00045 12.500 .924170 .00045 12.600 .929550 .00045 12.700 .935530 .00044 12.800 .941100 .00046 12.900 .945310 .00046 13.000 .951170 .00044 13.100 .956790 .00046 13.200 .963200 .00044 13.300 .967930 .00045 13.400 .971790 .00044 13.500 .978800 .00046 13.600 .982830 .00045 13.700 .987820 .00045 13.800 .993400 .00046 13.900 .998350 .00044 14.001 .003430 .00044 14.101 .008070 .00045 14.201 .012580 .00045 14.301 .017430 .00043
14.600 .999290 .00044 14.701 .002510 .00044 14.801 .007540 .00043 14.901 .012910 .00044 15.001 .016290 .00043 15.101 .020520 .00043
14.200 .995390 .00043 14.300 .999100 .00044 14.401 .003120 .00045 14.501 .009220 .00044 14.601 .012760 .00043 14.701 .017740 .00043
40

12.000 .917340 .00045 12.100 .924110 .00047 12.200 .928400 .00045 12.300 .935050 .00046 12.400 .941430 .00046 12.500 .946950 .00047 12.600 .953580 .00046 12.700 .958440 .00047 12.800 .964150 .00046 12.900 .970170 .00048 13.000 .974300 .00045 13.100 .979920 .00047 13.200 .986090 .00045 13.300 .991350 .00047 13.400 .995780 .00046 13.501 .002050 .00046 13.601 .007230 .00045 13.701 .012690 .00046 13.801 .017700 .00047 13.901 .022550 .00045
50

11.500 .918510 .00049 11.550 .921590 .00046 11.600 .924540 .00046 11.650 .928540 .00046 11.700 .932330 .00047 11.750 .934520 .00047 11.800 .937380 .00046 11.850 .940420 .00047 11.900 .943260 .00048 11.950 .947230 .00048 12.000 .950220 .00046 12.050 .954480 .00048 12.100 .956660 .00048 12.150 .960610 .00050 12.200 .962650 .00048 12.250 .965030 .00048 12.300 .969100 .00048 12.350 .971630 .00047 12.400 .974970 .00048 12.450 .978140 .00047 12.500 .981360 .00049 12.550 .983640 .00048 12.600 .986580 .00047 12.650 .989400 .00049 12.700 .992310 .00047 12.750 .995220 .00047 12.800 .998010 .00048 12.851 .000960 .00048 12.901 .003590 .00047 12.951 .006920 .00047 13.001 .008730 .00047 13.051 .012410 .00047 13.101 .014820 .00046 13.151 .017480 .00047 13.201 .020950 .00047 
60

11.200 .919250 .00048 11.250 .922740 .00049 11.300 .926190 .00048 11.350 .929800 .00049 11.400 .932680 .00049 11.450 .935760 .00047 11.500 .938680 .00049 11.550 .942420 .00050 11.600 .945380 .00049 11.650 .948800 .00048 11.700 .952660 .00049 11.750 .955930 .00049 11.800 .958740 .00048 11.850 .961340 .00049 11.900 .965960 .00049 11.950 .968940 .00049 12.000 .971510 .00050 12.050 .975310 .00049 12.100 .977730 .00049 12.150 .981450 .00047 12.200 .983330 .00049 12.250 .987250 .00049 12.300 .989820 .00050 12.350 .993470 .00049 12.400 .995950 .00050 12.450 .998660 .00048 12.501 .002590 .00048 12.551 .005350 .00049 12.601 .008080 .00050 12.651 .011230 .00049 12.701 .014630 .00049 12.751 .017330 .00049 12.801 .019700 .00048

90

10.700 .916680 .00051 10.750 .920460 .00049 10.800 .923040 .00051 10.850 .927240 .00051 10.900 .929660 .00050 10.950 .933950 .00049 11.000 .938000 .00050 11.050 .941390 .00050 11.100 .944370 .00050 11.150 .947990 .00050 11.200 .950900 .00049 11.250 .954660 .00050 11.300 .958450 .00050
70

11.000 .919810 .00049 11.050 .924660 .00050 11.100 .927820 .00049 11.150 .931230 .00049 11.200 .934010 .00050 11.250 .936800 .00049 11.300 .940120 .00049 11.350 .944170 .00049 11.400 .948170 .00050 11.450 .951490 .00050 11.500 .953870 .00050 11.550 .958210 .00050 11.600 .961350 .00047 11.650 .965090 .00049 11.700 .966830 .00050 11.750 .970750 .00049 11.800 .975750 .00050 11.850 .977720 .00049 11.900 .981040 .00049 11.950 .984370 .00049 12.000 .986610 .00049 12.050 .989930 .00049 12.100 .992240 .00053 12.150 .995970 .00051 12.200 .998330 .00050 12.251 .001340 .00049 12.301 .005010 .00048 12.351 .008230 .00049 12.401 .010970 .00049 12.451 .015170 .00049 12.501 .017950 .00050 12.551 .020750 .00050 12.601 .023250 .00049
80

10.800 .916210 .00050 10.850 .919500 .00051 10.900 .922570 .00050 10.950 .926780 .00049 11.000 .930470 .00048 11.050 .934240 .00049 11.100 .936970 .00050 11.150 .940850 .00050 11.200 .945130 .00050 11.250 .947740 .00050 11.300 .950620 .00050 11.350 .954470 .00050 11.400 .957880 .00049 11.450 .961290 .00049 11.500 .964510 .00050 11.550 .968450 .00049 11.600 .971450 .00049 11.650 .974290 .00051 11.700 .978220 .00050 11.750 .981180 .00050 11.800 .984750 .00049 11.850 .987470 .00050 11.900 .991000 .00049 11.950 .994970 .00050 12.000 .997250 .00048 12.050 .999690 .00049 12.101 .002450 .00051 12.151 .007490 .00049 12.201 .010390 .00050 12.251 .011900 .00051 12.301 .015620 .00050 12.351 .018540 .00050 12.401 .022010 .00050
10.700 .920790 .00051 10.750 .924580 .00051 10.800 .928710 .00050 10.850 .933290 .00051 10.900 .935340 .00050 10.950 .938180 .00050 11.000 .942900 .00050 11.050 .947160 .00051 11.100 .950960 .00050 11.150 .953020 .00050 11.200 .956580 .00049 11.250 .960700 .00050 11.300 .965010 .00051
120

10.600 .920450 .00052 10.650 .924170 .00051 10.700 .927240 .00050 10.750 .931510 .00051 10.800 .934650 .00050 10.850 .937990 .00051 10.900 .941900 .00051 10.950 .945920 .00050 11.000 .948970 .00052 11.050 .952240 .00051 11.100 .955980 .00052 11.150 .959390 .00050 11.200 .963350 .00051 
11.350 .961920 .00050 11.400 .964710 .00050 11.450 .968640 .00050 11.500 .973450 .00051 11.550 .975380 .00049 11.600 .979170 .00050 11.650 .981590 .00050 11.700 .985960 .00050 11.750 .988850 .00049 11.800 .991910 .00051 11.850 .995530 .00051 11.900 .998170 .00051 11.951 .001510 .00052 12.001 .004280 .00050 12.051 .007580 .00051 12.101 .011360 .00050 12.151 .013620 .00048 12.201 .018010 .00051 12.251 .019690 .00050 12.301 .023210 .00051
11.350 .967320 .00051 11.400 .971360 .00048 11.450 .974860 .00051 11.500 .977620 .00050 11.550 .979970 .00051 11.600 .983210 .00050 11.650 .987530 .00049 11.700 .990590 .00051 11.750 .994200 .00051 11.800 .996370 .00050 11.850 .999850 .00050 11.901 .003320 .00049 11.951 .005910 .00050 12.001 .008940 .00049 12.051 .013590 .00050 12.101 .017190 .00051 12.151 .020190 .00050 12.201 .021610 .00051
11.250 .966640 .00050 11.300 .969740 .00052 11.350 .974070 .00050 11.400 .977090 .00050 11.450 .980460 .00051 11.500 .982810 .00050 11.550 .986930 .00051 11.600 .990410 .00052 11.650 .992750 .00050 11.700 .996220 .00051 11.751 .000110 .00050 11.801 .003420 .00052 11.851 .006150 .00050 11.901 .009630 .00050 11.951 .013520 .00051 12.001 .016840 .00051 12.051 .020180 .00051 12.101 .022180 .00051
130

10.600 .923380 .00052 10.650 .926330 .00050 10.700 .930360 .00051 10.750 .932960 .00052 10.800 .937920 .00051 10.850 .941890 .00053 10.900 .944950 .00051 10.950 .948020 .00051 11.000 .951380 .00051 11.050 .955860 .00050 11.100 .958360 .00050 11.150 .962330 .00051 11.200 .967070 .00052 11.250 .969520 .00052 11.300 .971630 .00053 11.350 .976890 .00051 11.400 .978730 .00051 11.450 .983890 .00051 11.500 .986740 .00051 11.550 .990300 .00052 11.600 .993770 .00050 11.650 .996940 .00051 11.701 .000010 .00051 11.751 .002650 .00053 11.801 .006680 .00051 11.851 .008960 .00050 11.901 .012300 .00052
150

10.500 .917550 .00052 10.550 .920530 .00050 10.600 .925290 .00051 10.650 .928600 .00051 10.700 .931780 .00052 10.750 .936090 .00052 10.800 .939720 .00051 10.850 .943300 .00051 10.900 .946340 .00052 10.950 .949750 .00050 11.000 .954180 .00051 11.050 .957790 .00051 11.100 .960790 .00051 11.150 .964790 .00052 11.200 .967640 .00051 11.250 .971160 .00051 11.300 .974560 .00050 11.350 .978360 .00051 11.400 .981110 .00051 11.450 .985520 .00051 11.500 .988560 .00051 11.550 .990620 .00051 11.600 .994920 .00052 11.650 .999160 .00051 11.701 .001280 .00051 11.751 .004840 .00051 11.801 .007650 .00051
200

10.500 .917870 .00050 10.550 .921910 .00052 10.600 .925730 .00053 10.650 .929770 .00051 10.700 .932930 .00051 10.750 .936300 .00051 10.800 .939530 .00051 10.850 .943830 .00050 10.900 .947380 .00050 10.950 .950910 .00051 11.000 .953620 .00051 11.050 .957210 .00051 11.100 .961910 .00050 11.150 .965810 .00053 11.200 .968760 .00052 11.250 .971960 .00051 11.300 .975960 .00050 11.350 .978830 .00051 11.400 .981580 .00051 11.450 .985340 .00050 11.500 .988410 .00052 11.550 .992470 .00051 11.600 .994790 .00050 11.650 .999110 .00051 11.701 .002420 .00050 11.751 .004650 .00052 11.801 .007940 .00051 
11.951 .016340 .00052 12.001 .020290 .00052 12.051 .022340 .00051 12.101 .025260 .00051

\section{9}

10.600 .922560 .00052 10.650 .925490 .00050 10.700 .929420 .00050 10.750 .933600 .00051 10.800 .935730 .00051 10.850 .939830 .00051 10.900 .943580 .00050 10.950 .948050 .00052 11.000 .950000 .00051 11.050 .954480 .00053 11.100 .958140 .00049 11.150 .961270 .00051 11.200 .964610 .00051 11.250 .968550 .00052 11.300 .972040 .00050 11.350 .974850 .00051 11.400 .978160 .00052 11.450 .982240 .00051 11.500 .985410 .00051 11.550 .988680 .00054 11.600 .992990 .00052 11.650 .995490 .00052 11.700 .999010 .00051 11.751 .001440 .00051 11.801 .004980 .00052 11.851 .007990 .00052 11.901 .012030 .00050 11.951 .015590 .00051 12.001 .018170 .00050 12.051 .020830 .00051 12.101 .024410 .00051

478 11.100 .919370 .00051 11.150 .922360 .00051 11.200 .925840 .00051 11.250 .929710 .00049 11.300 .932900 .00052 11.350 .936570 .00051 11.400 .941760 .00050 11.450 .943960 .00050
11.851 .011130 .00051 11.901 .014280 .00051 11.951 .017530 .00051 12.001 .021800 .00050
11.851 .011940 .00052 11.901 .016300 .00051 11.951 .018830 .00051 12.001 .021610 .00051
10.600 .920510 .00050 10.650 .924280 .00051 10.700 .927570 .00051 10.750 .932230 .00050 10.800 .936380 .00051 10.850 .938650 .00049 10.900 .943450 .00050 10.950 .945870 .00053 11.000 .949490 .00052 11.050 .953610 .00050 11.100 .956450 .00050 11.150 .960360 .00050 11.200 .963040 .00051 11.250 .967440 .00050 11.300 .971390 .00049 11.350 .974620 .00051 11.400 .978690 .00052 11.450 .981520 .00050 11.500 .983330 .00052 11.550 .987600 .00051 11.600 .991150 .00050 11.650 .993960 .00051 11.700 .997140 .00050 11.751 .000680 .00051 11.801 .003780 .00051 11.851 .006620 .00051 11.901 .009990 .00052 11.951 .013420 .00051 12.001 .017350 .00052 12.051 .019790 .00052 12.101 .022850 .00051
359

10.800 .921510 .00052 10.850 .924700 .00051 10.900 .928330 .00049 10.950 .932450 .00052 11.000 .936330 .00050 11.050 .939490 .00053 11.100 .942780 .00051 11.250 .952950 .00052 11.300 .956530 .00051 11.350 .959330 .00051 11.400 .963400 .00053 11.450 .966600 .00050 11.500 .969510 .00052 11.550 .973410 .00052 11.600 .976410 .00051 11.650 .979470 .00050 11.700 .982530 .00051 11.750 .986350 .00052 11.800 .989630 .00051 11.850 .993130 .00050 11.900 .996020 .00051 11.950 .999090 .00053 12.001 .002110 .00051 12.051 .004560 .00051 12.101 .009670 .00051 12.151 .012850 .00051 12.201 .015820 .00052 12.251 .018990 .00050 12.301 .020940 .00050
11.200 .920770 .00051 11.250 .923520 .00052 11.300 .926610 .00052 11.350 .929500 .00050 11.400 .933470 .00051 11.450 .936200 .00051 11.500 .940800 .00050 11.550 .943290 .00051
600

11.800 .920650 .00049 11.850 .923730 .00050 11.900 .927270 .00052 11.950 .929550 .00051 12.000 .933730 .00049 12.050 .936420 .00049 12.100 .940840 .00049 12.150 .944300 .00051 
11.500 .946470 .00051 11.550 .949740 .00050 11.600 .954210 .00051 11.650 .956580 .00051 11.700 .959130 .00052 11.750 .963090 .00052 11.800 .966160 .00050 11.850 .970680 .00051 11.900 .973330 .00051 11.950 .976180 .00050 12.000 .980110 .00051 12.050 .982500 .00053 12.100 .986220 .00051 12.150 .989480 .00052 12.200 .991800 .00052 12.250 .995730 .00051 12.300 .998610 .00051 12.351 .001670 .00052 12.401 .005230 .00050 12.451 .008870 .00052 12.501 .011120 .00050 12.551 .015330 .00050 12.601 .016760 .00050 12.651 .020180 .00051 12.701 .023450 .00051

650

12.400 .921570 .00051 12.450 .922880 .00052 12.500 .926370 .00050 12.550 .928920 .00050 12.600 .932920 .00050 12.650 .935870 .00050 12.700 .939180 .00051 12.750 .941450 .00051 12.800 .945110 .00050 12.850 .947670 .00050 12.900 .950510 .00051 12.950 .952950 .00051 13.000 .956410 .00050 13.050 .959600 .00050 13.100 .962700 .00051 13.150 .965720 .00051 13.200 .968360 .00051 13.250 .971420 .00052 13.300 .973710 .00050 13.350 .979150 .00050 13.400 .980030 .00052 13.450 .983690 .00052
11.600 .947320 .00050 11.650 .948540 .00051 11.700 .952840 .00052 11.750 .956970 .00051 11.800 .960210 .00051 11.850 .963070 .00052 11.900 .967330 .00050 11.950 .970040 .00052 12.000 .971640 .00051 12.050 .977050 .00049 12.100 .979260 .00052 12.150 .982460 .00052 12.200 .986580 .00051 12.250 .989660 .00050 12.300 .991380 .00050 12.350 .996110 .00051 12.400 .998790 .00051 12.451 .001490 .00050 12.501 .005150 .00052 12.551 .007430 .00050 12.601 .010790 .00053 12.651 .013990 .00052 12.701 .016490 .00052 12.751 .020610 .00053 12.801 .022580 .00052
12.200 .946370 .00050 12.250 .949790 .00051 12.300 .952240 .00050 12.350 .955580 .00049 12.400 .958700 .00050 12.450 .962710 .00051 12.500 .964880 .00050 12.550 .967690 .00051 12.600 .971030 .00052 12.650 .975400 .00050 12.700 .976860 .00050 12.750 .980390 .00051 12.800 .984060 .00051 12.850 .986960 .00051 12.900 .989490 .00052 12.950 .992760 .00050 13.000 .995550 .00051 13.050 .999200 .00051 13.101 .000170 .00050 13.151 .004140 .00052 13.201 .007360 .00050 13.251 .010560 .00051 13.301 .013750 .00051 13.351 .016400 .00052 13.401 .019060 .00052
12.600 .922510 .00051 12.650 .925570 .00051 12.700 .927510 .00052 12.750 .930730 .00053 12.800 .933850 .00050 12.850 .937370 .00051 12.900 .940020 .00051 12.950 .943130 .00051 13.000 .946580 .00051 13.050 .949620 .00051 13.100 .951150 .00052 13.150 .955020 .00051 13.200 .957570 .00051 13.250 .961610 .00050 13.300 .963230 .00052 13.350 .967110 .00050 13.400 .969300 .00050 13.450 .971710 .00050 13.500 .974880 .00051 13.550 .977560 .00052 13.600 .981520 .00053 13.650 .984020 .00052
670

12.800 .921670 .00049 12.850 .923850 .00051 12.900 .927690 .00050 12.950 .929540 .00052 13.000 .932330 .00052 13.050 .935350 .00050 13.100 .939210 .00049 13.150 .941660 .00052 13.200 .944670 .00050 13.250 .946990 .00051 13.300 .950300 .00050 13.350 .954230 .00050 13.400 .956620 .00051 13.450 .959540 .00050 13.500 .962710 .00050 13.550 .965410 .00052 13.600 .967530 .00052 13.650 .969920 .00051 13.700 .973470 .00050 13.750 .976860 .00051 13.800 .979930 .00051 13.850 .981390 .00051 
13.500 .987090 .00051 13.550 .988940 .00051 13.600 .992650 .00051 13.650 .994240 .00051 13.700 .997440 .00052 13.750 .999680 .00051 13.801 .003000 .00052 13.851 .006110 .00052 13.901 .008990 .00052 13.951 .011710 .00050 14.001 .014530 .00052 14.051 .017750 .00050 14.101 .019870 .00051
13.700 .987240 .00052 13.750 .989150 .00051 13.800 .992030 .00051 13.850 .994970 .00051 13.900 .998160 .00051 13.951 .001140 .00050 14.001 .003590 .00050 14.051 .006630 .00052 14.101 .008490 .00051 14.151 .012550 .00052 14.201 .014120 .00050 14.251 .017180 .00051 14.301 .019980 .00051
13.900 .984860 .00050 13.950 .988050 .00051 14.000 .991500 .00051 14.050 .993900 .00050 14.100 .995690 .00052 14.150 .998190 .00052 14.201 .001830 .00049 14.251 .005560 .00050 14.301 .007080 .00052 14.351 .009490 .00051 14.401 .012750 .00052 14.451 .015110 .00050 14.501 .017490 .00053
680

13.100 .921620 .00050 13.200 .927460 .00051 13.300 .933020 .00050 13.400 .938110 .00053 13.500 .943860 .00051 13.600 .950210 .00050 13.700 .957390 .00050 13.800 .962010 .00050 13.900 .967680 .00050 14.000 .973340 .00051 14.100 .978940 .00050 14.200 .983930 .00049 14.300 .989630 .00052 14.400 .995140 .00051 14.501 .000730 .00051 14.601 .005610 .00050 14.701 .011220 .00051 14.801 .016300 .00051 14.901 .022100 .00051
690

13.500 .917940 .00050 13.600 .923910 .00051 13.700 .929150 .00051 13.800 .936450 .00051 13.900 .941100 .00052 14.000 .946680 .00051 14.100 .951660 .00050 14.200 .956690 .00051 14.300 .963470 .00051 14.400 .968300 .00052 14.500 .972970 .00052 14.600 .978500 .00050 14.700 .984500 .00052 14.800 .988930 .00050 14.900 .996180 .00050 15.001 .000270 .00051 15.101 .005770 .00050 15.201 .010420 .00051 15.301 .016140 .00052 15.401 .019980 .00053
698

14.300 .917070 .00051 14.400 .921830 .00051 14.500 .928500 .00051 14.600 .933720 .00052 14.700 .938800 .00051 14.800 .944140 .00049 14.900 .949010 .00051 15.000 .954570 .00051 15.100 .959040 .00051 15.200 .964270 .00051 15.300 .970400 .00051 15.400 .974660 .00049 15.500 .979000 .00051 15.600 .985370 .00050 15.700 .989770 .00051 15.800 .994080 .00050 15.900 .998630 .00052 16.001 .004750 .00052 16.101 .009470 .00050 16.201 .014080 .00051 16.301 .019090 .00050 
Pun43-sp-1000-10

12

20.700 .925390 .00029

21.000 .930800 .00029

21.300 .936430 .00028

21.600 .941120 .00028

21.900 .946080 .00029

22.200 .951630 .00028

22.500 .954800 .00029

22.800 .959980 .00027

23.100 .964630 .00028

23.400 .968920 .00028

23.700 .973240 .00028

24.000 .977460 .00027

24.300 .981290 .00028

24.600 .985720 .00028

24.900 .989130 .00028

25.200 .993110 .00027

25.500 .996470 .00028

25.800 .999450 .00027

26.101 .003850 .00027

26.401 .006880 .00027

26.701 .010310 .00027

26.901 .012730 .00027
15

16.900 .922630 .00032

17.100 .928500 .00033

17.300 .933870 .00032

17.500 .938960 .00032

17.700 .944170 .00032

17.900 .949130 .00033

18.100 .954900 .00033

18.300 .959880 .00032

18.500 .963970 .00031

18.700 .968740 .00031

18.900 .973460 .00032

19.100 .977160 .00032

19.300 .982140 .00031

19.500 .986660 .00032

19.700 .990030 .00031

19.900 .995440 .00032

20.100 .999130 .00031

20.301 .002830 .00032

20.501 .006690 .00031

20.701 .010730 .00030

20.901 .015020 .00032
25

12.700 .922320 .00039

12.800 .927550 .00039

12.900 .932350 .00039

13.000 .936120 .00040

13.100 .941480 .00038

13.200 .945250 .00039

13.300 .950080 .00039

13.400 .954660 .00040

13.500 .958760 .00039

13.600 .963550 .00039

13.700 .968470 .00040

13.800 .971960 .00039

13.900 .976000 .00039

14.000 .980280 .00040

14.100 .985220 .00039

14.200 .988550 .00039
30

11.800 .919520 .00041

11.900 .925860 .00041

12.000 .930190 .00042

12.100 .936120 .00040

12.200 .940810 .00040

12.300 .946590 .00041

12.400 .950950 .00041

12.500 .956610 .00042

12.600 .961930 .00039

12.700 .966330 .00042

12.800 .970790 .00041

12.900 .975590 .00040

13.000 .980600 .00041

13.100 .984970 .00041

13.200 .989440 .00042

13.300 .994250 .00040
20

14.100 .922610 .00036

14.200 .926560 .00037

14.300 .930890 .00037

14.400 .934650 .00038

14.500 .938380 .00036

14.600 .941720 .00036

14.700 .945510 .00036

14.800 .949670 .00037

14.900 .952820 .00035

15.000 .955940 .00035

15.100 .960040 .00035

15.200 .963370 .00036

15.300 .967640 .00035

15.400 .971540 .00036

15.500 .974140 .00035

15.600 .977190 .00036

15.700 .981490 .00035

15.800 .984780 .00037

15.900 .987770 .00036

16.000 .991300 .00036

16.100 .994310 .00035

16.200 .996960 .00035

16.300 .999550 .00036

16.401 .003760 .00037

16.501 .006760 .00036

16.601 .009410 .00036

16.701 .012390 .00036

16.801 .015510 .00037

32

11.600 .923220 .00041

11.700 .927520 .00041

11.800 .933330 .00041

11.900 .939490 .00041

12.000 .944400 .00042

12.100 .948910 .00041

12.200 .954210 .00040

12.300 .960180 .00041

12.400 .965540 .00040

12.500 .970540 .00041

12.600 .975660 .00042

12.700 .979930 .00041

12.800 .984720 .00040

12.900 .989100 .00040

13.000 .994800 .00041

13.100 .998980 .00042 
14.300 .992280 .00039 14.400 .996590 .00039 14.500 .999930 .00038 14.601 .005290 .00038 14.701 .008330 .00040 14.801 .012500 .00040 14.901 .016090 .00038 15.001 .019990 .00037
13.400 .998770 .00040 13.501 .003730 .00040 13.601 .007720 .00040 13.701 .012510 .00041 13.801 .016220 .00040 13.901 .021420 .00040
13.201 .004110 .00041 13.301 .009790 .00042 13.401 .013560 .00041 13.501 .017680 .00041 13.601 .022890 .00042
35

11.300 .922750 .00042 11.400 .929620 .00042 11.500 .935040 .00042 11.600 .939810 .00043 11.700 .946720 .00042 11.800 .950710 .00042 11.900 .957030 .00042 12.000 .961780 .00042 12.100 .967420 .00042 12.200 .972810 .00042 12.300 .978120 .00042 12.400 .983210 .00042 12.500 .988490 .00042 12.600 .994210 .00041 12.700 .998570 .00041 12.801 .003630 .00042 12.901 .009030 .00041 13.001 .013190 .00042 13.101 .018650 .00041
40 10.900 .924460 .00042 10.950 .926900 .00042 11.000 .929830 .00042 11.050 .932340 .00043 11.100 .936540 .00043 11.150 .939660 .00044 11.200 .941770 .00041 11.250 .944330 .00042 11.300 .947680 .00043 11.350 .950010 .00042 11.400 .953780 .00043 11.450 .956570 .00043 11.500 .959830 .00043 11.550 .961800 .00042 11.600 .965570 .00043 11.650 .967650 .00043 11.700 .970150 .00043 11.750 .974320 .00042 11.800 .976640 .00043 11.850 .979070 .00044 11.900 .982490 .00042 11.950 .984110 .00043 12.000 .987850 .00042 12.050 .990530 .00044 12.100 .992490 .00043 12.150 .994630 .00043 12.200 .998390 .00043 12.251 .000640 .00043 12.301 .004060 .00043 12.351 .005860 .00042 12.401 .008760 .00043 12.451 .010230 .00042 12.501 .014190 .00043 12.551 .016910 .00043 12.601 .019640 .00043
50

10.300 .920450 .00045 10.350 .924380 .00044 10.400 .927510 .00044 10.450 .929760 .00045 10.500 .933860 .00045 10.550 .937320 .00044 10.600 .939960 .00045 10.650 .943580 .00044 10.700 .946210 .00044 10.750 .950550 .00043 10.800 .953100 .00045 10.850 .956800 .00044 10.900 .959030 .00046 10.950 .962020 .00045 11.000 .966220 .00044 11.050 .969230 .00045 11.100 .971510 .00044 11.150 .975130 .00044 11.200 .978520 .00045 11.250 .980230 .00045 11.300 .983020 .00044 11.350 .986810 .00044 11.400 .990960 .00045 11.450 .993750 .00043 11.500 .995500 .00044 11.550 .998500 .00043 11.601 .001280 .00045 11.651 .003980 .00044 11.701 .006710 .00045 11.751 .010250 .00045 11.801 .013110 .00044 11.851 .015410 .00043 11.901 .018110 .00044 
60

10.000 .923150 .00046 10.050 .926770 .00045 10.100 .930110 .00045 10.150 .933250 .00044 10.200 .936300 .00044 10.250 .940330 .00045 10.300 .943950 .00045 10.350 .947130 .00046 10.400 .950390 .00045 10.450 .954790 .00046 10.500 .958010 .00045 10.550 .959480 .00047 10.600 .963280 .00045 10.650 .966460 .00044 10.700 .969860 .00044 10.750 .973660 .00045 10.800 .977160 .00045 10.850 .979320 .00045 10.900 .982500 .00046 10.950 .985590 .00045 11.000 .988710 .00045 11.050 .992220 .00043 11.100 .994360 .00045 11.150 .998940 .00046 11.201 .000730 .00046 11.251 .004130 .00045 11.301 .006960 .00045 11.351 .009980 .00046 11.401 .012880 .00046 11.451 .015890 .00046 11.501 .019360 .00046

90

9.400 .917210 .00046 9.450 .921480 .00045 9.500 .925070 .00046 9.550 .929210 .00046 9.600 .932790 .00047 9.650 .936200 .00048 9.700 .939300 .00047 9.750 .943190 .00047 9.800 .946830 .00046 9.850 .951040 .00046 9.900 .954380 .00046 9.950 .957080 .00046 10.000 .960670 .00046 10.050 .964460 .00045 10.100 .968020 .00047
70

9.700 .917580 .00046

9.750 .922160 .00046

9.800 .925530 .00045

9.850 .930020 .00046

9.900 .932690 .00046

9.950 .936300 .00046

10.000 .940040 .00045

10.050 .943370 .00045

10.100 .946470 .00045

10.150 .950720 .00044

10.200 .953900 .00045

10.250 .957620 .00047

10.300 .960750 .00045

10.350 .963480 .00045

10.400 .967460 .00045

10.450 .971080 .00045

10.500 .973770 .00047

10.550 .977170 .00045

10.600 .979970 .00046

10.700 .986860 .00045

10.750 .990140 .00045

10.800 .993080 .00047

10.850 .996180 .00046

10.901 .000160 .00047

10.951 .003570 .00046

11.001 .006200 .00047

11.051 .009030 .00046

11.101 .012440 .00045

11.151 .014480 .00046

11.201 .018290 .00044
80

9.500 .915700 .00047

9.550 .920180 .00046

9.600 .923070 .00045

9.650 .926210 .00045

9.700 .930810 .00045

9.750 .934620 .00046

9.800 .937530 .00045

9.850 .941490 .00046

9.900 .944650 .00046

9.950 .949490 .00045

10.000 .952380 .00047

10.050 .955400 .00044

10.100 .958240 .00047

10.150 .962660 .00045

10.200 .965200 .00047

10.250 .969160 .00047

10.300 .972000 .00044

10.350 .976010 .00045

10.400 .979640 .00047

10.450 .982310 .00047

10.500 .985630 .00047

10.550 .989010 .00046

10.600 .993270 .00046

10.650 .995090 .00045

10.700 .998840 .00046

10.751 .002650 .00047

10.801 .005550 .00047

10.851 .007730 .00047

10.901 .011160 .00048

10.951 .014670 .00047

11.001 .017850 .00047

\section{0}

9.300 .916220 .00046

9.350 .920460 .00047

9.400 .923860 .00045

9.450 .927240 .00047

9.500 .931640 .00047

9.550 .935290 .00047

9.600 .938110 .00046

9.650 .942120 .00047

9.700 .946570 .00047

9.750 .949860 .00047

9.800 .953500 .00048

9.850 .957130 .00045

9.900 .960540 .00046

9.950 .964300 .00046

10.000 .967910 .00046
9.200 .917290 .00047

9.250 .921580 .00046

9.300 .925790 .00047

9.350 .929000 .00046

9.400 .933180 .00048

9.450 .937040 .00047

9.500 .940520 .00046

9.550 .944040 .00046

9.600 .947430 .00046

9.650 .951620 .00048

9.700 .955040 .00047

9.750 .958870 .00047

9.800 .962360 .00048

9.850 .965850 .00048

9.900 .969420 .00046 
10.150 .972050 .00045 10.200 .973750 .00047 10.250 .977570 .00047 10.300 .981390 .00048 10.350 .984590 .00048 10.400 .988300 .00047 10.450 .991380 .00047 10.500 .994580 .00046 10.550 .998230 .00046 10.601 .001490 .00046 10.651 .004330 .00047 10.701 .008400 .00047 10.751 .011070 .00047 10.801 .015530 .00046 10.851 .016490 .00048 10.901 .020580 .00048

130

9.200 .921530 .00047

9.250 .925380 .00048

9.300 .928800 .00047

9.350 .933280 .00046

9.400 .936210 .00046

9.450 .939930 .00046

9.500 .944160 .00048

9.550 .947750 .00048

9.600 .950340 .00047

9.650 .954840 .00047

9.700 .958440 .00047

9.750 .962120 .00047

9.800 .966000 .00047

9.900 .972150 .00046

9.950 .976050 .00046

10.000 .979710 .00046

10.050 .982620 .00047

10.100 .985440 .00046

10.150 .989620 .00048

10.200 .993130 .00048

10.250 .997370 .00047

10.301 .000450 .00047

10.351 .004210 .00049

10.401 .007490 .00047

10.451 .010930 .00047

10.501 .012950 .00048

10.551 .016210 .00046

10.601 .020100 .00048
10.050 .970240 .00047

10.100 .974440 .00047

10.150 .978420 .00048

10.200 .980580 .00048

10.250 .983950 .00047

10.300 .988470 .00047

10.350 .991820 .00047

10.400 .994660 .00047

10.450 .998080 .00048

10.501 .001180 .00046

10.551 .004000 .00047

10.601 .008070 .00047

10.651 .011780 .00047

10.701 .014750 .00046

10.751 .017860 .00048

10.801 .020900 .00047
9.950 .972210 .00047

10.000 .976400 .00046

10.050 .979440 .00048

10.100 .982680 .00045

10.150 .986440 .00047

10.200 .989230 .00047

10.250 .993750 .00048

10.300 .997070 .00047

10.351 .000350 .00047

10.401 .003170 .00048

10.451 .006670 .00048

10.501 .010240 .00047

10.551 .012950 .00047

10.601 .016470 .00046

10.651 .020060 .00046

10.701 .023260 .00047

\begin{tabular}{|c|c|}
\hline 150 & 200 \\
\hline 9.100 .918660 .00047 & 9.100 .922780 .00047 \\
\hline 9.150 .921240 .00048 & 9.150 .925350 .00046 \\
\hline 9.200 .925670 .00046 & 9.200 .930850 .00046 \\
\hline 9.250 .929910 .00046 & 9.250 .933940 .00048 \\
\hline 9.300 .932970 .00048 & 9.300 .937060 .00047 \\
\hline .350 .936820 .00046 & 9.350 .940950 .00047 \\
\hline 9.400 .940150 .00048 & 9.400 .944780 .00046 \\
\hline 9.450 .945130 .00046 & 9.450 .948480 .00047 \\
\hline 9.500 .947440 .00047 & 9.500 .952270 .00050 \\
\hline 9.550 .951900 .00048 & 9.550 .955780 .00047 \\
\hline 9.600 .955920 .00047 & 9.600 .959610 .00047 \\
\hline 90.00046 & 9.650 .962540 .00047 \\
\hline 0.00047 & 9.700 .967160 .00048 \\
\hline 9.750 .965760 .00048 & 9.750 .970280 .00047 \\
\hline 9.800 .969360 .00049 & 9.800 .973030 .00047 \\
\hline 9.850 .974810 .00046 & 9.850 .976940 .00048 \\
\hline 9.900 .976230 .00047 & 9.900 .980860 .00048 \\
\hline 0.00048 & 9.950 .984250 .00047 \\
\hline 0.00047 & 10.000 .987400 .00048 \\
\hline 10.050 .98 & 10.050 .991110 .00048 \\
\hline 10.100 .990840 .00046 & 10.100 .994400 .00046 \\
\hline 10.150 .994290 .00046 & 10.150 .998170 .00047 \\
\hline 10.200 .998020 .00047 & 10.201 .000950 .00047 \\
\hline 0.00047 & 10.251 .005210 .00048 \\
\hline 10.301 .004 & 10.301 .008810 .00047 \\
\hline 10.351 .007630 .00047 & 10.351 .010680 .00047 \\
\hline 10.401 .011280 .00047 & 10.401 .014880 .00047 \\
\hline 10.451 .01 & 10.451 .017660 .00048 \\
\hline 10.501 .0175 & 10.501 .020890 .00047 \\
\hline
\end{tabular}


239

9.100 .922410 .00047

9.150 .925610 .00048

9.200 .929580 .00047

9.250 .932480 .00048

9.300 .937220 .00047

9.350 .941080 .00047

9.400 .944700 .00048

9.450 .948070 .00047

9.500 .950870 .00047

9.550 .955030 .00047

9.600 .958940 .00049

9.650 .962700 .00047

9.700 .965220 .00046

9.750 .969480 .00047

9.800 .973920 .00047

9.850 .975440 .00047

9.900 .980230 .00048

9.950 .983370 .00049

10.000 .986330 .00047

10.050 .990890 .00047

10.100 .994420 .00047

10.150 .996120 .00047

10.201 .000700 .00046

10.251 .003260 .00048

10.301 .007170 .00047

10.351 .010310 .00047

10.401 .013110 .00048

10.451 .016580 .00048

10.501 .020610 .00048
250

9.100 .921290 .00046

9.150 .924680 .00048

9.200 .928440 .00047

9.250 .933070 .00047

9.300 .937230 .00048

9.350 .939470 .00046

9.400 .943510 .00047

9.500 .951480 .00048

9.550 .954730 .00048

9.600 .957980 .00047

9.650 .961910 .00047

9.700 .964870 .00046

9.750 .968510 .00047

9.800 .972000 .00048

9.850 .975120 .00048

9.900 .979770 .00047

9.950 .982160 .00046

10.000 .986420 .00046

10.050 .989430 .00047

10.100 .993000 .00047

10.150 .995690 .00048

10.201 .000090 .00048

10.251 .003570 .00049

10.301 .006420 .00046

10.351 .009230 .00048

10.401 .012010 .00048

10.451 .016420 .00047

10.501 .019090 .00047
9.450 .948230 .00046

359

9.200 .920150 .00047

9.250 .924350 .00047

9.300 .927770 .00047

9.350 .930680 .00047

9.400 .935330 .00047

9.450 .939090 .00048

9.500 .941710 .00048

9.550 .945950 .00046

9.600 .948830 .00048

9.650 .952460 .00048

9.700 .956060 .00047

9.750 .959500 .00047

9.800 .963090 .00047

9.850 .966620 .00048

9.900 .970390 .00048

9.950 .973260 .00047

10.000 .976960 .00048

10.050 .981080 .00049

10.100 .983640 .00048

10.150 .987380 .00048

10.200 .990550 .00048

10.250 .993150 .00047

10.300 .996290 .00046

10.351 .000130 .00048

10.401 .003410 .00048

10.451 .006510 .00048

10.501 .009670 .00049

10.551 .012680 .00047

10.601 .016320 .00046

10.651 .019670 .00047

10.701 .022950 .00046

500

9.500 .919600 .00048

9.550 .923070 .00048

9.600 .927940 .00047

9.650 .931050 .00049

9.700 .934070 .00047

9.750 .937750 .00046

9.800 .941020 .00047

9.850 .944090 .00046

9.900 .948440 .00048

9.950 .951790 .00048

10.000 .954210 .00047

10.050 .957220 .00047

10.100 .961840 .00047

10.150 .965090 .00048
600

10.000 .924100 .00046

10.050 .925940 .00048

10.100 .931160 .00046

10.150 .934180 .00046

10.200 .937520 .00047

10.250 .940710 .00046

10.300 .944580 .00047

10.350 .947230 .00048

10.400 .950350 .00046

10.450 .953180 .00047

10.500 .956520 .00046

10.550 .959960 .00047

10.600 .962620 .00047

10.650 .967000 .00048 
10.100 .966560 .00047

10.150 .969180 .00048

10.200 .973440 .00047

10.250 .976710 .00047

10.300 .979100 .00048

10.350 .982680 .00048

10.400 .986430 .00048

10.450 .989450 .00047

10.500 .992490 .00047

10.550 .994920 .00047

10.600 .999430 .00049

10.651 .002020 .00047

10.701 .004180 .00048

10.751 .008260 .00046

10.801 .010970 .00047

10.851 .014200 .00048

10.901 .018000 .00047

650

10.400 .920380 .00046 10.450 .923760 .00047 10.500 .926310 .00045 10.550 .929340 .00048 10.600 .932540 .00048 10.650 .936450 .00045 10.700 .939580 .00046 10.750 .942230 .00047 10.800 .945500 .00047 10.850 .948310 .00047 10.900 .952240 .00048 10.950 .953780 .00047 11.000 .958200 .00047 11.050 .960300 .00047 11.100 .964680 .00046 11.150 .966120 .00047 11.200 .969190 .00048 11.250 .973230 .00047 11.300 .975870 .00046 11.350 .978050 .00047 11.400 .981650 .00047 11.450 .984500 .00047 11.500 .986820 .00048 11.550 .989650 .00047 11.600 .993260 .00048 11.650 .996090 .00047 11.700 .998270 .00048 11.751 .001500 .00047 11.801 .003930 .00047
10.200 .968760 .00047 10.250 .971560 .00048 10.300 .975200 .00047 10.350 .977680 .00049 10.400 .981790 .00047 10.450 .984450 .00047 10.500 .987550 .00047 10.550 .990410 .00048 10.600 .993170 .00046 10.650 .997780 .00047 10.701 .001090 .00048 10.751 .003020 .00047 10.801 .006590 .00049 10.851 .008310 .00047 10.901 .012820 .00048 10.951 .015410 .00048 11.001 .019090 .00048 11.051 .022130 .00048
10.700 .969640 .00046 10.750 .971780 .00047 10.800 .975580 .00047 10.850 .977790 .00047 10.900 .981840 .00048 10.950 .985110 .00046 11.000 .987390 .00048 11.050 .990690 .00047 11.100 .993820 .00048 11.150 .995800 .00049 11.200 .998500 .00049 11.251 .001880 .00048 11.301 .005750 .00048 11.351 .008720 .00047 11.401 .010800 .00046 11.451 .014190 .00049 11.501 .017390 .00049
660

10.600 .924830 .00046 10.650 .927800 .00046 10.700 .931820 .00047 10.750 .934670 .00047 10.800 .936050 .00047 10.850 .939390 .00047 10.900 .942790 .00047 10.950 .945830 .00046 11.000 .948910 .00048 11.050 .952280 .00048 11.100 .953430 .00045 11.150 .958380 .00046 11.200 .961000 .00048 11.250 .963230 .00047 11.300 .967110 .00047 11.350 .969450 .00047 11.400 .972780 .00047 11.450 .975880 .00047 11.500 .978700 .00047 11.550 .981580 .00048 11.600 .983890 .00047 11.650 .987180 .00047 11.850 .998530 .00047 11.901 .001480 .00048 11.951 .004370 .00048 12.001 .006450 .00048 12.051 .009350 .00046 12.101 .012730 .00048 12.151 .014730 .00047
670

10.700 .920930 .00047 10.750 .923790 .00046 10.800 .927280 .00046 10.850 .929900 .00046 10.900 .933410 .00048 10.950 .936100 .00047 11.000 .939410 .00048 11.050 .941550 .00048 11.100 .945460 .00047 11.300 .957120 .00046 11.350 .959380 .00047 11.400 .962800 .00048 11.450 .965220 .00049 11.500 .968970 .00048 11.550 .970290 .00047 11.600 .974250 .00047 11.650 .976860 .00046 11.700 .979540 .00048 11.750 .982930 .00048 11.800 .985460 .00049 11.850 .987830 .00047 11.900 .989870 .00047 11.950 .993750 .00049 12.000 .996430 .00047 12.050 .999030 .00048 12.101 .001850 .00047 12.151 .003960 .00047 12.201 .008590 .00048 12.251 .010570 .00048 
11.851 .007150 .00048 11.901 .009660 .00047 11.951 .012300 .00047 12.001 .015430 .00047 12.051 .017830 .00047 12.101 .020800 .00048

680

10.900 .920210 .00048 11.000 .925890 .00047 11.100 .932780 .00048 11.200 .936870 .00046 11.300 .943900 .00049 11.400 .949570 .00047 11.500 .955470 .00046 11.600 .961140 .00047 11.700 .966100 .00047 11.800 .971400 .00047 11.900 .977340 .00048 12.000 .982840 .00048 12.100 .988550 .00046 12.200 .994350 .00047 12.300 .999450 .00047 12.401 .004750 .00047 12.501 .009800 .00046 12.601 .015010 .00049 12.701 .020230 .00048
12.201 .016370 .00047

12.251 .020250 .00047 12.301 .022800 .00048
12.301 .013050 .00048

12.351 .015130 .00047 12.401 .017010 .00047
690 11.200 .917490 .00048 11.300 .923260 .00047 11.400 .929270 .00047 11.500 .935370 .00046 11.600 .940240 .00046 11.700 .946020 .00046 11.800 .951690 .00047 11.900 .957660 .00047 12.000 .963140 .00048 12.100 .968160 .00048 12.200 .973900 .00047 12.300 .978300 .00047 12.400 .983990 .00047 12.500 .989560 .00047 12.600 .995350 .00046 12.700 .999090 .00048 12.801 .005400 .00047 12.901 .009650 .00047 13.001 .015960 .00047 13.101 .020250 .00047
698

11.900 .923430 .00048 12.000 .928270 .00046 12.100 .933000 .00047 12.200 .938170 .00046 12.300 .944820 .00047 12.400 .949940 .00046 12.500 .954060 .00047 12.600 .960280 .00049 12.700 .964580 .00047 12.800 .969120 .00046 12.900 .975050 .00047 13.000 .980190 .00047 13.100 .984570 .00047 13.200 .990570 .00048 13.300 .994570 .00046 13.400 .999790 .00047 13.501 .004020 .00047 13.601 .009480 .00047 13.701 .014000 .00046 13.801 .018970 .00047 
Pun46-sp-1000-00

12

26.100 .926940 .00030

26.500 .932200 .00030

26.900 .938580 .00029

27.300 .944110 .00030

27.700 .947780 .00030

28.100 .953840 .00029

28.500 .958300 .00029

28.900 .962650 .00029

29.300 .967860 .00028

29.700 .972450 .00028

30.100 .976540 .00029

30.500 .980300 .00028

30.900 .984620 .00029

31.300 .987670 .00028

31.700 .992520 .00027

32.100 .995970 .00028

32.500 .999670 .00027

32.901 .002680 .00027

33.301 .006430 .00027

33.701 .009000 .00026

34.001 .011710 .00027
21.300 .922700 .00035

21.500 .927400 .00035

21.700 .932230 .00035

21.900 .936560 .00034

22.100 .941360 .00034

22.300 .944870 .00034

22.500 .949660 .00034

22.700 .953950 .00034

22.900 .958150 .00034

23.100 .961660 .00034

23.300 .965720 .00033

23.500 .970000 .00034

23.700 .972870 .00034

23.900 .976990 .00034

24.100 .981470 .00033

24.300 .984170 .00034

24.500 .988290 .00033

24.700 .991790 .00034

24.900 .996150 .00032

25.100 .998600 .00033

25.301 .002120 .00033

25.501 .005090 .00033

25.701 .007990 .00032

25.901 .011380 .00033

26.101 .015160 .00031
25

16.400 .920770 .00043

16.500 .925240 .00043

16.600 .928250 .00043

16.700 .933690 .00043

16.800 .937900 .00043

16.900 .941160 .00042

17.000 .945450 .00043

17.100 .950380 .00042

17.200 .953660 .00042

17.300 .956790 .00044

17.400 .962000 .00042
30

15.500 .921680 .00045

15.600 .925790 .00045

15.700 .931180 .00045

15.800 .935820 .00045

15.900 .941910 .00046

16.000 .945680 .00045

16.100 .951430 .00045

16.200 .955530 .00044

16.300 .959480 .00044

16.400 .963780 .00045

16.500 .969470 .00044
20

18.000 .921470 .00041

18.100 .925240 .00039

18.200 .927820 .00040

18.300 .932790 .00041

18.400 .935140 .00040

18.500 .938280 .00039

18.600 .942580 .00039

18.700 .946040 .00039

18.800 .949160 .00039

18.900 .951250 .00038

19.000 .954260 .00040

19.100 .957750 .00039

19.200 .961160 .00039

19.300 .965030 .00039

19.400 .968110 .00038

19.500 .970440 .00039

19.600 .973680 .00038

19.700 .976160 .00040

19.800 .979970 .00039

19.900 .981150 .00038

20.000 .985120 .00038

20.100 .988240 .00039

20.200 .990130 .00040

20.300 .992560 .00038

20.400 .996040 .00038

20.500 .998760 .00040

20.601 .002180 .00038

20.701 .003740 .00039

20.801 .006540 .00039

20.901 .009330 .00039

21.001 .011840 .00038

21.101 .014800 .00038

21.201 .017370 .00037

32

15.200 .920850 .00045 15.300 .925490 .00046 15.400 .930210 .00045 15.500 .935650 .00047 15.600 .940170 .00044 15.700 .945350 .00046 15.800 .948920 .00046 15.900 .955010 .00045 16.000 .959690 .00045 16.100 .963640 .00046 16.200 .968350 .00045 
17.500 .965620 .00043 17.600 .968950 .00042 17.700 .972280 .00042 17.800 .977030 .00041 17.900 .980460 .00042 18.000 .984280 .00041 18.100 .987090 .00042 18.200 .991390 .00041 18.300 .995510 .00043 18.400 .998240 .00043 18.501 .001520 .00041 18.601 .006010 .00041 18.701 .009060 .00042 18.801 .012410 .00041 18.901 .016270 .00041 19.001 .018590 .00042
16.600 .971550 .00044 16.700 .976600 .00044 16.800 .980370 .00044 16.900 .984400 .00044 17.000 .989320 .00044 17.100 .992990 .00045 17.200 .997410 .00045 17.301 .001960 .00045 17.401 .005480 .00044 17.501 .008880 .00045 17.601 .014170 .00044 17.701 .017530 .00044
16.300 .972470 .00046 16.400 .978400 .00046 16.500 .982170 .00044 16.600 .985290 .00046 16.700 .990630 .00044 16.800 .995150 .00045 16.900 .999620 .00045 17.001 .003730 .00046 17.101 .007720 .00045 17.201 .012410 .00045 17.301 .015870 .00044 17.401 .019190 .00044
35

14.800 .917380 .00047 14.900 .923290 .00046 15.000 .928120 .00047 15.100 .933590 .00046 15.200 .938430 .00046 15.300 .943350 .00046 15.400 .948220 .00046 15.500 .952960 .00045 15.600 .957460 .00045 15.700 .964350 .00046 15.800 .967580 .00047 15.900 .972510 .00047 16.000 .977530 .00048 16.100 .981770 .00046 16.200 .987100 .00046 16.400 .996150 .00047 16.501 .000080 .00047 16.601 .004590 .00046 16.701 .009440 .00046 16.801 .013900 .00046 16.901 .017620 .00046
60

13.500 .920480 .00049 13.550 .922800 .00049 13.600 .926030 .00051 13.650 .929610 .00049 13.700 .932460 .00052 13.750 .936510 .00050
40

14.400 .919600 .00049 14.500 .925180 .00047 14.600 .930630 .00048 14.700 .935670 .00048 14.800 .941310 .00048 14.900 .946520 .00046 15.000 .951970 .00048 15.100 .956660 .00048 15.200 .963940 .00047 15.300 .967870 .00048 15.400 .972540 .00048 15.500 .978350 .00048 15.600 .982600 .00048 15.700 .987210 .00048 15.800 .992600 .00047 15.900 .996730 .00049 16.001 .002420 .00048 16.101 .005230 .00046 16.201 .011140 .00048 16.301 .016270 .00048 16.401 .020290 .00048
50

13.800 .917530 .00049 13.900 .923440 .00049 14.000 .930060 .00051 14.100 .935840 .00051 14.200 .941030 .00050 14.300 .947070 .00050 14.400 .953220 .00047 14.500 .958870 .00047 14.600 .964680 .00051 14.700 .968910 .00049 14.800 .975070 .00050 14.900 .980700 .00049 15.000 .986820 .00049 15.100 .990830 .00048 15.200 .996160 .00048 15.301 .001880 .00049 15.401 .006670 .00049 15.501 .010970 .00050 15.601 .018340 .00048 15.701 .022870 .00049
70

13.300 .919790 .00050 13.350 .924390 .00051 13.400 .927200 .00051 13.450 .930350 .00050 13.500 .934470 .00051 13.550 .936570 .00052
80

13.100 .917080 .00052 13.150 .920340 .00052 13.200 .923520 .00051 13.250 .927490 .00051 13.300 .930700 .00052 13.350 .933890 .00052 
13.800 .938860 .00051

13.850 .942490 .00050

13.900 .944140 .00049

13.950 .948400 .00050

14.000 .950240 .00050

14.050 .953610 .00050

14.100 .956590 .00050

14.150 .960070 .00050

14.200 .963810 .00050

14.250 .965750 .00049

14.300 .969270 .00050

14.350 .972290 .00051

14.400 .974330 .00051

14.450 .977700 .00051

14.500 .979550 .00050

14.550 .982100 .00051

14.600 .985800 .00051

14.650 .987690 .00051

14.700 .992000 .00050

14.750 .994580 .00049

14.800 .996980 .00050

14.850 .999730 .00050

14.901 .002710 .00052

14.951 .005480 .00051

15.001 .008090 .00050

15.051 .011850 .00051

15.101 .013440 .00049

15.151 .016520 .00049

15.201 .019780 .00051
13.600 .939670 .00050

13.650 .942840 .00051

13.700 .944350 .00051

13.750 .948980 .00052

13.800 .951700 .00051

13.850 .956250 .00052

13.900 .959520 .00051

13.950 .960820 .00051

14.000 .964470 .00050

14.050 .967930 .00050

14.100 .971500 .00052

14.150 .973680 .00053

14.200 .975850 .00049

14.250 .979800 .00052

14.300 .982920 .00052

14.350 .985950 .00050

14.400 .987800 .00050

14.450 .992090 .00052

14.500 .994070 .00052

14.550 .997160 .00050

14.601 .000040 .00051

14.651 .002750 .00050

14.701 .005530 .00050

14.751 .008700 .00050

14.801 .011770 .00052

14.851 .014320 .00051

14.901 .017220 .00051

14.951 .019990 .00050

15.001 .022220 .00051
13.400 .937460 .00051

13.450 .940670 .00050

13.500 .943800 .00050

13.550 .946550 .00052

13.600 .949490 .00051

13.650 .952730 .00050

13.700 .955220 .00051

13.750 .959740 .00051

13.800 .963180 .00052

13.850 .965430 .00050

13.900 .969080 .00052

13.950 .972390 .00051

14.000 .974260 .00051

14.050 .977260 .00052

14.100 .981250 .00052

14.150 .984690 .00052

14.200 .987540 .00051

14.250 .988970 .00050

14.300 .992620 .00052

14.350 .996140 .00051

14.400 .998820 .00052

14.451 .001990 .00051

14.501 .005480 .00052

14.551 .007350 .00053

14.601 .009910 .00052

14.651 .012820 .00052

14.701 .015780 .00051

14.751 .019800 .00052

14.801 .021710 .00053
90

13.000 .917550 .00052

13.050 .921510 .00052

13.100 .925410 .00052

13.150 .927990 .00051

13.200 .931890 .00051

13.250 .934280 .00052

13.300 .937510 .00052

13.350 .941070 .00052

13.400 .945590 .00052

13.450 .947920 .00052

13.500 .950390 .00052

13.550 .953600 .00052

13.600 .957550 .00053

13.650 .959340 .00052

13.700 .963580 .00052

13.750 .966600 .00053

13.800 .968850 .00053

13.850 .972610 .00053
100

12.900 .916790 .00053

12.950 .919100 .00052

13.000 .921990 .00053

13.050 .926270 .00053

13.100 .929040 .00051

13.150 .932810 .00052

13.200 .937060 .00051

13.250 .939230 .00053

13.300 .942810 .00050

13.350 .945110 .00052

13.400 .948710 .00055

13.450 .952590 .00052

13.500 .956910 .00052

13.550 .958690 .00050

13.600 .962420 .00052

13.650 .965950 .00052

13.700 .968420 .00051

13.750 .972090 .00052
120

12.900 .919630 .00051

12.950 .924320 .00053

13.000 .926440 .00053

13.050 .931230 .00053

13.100 .934970 .00053

13.150 .937840 .00053

13.200 .941670 .00051

13.250 .944830 .00051

13.300 .947550 .00052

13.350 .950070 .00052

13.400 .953890 .00055

13.450 .958140 .00052

13.500 .962000 .00053

13.550 .963840 .00053

13.600 .968110 .00053

13.650 .969460 .00052

13.700 .973800 .00053

13.750 .978060 .00052 
13.900 .975560 .00052 13.950 .979100 .00053 14.000 .982570 .00050 14.050 .986280 .00052 14.100 .988130 .00053 14.150 .991490 .00051 14.200 .994740 .00052 14.250 .996630 .00052 14.301 .000460 .00052 14.351 .002980 .00050 14.401 .005770 .00052 14.451 .008740 .00052 14.501 .012180 .00052 14.551 .014680 .00051 14.601 .018310 .00052 14.651 .020190 .00052 14.701 .024140 .00052
13.800 .975340 .00052 13.850 .977800 .00052 13.900 .981630 .00052 13.950 .984450 .00052 14.000 .987360 .00053 14.050 .990380 .00053 14.100 .995380 .00051 14.150 .996990 .00053 14.201 .000220 .00052 14.251 .003090 .00054 14.301 .006530 .00052 14.351 .008830 .00052 14.401 .013110 .00052 14.451 .014700 .00052 14.501 .017100 .00051 14.551 .021520 .00052 14.601 .023580 .00053
13.800 .980190 .00052 13.850 .984090 .00052 13.900 .986840 .00052 13.950 .988500 .00052 14.000 .992280 .00053 14.050 .995660 .00053 14.100 .998850 .00053 14.151 .001190 .00053 14.201 .004700 .00053 14.251 .008410 .00053 14.301 .010670 .00053 14.351 .013680 .00052 14.401 .016770 .00052 14.451 .019270 .00051 14.501 .022510 .00052
130

12.800 .915190 .00053 12.850 .919140 .00051 12.900 .922100 .00051 12.950 .925700 .00054 13.000 .929970 .00052 13.050 .932170 .00052 13.100 .936230 .00052 13.150 .939280 .00052 13.200 .943280 .00053 13.250 .946650 .00053 13.300 .949490 .00053 13.350 .953500 .00053 13.400 .955970 .00052 13.450 .959090 .00053 13.500 .963030 .00053 13.550 .965050 .00053 13.600 .968170 .00053 13.650 .971780 .00053 13.700 .975100 .00055 13.750 .979450 .00053 13.800 .982010 .00052 13.850 .984330 .00054 13.900 .987610 .00053 13.950 .991720 .00052 14.000 .994360 .00054 14.050 .998030 .00052 14.101 .000640 .00052 14.151 .003470 .00052 14.201 .006700 .00052 14.251 .010710 .00053
150

12.800 .916550 .00053 12.850 .920170 .00052 12.900 .924140 .00053 12.950 .928040 .00052 13.000 .930730 .00051 13.050 .933760 .00052 13.100 .938590 .00052 13.150 .940450 .00052 13.200 .944280 .00053 13.250 .947800 .00053 13.300 .951270 .00053 13.350 .954430 .00052 13.400 .958400 .00053 13.450 .961440 .00053 13.500 .963660 .00052 13.550 .967950 .00052 13.600 .970250 .00053 13.650 .974810 .00052 13.700 .976910 .00052 13.750 .980940 .00053 13.800 .983400 .00051 13.850 .986860 .00052 13.900 .989080 .00052 13.950 .993310 .00052 14.000 .996870 .00052 14.050 .999670 .00053 14.101 .001860 .00053 14.151 .005630 .00052 14.201 .008820 .00052 14.251 .012100 .00052
200

12.800 .914870 .00054 12.850 .917940 .00052 12.900 .922070 .00052 12.950 .924280 .00053 13.000 .927760 .00052 13.050 .931790 .00054 13.100 .934860 .00053 13.150 .938170 .00053 13.200 .942670 .00053 13.250 .945270 .00052 13.300 .948610 .00052 13.350 .952700 .00055 13.400 .956050 .00053 13.450 .958900 .00053 13.500 .962200 .00052 13.550 .966330 .00054 13.600 .969040 .00051 13.650 .971690 .00053 13.700 .975040 .00054 13.750 .977570 .00053 13.800 .981460 .00053 13.850 .984230 .00053 13.900 .987800 .00054 13.950 .990660 .00052 14.000 .993830 .00053 14.050 .997020 .00051 14.100 .999990 .00053 14.151 .003550 .00052 14.201 .005200 .00053 14.251 .008680 .00054 
14.301 .012870 .00052 14.351 .016450 .00053 14.401 .019920 .00052
14.301 .014000 .00053 14.351 .018400 .00052 14.401 .020340 .00051
14.301 .012040 .00051 14.351 .015070 .00052 14.401 .018050 .00052
239

12.900 .918160 .00051 13.000 .924130 .00053 13.050 .928690 .00054 13.100 .932180 .00053 13.150 .934400 .00050 13.200 .938810 .00051 13.250 .941120 .00051 13.300 .944770 .00052 13.350 .948530 .00052 13.400 .950540 .00052 13.450 .955490 .00052 13.500 .957620 .00052 13.550 .962520 .00053 13.600 .963930 .00053 13.650 .967650 .00053 13.700 .970280 .00052 13.750 .974210 .00053 13.800 .977250 .00053 13.850 .981660 .00054 13.900 .983460 .00053 13.950 .986530 .00051 14.000 .990210 .00053 14.050 .991690 .00052 14.100 .996750 .00052 14.150 .998390 .00051 14.201 .002600 .00052 14.251 .005440 .00054 14.301 .008610 .00053 14.351 .010980 .00052 14.401 .013800 .00053 14.451 .017700 .00053 14.501 .020280 .00052 14.551 .023180 .00051 14.601 .025730 .00052

478 13.400 .919570 .00051 13.450 .921790 .00053 13.500 .924940 .00052 13.550 .928120 .00050 13.600 .932460 .00052 13.650 .934560 .00052 13.700 .938530 .00051
250

12.900 .916100 .00052 12.950 .919540 .00053 13.000 .923440 .00053 13.050 .926940 .00053 13.100 .930230 .00053 13.150 .933090 .00052 13.200 .937870 .00053 13.250 .941560 .00053 13.300 .942850 .00052 13.350 .946770 .00054 13.400 .950380 .00052 13.450 .953720 .00053 13.500 .956830 .00052 13.550 .959400 .00051 13.600 .963010 .00054 13.650 .966310 .00053 13.700 .969280 .00052 13.750 .973030 .00053 13.800 .975690 .00054 13.850 .979460 .00052 13.900 .981510 .00053 13.950 .985130 .00053 14.000 .988700 .00052 14.050 .991800 .00052 14.100 .994640 .00052 14.150 .997430 .00053 14.201 .000810 .00053 14.251 .004020 .00052 14.301 .006810 .00052 14.351 .010360 .00052 14.401 .012900 .00054 14.451 .015550 .00051 14.501 .018590 .00053
359

13.200 .922050 .00052 13.250 .924950 .00051 13.300 .927060 .00052 13.350 .931820 .00051 13.400 .934670 .00052 13.450 .937100 .00052 13.500 .941380 .00052 13.550 .944910 .00052 13.600 .947030 .00050 13.650 .950850 .00052 13.700 .955330 .00053 13.750 .956300 .00052 13.800 .960290 .00051 13.850 .964690 .00052 13.900 .965700 .00052 13.950 .970590 .00052 14.000 .973260 .00052 14.050 .976120 .00051 14.100 .979130 .00053 14.150 .982350 .00052 14.200 .985960 .00051 14.250 .989050 .00053 14.300 .991880 .00053 14.350 .996300 .00051 14.400 .997260 .00052 14.451 .001150 .00053 14.501 .003100 .00053 14.551 .007230 .00052 14.601 .009760 .00051 14.651 .013720 .00052 14.701 .015670 .00051 14.751 .017740 .00051 14.801 .022100 .00053
13.500 .921790 .00053 13.550 .925100 .00051 13.600 .927420 .00051 13.650 .931940 .00052 13.700 .935650 .00052 13.750 .938320 .00053 13.800 .941430 .00052
600

13.700 .915590 .00053 13.800 .922740 .00051 13.900 .928820 .00052 14.000 .936320 .00051 14.100 .941850 .00050 14.200 .947040 .00053 14.300 .952650 .00051 
13.750 .940430 .00051 13.800 .945430 .00051 13.850 .948180 .00051 13.900 .950940 .00053 13.950 .954210 .00051 14.000 .958180 .00052 14.050 .961320 .00050 14.100 .963970 .00052 14.150 .967100 .00050 14.200 .970300 .00052 14.250 .972520 .00051 14.300 .976060 .00052 14.350 .979840 .00051 14.400 .983310 .00052 14.450 .985450 .00052 14.500 .988910 .00052 14.550 .992410 .00052 14.600 .994940 .00053 14.650 .997660 .00050 14.701 .000530 .00052 14.751 .003200 .00054 14.801 .007080 .00053 14.851 .009660 .00053 14.901 .013050 .00052 14.951 .014780 .00052 15.001 .018190 .00052 15.051 .021580 .00052 15.101 .023510 .00051
13.850 .944180 .00050 13.900 .947710 .00052 13.950 .951220 .00052 14.000 .953950 .00052 14.050 .957430 .00052 14.100 .961390 .00053 14.150 .962730 .00052 14.200 .965300 .00052 14.250 .968900 .00051 14.300 .973010 .00050 14.350 .976230 .00053 14.400 .979070 .00052 14.450 .982620 .00051 14.500 .984710 .00051 14.550 .987740 .00052 14.600 .990770 .00051 14.650 .994350 .00052 14.700 .997250 .00051 14.750 .999680 .00052 14.801 .003090 .00052 14.851 .006820 .00053 14.901 .008820 .00051 14.951 .011620 .00052 15.001 .014470 .00051 15.051 .017880 .00051 15.101 .019880 .00052
14.400 .960150 .00050 14.500 .966230 .00051 14.600 .973110 .00052 14.700 .978260 .00051 14.800 .986300 .00051 14.900 .990920 .00050 15.000 .997120 .00050 15.101 .001610 .00051 15.201 .008510 .00052 15.301 .014450 .00051 15.401 .019880 .00053 15.501 .025610 .00051
650

13.900 .917220 .00051 14.000 .922570 .00051 14.100 .930590 .00050 14.200 .934480 .00051 14.300 .942880 .00051 14.500 .955310 .00052 14.600 .960230 .00051 14.700 .965370 .00052 14.800 .973260 .00052 14.900 .978640 .00051 15.000 .985910 .00052 15.100 .990880 .00052 15.200 .996410 .00051 15.301 .002460 .00053 15.401 .008240 .00051 15.501 .012880 .00052 15.601 .020230 .00051 15.701 .025270 .00050
700

14.200 .919180 .00054 14.250 .922650 .00051 14.300 .925420 .00052 14.350 .929060 .00054 14.400 .931500 .00051 14.450 .935000 .00051 14.500 .937810 .00051 14.550 .941540 .00053 14.600 .944080 .00051 14.650 .946980 .00052 14.700 .950890 .00051 14.750 .954400 .00051 14.800 .955980 .00051 14.850 .958660 .00051 14.900 .962520 .00051 14.950 .965210 .00051 15.000 .968490 .00050 15.050 .970750 .00052 15.100 .975050 .00052
750

14.500 .915160 .00050 14.600 .920740 .00050 14.700 .928260 .00050 14.800 .934830 .00053 14.900 .940920 .00050 15.000 .946030 .00052 15.100 .952240 .00052 15.200 .958540 .00050 15.300 .964440 .00052 15.400 .970800 .00052 15.500 .976060 .00052 15.600 .981490 .00052 15.700 .987530 .00051 15.800 .993640 .00051 15.900 .998160 .00051 16.001 .003440 .00052 16.101 .009120 .00051 16.201 .015680 .00051 16.301 .021150 .00051 
15.150 .977890 .00053

15.200 .980230 .00052

15.250 .983770 .00052

15.300 .986590 .00049

15.350 .988310 .00054

15.400 .992690 .00050

15.450 .994480 .00052

15.500 .997840 .00051

15.551 .000560 .00050

15.601 .002750 .00052

15.651 .006250 .00053

15.701 .008740 .00052

15.751 .011870 .00052

15.801 .015430 .00051

15.851 .017610 .00051

15.901 .020010 .00052
760

14.600 .917080 .00051

14.700 .923300 .00050

14.800 .928710 .00051

14.900 .934900 .00051

15.000 .940720 .00050

15.100 .947770 .00051

15.200 .953800 .00052

15.300 .959900 .00051

15.400 .965350 .00051

15.500 .971740 .00052

15.600 .976940 .00053

15.700 .982140 .00051

15.800 .988580 .00052

15.900 .993750 .00052

16.000 .999380 .00050

16.101 .005160 .00052

16.201 .011010 .00051

16.301 .016770 .00051

16.401 .021460 .00050
765

14.600 .913190 .00050

14.700 .920200 .00051

14.800 .926710 .00051

14.900 .932870 .00049

15.000 .938530 .00051

15.100 .944720 .00052

15.200 .950610 .00052

15.300 .955930 .00052

15.400 .962300 .00050

15.500 .968260 .00051

15.600 .973680 .00050

15.700 .979310 .00050

15.800 .985000 .00051

15.900 .990200 .00051

16.000 .997780 .00051

16.101 .002860 .00052

16.201 .007970 .00051

16.301 .013530 .00050

16.401 .018780 .00051
770

14.700 .916570 .00051

14.800 .922350 .00052

14.900 .928560 .00051

15.000 .935370 .00051

15.100 .941680 .00052

15.200 .946760 .00050

15.300 .952520 .00051

15.400 .959240 .00050

15.500 .964370 .00050

15.600 .970800 .00051

15.700 .977560 .00052

15.800 .982310 .00050

15.900 .987160 .00049

16.000 .992840 .00051

16.100 .999710 .00051

16.201 .004850 .00051

16.301 .010340 .00052

16.401 .015600 .00052

16.501 .020130 .00051 


\section{5}

14.700 .914740 .00051

14.800 .920430 .00051

14.900 .927040 .00052

15.000 .933830 .00052

15.100 .938690 .00052

15.200 .944410 .00052

15.300 .951960 .00050

15.400 .957220 .00052

15.500 .963060 .00051

15.600 .968420 .00050

15.700 .974050 .00052

15.800 .979950 .00051

15.900 .984670 .00051

16.000 .990980 .00052

16.100 .996900 .00051

16.201 .003750 .00051

16.301 .007370 .00050

16.401 .014160 .00053

16.501 .018900 .00052

16.601 .023700 .00050 
Pun46-sp-1000-01

12

24.400 .926040 .00029

24.800 .931020 .00029

25.200 .936470 .00029

25.600 .942120 .00029

26.000 .946730 .00028

26.400 .952310 .00028

26.800 .956660 .00029

27.200 .961850 .00028

27.600 .966840 .00027

28.000 .969840 .00027

28.400 .975020 .00027

28.800 .979820 .00027

29.200 .983250 .00027

29.600 .987440 .00027

30.000 .990920 .00028

30.400 .994630 .00028

30.800 .998520 .00027

31.201 .001110 .00026

31.601 .004960 .00027

32.001 .007610 .00026

32.401 .010920 .00027

32.601 .013310 .00027
25

14.900 .923570 .00040 15.000 .928070 .00042 15.100 .932610 .00039 15.200 .936010 .00040 15.300 .940380 .00042 15.400 .944720 .00040 15.500 .948310 .00041 15.600 .952780 .00042 15.700 .956630 .00041 15.800 .960360 .00041 15.900 .964210 .00043
19.800 .924390 .00033

20.000 .929050 .00033

20.200 .933620 .00034

20.400 .938410 .00033

20.600 .942880 .00034

20.800 .947390 .00034

21.000 .950890 .00033

21.200 .955480 .00033

21.400 .959180 .00034

21.600 .963310 .00033

21.800 .967580 .00034

22.000 .971260 .00032

22.200 .975410 .00032

22.400 .979090 .00032

22.600 .983230 .00033

22.800 .985870 .00033

23.000 .989280 .00032

23.200 .992410 .00032

23.400 .996150 .00033

23.600 .999610 .00031

23.801 .002890 .00033

24.001 .005880 .00032

24.201 .009330 .00032

24.401 .012180 .00031

24.501 .014120 .00032
20

16.400 .921010 .00038

16.500 .924210 .00038

16.600 .927930 .00039

16.700 .931540 .00038

16.800 .934470 .00038

16.900 .937230 .00038

17.000 .940460 .00038

17.100 .943730 .00039

17.200 .947950 .00039

17.300 .950910 .00037

17.400 .953370 .00039

17.500 .957600 .00038

17.600 .960700 .00039

17.700 .964090 .00037

17.800 .967030 .00038

17.900 .969460 .00037

18.000 .973360 .00038

18.100 .975770 .00039

18.200 .978270 .00038

18.300 .980650 .00037

18.400 .984100 .00039

18.500 .987340 .00036

18.600 .990040 .00037

18.700 .993120 .00037

18.800 .995160 .00038

18.900 .998870 .00038

19.001 .000960 .00038

19.101 .004050 .00037

19.201 .006220 .00037

19.301 .009010 .00037

19.401 .012320 .00037

19.501 .014480 .00037

19.601 .017040 .00037

30

32

13.900 .921610 .00042

14.000 .925670 .00043

14.100 .930930 .00043

14.200 .935510 .00043

14.300 .939950 .00044

14.400 .944840 .00043

14.500 .948840 .00043

14.600 .954470 .00042

14.700 .957560 .00042

14.800 .962530 .00041

14.900 .968500 .00043
13.600 .920140 .00043

13.700 .924620 .00043

13.800 .930370 .00044

13.900 .934550 .00044

14.000 .940250 .00045

14.100 .943920 .00045

14.200 .949620 .00045

14.300 .953970 .00044

14.400 .958930 .00044

14.500 .963620 .00044

14.600 .967250 .00044 
16.000 .968380 .00041 16.100 .971890 .00040 16.200 .975660 .00041 16.300 .979440 .00042 16.400 .983670 .00041 16.500 .986140 .00041 16.600 .990520 .00040 16.700 .993400 .00041 16.800 .996490 .00040 16.901 .001850 .00041 17.001 .004800 .00042 17.101 .008620 .00040 17.201 .011720 .00041 17.301 .014450 .00040
15.000 .971900 .00043 15.100 .975810 .00042 15.200 .980190 .00042 15.300 .984450 .00044 15.400 .988020 .00043 15.500 .993250 .00044 15.600 .996480 .00042 15.701 .001830 .00043 15.801 .004860 .00043 15.901 .009610 .00043 16.001 .013290 .00043 16.101 .016240 .00042 16.201 .020100 .00042
14.700 .972200 .00044 14.900 .980770 .00044 15.000 .985680 .00044 15.100 .989750 .00044 15.200 .994230 .00045 15.300 .998810 .00043 15.401 .002540 .00043 15.501 .007660 .00046 15.601 .010820 .00045 15.701 .015520 .00042 15.801 .019730 .00042
35

13.300 .922180 .00044 13.400 .926850 .00045 13.500 .932860 .00044 13.600 .938220 .00044 13.700 .942940 .00045 13.800 .948620 .00043 13.900 .952620 .00045 14.000 .957260 .00044 14.100 .962690 .00045 14.200 .967610 .00044 14.300 .972280 .00044 14.400 .977330 .00044 14.500 .982090 .00045 14.600 .985120 .00045 14.700 .990370 .00045 14.800 .994410 .00046 14.901 .000000 .00045 15.001 .003780 .00043 15.101 .008610 .00044 15.201 .012990 .00045 15.301 .018050 .00045

60 11.900 .919310 .00047 11.950 .922170 .00048 12.000 .925490 .00048 12.050 .928870 .00049 12.100 .931680 .00049 12.150 .935990 .00048 12.200 .937880 .00049 12.250 .941820 .00048
40

50

12.800 .917930 .00044 12.900 .923740 .00046 13.000 .929800 .00047 13.100 .934890 .00046 13.200 .939710 .00046 13.300 .946040 .00046 13.400 .951810 .00045 13.500 .955890 .00045 13.600 .961560 .00045 13.700 .967400 .00046 13.800 .972070 .00045 13.900 .976920 .00046 14.000 .981530 .00044 14.100 .986780 .00045 14.200 .992540 .00047 14.300 .997170 .00045 14.401 .001600 .00045 14.501 .006200 .00044 14.601 .011260 .00047 14.701 .016180 .00046 14.801 .020190 .00048

12.200 .916420 .00047 12.300 .922750 .00047 12.400 .928460 .00049 12.500 .935710 .00048 12.600 .940660 .00047 12.700 .945970 .00048 12.800 .952850 .00049 12.900 .957920 .00048 13.000 .964450 .00047 13.100 .968980 .00046 13.200 .974710 .00048 13.300 .979780 .00048 13.400 .985160 .00046 13.500 .991220 .00049 13.600 .996460 .00047 13.701 .001760 .00048 13.801 .006350 .00049 13.901 .011280 .00047 14.001 .016560 .00047 14.101 .023450 .00046

70

11.600 .914150 .00050 11.700 .921180 .00049 11.800 .927570 .00049 11.900 .933600 .00049 12.000 .940110 .00048 12.100 .945630 .00049 12.200 .952820 .00050 12.300 .957560 .00049
80

11.500 .917190 .00051 11.550 .920260 .00049 11.600 .924190 .00049 11.650 .926850 .00051 11.700 .931270 .00050 11.750 .933860 .00049 11.800 .937650 .00050 11.850 .940510 .00049 
12.300 .944730 .00048

12.350 .947070 .00047

12.400 .949900 .00048

12.450 .953500 .00049

12.500 .956340 .00049

12.550 .959770 .00048

12.600 .962220 .00048

12.650 .964980 .00049

12.700 .968360 .00048

12.750 .971640 .00048

12.800 .973620 .00047

12.850 .976420 .00049

12.900 .980170 .00049

12.950 .983410 .00049

13.000 .985840 .00049

13.050 .988710 .00049

13.100 .991330 .00048

13.150 .994470 .00049

13.200 .997670 .00047

13.251 .000040 .00049

13.301 .003120 .00049

13.351 .005540 .00049

13.401 .008310 .00048

13.451 .010270 .00048

13.501 .014010 .00049

13.551 .016460 .00048

13.601 .019950 .00048

90

11.400 .917480 .00050

11.450 .920990 .00050

11.500 .925250 .00050

11.550 .928100 .00051

11.600 .931130 .00050

11.650 .935110 .00050

11.700 .938330 .00050

11.750 .941900 .00048

11.800 .944490 .00050

11.850 .948830 .00049

11.900 .951280 .00051

11.950 .954390 .00049

12.000 .957970 .00051

12.050 .960000 .00049

12.100 .964360 .00049

12.150 .968460 .00050

12.200 .969670 .00050

12.250 .973170 .00051

12.300 .976220 .00049

12.350 .980150 .00049
12.400 .965320 .00049

12.500 .971260 .00050

12.600 .975980 .00050

12.700 .982270 .00050

12.800 .987980 .00050

12.900 .994790 .00050

13.000 .999060 .00049

13.101 .005920 .00048

13.201 .011460 .00049

13.301 .016470 .00050

13.401 .022900 .00051
11.900 .944620 .00050

11.950 .946210 .00050

12.000 .950600 .00050

12.050 .953900 .00050

12.100 .956730 .00050

12.150 .960150 .00049

12.200 .962860 .00050

12.250 .966990 .00049

12.300 .969090 .00049

12.350 .972620 .00051

12.400 .975340 .00050

12.450 .977700 .00048

12.500 .981510 .00048

12.550 .984180 .00050

12.600 .987630 .00048

12.650 .990060 .00050

12.700 .992760 .00049

12.750 .995710 .00048

12.800 .999400 .00049

12.851 .002100 .00049

12.901 .004540 .00050

12.951 .007200 .00050

13.001 .010630 .00051

13.051 .014910 .00049

13.101 .017280 .00048

13.151 .018980 .00050

13.201 .022600 .00051

100

11.300 .916510 .00051

11.350 .920570 .00050

11.400 .923630 .00048

11.450 .926990 .00050

11.500 .930260 .00051

11.550 .933520 .00049

11.600 .936230 .00049

11.650 .940510 .00050

11.700 .943460 .00050

11.750 .946990 .00049

11.800 .949570 .00050

11.850 .953940 .00050

11.900 .957590 .00052

11.950 .959620 .00050

12.000 .963100 .00050

12.050 .967250 .00051

12.100 .970000 .00049

12.150 .972540 .00049

12.200 .976470 .00050

12.250 .979210 .00050
120

11.350 .925700 .00052 11.400 .928660 .00051

11.450 .931550 .00050

11.500 .935910 .00052

11.550 .938490 .00052

11.600 .942540 .00049

11.650 .946970 .00049

11.700 .948110 .00050

11.750 .952190 .00052

11.800 .955650 .00050

11.850 .959210 .00050 11.900 .962600 .00051 11.950 .965120 .00052 12.000 .968620 .00051 12.050 .970890 .00050 12.100 .975230 .00052 12.150 .978570 .00051 12.200 .981410 .00051 12.250 .983300 .00050 12.300 .988370 .00051 
12.400 .982670 .00050

12.450 .985470 .00051

12.500 .989280 .00050

12.550 .991070 .00049

12.600 .994760 .00050

12.650 .998390 .00051

12.701 .000790 .00050

12.751 .004570 .00051

12.801 .007470 .00050

12.851 .009560 .00050

12.901 .012700 .00050

12.951 .015380 .00050

13.001 .018460 .00050

13.051 .021790 .00050

13.101 .023970 .00049

130

11.200 .917440 .00051

11.250 .920390 .00051

11.300 .924680 .00050

11.350 .927270 .00049

11.400 .930790 .00051

11.450 .934280 .00051

11.500 .937950 .00050

11.550 .941100 .00050

11.600 .945060 .00050

11.650 .947960 .00050

11.700 .951970 .00052

11.750 .954410 .00051

11.800 .959030 .00051

11.850 .961050 .00050

11.900 .965140 .00050

11.950 .966600 .00050

12.000 .970900 .00052

12.050 .974070 .00050

12.100 .976630 .00051

12.150 .980030 .00053

12.200 .982950 .00052

12.250 .986770 .00051

12.300 .989320 .00051

12.350 .993050 .00051

12.400 .995520 .00049

12.450 .999100 .00050

12.501 .002440 .00049

12.551 .004520 .00050

12.601 .007940 .00051

12.651 .011130 .00051

12.701 .014460 .00051

12.751 .015660 .00051

12.801 .019650 .00052
12.300 .981980 .00051

12.350 .985100 .00052

12.400 .988120 .00050

12.450 .991440 .00051

12.500 .994210 .00051

12.550 .997390 .00051

12.601 .000090 .00051

12.651 .003720 .00051

12.701 .005910 .00050

12.751 .009220 .00050

12.801 .012520 .00049

12.851 .014790 .00050

12.901 .018410 .00050

12.951 .020720 .00049

13.001 .024070 .00050
12.350 .990870 .00049

12.400 .993980 .00052

12.450 .996460 .00049

12.500 .999530 .00052

12.551 .003230 .00050

12.601 .006250 .00050

12.651 .008730 .00050

12.701 .011590 .00050

12.751 .014280 .00050

12.801 .017560 .00053

12.851 .021010 .00050

12.901 .023660 .00052
150

11.200 .919310 .00052

11.250 .922870 .00049

11.300 .926450 .00051

11.350 .929210 .00051

11.400 .933130 .00051

11.450 .937030 .00050

11.500 .941140 .00050

11.550 .942430 .00051

11.600 .945570 .00050

11.650 .949600 .00051

11.700 .953340 .00051

11.750 .956460 .00051

11.800 .959190 .00051

11.850 .962910 .00050

11.900 .966440 .00051

11.950 .970250 .00051

12.000 .973070 .00051

12.050 .975650 .00049

12.100 .977920 .00051

12.150 .981780 .00049

12.200 .985530 .00052

12.250 .988630 .00051

12.300 .991630 .00050

12.350 .994850 .00050

12.400 .998300 .00051

12.451 .000550 .00052

12.501 .003820 .00051

12.551 .008160 .00050

12.601 .010350 .00052

12.651 .013270 .00051

12.701 .016010 .00051

12.751 .020120 .00051

12.801 .022050 .00052
200

11.200 .918430 .00050

11.250 .921490 .00050

11.300 .924780 .00051

11.350 .928250 .00050

11.400 .931740 .00052

11.450 .935230 .00051

11.500 .939570 .00050

11.550 .942860 .00050

11.600 .945620 .00051

11.650 .948180 .00051

11.700 .950900 .00050

11.750 .955590 .00051

11.800 .958600 .00052

11.850 .960470 .00050

11.900 .964590 .00052

11.950 .968180 .00051

12.000 .971920 .00051

12.050 .974090 .00051

12.100 .977960 .00051

12.150 .981350 .00051

12.200 .984070 .00051

12.250 .987110 .00051

12.300 .990550 .00051

12.350 .993470 .00052

12.400 .997520 .00052

12.451 .000900 .00051

12.501 .002930 .00051

12.551 .005860 .00052

12.601 .009260 .00051

12.651 .012080 .00050

12.701 .014270 .00051

12.751 .018490 .00052

12.801 .021060 .00051 
239

11.300 .921710 .00050 11.350 .924860 .00050 11.400 .926910 .00050 11.450 .931750 .00051 11.500 .935160 .00050 11.550 .938250 .00051 11.600 .940860 .00052 11.650 .944970 .00051 11.700 .948490 .00052 11.750 .952100 .00052 11.800 .955350 .00051 11.850 .957890 .00052 11.900 .960430 .00052 11.950 .963570 .00052 12.000 .967920 .00050 12.050 .971320 .00052 12.100 .973740 .00049 12.150 .976550 .00051 12.200 .980270 .00051 12.250 .984380 .00050 12.300 .986350 .00051 12.350 .990050 .00052 12.400 .992930 .00051 12.450 .995400 .00051 12.500 .998460 .00051 12.551 .001880 .00052 12.601 .004740 .00052 12.651 .007820 .00052 12.701 .011190 .00051 12.751 .013740 .00051 12.801 .016910 .00050 12.851 .020320 .00050 12.901 .023260 .00052

\section{8}

11.700 .916870 .00050 11.750 .921570 .00051 11.800 .924030 .00052 11.850 .926670 .00052 11.900 .931350 .00051 11.950 .934050 .00050 12.000 .937890 .00050 12.050 .940320 .00050 12.100 .943050 .00051 12.150 .947510 .00051
250

11.300 .920360 .00050 11.350 .922650 .00051 11.400 .927600 .00051 11.450 .930720 .00050 11.500 .934390 .00051 11.550 .936400 .00050 11.600 .940580 .00050 11.650 .944130 .00051 11.700 .947380 .00052 11.750 .949540 .00050 11.800 .953510 .00050 11.850 .957360 .00051 11.900 .961400 .00052 11.950 .963680 .00052 12.000 .966160 .00051 12.050 .969940 .00051 12.100 .972820 .00052 12.150 .975930 .00051 12.200 .978150 .00050 12.250 .982820 .00050 12.300 .985610 .00051 12.350 .988350 .00052 12.400 .991580 .00052 12.450 .994320 .00050 12.500 .997130 .00051 12.551 .001170 .00049 12.601 .005440 .00050 12.651 .006260 .00049 12.701 .010080 .00051 12.751 .012670 .00051 12.801 .016120 .00050 12.851 .018890 .00051 12.901 .021930 .00049
359

11.500 .920100 .00051 11.550 .922620 .00050 11.600 .925760 .00051 11.650 .929840 .00051 11.700 .932600 .00052 11.750 .935630 .00051 11.800 .939570 .00049 11.850 .942110 .00051 11.900 .945650 .00052 11.950 .949380 .00052 12.000 .951970 .00052 12.050 .955210 .00051 12.100 .959420 .00050 12.150 .962350 .00050 12.200 .964470 .00051 12.250 .967790 .00051 12.300 .970880 .00050 12.350 .974430 .00050 12.400 .977020 .00051 12.450 .980200 .00051 12.500 .983590 .00050 12.550 .986680 .00051 12.600 .990020 .00051 12.650 .992640 .00051 12.700 .995750 .00051 12.750 .998650 .00050 12.801 .000670 .00051 12.851 .004390 .00052 12.901 .006990 .00051 12.951 .011070 .00052 13.001 .013780 .00051 13.051 .016390 .00051 13.101 .019860 .00052
500

11.800 .921840 .00051 11.850 .924050 .00051 11.900 .927620 .00050 11.950 .931310 .00049 12.000 .933970 .00050 12.050 .937250 .00051 12.100 .940450 .00051 12.150 .942530 .00051 12.200 .946380 .00051 12.250 .949140 .00052
600

12.100 .922510 .00050 12.150 .925830 .00050 12.200 .927840 .00050 12.250 .932180 .00049 12.300 .935000 .00051 12.350 .939070 .00050 12.400 .940980 .00051 12.450 .945240 .00049 12.500 .946930 .00050 12.550 .950750 .00050 
12.200 .948650 .00052

12.250 .953020 .00052

12.300 .955870 .00051

12.350 .958770 .00051

12.400 .961960 .00052

12.450 .967020 .00051

12.500 .968490 .00051

12.550 .971840 .00051

12.600 .975050 .00051

12.650 .978130 .00050

12.700 .981570 .00052

12.750 .984340 .00051

12.800 .987290 .00051

12.850 .991430 .00052

12.900 .993060 .00051

12.950 .996340 .00052

13.000 .998910 .00052

13.051 .001280 .00050

13.101 .004840 .00052

13.151 .008040 .00051

13.201 .011170 .00051

13.251 .013140 .00053

13.301 .016300 .00049

13.351 .020060 .00053

13.401 .021730 .00051
12.300 .953030 .00052

12.350 .955320 .00050

12.400 .958810 .00051

12.450 .962700 .00051

12.500 .965400 .00052

12.550 .968420 .00050

12.600 .972160 .00050

12.650 .974520 .00051

12.700 .977380 .00052

12.750 .981330 .00051

12.800 .984260 .00050

12.850 .987370 .00049

12.900 .989540 .00051

12.950 .992530 .00052

13.000 .995660 .00051

13.050 .998080 .00051

13.101 .001730 .00051

13.151 .004160 .00051

13.201 .006260 .00052

13.251 .009780 .00052

13.301 .012890 .00050

13.351 .015150 .00052

13.401 .019410 .00051

13.451 .021590 .00052

13.501 .025690 .00052
12.600 .953680 .00050

12.650 .957250 .00052

12.700 .960750 .00052

12.750 .963420 .00050

12.800 .966240 .00051

12.850 .969380 .00052

12.900 .972400 .00051

12.950 .974770 .00052

13.000 .978440 .00052

13.050 .981440 .00050

13.100 .983500 .00052

13.150 .986920 .00051

13.200 .990230 .00051

13.250 .993440 .00051

13.300 .995500 .00054

13.350 .999070 .00051

13.401 .001670 .00050

13.451 .005070 .00051

13.501 .007830 .00050

13.551 .010190 .00050

13.601 .012890 .00051

13.651 .016610 .00050

13.701 .018090 .00051

13.751 .021000 .00052

13.801 .024830 .00052
650

12.200 .918180 .00050

12.300 .924240 .00050

12.400 .930410 .00051

12.500 .937670 .00050

12.600 .942800 .00050

12.700 .949570 .00051

12.800 .955690 .00052

12.900 .961150 .00050

13.000 .968110 .00050

13.100 .972650 .00051

13.200 .979180 .00051

13.300 .985440 .00050

13.400 .991280 .00051

13.500 .996870 .00051

13.601 .003300 .00051

13.701 .008570 .00050

13.801 .014370 .00052

13.901 .019730 .00051

14.001 .025510 .00052
700

12.500 .921640 .00051

12.550 .925500 .00051

12.600 .926990 .00050

12.650 .931210 .00051

12.700 .934500 .00049

12.750 .936590 .00051

12.800 .940520 .00052

12.850 .943060 .00051

12.900 .945860 .00052

12.950 .949840 .00050

13.000 .952580 .00050

13.050 .955940 .00051

13.100 .957970 .00050

13.150 .961990 .00049

13.200 .964190 .00050

13.250 .967730 .00050

13.300 .970460 .00050

13.350 .974080 .00051

13.400 .975210 .00050

13.450 .978730 .00050

13.500 .981890 .00053

13.550 .984580 .00052
750

12.800 .920150 .00052 12.900 .927060 .00049 13.000 .933250 .00050 13.100 .939570 .00051

13.200 .944220 .00050 13.300 .950600 .00051 13.400 .955620 .00051 13.500 .962780 .00049 13.600 .968300 .00051 13.700 .974690 .00051 13.800 .979230 .00049 13.900 .985900 .00051 14.000 .991320 .00052 14.100 .996450 .00052 14.201 .001560 .00052 14.301 .008040 .00051 14.401 .012360 .00051 14.501 .018960 .00051 14.601 .024030 .00050 
13.600 .988190 .00051

13.650 .990730 .00051

13.700 .993660 .00051

13.750 .995720 .00051

13.800 .999370 .00051

13.851 .002240 .00050

13.901 .005560 .00051

13.951 .007920 .00051

14.001 .010740 .00052

14.051 .012870 .00049

14.101 .014970 .00050

14.151 .018890 .00050

14.201 .022650 .00050

760

12.900 .922410 .00050

12.950 .924890 .00051

13.000 .928280 .00051

13.050 .931430 .00051

13.100 .934220 .00050

13.150 .937680 .00051

13.200 .940750 .00051

13.250 .943470 .00050

13.300 .946360 .00050

13.350 .949840 .00050

13.400 .952550 .00051

13.450 .955460 .00050

13.500 .957500 .00051

13.550 .960650 .00050

13.600 .963630 .00050

13.650 .966850 .00050

13.700 .968990 .00051

13.750 .972760 .00051

13.800 .975490 .00051

13.850 .977820 .00050

13.900 .981300 .00049

13.950 .984350 .00050

14.000 .986150 .00051

14.050 .989080 .00050

14.100 .991730 .00050

14.150 .994450 .00049

14.200 .997370 .00052

14.251 .001000 .00051

14.301 .003540 .00052

14.351 .005870 .00049

14.401 .007970 .00051

14.451 .011420 .00051

14.501 .014080 .00050

14.551 .017240 .00051

14.601 .018710 .00051
765

12.900 .921170 .00050

13.000 .925270 .00051

13.100 .930980 .00051

13.200 .937360 .00051

13.300 .943240 .00051

13.400 .948970 .00049

13.500 .955500 .00050

13.600 .961200 .00051

13.700 .966950 .00051

13.800 .972430 .00050

13.900 .978880 .00050

14.000 .984160 .00050

14.100 .988440 .00049

14.200 .995330 .00051

14.300 .999920 .00050

14.401 .007050 .00052

14.501 .011640 .00050

14.601 .016060 .00051

14.701 .022500 .00052
770

12.900 .915790 .00050

13.000 .922510 .00049

13.100 .929150 .00049

13.200 .935340 .00050

13.300 .940620 .00050

13.400 .945890 .00050

13.500 .952630 .00050

13.600 .959140 .00051

13.700 .964100 .00051

13.800 .969350 .00051

13.900 .975210 .00050

14.000 .980120 .00053

14.100 .985960 .00050

14.200 .993410 .00051

14.300 .997990 .00050

14.401 .002990 .00051

14.501 .008990 .00051

14.601 .014060 .00051

14.701 .018800 .00051 
775

13.000 .921070 .00051

13.100 .927030 .00049

13.200 .933180 .00051

13.300 .938750 .00050

13.400 .944770 .00052

13.500 .951490 .00050

13.600 .955980 .00051

13.700 .961750 .00051

13.800 .969310 .00050

13.900 .973850 .00051

14.000 .979560 .00050

14.100 .985050 .00052

14.200 .990340 .00051

14.300 .996350 .00050

14.401 .000980 .00051

14.501 .007350 .00051

14.601 .013550 .00050

14.701 .017420 .00051

14.801 .022100 .00051 
Pun46-sp-1000-10

12

23.400 .928400 .00028 23.800 .933870 .00028

24.200 .939040 .00028

24.600 .945110 .00027

25.000 .949600 .00027

25.400 .953990 .00027

25.800 .958880 .00028

26.200 .964270 .00025

26.600 .968790 .00027

27.000 .973510 .00027

27.400 .977440 .00026

27.800 .981520 .00026

28.200 .984990 .00026

28.600 .989320 .00025

29.000 .992800 .00026

29.400 .996580 .00027

29.800 .999850 .00026

30.201 .004090 .00026

30.601 .006790 .00026

31.001 .009680 .00026

31.401 .013230 .00025

31.601 .015150 .00025
18.600 .924580 .00031

18.800 .928540 .00030

19.000 .934650 .00031

19.200 .937960 .00032

19.400 .942780 .00032

19.600 .946450 .00032

19.800 .951500 .00031

20.000 .955560 .00031

20.200 .959080 .00032

20.400 .963380 .00031

20.600 .967180 .00031

20.800 .971490 .00030

21.000 .974580 .00031

21.200 .979280 .00030

21.400 .983090 .00032

21.600 .986140 .00031

21.800 .990070 .00030

22.000 .993340 .00030

22.200 .996140 .00031

22.401 .000020 .00030

22.601 .003230 .00030

22.801 .007120 .00031

23.001 .009160 .00030

23.201 .013490 .00030

23.301 .014480 .00030
25

13.600 .921400 .00038

13.700 .925510 .00040

13.800 .929960 .00038

13.900 .933160 .00039

14.000 .937880 .00039

14.100 .942250 .00040

14.200 .945970 .00039

14.300 .950580 .00039

14.400 .954400 .00038

14.500 .958210 .00039

14.600 .961650 .00038

14.700 .965910 .00038
30

12.700 .923310 .00040

12.800 .927840 .00040

12.900 .933490 .00041

13.000 .938300 .00039

13.100 .942940 .00039

13.200 .947760 .00040

13.300 .952380 .00040

13.400 .956680 .00040

13.500 .961670 .00041

13.600 .965980 .00039

13.700 .970790 .00040

13.800 .974530 .00041
20

15.300 .924510 .00037

15.400 .927990 .00036

15.500 .931970 .00036

15.600 .934960 .00036

15.700 .938630 .00036

15.800 .941580 .00037

15.900 .945440 .00036

16.000 .948890 .00036

16.100 .952320 .00037

16.200 .954310 .00036

16.300 .957500 .00035

16.400 .960920 .00036

16.500 .964190 .00036

16.600 .966970 .00036

16.700 .969830 .00035

16.800 .973400 .00034

16.900 .976470 .00035

17.000 .979430 .00035

17.100 .982460 .00035

17.200 .985190 .00036

17.300 .987920 .00035

17.400 .990970 .00035

17.500 .993740 .00037

17.600 .997080 .00036

17.700 .999460 .00036

17.801 .001970 .00034

17.901 .005100 .00035

18.001 .007310 .00035

18.101 .009280 .00035

18.201 .012600 .00036

18.301 .014960 .00035

18.401 .017710 .00036

32

12.400 .923950 .00040

12.500 .927450 .00041

12.600 .933340 .00041

12.700 .938070 .00041

12.800 .942150 .00041

12.900 .947340 .00041

13.000 .951670 .00041

13.100 .957500 .00041

13.200 .961810 .00041

13.300 .967400 .00041

13.400 .971180 .00042

13.500 .976270 .00041 
14.800 .969590 .00037 14.900 .974500 .00039 15.000 .977120 .00038 15.100 .980860 .00038 15.200 .984670 .00039 15.300 .988450 .00038 15.400 .993250 .00038 15.500 .996550 .00038 15.600 .999440 .00038 15.701 .003080 .00038 15.801 .005790 .00038 15.901 .009700 .00038 16.001 .013560 .00037 16.101 .016690 .00038
13.900 .978780 .00039 14.000 .982560 .00041 14.100 .986880 .00040 14.200 .991160 .00040 14.300 .994790 .00040 14.401 .000440 .00040 14.501 .004800 .00040 14.601 .008250 .00041 14.701 .012300 .00040 14.801 .015620 .00039 14.901 .019440 .00041
13.600 .980430 .00041 13.700 .983640 .00042 13.800 .989180 .00041 13.900 .992480 .00042 14.000 .997240 .00040 14.101 .003070 .00042 14.201 .005400 .00041 14.301 .010900 .00040 14.401 .014820 .00041 14.501 .018700 .00040
35

12.000 .920680 .00042

12.100 .926230 .00042

12.200 .930920 .00041

12.300 .935860 .00041

12.400 .940900 .00041

12.500 .946290 .00042

12.600 .951480 .00041

12.700 .956350 .00043

12.800 .961450 .00043

12.900 .965640 .00042

13.000 .972070 .00041

13.100 .976080 .00042

13.200 .981550 .00041

13.300 .985760 .00041

13.400 .990480 .00043

13.500 .994870 .00040

13.600 .999400 .00042

13.701 .003490 .00042

13.801 .008360 .00041

13.901 .012250 .00041

14.001 .017000 .00041
40

11.600 .924370 .00042

11.700 .928820 .00043

11.800 .935590 .00043

11.900 .939890 .00042

12.000 .945910 .00042

12.100 .949970 .00042

12.200 .956000 .00042

12.300 .961200 .00043

12.400 .966470 .00042

12.500 .971780 .00043

12.600 .977000 .00042

12.700 .982440 .00044

12.800 .986950 .00042

12.900 .992520 .00041

13.000 .997310 .00044

13.101 .001910 .00042

13.201 .007000 .00043

13.301 .011310 .00043

13.401 .015330 .00042

13.501 .021510 .00043
60

10.600 .923060 .00045 10.650 .925140 .00045 10.700 .930120 .00045 10.750 .932020 .00045 10.800 .935660 .00045 10.850 .938330 .00044 10.900 .942140 .00045 10.950 .944140 .00045
70

10.300 .919270 .00046 10.350 .921370 .00046 10.400 .925670 .00045 10.450 .928750 .00045 10.500 .932410 .00046 10.550 .935960 .00045 10.600 .937970 .00046 10.650 .942440 .00045
50

10.900 .917230 .00044 11.000 .925460 .00044 11.100 .931090 .00045 11.200 .935880 .00043 11.300 .942670 .00044 11.400 .947870 .00044 11.500 .953470 .00044 11.600 .959960 .00045 11.700 .965740 .00045 11.800 .971590 .00044 11.900 .976940 .00043 12.000 .982070 .00043 12.100 .987380 .00043 12.200 .993550 .00043 12.300 .998650 .00044 12.401 .004230 .00044 12.501 .009500 .00044 12.601 .014100 .00044 12.701 .019870 .00044 
11.000 .947770 .00045 11.050 .950470 .00045 11.100 .953730 .00044 11.150 .957240 .00045 11.200 .961100 .00045 11.250 .963710 .00045 11.300 .965340 .00045 11.350 .969430 .00044 11.400 .971070 .00044 11.450 .975270 .00044 11.500 .977870 .00044 11.550 .980980 .00045 11.600 .983780 .00045 11.650 .987080 .00044 11.700 .989170 .00045 11.750 .992870 .00044 11.800 .995340 .00046 11.850 .997770 .00045 11.901 .000220 .00045 11.951 .003160 .00044 12.001 .006440 .00045 12.051 .009570 .00044 12.101 .012340 .00045 12.151 .015810 .00044 12.201 .018010 .00046

90

10.000 .918700 .00046 10.050 .923030 .00046 10.100 .925270 .00044 10.150 .929310 .00046 10.200 .933190 .00046 10.250 .936590 .00045 10.300 .939380 .00046 10.350 .943010 .00046 10.400 .946230 .00045 10.450 .949850 .00046 10.500 .953090 .00047 10.550 .957080 .00047 10.600 .959690 .00045 10.650 .963350 .00046 10.700 .966150 .00046 10.750 .969250 .00047 10.800 .973360 .00045 10.850 .975990 .00047 10.900 .978530 .00046 10.950 .982380 .00047
10.700 .945030 .00045 10.750 .948370 .00047 10.800 .950560 .00046 10.850 .954290 .00045 10.900 .957690 .00045 10.950 .959990 .00045 11.000 .964050 .00046 11.050 .967040 .00045 11.100 .970090 .00045 11.150 .973330 .00045 11.200 .975480 .00044 11.250 .979030 .00044 11.300 .982520 .00046 11.350 .985160 .00045 11.400 .988110 .00047 11.450 .991570 .00047 11.500 .993310 .00046 11.550 .995750 .00046 11.600 .999730 .00046 11.651 .002720 .00046 11.701 .006320 .00046 11.751 .008610 .00045 11.801 .010350 .00047 11.851 .014700 .00045 11.901 .017690 .00047 11.951 .019790 .00046 12.001 .023060 .00045
10.600 .950360 .00046 10.650 .954020 .00046 10.700 .957070 .00048 10.750 .960480 .00046 10.800 .963420 .00045 10.850 .967170 .00046 10.900 .970010 .00046 10.950 .973020 .00046 11.000 .976360 .00046 11.050 .978740 .00045 11.100 .982170 .00046 11.150 .984490 .00047 11.200 .988400 .00045 11.250 .992030 .00047 11.300 .994220 .00046 11.350 .997840 .00046 11.401 .000140 .00045 11.451 .003350 .00045 11.501 .006110 .00045 11.551 .008900 .00047 11.601 .012820 .00045 11.651 .016380 .00046 11.701 .017820 .00046
9.900 .918250 .00046 9.950 .922270 .00046 10.000 .926290 .00045 10.050 .930310 .00045 10.100 .933450 .00046 10.150 .936200 .00046 10.200 .940480 .00047 10.250 .942350 .00046 10.300 .946100 .00047 10.350 .949090 .00045 10.400 .953180 .00046 10.450 .955620 .00045 10.500 .959790 .00047 10.550 .962590 .00047 10.600 .965980 .00046 10.650 .969690 .00045 10.700 .973620 .00046 10.750 .975920 .00047 10.800 .979460 .00045 10.850 .982480 .00045
120

9.800 .921650 .00046 9.850 .924770 .00046 9.900 .927440 .00046 9.950 .930700 .00048 10.000 .933750 .00046 10.050 .937590 .00047 10.100 .940290 .00046 10.150 .944080 .00046 10.200 .948410 .00046 10.250 .951350 .00048 10.300 .954280 .00047 10.350 .957290 .00047 10.400 .961960 .00048 10.450 .964710 .00046 10.500 .968170 .00047 10.550 .970850 .00047 10.600 .974350 .00046 10.650 .977650 .00047 10.700 .980160 .00047 10.750 .984050 .00046 
11.000 .985840 .00045 11.050 .988480 .00046 11.100 .991440 .00045 11.150 .995090 .00047 11.200 .997530 .00047 11.251 .000630 .00047 11.301 .002940 .00046 11.351 .007260 .00046 11.401 .009710 .00047 11.451 .012530 .00046 11.501 .015760 .00045 11.551 .017870 .00047 11.601 .020680 .00046
10.900 .985520 .00048 10.950 .988060 .00046 11.000 .991960 .00046 11.050 .994620 .00046 11.100 .997700 .00046 11.151 .000970 .00046 11.201 .003900 .00045 11.251 .007670 .00047 11.301 .010600 .00046 11.351 .012850 .00047 11.401 .016860 .00046 11.451 .018770 .00045 11.501 .022150 .00046
10.800 .987630 .00046 10.850 .990260 .00047 10.900 .993110 .00048 10.950 .997070 .00047 11.000 .999120 .00047 11.051 .003140 .00046 11.101 .005990 .00047 11.151 .009720 .00047 11.201 .012710 .00048 11.251 .015970 .00049 11.301 .017330 .00046
130

9.800 .923100 .00048

9.850 .926640 .00046

9.900 .930160 .00046

9.950 .933620 .00046 10.000 .937500 .00046 10.050 .940910 .00048 10.100 .944440 .00047 10.150 .948490 .00045 10.200 .951380 .00047 10.250 .953680 .00046 10.300 .958350 .00046 10.350 .961170 .00047 10.400 .964410 .00047 10.450 .967960 .00047 10.500 .971380 .00047 10.550 .974590 .00048 10.600 .978060 .00047 10.650 .980850 .00047 10.700 .983570 .00046 10.750 .987740 .00048 10.800 .990040 .00047 10.850 .993120 .00046 10.900 .996150 .00046 10.950 .999440 .00046 11.001 .002490 .00047 11.051 .006310 .00047 11.101 .008270 .00047 11.151 .012450 .00047 11.201 .014710 .00047 11.251 .018870 .00048 11.301 .021810 .00048
150

9.700 .920670 .00047

9.750 .924260 .00046

9.800 .927750 .00046

9.850 .930920 .00047

9.900 .934360 .00047

9.950 .938880 .00047

10.000 .942190 .00047

10.050 .944150 .00046

10.100 .948230 .00048

10.150 .951820 .00047

10.200 .955760 .00048

10.250 .958010 .00048

10.300 .961520 .00048

10.350 .965020 .00047

10.400 .968210 .00045

10.450 .971950 .00046

10.500 .974470 .00047

10.550 .977750 .00046

10.600 .981950 .00046

10.650 .984120 .00048

10.700 .988130 .00046

10.750 .990660 .00046

10.800 .993300 .00047

10.850 .997190 .00048

10.901 .000360 .00047

10.951 .003020 .00046

11.001 .007170 .00047

11.051 .008770 .00046

11.101 .013760 .00046

11.151 .016600 .00046

11.201 .019150 .00048
200

9.600 .917300 .00045

9.650 .919800 .00048

9.700 .924640 .00046

9.750 .927410 .00049

9.800 .931270 .00047

9.850 .934150 .00047

9.900 .937570 .00047

9.950 .940610 .00046

10.000 .944840 .00047

10.050 .947530 .00047

10.100 .950580 .00047

10.150 .955050 .00047

10.200 .958310 .00046

10.250 .961220 .00047

10.300 .964230 .00047

10.350 .967520 .00046

10.400 .971210 .00049

10.450 .974620 .00047

10.500 .977510 .00047

10.550 .980800 .00047

10.600 .983650 .00047

10.650 .988300 .00048

10.700 .990660 .00047

10.750 .994000 .00048

10.800 .996140 .00048

10.851 .000150 .00047

10.901 .003070 .00047

10.951 .005480 .00047

11.001 .009370 .00049

11.051 .011570 .00047

11.101 .015010 .00047

11.151 .018010 .00048

11.201 .021180 .00046 


\begin{abstract}
239
9.700 .923520 .00046

9.750 .925450 .00046

9.800 .929750 .00046

9.850 .933300 .00046

9.900 .935890 .00047

9.950 .939630 .00046

10.000 .942940 .00046

10.050 .946530 .00048

10.100 .951120 .00047

10.150 .953990 .00046

10.200 .957600 .00047

10.250 .960940 .00045

10.300 .963480 .00045

10.350 .965910 .00046

10.400 .970390 .00048

10.450 .973000 .00046

10.500 .976260 .00047

10.550 .979970 .00048

10.600 .982890 .00046

10.650 .985710 .00046

10.700 .989000 .00048

10.750 .992390 .00047

10.800 .994910 .00046

10.850 .999180 .00048

10.901 .001560 .00048

10.951 .004200 .00047

11.001 .007220 .00048

11.051 .011410 .00048

11.101 .013650 .00046

11.151 .016710 .00049

11.201 .020680 .00049
\end{abstract}

250

9.700 .922340 .00046

9.750 .925020 .00048

9.800 .929300 .00046

9.850 .932180 .00048

9.900 .936010 .00047

9.950 .940030 .00047

10.000 .942800 .00046

10.050 .946330 .00046

10.100 .949010 .00046

10.150 .952370 .00047

10.200 .955640 .00047

10.250 .960010 .00048

10.300 .962680 .00047

10.350 .966530 .00047

10.400 .968820 .00047

10.450 .972460 .00045

10.500 .975600 .00048

10.550 .979320 .00047

10.600 .982570 .00047

10.650 .985430 .00047

10.700 .988600 .00047

10.750 .991980 .00046

10.800 .995020 .00048

10.850 .997700 .00046

10.901 .000850 .00046

10.951 .004390 .00047

11.001 .007490 .00048

11.051 .009660 .00048

11.101 .013140 .00048

11.151 .016750 .00047

11.201 .019030 .00047
478

10.000 .924070 .00047

10.050 .926550 .00049

10.100 .929780 .00046

10.150 .934140 .00046

10.200 .935990 .00048

10.250 .940230 .00048

10.300 .943230 .00047

10.350 .947200 .00047

10.400 .950130 .00048

10.450 .952990 .00047

10.500 .955180 .00046
500

10.000 .920890 .00047

10.050 .924220 .00046

10.100 .927540 .00046

10.150 .930050 .00047

10.200 .934900 .00047

10.250 .937650 .00047

10.300 .941060 .00047

10.350 .944080 .00047

10.400 .947340 .00047

10.450 .950490 .00047

10.500 .953440 .00047
359

9.800 .920520 .00046

9.850 .923420 .00047

9.900 .926900 .00048

9.950 .930510 .00048

10.000 .934530 .00046

10.050 .937690 .00047

10.100 .940490 .00047

10.150 .943960 .00047

10.200 .946870 .00047

10.250 .951080 .00047

10.300 .954190 .00047

10.350 .957220 .00047

10.400 .960600 .00049

10.450 .963680 .00047

10.500 .966830 .00047

10.550 .970160 .00047

10.600 .972540 .00047

10.650 .976750 .00047

10.700 .981280 .00047

10.750 .982740 .00047

10.800 .985630 .00047

10.850 .988680 .00048

10.900 .991730 .00048

10.950 .995150 .00047

11.000 .998880 .00049

11.051 .000290 .00047

11.101 .003750 .00047

11.151 .006740 .00045

11.201 .009850 .00047

11.251 .012560 .00047

11.301 .015800 .00046

11.351 .018110 .00047

11.401 .021580 .00048

600

10.200 .920560 .00047

10.250 .922660 .00048

10.300 .926810 .00046

10.350 .931280 .00048

10.400 .933360 .00046

10.450 .936810 .00046

10.500 .939830 .00048

10.550 .943390 .00046

10.600 .945250 .00048

10.650 .948650 .00049

10.700 .952540 .00048 
10.550 .959810 .00047 10.600 .962340 .00047 10.650 .965170 .00047 10.700 .968660 .00047 10.750 .971130 .00047 10.800 .975260 .00047 10.850 .978220 .00046 10.900 .981860 .00048 10.950 .984070 .00048 11.000 .987330 .00047 11.050 .990350 .00046 11.100 .993420 .00048 11.150 .996260 .00047 11.200 .998890 .00047 11.251 .002700 .00047 11.301 .005440 .00047 11.351 .008340 .00048 11.401 .011740 .00047 11.451 .013100 .00048 11.501 .016270 .00049 11.551 .020240 .00048 11.601 .022600 .00047
10.550 .956400 .00047 10.600 .959430 .00047 10.650 .961690 .00047 10.700 .965480 .00048 10.750 .968660 .00047 10.800 .972060 .00047 10.850 .975690 .00048 10.900 .979440 .00047 10.950 .981030 .00046 11.000 .984760 .00047 11.050 .987050 .00048 11.100 .990800 .00047 11.150 .993250 .00049 11.200 .996580 .00047 11.251 .000070 .00047 11.301 .002840 .00047 11.351 .004970 .00046 11.401 .007420 .00047 11.451 .010610 .00048 11.501 .015120 .00046 11.551 .017900 .00049 11.601 .020100 .00048
10.750 .955410 .00047 10.800 .958510 .00047 10.850 .961220 .00047 10.900 .964990 .00047 10.950 .967340 .00047 11.000 .970610 .00047 11.050 .975130 .00049 11.100 .976580 .00047 11.150 .980720 .00047 11.200 .982730 .00047 11.250 .985410 .00047 11.300 .988710 .00048 11.350 .991140 .00047 11.400 .994840 .00047 11.450 .997370 .00046 11.501 .000990 .00047 11.551 .003210 .00046 11.601 .006770 .00047 11.651 .009930 .00047 11.701 .011260 .00048 11.751 .015310 .00047 11.801 .017210 .00047
650

10.300 .918820 .00047 10.350 .922090 .00048 10.400 .925570 .00046 10.450 .928540 .00047 10.500 .930710 .00046 10.550 .934710 .00046 10.600 .938100 .00045 10.650 .941000 .00048 10.700 .944590 .00047 10.750 .946530 .00047 10.800 .950350 .00047 10.850 .953260 .00048 10.900 .956190 .00047 10.950 .960020 .00047 11.000 .961920 .00048 11.050 .965330 .00048 11.100 .968420 .00047 11.150 .970660 .00047 11.200 .974390 .00048 11.250 .977370 .00046 11.300 .980220 .00048 11.350 .983500 .00047 11.400 .986190 .00047 11.450 .989230 .00049 11.500 .991410 .00047
700

10.500 .919860 .00047 10.550 .922580 .00046 10.600 .926120 .00046 10.650 .928600 .00047 10.700 .932690 .00048 10.750 .935170 .00047 10.800 .938620 .00048 10.850 .941190 .00048 10.900 .944520 .00047 10.950 .947890 .00047 11.000 .950250 .00047 11.050 .953060 .00047 11.100 .956420 .00045 11.150 .959750 .00047 11.200 .962300 .00046 11.250 .965820 .00046 11.300 .967580 .00047 11.350 .971800 .00048 11.400 .975060 .00048 11.450 .977250 .00046 11.500 .979920 .00048 11.550 .983140 .00046 11.600 .985850 .00047 11.650 .988500 .00047 11.700 .991230 .00046
750

10.700 .916540 .00049 10.800 .923660 .00047 10.900 .929770 .00048 11.000 .934710 .00047 11.100 .941090 .00046 11.200 .947050 .00047 11.300 .952520 .00046 11.400 .959090 .00046 11.500 .963840 .00047 11.600 .970440 .00047 11.700 .975080 .00047 11.800 .981250 .00047 11.900 .987310 .00046 12.000 .992650 .00048 12.100 .998510 .00047 12.201 .003740 .00047 12.301 .009910 .00049 12.401 .015400 .00047 12.501 .019900 .00047 
11.550 .993990 .00048 11.600 .998110 .00048 11.651 .000420 .00048 11.701 .003890 .00048 11.751 .006290 .00048 11.801 .009110 .00048 11.851 .011720 .00047 11.901 .015050 .00047 11.951 .017240 .00047 12.001 .020680 .00048
11.750 .994520 .00047 11.800 .998080 .00048 11.851 .000290 .00048 11.901 .003150 .00047 11.951 .005810 .00048 12.001 .008900 .00048 12.051 .011020 .00047 12.101 .013780 .00048 12.151 .017020 .00047 12.201 .019590 .00047
760

10.800 .920020 .00047 10.900 .924960 .00047 11.000 .932360 .00047 11.100 .937380 .00047 11.200 .943330 .00048 11.300 .950040 .00047 11.400 .956230 .00047 11.500 .961130 .00047 11.600 .967060 .00046 11.700 .972810 .00047 11.800 .978540 .00048 11.900 .983850 .00048 12.000 .988970 .00048 12.100 .994060 .00047 12.201 .000110 .00047 12.301 .006280 .00046 12.401 .010550 .00048 12.501 .015690 .00049 12.601 .021520 .00048
765
10.900 .923560 .00047 10.950 .926550 .00047 11.000 .929490 .00046 11.050 .932370 .00047 11.100 .935890 .00047 11.150 .938640 .00049 11.200 .940750 .00046 11.250 .944320 .00047 11.300 .947460 .00048 11.350 .949880 .00047 11.400 .952910 .00048 11.450 .955780 .00047 11.500 .959000 .00046 11.550 .961120 .00047 11.600 .965770 .00048 11.650 .968030 .00047 11.700 .970540 .00048 11.750 .972680 .00047 11.800 .976280 .00048 11.850 .978840 .00047 11.900 .981070 .00048 11.950 .984730 .00048 12.000 .988260 .00046 12.050 .990640 .00048 12.100 .993160 .00047 12.150 .995900 .00046 12.200 .998390 .00047 12.251 .000400 .00048 12.301 .002960 .00048 12.351 .005950 .00048 12.401 .009720 .00048 12.451 .011920 .00046 12.501 .014260 .00048 12.551 .018090 .00049 12.601 .019700 .00047
770

10.900 .921130 .00047 10.950 .924760 .00046 11.000 .927190 .00047 11.050 .930810 .00046 11.100 .932570 .00046 11.150 .936630 .00046 11.200 .939290 .00045 11.250 .942200 .00047 11.300 .945480 .00047 11.350 .947940 .00048 11.400 .950030 .00047 11.450 .953400 .00046 11.500 .956640 .00047 11.550 .959710 .00046 11.600 .962890 .00047 11.650 .964680 .00048 11.700 .967800 .00047 11.750 .972190 .00046 11.800 .974610 .00047 11.850 .976350 .00047 11.900 .979690 .00048 11.950 .982030 .00048 12.000 .985380 .00047 12.050 .988080 .00048 12.100 .991120 .00047 12.150 .994270 .00048 12.200 .995760 .00049 12.250 .998460 .00047 12.301 .000380 .00046 12.351 .004600 .00047 12.401 .006720 .00047 12.451 .009240 .00047 12.501 .012490 .00048 12.551 .014570 .00047 12.601 .017130 .00047 
775

10.900 .919310 .00047

11.000 .925880 .00047

11.100 .932800 .00048

11.200 .937900 .00046

11.300 .944000 .00048

11.400 .950310 .00047

11.500 .955170 .00047

11.600 .960880 .00046

11.700 .966200 .00048

11.800 .972000 .00047

11.900 .978740 .00047

12.000 .984380 .00048

12.100 .989560 .00048

12.200 .995080 .00047

12.301 .000120 .00046

12.401 .005420 .00045

12.501 .011400 .00048

12.601 .016150 .00047

12.701 .021180 .00046

0

8


Pun40-sp-0970-00

12

22.100 .924350 .00030

22.300 .927780 .00031

22.500 .931680 .00030

22.700 .935960 .00030

22.900 .939070 .00030

23.100 .943300 .00030

23.300 .946800 .00030

23.500 .949650 .00029

23.700 .954020 .00029

23.900 .957260 .00030

24.100 .960350 .00029

24.300 .963470 .00029

24.500 .966700 .00030

24.700 .969560 .00029

24.900 .973340 .00029

25.100 .976430 .00029

25.300 .979710 .00029

25.500 .982280 .00029

25.700 .985310 .00028

26.100 .991230 .00029

26.300 .993720 .00028

26.500 .996280 .00029

26.700 .998800 .00028

26.901 .001230 .00028

27.101 .004950 .00028

27.301 .007890 .00027

27.501 .009680 .00028

27.701 .011900 .00028

25

15.000 .920390 .00041

15.100 .924990 .00042

15.200 .930040 .00042

15.300 .934750 .00041

15.400 .940090 .00042

15.500 .943970 .00042

15.600 .947800 .00040

15.700 .953130 .00041

15.800 .957250 .00041

15.900 .961570 .00042

16.000 .965360 .00042

16.100 .970180 .00042

16.200 .974470 .00041

16.300 .978270 .00041

16.400 .983170 .00041

16.500 .987050 .00041
15

18.800 .922710 .00034

19.000 .929010 .00035

19.200 .933800 .00034

19.400 .939790 .00033

19.800 .950320 .00033

20.000 .955380 .00034

20.200 .961750 .00034

20.400 .966330 .00034

20.600 .970720 .00034

20.800 .976020 .00034

21.000 .981070 .00033

21.200 .985000 .00033

21.400 .990020 .00033

21.600 .994640 .00033

21.800 .998140 .00033

22.001 .003340 .00033

22.201 .007880 .00033

22.401 .010740 .00033

22.601 .015060 .00032

22.801 .019750 .00033

30

14.200 .918000 .00044

14.300 .922960 .00043

14.400 .928110 .00043

14.500 .933380 .00043

14.600 .939220 .00044

14.700 .943810 .00043

14.800 .948990 .00044

14.900 .953900 .00044

15.000 .958000 .00044

15.100 .963360 .00044

15.200 .968450 .00044

15.300 .973890 .00045

15.400 .977220 .00043

15.500 .983260 .00043

15.600 .986300 .00043

15.700 .991970 .00043
20

16.300 .923180 .00039

16.400 .927090 .00039

16.500 .930550 .00038

16.600 .934540 .00039

16.700 .939510 .00039

16.800 .941990 .00040

16.900 .945810 .00037

17.000 .949600 .00038

17.100 .953390 .00038

17.200 .956460 .00039

17.300 .960330 .00038

17.400 .964380 .00038

17.500 .968470 .00040

17.600 .971060 .00038

17.700 .974360 .00038

17.800 .979780 .00039

17.900 .981230 .00039

18.000 .985490 .00037

18.100 .988420 .00038

18.200 .992000 .00037

18.300 .995620 .00038

18.400 .997560 .00038

18.501 .001080 .00039

18.601 .003790 .00038

18.701 .008250 .00038

18.801 .011970 .00038

19.001 .016710 .00038

32

14.000 .918330 .00044

14.100 .924640 .00044

14.200 .930520 .00044

14.300 .936030 .00045

14.400 .939770 .00043

14.500 .945390 .00044

14.600 .951660 .00045

14.700 .957040 .00044

14.800 .961410 .00043

14.900 .965650 .00044

15.000 .971530 .00043

15.100 .976440 .00043

15.200 .981630 .00044

15.300 .985510 .00043

15.400 .991060 .00044

15.500 .995270 .00043 
16.600 .991530 .00040 16.700 .995820 .00041 16.800 .999100 .00040 16.901 .003310 .00042 17.001 .007200 .00041 17.101 .010380 .00041 17.201 .013870 .00041 17.301 .018430 .00041

35

13.700 .917620 .00045 13.800 .922710 .00045 13.900 .928610 .00045 14.000 .934550 .00044 14.100 .940460 .00043 14.200 .945360 .00044 14.300 .950840 .00045 14.400 .956450 .00046 14.500 .961340 .00044 14.600 .966800 .00045 14.700 .972170 .00044 14.800 .977770 .00044 14.900 .981970 .00044 15.000 .987260 .00045 15.100 .992680 .00044 15.200 .997400 .00044 15.301 .001560 .00045 15.401 .007800 .00045 15.501 .011790 .00045 15.601 .017200 .00045

60 12.600 .915770 .00048 12.650 .919000 .00048 12.700 .923210 .00048 12.750 .925200 .00047 12.800 .928780 .00047 12.850 .932560 .00048 12.900 .935510 .00048 12.950 .937210 .00047 13.000 .941400 .00049 13.050 .945110 .00048 13.100 .948280 .00048 13.250 .957790 .00047 13.300 .960320 .00049 13.350 .963800 .00049 13.400 .967250 .00050
15.800 .996750 .00044 15.901 .000130 .00043 16.001 .005160 .00044 16.101 .010000 .00042 16.201 .014660 .00042 16.301 .018380 .00043
15.601 .000110 .00044 15.701 .004550 .00043 15.801 .009740 .00043 15.901 .013910 .00044 16.001 .018660 .00044
40

13.400 .920680 .00045

13.500 .926670 .00047

13.600 .933660 .00045

13.700 .938700 .00046

13.800 .944160 .00046

13.900 .950360 .00046

14.000 .955610 .00047

14.100 .960170 .00046

14.200 .966780 .00046

14.300 .972480 .00047

14.400 .977490 .00046

14.500 .983170 .00046

14.600 .988900 .00045

14.700 .993810 .00046

14.800 .998210 .00047

14.901 .004660 .00046

15.001 .008280 .00045

15.101 .014340 .00045

15.201 .019140 .00047

15.301 .023980 .00046
50

12.900 .917230 .00047

13.000 .923870 .00048

13.100 .930280 .00047

13.200 .936110 .00047

13.300 .942770 .00046

13.400 .948610 .00048

13.500 .955160 .00048

13.600 .960630 .00048

13.700 .966480 .00046

13.800 .973570 .00048

13.900 .978220 .00047

14.000 .983800 .00046

14.100 .989280 .00047

14.200 .995290 .00048

14.601 .018020 .00047

14.701 .023160 .00048
12.500 .920380 .00047

12.550 .922070 .00048

12.600 .926500 .00049

12.650 .928550 .00049

12.700 .932570 .00050

12.750 .935810 .00049

12.800 .940280 .00050

12.850 .942570 .00048

12.900 .946540 .00049

12.950 .949250 .00050

13.000 .952690 .00050

13.050 .955870 .00051

13.100 .958630 .00048

13.150 .961560 .00048

13.200 .965360 .00048
80

12.400 .919950 .00050

12.450 .924240 .00049

12.500 .926500 .00049

12.550 .930580 .00049

12.600 .934150 .00049

12.650 .936570 .00049

12.700 .940050 .00050

12.750 .943240 .00048

12.800 .946500 .00050 12.850 .949990 .00048 12.900 .953560 .00050 12.950 .956990 .00049 13.000 .959400 .00050 13.050 .963760 .00051 13.100 .966290 .00049 
13.450 .969890 .00048 13.500 .972750 .00048 13.550 .976370 .00048 13.600 .979430 .00048 13.650 .981460 .00048 13.700 .984480 .00048 13.750 .987970 .00048 13.800 .990460 .00050 13.850 .993600 .00048 13.900 .996570 .00048 13.950 .999220 .00049 14.001 .002320 .00050 14.051 .005760 .00048 14.101 .008320 .00048 14.151 .011320 .00049 14.201 .013730 .00047 14.251 .016950 .00049 14.301 .019780 .00048
13.250 .967560 .00049 13.300 .970930 .00048 13.350 .974610 .00047 13.400 .976950 .00049 13.450 .981590 .00050 13.500 .983580 .00049 13.550 .986270 .00050 13.600 .989060 .00049 13.650 .992990 .00049 13.700 .994680 .00049 13.750 .998790 .00049 13.801 .001290 .00049 13.851 .003080 .00049 13.901 .006950 .00049 13.951 .009950 .00049 14.001 .012440 .00050 14.051 .016380 .00050 14.101 .019170 .00049
13.150 .969410 .00049 13.200 .971920 .00050 13.250 .975660 .00049 13.300 .978730 .00050 13.350 .981280 .00051 13.400 .984360 .00049 13.450 .988600 .00048 13.500 .990980 .00050 13.550 .994060 .00050 13.600 .996540 .00051 13.651 .000070 .00049 13.701 .003540 .00050 13.751 .006230 .00049 13.801 .009270 .00051 13.851 .012560 .00050 13.901 .014830 .00050 13.951 .018210 .00050 14.001 .020890 .00049
90

12.300 .918290 .00048

12.350 .921700 .00050

12.400 .924340 .00049

12.450 .927980 .00049

12.500 .931870 .00051

12.550 .935780 .00050

12.600 .939270 .00051

12.650 .942010 .00049

12.700 .945420 .00050

12.750 .948970 .00048

12.800 .951410 .00051

12.850 .954960 .00049

12.900 .959200 .00050

12.950 .961420 .00050

13.000 .964820 .00048

13.050 .966850 .00049

13.100 .971670 .00049

13.150 .974650 .00049

13.200 .976820 .00050

13.250 .980410 .00049

13.300 .983760 .00050

13.350 .987010 .00050

13.400 .990410 .00049

13.450 .993290 .00049

13.500 .995670 .00050

13.550 .998830 .00051

13.601 .002690 .00049

13.651 .005940 .00049

13.701 .008800 .00049
100

12.300 .921380 .00050

12.350 .925470 .00049

12.400 .928190 .00049

12.450 .932730 .00050

12.500 .936160 .00051

12.550 .938500 .00050

12.600 .942580 .00050

12.650 .945020 .00051

12.700 .949120 .00050

12.750 .952390 .00049

12.800 .956200 .00050

12.850 .958800 .00049

12.900 .961040 .00049

12.950 .965300 .00051

13.000 .967920 .00050

13.050 .972010 .00051

13.100 .975420 .00049

13.150 .978000 .00052

13.200 .980670 .00050

13.250 .983280 .00050

13.300 .987220 .00050

13.350 .990420 .00049

13.400 .993190 .00051

13.450 .996500 .00050

13.500 .998970 .00050

13.551 .002100 .00051

13.601 .005610 .00050

13.651 .008140 .00051

13.701 .011540 .00050
120

12.200 .917300 .00051

12.250 .920830 .00050

12.300 .924610 .00050

12.350 .927370 .00049

12.400 .931130 .00050

12.450 .934620 .00050

12.500 .937810 .00050

12.550 .940550 .00050

12.600 .943010 .00051

12.650 .947310 .00050

12.700 .950870 .00050

12.750 .954740 .00050

12.800 .957350 .00050

12.850 .960670 .00049

12.900 .962200 .00051

12.950 .966640 .00050

13.000 .969550 .00049

13.050 .973820 .00051

13.100 .975520 .00051

13.150 .978770 .00051

13.200 .982150 .00051

13.250 .985560 .00049

13.300 .988820 .00050

13.350 .991700 .00050

13.400 .995730 .00051

13.450 .997980 .00049

13.501 .000860 .00050

13.551 .002730 .00049

13.601 .006980 .00049 
13.751 .011780 .00050 13.801 .014150 .00050 13.851 .016580 .00050 13.901 .020650 .00050
13.751 .014160 .00051 13.801 .017470 .00050 13.851 .019640 .00049 13.901 .022470 .00050
130

12.200 .918420 .00051 12.250 .922480 .00051 12.300 .925010 .00049 12.350 .930040 .00048 12.400 .932880 .00048 12.450 .935490 .00049 12.500 .939410 .00049 12.550 .942240 .00050 12.600 .945320 .00050 12.650 .948910 .00050 12.700 .951250 .00051 12.750 .955800 .00048 12.800 .958600 .00051 12.850 .962390 .00051 12.900 .965630 .00050 12.950 .968840 .00050 13.000 .970430 .00049 13.050 .974100 .00050 13.100 .978120 .00050 13.150 .981830 .00049 13.200 .983700 .00049 13.250 .987290 .00052 13.300 .991170 .00051 13.350 .994630 .00050 13.400 .995990 .00050 13.450 .999880 .00049 13.501 .002310 .00049 13.551 .005050 .00051 13.601 .008920 .00049 13.651 .011980 .00050 13.701 .015220 .00050 13.751 .018080 .00053 13.801 .020890 .00050
13.651 .009900 .00050

13.701 .013450 .00050

13.751 .016330 .00050

13.801 .019340 .00050 13.851 .021990 .00050 13.901 .025050 .00050

200

12.300 .919900 .00049

12.350 .924750 .00050

12.400 .927810 .00049

12.450 .931690 .00050

12.500 .934480 .00049

12.550 .937320 .00051

12.600 .940890 .00050

12.650 .944080 .00049

12.700 .946610 .00051

12.750 .949990 .00051

12.800 .953630 .00050

12.850 .956840 .00049

12.900 .960140 .00050

12.950 .963110 .00050

13.000 .966090 .00051

13.050 .968740 .00051

13.100 .971300 .00050

13.150 .975820 .00050

13.200 .978660 .00049

13.250 .981750 .00051

13.300 .984760 .00049

13.350 .987820 .00050

13.400 .989700 .00048

13.450 .993870 .00050

13.500 .996090 .00051

13.550 .999820 .00049

13.601 .003490 .00051

13.651 .005320 .00049

13.701 .007740 .00050

13.751 .011060 .00049

13.801 .014500 .00050

13.851 .017140 .00051

13.901 .019690 .00052

\section{9}

12.400 .923290 .00049

12.450 .925120 .00050

12.500 .928860 .00049

12.550 .932050 .00049
250

12.400 .920960 .00049

12.500 .928600 .00049

12.550 .931560 .00051

12.600 .934470 .00050
359

12.600 .919450 .00050 12.650 .923700 .00049 12.700 .925660 .00049 12.750 .929180 .00049 
12.600 .936780 .00049

12.650 .939160 .00050

12.700 .942950 .00050

12.750 .944940 .00049

12.800 .948620 .00049

12.850 .951160 .00050

12.900 .955670 .00049

12.950 .957680 .00050

13.000 .960770 .00049

13.050 .964700 .00050

13.100 .966940 .00050

13.150 .970620 .00051

13.200 .972720 .00050

13.250 .976030 .00049

13.300 .979660 .00051

13.350 .982830 .00049

13.400 .985600 .00049

13.450 .988560 .00050

13.500 .990810 .00050

13.550 .994510 .00048

13.600 .996680 .00049

13.650 .999590 .00051

13.701 .002040 .00050

13.751 .005170 .00051

13.801 .008700 .00051

13.851 .011440 .00049

13.901 .014140 .00051

13.951 .017490 .00049

14.001 .018440 .00051

14.051 .023220 .00049

14.101 .024950 .00049

\section{8}

12.900 .921420 .00049

12.950 .924320 .00050

13.000 .927990 .00049

13.050 .930900 .00050

13.100 .934350 .00049

13.150 .936820 .00048

13.200 .940420 .00048

13.250 .942300 .00049

13.300 .945720 .00048

13.350 .948390 .00050

13.400 .952050 .00049

13.450 .954610 .00049

13.500 .957330 .00050

13.550 .960600 .00049

13.600 .963170 .00049

13.650 .965630 .00050

13.700 .969320 .00048

13.750 .972290 .00049
12.650 .937080 .00050

12.700 .939900 .00049

12.750 .943640 .00050

12.800 .947070 .00051

12.850 .949070 .00049

12.900 .953460 .00049

12.950 .956460 .00049

13.000 .959420 .00051

13.050 .962360 .00049

13.100 .965980 .00049

13.150 .968630 .00050

13.200 .970870 .00050

13.250 .974910 .00050

13.300 .978870 .00049

13.350 .980270 .00049

13.400 .982490 .00050

13.450 .986220 .00051

13.500 .989610 .00051

13.550 .992150 .00049

13.650 .998790 .00049

13.701 .001530 .00050

13.751 .004090 .00049

13.801 .007320 .00051

13.851 .010600 .00050

13.901 .012950 .00050

13.951 .013810 .00052

14.001 .018080 .00050

12.800 .932750 .00048

12.850 .936370 .00049

12.900 .938040 .00048

12.950 .941800 .00049

13.000 .945160 .00049

13.050 .948220 .00049

13.100 .950580 .00051

13.150 .954370 .00049

13.200 .956280 .00048

13.250 .960020 .00049

13.300 .962640 .00050

13.350 .967200 .00049

13.400 .968940 .00050

13.450 .972290 .00049

13.500 .974200 .00048

13.550 .977550 .00050

13.600 .980210 .00048

13.650 .982300 .00050

13.700 .986310 .00047

13.750 .988880 .00050

13.800 .991950 .00049

13.850 .994630 .00048

13.900 .997200 .00050

13.951 .000520 .00049

14.001 .002350 .00050

14.051 .005540 .00050

14.101 .007730 .00049

14.151 .011140 .00050

14.201 .014010 .00050

14.251 .015750 .00049

14.301 .018900 .00049

\section{0}

12.900 .918750 .00047

13.000 .924240 .00048

13.100 .930390 .00049

13.200 .937380 .00048

13.300 .942020 .00049

13.400 .947950 .00050

13.500 .953800 .00050

13.600 .958900 .00049

13.800 .969620 .00049

13.900 .976840 .00050

14.000 .981780 .00050

14.100 .987520 .00049

14.200 .991990 .00048

14.300 .997170 .00049

14.401 .003320 .00049

14.501 .008540 .00048

14.601 .012800 .00049

14.701 .019020 .00049
600

13.300 .917800 .00048

13.400 .922170 .00048

13.500 .929170 .00050

13.700 .940970 .00048

13.800 .946220 .00049

13.900 .950430 .00048

14.000 .956590 .00049

14.100 .962860 .00048

14.200 .968050 .00048

14.300 .974930 .00049

14.400 .979260 .00048

14.500 .984010 .00048

14.600 .988060 .00048

14.700 .994480 .00048

15.001 .009750 .00048

15.101 .014030 .00049

15.201 .019600 .00047 
13.800 .974880 .00049 13.850 .977200 .00049 13.900 .979810 .00049 13.950 .982310 .00049 14.000 .985710 .00050 14.050 .988210 .00049 14.100 .991890 .00048 14.150 .993510 .00049 14.200 .995680 .00048 14.251 .000000 .00049 14.301 .001500 .00049 14.351 .003870 .00050 14.401 .007570 .00049 14.451 .009520 .00050 14.501 .012490 .00049 14.551 .015170 .00048 14.601 .017860 .00050

650

13.800 .927460 .00049 13.900 .932790 .00048 14.000 .938430 .00047 14.100 .943140 .00047 14.200 .949720 .00047 14.300 .955000 .00047 14.400 .959750 .00048 14.500 .965980 .00049 14.600 .971010 .00047 14.700 .976240 .00048 14.800 .980740 .00049 14.900 .986310 .00049 15.000 .990550 .00048 15.100 .996980 .00049 15.201 .001190 .00048 15.301 .007380 .00048 15.401 .010870 .00048 15.501 .016550 .00048 15.601 .021910 .00048

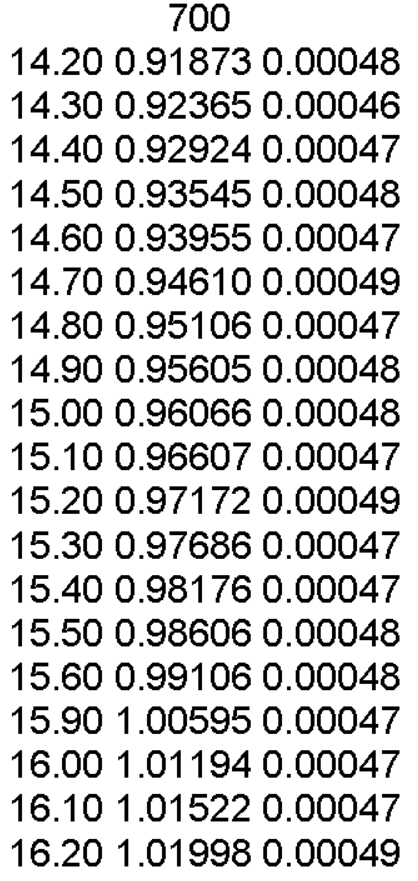

754

752

15.800 .917680 .00049 15.900 .923480 .00046 16.000 .927380 .00049
14.200 .918730 .00048 14.300 .923650 .00046 14.400 .929240 .00047 14.500 .935450 .00048 14.600 .939550 .00047 14.700 .946100 .00049 14.800 .951060 .00047 14.900 .956050 .00048 15.000 .960660 .00048 15.100 .966070 .00047 15.200 .971720 .00049 15.300 .976860 .00047 15.400 .981760 .00047 15.500 .986060 .00048 15.600 .991060 .00048 15.901 .005950 .00047 16.101 .015220 .00047 16.201 .019980 .00049

\begin{abstract}
750
15.600 .916590 .00048 15.700 .922360 .00046 15.800 .926980 .00047 15.900 .932000 .00047 16.000 .937280 .00047 16.100 .941700 .00048 16.200 .945580 .00046 16.300 .951940 .00047 16.400 .956100 .00046 16.500 .960750 .00048 16.600 .964520 .00047 16.700 .969880 .00047 16.800 .974140 .00046 16.900 .980160 .00046 17.000 .985300 .00047 17.100 .988410 .00047 17.200 .993460 .00047 17.300 .996790 .00047 17.401 .001850 .00047 17.501 .006240 .00047 17.601 .011260 .00047 17.701 .015370 .00047 17.801 .018460 .00048
\end{abstract}

\section{5}

16.300 .916640 .00047 16.400 .921600 .00047 16.500 .927370 .00046 
16.100 .932510 .00046 16.200 .936660 .00048 16.300 .942400 .00048 16.400 .946400 .00047 16.500 .951590 .00049 16.600 .956350 .00048 16.700 .960710 .00047 16.800 .965760 .00048 16.900 .970720 .00047 17.200 .983760 .00048 17.300 .988880 .00048 17.400 .992880 .00047 17.500 .997720 .00046 17.601 .001930 .00048 17.701 .005470 .00049 17.801 .010730 .00047 17.901 .015020 .00047 18.001 .018990 .00048

Pun40-sp-0970-01

12

20.700 .926320 .00030 20.900 .930060 .00029 21.100 .933410 .00029 21.300 .937840 .00029 21.500 .941330 .00029 21.700 .945280 .00030 21.900 .948570 .00029 22.100 .952450 .00029 22.300 .954740 .00028 22.500 .958940 .00028 22.700 .962260 .00029 22.900 .966240 .00029 23.100 .968810 .00029 23.300 .972180 .00029 23.500 .975150 .00028 23.700 .978120 .00027 23.900 .981070 .00029 24.100 .984480 .00028 24.300 .987090 .00027 24.500 .989590 .00027 24.700 .992230 .00027 24.900 .995490 .00028 25.100 .997460 .00028 25.301 .000080 .00027 25.501 .003370 .00027 25.701 .005670 .00029
16.400 .934040 .00046 16.500 .938440 .00047 16.600 .942680 .00048 16.700 .948150 .00046 16.800 .953620 .00047 16.900 .957360 .00045 17.000 .962650 .00046 17.100 .966160 .00047 17.200 .971410 .00047 17.300 .976830 .00048 17.400 .979980 .00047 17.500 .984710 .00046 17.600 .989190 .00048 17.700 .993460 .00047 17.800 .997760 .00048 17.901 .001650 .00047 18.001 .005750 .00047 18.101 .011320 .00046 18.201 .015000 .00047 18.301 .019220 .00047

15

17.300 .922530 .00034 17.500 .928600 .00033 17.700 .934660 .00033 17.900 .940510 .00034 18.100 .945730 .00033 18.300 .951010 .00034 18.500 .956250 .00034 18.700 .961140 .00033 18.900 .966060 .00033 19.100 .971600 .00033 19.300 .976660 .00034 19.500 .980660 .00032 19.700 .985000 .00032 19.900 .990600 .00032 20.100 .994210 .00032 20.300 .999050 .00032 20.501 .002820 .00032 20.701 .007970 .00032 20.901 .011830 .00032 21.101 .016690 .00032
16.600 .931740 .00048 16.700 .936190 .00045 16.800 .940640 .00047 16.900 .945920 .00048 17.000 .950610 .00047 17.100 .954590 .00047 17.200 .959040 .00048 17.300 .963790 .00048 17.400 .968840 .00046 17.500 .973470 .00047 17.600 .976300 .00047 17.700 .981080 .00046 17.800 .986100 .00045 17.900 .990300 .00047 18.000 .995270 .00048 18.100 .998990 .00047 18.201 .002250 .00047 18.301 .007480 .00046 18.401 .011250 .00047 18.501 .014820 .00046 18.601 .019460 .00048
20

0.922630 .00037 14.900 .926200 .00036 15.000 .930810 .00038 15.100 .935290 .00038 15.300 .942640 .00038 15.400 .946410 .00037 15.500 .950190 .00038 15.600 .954110 .00037 $\begin{array}{llll}15.70 & 0.95758 & 0.00037\end{array}$ 15.800 .960980 .00038 $\begin{array}{lll}15.90 & 0.964580 .00037\end{array}$ 16.000 .967800 .00037 16.100 .971250 .00037 16.200 .975620 .00037 16.300 .978160 .00037 16.400 .982480 .00038 16.500 .985350 .00037 16.600 .988890 .00037 16.700 .992370 .00037 16.800 .995810 .00038 16.900 .998620 .00037 17.001 .002090 .00037 17.101 .005190 .00037 17.201 .008520 .00037 17.301 .011180 .00037 17.401 .014990 .00037 
25.901 .008760 .00027

26.101 .011140 .00027

26.301 .013060 .00027

25

13.500 .920520 .00041

13.600 .925850 .00039

13.700 .930760 .00040

13.800 .935310 .00040

13.900 .940550 .00040

14.000 .943810 .00041

14.100 .948250 .00040

14.200 .953580 .00040

14.300 .957370 .00040

14.400 .961840 .00040

14.500 .966170 .00040

14.600 .971340 .00039

14.700 .975830 .00040

14.800 .979260 .00039

14.900 .983410 .00040

15.000 .987660 .00039

15.100 .991540 .00040

15.200 .995440 .00040

15.300 .999460 .00039

15.401 .002700 .00039

15.501 .007810 .00039

15.601 .011690 .00039

15.701 .015490 .00039

15.801 .018920 .00040

35

12.300 .924400 .00043

12.400 .930230 .00043

12.500 .935920 .00044

12.600 .940920 .00043

12.700 .946880 .00042

12.800 .952290 .00043

12.900 .957370 .00043

13.000 .962260 .00043

13.100 .968160 .00043

13.200 .972770 .00043

13.300 .978210 .00043

13.400 .983100 .00043

13.500 .989520 .00043

13.600 .992930 .00043

13.700 .998210 .00043

13.801 .003370 .00043
17.501 .018270 .00037

30

32

12.700 .918520 .00042

12.800 .923780 .00042

12.900 .929620 .00041

13.000 .934630 .00042

13.100 .939240 .00041

13.200 .945300 .00043

13.300 .949900 .00041

13.400 .955450 .00041

13.500 .959320 .00042

13.600 .964490 .00042

13.700 .969680 .00042

13.800 .973440 .00042

13.900 .979090 .00040

14.000 .983730 .00042

14.100 .987800 .00043

14.200 .992440 .00042

14.300 .996960 .00041

14.401 .001180 .00041

14.501 .006450 .00042

14.601 .011670 .00042

14.701 .015250 .00043

14.801 .019600 .00042

12.500 .920340 .00043

12.600 .925170 .00043

12.700 .930740 .00042

12.800 .936580 .00042

12.900 .941710 .00041

13.000 .947260 .00042

13.100 .951770 .00042

13.200 .957190 .00042

13.300 .962220 .00043

13.400 .966220 .00042

13.500 .972180 .00042

13.600 .977320 .00043

13.700 .982550 .00042

13.800 .987110 .00044

13.900 .991190 .00043

14.000 .996770 .00042

14.101 .001060 .00042

14.201 .005380 .00042

14.301 .010140 .00042

14.401 .015470 .00043

14.501 .018490 .00042
11.900 .921810 .00046

12.000 .927730 .00045

12.100 .934410 .00045

12.200 .939530 .00044

12.300 .944990 .00045

12.400 .950930 .00043

12.500 .957230 .00045

12.600 .962890 .00044

12.700 .968000 .00044

12.800 .973350 .00044

12.900 .978840 .00044

13.000 .984550 .00044

13.100 .989970 .00044

13.200 .995510 .00045

13.300 .999720 .00043

13.401 .005950 .00044
50

11.400 .918640 .00045 11.450 .922530 .00045 11.500 .925130 .00044 11.550 .929830 .00046 11.600 .931480 .00044 11.650 .934600 .00045 11.700 .938240 .00046 11.750 .941130 .00045 11.800 .944110 .00045 11.850 .947770 .00046 11.900 .950480 .00046 11.950 .953600 .00046 12.000 .956250 .00046 12.050 .959480 .00045 12.100 .963240 .00045 12.300 .974340 .00045 
13.901 .008080 .00041 14.001 .012130 .00043 14.101 .017800 .00043 14.201 .022220 .00043
13.501 .010520 .00044 13.601 .015600 .00044 13.701 .020340 .00043
60

11.100 .916760 .00046 11.150 .920360 .00046 11.200 .924870 .00047 11.250 .926440 .00047 11.300 .930400 .00046 11.350 .933100 .00046 11.400 .936990 .00045 11.450 .940940 .00047 11.500 .943820 .00045 11.550 .947140 .00046 11.600 .949940 .00046 11.650 .953330 .00046 11.700 .955880 .00046 11.750 .959430 .00047 11.800 .962670 .00047 11.850 .965940 .00047 11.900 .969730 .00048 11.950 .971630 .00047 12.000 .974830 .00046 12.050 .977860 .00046 12.100 .981270 .00045 12.150 .983680 .00047 12.200 .986940 .00046 12.250 .989610 .00047 12.300 .992740 .00046 12.350 .995520 .00046 12.400 .999820 .00047 12.451 .001960 .00047 12.501 .003600 .00046 12.551 .006340 .00048 12.601 .011730 .00045 12.651 .012990 .00046 12.701 .016000 .00047
70

11.000 .920480 .00048 11.050 .925330 .00048 11.100 .928510 .00047 11.150 .931410 .00046 11.200 .935390 .00047 11.250 .937950 .00048 11.300 .942020 .00048 11.350 .944850 .00047 11.400 .948490 .00048 11.450 .951560 .00047 11.500 .954880 .00047 11.550 .958070 .00047 11.600 .960880 .00046 11.650 .964740 .00047 11.700 .966910 .00045 11.750 .969910 .00048 11.800 .973100 .00047 11.850 .976620 .00045 11.900 .979570 .00048 11.950 .982340 .00046 12.000 .985630 .00047 12.050 .988850 .00046 12.100 .992550 .00046 12.150 .995060 .00047 12.200 .997750 .00047 12.251 .001090 .00046 12.301 .003420 .00048 12.351 .006670 .00047 12.401 .009980 .00047 12.451 .011900 .00048 12.501 .015660 .00047 12.551 .019010 .00048 12.601 .020370 .00048
12.350 .977050 .00046 12.400 .979590 .00045 12.450 .983210 .00046 12.500 .985640 .00045 12.550 .989130 .00044 12.600 .990400 .00046 12.650 .994170 .00044 12.700 .997790 .00046 12.751 .000400 .00045 12.801 .002690 .00046 12.851 .005410 .00045 12.901 .007480 .00046 13.001 .012810 .00045 13.051 .016790 .00047 13.101 .019030 .00044

80

10.800 .915360 .00047 10.850 .919860 .00048 10.900 .921840 .00048 10.950 .926450 .00048 11.000 .929470 .00047 11.050 .932970 .00047 11.100 .936530 .00047 11.150 .938840 .00046 11.200 .942040 .00047 11.250 .946220 .00049 11.300 .948900 .00047 11.350 .952530 .00048 11.400 .956230 .00047 11.450 .959420 .00048 11.500 .961930 .00047 11.550 .965580 .00048 11.600 .969040 .00048 11.650 .972150 .00048 11.700 .975530 .00049 11.750 .978370 .00048 11.800 .981570 .00049 11.850 .984840 .00047 11.900 .987870 .00048 11.950 .991230 .00048 12.000 .993940 .00049 12.050 .996570 .00047 12.101 .000190 .00046 12.151 .003320 .00047 12.201 .005990 .00048 12.251 .008860 .00047 12.301 .011890 .00048 12.351 .015250 .00049 12.401 .017880 .00046 
12.751 .018320 .00046

12.801 .021610 .00047

90

10.800 .921140 .00049 10.850 .924500 .00049 10.900 .927910 .00047 10.950 .931490 .00048 11.000 .934510 .00047 11.050 .938420 .00050 11.100 .940680 .00047 11.150 .944530 .00047 11.200 .947930 .00048 11.250 .952210 .00047 11.300 .954280 .00048 11.350 .957910 .00049 11.400 .961090 .00048 11.450 .964670 .00049 11.500 .967810 .00048 11.550 .971750 .00048 11.600 .974480 .00048 11.650 .977200 .00048 11.700 .980450 .00047 11.750 .983160 .00049 11.800 .985420 .00049 11.850 .989230 .00047 11.900 .993590 .00049 11.950 .996230 .00047 12.000 .999710 .00049 12.051 .001470 .00049 12.101 .004350 .00047 12.151 .008480 .00048 12.201 .010700 .00049 12.251 .013390 .00049 12.301 .017510 .00048 12.351 .019370 .00049 12.401 .023180 .00048

130

10.700 .922290 .00049 10.750 .925470 .00046 10.800 .929090 .00048 10.850 .932910 .00047 10.900 .935840 .00049 10.950 .939290 .00048 11.000 .942630 .00047 11.050 .945560 .00048
100

10.700 .917210 .00048

10.750 .920620 .00047

10.800 .924650 .00048

10.850 .928250 .00048

10.900 .931430 .00049

10.950 .935170 .00048

11.000 .938370 .00048

11.050 .942180 .00046

11.100 .945450 .00048

11.150 .947930 .00047

11.200 .951230 .00048

11.250 .955670 .00049

11.300 .958530 .00048

11.350 .961910 .00049

11.400 .964910 .00047

11.450 .968960 .00049

11.500 .971870 .00049

11.550 .973760 .00049

11.600 .976700 .00047

11.650 .980390 .00048

11.700 .984170 .00048

11.750 .986540 .00049

11.800 .990810 .00049

11.850 .992560 .00048

11.900 .996020 .00048

11.950 .998440 .00049

12.001 .001190 .00048

12.051 .004890 .00048

12.101 .008350 .00049

12.151 .011240 .00049

12.201 .013650 .00049

12.251 .018410 .00048

12.301 .019530 .00047
120

10.700 .920020 .00048

10.750 .923840 .00048

10.800 .927450 .00047

10.850 .931180 .00048

10.900 .934640 .00048

10.950 .937950 .00049

11.000 .941130 .00047

11.050 .943390 .00048

11.100 .947180 .00049

11.150 .950800 .00049

11.200 .954080 .00047

11.250 .957300 .00049

11.300 .960330 .00048

11.350 .965090 .00048

11.400 .967860 .00048

11.450 .970020 .00048

11.500 .973310 .00049

11.550 .976170 .00049

11.600 .979990 .00049

11.650 .983370 .00048

11.700 .985550 .00047

11.750 .989280 .00048

11.800 .991710 .00049

11.850 .995640 .00048

11.900 .997850 .00049

11.951 .001570 .00049

12.001 .004860 .00050

12.051 .007670 .00049

12.101 .010670 .00048

12.151 .013700 .00048

12.201 .016480 .00048

12.251 .019280 .00048

12.301 .022600 .00049
10.700 .922290 .00048

10.750 .924990 .00048

10.800 .929070 .00048

10.850 .932260 .00050

10.900 .935140 .00050

10.950 .938880 .00048

11.000 .941260 .00048

11.100 .948230 .00048
200

10.700 .917560 .00050 10.750 .921750 .00049 10.800 .924810 .00050 10.850 .927160 .00050 10.900 .931440 .00048 10.950 .935700 .00049 11.000 .937390 .00048 11.050 .940850 .00048 
11.100 .949020 .00047 11.150 .952950 .00047 11.200 .955530 .00048 11.250 .959310 .00048 11.300 .962550 .00047 11.350 .964800 .00047 11.400 .968450 .00049 11.450 .972520 .00048 11.500 .974170 .00048 11.550 .978580 .00049 11.600 .981510 .00049 11.650 .985530 .00048 11.700 .987680 .00049 11.750 .990120 .00049 11.800 .994130 .00049 11.850 .997030 .00048 12.101 .011960 .00048 12.151 .014600 .00047 12.201 .018120 .00049

239

10.800 .920480 .00047 10.850 .923060 .00048 10.900 .927620 .00049 10.950 .930320 .00049 11.000 .933070 .00048 11.050 .936360 .00048 11.100 .939970 .00047 11.150 .942820 .00049 11.200 .946200 .00047 11.250 .949530 .00049 11.300 .951790 .00050 11.350 .955500 .00050 11.400 .957760 .00049 11.450 .962330 .00049 11.500 .964650 .00048 11.550 .967750 .00046 11.600 .970960 .00049 11.650 .973640 .00049 11.700 .978180 .00050 11.750 .980810 .00049
11.150 .951770 .00047 11.200 .955950 .00049 11.250 .958540 .00049 11.300 .962050 .00050 11.350 .965250 .00049 11.400 .967010 .00048 11.450 .971880 .00048 11.500 .974910 .00050 11.550 .976350 .00049 11.600 .980780 .00048 11.650 .983720 .00048 11.700 .986630 .00049 11.750 .989700 .00049 11.800 .994050 .00049 11.850 .995900 .00048 11.900 .998950 .00049 11.951 .001650 .00048 12.001 .004840 .00049 12.051 .008170 .00049 12.101 .011590 .00049 12.151 .014150 .00048 12.201 .017340 .00048 12.251 .020430 .00049 12.301 .022870 .00050

250

10.800 .918790 .00047 10.850 .922050 .00048 10.900 .924720 .00049 10.950 .928110 .00048 11.000 .930750 .00049 11.050 .935040 .00048 11.100 .937920 .00048 11.150 .940680 .00049 11.200 .944300 .00049 11.250 .947000 .00048 11.300 .950690 .00048 11.350 .953420 .00049 11.400 .956680 .00048 11.500 .963210 .00049 11.550 .966380 .00048 11.600 .969820 .00048 11.650 .972220 .00049 11.700 .976330 .00049 11.750 .978360 .00049 11.800 .980960 .00049
11.100 .944570 .00049 11.150 .946630 .00048 11.200 .950710 .00048 11.250 .954850 .00049 11.300 .956660 .00048 11.350 .959470 .00050 11.400 .963530 .00048 11.450 .966450 .00048 11.500 .969550 .00048 11.550 .973070 .00048 11.600 .975360 .00047 11.650 .979160 .00049 11.700 .982550 .00048 11.750 .985300 .00049 11.800 .987770 .00050 11.850 .991040 .00048 11.900 .994450 .00048 11.950 .997440 .00047 12.000 .999620 .00048 12.051 .003090 .00049 12.101 .006410 .00048 12.151 .008940 .00049 12.201 .011800 .00049 12.251 .014810 .00050 12.301 .017760 .00047 12.351 .019770 .00048 12.401 .022850 .00049
359
11.000 .916830 .00049 11.100 .923130 .00046 11.200 .930690 .00048 11.300 .936420 .00049 11.400 .943190 .00049 11.500 .949860 .00048 11.600 .954430 .00048 11.700 .960840 .00048 11.800 .966340 .00049 11.900 .971860 .00048 12.000 .978780 .00048 12.100 .983730 .00048 12.200 .989760 .00048 12.300 .995170 .00049 12.401 .000030 .00048 12.501 .006340 .00049 12.601 .011750 .00049 12.701 .016950 .00048 12.801 .022510 .00048 
11.800 .983590 .00049 11.850 .986190 .00048 11.900 .989210 .00050 11.950 .991670 .00048 12.050 .997810 .00049 12.101 .000410 .00049 12.151 .003250 .00049 12.201 .005790 .00049 12.251 .008940 .00048 12.301 .011790 .00049 12.351 .015180 .00048 12.401 .018030 .00049 12.451 .020990 .00050 12.501 .023470 .00048
11.850 .985460 .00048 11.900 .986830 .00048 11.950 .989440 .00049 12.000 .993470 .00048 12.050 .996070 .00048 12.100 .999850 .00048 12.151 .001230 .00049 12.201 .004110 .00048 12.251 .007950 .00048 12.301 .010820 .00047 12.351 .013540 .00049 12.401 .015870 .00048 12.451 .019220 .00049 12.501 .021480 .00049
478 11.300 .919310 .00047 11.400 .925110 .00049 11.500 .931430 .00049 11.600 .937910 .00047 11.700 .942460 .00048 11.800 .949050 .00049 11.900 .955120 .00048 12.000 .961240 .00048 12.100 .966850 .00048 12.200 .971500 .00050 12.300 .977960 .00049 12.400 .983170 .00046 12.500 .989080 .00049 12.600 .994550 .00048 12.700 .998890 .00048 12.801 .004660 .00048 12.901 .009540 .00048 13.001 .015510 .00047 13.101 .019970 .00047
500 11.400 .921410 .00047 11.450 .925420 .00047 11.500 .928350 .00048 11.550 .931230 .00048 11.600 .934240 .00048 11.650 .936710 .00048 11.700 .939810 .00048 11.750 .941750 .00048 11.800 .945260 .00048 11.850 .949140 .00048 11.900 .951650 .00047 11.950 .954480 .00049 12.000 .957500 .00050 12.050 .961060 .00049 12.100 .963380 .00048 12.150 .966510 .00049 12.200 .968200 .00047 12.250 .971100 .00049 12.300 .974740 .00049 12.350 .977490 .00048 12.400 .979310 .00049 12.450 .982760 .00048 12.500 .985770 .00049 12.550 .987330 .00048 12.600 .991400 .00047 12.650 .993850 .00048 12.700 .996120 .00049 12.801 .000410 .00047 12.851 .003470 .00049 12.901 .006550 .00049 12.951 .009000 .00048 13.001 .011990 .00048 13.051 .013380 .00048
600 11.800 .922330 .00047 11.900 .928890 .00047 12.000 .934510 .00048 12.100 .940660 .00047 12.200 .945830 .00047 12.400 .957620 .00047 12.500 .962340 .00049 12.600 .967720 .00047 12.700 .973550 .00047 12.800 .977680 .00048 12.900 .983360 .00049 13.000 .989150 .00047 13.100 .994880 .00048 13.200 .999270 .00048 13.301 .004420 .00048 13.401 .009480 .00048 13.601 .019490 .00049 
CHPRC-01552, Revision 0

13.101 .016460 .00047

650

12.000 .917860 .00049

12.100 .923090 .00049

12.200 .929270 .00047

12.400 .939880 .00048

12.500 .945940 .00047

12.600 .950960 .00048

12.700 .956770 .00048

12.800 .961790 .00048

12.900 .967260 .00048

13.000 .971510 .00048

13.100 .977510 .00047

13.200 .982150 .00048

13.300 .988980 .00049

13.400 .992630 .00048

13.500 .997980 .00048

13.601 .002170 .00048

13.701 .008700 .00047

13.801 .012310 .00048

13.901 .017440 .00049
700

12.500 .917590 .00047

12.600 .923670 .00047

12.700 .929880 .00047

12.800 .934540 .00048

12.900 .939280 .00047

13.000 .944340 .00048

13.100 .949690 .00047

13.200 .955310 .00047

13.300 .959920 .00047

13.500 .972390 .00047

13.600 .975730 .00048

13.700 .980380 .00048

13.800 .985620 .00048

13.900 .990870 .00047

14.000 .996160 .00048

14.101 .000020 .00046

14.301 .009880 .00048

14.401 .014330 .00047

14.501 .018760 .00047
752

14.000 .922060 .00047

14.100 .927800 .00047

14.200 .931870 .00048

14.300 .937670 .00047

14.400 .941380 .00046

14.500 .946100 .00046

14.600 .950910 .00046

14.700 .955700 .00046

14.800 .960120 .00047

14.900 .964640 .00046

15.000 .970330 .00046

15.100 .973940 .00046

15.200 .979450 .00047

15.300 .984050 .00047

15.701 .001190 .00046

15.801 .005650 .00047

15.901 .009530 .00048

16.001 .014070 .00048

16.101 .017020 .00047
754

14.200 .921660 .00047

14.300 .925610 .00047

14.400 .930300 .00048

14.500 .934410 .00046

14.600 .939920 .00046

14.700 .944440 .00048

14.800 .949520 .00046

14.900 .953830 .00047

15.000 .957480 .00047

15.100 .963250 .00046

15.200 .967590 .00047

15.300 .971480 .00048

15.400 .977150 .00048

15.500 .980340 .00047

15.600 .984330 .00047

15.700 .990010 .00047

15.800 .993950 .00048

15.900 .998130 .00047

16.001 .002670 .00047
750

13.800 .920460 .00047

13.900 .925860 .00046

14.000 .929910 .00046

14.100 .935110 .00046

14.200 .939800 .00047

14.300 .945080 .00047

14.400 .949230 .00048

14.500 .954470 .00047

14.600 .958700 .00046

14.700 .963830 .00047

14.800 .968820 .00046

14.900 .974050 .00047

15.000 .978390 .00047

15.100 .981550 .00048

15.200 .986750 .00046

15.300 .990470 .00047

15.400 .995070 .00047

15.501 .000250 .00047

15.601 .003690 .00047

15.701 .008380 .00047

15.801 .013020 .00047

15.901 .017510 .00049

16.001 .020770 .00046

755

14.200 .909940 .00046

14.300 .914990 .00046

14.400 .919720 .00047

14.500 .925240 .00048

14.600 .929700 .00046

14.700 .934900 .00047

14.800 .939570 .00047

14.900 .943290 .00046

15.000 .948130 .00047

15.100 .953360 .00047

15.200 .956540 .00047

15.300 .961570 .00047

15.400 .967090 .00047

15.500 .970950 .00048

15.600 .975180 .00047

15.700 .979580 .00047

15.800 .983670 .00047

15.900 .988420 .00048

16.000 .991990 .00047 
16.101 .006870 .00046 16.201 .011120 .00047 16.301 .015030 .00049 16.401 .018620 .00047
Pun40-sp-0970-10

12

19.700 .928190 .00028 19.900 .931550 .00028

20.100 .935990 .00028 20.300 .939970 .00028 20.500 .943300 .00028 20.700 .946610 .00028 20.900 .950300 .00028 21.100 .953840 .00028 21.300 .957880 .00028 21.500 .959690 .00028 21.700 .964470 .00027 21.900 .967290 .00027 22.100 .970400 .00028 22.300 .974380 .00027 22.500 .976340 .00027 22.700 .979640 .00027 22.900 .983400 .00027 23.100 .985530 .00027 23.300 .988720 .00027 23.700 .993910 .00026 23.900 .997380 .00027 24.301 .001700 .00025 24.501 .004580 .00026 24.701 .007940 .00026 24.901 .009820 .00026 25.101 .012240 .00027
15

16.300 .925600 .00032 16.400 .927940 .00032 16.500 .931900 .00032 16.600 .934260 .00031 16.700 .937220 .00031 16.800 .939840 .00032 16.900 .942900 .00032 17.000 .944810 .00030 17.100 .947910 .00031 17.200 .951130 .00031 17.300 .953600 .00031 17.400 .955770 .00032 17.500 .958390 .00031 17.600 .961170 .00032 17.700 .964000 .00031 17.800 .966720 .00032 17.900 .969750 .00031 18.000 .971800 .00031 18.100 .973980 .00031 18.200 .975730 .00031 18.300 .978080 .00031 18.400 .980820 .00031 18.500 .983330 .00031 18.600 .985290 .00031 18.700 .987550 .00030 18.800 .989530 .00030 18.900 .992680 .00030 19.000 .994920 .00030 19.100 .996670 .00031 19.200 .998910 .00029 19.301 .001500 .00031 19.401 .003690 .00030 19.501 .005320 .00029 19.601 .008080 .00030 19.701 .010440 .00030 19.801 .012180 .00031 19.901 .014230 .00031 20.001 .015990 .00031
16.100 .996390 .00046 16.201 .000690 .00047 16.301 .004690 .00047 16.401 .009390 .00048 16.501 .012360 .00047 16.601 .018090 .00048 16.701 .021760 .00047

20

13.600 .919180 .00034 13.700 .922580 .00036 13.800 .926540 .00036 13.900 .930410 .00036 14.000 .935400 .00035 14.100 .938680 .00034 14.200 .942770 .00036 14.300 .946790 .00035 14.400 .950640 .00035 14.500 .953010 .00035 14.600 .958150 .00035 14.700 .961880 .00034 14.800 .965700 .00035 14.900 .968980 .00035 15.000 .973000 .00035 15.100 .975810 .00035 15.200 .979550 .00035 15.300 .982920 .00035 15.400 .986460 .00035 15.500 .989580 .00034 15.600 .993060 .00035 15.700 .996580 .00035 15.801 .000450 .00035 15.901 .002840 .00035 16.001 .006970 .00036 16.101 .009780 .00034 16.201 .012230 .00035 16.301 .015450 .00034 
25

12.400 .921600 .00038

12.500 .927230 .00037

12.600 .931960 .00038

12.700 .936360 .00038

12.800 .941470 .00038

12.900 .945460 .00037

13.000 .949720 .00038

13.100 .955440 .00038

13.200 .959320 .00037

13.300 .964370 .00038

13.400 .968580 .00037

13.500 .972490 .00037

13.600 .977190 .00037

13.700 .981500 .00037

13.800 .986160 .00037

13.900 .988870 .00038

14.000 .993790 .00036

14.100 .997240 .00037

14.201 .001510 .00037

14.301 .005630 .00037

14.401 .008920 .00037

14.501 .013260 .00038

14.601 .017840 .00036
30

11.600 .921380 .00039

11.700 .926390 .00040

11.800 .932000 .00039

11.900 .937240 .00039

12.000 .941850 .00039

12.100 .947350 .00039

12.200 .952940 .00039

12.300 .957740 .00039

12.400 .962560 .00039

12.500 .967160 .00039

12.600 .972760 .00038

12.700 .977220 .00039

12.800 .981880 .00039

12.900 .986820 .00039

13.000 .991220 .00039

13.100 .996260 .00039

13.201 .000550 .00038

13.301 .004720 .00039

13.401 .009520 .00039

13.501 .014760 .00039

13.601 .018840 .00039
32

11.400 .922860 .00040

11.500 .927870 .00039

11.600 .933340 .00040

11.700 .938610 .00039

11.800 .944830 .00040

11.900 .949520 .00039

12.000 .954930 .00040

12.100 .959940 .00040

12.200 .965660 .00038

12.300 .970040 .00039

12.400 .975660 .00039

12.500 .980510 .00040

12.600 .985990 .00040

12.700 .990140 .00040

12.800 .994640 .00040

12.900 .999450 .00040

13.001 .003890 .00040

13.101 .009330 .00040

13.201 .014360 .00039

13.301 .017750 .00039
35

11.100 .921620 .00040 11.200 .927980 .00041 11.300 .933420 .00041 11.400 .939200 .00041 11.500 .944990 .00040 11.600 .950140 .00039 11.700 .955720 .00040 11.800 .961090 .00040 11.900 .966790 .00040 12.000 .972370 .00041 12.100 .976870 .00041 12.200 .982590 .00039 12.300 .987160 .00040 12.400 .993250 .00040 12.500 .998040 .00039 12.601 .002900 .00040 12.701 .006970 .00041 12.801 .012420 .00041 12.901 .017590 .00039 13.001 .021930 .00041
40

10.700 .920780 .00041 10.800 .926910 .00041 10.900 .933310 .00041 11.000 .938170 .00042 11.100 .944710 .00040 11.200 .949510 .00041 11.300 .957130 .00041 11.400 .962420 .00042 11.500 .967110 .00043 11.600 .973380 .00042 11.700 .979050 .00041 11.800 .984080 .00041 11.900 .990430 .00043 12.000 .995790 .00042 12.100 .999680 .00040 12.201 .005480 .00041 12.301 .011260 .00039 12.401 .016080 .00041 12.501 .022360 .00041
50

10.300 .925510 .00043 10.350 .929250 .00042 10.550 .942270 .00042 10.600 .945230 .00042 10.650 .948410 .00042 10.700 .952510 .00043 10.750 .955020 .00043 10.800 .957160 .00043 10.850 .960670 .00043 10.900 .963790 .00041 10.950 .967140 .00042 11.000 .970520 .00043 11.050 .973410 .00042 11.100 .975470 .00042 11.150 .978870 .00042 11.250 .984820 .00042 11.300 .988080 .00042 11.350 .991360 .00042 11.400 .993800 .00042 11.450 .995870 .00043 11.500 .999600 .00042 11.551 .001860 .00042 
CHPRC-01552, Revision 0

11.601 .005520 .00043

11.651 .007670 .00043

11.701 .011020 .00044

11.751 .012470 .00042

11.801 .016390 .00043

11.851 .019280 .00042

11.901 .021820 .00041

60

9.900 .919140 .00043

9.950 .923340 .00045

10.000 .926960 .00043

10.050 .930500 .00043

10.100 .934430 .00043

10.150 .936040 .00042

10.200 .939780 .00044

10.250 .943140 .00044

10.300 .946690 .00043

10.350 .950240 .00042

10.400 .952630 .00044

10.450 .956730 .00043

10.500 .959780 .00042

10.550 .963070 .00043

10.600 .965890 .00043

10.650 .968690 .00044

10.700 .971520 .00043

10.750 .974960 .00043

10.800 .978310 .00042

10.850 .980890 .00044

10.900 .984200 .00043

10.950 .987670 .00042

11.000 .990280 .00044

11.050 .993140 .00042

11.100 .996900 .00043

11.150 .999410 .00043

11.201 .002340 .00044

11.251 .005640 .00043

11.301 .008430 .00043

11.351 .011380 .00044

11.401 .015010 .00044

11.451 .017430 .00043

11.501 .020440 .00043
70

9.700 .918730 .00044

9.750 .923080 .00045

9.800 .926160 .00043

9.850 .929020 .00044

9.900 .932190 .00043

9.950 .936410 .00044

10.000 .939600 .00044

10.050 .943210 .00045

10.100 .946530 .00044

10.150 .949450 .00044

10.200 .953140 .00044

10.250 .956990 .00045

10.300 .959820 .00043

10.350 .963140 .00044

10.400 .966410 .00043

10.450 .969610 .00045

10.500 .973660 .00043

10.550 .976940 .00043

10.600 .978390 .00044

10.650 .982350 .00044

10.700 .985520 .00043

10.750 .988210 .00043

10.800 .990990 .00044

10.850 .994490 .00044

10.900 .997820 .00044

10.951 .000120 .00044

11.001 .004090 .00044

11.051 .006530 .00044

11.101 .009450 .00044

11.151 .012280 .00044

11.201 .015630 .00044

11.251 .019210 .00043

11.301 .020960 .00044
80

9.500 .914540 .00044

9.550 .919040 .00044

9.600 .921010 .00044

9.650 .925930 .00044

9.700 .929250 .00045

9.750 .933090 .00043

9.800 .936120 .00043

9.850 .939300 .00041

9.900 .942740 .00044

9.950 .946530 .00044

10.000 .949250 .00046

10.050 .952790 .00045

10.100 .956670 .00045

10.150 .959390 .00043

10.200 .962540 .00046

10.250 .966860 .00043

10.300 .970320 .00044

10.350 .971780 .00045

10.400 .976710 .00043

10.450 .979680 .00045

10.500 .983480 .00044

10.550 .984890 .00044

10.600 .988040 .00045

10.650 .991310 .00045

10.700 .994500 .00044

10.750 .996810 .00044

10.801 .002010 .00044

10.851 .004020 .00042

10.901 .006960 .00044

10.951 .010670 .00044

11.001 .013130 .00044

11.051 .016220 .00044

11.101 .019340 .00045
90

9.500 .922070 .00044 9.550 .925290 .00044 9.600 .928900 .00045
100

9.400 .920540 .00044

9.450 .922780 .00044

9.500 .926540 .00045
120

9.300 .916620 .00044

9.350 .920840 .00045

9.400 .923980 .00045 
9.650 .933220 .00044

9.700 .935960 .00045

9.750 .938930 .00045

9.800 .942350 .00045

9.850 .946980 .00045

9.900 .948930 .00043

9.950 .952940 .00044

10.000 .956380 .00044

10.050 .959620 .00044

10.100 .962870 .00044

10.150 .966700 .00045

10.200 .969850 .00045

10.250 .973780 .00044

10.300 .976000 .00044

10.350 .978880 .00045

10.400 .982030 .00045

10.450 .985950 .00045

10.500 .988960 .00044

10.550 .991980 .00044

10.600 .995620 .00044

10.650 .998820 .00044

10.701 .001440 .00044

10.751 .003770 .00045

10.801 .006650 .00044

10.851 .010260 .00044

10.901 .013210 .00045

10.951 .016570 .00045

11.001 .018860 .00045
9.550 .930570 .00045

9.600 .932770 .00044

9.650 .937190 .00045

9.700 .941180 .00045

9.750 .944210 .00045

9.800 .948300 .00044

9.850 .950980 .00045

9.900 .953550 .00044

9.950 .957600 .00045

10.000 .960860 .00045

10.050 .964530 .00045

10.100 .967150 .00046

10.150 .971750 .00046

10.200 .974750 .00044

10.250 .978660 .00046

10.300 .981320 .00045

10.350 .982750 .00045

10.400 .986870 .00045

10.450 .990190 .00045

10.500 .993950 .00044

10.550 .996740 .00045

10.600 .999260 .00045

10.651 .002740 .00043

10.701 .005960 .00045

10.751 .008990 .00044

10.801 .012420 .00045

10.851 .015100 .00045

10.901 .018170 .00044
9.450 .928430 .00045

9.500 .932100 .00045

9.550 .935400 .00044

9.600 .938630 .00045

9.650 .942440 .00045

9.700 .946070 .00044

9.750 .949600 .00043

9.800 .952870 .00045

9.850 .956080 .00047

9.900 .959680 .00045

9.950 .962430 .00044

10.000 .966560 .00044

10.050 .969440 .00045

10.100 .972850 .00044

10.150 .976270 .00045

10.200 .978660 .00045

10.250 .982710 .00045

10.300 .985320 .00046

10.350 .988630 .00045

10.400 .992590 .00044

10.450 .995050 .00045

10.500 .998150 .00045

10.551 .000860 .00045

10.601 .003990 .00045

10.651 .007940 .00046

10.701 .010480 .00045

10.751 .013250 .00045

10.801 .016320 .00045

10.851 .020440 .00045

10.901 .023100 .00045

150

9.200 .914290 .00045

9.250 .919230 .00044

9.300 .921890 .00045

9.400 .929700 .00045

9.450 .932910 .00045

9.500 .937000 .00045

9.550 .939730 .00045

9.600 .943200 .00045

9.650 .947280 .00047

9.700 .949870 .00046

9.750 .953950 .00045

9.800 .957490 .00045

9.850 .959490 .00045

9.900 .963820 .00045

9.950 .966670 .00045

10.000 .969650 .00045

10.050 .973870 .00044

\begin{abstract}
200
9.300 .922720 .00045

9.350 .926160 .00044

9.400 .929830 .00044

9.450 .932720 .00044

9.500 .935330 .00046

9.550 .938630 .00045

9.600 .942840 .00045

9.650 .946350 .00046

9.700 .949350 .00045

9.750 .953200 .00046

9.800 .956090 .00045

9.850 .959110 .00045

9.900 .962250 .00044

9.950 .965960 .00044

10.000 .968920 .00045

10.050 .971910 .00046

10.100 .975340 .00045
\end{abstract}


10.200 .981220 .00044 10.400 .993860 .00044 10.450 .998300 .00045 10.501 .000800 .00045 10.551 .004520 .00044 10.601 .007060 .00045 10.651 .010960 .00045 10.701 .012740 .00045 10.751 .016290 .00045 10.801 .018350 .00044

239

9.300 .920920 .00045 9.350 .923930 .00045 9.400 .927710 .00044 9.450 .930370 .00044 9.500 .933450 .00044 9.550 .935800 .00044 9.600 .941200 .00045 9.650 .944000 .00044 9.700 .947040 .00045 9.750 .951020 .00044 9.800 .953600 .00044 9.850 .956150 .00045 9.900 .961220 .00044 9.950 .962640 .00045 10.000 .965860 .00044 10.050 .970050 .00045 10.100 .972990 .00044 10.150 .976690 .00045 10.200 .978410 .00045 10.250 .981940 .00045 10.300 .985180 .00045 10.350 .987990 .00046 10.450 .994230 .00045 10.500 .996660 .00046 10.551 .000820 .00045 10.601 .002980 .00047 10.651 .005830 .00045 10.701 .008860 .00046 10.751 .011920 .00045 10.801 .014930 .00044 10.851 .017040 .00045 10.901 .020620 .00045
10.100 .976520 .00045 10.150 .979900 .00045 10.200 .982390 .00045 10.250 .985400 .00045 10.300 .989600 .00044 10.350 .993000 .00045 10.400 .995610 .00044 10.450 .998890 .00045 10.501 .001580 .00045 10.551 .004790 .00046 10.601 .007760 .00045 10.651 .010470 .00045 10.701 .013770 .00045 10.751 .016650 .00046 10.801 .019320 .00045

250

9.300 .919550 .00044 9.350 .922430 .00046 9.400 .926170 .00045 9.450 .929120 .00044 9.500 .933660 .00045 9.550 .936820 .00045 9.600 .938900 .00045 9.650 .942680 .00045 9.700 .946720 .00045 9.750 .948750 .00045 9.800 .952660 .00045 9.850 .956130 .00045 9.900 .959750 .00046 9.950 .962240 .00045 10.000 .964680 .00045 10.050 .968330 .00044 10.100 .971400 .00044 10.150 .974630 .00045 10.200 .977960 .00045 10.250 .980730 .00044 10.300 .983460 .00045 10.350 .987130 .00046 10.400 .990430 .00045 10.450 .992510 .00045 10.500 .996040 .00044 10.550 .999320 .00045 10.601 .001480 .00046 10.651 .004810 .00046 10.701 .007610 .00046 10.751 .011040 .00045 10.801 .013790 .00046 10.851 .016380 .00044 10.901 .018590 .00045
10.150 .978540 .00047 10.200 .981140 .00046 10.250 .985200 .00046 10.300 .987530 .00045 10.350 .990660 .00044 10.400 .993660 .00044 10.450 .997640 .00045 10.500 .999970 .00045 10.551 .002940 .00044 10.601 .006820 .00044 10.651 .009350 .00045 10.701 .011490 .00044 10.751 .015650 .00046 10.801 .017810 .00046

\begin{tabular}{|c|}
\hline $\begin{array}{ccc}3 & 359 \\
9.50 & 0.92345 & 0.00044 \\
9.55 & 0.92679 & 0.00044 \\
9.60 & 0.92896 & 0.00046 \\
9.65 & 0.93337 & 0.00045 \\
9.70 & 0.93538 & 0.00044 \\
9.75 & 0.93887 & 0.00045 \\
9.80 & 0.94226 & 0.00044 \\
9.85 & 0.94618 & 0.00045 \\
9.90 & 0.94853 & 0.00044 \\
9.95 & 0.95172 & 0.00044 \\
10.00 & 0.95444 & 0.00047 \\
10.05 & 0.95813 & 0.00046 \\
10.10 & 0.96150 & 0.00044 \\
10.15 & 0.96463 & 0.00046 \\
10.20 & 0.96668 & 0.00044 \\
10.25 & 0.97036 & 0.00046 \\
10.30 & 0.97307 & 0.00045 \\
10.35 & 0.97566 & 0.00045 \\
10.40 & 0.97966 & 0.00045 \\
10.45 & 0.98267 & 0.00044 \\
10.50 & 0.98431 & 0.00045 \\
10.55 & 0.98719 & 0.00044 \\
10.60 & 0.99098 & 0.00045 \\
10.65 & 0.99364 & 0.00046 \\
10.70 & 0.99537 & 0.00045 \\
10.75 & 0.99882 & 0.00044 \\
10.80 & 1.00236 & 0.00045 \\
10.85 & 1.00539 & 0.00045 \\
10.90 & 1.00776 & 0.00046 \\
10.95 & 1.00988 & 0.00047 \\
11.00 & 1.01344 & 0.00045 \\
11.05 & 1.01514 & 0.00045 \\
11.10 & 1.01915 & 0.00045\end{array}$ \\
\hline
\end{tabular}

F-86 
478

9.600 .916200 .00044

9.700 .922630 .00044

9.800 .929270 .00045

9.900 .935380 .00045

10.000 .941160 .00044

10.100 .947940 .00045

10.200 .953660 .00046

10.300 .959090 .00044

10.400 .965680 .00046

10.500 .970760 .00045

10.600 .976320 .00045

10.700 .982560 .00044

10.800 .987160 .00045

10.900 .993720 .00046

11.000 .998700 .00045

11.101 .003820 .00046

11.201 .008940 .00045

11.301 .014680 .00044

11.401 .019840 .00044

\section{0}

10.300 .925260 .00045 10.400 .930390 .00045 10.500 .935880 .00044 10.600 .941510 .00045 10.700 .947160 .00045 10.800 .952890 .00045 10.900 .957900 .00046 11.000 .964060 .00044 11.100 .968580 .00045 11.200 .974520 .00044
500

9.700 .919840 .00044

9.750 .923560 .00045

9.800 .926090 .00045

9.850 .929590 .00045

9.900 .932910 .00045

9.950 .934990 .00045

10.000 .938590 .00044

10.050 .942270 .00043

10.100 .944680 .00046

10.150 .947560 .00044

10.200 .950720 .00045

10.250 .953800 .00045

10.300 .955360 .00044

10.350 .959400 .00045

10.500 .967530 .00045

10.550 .971460 .00045

10.600 .973120 .00045

10.650 .976640 .00045

10.700 .978720 .00045

10.750 .982650 .00044

10.800 .984020 .00046

10.850 .988120 .00046

10.900 .990380 .00044

10.950 .993230 .00044

11.000 .995830 .00046

11.050 .998570 .00045

11.101 .001400 .00046

11.151 .004150 .00044

11.201 .006720 .00045

11.251 .008970 .00044

11.301 .011360 .00045

11.351 .014730 .00045

11.401 .017090 .00046

700

10.700 .925710 .00044

10.800 .930400 .00044

10.900 .936440 .00045

11.000 .942080 .00044

11.100 .946910 .00045

11.200 .953260 .00043

11.300 .957480 .00044

11.500 .968010 .00044

11.600 .973570 .00045

11.700 .977830 .00044
600

10.000 .920530 .00044

10.100 .926000 .00044

10.200 .932000 .00045

10.300 .938380 .00044

10.400 .943930 .00045

10.700 .960880 .00044

10.800 .966660 .00047

10.900 .971690 .00045

11.000 .976970 .00044

11.200 .987690 .00044

11.300 .993390 .00045

11.400 .998250 .00044

11.501 .004580 .00044

11.601 .008500 .00046

11.701 .014230 .00045

11.801 .018910 .00044 
11.300 .980020 .00044 11.400 .984150 .00046 11.500 .990550 .00045 11.600 .995810 .00045 11.700 .999080 .00044 11.801 .005770 .00045 11.901 .010290 .00045 12.001 .015950 .00044

752

11.700 .920650 .00044 11.800 .926070 .00043 11.900 .931170 .00044 12.000 .935430 .00044 12.100 .940520 .00044 12.500 .959780 .00044 12.600 .964350 .00043 12.700 .969340 .00045 12.800 .973360 .00044 12.900 .978750 .00044 13.000 .982330 .00044 13.100 .987400 .00044 13.200 .991780 .00044 13.300 .995500 .00044 13.400 .999480 .00044 13.501 .004610 .00045 13.601 .009370 .00044 13.701 .012660 .00045 13.801 .017490 .00045
11.800 .982770 .00044 11.900 .987530 .00044 12.000 .993310 .00044 12.100 .998810 .00045 12.201 .002160 .00044 12.301 .007330 .00045 12.401 .012370 .00043 12.501 .016970 .00044 12.601 .021810 .00045
12.600 .969630 .00044

12.700 .974990 .00044

12.800 .979430 .00045

12.900 .984010 .00044

13.000 .988370 .00045

13.100 .992750 .00045

13.200 .997620 .00044

13.301 .001360 .00044

13.401 .006680 .00043 13.501 .010210 .00045 13.601 .014840 .00045 13.701 .019650 .00045

754

11.900 .922040 .00043 12.000 .928070 .00044 12.100 .931590 .00044 12.200 .936570 .00045 12.300 .941220 .00044 12.400 .945350 .00044 12.500 .951280 .00043 12.600 .955470 .00044 12.700 .959780 .00044 12.800 .964920 .00045 12.900 .969240 .00046 13.000 .973480 .00044 13.100 .978360 .00045 13.200 .983030 .00044 13.300 .986900 .00045 13.400 .991710 .00045 13.500 .996000 .00043 13.601 .000740 .00044 13.701 .004460 .00044 13.801 .009120 .00044 13.901 .012970 .00045 14.001 .016310 .00044 14.101 .020610 .00045
755

12.100 .924400 .00044 12.200 .929370 .00045 12.300 .934100 .00043 12.400 .937710 .00043 12.500 .943210 .00044 12.600 .947100 .00044 12.700 .952090 .00044 12.800 .956500 .00044 12.900 .962270 .00044 13.000 .965920 .00043 13.100 .970130 .00044 13.200 .974750 .00044 13.300 .978760 .00044 13.400 .983460 .00044 13.500 .988110 .00044 13.600 .991840 .00045 13.700 .996100 .00044 13.801 .000070 .00045 13.901 .005040 .00045 14.001 .008290 .00044 14.101 .013260 .00045 14.201 .016810 .00045 
Pun43-sp-0970-00

12

24.600 .925430 .00029

24.900 .929690 .00031

25.200 .934590 .00030

25.500 .938510 .00029

25.800 .942980 .00029

26.100 .947160 .00029

26.400 .951810 .00030

26.700 .955590 .00028

27.000 .959540 .00030

27.300 .963300 .00029

27.600 .967150 .00029

27.900 .970960 .00029

28.200 .973910 .00028

28.500 .977090 .00029

28.800 .981280 .00029

29.100 .983440 .00029

29.400 .987540 .00027

29.700 .990900 .00028

30.000 .993580 .00028

30.300 .996900 .00028

30.600 .999200 .00028

30.901 .002090 .00028

31.201 .005020 .00027

31.501 .007980 .00028

31.801 .010330 .00028

32.101 .012610 .00027

32.201 .014080 .00028
15

20.400 .923270 .00035

20.600 .928070 .00035

20.800 .934050 .00035

21.000 .938210 .00035

21.200 .943020 .00034

21.400 .947060 .00034

21.600 .952670 .00035

21.800 .956220 .00033

22.000 .960300 .00034

22.200 .964120 .00034

22.400 .968940 .00034

22.600 .972540 .00035

22.800 .976890 .00034

23.000 .980440 .00033

23.200 .984040 .00033

23.400 .988580 .00033

23.600 .991810 .00034

23.800 .996250 .00034

24.000 .999170 .00033

24.201 .002820 .00033

24.401 .006550 .00033

24.601 .009540 .00033

24.801 .012950 .00033

25.001 .015730 .00034
25

16.000 .926480 .00042

16.100 .930580 .00044

16.200 .935490 .00043

16.300 .939510 .00043

16.400 .943400 .00043

16.500 .948400 .00043

16.600 .952250 .00043

16.700 .955480 .00043

16.800 .960480 .00044

16.900 .962920 .00042

17.000 .967630 .00043

17.100 .971870 .00042
30

15.000 .921440 .00045

15.100 .925230 .00045

15.200 .929960 .00046

15.300 .935200 .00045

15.400 .939410 .00046

15.500 .944870 .00043

15.600 .949920 .00045

15.700 .953120 .00046

15.800 .958680 .00045

15.900 .962610 .00045

16.000 .967660 .00044

16.100 .970530 .00045
20

17.300 .921040 .00040

17.400 .924150 .00040

17.500 .927640 .00040

17.600 .931180 .00041

17.700 .934540 .00040

17.800 .937650 .00040

17.900 .942050 .00039

18.100 .948160 .00040

18.200 .952150 .00039

18.300 .954020 .00038

18.400 .957920 .00038

18.500 .960730 .00038

18.600 .964850 .00039

18.700 .967750 .00041

18.800 .970780 .00040

18.900 .973300 .00039

19.000 .976190 .00039

19.100 .980010 .00039

19.200 .981890 .00040

19.300 .985930 .00039

19.400 .987860 .00040

19.500 .991990 .00038

19.600 .994070 .00039

19.700 .996700 .00039

19.801 .000200 .00039

19.901 .003110 .00039

20.001 .005240 .00038

20.101 .008310 .00039

20.201 .011040 .00038

20.301 .012500 .00039

20.401 .016350 .00037

20.501 .018440 .00038

32

14.700 .917930 .00046

14.800 .923360 .00045

14.900 .928390 .00045

15.000 .933120 .00044

15.100 .938510 .00045

15.200 .942860 .00045

15.300 .947910 .00044

15.400 .952080 .00046

15.500 .956910 .00044

15.600 .962590 .00046

15.700 .966860 .00045

15.800 .971490 .00045 
17.200 .975390 .00042 17.300 .978900 .00043 17.400 .981650 .00042 17.500 .986300 .00043 17.600 .989630 .00042 17.700 .994470 .00043 17.800 .998020 .00042 17.901 .001240 .00043 18.001 .004530 .00042 18.101 .007200 .00042 18.201 .011230 .00042 18.301 .015350 .00043 18.401 .019170 .00042
16.200 .975940 .00044 16.300 .980280 .00045 16.400 .984910 .00043 16.500 .988240 .00044 16.600 .993150 .00044 16.801 .001390 .00046 16.901 .005420 .00044 17.001 .009390 .00044 17.101 .013110 .00043 17.201 .016410 .00043 17.301 .020310 .00044
15.900 .975600 .00046 16.000 .980640 .00045 16.100 .984710 .00045 16.200 .989410 .00046 16.300 .993130 .00045 16.400 .997790 .00045 16.501 .002090 .00045 16.601 .006090 .00046 16.701 .010290 .00047 16.801 .013720 .00045 16.901 .019030 .00045
35

14.400 .919160 .00047 14.500 .923550 .00047 14.600 .928980 .00047 14.800 .938600 .00046 14.900 .944420 .00047 15.000 .949930 .00046 15.100 .955570 .00046 15.200 .959580 .00045 15.300 .964630 .00045 15.400 .968930 .00047 15.500 .973520 .00047 15.600 .977790 .00047 15.700 .983470 .00046 15.800 .988680 .00047 15.900 .992480 .00045 16.000 .997960 .00045 16.100 .999790 .00046 16.201 .006090 .00047 16.301 .010110 .00046 16.401 .014610 .00045 16.501 .019210 .00046

60

13.200 .917280 .00051 13.300 .923650 .00050 13.400 .929870 .00051 13.600 .942790 .00049 13.700 .947400 .00050 13.800 .954600 .00050 13.900 .959730 .00052 14.000 .965920 .00051 14.100 .970960 .00052
40

14.000 .917660 .00048

14.100 .923410 .00047

14.200 .929140 .00048

14.300 .934900 .00049

14.400 .940250 .00047

14.500 .945280 .00047

14.600 .950350 .00048

14.700 .956890 .00048

14.800 .960860 .00047

14.900 .965690 .00047

15.000 .972020 .00047

15.100 .976200 .00047

15.200 .980790 .00047

15.300 .986240 .00047

15.400 .992020 .00048

15.500 .996940 .00048

15.601 .001110 .00046

15.701 .006010 .00049

15.801 .010810 .00047

15.901 .013940 .00047

16.001 .020460 .00048
50

13.500 .917610 .00051

13.600 .923920 .00050

13.700 .930650 .00051

13.800 .936150 .00049

13.900 .942070 .00049

14.000 .948190 .00049

14.100 .952480 .00049

14.200 .958460 .00049

14.300 .964330 .00051

14.400 .969600 .00049

14.600 .981010 .00049

14.700 .985970 .00050

14.800 .991080 .00050

14.900 .997890 .00048

15.001 .001330 .00051

15.101 .007080 .00049

15.201 .012920 .00047

15.301 .015800 .00051

15.401 .022300 .00049
70

13.000 .917170 .00050

13.100 .923760 .00052

13.200 .928870 .00052

13.300 .935950 .00052

13.400 .941730 .00050

13.500 .947420 .00051

13.600 .954270 .00052

13.700 .959970 .00050

13.800 .965440 .00051
80

12.900 .917020 .00052 12.950 .921000 .00051 13.000 .923800 .00052 13.050 .926720 .00051 13.100 .930320 .00050 13.150 .934390 .00050 13.200 .937040 .00052 13.250 .939780 .00051 13.300 .943500 .00053 
14.200 .976440 .00050 14.300 .982510 .00051 14.400 .988660 .00050 14.500 .993080 .00052 14.600 .998860 .00050 14.701 .005280 .00050 14.801 .010050 .00050 14.901 .014610 .00051 15.001 .021650 .00049
13.900 .971000 .00053 14.000 .978200 .00051 14.100 .983760 .00050 14.200 .989290 .00051 14.300 .994560 .00052 14.401 .000190 .00050 14.501 .006890 .00050 14.601 .011770 .00051 14.701 .017300 .00051 14.801 .022240 .00050
13.350 .945810 .00053 13.400 .950230 .00052 13.450 .951470 .00051 13.500 .954760 .00052 13.550 .958640 .00050 13.600 .961510 .00052 13.650 .964520 .00052 13.700 .967250 .00051 13.750 .970390 .00051 13.800 .972230 .00051 13.850 .976250 .00050 13.900 .980640 .00051 13.950 .982840 .00051 14.000 .984830 .00052 14.050 .987500 .00051 14.100 .990140 .00052 14.150 .994180 .00052 14.200 .997370 .00052 14.250 .999210 .00051 14.301 .002360 .00051 14.351 .005810 .00051 14.401 .008320 .00051 14.451 .010860 .00051 14.501 .012870 .00052 14.551 .015270 .00051 14.601 .019200 .00051

90

12.900 .921640 .00052 12.950 .925210 .00051 13.000 .927790 .00052 13.050 .931190 .00053 13.100 .933780 .00051 13.150 .937710 .00053 13.200 .940250 .00051 13.250 .944550 .00053 13.300 .948660 .00051 13.350 .951450 .00052 13.400 .953850 .00052 13.450 .956160 .00053 13.500 .959110 .00052 13.550 .962510 .00052 13.600 .966550 .00050 13.650 .968650 .00054 13.700 .971510 .00052 13.750 .975000 .00051 13.800 .977320 .00053 13.850 .981220 .00053 13.900 .983500 .00053
100

12.900 .924620 .00053 12.950 .927330 .00052 13.050 .935310 .00052 13.100 .937330 .00051 13.150 .941040 .00052 13.200 .943520 .00053 13.250 .947080 .00052 13.300 .948660 .00053 13.350 .952340 .00052 13.400 .955790 .00053 13.450 .959800 .00051 13.500 .962100 .00053 13.550 .965180 .00053 13.650 .971030 .00053 13.700 .974960 .00051 13.750 .977240 .00054 13.800 .980950 .00053 13.850 .983830 .00052 13.900 .986240 .00052 13.950 .989120 .00051 14.000 .992000 .00053
120

12.800 .918660 .00053 12.850 .922770 .00052 12.900 .925070 .00051 12.950 .928980 .00052 13.000 .931960 .00052 13.050 .936260 .00053 13.100 .937650 .00052 13.150 .941100 .00051 13.200 .945070 .00054 13.250 .947690 .00051 13.300 .950480 .00052 13.350 .953990 .00053 13.400 .957960 .00053 13.450 .960990 .00052 13.500 .963000 .00054 13.550 .966610 .00052 13.600 .969270 .00054 13.650 .973130 .00052 13.700 .976160 .00053 13.750 .978370 .00053 13.800 .982280 .00052 
13.950 .987740 .00052 14.000 .990500 .00052 14.050 .992470 .00053 14.100 .995350 .00052 14.150 .998140 .00051 14.201 .000730 .00054 14.251 .004150 .00052 14.301 .006710 .00051 14.351 .009460 .00051 14.401 .011920 .00051 14.451 .014590 .00051 14.501 .017890 .00052 14.551 .019900 .00053 14.601 .023030 .00052

130

12.850 .923390 .00052 12.900 .925950 .00052 12.950 .930470 .00052 13.000 .932770 .00053 13.050 .936320 .00052 13.100 .938810 .00053 13.150 .943800 .00053 13.200 .945700 .00051 13.250 .949750 .00051 13.300 .952690 .00051 13.350 .954870 .00052 13.400 .957970 .00053 13.450 .960890 .00052 13.500 .963980 .00053 13.550 .966650 .00053 13.600 .970110 .00053 13.650 .973960 .00054 13.700 .976950 .00053 13.750 .979350 .00053 13.800 .981980 .00052 13.850 .984210 .00052 13.900 .988920 .00052 13.950 .991610 .00054 14.000 .993370 .00052 14.050 .997770 .00052 14.100 .999690 .00055 14.151 .002190 .00051 14.201 .006040 .00053 14.251 .008290 .00052 14.301 .010470 .00055 14.351 .014170 .00052 14.401 .016330 .00053 14.451 .019650 .00052 14.501 .021060 .00052
14.050 .995050 .00051 14.100 .997110 .00052 14.151 .001060 .00053 14.201 .003360 .00053 14.251 .006810 .00053 14.301 .009160 .00052 14.351 .013480 .00052 14.401 .014830 .00052 14.451 .017030 .00052 14.501 .020020 .00052

150

12.800 .918210 .00052 12.850 .921090 .00052 12.900 .923350 .00053 12.950 .928250 .00053 13.000 .930640 .00052 13.050 .932970 .00053 13.100 .936770 .00050 13.150 .940440 .00053 13.200 .943840 .00052 13.250 .946070 .00052 13.300 .948500 .00051 13.350 .952690 .00053 13.400 .955490 .00052 13.500 .962460 .00052 13.550 .965160 .00053 13.600 .967950 .00053 13.650 .971160 .00052 13.700 .974320 .00054 13.750 .977080 .00053 13.800 .979280 .00054 13.850 .982100 .00052 13.900 .984810 .00052 13.950 .988270 .00051 14.000 .991110 .00052 14.050 .994360 .00054 14.100 .996420 .00053 14.150 .999530 .00052 14.201 .002070 .00054 14.251 .005750 .00052 14.301 .008140 .00052 14.351 .010820 .00052 14.401 .013550 .00052 14.451 .016100 .00053 14.501 .019570 .00053
13.850 .985130 .00052 13.900 .986980 .00052 13.950 .990120 .00053 14.000 .992060 .00052 14.050 .995060 .00053 14.100 .997900 .00051 14.151 .001510 .00052 14.201 .003460 .00051 14.251 .007170 .00052 14.301 .009090 .00052 14.351 .013050 .00053 14.401 .015360 .00052 14.451 .018980 .00050 14.501 .020050 .00052

200

12.900 .915890 .00053 13.000 .922400 .00053 13.100 .929380 .00052 13.200 .935180 .00052 13.300 .940180 .00053 13.400 .947740 .00052 13.500 .952100 .00054 13.600 .959000 .00051 13.700 .964970 .00053 13.800 .971230 .00052 13.900 .976960 .00053 14.000 .981850 .00053 14.100 .987320 .00052 14.200 .993480 .00052 14.300 .998890 .00053 14.401 .004450 .00052 14.501 .010210 .00052 14.601 .015140 .00052 14.701 .020850 .00052 
239

13.100 .919090 .00053 13.200 .925390 .00051 13.300 .932870 .00052 13.400 .938050 .00052 13.500 .944000 .00052 13.600 .950020 .00052 13.700 .954870 .00054 13.800 .961470 .00053 13.900 .967110 .00053 14.000 .972460 .00051 14.100 .978000 .00051 14.200 .983660 .00053 14.300 .989460 .00052 14.400 .994900 .00052 14.501 .001080 .00052 14.601 .004880 .00052 14.701 .010180 .00052 14.801 .016410 .00051 14.901 .021100 .00052 15.001 .026500 .00051
250

13.100 .917510 .00053

13.200 .923150 .00051

13.300 .929780 .00052

13.400 .934910 .00053

13.500 .941750 .00052

13.600 .947340 .00052

13.700 .952780 .00053

13.800 .958720 .00051

13.900 .964800 .00050

14.000 .970400 .00051

14.100 .974970 .00052

14.200 .981650 .00051

14.300 .986350 .00052

14.400 .991710 .00053

14.500 .998460 .00051

14.601 .003120 .00054

14.701 .008400 .00052

14.801 .013750 .00051

14.901 .017600 .00052

15.001 .024340 .00052
478

14.100 .920160 .00051 14.200 .926560 .00051 14.300 .932200 .00051 14.400 .936500 .00051 14.500 .942670 .00051 14.600 .947550 .00052 14.700 .953290 .00051 14.800 .959100 .00050 14.900 .963180 .00051 15.000 .969490 .00052 15.100 .973800 .00051 15.200 .979310 .00050 15.300 .983320 .00051 15.400 .989570 .00051 15.500 .993850 .00052 15.600 .998720 .00051 15.701 .002900 .00051 15.801 .007270 .00051 15.901 .014160 .00049 16.001 .017040 .00051 16.101 .022540 .00053
359

13.600 .922460 .00051

13.700 .927030 .00051

13.800 .933570 .00051

13.900 .939870 .00051

14.000 .944640 .00052

14.100 .950530 .00052

14.200 .956250 .00051

14.300 .961760 .00052

14.400 .967820 .00051

14.500 .972770 .00052 14.600 .977390 .00051 14.700 .983410 .00052 14.800 .987910 .00052 14.900 .993400 .00051 15.000 .997720 .00052 15.101 .003950 .00053 15.201 .008450 .00052 15.301 .014300 .00051 15.401 .018480 .00052
500
14.200 .919290 .00051 14.300 .924390 .00050 14.400 .929950 .00051 14.500 .935080 .00051 14.600 .940860 .00052 14.700 .947460 .00051 14.800 .951200 .00050 15.100 .967470 .00050 15.200 .972330 .00051 15.300 .976820 .00050 15.400 .982300 .00051 15.500 .986140 .00051 15.600 .990710 .00052 15.700 .996830 .00049 15.801 .001030 .00051 15.901 .005770 .00051 16.001 .009930 .00051 16.101 .015280 .00051 16.201 .019930 .00049

\begin{abstract}
600
15.000 .920680 .00051 15.100 .925320 .00050 15.200 .931230 .00050 15.300 .934790 .00049 15.400 .939930 .00051 15.500 .945200 .00048 15.600 .950160 .00051 15.700 .954170 .00051 15.800 .960130 .00050 15.900 .964660 .00050 16.000 .970870 .00050 16.100 .973490 .00050 16.200 .979100 .00050 16.300 .983970 .00050 16.400 .988510 .00052 16.500 .992500 .00050 16.600 .997340 .00050 16.701 .001510 .00050 16.801 .006140 .00051 16.901 .010540 .00049 17.001 .015960 .00050 17.101 .019550 .00050
\end{abstract}


650

15.800 .921500 .00050 15.900 .926330 .00050 16.000 .931810 .00049 16.100 .936340 .00050 16.200 .940940 .00050 16.300 .945440 .00050 16.400 .949700 .00049 16.500 .955520 .00050 16.600 .960540 .00051 16.700 .964010 .00050 16.800 .969560 .00050 16.900 .973470 .00050 17.000 .978410 .00049 17.100 .982680 .00050 17.200 .986130 .00050 17.300 .991240 .00050 17.400 .995150 .00049 17.500 .999810 .00051 17.601 .004510 .00049 17.701 .009070 .00050 17.801 .013420 .00050 17.901 .017960 .00049 18.001 .021050 .00050

680

16.700 .923270 .00049 16.800 .928380 .00049 16.900 .932660 .00049 17.000 .937660 .00049 17.300 .950230 .00049 17.400 .954840 .00049 17.500 .960690 .00050 17.600 .964760 .00050 17.700 .969320 .00048 17.800 .972660 .00049 17.900 .977200 .00049 18.000 .981220 .00048 18.100 .986260 .00049 18.200 .989210 .00050 18.300 .994850 .00051 18.400 .998580 .00049 18.501 .002090 .00052 18.601 .006280 .00049 18.701 .010930 .00050 18.801 .013550 .00050 18.901 .018400 .00050
660

16.000 .920680 .00050

16.100 .924560 .00049

16.200 .929100 .00049

16.300 .934350 .00050

16.400 .939530 .00049

16.500 .943750 .00050

16.600 .949070 .00051

16.700 .953780 .00049

16.800 .958540 .00049

16.900 .963050 .00049

17.000 .967080 .00050

17.100 .972790 .00049

17.200 .976360 .00049

17.300 .980330 .00049

17.400 .984060 .00050

17.500 .989610 .00050

17.600 .994320 .00050

17.700 .998020 .00049

17.801 .003820 .00051

17.901 .007180 .00050

18.001 .010970 .00050

18.101 .015330 .00050

18.201 .018790 .00050

18.301 .023060 .00049
670

16.300 .921860 .00049

16.400 .926040 .00050

16.500 .930320 .00050

16.600 .935670 .00050

16.700 .941240 .00051

16.800 .946080 .00050

16.900 .949640 .00049

17.000 .954050 .00049

17.100 .959450 .00049

17.200 .963510 .00050

17.300 .967590 .00050

17.400 .972750 .00049

17.500 .976680 .00051

17.600 .981080 .00050

17.700 .987190 .00050

17.800 .989530 .00049

17.900 .993940 .00050

18.000 .998490 .00050

18.101 .003360 .00050

18.201 .007310 .00051

18.301 .010830 .00051

18.401 .015410 .00050

18.501 .018980 .00050

18.601 .022490 .00049
17.100 .916000 .00049

17.200 .920560 .00050

17.300 .927150 .00050

17.400 .930700 .00050

17.500 .934700 .00050

17.600 .939540 .00050

17.700 .944360 .00048

17.800 .947910 .00049

17.900 .951750 .00049

18.000 .956810 .00050

18.100 .961790 .00049

18.200 .965120 .00049

18.300 .969540 .00049

18.400 .973240 .00051

18.500 .977870 .00052

18.600 .984010 .00049

18.700 .986440 .00050

18.800 .990490 .00049

18.900 .994790 .00049

19.000 .998060 .00050

19.101 .001560 .00049
698

18.200 .919710 .00048

18.300 .923650 .00049

18.400 .928650 .00048

18.500 .932760 .00048

18.600 .936960 .00051

18.700 .941970 .00048

18.800 .945640 .00049

18.900 .949860 .00048

19.000 .954590 .00049

19.100 .958480 .00049

19.200 .962200 .00048

19.300 .966690 .00049

19.400 .970690 .00049

19.500 .973470 .00050

19.600 .977350 .00049

19.700 .982120 .00050

19.800 .986480 .00050

19.900 .990150 .00048

20.000 .994220 .00048

20.100 .997030 .00050

20.201 .000480 .00049 
19.001 .022960 .00049

Pun43-sp-0970-01

12

23.000 .924380 .00030

23.300 .929100 .00030

23.600 .933960 .00030

23.900 .937410 .00029

24.200 .942100 .00030

24.500 .946330 .00029

24.800 .950160 .00029

25.100 .954460 .00029

25.400 .958360 .00030

25.700 .961930 .00029

26.000 .965910 .00028

26.300 .969340 .00029

26.600 .973090 .00027

26.900 .976710 .00028

27.200 .981010 .00028

27.500 .983860 .00028

27.800 .986530 .00028

28.100 .989900 .00028

28.400 .992560 .00027

28.700 .995460 .00028

29.000 .998660 .00028

29.301 .001550 .00027

29.601 .004020 .00027

29.901 .007240 .00027

30.201 .010260 .00027

30.501 .011980 .00026

30.601 .013840 .00026
19.201 .007710 .00051

19.301 .010180 .00049

19.401 .014010 .00050

19.501 .016420 .00050

19.601 .021960 .00049
20.301 .004840 .00048

20.401 .008780 .00049

20.501 .012600 .00049

20.601 .015880 .00049

20.701 .019770 .00050

\section{5}

18.900 .924630 .00035

19.100 .929290 .00034

19.300 .934800 .00034

19.500 .938700 .00034

19.700 .943770 .00033

19.900 .948200 .00033

20.100 .952070 .00033

20.300 .956990 .00033

20.500 .960570 .00034

20.700 .965020 .00035

20.900 .969750 .00032

21.100 .973730 .00031

21.300 .977630 .00033

21.500 .981150 .00033

21.700 .985390 .00033

21.900 .989220 .00033

22.100 .992990 .00033

22.300 .996680 .00032

22.500 .999810 .00034

22.701 .003420 .00033

22.901 .007780 .00032

23.101 .010890 .00032

23.301 .013540 .00032

23.401 .014390 .00032
25

14.300 .919430 .00042 14.400 .923450 .00041 14.500 .929040 .00042 14.600 .932670 .00041 14.700 .937330 .00042 14.800 .940580 .00041 14.900 .944760 .00041 15.000 .948560 .00041
30

13.500 .923170 .00042 13.600 .927090 .00043 13.700 .932250 .00044 13.800 .936890 .00044 13.900 .941860 .00044 14.000 .945980 .00044 14.100 .949720 .00043 14.200 .954750 .00042
20

15.900 .925370 .00038 16.000 .928400 .00039 16.100 .931460 .00039 16.200 .936230 .00038 16.300 .939000 .00038 16.400 .942920 .00039 16.500 .945120 .00039 16.600 .949160 .00039 16.700 .952080 .00040 16.900 .958400 .00038 17.000 .961630 .00038 17.100 .964790 .00038 17.200 .968380 .00038 17.300 .971200 .00038 17.400 .974820 .00038 17.500 .977190 .00038 17.600 .980660 .00037 17.700 .983780 .00039 17.800 .986170 .00038 17.900 .990230 .00038 18.000 .992390 .00039 18.100 .995930 .00038 18.200 .997470 .00037 18.301 .000720 .00036 18.401 .002850 .00038 18.501 .005190 .00038 18.601 .009140 .00036 18.701 .012620 .00037 18.801 .014540 .00037 18.901 .017110 .00037

32

13.200 .920220 .00044 13.300 .924700 .00043 13.400 .930380 .00043 13.500 .935010 .00044 13.600 .939680 .00044 13.700 .944690 .00045 13.800 .949540 .00044 13.900 .954240 .00045 
15.100 .952960 .00040 15.200 .957370 .00041 15.300 .960320 .00041 15.400 .964650 .00041 15.500 .968840 .00040 15.600 .972290 .00040 15.700 .976460 .00041 15.800 .980710 .00041 15.900 .983340 .00042 16.000 .987340 .00040 16.100 .992080 .00041 16.200 .995640 .00042 16.300 .999250 .00041 16.401 .001900 .00042 16.501 .005750 .00040 16.601 .009330 .00040 16.701 .012490 .00040 16.801 .015620 .00040 16.901 .019270 .00041
14.300 .960130 .00044 14.400 .964540 .00042 14.500 .968890 .00043 14.600 .973510 .00043 14.700 .977640 .00043 14.800 .982340 .00042 14.900 .986130 .00043 15.000 .990340 .00042 15.100 .994610 .00043 15.200 .998300 .00043 15.301 .002030 .00044 15.401 .006840 .00043 15.501 .010840 .00043 15.601 .014920 .00042 15.701 .018320 .00041
14.000 .959950 .00045 14.100 .963930 .00044 14.200 .968550 .00045 14.300 .973820 .00044 14.400 .977090 .00044 14.500 .982520 .00045 14.600 .986910 .00043 14.700 .991490 .00044 14.800 .995230 .00042 14.900 .999580 .00044 15.001 .003530 .00045 15.101 .007530 .00044 15.201 .012160 .00043 15.301 .016520 .00045 15.401 .020730 .00044
35

12.900 .920550 .00045 13.000 .925860 .00044 13.100 .932480 .00044 13.200 .936400 .00045 13.300 .941820 .00046 13.400 .946480 .00044 13.500 .951370 .00044 13.600 .956870 .00045 13.700 .961060 .00043 13.800 .966010 .00044 13.900 .971430 .00044 14.000 .976420 .00045 14.100 .980500 .00045 14.200 .986420 .00045 14.300 .989420 .00044 14.400 .994790 .00045 14.500 .998800 .00044 14.601 .004120 .00044 14.701 .007500 .00046 14.801 .011500 .00046 14.901 .016050 .00044 15.001 .021360 .00045
40

12.500 .920560 .00046 12.600 .924950 .00047 12.700 .931770 .00046 12.800 .936540 .00045 12.900 .941860 .00045 13.000 .948060 .00047 13.100 .953460 .00046 13.200 .957750 .00045 13.300 .963990 .00045 13.400 .968220 .00045 13.500 .973470 .00046 13.600 .979520 .00046 13.700 .984130 .00047 13.800 .989100 .00044 13.900 .993130 .00047 14.000 .997860 .00046 14.101 .003530 .00048 14.201 .007940 .00045 14.301 .012850 .00045 14.401 .017140 .00045
50

12.000 .921090 .00048 12.100 .927280 .00048 12.200 .933160 .00047 12.300 .939060 .00048 12.400 .944210 .00048 12.500 .950120 .00048 12.600 .956390 .00047 12.700 .961280 .00049 12.800 .967800 .00047 12.900 .972590 .00047 13.000 .977550 .00046 13.100 .984050 .00047 13.200 .989460 .00047 13.300 .993880 .00048 13.400 .999290 .00047 13.501 .004860 .00046 13.601 .009470 .00047 13.701 .016090 .00049 13.801 .019500 .00047 
60

11.700 .920830 .00049 11.750 .923880 .00049 11.800 .927080 .00048 11.850 .930300 .00049 11.950 .937050 .00048 12.000 .939530 .00048 12.050 .943030 .00049 12.100 .945870 .00049 12.150 .947580 .00048 12.200 .951870 .00047 12.250 .955580 .00048 12.300 .956960 .00048 12.350 .961190 .00049 12.400 .963610 .00049 12.450 .966110 .00048 12.500 .968870 .00049 12.550 .972050 .00047 12.600 .974760 .00050 12.650 .977710 .00048 12.700 .980650 .00049 12.750 .982480 .00049 12.800 .986440 .00049 12.850 .988400 .00050 12.900 .990760 .00049 12.950 .994220 .00046 13.000 .997010 .00049 13.051 .000270 .00049 13.101 .002520 .00049 13.151 .005350 .00049 13.201 .007760 .00049 13.251 .010040 .00049 13.301 .013420 .00049 13.351 .016300 .00047 13.401 .018070 .00049

90

11.300 .920100 .00049 11.350 .923090 .00049 11.400 .926540 .00051 11.450 .929700 .00051 11.500 .933380 .00049 11.550 .936450 .00050 11.600 .939700 .00051 11.650 .943030 .00050 11.700 .946000 .00051 11.750 .949030 .00050
70

11.500 .920450 .00048 11.550 .924110 .00049 11.600 .926670 .00049 11.650 .930120 .00049 11.700 .933060 .00050 11.800 .939360 .00049 11.850 .943060 .00049 11.900 .945080 .00050 11.950 .948520 .00049 12.000 .952220 .00049 12.050 .954950 .00049 12.100 .958560 .00049 12.150 .960680 .00050 12.200 .963380 .00049 12.250 .966540 .00049 12.300 .969760 .00048 12.350 .973300 .00049 12.400 .975710 .00050 12.450 .979090 .00050 12.500 .980750 .00050 12.550 .984280 .00049 12.600 .986550 .00049 12.650 .990380 .00049 12.700 .991700 .00049 12.750 .995970 .00050 12.800 .999070 .00050 12.851 .001430 .00051 12.901 .003990 .00050 12.951 .006150 .00050 13.001 .009190 .00048 13.051 .012780 .00050 13.101 .014320 .00050 13.151 .017640 .00049 13.201 .021360 .00048

80

11.400 .921720 .00049 11.450 .924680 .00048 11.500 .927740 .00048 11.550 .931870 .00050 11.600 .933460 .00049 11.650 .937780 .00051 11.700 .940980 .00049 11.750 .944020 .00049 11.800 .947260 .00050 11.850 .950040 .00050 11.950 .956670 .00049 12.000 .959350 .00049 12.050 .962840 .00048 12.100 .965630 .00048 12.150 .968430 .00050 12.200 .971060 .00050 12.250 .974390 .00051 12.300 .978460 .00049 12.350 .979400 .00049 12.400 .984280 .00049 12.450 .986420 .00050 12.500 .989050 .00051 12.550 .992140 .00050 12.600 .995120 .00049 12.650 .997510 .00049 12.701 .000150 .00048 12.751 .002230 .00052 12.801 .006240 .00050 12.851 .008420 .00050 12.901 .012270 .00049 12.951 .014640 .00052 13.001 .016350 .00049

100

11.200 .916740 .00050 11.250 .920370 .00050 11.300 .922690 .00051 11.350 .926860 .00050 11.400 .930170 .00051 11.450 .932860 .00048 11.500 .935660 .00049 11.550 .939330 .00050 11.600 .941760 .00049 11.650 .944220 .00051
120

11.200 .918450 .00050 11.250 .921230 .00051 11.300 .924460 .00052 11.350 .927930 .00050 11.400 .930770 .00050 11.450 .934270 .00051 11.500 .937570 .00051 11.550 .939660 .00050 11.650 .946000 .00051 11.700 .948990 .00051 
11.800 .950620 .00050 11.850 .954820 .00050 11.900 .958610 .00050 11.950 .960570 .00050 12.050 .967380 .00050 12.100 .970170 .00049 12.150 .972830 .00051 12.200 .976820 .00049 12.300 .982120 .00050 12.350 .985660 .00050 12.400 .987310 .00049 12.450 .991040 .00050 12.500 .992800 .00049 12.550 .996790 .00049 12.600 .999380 .00050 12.651 .001900 .00050 12.701 .005440 .00050 12.751 .007860 .00051 12.801 .010130 .00050 12.851 .012370 .00050 12.901 .016140 .00051 12.951 .019650 .00049 13.001 .021450 .00051

130

11.200 .918780 .00050 11.250 .921630 .00050 11.300 .925170 .00050 11.350 .928940 .00050 11.400 .932060 .00050 11.450 .935100 .00053 11.500 .938810 .00051 11.550 .941170 .00050 11.600 .945600 .00050 11.650 .947170 .00051 11.700 .950300 .00051 11.750 .953830 .00052 11.800 .956900 .00051 11.850 .960330 .00051 11.900 .962960 .00051 11.950 .966180 .00051 12.000 .968830 .00051 12.050 .972150 .00052 12.100 .975290 .00050 12.150 .978780 .00051 12.200 .981920 .00049 12.250 .984910 .00049 12.300 .986910 .00050
11.700 .947770 .00050 11.750 .952120 .00051 11.800 .954940 .00050 11.850 .957710 .00050 11.900 .961330 .00050 11.950 .963530 .00051 12.000 .966500 .00051 12.050 .969530 .00051 12.100 .972940 .00051 12.150 .975610 .00049 12.200 .979390 .00049 12.250 .982250 .00053 12.300 .984870 .00048 12.350 .988270 .00050 12.400 .991560 .00049 12.450 .993560 .00051 12.500 .996450 .00050 12.550 .999120 .00051 12.601 .002690 .00050 12.651 .005750 .00050 12.751 .010360 .00049 12.801 .013970 .00050 12.851 .015260 .00051 12.901 .019140 .00050
11.750 .952720 .00050 11.800 .956160 .00051 11.850 .959260 .00050 11.900 .961950 .00049 11.950 .965560 .00051 12.000 .967640 .00050 12.050 .971240 .00050 12.100 .974190 .00051 12.150 .977370 .00051 12.200 .980200 .00051 12.250 .983630 .00050 12.300 .984990 .00051 12.350 .988720 .00049 12.400 .992250 .00050 12.450 .994720 .00052 12.500 .997440 .00051 12.550 .999410 .00051 12.601 .003170 .00052 12.651 .005290 .00052 12.701 .008780 .00050 12.751 .012290 .00051 12.801 .014800 .00050 12.851 .016770 .00051 12.901 .020760 .00052
150
200
11.200 .917180 .00052 11.250 .919860 .00048 11.300 .924370 .00049 11.350 .927130 .00051 11.400 .929690 .00050 11.450 .933720 .00050 11.500 .936300 .00050 11.550 .940320 .00051 11.600 .941860 .00052 11.650 .945800 .00050 11.700 .949210 .00052 11.750 .951740 .00050 11.800 .954140 .00050 11.850 .958490 .00052 11.900 .961420 .00051 11.950 .963800 .00050 12.000 .966580 .00051 12.050 .969070 .00050 12.100 .974180 .00050 12.150 .976430 .00051 12.200 .978820 .00052 12.250 .982220 .00049 12.300 .985900 .00050 
12.350 .990030 .00051

12.400 .992900 .00050

12.450 .995960 .00050

12.500 .998490 .00050

12.551 .001730 .00052

12.601 .004070 .00051

12.651 .007190 .00051

12.701 .009220 .00050

12.751 .013850 .00052

12.801 .015490 .00050

12.851 .018170 .00050

12.901 .020420 .00050
12.350 .987070 .00053

12.400 .990810 .00051

12.450 .993140 .00051

12.500 .996350 .00052

12.551 .000010 .00051

12.601 .001930 .00052

12.651 .005450 .00051

12.701 .007710 .00050

12.751 .010150 .00051

12.801 .012870 .00050

12.851 .016230 .00051

12.901 .020210 .00050
12.550 .990500 .00050 12.600 .993470 .00051 12.650 .996320 .00052 12.701 .000100 .00051 12.751 .001130 .00051 12.801 .004790 .00051 12.851 .007330 .00051 12.901 .010350 .00052 12.951 .012030 .00050 13.001 .015230 .00052 13.051 .018140 .00052 13.101 .020630 .00051
239

11.500 .920980 .00051 11.550 .923490 .00051 11.600 .926340 .00051 11.650 .929760 .00051 11.700 .932400 .00050 11.750 .935840 .00051 11.800 .939310 .00051 11.850 .942140 .00050 11.900 .945110 .00050 11.950 .948260 .00051 12.000 .950850 .00053 12.050 .953880 .00050 12.100 .956810 .00050 12.150 .959170 .00050 12.200 .962590 .00050 12.250 .966010 .00049 12.300 .967890 .00050 12.350 .970840 .00051 12.400 .973960 .00051 12.450 .976870 .00053 12.500 .980040 .00053 12.550 .982570 .00051 12.600 .984790 .00050 12.650 .987530 .00050 12.700 .990650 .00052

12.750 .993080 .00050

12.800 .996620 .00052 12.850 .998590 .00048 12.901 .001880 .00051 12.951 .003900 .00052 13.001 .006820 .00051 13.051 .008800 .00050 13.101 .011840 .00050 13.151 .014640 .00050 13.201 .016870 .00052
250

11.600 .924630 .00051 11.650 .928570 .00051 11.700 .929510 .00052 11.750 .934370 .00051 11.800 .936330 .00051 11.900 .942420 .00051 11.950 .945950 .00050 12.000 .948100 .00051 12.050 .950290 .00049 12.100 .954830 .00051 12.250 .963200 .00049 12.300 .966370 .00051 12.350 .969140 .00051 12.400 .971060 .00049 12.450 .974090 .00050 12.500 .976400 .00051 12.550 .978160 .00049 12.600 .983090 .00050 12.650 .983730 .00051 12.700 .987830 .00050 12.750 .991470 .00050 12.800 .994020 .00051 12.850 .996190 .00052 12.900 .998680 .00050 12.951 .000920 .00053 13.001 .003930 .00051 13.051 .007470 .00051 13.101 .010130 .00050 13.151 .011460 .00050 13.201 .013770 .00050
359

12.000 .924510 .00051 12.100 .930150 .00051 12.200 .936160 .00050 12.300 .941560 .00052 12.400 .946810 .00051 12.500 .953590 .00050 12.600 .958400 .00051 12.700 .964210 .00050 12.800 .968870 .00049 12.900 .974640 .00050 13.000 .978960 .00051 13.100 .985200 .00051 13.200 .990250 .00051 13.300 .995630 .00051 13.401 .000790 .00050 13.501 .006250 .00049 13.601 .010420 .00051 13.701 .015980 .00050 13.801 .020790 .00050 
478

12.400 .919520 .00050

12.500 .924880 .00050

12.600 .931490 .00049

12.700 .935930 .00052

12.800 .941220 .00050

12.900 .946360 .00051

13.000 .952710 .00052

13.100 .956920 .00050

13.200 .963060 .00052

13.300 .967460 .00049

13.400 .972580 .00052

13.500 .977910 .00050

13.600 .982590 .00050

13.700 .987700 .00050

13.800 .991520 .00049

13.900 .997470 .00050

14.001 .002630 .00051

14.101 .007610 .00050

14.201 .012440 .00050

14.301 .016650 .00051

14.401 .021400 .00051
500

12.600 .923090 .00051

12.700 .928910 .00050

12.800 .934670 .00052

12.900 .941030 .00051

13.000 .944140 .00051

13.100 .951180 .00050

13.200 .955900 .00050

13.300 .961110 .00050

13.400 .965080 .00049

13.500 .971600 .00049

13.600 .975190 .00050

13.700 .981970 .00051

13.800 .985970 .00049

13.900 .989860 .00050

14.000 .996390 .00049

14.101 .001040 .00050

14.201 .004800 .00050

14.301 .009890 .00049

14.401 .014800 .00050

14.501 .019630 .00050
650

14.000 .923380 .00049

14.100 .928090 .00048

14.200 .933550 .00048

14.300 .937400 .00051

14.400 .942540 .00050

14.500 .947690 .00049

14.600 .952430 .00050

14.700 .956520 .00049

14.800 .962090 .00050

14.900 .966400 .00050

15.000 .970460 .00050

15.100 .974720 .00049

15.200 .978870 .00049

15.300 .983540 .00050

15.400 .988670 .00050

15.701 .001510 .00049

15.801 .005020 .00051

15.901 .010480 .00050

16.001 .013600 .00050
660

14.100 .918720 .00049

14.200 .922560 .00049

14.300 .928030 .00049

14.400 .933080 .00050

14.500 .937760 .00049

14.600 .942350 .00048

14.700 .947140 .00050

14.800 .952090 .00050

14.900 .956880 .00050

15.000 .960920 .00051

15.100 .965850 .00049

15.200 .970030 .00050

15.300 .975050 .00049

15.400 .979280 .00050

15.500 .983670 .00050

15.600 .987540 .00048

15.700 .991390 .00050

15.800 .997290 .00050

15.901 .001420 .00048
600

13.200 .918060 .00048

13.300 .922530 .00049

13.400 .928600 .00049

13.500 .934310 .00049

13.600 .938050 .00051

13.700 .943290 .00050

13.800 .949110 .00050

13.900 .953800 .00050

14.000 .958050 .00049

14.100 .963460 .00050

14.200 .968380 .00049

14.300 .973900 .00050

14.400 .977140 .00051

14.500 .981190 .00050

14.600 .986330 .00049

14.700 .989590 .00050

14.800 .995220 .00050

14.900 .999520 .00047

15.001 .004670 .00049

15.101 .008240 .00050

15.201 .013270 .00049

15.301 .017010 .00051

15.401 .022650 .00051

15.501 .025720 .00051

\section{0}

14.400 .921630 .00050

14.500 .926340 .00048

14.600 .930490 .00050

14.700 .935880 .00050

14.800 .940770 .00049

14.900 .944570 .00049

15.000 .948360 .00048

15.100 .953820 .00049

15.200 .959780 .00049

15.300 .963390 .00049

15.400 .967680 .00049

15.500 .972210 .00049

15.600 .976240 .00048

15.700 .981530 .00049

15.800 .985320 .00049

15.900 .989390 .00049

16.000 .993080 .00050

16.100 .997790 .00049

16.201 .001590 .00051 
$16.101 .019230 .00048 \quad 16.001 .005010 .00048$

680

14.700 .920340 .00048 14.800 .925300 .00048 14.900 .929360 .00050 15.000 .934970 .00049 15.100 .939060 .00051 15.200 .943450 .00050 15.300 .948100 .00050 15.400 .953760 .00048 15.500 .956500 .00049 15.600 .961910 .00049 15.700 .967350 .00049 15.800 .970010 .00051 15.900 .974770 .00049 16.000 .978410 .00049 16.100 .983400 .00049 16.200 .986570 .00048 16.300 .991930 .00049 16.400 .994760 .00050 16.500 .999880 .00050 16.601 .003930 .00049 16.701 .007670 .00048 16.801 .011220 .00049 16.901 .016310 .00048 17.001 .020540 .00050 17.101 .023410 .00050 17.201 .027280 .00051 17.301 .031460 .00049 17.401 .035370 .00049 17.501 .038060 .00049 17.601 .041190 .00049 16.101 .008070 .00050 16.201 .012970 .00051 16.301 .016880 .00050 16.401 .021360 .00049

16.301 .006220 .00050 16.401 .010100 .00049 16.501 .014620 .00049 16.601 .018130 .00050

690

15.200 .922080 .00050 15.300 .926520 .00049 15.400 .930270 .00049 15.500 .934920 .00049 15.600 .939590 .00050 15.700 .943820 .00050 15.800 .947760 .00049 15.900 .953740 .00048 16.000 .955870 .00049 16.100 .961370 .00049 16.200 .965370 .00048 16.300 .969160 .00050 16.400 .973840 .00049 16.500 .978830 .00048 16.600 .982360 .00050 16.700 .984900 .00049 16.800 .990940 .00051 16.900 .994500 .00049 17.000 .998570 .00050 17.101 .002780 .00048 17.201 .005390 .00050 17.301 .010370 .00049 17.401 .014020 .00049 17.501 .017410 .00050 17.601 .021140 .00049
698

16.100 .921480 .00048 16.200 .926090 .00049 16.300 .930050 .00048 16.400 .934840 .00050 16.500 .938800 .00049 16.600 .942580 .00049 16.700 .946610 .00048 16.800 .950740 .00049 16.900 .954740 .00049 17.000 .958890 .00048 17.100 .963410 .00050 17.200 .966470 .00049 17.300 .970390 .00049 17.400 .974760 .00049 17.500 .979750 .00049 17.600 .982880 .00049 17.700 .986710 .00049 17.800 .990870 .00050 17.900 .994570 .00050 18.000 .998660 .00049 18.101 .001300 .00049 18.201 .005300 .00049 18.301 .009310 .00052 18.401 .012610 .00050 18.501 .016650 .00051 
Pun43-sp-0970-10

12

22.100 .927370 .00028

22.400 .932280 .00028

22.700 .936680 .00028

23.000 .941170 .00028

23.300 .945940 .00027

23.600 .949950 .00027

23.900 .953720 .00027

24.200 .957260 .00028

24.500 .961550 .00027

24.800 .965350 .00027

25.100 .969290 .00028

25.400 .972420 .00027

25.700 .975780 .00028

26.000 .978830 .00027

26.300 .983020 .00027

26.600 .986130 .00026

26.900 .989000 .00026

27.200 .991970 .00026

27.500 .995930 .00027

27.800 .999010 .00026

28.101 .001430 .00026

28.401 .003700 .00025

28.701 .007370 .00026

29.001 .009510 .00026

29.301 .012120 .00026

29.401 .013570 .00025
15

17.800 .925620 .00032

18.000 .929870 .00032

18.200 .935320 .00032

18.400 .940210 .00032

18.600 .944060 .00032

18.800 .949520 .00031

19.000 .953120 .00032

19.200 .957090 .00032

19.400 .962040 .00032

19.600 .967100 .00031

19.800 .970160 .00031

20.000 .975290 .00031

20.200 .978530 .00031

20.400 .981900 .00031

20.600 .986250 .00030

20.800 .990290 .00031

21.000 .993000 .00031

21.200 .997770 .00030

21.401 .000700 .00031

21.601 .004410 .00031

21.801 .008010 .00031

22.001 .011270 .00031

22.201 .014420 .00030
25

13.200 .922950 .00038

13.300 .927080 .00039

13.500 .935910 .00037

13.600 .939730 .00038

13.700 .944250 .00038

13.800 .947420 .00039

13.900 .952220 .00039

14.000 .956650 .00039

14.100 .960170 .00038

14.200 .964220 .00038

14.300 .967880 .00039

14.400 .971640 .00039

14.500 .976380 .00039

14.600 .979890 .00038

14.700 .984190 .00039

14.800 .988310 .00040
30

12.300 .922560 .00040

12.400 .926680 .00041

12.500 .931570 .00041

12.600 .936960 .00040

12.700 .940860 .00040

12.800 .946510 .00042

12.900 .950750 .00040

13.000 .955080 .00041

13.100 .959960 .00041

13.200 .964090 .00040

13.300 .968580 .00040

13.400 .973760 .00041

13.500 .978970 .00040

13.600 .981490 .00041

13.700 .986450 .00041

13.800 .992020 .00040
20

14.700 .922820 .00036

14.800 .926690 .00037

14.900 .929880 .00036

15.000 .933620 .00036

15.100 .936680 .00036

15.200 .940870 .00036

15.300 .944880 .00036

15.400 .947530 .00035

15.500 .950810 .00037

15.700 .957760 .00036

15.800 .961070 .00037

15.900 .964110 .00037

16.000 .967300 .00036

16.100 .970990 .00035

16.200 .972800 .00036

16.300 .976830 .00036

16.400 .979480 .00035

16.500 .982000 .00035

16.700 .988260 .00036

16.800 .991710 .00036

16.900 .994570 .00036

17.000 .997530 .00035

17.101 .000340 .00036

17.201 .003430 .00035

17.301 .006010 .00035

17.401 .009230 .00035

17.501 .011260 .00035

17.601 .014350 .00036

32

12.100 .925240 .00041

12.200 .930740 .00040

12.300 .935160 .00040

12.400 .939770 .00042

12.500 .945430 .00042

12.600 .950750 .00041

12.700 .954840 .00043

12.800 .959760 .00041

12.900 .965400 .00041

13.000 .970300 .00040

13.100 .973900 .00041

13.200 .978960 .00041

13.300 .983050 .00040

13.400 .987000 .00041

13.500 .991140 .00042

13.600 .996640 .00040 
14.900 .990880 .00039 15.000 .995060 .00039 15.100 .999000 .00038 15.201 .001980 .00039 15.301 .006010 .00037 15.401 .009810 .00038 15.501 .013330 .00039 15.601 .016210 .00038
13.900 .995160 .00041 14.000 .998670 .00042 14.101 .003310 .00041 14.201 .006930 .00042 14.301 .012010 .00041 14.401 .014860 .00040 14.501 .019280 .00040
13.701 .000330 .00041 13.801 .005440 .00041 13.901 .008370 .00043 14.001 .013710 .00041 14.101 .017010 .00040 14.201 .022400 .00040
35

11.700 .921690 .00042 11.800 .926550 .00042 11.900 .931760 .00042 12.000 .937290 .00042 12.100 .943130 .00042 12.200 .948250 .00043 12.300 .952260 .00043 12.400 .957900 .00042 12.500 .962900 .00042 12.600 .967760 .00041 12.700 .972420 .00042 12.800 .976880 .00041 12.900 .981830 .00043 13.000 .987110 .00041 13.100 .991720 .00041 13.200 .996360 .00042 13.301 .000590 .00040 13.401 .005100 .00042 13.501 .010290 .00042 13.601 .014250 .00041 13.701 .019240 .00042

60

10.400 .921170 .00044 10.450 .924890 .00045 10.500 .926280 .00044 10.550 .930130 .00044 10.600 .932510 .00044 10.650 .936600 .00044 10.700 .939160 .00046 10.750 .942040 .00045 10.800 .945820 .00045 10.900 .951680 .00047 10.950 .953840 .00046 11.000 .958150 .00044 11.050 .960710 .00046 11.100 .964560 .00044
40

11.300 .921740 .00042 11.400 .927470 .00044 11.500 .933810 .00043 11.600 .938750 .00042 11.700 .943950 .00041 11.800 .949740 .00043 11.900 .955100 .00042 12.000 .960660 .00043 12.100 .965390 .00042 12.200 .971120 .00043 12.300 .975910 .00043 12.400 .980940 .00043 12.500 .985950 .00043 12.600 .991530 .00044 12.700 .996630 .00043 12.801 .000770 .00043 12.901 .006590 .00042 13.001 .010530 .00043 13.101 .015230 .00044 13.201 .020280 .00042
50

10.700 .919190 .00043 10.800 .925380 .00043 10.900 .930820 .00044 11.000 .936140 .00044 11.100 .942990 .00044 11.200 .949340 .00043 11.300 .954870 .00045 11.500 .965570 .00045 11.600 .971550 .00044 11.700 .976390 .00044 11.800 .982400 .00045 11.900 .987560 .00044 12.000 .992910 .00044 12.100 .998700 .00044 12.201 .004640 .00044 12.301 .009100 .00044 12.401 .014600 .00044 12.501 .018940 .00043
10.200 .922300 .00047 10.250 .925700 .00046 10.300 .928180 .00045 10.350 .931490 .00045 10.400 .934870 .00045 10.450 .939010 .00046 10.500 .941580 .00046 10.550 .943920 .00046 10.600 .948110 .00045 10.650 .951040 .00046 10.700 .953500 .00045 10.750 .956160 .00045 10.800 .959380 .00045 10.850 .962540 .00045
80

10.100 .925340 .00045 10.150 .928620 .00045 10.200 .931610 .00046 10.250 .934240 .00045 10.300 .938440 .00045 10.350 .941310 .00046 10.400 .944190 .00046 10.450 .948240 .00047 10.500 .950520 .00046 10.550 .953700 .00045 10.600 .957230 .00047 10.650 .959860 .00046 10.750 .966890 .00045 10.800 .969160 .00046 
11.150 .967350 .00046 11.200 .969330 .00046 11.250 .972630 .00044 11.300 .975330 .00047 11.350 .978590 .00046 11.400 .981170 .00046 11.450 .983890 .00044 11.500 .986550 .00045 11.550 .989390 .00045 11.600 .992760 .00046 11.650 .994930 .00044 11.700 .997840 .00045 11.751 .001220 .00045 11.801 .003250 .00045 11.851 .006350 .00046 11.901 .008660 .00044 11.951 .011960 .00045 12.001 .014440 .00044 12.051 .016960 .00045 12.101 .019410 .00046
10.900 .965820 .00045 10.950 .969040 .00045 11.000 .971380 .00045 11.050 .975460 .00045 11.100 .978230 .00046 11.150 .981100 .00046 11.200 .983580 .00046 11.250 .986170 .00046 11.300 .989290 .00045 11.350 .992710 .00045 11.400 .994920 .00046 11.450 .998650 .00045 11.501 .001010 .00045 11.551 .004390 .00046 11.601 .006720 .00046 11.651 .009650 .00044 11.701 .011870 .00044 11.751 .015410 .00045 11.801 .017590 .00046
10.850 .972110 .00046 10.900 .975750 .00046 10.950 .978610 .00047 11.000 .982130 .00047 11.050 .983750 .00046 11.100 .988270 .00046 11.150 .990760 .00046 11.200 .993310 .00046 11.250 .997060 .00046 11.300 .998170 .00045 11.351 .001600 .00046 11.401 .004900 .00045 11.451 .008310 .00046 11.501 .009820 .00047 11.551 .013010 .00046 11.601 .016560 .00045 11.651 .019720 .00047 11.701 .022160 .00046

\section{0}

10.000 .925170 .00046 10.050 .927880 .00046 10.100 .931320 .00045 10.150 .935750 .00046 10.200 .937450 .00047 10.250 .941260 .00046 10.300 .944210 .00047 10.350 .947340 .00045 10.400 .951260 .00046 10.450 .953990 .00045 10.500 .956140 .00047 10.550 .960040 .00047 10.600 .962830 .00046 10.650 .966990 .00046 10.700 .970280 .00046 10.750 .972370 .00047 10.800 .976410 .00046 10.850 .979110 .00047 10.900 .981680 .00047 10.950 .984970 .00047 11.000 .987160 .00046 11.050 .989730 .00046 11.100 .994010 .00046 11.150 .996220 .00046 11.200 .999060 .00047 11.251 .002730 .00045 11.301 .006060 .00047
100

9.900 .922490 .00045 9.950 .926040 .00046 10.000 .929200 .00047 10.050 .933320 .00047 10.100 .935430 .00048 10.150 .939230 .00047 10.200 .942320 .00046 10.250 .946460 .00047 10.300 .949940 .00047 10.350 .952430 .00046 10.400 .955460 .00046 10.450 .958660 .00047 10.500 .961660 .00045 10.550 .965420 .00047 10.600 .968160 .00046 10.650 .970080 .00046 10.700 .973790 .00047 10.750 .976960 .00047 10.800 .979670 .00047 10.850 .983310 .00047 10.900 .986680 .00046 10.950 .989550 .00047 11.000 .992350 .00046 11.050 .994740 .00047 11.100 .997350 .00046 11.151 .001660 .00047 11.201 .005030 .00046
120

9.800 .920550 .00047 9.850 .923750 .00046 9.900 .927600 .00047 9.950 .929420 .00048 10.000 .933910 .00046 10.050 .937260 .00047 10.100 .940640 .00047 10.150 .943680 .00047 10.200 .947680 .00047 10.250 .950690 .00047 10.300 .954000 .00046 10.350 .956490 .00048 10.400 .959150 .00048 10.450 .962890 .00046 10.500 .965130 .00047 10.550 .968340 .00046 10.600 .972440 .00047 10.650 .975260 .00049 10.700 .977810 .00047 10.750 .982120 .00047 10.800 .983960 .00046 10.850 .987240 .00045 10.900 .989910 .00047 10.950 .992900 .00046 11.000 .996350 .00046 11.050 .998830 .00047 11.101 .002450 .00047 
11.351 .007540 .00047 11.401 .010320 .00046 11.451 .014340 .00046 11.501 .016860 .00046 11.551 .019650 .00046

130

9.700 .916390 .00046

9.750 .918840 .00046

9.800 .923000 .00046

9.850 .925840 .00047

9.900 .929530 .00047

9.950 .932700 .00046

10.000 .935990 .00045

10.050 .938790 .00047

10.150 .946020 .00048

10.200 .948730 .00046

10.250 .951740 .00047

10.300 .955610 .00047

10.350 .959230 .00048

10.400 .960860 .00048

10.450 .964640 .00047

10.500 .967480 .00047

10.550 .971030 .00047

10.600 .974450 .00046

10.650 .976740 .00046

10.700 .980270 .00047

10.750 .982090 .00047

10.800 .985830 .00048

10.850 .989260 .00047

10.900 .991950 .00049

10.950 .994450 .00048

11.000 .997130 .00047

11.051 .001190 .00047

11.101 .003350 .00047

11.151 .006440 .00047

11.201 .009200 .00047

11.251 .012180 .00047

11.301 .014550 .00046

11.351 .017740 .00047

11.401 .020950 .00047
11.251 .006210 .00046 11.301 .010960 .00045 11.351 .011970 .00047 11.401 .015200 .00047 11.451 .017530 .00047 11.501 .020510 .00046
11.151 .004880 .00048 11.201 .006670 .00048 11.251 .010130 .00046 11.301 .013500 .00047 11.351 .016280 .00047 11.401 .018210 .00047
150

9.800 .922990 .00048 9.850 .926540 .00046 9.900 .929840 .00046 9.950 .932770 .00047 10.000 .936060 .00047 10.050 .939980 .00047 10.100 .944290 .00047 10.150 .945470 .00047 10.200 .949810 .00046 10.250 .952730 .00047 10.300 .954880 .00046 10.350 .958620 .00048 10.400 .961200 .00047 10.450 .964930 .00047 10.500 .967850 .00047 10.550 .971030 .00048 10.600 .973520 .00048 10.650 .978400 .00046 10.700 .980100 .00048 10.750 .983130 .00047 10.800 .986230 .00047 10.850 .989770 .00048 10.900 .992320 .00048 10.950 .994900 .00046 11.000 .997550 .00047 11.051 .000120 .00046 11.101 .004430 .00046 11.151 .006170 .00047 11.201 .008950 .00049 11.251 .012220 .00046 11.301 .015530 .00048 11.351 .017040 .00046 11.401 .021140 .00047

\section{0}

9.800 .921160 .00048 9.850 .923900 .00046 9.900 .927270 .00047 9.950 .929900 .00048 10.000 .933540 .00046 10.050 .937070 .00047 10.100 .940380 .00047 10.150 .943960 .00048 10.200 .945540 .00047 10.250 .949660 .00048 10.300 .951960 .00047 10.350 .955450 .00045 10.400 .957780 .00047 10.450 .960380 .00048 10.500 .963740 .00047 10.550 .967210 .00047 10.600 .970430 .00047 10.650 .973930 .00047 10.700 .976050 .00048 10.750 .979130 .00048 10.800 .982130 .00048 10.850 .984600 .00047 10.900 .987800 .00047 10.950 .990740 .00047 11.000 .994540 .00046 11.050 .995810 .00048 11.101 .000190 .00048 11.151 .000920 .00049 11.201 .005130 .00048 11.251 .007610 .00047 11.301 .010190 .00045 11.351 .013560 .00047 11.401 .016100 .00048 11.451 .017980 .00046 11.501 .021490 .00047 
239

9.900 .921660 .00046

9.950 .925430 .00047

10.000 .927810 .00046

10.050 .930650 .00046

10.100 .933510 .00047

10.150 .937700 .00047

10.200 .940260 .00047

10.250 .943110 .00047

10.300 .946580 .00047

10.350 .949840 .00047

10.400 .952290 .00047

10.450 .955670 .00049

10.500 .958380 .00047

10.550 .961690 .00046

10.600 .964010 .00047

10.650 .967500 .00048

10.700 .970160 .00048

10.750 .973130 .00047

10.800 .975400 .00046

10.850 .979220 .00047

10.900 .982070 .00047

10.950 .984980 .00048

11.000 .986800 .00047

11.050 .989850 .00045

11.100 .993270 .00048

11.150 .995520 .00048

11.200 .999230 .00048

11.251 .001020 .00049

11.301 .004740 .00048

11.351 .006940 .00047

11.401 .009000 .00048

11.451 .013170 .00047

11.501 .015130 .00047

11.551 .017230 .00049

11.601 .020450 .00048

478

10.600 .923930 .00046

10.700 .929380 .00047

10.800 .935000 .00047

10.900 .940060 .00048

11.000 .945910 .00048

11.100 .950530 .00047

11.200 .956390 .00047

11.300 .961750 .00048

11.400 .966790 .00046

11.500 .972360 .00046

11.600 .977570 .00047
250

10.050 .929450 .00048

10.100 .931930 .00047

10.150 .935670 .00046

10.200 .939680 .00046

10.250 .942240 .00047

10.300 .945430 .00046

10.350 .948230 .00047

10.400 .950280 .00045

10.450 .954050 .00047

10.500 .957280 .00046

10.550 .959470 .00048

10.600 .962350 .00048

10.650 .965470 .00046

10.700 .968900 .00047

10.750 .970600 .00046

10.800 .974530 .00049

10.850 .977880 .00047

10.900 .980420 .00046

10.950 .983390 .00048

11.000 .985900 .00048

11.050 .987490 .00047

11.100 .990510 .00048

11.150 .993950 .00047

11.200 .997260 .00046

11.251 .000580 .00047

11.301 .001600 .00048

11.351 .005330 .00047

11.401 .007530 .00047

11.451 .010510 .00047

11.501 .012090 .00046

11.551 .015870 .00047
359

10.300 .927290 .00046

10.400 .933180 .00047

10.500 .939480 .00047

10.600 .945060 .00046

10.700 .950690 .00046

10.800 .956450 .00047

10.900 .962130 .00047

11.000 .966460 .00046

11.100 .972350 .00048

11.200 .978310 .00047

11.300 .983860 .00048

11.400 .989550 .00049

11.500 .993810 .00049

11.600 .999200 .00048

11.701 .004840 .00048

11.801 .009660 .00049

11.901 .015010 .00047

12.001 .019940 .00047
10.700 .923290 .00047

10.800 .928830 .00047

10.900 .934920 .00046

11.000 .939800 .00046

11.100 .945230 .00047

11.200 .950250 .00047

11.300 .955510 .00047

11.400 .960270 .00045

11.500 .966660 .00046

11.600 .971670 .00046

11.700 .976210 .00047
600

11.200 .920160 .00046 11.300 .926520 .00046 11.400 .930390 .00047 11.500 .935980 .00047 11.600 .941220 .00046 11.700 .946570 .00048 11.800 .951420 .00047 11.900 .955910 .00047 12.000 .961920 .00047 12.100 .966160 .00046 12.200 .970580 .00048 
11.700 .981740 .00045 11.800 .986680 .00048 11.900 .991860 .00047 12.000 .996510 .00048 12.101 .002020 .00048 12.201 .007270 .00047 12.301 .011570 .00045 12.401 .016320 .00047 12.501 .020500 .00048
11.800 .982120 .00047 11.900 .986800 .00047 12.000 .990930 .00046 12.100 .997030 .00047 12.201 .001540 .00048 12.301 .006530 .00047 12.401 .010580 .00046 12.501 .014610 .00048 12.601 .019460 .00048
650

11.800 .923690 .00048 11.900 .929600 .00046 12.000 .933560 .00045 12.100 .938640 .00046 12.200 .942950 .00049 12.300 .948500 .00046 12.400 .953920 .00046 12.500 .957730 .00047 12.600 .962380 .00046 12.700 .966700 .00046 12.800 .971440 .00046 12.900 .975860 .00045 13.000 .981200 .00048 13.100 .984320 .00046 13.200 .989310 .00046 13.300 .993850 .00045 13.400 .998260 .00046 13.501 .002570 .00046 13.601 .005810 .00046 13.701 .011690 .00046 13.801 .014820 .00047 13.901 .019390 .00047
12.300 .975280 .00047 12.400 .980780 .00047 12.500 .985240 .00047 12.600 .989000 .00047 12.700 .994700 .00046 12.800 .998080 .00047 12.901 .003460 .00048 13.001 .007940 .00047 13.101 .011970 .00046 13.201 .016910 .00047 13.301 .021250 .00048

660

11.900 .921480 .00047 12.000 .926820 .00046 12.100 .932010 .00045 12.200 .935810 .00047 12.300 .940920 .00047 12.400 .946760 .00046 12.500 .949650 .00047 12.600 .955260 .00047 12.700 .960230 .00046 12.800 .964310 .00045 12.900 .968060 .00048 13.000 .973360 .00046 13.100 .977100 .00045 13.200 .982480 .00045 13.500 .994920 .00047 13.600 .999660 .00045 13.701 .004220 .00047 13.801 .007600 .00046 13.901 .012590 .00046 14.001 .015940 .00047

680

12.300 .919730 .00046 12.400 .924940 .00045 12.500 .929160 .00045 12.600 .934630 .00047 12.700 .938310 .00047 12.800 .943820 .00047 12.900 .947740 .00046 13.000 .952360 .00045 13.100 .957180 .00046
690

12.700 .922400 .00046 12.800 .926160 .00047 12.900 .930250 .00046 13.000 .935700 .00046 13.100 .939950 .00046 13.200 .943730 .00046 13.300 .947830 .00046 13.400 .952710 .00046 13.500 .957020 .00047
670

12.100 .922610 .00046 12.200 .926620 .00046 12.300 .932320 .00047 12.400 .937190 .00045 12.500 .941410 .00046 12.600 .945870 .00047 12.700 .950430 .00047 12.800 .955250 .00044 12.900 .958840 .00046 13.000 .964200 .00047 13.100 .968800 .00047 13.200 .973590 .00047 13.300 .977870 .00046 13.400 .981410 .00047 13.500 .986160 .00046 13.600 .990350 .00046 13.700 .994430 .00046 13.800 .998440 .00047 13.901 .003800 .00046 14.001 .007150 .00047 14.101 .010930 .00046 14.201 .014630 .00047 14.301 .019580 .00047

698

13.400 .922770 .00045 13.500 .927340 .00045 13.600 .931480 .00048 13.800 .939690 .00045 13.900 .942900 .00046 14.000 .947430 .00046 14.100 .951060 .00045 14.200 .955640 .00046 14.300 .959700 .00046 
13.200 .960500 .00045 13.300 .965180 .00046 13.400 .969610 .00046 13.500 .973240 .00045 13.600 .978530 .00046 13.700 .982240 .00045 13.800 .986690 .00047 13.900 .990810 .00046 14.000 .994850 .00047 14.100 .999810 .00047 14.201 .003420 .00047 14.301 .007110 .00047 14.401 .011790 .00046 14.501 .015750 .00048 14.601 .018970 .00046
13.600 .960980 .00045

13.700 .965160 .00045

13.800 .970390 .00047

13.900 .973990 .00046

14.000 .978030 .00046

14.100 .982560 .00046

14.200 .986260 .00046

14.300 .990140 .00046

14.400 .993770 .00048

14.500 .997880 .00047

14.601 .002320 .00045

14.701 .005920 .00046

14.801 .010340 .00046

14.901 .014590 .00046
14.400 .963970 .00046

14.500 .967400 .00048

14.600 .971450 .00045

14.700 .975220 .00046

14.800 .979970 .00045

14.900 .982910 .00047

15.000 .986830 .00046

15.100 .990690 .00046

15.200 .995280 .00047

15.300 .998670 .00046

15.401 .002620 .00047

15.501 .005320 .00047

15.601 .009590 .00046

15.701 .012210 .00047

15.801 .017270 .00047 
Pun46-sp-0970-00

12

28.700 .938420 .00029

29.200 .943940 .00028

29.700 .949180 .00027

30.200 .954700 .00027

30.700 .959390 .00028

31.200 .964110 .00027

31.700 .968770 .00027

32.200 .972720 .00028

32.700 .976790 .00027

33.200 .981540 .00027

33.700 .985140 .00027

34.200 .988700 .00026

34.700 .993510 .00027

35.200 .996630 .00026

35.700 .999970 .00025

36.201 .003240 .00026

36.701 .006940 .00026

37.201 .009410 .00026

37.701 .012830 .00026
15

22.400 .925120 .00034

22.600 .929200 .00034

22.800 .933040 .00034

23.000 .937090 .00034

23.200 .940390 .00035

23.400 .944730 .00033

23.600 .948960 .00034

23.800 .952040 .00033

24.000 .956540 .00033

24.200 .958850 .00034

24.400 .963230 .00033

24.600 .967340 .00033

24.800 .970680 .00033

25.000 .973990 .00033

25.200 .976470 .00033

25.400 .980540 .00034

25.600 .982640 .00033

25.800 .986490 .00033

26.000 .989440 .00033

26.200 .992070 .00032

26.400 .995430 .00031

26.600 .999080 .00032

26.801 .001140 .00032

27.001 .003860 .00032

27.201 .007200 .00032

27.401 .009130 .00032

27.601 .012290 .00032

27.701 .013820 .00032
25

17.000 .921900 .00041

17.100 .925530 .00043

17.200 .928600 .00042

17.300 .932800 .00042

17.400 .937300 .00042

17.500 .940730 .00042

17.600 .943080 .00040

17.700 .947280 .00042

18.300 .969120 .00040
30

16.000 .920920 .00046

16.100 .923880 .00044

16.200 .927770 .00045

16.300 .933560 .00044

16.400 .936400 .00045

16.500 .942260 .00045

16.600 .945620 .00045

16.700 .949710 .00043

16.800 .954670 .00044
20

18.800 .924220 .00039

18.900 .928980 .00039

19.000 .930830 .00039

19.100 .934010 .00038

19.200 .937250 .00039

19.300 .939410 .00039

19.400 .942300 .00038

19.500 .946150 .00039

19.600 .949320 .00038

19.700 .951700 .00039

19.800 .955110 .00039

19.900 .957570 .00040

20.000 .960020 .00039

20.100 .963220 .00038

20.200 .965310 .00038

20.300 .968680 .00038

20.400 .970670 .00037

20.500 .973380 .00038

20.600 .976120 .00037

20.700 .979560 .00038

20.800 .981750 .00038

20.900 .984140 .00037

21.000 .986750 .00037

21.100 .989420 .00039

21.200 .991420 .00037

21.300 .994870 .00038

21.400 .997640 .00037

21.500 .999480 .00038

21.601 .001960 .00038

21.701 .003730 .00038

21.901 .008740 .00040

22.001 .010630 .00036

22.101 .013320 .00037

22.201 .015830 .00037

22.301 .018910 .00037

32

15.800 .923820 .00045

15.900 .927820 .00045

16.000 .932470 .00045

16.100 .936750 .00046

16.200 .942210 .00045

16.300 .947010 .00045

16.400 .950700 .00044

16.500 .954700 .00046

16.600 .960050 .00043 
18.400 .972170 .00042 18.500 .975230 .00041 18.600 .979180 .00041 18.700 .982640 .00041 18.800 .985230 .00042 18.900 .989340 .00042 19.000 .992580 .00042 19.100 .996160 .00042 19.200 .998110 .00041 19.301 .001620 .00041 19.401 .005000 .00042 19.501 .008260 .00040 19.601 .011060 .00041 19.701 .014120 .00041 19.801 .018160 .00041

35

15.400 .921800 .00047 15.500 .925470 .00046 16.000 .949860 .00046 16.100 .954320 .00047 16.200 .958650 .00046 16.300 .962780 .00045 16.400 .967070 .00046 16.500 .971110 .00045 16.600 .976510 .00046 16.700 .980540 .00046 16.800 .986260 .00045 17.000 .993710 .00046 17.100 .998150 .00045 17.201 .000820 .00045 17.301 .005360 .00044 17.401 .009590 .00046 17.501 .014000 .00046 17.601 .017050 .00046 17.701 .021520 .00046
16.900 .957380 .00044 17.000 .961950 .00043 17.100 .967250 .00044 17.200 .970700 .00043 17.300 .974070 .00045 17.400 .978850 .00044 17.500 .981960 .00044 17.600 .986750 .00043 17.700 .990070 .00044 17.800 .993380 .00044 17.900 .997210 .00044 18.001 .000890 .00044 18.101 .004260 .00045 18.201 .007760 .00045 18.301 .012160 .00044 18.401 .014530 .00046 18.501 .018710 .00043
16.700 .963910 .00046 16.800 .967970 .00046 16.900 .971830 .00045 17.000 .975980 .00046 17.100 .979120 .00045 17.200 .983250 .00045 17.300 .987950 .00044 17.400 .992310 .00045 17.500 .995270 .00043 17.600 .999810 .00044 17.701 .004110 .00045 17.801 .007160 .00045 17.901 .010920 .00046 18.001 .014880 .00045 18.101 .018900 .00045
14.900 .918660 .00048 15.000 .923780 .00047 15.100 .927940 .00047 15.200 .934030 .00046 15.300 .938260 .00048 15.400 .944240 .00048 15.500 .948780 .00049 15.600 .953220 .00047 15.700 .958100 .00046 15.800 .963630 .00046 15.900 .967800 .00047 16.000 .972230 .00048 16.100 .977620 .00046 16.200 .981580 .00048 16.300 .986670 .00047 16.801 .007280 .00047 17.101 .019600 .00046

60 14.000 .917930 .00049 14.100 .923440 .00048 14.200 .929410 .00051 14.300 .934560 .00048 14.400 .940840 .00051 14.500 .945870 .00049
70

13.900 .922170 .00051 14.000 .927500 .00051 14.100 .933690 .00050 14.200 .940400 .00051 14.300 .945340 .00051 14.400 .950240 .00051
50

14.400 .921970 .00049 14.500 .927520 .00050 14.700 .938320 .00049 14.800 .943420 .00048 14.900 .948690 .00048 15.000 .953100 .00049 15.100 .958620 .00049 15.200 .964530 .00049 15.300 .969330 .00049 15.400 .974420 .00048 15.500 .980050 .00049 15.600 .984110 .00049 15.700 .988910 .00047 15.800 .993220 .00048 15.900 .999560 .00048 16.001 .003970 .00049 16.101 .008010 .00048 16.201 .012900 .00049 16.301 .017120 .00047 16.401 .022080 .00048

\section{0}

13.800 .923270 .00052 13.900 .929690 .00051 14.000 .935150 .00051 14.200 .946780 .00050 14.300 .952260 .00051 14.400 .959280 .00051 
14.600 .950840 .00049 14.700 .955240 .00050 15.000 .972770 .00050 15.100 .977400 .00051 15.200 .983940 .00049 15.300 .987900 .00049 15.400 .993310 .00050 15.500 .998070 .00050 15.601 .002240 .00052 15.701 .009200 .00050 15.801 .013290 .00050 15.901 .018010 .00051

90

13.700 .921540 .00050 13.800 .928330 .00051 13.900 .933310 .00050 14.000 .939350 .00050 14.100 .944250 .00051 14.200 .951460 .00052 14.300 .956950 .00050 14.400 .963330 .00051 14.500 .967560 .00052 14.600 .973220 .00053 14.700 .979300 .00050 14.800 .984790 .00051 14.900 .991390 .00051 15.000 .995930 .00051 15.501 .021900 .00050

130

13.600 .918310 .00051 13.700 .923310 .00051 13.800 .931030 .00051 13.900 .935330 .00053 14.000 .941590 .00051 14.100 .947340 .00051 14.200 .954110 .00053 14.300 .959320 .00052 14.400 .964430 .00053 14.500 .971030 .00054 14.800 .987430 .00053 14.900 .992470 .00051 15.000 .998250 .00051
14.500 .956790 .00051 14.600 .961530 .00050 14.700 .967670 .00052 14.800 .972360 .00052 14.900 .977140 .00048 15.000 .984450 .00049 15.100 .988000 .00051 15.200 .994080 .00050 15.300 .999150 .00051 15.401 .004360 .00051 15.501 .009310 .00052 15.601 .013750 .00051 15.701 .018720 .00051
14.500 .963390 .00051 14.600 .968470 .00051 14.700 .974670 .00051 14.800 .979850 .00052 14.900 .986090 .00051 15.000 .991900 .00052 15.100 .996370 .00052 15.201 .001270 .00051 15.301 .007190 .00050 15.401 .011410 .00052 15.501 .015860 .00051 15.601 .022190 .00050
100

13.600 .917780 .00051 13.700 .924230 .00053 13.900 .936020 .00053 14.000 .942480 .00051 14.100 .948650 .00052 14.200 .955340 .00052 14.300 .959690 .00052 14.400 .966250 .00052 14.500 .971120 .00052 14.600 .976820 .00053 14.700 .982330 .00051 14.800 .988400 .00051 14.900 .992920 .00052 15.000 .997840 .00051 15.101 .003550 .00052 15.201 .008710 .00053 15.301 .014200 .00052

150

13.600 .915340 .00051 13.700 .921710 .00054 13.800 .927510 .00051 13.900 .934180 .00051 14.000 .939950 .00052 14.100 .944670 .00052 14.200 .952630 .00051 14.300 .955830 .00053 14.400 .962220 .00052 14.500 .968730 .00054 14.600 .973400 .00053 14.700 .979800 .00052 14.800 .983830 .00053
120

13.600 .918980 .00052 13.700 .924240 .00050 13.800 .931290 .00053 13.900 .936440 .00052 14.000 .942100 .00053 14.100 .948250 .00052 14.200 .953950 .00052 14.300 .959720 .00052 14.400 .965470 .00052 14.500 .970630 .00052 14.700 .981490 .00052 14.800 .986820 .00052 14.900 .991400 .00051 15.000 .997500 .00051 15.101 .002910 .00051 15.201 .008940 .00053 15.301 .013480 .00052

\section{0}

13.800 .917270 .00052 13.900 .922470 .00052 14.000 .928290 .00053 14.100 .935350 .00051 14.200 .939980 .00051 14.300 .944850 .00052 14.400 .950940 .00052 14.500 .956610 .00050 14.600 .963480 .00051 14.700 .967670 .00052 14.800 .973090 .00052 14.900 .978720 .00052 15.000 .984550 .00051 
15.101 .002990 .00051 15.201 .006970 .00053 15.301 .013350 .00052 15.401 .018370 .00052 15.501 .023770 .00051 15.601 .028190 .00051 15.701 .032900 .00052 15.801 .038430 .00052 15.901 .041930 .00050 16.001 .047510 .00052 16.101 .053330 .00052
239

14.000 .918990 .00051 14.050 .922890 .00051 14.100 .925500 .00051 14.150 .927710 .00051 14.200 .930360 .00052 14.250 .933610 .00052 14.300 .935940 .00053 14.350 .938700 .00052 14.400 .942830 .00052 14.450 .945370 .00051 14.550 .950730 .00051 14.600 .952360 .00052 14.650 .955720 .00051 14.700 .958770 .00051 14.750 .960340 .00051 14.800 .964450 .00052 14.850 .966520 .00052 14.900 .969420 .00052 14.950 .972010 .00052 15.000 .974500 .00052 15.050 .976650 .00053 15.100 .979930 .00050 15.150 .981990 .00052 15.200 .983180 .00052 15.250 .986850 .00052 15.300 .990170 .00052 15.350 .993190 .00051 15.400 .994590 .00051 15.450 .997880 .00053 15.500 .998820 .00053 15.551 .001580 .00052 15.601 .004820 .00052 15.651 .006380 .00051 15.701 .010240 .00052
14.900 .990110 .00054 15.101 .001530 .00053 15.201 .005290 .00052 15.401 .015160 .00052 15.501 .020550 .00053
15.100 .989110 .00053 15.200 .993900 .00051 15.300 .998140 .00052 15.401 .004800 .00052 15.501 .009520 .00052 15.601 .014680 .00053 15.701 .019500 .00052 15.801 .025300 .00051 15.901 .029390 .00052 16.001 .034840 .00052

250

14.000 .916490 .00052 14.100 .922840 .00051 14.200 .928560 .00051 14.300 .934690 .00052 14.400 .939590 .00051 14.500 .945890 .00051 14.600 .951090 .00051 14.700 .956060 .00050 14.800 .961260 .00053 14.900 .965980 .00051 15.000 .971070 .00053 15.100 .976660 .00052 15.200 .982040 .00050 15.300 .986680 .00050 15.400 .992690 .00052 15.500 .997730 .00053 15.601 .001520 .00051 15.701 .007850 .00052 15.801 .012010 .00050 15.901 .017020 .00052 16.001 .020850 .00052 16.101 .026360 .00053 16.201 .029720 .00051 16.301 .034750 .00049 16.401 .040030 .00050 16.501 .044450 .00050 16.601 .048980 .00052 16.701 .053600 .00053 16.801 .057310 .00053
359

14.500 .921450 .00050 14.600 .926300 .00050 14.700 .931500 .00051 14.800 .937050 .00050 14.900 .942370 .00052 15.000 .947080 .00050 15.100 .951730 .00052 15.200 .957250 .00051 15.300 .962770 .00051 15.400 .967660 .00053 15.500 .972650 .00050 15.600 .977870 .00050 15.700 .982870 .00050 15.800 .987220 .00051 15.900 .992860 .00051 16.000 .996690 .00052 16.101 .001110 .00052 16.201 .005280 .00050 16.301 .011230 .00052 
478

14.900 .922320 .00050 15.000 .927500 .00050 15.100 .932700 .00050 15.200 .937900 .00050 15.300 .942650 .00051 15.400 .946430 .00052 15.600 .958450 .00048 15.700 .962420 .00051 15.800 .967300 .00051 15.900 .971010 .00050 16.000 .977200 .00050 16.100 .980870 .00051 16.200 .986620 .00052 16.300 .990690 .00051 16.400 .995230 .00051 16.500 .999360 .00051 16.601 .003620 .00050 16.701 .008320 .00049 16.801 .013730 .00051 16.901 .017760 .00052 17.001 .022370 .00049

\section{0}

15.500 .920100 .00049 15.600 .925250 .00050 15.700 .929620 .00049 15.800 .934860 .00050 15.900 .940220 .00049 16.000 .944760 .00050 16.100 .950430 .00050 16.200 .955220 .00050 16.300 .959740 .00050 16.400 .963910 .00049 16.500 .968290 .00050 16.600 .973720 .00049
500

15.000 .921820 .00050

15.100 .928400 .00049

15.200 .933790 .00050

15.300 .937740 .00051

15.400 .943590 .00051

15.500 .948640 .00050

15.600 .953930 .00049

15.700 .958440 .00051

15.800 .962870 .00051

15.900 .968880 .00052

16.000 .973160 .00050

16.100 .977920 .00049

16.200 .982380 .00051

16.300 .987160 .00051

16.400 .990530 .00050

16.500 .995730 .00051

16.601 .000070 .00049

16.701 .005220 .00050

16.801 .009700 .00049

16.901 .014080 .00051

17.001 .017980 .00050

17.101 .023280 .00050

17.201 .025980 .00050

17.301 .031160 .00051

17.401 .034690 .00051

17.501 .039340 .00051

17.601 .043450 .00051

17.701 .048970 .00050

17.801 .051730 .00050

17.901 .056230 .00050

18.001 .059970 .00049

18.101 .063880 .00052

18.201 .067620 .00050

700

15.800 .921160 .00051

15.900 .925640 .00050

16.000 .931050 .00050

16.100 .935610 .00050

16.200 .941070 .00052

16.300 .947030 .00049

16.400 .950490 .00050

16.500 .955930 .00051

16.600 .960590 .00050

16.700 .965450 .00049

16.800 .969940 .00050

16.900 .973740 .00051
600

15.300 .919520 .00050

15.400 .925980 .00051

15.500 .930690 .00050

15.600 .936110 .00050

15.700 .939970 .00051

15.800 .945320 .00050

15.900 .950430 .00050

16.000 .955380 .00049

16.100 .959840 .00050

16.200 .964030 .00050

16.300 .969610 .00049

16.400 .973170 .00049

16.500 .978440 .00049

16.600 .984030 .00050

16.700 .987010 .00051

16.800 .992760 .00050

16.900 .996610 .00049

17.001 .000220 .00049

17.101 .006190 .00050

17.201 .009800 .00050

17.301 .014710 .00050

17.401 .018430 .00050

17.501 .022910 .00051 
16.700 .977750 .00049 16.800 .982060 .00050 16.900 .987340 .00050 17.000 .991290 .00049 17.100 .995530 .00050 17.200 .999420 .00049 17.301 .004280 .00049 17.401 .007840 .00050 17.501 .012710 .00050 17.601 .016820 .00050 17.701 .022120 .00050
17.000 .977830 .00049 17.100 .983080 .00050 17.200 .987780 .00050 17.300 .991250 .00051 17.400 .995980 .00049 17.501 .000580 .00050 17.601 .003730 .00050 17.701 .008900 .00050 17.801 .012960 .00051 17.901 .016810 .00049 18.001 .020860 .00051
760

16.200 .919240 .00050 16.300 .924730 .00049 16.400 .928290 .00049 16.500 .933830 .00049 16.600 .939120 .00050 16.700 .942750 .00049 16.800 .947720 .00049 16.900 .952550 .00049 17.000 .957730 .00049 17.100 .961530 .00049 17.200 .966770 .00050 17.300 .971070 .00048 17.400 .975910 .00049 17.500 .979520 .00049 17.600 .983810 .00048 17.700 .988200 .00049 17.800 .991730 .00049 17.900 .996940 .00049 18.001 .001510 .00050 18.101 .005630 .00048 18.201 .008640 .00048 18.301 .013790 .00050 18.401 .017140 .00049 18.501 .022470 .00050

17.300 .974820 .00051

17.400 .978940 .00049

17.500 .983990 .00049

17.600 .988330 .00050

17.700 .991980 .00049

17.800 .995840 .00048

17.901 .000130 .00049 18.001 .005380 .00048 18.101 .007650 .00050 18.201 .013330 .00050 18.301 .017940 .00048 18.401 .021740 .00050 18.501 .024640 .00051 18.601 .028570 .00050 18.701 .033530 .00050 18.801 .037730 .00051 18.901 .041080 .00048 19.001 .045060 .00050 19.101 .049390 .00048 19.201 .052670 .00049 19.301 .056880 .00050 19.401 .060940 .00050 19.501 .063810 .00052

765

16.300 .921580 .00049 16.400 .926780 .00048 16.500 .930650 .00049 16.600 .935590 .00048 16.700 .940790 .00049 16.800 .945870 .00048 16.900 .949860 .00050 17.000 .954620 .00050 17.100 .959200 .00049 17.200 .963690 .00048 17.300 .968160 .00051 17.400 .973160 .00050 17.500 .976580 .00051 17.600 .981830 .00050 17.700 .985560 .00050 17.800 .989850 .00048 17.900 .994580 .00050 18.000 .998330 .00049 18.101 .003040 .00050 18.201 .006630 .00050 18.301 .010980 .00049 18.501 .018640 .00050 18.601 .023950 .00049
770

16.300 .918750 .00048 16.400 .924860 .00050 16.500 .928670 .00050 16.600 .933530 .00049 16.700 .938340 .00049 16.800 .943220 .00049 16.900 .948270 .00050 17.000 .952170 .00048 17.100 .956320 .00048 17.200 .961730 .00048 17.300 .965330 .00049 17.400 .970180 .00051 17.500 .974340 .00048 17.600 .978750 .00050 17.700 .984290 .00048 17.800 .987800 .00048 17.900 .992300 .00050 18.000 .997090 .00049 18.101 .000240 .00049 18.201 .004410 .00049 18.301 .009320 .00050 18.501 .017500 .00049 18.601 .021520 .00048 
775

16.300 .917440 .00049 16.400 .922360 .00050 16.500 .926400 .00048 16.600 .931570 .00049 16.700 .936570 .00050 16.800 .941370 .00049 16.900 .945740 .00050 17.000 .951090 .00049 17.100 .955570 .00049 17.200 .959770 .00050 17.300 .963290 .00050 17.400 .968990 .00051 17.600 .977630 .00049 17.700 .981270 .00050 17.800 .986410 .00048 17.900 .991270 .00050 18.000 .995550 .00048 18.100 .998820 .00049 18.201 .002750 .00050 18.301 .006970 .00049 18.401 .012040 .00050 18.501 .016100 .00049

\section{Pun46-sp-0970-01}

12

27.200 .938690 .00028

27.700 .944740 .00028

28.200 .949470 .00029

28.700 .955530 .00027

29.200 .959540 .00026

29.700 .964980 .00027

30.200 .969310 .00027

30.700 .973930 .00028

31.200 .978210 .00027

31.700 .982360 .00026

32.200 .985660 .00025

32.700 .989930 .00026

33.200 .993790 .00027

33.700 .997150 .00026

34.201 .000980 .00025

34.701 .003880 .00026

35.201 .007340 .00026

35.701 .010360 .00024 36.201 .014140 .00025

\section{0}

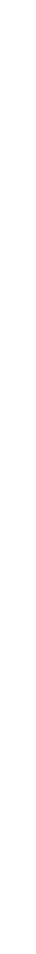


24.900 .996780 .00031 25.100 .999710 .00031 25.301 .001970 .00032 25.501 .004770 .00031 25.701 .007680 .00031 25.901 .010700 .00031 26.101 .013910 .00031 26.201 .015270 .00030

25

15.500 .923970 .00040 15.600 .928370 .00040 15.700 .931460 .00042 15.800 .936020 .00040 15.900 .939230 .00040 16.600 .964970 .00040 16.700 .968740 .00040 16.800 .972020 .00039 16.900 .974830 .00040 17.000 .978780 .00040 17.100 .982060 .00041 17.200 .985060 .00040 17.300 .988820 .00040 17.400 .992590 .00040 17.500 .995640 .00041 17.600 .998640 .00039 17.701 .000950 .00040 17.801 .004480 .00040 17.901 .007930 .00039 18.001 .011390 .00039 18.101 .013480 .00038 18.201 .016260 .00040
30

14.500 .923830 .00043 14.600 .927670 .00042 14.700 .932930 .00043 14.800 .936660 .00042 14.900 .941870 .00043 15.000 .944920 .00042 15.100 .948900 .00042 15.200 .953090 .00043 15.300 .957290 .00043 15.400 .961010 .00042 15.500 .965780 .00043 15.600 .970290 .00043 15.700 .974160 .00041 15.800 .978050 .00043 15.900 .981590 .00044 16.000 .985420 .00042 16.100 .989080 .00043 16.200 .993250 .00042 16.300 .996190 .00042 16.401 .000600 .00042 16.501 .004520 .00043 16.601 .007510 .00043 16.701 .011210 .00042 16.801 .014990 .00042 16.901 .018810 .00042
19.200 .981690 .00038 19.300 .984610 .00037 19.400 .986280 .00037 19.500 .988890 .00036 19.600 .991940 .00037 19.700 .993270 .00037 19.800 .996620 .00036 19.900 .998740 .00037 20.001 .001680 .00036 20.101 .003950 .00036 20.201 .006620 .00036 20.301 .008170 .00037 20.401 .010730 .00036 20.501 .013030 .00036 20.601 .015230 .00035 20.701 .017770 .00036

32

14.200 .923020 .00043 14.300 .928520 .00042 14.400 .932190 .00043 14.500 .936390 .00043 14.600 .941840 .00045 14.700 .945560 .00043 14.800 .950590 .00044 14.900 .955400 .00043 15.000 .959370 .00043 15.100 .963210 .00042 15.200 .966970 .00043 15.300 .970800 .00044 15.400 .974440 .00044 15.500 .979700 .00043 15.600 .983750 .00043 15.700 .987630 .00044 15.800 .991220 .00044 15.900 .995620 .00043 16.101 .003420 .00042 16.201 .006520 .00043 16.301 .010740 .00043 16.401 .015030 .00043 
35

14.100 .935000 .00044 14.200 .940040 .00045 14.300 .945210 .00046 14.400 .948930 .00045 14.500 .953700 .00045 14.600 .958130 .00044 14.700 .962650 .00046 14.800 .967620 .00045 14.900 .972390 .00044 15.000 .974770 .00044 15.100 .980370 .00045 15.200 .983520 .00044 15.300 .988890 .00044 15.400 .992210 .00045 15.500 .996250 .00043 15.601 .000790 .00046 15.701 .005640 .00044 15.801 .008630 .00045 15.901 .013400 .00043 16.001 .017260 .00045 16.101 .020450 .00043
40

13.300 .917650 .00045

13.400 .923330 .00045

13.500 .928150 .00046

13.600 .933940 .00046

13.700 .937810 .00045

13.800 .943530 .00045

13.900 .949400 .00045

14.000 .953760 .00046

14.100 .957600 .00047

14.200 .962960 .00045

14.300 .967670 .00044

14.800 .990330 .00046

15.101 .003490 .00045

15.201 .007370 .00046

15.301 .011460 .00045

15.501 .020020 .00045
60

12.500 .922600 .00048 12.600 .928940 .00047 12.700 .934950 .00047 12.800 .940720 .00048 13.100 .957620 .00048 13.200 .963000 .00048 13.300 .967800 .00047 13.400 .973460 .00047 13.500 .977920 .00049 13.600 .982930 .00049 13.700 .988280 .00049 13.800 .993620 .00049 13.900 .998700 .00047 14.001 .005270 .00049 14.101 .009090 .00049 14.201 .013910 .00047 14.301 .018830 .00047
70

12.300 .922700 .00049

12.400 .928240 .00049

12.500 .934770 .00049

12.600 .940890 .00048

12.700 .946150 .00049

12.800 .951300 .00048

12.900 .958110 .00050

13.000 .962730 .00049

13.100 .967240 .00050

13.200 .973540 .00048

13.300 .979960 .00048

13.400 .983640 .00049

13.500 .990090 .00049

13.600 .994080 .00050

13.701 .000790 .00049

13.801 .005210 .00049

13.901 .010150 .00047

14.001 .015530 .00049

14.101 .019940 .00048
50

12.800 .921510 .00047

12.900 .927230 .00047

13.000 .933260 .00047

13.100 .937770 .00048

13.200 .944260 .00047

13.300 .948910 .00048

13.400 .954150 .00047

13.500 .960330 .00047

13.600 .964210 .00048

13.700 .969740 .00046

13.800 .974230 .00048

13.900 .978310 .00049

14.100 .989580 .00048

14.200 .994430 .00046

14.300 .998290 .00048

14.401 .003730 .00046

14.501 .008380 .00047

14.601 .013660 .00047

14.701 .017160 .00048
80
12.100 .918600 .00050
12.200 .924810 .00050
12.300 .931380 .00049
12.400 .936360 .00048
12.500 .942240 .00050
12.600 .947290 .00048
12.700 .954020 .00050
12.800 .958880 .00049
12.900 .964900 .00047
13.000 .971050 .00049
13.100 .976090 .00049
13.200 .980880 .00049
13.300 .987050 .00049
13.400 .991920 .00049
13.500 .998740 .00051
13.601 .002450 .00049
13.701 .007710 .00051
13.801 .013360 .00049
13.901 .018210 .00051 
90

12.100 .922690 .00050

12.200 .928760 .00049

12.300 .936200 .00049

12.400 .941560 .00050

12.500 .947110 .00048

12.600 .952980 .00050

12.700 .960210 .00050

12.800 .964380 .00050

12.900 .970290 .00051

13.400 .996580 .00050

13.501 .002210 .00049

13.601 .007330 .00050

13.701 .013520 .00050

13.801 .017900 .00050
130

12.000 .921760 .00050

12.100 .927200 .00049

12.200 .932610 .00051

12.300 .937780 .00049

12.400 .945000 .00050

12.500 .951270 .00050

12.600 .957330 .00052

12.700 .962370 .00049

12.800 .968610 .00050

12.900 .973070 .00051

13.000 .979480 .00051

13.100 .985580 .00049

13.200 .989900 .00050

13.300 .994920 .00050

13.401 .001200 .00050

13.501 .006000 .00051

13.601 .010230 .00050

13.701 .015770 .00050

13.801 .020020 .00051
100

12.000 .920630 .00051

12.100 .926810 .00049

12.200 .931620 .00050

12.300 .938350 .00051

12.400 .943500 .00050

12.500 .950100 .00051

12.600 .955450 .00051

12.700 .961120 .00050

12.800 .967060 .00050

12.900 .972550 .00050

13.000 .977830 .00049

13.100 .983880 .00050

13.200 .989040 .00051

13.300 .994700 .00051

13.400 .999550 .00051

13.501 .005620 .00049

13.601 .010920 .00051

13.701 .015670 .00050

13.801 .020200 .00050
120

12.000 .920120 .00050

12.100 .926880 .00051

12.200 .932960 .00048

12.300 .938480 .00048

12.400 .945320 .00051

12.500 .952160 .00050

12.600 .956420 .00051

12.700 .962460 .00050

12.800 .968360 .00051

12.900 .973590 .00051

13.000 .978870 .00050

13.100 .984270 .00050

13.200 .990950 .00050

13.300 .995350 .00051

13.400 .999610 .00051

13.501 .004560 .00051

13.601 .010650 .00050

13.701 .016340 .00049

13.801 .021040 .00050
150

12.000 .919280 .00051

12.050 .922000 .00052

12.100 .924890 .00049

12.150 .927710 .00050

12.200 .931450 .00049

12.250 .933710 .00050

12.300 .936880 .00050

12.350 .939200 .00050

12.450 .946270 .00052

12.500 .948360 .00051

12.550 .952150 .00050

12.600 .953120 .00050

12.650 .957210 .00050

12.700 .961100 .00051

12.750 .962220 .00050

12.800 .965170 .00050

12.850 .967980 .00051

12.900 .971090 .00050

12.950 .973330 .00050

13.000 .976240 .00051

13.050 .978950 .00050

13.100 .982160 .00051

13.150 .986000 .00051

13.200 .987160 .00050

13.250 .989270 .00049

13.300 .991700 .00050

13.350 .996390 .00051
200

12.200 .921370 .00052

12.300 .928300 .00049

12.400 .933190 .00050

12.500 .938370 .00049

12.600 .944990 .00050

12.700 .949100 .00050

12.800 .955270 .00050

12.900 .960940 .00052

13.000 .966720 .00050

13.100 .971860 .00052

13.200 .977130 .00052

13.300 .982520 .00050

13.400 .987420 .00051

13.500 .992500 .00051

13.600 .997800 .00051

13.701 .003520 .00050

13.801 .007540 .00050

13.901 .013100 .00052

14.001 .019340 .00050 
13.400 .997340 .00051

13.451 .000360 .00051

13.501 .003330 .00051

13.551 .006550 .00050

13.601 .008650 .00051

13.651 .010140 .00050

13.701 .012880 .00051
239

12.300 .919150 .00050

12.400 .924920 .00049

12.500 .930760 .00051

12.600 .937090 .00050

12.700 .942150 .00051

12.800 .947680 .00050

12.900 .952060 .00049

13.000 .958080 .00051

13.100 .963200 .00051

13.200 .968500 .00051

13.300 .974180 .00051

13.400 .979370 .00051

13.500 .984630 .00051

13.600 .989390 .00050

13.700 .994880 .00050

13.800 .999230 .00049

13.901 .004110 .00050

14.001 .009010 .00050

14.101 .013110 .00052

14.201 .018260 .00050

14.301 .022970 .00052

14.401 .028660 .00050

14.501 .031780 .00051

14.601 .037100 .00050

14.701 .041760 .00051

14.801 .046070 .00051

14.901 .050990 .00050

15.001 .055550 .00050

15.101 .059090 .00049

15.201 .064910 .00052

15.301 .068020 .00051
478

13.100 .917490 .00052 13.300 .927980 .00049 13.400 .934610 .00051 13.500 .938530 .00051 13.700 .947900 .00048
250

12.400 .922090 .00050

12.500 .927710 .00051

12.600 .934250 .00050

12.700 .938110 .00049

12.900 .951010 .00050

13.000 .954890 .00051

13.100 .960950 .00050

13.200 .966100 .00050

13.300 .971300 .00050

13.400 .976450 .00051

13.500 .981840 .00050

13.600 .987020 .00051

13.700 .991930 .00052

13.800 .996330 .00049

13.901 .001200 .00049

14.001 .005850 .00051

14.101 .011650 .00050

14.201 .016090 .00051
359

12.800 .921560 .00049

12.900 .926170 .00052

13.000 .932490 .00051

13.100 .937020 .00050

13.200 .943790 .00051

13.300 .947430 .00051

13.400 .952810 .00051

13.500 .958220 .00052

13.600 .962470 .00050

13.700 .967780 .00051

14.000 .983410 .00049

14.100 .987360 .00050

14.200 .991390 .00050

14.300 .996740 .00051

14.501 .006900 .00052

14.601 .010630 .00051

14.701 .014850 .00049

14.801 .018950 .00049
13.200 .919340 .00049

13.300 .924550 .00048

13.400 .929490 .00050

13.500 .935020 .00050

13.600 .939010 .00051
600

13.400 .912080 .00048

13.500 .917260 .00049

13.600 .921330 .00049

13.700 .926230 .00049

13.800 .931710 .00050 
13.800 .953090 .00051 13.900 .957370 .00050 14.000 .963230 .00051 14.100 .967470 .00051 14.200 .972480 .00049 14.300 .977840 .00050 14.400 .982670 .00050 14.500 .986180 .00050 14.600 .990690 .00050 14.700 .995540 .00051 14.801 .001030 .00050 14.901 .006180 .00050 15.001 .008950 .00049 15.101 .014170 .00049 15.201 .017120 .00049
13.800 .951060 .00049 13.900 .953550 .00050 14.000 .960390 .00049 14.100 .963890 .00050 14.200 .969100 .00049 14.300 .973080 .00050 14.400 .978780 .00049 14.500 .982300 .00051 14.600 .987480 .00051 14.700 .992940 .00049 14.800 .996410 .00050 14.901 .001650 .00049 15.001 .006770 .00050 15.101 .010120 .00049 15.201 .013730 .00050 15.301 .018620 .00051
650

13.800 .922700 .00049 13.900 .926340 .00050 14.000 .932520 .00049 14.100 .938270 .00049 14.200 .942330 .00050 14.300 .947110 .00049 14.400 .951390 .00050 14.500 .955690 .00049 14.600 .960980 .00050 14.700 .965340 .00049 14.800 .969740 .00049 14.900 .974890 .00050 15.000 .979600 .00049 15.100 .983440 .00051 15.200 .988470 .00048 15.300 .991860 .00049 15.400 .996810 .00048 15.501 .000740 .00049 15.601 .004820 .00050 15.701 .009520 .00049 15.801 .015100 .00051
700

14.000 .919300 .00049

14.100 .924200 .00049

14.200 .928480 .00050

14.300 .935100 .00048

14.400 .939000 .00049

14.500 .944050 .00048

14.600 .948400 .00050

14.700 .952440 .00050

14.800 .957820 .00047

14.900 .963330 .00048

15.000 .967350 .00050

15.100 .970650 .00048

15.200 .975140 .00050

15.300 .980100 .00050

15.400 .984990 .00050

15.500 .989030 .00049

15.600 .993210 .00050

15.700 .998100 .00048

15.801 .001610 .00049

15.901 .006570 .00051

16.001 .011320 .00050

16.101 .013610 .00049

16.201 .018830 .00048
13.900 .937510 .00048

14.000 .942170 .00051

14.100 .945380 .00050

14.200 .951630 .00050

14.300 .955850 .00050

14.400 .960120 .00052

14.500 .965080 .00050

14.600 .971070 .00051

14.700 .973870 .00049

14.800 .979330 .00048

14.900 .983960 .00048

15.000 .988480 .00051

15.100 .993400 .00051

15.200 .998170 .00048

15.301 .001830 .00051

15.401 .006680 .00049

15.501 .010460 .00050

15.601 .015150 .00049

15.701 .019030 .00049

750

14.300 .917520 .00049

14.400 .922650 .00048

14.500 .928510 .00050

14.600 .932220 .00050

14.700 .936540 .00048

14.800 .941500 .00048

14.900 .946040 .00050

15.100 .955920 .00048

15.200 .960350 .00050

15.300 .964870 .00050

15.400 .968770 .00049

15.500 .972730 .00050

15.600 .978360 .00050

15.700 .981830 .00049

15.800 .986760 .00049

15.900 .990450 .00048

16.000 .996100 .00051

16.100 .999420 .00049

16.201 .003530 .00050

16.301 .007370 .00049

16.401 .011770 .00048

16.501 .016090 .00049 
760

14.400 .918660 .00050

14.500 .923650 .00049

14.600 .928780 .00049

14.700 .932460 .00050

14.800 .937290 .00050

14.900 .941950 .00050

15.000 .947390 .00050

15.100 .952000 .00049

15.200 .957160 .00050

15.300 .961160 .00049

15.400 .966010 .00049

15.500 .969770 .00048

15.600 .974290 .00048

15.700 .978450 .00050

15.800 .982830 .00047

15.900 .987000 .00050

16.000 .991170 .00050

16.100 .995640 .00051

16.201 .000350 .00050

16.301 .004400 .00049

16.401 .007950 .00050

16.501 .012220 .00051

16.601 .016480 .00049

16.701 .020880 .00049

775

14.500 .917770 .00049

14.600 .922980 .00047

14.700 .926490 .00048

14.800 .932590 .00048

14.900 .937640 .00048

15.000 .941390 .00048

15.100 .945360 .00049

15.200 .951080 .00049

15.300 .954730 .00048

15.400 .960490 .00048

15.500 .964200 .00050

15.600 .968860 .00049

15.700 .972770 .00050

15.800 .977130 .00049

15.900 .981760 .00048

16.000 .986280 .00048

16.100 .989810 .00049

16.200 .995250 .00048

16.300 .998870 .00050

16.401 .002520 .00049

16.501 .008080 .00049

16.601 .011010 .00049

16.701 .015340 .00050
765

14.500 .921870 .00048

14.600 .926830 .00049

14.700 .931220 .00049

14.800 .936670 .00049

14.900 .940960 .00050

15.000 .944860 .00049

15.100 .949970 .00049

15.200 .955480 .00048

15.300 .958930 .00049

15.400 .963600 .00051

15.500 .969100 .00048

15.600 .972100 .00048

15.700 .976910 .00049

15.800 .981120 .00049

15.900 .985510 .00050

16.000 .988510 .00048

16.100 .994130 .00051

16.200 .997660 .00049

16.301 .002450 .00048

16.401 .006260 .00050

16.501 .010990 .00048

16.601 .014390 .00051

16.701 .018920 .00050

0
770

14.500 .919350 .00048

14.600 .924690 .00050

14.800 .933330 .00050

14.900 .937550 .00048

15.000 .942820 .00049

15.100 .947210 .00048

15.200 .951290 .00049

15.300 .955570 .00049

15.400 .960720 .00048

15.500 .965130 .00051

15.600 .969940 .00048

15.700 .974730 .00048

15.800 .978530 .00049

15.900 .983090 .00049

16.000 .987330 .00050

16.100 .991110 .00048

16.200 .995410 .00048

16.300 .999840 .00049

16.401 .004290 .00048

16.501 .009100 .00048

16.601 .011870 .00050

16.701 .016090 .00048

16.801 .020020 .00049

16.901 .024440 .00049

17.001 .028050 .00049

17.101 .032320 .00049 
Pun46-sp-0970-10

12

26.000 .939070 .00027

26.500 .944080 .00027

27.000 .948900 .00027

27.500 .955570 .00026

28.000 .959880 .00026

28.500 .964060 .00026

29.000 .968880 .00026

29.500 .973620 .00025

30.000 .977600 .00025

30.500 .981660 .00024

31.000 .985990 .00025

31.500 .990040 .00026

32.000 .993750 .00025

32.500 .997480 .00025

33.001 .000630 .00025

33.501 .003890 .00024

34.001 .007240 .00025

34.501 .010270 .00024

35.001 .013750 .00025
19.600 .924860 .00031

19.800 .928170 .00032

20.000 .933170 .00032

20.200 .937560 .00031

20.400 .941500 .00030

20.600 .944400 .00031

20.800 .948180 .00031

21.000 .952760 .00030

21.200 .956300 .00031

21.400 .959710 .00031

21.600 .963130 .00031

21.800 .966490 .00031

22.000 .970140 .00030

22.400 .975900 .00030

22.600 .980360 .00030

22.800 .983030 .00030

23.000 .986880 .00031

23.200 .989140 .00029

23.400 .992990 .00030

23.600 .996220 .00030

23.800 .998220 .00030

24.001 .000750 .00030

24.201 .003690 .00030

24.401 .007230 .00030

24.601 .009660 .00029

24.801 .012400 .00029

24.901 .013830 .00029
25

14.200 .921940 .00038 14.300 .926850 .00037 14.400 .929710 .00038 14.500 .934500 .00039 14.600 .938330 .00039 14.700 .940800 .00038 14.800 .945160 .00038 15.400 .966870 .00038 15.500 .970980 .00038
30

13.200 .922600 .00040 13.300 .927090 .00040 13.400 .931090 .00040 13.500 .936190 .00041 13.600 .940150 .00039 13.700 .943920 .00040 13.800 .948800 .00040 13.900 .953260 .00041 14.000 .957280 .00040
20

16.000 .925360 .00036 16.100 .928210 .00036 16.200 .930990 .00036 16.300 .934700 .00036 16.400 .937190 .00036 16.500 .940240 .00036 16.600 .943250 .00036 16.700 .946790 .00035 16.800 .949060 .00035 16.900 .953160 .00035 17.000 .955490 .00036 17.100 .958630 .00036 17.200 .960950 .00035 17.300 .963900 .00035 17.400 .966880 .00035 17.500 .968650 .00035 17.600 .971960 .00035 17.700 .974610 .00035 17.800 .977060 .00034 17.900 .979440 .00035 18.000 .982400 .00035 18.100 .984580 .00035 18.200 .987090 .00035 18.300 .990040 .00034 18.400 .992580 .00034 18.500 .995010 .00034 18.600 .998230 .00034 18.701 .000440 .00035 18.801 .002520 .00034 18.901 .004320 .00035 19.001 .007510 .00033 19.101 .010580 .00035 19.201 .011800 .00034 19.301 .014830 .00035

32

12.900 .921950 .00040 13.000 .927280 .00040 13.100 .931530 .00040 13.200 .937040 .00041 13.300 .940450 .00041 13.400 .945040 .00041 13.500 .949130 .00041 13.600 .953810 .00041 13.700 .958250 .00041 
15.600 .974040 .00038 15.700 .977280 .00038 15.800 .980440 .00039 15.900 .984610 .00038 16.000 .987410 .00038 16.100 .991490 .00037 16.200 .994570 .00038 16.300 .997480 .00037 16.401 .000600 .00038 16.501 .003600 .00037 16.601 .007090 .00038 16.701 .010640 .00038 16.801 .013890 .00038 16.901 .017150 .00038

35

12.500 .920220 .00043 13.000 .944220 .00041 13.100 .949250 .00041 13.200 .953930 .00041 13.300 .957900 .00042 13.400 .963120 .00042 13.500 .967230 .00041 13.600 .972680 .00041 13.700 .975840 .00041 13.900 .984890 .00041 14.000 .990200 .00041 14.100 .993940 .00042 14.200 .996570 .00042 14.301 .001300 .00041 14.401 .006310 .00041 14.501 .009720 .00041 14.601 .013270 .00040 14.701 .018220 .00042

60

11.100 .922440 .00046 11.200 .928790 .00044 11.300 .934240 .00045 11.400 .939860 .00044 11.500 .944890 .00043 11.600 .951420 .00044 11.700 .956750 .00044 11.800 .962310 .00044 11.900 .968610 .00046
14.100 .961690 .00040 14.200 .965640 .00040 14.300 .969110 .00039 14.400 .974020 .00041 14.500 .977780 .00041 14.600 .980660 .00040 14.700 .985010 .00041 14.800 .989190 .00040 14.900 .993270 .00040 15.000 .996660 .00039 15.101 .000230 .00040 15.201 .003750 .00039 15.301 .007060 .00040 15.401 .010740 .00040 15.501 .014900 .00040 15.601 .017480 .00040

\section{0}

12.100 .923120 .00042 12.200 .928580 .00042 12.300 .935160 .00042 12.400 .939900 .00041 12.500 .944370 .00043 12.600 .950140 .00042 12.700 .953870 .00042 12.800 .958710 .00042 12.900 .963860 .00042 13.000 .968820 .00041 13.100 .972500 .00044 13.200 .977710 .00041 13.300 .982230 .00043 13.801 .004920 .00043 14.101 .018330 .00043

.

13.800 .962370 .00040 13.900 .966560 .00040 14.000 .971050 .00041 14.100 .975040 .00042 14.200 .979040 .00041 14.300 .983050 .00041 14.400 .987060 .00040 14.500 .991220 .00041 14.600 .995390 .00042 14.700 .998590 .00040 14.801 .003240 .00040 14.901 .006360 .00040 15.001 .010160 .00041 15.101 .014320 .00040 15.201 .017920 .00039
50

11.500 .924790 .00044 11.600 .929350 .00044 11.800 .941830 .00044 11.900 .946670 .00045 12.000 .951910 .00044 12.100 .957460 .00043 12.200 .962700 .00045 12.300 .967880 .00043 12.400 .972710 .00044 12.500 .977560 .00044 12.600 .982830 .00045 12.700 .987120 .00044 12.800 .992560 .00044 12.900 .997060 .00044 13.001 .002080 .00044 13.101 .006690 .00043 13.201 .011580 .00044 13.301 .016900 .00044

70

10.800 .917660 .00045 10.900 .923950 .00045 11.000 .929710 .00045 11.100 .935750 .00044 11.200 .941890 .00045 11.300 .946700 .00045 11.400 .953460 .00045 11.500 .959120 .00045 11.600 .964290 .00045
80

10.700 .921810 .00045 10.800 .927490 .00045 10.900 .933920 .00046 11.000 .939320 .00046 11.100 .946000 .00046 11.200 .951870 .00047 11.400 .962840 .00046 11.500 .969290 .00046 11.600 .973950 .00046 
12.000 .972800 .00045

12.300 .988430 .00044

12.400 .994800 .00045

12.500 .998230 .00046

12.601 .004600 .00045

12.701 .008530 .00045

12.801 .013450 .00044

12.901 .018870 .00045

90

10.500 .915000 .00046

10.600 .922200 .00045

10.700 .928670 .00046

10.800 .934060 .00046

10.900 .939820 .00046

11.000 .947100 .00046

11.100 .951610 .00046

11.200 .958350 .00045

11.300 .963900 .00046

11.400 .969740 .00044

11.500 .974860 .00046

11.600 .981100 .00047

11.700 .984900 .00046

11.800 .991880 .00047

11.900 .996550 .00047

12.301 .017720 .00047

12.401 .023470 .00046
11.700 .969670 .00046

11.800 .974780 .00046

11.900 .981220 .00044

12.000 .986300 .00045

12.100 .992010 .00045

12.200 .996310 .00046

12.301 .002270 .00045

12.401 .007100 .00045

12.501 .011760 .00045

12.601 .018740 .00045

12.701 .022640 .00045
11.700 .980080 .00046

11.800 .985050 .00046

11.900 .990110 .00046

12.000 .996170 .00045

12.101 .000930 .00046

12.301 .012890 .00046

12.401 .017160 .00046

12.501 .022820 .00046
100

10.500 .919990 .00048

10.550 .924350 .00046

10.600 .927520 .00047

10.650 .929650 .00046

10.700 .932650 .00047

10.750 .934960 .00046

10.800 .939030 .00046

10.850 .941780 .00046

10.900 .945020 .00046

10.950 .947750 .00047

11.000 .949740 .00046

11.050 .953610 .00047

11.100 .956190 .00047

11.150 .958810 .00044

11.200 .963510 .00047

11.250 .964920 .00047

11.350 .971040 .00045

11.400 .973740 .00046

11.500 .978920 .00045

11.550 .982520 .00046

11.600 .985140 .00046

11.650 .987220 .00047

11.700 .990660 .00047

11.750 .993700 .00046

11.800 .996400 .00046

11.901 .001530 .00047

11.951 .004670 .00047

12.001 .006840 .00047

12.051 .009560 .00045

12.101 .012240 .00048

12.151 .013790 .00047

12.201 .016620 .00046
120

10.400 .918390 .00047

10.450 .921070 .00047

10.500 .923660 .00045

10.550 .927290 .00047

10.600 .931090 .00045

10.650 .933310 .00046

10.700 .936280 .00046

10.750 .940280 .00046

10.800 .941920 .00046

10.850 .945040 .00046

10.900 .948620 .00046

10.950 .950800 .00047

11.000 .954680 .00047

11.050 .956950 .00045

11.100 .960310 .00046

11.150 .963130 .00045

11.200 .965740 .00047

11.250 .968460 .00047

11.300 .970170 .00047

11.350 .974920 .00049

11.400 .978370 .00047

11.450 .978880 .00047

11.500 .982970 .00046

11.550 .986710 .00048

11.600 .988990 .00046

11.650 .991490 .00047

11.700 .993680 .00046

11.750 .996230 .00046

11.800 .999550 .00047

11.851 .002430 .00046

11.901 .003800 .00046

11.951 .007540 .00047

12.001 .009780 .00047

12.051 .012240 .00047

12.101 .014770 .00047 
130

10.400 .919620 .00047 10.500 .925800 .00046 10.600 .931120 .00045 10.700 .938120 .00047 10.800 .943340 .00047 10.900 .949950 .00048 11.000 .956300 .00045 11.100 .962060 .00048 11.200 .967020 .00047 11.300 .973390 .00046 11.400 .977910 .00047 11.500 .983120 .00047 11.600 .988570 .00047 11.700 .995310 .00047 11.801 .000800 .00047 11.901 .005550 .00046 12.001 .010800 .00046 12.101 .016310 .00045 12.201 .020960 .00046

239

10.600 .921850 .00047 10.700 .927820 .00045 10.900 .939690 .00047 11.000 .945120 .00045 11.100 .950770 .00047 11.200 .956840 .00047 11.300 .961340 .00046 11.400 .966900 .00046 11.500 .972360 .00047 11.600 .978320 .00048 11.700 .982140 .00048 11.800 .988370 .00046 11.900 .993010 .00047 12.000 .998580 .00046 12.101 .004120 .00048 12.201 .009230 .00047 12.301 .013710 .00048 12.401 .018130 .00046
150

10.500 .927290 .00046

10.600 .931570 .00046

10.700 .938450 .00046

10.800 .943420 .00045

10.900 .949610 .00047

11.000 .956340 .00045

11.100 .962320 .00046

11.200 .968080 .00046

11.300 .972930 .00047

11.400 .978080 .00047

11.500 .983670 .00048

11.600 .989050 .00046

11.700 .994690 .00045

11.800 .999960 .00047

11.901 .005320 .00046

12.001 .011040 .00047

12.101 .015340 .00046

12.201 .021080 .00047

12.301 .025990 .00046

12.401 .031530 .00046

12.501 .036140 .00046
200

10.400 .915630 .00046 10.500 .921850 .00047 10.600 .927540 .00046 10.700 .934160 .00046 10.800 .939710 .00045 10.900 .945330 .00049 11.000 .951030 .00047 11.100 .955910 .00047 11.200 .962480 .00047 11.300 .967080 .00047 11.400 .972740 .00047 11.500 .977570 .00047 11.600 .984710 .00048 11.700 .989450 .00048 11.800 .994700 .00047 11.901 .000190 .00047 12.101 .009750 .00047 12.201 .014570 .00047 12.301 .018810 .00047
10.600 .920510 .00047 10.700 .926330 .00047 10.800 .931620 .00047 10.900 .937850 .00048 11.000 .943350 .00047 11.100 .949530 .00046 11.200 .954610 .00047 11.300 .959620 .00048 11.400 .965910 .00048 11.500 .970170 .00046 11.600 .975660 .00048 11.700 .980540 .00048 11.800 .984940 .00048 11.900 .991480 .00048 12.000 .996720 .00047 12.101 .001510 .00046 12.201 .006740 .00047 12.301 .010790 .00046 12.401 .016500 .00047 12.501 .020940 .00046
359

10.900 .921130 .00046 11.000 .926530 .00047 11.100 .931820 .00047 11.200 .936840 .00046 11.300 .942150 .00047 11.400 .947240 .00046 11.500 .952730 .00046 11.600 .957760 .00045 11.700 .962530 .00047 11.800 .969120 .00048 11.900 .973570 .00048 12.000 .977890 .00048 12.100 .982590 .00046 12.200 .987950 .00048 12.300 .992630 .00045 12.400 .997850 .00048 12.501 .002200 .00047 12.601 .006300 .00045 12.701 .011450 .00046 
478

11.200 .922270 .00046 11.400 .933140 .00047 11.600 .943490 .00047 11.700 .948120 .00047 11.800 .952710 .00047 11.900 .957140 .00047 12.100 .967460 .00046 12.200 .971910 .00046 12.300 .976920 .00045 12.400 .981310 .00047 12.500 .986430 .00046 12.600 .990790 .00046 12.700 .995730 .00047 12.801 .001510 .00047 12.901 .004230 .00048 13.001 .009490 .00047
650

11.700 .922620 .00047 11.800 .926880 .00046 11.900 .933030 .00046 12.000 .937930 .00046 12.100 .943230 .00046 12.200 .946500 .00046 12.300 .952270 .00047 12.400 .957010 .00046 12.500 .961910 .00046 12.600 .966080 .00046 12.700 .970850 .00048 12.800 .974980 .00046 12.900 .980410 .00046 13.000 .984380 .00046 13.100 .987700 .00047 13.200 .993630 .00047 13.300 .997440 .00046 13.401 .001530 .00046 13.501 .006480 .00046 13.601 .010810 .00046 13.701 .014390 .00046 13.801 .018720 .00046
500

11.200 .918830 .00047 11.300 .924700 .00047 11.400 .930050 .00047 11.500 .933570 .00048 11.600 .938700 .00047 11.700 .944870 .00046 11.800 .948640 .00047 11.900 .954670 .00047 12.000 .959700 .00046 12.100 .964540 .00046 12.200 .969390 .00048 12.300 .973420 .00045 12.400 .978310 .00046 12.500 .982780 .00046 12.600 .987430 .00047 12.700 .992160 .00047 12.800 .997380 .00047 12.901 .001560 .00047 13.001 .006540 .00047 13.101 .010210 .00046 13.201 .015220 .00047 13.301 .018750 .00046
600

11.500 .920940 .00046 11.600 .925420 .00046 11.700 .931070 .00046 11.800 .935770 .00046 11.900 .939830 .00046 12.000 .945860 .00046 12.100 .950160 .00046 12.300 .960340 .00045 12.400 .965850 .00046 12.500 .969620 .00045 12.600 .974630 .00046 12.700 .978590 .00045 12.800 .983240 .00046 12.900 .987550 .00048 13.000 .992170 .00047 13.100 .995900 .00046 13.201 .001490 .00048 13.301 .006110 .00045 13.401 .009640 .00047 13.501 .013620 .00046
11.900 .922550 .00047 12.000 .927490 .00047 12.100 .932890 .00045 12.200 .937320 .00047 12.300 .941380 .00045 12.400 .945850 .00047 12.500 .951330 .00047 12.600 .956340 .00048 12.700 .960810 .00046 12.800 .966060 .00046 12.900 .970070 .00046 13.000 .973720 .00046 13.100 .978930 .00046 13.200 .982930 .00046 13.300 .988020 .00045 13.400 .992440 .00047 13.500 .995970 .00046 13.601 .000150 .00046 13.701 .004560 .00047 13.801 .008830 .00045 13.901 .013470 .00047 14.001 .017830 .00047 14.101 .021400 .00045

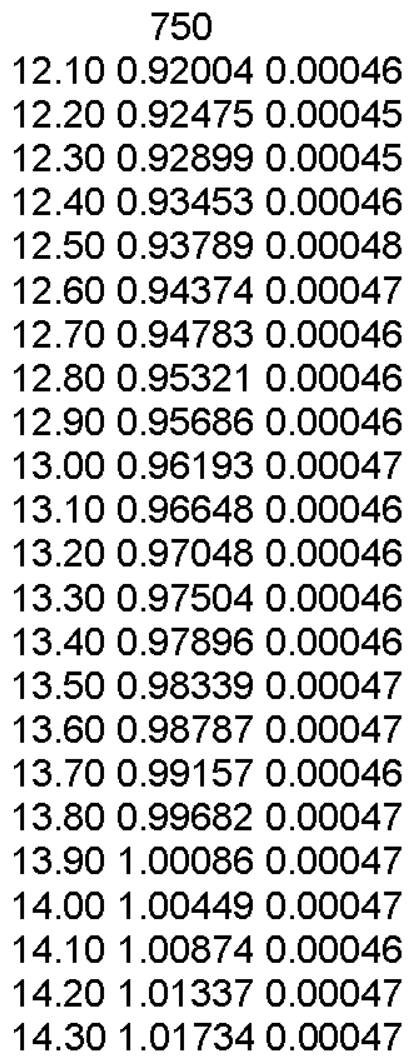


760

12.200 .921100 .00046

12.300 .926070 .00047

12.400 .931120 .00046

12.500 .935670 .00046

12.600 .940500 .00045

12.700 .944750 .00047

12.800 .948870 .00046

12.900 .953920 .00046

13.000 .958190 .00046

13.100 .961950 .00047

13.200 .967450 .00046

13.300 .972460 .00047

13.400 .976670 .00045

13.500 .980140 .00046

13.600 .984780 .00047

13.700 .989980 .00045

13.800 .993380 .00047

13.900 .996950 .00046

14.001 .002250 .00047

14.101 .006520 .00046

14.201 .010000 .00046

14.301 .013610 .00046

14.401 .017870 .00047
765

12.200 .919370 .00046

12.300 .923750 .00046

12.500 .933320 .00046

12.600 .938250 .00047

12.700 .944150 .00046

12.800 .948360 .00046

12.900 .952530 .00047

13.000 .956460 .00046

13.100 .961570 .00047

13.200 .965770 .00045

13.300 .970480 .00046

13.400 .974610 .00046

13.500 .978470 .00045

13.600 .983100 .00048

13.700 .987320 .00046

13.800 .991310 .00046

13.900 .995600 .00046

14.000 .999800 .00046

14.101 .005190 .00046

14.201 .008480 .00045

14.301 .012910 .00045

14.401 .016290 .00046
770

12.300 .922950 .00047

12.400 .926860 .00045

12.500 .931800 .00045

12.600 .936640 .00046

12.700 .941230 .00046

12.800 .946220 .00047

12.900 .950460 .00046

13.000 .954960 .00047

13.100 .959880 .00046

13.200 .963710 .00046

13.300 .968580 .00046

13.400 .973570 .00046

13.500 .976970 .00047

13.600 .981500 .00047

13.700 .985410 .00047

13.800 .989630 .00048

13.900 .994640 .00047

14.000 .998780 .00047

14.101 .003230 .00045

14.201 .007480 .00045

14.301 .011000 .00047

14.401 .014310 .00047
775

12.300 .921050 .00046

12.400 .925740 .00046

12.500 .930620 .00047

12.600 .935030 .00045

12.700 .940310 .00046

12.800 .945510 .00047

12.900 .949290 .00046

13.000 .953680 .00046

13.100 .957410 .00046

13.200 .963390 .00047

13.300 .967870 .00046

13.400 .971660 .00048

13.500 .975950 .00046

13.600 .979470 .00046

13.700 .985180 .00046

13.800 .988650 .00045

13.900 .993200 .00047

14.000 .997550 .00045

14.101 .001090 .00047

14.201 .005680 .00046

14.301 .009790 .00046

14.401 .013550 .00046

14.501 .017590 .00046
0

F-127 
Pun40-sp-0950-00

12

22.900 .925550 .00031

23.200 .930480 .00032

23.500 .935660 .00031

23.800 .941130 .00032

24.100 .945810 .00031

24.400 .950900 .00030

24.700 .955830 .00031

25.000 .960150 .00031

25.300 .964730 .00031

25.600 .969090 .00030

25.900 .973290 .00030

26.200 .976560 .00030

26.800 .985560 .00029

27.100 .988950 .00030

27.400 .993830 .00028

27.700 .997020 .00029

28.001 .000220 .00029

28.301 .003730 .00030

28.601 .006550 .00029

28.901 .010830 .00029

29.201 .013080 .00029
15

19.300 .922770 .00036

19.500 .928420 .00037

19.700 .933940 .00036

19.900 .939160 .00036

20.100 .944690 .00034

20.300 .950160 .00035

20.500 .954180 .00036

20.700 .959110 .00035

20.900 .963970 .00036

21.100 .968830 .00034

21.300 .973230 .00035

21.500 .976950 .00035

21.700 .981290 .00035

21.900 .984990 .00034

22.100 .989720 .00035

22.300 .994240 .00035

22.500 .997980 .00034

22.701 .002190 .00035

22.901 .006350 .00034

23.101 .010420 .00034

23.301 .013790 .00034

23.401 .016010 .00035
25

15.300 .920170 .00043

15.400 .924620 .00043

15.500 .928320 .00044

15.600 .934010 .00044

15.700 .937110 .00043

15.800 .942440 .00045

15.900 .946280 .00045

16.000 .951310 .00043

16.100 .955240 .00042

16.200 .959290 .00044

16.300 .963880 .00043

16.400 .967210 .00044

16.500 .971840 .00043

16.600 .975240 .00043

16.700 .980140 .00043

16.800 .983680 .00042
30

14.500 .918640 .00045

14.600 .923440 .00046

14.700 .929350 .00046

14.800 .933740 .00045

14.900 .939150 .00046

15.000 .944110 .00045

15.100 .948120 .00045

15.200 .954070 .00047

15.300 .958300 .00047

15.400 .963080 .00045

15.500 .968100 .00044

15.600 .971970 .00046

15.700 .976440 .00047

15.800 .980630 .00045

15.900 .985280 .00045

16.000 .990190 .00045
20

16.700 .924020 .00041

16.800 .927150 .00041

16.900 .930920 .00041

17.000 .934950 .00041

17.100 .938420 .00041

17.200 .943040 .00040

17.300 .945860 .00040

17.400 .949710 .00041

17.500 .953670 .00040

17.600 .957210 .00041

17.700 .960580 .00041

17.800 .963080 .00041

17.900 .967180 .00040

18.000 .970540 .00040

18.100 .973840 .00041

18.200 .975980 .00040

18.300 .979240 .00039

18.400 .983490 .00039

18.500 .986780 .00040

18.600 .989340 .00039

18.700 .991870 .00039

18.900 .998580 .00038

19.001 .001920 .00040

19.101 .005520 .00039

19.201 .007620 .00040

19.301 .010510 .00038

19.401 .013340 .00038

19.501 .016250 .00040

32

14.300 .920210 .00047

14.400 .925550 .00046

14.500 .930170 .00046

14.600 .935100 .00047

14.700 .941070 .00047

14.800 .946130 .00046

14.900 .951060 .00048

15.000 .955430 .00046

15.100 .960020 .00046

15.200 .965230 .00045

15.300 .969850 .00047

15.400 .975790 .00044

15.500 .979110 .00046

15.600 .983450 .00046

15.700 .988880 .00047

15.800 .994000 .00045 
16.900 .986580 .00043 17.000 .991250 .00043 17.100 .995400 .00042 17.200 .998900 .00043 17.301 .003280 .00044 17.401 .006240 .00044 17.501 .009060 .00043 17.601 .014010 .00043 17.701 .016920 .00043
16.100 .993720 .00046 16.200 .997880 .00045 16.301 .002950 .00045 16.401 .006050 .00045 16.501 .012030 .00045 16.601 .014810 .00046 16.701 .019080 .00045
15.900 .997010 .00045 16.001 .001290 .00046 16.101 .006320 .00043 16.201 .010200 .00045 16.301 .014080 .00046 16.401 .019530 .00046 16.501 .023100 .00046
35

14.000 .919860 .00047 14.100 .925370 .00047 14.200 .931160 .00047 14.300 .935520 .00047 14.400 .940530 .00048 14.500 .945740 .00047 14.600 .951070 .00047 14.700 .955870 .00047 14.800 .962410 .00046 15.000 .972290 .00045 15.100 .975680 .00046 15.200 .980180 .00047 15.300 .985340 .00048 15.400 .990670 .00046 15.500 .994930 .00047 15.701 .003350 .00046 15.801 .008810 .00047 15.901 .013850 .00046 16.001 .019220 .00046

60

12.900 .917940 .00051 13.000 .923980 .00049 13.100 .930130 .00050 13.200 .936800 .00052 13.300 .942720 .00049 13.400 .948980 .00050 13.500 .954990 .00050 13.600 .961650 .00051 13.700 .967760 .00051 13.800 .972360 .00050 13.900 .978860 .00052 14.000 .984050 .00050 14.100 .990480 .00051 14.200 .994660 .00050
40

13.600 .916660 .00047 13.700 .922620 .00048 13.800 .927770 .00048 13.900 .933410 .00048 14.000 .939450 .00048 14.100 .944980 .00049 14.300 .956200 .00049 14.400 .961180 .00049 14.500 .966440 .00048 14.600 .972760 .00048 14.700 .976950 .00048 14.800 .980780 .00048 14.900 .986540 .00049 15.000 .991880 .00049 15.100 .997620 .00048 15.201 .001810 .00048 15.301 .006800 .00047 15.401 .011080 .00048 15.501 .016810 .00047 15.601 .020430 .00049

70

12.800 .921350 .00052 12.850 .925210 .00052 12.900 .927970 .00051 12.950 .931300 .00051 13.000 .933590 .00051 13.050 .936630 .00052 13.100 .940510 .00051 13.150 .942960 .00049 13.200 .946750 .00052 13.250 .950750 .00052 13.300 .953210 .00051 13.350 .956370 .00052 13.400 .959430 .00052 13.450 .962060 .00051
50

13.200 .919060 .00051 13.300 .926020 .00050 13.400 .932120 .00050 13.500 .937380 .00050 13.600 .944180 .00049 13.700 .950640 .00051 13.800 .956350 .00048 13.900 .960640 .00049 14.000 .966640 .00049 14.100 .971680 .00049 14.200 .978740 .00049 14.300 .983180 .00050 14.400 .989980 .00051 14.500 .993790 .00050 14.600 .999210 .00048 14.701 .004980 .00049 14.801 .010100 .00048 14.901 .014510 .00048 15.001 .019760 .00050 
14.301 .001070 .00050 14.401 .006210 .00051 14.501 .011400 .00051 14.601 .017220 .00051 14.701 .022020 .00051
13.500 .965110 .00051

13.550 .967340 .00050 13.600 .970500 .00051 13.650 .973940 .00051 13.700 .977210 .00051 13.750 .979110 .00053 13.800 .981460 .00052 13.850 .984820 .00052 13.900 .989260 .00051 13.950 .990690 .00050 14.000 .994310 .00052 14.050 .997150 .00050 14.101 .000200 .00052 14.151 .001460 .00051 14.201 .004930 .00052 14.251 .008230 .00052 14.301 .010970 .00051 14.351 .013130 .00052 14.401 .015430 .00053 14.451 .019340 .00049 14.501 .020490 .00051

\section{0}

12.600 .919390 .00052

12.650 .922900 .00052

12.700 .925950 .00050

12.750 .929090 .00052

12.800 .932860 .00054

12.850 .935370 .00051

12.900 .937840 .00053

12.950 .942180 .00051

13.000 .944100 .00051

13.050 .947720 .00052

13.100 .951990 .00052

13.150 .954760 .00053

13.200 .956780 .00053

13.250 .960170 .00052

13.300 .962800 .00051

13.350 .967310 .00051

13.400 .969050 .00050

13.450 .972390 .00053

13.500 .974430 .00051

13.550 .978040 .00052

13.600 .981260 .00052

13.650 .984120 .00051

13.700 .985960 .00054

13.750 .989980 .00051

13.800 .993900 .00052

13.850 .995160 .00052
100

12.600 .921670 .00053

12.650 .924850 .00053

12.700 .927810 .00052

12.750 .931440 .00051

12.800 .935520 .00052

12.850 .938150 .00051

12.900 .941290 .00053

12.950 .944710 .00052

13.000 .947240 .00053

13.050 .950300 .00054

13.100 .953220 .00053

13.150 .957310 .00052

13.200 .959510 .00053

13.250 .963590 .00053

13.300 .965540 .00053

13.350 .968950 .00052

13.400 .971760 .00051

13.450 .973960 .00052

13.500 .977370 .00053

13.550 .980660 .00052

13.600 .982780 .00052

13.650 .986450 .00052

13.700 .989610 .00053

13.750 .991740 .00052

13.800 .995270 .00052

13.850 .997390 .00053
14.001 .000250 .00051

14.101 .005580 .00053

14.201 .010800 .00052

14.301 .016690 .00053

14.401 .021230 .00053
120

12.500 .916380 .00054 12.550 .920040 .00053 12.600 .923940 .00052 12.650 .925580 .00053 12.700 .929780 .00053 12.750 .932530 .00052 12.800 .936250 .00052 12.850 .939050 .00051 12.900 .942060 .00052 12.950 .945760 .00053 13.000 .949360 .00053 13.050 .951790 .00051 13.100 .954290 .00054 13.150 .957910 .00053 13.200 .960500 .00052 13.250 .964310 .00052 13.300 .967400 .00053 13.350 .969290 .00054 13.400 .973260 .00051 13.450 .975150 .00053 13.500 .978280 .00052 13.550 .980590 .00053 13.600 .985000 .00053 13.650 .986820 .00052 13.700 .989790 .00052 13.750 .993640 .00052 
13.900 .998150 .00053 13.951 .000640 .00052 14.001 .004460 .00050 14.051 .007200 .00051 14.101 .010810 .00052 14.151 .014390 .00052 14.201 .015370 .00052 14.251 .019000 .00052 14.301 .020950 .00053
13.901 .000620 .00053 13.951 .003790 .00053 14.001 .006770 .00052 14.051 .009190 .00053 14.101 .013530 .00052 14.151 .015390 .00052 14.201 .017650 .00055 14.251 .019140 .00052 14.301 .022800 .00051
13.800 .995450 .00054 13.850 .998380 .00053 13.901 .002160 .00052 13.951 .004160 .00052 14.001 .007460 .00053 14.051 .011620 .00052 14.101 .014120 .00053 14.151 .016230 .00053 14.201 .018720 .00052
130

12.500 .918010 .00052 12.550 .920100 .00054 12.600 .923970 .00053 12.650 .927500 .00052 12.700 .930640 .00052 12.750 .933040 .00054 12.800 .937620 .00053 12.850 .940080 .00054 12.900 .943720 .00052 13.000 .949350 .00054 13.100 .955530 .00052 13.150 .958480 .00052 13.200 .960780 .00052 13.250 .964780 .00053 13.300 .966710 .00052 13.350 .971260 .00053 13.400 .972630 .00053 13.450 .976620 .00053 13.500 .980140 .00052 13.550 .982410 .00052 13.600 .985430 .00051 13.650 .988550 .00053 13.700 .990860 .00052 13.750 .993830 .00053 13.800 .996970 .00052 13.851 .000590 .00051 13.901 .002080 .00053 13.951 .005110 .00054 14.001 .007850 .00053 14.051 .011480 .00053 14.101 .012750 .00053 14.151 .015960 .00054 14.201 .018670 .00052
150

12.500 .914730 .00051

12.600 .921710 .00053

12.700 .930150 .00053

12.800 .935120 .00052

12.900 .942240 .00052

13.000 .948040 .00054

13.100 .953470 .00052

13.200 .960070 .00053

13.300 .965250 .00053

13.400 .970860 .00052

13.500 .976560 .00053

13.600 .983550 .00052

13.700 .988750 .00052

13.800 .995270 .00053

13.901 .000480 .00052

14.001 .006480 .00053

14.101 .011200 .00052

14.201 .016880 .00052

14.301 .022040 .00052
200

12.600 .917270 .00053

12.700 .922260 .00052

12.800 .929960 .00053

12.900 .935500 .00053

13.000 .941220 .00052

13.100 .946920 .00052

13.200 .953890 .00052

13.300 .959630 .00053

13.400 .964740 .00053

13.600 .976260 .00052

13.700 .981900 .00052

13.800 .985310 .00053

13.900 .993170 .00054

14.000 .998730 .00054

14.101 .003790 .00053

14.201 .010370 .00052

14.301 .015120 .00053

14.401 .019960 .00053 
239

12.700 .916790 .00052

12.800 .923690 .00052

12.900 .930420 .00051

13.000 .935400 .00052

13.100 .941270 .00053

13.200 .946750 .00052

13.300 .953430 .00050

13.400 .959520 .00052

13.500 .963590 .00052

13.600 .970660 .00052

13.700 .976230 .00052

13.800 .981870 .00052

13.900 .987500 .00053

14.000 .992340 .00052

14.100 .997450 .00053

14.201 .003550 .00053

14.301 .008160 .00053

14.401 .013210 .00051

14.501 .019460 .00054
250

12.800 .920770 .00053

12.900 .926900 .00052

13.000 .934110 .00053

13.100 .939320 .00053

13.200 .945060 .00052

13.300 .951710 .00052

13.400 .956440 .00052

13.500 .962090 .00052

13.600 .967060 .00051

13.700 .973440 .00052

13.800 .979650 .00052

13.900 .984840 .00052

14.000 .990750 .00050

14.100 .996400 .00051

14.200 .999980 .00052

14.301 .006070 .00051

14.401 .011850 .00051

14.501 .016080 .00052

14.601 .020360 .00053
478

13.300 .918550 .00051

13.400 .922460 .00052

13.500 .929200 .00051

13.600 .934440 .00051

13.700 .939950 .00049

13.800 .945810 .00051

13.900 .950200 .00051

14.000 .955650 .00050

14.100 .960520 .00050

14.200 .967270 .00052

14.300 .971290 .00050

14.400 .976870 .00050

14.500 .981670 .00051

14.600 .986080 .00051

14.700 .991570 .00050

14.800 .996740 .00051

14.901 .001050 .00050

15.001 .005680 .00052

15.101 .010680 .00051

15.201 .015320 .00050

15.301 .020430 .00050
500

13.400 .919290 .00051

13.500 .924770 .00051

13.600 .930660 .00050

13.700 .936220 .00052

13.800 .941670 .00052

13.900 .946520 .00051

14.000 .953140 .00051

14.100 .956640 .00053

14.200 .962310 .00052

14.300 .967690 .00053

14.400 .973800 .00051

14.500 .977170 .00050

14.600 .983320 .00050

14.700 .987530 .00050

14.800 .992040 .00051

14.900 .998010 .00052

15.001 .002550 .00051

15.101 .007530 .00051

15.201 .011420 .00053

15.301 .016580 .00051

15.401 .020490 .00050
359

13.200 .930970 .00052

13.300 .935040 .00051

13.400 .940040 .00051

13.500 .946380 .00052

13.600 .952520 .00053

13.700 .957980 .00051

13.800 .963000 .00051

13.900 .967990 .00051

14.000 .973890 .00052

14.100 .979150 .00051

14.200 .984660 .00051

14.300 .988440 .00051

14.400 .993590 .00051

14.500 .999130 .00051

14.601 .004310 .00051

14.701 .009170 .00050

14.801 .013800 .00052

14.901 .018980 .00052

\section{0}

13.900 .922990 .00050 14.000 .928160 .00051 14.100 .933490 .00049 14.200 .939180 .00050 14.300 .944200 .00050 14.400 .948900 .00050 14.500 .954180 .00050 14.600 .959220 .00050 14.700 .963940 .00050 14.800 .968830 .00049 14.900 .973850 .00051 15.000 .979370 .00049 15.100 .984780 .00051 15.200 .989170 .00050 15.300 .993100 .00051 15.400 .997610 .00052 15.501 .003440 .00051 15.601 .006660 .00051 15.701 .011950 .00050 15.801 .016600 .00051 15.901 .020320 .00050 
650

14.200 .919730 .00050 14.300 .926130 .00049 14.400 .931240 .00050 14.500 .936650 .00049 14.600 .941910 .00051 14.700 .947570 .00051 14.800 .952520 .00051 14.900 .957190 .00050 15.000 .960400 .00049 15.100 .966520 .00051 15.200 .970650 .00050 15.300 .974930 .00051 15.400 .980330 .00049 15.500 .985680 .00050 15.600 .990360 .00051 15.800 .999810 .00050 15.901 .003580 .00051 16.001 .008260 .00050 16.101 .012430 .00051 16.201 .016730 .00052
700

14.800 .922540 .00050

14.900 .927230 .00049

15.000 .932400 .00051

15.100 .937450 .00049

15.200 .942450 .00049

15.300 .947730 .00051

15.400 .951380 .00049

15.500 .956440 .00051

15.600 .961300 .00049

15.700 .965970 .00049

15.800 .972210 .00050

15.900 .975010 .00050

16.000 .979560 .00051

16.100 .984310 .00050

16.300 .993500 .00048

16.400 .998080 .00049

16.501 .003000 .00049

16.601 .006670 .00049

16.801 .015470 .00050

16.901 .019030 .00050
752

16.400 .919830 .00048 16.500 .924010 .00049 16.600 .928920 .00047 16.700 .933470 .00050 16.800 .937290 .00049 16.900 .942480 .00049 17.000 .946300 .00049 17.100 .950970 .00049 17.200 .955760 .00049 17.300 .960890 .00050 17.400 .964060 .00049 17.500 .968570 .00050 17.600 .972390 .00049 17.700 .976570 .00050 17.800 .981230 .00049 17.900 .984890 .00049 18.000 .989370 .00048 18.100 .993340 .00049 18.200 .997480 .00049 18.301 .000570 .00049 18.401 .004190 .00051 18.501 .009190 .00049
754

16.800 .925570 .00050 16.900 .929490 .00048 17.000 .935350 .00049 17.100 .938980 .00048 17.200 .944700 .00048 17.300 .948560 .00050 17.400 .953250 .00050 17.500 .957540 .00050 17.600 .960940 .00050 17.700 .965090 .00049 17.800 .968780 .00049 17.900 .973940 .00048 18.000 .978280 .00049 18.100 .981700 .00049 18.200 .985310 .00049 18.300 .988910 .00049 18.400 .993500 .00049 18.500 .997990 .00050 18.601 .001480 .00048 18.701 .005780 .00049 18.801 .010230 .00049 18.901 .014520 .00048
750

16.300 .924400 .00048

16.400 .928790 .00049

16.500 .932170 .00049

16.600 .936650 .00050

16.700 .942090 .00048

16.800 .946290 .00049 16.900 .950340 .00048 17.000 .955410 .00049 17.100 .960220 .00051 17.200 .963210 .00047 17.300 .967770 .00049 17.400 .972030 .00050 17.500 .978170 .00049 17.600 .979790 .00048 17.700 .984670 .00050 17.800 .989920 .00048 17.900 .993270 .00050 18.000 .996610 .00048 18.101 .000790 .00049 18.201 .005860 .00050 18.301 .009560 .00049 18.401 .013090 .00049 18.501 .016370 .00048 18.601 .021420 .00049

755

16.900 .920040 .00049 17.000 .923960 .00049 17.100 .928910 .00050 17.200 .933420 .00049 17.300 .936580 .00049 17.400 .941420 .00050 17.500 .946460 .00050 17.600 .950520 .00049 17.700 .953880 .00048 17.800 .957870 .00049 17.900 .963290 .00048 18.000 .967220 .00049 18.100 .971100 .00050 18.200 .974170 .00048 18.300 .977910 .00050 18.400 .983560 .00049 18.500 .986460 .00049 18.600 .991550 .00049 18.700 .994800 .00047 18.800 .998230 .00049 18.901 .002710 .00049 19.001 .006300 .00048 
CHPRC-01552, Revision 0

18.601 .012860 .00050

18.701 .016180 .00049

18.801 .020690 .00048
19.001 .017190 .00049

19.101 .021240 .00049

19.101 .010610 .00049

19.201 .014000 .00049

19.301 .018000 .00049 
Pun40-sp-0950-01

12

21.400 .924900 .00030

21.700 .931060 .00031

22.000 .935640 .00030

22.300 .941790 .00030

22.600 .946230 .00030

22.900 .950980 .00029

23.200 .955810 .00030

23.500 .959790 .00029

23.800 .964890 .00029

24.100 .969160 .00030

24.400 .973500 .00030

24.700 .977960 .00028

25.000 .981840 .00029

25.300 .985850 .00029

25.600 .989710 .00029

25.900 .992890 .00029

26.200 .997680 .00028

26.501 .000060 .00028

26.801 .003640 .00027

27.101 .007360 .00028

27.401 .010290 .00028

27.701 .013910 .00028

27.801 .014560 .00028
15

17.800 .923270 .00035 18.000 .927880 .00035 18.200 .933910 .00035 18.400 .939180 .00035 18.600 .943640 .00034 18.800 .948200 .00035 19.000 .954310 .00034 19.200 .959510 .00033 19.400 .964500 .00034 19.600 .968590 .00033 19.800 .973630 .00035 20.000 .977930 .00034 20.200 .981770 .00034 20.400 .986610 .00034 20.600 .990340 .00035 20.800 .994590 .00033 21.000 .998240 .00035 21.201 .002190 .00032 21.401 .006290 .00034 21.601 .010890 .00033 21.801 .013700 .00032
25

13.800 .921520 .00043 13.900 .924460 .00042 14.000 .930070 .00042 14.100 .933870 .00042 14.200 .938170 .00042 14.300 .942670 .00042 14.400 .947710 .00042 14.500 .952000 .00043 14.600 .955600 .00042 14.700 .960320 .00041 14.800 .963860 .00042 14.900 .969070 .00040 15.000 .972390 .00040 15.100 .975910 .00041 15.200 .980800 .00042
30
13.000 .920260 .00044

13.100 .924860 .00043

13.200 .929390 .00044

13.300 .934690 .00044

13.400 .939630 .00044

13.500 .943950 .00043

13.600 .949440 .00044

13.700 .954010 .00043

13.800 .959460 .00044

13.900 .963620 .00044

14.000 .968330 .00043

14.200 .977800 .00044

14.300 .982400 .00043

14.400 .986630 .00043

14.500 .990830 .00043
20

15.100 .920470 .00040

15.200 .924190 .00040

15.300 .927990 .00039

15.400 .932090 .00038

15.500 .935200 .00039

15.600 .938720 .00038

15.700 .943170 .00039

15.800 .946600 .00040

16.000 .953720 .00039

16.100 .956890 .00039

16.200 .960910 .00039

16.300 .963770 .00039

16.400 .966790 .00040

16.500 .970600 .00040

16.600 .974430 .00038

16.700 .977340 .00038

16.800 .980040 .00038

16.900 .983860 .00038

17.000 .986980 .00038

17.100 .990380 .00038

17.200 .993830 .00037

17.300 .996620 .00038

17.400 .999280 .00039

17.501 .001260 .00038

17.601 .004450 .00039

17.701 .008260 .00038

17.801 .011600 .00038

17.901 .014530 .00038

18.001 .017930 .00038

32

12.800 .921030 .00044

12.900 .926410 .00045

13.000 .931290 .00045

13.100 .937080 .00045

13.200 .941310 .00044

13.300 .947120 .00044

13.400 .952440 .00045

13.500 .956440 .00045

13.600 .961580 .00045

13.700 .965450 .00044

13.800 .971390 .00043

13.900 .975870 .00045

14.000 .980250 .00043

14.100 .985680 .00044

14.200 .988910 .00044 
15.300 .984650 .00042 15.400 .987940 .00041 15.500 .991910 .00041 15.600 .996190 .00041 15.700 .999770 .00041 15.801 .004020 .00041 15.901 .007030 .00042 16.001 .010760 .00041 16.101 .014250 .00041 16.201 .018020 .00040
14.600 .994090 .00044 14.700 .999430 .00043 14.801 .003450 .00044 14.901 .007420 .00043 15.001 .011290 .00045 15.101 .017100 .00045 15.201 .020280 .00044
14.300 .993780 .00043 14.501 .002870 .00045 14.601 .007230 .00044 14.701 .011790 .00044 14.801 .015790 .00044 14.901 .019850 .00044
35

12.500 .920360 .00045 12.600 .926120 .00046 12.700 .930840 .00044 12.900 .941700 .00046 13.000 .947320 .00044 13.100 .953100 .00045 13.200 .957270 .00046 13.300 .963170 .00045 13.400 .967360 .00046 13.500 .973490 .00045 13.600 .976280 .00045 13.700 .982270 .00046 13.800 .986650 .00045 14.000 .996790 .00046 14.101 .002200 .00044 14.201 .005680 .00045 14.301 .010410 .00045 14.401 .014290 .00045 14.501 .019190 .00045

60

11.400 .919700 .00049 11.450 .922710 .00047 11.500 .926600 .00049 11.550 .929470 .00048 11.600 .932090 .00049 11.650 .935560 .00049 11.700 .939230 .00049 11.750 .942570 .00048 11.800 .944320 .00049 11.850 .947420 .00049 11.900 .950990 .00048 11.950 .953200 .00048 12.000 .957000 .00049
40

12.100 .918190 .00046 12.200 .924280 .00046 12.300 .929480 .00045 12.400 .935350 .00045 12.500 .940330 .00046 12.600 .945210 .00046 12.700 .951650 .00046 12.800 .957030 .00047 12.900 .962870 .00045 13.000 .967730 .00045 13.100 .973610 .00047 13.200 .977670 .00046 13.300 .983540 .00046 13.400 .988290 .00045 13.500 .994550 .00045 13.600 .998210 .00046 13.701 .003330 .00045 13.801 .008850 .00048 13.901 .012240 .00045 14.001 .017110 .00045

70

11.200 .917590 .00051 11.250 .921080 .00050 11.300 .924150 .00051 11.350 .928240 .00050 11.400 .929870 .00050 11.450 .934180 .00049 11.500 .937060 .00049 11.550 .939040 .00048 11.600 .942930 .00049 11.650 .946410 .00049 11.700 .949170 .00049 11.750 .952930 .00049 11.800 .956080 .00049
50

11.700 .921420 .00049 11.800 .928110 .00047 11.900 .934050 .00047 12.000 .940430 .00048 12.100 .945730 .00049 12.200 .950740 .00048 12.300 .957530 .00047 12.400 .963310 .00049 12.500 .969170 .00047 12.600 .974140 .00048 12.700 .979720 .00048 12.800 .984390 .00048 12.900 .991270 .00048 13.000 .996070 .00049 13.101 .001840 .00046 13.201 .007160 .00050 13.301 .011710 .00048 13.401 .016280 .00049 13.501 .022160 .00047

80

11.100 .917770 .00050 11.150 .920320 .00051 11.200 .924550 .00050 11.250 .927960 .00050 11.300 .931400 .00050 11.350 .933910 .00050 11.400 .938180 .00049 11.450 .941770 .00051 11.500 .943660 .00050 11.550 .946490 .00050 11.600 .949780 .00048 11.650 .952980 .00050 11.700 .957480 .00048 
12.050 .959510 .00049

12.100 .963250 .00050

12.150 .966560 .00049

12.200 .968990 .00050

12.250 .971640 .00049

12.300 .974480 .00048

12.350 .977670 .00049

12.400 .979630 .00050

12.450 .983280 .00050

12.500 .986490 .00050

12.550 .987680 .00049

12.600 .991870 .00048

12.650 .995710 .00049

12.700 .997830 .00049

12.750 .999930 .00049

12.801 .003000 .00047

12.851 .005040 .00049

12.901 .008580 .00048

12.951 .011380 .00049

13.001 .014210 .00047

13.051 .016030 .00049

13.101 .018320 .00051

\section{0}

11.100 .923430 .00050

11.150 .924840 .00049

11.200 .928250 .00051

11.250 .931750 .00052

11.300 .935480 .00051

11.350 .938890 .00051

11.400 .941050 .00049

11.450 .944910 .00050

11.500 .947980 .00051

11.550 .951370 .00049

11.600 .953630 .00050

11.650 .957100 .00049

11.700 .960560 .00050

11.750 .964000 .00051

11.800 .967200 .00050

11.850 .970560 .00050

11.900 .973160 .00050

11.950 .975530 .00049

12.000 .978300 .00051

12.050 .981750 .00049

12.100 .984410 .00051

12.150 .986810 .00049

12.200 .989680 .00050

12.250 .992810 .00050

12.300 .996340 .00051
11.850 .958950 .00048

11.900 .960700 .00050

11.950 .963820 .00050

12.000 .967240 .00048

12.050 .970190 .00049

12.100 .973240 .00048

12.150 .976980 .00050

12.200 .977840 .00049

12.250 .981480 .00049

12.300 .985250 .00049

12.350 .988100 .00050

12.400 .990830 .00048

12.450 .994010 .00048

12.500 .996090 .00051

12.550 .998480 .00051

12.601 .001170 .00051

12.651 .004510 .00050

12.701 .006930 .00048

12.751 .010060 .00048

12.801 .013190 .00048

12.851 .016190 .00050

12.901 .018020 .00051
11.800 .962310 .00051

11.850 .964950 .00049

11.900 .967580 .00050

11.950 .971010 .00050

12.000 .973570 .00050

12.050 .978050 .00049

12.100 .980040 .00050

12.150 .982690 .00049

12.200 .985420 .00050

12.250 .989220 .00050

12.300 .991800 .00050

12.350 .994650 .00050

12.400 .998140 .00050

12.451 .000320 .00051

12.501 .002780 .00050

12.551 .005910 .00051

12.601 .008460 .00050

12.651 .011480 .00048

12.701 .013020 .00050

12.751 .016240 .00049

12.801 .020100 .00050
11.000 .918420 .00051

11.050 .921370 .00049

11.100 .924440 .00050

11.150 .928280 .00049

11.200 .931870 .00050

11.250 .935240 .00050

11.300 .938260 .00052

11.350 .941270 .00051

11.400 .944500 .00050

11.450 .947070 .00050

11.500 .950100 .00052

11.550 .953800 .00050

11.600 .957950 .00051

11.650 .959850 .00050

11.700 .962530 .00048

11.750 .965890 .00049

11.800 .968900 .00051

11.850 .971740 .00050

11.900 .974970 .00050 11.950 .979160 .00051 12.000 .982360 .00051

12.050 .983780 .00049

12.150 .989480 .00051

12.200 .993390 .00050

12.250 .996410 .00052
120

11.000 .920370 .00051 11.050 .922910 .00049 11.100 .926470 .00051 11.150 .930880 .00050 11.200 .933860 .00052 11.250 .936750 .00051 11.300 .939210 .00050 11.350 .943780 .00051 11.400 .946010 .00050 11.450 .948580 .00051 11.500 .952610 .00051 11.550 .955350 .00050 11.600 .957890 .00051 11.650 .962070 .00051 11.700 .964550 .00051 11.750 .967660 .00051 11.800 .969980 .00051 11.850 .972840 .00052 11.900 .976660 .00051 11.950 .979390 .00052 12.000 .982980 .00052 12.050 .985730 .00052 12.100 .987900 .00050 12.150 .991830 .00051 12.200 .994760 .00050 
12.350 .998470 .00050

12.401 .001560 .00050

12.451 .004520 .00049

12.501 .007100 .00050

12.551 .009680 .00050

12.601 .013160 .00050

12.651 .015020 .00051

12.701 .019310 .00051
12.300 .999160 .00052

12.351 .001720 .00050

12.401 .004800 .00051

12.451 .007440 .00051

12.551 .012900 .00050

12.651 .018330 .00050

12.701 .020410 .00051
130

11.000 .921130 .00051 11.050 .924500 .00052 11.100 .928710 .00053 11.150 .931090 .00049 11.200 .934210 .00049 11.250 .936880 .00051 11.300 .940360 .00051 11.350 .944270 .00050 11.400 .947010 .00052 11.450 .949980 .00049 11.500 .953100 .00050 11.600 .959190 .00050 11.650 .962640 .00051 11.700 .965670 .00051 11.750 .967600 .00051 11.800 .971810 .00051 11.850 .974320 .00052 11.900 .978630 .00051 11.950 .980080 .00051 12.000 .982160 .00051 12.050 .985190 .00050 12.100 .988370 .00050 12.150 .990730 .00050 12.200 .994490 .00051 12.300 .999450 .00052 12.351 .003780 .00051 12.401 .006560 .00051 12.451 .009130 .00051 12.501 .012070 .00051 12.551 .013750 .00052 12.601 .017780 .00051 12.651 .020430 .00051 12.701 .022200 .00051
150

11.000 .919400 .00050 11.050 .923470 .00052 11.100 .925580 .00052 11.150 .928870 .00050 11.200 .932990 .00050 11.250 .935670 .00049 11.300 .938410 .00051 11.350 .942630 .00051 11.400 .944420 .00052 11.450 .947320 .00050 11.500 .951100 .00051 11.550 .954350 .00051 11.600 .957720 .00051 11.650 .960430 .00050 11.700 .963160 .00051 11.750 .966500 .00050 11.800 .969940 .00052 11.850 .972490 .00051 11.900 .975670 .00051 11.950 .978860 .00052 12.050 .984000 .00051 12.100 .987200 .00050 12.150 .989610 .00051 12.200 .993300 .00050 12.250 .996620 .00050 12.300 .999050 .00051 12.351 .000780 .00051 12.401 .003590 .00051 12.451 .006900 .00050 12.501 .009990 .00051 12.551 .012660 .00052 12.601 .014780 .00051 12.651 .016990 .00050 12.701 .021470 .00051
12.250 .997300 .00052 12.301 .000170 .00051 12.351 .003860 .00052 12.401 .006410 .00051 12.451 .008770 .00051 12.501 .010700 .00051 12.551 .014200 .00052 12.601 .017920 .00052 12.651 .019210 .00050 12.701 .021790 .00052

200

11.100 .920130 .00052 11.150 .924380 .00050 11.200 .926530 .00051 11.250 .930030 .00050 11.300 .933430 .00051 11.350 .936820 .00050 11.400 .939560 .00051 11.450 .942260 .00051 11.500 .945190 .00050 11.550 .947170 .00051 11.600 .951400 .00051 11.650 .954700 .00050 11.700 .957280 .00050 11.750 .959870 .00051 11.800 .962940 .00051 11.850 .966450 .00049 11.900 .970440 .00050 11.950 .971450 .00051 12.000 .974480 .00052 12.050 .977720 .00051 12.100 .980490 .00053 12.150 .983190 .00052 12.200 .987250 .00051 12.250 .989630 .00052 12.300 .991850 .00052 12.350 .995760 .00051 12.400 .997430 .00051 12.451 .000290 .00051 12.501 .002330 .00051 12.551 .004620 .00051 12.601 .007840 .00052 12.651 .011120 .00051 12.701 .013970 .00052 12.751 .017280 .00050 12.801 .018600 .00050 
239

11.200 .921380 .00051

11.250 .923500 .00051

11.300 .926930 .00051

11.350 .930150 .00050

11.400 .933590 .00051

11.450 .936420 .00050

11.500 .939920 .00051

11.550 .942080 .00051

11.600 .945420 .00050

11.650 .948370 .00049

11.700 .951600 .00049

11.750 .954540 .00051

11.800 .957530 .00050

11.850 .960290 .00051

11.900 .962540 .00051

11.950 .966230 .00052

12.000 .968780 .00051

12.050 .971000 .00052

12.100 .974260 .00053

12.150 .976990 .00051

12.200 .979650 .00049

12.250 .982600 .00050

12.300 .985290 .00051

12.350 .988410 .00052

12.400 .991190 .00051

12.450 .993170 .00050

12.500 .996060 .00051

12.550 .999780 .00052

12.601 .002200 .00052

12.651 .004360 .00052

12.701 .007130 .00051

12.751 .009580 .00050

12.801 .012200 .00051

12.851 .014980 .00050

12.901 .017040 .00050
478

11.800 .921450 .00050 11.900 .928230 .00051

12.000 .932390 .00050

12.100 .938110 .00050

12.300 .949190 .00050

12.400 .955420 .00049

12.500 .960140 .00050

12.600 .965280 .00050

12.700 .970680 .00052

12.900 .980410 .00050
250

11.300 .924990 .00052

11.400 .931790 .00051

11.500 .938210 .00051

11.600 .943140 .00052

11.700 .950060 .00050

11.800 .955010 .00051

11.900 .960230 .00052

12.000 .965530 .00051

12.100 .973100 .00050

12.200 .977560 .00051

12.300 .982990 .00049

12.400 .988920 .00052

12.500 .994830 .00052

12.600 .999710 .00050

12.701 .004100 .00050

12.801 .010520 .00051

12.901 .016380 .00051
359

11.500 .922240 .00051

11.600 .926780 .00050

11.700 .933990 .00050

11.800 .939080 .00050

11.900 .945790 .00049

12.000 .950370 .00052

12.100 .956170 .00050

12.200 .962150 .00050

12.300 .966690 .00051

12.400 .972410 .00050

12.500 .977480 .00050

12.600 .982220 .00050

12.700 .988040 .00049

12.800 .992970 .00050

12.900 .997460 .00051

13.001 .002500 .00051

13.101 .009090 .00050

13.201 .013260 .00050

13.301 .018790 .00049
500

11.900 .923400 .00050 12.000 .928300 .00051

12.100 .934460 .00050

12.200 .940020 .00052

12.300 .946140 .00052

12.400 .950400 .00050

12.600 .961320 .00050

12.700 .966410 .00050

12.800 .972280 .00048

12.900 .975980 .00051
600

12.300 .923220 .00049

12.400 .928230 .00050

12.500 .934060 .00051

12.600 .938300 .00048

12.700 .944660 .00048

12.800 .950280 .00050

12.900 .954830 .00049

13.000 .959560 .00049

13.100 .964800 .00049

13.200 .970080 .00049 
13.000 .985540 .00049 13.100 .990030 .00049 13.200 .994250 .00050 13.301 .000610 .00050 13.401 .005620 .00050 13.501 .009720 .00050 13.601 .014180 .00051

650

12.600 .923710 .00049 12.700 .928540 .00049 12.800 .933850 .00049 12.900 .938610 .00050 13.000 .943080 .00049 13.100 .949250 .00049 13.200 .954020 .00048 13.300 .958720 .00051 13.400 .964170 .00049 13.500 .967950 .00049 13.600 .972380 .00049 13.700 .978220 .00050 13.800 .981800 .00049 13.900 .986570 .00049 14.000 .991380 .00050 14.100 .996580 .00051 14.201 .001940 .00051 14.301 .004470 .00049 14.401 .009580 .00050 14.501 .013890 .00049 14.601 .018650 .00049

752

14.500 .919410 .00049 14.600 .924410 .00049 14.700 .928090 .00048 14.800 .933450 .00050 14.900 .936820 .00049 15.000 .942660 .00050 15.100 .944920 .00049 15.200 .951260 .00049 15.300 .954890 .00048 15.400 .959950 .00049 15.500 .963980 .00048
13.000 .980910 .00050 13.100 .986650 .00051 13.200 .991490 .00049 13.300 .995940 .00050 13.401 .001700 .00052 13.501 .005500 .00050 13.601 .010460 .00051 13.701 .014750 .00050 13.801 .019950 .00050

700

13.100 .921530 .00050 13.300 .932050 .00048 13.400 .936880 .00049 13.500 .942980 .00049 13.600 .946900 .00048 13.700 .951750 .00050 13.800 .956940 .00048 13.900 .962210 .00050 14.000 .966360 .00048 14.100 .970520 .00048 14.200 .975520 .00048 14.300 .979300 .00048 14.400 .984050 .00050 14.500 .989420 .00049 14.600 .992980 .00050 14.700 .998260 .00047 14.801 .002190 .00049 14.901 .006810 .00050 15.001 .009870 .00049 15.101 .014310 .00049 15.201 .019300 .00050

754

14.700 .918640 .00048 14.800 .922780 .00048 14.900 .927020 .00049 15.000 .932230 .00048 15.100 .935830 .00049 15.200 .941040 .00050 15.300 .945200 .00047 15.400 .948640 .00049 15.500 .953500 .00048 15.600 .958140 .00050 15.700 .962710 .00050
13.300 .974030 .00049 13.400 .979400 .00051 13.500 .984850 .00050 13.600 .988520 .00050 13.700 .993060 .00050 13.800 .997430 .00050 13.901 .002760 .00050 14.001 .006990 .00051 14.101 .011770 .00050 14.201 .016310 .00049 14.301 .021010 .00050

\begin{abstract}
750
14.400 .922170 .00048 14.500 .927830 .00048 14.600 .931010 .00049 14.700 .935780 .00050 14.800 .941440 .00049 14.900 .944800 .00051 15.000 .949850 .00047 15.100 .954430 .00048 15.200 .957810 .00049 15.300 .962840 .00048 15.400 .966690 .00049 15.700 .979540 .00050 15.800 .984370 .00049 16.000 .991810 .00049 16.100 .996120 .00048 16.200 .999960 .00049 16.301 .003900 .00048 16.401 .007920 .00049 16.501 .011870 .00049 16.601 .015790 .00048
\end{abstract}

755

15.100 .926440 .00046 15.200 .931970 .00047 15.300 .935570 .00048 15.400 .939360 .00048 15.500 .944680 .00048 15.600 .947380 .00048 15.700 .952400 .00049 15.800 .957710 .00048 15.900 .961520 .00049 16.000 .965830 .00048 16.100 .969730 .00048 
15.600 .967960 .00048 15.700 .972540 .00048 15.800 .975310 .00048 15.900 .980070 .00050 16.000 .984000 .00048 16.100 .988920 .00049 16.200 .992850 .00049 16.300 .997360 .00050 16.400 .999870 .00049 16.501 .004660 .00050 16.601 .007090 .00047 16.701 .013240 .00049 16.801 .015920 .00049 16.901 .020210 .00050 Pun40-sp-0950-10

12

20.300 .925380 .00029 20.600 .930790 .00028 20.900 .936190 .00029 21.200 .941780 .00029 21.500 .946080 .00029 21.800 .951310 .00028 22.100 .956090 .00028 22.400 .960820 .00029 22.700 .965910 .00028 23.000 .969580 .00028 23.300 .973470 .00028 23.600 .977570 .00028 23.900 .981980 .00028 24.200 .985700 .00028 24.500 .989880 .00028 24.800 .993540 .00028 25.100 .997350 .00028 25.401 .000010 .00028 25.701 .003940 .00026 26.001 .007780 .00027 26.301 .010900 .00026 26.501 .012670 .00026
15.800 .966350 .00048 15.900 .970460 .00049 16.000 .975230 .00049 16.100 .978250 .00047 16.200 .983370 .00049 16.400 .991080 .00049 16.500 .994990 .00049 16.600 .998930 .00047 16.701 .002240 .00048 16.801 .006520 .00049 16.901 .010290 .00050 17.001 .014070 .00049 17.101 .017490 .00047
16.200 .973080 .00050 16.300 .976210 .00048 16.400 .981110 .00049 16.500 .984900 .00048 16.600 .988860 .00048 16.700 .992280 .00049 16.800 .996710 .00049 16.901 .000400 .00048 17.001 .005080 .00049 17.101 .008590 .00048 17.201 .012530 .00049 17.301 .016090 .00050 17.401 .019110 .00048
15

16.800 .926050 .00033 17.000 .931530 .00033 17.200 .936940 .00032 17.400 .942260 .00032 17.600 .946570 .00033 17.800 .952190 .00032 18.000 .957340 .00032 18.200 .961440 .00033 18.400 .966380 .00033 18.600 .969890 .00033 18.800 .975060 .00032 19.000 .980330 .00032 19.200 .984620 .00033 19.400 .988820 .00031 19.600 .992720 .00031 19.800 .996590 .00032 20.001 .000980 .00031 20.201 .004900 .00032 20.401 .008520 .00032 20.601 .012820 .00032 20.701 .014400 .00031
20

14.000 .920700 .00037 14.100 .924860 .00036 14.200 .928900 .00037 14.300 .932870 .00037 14.400 .936890 .00037 14.500 .940300 .00038 14.600 .944760 .00037 14.700 .947350 .00037 14.800 .950600 .00036 14.900 .954650 .00036 15.000 .957960 .00037 15.100 .962520 .00035 15.200 .965290 .00037 15.300 .968720 .00037 15.400 .971730 .00035 15.500 .975510 .00038 15.600 .978480 .00036 15.700 .981520 .00037 15.800 .984810 .00037 15.900 .988050 .00037 16.000 .991160 .00036 16.100 .994510 .00036 16.200 .997710 .00035 16.301 .000590 .00035 16.401 .003370 .00035 16.501 .006720 .00036 16.601 .009830 .00037 16.701 .013110 .00037 16.801 .014440 .00036 
25

12.700 .921980 .00039

12.800 .926490 .00039

12.900 .931450 .00041

13.000 .935960 .00039

13.100 .940850 .00039

13.200 .944860 .00039

13.300 .949380 .00039

13.400 .953730 .00040

13.500 .957480 .00039

13.600 .962560 .00040

13.700 .966650 .00039

13.800 .970210 .00038

13.900 .974090 .00040

14.000 .978880 .00038

14.100 .982380 .00039

14.200 .987020 .00039

14.300 .990550 .00039

14.400 .994620 .00038

14.500 .998230 .00039

14.601 .002370 .00039

14.701 .005920 .00039

14.801 .008770 .00040

14.901 .013890 .00038

15.001 .017160 .00039
30

11.900 .922350 .00041

12.000 .927710 .00041

12.100 .932360 .00041

12.200 .937880 .00041

12.300 .943260 .00042

12.400 .947970 .00040

12.500 .952120 .00041

12.600 .957410 .00040

12.700 .962030 .00042

12.800 .965740 .00041

12.900 .971570 .00040

13.000 .976370 .00042

13.100 .981200 .00040

13.200 .984970 .00041

13.300 .989290 .00041

13.400 .993610 .00039

13.500 .998190 .00040

13.601 .003150 .00040

13.701 .007440 .00041

13.801 .010500 .00042

13.901 .015320 .00040

14.001 .020320 .00042
32

11.600 .918090 .00042

11.700 .924330 .00042

11.800 .929880 .00042

11.900 .934820 .00041

12.000 .939650 .00040

12.100 .944680 .00041

12.200 .950780 .00042

12.300 .955820 .00041

12.400 .959420 .00042

12.500 .965200 .00042

12.600 .969610 .00042

12.700 .974760 .00042

12.800 .979210 .00041

12.900 .985100 .00041

13.000 .988970 .00041

13.100 .994210 .00041

13.200 .998600 .00042

13.301 .002120 .00041

13.401 .007950 .00042

13.501 .011900 .00041

13.701 .020090 .00040
35

11.300 .919330 .00041

11.400 .924520 .00042

11.500 .930690 .00043

11.600 .935610 .00042

11.700 .940710 .00042

11.800 .946410 .00043

11.900 .952910 .00043

12.000 .956890 .00042

12.100 .961720 .00041

12.200 .966450 .00040

12.300 .972530 .00041

12.400 .977370 .00043

12.500 .981990 .00042

12.600 .985890 .00042

12.700 .992020 .00042

12.800 .996040 .00042

12.901 .001230 .00044

13.001 .006160 .00042

13.201 .015380 .00043

13.301 .020040 .00042
40

10.900 .917660 .00043

11.000 .924280 .00043

11.100 .929500 .00043

11.200 .934060 .00042

11.300 .940840 .00043

11.400 .946090 .00043

11.500 .951770 .00044

11.600 .957760 .00042

11.700 .964010 .00043

11.800 .968180 .00042

11.900 .972790 .00043

12.000 .978850 .00044

12.100 .983980 .00043

12.200 .988710 .00043

12.300 .993860 .00043

12.400 .999590 .00042

12.501 .005230 .00043

12.601 .009120 .00044

12.701 .014390 .00043

12.801 .019380 .00043
50

10.500 .923170 .00044

10.550 .926640 .00044

10.600 .929160 .00043

10.650 .933910 .00044

10.700 .936670 .00044

10.750 .937930 .00045

10.800 .942240 .00043

10.850 .944890 .00044

10.900 .948360 .00046

10.950 .951200 .00044

11.000 .953900 .00045

11.100 .959480 .00045

11.150 .962390 .00045

11.200 .964870 .00043

11.250 .967960 .00044

11.300 .971290 .00045

11.350 .974310 .00045

11.400 .977040 .00043

11.450 .980150 .00045

11.500 .981710 .00045 
11.550 .984570 .00045

11.600 .987620 .00045

11.650 .990380 .00044

11.700 .994260 .00044

11.750 .995700 .00045

11.800 .998420 .00044

11.851 .001330 .00045

11.901 .004330 .00045

11.951 .008000 .00045

12.001 .009840 .00044

12.051 .012060 .00044

12.101 .015890 .00045

12.151 .017390 .00044

12.201 .020440 .00045

60

10.100 .917580 .00044

10.150 .920580 .00045

10.200 .923500 .00046

10.250 .927450 .00043

10.300 .930510 .00046

10.350 .932880 .00045

10.400 .936680 .00045

10.450 .940640 .00044

10.500 .942280 .00046

10.550 .945150 .00046

10.600 .949620 .00045

10.650 .952230 .00045

10.700 .955150 .00044

10.750 .958280 .00045

10.800 .961280 .00045

10.850 .965100 .00045

10.900 .967760 .00046

10.950 .970490 .00044

11.000 .973710 .00047

11.050 .976150 .00044

11.100 .978390 .00047

11.150 .982800 .00045

11.200 .984130 .00046

11.250 .989330 .00045

11.300 .990690 .00046

11.350 .993950 .00045

11.400 .997000 .00045

11.450 .999560 .00045

11.501 .002370 .00044

11.601 .007020 .00046

11.651 .010110 .00045

11.701 .013400 .00045

11.751 .015840 .00045
70

9.900 .916460 .00045

9.950 .920050 .00044

10.000 .923940 .00046

10.050 .926850 .00047

10.100 .930070 .00045

10.150 .932480 .00046

10.200 .936320 .00047

10.250 .939610 .00046

10.300 .942370 .00045

10.350 .945260 .00048

10.400 .949310 .00046

10.450 .952360 .00046

10.500 .955460 .00046

10.550 .958890 .00045

10.600 .960640 .00047

10.650 .965030 .00047

10.700 .967830 .00047

10.750 .971550 .00046

10.800 .973770 .00046

10.850 .976890 .00046

10.900 .979660 .00046

10.950 .981580 .00045

11.000 .985090 .00046

11.050 .988540 .00047

11.100 .991720 .00046

11.150 .994410 .00046

11.200 .996990 .00046

11.250 .999800 .00045

11.301 .002770 .00045

11.351 .005640 .00047

11.401 .009330 .00046

11.451 .011400 .00045

11.501 .013630 .00045
80

9.800 .918910 .00045

9.850 .921740 .00047

9.900 .925290 .00046

9.950 .927970 .00046

10.000 .932340 .00046

10.050 .934970 .00047

10.100 .938540 .00046

10.150 .941820 .00046

10.200 .945170 .00045

10.250 .948630 .00047

10.300 .951300 .00048

10.350 .954320 .00045

10.400 .957860 .00047

10.450 .960650 .00047

10.500 .963910 .00044

10.550 .966260 .00047

10.600 .970890 .00046

10.650 .973570 .00047

10.700 .976280 .00047

10.750 .979950 .00045

10.800 .982120 .00047

10.850 .986390 .00047

10.900 .988220 .00046

10.950 .991660 .00046

11.000 .994130 .00046

11.050 .997190 .00047

11.100 .999460 .00047

11.151 .002460 .00047

11.201 .005400 .00046

11.251 .008310 .00047

11.301 .012630 .00047

11.351 .014970 .00046

11.401 .017060 .00046 
90

9.700 .918640 .00047

9.750 .920860 .00047

9.800 .924960 .00046

9.900 .931780 .00046

9.950 .935110 .00048

10.000 .938270 .00046

10.050 .941830 .00047

10.100 .944710 .00047

10.150 .947980 .00047

10.200 .950480 .00047

10.250 .954060 .00046

10.300 .957980 .00047

10.350 .961380 .00047

10.400 .962870 .00046

10.450 .967390 .00046

10.500 .969560 .00047

10.550 .973010 .00047

10.600 .976200 .00046

10.650 .979130 .00046

10.700 .982740 .00046

10.750 .985780 .00048

10.800 .987830 .00046

10.850 .990700 .00048

10.900 .994300 .00047

10.950 .995640 .00048

11.001 .000170 .00046

11.051 .002400 .00047

11.101 .005250 .00046

11.151 .008830 .00048

11.201 .010580 .00047

11.251 .014370 .00046

11.301 .017480 .00047
100

9.700 .922590 .00045

9.750 .926100 .00046

9.800 .928520 .00046

9.850 .931580 .00046

9.900 .935500 .00047

9.950 .939210 .00046

10.000 .941650 .00046

10.050 .945870 .00045

10.100 .949700 .00047

10.150 .951970 .00046

10.200 .955530 .00047

10.250 .958560 .00047

10.300 .961060 .00047

10.350 .964250 .00046

10.400 .967460 .00046

10.450 .970760 .00047

10.500 .974570 .00048

10.550 .977350 .00047

10.600 .979930 .00047

10.700 .985930 .00046

10.800 .991510 .00047

10.850 .994490 .00047

10.900 .996360 .00046

11.001 .003020 .00047

11.051 .006860 .00047

11.101 .009640 .00047

11.151 .012650 .00047

11.201 .014490 .00047

11.251 .017880 .00048

11.301 .021050 .00047
120

9.650 .923350 .00046

9.700 .926600 .00049

9.750 .929800 .00048

9.800 .932500 .00047

9.850 .936840 .00046

9.900 .939360 .00046

9.950 .943240 .00046

10.050 .949590 .00046

10.100 .953110 .00046

10.150 .955730 .00047

10.200 .958500 .00048

10.250 .962080 .00047

10.300 .966960 .00047

10.350 .968630 .00046

10.400 .971220 .00048

10.450 .974790 .00047

10.500 .978050 .00047

10.550 .981180 .00045

10.600 .984370 .00047

10.650 .987200 .00047

10.700 .990200 .00049

10.750 .993020 .00047

10.800 .996140 .00047

10.850 .998840 .00047

10.901 .001740 .00049

10.951 .004110 .00047

11.001 .007160 .00045

11.051 .010330 .00046

11.101 .013060 .00047

11.151 .015580 .00048

11.201 .019140 .00048
130

9.600 .922990 .00047

9.650 .925720 .00046

9.700 .928370 .00048

9.750 .932620 .00047

9.800 .935470 .00047

9.850 .937880 .00046

9.900 .941930 .00047

9.950 .945140 .00048

10.000 .949170 .00046
150

9.600 .922710 .00047

9.650 .926340 .00047

9.700 .929490 .00047

9.750 .932540 .00047

9.800 .935710 .00047

9.850 .938520 .00047

9.900 .943610 .00046

9.950 .945460 .00045

10.000 .949890 .00048
200

9.600 .920630 .00047

9.650 .925040 .00047

9.700 .927880 .00048

9.750 .931850 .00048

9.800 .934470 .00048

9.850 .936920 .00046

9.900 .939810 .00047

9.950 .942600 .00048

10.000 .946610 .00046 
10.050 .951160 .00047 10.100 .954650 .00048 10.150 .958000 .00048 10.200 .961680 .00047 10.250 .963440 .00046 10.300 .967330 .00046 10.350 .970410 .00047 10.400 .972840 .00046 10.450 .976710 .00047 10.500 .979950 .00047 10.550 .981680 .00046 10.600 .985440 .00047 10.650 .988690 .00048 10.700 .991400 .00047 10.750 .993100 .00047 10.800 .997240 .00048 10.850 .999900 .00047 10.901 .003280 .00047 10.951 .005850 .00046 11.001 .008480 .00047 11.051 .011470 .00048 11.101 .014020 .00046 11.151 .017490 .00047 11.201 .020100 .00047
10.050 .952410 .00048 10.100 .955430 .00047 10.150 .959000 .00047 10.200 .961510 .00047 10.250 .964850 .00047 10.300 .968100 .00047 10.350 .971110 .00047 10.400 .972720 .00047 10.450 .977480 .00048 10.500 .979670 .00046 10.550 .982780 .00049 10.600 .984610 .00048 10.650 .988320 .00047 10.700 .991270 .00047 10.750 .994450 .00049 10.800 .997110 .00047 10.851 .000890 .00047 10.901 .001790 .00047 10.951 .007040 .00048 11.001 .008690 .00047 11.051 .011270 .00047 11.101 .014730 .00047 11.151 .017170 .00047 11.201 .020700 .00048
10.050 .949650 .00048 10.100 .952720 .00048 10.150 .956000 .00047 10.200 .958680 .00046 10.250 .962600 .00048 10.300 .964320 .00047 10.350 .967940 .00046 10.400 .970720 .00047 10.450 .973740 .00047 10.500 .976940 .00047 10.550 .979680 .00048 10.600 .982740 .00047 10.650 .986040 .00047 10.700 .988880 .00048 10.750 .991490 .00048 10.800 .994170 .00046 10.850 .997130 .00046 10.900 .999930 .00048 10.951 .002800 .00049 11.051 .008180 .00047 11.101 .009870 .00047 11.151 .013780 .00047 11.201 .016500 .00049 11.251 .018230 .00046 11.301 .021720 .00048

239

9.700 .924620 .00047 9.750 .927820 .00045 9.800 .930340 .00047 9.850 .933840 .00047 9.900 .937100 .00047 9.950 .940700 .00047 10.000 .944500 .00049 10.050 .945700 .00049 10.100 .948940 .00048 10.150 .952990 .00048 10.200 .955780 .00047 10.250 .957700 .00047 10.300 .961320 .00047 10.350 .964810 .00047 10.400 .967410 .00046 10.450 .969060 .00047 10.500 .973440 .00048 10.550 .976330 .00047 10.600 .978510 .00047 10.650 .981870 .00046 10.700 .983330 .00047 10.750 .986990 .00047

\begin{tabular}{|c|c|}
\hline 250 & 359 \\
\hline 9.600 .917740 .00047 & 9.900 .923450 .00047 \\
\hline 9.700 .923760 .00047 & 10.000 .930420 .00047 \\
\hline 800.930020 .00048 & 10.100 .936760 .00047 \\
\hline .900 .935960 .00047 & 10.200 .942640 .00047 \\
\hline .942230 .00045 & 10.300 .947370 .00046 \\
\hline 0.947880 .00047 & 10.400 .953860 .00047 \\
\hline 00.953360 .00048 & 10.500 .958570 .00048 \\
\hline 0.958550 .00048 & 10.600 .964760 .00045 \\
\hline 10.400 .965040 .00048 & 10.700 .970850 .00047 \\
\hline 0.970990 .00047 & 10.800 .975590 .00048 \\
\hline 7430.00046 & 10.900 .981360 .00048 \\
\hline 0.982470 .00046 & 11.000 .986880 .00047 \\
\hline 10.800 .987170 .00047 & 11.100 .991620 .00046 \\
\hline 10.900 .995410 .00046 & 11.200 .996850 .00048 \\
\hline .001 .000460 .00048 & 11.301 .002290 .00048 \\
\hline 1.004310 .00047 & 11.401 .006940 .00046 \\
\hline 1.009570 .0004 & 11.501 .011990 .00046 \\
\hline 1.016300 .000 & 11.601 .016150 .00049 \\
\hline 0260.00048 & 2150.00048 \\
\hline
\end{tabular}


10.800 .990130 .00048 10.850 .992020 .00047 10.900 .995390 .00047 10.950 .997990 .00048 11.001 .001060 .00048 11.051 .003750 .00046 11.101 .005580 .00047 11.151 .009540 .00047 11.201 .011480 .00047 11.251 .014580 .00047 11.301 .016680 .00048

478

10.100 .921830 .00047 10.200 .928280 .00047 10.300 .934900 .00046 10.400 .939080 .00047 10.500 .945630 .00047 10.600 .950300 .00047 10.700 .955620 .00047 10.800 .960430 .00047 10.900 .966730 .00048 11.000 .971670 .00047 11.100 .977080 .00047 11.200 .981650 .00047 11.300 .987880 .00047 11.400 .992670 .00047 11.500 .998160 .00047 11.601 .002160 .00049 11.701 .007560 .00047 11.901 .016170 .00047

650

10.700 .921900 .00047 10.800 .927070 .00047 10.900 .931840 .00047 11.000 .937760 .00045 11.100 .944100 .00046 11.200 .948040 .00046 11.300 .953480 .00047 11.400 .957560 .00046 11.500 .962810 .00048 11.600 .968540 .00048 11.700 .973050 .00046 11.800 .976910 .00048
500

10.100 .918940 .00046

10.200 .925010 .00047

10.300 .930690 .00046

10.400 .936280 .00047

10.500 .941390 .00046

10.700 .952230 .00046

10.800 .958400 .00049

10.900 .964570 .00048

11.000 .969030 .00048

11.100 .974410 .00047

11.200 .978870 .00046

11.300 .983900 .00046

11.400 .989750 .00047

11.500 .993540 .00046

11.600 .999070 .00048

11.701 .003930 .00048

11.801 .009660 .00047

11.901 .013780 .00046

12.001 .018630 .00048

700

11.100 .921670 .00045

11.200 .927630 .00045

11.300 .932320 .00046

11.400 .937120 .00045

11.500 .943370 .00046

11.600 .947470 .00046

11.700 .952120 .00046

11.800 .957110 .00046

11.900 .962020 .00045

12.000 .966530 .00046

12.100 .970690 .00047

12.200 .975820 .00046
600

10.500 .924050 .00047

10.600 .929310 .00048

10.700 .935760 .00046

10.800 .940480 .00048

10.900 .945940 .00046

11.000 .950100 .00048

11.100 .955780 .00046

11.200 .960680 .00047

11.300 .965620 .00047

11.400 .971630 .00047

11.500 .976400 .00047

11.600 .980690 .00047

11.700 .986250 .00047

11.800 .990550 .00046

11.900 .995790 .00047

12.001 .000040 .00048

12.101 .005370 .00047

12.201 .009750 .00045

12.301 .014490 .00046

12.401 .018390 .00046

750

12.100 .922640 .00046

12.200 .926580 .00045

12.300 .932040 .00046

12.400 .936120 .00045

12.500 .941400 .00046

12.600 .944900 .00046

12.700 .950120 .00046

12.800 .953770 .00046

12.900 .957960 .00047

13.000 .963560 .00046

13.100 .966500 .00047

13.200 .972080 .00046 
11.900 .983430 .00045 12.000 .987670 .00046 12.100 .992100 .00048 12.200 .996810 .00046 12.301 .000980 .00048 12.401 .005380 .00045 12.501 .010780 .00047 12.601 .014870 .00047 12.701 .018650 .00048
12.300 .980030 .00047 12.400 .985170 .00046 12.500 .989560 .00048 12.600 .993410 .00046 12.700 .998460 .00048 12.801 .002080 .00047 12.901 .007390 .00047 13.001 .011320 .00046 13.101 .015890 .00046
752

12.200 .922010 .00045 12.300 .925550 .00046 12.400 .930280 .00045 12.500 .933880 .00045 12.600 .939540 .00045 12.700 .943540 .00047 12.800 .948240 .00047 12.900 .952660 .00048 13.000 .956780 .00046 13.100 .961590 .00047 13.200 .965930 .00047 13.300 .970510 .00046 13.400 .974860 .00045 13.500 .977760 .00047 13.600 .982600 .00046 13.700 .986390 .00045 13.800 .990220 .00047 13.900 .993700 .00046 14.000 .998370 .00047 14.201 .006990 .00047 14.301 .009720 .00045 14.401 .013980 .00046 14.501 .018260 .00046

Pun40-icyl-1000-00

12

15.700 .923330 .00032 15.900 .929340 .00032 16.100 .934710 .00032 16.300 .940230 .00030 16.500 .945710 .00032 16.700 .951460 .00031 16.900 .956160 .00031 17.100 .961920 .00030

13.300 .975710 .00047 13.400 .980750 .00045 13.500 .983870 .00045 13.600 .988020 .00046 13.700 .991690 .00047 13.800 .996200 .00045 13.901 .000380 .00045 14.001 .004200 .00046 14.101 .007960 .00047 14.201 .012560 .00046 14.301 .015960 .00047

754

12.400 .922670 .00046 12.500 .928240 .00046 12.600 .931190 .00045 12.700 .935780 .00046 12.800 .940150 .00045 12.900 .944670 .00045 13.000 .948960 .00046 13.100 .953200 .00045 13.200 .958080 .00046 13.300 .961900 .00046 13.400 .966110 .00046 13.500 .970240 .00046 13.600 .974770 .00045 13.700 .979120 .00045 13.800 .982250 .00047 13.900 .986280 .00047 14.000 .991640 .00047 14.100 .994200 .00045 14.200 .998820 .00046 14.301 .001510 .00045 14.401 .005900 .00046 14.501 .010280 .00046 14.601 .013460 .00046 14.701 .017060 .00045
755

12.600 .923740 .00044 12.700 .928170 .00046 12.800 .932740 .00046 12.900 .938070 .00047 13.000 .942000 .00045 13.100 .945720 .00045 13.200 .951050 .00047 13.300 .954400 .00045 13.400 .959540 .00046 13.500 .962700 .00046 13.600 .967480 .00046 13.700 .971530 .00046 13.800 .975500 .00045 13.900 .978990 .00045 14.000 .983130 .00046 14.100 .987360 .00046 14.200 .991590 .00046 14.300 .995180 .00046 14.400 .998210 .00047 14.501 .003210 .00046 14.701 .010020 .00045 14.801 .013940 .00046 14.901 .017960 .00045
15

13.400 .920210 .00035 13.500 .924830 .00036 13.600 .929500 .00036 13.700 .933210 .00035 13.800 .937410 .00036 13.900 .941450 .00036 14.000 .945640 .00034 14.100 .949090 .00035
20

11.600 .918170 .00040 11.700 .924400 .00040 11.800 .930570 .00040 11.900 .935380 .00040 12.000 .942260 .00040 12.100 .946800 .00040 12.200 .952760 .00040 12.300 .957540 .00040 
17.300 .966210 .00029 17.500 .971550 .00030 17.700 .976130 .00031 17.900 .980310 .00030 18.100 .984470 .00030 18.300 .989410 .00030 18.500 .993190 .00029 18.700 .997980 .00029 18.901 .001540 .00029 19.101 .005750 .00030 19.301 .010020 .00029 19.501 .012840 .00030
25

10.700 .918370 .00042 10.750 .921870 .00043 10.800 .924920 .00043 10.850 .928520 .00043 10.900 .931830 .00043 10.950 .935520 .00044 11.000 .938540 .00042 11.050 .941840 .00042 11.100 .945210 .00043 11.150 .949140 .00042 11.200 .951240 .00043 11.250 .954660 .00044 11.300 .957710 .00042 11.350 .961780 .00042 11.400 .964590 .00043 11.450 .966510 .00042 11.500 .969660 .00042 11.550 .973050 .00041 11.600 .975880 .00042 11.650 .978250 .00041 11.700 .982550 .00043 11.750 .984450 .00042 11.800 .988360 .00040 11.850 .991160 .00043 11.900 .994020 .00041 11.950 .996790 .00042 12.000 .999950 .00043
14.200 .952770 .00035 14.300 .956390 .00035 14.400 .960640 .00034 14.500 .963910 .00034 14.600 .968120 .00036 14.700 .971220 .00034 14.800 .975280 .00036 14.900 .978180 .00035 15.000 .981860 .00036 15.100 .985870 .00036 15.200 .988620 .00035 15.300 .992350 .00034 15.400 .995660 .00033 15.500 .997280 .00034 15.601 .000570 .00034 15.701 .004280 .00034 15.801 .008030 .00035 15.901 .011090 .00034 16.001 .014870 .00033 16.101 .016640 .00035
12.400 .962230 .00040 12.500 .967700 .00040 12.600 .972860 .00040 12.700 .977160 .00040 12.800 .983350 .00040 12.900 .987900 .00040 13.000 .992060 .00039 13.100 .996260 .00040 13.201 .001780 .00039 13.301 .006260 .00040 13.401 .011280 .00038 13.501 .014880 .00039 13.601 .019310 .00040
10.200 .922770 .00046 10.250 .926500 .00044 10.300 .930040 .00045 10.350 .933820 .00045 10.400 .938170 .00044 10.450 .941860 .00045 10.500 .944820 .00045 10.550 .947720 .00045 10.600 .951890 .00045 10.650 .955930 .00044 10.700 .958600 .00046 10.750 .962410 .00044 10.800 .965740 .00044 10.850 .969090 .00044 10.900 .972460 .00044 10.950 .976470 .00045 11.000 .979810 .00044 11.050 .982730 .00045 11.100 .986140 .00045 11.150 .989400 .00044 11.200 .992780 .00044 11.250 .995270 .00044 11.300 .998460 .00044 11.351 .002810 .00045 11.401 .005960 .00045 11.451 .008450 .00044 11.501 .012270 .00043
32

10.000 .919740 .00045 10.050 .923410 .00046 10.100 .928250 .00045 10.150 .931380 .00045 10.200 .934910 .00044 10.250 .938790 .00045 10.300 .942880 .00045 10.350 .946850 .00045 10.400 .950600 .00045 10.450 .954320 .00044 10.500 .958300 .00044 10.550 .960220 .00046 10.600 .963660 .00045 10.650 .967580 .00044 10.700 .971400 .00046 10.750 .974930 .00045 10.800 .978870 .00046 10.850 .982360 .00044 10.900 .985400 .00044 10.950 .988960 .00045 11.000 .993140 .00046 11.050 .995890 .00045 11.101 .000160 .00045 11.151 .002800 .00046 11.201 .005820 .00045 11.251 .009780 .00045 11.301 .012940 .00046 
12.051 .002980 .00042 12.101 .005470 .00043 12.151 .007210 .00042 12.201 .010980 .00043 12.251 .014610 .00043 12.301 .016340 .00043 12.351 .020410 .00042 12.401 .022440 .00041

35

9.800 .919520 .00046 9.850 .924800 .00046 9.900 .928200 .00047 9.950 .932450 .00046 10.000 .935810 .00046 10.050 .940260 .00047 10.100 .943840 .00048 10.150 .948300 .00045 10.200 .951790 .00046 10.250 .955390 .00047 10.300 .958770 .00047 10.350 .963500 .00048 10.400 .966820 .00047 10.450 .970940 .00046 10.500 .973860 .00045 10.550 .977700 .00045 10.600 .982540 .00046 10.650 .986010 .00046 10.700 .989140 .00045 10.750 .992500 .00046 10.800 .996340 .00045 10.851 .000050 .00045 10.901 .003690 .00047 10.951 .006940 .00046 11.001 .009690 .00046 11.051 .013240 .00047 11.101 .016910 .00046 11.151 .019110 .00046 11.201 .023490 .00046
11.551 .014940 .00044 11.601 .019050 .00045
11.351 .016450 .00046 11.401 .018410 .00045
40

9.500 .916880 .00046 9.550 .921680 .00046 9.600 .925970 .00048 9.650 .930480 .00047 9.700 .934860 .00047 9.750 .938310 .00047 9.800 .942860 .00048 9.850 .946640 .00047 9.900 .951040 .00047 9.950 .955370 .00047 10.000 .958330 .00047 10.050 .962580 .00047 10.100 .967130 .00046 10.150 .971290 .00047 10.200 .974840 .00046 10.250 .978300 .00048 10.300 .983300 .00047 10.350 .986370 .00046 10.400 .989730 .00046 10.450 .994230 .00047 10.500 .998240 .00046 10.551 .001430 .00047 10.601 .004550 .00046 10.651 .008170 .00047 10.701 .012330 .00048 10.751 .016610 .00047 10.801 .018860 .00047
50

9.100 .913130 .00049 9.150 .917070 .00048 9.200 .922360 .00049 9.250 .926690 .00049 9.300 .932330 .00047 9.350 .935880 .00049 9.400 .940200 .00048 9.450 .944410 .00048 9.500 .948680 .00049 9.550 .952850 .00047 9.600 .957160 .00048 9.650 .961760 .00047 9.700 .966420 .00049 9.750 .970580 .00049 9.800 .974680 .00049 9.850 .978910 .00049 9.900 .983100 .00049 9.950 .986410 .00048 10.000 .991760 .00049 10.050 .995550 .00049 10.100 .998850 .00047 10.151 .003550 .00047 10.201 .007190 .00049 10.251 .010610 .00049 10.301 .014890 .00049 10.351 .019060 .00049 10.401 .022870 .00048
60

8.900 .914060 .00049 8.950 .919950 .00050 9.000 .924030 .00050 9.050 .928470 .00049 9.100 .932740 .00050 9.150 .938510 .00049
70

8.800 .918330 .00049 8.850 .923560 .00049 8.900 .927950 .00049 8.950 .932300 .00050 9.000 .937400 .00049 9.050 .942580 .00049
80

8.700 .918220 .00050 8.750 .924250 .00050 8.800 .928730 .00052 8.850 .933270 .00052 8.900 .937640 .00051 8.950 .944120 .00051 
9.200 .943090 .00047

9.250 .947750 .00050

9.300 .952100 .00049

9.350 .956490 .00049

9.400 .961030 .00049

9.450 .965790 .00050

9.500 .970840 .00050

9.550 .974360 .00049

9.600 .978790 .00049

9.650 .982900 .00049

9.700 .988130 .00049

9.750 .990910 .00048

9.800 .995660 .00049

9.850 .999970 .00049

9.901 .004480 .00047

9.951 .008850 .00050

10.001 .012390 .00050

10.051 .016590 .00050

10.101 .020940 .00049
9.100 .947000 .00050

9.150 .951330 .00049

9.200 .956640 .00049

9.250 .961420 .00049

9.300 .965890 .00049

9.350 .970730 .00052

9.400 .974460 .00051

9.450 .979950 .00051

9.500 .984490 .00049

9.550 .988220 .00050

9.600 .992810 .00050

9.650 .996640 .00050

9.701 .002420 .00049

9.751 .005800 .00051

9.801 .010200 .00051

9.851 .014090 .00051

9.901 .018850 .00050

9.951 .023310 .00050

10.001 .026580 .00049
9.000 .948100 .00051

9.050 .953300 .00050

9.100 .958790 .00050

9.150 .962720 .00050

9.200 .966160 .00049

9.250 .972430 .00050

9.300 .976540 .00050

9.350 .981390 .00051

9.400 .984890 .00050

9.450 .988900 .00051

9.500 .994700 .00048

9.550 .998700 .00050

9.601 .002970 .00051

9.651 .007840 .00050

9.701 .012270 .00050

9.751 .016990 .00050

9.801 .021270 .00050
90

8.600 .916080 .00050

8.650 .920950 .00051

8.700 .926490 .00051

8.750 .932010 .00052

8.800 .937330 .00052

8.850 .941090 .00051

8.900 .946030 .00051

8.950 .951260 .00051

9.000 .954850 .00051

9.050 .960060 .00051

9.100 .966300 .00051

9.150 .969760 .00051

9.200 .974540 .00051

9.250 .979450 .00050

9.300 .983870 .00049

9.350 .988040 .00052

9.400 .993100 .00051

9.450 .997130 .00050

9.501 .001980 .00050

9.551 .006690 .00049

9.601 .010130 .00050

9.651 .015110 .00052

9.701 .019560 .00051

9.751 .025440 .00050

9.801 .028570 .00050
100

8.500 .911260 .00052

8.550 .916430 .00051

8.600 .921810 .00052

8.650 .927910 .00050

8.700 .932720 .00050

8.750 .936920 .00050

8.800 .942110 .00051

8.850 .946420 .00052

8.900 .952040 .00051

8.950 .957110 .00050

9.000 .961210 .00050

9.050 .966240 .00052

9.100 .971340 .00049

9.150 .975890 .00051

9.200 .980400 .00052

9.250 .985130 .00052

9.300 .989570 .00050

9.350 .994750 .00051

9.400 .998320 .00051

9.451 .004660 .00051

9.501 .007370 .00050

9.551 .012780 .00050

9.601 .016600 .00052

9.651 .022120 .00051

9.701 .025600 .00051
120

8.500 .918830 .00050

8.550 .924780 .00051

8.600 .929640 .00051

8.650 .933160 .00050

8.700 .938620 .00051

8.750 .944580 .00050

8.800 .949470 .00051

8.850 .954690 .00052

8.900 .957860 .00050

8.950 .965100 .00051

9.000 .968530 .00051

9.050 .974740 .00051

9.100 .977940 .00051

9.150 .982630 .00052

9.200 .988930 .00052

9.250 .993380 .00052

9.300 .997620 .00051

9.351 .002160 .00053

9.401 .005720 .00051

9.451 .010390 .00051

9.501 .015300 .00050

9.551 .019190 .00052

9.601 .023610 .00052 
130

8.400 .912160 .00051 8.450 .917910 .00051 8.500 .923240 .00051 8.550 .927500 .00053 8.600 .933090 .00050 8.650 .938040 .00051 8.700 .943470 .00052 8.750 .948710 .00051 8.800 .952780 .00052 8.850 .958690 .00051 8.900 .962520 .00051 8.950 .968480 .00052 9.000 .972850 .00052 9.050 .977140 .00052 9.100 .982630 .00050 9.150 .986540 .00051 9.200 .991240 .00052 9.250 .996390 .00050 9.301 .000560 .00051 9.351 .005110 .00051 9.401 .009350 .00052 9.451 .014990 .00051 9.501 .019830 .00052 9.551 .023760 .00050 9.601 .028970 .00051
150

8.400 .915610 .00050

8.450 .920580 .00050

8.500 .926180 .00052

8.550 .931330 .00052

8.600 .936590 .00052

8.650 .941420 .00049

8.700 .946230 .00052

8.750 .950980 .00052

8.800 .956230 .00052

8.850 .960500 .00052

8.900 .966800 .00050

8.950 .971180 .00053

9.000 .975790 .00052

9.050 .980730 .00051

9.100 .984790 .00051

9.150 .989620 .00051

9.200 .994860 .00052

9.250 .999170 .00051

9.301 .004090 .00051

9.351 .008810 .00051

9.401 .013050 .00051

9.451 .017600 .00052

9.501 .022740 .00052
200

8.400 .920800 .00050

8.450 .925450 .00051

8.500 .930520 .00054

8.550 .935640 .00051

8.600 .940590 .00051

8.650 .946080 .00053

8.700 .951090 .00052

8.750 .956490 .00051

8.800 .960750 .00051

8.850 .965010 .00050

8.900 .970640 .00050

8.950 .974940 .00052

9.000 .980500 .00050

9.050 .985090 .00052

9.100 .989760 .00052

9.150 .994000 .00051

9.200 .999390 .00050

9.251 .003870 .00052

9.301 .008490 .00050

9.351 .013050 .00051

9.401 .015980 .00052

9.451 .021190 .00051

9.501 .025290 .00052
239

8.400 .921310 .00050

8.450 .926150 .00052

8.500 .931180 .00051

8.550 .937660 .00052

8.600 .941550 .00052

8.650 .946720 .00051

8.700 .952290 .00051

8.750 .956480 .00051

8.800 .960840 .00052

8.850 .965260 .00052

8.900 .971190 .00051

8.950 .975330 .00050

9.000 .980480 .00050

9.050 .985880 .00050

9.100 .989950 .00051

9.150 .995000 .00051

9.201 .000260 .00051

9.251 .004370 .00051

9.301 .008840 .00053

9.351 .012690 .00052

9.401 .017330 .00049
250

8.400 .920720 .00052

8.450 .926180 .00050

8.500 .930490 .00051

8.550 .935870 .00050

8.600 .942000 .00052

8.650 .946210 .00050

8.700 .950640 .00052

8.750 .956560 .00052

8.800 .961000 .00051

8.850 .965840 .00051

8.900 .970860 .00052

8.950 .975100 .00051

9.000 .981030 .00052

9.050 .985270 .00050

9.100 .991140 .00052

9.150 .995080 .00050

9.200 .998850 .00051

9.251 .004310 .00051

9.301 .008350 .00052

9.351 .013800 .00052

9.401 .018130 .00051
359

8.400 .916670 .00050

8.450 .922360 .00051

8.500 .927420 .00050

8.550 .932300 .00052

8.600 .936730 .00052

8.650 .941700 .00050

8.700 .947320 .00052

8.750 .952090 .00051

8.800 .956940 .00051

8.850 .961860 .00051

8.900 .966240 .00052

8.950 .971720 .00050

9.000 .977030 .00051

9.050 .980950 .00052

9.100 .986120 .00051

9.150 .989830 .00051

9.200 .995330 .00050

9.251 .000460 .00051

9.301 .004460 .00052

9.351 .008750 .00052

9.401 .013330 .00051 
9.451 .021720 .00053

9.501 .026470 .00050
9.451 .021170 .00052

9.501 .026860 .00051
9.451 .017970 .00052

9.501 .022260 .00050
478

8.500 .916860 .00052

8.550 .922120 .00050

8.600 .926400 .00051

8.650 .932260 .00051

8.700 .935560 .00050

8.750 .941290 .00051

8.800 .946730 .00050

8.850 .950610 .00051

8.900 .956420 .00050

8.950 .960890 .00051

9.000 .966290 .00050

9.050 .970930 .00051

9.100 .975620 .00051

9.150 .980010 .00052

9.200 .984810 .00052

9.250 .989300 .00052

9.300 .994740 .00052

9.350 .998920 .00051

9.401 .002090 .00052

9.451 .006880 .00051

9.501 .013230 .00052

9.551 .017540 .00049

9.601 .021090 .00053

650

9.000 .919310 .00049

9.050 .923330 .00050

9.100 .928970 .00050

9.150 .934230 .00049

9.200 .938180 .00050

9.250 .943660 .00051

9.300 .948220 .00051

9.350 .952770 .00052

9.400 .957430 .00051

9.450 .961200 .00050

9.500 .966050 .00050

9.550 .970390 .00050

9.600 .973590 .00052

9.650 .979520 .00051

9.700 .983710 .00049

9.750 .988580 .00050
500

8.500 .913930 .00051

8.550 .918010 .00050

8.600 .923150 .00051

8.650 .928960 .00051

8.700 .933930 .00051

8.750 .939040 .00051

8.800 .943500 .00051

8.850 .948730 .00052

8.900 .953060 .00051

8.950 .958360 .00051

9.000 .962690 .00051

9.050 .967940 .00050

9.100 .972450 .00050

9.150 .977720 .00049

9.200 .982930 .00050

9.250 .986760 .00053

9.300 .989850 .00051

9.350 .995250 .00049

9.401 .000130 .00050

9.451 .005440 .00051

9.501 .009250 .00052

9.551 .013250 .00052

9.601 .018140 .00052

9.651 .021930 .00051

9.701 .026660 .00052

700

9.300 .912900 .00050

9.350 .918830 .00048

9.400 .922220 .00050

9.450 .926980 .00050

9.500 .931740 .00050

9.550 .936400 .00049

9.600 .940760 .00049

9.650 .946670 .00049

9.700 .949810 .00050

9.750 .954230 .00050

9.800 .958830 .00050

9.850 .962540 .00049

9.900 .967050 .00051

9.950 .971560 .00051

10.000 .976710 .00051

10.050 .980250 .00051
600

8.800 .919590 .00052

8.850 .924640 .00050

8.900 .929290 .00050

8.950 .935200 .00050

9.000 .939510 .00052

9.050 .944340 .00049

9.100 .948330 .00051

9.150 .954010 .00050

9.200 .958470 .00051

9.250 .962890 .00050

9.300 .966450 .00051

9.350 .972040 .00050

9.400 .976680 .00049

9.450 .981590 .00051

9.500 .986190 .00051

9.550 .989670 .00052

9.600 .994680 .00050

9.650 .999350 .00051

9.701 .004200 .00052

9.751 .007430 .00051

9.801 .011750 .00051

9.851 .017520 .00049

9.901 .020790 .00050

9.951 .024990 .00051

10.001 .029330 .00050

\section{0}

10.300 .916110 .00051

10.350 .919670 .00049

10.400 .924150 .00051

10.450 .928580 .00049

10.500 .932440 .00050

10.550 .936820 .00050

10.600 .940990 .00051

10.650 .944590 .00050

10.700 .948470 .00049

10.750 .952200 .00049

10.800 .957640 .00050

10.850 .961660 .00051

10.900 .965280 .00050

10.950 .969200 .00050

11.000 .973450 .00051

11.050 .978110 .00051 
9.800 .993670 .00051

9.850 .996960 .00051

9.901 .001000 .00050

9.951 .006470 .00050 10.001 .010770 .00050 10.051 .013810 .00051 10.101 .018760 .00050 10.151 .022960 .00052 10.201 .025920 .00051

752

10.400 .913700 .00051 10.450 .917480 .00050 10.500 .922020 .00049 10.550 .926000 .00049 10.600 .930380 .00050 10.650 .934180 .00050 10.700 .938910 .00051 10.750 .942370 .00051 10.800 .947670 .00051 10.850 .950130 .00050 10.900 .954470 .00050 10.950 .958610 .00050 11.000 .963230 .00051 11.050 .966990 .00052 11.100 .971550 .00051 11.150 .974140 .00051 11.200 .978170 .00051 11.250 .983150 .00050 11.300 .987690 .00050 11.350 .989580 .00051 11.400 .994000 .00051 11.450 .997650 .00050 11.501 .001480 .00050 11.551 .004490 .00051 11.601 .009340 .00052 11.651 .013140 .00051 11.701 .016140 .00050 11.751 .020820 .00049 11.801 .024040 .00051
10.100 .984800 .00052

10.150 .989220 .00050

10.200 .993040 .00051

10.250 .997450 .00051

10.301 .001880 .00050

10.351 .006100 .00050 10.401 .010170 .00051 10.451 .014830 .00052 10.501 .017870 .00050 10.551 .022500 .00051 10.601 .025400 .00052

754

10.600 .916220 .00049 10.650 .920110 .00049 10.700 .925100 .00051 10.750 .928610 .00051 10.800 .934020 .00051 10.850 .936340 .00050 10.900 .941180 .00050 10.950 .945120 .00049 11.000 .947650 .00050 11.050 .951930 .00050 11.100 .956550 .00050 11.150 .959560 .00050 11.200 .964670 .00052 11.250 .969580 .00050 11.300 .971780 .00050 11.350 .976490 .00051 11.400 .979980 .00050 11.450 .983560 .00049 11.500 .987530 .00050 11.550 .991540 .00050 11.600 .994540 .00049 11.650 .998900 .00050 11.701 .002410 .00051 11.751 .006460 .00049 11.801 .010060 .00050 11.851 .013860 .00050 11.901 .017380 .00050 11.951 .020860 .00050 12.001 .024240 .00050
11.100 .980660 .00051

11.150 .985090 .00050

11.200 .988840 .00049

11.250 .992890 .00050 11.300 .996190 .00051 11.351 .000180 .00051 11.401 .004740 .00050 11.451 .008320 .00051 11.501 .011280 .00049 11.551 .015740 .00049 11.601 .019740 .00050 11.651 .022230 .00050 11.701 .027290 .00050

755

10.800 .916450 .00049 10.850 .920560 .00051 10.900 .924810 .00050 10.950 .928390 .00049 11.000 .933700 .00050 11.050 .937050 .00050 11.100 .940690 .00050 11.150 .944840 .00049 11.200 .948770 .00050 11.250 .952170 .00050 11.300 .956280 .00049 11.350 .959750 .00051 11.400 .963540 .00050 11.450 .968220 .00050 11.500 .971790 .00051 11.550 .975080 .00049 11.600 .979780 .00051 11.650 .982100 .00050 11.700 .987040 .00050 11.750 .990560 .00049 11.800 .993680 .00049 11.850 .997870 .00050 11.901 .002380 .00050 11.951 .004850 .00051 12.001 .008560 .00048 12.051 .012930 .00050 12.101 .016340 .00048 12.151 .019170 .00049 12.201 .023720 .00051 
Pun40-icyl-1000-01

12

14.200 .924200 .00031 14.400 .930050 .00031 14.600 .935120 .00030 14.800 .940550 .00030 15.000 .946620 .00029 15.200 .951600 .00030 15.400 .956940 .00031 15.600 .962050 .00030 15.800 .966500 .00030 16.000 .971800 .00029 16.200 .976480 .00029 16.400 .980940 .00030 16.600 .984870 .00029 16.800 .989470 .00030 17.000 .994130 .00029 17.200 .998100 .00029 17.401 .001890 .00029 17.601 .005680 .00028 17.801 .009750 .00028 18.001 .013730 .00028 18.101 .015200 .00028

25

9.200 .919490 .00040

9.250 .923160 .00041

9.300 .926420 .00042

9.350 .929010 .00042

9.400 .932840 .00041

9.450 .935980 .00041

9.500 .939770 .00040

9.550 .943230 .00041

9.600 .946140 .00041

9.650 .949740 .00041

9.700 .952410 .00041

9.750 .955420 .00041

9.800 .958860 .00042

9.850 .961880 .00041

9.900 .965450 .00042 9.950 .968910 .00040 10.000 .971000 .00041
15

11.900 .920880 .00034 12.000 .925140 .00034

12.100 .929260 .00035

12.200 .933970 .00034

12.300 .937960 .00034

12.400 .942150 .00034

12.500 .945230 .00035

12.600 .949340 .00033

12.700 .953520 .00034

12.800 .957010 .00034

12.900 .960870 .00034

13.000 .964720 .00034

13.100 .968600 .00033

13.200 .971110 .00035

13.300 .975080 .00034

13.400 .979110 .00033

13.500 .981900 .00033

13.600 .985670 .00034

13.700 .988950 .00033

13.800 .992690 .00033

13.900 .996430 .00033

14.000 .998440 .00033

14.101 .002090 .00033

14.201 .005650 .00032

14.301 .008330 .00033

14.401 .011810 .00033

14.501 .014780 .00033

30

8.700 .923550 .00042

8.750 .928200 .00043

8.800 .930720 .00043

8.850 .935410 .00043

8.900 .938000 .00043

8.950 .942670 .00044

9.000 .946100 .00043

9.050 .949190 .00043

9.100 .952700 .00042

9.150 .956720 .00043

9.200 .959510 .00043

9.250 .963040 .00042

9.300 .967690 .00043

9.350 .971090 .00042

9.400 .974720 .00044

9.450 .977140 .00044

9.500 .980150 .00043
20

10.100 .918710 .00038

10.200 .924820 .00038

10.300 .930540 .00038

10.400 .937050 .00038

10.500 .941860 .00039

10.600 .947330 .00038

10.700 .952710 .00038

10.800 .958750 .00038

10.900 .963000 .00038

11.000 .968780 .00040

11.100 .973890 .00038

11.200 .978250 .00038

11.300 .983260 .00039

11.400 .988360 .00037

11.500 .993680 .00038

11.600 .997840 .00039

11.701 .003040 .00036

11.801 .007130 .00038

11.901 .012110 .00038

12.001 .016320 .00038
8.500 .920900 .00044

8.550 .924960 .00043

8.600 .929420 .00042

8.650 .932720 .00044

8.700 .936710 .00044

8.750 .940130 .00043

8.800 .944160 .00044

8.850 .947180 .00043

8.900 .952480 .00043

8.950 .954990 .00045

9.000 .958420 .00043

9.050 .962450 .00042

9.100 .966020 .00045

9.150 .969570 .00044

9.200 .973960 .00042

9.250 .976550 .00044

9.300 .980710 .00042 
10.050 .974080 .00041

10.100 .977950 .00042

10.150 .980680 .00041

10.200 .983020 .00042

10.250 .986120 .00041

10.300 .989520 .00041

10.350 .992100 .00041

10.400 .995610 .00042

10.450 .997610 .00040

10.501 .001070 .00041

10.551 .004200 .00042

10.601 .006160 .00041

10.651 .009440 .00040

10.701 .011850 .00041

10.751 .014440 .00042

10.801 .018060 .00042
9.550 .983940 .00043

9.600 .986710 .00042

9.650 .990920 .00043

9.700 .994850 .00043

9.750 .997460 .00043

9.800 .999820 .00043

9.851 .003870 .00043

9.901 .007070 .00041

9.951 .010810 .00043

10.001 .013520 .00043

10.051 .017220 .00042

10.101 .019290 .00043
9.350 .983380 .00044

9.400 .987290 .00043

9.450 .990040 .00042

9.500 .994130 .00043

9.550 .997650 .00044

9.601 .000790 .00044

9.651 .004250 .00043

9.701 .007660 .00043

9.751 .010440 .00042

9.801 .014740 .00043

9.851 .017740 .00041

9.901 .020200 .00044
35

8.300 .921650 .00045

8.350 .925020 .00045

8.400 .930290 .00044

8.450 .933680 .00044

8.500 .937290 .00044

8.550 .941750 .00044

8.600 .944960 .00045

8.650 .949880 .00043

8.700 .952780 .00044

8.750 .956720 .00045

8.800 .961030 .00045

8.850 .965080 .00044

8.900 .968440 .00045

8.950 .972760 .00043

9.000 .976630 .00044

9.050 .979260 .00045

9.100 .983160 .00044

9.150 .986890 .00045

9.200 .990480 .00044

9.250 .994010 .00044

9.300 .997570 .00045

9.351 .001390 .00044

9.401 .005260 .00045

9.451 .008670 .00044

9.501 .011390 .00044

9.551 .015020 .00044

9.601 .017620 .00044
40

8.000 .918930 .00043

8.050 .923320 .00046

8.100 .927600 .00044

8.150 .931560 .00046

8.200 .936230 .00045

8.250 .940090 .00045

8.300 .945230 .00045

8.350 .949070 .00045

8.400 .953330 .00047

8.450 .956850 .00046

8.500 .960310 .00045

8.550 .964940 .00045

8.600 .968450 .00045

8.650 .972010 .00045

8.700 .976880 .00046

8.750 .979340 .00045

8.800 .984660 .00045

8.850 .987540 .00046

8.900 .991980 .00046

8.950 .995970 .00045

9.000 .999640 .00045

9.051 .003270 .00045

9.101 .007090 .00045

9.151 .010850 .00046

9.201 .013950 .00046

9.251 .019100 .00046

9.301 .021610 .00044
50

7.600 .914470 .00045

7.650 .919480 .00046

7.700 .923670 .00046

7.750 .929290 .00046

7.800 .933460 .00047

7.850 .937680 .00046

7.900 .942060 .00046

7.950 .946090 .00046

8.000 .951970 .00047

8.050 .955240 .00046

8.100 .960360 .00047

8.150 .964530 .00047

8.200 .968530 .00046

8.250 .972630 .00046

8.300 .977380 .00045

8.350 .982330 .00045

8.400 .984960 .00045

8.450 .989190 .00046

8.500 .993420 .00047

8.550 .997840 .00047

8.601 .002340 .00047

8.651 .005840 .00046

8.701 .010070 .00046

8.751 .014060 .00047

8.801 .017470 .00048

8.851 .021750 .00045

8.901 .024410 .00046 
60

7.400 .917110 .00047

7.450 .922130 .00048

7.500 .926540 .00048

7.550 .932310 .00048

7.600 .936030 .00047

7.650 .940670 .00048

7.700 .945600 .00049

7.750 .951060 .00048

7.800 .954650 .00048

7.850 .959670 .00047

7.900 .963920 .00047

7.950 .968050 .00045

8.000 .972390 .00046

8.050 .977520 .00047

8.100 .981600 .00048

8.150 .985640 .00047

8.200 .990480 .00049

8.250 .993510 .00047

8.300 .998700 .00049

8.351 .002990 .00048

8.401 .006210 .00047

8.451 .011340 .00047

8.501 .015380 .00047

8.551 .019770 .00047

8.601 .023950 .00049

90

7.100 .920170 .00050

7.150 .925470 .00049

7.200 .930150 .00049

7.250 .936230 .00048

7.300 .939370 .00047

7.350 .944620 .00048

7.400 .950270 .00048

7.450 .954390 .00048

7.500 .959800 .00048

7.550 .964260 .00049

7.600 .969170 .00050

7.650 .974020 .00048

7.700 .979120 .00049

7.750 .982430 .00049

7.800 .987400 .00049

7.850 .993280 .00049

7.900 .997160 .00049

7.951 .001660 .00050

8.001 .006410 .00049

8.051 .010340 .00049

8.101 .014380 .00050

8.151 .019140 .00049

8.201 .023560 .00048
70

7.300 .921740 .00047

7.350 .925930 .00050

7.400 .931380 .00046

7.450 .935780 .00047

7.500 .940600 .00047

7.550 .945290 .00048

7.600 .950800 .00048

7.650 .954050 .00047

7.700 .959650 .00048

7.750 .965110 .00049

7.800 .968950 .00049

7.850 .973650 .00048

7.900 .978460 .00048

7.950 .982420 .00049

8.000 .986750 .00048

8.050 .991260 .00048

8.100 .996190 .00048

8.150 .999770 .00049

8.201 .005380 .00048

8.251 .009490 .00048

8.301 .012390 .00047

8.351 .017030 .00048

8.401 .022420 .00046
80

7.200 .922110 .00048

7.250 .926830 .00048

7.300 .932260 .00049

7.350 .936710 .00048

7.400 .941230 .00048

7.450 .946610 .00048

7.500 .951170 .00049

7.550 .956450 .00048

7.600 .961220 .00048

7.650 .965840 .00048

7.700 .970420 .00048

7.750 .975490 .00049

7.800 .979280 .00049

7.850 .984230 .00049

7.900 .988300 .00047

7.950 .993480 .00048

8.000 .997930 .00048

8.051 .003020 .00048

8.101 .007240 .00049

8.151 .011210 .00047

8.201 .015810 .00048

8.251 .020200 .00048

8.301 .023980 .00049
100

7.000 .915260 .00049

7.050 .921000 .00049

7.100 .925480 .00049

7.150 .931690 .00048

7.200 .935800 .00048

7.250 .940940 .00050

7.300 .946270 .00050

7.350 .950700 .00047

7.400 .955330 .00050

7.450 .960780 .00048

7.500 .964800 .00049

7.550 .971180 .00049

7.600 .975120 .00049

7.650 .979730 .00048

7.700 .984040 .00051

7.750 .989120 .00051

7.800 .994780 .00049

7.850 .998570 .00049

7.901 .002220 .00049

7.951 .006000 .00048

8.001 .012090 .00049

8.051 .016580 .00049

8.101 .021040 .00050
120

7.000 .923380 .00049

7.050 .927990 .00050

7.100 .934030 .00050

7.150 .938850 .00049

7.200 .943500 .00049

7.250 .948600 .00049

7.300 .953060 .00050

7.350 .958440 .00048

7.400 .963320 .00049

7.450 .968050 .00050

7.500 .972860 .00050

7.550 .978040 .00050

7.600 .983270 .00048

7.650 .988210 .00049

7.700 .992350 .00050

7.750 .996980 .00049

7.801 .002270 .00050

7.851 .006730 .00048

7.901 .011210 .00048

7.951 .015720 .00051

8.001 .019810 .00049 
130

6.900 .916340 .00049

6.950 .922250 .00049

7.000 .926060 .00048

7.050 .932760 .00049

7.100 .936160 .00049

7.150 .941570 .00050

7.200 .947030 .00049

7.250 .952340 .00049

7.300 .956600 .00050

7.350 .962300 .00049

7.400 .967390 .00050

7.450 .971120 .00049

7.500 .977050 .00052

7.550 .981740 .00049

7.600 .985950 .00050

7.650 .990230 .00049

7.700 .995810 .00050

7.751 .000230 .00050

7.801 .004690 .00048

7.851 .009520 .00050

7.901 .015040 .00048

7.951 .018690 .00050

8.001 .022860 .00048
150

6.900 .920230 .00048

6.950 .926170 .00049

7.000 .931300 .00049

7.050 .936890 .00050

7.100 .940840 .00051

7.150 .946200 .00048

7.200 .951410 .00049

7.250 .956390 .00050

7.300 .960770 .00050

7.350 .965640 .00049

7.400 .970420 .00051

7.450 .975370 .00049

7.500 .980450 .00049

7.550 .985920 .00048

7.600 .989390 .00050

7.650 .993850 .00051

7.700 .999460 .00051

7.751 .003040 .00049

7.801 .008220 .00050

7.851 .013590 .00050

7.901 .016270 .00049

7.951 .021240 .00050

8.001 .026810 .00049
200

6.800 .915190 .00049

6.850 .919410 .00050

6.900 .924550 .00048

6.950 .929880 .00051

7.000 .935790 .00049

7.050 .940190 .00049

7.100 .945510 .00050

7.150 .950570 .00050

7.200 .955670 .00049

7.250 .960310 .00050

7.300 .965800 .00049

7.350 .970180 .00050

7.400 .975050 .00051

7.450 .979400 .00049

7.500 .983720 .00050

7.550 .990230 .00050

7.600 .993250 .00051

7.650 .999630 .00049

7.701 .002840 .00049

7.751 .007550 .00050

7.801 .013540 .00050

7.851 .016890 .00050

7.901 .021120 .00051
239

6.800 .914710 .00049

6.850 .920080 .00049

6.900 .925860 .00046

6.950 .930260 .00050

7.000 .936380 .00048

7.050 .940650 .00049

7.100 .945540 .00051

7.150 .951260 .00049

7.200 .955760 .00050

7.250 .961500 .00050

7.300 .965470 .00049

7.350 .970470 .00050

7.400 .976300 .00049

7.450 .980550 .00049

7.500 .986230 .00050

7.550 .989880 .00049

7.600 .994950 .00050

7.650 .999440 .00050

7.701 .004980 .00049

7.751 .008510 .00051

7.801 .014030 .00050

7.851 .017600 .00050

7.901 .022960 .00051
250

6.800 .915060 .00049

6.850 .920150 .00049

6.900 .924920 .00051

6.950 .930660 .00049

7.000 .935460 .00048

7.050 .941170 .00050

7.100 .945490 .00050

7.150 .949750 .00050

7.200 .956300 .00049

7.250 .960590 .00049

7.300 .965870 .00050

7.350 .969890 .00048

7.400 .974080 .00051

7.450 .980500 .00051

7.500 .984870 .00048

7.550 .988860 .00050

7.600 .993770 .00048

7.650 .998730 .00049

7.701 .003180 .00050

7.751 .008570 .00049

7.801 .012950 .00049

7.851 .017340 .00050

7.901 .021830 .00051
359

6.900 .921980 .00050

6.950 .926890 .00050

7.000 .932040 .00051

7.050 .937020 .00048

7.100 .942120 .00049

7.150 .945750 .00050

7.200 .951920 .00049

7.250 .956040 .00050

7.300 .960470 .00050

7.350 .965920 .00049

7.400 .971920 .00051

7.450 .975910 .00050

7.500 .980350 .00048

7.550 .985700 .00051

7.600 .989480 .00050

7.650 .994610 .00049

7.700 .999490 .00050

7.751 .004060 .00051

7.801 .008160 .00050

7.851 .012750 .00050

7.901 .017180 .00051

7.951 .022110 .00051

8.001 .026360 .00050 
478

7.000 .921440 .00049

7.050 .927100 .00050

7.100 .931860 .00050

7.150 .936590 .00049

7.200 .941490 .00050

7.250 .945930 .00050

7.300 .950690 .00050

7.350 .957200 .00050

7.400 .961410 .00050

7.450 .966110 .00050

7.500 .970140 .00051

7.550 .975810 .00051

7.600 .981010 .00050

7.650 .983470 .00049

7.700 .988780 .00051

7.750 .993850 .00049

7.800 .998350 .00049

7.851 .002820 .00048

7.901 .008080 .00051

7.951 .012020 .00051

8.001 .016420 .00050

8.051 .020450 .00050

8.101 .025490 .00050

650

7.400 .919740 .00049

7.450 .925410 .00049

7.500 .930380 .00050

7.550 .934890 .00050

7.600 .940130 .00050

7.650 .944550 .00051

7.700 .948770 .00050

7.750 .953720 .00049

7.800 .958230 .00048

7.850 .963070 .00049

7.900 .968070 .00050

7.950 .972480 .00049 8.000 .975650 .00051

8.050 .981100 .00050

8.100 .985040 .00048

8.150 .989580 .00050

8.200 .994340 .00050

8.250 .998880 .00050

8.301 .003460 .00049

8.351 .006540 .00050

8.401 .011660 .00052
500

7.000 .918310 .00050

7.050 .923840 .00050

7.100 .928640 .00050

7.150 .933470 .00047

7.200 .938890 .00049

7.250 .944360 .00050

7.300 .948900 .00051

7.350 .953850 .00051

7.400 .957970 .00050

7.450 .962990 .00050

7.500 .967800 .00049

7.550 .971860 .00049

7.600 .977070 .00050

7.650 .982660 .00050

7.700 .986220 .00049

7.750 .990270 .00050

7.800 .995310 .00049

7.850 .999920 .00049

7.901 .004690 .00050

7.951 .009610 .00050

8.001 .014120 .00050

8.051 .018200 .00049

8.101 .022100 .00050
600

7.200 .918130 .00051

7.250 .923510 .00051

7.300 .927880 .00049

7.400 .938090 .00049

7.450 .941950 .00050

7.500 .947810 .00050

7.550 .952270 .00050

7.600 .956330 .00049

7.650 .960630 .00050

7.700 .966470 .00051

7.750 .970720 .00049

7.800 .974940 .00051

7.850 .979760 .00048

7.900 .983860 .00050

7.950 .988430 .00049

8.000 .992900 .00050

8.050 .998000 .00049

8.101 .001480 .00048

8.151 .005920 .00050

8.201 .010550 .00049

8.251 .015460 .00050

8.301 .019900 .00050
7.700 .919750 .00049

7.750 .925190 .00049

7.800 .929890 .00049

7.850 .934330 .00050

7.900 .939130 .00050

7.950 .944170 .00050

8.000 .948200 .00049

8.050 .951970 .00049

8.100 .956340 .00050

8.150 .961270 .00049

8.200 .965170 .00050

8.250 .969390 .00050

8.300 .974750 .00049

8.350 .979470 .00051

8.400 .982410 .00050

8.450 .986080 .00050

8.500 .991540 .00051

8.550 .995040 .00050

8.600 .999300 .00050

8.651 .002590 .00050

8.701 .007390 .00049
750

8.500 .920890 .00050

8.550 .924520 .00050

8.600 .929020 .00049

8.650 .932580 .00048

8.700 .936930 .00050

8.750 .941370 .00049

8.800 .945820 .00052

8.850 .950350 .00049

8.900 .954030 .00050

8.950 .958310 .00050

9.000 .961830 .00051

9.050 .965690 .00050

9.100 .969580 .00050

9.150 .972770 .00050

9.200 .977120 .00049

9.250 .981530 .00050

9.300 .985900 .00050

9.350 .990820 .00052

9.400 .992860 .00049

9.450 .996860 .00049

9.501 .000620 .00049 
8.451 .015900 .00051

8.501 .020260 .00051

8.551 .023930 .00051

8.601 .027890 .00050
8.751 .011910 .00051

8.801 .016140 .00050

8.851 .019310 .00051

8.901 .025040 .00049
752

8.600 .920440 .00048 8.650 .924290 .00051

8.700 .928610 .00050 8.750 .933250 .00051 8.800 .937690 .00049 8.850 .941440 .00050 8.900 .945170 .00048 8.950 .949630 .00049 9.000 .953400 .00049 9.050 .956510 .00050 9.100 .961610 .00049 9.150 .964650 .00049 9.200 .968730 .00050 9.250 .973350 .00050 9.300 .977260 .00049 9.350 .980390 .00050 9.400 .985930 .00050 9.450 .988280 .00051 9.500 .992180 .00050 9.550 .996270 .00049 9.600 .999570 .00050 9.651 .002820 .00050 9.701 .007590 .00050 9.751 .010700 .00050 9.801 .014010 .00050 9.851 .017830 .00050 9.901 .021540 .00048
754

8.700 .917740 .00049 8.750 .921360 .00048 8.800 .926560 .00049 8.850 .929230 .00051 8.900 .933890 .00050 8.950 .938120 .00048 9.000 .942100 .00049 9.050 .946110 .00051 9.100 .950390 .00049 9.150 .953470 .00050 9.200 .957540 .00049 9.250 .961470 .00050 9.300 .964430 .00050 9.350 .969260 .00048 9.400 .972240 .00050 9.450 .977200 .00049 9.500 .980640 .00050 9.550 .984310 .00051 9.600 .987520 .00050 9.650 .992330 .00048 9.700 .996020 .00050 9.750 .999020 .00051 9.801 .002630 .00050 9.851 .006930 .00050 9.901 .010070 .00049 9.951 .013820 .00050 10.001 .017160 .00051 10.051 .020560 .00050 10.101 .024430 .00048
9.551 .004460 .00051 9.601 .007760 .00049 9.651 .011040 .00050 9.701 .014380 .00049 9.751 .018290 .00050 9.801 .021940 .00049

755

8.900 .921580 .00049 8.950 .924940 .00051 9.000 .929340 .00048 9.050 .933060 .00050 9.100 .937510 .00052 9.150 .940220 .00051 9.200 .944760 .00049 9.250 .948330 .00051 9.300 .952530 .00050 9.350 .957350 .00048 9.400 .960150 .00048 9.450 .963590 .00049 9.500 .967860 .00049 9.550 .971330 .00050 9.600 .975550 .00049 9.650 .978440 .00050 9.700 .983340 .00050 9.750 .987440 .00051 9.800 .990020 .00049 9.850 .993500 .00050 9.900 .998250 .00050 9.951 .000910 .00050 10.001 .003700 .00050 10.051 .007780 .00050 10.101 .012200 .00050 10.151 .015620 .00049 10.201 .019590 .00050 
Pun40-icyl-1000-10

12

13.100 .923580 .00028

13.300 .928910 .00030

13.500 .934900 .00029

13.700 .940300 .00029

13.900 .946410 .00029

14.100 .951670 .00028

14.300 .956680 .00028

14.500 .961800 .00029

14.700 .966550 .00028

14.900 .971750 .00028

15.100 .975750 .00028

15.300 .980200 .00028

15.500 .984950 .00028

15.700 .989420 .00028

15.900 .993760 .00028

16.100 .997890 .00027

16.301 .001790 .00027

16.501 .006100 .00027

16.701 .010080 .00027

16.901 .014120 .00027

\section{5}

8.100 .921930 .00038

8.150 .924850 .00038

8.200 .928580 .00040

8.250 .931330 .00038

8.300 .935160 .00040

8.350 .938640 .00038

8.400 .941500 .00038

8.450 .945140 .00038

8.500 .948560 .00039

8.550 .951520 .00038

8.600 .954670 .00038

8.650 .957910 .00037

8.700 .961250 .00038

8.750 .964800 .00038

8.800 .967950 .00037

8.850 .971050 .00039

8.900 .974440 .00038
15

10.900 .925590 .00032

11.000 .929070 .00032

11.100 .934070 .00032

11.200 .937500 .00033

11.300 .941470 .00033

11.400 .946360 .00032

11.500 .950200 .00032

11.600 .953470 .00033

11.700 .957270 .00032

11.800 .960770 .00032

11.900 .965140 .00032

12.000 .968340 .00032

12.100 .972120 .00032

12.200 .975110 .00032

12.300 .979010 .00031

12.400 .983310 .00032

12.500 .986010 .00032

12.600 .989570 .00031

12.700 .992900 .00030

12.800 .995620 .00031

12.900 .999160 .00030

13.001 .002390 .00031

13.101 .006000 .00031

13.201 .008150 .00032

13.301 .011690 .00031

13.401 .015220 .00031

13.501 .018390 .00031
20

9.000 .920500 .00037

9.100 .926380 .00035

9.200 .931990 .00035

9.300 .937390 .00036

9.400 .942540 .00036

9.500 .948630 .00037

9.600 .953690 .00036

9.700 .959550 .00036

9.800 .965090 .00035

9.900 .969800 .00035

10.000 .975020 .00035

10.100 .979640 .00035

10.200 .984740 .00036

10.300 .990350 .00036

10.400 .995620 .00035

10.500 .999820 .00035

10.601 .003670 .00036

10.701 .010050 .00036

10.801 .013800 .00035

10.901 .018330 .00035
30

7.500 .919920 .00040

7.550 .922300 .00039

7.600 .927610 .00041

7.650 .930520 .00040

7.700 .935460 .00040

7.750 .938930 .00041

7.800 .942670 .00040

7.850 .946400 .00040

7.900 .949750 .00039

7.950 .953930 .00041

8.000 .956570 .00039

8.050 .960670 .00040

8.100 .964950 .00040

8.150 .967910 .00040

8.200 .970940 .00040

8.250 .974840 .00041

8.300 .978530 .00040
32

7.300 .917020 .00040

7.350 .920520 .00041

7.400 .925060 .00040

7.450 .929290 .00039

7.500 .933050 .00040

7.550 .937300 .00041

7.600 .941430 .00040

7.650 .945110 .00039

7.750 .951680 .00040

7.800 .956580 .00039

7.850 .960130 .00040

7.900 .964000 .00041

7.950 .967340 .00041

8.000 .971200 .00040

8.050 .975370 .00039

8.100 .978320 .00040

8.150 .981450 .00040 


\begin{abstract}
8.950 .976970 .00039 9.000 .980540 .00039 9.050 .983320 .00038 9.100 .986810 .00037 9.150 .989560 .00037 9.200 .992190 .00039 9.250 .996250 .00039 9.300 .998500 .00037 9.351 .000700 .00037 9.401 .004090 .00037 9.451 .007310 .00038 9.501 .010390 .00038 9.551 .012950 .00038 9.601 .015420 .00037 9.651 .018210 .00038 9.701 .021350 .00037
\end{abstract}

35

7.100 .918050 .00041 7.150 .923260 .00040 7.200 .926700 .00041 7.250 .931230 .00041 7.300 .935450 .00041 7.350 .938410 .00040 7.400 .943010 .00041 7.450 .947340 .00040 7.500 .950950 .00040 7.550 .955230 .00041 7.600 .959610 .00040 7.650 .962930 .00040 7.700 .966990 .00041 7.750 .970470 .00039 7.800 .974440 .00042 7.850 .977980 .00041 7.900 .981990 .00041 7.950 .986160 .00041 8.050 .992290 .00041 8.151 .000620 .00040 8.201 .004460 .00041 8.251 .007600 .00041 8.301 .010440 .00041 8.351 .014600 .00040 8.401 .017870 .00040
8.350 .981610 .00039

8.400 .985670 .00040

8.450 .988770 .00040

8.500 .991800 .00040

8.550 .994640 .00040

8.600 .998170 .00039

8.651 .001040 .00039

8.701 .005490 .00040

8.751 .008650 .00039

8.801 .011810 .00039

8.851 .014910 .00040

8.901 .018330 .00040
8.300 .992510 .00040

8.350 .996020 .00040

8.501 .006690 .00039

8.551 .009610 .00040

8.601 .012860 .00039

8.651 .015560 .00041

8.701 .019590 .00041

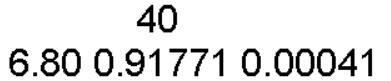

6.850 .920900 .00041

6.900 .926370 .00042

6.950 .929850 .00041

7.000 .935020 .00041

7.050 .939230 .00042

7.100 .943260 .00041

7.150 .947350 .00043

7.200 .951560 .00041

7.250 .955460 .00042

7.300 .959680 .00042

7.350 .963560 .00041

7.400 .967860 .00041

7.450 .972100 .00042

7.500 .976880 .00041

7.550 .979730 .00043

7.600 .984870 .00042

7.650 .987900 .00043

7.700 .993050 .00041

7.750 .996100 .00043

7.800 .999500 .00041

7.851 .003750 .00042

7.901 .007120 .00043

7.951 .011760 .00042

8.001 .014790 .00042

8.051 .018570 .00042

8.101 .021900 .00041
50

6.400 .914830 .00043

6.450 .921320 .00042

6.500 .926440 .00042

6.550 .930250 .00042

6.600 .933780 .00042

6.650 .938300 .00043

6.700 .944140 .00043

6.750 .948210 .00042

6.800 .952920 .00043

6.850 .957770 .00044

6.900 .961110 .00043

6.950 .967080 .00042

7.000 .969640 .00043

7.050 .974620 .00043

7.100 .979510 .00043

7.150 .983910 .00043

7.200 .987410 .00042

7.250 .991530 .00043

7.300 .996590 .00043

7.351 .000620 .00044

7.401 .004410 .00042

7.451 .008530 .00042

7.501 .012280 .00043

7.551 .016500 .00042

7.601 .020200 .00043 
60

6.200 .919820 .00043 6.250 .925170 .00043 6.300 .929890 .00045 6.350 .935170 .00044 6.400 .940430 .00043 6.450 .944410 .00043 6.500 .949300 .00044 6.550 .954280 .00043 6.600 .958700 .00043 6.650 .963940 .00043 6.700 .967160 .00044 6.750 .973100 .00043 6.800 .977750 .00043 6.850 .981710 .00044 6.900 .986260 .00045 6.950 .990820 .00043 7.000 .994420 .00045 7.050 .998980 .00044 7.101 .004480 .00044 7.151 .007970 .00044 7.201 .012410 .00045 7.251 .016530 .00043 7.301 .020790 .00043

90

5.800 .918600 .00045 5.850 .924100 .00044 5.900 .929580 .00045 5.950 .933620 .00045 6.000 .940440 .00044 6.050 .944600 .00044 6.100 .950330 .00045 6.150 .954870 .00045 6.200 .959280 .00045 6.250 .965310 .00045 6.300 .969830 .00044 6.350 .974670 .00046 6.400 .979350 .00045 6.450 .984490 .00045 6.500 .988060 .00044 6.550 .994170 .00045 6.600 .998750 .00046 6.651 .003060 .00045 6.701 .007090 .00045 6.751 .012680 .00045 6.801 .017130 .00044 6.851 .021410 .00045 6.901 .025970 .00045
70

6.000 .916960 .00044

6.050 .922010 .00043

6.100 .926520 .00044

6.150 .932170 .00044

6.200 .937070 .00045

6.250 .942570 .00045

6.300 .946920 .00045

6.350 .952130 .00043

6.400 .956780 .00046

6.450 .961310 .00043

6.500 .965570 .00044

6.550 .970930 .00045

6.600 .975700 .00044

6.650 .980050 .00043

6.700 .984930 .00044

6.750 .989320 .00044

6.800 .993790 .00045

6.850 .999120 .00044

6.901 .002570 .00043

6.951 .008190 .00043

7.001 .011210 .00044

7.051 .015800 .00044

7.101 .020480 .00043
80

5.900 .919850 .00045

5.950 .924570 .00044

6.000 .930200 .00045

6.050 .935570 .00045

6.100 .939780 .00045

6.150 .945360 .00045

6.200 .949420 .00045

6.250 .954280 .00044

6.300 .960970 .00045

6.350 .964750 .00045

6.400 .969330 .00045

6.450 .974510 .00044

6.500 .979750 .00044

6.550 .984330 .00045

6.600 .988610 .00046

6.650 .994230 .00045

6.700 .997470 .00046

6.751 .002590 .00044

6.801 .006610 .00044

6.851 .010780 .00044

6.901 .016610 .00046

6.951 .020320 .00044

7.001 .025390 .00045
5.700 .916000 .00044 5.750 .921380 .00045 5.800 .926390 .00045 5.850 .931720 .00044 5.900 .937540 .00045 5.950 .942130 .00045 6.000 .947720 .00045 6.050 .952140 .00045 6.100 .958110 .00045 6.150 .962280 .00044 6.200 .967610 .00045 6.250 .973320 .00044 6.300 .978090 .00045 6.350 .982470 .00045 6.400 .986620 .00046 6.450 .991690 .00045 6.500 .996770 .00044 6.551 .001850 .00044 6.601 .006390 .00046 6.651 .010340 .00046 6.701 .014820 .00047 6.751 .020500 .00044 6.801 .024190 .00045
120

5.600 .915690 .00044 5.650 .921700 .00046 5.700 .927000 .00043 5.750 .932270 .00044 5.800 .937510 .00045 5.850 .942920 .00047 5.900 .947560 .00045 5.950 .953610 .00044 6.000 .958330 .00047 6.050 .962890 .00045 6.100 .968740 .00046 6.150 .972840 .00045 6.200 .978150 .00046 6.250 .982960 .00045 6.300 .987580 .00046 6.350 .992850 .00045 6.400 .997820 .00046 6.451 .002890 .00045 6.501 .006850 .00046 6.551 .011890 .00045 6.601 .016850 .00045 6.651 .021560 .00045 6.701 .026230 .00046 
130

5.600 .921010 .00045 5.650 .926570 .00046 5.700 .931700 .00046 5.750 .937290 .00045 5.800 .941850 .00045 5.850 .947480 .00045 5.900 .952740 .00045 5.950 .958130 .00045 6.000 .963800 .00046 6.050 .968250 .00044 6.100 .973930 .00045 6.150 .977870 .00045 6.200 .983070 .00046 6.250 .987660 .00045 6.300 .993290 .00047 6.350 .997000 .00046 6.401 .002550 .00046 6.451 .007400 .00045 6.501 .012630 .00045 6.551 .017190 .00044 6.601 .021340 .00046

239

5.400 .919050 .00046 5.450 .923920 .00044 5.500 .928460 .00046 5.550 .934590 .00045 5.600 .939360 .00045 5.650 .944990 .00045 5.700 .950620 .00045 5.750 .955160 .00045 5.800 .960230 .00044 5.850 .965680 .00046 5.900 .970810 .00045 5.950 .975590 .00046 6.000 .980870 .00047 6.050 .985610 .00044 6.100 .990580 .00046 6.150 .996130 .00046 6.201 .000630 .00047 6.251 .004640 .00044 6.301 .009530 .00046 6.351 .014500 .00047 6.401 .018280 .00046
150

5.500 .916790 .00046

5.550 .922280 .00045

5.600 .928320 .00046

5.650 .933410 .00045

5.700 .937400 .00046

5.750 .943690 .00045

5.800 .948180 .00046

5.850 .953350 .00046

5.900 .958680 .00045

5.950 .964460 .00045

6.000 .968080 .00045

6.050 .974650 .00045

6.100 .978950 .00046

6.150 .983930 .00046

6.200 .988820 .00046

6.250 .993520 .00044

6.300 .999270 .00044

6.351 .003060 .00046

6.401 .008110 .00046

6.451 .012240 .00045

6.501 .018140 .00046
200

5.400 .914500 .00045

5.450 .919510 .00045

5.500 .925930 .00045

5.550 .931600 .00045

5.600 .935900 .00046

5.650 .941320 .00045

5.700 .946830 .00045

5.750 .951810 .00045

5.800 .956620 .00044

5.850 .962450 .00046

5.900 .967720 .00046

5.950 .972910 .00045

6.000 .977170 .00046

6.050 .982640 .00045

6.100 .987210 .00046

6.150 .991910 .00046

6.200 .997660 .00046

6.251 .001560 .00045

6.301 .007160 .00046

6.351 .011700 .00046

6.401 .016120 .00046
250
5.400 .918670 .00044

5.450 .924820 .00044

5.500 .929940 .00044

5.550 .934810 .00047

5.600 .940270 .00045

5.650 .945490 .00046

5.700 .950990 .00045

5.750 .955540 .00045

5.800 .960540 .00046

5.850 .966420 .00045

5.900 .971070 .00046

5.950 .976190 .00044

6.000 .981710 .00045

6.050 .986610 .00046

6.100 .991040 .00046

6.150 .996200 .00047

6.201 .000420 .00046

6.251 .004710 .00046

6.301 .009630 .00045

6.351 .014890 .00048

6.401 .020110 .00045
359

5.400 .920310 .00046

5.450 .924770 .00045

5.500 .931400 .00045

5.550 .936520 .00045

5.600 .941550 .00046

5.650 .946170 .00045

5.700 .951510 .00046

5.750 .956830 .00044

5.800 .961780 .00045

5.850 .967250 .00046

5.900 .971950 .00045

5.950 .976600 .00045

6.000 .982260 .00045

6.050 .986000 .00046

6.100 .991380 .00046

6.150 .996030 .00046

6.201 .000630 .00046

6.251 .006150 .00045

6.301 .010990 .00045

6.351 .014610 .00047

6.401 .019590 .00046 
478

5.400 .914940 .00048 5.450 .921210 .00046 5.500 .925900 .00046 5.550 .931600 .00046 5.600 .936730 .00046 5.650 .941030 .00046 5.700 .946750 .00046 5.750 .951180 .00047 5.800 .956320 .00048 5.850 .961450 .00046 5.900 .965890 .00046 5.950 .970860 .00046 6.000 .975720 .00046 6.050 .980610 .00044 6.100 .985060 .00046 6.150 .990510 .00046 6.200 .994550 .00046 6.250 .999730 .00046 6.301 .003300 .00046 6.351 .008340 .00045 6.401 .013220 .00045 6.451 .018100 .00046 6.501 .022670 .00046

650

5.700 .919230 .00046 5.750 .923970 .00045 5.800 .928300 .00047 5.850 .933520 .00045 5.900 .938620 .00047 5.950 .942680 .00046 6.000 .947730 .00046 6.050 .952780 .00045 6.100 .957670 .00046 6.150 .962440 .00046 6.200 .967070 .00045 6.250 .970850 .00045 6.300 .975360 .00045 6.350 .980620 .00046 6.400 .984950 .00046 6.450 .990290 .00046 6.500 .994150 .00045 6.550 .998210 .00047 6.601 .002550 .00046 6.651 .006570 .00047 6.701 .010900 .00046 6.751 .016530 .00047
500

5.500 .924020 .00046

5.550 .929090 .00046

5.600 .934460 .00047

5.650 .939130 .00045

5.700 .944420 .00045

5.750 .949540 .00046

5.800 .954750 .00046

5.850 .959310 .00046

5.900 .964450 .00047

5.950 .968780 .00046

6.000 .974090 .00046

6.050 .980190 .00045

6.100 .983960 .00047

6.150 .988720 .00046

6.200 .993260 .00045

6.250 .997300 .00047

6.301 .002720 .00045

6.351 .007280 .00046

6.401 .010720 .00046

6.451 .016690 .00044

6.501 .020220 .00046

700

5.900 .919040 .00046 5.950 .924050 .00046 6.000 .927800 .00045 6.050 .932770 .00045 6.100 .937460 .00047 6.150 .942510 .00046 6.200 .947450 .00046 6.250 .951430 .00047 6.300 .955330 .00047 6.350 .959680 .00047 6.400 .964540 .00046 6.450 .969690 .00048 6.500 .973230 .00046 6.550 .978470 .00046 6.600 .981850 .00045 6.650 .985680 .00046 6.700 .991690 .00046 6.750 .994930 .00046 6.800 .998970 .00046 6.851 .002740 .00045 6.901 .008330 .00045 6.951 .011920 .00046
600

5.600 .920940 .00046 5.650 .925560 .00047 5.700 .931100 .00046 5.750 .935470 .00046 5.800 .940210 .00045 5.850 .945150 .00045 5.900 .949920 .00046 5.950 .955600 .00046 6.000 .960930 .00045 6.050 .964010 .00046 6.100 .969720 .00045 6.150 .973890 .00047 6.200 .980080 .00046 6.250 .984230 .00046 6.300 .988010 .00047 6.350 .992150 .00045 6.400 .997990 .00046 6.451 .001300 .00047 6.501 .005190 .00046 6.551 .010270 .00046 6.601 .014820 .00046 6.651 .019060 .00046 6.701 .022930 .00047 
6.801 .019840 .00047

752

6.500 .918180 .00044

6.550 .922510 .00046

6.600 .926360 .00046

6.650 .931270 .00047

6.700 .935020 .00046

6.750 .938710 .00046

6.800 .943330 .00046

6.850 .947430 .00047

6.900 .951020 .00046

6.950 .955310 .00046

7.000 .958970 .00046

7.050 .963520 .00046

7.100 .967150 .00046

7.150 .971310 .00044

7.200 .976200 .00047

7.250 .978670 .00046

7.300 .982670 .00046

7.350 .986930 .00047

7.400 .989980 .00046

7.450 .993740 .00046

7.500 .998070 .00047

7.551 .001810 .00046

7.601 .005410 .00046

7.651 .009920 .00046

7.701 .013300 .00047

7.751 .017030 .00046

7.801 .020040 .00046

7.500 .990770 .00046
7.001 .015730 .00047

7.051 .019520 .00047

7.101 .023370 .00046

7.501 .004530 .00047

7.551 .008220 .00046

7.601 .011660 .00047

7.651 .016300 .00046

7.701 .019200 .00046

754

6.600 .918610 .00045

6.650 .923130 .00045

6.700 .927970 .00046

6.750 .932250 .00045

6.800 .935160 .00045

6.850 .939760 .00046

6.900 .943400 .00046

6.950 .947710 .00047

7.000 .951500 .00046

7.050 .956120 .00046

7.100 .959160 .00048

7.150 .963090 .00046

7.200 .967340 .00045

7.250 .971790 .00044

7.300 .974330 .00047

7.350 .978480 .00046

7.400 .982200 .00045

7.450 .985800 .00047

7.550 .994660 .00046

7.600 .997310 .00047

7.651 .001720 .00046

7.701 .004160 .00046

7.751 .008970 .00046

7.801 .012450 .00046

7.851 .015890 .00047

7.901 .020070 .00046
755

6.700 .919120 .00046

6.750 .922980 .00044

6.800 .927250 .00047

6.850 .931090 .00046

6.900 .935160 .00046

6.950 .938150 .00047

7.000 .942700 .00046

7.050 .946190 .00046

7.100 .950150 .00047

7.150 .954220 .00046

7.200 .957920 .00045

7.250 .961740 .00045

7.300 .966150 .00047

7.350 .970010 .00045

7.400 .974460 .00046

7.450 .976710 .00045

7.500 .981000 .00046

7.550 .984360 .00046

7.600 .988040 .00045

7.650 .991840 .00046

7.700 .995310 .00046

7.750 .999690 .00046

7.801 .003060 .00046

7.851 .005720 .00047

7.901 .010510 .00047

7.951 .013490 .00046

8.001 .017240 .00046 
Pun43-icyl-1000-00

12

17.400 .924630 .00029

17.600 .929990 .00030

17.800 .934030 .00030

18.000 .938310 .00030

18.200 .943460 .00029

18.400 .947490 .00029

18.600 .952110 .00029

18.800 .956190 .00028

19.000 .959380 .00029

19.200 .963860 .00028

19.400 .967660 .00029

19.600 .971210 .00029

19.800 .974420 .00029

20.000 .978240 .00028

20.200 .981560 .00029

20.400 .984360 .00028

20.600 .988240 .00028

20.800 .991790 .00029

21.000 .994770 .00028

21.200 .998110 .00028

21.401 .000710 .00029

21.601 .004410 .00027

21.801 .007040 .00027

22.001 .009340 .00028

22.201 .013020 .00028

22.301 .013560 .00028

25

11.300 .918690 .00043

11.400 .924690 .00043

11.500 .930340 .00042

11.600 .937200 .00042

11.700 .942720 .00041

11.800 .948700 .00042

12.100 .965950 .00042

12.200 .971640 .00042

12.300 .977490 .00042

12.400 .982230 .00041

12.500 .988080 .00043
15

14.500 .921090 .00034

14.600 .924870 .00035

14.700 .928350 .00034

14.800 .932050 .00034

14.900 .935730 .00034

15.000 .938790 .00035

15.100 .942900 .00034

15.200 .945940 .00034

15.300 .949090 .00034

15.400 .952400 .00034

15.500 .955900 .00034

15.600 .959000 .00034

15.700 .962400 .00033

15.800 .965400 .00034

15.900 .968390 .00033

16.000 .970770 .00033

16.100 .974230 .00034

16.200 .977090 .00033

16.300 .979860 .00033

16.400 .983160 .00033

16.500 .986030 .00034

16.600 .989030 .00033

16.700 .991120 .00033

16.800 .994640 .00033

16.900 .996720 .00033

17.000 .999390 .00033

17.101 .002640 .00033

17.201 .004800 .00032

17.301 .007080 .00033

17.401 .009840 .00032

17.501 .012190 .00033

17.601 .015620 .00032

17.701 .016690 .00032

30

10.700 .919860 .00044

10.750 .924150 .00044

10.800 .927080 .00043

10.850 .930150 .00045

10.900 .934910 .00044

10.950 .937380 .00043

11.000 .940680 .00044

11.050 .944130 .00043

11.100 .948070 .00044

11.150 .950600 .00044

11.200 .954240 .00043
20

12.400 .921440 .00039

12.500 .926720 .00038

12.600 .932490 .00040

12.700 .937680 .00039

12.800 .942240 .00039

12.900 .946340 .00038

13.000 .951730 .00039

13.100 .956480 .00037

13.200 .961230 .00038

13.300 .965300 .00039

13.400 .969940 .00040

13.500 .975150 .00037

13.600 .979070 .00038

13.700 .983110 .00038

13.800 .987260 .00038

13.900 .991520 .00039

14.000 .996450 .00038

14.101 .000090 .00038

14.201 .004060 .00038

14.301 .007820 .00037

14.401 .012290 .00038

14.501 .016310 .00037

14.601 .020410 .00038 
12.600 .992480 .00040

12.700 .998470 .00041

12.801 .003560 .00041

12.901 .008340 .00040

13.001 .013480 .00041

13.101 .018370 .00041
11.250 .957100 .00045 11.300 .960500 .00044 11.350 .962850 .00043 11.400 .967000 .00044 11.450 .970420 .00043 11.500 .973880 .00043 11.550 .977110 .00044 11.600 .979640 .00044 11.650 .981710 .00045 11.700 .985930 .00044 11.750 .988950 .00043 11.800 .991310 .00044 11.850 .995120 .00043 11.900 .998120 .00044 11.951 .001110 .00042 12.001 .003460 .00043 12.051 .006810 .00044 12.101 .009710 .00043 12.151 .012940 .00044 12.201 .015220 .00044 12.251 .017470 .00043 12.301 .020860 .00043
11.050 .957750 .00044 11.100 .960780 .00045 11.150 .964980 .00044 11.200 .967790 .00044 11.250 .970810 .00045 11.300 .974430 .00043 11.350 .977510 .00044 11.400 .980950 .00045 11.450 .984290 .00045 11.500 .987620 .00045 11.550 .989470 .00044 11.600 .993790 .00044 11.650 .995540 .00044 11.701 .000070 .00044 11.751 .002650 .00044 11.801 .006090 .00044 11.851 .008350 .00045 11.901 .011860 .00043 11.951 .014230 .00044 12.001 .017330 .00046
35

10.300 .921350 .00046 10.350 .925010 .00045 10.400 .928890 .00044 10.450 .932220 .00047 10.500 .936210 .00046 10.550 .940400 .00045 10.600 .942490 .00046 10.650 .948110 .00045 10.700 .951010 .00045 10.750 .954860 .00044 10.800 .957460 .00046 10.850 .961550 .00046 10.900 .965030 .00045 10.950 .969050 .00045 11.000 .972320 .00047 11.050 .975470 .00045 11.100 .978520 .00045 11.150 .982200 .00045 11.200 .985140 .00046 11.250 .989730 .00045 11.300 .991910 .00045 11.350 .994990 .00044 11.400 .997960 .00045 11.451 .001450 .00045 11.501 .005030 .00046
40

10.000 .920320 .00046 10.050 .925140 .00046 10.100 .928360 .00047 10.150 .932260 .00047 10.200 .936770 .00047 10.250 .940710 .00048 10.300 .944380 .00047 10.350 .948520 .00047 10.400 .951730 .00048 10.450 .956200 .00046 10.500 .959110 .00047 10.550 .964270 .00046 10.600 .967300 .00047 10.650 .971270 .00047 10.700 .973790 .00045 10.750 .977270 .00046 10.800 .980790 .00046 10.850 .985200 .00047 10.900 .989310 .00046 10.950 .991870 .00046 11.000 .994940 .00045 11.050 .999580 .00046 11.101 .003230 .00046 11.151 .006020 .00046 11.201 .008750 .00047
50

9.600 .919700 .00048 9.650 .924580 .00048 9.700 .929770 .00049 9.750 .933340 .00048 9.800 .937210 .00048 9.850 .942120 .00047 9.900 .945710 .00048 9.950 .950610 .00047 10.000 .953820 .00046 10.050 .958640 .00047 10.100 .962900 .00048 10.150 .966510 .00048 10.200 .970260 .00048 10.250 .974740 .00048 10.300 .978840 .00049 10.350 .982480 .00048 10.400 .985130 .00048 10.450 .989220 .00047 10.500 .993960 .00048 10.550 .998270 .00047 10.601 .000820 .00047 10.651 .004790 .00049 10.701 .007970 .00047 10.751 .012130 .00048 10.801 .016140 .00049 
11.551 .009050 .00046 11.601 .011710 .00045 11.651 .014710 .00045 11.701 .018210 .00046

60

9.300 .914550 .00048 9.350 .918470 .00049 9.400 .923850 .00049 9.450 .928960 .00049 9.500 .932490 .00048 9.550 .937150 .00049 9.600 .942080 .00048 9.650 .946190 .00049 9.700 .951080 .00049 9.750 .953990 .00048 9.800 .958850 .00049 9.850 .963400 .00049 9.900 .967250 .00048 9.950 .971400 .00049 10.000 .975560 .00050 10.050 .979740 .00050 10.100 .983910 .00048 10.150 .987210 .00049 10.200 .991640 .00048 10.250 .995370 .00048 10.301 .000060 .00049 10.351 .003980 .00050 10.401 .007040 .00049 10.451 .011630 .00047 10.501 .015730 .00048 10.551 .019080 .00049 10.601 .022760 .00048

\section{0}

9.000 .918490 .00050 9.050 .923860 .00051 9.100 .928950 .00050 9.150 .933540 .00051 9.200 .937350 .00050 9.250 .943030 .00050 9.300 .947140 .00051 9.350 .951630 .00049 9.400 .956060 .00050 9.450 .961300 .00052 9.500 .965800 .00050 9.550 .970650 .00051
11.251 .013570 .00045 11.301 .016140 .00046 11.351 .019290 .00046 11.401 .022680 .00045
10.851 .019530 .00048 10.901 .023540 .00047
70

9.200 .921710 .00050 9.250 .925510 .00050 9.300 .929410 .00049 9.350 .933820 .00051 9.400 .938700 .00049 9.450 .943470 .00049 9.500 .948150 .00048 9.550 .953090 .00050 9.600 .957170 .00048 9.650 .960570 .00049 9.700 .965550 .00050 9.750 .969840 .00047 9.800 .974510 .00049 9.850 .978160 .00049 9.900 .983520 .00050 9.950 .986960 .00050 10.000 .991070 .00051 10.050 .994820 .00051 10.100 .998760 .00049 10.151 .003360 .00049 10.201 .007970 .00049 10.251 .011950 .00050 10.301 .014080 .00049 10.351 .020520 .00048 10.401 .023300 .00050

100 8.900 .914920 .00050 8.950 .919250 .00050 9.000 .924760 .00051 9.050 .929560 .00052 9.100 .934300 .00052 9.150 .938190 .00051 9.200 .942460 .00051 9.250 .947880 .00049 9.300 .952610 .00051 9.350 .956490 .00051 9.400 .960910 .00052 9.450 .966150 .00050
80

9.000 .912700 .00051 9.050 .916670 .00049 9.100 .920920 .00049 9.150 .926780 .00050 9.200 .931070 .00050 9.250 .935590 .00050 9.300 .940740 .00051 9.350 .944860 .00050 9.400 .949190 .00051 9.450 .954100 .00050 9.500 .958240 .00051 9.550 .962250 .00049 9.600 .966700 .00050 9.650 .970490 .00050 9.700 .976180 .00050 9.750 .980610 .00051 9.800 .984700 .00051 9.850 .988350 .00050 9.900 .992920 .00049 9.950 .997230 .00052 10.001 .001630 .00051 10.051 .005870 .00050 10.101 .009140 .00050 10.151 .014340 .00050 10.201 .017850 .00050 10.251 .022430 .00050 10.301 .026440 .00049

120

8.900 .921130 .00051 8.950 .925700 .00050 9.000 .930010 .00051 9.050 .935070 .00051 9.100 .940130 .00050 9.150 .944990 .00050 9.200 .949490 .00051 9.250 .954280 .00050 9.300 .958580 .00052 9.350 .961960 .00051 9.400 .966420 .00050 9.450 .971670 .00050 
9.600 .974410 .00050

9.650 .978990 .00050

9.700 .983000 .00051

9.750 .987500 .00050

9.800 .991830 .00051

9.850 .996400 .00050

9.901 .000300 .00049

9.951 .004270 .00050

10.001 .008880 .00051

10.051 .012470 .00050

10.101 .018000 .00050

10.151 .021190 .00051

10.201 .025190 .00051
9.500 .971410 .00051

9.550 .975130 .00052

9.600 .980190 .00049

9.650 .984820 .00050

9.700 .988830 .00050

9.750 .992270 .00050

9.800 .997460 .00051

9.851 .000230 .00051

9.901 .006230 .00052

9.951 .010370 .00051

10.001 .013430 .00050

10.051 .019810 .00051

10.101 .022450 .00051
9.500 .976800 .00051

9.550 .980230 .00050

9.600 .984860 .00052

9.650 .989620 .00051

9.700 .994970 .00052

9.750 .999090 .00050

9.801 .004210 .00051

9.851 .008210 .00051

9.901 .012080 .00051

9.951 .015720 .00050

10.001 .020440 .00051
130

8.800 .913990 .00052

8.850 .918790 .00052

8.900 .922420 .00052

8.950 .928520 .00052

9.000 .933450 .00051

9.050 .938130 .00049

9.100 .943240 .00051

9.150 .947810 .00051

9.200 .951520 .00051

9.250 .956210 .00050

9.300 .961540 .00050

9.350 .965720 .00050

9.400 .970500 .00051

9.450 .975640 .00051

9.500 .979380 .00050

9.550 .983020 .00051

9.600 .988220 .00050

9.650 .993070 .00051

9.700 .997280 .00051

9.751 .002210 .00050

9.801 .005510 .00051

9.851 .010230 .00050

9.901 .015720 .00051

9.951 .018380 .00050

10.001 .021890 .00052
150

8.800 .915400 .00052

8.850 .920380 .00052

8.900 .925460 .00052

8.950 .929920 .00051

9.000 .934500 .00050

9.050 .939040 .00051

9.100 .944390 .00051

9.150 .949140 .00050

9.200 .954350 .00051

9.250 .958070 .00052

9.300 .963930 .00052

9.350 .967350 .00052

9.400 .972690 .00050

9.450 .976790 .00051

9.500 .981930 .00053

9.550 .985940 .00050

9.600 .990240 .00051

9.650 .994790 .00051

9.700 .999390 .00051

9.751 .002880 .00052

9.801 .007560 .00053

9.851 .012220 .00052

9.901 .016070 .00051

9.951 .020920 .00049

10.001 .025160 .00052
200

8.800 .915480 .00050

8.850 .920830 .00052

8.900 .925510 .00049

8.950 .929730 .00052

9.000 .935320 .00052

9.050 .940350 .00053

9.100 .944290 .00051

9.150 .949300 .00051

9.200 .953200 .00050

9.250 .958660 .00051

9.300 .964060 .00050

9.350 .968040 .00050

9.400 .972870 .00052

9.450 .977570 .00051

9.500 .981850 .00050

9.550 .985890 .00051

9.600 .991160 .00050

9.650 .994590 .00052

9.700 .999650 .00051

9.751 .004520 .00051

9.801 .008010 .00052

9.851 .011710 .00052

9.901 .016020 .00052

9.951 .020190 .00052

10.001 .024360 .00052
239

8.800 .912160 .00052 8.850 .916320 .00050 8.900 .921730 .00051 8.950 .926890 .00050 9.000 .931920 .00052
250

8.900 .920390 .00051

8.950 .925000 .00052

9.000 .930500 .00050

9.050 .935210 .00049

9.100 .939660 .00052
359

9.000 .914940 .00050

9.050 .919800 .00050

9.100 .924050 .00052

9.150 .929570 .00051

9.200 .934510 .00051 
9.050 .935960 .00050

9.100 .941230 .00050

9.150 .945810 .00051

9.200 .951000 .00051

9.250 .954390 .00050

9.300 .959710 .00050

9.350 .964760 .00051

9.400 .969770 .00051

9.450 .972490 .00051

9.500 .977190 .00050

9.550 .982310 .00052

9.600 .986100 .00052

9.650 .991460 .00052

9.700 .995850 .00052

9.751 .000350 .00050

9.801 .004310 .00051

9.851 .009010 .00051

9.901 .013690 .00051

9.951 .017520 .00050

10.001 .021180 .00052
9.150 .943970 .00049

9.200 .948980 .00051

9.250 .954510 .00052

9.300 .958860 .00051

9.350 .962660 .00051

9.400 .967500 .00052

9.450 .973430 .00050

9.500 .976950 .00050

9.550 .981580 .00051

9.600 .986620 .00051

9.650 .989680 .00052

9.700 .994060 .00050

9.750 .999090 .00051

9.801 .003450 .00051

9.851 .007220 .00050

9.901 .011710 .00050

9.951 .014940 .00051

10.001 .019310 .00051

10.051 .024700 .00051

10.101 .028270 .00050
9.250 .938710 .00050

9.300 .943450 .00051

9.350 .948040 .00051

9.400 .951560 .00051

9.450 .957610 .00051

9.500 .961450 .00052

9.550 .965490 .00050

9.600 .970780 .00051

9.650 .974940 .00051

9.700 .979790 .00052

9.750 .983490 .00050

9.800 .988410 .00051

9.850 .992640 .00051

9.900 .997070 .00051

9.951 .000680 .00050

10.001 .005120 .00051

10.051 .008870 .00051

10.101 .012960 .00050

10.151 .017900 .00051

10.201 .022380 .00051
478

9.300 .918530 .00052

9.350 .923220 .00050

9.400 .926170 .00050

9.450 .932810 .00050

9.500 .936620 .00052

9.550 .941330 .00051

9.600 .946170 .00051

9.650 .950390 .00051

9.700 .953670 .00050

9.750 .959460 .00051

9.800 .963520 .00051

9.850 .967020 .00050

9.900 .972660 .00051

9.950 .976590 .00050

10.000 .981480 .00051

10.050 .985560 .00052

10.100 .989300 .00052

10.150 .992280 .00051

10.200 .997630 .00051

10.251 .002200 .00051

10.301 .005350 .00050

10.351 .008800 .00050

10.401 .014330 .00050

10.451 .017950 .00051

10.501 .021340 .00050
500

9.400 .919760 .00050

9.450 .924390 .00049

9.500 .928790 .00050

9.550 .933470 .00051

9.600 .937720 .00050

9.650 .942200 .00051

9.700 .947780 .00051

9.750 .951500 .00050

9.800 .955920 .00050

9.850 .961310 .00050

9.900 .965290 .00051

9.950 .969940 .00051

10.000 .973050 .00050

10.050 .977430 .00051

10.100 .981510 .00051

10.150 .985700 .00049

10.200 .990870 .00050

10.250 .995430 .00052

10.300 .998770 .00051

10.351 .002470 .00050

10.401 .005470 .00051

10.451 .010530 .00051

10.501 .015620 .00051

10.551 .018630 .00051

10.601 .023540 .00052
600

9.900 .920990 .00052

9.950 .924930 .00050

10.000 .929790 .00050

10.050 .933240 .00052

10.100 .938190 .00050

10.150 .942090 .00050

10.200 .947060 .00051

10.250 .950460 .00049

10.300 .955290 .00052

10.350 .959370 .00051

10.400 .963750 .00050

10.450 .967270 .00050

10.500 .970490 .00051

10.550 .976130 .00051

10.600 .979430 .00049

10.650 .984020 .00051

10.700 .988250 .00051

10.750 .992090 .00052

10.800 .996370 .00051

10.850 .999250 .00050

10.901 .004200 .00051

10.951 .007390 .00051

11.001 .010960 .00052

11.051 .015200 .00052

11.101 .019730 .00052 
650

10.300 .910710 .00050 10.350 .914540 .00050 10.400 .919300 .00050 10.450 .924520 .00050 10.500 .927590 .00050 10.550 .932940 .00048 10.600 .935840 .00050 10.650 .941730 .00050 10.700 .944820 .00049 10.750 .948300 .00051 10.800 .952760 .00051 10.850 .956570 .00051 10.900 .960620 .00052 10.950 .964250 .00050 11.000 .968640 .00050 11.050 .973260 .00050 11.100 .977110 .00050 11.150 .980510 .00050 11.200 .984530 .00050 11.250 .988510 .00050 11.300 .991960 .00050 11.350 .995310 .00049 11.400 .999200 .00050 11.451 .004370 .00049 11.501 .006910 .00052 11.551 .010120 .00050 11.601 .013810 .00051 11.651 .018360 .00049 11.701 .021280 .00050

680

11.000 .921750 .00050 11.050 .925000 .00049 11.100 .929040 .00048 11.150 .932230 .00049 11.200 .936410 .00050 11.250 .940980 .00050 11.300 .944740 .00049 11.350 .948550 .00051 11.400 .953250 .00050 11.450 .956640 .00051 11.500 .961390 .00050 11.550 .964610 .00051 11.600 .967740 .00049 11.650 .971650 .00050 11.700 .975060 .00049 11.750 .978210 .00050
660

10.500 .915880 .00050

10.550 .919910 .00050

10.600 .923700 .00048

10.650 .927510 .00050

10.700 .932740 .00050

10.750 .935910 .00053

10.800 .940100 .00050

10.850 .944060 .00050

10.900 .949500 .00051

10.950 .952130 .00051

11.000 .955510 .00049

11.050 .961090 .00050

11.100 .963750 .00048

11.150 .968230 .00049

11.200 .971630 .00050

11.250 .975320 .00051

11.300 .979390 .00049

11.350 .984590 .00050

11.400 .987660 .00050

11.450 .991290 .00050

11.500 .994470 .00051

11.550 .997990 .00051

11.601 .002490 .00051

11.651 .005550 .00050

11.701 .009420 .00050

11.751 .012980 .00050

11.801 .016280 .00050

11.851 .021350 .00050

11.901 .024660 .00050
670

10.700 .916580 .00050

10.750 .920500 .00050

10.800 .925410 .00050

10.850 .928770 .00051

10.900 .932540 .00049

10.950 .936930 .00049

11.000 .941950 .00051

11.050 .946020 .00051

11.100 .949070 .00051

11.150 .952800 .00050

11.200 .957440 .00052

11.250 .960490 .00051

11.300 .964890 .00049

11.350 .969210 .00050

11.400 .971250 .00051

11.450 .977590 .00050

11.500 .980600 .00049

11.550 .983940 .00050

11.600 .987280 .00051

11.650 .991790 .00051

11.700 .994780 .00051

11.750 .998380 .00051

11.801 .002400 .00050

11.851 .006230 .00051

11.901 .009220 .00051

11.951 .013620 .00050

12.001 .016880 .00051

12.051 .020210 .00051

12.101 .025030 .00050
11.300 .914430 .00051

11.350 .918360 .00050

11.400 .923180 .00050

11.450 .926210 .00050

11.500 .929730 .00050

11.550 .933830 .00050

11.600 .937290 .00050

11.650 .941950 .00049

11.700 .945790 .00052

11.750 .948860 .00050

11.800 .952560 .00051

11.850 .956310 .00050

11.900 .959360 .00051

11.950 .963790 .00050

12.000 .968050 .00049

12.050 .971220 .00049
698

12.100 .922020 .00050

12.150 .925420 .00050

12.200 .928560 .00050

12.250 .932950 .00050

12.300 .936950 .00049

12.350 .939760 .00050

12.400 .943630 .00051

12.450 .947220 .00051

12.500 .950320 .00049

12.550 .954320 .00050

12.600 .958230 .00050

12.650 .960870 .00048

12.700 .964830 .00050

12.750 .967740 .00050

12.800 .971630 .00049

12.850 .973890 .00050 
11.800 .983400 .00050 11.850 .986530 .00049 11.900 .990020 .00050 11.950 .993390 .00050 12.000 .997750 .00051 12.051 .000640 .00050 12.101 .004000 .00049 12.151 .007560 .00051 12.201 .012210 .00050 12.251 .016130 .00053 12.301 .018490 .00049 12.351 .022370 .00050 12.401 .025650 .00050
12.100 .975390 .00051

12.150 .978190 .00050 12.200 .981960 .00050 12.250 .985210 .00049 12.300 .989210 .00050 12.350 .992040 .00050 12.400 .995230 .00051 12.450 .999780 .00051 12.501 .002300 .00049 12.551 .005450 .00051 12.601 .010330 .00049 12.651 .013390 .00050 12.701 .016650 .00050 12.751 .020260 .00050 12.801 .023100 .00051
12.900 .978260 .00050 12.950 .981450 .00050 13.000 .985210 .00051 13.050 .988630 .00051 13.100 .991200 .00051 13.150 .995080 .00050 13.200 .997480 .00051 13.251 .001300 .00049 13.301 .004430 .00051 13.351 .007850 .00051 13.401 .011260 .00050 13.451 .014730 .00049 13.501 .017960 .00050 13.551 .020680 .00049 13.601 .023570 .00051

Pun43-icyl-1000-01

12

15.800 .922870 .00030 16.000 .928310 .00029 16.200 .932950 .00028 16.400 .937250 .00029 16.600 .941470 .00029 16.800 .945920 .00029 17.000 .950440 .00028 17.200 .954670 .00029 17.400 .957940 .00029 17.600 .962590 .00029 17.800 .965990 .00028 18.000 .970010 .00028 18.200 .973430 .00028 18.400 .976530 .00028 18.600 .981120 .00028 18.800 .984020 .00027 19.000 .987370 .00028 19.200 .990840 .00028 19.400 .993480 .00027 19.600 .997850 .00028 19.801 .000070 .00028 20.001 .003140 .00027 20.201 .006450 .00027 20.401 .008800 .00027 20.601 .012180 .00027 20.701 .013580 .00028
15

13.000 .922840 .00033

13.100 .926710 .00033

13.200 .930030 .00033

13.300 .933770 .00033

13.400 .936760 .00034

13.500 .940480 .00033

13.600 .943710 .00033

13.700 .947410 .00033

13.800 .950120 .00033

13.900 .954020 .00033

14.000 .957460 .00033

14.100 .959470 .00032

14.200 .963290 .00033

14.300 .966000 .00032

14.400 .968800 .00033

14.500 .972170 .00033

14.600 .975100 .00032

14.700 .978380 .00032

14.800 .981460 .00033

14.900 .983560 .00032

15.000 .986720 .00033

15.100 .989730 .00032

15.200 .992360 .00032

15.300 .995240 .00032

15.400 .997720 .00032

15.501 .000800 .00032

15.601 .002710 .00032

15.701 .005670 .00032

15.801 .008720 .00031

15.901 .010510 .00032

16.001 .013490 .00031

16.101 .015330 .00032
20

10.900 .923430 .00037

11.000 .928580 .00037

11.100 .934180 .00037

11.200 .939190 .00037

11.300 .943820 .00038

11.400 .948980 .00038

$\begin{array}{lll}11.50 & 0.95386 & 0.00037\end{array}$

11.600 .958050 .00037

11.700 .963270 .00036

11.800 .967420 .00038

11.900 .971660 .00038

12.000 .976210 .00036

12.100 .980110 .00038

12.200 .984560 .00038

12.300 .989100 .00037

12.400 .993090 .00037

12.500 .997660 .00037

12.601 .001440 .00037

12.701 .006440 .00036

12.801 .009930 .00037

12.901 .013980 .00036

13.001 .017730 .00036 
25

9.800 .920930 .00041

9.900 .926470 .00039

10.000 .932810 .00041

10.100 .938900 .00040

10.200 .944780 .00040

10.300 .950910 .00040

10.400 .956940 .00039

10.500 .962440 .00041

10.600 .968570 .00040

10.700 .973650 .00041

10.800 .978330 .00040

10.900 .984780 .00039

11.000 .989230 .00039

11.100 .995150 .00039

11.201 .000330 .00040

11.301 .005230 .00040

11.401 .009750 .00041

11.501 .015840 .00039

11.601 .020000 .00039

\section{5}

8.700 .917050 .00043 8.750 .921200 .00044 8.800 .924520 .00043 8.850 .927810 .00043 8.900 .932310 .00044 8.950 .935600 .00044 9.000 .938990 .00044 9.050 .943340 .00043 9.100 .947700 .00043 9.150 .950220 .00043 9.200 .954610 .00044 9.250 .956510 .00044
30

9.200 .922700 .00043

9.250 .926400 .00042

9.300 .929530 .00043

9.350 .933850 .00043

9.400 .936450 .00042

9.450 .940940 .00042

9.500 .944110 .00043

9.550 .946430 .00042

9.600 .950460 .00041

9.650 .953730 .00042

9.700 .956720 .00042

9.750 .959590 .00042

9.800 .963430 .00042

9.850 .966960 .00042

9.900 .969330 .00043

9.950 .972110 .00042

10.000 .976260 .00043

10.050 .979450 .00041

10.100 .981990 .00041

10.150 .985040 .00041

10.200 .987990 .00041

10.250 .991750 .00041

10.300 .993730 .00041

10.350 .996230 .00042

10.401 .001600 .00042

10.451 .002980 .00043

10.501 .005720 .00041

10.551 .009330 .00043

10.601 .012030 .00042

10.651 .015290 .00042

10.701 .017140 .00042
32

9.000 .921500 .00044

9.050 .925990 .00043

9.100 .929390 .00042

9.150 .932860 .00042

9.200 .936510 .00043

9.250 .940180 .00042

9.300 .943440 .00042

9.350 .946740 .00043

9.400 .950550 .00042

9.450 .953070 .00043

9.500 .957260 .00042

9.550 .959850 .00043

9.600 .963260 .00042

9.650 .967250 .00043

9.700 .971400 .00043

9.750 .973390 .00043

9.800 .977090 .00042

9.850 .979850 .00044

9.900 .983870 .00042

9.950 .986610 .00043

10.000 .989440 .00042

10.050 .992740 .00043

10.100 .995640 .00043

10.150 .999060 .00043

10.201 .001820 .00042

10.251 .004590 .00042

10.301 .008120 .00043

10.351 .010790 .00042

10.401 .014630 .00043

10.451 .016850 .00043

10.501 .021190 .00043
40

8.400 .916720 .00043

8.450 .920530 .00044

8.500 .924780 .00045

8.550 .928160 .00044

8.600 .932290 .00045

8.650 .936010 .00044

8.700 .940430 .00046

8.750 .944920 .00046

8.800 .947790 .00044

8.850 .951470 .00045

8.900 .955160 .00044

8.950 .959300 .00045
50

8.000 .916910 .00045 8.050 .920610 .00046

8.100 .925310 .00046

8.150 .929180 .00045

8.200 .933390 .00047

8.250 .937460 .00046

8.300 .941710 .00045

8.350 .945910 .00046

8.400 .950510 .00046

8.450 .954660 .00045

8.500 .958170 .00046

8.550 .962170 .00046 
9.300 .960820 .00043

9.350 .964220 .00043

9.400 .968600 .00044

9.450 .972270 .00044

9.500 .974560 .00045

9.550 .978280 .00043

9.600 .982360 .00043

9.650 .985440 .00043

9.700 .987280 .00044

9.750 .991110 .00044

9.800 .993970 .00042

9.850 .999070 .00043

9.901 .001420 .00043

9.951 .004770 .00044

10.001 .007980 .00043

10.051 .011220 .00042

10.101 .013700 .00044

10.151 .017080 .00043

10.201 .020610 .00042
9.000 .963390 .00045

9.050 .965480 .00047

9.100 .970470 .00044

9.150 .974390 .00043

9.200 .977500 .00044

9.250 .981870 .00045

9.300 .985360 .00044

9.350 .989000 .00045

9.400 .992100 .00045

9.450 .995440 .00044

9.500 .999190 .00045

9.551 .002680 .00045

9.601 .006790 .00045

9.651 .008810 .00044

9.701 .012160 .00044

9.751 .016770 .00045

9.801 .020340 .00044
8.600 .967210 .00046

8.650 .970030 .00046

8.700 .973680 .00047

8.750 .978570 .00046

8.800 .982520 .00047

8.850 .986630 .00046

8.900 .990140 .00046

8.950 .994670 .00047

9.000 .997180 .00047

9.051 .000880 .00046

9.101 .005120 .00046

9.151 .009100 .00048

9.201 .011740 .00045

9.251 .016420 .00046

9.301 .019570 .00046
60

7.800 .919970 .00047

7.850 .924220 .00047

7.900 .928560 .00047

7.950 .933450 .00047

8.000 .937070 .00046

8.050 .941680 .00047

8.100 .946470 .00046

8.150 .950210 .00048

8.200 .955250 .00048

8.250 .958290 .00047

8.300 .963320 .00046

8.350 .967360 .00049

8.400 .972270 .00046

8.450 .976210 .00046

8.500 .980630 .00046

8.550 .984190 .00046

8.600 .988170 .00046

8.650 .992730 .00048

8.700 .996460 .00047

8.751 .000210 .00047

8.801 .003910 .00047

8.851 .007850 .00046

8.901 .012550 .00047

8.951 .015650 .00047

9.001 .019680 .00048
70

7.600 .915950 .00048

7.650 .922360 .00048

7.700 .926550 .00049

7.750 .930140 .00047

7.800 .935000 .00047

7.850 .939490 .00049

7.900 .943890 .00046

7.950 .948360 .00047

8.000 .952650 .00046

8.050 .957240 .00047

8.100 .962230 .00047

8.150 .966350 .00048

8.200 .970650 .00046

8.250 .975350 .00046

8.300 .979240 .00047

8.350 .983110 .00047

8.400 .988280 .00047

8.450 .991990 .00049

8.500 .995730 .00047

8.551 .000000 .00049

8.601 .004460 .00049

8.651 .008920 .00048

8.701 .012610 .00047

8.751 .015970 .00048

8.801 .019550 .00048
80

7.500 .918140 .00047

7.550 .921770 .00048

7.600 .926860 .00048

7.650 .931950 .00048

7.700 .937010 .00048

7.750 .940740 .00049

7.800 .946150 .00050

7.850 .949880 .00048

7.900 .954800 .00048

7.950 .958690 .00048

8.000 .964650 .00049

8.050 .968230 .00049

8.100 .972920 .00048

8.150 .976450 .00049

8.200 .980890 .00047

8.250 .986330 .00047

8.300 .989500 .00048

8.350 .994740 .00049

8.400 .998010 .00049

8.451 .001820 .00046

8.501 .007440 .00048

8.551 .011110 .00046

8.601 .014310 .00049

8.651 .019470 .00049

8.701 .023720 .00047 
90

7.400 .915340 .00048

7.450 .920190 .00048

7.500 .925050 .00048

7.550 .929890 .00048

7.600 .934230 .00046

7.650 .938760 .00049

7.700 .943180 .00048

7.750 .949110 .00049

7.800 .953510 .00047

7.850 .958390 .00049

7.900 .961900 .00048

7.950 .966400 .00049

8.000 .971710 .00049

8.050 .976440 .00046

8.100 .980580 .00048

8.150 .985020 .00049

8.200 .988880 .00049

8.250 .993780 .00049

8.300 .996910 .00048

8.351 .002400 .00049

8.401 .005920 .00048

8.451 .010570 .00048

8.501 .015360 .00047

8.551 .018630 .00049

8.601 .022490 .00048
100

7.400 .920860 .00049

7.450 .926020 .00048

7.500 .930210 .00049

7.550 .935290 .00049

7.600 .939790 .00049

7.650 .944760 .00049

7.700 .949510 .00047

7.750 .954170 .00048

7.800 .958050 .00048

7.850 .963700 .00049

7.900 .967650 .00049

7.950 .972490 .00048

8.000 .977120 .00049

8.050 .981570 .00048

8.100 .986060 .00047

8.150 .990700 .00049

8.200 .994710 .00049

8.250 .997970 .00049

8.301 .003370 .00048

8.351 .007750 .00049

8.401 .011500 .00049

8.451 .015310 .00048

8.501 .020020 .00049
130

7.300 .921410 .00049

7.350 .925740 .00049

7.400 .930780 .00049

7.450 .935110 .00050

7.500 .940130 .00048

7.550 .945180 .00049

7.600 .950140 .00050

7.650 .954490 .00049

7.700 .959310 .00050

7.750 .963470 .00048

7.800 .968510 .00048

7.850 .973060 .00049

7.900 .978040 .00049

7.950 .982630 .00049

8.000 .986530 .00050

8.050 .991340 .00049

8.100 .995180 .00051

8.150 .999880 .00050

8.201 .004500 .00050

8.251 .008050 .00048

8.301 .012560 .00049
150
7.200 .912100 .00048

7.250 .918850 .00048

7.300 .922980 .00048

7.350 .928000 .00048

7.400 .933260 .00049

7.450 .938370 .00047

7.500 .942090 .00050

7.550 .947720 .00048

7.600 .951970 .00049

7.650 .956550 .00049

7.700 .960790 .00049

7.750 .966830 .00048

7.800 .970690 .00050

7.850 .975430 .00049

7.900 .980920 .00050

7.950 .984740 .00050

8.000 .989110 .00051

8.050 .993720 .00048

8.100 .997380 .00048

8.151 .002130 .00050

8.201 .006930 .00048
120

7.300 .918170 .00050

7.350 .923170 .00050

7.400 .927430 .00049

7.450 .932270 .00049

7.500 .937850 .00048

7.550 .942040 .00048

7.600 .946200 .00049

7.650 .951160 .00051

7.700 .956660 .00050

7.750 .961420 .00049

7.800 .965740 .00048

7.850 .969600 .00050

7.900 .974590 .00049

7.950 .980220 .00049

8.000 .983110 .00049

8.050 .987720 .00048

8.100 .993690 .00050

8.150 .997670 .00050

8.201 .001220 .00049

8.251 .005560 .00049

8.301 .009540 .00049

8.351 .013770 .00050

8.401 .018770 .00049

8.451 .022420 .00049

8.501 .027070 .00049

200

7.200 .913720 .00051

7.250 .919930 .00049

7.300 .924170 .00048

7.350 .930030 .00048

7.400 .934390 .00050

7.450 .939130 .00049

7.500 .944300 .00050

7.550 .948630 .00050

7.600 .952940 .00048

7.650 .958060 .00050

7.700 .962820 .00049

7.750 .968110 .00049

7.800 .972130 .00051

7.850 .976350 .00050

7.900 .981090 .00049

7.950 .985730 .00049

8.000 .989820 .00048

8.050 .994000 .00050

8.100 .998470 .00049

8.151 .002690 .00049

8.201 .006960 .00050 
8.351 .017370 .00050 8.401 .020720 .00049

239

7.300 .921290 .00049 7.350 .925970 .00051

7.400 .931480 .00050 7.450 .937030 .00049 7.500 .940530 .00049 7.550 .946170 .00049 7.600 .950630 .00050 7.650 .955220 .00049 7.700 .959360 .00049 7.750 .964470 .00050 7.800 .969230 .00049 7.850 .972630 .00050 7.900 .978490 .00050 7.950 .982710 .00050 8.000 .986920 .00049 8.050 .990790 .00050 8.100 .995730 .00051 8.151 .000440 .00049 8.201 .004500 .00050 8.251 .008990 .00052 8.301 .013360 .00049 8.351 .017420 .00049 8.401 .021020 .00050

\section{8}

7.600 .914200 .00050 7.650 .919330 .00050 7.700 .923790 .00049 7.750 .927700 .00050 7.800 .933160 .00049 7.850 .937960 .00049 7.900 .941510 .00050 7.950 .945370 .00050 8.000 .950630 .00049 8.050 .954510 .00052 8.100 .959460 .00050 8.150 .964730 .00050 8.200 .968660 .00048 8.250 .972600 .00050
8.251 .011380 .00049

8.301 .015660 .00050

8.351 .018310 .00051

8.401 .023850 .00050
8.251 .012150 .00052 8.301 .016370 .00049 8.351 .019630 .00050 8.401 .024680 .00050
7.300 .921040 .00049

7.350 .926320 .00050

7.400 .930480 .00048

7.450 .935020 .00049

7.500 .940180 .00049

7.550 .944760 .00049

7.600 .949210 .00050

7.650 .953840 .00050

7.700 .958790 .00048

7.750 .964370 .00048

7.800 .967790 .00050

7.850 .973030 .00049

7.900 .977340 .00051

7.950 .982210 .00049 8.000 .985890 .00049 8.050 .990380 .00050 8.100 .994100 .00052 8.151 .000100 .00048 8.201 .003000 .00050 8.251 .007590 .00050 8.301 .012040 .00050 8.351 .016400 .00049 8.401 .019790 .00050
359

7.400 .917510 .00050 7.450 .922030 .00050 7.500 .926330 .00050 7.550 .931070 .00049 7.600 .936060 .00048 7.650 .940720 .00050 7.700 .945000 .00049 7.750 .949930 .00051 7.800 .954800 .00050 7.850 .958570 .00048 7.900 .962990 .00050 7.950 .968350 .00050 8.000 .972710 .00050 8.050 .976610 .00050 8.100 .981050 .00051 8.150 .984960 .00049 8.200 .990110 .00049 8.250 .995020 .00050 8.300 .998660 .00049 8.351 .003540 .00049 8.401 .006650 .00050 8.451 .011720 .00049 8.501 .015210 .00051 8.551 .019390 .00050 8.601 .023550 .00049

600

7.700 .917920 .00050 7.750 .921090 .00049 7.800 .926650 .00051 7.850 .930920 .00049 7.900 .935430 .00049 7.950 .939200 .00050 8.000 .943800 .00049 8.050 .948390 .00049 8.100 .953240 .00051 8.150 .957880 .00050 8.200 .962710 .00050 8.250 .966830 .00049 8.300 .970740 .00049 8.350 .973620 .00048
8.100 .916380 .00050 8.150 .920300 .00050 8.200 .924420 .00049 8.250 .929400 .00051 8.300 .933320 .00049 8.350 .937080 .00049 8.400 .941550 .00049 8.450 .946110 .00051 8.500 .949820 .00048 8.550 .954360 .00049 8.600 .958570 .00050 8.650 .962800 .00050 8.700 .967140 .00050 8.750 .970450 .00050 
8.300 .976450 .00049

8.350 .981500 .00049

8.400 .985470 .00049

8.450 .989440 .00048

8.500 .993300 .00050

8.550 .998050 .00049

8.601 .003040 .00052

8.651 .007190 .00049

8.701 .009760 .00050

8.751 .014090 .00049

8.801 .018300 .00050

8.851 .022720 .00050

8.901 .025950 .00048
8.400 .979040 .00049

8.450 .982860 .00049

8.500 .988000 .00050

8.550 .991490 .00052

8.600 .995420 .00051

8.651 .000510 .00049

8.701 .004180 .00049

8.751 .007510 .00050

8.801 .012730 .00050

8.851 .016540 .00049

8.901 .020060 .00050

\section{0}

8.600 .923200 .00049

8.650 .926090 .00049

8.700 .930920 .00050

8.750 .933990 .00049

8.800 .939240 .00048

8.850 .942000 .00050

8.900 .945920 .00050

8.950 .950840 .00050

9.000 .955060 .00050

9.050 .958360 .00048

9.100 .962390 .00049

9.150 .966480 .00050

9.200 .970460 .00049

9.250 .974090 .00049

9.300 .978060 .00048

9.350 .981850 .00049

9.400 .986170 .00049

9.450 .989210 .00050

9.500 .993450 .00051

9.550 .997820 .00050

9.601 .001940 .00050

9.651 .004330 .00048

9.701 .007960 .00050

9.751 .012080 .00049

9.801 .016580 .00049

9.851 .020030 .00049

9.901 .022820 .00049
660

8.700 .919730 .00050

8.750 .924420 .00049

8.800 .928010 .00050

8.850 .933290 .00049

8.900 .936520 .00051

8.950 .940130 .00050

9.000 .945240 .00048

9.050 .948840 .00049

9.100 .952140 .00049

9.150 .956940 .00050

9.200 .959500 .00050

9.250 .964560 .00051

9.300 .968660 .00049

9.350 .971470 .00050

9.400 .976150 .00051

9.450 .978750 .00050

9.500 .982760 .00050

9.550 .986670 .00051

9.600 .990990 .00050

9.650 .994880 .00050

9.700 .997340 .00050

9.751 .001510 .00049

9.801 .005870 .00050

9.851 .010080 .00049

9.901 .012370 .00049

9.951 .015790 .00049 10.001 .019480 .00047
8.800 .974840 .00050

8.850 .979120 .00050

8.900 .982940 .00050

8.950 .987360 .00051

9.000 .991030 .00050

9.050 .994190 .00051

9.100 .998880 .00050

9.151 .002870 .00050

9.201 .006380 .00050

9.251 .010520 .00050

9.301 .013480 .00049

9.351 .018010 .00049

9.401 .023020 .00051

670

8.800 .916020 .00049

8.850 .919280 .00047

8.900 .924400 .00048

8.950 .928550 .00049

9.000 .932820 .00050

9.050 .936660 .00048

9.100 .939980 .00049

9.150 .944230 .00050

9.200 .947460 .00051

9.250 .951510 .00050

9.300 .954590 .00048

9.350 .959140 .00050

9.400 .963600 .00051

9.450 .966940 .00050

9.500 .970360 .00050

9.550 .974870 .00049

9.600 .978320 .00050

9.650 .982730 .00050

9.700 .985330 .00050

9.750 .989950 .00049

9.800 .992900 .00049

9.850 .996550 .00051

9.901 .000730 .00049

9.951 .003980 .00050

10.001 .007430 .00050

10.051 .011420 .00050

10.101 .015280 .00052

10.151 .018140 .00049

10.201 .022360 .00049 
680

9.100 .924890 .00049

9.150 .927580 .00050

9.200 .931510 .00049

9.250 .935560 .00049

9.300 .939010 .00050

9.350 .943550 .00048

9.400 .946900 .00049

9.450 .950820 .00050

9.500 .954270 .00048

9.550 .958370 .00050

9.600 .962200 .00049

9.650 .966150 .00048

9.700 .969660 .00050

9.750 .973260 .00049

9.800 .976590 .00048

9.850 .980440 .00049

9.900 .985480 .00050

9.950 .987620 .00050

10.000 .990970 .00049

10.050 .994960 .00050

10.100 .998590 .00050

10.151 .001950 .00049

10.201 .006200 .00049

10.251 .008670 .00050

10.301 .013040 .00048

10.351 .016200 .00048

10.401 .019110 .00048

Pun43-icyl-1000-10

12

14.700 .923950 .00029

14.900 .929440 .00028

15.100 .933240 .00028

15.300 .938040 .00027

15.500 .942610 .00027

15.700 .946590 .00028

15.900 .950760 .00028

16.100 .954930 .00027

16.300 .958640 .00028

16.500 .962930 .00027

16.700 .967000 .00027

16.900 .970630 .00028

17.100 .974060 .00026

17.300 .977450 .00027

17.500 .981190 .00027
690

9.400 .922030 .00049

9.450 .926340 .00049

9.500 .929810 .00049

9.550 .934040 .00049

9.600 .937900 .00050

9.650 .941450 .00050

9.700 .944770 .00050

9.750 .948430 .00051

9.800 .951980 .00051

9.850 .955900 .00049

9.900 .959380 .00050

9.950 .963230 .00050

10.000 .965780 .00049

10.050 .970870 .00050

10.100 .974610 .00049

10.150 .976810 .00049

10.200 .980180 .00051

10.250 .985060 .00050

10.300 .986940 .00051

10.350 .991400 .00050

10.400 .995670 .00048

10.450 .998120 .00050

10.501 .001410 .00048

10.551 .005100 .00050

10.601 .007820 .00050

10.651 .011940 .00049

10.701 .015240 .00052

10.751 .019120 .00052

10.801 .021810 .00050

15

11.900 .924240 .00032

12.000 .928020 .00031

12.100 .931540 .00032

12.200 .934320 .00030

12.300 .939070 .00031

12.400 .941720 .00031

12.500 .945270 .00032

13.100 .964590 .00031

13.200 .967580 .00031

13.300 .971110 .00031

13.400 .974270 .00032

13.500 .976900 .00031

13.600 .980060 .00031

13.700 .982450 .00031

13.800 .985760 .00030
698

9.900 .916250 .00049

9.950 .920140 .00048

10.000 .923170 .00050

10.050 .926860 .00051

10.100 .930670 .00050

10.150 .933720 .00050

10.200 .938130 .00048

10.250 .941310 .00050

10.300 .944860 .00049

10.350 .948110 .00051

10.400 .951480 .00050

10.450 .955850 .00050

10.500 .958370 .00050

10.550 .961280 .00048

10.600 .965430 .00048

10.650 .968870 .00049

10.700 .972330 .00049

10.750 .975530 .00049

10.800 .978700 .00050

10.850 .981990 .00052

10.900 .985100 .00050

10.950 .988650 .00050

11.000 .992540 .00050

11.050 .995920 .00050

11.100 .998510 .00049

11.151 .002700 .00050

11.201 .005210 .00051

11.251 .007880 .00050

11.301 .010830 .00049

11.351 .014320 .00049

11.401 .017300 .00050

\section{F-178

\section{0}

9.700 .921240 .00035

9.800 .926590 .00035

9.900 .931690 .00035

10.000 .936930 .00035

10.100 .941110 .00034

10.200 .947020 .00036

10.300 .951610 .00035

10.400 .956070 .00036

10.500 .961230 .00035

10.600 .965170 .00034

10.700 .970890 .00036

10.800 .975140 .00035

10.900 .979050 .00035

11.000 .983940 .00035

11.100 .987540 .00035 
17.700 .984550 .00026 17.900 .988020 .00026 18.100 .991380 .00026 18.300 .995360 .00027 18.500 .997740 .00027 18.701 .000790 .00025 18.901 .003890 .00026 19.101 .006780 .00026 19.301 .009390 .00026 19.501 .012330 .00025 19.601 .014430 .00024
13.900 .988390 .00029 14.000 .991700 .00030 14.100 .993810 .00030 14.200 .996440 .00030 14.300 .998690 .00030 14.401 .001510 .00030 14.501 .004830 .00030 14.601 .007380 .00030 14.701 .009870 .00030 14.801 .012310 .00031 14.901 .014560 .00030
11.200 .991890 .00034 11.300 .996740 .00034 11.401 .000780 .00035 11.501 .004680 .00035 11.601 .009050 .00035 11.701 .013390 .00035 11.801 .016490 .00035
25

8.600 .919480 .00037 8.700 .925280 .00038 8.800 .931880 .00037 8.900 .937530 .00037 9.000 .943790 .00037 9.100 .949930 .00038 9.200 .955770 .00037 9.300 .961330 .00038 9.400 .967920 .00038 9.500 .972250 .00037 9.600 .978400 .00037 9.700 .984160 .00037 9.800 .989370 .00038 9.900 .994490 .00037 10.000 .999860 .00038 10.101 .005160 .00038 10.201 .010520 .00037 10.301 .014930 .00037 10.401 .020520 .00036
30

8.000 .923110 .00040 8.050 .925800 .00039

8.100 .929880 .00039 8.150 .933150 .00038 8.200 .936410 .00039 8.250 .940370 .00039 8.300 .943320 .00039 8.350 .946890 .00039 8.400 .950210 .00038 8.450 .954370 .00039 8.500 .957650 .00038 8.550 .960420 .00038 8.600 .964090 .00039 8.650 .967180 .00040 8.700 .970010 .00039 8.750 .973810 .00039 8.800 .976020 .00039 8.850 .979820 .00039 8.900 .982450 .00040 8.950 .986120 .00040 9.000 .989450 .00039 9.050 .991860 .00040 9.100 .995000 .00039 9.150 .997830 .00039 9.201 .001220 .00038 9.251 .003870 .00038 9.301 .007120 .00039 9.351 .009920 .00039 9.401 .012500 .00038 9.451 .016220 .00039 9.501 .017530 .00038
32

7.800 .922140 .00039

7.850 .925550 .00039

7.900 .929870 .00040

7.950 .933660 .00040

8.000 .936430 .00040

8.050 .940200 .00039

8.100 .943770 .00040

8.150 .948320 .00039

8.200 .950470 .00040

8.250 .954720 .00040

8.300 .958300 .00039

8.350 .961280 .00040

8.400 .964400 .00039

8.450 .967960 .00039

8.500 .971270 .00040

8.550 .974400 .00038

8.600 .978000 .00040

8.650 .981480 .00039

8.700 .984270 .00040

8.750 .989010 .00040

8.800 .991080 .00041

8.850 .994580 .00039

8.900 .997650 .00039

8.951 .001180 .00038

9.001 .003490 .00039

9.051 .007250 .00040

9.101 .010040 .00041

9.151 .013340 .00040

9.201 .015920 .00040

9.251 .018220 .00039

9.301 .022190 .00040 
35

7.500 .917520 .00041

7.550 .921730 .00041

7.600 .925910 .00040

7.650 .929420 .00041

7.700 .933510 .00040

7.750 .936270 .00040

7.800 .940750 .00040

7.850 .944000 .00040

7.900 .947300 .00039

7.950 .951890 .00040

8.000 .955880 .00041

8.050 .958750 .00040

8.100 .962480 .00041

8.150 .966600 .00040

8.200 .970340 .00040

8.250 .974150 .00040

8.300 .976390 .00041

8.450 .988190 .00041

8.500 .990450 .00041

8.550 .994040 .00040

8.600 .997210 .00041

8.651 .000150 .00040

8.701 .003390 .00041

8.801 .010910 .00040

8.851 .013670 .00039

60

6.500 .918270 .00043

6.550 .922520 .00044

6.600 .927660 .00043

6.650 .932120 .00043

6.700 .936950 .00044

6.750 .941060 .00043

6.800 .945300 .00044

6.850 .949870 .00043

6.900 .953980 .00042

6.950 .959030 .00043

7.000 .963580 .00043

7.050 .967340 .00044

7.100 .972000 .00043

7.150 .975350 .00043

7.200 .979840 .00043

7.250 .984600 .00043

7.300 .988230 .00044

7.350 .992890 .00043

7.400 .996840 .00043

7.451 .000910 .00044

7.501 .004700 .00043

7.551 .009290 .00044

7.601 .011950 .00043
40

7.400 .934370 .00040

7.450 .938820 .00040

7.500 .941960 .00041

7.550 .947140 .00042

7.600 .950710 .00041

7.650 .954930 .00041

7.700 .958700 .00040

7.750 .962200 .00040

7.800 .965690 .00041

7.850 .970490 .00041

7.900 .974300 .00040

7.950 .977910 .00041

8.000 .981050 .00041

8.050 .984690 .00041

8.100 .988800 .00041

8.150 .990380 .00041

8.200 .995290 .00042

8.251 .000360 .00041

8.301 .002280 .00041

8.351 .006230 .00039

8.401 .009750 .00041

8.451 .013140 .00041

8.501 .016810 .00041

\section{0}

6.300 .917850 .00043

6.350 .921900 .00045

6.400 .927000 .00043

6.450 .931000 .00043

6.500 .936260 .00044

6.550 .941300 .00045

6.600 .945260 .00044

6.650 .949970 .00044

6.700 .954240 .00043

6.750 .960210 .00043

6.800 .963380 .00044

6.850 .968110 .00043

6.900 .972590 .00044

6.950 .976920 .00043

7.000 .981450 .00043

7.050 .985890 .00044

7.100 .989570 .00043

7.150 .993520 .00044

7.200 .998960 .00044

7.251 .002940 .00043

7.301 .007310 .00045

7.351 .011130 .00044

7.401 .015140 .00043
50

6.800 .921100 .00042

6.850 .925950 .00042

6.900 .930360 .00043

6.950 .934950 .00042

7.000 .938980 .00042

7.050 .943410 .00042

7.100 .946050 .00042

7.150 .951030 .00042

7.200 .955920 .00042

7.250 .960260 .00043

7.300 .963910 .00042

7.350 .968150 .00041

7.400 .972110 .00043

7.450 .976020 .00042

7.500 .980580 .00043

7.550 .984740 .00043

7.600 .987800 .00042

7.650 .991520 .00043

7.700 .995580 .00042

7.750 .999450 .00044

7.801 .004060 .00042

7.851 .007400 .00041

7.901 .010920 .00042

7.951 .014360 .00042

8.001 .018790 .00042

80

6.200 .920310 .00046

6.250 .923900 .00042

6.300 .930420 .00044

6.350 .934350 .00043

6.400 .939330 .00045

6.450 .944290 .00044

6.500 .949350 .00045

6.550 .953940 .00044

6.600 .958210 .00044

6.650 .963160 .00044

6.700 .967550 .00044

6.750 .971610 .00044

6.800 .976400 .00045

6.850 .980990 .00045

6.900 .985420 .00044

6.950 .989760 .00044

7.000 .994500 .00043

7.050 .998360 .00045

7.101 .003470 .00045

7.151 .007780 .00044

7.201 .010920 .00044

7.251 .015500 .00044

7.301 .019790 .00044 
7.651 .017180 .00044 7.701 .020610 .00043
7.451 .019150 .00044

7.501 .023420 .00044
90

6.100 .919270 .00045 6.150 .924720 .00045 6.200 .930430 .00044 6.250 .934710 .00043 6.300 .939910 .00044 6.350 .944420 .00045 6.400 .949440 .00045 6.450 .954920 .00044 6.500 .958640 .00044 6.550 .963800 .00045 6.600 .968290 .00046 6.650 .972620 .00043 6.700 .976720 .00044 6.750 .981570 .00044 6.800 .986130 .00044 6.850 .990270 .00044 6.900 .994780 .00045 6.950 .999990 .00045 7.001 .003780 .00045 7.051 .008050 .00043 7.101 .013480 .00044 7.151 .016710 .00044 7.201 .021210 .00045

130

5.900 .922090 .00045 5.950 .927400 .00046 6.000 .932330 .00044 6.050 .937410 .00045 6.100 .941440 .00045 6.150 .947320 .00045 6.200 .952300 .00045 6.250 .957340 .00045 6.300 .961670 .00044 6.350 .966230 .00047 6.400 .971590 .00047 6.450 .976070 .00045 6.500 .980970 .00044 6.550 .984880 .00045 6.600 .990030 .00046 6.650 .993770 .00045 6.700 .998360 .00046 6.751 .002970 .00045
100

6.000 .917940 .00044

6.050 .922030 .00044

6.100 .928140 .00045

6.150 .933060 .00044

6.200 .937550 .00045

6.250 .943020 .00045

6.300 .946810 .00045

6.350 .951630 .00045

6.400 .956600 .00045

6.450 .960410 .00045

6.500 .965620 .00045

6.550 .971000 .00045

6.600 .975520 .00044

6.650 .980710 .00045

6.700 .985160 .00045

6.750 .989420 .00045

6.800 .994070 .00045

6.850 .998270 .00044

6.901 .002320 .00043

6.951 .007110 .00045

7.001 .011170 .00044

7.051 .015680 .00044

7.101 .020050 .00044
120

5.900 .917290 .00045 5.950 .923540 .00044 6.000 .928070 .00044 6.050 .932400 .00045 6.100 .937760 .00045 6.150 .942230 .00045 6.200 .946890 .00045 6.250 .952810 .00045 6.300 .957220 .00045 6.350 .962620 .00045 6.400 .966140 .00045 6.450 .971450 .00046 6.500 .975910 .00046 6.550 .980580 .00045 6.600 .985810 .00045 6.650 .989900 .00045 6.700 .994130 .00044 6.750 .998610 .00045 6.801 .003760 .00045 6.851 .007700 .00044 6.901 .012520 .00044 6.951 .017000 .00046 7.001 .020820 .00046 $\begin{array}{cc}150 \\ 5.80 & 0.916930 .00045\end{array}$

5.850 .922530 .00044

5.900 .926860 .00045

5.950 .932600 .00045

6.000 .937200 .00046

6.050 .942290 .00044

6.100 .946930 .00046

6.150 .951770 .00046

6.200 .957190 .00045

6.250 .962020 .00047

6.300 .966640 .00046

6.350 .971390 .00047

6.400 .976060 .00046

6.450 .980910 .00045

6.500 .985450 .00046

6.550 .989690 .00044

6.600 .994770 .00046

6.650 .999430 .00045
200

5.800 .923780 .00046

5.850 .928780 .00045

5.900 .934190 .00046

5.950 .939000 .00044

6.000 .944030 .00044

6.050 .948000 .00045

6.100 .953950 .00046

6.150 .959460 .00045

6.200 .963570 .00045

6.250 .968170 .00046

6.300 .972750 .00044

6.350 .977200 .00044

6.400 .983500 .00045

6.450 .987460 .00046

6.500 .992560 .00045

6.550 .996110 .00045

6.601 .000230 .00047

6.651 .005860 .00046 
6.801 .007460 .00044 6.851 .011900 .00045 6.901 .016190 .00046 6.951 .020860 .00045 7.001 .025900 .00044

\section{9}

5.700 .914990 .00045 5.750 .920260 .00046 5.800 .924540 .00044 5.850 .930240 .00045 5.900 .934800 .00046 5.950 .940320 .00044 6.000 .944860 .00045 6.050 .949070 .00045 6.100 .953870 .00047 6.150 .960070 .00046 6.200 .964110 .00046 6.250 .968690 .00045 6.300 .973420 .00045 6.350 .978360 .00046 6.400 .982760 .00048 6.450 .987490 .00046 6.500 .991710 .00046 6.550 .996220 .00045 6.601 .001280 .00045 6.651 .004870 .00046 6.701 .010020 .00046 6.751 .012590 .00045 6.801 .018820 .00045

478

5.900 .916300 .00046 5.950 .921170 .00045 6.000 .925490 .00046 6.050 .929740 .00045 6.100 .935510 .00046 6.150 .939540 .00046 6.200 .944570 .00045 6.250 .948880 .00047 6.300 .953090 .00045 6.350 .958890 .00046 6.400 .962260 .00044 6.450 .967320 .00046 6.500 .971260 .00046
6.701 .004330 .00045

6.751 .008650 .00047

6.801 .012040 .00046

6.851 .016970 .00046

6.901 .021990 .00045
6.701 .009140 .00046

6.751 .013590 .00045

6.801 .017580 .00045
250

5.700 .915650 .00046

5.750 .918890 .00046

5.800 .924540 .00044

5.850 .929410 .00045

5.900 .934970 .00046

5.950 .939730 .00045

6.000 .946080 .00047

6.050 .949430 .00046

6.100 .954480 .00047

6.150 .959610 .00045

6.200 .964400 .00045

6.250 .968540 .00046

6.300 .973250 .00045

6.350 .978500 .00045

6.400 .983020 .00046

6.450 .986890 .00046

6.500 .992190 .00045

6.550 .996520 .00046

6.601 .000690 .00045

6.651 .004800 .00046

6.701 .009400 .00046

6.751 .013880 .00046

6.801 .017700 .00046

6.851 .022270 .00046

6.901 .026990 .00046
359

5.800 .919180 .00047 5.850 .923640 .00045 5.900 .929730 .00046 5.950 .935140 .00045 6.000 .938770 .00045 6.050 .943540 .00045 6.100 .949220 .00047 6.150 .953460 .00045 6.200 .957600 .00046 6.250 .962540 .00046 6.300 .967630 .00045 6.350 .971770 .00046 6.400 .975960 .00046 6.450 .980260 .00047 6.500 .984790 .00045 6.550 .989060 .00046 6.600 .993850 .00046 6.650 .998640 .00045 6.701 .003520 .00046 6.751 .007280 .00046 6.801 .011340 .00045 6.851 .015230 .00046 6.901 .020050 .00045
500

6.000 .921240 .00046

6.050 .925840 .00046

6.100 .930560 .00046

6.150 .935300 .00045

6.200 .940170 .00046

6.250 .944460 .00048

6.300 .949020 .00045

6.350 .953680 .00046

6.400 .958500 .00045

6.450 .962350 .00046

6.500 .967300 .00046

6.550 .971590 .00045

6.600 .974800 .00046
600

6.300 .923120 .00045

6.350 .927820 .00045

6.400 .931750 .00045

6.450 .935770 .00046

6.500 .940670 .00046

6.550 .944190 .00046

6.600 .949170 .00047

6.650 .954130 .00046

6.700 .957870 .00046

6.750 .961730 .00047

6.800 .966290 .00045

6.850 .970270 .00045

6.900 .974370 .00047 
6.550 .975510 .00044 6.600 .980100 .00045 6.650 .984500 .00045 6.700 .988510 .00046 6.750 .992900 .00047 6.800 .995970 .00046 6.851 .000860 .00045 6.901 .005940 .00046 6.951 .008780 .00046 7.001 .013850 .00047 7.051 .017850 .00046 7.101 .022660 .00046

650

6.500 .915500 .00046 6.550 .919580 .00045 6.600 .924520 .00045 6.650 .928870 .00045 6.700 .933290 .00045 6.750 .936990 .00045 6.800 .940600 .00045 6.850 .944770 .00046 6.900 .948580 .00046 6.950 .953090 .00045 7.000 .956450 .00046 7.050 .960840 .00047 7.100 .964790 .00048 7.150 .968710 .00045 7.200 .973000 .00046 7.250 .976800 .00045 7.300 .980390 .00046 7.350 .984160 .00047 7.400 .987850 .00044 7.450 .991690 .00047 7.500 .995380 .00046 7.550 .998880 .00045 7.601 .002710 .00046 7.651 .006760 .00047 7.701 .010790 .00046 7.751 .013880 .00046 7.801 .017940 .00045

\section{0}

6.900 .921140 .00047 6.950 .925320 .00046 7.000 .930750 .00046 7.050 .933620 .00045
6.650 .980100 .00046

6.700 .983480 .00046

6.750 .987590 .00045

6.800 .992260 .00045

6.850 .996420 .00046

6.901 .000240 .00047

6.951 .004680 .00046

7.001 .009730 .00047

7.051 .014430 .00046

7.101 .018060 .00047
6.950 .978060 .00045

7.000 .981550 .00045

7.050 .985890 .00047

7.100 .990840 .00046

7.150 .994150 .00047

7.200 .998350 .00046

7.251 .002200 .00046

7.301 .006800 .00046

7.351 .010670 .00046

7.401 .013810 .00045

7.451 .017700 .00047

7.501 .022230 .00047

\section{0}

6.600 .916790 .00046

6.650 .921290 .00046

6.700 .925330 .00044

6.750 .929820 .00046

6.800 .933190 .00046

6.850 .937600 .00047

6.900 .941600 .00046

6.950 .945040 .00045

7.000 .949590 .00047

7.050 .952970 .00046

7.100 .958090 .00046

7.150 .961310 .00045

7.200 .964670 .00047

7.250 .969230 .00046

7.300 .972980 .00046

7.350 .977580 .00046

7.400 .980610 .00046

7.450 .985060 .00047

7.500 .987250 .00047

7.550 .991440 .00047

7.600 .995750 .00047

7.650 .998870 .00046

7.701 .002910 .00046

7.751 .006560 .00047

7.801 .010060 .00047

7.851 .013020 .00047

7.901 .017510 .00047
670

6.800 .925130 .00047

6.850 .929340 .00045

6.900 .933430 .00043

6.950 .936760 .00046

7.000 .940640 .00045

7.050 .944950 .00046

7.100 .949290 .00045

7.150 .952910 .00046

7.200 .956460 .00046

7.250 .960950 .00046

7.300 .964890 .00046

7.350 .968200 .00047

7.400 .971570 .00046

7.450 .975880 .00047

7.500 .979650 .00045

7.550 .982780 .00047

7.600 .986400 .00045

7.650 .990630 .00046

7.700 .994050 .00047

7.750 .998310 .00046

7.801 .001100 .00045

7.851 .004940 .00047

7.901 .008640 .00045

7.951 .012110 .00047

8.001 .015620 .00047

8.051 .018870 .00045

8.101 .022110 .00046
690

7.100 .920290 .00047

7.150 .923570 .00047

7.200 .928380 .00046

7.250 .931620 .00045
698

7.500 .921700 .00045 7.550 .924670 .00046 7.600 .928570 .00045 7.650 .931690 .00045 
7.100 .937910 .00045 7.150 .940950 .00046 7.200 .945110 .00046 7.250 .949200 .00047 7.300 .951920 .00045 7.350 .957210 .00046 7.400 .960370 .00046 7.450 .964170 .00046 7.500 .968070 .00046 7.550 .971660 .00045 7.600 .975680 .00047 7.650 .978230 .00045 7.700 .982040 .00046 7.750 .985250 .00046 7.800 .989790 .00047 7.850 .993520 .00046 7.900 .997200 .00045 7.950 .998920 .00047 8.001 .004030 .00047 8.051 .008270 .00047 8.101 .011470 .00046 8.151 .014360 .00047 8.201 .017620 .00046
7.300 .936030 .00046

7.350 .939840 .00046

7.400 .943440 .00045

7.450 .946420 .00045

7.500 .950450 .00046

7.550 .954900 .00045

7.600 .957320 .00046

7.650 .960750 .00046

7.700 .965160 .00046

7.750 .968860 .00045

7.800 .971690 .00046

7.850 .975160 .00046

7.900 .978980 .00046

7.950 .982760 .00047

8.000 .987550 .00046

8.050 .989460 .00046

8.100 .992640 .00046

8.150 .996780 .00046

8.200 .999110 .00046

8.251 .003350 .00046

8.301 .005920 .00046

8.351 .009590 .00045

8.401 .013220 .00045

8.451 .016420 .00046

8.501 .019640 .00046
7.700 .935460 .00045

7.750 .939580 .00045

7.800 .941520 .00046

7.850 .945440 .00046

7.900 .949410 .00046

7.950 .952700 .00045

8.000 .955980 .00045

8.050 .958620 .00045

8.100 .962480 .00046

8.150 .965460 .00045

8.200 .969540 .00047

8.250 .973380 .00046

8.300 .975630 .00047

8.350 .979630 .00047

8.400 .983010 .00045

8.450 .986380 .00046

8.500 .989440 .00046

8.550 .992280 .00045

8.600 .996310 .00047

8.650 .999390 .00047

8.701 .002990 .00047

8.751 .005480 .00047

8.801 .007770 .00047

8.851 .011600 .00047

8.901 .014920 .00045

8.951 .017540 .00047

9.001 .021070 .00045

Pun46-icyl-1000-00

12

19.400 .924680 .00029

19.700 .930750 .00030

20.000 .936220 .00028

20.300 .941340 .00029

20.600 .946260 .00029

20.900 .951940 .00028

21.200 .956630 .00029

21.500 .960880 .00028

21.800 .965590 .00029

22.100 .970110 .00027

22.400 .974180 .00028

22.700 .978230 .00027

23.000 .982100 .00027

23.300 .986550 .00027

23.600 .989610 .00027

23.900 .993530 .00027

24.200 .997050 .00026

24.501 .000530 .00026

24.801 .003970 .00027

25.101 .007240 .00026
15

15.900 .923590 .00034 16.000 .927080 .00033

16.100 .930810 .00034

16.200 .933320 .00033

16.300 .935730 .00033

16.400 .938400 .00034

16.500 .942130 .00034

16.600 .944740 .00033

16.700 .947380 .00034

16.800 .950710 .00033

16.900 .953370 .00034

17.000 .956510 .00033

17.100 .958340 .00033

17.200 .961500 .00032

17.300 .964510 .00032

17.400 .966930 .00033

17.500 .969170 .00032

17.600 .971320 .00033

17.700 .974310 .00033

17.800 .976050 .00033
20

13.500 .929140 .00039

13.600 .933470 .00038

13.700 .937620 .00038

13.800 .942190 .00037

13.900 .946130 .00038

14.000 .950390 .00037

14.100 .955480 .00038

14.200 .958770 .00039

14.300 .963180 .00038

14.400 .967410 .00038

14.500 .970190 .00038

14.600 .975220 .00037

14.700 .978530 .00038

14.800 .981990 .00037

14.900 .985940 .00037

15.000 .989510 .00038

15.100 .993780 .00038

15.200 .997780 .00037

15.301 .001090 .00037

15.401 .003860 .00036 
25.401 .010140 .00026 25.701 .012540 .00026 25.801 .013800 .00026

25

12.100 .919660 .00041

12.200 .925180 .00041

12.300 .929950 .00040

12.400 .936200 .00042

12.500 .941360 .00041

12.600 .947030 .00042

12.700 .951350 .00041

12.800 .957090 .00042

12.900 .962790 .00040

13.000 .967620 .00041

13.100 .971820 .00041

13.300 .982620 .00040

13.400 .986350 .00040

13.500 .992090 .00040

13.600 .996380 .00041

13.801 .005230 .00041

13.901 .010200 .00041

14.001 .014250 .00039

14.101 .019320 .00040
17.900 .979100 .00032 18.000 .981290 .00031 18.100 .984380 .00032 18.200 .986520 .00032 18.300 .989080 .00032 18.400 .991130 .00031 18.500 .992970 .00032 18.600 .994760 .00031 18.700 .997710 .00031 18.800 .999440 .00033 18.901 .002340 .00032 19.001 .004270 .00032 19.101 .006050 .00032 19.201 .007730 .00033 19.301 .010330 .00032 19.401 .012670 .00031 19.501 .015150 .00032

30 11.400 .918850 .00043 11.500 .925380 .00044 11.550 .928490 .00044 11.600 .931930 .00043 11.650 .935010 .00043 11.700 .938540 .00042 11.750 .940990 .00043 11.800 .944970 .00044 11.850 .947170 .00044 11.900 .950800 .00044 11.950 .953350 .00044 12.000 .957160 .00043 12.050 .959130 .00044 12.100 .962050 .00043 12.150 .964910 .00043 12.200 .968170 .00044 12.250 .970850 .00043 12.300 .974230 .00043 12.350 .976120 .00043 12.400 .979220 .00044 12.450 .982430 .00045 12.500 .985090 .00043 12.550 .988060 .00043 12.600 .989990 .00044 12.650 .993060 .00042 12.700 .995880 .00042 12.750 .998660 .00043 12.801 .002070 .00043 12.851 .003890 .00043 12.901 .006860 .00043 12.951 .009930 .00043
15.501 .008190 .00036 15.601 .011360 .00037 15.701 .014690 .00037 15.801 .018530 .00037
32

11.200 .920730 .00044 11.350 .929890 .00044 11.400 .933660 .00043 11.450 .936030 .00043 11.500 .939600 .00045 11.550 .942280 .00043 11.600 .945930 .00043 11.650 .950080 .00044 11.700 .951520 .00044 11.750 .954600 .00044 11.800 .958420 .00045 11.900 .964360 .00043 12.000 .969920 .00045 12.050 .973590 .00044 12.100 .975790 .00044 12.150 .980140 .00043 12.200 .982380 .00045 12.250 .984810 .00043 12.300 .987650 .00044 12.350 .990250 .00044 12.400 .993760 .00044 12.450 .996240 .00043 12.500 .998880 .00044 12.551 .002420 .00042 12.601 .004230 .00043 12.651 .008340 .00044 12.701 .011060 .00044 12.751 .012820 .00043 12.801 .015990 .00044 12.851 .016980 .00044 12.901 .020980 .00043 
13.001 .012570 .00043

13.051 .014330 .00042

13.101 .017140 .00042
35

10.900 .916750 .00046 10.950 .921300 .00043 11.000 .923480 .00044 11.050 .927160 .00045 11.100 .930130 .00046 11.150 .933970 .00046 11.200 .936620 .00045 11.250 .941070 .00045 11.300 .943820 .00045 11.350 .947010 .00045 11.400 .950220 .00044 11.450 .954180 .00044 11.500 .957230 .00044 11.550 .960250 .00044 11.600 .963570 .00045 11.650 .966370 .00045 11.700 .970200 .00044 11.750 .972640 .00044 11.800 .976490 .00044 11.850 .978810 .00045 11.900 .982690 .00045 11.950 .985420 .00045 12.000 .988860 .00045 12.050 .991800 .00044 12.100 .994050 .00046 12.150 .997510 .00044 12.201 .000580 .00045 12.251 .002620 .00043 12.301 .006520 .00044 12.351 .009630 .00044 12.401 .012640 .00044 12.451 .014780 .00043 12.501 .017500 .00043
40

10.600 .918890 .00045 10.650 .922140 .00045 10.700 .925040 .00047 10.750 .929980 .00046 10.800 .933760 .00046 10.850 .937640 .00045 10.900 .940660 .00044 10.950 .943390 .00046 11.000 .947810 .00046 11.050 .950930 .00044 11.100 .954800 .00046 11.150 .958190 .00046 11.200 .961670 .00046 11.250 .964790 .00044 11.300 .968690 .00046 11.400 .975140 .00046 11.450 .979210 .00045 11.500 .981580 .00045 11.550 .985030 .00046 11.600 .988260 .00046 11.650 .991900 .00045 11.750 .998910 .00046 11.801 .001290 .00045 11.851 .004740 .00047 11.901 .007370 .00045 11.951 .011210 .00045 12.001 .013400 .00045 12.051 .016590 .00045 12.101 .019260 .00046
50

10.200 .921880 .00048 10.250 .925930 .00047 10.300 .929490 .00048 10.350 .934060 .00046 10.400 .937110 .00047 10.450 .940710 .00046 10.500 .944860 .00046 10.550 .949420 .00047 10.600 .953420 .00047 10.650 .956300 .00047 10.750 .964100 .00048 10.800 .966590 .00047 10.850 .970710 .00048 10.900 .974630 .00047 10.950 .978210 .00046 11.000 .983270 .00048 11.050 .986080 .00047 11.100 .990530 .00047 11.150 .994100 .00048 11.200 .995970 .00048 11.251 .000840 .00048 11.301 .004060 .00046 11.351 .007380 .00047 11.401 .010090 .00047 11.451 .014550 .00048 11.501 .017140 .00048 11.551 .020840 .00046 11.601 .023360 .00046
60

9.900 .918320 .00048 9.950 .922920 .00050 10.000 .927130 .00049 10.100 .935090 .00051 10.150 .938170 .00047 10.200 .943210 .00049 10.300 .951460 .00048
70

9.700 .915050 .00050 9.750 .919060 .00050 9.800 .923580 .00050 9.850 .927300 .00049 9.900 .931980 .00049 9.950 .936610 .00049 10.000 .940300 .00050
80

9.600 .916220 .00050 9.650 .920750 .00050 9.750 .930070 .00050 9.800 .933910 .00050 9.850 .937760 .00050 9.900 .942410 .00050 10.000 .951000 .00049 
10.350 .955810 .00048 10.400 .958560 .00048 10.450 .963350 .00050 10.500 .966840 .00049 10.550 .970400 .00050 10.600 .974680 .00049 10.650 .977970 .00048 10.700 .982310 .00048 10.750 .985440 .00048 10.800 .989690 .00048 10.850 .993750 .00048 10.900 .996780 .00050 10.951 .001150 .00049 11.001 .005310 .00047 11.051 .008500 .00048 11.101 .012140 .00048 11.151 .015070 .00049 11.201 .019400 .00049
10.100 .948930 .00049 10.150 .953170 .00049 10.200 .957300 .00049 10.250 .960500 .00049 10.300 .965680 .00049 10.350 .969430 .00048 10.400 .972940 .00047 10.450 .976770 .00050 10.500 .982380 .00050 10.550 .984860 .00048 10.600 .988480 .00050 10.650 .992950 .00049 10.751 .000240 .00050 10.851 .008200 .00049 10.901 .010890 .00049 11.001 .019480 .00049
10.100 .958680 .00051 10.250 .971980 .00050 10.300 .975340 .00050 10.350 .980100 .00050 10.400 .982990 .00050 10.450 .988280 .00049 10.500 .992240 .00050 10.601 .000350 .00047 10.701 .006770 .00052 10.801 .015030 .00050 10.851 .019230 .00051 10.901 .022740 .00049

\section{0}

9.550 .918800 .00050 9.600 .924050 .00051 9.650 .928040 .00050 9.700 .932620 .00048 9.750 .936190 .00050 9.800 .940910 .00050 9.850 .944850 .00050 9.900 .950340 .00049 9.950 .953780 .00050 10.000 .958040 .00049 10.050 .962320 .00050 10.100 .966520 .00051 10.150 .970560 .00051 10.200 .975650 .00051 10.250 .979850 .00050 10.300 .983030 .00051 10.350 .987740 .00052 10.400 .991260 .00051 10.450 .994600 .00051 10.500 .999330 .00050 10.551 .003130 .00049 10.601 .007820 .00051 10.701 .013900 .00050 10.751 .019490 .00049 10.801 .022250 .00050
100

9.500 .919910 .00051 9.550 .923870 .00051 9.600 .928800 .00050 9.650 .933730 .00052 9.700 .937030 .00051 9.750 .943130 .00050 9.800 .945800 .00051 9.850 .950720 .00051 9.900 .954750 .00052 9.950 .960020 .00049 10.000 .963310 .00050 10.050 .968530 .00051 10.150 .976540 .00049 10.200 .980170 .00051 10.250 .984580 .00051 10.300 .988530 .00052 10.350 .994220 .00050 10.400 .996200 .00049 10.451 .000190 .00050 10.501 .004590 .00051 10.551 .008650 .00051 10.601 .012350 .00050 10.651 .016470 .00051 10.701 .020510 .00050
120

9.400 .915260 .00050 9.450 .920290 .00052 9.500 .925160 .00050 9.550 .928690 .00050 9.600 .934570 .00050 9.650 .938830 .00051 9.750 .947760 .00051 9.800 .951270 .00051 9.850 .956260 .00050 9.900 .960120 .00050 10.000 .968510 .00049 10.050 .973370 .00051 10.100 .977220 .00050 10.150 .981850 .00051 10.200 .985960 .00050 10.250 .990190 .00051 10.300 .994580 .00051 10.350 .997990 .00052 10.401 .002790 .00051 10.451 .006470 .00049 10.501 .009850 .00051 10.551 .013050 .00050 10.601 .018160 .00051 10.651 .022080 .00052 10.701 .025480 .00050 
130

9.400 .917400 .00051

9.450 .922650 .00050

9.500 .927190 .00051

9.550 .932050 .00052

9.600 .936260 .00052

9.650 .941000 .00050

9.700 .945870 .00051

9.750 .950070 .00052

9.800 .954320 .00052

9.850 .958030 .00051

9.900 .962310 .00050

9.950 .966520 .00050

10.000 .971270 .00050

10.050 .975330 .00051

10.100 .979760 .00051

10.150 .983370 .00051

10.200 .987950 .00051

10.250 .992330 .00052

10.300 .996370 .00050

10.351 .000320 .00050

10.401 .004360 .00050

10.451 .007240 .00051

10.501 .011560 .00050

10.551 .016190 .00050

10.601 .020460 .00050

10.651 .024300 .00051

10.701 .028060 .00051
150

9.400 .919790 .00049

9.450 .924930 .00051

9.500 .928550 .00051

9.550 .933010 .00051

9.600 .937880 .00049

9.650 .942460 .00051

9.700 .947330 .00051

9.750 .951050 .00052

9.800 .955460 .00050

9.850 .959600 .00051

9.900 .962980 .00051

9.950 .968820 .00052

10.000 .973250 .00051

10.050 .976970 .00051

10.100 .981660 .00051

10.150 .985760 .00051

10.200 .989580 .00051

10.250 .993970 .00050

10.300 .998270 .00050

10.351 .002530 .00052

10.401 .005360 .00051

10.451 .009900 .00051

10.501 .013320 .00051

10.551 .017560 .00051

10.601 .021750 .00051
239

9.400 .913270 .00050

9.450 .917570 .00049

9.500 .923110 .00051

9.550 .926790 .00052

9.600 .932740 .00050

9.650 .936880 .00051

9.700 .942900 .00051

9.750 .945400 .00052

9.800 .949630 .00050

9.850 .954710 .00050

9.900 .958750 .00051

9.950 .963810 .00051

10.000 .967110 .00052

10.050 .971100 .00051

10.100 .976310 .00051

10.150 .979650 .00051

10.200 .983810 .00050

10.250 .988360 .00051

10.300 .991940 .00050
250

9.500 .921880 .00052

9.550 .925970 .00051

9.600 .930380 .00051

9.650 .935400 .00050

9.700 .939280 .00051

9.750 .944350 .00050

9.800 .947660 .00052

9.850 .953570 .00050

9.900 .957670 .00052

9.950 .961280 .00051

10.000 .965300 .00050

10.050 .970700 .00051

10.100 .974340 .00051

10.150 .978040 .00051

10.200 .982210 .00050

10.250 .986660 .00052

10.300 .991990 .00051

10.400 .998830 .00051

10.451 .003620 .00050
200

9.400 .918140 .00051

9.450 .923110 .00052

9.500 .926640 .00051

9.550 .931440 .00051

9.600 .935750 .00050

9.650 .940250 .00051

9.700 .945280 .00051

9.750 .949270 .00051

9.800 .954370 .00051

9.850 .958250 .00052

9.900 .962290 .00051

9.950 .967120 .00052

10.000 .971120 .00051

10.050 .975600 .00051

10.100 .979800 .00051

10.150 .983940 .00051

10.200 .987250 .00051

10.250 .992230 .00051

10.300 .995940 .00051

10.351 .000220 .00050

10.401 .003740 .00052

10.451 .008660 .00051

10.501 .012170 .00051

10.551 .015830 .00050

10.601 .021400 .00051

10.651 .025200 .00050

10.701 .028200 .00052

359

9.600 .914360 .00049

9.650 .920250 .00050

9.700 .924610 .00051

9.750 .928520 .00050

9.800 .933200 .00051

9.850 .937590 .00050

9.900 .941380 .00051

9.950 .946100 .00052

10.000 .950310 .00051

10.050 .955200 .00049

10.100 .958410 .00052

10.150 .962980 .00051

10.250 .971640 .00051

10.350 .980160 .00052

10.450 .989290 .00051

10.500 .991700 .00051

10.550 .995930 .00052

10.601 .000130 .00050

10.651 .003570 .00052 
10.350 .995610 .00050 10.401 .000370 .00051 10.451 .004990 .00050 10.501 .008320 .00050 10.551 .012740 .00051 10.601 .016510 .00051 10.651 .021020 .00051 10.701 .024800 .00050
10.501 .007060 .00050 10.551 .011340 .00050 10.601 .015480 .00051 10.651 .019490 .00050 10.701 .022870 .00051
10.701 .008700 .00051 10.851 .019840 .00050 10.901 .023630 .00051
478

9.800 .917030 .00049 9.850 .921650 .00050 9.900 .926300 .00051 9.950 .930340 .00053 10.000 .934740 .00052 10.050 .939580 .00050 10.100 .942900 .00051 10.150 .947340 .00050 10.200 .951980 .00049 10.250 .956460 .00051 10.300 .960630 .00052 10.350 .965070 .00051 10.400 .969100 .00049 10.450 .974080 .00052 10.500 .976840 .00050 10.550 .981690 .00049 10.600 .984940 .00050 10.650 .989410 .00051 10.700 .992790 .00050 10.750 .997750 .00049 10.801 .000880 .00050 10.851 .004400 .00051 10.901 .008090 .00051 10.951 .012640 .00050 11.001 .016530 .00050 11.051 .020420 .00050 11.101 .024750 .00049
500

9.900 .922390 .00050 9.950 .927330 .00049 10.000 .931210 .00048 10.050 .935490 .00050 10.100 .939390 .00051 10.150 .943880 .00051 10.200 .947950 .00049 10.250 .952260 .00050 10.300 .957440 .00051 10.350 .960450 .00050 10.400 .965000 .00050 10.450 .969140 .00050 10.500 .973210 .00050 10.550 .977090 .00051 10.600 .981310 .00051 10.650 .986050 .00050 10.700 .989870 .00050 10.750 .993110 .00050 10.800 .997880 .00049 10.851 .002270 .00050 10.901 .005180 .00051 10.951 .009590 .00050 11.001 .014590 .00049 11.051 .016920 .00050 11.101 .020740 .00052
650

10.200 .918040 .00050 10.250 .922120 .00049 10.400 .934630 .00049 10.450 .939540 .00049 10.500 .943310 .00050 10.550 .948600 .00051 10.600 .951770 .00050 10.650 .956530 .00050
700

10.400 .918200 .00052 10.450 .922430 .00049 10.500 .926420 .00050 10.550 .930980 .00050 10.600 .935490 .00050 10.650 .939400 .00052 10.700 .943080 .00051 10.750 .947790 .00051
600

10.100 .920880 .00052 10.150 .925740 .00050 10.200 .929970 .00050 10.250 .933880 .00049 10.300 .937180 .00051 10.350 .941680 .00051 10.400 .946000 .00050 10.450 .950280 .00051 10.500 .955130 .00051 10.550 .958470 .00050 10.600 .962890 .00051 10.650 .967170 .00050 10.700 .971390 .00049 10.750 .975620 .00051 10.800 .978710 .00049 10.850 .982360 .00049 10.900 .986940 .00050 10.950 .989710 .00051 11.000 .995750 .00050 11.050 .998870 .00050 11.101 .002400 .00051 11.151 .006400 .00049 11.201 .010510 .00050 11.251 .013290 .00050 11.351 .022470 .00050 11.401 .024080 .00052 
10.700 .959400 .00049

10.750 .964290 .00050

10.800 .967220 .00050

10.850 .972080 .00051

10.900 .975840 .00050

10.950 .978340 .00050

11.000 .983740 .00051

11.050 .987070 .00051

11.100 .991400 .00050

11.150 .995570 .00051

11.200 .998750 .00051

11.251 .002050 .00050

11.301 .006850 .00050

11.351 .010050 .00051

11.401 .014370 .00052

11.451 .018070 .00052

11.501 .020890 .00050

760

10.700 .915890 .00051

10.750 .920450 .00048

10.800 .924540 .00048

10.850 .928410 .00050

10.900 .933660 .00050

10.950 .935960 .00049

11.000 .940970 .00050

11.050 .945000 .00049

11.100 .948770 .00049

11.150 .952720 .00049

11.200 .956450 .00050

11.250 .959930 .00050

11.300 .964390 .00051

11.350 .968820 .00050

11.450 .975620 .00050

11.500 .979700 .00050

11.550 .983440 .00050

11.600 .987320 .00050

11.650 .990530 .00050

11.700 .994470 .00049

11.750 .998940 .00049

11.801 .002640 .00050

11.851 .006200 .00049

11.951 .012020 .00050

12.001 .017470 .00049

12.051 .019800 .00050

12.101 .023720 .00050
10.800 .951780 .00050

10.850 .955430 .00049

10.900 .959440 .00048

10.950 .963980 .00050

11.000 .967310 .00050

11.050 .970840 .00049

11.100 .976070 .00050

11.150 .979790 .00052

11.200 .982920 .00051

11.250 .986300 .00050

11.300 .991200 .00050

11.350 .994360 .00051

11.400 .998380 .00051

11.451 .002240 .00050

11.501 .005460 .00052

11.551 .009030 .00051

11.601 .013780 .00052

11.651 .017730 .00050

11.701 .020820 .00050

765

10.800 .920920 .00050

10.950 .933740 .00050

11.000 .938010 .00048

11.100 .946030 .00050

11.150 .949940 .00051

11.200 .953910 .00050

11.250 .957420 .00051

11.300 .961650 .00051

11.350 .966680 .00051

11.400 .969450 .00049

11.450 .973430 .00049

11.500 .976340 .00051

11.600 .984290 .00050

11.650 .988150 .00050

11.700 .992180 .00050

11.750 .995260 .00048

11.800 .999660 .00050

11.851 .002410 .00050

11.901 .005950 .00050

11.951 .010730 .00050

12.001 .014240 .00050

12.051 .017820 .00051

12.101 .020790 .00049
11.100 .952780 .00050

11.150 .956900 .00051

11.200 .961130 .00050

11.250 .965320 .00049

11.300 .968730 .00049

11.350 .972870 .00051

11.400 .976670 .00050

11.450 .981200 .00050

11.500 .984560 .00049 11.600 .992300 .00048 11.650 .996090 .00051 11.751 .003770 .00050 11.801 .006830 .00050 11.851 .010490 .00050 11.901 .015890 .00050 11.951 .017360 .00050 12.001 .021530 .00050
770

10.800 .918420 .00050

10.850 .922680 .00049

10.900 .926050 .00050

10.950 .930090 .00050

11.000 .935330 .00051

11.050 .937930 .00050

11.100 .942730 .00049

11.150 .946660 .00049

11.200 .950830 .00049

11.250 .953870 .00049

11.300 .958890 .00050

11.350 .961960 .00051

11.400 .965480 .00050

11.450 .970690 .00048

11.500 .973180 .00050

11.550 .977560 .00049

11.600 .981980 .00051

11.650 .985180 .00050

11.700 .988800 .00049

11.750 .992320 .00050

11.800 .996440 .00050

11.851 .000120 .00050

11.901 .004280 .00051

11.951 .007420 .00051

12.001 .010030 .00050

12.051 .015060 .00050

12.101 .017810 .00051

12.151 .021370 .00050

12.201 .025050 .00050 
775

10.800 .916240 .00049 10.850 .921510 .00049 10.900 .925240 .00050 10.950 .928420 .00049 11.000 .932430 .00049 11.050 .936490 .00049 11.100 .941200 .00050 11.150 .945520 .00049 11.200 .947900 .00050 11.250 .953090 .00050 11.300 .956350 .00051 11.350 .960720 .00050 11.400 .963000 .00050 11.450 .967900 .00050 11.500 .972550 .00050 11.550 .975120 .00051 11.600 .979720 .00050 11.650 .983250 .00050 11.700 .986970 .00052 11.750 .990120 .00049 11.800 .994330 .00051 11.850 .998440 .00050 11.901 .000860 .00049 11.951 .006830 .00049 12.001 .009120 .00051 12.051 .012750 .00051 12.101 .016290 .00050 12.151 .019870 .00051 12.201 .022910 .00049

Pun46-icyl-1000-01

12

17.900 .926620 .00029 18.200 .932030 .00028 18.500 .938020 .00028 18.800 .943260 .00028 19.100 .947690 .00028 19.400 .953290 .00027 19.700 .957760 .00027 20.000 .962490 .00027 20.300 .966920 .00026 20.600 .971190 .00026 20.900 .974850 .00027 21.200 .979220 .00027 21.500 .983000 .00026 21.800 .987370 .00027
0

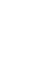


22.100 .990220 .00026 22.400 .994480 .00026 22.700 .998060 .00026 23.001 .001040 .00026 23.301 .004750 .00026 23.601 .007770 .00026 23.901 .010790 .00025 24.101 .013180 .00026

25

10.600 .923480 .00039 10.700 .929590 .00040 10.800 .935130 .00039 10.900 .940670 .00040 11.000 .945990 .00040 11.100 .951300 .00038 11.200 .956950 .00040 11.300 .960830 .00039 11.400 .966510 .00040 11.500 .971770 .00038 11.600 .976720 .00039 11.700 .981660 .00040 11.800 .986830 .00040 11.900 .990900 .00039 12.000 .996090 .00039 12.101 .000020 .00040 12.201 .005470 .00039 12.401 .014120 .00039 12.501 .018330 .00039
15.700 .962990 .00032 15.800 .965630 .00031 15.900 .968740 .00032 16.000 .971000 .00031 16.100 .973070 .00031 16.200 .976080 .00030 16.300 .978280 .00031 16.400 .980840 .00031 16.500 .984150 .00031 16.600 .985230 .00032 16.700 .988670 .00032 16.800 .990230 .00031 16.900 .993270 .00031 17.000 .995080 .00031 17.100 .997120 .00032 17.200 .999740 .00031 17.301 .001910 .00031 17.401 .003840 .00031 17.501 .005260 .00032 17.601 .008060 .00032 17.701 .009720 .00031 17.801 .012260 .00030
13.300 .985920 .00036 13.400 .989220 .00037 13.500 .992650 .00036 13.600 .997540 .00036 13.701 .000130 .00036 13.801 .004000 .00037 14.001 .011140 .00036 14.101 .013890 .00036 14.201 .017810 .00037

\section{0}

9.800 .918250 .00040 9.850 .921610 .00041 9.950 .928190 .00043 10.000 .930920 .00041 10.050 .934750 .00042 10.100 .937260 .00042 10.150 .940970 .00042 10.200 .943650 .00041 10.250 .946290 .00041 10.300 .949960 .00041 10.350 .952630 .00040 10.400 .955860 .00041 10.450 .959050 .00042 10.500 .961330 .00041 10.550 .964540 .00042 10.600 .966860 .00041 10.650 .970250 .00041 10.700 .973150 .00041 10.750 .976120 .00041 10.800 .979220 .00042 10.850 .981640 .00042 10.900 .984590 .00042 10.950 .987260 .00041 11.000 .990550 .00041 11.050 .993660 .00043
32

9.650 .922810 .00043 9.750 .928410 .00041 9.800 .932430 .00042 9.850 .934770 .00042 9.900 .938780 .00042 9.950 .941820 .00043 10.000 .945520 .00042 10.050 .947470 .00042 10.100 .950810 .00042 10.150 .954340 .00042 10.200 .957750 .00042 10.250 .960510 .00042 10.300 .963080 .00042 10.350 .967790 .00042 10.400 .970010 .00043 10.450 .973330 .00043 10.500 .976140 .00042 10.550 .978160 .00043 10.600 .982680 .00043 10.650 .984210 .00042 10.700 .988170 .00042 10.750 .990090 .00041 10.800 .993190 .00043 10.850 .995630 .00042 10.900 .997850 .00042 
11.100 .995840 .00042 11.150 .998320 .00041 11.201 .001160 .00042 11.251 .004320 .00041 11.301 .006270 .00042 11.351 .008690 .00041 11.401 .011960 .00041 11.451 .013740 .00041 11.501 .016400 .00041
10.951 .001790 .00042 11.001 .004390 .00042 11.051 .006890 .00044 11.101 .010490 .00042 11.151 .012320 .00042 11.201 .016170 .00042 11.251 .017740 .00042 11.301 .021240 .00041
35

9.400 .922670 .00044 9.450 .927030 .00044 9.500 .929970 .00043 9.550 .933300 .00043 9.600 .936710 .00045 9.650 .941090 .00044 9.700 .943080 .00044 9.750 .946700 .00042 9.800 .950290 .00044 9.850 .953830 .00041 9.900 .956990 .00044 9.950 .960100 .00042 10.000 .963380 .00043 10.050 .966820 .00044 10.100 .970000 .00042 10.150 .972650 .00043 10.200 .976540 .00043 10.250 .978600 .00043 10.300 .982550 .00042 10.350 .985200 .00043 10.400 .988100 .00043 10.450 .990640 .00042 10.500 .994000 .00044 10.550 .997100 .00043 10.600 .999930 .00041 10.651 .003040 .00043 10.701 .004760 .00043 10.751 .009160 .00044 10.801 .012060 .00043 10.851 .014360 .00043 10.901 .017490 .00042
40

9.000 .918030 .00045 9.050 .921800 .00043 9.100 .926330 .00044 9.150 .929290 .00043 9.200 .933350 .00045 9.250 .936060 .00044 9.300 .940390 .00046 9.350 .943070 .00044 9.400 .948070 .00043 9.450 .951320 .00043 9.500 .955030 .00044 9.550 .957930 .00044 9.600 .961460 .00045 9.700 .968120 .00043 9.800 .974270 .00043 9.850 .978110 .00045 9.900 .982080 .00043 9.950 .984870 .00044 10.000 .988610 .00044 10.050 .991040 .00044 10.150 .998510 .00044 10.201 .001770 .00046 10.301 .007490 .00044 10.351 .011150 .00044 10.401 .013860 .00045 10.451 .016300 .00045 10.501 .019010 .00045
50

8.600 .921460 .00047 8.650 .925000 .00045 8.700 .928980 .00047 8.750 .933980 .00047 8.800 .936660 .00045 8.850 .941140 .00045 8.900 .945310 .00045 9.000 .952550 .00045 9.050 .956380 .00046 9.100 .959540 .00045 9.150 .964040 .00046 9.200 .967420 .00046 9.250 .972370 .00045 9.300 .974880 .00046 9.350 .978970 .00046 9.400 .982090 .00046 9.450 .986630 .00045 9.500 .989630 .00046 9.550 .994080 .00045 9.600 .996820 .00046 9.651 .000360 .00044 9.701 .003580 .00047 9.751 .006640 .00045 9.801 .010920 .00045 9.851 .014100 .00044 9.901 .017100 .00046 
60

8.300 .918610 .00048 8.350 .923230 .00046 8.400 .927010 .00046 8.500 .935680 .00045 8.550 .939200 .00047 8.600 .944810 .00047 8.650 .946970 .00049 8.700 .950710 .00047 8.750 .955650 .00045 8.800 .959960 .00047 8.850 .963330 .00047 8.900 .967810 .00046 8.950 .971930 .00047 9.000 .974750 .00047 9.050 .979540 .00048 9.100 .982530 .00047 9.150 .986550 .00046 9.200 .990740 .00046 9.250 .993390 .00047 9.300 .997250 .00046 9.351 .001280 .00046 9.401 .005630 .00047 9.451 .008810 .00046 9.501 .012930 .00045 9.551 .015340 .00046 9.601 .018860 .00045

\section{0}

7.900 .916560 .00049 7.950 .920890 .00048 8.000 .925730 .00047 8.050 .929550 .00047 8.100 .934840 .00048 8.150 .938070 .00048 8.200 .943050 .00048 8.250 .947570 .00048 8.300 .951020 .00049 8.350 .956110 .00048 8.400 .960350 .00048 8.450 .964580 .00049 8.500 .968650 .00048 8.550 .973190 .00050 8.600 .977250 .00049 8.650 .979930 .00048 8.700 .985330 .00048 8.750 .989450 .00047
70

8.100 .915460 .00048

8.150 .920110 .00049

8.200 .924530 .00047

8.250 .927570 .00046

8.300 .933510 .00048

8.350 .937280 .00047

8.400 .942310 .00047

8.450 .946030 .00047

8.500 .950590 .00047

8.550 .954470 .00047

8.600 .958370 .00046

8.650 .962540 .00047

8.700 .966310 .00048

8.750 .970670 .00047

8.800 .974500 .00048

8.850 .978320 .00048

8.900 .982380 .00047

8.950 .986350 .00047

9.000 .990200 .00049

9.050 .993000 .00048

9.100 .998130 .00049

9.201 .005690 .00048

9.251 .008870 .00047

9.351 .016360 .00048

9.401 .020340 .00048
80

8.000 .917150 .00048

8.050 .922240 .00048

8.100 .927070 .00047

8.150 .931150 .00049

8.200 .935420 .00048

8.250 .939830 .00048

8.300 .943600 .00047

8.350 .948120 .00048

8.400 .952770 .00048

8.450 .956370 .00049

8.500 .961860 .00047

8.650 .972990 .00048

8.700 .978100 .00046

8.750 .980280 .00049

8.800 .985300 .00048

8.850 .988670 .00047

8.950 .997510 .00048

9.051 .004350 .00048

9.151 .011970 .00047

9.201 .016730 .00047

9.251 .019510 .00049

9.301 .023890 .00048
100

7.900 .921650 .00049

7.950 .926780 .00048

8.000 .931290 .00048

8.050 .935940 .00048

8.100 .939320 .00047

8.150 .944140 .00049

8.200 .949130 .00047

8.250 .952990 .00047

8.300 .957100 .00047

8.350 .961100 .00049

8.400 .966080 .00048

8.500 .974050 .00047

8.550 .978230 .00048

8.600 .982550 .00048

8.650 .987050 .00050

8.700 .991630 .00050

8.750 .994050 .00049

8.800 .998760 .00048
120

7.800 .919290 .00049

7.850 .923920 .00049

7.900 .928150 .00050

7.950 .932080 .00050

8.050 .941290 .00048

8.100 .945600 .00049

8.150 .950150 .00048

8.200 .954970 .00049

8.300 .963780 .00048

8.350 .968210 .00050

8.400 .971410 .00049

8.450 .976880 .00048

8.500 .981120 .00049

8.550 .984960 .00048

8.600 .989200 .00048

8.650 .992290 .00049

8.700 .996540 .00049

8.751 .001530 .00048 
8.800 .992950 .00048 8.850 .997010 .00047 8.901 .001320 .00049 8.951 .004450 .00048 9.051 .011870 .00048 9.101 .016730 .00049 9.151 .020750 .00049 9.201 .024930 .00049

130

7.800 .921390 .00050 7.850 .925590 .00047 7.900 .930400 .00049 7.950 .935010 .00048 8.000 .939580 .00048 8.050 .943990 .00049 8.100 .948270 .00050 8.150 .952120 .00049 8.200 .956600 .00049 8.250 .961380 .00048 8.300 .965780 .00050 8.350 .969590 .00049 8.400 .974110 .00050 8.450 .978780 .00049 8.500 .983190 .00049 8.550 .986510 .00048 8.600 .991330 .00049 8.650 .994940 .00050 8.700 .998880 .00049 8.751 .002890 .00050 8.801 .007550 .00049 8.851 .011150 .00048 8.901 .015460 .00048 8.951 .019350 .00050 9.001 .022750 .00050

\section{9}

7.800 .920110 .00048 7.850 .925000 .00050 7.900 .929370 .00049 7.950 .932440 .00049 8.000 .937720 .00049 8.050 .942360 .00050 8.100 .946310 .00050 8.150 .950840 .00049
8.851 .003700 .00048 8.901 .006980 .00048 8.951 .011230 .00049 9.001 .015480 .00048 9.051 .018350 .00049 9.101 .021980 .00048
8.801 .004730 .00048 8.851 .008710 .00050 8.901 .013330 .00049 8.951 .016940 .00050 9.001 .020430 .00050
150
7.700 .914330 .00048

7.750 .919070 .00050

7.800 .924110 .00049

7.850 .928420 .00049

7.900 .932440 .00049

7.950 .936920 .00049 8.000 .941240 .00049 8.050 .946320 .00049 8.100 .950790 .00049 8.150 .955010 .00049 8.200 .959880 .00048 8.250 .964120 .00049 8.300 .967310 .00049 8.350 .971540 .00050 8.400 .976160 .00048 8.450 .980380 .00049 8.500 .985060 .00048 8.550 .989430 .00048 8.600 .994200 .00050 8.650 .997660 .00049 8.701 .001690 .00050 8.751 .005730 .00050 8.801 .008280 .00048 8.851 .013200 .00051 8.901 .016690 .00049 8.951 .021720 .00049 9.001 .024960 .00049
200

7.800 .923370 .00050 7.850 .927400 .00047 7.900 .931920 .00048 7.950 .936120 .00050 8.000 .941060 .00049 8.050 .945470 .00050 8.100 .949260 .00049 8.150 .954390 .00049 8.200 .958300 .00049 8.250 .963160 .00049 8.300 .967910 .00050 8.350 .971540 .00049 8.400 .975950 .00050 8.450 .979350 .00049 8.500 .984450 .00049 8.550 .988080 .00048 8.600 .993030 .00048 8.650 .996470 .00049 8.701 .001150 .00050 8.751 .004740 .00048 8.801 .008720 .00049 8.851 .012640 .00049 8.901 .016470 .00049 8.951 .020710 .00050 9.001 .024240 .00050
250

7.800 .919040 .00048 7.850 .922610 .00049 7.900 .928480 .00050 7.950 .932270 .00049 8.000 .937130 .00050 8.050 .940430 .00048 8.100 .946260 .00050 8.150 .950350 .00051
359

7.900 .913710 .00050 7.950 .919310 .00049 8.000 .923000 .00049 8.050 .928560 .00048 8.100 .932030 .00049 8.150 .936170 .00049 8.200 .940010 .00050 8.250 .945370 .00049 
8.200 .955360 .00048

8.250 .960050 .00049

8.300 .964330 .00051

8.350 .968440 .00051

8.400 .973390 .00049

8.450 .976810 .00050

8.500 .981370 .00050

8.550 .984920 .00050

8.600 .989530 .00049

8.650 .993690 .00049

8.700 .997880 .00051

8.751 .001730 .00051

8.801 .005710 .00049

8.851 .010070 .00049

8.901 .013800 .00050

8.951 .017040 .00048

9.001 .021000 .00050
8.200 .955170 .00049

8.250 .959030 .00049

8.300 .963590 .00049

8.350 .967060 .00050

8.400 .971890 .00049

8.450 .976140 .00049

8.500 .980260 .00049

8.550 .983860 .00051

8.600 .988290 .00050

8.650 .992520 .00049

8.700 .996110 .00050

8.801 .004680 .00048

8.851 .008930 .00050

8.901 .012360 .00050

8.951 .016530 .00049

9.001 .020060 .00048
8.300 .950980 .00048

8.350 .954250 .00049

8.400 .957360 .00051

8.500 .966330 .00049

8.600 .974020 .00050

8.700 .983340 .00049

8.800 .990270 .00051

8.850 .995510 .00050

8.900 .999670 .00049

8.951 .003020 .00049

9.001 .006620 .00052

9.051 .011060 .00049

9.201 .022370 .00052
478

8.100 .917890 .00049

8.150 .922660 .00050

8.200 .926150 .00051

8.250 .930110 .00049

8.300 .935580 .00050

8.350 .939140 .00048

8.400 .943210 .00050

8.450 .947670 .00050

8.500 .952260 .00049

8.550 .955830 .00049

8.600 .959750 .00049

8.650 .963660 .00049

8.700 .969360 .00051

8.750 .973720 .00049

8.800 .977200 .00049

8.850 .981200 .00051

8.900 .985790 .00049

8.950 .989160 .00049

9.000 .992180 .00049

9.050 .996630 .00050

9.101 .001060 .00049

9.151 .003890 .00050

9.201 .008890 .00049

9.251 .012550 .00050

9.301 .015390 .00049

9.351 .020120 .00050

9.401 .024070 .00051
500

8.200 .923550 .00050

8.250 .927150 .00049

8.300 .931630 .00049

8.350 .935320 .00049

8.400 .940600 .00050

8.450 .945140 .00050

8.500 .949200 .00049

8.550 .954020 .00051

8.600 .956810 .00050

8.650 .961020 .00051

8.700 .965760 .00049

8.750 .968990 .00049

8.800 .972440 .00050

8.850 .978170 .00049

8.900 .981660 .00052

8.950 .985580 .00050

9.000 .990590 .00049

9.050 .994330 .00050

9.100 .997530 .00051

9.151 .000950 .00049

9.201 .005110 .00050

9.251 .008140 .00051

9.301 .012610 .00050

9.351 .016760 .00051

9.401 .021040 .00049
600

8.300 .914820 .00050

8.350 .919500 .00049

8.400 .922900 .00049

8.450 .927810 .00050

8.500 .931630 .00050

8.550 .936170 .00049

8.600 .939930 .00049

8.650 .944420 .00048

8.700 .948960 .00049

8.750 .952810 .00050

8.800 .956090 .00050

8.850 .960550 .00049

8.900 .964850 .00047

8.950 .968400 .00049

9.000 .971800 .00049

9.050 .976860 .00051

9.100 .979870 .00049

9.150 .983880 .00049

9.200 .988220 .00048

9.250 .991420 .00051

9.300 .996950 .00049

9.350 .999190 .00051

9.401 .003620 .00050

9.451 .007130 .00049

9.501 .011270 .00050

9.601 .018640 .00050 
650

8.500 .921580 .00050 8.550 .925740 .00051 8.600 .929440 .00051 8.650 .933560 .00050 8.700 .937730 .00050 8.750 .942070 .00050 8.800 .946610 .00048 8.850 .950020 .00050 8.900 .953520 .00049 8.950 .958770 .00050 9.000 .963070 .00049 9.050 .966860 .00049 9.100 .970830 .00050 9.150 .974350 .00051 9.200 .978070 .00049 9.300 .986690 .00050 9.350 .989320 .00049 9.400 .993410 .00049 9.450 .998000 .00049 9.501 .001270 .00050 9.551 .005300 .00050 9.601 .009070 .00050 9.651 .013180 .00049 9.701 .016200 .00050 9.751 .020320 .00050 9.801 .023840 .00051

760

8.900 .916980 .00049 8.950 .920700 .00050 9.000 .924980 .00050 9.050 .928040 .00049 9.100 .932170 .00048 9.150 .936730 .00049 9.200 .940800 .00051 9.250 .944660 .00049 9.300 .948010 .00049 9.350 .952250 .00049 9.400 .955950 .00049 9.450 .959870 .00050 9.500 .965160 .00050 9.550 .968340 .00050 9.600 .971930 .00049 9.650 .975260 .00048 9.700 .979460 .00049
700

8.700 .923100 .00048

8.750 .927740 .00047

8.800 .931330 .00051

8.850 .935620 .00049

8.900 .939350 .00049

8.950 .943660 .00049

9.000 .948970 .00049

9.050 .952710 .00050

9.100 .955540 .00049

9.150 .960000 .00049

9.200 .963970 .00051

9.250 .967870 .00049

9.300 .971740 .00049

9.350 .975770 .00051

9.400 .979270 .00049

9.450 .982720 .00049

9.500 .987190 .00050

9.550 .990740 .00049

9.600 .994340 .00049

9.650 .997660 .00049

9.701 .001090 .00050

9.751 .005470 .00049

9.801 .008830 .00050

9.851 .013380 .00049

9.901 .016300 .00049

9.951 .019920 .00049

10.001 .024350 .00051
750

8.900 .921340 .00050

8.950 .925450 .00048

9.000 .928660 .00050

9.050 .933520 .00049

9.100 .937230 .00050

9.150 .941020 .00051

9.200 .944850 .00050

9.250 .949030 .00052

9.300 .953080 .00049

9.350 .957300 .00050

9.400 .960860 .00050

9.450 .964750 .00050

9.500 .967790 .00051

9.550 .972260 .00049

9.600 .976110 .00048

9.650 .979940 .00050

9.700 .983660 .00049

9.750 .986680 .00050

9.850 .994250 .00050

9.900 .997810 .00049

10.001 .005570 .00049 10.051 .008590 .00050 10.101 .013230 .00050 10.151 .016040 .00051 10.201 .019220 .00050
8.900 .914060 .00050

8.950 .918650 .00050

9.000 .922010 .00049

9.050 .927050 .00050

9.200 .939060 .00049

9.250 .941730 .00050

9.300 .945440 .00050

9.350 .950030 .00050

9.400 .953470 .00049

9.450 .956930 .00049

9.500 .961950 .00050

9.550 .965360 .00048

9.600 .970030 .00049

9.650 .972920 .00050

9.700 .976560 .00049

9.750 .980260 .00049

9.800 .983690 .00050
770

9.000 .918890 .00049

9.050 .923530 .00050

9.100 .927890 .00050

9.150 .931280 .00049

9.200 .936340 .00049

9.250 .939540 .00050

9.350 .947430 .00050

9.400 .950480 .00048

9.450 .954760 .00049

9.500 .959250 .00049

9.550 .962400 .00049 9.600 .966270 .00049 9.650 .969610 .00050 9.750 .978240 .00050 9.800 .981460 .00049 9.850 .985000 .00050 9.900 .989270 .00050 


\begin{abstract}
9.750 .983290 .00050
9.800 .987030 .00051

9.850 .990160 .00049

9.900 .994120 .00051

9.950 .997710 .00050

10.001 .000830 .00050

10.051 .005450 .00050

10.101 .007170 .00050

10.151 .012690 .00049

10.201 .014930 .00050

10.301 .022530 .00050
\end{abstract}

9.850 .987740 .00048

9.900 .991530 .00052

9.950 .994990 .00049

10.000 .997990 .00050

10.051 .001440 .00051

10.101 .005910 .00050

10.151 .009850 .00049

10.201 .012890 .00051

10.251 .016240 .00050

10.301 .019800 .00048
9.950 .991470 .00049

10.000 .995850 .00049

10.050 .998210 .00050

10.101 .003120 .00050

10.151 .006390 .00050

10.201 .010010 .00050

10.251 .014410 .00051

10.301 .016990 .00049

10.351 .021020 .00051

10.401 .023900 .00050
775

9.000 .918210 .00050

9.050 .920890 .00049

9.100 .925700 .00051

9.150 .930150 .00049

9.250 .938350 .00050

9.300 .941300 .00050

9.350 .945870 .00050

9.400 .948970 .00048

9.450 .953830 .00050

9.500 .958160 .00050

9.550 .961310 .00050

9.600 .964480 .00049

9.650 .967970 .00051

9.700 .971310 .00050

9.750 .975810 .00049

9.800 .980280 .00050

9.850 .982920 .00050

9.900 .986910 .00048

9.950 .990390 .00049

10.000 .994280 .00048

10.050 .997860 .00050

10.101 .001940 .00050

10.151 .004890 .00051

10.201 .008690 .00050

10.251 .012810 .00049

10.301 .015190 .00052

10.351 .018800 .00051

10.401 .023540 .00051

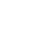


Pun46-icyl-1000-10

12

16.700 .926430 .00027

17.000 .931850 .00026

17.300 .937240 .00026

17.600 .942940 .00026

17.900 .947030 .00026

18.200 .952670 .00027

18.500 .957860 .00026

18.800 .962470 .00026

19.100 .966240 .00026

19.400 .971250 .00026

19.700 .975110 .00025

20.000 .979380 .00026

20.300 .983280 .00026

20.600 .987140 .00026

20.900 .991010 .00025

21.200 .994600 .00024

21.500 .998370 .00025

21.801 .001120 .00025

22.101 .005190 .00024

22.401 .007570 .00024

22.701 .011570 .00024

22.901 .013000 .00024
13.100 .923510 .00030

13.200 .925840 .00031

13.300 .929720 .00030

13.400 .932780 .00031

13.500 .935850 .00032

13.600 .938100 .00031

13.700 .941790 .00031

13.800 .944690 .00030

13.900 .947770 .00031

14.000 .950410 .00029

14.100 .952360 .00030

14.200 .955760 .00029

14.300 .957850 .00030

14.400 .961520 .00030

14.500 .963170 .00030

14.600 .965950 .00030

14.700 .968980 .00030

14.800 .971590 .00030

14.900 .973950 .00031

15.000 .976160 .00028

15.100 .979310 .00030

15.200 .981560 .00029

15.300 .983810 .00029

15.400 .987040 .00030

15.500 .988570 .00030

15.600 .991280 .00029

15.700 .993310 .00029

15.800 .995570 .00029

15.900 .997720 .00030

16.000 .999870 .00030

16.101 .002220 .00029

16.201 .004330 .00029

16.301 .006070 .00029

16.401 .008550 .00029

16.501 .010400 .00030

16.601 .012440 .00028

16.701 .014510 .00029

16.801 .016260 .00029
10.600 .924730 .00035

10.700 .929290 .00034

10.800 .933440 .00035

10.900 .938170 .00034

11.300 .955570 .00035

11.400 .959600 .00035

11.500 .963750 .00034

11.600 .968100 .00034

11.700 .971490 .00035

11.800 .976140 .00033

11.900 .979260 .00034

12.000 .983540 .00035

12.100 .986830 .00033

12.200 .991130 .00034

12.300 .994790 .00035

12.400 .998070 .00034

12.501 .002310 .00033

12.601 .005880 .00033

12.701 .008570 .00034

12.801 .012190 .00033

12.901 .015930 .00033
25

9.300 .920790 .00037

9.400 .926020 .00037

9.500 .931890 .00036

9.600 .938140 .00036

9.700 .942960 .00037

9.800 .949150 .00037
30

8.600 .923000 .00038

8.650 .925790 .00038

8.700 .929180 .00039

8.750 .932150 .00039

8.850 .938740 .00039

8.900 .942030 .00039
32

8.300 .917580 .00039

8.350 .920960 .00039

8.400 .923800 .00039

8.450 .927800 .00039

8.500 .931000 .00039

8.550 .934390 .00038 
9.900 .953470 .00037 10.000 .959460 .00037 10.100 .964380 .00037 10.200 .969210 .00037 10.300 .974190 .00037 10.400 .979250 .00037 10.500 .984540 .00037 10.600 .988720 .00037 10.700 .993740 .00037 10.800 .998920 .00036 10.901 .003490 .00037 11.001 .007520 .00038 11.201 .016770 .00036
8.950 .945460 .00040

9.000 .948050 .00039

9.100 .953910 .00039

9.150 .957270 .00038

9.200 .960430 .00039

9.250 .963410 .00039

9.300 .966260 .00038

9.350 .968570 .00038

9.400 .971670 .00038

9.450 .975620 .00039

9.500 .978430 .00038

9.550 .980700 .00038

9.600 .983320 .00037

9.650 .986830 .00038

9.700 .988680 .00038

9.750 .991570 .00039

9.800 .994400 .00038

9.850 .997350 .00038

9.901 .000690 .00038

9.951 .003020 .00037

10.001 .005930 .00038

10.051 .009030 .00037

10.101 .010110 .00039

10.151 .013180 .00038

10.201 .016560 .00038

10.251 .018910 .00038

10.301 .021140 .00039
8.600 .937010 .00039

8.700 .943850 .00039

8.750 .947590 .00038

8.850 .952680 .00039

8.900 .956910 .00038

8.950 .959890 .00038

9.000 .963580 .00039

9.050 .966420 .00040

9.100 .968380 .00040

9.150 .971950 .00039

9.250 .977540 .00039

9.300 .980740 .00039

9.350 .984070 .00039

9.400 .986890 .00039

9.500 .992620 .00038

9.550 .996100 .00039

9.600 .998420 .00039

9.651 .001160 .00039

9.701 .004560 .00040

9.751 .007020 .00038

9.801 .009830 .00038

9.851 .012520 .00038

9.901 .015260 .00040

9.951 .017860 .00040

10.001 .020540 .00039
35

8.100 .922200 .00039 8.150 .926010 .00041 8.200 .929620 .00039 8.250 .932880 .00039 8.300 .936260 .00039 8.350 .939720 .00041 8.400 .942950 .00040 8.450 .946670 .00040 8.500 .949630 .00040 8.550 .953410 .00040 8.600 .956390 .00040 8.650 .960040 .00039 8.700 .963520 .00040 8.750 .966680 .00040 8.800 .969560 .00040 8.850 .973450 .00040 8.900 .976670 .00039 8.950 .979510 .00039 9.000 .982060 .00040 9.050 .984860 .00039
40

7.700 .918240 .00040

7.750 .922510 .00041

7.800 .926340 .00040

7.850 .930540 .00041

7.900 .933300 .00041

7.950 .938050 .00041

8.000 .941430 .00041

8.050 .944870 .00040

8.100 .948610 .00040

8.150 .951800 .00040

8.200 .955180 .00041

8.250 .959590 .00040

8.300 .962280 .00040

8.350 .965730 .00040

8.400 .969240 .00040

8.450 .972780 .00041

8.500 .975970 .00040

8.550 .980120 .00040

8.600 .983280 .00041

8.650 .986850 .00040
50

7.300 .925180 .00043

7.350 .929130 .00042

7.400 .932740 .00043

7.450 .937540 .00042

7.500 .941470 .00042

7.550 .944920 .00042

7.650 .952150 .00043

7.700 .956060 .00043

7.750 .959770 .00041

7.800 .964650 .00042

7.850 .967130 .00041

7.900 .971620 .00042

7.950 .975290 .00042

8.000 .978810 .00042

8.050 .982210 .00043

8.100 .986150 .00041

8.150 .990380 .00042

8.200 .994180 .00044

8.250 .997460 .00042

8.301 .000910 .00043 
9.100 .988300 .00040 9.150 .991660 .00040 9.200 .995330 .00040 9.250 .997670 .00041 9.301 .000060 .00041 9.351 .004000 .00039 9.401 .006860 .00040 9.451 .009590 .00038 9.501 .011930 .00040 9.551 .015110 .00039 9.601 .018640 .00040

60

7.000 .923680 .00044 7.050 .928500 .00043 7.100 .932720 .00043 7.150 .937150 .00043 7.200 .941990 .00044 7.250 .945350 .00042 7.300 .949320 .00043 7.350 .953980 .00043 7.400 .957160 .00043 7.450 .962000 .00043 7.500 .966570 .00042 7.600 .974590 .00042 7.650 .977420 .00042 7.700 .981310 .00043 7.750 .985260 .00044 7.800 .989290 .00043 7.850 .993350 .00043 7.900 .996190 .00043 7.951 .000900 .00044 8.101 .011770 .00042 8.151 .014950 .00043 8.201 .018830 .00043

90

6.500 .919830 .00044 6.550 .924670 .00044 6.600 .929110 .00044 6.650 .934280 .00044 6.750 .943680 .00044 6.850 .951500 .00044 7.000 .965590 .00045 7.050 .969070 .00045
8.700 .989700 .00040

8.750 .992730 .00041

8.800 .996010 .00040 8.850 .998510 .00041 8.901 .002280 .00041 8.951 .005670 .00041 9.001 .009040 .00041 9.101 .015780 .00040 9.151 .018730 .00040
8.351 .003510 .00043 8.451 .011700 .00043 8.501 .014770 .00042 8.601 .021090 .00041
70

6.800 .924120 .00044

6.850 .928710 .00043

6.900 .932900 .00044

6.950 .938600 .00042

7.000 .941870 .00043

7.050 .946080 .00044

7.100 .950650 .00044

7.150 .955310 .00043

7.200 .958850 .00043

7.250 .962990 .00044

7.300 .967120 .00043

7.350 .970260 .00044

7.450 .979590 .00045

7.500 .983400 .00045

7.550 .986630 .00044

7.600 .990570 .00044

7.650 .994940 .00044

7.700 .999100 .00043

7.751 .003470 .00043

7.801 .006400 .00043

7.851 .010580 .00044

7.901 .014510 .00044

7.951 .017380 .00044

8.001 .022150 .00044
80

6.600 .918830 .00044

6.650 .924240 .00043

6.700 .928160 .00044

6.800 .937470 .00044

6.850 .942020 .00044

6.900 .946580 .00044

6.950 .950680 .00045

7.000 .954090 .00043

7.050 .958500 .00044

7.100 .964300 .00045

7.150 .967320 .00044

7.200 .971660 .00043

7.250 .976100 .00044

7.300 .980620 .00045

7.350 .984000 .00045

7.400 .988330 .00044

7.450 .992760 .00044

7.500 .996160 .00044

7.551 .000440 .00044

7.651 .007720 .00044

7.701 .012430 .00044

7.751 .016720 .00044
100

6.400 .919510 .00044

6.450 .922300 .00044

6.500 .927550 .00044

6.550 .932990 .00046

6.600 .937110 .00044

6.650 .941430 .00043

6.700 .946090 .00044

6.750 .950370 .00043
120

6.300 .918380 .00044 6.350 .923230 .00044 6.400 .928280 .00044 6.450 .933450 .00045 6.500 .937420 .00045 6.550 .942720 .00044 6.600 .946080 .00045 6.650 .951290 .00045 
7.100 .973530 .00044 7.150 .977070 .00045 7.200 .982110 .00044 7.250 .985070 .00045 7.300 .990130 .00045 7.400 .998030 .00044 7.501 .006100 .00044 7.551 .010620 .00044 7.601 .013860 .00045 7.651 .017880 .00044

130

6.300 .923580 .00044 6.350 .927560 .00045 6.400 .931510 .00045 6.450 .936570 .00045 6.500 .941750 .00045 6.550 .945550 .00045 6.600 .950070 .00046 6.650 .954970 .00044 6.700 .959450 .00045 6.750 .964390 .00044 6.850 .973000 .00045 6.900 .977040 .00044 6.950 .981200 .00044 7.000 .984980 .00044 7.050 .990050 .00045 7.150 .998060 .00045 7.201 .001990 .00044 7.251 .006590 .00045 7.301 .010120 .00045 7.351 .014310 .00044 7.401 .019290 .00045
6.800 .954690 .00045

6.850 .960080 .00043

6.900 .963260 .00046

6.950 .967940 .00044

7.000 .972300 .00044

7.050 .976030 .00044

7.100 .980670 .00044

7.150 .984590 .00045

7.200 .988380 .00044

7.250 .993150 .00045

7.300 .997350 .00044

7.351 .002130 .00043

7.401 .005630 .00045

7.451 .009370 .00046

7.501 .013360 .00043

7.551 .018050 .00045
6.700 .955690 .00044 6.750 .960630 .00045 6.800 .964960 .00045 6.850 .968540 .00045 6.900 .972340 .00045 6.950 .977290 .00046 7.000 .981970 .00047 7.100 .990580 .00045 7.150 .994820 .00045 7.200 .998930 .00045 7.251 .002860 .00044 7.301 .006870 .00044 7.351 .010510 .00044 7.401 .014930 .00044 7.451 .018370 .00044 7.501 .022500 .00046
150

6.200 .919020 .00043

6.250 .923090 .00044

6.300 .927470 .00045

6.350 .932730 .00044

6.400 .937090 .00045

6.450 .941330 .00045

6.500 .946560 .00045

6.550 .950390 .00046

6.600 .955910 .00046

6.650 .960300 .00044

6.700 .964480 .00045

6.750 .969160 .00046

6.800 .973120 .00045

6.850 .977310 .00046

6.900 .981520 .00044

6.950 .985830 .00046

7.000 .989190 .00046

7.050 .994600 .00045

7.100 .998590 .00044

7.151 .002560 .00045

7.201 .006840 .00045

7.251 .010750 .00046

7.301 .014820 .00045

7.351 .019210 .00046

7.401 .023640 .00044
200

6.200 .923420 .00044 6.250 .929410 .00045 6.300 .933020 .00046 6.350 .937460 .00045 6.400 .942790 .00044 6.450 .946860 .00045 6.500 .951750 .00044 6.550 .955770 .00046 6.600 .960550 .00045 6.650 .964420 .00045 6.700 .968880 .00046 6.750 .974250 .00046 6.800 .978330 .00045 6.850 .982630 .00046 6.900 .986510 .00046 6.950 .990560 .00046 7.000 .995480 .00045 7.050 .999100 .00046 7.101 .003670 .00045 7.151 .007510 .00044 7.201 .012010 .00046 7.251 .015050 .00046 7.301 .019970 .00044 
239

6.200 .924630 .00045

6.250 .929360 .00044

6.300 .933640 .00045

6.350 .938230 .00046

6.400 .942980 .00046

6.450 .946980 .00045

6.500 .952510 .00046

6.550 .956230 .00044

6.600 .961170 .00047

6.650 .964900 .00046

6.700 .970120 .00045

6.750 .973720 .00044

6.800 .978270 .00047

6.850 .982870 .00045

6.900 .985520 .00046

6.950 .991060 .00045

7.000 .995330 .00045

7.050 .998780 .00045

7.101 .003150 .00046

7.151 .007750 .00046

7.201 .012050 .00046

7.251 .015890 .00045

7.301 .019570 .00045
250

6.200 .924510 .00044

6.250 .928380 .00046

6.300 .933690 .00047

6.350 .938460 .00046

6.400 .942130 .00045

6.450 .947450 .00047

6.500 .952160 .00046

6.550 .956030 .00046

6.600 .960410 .00044

6.650 .965150 .00044

6.700 .969140 .00043

6.750 .974160 .00046

6.800 .978950 .00044

6.850 .982430 .00045

6.900 .986700 .00045

6.950 .989940 .00046

7.000 .994750 .00045

7.050 .998990 .00045

7.101 .002940 .00047

7.151 .006920 .00045

7.201 .011390 .00045

7.251 .014950 .00046

7.301 .019460 .00045
359

6.200 .919590 .00045

6.250 .923020 .00046

6.300 .928700 .00046

6.350 .932300 .00045

6.400 .937770 .00045

6.450 .941940 .00045

6.500 .946750 .00045

6.550 .950800 .00045

6.600 .954130 .00045

6.650 .958760 .00046

6.700 .964430 .00046

6.750 .967690 .00045

6.800 .972360 .00045

6.850 .975290 .00047

6.900 .979980 .00045

6.950 .984450 .00046

7.000 .988740 .00047

7.050 .992880 .00046

7.100 .996920 .00045

7.151 .000980 .00045

7.201 .005300 .00046

7.251 .008780 .00046

7.301 .011540 .00046

7.351 .016610 .00046

7.401 .020260 .00045

478

6.300 .919430 .00045

6.350 .924330 .00045

6.400 .928750 .00046

6.450 .932870 .00045

6.500 .937830 .00047

6.550 .941560 .00045

6.600 .945590 .00046

6.650 .950140 .00046

6.700 .955000 .00045

6.750 .958260 .00046

6.800 .963560 .00045

6.850 .967410 .00046

6.900 .970870 .00046

6.950 .975380 .00044

7.000 .979440 .00045

7.050 .983210 .00045

7.100 .987120 .00045

7.150 .991810 .00046

7.200 .995250 .00045

7.250 .999800 .00047

7.301 .003860 .00046
500
6.300 .918120 .00045
6.350 .921820 .00046
6.400 .926410 .00045
6.450 .930950 .00045
6.500 .935530 .00046
6.550 .939310 .00046
6.600 .945120 .00045
6.650 .948170 .00045
6.700 .951760 .00046
6.750 .956450 .00045
6.800 .960470 .00046
6.850 .964900 .00047
6.900 .968550 .00046
6.950 .973820 .00046
7.000 .978290 .00046
7.050 .980670 .00045
7.100 .985110 .00047
7.150 .990100 .00046
7.200 .993390 .00046
7.250 .996870 .00046
7.301 .000830 .00046
600
6.500 .923830 .00046
6.600 .933010 .00045
6.650 .935900 .00045
6.700 .940570 .00046
6.750 .944410 .00045
6.800 .949360 .00045
6.850 .953350 .00046
7.050 .969490 .00046
7.100 .973950 .00047
7.150 .977200 .00046
7.200 .981320 .00046
7.250 .984860 .00045
7.300 .988010 .00045
7.350 .992830 .00047
7.400 .996850 .00044
7.451 .001290 .00045
7.501 .004700 .00045
7.551 .008860 .00044
7.601 .012550 .00045
7.651 .016600 .00045
7.701 .019830 .00046 
7.351 .006890 .00046 7.401 .010700 .00046 7.451 .014530 .00046 7.501 .019010 .00046

650

6.500 .916810 .00045 6.550 .921780 .00046 6.600 .924990 .00044 6.650 .929700 .00047 6.700 .934070 .00045 6.750 .938000 .00046 6.800 .942230 .00045 6.850 .946510 .00046 6.900 .950610 .00047 6.950 .955200 .00046 7.000 .958140 .00046 7.050 .961960 .00046 7.100 .966200 .00046 7.150 .969670 .00047 7.200 .974260 .00045 7.250 .977860 .00047 7.300 .981890 .00047 7.350 .986620 .00046 7.400 .989280 .00047 7.450 .993040 .00047 7.500 .997890 .00046 7.551 .000950 .00045 7.601 .005700 .00045 7.651 .008900 .00045 7.701 .011700 .00047 7.751 .015560 .00046 7.801 .020720 .00046

760

6.950 .928110 .00045 7.000 .932070 .00047 7.050 .935620 .00045 7.100 .940160 .00045 7.150 .943330 .00046 7.200 .947800 .00045 7.250 .951490 .00047 7.300 .955340 .00048 7.350 .958440 .00046 7.400 .963030 .00045 7.450 .966450 .00047
7.351 .005800 .00045

7.401 .008550 .00044

7.451 .013320 .00047

7.551 .019850 .00046

7.601 .023520 .00046

700

6.700 .923920 .00045

6.750 .928130 .00046

6.800 .932350 .00046

6.850 .936060 .00045

6.900 .940310 .00044

6.950 .944510 .00046

7.000 .948620 .00046

7.050 .952250 .00046

7.100 .955850 .00046

7.150 .960410 .00046

7.200 .963730 .00047

7.250 .967850 .00046

7.300 .971780 .00045

7.350 .975950 .00046

7.400 .978990 .00047

7.450 .983670 .00047

7.500 .987270 .00045

7.550 .991160 .00046

7.600 .994020 .00047

7.650 .998450 .00046

7.701 .001790 .00046

7.751 .005240 .00045

7.801 .009290 .00046

7.851 .012650 .00046

7.901 .015740 .00046
750

6.800 .919190 .00046 6.850 .922460 .00045 6.900 .926740 .00043 6.950 .931050 .00046 7.000 .934730 .00045 7.050 .938780 .00046 7.100 .942230 .00046 7.150 .946490 .00046 7.200 .950730 .00045 7.250 .954670 .00046 7.300 .958160 .00045 7.350 .962300 .00045 7.400 .965610 .00047 7.450 .970690 .00045 7.500 .973690 .00047 7.550 .977650 .00046 7.600 .980750 .00046 7.650 .984320 .00048 7.700 .988020 .00046 7.750 .991870 .00046 7.800 .995760 .00046 7.850 .999100 .00047 7.901 .002650 .00046 7.951 .006070 .00046 8.001 .011710 .00046 8.101 .016410 .00046
6.900 .922080 .00044 6.950 .925240 .00045 7.000 .929620 .00046 7.050 .933510 .00046 7.100 .938730 .00046 7.150 .942610 .00045 7.200 .945670 .00046 7.250 .949170 .00046 7.300 .952530 .00046 7.350 .957980 .00046 7.400 .961460 .00045
770

6.900 .919940 .00045 6.950 .924250 .00046 7.000 .928140 .00045 7.050 .932240 .00046 7.100 .936410 .00046 7.150 .940100 .00046 7.200 .944410 .00046 7.250 .947860 .00045 7.300 .951900 .00045 7.350 .955460 .00045 7.400 .958720 .00047 
7.500 .969440 .00046

7.550 .974010 .00047

7.600 .977430 .00046

7.650 .981100 .00046

7.700 .985350 .00046

7.750 .989040 .00045

7.800 .991970 .00046

7.850 .996750 .00047

7.900 .999440 .00047

7.951 .002580 .00046

8.001 .006540 .00046

8.051 .009970 .00047

8.101 .013360 .00046

8.151 .017370 .00045

8.201 .020530 .00046

775

6.900 .918740 .00045

7.000 .926510 .00046

7.050 .930590 .00047

7.100 .935080 .00047

7.150 .939020 .00046

7.200 .942800 .00046

7.250 .946870 .00046

7.300 .950360 .00046

7.350 .954300 .00046

7.400 .957250 .00045

7.450 .961670 .00045

7.500 .965580 .00046

7.550 .969800 .00046

7.600 .972520 .00044

7.650 .976790 .00046

7.700 .979930 .00045

7.750 .983620 .00045

7.800 .987540 .00046

7.850 .990490 .00046

7.900 .994070 .00048

7.950 .998030 .00044

8.001 .003210 .00045

8.051 .005460 .00046

8.101 .009200 .00046

8.151 .012340 .00045

8.201 .016080 .00047
7.450 .964800 .00046

7.500 .968830 .00045

7.550 .972100 .00046

7.600 .976130 .00046

7.650 .979160 .00046

7.700 .984140 .00044

7.750 .986880 .00045

7.800 .990100 .00047

7.850 .993630 .00046

7.900 .997240 .00046

7.951 .000820 .00046

8.001 .004970 .00044

8.051 .008580 .00046

8.101 .011890 .00046

8.151 .015880 .00046

8.201 .019000 .00046
7.450 .963560 .00046

7.500 .966500 .00046

7.550 .970760 .00047

7.600 .974590 .00046

7.650 .976960 .00045

7.700 .980890 .00047

7.750 .985320 .00046

7.800 .988780 .00045

7.850 .992120 .00047

7.900 .995580 .00046

7.950 .999140 .00046

8.001 .002640 .00045

8.051 .006030 .00046

8.101 .010060 .00046

8.201 .017930 .00047

0 
Pun40-icyl-0970-00

12

16.400 .921790 .00032 16.600 .927420 .00031 16.800 .932880 .00031 17.000 .937370 .00031 17.200 .942380 .00031 17.400 .947810 .00031 17.600 .952040 .00030 17.800 .956310 .00030 18.000 .960500 .00030 18.200 .964730 .00030 18.400 .969510 .00029 18.600 .973380 .00030 18.800 .977980 .00029 19.000 .981190 .00029 19.200 .985330 .00029 19.400 .988830 .00030 19.600 .992660 .00029 19.800 .995700 .00029 20.000 .999170 .00029 20.201 .003130 .00029 20.401 .006430 .00028 20.601 .009730 .00029 20.801 .012940 .00028

25

11.100 .921990 .00042 11.150 .926200 .00043 11.200 .928450 .00041 11.250 .931520 .00044 11.300 .935250 .00042 11.350 .937610 .00043 11.400 .941060 .00042 11.450 .943870 .00042 11.500 .946680 .00043 11.550 .949860 .00042 11.600 .952620 .00041 11.650 .955320 .00042 11.700 .958400 .00042 11.750 .961980 .00041
15

13.900 .920970 .00034

14.000 .923680 .00035

14.100 .927710 .00034

14.200 .932510 .00035

14.300 .935450 .00033

14.400 .938770 .00035

14.500 .943000 .00034

14.600 .947030 .00034

14.700 .949700 .00036

14.800 .953470 .00034

14.900 .956930 .00035

15.000 .960370 .00035

15.100 .962870 .00034

15.200 .966880 .00034

15.300 .970580 .00035

15.400 .973430 .00035

15.500 .976390 .00034

15.600 .979060 .00034

15.700 .983240 .00034

15.800 .986520 .00034

15.900 .988830 .00034

16.000 .991080 .00033

16.100 .994710 .00034

16.200 .997970 .00034

16.301 .000410 .00034

16.401 .003040 .00032

16.501 .005820 .00034

16.601 .008880 .00034

16.701 .011590 .00034

16.801 .013880 .00034

16.901 .017280 .00033

30

10.500 .920450 .00044

10.550 .924170 .00044

10.600 .927960 .00044

10.650 .931180 .00045

10.700 .934750 .00043

10.750 .938700 .00044

10.800 .941530 .00042

10.850 .945270 .00044

10.900 .948540 .00044

10.950 .952230 .00045

11.000 .955090 .00046

11.050 .957310 .00044

11.100 .962140 .00044

11.150 .964350 .00043
20

12.000 .919330 .00039

12.100 .924320 .00040

12.200 .929490 .00039

12.300 .935270 .00039

12.400 .940220 .00039

12.500 .945210 .00040

12.600 .949890 .00040

12.700 .954570 .00040

12.800 .960150 .00039

12.900 .963600 .00038

13.000 .969240 .00038

13.100 .974000 .00039

13.200 .978800 .00039

13.300 .982810 .00038

13.400 .987300 .00039

13.500 .991870 .00038

13.600 .995990 .00039

13.701 .000530 .00039

13.801 .003620 .00037

13.901 .008980 .00038

14.001 .013110 .00038

14.101 .017330 .00038 
11.800 .963650 .00041 11.850 .966300 .00041 11.900 .970730 .00042 11.950 .972880 .00042 12.000 .974960 .00041 12.050 .978050 .00041 12.100 .980800 .00042 12.150 .984050 .00041 12.200 .985890 .00042 12.250 .989070 .00041 12.300 .992130 .00042 12.350 .994730 .00041 12.400 .998300 .00041 12.451 .000380 .00040 12.501 .002900 .00041 12.551 .005180 .00041 12.601 .008160 .00042 12.651 .011060 .00042 12.701 .013040 .00042 12.751 .015020 .00042 12.801 .017570 .00042

35

10.100 .919660 .00045 10.150 .922840 .00046 10.200 .926790 .00045 10.250 .931510 .00046 10.300 .933780 .00044 10.350 .937000 .00045 10.400 .941590 .00046 10.450 .944680 .00046 10.500 .949230 .00044 10.550 .951860 .00045 10.600 .955920 .00045 10.650 .959000 .00045 10.700 .962790 .00046 10.750 .966280 .00046 10.800 .969970 .00046 10.850 .973680 .00045 10.900 .976800 .00045 10.950 .980070 .00046 11.000 .983860 .00045 11.050 .986310 .00046 11.100 .990140 .00045 11.150 .992720 .00046 11.200 .996460 .00045 11.250 .999890 .00046 11.301 .003030 .00045 11.351 .005400 .00046 11.401 .009510 .00045
11.200 .967790 .00044 11.250 .970680 .00044 11.300 .973030 .00044 11.350 .977260 .00044 11.400 .980090 .00044 11.450 .983280 .00045 11.500 .986370 .00043 11.550 .989730 .00045 11.600 .992450 .00044 11.650 .994920 .00045 11.700 .998520 .00044 11.751 .001700 .00044 11.801 .004050 .00044 11.851 .007790 .00044 11.901 .010170 .00043 11.951 .013410 .00044 12.001 .015640 .00044 12.051 .019360 .00045 12.101 .021820 .00043
11.000 .967030 .00045 11.050 .970490 .00044 11.100 .974190 .00045 11.150 .977440 .00044 11.200 .979700 .00045 11.250 .983450 .00044 11.300 .987170 .00045 11.350 .990100 .00045 11.400 .992820 .00045 11.450 .995980 .00044 11.500 .999410 .00045 11.551 .003060 .00045 11.601 .005590 .00045 11.651 .009080 .00045 11.701 .011220 .00046 11.751 .014440 .00045 11.801 .016460 .00045
40

9.800 .915770 .00048 9.850 .921460 .00047 9.900 .924660 .00047 9.950 .929200 .00047 10.000 .933560 .00048 10.050 .937220 .00046 10.100 .940510 .00048 10.150 .943390 .00046 10.200 .948090 .00047 10.250 .952230 .00047 10.300 .955950 .00047 10.350 .959180 .00046 10.400 .962400 .00045 10.450 .966910 .00047 10.500 .970100 .00047 10.550 .974230 .00047 10.600 .977230 .00048 10.650 .981480 .00047 10.700 .983980 .00046 10.750 .988810 .00046 10.800 .991320 .00047 10.850 .995180 .00046 10.900 .998770 .00045 10.951 .002430 .00046 11.001 .006110 .00047 11.051 .009600 .00045 11.101 .012150 .00046
50

9.500 .920240 .00047 9.550 .924520 .00046 9.600 .928900 .00049 9.650 .932890 .00047 9.700 .936550 .00048 9.750 .940950 .00048 9.800 .945470 .00048 9.850 .949280 .00049 9.900 .953140 .00047 9.950 .957520 .00048 10.000 .961150 .00048 10.050 .964940 .00048 10.100 .969050 .00048 10.150 .973090 .00049 10.200 .977410 .00048 10.250 .980780 .00048 10.300 .984010 .00048 10.350 .988320 .00048 10.400 .992470 .00047 10.450 .996470 .00049 10.500 .999420 .00048 10.551 .003240 .00048 10.601 .006100 .00049 10.651 .009620 .00048 10.701 .014380 .00048 10.751 .017590 .00048 10.801 .021400 .00049 
CHPRC-01552, Revision 0

11.451 .012510 .00045 11.501 .015450 .00046 11.551 .018300 .00045 11.601 .021370 .00046
11.151 .014810 .00047 11.201 .018610 .00047
60

9.300 .922120 .00048 9.350 .924880 .00048 9.400 .930120 .00048 9.450 .934180 .00049 9.500 .938880 .00050 9.550 .943310 .00049 9.600 .947470 .00048 9.650 .951350 .00049 9.700 .955710 .00050 9.750 .960010 .00051 9.800 .963380 .00048 9.850 .967360 .00048 9.900 .972170 .00048 9.950 .975860 .00049 10.000 .979800 .00050 10.050 .983680 .00051 10.100 .987310 .00049 10.150 .990870 .00050 10.200 .995230 .00050 10.250 .998900 .00049 10.301 .003460 .00049 10.351 .006390 .00049 10.401 .010490 .00049 10.451 .014750 .00050 10.501 .017780 .00049 10.551 .022890 .00051 10.601 .025860 .00049
70

9.100 .914180 .00050 9.150 .918670 .00050 9.200 .922900 .00049 9.250 .927240 .00051 9.300 .932400 .00049 9.350 .936430 .00050 9.400 .940750 .00049 9.450 .944840 .00050 9.500 .949580 .00050 9.550 .954400 .00050 9.600 .957570 .00049 9.650 .961370 .00049 9.700 .966020 .00050 9.750 .970790 .00051 9.800 .974600 .00050 9.850 .978350 .00049 9.900 .982620 .00050 9.950 .986350 .00049 10.000 .990840 .00049 10.050 .995080 .00049 10.100 .998120 .00050 10.151 .001840 .00050 10.201 .006290 .00051 10.251 .010730 .00049 10.301 .014780 .00050 10.351 .018170 .00052 10.401 .021440 .00049
80

9.000 .912590 .00051 9.050 .917570 .00050 9.100 .922230 .00050 9.150 .926990 .00050 9.200 .931770 .00050 9.250 .935880 .00050 9.300 .939840 .00050 9.350 .944650 .00051 9.400 .949400 .00051 9.450 .952860 .00050 9.500 .957660 .00050 9.550 .961600 .00050 9.600 .965750 .00051 9.650 .969710 .00051 9.700 .974260 .00050 9.750 .978170 .00050 9.800 .982580 .00052 9.850 .985120 .00050 9.900 .990630 .00051 9.950 .994680 .00051 10.000 .998010 .00050 10.051 .003390 .00051 10.101 .006350 .00051 10.151 .010270 .00052 10.201 .015580 .00049 10.251 .018300 .00050 10.301 .022360 .00051
90

9.000 .918450 .00051 9.050 .922390 .00052 9.100 .926570 .00051 9.150 .932990 .00051 9.200 .935910 .00051 9.250 .940620 .00050 9.300 .944760 .00051 9.350 .949140 .00050 9.400 .954510 .00049 9.450 .957950 .00052 9.500 .962400 .00051 9.550 .967020 .00051
100

9.000 .921100 .00051 9.050 .925960 .00052 9.100 .931330 .00049 9.150 .935120 .00050 9.200 .939360 .00050 9.250 .944450 .00051 9.300 .948480 .00051 9.350 .953340 .00051 9.400 .955900 .00051 9.450 .960980 .00052 9.500 .965780 .00051 9.550 .970810 .00051
120

9.000 .924280 .00051 9.050 .928040 .00051 9.100 .932120 .00051 9.150 .938280 .00050 9.200 .941810 .00050 9.250 .947330 .00051 9.300 .950900 .00051 9.350 .955210 .00052 9.400 .959950 .00050 9.450 .963840 .00050 9.500 .968850 .00050 9.550 .972620 .00051 
9.600 .970080 .00051

9.650 .975220 .00050

9.700 .979150 .00051

9.750 .983890 .00052

9.800 .987670 .00051

9.850 .992170 .00051

9.900 .995750 .00049

9.950 .999740 .00050

10.001 .003120 .00049

10.051 .006590 .00051

10.101 .011930 .00051

10.151 .015470 .00049

10.201 .019500 .00050

10.251 .022850 .00050

10.301 .026520 .00053
9.600 .973510 .00051

9.650 .978440 .00050

9.700 .981870 .00052

9.750 .986220 .00050

9.800 .990320 .00051

9.850 .995600 .00051

9.900 .998200 .00050

9.951 .002240 .00051

10.001 .007330 .00050

10.051 .010050 .00051

10.101 .014420 .00050

10.151 .018710 .00052

10.201 .022840 .00051
9.600 .977140 .00051

9.650 .981110 .00051

9.700 .984480 .00050

9.750 .987890 .00052

9.800 .992760 .00052

9.850 .996740 .00050

9.901 .000750 .00051

9.951 .005080 .00049 10.001 .008140 .00051 10.051 .013420 .00053 10.101 .016500 .00050 10.151 .021470 .00051 10.201 .025060 .00052
130

8.900 .916560 .00051

8.950 .920760 .00052

9.000 .925570 .00050

9.050 .931400 .00050

9.150 .938940 .00051

9.200 .942940 .00050

9.250 .948400 .00053

9.300 .952690 .00052

9.350 .955530 .00052

9.400 .961990 .00051

9.450 .965700 .00052

9.500 .970730 .00051

9.550 .974090 .00052

9.600 .978140 .00051

9.650 .982930 .00052

9.700 .986810 .00051

9.750 .990520 .00052

9.800 .994360 .00052

9.850 .998180 .00051

9.901 .002870 .00051

9.951 .006320 .00051

10.001 .010920 .00049

10.051 .014140 .00052

10.101 .018940 .00050
150

8.900 .916280 .00050

8.950 .920310 .00050

9.000 .924990 .00051

9.050 .929360 .00051

9.100 .934460 .00051

9.150 .939070 .00052

9.200 .943370 .00049

9.250 .947910 .00053

9.300 .952350 .00052

9.350 .955330 .00050

9.400 .960010 .00052

9.450 .964310 .00051

9.500 .969190 .00051

9.550 .972380 .00051

9.600 .976750 .00053

9.700 .986070 .00050

9.750 .988930 .00051

9.800 .993520 .00052

9.850 .997760 .00051

9.901 .000730 .00050

9.951 .005120 .00051

10.001 .009410 .00051

10.101 .017380 .00049

10.151 .020770 .00050

10.201 .025360 .00051
200

9.000 .922740 .00050

9.050 .926610 .00051

9.100 .930550 .00052

9.150 .935160 .00050

9.200 .939100 .00050

9.250 .943130 .00049

9.300 .946940 .00051

9.350 .952270 .00051

9.400 .957000 .00051

9.450 .961060 .00051

9.500 .964790 .00050

9.550 .968570 .00051

9.600 .972690 .00052

9.650 .976510 .00050

9.700 .981100 .00051

9.750 .986000 .00052

9.800 .989190 .00050

9.850 .991830 .00051

9.900 .997710 .00051

9.951 .000190 .00051

10.001 .004930 .00051

10.051 .007760 .00051

10.101 .011450 .00051

10.151 .015260 .00051

10.201 .019880 .00052
239

9.000 .917380 .00052

9.050 .921410 .00049

9.100 .925560 .00051
250

9.000 .914540 .00050

9.050 .920030 .00052

9.100 .923730 .00051
359

9.200 .918130 .00051

9.250 .923260 .00051

9.300 .927470 .00049 
9.150 .929860 .00052

9.200 .934520 .00051

9.250 .938700 .00050

9.300 .941470 .00050

9.350 .947900 .00051

9.400 .952160 .00053

9.450 .956470 .00051

9.500 .960020 .00050

9.550 .963180 .00051

9.600 .967880 .00051

9.650 .971650 .00053

9.700 .976300 .00051

9.750 .980640 .00053

9.800 .984130 .00050

9.850 .988250 .00051

9.900 .992430 .00051

9.950 .995890 .00052

10.000 .999260 .00051

10.051 .003600 .00051

10.151 .010190 .00051

10.201 .014160 .00050

10.251 .018710 .00050

10.301 .021330 .00051

478

9.400 .917660 .00050

9.450 .922640 .00049

9.500 .925790 .00050

9.550 .931320 .00049

9.600 .934490 .00049

9.650 .938630 .00051

9.700 .943250 .00050

9.750 .946980 .00049

9.800 .950740 .00052

9.850 .953870 .00050

9.900 .957720 .00050

9.950 .961120 .00051

10.000 .965640 .00051

10.050 .968680 .00050

10.100 .973400 .00050

10.150 .977020 .00048

10.200 .979620 .00049

10.250 .983280 .00050

10.300 .987440 .00051

10.350 .992400 .00051

10.400 .994820 .00049

10.450 .998100 .00050
9.150 .928770 .00051

9.250 .937220 .00051

9.300 .941330 .00051

9.350 .945630 .00052

9.400 .948890 .00051

9.450 .953440 .00052

9.500 .957980 .00051

9.550 .961430 .00052

9.600 .966880 .00052

9.650 .971020 .00049

9.700 .973770 .00049

9.750 .978130 .00050

9.800 .982230 .00052

9.850 .985490 .00051

9.900 .989690 .00051

9.950 .993060 .00050

10.000 .997040 .00051

10.051 .001680 .00051

10.101 .004950 .00052

10.151 .008880 .00052

10.201 .012080 .00052

10.251 .015960 .00052

10.301 .020040 .00051

10.351 .023140 .00051

10.401 .027700 .00052

\section{0}

9.500 .922920 .00050

9.550 .926920 .00049

9.600 .930540 .00050

9.650 .935160 .00050

9.700 .938840 .00049

9.750 .942490 .00051

9.800 .946560 .00051

9.850 .950780 .00050

9.900 .954430 .00049

9.950 .958640 .00049

10.000 .961920 .00050

10.050 .965870 .00050

10.100 .969220 .00050

10.150 .973150 .00052

10.200 .976640 .00049

10.250 .980280 .00051

10.300 .982960 .00051

10.350 .987030 .00049

10.400 .991320 .00049

10.450 .995320 .00050

10.500 .997440 .00050

10.551 .001840 .00050
9.350 .931870 .00049

9.400 .935740 .00050

9.450 .939820 .00050

9.500 .943580 .00051

9.650 .955840 .00051

9.700 .958970 .00050

9.750 .964590 .00051

9.800 .966900 .00051

9.850 .970790 .00050

9.900 .974450 .00050

9.950 .978340 .00049

10.000 .983660 .00051

10.050 .986460 .00051

10.100 .990840 .00049

10.150 .993600 .00050

10.200 .997850 .00051

10.251 .000550 .00050

10.301 .004760 .00050

10.351 .007320 .00050

10.401 .011670 .00050

10.451 .015390 .00050

10.501 .019020 .00051

\section{0}

9.800 .922970 .00049

9.850 .925890 .00050

9.900 .929850 .00049

9.950 .933120 .00049

10.000 .937690 .00052

10.050 .941820 .00050

10.100 .944710 .00050

10.150 .949020 .00050

10.200 .952190 .00051

10.250 .957460 .00048

10.300 .960100 .00051

10.350 .963080 .00051

10.400 .967170 .00049

10.450 .970290 .00050

10.500 .975010 .00050

10.550 .978200 .00049

10.600 .980650 .00049

10.650 .984940 .00051

10.700 .987460 .00051

10.750 .991870 .00050

10.800 .994910 .00049

10.850 .997890 .00048 
10.501 .001610 .00050 10.551 .006000 .00050 10.601 .008140 .00048 10.651 .012800 .00051 10.701 .015810 .00051 10.751 .019060 .00050 10.801 .022480 .00049
10.601 .004220 .00050 10.651 .009280 .00049 10.701 .011920 .00050 10.751 .015920 .00050 10.801 .017930 .00050 10.851 .022370 .00051 10.901 .025180 .00050
10.901 .003030 .00050 10.951 .005510 .00050 11.001 .007780 .00049 11.051 .011310 .00050 11.101 .015390 .00050 11.151 .018240 .00050 11.201 .021820 .00049
650

10.000 .920020 .00048 10.100 .926790 .00049 10.150 .929860 .00049 10.200 .933960 .00049 10.250 .938600 .00049 10.300 .941910 .00049 10.350 .944170 .00050 10.400 .949170 .00049 10.450 .952940 .00049 10.500 .955980 .00049 10.550 .960460 .00049 10.600 .962500 .00048 10.650 .966710 .00049 10.700 .971000 .00050 10.750 .973610 .00049 10.800 .978020 .00049 10.850 .979920 .00049 10.900 .983590 .00049 10.950 .987390 .00050 11.000 .990690 .00049 11.050 .993920 .00051 11.100 .997280 .00050 11.151 .001100 .00049 11.201 .004060 .00049 11.251 .007760 .00049 11.301 .011080 .00050 11.351 .013630 .00049 11.401 .016600 .00051 11.451 .019730 .00049 11.501 .022880 .00050
700

10.400 .917950 .00049 10.450 .921370 .00049 10.500 .925050 .00049 10.550 .929780 .00048 10.600 .932550 .00050 10.650 .936360 .00048 10.700 .940650 .00049 10.750 .943350 .00049 10.800 .947490 .00049 10.850 .950790 .00049 10.900 .954770 .00051 10.950 .957350 .00048 11.000 .961430 .00049 11.050 .966130 .00048 11.100 .967980 .00051 11.150 .972270 .00049 11.200 .974450 .00050 11.250 .978000 .00050 11.300 .982090 .00050 11.350 .984130 .00050 11.400 .988290 .00049 11.450 .991330 .00049 11.500 .993870 .00049 11.550 .998390 .00050 11.601 .000870 .00049 11.651 .003870 .00049 11.751 .009280 .00048 11.801 .013490 .00048 11.851 .017090 .00050 11.901 .019370 .00049
750

11.400 .914720 .00049 11.450 .917780 .00050 11.500 .921600 .00050 11.550 .924630 .00048 11.600 .928500 .00048 11.650 .931450 .00047 11.700 .935300 .00048 11.750 .938210 .00049 11.800 .941070 .00049 11.850 .944460 .00048 11.900 .947300 .00049 11.950 .950560 .00048 12.000 .953850 .00049 12.050 .957190 .00048 12.100 .961320 .00048 12.150 .962900 .00048 12.200 .966480 .00048 12.250 .969870 .00048 12.300 .972160 .00048 12.350 .975770 .00048 12.400 .979320 .00049 12.450 .982600 .00050 12.500 .984290 .00049 12.550 .988000 .00049 12.600 .991080 .00049 12.650 .993450 .00050 12.700 .996870 .00048 12.750 .999680 .00049 12.801 .002120 .00048 12.851 .004980 .00049 12.901 .008350 .00049 12.951 .011660 .00049 13.001 .013680 .00048 13.051 .017530 .00050 13.101 .019220 .00050 
752

11.600 .918270 .00047 11.650 .922200 .00048 11.700 .925480 .00048 11.750 .929880 .00049 11.800 .932580 .00048 11.850 .934860 .00049 11.900 .938210 .00047 11.950 .942950 .00049 12.000 .945630 .00049 12.050 .947890 .00048 12.100 .951980 .00049 12.150 .954290 .00048 12.200 .957890 .00049 12.250 .961570 .00049 12.300 .963390 .00050 12.350 .965990 .00050 12.400 .970440 .00047 12.450 .972930 .00049 12.500 .976300 .00048 12.550 .978620 .00049 12.600 .982140 .00049 12.650 .984700 .00049 12.700 .988680 .00048 12.750 .991440 .00048 12.800 .993450 .00048 12.850 .997130 .00048 12.900 .999870 .00049 12.951 .002240 .00049 13.001 .005770 .00049 13.051 .008310 .00049 13.101 .010610 .00048 13.151 .014030 .00048 13.201 .016750 .00050 13.251 .018710 .00050 13.301 .022110 .00048
754

11.800 .919290 .00048 11.850 .922520 .00048 11.900 .926050 .00047 11.950 .928870 .00049 12.000 .931920 .00048 12.050 .934780 .00048 12.100 .938460 .00047 12.150 .941330 .00049 12.200 .944810 .00048 12.250 .947860 .00048 12.300 .951710 .00049 12.350 .955080 .00049 12.400 .957630 .00048 12.450 .959530 .00049 12.500 .963240 .00049 12.550 .966020 .00049 12.600 .970070 .00048 12.650 .971700 .00050 12.700 .975250 .00050 12.750 .978870 .00050 12.800 .981130 .00048 12.850 .985030 .00048 12.900 .987220 .00048 12.950 .990690 .00048 13.000 .992380 .00049 13.050 .996130 .00049 13.100 .998500 .00048 13.151 .002230 .00049 13.201 .003980 .00049 13.251 .006810 .00049 13.301 .010600 .00050 13.351 .011890 .00048 13.401 .016070 .00050 13.451 .018100 .00048 13.501 .020610 .00049
755

12.000 .920620 .00049

12.050 .925200 .00048

12.100 .925770 .00049

12.150 .930260 .00049

12.200 .933030 .00050

12.250 .935630 .00049

12.300 .939040 .00048

12.350 .943420 .00049

12.400 .945160 .00049

12.450 .950000 .00048

12.500 .951850 .00048

12.550 .954930 .00049

12.600 .958580 .00048

12.650 .961550 .00048

12.700 .963540 .00048

12.750 .967750 .00048

12.800 .969440 .00047

12.850 .972730 .00048

12.900 .975650 .00050

12.950 .978490 .00048

13.000 .982850 .00049

13.050 .985080 .00048

13.100 .986870 .00048

13.150 .990340 .00049

13.200 .992630 .00050

13.250 .996680 .00048

13.300 .998830 .00048

13.351 .002140 .00048

13.401 .003990 .00050

13.451 .007180 .00047

13.501 .009870 .00047

13.551 .011960 .00048

13.601 .014750 .00050

13.651 .018380 .00049

13.701 .020930 .00049 
Pun40-icyl-0970-01

12

15.000 .924940 .00029

15.200 .930670 .00029

15.400 .934960 .00031

15.600 .939940 .00030

15.800 .945190 .00029

16.000 .949360 .00029

16.200 .954150 .00029

16.400 .958380 .00029

16.600 .963370 .00029

16.800 .967540 .00030

17.000 .971530 .00029

17.200 .975780 .00028

17.400 .979280 .00028

17.600 .983680 .00028

17.800 .987180 .00029

18.000 .991000 .00029

18.200 .995050 .00029

18.400 .997650 .00028

18.601 .001880 .00028

18.801 .004990 .00028

19.001 .008490 .00028

19.201 .011470 .00027

19.401 .014640 .00027
25

9.600 .923410 .00040

9.650 .926760 .00041

9.700 .930020 .00040

9.750 .932880 .00040

9.800 .935530 .00040

9.850 .939090 .00041

9.900 .942550 .00042

9.950 .946190 .00040

10.000 .948040 .00041

10.050 .950940 .00042

10.100 .954520 .00042

10.150 .957340 .00041

10.200 .959890 .00040

10.250 .962650 .00040

10.300 .965780 .00041
15

12.500 .924340 .00035

12.600 .928280 .00034

12.700 .932290 .00035

12.800 .935290 .00034

12.900 .940030 .00033

13.000 .943540 .00034

13.100 .946730 .00033

13.200 .950480 .00035

13.300 .954150 .00034

13.400 .957070 .00033

13.500 .960400 .00033

13.600 .963830 .00034

13.700 .967260 .00034

13.800 .970160 .00034

13.900 .974070 .00034

14.000 .976940 .00034

14.100 .980590 .00033

14.200 .983500 .00032

14.300 .986240 .00033

14.400 .989230 .00033

14.500 .992700 .00033

14.600 .995450 .00032

14.700 .998110 .00033

14.801 .001010 .00032

14.901 .003710 .00032

15.001 .007030 .00032

15.101 .009500 .00033

15.201 .011320 .00032

15.301 .014070 .00032
20

10.500 .920020 .00038

10.600 .925480 .00038

10.700 .930630 .00038

10.800 .935600 .00038

10.900 .940950 .00037

11.000 .946030 .00037

11.100 .951350 .00038

11.200 .955410 .00038

11.300 .960080 .00038

11.400 .965690 .00037

11.500 .969810 .00038

11.600 .974110 .00037

11.700 .980230 .00037

11.800 .983910 .00037

11.900 .988280 .00038

12.000 .993060 .00038

12.100 .997160 .00038

12.201 .001420 .00038

12.301 .004810 .00036

12.401 .010270 .00038

12.501 .014010 .00038

12.601 .018290 .00038
30

9.000 .922190 .00042

9.050 .925530 .00044

9.100 .929090 .00042

9.150 .932840 .00042

9.200 .936170 .00041

9.250 .939740 .00042

9.300 .942830 .00042

9.350 .946680 .00043

9.400 .949310 .00041

9.450 .953090 .00044

9.500 .955680 .00041

9.550 .960390 .00044

9.600 .962380 .00042

9.650 .966130 .00043

9.700 .969620 .00042
32

8.800 .919750 .00043

8.850 .923820 .00043

8.900 .926960 .00043

8.950 .930570 .00044

9.000 .934870 .00043

9.050 .937800 .00043

9.100 .941960 .00043

9.150 .945030 .00042

9.200 .949080 .00043

9.250 .951860 .00043

9.300 .955230 .00043

9.350 .958100 .00043

9.400 .961020 .00043

9.450 .966140 .00044

9.500 .968580 .00044 
10.350 .967940 .00040 10.400 .970990 .00041 10.450 .973860 .00040 10.500 .976760 .00041 10.550 .979910 .00040 10.600 .982880 .00040 10.650 .984930 .00039 10.700 .988870 .00040 10.750 .990730 .00040 10.800 .993880 .00040 10.850 .995680 .00041 10.900 .998720 .00042 10.951 .001340 .00041 11.001 .003890 .00040 11.051 .006640 .00041 11.101 .008960 .00042 11.151 .011710 .00041 11.201 .013530 .00040 11.251 .016560 .00041 11.301 .019130 .00039
9.750 .972430 .00043 9.800 .976120 .00042 9.850 .978350 .00042 9.900 .981860 .00042 9.950 .984510 .00044 10.000 .988760 .00042 10.050 .990630 .00043 10.100 .993790 .00041 10.150 .996630 .00043 10.201 .000460 .00041 10.251 .002880 .00041 10.301 .006420 .00043 10.351 .009030 .00042 10.401 .011830 .00044 10.451 .014680 .00042 10.501 .017380 .00042 10.551 .020390 .00042 10.601 .023700 .00043
9.550 .972430 .00044

9.600 .975280 .00043 9.650 .978990 .00042 9.700 .982070 .00044 9.750 .985110 .00043 9.800 .988550 .00043 9.850 .991510 .00043 9.900 .994360 .00043 9.950 .998070 .00042 10.001 .000740 .00042 10.051 .003370 .00042 10.101 .007130 .00043 10.151 .009710 .00043 10.201 .012780 .00043 10.251 .015900 .00042 10.301 .018770 .00043
35

8.600 .920740 .00045 8.650 .924920 .00044 8.700 .928640 .00043 8.750 .931820 .00043 8.800 .935620 .00043 8.850 .939680 .00044 8.900 .943340 .00044 8.950 .946450 .00044 9.000 .950060 .00043 9.050 .953560 .00044 9.100 .957430 .00044 9.150 .960720 .00044 9.200 .964700 .00044 9.250 .968400 .00043 9.300 .972190 .00044 9.350 .975400 .00044 9.400 .978130 .00043 9.450 .981630 .00045 9.500 .984530 .00044 9.550 .988270 .00043 9.600 .991740 .00045 9.650 .995870 .00043 9.700 .999140 .00044 9.751 .001790 .00043 9.801 .004270 .00043 9.851 .008360 .00044 9.901 .011190 .00044
40

8.300 .918940 .00045

8.350 .923200 .00045

8.400 .926910 .00044

8.450 .931970 .00044

8.500 .935710 .00044

8.550 .938250 .00045

8.600 .942350 .00045

8.650 .947150 .00045

8.700 .950130 .00043

8.750 .954580 .00045

8.800 .957950 .00045

8.850 .961920 .00045

8.900 .964800 .00045

8.950 .969630 .00044

9.000 .972340 .00046

9.050 .975710 .00044

9.100 .980110 .00044

9.150 .982840 .00045

9.200 .986810 .00043

9.250 .990930 .00045

9.300 .993870 .00045

9.350 .997290 .00045

9.401 .001120 .00045

9.451 .003850 .00044

9.501 .008520 .00045

9.551 .011050 .00045

9.601 .014360 .00045
50

7.900 .914570 .00046

7.950 .918890 .00049

8.000 .922980 .00047

8.050 .928260 .00046

8.100 .932580 .00046

8.150 .935380 .00046

8.200 .940190 .00046

8.250 .943460 .00047

8.300 .948290 .00046

8.350 .952720 .00045

8.400 .955880 .00044

8.450 .960790 .00046

8.500 .964660 .00046

8.550 .968290 .00045

8.600 .972140 .00047

8.650 .975490 .00046

8.700 .980450 .00046

8.750 .984220 .00048

8.800 .987470 .00045

8.850 .991390 .00045

8.900 .994080 .00047

8.950 .999190 .00045

9.001 .002200 .00047

9.051 .006730 .00046

9.101 .009660 .00047

9.151 .012810 .00047

9.201 .016480 .00047 
9.951 .014350 .00045 10.001 .016850 .00044 10.051 .020370 .00044 10.101 .024220 .00043
9.651 .017930 .00044 9.701 .021690 .00045
9.251 .019950 .00045

9.301 .024150 .00048
60

7.700 .915020 .00046 7.750 .920150 .00046 7.800 .925010 .00046 7.850 .929020 .00048 7.900 .932850 .00048 7.950 .937920 .00046 8.000 .941780 .00048 8.050 .946410 .00048 8.100 .950850 .00046 8.150 .955350 .00047 8.200 .958810 .00047 8.250 .962470 .00047 8.300 .966890 .00047 8.350 .971080 .00048 8.400 .975750 .00047 8.450 .979090 .00047 8.500 .982680 .00049 8.550 .986930 .00046 8.600 .990970 .00048 8.650 .995100 .00048 8.700 .999150 .00049 8.751 .002800 .00047 8.801 .007230 .00046 8.851 .009970 .00048 8.901 .013240 .00047 8.951 .017550 .00046 9.001 .022210 .00047

90

7.500 .923050 .00048 7.550 .927730 .00049 7.600 .931720 .00048 7.650 .936580 .00049 7.700 .941690 .00049 7.750 .945170 .00050 7.800 .949590 .00049 7.850 .953090 .00049 7.900 .959060 .00049 7.950 .963670 .00050 8.000 .967610 .00050 8.050 .970590 .00049
70

7.600 .917230 .00049

7.650 .922290 .00047

7.700 .926730 .00048

7.750 .931260 .00047

7.800 .937270 .00048

7.850 .940380 .00048

7.900 .944930 .00048

7.950 .949100 .00048

8.000 .953760 .00049

8.050 .958470 .00048

8.100 .961610 .00047

8.150 .966370 .00048

8.200 .969490 .00047

8.250 .974890 .00048

8.300 .978290 .00048

8.350 .982850 .00048

8.400 .986640 .00048

8.450 .991340 .00048

8.500 .995890 .00048

8.550 .997620 .00048

8.601 .002920 .00049

8.651 .006220 .00049

8.701 .010870 .00048

8.751 .014370 .00048

8.801 .018630 .00048

100

7.400 .917460 .00049

7.450 .921740 .00049

7.500 .926880 .00049

7.550 .931630 .00048

7.600 .934910 .00049

7.650 .940860 .00050

7.700 .944630 .00049

7.750 .948340 .00049

7.800 .953660 .00049

7.850 .958120 .00048

7.900 .962160 .00048

7.950 .966480 .00049
80

7.500 .917630 .00049

7.550 .922940 .00048

7.600 .926770 .00048

7.650 .931160 .00048

7.700 .935460 .00047

7.750 .939810 .00048

7.800 .944550 .00048

7.850 .948470 .00048

7.900 .953090 .00048

7.950 .957280 .00048

8.000 .962410 .00047

8.050 .966020 .00049

8.100 .969960 .00049

8.150 .974160 .00049

8.200 .977870 .00050

8.250 .983300 .00049

8.300 .985290 .00048

8.350 .991810 .00050

8.400 .995870 .00048

8.450 .999140 .00049

8.501 .003310 .00048

8.551 .007360 .00048

8.601 .010050 .00050

8.651 .014350 .00048

8.701 .018840 .00049

8.751 .022330 .00048

8.801 .026540 .00048
7.400 .920960 .00049

7.450 .925040 .00048

7.500 .929860 .00050

7.550 .935030 .00049

7.600 .939120 .00051

7.650 .944290 .00050

7.700 .948060 .00049

7.750 .951820 .00050

7.800 .956500 .00049

7.850 .961210 .00048

7.900 .965770 .00049

7.950 .968570 .00050 
8.100 .975610 .00048 8.200 .983610 .00050 8.250 .988290 .00048 8.300 .992300 .00049 8.350 .995940 .00049 8.401 .000330 .00048 8.451 .003980 .00048 8.501 .008290 .00049 8.551 .013030 .00048 8.601 .016420 .00049 8.651 .020350 .00049 8.701 .024220 .00049

130

7.400 .922190 .00049 7.450 .927140 .00049 7.500 .930880 .00049 7.550 .935920 .00050 7.600 .941210 .00049 7.650 .944420 .00050 7.700 .949330 .00048 7.750 .953630 .00050 7.800 .958200 .00048 7.850 .963180 .00050 7.900 .967420 .00049 7.950 .970670 .00049 8.000 .975470 .00048 8.050 .979130 .00051 8.100 .983780 .00050 8.150 .987060 .00049 8.200 .992440 .00050 8.250 .996220 .00049 8.300 .999900 .00049 8.351 .004220 .00049 8.401 .007880 .00049 8.451 .012140 .00049 8.501 .015000 .00048 8.551 .021400 .00048 8.601 .024620 .00049

\section{9}

7.500 .922850 .00049 7.550 .928060 .00049 7.600 .931820 .00048 7.650 .936000 .00049 7.700 .941000 .00049
8.000 .970790 .00049

8.050 .975240 .00049

8.100 .979220 .00049

8.150 .983130 .00050

8.200 .986930 .00049

8.250 .992280 .00049

8.300 .996210 .00049

8.350 .999480 .00050

8.401 .004280 .00048

8.451 .007690 .00048

8.501 .012130 .00050

8.551 .016090 .00050

8.601 .020050 .00050
8.000 .972850 .00049

8.050 .978180 .00048

8.100 .981560 .00049

8.150 .985610 .00049

8.200 .989420 .00049

8.250 .993570 .00050

8.300 .997970 .00049

8.351 .003450 .00048

8.401 .006500 .00049 8.451 .009980 .00049 8.501 .014280 .00048 8.551 .017500 .00049 8.601 .022520 .00049
150
7.400 .922150 .00048

7.450 .926030 .00049

7.500 .931350 .00050

7.550 .935560 .00049

7.600 .939760 .00049

7.650 .945390 .00050

7.700 .950230 .00049

7.750 .953270 .00050

7.800 .959100 .00050

7.850 .961830 .00049

7.900 .966290 .00050

7.950 .970360 .00051

8.000 .974740 .00048

8.050 .978900 .00049

8.100 .983550 .00049

8.150 .987830 .00048

8.200 .990340 .00050

8.250 .995010 .00049

8.300 .999380 .00047

8.351 .003480 .00049

8.401 .007110 .00050

8.451 .011610 .00050

8.501 .014760 .00049

8.551 .019150 .00051

8.601 .022690 .00049
200

7.400 .918540 .00049

7.450 .924570 .00050

7.500 .927630 .00049

7.550 .932900 .00050

7.600 .937400 .00050

7.650 .941430 .00050

7.700 .945380 .00048

7.750 .950390 .00049

7.800 .954950 .00050

7.850 .958290 .00050

7.900 .961410 .00049

7.950 .967510 .00050

8.000 .970830 .00050

8.050 .975170 .00049

8.100 .978260 .00048

8.150 .982470 .00051

8.200 .986460 .00049

8.250 .990690 .00050

8.300 .994930 .00050

8.350 .999190 .00048

8.401 .002370 .00050

8.451 .006550 .00051

8.501 .010530 .00049

8.551 .014740 .00050
250

7.500 .921840 .00050

7.550 .926020 .00049

7.600 .930440 .00049

7.650 .936260 .00049

7.700 .939220 .00051
359

7.600 .917070 .00050 7.650 .921300 .00049 7.700 .924900 .00050 7.750 .929550 .00049 7.800 .933610 .00051 
7.750 .946070 .00048

7.800 .948700 .00050

7.850 .954830 .00049

7.900 .957590 .00050

7.950 .961970 .00049

8.000 .965880 .00049

8.050 .970070 .00049

8.100 .974570 .00049

8.150 .978280 .00051

8.200 .982910 .00051

8.250 .985880 .00049

8.300 .989750 .00049

8.350 .993760 .00049

8.400 .997410 .00049

8.451 .001420 .00050

8.501 .004590 .00050

8.551 .009300 .00049

8.601 .012930 .00051

8.651 .016940 .00049

8.701 .020290 .00049

\section{8}

7.800 .917340 .00049

7.850 .920910 .00049

7.900 .925700 .00050

7.950 .928480 .00049

8.000 .934100 .00049

8.050 .938270 .00048

8.100 .940810 .00050

8.150 .945470 .00050

8.200 .949530 .00049

8.250 .952460 .00049

8.300 .957660 .00048

8.350 .959840 .00048

8.400 .963510 .00050

8.450 .968450 .00049

8.500 .971790 .00048

8.550 .974930 .00049

8.600 .979530 .00048

8.650 .982830 .00050

8.700 .985960 .00049

8.750 .990460 .00049

8.800 .994030 .00048

8.850 .997260 .00050

8.901 .001180 .00049

8.951 .004130 .00049

9.001 .007870 .00050

9.051 .011250 .00049

9.101 .015210 .00049
7.750 .944440 .00049

7.800 .948470 .00048

7.850 .952550 .00049

7.900 .955990 .00050

7.950 .960620 .00050

8.000 .964170 .00050

8.050 .968890 .00051

8.100 .972740 .00050

8.150 .976350 .00050

8.200 .979380 .00050

8.250 .984210 .00050

8.300 .989310 .00051

8.350 .991770 .00050

8.400 .996610 .00049

8.450 .999950 .00049

8.501 .004530 .00049

8.601 .010620 .00049

8.651 .015870 .00048

8.701 .018430 .00049
7.900 .943050 .00049

7.950 .947100 .00048

8.000 .949890 .00048

8.050 .953980 .00050

8.100 .959060 .00048

8.150 .961790 .00049

8.200 .966250 .00049

8.250 .969290 .00049

8.300 .973840 .00049

8.350 .976960 .00050

8.400 .981060 .00050

8.450 .985310 .00048

8.500 .989200 .00049

8.550 .992680 .00049

8.600 .996760 .00050

8.651 .000330 .00049

8.701 .003550 .00049

8.751 .007280 .00048

8.801 .010500 .00049

8.851 .014640 .00050

8.901 .018370 .00050

$$
500
$$

7.900 .921230 .00048

7.950 .925600 .00049

8.000 .930690 .00048

8.050 .933720 .00048

8.100 .937640 .00049

8.150 .941650 .00050

8.200 .946210 .00050

8.250 .949280 .00050

8.300 .953750 .00048

8.350 .956610 .00049

8.400 .960450 .00049

8.450 .964750 .00049

8.500 .968560 .00048

8.550 .972440 .00049

8.600 .976220 .00048

8.650 .979090 .00049

8.700 .983060 .00051

8.750 .986430 .00050

8.800 .991000 .00049

8.850 .994210 .00049

8.900 .998470 .00050

8.951 .000040 .00050

9.001 .005260 .00049

9.051 .008110 .00049

9.101 .011140 .00050

9.151 .015070 .00048

9.201 .017990 .00051
600

8.200 .924230 .00047

8.250 .927680 .00049

8.300 .931300 .00050

8.400 .939190 .00050

8.450 .942540 .00051

8.500 .946600 .00048

8.550 .950830 .00050

8.600 .954500 .00050

8.650 .957480 .00051

8.700 .961280 .00048

8.750 .964340 .00049

8.800 .969070 .00049

8.850 .971990 .00050

8.900 .974840 .00049

8.950 .979410 .00049

9.000 .982310 .00051

9.050 .986320 .00051

9.100 .990480 .00048

9.150 .992910 .00049

9.200 .995750 .00048

9.250 .999340 .00051

9.301 .003230 .00049

9.351 .006340 .00049

9.401 .008440 .00049

9.451 .013430 .00050

9.501 .015680 .00049
8.350 .934270 .00050 
9.251 .020830 .00049

9.301 .024990 .00049

650

8.400 .922370 .00050

8.450 .926620 .00050

8.500 .930240 .00048

8.550 .934520 .00050

8.600 .937470 .00047

8.650 .941110 .00048

8.700 .944900 .00049

8.750 .948900 .00048

8.800 .952690 .00050

8.850 .954550 .00048

8.900 .959280 .00050

8.950 .963810 .00048

9.000 .965740 .00050

9.050 .969760 .00048

9.100 .973500 .00049

9.150 .976890 .00048

9.200 .980990 .00048

9.250 .983360 .00048

9.300 .987140 .00049

9.350 .991060 .00049

9.400 .994090 .00048

9.450 .996200 .00049

9.501 .000400 .00049

9.551 .002860 .00050

9.601 .005860 .00049

9.651 .010060 .00048

9.701 .013000 .00050

9.751 .016760 .00049

9.801 .019800 .00049

\section{2}

9.700 .919020 .00048 9.750 .922720 .00049 9.800 .925930 .00048 9.850 .928820 .00048 9.900 .932180 .00049 9.950 .935570 .00050
9.551 .019430 .00048

9.601 .021240 .00049
700

8.700 .918690 .00050

8.750 .921980 .00048

8.800 .926650 .00049

8.850 .930170 .00049

8.900 .933870 .00050

8.950 .936830 .00047

9.000 .940960 .00048

9.050 .944160 .00048

9.100 .949050 .00048

9.150 .951270 .00050

9.200 .954690 .00048

9.250 .958820 .00048

9.300 .961430 .00049

9.350 .965050 .00048

9.400 .967690 .00050

9.450 .971440 .00049

9.500 .975220 .00049

9.550 .977880 .00049

9.600 .980790 .00048

9.650 .984070 .00050

9.750 .990880 .00049

9.800 .993930 .00050

9.850 .997530 .00048

9.901 .001070 .00048

9.951 .004380 .00047

10.001 .006990 .00048

10.051 .009930 .00049

10.101 .013450 .00049

10.151 .015500 .00049
10.201 .019490 .00050

750

9.600 .920270 .00049

9.650 .923010 .00049

9.700 .926420 .00049

9.750 .929430 .00048

9.800 .932760 .00050

9.850 .936140 .00048

9.900 .939360 .00048

9.950 .942280 .00049

10.000 .946570 .00048

10.050 .949320 .00048

10.100 .952490 .00048

10.150 .955560 .00049

10.200 .959710 .00049

10.250 .962460 .00049

10.300 .964570 .00048

10.350 .968460 .00048

10.400 .971110 .00047

10.450 .973780 .00048

10.500 .976900 .00047

10.550 .980000 .00048

10.600 .983430 .00047

10.650 .985950 .00048

10.700 .989410 .00049

10.750 .991590 .00049

10.800 .993870 .00048

10.850 .997580 .00048

10.901 .001140 .00048

10.951 .003700 .00049

11.001 .005890 .00048

11.051 .009550 .00050

11.101 .012020 .00048

11.151 .015580 .00049

11.201 .017360 .00050

11.251 .019660 .00048

11.301 .023380 .00048

\section{4}

9.900 .920910 .00049

9.950 .924890 .00048

10.000 .928580 .00048

10.050 .931180 .00048

10.100 .933680 .00048

10.150 .936900 .00048

\section{5}

10.000 .918490 .00048

10.050 .921080 .00048

10.100 .924510 .00049

10.150 .927210 .00047

10.200 .931290 .00048

10.250 .934040 .00048 
10.000 .938640 .00048 10.050 .942030 .00048 10.100 .944740 .00048 10.200 .951350 .00049 10.250 .954910 .00047 10.300 .957340 .00048 10.350 .960010 .00049 10.400 .963510 .00049 10.450 .965820 .00050 10.500 .969810 .00049 10.550 .972640 .00049 10.600 .975150 .00048 10.650 .979240 .00050 10.700 .981990 .00048 10.750 .984030 .00049 10.800 .986950 .00049 10.850 .989690 .00047 10.900 .993380 .00049 10.950 .997210 .00049 11.000 .999550 .00048 11.051 .001930 .00047 11.101 .004360 .00049 11.151 .007660 .00048 11.201 .010350 .00049 11.251 .012690 .00049 11.301 .016220 .00049 11.351 .019570 .00049 11.401 .021460 .00048

Pun40-icyl-0970-10 12

13.900 .924540 .00029 14.100 .930480 .00028 14.300 .935210 .00029 14.500 .939630 .00028 14.700 .945380 .00028 14.900 .949970 .00028 15.100 .954530 .00028 15.300 .959090 .00027 15.500 .963320 .00028 15.700 .967720 .00028 15.900 .972300 .00028 16.100 .975890 .00028 16.300 .980030 .00027 16.500 .984570 .00027 16.700 .987390 .00027 16.900 .991520 .00027 17.100 .994600 .00027 17.300 .998590 .00027
10.200 .941100 .00048 10.250 .944320 .00048 10.300 .946820 .00048 10.350 .950240 .00048 10.400 .953560 .00048 10.450 .955150 .00048 10.500 .958170 .00048 10.550 .961330 .00047 10.600 .964860 .00049 10.650 .968490 .00049 10.700 .970910 .00048 10.750 .974120 .00049 10.800 .976690 .00048 10.850 .980010 .00050 10.900 .982470 .00049 10.950 .985410 .00050 11.000 .988850 .00048 11.050 .991170 .00049 11.100 .993870 .00049 11.150 .997820 .00050 11.201 .000320 .00048 11.251 .002000 .00049 11.301 .005640 .00049 11.351 .007750 .00049 11.401 .011000 .00048 11.451 .013700 .00049 11.501 .016640 .00050 11.551 .018880 .00049 11.601 .022100 .00049
10.300 .937260 .00050 10.350 .940360 .00048 10.400 .943480 .00049 10.450 .946130 .00049 10.500 .950080 .00048 10.550 .952550 .00051 10.600 .955650 .00048 10.650 .958490 .00049 10.700 .961080 .00047 10.750 .964680 .00047 10.800 .967990 .00048 10.850 .969940 .00048 10.900 .974360 .00047 10.950 .976460 .00047 11.000 .979920 .00049 11.050 .982630 .00048 11.100 .983990 .00050 11.150 .987470 .00049 11.200 .991340 .00048 11.250 .992860 .00049 11.300 .995830 .00048 11.351 .000180 .00047 11.401 .001340 .00048 11.451 .004350 .00050 11.501 .007540 .00048 11.551 .010180 .00049 11.601 .012660 .00049 11.651 .015830 .00048 11.701 .018860 .00048
11.400 .924960 .00032 11.500 .929180 .00032 11.600 .932380 .00032 11.700 .936200 .00032 11.800 .939840 .00032 11.900 .943390 .00031 12.000 .946770 .00032 12.100 .950150 .00031 12.200 .953500 .00032 12.300 .957030 .00032 12.400 .961080 .00031 12.500 .964130 .00031 12.600 .967910 .00032 12.700 .970620 .00033 12.800 .975010 .00030 12.900 .977940 .00030 13.000 .981080 .00031 13.100 .984300 .00031
20

9.400 .921050 .00035 9.500 .926470 .00036 9.600 .931730 .00036 9.700 .937090 .00035 9.800 .943430 .00035 9.900 .947230 .00036 10.000 .952730 .00036 10.100 .957160 .00035 10.200 .961950 .00035 10.300 .966760 .00035 10.400 .971920 .00036 10.500 .977060 .00035 10.600 .981060 .00035 10.700 .985420 .00034 10.800 .989980 .00036 10.900 .995090 .00035 11.000 .998850 .00036 11.101 .004230 .00035 
17.501 .001900 .00026 17.701 .005410 .00026 17.901 .008610 .00026 18.101 .011850 .00027 18.201 .012810 .00026

25

8.400 .919850 .00038 8.450 .923280 .00037 8.500 .926190 .00039 8.550 .929100 .00039 8.600 .932210 .00037 8.650 .935540 .00038 8.700 .939580 .00037 8.750 .942620 .00037 8.800 .944720 .00037 8.850 .947880 .00038 8.900 .951320 .00038 8.950 .954730 .00038 9.000 .957770 .00038 9.050 .959660 .00037 9.100 .963410 .00039 9.150 .966560 .00038 9.200 .969030 .00039 9.250 .972400 .00037 9.300 .974700 .00038 9.350 .977080 .00039 9.400 .980040 .00037 9.450 .983020 .00038 9.500 .985670 .00038 9.550 .988460 .00038 9.600 .991800 .00038 9.650 .994560 .00038 9.700 .996530 .00038 9.750 .999650 .00037 9.801 .001940 .00038 9.851 .004550 .00039 9.901 .007490 .00038 9.951 .010470 .00037 10.001 .012400 .00038 10.051 .014660 .00038 10.101 .017780 .00038
13.200 .986770 .00032 13.300 .989430 .00031 13.400 .993050 .00031 13.500 .995930 .00031 13.600 .998970 .00031 13.701 .002370 .00031 13.801 .004470 .00031 13.901 .007080 .00030 14.001 .009960 .00030 14.101 .012110 .00031 14.201 .015250 .00030

11.201 .008430 .00035 11.301 .011870 .00035 11.401 .016260 .00034
30

7.800 .919550 .00040 7.850 .922640 .00039 7.900 .926990 .00039 7.950 .930880 .00038 8.000 .933220 .00039 8.050 .937800 .00039 8.100 .941010 .00039 8.150 .944640 .00038 8.200 .947940 .00040 8.250 .950210 .00039 8.300 .954480 .00040 8.350 .957780 .00039 8.400 .960860 .00039 8.450 .964570 .00040 8.500 .967770 .00040 8.550 .971340 .00040 8.600 .974510 .00039 8.650 .977930 .00039 8.700 .980300 .00039 8.750 .983630 .00039 8.800 .987410 .00039 8.850 .990260 .00038 8.900 .993390 .00039 8.950 .996220 .00039 9.001 .000010 .00039 9.051 .002560 .00039 9.101 .005600 .00040 9.151 .007480 .00039 9.201 .011760 .00039 9.251 .014000 .00040 9.301 .017360 .00039
32

7.600 .918000 .00039 7.650 .921120 .00040 7.700 .925420 .00039 7.750 .929380 .00040 7.800 .932610 .00039 7.850 .936160 .00040 7.900 .940080 .00039 7.950 .944340 .00040 8.000 .947520 .00040 8.050 .950460 .00039 8.100 .953970 .00040 8.150 .957530 .00040 8.200 .961890 .00040 8.250 .964080 .00040 8.300 .968250 .00040 8.350 .971680 .00040 8.400 .974830 .00040 8.450 .978580 .00040 8.500 .981730 .00039 8.550 .984870 .00039 8.600 .987930 .00040 8.650 .990940 .00040 8.700 .994510 .00040 8.750 .997600 .00040 8.801 .000880 .00040 8.851 .003750 .00040 8.901 .007370 .00040 8.951 .011020 .00041 9.001 .013720 .00040 9.051 .016860 .00040 9.101 .019530 .00040 
35

7.400 .919360 .00040

7.450 .923310 .00041

7.500 .927260 .00041

7.550 .931340 .00040

7.600 .935500 .00040

7.650 .938910 .00041

7.700 .942870 .00040

7.750 .947080 .00041

7.800 .950580 .00040

7.850 .953940 .00039

7.900 .957530 .00041

7.950 .961300 .00041

8.000 .965090 .00040

8.050 .968400 .00041

8.100 .971200 .00040

8.150 .975060 .00041

8.200 .978600 .00041

8.250 .981620 .00040

8.300 .985610 .00040

8.350 .987980 .00041

8.400 .992620 .00041

8.450 .995700 .00040

8.500 .999080 .00041

8.551 .002590 .00041

8.601 .005290 .00040

8.651 .008960 .00040

8.701 .012050 .00040

8.751 .014870 .00040

8.801 .019060 .00041

60

6.500 .921510 .00043

6.550 .925850 .00044

6.600 .930330 .00044

6.650 .935820 .00043

6.700 .939760 .00043

6.750 .943400 .00044

6.800 .948480 .00045

6.850 .952660 .00044

6.900 .957590 .00044

6.950 .961270 .00044

7.000 .964900 .00043

7.050 .970140 .00044

7.100 .973200 .00043

7.150 .977160 .00044 7.200 .981670 .00042
40

7.100 .918700 .00042

7.150 .923190 .00042

7.200 .926980 .00043

7.250 .931420 .00041

7.300 .935260 .00040

7.350 .939870 .00041

7.400 .943800 .00041

7.450 .946950 .00042

7.500 .950800 .00042

7.550 .954650 .00042

7.600 .959090 .00042

7.650 .962940 .00041

7.700 .966440 .00042

7.750 .969480 .00041

7.800 .973590 .00042

7.850 .977190 .00042

7.900 .981110 .00042

7.950 .985080 .00042

8.000 .988920 .00042

8.050 .992150 .00041

8.100 .995800 .00042

8.150 .999600 .00043

8.201 .002640 .00042

8.251 .005660 .00041

8.301 .009850 .00043

8.351 .012550 .00041

8.401 .016690 .00042

8.451 .019300 .00041

8.501 .023610 .00043
50

6.700 .917730 .00043

6.750 .921810 .00043

6.800 .925860 .00043

6.850 .931060 .00043

6.900 .935080 .00043

6.950 .938980 .00043

7.000 .942770 .00043

7.050 .947300 .00042

7.100 .951910 .00044

7.150 .955650 .00042

7.200 .960400 .00042

7.250 .963960 .00043

7.300 .967960 .00043

7.350 .972060 .00043

7.400 .977460 .00042

7.450 .979900 .00042

7.500 .984160 .00043

7.550 .987110 .00044

7.600 .992360 .00042

7.650 .995470 .00043

7.700 .999980 .00043

7.751 .004060 .00043

7.801 .007000 .00045

7.851 .010790 .00043

7.901 .015060 .00043

7.951 .017780 .00043

8.001 .022220 .00043
70

6.300 .918310 .00044

6.350 .922540 .00046

6.400 .926700 .00044

6.450 .931010 .00045

6.500 .935780 .00043

6.550 .939890 .00045

6.600 .945220 .00045

6.650 .949560 .00043

6.700 .953520 .00044

6.750 .958990 .00043

6.800 .961780 .00044

6.850 .967190 .00045

6.900 .970630 .00044

6.950 .975440 .00044

7.000 .979850 .00045
80

6.200 .918240 .00045

6.250 .923300 .00044

6.300 .927750 .00044

6.350 .931970 .00045

6.400 .938380 .00044

6.450 .942140 .00043

6.500 .946310 .00044 6.550 .952040 .00045 6.600 .956480 .00045 6.650 .960150 .00043 6.700 .963950 .00044 6.750 .968790 .00045 6.800 .973420 .00045 6.850 .977400 .00045 6.900 .982310 .00045 
7.250 .986140 .00045

7.300 .990640 .00042

7.350 .993720 .00043

7.400 .997750 .00045

7.451 .002110 .00043

7.501 .006470 .00043

7.551 .010280 .00044

7.601 .014300 .00043

7.651 .017860 .00043

7.701 .021340 .00044

90

6.100 .916750 .00045

6.150 .921990 .00044

6.200 .926550 .00045

6.250 .931160 .00044

6.300 .935770 .00044

6.350 .940870 .00045

6.400 .945100 .00044

6.450 .949900 .00043

6.500 .954850 .00046

6.550 .958630 .00045

6.600 .962740 .00046

6.650 .967150 .00044

6.700 .972320 .00045

6.750 .976320 .00046

6.800 .981360 .00044

6.850 .984830 .00046

6.900 .989190 .00045

6.950 .993090 .00045

7.000 .997460 .00045

7.051 .001240 .00045

7.101 .005270 .00045

7.151 .009420 .00045

7.201 .013770 .00045

7.251 .017920 .00045

7.301 .021420 .00045

130

6.100 .933460 .00046

6.150 .937540 .00046

6.200 .942830 .00044

6.250 .947460 .00045

6.300 .952130 .00044

6.350 .956010 .00045

6.400 .960860 .00045

6.450 .965520 .00045
7.050 .983560 .00043

7.100 .987900 .00044

7.150 .991580 .00043

7.200 .996570 .00044

7.250 .999760 .00046

7.301 .004150 .00045

7.351 .008230 .00044

7.401 .011820 .00046

7.451 .016410 .00045

7.501 .019830 .00045
6.950 .986340 .00045

7.000 .990800 .00045

7.050 .994410 .00045

7.100 .998720 .00044

7.151 .002770 .00045

7.201 .006560 .00044

7.251 .010130 .00045

7.301 .015270 .00044

7.351 .018080 .00045

7.401 .022170 .00045
100

6.200 .931650 .00044

6.250 .937260 .00043

6.300 .942000 .00046

6.350 .946840 .00045

6.400 .951130 .00045

6.450 .955200 .00045

6.500 .960660 .00045

6.550 .964840 .00046

6.600 .969290 .00045

6.650 .973380 .00044

6.700 .977260 .00045

6.750 .981620 .00044

6.800 .986570 .00045

6.850 .990530 .00045

6.900 .994990 .00045

6.950 .999650 .00045

7.001 .002650 .00045

7.051 .006970 .00044

7.101 .012180 .00045
120

6.000 .920000 .00045

6.050 .924350 .00047

6.100 .930120 .00045

6.150 .934740 .00044

6.200 .938930 .00045

6.250 .943930 .00046

6.300 .949010 .00046

6.350 .952320 .00045

6.400 .957080 .00045

6.450 .962650 .00045

6.500 .966310 .00045

6.550 .972020 .00045

6.600 .975630 .00046

6.650 .980400 .00046

6.700 .983760 .00043

6.750 .988440 .00045

6.800 .992760 .00044

6.850 .996240 .00046

6.901 .001230 .00046
150

5.900 .917680 .00046

5.950 .921170 .00045

6.000 .926620 .00046

6.050 .931210 .00045

6.100 .935790 .00046

6.150 .940840 .00045

6.200 .944760 .00044

6.250 .948630 .00046
200

5.900 .919320 .00046 5.950 .923730 .00046 6.000 .928700 .00045 6.050 .932940 .00046 6.100 .937550 .00046 6.150 .942080 .00046 6.200 .946450 .00045 6.250 .951160 .00046 
6.500 .970020 .00046

6.550 .973880 .00047

6.600 .978440 .00045

6.650 .983260 .00045

6.700 .986970 .00045

6.750 .991380 .00046

6.800 .996040 .00045

6.850 .999250 .00045

6.901 .004510 .00045
6.300 .954050 .00045

6.350 .959520 .00045

6.400 .962050 .00046

6.450 .967780 .00045

6.500 .971800 .00045

6.550 .976530 .00045

6.600 .981510 .00044

6.650 .984680 .00045

6.700 .988820 .00046

6.750 .992720 .00047

6.800 .998220 .00046

6.851 .000850 .00046

6.901 .004970 .00048

6.951 .010260 .00045

7.001 .013130 .00044
6.300 .955050 .00046

6.350 .960210 .00046

6.400 .964160 .00048

6.450 .967700 .00047

6.500 .973220 .00044

6.550 .976480 .00045

6.600 .980580 .00046

6.650 .985000 .00046

6.700 .990410 .00046

6.750 .994270 .00046

6.800 .998260 .00045

6.851 .001680 .00046

6.901 .005790 .00046

6.951 .009060 .00046

7.001 .013160 .00046

7.051 .017660 .00045

7.101 .021310 .00045

359

239

250

5.900 .917870 .00046

5.950 .922210 .00045

6.000 .927080 .00045

6.050 .931600 .00046

6.100 .935850 .00045

6.150 .940530 .00046

6.200 .944400 .00047

6.250 .949380 .00045

6.300 .953260 .00046

6.350 .958030 .00045

6.400 .962140 .00046

6.450 .966470 .00045

6.500 .970470 .00046

6.550 .974120 .00046

6.600 .978700 .00047

6.650 .981850 .00047

6.700 .986730 .00045

6.750 .990740 .00045

6.800 .994620 .00045

6.850 .998380 .00046

6.901 .002940 .00045

6.951 .006880 .00044

7.001 .011150 .00046

7.051 .014790 .00045

7.101 .018250 .00045
6.000 .919410 .00045

6.050 .923440 .00045

6.100 .927710 .00045

6.150 .932750 .00044

6.200 .936280 .00045

6.250 .941120 .00045

6.300 .945510 .00045

6.350 .949550 .00046

6.400 .952890 .00046

6.450 .957130 .00045

6.500 .962220 .00044

6.550 .966090 .00045

6.600 .970060 .00046

6.650 .973840 .00047

6.700 .977210 .00043

6.750 .982240 .00047

6.800 .985690 .00047

6.850 .988920 .00046

6.900 .993220 .00045

6.950 .996900 .00045

7.001 .001670 .00046

7.051 .004960 .00046

7.101 .008850 .00045

7.151 .012070 .00045

7.201 .015790 .00046 
478

6.100 .918280 .00046 6.150 .921300 .00046 6.200 .925180 .00045 6.250 .929640 .00046 6.300 .933550 .00044 6.350 .937880 .00046 6.400 .941400 .00045 6.450 .945920 .00047 6.500 .950780 .00046 6.550 .954320 .00046 6.600 .957610 .00046 6.650 .961840 .00045 6.700 .966210 .00045 6.750 .969640 .00044 6.800 .973550 .00045 6.850 .977260 .00046 6.900 .982260 .00047 6.950 .984650 .00045 7.000 .988560 .00046 7.050 .992450 .00045 7.100 .996480 .00047 7.150 .999810 .00045 7.201 .003160 .00045 7.251 .006410 .00046 7.301 .010090 .00047

650

6.500 .919510 .00045 6.550 .924060 .00045 6.600 .928200 .00046 6.650 .931110 .00044 6.700 .936000 .00045 6.750 .938670 .00044 6.800 .942980 .00045 6.850 .946920 .00045 6.900 .951430 .00045 6.950 .953900 .00045 7.000 .958020 .00045 7.050 .961100 .00045 7.100 .964950 .00046 7.150 .967690 .00046 7.200 .972240 .00045 7.250 .975660 .00045 7.300 .978590 .00046 7.350 .981960 .00046 7.400 .986020 .00046
500

6.200 .923200 .00045

6.250 .927320 .00045

6.300 .932410 .00046

6.350 .935460 .00045

6.400 .939640 .00046

6.450 .944140 .00046

6.500 .947920 .00046

6.550 .951750 .00047

6.600 .954830 .00047

6.650 .960680 .00045

6.700 .964020 .00046

6.750 .967790 .00046

6.800 .971270 .00045

6.850 .974080 .00045

6.900 .977630 .00046

6.950 .982150 .00046

7.000 .986000 .00046

7.050 .990150 .00045

7.100 .993910 .00045

7.150 .996500 .00046

7.201 .000780 .00047

7.251 .004900 .00047

7.301 .007740 .00046

7.351 .010410 .00045

7.401 .014730 .00046

7.451 .018110 .00045

7.501 .021370 .00045

700

6.800 .924600 .00046

6.850 .927460 .00044

6.900 .931390 .00044

6.950 .936080 .00044

7.000 .938830 .00045

7.050 .941710 .00047

7.100 .946370 .00045

7.150 .950160 .00047

7.200 .952780 .00045

7.250 .956640 .00044

7.300 .959570 .00045

7.350 .962970 .00045

7.400 .966630 .00044

7.450 .970040 .00047

7.500 .973550 .00046

7.550 .977150 .00045

7.600 .980620 .00046

7.650 .983280 .00046

7.700 .987040 .00047
600

7.200 .983940 .00045

7.220 .986020 .00045

7.240 .986810 .00047

7.260 .988890 .00044

7.280 .990560 .00047

7.300 .990920 .00045

7.320 .992400 .00046

7.340 .994450 .00046

7.360 .994760 .00047

7.380 .995270 .00047

7.400 .997620 .00047

7.420 .999170 .00044

7.441 .000210 .00045

7.461 .002400 .00046

7.481 .003790 .00045

7.501 .004380 .00045 \begin{tabular}{ccc}
\multicolumn{1}{c}{750} \\
7.40 & 0.92242 & 0.00045 \\
7.45 & 0.92535 & 0.00045 \\
7.50 & 0.92996 & 0.00046 \\
7.55 & 0.93309 & 0.00044 \\
7.60 & 0.93610 & 0.00045 \\
7.65 & 0.93848 & 0.00045 \\
7.70 & 0.94190 & 0.00046 \\
7.75 & 0.94538 & 0.00045 \\
7.80 & 0.94892 & 0.00045 \\
7.85 & 0.95170 & 0.00047 \\
7.90 & 0.95465 & 0.00046 \\
7.95 & 0.95793 & 0.00044 \\
8.00 & 0.96123 & 0.00044 \\
8.05 & 0.96454 & 0.00046 \\
8.10 & 0.96800 & 0.00045 \\
8.15 & 0.97049 & 0.00046 \\
8.20 & 0.97439 & 0.00045 \\
8.25 & 0.97607 & 0.00045 \\
8.30 & 0.98019 & 0.00045
\end{tabular}

8.300 .980190 .00045 
7.450 .989730 .00047

7.500 .992510 .00046

7.550 .995980 .00045

7.600 .999120 .00047

7.651 .003180 .00044

7.701 .006490 .00045
7.750 .990030 .00046

7.800 .993930 .00045

7.850 .996800 .00045

7.901 .000500 .00046

7.951 .002830 .00045

8.001 .005900 .00046

8.051 .009900 .00045

8.101 .012560 .00046

8.151 .015300 .00048

8.201 .018260 .00046
8.350 .982940 .00046

8.400 .986710 .00046

8.450 .988960 .00047

8.500 .991950 .00044

8.550 .995260 .00045

8.600 .997580 .00046

8.651 .001110 .00046

8.751 .007110 .00044

8.801 .008870 .00047

8.851 .012360 .00045

8.901 .015230 .00046

8.951 .018120 .00046

9.001 .020620 .00046

\section{5}

7.700 .922730 .00044

7.800 .929160 .00043

7.900 .936280 .00045

8.000 .941240 .00045

8.100 .948560 .00046

8.200 .954430 .00045

8.300 .959800 .00045

8.400 .966780 .00044

8.500 .971890 .00047

8.600 .977280 .00044

8.700 .983990 .00046

8.800 .988630 .00044

8.900 .995410 .00045

9.001 .000710 .00046

9.101 .005760 .00047

9.201 .011840 .00045

9.301 .016610 .00046

9.401 .021920 .00045

9.501 .026950 .00046

9.601 .032700 .00046

9.701 .038120 .00045

9.801 .043850 .00044

9.901 .048200 .00046 
Pun43-icyl-0970-00

12

18.400 .925560 .00030 18.600 .929900 .00029 18.800 .933230 .00029 19.000 .937560 .00029 19.400 .945200 .00029 19.600 .948170 .00029 19.800 .952510 .00028 20.000 .955550 .00029 20.200 .959020 .00029 20.400 .962690 .00028 20.600 .965490 .00028 20.800 .969030 .00028 21.000 .972510 .00027 21.200 .975370 .00028 21.400 .978050 .00027 21.600 .981200 .00028 21.800 .984030 .00027 22.000 .986180 .00028 22.200 .989150 .00028 22.400 .991910 .00027 22.600 .994620 .00027 22.800 .997510 .00027 23.000 .999670 .00027 23.201 .002560 .00027 23.401 .005170 .00028 23.601 .007380 .00027 23.801 .009840 .00026 24.001 .012010 .00026 24.101 .013010 .00026

25

11.700 .918890 .00043 11.800 .925450 .00041 11.900 .931830 .00040 12.000 .936620 .00041 12.100 .940880 .00040 12.200 .947590 .00043 12.300 .952280 .00041 12.400 .958290 .00042 12.500 .962950 .00041
15

15.200 .924450 .00034 15.300 .927480 .00034 15.400 .930900 .00033 15.500 .934390 .00034 15.600 .936170 .00033 15.700 .940260 .00034 15.800 .943440 .00033 15.900 .945940 .00033 16.000 .949090 .00034 16.100 .952300 .00033 16.200 .954860 .00034 16.300 .957860 .00033 16.400 .960630 .00033 16.500 .963070 .00033 16.600 .967210 .00034 16.700 .968600 .00033 16.800 .971280 .00032 16.900 .973900 .00033 17.000 .976840 .00033 17.100 .979970 .00033 17.200 .982120 .00032 17.300 .984000 .00032 17.400 .987030 .00032 17.500 .989010 .00033 17.600 .991810 .00033 17.700 .994530 .00031 17.800 .996480 .00032 17.900 .998960 .00032 18.001 .001220 .00032 18.101 .003740 .00032 18.201 .005500 .00032 18.301 .007930 .00032 18.401 .010550 .00032 18.501 .012560 .00031 18.601 .015550 .00032

30

11.000 .916050 .00044 11.100 .923270 .00044 11.200 .929350 .00043 11.300 .935130 .00044 11.400 .942300 .00043 11.500 .948120 .00043 11.600 .953990 .00042 11.700 .958990 .00044 11.800 .965140 .00043
20

12.800 .919900 .00038 12.900 .923590 .00038 13.000 .928530 .00039 13.100 .933330 .00039 13.200 .938200 .00038 13.300 .942540 .00038 13.400 .946670 .00038 13.500 .950590 .00038 13.600 .956190 .00039 13.700 .959590 .00038 13.800 .963490 .00038 13.900 .968270 .00038 14.000 .972130 .00037 14.100 .976310 .00039 14.200 .980430 .00037 14.300 .983830 .00038 14.400 .988520 .00039 14.500 .991910 .00039 14.600 .995410 .00038 14.700 .999250 .00037 14.801 .003420 .00037 14.901 .006430 .00037 15.001 .010500 .00036 15.101 .013700 .00036 15.201 .017600 .00037 
12.600 .968220 .00042

12.700 .973460 .00040

12.800 .978230 .00041

13.000 .988410 .00042

13.100 .993180 .00041

13.200 .997760 .00040

13.301 .002680 .00042

13.401 .007330 .00040

13.501 .011530 .00042

13.601 .015910 .00040

13.701 .020470 .00041
12.900 .982820 .00040
11.900 .970980 .00043

12.000 .977890 .00043

12.100 .983100 .00043

12.200 .989650 .00042

12.400 .999950 .00042

12.501 .004720 .00043

12.601 .010530 .00043

12.701 .015400 .00042

12.801 .020180 .00043
11.250 .945240 .00044

11.300 .948110 .00043

11.350 .951390 .00044

11.400 .954770 .00044

11.450 .957510 .00044

11.500 .961470 .00044

11.550 .964280 .00045

11.600 .966740 .00045

11.650 .970290 .00044

11.700 .972690 .00044

11.750 .975980 .00044

11.800 .979180 .00044

11.850 .982600 .00044

11.900 .984480 .00044

11.950 .988660 .00043

12.000 .990650 .00044

12.050 .994430 .00043

12.100 .996090 .00044

12.150 .999500 .00044

12.201 .001840 .00044

12.251 .004040 .00044

12.301 .007340 .00044

12.351 .010450 .00043

12.401 .012810 .00043

12.451 .015110 .00043

12.501 .018760 .00043

35

10.600 .917290 .00045 10.650 .921370 .00046 10.700 .925110 .00045 10.750 .927700 .00046 10.800 .931040 .00047 10.850 .935280 .00044 10.900 .938600 .00045 10.950 .941650 .00045 11.000 .945510 .00045 11.050 .948400 .00045 11.100 .952330 .00045 11.150 .955210 .00045 11.200 .958910 .00045 11.250 .961460 .00044 11.300 .964530 .00044 11.350 .968510 .00045 11.400 .969990 .00044 11.450 .974440 .00044 11.500 .977230 .00045 11.550 .980590 .00045 11.600 .984020 .00045

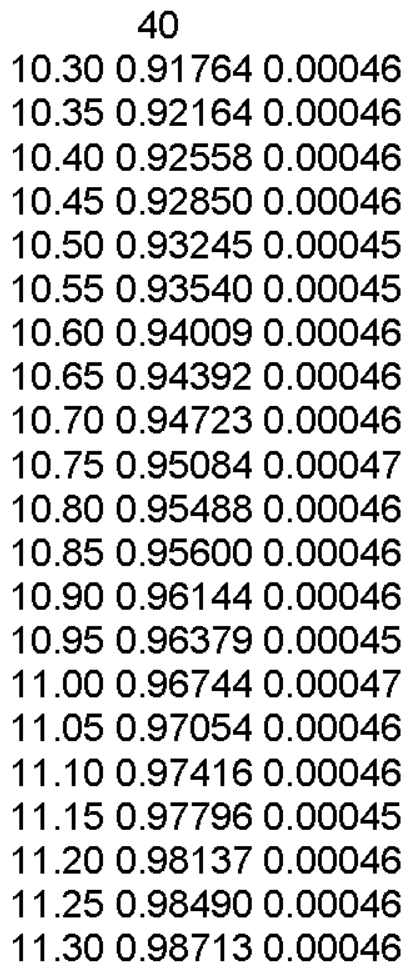

50

10.000 .924620 .00048 10.050 .928790 .00047 10.100 .931810 .00047 10.150 .935580 .00047 10.200 .939930 .00047 10.250 .944670 .00049 10.300 .948430 .00048 10.350 .951460 .00047 10.400 .954310 .00047 10.450 .958870 .00048 10.500 .961280 .00047 10.550 .965680 .00048 10.600 .970660 .00049 10.650 .973650 .00048 10.700 .977030 .00047 10.750 .980310 .00046 10.800 .984270 .00046 10.850 .987510 .00048 10.900 .991510 .00047 10.950 .994390 .00048 11.000 .998170 .00046 
11.650 .986510 .00045 11.700 .989810 .00043 11.750 .993070 .00045 11.800 .996990 .00046 11.850 .998980 .00044 11.901 .002340 .00044 11.951 .005470 .00045 12.001 .008260 .00044 12.051 .010020 .00045 12.101 .014170 .00043 12.151 .016610 .00046 12.201 .019940 .00045
11.350 .991050 .00047 11.400 .993860 .00045 11.450 .996270 .00045 11.500 .999850 .00048 11.551 .003060 .00047 11.601 .007110 .00045 11.651 .008900 .00045 11.701 .012300 .00046 11.751 .015480 .00046 11.801 .018800 .00046
11.051 .001410 .00048 11.101 .005330 .00046 11.151 .008470 .00047 11.201 .012610 .00049 11.251 .015320 .00048 11.301 .017620 .00047
60

9.700 .917500 .00048

9.750 .922730 .00048

9.800 .926710 .00046

9.850 .930830 .00049

9.900 .934450 .00049

9.950 .939140 .00049 10.000 .942790 .00049 10.050 .947180 .00048 10.100 .950320 .00047 10.150 .953680 .00049 10.200 .959000 .00049 10.250 .962860 .00049 10.300 .965920 .00048 10.350 .968880 .00049 10.400 .973860 .00048 10.450 .977660 .00048 10.550 .985310 .00049 10.600 .987080 .00048 10.650 .992200 .00050 10.700 .996430 .00049 10.751 .000390 .00048 10.801 .002620 .00049 10.851 .006470 .00049 10.951 .013250 .00049 11.001 .016780 .00049 11.051 .020210 .00049 11.101 .024680 .00048

90

9.400 .916090 .00049 9.450 .920680 .00050 9.500 .925760 .00050
70

9.500 .913500 .00050 9.550 .917980 .00048 9.600 .922570 .00049 9.650 .926080 .00050 9.700 .930350 .00049 9.750 .934690 .00049 9.800 .938690 .00049 9.850 .943060 .00050 9.900 .947740 .00048 9.950 .951330 .00049 10.000 .954730 .00051 10.100 .963630 .00049 10.150 .967670 .00049 10.200 .971290 .00050 10.250 .974570 .00049 10.300 .978050 .00051 10.350 .982120 .00050 10.400 .986370 .00048 10.450 .990090 .00050 10.500 .993610 .00048 10.550 .997870 .00049 10.601 .000300 .00048 10.651 .004990 .00050 10.701 .008750 .00050 10.751 .011270 .00050 10.801 .015060 .00048 10.851 .017980 .00049 10.901 .021870 .00049
80

9.500 .921020 .00050

9.550 .925110 .00049

9.600 .929400 .00049

9.650 .934020 .00050

9.700 .938330 .00049

9.750 .941660 .00051

9.800 .946170 .00050

9.850 .951280 .00050

9.900 .953850 .00050

9.950 .959660 .00050

10.000 .962880 .00049 10.050 .965370 .00051 10.100 .971000 .00050 10.150 .975270 .00049 10.200 .979130 .00050 10.250 .982100 .00050 10.300 .985910 .00050 10.350 .990180 .00051 10.400 .993280 .00050 10.450 .997730 .00050 10.501 .000200 .00050 10.551 .003660 .00050 10.601 .008190 .00048 10.651 .011310 .00049 10.701 .016410 .00051 10.751 .019540 .00049 10.801 .022410 .00049
100

9.400 .920280 .00051

9.450 .923780 .00051

9.500 .927800 .00050
120

9.400 .921600 .00050

9.450 .924600 .00050

9.500 .929360 .00051 
9.550 .930020 .00049

9.600 .933990 .00050

9.650 .937850 .00051

9.700 .942840 .00050

9.750 .946170 .00050

9.800 .950370 .00051

9.850 .955160 .00050

9.900 .958950 .00050

9.950 .963450 .00050

10.000 .966670 .00049

10.050 .971830 .00051

10.100 .974640 .00052

10.150 .978540 .00050

10.200 .982420 .00050

10.250 .986630 .00049

10.300 .990050 .00051

10.350 .994230 .00050

10.400 .998260 .00049

10.451 .001690 .00049

10.501 .005440 .00050

10.551 .009090 .00051

10.601 .012720 .00050

10.651 .016620 .00052

10.701 .020310 .00051
9.550 .933650 .00052

9.600 .936340 .00050

9.650 .941340 .00049

9.700 .945360 .00050

9.750 .950060 .00050

9.800 .953470 .00051

9.850 .958630 .00051

9.900 .961370 .00049

9.950 .965470 .00050

10.000 .970970 .00052

10.050 .973900 .00052

10.100 .978310 .00050

10.150 .981300 .00050

10.200 .986390 .00051

10.250 .989620 .00050

10.300 .993630 .00050

10.350 .996600 .00049

10.401 .000900 .00051

10.451 .004590 .00051

10.501 .008380 .00051

10.551 .011970 .00052

10.601 .016100 .00051

10.651 .019560 .00050

10.701 .022990 .00049
9.550 .933540 .00051

9.600 .938470 .00051

9.650 .942180 .00049

9.700 .947110 .00050

9.750 .949990 .00049

9.800 .954400 .00051

9.850 .958740 .00051

9.900 .963290 .00051

9.950 .967230 .00051

10.000 .971200 .00052

10.050 .974770 .00050

10.100 .978250 .00051

10.150 .983100 .00050

10.200 .985220 .00052

10.250 .990300 .00051

10.300 .993960 .00052

10.401 .001760 .00052

10.451 .006420 .00052

10.501 .009800 .00050

10.551 .012750 .00049

10.601 .016750 .00050

10.651 .020910 .00050

10.701 .024600 .00050
130

9.400 .921840 .00051

9.450 .927120 .00050

9.500 .930300 .00049

9.550 .934430 .00050

9.600 .939040 .00051

9.650 .942860 .00050

9.700 .946660 .00049

9.750 .952920 .00050

9.800 .955410 .00052

9.850 .959200 .00050

9.900 .963440 .00051

9.950 .967900 .00050

10.000 .972220 .00052 10.050 .975370 .00051 10.100 .979620 .00051 10.150 .983180 .00050 10.200 .987260 .00051 10.250 .991270 .00050 10.300 .994690 .00052 10.350 .998360 .00051 10.401 .002720 .00051 10.451 .005930 .00051 10.501 .010610 .00051
150

9.450 .924500 .00051

9.500 .929010 .00052

9.550 .933280 .00051

9.600 .936880 .00050

9.700 .945580 .00049

9.750 .949820 .00049

9.800 .953520 .00050

9.850 .958180 .00049

9.900 .960730 .00051

9.950 .965360 .00051

10.000 .969580 .00050

10.050 .973680 .00049

10.100 .977640 .00052

10.150 .981540 .00052

10.200 .985230 .00051

10.250 .989250 .00051

10.300 .992500 .00051

10.350 .996340 .00051

10.401 .000570 .00052

10.451 .003380 .00051

10.501 .006900 .00051

10.551 .010870 .00052

10.601 .016820 .00051 $\begin{array}{rrr} & 200 \\ 9.50 & 0.92038 & 0.00051 \\ 9.55 & 0.92459 & 0.00051 \\ 9.60 & 0.92877 & 0.00051 \\ 9.65 & 0.93327 & 0.00050 \\ 9.70 & 0.93759 & 0.00052 \\ 9.75 & 0.94165 & 0.00051 \\ 9.80 & 0.94477 & 0.00051 \\ 9.85 & 0.94938 & 0.00048 \\ 9.90 & 0.95303 & 0.00052 \\ 9.95 & 0.95688 & 0.00050 \\ 10.00 & 0.96134 & 0.00050 \\ 10.05 & 0.96519 & 0.00051 \\ 10.10 & 0.96976 & 0.00050 \\ 10.15 & 0.97281 & 0.00052 \\ 10.20 & 0.97633 & 0.00050 \\ 10.25 & 0.97957 & 0.00052 \\ 10.30 & 0.98435 & 0.00051 \\ 10.35 & 0.98776 & 0.00051 \\ 10.40 & 0.99150 & 0.00051 \\ 10.45 & 0.99602 & 0.00050 \\ 10.50 & 0.99881 & 0.00050 \\ 10.55 & 1.00193 & 0.00051 \\ 10.60 & 1.00616 & 0.00050\end{array}$ 
10.551 .014680 .00051 10.601 .017230 .00051 10.651 .020710 .00053 10.701 .024720 .00051
10.651 .018830 .00051 10.701 .021820 .00051
10.651 .010700 .00051

10.701 .013160 .00052

10.751 .017160 .00051
239

9.600 .920040 .00052 9.650 .925400 .00051

9.700 .927660 .00050

9.750 .932670 .00051

9.800 .935870 .00050

9.850 .939690 .00052

9.900 .943700 .00051

9.950 .947700 .00053

10.000 .951570 .00050

10.050 .956150 .00051

10.100 .959630 .00051

10.150 .963700 .00049

10.200 .967230 .00052

10.250 .971270 .00051

10.300 .975410 .00050

10.350 .978770 .00052

10.400 .982260 .00051

10.450 .984800 .00051

10.500 .989690 .00051

10.550 .993080 .00051

10.600 .996980 .00050

10.651 .000660 .00052

10.701 .004890 .00051

10.751 .008290 .00051

10.801 .011020 .00051

10.851 .013910 .00049

10.901 .019100 .00051

10.951 .021490 .00050

11.001 .024370 .00050
250

9.600 .917310 .00051

9.650 .922380 .00051

9.700 .925240 .00050

9.750 .929520 .00051

9.800 .934280 .00051

9.850 .937050 .00051

9.900 .942100 .00050

9.950 .944700 .00053

10.000 .949620 .00052

10.050 .953840 .00051

10.100 .957220 .00053

10.150 .961350 .00051

10.200 .965900 .00051

10.250 .968860 .00050

10.300 .972390 .00050

10.350 .975830 .00052

10.400 .979300 .00051

10.450 .982860 .00050

10.500 .986730 .00051

10.550 .991220 .00051

10.600 .994710 .00051

10.650 .998110 .00051

10.701 .001240 .00051

10.751 .005070 .00052

10.801 .008490 .00049

10.851 .012540 .00051

10.901 .014930 .00051

10.951 .018420 .00051

11.001 .022170 .00050
478

10.300 .918170 .00049 10.350 .922230 .00050 10.400 .926060 .00049 10.450 .929330 .00049 10.500 .932770 .00049 10.550 .936700 .00049 10.600 .940030 .00048 10.650 .943850 .00048

\section{0}

10.400 .918470 .00050 10.450 .921790 .00048 10.500 .925250 .00050 10.550 .928930 .00050 10.600 .932130 .00050 10.650 .937160 .00050 10.700 .940070 .00051 10.750 .943240 .00049

\section{9}

9.900 .917410 .00050 9.950 .920990 .00051 10.000 .924700 .00049 10.050 .928720 .00050 10.100 .933180 .00052 10.150 .936320 .00050 10.200 .939750 .00049 10.250 .943140 .00048 10.300 .947190 .00050 10.350 .950760 .00050 10.400 .955090 .00052 10.450 .959120 .00051 10.500 .963210 .00050 10.550 .965730 .00050 10.600 .969780 .00051 10.650 .972380 .00050 10.700 .976440 .00050 10.750 .979920 .00050 10.800 .983850 .00050 10.850 .987760 .00050 10.900 .990310 .00051 10.950 .993750 .00050 11.000 .996190 .00050 11.051 .000970 .00051 11.101 .004040 .00050 11.151 .006920 .00050 11.201 .010110 .00049 11.251 .013660 .00049 11.301 .017030 .00050 11.351 .019930 .00050 11.401 .023050 .00049

600

11.000 .919800 .00049 11.050 .923980 .00049 11.100 .926740 .00049 11.150 .928690 .00049 11.200 .932980 .00047 11.250 .936550 .00048 11.300 .939640 .00050 11.350 .944020 .00049 
10.700 .946400 .00049

10.750 .950420 .00050

10.800 .953510 .00051

10.850 .957830 .00050

10.900 .960440 .00048

10.950 .964840 .00048

11.000 .967400 .00051

11.050 .971090 .00049

11.100 .974180 .00049

11.150 .978500 .00048

11.200 .981440 .00050

11.250 .984710 .00049

11.300 .987760 .00051

11.350 .990810 .00049

11.400 .994940 .00048

11.450 .997800 .00051

11.501 .000740 .00049

11.601 .007340 .00049

11.651 .011070 .00049

11.701 .013470 .00050

11.751 .016390 .00050

11.801 .020170 .00050

650

11.650 .925430 .00048

11.700 .927930 .00049

11.750 .932220 .00048

11.800 .934720 .00048

11.850 .937860 .00049

11.900 .941390 .00049

11.950 .944220 .00050

12.000 .947230 .00048

12.050 .950860 .00050

12.100 .954130 .00049

12.150 .956820 .00047

12.200 .959680 .00050

12.250 .963230 .00050

12.300 .966330 .00050

12.550 .980800 .00047

12.600 .984220 .00049

12.650 .987350 .00048

12.700 .989690 .00049

12.750 .992880 .00049

12.800 .995210 .00051

12.850 .998960 .00048

12.901 .001560 .00051

12.951 .004200 .00048
10.800 .946470 .00052

10.850 .950990 .00051

10.900 .954160 .00050

10.950 .956550 .00050

11.000 .960430 .00050

11.100 .967350 .00050

11.150 .970630 .00050

11.200 .974130 .00049

11.250 .977140 .00050

11.300 .980840 .00049

11.350 .984220 .00048

11.400 .986610 .00048

11.450 .990320 .00051

11.500 .993860 .00050

11.550 .996520 .00049

11.600 .998950 .00050

11.651 .002700 .00051

11.701 .006150 .00050

11.751 .009510 .00049

11.801 .012590 .00049

11.851 .014990 .00049

11.901 .018200 .00049

11.951 .021270 .00050

12.001 .024420 .00050
11.400 .946630 .00048

11.450 .950140 .00048

11.500 .953470 .00050

11.550 .956600 .00050

11.600 .958710 .00049

11.650 .962820 .00051

11.700 .965230 .00049

11.750 .969100 .00049

11.800 .973190 .00049

11.850 .975850 .00049

11.900 .980040 .00050

11.950 .981320 .00049

12.000 .984770 .00049

12.050 .988460 .00051

12.100 .990740 .00050

12.200 .997790 .00049

12.251 .000470 .00048

12.301 .003150 .00050

12.351 .006140 .00050

12.401 .010110 .00051

12.451 .011880 .00051

12.501 .014360 .00047

12.551 .017090 .00049

12.601 .019480 .00049
660
11.700 .917210 .00048

11.750 .920300 .00049

11.800 .923880 .00049

11.850 .926960 .00049

11.900 .931000 .00050

11.950 .933250 .00048

12.000 .937000 .00049

12.050 .939790 .00048

12.100 .942710 .00048

12.150 .946000 .00049

12.200 .948850 .00048

12.250 .952750 .00050

12.300 .955570 .00048

12.350 .959270 .00048

12.400 .962010 .00050

12.450 .964190 .00048

12.500 .967250 .00049

12.550 .970570 .00047

12.600 .974240 .00049

12.650 .977290 .00047

12.700 .979350 .00046

12.750 .981890 .00048

12.800 .985600 .00048
670

12.000 .923110 .00048

12.050 .927110 .00046

12.100 .930590 .00048

12.150 .933830 .00049

12.250 .939810 .00049

12.300 .943500 .00050

12.350 .946170 .00049

12.400 .949540 .00047

12.450 .951220 .00047

12.500 .955260 .00050

12.550 .959100 .00048

12.600 .960560 .00048

12.650 .963630 .00047

12.700 .966590 .00049

12.750 .971150 .00048

12.850 .975410 .00049

12.900 .978990 .00048

12.950 .981320 .00048

13.050 .987510 .00048

13.150 .992430 .00048

13.200 .996110 .00048

13.250 .998980 .00049

13.301 .001100 .00049 
13.001 .007270 .00049 13.051 .010060 .00048 13.101 .013370 .00049 13.151 .015050 .00049 13.201 .017050 .00049

680

12.200 .919710 .00049 12.300 .925830 .00049 12.400 .931670 .00049 12.500 .937500 .00048 12.600 .945110 .00048 12.800 .956350 .00049 12.900 .961440 .00048 13.000 .967710 .00048 13.100 .973920 .00048 13.200 .979880 .00050 13.300 .985360 .00047 13.400 .989910 .00046 13.500 .996820 .00049 13.601 .000300 .00049 13.701 .006960 .00047 13.801 .011970 .00048 13.901 .018080 .00050 14.001 .022750 .00047
12.850 .988580 .00050 12.900 .991900 .00049 13.000 .995830 .00048 13.051 .000030 .00050 13.101 .003410 .00049 13.151 .005720 .00048 13.201 .008370 .00049 13.251 .010420 .00048 13.301 .014190 .00049 13.351 .015670 .00048 13.401 .020000 .00050
13.351 .004780 .00048 13.401 .007090 .00048 13.451 .009650 .00048 13.501 .012100 .00048 13.551 .014860 .00048 13.601 .017770 .00049
690

12.600 .919770 .00049 12.700 .925590 .00049 12.800 .931530 .00048 13.000 .942930 .00049 13.100 .948850 .00048 13.200 .955240 .00048 13.300 .960700 .00049 13.400 .966300 .00048 13.500 .972130 .00047 13.600 .976680 .00049 13.700 .982490 .00049 13.800 .989240 .00048 13.900 .994840 .00049 14.000 .998630 .00049 14.101 .005130 .00047 14.201 .010100 .00049 14.301 .014450 .00048 14.401 .019600 .00048
Pun43-icyl-0970-01

12

16.900 .926500 .00028 17.100 .930360 .00029 17.300 .934040 .00029 17.500 .938310 .00029 17.900 .946100 .00028 18.100 .949100 .00028 18.300 .953140 .00029 18.500 .956380 .00027 18.700 .959960 .00028 18.900 .963430 .00027 19.100 .965870 .00027 19.300 .969840 .00027
15

13.600 .922790 .00034

13.700 .925580 .00032

13.800 .929260 .00032

13.900 .931890 .00032

14.000 .934690 .00032

14.100 .938530 .00033

14.200 .941730 .00032

14.300 .944850 .00032

14.400 .947660 .00033

14.500 .950300 .00032

14.600 .953220 .00032

14.700 .955980 .00032
698

13.400 .921730 .00048 13.500 .927600 .00048 13.600 .933040 .00047 13.700 .938690 .00047 13.800 .944560 .00047 13.900 .950270 .00048 14.000 .955460 .00049 14.100 .960920 .00048 14.200 .966040 .00048 14.300 .971580 .00047 14.400 .976860 .00050 14.500 .982780 .00048 14.600 .986960 .00049 14.700 .992700 .00048 14.800 .997110 .00048 14.901 .002610 .00048 15.001 .006520 .00049 15.101 .011920 .00049 15.201 .017330 .00048 15.301 .021790 .00049

20

11.300 .921760 .00037 11.400 .925650 .00038 11.500 .930540 .00037 11.600 .935310 .00039 11.700 .939700 .00037 11.800 .943880 .00037 11.900 .948510 .00037 12.000 .952960 .00036 12.100 .956700 .00037 12.200 .962050 .00036 12.300 .965620 .00036 12.400 .969880 .00037 
19.500 .972610 .00028 19.700 .975800 .00027 19.900 .978270 .00026 20.100 .981380 .00027 20.300 .983960 .00027 20.500 .987100 .00027 20.700 .989510 .00027 20.900 .992960 .00027 21.100 .995050 .00027 21.300 .997960 .00026 21.501 .000390 .00026 21.701 .003100 .00027 21.901 .005300 .00026 22.101 .007460 .00026 22.301 .010260 .00025 22.501 .012670 .00026
14.800 .959080 .00031 14.900 .961260 .00032 15.000 .964460 .00031 15.100 .966810 .00032 15.200 .969550 .00032 15.300 .971970 .00031 15.400 .975360 .00032 15.500 .977760 .00031 15.600 .980260 .00032 15.700 .983130 .00031 15.800 .984910 .00033 15.900 .988160 .00031 16.000 .990260 .00032 16.100 .992820 .00031 16.200 .995160 .00032 16.300 .997930 .00031 16.400 .999650 .00031 16.501 .002110 .00032 16.601 .004650 .00031 16.701 .007040 .00032 16.801 .009160 .00031 16.901 .011420 .00031 17.001 .013610 .00030 17.101 .015350 .00031

25

10.200 .921550 .00040 10.300 .927280 .00040 10.400 .933730 .00041 10.500 .938630 .00039 10.600 .944190 .00039 10.700 .950220 .00040 10.800 .954890 .00040 10.900 .960250 .00040 11.000 .965010 .00040 11.100 .970370 .00039 11.200 .975950 .00040 11.300 .980600 .00039 11.400 .985310 .00039 11.500 .991010 .00041 11.600 .995280 .00040 11.701 .000720 .00040 11.801 .004140 .00040 11.901 .009390 .00039 12.001 .013510 .00040 12.101 .017920 .00039
30

9.500 .918930 .00042 9.550 .923170 .00042 9.600 .925690 .00041 9.650 .928630 .00042 9.700 .931780 .00041 9.750 .935380 .00042 9.800 .938320 .00042 9.850 .941640 .00043 9.900 .944430 .00041 9.950 .947660 .00042 10.000 .950480 .00042 10.050 .953800 .00042 10.100 .957210 .00041 10.150 .960410 .00042 10.200 .962650 .00041 10.250 .965350 .00042 10.300 .968400 .00042 10.350 .971180 .00041 10.500 .979860 .00042 10.550 .983390 .00042 10.600 .985130 .00042 10.650 .988060 .00041 10.700 .990460 .00042
12.500 .974100 .00037 12.600 .977270 .00037 12.700 .982570 .00036 12.800 .985620 .00037 12.900 .989490 .00037 13.000 .993690 .00036 13.100 .997400 .00036 13.201 .000860 .00036 13.301 .004220 .00036 13.401 .008130 .00036 13.501 .012260 .00036 13.601 .015530 .00036 13.701 .019140 .00036

\begin{abstract}
32
9.300 .918180 .00042 9.350 .922190 .00043 9.400 .925390 .00043 9.450 .929140 .00042 9.500 .932370 .00042 9.550 .935290 .00043 9.600 .939170 .00043 9.650 .941760 .00043 9.700 .945110 .00041 9.750 .948340 .00042 9.800 .951090 .00043 9.850 .953940 .00042 9.900 .957120 .00042 9.950 .961290 .00043 10.000 .963840 .00042 10.050 .966530 .00042 10.100 .970000 .00043 10.150 .973220 .00043 10.200 .975010 .00041 10.250 .979000 .00043 10.300 .982740 .00043 10.350 .984570 .00044 10.400 .986830 .00042
\end{abstract}


35

9.100 .921890 .00043

9.150 .925220 .00043

9.200 .927600 .00044

9.250 .931660 .00043

9.300 .935540 .00043

9.350 .938780 .00043

9.400 .941900 .00044

9.450 .944980 .00043

9.500 .948820 .00043

9.550 .951240 .00043

9.600 .954890 .00044

9.650 .958590 .00042

9.700 .961370 .00044

9.750 .964770 .00043

9.800 .968150 .00044

9.850 .971220 .00041

9.900 .974610 .00042

9.950 .977120 .00044

10.000 .981230 .00044

10.050 .983530 .00043

10.100 .987160 .00043

10.150 .990170 .00043

10.200 .992780 .00043

10.250 .995440 .00043

10.300 .999030 .00043

10.351 .000940 .00044

10.401 .004230 .00044

10.451 .007270 .00044

10.501 .010770 .00043

10.551 .013720 .00043

10.601 .016480 .00043
10.750 .994980 .00043 10.800 .996820 .00042 10.850 .998930 .00041 10.901 .002230 .00041 10.951 .004340 .00041 11.001 .006840 .00042 11.051 .010410 .00042 11.101 .012860 .00042 11.151 .014970 .00041 11.201 .018670 .00042
10.450 .990070 .00042

10.500 .992770 .00043

10.550 .996690 .00042

10.600 .999120 .00042

10.651 .001870 .00043

10.701 .005280 .00042

10.751 .007860 .00042

10.801 .010860 .00042

10.851 .013280 .00043

10.901 .015230 .00042

10.951 .018700 .00042

11.001 .021210 .00042

50

8.800 .922520 .00044

8.850 .925630 .00045

8.900 .928790 .00045

8.950 .932750 .00045

9.000 .936770 .00045

9.050 .939840 .00044

9.100 .942730 .00045

9.150 .947630 .00044

9.200 .951600 .00045

9.250 .954100 .00045

9.300 .956980 .00044

9.350 .960410 .00043

9.400 .964130 .00044

9.450 .967650 .00044

9.500 .971660 .00043

9.550 .974410 .00045

9.600 .978570 .00045

9.650 .981820 .00045

9.700 .984440 .00045

9.750 .986980 .00043

9.800 .991210 .00045

9.850 .994210 .00045

9.900 .997970 .00044

9.951 .000900 .00045

10.001 .004440 .00043

10.051 .006660 .00044

10.101 .009810 .00043

10.151 .013600 .00043

10.201 .015660 .00043

10.251 .019550 .00044

10.301 .021720 .00044
8.400 .921260 .00046 8.450 .926210 .00046 8.500 .928580 .00045 8.550 .933380 .00047 8.600 .937940 .00045 8.650 .940630 .00046 8.700 .944110 .00045 8.750 .948960 .00047 8.800 .951910 .00046 8.850 .956160 .00045 8.900 .960000 .00046 8.950 .963620 .00046 9.000 .966840 .00047 9.050 .970950 .00046 9.100 .974790 .00047 9.150 .978150 .00048 9.200 .981400 .00046 9.250 .985000 .00046 9.300 .988860 .00046 9.350 .991920 .00047 9.400 .995500 .00045 9.450 .998850 .00045 9.501 .002110 .00046 9.551 .006480 .00047 9.601 .009000 .00047 9.651 .012470 .00046 9.701 .015550 .00045 9.751 .019440 .00046 9.801 .022070 .00047 
60

8.100 .915250 .00047 8.150 .919940 .00047 8.200 .923850 .00048 8.250 .927500 .00047 8.300 .932290 .00046 8.350 .935720 .00046 8.400 .940190 .00047 8.450 .943790 .00047 8.500 .947390 .00046 8.550 .952440 .00046 8.600 .956010 .00047 8.650 .959510 .00046 8.700 .963700 .00046 8.750 .967530 .00047 8.800 .971600 .00048 8.850 .974500 .00047 8.900 .980450 .00046 8.950 .983330 .00047 9.000 .985970 .00046 9.050 .989790 .00046 9.100 .993040 .00047 9.150 .996660 .00048 9.201 .000790 .00047 9.251 .004120 .00048 9.301 .008490 .00045 9.351 .011170 .00046 9.401 .014350 .00046 9.451 .018570 .00045 9.501 .022100 .00047

\section{0}

7.800 .915250 .00048 7.850 .920040 .00049 7.900 .925060 .00048 7.950 .928480 .00048 8.000 .932580 .00047 8.050 .936960 .00048 8.100 .940810 .00048 8.150 .944920 .00047 8.200 .949080 .00049 8.250 .953970 .00048 8.300 .957510 .00047 8.350 .961810 .00048 8.400 .965930 .00047 8.450 .969290 .00049 8.500 .973650 .00049 8.550 .977950 .00048 8.600 .981870 .00048
70

8.000 .918820 .00048

8.050 .923150 .00047

8.100 .927840 .00047

8.150 .932190 .00047

8.200 .936110 .00048

8.250 .940600 .00049

8.300 .944050 .00046

8.350 .949080 .00047

8.400 .953040 .00047

8.450 .956930 .00048

8.500 .959980 .00046

8.550 .964380 .00049

8.600 .968100 .00048

8.650 .972720 .00049

8.700 .977340 .00046

8.750 .980050 .00047

8.800 .983980 .00048

8.850 .988040 .00048

8.900 .991330 .00047

8.950 .995250 .00047

9.000 .998980 .00046

9.051 .003410 .00047

9.101 .007250 .00049

9.151 .008860 .00047

9.201 .013260 .00047

9.251 .016980 .00047

9.301 .021350 .00047
80

7.900 .917850 .00048

7.950 .924290 .00049

8.000 .927930 .00048

8.050 .932150 .00048

8.100 .936190 .00049

8.150 .940450 .00047

8.200 .944750 .00048

8.250 .948030 .00048

8.300 .952430 .00048

8.350 .956890 .00048

8.400 .959790 .00047

8.450 .963760 .00048

8.500 .968610 .00050

8.550 .973520 .00048

8.600 .975890 .00048

8.650 .980740 .00049

8.700 .985000 .00049

8.750 .987550 .00048

8.800 .991980 .00047

8.850 .995780 .00048

8.900 .999240 .00048

8.951 .003280 .00047 9.001 .006870 .00048 9.051 .010270 .00048 9.101 .014550 .00049 9.151 .017630 .00048 9.201 .021620 .00049
100

7.800 .917870 .00049

7.850 .923900 .00049

7.900 .927360 .00049

7.950 .931750 .00049

8.000 .935550 .00049

8.050 .939760 .00049

8.100 .944370 .00048

8.150 .948520 .00049

8.200 .952160 .00048

8.250 .956900 .00048

8.300 .960490 .00048

8.350 .964290 .00050

8.400 .969070 .00048

8.450 .973600 .00050

8.500 .976380 .00048

8.550 .981010 .00050

8.600 .984960 .00048
120

7.800 .920730 .00048

7.850 .924410 .00050

7.900 .929760 .00048

7.950 .933650 .00047

8.000 .938490 .00047

8.050 .941980 .00050

8.100 .946610 .00050

8.150 .951310 .00050

8.200 .955230 .00048

8.250 .959030 .00049

8.300 .962780 .00048

8.350 .966750 .00049

8.400 .971190 .00049

8.450 .975070 .00050

8.500 .979110 .00048

8.600 .986260 .00049

8.650 .990020 .00050 
8.650 .985040 .00048

8.700 .989670 .00048

8.750 .993390 .00048

8.800 .996310 .00048

8.851 .001270 .00048

8.901 .004910 .00048

8.951 .008750 .00048

9.001 .011800 .00050

9.051 .014900 .00048

9.101 .019100 .00049

130

7.800 .922370 .00048

7.850 .925770 .00048

7.900 .931020 .00049

7.950 .934020 .00049

8.000 .939530 .00049

8.050 .943410 .00049

8.100 .947200 .00048

8.150 .951830 .00047

8.200 .956330 .00048

8.250 .959770 .00049

8.300 .964160 .00048

8.350 .967400 .00048

8.400 .971940 .00047

8.450 .975990 .00049

8.500 .979630 .00049

8.550 .983820 .00048

8.600 .988130 .00050

8.650 .991320 .00050

8.750 .999070 .00050

8.801 .003720 .00048

8.851 .006640 .00050

8.901 .009920 .00048

8.951 .013970 .00049

239

8.000 .923260 .00050

8.050 .927440 .00051

8.100 .930550 .00048

8.150 .935300 .00049

8.200 .939090 .00049

8.250 .943290 .00049

8.300 .947200 .00049
8.650 .988750 .00050

8.700 .992740 .00048

8.750 .996880 .00049

8.801 .000530 .00048

8.851 .003670 .00048

8.901 .007840 .00049

8.951 .011060 .00048

9.001 .015800 .00049

9.051 .018380 .00048

9.101 .022400 .00049
8.700 .994200 .00048

8.750 .998200 .00048

8.851 .005150 .00050

8.901 .009000 .00048

8.951 .011940 .00050

9.001 .017560 .00049

9.051 .021000 .00048

9.101 .024010 .00048

200
7.900 .921550 .00049

7.800 .921010 .00051

7.850 .924760 .00051

7.900 .929580 .00050

7.950 .933810 .00051

8.050 .942260 .00050

8.100 .946390 .00050

8.150 .949740 .00049

8.200 .954310 .00048

8.250 .958250 .00049

8.300 .961740 .00050

8.350 .966770 .00049

8.400 .969450 .00049

8.450 .974480 .00049

8.500 .977820 .00049

8.550 .981830 .00048

8.600 .986070 .00049

8.650 .989800 .00050

8.700 .993550 .00049

8.750 .997490 .00050

8.801 .000570 .00049

8.851 .005290 .00048

8.901 .008600 .00050

8.951 .011440 .00047

9.001 .015100 .00049

9.051 .019580 .00050

9.101 .022570 .00048
7.950 .926470 .00049

8.000 .930580 .00048

8.050 .934680 .00048

8.100 .939600 .00050

8.150 .943080 .00050

8.200 .947210 .00049

8.250 .951340 .00049

8.300 .954900 .00050

8.350 .959130 .00049

8.400 .963050 .00049

8.450 .967440 .00049

8.500 .970520 .00050

8.550 .974630 .00049

8.600 .978450 .00049

8.650 .982370 .00048

8.700 .985390 .00049

8.750 .989080 .00050

8.800 .994000 .00050

8.850 .996780 .00050

8.901 .000260 .00049

8.951 .004040 .00050

9.001 .007390 .00051

9.051 .010460 .00049

9.151 .017720 .00049

9.201 .021900 .00049
250

8.000 .920220 .00050

8.050 .924440 .00049

8.100 .928740 .00051

8.150 .932550 .00050

8.200 .937170 .00049

8.250 .940910 .00051

8.300 .945210 .00049
359

8.300 .922320 .00048 8.350 .925850 .00048 8.400 .929460 .00050 8.450 .933210 .00049 8.500 .937190 .00050 8.550 .941490 .00050 8.600 .944650 .00050 


\begin{tabular}{|c|c|}
\hline & \\
\hline & \\
\hline & \\
\hline & \\
\hline & \\
\hline & 8 \\
\hline 20.000 & 00047 \\
\hline 91 & 8.7 \\
\hline & \\
\hline & \\
\hline & 8617 \\
\hline 190 ח & $99289 \mathrm{C}$ \\
\hline 5930.0 & 0.996650 .0004 \\
\hline 00 & 00041 \\
\hline & 9.1 \\
\hline מחת & 9.1 \\
\hline .000 & 1.011420 .0004 \\
\hline 019 & 1.014140 .0005 \\
\hline & 9.301 .017560 .0004 \\
\hline & \\
\hline
\end{tabular}

478

8.600 .917370 .00049

8.650 .921870 .00049

8.700 .925700 .00048

8.750 .928550 .00049

8.800 .932710 .00048

8.850 .935090 .00050

8.900 .939770 .00047

8.950 .942930 .00047

9.000 .946620 .00049

9.050 .950720 .00048

9.100 .953650 .00050

9.150 .957340 .00048

9.200 .959890 .00050

9.250 .964120 .00049

9.300 .967960 .00049

9.350 .971180 .00049

9.400 .974590 .00050

9.450 .977270 .00050

9.500 .980760 .00048

9.550 .984030 .00050

9.600 .987380 .00048

9.650 .990520 .00049

9.700 .993090 .00048

9.750 .996980 .00049

9.801 .000450 .00049
8.650 .948730 .00049

8.700 .951750 .00050

8.750 .955130 .00048

8.800 .959000 .00049

8.850 .963470 .00050

8.900 .966630 .00048

8.950 .969970 .00050

9.000 .974540 .00049

9.050 .977800 .00051

9.100 .981040 .00050

9.150 .983080 .00049

9.200 .987900 .00048

9.250 .990790 .00050

9.300 .994150 .00049

9.350 .997890 .00049

9.401 .001040 .00050

9.451 .003460 .00050

9.501 .007130 .00050

9.551 .012010 .00049

9.601 .014680 .00049

9.651 .017590 .00053

9.701 .021120 .00048

$\begin{array}{rrr} & 600 \\ 9.30 & 0.92475 & 0.00049 \\ 9.35 & 0.92759 & 0.00049 \\ 9.40 & 0.93131 & 0.00049 \\ 9.45 & 0.93452 & 0.00049 \\ 9.50 & 0.93848 & 0.00049 \\ 9.55 & 0.94053 & 0.00048 \\ 9.60 & 0.94463 & 0.00047 \\ 9.70 & 0.95247 & 0.00048 \\ 9.75 & 0.95439 & 0.00049 \\ 9.80 & 0.95822 & 0.00048 \\ 9.85 & 0.96096 & 0.00048 \\ 9.90 & 0.96361 & 0.00051 \\ 9.95 & 0.96791 & 0.00049 \\ 10.00 & 0.97003 & 0.00049 \\ 10.05 & 0.97360 & 0.00050 \\ 10.10 & 0.97777 & 0.00049 \\ 10.15 & 0.97954 & 0.00047 \\ 10.20 & 0.98330 & 0.00048 \\ 10.25 & 0.98563 & 0.00048 \\ 10.30 & 0.98912 & 0.00049 \\ 10.35 & 0.99135 & 0.00049 \\ 10.40 & 0.99521 & 0.00049 \\ 10.45 & 0.99817 & 0.00049 \\ 10.50 & 1.00101 & 0.00047 \\ 10.55 & 1.00382 & 0.00049\end{array}$


9.851 .003360 .00050

9.901 .006810 .00049

9.951 .009440 .00048 10.001 .012990 .00050 10.051 .015500 .00050 10.101 .018300 .00049
10.001 .006560 .00049 10.051 .009600 .00050 10.101 .012030 .00049 10.151 .015220 .00049 10.201 .018080 .00050
10.601 .006810 .00049 10.651 .009790 .00049 10.701 .012600 .00049 10.751 .015180 .00049 10.801 .019120 .00048
650

9.700 .918370 .00048 9.750 .921460 .00049 9.800 .925130 .00049 9.850 .928920 .00047 9.900 .931400 .00049 9.950 .934690 .00048 10.050 .941290 .00050 10.100 .944470 .00049 10.150 .947530 .00048 10.200 .950140 .00049 10.250 .953620 .00050 10.300 .956220 .00048 10.350 .959850 .00047 10.400 .963370 .00049 10.450 .966030 .00049 10.500 .969140 .00049 10.550 .971850 .00048 10.600 .975160 .00050 10.650 .979120 .00048 10.700 .980830 .00049 10.800 .987360 .00048 10.900 .992080 .00050 10.950 .995280 .00049 11.000 .998260 .00049 11.101 .003490 .00050 11.151 .006240 .00049 11.201 .010260 .00049 11.251 .011800 .00050 11.301 .014900 .00048 11.351 .017140 .00049 11.401 .019990 .00047

680 10.300 .922580 .00049 10.350 .925900 .00047 10.400 .929030 .00048 10.450 .932390 .00049
660

9.900 .923320 .00048 9.950 .925640 .00048 10.000 .929650 .00048 10.050 .933050 .00049 10.100 .936020 .00049 10.150 .939330 .00047 10.200 .941470 .00049 10.250 .945310 .00048 10.300 .947250 .00049 10.350 .950360 .00048 10.400 .954760 .00048 10.450 .956210 .00048 10.500 .960200 .00049 10.550 .963070 .00049 10.600 .965690 .00048 10.650 .969390 .00047 10.700 .972760 .00047 10.750 .975080 .00049 10.800 .977760 .00047 10.850 .981460 .00048 10.900 .983400 .00048 10.950 .986570 .00048 11.000 .989470 .00050 11.050 .992310 .00049 11.100 .994970 .00048 11.150 .998010 .00049 11.201 .001100 .00048 11.251 .004290 .00049 11.301 .006110 .00048 11.351 .009610 .00049 11.401 .011810 .00049 11.451 .014750 .00048 11.501 .017480 .00049
670

10.000 .917680 .00049 10.050 .921760 .00048 10.100 .924640 .00049 10.150 .927280 .00048 10.200 .931020 .00047 10.250 .933590 .00048 10.300 .937080 .00047 10.350 .940540 .00048 10.400 .943430 .00047 10.450 .946320 .00047 10.500 .949000 .00050 10.550 .952410 .00049 10.600 .955290 .00048 10.650 .957910 .00049 10.700 .961270 .00048 10.750 .963880 .00048 10.800 .967900 .00048 10.850 .970430 .00049 10.900 .972860 .00049 10.950 .975690 .00047 11.000 .978710 .00050 11.050 .982420 .00049 11.100 .984230 .00049 11.150 .986560 .00048 11.200 .989530 .00049 11.350 .997790 .00049 11.451 .004350 .00049 11.501 .005980 .00049 11.551 .009940 .00048 11.601 .012330 .00048 11.651 .014500 .00049 11.701 .017460 .00048
690

10.600 .920770 .00048 10.700 .926160 .00049 10.800 .931410 .00046 10.900 .937740 .00049
698 11.300 .923700 .00047 11.400 .929280 .00049 11.500 .935170 .00048 11.600 .940160 .00048 
10.500 .934620 .00049 10.550 .937760 .00048 10.600 .940610 .00049 10.650 .943990 .00049 10.700 .946920 .00049 10.750 .949850 .00048 10.850 .955690 .00048 10.900 .959560 .00048 10.950 .961670 .00048 11.000 .965280 .00050 11.050 .967230 .00048 11.100 .970880 .00049 11.150 .972990 .00048 11.200 .975350 .00049 11.250 .978250 .00048 11.300 .982180 .00049 11.350 .984460 .00048 11.400 .987760 .00048 11.450 .989660 .00047 11.500 .991290 .00050 11.600 .997730 .00050 11.651 .000730 .00048 11.701 .002620 .00048 11.751 .005640 .00049 11.801 .008960 .00049 11.851 .011530 .00047 11.901 .014400 .00048 11.951 .015910 .00046 12.001 .018700 .00048

Pun43-icyl-0970-10

12

15.700 .925540 .00027 15.900 .929820 .00027 16.300 .937100 .00027 16.500 .940960 .00027 16.700 .944720 .00027 16.900 .947950 .00027 17.100 .952060 .00027 17.300 .955560 .00028 17.500 .959150 .00027 17.700 .962760 .00027 17.900 .966140 .00026 18.100 .969160 .00026 18.300 .971800 .00025 18.500 .975340 .00026 18.700 .977590 .00027 18.900 .981080 .00026 19.100 .984160 .00026
11.000 .943840 .00047 11.100 .949950 .00049 11.200 .954160 .00046 11.300 .961310 .00047 11.400 .966880 .00048 11.500 .971720 .00050 11.600 .976770 .00048 11.700 .982370 .00049 11.800 .989060 .00049 11.900 .993260 .00049 12.000 .999350 .00048 12.101 .003850 .00049 12.201 .008710 .00049 12.301 .014480 .00049 12.401 .019660 .00049
11.700 .946050 .00047 11.800 .951930 .00048 11.900 .956990 .00048 12.000 .961810 .00048 12.100 .966990 .00048 12.200 .972030 .00047 12.300 .978160 .00047 12.400 .982850 .00049 12.500 .987970 .00048 12.600 .992990 .00048 12.700 .997450 .00049 12.801 .001890 .00049 12.901 .008330 .00048 13.001 .012360 .00049 13.101 .017010 .00050
12.600 .926740 .00030

12.700 .930570 .00031

12.800 .933600 .00030

12.900 .937020 .00032

13.000 .940500 .00031

13.100 .942900 .00031

13.200 .946220 .00030

13.300 .948330 .00031

13.400 .952240 .00031

13.500 .954240 .00031

13.600 .957150 .00029

13.700 .960300 .00030

13.800 .963190 .00030

13.900 .966110 .00030

14.000 .968250 .00030

14.100 .971850 .00030

14.200 .973860 .00030
20

10.100 .919810 .00035

10.200 .923700 .00034

10.300 .929350 .00035

10.400 .933500 .00034

10.500 .938230 .00034

10.600 .942760 .00035

10.700 .947520 .00035

10.800 .951860 .00036

10.900 .956210 .00034

11.000 .960670 .00034

11.100 .965180 .00034

11.200 .968800 .00034 11.300 .973390 .00035 11.400 .977520 .00034 11.500 .981100 .00035 11.600 .985250 .00034 11.700 .990120 .00035 
19.300 .986540 .00026 19.500 .989220 .00026 19.700 .992760 .00026 19.900 .995160 .00025 20.100 .996970 .00025 20.300 .999590 .00025 20.501 .002260 .00026 20.701 .005180 .00025 20.901 .007410 .00024 21.101 .009750 .00025 21.301 .012700 .00024 21.401 .013070 .00025
25

9.000 .920910 .00037 9.100 .926270 .00037 9.200 .933110 .00038 9.300 .938510 .00038 9.400 .943880 .00036 9.500 .949430 .00037 9.600 .955430 .00037 9.700 .960330 .00037 9.800 .965060 .00037 9.900 .970720 .00037 10.000 .976090 .00037 10.100 .980460 .00037 10.200 .985810 .00037 10.300 .990720 .00037 10.400 .996030 .00036 10.501 .000210 .00037 10.601 .004980 .00036 10.701 .009470 .00037 10.801 .015160 .00038 10.901 .019060 .00035
14.300 .976460 .00030 14.400 .979010 .00030 14.500 .981630 .00031 14.600 .984130 .00030 14.700 .987120 .00029 14.800 .989420 .00030 14.900 .991620 .00030 15.000 .994310 .00030 15.100 .997420 .00029 15.200 .999400 .00030 15.301 .001990 .00029 15.401 .003750 .00030 15.501 .005640 .00030 15.601 .008120 .00030 15.701 .010320 .00029 15.801 .012800 .00029 15.901 .015080 .00029

$$
30
$$

8.300 .919930 .00039

8.350 .923430 .00039

8.400 .925530 .00038

8.450 .929750 .00039

8.500 .932210 .00040

8.550 .936770 .00039

8.600 .939580 .00037

8.650 .941920 .00039

8.700 .945420 .00040

8.750 .947920 .00037

8.800 .951380 .00038

8.850 .954400 .00039

8.900 .958220 .00040

8.950 .960550 .00038

9.100 .969800 .00038

9.150 .972490 .00037

9.200 .975720 .00038

9.250 .978950 .00039

9.300 .980750 .00039

9.350 .984330 .00039

9.400 .987380 .00039

9.450 .989670 .00039

9.500 .993140 .00039

9.550 .995810 .00039

9.600 .997980 .00037

9.651 .001450 .00040

9.701 .004130 .00039

9.751 .006610 .00040

9.801 .008680 .00038

9.851 .011740 .00038

9.901 .014300 .00039
11.800 .992600 .00035 11.900 .996720 .00035 12.001 .000380 .00033 12.101 .003760 .00034 12.201 .007790 .00033 12.301 .010770 .00034 12.401 .014810 .00034 12.501 .018390 .00034

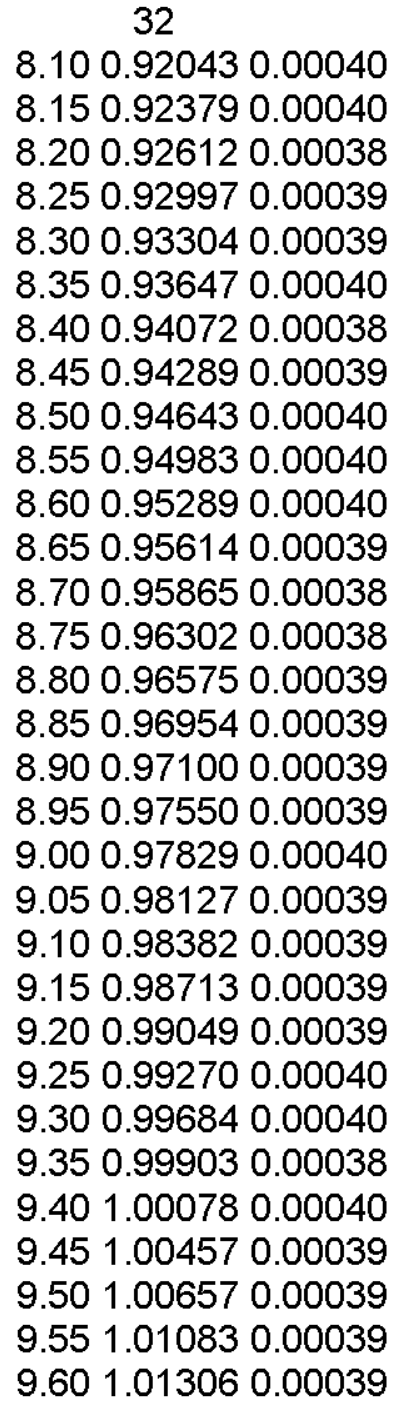


$\begin{array}{rrr}9.951 .017880 .00039 & 9.651 .015370 .00039 \\ 10.001 .020200 .00039 & 9.701 .018450 .00040\end{array}$
35

7.900 .923040 .00040

7.950 .927040 .00040

8.000 .929850 .00040

8.050 .934390 .00041

8.100 .937410 .00039

8.150 .940870 .00039

8.200 .944500 .00039

8.250 .948290 .00041

8.300 .950850 .00039

8.350 .955450 .00039

8.400 .957800 .00041

8.450 .961230 .00039

8.500 .964610 .00039

8.550 .968230 .00040

8.600 .971230 .00040

8.650 .974950 .00040

8.700 .977490 .00040

8.750 .980450 .00040

8.800 .983840 .00040

8.850 .987260 .00039

8.900 .990370 .00040

8.950 .993090 .00040

9.000 .996710 .00040

9.050 .999520 .00041

9.101 .002070 .00039

9.151 .005440 .00040

9.201 .008100 .00041

9.251 .011080 .00040

9.301 .013640 .00040

9.351 .016990 .00040

9.401 .019640 .00040

\section{0}

6.800 .916860 .00042

6.850 .922110 .00044

6.900 .925020 .00043

6.950 .929530 .00042

7.050 .938220 .00044

7.100 .941330 .00043

7.150 .946470 .00044

7.200 .950050 .00043

7.250 .954280 .00043

7.300 .957520 .00045
40

7.500 .917890 .00041

7.550 .921120 .00040

7.600 .925220 .00041

7.650 .929430 .00042

7.700 .934030 .00041

7.750 .936400 .00041

7.800 .940090 .00040

7.850 .943940 .00040

7.900 .947270 .00040

7.950 .951770 .00041

8.000 .954970 .00042

8.050 .958800 .00040

8.100 .962870 .00041

8.150 .964910 .00040

8.200 .968150 .00040

8.250 .972920 .00041

8.300 .976020 .00042

8.350 .979640 .00040

8.400 .982670 .00040

8.450 .985590 .00040

8.500 .989410 .00043

8.550 .991200 .00042

8.600 .995970 .00042

8.650 .999220 .00041

8.701 .002140 .00040

8.751 .004650 .00041

8.801 .007820 .00041

8.851 .011560 .00041

8.901 .015530 .00041

8.951 .018030 .00041

9.001 .021400 .00042
50

7.100 .920370 .00042

7.150 .924690 .00042

7.200 .928050 .00042

7.250 .933110 .00042

7.300 .936880 .00042

7.350 .939640 .00042

7.400 .943610 .00042

7.450 .948060 .00042

7.500 .952430 .00043

7.550 .955180 .00042

7.600 .959290 .00043

7.650 .963620 .00043

7.700 .966470 .00042

7.750 .970660 .00042

7.800 .975090 .00042

7.850 .978250 .00043

7.900 .981620 .00042

7.950 .985560 .00043

8.000 .988640 .00043

8.050 .992700 .00042

8.100 .997060 .00042

8.150 .999660 .00043

8.201 .003230 .00042

8.251 .006900 .00042

8.301 .009900 .00042

8.351 .014030 .00043

8.401 .016750 .00043

8.451 .020930 .00043

8.501 .024130 .00044
6.700 .923590 .00043

6.750 .928190 .00042

6.800 .931850 .00044

6.850 .936570 .00043

6.900 .940830 .00042

6.950 .944970 .00043

7.000 .949840 .00043

7.050 .953350 .00042

7.100 .957070 .00043

7.150 .961620 .00044
80

6.500 .916770 .00043 6.550 .921420 .00045 6.600 .924640 .00044 6.650 .929660 .00043 6.700 .932910 .00044 6.750 .938920 .00043 6.800 .942650 .00043 6.850 .946590 .00046 6.900 .950710 .00044 6.950 .956050 .00045 
7.350 .961430 .00043 7.400 .965330 .00042 7.450 .969860 .00043 7.500 .973990 .00044 7.550 .977710 .00043 7.600 .981240 .00042 7.650 .985690 .00043 7.700 .989040 .00043 7.750 .993350 .00043 7.800 .996820 .00042 7.851 .000580 .00043 7.901 .004260 .00043 7.951 .007550 .00042 8.001 .011030 .00044 8.051 .014280 .00044 8.101 .018870 .00044

\section{0}

6.400 .915210 .00045 6.450 .919930 .00044 6.500 .924300 .00044 6.550 .928530 .00045 6.600 .932930 .00044 6.650 .937770 .00044 6.700 .941500 .00045 6.750 .946200 .00044 6.800 .949850 .00043 6.850 .954740 .00045 6.900 .958590 .00044 6.950 .962970 .00044 7.000 .967190 .00045 7.050 .970640 .00044 7.100 .974690 .00044 7.150 .979520 .00044 7.200 .983300 .00045 7.250 .987030 .00045 7.300 .991310 .00044 7.350 .994130 .00046 7.400 .998880 .00044 7.451 .002770 .00047 7.501 .006810 .00044 7.551 .011070 .00044 7.601 .014350 .00044 7.651 .018020 .00044 7.701 .021650 .00044
7.200 .965770 .00044

7.250 .970010 .00043

7.300 .974340 .00043

7.350 .977160 .00044

7.400 .981550 .00044

7.450 .985140 .00044

7.500 .989090 .00044

7.550 .992900 .00043

7.600 .996900 .00044

7.651 .000500 .00043

7.701 .004890 .00044

7.751 .008610 .00045

7.801 .011250 .00044

7.851 .015600 .00044

7.901 .019940 .00045
7.000 .959900 .00044

7.050 .964100 .00044

7.100 .967480 .00044

7.150 .972450 .00043

7.200 .974960 .00043

7.250 .980520 .00043

7.300 .984170 .00045

7.350 .987100 .00046

7.400 .992380 .00045

7.450 .995950 .00045

7.501 .000000 .00043

7.551 .003640 .00044

7.601 .006980 .00046

7.651 .010620 .00045

7.701 .014590 .00044

7.751 .017940 .00045

7.801 .022230 .00044

120

6.400 .920610 .00045

6.450 .925630 .00043

6.500 .929620 .00045

6.550 .934380 .00045

6.600 .938840 .00046

6.650 .943440 .00044

6.700 .947140 .00043

6.750 .951360 .00045

6.800 .955400 .00045

6.850 .960250 .00044

6.900 .963810 .00045

6.950 .967750 .00046

7.000 .972760 .00044

7.050 .976830 .00045

7.100 .980880 .00046

7.150 .984760 .00045

7.200 .988340 .00044

7.250 .992470 .00044

7.300 .995740 .00044

7.350 .999630 .00045

7.401 .003970 .00045

7.451 .008440 .00045

7.501 .010670 .00045

7.551 .015570 .00045

7.601 .019550 .00045
6.300 .918310 .00044

6.350 .922460 .00045

6.400 .927380 .00044

6.450 .930300 .00044

6.500 .936160 .00044

6.550 .939750 .00045

6.600 .944550 .00045

6.650 .949140 .00046

6.700 .952410 .00046

6.750 .957630 .00046

6.800 .961500 .00045

6.850 .967110 .00046

6.900 .969030 .00045

6.950 .973730 .00046

7.000 .978100 .00046

7.050 .981780 .00046

7.100 .985240 .00045

7.200 .994440 .00045

7.250 .998340 .00045

7.301 .001830 .00046

7.351 .005860 .00045

7.401 .009520 .00046

7.451 .013350 .00046

7.501 .016700 .00046 
130

6.300 .921090 .00046

6.350 .925400 .00046

6.400 .929930 .00043

6.450 .933890 .00045

6.500 .938590 .00047

6.550 .942050 .00043

6.600 .946750 .00046

6.650 .952010 .00046

6.700 .955350 .00045

6.750 .961200 .00044

6.800 .965120 .00045

6.850 .968610 .00045

6.900 .971990 .00045

6.950 .976100 .00045

7.000 .980120 .00044

7.050 .985050 .00046

7.100 .988750 .00044

7.150 .992200 .00044

7.200 .996530 .00045

7.250 .999660 .00045

7.301 .003640 .00044

7.351 .007610 .00046

7.401 .011470 .00046

7.451 .015610 .00046
150

6.300 .922580 .00045

6.350 .927190 .00044

6.400 .932670 .00044

6.450 .935570 .00046

6.500 .940320 .00044

6.600 .948880 .00045

6.650 .953570 .00046

6.700 .957510 .00045

6.750 .961640 .00045

6.800 .964870 .00046

6.850 .970070 .00046

6.900 .973860 .00046

6.950 .977450 .00046

7.000 .981140 .00046

7.050 .986240 .00046

7.100 .989010 .00046

7.150 .993250 .00046

7.200 .997180 .00046

7.251 .000680 .00044

7.301 .005530 .00045

7.351 .007830 .00045

7.401 .012010 .00045

7.451 .016080 .00045

7.501 .020250 .00044
200

6.300 .922540 .00044

6.350 .926910 .00046

6.400 .931390 .00045

6.450 .934710 .00045

6.500 .939370 .00045

6.550 .943760 .00046

6.600 .947730 .00045

6.650 .951760 .00045

6.700 .955850 .00046

6.750 .960140 .00047

6.800 .964170 .00046

6.850 .968130 .00046

6.900 .972210 .00044

6.950 .975320 .00045

7.000 .980280 .00046

7.050 .984070 .00045

7.100 .987230 .00045

7.150 .991640 .00045

7.200 .994210 .00044

7.250 .999760 .00046

7.301 .002610 .00045

7.351 .006860 .00046

7.401 .010730 .00046

7.451 .014640 .00045

7.501 .017530 .00046
239

6.350 .923580 .00045

6.400 .927350 .00044

6.500 .935900 .00045

6.550 .940500 .00044

6.600 .943700 .00045

6.650 .948330 .00044

6.700 .951550 .00045

6.750 .955230 .00045

6.800 .959740 .00046

6.850 .964420 .00045

6.900 .967910 .00046

6.950 .971970 .00044

7.000 .975460 .00044

7.050 .979270 .00045

7.100 .982710 .00045

7.150 .986640 .00045

7.200 .991180 .00045

7.250 .994420 .00047

7.300 .998030 .00046

7.351 .001080 .00045

7.401 .004870 .00045
250

6.400 .926340 .00047

6.450 .929400 .00046

6.500 .935300 .00045

6.550 .938550 .00045

6.600 .942780 .00046

6.650 .946170 .00046

6.700 .950850 .00046

6.750 .954560 .00045

6.800 .957690 .00046

6.850 .962620 .00045

6.900 .965820 .00046

6.950 .969850 .00045

7.000 .974170 .00046

7.050 .976990 .00045

7.100 .981790 .00046

7.150 .985200 .00045

7.200 .989780 .00047

7.250 .992980 .00046

7.300 .996760 .00045

7.351 .000820 .00047

7.401 .003430 .00046
359

6.500 .920540 .00046

6.550 .924190 .00045

6.600 .928380 .00045

6.650 .932780 .00047

6.700 .936570 .00047

6.750 .940740 .00047

6.800 .944400 .00046

6.850 .947750 .00045

6.900 .951370 .00045

6.950 .955380 .00044

7.000 .958980 .00046

7.050 .963190 .00045

7.100 .967020 .00045

7.150 .970440 .00046

7.200 .974300 .00045

7.250 .977390 .00045

7.300 .980880 .00046

7.350 .984740 .00046

7.400 .988010 .00047

7.450 .991130 .00046

7.500 .995410 .00046 
7.451 .007890 .00046 7.501 .012990 .00044 7.551 .015980 .00045 7.601 .019780 .00045
7.451 .008350 .00045

7.551 .014890 .00044

7.601 .017970 .00047
7.550 .997550 .00046

7.601 .001910 .00045

7.651 .005610 .00048

7.701 .008340 .00045

7.751 .011330 .00046

7.801 .015170 .00046

7.851 .019470 .00045

7.901 .022050 .00045

478

6.800 .925200 .00045 6.850 .928960 .00045 6.900 .932310 .00045 6.950 .936360 .00046 7.000 .940630 .00045 7.050 .944100 .00046 7.100 .947700 .00047 7.150 .950210 .00045 7.200 .955010 .00046 7.250 .958110 .00044 7.300 .961390 .00045 7.350 .965030 .00046 7.400 .967700 .00045 7.450 .972260 .00045 7.500 .975020 .00044 7.550 .978340 .00045 7.600 .981680 .00045 7.650 .985210 .00047 7.700 .988680 .00046 7.800 .995170 .00047 7.850 .997600 .00046 7.901 .001390 .00047 7.951 .004840 .00046 8.001 .008010 .00046 8.051 .011580 .00045 8.101 .014990 .00045 8.151 .017400 .00047 8.201 .020480 .00045

650

7.500 .920460 .00045 7.550 .924690 .00046 7.600 .927190 .00045 7.650 .930100 .00043 7.700 .934310 .00045
500

6.800 .921130 .00046

6.850 .925520 .00044

6.900 .928490 .00045

6.950 .931560 .00047

7.000 .936110 .00045

7.050 .938900 .00044

7.100 .942240 .00046

7.150 .946000 .00046

7.250 .952960 .00045

7.300 .956720 .00045

7.350 .960900 .00045

7.400 .963200 .00044

7.450 .967370 .00045

7.500 .970140 .00046

7.550 .972980 .00045

7.600 .976780 .00045

7.650 .980600 .00047

7.700 .984020 .00046

7.750 .987770 .00045

7.800 .990710 .00046

7.850 .993610 .00045

7.900 .996470 .00045

7.950 .999640 .00045

8.001 .002670 .00046

8.051 .006310 .00046

8.101 .008980 .00046

8.151 .012730 .00045

8.201 .015510 .00047

8.251 .018080 .00046

8.301 .020990 .00045
600

7.200 .923460 .00046 7.250 .927390 .00045 7.300 .930080 .00046 7.350 .933650 .00046 7.400 .937680 .00045 7.450 .940770 .00046 7.500 .944500 .00046 7.550 .946860 .00045 7.600 .950810 .00044 7.650 .952830 .00045 7.700 .957550 .00046 7.750 .961070 .00045 7.800 .963670 .00044 7.850 .967350 .00046 8.000 .976070 .00046 8.050 .979170 .00045 8.100 .982320 .00046 8.150 .985530 .00044 8.200 .988940 .00046 8.300 .994830 .00045 8.350 .996960 .00044 8.401 .000710 .00046 8.451 .003940 .00045 8.501 .006930 .00044 8.551 .009690 .00046 8.601 .012040 .00045 8.651 .015670 .00046 8.701 .018980 .00046
660

7.650 .923700 .00044 7.700 .926930 .00045 7.750 .930570 .00044 7.850 .936820 .00046 7.900 .939140 .00045

\section{0}

7.800 .926420 .00047 7.850 .927960 .00045 7.900 .931930 .00045 7.950 .934880 .00045 8.000 .937800 .00045 
7.750 .937470 .00044 7.800 .940190 .00045 7.850 .943630 .00045 7.900 .946220 .00045 7.950 .949930 .00045 8.000 .952510 .00044 8.050 .955120 .00045 8.100 .958620 .00044 8.150 .961410 .00045 8.200 .965130 .00046 8.250 .968380 .00045 8.350 .973200 .00046 8.400 .976730 .00044 8.450 .980500 .00045 8.500 .982370 .00046 8.550 .985080 .00045 8.600 .988680 .00045 8.650 .991610 .00045 8.700 .994800 .00045 8.750 .997260 .00045 8.800 .999910 .00046 8.851 .003190 .00045 8.901 .005810 .00046 8.951 .009610 .00046 9.001 .011430 .00045 9.051 .014730 .00045 9.101 .016400 .00044 9.201 .022980 .00045

680

7.900 .922290 .00044 8.150 .936560 .00043 8.250 .942980 .00046 8.300 .946250 .00044 8.350 .949160 .00045 8.400 .952310 .00045 8.450 .954750 .00045 8.500 .958030 .00045 8.550 .961200 .00046 8.600 .963500 .00045 8.650 .966860 .00045 8.700 .970580 .00045 8.750 .972400 .00046 8.800 .975620 .00043 8.850 .977820 .00046 8.900 .981320 .00045 8.950 .983870 .00045 9.050 .990140 .00046 9.100 .992350 .00046
8.000 .946150 .00044 8.050 .949410 .00045 8.100 .952240 .00045 8.150 .955790 .00045 8.200 .958230 .00046 8.250 .961310 .00045 8.300 .964750 .00046 8.350 .967280 .00046 8.400 .971180 .00045 8.450 .973640 .00045 8.500 .976190 .00045 8.550 .978490 .00044 8.600 .982170 .00044 8.650 .984350 .00045 8.700 .987630 .00045 8.750 .990660 .00045 8.800 .993150 .00044 8.850 .996630 .00046 8.900 .999300 .00045 8.951 .001850 .00045 9.001 .004320 .00045 9.051 .007480 .00045 9.101 .010920 .00045 9.151 .012750 .00045 9.201 .015290 .00045 9.251 .018170 .00045 9.301 .021000 .00045
8.050 .940750 .00045 8.100 .944830 .00044 8.150 .947550 .00046 8.200 .950760 .00046 8.250 .953280 .00044 8.300 .956280 .00046 8.350 .959440 .00045 8.400 .962510 .00046 8.450 .965140 .00045 8.500 .968670 .00046 8.550 .971030 .00045 8.600 .973370 .00046 8.650 .977980 .00047 8.700 .980370 .00045 8.750 .981990 .00044 8.800 .985800 .00044 8.850 .988810 .00044 8.900 .991510 .00046 8.950 .994600 .00045 9.000 .997030 .00045 9.051 .000190 .00046 9.101 .001690 .00045 9.151 .005240 .00047 9.201 .007650 .00046 9.251 .010290 .00044 9.301 .013620 .00045 9.351 .015350 .00044 9.401 .018450 .00045
698

8.200 .925310 .00046 8.250 .928180 .00044 8.300 .931600 .00046 8.400 .937240 .00044 8.450 .939750 .00045 8.500 .943380 .00044 8.550 .946220 .00046 8.600 .948370 .00044 8.700 .954890 .00045 8.750 .957320 .00045 8.800 .960210 .00045 8.850 .963120 .00044 8.900 .966110 .00045 8.950 .968890 .00045 9.000 .971000 .00045 9.050 .974360 .00045 9.100 .977740 .00045 9.150 .979680 .00045 9.200 .982630 .00045 
9.150 .994760 .00045 9.200 .997400 .00045 9.251 .000340 .00046 9.301 .002760 .00045 9.351 .005380 .00044 9.401 .008690 .00046 9.451 .009930 .00045 9.501 .012390 .00044 9.551 .016960 .00047 9.601 .019620 .00045

Pun46-icyl-0970-00

12

20.700 .925880 .00028 21.000 .930420 .00028 21.300 .935110 .00028 21.600 .940200 .00027 21.900 .943770 .00028 22.200 .948290 .00028 22.500 .953060 .00028 22.800 .956670 .00028 23.100 .960420 .00027 23.400 .963640 .00027 23.700 .967780 .00027 24.000 .971280 .00027 24.300 .974310 .00026 24.600 .977420 .00026 24.900 .980990 .00026 25.200 .984160 .00027 25.500 .987590 .00026 25.800 .990900 .00026 26.100 .993440 .00025 26.400 .996000 .00025 26.700 .998780 .00026 27.001 .001460 .00025 27.301 .004430 .00026 27.601 .006730 .00025 27.901 .009360 .00025 28.201 .011570 .00025 28.401 .013300 .00025
9.250 .984800 .00044

9.300 .988280 .00045

9.350 .990290 .00045

9.400 .993500 .00046

9.450 .995570 .00046

9.500 .999030 .00045

9.551 .000560 .00045

9.601 .003790 .00045

9.651 .006420 .00045

9.701 .008140 .00044

9.751 .011140 .00045

9.801 .014310 .00045

9.851 .017080 .00044

9.901 .017910 .00046

15

16.600 .922310 .00033

16.800 .927210 .00032

17.000 .933230 .00033

17.200 .938590 .00032

17.400 .943800 .00033

17.600 .948610 .00033

17.800 .952760 .00032

18.000 .958300 .00033

18.200 .962630 .00032

18.400 .967630 .00032

18.600 .971080 .00031

18.800 .976280 .00031

19.000 .980460 .00031

19.200 .984890 .00031

19.400 .989090 .00032

19.600 .992510 .00031

19.800 .997390 .00031

20.001 .000070 .00031

20.201 .004020 .00030

20.401 .007290 .00030

20.601 .011080 .00030

20.801 .014550 .00031
20

13.900 .922880 .00037

14.000 .926400 .00038

14.100 .930660 .00038

14.200 .934450 .00038

14.300 .938970 .00037

14.400 .942610 .00039

14.500 .945720 .00037

14.600 .950450 .00037

14.700 .953940 .00037

14.800 .957470 .00037

14.900 .961520 .00037

15.000 .965270 .00037

15.100 .969700 .00038

15.200 .973680 .00037

15.300 .976200 .00037

15.400 .979710 .00038

15.500 .982480 .00038

15.600 .986540 .00036

15.700 .988920 .00037

15.800 .992640 .00037

15.900 .996200 .00036

16.000 .999630 .00037

16.101 .003050 .00037

16.201 .005540 .00037

16.301 .008770 .00036

16.401 .012520 .00036

16.501 .014410 .00036

16.601 .018460 .00036 
25

12.600 .921950 .00040

12.700 .927220 .00041

12.800 .932210 .00042

12.900 .936710 .00041

13.000 .942150 .00041

13.100 .946510 .00041

13.200 .951600 .00040

13.300 .956750 .00040

13.400 .961540 .00041

13.500 .965650 .00040

13.600 .971220 .00040

13.700 .975390 .00040

13.800 .979810 .00040

13.900 .983660 .00040

14.000 .988780 .00041

14.100 .992630 .00040

14.200 .997320 .00040

14.301 .001240 .00041

14.401 .004620 .00039

14.501 .009180 .00040

14.601 .013680 .00040

14.701 .017280 .00040
30

11.800 .919220 .00042

11.900 .925060 .00043

12.000 .930200 .00043

12.100 .935570 .00042

12.200 .941780 .00043

12.300 .947040 .00042

12.400 .953170 .00043

12.500 .958490 .00042

12.600 .963280 .00043

12.700 .968710 .00043

12.800 .974010 .00042

12.900 .979260 .00042

13.000 .984520 .00042

13.100 .989050 .00043

13.200 .994140 .00042

13.300 .998910 .00042

13.401 .003350 .00042

13.501 .008740 .00042

13.601 .013860 .00042

13.701 .017720 .00043
32

11.600 .919630 .00042

11.700 .925800 .00043

11.800 .931480 .00043

11.900 .937970 .00044

12.000 .943910 .00045

12.100 .949180 .00045

12.200 .954560 .00043

12.300 .960010 .00044

12.400 .966570 .00044

12.500 .971090 .00044

12.600 .976910 .00042

12.700 .981770 .00043

12.800 .987150 .00043

12.900 .993080 .00043

13.000 .998940 .00043

13.101 .003490 .00044

13.201 .007960 .00042

13.301 .013170 .00042

13.401 .017640 .00043
35

11.400 .923280 .00045

11.450 .926580 .00044

11.500 .930290 .00045

11.550 .933180 .00044

11.600 .937510 .00046

11.650 .939510 .00045

11.700 .942020 .00044

11.750 .945880 .00044

11.800 .948690 .00045

11.850 .951150 .00044

11.900 .955070 .00045

11.950 .957740 .00044

12.000 .960660 .00044

12.050 .963370 .00046

12.100 .966690 .00043

12.150 .968950 .00043

12.200 .971750 .00044

12.250 .974900 .00044

12.300 .977880 .00044

12.350 .981100 .00044

12.400 .983370 .00044

12.450 .986360 .00044
40

11.000 .918940 .00045 11.050 .923180 .00045

11.100 .926580 .00045

11.150 .929550 .00046

11.200 .933040 .00047

11.250 .935900 .00046

11.300 .939780 .00046

11.350 .942630 .00045

11.400 .945750 .00047

11.450 .949020 .00046

11.500 .952950 .00046

11.550 .955610 .00045

11.600 .958940 .00046

11.650 .961770 .00046

11.700 .965120 .00045

11.750 .968720 .00046

11.800 .971720 .00045

11.850 .974480 .00046

11.900 .977180 .00045

11.950 .979130 .00046

12.000 .982940 .00045

12.050 .986020 .00045
50

10.600 .922150 .00048 10.650 .925910 .00047 10.700 .928940 .00047 10.750 .932320 .00047 10.800 .935720 .00046 10.850 .939150 .00047 10.900 .943800 .00047 10.950 .947590 .00048 11.000 .950370 .00047 11.050 .954130 .00048 11.100 .957670 .00047 11.150 .960860 .00046 11.200 .964530 .00047 11.250 .966610 .00048 11.300 .971300 .00047 11.350 .973270 .00047 11.400 .978040 .00046 11.450 .980320 .00047 11.500 .983790 .00047 11.550 .987300 .00047 11.600 .990570 .00048 11.650 .993490 .00045 
12.500 .990140 .00044

12.550 .991440 .00043

12.600 .994030 .00043

12.650 .997060 .00044

12.700 .999790 .00044

12.751 .001980 .00044

12.801 .005340 .00044

12.851 .007300 .00044

12.901 .010440 .00044

12.951 .012780 .00043

13.001 .015580 .00043

13.051 .018540 .00044

13.101 .020720 .00043
12.100 .989930 .00046

12.150 .992160 .00046

12.200 .995130 .00044

12.250 .998240 .00045

12.301 .000950 .00045

12.351 .004750 .00043

12.401 .007550 .00045

12.451 .009760 .00046

12.501 .012170 .00043

12.551 .015820 .00046

12.601 .017500 .00045

12.651 .020680 .00044

12.701 .023190 .00045
11.700 .997090 .00048

11.750 .999570 .00046

11.801 .002860 .00047

11.851 .006610 .00048

11.901 .009160 .00048

11.951 .011430 .00046

12.001 .015120 .00046

12.051 .019200 .00047

12.101 .021320 .00046
60

10.300 .917310 .00048

10.350 .922640 .00049

10.400 .925320 .00048

10.450 .928350 .00050

10.500 .933280 .00047

10.550 .936390 .00047

10.600 .940990 .00048

10.650 .943890 .00049

10.700 .947670 .00047

10.750 .951670 .00047

10.800 .954750 .00049

10.850 .957380 .00049

10.900 .961820 .00049

10.950 .966590 .00048

11.000 .969030 .00050

11.050 .972880 .00048

11.100 .975290 .00049

11.150 .978790 .00049

11.200 .982560 .00047

11.250 .987530 .00048

11.300 .989020 .00048

11.350 .992970 .00047

11.400 .996650 .00049

11.451 .000110 .00047

11.501 .003020 .00049

11.551 .005250 .00048

11.601 .010220 .00048

11.651 .012870 .00049

11.701 .016090 .00047

11.751 .018410 .00050

11.801 .023070 .00046
70

10.200 .920210 .00048

10.250 .924490 .00051

10.300 .928320 .00048

10.350 .932070 .00049

10.400 .936830 .00049

10.450 .939890 .00046

10.500 .944260 .00048

10.550 .947600 .00049

10.600 .951670 .00049

10.650 .954110 .00050

10.700 .959100 .00048

10.750 .963350 .00050

10.800 .965130 .00049

10.850 .968920 .00049

10.900 .972930 .00049

10.950 .976640 .00051

11.000 .980390 .00049

11.050 .983720 .00050

11.100 .987560 .00048

11.150 .990260 .00048

11.200 .993530 .00049

11.250 .997130 .00049

11.301 .001340 .00049

11.351 .004770 .00048

11.401 .007830 .00048

11.451 .011090 .00049

11.501 .014760 .00049

11.551 .017480 .00048

11.601 .020330 .00050
80

10.100 .920340 .00050

10.150 .924270 .00048

10.200 .928700 .00050

10.250 .932280 .00049

10.300 .936540 .00050

10.350 .940240 .00050

10.400 .943780 .00049

10.450 .947800 .00049

10.500 .951820 .00049

10.550 .954670 .00048

10.600 .959690 .00050

10.650 .962350 .00050

10.700 .966760 .00051

10.750 .969840 .00049

10.800 .973620 .00050

10.850 .976330 .00050

10.900 .981330 .00049

10.950 .983960 .00051

11.000 .987530 .00050

11.050 .992410 .00050

11.100 .994610 .00050

11.150 .998090 .00050

11.201 .002690 .00050

11.251 .005540 .00049

11.301 .008330 .00049

11.351 .011970 .00049

11.401 .015950 .00048

11.451 .018290 .00049

11.501 .021640 .00050 
90

10.000 .917310 .00051 10.050 .921040 .00050 10.100 .925200 .00050 10.150 .927860 .00049 10.200 .933010 .00049 10.250 .935940 .00051 10.300 .940110 .00050 10.350 .944680 .00049 10.400 .948010 .00050 10.450 .951460 .00049 10.500 .956510 .00050 10.550 .960310 .00048 10.600 .962780 .00050 10.650 .967010 .00050 10.700 .970700 .00050 10.750 .974240 .00050 10.800 .978420 .00048 10.850 .981950 .00049 10.900 .985300 .00049 10.950 .988390 .00050 11.000 .992320 .00048 11.050 .996990 .00048 11.100 .999950 .00050 11.151 .003210 .00049 11.201 .004840 .00049 11.251 .010560 .00049 11.301 .013560 .00049 11.351 .016370 .00051 11.401 .020420 .00051

130

10.000 .919780 .00051 10.050 .924310 .00050 10.100 .927480 .00049 10.150 .931930 .00050 10.200 .936280 .00050 10.250 .940470 .00051 10.300 .943570 .00051 10.350 .947660 .00051 10.400 .952450 .00050 10.450 .954520 .00051 10.500 .958630 .00050 10.550 .962960 .00051 10.600 .966340 .00050
100

9.900 .912200 .00052 9.950 .916100 .00048 10.000 .919800 .00049 10.050 .924640 .00051 10.100 .928550 .00050 10.150 .931810 .00050 10.200 .936130 .00050 10.250 .940370 .00051 10.300 .944320 .00050 10.350 .947670 .00051 10.400 .949960 .00051 10.450 .956000 .00051 10.500 .958820 .00051 10.550 .962960 .00049 10.600 .966800 .00049 10.650 .970610 .00050 10.700 .973770 .00049 10.750 .978270 .00050 10.800 .981880 .00050 10.850 .984580 .00050 10.900 .988620 .00049 10.950 .992570 .00050 11.000 .995030 .00051 11.050 .999030 .00051 11.101 .002960 .00049 11.151 .005830 .00051 11.201 .009300 .00051 11.251 .013500 .00050 11.301 .015790 .00049 11.351 .019840 .00050 11.401 .023070 .00049

150

10.000 .917470 .00051 10.050 .921650 .00050 10.100 .925360 .00052 10.150 .929080 .00049 10.200 .934360 .00050 10.250 .937570 .00051 10.300 .941100 .00049 10.350 .945170 .00050 10.400 .948830 .00052 10.450 .952520 .00052 10.500 .956250 .00050 10.550 .959200 .00050 10.600 .964830 .00052
120

10.000 .920020 .00051 10.050 .924150 .00050 10.100 .927970 .00051 10.150 .931770 .00051 10.200 .935600 .00051 10.250 .940130 .00050 10.300 .944280 .00050 10.350 .947870 .00052 10.400 .951280 .00048 10.450 .955420 .00050 10.500 .958570 .00050 10.550 .963250 .00051 10.600 .966530 .00050 10.650 .969410 .00051 10.700 .974260 .00050 10.750 .978630 .00051 10.800 .981600 .00050 10.850 .985590 .00049 10.900 .988180 .00050 10.950 .991780 .00049 11.000 .995960 .00052 11.050 .999850 .00051 11.101 .001780 .00052 11.151 .006030 .00050 11.201 .009440 .00052 11.251 .013590 .00050 11.301 .016030 .00050 11.351 .019370 .00051 11.401 .022910 .00050

200

10.100 .914880 .00049 10.150 .919660 .00050 10.200 .922300 .00049 10.250 .927580 .00051 10.300 .932050 .00051 10.350 .934990 .00051 10.400 .938670 .00050 10.450 .942000 .00050 10.500 .946440 .00049 10.550 .950060 .00049 10.600 .953110 .00051 10.650 .958680 .00051 10.700 .960660 .00051 
10.650 .970410 .00051

10.700 .974430 .00049

10.750 .977010 .00052

10.800 .981380 .00053

10.850 .984750 .00051

10.900 .987730 .00050

10.950 .991420 .00049

11.000 .995670 .00052

11.051 .000150 .00051

11.101 .001900 .00052

11.151 .005730 .00049

11.201 .010290 .00050

11.251 .013210 .00052

11.301 .016150 .00051

11.351 .018970 .00050

11.401 .023860 .00050
10.650 .967590 .00050

10.700 .971600 .00050

10.750 .975260 .00050

10.800 .979100 .00050

10.850 .982670 .00049

10.900 .985470 .00050

10.950 .988810 .00050

11.000 .992630 .00052

11.050 .996650 .00051

11.101 .000130 .00050

11.151 .002610 .00050

11.201 .007090 .00050

11.251 .010160 .00050

11.301 .013330 .00053

11.351 .017610 .00049

11.401 .020520 .00052
239

10.300 .921790 .00050

10.350 .925660 .00051

10.400 .929380 .00049

10.450 .933790 .00050

10.500 .937270 .00049

10.550 .939980 .00051

10.600 .944040 .00049

10.650 .947850 .00050

10.700 .951360 .00050

10.750 .954610 .00051

10.800 .958330 .00049

10.850 .962270 .00049

10.900 .966420 .00051

10.950 .969950 .00050

11.000 .973110 .00052

11.050 .975500 .00050

11.100 .979200 .00050

11.150 .983090 .00051

11.200 .986070 .00050

11.250 .991100 .00051

11.300 .993240 .00052

11.350 .996960 .00049

11.400 .999750 .00050

11.451 .003780 .00051

11.501 .006440 .00051

11.551 .009600 .00050

11.601 .012940 .00051

11.651 .015460 .00050

11.701 .019280 .00049
250

10.300 .919580 .00050

10.350 .923330 .00049

10.400 .926980 .00050

10.450 .930830 .00052

10.500 .934450 .00050

10.550 .939310 .00051

10.600 .941730 .00051

10.650 .946020 .00049

10.700 .948840 .00050

10.750 .952580 .00051

10.800 .955100 .00051

10.850 .959910 .00050

10.900 .963090 .00051

10.950 .966320 .00050

11.000 .969450 .00052

11.050 .975050 .00052

11.100 .977810 .00049

11.150 .981190 .00051

11.200 .983490 .00049

11.250 .987550 .00051

11.300 .991010 .00050

11.350 .994160 .00051

11.400 .996760 .00051

11.451 .000180 .00049

11.501 .002990 .00051

11.551 .006880 .00049

11.601 .010460 .00050

11.651 .013580 .00050

11.701 .016150 .00051

11.751 .019120 .00051

11.801 .022380 .00050
10.750 .963850 .00050

10.800 .967170 .00050

10.850 .971830 .00050

10.900 .976550 .00050

10.950 .978660 .00051

11.000 .981570 .00050

11.050 .984980 .00051

11.100 .989300 .00051

11.150 .992530 .00050

11.200 .996320 .00051

11.250 .999750 .00050

11.301 .003190 .00050

11.351 .005210 .00049

11.401 .009000 .00051

11.451 .013000 .00050

11.501 .016480 .00051

11.551 .020100 .00051

11.601 .021510 .00051

\section{9}

10.600 .917750 .00050

10.650 .921580 .00049

10.700 .924890 .00051

10.750 .927470 .00050

10.800 .931850 .00050

10.850 .934770 .00050

10.900 .939750 .00050

10.950 .942580 .00049

11.000 .946390 .00049

11.050 .949340 .00050

11.100 .953380 .00050

11.150 .956300 .00049

11.200 .960470 .00049

11.250 .963550 .00050

11.300 .965600 .00049

11.350 .970010 .00050

11.400 .972980 .00051

11.450 .976340 .00050

11.500 .979320 .00048

11.550 .982540 .00048

11.600 .985410 .00049

11.650 .988500 .00050

11.700 .992780 .00050

11.750 .995370 .00050

11.800 .998570 .00052

11.851 .000750 .00049

11.901 .004610 .00050

11.951 .007350 .00050

12.001 .010500 .00050

12.051 .013600 .00049

12.101 .016800 .00051 
12.151 .019200 .00051

12.201 .023550 .00051

478

10.900 .918450 .00048 10.950 .922130 .00048 11.000 .926070 .00050 11.050 .928800 .00049 11.100 .932860 .00050 11.150 .936020 .00050 11.200 .940080 .00048 11.250 .942580 .00049 11.300 .946080 .00049 11.350 .948680 .00050 11.400 .952700 .00051 11.450 .955960 .00049 11.500 .959250 .00051 11.550 .962200 .00050 11.600 .965730 .00050 11.650 .969300 .00049 11.700 .972150 .00049 11.750 .975590 .00050 11.800 .978470 .00049 11.850 .981850 .00048 11.900 .984500 .00050 11.950 .988230 .00050 12.000 .990660 .00050 12.050 .994120 .00050 12.100 .997340 .00050 12.150 .998990 .00049 12.201 .003450 .00050 12.251 .005950 .00049 12.301 .008170 .00050 12.351 .011490 .00050 12.401 .014400 .00048 12.451 .017810 .00051 12.501 .020580 .00048
500

11.000 .922190 .00049 11.050 .925570 .00049 11.100 .929090 .00051 11.150 .932900 .00048 11.200 .936050 .00050 11.250 .939170 .00049 11.300 .941590 .00049 11.350 .945930 .00049 11.400 .948550 .00049 11.450 .951660 .00048 11.500 .956280 .00051 11.550 .958710 .00049 11.600 .962130 .00048 11.650 .965660 .00049 11.700 .968560 .00050 11.750 .971260 .00050 11.800 .975440 .00048 11.850 .978870 .00049 11.900 .981890 .00049 11.950 .984030 .00049 12.000 .988160 .00050 12.050 .989810 .00050 12.100 .993380 .00048 12.150 .996810 .00049 12.200 .999080 .00050 12.251 .002380 .00050 12.301 .006520 .00049 12.351 .008150 .00050 12.401 .011710 .00051 12.451 .013440 .00048 12.501 .016990 .00050 12.551 .019820 .00049 12.601 .023230 .00050
650

11.400 .920650 .00049 11.450 .924540 .00047 11.500 .927790 .00048 11.550 .930560 .00051 11.600 .934810 .00048 11.650 .937680 .00049
700

11.600 .921120 .00050 11.650 .924860 .00047 11.700 .927290 .00048 11.750 .929570 .00049 11.800 .933250 .00050 11.850 .936810 .00049
600

11.200 .917320 .00051 11.250 .920820 .00048 11.300 .924100 .00050 11.350 .927540 .00049 11.400 .932240 .00049 11.450 .934900 .00049 11.500 .937180 .00048 11.550 .940930 .00049 11.600 .944640 .00050 11.650 .947960 .00048 11.700 .950350 .00048 11.750 .953520 .00049 11.800 .957090 .00049 11.850 .960490 .00050 11.900 .963280 .00049 11.950 .966730 .00049 12.000 .970540 .00048 12.050 .972610 .00049 12.100 .975620 .00048 12.150 .978130 .00049 12.200 .982800 .00050 12.250 .984650 .00050 12.300 .988030 .00049 12.350 .990610 .00050 12.400 .992830 .00050 12.450 .996860 .00048 12.500 .999130 .00048 12.551 .003220 .00048 12.601 .005650 .00048 12.651 .008110 .00049 12.701 .010310 .00048 12.751 .013690 .00050 12.801 .017000 .00049 12.851 .019450 .00049 12.901 .022660 .00048

\section{0}

11.800 .914690 .00047 11.850 .919460 .00050 11.900 .922480 .00049 11.950 .925580 .00048 12.000 .929610 .00048 12.050 .931750 .00048 
11.700 .940820 .00049 11.750 .943270 .00049 11.800 .947390 .00048 11.850 .949710 .00048 11.900 .953560 .00048 11.950 .957220 .00049 12.000 .959750 .00049 12.050 .962780 .00048 12.100 .965750 .00049 12.150 .969540 .00048 12.200 .972130 .00049 12.250 .974180 .00049 12.300 .978630 .00048 12.350 .980660 .00049 12.400 .984580 .00049 12.450 .987590 .00048 12.500 .989790 .00048 12.550 .993460 .00048 12.600 .995710 .00047 12.650 .998530 .00049 12.701 .001980 .00048 12.751 .004530 .00049 12.801 .006900 .00048 12.851 .010270 .00049 12.901 .013030 .00049 12.951 .016110 .00049 13.001 .018250 .00049

760

11.900 .918270 .00048 11.950 .921700 .00049 12.000 .924470 .00049 12.050 .928680 .00048 12.100 .930300 .00049 12.150 .934960 .00046 12.200 .937410 .00047 12.250 .940000 .00049 12.300 .943040 .00046 12.350 .947600 .00046 12.400 .951140 .00048 12.450 .954200 .00049 12.500 .955510 .00047 12.550 .959810 .00048 12.600 .962260 .00049 12.650 .966020 .00048 12.700 .969040 .00048 12.750 .971600 .00047 12.800 .973440 .00048
11.900 .940100 .00050 11.950 .942480 .00049 12.000 .946220 .00048 12.050 .948940 .00049 12.100 .952530 .00048 12.150 .955040 .00049 12.200 .958450 .00049 12.250 .962600 .00047 12.300 .964920 .00048 12.350 .967800 .00049 12.400 .971580 .00047 12.450 .974120 .00048 12.500 .977940 .00049 12.550 .979850 .00049 12.600 .983670 .00048 12.650 .985950 .00049 12.700 .988900 .00048 12.750 .991340 .00048 12.800 .994670 .00047 12.850 .996980 .00048 12.901 .000520 .00049 12.951 .003440 .00049 13.001 .005380 .00048 13.051 .008900 .00047 13.101 .010970 .00048 13.151 .013470 .00049 13.201 .016450 .00048 13.251 .020610 .00048 13.301 .022960 .00048

765

11.900 .915300 .00046 12.000 .922660 .00048 12.100 .929110 .00048 12.200 .934780 .00049 12.300 .941990 .00048 12.400 .947710 .00049 12.500 .953720 .00049 12.600 .959930 .00048 12.700 .964490 .00048 12.800 .972000 .00049 12.900 .977630 .00049 13.000 .982770 .00047 13.100 .990330 .00048 13.200 .995670 .00047 13.301 .000660 .00047 13.401 .007000 .00048 13.501 .011280 .00049 13.601 .016400 .00049 13.701 .022470 .00049
12.100 .934480 .00047 12.150 .938270 .00049 12.200 .941710 .00047 12.250 .944520 .00048 12.300 .947410 .00049 12.350 .951840 .00048 12.400 .954110 .00049 12.450 .956820 .00046 12.500 .959520 .00048 12.550 .963320 .00047 12.600 .966070 .00048 12.650 .968030 .00048 12.700 .972410 .00047 12.750 .975480 .00048 12.800 .978580 .00049 12.850 .981370 .00048 12.900 .983180 .00048 12.950 .987150 .00048 13.000 .989970 .00048 13.050 .992450 .00049 13.100 .994980 .00049 13.150 .998550 .00049 13.201 .000600 .00048 13.251 .003850 .00048 13.301 .006020 .00048 13.351 .009340 .00049 13.401 .011810 .00048 13.451 .014910 .00047 13.501 .017300 .00048

770

12.000 .920170 .00046 12.050 .923040 .00049 12.100 .925960 .00048 12.150 .929550 .00049 12.200 .932770 .00047 12.250 .935850 .00047 12.300 .939200 .00049 12.350 .942120 .00049 12.400 .945940 .00048 12.450 .948500 .00049 12.500 .951020 .00047 12.550 .954080 .00048 12.600 .957520 .00048 12.650 .960720 .00047 12.700 .963200 .00048 12.750 .967240 .00047 12.800 .969130 .00049 12.850 .972220 .00050 12.900 .974650 .00050 
12.850 .976640 .00049

12.900 .979820 .00048

12.950 .983350 .00049

13.000 .985880 .00047

13.050 .989280 .00049

13.100 .990890 .00048

13.150 .994540 .00050

13.200 .997370 .00048

13.251 .000570 .00048

13.301 .003210 .00049

13.351 .005010 .00048

13.401 .008290 .00049

13.451 .011240 .00048

13.501 .013590 .00048

13.551 .016660 .00048

13.601 .019150 .00048

775

12.000 .918990 .00048

12.050 .921380 .00047

12.100 .925100 .00048

12.150 .928620 .00048

12.200 .931560 .00048

12.250 .934740 .00048

12.300 .937470 .00047

12.350 .940660 .00048

12.400 .944130 .00048

12.450 .947410 .00048

12.500 .949990 .00048

12.550 .952270 .00048

12.600 .955930 .00048

12.650 .959840 .00046

12.700 .961740 .00048

12.750 .965160 .00048

12.800 .968500 .00048

12.850 .971550 .00048

12.900 .973440 .00047

12.950 .976630 .00047

13.000 .979460 .00049

13.050 .983060 .00048

13.100 .985120 .00047

13.150 .988440 .00048

13.200 .991290 .00049

13.250 .993430 .00049

13.300 .996190 .00048

13.350 .998530 .00048

13.401 .002460 .00048

13.451 .005460 .00049

13.501 .007760 .00049

13.551 .011350 .00049

13.601 .013340 .00049
12.950 .978540 .00049

13.000 .980220 .00048

13.050 .983860 .00049

13.100 .986650 .00049

13.150 .989750 .00048

13.200 .992820 .00050

13.250 .994680 .00047

13.300 .997320 .00048

13.351 .000720 .00048

13.401 .004030 .00048

13.451 .007630 .00047

13.501 .009910 .00049

13.551 .010940 .00048

13.601 .014290 .00048

13.651 .018460 .00047

13.701 .020660 .00049

0 
13.651 .016220 .00048

13.701 .019180 .00048

Pun46-icyl-0970-01

12

19.200 .926480 .00027 19.500 .931190 .00028 19.800 .935980 .00028 20.100 .940560 .00028 20.400 .945470 .00027 20.700 .949200 .00027 21.000 .953860 .00026 21.300 .957830 .00027 21.600 .960850 .00026 21.900 .965060 .00026 22.200 .968770 .00026 22.500 .972060 .00026 22.800 .975580 .00026 23.100 .979050 .00026 23.400 .982240 .00025 23.700 .984990 .00026 24.000 .987600 .00025 24.300 .991590 .00026 24.600 .994110 .00025 24.900 .997010 .00026 25.201 .000020 .00025 25.501 .002600 .00025 25.801 .004870 .00025 26.101 .007930 .00024 26.401 .009810 .00024 26.701 .012710 .00024 26.801 .012670 .00023

25

11.000 .921310 .00040 11.100 .927240 .00040 11.200 .931720 .00039 11.300 .936520 .00038 11.400 .941780 .00039 11.500 .946840 .00039 11.600 .951780 .00039 11.700 .956340 .00040 11.800 .961390 .00038 11.900 .965110 .00040 12.000 .970380 .00040 12.100 .975650 .00039 12.200 .979730 .00038 12.300 .982740 .00039
15

15.100 .924420 .00032 15.300 .930600 .00031 15.500 .934950 .00032 15.700 .940780 .00032 15.900 .945790 .00032 16.100 .950180 .00031 16.300 .955220 .00032 16.500 .960540 .00031 16.700 .964540 .00031 16.900 .969180 .00030 17.100 .973520 .00030 17.300 .977830 .00031 17.500 .982220 .00030 17.700 .986270 .00030 17.900 .990400 .00030 18.100 .994060 .00030 18.300 .998150 .00031 18.501 .002360 .00030 18.701 .006290 .00030 18.901 .009080 .00030 19.101 .012740 .00030 19.201 .014390 .00029

30

10.300 .924120 .00042 10.400 .929810 .00040 10.500 .935910 .00042 10.600 .940450 .00041 10.700 .947350 .00041 10.800 .952520 .00040 10.900 .957890 .00043 11.000 .963670 .00041 11.100 .968670 .00041 11.200 .974590 .00040 11.300 .979250 .00042 11.400 .983430 .00041 11.500 .988930 .00041 11.600 .995090 .00042
20

12.300 .922070 .00036 12.400 .925990 .00036 12.500 .930230 .00036 12.600 .934330 .00037 12.700 .937850 .00036 12.800 .942230 .00036 12.900 .946460 .00037 13.000 .949450 .00036 13.100 .953730 .00036 13.200 .957760 .00036 13.300 .961790 .00036 13.400 .964630 .00036 13.500 .969480 .00036 13.600 .971930 .00035 13.700 .975930 .00035 13.800 .979780 .00035 13.900 .982460 .00037 14.000 .986300 .00035 14.100 .990080 .00036 14.200 .992550 .00036 14.300 .995890 .00035 14.400 .998580 .00035 14.501 .002380 .00036 14.601 .005070 .00036 14.701 .008290 .00036 14.801 .012230 .00035 14.901 .014300 .00035

32

10.000 .919390 .00042 10.100 .925610 .00044 10.200 .931250 .00043 10.300 .937950 .00041 10.400 .943630 .00042 10.500 .948840 .00040 10.600 .954610 .00042 10.700 .960700 .00042 10.800 .965960 .00042 10.900 .971250 .00041 11.000 .976790 .00041 11.100 .983160 .00043 11.200 .987390 .00042 11.300 .992410 .00041 
12.400 .988050 .00038

12.500 .993310 .00039

12.600 .995950 .00039

12.701 .001200 .00038

12.801 .005140 .00039

12.901 .009730 .00038

13.001 .012550 .00039

13.101 .017360 .00039
11.700 .998650 .00041 11.801 .003810 .00041 11.901 .009060 .00040 12.001 .014170 .00040 12.101 .017980 .00040
11.400 .997880 .00041 11.501 .003130 .00040 11.601 .007660 .00041 11.701 .014030 .00042 11.801 .018300 .00043
35

9.800 .923840 .00042 9.850 .927330 .00043 9.900 .929840 .00043 9.950 .932990 .00042 10.000 .936040 .00043 10.050 .939950 .00043 10.100 .942400 .00042 10.150 .945500 .00042 10.200 .948010 .00043 10.250 .951850 .00043 10.300 .954580 .00042 10.350 .957060 .00043 10.400 .960750 .00043 10.450 .963670 .00043 10.500 .966220 .00043 10.550 .968960 .00042 10.600 .972220 .00044 10.650 .974670 .00043 10.700 .977840 .00043 10.750 .980800 .00041 10.800 .983440 .00042 10.850 .986550 .00042 10.900 .989000 .00043 10.950 .991080 .00043 11.000 .994370 .00043 11.050 .997600 .00043 11.101 .000340 .00041 11.151 .003330 .00042 11.201 .005550 .00041 11.251 .007750 .00042 11.301 .010260 .00043 11.351 .013190 .00041 11.401 .015720 .00044 11.451 .018610 .00042 11.501 .021340 .00042
40

9.400 .920250 .00044 9.450 .922950 .00043 9.500 .927450 .00045 9.550 .929410 .00044 9.600 .933040 .00043 9.650 .936300 .00044 9.750 .942950 .00044 9.800 .946650 .00045 9.850 .949550 .00044 9.900 .952930 .00044 9.950 .955820 .00043 10.000 .959900 .00044 10.050 .963060 .00044 10.100 .965600 .00044 10.150 .968210 .00044 10.200 .972170 .00044 10.250 .975070 .00044 10.300 .977960 .00044 10.350 .980690 .00044 10.400 .984700 .00044 10.450 .986630 .00044 10.500 .989750 .00043 10.550 .991910 .00045 10.600 .995390 .00042 10.650 .998330 .00044 10.701 .001890 .00043 10.751 .004620 .00043 10.801 .007910 .00044 10.851 .010120 .00043 10.901 .012700 .00044 10.951 .014670 .00044 11.001 .018270 .00043
50

9.000 .923260 .00044 9.050 .925500 .00044 9.100 .930100 .00046 9.150 .933690 .00045 9.200 .936610 .00046 9.250 .940780 .00045 9.300 .944560 .00044 9.350 .947520 .00044 9.400 .951040 .00046 9.450 .954270 .00046 9.500 .958510 .00046 9.550 .961460 .00045 9.600 .964340 .00045 9.650 .968250 .00046 9.700 .971600 .00046 9.750 .974370 .00046 9.800 .977930 .00045 9.850 .981730 .00045 9.900 .984120 .00044 9.950 .987880 .00045 10.000 .991120 .00044 10.050 .994590 .00046 10.100 .997880 .00045 10.151 .001950 .00045 10.201 .002990 .00045 10.251 .007030 .00045 10.301 .010010 .00046 10.351 .013220 .00047 10.401 .015970 .00044 10.451 .019030 .00044 10.501 .021470 .00044 
60

8.700 .919260 .00047 8.750 .923060 .00046 8.800 .926560 .00047 8.850 .931050 .00047 8.900 .935440 .00046 8.950 .938640 .00047 9.000 .940910 .00046 9.050 .945780 .00046 9.100 .948810 .00047 9.150 .952640 .00045 9.200 .956540 .00048 9.250 .958960 .00046 9.300 .963770 .00046 9.350 .966960 .00046 9.400 .970590 .00044 9.450 .973880 .00047 9.500 .977070 .00047 9.550 .981480 .00048 9.600 .984910 .00046 9.650 .987940 .00045 9.700 .991620 .00046 9.750 .994580 .00046 9.800 .997140 .00045 9.851 .001930 .00046 9.901 .004750 .00047 9.951 .007640 .00045 10.001 .010340 .00045 10.051 .014640 .00047 10.101 .017880 .00046 10.151 .020650 .00047 10.201 .022650 .00046

90

8.400 .920520 .00047 8.450 .924240 .00048 8.500 .927350 .00048 8.550 .930980 .00048 8.600 .936340 .00047 8.650 .939980 .00049 8.700 .943250 .00047 8.750 .947640 .00048 8.800 .951430 .00048 8.850 .954520 .00047 8.900 .958460 .00049 8.950 .962360 .00049 9.000 .966520 .00048
70

8.500 .914940 .00048

8.550 .919130 .00047

8.600 .922480 .00046

8.650 .926890 .00047

8.700 .930450 .00048

8.750 .934220 .00047

8.800 .939120 .00047

8.850 .942120 .00047

8.900 .945890 .00045

8.950 .949770 .00047

9.000 .952690 .00047

9.050 .957360 .00047

9.100 .960530 .00047

9.150 .963810 .00046

9.200 .967570 .00047

9.250 .971350 .00047

9.300 .975600 .00048

9.350 .979010 .00047

9.400 .982310 .00049

9.450 .986150 .00047

9.500 .989720 .00047

9.550 .992060 .00046

9.600 .996290 .00046

9.650 .998550 .00047

9.701 .003930 .00047

9.751 .006350 .00048

9.801 .009280 .00047

9.851 .012540 .00047

9.901 .016020 .00048

9.951 .019980 .00047

10.001 .023360 .00049
80

8.400 .915100 .00046

8.450 .918810 .00047

8.500 .922370 .00047

8.550 .927390 .00048

8.600 .931780 .00047

8.650 .935580 .00046

8.700 .939310 .00049

8.750 .942650 .00047

8.800 .946800 .00048

8.850 .950680 .00047

8.900 .955120 .00048

8.950 .957530 .00047

9.000 .961240 .00046

9.050 .964700 .00047

9.100 .968570 .00048

9.150 .972340 .00048

9.200 .975720 .00047

9.250 .980420 .00047

9.300 .982920 .00047

9.350 .986670 .00048

9.400 .990990 .00048

9.450 .993580 .00048

9.500 .997710 .00048

9.551 .000720 .00047

9.601 .005030 .00048

9.651 .007320 .00049

9.701 .012140 .00049

9.751 .014070 .00047

9.801 .017090 .00048
100

8.400 .922870 .00049

8.450 .927390 .00049

8.500 .931000 .00047

8.550 .935290 .00050

8.600 .939550 .00048

8.650 .943830 .00047

8.700 .947230 .00049

8.750 .950570 .00048

8.800 .954400 .00048

8.850 .958670 .00047

8.900 .962850 .00050

8.950 .966170 .00049

9.000 .969710 .00048
120

8.300 .916710 .00048

8.350 .920570 .00050

8.400 .925260 .00049

8.450 .928720 .00049

8.500 .932620 .00049

8.550 .937450 .00048

8.600 .940260 .00048

8.650 .944210 .00048

8.700 .948500 .00051

8.750 .952890 .00049

8.800 .956510 .00050

8.850 .960020 .00049

8.900 .962650 .00048 
9.050 .969940 .00048 9.100 .973320 .00048 9.150 .977420 .00049 9.200 .981230 .00047 9.250 .985470 .00048 9.300 .988710 .00048 9.350 .992060 .00049 9.400 .996630 .00048 9.450 .998020 .00048 9.501 .002200 .00047 9.551 .006030 .00049 9.601 .008780 .00049 9.651 .012180 .00049 9.701 .016220 .00048 9.751 .019180 .00049 9.801 .023410 .00048

130

8.400 .925790 .00049 8.450 .929520 .00049 8.500 .932170 .00049 8.550 .937530 .00048 8.600 .941310 .00048 8.650 .944470 .00049 8.700 .948140 .00048 8.750 .952750 .00049 8.800 .956320 .00049 8.850 .959560 .00049 8.900 .963570 .00049 8.950 .968110 .00048 9.000 .971280 .00049 9.050 .975350 .00049 9.100 .977950 .00048 9.150 .982930 .00049 9.200 .985900 .00050 9.250 .989600 .00049 9.300 .991870 .00047 9.350 .995860 .00048 9.401 .000450 .00048 9.451 .002730 .00048 9.501 .005740 .00050 9.551 .010170 .00048 9.601 .012810 .00049 9.651 .016620 .00049 9.701 .020530 .00048
9.050 .973770 .00048

9.100 .978060 .00048

9.150 .981350 .00049

9.200 .983830 .00049

9.250 .987840 .00047

9.300 .991550 .00048

9.350 .994670 .00049

9.400 .998710 .00049

9.451 .002860 .00049

9.501 .006130 .00049

9.551 .009560 .00049

9.601 .012190 .00049

9.651 .016020 .00049

9.701 .019780 .00048

8.950 .967710 .00050 9.000 .970630 .00048 9.050 .974380 .00049 9.100 .977810 .00049 9.150 .982630 .00047 9.200 .985870 .00048 9.250 .989070 .00048 9.300 .992390 .00048 9.350 .997050 .00049 9.400 .999370 .00049 9.451 .002760 .00049 9.501 .006410 .00050 9.551 .010010 .00049 9.601 .013040 .00049 9.651 .016100 .00049 9.701 .020880 .00049

150

8.300 .915430 .00048

8.350 .919860 .00049

8.400 .923510 .00048

8.450 .927230 .00048

8.500 .931950 .00048

8.550 .935050 .00049

8.600 .938410 .00048

8.650 .942890 .00049

8.700 .945690 .00050

8.750 .950170 .00049

8.800 .953470 .00050

8.850 .958460 .00048

8.900 .961300 .00049

8.950 .965410 .00050

9.000 .969370 .00049

9.050 .972550 .00049

9.100 .976410 .00049

9.150 .980410 .00050

9.200 .983960 .00048

9.250 .987760 .00049

9.300 .990400 .00050

9.350 .994720 .00051

9.400 .997240 .00049

9.451 .001600 .00049

9.501 .005290 .00048

9.551 .008140 .00049

9.601 .011230 .00049

9.651 .014710 .00049

9.701 .018220 .00050
200

8.500 .922740 .00049 8.550 .925870 .00049 8.600 .930070 .00048 8.650 .934690 .00048 8.700 .937990 .00048 8.750 .941160 .00051 8.800 .945340 .00048 8.850 .949150 .00048 8.900 .952610 .00050 8.950 .956090 .00050 9.000 .959860 .00050 9.050 .963280 .00049 9.100 .965880 .00048 9.150 .970500 .00049 9.200 .974570 .00049 9.250 .978020 .00050 9.300 .982520 .00049 9.350 .984400 .00049 9.400 .988850 .00050 9.450 .991730 .00049 9.500 .995650 .00049 9.550 .997940 .00048 9.601 .002690 .00048 9.651 .005350 .00049 9.701 .008220 .00049 9.751 .012020 .00048 9.801 .014650 .00047 9.851 .017630 .00048 9.901 .021650 .00049 
239

8.600 .922130 .00048

8.650 .925570 .00048

8.700 .929140 .00048

8.750 .933400 .00049

8.800 .936530 .00050

8.850 .941320 .00048

8.900 .943920 .00049

8.950 .948470 .00050

9.000 .952320 .00051

9.050 .955540 .00049

9.100 .957930 .00049

9.150 .962380 .00049

9.200 .966580 .00050

9.250 .969950 .00048

9.300 .972800 .00050

9.350 .977380 .00050

9.400 .979520 .00049

9.450 .982530 .00049

9.500 .986120 .00048

9.550 .989590 .00049

9.600 .993370 .00049

9.650 .996400 .00049

9.700 .999400 .00049

9.751 .003650 .00048

9.801 .006560 .00050

9.851 .009520 .00048

9.901 .013670 .00049

9.951 .015840 .00050

10.001 .019190 .00049

478

9.200 .921520 .00048

9.250 .924330 .00050

9.300 .928200 .00049

9.350 .931800 .00049

9.400 .935800 .00049

9.450 .938450 .00049

9.500 .942270 .00049

9.550 .944780 .00049

9.600 .948760 .00049

9.650 .951580 .00049

9.700 .954920 .00048

9.750 .959220 .00047

9.800 .962500 .00051
250

8.600 .920640 .00050

8.650 .922810 .00048

8.700 .927670 .00050

8.750 .931290 .00049

8.800 .934900 .00048

8.850 .938720 .00049

8.900 .941640 .00048

8.950 .945630 .00049

9.000 .950240 .00050

9.050 .952500 .00049

9.100 .955710 .00050

9.150 .960350 .00048

9.200 .963270 .00049

9.250 .966240 .00049

9.300 .970510 .00048

9.350 .974120 .00048

9.400 .977340 .00048

9.450 .980470 .00049

9.500 .984660 .00049

9.550 .987150 .00049

9.600 .990520 .00049

9.650 .993410 .00050

9.700 .997070 .00050

9.751 .000940 .00049

9.801 .002810 .00048

9.851 .006740 .00049

9.901 .010350 .00049

9.951 .013520 .00050

10.001 .016410 .00048

10.051 .019060 .00049

10.101 .023130 .00048
359

8.900 .919140 .00049

8.950 .923270 .00049

9.000 .926990 .00049

9.050 .930430 .00050

9.100 .934600 .00048

9.150 .937060 .00048

9.200 .940480 .00049

9.250 .944170 .00049

9.300 .946840 .00049

9.350 .950510 .00050

9.400 .954650 .00050

9.450 .958430 .00049

9.500 .961810 .00048

9.550 .964920 .00049

9.600 .967180 .00049

9.650 .970330 .00051

9.700 .974600 .00049

9.750 .977990 .00050

9.800 .979820 .00048

9.850 .983940 .00049

9.900 .987100 .00049

9.950 .990390 .00050

10.000 .994270 .00049

10.050 .996560 .00049

10.100 .999840 .00050

10.151 .002120 .00049

10.201 .005600 .00049

10.251 .008570 .00050

10.301 .012160 .00049

10.351 .015030 .00048

10.401 .017540 .00048
9.300 .925860 .00049

9.350 .928120 .00049

9.400 .931290 .00048

9.450 .935080 .00048

9.500 .938160 .00049

9.550 .941670 .00048

9.600 .945100 .00049

9.650 .948540 .00050

9.700 .950900 .00049

9.750 .954560 .00049

9.800 .958370 .00049

9.850 .961290 .00048

9.900 .964120 .00048
600

9.500 .921660 .00049

9.550 .923200 .00047

9.600 .927750 .00050

9.650 .930820 .00049

9.700 .933810 .00049

9.750 .937630 .00048

9.800 .941310 .00048

9.850 .944940 .00048

9.900 .948370 .00048

9.950 .949980 .00049

10.000 .953950 .00048

10.050 .957350 .00048

10.100 .960470 .00049 
9.850 .964830 .00048

9.900 .967580 .00048

9.950 .970620 .00050

10.000 .974360 .00049

10.050 .977920 .00049

10.100 .980510 .00049

10.150 .982900 .00050

10.200 .986900 .00049

10.250 .990150 .00049

10.300 .992630 .00049

10.350 .995860 .00048

10.400 .999140 .00048

10.451 .001610 .00049

10.501 .004770 .00050

10.551 .007570 .00049

10.601 .010180 .00050

10.651 .013230 .00049

10.701 .016030 .00050

10.751 .018240 .00049

10.801 .022680 .00047
9.950 .967150 .00048

10.000 .970310 .00048

10.050 .973730 .00049

10.100 .976850 .00049

10.150 .980030 .00048

10.200 .982750 .00049

10.250 .987030 .00049

10.300 .989480 .00049

10.350 .992140 .00048

10.400 .994810 .00049

10.450 .997970 .00048

10.501 .001120 .00049

10.551 .004160 .00049

10.601 .007230 .00049

10.651 .009760 .00050

10.701 .012100 .00049

10.751 .015240 .00048

10.801 .017220 .00050
650

9.600 .918890 .00047

9.650 .921710 .00049

9.700 .925280 .00048

9.750 .929120 .00050

9.800 .931810 .00048

9.850 .935840 .00048

9.900 .938400 .00048

9.950 .941580 .00048

10.000 .944910 .00046

10.050 .947160 .00048

10.100 .951090 .00048

10.150 .953750 .00048

10.200 .956780 .00049

10.250 .961010 .00048

10.300 .963380 .00049

10.350 .966320 .00049

10.400 .970030 .00048

10.450 .971740 .00049

10.500 .974900 .00047

10.550 .978650 .00047

10.600 .981620 .00048

10.650 .983700 .00047

10.700 .987630 .00047

10.750 .989940 .00049

10.800 .993380 .00049

10.850 .997090 .00049

10.900 .998470 .00048
700

9.800 .919870 .00049

9.850 .923210 .00049

9.900 .926870 .00049

9.950 .928460 .00048

10.000 .931390 .00049

10.050 .936320 .00048

10.100 .939070 .00047

10.150 .941620 .00047

10.200 .945030 .00049

10.250 .948740 .00048

10.300 .951200 .00048

10.350 .953510 .00048

10.400 .957560 .00047

10.450 .959740 .00049

10.500 .963140 .00049

10.550 .966370 .00048

10.600 .969080 .00047

10.650 .972130 .00048

10.700 .975850 .00049

10.750 .978990 .00047

10.800 .981290 .00048

10.850 .984000 .00047

10.900 .987540 .00049

10.950 .989560 .00049

11.000 .992030 .00049

11.050 .995310 .00049

11.100 .998500 .00048
10.150 .963580 .00047

10.200 .966300 .00050

10.250 .969090 .00048

10.300 .972360 .00047

10.350 .976220 .00048

10.400 .977780 .00048

10.450 .982720 .00049

10.500 .984500 .00048

10.550 .987200 .00047

10.600 .989980 .00047

10.650 .992370 .00050

10.700 .996240 .00049

10.750 .999580 .00047

10.801 .002420 .00050

10.851 .005030 .00048

10.901 .007850 .00048

10.951 .010240 .00048

11.001 .013870 .00048

11.051 .016410 .00050

11.101 .018850 .00047

750

10.100 .922920 .00047

10.150 .925940 .00047

10.200 .929310 .00048

10.250 .932520 .00047

10.300 .936600 .00048

10.350 .938370 .00048

10.400 .942360 .00048

10.450 .944870 .00048

10.500 .947140 .00048

10.550 .950860 .00049

10.600 .953730 .00050

10.650 .957060 .00047

10.700 .960210 .00046

10.750 .963580 .00048

10.800 .966460 .00048

10.850 .970600 .00048

10.900 .971850 .00047

10.950 .974860 .00048

11.000 .977180 .00049

11.050 .980770 .00047

11.100 .983210 .00048

11.150 .986220 .00048

11.200 .989540 .00048

11.250 .992080 .00048

11.300 .994660 .00048

11.350 .997710 .00047

11.401 .000630 .00048 
10.951 .001250 .00049 11.001 .004630 .00049 11.051 .007840 .00049 11.101 .010240 .00049 11.151 .013120 .00050 11.201 .015150 .00050

760

10.100 .919240 .00048 10.150 .922770 .00048 10.200 .926030 .00047 10.250 .928870 .00047 10.300 .932490 .00049 10.350 .936210 .00047 10.400 .938870 .00049 10.450 .941680 .00047 10.500 .944400 .00049 10.550 .948260 .00049 10.600 .949810 .00046 10.650 .954260 .00048 10.700 .956640 .00047 10.750 .959800 .00049 10.800 .962390 .00048 10.850 .965620 .00048 10.900 .968640 .00048 10.950 .971220 .00048 11.000 .974090 .00048 11.050 .977270 .00047 11.100 .980180 .00049 11.150 .982390 .00048 11.200 .985980 .00048 11.250 .988030 .00048 11.300 .992710 .00048 11.350 .994690 .00048 11.400 .997150 .00049 11.451 .000170 .00048 11.501 .002760 .00048 11.551 .005820 .00048 11.601 .008760 .00049 11.651 .010690 .00047 11.701 .013970 .00049 11.751 .016110 .00047 11.801 .018660 .00049
11.151 .001220 .00050 11.201 .003990 .00049 11.251 .005980 .00048 11.301 .009050 .00049 11.351 .012500 .00048 11.401 .015010 .00050 11.451 .018160 .00048 11.501 .020450 .00049
11.451 .003010 .00048 11.501 .006100 .00049 11.551 .008440 .00048 11.601 .011420 .00049 11.651 .014730 .00049 11.701 .017350 .00048 11.751 .019380 .00048 11.801 .021990 .00048
765
10.200 .923730 .00048 10.250 .926830 .00047 10.300 .929980 .00047 10.350 .933250 .00049 10.400 .935210 .00049 10.450 .938590 .00047 10.500 .942980 .00047 10.550 .946140 .00048 10.600 .948990 .00047 10.650 .952140 .00048 10.700 .955000 .00048 10.750 .957060 .00049 10.800 .960710 .00048 10.850 .963410 .00049 10.900 .966480 .00048 10.950 .970490 .00049 11.000 .973040 .00049 11.050 .975390 .00048 11.100 .978170 .00047 11.150 .980660 .00049 11.200 .983200 .00049 11.250 .987330 .00049 11.300 .988490 .00049 11.350 .992160 .00049 11.400 .994810 .00049 11.450 .997300 .00049 11.501 .000040 .00047 11.551 .003790 .00048 11.601 .006070 .00048 11.651 .008920 .00048 11.701 .011640 .00048 11.751 .014510 .00049 11.801 .016760 .00048 11.851 .019720 .00049 11.901 .021610 .00048
770

10.200 .922100 .00048 10.250 .924630 .00047 10.300 .928310 .00046 10.350 .930310 .00048 10.400 .932850 .00050 10.450 .936740 .00048 10.500 .939740 .00048 10.550 .942900 .00049 10.600 .946230 .00049 10.650 .949610 .00050 10.700 .952960 .00048 10.750 .954670 .00049 10.800 .959450 .00048 10.850 .961590 .00046 10.900 .964350 .00048 10.950 .966910 .00048 11.000 .969520 .00049 11.050 .973220 .00048 11.100 .975070 .00048 11.150 .979040 .00049 11.200 .981820 .00048 11.250 .984120 .00048 11.300 .987660 .00047 11.350 .989780 .00050 11.400 .992450 .00049 11.450 .995990 .00049 11.500 .998530 .00047 11.551 .000960 .00049 11.601 .003900 .00048 11.651 .006330 .00050 11.701 .009730 .00046 11.751 .011690 .00049 11.801 .014870 .00047 11.851 .017550 .00047 11.901 .020480 .00051 


\section{5}

10.200 .920990 .00049

10.250 .923090 .00049

10.300 .926100 .00048

10.350 .929460 .00048

10.400 .932710 .00047

10.450 .936480 .00047

10.500 .939200 .00049

10.550 .942060 .00047

10.600 .944160 .00048

10.650 .947200 .00048

10.700 .951890 .00048

10.750 .954820 .00047

10.800 .958020 .00048

10.850 .960210 .00048

10.900 .962640 .00048

10.950 .965820 .00048

11.000 .968590 .00048

11.050 .972250 .00047

11.100 .974730 .00047

11.150 .977290 .00047

11.200 .980420 .00049

11.250 .984050 .00048

11.300 .986780 .00049

11.350 .989990 .00048

11.400 .991450 .00049

11.450 .994800 .00046

11.500 .997350 .00048

11.550 .999450 .00049

11.601 .002690 .00049

11.651 .005150 .00048

11.701 .008950 .00050

11.751 .010480 .00048

11.801 .012890 .00048

11.851 .015980 .00047

11.901 .018860 .00047

Pun46-icyl-0970-10

12

17.900 .925670 .00026

18.200 .930430 .00027

18.500 .935040 .00026

18.800 .939660 .00026

19.100 .943600 .00026

19.400 .948540 .00026

19.700 .951820 .00025

20.000 .956560 .00025

0

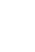

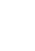

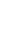


20.300 .960560 .00025 20.600 .964650 .00025 20.900 .967550 .00025 21.200 .970840 .00024 21.500 .974470 .00024 21.800 .978240 .00024 22.100 .981610 .00025 22.400 .984610 .00024 22.700 .988030 .00025 23.000 .990460 .00024 23.300 .993460 .00024 23.600 .996220 .00025 23.900 .999400 .00024 24.201 .002480 .00024 24.501 .004260 .00023 24.801 .006640 .00023 25.101 .009520 .00024 25.401 .011340 .00024 25.601 .013810 .00023
25

9.700 .919670 .00037 9.800 .925220 .00037 9.900 .929970 .00038 10.000 .935110 .00037 10.100 .940400 .00036 10.200 .945540 .00037 10.300 .949660 .00036 10.400 .954760 .00036 10.500 .959260 .00036 10.600 .964660 .00037 10.700 .968360 .00036 10.800 .974050 .00037 10.900 .977990 .00036 11.000 .982620 .00035 11.100 .986920 .00036 11.200 .991100 .00037 11.300 .996050 .00036 11.400 .999310 .00037 11.501 .003660 .00035 11.601 .008190 .00037 11.701 .011270 .00037 11.801 .015990 .00036
15.500 .965600 .00029 15.700 .969510 .00029 15.900 .974350 .00029 16.100 .979170 .00029 16.300 .982410 .00030 16.500 .987030 .00030 16.700 .990800 .00029 16.900 .995070 .00029 17.100 .998810 .00029 17.301 .002380 .00027 17.501 .005640 .00029 17.701 .009560 .00028 17.901 .013430 .00028 18.001 .014950 .00028
11.900 .954710 .00035 12.000 .959270 .00034 12.100 .963500 .00034 12.200 .967200 .00033 12.300 .970380 .00034 12.400 .973690 .00033 12.500 .977510 .00034 12.600 .981260 .00034 12.700 .984480 .00034 12.800 .987120 .00033 12.900 .991360 .00033 13.000 .994520 .00033 13.100 .997240 .00034 13.201 .000920 .00033 13.301 .004490 .00034 13.401 .007510 .00032 13.501 .010310 .00033 13.601 .013850 .00033 13.701 .016420 .00033

32

9.000 .923300 .00039 9.100 .929130 .00038 9.200 .935770 .00040 9.300 .940680 .00038 9.400 .945740 .00039 9.500 .952370 .00039 9.600 .957660 .00039 9.700 .962970 .00038 9.800 .968700 .00038 9.900 .974440 .00038 10.000 .979170 .00038 10.100 .984990 .00037 10.200 .989510 .00038 10.300 .994190 .00039 10.400 .998690 .00037 10.501 .004660 .00038 10.601 .008750 .00037 10.701 .013150 .00038 10.801 .018270 .00039
8.700 .918690 .00038 8.800 .925830 .00039 8.900 .931330 .00040 9.000 .937130 .00038 9.100 .943530 .00039 9.200 .949880 .00040 9.300 .955100 .00039 9.400 .961080 .00040 9.500 .965820 .00039 9.700 .977730 .00038 9.800 .982770 .00038 9.900 .987720 .00039 10.000 .993410 .00038 10.100 .998530 .00038 10.201 .003700 .00039 10.301 .008900 .00039 10.401 .013710 .00039 10.501 .018190 .00039 9.600 .972500 .00039 
35

8.500 .924240 .00039

8.550 .926960 .00041

8.600 .931240 .00040

8.650 .933750 .00039

8.700 .937060 .00040

8.750 .939750 .00039

8.800 .943140 .00039

8.850 .946260 .00040

8.900 .949620 .00040

8.950 .953000 .00040

9.000 .955230 .00040

9.050 .959270 .00039

9.100 .961950 .00039

9.150 .965040 .00038

9.200 .967320 .00040

9.250 .970160 .00041

9.300 .973040 .00039

9.350 .976650 .00040

9.400 .979440 .00041

9.450 .982810 .00039

9.500 .984380 .00040

9.550 .987260 .00039

9.600 .990480 .00040

9.650 .993910 .00040

9.700 .995780 .00041

9.750 .999150 .00039

9.801 .002040 .00039

9.851 .003780 .00041

9.901 .006410 .00039

9.951 .009730 .00041

10.001 .012470 .00039

10.101 .017840 .00040
40

8.100 .921790 .00041

8.150 .925430 .00040

8.200 .928950 .00040

8.250 .932290 .00040

8.300 .935410 .00041

8.350 .938480 .00041

8.450 .945400 .00040

8.500 .949320 .00041

8.550 .952090 .00040

8.600 .955720 .00041

8.650 .959100 .00041

8.700 .961370 .00040

8.800 .968580 .00040

8.850 .971370 .00040

8.950 .977000 .00041

9.000 .980280 .00041

9.050 .983910 .00041

9.100 .986670 .00041

9.150 .988900 .00039

9.200 .992480 .00040

9.250 .995180 .00040

9.300 .998110 .00041

9.351 .001470 .00041

9.401 .004830 .00041

9.451 .007000 .00041

9.501 .010050 .00041

9.551 .013720 .00040

9.601 .015240 .00040

9.651 .018130 .00040

9.701 .021820 .00040
50

7.600 .919750 .00042

7.650 .923870 .00041

7.700 .927680 .00042

7.750 .931810 .00042

7.800 .934590 .00041

7.850 .938140 .00042

7.900 .942780 .00042

7.950 .946260 .00043

8.000 .950010 .00042

8.050 .952190 .00043

8.100 .956690 .00043

8.150 .960820 .00041

8.200 .963890 .00043

8.250 .967220 .00041

8.300 .970130 .00042

8.350 .973500 .00042

8.400 .977060 .00041

8.450 .980670 .00042

8.500 .983380 .00042

8.550 .986600 .00041

8.600 .990120 .00043

8.650 .993990 .00042

8.700 .996430 .00041

8.750 .999290 .00042

8.801 .002840 .00041

8.851 .005920 .00041

8.901 .010100 .00043

8.951 .012560 .00042

9.001 .015720 .00042
60

7.300 .918990 .00043

7.350 .923060 .00044

7.400 .926990 .00044

7.450 .930950 .00042

7.500 .935000 .00042

7.550 .938630 .00042

7.600 .943220 .00043

7.650 .947420 .00043

7.700 .950270 .00043

7.750 .953640 .00043

7.800 .957840 .00044

7.850 .960840 .00043
70

7.100 .918640 .00043

7.150 .922480 .00042

7.200 .926410 .00042

7.250 .930100 .00044

7.300 .934130 .00045

7.350 .937680 .00043

7.400 .941930 .00043

7.450 .946010 .00045

7.500 .949360 .00043

7.550 .953620 .00044

7.600 .957560 .00042

7.650 .960780 .00044
80

7.000 .921010 .00043

7.050 .924580 .00043

7.100 .928800 .00044

7.150 .933300 .00044

7.200 .936910 .00044

7.250 .940660 .00044

7.300 .944620 .00044

7.350 .948680 .00044

7.400 .952660 .00044

7.450 .956440 .00043

7.500 .959440 .00045

7.550 .964510 .00044 
7.900 .964550 .00043 7.950 .968240 .00044 8.000 .971850 .00043 8.050 .975650 .00043 8.100 .978400 .00042 8.150 .982810 .00042 8.200 .986320 .00042 8.250 .989230 .00042 8.300 .993170 .00042 8.350 .996470 .00043 8.400 .999080 .00042 8.451 .002200 .00043 8.501 .005890 .00043 8.551 .009450 .00043 8.601 .012480 .00044 8.651 .016490 .00042 8.701 .019690 .00042

\section{0}

6.900 .919950 .00044 6.950 .924660 .00044 7.000 .928780 .00043 7.050 .932510 .00045 7.100 .937010 .00044 7.150 .939890 .00043 7.200 .944180 .00043 7.250 .947980 .00043 7.300 .951620 .00045 7.350 .955410 .00044 7.400 .960520 .00045 7.450 .963690 .00045 7.500 .967020 .00045 7.550 .971480 .00044 7.600 .975870 .00045 7.650 .978940 .00044 7.700 .982000 .00044 7.750 .986500 .00045 7.800 .989940 .00045 7.850 .994350 .00044 7.900 .997480 .00044 7.951 .000440 .00045 8.001 .004110 .00044 8.051 .007600 .00044 8.101 .010350 .00044 8.151 .014380 .00045 8.201 .017670 .00046
7.700 .964340 .00044

7.750 .968100 .00043

7.800 .971980 .00043

7.850 .975880 .00044

7.900 .979810 .00044

7.950 .983440 .00044 8.000 .986510 .00043 8.050 .990160 .00043 8.100 .992460 .00044 8.150 .997120 .00044 8.200 .999120 .00043 8.251 .003330 .00044 8.301 .007080 .00043 8.351 .011170 .00044 8.401 .013990 .00044 8.451 .017610 .00044 8.501 .020550 .00044
7.600 .967630 .00043 7.650 .971870 .00043 7.700 .975570 .00044 7.750 .979070 .00045 7.800 .982490 .00043 7.850 .986300 .00044 7.900 .990010 .00045 7.950 .993520 .00043 8.000 .996790 .00044 8.050 .999870 .00043 8.101 .003770 .00043 8.151 .007710 .00045 8.201 .010930 .00044 8.251 .013800 .00045 8.301 .018310 .00043
100

6.800 .917820 .00043

6.850 .922050 .00045

6.900 .925460 .00044

6.950 .929330 .00044

7.000 .934540 .00045

7.050 .937870 .00045

7.100 .941780 .00045

7.150 .946110 .00045

7.200 .949650 .00045

7.250 .953080 .00045

7.300 .956860 .00045

7.350 .961870 .00046

7.400 .965800 .00046

7.450 .969450 .00045

7.500 .973500 .00045

7.550 .977190 .00044

7.600 .979920 .00045

7.650 .983930 .00045

7.700 .987320 .00044

7.750 .991340 .00045

7.800 .994550 .00043

7.850 .998200 .00045

7.901 .001820 .00044

7.951 .005760 .00045 8.001 .008980 .00045 8.051 .013230 .00045

8.101 .015800 .00046 8.151 .019190 .00045 8.201 .022910 .00045
120

6.800 .923440 .00043 6.850 .927650 .00045 6.900 .930820 .00045 6.950 .936070 .00043 7.000 .939730 .00044 7.050 .943070 .00044 7.100 .946920 .00045 7.150 .949640 .00045 7.200 .955570 .00044 7.250 .958270 .00045 7.300 .961480 .00044 7.350 .966980 .00047 7.400 .969740 .00044 7.450 .973560 .00046 7.500 .977040 .00045 7.550 .981640 .00043 7.600 .985390 .00044 7.650 .988370 .00045 7.700 .992380 .00045 7.750 .995810 .00043 7.801 .000130 .00045 7.851 .003600 .00046 7.901 .007120 .00044 7.951 .010170 .00045 8.001 .012950 .00046 8.051 .017110 .00045 8.101 .020580 .00045 
130

6.800 .925300 .00044

6.850 .929380 .00044

6.900 .933280 .00045

6.950 .937070 .00045

7.000 .941200 .00045

7.050 .945980 .00044

7.100 .949540 .00045

7.150 .952860 .00045

7.200 .957400 .00044

7.250 .960570 .00046

7.300 .964270 .00044

7.350 .967390 .00045

7.400 .971520 .00045

7.450 .975970 .00045

7.500 .980730 .00045

7.550 .983250 .00044

7.600 .986590 .00045

7.650 .990050 .00045

7.700 .994090 .00044

7.750 .997130 .00045

7.801 .001130 .00044

7.851 .004880 .00045

7.901 .008830 .00045

7.951 .011660 .00045

8.001 .016780 .00045

239

6.800 .920990 .00046

6.850 .924140 .00045

6.900 .928690 .00045

6.950 .932760 .00045

7.000 .936390 .00045

7.050 .940120 .00045

7.100 .944400 .00045

7.150 .946960 .00044

7.200 .951140 .00046

7.250 .955030 .00044

7.300 .958680 .00045

7.350 .962100 .00043

7.400 .966520 .00047

7.450 .968960 .00044 7.500 .973200 .00045
150

6.700 .917940 .00045

6.750 .922430 .00046

6.800 .926820 .00044

6.850 .931130 .00045

6.900 .935140 .00045

6.950 .938840 .00044

7.000 .942800 .00045

7.050 .947050 .00044

7.100 .950390 .00046

7.150 .954580 .00046

7.200 .957760 .00045

7.250 .961500 .00044

7.300 .965380 .00046

7.350 .968570 .00045

7.400 .973280 .00045

7.450 .977460 .00044

7.500 .980190 .00045

7.550 .984620 .00045

7.600 .988960 .00044

7.650 .991540 .00046

7.700 .995060 .00045

7.750 .998990 .00045

7.801 .001270 .00044

7.851 .005990 .00045

7.901 .008850 .00046

7.951 .012390 .00045

8.001 .016420 .00045

8.051 .019990 .00045

8.101 .023110 .00046
200

6.800 .925350 .00046

6.850 .928890 .00045

6.900 .933340 .00046

6.950 .935740 .00045

7.000 .940330 .00045

7.050 .943410 .00044

7.100 .947890 .00045

7.150 .951460 .00044

7.200 .955730 .00045

7.250 .959180 .00045

7.300 .963250 .00046

7.350 .966330 .00046

7.400 .970220 .00045

7.450 .973330 .00046

7.500 .977880 .00045

7.550 .981070 .00046

7.600 .984370 .00045

7.650 .987690 .00046

7.700 .991570 .00046

7.750 .995050 .00046

7.800 .998300 .00046

7.851 .001910 .00045

7.901 .005920 .00045

7.951 .008610 .00045

8.001 .011420 .00046

8.051 .015690 .00046

8.101 .017940 .00046
6.800 .919850 .00046

6.850 .923690 .00044

6.900 .927820 .00046

6.950 .930560 .00045

7.000 .935080 .00045

7.050 .939040 .00045

7.100 .942590 .00044

7.150 .946250 .00045

7.200 .949920 .00046

7.250 .952890 .00045

7.300 .957330 .00044

7.350 .960700 .00045

7.400 .963890 .00045

7.450 .967740 .00046

7.500 .971840 .00046
359

7.000 .920940 .00045

7.050 .925420 .00044

7.100 .928530 .00045

7.150 .932180 .00046

7.200 .935810 .00045

7.250 .939100 .00045

7.300 .943430 .00045

7.350 .945980 .00046

7.400 .950040 .00047

7.450 .952170 .00046

7.500 .956770 .00045

7.550 .959710 .00045

7.600 .962710 .00045

7.650 .966870 .00046

7.700 .969970 .00045 
7.550 .976100 .00046 7.600 .979880 .00045 7.650 .983220 .00046 7.700 .986410 .00045 7.750 .990500 .00045 7.800 .993330 .00044 7.850 .995930 .00044 7.901 .001050 .00045 7.951 .002820 .00045 8.001 .006380 .00046 8.051 .009770 .00045 8.101 .013830 .00045 8.151 .016960 .00045 8.201 .019770 .00044
7.550 .974280 .00046

7.600 .978140 .00046

7.650 .981550 .00046

7.700 .985100 .00044

7.750 .989320 .00046

7.800 .991620 .00045

7.850 .995490 .00047

7.900 .998040 .00046

7.951 .002110 .00045

8.001 .005690 .00044

8.051 .008370 .00046

8.101 .011230 .00046

8.151 .014630 .00045

8.201 .018310 .00044
7.750 .974250 .00045

7.800 .977370 .00045

7.850 .980780 .00046

7.900 .983670 .00047

7.950 .985730 .00046

8.000 .989570 .00045

8.050 .992350 .00045

8.100 .996150 .00046

8.150 .998930 .00045

8.201 .002380 .00046

8.251 .005700 .00045

8.301 .008850 .00046

8.351 .010840 .00046

8.401 .014780 .00046

8.451 .017440 .00045

8.501 .021000 .00046
478

7.200 .923030 .00046

7.250 .926500 .00046

7.300 .928940 .00046

7.350 .933190 .00045

7.400 .936840 .00045

7.450 .940090 .00045

7.500 .943270 .00045

7.550 .946200 .00045

7.600 .949620 .00045

7.650 .953350 .00045

7.700 .956090 .00046

7.750 .958960 .00045

7.800 .963500 .00044

7.850 .966410 .00045

7.900 .969560 .00045

7.950 .973160 .00047

8.000 .975130 .00047

8.050 .979060 .00046

8.100 .982240 .00046

8.150 .984550 .00045

8.200 .987890 .00045

8.250 .991170 .00045

8.300 .994690 .00045

8.350 .997170 .00044

8.401 .000850 .00045

8.451 .002380 .00046

8.501 .005500 .00047

8.551 .009340 .00045

8.601 .012190 .00046

8.651 .015030 .00046

8.701 .017800 .00045
500

7.200 .919460 .00044

7.250 .922850 .00045

7.300 .926450 .00045

7.350 .930140 .00045

7.400 .933650 .00047

7.450 .937460 .00045

7.500 .941010 .00044

7.550 .943780 .00045

7.600 .947160 .00045

7.650 .950550 .00046

7.700 .954560 .00045

7.750 .956960 .00045

7.800 .961570 .00046

7.850 .962700 .00045

7.900 .966660 .00046

7.950 .969690 .00046

8.000 .973450 .00044

8.050 .976320 .00044

8.100 .979020 .00045

8.150 .983100 .00046

8.200 .985180 .00046

8.250 .989130 .00046

8.300 .991820 .00045

8.350 .994990 .00047

8.400 .998350 .00045

8.451 .000270 .00045

8.501 .004690 .00045

8.551 .007200 .00046

8.601 .009080 .00045

8.651 .013540 .00045

8.701 .015930 .00046
600

7.400 .921720 .00045

7.450 .924910 .00046

7.500 .927980 .00045

7.550 .930690 .00045

7.600 .935180 .00044

7.650 .938020 .00046

7.700 .942140 .00045

7.750 .944070 .00046

7.800 .948280 .00045

7.850 .951620 .00046

7.900 .954360 .00046

7.950 .957710 .00045

8.000 .959720 .00044

8.050 .963840 .00045

8.100 .966050 .00045

8.150 .969370 .00045

8.200 .972830 .00047

8.250 .977000 .00045

8.300 .978770 .00045

8.350 .982870 .00046

8.400 .984140 .00043

8.450 .988030 .00046

8.500 .990910 .00044

8.550 .994540 .00044

8.600 .996250 .00046

8.650 .999970 .00046

8.701 .001940 .00045

8.751 .005810 .00047

8.801 .008610 .00045

8.851 .010880 .00046

8.901 .014280 .00045 
8.751 .018100 .00046 8.801 .021250 .00045
8.951 .016410 .00045 9.001 .018930 .00045
650

7.500 .921580 .00044 7.550 .924830 .00045 7.600 .928380 .00045 7.650 .931800 .00045 7.700 .934080 .00045 7.750 .937810 .00045 7.800 .940520 .00046 7.850 .944340 .00045 7.900 .947710 .00046 7.950 .950120 .00045 8.000 .953630 .00045 8.050 .957630 .00046 8.100 .959160 .00047 8.150 .962530 .00045 8.200 .965880 .00045 8.250 .969180 .00046 8.300 .971520 .00046 8.350 .974870 .00045 8.400 .977930 .00046 8.450 .980730 .00045 8.500 .984190 .00046 8.550 .987620 .00045 8.600 .989560 .00045 8.650 .993350 .00046 8.700 .996430 .00046 8.750 .998190 .00045 8.801 .002140 .00046 8.851 .004910 .00044 8.901 .006960 .00045 8.951 .009990 .00046 9.001 .013560 .00046 9.051 .015520 .00045 9.101 .018430 .00045

760

7.800 .917720 .00044 7.850 .920360 .00044 7.900 .923940 .00044 7.950 .927580 .00045 8.000 .931220 .00045 8.050 .933340 .00046
700

7.600 .919990 .00045

7.650 .922140 .00044

7.700 .925370 .00046

7.750 .928250 .00044

7.800 .931660 .00045

7.850 .934050 .00044

7.900 .937870 .00045

7.950 .941230 .00046

8.000 .944810 .00046

8.050 .947470 .00045

8.100 .951320 .00045

8.150 .953570 .00046

8.200 .956850 .00045

8.250 .959890 .00045

8.300 .963370 .00045

8.350 .965790 .00044

8.400 .970310 .00043

8.450 .971990 .00045

8.500 .974370 .00046

8.550 .977320 .00045

8.600 .981200 .00045

8.650 .983550 .00045

8.700 .986780 .00044

8.750 .989250 .00045

8.800 .992330 .00044

8.850 .996110 .00044

8.900 .998380 .00046

8.951 .001430 .00046

9.001 .003610 .00046

9.051 .006760 .00046

9.101 .009600 .00046

9.151 .011770 .00044

9.201 .014750 .00045

9.251 .017300 .00045

9.301 .020780 .00045
750

7.800 .921170 .00046

7.850 .923350 .00045

7.900 .926750 .00045

7.950 .930290 .00044

8.000 .933680 .00046

8.050 .936430 .00046

8.100 .939660 .00045

8.150 .942790 .00044

8.200 .945870 .00046

8.250 .948920 .00046

8.300 .951650 .00045

8.350 .954570 .00044

8.400 .957760 .00046

8.450 .960760 .00044

8.500 .963570 .00045

8.550 .966300 .00045

8.600 .970070 .00045

8.650 .972690 .00045

8.700 .975110 .00044

8.750 .978280 .00046

8.800 .980420 .00044

8.850 .983850 .00045

8.900 .987500 .00045

8.950 .989090 .00046

9.000 .992070 .00045

9.050 .995090 .00046

9.100 .998170 .00045

9.150 .999910 .00046

9.201 .002670 .00045

9.251 .005480 .00046

9.301 .008240 .00044

9.351 .011560 .00045

9.401 .013810 .00045

9.451 .016770 .00045

9.501 .019840 .00045
765

7.900 .923400 .00044 7.950 .925230 .00045 8.000 .928790 .00044 8.050 .931940 .00045 8.100 .935290 .00046 8.150 .937940 .00046
770

7.900 .920990 .00045 7.950 .925310 .00044 8.000 .928610 .00045 8.050 .930360 .00044 8.100 .933110 .00044 8.150 .935850 .00044 
8.100 .936840 .00045 8.150 .939850 .00045 8.200 .943080 .00045 8.250 .946110 .00045 8.300 .948620 .00045 8.350 .952200 .00045 8.400 .954400 .00043 8.450 .958260 .00046 8.500 .960560 .00045 8.550 .964080 .00045 8.600 .967140 .00045 8.650 .969160 .00045 8.700 .972930 .00044 8.750 .975810 .00046 8.800 .978520 .00045 8.850 .980790 .00044 8.900 .983900 .00045 8.950 .986930 .00045 9.000 .988600 .00044 9.050 .992710 .00044 9.100 .995510 .00045 9.150 .998030 .00045 9.201 .000880 .00044 9.251 .003850 .00045 9.301 .006030 .00046 9.351 .009070 .00045 9.401 .011880 .00045 9.451 .014240 .00047 9.501 .017760 .00045

\section{5}

7.900 .920300 .00045 7.950 .923160 .00044 8.000 .926560 .00044 8.050 .930620 .00046 8.100 .932920 .00045 8.150 .936250 .00045 8.200 .938590 .00043 8.250 .942350 .00046 8.300 .945400 .00045 8.350 .948000 .00045 8.400 .950930 .00045 8.450 .954080 .00044 8.500 .956880 .00046 8.550 .959670 .00045 8.600 .963760 .00045 8.650 .965770 .00045 8.700 .968450 .00045 8.750 .971380 .00045
8.200 .942270 .00046

8.250 .944620 .00046

8.300 .947460 .00044

8.350 .950450 .00044

8.400 .953500 .00046

8.450 .955710 .00044

8.500 .960140 .00046

8.550 .961820 .00045

8.600 .965160 .00047 8.650 .968210 .00045

8.700 .971970 .00044

8.750 .974200 .00045

8.800 .976700 .00046

8.850 .979440 .00045

8.900 .982700 .00046

8.950 .985360 .00045

9.000 .988280 .00045

9.050 .991970 .00046

9.100 .992930 .00045

9.150 .996690 .00045

9.200 .998570 .00046

9.251 .001050 .00045

9.301 .004290 .00045

9.351 .006940 .00046

9.401 .010500 .00044

9.451 .012510 .00045

9.501 .015410 .00045
8.200 .939870 .00045

8.250 .943170 .00044

8.300 .946090 .00044

8.350 .949730 .00046

8.400 .952110 .00046

8.450 .954620 .00045

8.500 .957460 .00045

8.550 .960920 .00046

8.600 .963040 .00046

8.650 .966980 .00044

8.700 .969500 .00046

8.750 .971610 .00045

8.800 .975870 .00046

8.850 .977900 .00045

8.900 .980860 .00044

8.950 .983770 .00045

9.000 .986890 .00046

9.050 .988910 .00044

9.100 .992030 .00045

9.150 .994710 .00046

9.200 .997770 .00044

9.250 .999900 .00044

9.301 .003040 .00045

9.351 .005900 .00045

9.401 .008090 .00044

9.451 .011270 .00045

9.501 .014140 .00045

9.551 .016700 .00046

9.601 .019090 .00046

\section{0}


8.800 .975080 .00044 8.850 .977100 .00045 8.900 .980680 .00046 8.950 .982370 .00045 9.000 .985530 .00045 9.050 .988680 .00045 9.100 .990210 .00045 9.150 .993830 .00045 9.200 .997100 .00044 9.250 .999090 .00044 9.301 .002140 .00045 9.351 .004850 .00044 9.401 .007720 .00044 9.451 .010150 .00044 9.501 .012750 .00045 9.551 .015710 .00045 9.601 .018340 .00045

Pun40-pl-1000-00

12

9.600 .921940 .00030

9.700 .926560 .00030

9.800 .930960 .00030

9.900 .935540 .00030

10.000 .939800 .00029

10.100 .944060 .00029

10.200 .948390 .00030

10.300 .952320 .00029

10.400 .955980 .00030

10.500 .960060 .00029

10.600 .963830 .00028

10.700 .968170 .00029

10.800 .971580 .00029

10.900 .975560 .00029

11.000 .978980 .00029

11.100 .982070 .00028

11.200 .985430 .00028

11.300 .988860 .00029

11.400 .992380 .00029

11.500 .995750 .00028

11.600 .998540 .00028

11.701 .002110 .00028

11.801 .004540 .00028

11.901 .008280 .00028

12.001 .011140 .00029

12.101 .013670 .00028
15

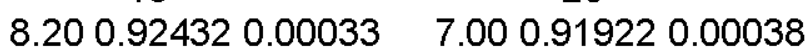
$\begin{array}{llll}8.250 .92720 & 0.00033 & 7.050 .92399 & 0.00038\end{array}$ $8.300 .930130 .00032 \quad 7.100 .928460 .00038$ $8.350 .933970 .00034 \quad 7.150 .933450 .00039$

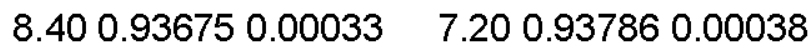
$8.450 .939890 .00033 \quad 7.250 .941520 .00037$ $8.500 .942680 .00034 \quad 7.300 .946050 .00038$ $\begin{array}{llll}8.550 .946390 .00035 & 7.350 .950310 .00038\end{array}$ $\begin{array}{llll}8.60 & 0.949210 .00034 & 7.40 & 0.955060 .00038\end{array}$ $\begin{array}{lll}8.650 .952390 .00033 & 7.450 .958460 .00039\end{array}$ $\begin{array}{lllll}8.70 & 0.955180 .00033 & 7.50 & 0.96281 & 0.00037\end{array}$ $\begin{array}{lll}8.750 .958460 .00033 & 7.550 .966350 .00038\end{array}$ $\begin{array}{llll}8.80 & 0.961530 .00033 & 7.60 & 0.970420 .00038\end{array}$ $\begin{array}{lll}8.850 .963690 .00033 & 7.650 .974970 .00038\end{array}$

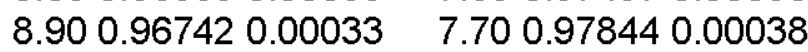
$\begin{array}{llll}8.950 .96946 & 0.00033 & 7.750 .98248 & 0.00037\end{array}$ $9.000 .972730 .00034 \quad 7.800 .986420 .00038$ $\begin{array}{lll}9.050 .975650 .00033 & 7.850 .99010 & 0.00037\end{array}$ $9.100 .977960 .00033 \quad 7.900 .994530 .00038$ $9.150 .980890 .00034 \quad 7.950 .997270 .00037$ $9.200 .983360 .00033 \quad 8.001 .001430 .00038$ $9.250 .986790 .00032 \quad 8.051 .005020 .00037$ $9.300 .988980 .00034 \quad 8.101 .008290 .00036$ $9.350 .990680 .00032 \quad 8.151 .012590 .00036$ $9.400 .994080 .00033 \quad 8.201 .015450 .00037$ $9.450 .996720 .00032 \quad 8.251 .019210 .00036$

$9.500 .998800 .00034 \quad 8.301 .021790 .00038$ 9.551 .002120 .00032 9.601 .003860 .00032 9.651 .006180 .00032 
9.701 .008190 .00032

9.751 .011320 .00033

9.801 .013870 .00032

9.851 .015220 .00033

9.901 .018370 .00032
25

6.400 .917190 .00042 6.450 .922260 .00041 6.500 .928560 .00041 6.550 .932970 .00041 6.600 .938540 .00040 6.650 .943350 .00041 6.700 .949060 .00040 6.750 .953800 .00041 6.800 .958930 .00040 6.850 .963560 .00040 6.900 .969020 .00039 6.950 .973690 .00041 7.000 .978200 .00040 7.050 .983320 .00039 7.100 .987080 .00041 7.150 .992170 .00040 7.200 .996760 .00041 7.251 .001370 .00040 7.301 .005520 .00041 7.351 .009480 .00040 7.401 .014460 .00040 7.451 .019000 .00041 7.501 .023260 .00040

35

5.800 .916550 .00043 5.850 .922530 .00043 5.900 .928820 .00044 5.950 .935040 .00045 6.000 .941850 .00044 6.050 .947230 .00044 6.100 .953730 .00042 6.150 .960090 .00044 6.200 .965630 .00043 6.250 .971530 .00044 6.300 .977100 .00043 6.350 .983710 .00042 6.400 .988880 .00044 6.450 .994240 .00044 6.500 .999440 .00043
30

6.000 .911740 .00042 6.050 .918090 .00043

6.100 .923940 .00042

6.150 .929880 .00043

6.200 .935650 .00042

6.250 .941090 .00042

6.300 .947490 .00043

6.350 .953050 .00042

6.400 .958210 .00041

6.450 .963660 .00042

6.500 .969460 .00042

6.550 .975030 .00042

6.600 .980630 .00041

6.650 .985140 .00043

6.700 .990520 .00041

6.750 .997170 .00042

6.801 .000880 .00043

6.851 .006330 .00041

6.901 .011240 .00041

6.951 .015710 .00043

7.001 .020280 .00042
32

5.900 .912330 .00043 5.950 .917840 .00043 6.000 .924750 .00044 6.050 .930780 .00043 6.100 .936110 .00043 6.150 .943300 .00043 6.200 .948780 .00041 6.250 .955070 .00044 6.300 .959580 .00043 6.350 .966030 .00043 6.400 .971220 .00043 6.450 .977250 .00043 6.500 .982480 .00042 6.550 .988610 .00043 6.600 .993070 .00043 6.650 .999410 .00042 6.701 .004320 .00043 6.751 .009160 .00042 6.801 .014780 .00042 6.851 .020430 .00043 6.901 .025210 .00043
40

5.600 .911940 .00043

5.650 .918520 .00045

5.700 .925800 .00045

5.750 .932870 .00045

5.800 .939190 .00044

5.850 .945740 .00045

5.900 .952350 .00044

5.950 .958780 .00046

6.000 .964800 .00044

6.050 .971380 .00043

6.100 .977290 .00045

6.150 .983890 .00044

6.200 .990580 .00045

6.250 .995800 .00043

6.301 .001750 .00045
50

5.400 .915320 .00044 5.450 .922480 .00046 5.500 .930620 .00047 5.550 .937800 .00046 5.600 .944650 .00046 5.650 .952400 .00047 5.700 .958820 .00047 5.750 .966310 .00044 5.800 .972440 .00046 5.850 .979130 .00047 5.900 .985220 .00046 5.950 .992090 .00044 6.000 .998240 .00045 6.051 .004130 .00045 6.101 .011690 .00045 
6.551 .005460 .00042 6.601 .011790 .00043 6.651 .016190 .00044 6.701 .022510 .00044

60

5.200 .907630 .00047 5.250 .914770 .00047 5.300 .922880 .00047 5.350 .930060 .00047 5.400 .938310 .00047 5.450 .945010 .00046 5.500 .951490 .00046 5.550 .959260 .00047 5.600 .966340 .00045 5.650 .974200 .00047 5.700 .979870 .00046 5.750 .986870 .00048 5.800 .993060 .00046 5.851 .000380 .00047 5.901 .007660 .00049 5.951 .013880 .00048 6.001 .020610 .00047

\section{0}

5.000 .908710 .00047 5.050 .917500 .00050 5.100 .924750 .00048 5.150 .932780 .00048 5.200 .939540 .00050 5.250 .948920 .00049 5.300 .955350 .00047 5.350 .963750 .00049 5.400 .971270 .00049 5.450 .978650 .00049 5.500 .987540 .00047 5.550 .992960 .00048 5.600 .999700 .00048 5.651 .007220 .00048 5.701 .015450 .00048 5.751 .021250 .00048 5.801 .028660 .00048
6.351 .007710 .00044

6.401 .013440 .00044

6.451 .019260 .00045

6.501 .025250 .00044
6.151 .017500 .00046

6.201 .023860 .00047
70

5.100 .905420 .00047

5.150 .914280 .00048

5.200 .921400 .00047

5.250 .929750 .00049

5.300 .937250 .00047

5.350 .943940 .00045

5.400 .952170 .00048

5.450 .959540 .00046

5.500 .967010 .00047

5.550 .973940 .00048

5.600 .980970 .00048

5.650 .988630 .00049

5.700 .994910 .00046

5.751 .001580 .00047

5.801 .009580 .00048

5.851 .015930 .00047

5.901 .022900 .00047
80

5.100 .916570 .00047 5.150 .924200 .00048 5.200 .932150 .00048 5.250 .940080 .00048 5.300 .948300 .00048 5.350 .955530 .00047 5.400 .963540 .00050 5.450 .970300 .00048 5.500 .978100 .00049 5.550 .985070 .00047 5.600 .992680 .00048 5.650 .999110 .00047 5.701 .005760 .00048 5.751 .013430 .00047 5.801 .020270 .00049
5.000 .914240 .00049 5.050 .922860 .00048 5.100 .931080 .00048 5.150 .938890 .00051 5.200 .947030 .00048 5.250 .955240 .00049 5.300 .961880 .00049 5.350 .970160 .00048 5.400 .976730 .00049 5.450 .985300 .00048 5.500 .991510 .00050 5.550 .999790 .00048 5.601 .006600 .00047 5.651 .012990 .00049 5.701 .021420 .00049
120

4.900 .906080 .00048 4.950 .914250 .00051 5.000 .922920 .00049 5.050 .930990 .00047 5.100 .938510 .00050 5.150 .946210 .00049 5.200 .954900 .00049 5.250 .962380 .00049 5.300 .969690 .00049 5.350 .977200 .00049 5.400 .985580 .00049 5.450 .992730 .00048 5.500 .999810 .00049 5.551 .008070 .00050 5.601 .014300 .00048 5.651 .022060 .00048 5.701 .028690 .00048 
130

4.900 .909850 .00047 4.950 .918500 .00049 5.000 .925980 .00049 5.050 .934290 .00050 5.100 .942570 .00048 5.150 .950600 .00048 5.200 .958730 .00048 5.250 .966640 .00048 5.300 .973840 .00048 5.350 .981700 .00049 5.400 .987980 .00049 5.450 .996410 .00049 5.501 .004640 .00049 5.551 .010680 .00050 5.601 .018700 .00049 5.651 .025570 .00049 5.701 .031520 .00049

\section{9}

4.900 .919640 .00048 4.950 .927550 .00048 5.000 .936050 .00049 5.050 .945460 .00050 5.100 .952280 .00049 5.150 .959780 .00048 5.200 .969050 .00051 5.250 .975000 .00048 5.300 .983570 .00049 5.350 .990750 .00050 5.400 .998170 .00049 5.451 .006120 .00050 5.501 .013130 .00050 5.551 .019930 .00050 5.601 .027470 .00051

\section{8}

4.900 .906120 .00048 4.950 .914000 .00048 5.000 .922830 .00050 5.050 .930600 .00050 5.100 .938210 .00049 5.150 .946150 .00047 5.200 .954280 .00049 5.250 .961700 .00049
150

4.900 .912610 .00048

4.950 .921930 .00051

5.000 .930020 .00050

5.050 .938570 .00049

5.100 .946100 .00050

5.150 .954130 .00049

5.200 .961710 .00049

5.250 .970270 .00050

5.300 .977460 .00049

5.350 .985770 .00050

5.400 .992050 .00049

5.451 .000140 .00049

5.501 .007550 .00047

5.551 .014860 .00049

5.601 .020980 .00048
200

4.900 .917870 .00049 4.950 .927680 .00048 5.000 .935310 .00048 5.050 .942750 .00049 5.100 .951330 .00051 5.150 .959370 .00050 5.200 .967510 .00049 5.250 .973500 .00051 5.300 .982080 .00048 5.350 .989100 .00051 5.400 .996700 .00049 5.451 .005810 .00049 5.501 .012690 .00050 5.551 .019620 .00050 5.601 .025970 .00050
4.900 .918460 .00050 4.950 .927710 .00050 5.000 .935780 .00049 5.050 .944370 .00048 5.100 .952470 .00049 5.150 .960410 .00049 5.200 .967700 .00049 5.250 .975620 .00049 5.300 .983600 .00050 5.350 .990950 .00048 5.400 .997610 .00049 5.451 .005970 .00050 5.501 .012490 .00049 5.551 .019840 .00050 5.601 .027830 .00049
359

4.900 .916330 .00050 4.950 .924010 .00050 5.000 .932770 .00049 5.050 .940660 .00049 5.100 .947950 .00050 5.150 .957170 .00049 5.200 .964440 .00050 5.250 .972270 .00049 5.300 .979020 .00049 5.350 .987270 .00047 5.400 .994810 .00049 5.451 .001230 .00049 5.501 .008440 .00049 5.551 .016250 .00048 5.601 .023180 .00048
500

5.000 .918830 .00050 5.050 .927540 .00048 5.100 .935670 .00049 5.150 .944220 .00049 5.200 .951330 .00049 5.250 .958440 .00048 5.300 .967150 .00049 5.350 .974600 .00049
600

5.100 .912280 .00050 5.150 .919880 .00050 5.200 .928420 .00048 5.250 .935390 .00049 5.300 .943590 .00048 5.350 .950730 .00049 5.400 .958200 .00049 5.450 .965710 .00050 
5.300 .969170 .00049 5.350 .976860 .00050 5.400 .984600 .00049 5.450 .991740 .00048 5.500 .998840 .00049 5.551 .005470 .00049 5.601 .014290 .00050 5.651 .020330 .00050 5.701 .026530 .00049

\section{0}

5.200 .908810 .00049 5.300 .923980 .00049 5.350 .932160 .00049 5.400 .940820 .00049 5.450 .947040 .00047 5.500 .954920 .00049 5.550 .962320 .00048 5.600 .968840 .00050 5.650 .977250 .00049 5.700 .984320 .00049 5.750 .990480 .00049 5.800 .997180 .00050 5.851 .004390 .00051 5.901 .011380 .00048 5.951 .017630 .00048 6.001 .024990 .00050

752

6.100 .915410 .00048 6.150 .921320 .00050 6.200 .927960 .00048 6.250 .935160 .00046 6.300 .941620 .00049 6.350 .947590 .00049 6.400 .953610 .00049 6.450 .959980 .00048 6.500 .967260 .00049 6.550 .973550 .00047 6.600 .980410 .00048 6.650 .985320 .00048 6.700 .991880 .00049 6.750 .997550 .00048 6.801 .003330 .00049
5.400 .981790 .00050

5.450 .988600 .00049

5.500 .996880 .00048

5.551 .003900 .00049

5.601 .010760 .00049

5.651 .017340 .00049

5.701 .024410 .00049

700

5.500 .922680 .00048

5.550 .929040 .00048

5.600 .936550 .00047

5.650 .943800 .00049

5.700 .951010 .00049

5.750 .958770 .00049

5.800 .966130 .00049

5.850 .971860 .00050

5.900 .979530 .00048

5.950 .985520 .00049

6.000 .993040 .00049

6.050 .998660 .00050

6.101 .006700 .00050

6.151 .012510 .00048

6.201 .019120 .00049
5.500 .974470 .00050

5.550 .979770 .00050

5.600 .987560 .00048

5.650 .994810 .00050

5.701 .001710 .00049

5.751 .009950 .00049

5.801 .014980 .00048

5.851 .022930 .00048

5.901 .030170 .00048

750

6.000 .910820 .00049

6.050 .917460 .00047

6.100 .924930 .00049

6.150 .931050 .00049

6.200 .938510 .00048

6.250 .944140 .00048

6.300 .951030 .00049

6.350 .957870 .00050

6.400 .964290 .00049

6.450 .970400 .00049

6.500 .977160 .00049

6.550 .983250 .00048

6.600 .989260 .00049

6.650 .995770 .00048

6.701 .001980 .00050

6.751 .007960 .00048

6.801 .012980 .00049

6.851 .019230 .00048

6.901 .025520 .00049

754

755

6.200 .914020 .00048

6.250 .922220 .00049

6.300 .926820 .00046

6.350 .934580 .00048

6.400 .940580 .00049

6.450 .947610 .00049

6.500 .953920 .00048

6.550 .960270 .00048

6.600 .966650 .00048

6.650 .972970 .00048

6.700 .978370 .00050

6.750 .984990 .00048

6.800 .990590 .00048

6.850 .996340 .00050

6.901 .002790 .00049 $\begin{array}{lll}6.30 & 0.91307 & 0.00048 \\ 6.35 & 0.91936 & 0.00049 \\ 6.40 & 0.92647 & 0.00047 \\ 6.45 & 0.93210 & 0.00048 \\ 6.50 & 0.93905 & 0.00050 \\ 6.55 & 0.94567 & 0.00047 \\ 6.60 & 0.95161 & 0.00049 \\ 6.65 & 0.95719 & 0.00049 \\ 6.70 & 0.96331 & 0.00048 \\ 6.75 & 0.96993 & 0.00048 \\ 6.80 & 0.97599 & 0.00048 \\ 6.85 & 0.98150 & 0.00049 \\ 6.90 & 0.98774 & 0.00049 \\ 6.95 & 0.99438 & 0.00050 \\ 7.00 & 0.99955 & 0.00050\end{array}$ 
CHPRC-01552, Revision 0

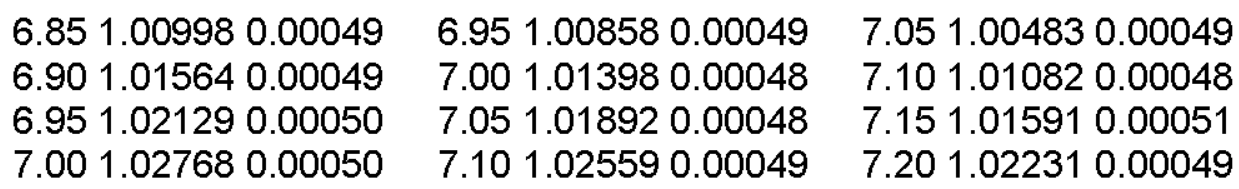


Pun40-pl-1000-01

12

8.100 .922750 .00029

8.200 .927890 .00029

8.300 .932790 .00029

8.400 .936470 .00029

8.500 .940600 .00029

8.600 .945370 .00029

8.700 .949850 .00029

8.800 .953000 .00029

8.900 .957020 .00028

9.000 .961200 .00028

9.100 .964830 .00028

9.200 .969030 .00028

9.300 .972280 .00028

9.400 .975730 .00029

9.500 .979630 .00029

9.600 .983330 .00027

9.700 .986590 .00027

9.800 .989630 .00027

9.900 .993700 .00027

10.000 .996680 .00028

10.100 .999900 .00027

10.201 .002740 .00028

10.301 .005670 .00027

10.401 .009010 .00027

10.501 .011550 .00028

10.601 .015180 .00027
6.600 .918470 .00034

6.650 .921970 .00032

6.700 .925650 .00032

6.750 .929030 .00033

6.800 .932080 .00033

6.850 .934840 .00032

6.900 .937900 .00032

6.950 .941840 .00033

7.000 .944500 .00033

7.050 .948020 .00033

7.100 .950740 .00032

7.150 .953650 .00033

7.200 .956510 .00031

7.250 .959910 .00032

7.300 .962270 .00032

7.350 .965770 .00032

7.400 .968390 .00032

7.450 .970770 .00032

7.500 .974310 .00032

7.550 .976230 .00032

7.600 .979570 .00032

7.650 .982230 .00032

7.700 .984740 .00032

7.750 .987480 .00032

7.800 .990280 .00031

7.850 .992770 .00031

7.900 .995320 .00032

7.950 .997180 .00032

8.000 .999540 .00031

8.051 .002500 .00032

8.101 .005070 .00032

8.151 .008070 .00032

8.201 .010120 .00032

8.251 .011980 .00031

8.301 .014230 .00031
20

$\begin{array}{lll}5.50 & 0.92182 & 0.00037\end{array}$

5.550 .925850 .00038

5.600 .930630 .00036

5.650 .935060 .00036

5.700 .939140 .00037

5.750 .943240 .00036

5.800 .948470 .00036

5.850 .952330 .00037

5.900 .956710 .00036

5.950 .960210 .00036

6.000 .964160 .00036

6.050 .968610 .00036

6.100 .972660 .00036

6.150 .977080 .00036

6.200 .981220 .00035

6.250 .984400 .00036

6.300 .987750 .00035

6.350 .992080 .00036

6.400 .995930 .00036

6.450 .999540 .00036

6.501 .003240 .00035

6.551 .006540 .00036

6.601 .010690 .00036

6.651 .014070 .00035

6.701 .017920 .00035

\section{5}

4.900 .920200 .00038

4.950 .925070 .00039

5.000 .930700 .00039

5.050 .935420 .00039

5.100 .941220 .00040

5.150 .946340 .00039

5.200 .951680 .00038

5.250 .956370 .00039

5.300 .961600 .00040
30

4.500 .915410 .00042

4.550 .921570 .00040

4.600 .927250 .00040

4.650 .932950 .00042

4.700 .938430 .00040

4.750 .944750 .00041

4.800 .950070 .00041

4.850 .955880 .00040

4.900 .962390 .00041
32

4.400 .916330 .00042

4.450 .921880 .00040

4.500 .928470 .00041

4.550 .934170 .00041

4.600 .940890 .00042

4.650 .947130 .00040

4.700 .952690 .00040

4.750 .957470 .00040

4.800 .964210 .00040 
5.350 .966820 .00039 5.400 .972030 .00039 5.450 .976450 .00039 5.500 .980790 .00038 5.550 .985470 .00038 5.600 .990230 .00038 5.650 .995140 .00039 5.700 .999740 .00038 5.751 .004220 .00038 5.801 .008550 .00038 5.851 .013240 .00039 5.901 .016870 .00037

35

4.300 .919910 .00042 4.350 .927160 .00042 4.400 .933170 .00043 4.450 .939590 .00041 4.500 .945410 .00041 4.550 .951640 .00042 4.600 .957740 .00041 4.650 .964580 .00043 4.700 .970510 .00041 4.750 .976500 .00042 4.800 .981960 .00042 4.850 .987740 .00043 4.900 .993200 .00041 4.950 .998900 .00042 5.001 .004680 .00042 5.051 .010110 .00043 5.101 .015510 .00042 5.151 .021500 .00042 5.201 .025780 .00041

60

3.700 .915900 .00045 3.750 .921640 .00046 3.800 .929720 .00044 3.850 .938070 .00045 3.900 .945080 .00046 3.950 .952770 .00045 4.000 .959810 .00045 4.050 .966680 .00044 4.100 .974030 .00045 4.150 .979950 .00046 4.200 .987540 .00046 4.250 .994930 .00044
4.950 .966960 .00041 5.000 .972300 .00042 5.100 .983540 .00041 5.150 .989270 .00041 5.200 .994030 .00041 5.251 .000260 .00040 5.301 .004220 .00040 5.351 .009450 .00041 5.401 .013490 .00040 5.451 .019240 .00041 5.501 .023930 .00040 5.050 .979190 .00040

4.850 .970400 .00041 4.900 .974600 .00041 4.950 .981650 .00041 5.000 .985760 .00042 5.050 .992090 .00041 5.100 .997410 .00041 5.151 .003080 .00042 5.201 .007920 .00041 5.251 .013130 .00040 5.301 .018960 .00042

50

4.100 .916170 .00043

4.150 .924520 .00043

4.200 .930980 .00041

4.250 .937610 .00043

4.300 .943640 .00042

4.350 .951240 .00044

4.400 .956760 .00044

4.450 .963200 .00043

4.500 .969250 .00045

4.550 .976660 .00043

4.600 .982500 .00042

4.650 .989220 .00043

4.700 .994600 .00041

4.751 .000330 .00042

4.801 .005720 .00042

4.851 .011790 .00043

4.901 .018160 .00042

3.900 .922520 .00044 3.950 .930060 .00044 4.000 .936890 .00044 4.050 .943820 .00044 4.100 .951480 .00044 4.150 .957410 .00045 4.200 .964670 .00044 4.250 .971350 .00043 4.300 .978330 .00043 4.350 .984500 .00044 4.400 .991970 .00044 4.450 .998470 .00044 4.501 .004690 .00043 4.551 .011170 .00044 4.601 .017030 .00044 4.651 .023130 .00044 4.701 .029880 .00045
3.600 .913760 .00045 3.650 .921780 .00045 3.700 .929900 .00046 3.750 .938560 .00046 3.800 .944810 .00046 3.850 .952410 .00046 3.900 .960780 .00045 3.950 .967440 .00046 4.000 .974810 .00046 4.050 .982980 .00046 4.100 .988810 .00046 4.150 .996340 .00046
80

3.500 .909260 .00047 3.550 .918080 .00046 3.600 .925750 .00047 3.650 .933210 .00044 3.700 .941340 .00046 3.750 .949300 .00047 3.800 .956440 .00046 3.850 .965280 .00046 3.900 .971360 .00046 3.950 .979920 .00047 4.000 .986950 .00045 4.050 .993950 .00046 
4.301 .002260 .00045 4.351 .006890 .00046 4.401 .014810 .00046 4.451 .021210 .00045 4.501 .027550 .00045

90

3.500 .918590 .00046 3.550 .926870 .00047 3.600 .934280 .00047 3.650 .942880 .00047 3.700 .949800 .00047 3.750 .957810 .00047 3.800 .965220 .00048 3.850 .973400 .00046 3.900 .980230 .00047 3.950 .988580 .00047 4.000 .995630 .00047 4.051 .002740 .00047 4.101 .009580 .00046 4.151 .017130 .00047 4.201 .023930 .00046

130

3.400 .921420 .00045 3.450 .929710 .00047 3.500 .938120 .00047 3.550 .945760 .00047 3.600 .953740 .00045 3.650 .961200 .00048 3.700 .969860 .00048 3.750 .977710 .00048 3.800 .986210 .00046 3.850 .992660 .00046 3.900 .998520 .00047 3.951 .006000 .00047 4.001 .014270 .00047 4.051 .021390 .00047 4.101 .028980 .00048

239

3.300 .916330 .00049 3.350 .924180 .00047 3.400 .932230 .00048 3.450 .940960 .00049
4.201 .002660 .00046

4.251 .009900 .00047

4.301 .017170 .00044

4.351 .023670 .00047

4.401 .029170 .00046
4.101 .000400 .00047

4.151 .007090 .00045

4.201 .014470 .00046

4.251 .022080 .00047

4.301 .028210 .00046
100

3.500 .925320 .00047 3.550 .932840 .00047 3.600 .940330 .00047 3.650 .947950 .00047 3.700 .956720 .00046 3.750 .964620 .00046 3.800 .972950 .00046 3.850 .980450 .00047 3.900 .987640 .00048 3.950 .994080 .00048 4.001 .002280 .00047 4.051 .008790 .00047 4.101 .015790 .00045 4.151 .022980 .00047 4.201 .030450 .00047
120

3.400 .917340 .00047 3.450 .925970 .00048 3.500 .933850 .00047 3.550 .942090 .00048 3.600 .950320 .00047 3.650 .957830 .00048 3.700 .965830 .00047 3.750 .973540 .00047 3.800 .980770 .00047 3.850 .988970 .00046 3.900 .996140 .00047 3.951 .003230 .00046 4.001 .010430 .00049 4.051 .018420 .00046 4.101 .024250 .00047
3.300 .908030 .00047 3.350 .917760 .00047 3.400 .925590 .00047 3.450 .933930 .00046 3.500 .942670 .00048 3.550 .949850 .00047 3.600 .958030 .00046 3.650 .966410 .00047 3.700 .974010 .00046 3.750 .981760 .00047 3.800 .989060 .00048 3.850 .996990 .00047 3.901 .003890 .00048 3.951 .011100 .00047 4.001 .018700 .00048
200

3.300 .914530 .00049 3.350 .923240 .00048 3.400 .931430 .00047 3.450 .939790 .00049 3.500 .947480 .00047 3.550 .955720 .00048 3.600 .963590 .00048 3.650 .971860 .00048 3.700 .979570 .00049 3.750 .986800 .00048 3.800 .994150 .00047 3.851 .002780 .00047 3.901 .008700 .00048 3.951 .017000 .00047 4.001 .024080 .00048
250

3.300 .916520 .00048 3.350 .924760 .00046 3.400 .933060 .00047 3.450 .941250 .00048
359

3.300 .914080 .00047 3.350 .921400 .00047 3.400 .930710 .00048 3.450 .938400 .00048 
3.500 .949390 .00048 3.550 .956550 .00048 3.600 .964460 .00049 3.650 .972640 .00048 3.700 .980750 .00046 3.750 .987240 .00050 3.800 .995910 .00047 3.851 .003330 .00047 3.901 .010140 .00047 3.951 .018030 .00048 4.001 .024920 .00048

478

3.400 .921250 .00047 3.450 .928980 .00047 3.500 .937220 .00047 3.550 .945900 .00048 3.600 .952340 .00048 3.650 .960770 .00048 3.700 .968340 .00047 3.750 .976160 .00048 3.800 .983190 .00047 3.850 .989970 .00048 3.900 .997720 .00048 3.951 .004470 .00048 4.001 .012150 .00048 4.051 .018630 .00049 4.101 .025730 .00048

650

3.600 .918610 .00047 3.650 .925920 .00049 3.700 .933070 .00047 3.750 .941530 .00047 3.850 .956330 .00048 3.900 .962850 .00048 3.950 .969660 .00048 4.000 .977210 .00047 4.050 .985130 .00048 4.100 .991690 .00050 4.150 .998420 .00048 4.201 .005560 .00049 4.251 .011350 .00048 4.301 .017960 .00050
3.500 .949320 .00047

3.550 .957530 .00048

3.600 .964580 .00047

3.650 .972750 .00047

3.700 .980270 .00046

3.750 .987940 .00047

3.800 .995260 .00046

3.851 .002950 .00048

3.901 .010250 .00048

3.951 .017870 .00049

4.001 .024210 .00048
3.500 .946340 .00048

3.550 .954140 .00048

3.600 .960940 .00047

3.650 .969400 .00049

3.700 .977060 .00046

3.750 .984570 .00049

3.800 .992020 .00048 3.850 .999530 .00049 3.901 .006870 .00049 3.951 .013950 .00047 4.001 .020370 .00049

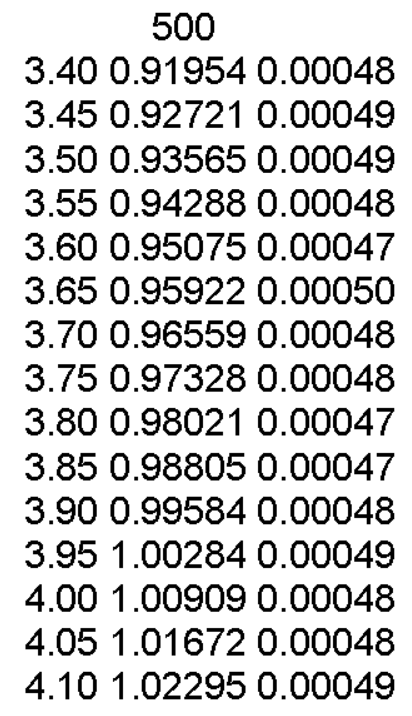

700

3.700 .909690 .00048

3.750 .917510 .00047

3.800 .925360 .00048

3.850 .931610 .00048

3.900 .938400 .00048

3.950 .946420 .00048

4.000 .953270 .00049

4.050 .960110 .00050

4.100 .967020 .00048

4.150 .974640 .00049

4.200 .981340 .00049

4.250 .987520 .00049

4.300 .993630 .00049

4.351 .000310 .00050

4.401 .007300 .00048

4.451 .013470 .00048

4.501 .019920 .00049
600

3.500 .916870 .00049 3.550 .925960 .00049 3.600 .932420 .00049 3.650 .940570 .00046 3.700 .948430 .00047 3.750 .955500 .00048 3.800 .963160 .00048 3.850 .969860 .00047 3.900 .977450 .00049 3.950 .984960 .00047 4.000 .991550 .00048 4.050 .998760 .00049 4.101 .005300 .00048 4.151 .013250 .00049 4.201 .019160 .00047 $\begin{array}{ccc} & 750 \\ 4.20 & 0.92173 & 0.00047 \\ 4.25 & 0.92905 & 0.00049 \\ 4.30 & 0.93532 & 0.00048 \\ 4.35 & 0.94162 & 0.00049 \\ 4.40 & 0.94764 & 0.00048 \\ 4.45 & 0.95416 & 0.00050 \\ 4.50 & 0.96036 & 0.00048 \\ 4.55 & 0.96682 & 0.00049 \\ 4.60 & 0.97310 & 0.00048 \\ 4.65 & 0.97929 & 0.00049 \\ 4.70 & 0.98511 & 0.00048 \\ 4.75 & 0.99071 & 0.00047 \\ 4.80 & 0.99771 & 0.00048 \\ 4.85 & 1.00333 & 0.00048 \\ 4.90 & 1.00962 & 0.00049 \\ 4.95 & 1.01412 & 0.00047 \\ 5.00 & 1.02073 & 0.00050\end{array}$ 
752

4.200 .914760 .00047 4.250 .921050 .00049 4.300 .927680 .00048 4.350 .934670 .00047 4.400 .941060 .00049 4.450 .947200 .00047 4.500 .953650 .00048 4.550 .959850 .00049 4.600 .966020 .00048 4.650 .971660 .00048 4.700 .977990 .00048 4.750 .984340 .00049 4.800 .989570 .00049 4.850 .995780 .00047 4.901 .001740 .00047 4.951 .008340 .00047 5.001 .012990 .00048 5.051 .019160 .00048 5.101 .025270 .00049

Pun40-pl-1000-10
754

4.300 .918180 .00047

4.350 .925360 .00047

4.400 .931930 .00047

4.450 .937960 .00048

4.500 .944090 .00047

4.550 .950030 .00049

4.600 .956700 .00048

4.650 .962540 .00047

4.700 .968960 .00048

4.750 .974420 .00048

4.800 .980840 .00050

4.850 .986370 .00048

4.900 .992240 .00048

4.950 .998680 .00048

5.001 .002830 .00049

5.051 .009310 .00048

5.101 .015260 .00049
12

7.000 .922550 .00028

7.100 .927180 .00026

7.200 .931670 .00027

7.300 .936610 .00026

7.400 .940550 .00027

7.500 .944840 .00027

7.600 .949160 .00027

7.700 .953530 .00027

7.800 .957500 .00027

7.900 .961200 .00027

8.000 .964970 .00026

8.100 .969160 .00026

8.200 .972570 .00027

8.300 .976760 .00026

8.400 .979810 .00026

8.500 .983570 .00027

8.600 .986930 .00026

8.700 .990560 .00026

8.800 .993640 .00026

8.900 .996890 .00025

9.001 .000310 .00025

9.101 .003390 .00026

9.201 .005900 .00025
15

5.600 .925580 .00031 5.650 .929080 .00031 5.700 .932550 .00031 5.750 .935580 .00030 5.800 .938780 .00030 5.850 .942100 .00030 5.900 .945120 .00030 5.950 .948380 .00031 6.000 .951290 .00030 6.050 .954280 .00030 6.100 .957940 .00030 6.150 .960220 .00029 6.200 .963440 .00030 6.250 .966410 .00030 6.300 .968850 .00029 6.350 .972010 .00031 6.400 .974750 .00030 6.450 .978110 .00030 6.500 .980360 .00029 6.550 .982940 .00029 6.600 .985780 .00029 6.650 .988280 .00029 6.700 .991540 .00030
755

4.400 .920510 .00048 4.450 .927210 .00049 4.500 .933470 .00049 4.550 .938780 .00049 4.600 .946410 .00048 4.650 .951720 .00049 4.700 .957550 .00048 4.750 .964070 .00048 4.800 .969450 .00050 4.850 .975560 .00047 4.900 .981330 .00049 4.950 .987700 .00047 5.000 .993180 .00049 5.050 .998790 .00048 5.101 .004750 .00049 5.151 .010180 .00050 5.201 .015670 .00047 5.251 .021500 .00049 5.301 .026330 .00048 
9.301 .009340 .00025 9.401 .012920 .00026 9.501 .015130 .00025

25

3.800 .925310 .00036 3.850 .930980 .00036 3.900 .935830 .00036 3.950 .941740 .00035 4.000 .946630 .00036 4.050 .951710 .00035 4.100 .957490 .00035 4.150 .961470 .00036 4.200 .966830 .00035 4.250 .971730 .00035 4.300 .977010 .00036 4.350 .982520 .00035 4.400 .986890 .00036 4.450 .991860 .00036 4.500 .996650 .00036 4.551 .000790 .00035 4.601 .005040 .00036 4.651 .010040 .00035 4.701 .015230 .00035 4.751 .018800 .00036 4.801 .023040 .00035

35

3.100 .917820 .00038 3.150 .923760 .00038 3.200 .931480 .00039 3.250 .937500 .00039 3.300 .943900 .00039 3.350 .950550 .00039 3.400 .957400 .00039 3.450 .963220 .00038 3.500 .969160 .00038 3.550 .975410 .00040 3.600 .982270 .00038 3.650 .987590 .00038
6.750 .993210 .00029

6.800 .996130 .00029

6.850 .999330 .00029

6.901 .001830 .00029

6.951 .003970 .00028

7.001 .006320 .00029

7.051 .009060 .00029

7.101 .011640 .00030

7.151 .013700 .00030

7.201 .015520 .00028

30

3.400 .923490 .00037

3.450 .929170 .00037

3.500 .935350 .00038

3.550 .941610 .00037

3.600 .947500 .00038

3.650 .953460 .00037

3.700 .959370 .00038

3.750 .964960 .00037

3.800 .970580 .00038

3.850 .976650 .00037

3.900 .981420 .00037

3.950 .987270 .00037

4.000 .991770 .00038

4.050 .997860 .00038

4.101 .002740 .00037

4.151 .008640 .00037

4.201 .012730 .00037

4.251 .018670 .00038

4.301 .023270 .00037

5.551 .017590 .00033

5.601 .021630 .00032
32

3.300 .924990 .00038

3.350 .931060 .00037

3.400 .937770 .00036

3.450 .943630 .00037

3.500 .950120 .00038

3.550 .956240 .00038

3.600 .961740 .00038

3.650 .968530 .00037

3.700 .973980 .00037

3.750 .979640 .00037

3.800 .985120 .00037

3.850 .991240 .00036

3.900 .996600 .00038

3.951 .002630 .00038

4.001 .007710 .00038

4.051 .012190 .00036

4.101 .018120 .00038
2.900 .916290 .00039

2.950 .923820 .00040

3.000 .930710 .00040

3.050 .937570 .00039

3.100 .944530 .00039

3.150 .951350 .00040

3.200 .958680 .00039

3.250 .965370 .00039

3.300 .971450 .00040

3.350 .978100 .00039

3.400 .984500 .00039

3.450 .990700 .00039
50

2.600 .910640 .00040 2.650 .918940 .00039 2.700 .926580 .00040 2.750 .934620 .00041 2.800 .941890 .00042 2.850 .948620 .00039 2.900 .956020 .00041 2.950 .963620 .00041 3.000 .970110 .00041 3.050 .977910 .00040 3.100 .984700 .00042 3.150 .991980 .00042 
3.700 .993800 .00038 3.750 .999230 .00038 3.801 .005120 .00038 3.851 .010400 .00039 3.901 .016530 .00038 3.951 .021610 .00039 4.001 .028360 .00039
3.500 .996200 .00039

3.551 .003230 .00038

3.601 .009080 .00039

3.651 .015560 .00039

3.701 .021040 .00039
3.200 .997990 .00041

3.251 .004900 .00041

3.301 .011460 .00040

3.351 .017670 .00040

3.401 .024860 .00041
60

2.500 .923120 .00041 2.550 .931300 .00041 2.600 .939140 .00041 2.650 .946980 .00041 2.700 .954130 .00041 2.750 .961390 .00041 2.800 .969750 .00041 2.850 .977710 .00042 2.900 .984040 .00041 2.950 .991570 .00041 3.000 .997850 .00041 3.051 .005590 .00040 3.101 .012780 .00041 3.151 .019040 .00040 3.201 .025350 .00041

90

2.200 .922040 .00042 2.250 .930110 .00042 2.300 .937830 .00043 2.350 .947590 .00042 2.400 .954830 .00040 2.450 .962790 .00043 2.500 .970790 .00043 2.550 .978300 .00042 2.600 .986630 .00043 2.650 .994100 .00042 2.701 .001190 .00042 2.751 .009680 .00042 2.801 .017000 .00042
70

2.300 .910190 .00041

2.350 .918760 .00041

2.400 .926390 .00042

2.450 .935390 .00041

2.500 .942720 .00043

2.550 .950730 .00043

2.600 .959380 .00042

2.650 .966310 .00041

2.700 .974430 .00042

2.750 .982070 .00041

2.800 .989600 .00042

2.850 .996970 .00042

2.901 .003490 .00041

2.951 .010340 .00041

3.001 .018110 .00042
80

2.300 .925800 .00041

2.350 .934740 .00043

2.400 .942220 .00043

2.450 .951260 .00042

2.500 .958730 .00043

2.550 .966890 .00043

2.600 .974250 .00042

2.650 .982020 .00041

2.700 .989680 .00042

2.750 .997190 .00041

2.801 .005270 .00042

2.851 .012060 .00042

2.901 .018970 .00041
2.100 .913880 .00044

2.150 .923490 .00043

2.200 .931580 .00042

2.250 .939950 .00042

2.300 .948730 .00041

2.350 .956530 .00043

2.400 .964620 .00043

2.450 .972870 .00042

2.500 .980710 .00042

2.550 .988570 .00041

2.600 .995830 .00043

2.651 .003780 .00043

2.701 .011160 .00043

2.751 .018600 .00042

2.801 .026300 .00042
120

2.000 .911010 .00043

2.050 .919950 .00043

2.100 .928840 .00043

2.150 .937800 .00043

2.200 .946080 .00043

2.250 .954840 .00043

2.300 .962980 .00042

2.350 .970450 .00043

2.400 .978590 .00043

2.450 .987620 .00043

2.500 .994800 .00042

2.551 .002690 .00042

2.601 .010780 .00043

2.651 .017920 .00043

2.701 .024720 .00042 
130

2.000 .917620 .00043

2.050 .926380 .00043

2.100 .935690 .00042

2.150 .944420 .00042

2.200 .952280 .00043

2.250 .960680 .00043

2.300 .968690 .00042

2.350 .976730 .00044

2.400 .984520 .00043

2.450 .992710 .00042

2.501 .000210 .00042

2.551 .009050 .00044

2.601 .015620 .00042

239

1.800 .913040 .00042

1.850 .921740 .00044

1.900 .930930 .00042

1.950 .938860 .00044

2.000 .947720 .00043

2.050 .955690 .00043

2.100 .964010 .00043

2.150 .972910 .00043

2.200 .980620 .00044

2.250 .989010 .00042

2.300 .996250 .00044

2.351 .004220 .00043

2.401 .012280 .00043

2.451 .019030 .00044

2.501 .026640 .00044

478

1.800 .923770 .00043

1.850 .931890 .00044

1.900 .940160 .00043

1.950 .948380 .00043

2.000 .956360 .00044

2.050 .964360 .00044

2.100 .972300 .00044

2.150 .980330 .00044

2.200 .987100 .00043

2.250 .994850 .00045

2.301 .003060 .00045

2.351 .010240 .00043
150

1.900 .908750 .00042

1.950 .917620 .00043

2.000 .927390 .00042

2.050 .935880 .00043

2.100 .943940 .00043

2.150 .952630 .00042

2.200 .961040 .00043

2.250 .969600 .00044

2.300 .977750 .00044

2.350 .985390 .00044

2.400 .993240 .00044

2.451 .001130 .00044

2.501 .009160 .00044

2.551 .017060 .00042

2.601 .023410 .00044
200

1.900 .923550 .00043

1.950 .932900 .00042

2.000 .940930 .00044

2.050 .949710 .00043

2.100 .957820 .00043

2.150 .965860 .00044

2.200 .974130 .00042

2.250 .982150 .00043

2.300 .991260 .00043

2.350 .998730 .00042

2.401 .005640 .00043

2.451 .013690 .00043

2.501 .021370 .00044
250

1.800 .914110 .00043

1.850 .923210 .00041

1.900 .932030 .00044

1.950 .940270 .00043

2.000 .948570 .00044

2.050 .956950 .00043

2.100 .965470 .00044

2.150 .974030 .00043

2.200 .981640 .00043

2.250 .989590 .00043

2.300 .997700 .00044

2.351 .005950 .00042

2.401 .013690 .00045

2.451 .020520 .00043

2.501 .027970 .00044
359

1.800 .922950 .00044

1.850 .930930 .00043

1.900 .939550 .00043

1.950 .947510 .00043

2.000 .956160 .00044

2.050 .964600 .00044

2.100 .971470 .00043

2.150 .979260 .00043

2.200 .988440 .00043

2.250 .995540 .00044

2.301 .003900 .00044

2.351 .009860 .00043

2.401 .018360 .00043
500

1.800 .923880 .00043

1.850 .931600 .00043

1.900 .939860 .00044

1.950 .948020 .00044

2.000 .955700 .00043

2.050 .963640 .00043

2.100 .971890 .00043

2.150 .979240 .00043

2.200 .986970 .00044

2.250 .994360 .00042

2.301 .001910 .00044

2.351 .009380 .00043
600

1.800 .917200 .00044

1.850 .925790 .00043

1.900 .933690 .00043

1.950 .940990 .00043

2.000 .949150 .00044

2.050 .956020 .00044

2.100 .963240 .00044

2.150 .972060 .00046

2.200 .979050 .00045

2.250 .986440 .00044

2.300 .993190 .00044

2.351 .000670 .00043 
CHPRC-01552, Revision 0

$2.401 .016850 .00044 \quad 2.401 .016270 .00045 \quad 2.401 .008240 .00043$

2.451 .015020 .00043

2.501 .021740 .00045

650

700

750

1.800 .911000 .00044

1.900 .915350 .00042

1.950 .923770 .00044

1.850 .919430 .00043

1.900 .927000 .00043

2.000 .931240 .00043

1.950 .934640 .00042

2.050 .937620 .00044

2.000 .942350 .00043

2.100 .945460 .00045

2.050 .950200 .00044

2.150 .952250 .00043

2.100 .957090 .00044

2.200 .960050 .00044

2.150 .965070 .00044

2.250 .967040 .00044

2.200 .971900 .00043

2.250 .978660 .00044

2.300 .974370 .00044

2.350 .980240 .00043

2.300 .986050 .00043

2.350 .993870 .00045

2.401 .000510 .00045

2.451 .007490 .00045

2.501 .014500 .00044

2.551 .021430 .00044

2.601 .028340 .00043

2.400 .987880 .00044

2.501 .000000 .00043

2.551 .007470 .00045

2.601 .013490 .00045

2.651 .020150 .00045

2.701 .026780 .00045

2.100 .917010 .00043

2.150 .923420 .00045

2.200 .929840 .00043

2.250 .937160 .00044

2.300 .943310 .00045

2.350 .950280 .00046

2.400 .955690 .00044

2.450 .962740 .00044

2.500 .967940 .00044

2.550 .975490 .00044

2.600 .981330 .00043

2.650 .987300 .00044

2.700 .993950 .00044

2.750 .999210 .00044

2.801 .005480 .00046

2.851 .010300 .00044

2.901 .016670 .00044

752

754

755

2.200 .925610 .00044

2.250 .933510 .00043

2.300 .939350 .00043

2.350 .945990 .00045

2.400 .952380 .00043

2.450 .958920 .00044

2.500 .965040 .00043

2.550 .971560 .00044

2.600 .977510 .00044

2.650 .983580 .00045

2.700 .988500 .00044

2.750 .995420 .00043

2.801 .000560 .00044

2.851 .007470 .00045

2.901 .013120 .00044

2.951 .018220 .00044

3.001 .024290 .00044

2.200 .922180 .00044

2.250 .928430 .00042

2.300 .935080 .00045

2.350 .940920 .00045

2.400 .947900 .00044

2.450 .953430 .00042

2.500 .959750 .00044

2.550 .966150 .00044

2.600 .972500 .00044

2.650 .977850 .00044

2.700 .983890 .00045

2.750 .990080 .00044

2.800 .996200 .00045

2.851 .001580 .00043

2.901 .007210 .00043

2.951 .013090 .00045

3.001 .018260 .00046

2.200 .916860 .00043

2.250 .923050 .00044

2.300 .928990 .00043

2.350 .935810 .00044

2.400 .942460 .00044

2.450 .948560 .00045

2.500 .953380 .00044

2.550 .960450 .00045

2.600 .966310 .00043

2.650 .972230 .00045

2.700 .977840 .00043

2.750 .984370 .00044

2.800 .989790 .00043

2.850 .995210 .00045

2.901 .002030 .00044

2.951 .006580 .00044

3.001 .012260 .00045

3.051 .017300 .00045

3.101 .022720 .00045 
Pun43-pl-1000-00

12

10.700 .923780 .00029 10.800 .927550 .00028 10.900 .931420 .00029 11.000 .934590 .00028 11.100 .938750 .00028 11.200 .942320 .00028 11.300 .945570 .00029 11.400 .948490 .00028 11.500 .951510 .00027 11.600 .954950 .00028 11.700 .957580 .00028 11.800 .960980 .00028 11.900 .964160 .00028 12.000 .966420 .00027 12.100 .970010 .00028 12.200 .972670 .00027 12.300 .975590 .00028 12.400 .978370 .00027 12.500 .981200 .00027 12.600 .983460 .00027 12.700 .986910 .00026 12.800 .989120 .00027 12.900 .991730 .00027 13.000 .994230 .00027 13.100 .996110 .00027 13.200 .998600 .00027 13.301 .000640 .00026 13.401 .003810 .00027 13.501 .005790 .00025 13.601 .007450 .00026 13.701 .010390 .00026 13.801 .012780 .00026 13.901 .014160 .00025
15

8.800 .918790 .00033

8.900 .924330 .00032

9.000 .929860 .00032

9.100 .935110 .00032

9.200 .940690 .00033

9.300 .945280 .00032

9.400 .950390 .00033

9.500 .956230 .00031

9.600 .960650 .00032

9.700 .965480 .00032

9.800 .970510 .00031

9.900 .974480 .00031

10.000 .979560 .00031

10.100 .984350 .00032

10.200 .988940 .00031

10.300 .993280 .00031

10.400 .996800 .00031

10.501 .001460 .00031

10.601 .005270 .00031

10.701 .009390 .00031

10.801 .013160 .00030

10.901 .017810 .00031
7.500 .921910 .00037

7.550 .925680 .00037

7.600 .930350 .00038

7.650 .934570 .00038

7.700 .937900 .00037

7.750 .941190 .00037

7.800 .945720 .00037

7.850 .949330 .00037

7.900 .953470 .00035

7.950 .956510 .00037

8.000 .959900 .00037

8.050 .964370 .00037

8.100 .967510 .00037

8.150 .970440 .00037

8.200 .974240 .00037

8.250 .978260 .00036

8.300 .981660 .00037

8.350 .984730 .00036

8.400 .988410 .00036

8.450 .992010 .00036

8.500 .995030 .00036

8.550 .998280 .00036

8.601 .001230 .00035

8.651 .004150 .00037

8.701 .008310 .00036

8.751 .010650 .00036

8.801 .014680 .00036

8.851 .017320 .00036

8.901 .020050 .00036
25

6.800 .918540 .00040

6.850 .923780 .00040

6.900 .928590 .00040

6.950 .934260 .00039

7.000 .937870 .00041

7.050 .943130 .00040

7.100 .947860 .00038

7.150 .952140 .00039

7.200 .956210 .00040

7.250 .960580 .00041
30

6.400 .919340 .00042

6.450 .925930 .00042

6.500 .930210 .00041

6.550 .936150 .00041

6.600 .941190 .00042

6.650 .946840 .00041

6.700 .951800 .00042

6.750 .956720 .00041

6.800 .961920 .00041

6.850 .966820 .00043
32

6.300 .922320 .00042

6.350 .927940 .00043

6.400 .932440 .00041

6.450 .938800 .00042

6.500 .944040 .00043

6.550 .949850 .00042

6.600 .955720 .00042

6.650 .960260 .00043

6.700 .965140 .00043

6.750 .971000 .00041 
7.300 .965910 .00040 7.350 .969950 .00039 7.400 .974370 .00039 7.450 .978370 .00039 7.500 .983280 .00040 7.550 .987200 .00038 7.600 .991020 .00039 7.650 .995160 .00040 7.700 .999370 .00039 7.751 .003290 .00039 7.801 .008030 .00038 7.851 .011120 .00039 7.901 .014900 .00039 7.951 .018820 .00038 8.001 .022840 .00038

35

6.100 .915600 .00043 6.150 .921780 .00043 6.200 .928000 .00043 6.250 .933490 .00044 6.300 .939770 .00043 6.350 .944860 .00043 6.400 .950750 .00042 6.450 .957010 .00043 6.500 .961890 .00043 6.550 .967600 .00044 6.600 .973060 .00043 6.650 .979320 .00044 6.700 .983370 .00044 6.750 .989130 .00043 6.800 .993670 .00043 6.850 .999400 .00042 6.901 .004670 .00043 6.951 .009400 .00043 7.001 .014650 .00042 7.051 .019120 .00043 7.101 .024500 .00042

\section{0}

5.500 .914760 .00047 5.550 .921700 .00046 5.600 .929490 .00046 5.650 .935030 .00048 5.700 .942630 .00046 5.750 .949520 .00047 5.800 .956800 .00045
6.900 .971930 .00040

6.950 .976790 .00041

7.000 .981480 .00042

7.050 .986890 .00040

7.100 .992090 .00040

7.150 .996130 .00040

7.201 .000530 .00041

7.251 .005630 .00041

7.301 .010490 .00043

7.351 .015160 .00041

7.401 .019640 .00042
6.800 .976110 .00042 6.850 .981090 .00041 6.900 .985650 .00041 6.950 .990970 .00043 7.000 .995970 .00042 7.051 .001220 .00041 7.101 .006350 .00041 7.151 .010640 .00042 7.201 .014560 .00043 7.251 .020070 .00042 7.301 .024780 .00042
40

5.900 .913740 .00044 5.950 .920540 .00045 6.000 .927020 .00044 6.050 .932440 .00045 6.100 .939110 .00045 6.150 .945340 .00044 6.200 .951390 .00045 6.250 .957310 .00045 6.300 .962800 .00044 6.350 .969570 .00044 6.400 .975050 .00044 6.450 .980600 .00046 6.500 .986350 .00044 6.550 .991890 .00043 6.600 .997570 .00045 6.651 .003540 .00044 6.701 .007860 .00044 6.751 .014140 .00044 6.801 .018720 .00044
50

5.700 .921160 .00047 5.750 .928180 .00045 5.800 .934780 .00045 5.850 .942060 .00045 5.900 .947830 .00047 5.950 .954600 .00045 6.000 .961700 .00045 6.050 .967650 .00046 6.100 .973410 .00046 6.150 .980550 .00046 6.200 .985770 .00046 6.250 .991590 .00045 6.300 .998190 .00045 6.351 .004600 .00046 6.401 .010460 .00044 6.451 .015350 .00045 6.501 .021570 .00045
70

5.400 .915350 .00047 5.450 .923590 .00047 5.500 .930130 .00047 5.550 .937950 .00048 5.600 .945190 .00047 5.650 .952300 .00048 5.700 .960680 .00047
80

5.300 .911330 .00047 5.350 .919790 .00048 5.400 .925480 .00047 5.450 .933610 .00049 5.500 .941820 .00047 5.550 .947600 .00047 5.600 .955220 .00048 
5.850 .962710 .00047

5.900 .970580 .00047

5.950 .977210 .00047

6.000 .983700 .00046

6.050 .989490 .00046

6.100 .995690 .00048

6.151 .002030 .00045

6.201 .009200 .00047

6.251 .014710 .00046

6.301 .020630 .00047

90

5.300 .918720 .00048

5.350 .925780 .00048

5.400 .933320 .00049

5.450 .941880 .00048

5.500 .949270 .00048

5.550 .956370 .00048

5.600 .962850 .00047

5.650 .970660 .00049

5.700 .978730 .00048

5.750 .984330 .00047

5.800 .991610 .00047

5.850 .998360 .00048

5.901 .005000 .00047

5.951 .011150 .00047

6.001 .018140 .00048

6.051 .024640 .00047

6.101 .030080 .00046

130

5.200 .917910 .00047

5.250 .925910 .00049

5.300 .934080 .00049

5.350 .941850 .00048

5.400 .949910 .00048

5.450 .956850 .00049

5.500 .964000 .00049

5.550 .971480 .00049

5.600 .978690 .00047

5.650 .986580 .00048

5.700 .992920 .00049

5.751 .000320 .00049

5.801 .006850 .00049

5.851 .013330 .00049

5.901 .020050 .00048
5.750 .966140 .00048

5.800 .971960 .00047

5.850 .980010 .00047

5.900 .986690 .00047

5.950 .992550 .00046

6.000 .999890 .00046

6.051 .006120 .00047

6.101 .012110 .00047

6.151 .018250 .00048

6.201 .024770 .00047
5.650 .962850 .00046

5.700 .969770 .00047

5.750 .975710 .00049

5.800 .983910 .00045

5.850 .989960 .00048

5.900 .996450 .00049

5.951 .003390 .00048

6.001 .010060 .00049

6.051 .015740 .00049

6.101 .023000 .00049
100

5.200 .908130 .00048

5.250 .916560 .00048

5.300 .924600 .00049

5.350 .932120 .00049

5.400 .939640 .00049

5.450 .946790 .00049

5.500 .954410 .00050

5.550 .962920 .00048

5.600 .969270 .00049

5.650 .976760 .00049

5.700 .982790 .00049

5.750 .990260 .00048

5.800 .997620 .00047

5.851 .003790 .00049

5.901 .010090 .00048

5.951 .017340 .00048

6.001 .025370 .00046
120

5.200 .915210 .00049

5.250 .922930 .00049

5.300 .930340 .00047

5.350 .938940 .00049

5.400 .946490 .00048

5.450 .953490 .00049

5.500 .961220 .00049

5.550 .967920 .00049

5.600 .976300 .00049

5.650 .982360 .00049

5.700 .988950 .00050

5.750 .996840 .00048

5.801 .003690 .00048

5.851 .010470 .00048

5.901 .017350 .00048

5.951 .023920 .00048

6.001 .031170 .00049
5.200 .921380 .00049

5.250 .928900 .00049

5.300 .936350 .00048

5.350 .943940 .00049

5.400 .951490 .00050

5.450 .958720 .00049

5.500 .965460 .00049

5.550 .973300 .00050

5.600 .981820 .00049

5.650 .988210 .00050

5.700 .995380 .00050

5.751 .002510 .00049

5.801 .008880 .00050

5.851 .015850 .00049

5.901 .022650 .00048
200

5.200 .922600 .00048

5.250 .929840 .00049

5.300 .937810 .00049

5.350 .944830 .00049

5.400 .951950 .00049

5.450 .960130 .00047

5.500 .967360 .00049

5.550 .975290 .00049

5.600 .981990 .00048

5.650 .989470 .00050

5.700 .995620 .00050

5.751 .002590 .00050

5.801 .010480 .00049

5.851 .016690 .00049

5.901 .022850 .00048 
239

5.200 .919210 .00047 5.250 .925480 .00048 5.300 .933850 .00048 5.350 .941020 .00049 5.400 .949070 .00049 5.450 .956750 .00049 5.500 .964900 .00049 5.550 .971390 .00049 5.600 .978600 .00050 5.650 .985610 .00050 5.700 .993700 .00048 5.750 .999860 .00049 5.801 .007570 .00048 5.851 .013740 .00050 5.901 .020740 .00049

478

5.400 .909970 .00049 5.450 .917800 .00048 5.500 .924300 .00047 5.550 .932670 .00049 5.600 .939580 .00050 5.650 .946320 .00048 5.700 .953560 .00049 5.750 .960520 .00048 5.800 .967410 .00047 5.850 .975720 .00049 5.900 .981640 .00049 5.950 .987950 .00050 6.000 .995060 .00049 6.051 .002000 .00049 6.101 .007650 .00049 6.151 .015040 .00049 6.201 .021460 .00049
250

5.200 .918720 .00049

5.250 .925480 .00047

5.300 .933450 .00049

5.350 .940820 .00048

5.400 .948460 .00050

5.450 .956370 .00049

5.500 .962890 .00050

5.550 .971040 .00050

5.600 .977120 .00048

5.650 .984520 .00050

5.700 .991030 .00047

5.750 .998440 .00051

5.801 .005320 .00049

5.851 .012520 .00049

5.901 .018960 .00049

5.951 .025810 .00049

6.001 .032720 .00048

500

5.500 .916830 .00049

5.550 .924730 .00050

5.600 .931700 .00048

5.650 .939700 .00050

5.700 .946910 .00048

5.750 .952920 .00049

5.800 .960320 .00050

5.850 .967980 .00048

5.900 .975260 .00050

5.950 .980310 .00049

6.000 .988880 .00049

6.050 .994550 .00048

6.101 .001160 .00049

6.151 .008250 .00048

6.201 .015570 .00049

6.251 .019710 .00050

6.301 .027000 .00049
359

5.300 .918150 .00050

5.350 .926270 .00048

5.400 .933690 .00048

5.450 .941140 .00049

5.500 .948230 .00047

5.550 .955880 .00049

5.600 .963750 .00049

5.650 .970530 .00049

5.700 .976930 .00049

5.750 .985000 .00047

5.800 .991560 .00048

5.850 .998310 .00050

5.901 .004790 .00049

5.951 .011320 .00049

6.001 .018380 .00049

6.051 .024850 .00049

6.101 .031940 .00050

600

5.800 .917720 .00047

5.850 .925290 .00048

5.900 .932040 .00050

5.950 .939630 .00050

6.000 .945870 .00049

6.050 .953600 .00049

6.100 .958830 .00048

6.150 .966440 .00048

6.200 .972250 .00049

6.250 .979100 .00050

6.300 .985880 .00049

6.350 .992390 .00047

6.400 .998110 .00048

6.451 .004120 .00048

6.501 .011610 .00049

6.551 .016570 .00049

6.601 .022890 .00049
650

6.100 .918070 .00049 6.150 .924560 .00047 6.200 .930760 .00048 6.250 .937680 .00048 6.300 .944610 .00049 6.350 .951070 .00049
660

6.200 .919970 .00048

6.250 .925930 .00048

6.300 .933310 .00048

6.350 .939750 .00048

6.400 .944780 .00049

6.450 .952390 .00049
670

6.300 .918280 .00050 6.350 .924910 .00048 6.400 .930830 .00048 6.450 .937620 .00048 6.500 .943700 .00048 6.550 .949840 .00049 
6.400 .958160 .00047 6.450 .963420 .00048 6.500 .969760 .00048 6.550 .976730 .00049 6.600 .982630 .00049 6.650 .988340 .00049 6.700 .995040 .00050 6.751 .000730 .00048 6.801 .007050 .00049 6.851 .012430 .00048 6.901 .018220 .00050 6.951 .023950 .00049 7.001 .030690 .00049

680

6.400 .912080 .00048 6.450 .918930 .00047 6.500 .924810 .00049 6.550 .931300 .00048 6.600 .937070 .00049 6.650 .943720 .00047 6.700 .950060 .00049 6.750 .955370 .00050 6.800 .961740 .00047 6.850 .968170 .00049 6.900 .974350 .00049 6.950 .980400 .00049 7.000 .986640 .00048 7.050 .991750 .00048 7.100 .996640 .00049 7.151 .002730 .00049 7.201 .008340 .00049 7.251 .014320 .00049 7.301 .020530 .00050
6.500 .958330 .00049

6.550 .964670 .00049

6.600 .971550 .00050

6.650 .976360 .00048

6.700 .982300 .00048

6.750 .989080 .00049 6.800 .994560 .00047 6.851 .000490 .00048 6.901 .006540 .00048 6.951 .013090 .00049 7.001 .019010 .00049 7.051 .023670 .00048 7.101 .028730 .00049
6.600 .956750 .00047 6.650 .963340 .00049 6.700 .968080 .00050 6.750 .973870 .00048 6.800 .980960 .00050 6.850 .986990 .00049 6.900 .992030 .00049 6.950 .997780 .00050 7.001 .004210 .00048 7.051 .010760 .00048 7.101 .015140 .00048 7.151 .021290 .00048 7.201 .026930 .00050
6.700 .920690 .00048

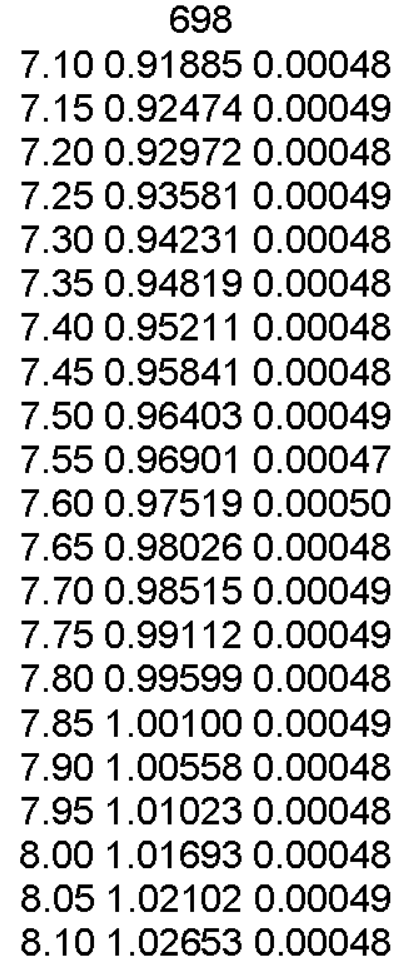
6.750 .927260 .00049
$\begin{array}{lll}6.80 & 0.933810 .00047\end{array}$
6.850 .939800 .00048
6.900 .945670 .00049
6.950 .951420 .00049
7.000 .957240 .00048
7.050 .962650 .00047
7.100 .968580 .00050
7.150 .973610 .00049
7.200 .979970 .00050
7.250 .987010 .00048
7.300 .991980 .00049
7.350 .996460 .00049
7.401 .002040 .00050
7.451 .009270 .00050
7.501 .013700 .00048
7.551 .019210 .00048
7.601 .024830 .00049 
Pun43-pl-1000-01

12

9.200 .926070 .00028

9.300 .929610 .00027

9.400 .932890 .00028

9.500 .936620 .00028

9.600 .939840 .00028

9.700 .943580 .00027

9.800 .946690 .00027

9.900 .949800 .00027

10.000 .952690 .00027

10.100 .956280 .00027

10.200 .959170 .00027

10.300 .962370 .00026

10.400 .965270 .00026

10.500 .968410 .00026

10.600 .971530 .00027

10.700 .974110 .00026

10.800 .976680 .00026

10.900 .979370 .00026

11.000 .982270 .00027

11.100 .984960 .00027

11.200 .987720 .00026

11.300 .990110 .00025

11.400 .992250 .00026

11.500 .994690 .00025

11.600 .997730 .00026

11.701 .000010 .00025

11.801 .002310 .00026

11.901 .004030 .00025

12.001 .006830 .00026

12.101 .009070 .00026

12.201 .010800 .00025

12.301 .012980 .00025
15

7.300 .920910 .00031

7.400 .926660 .00033

7.500 .932500 .00032

7.600 .937760 .00031

7.700 .943020 .00031

7.800 .947860 .00031

7.900 .953180 .00031

8.000 .958370 .00030

8.100 .963070 .00031

8.200 .968040 .00030

8.300 .972250 .00030

8.400 .978290 .00030

8.500 .982070 .00031

8.600 .986500 .00030

8.700 .990740 .00031

8.800 .994940 .00031

8.900 .999220 .00030

9.001 .003050 .00031

9.101 .007400 .00030

9.201 .011160 .00031

9.301 .015310 .00030

\section{5}

5.300 .923300 .00037

5.350 .928010 .00038

5.400 .933910 .00039

5.450 .938920 .00038

5.500 .943350 .00038

5.550 .947510 .00039

5.600 .952570 .00038

5.650 .957110 .00037

5.700 .961780 .00037

5.750 .966130 .00037

5.800 .969840 .00038

5.850 .974240 .00037
30

4.800 .913510 .00041

4.850 .919250 .00040

4.900 .924700 .00040

4.950 .930640 .00040

5.000 .935770 .00040

5.050 .942120 .00040

5.100 .947200 .00041

5.150 .952100 .00041

5.200 .957730 .00040

5.250 .962020 .00040

5.300 .967830 .00041

5.350 .972740 .00039
20

5.900 .917490 .00036

5.950 .921290 .00037

6.000 .925710 .00036

6.050 .928980 .00036

6.100 .933970 .00036

6.150 .937540 .00035

6.200 .941340 .00036

6.250 .945480 .00035

6.300 .948920 .00036

6.350 .952490 .00036

6.400 .956220 .00036

6.450 .959840 .00035

6.500 .963930 .00035

6.550 .967290 .00036

6.600 .971330 .00034

6.650 .974170 .00035

6.700 .977790 .00035

6.750 .981710 .00035

6.800 .984440 .00035

6.850 .988400 .00036

6.900 .992290 .00035

6.950 .995630 .00035

7.000 .997360 .00035

7.051 .001350 .00035

7.101 .004580 .00035

7.151 .007620 .00035

7.201 .011060 .00036

7.251 .014150 .00035

7.301 .016720 .00035 
5.900 .978870 .00037 5.950 .983250 .00038 6.000 .987420 .00039 6.050 .991770 .00038 6.100 .996190 .00038 6.150 .999900 .00037 6.201 .004670 .00038 6.251 .008270 .00037 6.301 .011950 .00038 6.351 .015770 .00038 6.401 .019510 .00037

35

4.600 .922530 .00042 4.650 .928520 .00041 4.700 .934770 .00042 4.750 .940830 .00040 4.800 .946550 .00040 4.850 .951560 .00041 4.900 .956790 .00042 4.950 .962920 .00040 5.000 .968410 .00040 5.050 .974200 .00041 5.100 .979500 .00041 5.150 .984670 .00041 5.200 .989380 .00042 5.250 .994830 .00042 5.301 .000780 .00041 5.351 .005340 .00041 5.401 .010780 .00040 5.451 .014990 .00042 5.501 .020750 .00041
5.400 .977600 .00040

5.450 .982040 .00040 5.500 .986910 .00041 5.550 .992160 .00039 5.600 .997030 .00040 5.651 .001220 .00040 5.701 .005350 .00040 5.751 .010620 .00041 5.801 .015500 .00041 5.851 .019480 .00040 5.901 .023430 .00040
5.300 .980940 .00041 5.350 .985950 .00040 5.400 .992000 .00040 5.450 .996770 .00040 5.501 .001880 .00041 5.551 .006640 .00040 5.601 .011190 .00041 5.651 .015880 .00040 5.701 .020520 .00040
40

4.400 .921590 .00043

4.450 .928480 .00043

4.500 .934790 .00043

4.550 .941120 .00042

4.600 .946140 .00044

4.650 .952580 .00042

4.700 .959190 .00042

4.750 .964660 .00042

4.800 .970610 .00042

4.850 .976280 .00042

4.900 .981730 .00042

4.950 .988590 .00042

5.000 .993330 .00041

5.050 .998850 .00040

5.101 .004080 .00043

5.151 .009850 .00041

5.201 .014330 .00043

5.251 .020570 .00042

5.301 .025960 .00042
60

2.200 .604840 .00041

2.220 .608570 .00042

2.240 .612910 .00042

2.260 .617110 .00041

2.280 .622110 .00043

2.300 .625530 .00043
70

3.800 .912280 .00045 3.850 .919600 .00046 3.900 .927310 .00046 3.950 .934800 .00046 4.000 .941940 .00044 4.050 .949170 .00046
50

3.100 .760350 .00043 3.120 .763630 .00044 3.140 .767200 .00043 3.160 .770450 .00043 3.180 .773820 .00044 3.200 .777010 .00043 3.220 .780570 .00043 3.240 .784430 .00043 3.260 .787270 .00043 3.280 .790550 .00044 3.300 .793850 .00043 3.320 .797810 .00044 3.340 .800870 .00044 3.360 .803670 .00043 3.380 .807180 .00044 3.400 .810820 .00042 3.420 .814490 .00044 3.440 .816990 .00043 3.460 .821240 .00044 3.480 .823570 .00044 3.500 .826810 .00042 3.520 .829940 .00043 3.540 .833480 .00042 3.560 .836860 .00044 3.580 .840530 .00045 3.600 .842510 .00043

80
3.800 .924220 .00046 3.850 .931080 .00045 3.900 .939150 .00043 3.950 .945540 .00046 4.000 .953590 .00045 4.050 .960640 .00046 
2.320 .629950 .00042 2.340 .633950 .00043 2.360 .638510 .00042 2.380 .642690 .00042 2.400 .647270 .00041 2.420 .651420 .00042 2.440 .655220 .00043 2.460 .659700 .00042 2.480 .662840 .00042 2.500 .667730 .00043

90

3.700 .916990 .00045 3.750 .924420 .00047 3.800 .932290 .00046 3.850 .939120 .00047 3.900 .947400 .00046 3.950 .954660 .00046 4.000 .961860 .00047 4.050 .968980 .00046 4.100 .975740 .00045 4.150 .982660 .00046 4.200 .990230 .00047 4.250 .997660 .00046 4.301 .003880 .00045 4.351 .010990 .00046 4.401 .017420 .00047

130

3.600 .918840 .00045 3.650 .927350 .00047 3.700 .934700 .00047 3.750 .942860 .00048 3.800 .950290 .00047 3.850 .958010 .00046 3.900 .964420 .00047 3.950 .972200 .00049 4.000 .979410 .00047 4.050 .986730 .00047 4.100 .994540 .00046 4.151 .000480 .00046 4.201 .007820 .00046 4.251 .014700 .00047 4.301 .020700 .00047
4.100 .956030 .00045

4.150 .962830 .00045 4.200 .969960 .00046 4.250 .977470 .00046 4.300 .983910 .00045 4.350 .989910 .00046 4.400 .996130 .00044 4.451 .002570 .00046 4.501 .010130 .00045 4.551 .015570 .00044 4.601 .022770 .00047
4.100 .967590 .00046 4.150 .974810 .00045 4.200 .981310 .00046 4.250 .987890 .00046 4.300 .995900 .00046 4.351 .001990 .00045 4.401 .009340 .00045 4.451 .014870 .00047 4.501 .020820 .00046
100

3.700 .922660 .00046

3.750 .930570 .00046

3.800 .938980 .00046

3.850 .946630 .00046

3.900 .953270 .00046

3.950 .961170 .00047

4.000 .967900 .00048

4.050 .975420 .00045

4.100 .981940 .00047

4.150 .989450 .00045

4.200 .997520 .00046

4.251 .003350 .00046

4.301 .009890 .00047

4.351 .016370 .00047

4.401 .023640 .00046
120

3.600 .915760 .00047 3.650 .923350 .00048 3.700 .931770 .00046 3.750 .938610 .00046 3.800 .946410 .00047 3.850 .954190 .00047 3.900 .960890 .00047 3.950 .968390 .00048 4.000 .976740 .00045 4.050 .983050 .00048 4.100 .990730 .00047 4.150 .997120 .00046 4.201 .004580 .00047 4.251 .011690 .00048 4.301 .018830 .00048
3.600 .922300 .00047

3.650 .930220 .00048

3.700 .938280 .00048

3.750 .946200 .00048

3.800 .953670 .00048

3.850 .960790 .00047

3.900 .967870 .00048 3.950 .975510 .00047 4.000 .982710 .00047 4.050 .989720 .00048 4.100 .996010 .00047 4.151 .003390 .00048 4.201 .011640 .00048 4.251 .017310 .00046 4.301 .025070 .00046
200

3.500 .910770 .00047 3.550 .918350 .00047 3.600 .925850 .00046 3.650 .932430 .00047 3.700 .940790 .00048 3.750 .949110 .00047 3.800 .955610 .00048 3.850 .963140 .00047 3.900 .970920 .00049 3.950 .977920 .00047 4.000 .984760 .00047 4.050 .992670 .00047 4.100 .999030 .00047 4.151 .006490 .00047 4.201 .013650 .00048 4.251 .019350 .00047 4.301 .026020 .00047 
239

3.600 .923930 .00048 3.650 .932110 .00046 3.700 .939230 .00047 3.750 .946360 .00048 3.800 .954390 .00047 3.850 .961570 .00049 3.900 .969060 .00046 3.950 .976360 .00047 4.000 .982660 .00047 4.050 .989990 .00047 4.100 .997810 .00049 4.151 .004420 .00048 4.201 .011150 .00047 4.251 .018350 .00049 4.301 .024740 .00047

\section{8}

3.800 .923680 .00048 3.850 .932440 .00049 3.900 .939360 .00048 3.950 .945680 .00048 4.000 .953670 .00048 4.050 .960360 .00048 4.100 .966860 .00047 4.150 .973610 .00049 4.200 .980460 .00048 4.250 .987590 .00047 4.300 .992890 .00047 4.351 .000520 .00048 4.401 .006520 .00047 4.451 .014060 .00047 4.501 .019190 .00049
250

3.600 .923890 .00048

3.650 .931080 .00047

3.700 .938500 .00047

3.750 .946390 .00047

3.800 .953220 .00047

3.850 .960470 .00048

3.900 .968900 .00047

3.950 .974800 .00047

4.000 .982920 .00047

4.050 .989790 .00046

4.100 .996950 .00049

4.151 .003530 .00047

4.201 .010900 .00048

4.251 .017040 .00048

4.301 .022950 .00047
359

3.600 .912490 .00046 3.650 .920500 .00047 3.700 .928570 .00049 3.750 .935580 .00048 3.800 .943030 .00048 3.850 .950720 .00048 3.900 .957930 .00047 3.950 .965390 .00049 4.000 .971760 .00048 4.050 .979300 .00046 4.100 .984910 .00048 4.150 .992540 .00048 4.200 .999910 .00047 4.251 .006510 .00047 4.301 .011820 .00049 4.351 .019600 .00048 4.401 .025160 .00048

\begin{tabular}{|c|}
\hline $\begin{array}{llll}2.82 & 0.73330 & 0.0004 \\
2.84 & 0.73641 & 0.0004 \\
2.86 & 0.73912 & 0.0004 \\
2.88 & 0.74344 & 0.0004 \\
2.90 & 0.74634 & 0.0004 \\
2.92 & 0.75041 & 0.0004 \\
2.94 & 0.75337 & 0.0004 \\
2.96 & 0.75656 & 0.0004 \\
2.98 & 0.76022 & 0.0004 \\
3.00 & 0.76333 & 0.0004 \\
3.02 & 0.76671 & 0.0004 \\
3.04 & 0.76978 & 0.0004\end{array}$ \\
\hline
\end{tabular}


650

3.500 .814500 .00047

3.550 .821590 .00047

3.600 .827830 .00048

3.650 .835530 .00047

3.700 .843000 .00049

3.750 .849730 .00047

3.800 .857160 .00048

3.850 .863760 .00047

3.900 .871150 .00048

3.950 .878440 .00048

4.000 .884750 .00048

4.050 .892030 .00047

4.100 .899300 .00047
680

4.500 .918620 .00048 4.550 .924940 .00047 4.600 .929860 .00048 4.650 .936090 .00048 4.700 .942370 .00047 4.750 .948700 .00049 4.800 .955390 .00047 4.850 .960170 .00047 4.900 .966950 .00049 4.950 .971240 .00048 5.000 .977980 .00049 5.050 .983610 .00049 5.100 .989390 .00049 5.150 .994950 .00048 5.201 .001350 .00049 5.251 .005950 .00049 5.301 .011600 .00048 5.351 .016910 .00049 $\begin{array}{lll}5.40 & 1.02300 & 0.00047\end{array}$
660

4.350 .922790 .00047

4.400 .930540 .00047

4.450 .936330 .00049

4.500 .942670 .00048

4.550 .948450 .00047

4.600 .954630 .00048

4.650 .961430 .00048

4.700 .966190 .00047

4.750 .973120 .00048

4.800 .979310 .00049

4.850 .984940 .00048

4.900 .991190 .00048

4.950 .995830 .00048

5.001 .002470 .00048

5.051 .007800 .00050

5.101 .014190 .00049

5.151 .019230 .00048

5.201 .025430 .00047
4.300 .917010 .00048

670

4.400 .919030 .00048

4.450 .925660 .00048

4.500 .932000 .00047

4.550 .938060 .00048

4.600 .944380 .00049

4.650 .950660 .00049

4.700 .956370 .00048

4.750 .963160 .00048

4.800 .968600 .00047

4.850 .974930 .00049

4.900 .980830 .00049

4.950 .986000 .00049

5.000 .991390 .00049 5.050 .997750 .00049 5.101 .003410 .00049 5.151 .009530 .00048 5.201 .014510 .00049 5.251 .020470 .00048 5.301 .025050 .00048

698

4.700 .921760 .00049

4.750 .926910 .00049

4.800 .934500 .00047

4.850 .939360 .00048

4.900 .945600 .00049

4.950 .951570 .00050

5.000 .956570 .00048

5.050 .962590 .00048

5.100 .967770 .00047

5.150 .973980 .00049

5.200 .978890 .00048

5.250 .985290 .00048

5.300 .990120 .00047

5.350 .995620 .00048

5.401 .001510 .00047

5.451 .006900 .00048

5.501 .010650 .00047

5.551 .017860 .00049

5.601 .022740 .00048
5.000 .920550 .00047 5.050 .926900 .00047 5.100 .931530 .00050 5.150 .937190 .00048 5.200 .943020 .00047 5.250 .947890 .00048 5.300 .953150 .00048 5.350 .959350 .00047 5.400 .964020 .00048 5.450 .968740 .00048 5.500 .975190 .00049 5.550 .979880 .00048 5.600 .985850 .00050 5.650 .991570 .00048 5.700 .994130 .00048 5.751 .000200 .00049 5.801 .005520 .00048 5.851 .010370 .00050 5.901 .015710 .00049 5.951 .021050 .00048 6.001 .025500 .00047 
Pun43-pl-1000-10

12

8.000 .923840 .00026

8.100 .927480 .00025

8.200 .930720 .00026

8.300 .934500 .00026

8.400 .938090 .00025

8.500 .941390 .00026

8.600 .944690 .00026

8.700 .947950 .00026

8.800 .951730 .00025

8.900 .954630 .00026

9.000 .957880 .00026

9.100 .961040 .00025

9.200 .964340 .00025

9.300 .966670 .00025

9.400 .969740 .00025

9.500 .972630 .00025

9.600 .975340 .00025

9.700 .978070 .00025

9.800 .980820 .00025

9.900 .983920 .00025

10.000 .986680 .00024

10.100 .989090 .00025

10.200 .992020 .00025

10.300 .994280 .00024

10.400 .996690 .00024

10.500 .998000 .00025

10.601 .001110 .00025

10.701 .003540 .00024

10.801 .005750 .00024

10.901 .007970 .00024

11.001 .010560 .00024

11.101 .012610 .00024

11.201 .015060 .00024
15

6.200 .923750 .00030

6.300 .929470 .00030

6.400 .935120 .00029

6.500 .940630 .00029

6.600 .946300 .00028

6.700 .951020 .00029

6.800 .956440 .00028

6.900 .960790 .00029

7.000 .966210 .00028

7.100 .971070 .00029

7.200 .975300 .00029

7.300 .979750 .00029

7.400 .984900 .00029

7.500 .989380 .00028

7.600 .992960 .00028

7.700 .997920 .00028

7.801 .001900 .00028

7.901 .006120 .00028

8.001 .010300 .00028

8.101 .014200 .00027
25

4.100 .922390 .00036 4.150 .927600 .00036 4.200 .932410 .00036 4.250 .937810 .00035 4.300 .943200 .00034 4.350 .947650 .00035 4.400 .952310 .00034 4.450 .956890 .00035 4.500 .961710 .00035 4.550 .965460 .00035 4.600 .970900 .00034
30

3.600 .915170 .00036

3.650 .920800 .00036

3.700 .925590 .00037

3.750 .932670 .00037

3.800 .937730 .00037

3.850 .943530 .00037

3.900 .948880 .00037

3.950 .953630 .00036

4.000 .958280 .00037

4.050 .963910 .00037

4.100 .970210 .00037
20

4.800 .923160 .00033

4.850 .927120 .00032

4.900 .931520 .00033

4.950 .935660 .00034

5.000 .939380 .00032

5.050 .943350 .00032

5.100 .947280 .00033

5.150 .951200 .00034

5.200 .955040 .00033

5.250 .958310 .00034

5.300 .962020 .00033

5.350 .966150 .00033

5.400 .969920 .00032

5.450 .973700 .00032

5.500 .976250 .00032

5.550 .980270 .00033

5.600 .983540 .00031

5.650 .986790 .00033

5.700 .990280 .00033

5.750 .993800 .00033

5.800 .996870 .00033

5.851 .000280 .00032

5.901 .003340 .00031

5.951 .007010 .00032

6.001 .010290 .00033

6.051 .013470 .00033

6.101 .016120 .00032 
4.650 .975190 .00035 4.700 .979060 .00035 4.750 .983760 .00035 4.800 .987760 .00035 4.850 .992110 .00035 4.900 .996500 .00034 4.951 .001080 .00035 5.001 .005690 .00036 5.051 .008360 .00035 5.101 .012890 .00035 5.151 .016740 .00035 5.201 .020390 .00035

35

3.300 .913420 .00037 3.350 .919850 .00037 3.400 .925800 .00037 3.450 .931270 .00038 3.500 .938480 .00038 3.550 .943530 .00037 3.600 .949920 .00037 3.650 .955580 .00038 3.700 .960990 .00037 3.750 .967160 .00038 3.800 .972860 .00038 3.850 .978320 .00038 3.900 .984180 .00038 3.950 .989530 .00037 4.000 .994460 .00037 4.051 .000200 .00037 4.101 .005570 .00039 4.151 .010560 .00037 4.201 .015730 .00037

60

2.600 .911100 .00040 2.650 .919540 .00041

2.700 .926700 .00040

2.750 .934840 .00041

2.800 .941870 .00041

2.850 .948330 .00042

2.900 .955750 .00040

2.950 .962510 .00040 3.000 .969760 .00042 3.050 .977500 .00040 3.100 .983500 .00041 3.150 .989940 .00042
4.150 .974900 .00037

4.200 .979490 .00037

4.250 .985000 .00037

4.300 .989550 .00036

4.350 .994370 .00037

4.400 .999980 .00036

4.451 .003440 .00036

4.501 .009210 .00036

4.551 .013750 .00036

4.601 .018600 .00036
4.050 .979920 .00037

4.100 .985010 .00037

4.150 .990120 .00036

4.200 .995550 .00036

4.251 .000540 .00037

4.301 .005420 .00037

4.351 .009750 .00036

4.401 .014470 .00038

4.451 .019940 .00038

4.501 .024710 .00037
40
3.100 .914930 .00039

3.150 .921480 .00039

3.200 .927950 .00038

3.250 .934060 .00039

3.300 .940720 .00039

3.350 .947190 .00038

3.400 .953080 .00039

3.450 .959290 .00038

3.500 .965100 .00039

3.550 .971610 .00039

3.600 .978380 .00039

3.650 .983550 .00039

3.700 .989950 .00038

3.750 .995050 .00040

3.801 .001750 .00039

3.851 .006000 .00039

3.901 .011970 .00038

3.951 .017340 .00038

4.001 .022910 .00039
50

2.800 .913570 .00040 2.850 .921570 .00040 2.900 .927070 .00040 2.950 .935140 .00040 3.000 .941700 .00040 3.050 .948960 .00040 3.100 .955010 .00040 3.150 .961920 .00040 3.200 .969450 .00039 3.250 .975870 .00040 3.300 .981270 .00040 3.350 .988560 .00040 3.400 .993900 .00040 3.451 .000380 .00041 3.501 .006630 .00039 3.551 .013210 .00040 3.601 .018980 .00040
70

2.500 .917310 .00040

2.550 .925180 .00042

2.600 .933390 .00042

2.650 .939610 .00041

2.700 .947630 .00041

2.750 .955200 .00041

2.800 .961990 .00042

2.850 .969930 .00041

2.900 .977500 .00042

2.950 .983510 .00042

3.000 .990210 .00042

3.050 .997420 .00041
80

2.400 .916800 .00041 2.450 .925380 .00042 2.500 .932860 .00042 2.550 .940120 .00041 2.600 .947960 .00042 2.650 .956430 .00042 2.700 .962850 .00042 2.750 .970760 .00044 2.800 .978110 .00041 2.850 .984470 .00042 2.900 .992170 .00042 2.950 .999400 .00042 
3.200 .995910 .00040 3.251 .003830 .00041 3.301 .009280 .00041 3.351 .015440 .00041 3.401 .022060 .00040
3.101 .003610 .00041

3.151 .011020 .00041

3.201 .017110 .00042
3.001 .005250 .00041

3.051 .013160 .00041

3.101 .018860 .00041

90

2.300 .913090 .00042

2.350 .920710 .00041

2.400 .930090 .00042

2.450 .936840 .00043

2.500 .944430 .00043

2.550 .952980 .00042

2.600 .959810 .00041

2.650 .968150 .00042

2.700 .974290 .00042

2.750 .982610 .00043

2.800 .989980 .00041

2.850 .997160 .00042

2.901 .003630 .00042

2.951 .010320 .00044

3.001 .018240 .00042

100

2.300 .923210 .00041

2.350 .931240 .00041

2.400 .939290 .00042

2.450 .946690 .00043

2.500 .954920 .00043

2.550 .961770 .00042

2.600 .970440 .00042

2.650 .976160 .00043

2.700 .984280 .00042

2.750 .991700 .00042

2.800 .999040 .00042

2.851 .006770 .00042

2.901 .013590 .00043

2.951 .020050 .00042

3.001 .026420 .00042

120

2.200 .920800 .00042

2.250 .929040 .00043

2.300 .937370 .00042

2.350 .945180 .00043

2.400 .953080 .00042

2.450 .960360 .00043

2.500 .968880 .00043

2.550 .977000 .00042

2.600 .984100 .00043

2.650 .991460 .00043

2.700 .998360 .00043

2.751 .005950 .00043

2.801 .012360 .00043

2.851 .020210 .00043

2.901 .026650 .00043

130

2.100 .910510 .00041

2.150 .918990 .00043

2.200 .927800 .00043

2.250 .935280 .00042

2.300 .942630 .00044

2.350 .950180 .00042

2.400 .959250 .00042

2.450 .966260 .00042

2.500 .973840 .00043

2.550 .981970 .00042

2.600 .989020 .00044

2.650 .996890 .00043

2.701 .003520 .00043

2.751 .011220 .00043

2.801 .018620 .00042

$\begin{array}{ccc} & 150 \\ 2.10 & 0.91858 & 0.00043 \\ 2.15 & 0.92747 & 0.00042 \\ 2.20 & 0.93524 & 0.00042 \\ 2.25 & 0.94309 & 0.00042 \\ 2.30 & 0.95142 & 0.00043 \\ 2.35 & 0.95928 & 0.00043 \\ 2.40 & 0.96679 & 0.00042 \\ 2.45 & 0.97456 & 0.00041 \\ 2.50 & 0.98159 & 0.00042 \\ 2.55 & 0.98949 & 0.00042 \\ 2.60 & 0.99726 & 0.00043 \\ 2.65 & 1.00388 & 0.00043 \\ 2.70 & 1.01127 & 0.00042 \\ 2.75 & 1.01779 & 0.00042 \\ 2.80 & 1.02507 & 0.00043\end{array}$

200

2.000 .915460 .00042

2.050 .923870 .00042

2.100 .931530 .00044

2.150 .939800 .00043

2.200 .948010 .00042

2.250 .956080 .00043

2.300 .963740 .00042

2.350 .970920 .00042

2.400 .977970 .00044

2.450 .986660 .00043

2.500 .993730 .00044

2.551 .001110 .00043

2.601 .008460 .00043

2.651 .015010 .00044

2.701 .022050 .00042

239

2.000 .921570 .00043

2.050 .929500 .00043

2.100 .936830 .00044

2.150 .945140 .00042
250

2.000 .922090 .00042

2.050 .929850 .00042

2.100 .938420 .00042

2.150 .945720 .00043
359

2.000 .925340 .00043 2.050 .933620 .00043 2.100 .941820 .00044 2.150 .949140 .00044 
2.200 .952980 .00044 2.250 .960600 .00043 2.300 .968000 .00042 2.350 .975430 .00042 2.400 .983190 .00044 2.450 .990250 .00043 2.500 .996970 .00043 2.551 .004970 .00044 2.601 .011470 .00043 2.651 .018330 .00044 2.701 .025600 .00044

478

2.000 .921590 .00043 2.050 .929510 .00044 2.100 .936680 .00043 2.150 .943480 .00044 2.200 .950460 .00044 2.250 .957550 .00044 2.300 .964420 .00043 2.350 .972140 .00043 2.400 .978680 .00043 2.450 .985850 .00044 2.500 .992520 .00045 2.550 .999530 .00044 2.601 .007010 .00045 2.651 .012290 .00043 2.701 .018930 .00043

650

2.200 .919730 .00044 2.250 .925920 .00043 2.300 .932240 .00042 2.350 .938550 .00043 2.400 .945160 .00044 2.450 .951800 .00043 2.500 .957590 .00043 2.550 .964440 .00045 2.600 .970590 .00044 2.650 .976370 .00044 2.700 .982130 .00043 2.750 .988580 .00046 2.800 .995020 .00043 2.850 .999750 .00044 2.901 .005790 .00044
2.200 .952990 .00043

2.250 .961300 .00043

2.300 .969070 .00043

2.350 .975740 .00042

2.400 .983220 .00042

2.450 .990890 .00042

2.500 .998440 .00044

2.551 .004690 .00044

2.601 .012490 .00043

2.651 .018930 .00043

2.701 .026460 .00043
2.200 .956190 .00042

2.250 .963540 .00043

2.300 .971480 .00042

2.350 .977520 .00044

2.400 .984950 .00044

2.450 .992430 .00043

2.500 .999850 .00042

2.551 .006060 .00043

2.601 .012470 .00044 2.651 .018800 .00043 2.701 .026090 .00043
500

2.000 .919630 .00044

2.050 .927610 .00043

2.100 .934410 .00043

2.150 .941010 .00043

2.200 .948970 .00044

2.250 .955050 .00045

2.300 .963300 .00043

2.350 .969800 .00042

2.400 .976200 .00043

2.450 .982990 .00044

2.500 .989410 .00044

2.550 .995920 .00045

2.601 .003630 .00042

2.651 .010400 .00044

2.701 .015530 .00044
600

2.100 .919730 .00042 2.150 .927530 .00044 2.200 .934000 .00043 2.250 .940560 .00043 2.300 .948150 .00043 2.350 .954470 .00043 2.400 .961260 .00044 2.450 .967870 .00044 2.500 .974260 .00044 2.550 .980900 .00043 2.600 .985990 .00044 2.650 .992980 .00044 2.700 .999220 .00044 2.751 .004910 .00045 2.801 .012000 .00044 2.851 .017390 .00042 2.901 .022960 .00045

660

2.200 .915440 .00044

2.250 .921510 .00042

2.300 .928340 .00043

2.350 .934480 .00045

2.400 .941180 .00042

2.450 .946850 .00043

2.500 .952530 .00044

2.550 .959690 .00044

2.600 .964680 .00043

2.650 .971260 .00044

2.700 .977510 .00044

2.750 .982780 .00044

2.800 .989150 .00043

2.850 .995980 .00044

2.901 .000560 .00044
670

2.300 .923330 .00043

2.350 .929530 .00043

2.400 .934900 .00043

2.450 .941580 .00044

2.500 .947950 .00044

2.550 .954120 .00045

2.600 .960100 .00045

2.650 .965830 .00045

2.700 .971770 .00043

2.750 .977300 .00044

2.800 .983850 .00045

2.850 .989250 .00045 2.900 .994520 .00044 2.951 .001260 .00045 3.001 .006710 .00043 
2.951 .012270 .00044 3.001 .016800 .00043

680

2.300 .916990 .00044 2.350 .921990 .00043 2.400 .928470 .00043 2.450 .934370 .00043 2.500 .940340 .00044 2.550 .946410 .00044 2.600 .951440 .00043 2.650 .958480 .00044 2.700 .964450 .00044 2.750 .971230 .00046 2.800 .976080 .00044 2.850 .981740 .00042 2.900 .986720 .00044 2.950 .992930 .00044 3.000 .998720 .00044 3.051 .003960 .00043 3.101 .009700 .00044 3.151 .015020 .00043 3.201 .020000 .00044

Pun46-pl-1000-00

12

12.000 .925330 .00028

12.200 .931000 .00028

12.400 .936520 .00027

12.600 .941880 .00027

12.800 .947260 .00027

13.000 .952410 .00027

13.200 .957430 .00027

13.400 .961960 .00026

13.600 .966860 .00027

13.800 .970670 .00026

14.000 .975580 .00026

14.200 .979330 .00026

14.400 .983740 .00026 14.600 .987380 .00025 14.800 .991620 .00026 15.000 .995070 .00025 15.200 .998700 .00026 15.401 .002180 .00026 15.601 .005710 .00025
2.951 .006610 .00043

3.001 .011720 .00043

3.051 .017080 .00044

3.101 .023410 .00044
3.051 .012110 .00044

3.101 .017490 .00044

698

2.400 .918290 .00045

2.450 .924250 .00042

2.500 .930150 .00043

2.550 .936040 .00044

2.600 .942400 .00044

2.650 .947260 .00042

2.700 .953540 .00043

2.750 .959320 .00044

2.800 .964370 .00044

2.850 .970240 .00045

2.900 .975480 .00043

2.950 .980080 .00044

3.000 .986400 .00044

3.050 .992060 .00044

3.100 .997040 .00044

3.151 .003220 .00045

3.201 .008620 .00044

3.251 .012940 .00045

3.301 .017950 .00045
2.600 .923220 .00043

2.650 .929610 .00044

2.700 .933230 .00043

2.750 .940030 .00044

2.800 .945530 .00043

2.850 .950600 .00045

2.900 .955910 .00043

2.950 .960910 .00044

3.000 .967040 .00044

3.050 .970980 .00043

3.100 .976770 .00044

3.150 .981750 .00046

3.200 .987230 .00044

3.250 .992380 .00044

3.300 .997120 .00045

3.351 .001690 .00045

3.401 .007660 .00044

3.451 .012910 .00045

3.501 .016250 .00044
9.700 .921800 .00032
9.800 .926690 .00032
9.900 .931840 .00032
10.000 .936440 .00032
10.100 .941210 .00032
10.200 .945310 .00032
10.300 .950070 .00031
10.400 .953690 .00031
10.500 .957900 .00031
10.600 .962120 .00031
10.700 .966830 .00031
10.800 .970220 .00031
10.900 .973930 .00030
11.000 .978160 .00031
11.100 .981610 .00032
11.200 .985160 .00031
11.300 .989020 .00031
11.400 .992810 .00030
11.500 .996200 .00030
8.100 .921840 .00037
8.150 .925250 .00036
8.200 .928910 .00037
8.250 .932710 .00036
8.300 .935860 .00037
8.350 .939890 .00035
8.400 .942680 .00036
8.450 .946160 .00037
8.500 .949710 .00036
8.550 .952080 .00035
8.600 .956590 .00036
8.650 .959550 .00035
8.700 .961870 .00036
8.750 .965490 .00034
8.800 .968720 .00036
8.850 .971490 .00036
8.900 .974680 .00036
8.950 .978720 .00035
9.000 .980480 .00036 
15.801 .008940 .00024 16.001 .011930 .00025 16.101 .013700 .00025
11.601 .000030 .00030 11.701 .002710 .00031 11.801 .006130 .00030 11.901 .009980 .00029 12.001 .013100 .00029 12.101 .016160 .00029
9.050 .983740 .00036 9.100 .987160 .00035 9.150 .989650 .00037 9.200 .992990 .00035 9.250 .995840 .00036 9.300 .999030 .00036 9.351 .001360 .00036 9.401 .004300 .00035 9.451 .007000 .00035 9.501 .009980 .00036 9.551 .012680 .00035 9.601 .015240 .00035 9.651 .017120 .00034 9.701 .020270 .00035
25

7.300 .920020 .00039 7.350 .923420 .00039 7.400 .928060 .00039 7.450 .932340 .00039 7.500 .936800 .00040 7.550 .940620 .00038 7.600 .944960 .00039 7.650 .949350 .00039 7.700 .953010 .00039 7.750 .957850 .00039 7.800 .961160 .00039 7.850 .965520 .00037 7.900 .969200 .00039 7.950 .973270 .00040 8.000 .978050 .00039 8.050 .981430 .00038 8.100 .985040 .00040 8.150 .988920 .00039 8.200 .992830 .00038 8.250 .996400 .00038 8.301 .000030 .00039 8.351 .003400 .00037 8.401 .006490 .00038 8.451 .011110 .00038 8.501 .014130 .00038 8.551 .017900 .00039 8.601 .020420 .00039
30

6.800 .914880 .00041

6.850 .919310 .00040

6.900 .924400 .00042

6.950 .929870 .00040

7.000 .934710 .00041

7.050 .939410 .00042

7.100 .944560 .00041

7.150 .949130 .00040

7.200 .953860 .00041

7.250 .958600 .00041

7.300 .962960 .00042

7.350 .967140 .00041

7.400 .972460 .00041

7.450 .976780 .00041

7.500 .980990 .00042

7.550 .986090 .00041

7.600 .989500 .00041

7.650 .994340 .00040

7.700 .998360 .00041

7.751 .003080 .00042

7.801 .007140 .00041

7.851 .010310 .00041

7.901 .015470 .00041

7.951 .018930 .00039 8.001 .023050 .00041
32

6.700 .918270 .00042

6.750 .923510 .00042

6.800 .928090 .00042

6.850 .933210 .00041

6.900 .938070 .00041

6.950 .943560 .00041

7.000 .948410 .00041

7.050 .953670 .00042

7.100 .958390 .00042

7.150 .963770 .00042

7.200 .967800 .00041

7.250 .973360 .00041

7.300 .976980 .00041

7.350 .982180 .00042

7.400 .986720 .00041

7.450 .990570 .00041

7.500 .995600 .00041

7.551 .000120 .00041

7.601 .004680 .00042

7.651 .008210 .00041

7.701 .013290 .00041

7.751 .017160 .00043

7.801 .021860 .00040 
35

6.500 .913730 .00042

6.550 .919310 .00043

6.600 .925310 .00041

6.650 .929990 .00043

6.700 .936120 .00044

6.750 .941800 .00043

6.800 .946260 .00043

6.850 .951090 .00043

6.900 .957200 .00042

6.950 .962020 .00042

7.000 .967120 .00041

7.050 .971130 .00043

7.100 .976930 .00042

7.150 .981660 .00043

7.200 .986020 .00041

7.250 .991210 .00041

7.300 .995420 .00042

7.351 .000220 .00043

7.401 .005440 .00041

7.451 .009800 .00043

7.501 .014180 .00042

7.551 .018890 .00043

7.601 .024130 .00044
40

6.300 .915050 .00043

6.350 .920400 .00043

6.400 .926900 .00044

6.450 .932440 .00043

6.500 .937990 .00044

6.550 .944350 .00044

6.600 .949000 .00045

6.650 .955640 .00044

6.700 .961230 .00044

6.750 .965940 .00042

6.800 .971550 .00043

6.850 .976420 .00045

6.900 .981860 .00044

6.950 .987070 .00044

7.000 .991800 .00043

7.050 .997520 .00043

7.101 .002270 .00045

7.151 .007450 .00044

7.201 .011670 .00043

7.251 .016710 .00044

7.301 .021900 .00045
50

6.000 .911780 .00045

6.050 .918870 .00045

6.100 .925460 .00046

6.150 .931080 .00046

6.200 .937570 .00044

6.250 .943510 .00046

6.300 .950570 .00045

6.350 .956050 .00045

6.400 .961830 .00046

6.450 .968070 .00044

6.500 .973680 .00045

6.550 .979690 .00045

6.600 .985170 .00045

6.650 .990500 .00045

6.700 .996720 .00044

6.751 .001600 .00045

6.801 .007350 .00045

6.851 .012750 .00045

6.901 .018300 .00044

6.951 .023410 .00045

7.001 .028900 .00044
60

5.900 .921340 .00045

5.950 .927700 .00045

6.000 .934440 .00045

6.050 .940600 .00047

6.100 .947260 .00046

6.150 .953630 .00045

6.200 .960080 .00045

6.250 .966230 .00046

6.300 .973240 .00047

6.350 .979390 .00047

6.400 .984990 .00046

6.450 .990020 .00046

6.500 .997110 .00045

6.551 .002490 .00046

6.601 .007850 .00046

6.651 .013850 .00047

6.701 .019800 .00046

6.751 .025660 .00046 6.801 .030040 .00046
70

5.700 .908350 .00047

5.750 .915680 .00047

5.800 .922020 .00047

5.850 .929280 .00047

5.900 .935810 .00047

5.950 .942540 .00047

6.000 .949920 .00046

6.050 .956190 .00047

6.100 .962720 .00048

6.150 .968320 .00048

6.200 .975220 .00046

6.250 .981820 .00047

6.300 .987630 .00047

6.350 .993470 .00048

6.400 .999940 .00047

6.451 .004520 .00047

6.501 .011930 .00047

6.551 .018090 .00047

6.601 .022960 .00047
80

5.700 .919840 .00047

5.750 .925580 .00046

5.800 .932750 .00045

5.850 .940140 .00047

5.900 .947000 .00049

5.950 .953110 .00048

6.000 .959910 .00048

6.050 .966740 .00047

6.100 .972680 .00046

6.150 .979720 .00048

6.200 .986130 .00047

6.250 .992770 .00048

6.300 .999090 .00048

6.351 .004810 .00047

6.401 .011320 .00048

6.451 .016650 .00047

6.501 .022700 .00047

6.551 .028230 .00047

6.601 .035030 .00047 
90

5.600 .912340 .00048 5.650 .920080 .00048 5.700 .927370 .00047 5.750 .934150 .00047 5.800 .940500 .00048 5.850 .947370 .00047 5.900 .954400 .00046 5.950 .960780 .00047 6.000 .968230 .00049 6.050 .974460 .00046 6.100 .980230 .00049 6.150 .987560 .00047 6.200 .993930 .00048 6.251 .000060 .00047 6.301 .006850 .00048 6.351 .012540 .00048 6.401 .017600 .00047 6.451 .025410 .00046 6.501 .030830 .00047

130

5.500 .912240 .00048 5.550 .918020 .00049 5.600 .927250 .00049 5.650 .933290 .00048 5.700 .940710 .00049 5.750 .947270 .00048 5.800 .955230 .00047 5.850 .961830 .00048 5.900 .968200 .00048 5.950 .975450 .00049 6.000 .982280 .00048 6.050 .989040 .00048 6.100 .995260 .00049 6.151 .001440 .00048 6.201 .007910 .00049 6.251 .013660 .00049 6.301 .019850 .00048 6.351 .026630 .00049 6.401 .032900 .00049

239

5.500 .908170 .00049 5.550 .917010 .00047 5.600 .923810 .00049 5.650 .931990 .00048
100

5.500 .903320 .00047

5.550 .911030 .00048

5.600 .918860 .00047

5.650 .925520 .00048

5.700 .932530 .00048

5.750 .939820 .00047

5.800 .946840 .00047

5.850 .953700 .00046

5.900 .960380 .00048

5.950 .967000 .00046

6.000 .973540 .00048

6.050 .979820 .00049

6.100 .986440 .00048

6.150 .993840 .00048

6.200 .999550 .00048

6.251 .006240 .00048

6.301 .011980 .00048

6.351 .018550 .00047

6.401 .024450 .00047
120

5.500 .909940 .00048

5.550 .916800 .00050

5.600 .924420 .00047

5.650 .931210 .00049

5.700 .938320 .00048

5.750 .945670 .00048

5.800 .952750 .00049

5.850 .960000 .00047

5.900 .966270 .00047

5.950 .973250 .00048

6.000 .980560 .00048

6.050 .986160 .00047

6.100 .992720 .00048

6.150 .999290 .00049

6.201 .005530 .00049

6.251 .012250 .00049

6.301 .018740 .00048

6.351 .024690 .00048

6.401 .030600 .00049
5.400 .899100 .00049

5.450 .906090 .00048

5.500 .913890 .00049

5.550 .920970 .00049

5.600 .928230 .00049

5.650 .935020 .00049

5.700 .942660 .00049

5.750 .950100 .00048

5.800 .956670 .00049

5.850 .963910 .00049

5.900 .970640 .00048

5.950 .977620 .00048

6.000 .984230 .00049

6.050 .991350 .00049

6.100 .997980 .00048

6.151 .004480 .00048

6.201 .010710 .00048

6.251 .016750 .00048

6.301 .022540 .00048
200

5.500 .913160 .00049

5.550 .919680 .00050

5.600 .927620 .00048

5.650 .934720 .00048

5.700 .941960 .00048

5.750 .948430 .00049

5.800 .955510 .00049

5.850 .963140 .00049

5.900 .969320 .00049

5.950 .977130 .00049

6.000 .982860 .00050

6.050 .989420 .00049

6.100 .995910 .00048

6.151 .003280 .00050

6.201 .009660 .00048

6.251 .016180 .00051

6.301 .021950 .00047

6.351 .028420 .00050

6.401 .033950 .00049
250

5.500 .908090 .00049

5.550 .914960 .00049

5.600 .923570 .00050

5.650 .929660 .00048
359

5.600 .909090 .00048 5.650 .916420 .00049 5.700 .922840 .00050 5.750 .929220 .00050 
5.700 .939100 .00048 5.750 .945280 .00048 5.800 .952570 .00048 5.850 .960090 .00050 5.900 .966670 .00049 5.950 .973290 .00047 6.000 .979000 .00048 6.050 .986480 .00050 6.100 .992970 .00048 6.150 .999700 .00049 6.201 .004970 .00048 6.251 .011750 .00049 6.301 .018620 .00049 6.351 .024490 .00049 6.401 .030270 .00048

478

5.700 .908540 .00049 5.750 .914670 .00049 5.800 .921510 .00048 5.850 .928730 .00048 5.900 .935340 .00048 5.950 .941980 .00049 6.000 .949460 .00048 6.050 .956090 .00047 6.100 .962230 .00048 6.200 .976180 .00051 6.250 .982240 .00048 6.300 .987920 .00048 6.350 .994350 .00049 6.401 .000710 .00048 6.451 .007410 .00049 6.501 .013370 .00048 6.551 .019490 .00048 6.601 .026080 .00048

\section{0}

6.000 .915500 .00049 6.050 .922680 .00046 6.100 .929640 .00049 6.150 .935610 .00049 6.200 .943090 .00048 6.250 .949120 .00049 6.300 .955830 .00049 6.350 .962180 .00049 6.400 .968780 .00048
5.700 .936820 .00048

5.750 .944280 .00048

5.800 .951540 .00048

5.850 .958480 .00049

5.900 .965250 .00048

5.950 .972620 .00048

6.000 .979180 .00048

6.050 .985230 .00049

6.100 .991800 .00049

6.150 .998060 .00049

6.201 .004640 .00050

6.251 .010330 .00050

6.301 .016900 .00050

6.351 .023380 .00050

6.401 .029740 .00048
5.800 .937190 .00049

5.850 .943770 .00049

5.900 .950600 .00048

5.950 .957590 .00049

6.000 .964590 .00048

6.050 .971320 .00046

6.100 .978380 .00051

6.150 .982920 .00048

6.200 .990990 .00049

6.250 .996550 .00047

6.301 .003110 .00050

6.351 .009560 .00049

6.401 .016100 .00050

6.451 .021830 .00049

6.501 .027950 .00048

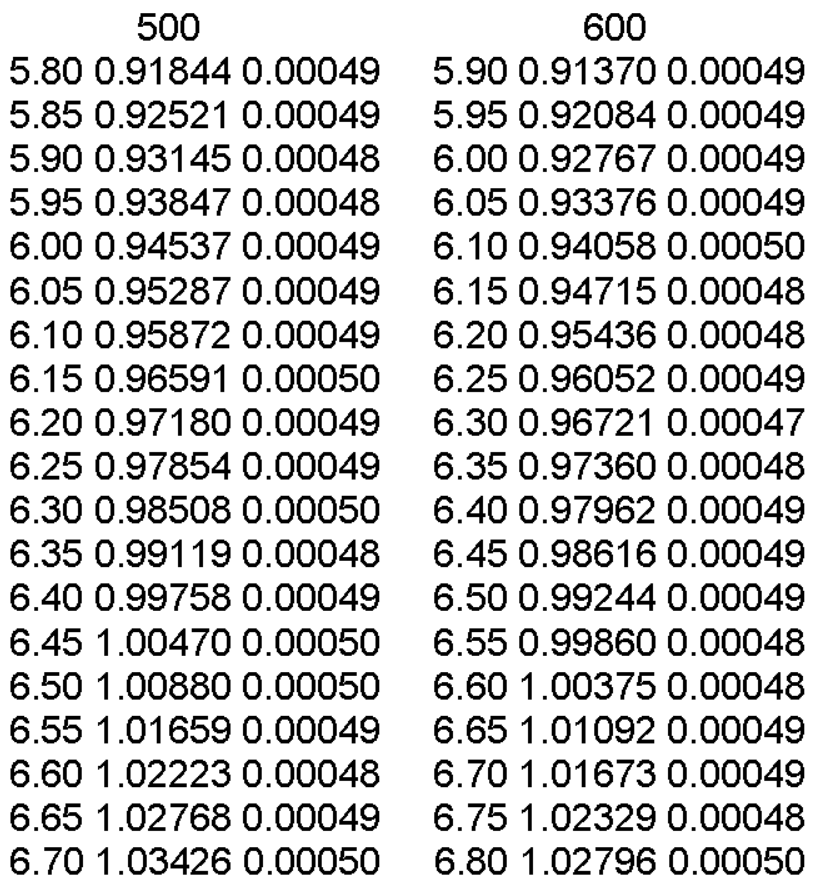

700

6.100 .913510 .00048

6.150 .920580 .00049

6.200 .926640 .00049

6.250 .933090 .00047

6.300 .939360 .00049

6.350 .946570 .00049

6.400 .952040 .00049

6.450 .959800 .00049

6.500 .965520 .00048
750

6.300 .918700 .00047 6.350 .925130 .00049 6.400 .931450 .00048 6.450 .938150 .00046 6.500 .943950 .00048 6.550 .951310 .00049 6.600 .956860 .00049 6.650 .962460 .00047 6.700 .970180 .00049 
6.450 .974650 .00048

6.500 .981880 .00048

6.550 .987320 .00048

6.600 .993630 .00049

6.650 .999400 .00049

6.701 .006020 .00050

6.751 .011470 .00050

6.801 .018050 .00046

6.851 .022860 .00047

6.901 .029740 .00048

760

6.300 .913790 .00048 6.350 .920470 .00048

6.400 .926270 .00048

6.450 .933590 .00048

6.500 .940060 .00050

6.550 .945480 .00048

6.600 .952560 .00047

6.650 .959260 .00048

6.700 .964980 .00048

6.750 .970810 .00048

6.800 .977160 .00047

6.850 .982860 .00048

6.900 .988510 .00050

6.950 .994730 .00049

7.000 .999880 .00048

7.051 .005750 .00050

7.101 .012240 .00049

7.151 .017450 .00049

7.201 .023820 .00048

\section{5}

6.400 .919090 .00050

6.450 .925020 .00048

6.500 .932390 .00049

6.550 .937510 .00047

6.600 .944780 .00049

6.650 .950370 .00048

6.700 .956890 .00046

6.750 .963360 .00049

6.800 .968790 .00049

6.850 .974050 .00048

6.900 .981600 .00049

6.950 .986490 .00049

7.000 .992790 .00048

7.050 .997760 .00048
6.550 .972950 .00048

6.600 .977860 .00047

6.650 .983920 .00049

6.700 .990590 .00049

6.750 .996380 .00047

6.801 .002290 .00048

6.851 .008530 .00049

6.901 .014230 .00049

6.951 .019390 .00050

7.001 .026140 .00049
6.750 .976640 .00048

6.800 .981900 .00049

6.850 .986980 .00049

6.900 .994540 .00048

6.950 .998240 .00048

7.001 .005600 .00047

7.051 .010470 .00048

7.101 .017110 .00048

7.151 .022670 .00049

7.201 .027720 .00049
765

6.400 .923900 .00048

6.450 .931120 .00048

6.500 .936940 .00048

6.550 .942690 .00048

6.600 .949200 .00048

6.650 .955570 .00047

6.700 .961350 .00047

6.750 .967950 .00049

6.800 .974570 .00049

6.850 .979760 .00048

6.900 .986690 .00049

6.950 .992390 .00049

7.000 .997500 .00050

7.051 .003320 .00048

7.101 .009650 .00049

7.151 .016000 .00049

7.201 .020000 .00050
770

6.400 .920840 .00048

6.450 .927980 .00049

6.500 .934150 .00049

6.550 .940730 .00048

6.600 .946940 .00047

6.650 .953140 .00047

6.700 .959060 .00048

6.750 .965360 .00048

6.800 .970220 .00050

6.850 .977390 .00048

6.900 .982470 .00049

6.950 .988840 .00048

7.000 .994810 .00049

7.051 .000910 .00048

7.101 .006470 .00049

7.151 .011720 .00049

7.201 .017300 .00049

7.251 .023290 .00049

7.301 .028930 .00048 
7.101 .004830 .00048

7.151 .010900 .00049

7.201 .016050 .00048

7.251 .021090 .00048

7.301 .026520 .00049

Pun46-pl-1000-01

12

10.400 .924560 .00027

10.600 .930580 .00027

10.800 .936050 .00027

11.000 .941660 .00026

11.200 .946480 .00026

11.400 .952190 .00026

11.600 .956720 .00026

11.800 .962150 .00027

12.000 .966090 .00026

12.200 .970560 .00025

12.400 .974880 .00026

12.600 .979550 .00025

12.800 .983270 .00026

13.000 .987340 .00025

13.200 .991110 .00025

13.400 .994700 .00024

13.600 .998370 .00025

13.801 .002450 .00024

14.001 .005430 .00024

14.201 .008610 .00025

14.401 .012260 .00025

14.501 .013130 .00024

25

5.700 .919980 .00038

5.750 .923180 .00037

5.800 .927560 .00038

5.850 .932550 .00037

5.900 .936470 .00038

5.950 .940560 .00038
15

8.200 .926740 .00031

8.300 .931170 .00030

8.400 .935880 .00032

8.500 .940620 .00030

8.600 .944330 .00030

8.700 .949010 .00031

8.800 .953820 .00030

8.900 .957550 .00030

9.000 .961490 .00031

9.100 .965730 .00030

9.200 .969720 .00030

9.300 .973390 .00031

9.400 .977400 .00029

9.500 .981420 .00030

9.600 .985130 .00030

9.700 .988840 .00030

9.800 .992140 .00030

9.900 .995800 .00029

10.000 .999130 .00029

10.101 .002720 .00030

10.201 .006250 .00029

10.301 .009040 .00028

10.401 .012430 .00029

10.501 .015680 .00029

30

5.300 .924440 .00039

5.350 .929430 .00039

5.400 .934900 .00040

5.450 .939530 .00041

5.500 .944260 .00040

5.550 .949700 .00040
20

6.500 .921680 .00036

6.550 .923920 .00035

6.600 .928780 .00034

6.650 .932140 .00035

6.700 .935810 .00035

6.750 .938020 .00035

6.800 .942550 .00035

6.850 .946310 .00034

6.900 .948920 .00035

6.950 .952170 .00034

7.000 .955220 .00035

7.050 .959040 .00034

7.100 .961980 .00034

7.150 .965220 .00035

7.200 .968070 .00035

7.250 .971420 .00034

7.300 .974340 .00034

7.350 .977720 .00034

7.400 .980720 .00033

7.450 .983690 .00034

7.500 .986380 .00035

7.550 .989430 .00034

7.600 .993100 .00035

7.650 .995720 .00035

7.700 .998150 .00034

7.751 .000330 .00034

7.801 .003830 .00034

7.851 .006360 .00034

7.901 .008610 .00034

7.951 .012450 .00034

8.001 .015080 .00034

32

5.100 .917190 .00040

5.150 .923580 .00040

5.200 .928540 .00040

5.250 .933560 .00040

5.300 .939900 .00040

5.350 .943180 .00041 
6.000 .945410 .00038 6.050 .948800 .00037 6.100 .953500 .00038 6.150 .957710 .00037 6.200 .961480 .00037 6.250 .965090 .00037 6.300 .969440 .00037 6.350 .973240 .00038 6.400 .977170 .00039 6.450 .980780 .00037 6.500 .984660 .00037 6.550 .988320 .00037 6.600 .992850 .00038 6.650 .995620 .00036 6.700 .999380 .00037 6.751 .003280 .00037 6.801 .007100 .00038 6.851 .010310 .00038 6.901 .013860 .00037 6.951 .016920 .00037 7.001 .020940 .00037

\section{5}

4.900 .914370 .00041 4.950 .919780 .00041 5.000 .925880 .00040 5.050 .931380 .00041 5.100 .936400 .00040 5.150 .940850 .00041 5.200 .946610 .00040 5.250 .952930 .00042 5.300 .957930 .00041 5.350 .962440 .00040 5.400 .967730 .00041 5.450 .972550 .00041 5.500 .977080 .00041 5.550 .981870 .00041 5.600 .987050 .00040 5.650 .992690 .00041 5.700 .997140 .00041 5.751 .002510 .00041 5.801 .005510 .00040 5.851 .010820 .00040 5.901 .015320 .00039 5.951 .019620 .00041 6.001 .024160 .00041
5.600 .953440 .00039

5.650 .959230 .00040

5.700 .963280 .00039

5.750 .967840 .00039

5.800 .972020 .00039

5.850 .977130 .00038

5.900 .981320 .00040

5.950 .986260 .00039

6.000 .990290 .00039

6.050 .994160 .00040

6.100 .998360 .00039

6.151 .002930 .00040

6.201 .007010 .00039

6.251 .010530 .00039

6.301 .015450 .00039

6.351 .019190 .00039

6.401 .022800 .00039
5.400 .948490 .00041 5.450 .954340 .00039 5.500 .958890 .00040 5.550 .963990 .00040 5.600 .967300 .00040 5.650 .973300 .00040 5.700 .978270 .00041 5.750 .982460 .00040 5.800 .986440 .00040 5.850 .991480 .00040 5.900 .995780 .00041 5.951 .000090 .00040 6.001 .004010 .00039 6.051 .009660 .00039 6.101 .013990 .00041 6.151 .017280 .00039 6.201 .021660 .00040
4.700 .916070 .00042
50
4.750 .922140 .00042 4.800 .927690 .00042 4.850 .933840 .00043 4.900 .939770 .00042 4.950 .945170 .00041 5.000 .950260 .00042 5.050 .956230 .00041 5.100 .962520 .00041 5.150 .966450 .00042 5.200 .972180 .00042 5.250 .977610 .00042 5.300 .982800 .00042 5.350 .987580 .00041 5.400 .993160 .00041 5.450 .998040 .00042 5.501 .002800 .00040 $\begin{array}{lll}4.50 & 0.92763 & 0.00044 \\ 4.55 & 0.93307 & 0.00044 \\ 4.60 & 0.94012 & 0.00043 \\ 4.65 & 0.94571 & 0.00044 \\ 4.70 & 0.95159 & 0.00043 \\ 4.75 & 0.95712 & 0.00042 \\ 4.80 & 0.96410 & 0.00044 \\ 4.85 & 0.96932 & 0.00044 \\ 4.90 & 0.97605 & 0.00044 \\ 4.95 & 0.98121 & 0.00043 \\ 5.00 & 0.98725 & 0.00043 \\ 5.05 & 0.99237 & 0.00043 \\ 5.10 & 0.99802 & 0.00042 \\ 5.15 & 1.00365 & 0.00043 \\ 5.20 & 1.00926 & 0.00045 \\ 5.25 & 1.01473 & 0.00043 \\ 5.30 & 1.01973 & 0.00042\end{array}$ 5.551 .007890 .00042 5.601 .013200 .00043 5.651 .018200 .00041 5.701 .022660 .00042 
60

4.300 .924790 .00044 4.350 .931190 .00044 4.400 .937640 .00044 4.450 .944610 .00045 4.500 .951280 .00045 4.550 .957250 .00045 4.600 .962820 .00045 4.650 .969080 .00045 4.700 .975300 .00044 4.750 .981510 .00044 4.800 .987920 .00044 4.850 .993110 .00045 4.900 .999130 .00043 4.951 .005720 .00045 5.001 .010200 .00044 5.051 .016850 .00044 5.101 .021340 .00044

\section{0}

4.000 .918540 .00045 4.050 .925980 .00046 4.100 .933760 .00045 4.150 .939180 .00046 4.200 .946820 .00046 4.250 .954310 .00046 4.300 .959850 .00047 4.350 .967200 .00047 4.400 .974100 .00046 4.450 .979560 .00047 4.500 .986070 .00046 4.550 .992910 .00047 4.600 .999190 .00046 4.651 .005790 .00047 4.701 .011680 .00045 4.751 .018440 .00045 4.801 .023870 .00046

130

3.900 .921230 .00047 3.950 .927980 .00047 4.000 .935400 .00046 4.050 .942060 .00048 4.100 .949180 .00047 4.150 .956200 .00046
70

4.200 .927040 .00044

4.250 .933550 .00045

4.300 .941190 .00045

4.350 .946790 .00044

4.400 .953090 .00045

4.450 .959990 .00045

4.500 .966620 .00044

4.550 .972060 .00045

4.600 .978730 .00046

4.650 .984930 .00045

4.700 .992110 .00045

4.750 .997880 .00044

4.801 .003490 .00044

4.851 .009000 .00046

4.901 .014970 .00046

4.951 .020350 .00044

5.001 .026190 .00045
80

4.100 .924380 .00045

4.150 .931150 .00046

4.200 .938290 .00044

4.250 .945230 .00045

4.300 .951890 .00045

4.350 .957930 .00046

4.400 .965470 .00047

4.450 .971510 .00046

4.500 .977990 .00045

4.550 .984410 .00045

4.600 .990920 .00045

4.650 .997500 .00045

4.701 .003840 .00046

4.751 .009620 .00045

4.801 .015520 .00045

4.851 .021290 .00045

4.901 .027110 .00045
100

3.900 .910730 .00045

3.950 .918430 .00046

4.000 .924860 .00046

4.050 .931890 .00046

4.100 .939410 .00046

4.150 .946180 .00046

4.200 .953420 .00047

4.250 .960030 .00046

4.300 .967300 .00046

4.350 .973270 .00048

4.400 .979570 .00046

4.450 .986340 .00046

4.500 .992550 .00046

4.550 .998970 .00047

4.601 .005180 .00047

4.651 .012290 .00046

4.701 .018750 .00046

4.751 .024000 .00045

4.801 .029670 .00046
120

3.900 .917780 .00047 3.950 .925710 .00048 4.000 .932330 .00046 4.050 .939630 .00046 4.100 .946500 .00048 4.150 .953310 .00047 4.200 .961140 .00048 4.250 .967220 .00046 4.300 .974370 .00047 4.350 .981190 .00047 4.400 .987420 .00045 4.450 .993710 .00045 4.500 .999470 .00046 4.551 .007860 .00047 4.601 .012420 .00046 4.651 .019470 .00046 4.701 .025390 .00047
150

3.900 .924060 .00047 3.950 .930510 .00047 4.000 .938640 .00046 4.050 .945320 .00046 4.100 .952840 .00048 4.150 .959820 .00047
200

3.800 .911920 .00046 3.850 .919000 .00047 3.900 .925340 .00046 3.950 .932480 .00048 4.000 .940100 .00046 4.050 .947960 .00047 
4.200 .962950 .00047

4.250 .970540 .00047

4.300 .976860 .00047

4.350 .983570 .00047

4.400 .990210 .00046

4.450 .996480 .00046

4.501 .003230 .00046

4.551 .009270 .00048

4.601 .015970 .00047

4.651 .022070 .00047

4.701 .028480 .00046

239

3.900 .923550 .00048

3.950 .930900 .00047

4.000 .937800 .00047

4.050 .945370 .00048

4.100 .951920 .00047

4.150 .958800 .00048

4.200 .965230 .00047

4.250 .972710 .00046

4.300 .979320 .00048

4.350 .984960 .00047

4.400 .992640 .00048

4.450 .998740 .00048

4.501 .004920 .00048

4.551 .011160 .00047

4.601 .017190 .00047

4.651 .024280 .00047

4.701 .030320 .00047

\section{8}

4.000 .913440 .00048

4.050 .920430 .00047

4.100 .927290 .00048

4.150 .934430 .00048

4.200 .941400 .00047

4.250 .947740 .00048

4.300 .954330 .00048

4.350 .959760 .00048

4.400 .966750 .00049

4.450 .973370 .00048

4.500 .979290 .00047

4.550 .987030 .00048

4.600 .991980 .00047
4.200 .966820 .00047

4.250 .973240 .00048

4.300 .978790 .00047

4.350 .986290 .00047

4.400 .993160 .00046

4.450 .999620 .00047

4.501 .006510 .00048

4.551 .012430 .00048

4.601 .018830 .00047

4.651 .025020 .00046

4.701 .030250 .00046
4.100 .953900 .00046

4.150 .961140 .00047

4.200 .968180 .00046

4.250 .974520 .00047

4.300 .980400 .00047

4.350 .988280 .00047

4.400 .993820 .00047

4.451 .001290 .00047

4.501 .007270 .00046

4.551 .013620 .00048

4.601 .019980 .00047
250

3.950 .929260 .00048

4.000 .936650 .00048

4.050 .943350 .00048

4.100 .951050 .00049

4.150 .958230 .00047

4.200 .965110 .00047

4.250 .971250 .00048

4.300 .978450 .00047

4.350 .984820 .00048

4.400 .991720 .00047

4.450 .997410 .00050

4.501 .003120 .00048

4.551 .011070 .00048

4.601 .016420 .00048

4.651 .022820 .00048

4.701 .029030 .00047
3.900 .922180 .00047

359

3.900 .911730 .00048

3.950 .918320 .00049

4.000 .925600 .00047

4.050 .933110 .00048

4.100 .939130 .00047

4.150 .947020 .00049

4.200 .953910 .00048

4.250 .960200 .00046

4.300 .967090 .00048

4.350 .973120 .00048

4.400 .979510 .00049

4.450 .986400 .00048

4.500 .993080 .00048

4.550 .998220 .00047

4.601 .005950 .00048

4.651 .011240 .00046

4.701 .016830 .00047

4.751 .023420 .00048

4.801 .029290 .00048

500

4.100 .924690 .00048

4.150 .931400 .00047

4.200 .938200 .00049

4.250 .944720 .00048

4.300 .951870 .00048

4.350 .957430 .00048

4.400 .965030 .00048

4.450 .970400 .00049

4.500 .977080 .00048

4.550 .983260 .00049

4.600 .988720 .00049

4.650 .995760 .00048

4.701 .001260 .00048
600

4.200 .923520 .00047

4.250 .929710 .00047

4.350 .942070 .00046

4.400 .948840 .00049

4.450 .954890 .00048

4.500 .961590 .00048

4.550 .968220 .00048

4.600 .974220 .00049

4.650 .979630 .00049

4.700 .987490 .00049

4.750 .992410 .00048

4.800 .998680 .00048

4.851 .004040 .00047 
4.650 .998670 .00048 4.701 .004670 .00048 4.751 .010570 .00047 4.801 .017150 .00047 4.851 .022650 .00047 4.901 .028490 .00047

\section{0}

4.300 .926820 .00048 4.350 .932910 .00048 4.400 .940460 .00047 4.450 .945410 .00049 4.500 .952760 .00049 4.550 .958310 .00048 4.600 .965110 .00049 4.650 .970650 .00049 4.700 .977790 .00048 4.750 .983020 .00048 4.800 .990260 .00048 4.850 .995700 .00048 4.901 .001560 .00048 4.951 .006230 .00047 5.001 .013450 .00049 5.051 .018750 .00048 5.101 .024640 .00049

760

4.500 .919620 .00046 4.550 .925410 .00047 4.600 .932290 .00048 4.650 .938340 .00047 4.700 .943680 .00049 4.750 .950940 .00048 4.800 .956610 .00047 4.850 .962850 .00048 4.900 .968810 .00049 4.950 .975340 .00048 5.000 .980630 .00047 5.050 .986140 .00048 5.100 .991650 .00048 5.150 .997480 .00048 5.201 .003070 .00048 5.251 .008690 .00048 5.301 .015020 .00049 5.351 .020520 .00049 $\begin{array}{lll}5.40 & 1.02548 & 0.00049\end{array}$
4.751 .006830 .00048 4.801 .014160 .00048 4.851 .020270 .00048 4.901 .025080 .00049
4.901 .010660 .00049 4.951 .017420 .00048 5.001 .021920 .00048
4.300 .913680 .00048 4.350 .919810 .00048 4.400 .927010 .00049 4.450 .933030 .00049 4.500 .939450 .00048 4.550 .945600 .00048 4.600 .952630 .00048 4.650 .958820 .00048 4.700 .965400 .00047 4.750 .970020 .00047 4.800 .976610 .00050 4.850 .982660 .00049 4.900 .988940 .00049 4.950 .995140 .00047 5.001 .001160 .00049 5.051 .005670 .00047 5.101 .012070 .00048 5.151 .017220 .00049 5.201 .022820 .00048

\section{5}

4.500 .917330 .00047 4.550 .924140 .00048 4.600 .930350 .00048 4.650 .935060 .00047 4.700 .942180 .00049 4.750 .948990 .00048 4.800 .954440 .00049 4.850 .960020 .00048 4.900 .966120 .00048 4.950 .971760 .00049 5.000 .978180 .00048 5.050 .983550 .00049 5.100 .989200 .00048 5.150 .995830 .00047 5.201 .001300 .00049 5.251 .006330 .00050 5.301 .011550 .00049 5.351 .017460 .00048 5.401 .022850 .00048
750

4.500 .923160 .00047 4.550 .929430 .00048 4.600 .936360 .00047 4.650 .942020 .00048 4.700 .947590 .00048 4.750 .954700 .00047 4.800 .960480 .00048 4.850 .966030 .00049 4.900 .971810 .00048 4.950 .978560 .00047 5.000 .984440 .00049 5.050 .989790 .00048 5.100 .995600 .00048 5.151 .000950 .00049 5.201 .006770 .00048 5.251 .012820 .00048 5.301 .017510 .00048

\section{0}

4.500 .914980 .00048 4.550 .921810 .00047 4.600 .927550 .00047 4.650 .933140 .00047 4.700 .939730 .00048 4.750 .946110 .00047 4.800 .951940 .00048 4.850 .957890 .00050 4.900 .964560 .00047 4.950 .970150 .00047 5.000 .975480 .00048 5.050 .981650 .00049 5.100 .987550 .00049 5.150 .992640 .00050 5.200 .999330 .00048 5.251 .003130 .00049 5.301 .009670 .00048 5.351 .014840 .00050 5.401 .020020 .00048 5.451 .026230 .00049 5.501 .031300 .00049 


\section{5}

4.600 .925100 .00049

4.650 .932780 .00048

4.700 .938140 .00048

4.750 .943890 .00048

4.800 .950000 .00048

4.850 .956560 .00047

4.900 .961970 .00049

4.950 .968060 .00048

5.000 .974170 .00048

5.050 .980110 .00049

5.100 .986240 .00048

5.150 .991010 .00047

5.200 .996620 .00047

5.251 .002130 .00048

5.301 .008320 .00049

5.351 .012850 .00047

5.401 .018390 .00048
0 
6.551 .004020 .00032

6.601 .006600 .00031

6.651 .008920 .00032

6.701 .012420 .00032

6.751 .015020 .00031

6.801 .016890 .00032

25

4.500 .924020 .00035 4.550 .928700 .00035 4.600 .933250 .00034 4.650 .937120 .00035 4.700 .942150 .00034 4.750 .945970 .00034 4.800 .949950 .00035 4.850 .954510 .00035 4.900 .958610 .00034 4.950 .962160 .00035 5.000 .966720 .00034 5.050 .971050 .00035 5.100 .974270 .00034 5.150 .979140 .00034 5.200 .982010 .00034 5.250 .986840 .00034 5.300 .991100 .00034 5.350 .993730 .00035 5.400 .997510 .00034 5.451 .001290 .00034 5.501 .004980 .00034 5.551 .008880 .00034 5.601 .012040 .00035 5.651 .015610 .00033 5.701 .019760 .00034

35

3.700 .925710 .00037 3.750 .931350 .00037 3.800 .936390 .00037 3.850 .941700 .00038 3.900 .946840 .00038 3.950 .952200 .00038 4.000 .958500 .00039 4.050 .962740 .00039 4.100 .968990 .00037 4.150 .973340 .00037 4.200 .978850 .00037 4.250 .982940 .00037
30

4.000 .922540 .00037 4.050 .927540 .00036

4.100 .932760 .00036

4.150 .937670 .00037

4.200 .942800 .00037

4.250 .948240 .00035

4.300 .952610 .00036

4.350 .957570 .00035

4.400 .961810 .00036

4.450 .967020 .00036

4.500 .971010 .00036

4.550 .976080 .00037

4.600 .981120 .00036

4.650 .985180 .00035

4.700 .989810 .00036

4.750 .993780 .00036 4.800 .998560 .00037 4.851 .001930 .00035 4.901 .006740 .00036 4.951 .010990 .00036 5.001 .015380 .00036
32

3.800 .916610 .00037 3.850 .922160 .00036 3.900 .928530 .00037 3.950 .932950 .00037 4.000 .937960 .00037 4.050 .942890 .00037 4.100 .948700 .00037 4.150 .953470 .00037 4.200 .958040 .00036 4.250 .962830 .00036 4.300 .967720 .00037 4.350 .973050 .00038 4.400 .977910 .00038 4.450 .982880 .00036 4.500 .986660 .00037 4.550 .991780 .00036 4.600 .996430 .00036 4.651 .001560 .00036 4.701 .005570 .00037 4.751 .009690 .00037 4.801 .014290 .00037 4.851 .017770 .00036 4.901 .022210 .00037
40

3.400 .918900 .00037

3.450 .923910 .00038

3.500 .930720 .00039

3.550 .936930 .00040

3.600 .942010 .00039

3.650 .948310 .00039

3.700 .954060 .00038

3.750 .959220 .00038

3.800 .964260 .00038

3.850 .970840 .00038

3.900 .976100 .00039

3.950 .981140 .00039
50

3.100 .921430 .00040 3.150 .927910 .00040 3.200 .934800 .00039 3.250 .940850 .00038 3.300 .947930 .00039 3.350 .953730 .00040 3.400 .960090 .00040 3.450 .966070 .00040 3.500 .972160 .00040 3.550 .978010 .00039 3.600 .983410 .00040 3.650 .989190 .00039 
4.300 .988580 .00037 4.350 .992920 .00038 4.400 .998270 .00037 4.451 .002950 .00037 4.501 .007620 .00037 4.551 .012060 .00038 4.601 .017030 .00037

60

2.900 .923300 .00040 2.950 .929920 .00040 3.000 .936440 .00040 3.050 .943520 .00040 3.100 .950210 .00040 3.150 .956540 .00040 3.200 .962960 .00040 3.250 .969500 .00041 3.300 .975390 .00040 3.350 .981060 .00041 3.400 .987680 .00040 3.450 .994250 .00041 3.501 .000160 .00040 3.551 .005610 .00039 3.601 .012010 .00041 3.651 .017180 .00040 3.701 .023260 .00040

90

2.600 .928180 .00042

2.650 .936470 .00042

2.700 .942940 .00041

2.750 .950340 .00042

2.800 .956750 .00042

2.850 .964360 .00042

2.900 .971460 .00041

2.950 .976690 .00043 3.000 .984250 .00042 3.050 .990090 .00042 3.100 .997200 .00041 3.151 .004520 .00042 3.201 .009600 .00042 3.251 .016450 .00042 3.301 .021920 .00043
4.000 .986650 .00038 4.050 .992270 .00039 4.100 .996280 .00038 4.151 .001940 .00038 4.201 .007470 .00038 4.251 .012280 .00039 4.301 .017000 .00038 4.351 .021890 .00038 4.401 .027040 .00038
3.700 .994970 .00039 3.751 .000300 .00039 3.801 .006680 .00040 3.851 .011780 .00040 3.901 .016610 .00040
2.700 .915700 .00041

2.750 .922250 .00041

2.800 .929310 .00041

2.850 .936960 .00041

2.900 .943620 .00041

2.950 .950670 .00041 3.000 .957140 .00041 3.050 .964350 .00040 3.100 .969910 .00041 3.150 .976060 .00042 3.200 .982710 .00041 3.250 .989000 .00042 3.300 .995740 .00041 3.351 .002970 .00041 3.401 .008310 .00041 3.451 .014110 .00041 3.501 .019450 .00042
80

2.600 .916140 .00040 2.650 .923750 .00041 2.700 .930910 .00042 2.750 .937660 .00042 2.800 .945100 .00041 2.850 .951330 .00039 2.900 .958880 .00040 2.950 .965800 .00042 3.000 .971900 .00043 3.050 .979060 .00040 3.100 .985760 .00040 3.150 .992130 .00042 3.200 .998170 .00042 3.251 .004680 .00042 3.301 .011670 .00042 3.351 .017180 .00041 3.401 .023610 .00042
100

2.500 .923010 .00042

2.550 .931440 .00042

2.600 .937800 .00042

2.650 .945470 .00041

2.700 .952740 .00042

2.750 .960070 .00041

2.800 .967030 .00041

2.850 .973750 .00042

2.900 .980150 .00041

2.950 .986930 .00042 3.000 .993550 .00042 3.051 .000310 .00042 3.101 .007210 .00042 3.151 .012700 .00041 3.201 .019720 .00041
120

2.400 .922670 .00042 2.450 .930430 .00043 2.500 .937260 .00042 2.550 .944880 .00042 2.600 .951270 .00042 2.650 .958600 .00042 2.700 .966130 .00042 2.750 .972770 .00042 2.800 .980360 .00042 2.850 .986850 .00042 2.900 .993580 .00043 2.950 .999670 .00041 3.001 .006690 .00043 3.051 .013130 .00043 3.101 .019250 .00042 
130

2.400 .928560 .00042

2.450 .935940 .00042

2.500 .942150 .00043

2.550 .949860 .00042

2.600 .957360 .00045

2.650 .964870 .00042

2.700 .971150 .00043

2.750 .978700 .00042

2.800 .985220 .00041

2.850 .991350 .00043

2.900 .998570 .00042

2.951 .005410 .00041

3.001 .011310 .00042

3.051 .018520 .00041

3.101 .024280 .00043
150

2.300 .921380 .00042

2.350 .929100 .00043

2.400 .935820 .00043

2.450 .943320 .00043

2.500 .950480 .00043

2.550 .957200 .00042

2.600 .965420 .00042

2.650 .971870 .00042

2.700 .979410 .00042

2.750 .986180 .00044

2.800 .991930 .00043

2.851 .000260 .00042

2.901 .005870 .00042

2.951 .012500 .00042

3.001 .018940 .00042
200

2.200 .918120 .00043

2.250 .925930 .00043

2.300 .933060 .00042

2.350 .940100 .00043

2.400 .947660 .00043

2.450 .954360 .00042

2.500 .961570 .00044

2.550 .968400 .00042

2.600 .975630 .00043

2.650 .981870 .00044

2.700 .989350 .00043

2.750 .995460 .00044

2.801 .003300 .00041

2.851 .008820 .00043

2.901 .016030 .00044

239

250

359
2.200 .926870 .00044

2.200 .923040 .00042

2.250 .930450 .00043

2.300 .936960 .00043

2.350 .945320 .00043

2.400 .951540 .00042

2.450 .958850 .00044

2.500 .965610 .00043

2.550 .972980 .00043

2.600 .979590 .00043

2.650 .986100 .00044

2.700 .991870 .00042

2.750 .999020 .00043

2.801 .005880 .00043

2.851 .012100 .00043

2.901 .018800 .00044

2.200 .924180 .00043

2.250 .931250 .00042

2.300 .938050 .00042

2.350 .945140 .00043

2.400 .952360 .00043

2.450 .959590 .00042

2.500 .966830 .00044

2.550 .973310 .00043

2.600 .980600 .00044

2.650 .987190 .00043

2.700 .993820 .00043

2.751 .001020 .00044

2.801 .006100 .00043

2.851 .012670 .00043

2.901 .019360 .00043

2.250 .934610 .00043

2.300 .940560 .00043

2.350 .948200 .00044

2.400 .954590 .00043

2.450 .961640 .00043

2.500 .967930 .00044

2.550 .974840 .00042

2.600 .981500 .00044

2.650 .987370 .00043

2.700 .994800 .00044

2.751 .000610 .00045

2.801 .006730 .00044

2.851 .012220 .00043

2.901 .019360 .00044

478

500

600

2.200 .925300 .00042

2.250 .932520 .00044

2.300 .939010 .00044

2.350 .946400 .00043

2.400 .952490 .00044

2.450 .959280 .00045

2.500 .965770 .00044

2.550 .972820 .00044

2.600 .978810 .00045

2.650 .985260 .00043

2.700 .990680 .00044

2.200 .924780 .00042

2.250 .931010 .00043

2.300 .938630 .00044

2.350 .944900 .00043

2.400 .951780 .00043

2.450 .958080 .00044

2.500 .963860 .00044

2.550 .970880 .00043

2.600 .978000 .00045

2.650 .983320 .00044

2.700 .989880 .00044

2.200 .919220 .00043

2.250 .926230 .00042

2.300 .932540 .00044

2.350 .939610 .00043

2.400 .946280 .00044

2.450 .952360 .00044

2.500 .957870 .00043

2.550 .964440 .00044

2.600 .970640 .00044

2.650 .977530 .00043

2.700 .983750 .00044 
2.750 .996830 .00043 2.801 .003820 .00044 2.851 .009900 .00045 2.901 .015160 .00044 2.951 .021680 .00044 3.001 .027370 .00043

650

2.300 .929650 .00042 2.350 .935690 .00043 2.400 .942020 .00043 2.450 .947490 .00044 2.500 .954300 .00042 2.550 .960630 .00044 2.600 .966510 .00043 2.650 .972760 .00045 2.700 .978910 .00043 2.750 .984570 .00044 2.800 .990720 .00044 2.850 .996760 .00044 2.901 .002930 .00043 2.951 .008170 .00045 3.001 .013980 .00044 3.051 .020440 .00044 3.101 .025000 .00044

760

2.400 .927050 .00042 2.450 .931830 .00043 2.500 .938240 .00044 2.550 .944210 .00043 2.600 .950120 .00044 2.650 .956090 .00044 2.700 .962100 .00045 2.750 .967730 .00043 2.800 .973920 .00045 2.850 .979470 .00045 2.900 .984910 .00043 2.950 .990200 .00044 3.000 .996230 .00043 3.051 .002200 .00045 3.101 .006940 .00046 3.151 .011690 .00043 3.201 .018550 .00043
2.750 .996120 .00043

2.801 .001930 .00044

2.851 .008030 .00043

2.901 .014910 .00044

2.951 .020780 .00043

3.001 .025860 .00045
2.750 .989980 .00045

2.800 .995050 .00044

2.851 .000980 .00043

2.901 .007670 .00042

2.951 .013380 .00043

3.001 .018390 .00043
2.300 .923420 .00043

2.350 .929920 .00043

2.400 .935320 .00043

2.450 .941960 .00044

2.500 .948540 .00045

2.550 .954200 .00042

2.650 .966290 .00044

2.700 .971990 .00044

2.750 .978230 .00044

2.800 .984240 .00044

2.850 .989860 .00044

2.900 .995120 .00043

2.951 .001850 .00044

3.001 .007220 .00045

3.051 .012020 .00045

3.101 .018320 .00044
750

2.400 .928490 .00044 2.450 .933790 .00044 2.500 .940080 .00043 2.550 .946550 .00044 2.600 .951910 .00043 2.650 .958540 .00044 2.700 .964230 .00045 2.750 .969820 .00044 2.800 .975620 .00044 2.850 .981160 .00044 2.900 .986850 .00045 2.950 .992970 .00044 3.000 .997890 .00043 3.051 .004100 .00043 3.101 .008860 .00044 3.151 .014680 .00044 3.201 .020370 .00046

765

2.400 .926490 .00042

2.450 .931440 .00043

2.500 .937200 .00043

2.550 .943350 .00044

2.600 .949830 .00044

2.650 .954750 .00043

2.700 .960250 .00044

2.750 .967080 .00043

2.800 .972150 .00043

2.850 .978010 .00046

2.900 .983340 .00044

2.950 .989670 .00045

3.000 .994640 .00044

3.051 .000140 .00042

3.101 .005600 .00043

3.151 .011730 .00043

3.201 .016470 .00044
770

2.400 .924060 .00044

2.450 .930000 .00042

2.500 .936620 .00043

2.550 .941460 .00043

2.600 .948380 .00045

2.650 .954180 .00045

2.700 .959470 .00045

2.750 .966030 .00044

2.800 .970340 .00043

2.850 .976180 .00045

2.900 .982370 .00045

2.950 .988370 .00044 3.000 .993790 .00044 3.050 .998280 .00044 3.101 .004610 .00043 3.151 .008700 .00044 3.201 .015470 .00044 3.251 .020480 .00044 3.301 .025050 .00045 
775

2.400 .923930 .00042

2.450 .929840 .00044

2.500 .935440 .00045

2.550 .941630 .00045

2.600 .947270 .00044

2.650 .952780 .00043

2.700 .958530 .00043

2.750 .965290 .00045

2.800 .970720 .00044

2.850 .975760 .00043

2.900 .981680 .00044

2.950 .987460 .00045

3.000 .992620 .00045

3.050 .998140 .00043

3.101 .003080 .00044

3.151 .009120 .00044

3.201 .014190 .00045

3.251 .019320 .00045

3.301 .025100 .00045

Pun40-pl-0970-00

12

10.100 .923490 .00029

10.200 .927350 .00029

10.300 .930970 .00030

10.400 .934370 .00030

10.500 .938890 .00029

10.600 .942700 .00029

10.700 .946570 .00029

10.800 .950230 .00029

10.900 .953750 .00028

11.000 .956840 .00028

11.100 .960480 .00028

11.200 .964140 .00027

11.300 .966790 .00029

11.400 .969990 .00028

11.500 .973580 .00027

11.600 .976420 .00028

11.700 .980260 .00028

11.800 .982540 .00028

11.900 .985880 .00028

12.000 .988330 .00028

12.100 .991640 .00027

12.200 .994240 .00027

12.300 .997830 .00028

12.400 .999770 .00028

\section{0}


12.501 .002410 .00027

12.601 .005930 .00027

12.701 .007750 .00027

12.801 .010390 .00027

12.901 .012650 .00027

13.001 .015340 .00027
8.401 .005240 .00037

8.451 .008400 .00036

8.501 .011170 .00037

8.551 .014750 .00035

8.601 .017520 .00036
25

6.600 .915940 .00040 6.650 .920630 .00041

6.700 .925320 .00040 6.750 .930190 .00040 6.800 .934900 .00041 6.850 .940210 .00040 6.900 .945520 .00039 6.950 .949520 .00040 7.000 .954340 .00041 7.050 .958750 .00039 7.100 .963950 .00040 7.150 .968900 .00040 7.200 .971900 .00040 7.250 .977230 .00039 7.300 .980670 .00040 7.350 .986130 .00040 7.400 .989900 .00039 7.450 .994330 .00039 7.500 .998180 .00039 7.551 .001520 .00039 7.601 .006510 .00040 7.651 .010910 .00040 7.701 .014800 .00040 7.751 .018310 .00039 $\begin{array}{llll}7.80 & 1.02238 & 0.00038\end{array}$

35

6.000 .916810 .00043 6.050 .923300 .00044 6.100 .928230 .00043 6.150 .934650 .00042 6.200 .940180 .00043 6.250 .946300 .00042 6.300 .952490 .00043 6.350 .957540 .00044 6.400 .963100 .00043 6.450 .968330 .00043 6.500 .973690 .00042 6.550 .979590 .00044
30

6.300 .923100 .00042

6.350 .928470 .00043

6.400 .934260 .00042

6.450 .940090 .00042

6.500 .944990 .00042

6.550 .949910 .00043

6.600 .955360 .00041

6.650 .960730 .00042

6.700 .965080 .00043

6.750 .970620 .00042

6.800 .975490 .00041

6.850 .980410 .00041

6.900 .985910 .00040

6.950 .990640 .00040

7.000 .994510 .00042

7.051 .000040 .00043

7.101 .003920 .00042

7.151 .008560 .00042

7.201 .014020 .00042

7.251 .018050 .00042

7.301 .023080 .00042
32

6.100 .912460 .00042 6.150 .918630 .00043 6.200 .924140 .00043 6.250 .929810 .00043 6.300 .935800 .00044 6.350 .941220 .00043 6.400 .946690 .00042 6.450 .951610 .00042 6.500 .957020 .00042 6.550 .963560 .00043 6.600 .968070 .00042 6.650 .973310 .00042 6.700 .978680 .00042 6.750 .983430 .00042 6.800 .988860 .00042 6.850 .993850 .00043 6.900 .998730 .00042 6.951 .004000 .00042 7.001 .007980 .00042 7.051 .012920 .00041 7.101 .017850 .00042
5.800 .913860 .00045

5.850 .919800 .00044

5.900 .925640 .00044

5.950 .932830 .00045

6.000 .938980 .00045

6.050 .945000 .00045

6.100 .950410 .00044

6.150 .956810 .00046

6.200 .962360 .00042

6.250 .968300 .00043

6.300 .974070 .00044

6.350 .980110 .00045
50

5.600 .916050 .00046 5.650 .923360 .00046 5.700 .929860 .00046 5.750 .935790 .00046 5.800 .943730 .00045 5.850 .949820 .00046 5.900 .955640 .00046 5.950 .961930 .00046 6.000 .968320 .00045 6.050 .974950 .00045 6.100 .980180 .00045 6.150 .986600 .00047 
6.600 .984910 .00044 6.650 .989450 .00043 6.700 .995080 .00044 6.750 .999570 .00043 6.801 .005210 .00044 6.851 .009630 .00042 6.901 .015400 .00043 6.951 .019260 .00044 7.001 .024180 .00041

60

5.500 .921730 .00046 5.550 .928810 .00047 5.600 .935600 .00047 5.650 .941300 .00045 5.700 .949330 .00046 5.750 .956030 .00048 5.800 .962690 .00047 5.850 .968460 .00047 5.900 .975730 .00047 5.950 .981560 .00046 6.000 .987830 .00046 6.050 .993680 .00047 6.100 .999900 .00045 6.151 .007280 .00047 6.201 .011620 .00047 6.251 .017800 .00046 6.301 .023000 .00047

\section{0}

5.300 .918490 .00047 5.350 .925720 .00048 5.400 .932570 .00047 5.450 .939510 .00049 5.500 .947300 .00047 5.550 .954370 .00047 5.600 .960020 .00049 5.650 .967250 .00050 5.700 .974470 .00047 5.750 .981520 .00049 5.800 .988180 .00048 5.850 .994810 .00049 5.901 .000380 .00048 5.951 .006770 .00049 6.001 .013170 .00047 6.051 .019710 .00048 6.101 .024890 .00049
6.400 .985500 .00044

6.450 .991060 .00045

6.500 .996580 .00044

6.551 .001800 .00044

6.601 .007320 .00044

6.651 .012860 .00044

6.701 .017370 .00044

6.751 .023030 .00044 6.801 .028580 .00043
6.200 .992170 .00044 6.250 .998030 .00047 6.301 .004900 .00046 6.351 .010010 .00045 6.401 .015090 .00047 6.451 .021370 .00047 6.501 .027460 .00046

80

5.400 .918960 .00047 5.450 .925900 .00047 5.500 .932810 .00047 5.550 .940260 .00047 5.600 .947860 .00048 5.650 .954460 .00048 5.700 .960370 .00047 5.750 .967200 .00047 5.800 .973170 .00047 5.850 .980710 .00049 5.900 .986730 .00048 5.950 .993680 .00047 6.000 .999030 .00048 6.051 .005730 .00047 6.101 .012130 .00049 6.151 .017140 .00048 6.201 .023640 .00046

100

5.200 .907500 .00049 5.250 .914280 .00048 5.300 .921760 .00047 5.350 .929670 .00048 5.400 .936410 .00049 5.450 .943840 .00049 5.500 .950350 .00048 5.550 .958080 .00049 5.600 .963860 .00049 5.650 .971300 .00049 5.700 .977880 .00048 5.901 .004890 .00048 5.951 .010160 .00049 6.001 .016540 .00048 6.051 .022610 .00047 6.101 .028080 .00048
5.300 .912800 .00048 5.350 .920200 .00047 5.400 .926170 .00047 5.450 .934620 .00047 5.500 .941280 .00048 5.550 .948190 .00049 5.600 .955550 .00048 5.650 .961910 .00049 5.700 .968660 .00047 5.750 .975940 .00047 5.800 .981980 .00046 5.850 .988670 .00047 5.900 .994630 .00047 5.951 .002500 .00047 6.001 .007360 .00047 6.051 .013840 .00047 6.101 .020560 .00047

\section{0}

5.200 .910350 .00048 5.250 .917090 .00049 5.300 .925550 .00049 5.350 .932620 .00048 5.400 .939280 .00049 5.450 .946750 .00048 5.500 .953810 .00048 5.550 .959730 .00048 5.600 .967530 .00049 5.650 .973950 .00047 5.700 .981240 .00049 5.750 .987700 .00049 5.800 .993620 .00048 5.851 .000740 .00049 5.901 .007070 .00049 5.951 .013760 .00050 6.001 .019850 .00050 
130

5.200 .912720 .00049 5.250 .920380 .00049 5.300 .927560 .00050 5.350 .934340 .00050 5.400 .941120 .00048 5.450 .947930 .00048 5.500 .955780 .00050 5.550 .962480 .00048 5.600 .969350 .00049 5.650 .976700 .00048 5.700 .982600 .00049 5.750 .988610 .00048 5.800 .995870 .00049 5.851 .001930 .00049 5.901 .008570 .00048 5.951 .014790 .00048 6.001 .020940 .00048
150

5.200 .913030 .00048

5.250 .919100 .00049

5.300 .926550 .00049

5.350 .934760 .00047

5.400 .941020 .00048

5.450 .947890 .00049

5.500 .955460 .00049

5.550 .961810 .00048

5.600 .968570 .00050

5.650 .975320 .00049

5.700 .981570 .00047

5.750 .988360 .00048

5.800 .994300 .00049

5.851 .001470 .00048

5.901 .007680 .00048

5.951 .013400 .00048

6.001 .020000 .00050
239

5.300 .919750 .00050 5.350 .926330 .00048 5.400 .934560 .00048 5.450 .940360 .00049 5.500 .946900 .00050 5.550 .953240 .00048 5.600 .960270 .00049 5.650 .966590 .00049 5.700 .973270 .00050 5.750 .979470 .00049 5.800 .986090 .00049 5.850 .991900 .00049 5.900 .998820 .00047 5.951 .004300 .00049 6.001 .009810 .00049 6.051 .017620 .00050 6.101 .022040 .00050
250

5.300 .918190 .00048 5.350 .924750 .00049 5.400 .931860 .00049 5.450 .938190 .00049 5.500 .944830 .00050 5.550 .951740 .00049 5.600 .958450 .00048 5.650 .965160 .00049 5.700 .970690 .00050 5.750 .977910 .00049 5.800 .984680 .00049 5.850 .990230 .00049 5.900 .996910 .00049 5.951 .002860 .00049 6.001 .008600 .00048 6.051 .014580 .00048 6.101 .019820 .00050
200

5.200 .909360 .00049

5.250 .916710 .00049

5.300 .924250 .00047

5.350 .930560 .00050

5.400 .938060 .00048

5.450 .944960 .00049

5.500 .952130 .00048

5.550 .958250 .00049

5.600 .965250 .00048

5.650 .972190 .00048

5.700 .977920 .00049

5.750 .984400 .00050

5.800 .989930 .00048

5.850 .996220 .00049

5.901 .003820 .00048

5.951 .009070 .00050

6.001 .016380 .00049

6.051 .021210 .00049

6.101 .027360 .00048

5.400 .918040 .00049

5.450 .925230 .00048

5.500 .931760 .00049

5.550 .938630 .00050

5.600 .944510 .00049

5.650 .950890 .00047

5.700 .956800 .00047

5.750 .963790 .00048

5.800 .969550 .00049

5.850 .976000 .00050

5.900 .981590 .00049

5.950 .988950 .00048

6.000 .994420 .00048

6.050 .999870 .00049

6.101 .005320 .00048

6.151 .010760 .00049

6.201 .017260 .00049

6.251 .021980 .00048

6.301 .027610 .00048 
478

5.500 .915080 .00048

5.550 .922080 .00048

5.600 .928480 .00050

5.650 .934120 .00050

5.700 .940550 .00048

5.750 .946670 .00047

5.800 .952800 .00049

5.850 .959450 .00048

5.900 .964830 .00049

5.950 .970590 .00049

6.000 .976480 .00049

6.050 .983550 .00049

6.100 .989120 .00047

6.301 .011370 .00048

6.351 .015610 .00049

6.401 .021210 .00049
650

5.900 .920860 .00049

5.950 .927010 .00047

6.000 .932140 .00047

6.050 .938330 .00048

6.100 .944130 .00049

6.150 .950050 .00047

6.200 .955470 .00048

6.250 .961510 .00048

6.300 .966760 .00048

6.350 .972860 .00049

6.400 .978720 .00048

6.450 .983270 .00048

6.500 .989180 .00048

6.701 .010840 .00049

6.751 .015990 .00048

6.801 .019110 .00049
500

5.500 .911070 .00048

5.550 .917770 .00048

5.600 .923860 .00050

5.650 .931080 .00048

5.700 .936860 .00049

5.750 .943980 .00050

5.800 .949720 .00048

5.850 .955930 .00048

5.900 .961170 .00047

5.950 .967880 .00049

6.000 .973880 .00049

6.050 .979020 .00048

6.100 .984770 .00048

6.150 .990590 .00049

6.200 .997370 .00050

6.251 .001650 .00049

6.301 .008350 .00049

6.351 .013270 .00048

6.401 .017590 .00048
600

5.700 .913490 .00047

5.750 .920230 .00048

5.800 .926430 .00049

5.850 .932750 .00048

5.900 .938050 .00048

5.950 .943530 .00048

6.000 .949900 .00048

6.050 .956450 .00049

6.100 .961960 .00049

6.150 .966310 .00047

6.200 .973130 .00048

6.250 .979110 .00049

6.300 .984690 .00047

6.350 .990820 .00049

6.400 .994950 .00047

6.451 .001200 .00047

6.501 .005730 .00048

6.551 .011210 .00049

6.601 .016630 .00046

6.651 .021240 .00047

6.701 .026540 .00048

750

6.100 .915280 .00046

6.150 .921600 .00048

6.200 .926700 .00046

6.250 .932970 .00048

6.300 .938710 .00048

6.350 .944110 .00048

6.400 .948710 .00046

6.450 .955360 .00048

6.500 .960550 .00048

6.550 .965360 .00049

6.600 .971150 .00047

6.650 .976870 .00048

6.700 .981410 .00048

6.750 .987190 .00048

6.800 .992680 .00048

6.850 .996790 .00047

6.901 .003000 .00048

6.951 .007890 .00048

7.001 .012000 .00048

7.051 .017010 .00048

7.101 .023070 .00047
6.700 .913320 .00047

6.750 .918310 .00046

6.800 .924200 .00047

6.850 .929250 .00046

6.900 .933320 .00048

6.950 .938890 .00047

7.000 .943950 .00047

7.050 .948980 .00047

7.100 .954670 .00048

7.150 .960110 .00046

7.200 .965290 .00046

7.250 .969260 .00047

7.300 .973860 .00048

7.350 .979410 .00047

7.400 .983580 .00048

7.450 .988270 .00049

7.500 .992850 .00048

7.550 .998000 .00047

7.601 .002080 .00048

7.651 .007770 .00046

7.701 .011440 .00046

7.751 .015380 .00048

7.801 .020160 .00048 
752

6.800 .914330 .00047 6.850 .920130 .00047 6.900 .924960 .00048 6.950 .930600 .00046 7.000 .935190 .00048 7.050 .941330 .00047

7.100 .945460 .00047

7.150 .950140 .00047

7.200 .955940 .00048

7.250 .960250 .00046

7.300 .965830 .00046

7.350 .970210 .00048

7.400 .975880 .00047

7.450 .981070 .00048

7.500 .985090 .00047

7.550 .989410 .00047

7.600 .995020 .00048

7.650 .999450 .00048

7.701 .003420 .00048

7.751 .007170 .00047

7.801 .011770 .00048

7.851 .016420 .00047

7.901 .021410 .00048
754

6.900 .913390 .00045

6.950 .918000 .00046

7.000 .923080 .00047

7.050 .929030 .00048

7.100 .934720 .00048

7.150 .939040 .00047

7.200 .944390 .00048

7.250 .949380 .00048

7.300 .953730 .00046

7.350 .958140 .00047

7.400 .963660 .00047

7.450 .968620 .00048

7.500 .972400 .00046

7.550 .977940 .00047

7.600 .981850 .00048

7.650 .986560 .00046

7.700 .990710 .00047

7.750 .995430 .00047

7.801 .000820 .00048

7.851 .005260 .00047

7.901 .009230 .00048

7.951 .014110 .00047

8.001 .017640 .00048
755

7.100 .923540 .00046

7.150 .927950 .00047

7.200 .932960 .00048

7.250 .938290 .00047

7.300 .943270 .00047

7.350 .947990 .00046

7.400 .953220 .00047

7.450 .956770 .00046

7.500 .963390 .00047

7.550 .966580 .00048

7.600 .971900 .00048

7.650 .977190 .00048

7.700 .980710 .00047

7.750 .986250 .00048

7.800 .990470 .00047

7.850 .994650 .00047

7.900 .998940 .00048

7.951 .003590 .00047

8.001 .007780 .00047

8.051 .013080 .00047

8.101 .016800 .00047

8.151 .021110 .00047

8.201 .024800 .00047 
Pun40-pl-0970-01

12

8.600 .924370 .00028

8.700 .928430 .00029

8.800 .931640 .00028

8.900 .935800 .00028

9.000 .939340 .00028

9.100 .943840 .00029

9.200 .947600 .00028

9.300 .950610 .00027

9.400 .954270 .00028

9.500 .957450 .00027

9.600 .961000 .00028

9.700 .964160 .00027

9.800 .967620 .00028

9.900 .971040 .00028

10.000 .973850 .00027

10.100 .977040 .00027

10.200 .980390 .00027

10.300 .983690 .00027

10.400 .986030 .00028

10.500 .989460 .00027

10.600 .991730 .00027

10.700 .994910 .00027

10.800 .997570 .00027

10.901 .001050 .00027

11.001 .003190 .00027

11.101 .005900 .00027

11.201 .008190 .00027

11.301 .010580 .00026

11.401 .012720 .00026

11.501 .016060 .00026
15

7.000 .923850 .00032

7.100 .929540 .00031

7.200 .934970 .00033

7.300 .941080 .00033

7.400 .947410 .00032

7.500 .952420 .00031

7.600 .957740 .00031

7.700 .962430 .00033

7.800 .967670 .00033

7.900 .972680 .00031

8.000 .977490 .00032

8.100 .982960 .00030

8.200 .988470 .00031

8.300 .992630 .00031

8.400 .996840 .00030

8.501 .001460 .00031

8.601 .005620 .00030

8.701 .010260 .00030

8.801 .014240 .00031
25

5.100 .918260 .00039

5.150 .923610 .00038

5.200 .929140 .00038

5.250 .933260 .00038

5.300 .937840 .00038

5.350 .943340 .00038

5.400 .948210 .00037

5.450 .953440 .00038

5.500 .958070 .00038

5.550 .962420 .00038

5.600 .966700 .00039

5.650 .971830 .00039

5.700 .975560 .00038

5.750 .980390 .00038
30

4.700 .915410 .00039

4.750 .921240 .00039

4.800 .926730 .00041

4.850 .932380 .00041

4.900 .937450 .00040

4.950 .942920 .00040

5.000 .948600 .00040

5.050 .953720 .00040

5.100 .959380 .00040

5.150 .964280 .00041

5.200 .969510 .00041

5.250 .973470 .00040

5.300 .980180 .00040

5.350 .984560 .00039
20

5.700 .917650 .00037

5.750 .922070 .00037

5.800 .926530 .00035

5.850 .929980 .00036

5.900 .934200 .00036

5.950 .938230 .00036

6.000 .941640 .00036

6.050 .946210 .00037

6.100 .950450 .00036

6.150 .954220 .00036

6.200 .958150 .00035

6.250 .962140 .00035

6.300 .965230 .00036

6.350 .968930 .00035

6.400 .972970 .00035

6.450 .976860 .00036

6.500 .980020 .00036

6.550 .983500 .00035

6.600 .987190 .00036

6.650 .990450 .00036

6.700 .994320 .00035

6.750 .997050 .00036

6.801 .000660 .00034

6.851 .004360 .00035

6.901 .007530 .00035

6.951 .010860 .00035

7.001 .014040 .00036

7.051 .017680 .00035

7.101 .019910 .00034

\section{2}

4.600 .917100 .00041

4.650 .923480 .00041

4.700 .928210 .00041

4.750 .934370 .00041

4.800 .939220 .00040

4.850 .945850 .00040

4.900 .950450 .00041

4.950 .956520 .00040

5.000 .961720 .00041

5.050 .968030 .00041

5.100 .972160 .00042

5.150 .977540 .00040

5.200 .982430 .00041

5.250 .987610 .00041 
5.800 .985020 .00039 5.850 .988900 .00038 5.900 .993030 .00038 5.950 .997330 .00039 6.001 .001370 .00039 6.051 .004730 .00038 6.101 .009350 .00039 6.151 .013360 .00039 6.201 .017320 .00037
5.400 .988700 .00042

5.450 .994730 .00040

5.500 .999160 .00041

5.551 .003250 .00040

5.601 .008420 .00039

5.651 .012660 .00040

5.701 .016710 .00040
5.300 .992280 .00041 5.350 .997300 .00041 5.401 .003080 .00040 5.451 .006550 .00040 5.501 .012250 .00040 5.551 .017310 .00040 5.601 .021250 .00040
35

4.500 .921630 .00042 4.550 .927100 .00042 4.600 .933450 .00042 4.650 .938860 .00042 4.700 .944710 .00043 4.750 .951280 .00041 4.800 .957430 .00042 4.850 .962440 .00042 4.900 .967990 .00042 4.950 .972800 .00041 5.000 .978500 .00041 5.050 .983890 .00041 5.100 .989070 .00041 5.150 .995080 .00040 5.200 .999480 .00042 5.251 .004900 .00041 5.301 .010400 .00041 5.351 .015320 .00042 5.401 .020060 .00041

60

3.900 .915390 .00046 3.950 .922330 .00045 4.000 .929760 .00045 4.050 .936030 .00045 4.100 .943080 .00045 4.150 .950800 .00044 4.200 .957070 .00045 4.250 .963800 .00046 4.300 .970360 .00044 4.350 .976920 .00045 4.400 .983020 .00046 4.450 .989700 .00044 4.500 .996710 .00046 4.551 .001730 .00044 4.601 .007490 .00045
40

4.300 .919700 .00041 4.350 .925830 .00043 4.400 .931810 .00043 4.450 .938180 .00043 4.500 .945010 .00043 4.550 .950350 .00043 4.600 .956620 .00043 4.650 .962300 .00042 4.700 .967960 .00043 4.750 .973470 .00043 4.800 .980060 .00043 4.850 .985380 .00042 4.900 .991420 .00043 4.950 .996590 .00042 5.001 .001600 .00044 5.051 .007740 .00043 5.101 .013080 .00042 5.151 .018220 .00043 5.201 .022940 .00043
50

4.100 .923330 .00044 4.150 .930360 .00045 4.200 .936910 .00044 4.250 .943150 .00044 4.300 .950480 .00044 4.350 .955610 .00045 4.400 .963220 .00045 4.450 .969340 .00043 4.500 .975690 .00044 4.550 .981160 .00044 4.600 .987320 .00044 4.650 .993600 .00043 4.700 .999280 .00045 4.751 .005780 .00045 4.801 .011050 .00043 4.851 .016540 .00046 4.901 .022020 .00043
70

3.800 .913290 .00045 3.850 .921770 .00046 3.900 .927770 .00046 3.950 .935510 .00046 4.000 .941760 .00045 4.050 .948950 .00046 4.100 .956340 .00045 4.150 .963010 .00045 4.200 .969300 .00046 4.250 .975720 .00046 4.300 .983000 .00045 4.350 .988380 .00046 4.400 .995190 .00045 4.451 .002220 .00044 4.501 .007540 .00048
80

3.800 .922960 .00046 3.850 .929660 .00047 3.900 .938090 .00046 3.950 .944560 .00045 4.000 .951910 .00046 4.050 .958750 .00045 4.100 .965130 .00047 4.150 .971600 .00046 4.200 .979320 .00047 4.250 .985570 .00047 4.300 .992710 .00046 4.350 .998580 .00047 4.401 .004480 .00047 4.451 .011460 .00046 4.501 .016290 .00047 
4.651 .013300 .00045

4.701 .019660 .00044

90

3.700 .914620 .00047

3.750 .923340 .00047

3.800 .929900 .00047

3.850 .937220 .00047

3.900 .944780 .00045

3.950 .951500 .00046

4.000 .957350 .00047

4.050 .964870 .00046

4.100 .971340 .00048

4.150 .978430 .00046

4.200 .985380 .00047

4.250 .992100 .00047

4.300 .998100 .00047

4.351 .004030 .00046

4.401 .010730 .00047

4.451 .017540 .00047

4.501 .022910 .00046

130

3.600 .911010 .00046

3.650 .918480 .00048

3.700 .925870 .00047

3.750 .933130 .00046

3.800 .940310 .00046

3.850 .948110 .00047

3.900 .954620 .00046

3.950 .961420 .00046

4.000 .968250 .00047

4.050 .975890 .00047

4.100 .981530 .00045

4.150 .988320 .00047

4.200 .994370 .00048

4.251 .001320 .00049

4.301 .007190 .00048

4.351 .014550 .00048

4.401 .020590 .00049

239

3.700 .920690 .00048 3.750 .928470 .00047 3.800 .935820 .00048
4.551 .014500 .00046

4.601 .020890 .00045

100

3.700 .919440 .00047

3.750 .926930 .00046

3.950 .954770 .00048

4.000 .962890 .00045

4.050 .969660 .00047

4.100 .974940 .00047

4.150 .982090 .00047

4.200 .989820 .00047

4.250 .996210 .00046

4.301 .002230 .00047

4.351 .008530 .00047

4.401 .015160 .00047

4.451 .021260 .00047

4.501 .027290 .00048
120

3.700 .924030 .00045

3.750 .931580 .00046

3.800 .937790 .00047

3.850 .945470 .00045

3.900 .952280 .00046

3.950 .959400 .00047

4.000 .966680 .00047

4.050 .973060 .00046

4.100 .979680 .00047

4.150 .986430 .00047

4.200 .992750 .00047

4.250 .999880 .00047

4.301 .005520 .00045

4.351 .012850 .00048

4.401 .017640 .00047
3.600 .912140 .00047

3.650 .918950 .00047

3.700 .926380 .00048

3.750 .934070 .00046

3.800 .940770 .00047

3.850 .948670 .00047

3.900 .955520 .00048

3.950 .961670 .00046

4.000 .969020 .00048

4.050 .975060 .00049

4.100 .981980 .00047

4.150 .988530 .00047

4.200 .995180 .00046

4.251 .001670 .00047

4.301 .007450 .00047

4.351 .014300 .00047

4.401 .019860 .00047
200

3.600 .909900 .00048

3.650 .917130 .00047

3.700 .924850 .00047

3.750 .932230 .00048

3.800 .938660 .00047

3.850 .945570 .00049

3.900 .953460 .00048

3.950 .959240 .00048

4.000 .965670 .00048

4.050 .972850 .00047

4.100 .978790 .00046

4.150 .985890 .00047

4.200 .991560 .00049

4.250 .997350 .00047

4.301 .004770 .00048

4.351 .010780 .00047

4.401 .016060 .00047
250

3.700 .919330 .00047

3.750 .926430 .00048

3.800 .933420 .00047
359

3.800 .921430 .00049 3.850 .928160 .00048 3.900 .934680 .00047 
3.850 .941120 .00048 3.900 .948280 .00047 3.950 .955180 .00047 4.000 .962100 .00047 4.050 .968260 .00047 4.100 .974560 .00047 4.150 .981060 .00047 4.200 .987650 .00048 4.250 .993290 .00048 4.301 .000170 .00047 4.351 .005730 .00047 4.401 .012010 .00047 4.451 .017680 .00048 4.501 .023770 .00047

478

3.900 .918460 .00048 3.950 .925390 .00047 4.000 .932220 .00047 4.050 .938260 .00048 4.100 .944240 .00048 4.150 .950340 .00048 4.200 .956330 .00047 4.250 .962470 .00048 4.300 .968960 .00049 4.350 .973580 .00048 4.400 .980020 .00048 4.450 .985710 .00048 4.500 .992460 .00046 4.550 .996120 .00048 4.601 .003410 .00048 4.651 .007780 .00048 4.701 .012990 .00048 4.751 .019030 .00048 4.801 .024140 .00047

\section{0}

4.200 .919390 .00048 4.250 .926110 .00047 4.300 .932210 .00046 4.350 .937040 .00047 4.400 .943540 .00047 4.450 .948880 .00047 4.500 .954430 .00048 4.550 .959780 .00048 4.600 .965530 .00048 4.650 .970610 .00046
3.850 .940370 .00046

3.900 .947300 .00048

3.950 .953440 .00049

4.000 .960990 .00048

4.050 .967450 .00048

4.100 .973630 .00047

4.150 .979020 .00048

4.200 .987330 .00048

4.250 .992830 .00048

4.300 .998230 .00048

4.351 .004520 .00048

4.401 .010490 .00048

4.451 .015790 .00048

4.501 .021580 .00048
3.950 .941800 .00048

4.000 .947650 .00047

4.050 .953410 .00048

4.250 .978680 .00049

4.300 .984700 .00046

4.350 .990600 .00048

4.400 .995740 .00048

4.451 .003040 .00048

4.501 .008180 .00047

4.551 .012690 .00047

4.601 .019190 .00047
500

3.900 .917000 .00048

3.950 .921940 .00047

4.000 .928610 .00048

4.050 .935290 .00048

4.100 .942230 .00048

4.150 .947440 .00047

4.200 .953660 .00048

4.250 .959270 .00047

4.300 .966010 .00048

4.350 .971220 .00047

$4.40 \quad 0.977020 .00047$

4.450 .981810 .00048

4.500 .988200 .00048

4.550 .993510 .00048

4.600 .999370 .00047

4.651 .005180 .00048

4.701 .010610 .00049

4.751 .016140 .00046

4.801 .021270 .00047
600

4.100 .921510 .00049 4.300 .945180 .00048 4.350 .950940 .00048 4.400 .957310 .00048 4.450 .962820 .00046 4.500 .968250 .00048 4.550 .974770 .00047 4.600 .979920 .00048 4.650 .984830 .00048 4.700 .991220 .00048 4.750 .995700 .00048 4.801 .002460 .00048 4.851 .006080 .00047 4.901 .011720 .00047 4.951 .017260 .00048 5.001 .021020 .00048
700

4.400 .921230 .00047 4.450 .927240 .00048 4.500 .931940 .00045 4.550 .938640 .00047 4.600 .943580 .00047 4.650 .948850 .00047 4.700 .954540 .00048 4.750 .959630 .00048 4.800 .964640 .00047 4.850 .969870 .00048
750

4.900 .923050 .00047 4.950 .927760 .00048 5.000 .933350 .00048 5.050 .938930 .00047 5.100 .943060 .00046 5.150 .948840 .00047 5.200 .953690 .00046 5.250 .958050 .00048 5.300 .962700 .00048 5.350 .967920 .00047 
4.700 .976890 .00046 4.750 .982060 .00047 4.800 .986320 .00047 4.850 .992510 .00046 4.900 .998100 .00046 4.951 .002780 .00048 5.001 .008350 .00048 5.051 .012770 .00047 5.101 .018100 .00048

752

4.900 .916580 .00047 4.950 .922210 .00047 5.000 .926720 .00048 5.050 .931900 .00048 5.100 .936770 .00047 5.150 .942230 .00046 5.200 .946710 .00047 5.250 .951680 .00046 5.300 .957080 .00047 5.350 .961900 .00047 5.400 .966280 .00048 5.450 .971290 .00048 5.500 .975800 .00047 5.550 .981080 .00047 5.600 .984560 .00047 5.650 .990160 .00047 5.700 .995370 .00047 5.750 .998650 .00046 5.801 .003570 .00047 5.851 .007960 .00046 5.901 .012750 .00047 5.951 .017070 .00046 6.001 .020980 .00047

Pun40-pl-0970-10

12

7.500 .924320 .00027 7.600 .928130 .00027

7.700 .932240 .00026

7.800 .936560 .00026

7.900 .939830 .00027 8.000 .943790 .00026 8.100 .947820 .00026 8.200 .951210 .00026
4.900 .975220 .00047 4.950 .980430 .00048 5.000 .985520 .00046 5.050 .991050 .00046 5.100 .995280 .00046 5.151 .000690 .00049 5.201 .005360 .00047 5.251 .011170 .00048 5.301 .015950 .00049 5.351 .020590 .00048 5.401 .025260 .00046

754

5.000 .918700 .00047 5.050 .923460 .00047 5.100 .928200 .00046 5.150 .933400 .00047 5.200 .937750 .00047 5.250 .942740 .00048 5.300 .948230 .00047 5.350 .952150 .00047 5.400 .957560 .00045 5.450 .962110 .00049 5.500 .968320 .00046 5.550 .971330 .00046 5.600 .975940 .00047 5.650 .981060 .00048 5.700 .984540 .00048 5.750 .990090 .00047 5.800 .994600 .00047 5.850 .998730 .00047 5.901 .003590 .00047 5.951 .007970 .00046 6.001 .012140 .00046 6.051 .016740 .00048 6.101 .020840 .00046
5.400 .972990 .00048 5.450 .978020 .00046 5.500 .982540 .00047 5.550 .987460 .00048 5.600 .990970 .00047 5.650 .996320 .00047 5.701 .000580 .00045 5.751 .005800 .00047 5.801 .010420 .00048 5.851 .014830 .00048 5.901 .018310 .00048
5.100 .919990 .00046 5.150 .924910 .00048 5.200 .929820 .00046 5.250 .935300 .00049 5.300 .940530 .00047 5.350 .944480 .00047 5.400 .950040 .00047 5.450 .953610 .00048 5.500 .958550 .00048 5.550 .963400 .00047 5.600 .968860 .00048 5.650 .972250 .00047 5.700 .977670 .00046 5.750 .981930 .00047 5.800 .986750 .00047 5.850 .990230 .00048 5.900 .995990 .00047 5.950 .999630 .00047 6.001 .003360 .00047 6.051 .008290 .00046 6.101 .013150 .00046 6.151 .017050 .00047 6.201 .020460 .00048
15

5.900 .925000 .00031 6.000 .930760 .00030 6.100 .936440 .00031 6.200 .942750 .00030 6.300 .948280 .00030 6.400 .954120 .00030 6.500 .959150 .00030 6.600 .964180 .00029
20

4.600 .920510 .00034 4.650 .925010 .00034 4.700 .929150 .00032 4.750 .932680 .00033 4.800 .937150 .00033 4.850 .941350 .00033 4.900 .945580 .00034 4.950 .949910 .00034 
8.300 .955050 .00026 8.400 .958040 .00026 8.500 .961410 .00026 8.600 .965260 .00026 8.700 .967980 .00026 8.800 .971760 .00027 8.900 .975040 .00026 9.000 .977880 .00025 9.100 .981130 .00026 9.200 .984430 .00026 9.300 .987500 .00026 9.400 .989670 .00026 9.500 .992490 .00026 9.600 .995390 .00025 9.700 .998160 .00026 9.801 .001180 .00025 9.901 .003920 .00025 10.001 .006750 .00025 10.101 .008870 .00025 10.201 .011540 .00025 10.301 .014270 .00024
6.700 .969220 .00028

6.800 .974070 .00030

6.900 .979430 .00030

7.000 .984200 .00029

7.100 .989330 .00029

7.200 .994140 .00029

7.300 .998580 .00029

7.401 .002760 .00030

7.501 .007480 .00029

7.601 .011420 .00030

7.701 .016130 .00029
25

4.000 .925230 .00035 4.050 .929420 .00036 4.100 .934780 .00035 4.150 .939820 .00035 4.200 .944800 .00035 4.250 .949660 .00035 4.300 .954280 .00035 4.350 .959530 .00036 4.400 .963970 .00036 4.450 .968880 .00036 4.500 .973170 .00036 4.550 .977680 .00036 4.600 .981910 .00037 4.650 .987490 .00036 4.700 .991810 .00036 4.750 .994910 .00034 4.801 .000000 .00036 4.851 .004390 .00035 4.901 .008000 .00035 4.951 .012250 .00036 5.001 .016370 .00035
5.000 .953380 .00033 5.050 .957750 .00034 5.100 .961490 .00033 5.150 .965760 .00033 5.200 .968710 .00033 5.250 .972370 .00033 5.300 .976660 .00033 5.350 .980520 .00032 5.400 .983200 .00033 5.450 .987610 .00033 5.500 .990510 .00033 5.550 .994540 .00032 5.600 .997520 .00032 5.651 .001360 .00033 5.701 .004750 .00032 5.751 .008060 .00034 5.801 .010610 .00032 5.851 .014530 .00032 5.901 .017950 .00032
3.600 .925200 .00037 3.650 .929920 .00037 3.700 .935870 .00038 3.750 .942180 .00037 3.800 .946880 .00037 3.850 .952950 .00037 3.900 .958100 .00037 3.950 .963340 .00038 4.000 .968730 .00038 4.050 .974000 .00037 4.100 .978980 .00036 4.150 .984040 .00037 4.200 .988870 .00037 4.250 .994310 .00037 4.300 .998370 .00037 4.351 .004120 .00036 4.401 .007600 .00036 4.451 .013800 .00037 4.501 .017710 .00037
32

3.400 .915580 .00037 3.450 .921360 .00036 3.500 .927810 .00038 3.550 .933530 .00038 3.600 .939040 .00036 3.650 .944650 .00038 3.700 .951280 .00038 3.750 .956280 .00038 3.800 .961680 .00038 3.850 .967280 .00037 3.900 .972720 .00038 3.950 .978110 .00036 4.000 .983760 .00039 4.050 .988170 .00038 4.100 .993640 .00037 4.150 .998220 .00037 4.201 .004490 .00038 4.251 .008420 .00038 4.301 .013360 .00038 4.351 .018900 .00037 4.401 .023270 .00037 
35

3.300 .921820 .00038

3.350 .927850 .00038

3.400 .933820 .00038

3.450 .940250 .00037

3.500 .946060 .00039

3.550 .952050 .00038

3.600 .958610 .00038

3.650 .963000 .00040

3.700 .970060 .00037

3.750 .974990 .00039

3.800 .980600 .00039

3.850 .986360 .00039

3.900 .991530 .00038

3.950 .996620 .00039

4.001 .002140 .00037

4.051 .007390 .00039

4.101 .013160 .00038

4.151 .017840 .00037

4.201 .022420 .00039
40

3.100 .921110 .00039

3.150 .927450 .00039

3.200 .934380 .00039

3.250 .941570 .00038

3.300 .947410 .00040

3.350 .953490 .00039

3.400 .959690 .00039

3.450 .966090 .00039

3.500 .971530 .00039

3.550 .978260 .00038

3.600 .983860 .00040

3.650 .989650 .00039

3.700 .995350 .00038

3.751 .001450 .00039

3.801 .006170 .00039

3.851 .012480 .00040

3.901 .018410 .00039
50

2.800 .916750 .00041

2.850 .924030 .00039

2.900 .930980 .00040

2.950 .938430 .00041

3.000 .944640 .00041

3.050 .951680 .00041

3.100 .958650 .00040

3.150 .965400 .00040

3.200 .971220 .00040

3.250 .977690 .00041

3.300 .984560 .00041

3.350 .990280 .00039

3.400 .996510 .00039

3.451 .003050 .00040

3.501 .008510 .00040

3.551 .014460 .00040

3.601 .020510 .00040
60

2.600 .913070 .00042

2.650 .920390 .00040

2.700 .928300 .00041

2.750 .935220 .00042

2.800 .943020 .00041

2.850 .950330 .00041

2.900 .956320 .00040

2.950 .963840 .00041

3.000 .970460 .00041

3.050 .976950 .00042

3.100 .983630 .00041

3.150 .990070 .00040

3.200 .997130 .00041

3.251 .003160 .00040

3.301 .009790 .00041

3.351 .015280 .00041

3.401 .021760 .00041
70

2.500 .916010 .00041

2.550 .923490 .00041

2.600 .931080 .00042

2.650 .938870 .00041

2.700 .946040 .00042

2.750 .952420 .00042

2.800 .959910 .00041

2.850 .967920 .00041

2.900 .973930 .00041

2.950 .981460 .00040

3.000 .988200 .00041

3.050 .994450 .00041

3.101 .000810 .00041

3.151 .008100 .00041

3.201 .013540 .00040

3.251 .019340 .00041

3.301 .026420 .00041
80

2.400 .914220 .00041

2.450 .923020 .00041

2.500 .929720 .00043

2.550 .936330 .00041

2.600 .945210 .00042

2.650 .952720 .00043

2.700 .959220 .00041

2.750 .966350 .00043

2.800 .973750 .00042

2.850 .980360 .00041

2.900 .987640 .00043

2.950 .994350 .00042

3.001 .000820 .00043

3.051 .007520 .00042

3.101 .013460 .00044

3.151 .020950 .00043

3.201 .026530 .00042
90

2.400 .925270 .00042

2.450 .932470 .00043

2.500 .940320 .00042

2.550 .947750 .00042

2.600 .955440 .00042

2.650 .962460 .00041
100

2.300 .917180 .00042

2.350 .925670 .00042

2.400 .933660 .00042

2.450 .940640 .00042

2.500 .948120 .00043

2.550 .955540 .00043
120

2.200 .912890 .00043

2.250 .921840 .00042

2.300 .928980 .00044

2.350 .937160 .00043

2.400 .943430 .00042

2.450 .951740 .00043 
2.700 .969010 .00044 2.750 .976550 .00042 2.800 .983900 .00042 2.850 .990530 .00042 2.900 .997150 .00044 2.951 .003540 .00043 3.001 .009460 .00041 3.051 .017080 .00041 3.101 .023650 .00042

130

2.200 .917590 .00044 2.250 .926160 .00043 2.300 .933230 .00044 2.350 .940320 .00043 2.400 .948760 .00043 2.450 .956330 .00043 2.500 .963370 .00044 2.550 .970090 .00043 2.600 .977180 .00042 2.650 .984880 .00042 2.700 .991580 .00043 2.750 .998590 .00045 2.801 .004890 .00043 2.851 .011680 .00043 2.901 .017280 .00043

239

2.100 .920870 .00043 2.150 .928080 .00043 2.200 .935190 .00044 2.250 .941940 .00043 2.300 .950060 .00044 2.350 .956420 .00042 2.400 .963750 .00043 2.450 .969930 .00044 2.500 .977260 .00044 2.550 .982960 .00044 2.600 .989850 .00043 2.650 .997130 .00045 2.701 .001730 .00043 2.751 .009660 .00044 2.801 .016100 .00044
2.600 .962760 .00043

2.650 .970380 .00043

2.850 .997650 .00043

2.901 .005070 .00041

2.951 .011110 .00043

3.001 .018700 .00043
2.500 .958650 .00043

2.550 .966250 .00042

2.600 .972960 .00043

2.650 .980540 .00043

2.700 .986980 .00044

2.750 .993660 .00042

2.801 .000770 .00043

2.851 .007350 .00044

2.901 .014410 .00043

2.951 .021260 .00042

3.001 .027100 .00043

150

2.200 .924420 .00044

2.250 .931720 .00044

2.300 .939770 .00042

2.350 .947750 .00043

2.400 .954110 .00044

2.450 .961520 .00043

2.500 .968670 .00043

2.550 .974990 .00043

2.600 .982080 .00044

2.650 .990000 .00043

2.700 .996360 .00044

2.751 .003400 .00044

2.801 .009750 .00044

2.851 .016240 .00044

2.901 .022530 .00044
200

2.100 .918130 .00043

2.150 .925600 .00042

2.200 .931960 .00044

2.250 .940770 .00043

2.300 .947620 .00044

2.350 .954240 .00042

2.400 .961500 .00042

2.450 .967370 .00044

2.500 .974250 .00043

2.550 .982210 .00043

2.600 .988190 .00042

2.650 .995760 .00044

2.701 .002640 .00044

2.751 .008220 .00043

2.801 .014350 .00044
250
359
2.100 .921080 .00044
2.150 .927800 .00044
2.200 .934840 .00043
2.250 .942400 .00042
2.300 .949620 .00043
2.350 .957270 .00044
2.400 .963580 .00045
2.450 .969830 .00043
2.500 .976640 .00043
2.550 .983890 .00045
2.600 .989540 .00044
2.650 .996440 .00044
2.701 .002700 .00043
2.751 .009260 .00044
2.801 .015860 .00044
2.100 .921000 .00043
2.150 .927840 .00044
2.200 .935050 .00044
2.250 .941030 .00043
2.300 .948310 .00043
2.350 .954570 .00044
2.400 .961870 .00043
2.450 .968270 .00043
2.500 .974510 .00043
2.550 .979600 .00044
2.600 .986120 .00043
2.650 .992950 .00042
2.700 .999020 .00044
2.751 .004460 .00045
2.801 .010880 .00045
2.851 .016730 .00044
2.901 .022400 .00044 
478

2.100 .916190 .00044 2.150 .923090 .00043 2.200 .929750 .00043 2.250 .936120 .00043 2.300 .942240 .00043 2.350 .949680 .00043 2.400 .956250 .00044 2.450 .961870 .00044 2.500 .967580 .00043 2.700 .991330 .00043 2.750 .998070 .00044 2.801 .004050 .00043 2.851 .009310 .00044 2.901 .014320 .00044

650

2.300 .924630 .00043 2.350 .929680 .00043 2.400 .935980 .00044 2.450 .941810 .00043 2.500 .947650 .00043 2.550 .953860 .00043 2.750 .977030 .00044 2.800 .982050 .00044 2.850 .988240 .00044 2.900 .992270 .00044 2.950 .998260 .00042 3.001 .003580 .00044 3.051 .009040 .00043 3.101 .013780 .00044 3.151 .019080 .00043 3.201 .024130 .00043
500

2.100 .915230 .00042

2.150 .921180 .00043

2.200 .928450 .00043

2.250 .935400 .00044

2.300 .942000 .00044

2.350 .948700 .00045

2.400 .953710 .00043

2.450 .960710 .00045

2.500 .966310 .00045

2.550 .972410 .00043

2.600 .978830 .00045

2.650 .984170 .00045

2.700 .989670 .00044

2.750 .996250 .00044

2.801 .002320 .00045

2.851 .007370 .00043

2.901 .012960 .00045

2.951 .018460 .00044

3.001 .023830 .00044
600

2.200 .919280 .00043

2.250 .925930 .00043

2.300 .931540 .00044

2.350 .937770 .00043

2.400 .943520 .00043

2.450 .950260 .00043

2.500 .956590 .00044

2.550 .961850 .00044

2.600 .968060 .00044

2.650 .973940 .00044

2.700 .979300 .00044

2.750 .985280 .00043

2.800 .990200 .00045

2.850 .995390 .00044

2.901 .000460 .00044

2.951 .006190 .00044

3.001 .011650 .00044

3.051 .017760 .00044

3.101 .022420 .00045

750

2.400 .924220 .00043

2.450 .930460 .00043

2.500 .935550 .00044

2.550 .941750 .00044

2.600 .947260 .00044

2.650 .952850 .00043

2.700 .959230 .00043

2.750 .963180 .00043

2.800 .970150 .00043

2.850 .974460 .00044

2.900 .979160 .00045

2.950 .985010 .00043

3.000 .990650 .00044

3.050 .995110 .00045

3.101 .000110 .00043

3.151 .005880 .00043

3.201 .010700 .00045

3.251 .015550 .00044

3.301 .020200 .00045
2.600 .918510 .00043

2.650 .924350 .00044

2.700 .928770 .00043

2.750 .933980 .00043

2.800 .939890 .00043

2.850 .945170 .00043

2.900 .949450 .00043

2.950 .954600 .00044

3.000 .959430 .00043

3.050 .965310 .00045

3.100 .969620 .00043

3.150 .973590 .00044

3.200 .978650 .00044

3.250 .983520 .00044

3.300 .987950 .00044

3.350 .992830 .00044

3.400 .997660 .00044

3.451 .001200 .00044

3.501 .006710 .00044

3.551 .011130 .00043

3.601 .015420 .00045 
752

2.700 .925660 .00044

2.750 .930800 .00043

2.800 .936400 .00043

2.850 .940740 .00045

2.900 .946060 .00042

2.950 .951420 .00042

3.000 .956410 .00045

3.050 .961830 .00045

3.100 .965340 .00044

3.150 .969990 .00043

3.200 .974760 .00044

3.250 .980380 .00043

3.300 .984710 .00043

3.350 .989060 .00044

3.400 .994140 .00043

3.450 .998520 .00044

3.501 .003510 .00045

3.551 .006650 .00044

3.601 .012040 .00043

3.651 .016210 .00043

3.701 .020770 .00044
754

2.700 .921230 .00043

2.750 .926260 .00044

2.800 .931460 .00043

2.850 .936060 .00044

2.900 .941660 .00043

2.950 .946140 .00044

3.000 .951220 .00043

3.050 .954960 .00043

3.100 .960390 .00043

3.150 .965320 .00044

3.200 .969880 .00043

3.250 .974340 .00044

3.300 .979640 .00044

3.350 .983750 .00045

3.400 .988410 .00042

3.450 .992900 .00044

3.500 .998020 .00045

3.551 .002130 .00044

3.601 .007050 .00044

3.651 .010670 .00044

3.701 .015660 .00043
755

2.700 .916830 .00043

2.750 .921270 .00042

2.800 .926620 .00044

2.850 .932200 .00043

2.900 .936630 .00044

2.950 .941690 .00043

3.000 .946360 .00042

3.050 .951320 .00043

3.100 .956660 .00042

3.150 .960370 .00044

3.200 .965250 .00044

3.250 .970560 .00044

3.300 .975100 .00043

3.350 .979930 .00043

3.400 .983030 .00043

3.450 .988000 .00044

3.500 .992600 .00044

3.550 .997010 .00043

3.601 .000870 .00043

3.651 .005550 .00043

3.701 .009980 .00043

3.751 .014300 .00044

3.801 .018710 .00044
U5o2N20-sp-1000-00

12

24.200 .777120 .00025

25.800 .796000 .00024

27.400 .814340 .00024

29.000 .829780 .00023

30.600 .843450 .00023

32.200 .855610 .00022

33.800 .866540 .00022

35.400 .875310 .00022

37.000 .884690 .00021

38.600 .891680 .00020

40.200 .898670 .00020

41.800 .905490 .00020

43.400 .911140 .00019

45.000 .915330 .00019

46.600 .920660 .00018

48.200 .925280 .00018

49.800 .929150 .00017

51.400 .931980 .00017

53.000 .935820 .00017

54.600 .938340 .00017

56.200 .941810 .00017

57.400 .943940 .00017
15

29.100 .927780 .00026

29.700 .934710 .00026

30.300 .940080 .00025

30.900 .944710 .00026

31.500 .949680 .00024

32.100 .954550 .00024

32.700 .959150 .00025

33.300 .962880 .00024

33.900 .968320 .00023

34.500 .972000 .00024

35.100 .975580 .00024

35.700 .979730 .00023

36.300 .983400 .00023

36.900 .986560 .00023

37.500 .989930 .00021

38.100 .992850 .00023

38.700 .996240 .00023

39.300 .998890 .00022

39.901 .001940 .00022

40.501 .004050 .00022

41.101 .006760 .00022

41.301 .008000 .00021
20

21.000 .924200 .00033

21.200 .929490 .00033

21.400 .933850 .00032

21.600 .937700 .00033

21.800 .941540 .00033

22.000 .945210 .00033

22.200 .950870 .00032

22.400 .953750 .00032

22.600 .957680 .00032

22.800 .961890 .00033

23.000 .964880 .00032

23.200 .968780 .00032

23.400 .972950 .00031

23.600 .976000 .00031

23.800 .980500 .00032

24.000 .982880 .00032

24.200 .986810 .00032

24.400 .989980 .00031

24.600 .992930 .00031

24.800 .995860 .00031

25.000 .998910 .00031

25.201 .001970 .00031

25.401 .005300 .00031 
25.601 .007490 .00030

25.801 .011770 .00030

26.001 .014290 .00030

25

18.000 .924540 .00037

18.100 .927960 .00037

18.200 .930360 .00036

18.300 .933190 .00037

18.400 .936940 .00037

18.500 .939960 .00036

18.600 .943400 .00038

18.700 .946130 .00037

18.800 .948870 .00038

18.900 .952010 .00036

19.000 .954380 .00038

19.100 .957570 .00036

19.200 .961030 .00037

19.300 .963950 .00038

19.400 .966170 .00037

19.500 .969100 .00036

19.600 .971860 .00036

19.700 .974460 .00037

19.800 .977540 .00036

19.900 .979680 .00037

20.000 .982590 .00035

20.100 .985130 .00035

20.200 .987800 .00035

20.300 .990260 .00037

20.400 .992890 .00035

20.500 .995620 .00036

20.600 .998860 .00035

20.700 .999710 .00036

20.801 .003240 .00037

20.901 .005580 .00037

21.001 .007190 .00036

21.101 .009980 .00035

21.201 .012290 .00035

21.301 .015190 .00036

21.401 .016070 .00035
30

16.300 .921720 .00041

16.400 .925050 .00039

16.500 .929390 .00040

16.600 .932600 .00040

16.700 .936550 .00040

16.800 .940180 .00041

16.900 .945000 .00040

17.000 .948670 .00040

17.100 .951740 .00041

17.200 .955770 .00040

17.300 .959700 .00040

17.400 .962740 .00040

17.500 .965670 .00040

17.600 .970250 .00040

17.700 .973260 .00039

17.800 .977650 .00038

17.900 .981200 .00039

18.000 .984150 .00039

18.100 .986360 .00039

18.200 .990150 .00038

18.300 .993660 .00040

18.400 .997310 .00039

18.501 .000410 .00040

18.601 .004100 .00039

18.701 .007320 .00038

18.801 .009660 .00039

18.901 .012780 .00040

19.001 .015780 .00038

19.101 .018740 .00039
32

15.800 .919630 .00041

15.900 .924210 .00042 16.000 .927690 .00041

16.100 .931910 .00041

16.200 .935650 .00041

16.300 .940740 .00041

16.400 .945010 .00041

16.500 .948900 .00040

16.600 .952830 .00041

16.700 .955460 .00042

16.800 .961150 .00041

16.900 .963830 .00040

17.000 .967460 .00040

17.100 .971590 .00040

17.200 .975880 .00042

17.300 .979370 .00040

17.400 .982740 .00041

17.500 .986000 .00040

17.600 .990550 .00040

17.700 .993460 .00041

17.800 .996770 .00040

17.901 .000290 .00040

18.001 .003790 .00041

18.101 .007610 .00041

18.201 .011330 .00041

18.301 .014050 .00040

18.401 .017740 .00039
35

15.300 .922970 .00041

15.400 .927710 .00042

15.500 .932080 .00041

15.600 .936290 .00041

15.700 .940830 .00042
40

14.500 .918960 .00044 14.600 .923800 .00045 14.700 .929170 .00046 14.800 .934580 .00043 14.900 .939190 .00045
50

13.600 .921840 .00047 13.700 .926880 .00047 13.800 .933840 .00044 13.900 .938870 .00046 14.000 .945070 .00047 
15.800 .944840 .00042 15.900 .950110 .00043 16.000 .953030 .00042 16.100 .957980 .00043 16.200 .962900 .00042 16.300 .966420 .00041 16.400 .971500 .00042 16.500 .975440 .00041 16.600 .978630 .00042 16.700 .983280 .00041 16.800 .986260 .00041 16.900 .991380 .00041 17.000 .994670 .00041 17.100 .998820 .00041 17.201 .002090 .00041 17.301 .005490 .00042 17.401 .010350 .00043 17.501 .013970 .00041 17.601 .017200 .00041
15.000 .945090 .00044 15.100 .949760 .00045 15.200 .954640 .00045 15.300 .958900 .00046 15.400 .962320 .00044 15.500 .968720 .00044 15.600 .972810 .00044 15.700 .977700 .00044 15.800 .982330 .00044 15.900 .986580 .00044 16.000 .991120 .00044 16.100 .995220 .00044 16.200 .999770 .00044 16.301 .004940 .00044 16.401 .009000 .00044 16.501 .011220 .00043 16.601 .015950 .00043 16.701 .020970 .00043
14.100 .949530 .00047 14.200 .955450 .00046 14.300 .962190 .00047 14.400 .967690 .00045 14.500 .972150 .00046 14.600 .977640 .00047 14.700 .983500 .00046 14.800 .988440 .00047 14.900 .993530 .00048 15.000 .997370 .00047 15.101 .003910 .00047 15.201 .009080 .00047 15.301 .013660 .00045 15.401 .018770 .00046
60

12.900 .914350 .00048 13.000 .921260 .00048 13.100 .928570 .00049 13.200 .934210 .00048 13.300 .941900 .00048 13.400 .946580 .00048 13.500 .952540 .00050 13.600 .959800 .00048 13.700 .965290 .00049 13.800 .971720 .00049 13.900 .978450 .00048 14.000 .983220 .00049 14.100 .989270 .00048 14.200 .994420 .00049 14.300 .999700 .00050 14.401 .005670 .00048 14.501 .012070 .00047 14.601 .017470 .00049 14.701 .022530 .00049
70

12.500 .915120 .00050 12.550 .918320 .00050 12.600 .922610 .00049 12.650 .926550 .00049 12.700 .930180 .00050 12.750 .932030 .00050 12.800 .935460 .00051 12.850 .940040 .00050 12.900 .942270 .00050 12.950 .945590 .00050 13.000 .949500 .00051 13.050 .952180 .00048 13.100 .956320 .00052 13.150 .959010 .00051 13.200 .962760 .00050 13.250 .965750 .00051 13.300 .968500 .00050 13.350 .971140 .00051 13.400 .974610 .00051 13.450 .978020 .00050 13.500 .980450 .00050 13.550 .984380 .00050 13.600 .987960 .00050 13.650 .991260 .00050 13.700 .993520 .00050 13.750 .996260 .00050 13.800 .999080 .00050 13.851 .003000 .00048
80

12.200 .915230 .00051 12.250 .919320 .00052 12.300 .922060 .00051 12.350 .925990 .00050 12.400 .930320 .00051 12.450 .933730 .00051 12.500 .937040 .00051 12.550 .940580 .00052 12.600 .943580 .00051 12.650 .947880 .00051 12.700 .950240 .00049 12.750 .954490 .00051 12.800 .958770 .00052 12.850 .961280 .00050 12.900 .964140 .00052 12.950 .968010 .00052 13.000 .970720 .00052 13.050 .974020 .00050 13.100 .978360 .00050 13.150 .980530 .00050 13.200 .983820 .00051 13.250 .986630 .00053 13.300 .991640 .00051 13.350 .994020 .00050 13.400 .997510 .00052 13.450 .999990 .00049 13.501 .003260 .00052 13.551 .006500 .00051 
13.901 .005760 .00049 13.951 .008270 .00049 14.001 .011840 .00049 14.051 .014340 .00049 14.101 .017900 .00051 14.151 .019900 .00050 14.201 .023990 .00049
13.601 .010740 .00051 13.651 .013370 .00051 13.701 .015670 .00052 13.751 .018510 .00051 13.801 .021680 .00051
90

12.000 .917130 .00052 12.050 .920400 .00053 12.100 .925070 .00051 12.150 .927720 .00052 12.200 .931160 .00052 12.250 .935430 .00053 12.300 .938420 .00051 12.350 .942510 .00052 12.400 .946300 .00052 12.450 .950410 .00053 12.500 .954100 .00050 12.550 .957490 .00051 12.600 .960900 .00052 12.650 .964780 .00052 12.700 .967040 .00053 12.750 .971110 .00053 12.800 .973000 .00054 12.850 .977320 .00052 12.900 .981140 .00051 12.950 .985470 .00052 13.000 .988130 .00052 13.050 .991520 .00051 13.100 .994980 .00051 13.150 .997380 .00052 13.201 .001230 .00052 13.251 .004650 .00052 13.301 .007940 .00052 13.351 .010470 .00051 13.401 .014250 .00051 13.451 .017560 .00051 13.501 .021090 .00051
100

11.800 .914480 .00052 11.850 .918360 .00053 11.900 .922340 .00052 11.950 .925830 .00053 12.000 .929650 .00051 12.050 .933730 .00053 12.100 .936980 .00054 12.150 .942070 .00053 12.200 .945320 .00052 12.250 .948600 .00053 12.300 .951170 .00053 12.350 .956320 .00052 12.400 .959900 .00052 12.450 .963110 .00053 12.500 .966660 .00052 12.550 .970070 .00054 12.600 .973450 .00054 12.650 .977190 .00053 12.700 .980840 .00051 12.750 .983900 .00052 12.800 .987890 .00052 12.850 .990950 .00053 12.900 .994770 .00053 12.950 .998430 .00053 13.001 .002950 .00052 13.051 .004090 .00052 13.101 .008480 .00052 13.151 .012550 .00052 13.201 .015290 .00053 13.251 .018750 .00053 13.301 .021630 .00052
130 11.500 .919410 .00053 11.550 .922550 .00054 11.600 .926990 .00053 11.650 .931110 .00055 11.700 .934930 .00054
150 11.300 .914180 .00054 11.350 .917780 .00053 11.400 .922920 .00054 11.450 .927080 .00052 11.500 .932610 .00053
118

11.600 .916800 .00054 11.650 .920900 .00053 11.700 .924180 .00054 11.750 .928330 .00053 11.800 .932030 .00054 11.850 .935830 .00053 11.900 .939830 .00054 11.950 .944210 .00053 12.000 .949050 .00053 12.050 .952240 .00054 12.100 .955730 .00055 12.150 .959860 .00053 12.200 .964220 .00054 12.250 .966550 .00054 12.300 .970490 .00053 12.350 .974640 .00053 12.400 .978480 .00053 12.450 .981270 .00054 12.500 .986180 .00054 12.550 .989530 .00053 12.600 .992110 .00054 12.650 .997520 .00052 12.700 .999570 .00054 12.751 .003380 .00054 12.801 .007060 .00056 12.851 .010900 .00054 12.901 .014110 .00056 12.951 .018100 .00053 13.001 .021010 .00054

\section{0}

11.100 .915810 .00053 11.150 .920010 .00053 11.200 .924860 .00057 11.250 .927580 .00055 11.300 .933600 .00056 
11.750 .937900 .00054 11.800 .943080 .00055 11.850 .947260 .00052 11.900 .950970 .00053 11.950 .954940 .00054 12.000 .958290 .00053 12.050 .961780 .00053 12.100 .966480 .00053 12.150 .970160 .00053 12.200 .973900 .00055 12.250 .977120 .00054 12.300 .980850 .00054 12.350 .985010 .00056 12.400 .988730 .00054 12.450 .992780 .00053 12.500 .996240 .00054 12.551 .000870 .00054 12.601 .002790 .00053 12.651 .006880 .00055 12.701 .010350 .00053 12.751 .014340 .00055 12.801 .018860 .00053 12.851 .021050 .00054 12.901 .024510 .00054

235

11.000 .913330 .00056 11.050 .917830 .00056 11.100 .921430 .00056 11.150 .927080 .00055 11.200 .930230 .00056 11.250 .934480 .00055 11.300 .939120 .00055 11.350 .943820 .00055 11.400 .946710 .00054 11.450 .951940 .00055 11.500 .955660 .00056 11.550 .960060 .00056 11.600 .964180 .00056 11.650 .968640 .00055 11.700 .972730 .00055 11.750 .976120 .00056 11.800 .979550 .00056 11.850 .983380 .00056 11.900 .988070 .00054 11.950 .991440 .00055 12.000 .994960 .00057 12.051 .000330 .00056 12.101 .003880 .00057
11.550 .936160 .00055 11.600 .939800 .00054 11.650 .944150 .00054 11.700 .947250 .00056 11.750 .951320 .00054 11.800 .955330 .00056 11.850 .959490 .00053 11.900 .963400 .00055 11.950 .967050 .00055 12.000 .971510 .00057 12.050 .974860 .00056 12.100 .979310 .00054 12.150 .982430 .00055 12.200 .985700 .00055 12.250 .989860 .00055 12.300 .992630 .00057 12.350 .998050 .00055 12.401 .001420 .00054 12.451 .005060 .00054 12.501 .008000 .00054 12.551 .012920 .00055 12.601 .016770 .00054 12.651 .020960 .00054 12.701 .023580 .00053
11.350 .937300 .00054 11.400 .941290 .00055 11.450 .945000 .00055 11.500 .949610 .00055 11.550 .952900 .00056 11.600 .959050 .00055 11.650 .962380 .00056 11.700 .966240 .00054 11.750 .969270 .00055 11.800 .972330 .00055 11.850 .976990 .00056 11.900 .980740 .00055 11.950 .984390 .00056 12.000 .989170 .00056 12.050 .993250 .00057 12.100 .997090 .00056 12.151 .000620 .00055 12.201 .004260 .00057 12.251 .007790 .00054 12.301 .012350 .00054 12.351 .015950 .00054 12.401 .019600 .00056 12.451 .023120 .00054 12.501 .027180 .00055
250 353
11.000 .915560 .00056 11.050 .920140 .00054 11.100 .924260 .00056 11.150 .926970 .00056 11.200 .931890 .00056 11.250 .936780 .00056 11.300 .941190 .00056 11.350 .945620 .00056 11.450 .952670 .00055 11.500 .957820 .00054 11.550 .961380 .00057 11.600 .965600 .00056 11.650 .969290 .00055 11.700 .973190 .00056 11.750 .977170 .00057 11.800 .982190 .00056 11.850 .985330 .00056 11.900 .990250 .00056 11.950 .993790 .00057 12.000 .997810 .00056 12.051 .001610 .00057 12.101 .005000 .00055 12.151 .010640 .00055 
12.151 .007270 .00054 12.201 .010340 .00055 12.251 .015080 .00057 12.301 .017910 .00055 12.351 .022850 .00055 12.401 .025990 .00056

470

11.000 .913450 .00056 11.050 .916820 .00055 11.100 .921190 .00054 11.150 .925420 .00054 11.200 .929810 .00055 11.250 .933640 .00056 11.300 .937270 .00055 11.350 .941560 .00056 11.400 .945880 .00055 11.450 .948550 .00056 11.500 .954920 .00054 11.550 .958800 .00055 11.600 .962560 .00054 11.650 .965980 .00056 11.700 .970540 .00055 11.750 .973800 .00053 11.800 .978170 .00057 11.850 .982840 .00056 11.900 .986550 .00056 11.950 .988820 .00055 12.000 .993490 .00056 12.050 .996890 .00055 12.101 .000770 .00056 12.151 .004320 .00053 12.201 .009070 .00056 12.251 .012510 .00055 12.301 .015560 .00055 12.351 .019480 .00055 12.401 .024480 .00055

605

11.200 .916430 .00054 11.250 .920700 .00055 11.300 .923490 .00054 11.350 .927950 .00055 11.400 .932370 .00054 11.450 .935730 .00054 11.500 .940130 .00055 11.550 .944550 .00055 11.600 .947260 .00055 11.650 .952610 .00053
12.201 .014040 .00054

12.251 .015860 .00057

12.301 .021090 .00057

500

11.100 .919420 .00055 11.150 .923510 .00055 11.200 .927980 .00054 11.250 .932480 .00055 11.300 .936110 .00054 11.350 .939760 .00054 11.400 .942100 .00055 11.450 .947750 .00055 11.500 .952040 .00055 11.550 .956970 .00056 11.600 .960900 .00055 11.650 .963680 .00057 11.700 .967440 .00055 11.750 .972440 .00055 11.800 .976480 .00055 11.850 .978930 .00054 11.900 .983340 .00054 11.950 .987530 .00055 12.000 .990610 .00055 12.050 .995230 .00055 12.100 .999520 .00055 12.151 .001770 .00056 12.201 .005490 .00056 12.251 .009980 .00055 12.301 .013200 .00056 12.351 .017110 .00057 12.401 .020410 .00054

610 11.200 .914990 .00055 11.250 .919130 .00055 11.300 .923740 .00055 11.350 .926590 .00054 11.400 .931710 .00054 11.450 .935460 .00053 11.500 .939630 .00055 11.550 .943510 .00055 11.600 .947220 .00054 11.650 .951550 .00056
12.151 .010760 .00057

12.201 .013670 .00055

12.251 .017820 .00058

12.301 .022960 .00056

11.200 .916210 .00054 11.250 .920330 .00053 11.300 .924850 .00055 11.350 .928810 .00054 11.400 .933140 .00055 11.450 .936000 .00056 11.500 .941470 .00055 11.550 .943750 .00054 11.600 .947830 .00052 11.650 .952450 .00055 11.700 .956360 .00054 11.750 .959820 .00054 11.800 .963410 .00054 11.850 .966970 .00053 11.900 .970190 .00052 11.950 .975220 .00056 12.000 .979390 .00054 12.050 .982450 .00055 12.100 .986570 .00054 12.150 .990300 .00054 12.200 .994110 .00054 12.250 .997690 .00055 12.301 .000900 .00054 12.351 .005000 .00055 12.401 .009120 .00054 12.451 .012370 .00055 12.501 .016350 .00054 12.551 .017960 .00055 12.601 .024270 .00053

615

11.200 .915490 .00056 11.250 .919080 .00054 11.300 .924250 .00055 11.350 .927620 .00054 11.400 .932130 .00055 11.450 .935800 .00055 11.500 .937920 .00053 11.550 .942860 .00055 11.600 .947090 .00054 11.650 .950520 .00054 
11.700 .956490 .00053 11.750 .960360 .00054 11.800 .963930 .00055 11.850 .966280 .00055 11.900 .972470 .00054 11.950 .975750 .00056 12.000 .978800 .00053 12.050 .982030 .00055 12.100 .986520 .00053 12.150 .990070 .00054 12.200 .993840 .00054 12.250 .997770 .00055 12.300 .999780 .00055 12.351 .004460 .00056 12.401 .008240 .00054 12.451 .010910 .00054 12.501 .016190 .00053 12.551 .019710 .00056 12.601 .022530 .00055

\section{0}

11.200 .912750 .00054 11.250 .918390 .00054 11.300 .921850 .00054 11.350 .925640 .00054 11.400 .930550 .00053 11.450 .932820 .00054 11.500 .938130 .00054 11.550 .941750 .00055 11.650 .949370 .00055 11.700 .954230 .00054 11.750 .958660 .00054 11.800 .961340 .00054 11.850 .964620 .00053 11.900 .968600 .00054 11.950 .972600 .00053 12.000 .977150 .00054 12.050 .980680 .00055 12.100 .984520 .00054 12.150 .987340 .00056 12.200 .991120 .00055 12.250 .995380 .00055 12.300 .999170 .00054 12.351 .002280 .00055 12.401 .005560 .00053 12.451 .009550 .00054 12.501 .011840 .00054 12.551 .016130 .00056 12.601 .019960 .00056
11.700 .956420 .00056 11.750 .959190 .00054 11.800 .962500 .00054 11.850 .966970 .00055 11.900 .970730 .00054 11.950 .974400 .00054 12.000 .978720 .00055 12.050 .981300 .00055 12.100 .985640 .00054 12.150 .989480 .00055 12.200 .993600 .00054 12.250 .997110 .00055 12.300 .999810 .00055 12.351 .004480 .00056 12.401 .007960 .00055 12.451 .012090 .00054 12.501 .014300 .00054 12.551 .018960 .00055 12.601 .021400 .00054

\section{5}

11.200 .914030 .00053 11.250 .917340 .00055 11.300 .922190 .00053 11.350 .925490 .00053 11.400 .929100 .00056 11.450 .933700 .00055 11.500 .937140 .00053 11.550 .941320 .00054 11.600 .945550 .00055 11.650 .949550 .00053 11.700 .952880 .00053 11.750 .957220 .00055 11.800 .962210 .00056 11.850 .964480 .00053 11.900 .968010 .00054 11.950 .972620 .00055 12.000 .975030 .00054 12.050 .980140 .00055 12.100 .983100 .00055 12.150 .988540 .00054 12.200 .991270 .00053 12.250 .995220 .00054 12.300 .997460 .00055 12.351 .001900 .00054 12.401 .005540 .00056 12.451 .009170 .00055 12.501 .012870 .00054 12.551 .015840 .00056 12.601 .020900 .00054
11.700 .954420 .00054 11.750 .959430 .00054 11.800 .961760 .00054 11.850 .966150 .00054 11.900 .970400 .00054 11.950 .975620 .00055 12.000 .978520 .00053 12.050 .982660 .00055 12.100 .984880 .00055 12.150 .989840 .00054 12.200 .993740 .00054 12.250 .997120 .00053 12.301 .000670 .00054 12.351 .003880 .00054 12.401 .007680 .00056 12.451 .010700 .00054 12.501 .015200 .00055 12.551 .018370 .00054 12.601 .021280 .00053

\section{9}

11.300 .920790 .00053 11.350 .924390 .00055 11.400 .928130 .00055 11.450 .932510 .00053 11.500 .937220 .00055 11.550 .940940 .00052 11.600 .945040 .00054 11.650 .948280 .00054 11.700 .951870 .00054 11.750 .956380 .00054 11.800 .960530 .00054 11.850 .964430 .00051 11.900 .968760 .00054 11.950 .971700 .00054 12.000 .975570 .00054 12.050 .978850 .00054 12.100 .982580 .00055 12.150 .985780 .00056 12.200 .988890 .00054 12.250 .994210 .00054 12.300 .997240 .00054 12.351 .002300 .00054 12.401 .004270 .00055 12.451 .008000 .00055 12.501 .012830 .00056 12.551 .015320 .00055 12.601 .019530 .00055 
U5o2N20-sp-1000-01

12

38.800 .899270 .00020

39.700 .902820 .00019

40.600 .906070 .00019

41.500 .909420 .00018

42.400 .912520 .00019

43.300 .915710 .00019

44.200 .917810 .00019

45.100 .920670 .00018

46.000 .922960 .00017

46.900 .925440 .00018

47.800 .927510 .00018

48.700 .929250 .00018

49.600 .931860 .00017

50.500 .933180 .00017

51.400 .935800 .00016

52.300 .936670 .00016

53.200 .938790 .00017

54.100 .940590 .00017

55.000 .942570 .00017

55.900 .943280 .00016

56.800 .945030 .00016

57.000 .945030 .00016
15

27.500 .927510 .00025

28.100 .932780 .00025

28.700 .938290 .00025

29.300 .943580 .00025

29.900 .948790 .00024

30.500 .954520 .00025

31.100 .958810 .00024

31.700 .963080 .00024

32.300 .967120 .00023

32.900 .971840 .00023

33.500 .975020 .00023

34.100 .979020 .00023

34.700 .982590 .00023

35.300 .986400 .00023

35.900 .989100 .00022

36.500 .993830 .00021

37.100 .995520 .00022

37.700 .998650 .00022

38.301 .001340 .00022

38.901 .004260 .00021

39.401 .006240 .00021
25

16.400 .921840 .00036

16.500 .924770 .00037

16.600 .927490 .00036

16.700 .931260 .00036

16.800 .934150 .00035

16.900 .936480 .00036

17.000 .940400 .00035

17.100 .943410 .00035

17.200 .947060 .00035

17.300 .949400 .00036

17.400 .952150 .00036

17.500 .955530 .00036

17.600 .958360 .00035

17.700 .961670 .00035

17.800 .963920 .00036

17.900 .966700 .00035
30

14.800 .921160 .00038

14.900 .925360 .00038

15.000 .929880 .00039

15.100 .933200 .00038

15.200 .937270 .00038

15.300 .941670 .00038

15.400 .945130 .00039

15.500 .948320 .00037

15.600 .952900 .00038

15.700 .956570 .00037

15.800 .960530 .00037

15.900 .963500 .00038

16.000 .967040 .00038

16.100 .970610 .00038

16.200 .973980 .00038

16.300 .977350 .00039
20

19.500 .924910 .00031

19.700 .929610 .00032

19.900 .933470 .00032

20.100 .937360 .00032

20.300 .941830 .00032

20.500 .946010 .00032

20.700 .950560 .00032

20.900 .954140 .00031

21.100 .957600 .00030

21.300 .961690 .00031

21.500 .966280 .00031

21.700 .968930 .00031

21.900 .972220 .00031

22.100 .976550 .00031

22.300 .979970 .00030

22.500 .983430 .00029

22.700 .987010 .00031

22.900 .989740 .00030

23.100 .992910 .00031

23.300 .995890 .00030

23.500 .999480 .00030

23.701 .002870 .00030

23.901 .005060 .00030

24.101 .008320 .00030

24.301 .011330 .00030

24.501 .014700 .00030

24.601 .015400 .00029

32

14.300 .919200 .00040

14.400 .923910 .00039

14.500 .928740 .00040

14.600 .932970 .00039

14.700 .936450 .00039

14.800 .941220 .00039

14.900 .944420 .00040

15.000 .949040 .00039

15.100 .952970 .00038

15.200 .957640 .00039

15.300 .960640 .00039

15.400 .964700 .00040

15.500 .967980 .00039

15.600 .972920 .00039

15.700 .976670 .00040

15.800 .979640 .00039 
18.000 .969290 .00035 18.100 .972720 .00037 18.200 .974750 .00035 18.300 .978540 .00034 18.400 .980940 .00036 18.500 .983230 .00036 18.600 .986310 .00034 18.700 .987860 .00034 18.800 .990340 .00035 18.900 .992990 .00036 19.000 .995530 .00035 19.100 .998360 .00034 19.201 .001120 .00035 19.301 .003190 .00034 19.401 .005420 .00033 19.501 .008560 .00035 19.601 .010930 .00034 19.701 .012660 .00034 19.801 .015170 .00035
16.400 .981510 .00039 16.500 .984070 .00038 16.600 .987850 .00037 16.700 .990660 .00038 16.800 .994750 .00037 16.900 .997100 .00038 17.001 .000870 .00037 17.101 .003710 .00037 17.201 .008160 .00036 17.301 .010310 .00037 17.401 .013430 .00039 17.501 .016840 .00037
15.900 .983050 .00038 16.000 .987200 .00038 16.100 .991270 .00040 16.200 .993980 .00038 16.300 .998050 .00039 16.401 .001040 .00039 16.501 .004920 .00040 16.601 .008120 .00038 16.701 .011080 .00037 16.801 .014360 .00038 16.901 .018730 .00040
35

13.700 .918760 .00041 13.800 .922510 .00040 13.900 .928100 .00041 14.000 .932230 .00041 14.100 .937220 .00041 14.200 .941100 .00039 14.300 .946310 .00041 14.400 .950570 .00040 14.500 .955110 .00039 14.600 .958760 .00040 14.700 .963930 .00041 14.800 .966900 .00041 14.900 .971850 .00041 15.000 .975510 .00039 15.100 .979850 .00041 15.200 .983520 .00040 15.300 .987370 .00039 15.400 .991160 .00041 15.500 .996030 .00041 15.600 .999800 .00040 15.701 .004010 .00041 15.801 .006640 .00040 15.901 .011170 .00040 16.001 .014150 .00039 16.101 .017780 .00040
40

13.000 .919770 .00042 13.100 .923320 .00042 13.200 .930120 .00043 13.300 .935030 .00043 13.400 .939950 .00042 13.500 .945770 .00042 13.600 .950020 .00043 13.700 .954680 .00042 13.800 .959330 .00042 13.900 .964220 .00042 14.000 .969850 .00042 14.100 .973190 .00042 14.200 .978850 .00043 14.300 .983520 .00042 14.400 .986970 .00043 14.500 .991190 .00044 14.600 .996380 .00042 14.700 .999820 .00042 14.801 .004410 .00042 14.901 .009050 .00040 15.001 .013250 .00042 15.101 .017470 .00042
50

12.000 .916250 .00045 12.100 .922900 .00044 12.200 .928330 .00045 12.300 .934070 .00045 12.400 .938900 .00045 12.500 .945070 .00045 12.600 .951630 .00045 12.700 .956030 .00045 12.800 .963010 .00045 12.900 .968970 .00045 13.000 .972730 .00044 13.100 .979290 .00045 13.200 .984060 .00045 13.300 .989330 .00045 13.400 .993910 .00044 13.500 .999570 .00044 13.601 .004700 .00044 13.701 .009570 .00044 13.801 .014680 .00044 13.901 .019190 .00045 
60

11.400 .915890 .00047 11.500 .921450 .00048 11.600 .929850 .00046 11.700 .934870 .00046 11.800 .941390 .00047 11.900 .948210 .00045 12.000 .953930 .00046 12.100 .959820 .00047 12.200 .965350 .00048 12.300 .972470 .00048 12.400 .978340 .00046 12.500 .984940 .00046 12.600 .989550 .00047 12.700 .996240 .00046 12.801 .000770 .00047 12.901 .005820 .00047 13.001 .011690 .00048 13.101 .017660 .00046 13.201 .022990 .00046

\section{0}

10.500 .918120 .00049 10.550 .921860 .00050 10.600 .926210 .00048 10.650 .930330 .00050 10.700 .932510 .00049 10.750 .937440 .00050 10.800 .940300 .00049 10.850 .943940 .00050 10.900 .947680 .00050 10.950 .950580 .00050 11.000 .954570 .00050 11.050 .958750 .00049 11.100 .961950 .00049
70

11.000 .916100 .00047 11.050 .919710 .00049 11.100 .924250 .00048 11.150 .926150 .00049 11.200 .930000 .00048 11.250 .933680 .00047 11.300 .936750 .00048 11.350 .939690 .00048 11.400 .943090 .00048 11.450 .947330 .00049 11.500 .950690 .00047 11.550 .953370 .00048 11.600 .956660 .00048 11.650 .960550 .00048 11.700 .963100 .00048 11.750 .966330 .00047 11.800 .969800 .00048 11.850 .973340 .00047 11.900 .976740 .00049 11.950 .979950 .00047 12.000 .983070 .00049 12.050 .986050 .00048 12.100 .988130 .00049 12.150 .991600 .00048 12.200 .994350 .00048 12.250 .998500 .00046 12.301 .001060 .00048 12.351 .003950 .00048 12.401 .006380 .00048 12.451 .009740 .00048 12.501 .012610 .00048 12.551 .016540 .00050 12.601 .018450 .00047

100

10.300 .916390 .00050 10.350 .919410 .00051 10.400 .923450 .00050 10.450 .927160 .00048 10.500 .931620 .00050 10.550 .935220 .00051 10.600 .939200 .00051 10.650 .942360 .00050 10.700 .947500 .00051 10.750 .949730 .00051 10.800 .953620 .00050 10.850 .957690 .00052 10.900 .961380 .00049
80

10.700 .916120 .00049

10.750 .918880 .00049

10.800 .923100 .00047

10.850 .926230 .00049

10.900 .931440 .00049

10.950 .934990 .00048

11.000 .938530 .00050 11.050 .942700 .00047 11.100 .944750 .00049 11.150 .948540 .00050 11.200 .951530 .00049 11.250 .955890 .00049 11.300 .958070 .00049 11.350 .961720 .00048 11.400 .965930 .00048 11.450 .968810 .00048 11.500 .972920 .00049 11.550 .975050 .00049 11.600 .979960 .00050 11.650 .981210 .00049 11.700 .986180 .00048 11.750 .989130 .00048 11.800 .991840 .00049 11.850 .995670 .00048 11.900 .998870 .00049 11.951 .001170 .00048 12.001 .005700 .00050 12.051 .008460 .00049 12.101 .010870 .00049 12.151 .014700 .00050 12.201 .017150 .00050 12.251 .021460 .00048 12.301 .023890 .00047

118

10.100 .919720 .00051 10.150 .922010 .00053 10.200 .926470 .00051 10.250 .930330 .00051 10.300 .934890 .00052 10.350 .938320 .00051 10.400 .941900 .00050 10.450 .946300 .00050 10.500 .950130 .00051 10.550 .954380 .00052 10.600 .957970 .00052 10.650 .962090 .00050 10.700 .966030 .00053 
11.150 .964990 .00050 11.200 .968360 .00049 11.250 .971590 .00050 11.300 .976240 .00049 11.350 .979170 .00049 11.400 .982830 .00050 11.450 .987080 .00050 11.500 .989920 .00048 11.550 .994380 .00052 11.600 .995350 .00049 11.650 .999930 .00050 11.701 .002340 .00050 11.751 .006380 .00051 11.801 .009690 .00050 11.851 .012950 .00051 11.901 .016490 .00049 11.951 .019320 .00049 12.001 .021680 .00050
10.950 .965080 .00051 11.000 .969290 .00051 11.050 .972620 .00050 11.100 .976080 .00050 11.150 .980320 .00049 11.200 .983720 .00050 11.250 .986260 .00050 11.300 .990130 .00052 11.350 .992560 .00051 11.400 .995440 .00051 11.450 .999890 .00050 11.501 .003370 .00052 11.551 .006740 .00051 11.601 .010440 .00050 11.651 .014490 .00050 11.701 .016070 .00051 11.751 .020060 .00052 11.801 .023980 .00051
10.750 .969960 .00054 10.800 .972200 .00051 10.850 .977590 .00050 10.900 .981490 .00051 10.950 .983010 .00050 11.000 .987790 .00051 11.050 .991760 .00052 11.100 .995430 .00052 11.150 .999020 .00051 11.201 .002930 .00052 11.251 .005340 .00052 11.301 .009440 .00052 11.351 .012310 .00051 11.401 .016450 .00052 11.451 .019550 .00053 11.501 .023620 .00052
130

10.000 .920630 .00051 10.050 .925340 .00052 10.100 .928420 .00053 10.150 .932770 .00052 10.200 .937210 .00051 10.250 .941020 .00051 10.300 .945100 .00053 10.350 .949570 .00052 10.400 .953010 .00051 10.450 .956500 .00050 10.500 .960380 .00052 10.550 .964170 .00052 10.600 .968860 .00051 10.650 .971690 .00052 10.700 .976080 .00052 10.750 .978170 .00052 10.800 .983110 .00052 10.850 .987450 .00053 10.900 .990370 .00051 10.950 .994070 .00052 11.000 .997510 .00051 11.051 .000430 .00052 11.101 .005060 .00051 11.151 .009490 .00050 11.201 .012780 .00051 11.251 .016560 .00050 11.301 .019980 .00052
150

9.800 .916030 .00051 9.850 .921550 .00052 9.900 .924460 .00051 9.950 .928810 .00054 10.000 .933020 .00051 10.050 .938010 .00051 10.100 .942350 .00053 10.150 .945670 .00052 10.200 .949790 .00052 10.250 .953670 .00053 10.300 .958070 .00053 10.350 .961270 .00052 10.400 .965710 .00051 10.450 .968990 .00052 10.500 .973000 .00052 10.550 .976310 .00051 10.600 .980950 .00051 10.650 .984980 .00054 10.700 .988510 .00052 10.750 .992760 .00052 10.800 .995760 .00052 10.850 .999830 .00053 10.901 .003780 .00053 10.951 .007820 .00053 11.001 .011040 .00053 11.051 .015160 .00054 11.101 .017120 .00054 11.151 .021000 .00054 11.201 .024980 .00053
200

9.600 .918620 .00052 9.650 .921720 .00053 9.700 .925920 .00053 9.750 .931450 .00053 9.800 .935390 .00051 9.850 .939370 .00054 9.900 .943170 .00052 9.950 .946980 .00054 10.000 .951520 .00053 10.050 .955800 .00053 10.100 .959870 .00053 10.150 .963900 .00054 10.200 .967610 .00053 10.250 .971850 .00052 10.300 .975310 .00053 10.350 .979780 .00054 10.400 .985010 .00053 10.450 .988730 .00054 10.500 .990780 .00055 10.550 .995600 .00052 10.600 .999160 .00053 10.651 .002250 .00054 10.701 .007280 .00053 10.751 .011020 .00053 10.801 .014800 .00053 10.851 .018070 .00051 10.901 .021690 .00053 
235

9.500 .915510 .00054

9.550 .920430 .00054

9.600 .926010 .00053

9.650 .928540 .00053

9.700 .933070 .00053

9.750 .937050 .00053

9.800 .941480 .00052

9.850 .945930 .00053

9.900 .950880 .00054

9.950 .954510 .00053

10.000 .958430 .00053

10.050 .963170 .00052

10.100 .966140 .00053

10.150 .971670 .00052

10.200 .975360 .00053

10.250 .979150 .00054

10.300 .982920 .00052

10.350 .986560 .00054

10.400 .990200 .00053

10.450 .994890 .00052

10.500 .998170 .00054

10.551 .002450 .00053

10.601 .006430 .00053

10.651 .009090 .00053

10.701 .013490 .00054

10.801 .021710 .00054

470

9.500 .916320 .00053

9.550 .920650 .00053

9.600 .924350 .00054

9.650 .929610 .00053

9.700 .932970 .00054

9.750 .936900 .00052

9.800 .942570 .00053

9.850 .945280 .00055

9.900 .949830 .00055

9.950 .953710 .00054

10.000 .959100 .00054

10.050 .961870 .00053

10.100 .965310 .00053

10.150 .969870 .00053

10.200 .974320 .00054

10.250 .976860 .00054
250

9.500 .917720 .00054

9.550 .922650 .00052

9.600 .926670 .00054

9.650 .930910 .00053

9.700 .935950 .00053

9.750 .938890 .00054

9.800 .943810 .00054

9.850 .947780 .00051

9.900 .951640 .00054

9.950 .955730 .00053

10.000 .960070 .00053

10.050 .964380 .00053

10.100 .968370 .00055

10.150 .973130 .00053

10.200 .976560 .00054

10.250 .981170 .00055

10.300 .984700 .00053

10.350 .988780 .00055

10.400 .991400 .00053

10.450 .995820 .00055

10.501 .000200 .00054

10.551 .004810 .00055

10.601 .008290 .00053

10.651 .012210 .00055

10.701 .015190 .00053

10.751 .018780 .00052

10.801 .023470 .00053
353

9.500 .921980 .00054

9.550 .925360 .00052

9.600 .930080 .00054

9.650 .934050 .00053

9.700 .938990 .00054

9.750 .942620 .00053

9.800 .946460 .00054

9.850 .951310 .00053

9.900 .954850 .00054

9.950 .957650 .00054

10.000 .963220 .00054

10.050 .967740 .00054

10.100 .972760 .00054

10.150 .975660 .00053

10.200 .980260 .00055

10.250 .983530 .00054

10.300 .987520 .00053

10.350 .992250 .00053

10.400 .995100 .00054

10.450 .999260 .00052

10.501 .002730 .00055

10.551 .007840 .00053

10.601 .010630 .00054

10.651 .014550 .00055

10.701 .017900 .00054

10.751 .022130 .00054

10.801 .025790 .00053
500

9.500 .914930 .00051

9.550 .918840 .00052

9.600 .922750 .00054

9.650 .926650 .00052

9.700 .931350 .00053

9.750 .935600 .00053

9.800 .938950 .00055

9.850 .943340 .00051

9.900 .947450 .00054

9.950 .952100 .00053

10.000 .956030 .00052

10.050 .959530 .00052

10.100 .964210 .00053

10.150 .967740 .00053

10.200 .973030 .00053

10.250 .976520 .00052
600

9.700 .920810 .00054

9.750 .924630 .00052

9.800 .928570 .00052

9.850 .933290 .00053

9.900 .937470 .00053

9.950 .940230 .00051

10.000 .945160 .00054

10.050 .948320 .00052

10.100 .953320 .00052

10.150 .956650 .00053

10.200 .959920 .00052

10.250 .964160 .00052

10.300 .968360 .00052

10.350 .971550 .00053

10.400 .976270 .00053

10.450 .979350 .00053 
10.300 .982520 .00054 10.350 .985980 .00054 10.400 .989720 .00055 10.450 .992710 .00054 10.500 .997530 .00053 10.551 .001620 .00052 10.601 .005120 .00053 10.651 .007820 .00054 10.701 .010810 .00054 10.751 .015570 .00053 10.801 .020600 .00056 10.851 .022940 .00056 10.901 .026800 .00055
10.300 .980190 .00053 10.350 .983800 .00053 10.400 .987020 .00055 10.450 .991830 .00053 10.500 .994160 .00052 10.550 .996920 .00054 10.601 .002840 .00052 10.651 .005890 .00053 10.701 .009790 .00055 10.751 .013250 .00052 10.801 .015720 .00054 10.851 .021050 .00053 10.901 .024440 .00055
10.500 .984540 .00054 10.550 .987430 .00055 10.600 .991420 .00055 10.650 .994290 .00053 10.700 .996900 .00054 10.751 .002300 .00053 10.801 .005800 .00052 10.851 .010410 .00053 10.901 .012970 .00053 10.951 .016570 .00052 11.001 .020140 .00053
605

9.700 .920250 .00052 9.750 .924260 .00052 9.800 .928500 .00054 9.850 .932510 .00053 9.900 .936950 .00052 9.950 .941020 .00052 10.000 .943680 .00052 10.050 .948760 .00054 10.100 .952360 .00054 10.150 .955900 .00053 10.200 .960650 .00052 10.250 .963910 .00053 10.300 .968460 .00052 10.350 .971150 .00054 10.400 .976270 .00052 10.450 .979660 .00054 10.500 .983570 .00053 10.550 .987390 .00053 10.600 .991880 .00055 10.650 .995050 .00053 10.700 .998280 .00053 10.751 .002080 .00054 10.801 .005870 .00055 10.851 .008350 .00054 10.901 .013470 .00054 10.951 .016680 .00053 11.001 .020670 .00053
610

9.700 .920110 .00053

9.750 .924600 .00052

9.800 .929140 .00053

9.850 .931060 .00053

9.900 .936230 .00052

9.950 .940190 .00053

10.000 .945300 .00052 10.050 .948730 .00051 10.100 .951980 .00054 10.150 .955400 .00053 10.200 .960280 .00053 10.250 .963420 .00053 10.300 .967290 .00054 10.350 .970590 .00054 10.400 .974200 .00054 10.450 .979560 .00052 10.500 .983360 .00052 10.550 .986040 .00052 10.600 .989680 .00054 10.650 .993570 .00052 10.700 .996530 .00053 10.751 .001150 .00055 10.801 .004480 .00053 10.851 .008830 .00052 10.901 .011780 .00053 10.951 .015700 .00054 11.001 .019480 .00053 11.051 .023120 .00053 11.101 .026540 .00056
615

9.700 .919530 .00052 9.750 .924220 .00052 9.800 .928490 .00053 9.850 .931990 .00053 9.900 .935740 .00051 9.950 .940130 .00053 10.000 .943870 .00053 10.050 .948130 .00055 10.100 .952380 .00053 10.150 .955030 .00054 10.200 .958960 .00052 10.250 .963790 .00051 10.300 .966560 .00053 10.350 .970900 .00052 10.400 .975020 .00052 10.450 .978780 .00054 10.500 .981860 .00051 10.550 .986320 .00051 10.600 .990250 .00053 10.650 .993170 .00053 10.700 .996130 .00054 10.751 .000150 .00055 10.801 .003460 .00052 10.851 .007970 .00053 10.901 .011520 .00053 10.951 .015490 .00054 11.001 .019290 .00052 
620

9.700 .919440 .00052

9.750 .922400 .00052

9.800 .926070 .00052

9.900 .934800 .00051

9.950 .938970 .00052

10.000 .942110 .00052

10.050 .946460 .00053

10.100 .949890 .00053

10.150 .955850 .00053

10.200 .958160 .00052

10.250 .961430 .00054

10.300 .966010 .00052

10.350 .969640 .00052

10.400 .974100 .00053

10.450 .976170 .00052

10.500 .981140 .00054

10.550 .984430 .00053

10.600 .988800 .00054

10.650 .992490 .00053

10.700 .996180 .00054

10.750 .999690 .00055

10.801 .002870 .00053

10.851 .006720 .00053

10.901 .011120 .00053

10.951 .013980 .00054

11.001 .016960 .00052
625

9.700 .917210 .00051

9.750 .921960 .00053

9.800 .926590 .00052

9.850 .930650 .00052

9.900 .933720 .00054

9.950 .938360 .00054

10.000 .941830 .00054

10.050 .945810 .00053

10.100 .949610 .00051

10.150 .954250 .00052

10.200 .958090 .00053

10.250 .961740 .00053

10.300 .965870 .00052

10.350 .970150 .00053

10.400 .972580 .00052

10.450 .976540 .00053

10.500 .981120 .00052

10.550 .984540 .00052

10.600 .988250 .00053

10.650 .991650 .00053

10.700 .995970 .00054

10.750 .999600 .00052

10.801 .002840 .00053

10.851 .006630 .00054

10.901 .009610 .00054

10.951 .013370 .00051

11.001 .016870 .00051

11.051 .020820 .00054

11.101 .023350 .00053
629

9.700 .917880 .00053

9.750 .921630 .00053

9.800 .926620 .00052

9.850 .930390 .00052

9.900 .933430 .00054

9.950 .936930 .00052

10.000 .942060 .00054

10.050 .945540 .00053

10.100 .949470 .00053

10.150 .952530 .00052

10.200 .957200 .00053

10.250 .960220 .00052

10.300 .965060 .00051

10.350 .968200 .00052

10.400 .972720 .00054

10.450 .976230 .00052

10.500 .980460 .00052

10.550 .984030 .00053

10.600 .986900 .00053

10.650 .990390 .00053

10.700 .994790 .00054

10.750 .998120 .00053

10.801 .001930 .00052

10.851 .005720 .00054

10.901 .009100 .00052

10.951 .013570 .00054

11.001 .015870 .00052

11.051 .019700 .00053

11.101 .022040 .00053 
U5o2N20-sp-1000-10

12

41.000 .911440 .00018 41.700 .913860 .00018 42.400 .915810 .00018 43.100 .917480 .00018 43.800 .920240 .00018 44.500 .922010 .00017 45.200 .923390 .00017 45.900 .926140 .00017 46.600 .927220 .00017 47.300 .929280 .00017 48.000 .930620 .00016 48.700 .931880 .00018 49.400 .933250 .00017 50.100 .935000 .00017 50.800 .936420 .00016 51.500 .937550 .00017 52.200 .938950 .00016 52.900 .940210 .00016 53.600 .941710 .00016 54.300 .942100 .00016 55.000 .943790 .00016 55.700 .944920 .00015 56.000 .945260 .00016
15

26.300 .925780 .00024 26.900 .931870 .00024 27.500 .938130 .00024 28.100 .943660 .00023 28.700 .947770 .00023 29.300 .953310 .00023 29.900 .957100 .00024 30.500 .962210 .00023 31.100 .966600 .00022 31.700 .971070 .00022 32.300 .974210 .00023 32.900 .979270 .00021 33.500 .981850 .00022 34.100 .984820 .00022 34.700 .988880 .00021 35.300 .991970 .00021 35.900 .995460 .00021 36.500 .998650 .00021 37.101 .000670 .00021 37.701 .003940 .00020 38.301 .006670 .00020 38.701 .008120 .00020
25

15.400 .923490 .00034 15.500 .927460 .00033 15.600 .930310 .00034 15.700 .933590 .00032 15.800 .936930 .00034 15.900 .939610 .00034 16.000 .942800 .00034 16.100 .946090 .00034 16.200 .948990 .00034 16.300 .951870 .00034 16.400 .955080 .00033 16.500 .957430 .00034 16.600 .961460 .00034 16.700 .963680 .00033 16.800 .966690 .00033 16.900 .968740 .00034 17.000 .971660 .00033 17.100 .974900 .00033
30

13.800 .924190 .00036 13.900 .928830 .00036 14.000 .933090 .00037 14.100 .936710 .00036 14.200 .940700 .00037 14.300 .944720 .00037 14.400 .949100 .00036 14.500 .953370 .00036 14.600 .955750 .00036 14.700 .960040 .00037 14.800 .963360 .00035 14.900 .966810 .00036 15.000 .969410 .00036 15.100 .973960 .00035 15.200 .977030 .00036 15.300 .980360 .00036 15.400 .984250 .00035 15.500 .988000 .00036
20

18.400 .924110 .00031 18.600 .928720 .00029 18.800 .932670 .00029 19.000 .938130 .00030 19.200 .941450 .00030 19.400 .945780 .00029 19.600 .949520 .00030 19.800 .953350 .00029 20.000 .958000 .00030 20.200 .961560 .00030 20.400 .965560 .00029 20.600 .968570 .00029 20.800 .972990 .00029 21.000 .975890 .00029 21.200 .979550 .00028 21.400 .982650 .00028 21.600 .985550 .00029 21.800 .989270 .00028 22.000 .993240 .00029 22.200 .996180 .00028 22.400 .999180 .00029 22.601 .002610 .00028 22.801 .005710 .00029 23.001 .009240 .00029 23.201 .011590 .00028 23.401 .014490 .00029

32

13.300 .924310 .00038 13.400 .927800 .00037 13.500 .931890 .00037 13.600 .936510 .00037 13.700 .940950 .00037 13.800 .945240 .00038 13.900 .948550 .00037 14.000 .952670 .00037 14.100 .957470 .00038 14.200 .960940 .00037 14.300 .963940 .00037 14.400 .968730 .00037 14.500 .972860 .00037 14.600 .976610 .00037 14.700 .979570 .00037 14.800 .983690 .00037 14.900 .986710 .00036 15.000 .990760 .00037 
17.200 .977240 .00034 17.300 .979590 .00033 17.400 .982890 .00033 17.500 .984920 .00033 17.600 .988240 .00033 17.700 .990600 .00033 17.800 .992760 .00033 17.900 .995950 .00033 18.000 .998440 .00033 18.101 .000790 .00033 18.201 .003170 .00032 18.301 .006100 .00032 18.401 .007890 .00032 18.501 .009960 .00033 18.601 .012750 .00032 18.701 .015390 .00032

35 12.700 .922490 .00037 12.800 .927770 .00039 12.900 .932280 .00040 13.000 .936340 .00038 13.100 .940810 .00039 13.200 .945750 .00037 13.300 .950130 .00038 13.400 .954660 .00038 13.500 .958770 .00039 13.600 .963830 .00038 13.700 .967930 .00037 13.800 .971660 .00038 13.900 .975970 .00038 14.000 .979750 .00038 14.100 .983620 .00038 14.200 .987410 .00038 14.300 .992450 .00038 14.400 .995900 .00037 14.501 .000600 .00037 14.601 .002930 .00038 14.701 .006760 .00038 14.801 .010650 .00039 14.901 .014380 .00036 15.001 .019060 .00038
15.600 .991460 .00036 15.700 .994730 .00037 15.800 .997460 .00036 15.901 .001150 .00034 16.001 .003840 .00035 16.101 .007510 .00035 16.201 .010090 .00035 16.301 .013080 .00036 16.401 .016720 .00036
15.100 .994630 .00036 15.200 .997740 .00035 15.301 .001960 .00038 15.401 .005720 .00036 15.501 .008310 .00037 15.601 .012010 .00037 15.701 .015720 .00037 15.801 .018550 .00036
40

11.900 .919510 .00041 12.000 .924820 .00039 12.100 .930340 .00040 12.200 .935220 .00040 12.300 .939650 .00039 12.400 .945100 .00039 12.500 .949690 .00038 12.600 .954810 .00039 12.700 .959630 .00039 12.800 .964940 .00038 12.900 .969580 .00039 13.000 .974810 .00040 13.100 .978380 .00040 13.200 .982580 .00039 13.300 .987900 .00039 13.400 .992040 .00039 13.500 .996500 .00039 13.601 .001250 .00039 13.701 .005630 .00038 13.801 .010010 .00039 13.901 .014480 .00040 14.001 .018900 .00038
50

11.000 .923080 .00042 11.100 .929210 .00042 11.200 .934320 .00042 11.300 .940380 .00043 11.400 .946310 .00042 11.500 .953000 .00042 11.600 .957650 .00042 11.700 .963940 .00041 11.800 .968840 .00042 11.900 .974440 .00041 12.000 .979290 .00041 12.100 .985080 .00043 12.200 .990190 .00041 12.300 .996140 .00042 12.401 .002200 .00042 12.501 .006470 .00041 12.601 .011390 .00041 12.701 .015520 .00041 12.801 .020930 .00042
60

10.300 .917320 .00043 10.350 .920680 .00043 10.400 .923810 .00043
70

9.900 .918750 .00044 9.950 .923180 .00044 10.000 .925830 .00044
80

9.600 .919420 .00045 9.650 .923260 .00045 9.700 .927200 .00045 
10.450 .927240 .00043 10.500 .930840 .00042 10.550 .933820 .00044 10.600 .937450 .00043 10.650 .940340 .00042 10.700 .943450 .00043 10.750 .946070 .00043 10.800 .950420 .00044 10.850 .953530 .00044 10.900 .956560 .00043 10.950 .958730 .00043 11.000 .962200 .00043 11.050 .965700 .00043 11.100 .968910 .00044 11.150 .971400 .00044 11.200 .974620 .00043 11.250 .978050 .00044 11.300 .981140 .00043 11.350 .983920 .00044 11.400 .987270 .00044 11.450 .990480 .00042 11.500 .992090 .00043 11.550 .995570 .00044 11.600 .998490 .00044 11.651 .002120 .00044 11.701 .003580 .00044 11.751 .007370 .00043 11.801 .009220 .00043 11.851 .013270 .00043 11.901 .014940 .00043 11.951 .018040 .00044 12.001 .020360 .00044
10.050 .929150 .00044 10.100 .933300 .00044 10.150 .936580 .00046 10.200 .939240 .00045 10.250 .943250 .00045 10.300 .947420 .00044 10.350 .949860 .00044 10.400 .953860 .00045 10.450 .957010 .00046 10.500 .959660 .00046 10.550 .963300 .00044 10.600 .966390 .00044 10.650 .970030 .00045 10.750 .975750 .00045 10.800 .979890 .00044 10.850 .982610 .00046 10.900 .986570 .00046 10.950 .988940 .00044 11.000 .992470 .00044 11.050 .996080 .00043 11.100 .998570 .00044 11.151 .001370 .00045 11.201 .004780 .00045 11.251 .007830 .00046 11.301 .009900 .00045 11.351 .013820 .00043 11.401 .016940 .00044 11.451 .019960 .00044 11.501 .022740 .00044
9.750 .930090 .00044 9.800 .934740 .00045 9.850 .938320 .00044 9.900 .941980 .00045 9.950 .945350 .00045 10.000 .948720 .00046 10.050 .952200 .00045 10.100 .956330 .00046 10.150 .959000 .00046 10.200 .963170 .00047 10.250 .967530 .00046 10.300 .970320 .00046 10.350 .974660 .00047 10.400 .976860 .00045 10.450 .980580 .00045 10.500 .983100 .00045 10.550 .987500 .00044 10.600 .990190 .00045 10.650 .993300 .00045 10.700 .996700 .00046 10.751 .000660 .00046 10.801 .003670 .00046 10.851 .006690 .00045 10.901 .010050 .00045 10.951 .013800 .00044 11.001 .016900 .00046 11.051 .019850 .00047 11.101 .023110 .00045
90

9.400 .922590 .00045

9.450 .926860 .00046

9.500 .929260 .00046

9.550 .933950 .00046

9.600 .937830 .00046

9.650 .942240 .00044

9.700 .944590 .00046

9.750 .949680 .00046

9.800 .952970 .00047

9.850 .956210 .00047

9.900 .960340 .00046

9.950 .964300 .00046 10.000 .967320 .00048 10.050 .970980 .00046 10.100 .974540 .00047
100

9.200 .921900 .00046

9.250 .924880 .00046

9.300 .928440 .00047

9.350 .932750 .00046

9.400 .937420 .00047

9.450 .940510 .00047

9.500 .944710 .00046 9.550 .948870 .00047 9.600 .951990 .00046 9.650 .955640 .00045 9.700 .959990 .00046 9.750 .963470 .00047 9.800 .967150 .00046 9.850 .971400 .00047 9.900 .975210 .00045
118

8.900 .917350 .00047 8.950 .921610 .00047 9.000 .926660 .00046 9.050 .929220 .00048 9.100 .934700 .00047 9.150 .938240 .00046 9.200 .942830 .00048 9.250 .945990 .00047 9.300 .949960 .00046 9.350 .954210 .00048 9.400 .957730 .00048 9.450 .961470 .00048 9.500 .965160 .00049 9.550 .969190 .00048 9.600 .973260 .00047 
10.150 .978480 .00047

10.200 .981150 .00046

10.250 .985380 .00047

10.300 .989500 .00046

10.350 .993320 .00046

10.400 .995920 .00045

10.450 .998490 .00047

10.501 .003530 .00046

10.551 .006290 .00047

10.601 .008760 .00047

10.651 .012960 .00046

10.701 .016250 .00046

10.751 .018570 .00047

10.801 .021470 .00047
9.950 .979080 .00046 10.000 .982010 .00045 10.050 .985020 .00048 10.100 .989900 .00047 10.150 .992700 .00046 10.200 .996280 .00046 10.250 .999000 .00047 10.301 .003730 .00046 10.351 .007160 .00048 10.401 .010130 .00045 10.451 .014680 .00047 10.501 .016780 .00047 10.551 .020390 .00047 10.601 .024890 .00048
9.650 .976050 .00047

9.700 .980790 .00048 9.750 .984330 .00048 9.800 .987310 .00048 9.850 .993030 .00048 9.900 .996070 .00048 9.950 .999470 .00048 10.001 .002290 .00047 10.051 .007420 .00048 10.101 .010520 .00047 10.151 .013690 .00048 10.201 .017600 .00047 10.251 .021130 .00048 10.301 .024150 .00048
130

8.800 .921020 .00048

8.850 .925080 .00048

8.900 .929180 .00047

8.950 .933650 .00049

9.000 .937920 .00048

9.050 .940660 .00048

9.100 .945480 .00047

9.150 .948970 .00049

9.200 .952800 .00049

9.250 .957700 .00048

9.300 .962330 .00047

9.350 .965780 .00047

9.400 .969360 .00048

9.450 .973190 .00048

9.500 .977010 .00048

9.550 .981340 .00048

9.600 .985010 .00048

9.650 .988410 .00047

9.700 .992430 .00048

9.750 .995890 .00049

9.801 .000320 .00047

9.851 .004240 .00048

9.901 .007160 .00049

9.951 .010640 .00048 10.001 .015250 .00048 10.051 .018760 .00050 10.101 .021570 .00049
150

8.600 .918130 .00048 8.650 .922270 .00048 8.700 .926480 .00047 8.750 .930970 .00049 8.800 .934120 .00048 8.850 .938820 .00049 8.900 .943310 .00048 8.950 .947470 .00048 9.000 .951500 .00049 9.050 .955050 .00049 9.100 .959590 .00048 9.150 .964000 .00049 9.200 .967720 .00048 9.250 .971800 .00048 9.300 .975700 .00048 9.350 .979620 .00048 9.400 .984120 .00048 9.450 .987720 .00048 9.500 .991550 .00049 9.550 .995170 .00049 9.600 .998660 .00049 9.651 .003460 .00048 9.701 .007000 .00049 9.751 .010070 .00049 9.801 .012810 .00049 9.851 .017270 .00049 9.901 .022030 .00048
200

8.400 .922580 .00048

8.450 .926910 .00048

8.500 .930950 .00047

8.550 .935020 .00049

8.600 .939680 .00049

8.650 .944490 .00047

8.700 .948480 .00048

8.750 .952970 .00048

8.800 .956600 .00049

8.850 .960290 .00049

8.900 .965220 .00050

8.950 .969630 .00049

9.000 .973600 .00049

9.050 .977500 .00049

9.100 .981640 .00049

9.150 .985810 .00049

9.200 .989860 .00049

9.250 .993590 .00049

9.300 .997090 .00048

9.351 .001700 .00050

9.401 .005750 .00047

9.451 .009340 .00048

9.501 .013380 .00048

9.551 .017560 .00050

9.601 .022010 .00049 
235

8.300 .922350 .00047 8.350 .926730 .00049 8.400 .931460 .00048 8.450 .935620 .00050 8.500 .939900 .00049 8.550 .944640 .00050 8.650 .952070 .00048 8.700 .956520 .00049 8.750 .961120 .00049 8.800 .965400 .00050 8.850 .969440 .00050 8.900 .973870 .00049 8.950 .978340 .00048 9.000 .982850 .00049 9.050 .985540 .00049 9.100 .990860 .00049 9.150 .993650 .00049 9.200 .998060 .00048 9.251 .002240 .00049 9.301 .006550 .00049 9.351 .010540 .00049 9.401 .013760 .00049 9.451 .018070 .00049 9.501 .020900 .00051

\section{0}

8.100 .915710 .00048

8.150 .921220 .00049

8.200 .923720 .00049

8.250 .928850 .00048

8.300 .932930 .00049

8.350 .937620 .00050

8.400 .941200 .00049

8.450 .945410 .00050

8.500 .949700 .00048

8.550 .954960 .00048 8.600 .958010 .00051 8.650 .962600 .00048 8.700 .967500 .00050 8.750 .970890 .00049 8.800 .975090 .00050 8.850 .978260 .00049 8.900 .982810 .00049 8.950 .986230 .00049 9.000 .990720 .00050
250

8.200 .916270 .00048

8.250 .921150 .00049

8.300 .925110 .00049

8.350 .929370 .00049

8.400 .933710 .00049

8.450 .938140 .00050

8.500 .943400 .00050

8.550 .946680 .00050

8.600 .951740 .00049

8.650 .954420 .00049

8.700 .959420 .00050

8.750 .964050 .00049

8.800 .967780 .00048

8.850 .972400 .00050

8.900 .976190 .00051

8.950 .980240 .00050

9.000 .984240 .00049

9.050 .988360 .00049

9.100 .993550 .00048

9.150 .996400 .00049

9.201 .001210 .00051

9.251 .005350 .00049

9.301 .009510 .00051

9.351 .013080 .00050

9.401 .016860 .00048

9.451 .019210 .00050

9.501 .024250 .00049
353

8.100 .916370 .00048

8.150 .920890 .00049

8.200 .924640 .00049

8.250 .930160 .00050

8.300 .933650 .00048

8.350 .938490 .00050

8.400 .941740 .00051

8.450 .945970 .00050

8.500 .951290 .00049

8.550 .955340 .00050

8.600 .959270 .00049

8.650 .964060 .00050

8.700 .967760 .00048

8.750 .971370 .00049

8.800 .976180 .00050

8.850 .980170 .00051

8.900 .984920 .00050

8.950 .988620 .00050

9.000 .991620 .00051

9.050 .996170 .00050

9.101 .000380 .00050

9.151 .004680 .00050

9.201 .008420 .00050

9.251 .013000 .00051

9.301 .016800 .00049

9.351 .020030 .00050

9.401 .023980 .00050
500
8.100 .914630 .00048

8.150 .918940 .00049

8.200 .923800 .00048

8.250 .928210 .00050

8.300 .932470 .00049

8.350 .936930 .00050

8.400 .940680 .00048

8.450 .945120 .00048

8.500 .949220 .00050

8.550 .952290 .00050

8.600 .958180 .00049

8.650 .961920 .00049

8.700 .966140 .00051

8.750 .969880 .00050

8.800 .973440 .00049

8.850 .977670 .00049

8.900 .981180 .00050

8.950 .985290 .00049

9.000 .989350 .00049
600

8.200 .917710 .00047

8.250 .921310 .00050

8.300 .925470 .00048

8.350 .929320 .00049

8.400 .934480 .00048

8.450 .937440 .00049

8.500 .942830 .00049

8.550 .945590 .00048

8.600 .950860 .00050

8.650 .954240 .00049

8.700 .958560 .00049

8.750 .963360 .00050

8.800 .966330 .00049

8.850 .970710 .00048

8.900 .974680 .00050

8.950 .978190 .00051

9.000 .982620 .00048

9.050 .986130 .00049

9.100 .989860 .00048 
9.050 .994670 .00049 9.100 .999840 .00050 9.151 .002470 .00050 9.201 .006040 .00051 9.251 .010290 .00050 9.301 .013940 .00050 9.351 .016970 .00050 9.401 .021820 .00050

\section{5}

8.200 .917500 .00048 8.250 .920660 .00049 8.300 .925600 .00048 8.350 .929740 .00050 8.400 .934160 .00048 8.450 .937400 .00049 8.500 .942380 .00048 8.550 .945910 .00049 8.600 .950040 .00048 8.650 .953340 .00048 8.700 .958140 .00048 8.750 .962760 .00049 8.800 .966390 .00048 8.850 .970870 .00049 8.900 .973980 .00049 8.950 .977800 .00049 9.000 .981870 .00050 9.050 .985650 .00050 9.100 .989410 .00048 9.150 .993070 .00049 9.200 .997040 .00048 9.251 .000440 .00049 9.301 .004350 .00049 9.351 .007960 .00049 9.401 .012040 .00049 9.451 .015330 .00050 9.501 .018830 .00050 9.551 .022970 .00050 9.601 .026630 .00050

\section{0}

8.200 .915710 .00049 8.250 .920240 .00048 8.300 .924140 .00048 8.350 .928710 .00049 8.400 .932720 .00049 8.450 .935910 .00049
9.050 .994030 .00050

9.100 .997530 .00049

9.151 .001450 .00049

9.201 .005430 .00051

9.251 .009610 .00051

9.301 .013270 .00048

9.351 .017150 .00050

9.401 .020780 .00049
9.150 .994120 .00051 9.200 .997480 .00050 9.251 .001350 .00049 9.301 .004860 .00050 9.351 .009130 .00049 9.401 .013040 .00050 9.451 .016030 .00048 9.501 .019840 .00051
610

8.200 .917620 .00049

8.250 .921620 .00048

8.300 .924640 .00048

8.350 .929160 .00049

8.400 .933260 .00048

8.450 .937390 .00050

8.500 .941840 .00049

8.550 .945360 .00050

8.600 .949050 .00050

8.650 .954600 .00048

8.700 .958940 .00048

8.750 .962050 .00050

8.800 .965810 .00048

8.850 .970890 .00049

8.900 .973800 .00048

8.950 .977070 .00049

9.000 .982150 .00048

9.050 .984520 .00050

9.100 .988730 .00049

9.150 .993590 .00050

9.200 .996870 .00049

9.251 .000330 .00050

9.301 .005200 .00050

9.351 .008320 .00048

9.401 .011470 .00050

9.451 .015440 .00048

9.501 .019420 .00049
615

8.200 .916940 .00049 8.250 .920070 .00049 8.300 .924820 .00049 8.350 .929310 .00051 8.400 .933140 .00048 8.450 .937080 .00049 8.500 .941290 .00049 8.550 .945240 .00048 8.600 .949970 .00048 8.650 .953680 .00049 8.700 .957720 .00050 8.750 .961660 .00050 8.800 .966380 .00049 8.850 .969780 .00049 8.900 .973030 .00049 8.950 .977330 .00049 9.000 .980940 .00049 9.050 .985670 .00049 9.100 .988800 .00050 9.150 .992480 .00050 9.200 .996330 .00049 9.251 .001280 .00049 9.301 .003210 .00051 9.351 .007350 .00050 9.401 .012600 .00049 9.451 .015390 .00050 9.501 .018780 .00049
625

8.200 .914590 .00049

8.250 .920460 .00050

8.300 .924500 .00050

8.350 .927860 .00048

8.400 .932340 .00049

8.450 .936240 .00048
629

8.300 .922840 .00049 8.350 .927170 .00049 8.400 .931720 .00048 8.450 .935780 .00048 8.500 .939820 .00048 8.550 .944080 .00047 


\begin{tabular}{|c|c|}
\hline 3.500 .940750 .00049 & 8.500 \\
\hline .550 .943900 .00048 & 0.944070 .0004 \\
\hline 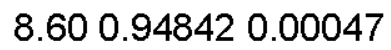 & 0004 \\
\hline 050 & 0004 \\
\hline 0049 & 004 \\
\hline 0048 & 004 \\
\hline 0049 & .9650 \\
\hline 050 & .0004 \\
\hline 48 & 8 \\
\hline & \\
\hline 49 & 0049 \\
\hline 048 & .00050 \\
\hline .98874 & 860.0004 \\
\hline 0.991190 & 9.150 .992140 .0004 \\
\hline 0050 & 9.200 .996510 .0004 \\
\hline 05 & 9.2 \\
\hline 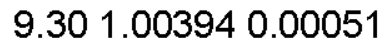 & 9.3 \\
\hline .006750 .0 & 006700.0004 \\
\hline 1.011080 .00049 & 011460.0004 \\
\hline 013510.00048 & 013670.0004 \\
\hline 018010 & (OnOS \\
\hline & $21<$ \\
\hline $025 t$ & .025150 .00 \\
\hline
\end{tabular}

U5o2N23-sp-1000-00

12

47.400 .874300 .00019 48.300 .876710 .00019 49.200 .879590 .00018 50.100 .881570 .00018 51.000 .883650 .00018 51.900 .885930 .00017 52.800 .887310 .00017 53.700 .889570 .00017 54.600 .890870 .00017 55.500 .892380 .00017 56.400 .893720 .00016 57.300 .895580 .00017 58.200 .897110 .00017 59.100 .898580 .00017 60.000 .899530 .00016 60.900 .900620 .00017 61.800 .901980 .00016 62.700 .903200 .00016 63.600 .904500 .00016 64.500 .904930 .00016 65.400 .906310 .00016 66.300 .907500 .00016 66.500 .907770 .00015
35.800 .932140 .00023 36.600 .937640 .00023 37.400 .940830 .00023 38.200 .945220 .00023 39.000 .949520 .00022 39.800 .952950 .00022 40.600 .956600 .00022 41.400 .959780 .00022 42.200 .963120 .00021 43.000 .966240 .00021 43.800 .969390 .00021 44.600 .972430 .00021 45.400 .974500 .00021 46.200 .977360 .00020 47.000 .979740 .00020 47.800 .982060 .00020 48.600 .984050 .00020 49.400 .986550 .00020 50.200 .988820 .00020 51.000 .990290 .00020 51.800 .992520 .00019 52.200 .992920 .00019
8.600 .948440 .00049 8.650 .950710 .00050 8.700 .955090 .00048 8.750 .959090 .00049 8.800 .964420 .00050 8.850 .968000 .00048 8.900 .971990 .00048 8.950 .975870 .00051 9.000 .979060 .00049 9.050 .982970 .00048 9.100 .987980 .00048 9.150 .990430 .00048 9.200 .995300 .00049 9.250 .999110 .00048 9.301 .002220 .00049 9.351 .005970 .00049 9.401 .009370 .00048 9.451 .013340 .00049 9.501 .016490 .00048 9.551 .020260 .00049 9.601 .024290 .00049
20

23.500 .926670 .00031 23.800 .931240 .00031 24.100 .937740 .00030 24.400 .942010 .00032 24.700 .946880 .00030 25.000 .951210 .00030 25.300 .956390 .00031 25.600 .960390 .00030 25.900 .965440 .00030 26.200 .969360 .00030 26.500 .973910 .00030 26.800 .977670 .00030 27.100 .981020 .00029 27.400 .985230 .00029 27.700 .988470 .00030 28.000 .992530 .00029 28.300 .995520 .00029 28.600 .999190 .00029 28.901 .003100 .00029 29.201 .005800 .00028 29.501 .009510 .00028 29.801 .012100 .00028 30.001 .014500 .00028 
25

19.600 .925020 .00036 19.800 .929020 .00035 20.000 .935880 .00034 20.200 .940630 .00035 20.400 .946010 .00036 20.600 .950160 .00035 20.800 .955510 .00034 21.000 .960190 .00036 21.200 .964730 .00035 21.400 .969440 .00034 21.600 .974010 .00034 21.800 .978670 .00035 22.000 .983180 .00035 22.200 .987200 .00035 22.400 .991200 .00034 22.600 .995860 .00034 22.800 .999870 .00034 23.001 .003600 .00034 23.201 .008000 .00033 23.401 .011070 .00033 23.601 .015290 .00034 23.701 .017530 .00033

35

16.300 .920710 .00041 16.400 .923880 .00041 16.500 .928880 .00041 16.600 .933100 .00041 16.700 .936760 .00041 16.800 .941750 .00041 16.900 .945090 .00042 17.000 .947780 .00041 17.100 .952020 .00041 17.200 .956800 .00040 17.300 .959960 .00042
30

32

17.600 .923290 .00039 17.700 .926130 .00038 17.800 .930630 .00040 17.900 .935000 .00038 18.000 .937820 .00039 18.100 .940460 .00039 18.200 .943350 .00039 18.300 .946890 .00040 18.400 .950470 .00038 18.500 .953950 .00038 18.600 .957010 .00038 18.700 .960100 .00038 18.800 .963740 .00039 18.900 .966030 .00039 19.000 .968970 .00038 19.100 .972170 .00038 19.200 .975230 .00038 19.300 .977600 .00039 19.400 .981310 .00038 19.500 .984760 .00038 19.600 .986440 .00037 19.700 .989960 .00039 19.800 .993520 .00037 19.900 .995230 .00039 20.000 .998150 .00038 20.101 .001060 .00039 20.201 .003550 .00037 20.301 .005730 .00038 20.401 .008870 .00037 20.501 .010770 .00038 20.601 .013910 .00038 20.701 .016410 .00037

40

15.500 .921820 .00043 15.600 .927070 .00042 15.700 .931750 .00042 15.800 .936110 .00043 15.900 .940930 .00043 16.000 .944750 .00044 16.100 .949570 .00043 16.200 .953620 .00045 16.300 .958550 .00043 16.400 .962560 .00043 16.500 .966000 .00043
17.000 .921480 .00040 17.100 .924940 .00040 17.200 .928520 .00040 17.300 .933220 .00040 17.400 .937200 .00039 17.500 .940510 .00040 17.600 .943800 .00040 17.700 .947080 .00039 17.800 .950330 .00040 17.900 .955170 .00039 18.000 .957260 .00040 18.100 .959990 .00039 18.200 .964600 .00038 18.300 .967140 .00039 18.400 .970740 .00039 18.500 .974110 .00039 18.600 .977620 .00040 18.700 .979840 .00038 18.800 .983890 .00039 18.900 .986130 .00040 19.000 .989740 .00039 19.100 .993030 .00039 19.200 .996150 .00039 19.300 .999460 .00038 19.401 .002570 .00038 19.501 .005470 .00039 19.601 .007630 .00039 19.701 .011380 .00040 19.801 .014720 .00039 19.901 .016690 .00039 
17.400 .964050 .00040 17.500 .967590 .00040 17.600 .970840 .00041 17.700 .973740 .00041 17.800 .977850 .00041 17.900 .982030 .00040 18.000 .985230 .00041 18.100 .988690 .00041 18.200 .992010 .00041 18.300 .994880 .00041 18.400 .998520 .00040 18.501 .002720 .00041 18.601 .005080 .00040 18.701 .008550 .00040 18.801 .011850 .00040 18.901 .015610 .00040 19.001 .018220 .00040
16.600 .970540 .00042 16.700 .975240 .00042 16.800 .978470 .00043 16.900 .982850 .00043 17.000 .986520 .00042 17.100 .990790 .00043 17.200 .994060 .00043 17.300 .998100 .00043 17.401 .002390 .00041 17.501 .005890 .00041 17.601 .009650 .00043 17.701 .014850 .00042 17.801 .017070 .00042 17.901 .020520 .00041
15.500 .977710 .00046 15.600 .982870 .00043 15.700 .988040 .00045 15.800 .991980 .00046 15.900 .996610 .00046 16.001 .000950 .00046 16.101 .006550 .00046 16.201 .009840 .00047 16.301 .015760 .00046 16.401 .019720 .00046

\section{0}

13.700 .919850 .00048 13.800 .925570 .00049 13.900 .931530 .00048 14.000 .938230 .00048 14.100 .944010 .00049 14.200 .948520 .00047 14.300 .954820 .00048 14.400 .960420 .00048 14.500 .964950 .00048 14.600 .972030 .00049 14.700 .976110 .00048 14.800 .981370 .00048 14.900 .988200 .00049 15.000 .993060 .00048 15.100 .998140 .00047 15.201 .004120 .00048 15.301 .008620 .00049 15.401 .013580 .00049 15.501 .018760 .00048
70

13.200 .916200 .00049 13.300 .923450 .00049 13.400 .929690 .00049 13.500 .935670 .00050 13.600 .942430 .00050 13.700 .948550 .00049 13.800 .954700 .00050 13.900 .961370 .00050 14.000 .967210 .00049 14.100 .974310 .00050 14.200 .978530 .00050 14.300 .984190 .00049 14.400 .989780 .00048 14.500 .994970 .00049 14.601 .001360 .00049 14.701 .006660 .00050 14.801 .012670 .00050 14.901 .018230 .00051 15.001 .022780 .00049
80

12.900 .917960 .00051 12.950 .922400 .00048 13.000 .925440 .00050 13.050 .929090 .00050 13.100 .932150 .00051 13.150 .934930 .00050 13.200 .939680 .00052 13.250 .942280 .00050 13.300 .945890 .00052 13.350 .947960 .00050 13.400 .951740 .00051 13.450 .955550 .00051 13.500 .958210 .00050 13.550 .961580 .00051 13.600 .964870 .00050 13.650 .966780 .00050 13.700 .970770 .00051 13.750 .973310 .00051 13.800 .976820 .00050 13.850 .979860 .00051 13.900 .983550 .00052 13.950 .985570 .00051 14.000 .988580 .00050 14.050 .991180 .00050 14.100 .994500 .00053 14.150 .997690 .00051 14.201 .000570 .00052 14.251 .003740 .00051 14.301 .006000 .00050 14.351 .009400 .00052 
CHPRC-01552, Revision 0

14.401 .012610 .00050

14.451 .015740 .00050

14.501 .018340 .00051

14.551 .021960 .00051

14.601 .024260 .00052

90

12.600 .915570 .00051

12.650 .918900 .00051

12.700 .923110 .00050

12.750 .925420 .00053

12.800 .929900 .00051

12.850 .932890 .00050

12.900 .936860 .00051

12.950 .939110 .00052

13.000 .943150 .00052

13.050 .947870 .00051

13.100 .950040 .00050

13.150 .952820 .00050

13.200 .956730 .00051

13.250 .960530 .00052

13.300 .963210 .00051

13.350 .967110 .00052

13.400 .970170 .00051

13.450 .973900 .00051

13.500 .976220 .00052

13.550 .980090 .00050

13.600 .983680 .00052

13.650 .985400 .00052

13.700 .989990 .00051

13.750 .991790 .00052

13.800 .995330 .00052

13.850 .998880 .00052

13.901 .000980 .00052

13.951 .004160 .00051

14.001 .007250 .00052

14.051 .011290 .00051

14.101 .014240 .00051

14.151 .016830 .00050

14.201 .018280 .00051
100

12.500 .921900 .00052

12.550 .925300 .00051

12.600 .929610 .00052

12.650 .933210 .00053

12.700 .936910 .00053

12.750 .940250 .00051

12.800 .944340 .00052

12.850 .946300 .00052

12.900 .950660 .00051

12.950 .954490 .00053

13.000 .957110 .00051

13.050 .960230 .00052

13.100 .963950 .00051

13.150 .967400 .00052

13.200 .971220 .00052

13.250 .974420 .00053

13.300 .977120 .00051

13.350 .981460 .00053

13.400 .984220 .00051

13.450 .986130 .00052

13.500 .989910 .00051

13.550 .993760 .00051

13.600 .996720 .00052

13.650 .999850 .00053

13.701 .003620 .00052

13.751 .007200 .00052

13.801 .009060 .00051

13.851 .013540 .00052

13.901 .015720 .00053

13.951 .018440 .00053

14.001 .021860 .00053
118

12.200 .918560 .00053

12.250 .922130 .00052

12.300 .925530 .00053

12.350 .929280 .00053

12.400 .932610 .00052

12.450 .936750 .00052

12.500 .940730 .00053

12.550 .944320 .00053

12.600 .946910 .00053

12.650 .951510 .00053

12.700 .954850 .00053

12.750 .958730 .00053

12.800 .961370 .00054

12.850 .964770 .00053

12.900 .968850 .00053

12.950 .972880 .00054

13.000 .975230 .00054

13.050 .979840 .00052

13.100 .982030 .00051

13.150 .986460 .00053

13.200 .989640 .00053

13.250 .993300 .00054

13.300 .996570 .00053

13.350 .999100 .00054

13.401 .002830 .00053

13.451 .005690 .00053

13.501 .010050 .00053

13.551 .012970 .00052

13.601 .015900 .00055

13.651 .020690 .00053

13.701 .022110 .00053
130

12.000 .911950 .00053

12.050 .916510 .00054

12.100 .921080 .00051

12.150 .922810 .00053

12.200 .928320 .00052
150

11.900 .917210 .00055 11.950 .919960 .00054 12.000 .924530 .00054 12.050 .927360 .00055 12.100 .931840 .00054
200

11.700 .917600 .00055

11.750 .921330 .00057

11.800 .925010 .00056

11.850 .929560 .00054

11.900 .934110 .00054 
12.250 .931570 .00054

12.300 .935450 .00055

12.350 .939370 .00052

12.400 .942580 .00053

12.450 .946790 .00053

12.500 .950540 .00054

12.550 .954810 .00053

12.600 .956960 .00053

12.650 .960630 .00053

12.700 .965150 .00055

12.750 .969070 .00054

12.800 .970870 .00053

12.850 .976080 .00053

12.900 .978990 .00052

12.950 .982100 .00053

13.000 .986320 .00054

13.050 .989720 .00053

13.100 .993200 .00055

13.150 .996890 .00053

13.200 .999590 .00053

13.251 .003350 .00054

13.301 .006160 .00053

13.351 .010610 .00055

13.401 .013790 .00054

13.451 .016410 .00054

13.501 .020410 .00054
12.150 .936470 .00055

12.200 .940690 .00054

12.250 .943350 .00054

12.300 .946510 .00054

12.350 .951120 .00054

12.400 .955240 .00054

12.450 .957940 .00054

12.500 .962440 .00055

12.550 .965260 .00054

12.600 .970000 .00054

12.650 .973730 .00055

12.700 .976910 .00054

12.750 .980840 .00054

12.800 .984240 .00055

12.850 .987940 .00053

12.900 .991150 .00054

12.950 .994720 .00055

13.000 .997540 .00055

13.051 .002270 .00054

13.101 .006320 .00054

13.151 .008420 .00055

13.201 .011430 .00054

13.251 .015650 .00053

13.301 .018730 .00056

13.351 .021610 .00053

13.401 .025520 .00055
11.950 .937900 .00054

12.000 .942390 .00055

12.050 .945470 .00055

12.100 .949480 .00054

12.150 .954110 .00056

12.200 .957080 .00056

12.250 .960520 .00056

12.300 .964230 .00055

12.350 .968740 .00056

12.400 .973200 .00054

12.450 .976140 .00054

12.500 .979720 .00053

12.550 .984430 .00054

12.600 .987500 .00054

12.650 .991440 .00054

12.700 .995100 .00057

12.750 .997320 .00054

12.801 .002250 .00056

12.851 .005000 .00053

12.901 .008330 .00055

12.951 .011720 .00054

13.001 .015450 .00055

13.051 .019180 .00054

13.101 .022060 .00054
235

11.600 .914810 .00054 11.650 .920130 .00056 11.700 .922210 .00054 11.750 .926450 .00054 11.800 .930420 .00055 11.850 .935040 .00055 11.900 .939800 .00054 11.950 .942530 .00055 12.000 .945830 .00055 12.050 .950830 .00056 12.100 .954690 .00056 12.150 .958460 .00056 12.200 .961700 .00054 12.250 .966260 .00055 12.300 .970620 .00055 12.350 .974980 .00057 12.400 .977070 .00055 12.450 .981990 .00057 12.500 .986360 .00054 12.550 .988710 .00053 12.600 .992120 .00055
250

11.600 .916160 .00055 11.650 .920640 .00054 11.700 .923950 .00055 11.750 .929120 .00054 11.800 .932930 .00055 11.850 .936510 .00054 11.900 .941290 .00054 11.950 .943290 .00054 12.000 .949180 .00057 12.050 .953790 .00056 12.100 .956510 .00054 12.150 .960620 .00055 12.200 .964310 .00056 12.250 .967220 .00055 12.300 .971880 .00056 12.350 .976070 .00057 12.400 .979750 .00057 12.450 .983030 .00056 12.500 .986640 .00055 12.550 .989670 .00054 12.600 .994440 .00056
353

11.600 .914960 .00055 11.650 .919210 .00056 11.750 .927150 .00055 11.800 .931100 .00055 11.850 .934930 .00055 11.900 .938610 .00055 11.950 .942730 .00056 12.000 .946690 .00054 12.050 .951720 .00055 12.100 .954820 .00054 12.150 .958890 .00055 12.200 .962480 .00055 12.250 .966800 .00054 12.300 .970660 .00057 12.350 .972430 .00056 12.400 .976840 .00054 12.450 .981600 .00055 12.500 .983970 .00056 12.550 .988590 .00056 12.600 .992420 .00056 12.650 .995090 .00056 
12.650 .997210 .00056

12.701 .000200 .00055

12.751 .003490 .00055

12.801 .006970 .00056

12.851 .010050 .00054

12.901 .013980 .00055

12.951 .018270 .00054

13.001 .021130 .00055

13.051 .025130 .00057

13.101 .027860 .00054
12.650 .998640 .00056

12.701 .001280 .00056

12.751 .004670 .00055

12.801 .009640 .00054

12.851 .011270 .00055

12.901 .016430 .00054

12.951 .018800 .00057

13.001 .022020 .00056
12.701 .000620 .00056

12.751 .004010 .00056

12.801 .008150 .00054

12.851 .010410 .00058

12.901 .014250 .00055

12.951 .017860 .00056

13.001 .021220 .00057
470

11.800 .919720 .00055 11.850 .924310 .00055 11.900 .927250 .00054 11.950 .931210 .00053 12.000 .935240 .00054 12.050 .938690 .00054 12.100 .942870 .00054 12.150 .945960 .00055 12.200 .950300 .00056 12.250 .954240 .00054 12.300 .958750 .00055 12.350 .961180 .00057 12.400 .965680 .00055 12.450 .969780 .00055 12.500 .973650 .00054 12.550 .976730 .00056 12.600 .979930 .00055 12.650 .983550 .00054 12.700 .986770 .00055 12.750 .990680 .00054 12.800 .994400 .00055 12.850 .998560 .00055 12.901 .001370 .00054 12.951 .004410 .00054 13.001 .008940 .00055 13.051 .011580 .00057 13.101 .015200 .00055 13.151 .017760 .00054 13.201 .021770 .00055
500

11.800 .914650 .00055 11.850 .918950 .00053 11.900 .921990 .00054 11.950 .927820 .00053 12.000 .930320 .00055 12.050 .934810 .00054 12.100 .938900 .00054 12.150 .942300 .00056 12.200 .946190 .00054 12.250 .949350 .00057 12.300 .953080 .00054 12.350 .957410 .00055 12.400 .959550 .00054 12.450 .964730 .00057 12.500 .968570 .00053 12.550 .970180 .00054 12.600 .976230 .00055 12.650 .978610 .00055 12.700 .981270 .00054 12.750 .984740 .00055 12.800 .990660 .00057 12.850 .992310 .00054 12.900 .997450 .00054 12.950 .999460 .00054 13.001 .003100 .00055 13.051 .006840 .00055 13.101 .010410 .00056 13.151 .014750 .00055 13.201 .016660 .00056 13.251 .021140 .00056 13.301 .024440 .00057
510 11.900 .921210 .00053 11.950 .924580 .00054 12.000 .928420 .00054 12.050 .933010 .00054 12.100 .937590 .00054 12.150 .939420 .00053 12.200 .944000 .00054 12.250 .947220 .00054 12.300 .951740 .00053 12.350 .955300 .00055 12.400 .959420 .00054 12.450 .962230 .00055 12.500 .966490 .00054 12.550 .970240 .00054 12.600 .973560 .00054 12.650 .976460 .00054 12.700 .979890 .00054 12.750 .984120 .00055 12.800 .987570 .00055 12.850 .990380 .00053 12.900 .995050 .00053 12.950 .998800 .00054 13.001 .000750 .00053 13.051 .005600 .00055 13.101 .008990 .00055 13.151 .012860 .00055 13.201 .016110 .00053 13.251 .017970 .00055 13.301 .021980 .00054 
520

11.900 .919390 .00054 11.950 .924210 .00054 12.000 .927710 .00053 12.050 .931460 .00054 12.100 .935210 .00054 12.150 .938760 .00056 12.200 .942900 .00053 12.250 .945810 .00055 12.300 .949210 .00054 12.350 .953390 .00054 12.400 .958160 .00055 12.450 .960950 .00055 12.500 .964920 .00053 12.550 .967200 .00053 12.600 .972260 .00055 12.650 .974300 .00055 12.700 .978720 .00054 12.750 .982250 .00054 12.800 .986150 .00054 12.850 .989220 .00055 12.900 .992370 .00054 12.950 .996290 .00054 13.001 .000270 .00056 13.051 .003190 .00053 13.101 .006000 .00054 13.151 .010020 .00054 13.201 .015090 .00054 13.251 .017790 .00054 13.301 .020380 .00054 13.351 .024480 .00056 13.401 .027770 .00055

\section{0}

11.900 .914520 .00054 11.950 .918920 .00053 12.000 .922780 .00054 12.050 .925720 .00054 12.100 .929800 .00052 12.150 .934070 .00054 12.200 .936040 .00053 12.250 .941050 .00053 12.300 .945440 .00054 12.350 .948720 .00055 12.400 .952760 .00055 12.450 .955440 .00054 12.500 .958990 .00054 12.550 .964060 .00053 12.600 .966200 .00055 12.650 .971330 .00054 12.700 .974590 .00055
530

11.900 .918450 .00055 11.950 .922400 .00056 12.000 .925450 .00054 12.050 .929300 .00054 12.100 .933370 .00055 12.150 .938030 .00054 12.200 .940500 .00055 12.250 .944470 .00056 12.300 .947980 .00053 12.350 .952170 .00054 12.400 .955110 .00055 12.450 .959790 .00054 12.500 .963580 .00054 12.550 .966650 .00056 12.600 .969640 .00055 12.650 .973700 .00055 12.700 .976940 .00056 12.750 .981070 .00055 12.800 .984230 .00055 12.850 .987730 .00054 12.900 .991550 .00056 12.950 .995210 .00055 13.000 .997930 .00056 13.051 .001890 .00054 13.101 .005060 .00054 13.151 .008480 .00054 13.201 .011810 .00054 13.251 .015530 .00054 13.301 .018010 .00055 13.351 .022550 .00055 13.401 .024680 .00055 560

12.000 .921670 .00055 12.050 .924400 .00055 12.100 .929200 .00052 12.150 .931510 .00055 12.200 .936060 .00055 12.250 .939970 .00053 12.300 .942620 .00054 12.350 .947510 .00054 12.400 .950340 .00055 12.450 .953710 .00054 12.500 .957750 .00054 12.550 .961690 .00056 12.600 .965030 .00054 12.650 .968710 .00052 12.700 .971790 .00056 12.750 .975530 .00055 12.800 .978960 .00053
540

11.900 .917060 .00053 11.950 .919450 .00054 12.000 .924320 .00055 12.050 .927100 .00054 12.100 .931780 .00054 12.150 .935680 .00055 12.200 .938700 .00053 12.250 .943530 .00055 12.300 .947210 .00056 12.350 .950980 .00054 12.400 .953370 .00053 12.450 .957420 .00053 12.500 .960990 .00056 12.550 .964940 .00054 12.600 .968410 .00054 12.650 .973440 .00055 12.700 .975070 .00053 12.750 .979260 .00055 12.800 .983700 .00053 12.850 .986860 .00055 12.900 .990350 .00053 12.950 .992410 .00055 13.000 .995600 .00055 13.051 .000200 .00055 13.101 .003360 .00054 13.151 .008270 .00054 13.201 .010010 .00055 13.251 .013920 .00054 13.301 .016890 .00054 13.351 .020930 .00054 13.401 .023340 .00056 
12.750 .977700 .00055

12.800 .981800 .00056

12.850 .984710 .00055

12.900 .988350 .00055

12.950 .990740 .00055

13.000 .995710 .00054

13.050 .997420 .00056

13.101 .001510 .00054

13.151 .004810 .00053

13.251 .011030 .00054

13.301 .015760 .00054

13.351 .019170 .00055

13.401 .021340 .00055

U5o2N23-sp-1000-01

12

33.400 .825970 .00021

33.600 .827470 .00020

33.800 .828010 .00021

34.000 .829460 .00021

34.200 .830110 .00021

34.400 .831440 .00021

34.600 .832770 .00021

34.800 .834110 .00020

35.000 .834970 .00020

35.200 .835670 .00021

35.400 .836950 .00020

35.600 .838320 .00020

35.800 .838840 .00020

36.000 .839830 .00020

36.200 .839830 .00020

36.400 .842030 .00020

36.600 .842260 .00020

36.800 .843390 .00020

37.000 .844220 .00020

37.200 .845520 .00020

37.400 .846420 .00020

37.500 .846800 .00020
12.850 .982230 .00054

12.900 .985480 .00053

12.950 .989570 .00054

13.000 .992160 .00054

13.050 .996330 .00054

13.101 .000710 .00054

13.151 .002710 .00055

13.201 .006050 .00054

13.251 .009620 .00054

13.301 .013660 .00056

13.351 .015860 .00053

13.401 .020160 .00052

13.451 .022080 .00054

13.501 .025880 .00055

15

31.600 .914880 .00024

31.650 .915380 .00024

31.700 .915910 .00024

31.750 .915660 .00023

31.800 .916130 .00024

31.850 .916960 .00023

31.900 .916940 .00023

31.950 .917410 .00023

32.000 .917670 .00023

32.050 .918370 .00023

32.100 .918120 .00023

32.150 .918550 .00023

32.200 .918660 .00023

32.250 .919600 .00023

32.300 .919820 .00023

32.350 .919860 .00023

32.400 .920460 .00023

32.450 .920520 .00023

32.500 .921000 .00023

32.550 .920360 .00023

32.600 .921360 .00024

32.650 .922040 .00023

32.700 .922260 .00024

32.750 .922460 .00023

32.800 .923570 .00024

32.850 .922690 .00023

32.900 .923380 .00023

32.950 .923740 .00022

33.000 .924180 .00023
20

21.800 .924310 .00031

22.100 .929760 .00030

22.400 .933950 .00030

22.700 .940160 .00029

23.000 .944760 .00029

23.300 .949090 .00031

23.600 .953810 .00029

23.900 .957970 .00029

24.200 .963200 .00030

24.500 .967140 .00029

24.800 .971370 .00029

25.100 .974860 .00028

25.400 .979130 .00029

25.700 .983180 .00029

26.000 .985940 .00030

26.300 .990050 .00029

26.600 .993740 .00028

26.900 .997360 .00028

27.201 .001340 .00028

27.501 .004000 .00027

27.801 .007570 .00027

28.101 .010550 .00028

28.301 .012990 .00027 
25

18.000 .923130 .00035

18.200 .929180 .00034

18.400 .935170 .00034

18.600 .939590 .00034

18.800 .944050 .00034

19.000 .949950 .00035

19.200 .954350 .00033

19.400 .959570 .00035

19.600 .963570 .00035

19.800 .968910 .00035

20.000 .973020 .00034

20.200 .977520 .00034

20.400 .981710 .00032

20.600 .985920 .00033

20.800 .990360 .00033

21.000 .993860 .00032

21.200 .998520 .00033

21.401 .002880 .00033

21.601 .006450 .00034

21.801 .010150 .00033

22.001 .014300 .00033

22.101 .016600 .00033
35

14.800 .922750 .00039

14.900 .926660 .00040

15.000 .930200 .00040

15.100 .934920 .00039

15.200 .938050 .00038

15.300 .942760 .00039

15.400 .946910 .00041

15.500 .950230 .00040

15.600 .954340 .00039

15.700 .957780 .00039

15.800 .962400 .00038

15.900 .964890 .00040

16.000 .969270 .00038

16.100 .972590 .00040
30

16.000 .921940 .00037

16.100 .925460 .00038

16.200 .928890 .00037

16.300 .931290 .00037

16.400 .935780 .00036

16.500 .938930 .00037

16.600 .943220 .00037

16.700 .945740 .00038

16.800 .948720 .00037

16.900 .952390 .00036

17.000 .955550 .00037

17.100 .959010 .00037

17.200 .961580 .00037

17.300 .965180 .00038

17.400 .967960 .00037

17.500 .970520 .00038

17.600 .974510 .00037

17.700 .976880 .00038

17.800 .979800 .00038

17.900 .982480 .00037

18.000 .984920 .00038

18.100 .988370 .00037

18.200 .991150 .00037

18.300 .993700 .00036

18.400 .996620 .00036

18.500 .999160 .00037

18.601 .002550 .00036

18.701 .005780 .00036

18.801 .007980 .00036

18.901 .010420 .00038

19.001 .012600 .00036

19.101 .014990 .00037

40

13.900 .920140 .00042

14.000 .924520 .00042

14.100 .929190 .00042

14.200 .934600 .00043

14.300 .938360 .00041

14.400 .942700 .00041

14.500 .947400 .00042

14.600 .952030 .00041

14.700 .955860 .00042

14.800 .959620 .00041

14.900 .964290 .00041

15.000 .968540 .00041

15.100 .973120 .00041

15.200 .976660 .00042
32

15.500 .923570 .00039

15.600 .927310 .00039

15.700 .931440 .00038

15.800 .934120 .00038

15.900 .938300 .00039

16.000 .941640 .00038

16.100 .945650 .00038

16.200 .948850 .00038

16.300 .952620 .00039

16.400 .956560 .00039

16.500 .959280 .00038

16.600 .963160 .00037

16.700 .966170 .00038

16.800 .969020 .00038

16.900 .972980 .00038

17.000 .975520 .00037

17.100 .978990 .00037

17.200 .982320 .00038

17.300 .986040 .00038

17.400 .987940 .00037

17.500 .990930 .00038

17.600 .994820 .00038

17.700 .997400 .00039

17.801 .000360 .00037

17.901 .003800 .00038

18.001 .006690 .00038

18.101 .009830 .00038

18.201 .012540 .00036

18.301 .014880 .00037

18.401 .018070 .00038

50

12.800 .918950 .00044

12.900 .924080 .00044

13.000 .929490 .00044

13.100 .934540 .00043

13.200 .940170 .00045

13.300 .946020 .00043

13.400 .950420 .00045

13.500 .956210 .00045

13.600 .960710 .00044

13.700 .965640 .00044

13.800 .970730 .00045

13.900 .975340 .00043

14.000 .980150 .00043

14.100 .985520 .00043 
16.200 .977450 .00039 16.300 .979790 .00039 16.400 .982860 .00039 16.500 .987160 .00040 16.600 .990360 .00039 16.700 .994280 .00039 16.800 .996620 .00040 16.901 .000450 .00039 17.001 .003970 .00038 17.101 .007200 .00040 17.201 .010480 .00040 17.301 .014070 .00038 17.401 .017270 .00039

60

12.200 .923180 .00045 12.300 .929120 .00046 12.400 .935240 .00046 12.500 .940730 .00046 12.600 .946910 .00046 12.700 .951990 .00047 12.800 .958150 .00046 12.900 .963130 .00046 13.000 .969430 .00048 13.100 .974840 .00046 13.200 .979960 .00046 13.300 .985810 .00047 13.400 .991550 .00046 13.500 .995590 .00046 13.601 .000710 .00048 13.701 .006880 .00045 13.801 .010870 .00045 13.901 .015790 .00046 14.001 .020910 .00044
15.300 .982130 .00040 15.400 .984830 .00042 15.500 .988010 .00042 15.600 .992830 .00040 15.700 .997230 .00042 15.801 .001060 .00041 15.901 .004130 .00042 16.001 .008680 .00040 16.101 .012130 .00041 16.201 .015850 .00041 16.301 .020070 .00041
14.200 .989480 .00044 14.300 .995090 .00044 14.401 .000050 .00043 14.501 .004370 .00043 14.601 .008370 .00044 14.701 .013240 .00043 14.801 .017440 .00043 14.901 .022020 .00044
70
11.700 .921000 .00046 11.750 .923550 .00048 11.800 .925950 .00048 11.850 .929860 .00046 11.900 .933810 .00046 11.950 .937050 .00048 12.000 .939430 .00047 12.050 .942310 .00048 12.100 .945260 .00047 12.150 .947770 .00048 12.200 .951650 .00048 12.250 .955440 .00047 12.300 .958550 .00047 12.350 .961250 .00050 12.400 .963430 .00047 12.450 .966340 .00047 12.500 .969680 .00049 12.550 .972870 .00049 12.600 .974860 .00049 12.650 .978940 .00046 12.700 .981420 .00047 12.750 .983110 .00048 12.800 .987800 .00048 12.850 .990640 .00048 12.900 .993720 .00048 12.950 .996080 .00047 13.000 .998380 .00047 13.051 .001800 .00047 13.101 .004260 .00047 13.151 .007120 .00048 13.201 .009670 .00048 13.251 .013290 .00047 13.301 .015260 .00047 13.351 .016960 .00047 13.401 .021160 .00047
80

11.400 .922640 .00049 11.450 .925610 .00049 11.500 .929810 .00049 11.550 .932170 .00048 11.600 .936400 .00049 11.650 .940340 .00049 11.700 .942430 .00048 11.750 .945620 .00048 11.800 .949010 .00048 11.850 .951670 .00048 11.900 .955260 .00049 11.950 .959600 .00047 12.000 .962030 .00049 12.050 .964950 .00048 12.100 .967860 .00049 12.150 .971030 .00048 12.200 .974780 .00048 12.250 .978050 .00049 12.300 .980700 .00048 12.350 .984660 .00047 12.400 .986970 .00048 12.450 .989740 .00049 12.500 .992960 .00048 12.550 .995960 .00049 12.600 .999280 .00048 12.651 .002390 .00049 12.701 .004460 .00047 12.751 .008550 .00048 12.801 .010330 .00048 12.851 .012320 .00047 12.901 .015790 .00050 12.951 .018010 .00049 13.001 .023490 .00048 
90

11.100 .919810 .00049

11.150 .923620 .00049

11.200 .927280 .00050

11.250 .930470 .00049

11.300 .932960 .00048

11.350 .937680 .00047

11.400 .940600 .00048

11.450 .943890 .00049

11.500 .947570 .00049

11.550 .951460 .00049

11.600 .954600 .00050

11.650 .957870 .00048

11.700 .960970 .00049

11.750 .964010 .00050

11.800 .967210 .00049

11.850 .970610 .00050

11.900 .973930 .00049

11.950 .976820 .00049

12.000 .980270 .00050

12.050 .983010 .00048

12.100 .986500 .00048

12.150 .990090 .00049

12.200 .992780 .00050

12.250 .996180 .00049

12.300 .999240 .00049

12.351 .002250 .00049

12.401 .005290 .00049

12.451 .008820 .00049

12.501 .011620 .00049

12.551 .013680 .00050

12.601 .017380 .00048

12.651 .020530 .00049

12.701 .023880 .00048

130

10.500 .917310 .00052

10.550 .921140 .00051

10.600 .925380 .00050

10.650 .930010 .00051

10.700 .932630 .00050

10.750 .936680 .00050

10.800 .939320 .00050

10.850 .944700 .00052

10.900 .948630 .00050

10.950 .950480 .00052

11.000 .955210 .00051

11.050 .958700 .00051

11.100 .961860 .00051
100

10.900 .919330 .00049

10.950 .922630 .00049

11.000 .925980 .00050

11.050 .929700 .00051

11.100 .933210 .00050

11.150 .936760 .00049

11.200 .941700 .00050

11.250 .944250 .00050

11.300 .947610 .00050

11.350 .950990 .00049

11.400 .954770 .00049

11.450 .958470 .00050

11.500 .960780 .00052

11.550 .965420 .00049

11.600 .967650 .00050

11.650 .971320 .00050

11.700 .975040 .00050

11.750 .978420 .00050

11.800 .982100 .00052

11.850 .984710 .00049

11.900 .988090 .00050

11.950 .991300 .00050

12.000 .994570 .00050

12.050 .998100 .00050

12.101 .000710 .00051

12.151 .004170 .00051

12.201 .007730 .00050

12.251 .010260 .00049

12.301 .014230 .00049

12.351 .016750 .00049

12.401 .020570 .00051
118

10.700 .923750 .00050

10.750 .927120 .00051

10.800 .930800 .00049

10.850 .934790 .00051

10.900 .937510 .00049

10.950 .941460 .00050

11.000 .944410 .00050

11.050 .949300 .00049

11.100 .952560 .00051

11.150 .955650 .00050

11.200 .959430 .00050

11.250 .963710 .00052

11.300 .966480 .00052

11.350 .969660 .00052

11.400 .973230 .00051

11.450 .977790 .00052

11.500 .981530 .00051

11.550 .983720 .00053

11.600 .987440 .00051

11.650 .990810 .00051

11.700 .993750 .00051

11.750 .997330 .00051

11.801 .001170 .00052

11.851 .004250 .00053

11.901 .007040 .00051

11.951 .011500 .00051

12.001 .015080 .00050

12.051 .016480 .00051

12.101 .021280 .00051
10.400 .922620 .00052

10.450 .925670 .00052

10.500 .929470 .00052

10.550 .933980 .00052

10.600 .937830 .00053

10.650 .941240 .00052

10.700 .944470 .00052

10.750 .948340 .00052

10.800 .952000 .00052

10.850 .956920 .00052

10.900 .959890 .00053

10.950 .963940 .00052

11.000 .967270 .00053
200

10.200 .924210 .00053

10.250 .927720 .00052

10.300 .931140 .00053

10.350 .936010 .00051

10.400 .940260 .00053

10.450 .943770 .00052

10.500 .948190 .00053

10.550 .951560 .00052

10.600 .955440 .00052

10.650 .959200 .00051

10.700 .962330 .00052

10.750 .967330 .00052

10.800 .970800 .00053 
11.150 .965720 .00052 11.200 .968960 .00051 11.250 .973840 .00051 11.300 .977000 .00052 11.350 .980640 .00051 11.400 .984050 .00050 11.450 .987540 .00051 11.500 .990390 .00052 11.550 .994600 .00052 11.600 .997610 .00051 11.651 .000690 .00052 11.701 .004770 .00051 11.751 .007900 .00051 11.801 .011470 .00050 11.851 .015040 .00052 11.901 .016910 .00052 11.951 .021070 .00050 12.001 .023710 .00052
11.050 .971440 .00052 11.100 .974970 .00052 11.150 .978640 .00052 11.200 .981670 .00053 11.250 .985030 .00053 11.300 .988880 .00051 11.400 .996410 .00052 11.451 .000010 .00052 11.501 .003430 .00052 11.551 .007120 .00053 11.601 .010860 .00051 11.651 .013430 .00052 11.701 .016860 .00051 11.751 .019980 .00051 11.801 .024700 .00054
10.850 .975320 .00054 10.900 .977480 .00052 10.950 .982640 .00052 11.000 .984510 .00052 11.050 .989690 .00054 11.100 .993400 .00054 11.150 .996360 .00053 11.200 .999770 .00054 11.251 .004010 .00053 11.301 .007220 .00053 11.351 .010350 .00053 11.401 .014000 .00052 11.451 .018500 .00051 11.501 .021870 .00052
235

10.100 .921720 .00053 10.150 .925060 .00053 10.200 .928780 .00053 10.250 .932160 .00052 10.300 .938010 .00052 10.350 .941700 .00053 10.400 .945590 .00054 10.450 .948710 .00053 10.500 .953000 .00053 10.550 .957730 .00054 10.600 .961170 .00053 10.650 .965650 .00054 10.700 .968930 .00055 10.750 .972870 .00052 10.800 .975450 .00053 10.850 .980710 .00054 10.900 .983280 .00052 10.950 .986640 .00053 11.000 .991160 .00053 11.050 .994970 .00051 11.100 .998140 .00052 11.151 .001300 .00053 11.201 .006490 .00053 11.251 .009570 .00052 11.301 .013690 .00054 11.351 .016770 .00054 11.401 .020450 .00054
250

10.100 .923510 .00053

10.150 .926760 .00053

10.200 .931460 .00053

10.250 .936030 .00053

10.300 .939870 .00052

10.350 .942520 .00053

10.400 .946840 .00054

10.450 .951040 .00054

10.500 .955720 .00052

10.550 .959930 .00052

10.600 .962830 .00052

10.650 .966350 .00053

10.700 .970570 .00054

10.750 .973560 .00053

10.800 .977680 .00054

10.850 .981810 .00054

10.900 .984780 .00053

10.950 .989840 .00053

11.000 .992210 .00054

11.050 .996510 .00053

11.101 .000500 .00054

11.151 .004600 .00053

11.201 .008250 .00053

11.251 .010630 .00054

11.301 .015600 .00052

11.351 .017690 .00053

11.401 .022350 .00053
353

10.000 .915750 .00051 10.050 .919470 .00051 10.100 .923480 .00055 10.150 .927560 .00052 10.200 .930990 .00054 10.250 .934540 .00053 10.300 .939200 .00053 10.350 .943500 .00054 10.400 .946790 .00053 10.450 .951040 .00053 10.500 .955140 .00054 10.550 .959370 .00053 10.600 .962710 .00053 10.650 .965260 .00054 10.700 .970260 .00053 10.750 .973690 .00053 10.800 .977270 .00054 10.850 .980580 .00053 10.900 .984250 .00052 10.950 .988790 .00054 11.000 .993590 .00054 11.050 .996040 .00054 11.100 .999960 .00054 11.151 .003070 .00055 11.201 .006560 .00054 11.251 .010710 .00055 11.301 .014510 .00053 11.351 .017130 .00055 11.401 .021720 .00055 
470

10.200 .920430 .00054 10.250 .924400 .00052 10.300 .929020 .00053 10.350 .933000 .00052 10.400 .935900 .00053 10.450 .940010 .00054 10.500 .944350 .00052 10.550 .948510 .00053 10.600 .951190 .00053 10.650 .955480 .00052 10.700 .959010 .00052 10.750 .963690 .00053 10.800 .966070 .00052 10.850 .970690 .00054 10.900 .973770 .00054 10.950 .977550 .00054 11.000 .981130 .00053 11.050 .984520 .00052 11.100 .988500 .00054 11.150 .992680 .00054 11.200 .996080 .00053 11.250 .998900 .00052 11.301 .002460 .00053 11.351 .006210 .00055 11.401 .009850 .00053 11.451 .012540 .00052 11.501 .017350 .00052 11.551 .020260 .00054 11.601 .023070 .00053

520

10.300 .921570 .00053 10.350 .926040 .00050 10.400 .929080 .00053 10.450 .933760 .00054 10.500 .936840 .00055 10.550 .940080 .00052 10.600 .944050 .00052 10.650 .949280 .00053 10.700 .951320 .00051 10.750 .954880 .00053 10.800 .959280 .00051 10.850 .962980 .00052
500

10.200 .916570 .00053

10.250 .920460 .00052

10.300 .924650 .00053

10.350 .928790 .00053

10.400 .931470 .00053

10.450 .935040 .00052

10.500 .939950 .00052

10.550 .944000 .00053

10.600 .947180 .00052

10.650 .950740 .00053

10.700 .955320 .00055

10.750 .958720 .00053

10.800 .963590 .00054

10.850 .965620 .00053

10.900 .969950 .00052

10.950 .973090 .00053

11.000 .975770 .00053

11.050 .980500 .00052

11.100 .983750 .00052

11.150 .987280 .00054

11.200 .990690 .00052

11.250 .994960 .00055

11.300 .998510 .00053

11.351 .001060 .00051

11.401 .005260 .00055

11.451 .008930 .00053

11.501 .012050 .00052

11.551 .015170 .00051

11.601 .019250 .00053

11.651 .022060 .00055

11.701 .025240 .00055

530

10.300 .919680 .00053

10.350 .923900 .00052

10.400 .926620 .00051

10.450 .932190 .00052

10.500 .935850 .00052

10.550 .939130 .00053

10.600 .942360 .00052

10.650 .946540 .00051

10.700 .950590 .00053

10.750 .954480 .00053

10.800 .958260 .00053

10.850 .961800 .00054
510

10.300 .921890 .00052

10.350 .926670 .00053

10.400 .930890 .00053

10.450 .934020 .00054

10.500 .938610 .00053

10.550 .942450 .00052

10.600 .946490 .00052

10.650 .948710 .00052

10.700 .953750 .00053

10.750 .956710 .00052

10.800 .961270 .00053

10.850 .965240 .00051

10.900 .967900 .00053

10.950 .972350 .00054

11.000 .975200 .00054

11.050 .979180 .00053

11.100 .981880 .00053

11.150 .985840 .00053

11.200 .989460 .00053

11.250 .993810 .00052

11.300 .996470 .00054

11.350 .999340 .00054

11.401 .003830 .00052

11.451 .006750 .00054

11.501 .010870 .00052

11.551 .014600 .00053

11.601 .016710 .00054

11.651 .020730 .00053

11.701 .023460 .00053

540

10.300 .918480 .00052

10.350 .922600 .00052

10.400 .926110 .00051

10.450 .930010 .00052

10.500 .933620 .00052

10.550 .938410 .00054

10.600 .941190 .00053

10.650 .944240 .00054

10.700 .948650 .00052

10.750 .953080 .00054

10.850 .960130 .00053

10.900 .963840 .00053 
10.900 .967050 .00053 10.950 .970590 .00053 11.000 .973860 .00051 11.050 .977930 .00052 11.100 .981490 .00052 11.150 .984530 .00053 11.200 .988510 .00053 11.250 .991020 .00053 11.300 .994180 .00054 11.350 .997880 .00053 11.401 .002250 .00053 11.501 .008760 .00053 11.551 .012950 .00052 11.601 .015860 .00054 11.651 .017960 .00055 11.701 .022510 .00054

\section{0}

10.300 .917950 .00051 10.350 .921580 .00052 10.400 .923710 .00054 10.450 .928090 .00054 10.500 .932870 .00052 10.550 .936710 .00051 10.600 .938960 .00052 10.650 .943430 .00052 10.700 .946520 .00052 10.750 .951370 .00053 10.800 .954530 .00052 10.850 .958060 .00053 10.900 .961260 .00052 10.950 .965020 .00054 11.000 .969780 .00052 11.050 .972620 .00052 11.100 .976190 .00053 11.150 .979220 .00053 11.200 .983630 .00055 11.250 .986410 .00053 11.300 .989400 .00053 11.350 .993390 .00052 11.400 .997010 .00053 11.451 .001000 .00053 11.501 .003610 .00052 11.551 .007780 .00051 11.601 .010130 .00053 11.651 .013830 .00053 11.701 .017990 .00054 11.751 .021180 .00053 11.801 .023940 .00054
10.900 .965320 .00053 10.950 .968620 .00051 11.000 .972850 .00052 11.050 .974200 .00052 11.100 .978370 .00053 11.150 .982450 .00053 11.200 .987600 .00053 11.250 .990870 .00053 11.300 .993520 .00052 11.350 .996860 .00055 11.401 .000570 .00054 11.451 .004610 .00054 11.501 .008010 .00053 11.551 .011240 .00055 11.601 .013230 .00054 11.651 .017380 .00053 11.701 .020960 .00052

\section{0}

10.400 .923250 .00052 10.450 .926490 .00052 10.500 .930470 .00052 10.550 .935040 .00051 10.600 .939280 .00052 10.650 .942160 .00051 10.700 .945960 .00052 10.750 .948880 .00052 10.800 .952590 .00053 10.850 .956210 .00052 10.900 .959540 .00053 10.950 .963870 .00053 11.000 .967900 .00055 11.050 .971530 .00053 11.100 .973800 .00053 11.150 .978730 .00051 11.200 .980610 .00054 11.250 .984920 .00053 11.300 .988600 .00053 11.350 .991550 .00052 11.400 .996450 .00053 11.450 .999210 .00052 11.501 .002390 .00052 11.551 .004760 .00053 11.601 .008760 .00052 11.651 .012790 .00054 11.701 .015610 .00053 11.751 .019980 .00053 11.801 .022470 .00053
10.950 .966620 .00052 11.000 .970760 .00052 11.050 .973620 .00054 11.100 .978470 .00053 11.150 .982460 .00053 11.200 .984980 .00053 11.250 .988630 .00053 11.300 .992260 .00053 11.350 .995540 .00053 11.400 .998940 .00053 11.451 .001830 .00054 11.501 .004120 .00052 11.551 .008510 .00053 11.601 .013350 .00052 11.651 .016080 .00055 11.701 .019930 .00052 11.751 .021780 .00053 11.801 .026040 .00053 
U5o2N23-sp-1000-10

12

33.500 .832610 .00019

33.700 .833360 .00019

33.900 .835440 .00020

34.100 .835710 .00019

34.300 .837230 .00020

34.500 .837930 .00020

34.700 .838460 .00019

34.900 .839430 .00019

35.100 .840410 .00019

35.300 .841670 .00019

35.500 .842720 .00019

35.700 .844060 .00019

35.900 .844690 .00019

36.100 .845330 .00019

36.300 .846580 .00019

36.500 .847480 .00019

36.700 .848200 .00019

36.900 .848550 .00019

37.100 .849440 .00019

37.300 .850290 .00019

37.500 .851580 .00019

37.700 .852180 .00018

37.900 .852980 .00018

25

16.900 .924090 .00033

17.100 .930400 .00032

17.300 .934820 .00033

17.500 .939980 .00033

17.700 .945420 .00032

17.900 .950550 .00032

18.100 .955880 .00034

18.300 .960100 .00031

18.500 .964780 .00032

18.700 .969530 .00031

18.900 .974100 .00032

19.100 .978260 .00033

19.300 .983770 .00032

19.500 .987190 .00032

19.700 .991100 .00031

19.900 .995720 .00031

20.101 .000180 .00032

20.301 .004130 .00032
15

31.800 .924120 .00022

31.850 .924910 .00022

31.900 .924270 .00023

31.950 .924630 .00022

32.000 .925830 .00022

32.050 .925450 .00021

32.100 .925450 .00021

32.150 .926040 .00022

32.200 .926340 .00022

32.250 .926920 .00022

32.300 .927230 .00022

32.350 .926860 .00022

32.400 .928020 .00022

32.450 .928220 .00022

32.500 .928460 .00022

32.550 .928310 .00022

32.600 .928740 .00022

32.650 .928900 .00023

32.700 .929390 .00022

32.750 .929920 .00022

32.800 .930420 .00022

32.850 .930780 .00022

32.900 .930040 .00022

32.950 .931190 .00022

33.000 .931300 .00022
20

20.700 .924940 .00028

21.000 .929920 .00029

21.300 .934980 .00029

21.600 .940550 .00028

21.900 .944470 .00029

22.200 .949610 .00029

22.500 .954280 .00028

22.800 .959120 .00027

23.100 .962630 .00027

23.400 .967460 .00028

23.700 .971840 .00028

24.000 .975990 .00027

24.300 .979520 .00027

24.600 .983930 .00027

24.900 .987440 .00028

25.200 .990890 .00028

25.500 .994670 .00027

25.800 .997790 .00027

26.101 .001130 .00027

26.401 .004360 .00027

26.701 .007780 .00027

27.001 .011610 .00026

27.201 .013020 .00027
30

14.900 .923590 .00035

15.000 .926980 .00035

15.100 .930530 .00035

15.200 .933790 .00036

15.300 .936660 .00034

15.400 .940310 .00035

15.500 .943430 .00035

15.600 .947450 .00036

15.700 .949750 .00035

15.800 .953280 .00036

15.900 .956570 .00035

16.000 .960550 .00035

16.100 .963150 .00036

16.200 .966570 .00035

16.300 .968690 .00035

16.400 .972500 .00035

16.500 .975400 .00035

16.600 .978640 .00034
32

14.400 .925020 .00036

14.500 .928890 .00037

14.600 .933240 .00037

14.700 .936070 .00035

14.800 .940210 .00036

14.900 .944310 .00035

15.000 .947210 .00036

15.100 .950330 .00036

15.200 .954040 .00035

15.300 .957250 .00036

15.400 .960870 .00034

15.500 .964800 .00037

15.600 .968300 .00035

15.700 .970950 .00034

15.800 .975210 .00036

15.900 .977420 .00036

16.000 .981240 .00036

16.100 .984350 .00036 
20.501 .008490 .00032 20.701 .011340 .00031 20.901 .015770 .00031

35

13.600 .920000 .00039 13.700 .924450 .00038 13.800 .928770 .00038 13.900 .933120 .00037 14.000 .936980 .00037 14.100 .940660 .00038 14.200 .945380 .00038 14.300 .949080 .00038 14.400 .952920 .00037 14.500 .956190 .00037 14.600 .960290 .00036 14.700 .963820 .00038 14.800 .968170 .00037 14.900 .970880 .00039 15.000 .975750 .00037 15.100 .978200 .00037 15.200 .982550 .00036 15.300 .985840 .00037 15.400 .989600 .00037 15.500 .993170 .00037 15.600 .996170 .00037 15.700 .999760 .00037 15.801 .002870 .00037 15.901 .006310 .00038 16.001 .010370 .00037 16.101 .012670 .00037 16.201 .016720 .00037
16.700 .981640 .00036 16.800 .984460 .00034 16.900 .987110 .00035 17.000 .988860 .00035 17.100 .993510 .00035 17.200 .995950 .00035 17.300 .998650 .00035 17.401 .001210 .00034 17.501 .003960 .00034 17.601 .006450 .00034 17.701 .009250 .00035 17.801 .011650 .00034 17.901 .014890 .00034 18.001 .017380 .00035
16.200 .987290 .00038 16.300 .989860 .00036 16.400 .993470 .00035 16.500 .996460 .00035 16.600 .999440 .00035 16.701 .002450 .00036 16.801 .005480 .00036 16.901 .008450 .00035 17.001 .011750 .00036 17.101 .014830 .00033 17.201 .017430 .00036
40

12.800 .922090 .00040

12.900 .927940 .00038

13.000 .932780 .00038

13.100 .936210 .00039

13.200 .941260 .00039

13.300 .946180 .00039

13.400 .950310 .00039

13.500 .954980 .00038

13.600 .958970 .00040

13.700 .963690 .00039

13.800 .967130 .00038

13.900 .971510 .00039

14.000 .975590 .00039

14.100 .979300 .00039

14.200 .983840 .00038

14.300 .988560 .00038

14.400 .991920 .00038

14.500 .995820 .00038

14.600 .999670 .00038

14.701 .003330 .00039

14.801 .007680 .00039

14.901 .011540 .00039

15.001 .014490 .00037

15.101 .018510 .00038
50

11.700 .922390 .00041 11.800 .928440 .00040 11.900 .933140 .00040 12.000 .939870 .00041 12.100 .945030 .00042 12.200 .949260 .00042 12.300 .954930 .00040 12.400 .961540 .00041 12.500 .965190 .00041 12.600 .970370 .00041 12.700 .975260 .00042 12.800 .979600 .00041 12.900 .985130 .00041 13.000 .988860 .00041 13.100 .994870 .00042 13.200 .999620 .00040 13.301 .004170 .00040 13.401 .008130 .00041 13.501 .013020 .00042 13.601 .016710 .00042 
60

11.000 .921690 .00042

11.100 .927670 .00042

11.200 .934630 .00042

11.300 .940230 .00043

11.400 .945730 .00042

11.500 .952020 .00042

11.600 .957330 .00042

11.700 .963520 .00042

11.800 .968870 .00042

11.900 .975630 .00043

12.000 .979610 .00041

12.100 .985100 .00042

12.200 .991330 .00043

12.300 .996340 .00043

12.401 .000990 .00042

12.501 .006660 .00042

12.601 .010650 .00043

12.701 .017710 .00043

12.801 .022130 .00042
10.500 .920880 .00044

10.550 .923850 .00044

10.600 .927050 .00044

10.650 .930950 .00045

10.700 .933490 .00045

10.750 .936670 .00044

10.800 .940130 .00045

10.850 .943570 .00044

10.900 .946420 .00043

10.950 .950080 .00044

11.000 .953060 .00043

11.050 .956440 .00044

11.100 .958670 .00044

11.150 .961520 .00043

11.200 .964700 .00045

11.250 .968080 .00044

11.300 .970470 .00043

11.350 .973910 .00043

11.400 .977140 .00044

11.450 .980010 .00044

11.500 .982960 .00044

11.550 .985460 .00044

11.600 .988610 .00045

11.650 .992360 .00043

11.700 .995210 .00044

11.750 .997590 .00044

11.800 .999730 .00043

11.851 .004110 .00043

11.901 .006460 .00044

11.951 .008160 .00044

12.001 .011610 .00045

12.051 .013840 .00045

12.101 .017280 .00044

12.151 .019680 .00044

12.201 .022990 .00044
80

10.200 .923820 .00046

10.250 .927670 .00044

10.300 .930860 .00045

10.350 .934240 .00044

10.400 .937250 .00044

10.450 .940490 .00045

10.500 .943920 .00046

10.550 .947110 .00044

10.600 .951060 .00045

10.650 .954080 .00044

10.700 .957760 .00045

10.750 .960500 .00044

10.800 .963400 .00044

10.850 .966990 .00044

10.900 .969660 .00045

10.950 .973580 .00045

11.000 .976640 .00044

11.050 .979900 .00044

11.100 .982790 .00046

11.150 .985400 .00045

11.200 .989370 .00043

11.250 .991530 .00045

11.300 .994800 .00044

11.350 .997780 .00045

11.401 .001220 .00046

11.451 .004300 .00045

11.501 .006910 .00046

11.551 .010390 .00045

11.601 .013060 .00045

11.651 .016010 .00046

11.701 .018780 .00046
90

9.900 .921440 .00046

9.950 .925390 .00045

10.000 .928700 .00046

10.050 .933150 .00046

10.100 .936040 .00045

10.150 .940060 .00045

10.200 .942620 .00046

10.250 .946420 .00044

10.300 .949550 .00045

10.350 .953140 .00045

10.400 .956110 .00046
100

9.700 .921610 .00046

9.750 .925220 .00046

9.800 .929310 .00046

9.850 .932940 .00047

9.900 .936840 .00047

9.950 .939550 .00045

10.000 .943610 .00046

10.050 .947800 .00045

10.100 .951260 .00046

10.150 .954320 .00046

10.200 .957930 .00047
118

9.400 .920440 .00048

9.450 .923420 .00046

9.500 .927990 .00047

9.550 .931980 .00046

9.600 .935030 .00045

9.650 .938620 .00046

9.700 .943040 .00047

9.750 .945880 .00047

9.800 .950210 .00046

9.850 .953670 .00046

9.900 .958000 .00048 
10.450 .961020 .00045 10.500 .963500 .00045 10.550 .966740 .00046 10.600 .971100 .00045 10.650 .973820 .00044 10.700 .975910 .00044 10.750 .980400 .00044 10.800 .982860 .00046 10.850 .986150 .00046 10.900 .989340 .00046 10.950 .992910 .00045 11.000 .996390 .00047 11.050 .998880 .00046 11.101 .002380 .00045 11.151 .005600 .00046 11.201 .008270 .00045 11.251 .012690 .00046 11.301 .014950 .00046 11.351 .018350 .00048 11.401 .020920 .00046

130

9.300 .923550 .00048

9.350 .926680 .00047

9.400 .931230 .00048

9.450 .934590 .00047

9.500 .937480 .00048

9.550 .942660 .00048

9.600 .946130 .00048

9.650 .949650 .00046

9.700 .954180 .00047

9.750 .957440 .00047

9.800 .960550 .00047

9.850 .964670 .00047

9.900 .967410 .00048

9.950 .972070 .00048

10.000 .975630 .00047

10.050 .979790 .00047

10.100 .982590 .00047

10.150 .987210 .00047

10.200 .991060 .00045

10.250 .994280 .00048

10.300 .997730 .00048

10.351 .000270 .00047

10.401 .004090 .00048

10.451 .007420 .00048

10.501 .011590 .00048

10.551 .014170 .00047

10.601 .018000 .00046

10.651 .021280 .00048

10.701 .023820 .00048
10.250 .961410 .00047

10.300 .964430 .00048

10.350 .968020 .00047

10.400 .972010 .00045

10.450 .974830 .00045

10.500 .977690 .00047

10.550 .982070 .00046

10.600 .985620 .00045

10.650 .988520 .00045

10.700 .991170 .00045

10.750 .995340 .00048

10.800 .998420 .00046

10.851 .002500 .00047

10.901 .004550 .00046

10.951 .008460 .00047

11.001 .011300 .00046

11.051 .014320 .00047

11.101 .018750 .00047

150

9.100 .921980 .00047

9.150 .925080 .00047

9.200 .929440 .00047

9.250 .933310 .00049

9.300 .937130 .00047

9.350 .941370 .00047

9.400 .943970 .00047

9.450 .949700 .00048

9.500 .953270 .00049

9.550 .956450 .00049

9.600 .960580 .00047

9.650 .963440 .00047

9.700 .967120 .00049

9.750 .970940 .00049

9.800 .975460 .00047

9.850 .978360 .00049

9.900 .982750 .00047

9.950 .986670 .00047

10.000 .990330 .00048

10.050 .993790 .00049

10.100 .996640 .00048

10.151 .001160 .00048

10.201 .004940 .00048

10.251 .008050 .00048

10.301 .011810 .00048

10.351 .015280 .00048

10.401 .018260 .00047

10.451 .022190 .00051

10.501 .025260 .00049
9.950 .960330 .00048

10.000 .964990 .00047

10.050 .967440 .00048

10.100 .971600 .00047

10.150 .975680 .00048

10.200 .979300 .00046

10.250 .982770 .00047

10.300 .985740 .00046

10.350 .989730 .00046

10.400 .993840 .00047

10.450 .997660 .00047

10.500 .999500 .00047

10.551 .003900 .00046

10.601 .007330 .00048

10.651 .009280 .00048

10.701 .012400 .00047

10.751 .016910 .00046

10.801 .020210 .00047 
235

8.700 .918120 .00049

8.750 .922340 .00048

8.800 .927020 .00048

8.850 .929980 .00049

8.900 .935530 .00048

8.950 .938680 .00049

9.000 .943170 .00049

9.050 .945450 .00048

9.100 .950790 .00049

9.150 .954790 .00048

9.200 .959190 .00050

9.250 .962330 .00048

9.300 .967580 .00049

9.350 .970820 .00051

9.400 .974920 .00049

9.450 .978910 .00049

9.500 .983150 .00049

9.550 .986230 .00049

9.600 .990780 .00051

9.650 .993720 .00049

9.700 .997140 .00049

9.751 .001750 .00049

9.801 .004590 .00047

9.851 .007990 .00048

9.901 .012210 .00050

9.951 .016550 .00049

10.001 .019550 .00049
250

8.700 .920010 .00049

8.750 .924790 .00048

8.800 .928170 .00049

8.850 .933690 .00048

8.900 .937760 .00049

8.950 .941150 .00049

9.000 .945010 .00049

9.050 .949150 .00049

9.100 .953090 .00050

9.150 .957730 .00048

9.200 .961500 .00048

9.250 .964830 .00048

9.300 .970060 .00049

9.350 .974130 .00051

9.400 .977120 .00048

9.450 .981180 .00050

9.500 .984870 .00050

9.550 .988860 .00049

9.600 .993510 .00049

9.650 .996020 .00048

9.700 .999730 .00049

9.751 .003220 .00049

9.801 .007070 .00049

9.851 .010760 .00049

9.901 .014110 .00049

9.951 .018190 .00050

10.001 .022510 .00050
353

8.600 .919310 .00047

8.650 .921670 .00049

8.700 .926900 .00050

8.750 .930640 .00050

8.800 .935550 .00049

8.850 .939160 .00049

8.900 .942650 .00051

8.950 .947480 .00049

9.000 .950910 .00050

9.050 .955280 .00050

9.100 .959610 .00049

9.150 .963040 .00051

9.200 .967370 .00049

9.250 .970980 .00050

9.300 .973770 .00048

9.350 .978050 .00049

9.400 .982610 .00051

9.450 .986480 .00048

9.500 .989140 .00049

9.550 .993710 .00049

9.600 .996850 .00048

9.651 .001560 .00049

9.701 .004600 .00049

9.751 .008210 .00049

9.801 .012290 .00049

9.851 .015130 .00051

9.951 .022930 .00050

10.001 .026370 .00049
470

8.700 .922560 .00046

8.750 .926510 .00047

8.800 .930490 .00049

8.850 .933770 .00047

8.900 .938800 .00047

8.950 .942460 .00048

9.000 .945260 .00048

9.050 .950690 .00049

9.100 .954120 .00050

9.150 .957900 .00049

9.200 .962440 .00049

9.250 .965640 .00050

9.300 .969810 .00049

9.350 .972720 .00049

9.400 .977630 .00050

9.450 .980310 .00048

9.500 .983660 .00049

9.550 .986710 .00050
500

8.700 .920520 .00048

8.750 .923970 .00049

8.800 .928250 .00050

8.850 .931510 .00049

8.900 .935140 .00049

8.950 .939480 .00049

9.000 .943080 .00049

9.050 .946270 .00048

9.100 .951110 .00048

9.150 .955880 .00049

9.200 .958490 .00049

9.250 .962580 .00048

9.300 .965510 .00048

9.350 .970940 .00050

9.400 .974280 .00049

9.450 .978250 .00050

9.550 .985290 .00050

9.600 .988350 .00050
510

8.700 .918930 .00048

8.750 .922510 .00047

8.800 .926820 .00049

8.850 .930700 .00048

8.900 .933980 .00049

8.950 .938740 .00049

9.000 .942880 .00048

9.050 .946230 .00050

9.100 .950630 .00050

9.150 .953630 .00050

9.200 .958880 .00050

9.250 .961400 .00050

9.300 .965620 .00049

9.350 .969340 .00048

9.400 .972360 .00050

9.450 .976680 .00048

9.500 .979820 .00049

9.550 .983300 .00048 
9.600 .992520 .00049

9.650 .994990 .00049

9.700 .998960 .00048

9.751 .002810 .00048

9.801 .005080 .00051

9.851 .009570 .00048

9.901 .013320 .00049

9.951 .015980 .00050 10.001 .020270 .00048

\section{0}

8.700 .917410 .00049

8.750 .922230 .00048

8.800 .925380 .00050

8.850 .928820 .00049

8.900 .933700 .00050

8.950 .936750 .00048

9.000 .942550 .00050

9.050 .946060 .00049

9.100 .948430 .00049

9.150 .952340 .00049

9.200 .956490 .00049

9.250 .959990 .00048

9.300 .965360 .00048

9.350 .968160 .00050

9.400 .971290 .00049

9.450 .975300 .00050

9.500 .979730 .00049

9.550 .983180 .00049

9.600 .986630 .00049

9.650 .989500 .00049

9.700 .994280 .00048

9.750 .997380 .00048

9.801 .001990 .00049

9.851 .004060 .00049

9.901 .008020 .00050

9.951 .011370 .00050 10.001 .014570 .00048 10.051 .017490 .00050 10.101 .021830 .00049
9.650 .992220 .00049

9.700 .996160 .00049

9.750 .999660 .00050

9.801 .003260 .00050

9.851 .006640 .00051

9.901 .010870 .00049

9.951 .014340 .00050 10.001 .017410 .00050 10.051 .020650 .00048 10.101 .022870 .00050

530

8.700 .916870 .00049

8.750 .920010 .00049

8.800 .924850 .00048

8.850 .928220 .00050

8.900 .932560 .00049

8.950 .936840 .00049

9.000 .941390 .00048

9.050 .944780 .00048

9.100 .947890 .00048

9.150 .951860 .00049

9.200 .955720 .00050

9.250 .959580 .00048

9.300 .963320 .00049

9.350 .967580 .00048

9.400 .969300 .00049

9.450 .975110 .00048

9.500 .977790 .00048

9.550 .981690 .00049

9.600 .986280 .00049

9.650 .988290 .00049

9.700 .992410 .00049

9.750 .995460 .00048

9.800 .999860 .00049

9.851 .002980 .00049

9.901 .006790 .00049

9.951 .009660 .00050 10.001 .013850 .00051 10.051 .017670 .00050 10.101 .020110 .00048
9.600 .987500 .00049

9.650 .991080 .00050

9.700 .994340 .00048

9.750 .997280 .00048

9.801 .001810 .00049

9.851 .004590 .00049

9.901 .009750 .00049

9.951 .011760 .00049 10.001 .015620 .00049 10.051 .018860 .00050 10.101 .022080 .00050

540

8.700 .916420 .00049

8.750 .919390 .00049

8.800 .923870 .00047

8.850 .928040 .00050

8.900 .931210 .00049

8.950 .934930 .00049

9.000 .939660 .00050

9.050 .943850 .00049

9.100 .946330 .00048

9.150 .951490 .00048

9.200 .955580 .00048

9.250 .958490 .00049

9.300 .962240 .00049

9.350 .965610 .00049

9.400 .969320 .00048

9.450 .973410 .00049

9.600 .984380 .00049

9.650 .986930 .00047

9.700 .990770 .00050

9.750 .995420 .00048 9.800 .997760 .00050 9.851 .001960 .00049 9.901 .005460 .00050 9.951 .008320 .00049 10.001 .012050 .00048 10.051 .015480 .00050 10.101 .019340 .00050 
550

8.800 .922580 .00048

8.850 .926960 .00048

8.900 .930790 .00047

8.950 .933860 .00048

9.000 .938340 .00049

9.050 .942230 .00048

9.100 .945780 .00049

9.150 .949680 .00049

9.200 .953990 .00049

9.250 .957850 .00048

9.300 .961860 .00048

9.350 .964340 .00049

9.400 .968390 .00048

9.450 .972600 .00049

9.500 .976800 .00049

9.550 .979980 .00049

9.600 .982900 .00048

9.650 .986230 .00049

9.700 .989500 .00048

9.750 .993250 .00050

9.800 .997480 .00048

9.851 .001300 .00050

9.901 .004450 .00050

9.951 .007050 .00047

10.001 .011030 .00049

10.051 .014540 .00050

10.101 .017930 .00048

U5o2N26-sp-1000-00

12

64.300 .862950 .00016

65.200 .864780 .00016

66.100 .865760 .00016

67.000 .866620 .00016

67.900 .867810 .00016

68.800 .869090 .00015

69.700 .869750 .00016

70.600 .870060 .00015

71.500 .871000 .00016

72.400 .871570 .00015

73.300 .872600 .00016

74.200 .873600 .00015

75.100 .874450 .00015

76.000 .874740 .00015

76.900 .875500 .00014

77.800 .875840 .00015
560

8.800 .921830 .00048

8.850 .926020 .00048

8.900 .929520 .00048

8.950 .932830 .00049

9.000 .937910 .00048

9.050 .941500 .00048

9.100 .945240 .00048

9.150 .948940 .00049

9.200 .951960 .00049

9.250 .956600 .00050

9.300 .959810 .00048

9.350 .963620 .00048

9.400 .966620 .00050

9.450 .970660 .00049

9.500 .974860 .00048

9.550 .977770 .00049

9.600 .981600 .00049

9.650 .984570 .00048

9.700 .989600 .00049

9.750 .992350 .00050

9.800 .995570 .00050

9.850 .999710 .00049

9.901 .003060 .00050

9.951 .006070 .00049

10.001 .009340 .00049

10.051 .013610 .00050

10.101 .016350 .00050

10.151 .020890 .00051

10.201 .022710 .00050

15

37.700 .898600 .00022

38.400 .902610 .00022

39.100 .906100 .00022

39.800 .909680 .00022

40.500 .913430 .00022

41.200 .916150 .00022

41.900 .919120 .00021

42.600 .922050 .00021

43.300 .923800 .00021

44.000 .926980 .00021

44.700 .929280 .00021

45.400 .931900 .00021

46.100 .933890 .00020

46.800 .936220 .00020

47.500 .938880 .00020

48.200 .940200 .00020
20

26.500 .926900 .00029

27.000 .933510 .00030

27.500 .939480 .00029

28.000 .946290 .00029

28.500 .952150 .00028

29.000 .958160 .00029

29.500 .963190 .00029

30.000 .969240 .00028

30.500 .974100 .00028

31.000 .978580 .00028

31.500 .982960 .00027

32.000 .987630 .00027

32.500 .991980 .00027

33.000 .996770 .00028

33.500 .999870 .00027

34.001 .003900 .00026 
78.700 .876880 .00015 79.600 .876960 .00014 80.500 .877360 .00015 81.400 .878270 .00015 82.300 .879160 .00015 82.400 .879140 .00015

25

21.500 .923570 .00033 21.700 .927590 .00035 21.900 .933620 .00035 22.100 .936420 .00035 22.300 .941500 .00034 22.500 .945570 .00034 22.700 .949760 .00034 22.900 .954460 .00034 23.100 .957950 .00034 23.300 .962470 .00034 23.500 .965900 .00033 23.700 .970150 .00033 23.900 .973570 .00033 24.100 .977370 .00033 24.300 .980400 .00033 24.500 .983980 .00033 24.700 .987670 .00033 24.900 .990920 .00033 25.100 .993870 .00033 25.300 .997590 .00033 25.501 .000740 .00033 25.701 .003760 .00033 25.901 .007020 .00032 26.101 .010780 .00032 26.301 .013370 .00033 26.401 .015740 .00032
48.900 .942320 .00020 49.600 .944020 .00021 50.300 .946480 .00019 51.000 .947500 .00020 51.700 .949380 .00019 52.400 .950940 .00019 52.500 .951570 .00019
34.501 .008240 .00026 35.001 .012160 .00026 35.501 .015160 .00026 36.001 .018900 .00026 36.501 .022110 .00026
30

19.100 .923580 .00039 19.200 .925870 .00037 19.300 .928860 .00038 19.400 .932320 .00037 19.500 .934770 .00036 19.600 .937630 .00037 19.700 .940930 .00037 19.800 .943360 .00038 19.900 .946360 .00037 20.000 .949350 .00038 20.100 .951280 .00038 20.200 .954680 .00037 20.300 .957730 .00036 20.400 .960540 .00037 20.500 .963310 .00037 20.600 .964710 .00037 20.700 .968660 .00037 20.800 .970190 .00037 20.900 .973710 .00038 21.000 .976050 .00038 21.100 .977660 .00037 21.200 .980910 .00036 21.300 .983040 .00036 21.400 .985910 .00036 21.500 .988980 .00036 21.600 .990430 .00036 21.700 .992750 .00037 21.800 .995670 .00037 21.900 .996970 .00036 22.001 .000220 .00037 22.101 .002350 .00036 22.201 .005100 .00037 22.301 .006920 .00037 22.401 .009350 .00036 22.501 .011210 .00036 22.601 .013700 .00036 22.701 .015990 .00036 22.801 .018330 .00036
32

18.400 .921650 .00038 18.500 .925040 .00039 18.600 .928280 .00039 18.700 .931890 .00040 18.800 .934600 .00039 18.900 .937660 .00038 19.200 .947400 .00039 19.300 .950500 .00039 19.400 .952560 .00039 19.500 .956390 .00038 19.600 .958910 .00039 19.700 .961640 .00038 19.800 .965140 .00038 19.900 .968510 .00038 20.000 .971310 .00038 20.100 .973290 .00038 20.200 .976580 .00037 20.300 .978940 .00037 20.400 .982390 .00038 20.500 .984720 .00038 20.600 .987290 .00037 20.700 .989420 .00038 20.800 .992430 .00038 20.900 .995770 .00038 21.000 .997890 .00039 21.101 .000720 .00037 21.201 .002470 .00038 21.301 .005650 .00038 21.401 .008560 .00037 21.501 .010080 .00037 21.601 .011890 .00036 21.701 .015540 .00037 
35

17.600 .921370 .00041

17.700 .925760 .00040

17.800 .928110 .00040

17.900 .932130 .00041

18.000 .935480 .00040

18.100 .939070 .00041

18.200 .942470 .00039

18.300 .945440 .00040

18.400 .949130 .00041

18.500 .952950 .00039

18.600 .956310 .00039

18.700 .959120 .00040

18.800 .962070 .00040

18.900 .966000 .00041

19.000 .970030 .00039

19.100 .972450 .00040

19.200 .974710 .00038

19.300 .979070 .00039

19.400 .981170 .00040

19.500 .985000 .00040

19.600 .987220 .00039

19.700 .990560 .00040

19.800 .993450 .00039

19.900 .996020 .00041

20.000 .999720 .00039

20.101 .002060 .00041

20.301 .008520 .00038

20.401 .010860 .00039

20.501 .013450 .00040

20.601 .015880 .00040

60

14.600 .919090 .00048

14.700 .924830 .00048

14.800 .931320 .00047

14.900 .936310 .00048

15.000 .941490 .00047

15.100 .945830 .00047

15.200 .952130 .00046

15.300 .956990 .00046

15.400 .961550 .00048

15.500 .966280 .00047

15.600 .971520 .00049

15.700 .976760 .00047

15.800 .981450 .00046

15.900 .986020 .00047

16.000 .991850 .00046
40

16.600 .919740 .00042

16.700 .923820 .00042

16.800 .928440 .00043

16.900 .932790 .00042

17.000 .936140 .00043

17.100 .941380 .00042

17.200 .944430 .00042

17.300 .948220 .00041

17.400 .952580 .00041

17.500 .956070 .00043

17.600 .959460 .00042

17.700 .963710 .00043

17.800 .966970 .00041

17.900 .970660 .00041

18.000 .974810 .00042

18.100 .978640 .00042

18.200 .981330 .00041

18.300 .985270 .00042

18.400 .988420 .00042

18.500 .992320 .00041

18.600 .995890 .00041

18.700 .999680 .00042

18.801 .002110 .00042

18.901 .005480 .00041

19.001 .009180 .00042

19.101 .012180 .00041

19.201 .015950 .00041

19.301 .018550 .00041
50

15.400 .921870 .00045

15.500 .926680 .00045

15.600 .931490 .00046

15.700 .936540 .00046

15.800 .940270 .00046

15.900 .946490 .00045

16.000 .950490 .00044

16.100 .954560 .00045

16.200 .959760 .00046

16.300 .963520 .00045

16.400 .968950 .00047

16.500 .973400 .00045

16.600 .977940 .00045

16.700 .980790 .00045

16.800 .986260 .00044

16.900 .990290 .00043

17.000 .993770 .00045

17.100 .998630 .00045

17.201 .002920 .00045

17.301 .007300 .00044

17.401 .010820 .00045

17.501 .014950 .00044

17.601 .019670 .00045
14.100 .920240 .00048

14.300 .932120 .00049

14.400 .937140 .00050

14.500 .942470 .00050

14.600 .948440 .00050

14.700 .955580 .00048

14.800 .959080 .00050

14.900 .966110 .00048

15.000 .972210 .00047

15.100 .976850 .00048

15.200 .982050 .00048

15.300 .986970 .00049

15.400 .992350 .00049

15.500 .998640 .00049

15.601 .002150 .00049
80

13.700 .918870 .00048

13.800 .922090 .00051

13.900 .929150 .00049

14.000 .935350 .00050

14.100 .941850 .00050

14.200 .948460 .00050

14.300 .953950 .00050

14.400 .959530 .00051

14.600 .970530 .00049

14.700 .977200 .00050

14.800 .982520 .00050

14.900 .988200 .00049

15.000 .994000 .00051

15.100 .999530 .00050

15.201 .005100 .00049 
16.100 .995840 .00048 16.201 .001220 .00046 16.301 .004840 .00047 16.401 .009640 .00046 16.501 .014860 .00045 16.601 .019590 .00047
15.701 .007700 .00049 15.801 .011230 .00048 15.901 .018250 .00050
15.301 .010300 .00050 15.401 .015240 .00049 15.501 .020610 .00050
90

13.400 .915910 .00051 13.450 .919140 .00050 13.500 .921540 .00052 13.550 .925030 .00051 13.600 .927650 .00051 13.650 .931910 .00050 13.700 .934940 .00050 13.750 .937420 .00051 13.800 .940830 .00051 13.850 .944970 .00051 13.900 .947920 .00049 13.950 .949980 .00051 14.000 .953900 .00052 14.050 .957590 .00052 14.100 .959500 .00051 14.150 .962980 .00052 14.200 .965470 .00051 14.250 .968760 .00051 14.300 .971490 .00052 14.350 .974600 .00050 14.400 .979110 .00052 14.450 .980800 .00052 14.500 .983510 .00052 14.550 .986890 .00050 14.600 .989510 .00049 14.650 .993170 .00050 14.700 .996670 .00052 14.750 .997800 .00050 14.801 .000890 .00052 14.851 .003910 .00051 14.901 .007170 .00050 14.951 .009640 .00051 15.001 .011100 .00051 15.051 .015750 .00051 15.101 .017490 .00051
100

13.300 .923290 .00051 13.350 .926670 .00052 13.400 .929510 .00051 13.450 .933070 .00052 13.500 .935770 .00051 13.550 .940160 .00052 13.600 .941880 .00052 13.650 .946700 .00051 13.750 .951340 .00051 13.800 .954270 .00052 13.850 .958480 .00052 13.900 .962480 .00051 14.000 .966890 .00051 14.050 .971300 .00052 14.100 .973690 .00052 14.150 .977470 .00052 14.200 .979500 .00052 14.250 .984040 .00053 14.300 .987000 .00052 14.350 .989540 .00052 14.400 .991610 .00053 14.450 .996000 .00052 14.500 .998630 .00051 14.551 .001110 .00053 14.601 .004180 .00051 14.651 .006610 .00051 14.701 .010980 .00051 14.751 .013050 .00052 14.801 .015610 .00051 14.851 .019260 .00051 14.901 .021540 .00053
118

13.000 .921820 .00052 13.050 .925440 .00051 13.100 .927380 .00053 13.150 .931420 .00053 13.200 .934270 .00052 13.250 .938390 .00052 13.300 .940820 .00052 13.350 .944430 .00053 13.400 .949450 .00053 13.450 .951520 .00053 13.600 .960950 .00051 13.650 .963740 .00053 13.700 .968940 .00053 13.750 .971670 .00052 13.800 .974180 .00052 13.850 .976720 .00052 13.900 .981060 .00053 13.950 .984270 .00052 14.000 .986710 .00052 14.050 .990160 .00052 14.100 .992960 .00053 14.150 .996990 .00054 14.200 .999740 .00053 14.251 .002890 .00053 14.301 .006170 .00053 14.351 .008350 .00053 14.401 .011240 .00052 14.451 .014770 .00052 14.501 .018000 .00054 14.551 .021440 .00051 14.601 .024100 .00053 
130

12.850 .920940 .00053

12.900 .924230 .00052

12.950 .926230 .00053

13.000 .930390 .00053

13.050 .935170 .00055

13.100 .938180 .00052

13.150 .941590 .00052

13.200 .944770 .00053

13.250 .948180 .00053

13.300 .951010 .00055

13.350 .954550 .00053

13.400 .958330 .00053

13.450 .962870 .00052

13.500 .965580 .00054

13.550 .968440 .00054

13.600 .971470 .00055

13.650 .975700 .00053

13.700 .978040 .00053

13.750 .981790 .00053

13.800 .984340 .00053

13.850 .988440 .00055

13.900 .992030 .00053

13.950 .994600 .00052

14.000 .997560 .00053

14.050 .999840 .00053

14.101 .004580 .00054

14.151 .007260 .00052

14.201 .009770 .00052

14.251 .014130 .00054

14.301 .016230 .00054

14.351 .019280 .00054

14.401 .022880 .00053
12.800 .917600 .00054

150

12.700 .921440 .00054

12.750 .924620 .00053

12.800 .928820 .00054

12.850 .932000 .00055

12.900 .935990 .00053

12.950 .938270 .00054

13.000 .943060 .00052

13.050 .945960 .00054

13.100 .949960 .00054

13.150 .953710 .00054

13.200 .955980 .00055

13.250 .959760 .00054

13.300 .963320 .00054

13.350 .966630 .00053

13.400 .970790 .00054

13.450 .973610 .00053

13.500 .978090 .00054

13.550 .980520 .00054

13.600 .983980 .00055

13.650 .987420 .00053

13.700 .990630 .00055

13.750 .993930 .00054

13.800 .996330 .00054

13.851 .000340 .00054

13.901 .003880 .00054

13.951 .008040 .00056

14.001 .010040 .00053

14.051 .012320 .00054

14.101 .016980 .00055

14.151 .019550 .00054

14.201 .022970 .00053

235

12.400 .917560 .00055

12.450 .922220 .00054

12.500 .925890 .00057

12.550 .929230 .00056

12.600 .933380 .00052

12.650 .935840 .00056

12.700 .939980 .00054

12.750 .944260 .00053

12.800 .947500 .00054

12.900 .954660 .00054

12.950 .958400 .00054

13.000 .962070 .00055

13.050 .965250 .00055
250

12.400 .917880 .00054

12.450 .921440 .00054

12.500 .926010 .00054

12.550 .929710 .00054

12.600 .933350 .00055

12.650 .936820 .00055

12.700 .940140 .00054

12.750 .944500 .00055

12.800 .948380 .00055

12.850 .950720 .00055

12.900 .956260 .00054

12.950 .959360 .00054

13.000 .961760 .00053
200

12.400 .913660 .00055

12.450 .918220 .00054

12.500 .922610 .00057

12.550 .926610 .00055

12.600 .929230 .00054

12.650 .933000 .00056

12.700 .935730 .00055

12.750 .939770 .00056

12.800 .942440 .00055

12.850 .948050 .00055

12.900 .951880 .00054

12.950 .954610 .00055

13.000 .958010 .00054

13.050 .960840 .00055

13.100 .964850 .00055

13.150 .968580 .00055

13.200 .971990 .00055

13.250 .975770 .00054

13.300 .978620 .00055

13.350 .982410 .00056

13.400 .986150 .00055

13.450 .989040 .00056

13.500 .992230 .00054

13.550 .995320 .00055

13.600 .999890 .00055

13.651 .002490 .00054

13.701 .005510 .00053

13.751 .008760 .00054

13.801 .012490 .00053

13.851 .015540 .00055

13.901 .019540 .00054

13.951 .021960 .00055

14.001 .024720 .00056

\section{3}

12.500 .917690 .00054

12.550 .921380 .00053

12.600 .924340 .00055

12.650 .928920 .00055

12.750 .936080 .00054

12.800 .939860 .00055

12.850 .942280 .00055

12.900 .945730 .00056

12.950 .950340 .00056

13.000 .953570 .00055

13.050 .958140 .00055

13.100 .961860 .00057

13.150 .964220 .00055 
13.100 .968720 .00055 13.150 .972370 .00056 13.200 .976170 .00055 13.250 .980950 .00055 13.300 .982420 .00057 13.350 .986510 .00056 13.400 .990560 .00055 13.450 .993420 .00054 13.500 .997040 .00055 13.551 .000100 .00054 13.601 .003490 .00056 13.651 .007310 .00055 13.701 .010180 .00054 13.751 .012920 .00056 13.801 .016990 .00055 13.851 .019060 .00057 13.901 .023130 .00057

470

12.800 .916810 .00054 12.850 .921050 .00054 12.900 .924340 .00055 12.950 .927410 .00056 13.000 .930290 .00053 13.050 .933350 .00055 13.100 .937430 .00053 13.150 .940780 .00053 13.200 .944320 .00054 13.250 .948720 .00054 13.300 .952500 .00055 13.350 .955100 .00056 13.400 .958400 .00055 13.450 .961650 .00054 13.500 .964930 .00054 13.550 .967900 .00054 13.600 .971210 .00055 13.650 .975060 .00054 13.700 .977840 .00055 13.750 .981500 .00055 13.800 .985350 .00056 13.850 .988020 .00056 13.900 .991580 .00054 13.950 .994150 .00052 14.000 .997490 .00054 14.051 .000930 .00055 14.101 .004720 .00055 14.151 .008120 .00053 14.201 .009790 .00055
13.050 .966770 .00056

13.100 .969890 .00056

13.150 .974120 .00056

13.200 .977180 .00055

13.250 .979890 .00054

13.300 .983480 .00056

13.350 .987120 .00054

13.400 .990160 .00054

13.450 .993790 .00057

13.500 .995900 .00055

13.551 .000670 .00055

13.601 .004140 .00056

13.651 .007380 .00055

13.701 .009880 .00054

13.751 .014150 .00054

13.801 .017140 .00055

13.851 .019840 .00055

13.901 .023880 .00057
13.200 .967480 .00055

13.250 .971120 .00055

13.300 .976380 .00054

13.350 .978050 .00056

13.400 .981740 .00055

13.450 .985730 .00055

13.500 .988030 .00055

13.550 .992010 .00053

13.600 .995020 .00054

13.650 .997610 .00055

13.701 .001590 .00056

13.751 .004740 .00056

13.801 .008640 .00055

13.851 .011470 .00054

13.901 .014970 .00056

13.951 .018300 .00055

14.001 .021750 .00054
475

12.850 .919110 .00054

12.900 .922030 .00053

12.950 .926880 .00054

13.000 .928700 .00054

13.050 .932580 .00053

13.100 .936860 .00054

13.150 .939040 .00055

13.200 .942830 .00054

13.250 .947300 .00053

13.300 .950670 .00053

13.350 .952930 .00054

13.400 .956370 .00055

13.450 .959920 .00054

13.500 .963670 .00054

13.550 .966860 .00054

13.600 .969920 .00054

13.650 .973330 .00053

13.700 .977500 .00054

13.750 .979600 .00054

13.800 .982770 .00053

13.850 .986930 .00055

13.900 .989350 .00054

13.950 .993950 .00054

14.000 .996510 .00054

14.050 .998060 .00053

14.101 .003400 .00053

14.151 .004240 .00056

14.201 .008870 .00055

14.251 .011290 .00054
480

12.900 .921500 .00055

12.950 .923280 .00052

13.000 .928510 .00054

13.050 .931380 .00054

13.100 .934770 .00055

13.150 .938870 .00054

13.200 .942100 .00053

13.250 .945350 .00053

13.300 .947960 .00054

13.350 .952080 .00055

13.400 .955850 .00055

13.450 .959880 .00054

13.500 .961750 .00054

13.550 .965280 .00055

13.600 .968950 .00056

13.650 .972610 .00053

13.700 .976250 .00052

13.750 .977810 .00055

13.800 .981260 .00055

13.850 .984900 .00054

13.900 .988040 .00055

13.950 .991660 .00054

14.000 .994440 .00054

14.050 .998200 .00054

14.101 .001470 .00054

14.151 .004800 .00054

14.201 .007500 .00055

14.251 .009950 .00056

14.301 .014270 .00055 
14.251 .014390 .00055 14.301 .016010 .00055 14.351 .019570 .00054 14.401 .022040 .00056

486

12.900 .919040 .00053 12.950 .923020 .00053 13.000 .927190 .00052 13.050 .930840 .00054 13.100 .934420 .00054 13.150 .938040 .00053 13.200 .941860 .00055 13.250 .944590 .00053 13.300 .947980 .00053 13.350 .950770 .00055 13.400 .954690 .00055 13.450 .958610 .00055 13.500 .961080 .00055 13.550 .964940 .00054 13.600 .967820 .00053 13.650 .969360 .00055 13.700 .974400 .00056 13.750 .977820 .00054 13.800 .979960 .00055 13.850 .984510 .00054 13.900 .987730 .00054 13.950 .990410 .00053 14.000 .994510 .00053 14.050 .997920 .00055 14.100 .999380 .00056 14.151 .003370 .00053 14.201 .006320 .00055 14.251 .009950 .00055 14.301 .013080 .00054 14.351 .016010 .00056 14.401 .018050 .00054 14.451 .021940 .00054 14.501 .024910 .00055
14.301 .014230 .00055 14.351 .018590 .00054 14.401 .020890 .00054 14.451 .024210 .00054 14.501 .028200 .00055
14.351 .016060 .00053

14.401 .020480 .00055

\section{0}

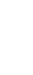


U5o2N26-sp-1000-01

12

66.100 .867230 .00016 67.100 .868540 .00015 68.100 .869580 .00016 69.100 .870360 .00016 70.100 .871560 .00016 71.100 .872050 .00015 72.100 .873490 .00015 73.100 .873930 .00015 74.100 .873990 .00014 75.100 .875120 .00015 76.100 .876320 .00015 77.100 .876410 .00015 78.100 .877550 .00015 79.100 .877690 .00015 80.100 .878270 .00015 81.100 .878790 .00014 82.100 .880100 .00014 83.100 .880600 .00015 84.100 .880390 .00014 85.100 .880890 .00014 86.100 .882010 .00014 86.200 .881920 .00015
15

41.000 .921880 .00022 41.500 .923620 .00021 42.000 .924460 .00020 42.500 .927000 .00021 43.000 .929160 .00021 43.500 .930710 .00021 44.000 .932030 .00020 44.500 .934190 .00020 45.000 .935350 .00020 45.500 .937050 .00020 46.000 .938120 .00021 46.500 .939970 .00020 47.000 .941370 .00020 47.500 .943470 .00019 48.000 .943890 .00020 48.500 .946110 .00019 49.000 .946900 .00020 49.500 .948170 .00020 50.000 .949290 .00019 50.500 .950780 .00019 51.000 .951480 .00019 51.400 .952950 .00019
25

19.900 .924400 .00034 20.100 .928430 .00032 20.300 .932580 .00034 20.500 .937190 .00032 20.700 .941780 .00034 20.900 .945980 .00033 21.100 .950140 .00033 21.300 .954410 .00033 21.500 .958740 .00033 21.700 .961320 .00032 21.900 .966190 .00033 22.100 .970280 .00033 22.300 .973680 .00033 22.500 .977580 .00032 22.700 .981300 .00033 23.100 .988480 .00031 23.300 .991020 .00032 23.500 .994510 .00032 23.700 .997940 .00032 23.901 .001180 .00032

\section{0}

17.500 .922890 .00037 17.600 .926000 .00037 17.700 .929230 .00037 17.800 .932070 .00036 17.900 .935520 .00036 18.000 .937580 .00036 18.100 .940530 .00036 18.200 .943980 .00037 18.300 .947010 .00036 18.400 .949310 .00036 18.500 .952260 .00036 18.600 .954630 .00037 18.700 .957740 .00035 18.800 .960250 .00036 18.900 .962270 .00035 19.000 .965170 .00036 19.100 .967860 .00036 19.200 .970890 .00035 19.300 .973780 .00035 19.400 .976280 .00036
20

24.800 .925800 .00029 25.200 .931020 .00028 25.600 .936410 .00028 26.000 .941830 .00028 26.400 .946100 .00029 26.800 .951110 .00028 27.600 .960450 .00027 28.000 .965080 .00027 28.400 .968840 .00028 28.800 .972350 .00028 29.200 .976500 .00027 29.600 .979630 .00027 30.000 .984020 .00027 30.400 .988160 .00027 30.800 .991360 .00026 31.200 .994960 .00027 31.600 .998250 .00026 32.001 .000770 .00027 32.401 .004410 .00026 32.801 .007790 .00025 33.201 .010850 .00026 33.601 .013710 .00026 34.001 .016860 .00025 34.401 .019500 .00025

\section{2}

16.800 .921750 .00037 16.900 .924990 .00036 17.000 .928900 .00038 17.100 .930420 .00037 17.200 .934740 .00037 17.300 .937340 .00037 17.400 .941340 .00037 17.500 .943660 .00038 17.600 .946480 .00037 17.700 .950130 .00038 17.800 .953080 .00038 17.900 .956730 .00037 18.000 .958940 .00036 18.100 .962100 .00038 18.200 .965010 .00036 18.300 .968380 .00038 18.400 .970540 .00038 18.500 .973520 .00038 18.600 .976220 .00038 18.700 .979440 .00038 
24.101 .004930 .00031 24.301 .007290 .00031 24.501 .010320 .00032 24.701 .012860 .00033 24.801 .014860 .00032
19.500 .978590 .00037 19.600 .981110 .00035 19.700 .983660 .00036 19.800 .985590 .00035 19.900 .988860 .00036 20.000 .991110 .00036 20.100 .993310 .00036 20.200 .995320 .00035 20.300 .997140 .00036 20.401 .000260 .00035 20.501 .002760 .00036 20.601 .005070 .00035 20.701 .007410 .00035 20.801 .010120 .00037 20.901 .011760 .00036 21.001 .013570 .00034
18.800 .981660 .00037 18.900 .985590 .00037 19.000 .986900 .00037 19.100 .990960 .00036 19.200 .992480 .00036 19.300 .995640 .00037 19.400 .997840 .00037 19.501 .000480 .00037 19.601 .003560 .00036 19.701 .005260 .00035 19.801 .007890 .00037 19.901 .010620 .00036 20.001 .013130 .00036 20.101 .015560 .00037
35

16.000 .921480 .00040 16.100 .924880 .00040 16.200 .929190 .00039 16.300 .931890 .00040 16.400 .934720 .00038 16.500 .939040 .00040 16.600 .942200 .00039 16.700 .945850 .00039 16.800 .949250 .00039 16.900 .952790 .00038 17.000 .956020 .00039 17.100 .960200 .00038 17.200 .962820 .00039 17.300 .966110 .00039 17.400 .969020 .00040 17.500 .972320 .00039 17.600 .975550 .00038 17.700 .978260 .00039 17.800 .982250 .00040 17.900 .985020 .00038 18.000 .987710 .00038 18.100 .990790 .00039 18.200 .993640 .00038 18.300 .996850 .00038 18.400 .999150 .00037 18.501 .002630 .00039 18.601 .005730 .00038 18.701 .008150 .00038 18.801 .010530 .00038 18.901 .013710 .00037 19.001 .016330 .00038
40

15.100 .923760 .00040 15.200 .928050 .00041 15.300 .932650 .00041 15.400 .937030 .00041 15.500 .940690 .00040 15.600 .944520 .00041 15.700 .948770 .00040 15.800 .952300 .00042 15.900 .955930 .00041 16.000 .960080 .00040 16.100 .963970 .00041 16.200 .967610 .00040 16.300 .971330 .00040 16.400 .974980 .00040 16.500 .978220 .00040 16.600 .981860 .00040 16.700 .985570 .00041 16.800 .988660 .00041 16.900 .992210 .00039 17.000 .995740 .00041 17.100 .999640 .00040 17.201 .002650 .00041 17.401 .010350 .00039 17.501 .012980 .00040 17.601 .015730 .00041
50 13.800 .922030 .00042 13.900 .927210 .00043 14.000 .932590 .00043 14.100 .936350 .00043 14.200 .941280 .00040 14.300 .946000 .00044 14.400 .951100 .00043 14.500 .955050 .00043 14.600 .959210 .00043 14.700 .965000 .00044 14.800 .968810 .00044 14.900 .973680 .00043 15.000 .977620 .00043 15.100 .982450 .00044 15.200 .986480 .00043 15.300 .990710 .00042 15.400 .994930 .00042 15.500 .998810 .00043 15.601 .003970 .00041 15.701 .007070 .00042 15.801 .011450 .00043 15.901 .015270 .00042 16.001 .019890 .00043 
60

13.000 .919540 .00045

13.100 .925040 .00046

13.200 .931300 .00047

13.300 .936640 .00045

13.400 .942100 .00045

13.500 .946860 .00046

13.600 .952880 .00046

13.700 .956990 .00045

13.800 .962020 .00046

13.900 .968360 .00045

14.000 .973110 .00046

14.100 .976970 .00044

14.200 .982180 .00046

14.300 .986360 .00046

14.400 .992360 .00045

14.500 .996970 .00044

14.601 .001740 .00045

14.701 .006410 .00044

14.801 .011020 .00044

14.901 .015330 .00045

15.001 .019550 .00045
70

12.500 .921300 .00046

12.600 .927180 .00046

12.700 .932850 .00047

12.800 .938610 .00047

12.900 .944080 .00047

13.000 .949650 .00048

13.100 .955810 .00046

13.200 .961700 .00046

13.300 .966130 .00047

13.400 .972150 .00046

13.500 .977140 .00047

13.600 .983050 .00048

13.700 .988340 .00046

13.800 .993300 .00047

13.900 .998180 .00047

14.001 .003630 .00047

14.101 .008930 .00047

14.201 .013390 .00047

14.301 .017330 .00047
80

12.100 .919190 .00048

12.200 .923980 .00048

12.300 .930780 .00049

12.400 .936660 .00048

12.500 .943400 .00049

12.600 .948610 .00048

12.700 .955000 .00047

12.800 .961100 .00048

12.900 .966470 .00048

13.000 .972710 .00048

13.100 .978850 .00048

13.200 .983640 .00048

13.300 .989230 .00046

13.400 .994430 .00049

13.501 .000210 .00047

13.601 .006140 .00048

13.701 .011010 .00048

13.801 .016310 .00048

13.901 .022200 .00048
90

11.800 .916940 .00049

11.850 .920030 .00050

11.900 .922440 .00048

11.950 .926100 .00050

12.000 .929920 .00049

12.050 .932370 .00048

12.100 .936910 .00047

12.150 .939200 .00049

12.200 .942690 .00049

12.250 .945670 .00049

12.300 .948080 .00048

12.350 .951260 .00050

12.400 .955090 .00049

12.450 .957850 .00050

12.500 .961140 .00048

12.550 .964460 .00047

12.600 .967270 .00049

12.650 .969960 .00048

12.700 .972080 .00050

12.750 .976430 .00049

12.800 .978690 .00049

12.850 .982100 .00048
100

11.600 .918260 .00050

11.650 .920920 .00049

11.700 .925310 .00049

11.750 .927830 .00050

11.800 .930900 .00050

11.850 .934580 .00051

11.900 .937350 .00050

11.950 .940320 .00049

12.000 .943940 .00051

12.050 .946150 .00050

12.100 .950550 .00049

12.150 .954190 .00048

12.200 .956940 .00048

12.250 .959680 .00051

12.300 .963310 .00050

12.350 .965650 .00050

12.400 .969800 .00050

12.450 .972140 .00049

12.500 .976000 .00051

12.550 .978520 .00049

12.600 .981810 .00049

12.650 .984830 .00050
118

11.300 .915840 .00050 11.350 .919710 .00051

11.400 .922080 .00050

11.450 .926100 .00051

11.500 .929100 .00050

11.550 .934350 .00051

11.600 .936870 .00050

11.650 .940420 .00051

11.700 .943790 .00050

11.750 .947100 .00050

11.800 .950950 .00052

11.850 .953870 .00049

11.900 .957310 .00049

11.950 .959370 .00050

12.000 .964160 .00049

12.050 .967270 .00052

12.100 .969570 .00050

12.150 .974330 .00050

12.200 .976880 .00050

12.250 .979360 .00051

12.300 .982100 .00050

12.350 .986650 .00050 
12.900 .985780 .00049 13.000 .989840 .00049 13.050 .994580 .00049 13.100 .996370 .00050 13.150 .999450 .00049 13.201 .002610 .00049 13.251 .005090 .00048 13.301 .007310 .00047 13.351 .010160 .00049 13.401 .012800 .00049 13.451 .016420 .00049 13.501 .019150 .00048

130

11.200 .918870 .00051 11.250 .922860 .00052 11.300 .926880 .00051 11.350 .930020 .00050 11.400 .932270 .00052 11.450 .937190 .00052 11.500 .939930 .00050 11.550 .942990 .00051 11.600 .945960 .00050 11.650 .949280 .00051 11.700 .953520 .00052 11.750 .957150 .00050 11.800 .960710 .00051 11.850 .964790 .00051 11.900 .968000 .00051 11.950 .969920 .00051 12.000 .973220 .00052 12.050 .976090 .00050 12.100 .980490 .00051 12.150 .983980 .00050 12.200 .987880 .00052 12.250 .990250 .00051 12.300 .993060 .00050 12.350 .997620 .00053 12.400 .999600 .00051 12.451 .002440 .00050 12.501 .006100 .00051 12.551 .008600 .00051 12.601 .012660 .00052 12.651 .015360 .00051 12.701 .017420 .00051
12.700 .988780 .00049

12.750 .990930 .00050

12.800 .994440 .00048

12.850 .996800 .00049

12.901 .000210 .00052

12.951 .002210 .00051

13.001 .005670 .00048

13.051 .007170 .00049

13.101 .011030 .00049

13.151 .013810 .00051

13.201 .017720 .00050

13.251 .019550 .00051

13.301 .022650 .00050
12.400 .989880 .00051 12.450 .991570 .00051 12.500 .996050 .00051 12.550 .999060 .00051 12.601 .000790 .00050 12.651 .004140 .00052 12.701 .006990 .00050 12.751 .009810 .00050 12.801 .013500 .00051 12.851 .016090 .00050 12.901 .019790 .00051
150

11.000 .916730 .00052 11.050 .919470 .00051 11.100 .923220 .00050 11.150 .928200 .00050 11.200 .930600 .00052 11.250 .933610 .00052 11.300 .938150 .00051 11.350 .941440 .00050 11.400 .945720 .00051 11.450 .947590 .00051 11.500 .952080 .00052 11.550 .955340 .00052 11.600 .959510 .00053 11.650 .962630 .00051 11.700 .966330 .00051 11.750 .968820 .00051 11.800 .972380 .00052 11.850 .975740 .00052 11.900 .979000 .00052 11.950 .983030 .00051 12.000 .986350 .00052 12.050 .989160 .00053 12.100 .992680 .00051 12.150 .996230 .00051 12.200 .999050 .00051 12.251 .003380 .00051 12.301 .006370 .00053 12.351 .009090 .00052 12.401 .012390 .00051 12.451 .015750 .00052 12.501 .017760 .00052
200

10.800 .917350 .00052 10.850 .921110 .00052 10.900 .925500 .00053 10.950 .927830 .00055 11.000 .933360 .00052 11.050 .936010 .00051 11.100 .938950 .00052 11.150 .942550 .00053 11.200 .946740 .00053 11.250 .950040 .00052 11.300 .953430 .00053 11.350 .958120 .00053 11.400 .960790 .00053 11.450 .965420 .00051 11.500 .967850 .00052 11.550 .971010 .00052 11.600 .975140 .00053 11.650 .978920 .00053 11.700 .982180 .00054 11.750 .984740 .00053 11.800 .988760 .00053 11.850 .992300 .00053 11.900 .995950 .00052 11.950 .998850 .00053 12.001 .001510 .00054 12.051 .005950 .00054 12.101 .008360 .00054 12.151 .011390 .00052 12.201 .016330 .00054 12.251 .018510 .00054 12.301 .021980 .00053 
235

10.800 .921560 .00052 10.850 .926210 .00051 10.900 .928480 .00053 10.950 .933200 .00052 11.000 .937070 .00052 11.050 .939740 .00052 11.100 .944170 .00053 11.150 .948530 .00054 11.200 .952200 .00052 11.250 .954970 .00052 11.300 .958410 .00052 11.350 .962710 .00053 11.400 .964840 .00052 11.450 .970370 .00054 11.500 .973310 .00052 11.550 .975720 .00053 11.600 .979270 .00053 11.650 .983000 .00053 11.700 .986650 .00052 11.750 .989630 .00052 11.800 .994100 .00053 11.850 .996680 .00051 11.901 .000240 .00053 11.951 .003730 .00055 12.001 .006910 .00053 12.051 .009380 .00055 12.101 .012580 .00054 12.151 .016770 .00053 12.201 .020150 .00053

470

11.100 .919630 .00051 11.150 .922140 .00053 11.200 .926090 .00053 11.250 .928500 .00053 11.300 .932470 .00054 11.350 .935510 .00053 11.400 .940280 .00053 11.450 .942880 .00052 11.500 .946010 .00052 11.550 .949010 .00053 11.600 .952430 .00053 11.650 .956210 .00052 11.700 .960190 .00054 11.750 .963910 .00052 11.800 .966700 .00052
250

10.700 .914670 .00053

10.750 .918900 .00054

10.800 .923020 .00054

10.850 .926010 .00052

10.900 .929840 .00054

10.950 .933810 .00053

11.000 .937310 .00055

11.050 .941760 .00053

11.100 .945220 .00051

11.150 .947100 .00054

11.200 .952650 .00054

11.250 .955190 .00053

11.300 .959280 .00052

11.350 .962470 .00052

11.400 .966470 .00055

11.450 .970320 .00052

11.500 .973890 .00053

11.550 .976600 .00052

11.600 .980220 .00054

11.650 .984110 .00053

11.700 .988170 .00054

11.750 .990490 .00052

11.800 .994220 .00052

11.850 .997780 .00053

11.901 .001120 .00053

11.951 .004810 .00053

12.001 .007640 .00053

12.051 .012170 .00055

12.101 .014670 .00054

12.151 .017270 .00053

12.201 .020730 .00053

475

11.100 .917720 .00053

11.150 .920820 .00051

11.200 .924280 .00052

11.250 .927400 .00053

11.300 .932210 .00054

11.350 .934300 .00051

11.400 .938840 .00053

11.450 .942620 .00052

11.500 .944690 .00052

11.550 .949010 .00052

11.600 .950920 .00053

11.650 .954460 .00053

11.700 .959060 .00052

11.750 .962030 .00052

11.800 .965890 .00052
353

10.800 .916710 .00052

10.850 .920250 .00053

10.900 .924820 .00052

10.950 .927540 .00054

11.000 .932240 .00052

11.050 .935170 .00053

11.100 .938310 .00052

11.150 .943390 .00052

11.200 .945520 .00051

11.250 .949390 .00054

11.300 .952640 .00052

11.350 .955730 .00053

11.400 .959810 .00053

11.450 .963090 .00052

11.500 .967640 .00053

11.550 .971340 .00051

11.600 .973470 .00052

11.650 .977480 .00053

11.700 .980020 .00054

11.750 .984150 .00053

11.800 .986720 .00054

11.850 .991410 .00053

11.900 .994050 .00053

11.950 .997040 .00053

12.001 .001150 .00054

12.051 .003770 .00053

12.101 .007520 .00054

12.151 .010720 .00052

12.201 .013560 .00054

12.251 .017260 .00054

12.301 .021290 .00054

480

11.100 .916130 .00051

11.150 .919340 .00052

11.200 .923090 .00053

11.250 .927480 .00054

11.300 .930530 .00053

11.350 .933770 .00052

11.400 .936920 .00054

11.450 .939770 .00053

11.500 .944500 .00052

11.550 .946430 .00053

11.600 .950400 .00053

11.650 .954870 .00055

11.700 .957040 .00053

11.750 .960330 .00054

11.800 .964760 .00053 
11.850 .970410 .00053 11.900 .973080 .00054 11.950 .976080 .00053 12.000 .980600 .00054 12.050 .983490 .00055 12.100 .986150 .00054 12.150 .989310 .00052 12.200 .992780 .00054 12.250 .995050 .00052 12.300 .998670 .00053 12.351 .001950 .00054 12.401 .006180 .00053 12.451 .008440 .00056 12.501 .011360 .00053 12.551 .014780 .00052 12.601 .016790 .00052 12.651 .021080 .00051 12.701 .022860 .00054
11.850 .968680 .00051 11.900 .972380 .00054 11.950 .975070 .00053 12.000 .979130 .00052 12.050 .981560 .00052 12.100 .985140 .00052 12.150 .988670 .00053 12.200 .991380 .00054 12.250 .994560 .00055 12.300 .998440 .00052 12.351 .002180 .00054 12.401 .004090 .00053 12.451 .007070 .00053 12.501 .011590 .00055 12.551 .014370 .00052 12.601 .016790 .00054 12.651 .018960 .00052 12.701 .022840 .00052
11.850 .967020 .00054 11.900 .972080 .00052 11.950 .974190 .00052 12.000 .977500 .00052 12.050 .980810 .00054 12.100 .984110 .00053 12.150 .987060 .00053 12.200 .991190 .00054 12.250 .993440 .00052 12.300 .996930 .00052 12.350 .999970 .00052 12.401 .003380 .00054 12.451 .005730 .00053 12.501 .008530 .00054 12.551 .012970 .00052 12.601 .015440 .00055 12.651 .018040 .00053 12.701 .022070 .00052
486

11.100 .914570 .00052 11.150 .918690 .00053 11.200 .921500 .00053 11.250 .925030 .00053 11.300 .928840 .00053 11.350 .933060 .00051 11.400 .936340 .00054 11.450 .939660 .00053 11.500 .942650 .00052 11.550 .945930 .00053 11.600 .949620 .00053 11.650 .952880 .00052 11.700 .956570 .00053 11.750 .959470 .00053 11.800 .963230 .00053 11.850 .967730 .00053 11.900 .969730 .00054 11.950 .972390 .00052 12.000 .976190 .00053 12.050 .979740 .00052 12.100 .982290 .00053 12.150 .986630 .00052 12.200 .988950 .00053 12.250 .992920 .00053 12.300 .995650 .00052 12.350 .998940 .00055 12.401 .002070 .00052 12.451 .005080 .00051 12.501 .009970 .00053

\section{0}


12.551 .011900 .00054

12.601 .014860 .00052

12.651 .017410 .00054

12.701 .021820 .00054

U5o2N26-sp-1000-10

12

65.300 .868290 .00015 66.300 .868770 .00015 67.300 .869530 .00015 68.300 .870490 .00015 69.300 .871570 .00015 70.300 .872640 .00015 71.300 .873030 .00015 72.300 .873430 .00015 73.300 .874820 .00015 74.300 .875640 .00015 75.300 .875930 .00014 76.300 .876930 .00014 77.300 .877930 .00014 78.300 .878410 .00014 79.300 .878390 .00014 80.300 .879260 .00015 81.300 .879690 .00014 82.300 .880760 .00014 83.300 .881440 .00014 84.300 .881280 .00014 85.300 .881890 .00014 85.700 .881900 .00014

25

18.700 .923940 .00032 18.900 .929010 .00031 19.100 .933520 .00032 19.300 .937380 .00032 19.500 .941870 .00031 19.700 .945680 .00031 19.900 .950100 .00032 20.100 .954160 .00032 20.300 .958420 .00031 20.500 .962670 .00030 20.700 .965750 .00032 20.900 .970140 .00031 21.100 .973600 .00030 21.300 .977740 .00030 21.500 .980810 .00030
15

35.000 .899560 .00021

35.800 .903200 .00021

36.600 .907990 .00021

37.400 .911980 .00021

38.200 .914470 .00021

39.000 .918700 .00020

39.800 .921360 .00021

40.600 .924970 .00020

41.400 .928090 .00020

42.200 .929870 .00020

43.000 .933280 .00019

43.800 .935410 .00020

44.600 .937790 .00020

45.400 .940040 .00020

46.200 .942670 .00019

47.000 .944770 .00019

47.800 .947410 .00018

48.600 .948520 .00018

49.400 .950980 .00019

50.200 .952150 .00019

51.000 .953880 .00019

51.800 .955910 .00018

51.900 .956080 .00019

30

16.300 .923830 .00036

16.400 .926300 .00034

16.500 .929290 .00034

16.600 .931720 .00034

16.700 .935410 .00034

16.800 .938860 .00034

16.900 .941210 .00034

17.000 .944220 .00034

17.100 .946890 .00035

17.200 .950300 .00035

17.300 .952960 .00033

17.400 .956110 .00034

17.500 .958260 .00034

17.600 .960730 .00034

17.700 .963460 .00034
20

23.600 .925280 .00028

24.000 .930530 .00028

24.400 .936540 .00028

24.800 .941560 .00028

25.200 .946190 .00027

25.600 .950810 .00027

26.000 .955910 .00026

26.400 .960200 .00027

26.800 .964360 .00025

27.600 .972670 .00026

28.000 .976970 .00026

28.400 .980680 .00026

28.800 .984210 .00026

29.200 .987710 .00025

29.600 .991500 .00026

30.000 .995080 .00025

30.400 .998260 .00025

30.801 .001410 .00025

31.201 .004680 .00025

31.601 .008690 .00024

32.001 .010720 .00025

32.401 .014050 .00025

32.501 .014370 .00024

32

15.600 .922580 .00035

15.700 .925610 .00036

15.800 .928810 .00036

15.900 .931980 .00035

16.000 .935150 .00035

16.100 .938460 .00036

16.200 .941990 .00035

16.300 .944600 .00034

16.400 .947440 .00036

16.500 .951080 .00035

16.600 .954080 .00034

16.700 .957030 .00035

16.800 .960320 .00036

16.900 .962090 .00034

17.000 .965760 .00035 
21.700 .984800 .00031

21.900 .988390 .00030

22.100 .991790 .00030

22.500 .997450 .00031

22.701 .002010 .00030

22.901 .004030 .00029

23.101 .007980 .00030

23.301 .011230 .00030

23.501 .013900 .00029
17.800 .966450 .00034

17.900 .969580 .00034

18.000 .971300 .00035

18.100 .973550 .00033

18.200 .976460 .00034

18.300 .979500 .00034

18.400 .981330 .00034

18.500 .984000 .00034

18.600 .986600 .00034

18.700 .989330 .00033

18.800 .991510 .00034

18.900 .994530 .00035

19.000 .996490 .00034

19.100 .997900 .00034

19.201 .001600 .00033

19.301 .003880 .00034

19.401 .005660 .00033

19.501 .008320 .00033

19.601 .009540 .00034

19.701 .012080 .00034

19.801 .014430 .00033

19.901 .017210 .00033

35

14.800 .921580 .00036

14.900 .926030 .00037

15.000 .928940 .00036

15.100 .933550 .00036

15.200 .936060 .00036

15.300 .939880 .00036

15.400 .943320 .00037

15.500 .946920 .00036

15.600 .949760 .00036

15.700 .953620 .00037

15.800 .957420 .00036

15.900 .961220 .00036

16.000 .963520 .00036

16.100 .966550 .00036

16.200 .970510 .00037

16.300 .974060 .00036

16.400 .976460 .00036

16.500 .980390 .00036

16.600 .982590 .00036

16.700 .985540 .00036

16.800 .988520 .00036

16.900 .992990 .00036

17.000 .994490 .00037

17.100 .998300 .00036

17.201 .000740 .00036

17.301 .003650 .00037
13.800 .921110 .00038

13.900 .925980 .00038

14.000 .929830 .00038

14.100 .933550 .00038

14.200 .937880 .00037

14.300 .942030 .00039

14.400 .946270 .00038

14.500 .950270 .00038

14.600 .953850 .00037

14.700 .957560 .00038

14.800 .961580 .00038

14.900 .965160 .00037

15.000 .968880 .00037

15.100 .973570 .00038

15.200 .976520 .00038

15.300 .980030 .00039

15.400 .983300 .00038

15.500 .987570 .00039

15.600 .991260 .00039

15.700 .994080 .00037

15.800 .997210 .00039

15.901 .000950 .00037

16.001 .004110 .00037

16.101 .008310 .00038

16.301 .014150 .00038

16.401 .017070 .00037
17.100 .968750 .00035

17.200 .972020 .00036

17.300 .974840 .00034

17.400 .977170 .00036

17.500 .979950 .00034

17.600 .983510 .00035

17.700 .985670 .00034

17.800 .988370 .00035

17.900 .990890 .00034

18.000 .993120 .00035

18.100 .996230 .00035

18.200 .999420 .00035

18.301 .001930 .00034

18.401 .003910 .00034

18.501 .007220 .00034

18.601 .009660 .00035

18.701 .010990 .00034

18.801 .013890 .00034

18.901 .016500 .00034
50

12.500 .919360 .00041

12.600 .924790 .00040

12.700 .929810 .00041

12.800 .934660 .00040

12.900 .939520 .00040

13.000 .944710 .00041

13.100 .948670 .00040

13.200 .953930 .00040

13.300 .958180 .00041

13.400 .962380 .00040

13.500 .967710 .00041

13.600 .971460 .00039

13.700 .975970 .00040

13.800 .981190 .00040

13.900 .985380 .00041

14.000 .989350 .00040

14.100 .993640 .00041

14.200 .998890 .00041

14.301 .002390 .00040

14.401 .006990 .00039

14.501 .010420 .00039

14.601 .014660 .00041

14.701 .019200 .00039 
17.401 .006730 .00036

17.501 .009450 .00036

17.601 .012280 .00035

17.701 .014670 .00036

60

11.700 .918080 .00041 11.800 .923790 .00042 11.900 .929140 .00042 12.000 .935010 .00043

12.100 .940270 .00042 12.200 .945330 .00041 12.300 .951950 .00042 12.400 .955780 .00043 12.500 .961760 .00042 12.600 .966730 .00041 12.700 .971430 .00042 12.800 .977340 .00042 12.900 .981110 .00042 13.000 .987000 .00042 13.100 .991510 .00042 13.200 .996380 .00042 13.301 .001180 .00044 13.401 .006370 .00043 13.501 .010870 .00041 13.601 .014960 .00042 13.701 .019620 .00042
70

80

$11.200 .919380 .00044 \quad 10.800 .919000 .00044$ $11.300 .926010 .00044 \quad 10.850 .921940 .00044$

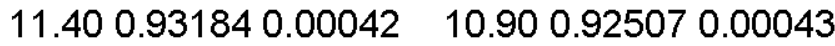
11.500 .938820 .00045 11.600 .943700 .00045 11.700 .948740 .00044 11.800 .955420 .00043 11.900 .961140 .00043 12.000 .967710 .00044 12.100 .971380 .00044 12.200 .976570 .00044 12.300 .981910 .00043 12.400 .987680 .00044 12.500 .993960 .00044 12.600 .998320 .00043 12.701 .004080 .00044 12.801 .009510 .00044 12.901 .014010 .00045 13.001 .019360 .00044

10.950 .928480 .00044 11.000 .931490 .00045 11.050 .934830 .00044 11.100 .937700 .00045 11.150 .940850 .00044 11.200 .943860 .00045 11.250 .946490 .00045 11.300 .950670 .00044 11.350 .952360 .00043 11.400 .955620 .00044 11.450 .958740 .00044 11.500 .960980 .00044 11.550 .964170 .00046 11.600 .968190 .00044 11.650 .970810 .00045 11.700 .973590 .00044 11.750 .975600 .00043 11.800 .978030 .00046 11.850 .982530 .00045 11.900 .985200 .00045 11.950 .987270 .00043 12.000 .990850 .00045 12.050 .993700 .00044 12.100 .996640 .00044 12.150 .999060 .00044 12.201 .002120 .00046 12.251 .004660 .00045 12.301 .006660 .00044 12.351 .010230 .00044 12.401 .012190 .00045 12.451 .014830 .00043 12.501 .018060 .00045

90 100 118 10.500 .918650 .00045 10.550 .921330 .00044 10.600 .924890 .00044 10.650 .928620 .00044 10.300 .919680 .00045
10.350 .923380 .00045 10.400 .926290 .00045 10.450 .929740 .00045
10.000 .919060 .00048 10.050 .923490 .00046 10.100 .927810 .00047 10.150 .930800 .00046 
10.700 .930990 .00045

10.750 .934850 .00046

10.800 .938320 .00045

10.850 .941180 .00044

10.900 .944360 .00045

10.950 .946840 .00045

11.000 .951380 .00045

11.050 .953150 .00043

11.100 .956060 .00044

11.150 .959380 .00045

11.200 .962820 .00045

11.250 .966550 .00046

11.300 .968620 .00046

11.350 .972450 .00045

11.400 .975320 .00045

11.450 .977830 .00045

11.500 .981410 .00045

11.550 .984020 .00045

11.600 .987160 .00045

11.650 .989420 .00046

11.700 .994020 .00045

11.750 .996110 .00045

11.800 .997850 .00044

11.851 .001590 .00045

11.901 .004350 .00045

11.951 .006700 .00045

12.001 .010970 .00046

12.051 .013500 .00046

12.101 .015600 .00046

12.151 .018940 .00045

12.201 .020920 .00046
10.500 .933750 .00045

10.550 .936820 .00046

10.600 .939460 .00045

10.650 .943660 .00047

10.700 .946850 .00045

10.750 .950690 .00046

10.800 .953230 .00046

10.850 .955770 .00046

10.900 .959140 .00046

10.950 .963430 .00046

11.000 .966290 .00046

11.050 .970110 .00045

11.100 .972530 .00048

11.150 .976010 .00046

11.200 .978540 .00047

11.250 .981720 .00046

11.300 .983780 .00046

11.350 .987970 .00046

11.400 .991700 .00046

11.450 .993520 .00047

11.500 .997140 .00046

11.551 .000660 .00046

11.601 .002490 .00046

11.651 .005840 .00045

11.701 .009000 .00046

11.751 .011990 .00045

11.801 .014860 .00046

11.851 .016840 .00046

11.901 .020500 .00045
10.200 .933700 .00049

10.250 .937550 .00046

10.300 .941100 .00046

10.350 .944230 .00046

10.400 .947340 .00046

10.450 .951890 .00046

10.500 .954280 .00046

10.550 .958680 .00046

10.600 .961580 .00047

10.650 .964040 .00046

10.700 .968090 .00045

10.750 .970960 .00046

10.800 .974050 .00047

10.850 .978290 .00046

10.900 .981060 .00047

10.950 .983990 .00046

11.000 .987640 .00048

11.050 .989860 .00047

11.100 .994080 .00047

11.150 .997230 .00047

11.201 .000080 .00046

11.251 .003280 .00046

11.301 .006980 .00048

11.351 .009400 .00047

11.401 .012840 .00048

11.451 .015300 .00046

11.501 .018980 .00047
130

9.800 .916850 .00047

9.850 .920480 .00047

9.900 .923980 .00046

9.950 .927960 .00046

10.000 .931020 .00046

10.050 .934140 .00045

10.100 .938180 .00047

10.150 .942380 .00046

10.200 .945010 .00047

10.250 .948160 .00047

10.300 .953180 .00047

10.350 .955370 .00047

10.400 .959550 .00046

10.450 .962130 .00047

10.500 .964720 .00049

10.550 .969180 .00048
150

9.600 .916470 .00047

9.650 .920710 .00047

9.700 .923720 .00046

9.750 .926410 .00048

9.800 .930470 .00047

9.850 .933920 .00048

9.900 .937710 .00048

9.950 .941960 .00047

10.000 .945240 .00047

10.050 .949250 .00048

10.100 .952000 .00047

10.150 .955600 .00047

10.200 .959520 .00047

10.250 .962670 .00050

10.300 .966100 .00047

10.350 .969940 .00047

\begin{abstract}
200
9.400 .920510 .00048

9.450 .924720 .00047

9.500 .928030 .00048

9.550 .932320 .00048

9.600 .936000 .00047

9.650 .939690 .00047

9.700 .943920 .00048

9.750 .946750 .00048

9.800 .950650 .00049

9.850 .953750 .00049

9.900 .958660 .00048

9.950 .962090 .00047

10.000 .965560 .00048

10.050 .969880 .00047

10.100 .973120 .00048

10.150 .975780 .00048
\end{abstract}


10.600 .972300 .00047 10.650 .976490 .00048 10.700 .979830 .00047 10.750 .982110 .00048 10.800 .985730 .00047 10.850 .989380 .00048 10.900 .991690 .00048 10.950 .996120 .00047 11.000 .998680 .00047 11.051 .002020 .00046 11.101 .005280 .00046 11.151 .008350 .00047 11.201 .012280 .00046 11.251 .015200 .00047 11.301 .018180 .00048
10.400 .973280 .00047 10.450 .976670 .00048 10.500 .980050 .00048 10.550 .983000 .00047 10.600 .986170 .00050 10.650 .990300 .00047 10.700 .993900 .00048 10.750 .997090 .00046 10.801 .000560 .00046 10.851 .003510 .00048 10.901 .007860 .00048 10.951 .010390 .00047 11.001 .013700 .00047 11.051 .016760 .00049 11.101 .019510 .00049
10.200 .979630 .00048 10.250 .983180 .00049 10.300 .986320 .00049 10.350 .989190 .00048 10.400 .993300 .00049 10.450 .996840 .00048 10.501 .000690 .00049 10.551 .003810 .00051 10.601 .007070 .00049 10.651 .009550 .00048 10.701 .013640 .00048 10.751 .016480 .00049 10.801 .020960 .00048
235

9.300 .920000 .00048 9.350 .924680 .00048 9.400 .927850 .00049 9.450 .931210 .00049 9.500 .935450 .00050 9.550 .939710 .00049 9.600 .942570 .00048 9.650 .947450 .00049 9.700 .949690 .00050 9.750 .954440 .00047 9.800 .957800 .00049 9.850 .961320 .00048 9.900 .965060 .00049 9.950 .968140 .00048 10.000 .973000 .00047 10.050 .976200 .00048 10.100 .978990 .00047 10.150 .983530 .00048 10.200 .986780 .00050 10.250 .990460 .00048 10.300 .993370 .00049 10.350 .997370 .00048 10.401 .000560 .00049 10.451 .003370 .00049 10.501 .006310 .00049 10.551 .011160 .00050 10.601 .013950 .00048 10.651 .018350 .00049 10.701 .020750 .00050
250

9.300 .922070 .00048

9.350 .926120 .00047

9.400 .930350 .00048

9.450 .933550 .00047

9.500 .937090 .00049

9.550 .941110 .00048

9.600 .945590 .00049

9.650 .948920 .00049

9.700 .952420 .00048

9.750 .955460 .00047

9.800 .960350 .00049

9.850 .964200 .00047

9.900 .967460 .00049

9.950 .969900 .00050

10.000 .974600 .00049

10.050 .977120 .00049

10.100 .979990 .00049

10.150 .985210 .00048

10.200 .988380 .00049

10.250 .991640 .00049

10.300 .995690 .00049

10.350 .998710 .00049

10.401 .001960 .00049

10.451 .004800 .00048

10.501 .008340 .00047

10.551 .012940 .00048 10.601 .015720 .00049 10.651 .018940 .00048 10.701 .022220 .00049
353

9.300 .922420 .00049

9.350 .926660 .00049

9.400 .931270 .00049

9.450 .934320 .00048

9.500 .938180 .00049

9.550 .941500 .00048

9.600 .946400 .00049

9.650 .949220 .00049

9.700 .952310 .00046

9.750 .956000 .00049

9.800 .959880 .00047

9.850 .963760 .00049

9.900 .967750 .00048

9.950 .970810 .00047

10.000 .974710 .00050

10.050 .977810 .00048

10.100 .982060 .00048

10.150 .984460 .00048

10.200 .987970 .00050

10.250 .991820 .00049

10.300 .994970 .00051

10.350 .998810 .00050

10.401 .003030 .00050

10.451 .005870 .00049

10.501 .007250 .00049 10.551 .012200 .00048 10.601 .014930 .00050 10.651 .018550 .00049 10.701 .021790 .00049 
470

9.400 .918250 .00048

9.450 .921990 .00048

9.500 .926380 .00048

9.550 .930160 .00048

9.600 .933890 .00048

9.650 .937290 .00048

9.700 .940060 .00048

9.750 .943540 .00049

9.800 .948070 .00047

9.850 .950560 .00048

9.900 .955130 .00048

9.950 .957080 .00048

10.000 .961430 .00048

10.050 .964840 .00048

10.100 .967890 .00049

10.150 .972190 .00048

10.200 .976320 .00049

10.250 .977870 .00048

10.300 .981290 .00048

10.350 .985180 .00049

10.400 .988430 .00048

10.450 .991980 .00051

10.500 .994420 .00048

10.550 .997550 .00049

10.601 .001690 .00049

10.651 .003840 .00048

10.701 .007810 .00049

10.751 .010580 .00050

10.801 .013890 .00048

10.851 .016680 .00049

10.901 .019250 .00049
475

9.400 .918060 .00048

9.450 .921810 .00047

9.500 .924920 .00048

9.550 .928230 .00049

9.600 .931950 .00049

9.650 .937230 .00049

9.700 .939730 .00048

9.750 .942480 .00048

9.800 .946300 .00047

9.850 .950180 .00047

9.900 .954210 .00047

9.950 .957610 .00048

10.000 .960030 .00048

10.050 .963650 .00048

10.100 .967390 .00050

10.150 .970310 .00049

10.200 .973720 .00047

10.250 .977270 .00049

10.300 .980030 .00048

10.350 .984240 .00049

10.400 .987700 .00048

10.450 .990560 .00050

10.500 .993580 .00049

10.550 .996140 .00049

10.601 .000760 .00048

10.651 .004040 .00050

10.701 .006420 .00049

10.751 .009400 .00049

10.801 .013470 .00050

10.851 .014910 .00049

10.901 .018510 .00050
480

9.400 .916020 .00048

9.450 .921730 .00048

9.500 .923860 .00047

9.550 .927660 .00049

9.600 .931890 .00048

9.650 .935250 .00049

9.700 .939050 .00048

9.750 .942110 .00048

9.800 .945190 .00048

9.850 .949740 .00047

9.900 .952280 .00047

9.950 .955570 .00047

10.000 .959510 .00049

10.050 .962080 .00049

10.100 .965890 .00047

10.150 .970040 .00049

10.200 .973620 .00049

10.250 .976760 .00049

10.300 .979080 .00048

10.350 .982960 .00049

10.400 .986900 .00048

10.450 .989840 .00048

10.500 .992600 .00048

10.550 .996320 .00048

10.600 .999160 .00049

10.651 .002220 .00051

10.701 .005440 .00048

10.751 .007690 .00048

10.801 .012380 .00049

10.851 .014950 .00051

10.901 .018540 .00049

10.951 .021690 .00049

11.001 .024920 .00048
486

9.500 .924880 .00047

9.550 .927070 .00049

9.600 .930800 .00047

9.650 .935170 .00048

9.700 .938110 .00048

9.750 .940810 .00051

9.800 .945650 .00049

9.850 .948960 .00049

9.900 .951540 .00049

9.950 .955540 .00048

10.000 .959080 .00049

10.050 .962620 .00047

10.100 .965440 .00049
0

F-387 
10.150 .969710 .00048

10.200 .972450 .00048

10.250 .974160 .00050

10.300 .979670 .00048

10.350 .982450 .00049

10.400 .985290 .00049

10.450 .989800 .00048

10.500 .993750 .00048

10.550 .995250 .00049

10.600 .998980 .00049

10.651 .002090 .00048

10.701 .005740 .00049

10.751 .008980 .00050

10.801 .011200 .00050

10.851 .014850 .00049

10.901 .017260 .00049

10.951 .020860 .00048

11.001 .024170 .00050

U5o2N20-sp-0935-00

12

69.000 .923440 .00015 71.300 .925550 .00015 73.600 .926970 .00014 75.900 .928620 .00014 78.200 .930540 .00014 80.500 .932020 .00013 82.800 .933200 .00014 85.100 .934390 .00013 87.400 .935830 .00014 89.700 .937010 .00013 92.000 .937890 .00013 94.300 .938490 .00013 96.600 .939380 .00013 98.900 .940200 .00012 101.200 .940990 .00012 103.500 .941490 .00012 105.800 .941790 .00012 108.100 .942690 .00012 110.400 .943490 .00012 112.700 .944390 .00012 115.000 .944880 .00012 115.700 .944700 .00012
15

31.600 .919530 .00024

32.100 .923520 .00023

32.600 .926830 .00024

33.100 .930870 .00024

33.600 .934430 .00023

34.100 .937770 .00023

34.600 .941280 .00023

35.100 .944050 .00023

35.600 .947310 .00024

36.100 .950500 .00023

36.600 .953070 .00022

37.100 .955770 .00022

37.600 .958120 .00022

38.100 .961040 .00022

38.600 .963270 .00022

39.100 .965680 .00022

39.600 .967940 .00022

40.100 .969830 .00022

40.600 .972060 .00021

41.100 .974560 .00021

41.400 .975660 .00021
20

22.400 .926830 .00032

22.700 .932590 .00032

23.000 .937850 .00031

23.300 .942100 .00031

23.600 .947450 .00030

23.900 .952800 .00031

24.200 .958030 .00030

24.500 .962100 .00030

24.800 .967140 .00030

25.100 .972090 .00030

25.400 .976550 .00030

25.700 .980680 .00030

26.000 .983840 .00030

26.300 .988800 .00029

26.600 .993150 .00029

26.900 .997230 .00028

27.201 .000900 .00029

27.501 .004710 .00029

27.801 .007840 .00029

28.101 .012090 .00028

28.301 .013770 .00028 
25

18.800 .924570 .00036 18.900 .926890 .00036 19.000 .929750 .00036 19.100 .932980 .00034 19.200 .935860 .00035 19.300 .938450 .00035 19.400 .940410 .00036 19.500 .943750 .00035 19.600 .946510 .00035 19.700 .948750 .00035 19.800 .952410 .00035 19.900 .954880 .00034 20.000 .956370 .00036 20.100 .960030 .00035 20.200 .962040 .00035 20.300 .964690 .00035 20.400 .966610 .00034 20.500 .970150 .00034 20.600 .972440 .00034 20.700 .974860 .00035 20.800 .976870 .00035 20.900 .978710 .00035 21.000 .982140 .00034 21.100 .983510 .00034 21.200 .986290 .00035 21.300 .988940 .00035 21.400 .990820 .00035 21.500 .993620 .00034 21.600 .995100 .00034 21.700 .996930 .00034 21.800 .999210 .00034 21.901 .001550 .00035 22.001 .004490 .00034 22.101 .006260 .00034 22.201 .007600 .00034 22.301 .010460 .00033 22.401 .012170 .00035 22.501 .014300 .00034
30

16.900 .921310 .00039

17.000 .925280 .00039

17.100 .929260 .00039

17.200 .933040 .00039

17.300 .936590 .00039

17.400 .939640 .00037

17.500 .943390 .00039

17.600 .946920 .00038

17.700 .949840 .00038

17.800 .953890 .00038

17.900 .957360 .00038

18.000 .959950 .00039

18.100 .963660 .00039

18.200 .967530 .00040

18.300 .970260 .00038

18.400 .973950 .00038

18.500 .976140 .00039

18.600 .980140 .00038

18.700 .983120 .00039

18.800 .986140 .00037

18.900 .988960 .00038

19.000 .991260 .00038

19.100 .994560 .00039

19.200 .997390 .00039

19.301 .001200 .00038

19.401 .003750 .00038

19.501 .007140 .00038

19.601 .009710 .00038

19.701 .012560 .00037

19.801 .015520 .00038
32

16.400 .922490 .00041

16.500 .926730 .00040

16.600 .930830 .00039

16.700 .935070 .00039

16.800 .938990 .00041

16.900 .942480 .00038

17.000 .945220 .00039

17.100 .950190 .00040

17.200 .952610 .00041

17.300 .956470 .00039

17.400 .960080 .00040

17.500 .963650 .00039

17.600 .968210 .00040

17.700 .970840 .00039

17.800 .974800 .00041

17.900 .977920 .00039

18.000 .981950 .00039

18.100 .984860 .00038

18.200 .988040 .00040

18.300 .991440 .00038

18.400 .994600 .00037

18.500 .998110 .00038

18.601 .001560 .00040

18.701 .004390 .00040

18.801 .007750 .00039

18.901 .010780 .00038

19.001 .014200 .00039

19.101 .016850 .00038

19.201 .019680 .00039
35

15.700 .920020 .00042 15.800 .925150 .00040 15.900 .929010 .00041 16.000 .933130 .00040 16.100 .936600 .00041 16.200 .940780 .00040 16.300 .946210 .00041 16.400 .949440 .00040
40

14.900 .919870 .00044 15.000 .925570 .00043 15.100 .930470 .00042 15.200 .934790 .00044 15.300 .939130 .00043 15.400 .943750 .00043 15.500 .948070 .00043 15.600 .953140 .00043
50

13.900 .921750 .00045 14.000 .927530 .00046 14.100 .933600 .00046 14.200 .937910 .00045 14.300 .943300 .00047 14.400 .949400 .00047 14.500 .954390 .00045 14.600 .959670 .00046 
16.500 .953760 .00041 16.600 .958240 .00042 16.700 .962390 .00041 16.800 .965560 .00042 16.900 .968640 .00040 17.000 .972620 .00041 17.100 .977060 .00040 17.200 .980980 .00040 17.300 .984520 .00040 17.400 .987620 .00039 17.500 .991270 .00041 17.600 .995230 .00040 17.700 .998700 .00041 17.801 .003090 .00041 17.901 .006650 .00040 18.001 .009900 .00040 18.101 .013440 .00039 18.201 .016440 .00040 18.301 .019420 .00040
15.700 .958000 .00043 15.800 .961720 .00043 15.900 .966830 .00042 16.000 .970090 .00043 16.100 .974940 .00043 16.200 .980080 .00044 16.300 .984040 .00044 16.400 .987930 .00043 16.500 .992160 .00043 16.600 .996460 .00042 16.700 .999770 .00043 16.801 .003820 .00042 16.901 .008240 .00042 17.001 .012150 .00043 17.101 .016660 .00043 17.201 .018940 .00042
14.700 .964890 .00047 14.800 .969620 .00046 14.900 .975180 .00046 15.000 .980420 .00046 15.100 .985680 .00046 15.200 .990710 .00045 15.300 .995650 .00046 15.401 .000230 .00045 15.501 .005490 .00046 15.601 .010370 .00046 15.701 .014190 .00046 15.801 .019910 .00045

\section{0}

13.200 .918690 .00047 13.300 .925310 .00048 13.400 .932550 .00048 13.500 .936470 .00047 13.600 .943690 .00047 13.700 .949740 .00047 13.800 .955580 .00048 13.900 .961880 .00047 14.000 .967510 .00048 14.100 .972960 .00047 14.200 .979580 .00047 14.300 .984460 .00048 14.400 .989290 .00048 14.500 .995390 .00047 14.601 .000520 .00048 14.701 .004710 .00046 14.801 .011180 .00046 14.901 .016440 .00047 15.001 .021560 .00047
70

12.800 .920250 .00049 12.850 .924510 .00050 12.900 .927110 .00049 12.950 .931460 .00048 13.000 .934950 .00049 13.050 .937240 .00049 13.100 .940590 .00049 13.150 .944790 .00050 13.200 .947660 .00049 13.250 .949550 .00049 13.300 .954490 .00050 13.350 .956720 .00048 13.400 .960190 .00050 13.450 .963830 .00049 13.500 .966240 .00050 13.550 .970210 .00050 13.600 .972570 .00049 13.650 .976640 .00049 13.700 .978500 .00049 13.750 .981960 .00048 13.800 .984190 .00049 13.850 .987890 .00049 13.900 .989700 .00049 13.950 .993480 .00049 14.000 .997580 .00050 14.050 .999250 .00049 14.101 .003180 .00048 14.151 .006650 .00048
80

12.400 .916770 .00051 12.450 .919500 .00051 12.500 .923050 .00050 12.550 .926840 .00051 12.600 .929790 .00049 12.650 .934690 .00051 12.700 .937240 .00051 12.750 .941350 .00051 12.800 .944800 .00050 12.850 .947550 .00051 12.900 .950630 .00049 12.950 .954020 .00051 13.000 .958310 .00051 13.050 .962060 .00049 13.100 .963380 .00049 13.150 .967550 .00050 13.200 .970690 .00051 13.250 .973330 .00051 13.300 .977000 .00050 13.350 .980710 .00051 13.400 .982700 .00051 13.450 .987430 .00051 13.500 .989740 .00050 13.550 .992280 .00051 13.600 .995860 .00050 13.650 .999780 .00050 13.701 .001670 .00050 13.751 .005830 .00050 
14.201 .007930 .00050 14.251 .011520 .00049 14.301 .013120 .00049 14.351 .016630 .00050 14.401 .019460 .00050

90

12.200 .919430 .00052

12.250 .922350 .00050

12.300 .925880 .00051

12.350 .929810 .00051

12.400 .932610 .00050

12.450 .936900 .00052

12.500 .941450 .00050

12.550 .943880 .00053

12.600 .947510 .00052

12.650 .951010 .00051

12.700 .955990 .00052

12.750 .958300 .00052

12.800 .961250 .00050

12.850 .964620 .00052

12.900 .969010 .00053

12.950 .971370 .00051

13.000 .975170 .00051

13.050 .978340 .00051

13.100 .981460 .00053

13.150 .984240 .00051

13.200 .987790 .00051

13.250 .991140 .00052

13.300 .993810 .00052

13.350 .998810 .00052

13.401 .000590 .00051

13.451 .004280 .00050

13.501 .007680 .00051

13.551 .010930 .00051

13.601 .013490 .00052

13.651 .016600 .00051

13.701 .019640 .00051

130

11.600 .916640 .00053 11.650 .919280 .00053 11.700 .924690 .00053 11.750 .927480 .00054 11.800 .933180 .00053
100

12.000 .918440 .00052

12.050 .922150 .00051

12.100 .925500 .00051

12.150 .928550 .00052

12.200 .933460 .00053

12.250 .936920 .00053

12.300 .940730 .00051

12.350 .943540 .00052

12.400 .946770 .00052

12.450 .951040 .00051

12.500 .953830 .00052

12.550 .957430 .00053

12.600 .960610 .00051

12.650 .965300 .00052

12.700 .967960 .00053

12.750 .971770 .00052

12.800 .974880 .00051

12.850 .978380 .00053

12.900 .982500 .00051

12.950 .985790 .00052

13.000 .988680 .00053

13.050 .991900 .00052

13.100 .996270 .00054

13.150 .999170 .00053

13.201 .002520 .00052

13.251 .005340 .00052

13.301 .008750 .00052

13.351 .013250 .00051

13.401 .015800 .00052

13.451 .018470 .00051

13.501 .022180 .00053
150

11.500 .919940 .00054 11.550 .923740 .00053 11.600 .927670 .00054 11.650 .930800 .00055 11.700 .935890 .00053
13.801 .007910 .00051

13.851 .011170 .00050

13.901 .013750 .00051

13.951 .017000 .00051

14.001 .020210 .00051

14.051 .022180 .00051

14.101 .025710 .00052

118

11.800 .921410 .00053

11.850 .925220 .00053

11.900 .929050 .00053

11.950 .933190 .00054

12.000 .937700 .00052

12.050 .940790 .00054

12.100 .945180 .00052

12.150 .948680 .00052

12.200 .952020 .00051

12.250 .955310 .00053

12.300 .959140 .00054

12.350 .962460 .00053

12.400 .966950 .00051

12.450 .970100 .00054

12.500 .972920 .00054

12.550 .977900 .00053

12.600 .982320 .00053

12.650 .985610 .00052

12.700 .988580 .00053

12.750 .991880 .00053

12.800 .995130 .00052

12.850 .999030 .00052

12.901 .000880 .00054

12.951 .006450 .00053

13.001 .009180 .00054

13.051 .011910 .00053

13.101 .016510 .00053

13.151 .018590 .00054

13.201 .023210 .00054
200
11.200 .914070 .00055
11.250 .918230 .00053
11.300 .921620 .00054
11.350 .927010 .00055
11.400 .930560 .00054 
11.850 .936440 .00054 11.900 .940050 .00055 11.950 .943370 .00052 12.000 .948390 .00054 12.050 .951170 .00054 12.100 .954370 .00052 12.150 .959590 .00052 12.200 .961970 .00056 12.250 .966640 .00055 12.300 .969710 .00052 12.350 .973500 .00053 12.400 .977670 .00053 12.450 .981390 .00052 12.500 .984850 .00054 12.550 .987510 .00053 12.600 .991570 .00054 12.650 .994900 .00054 12.700 .998790 .00053 12.751 .003850 .00053 12.801 .007030 .00054 12.851 .009880 .00055 12.901 .012370 .00053 12.951 .015250 .00053 13.001 .019870 .00054

235

11.200 .920050 .00054 11.250 .923930 .00056 11.300 .928600 .00055 11.350 .933300 .00056 11.400 .936480 .00054 11.450 .941210 .00054 11.500 .945140 .00056 11.650 .957970 .00055 11.800 .969650 .00054 11.850 .973460 .00056 11.900 .977890 .00053 11.950 .980730 .00055 12.000 .985480 .00056 12.050 .989830 .00055 12.100 .993100 .00056 12.150 .997320 .00057 12.201 .000970 .00056 12.251 .004020 .00055 12.301 .007630 .00055 12.351 .011670 .00055 12.401 .015440 .00056 12.451 .019160 .00056 12.501 .022920 .00056
11.750 .940460 .00054 11.800 .944290 .00053 11.850 .948730 .00054 11.900 .952520 .00054 11.950 .955490 .00053 12.000 .959840 .00054 12.050 .963340 .00054 12.100 .967280 .00054 12.150 .970450 .00053 12.200 .976030 .00055 12.250 .977940 .00055 12.300 .981910 .00053 12.350 .985720 .00055 12.400 .990070 .00055 12.450 .993080 .00055 12.500 .997360 .00054 12.551 .001470 .00054 12.601 .004340 .00055 12.651 .007780 .00054 12.701 .012190 .00054

$\begin{array}{lll} & 250 \\ 11.30 & 0.93000 & 0.00055 \\ 11.35 & 0.93492 & 0.00055 \\ 11.40 & 0.93901 & 0.00055 \\ 11.45 & 0.94340 & 0.00055 \\ 11.50 & 0.94750 & 0.00055 \\ 11.55 & 0.95134 & 0.00055 \\ 11.60 & 0.95506 & 0.00056 \\ 11.65 & 0.96013 & 0.00055 \\ 11.70 & 0.96383 & 0.00055 \\ 11.75 & 0.96679 & 0.00054 \\ 11.80 & 0.97084 & 0.00056 \\ 11.85 & 0.97442 & 0.00055 \\ 11.90 & 0.97920 & 0.00054 \\ 11.95 & 0.98280 & 0.00056 \\ 12.00 & 0.98626 & 0.00057 \\ 12.05 & 0.98989 & 0.00056 \\ 12.10 & 0.99451 & 0.00056 \\ 12.15 & 0.99839 & 0.00055 \\ 12.20 & 1.00192 & 0.00056 \\ 12.25 & 1.00590 & 0.00055 \\ 12.30 & 1.00886 & 0.00056 \\ 12.35 & 1.01338 & 0.00056 \\ 12.40 & 1.01714 & 0.00056\end{array}$
11.450 .935120 .00054 11.500 .939560 .00056 11.550 .943680 .00055 11.600 .947070 .00055 11.650 .950110 .00054 11.700 .955630 .00056 11.750 .958960 .00056 11.800 .962280 .00053 11.850 .968590 .00057 11.900 .972000 .00055 11.950 .975470 .00053 12.000 .978220 .00056 12.050 .982760 .00055 12.100 .986150 .00056 12.150 .989610 .00055 12.200 .993570 .00056 12.250 .997920 .00055 12.301 .000790 .00054 12.351 .003850 .00056 12.401 .008680 .00054 12.451 .012980 .00053 12.501 .016610 .00054 12.551 .019690 .00055 12.601 .023570 .00055
353
11.100 .915880 .00054 11.150 .921610 .00055 11.200 .925370 .00054 11.250 .929110 .00056 11.300 .933130 .00056 11.350 .936650 .00055 11.400 .940710 .00055 11.450 .946190 .00055 11.500 .950230 .00056 11.550 .953590 .00056 11.600 .956630 .00056 11.650 .962130 .00056 11.700 .965220 .00054 11.750 .970460 .00054 11.800 .973940 .00056 11.850 .976590 .00055 11.900 .980100 .00056 11.950 .985500 .00054 12.000 .988630 .00056 12.050 .993530 .00056 12.100 .997120 .00057 12.150 .999580 .00056 12.201 .003790 .00055 
CHPRC-01552, Revision 0

12.451 .020200 .00057 12.501 .023940 .00054
12.251 .007420 .00055 12.301 .011340 .00056 12.351 .014850 .00054 12.401 .018510 .00055 12.451 .023270 .00058 12.501 .027060 .00056
470

11.200 .917070 .00055 11.250 .921740 .00054 11.300 .926650 .00053 11.350 .929980 .00055 11.400 .936050 .00054 11.450 .938510 .00056 11.500 .942770 .00055 11.550 .946990 .00056 11.600 .950840 .00055 11.650 .956310 .00054 11.700 .959010 .00057 11.750 .962890 .00055 11.800 .966690 .00054 11.850 .971590 .00055 11.900 .974560 .00057 11.950 .978210 .00054 12.000 .981010 .00054 12.050 .985770 .00055 12.100 .988820 .00054 12.150 .993160 .00055 12.200 .996140 .00055 12.251 .001350 .00056 12.301 .003380 .00057 12.351 .007720 .00055 12.401 .011240 .00055 12.451 .014430 .00056 12.501 .018280 .00056 12.551 .022710 .00057 12.601 .025100 .00054

605 11.400 .918210 .00055 11.450 .922930 .00054 11.500 .926690 .00055 11.550 .930450 .00055 11.600 .935000 .00052 11.650 .937610 .00056
500

11.200 .913670 .00052 11.250 .918560 .00054 11.300 .922640 .00056 11.350 .926250 .00056 11.400 .931530 .00054 11.450 .934180 .00056 11.500 .938510 .00055 11.550 .942690 .00055 11.600 .946570 .00055 11.650 .949770 .00054 11.700 .955240 .00055 11.750 .958440 .00055 11.800 .962350 .00053 11.850 .965980 .00055 11.900 .970050 .00055 11.950 .972560 .00055 12.000 .975380 .00055 12.050 .980600 .00055 12.100 .984890 .00055 12.150 .987660 .00054 12.200 .992060 .00055 12.250 .995130 .00055 12.301 .000000 .00055 12.351 .002420 .00056 12.401 .006320 .00055 12.451 .010120 .00056 12.501 .014330 .00056 12.551 .016130 .00055 12.601 .021100 .00055 12.651 .023650 .00054 12.701 .027660 .00056

610 11.400 .918950 .00053 11.450 .921230 .00054 11.500 .925800 .00054 11.550 .930630 .00054 11.600 .933900 .00055 11.650 .938140 .00054
600

11.400 .919050 .00054 11.450 .922140 .00055 11.500 .927350 .00055 11.550 .932020 .00054 11.600 .933880 .00055 11.650 .937950 .00055 11.700 .941760 .00055 11.750 .945820 .00055 11.800 .950620 .00055 11.850 .953440 .00053 11.900 .958340 .00055 11.950 .960720 .00056 12.000 .964640 .00056 12.050 .968310 .00054 12.100 .972300 .00054 12.150 .975720 .00055 12.200 .979330 .00054 12.250 .983230 .00055 12.300 .986810 .00055 12.350 .991020 .00055 12.400 .995020 .00055 12.450 .998140 .00056 12.501 .001060 .00055 12.551 .005050 .00054 12.601 .008340 .00055 12.651 .011470 .00056 12.701 .015510 .00055 12.751 .019030 .00056 12.801 .022090 .00054 
11.700 .941150 .00054 11.750 .945590 .00055 11.800 .948980 .00055 11.850 .952620 .00055 11.900 .957820 .00054 11.950 .960720 .00054 12.000 .964420 .00055 12.050 .969870 .00055 12.100 .972020 .00055 12.150 .975290 .00055 12.200 .979700 .00053 12.250 .983050 .00053 12.300 .987510 .00053 12.350 .989540 .00055 12.400 .994320 .00056 12.450 .997000 .00055 12.501 .001210 .00055 12.551 .004440 .00056 12.601 .007370 .00056 12.651 .011200 .00055 12.701 .015970 .00055 12.751 .018680 .00055 12.801 .021480 .00053
620

11.400 .916240 .00053 11.450 .919720 .00054 11.500 .925060 .00055 11.550 .927660 .00054 11.600 .932770 .00055 11.650 .935700 .00054 11.700 .940380 .00053 11.750 .943740 .00053 11.800 .948010 .00053 11.850 .950390 .00054 11.900 .955180 .00055 11.950 .957380 .00053 12.000 .963000 .00056 12.050 .966770 .00053 12.100 .969780 .00055 12.150 .973580 .00054 12.200 .976290 .00054 12.250 .980640 .00054 12.300 .984150 .00054 12.350 .988920 .00055 12.400 .991700 .00055 12.450 .994510 .00054
11.700 .940920 .00053 11.750 .946230 .00054 11.800 .949380 .00053 11.850 .953130 .00054 11.900 .956990 .00055 11.950 .960210 .00053 12.000 .964960 .00054 12.050 .968420 .00054 12.100 .971600 .00055 12.150 .975640 .00055 12.200 .979560 .00053 12.250 .982160 .00055 12.300 .984780 .00054 12.350 .989840 .00055 12.400 .993710 .00054 12.450 .996320 .00053 12.501 .000080 .00054 12.551 .003950 .00054 12.601 .007090 .00054 12.651 .010720 .00056 12.701 .014390 .00055 12.751 .019610 .00057 12.801 .021700 .00055
11.700 .939590 .00052

11.750 .944460 .00053

11.800 .947950 .00055

11.850 .951960 .00054

11.900 .954510 .00054

11.950 .959420 .00055

12.000 .963310 .00054

12.050 .966240 .00056

12.100 .969890 .00054

12.150 .974280 .00052

12.200 .978170 .00055

12.250 .981500 .00055

12.300 .985290 .00055

12.350 .987200 .00052

12.400 .992780 .00054

12.450 .994740 .00054

12.500 .998970 .00054

12.551 .002470 .00054

12.601 .006080 .00055

12.651 .008730 .00053

12.701 .014550 .00055

12.751 .017500 .00055

12.801 .020210 .00055

12.851 .022720 .00055

12.901 .026780 .00056

625

11.400 .916280 .00054

11.450 .920170 .00054

11.500 .923540 .00054

11.550 .928200 .00055

11.600 .931630 .00055

11.650 .935680 .00053

11.700 .939350 .00055

11.750 .943630 .00055

11.800 .946940 .00052

11.850 .951520 .00053

11.900 .954350 .00054

11.950 .959000 .00055

12.000 .962050 .00054

12.050 .965760 .00054

12.100 .968990 .00054

12.150 .973830 .00053

12.200 .976850 .00054

12.250 .979990 .00054

12.300 .982970 .00054

12.350 .986470 .00053

12.400 .990600 .00053

12.450 .994940 .00053
629

11.400 .914780 .00056

11.450 .918650 .00055

11.500 .922800 .00054

11.550 .926480 .00054

11.600 .930930 .00052

11.650 .934110 .00053

11.700 .938030 .00053

11.750 .942540 .00054

11.800 .945540 .00055

11.850 .949570 .00054

11.900 .953800 .00054

11.950 .957250 .00053

12.000 .960570 .00056

12.050 .964580 .00054

12.100 .967870 .00054

12.150 .971940 .00054

12.200 .975620 .00054

12.250 .979850 .00054

12.300 .983350 .00055

12.350 .987230 .00054

12.400 .989290 .00054

12.450 .993930 .00055 
12.500 .998700 .00056

12.551 .002600 .00053

12.601 .005460 .00057

12.651 .010100 .00055

12.701 .012230 .00056

12.751 .015600 .00054

12.801 .020450 .00054
12.500 .998270 .00054

12.551 .001180 .00053

12.601 .005050 .00055

12.651 .008590 .00053

12.701 .010980 .00055

12.751 .015260 .00056

12.801 .018960 .00054
12.500 .997800 .00054

12.551 .000780 .00053

12.601 .005130 .00054

12.651 .007780 .00055

12.701 .010650 .00054

12.751 .015420 .00055

12.801 .018010 .00055

12.851 .021970 .00053

12.901 .024030 .00054

U5o2N20-sp-0935-01

12

74.700 .928570 .00014 79.200 .932130 .00014 83.700 .934480 .00014 88.200 .936550 .00013 92.700 .939020 .00013 97.200 .940210 .00013 101.700 .941680 .00013 106.200 .942990 .00012 110.700 .944650 .00012 115.200 .945080 .00012 119.700 .945690 .00012 124.200 .947020 .00012 128.700 .947380 .00012 133.200 .948090 .00011 137.700 .948300 .00011 142.200 .949400 .00011 146.700 .950060 .00011 151.200 .950440 .00011 155.700 .950560 .00011 160.200 .951070 .00011 164.700 .951340 .00011 166.100 .951670 .00011
15

30.900 .926020 .00024 31.900 .932860 .00023 32.900 .939900 .00022 33.900 .945920 .00022 34.900 .952360 .00021 35.900 .957690 .00021 36.900 .962200 .00021 37.900 .966950 .00021 38.900 .971450 .00021 39.900 .975820 .00020 40.900 .979530 .00020 41.900 .983280 .00020 42.900 .986020 .00020 43.900 .990190 .00020 44.900 .993780 .00020 45.900 .996560 .00019 46.900 .998850 .00019 47.901 .002070 .00019 48.901 .003690 .00018 49.901 .005910 .00018 50.901 .008700 .00018 51.901 .009830 .00018 52.401 .012090 .00018
20

20.800 .924720 .00030 21.000 .928920 .00030 21.200 .932260 .00030 21.400 .936130 .00030 21.600 .939910 .00031 21.800 .943390 .00031 22.000 .946390 .00030 22.200 .949410 .00030 22.400 .952480 .00030 22.600 .956860 .00030 22.800 .959640 .00029 23.000 .963020 .00030 23.200 .966360 .00029 23.400 .969560 .00029 23.600 .972240 .00030 23.800 .974940 .00030 24.000 .978340 .00030 24.200 .980770 .00029 24.400 .983930 .00029 24.600 .986090 .00029 24.800 .989200 .00029 25.000 .991670 .00028 25.200 .994880 .00029 25.400 .996930 .00028 25.600 .999470 .00028 25.801 .002430 .00028 26.001 .005340 .00028 26.201 .007120 .00028 26.401 .009710 .00027 26.501 .010770 .00028 
25

17.200 .921570 .00035 17.400 .927560 .00035 17.600 .933920 .00035 17.800 .938440 .00034 18.000 .944270 .00035 18.200 .949330 .00033 18.400 .954910 .00034 18.600 .960160 .00034 18.800 .965150 .00035 19.000 .970520 .00035 19.200 .974930 .00035 19.400 .980700 .00033 19.600 .984300 .00034 19.800 .989400 .00034 20.000 .993240 .00033 20.200 .998350 .00034 20.401 .002000 .00034 20.601 .006940 .00033 20.801 .010330 .00033 21.001 .014230 .00032

\section{5}

14.200 .921170 .00040 14.300 .925050 .00041 14.400 .929810 .00039 14.500 .933750 .00041 14.600 .938270 .00039 14.700 .941920 .00039 14.800 .946130 .00038 14.900 .951190 .00039 15.000 .954260 .00039 15.100 .957910 .00038 15.200 .961600 .00040 15.300 .966030 .00039 15.400 .969820 .00040 15.500 .973870 .00039 15.600 .978290 .00040 15.700 .981800 .00039
30

15.400 .922430 .00038 15.500 .926100 .00038 15.600 .929870 .00036 15.700 .933350 .00037 15.800 .937290 .00037 15.900 .941290 .00037 16.000 .943720 .00038 16.100 .947640 .00037 16.200 .950520 .00037 16.300 .954570 .00038 16.400 .957670 .00037 16.500 .961880 .00037 16.600 .964530 .00036 16.700 .967930 .00037 16.800 .970280 .00037 16.900 .974110 .00037 17.000 .977160 .00037 17.100 .980790 .00037 17.200 .983730 .00037 17.300 .986130 .00037 17.400 .989670 .00037 17.500 .992650 .00038 17.600 .995870 .00037 17.700 .998730 .00036 17.801 .001920 .00037 17.901 .004100 .00037 18.001 .007030 .00036 18.101 .009840 .00038 18.201 .012320 .00035 18.301 .015840 .00037

40

13.400 .920950 .00041 13.500 .925660 .00042 13.600 .930870 .00041 13.700 .934850 .00041 13.800 .940130 .00042 13.900 .944560 .00042 14.000 .948780 .00042 14.100 .954320 .00041 14.200 .958410 .00041 14.300 .962650 .00041 14.400 .966640 .00042 14.500 .970820 .00041 14.600 .976180 .00041 14.700 .980120 .00041 14.800 .984700 .00040 14.900 .988240 .00041
32

14.900 .923420 .00038 15.000 .927100 .00039 15.100 .930930 .00038 15.200 .934970 .00039 15.300 .938330 .00039 15.400 .943410 .00039 15.500 .947310 .00037 15.600 .950280 .00038 15.700 .953810 .00038 15.800 .957460 .00038 15.900 .961580 .00039 16.000 .965180 .00039 16.100 .967170 .00037 16.200 .971640 .00038 16.300 .975220 .00037 16.400 .977870 .00039 16.500 .982320 .00039 16.600 .985830 .00038 16.700 .988490 .00037 16.800 .992360 .00038 16.900 .995240 .00038 17.000 .998130 .00037 17.101 .001470 .00037 17.201 .004320 .00038 17.301 .008100 .00037 17.401 .010990 .00037 17.501 .014540 .00038 17.601 .016900 .00038

50

12.400 .922840 .00045 12.500 .927570 .00044 12.600 .933230 .00045 12.700 .939580 .00043 12.800 .945670 .00045 12.900 .949950 .00044 13.000 .955490 .00045 13.100 .961070 .00043 13.200 .965560 .00043 13.300 .971170 .00044 13.400 .976600 .00043 13.500 .981400 .00044 13.600 .986860 .00044 13.700 .992160 .00043 13.800 .995730 .00043 13.901 .000800 .00043 
15.800 .984730 .00039 15.900 .989160 .00038 16.000 .992100 .00041 16.100 .995270 .00039 16.200 .999440 .00040 16.301 .002940 .00038 16.401 .006790 .00039 16.501 .010060 .00039 16.601 .013130 .00038 16.701 .016820 .00039
15.000 .992730 .00041 15.100 .996900 .00042 15.201 .000930 .00040 15.301 .004900 .00042 15.401 .008730 .00040 15.501 .011910 .00041 15.601 .017010 .00041 15.701 .020120 .00040
14.001 .005500 .00044 14.101 .010900 .00044 14.201 .015520 .00044 14.301 .019410 .00043
60

11.700 .919550 .00046 11.800 .925830 .00045 11.900 .932720 .00047 12.000 .937700 .00046 12.100 .943870 .00045 12.200 .950260 .00045 12.300 .955990 .00046 12.400 .962370 .00046 12.500 .968940 .00046 12.600 .974270 .00045 12.700 .979900 .00047 12.800 .984820 .00046 12.900 .990430 .00046 13.000 .996330 .00046 13.101 .000660 .00046 13.201 .006670 .00046 13.301 .012260 .00046 13.401 .017790 .00047 13.501 .022990 .00046
70 11.300 .922140 .00046 11.350 .926000 .00047 11.400 .928580 .00047 11.450 .932820 .00048 11.500 .936350 .00048 11.550 .938940 .00047 11.600 .941780 .00046 11.650 .945180 .00045 11.700 .947780 .00046 11.750 .952090 .00047 11.800 .954700 .00047 11.850 .958650 .00047 11.900 .961060 .00047 11.950 .964630 .00048 12.000 .967870 .00048 12.050 .970910 .00048 12.100 .973730 .00045 12.150 .976830 .00046 12.200 .980400 .00046 12.250 .982970 .00047 12.300 .986100 .00046 12.350 .988610 .00046 12.400 .992660 .00048 12.450 .994810 .00046 12.500 .998180 .00048 12.551 .000900 .00047 12.601 .003870 .00047 12.651 .006660 .00046 12.701 .008710 .00047 12.751 .011910 .00047 12.801 .015160 .00048 12.851 .018120 .00047 12.901 .020470 .00048
80

10.900 .916730 .00047 10.950 .921470 .00048 11.000 .923780 .00047 11.050 .927370 .00048 11.100 .930960 .00047 11.150 .935500 .00049 11.200 .937680 .00048 11.250 .942170 .00047 11.300 .945220 .00047 11.350 .948500 .00048 11.400 .952420 .00047 11.450 .954500 .00047 11.500 .958670 .00050 11.550 .961880 .00048 11.600 .964780 .00048 11.650 .968100 .00048 11.700 .970890 .00047 11.750 .975670 .00050 11.800 .979040 .00048 11.850 .981880 .00049 11.900 .984230 .00048 11.950 .988890 .00047 12.000 .990590 .00048 12.050 .993540 .00050 12.100 .997000 .00048 12.151 .000930 .00049 12.201 .003800 .00049 12.251 .006620 .00048 12.301 .009590 .00047 12.351 .013500 .00048 12.401 .015470 .00049 12.451 .018530 .00047 12.501 .022090 .00047 
90

10.700 .919980 .00048

10.750 .924110 .00049

10.800 .926510 .00051

10.850 .930700 .00048

10.900 .934660 .00049

10.950 .938620 .00049

11.000 .941200 .00049

11.050 .945050 .00051

11.100 .949350 .00050

11.150 .952350 .00049

11.200 .956600 .00050

11.250 .958600 .00049

11.300 .962380 .00049

11.350 .965650 .00051

11.400 .969300 .00050

11.450 .973220 .00050

11.500 .976760 .00049

11.550 .979020 .00049

11.600 .982950 .00049

11.650 .986500 .00049

11.700 .990570 .00048

11.750 .993320 .00049

11.800 .995990 .00049

11.850 .999960 .00050

11.901 .002030 .00050

11.951 .006860 .00049

12.001 .009750 .00049

12.051 .012880 .00049

12.101 .015880 .00051

12.151 .017970 .00049

12.201 .022120 .00047

130

10.100 .918310 .00051

10.150 .922120 .00051

10.200 .925900 .00052

10.250 .930100 .00051

10.300 .934130 .00053

10.350 .937460 .00052

10.400 .941720 .00051

10.450 .946420 .00051

10.500 .950550 .00052

10.550 .953120 .00051

10.600 .956660 .00050

10.650 .960370 .00051

10.700 .964820 .00052

10.750 .968130 .00051

10.800 .971820 .00052
100

10.500 .919660 .00051

10.550 .923910 .00050

10.600 .927440 .00050

10.650 .930800 .00049

10.700 .933930 .00051

10.750 .938450 .00051

10.800 .942650 .00051

10.850 .944740 .00051

10.900 .948920 .00050

10.950 .952110 .00051

11.000 .957190 .00050

11.050 .959990 .00049

11.100 .963140 .00050

11.150 .966460 .00049

11.200 .970150 .00050

11.250 .973110 .00049

11.300 .977690 .00050

11.350 .981220 .00050

11.400 .984360 .00051

11.450 .987430 .00050

11.500 .990560 .00051

11.550 .993210 .00049

11.600 .997150 .00049

11.651 .001220 .00049

11.701 .004760 .00048

11.751 .007600 .00049

11.801 .011060 .00051

11.851 .014840 .00049

11.901 .017350 .00051

11.951 .019880 .00051

12.001 .024320 .00051
118

10.200 .916080 .00050

10.250 .920250 .00052

10.300 .923630 .00051

10.350 .927930 .00050

10.400 .931020 .00051

10.450 .935570 .00049

10.500 .940050 .00052

10.550 .942880 .00049

10.600 .945740 .00050

10.650 .951550 .00052

10.700 .954080 .00050

10.750 .958290 .00051

10.800 .961080 .00051

10.850 .965550 .00051

10.900 .968880 .00050

10.950 .971980 .00051

11.000 .976200 .00052

11.050 .980130 .00050

11.100 .983450 .00052

11.150 .986200 .00051

11.250 .994400 .00050

11.300 .997090 .00051

11.351 .000580 .00052

11.401 .004150 .00050

11.451 .007490 .00052

11.501 .011120 .00052

11.551 .015030 .00051

11.601 .017720 .00050

11.651 .021690 .00051

11.701 .024260 .00051
10.000 .922440 .00049

10.050 .926350 .00052

10.100 .929750 .00051

10.150 .935010 .00051

10.200 .937760 .00051

10.250 .942060 .00051

10.300 .946490 .00052

10.350 .950210 .00052

10.400 .953490 .00051

10.450 .957970 .00051

10.500 .962620 .00052

10.550 .966510 .00054

10.600 .969470 .00052

10.650 .973440 .00052

10.700 .976990 .00052
200

9.700 .916190 .00055

9.750 .921980 .00051

9.800 .924520 .00054

9.850 .929520 .00053

9.900 .933150 .00053

9.950 .938380 .00052

10.000 .941730 .00053

10.050 .945130 .00052

10.100 .948440 .00052

10.150 .954200 .00052

10.200 .957640 .00052

10.250 .960970 .00053

10.300 .965760 .00053

10.350 .968930 .00053

10.400 .973580 .00053 
10.850 .976260 .00052 10.900 .978880 .00052 10.950 .983230 .00051 11.000 .986580 .00053 11.050 .990130 .00051 11.100 .993570 .00052 11.150 .997610 .00051 11.201 .000420 .00052 11.251 .004620 .00051 11.301 .008480 .00052 11.351 .011670 .00051 11.401 .014190 .00052 11.451 .020100 .00051 11.501 .021520 .00052
10.750 .979900 .00053 10.800 .984540 .00051 10.850 .987530 .00051 10.900 .990730 .00052 10.950 .995290 .00052 11.000 .999220 .00051 11.051 .003500 .00053 11.101 .006730 .00052 11.151 .009550 .00051 11.201 .013810 .00052 11.251 .017500 .00052 11.301 .021150 .00050
10.450 .976910 .00053 10.500 .981510 .00052 10.550 .985120 .00052 10.600 .988320 .00054 10.650 .992310 .00053 10.700 .996220 .00054 10.750 .999200 .00054 10.801 .003630 .00053 10.851 .007910 .00053 10.901 .011480 .00053 10.951 .015300 .00053 11.001 .019210 .00053
235

9.700 .923440 .00052 9.750 .927860 .00053 9.800 .930730 .00053 9.850 .935750 .00052 9.900 .940310 .00053 9.950 .944160 .00053 10.000 .948130 .00055 10.050 .952890 .00053 10.100 .956600 .00052 10.150 .960920 .00051 10.200 .964700 .00053 10.250 .968890 .00053 10.300 .971780 .00054 10.350 .977030 .00054 10.400 .981130 .00053 10.450 .984070 .00053 10.500 .988110 .00054 10.550 .991950 .00053 10.600 .996040 .00054 10.650 .999680 .00054 10.701 .002170 .00051 10.751 .007050 .00054 10.801 .010410 .00052 10.851 .015110 .00053 10.901 .017730 .00054 10.951 .021690 .00052 11.001 .026240 .00053
250

9.600 .916630 .00052

9.650 .920140 .00052

9.700 .924460 .00052

9.750 .928930 .00054

9.800 .932590 .00054

9.850 .937380 .00053

9.900 .942180 .00052

9.950 .945840 .00054

10.000 .950320 .00053

10.050 .954940 .00052

10.100 .957430 .00053

10.150 .962610 .00054

10.200 .964520 .00054

10.250 .970860 .00054

10.300 .974840 .00053

10.350 .977530 .00054

10.400 .980750 .00053

10.450 .986250 .00053

10.500 .989620 .00054

10.550 .993260 .00055

10.600 .997250 .00054

10.651 .000930 .00054

10.701 .005310 .00053

10.751 .009230 .00053

10.801 .012160 .00053

10.851 .016560 .00052

10.901 .020310 .00055
353

9.600 .920360 .00053

9.650 .923820 .00052

9.700 .928690 .00054

9.750 .933440 .00052

9.800 .936840 .00053

9.850 .941390 .00053

9.900 .945080 .00054

9.950 .948480 .00053

10.000 .952960 .00054

10.050 .957840 .00053

10.100 .961280 .00054

10.150 .964660 .00053

10.200 .968880 .00052

10.250 .973450 .00054

10.300 .977090 .00053

10.350 .980340 .00054

10.400 .985250 .00053

10.450 .989160 .00053

10.500 .992640 .00052

10.550 .996790 .00055

10.601 .001800 .00054

10.651 .003740 .00054

10.701 .007950 .00053

10.751 .010700 .00053

10.801 .014980 .00053

10.851 .017560 .00052

10.901 .021560 .00054 
470

9.700 .923090 .00055

9.750 .926860 .00052

9.800 .930790 .00053

9.850 .935530 .00055

9.900 .939290 .00053

9.950 .942590 .00053

10.000 .946520 .00053

10.050 .951220 .00055

10.100 .954160 .00052

10.150 .958160 .00053

10.200 .961990 .00052

10.250 .966760 .00054

10.300 .970430 .00054

10.350 .973700 .00053

10.400 .978600 .00052

10.450 .981450 .00055

10.500 .985000 .00052

10.550 .989370 .00052

10.600 .993620 .00053

10.650 .996820 .00053

10.701 .001090 .00055

10.751 .003690 .00053

10.801 .007890 .00054

10.851 .011190 .00054

10.901 .016030 .00056

10.951 .019300 .00054

11.001 .022180 .00053
500

9.800 .927520 .00053

9.850 .931180 .00053

9.900 .935090 .00053

9.950 .938730 .00052

10.000 .942900 .00052

10.050 .947490 .00053

10.100 .951540 .00053

10.150 .954680 .00053

10.200 .957370 .00053

10.250 .962310 .00052

10.300 .965600 .00053

10.350 .969000 .00054

10.400 .973630 .00054

10.450 .977850 .00053

10.500 .981520 .00054

10.550 .985380 .00054

10.600 .988690 .00054

10.650 .992610 .00053

10.700 .996740 .00054

10.751 .000160 .00054

10.801 .003620 .00054

10.851 .007730 .00053

10.901 .010130 .00052

10.951 .015380 .00054

11.001 .017520 .00054

11.051 .020910 .00054

11.101 .024580 .00053
600

9.800 .916120 .00052

9.850 .920010 .00052

9.900 .924520 .00052

9.950 .926980 .00052

10.000 .931890 .00052

10.050 .935560 .00051

10.100 .940080 .00051

10.150 .943230 .00052

10.200 .947550 .00053

10.250 .950610 .00053

10.300 .955850 .00054

10.350 .958370 .00053

10.400 .962960 .00053

10.450 .966280 .00053

10.500 .971210 .00054

10.550 .973330 .00053

10.600 .977750 .00053

10.650 .981060 .00053

10.700 .985070 .00054

10.750 .988640 .00053

10.800 .992160 .00053

10.850 .996050 .00054

10.900 .999800 .00053

10.951 .002290 .00052

11.001 .006510 .00053

11.051 .009580 .00052

11.101 .013800 .00053

11.151 .016870 .00055

11.201 .020130 .00054

610

9.800 .915800 .00052

9.850 .919190 .00052

9.900 .923530 .00054

9.950 .926740 .00053

10.000 .931860 .00053

10.050 .935100 .00052

10.100 .938530 .00053

10.150 .942830 .00052

10.200 .946550 .00055

10.250 .950410 .00053

10.300 .953700 .00051

10.350 .957940 .00053

10.400 .962370 .00053

10.450 .965610 .00052

10.500 .968610 .00052

10.550 .972990 .00054

10.600 .976750 .00052
615

9.900 .922030 .00051

9.950 .924430 .00054

10.000 .930070 .00053

10.050 .933510 .00054

10.100 .936700 .00053

10.150 .941600 .00052

10.200 .945640 .00053

10.250 .948920 .00053

10.300 .952490 .00052

10.350 .957050 .00053

10.400 .961260 .00054

10.450 .963870 .00054

10.500 .968210 .00053

10.550 .971980 .00051

10.600 .974410 .00053

10.650 .978630 .00054

10.700 .982700 .00053 
10.650 .980550 .00053

10.700 .984810 .00053

10.750 .987660 .00053

10.800 .991480 .00053

10.850 .995590 .00053

10.900 .998180 .00052

10.951 .003110 .00052

11.001 .005400 .00053

11.051 .009320 .00053

11.101 .013690 .00052

11.151 .016670 .00054

11.201 .018900 .00051
620

9.900 .920800 .00050

9.950 .925080 .00054

10.000 .928590 .00050

10.050 .933490 .00054

10.100 .936740 .00052

10.150 .941480 .00052

10.200 .945320 .00052

10.250 .948940 .00051

10.300 .952890 .00052

10.350 .956500 .00054

10.400 .960290 .00053

10.450 .963120 .00054

10.500 .967080 .00053

10.550 .971850 .00054

10.600 .974670 .00052

10.650 .978880 .00053

10.700 .982010 .00054

10.750 .985660 .00052

10.800 .989920 .00052

10.850 .993860 .00053

10.900 .997800 .00054

10.951 .000070 .00055

11.001 .003840 .00054

11.051 .006440 .00054

11.101 .011600 .00053

11.151 .014680 .00053

11.201 .018010 .00053

11.251 .020550 .00053

11.301 .024090 .00054
10.650 .980710 .00052

10.700 .984110 .00053

10.750 .986820 .00051

10.800 .990650 .00052

10.850 .994900 .00052

10.900 .997230 .00055

10.951 .002580 .00054

11.001 .005610 .00053

11.051 .009150 .00054

11.101 .012610 .00054

11.151 .015540 .00054

11.201 .019190 .00052

11.251 .023380 .00053

11.301 .025490 .00051
10.750 .986050 .00053

10.800 .988840 .00052

10.850 .992900 .00054

10.900 .997110 .00052

10.951 .000030 .00053

11.001 .005180 .00054

11.051 .007000 .00053

11.101 .011160 .00052

11.151 .013630 .00052

11.201 .017100 .00052

11.251 .020870 .00054

11.301 .024500 .00054
625

9.900 .920980 .00053

9.950 .924930 .00052

10.000 .929180 .00053

10.050 .932990 .00054

10.100 .936560 .00053

10.150 .940030 .00054

10.200 .944400 .00053

10.250 .948680 .00053

10.300 .953070 .00052

10.350 .955200 .00053

10.400 .959430 .00051

10.450 .962760 .00052

10.500 .966870 .00051

10.550 .970630 .00052

10.600 .975310 .00053

10.650 .977440 .00051

10.700 .982260 .00052

10.750 .985030 .00054

10.800 .988740 .00052

10.850 .992550 .00053

10.900 .995820 .00053

10.950 .998920 .00052

11.001 .002990 .00053

11.051 .006570 .00056

11.101 .009900 .00052

11.151 .013010 .00054

11.201 .017410 .00052

11.251 .019890 .00052

11.301 .023740 .00053
629

9.900 .920590 .00052

9.950 .924410 .00053

10.000 .928730 .00052

10.050 .932000 .00053

10.100 .936000 .00053

10.150 .939220 .00052

10.200 .943310 .00053

10.250 .947060 .00053

10.300 .951520 .00053

10.350 .954860 .00054

10.400 .959050 .00053

10.450 .962150 .00054

10.500 .965760 .00052

10.550 .970160 .00052

10.600 .973610 .00053

10.650 .977660 .00054

10.700 .981340 .00052

10.750 .985180 .00052

10.800 .988570 .00053

10.850 .991720 .00053

10.900 .994730 .00053

10.950 .998110 .00054

11.001 .002270 .00052

11.051 .005770 .00054

11.101 .008570 .00054

11.151 .012980 .00052

11.201 .016260 .00054

11.251 .019360 .00053

11.301 .022470 .00053 
U5o2N20-sp-0935-10

12

69.900 .926500 .00014 72.400 .928240 .00014 74.900 .930290 .00014 77.400 .931270 .00014 79.900 .933190 .00013 82.400 .934170 .00014 84.900 .935670 .00013 87.400 .936650 .00013 89.900 .937690 .00012 92.400 .938900 .00012 94.900 .939920 .00012 97.400 .940880 .00012 99.900 .941980 .00012 102.400 .942150 .00012 104.900 .942680 .00012 107.400 .943740 .00012 109.900 .944000 .00012 112.400 .944980 .00012 114.900 .945520 .00011 117.400 .945920 .00012 119.900 .946790 .00012

25

16.200 .924280 .00032 16.300 .926600 .00032 16.400 .929410 .00034 16.500 .932030 .00033 16.600 .935730 .00033 16.700 .937750 .00034 16.800 .941310 .00031 16.900 .943500 .00032 17.000 .946510 .00033 17.100 .949770 .00033 17.200 .951830 .00032 17.300 .954150 .00033 17.400 .957540 .00033 17.500 .959560 .00032 17.600 .961780 .00032 17.700 .964430 .00032 17.800 .967070 .00032 17.900 .969860 .00032 18.000 .972390 .00032 18.100 .974340 .00031 18.200 .977410 .00031 18.300 .979260 .00032
15

30.100 .928540 .00022 31.100 .935270 .00022 32.100 .941270 .00022 33.100 .947630 .00021 34.100 .953520 .00021 35.100 .958940 .00021 36.100 .963450 .00020 37.100 .967820 .00021 38.100 .972970 .00020 39.100 .977380 .00019 40.100 .980590 .00020 41.100 .984350 .00019 42.100 .987920 .00020 43.100 .990950 .00019 44.100 .994480 .00019 45.100 .997290 .00019 46.101 .000700 .00019 47.101 .002030 .00018 48.101 .004970 .00018 49.101 .006860 .00017 50.101 .008600 .00018 50.901 .011130 .00018
20

19.700 .923510 .00028 20.000 .930430 .00029 20.300 .935580 .00029 20.600 .941090 .00029 20.900 .947020 .00029 21.200 .951400 .00028 21.500 .956710 .00028 21.800 .961460 .00028 22.100 .965780 .00028 22.400 .970570 .00028 22.700 .974900 .00028 23.000 .979980 .00028 23.300 .984610 .00027 23.600 .987770 .00028 23.900 .991990 .00028 24.200 .995230 .00027 24.501 .000100 .00027 24.801 .003290 .00026 25.101 .007580 .00026 25.401 .011300 .00026
14.300 .921490 .00036 14.400 .925920 .00035 14.500 .929800 .00036 14.600 .932190 .00036 14.700 .936590 .00035 14.800 .939820 .00036 14.900 .943450 .00035 15.000 .947300 .00035 15.100 .949950 .00034 15.200 .954680 .00035 15.300 .956850 .00035 15.400 .959830 .00035 15.500 .963810 .00035 15.600 .967520 .00036 15.700 .970840 .00035 15.800 .974000 .00035 15.900 .976660 .00035 16.000 .980470 .00036 16.100 .983320 .00035 16.200 .986220 .00036 16.300 .990110 .00034 16.400 .991870 .00034
32

13.800 .922260 .00036 13.900 .926700 .00036 14.000 .930330 .00036 14.100 .934950 .00036 14.200 .937980 .00036 14.300 .942200 .00036 14.400 .946030 .00036 14.500 .950200 .00036 14.600 .953430 .00036 14.700 .957690 .00036 14.800 .961310 .00037 14.900 .964490 .00036 15.000 .968210 .00036 15.100 .971410 .00036 15.200 .975860 .00036 15.300 .978620 .00035 15.400 .982700 .00035 15.500 .985430 .00037 15.600 .988450 .00036 15.700 .992510 .00036 15.800 .995130 .00035 15.900 .998980 .00035 
18.400 .981650 .00032 18.500 .984730 .00031 18.600 .986380 .00032 18.700 .988180 .00032 18.800 .991190 .00032 18.900 .993230 .00032 19.000 .995410 .00032 19.100 .997580 .00033 19.201 .000320 .00032 19.301 .001850 .00030 19.401 .004210 .00033 19.501 .006480 .00032 19.601 .008390 .00031 19.701 .010280 .00031 19.801 .013150 .00031 19.901 .014870 .00031

35 13.100 .921290 .00036 13.200 .924290 .00037 13.300 .928770 .00038 13.400 .933700 .00037 13.500 .937740 .00038 13.600 .942070 .00037 13.700 .945460 .00036 13.800 .950670 .00037 13.900 .953890 .00037 14.000 .958470 .00039 14.100 .962160 .00037 14.200 .966360 .00038 14.300 .969300 .00037 14.400 .973960 .00037 14.500 .977600 .00037 14.600 .981310 .00037 14.700 .985300 .00037 14.800 .988200 .00038 14.900 .992610 .00038 15.000 .996160 .00038 15.100 .999390 .00037 15.201 .003750 .00036 15.301 .006450 .00038 15.401 .009970 .00036 15.501 .013450 .00037 15.601 .016960 .00035
16.500 .995790 .00035 16.600 .998370 .00035 16.701 .001510 .00034 16.801 .003850 .00034 16.901 .007310 .00034 17.001 .010070 .00035 17.101 .013140 .00034 17.201 .016120 .00034
16.001 .002280 .00036 16.101 .005030 .00036 16.201 .008180 .00035 16.301 .012350 .00034 16.401 .015070 .00034 16.501 .017320 .00035
12.300 .920660 .00038

12.400 .925080 .00038

12.500 .931000 .00039

12.600 .935250 .00040

12.700 .939660 .00038

12.800 .944410 .00039

12.900 .949200 .00038

13.000 .954060 .00038

13.100 .958410 .00040

13.200 .963320 .00038

13.300 .967650 .00039

13.400 .971890 .00038

13.500 .975730 .00039

13.600 .981060 .00038

13.700 .984350 .00039

13.800 .989370 .00039

13.900 .993370 .00040

14.000 .997660 .00039

14.101 .001580 .00038

14.201 .005420 .00039

14.301 .009770 .00038

14.401 .013530 .00038

14.501 .018060 .00039
50

11.300 .922430 .00041 11.400 .928410 .00040 11.500 .933980 .00042 11.600 .939490 .00041 11.700 .946200 .00042 11.800 .951610 .00042 11.900 .957010 .00042 12.000 .962130 .00041 12.100 .967510 .00041 12.200 .973080 .00040 12.300 .976960 .00041 12.400 .983010 .00041 12.500 .988110 .00040 12.600 .992160 .00041 12.700 .997800 .00042 12.801 .003290 .00041 12.901 .007550 .00040 13.001 .012810 .00041 13.101 .017120 .00041 
60

10.600 .920670 .00043

10.650 .923660 .00043

10.700 .928010 .00043

10.750 .931160 .00044

10.800 .934400 .00043

10.850 .938210 .00042

10.900 .940600 .00043

10.950 .942870 .00042

11.000 .945930 .00041

11.050 .949090 .00042

11.100 .951870 .00043

11.150 .955440 .00043

11.200 .957840 .00042

11.250 .961180 .00044

11.300 .963810 .00042

11.350 .967900 .00042

11.400 .970060 .00043

11.450 .972870 .00042

11.500 .976770 .00042

11.550 .978700 .00043

11.600 .981830 .00043

11.650 .984340 .00042

11.700 .986540 .00043

11.750 .989760 .00043

11.800 .993120 .00043

11.850 .996170 .00042

11.900 .998670 .00042

11.951 .001020 .00042

12.001 .004320 .00043

12.051 .006730 .00042

12.101 .009440 .00043

12.151 .011600 .00043

12.201 .014100 .00042

12.251 .017240 .00044

12.301 .019820 .00043
70

10.100 .918070 .00045

10.150 .920990 .00045

10.200 .924440 .00044

10.250 .928390 .00044

10.300 .931590 .00042

10.350 .935100 .00043

10.400 .938170 .00043

10.450 .941430 .00044

10.500 .944550 .00045

10.550 .948270 .00044

10.600 .951820 .00043

10.650 .954050 .00044

10.700 .958120 .00043

10.750 .960880 .00043

10.800 .965040 .00043

10.850 .967150 .00044

10.900 .971030 .00044

10.950 .974290 .00044

11.000 .976390 .00044

11.050 .980110 .00044

11.100 .984540 .00045

11.150 .986900 .00045

11.200 .989880 .00043

11.250 .992380 .00044

11.300 .995340 .00043

11.350 .998830 .00046

11.401 .001190 .00044

11.451 .003650 .00044

11.501 .007250 .00044

11.551 .010760 .00044

11.601 .013310 .00044

11.651 .015710 .00045

11.701 .018260 .00045
80

9.800 .920980 .00045

9.850 .923120 .00045

9.900 .927340 .00045

9.950 .931320 .00045

10.000 .934710 .00045

10.050 .938260 .00044

10.100 .941790 .00045

10.150 .946440 .00046

10.200 .948980 .00045

10.250 .952830 .00045

10.300 .955660 .00044

10.350 .959210 .00045

10.400 .962920 .00044

10.450 .965300 .00046

10.500 .969410 .00045

10.550 .972450 .00045

10.600 .976160 .00046

10.650 .979010 .00045

10.700 .983120 .00044

10.750 .986280 .00045

10.800 .988710 .00045

10.850 .992070 .00045

10.900 .994700 .00046

10.950 .999180 .00045

11.001 .001620 .00045

11.051 .004700 .00045

11.101 .008560 .00045

11.151 .011130 .00045

11.201 .014030 .00044

11.251 .017360 .00045

11.301 .020090 .00044
90

9.500 .916890 .00046

9.550 .921010 .00044

9.600 .924690 .00046

9.650 .928620 .00045

9.700 .931640 .00046

9.750 .936220 .00045

9.800 .938230 .00045

9.850 .943670 .00047

9.900 .947300 .00046

9.950 .949810 .00045

10.000 .954460 .00046
100

9.300 .917160 .00046

9.350 .920460 .00046

9.400 .924410 .00046

9.450 .928130 .00046

9.500 .933310 .00047

9.550 .936030 .00047

9.600 .940490 .00047

9.650 .944450 .00046

9.700 .947260 .00046

9.750 .950010 .00046

9.800 .954150 .00045
118

9.000 .913810 .00047

9.050 .918060 .00046

9.100 .922360 .00046

9.150 .926300 .00045

9.200 .930410 .00046

9.250 .934430 .00046

9.300 .938040 .00047

9.350 .941800 .00047

9.400 .945820 .00047

9.450 .950220 .00048

9.500 .954080 .00046 
10.050 .956820 .00045 10.100 .960500 .00047 10.150 .964910 .00046 10.200 .967630 .00046 10.250 .970760 .00045 10.300 .973840 .00044 10.350 .978920 .00046 10.400 .981190 .00045 10.450 .985280 .00047 10.500 .988500 .00047 10.550 .992540 .00047 10.600 .995960 .00046 10.650 .997970 .00046 10.701 .001860 .00045 10.751 .005130 .00046 10.801 .008360 .00047 10.851 .011440 .00046 10.901 .014900 .00045 10.951 .017790 .00047 11.001 .019350 .00046

130

8.900 .917440 .00049 8.950 .922010 .00047 9.000 .926070 .00047 9.050 .928850 .00048 9.100 .933500 .00047 9.150 .938060 .00046 9.200 .942560 .00048 9.250 .946430 .00048 9.300 .950180 .00047 9.350 .953770 .00047 9.400 .957480 .00045 9.450 .960340 .00047 9.500 .965870 .00048 9.550 .969990 .00047 9.600 .973060 .00047 9.650 .976340 .00048 9.700 .980660 .00046 9.750 .984920 .00047 9.800 .987940 .00047 9.850 .992250 .00047 9.900 .995900 .00048 9.950 .997890 .00048 10.001 .002500 .00047 10.051 .006180 .00048 10.101 .010180 .00047 10.151 .012790 .00047 10.201 .017070 .00047 10.251 .021450 .00047 10.301 .024410 .00047
9.850 .958150 .00045

9.900 .961780 .00046

9.950 .965090 .00047

10.000 .969730 .00046

10.050 .972170 .00046

10.100 .976580 .00046

10.150 .979340 .00046

10.200 .983090 .00045

10.250 .986010 .00046

10.300 .990730 .00046

10.350 .993740 .00046

10.400 .997100 .00046

10.451 .000650 .00045

10.501 .004400 .00048

10.551 .008010 .00047

10.601 .010160 .00046

10.651 .014050 .00047

10.701 .018090 .00047
9.550 .957200 .00048

9.600 .960850 .00047

9.650 .965820 .00046

9.700 .969480 .00047

9.750 .972590 .00047

9.800 .977040 .00046

9.850 .979380 .00048

9.900 .983130 .00046

9.950 .987560 .00047 10.000 .992410 .00047 10.050 .993710 .00046 10.100 .997750 .00047 10.151 .001410 .00046 10.201 .005360 .00046 10.251 .008860 .00046 10.301 .012510 .00047 10.351 .015860 .00047 10.401 .018950 .00047
150

8.700 .914890 .00046

8.750 .918430 .00048

8.800 .922560 .00048

8.850 .928430 .00047

8.900 .931710 .00048

8.950 .935950 .00049

9.000 .939250 .00048

9.050 .944240 .00048

9.100 .948630 .00048

9.150 .952510 .00048

9.200 .957050 .00047

9.250 .960660 .00049

9.300 .964070 .00049

9.350 .968520 .00048

9.400 .971880 .00048

9.450 .975900 .00048

9.500 .980250 .00048

9.550 .982920 .00047

9.600 .987120 .00048

9.650 .991030 .00049

9.700 .994070 .00050

9.750 .999170 .00047

9.801 .002330 .00049

9.851 .005330 .00047

9.901 .009580 .00049

9.951 .013800 .00049 10.001 .016340 .00047 10.051 .022050 .00050 10.101 .024250 .00049
200

8.500 .920490 .00047 8.550 .924840 .00048 8.600 .930540 .00048 8.650 .933550 .00047 8.700 .938350 .00049 8.750 .941520 .00048 8.800 .946040 .00049 8.850 .950660 .00049 8.900 .954750 .00049 8.950 .958270 .00049 9.000 .962750 .00049 9.050 .967110 .00050 9.100 .970270 .00047 9.150 .975380 .00048 9.200 .978970 .00050 9.250 .983400 .00050 9.300 .987240 .00050 9.350 .990430 .00049 9.400 .994800 .00048 9.450 .998130 .00050 9.501 .002220 .00049 9.551 .006990 .00050 9.601 .009870 .00050 9.651 .013900 .00050 9.701 .017510 .00049 9.751 .021960 .00049 9.801 .024830 .00049 
235

8.400 .920380 .00049 8.450 .926460 .00048 8.500 .929650 .00048 8.550 .934280 .00048 8.600 .937490 .00047 8.650 .942310 .00049 8.700 .945650 .00049 8.750 .951040 .00048 8.800 .955740 .00048 8.850 .959950 .00048 8.900 .963800 .00049 8.950 .967850 .00049 9.000 .971900 .00050 9.050 .975650 .00049 9.100 .979770 .00048 9.150 .983960 .00049 9.200 .987190 .00048 9.250 .992370 .00051 9.300 .995430 .00049 9.350 .999800 .00049 9.401 .002860 .00049 9.451 .008160 .00049 9.501 .011070 .00050 9.551 .014470 .00048 9.601 .018820 .00049 9.651 .022030 .00048 9.701 .026110 .00049

\section{0}

8.300 .922820 .00051

8.350 .926260 .00049

8.400 .931970 .00048

8.450 .935540 .00049

8.500 .939650 .00050

8.550 .944730 .00050

8.600 .947920 .00049

8.650 .952010 .00050

8.700 .956260 .00048

8.750 .958940 .00050

8.800 .963730 .00049

8.850 .967380 .00049

8.900 .971040 .00049

8.950 .976910 .00051

9.000 .979680 .00048

9.050 .983780 .00048

9.100 .986720 .00051

9.150 .991240 .00049

9.200 .996830 .00048
250

8.300 .915520 .00048

8.350 .919460 .00049

8.400 .923740 .00048

8.450 .928630 .00049

8.500 .932460 .00050

8.550 .936740 .00049

8.600 .940870 .00049

8.650 .945540 .00049

8.700 .950000 .00049

8.750 .954190 .00051

8.800 .958840 .00048

8.850 .962130 .00050

8.900 .965480 .00050

8.950 .970640 .00051

9.000 .973680 .00049

9.050 .979230 .00050

9.100 .982160 .00048

9.150 .985990 .00049

9.200 .990690 .00049

9.250 .993960 .00049

9.300 .997790 .00050

9.351 .002680 .00048

9.401 .006700 .00050

9.451 .010290 .00048

9.501 .013450 .00049

9.551 .018240 .00049

9.601 .021460 .00049
353

8.200 .914310 .00049

8.250 .920280 .00050

8.300 .923020 .00048

8.350 .928300 .00049

8.400 .932500 .00049

8.450 .936570 .00049

8.500 .941330 .00049

8.550 .946140 .00048

8.600 .948990 .00050

8.650 .953440 .00049

8.700 .956830 .00049

8.750 .961500 .00050

8.800 .965590 .00049

8.850 .969460 .00049

8.900 .974220 .00050

8.950 .978710 .00050

9.000 .981690 .00049

9.050 .986780 .00050

9.100 .990110 .00050

9.150 .994040 .00048

9.200 .997810 .00049

9.251 .001680 .00049

9.301 .005790 .00050

9.351 .010650 .00049

9.401 .013760 .00050

9.451 .018090 .00050

9.501 .020850 .00050
500
8.300 .920210 .00048

8.350 .924830 .00049

8.400 .928460 .00050

8.450 .933020 .00050

8.500 .937090 .00049

8.550 .941290 .00052

8.600 .946270 .00048

8.650 .949580 .00050

8.700 .954270 .00048

8.750 .956540 .00049

8.800 .961780 .00049

8.850 .966100 .00050

8.900 .969410 .00050

8.950 .973640 .00049

9.000 .977790 .00050

9.050 .980820 .00049

9.100 .985040 .00050

9.150 .989320 .00048

9.200 .992990 .00049
600

8.400 .921740 .00048

8.450 .926330 .00051

8.500 .930290 .00047

8.550 .935090 .00048

8.600 .938010 .00048

8.650 .941600 .00049

8.700 .945830 .00050

8.750 .950250 .00049

8.800 .954570 .00050

8.850 .958570 .00049

8.900 .961570 .00050

8.950 .965900 .00050

9.000 .969960 .00048

9.050 .973190 .00050

9.100 .977380 .00049

9.150 .980760 .00049

9.200 .984700 .00050

9.250 .988990 .00048

9.300 .991170 .00049 
9.250 .999440 .00048 9.301 .002570 .00048 9.351 .007080 .00049 9.401 .010720 .00050 9.451 .015190 .00049 9.501 .018920 .00049

605

8.400 .921500 .00049 8.450 .925960 .00049 8.500 .930980 .00049 8.550 .933990 .00049 8.600 .937950 .00049 8.650 .942070 .00047 8.700 .946300 .00049 8.750 .949680 .00049 8.800 .953780 .00050 8.850 .956950 .00050 8.900 .961310 .00048 8.950 .965350 .00050 9.000 .968680 .00049 9.050 .974070 .00049 9.100 .977190 .00049 9.150 .981170 .00049 9.200 .984780 .00048 9.250 .987390 .00049 9.300 .991350 .00050 9.350 .994870 .00049 9.400 .999990 .00049 9.451 .002550 .00049 9.501 .006630 .00050 9.551 .009600 .00050 9.601 .013980 .00049 9.651 .017300 .00050 9.701 .022170 .00049

\section{0}

8.400 .921190 .00047 8.450 .925080 .00048 8.500 .929010 .00047 8.550 .932100 .00048 8.600 .936690 .00049 8.650 .940370 .00049
9.250 .996530 .00047

9.301 .000810 .00049

9.351 .003950 .00049

9.401 .007440 .00048

9.451 .012240 .00049

9.501 .015260 .00051

9.551 .018960 .00049

9.601 .022390 .00049
9.350 .996150 .00048

9.400 .998400 .00050

9.451 .002840 .00050

9.501 .006970 .00049

9.551 .010040 .00049

9.601 .014180 .00050 9.651 .017580 .00047 9.701 .020980 .00050
8.300 .913050 .00048

8.350 .917020 .00049

8.400 .921540 .00048

8.450 .924990 .00049

8.500 .929700 .00048

8.550 .933800 .00048

8.600 .938010 .00049

8.650 .942210 .00049

8.700 .945540 .00049

8.750 .949620 .00049

8.800 .953890 .00049

8.850 .957820 .00050

8.900 .961450 .00048

8.950 .965960 .00048

9.000 .969140 .00049

9.050 .973170 .00049

9.100 .976780 .00050

9.150 .979870 .00049

9.200 .984260 .00050

9.250 .988010 .00049

9.300 .990670 .00051

9.350 .994590 .00048

9.400 .999110 .00049

9.451 .001950 .00048

9.501 .006520 .00048

9.551 .010050 .00047

9.601 .013160 .00049

9.651 .017040 .00050

9.701 .020520 .00050
615

8.400 .921210 .00050 8.450 .924650 .00047 8.500 .928320 .00050 8.550 .933090 .00047 8.600 .935940 .00049 8.650 .940340 .00049 8.700 .944640 .00048 8.750 .949430 .00049 8.800 .952540 .00049 8.850 .956700 .00049 8.900 .959710 .00050 8.950 .964100 .00050 9.000 .968050 .00049 9.050 .972040 .00049 9.100 .976330 .00048 9.150 .980310 .00049 9.200 .983420 .00049 9.250 .986820 .00050 9.300 .990750 .00050 9.350 .994780 .00048 9.400 .997800 .00048 9.451 .000990 .00049 9.501 .005890 .00049 9.551 .009580 .00048 9.601 .012830 .00049 9.651 .015840 .00049 9.701 .020210 .00049
625

8.400 .920130 .00048

8.450 .924180 .00050

8.500 .927920 .00049

8.550 .931480 .00049

8.600 .935760 .00048

8.650 .940250 .00049
629

8.400 .918840 .00049 8.450 .923370 .00048 8.500 .926950 .00050 8.550 .932000 .00048 8.600 .936270 .00049 8.650 .938220 .00049 
8.700 .944320 .00048

8.750 .948190 .00048

8.800 .952340 .00049

8.850 .956740 .00049

8.900 .960260 .00049

8.950 .963300 .00050

9.000 .967850 .00049

9.050 .971610 .00050

9.100 .975420 .00051

9.150 .979220 .00049

9.200 .983310 .00049

9.250 .986410 .00047

9.300 .990000 .00049

9.350 .993260 .00049

9.400 .997260 .00048

9.451 .002020 .00048

9.501 .005750 .00050

9.551 .008890 .00049

9.601 .011170 .00048

9.651 .015600 .00050

9.701 .019140 .00050

U5o2N23-sp-0935-00

12

107.100 .895000 .00012

112.100 .895930 .00012

117.100 .897600 .00013

122.100 .898680 .00012

127.100 .899480 .00012

132.100 .900070 .00012

137.100 .900950 .00012

142.100 .901440 .00012

147.100 .901870 .00011

152.100 .902510 .00012

157.100 .902950 .00012

162.100 .903460 .00011

167.100 .903380 .00011

172.100 .903470 .00011

177.100 .904100 .00011

182.100 .904900 .00011

187.100 .904400 .00011

192.100 .905010 .00011

197.100 .905370 .00011

202.100 .905410 .00011

207.100 .905770 .00011 207.600 .905590 .00011
8.700 .944640 .00048

8.750 .949420 .00050

8.800 .952660 .00050

8.850 .956520 .00048

8.900 .959710 .00048

8.950 .964300 .00049

9.000 .968290 .00049

9.050 .971850 .00047

9.100 .975040 .00049

9.150 .979410 .00050

9.200 .982720 .00049

9.250 .985370 .00048

9.300 .989890 .00050

9.350 .993780 .00049

9.400 .997980 .00051

9.451 .001540 .00049

9.501 .004470 .00048

9.551 .008300 .00049

9.601 .011200 .00049

9.651 .014880 .00049

9.701 .018590 .00050
8.800 .951540 .00048

8.850 .954820 .00047

8.900 .959800 .00049

8.950 .961850 .00048

9.000 .966990 .00048

9.050 .970910 .00049

9.100 .974190 .00048

9.150 .978710 .00050

9.200 .982120 .00049

9.250 .985420 .00048

9.300 .989890 .00050

9.350 .992570 .00049

9.400 .995760 .00049

9.451 .000010 .00049

9.501 .003680 .00051

9.551 .008090 .00049

9.601 .011460 .00050

9.651 .014940 .00051

9.701 .017310 .00049
41.800 .928780 .00021

42.800 .933090 .00021

43.800 .936650 .00020

44.800 .939790 .00021

45.800 .942880 .00020

46.800 .945290 .00020

47.800 .949530 .00020

48.800 .952020 .00019

49.800 .953930 .00019

50.800 .956390 .00019

51.800 .958240 .00018

52.800 .961100 .00018

53.800 .962830 .00018

54.800 .965160 .00018

55.800 .967330 .00018

56.800 .969310 .00017

57.800 .970850 .00018

58.800 .972260 .00017

59.800 .973870 .00018

60.800 .974600 .00017

61.800 .976890 .00017

62.800 .978270 .00017

62.900 .978090 .00017
20

25.200 .926070 .00031 25.600 .931700 .00029 26.000 .937020 .00029 26.400 .943120 .00029 26.800 .947560 .00028 27.200 .953170 .00029 27.600 .958040 .00029 28.000 .963020 .00028 28.400 .967240 .00028 28.800 .972350 .00028 29.200 .976520 .00027 29.600 .980300 .00028 30.000 .984070 .00027 30.400 .988170 .00027 30.800 .991770 .00026 31.200 .995150 .00027 31.600 .999900 .00027 32.001 .002660 .00027 32.401 .006110 .00027 32.801 .009470 .00026 33.101 .011130 .00025 
25

20.500 .922600 .00034 20.700 .927590 .00034 20.900 .931480 .00035 21.100 .935940 .00034 21.300 .940740 .00035 21.500 .945290 .00034 21.700 .949810 .00033 21.900 .954000 .00034 22.100 .958430 .00033 22.300 .962460 .00034 22.500 .966430 .00034 22.700 .970550 .00033 22.900 .975220 .00033 23.100 .978450 .00034 23.300 .982860 .00033 23.500 .986590 .00033 23.700 .989850 .00034 23.900 .993810 .00033 24.100 .997290 .00033 24.301 .000870 .00032 24.501 .004070 .00032 24.701 .007140 .00033 24.901 .010940 .00032 25.101 .013830 .00032 25.201 .015380 .00032
35

16.900 .923190 .00040 17.000 .926820 .00039 17.100 .930460 .00039 17.200 .933770 .00041 17.300 .937670 .00040 17.400 .941800 .00041 17.500 .946480 .00040 17.700 .951250 .00040 17.800 .955280 .00041 17.900 .959480 .00041 18.000 .963120 .00041 18.100 .966760 .00039 18.200 .969670 .00040
30

18.300 .923720 .00038 18.400 .927270 .00038 18.500 .930540 .00038 18.600 .934100 .00037 18.700 .936360 .00038 18.800 .939200 .00036 19.000 .945400 .00038 19.200 .951260 .00037 19.300 .954880 .00037 19.400 .957330 .00037 19.500 .959880 .00037 19.600 .962440 .00037 19.700 .965240 .00036 19.800 .968450 .00036 19.900 .971600 .00037 20.100 .976240 .00036 20.200 .979680 .00037 20.300 .981800 .00037 20.600 .989300 .00037 20.700 .991340 .00037 20.800 .994550 .00037 20.900 .996750 .00037 21.000 .999660 .00037 21.101 .001700 .00036 21.201 .004440 .00037 21.301 .007120 .00036 21.401 .009040 .00036 21.501 .011460 .00036 21.601 .013420 .00035 21.701 .016230 .00035 21.801 .017940 .00035

40

15.900 .920420 .00042 16.000 .924740 .00043 16.100 .928830 .00042 16.200 .934520 .00043 16.300 .937420 .00042 16.400 .941830 .00041 16.500 .946060 .00042 16.600 .949570 .00042 16.700 .954370 .00042 16.800 .958820 .00041 16.900 .962050 .00041 17.000 .964980 .00042 17.100 .969700 .00042
32

17.600 .921030 .00039 17.700 .925020 .00040 17.800 .928430 .00039 17.900 .930720 .00038 18.000 .935610 .00038 18.100 .937400 .00039 18.200 .941310 .00039 18.300 .944300 .00039 18.400 .948550 .00038 18.500 .951200 .00038 18.600 .954280 .00039 18.700 .957840 .00039 18.800 .960520 .00039 18.900 .963980 .00039 19.000 .967160 .00039 19.100 .969340 .00038 19.200 .972270 .00038 19.300 .976320 .00039 19.400 .977810 .00037 19.500 .981270 .00038 19.700 .987560 .00038 19.800 .989920 .00038 19.900 .992480 .00038 20.000 .995510 .00037 20.100 .998380 .00037 20.201 .000850 .00037 20.501 .009090 .00038 20.601 .011540 .00037 20.701 .014320 .00037 20.801 .016680 .00036

50

14.800 .924980 .00045 14.900 .929180 .00045 15.000 .935030 .00046 15.100 .939980 .00045 15.200 .945270 .00044 15.300 .949670 .00044 15.400 .954290 .00045 15.500 .959610 .00045 15.700 .969360 .00045 15.900 .978760 .00044 16.000 .982710 .00046 16.100 .987550 .00044 16.200 .991700 .00044 
18.300 .973480 .00040 18.500 .980360 .00039 18.600 .982390 .00039 18.700 .985980 .00040 18.800 .989200 .00040 18.900 .992080 .00038 19.100 .998480 .00039 19.301 .005170 .00038 19.401 .007610 .00038 19.501 .010570 .00039 19.601 .013110 .00039 19.701 .016470 .00039

60

13.900 .915330 .00047 14.000 .921640 .00048 14.100 .928060 .00048 14.200 .933470 .00046 14.300 .938400 .00048 14.400 .943990 .00046 14.500 .948930 .00047 14.600 .955010 .00045 14.700 .960010 .00047 14.800 .965290 .00048 14.900 .970790 .00047 15.000 .976620 .00046 15.100 .980420 .00048 15.200 .985670 .00047 15.300 .991280 .00047 15.400 .997000 .00046 15.501 .001940 .00046 15.601 .005620 .00047 15.701 .011140 .00046 15.801 .016240 .00047 15.901 .019690 .00045
17.200 .973690 .00041

17.300 .977660 .00040

17.400 .981240 .00041

17.500 .985840 .00040

17.600 .989370 .00041

17.700 .992190 .00042

17.800 .995390 .00042

17.901 .000290 .00041

18.001 .003430 .00042

18.101 .006490 .00041

18.201 .010170 .00042

18.301 .013400 .00041

18.401 .017260 .00042

18.501 .020150 .00041

70

13.500 .921620 .00050

13.600 .927550 .00049

13.700 .933170 .00049

13.800 .939410 .00048

13.900 .945540 .00048

14.000 .951800 .00049

14.100 .956980 .00048

14.200 .961970 .00048

14.300 .968840 .00048

14.400 .973500 .00047

14.500 .980350 .00048

14.600 .986320 .00047

14.800 .996150 .00050

14.901 .001710 .00050

15.001 .007440 .00049

15.101 .012850 .00048

15.201 .017990 .00049

15.301 .022200 .00049

16.300 .996960 .00045

16.401 .000580 .00042

16.501 .004970 .00044

16.601 .009100 .00045

16.701 .014040 .00044

16.801 .017860 .00045

80
13.100 .918430 .00050

13.150 .921780 .00049

13.200 .923750 .00050

13.250 .928830 .00049

13.300 .931880 .00048

13.350 .935060 .00049

13.400 .938290 .00050

13.450 .940290 .00049

13.500 .944060 .00050

13.550 .946970 .00049

13.600 .950570 .00051

13.650 .953340 .00049

13.700 .957480 .00049

13.750 .959570 .00050

13.800 .962040 .00050

13.850 .965640 .00051

13.900 .968560 .00048

13.950 .971800 .00049

14.000 .974310 .00050

14.050 .977060 .00049

14.100 .981260 .00049

14.150 .983650 .00051

14.200 .986820 .00049

14.250 .989840 .00050

14.300 .992490 .00048

14.350 .994950 .00049

14.400 .998180 .00051

14.451 .002010 .00050

14.501 .003630 .00049

14.551 .006290 .00048

14.601 .010260 .00050

14.651 .011860 .00050

14.701 .015480 .00051

14.751 .017380 .00050

14.801 .019940 .00049 
90

12.800 .916020 .00051

12.850 .919690 .00050

12.900 .923590 .00050

12.950 .925760 .00049

13.000 .930450 .00051

13.050 .933540 .00050

13.100 .936960 .00051

13.150 .940250 .00052

13.200 .943340 .00050

13.250 .946780 .00050

13.300 .949340 .00050

13.350 .953110 .00050

13.400 .956520 .00051

13.450 .959290 .00049

13.500 .962920 .00050

13.550 .965790 .00050

13.650 .972090 .00051

13.750 .978390 .00049

13.800 .982470 .00051

13.850 .985300 .00051

13.900 .987080 .00051

13.950 .990900 .00051

14.000 .994970 .00051

14.050 .996330 .00050

14.101 .000770 .00049

14.151 .003070 .00051

14.201 .006200 .00052

14.251 .010430 .00052

14.301 .011490 .00051

14.351 .014910 .00050

14.401 .017420 .00051

14.451 .019970 .00050

14.501 .024070 .00052
130

12.200 .915870 .00054

12.250 .921180 .00053

12.300 .923570 .00053

12.350 .927600 .00052

12.400 .930100 .00053

12.450 .934900 .00052

12.500 .938570 .00054

12.550 .942090 .00052
100

12.600 .917730 .00050

12.650 .920420 .00052

12.700 .923880 .00053

12.750 .928780 .00050

12.800 .931230 .00051

12.850 .934530 .00051

12.900 .938390 .00052

12.950 .940350 .00050

13.000 .944320 .00051

13.050 .948710 .00052

13.100 .952280 .00051

13.150 .955000 .00051

13.200 .958780 .00050

13.250 .962410 .00052

13.300 .963880 .00051

13.350 .968310 .00052

13.400 .971130 .00053

13.450 .974530 .00052

13.500 .977330 .00051

13.550 .980210 .00051

13.600 .983580 .00052

13.650 .987880 .00050

13.700 .992230 .00052

13.750 .993090 .00052

13.800 .996590 .00051

13.851 .000600 .00051

13.901 .002620 .00053

13.951 .005850 .00051

14.001 .009080 .00052

14.051 .010980 .00050

14.101 .014820 .00052

14.151 .018250 .00051

14.201 .020770 .00052

14.251 .022920 .00052

14.301 .026900 .00052

150

12.000 .913340 .00053

12.050 .917650 .00052

12.100 .921440 .00054

12.150 .924500 .00054

12.200 .929700 .00053

12.250 .932860 .00052

12.300 .936100 .00053

12.350 .939900 .00054
118

12.300 .914230 .00052

12.350 .918550 .00052

12.400 .921460 .00053

12.450 .925270 .00053

12.500 .929240 .00053

12.550 .932110 .00054

12.600 .935280 .00054

12.650 .939830 .00052

12.700 .942610 .00053

12.750 .946580 .00052

12.800 .951160 .00052

12.850 .952950 .00052

12.900 .957040 .00052

12.950 .960710 .00053

13.000 .963920 .00052

13.050 .967710 .00053

13.100 .970300 .00053

13.150 .974880 .00054

13.200 .977450 .00053

13.250 .981330 .00052

13.300 .984230 .00052

13.350 .987380 .00053

13.400 .991040 .00053

13.450 .994120 .00051

13.500 .998310 .00053

13.551 .001240 .00053

13.601 .003590 .00054

13.651 .006760 .00052

13.701 .009240 .00051

13.751 .012130 .00053

13.801 .015470 .00053

13.851 .019260 .00052

13.901 .022130 .00053

200

11.800 .914740 .00056

11.850 .918190 .00054

11.900 .922770 .00054

11.950 .926870 .00054

12.050 .934240 .00054

12.100 .937920 .00054

12.150 .942840 .00054

12.200 .946690 .00054 
12.600 .945920 .00054

12.650 .950470 .00054

12.700 .953030 .00052

12.750 .956810 .00053

12.800 .960410 .00053

12.850 .963720 .00053

12.900 .966540 .00054

13.000 .973900 .00053

13.050 .976810 .00052

13.100 .979940 .00053

13.200 .987610 .00053

13.250 .992270 .00054

13.300 .994850 .00054

13.350 .997670 .00053

13.401 .001320 .00054

13.451 .005460 .00053

13.501 .007400 .00053

13.551 .011190 .00054

13.601 .014030 .00054

13.651 .017050 .00052

13.701 .020690 .00053

235

11.700 .913930 .00055

11.750 .917620 .00053

11.800 .921750 .00054

11.850 .925250 .00056

11.900 .929670 .00055

11.950 .933550 .00053

12.000 .937120 .00056

12.050 .939930 .00055

12.100 .945170 .00055

12.150 .949410 .00054

12.200 .952680 .00056

12.250 .956070 .00055

12.300 .959950 .00055

12.350 .962880 .00055

12.400 .968640 .00054

12.450 .971490 .00054

12.500 .974940 .00054

12.550 .977220 .00054

12.600 .981570 .00055

12.650 .986800 .00055

12.700 .989360 .00054

12.750 .993400 .00055

12.800 .995840 .00056

12.851 .000750 .00057
12.400 .942780 .00053

12.450 .946350 .00053

12.500 .951560 .00054

12.550 .954510 .00053

12.600 .958370 .00054

12.650 .961510 .00054

12.700 .965230 .00053

12.750 .969270 .00053

12.800 .972450 .00053

12.850 .975610 .00053

12.900 .980600 .00053

12.950 .981630 .00053

13.000 .987150 .00055

13.050 .990020 .00054

13.100 .992450 .00052

13.150 .997200 .00054

13.200 .999870 .00053

13.251 .003510 .00054

13.301 .007040 .00054

13.351 .010480 .00053

13.401 .013360 .00053

13.451 .016990 .00052

13.501 .020160 .00054
12.250 .949930 .00054

12.300 .953540 .00054

12.350 .957180 .00055

12.400 .961250 .00054

12.450 .964900 .00055

12.500 .969450 .00055

12.550 .972120 .00055

12.600 .976060 .00056

12.650 .979160 .00055

12.700 .983520 .00055

12.750 .986700 .00053

12.800 .990750 .00056

12.850 .993970 .00053

12.900 .997720 .00054

12.951 .000680 .00055

13.001 .004130 .00054

13.101 .012050 .00056

13.151 .014790 .00055

13.201 .017840 .00055

13.251 .021560 .00054

13.301 .024950 .00055
250
11.700 .913750 .00055

11.750 .917350 .00054

11.800 .921970 .00055

11.850 .925970 .00055

11.900 .928870 .00055

11.950 .933460 .00054

12.000 .937480 .00055

12.050 .941400 .00056

12.100 .944980 .00055

12.150 .949590 .00054

12.200 .952800 .00054

12.250 .957720 .00053

12.300 .959520 .00055

12.350 .964720 .00056

12.400 .967760 .00053

12.450 .970730 .00055

12.500 .975410 .00055

12.550 .979770 .00056

12.600 .983750 .00055

12.650 .986400 .00054

12.700 .989430 .00055

12.750 .992790 .00056

12.800 .996880 .00054

12.901 .004850 .00055
353

11.800 .920050 .00055

11.850 .925620 .00055

11.900 .929480 .00054

11.950 .932590 .00056

12.000 .936060 .00054

12.050 .940730 .00055

12.100 .943220 .00055

12.150 .946590 .00056

12.200 .951810 .00055

12.250 .956360 .00054

12.300 .958510 .00056

12.350 .962500 .00055

12.400 .965820 .00056

12.450 .970200 .00055

12.500 .974540 .00056

12.550 .977290 .00055

12.600 .981400 .00055

12.650 .984560 .00055

12.700 .988460 .00057

12.750 .991840 .00055

12.800 .995660 .00057

12.850 .999370 .00054

12.901 .003080 .00055

12.951 .006940 .00055 
12.901 .003460 .00055 12.951 .007380 .00056 13.001 .011710 .00056 13.051 .013610 .00055 13.101 .017950 .00056 13.151 .021990 .00053 13.201 .023870 .00054
12.951 .006630 .00057 13.001 .011650 .00055 13.051 .013900 .00055 13.101 .017870 .00054 13.151 .021320 .00056 13.201 .025220 .00053
13.001 .009910 .00056 13.051 .013310 .00054 13.101 .016720 .00057 13.151 .019610 .00054 13.201 .023320 .00055
470

11.900 .915970 .00055 11.950 .920040 .00053 12.050 .927330 .00053 12.100 .930970 .00055 12.150 .935350 .00054 12.200 .937970 .00053 12.250 .942510 .00054 12.300 .946630 .00055 12.350 .949670 .00054 12.400 .953170 .00055 12.450 .957380 .00054 12.500 .960980 .00054 12.600 .967680 .00054 12.650 .971160 .00054 12.700 .974790 .00054 12.750 .977530 .00054 12.800 .982680 .00055 12.850 .985120 .00055 12.900 .989340 .00055 12.950 .991640 .00055 13.000 .995420 .00056 13.050 .998870 .00055 13.101 .002660 .00054 13.151 .005570 .00054 13.201 .008460 .00054 13.251 .014100 .00056 13.301 .015820 .00054 13.351 .019550 .00056 13.401 .022170 .00054

520 12.000 .913870 .00053 12.100 .920970 .00054 12.200 .929360 .00055 12.250 .931820 .00054 12.300 .936020 .00055
500

12.000 .917640 .00053 12.050 .921110 .00053 12.100 .925090 .00056 12.150 .928560 .00055 12.200 .933410 .00055 12.250 .937640 .00055 12.300 .940080 .00055 12.350 .944070 .00055 12.400 .947480 .00054 12.450 .951260 .00054 12.500 .954840 .00055 12.550 .957600 .00055 12.600 .961960 .00054 12.650 .965090 .00053 12.700 .968640 .00056 12.750 .971880 .00055 12.800 .975160 .00056 12.850 .979770 .00056 12.900 .982860 .00054 12.950 .986250 .00056 13.000 .989810 .00055 13.050 .992410 .00055 13.100 .996450 .00054 13.151 .000280 .00056 13.201 .003090 .00054 13.251 .005770 .00053 13.301 .009460 .00053 13.351 .012850 .00054 13.401 .016810 .00054 13.451 .019970 .00056 13.501 .023240 .00055

530

12.100 .919300 .00053 12.150 .923810 .00053 12.250 .931130 .00053 12.300 .934590 .00054 12.350 .937760 .00053
510 12.000 .915550 .00055 12.050 .918930 .00055 12.100 .924180 .00055 12.150 .926640 .00055 12.200 .930590 .00055 12.250 .933750 .00054 12.300 .938570 .00055 12.350 .941440 .00055 12.400 .945540 .00052 12.450 .948940 .00054 12.550 .957020 .00054 12.600 .960550 .00056 12.650 .962850 .00053 12.700 .967930 .00055 12.750 .969850 .00055 12.800 .973240 .00054 12.850 .976900 .00056 12.900 .981530 .00054 13.000 .987870 .00055 13.050 .991080 .00055 13.100 .994040 .00054 13.150 .998340 .00055 13.201 .000820 .00054 13.251 .004610 .00053 13.301 .007370 .00053 13.351 .011140 .00054 13.401 .015430 .00055 13.451 .017980 .00056 13.501 .020140 .00054

540

12.100 .917570 .00053 12.150 .921270 .00053 12.300 .932010 .00054 12.350 .936600 .00054 12.400 .939800 .00054 
12.350 .940030 .00054

12.400 .945150 .00054

12.450 .947560 .00054

12.500 .951020 .00055

12.550 .954440 .00054

12.600 .957820 .00053

12.650 .961470 .00054

12.700 .965600 .00054

12.750 .969140 .00054

12.800 .972340 .00055

12.850 .974920 .00055

12.900 .979460 .00054

12.950 .983050 .00056

13.000 .987040 .00056

13.050 .989250 .00053

13.100 .992750 .00055

13.150 .994760 .00055

13.200 .999150 .00053

13.251 .003270 .00054

13.301 .006820 .00054

13.351 .009770 .00055

13.401 .012330 .00056

13.451 .016910 .00057

13.501 .019250 .00056

13.551 .022600 .00055

13.601 .024820 .00056
12.400 .942150 .00053

12.450 .946070 .00055

12.500 .949180 .00055

12.550 .952320 .00055

12.600 .956990 .00054

12.650 .959640 .00054

12.700 .963490 .00054

12.750 .966990 .00055

12.800 .970290 .00053

12.850 .973680 .00055

12.900 .976120 .00055

12.950 .981770 .00055

13.000 .984600 .00054

13.050 .986980 .00055

13.100 .991550 .00054

13.150 .994000 .00054

13.200 .997920 .00055

13.251 .000840 .00056

13.301 .003430 .00056

13.351 .007040 .00055

13.401 .010480 .00055

13.451 .012640 .00056

13.501 .018060 .00056

13.551 .020760 .00055

13.601 .024260 .00054
12.450 .944430 .00054

12.500 .946610 .00056

12.550 .951260 .00055

12.600 .954840 .00055

12.650 .957640 .00054

12.700 .962140 .00054

12.750 .965150 .00054

12.800 .968210 .00054

12.850 .971900 .00054

12.900 .976470 .00056

12.950 .978790 .00052

13.000 .982970 .00054

13.050 .985380 .00055

13.100 .988750 .00055

13.150 .993690 .00053

13.200 .995830 .00054

13.250 .999230 .00054

13.301 .002610 .00054

13.351 .005680 .00053

13.401 .008490 .00055

13.451 .011960 .00054

13.501 .016200 .00053

13.551 .018540 .00056

13.601 .021090 .00055

13.651 .024580 .00055

13.701 .027790 .00054
550

12.100 .915180 .00054

12.150 .920220 .00053

12.200 .923710 .00054

12.250 .926490 .00054

12.300 .930280 .00055

12.350 .935010 .00055

12.400 .938910 .00053

12.450 .942020 .00053

12.500 .946120 .00055

12.550 .947630 .00056

12.600 .953430 .00053

12.650 .956370 .00053

12.700 .959140 .00053

12.750 .961950 .00056

12.800 .967660 .00053

12.850 .970820 .00054

12.900 .973010 .00053

12.950 .976290 .00054

13.000 .979880 .00054

13.050 .983940 .00056

13.100 .987330 .00055

\section{0}

12.200 .921940 .00053

12.250 .925950 .00053

12.300 .929310 .00053

12.350 .932650 .00054

12.400 .936170 .00052

12.450 .940400 .00054

12.500 .943390 .00052

12.550 .948330 .00053

12.600 .950770 .00054

12.650 .953890 .00054

12.700 .958620 .00053

12.750 .960400 .00054

12.800 .964570 .00055

12.850 .969120 .00054

12.900 .971020 .00054

12.950 .975750 .00056

13.000 .978740 .00055

13.050 .982470 .00054

13.100 .985070 .00055

13.150 .988800 .00054

13.200 .993050 .00053 
13.150 .990410 .00054 13.200 .993660 .00055 13.250 .997360 .00055 13.301 .000670 .00054 13.351 .003500 .00055 13.401 .008020 .00055 13.451 .010660 .00054 13.501 .012990 .00056 13.551 .018010 .00054 13.601 .019330 .00054 13.651 .024040 .00053 13.701 .025680 .00054

U5o2N23-sp-0935-01

12 144.300 .901450 .00012 148.800 .901890 .00011 153.300 .902050 .00012 157.800 .903000 .00011 162.300 .903230 .00011 166.800 .903250 .00011 171.300 .904010 .00011 175.800 .904060 .00011 180.300 .904730 .00011 184.800 .905270 .00011 189.300 .904990 .00011 193.800 .905090 .00011 198.300 .905430 .00011 202.800 .905000 .00011 207.300 .905470 .00011 211.800 .905910 .00011 216.300 .906110 .00011 220.800 .906050 .00011 225.300 .906330 .00011 229.800 .906440 .00011 234.300 .906470 .00011 234.600 .906500 .00011

25

19.000 .923290 .00033 19.200 .928070 .00034 19.600 .938070 .00033 19.800 .942510 .00033 20.000 .947390 .00033 20.200 .951330 .00033 20.400 .956070 .00032 20.600 .959520 .00033
13.250 .995120 .00054 13.300 .999350 .00055 13.351 .001740 .00055 13.401 .005250 .00053 13.451 .008490 .00054 13.501 .011830 .00054 13.551 .014860 .00054 13.601 .017740 .00055
15
39.600 .926750 .00021 41.100 .932130 .00020 42.600 .937710 .00020 44.100 .942800 .00019 45.600 .946930 .00019 47.100 .951000 .00020 48.600 .954450 .00019 50.100 .958490 .00018 51.600 .961370 .00018 53.100 .964360 .00017 54.600 .967510 .00018 56.100 .969780 .00017 57.600 .972650 .00017 59.100 .975210 .00017 60.600 .976650 .00017 62.100 .978940 .00017 63.600 .980690 .00016 65.100 .982260 .00016 66.600 .984160 .00016 68.100 .985840 .00016 69.600 .987640 .00016 70.000 .987010 .00016
20

23.700 .927060 .00029 24.100 .932550 .00028 24.500 .938080 .00028 24.900 .943090 .00029 25.300 .948920 .00028 25.700 .953590 .00028 26.100 .958010 .00028 26.500 .963290 .00027 26.900 .967730 .00027 27.300 .971750 .00027 27.700 .976460 .00027 28.100 .980810 .00027 28.500 .984940 .00027 28.900 .988160 .00026 29.300 .992510 .00027 29.700 .996180 .00027 30.100 .999500 .00027 30.501 .003120 .00026 30.901 .006440 .00026 31.301 .009550 .00026 31.501 .010570 .00025
30

16.700 .922660 .00036 16.800 .925150 .00037 16.900 .928470 .00036 17.000 .931710 .00037 17.100 .934840 .00037 17.200 .937440 .00037 17.300 .940700 .00036 17.400 .943920 .00036
32

16.100 .922730 .00038 16.200 .926530 .00038 16.300 .929500 .00038 16.400 .933260 .00038 16.500 .936300 .00038 16.600 .940040 .00037 16.700 .942960 .00037 16.800 .947040 .00038 
20.800 .964770 .00032 21.000 .967580 .00032 21.200 .971970 .00033 21.400 .975230 .00032 21.600 .979080 .00033 21.800 .983580 .00032 22.000 .987370 .00032 22.200 .991140 .00032 22.400 .994990 .00032 22.600 .998570 .00032 22.801 .001810 .00032 23.001 .004540 .00031 23.201 .008790 .00031 23.401 .011890 .00031 23.601 .014270 .00031
17.600 .950710 .00036 17.800 .955950 .00036 17.900 .958910 .00037 18.000 .961930 .00036 18.100 .964170 .00036 18.200 .967390 .00036 18.300 .969920 .00036 18.400 .972100 .00035 18.600 .978120 .00035 18.700 .980670 .00036 18.800 .983990 .00037 19.100 .990980 .00037 19.200 .992970 .00035 19.300 .995940 .00036 19.400 .998480 .00035 19.601 .003550 .00034 19.701 .006580 .00035 19.801 .008300 .00035 19.901 .010270 .00035 20.001 .012080 .00035 20.101 .015080 .00035 20.201 .017530 .00036

35

15.400 .924370 .00037 15.500 .928620 .00041 15.600 .932300 .00039 15.700 .935720 .00039 15.800 .938880 .00039 15.900 .942660 .00039 16.000 .946920 .00039 16.100 .951050 .00038 16.200 .954110 .00039 16.300 .958410 .00040 16.400 .961600 .00038 16.500 .964520 .00038 16.600 .966860 .00038 16.700 .970990 .00038 16.900 .977560 .00038 17.000 .981010 .00038 17.100 .985040 .00037 17.200 .988470 .00038 17.300 .990900 .00036 17.500 .996950 .00038 17.601 .000600 .00039 17.701 .003760 .00038

14.400 .922410 .00042 14.500 .927190 .00041 14.600 .930950 .00041 14.700 .935590 .00042 14.800 .939080 .00042 14.900 .944460 .00041 15.000 .948110 .00041 15.100 .952930 .00040 15.200 .956380 .00041 15.300 .960260 .00040 15.400 .964520 .00041 15.500 .968610 .00040 15.600 .972410 .00040 15.700 .975600 .00040 15.800 .979450 .00039 15.900 .983470 .00040 16.000 .987300 .00040 16.100 .990410 .00039 16.200 .994690 .00041 16.300 .998450 .00039 16.601 .009210 .00040 16.701 .011860 .00040
16.900 .949970 .00038 17.000 .952210 .00037 17.100 .956900 .00037 17.200 .958730 .00037 17.300 .961950 .00037 17.400 .965310 .00038 17.500 .968320 .00038 17.600 .970890 .00037 17.700 .974620 .00037 17.800 .977930 .00037 17.900 .979960 .00037 18.000 .982640 .00036 18.100 .985320 .00037 18.200 .989000 .00036 18.300 .991630 .00037 18.400 .994120 .00036 18.500 .997300 .00037 18.600 .999700 .00036 18.701 .002550 .00036 18.801 .005090 .00036 18.901 .008380 .00036 19.001 .010660 .00036 19.101 .012700 .00036 19.201 .015230 .00037 19.301 .018550 .00035

\section{0}

13.200 .922730 .00043 13.300 .929080 .00042 13.400 .932220 .00043 13.500 .937330 .00042 13.600 .942850 .00044 13.700 .947140 .00044 13.800 .952350 .00043 13.900 .956930 .00043 14.000 .962080 .00043 14.100 .966580 .00043 14.200 .971750 .00044 14.300 .975440 .00044 14.400 .981440 .00043 14.500 .984760 .00042 14.600 .990040 .00042 14.700 .993670 .00043 14.800 .999220 .00043 14.901 .003200 .00044 15.001 .007110 .00043 15.101 .011790 .00043 15.301 .020240 .00041 
17.801 .006370 .00039 17.901 .009180 .00039 18.001 .011900 .00038 18.101 .014680 .00038 18.201 .017720 .00039
16.801 .015140 .00040 16.901 .018470 .00040
60

12.400 .918620 .00047 12.500 .924690 .00045 12.600 .931320 .00045 12.700 .936380 .00045 12.800 .942320 .00046 12.900 .946340 .00045 13.000 .952980 .00046 13.100 .957870 .00047 13.200 .962580 .00047 13.300 .969350 .00045 13.400 .973980 .00046 13.500 .978920 .00044 13.600 .984550 .00045 13.700 .988410 .00044 13.800 .993810 .00045 13.901 .000150 .00045 14.001 .004380 .00045 14.101 .008650 .00044 14.201 .014760 .00044 14.301 .017140 .00045
70 11.900 .918680 .00045 12.000 .925130 .00046 12.100 .931190 .00046 12.200 .937770 .00047 12.300 .942400 .00046 12.400 .948770 .00048 12.500 .954560 .00047 12.600 .960940 .00047 12.700 .966410 .00046 12.800 .971900 .00047 12.900 .977340 .00046 13.100 .988970 .00047 13.300 .999590 .00045 13.401 .004990 .00047 13.501 .011590 .00048 13.601 .014790 .00047 13.701 .021400 .00047
90 11.300 .921430 .00048 11.350 .923420 .00048 11.400 .927410 .00050 11.450 .930700 .00047
100

11.100 .920360 .00050 11.150 .924220 .00049 11.200 .928220 .00049 11.250 .931590 .00050
80

11.600 .921580 .00047 11.650 .925830 .00048 11.700 .929140 .00048 11.750 .931910 .00049 11.800 .935020 .00047 11.850 .938450 .00048 11.900 .941920 .00047 11.950 .944240 .00047 12.000 .947530 .00047 12.050 .950580 .00048 12.100 .953990 .00048 12.150 .957450 .00048 12.200 .959020 .00047 12.250 .963790 .00047 12.300 .966890 .00049 12.350 .968900 .00048 12.400 .972120 .00048 12.450 .975100 .00049 12.500 .979030 .00048 12.550 .981540 .00048 12.600 .983950 .00047 12.650 .986970 .00049 12.700 .989540 .00048 12.750 .992350 .00047 12.800 .996170 .00048 12.850 .998470 .00050 12.901 .002050 .00048 13.001 .006940 .00047 13.051 .009120 .00046 13.101 .012500 .00047 13.151 .015520 .00048 13.201 .018290 .00047 13.251 .021140 .00048 13.301 .024600 .00048

118

10.800 .918300 .00051 10.850 .922310 .00051 10.900 .925570 .00050 10.950 .929750 .00051 
11.550 .937240 .00047 11.600 .940740 .00051 11.650 .943490 .00050 11.700 .947680 .00049 11.750 .951550 .00048 11.800 .953420 .00051 11.850 .956430 .00049 11.900 .960040 .00050 11.950 .963160 .00049 12.050 .969720 .00048 12.100 .972700 .00050 12.150 .975740 .00049 12.200 .979180 .00049 12.250 .982680 .00050 12.300 .985640 .00049 12.350 .988700 .00050 12.400 .991790 .00048 12.450 .993440 .00047 12.500 .997380 .00049 12.551 .001040 .00048 12.601 .003390 .00049 12.651 .006280 .00049 12.701 .009920 .00048 12.751 .013060 .00048 12.801 .015230 .00050 12.851 .018100 .00049 12.901 .020330 .00047

130

10.700 .920210 .00050 10.750 .925510 .00052 10.800 .928710 .00051 10.850 .932870 .00052 10.900 .936090 .00051 10.950 .939770 .00051 11.000 .943140 .00049 11.050 .947380 .00052 11.100 .952150 .00052 11.150 .953750 .00052 11.200 .957790 .00050 11.250 .961250 .00051 11.300 .964160 .00051 11.350 .968000 .00050 11.400 .971960 .00050 11.450 .974520 .00049 11.500 .978300 .00051 11.550 .982810 .00051
11.300 .935270 .00049 11.350 .938080 .00048 11.400 .942610 .00049 11.450 .945040 .00050 11.500 .949610 .00049 11.550 .952460 .00048 11.600 .955320 .00049 11.650 .958070 .00049 11.700 .962770 .00049 11.750 .965170 .00050 11.800 .968400 .00050 11.850 .972030 .00049 11.900 .975780 .00049 11.950 .978300 .00049 12.000 .981880 .00049 12.050 .984260 .00051 12.100 .988050 .00050 12.150 .991050 .00050 12.200 .994360 .00051 12.250 .997170 .00050 12.300 .999830 .00049 12.351 .003230 .00051 12.401 .006210 .00050 12.451 .010430 .00048 12.501 .012660 .00049 12.551 .014790 .00050 12.601 .018840 .00049 12.651 .022160 .00049 12.701 .024900 .00050

50

10.500 .918820 .00053 10.550 .922250 .00050 10.600 .926150 .00051 10.650 .930800 .00052 10.700 .934250 .00051 10.750 .938690 .00050 10.800 .941670 .00052 10.850 .945750 .00051 10.900 .949000 .00051 10.950 .953370 .00052 11.000 .956170 .00050 11.050 .958960 .00052 11.100 .963490 .00050 11.150 .967210 .00051 11.200 .970980 .00052 11.250 .974240 .00051 11.300 .977550 .00049 11.350 .980830 .00052
11.000 .933040 .00050 11.050 .936530 .00051 11.100 .940050 .00049 11.150 .943600 .00049 11.200 .947410 .00050 11.250 .951590 .00051 11.300 .954700 .00050 11.350 .959080 .00050 11.400 .961580 .00050 11.450 .964780 .00050 11.500 .967350 .00049 11.550 .972110 .00051 11.600 .974920 .00052 11.650 .978210 .00050 11.700 .981550 .00051 11.750 .985750 .00049 11.800 .988350 .00051 11.850 .991780 .00051 11.900 .995580 .00051 11.950 .998880 .00049 12.001 .001880 .00052 12.051 .004760 .00052 12.101 .007600 .00052 12.151 .011020 .00049 12.201 .013420 .00052 12.251 .017950 .00051 12.301 .022090 .00050
200

10.200 .913200 .00050 10.250 .917710 .00052 10.300 .920590 .00052 10.350 .924500 .00051 10.400 .928660 .00053 10.450 .933370 .00052 10.500 .937370 .00052 10.550 .940840 .00052 10.600 .943520 .00052 10.650 .948650 .00052 10.700 .953230 .00052 10.750 .955350 .00051 10.800 .959070 .00053 10.850 .963850 .00054 10.900 .967340 .00053 10.950 .971750 .00051 11.000 .973940 .00052 11.050 .978500 .00053 
11.600 .985730 .00051 11.650 .988310 .00050 11.700 .991960 .00052 11.750 .995370 .00052 11.800 .998030 .00053 11.851 .002610 .00051 11.901 .005080 .00050 11.951 .009530 .00051 12.001 .012690 .00049 12.051 .014470 .00050 12.101 .019050 .00052 12.151 .021540 .00050 12.201 .024400 .00052
11.400 .984970 .00053 11.450 .988340 .00050 11.500 .992820 .00053 11.550 .994690 .00052 11.600 .998110 .00050 11.651 .002200 .00053 11.701 .005050 .00052 11.751 .009200 .00052 11.801 .012400 .00051 11.851 .014320 .00050 11.901 .019050 .00052 11.951 .021340 .00052 12.001 .024950 .00053
11.100 .982840 .00053 11.150 .986720 .00052 11.200 .988510 .00053 11.250 .992610 .00054 11.300 .995600 .00054 11.350 .999400 .00054 11.401 .003310 .00053 11.451 .006600 .00052 11.501 .010860 .00054 11.551 .013820 .00053 11.601 .017070 .00055 11.651 .020830 .00053 11.701 .023790 .00052
235

10.200 .919670 .00052 10.250 .924950 .00051 10.300 .927140 .00054 10.350 .931540 .00054 10.450 .939720 .00056 10.500 .943340 .00053 10.550 .947170 .00052 10.600 .951000 .00051 10.650 .953820 .00053 10.700 .958030 .00053 10.750 .961720 .00053 10.800 .966400 .00053 10.850 .969490 .00053 10.900 .973210 .00052 10.950 .977450 .00054 11.000 .980990 .00054 11.050 .985190 .00052 11.100 .989020 .00052 11.150 .991780 .00052 11.200 .995220 .00051 11.250 .999070 .00053 11.301 .003880 .00053 11.351 .005610 .00055 11.401 .010000 .00052 11.451 .012710 .00051 11.501 .016700 .00052 11.551 .020850 .00051 11.601 .023950 .00053
250

10.200 .921590 .00051

10.250 .925120 .00054

10.300 .928720 .00053

10.350 .931680 .00051

10.400 .936780 .00051

10.450 .940470 .00054

10.500 .944250 .00053

10.550 .948100 .00052

10.600 .952770 .00054

10.650 .955870 .00053

10.700 .959790 .00054

10.750 .963280 .00051

10.800 .967320 .00053

10.850 .971210 .00053

10.900 .975540 .00054

10.950 .977160 .00054

11.000 .981220 .00053

11.050 .985610 .00054

11.100 .989640 .00052

11.150 .992350 .00052

11.200 .996080 .00054

11.250 .998630 .00053

11.301 .002600 .00053

11.351 .007380 .00052

11.401 .011750 .00053

11.451 .014550 .00054

11.501 .016680 .00054

11.551 .020660 .00053

11.601 .024490 .00053
353

10.250 .925370 .00054

10.300 .928100 .00054

10.350 .932160 .00053

10.400 .936750 .00054

10.450 .941240 .00053

10.550 .947590 .00053

10.600 .950780 .00053

10.650 .956820 .00053

10.700 .960020 .00054

10.750 .961900 .00052

10.800 .966310 .00054

10.850 .970570 .00053

10.900 .974180 .00053

11.000 .981420 .00054

11.050 .985470 .00054

11.100 .989890 .00053

11.150 .992040 .00053

11.200 .996050 .00054

11.250 .999650 .00053

11.301 .004090 .00054

11.351 .005970 .00053

11.401 .008980 .00054

11.451 .013670 .00053

11.501 .016310 .00053

11.551 .020440 .00054

11.601 .023780 .00053 
470

10.300 .917870 .00053

10.350 .921680 .00052

10.400 .924970 .00052

10.450 .928710 .00053

10.500 .931990 .00052

10.550 .936420 .00053

10.650 .944170 .00055

10.700 .947320 .00053

10.750 .950620 .00052

10.800 .955060 .00052

10.850 .958840 .00053

10.900 .961950 .00055

10.950 .965130 .00052

11.000 .969640 .00053

11.050 .972280 .00055

11.100 .976600 .00052

11.150 .980320 .00053

11.200 .983360 .00054

11.250 .987390 .00053

11.300 .990940 .00054

11.350 .994140 .00054

11.400 .996640 .00054

11.450 .999870 .00054

11.501 .004280 .00054

11.551 .007160 .00053

11.601 .009570 .00052

11.651 .013440 .00052

11.701 .017530 .00052

11.751 .022010 .00052

11.801 .023680 .00052
500

10.400 .921030 .00053

10.450 .923580 .00052

10.500 .926830 .00052

10.550 .931170 .00052

10.600 .934460 .00053

10.650 .938670 .00053

10.700 .942210 .00054

10.750 .945440 .00055

10.800 .948790 .00053

10.850 .953380 .00052

10.900 .956460 .00053

10.950 .960420 .00052

11.000 .964370 .00052

11.050 .967850 .00054

11.100 .970980 .00054

11.150 .974450 .00053

11.250 .981690 .00053

11.300 .985570 .00054

11.350 .987780 .00053

11.400 .990540 .00052

11.450 .995420 .00054

11.500 .998680 .00052

11.551 .001420 .00054

11.601 .006020 .00054

11.651 .008600 .00053

11.701 .011540 .00052

11.751 .014830 .00053

11.801 .018750 .00053

11.851 .022400 .00052

11.901 .024010 .00053
510

10.400 .918560 .00053

10.450 .920200 .00053

10.500 .924950 .00052

10.550 .930650 .00054

10.600 .932690 .00053

10.650 .937300 .00053

10.700 .939680 .00053

10.750 .943630 .00054

10.800 .947970 .00052

10.850 .951800 .00051

10.900 .955290 .00053

10.950 .958150 .00053

11.050 .965670 .00053

11.150 .972400 .00053

11.200 .976790 .00054

11.300 .983400 .00053

11.350 .986890 .00054

11.400 .989500 .00053

11.450 .992970 .00053

11.500 .995910 .00053

11.550 .999740 .00053

11.601 .003660 .00055

11.651 .006870 .00053

11.701 .010580 .00054

11.751 .013810 .00052

11.801 .017650 .00053

11.851 .019930 .00053

11.901 .021580 .00053
520

10.500 .924080 .00053

10.550 .927040 .00052

10.600 .931840 .00053

10.650 .935260 .00053

10.700 .938570 .00052

10.750 .942560 .00053

10.800 .945930 .00052

10.850 .948960 .00052

10.900 .952670 .00054

10.950 .958010 .00053

11.050 .963730 .00053

11.100 .967470 .00052

11.150 .971660 .00052

11.200 .975250 .00053

11.250 .977730 .00053

11.300 .981710 .00054
530

10.500 .921760 .00053

10.550 .925590 .00051

10.600 .929150 .00053

10.650 .933650 .00053

10.700 .936470 .00053

10.750 .940920 .00052

10.800 .944960 .00052

10.900 .951470 .00054

10.950 .955210 .00053

11.000 .958230 .00053

11.050 .962050 .00053

11.100 .966210 .00052

11.150 .968580 .00053

11.200 .972710 .00051

11.250 .977400 .00054

11.300 .979820 .00054
540

10.500 .920620 .00052

10.550 .925290 .00052

10.600 .928030 .00052

10.650 .930900 .00053

10.700 .935550 .00052

10.750 .939600 .00051

10.800 .943550 .00051

10.850 .946590 .00053

10.900 .949260 .00052

10.950 .954040 .00052

11.000 .957390 .00053

11.050 .960830 .00052

11.150 .968780 .00052

11.200 .970890 .00052

11.250 .975050 .00053

11.300 .978190 .00053 
11.350 .986530 .00053 11.400 .987440 .00053 11.450 .991730 .00053 11.500 .995230 .00051 11.550 .998200 .00054 11.601 .002160 .00054 11.651 .005840 .00052 11.701 .008220 .00054 11.751 .012030 .00054 11.801 .015240 .00053 11.851 .019420 .00054 11.901 .021590 .00053
11.350 .982310 .00054 11.450 .988940 .00055 11.500 .993490 .00054 11.550 .996550 .00053 11.601 .000500 .00052 11.651 .002820 .00052 11.701 .006490 .00053 11.751 .009900 .00052 11.801 .013860 .00051 11.851 .017150 .00054 11.901 .020110 .00052
550

10.500 .919400 .00051 10.550 .923610 .00052 10.600 .927340 .00053 10.650 .930500 .00053 10.700 .934290 .00052 10.750 .936340 .00052 10.800 .941750 .00052 10.850 .945020 .00051 10.900 .948310 .00053 10.950 .951110 .00054 11.000 .956420 .00053 11.050 .958400 .00052 11.100 .962710 .00053 11.150 .966720 .00055 11.200 .970240 .00052 11.250 .972180 .00052 11.300 .976030 .00053 11.400 .983050 .00052 11.450 .987690 .00053 11.500 .990130 .00052 11.550 .992650 .00053 11.600 .995840 .00052 11.650 .999860 .00054 11.701 .003160 .00053 11.751 .007260 .00052 11.801 .009340 .00054 11.851 .013660 .00052 11.901 .016580 .00053 11.951 .019720 .00054 12.001 .023560 .00054
11.350 .982190 .00054 11.400 .984630 .00053 11.450 .987440 .00053 11.500 .991390 .00053 11.550 .995450 .00052 11.600 .998040 .00051 11.651 .001400 .00052 11.701 .005330 .00052 11.751 .009090 .00051 11.801 .012070 .00054 11.851 .014100 .00055 11.901 .018040 .00054

10.500 .917210 .00052 10.550 .922720 .00054 10.600 .924710 .00053 10.650 .927880 .00051 10.750 .936370 .00053 10.800 .939010 .00052 10.850 .944670 .00051 10.900 .947530 .00054 10.950 .950670 .00052 11.000 .954140 .00052 11.050 .956950 .00052 11.100 .961560 .00052 11.150 .964480 .00052 11.200 .968290 .00051 11.250 .970660 .00053 11.300 .974690 .00053 11.350 .979070 .00052 11.400 .981650 .00052 11.450 .984020 .00053 11.500 .988600 .00053 11.550 .991150 .00053 11.600 .995200 .00052 11.650 .998100 .00053 11.701 .000570 .00052 11.751 .005100 .00056 11.801 .007700 .00053 11.851 .011040 .00052 11.901 .014670 .00052 11.951 .018500 .00053 12.001 .021030 .00054 
U5o2N23-sp-0935-10

12

105.500 .894590 .00012 112.900 .897370 .00012 120.300 .897910 .00012 127.700 .898980 .00012 135.100 .900770 .00012 142.500 .901450 .00011 149.900 .902130 .00012 157.300 .902920 .00012 164.700 .903210 .00012 172.100 .903880 .00011 179.500 .904570 .00011 186.900 .904690 .00011 194.300 .905730 .00011 201.700 .905780 .00011 209.100 .905820 .00011 216.500 .906240 .00010 223.900 .906730 .00010 231.300 .906990 .00011 238.700 .907250 .00010 246.100 .906610 .00011 253.500 .907150 .00010 254.300 .907370 .00011

25

17.900 .923870 .00032 18.100 .928880 .00031 18.300 .933650 .00032 18.500 .938440 .00032 18.700 .943500 .00031 18.900 .948090 .00032 19.100 .951890 .00032 19.300 .956040 .00031 19.500 .960680 .00031 19.700 .965090 .00031 19.900 .969430 .00031 20.100 .972740 .00031 20.300 .977830 .00030 20.500 .980850 .00031 20.700 .984350 .00031 20.900 .988300 .00030 21.100 .992010 .00031 21.300 .995720 .00030 21.500 .998800 .00030 21.701 .002070 .00031 21.901 .005890 .00030 22.101 .009090 .00030
15

39.000 .928480 .00020 40.700 .935070 .00019 42.400 .940910 .00019 44.100 .946590 .00019 45.800 .950370 .00019 47.500 .955260 .00018 49.200 .958940 .00018 50.900 .962490 .00017 52.600 .965960 .00018 54.300 .969320 .00017 56.000 .972010 .00017 57.700 .974910 .00017 59.400 .977060 .00016 61.100 .979450 .00017 62.800 .981440 .00016 64.500 .982960 .00016 66.200 .985120 .00016 67.900 .986740 .00016 69.600 .988070 .00016 71.300 .989910 .00015 73.000 .990950 .00015 74.100 .992320 .00015
20

22.500 .925630 .00027 22.900 .931690 .00028 23.300 .937810 .00027 23.700 .942390 .00027 24.100 .948010 .00026 24.500 .952600 .00026 24.900 .957700 .00027 25.300 .962330 .00027 25.700 .966960 .00026 26.100 .972020 .00026 26.500 .976030 .00026 26.900 .980270 .00026 27.300 .983740 .00025 27.700 .989190 .00026 28.100 .991800 .00025 28.500 .995050 .00025 28.900 .999450 .00025 29.301 .002490 .00025 29.701 .006120 .00025 30.101 .008600 .00025 30.401 .011680 .00025
15.600 .923990 .00034 15.700 .926810 .00035 15.800 .929700 .00035 15.900 .932500 .00035 16.000 .936530 .00035 16.100 .938120 .00036 16.200 .942650 .00034 16.300 .945610 .00034 16.400 .947760 .00033 16.500 .951180 .00036 16.600 .953680 .00035 16.700 .957180 .00034 16.800 .960220 .00033 16.900 .962420 .00034 17.000 .965220 .00034 17.300 .974770 .00033 17.400 .977300 .00034 17.500 .979160 .00035 17.700 .983890 .00033 18.000 .992620 .00033 18.300 .999900 .00034 18.401 .002420 .00033
32

15.000 .923840 .00036 15.100 .927620 .00036 15.200 .930190 .00034 15.300 .934140 .00035 15.400 .937760 .00036 15.500 .941330 .00035 15.600 .944860 .00036 15.700 .948290 .00036 15.800 .951010 .00035 15.900 .954740 .00035 16.000 .957020 .00035 16.100 .961710 .00035 16.200 .963480 .00035 16.300 .966680 .00034 16.400 .969740 .00034 16.500 .972780 .00035 16.600 .975670 .00035 16.700 .978470 .00035 16.800 .982070 .00034 16.900 .984500 .00034 17.000 .987570 .00036 17.100 .989960 .00034 
22.301 .012450 .00029 22.401 .013500 .00030

35

14.200 .923050 .00036 14.300 .926580 .00036 14.400 .930990 .00036 14.500 .934230 .00037 14.600 .938050 .00037 14.700 .942130 .00036 14.800 .945210 .00036 14.900 .948500 .00036 15.000 .952600 .00037 15.100 .955510 .00036 15.200 .959610 .00036 15.300 .963280 .00036 15.400 .967120 .00037 15.500 .969720 .00037 15.700 .976700 .00035 15.800 .980160 .00035 15.900 .983250 .00036 16.000 .986450 .00035 16.100 .989970 .00036 16.200 .993320 .00036 16.300 .996490 .00037 16.601 .005790 .00035 16.701 .008530 .00035 16.801 .011380 .00035 17.001 .016890 .00035

60

11.300 .924030 .00041 11.400 .929190 .00042 11.500 .934860 .00042 11.600 .940880 .00042 11.700 .946910 .00041 11.800 .952930 .00042 11.900 .957730 .00043 12.000 .962430 .00041 12.200 .973380 .00041
18.501 .004900 .00032 18.601 .007530 .00034 18.701 .008960 .00033 18.901 .014800 .00033 19.001 .016220 .00033

40

13.200 .920490 .00038 13.300 .924820 .00038 13.400 .929510 .00038 13.500 .933510 .00038 13.600 .938110 .00038 13.700 .941520 .00038 13.800 .946330 .00039 13.900 .950510 .00039 14.000 .954820 .00038 14.100 .958690 .00039 14.200 .963020 .00038 14.300 .966820 .00038 14.400 .971120 .00038 14.500 .974250 .00038 14.600 .977680 .00038 14.700 .981800 .00036 14.800 .985900 .00037 14.900 .990020 .00038 15.000 .993020 .00037 15.100 .997170 .00038 15.201 .000900 .00039 15.401 .007390 .00037 15.501 .011300 .00038 15.601 .014510 .00038 15.701 .018550 .00038
17.200 .993110 .00034 17.300 .996300 .00034 17.400 .998810 .00035 17.501 .001410 .00034 17.601 .004220 .00034 17.701 .006850 .00033 17.801 .009980 .00035 17.901 .013040 .00034 18.001 .014420 .00034

50

12.000 .920120 .00040 12.100 .925840 .00040 12.200 .931550 .00041 12.300 .935950 .00040 12.400 .941580 .00039 12.500 .946030 .00041 12.600 .951370 .00039 12.700 .955990 .00040 12.800 .961340 .00040 12.900 .965940 .00039 13.000 .970320 .00040 13.100 .975540 .00040 13.200 .979760 .00041 13.300 .984880 .00041 13.400 .988930 .00039 13.500 .994030 .00041 13.600 .997410 .00040 13.701 .003000 .00040 13.801 .006270 .00040 13.901 .011680 .00041 14.001 .016420 .00041 14.101 .019750 .00040
10.800 .924590 .00043 10.850 .928250 .00042 10.900 .930510 .00043 10.950 .933410 .00043 11.000 .936000 .00042 11.050 .939890 .00045 11.100 .943260 .00043 11.150 .946460 .00043 11.200 .948550 .00044
80

10.400 .923530 .00045 10.450 .925560 .00046 10.500 .929560 .00045 10.550 .932900 .00045 10.600 .935920 .00044 10.650 .939680 .00045 10.700 .943030 .00045 10.750 .946670 .00044 10.800 .948960 .00045 
12.300 .978240 .00042

12.400 .984040 .00043

12.600 .994310 .00042

12.700 .999500 .00042

12.801 .004780 .00042

12.901 .009520 .00042

13.001 .014630 .00043

13.101 .018540 .00043
11.250 .952470 .00043 11.300 .954650 .00044 11.350 .958870 .00043 11.400 .961000 .00044 11.450 .964300 .00043 11.500 .966940 .00043 11.550 .970540 .00043 11.600 .972890 .00044 11.650 .975420 .00046 11.700 .977870 .00044 11.750 .981120 .00043 11.800 .984220 .00044 11.850 .987400 .00043 11.900 .989940 .00043 11.950 .993030 .00043 12.000 .995640 .00043 12.050 .998140 .00043 12.101 .000810 .00043 12.151 .002940 .00043 12.251 .009590 .00043 12.301 .011780 .00042 12.351 .014180 .00043

90

10.000 .914720 .00045 10.050 .919490 .00045 10.100 .922280 .00046 10.150 .925770 .00045 10.200 .929350 .00045 10.250 .933000 .00045 10.300 .936200 .00044 10.350 .939050 .00045 10.450 .946230 .00045 10.500 .949400 .00045 10.550 .952470 .00046 10.600 .956220 .00047 10.650 .958830 .00045 10.700 .962970 .00044 10.750 .966770 .00045 10.800 .969110 .00045 10.850 .972580 .00046 10.900 .975300 .00045 10.950 .978740 .00045 11.000 .982010 .00045 11.050 .984810 .00046 11.100 .988440 .00045 11.150 .990820 .00046

100

9.900 .923010 .00045 9.950 .926530 .00045 10.000 .931540 .00045 10.050 .934680 .00044 10.100 .938530 .00047 10.150 .942040 .00045 10.200 .944910 .00046 10.250 .948810 .00047 10.400 .958240 .00046 10.450 .961630 .00046 10.500 .965770 .00045 10.550 .968060 .00047 10.600 .972100 .00047 10.650 .975550 .00045 10.700 .978780 .00045 10.750 .982200 .00046 10.800 .984990 .00046 10.850 .988750 .00046 10.950 .995740 .00047 11.000 .997680 .00045 11.051 .001850 .00047 11.151 .007570 .00047
10.850 .951830 .00044 10.900 .954790 .00045 10.950 .958360 .00045 11.000 .961510 .00044 11.050 .964140 .00044 11.100 .967910 .00044 11.150 .969860 .00044 11.200 .973670 .00044 11.250 .976410 .00043 11.300 .980100 .00044 11.350 .983270 .00044 11.400 .985950 .00043 11.450 .988180 .00045 11.500 .991850 .00044 11.550 .993950 .00045 11.600 .997990 .00045 11.651 .000120 .00045 11.701 .003140 .00044 11.751 .006190 .00044 11.801 .009150 .00044 11.851 .011420 .00045 11.901 .014330 .00044 11.951 .017850 .00046 12.001 .021050 .00046 11.201 .011410 .00047

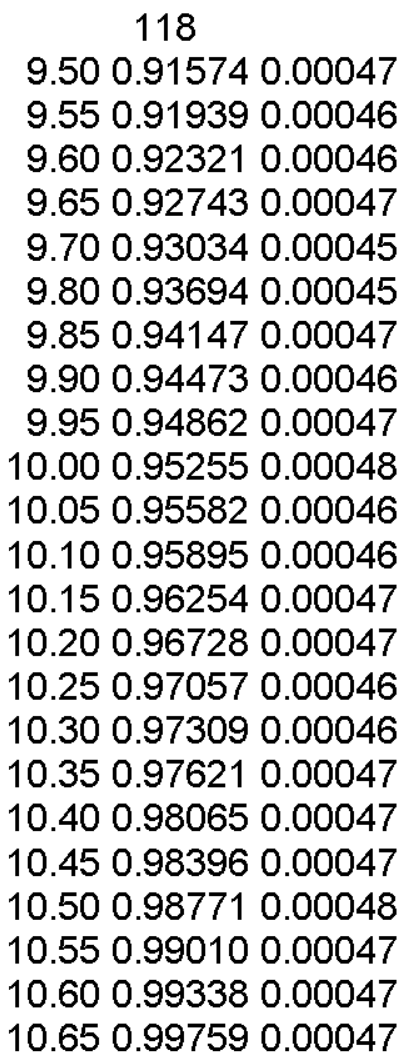


11.200 .994380 .00045 11.250 .996870 .00045 11.301 .000680 .00045 11.351 .003810 .00045 11.401 .006260 .00045 11.451 .009660 .00046 11.501 .012040 .00045 11.551 .014980 .00043 11.601 .018110 .00046
130

9.400 .919380 .00047 9.450 .922960 .00047 9.500 .926760 .00046 9.550 .930940 .00048 9.650 .939030 .00048 9.700 .941300 .00047 9.750 .946420 .00047 9.800 .948950 .00048 9.850 .952750 .00046 9.900 .956960 .00047 9.950 .959600 .00047 10.000 .963010 .00047 10.050 .968250 .00047 10.100 .971620 .00048 10.150 .974670 .00047 10.200 .978060 .00048 10.250 .981710 .00047 10.300 .984500 .00047 10.350 .988430 .00049 10.400 .991860 .00048 10.450 .995450 .00047 10.500 .998990 .00047 10.551 .002430 .00046 10.601 .005170 .00046 10.651 .009830 .00047 10.701 .012330 .00045 10.751 .014800 .00047 10.801 .019530 .00046

235

8.800 .915880 .00048 8.850 .920960 .00048 8.900 .924220 .00050 8.950 .929120 .00048 9.000 .933330 .00048
250

8.800 .918080 .00049 8.900 .926680 .00048 8.950 .931170 .00049 9.000 .935310 .00047 9.050 .939720 .00048
11.251 .014130 .00047 11.301 .017280 .00046 11.351 .020200 .00045 11.401 .023340 .00046

10.700 .999990 .00047 10.751 .003440 .00045 10.801 .007410 .00048 10.851 .010980 .00046 10.901 .013810 .00047 10.951 .017540 .00047 11.001 .019510 .00048

150

9.200 .918100 .00046 9.250 .921790 .00047 9.300 .926000 .00047 9.350 .929800 .00047 9.400 .934310 .00046 9.450 .937840 .00047 9.500 .942130 .00048 9.550 .944850 .00047 9.600 .949390 .00048 9.650 .952330 .00048 9.700 .955720 .00047 9.750 .960650 .00048 9.800 .963800 .00048 9.850 .968120 .00048 9.900 .970960 .00048 9.950 .973640 .00047 10.000 .978340 .00048 10.050 .981560 .00048 10.100 .985800 .00049 10.150 .988920 .00049 10.200 .992520 .00048 10.250 .996810 .00048 10.300 .999610 .00048 10.351 .002830 .00046 10.401 .007230 .00047 10.451 .009440 .00047 10.501 .013560 .00049 10.551 .016690 .00048 10.601 .019830 .00049
200

8.900 .916440 .00047 8.950 .919840 .00048 9.000 .924350 .00048 9.050 .928150 .00048 9.100 .931830 .00048 9.150 .935990 .00048 9.200 .940530 .00047 9.250 .944210 .00047 9.300 .948130 .00047 9.350 .951950 .00048 9.400 .955260 .00048 9.450 .959530 .00048 9.500 .963360 .00049 9.550 .967610 .00049 9.600 .970400 .00049 9.650 .974590 .00048 9.700 .978160 .00047 9.750 .980940 .00048 9.800 .985300 .00049 9.850 .988550 .00049 9.950 .997120 .00047 10.001 .000050 .00050 10.051 .004660 .00049 10.101 .007090 .00049 10.151 .011040 .00050 10.201 .014810 .00049 10.251 .017920 .00049 10.301 .021350 .00048
353

8.700 .916140 .00051 8.750 .921070 .00048 8.800 .925010 .00049 8.850 .928270 .00048 8.900 .932710 .00047 
9.050 .936930 .00048

9.100 .941030 .00049

9.150 .944280 .00049

9.200 .949420 .00050

9.250 .952700 .00049

9.300 .956970 .00050

9.350 .960920 .00049

9.400 .964330 .00049

9.450 .968670 .00049

9.500 .972310 .00049

9.550 .976110 .00047

9.600 .980980 .00047

9.650 .983460 .00049

9.700 .987100 .00048

9.750 .990990 .00048

9.800 .994810 .00049

10.001 .008580 .00051

10.051 .012120 .00051

10.101 .015710 .00049

10.151 .019550 .00050

10.201 .023680 .00049

470

8.800 .919990 .00049

8.850 .923370 .00048

8.900 .927480 .00048

8.950 .931380 .00049

9.000 .934290 .00049

9.050 .940310 .00049

9.100 .943010 .00049

9.150 .947440 .00048

9.200 .951210 .00048

9.250 .954310 .00048

9.300 .957920 .00049

9.350 .961990 .00049

9.400 .965250 .00048

9.450 .968320 .00049

9.500 .973190 .00049

9.550 .976880 .00049

9.600 .980080 .00049

9.650 .983460 .00049

9.700 .987500 .00049

9.750 .991130 .00049

9.800 .994000 .00050

9.901 .001490 .00049

9.951 .004300 .00050
9.100 .943050 .00049

9.150 .947150 .00049

9.200 .951000 .00048

9.250 .954750 .00049

9.300 .959470 .00049

9.350 .963080 .00049

9.400 .966300 .00049

9.450 .970180 .00050

9.500 .974450 .00048

9.550 .977950 .00049

9.600 .980750 .00048

9.650 .984320 .00048

9.700 .988850 .00049

9.750 .993000 .00049

9.800 .996100 .00049

9.851 .000320 .00050

9.901 .002960 .00050

9.951 .006920 .00050

10.001 .010390 .00048

10.051 .014270 .00050

10.101 .018410 .00050

10.151 .020830 .00050

10.201 .024470 .00048

500

8.800 .916940 .00049

8.850 .919880 .00048

8.900 .923870 .00050

8.950 .926580 .00048

9.000 .932430 .00050

9.050 .936360 .00049

9.100 .939360 .00049

9.150 .943750 .00048

9.200 .947790 .00050

9.250 .951230 .00049

9.300 .954400 .00050

9.350 .958080 .00049

9.400 .962030 .00048

9.450 .965620 .00050

9.500 .969030 .00050

9.550 .972420 .00049

9.600 .976120 .00048

9.650 .979260 .00049

9.700 .983150 .00049

9.750 .987240 .00051

9.800 .990090 .00049

9.850 .994770 .00049

9.900 .997610 .00049
8.950 .936000 .00049

9.000 .941780 .00049

9.050 .945140 .00050

9.100 .948750 .00049

9.150 .952150 .00050

9.200 .956360 .00048

9.250 .960300 .00049

9.300 .964280 .00049

9.350 .967560 .00049

9.400 .971750 .00051

9.450 .975120 .00048

9.500 .979840 .00050

9.550 .983590 .00049

9.600 .985810 .00048

9.650 .990540 .00050

9.700 .994690 .00049

9.750 .997830 .00049

9.801 .000730 .00048

9.851 .004260 .00051

9.901 .007910 .00050

9.951 .011870 .00049

10.001 .016220 .00049

10.051 .019760 .00049

10.101 .022130 .00050

510

8.800 .915500 .00049

8.850 .918950 .00048

8.900 .922640 .00048

8.950 .926250 .00048

9.050 .934400 .00048

9.100 .938000 .00049

9.150 .941680 .00048

9.200 .945470 .00048

9.250 .949830 .00048

9.300 .953670 .00049

9.350 .956060 .00049

9.400 .961060 .00049

9.450 .964000 .00047

9.500 .967640 .00050

9.550 .971280 .00049

9.600 .974740 .00049

9.650 .978310 .00049

9.700 .982400 .00048

9.750 .986230 .00050

9.800 .988630 .00048

9.850 .992660 .00048

9.900 .996270 .00048

9.950 .999440 .00049 
10.001 .008020 .00050 10.051 .012070 .00049 10.101 .015130 .00049 10.151 .017800 .00048 10.201 .021210 .00050

520

8.900 .922260 .00050 8.950 .924950 .00048 9.000 .929090 .00049 9.050 .932760 .00049 9.100 .936890 .00048 9.150 .941160 .00047 9.200 .945140 .00048 9.250 .947950 .00047 9.300 .952210 .00050 9.350 .956930 .00048 9.400 .959370 .00048 9.450 .962910 .00050 9.500 .966790 .00049 9.550 .970160 .00049 9.600 .974070 .00049 9.650 .977550 .00049 9.700 .980660 .00049 9.750 .984070 .00050 9.800 .988230 .00048 9.850 .991360 .00050 9.900 .995150 .00050 9.950 .998540 .00048 10.001 .002660 .00049 10.051 .005240 .00048 10.101 .008470 .00047 10.151 .012590 .00049 10.201 .014800 .00049 10.251 .019050 .00049 10.301 .022520 .00049
9.951 .000180 .00049 10.001 .003130 .00048 10.051 .006790 .00050 10.101 .010520 .00048 10.151 .014390 .00049 10.201 .017520 .00049 10.251 .020910 .00049 10.301 .024480 .00049
10.001 .002850 .00049 10.051 .007070 .00048 10.101 .009520 .00049 10.151 .013380 .00048 10.201 .016840 .00050 10.251 .018940 .00049 10.301 .023350 .00048
530

8.900 .920840 .00048 8.950 .925070 .00048 9.000 .928440 .00048 9.100 .936400 .00050 9.150 .939440 .00048 9.200 .942910 .00048 9.250 .947140 .00047 9.300 .950340 .00049 9.350 .954720 .00048 9.400 .957880 .00049 9.450 .961890 .00050 9.500 .965010 .00049 9.550 .968050 .00049 9.600 .972410 .00048 9.650 .976410 .00049 9.700 .979960 .00050 9.750 .982610 .00050 9.850 .991010 .00049 9.900 .994030 .00049 9.950 .996830 .00049 10.001 .000200 .00048 10.051 .004170 .00048 10.101 .007470 .00049 10.151 .010080 .00050 10.201 .014310 .00050 10.251 .017170 .00049 10.301 .021030 .00051
540

8.900 .919750 .00048 8.950 .924450 .00048 9.000 .927460 .00047 9.050 .930000 .00048 9.100 .934050 .00048 9.150 .939210 .00050 9.200 .942320 .00048 9.250 .945980 .00048 9.300 .949600 .00050 9.350 .953650 .00048 9.400 .956750 .00048 9.450 .960770 .00048 9.500 .964210 .00048 9.550 .968110 .00048 9.600 .971490 .00051 9.650 .975120 .00049 9.700 .979020 .00050 9.750 .981920 .00048 9.800 .985390 .00049 9.850 .988970 .00049 9.900 .992580 .00048 9.950 .996010 .00047 10.051 .002320 .00049 10.101 .006830 .00050 10.151 .010400 .00049 10.201 .013450 .00049 10.251 .016220 .00049 10.301 .019990 .00050
550

8.900 .918050 .00047 8.950 .922480 .00047 9.000 .925450 .00049 9.050 .930260 .00049 9.100 .933260 .00049 9.150 .936350 .00049
560

8.900 .917580 .00048

8.950 .921250 .00051

9.000 .924990 .00049

9.050 .929420 .00049

9.100 .933200 .00050

9.150 .936040 .00050 
9.200 .941010 .00049

9.250 .944700 .00049

9.300 .948820 .00049

9.350 .951790 .00049

9.400 .955430 .00049

9.450 .960140 .00048

9.500 .963110 .00049

9.550 .966580 .00048

9.650 .973630 .00049

9.700 .976960 .00049

9.750 .980440 .00048

9.800 .983670 .00048

9.850 .988930 .00049

9.900 .990400 .00049

9.950 .994890 .00048

10.000 .998570 .00049

10.051 .001480 .00049

10.101 .004980 .00048

10.151 .008460 .00050

10.201 .010830 .00050

10.251 .015290 .00049

10.301 .018790 .00050

10.351 .022080 .00049

10.401 .024240 .00048

\section{U5o2N26-sp-0935-00}

12

147.800 .861740 .00012 161.100 .862920 .00012 174.400 .864030 .00012 187.700 .864240 .00012 201.000 .865370 .00012 214.300 .866520 .00011 227.600 .866280 .00012 240.900 .866830 .00011 254.200 .866870 .00012 267.500 .867300 .00011 280.800 .867610 .00011 294.100 .867750 .00011 307.400 .868120 .00011 320.700 .868010 .00011 334.000 .868350 .00011 347.300 .868620 .00011 360.600 .868330 .00011 373.900 .868640 .00011 387.200 .868570 .00011 400.500 .868400 .00011 413.800 .869160 .00011 414.600 .868590 .00011
9.200 .940040 .00049

9.250 .943430 .00049

9.300 .947980 .00048

9.350 .951510 .00048

9.400 .955060 .00048

9.450 .958080 .00049

9.500 .962470 .00049

9.550 .964570 .00049

9.600 .968820 .00050

9.650 .972130 .00047

9.700 .975610 .00049

9.750 .980330 .00048

9.800 .982580 .00049

9.850 .987160 .00047

9.900 .988870 .00049

9.950 .993100 .00050

10.000 .996840 .00048

10.050 .999900 .00049

10.101 .003310 .00050

10.151 .006340 .00050

10.201 .010450 .00049

10.251 .014150 .00048

10.301 .016530 .00049

10.351 .019550 .00050

10.401 .023760 .00049

15

58.400 .929450 .00018

62.100 .934560 .00017

65.800 .940020 .00017

69.500 .943060 .00017

73.200 .947020 .00016

76.900 .949520 .00016

80.600 .952650 .00015

84.300 .955360 .00015

88.000 .957360 .00015

91.700 .959120 .00015

95.400 .960390 .00015

99.100 .961860 .00015

102.800 .963840 .00014

106.500 .965350 .00015

110.200 .965290 .00014

113.900 .966590 .00014

117.600 .967750 .00014

125.000 .969180 .00014

132.400 .970640 .00013

133.400 .970300 .00013
20

8.800 .926390 .00027

29.300 .931570 .00028

29.800 .936960 .00027

30.300 .942150 .00028

30.800 .947100 .00027

31.300 .951090 .00026

31.800 .955730 .00027

32.300 .960020 .00027

32.800 .964050 .00027

33.300 .967980 .00026

33.800 .972090 .00026

34.300 .975640 .00026

34.800 .979860 .00025

35.300 .982820 .00026

35.800 .986770 .00025

36.300 .989960 .00025

36.800 .992840 .00025

37.300 .995910 .00025

37.800 .998710 .00025

38.301 .001480 .00024

38.801 .004520 .00025

39.301 .006790 .00025 
39.801 .009720 .00024

40.301 .012480 .00024

40.401 .012070 .00024

25

30

32

22.700 .923770 .00034 23.000 .929680 .00033 23.300 .935320 .00033 23.600 .940790 .00033 23.900 .946610 .00032 24.200 .951380 .00032 24.500 .957700 .00033 24.800 .962350 .00032 25.100 .966400 .00031 25.400 .971690 .00032 25.700 .976290 .00031 26.000 .980720 .00032 26.300 .985460 .00031 26.600 .989490 .00032 26.900 .993770 .00031 27.200 .997630 .00031 27.501 .002470 .00031 27.801 .006420 .00031 28.101 .009820 .00030 28.401 .013700 .00030 28.501 .015220 .00030

19.900 .922420 .00037 20.100 .928640 .00036 20.300 .932980 .00037 20.500 .938670 .00036 20.700 .943360 .00037 20.900 .947970 .00036 21.100 .953470 .00035 21.300 .958320 .00036 21.500 .963540 .00036 21.700 .968460 .00034 21.900 .972170 .00035 22.100 .977590 .00036 22.300 .982190 .00035 22.500 .985990 .00035 22.700 .989450 .00037 22.900 .994090 .00035 23.100 .998330 .00036 23.301 .002600 .00035 23.501 .005910 .00035 23.701 .011450 .00035 23.901 .015080 .00034 24.001 .016640 .00035
19.100 .920670 .00038 19.200 .923310 .00038 19.300 .926790 .00037 19.400 .930480 .00037 19.500 .931890 .00039 19.600 .935570 .00037 19.700 .937580 .00038 19.800 .941540 .00038 19.900 .944510 .00037 20.000 .947360 .00037 20.100 .949640 .00038 20.200 .952230 .00037 20.300 .954750 .00037 20.400 .958650 .00037 20.500 .960230 .00038 20.600 .963540 .00036 20.700 .966150 .00036 20.800 .967990 .00036 20.900 .970810 .00038 21.000 .973720 .00038 21.100 .974850 .00036 21.200 .978060 .00037 21.300 .981330 .00037 21.400 .983110 .00037 21.500 .985520 .00037 21.600 .988340 .00036 21.700 .990230 .00037 21.900 .995100 .00036 22.000 .996900 .00036 22.100 .999290 .00036 22.201 .001590 .00037 22.301 .004740 .00036 22.401 .007190 .00036 22.501 .008890 .00037 22.601 .011330 .00035 22.701 .013220 .00036 22.801 .015230 .00036 
35

18.300 .923070 .00039 18.400 .926340 .00038 18.500 .929780 .00039 18.600 .932500 .00040 18.700 .936650 .00038 18.800 .939740 .00039 18.900 .941860 .00038 19.000 .946880 .00038 19.100 .949800 .00040 19.200 .952500 .00040 19.300 .955290 .00038 19.400 .957520 .00039 19.500 .960760 .00039 19.600 .964210 .00038 19.700 .966830 .00038 19.800 .969300 .00039 20.000 .976220 .00038 20.100 .978650 .00038 20.200 .981790 .00040 20.300 .983840 .00039 20.400 .986870 .00039 20.500 .989920 .00039 20.600 .992680 .00039 20.700 .995770 .00039 20.800 .998140 .00038 20.900 .999980 .00038 21.001 .002910 .00037 21.101 .006020 .00038 21.201 .007860 .00038 21.301 .011530 .00039 21.401 .013590 .00038 21.501 .015080 .00037
40

17.100 .919770 .00041

17.200 .923350 .00041

17.300 .927610 .00041

17.400 .931520 .00041

17.500 .934980 .00042

17.600 .938720 .00041

17.700 .942440 .00040

17.800 .946260 .00041

17.900 .949050 .00040

18.000 .953290 .00040

18.100 .956550 .00042

18.200 .959800 .00041

18.300 .963880 .00042

18.400 .966470 .00040

18.500 .970610 .00041

18.600 .974290 .00041

18.700 .977540 .00039

18.800 .981030 .00041

18.900 .982910 .00040

19.000 .986790 .00040

19.100 .990280 .00041

19.200 .993230 .00041

19.300 .996850 .00041

19.400 .999700 .00041

19.501 .003770 .00040

19.601 .006190 .00040

19.701 .008450 .00040

19.801 .011990 .00041

19.901 .014650 .00041

20.001 .018060 .00040
60

14.900 .919610 .00046 15.000 .924760 .00045 15.100 .929410 .00047 15.200 .934380 .00046 15.300 .939490 .00046 15.600 .954940 .00047 15.700 .959220 .00047 15.800 .964460 .00046 15.900 .968650 .00045 16.000 .974460 .00046 16.100 .978520 .00046 16.200 .983910 .00047 16.300 .987450 .00046 16.400 .992060 .00046
70

14.300 .915880 .00047

14.400 .921070 .00047

14.500 .927960 .00048

14.600 .932930 .00049

14.700 .938880 .00047

14.800 .944430 .00049

14.900 .949070 .00048

15.000 .955260 .00049

15.100 .961350 .00048

15.200 .965230 .00048

15.400 .976050 .00046

15.500 .981300 .00047

15.600 .986820 .00048

15.700 .991620 .00047
50

15.700 .917820 .00043

15.800 .922640 .00044

15.900 .927430 .00044

16.000 .930850 .00044

16.100 .935940 .00044

16.200 .940010 .00044

16.300 .945510 .00045

16.400 .949650 .00045

16.500 .954160 .00043

16.700 .962490 .00044

16.800 .967530 .00044

16.900 .970770 .00045

17.000 .975230 .00044

17.300 .987330 .00043

17.400 .991640 .00044

17.500 .995340 .00044

17.601 .000580 .00045

17.701 .003680 .00043

17.801 .007290 .00044

17.901 .010930 .00044 18.001 .014680 .00044 18.101 .018730 .00042 
16.500 .996750 .00045 16.601 .000790 .00045 16.701 .006000 .00045 16.801 .010440 .00047 16.901 .013820 .00045 17.001 .018930 .00047 17.101 .023170 .00046
15.800 .996180 .00048 15.901 .001930 .00048 16.001 .005600 .00048 16.101 .010580 .00048 16.201 .015080 .00048 16.301 .020160 .00048
90

13.800 .927820 .00050 13.900 .932800 .00051 14.000 .939940 .00050 14.100 .946260 .00050 14.200 .951680 .00051 14.300 .958610 .00049 14.400 .962360 .00050 14.500 .969160 .00052 14.600 .974230 .00050 14.800 .986520 .00050 14.900 .991790 .00049 15.000 .997330 .00049 15.101 .003250 .00049 15.201 .007760 .00049 15.301 .013170 .00049 15.401 .019440 .00051 15.501 .024740 .00051
100

13.500 .923430 .00050 13.550 .925820 .00051 13.600 .930190 .00051 13.650 .932310 .00052 13.700 .935630 .00051 13.750 .938970 .00051 13.800 .942650 .00051 13.850 .944350 .00051 13.900 .948310 .00051 13.950 .951770 .00049 14.000 .954770 .00050 14.050 .957030 .00052 14.100 .960770 .00050 14.150 .963460 .00051 14.200 .966660 .00050 14.250 .969550 .00051 14.300 .972810 .00051 14.350 .976130 .00052 14.400 .979220 .00051 14.450 .982450 .00051 14.500 .984430 .00052 14.550 .988860 .00051 14.600 .990350 .00051 14.650 .993690 .00051 14.700 .996230 .00052 14.750 .997750 .00049 14.901 .007270 .00051 14.951 .010770 .00051 15.001 .013360 .00051 15.051 .016190 .00052 15.101 .018410 .00051 15.151 .022340 .00051 15.201 .024630 .00052
118

13.100 .916700 .00052 13.150 .920210 .00051 13.200 .922460 .00052 13.250 .925940 .00052 13.300 .929180 .00052 13.350 .932430 .00052 13.400 .935750 .00051 13.450 .938860 .00052 13.500 .942830 .00052 13.550 .946210 .00051 13.600 .948470 .00053 13.650 .953470 .00052 13.700 .955290 .00052 13.750 .957690 .00051 13.800 .961300 .00053 13.850 .964450 .00050 13.900 .968520 .00051 13.950 .971050 .00054 14.000 .974720 .00051 14.050 .977770 .00051 14.200 .986020 .00052 14.250 .990700 .00051 14.300 .992930 .00052 14.350 .995650 .00052 14.400 .997470 .00051 14.551 .007730 .00052 14.601 .011780 .00052 14.651 .013450 .00052 14.701 .016810 .00052 14.751 .018780 .00053 14.801 .022680 .00053
130

13.000 .919270 .00052 13.050 .922530 .00051 13.100 .926370 .00054
150 12.950 .927390 .00053 13.000 .931610 .00052 13.050 .934440 .00055
200

12.600 .916970 .00055 12.650 .921040 .00054 12.700 .925080 .00054 
13.150 .929540 .00054

13.200 .932210 .00053

13.250 .936120 .00053

13.300 .939470 .00053

13.350 .942430 .00053

13.400 .946920 .00052

13.450 .948710 .00051

13.500 .952390 .00053

13.550 .956230 .00053

13.600 .958790 .00052

13.650 .962350 .00052

13.700 .965310 .00053

13.750 .969070 .00052

13.800 .970990 .00052

13.850 .975910 .00053

13.900 .978940 .00052

13.950 .981720 .00052

14.000 .985150 .00054

14.050 .987820 .00054

14.100 .990660 .00052

14.150 .995120 .00052

14.200 .998430 .00052

14.251 .000950 .00052

14.301 .003390 .00053

14.351 .006850 .00054

14.401 .009760 .00052

14.451 .011870 .00051

14.501 .015510 .00052

14.551 .019240 .00053

14.601 .021370 .00053

\section{5}

12.500 .914180 .00056

12.550 .917750 .00054

12.600 .921000 .00055

12.650 .925370 .00055

12.700 .929890 .00054

12.750 .933850 .00054

12.800 .936790 .00054

12.850 .940140 .00057

12.900 .943100 .00053

12.950 .947460 .00053

13.000 .950930 .00054

13.050 .953560 .00054

13.100 .958130 .00055

13.150 .961300 .00055

13.200 .965150 .00054

13.250 .968620 .00056

13.300 .971220 .00055
13.100 .937470 .00054

13.150 .941640 .00052

13.200 .944800 .00054

13.250 .947440 .00053

13.350 .954810 .00054

13.400 .956920 .00052

13.450 .960780 .00052

13.500 .965300 .00053

13.550 .968980 .00053

13.700 .977780 .00053

13.750 .981470 .00054

13.800 .984620 .00052

13.850 .987930 .00053

13.900 .990480 .00055

13.950 .995020 .00054

14.000 .998030 .00054

14.051 .000030 .00055

14.101 .003860 .00054

14.151 .007320 .00053

14.201 .010880 .00052

14.251 .012780 .00054

14.301 .016360 .00054

14.351 .018480 .00054
12.750 .927400 .00055

12.800 .931880 .00053

12.850 .935200 .00054

12.900 .938190 .00055

12.950 .943160 .00053

13.050 .950350 .00054

13.100 .952880 .00055

13.150 .957520 .00053

13.200 .959760 .00053

13.300 .967020 .00052

13.400 .974170 .00056

13.450 .977190 .00053

13.500 .981300 .00055

13.550 .984020 .00055

13.650 .990740 .00053

13.700 .993580 .00055

13.750 .996610 .00054

13.801 .001120 .00055

13.851 .002610 .00055

13.901 .006290 .00056

13.951 .010230 .00054

14.001 .013870 .00055

14.051 .017140 .00054

14.101 .018680 .00053

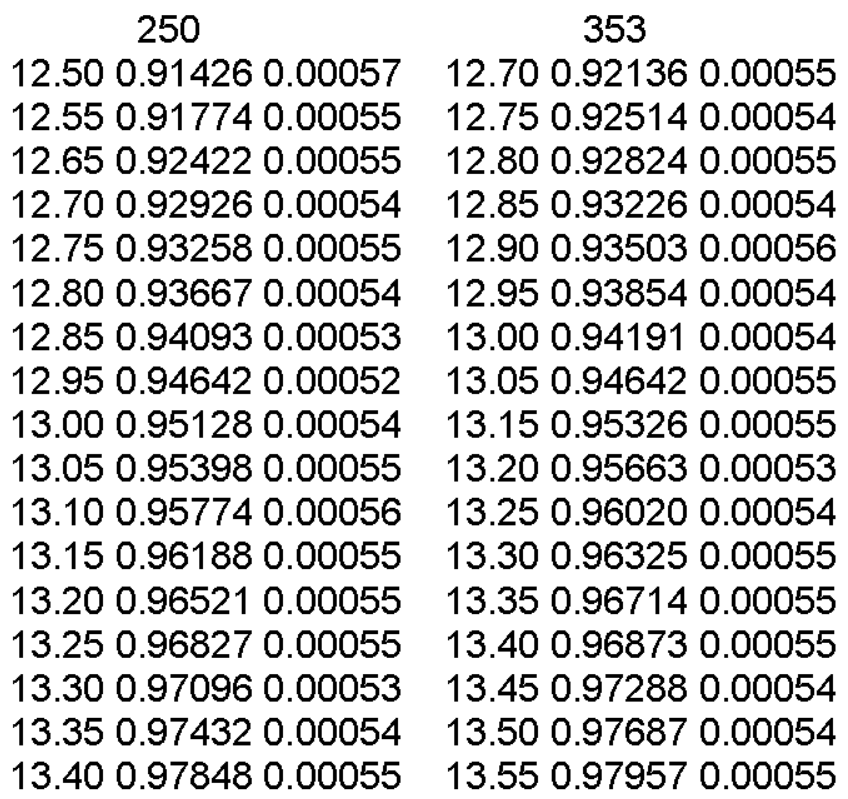

F-432 
13.350 .974970 .00056 13.400 .977960 .00056 13.450 .981680 .00054 13.500 .985330 .00055 13.550 .987990 .00053 13.600 .990850 .00055 13.650 .993990 .00053 13.700 .998400 .00055 13.751 .002090 .00055 13.801 .005920 .00055 13.851 .006640 .00055 13.901 .011390 .00056 13.951 .014870 .00055 14.001 .016890 .00055 14.051 .019890 .00054 14.101 .024500 .00053
13.450 .981870 .00054 13.500 .985730 .00053 13.550 .988730 .00057 13.600 .990940 .00053 13.650 .994530 .00054 13.700 .997840 .00056 13.751 .001920 .00056 13.801 .003940 .00055 13.851 .008500 .00056 13.901 .010900 .00055 13.951 .014570 .00053 14.001 .018600 .00055 14.051 .021780 .00054 14.101 .025280 .00054
13.600 .983360 .00056 13.650 .986200 .00055 13.700 .990600 .00055 13.750 .993530 .00054 13.800 .996100 .00056 13.850 .999570 .00053 13.901 .003910 .00055 13.951 .005660 .00054 14.001 .009970 .00056 14.051 .012700 .00057 14.101 .015260 .00054 14.151 .019870 .00054 14.201 .022620 .00054

\section{0}

13.000 .918720 .00053 13.050 .920860 .00053 13.150 .927200 .00053 13.200 .932080 .00054 13.250 .935950 .00054 13.300 .938370 .00053 13.350 .942450 .00055 13.400 .945700 .00053 13.500 .951590 .00055 13.550 .955730 .00054 13.650 .961740 .00054 13.700 .964670 .00055 13.750 .967910 .00054 13.800 .971990 .00054 13.900 .977500 .00054 13.950 .980400 .00052 14.000 .984650 .00054 14.050 .987880 .00056 14.100 .991850 .00054 14.150 .993210 .00054 14.200 .997220 .00054 14.251 .000000 .00056 14.301 .002470 .00056 14.351 .006240 .00053 14.451 .011990 .00054 14.501 .013570 .00055 14.551 .018440 .00054 14.601 .020530 .00055
475

13.100 .922020 .00054 13.150 .926790 .00054 13.200 .929590 .00054 13.250 .933480 .00054 13.350 .940120 .00054 13.400 .943990 .00055 13.450 .946200 .00053 13.500 .950840 .00054 13.550 .954090 .00053 13.600 .957140 .00054 13.650 .960410 .00053 13.700 .963320 .00054 13.750 .966360 .00056 13.800 .970200 .00052 13.850 .973800 .00054 13.950 .978850 .00055 14.000 .982500 .00054 14.050 .986020 .00054 14.100 .989090 .00054 14.150 .992700 .00054 14.200 .995940 .00054 14.250 .999360 .00056 14.301 .001890 .00052 14.351 .004590 .00054 14.401 .007580 .00054 14.451 .009880 .00053 14.501 .012690 .00056 14.551 .016730 .00054 14.601 .019560 .00054 14.651 .023380 .00055 14.701 .024750 .00053
480

13.150 .925410 .00054 13.200 .928530 .00054 13.300 .935800 .00054 13.350 .939720 .00053 13.400 .942580 .00054 13.450 .945100 .00054 13.550 .952300 .00055 13.600 .955340 .00055 13.650 .958520 .00054 13.700 .962200 .00054 13.750 .965440 .00054 13.800 .969120 .00056 13.850 .971620 .00054 13.900 .975690 .00055 13.950 .978280 .00053 14.000 .981290 .00053 14.050 .984240 .00055 14.100 .987110 .00054 14.150 .990680 .00053 14.200 .994950 .00054 14.250 .997580 .00056 14.301 .000860 .00054 14.351 .003170 .00055 14.401 .005440 .00054 14.451 .009030 .00054 14.501 .011600 .00055 14.551 .015150 .00055 14.601 .018780 .00054 14.651 .021550 .00054 14.701 .024520 .00055 
486

13.100 .919120 .00054

13.150 .922650 .00052

13.200 .926370 .00054

13.250 .930310 .00054

13.300 .933650 .00054

13.350 .937720 .00054

13.400 .940730 .00054

13.450 .942610 .00055

13.500 .947520 .00053

13.550 .950890 .00053

13.600 .953210 .00055

13.650 .956890 .00053

13.750 .963540 .00054

13.800 .966020 .00055

13.850 .970100 .00053

13.900 .972900 .00054

13.950 .976110 .00055

14.000 .979810 .00053

14.050 .982460 .00054

14.100 .985170 .00056

14.150 .988140 .00055

14.200 .991180 .00056

14.250 .994530 .00055

14.300 .999140 .00054

14.351 .000930 .00053

14.401 .003390 .00054

14.451 .007020 .00053

14.501 .010000 .00054

14.551 .013830 .00054

14.601 .016020 .00056

14.651 .018130 .00055

14.701 .020800 .00054

14.751 .025140 .00054

14.801 .027930 .00054 
U5o2N26-sp-0935-01

12

189.900 .864740 .00012 202.300 .865820 .00011 214.700 .866260 .00012 227.100 .866810 .00011 239.500 .866680 .00011 251.900 .866580 .00012 264.300 .867530 .00011 276.700 .867730 .00011 289.100 .867350 .00011 301.500 .868050 .00011 313.900 .867550 .00011 326.300 .868710 .00011 338.700 .868330 .00011 375.900 .867970 .00011 388.300 .868490 .00011 400.700 .868860 .00011 413.100 .868720 .00011 425.500 .868720 .00011 437.900 .869130 .00011 438.900 .868980 .00011
15

56.500 .928490 .00018 58.400 .931790 .00018 60.300 .934580 .00017 62.200 .936990 .00018 64.100 .939060 .00017 67.900 .943560 .00016 69.800 .945020 .00017 71.700 .946760 .00016 73.600 .949050 .00016 75.500 .950420 .00016 81.200 .954140 .00016 83.100 .955550 .00015 85.000 .956740 .00015 86.900 .957860 .00015 88.800 .959040 .00015 90.700 .959320 .00015 92.600 .960120 .00014 94.500 .960500 .00014 95.000 .960860 .00014
25

21.100 .923270 .00032 21.400 .929450 .00032 21.700 .935830 .00031 22.000 .940480 .00032 22.300 .945620 .00031 22.600 .952330 .00032 22.900 .956260 .00031 23.200 .962490 .00031 23.500 .967120 .00031 23.800 .971510 .00031 24.100 .976520 .00031 24.400 .980150 .00031 24.700 .985150 .00030 25.000 .990740 .00030 25.300 .994160 .00031 25.600 .998020 .00030 25.901 .001680 .00031 26.201 .006230 .00031 26.501 .010050 .00029 $\begin{array}{ccc}30 & \\ 18.30 & 0.92250 & 0.00037 \\ 18.50 & 0.92755 & 0.00035 \\ 18.70 & 0.93347 & 0.00036 \\ 18.90 & 0.93846 & 0.00036 \\ 19.10 & 0.94350 & 0.00036 \\ 19.30 & 0.94851 & 0.00035 \\ 19.50 & 0.95277 & 0.00035 \\ 19.70 & 0.95846 & 0.00034 \\ 19.90 & 0.96353 & 0.00035 \\ 20.10 & 0.96810 & 0.00034 \\ 20.30 & 0.97229 & 0.00034 \\ 20.50 & 0.97691 & 0.00035 \\ 20.70 & 0.98089 & 0.00034 \\ 20.90 & 0.98603 & 0.00035 \\ 21.10 & 0.98942 & 0.00034 \\ 21.30 & 0.99526 & 0.00035 \\ 21.50 & 0.99885 & 0.00035 \\ 21.70 & 1.00277 & 0.00035 \\ 21.90 & 1.00630 & 0.00034\end{array}$

32

17.600 .924120 .00036 17.700 .926610 .00035 17.800 .929410 .00036 17.900 .932800 .00037 18.000 .935950 .00037 18.100 .938250 .00036 18.200 .940740 .00037 18.400 .946960 .00036 18.500 .949940 .00036 18.600 .952900 .00036 18.700 .955230 .00035 18.800 .957120 .00037 18.900 .960450 .00035 19.000 .963970 .00036 19.100 .965990 .00035 19.300 .970470 .00036 19.400 .973440 .00036 19.500 .975770 .00036 19.600 .978760 .00036 
26.801 .013220 .00030 26.901 .015860 .00030
35

16.700 .923230 .00038 16.800 .926390 .00038 16.900 .929920 .00037 17.000 .933810 .00039 17.100 .935920 .00038 17.200 .939010 .00037 17.300 .942640 .00038 17.400 .946260 .00037 17.500 .949110 .00037 17.600 .952020 .00037 17.700 .955790 .00038 17.800 .958900 .00037 17.900 .961060 .00037 18.000 .964350 .00038 18.100 .967400 .00037 18.200 .970210 .00037 18.300 .972970 .00037 18.400 .976190 .00037 18.500 .978150 .00037 18.600 .981630 .00037 18.700 .983470 .00037 18.800 .986510 .00037 18.900 .989340 .00037 19.000 .993140 .00037 19.100 .995900 .00037 19.200 .998310 .00037 19.301 .000420 .00036 19.401 .002660 .00038 19.501 .005920 .00036 19.601 .008660 .00036 19.701 .010470 .00037 19.801 .013390 .00037 19.901 .015940 .00036 20.001 .018570 .00036
22.101 .010800 .00034 22.301 .014140 .00034 22.401 .016640 .00033
19.700 .980510 .00035

19.800 .983350 .00036 19.900 .985170 .00036 20.000 .988520 .00036 20.100 .990750 .00036 20.200 .992700 .00035 20.300 .994960 .00035 20.400 .997440 .00036 20.601 .001350 .00036 20.701 .004310 .00035 20.801 .006160 .00036 20.901 .008650 .00035 21.001 .010950 .00035 21.101 .013370 .00035 21.201 .015270 .00036

40 50 15.600 .923500 .00041 15.700 .927510 .00040 15.800 .931150 .00040 15.900 .934280 .00040 16.000 .938930 .00039 16.100 .942760 .00040 16.200 .946170 .00039 16.400 .953210 .00039 16.500 .956680 .00039 16.600 .960780 .00040 16.700 .963200 .00040 16.800 .966600 .00040 16.900 .970860 .00038 17.000 .973990 .00039 17.100 .976370 .00039 17.200 .980510 .00038 17.300 .984490 .00039 17.400 .987400 .00040 17.500 .990690 .00039 17.600 .993740 .00038 17.700 .997210 .00040 17.801 .000560 .00038 17.901 .002880 .00039 18.001 .005870 .00039 18.101 .009350 .00040 18.201 .012570 .00039 18.301 .015190 .00039
14.200 .922340 .00043 14.400 .931780 .00042 14.500 .937520 .00042 14.600 .941510 .00043 14.700 .945970 .00043 14.900 .954220 .00044 15.000 .958510 .00044 15.100 .962360 .00042 15.200 .966450 .00043 15.300 .971540 .00043 15.400 .974940 .00041 15.500 .979630 .00042 15.700 .987220 .00043 15.800 .991400 .00042 15.900 .994990 .00043 16.000 .999750 .00043 16.101 .003380 .00042 16.201 .007680 .00043 16.301 .011000 .00042 16.401 .014500 .00043 16.501 .019050 .00043 
60

13.300 .920300 .00045 13.400 .924190 .00045 13.500 .929800 .00046 13.600 .935210 .00046 13.700 .940360 .00045 14.000 .954930 .00044 14.100 .959560 .00045 14.200 .965310 .00044 14.300 .969690 .00045 14.400 .976140 .00045 14.500 .978460 .00045 14.600 .983820 .00044 14.700 .988390 .00046 14.800 .992080 .00044 14.900 .997460 .00043 15.001 .002000 .00044 15.201 .010860 .00046 15.301 .014420 .00043

90

12.100 .922130 .00049 12.150 .925360 .00048 12.200 .928150 .00048 12.250 .931130 .00049 12.300 .934860 .00048 12.350 .938290 .00047 12.400 .940190 .00048 12.450 .943870 .00048 12.500 .946870 .00048 12.550 .950310 .00048 12.600 .952990 .00049 12.650 .955570 .00049 12.700 .959550 .00049 12.750 .962360 .00049 12.800 .963720 .00048 12.850 .966890 .00049 12.900 .969730 .00047 12.950 .973180 .00047 13.000 .976570 .00048 13.050 .978440 .00049 13.100 .982230 .00048 13.150 .985760 .00048 13.350 .995910 .00048 13.400 .997970 .00048 13.451 .001010 .00049
70

12.700 .916530 .00046

12.800 .922530 .00047

12.900 .928750 .00047

13.000 .933620 .00045

13.100 .939930 .00046

13.400 .955790 .00047

13.600 .966590 .00045

13.700 .971700 .00046

13.800 .976580 .00046

13.900 .982070 .00046

14.000 .986600 .00045

14.100 .992680 .00045

14.200 .996870 .00047

14.301 .001610 .00046

14.401 .006850 .00048

14.501 .011370 .00046

14.601 .016370 .00046

14.701 .021750 .00047
80

12.300 .915090 .00049

12.400 .922370 .00047

12.500 .929450 .00046

12.700 .939450 .00048

12.900 .950290 .00048

13.000 .958000 .00047

13.100 .964300 .00048

13.200 .968590 .00047

13.300 .974800 .00048

13.400 .978890 .00047

13.500 .985220 .00048

13.600 .990760 .00047

13.700 .995380 .00047

13.801 .001070 .00047

13.901 .006780 .00048

14.001 .011390 .00047

14.101 .016640 .00047

14.201 .021190 .00047
11.800 .919130 .00050

11.850 .920580 .00049

11.900 .925100 .00048

11.950 .927210 .00048

12.050 .934080 .00049

12.100 .937040 .00050

12.150 .940690 .00050

12.200 .943760 .00049

12.250 .946430 .00050

12.300 .949510 .00049

12.350 .953530 .00048

12.400 .955100 .00047

12.450 .958320 .00050

12.500 .961510 .00050

12.550 .964820 .00050

12.600 .968730 .00049

12.650 .970290 .00049

12.700 .974140 .00048

12.800 .980470 .00048

12.850 .983160 .00049

12.900 .985930 .00049

12.950 .988920 .00050

13.000 .992430 .00049

13.050 .995390 .00051

13.100 .997910 .00050
118

11.700 .930790 .00050

11.750 .933790 .00049

11.850 .940200 .00050

11.900 .944270 .00049

11.950 .947240 .00050

12.000 .951240 .00049

12.050 .953930 .00051

12.100 .957300 .00050

12.150 .960360 .00050

12.200 .964480 .00049

12.250 .967410 .00051

12.300 .969790 .00048

12.350 .972740 .00049

12.400 .977200 .00050

12.450 .979570 .00049

12.500 .982870 .00051

12.550 .985260 .00051

12.600 .988210 .00050

12.650 .990220 .00050

12.700 .994410 .00050

12.750 .997780 .00050

12.800 .999780 .00050

12.851 .004680 .00050

12.901 .006970 .00050

12.951 .010620 .00051 
13.501 .004640 .00047 13.551 .006750 .00047 13.601 .010000 .00049 13.651 .012360 .00049 13.701 .015130 .00048 13.751 .017530 .00048 13.801 .020420 .00050

130

11.400 .920420 .00050 11.450 .924750 .00050 11.500 .928220 .00051 11.550 .931220 .00051 11.600 .934650 .00051 11.650 .938750 .00051 11.700 .942330 .00051 11.750 .945120 .00049 11.800 .946590 .00051 11.850 .951700 .00050 11.900 .953830 .00051 11.950 .958240 .00050 12.000 .961190 .00052 12.100 .967230 .00049 12.250 .976690 .00051 12.350 .982580 .00049 12.400 .986730 .00050 12.450 .988950 .00051 12.500 .992510 .00051 12.550 .996940 .00051 12.600 .999600 .00051 12.651 .002350 .00051 12.701 .005730 .00051 12.751 .008400 .00050 12.801 .012370 .00051 12.851 .014290 .00051 12.901 .016530 .00052 12.951 .020120 .00051 13.001 .022950 .00052

235 10.900 .918090 .00053 10.950 .921910 .00052 11.000 .925620 .00053
13.151 .000550 .00048 13.201 .003350 .00050 13.251 .005360 .00049 13.301 .008400 .00050 13.351 .011180 .00049 13.401 .015430 .00049 13.451 .017000 .00049 13.501 .019730 .00049

150 11.200 .918970 .00051 11.250 .922770 .00050 11.300 .925390 .00051 11.350 .930830 .00051 11.400 .933900 .00051 11.450 .937040 .00051 11.500 .940770 .00052 11.550 .943120 .00052 11.600 .947060 .00051 11.650 .950390 .00052 11.700 .954320 .00052 11.750 .957000 .00053 11.800 .961410 .00053 11.850 .963880 .00049 11.900 .968560 .00052 11.950 .970410 .00052 12.000 .973990 .00050 12.050 .977390 .00050 12.150 .983630 .00054 12.200 .987950 .00051 12.250 .990670 .00052 12.300 .992160 .00051 12.350 .995970 .00052 12.400 .999860 .00052 12.451 .002560 .00052 12.501 .007320 .00051 12.551 .009340 .00053 12.601 .012310 .00051 12.651 .015110 .00052 12.701 .018300 .00052 12.751 .021850 .00052 12.801 .024790 .00051

13.001 .012230 .00050 13.051 .016000 .00049 13.101 .018170 .00050

200 11.000 .921710 .00052 11.050 .923460 .00052 11.100 .928850 .00053 11.150 .932420 .00052 11.200 .934870 .00054 11.250 .940290 .00054 11.300 .942830 .00053 11.350 .945440 .00052 11.400 .950670 .00053 11.450 .952820 .00052 11.500 .956920 .00052 11.550 .961070 .00052 11.600 .963370 .00053 11.650 .966650 .00051 11.700 .971840 .00052 11.750 .974200 .00052 11.800 .977400 .00052 11.850 .980400 .00052 11.900 .983740 .00052 11.950 .988310 .00052 12.000 .990450 .00052 12.050 .994280 .00053 12.100 .996620 .00052 12.201 .004140 .00053 12.301 .010650 .00052 12.351 .013920 .00052 12.401 .017350 .00053 12.451 .019710 .00052 12.501 .022290 .00053

250 10.900 .919830 .00050 10.950 .923040 .00054 11.050 .930370 .00053
353 11.000 .920040 .00055 11.050 .924850 .00053 11.100 .927690 .00051 
11.050 .929580 .00053 11.100 .932460 .00053 11.150 .936330 .00052 11.200 .939910 .00052 11.300 .946970 .00052 11.350 .950290 .00052 11.400 .953900 .00052 11.450 .957290 .00052 11.500 .961340 .00052 11.550 .964470 .00052 11.600 .968140 .00053 11.650 .972500 .00053 11.750 .978800 .00052 11.850 .985100 .00052 11.900 .988300 .00053 11.950 .991470 .00054 12.000 .995460 .00052 12.050 .998060 .00052 12.101 .001350 .00051 12.151 .005240 .00050 12.251 .011330 .00053 12.301 .015170 .00052 12.351 .019150 .00053 12.401 .021480 .00053

470

11.300 .921450 .00053 11.350 .923060 .00053 11.400 .928630 .00052 11.450 .929630 .00053 11.500 .934190 .00052 11.550 .937430 .00053 11.600 .940180 .00052 11.650 .944300 .00052 11.700 .947070 .00054 11.750 .951210 .00053 11.800 .954170 .00053 11.850 .957560 .00053 11.900 .960740 .00052 11.950 .964470 .00054 12.000 .967420 .00053 12.050 .970960 .00052 12.100 .973730 .00052 12.150 .976560 .00052 12.200 .980890 .00053 12.250 .982310 .00054 12.300 .985050 .00053 12.350 .988660 .00053
11.150 .936400 .00052 11.200 .941130 .00052 11.250 .943170 .00053 11.300 .949000 .00053 11.350 .951370 .00053 11.450 .959390 .00052 11.500 .961900 .00052 11.550 .965930 .00053 11.600 .969630 .00052 11.650 .972000 .00054 11.700 .976850 .00052 11.750 .978790 .00051 11.800 .982550 .00051 11.850 .986030 .00052 11.900 .989600 .00053 11.950 .992950 .00053 12.000 .996100 .00053 12.051 .000870 .00054 12.101 .001820 .00053 12.151 .005610 .00052 12.201 .008520 .00055 12.251 .012990 .00052 12.301 .015580 .00052 12.351 .018950 .00055 12.401 .021530 .00054
11.150 .931690 .00053 11.200 .934770 .00053 11.250 .937810 .00053 11.300 .942570 .00052 11.400 .948590 .00054 11.450 .952250 .00053 11.500 .955600 .00052 11.700 .969280 .00053 11.750 .972330 .00054 11.800 .975400 .00053 11.850 .980090 .00055 11.950 .985790 .00054 12.000 .989750 .00052 12.050 .993420 .00052 12.100 .995320 .00053 12.150 .998810 .00054 12.201 .003330 .00053 12.251 .005290 .00051 12.301 .009000 .00052 12.351 .011680 .00053 12.401 .015300 .00053 12.501 .021130 .00052
475
480
11.300 .920230 .00053 11.350 .921830 .00053 11.400 .925760 .00053 11.450 .929930 .00051 11.500 .933590 .00053 11.550 .936280 .00052 11.600 .940070 .00052 11.650 .942970 .00052 11.700 .946850 .00053 11.750 .949610 .00054 11.800 .953230 .00053 11.850 .955800 .00052 11.900 .960150 .00055 11.950 .961590 .00053 12.000 .965470 .00052 12.100 .973140 .00052 12.150 .975090 .00054 12.200 .978670 .00054 12.250 .980620 .00053 12.300 .985640 .00053 12.350 .987460 .00052 12.400 .991340 .00052 
12.400 .993170 .00053

12.450 .996030 .00053

12.500 .999210 .00051

12.551 .001700 .00053

12.601 .005040 .00051

12.651 .007070 .00052

12.701 .011060 .00054

12.751 .014560 .00052

12.801 .016510 .00054

12.851 .020540 .00054

12.901 .022910 .00053
12.450 .994700 .00051

12.500 .998110 .00053

12.551 .000670 .00052

12.601 .003430 .00052

12.651 .005880 .00053

12.701 .008170 .00052

12.751 .011750 .00053

12.801 .016090 .00053

12.851 .019170 .00052

12.901 .021950 .00052
12.450 .993640 .00052

12.500 .996480 .00053

12.550 .999290 .00053

12.601 .002060 .00052

12.651 .005640 .00054

12.701 .008490 .00054

12.751 .011570 .00053

12.801 .013510 .00054

12.851 .017630 .00053

12.901 .019950 .00053

\section{6}

11.400 .922970 .00051

11.500 .929090 .00052

11.550 .932970 .00052

11.600 .936750 .00053

11.650 .939960 .00052

11.700 .942330 .00051

11.750 .944790 .00053

11.800 .949740 .00053

11.850 .953260 .00052

11.900 .955860 .00053

11.950 .958410 .00053

12.000 .962330 .00053

12.050 .966610 .00053

12.100 .969030 .00053

12.150 .973060 .00052

12.200 .975800 .00053

12.250 .978500 .00052

12.350 .985030 .00053

12.400 .988350 .00052

12.450 .991240 .00053

12.500 .994040 .00053

12.550 .997350 .00053

12.601 .000880 .00052

12.651 .003600 .00053

12.701 .005360 .00052

12.751 .009180 .00053

12.801 .012830 .00053

12.851 .015560 .00053

12.901 .018640 .00053

12.951 .020430 .00053

13.001 .025300 .00053

0 
U5o2N26-sp-0935-10

12

134.300 .860050 .00013 149.100 .861950 .00012 163.900 .863630 .00012 178.700 .864390 .00012 193.500 .864950 .00012 208.300 .865550 .00012 223.100 .865840 .00012 237.900 .866480 .00011 252.700 .867170 .00011 297.100 .867830 .00011 311.900 .868020 .00011 341.500 .867780 .00011 356.300 .868570 .00011 371.100 .868620 .00011 385.900 .868260 .00011 400.700 .868470 .00011 415.500 .868430 .00011 430.300 .869050 .00011 430.900 .869120 .00011

25

20.000 .925690 .00030 20.200 .929870 .00029 20.400 .933410 .00030 20.600 .937100 .00030 20.800 .940770 .00031 21.000 .944460 .00030 21.200 .947820 .00031 21.400 .952190 .00030 21.600 .955570 .00030 21.800 .958950 .00030 22.000 .961940 .00029 22.200 .965020 .00030 22.400 .968710 .00030 22.600 .971950 .00029 22.800 .975540 .00029 23.000 .978270 .00030 23.200 .981750 .00029 23.400 .984070 .00030 23.600 .987590 .00029 23.800 .990760 .00030 24.000 .993000 .00028 24.200 .996060 .00029 24.400 .997930 .00029
15

55.500 .929210 .00017 56.900 .931190 .00017 58.300 .933490 .00017 59.700 .934960 .00017 61.100 .937290 .00017 62.500 .939170 .00017 63.900 .940790 .00017 65.300 .942160 .00016 66.700 .943810 .00017 70.900 .947620 .00016 72.300 .949160 .00016 73.700 .949910 .00015 75.100 .950350 .00016 76.500 .952390 .00015 77.900 .953570 .00015 79.300 .953470 .00016 80.700 .954840 .00015 82.100 .955600 .00015 83.500 .956510 .00015 83.800 .956160 .00015

30

17.100 .923030 .00034 17.300 .927970 .00034 17.500 .933170 .00034 17.700 .938830 .00034 17.900 .943900 .00034 18.100 .948580 .00033 18.300 .953760 .00034 18.500 .959090 .00033 18.700 .964140 .00033 18.900 .968640 .00032 19.100 .973460 .00033 19.300 .977950 .00033 19.500 .981940 .00033 19.700 .987000 .00033 19.900 .991400 .00034 20.100 .995400 .00033 20.300 .998300 .00033 20.501 .003250 .00032 20.701 .007240 .00032 20.901 .011260 .00032 21.101 .015080 .00032 21.201 .016650 .00033
20

25.700 .922820 .00026 26.300 .929000 .00026 26.900 .936280 .00026 27.500 .941200 .00026 28.100 .948090 .00025 28.700 .952890 .00025 29.300 .957610 .00025 29.900 .963310 .00025 30.500 .968140 .00024 31.100 .973040 .00024 31.700 .977080 .00025 32.300 .982290 .00024 32.900 .986570 .00024 33.500 .989560 .00023 34.100 .993710 .00023 34.700 .996990 .00023 35.301 .000100 .00023 35.901 .004580 .00022 36.501 .007350 .00023 37.101 .010880 .00023 37.701 .012840 .00022

32

16.400 .924460 .00035 16.600 .929850 .00035 16.700 .933740 .00035 16.800 .936720 .00034 16.900 .938930 .00035 17.000 .942110 .00034 17.100 .945060 .00034 17.200 .947900 .00033 17.300 .950020 .00033 17.400 .953260 .00033 17.500 .956150 .00034 17.600 .958670 .00034 17.700 .961240 .00033 17.800 .964490 .00034 17.900 .966790 .00034 18.000 .968730 .00034 18.300 .977020 .00032 18.400 .979440 .00034 18.500 .981140 .00034 18.600 .984200 .00033 18.700 .986870 .00033 18.800 .989530 .00033 18.900 .991330 .00034 
24.601 .001860 .00029

24.801 .004470 .00029

25.001 .006780 .00029

25.201 .009740 .00028

25.401 .011390 .00029

25.601 .014400 .00029

35

15.500 .924220 .00036 15.600 .927290 .00035 15.700 .930830 .00036 15.800 .934550 .00036 15.900 .937690 .00036 16.000 .940710 .00037 16.100 .943760 .00036 16.200 .947320 .00035 16.300 .950820 .00035 16.400 .953220 .00035 16.500 .956230 .00034 16.600 .959400 .00035 16.700 .962510 .00034 16.800 .966330 .00036 16.900 .968790 .00036 17.000 .971130 .00036 17.100 .974150 .00036 17.200 .977530 .00035 17.300 .980090 .00034 17.400 .982700 .00035 17.500 .985580 .00035 17.600 .988870 .00036 17.700 .991280 .00035 17.800 .994120 .00034 17.900 .996120 .00035 18.000 .999920 .00035 18.101 .001590 .00035 18.201 .004550 .00034 18.301 .006590 .00034 18.401 .010230 .00035 18.501 .012420 .00034 18.601 .014880 .00035 18.701 .016970 .00035
19.000 .993650 .00034 19.100 .996470 .00034 19.200 .998080 .00034 19.301 .000570 .00034 19.401 .002840 .00034 19.501 .005770 .00033 19.601 .008290 .00033 19.701 .010980 .00033 19.801 .012080 .00032 19.901 .014470 .00034 20.001 .015990 .00033

40 50

$14.400 .924800 .00038 \quad 12.900 .920560 .00040$ $\begin{array}{lll}14.50 & 0.928520 .00038 & 13.000 .925420 .00040\end{array}$ $14.600 .932840 .00037 \quad 13.100 .930130 .00040$

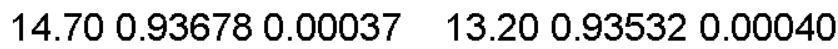

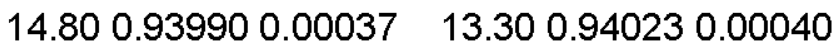
$14.900 .943950 .00037 \quad 13.400 .943040 .00040$

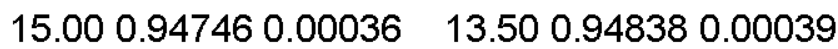

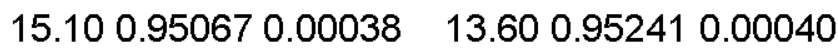
$15.200 .954840 .00037 \quad 13.700 .957070 .00040$

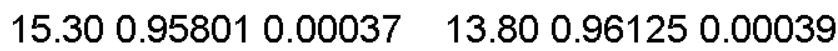

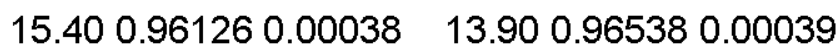
$\begin{array}{llll}15.50 & 0.964780 .00036 & 14.00 & 0.970440 .00040\end{array}$ $\begin{array}{llll}15.60 & 0.968470 .00037 & 14.10 & 0.973920 .00039\end{array}$

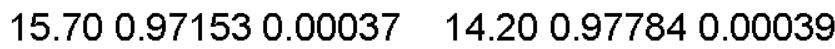

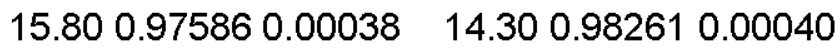

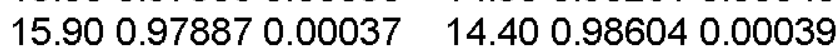

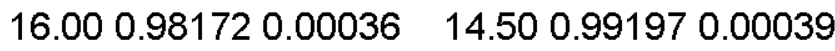

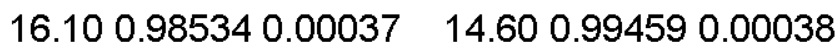
$16.200 .988840 .00037 \quad 14.700 .998590 .00040$ $16.300 .991350 .00037 \quad 14.801 .002860 .00040$

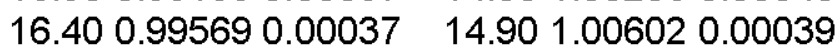

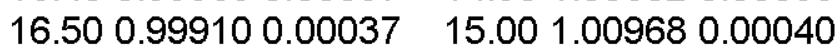
$16.601 .001610 .00036 \quad 15.101 .014180 .00039$ $\begin{array}{ll}16.701 .004650 .00036 & 15.201 .018570 .00039\end{array}$ 16.801 .008570 .00036 16.901 .010490 .00037 17.001 .013510 .00037 17.101 .015980 .00038 
60

12.100 .923140 .00043

12.200 .928480 .00041

12.300 .932910 .00041

12.400 .939520 .00043

12.500 .944610 .00041

12.600 .948770 .00043

12.700 .954040 .00041

12.800 .959690 .00042

12.900 .964040 .00043

13.000 .969060 .00041

13.100 .973580 .00043

13.300 .983600 .00042

13.400 .987600 .00041

13.500 .992230 .00042

13.600 .996790 .00043

13.701 .001590 .00042

13.801 .005680 .00042

13.901 .010620 .00042

14.101 .018750 .00041
70

11.600 .927880 .00042

11.700 .932540 .00042

11.800 .938890 .00043

11.900 .944970 .00043

12.000 .950660 .00042

12.100 .955960 .00044

12.200 .961160 .00042

12.400 .971450 .00042

12.500 .977240 .00042

12.600 .981480 .00043

12.700 .987060 .00043

13.001 .002350 .00043

13.101 .007290 .00042

13.201 .011580 .00042

13.301 .017040 .00042
90

10.700 .917380 .00044 10.800 .923470 .00046 10.900 .929430 .00045 11.000 .936360 .00045 11.100 .941630 .00044 11.200 .948800 .00046 11.300 .954810 .00045 11.400 .960840 .00044 11.500 .966230 .00043 11.600 .972300 .00045 11.700 .978050 .00046 11.800 .983510 .00044 11.900 .989440 .00044 12.000 .995760 .00045 12.201 .005690 .00046 12.301 .011750 .00045 12.401 .017800 .00045 12.501 .022400 .00043
100

10.500 .920280 .00046 10.550 .923160 .00044 10.650 .930110 .00045 10.700 .932280 .00045 10.750 .935820 .00044 10.800 .939660 .00046 10.850 .942460 .00046 10.900 .945290 .00044 10.950 .948430 .00045 11.000 .951950 .00046 11.050 .955450 .00045 11.150 .962210 .00046 11.200 .964940 .00046 11.250 .968370 .00044 11.300 .970640 .00046 11.350 .973050 .00046 11.400 .976370 .00045 11.450 .979880 .00045 11.500 .983770 .00046 11.550 .985980 .00046 11.600 .988760 .00045 11.650 .990930 .00045 11.700 .994390 .00046 11.750 .997820 .00045 11.801 .000690 .00046 11.851 .003590 .00046 11.901 .006370 .00045
80

11.200 .928260 .00045 11.300 .933590 .00044 11.400 .940280 .00044 11.500 .946340 .00045 11.600 .951830 .00045 11.700 .958020 .00044 11.800 .963870 .00043 11.900 .969850 .00043 12.000 .974620 .00043 12.100 .980680 .00044 12.200 .986130 .00044 12.300 .991100 .00044 12.400 .997340 .00045 12.601 .008020 .00043 12.701 .011180 .00044 12.801 .018580 .00044 12.901 .022250 .00043
118

10.200 .920910 .00047 10.250 .925030 .00045 10.300 .929460 .00046 10.350 .931770 .00046 10.400 .934840 .00047 10.450 .938120 .00047 10.500 .941890 .00047 10.550 .945490 .00047 10.600 .948370 .00046 10.650 .951830 .00046 10.700 .955170 .00047 10.750 .958170 .00047 10.800 .961860 .00046 10.850 .964800 .00045 10.900 .966720 .00045 10.950 .970800 .00045 11.050 .977260 .00046 11.100 .980140 .00047 11.150 .983670 .00045 11.200 .987990 .00047 11.250 .991120 .00047 11.300 .992830 .00046 11.350 .996730 .00046 11.400 .998850 .00046 11.451 .002210 .00047 11.501 .006240 .00048 11.551 .008540 .00047 
130

10.000 .918600 .00046 10.050 .922620 .00046 10.100 .926670 .00046 10.150 .928570 .00046 10.200 .932250 .00047 10.250 .936440 .00045 10.300 .939680 .00047 10.350 .942510 .00047 10.400 .946050 .00046 10.450 .950110 .00047 10.500 .953070 .00045 10.550 .957290 .00047 10.600 .960460 .00045 10.650 .963740 .00046 10.700 .966780 .00045 10.750 .970120 .00048 10.800 .973170 .00048 10.850 .976560 .00047 10.950 .983320 .00046 11.000 .986120 .00047 11.050 .989370 .00048 11.100 .992720 .00046 11.150 .994680 .00047 11.200 .997920 .00047 11.251 .001840 .00048 11.301 .005000 .00048 11.351 .007940 .00047 11.401 .011560 .00047 11.451 .013750 .00048 11.501 .017500 .00047 11.551 .019660 .00047 11.601 .022920 .00046
11.951 .009050 .00045 12.001 .012370 .00045 12.051 .015200 .00045 12.101 .016940 .00046

11.601 .011660 .00047 11.651 .013380 .00047 11.701 .017580 .00046 11.751 .020840 .00045 11.801 .022610 .00046

$\begin{array}{rrr} & 200 \\ 9.50 & 0.91763 & 0.00048 \\ 9.55 & 0.92177 & 0.00048 \\ 9.60 & 0.92500 & 0.00047 \\ 9.65 & 0.92896 & 0.00048 \\ 9.70 & 0.93232 & 0.00048 \\ 9.80 & 0.94063 & 0.00048 \\ 9.85 & 0.94299 & 0.00047 \\ 9.90 & 0.94705 & 0.00048 \\ 9.95 & 0.94974 & 0.00047 \\ 10.00 & 0.95364 & 0.00047 \\ 10.05 & 0.95744 & 0.00048 \\ 10.10 & 0.96032 & 0.00047 \\ 10.20 & 0.96697 & 0.00049 \\ 10.25 & 0.97117 & 0.00047 \\ 10.30 & 0.97469 & 0.00047 \\ 10.35 & 0.97903 & 0.00049 \\ 10.40 & 0.98125 & 0.00048 \\ 10.45 & 0.98587 & 0.00048 \\ 10.50 & 0.98934 & 0.00049 \\ 10.55 & 0.99229 & 0.00048 \\ 10.60 & 0.99572 & 0.00048 \\ 10.65 & 0.99757 & 0.00047 \\ 10.70 & 1.00180 & 0.00047 \\ 10.75 & 1.00627 & 0.00048 \\ 10.80 & 1.00801 & 0.00049 \\ 10.85 & 1.01239 & 0.00050 \\ 10.90 & 1.01580 & 0.00048 \\ 10.95 & 1.01758 & 0.00048 \\ 11.00 & 1.02139 & 0.00049\end{array}$

9.800 .919110 .00047 9.850 .922270 .00047 9.900 .925830 .00046 10.000 .932680 .00047 10.050 .937330 .00046 10.100 .940620 .00046 10.150 .943530 .00046 10.200 .947240 .00047 10.250 .950910 .00047 10.300 .954460 .00047 10.350 .956890 .00048 10.400 .960620 .00048 10.450 .964660 .00047 10.500 .967460 .00046 10.550 .970950 .00048 10.600 .975330 .00047 10.650 .977320 .00047 10.700 .981220 .00048 10.750 .984500 .00048 10.800 .987260 .00047 10.850 .991800 .00049 10.900 .993900 .00047 10.950 .997880 .00047 11.001 .000830 .00047 11.051 .004040 .00047 11.101 .007700 .00048 11.151 .010180 .00048 11.201 .013240 .00048 11.251 .016440 .00046 11.301 .019320 .00048
235

9.400 .916930 .00049 9.450 .920520 .00048 9.500 .924910 .00048 9.550 .928230 .00048 9.750 .942690 .00049 9.800 .947320 .00048
250

9.400 .919010 .00049 9.450 .922790 .00048 9.500 .925120 .00048 9.550 .930210 .00048 9.600 .932970 .00049 9.700 .940790 .00049
353

9.400 .919850 .00049 9.450 .924030 .00049 9.500 .927950 .00048 9.550 .931690 .00048 9.600 .934550 .00047 9.650 .938650 .00049 
9.850 .950760 .00049

9.900 .954040 .00050

9.950 .958200 .00049

10.000 .961430 .00048 10.050 .965280 .00047

10.100 .967280 .00048

10.150 .971980 .00049

10.200 .974400 .00049

10.250 .978630 .00049

10.300 .983010 .00048

10.350 .985120 .00049

10.400 .989320 .00050

10.450 .991470 .00048

10.500 .995370 .00049

10.601 .001580 .00047

10.651 .005500 .00049

10.701 .008040 .00048

10.751 .012530 .00049

10.801 .015550 .00048

10.851 .018060 .00049

10.901 .022210 .00050
9.750 .944400 .00049

9.800 .947540 .00048

9.850 .951790 .00048

9.900 .955570 .00048

9.950 .959450 .00049

10.050 .967200 .00049

10.100 .970150 .00049

10.150 .973020 .00049

10.250 .980520 .00048

10.300 .983140 .00048

10.350 .986840 .00048

10.400 .989450 .00049

10.450 .994000 .00049

10.500 .997490 .00049

10.551 .000030 .00048

10.601 .003790 .00049

10.701 .011430 .00049

10.751 .014180 .00049

10.801 .017620 .00047

10.851 .020540 .00050

10.901 .023510 .00049
9.700 .942900 .00048

9.750 .946250 .00049

9.800 .950150 .00049

9.850 .953550 .00049

9.900 .956370 .00049

9.950 .960370 .00049

10.000 .963960 .00049

10.050 .966950 .00050

10.100 .971080 .00049

10.150 .973350 .00049

10.250 .980920 .00048

10.300 .984590 .00049

10.400 .990200 .00049

10.450 .994250 .00049

10.500 .996410 .00050

10.601 .003790 .00049

10.651 .007120 .00048

10.701 .011180 .00049

10.751 .014480 .00050

10.901 .023770 .00050

\section{0}

9.600 .922100 .00048

9.750 .933160 .00048

9.800 .935900 .00048

9.950 .945990 .00049

10.000 .950260 .00047

10.050 .952510 .00049

10.200 .962720 .00048

10.250 .965350 .00049

10.300 .969730 .00049

10.350 .973720 .00048

10.400 .975960 .00048

10.450 .979620 .00049

10.500 .982760 .00049

10.550 .985250 .00048

10.600 .988570 .00050

10.650 .992870 .00050

10.700 .995760 .00048

10.750 .998560 .00048

10.801 .001770 .00049

10.851 .005290 .00049

10.901 .008020 .00048

10.951 .010530 .00049

11.001 .014670 .00050

11.051 .015970 .00048

11.101 .020410 .00049
475

9.650 .924320 .00047

9.700 .928290 .00048

9.750 .931520 .00049

9.800 .935310 .00050

9.850 .938290 .00049

9.900 .941090 .00047

9.950 .945520 .00049

10.000 .949270 .00048

10.050 .951940 .00049

10.100 .955060 .00048

10.150 .959330 .00048

10.200 .961580 .00050

10.250 .965200 .00049

10.300 .968590 .00048

10.350 .972070 .00047

10.400 .974860 .00049

10.450 .978550 .00048

10.500 .982860 .00049

10.550 .984030 .00050

10.600 .988060 .00049

10.650 .992190 .00047

10.700 .994380 .00049

10.750 .997930 .00049

10.801 .001130 .00049

10.851 .004400 .00049

10.901 .006650 .00048

\begin{abstract}
480
9.600 .920280 .00047

9.650 .923340 .00047

9.700 .927380 .00048

9.750 .930120 .00049

9.800 .934590 .00048

9.850 .937600 .00047

9.900 .940550 .00050

9.950 .944560 .00048

10.000 .948070 .00049

10.050 .951250 .00048

10.100 .954600 .00048

10.150 .957260 .00048

10.200 .961710 .00049

10.250 .964310 .00049

10.300 .967700 .00048

10.350 .970990 .00049

10.400 .973770 .00048

10.450 .977350 .00048

10.500 .980100 .00049

10.550 .983860 .00049

10.600 .986480 .00049

10.650 .990170 .00047

10.700 .992810 .00048

10.750 .997320 .00050

10.800 .999850 .00050

10.851 .001760 .00049
\end{abstract}


10.951 .009540 .00049 11.001 .012520 .00048 11.051 .015500 .00049 11.101 .019180 .00048
10.901 .005180 .00048

10.951 .008280 .00049

11.001 .012280 .00049

11.051 .015180 .00050

11.101 .017840 .00050

486

9.600 .919350 .00047

9.650 .921610 .00048

9.700 .926730 .00049

9.750 .930190 .00048

9.800 .933120 .00047

9.850 .936150 .00049

9.900 .939880 .00047

9.950 .943650 .00048

10.000 .946850 .00048

10.050 .949840 .00048

10.100 .952410 .00050

10.150 .957310 .00049

10.200 .959720 .00049

10.250 .962940 .00049

10.300 .965660 .00048

10.350 .969970 .00048

10.400 .972900 .00048

10.450 .976040 .00049

10.500 .979490 .00048

10.550 .982070 .00049

10.650 .989200 .00048

10.700 .991380 .00048

10.750 .994400 .00049

10.800 .999100 .00049

10.851 .001260 .00049

10.901 .005150 .00050

10.951 .007590 .00050

11.001 .009870 .00049

11.051 .013590 .00049

11.101 .016540 .00050

11.151 .019740 .00049

11.201 .021810 .00050

0

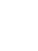

.

(2)


U5o2N20-icyl-1000-00

12

35.500 .921770 .00018 36.000 .923420 .00018 36.500 .924710 .00017 37.000 .926690 .00018 37.500 .927940 .00018 38.000 .929770 .00017 39.000 .932980 .00017 39.500 .933540 .00017 40.000 .935350 .00017 41.000 .937910 .00016 41.500 .939800 .00017 42.500 .941730 .00016 43.000 .942880 .00016 43.500 .943680 .00016 44.000 .944120 .00016 44.500 .945530 .00016 45.000 .946320 .00016 45.500 .947520 .00016 46.000 .948370 .00016 46.500 .949580 .00016

25

13.300 .921700 .00036 13.400 .925490 .00035 13.500 .930160 .00037 13.600 .933310 .00035 13.700 .938290 .00036 13.800 .941990 .00036 13.900 .945330 .00035 14.000 .949120 .00035 14.100 .953520 .00036 14.200 .957830 .00035 14.300 .961050 .00036 14.400 .964940 .00035 14.500 .968060 .00035 14.600 .972060 .00036 14.700 .975430 .00035 14.800 .979420 .00035 14.900 .982800 .00034 15.000 .986500 .00035 15.100 .989120 .00035 15.200 .992870 .00034 15.300 .996630 .00034 15.400 .999890 .00034
15

21.800 .927340 .00025

22.200 .933450 .00024

22.600 .937850 .00025

23.000 .942480 .00024

23.400 .946800 .00024

23.800 .951520 .00023

24.200 .955890 .00024

24.600 .959600 .00024

25.000 .964250 .00023

25.400 .967030 .00023

25.800 .971140 .00024

26.200 .974050 .00023

26.600 .977550 .00023

27.000 .980870 .00023

27.400 .983900 .00022

27.800 .986700 .00022

28.200 .989380 .00021

28.600 .992560 .00022

29.000 .995010 .00022

29.800 .999880 .00021

31.001 .007340 .00021

31.301 .008280 .00021
20

15.700 .925820 .00032 15.900 .931780 .00031 16.100 .937240 .00032 16.300 .942490 .00032 16.500 .947950 .00031 16.700 .953620 .00031 16.900 .958230 .00030 17.300 .968560 .00031 17.500 .972830 .00030 17.700 .977580 .00031 17.900 .982390 .00031 18.100 .986180 .00030 18.300 .991200 .00030 18.500 .995680 .00030 18.700 .999150 .00030 18.901 .002190 .00029 19.101 .007390 .00029 19.301 .011140 .00029 19.501 .014710 .00028
12.100 .922490 .00039 12.200 .926940 .00039 12.300 .932500 .00038 12.400 .937570 .00039 12.500 .943350 .00037 12.600 .948480 .00038 12.700 .952440 .00038 12.800 .957110 .00040 12.900 .962090 .00039 13.000 .966460 .00038 13.100 .971870 .00038 13.200 .975900 .00038 13.300 .980210 .00037 13.400 .985340 .00037 13.500 .989710 .00038 13.600 .994060 .00038 13.700 .998120 .00039 13.801 .002870 .00038 13.901 .006770 .00039 14.001 .010600 .00037 14.101 .014780 .00038 14.201 .019060 .00038
32

11.700 .919380 .00040 11.800 .925500 .00039 11.900 .930670 .00041 12.000 .935930 .00040 12.100 .941980 .00039 12.200 .946320 .00040 12.300 .952790 .00039 12.400 .957690 .00039 12.500 .962240 .00039 12.600 .967870 .00038 12.700 .971930 .00038 12.800 .977680 .00038 12.900 .982520 .00038 13.000 .987820 .00038 13.100 .991500 .00039 13.200 .996090 .00038 13.301 .001800 .00038 13.401 .005290 .00038 13.501 .009930 .00038 13.601 .015170 .00039 13.701 .019500 .00041 
15.501 .002590 .00034 15.601 .005800 .00034 15.701 .009020 .00033 15.801 .012350 .00035 15.901 .014650 .00034 16.001 .018300 .00034

35

11.300 .921250 .00041 11.400 .927470 .00041 11.500 .933880 .00040 11.600 .939800 .00040 11.700 .945750 .00042 11.800 .950960 .00040 11.900 .956470 .00040 12.000 .962610 .00040 12.100 .967630 .00040 12.200 .973500 .00041 12.300 .978300 .00040 12.400 .984550 .00040 12.500 .989330 .00039 12.600 .995070 .00040 12.700 .998770 .00040 12.801 .004050 .00040 12.901 .009950 .00039 13.001 .014620 .00041 13.101 .019100 .00040
40

10.700 .918910 .00044 10.750 .922170 .00041 10.800 .925370 .00043 10.850 .928930 .00044 10.900 .931880 .00043 10.950 .934950 .00042 11.000 .938690 .00042 11.050 .941410 .00043 11.100 .945240 .00043 11.150 .948950 .00042 11.200 .952100 .00043 11.250 .955120 .00043 11.300 .957670 .00043 11.350 .960940 .00042 11.400 .963560 .00042 11.450 .966740 .00042 11.500 .971030 .00042 11.550 .973600 .00042 11.600 .976380 .00044 11.650 .978820 .00041 11.700 .982870 .00043 11.750 .985270 .00042 11.800 .989000 .00042 11.850 .991340 .00043 11.900 .994710 .00042 11.950 .997230 .00042 12.000 .999010 .00041 12.051 .002730 .00042 12.101 .006190 .00042 12.151 .008890 .00043 12.201 .011430 .00043 12.251 .014580 .00040 12.301 .016810 .00042 12.351 .018860 .00042 12.401 .021720 .00043
50

10.000 .918710 .00044 10.050 .923490 .00046 10.100 .927320 .00045 10.150 .930920 .00045 10.200 .935600 .00045 10.250 .939070 .00045 10.300 .942480 .00044 10.350 .946760 .00045 10.400 .950500 .00044 10.450 .954380 .00045 10.500 .957260 .00045 10.600 .965090 .00045 10.650 .967970 .00046 10.700 .972660 .00045 10.750 .975410 .00045 10.800 .979150 .00045 10.850 .982390 .00044 10.900 .985320 .00046 10.950 .990360 .00045 11.000 .992900 .00044 11.050 .996450 .00046 11.100 .999270 .00045 11.151 .003230 .00045 11.201 .007180 .00046 11.251 .009720 .00046 11.301 .012920 .00044 11.351 .016520 .00043 11.401 .019410 .00046 
60

9.500 .915090 .00047

9.550 .920160 .00047

9.600 .924480 .00047

9.650 .928930 .00046

9.700 .932990 .00047

9.750 .936870 .00047

9.800 .941250 .00048

9.850 .945000 .00048

9.900 .949360 .00046

9.950 .954300 .00046

10.000 .958020 .00046

10.050 .961330 .00048

10.100 .966290 .00047

10.150 .969900 .00047

10.200 .974140 .00047

10.250 .978300 .00046

10.300 .981930 .00047

10.350 .985550 .00047

10.400 .989510 .00046

10.450 .992920 .00047

10.500 .997000 .00046

10.601 .004850 .00046

10.651 .008320 .00047

10.701 .012380 .00047

10.751 .015390 .00047

10.801 .018490 .00047

10.851 .022330 .00046

10.901 .026050 .00047
70

9.200 .916900 .00048

9.250 .921070 .00048

9.300 .925170 .00047

9.350 .929790 .00049

9.400 .934530 .00047

9.450 .938670 .00048

9.500 .943200 .00048

9.550 .947910 .00048

9.600 .951760 .00048

9.650 .956650 .00048

9.700 .960760 .00048

9.750 .965330 .00048

9.800 .969490 .00049

9.850 .973280 .00048

9.900 .978050 .00048

9.950 .981810 .00048

10.000 .985270 .00047

10.050 .990600 .00048

10.100 .994000 .00047

10.150 .998390 .00049

10.201 .002820 .00048

10.251 .006790 .00049

10.301 .010020 .00048

10.351 .014000 .00047

10.401 .017550 .00049

10.451 .021840 .00049

10.501 .025110 .00048
80

9.000 .920630 .00048

9.050 .925290 .00050

9.100 .928030 .00049

9.150 .933320 .00049

9.200 .939210 .00048

9.250 .942590 .00048

9.300 .947710 .00048

9.350 .952830 .00049

9.400 .956660 .00048

9.450 .960850 .00049

9.500 .965310 .00049

9.550 .970780 .00049

9.600 .975490 .00050

9.650 .979880 .00050

9.700 .983310 .00050

9.750 .988060 .00049

9.800 .991030 .00049

9.850 .996630 .00050

9.901 .001150 .00049

9.951 .004470 .00048

10.001 .008510 .00049

10.051 .013440 .00048

10.101 .017820 .00048

10.151 .022320 .00049

10.201 .025070 .00049
90

8.800 .917290 .00049

8.850 .921370 .00051

8.900 .927320 .00051

8.950 .930800 .00050

9.000 .936560 .00050

9.050 .941390 .00050

9.100 .946200 .00049

9.150 .950570 .00050

9.200 .956000 .00049

9.250 .959710 .00049

9.300 .965140 .00051

9.350 .969170 .00051

9.400 .973830 .00050

9.450 .977240 .00050

9.500 .983850 .00051

9.550 .987240 .00049

9.600 .992640 .00050

9.650 .996210 .00049
100

8.700 .919280 .00051

8.750 .924780 .00050

8.800 .929370 .00050

8.850 .935680 .00050

8.900 .939870 .00049

8.950 .945220 .00051

9.000 .949610 .00050

9.050 .954320 .00051

9.100 .959920 .00052

9.150 .964430 .00050

9.200 .969430 .00053

9.250 .973850 .00051

9.300 .978170 .00051

9.350 .984050 .00051

9.400 .987070 .00052

9.450 .991260 .00050

9.500 .996960 .00051

9.551 .001260 .00050
118

8.500 .918140 .00052

8.550 .922740 .00051

8.600 .928160 .00051

8.650 .932830 .00052

8.700 .937670 .00052

8.750 .944250 .00051

8.800 .949410 .00052

8.850 .953180 .00052

8.900 .959020 .00053

8.950 .962900 .00051

9.000 .968140 .00052

9.050 .972140 .00053

9.100 .978890 .00051

9.150 .983510 .00049

9.200 .988950 .00054

9.250 .992300 .00051

9.300 .998340 .00051

9.351 .003190 .00052 
9.701 .000220 .00050

9.751 .004740 .00050

9.801 .009900 .00049

9.851 .013980 .00050

9.901 .017050 .00050

9.951 .021420 .00050

10.001 .027200 .00050
9.601 .006270 .00050 9.651 .010950 .00051 9.701 .013870 .00052 9.751 .019560 .00049 9.801 .024150 .00050
9.401 .006870 .00051

9.451 .012260 .00052

9.501 .016720 .00052

9.551 .020530 .00051

9.601 .025260 .00051
130

8.400 .916680 .00052 8.450 .922620 .00051 8.500 .927520 .00052 8.550 .933750 .00053 8.600 .938180 .00052 8.650 .943200 .00053 8.750 .954120 .00053 8.800 .959310 .00050 8.850 .964290 .00051 8.900 .969210 .00052 8.950 .973560 .00051 9.000 .979380 .00052 9.050 .983980 .00052 9.100 .989360 .00054 9.150 .993640 .00052 9.200 .998310 .00053 9.301 .008140 .00051 9.351 .012760 .00051 9.401 .018540 .00052 9.451 .023290 .00053 9.501 .027420 .00054

235

8.100 .921230 .00053 8.150 .925570 .00053 8.200 .933220 .00056 8.250 .937590 .00054 8.350 .948820 .00055 8.400 .954010 .00054 8.450 .959450 .00053 8.500 .965240 .00055 8.550 .970800 .00055 8.600 .976610 .00056 8.650 .980970 .00054 8.700 .987090 .00054 8.750 .992530 .00055 8.800 .997790 .00053 8.851 .002840 .00054
150

8.300 .918820 .00051

8.350 .924070 .00053

8.400 .929790 .00054

8.450 .934260 .00052

8.500 .939910 .00053

8.550 .945180 .00053

8.600 .951310 .00053

8.650 .957720 .00052

8.700 .961520 .00054

8.750 .966530 .00052

8.800 .970280 .00052

8.850 .977570 .00052

8.900 .981860 .00053

8.950 .987240 .00053

9.000 .992650 .00052

9.050 .997550 .00052

9.101 .001740 .00052

9.151 .007240 .00051

9.201 .012200 .00053

9.251 .016790 .00054

9.301 .021230 .00055
200

8.100 .913690 .00052 8.150 .920110 .00051 8.200 .925630 .00053 8.250 .931360 .00051 8.300 .937310 .00054 8.350 .941950 .00053 8.400 .948350 .00053 8.450 .952270 .00053 8.500 .959360 .00052 8.550 .964530 .00053 8.600 .969160 .00053 8.650 .974360 .00052 8.700 .979740 .00053 8.750 .985640 .00053 8.800 .990130 .00053 8.850 .995280 .00053 8.901 .000280 .00054 8.951 .006640 .00053 9.001 .011170 .00052 9.051 .015110 .00051 9.101 .020690 .00053
8.050 .917340 .00055

8.100 .922860 .00054

8.150 .928300 .00054

8.200 .933850 .00052

8.250 .940210 .00054

8.300 .946040 .00054

8.350 .951660 .00054

8.450 .961690 .00053

8.500 .966850 .00053

8.550 .972150 .00053

8.650 .983010 .00054

8.800 .999170 .00054

8.851 .005060 .00053

8.901 .009120 .00054

8.951 .015220 .00055
353

8.000 .914550 .00054

8.050 .921540 .00053

8.100 .926230 .00053

8.150 .932130 .00053 8.200 .937280 .00054 8.250 .943040 .00053 8.300 .949390 .00054 8.350 .953780 .00053 8.400 .959820 .00053 8.450 .965360 .00053 8.500 .969860 .00054 8.550 .976550 .00054 8.600 .981650 .00056 8.650 .987280 .00055 8.700 .991430 .00053 
8.901 .006810 .00054 8.951 .013390 .00053 9.001 .017800 .00054 9.051 .022350 .00053 9.101 .027900 .00053

\section{0}

8.100 .921100 .00053 8.150 .927980 .00053 8.200 .933370 .00052 8.300 .943790 .00054 8.350 .949380 .00055 8.400 .953730 .00055 8.450 .958580 .00052 8.500 .965760 .00055 8.550 .970600 .00052 8.600 .975300 .00053 8.650 .981560 .00053 8.700 .985480 .00053 8.750 .991320 .00053 8.800 .995240 .00054 8.851 .000330 .00055 8.901 .005550 .00055 8.951 .010270 .00054 9.001 .015310 .00052 9.051 .019630 .00055 9.101 .025740 .00053

\section{5}

8.200 .918430 .00053 8.250 .923750 .00054 8.300 .930260 .00052 8.350 .934420 .00053 8.400 .940630 .00053 8.450 .945670 .00053 8.500 .950930 .00052 8.550 .955770 .00054 8.600 .961200 .00054 8.650 .966440 .00053 8.700 .971790 .00052 8.750 .975720 .00052 8.800 .981770 .00054 8.850 .987160 .00053 8.900 .991180 .00053 8.950 .996150 .00054 9.001 .000580 .00054
9.001 .019290 .00055 9.051 .024170 .00054 9.101 .030170 .00053

$$
500
$$

8.100 .919650 .00054 8.150 .924530 .00054 8.200 .931020 .00053 8.250 .935470 .00054 8.300 .941370 .00053 8.350 .948200 .00051 8.400 .951380 .00052 8.450 .957230 .00055 8.500 .962460 .00053 8.550 .967930 .00053 8.600 .972420 .00051 8.650 .978790 .00055 8.700 .983950 .00053 8.750 .989100 .00055 8.800 .994290 .00053 8.850 .998890 .00055 8.901 .004680 .00054 8.951 .008210 .00055 9.001 .013530 .00054 9.051 .017650 .00053 9.101 .024270 .00053
8.750 .997190 .00055 8.801 .001730 .00054 8.851 .005620 .00054 8.951 .016970 .00053 9.001 .021570 .00053

600

8.200 .919870 .00053 8.250 .924310 .00053 8.300 .930360 .00054 8.350 .935760 .00054 8.400 .941630 .00053 8.450 .945660 .00053 8.500 .951870 .00051 8.550 .956490 .00054 8.600 .961630 .00053 8.650 .966610 .00053 8.700 .972160 .00052 8.750 .977040 .00053 8.800 .982790 .00053 8.850 .986240 .00053 8.900 .992560 .00053 8.950 .996060 .00054 9.001 .001520 .00055 9.051 .007010 .00054 9.101 .011580 .00053 9.151 .015970 .00053 9.201 .019720 .00052
610
8.200 .918750 .00052

8.250 .924890 .00051

8.300 .929380 .00054

8.350 .934470 .00052

8.400 .939730 .00052

8.450 .944790 .00052

8.500 .950780 .00054

8.550 .956450 .00053 8.600 .961480 .00051 8.650 .966000 .00052 8.700 .971080 .00052 8.750 .977110 .00054 8.800 .980880 .00053 8.850 .985650 .00054 8.900 .992320 .00052 8.950 .996030 .00052 9.001 .000980 .00053
615

8.200 .918300 .00053 8.250 .922960 .00051 8.300 .928830 .00053 8.350 .934200 .00052 8.400 .940110 .00052 8.450 .944870 .00053 8.500 .949680 .00053 8.550 .955240 .00053 8.600 .960170 .00052 8.650 .965000 .00053 8.700 .970710 .00052 8.750 .974960 .00053 8.800 .980890 .00052 8.850 .985860 .00052 8.900 .990310 .00052 8.950 .995270 .00053 9.001 .000540 .00052 
9.051 .005310 .00053 9.101 .009890 .00053 9.151 .015490 .00052 9.201 .019990 .00052 9.251 .025190 .00052 9.301 .029720 .00053

\section{0}

8.200 .917040 .00050 8.250 .922010 .00052 8.300 .928170 .00053 8.350 .932600 .00053 8.400 .938780 .00053 8.500 .948480 .00053 8.550 .954990 .00051 8.600 .959380 .00051 8.800 .978680 .00052 8.850 .985180 .00053 8.900 .989570 .00053 8.950 .993260 .00054 9.000 .998500 .00053 9.051 .002970 .00053 9.101 .007940 .00053 9.151 .012670 .00052 9.201 .018930 .00053 9.251 .022470 .00054 9.301 .027160 .00052

U5o2N20-icyl-1000-01

12

35.300 .926350 .00017 35.900 .928330 .00017 36.500 .929620 .00018 37.100 .932020 .00017 37.700 .933150 .00017 38.900 .936110 .00016 39.500 .938260 .00016 40.100 .939200 .00016 41.300 .942490 .00016 41.900 .943800 .00016 43.100 .946130 .00015 43.700 .946800 .00015 44.300 .947960 .00015 44.900 .949180 .00016 45.500 .950690 .00015 46.100 .951340 .00015
9.051 .005570 .00052

9.101 .009910 .00054

9.151 .014770 .00053

9.201 .019650 .00054

9.251 .024810 .00054

9.301 .028760 .00052

625

8.200 .916810 .00054

8.250 .923180 .00051

8.300 .926850 .00053

8.350 .933440 .00052

8.400 .938870 .00053

8.450 .943630 .00053

8.500 .949220 .00052

8.550 .953620 .00052

8.600 .958560 .00051

8.650 .964770 .00053

8.700 .968490 .00053

8.750 .974560 .00051

8.800 .978090 .00053

8.850 .984240 .00053

8.900 .989890 .00051

8.950 .993990 .00053

9.000 .998650 .00051

9.051 .003480 .00052

9.101 .008440 .00052

9.151 .012540 .00054

9.201 .017910 .00055

9.051 .004980 .00051

9.101 .011080 .00052

9.151 .014440 .00053

9.201 .020270 .00054

629

8.250 .921490 .00053

8.300 .926270 .00052

8.350 .932550 .00050

8.400 .937680 .00050

8.500 .947990 .00053

8.550 .952780 .00051

8.600 .957060 .00053

8.650 .963310 .00052

8.700 .969010 .00053

8.750 .973380 .00053

8.800 .978160 .00053

8.850 .982970 .00053

8.900 .988620 .00054

8.950 .993120 .00054

9.000 .997910 .00054

9.051 .001920 .00052

9.101 .007570 .00052

9.151 .012260 .00053

9.201 .017330 .00053

9.251 .021470 .00053

9.301 .026220 .00052
20.300 .928330 .00025

20.800 .934510 .00024

21.300 .940190 .00024

21.800 .946010 .00024

22.300 .951820 .00024

22.800 .956610 .00023

23.300 .961730 .00024

23.800 .966600 .00022

24.300 .970870 .00022

24.800 .975150 .00022

25.300 .979230 .00022

26.300 .987220 .00021

26.800 .990550 .00022

27.300 .994170 .00022

27.800 .997100 .00021

28.300 .999870 .00021
20

14.300 .929170 .00031

14.900 .944860 .00030

15.100 .950830 .00030

15.300 .956390 .00030

15.500 .961810 .00030

15.700 .966190 .00029

15.900 .970630 .00030

16.100 .975310 .00030

16.300 .980270 .00030

16.500 .985040 .00029

16.900 .993180 .00029

17.100 .998400 .00029

17.301 .001690 .00029

17.501 .005170 .00029

17.701 .009660 .00029

17.901 .013650 .00029 
46.700 .952050 .00015 47.300 .953380 .00015 47.600 .953610 .00015

25

11.800 .922170 .00034 11.900 .926450 .00035 12.000 .930260 .00034 12.100 .934810 .00035 12.200 .937900 .00035 12.300 .942790 .00035 12.400 .947090 .00035 12.500 .950440 .00034 12.600 .954110 .00034 12.700 .957760 .00034 12.800 .962220 .00034 12.900 .965480 .00034 13.000 .969480 .00034 13.100 .973100 .00034 13.200 .976360 .00034 13.300 .980030 .00033 13.400 .983220 .00034 13.500 .987530 .00034 13.600 .990930 .00034 13.700 .993940 .00034 13.800 .996900 .00033 13.901 .000780 .00033 14.001 .003540 .00034 14.101 .006530 .00034 14.201 .009860 .00034 14.301 .013460 .00033 14.401 .015220 .00033

35

9.800 .922690 .00039

9.900 .929360 .00039 10.000 .935090 .00040 10.100 .940240 .00039 10.200 .946500 .00040 10.300 .952200 .00039 10.400 .958170 .00039 10.500 .963360 .00039 10.600 .969110 .00040 10.700 .975240 .00039 10.800 .980560 .00039 10.900 .985830 .00039
28.801 .003510 .00020 29.301 .006190 .00021 29.801 .008590 .00021 30.301 .012230 .00020

30

10.600 .923280 .00037 10.700 .928710 .00038 10.800 .933670 .00037 10.900 .938540 .00037 11.000 .943630 .00037 11.100 .948380 .00037 11.200 .953950 .00037 11.300 .958180 .00037 11.400 .962980 .00038 11.500 .967790 .00037 11.600 .972790 .00038 11.700 .978000 .00036 11.800 .981530 .00037 11.900 .986350 .00036 12.000 .990140 .00037 12.100 .994690 .00037 12.200 .999530 .00036 12.301 .003000 .00036 12.401 .008060 .00038 12.501 .011710 .00037 12.601 .015940 .00037
32

10.200 .920890 .00038 10.300 .925900 .00037 10.400 .931640 .00037 10.500 .937200 .00037 10.600 .942490 .00038 10.700 .948070 .00039 10.800 .953540 .00038 10.900 .959260 .00038 11.000 .963580 .00037 11.100 .969510 .00038 11.200 .973280 .00038 11.300 .978750 .00037 11.400 .982980 .00038 11.500 .988260 .00038 11.600 .992750 .00036 11.700 .996290 .00038 11.801 .002360 .00037 11.901 .006830 .00037 12.001 .011380 .00037 12.101 .015510 .00037
40
50
9.200 .919680 .00040
8.500 .920850 .00043
9.250 .923190 .00040
8.550 .924900 .00043
9.300 .927040 .00040
8.600 .928510 .00044
9.350 .929500 .00040
8.650 .932980 .00045
9.400 .933880 .00041
9.450 .936990 .00040
8.700 .936940 .00043
9.500 .940600 .00040
8.750 .939980 .00042
9.550 .943490 .00040
9.600 .945930 .00040
9.650 .949900 .00040
9.700 .952680 .00041
8.800 .944240 .00043
8.850 .948610 .00044
8.900 .952180 .00043
8.950 .955840 .00043
9.750 .956670 .00040 
11.000 .990400 .00040 11.100 .995570 .00039 11.201 .000830 .00038 11.301 .005590 .00038 11.401 .010160 .00038 11.501 .016240 .00037 11.601 .020830 .00038
9.800 .959080 .00041

9.850 .962480 .00041

9.900 .965140 .00040

9.950 .968030 .00041

10.000 .971370 .00040

10.050 .975020 .00040

10.100 .977910 .00041

10.150 .980410 .00041

10.200 .983950 .00040

10.250 .987140 .00041

10.300 .990390 .00041

10.350 .992250 .00041

10.400 .995800 .00040

10.450 .997430 .00040

10.501 .000970 .00040

10.551 .003960 .00041

10.601 .007060 .00039

10.651 .008910 .00041

10.701 .012570 .00040

10.751 .015150 .00040

10.801 .017600 .00041

\section{0}

8.000 .917550 .00044

8.050 .921760 .00045

8.100 .926760 .00045

8.150 .929870 .00044

8.200 .934690 .00044

8.250 .938740 .00046

8.300 .943170 .00045

8.350 .947650 .00046

8.400 .952250 .00044

8.450 .955600 .00047

8.500 .960740 .00044

8.550 .963930 .00043

8.600 .968570 .00046

8.650 .971440 .00045

8.700 .975320 .00044

8.750 .979480 .00046

8.800 .983460 .00045

8.850 .987880 .00045

8.900 .990990 .00045

8.950 .994850 .00044

9.000 .998560 .00045

9.051 .002760 .00045

9.101 .005970 .00045

9.151 .010550 .00045

9.201 .013990 .00045

9.251 .016470 .00044

9.301 .020740 .00044
70

7.700 .918880 .00046

7.750 .924150 .00046

7.800 .928380 .00047

7.850 .932780 .00045

7.900 .936900 .00045

7.950 .941640 .00045

8.000 .946200 .00046

8.050 .949820 .00046

8.100 .954480 .00046

8.150 .958940 .00045

8.200 .963150 .00045

8.250 .968410 .00047

8.300 .971880 .00045

8.350 .976600 .00046

8.400 .980380 .00046

8.450 .984310 .00047

8.500 .987930 .00046

8.550 .993570 .00046

8.600 .996340 .00046

8.651 .001520 .00046

8.701 .005050 .00046

8.751 .008660 .00046

8.801 .013540 .00045

8.851 .017030 .00046

8.901 .020160 .00047
9.100 .966280 .00044

9.150 .970470 .00044

9.200 .974520 .00043

9.250 .977480 .00043

9.300 .980710 .00045

9.350 .985050 .00043

9.400 .987340 .00042

9.450 .991400 .00043

9.500 .994720 .00044

9.550 .998410 .00045

9.601 .001580 .00043

9.651 .005060 .00043

9.751 .011840 .00044

9.801 .014780 .00043

9.851 .017670 .00042

9.901 .021260 .00043
80

7.500 .921840 .00047

7.550 .926980 .00047

7.600 .931300 .00046

7.650 .936020 .00048

7.700 .940980 .00046

7.750 .944870 .00047

7.800 .949450 .00048

7.850 .954720 .00047

7.900 .959960 .00047

7.950 .963290 .00046

8.000 .968300 .00047

8.050 .971980 .00049

8.100 .976920 .00048

8.150 .981730 .00046

8.200 .986170 .00048

8.250 .990910 .00048

8.300 .995430 .00047

8.350 .999730 .00047

8.401 .003610 .00047

8.451 .007890 .00048 8.501 .010990 .00047 8.551 .016010 .00048 8.601 .020640 .00047 
90

7.300 .919240 .00048 7.350 .924220 .00048 7.400 .929850 .00049 7.450 .933880 .00047 7.500 .939100 .00048 7.550 .944190 .00047 7.600 .948660 .00048 7.650 .953300 .00047 7.700 .958600 .00048 7.750 .962880 .00049 7.800 .968190 .00048 7.850 .971860 .00048 7.900 .977060 .00048 7.950 .981870 .00048 8.000 .986040 .00047 8.050 .991850 .00048 8.100 .995240 .00047 8.151 .000450 .00048 8.201 .003890 .00049 8.251 .008520 .00047 8.301 .012710 .00048 8.351 .017510 .00049 8.401 .020730 .00048

130

6.900 .921100 .00051

6.950 .926340 .00049

7.000 .931810 .00049

7.050 .936270 .00050

7.100 .941730 .00051

7.150 .946680 .00050

7.200 .952530 .00051

7.250 .958120 .00051

7.300 .962690 .00051

7.350 .968820 .00051

7.400 .974100 .00050

7.450 .978190 .00049

7.500 .982450 .00050

7.550 .987780 .00050

7.600 .992850 .00050

7.650 .998420 .00052

7.701 .002010 .00048

7.751 .007980 .00049

7.801 .012760 .00050

7.851 .017200 .00050 7.901 .021970 .00051
100

7.200 .922780 .00048

7.250 .927500 .00048

7.300 .932890 .00048

7.350 .938380 .00048

7.400 .942410 .00048

7.450 .947550 .00048

7.500 .953100 .00049

7.550 .957270 .00049

7.600 .962690 .00050

7.650 .966930 .00049

7.700 .972330 .00048

7.750 .977770 .00048

7.800 .981570 .00048

7.850 .986630 .00049

7.900 .991050 .00049

7.950 .996410 .00048

8.000 .999610 .00049

8.051 .004900 .00049

8.101 .009690 .00049

8.151 .013140 .00049

8.201 .017400 .00048

8.251 .022350 .00051

8.301 .027150 .00047
118

7.000 .921770 .00049

7.050 .925730 .00048

7.100 .931650 .00048

7.150 .937330 .00048

7.200 .941560 .00050

7.250 .946460 .00050

7.300 .952150 .00050

7.350 .957690 .00051

7.400 .961500 .00049

7.450 .967590 .00049

7.500 .972220 .00049

7.550 .977820 .00050

7.600 .982490 .00048

7.650 .987130 .00050

7.700 .993200 .00049

7.750 .996820 .00049

7.801 .001350 .00049

7.851 .007260 .00050

7.901 .010660 .00050

7.951 .015970 .00051

8.001 .019420 .00049
6.800 .921690 .00052

6.850 .927940 .00049

6.900 .933800 .00050

6.950 .938680 .00051

7.000 .943560 .00051

7.050 .949250 .00050

7.100 .954780 .00050

7.150 .960530 .00050

7.200 .965390 .00051

7.250 .969970 .00050

7.300 .975170 .00050

7.350 .981530 .00051

7.400 .986280 .00051

7.450 .991380 .00050

7.500 .996700 .00049

7.551 .000810 .00051

7.601 .005710 .00053

7.701 .016400 .00050

7.751 .020810 .00050

7.801 .026260 .00051
200

6.600 .918610 .00051

6.650 .924180 .00051

6.700 .930010 .00050

6.750 .937050 .00051

6.800 .941570 .00051

6.850 .946840 .00053

6.900 .953050 .00052

6.950 .958440 .00050

7.000 .963840 .00051

7.050 .969310 .00051

7.100 .974860 .00052

7.150 .979320 .00051

7.200 .985110 .00051

7.250 .989750 .00051

7.300 .994880 .00052

7.350 .999780 .00052

7.401 .005530 .00051

7.451 .010410 .00052

7.501 .014970 .00052

7.551 .020960 .00051

7.601 .025790 .00051 
235

6.500 .915210 .00052 6.550 .920410 .00049 6.600 .927150 .00050 6.650 .931960 .00051 6.700 .937650 .00052 6.750 .943880 .00051 6.800 .949560 .00051 6.850 .954800 .00052 6.900 .960710 .00052 6.950 .965480 .00050 7.000 .971550 .00053 7.050 .976130 .00051 7.100 .981270 .00052 7.150 .986830 .00052 7.200 .991740 .00052 7.250 .996420 .00052 7.301 .002870 .00051 7.351 .007900 .00051 7.401 .012600 .00052 7.451 .017970 .00051 7.501 .023380 .00051

\section{0}

6.500 .917870 .00052 6.550 .923010 .00052 6.600 .928650 .00052 6.650 .933690 .00050 6.700 .939780 .00052 6.750 .945710 .00051 6.800 .950910 .00051 6.850 .956270 .00051 6.900 .961450 .00052 6.950 .966320 .00052 7.000 .971300 .00052 7.050 .976490 .00052 7.100 .982420 .00051 7.150 .988060 .00052 7.200 .993060 .00052 7.250 .997820 .00053 7.301 .002910 .00054 7.351 .007880 .00052 7.401 .012690 .00051 7.451 .016860 .00050 7.501 .022690 .00051
250

6.500 .916990 .00052

6.550 .922380 .00051

6.600 .927470 .00051

6.650 .934580 .00051

6.700 .939240 .00052

6.750 .945150 .00050

6.800 .950520 .00050

6.850 .956480 .00053

6.900 .960970 .00051

7.000 .972490 .00052

7.050 .977720 .00052

7.100 .984120 .00052

7.150 .988810 .00053

7.200 .994070 .00051

7.250 .999530 .00051

7.301 .004190 .00051

7.351 .009810 .00052

7.401 .014070 .00051

7.451 .019620 .00053

7.501 .024760 .00051

500

6.500 .915690 .00051

6.550 .920370 .00051

6.600 .926720 .00052

6.650 .932740 .00050

6.700 .937280 .00053

6.750 .943100 .00053

6.800 .948880 .00051

6.850 .954200 .00050

6.900 .959890 .00052

6.950 .964330 .00053

7.000 .969860 .00051

7.050 .976030 .00050

7.100 .980760 .00052

7.150 .985500 .00053

7.200 .990840 .00051

7.250 .995410 .00052

7.301 .000100 .00052

7.351 .005370 .00053

7.401 .010390 .00053

7.451 .014450 .00052

7.501 .019750 .00052
353

6.500 .921400 .00050

6.550 .926570 .00052

6.600 .933020 .00051

6.650 .938960 .00051

6.700 .944700 .00051

6.750 .949380 .00052

6.850 .960510 .00054

6.900 .966110 .00051

6.950 .971640 .00053

7.000 .977420 .00053

7.050 .981660 .00053

7.100 .987530 .00051

7.150 .992450 .00051

7.251 .002800 .00053

7.301 .007600 .00052

7.351 .012810 .00052

7.451 .022840 .00053

\begin{tabular}{ccc}
\multicolumn{1}{c}{600} \\
6.60 & 0.91712 & 0.00051 \\
6.65 & 0.92290 & 0.00049 \\
6.70 & 0.92717 & 0.00050 \\
6.75 & 0.93278 & 0.00050 \\
6.80 & 0.93839 & 0.00052 \\
6.85 & 0.94462 & 0.00051 \\
6.90 & 0.94937 & 0.00051 \\
6.95 & 0.95411 & 0.00051 \\
7.00 & 0.96015 & 0.00052 \\
7.05 & 0.96491 & 0.00052 \\
7.10 & 0.97010 & 0.00051 \\
7.15 & 0.97573 & 0.00051 \\
7.20 & 0.97929 & 0.00050 \\
7.25 & 0.98496 & 0.00052 \\
7.30 & 0.99013 & 0.00052 \\
7.35 & 0.99507 & 0.00050 \\
7.40 & 1.00000 & 0.00050 \\
7.45 & 1.00381 & 0.00053 \\
7.50 & 1.01026 & 0.00051 \\
7.55 & 1.01393 & 0.00052 \\
7.60 & 1.01921 & 0.00051 \\
7.65 & 1.02383 & 0.00052 \\
7.70 & 1.02724 & 0.00051
\end{tabular} 
605

6.600 .915710 .00051

6.650 .921930 .00050

6.700 .928290 .00051

6.750 .933520 .00051

6.800 .938140 .00051

6.850 .942980 .00050

6.900 .948720 .00050

6.950 .954930 .00052

7.000 .959060 .00052

7.050 .964180 .00052

7.100 .970090 .00052

7.150 .975100 .00053

7.200 .979390 .00052

7.250 .984210 .00050

7.300 .989490 .00051

7.350 .993940 .00051

7.400 .998880 .00052

7.451 .004380 .00051

7.501 .008450 .00050

7.551 .013930 .00050

7.601 .018760 .00051

7.651 .023280 .00053

7.701 .027380 .00051

\section{0}

6.600 .915410 .00052

6.650 .919860 .00049

6.700 .925460 .00051

6.750 .931340 .00051

6.800 .936630 .00050

6.850 .941310 .00052

6.900 .946520 .00050

6.950 .952170 .00050

7.000 .957470 .00051

7.050 .962150 .00050

7.100 .967280 .00051

7.150 .972720 .00052

7.200 .978100 .00052

7.250 .982880 .00052

7.300 .987850 .00051

7.350 .992050 .00051

7.400 .996360 .00052

7.451 .001340 .00050

7.501 .007010 .00051

7.551 .012180 .00051

7.601 .016390 .00051

7.651 .020600 .00051

7.701 .026080 .00051
610

6.600 .916520 .00051

6.650 .922980 .00052

6.700 .926470 .00051

6.750 .932390 .00052

6.800 .938030 .00052

6.850 .944070 .00051

6.900 .949120 .00051

6.950 .953600 .00051

7.000 .957930 .00051

7.050 .963140 .00053

7.100 .968620 .00050

7.150 .973770 .00052

7.200 .978840 .00052

7.250 .984140 .00051

7.300 .989320 .00053

7.350 .994030 .00054

7.400 .999830 .00050

7.451 .004570 .00051

7.501 .008660 .00051

7.551 .013200 .00051

7.601 .018550 .00053

625

6.600 .915160 .00050

6.650 .919610 .00052

6.700 .925490 .00052

6.750 .930550 .00050

6.800 .936280 .00051

6.850 .941270 .00051

6.900 .946340 .00051

6.950 .951210 .00051

7.000 .956910 .00050

7.200 .976820 .00052

7.250 .982000 .00050

7.300 .987270 .00052

7.350 .991530 .00051

7.400 .996700 .00051

7.451 .001790 .00052

7.501 .006670 .00052

7.551 .012550 .00052

7.601 .016210 .00050

7.651 .021090 .00051

7.701 .025140 .00052
615

6.600 .916100 .00051

6.650 .920840 .00051

6.700 .926000 .00053

6.750 .932550 .00050

6.800 .937430 .00051

6.850 .943100 .00051

6.900 .949170 .00052

6.950 .953040 .00051

7.000 .957300 .00050

7.050 .964220 .00052

7.100 .968390 .00052

7.150 .973760 .00052

7.200 .979560 .00051

7.250 .983610 .00052

7.300 .988870 .00053

7.350 .993700 .00052

7.400 .998030 .00052

7.451 .003240 .00052

7.501 .008080 .00052

7.551 .013100 .00051

7.601 .017110 .00052

7.651 .022900 .00051

7.701 .027330 .00051

629
6.600 .915440 .00052

6.650 .919060 .00051

6.700 .924050 .00051

6.750 .929210 .00053

6.800 .935040 .00050

6.850 .940750 .00049

6.900 .945960 .00051

6.950 .950320 .00051

7.000 .956250 .00051

7.050 .961140 .00051

7.100 .966340 .00052

7.150 .970820 .00053

7.200 .976500 .00051

7.250 .981260 .00051

7.300 .985280 .00053

7.350 .991360 .00052

7.400 .995830 .00052

7.451 .001030 .00052

7.501 .006560 .00050

7.551 .010380 .00051

7.601 .015540 .00052

7.651 .020030 .00050

7.701 .025000 .00051 
U5o2N20-icyl-1000-10

12

32.100 .918770 .00017 32.600 .920820 .00017 33.100 .922410 .00017 34.100 .925860 .00017 34.600 .927350 .00017 35.100 .929060 .00016 36.100 .932360 .00016 36.600 .933600 .00016 37.100 .934760 .00016 37.600 .935690 .00016 38.100 .937070 .00016 38.600 .938570 .00016 39.100 .939430 .00016 40.100 .941750 .00016 40.600 .943020 .00015 41.100 .943820 .00016 41.600 .945050 .00016 42.100 .945720 .00015 42.600 .947180 .00015
15

19.100 .926570 .00023 19.600 .933410 .00023 20.100 .939240 .00022 20.600 .944270 .00023 21.100 .950340 .00023 21.600 .955660 .00023 22.100 .960950 .00022 22.600 .965110 .00022 23.600 .973950 .00021 24.100 .978590 .00022 24.600 .981980 .00022 25.100 .986270 .00021 25.600 .990060 .00021 26.100 .993370 .00021 26.600 .997200 .00021 27.101 .000470 .00021 27.601 .002710 .00020 28.101 .005580 .00020 28.601 .007690 .00020 29.101 .011270 .00020
25

10.800 .925750 .00032 10.900 .929330 .00033 11.000 .934280 .00033 11.100 .938030 .00031 11.200 .942810 .00033 11.300 .946250 .00033 11.400 .950960 .00032 11.500 .954730 .00031 11.600 .958330 .00031 11.700 .961670 .00033
30

9.500 .922390 .00035 9.600 .928540 .00034 9.700 .933120 .00035 9.800 .938850 .00035 9.900 .944030 .00034 10.000 .948940 .00034 10.100 .953940 .00035 10.200 .958760 .00035 10.300 .963940 .00034 10.400 .968980 .00035
20

13.100 .926110 .00029 13.200 .929390 .00028 13.400 .934750 .00029 13.700 .942760 .00028 13.800 .945360 .00028 13.900 .948500 .00029 14.000 .950890 .00028 14.100 .953600 .00028 14.200 .956010 .00028 14.300 .958060 .00030 14.400 .961200 .00028 14.500 .963390 .00028 14.700 .968870 .00028 14.900 .973580 .00028 15.000 .975460 .00029 15.100 .978380 .00029 15.200 .979840 .00028 15.300 .982470 .00028 15.400 .984720 .00028 15.500 .986890 .00028 15.600 .989420 .00028 15.700 .992190 .00027 15.800 .993660 .00028 15.900 .995380 .00027 16.000 .997710 .00028 16.100 .999800 .00027 16.201 .001410 .00027 16.301 .003500 .00027 16.401 .005400 .00026 16.501 .007820 .00028 16.601 .009840 .00028 16.701 .011390 .00028 16.801 .013820 .00027

$\begin{array}{ccc}32 & \\ 9.10 & 0.92145 & 0.00036 \\ 9.20 & 0.92625 & 0.00037 \\ 9.30 & 0.93234 & 0.00036 \\ 9.40 & 0.93787 & 0.00035 \\ 9.50 & 0.94307 & 0.00036 \\ 9.60 & 0.94834 & 0.00036 \\ 9.70 & 0.95457 & 0.00035 \\ 9.80 & 0.95922 & 0.00035 \\ 9.90 & 0.96453 & 0.00035 \\ 10.00 & 0.96891 & 0.00035\end{array}$

9.100 .921450 .00036 9.200 .926250 .00037 9.300 .932340 .00036 9.400 .937870 .00035 9.500 .943070 .00036 9.600 .948340 .00036 9.700 .954570 .00035 9.800 .959220 .00035 10.000 .968910 .00035 
11.800 .966340 .00032 11.900 .969550 .00032 12.000 .972830 .00032 12.100 .976410 .00032 12.200 .980510 .00032 12.300 .983540 .00032 12.400 .987090 .00031 12.500 .991070 .00031 12.600 .994440 .00031 12.700 .997460 .00032 12.801 .000140 .00032 12.901 .003800 .00033 13.001 .006990 .00032 13.101 .010190 .00032 13.201 .013150 .00032 13.301 .016900 .00030

35

8.700 .922970 .00037

8.750 .926440 .00037

8.800 .929540 .00037

8.850 .932340 .00036

8.900 .935600 .00038

8.950 .938450 .00037

9.000 .941180 .00036

9.050 .944380 .00037

9.100 .947080 .00036

9.150 .950890 .00037

9.200 .953030 .00036

9.250 .956340 .00036

9.300 .958310 .00037

9.350 .961790 .00037

9.400 .964280 .00036

9.450 .967490 .00037

9.500 .970550 .00036

9.550 .973380 .00037

9.600 .975490 .00036

9.650 .979360 .00037

9.700 .981680 .00036

9.750 .983440 .00037

9.800 .986750 .00037

9.850 .989420 .00037

9.900 .991450 .00036

9.950 .994230 .00036 10.000 .998160 .00035 10.050 .999960 .00036 10.101 .002060 .00036 10.151 .005280 .00036 10.201 .007290 .00036 10.251 .009400 .00036 10.301 .012290 .00036
10.500 .973800 .00034 10.600 .978290 .00034 10.700 .982590 .00035 10.800 .987260 .00035 10.900 .992670 .00034 11.000 .995510 .00035 11.101 .001060 .00034 11.201 .004520 .00034 11.301 .008280 .00034 11.401 .012450 .00034 11.501 .016940 .00033
10.100 .974990 .00036 10.200 .979350 .00035 10.300 .984670 .00034 10.400 .989010 .00034 10.500 .994510 .00035 10.600 .999360 .00036 10.701 .002860 .00035 10.801 .008710 .00035 10.901 .012170 .00035 11.001 .016920 .00035
40

8.100 .920700 .00038 8.150 .924680 .00039 8.200 .927250 .00039 8.250 .931010 .00039 8.300 .934690 .00039 8.350 .937860 .00038 8.400 .941830 .00038 8.450 .944650 .00039 8.500 .947300 .00039 8.550 .950640 .00038 8.600 .954240 .00038 8.650 .957980 .00038 8.700 .961120 .00038 8.750 .963940 .00037 8.800 .967150 .00038 8.850 .970430 .00037 8.900 .973730 .00037 8.950 .975950 .00037 9.000 .979580 .00038 9.050 .982420 .00039 9.100 .985920 .00038 9.150 .988830 .00039 9.200 .991970 .00038 9.250 .994040 .00038 9.300 .997940 .00038 9.351 .000770 .00038 9.401 .003220 .00037 9.451 .006520 .00038 9.501 .009450 .00038 9.551 .011650 .00038 9.601 .015260 .00037 9.651 .017150 .00038 9.701 .020650 .00039
50

7.400 .924300 .00039

7.450 .927410 .00040

7.500 .931540 .00040

7.550 .935600 .00040

7.600 .939070 .00041

7.650 .942910 .00039

7.700 .947740 .00040

7.750 .951220 .00040

7.800 .955250 .00040

7.850 .958180 .00040

7.900 .962600 .00038

8.000 .969780 .00040

8.050 .973580 .00040

8.100 .977400 .00039

8.150 .980730 .00040

8.200 .984780 .00041

8.250 .987280 .00040

8.300 .991250 .00040

8.350 .994720 .00040

8.400 .998200 .00041

8.451 .001420 .00040

8.501 .004850 .00040

8.551 .008100 .00040

8.601 .011580 .00040

8.651 .015570 .00040

8.701 .018640 .00040 
10.351 .014420 .00036

10.401 .016640 .00037
60

6.900 .921430 .00042 6.950 .926210 .00042 7.000 .930790 .00042 7.050 .934620 .00041 7.100 .938670 .00041 7.150 .943470 .00042 7.200 .948140 .00041 7.250 .951770 .00043 7.300 .956880 .00041 7.350 .960450 .00041 7.400 .965310 .00041 7.450 .968260 .00042 7.500 .972330 .00041 7.550 .976560 .00043 7.650 .984670 .00042 7.700 .988720 .00042 7.750 .992410 .00042 7.800 .995960 .00041 7.850 .999890 .00042 7.901 .004060 .00042 7.951 .007560 .00042 8.001 .011370 .00042 8.051 .014990 .00042 8.101 .019160 .00041

90

6.100 .917440 .00043 6.150 .922110 .00045 6.200 .927760 .00044 6.250 .932690 .00043 6.300 .938230 .00042 6.350 .943100 .00045 6.400 .947870 .00044 6.450 .953270 .00044 6.500 .957500 .00045 6.550 .961600 .00044 6.600 .968040 .00044 6.650 .972420 .00045 6.700 .977270 .00044 6.750 .981660 .00044 6.800 .985980 .00044 6.850 .992080 .00044 6.900 .995500 .00045
70

6.600 .924520 .00043

6.650 .928400 .00043

6.750 .938480 .00043

6.800 .942710 .00043

6.850 .947660 .00042

6.900 .951770 .00042

6.950 .956650 .00042

7.000 .960710 .00042

7.050 .965840 .00043

7.100 .969540 .00042

7.150 .974390 .00043

7.200 .978270 .00042

7.250 .982390 .00042

7.300 .987010 .00044

7.350 .991310 .00043

7.400 .994680 .00043

7.450 .999050 .00043

7.501 .002640 .00042

7.551 .007850 .00042

7.601 .011000 .00044

7.651 .015910 .00042

7.701 .019660 .00043

100

6.000 .923270 .00045

6.050 .926980 .00045

6.100 .932470 .00043

6.150 .938660 .00045

6.200 .942980 .00044

6.250 .948210 .00045

6.300 .953100 .00044

6.350 .958060 .00044

6.400 .964100 .00044

6.450 .968480 .00045

6.500 .972890 .00045

6.550 .978210 .00045

6.600 .983310 .00045

6.650 .988300 .00044

6.700 .992480 .00046

6.750 .997760 .00044

6.801 .002570 .00044
80

6.300 .919400 .00044 6.350 .923540 .00043 6.400 .929010 .00043 6.450 .934040 .00043 6.500 .938800 .00042 6.550 .943590 .00044 6.600 .947550 .00043 6.650 .953400 .00044 6.700 .957600 .00043 6.750 .963080 .00044 6.800 .966800 .00044 6.850 .971130 .00044 6.900 .976070 .00042 6.950 .980140 .00043 7.000 .985640 .00044 7.050 .989120 .00044 7.100 .994290 .00046 7.150 .998620 .00043 7.201 .003220 .00043 7.251 .007320 .00043 7.301 .011290 .00044 7.351 .015610 .00044 7.401 .021100 .00044

118

5.800 .922360 .00044 5.850 .928040 .00047 5.900 .934200 .00045 5.950 .939460 .00046 6.000 .944530 .00044 6.050 .950490 .00046 6.100 .955400 .00045 6.150 .960360 .00046 6.200 .965900 .00046 6.250 .970370 .00045 6.300 .975220 .00044 6.350 .980410 .00046 6.400 .985680 .00047 6.450 .991070 .00045 6.500 .995670 .00046 6.551 .000160 .00046 6.601 .005490 .00045 
6.950 .999760 .00045 7.001 .004680 .00044 7.051 .009040 .00045 7.101 .013310 .00045 7.151 .018430 .00044 7.201 .023360 .00043

130

5.700 .923500 .00045 5.750 .928980 .00045 5.800 .934110 .00045 5.850 .939670 .00046 5.900 .944970 .00047 5.950 .950590 .00047 6.000 .956970 .00044 6.100 .967210 .00046 6.150 .972020 .00046 6.200 .976920 .00044 6.250 .982690 .00046 6.300 .987720 .00047 6.350 .992520 .00046 6.400 .998410 .00045 6.451 .001720 .00046 6.501 .006620 .00047 6.551 .012730 .00045 6.601 .016840 .00047

\section{5}

5.200 .915120 .00047 5.250 .920960 .00047 5.300 .927520 .00048 5.350 .932870 .00047 5.400 .938500 .00047 5.450 .944490 .00047 5.500 .950530 .00047 5.550 .956080 .00047 5.600 .961830 .00046 5.650 .967460 .00047 5.700 .972440 .00047 5.750 .978280 .00048 5.800 .984250 .00048 5.850 .990080 .00047 5.900 .995660 .00047 5.950 .999890 .00047
6.851 .007040 .00045

6.901 .010970 .00045

6.951 .015590 .00044

7.001 .021370 .00045
6.651 .010040 .00047

6.701 .015000 .00044

6.751 .019630 .00044

6.801 .024300 .00046
150

5.500 .915470 .00046

5.550 .921120 .00047

5.600 .927610 .00045

5.650 .932750 .00047

5.700 .938970 .00045

5.750 .944840 .00048

5.800 .950610 .00046

5.850 .955570 .00046

5.900 .961110 .00046

5.950 .965440 .00046

6.000 .972040 .00046

6.050 .976980 .00046

6.100 .982640 .00046

6.150 .988250 .00046

6.200 .992820 .00047

6.250 .997900 .00044

6.301 .002630 .00046

6.351 .007200 .00046

6.401 .013160 .00046

6.451 .018190 .00047

6.501 .023270 .00045
200

5.300 .916430 .00046 5.350 .922160 .00048 5.400 .928480 .00046 5.450 .934630 .00047 5.500 .940150 .00047 5.550 .945830 .00048 5.600 .951620 .00047 5.650 .957720 .00046 5.700 .963140 .00046 5.750 .968260 .00046 5.800 .975330 .00047 5.850 .979530 .00047 5.900 .985440 .00047 5.950 .989840 .00046 6.000 .995700 .00048 6.051 .000830 .00047 6.101 .006180 .00048 6.151 .010880 .00047 6.201 .015760 .00047 6.251 .022280 .00046 6.301 .027000 .00046
5.200 .918530 .00046

5.250 .924130 .00048

5.300 .929310 .00047

5.350 .935560 .00047

5.400 .942230 .00047

5.450 .948880 .00048

5.500 .953380 .00049

5.550 .958880 .00047

5.600 .965040 .00047 5.650 .970790 .00048 5.700 .976580 .00048 5.750 .981200 .00047 5.800 .987420 .00048 5.850 .992370 .00047 5.900 .998090 .00046 5.951 .003130 .00047

\section{3}

5.100 .918220 .00048 5.150 .923970 .00048 5.200 .930850 .00047 5.250 .936110 .00048 5.300 .941660 .00048 5.350 .947290 .00047 5.400 .953220 .00048 5.450 .959070 .00047 5.500 .964470 .00047 5.550 .969850 .00048 5.600 .975510 .00047 5.650 .981210 .00048 5.700 .986480 .00049 5.750 .993110 .00047 5.800 .998700 .00047 5.851 .003160 .00048

$$
\text { F-461 }
$$


6.001 .006410 .00048 6.051 .010190 .00047 6.101 .016750 .00049 6.151 .019880 .00050 6.201 .027020 .00046

\section{0}

5.100 .921960 .00046 5.150 .927580 .00045 5.200 .932470 .00048 5.250 .938480 .00047 5.300 .944480 .00047 5.350 .949680 .00049 5.400 .955580 .00047 5.450 .960650 .00048 5.500 .966700 .00047 5.600 .978090 .00048 5.650 .982460 .00049 5.700 .987380 .00048 5.750 .993390 .00047 5.800 .998560 .00048 5.851 .003960 .00048 5.901 .008520 .00046 5.951 .014640 .00047 6.001 .019330 .00048

\section{5}

5.100 .916540 .00047 5.150 .921760 .00047 5.200 .927770 .00047 5.250 .933910 .00047 5.300 .938780 .00047 5.350 .944640 .00047 5.400 .949210 .00048 5.450 .954380 .00046 5.500 .959890 .00047 5.550 .966050 .00048 5.600 .971340 .00046 5.650 .976140 .00048 5.700 .981430 .00047 5.750 .986650 .00047 5.800 .992880 .00048 5.850 .997860 .00047 5.901 .001820 .00047 5.951 .006810 .00048 6.001 .011640 .00048 6.051 .017150 .00048 6.101 .021740 .00047
6.001 .008810 .00047 6.051 .013610 .00048 6.101 .017990 .00047

5.901 .008000 .00048 5.951 .014260 .00047 6.001 .018970 .00048
500

5.100 .921460 .00047 5.150 .926730 .00047 5.200 .932010 .00048 5.250 .937860 .00047 5.300 .943860 .00049 5.350 .949210 .00047 5.400 .954790 .00046 5.450 .959830 .00047 5.600 .976420 .00049 5.650 .982520 .00047 5.700 .987290 .00048 5.750 .992720 .00048 5.800 .998420 .00048 5.851 .003100 .00047 5.901 .007990 .00048 5.951 .013300 .00049 6.001 .018410 .00049
600

5.150 .922200 .00047 5.200 .928290 .00048 5.250 .933530 .00048 5.300 .938630 .00045 5.400 .949780 .00047 5.500 .960640 .00047 5.550 .965280 .00046 5.600 .970940 .00047 5.650 .976500 .00047 5.700 .982100 .00048 5.750 .987620 .00048 5.800 .992910 .00046 5.850 .997790 .00048 5.901 .002350 .00047 5.951 .007370 .00047 6.001 .012200 .00048 6.051 .016310 .00047 6.101 .021960 .00048
5.100 .915500 .00046 5.200 .926960 .00048 5.250 .933420 .00046 5.300 .938630 .00048 5.350 .944160 .00047 5.400 .949120 .00048 5.450 .954550 .00048 5.500 .959740 .00047 5.550 .965490 .00048 5.600 .970790 .00047 5.650 .975580 .00046 5.700 .980790 .00048 5.750 .986520 .00048 5.800 .992030 .00048 5.850 .997260 .00046 5.901 .001910 .00047 5.951 .007470 .00046 6.001 .011220 .00047 6.051 .017410 .00047 6.101 .020860 .00047
615

5.100 .916430 .00047 5.150 .921430 .00047 5.200 .926640 .00046 5.250 .932680 .00047 5.300 .938730 .00047 5.350 .944420 .00049 5.400 .949230 .00046 5.450 .954480 .00047 5.500 .959030 .00048 5.550 .965330 .00048 5.600 .971500 .00047 5.650 .975960 .00048 5.700 .981110 .00047 5.750 .986600 .00046 5.800 .991900 .00047 5.850 .997400 .00047 5.901 .001740 .00048 5.951 .006570 .00048 6.001 .011060 .00049 6.051 .016340 .00046 6.101 .021250 .00048 
620

5.100 .915130 .00046 5.150 .921070 .00047 5.200 .926330 .00047 5.250 .931520 .00047 5.300 .937570 .00049 5.350 .943300 .00045 5.400 .948990 .00047 5.450 .954630 .00046 5.500 .958630 .00048 5.550 .965120 .00048 5.600 .970050 .00046 5.650 .975900 .00047 5.700 .980530 .00048 5.750 .985200 .00047 5.800 .991170 .00048 5.850 .994920 .00048 5.901 .000560 .00048 5.951 .005780 .00047 6.001 .010990 .00046 6.051 .015530 .00048 6.101 .020550 .00046

U5o2N23-icyl-1000-00

12

41.100 .890180 .00017 41.900 .892540 .00016 42.700 .893760 .00016 43.500 .895610 .00016 44.300 .896810 .00017 45.100 .898910 .00015 45.900 .899860 .00016 46.700 .901630 .00016 47.500 .903280 .00015 48.300 .904280 .00015 49.100 .905440 .00015 49.900 .906350 .00015 50.700 .907510 .00016 51.500 .908950 .00015 52.300 .909720 .00015 53.100 .910660 .00015 53.900 .911310 .00015 54.700 .912890 .00015 55.500 .913540 .00015 56.300 .914070 .00015 57.100 .915090 .00015
625

5.100 .915490 .00048

5.150 .920380 .00047

5.200 .926230 .00046

5.250 .932490 .00048

5.300 .937980 .00047

5.350 .942780 .00047

5.400 .948880 .00048

5.450 .954110 .00047

5.500 .960760 .00047

5.550 .965270 .00048

5.600 .969030 .00047

5.650 .974540 .00049

5.700 .981120 .00049

5.750 .985500 .00047

5.800 .989820 .00047

5.850 .995670 .00047

5.901 .000710 .00048

5.951 .006230 .00048

6.001 .010880 .00048

6.051 .015430 .00046

6.101 .020540 .00048
629

5.100 .914890 .00048 5.150 .921100 .00048 5.200 .925970 .00046 5.250 .931220 .00047 5.300 .937590 .00046 5.350 .943270 .00048 5.400 .948160 .00046 5.450 .953740 .00047 5.500 .958520 .00047 5.550 .964580 .00047 5.600 .968760 .00048 5.650 .974730 .00048 5.700 .979710 .00047 5.750 .984170 .00047 5.800 .990720 .00047 5.850 .995390 .00048 5.901 .000210 .00047 5.951 .005090 .00049 6.001 .010140 .00048 6.051 .014890 .00047 6.101 .019410 .00048
26.300 .926890 .00023 27.300 .935210 .00022 28.300 .942250 .00022 29.300 .949050 .00022 30.300 .955300 .00021 31.300 .960510 .00021 32.300 .965810 .00020 33.300 .969990 .00020 34.300 .974540 .00020 35.300 .979100 .00020 36.300 .982620 .00020 37.300 .986490 .00019 38.300 .989700 .00019 39.300 .992630 .00019 40.300 .995880 .00018 41.300 .998550 .00018 42.301 .001050 .00018 43.301 .003500 .00018 44.301 .005630 .00018 45.301 .007960 .00018 46.301 .009250 .00018
20

17.500 .925380 .00032 17.700 .929780 .00030 17.900 .934730 .00030 18.100 .938830 .00031 18.300 .943190 .00030 18.500 .947400 .00030 18.700 .951760 .00030 18.900 .955010 .00029 19.100 .959890 .00030 19.300 .963580 .00029 19.500 .967150 .00029 19.700 .971010 .00030 19.900 .974160 .00028 20.100 .978120 .00028 20.300 .981060 .00028 20.500 .984630 .00029 20.700 .987620 .00028 20.900 .991950 .00028 21.100 .993980 .00030 21.300 .997000 .00028 21.501 .000160 .00027 
57.900 .916230 .00015 58.600 .916850 .00014
47.301 .011160 .00017 47.701 .012360 .00017
21.701 .003410 .00028 21.901 .005840 .00027 22.101 .008740 .00028 22.301 .011450 .00027 22.401 .013100 .00027

25 14.600 .924910 .00034 14.700 .928660 .00034 14.800 .932520 .00034 14.900 .935610 .00035 15.000 .938880 .00034 15.100 .942250 .00034 15.200 .946230 .00034 15.300 .949330 .00034 15.400 .951900 .00033 15.600 .958690 .00034 15.700 .962130 .00035 15.800 .965500 .00034 15.900 .968090 .00033 16.000 .970800 .00033 16.100 .973400 .00033 16.200 .977060 .00033 16.300 .979540 .00033 16.400 .983060 .00033 16.500 .986120 .00034 16.600 .988300 .00032 16.700 .991100 .00033 16.800 .993760 .00033 16.900 .996520 .00033 17.000 .999330 .00034 17.101 .002200 .00032 17.201 .004510 .00034 17.301 .007390 .00032 17.401 .009440 .00033 17.501 .012030 .00032 17.601 .014440 .00032

35

12.100 .921540 .00039 12.200 .927050 .00040 12.300 .932960 .00039 12.400 .937360 .00040 12.500 .943010 .00040 12.600 .947620 .00041 12.700 .952660 .00041 12.800 .957740 .00040
30

13.000 .921020 .00037 13.100 .925330 .00038 13.200 .929740 .00038 13.400 .938360 .00037 13.500 .943040 .00036 13.700 .951810 .00037 13.800 .955830 .00037 14.000 .964490 .00036 14.100 .967880 .00038 14.200 .972320 .00037 14.300 .975880 .00037 14.400 .979550 .00036 14.500 .983700 .00037 14.600 .987670 .00038 14.700 .990840 .00038 14.800 .995490 .00037 14.900 .998830 .00037 15.001 .002720 .00036 15.101 .006350 .00036 15.201 .010310 .00036 15.301 .012770 .00036 15.401 .016050 .00036
32

12.600 .921420 .00039 12.700 .925570 .00037 12.800 .931330 .00039 12.900 .935550 .00038 13.000 .940720 .00037 13.100 .946050 .00038 13.200 .949910 .00038 13.300 .954600 .00038 13.400 .959550 .00039 13.500 .963690 .00038 13.600 .967340 .00039 13.700 .971670 .00037 13.800 .977080 .00038 13.900 .980780 .00038 14.000 .985300 .00038 14.200 .993060 .00037 14.300 .997270 .00038 14.401 .001110 .00038 14.601 .008940 .00037 14.701 .012880 .00038 14.801 .015800 .00037
11.400 .918850 .00041 11.500 .925040 .00042 11.600 .930580 .00041 11.700 .936260 .00041 11.800 .941980 .00042 11.900 .948670 .00042 12.000 .954800 .00042 12.100 .959690 .00040
50

10.600 .919920 .00043 10.650 .923280 .00044 10.700 .927280 .00043 10.750 .930510 .00044 10.800 .934320 .00044 10.850 .937140 .00044 10.900 .941390 .00043 10.950 .943740 .00045 
12.900 .962500 .00039 13.000 .967410 .00040 13.100 .973470 .00038 13.200 .977030 .00040 13.300 .981850 .00039 13.400 .986740 .00039 13.500 .990990 .00039 13.600 .995470 .00039 13.701 .000460 .00038 13.801 .005450 .00039 13.901 .007890 .00039 14.001 .014590 .00038 14.101 .017110 .00039
12.200 .965570 .00041 12.300 .970680 .00041 12.400 .976390 .00042 12.500 .982090 .00041 12.600 .986820 .00041 12.700 .991190 .00041 12.800 .997240 .00040 12.901 .002080 .00041 13.001 .007070 .00040 13.101 .012370 .00040 13.201 .016960 .00041 13.301 .022770 .00040
11.000 .947750 .00045 11.050 .951130 .00044 11.100 .954390 .00045 11.150 .958120 .00045 11.200 .960970 .00044 11.250 .964420 .00044 11.300 .967690 .00044 11.350 .970920 .00043 11.400 .973550 .00044 11.450 .977630 .00043 11.500 .980320 .00043 11.550 .983870 .00045 11.600 .987600 .00045 11.650 .989980 .00044 11.700 .993020 .00044 11.750 .996290 .00043 11.800 .999620 .00044 11.851 .002100 .00044 11.901 .004410 .00045 11.951 .008890 .00045 12.001 .011430 .00043 12.051 .014430 .00044 12.101 .017680 .00043

60

10.100 .919960 .00046 10.150 .923530 .00046 10.200 .928700 .00047 10.250 .932240 .00046 10.300 .936270 .00047 10.350 .940180 .00046 10.400 .943940 .00046 10.450 .947250 .00046 10.500 .951770 .00046 10.550 .954880 .00046 10.600 .958680 .00046 10.650 .962280 .00045 10.700 .965540 .00047 10.750 .969230 .00046 10.800 .974550 .00045 10.850 .976330 .00046 10.900 .980830 .00046 10.950 .984070 .00046 11.000 .987730 .00044 11.050 .991800 .00045 11.100 .994260 .00046 11.201 .001730 .00045 11.251 .004010 .00046 11.301 .007310 .00047
70

9.700 .915310 .00047

9.750 .920580 .00047

9.800 .924060 .00048

9.850 .929340 .00048

9.900 .932710 .00048

10.000 .940540 .00048

10.050 .945440 .00048

10.100 .949300 .00047

10.150 .953460 .00048

10.200 .957370 .00047

10.250 .961480 .00048

10.300 .965120 .00048

10.350 .969510 .00047

10.400 .972920 .00048

10.450 .977520 .00047

10.500 .981200 .00047

10.550 .985180 .00047

10.600 .988370 .00048

10.650 .991610 .00047

10.700 .996390 .00048

10.750 .998750 .00048

10.801 .002980 .00049

10.851 .006930 .00047

10.901 .010410 .00047

$\begin{array}{rrr} & 80 \\ 9.50 & 0.92029 & 0.00048 \\ 9.55 & 0.92501 & 0.00048 \\ 9.60 & 0.92939 & 0.00049 \\ 9.65 & 0.93280 & 0.00049 \\ 9.70 & 0.93793 & 0.00048 \\ 9.75 & 0.94195 & 0.00049 \\ 9.80 & 0.94618 & 0.00049 \\ 9.85 & 0.95045 & 0.00049 \\ 9.90 & 0.95476 & 0.00048 \\ 9.95 & 0.95876 & 0.00050 \\ 10.00 & 0.96286 & 0.00049 \\ 10.05 & 0.96750 & 0.00047 \\ 10.10 & 0.97237 & 0.00049 \\ 10.15 & 0.97595 & 0.00049 \\ 10.20 & 0.98026 & 0.00050 \\ 10.25 & 0.98392 & 0.00048 \\ 10.30 & 0.98727 & 0.00051 \\ 10.40 & 0.99636 & 0.00048 \\ 10.45 & 1.00005 & 0.00048 \\ 10.50 & 1.00372 & 0.00047 \\ 10.55 & 1.00747 & 0.00048 \\ 10.60 & 1.01088 & 0.00049 \\ 10.65 & 1.01555 & 0.00048 \\ 10.70 & 1.01872 & 0.00049\end{array}$


11.351 .011540 .00046 11.401 .015000 .00046 11.451 .018780 .00046 11.501 .021270 .00046

90

9.300 .919550 .00049 9.350 .924540 .00050 9.400 .929630 .00050 9.450 .933300 .00050 9.500 .938620 .00051 9.550 .942910 .00050 9.600 .948120 .00048 9.650 .952460 .00050 9.700 .956600 .00049 9.750 .959770 .00051 9.800 .964980 .00050 9.850 .969440 .00050 9.900 .973540 .00050 9.950 .977790 .00049 10.000 .982200 .00049 10.100 .990580 .00049 10.150 .994160 .00050 10.200 .999450 .00048 10.251 .002560 .00048 10.301 .006610 .00052 10.351 .011400 .00048 10.401 .015270 .00047 10.451 .019400 .00049 10.501 .022910 .00049

130

8.800 .913980 .00051 8.850 .917950 .00051 8.900 .922900 .00051 8.950 .928350 .00051 9.000 .932990 .00053 9.050 .938000 .00053 9.100 .942620 .00051 9.150 .948550 .00050 9.200 .952210 .00052 9.250 .957280 .00051 9.300 .962500 .00053 9.350 .967090 .00053 9.400 .972520 .00051 9.450 .976660 .00051
10.951 .013590 .00048 11.001 .017700 .00046 11.051 .021450 .00046 11.101 .025480 .00047
10.751 .022860 .00049 10.801 .026690 .00048
100

9.100 .914190 .00050 9.150 .919270 .00050 9.200 .924350 .00048 9.250 .929340 .00049 9.300 .934150 .00050 9.350 .938380 .00050 9.400 .942940 .00050 9.450 .948660 .00052 9.500 .952150 .00051 9.550 .957080 .00049 9.600 .960920 .00051 9.650 .965720 .00050 9.700 .969650 .00050 9.750 .974560 .00050 9.800 .978780 .00051 9.850 .983480 .00049 9.900 .988290 .00051 9.950 .992960 .00051 10.000 .996290 .00050 10.051 .000430 .00050 10.101 .003800 .00050 10.151 .009300 .00050 10.201 .013330 .00050 10.251 .016710 .00049 10.301 .021660 .00049
118

8.900 .913260 .00051 8.950 .919140 .00051 9.000 .922700 .00050 9.050 .928360 .00051 9.100 .932910 .00050 9.150 .938470 .00051 9.200 .943350 .00051 9.250 .947880 .00051 9.300 .951900 .00052 9.350 .957830 .00052 9.400 .962070 .00051 9.450 .967160 .00049 9.500 .971250 .00052 9.550 .975540 .00051 9.600 .980630 .00054 9.650 .985040 .00051 9.700 .988850 .00050 9.750 .994340 .00050 9.800 .999090 .00052 9.851 .002510 .00052 9.901 .006840 .00051 9.951 .011930 .00053 10.001 .015860 .00049 10.051 .020090 .00051 10.101 .025170 .00053

$150 \quad 200$

8.700 .914380 .00053

8.750 .920690 .00051

8.800 .924900 .00052

8.850 .929890 .00051

8.900 .935230 .00052

8.950 .940470 .00052

9.000 .945980 .00052

9.050 .950340 .00053

9.100 .955320 .00052

9.150 .960800 .00052

9.200 .965370 .00051

9.250 .968530 .00053

9.350 .980150 .00052

8.600 .922270 .00054

8.650 .927320 .00054

8.700 .932050 .00053

8.750 .937710 .00052

8.800 .943450 .00052

8.900 .952880 .00053

8.950 .958050 .00051

9.000 .962930 .00052

9.050 .968220 .00051

9.100 .974100 .00055

9.150 .978170 .00052

9.200 .982960 .00053

9.250 .987550 .00052

9.400 .984750 .00053 
9.500 .981170 .00052

9.550 .985940 .00051

9.600 .990330 .00051

9.650 .994860 .00052

9.701 .000210 .00050

9.751 .004350 .00052

9.801 .009060 .00051

9.851 .013170 .00050

9.901 .017860 .00052

9.951 .021880 .00050
9.450 .990100 .00053

9.500 .993180 .00052

9.550 .998710 .00052

9.601 .002880 .00052

9.651 .008130 .00051

9.701 .012360 .00051

9.751 .016360 .00053

9.801 .022140 .00052
9.401 .002320 .00051 9.451 .006840 .00054 9.501 .012320 .00053 9.551 .016870 .00053 9.601 .022050 .00054
235

8.500 .916240 .00053 8.550 .922530 .00052 8.600 .928020 .00055 8.650 .933160 .00052 8.700 .937710 .00054 8.750 .943280 .00053 8.800 .947540 .00053 8.850 .953380 .00055 8.900 .959500 .00052 8.950 .964250 .00053 9.000 .969750 .00053 9.050 .973530 .00052 9.100 .978730 .00055 9.150 .984190 .00053 9.200 .988300 .00054 9.250 .993300 .00054 9.300 .997990 .00052 9.401 .007900 .00055 9.451 .012380 .00054 9.501 .017510 .00053 9.551 .021920 .00054 9.601 .027010 .00052

\section{0}

8.600 .917900 .00052 8.650 .922240 .00053 8.700 .927250 .00054 8.750 .932810 .00052 8.800 .937860 .00053 8.850 .943460 .00054 8.900 .948790 .00053 8.950 .952090 .00053 9.000 .957880 .00051 9.050 .962640 .00052 9.100 .968040 .00054
250

8.550 .923600 .00051 8.600 .929180 .00053

8.700 .939020 .00053

8.750 .945400 .00053

8.800 .949560 .00053

8.850 .955220 .00053

8.900 .959980 .00053

8.950 .965490 .00053

9.000 .970620 .00053

9.100 .981530 .00053

9.150 .985890 .00054

9.200 .991470 .00053

9.250 .995990 .00053

9.301 .000240 .00053

9.351 .004990 .00053

9.401 .010830 .00053

9.451 .014450 .00054

9.501 .019290 .00054

9.551 .023900 .00055

9.601 .027860 .00054
353

8.500 .917080 .00053 8.600 .928040 .00053 8.650 .934000 .00052 8.700 .938580 .00053 8.750 .944190 .00052 8.800 .948980 .00053 8.850 .953770 .00052 8.900 .960060 .00053 8.950 .964650 .00054 9.000 .968750 .00055 9.050 .974550 .00054 9.100 .979950 .00054 9.150 .984370 .00053 9.200 .989520 .00054 9.250 .995220 .00054 9.300 .999630 .00052 9.351 .004130 .00053 9.401 .008490 .00054 9.551 .022310 .00052 9.601 .028120 .00055
8.600 .913230 .00053

8.650 .917940 .00053

8.700 .922960 .00053

8.750 .927450 .00052

8.800 .933330 .00054 8.850 .937820 .00053 8.900 .943230 .00054 8.950 .947390 .00054 9.000 .954010 .00053 9.050 .958850 .00052 9.100 .963960 .00052
510

8.600 .911240 .00054 8.650 .915940 .00052 8.700 .922680 .00054 8.750 .928180 .00052 8.800 .932430 .00052 8.850 .937400 .00054 8.900 .941020 .00053 8.950 .946910 .00053 9.000 .952080 .00054 9.050 .956590 .00053 9.100 .962330 .00054 
9.150 .973590 .00053 9.200 .977950 .00051 9.250 .982000 .00053 9.300 .987800 .00053 9.350 .992530 .00053 9.400 .996180 .00053 9.451 .001960 .00054 9.551 .010610 .00054 9.601 .015820 .00054 9.651 .019030 .00054 9.701 .023070 .00054

520

8.700 .919940 .00051 8.750 .924730 .00052 8.850 .934830 .00052 8.900 .939780 .00054 8.950 .944690 .00055 9.000 .950190 .00051 9.050 .954650 .00053 9.100 .959680 .00053 9.150 .965720 .00054 9.200 .970010 .00053 9.250 .975080 .00053 9.300 .978480 .00052 9.350 .984000 .00054 9.400 .988490 .00053 9.450 .993290 .00053 9.500 .998510 .00053 9.551 .002540 .00052 9.601 .006640 .00053 9.651 .012340 .00052 9.701 .015340 .00054 9.751 .020180 .00053 9.801 .023980 .00052

\section{0}

8.700 .914550 .00052 8.750 .920950 .00052 8.800 .926040 .00051 8.850 .931430 .00051 8.950 .939570 .00053 9.000 .945560 .00054
9.200 .972130 .00051

9.250 .977750 .00053

9.300 .982010 .00052

9.350 .987480 .00053

9.400 .991120 .00053

9.450 .996860 .00053

9.501 .000780 .00054

9.551 .006070 .00051

9.601 .010670 .00053

9.651 .015200 .00054

9.701 .018890 .00053

9.751 .023400 .00052 9.801 .028090 .00053
9.150 .966050 .00052 9.200 .971490 .00051 9.250 .976300 .00053 9.300 .981210 .00052 9.350 .985590 .00053 9.400 .990090 .00054 9.450 .994990 .00053 9.500 .999130 .00054 9.551 .003510 .00053 9.601 .008640 .00053 9.651 .012830 .00053 9.701 .017920 .00054 9.751 .021750 .00055 9.801 .027670 .00055

530

8.700 .918840 .00053

8.750 .923190 .00051

8.800 .929210 .00051

8.850 .932950 .00051

8.900 .937450 .00054

8.950 .943780 .00053

9.000 .948960 .00052

9.050 .954230 .00051

9.100 .959800 .00053

9.150 .963320 .00052

9.200 .967940 .00054

9.250 .972520 .00051

9.300 .977750 .00052

9.350 .981630 .00052

9.400 .986810 .00052

9.450 .992340 .00051

9.500 .995460 .00053

9.551 .000710 .00052

9.601 .005280 .00052

9.651 .009240 .00053

9.701 .013590 .00053

9.751 .018820 .00054

9.801 .022760 .00055

\section{0}

8.700 .915730 .00053

8.750 .921420 .00053

8.800 .926920 .00051

8.850 .932150 .00052

8.900 .936910 .00051

8.950 .942940 .00052

9.000 .947880 .00052

9.050 .952570 .00052

9.100 .956520 .00053

9.150 .961880 .00053

9.200 .966390 .00054

9.250 .970700 .00052

9.300 .976140 .00053

9.350 .981100 .00052

9.400 .984520 .00052

9.450 .989050 .00052

9.500 .994180 .00052

9.550 .999410 .00051

9.601 .005520 .00052

9.651 .008960 .00053

9.751 .017320 .00054
560
8.700 .914620 .00051

8.750 .919080 .00052

8.800 .923980 .00053

8.850 .929210 .00051

8.900 .934100 .00053

8.950 .939140 .00051 
9.100 .955240 .00053

9.150 .959710 .00052

9.200 .964080 .00052

9.300 .974520 .00053

9.350 .978730 .00052

9.400 .983230 .00053

9.450 .988230 .00052

9.550 .997190 .00053

9.601 .001640 .00052

9.651 .006310 .00052

9.701 .010520 .00052

9.751 .015340 .00053

9.801 .019380 .00054

9.851 .023550 .00053

9.901 .028640 .00052

U5o2N23-icyl-1000-01

12

36.600 .882590 .00018 37.800 .886480 .00017 39.000 .888810 .00017 40.200 .891800 .00016 41.400 .894690 .00016 42.600 .897120 .00016 43.800 .899460 .00016 45.000 .901280 .00016 46.200 .903710 .00015 47.400 .905350 .00015 48.600 .906550 .00015 49.800 .908700 .00015 51.000 .909680 .00015 52.200 .911500 .00015 53.400 .913020 .00015 54.600 .914280 .00015 55.800 .915270 .00015 57.000 .916440 .00014 58.200 .917390 .00015 59.400 .918470 .00014 60.600 .919600 .00014 60.900 .919850 .00014
9.000 .942990 .00053

9.050 .949050 .00053

9.100 .954410 .00053

9.150 .958710 .00053

9.200 .963230 .00053

9.250 .967500 .00052

9.300 .972480 .00053

9.350 .977460 .00052

9.400 .982620 .00053

9.450 .986540 .00053

9.500 .990970 .00053

9.550 .995360 .00054

9.601 .000870 .00052

9.651 .005030 .00055

9.701 .007920 .00052

9.751 .013230 .00052

9.801 .018660 .00053

9.851 .023040 .00052

9.901 .026780 .00053
15

24.900 .928020 .00023 25.900 .935980 .00022 26.900 .943330 .00022 27.900 .949620 .00022 28.900 .956080 .00021 29.900 .961090 .00020 30.900 .966500 .00020 31.900 .971100 .00019 32.900 .975780 .00020 33.900 .979540 .00020 34.900 .983040 .00019 35.900 .987540 .00019 36.900 .990190 .00018 37.900 .993500 .00019 38.900 .996060 .00018 39.900 .998580 .00018 40.901 .000920 .00018 41.901 .003890 .00018 42.901 .005850 .00017 43.901 .008530 .00017 44.901 .009850 .00017 45.901 .011730 .00017 46.101 .011950 .00017
20

16.000 .926530 .00030 16.200 .931100 .00030 16.400 .935540 .00029 16.600 .940250 .00030 16.800 .944700 .00029 17.000 .948730 .00029 17.200 .952720 .00029 17.400 .956870 .00029 17.600 .960480 .00028 17.800 .964090 .00028 18.000 .967970 .00029 18.200 .971230 .00028 18.400 .975310 .00028 18.600 .978380 .00028 18.800 .982310 .00027 19.000 .985310 .00028 19.200 .988450 .00027 19.400 .991590 .00027 19.600 .994490 .00027 19.800 .998500 .00028 20.001 .001010 .00027 20.201 .004750 .00027 20.401 .007260 .00027 20.601 .009540 .00027 20.801 .011500 .00027 20.901 .013910 .00026 
25

13.000 .922390 .00034

13.100 .926440 .00033

13.300 .934130 .00033

13.400 .937440 .00034

13.500 .941080 .00034

13.600 .944000 .00033

13.700 .947610 .00034

13.900 .954050 .00033

14.000 .957430 .00032

14.100 .960490 .00033

14.200 .963340 .00032

14.300 .966540 .00032

14.400 .969460 .00033

14.500 .972920 .00032

14.600 .975520 .00032

14.700 .978540 .00033

14.800 .981230 .00032

14.900 .984590 .00032

15.000 .987170 .00032

15.100 .990300 .00033

15.200 .992520 .00033

15.300 .995470 .00033

15.400 .998650 .00032

15.501 .000210 .00032

15.601 .002750 .00032

15.701 .005530 .00032

15.801 .008790 .00032

15.901 .011360 .00032

16.001 .013820 .00032

16.101 .016040 .00031

35

10.500 .919110 .00039

10.600 .925080 .00039

10.700 .930500 .00038

10.800 .935870 .00038

10.900 .940100 .00039

11.000 .945920 .00038

11.100 .951400 .00039

11.200 .955770 .00038

11.300 .961050 .00038

11.400 .966080 .00038

11.500 .970730 .00039

11.600 .976550 .00037

11.700 .980090 .00037

11.800 .985280 .00038
30

11.500 .921970 .00037

11.600 .927860 .00035

11.700 .932030 .00036

11.800 .936640 .00036

11.900 .941140 .00035

12.100 .950570 .00037

12.200 .953900 .00036

12.300 .957720 .00035

12.400 .962710 .00035

12.500 .966470 .00036

12.600 .970730 .00035

12.700 .973500 .00036

12.800 .978630 .00035

12.900 .982590 .00035

13.000 .985550 .00036

13.100 .990150 .00036

13.200 .993250 .00035

13.300 .997460 .00034

13.401 .001270 .00034

13.501 .004790 .00036

13.601 .007990 .00035

13.701 .011370 .00035

13.801 .015600 .00034

13.901 .018240 .00035
32

11.100 .923620 .00037

11.200 .928890 .00038

11.300 .933640 .00037

11.400 .938630 .00038

11.500 .942740 .00037

11.600 .947810 .00037

11.700 .952510 .00037

11.800 .956740 .00037

11.900 .962060 .00036

12.000 .966620 .00037

12.100 .970330 .00036

12.200 .974910 .00036

12.300 .978670 .00037

12.400 .982640 .00037

12.500 .987430 .00037

12.600 .991790 .00036

12.700 .994600 .00036

12.800 .999660 .00036

12.901 .003440 .00035

13.001 .007470 .00037

13.101 .010560 .00037

13.201 .014500 .00035

13.301 .018090 .00037
40

9.900 .922050 .00041

10.000 .927400 .00041

10.100 .933610 .00041

10.200 .939650 .00039

10.300 .945580 .00040

10.400 .951410 .00041

10.500 .956980 .00040

10.600 .962750 .00040

10.700 .968380 .00040

10.800 .973980 .00040

10.900 .978550 .00040

11.000 .984830 .00040

11.100 .989730 .00040

11.200 .994880 .00039
50

9.000 .916150 .00044

9.050 .920410 .00042

9.100 .923690 .00042

9.150 .926950 .00042

9.200 .931340 .00042

9.250 .933560 .00044

9.300 .937460 .00042

9.350 .941470 .00042

9.400 .944830 .00043

9.450 .948320 .00044

9.500 .951510 .00041

9.550 .954460 .00043

9.600 .957630 .00042

9.650 .961140 .00044 
11.900 .989610 .00038 12.000 .994660 .00037

12.100 .998100 .00038

12.201 .003290 .00038

12.301 .007190 .00038

12.401 .011330 .00037

12.501 .015920 .00038

12.601 .019760 .00037
11.301 .000200 .00040 11.401 .004730 .00039 11.501 .010190 .00040 11.601 .015190 .00040 11.701 .019490 .00040
9.700 .965000 .00043

9.750 .968020 .00044

9.800 .971620 .00041

9.850 .975360 .00042

9.900 .977820 .00043

9.950 .981470 .00041

10.000 .984030 .00042

10.050 .987060 .00042

10.100 .990940 .00041

10.150 .993320 .00043

10.200 .996560 .00043

10.250 .999670 .00043

10.301 .002780 .00043

10.351 .006260 .00041

10.401 .009110 .00042

10.451 .011950 .00042

10.501 .015050 .00042

10.551 .017480 .00041

10.601 .020310 .00043

\section{0}

8.200 .921920 .00045

8.250 .925910 .00046

8.300 .929950 .00046

8.350 .933430 .00044

8.400 .938220 .00044

8.450 .942010 .00046

8.500 .946590 .00045

8.550 .950810 .00046

8.600 .954670 .00046

8.650 .958670 .00045

8.700 .961920 .00046

8.800 .970190 .00046

8.850 .974510 .00045

8.950 .981200 .00046

9.000 .986220 .00047

9.050 .989850 .00045

9.100 .993320 .00045

9.150 .997640 .00046

9.201 .000430 .00046

9.251 .004370 .00046

9.301 .008460 .00045

9.351 .011500 .00046

9.401 .016110 .00045

9.451 .019230 .00045

9.501 .023530 .00046
80

7.900 .916990 .00047

7.950 .921160 .00046

8.000 .926260 .00047

8.050 .931120 .00046

8.100 .935070 .00045

8.150 .939410 .00046

8.200 .943450 .00049

8.250 .947320 .00045

8.300 .952130 .00047

8.350 .956660 .00046

8.400 .960370 .00046

8.500 .968700 .00047

8.550 .974790 .00047

8.600 .977040 .00046

8.650 .981230 .00048

8.750 .989770 .00046

8.800 .993560 .00048

8.850 .998210 .00046

8.901 .001210 .00046

8.951 .006110 .00046 9.001 .008910 .00046 9.051 .013450 .00046 9.101 .017450 .00047 9.151 .020730 .00047 9.201 .024250 .00047 
90

7.700 .916780 .00047

7.750 .921300 .00048

7.800 .926600 .00047

7.850 .930500 .00047

7.900 .935560 .00048

7.950 .940050 .00048

8.000 .945300 .00047

8.050 .949150 .00049

8.150 .958780 .00048

8.200 .963090 .00047

8.250 .967070 .00048

8.300 .970810 .00047

8.350 .975030 .00048

8.400 .979120 .00049

8.450 .984050 .00048

8.500 .987130 .00048

8.550 .991620 .00048

8.600 .995580 .00047

8.650 .999930 .00049

8.701 .004680 .00048

8.751 .007670 .00047

8.801 .012720 .00049

8.851 .016000 .00046

8.901 .020490 .00047
100

7.600 .921340 .00050

7.650 .926620 .00049

7.700 .931000 .00048

7.750 .936000 .00048

7.800 .940440 .00048

7.850 .945500 .00048

7.900 .949480 .00047

7.950 .954970 .00049

8.000 .959330 .00047

8.050 .963120 .00049

8.100 .967990 .00048

8.150 .971830 .00048

8.200 .976410 .00049

8.250 .980340 .00048

8.300 .985440 .00049

8.350 .990490 .00047

8.400 .994790 .00048

8.450 .999660 .00049

8.501 .002020 .00049

8.551 .006370 .00048

8.601 .010790 .00049

8.651 .015170 .00047

8.701 .018740 .00047
118

7.400 .919400 .00050

7.450 .926090 .00049

7.500 .930400 .00049

7.550 .935850 .00049

7.600 .940710 .00049

7.650 .945480 .00048

7.700 .949700 .00049

7.750 .955670 .00048

7.800 .959420 .00048

7.850 .963730 .00048

7.900 .969290 .00047

7.950 .973660 .00050

8.000 .977670 .00050

8.050 .983580 .00048

8.100 .988270 .00047

8.150 .992560 .00050

8.200 .996420 .00049

8.251 .000740 .00049

8.301 .006240 .00049

8.351 .009880 .00048

8.401 .013710 .00049

8.451 .018520 .00050

8.501 .022260 .00050
130

7.300 .920960 .00048

7.350 .926810 .00050

7.400 .930590 .00050

7.450 .936890 .00049

7.500 .939650 .00050

7.550 .946270 .00049

7.600 .951000 .00049

7.650 .955180 .00050

7.700 .960880 .00050

7.750 .965450 .00049

7.800 .971340 .00050

7.850 .974770 .00049

7.900 .980460 .00048

7.950 .984810 .00049

8.000 .988870 .00049

8.050 .993960 .00049

8.100 .998890 .00050

8.151 .002760 .00050

8.201 .006660 .00049
150

7.100 .912300 .00048

7.150 .918390 .00049

7.200 .923210 .00048

7.250 .928110 .00051

7.300 .933890 .00050

7.350 .938630 .00050

7.400 .943570 .00049

7.450 .948740 .00050

7.500 .953220 .00050

7.550 .957470 .00049

7.600 .963380 .00049

7.650 .968090 .00051

7.700 .973000 .00049

7.800 .983200 .00050

7.900 .992390 .00050

7.950 .997490 .00050

8.001 .001080 .00050

8.051 .006260 .00049

8.101 .010100 .00052
200

7.000 .920350 .00050

7.050 .926840 .00051

7.100 .931500 .00050

7.150 .936400 .00049

7.200 .941330 .00051

7.350 .956860 .00050

7.400 .961930 .00051

7.450 .967600 .00050

7.500 .972190 .00049

7.550 .978450 .00052

7.600 .982260 .00051

7.650 .987050 .00051

7.700 .992910 .00050

7.750 .996940 .00050

7.801 .001620 .00051

7.851 .006280 .00051

7.901 .010580 .00053

7.951 .015940 .00050

8.001 .020630 .00051 
8.251 .009680 .00049

8.301 .016510 .00050

8.401 .024220 .00050
8.151 .015820 .00052

8.201 .019440 .00051
235

6.900 .916530 .00051

6.950 .921620 .00051

7.000 .926830 .00052

7.050 .931930 .00051

7.100 .938290 .00050

7.150 .943230 .00051

7.200 .947970 .00050

7.250 .952720 .00051

7.300 .958150 .00051

7.350 .963660 .00051

7.400 .969180 .00051

7.450 .974380 .00050

7.500 .978710 .00051

7.550 .982960 .00051

7.600 .989220 .00052

7.650 .993610 .00053

7.700 .998420 .00051

7.751 .003790 .00052

7.801 .007350 .00052

7.851 .012760 .00053

7.901 .017600 .00052

7.951 .022980 .00050

8.001 .025250 .00051
250

6.900 .918600 .00051

7.000 .928350 .00052

7.100 .938910 .00050

7.150 .944570 .00053

7.250 .954830 .00051

7.300 .960850 .00051

7.350 .965450 .00051

7.400 .970310 .00052

7.450 .975750 .00051

7.500 .980480 .00051

7.550 .984850 .00052

7.600 .990570 .00050

7.650 .995560 .00052

7.701 .000380 .00051

7.751 .004790 .00050

7.801 .009160 .00052

7.851 .015030 .00052

7.901 .019540 .00052

7.951 .024050 .00052

8.001 .028570 .00051
353

6.900 .919740 .00051

6.950 .925130 .00053

7.000 .929980 .00051

7.050 .934900 .00051

7.150 .946120 .00052

7.200 .950220 .00050

7.250 .956650 .00051

7.300 .961630 .00051

7.350 .966430 .00051

7.400 .971210 .00053

7.450 .975800 .00053

7.500 .980890 .00052

7.550 .984990 .00050

7.600 .990810 .00052

7.650 .995230 .00053

7.701 .001040 .00052

7.751 .005540 .00053

7.801 .010680 .00051

7.851 .014860 .00052

7.901 .019390 .00053
470

7.000 .920950 .00049

7.050 .926690 .00051

7.150 .935670 .00051

7.200 .942170 .00051

7.250 .946550 .00051

7.300 .950910 .00051

7.350 .955490 .00052

7.400 .961610 .00053

7.450 .966500 .00052

7.500 .971070 .00053

7.550 .975470 .00052

7.600 .980700 .00051

7.650 .985700 .00053

7.700 .990670 .00052

7.750 .995060 .00052

7.801 .000400 .00052

7.851 .004980 .00053
500

7.000 .917550 .00051

7.050 .922630 .00051

7.150 .932660 .00049

7.200 .937980 .00052

7.250 .943630 .00050

7.300 .947670 .00051

7.350 .952750 .00051

7.400 .957150 .00052

7.450 .961950 .00052

7.500 .967790 .00050

7.550 .972650 .00050

7.600 .976740 .00050

7.650 .981580 .00052

7.700 .986810 .00050

7.750 .991110 .00052

7.800 .996430 .00052

7.851 .000290 .00051
510

7.000 .915440 .00051

7.050 .920600 .00051

7.100 .925710 .00051

7.150 .931500 .00051

7.200 .937090 .00051

7.250 .941440 .00051

7.300 .946120 .00051

7.350 .951270 .00051

7.400 .956280 .00052

7.450 .959880 .00051

7.500 .965810 .00051

7.550 .970340 .00050

7.600 .975530 .00051

7.650 .980480 .00051

7.700 .984600 .00050

7.750 .989550 .00051

7.800 .994160 .00052 
7.901 .008700 .00050 7.951 .013560 .00052 8.001 .018240 .00052 8.051 .023310 .00052 8.101 .026840 .00052

\section{0}

7.000 .914170 .00051

7.050 .919810 .00049

7.100 .925080 .00049

7.150 .928650 .00051

7.200 .934990 .00052

7.250 .940040 .00052

7.300 .944760 .00051

7.350 .949820 .00050

7.400 .954530 .00051

7.450 .960880 .00051

7.500 .964860 .00051

7.550 .968620 .00051

7.600 .974410 .00052

7.650 .978760 .00052

7.700 .983700 .00052

7.750 .987100 .00051

7.850 .997180 .00051

7.901 .001800 .00050

7.951 .006120 .00052

8.001 .011980 .00051

8.051 .015230 .00051

8.101 .020500 .00051

550

7.100 .920010 .00050

7.150 .925170 .00051

7.200 .931650 .00051

7.250 .934460 .00051

7.300 .940570 .00051

7.350 .945090 .00051

7.400 .949720 .00051

7.450 .955070 .00051

7.500 .960800 .00051

7.550 .964840 .00050

7.600 .969750 .00051

7.700 .979060 .00051

7.800 .988640 .00051

7.850 .992420 .00051

7.900 .997380 .00051
7.901 .004390 .00051

8.001 .014510 .00051

8.051 .018650 .00052

8.101 .022380 .00052
7.901 .003310 .00052

7.951 .007810 .00051

8.001 .012460 .00052

8.051 .016940 .00051

8.101 .022240 .00052
530

7.000 .911890 .00051

7.050 .917970 .00051

7.100 .923850 .00052

7.150 .928180 .00052

7.200 .932980 .00051

7.300 .943040 .00051

7.350 .948070 .00051

7.400 .953490 .00052

7.450 .958440 .00051

7.500 .962820 .00051

7.550 .967140 .00052

7.600 .971940 .00052

7.650 .977260 .00053

7.700 .981990 .00052

7.750 .986480 .00051

7.800 .992280 .00050

7.850 .996100 .00052

7.901 .000830 .00052

7.951 .004260 .00052

8.001 .009700 .00051

8.051 .014130 .00052

8.101 .019410 .00051
540

7.100 .922390 .00052

7.150 .927110 .00050

7.200 .932510 .00051

7.250 .937510 .00051

7.300 .942880 .00051

7.350 .946940 .00050

7.400 .951580 .00051

7.450 .956860 .00051

7.500 .961420 .00053

7.550 .966870 .00051

7.600 .970980 .00051

7.650 .975110 .00052

7.700 .980680 .00052

7.750 .985420 .00050

7.800 .989990 .00053

7.850 .993530 .00052

7.900 .999250 .00052

7.951 .002910 .00051

8.001 .008310 .00052

8.051 .013010 .00051

8.101 .017020 .00053

8.151 .021420 .00052

8.201 .026240 .00052
560

7.100 .919680 .00050

7.150 .924470 .00051

7.200 .928400 .00051

7.300 .939980 .00051

7.350 .943430 .00051

7.400 .949160 .00051

7.450 .954640 .00051

7.550 .963770 .00051

7.600 .968160 .00050

7.650 .973020 .00050

7.700 .978630 .00052

7.750 .982170 .00050

7.800 .987350 .00051

7.850 .990960 .00051

7.900 .996230 .00052 
7.951 .001830 .00051 8.051 .010840 .00052 8.201 .022640 .00051

U5o2N23-icyl-1000-10

12

3.210 .197590 .00016

6.410 .425250 .00021

9.610 .577500 .00023

12.810 .674800 .00023

16.010 .739790 .00022

19.210 .784690 .00021

22.410 .816300 .00020

25.610 .839580 .00018

28.810 .857100 .00018

32.010 .871160 .00017

35.210 .881850 .00017

38.410 .890640 .00016

41.610 .897320 .00016

44.810 .902750 .00015

48.010 .907570 .00015

51.210 .911710 .00014

54.410 .914610 .00014

57.610 .917730 .00014

60.810 .920080 .00014

64.010 .922870 .00013

65.400 .923470 .00013

25

11.900 .925240 .00031

12.000 .928280 .00032

12.100 .931900 .00031

12.200 .935860 .00031

12.300 .938780 .00030

12.400 .942290 .00031

12.500 .945740 .00031

12.600 .949080 .00031

12.700 .952620 .00031

12.800 .955080 .00032
7.950 .999760 .00051

8.001 .003990 .00053

8.051 .009060 .00052

8.101 .012880 .00051

8.151 .018480 .00051

8.201 .022290 .00051

15

23.700 .927590 .00022

24.500 .934050 .00021

25.300 .940000 .00021

26.100 .945710 .00020

26.900 .950570 .00020

27.700 .955460 .00020

28.500 .960010 .00021

29.300 .964450 .00019

30.100 .967940 .00019

30.900 .971670 .00020

31.700 .975590 .00019

32.500 .978550 .00019

33.300 .981710 .00019

34.100 .984530 .00018

34.900 .987840 .00018

35.700 .990320 .00018

36.500 .992470 .00018

37.300 .994610 .00018

38.100 .996510 .00018

38.900 .999340 .00018

39.701 .001000 .00017

40.501 .003030 .00017

41.001 .003630 .00017

30

10.400 .925270 .00033

10.500 .930050 .00033

10.600 .934900 .00034

10.700 .938860 .00034

10.800 .943800 .00033

10.900 .948590 .00034

11.000 .951790 .00034

11.100 .956470 .00034

11.200 .960640 .00033

11.300 .964890 .00033
20

14.800 .925280 .00028 15.000 .929700 .00027 15.200 .933620 .00028 15.400 .939070 .00028 15.600 .943580 .00028 15.800 .947450 .00028 16.000 .951910 .00027 16.200 .955380 .00027 16.400 .959470 .00027 16.600 .963330 .00028 16.800 .967230 .00027 17.000 .970690 .00026 17.200 .973760 .00027 17.400 .977810 .00026 17.600 .981050 .00026 17.800 .984250 .00027 18.000 .987960 .00027 18.200 .991340 .00026 18.400 .993960 .00026 18.600 .997180 .00026 18.801 .000310 .00025 19.001 .003230 .00026 19.201 .005820 .00026 19.401 .008990 .00026 19.601 .012080 .00025 19.701 .013230 .00026

32

9.900 .921830 .00034 10.000 .926420 .00034 10.100 .931480 .00035 10.200 .936850 .00036 10.300 .941160 .00035 10.400 .946590 .00034 10.500 .951100 .00034 10.600 .955750 .00034 10.700 .960230 .00035 10.800 .965320 .00035 
13.000 .962440 .00031

13.100 .965550 .00031

13.200 .968700 .00031

13.300 .970830 .00031

13.400 .973810 .00031

13.500 .977550 .00031

13.600 .979810 .00031

13.700 .983270 .00031

13.800 .985490 .00030

13.900 .988730 .00030

14.000 .991570 .00031

14.100 .994330 .00030

14.200 .997110 .00030

14.301 .000750 .00031

14.401 .002650 .00031

14.501 .005410 .00030

14.601 .007680 .00030

14.701 .010050 .00030

14.801 .012880 .00030

14.901 .015650 .00030
11.400 .969240 .00034

11.600 .977190 .00032

11.700 .980910 .00033

11.900 .988010 .00033

12.000 .992740 .00034

12.100 .996730 .00034

12.201 .000000 .00032

12.301 .003280 .00034

12.401 .007490 .00033

12.501 .011080 .00034

12.601 .013960 .00032
10.900 .969230 .00035

11.000 .973810 .00034

11.100 .978020 .00033

11.300 .986200 .00034

11.400 .990110 .00035

11.500 .994930 .00035

11.600 .998790 .00034

11.701 .002720 .00035

11.801 .006350 .00033

11.901 .010440 .00035

12.001 .014500 .00033

12.101 .018100 .00035
35

9.400 .922960 .00036

9.500 .928810 .00036

9.600 .933590 .00036

9.700 .938580 .00035

9.800 .944400 .00035

9.900 .949210 .00035

10.000 .954980 .00036

10.100 .959810 .00036

10.200 .965190 .00035

10.300 .970350 .00035

10.400 .975040 .00034

10.500 .979050 .00036

10.600 .983730 .00036

10.700 .989250 .00035

10.800 .993700 .00035

10.900 .997620 .00035

11.001 .002500 .00036

11.101 .006770 .00035

11.201 .011490 .00035

11.301 .015140 .00035

11.401 .019840 .00035
40

8.700 .919540 .00037

8.750 .922900 .00037

8.800 .926350 .00037

8.850 .929010 .00037

8.900 .932390 .00038

8.950 .935820 .00037

9.000 .937800 .00037

9.050 .941510 .00036

9.100 .944190 .00037

9.150 .947090 .00037

9.200 .950420 .00037

9.250 .953910 .00037

9.300 .956440 .00037

9.350 .958630 .00037

9.400 .961260 .00036

9.450 .964060 .00037

9.500 .967600 .00036

9.550 .970270 .00037

9.600 .973210 .00037

9.650 .975930 .00038

9.700 .978500 .00037

9.750 .980430 .00037

9.800 .983960 .00037

9.850 .987100 .00037

9.900 .989230 .00036

9.950 .992320 .00037

10.000 .994590 .00037
50

7.900 .922790 .00039

7.950 .926860 .00040

8.000 .930190 .00039

8.050 .934300 .00039

8.100 .936840 .00039

8.150 .940480 .00039

8.200 .943650 .00040

8.250 .947740 .00039

8.300 .950860 .00039

8.350 .955300 .00039

8.400 .958170 .00040

8.450 .961280 .00039

8.500 .965460 .00039

8.550 .969520 .00040

8.600 .971540 .00039

8.650 .975100 .00040

8.700 .977900 .00041

8.750 .982210 .00040

8.800 .984950 .00040

8.850 .987660 .00039

8.900 .990910 .00040

8.950 .994020 .00040

9.000 .997520 .00040

9.051 .000280 .00040

9.101 .003920 .00039

9.151 .006510 .00039

9.201 .010030 .00039

$$
\text { F-476 }
$$


10.101 .000460 .00037

10.151 .002340 .00037

10.201 .005290 .00037

10.251 .007820 .00037

10.301 .010310 .00037

10.351 .013220 .00037

10.401 .014890 .00037
9.251 .012370 .00039

9.301 .015950 .00040

9.351 .018160 .00038

9.401 .022350 .00038
60

7.300 .917890 .00041

7.400 .925280 .00041

7.450 .929700 .00041

7.500 .933560 .00040

7.550 .937730 .00040

7.600 .940930 .00042

7.650 .945770 .00041

7.700 .948840 .00042

7.750 .953370 .00041

7.800 .957480 .00041

7.850 .961930 .00041

7.900 .964370 .00040

7.950 .969020 .00041

8.000 .971520 .00041

8.050 .975700 .00040

8.100 .979990 .00041

8.150 .982410 .00041

8.200 .987530 .00042

8.250 .990220 .00041

8.300 .993730 .00041

8.350 .997710 .00041

8.401 .001950 .00041

8.451 .004660 .00041

8.501 .007440 .00042

8.551 .011710 .00040

8.601 .014640 .00041

8.651 .017680 .00041

8.701 .021360 .00041
70

7.000 .922890 .00043

7.050 .928040 .00043

7.100 .931710 .00042

7.150 .936930 .00043

7.200 .941040 .00042

7.250 .944340 .00042

7.300 .948720 .00041

7.350 .953440 .00041

7.400 .957400 .00043

7.450 .961320 .00042

7.500 .965810 .00042

7.550 .968920 .00042

7.600 .973180 .00042

7.650 .977100 .00043

7.700 .982020 .00041

7.750 .985280 .00041

7.800 .989150 .00042

7.850 .992850 .00042

7.900 .996500 .00042

8.001 .003950 .00042

8.051 .007900 .00043

8.101 .012110 .00041

8.151 .014870 .00042

8.201 .019190 .00042
80

6.700 .919870 .00044

6.800 .929880 .00044

6.850 .933820 .00042

6.900 .938460 .00042

6.950 .943490 .00043

7.000 .947080 .00042

7.050 .951480 .00044

7.100 .957060 .00043

7.150 .960780 .00043

7.200 .964660 .00043

7.300 .973790 .00043

7.350 .976940 .00042

7.400 .982300 .00041

7.450 .985720 .00042

7.500 .989810 .00043

7.550 .993780 .00044

7.600 .998090 .00043

7.651 .002090 .00042

7.701 .005090 .00043

7.751 .010100 .00044

7.801 .014250 .00043

7.851 .018200 .00044

7.901 .021820 .00042
90

6.500 .921530 .00044

6.550 .925850 .00043

6.600 .930340 .00044

6.650 .935720 .00043

6.700 .939840 .00043

6.750 .944760 .00045

6.800 .949720 .00045

6.850 .954590 .00044
100

6.300 .917610 .00044

6.400 .926480 .00044

6.450 .932070 .00045

6.500 .937390 .00044

6.550 .941860 .00045

6.600 .946390 .00045

6.650 .951940 .00044

6.700 .955920 .00045
118

6.150 .924790 .00045

6.200 .930080 .00044

6.250 .934230 .00045

6.300 .939190 .00044

6.350 .944500 .00045

6.400 .949490 .00044

6.450 .955070 .00044

6.500 .959200 .00046 
6.900 .959100 .00044

6.950 .962700 .00044

7.000 .967350 .00044

7.050 .971590 .00042

7.100 .975830 .00044

7.150 .981040 .00043

7.200 .985250 .00044

7.250 .989120 .00043

7.300 .993610 .00044

7.350 .997980 .00045

7.401 .001920 .00044

7.451 .006010 .00044

7.501 .011220 .00043

7.551 .014680 .00044

7.601 .018630 .00044

130

6.000 .920480 .00045

6.050 .926420 .00045

6.100 .931020 .00044

6.150 .935680 .00046

6.200 .941260 .00045

6.250 .946220 .00044

6.300 .951100 .00044

6.350 .956020 .00045

6.400 .962060 .00043

6.450 .965490 .00046

6.500 .971170 .00046

6.550 .975970 .00044

6.600 .980800 .00045

6.650 .986050 .00045

6.700 .990560 .00045

6.750 .995230 .00045

6.800 .999380 .00045

6.851 .004540 .00046

6.901 .008820 .00045

6.951 .013360 .00046

7.001 .018120 .00045

\section{5}

5.500 .913720 .00046 5.550 .920790 .00046 5.600 .925580 .00047 5.650 .932310 .00046 5.750 .942100 .00047
6.750 .961460 .00044

6.800 .965660 .00044

6.850 .969530 .00043

6.900 .974540 .00045

6.950 .979000 .00045

7.000 .983250 .00045

7.050 .988760 .00044

7.100 .993210 .00042

7.150 .996920 .00044

7.201 .001220 .00045

7.251 .006120 .00044

7.301 .010360 .00044

7.351 .014640 .00045

7.401 .017580 .00045
6.550 .963970 .00044

6.600 .968880 .00045

6.650 .974280 .00043

6.700 .978790 .00045

6.750 .982930 .00045

6.800 .987850 .00047

6.850 .992210 .00044

6.900 .997850 .00044

6.951 .001790 .00045

7.001 .005950 .00045

7.051 .011030 .00045

7.101 .015170 .00046

7.151 .019280 .00046

7.201 .023630 .00045
150
5.800 .913840 .00044

5.850 .920090 .00044

5.900 .924460 .00044

5.950 .930270 .00045

6.000 .935550 .00046

6.050 .941150 .00045

6.100 .945810 .00046

6.150 .950540 .00045

6.200 .956720 .00048

6.250 .961400 .00045

6.300 .966380 .00046

6.350 .971030 .00046

6.400 .976620 .00046

6.450 .980740 .00046

6.500 .986540 .00047

6.550 .991100 .00045

6.600 .995960 .00047

6.651 .000600 .00045

6.701 .005120 .00044

6.751 .010290 .00046

6.801 .015050 .00046

6.851 .018660 .00045

6.901 .023450 .00046
200

5.600 .915970 .00047

5.650 .921050 .00046

5.700 .926670 .00047

5.750 .932450 .00047

5.800 .937300 .00047

5.850 .944390 .00047

5.900 .948090 .00047

5.950 .953370 .00046

6.000 .960280 .00046

6.050 .965700 .00046

6.100 .970000 .00046

6.150 .976020 .00048

6.200 .980450 .00047

6.250 .984810 .00047

6.300 .990300 .00047

6.350 .995610 .00046

6.401 .000250 .00047

6.451 .005520 .00048

6.501 .010440 .00046

6.551 .015330 .00047

6.601 .019220 .00045
250

5.550 .923480 .00048

5.600 .928890 .00046

5.650 .934590 .00048

5.700 .939850 .00047

5.750 .946110 .00047
353

5.400 .915570 .00047 5.500 .927220 .00046 5.550 .932970 .00047 5.600 .938340 .00047 5.650 .944070 .00047 
5.850 .953590 .00048 5.900 .958700 .00046 5.950 .963940 .00047 6.000 .969080 .00048 6.050 .974120 .00048 6.100 .978990 .00046 6.150 .983770 .00046 6.200 .989070 .00046 6.250 .994270 .00046 6.300 .999700 .00047 6.351 .004460 .00047 6.401 .010050 .00046 6.451 .013970 .00048 6.501 .018970 .00047

470

5.400 .915560 .00047 5.450 .921120 .00046 5.500 .926780 .00047 5.550 .932200 .00046 5.600 .936680 .00045 5.650 .942180 .00046 5.700 .948030 .00047 5.750 .952530 .00048 5.800 .958620 .00047 5.850 .962830 .00046 5.900 .968260 .00047 5.950 .972750 .00047 6.000 .977630 .00048 6.050 .982920 .00046 6.100 .987740 .00047 6.150 .992750 .00047 6.200 .996840 .00047 6.251 .002340 .00048 6.301 .007740 .00048 6.351 .011280 .00047 6.401 .018030 .00048 6.451 .021240 .00046 6.501 .025780 .00047

\section{0}

5.500 .922630 .00048 5.550 .928290 .00045 5.600 .933950 .00047 5.700 .944160 .00047 5.750 .949370 .00046 5.800 .954410 .00047
5.800 .949760 .00048

5.850 .956510 .00049

5.900 .961780 .00046

5.950 .966220 .00047

6.000 .972340 .00046

6.050 .976850 .00047

6.100 .981520 .00047

6.150 .987750 .00047

6.200 .992820 .00047

6.250 .997380 .00048

6.301 .002150 .00048

6.351 .007280 .00047

6.401 .011930 .00047

6.451 .016960 .00047
5.700 .949950 .00046

5.800 .959980 .00046 5.850 .964970 .00049 5.900 .970370 .00048 5.950 .975530 .00049 6.000 .980280 .00047 6.050 .985510 .00047 6.100 .990330 .00048 6.150 .996350 .00047 6.201 .000030 .00048 6.251 .005710 .00047 6.301 .010650 .00048 6.351 .015740 .00048 6.401 .019900 .00047
500

5.450 .919110 .00047

5.500 .924410 .00047

5.600 .934280 .00046

5.650 .940860 .00048

5.700 .945520 .00049

5.750 .950460 .00046

5.800 .956220 .00048

5.850 .960620 .00048

5.900 .966270 .00047

5.950 .971140 .00049

6.000 .975100 .00048

6.050 .980720 .00048

6.100 .986050 .00047

6.150 .990390 .00047

6.200 .996240 .00047

6.251 .000670 .00049

6.301 .005510 .00047

6.351 .009290 .00047

6.401 .014260 .00047

6.451 .018610 .00047

6.501 .022690 .00048
510

5.400 .912800 .00047 5.450 .918160 .00046 5.500 .923810 .00048 5.600 .934610 .00047 5.650 .939700 .00047 5.700 .945330 .00047 5.750 .951100 .00047 5.800 .954910 .00046 5.850 .959880 .00046 5.900 .964630 .00047 5.950 .969940 .00048 6.000 .974260 .00048 6.050 .979430 .00047 6.100 .984990 .00047 6.150 .989540 .00047 6.200 .994020 .00048 6.250 .999700 .00048 6.301 .004410 .00047 6.351 .008350 .00048 6.401 .013580 .00047 6.451 .017370 .00047 6.501 .022280 .00047
530

5.500 .922250 .00046 5.550 .927270 .00048 5.600 .932750 .00046 5.650 .937810 .00047 5.700 .943640 .00047 5.750 .948300 .00048
540

5.500 .921930 .00046 5.550 .926870 .00046 5.600 .932460 .00046 5.650 .936910 .00047 5.700 .942600 .00047 5.750 .947650 .00047 
5.850 .959310 .00048 5.900 .964550 .00048 5.950 .969230 .00047 6.000 .974490 .00046 6.050 .979640 .00047 6.100 .984370 .00046 6.150 .989150 .00048 6.200 .994640 .00049 6.250 .998810 .00047 6.301 .003360 .00048 6.351 .006970 .00048 6.401 .012490 .00046 6.451 .016590 .00048 6.501 .022140 .00047
5.800 .954340 .00046 5.850 .958650 .00048 5.950 .968310 .00047 6.000 .973330 .00047 6.050 .977840 .00046 6.100 .983170 .00045 6.150 .988580 .00046 6.200 .992380 .00048 6.250 .997800 .00048 6.301 .002020 .00046 6.351 .005940 .00048 6.401 .011040 .00047 6.451 .015650 .00047 6.501 .021020 .00046
5.800 .952250 .00047 5.850 .958150 .00046 5.900 .962000 .00047 5.950 .967330 .00047 6.000 .972220 .00047 6.050 .977430 .00047 6.100 .982180 .00045 6.150 .987250 .00047 6.200 .991980 .00049 6.250 .996540 .00049 6.301 .001170 .00048 6.351 .006420 .00048 6.401 .010720 .00046 6.451 .015740 .00048 6.501 .020450 .00047
550

5.500 .921220 .00047 5.550 .927040 .00048 5.600 .930940 .00047 5.650 .936490 .00047 5.700 .942230 .00046 5.750 .946610 .00046 5.800 .952430 .00048 5.850 .956910 .00047 5.950 .967040 .00048 6.000 .971840 .00046 6.050 .977100 .00047 6.100 .980470 .00047 6.150 .987010 .00047 6.200 .990990 .00048 6.250 .995490 .00048 6.301 .000110 .00046 6.351 .004440 .00047 6.401 .009310 .00049 6.451 .015250 .00047 6.501 .018770 .00048
560

5.500 .919680 .00047

5.550 .925600 .00047

5.600 .930020 .00046

5.650 .936090 .00046

5.700 .940200 .00047

5.750 .945860 .00048 5.800 .950330 .00047 5.850 .956310 .00048 5.900 .960990 .00047 5.950 .965780 .00046 6.000 .971490 .00047 6.050 .976040 .00048 6.100 .979620 .00048 6.150 .985550 .00047 6.200 .990450 .00048 6.250 .994870 .00048 6.300 .999840 .00046 6.351 .004930 .00046 6.401 .008890 .00047 6.451 .013590 .00046 6.501 .018130 .00047 
U5o2N26-icyl-1000-00

12

54.700 .872110 .00015 55.600 .872670 .00015 56.500 .873410 .00015 57.400 .874670 .00015 58.300 .875690 .00015 59.200 .876290 .00015 60.100 .877000 .00015 61.000 .877610 .00015 61.900 .878660 .00015 62.800 .879190 .00014 63.700 .879610 .00014 64.600 .880650 .00014 65.500 .880810 .00014 66.400 .882010 .00014 67.300 .882030 .00014 68.200 .882450 .00014 69.100 .883400 .00014 70.000 .884130 .00014 70.900 .884520 .00014 71.800 .884880 .00014 72.700 .885480 .00014

25

16.000 .923440 .00033 16.100 .925450 .00033 16.200 .929280 .00034 16.300 .932690 .00032 16.400 .934780 .00033 16.500 .938330 .00033 16.600 .940560 .00034 16.700 .943530 .00033 16.800 .946940 .00032 16.900 .948850 .00033 17.000 .952120 .00033 17.100 .954370 .00033 17.200 .957030 .00033 17.300 .960110 .00033 17.400 .962300 .00033 17.500 .964860 .00032 17.600 .967590 .00033 17.700 .969620 .00032 17.800 .972120 .00032 17.900 .974590 .00032
15

32.100 .921400 .00021

32.600 .923550 .00020

33.100 .926490 .00021

33.600 .928700 .00020

34.100 .930820 .00020

34.600 .933070 .00020

35.100 .935280 .00020

35.600 .937280 .00020

36.100 .939370 .00019

36.600 .940680 .00020

37.100 .942650 .00019

37.600 .944540 .00019

38.100 .946360 .00019

38.600 .948320 .00019

39.100 .948960 .00019

39.600 .950690 .00018

40.100 .952350 .00019

40.600 .953670 .00019

41.100 .955200 .00018

41.600 .956520 .00019

42.100 .958560 .00018

42.500 .959260 .00018

30

14.200 .922740 .00037

14.300 .927670 .00037

14.400 .931090 .00037

14.500 .935170 .00036

14.600 .938460 .00037

14.700 .943100 .00036

14.800 .946410 .00036

14.900 .949560 .00035

15.000 .953700 .00036

15.100 .956660 .00035

15.200 .960830 .00036

15.300 .963800 .00036

15.400 .968030 .00036

15.500 .971110 .00036

15.600 .974750 .00037

15.700 .977520 .00036

15.800 .981360 .00036

15.900 .984670 .00035

16.000 .987890 .00035

16.100 .991120 .00034
20

19.700 .924180 .00029

20.000 .930120 .00028

20.300 .934770 .00028

20.600 .940040 .00028

20.900 .944720 .00028

21.200 .949510 .00027

21.500 .954790 .00027

21.800 .959130 .00027

22.100 .963320 .00027

22.400 .966910 .00026

22.700 .971540 .00028

23.000 .974880 .00027

23.300 .979160 .00027

23.600 .982710 .00028

23.900 .986730 .00027

24.200 .990540 .00026

24.500 .993160 .00026

24.800 .996680 .00027

25.100 .999780 .00026

25.401 .002830 .00026

25.701 .006300 .00026

26.001 .009080 .00026

26.301 .011530 .00025

26.501 .014160 .00025

32

13.700 .923750 .00036

13.800 .927230 .00037

13.900 .931900 .00038

14.000 .935730 .00037

14.100 .939870 .00037

14.200 .943980 .00037

14.300 .947740 .00037

14.400 .951840 .00037

14.500 .956340 .00038

14.600 .960680 .00038

14.700 .963740 .00037

14.800 .968040 .00037

14.900 .971640 .00037

15.000 .975380 .00036

15.100 .979380 .00038

15.200 .982630 .00038

15.300 .985660 .00037

15.400 .989090 .00037

15.500 .993250 .00036

15.600 .996160 .00036 
18.000 .977490 .00033 18.100 .978830 .00033 18.200 .981750 .00032 18.300 .984620 .00031 18.400 .986410 .00033 18.500 .988320 .00032 18.600 .990510 .00032 18.700 .993080 .00032 18.800 .994680 .00031 18.900 .997310 .00032 19.000 .999690 .00030 19.101 .001580 .00032 19.201 .004480 .00032 19.301 .005980 .00031 19.401 .007920 .00032 19.501 .010320 .00031 19.601 .012170 .00030 19.701 .014650 .00031

\section{5}

13.000 .917880 .00038 13.100 .923150 .00039 13.200 .928290 .00039 13.300 .932770 .00039 13.400 .937160 .00039 13.500 .942070 .00039 13.600 .946600 .00039 13.700 .950720 .00039 13.800 .954890 .00037 13.900 .959480 .00038 14.000 .963870 .00039 14.100 .968420 .00038 14.200 .972400 .00038 14.300 .976200 .00039 14.400 .980330 .00039 14.500 .985360 .00039 14.600 .989460 .00039 14.700 .992650 .00038 14.800 .996550 .00037 14.901 .000490 .00037 15.001 .004440 .00038 15.101 .008000 .00039 15.201 .011330 .00038 15.301 .014890 .00037 15.401 .019400 .00038
16.200 .993140 .00035 16.300 .997540 .00036 16.400 .999940 .00035 16.501 .003300 .00036 16.601 .005870 .00035 16.701 .008600 .00035 16.801 .011920 .00035 16.901 .014650 .00035 17.001 .018180 .00035
15.701 .000470 .00036 15.801 .003590 .00037 15.901 .006300 .00037 16.001 .010250 .00036 16.101 .012870 .00036 16.201 .016580 .00035
40
12.300 .920780 .00042 12.400 .925630 .00040 12.500 .931030 .00040 12.600 .935990 .00041 12.700 .941680 .00040 12.800 .946820 .00040 12.900 .952260 .00040 13.000 .957290 .00042 13.100 .962070 .00040 13.200 .967380 .00041 13.300 .971390 .00041 13.400 .976120 .00040 13.500 .981390 .00040 13.600 .986270 .00041 13.700 .990850 .00041 13.800 .994980 .00040 13.901 .000460 .00039 14.001 .004620 .00040 14.101 .008830 .00040 14.201 .012620 .00039 14.301 .017210 .00039
50

11.400 .923600 .00043 11.450 .927010 .00044 11.500 .929590 .00044 11.550 .932530 .00044 11.600 .936260 .00044 11.650 .938220 .00043 11.700 .942380 .00044 11.750 .945120 .00044 11.800 .948290 .00043 11.850 .951270 .00043 11.900 .953920 .00045 11.950 .956870 .00043 12.000 .960740 .00043 12.050 .963890 .00042 12.100 .966450 .00044 12.150 .969180 .00042 12.200 .972450 .00043 12.250 .975010 .00044 12.300 .978080 .00043 12.350 .980910 .00044 12.400 .984660 .00044 12.450 .986630 .00043 12.500 .989270 .00043 12.550 .991520 .00043 12.600 .995780 .00043 12.650 .998280 .00043 12.701 .000190 .00044 12.751 .002930 .00043 12.801 .005660 .00043 
CHPRC-01552, Revision 0

12.851 .008200 .00043

12.901 .010980 .00043

12.951 .014070 .00043

13.001 .016600 .00043

13.051 .018110 .00043

13.101 .021800 .00044

60

10.800 .921550 .00045 10.850 .924710 .00045 10.900 .928280 .00046 10.950 .932710 .00045 11.000 .936490 .00046 11.050 .938630 .00045 11.100 .942760 .00045 11.150 .946380 .00047 11.200 .950030 .00044 11.250 .953280 .00044 11.300 .957020 .00046 11.350 .959950 .00045 11.400 .963160 .00045 11.450 .966890 .00045 11.500 .969240 .00047 11.550 .973630 .00045 11.600 .977420 .00046 11.650 .981090 .00046 11.700 .983230 .00046 11.750 .986280 .00045 11.800 .989860 .00045 11.850 .992530 .00045 11.900 .996190 .00045 11.950 .999120 .00047 12.001 .002870 .00046 12.051 .005140 .00045 12.101 .008220 .00045 12.151 .011640 .00045 12.201 .014110 .00046 12.251 .017300 .00046 12.301 .020470 .00045

\section{0}

10.400 .921420 .00048

10.450 .924660 .00047

10.500 .929010 .00047

10.550 .932360 .00047

10.600 .935870 .00048

10.650 .939240 .00048

10.700 .944240 .00046

10.750 .948480 .00047

10.800 .951270 .00046

10.850 .954840 .00046

10.900 .959610 .00048

10.950 .962540 .00048

11.000 .965820 .00046

11.050 .968940 .00048

11.100 .973350 .00048

11.150 .977480 .00047

11.200 .980920 .00048

11.250 .983420 .00047

11.300 .986730 .00047

11.350 .991300 .00046

11.400 .994550 .00047

11.450 .997550 .00048

11.501 .000900 .00047

11.551 .004280 .00047

11.601 .007720 .00047

11.651 .010950 .00046

11.701 .014280 .00047

11.751 .017390 .00047

11.801 .020760 .00046
80

10.100 .918360 .00049

10.150 .923480 .00049

10.200 .926780 .00048

10.250 .930860 .00047

10.300 .934480 .00048

10.350 .939460 .00049

10.400 .942050 .00048

10.450 .946870 .00048

10.500 .951200 .00049

10.550 .953670 .00048

10.600 .957870 .00049

10.650 .962450 .00048

10.700 .966600 .00048

10.750 .970940 .00049

10.800 .974190 .00045

10.850 .977660 .00049

10.900 .981390 .00047

10.950 .985110 .00047

11.000 .989460 .00050

11.050 .992320 .00047

11.100 .995040 .00049

11.150 .999900 .00046

11.201 .003410 .00049

11.251 .006660 .00048

11.301 .011420 .00048

11.351 .014380 .00048

11.401 .017570 .00049

11.451 .020330 .00049

11.501 .024750 .00048
90

9.900 .919370 .00048

9.950 .923980 .00047

10.000 .927790 .00050

10.050 .933330 .00049

10.100 .936070 .00048

10.150 .941220 .00049
100

9.700 .915440 .00048

9.750 .920140 .00051

9.800 .925390 .00050

9.850 .929170 .00051

9.900 .933090 .00050

9.950 .938100 .00050
118

9.500 .916010 .00051

9.550 .921490 .00049

9.600 .926680 .00051

9.650 .930040 .00051

9.700 .934620 .00051

9.750 .940150 .00051 
10.200 .944970 .00049

10.250 .948140 .00047

10.300 .952000 .00049

10.350 .957250 .00048

10.400 .961170 .00050

10.450 .964750 .00049

10.500 .968880 .00049

10.550 .973070 .00050

10.600 .976720 .00049

10.650 .980550 .00048

10.700 .984570 .00047

10.750 .989730 .00049

10.800 .992600 .00048

10.850 .995630 .00047

10.900 .999040 .00048

10.951 .003660 .00050

11.001 .007070 .00050

11.051 .010800 .00049

11.101 .015210 .00049

11.151 .018310 .00050

11.201 .022230 .00048
10.000 .941860 .00050

10.050 .946560 .00049

10.100 .951110 .00050

10.150 .953710 .00051

10.200 .958690 .00049

10.250 .963080 .00049

10.300 .967060 .00050

10.350 .971230 .00050

10.400 .976450 .00050

10.450 .979510 .00049

10.500 .983190 .00050

10.550 .988240 .00049

10.600 .991980 .00051

10.650 .995390 .00049

10.700 .998960 .00051

10.751 .003390 .00050

10.801 .007010 .00049

10.851 .010960 .00049

10.901 .015220 .00048

10.951 .019680 .00051

11.001 .021640 .00050
9.800 .943260 .00051

9.850 .948720 .00052

9.900 .952470 .00051

9.950 .958250 .00049

10.000 .961410 .00050

10.050 .965460 .00050

10.100 .969910 .00053

10.150 .974520 .00048

10.200 .978390 .00052

10.250 .982260 .00051

10.300 .986570 .00051

10.350 .991650 .00050

10.400 .995740 .00050

10.450 .999040 .00050

10.501 .004370 .00051

10.551 .007580 .00051

10.601 .011680 .00052

10.651 .015550 .00050

10.701 .019250 .00050

10.751 .023180 .00051

10.801 .027340 .00051
130

9.400 .916860 .00050

9.450 .922040 .00051

9.500 .926640 .00051

9.550 .930840 .00051

9.600 .935970 .00052

9.650 .940590 .00050

9.700 .945190 .00051

9.750 .949420 .00051

9.800 .954240 .00051

9.850 .958030 .00052

9.900 .962910 .00051

9.950 .967720 .00051

10.000 .972460 .00053

10.050 .974710 .00051

10.100 .980130 .00052

10.150 .983150 .00051

10.200 .988820 .00052

10.250 .992530 .00053

10.300 .996910 .00051

10.351 .001620 .00051

10.401 .006050 .00051

10.451 .009730 .00050

10.501 .013950 .00051

10.551 .018860 .00052

10.601 .020610 .00051
150

9.300 .919920 .00051

9.350 .924430 .00051

9.400 .929560 .00051

9.450 .933600 .00051

9.500 .938500 .00052

9.550 .943560 .00052

9.600 .947810 .00051

9.650 .952670 .00052

9.700 .956740 .00053

9.750 .961240 .00052

9.800 .966450 .00052

9.850 .971590 .00052

9.900 .975560 .00052

9.950 .979750 .00052

10.000 .984750 .00050

10.050 .988210 .00054

10.100 .993760 .00052

10.150 .997760 .00051

10.201 .000820 .00051

10.251 .005220 .00051

10.301 .010560 .00052

10.351 .014720 .00053

10.401 .018480 .00053

10.451 .022240 .00052

10.501 .026320 .00051
200

9.100 .915450 .00052

9.150 .920330 .00052

9.200 .925350 .00053

9.250 .929940 .00052

9.300 .935070 .00052

9.350 .939310 .00054

9.400 .945010 .00054

9.450 .949020 .00053

9.500 .953590 .00053

9.550 .958380 .00053

9.600 .963420 .00054

9.650 .968760 .00052

9.700 .973470 .00054

9.750 .977070 .00055

9.800 .981850 .00054

9.850 .987230 .00053

9.900 .990800 .00054

9.950 .995040 .00052

10.001 .001170 .00053

10.051 .004370 .00052

10.101 .008880 .00052

10.151 .013240 .00052

10.201 .017250 .00055

10.251 .022790 .00053

10.301 .026150 .00054 


\section{5}

9.100 .919700 .00053 9.150 .923760 .00052 9.200 .929880 .00052 9.250 .934530 .00051 9.300 .939590 .00051 9.350 .944290 .00054 9.400 .949340 .00053 9.450 .954130 .00052 9.500 .959610 .00052 9.550 .963140 .00051 9.600 .967820 .00052 9.650 .972700 .00054 9.700 .976470 .00054 9.750 .981690 .00052 9.800 .986770 .00054 9.850 .991350 .00054 9.900 .996350 .00053 9.951 .000300 .00052 10.001 .004660 .00052 10.051 .009320 .00052 10.101 .012710 .00053 10.151 .017780 .00053 10.201 .022210 .00054
250

9.100 .920030 .00053

9.150 .924800 .00053

9.200 .930090 .00052

9.250 .934510 .00053

9.300 .939950 .00053

9.350 .944580 .00052

9.400 .948830 .00052

9.450 .954090 .00055

9.500 .958620 .00052

9.550 .963620 .00054

9.600 .968200 .00053

9.650 .973310 .00055

9.700 .977950 .00052

9.750 .981730 .00054

9.800 .986460 .00054

9.850 .991530 .00055

9.900 .996350 .00054

9.951 .001120 .00054 10.001 .005210 .00054 10.051 .009470 .00053 10.101 .013960 .00054 10.151 .017940 .00054 10.201 .022230 .00052
353

9.100 .912430 .00052 9.150 .916230 .00054 9.200 .922720 .00053 9.250 .926930 .00052 9.300 .932220 .00052 9.350 .937300 .00051 9.400 .940880 .00054 9.450 .946140 .00053 9.500 .951600 .00053 9.550 .956340 .00052 9.600 .960520 .00054 9.650 .965150 .00053 9.700 .968960 .00055 9.750 .974670 .00052 9.800 .979460 .00054 9.850 .983460 .00054 9.900 .988300 .00053 9.950 .992350 .00053 10.000 .996960 .00053 10.051 .002560 .00054 10.101 .005980 .00054 10.151 .010470 .00051 10.201 .014230 .00054 10.251 .018960 .00053 10.301 .023860 .00055

475

9.400 .917450 .00052

9.450 .923150 .00052

9.500 .928290 .00053

9.550 .931280 .00053

9.600 .936940 .00052

9.650 .940970 .00054

9.700 .945920 .00052

9.750 .949700 .00052

9.800 .955080 .00051

9.850 .959350 .00054

9.900 .964600 .00052

9.950 .967370 .00052

10.000 .972800 .00052 10.050 .977150 .00053 10.100 .981150 .00054 10.150 .986020 .00053 10.200 .990210 .00053 10.250 .993820 .00053 10.300 .998660 .00052
480

9.400 .916650 .00051

9.450 .920990 .00053

9.500 .925690 .00052

9.550 .930930 .00051

9.600 .935150 .00052

9.650 .939990 .00052

9.700 .944980 .00051

9.750 .949470 .00052

9.800 .954170 .00053 9.850 .958360 .00052 9.900 .962100 .00052 9.950 .966660 .00053

10.000 .971260 .00053 10.050 .976450 .00052 10.100 .980350 .00053 10.150 .982900 .00052 10.200 .988970 .00052 10.250 .992890 .00054 10.300 .997530 .00052 
10.351 .003670 .00053 10.401 .007980 .00053 10.451 .012210 .00053 10.501 .016740 .00054 10.551 .020590 .00052 10.601 .025490 .00053
10.351 .002650 .00052 10.401 .007480 .00053 10.451 .010940 .00052 10.501 .015080 .00054 10.551 .018650 .00052 10.601 .023530 .00051
10.351 .001140 .00053

10.401 .006060 .00051

10.451 .009990 .00053 10.501 .013830 .00054 10.551 .018470 .00053 10.601 .022130 .00053

486

9.400 .915590 .00053

9.450 .921330 .00050

9.500 .926280 .00052

9.550 .930400 .00054

9.600 .934150 .00051

9.650 .939210 .00052

9.700 .943450 .00053

9.750 .948390 .00052

9.800 .952070 .00052

9.850 .956870 .00052

9.900 .961280 .00055

9.950 .965620 .00054

10.000 .971780 .00053

10.050 .974580 .00052

10.100 .978830 .00053

10.150 .983370 .00053

10.200 .988110 .00053

10.250 .991940 .00054

10.300 .995840 .00052

10.351 .000690 .00052

10.401 .004170 .00053

10.451 .008770 .00053

10.501 .012730 .00052

10.551 .016950 .00053

10.601 .021330 .00051 
U5o2N26-icyl-1000-01

12

55.800 .874210 .00014 56.800 .875420 .00014 57.800 .876780 .00014 58.800 .877270 .00015 59.800 .878460 .00014 60.800 .878740 .00014 61.800 .879780 .00014 62.800 .880500 .00014 63.800 .881190 .00014 64.800 .881890 .00014 65.800 .882280 .00014 66.800 .883320 .00014 67.800 .883790 .00014 68.800 .884270 .00014 69.800 .884920 .00014 70.800 .885540 .00014 71.800 .885750 .00014 72.800 .886160 .00014 73.800 .886610 .00014 74.800 .886850 .00014 75.800 .887600 .00013

25

14.400 .923630 .00033 14.600 .929830 .00032 14.800 .935050 .00031 15.000 .940730 .00032 15.200 .946720 .00031 15.400 .952020 .00032 15.600 .956960 .00031 15.800 .962730 .00031 16.000 .967890 .00031 16.200 .972550 .00030 16.400 .976870 .00032 16.600 .982510 .00031 16.800 .987400 .00031 17.000 .990750 .00031 17.200 .995960 .00031 17.400 .999750 .00031 17.601 .004210 .00031 17.801 .008350 .00031 18.001 .011820 .00031 18.201 .016650 .00030
15

38.900 .953610 .00019 41.800 .961070 .00018 44.700 .967000 .00018 47.600 .972370 .00017 50.500 .977120 .00016 53.400 .981040 .00017 56.300 .984310 .00015 59.200 .987270 .00016 62.100 .990010 .00015 65.000 .991570 .00015 67.900 .993800 .00015 70.800 .995110 .00015 73.700 .997240 .00014 76.600 .998380 .00014 79.500 .999790 .00015 82.401 .001370 .00014 85.301 .001780 .00014 88.201 .002770 .00014 91.101 .003210 .00013 94.001 .004460 .00013 96.901 .005160 .00013 97.501 .005160 .00014
20

18.200 .926150 .00028 18.500 .931900 .00027 18.800 .936470 .00028 19.100 .941530 .00027 19.400 .946440 .00027 19.700 .951360 .00028 20.000 .956100 .00027 20.300 .959910 .00027 20.600 .964480 .00027 20.900 .969060 .00026 21.200 .973170 .00027 21.500 .976610 .00027 21.800 .980420 .00026 22.100 .984130 .00026 22.400 .987850 .00027 22.700 .991030 .00026 23.000 .994890 .00026 23.300 .997750 .00025 23.601 .000820 .00026 23.901 .003770 .00025 24.201 .007330 .00025 24.501 .009900 .00026 24.801 .012730 .00025 24.901 .013740 .00026

30

12.600 .923040 .00034 12.700 .927490 .00035 12.800 .930980 .00035 12.900 .934670 .00035 13.000 .938780 .00036 13.100 .942940 .00035 13.200 .946900 .00035 13.300 .949960 .00035 13.400 .954030 .00035 13.500 .957370 .00035 13.600 .961270 .00035 13.700 .964890 .00035 13.800 .967770 .00035 13.900 .970850 .00034 14.000 .974540 .00034 14.100 .978470 .00034 14.200 .980930 .00035 14.300 .984650 .00034 14.400 .987750 .00035 14.500 .990860 .00035
32

12.100 .922840 .00036 12.200 .928350 .00036 12.300 .932100 .00037 12.400 .936180 .00036 12.500 .940620 .00036 12.600 .944340 .00036 12.700 .948410 .00036 12.800 .952000 .00036 12.900 .956420 .00035 13.000 .960020 .00036 13.100 .964310 .00036 13.200 .967690 .00036 13.300 .971980 .00036 13.400 .975600 .00035 13.500 .978810 .00036 13.600 .981810 .00036 13.700 .985960 .00035 13.800 .989960 .00036 13.900 .993570 .00035 14.000 .996600 .00036 
14.600 .994570 .00034 14.700 .997930 .00034 14.801 .000250 .00034 14.901 .003670 .00034 15.001 .006680 .00034 15.101 .009430 .00034 15.201 .012260 .00034 15.301 .015020 .00034
14.101 .000790 .00035 14.201 .002940 .00035 14.301 .006210 .00035 14.401 .010180 .00036 14.501 .013470 .00035 14.601 .016280 .00035
35

11.500 .923970 .00037 11.600 .928060 .00038 11.700 .932400 .00037 11.800 .938040 .00037 11.900 .942400 .00039 12.000 .947100 .00037 12.100 .950400 .00038 12.200 .955300 .00038 12.300 .960010 .00038 12.400 .964050 .00038 12.500 .969730 .00037 12.600 .972950 .00036 12.700 .977290 .00037 12.800 .981150 .00037 12.900 .984530 .00037 13.000 .988570 .00037 13.100 .993290 .00037 13.200 .996630 .00037 13.301 .000910 .00038 13.401 .004380 .00035 13.501 .008100 .00037 13.601 .011190 .00037 13.701 .015190 .00037
40

10.700 .920440 .00038 10.800 .926600 .00038 10.900 .930930 .00039 11.000 .936590 .00038 11.100 .942590 .00040 11.200 .947480 .00039 11.300 .952580 .00039 11.400 .957280 .00038 11.500 .963210 .00039 11.600 .967430 .00039 11.700 .972270 .00039 11.800 .977520 .00039 11.900 .982690 .00039 12.000 .986580 .00039 12.100 .990480 .00038 12.200 .995460 .00039 12.300 .999980 .00038 12.401 .005050 .00040 12.501 .008870 .00039 12.601 .013650 .00039 12.701 .017490 .00039
50

9.700 .916920 .00042 9.800 .922950 .00042 9.900 .930170 .00042 10.000 .936270 .00043 10.100 .942840 .00042 10.200 .948650 .00041 10.300 .955220 .00041 10.400 .961170 .00042 10.500 .967030 .00042 10.600 .973510 .00041 10.700 .978670 .00041 10.800 .984560 .00041 10.900 .990420 .00042 11.000 .995030 .00041 11.101 .001120 .00041 11.201 .007190 .00041 11.301 .011440 .00042 11.401 .017230 .00042 11.501 .023100 .00043
60

9.200 .923120 .00045 9.250 .925200 .00044 9.300 .929670 .00044 9.350 .933380 .00042 9.400 .937220 .00044 9.450 .940090 .00043 9.500 .944290 .00044 9.550 .947540 .00043 9.600 .950580 .00043 9.650 .954260 .00044 9.700 .957610 .00043 9.750 .961440 .00044
70

8.800 .921430 .00045 8.850 .926140 .00045 8.900 .930200 .00046 8.950 .934390 .00044 9.000 .938270 .00045 9.050 .941180 .00045 9.100 .945150 .00045 9.150 .949670 .00045 9.200 .953180 .00045 9.250 .956040 .00045 9.300 .960410 .00045 9.350 .964590 .00045
80

8.500 .920350 .00047 8.550 .924280 .00047 8.600 .928920 .00046 8.650 .932240 .00046 8.700 .935960 .00045 8.750 .940810 .00046 8.800 .945000 .00046 8.850 .948970 .00047 8.900 .952520 .00046 8.950 .956050 .00045 9.000 .960650 .00046 9.050 .964060 .00046 
9.800 .965400 .00044

9.850 .967970 .00044

9.900 .971350 .00043

9.950 .974050 .00043

10.000 .977450 .00044

10.050 .981640 .00043

10.100 .984550 .00044

10.150 .987380 .00043

10.200 .990900 .00043

10.250 .994180 .00044

10.300 .996530 .00043

10.351 .000740 .00043

10.401 .002990 .00045

10.451 .006920 .00043

10.501 .008790 .00043

10.551 .012020 .00043

10.601 .015800 .00042

10.651 .017920 .00043

10.701 .020950 .00044
9.400 .967110 .00045

9.450 .970570 .00045

9.500 .974640 .00046

9.550 .978080 .00046

9.600 .981510 .00044

9.650 .985150 .00045

9.700 .988310 .00046

9.750 .991780 .00046

9.800 .995870 .00045

9.850 .998530 .00046

9.901 .002620 .00046

9.951 .005470 .00046

10.001 .009860 .00043

10.051 .012390 .00045

10.101 .015250 .00044

10.151 .019070 .00046

10.201 .021580 .00046
9.100 .967610 .00047

9.150 .972240 .00046

9.200 .975620 .00045

9.250 .979960 .00047

9.300 .983350 .00045

9.350 .987760 .00046

9.400 .991360 .00048

9.450 .994350 .00046

9.500 .998420 .00047

9.551 .001370 .00046

9.601 .004700 .00046

9.651 .008860 .00046

9.701 .012100 .00047

9.751 .016020 .00046

9.801 .019860 .00046
90

8.300 .921670 .00047

8.350 .926600 .00047

8.400 .930660 .00047

8.450 .934770 .00047

8.500 .938660 .00048

8.550 .942260 .00047

8.600 .947090 .00048

8.650 .950910 .00045

8.700 .955810 .00048

8.750 .959400 .00045

8.800 .963290 .00048

8.850 .967280 .00047

8.900 .970770 .00047

8.950 .974730 .00048

9.000 .980100 .00047

9.050 .983810 .00046

9.100 .986680 .00047

9.150 .991230 .00049

9.200 .994090 .00046

9.250 .997860 .00048

9.301 .002370 .00048

9.351 .006190 .00048

9.401 .009410 .00048

9.451 .013190 .00045

9.501 .017440 .00047

9.551 .020190 .00047

9.601 .024740 .00047
100

8.100 .918510 .00049

8.150 .923620 .00048

8.200 .927800 .00047

8.250 .932200 .00048

8.300 .935820 .00048

8.350 .940640 .00047

8.400 .944290 .00049

8.450 .949230 .00047

8.500 .952570 .00048

8.550 .957240 .00048

8.600 .961470 .00048

8.650 .965380 .00048

8.700 .969670 .00048

8.750 .973960 .00049

8.800 .978080 .00049

8.850 .982640 .00047

8.900 .986410 .00049

8.950 .989270 .00049

9.000 .993420 .00047

9.050 .998240 .00047

9.101 .001900 .00048

9.151 .006900 .00048

9.201 .009450 .00048

9.251 .013680 .00048

9.301 .017020 .00048

9.351 .021640 .00049

9.401 .025060 .00047
118

7.900 .919040 .00049

7.950 .924870 .00048

8.000 .929130 .00049

8.050 .933580 .00049

8.100 .937490 .00048

8.150 .942530 .00048

8.200 .947100 .00050

8.250 .952260 .00050

8.300 .955460 .00048

8.350 .960020 .00048

8.400 .964660 .00049

8.450 .968770 .00048

8.500 .973080 .00050

8.550 .977570 .00048

8.600 .982240 .00048

8.650 .986400 .00050

8.700 .989780 .00048

8.750 .994460 .00050

8.800 .998490 .00049

8.851 .002290 .00049

8.901 .006280 .00049

8.951 .011130 .00050

9.001 .014440 .00048

9.051 .018030 .00051

9.101 .022900 .00048 
130

7.800 .920800 .00048

7.850 .924850 .00048

7.900 .929570 .00048

7.950 .934140 .00049

8.000 .939010 .00050

8.050 .942940 .00051

8.100 .948520 .00049

8.150 .952260 .00050

8.200 .957750 .00049

8.250 .961810 .00050

8.300 .966950 .00050

8.350 .970410 .00048

8.400 .976020 .00050

8.450 .979200 .00048

8.500 .984310 .00049

8.550 .988520 .00048

8.600 .992000 .00049

8.650 .995260 .00049

8.701 .000000 .00048

8.751 .003800 .00050

8.801 .009350 .00050

8.851 .012910 .00048

8.901 .017380 .00049

8.951 .021900 .00050

9.001 .025640 .00051
150

7.600 .913530 .00049

7.650 .918500 .00050

7.700 .923300 .00050

7.750 .928780 .00049

7.800 .933430 .00051

7.850 .936740 .00049

7.900 .942750 .00049

7.950 .946200 .00049

8.000 .951510 .00050

8.050 .956300 .00049

8.100 .961360 .00049

8.150 .964810 .00051

8.200 .970470 .00051

8.250 .974670 .00049

8.300 .979430 .00049

8.350 .983750 .00049

8.400 .988400 .00051

8.450 .992340 .00050

8.500 .997120 .00051

8.551 .001020 .00049

8.601 .004820 .00051

8.651 .007840 .00051

8.701 .013100 .00051

8.751 .017760 .00051

8.801 .021500 .00050
200

7.500 .920940 .00051

7.550 .926090 .00049

7.600 .930880 .00049

7.650 .936040 .00050

7.700 .940300 .00050

7.750 .944220 .00052

7.800 .949550 .00050

7.850 .955680 .00049

7.900 .959950 .00050

7.950 .964270 .00051

8.000 .969870 .00051

8.050 .973840 .00052

8.100 .978830 .00050

8.150 .982760 .00052

8.200 .987210 .00050

8.250 .991940 .00050

8.300 .996130 .00050

8.351 .000390 .00052

8.401 .005200 .00053

8.451 .009250 .00051

8.501 .013690 .00050

8.551 .017620 .00051

8.601 .022940 .00050
235

7.400 .915570 .00051

7.450 .921290 .00050

7.500 .927300 .00052

7.550 .930470 .00050

7.600 .935140 .00051

7.650 .941900 .00051

7.700 .945460 .00050

7.750 .950270 .00049

7.800 .955250 .00051

7.850 .959880 .00050

7.900 .964650 .00051

7.950 .969110 .00050

8.000 .973400 .00052

8.050 .979690 .00052

8.100 .983540 .00051

8.150 .987550 .00052

8.200 .991410 .00051

8.250 .996810 .00051

8.301 .001700 .00051
250

7.400 .918130 .00051

7.450 .922440 .00051

7.500 .926760 .00052

7.550 .932380 .00050

7.600 .936930 .00051

7.650 .942250 .00051

7.700 .946370 .00051

7.750 .951820 .00051

7.800 .955450 .00051

7.850 .960950 .00051

7.900 .965760 .00050

7.950 .969940 .00051

8.000 .975160 .00050

8.050 .979210 .00050

8.100 .984500 .00052

8.150 .988700 .00052

8.200 .993810 .00051

8.250 .997400 .00050

8.301 .002620 .00050
353

7.400 .912770 .00052

7.450 .916990 .00051

7.500 .922770 .00050

7.550 .927510 .00052

7.600 .932240 .00051

7.650 .937180 .00050

7.700 .941470 .00051

7.750 .946930 .00052

7.800 .951360 .00052

7.850 .955580 .00051

7.900 .961440 .00051

7.950 .966110 .00051

8.000 .970950 .00051

8.050 .974290 .00051

8.100 .979180 .00052

8.150 .984140 .00050

8.200 .987780 .00052

8.250 .993210 .00052

8.300 .997790 .00053 
8.351 .006160 .00051 8.401 .009670 .00051 8.451 .014990 .00052 8.501 .018560 .00052
8.351 .006990 .00051

8.401 .011640 .00052

8.451 .015280 .00051

8.501 .020220 .00051

\section{0}

7.700 .923420 .00051

7.750 .927320 .00051

7.800 .932360 .00051

7.850 .936830 .00052

7.900 .941520 .00051

7.950 .946760 .00050

8.000 .951080 .00051

8.050 .955040 .00051

8.100 .959960 .00052

8.150 .964040 .00052

8.200 .967520 .00053

8.250 .973280 .00051

8.300 .977810 .00050

8.350 .981990 .00053

8.400 .986600 .00051

8.450 .990880 .00051

8.500 .994540 .00051

8.550 .999320 .00051

8.601 .003000 .00051

8.651 .007260 .00052

8.701 .011290 .00052

8.751 .014730 .00050

8.801 .019020 .00052
475

7.700 .922200 .00050

7.750 .926150 .00051

7.800 .931450 .00050

7.850 .936130 .00050

7.900 .940470 .00052

7.950 .944900 .00050

8.000 .950290 .00049

8.050 .955070 .00051

8.100 .958540 .00052

8.150 .963110 .00052

8.200 .966950 .00051

8.250 .972020 .00051

8.300 .976380 .00052

8.350 .980900 .00053

8.400 .985400 .00051

8.450 .989010 .00052

8.500 .993170 .00050

8.550 .997280 .00052

8.601 .002260 .00052

8.651 .005630 .00052

8.701 .010400 .00049

8.751 .013820 .00052

8.801 .017720 .00051
8.351 .001260 .00052

8.401 .006300 .00051

8.451 .009770 .00052

8.501 .014300 .00052

8.551 .017880 .00050

8.601 .022400 .00052

480

7.700 .921090 .00050

7.750 .926230 .00051

7.800 .929980 .00050

7.850 .934890 .00050

7.900 .939230 .00051

7.950 .943440 .00051

8.000 .948920 .00050

8.050 .953570 .00050

8.100 .957180 .00051

8.150 .961660 .00052

8.200 .965970 .00051

8.250 .971540 .00051

8.300 .975190 .00051

8.350 .979380 .00051

8.400 .983880 .00050

8.450 .987930 .00052

8.500 .992560 .00051

8.550 .997480 .00053

8.601 .000360 .00054

8.651 .004480 .00052

8.701 .007840 .00052

8.751 .012800 .00050

8.801 .016450 .00051

8.851 .020420 .00051

8.901 .024840 .00050
486

7.700 .920790 .00050

7.750 .924740 .00052

7.800 .929180 .00049

7.850 .933770 .00051

7.900 .938300 .00052

7.950 .944760 .00051

8.000 .947800 .00052

8.050 .951870 .00052

8.100 .957170 .00051

8.150 .960820 .00052

8.200 .965580 .00052

8.250 .969910 .00051 
8.300 .973360 .00051

8.350 .978440 .00053

8.400 .983390 .00050

8.450 .987700 .00051

8.500 .992270 .00052

8.550 .995550 .00051

8.601 .000070 .00052

8.651 .004280 .00052

8.701 .007800 .00052

8.751 .012080 .00051

8.801 .015860 .00050

8.851 .020160 .00051

8.901 .024910 .00050

U5o2N26-icyl-1000-10

12

52.300 .872310 .00014 53.800 .874440 .00015 55.300 .875370 .00014 56.800 .876480 .00014 58.300 .878090 .00014 59.800 .878990 .00014 61.300 .880130 .00014 62.800 .881220 .00014 64.300 .882380 .00014 65.800 .883130 .00014 67.300 .884200 .00014 68.800 .885030 .00013 70.300 .885350 .00014 71.800 .886480 .00013 73.300 .887110 .00013 74.800 .887630 .00013 76.300 .888240 .00013 77.800 .889320 .00013 79.300 .889240 .00013 80.800 .889760 .00013 82.300 .890200 .00013 83.400 .890530 .00013
15

29.000 .919560 .00019 29.200 .920980 .00020 29.400 .922240 .00020 29.600 .923020 .00019 29.800 .924160 .00020 30.000 .924830 .00019 30.200 .925890 .00020 30.400 .926530 .00019 30.600 .927610 .00020 30.800 .928910 .00020 31.000 .929350 .00020 31.200 .930170 .00019 31.400 .931350 .00020 31.600 .932160 .00019 31.800 .933030 .00019 32.000 .934110 .00019 32.200 .934850 .00019 32.400 .935540 .00018 32.600 .936400 .00019 32.800 .936840 .00019 33.000 .937730 .00019 33.200 .938590 .00019 33.400 .939510 .00019 33.600 .940320 .00018 33.800 .940680 .00019 34.000 .941970 .00019 34.200 .942740 .00018 34.300 .942580 .00019
20

17.000 .926760 .00026 17.300 .931470 .00027 17.600 .936860 .00026 17.900 .941950 .00026 18.200 .946810 .00026 18.500 .951460 .00026 18.800 .955670 .00026 19.100 .960030 .00026 19.400 .964730 .00026 19.700 .969350 .00026 20.000 .973000 .00026 20.300 .977130 .00025 20.600 .980750 .00025 20.900 .983910 .00025 21.200 .987920 .00025 21.500 .991640 .00024 21.800 .994750 .00025 22.100 .997860 .00025 22.401 .000990 .00024 22.701 .004880 .00024 23.001 .007020 .00025 23.301 .010150 .00024 23.601 .012840 .00024 
25

13.200 .923560 .00030 13.300 .926810 .00032 13.400 .929850 .00030 13.500 .932610 .00030 13.600 .935920 .00030 13.700 .938760 .00030 13.800 .941170 .00031 13.900 .944040 .00030 14.000 .947260 .00030 14.100 .949500 .00031 14.200 .952550 .00031 14.300 .954720 .00030 14.400 .957880 .00030 14.500 .960540 .00029 14.600 .963570 .00030 14.700 .965650 .00030 14.800 .967850 .00029 14.900 .970820 .00030 15.000 .972480 .00030 15.100 .975700 .00030 15.200 .977520 .00031 15.300 .980200 .00029 15.400 .982570 .00030 15.500 .984550 .00030 15.600 .987180 .00030 15.700 .989810 .00029 15.800 .991640 .00029 15.900 .994170 .00029 16.000 .995940 .00029 16.100 .998450 .00029 16.201 .000440 .00029 16.301 .002740 .00030 16.401 .004300 .00029 16.501 .006460 .00030 16.601 .008570 .00029 16.701 .010710 .00030 16.801 .012890 .00029 16.901 .014940 .00028
30

11.400 .923980 .00034 11.500 .928800 .00034 11.600 .932760 .00032 11.700 .937100 .00033 11.800 .939840 .00033 11.900 .944100 .00033 12.000 .948570 .00034 12.100 .951100 .00033 12.200 .955340 .00033 12.300 .958950 .00033 12.400 .962410 .00033 12.500 .965720 .00032 12.600 .969160 .00034 12.700 .972340 .00033 12.800 .975960 .00032 12.900 .979360 .00033 13.000 .983190 .00032 13.100 .986010 .00032 13.200 .988890 .00033 13.300 .992600 .00032 13.400 .995440 .00033 13.500 .998770 .00032 13.601 .001160 .00032 13.701 .005120 .00032 13.801 .007590 .00033 13.901 .010510 .00032 14.001 .013320 .00033 14.101 .016440 .00032
32

10.900 .924900 .00034

11.000 .929130 .00034

11.100 .933340 .00034

11.200 .937820 .00034

11.300 .941800 .00033

11.400 .945910 .00033

11.500 .949940 .00033

11.600 .954220 .00033

11.700 .958360 .00033

11.800 .961430 .00033

11.900 .965520 .00034

12.000 .969860 .00034

12.100 .973390 .00033

12.200 .977010 .00034

12.300 .979980 .00034

12.400 .984600 .00033

12.500 .987700 .00034

12.600 .990800 .00033

12.700 .995070 .00033

12.800 .999050 .00032

12.901 .001980 .00033

13.001 .004810 .00033

13.101 .008450 .00033

13.201 .011720 .00033

13.301 .015010 .00033
35

10.300 .925220 .00035 10.400 .930080 .00035 10.500 .934490 .00036 10.600 .939190 .00034 10.700 .943820 .00036 10.800 .948310 .00035 10.900 .952800 .00034 11.000 .957250 .00036
40

9.500 .922860 .00036 9.600 .928710 .00037 9.700 .934510 .00037 9.800 .939440 .00037 9.900 .945060 .00036 10.000 .950180 .00037 10.100 .955550 .00036 10.200 .960450 .00036
50

8.500 .922060 .00038 8.550 .924980 .00038 8.600 .928980 .00038 8.650 .931900 .00039 8.700 .934820 .00039 8.750 .937480 .00039 8.800 .940850 .00039 8.850 .944840 .00039 
11.100 .961990 .00034 11.200 .967050 .00034 11.300 .970890 .00035 11.400 .975310 .00035 11.500 .978990 .00035 11.600 .982760 .00034 11.700 .986960 .00035 11.800 .991310 .00035 11.900 .994520 .00034 12.000 .998940 .00035 12.101 .003170 .00035 12.201 .006480 .00034 12.301 .010980 .00034 12.401 .014180 .00035 12.501 .017320 .00035
10.300 .965680 .00036 10.400 .970380 .00037 10.500 .974910 .00036 10.600 .979570 .00037 10.700 .985450 .00036 10.800 .989240 .00036 10.900 .993470 .00036 11.000 .998870 .00035 11.101 .003140 .00036 11.201 .006820 .00036 11.301 .012430 .00036 11.401 .016360 .00036
8.900 .947570 .00039 8.950 .950340 .00038 9.000 .953900 .00038 9.050 .956680 .00038 9.100 .960440 .00039 9.150 .962830 .00038 9.200 .965990 .00039 9.250 .968660 .00038 9.300 .972220 .00039 9.350 .974660 .00039 9.400 .977590 .00038 9.450 .980890 .00039 9.500 .983180 .00038 9.550 .986010 .00038 9.600 .989660 .00039 9.650 .992310 .00038 9.700 .994940 .00038 9.750 .997740 .00040 9.801 .000520 .00038 9.851 .002790 .00039 9.901 .005890 .00037 9.951 .009390 .00038 10.001 .012170 .00038 10.051 .013660 .00037 10.101 .016380 .00038
60

7.900 .922380 .00041 7.950 .925740 .00040 8.000 .929620 .00040 8.050 .932340 .00042 8.100 .936760 .00040 8.150 .939260 .00040 8.200 .943340 .00040 8.250 .947430 .00040 8.300 .950550 .00041 8.350 .953940 .00039 8.400 .957700 .00040 8.450 .961600 .00040 8.500 .965410 .00040 8.550 .967840 .00040 8.600 .971480 .00040 8.650 .974050 .00041 8.700 .977360 .00040 8.750 .980850 .00041 8.800 .984370 .00040 8.850 .987670 .00039 8.900 .991180 .00041 8.950 .993710 .00040
70

7.500 .922890 .00041

7.550 .926580 .00040

7.600 .930340 .00042

7.650 .934580 .00041

7.700 .938580 .00041

7.750 .942080 .00042

7.800 .946540 .00042

7.850 .950760 .00041

7.900 .954350 .00043

7.950 .957630 .00042

8.000 .961570 .00041

8.050 .965540 .00041

8.100 .968450 .00041

8.150 .972040 .00042

8.200 .975100 .00040

8.250 .979850 .00042

8.300 .982720 .00041

8.350 .986440 .00042

8.400 .990200 .00042

8.450 .993430 .00040

8.500 .996720 .00042

8.551 .000620 .00041
80

7.200 .923250 .00043

7.250 .926560 .00042

7.300 .931460 .00042

7.350 .935850 .00042

7.400 .939100 .00042

7.450 .943550 .00042

7.500 .947600 .00043

7.550 .951810 .00042

7.600 .955200 .00042

7.650 .959200 .00043

7.700 .963470 .00043

7.750 .966790 .00043

7.800 .970840 .00044

7.850 .974760 .00042

7.900 .979010 .00043

7.950 .982160 .00041

8.000 .986610 .00042

8.050 .989570 .00043

8.100 .994220 .00042

8.150 .997610 .00043

8.201 .001070 .00042

8.251 .004270 .00044 
9.000 .996840 .00040 9.051 .000340 .00040 9.101 .003850 .00039 9.151 .007040 .00040 9.201 .010460 .00041 9.251 .012840 .00039 9.301 .016250 .00040 9.351 .018790 .00041 9.401 .021710 .00039

90

6.900 .916290 .00042 6.950 .920070 .00043 7.000 .925100 .00044 7.050 .930360 .00043 7.100 .932890 .00043 7.150 .938170 .00043 7.200 .942700 .00044 7.250 .947130 .00043 7.300 .950290 .00043 7.350 .953970 .00045 7.400 .959180 .00044 7.450 .963260 .00042 7.500 .967590 .00044 7.550 .970950 .00044 7.600 .975460 .00044 7.650 .979370 .00042 7.700 .983100 .00043 7.750 .987290 .00043 7.800 .991080 .00044 7.850 .995390 .00043 7.900 .999190 .00043 7.951 .003120 .00044 8.001 .006040 .00043 8.051 .010600 .00043 8.101 .014210 .00044 8.151 .017940 .00044 8.201 .021520 .00044

130

6.400 .920630 .00046 6.450 .925370 .00045 6.500 .928900 .00044 6.550 .933670 .00044 6.600 .939020 .00045 6.650 .944080 .00044 6.700 .948830 .00045
8.601 .004360 .00041

8.651 .007400 .00041

8.701 .010110 .00040

8.751 .014030 .00042

8.801 .017620 .00041
8.301 .007790 .00042 8.351 .011980 .00044 8.401 .015060 .00042 8.451 .019380 .00043 8.501 .021750 .00042
100

6.800 .923240 .00043

6.850 .926930 .00045

6.900 .932160 .00043

6.950 .936450 .00044

7.000 .941080 .00044

7.050 .945570 .00043

7.100 .950030 .00044

7.150 .953320 .00044

7.200 .958680 .00046

7.250 .962950 .00045

7.300 .965950 .00043

7.350 .971500 .00043

7.400 .975040 .00042

7.450 .979750 .00044

7.500 .982960 .00044

7.550 .988350 .00043

7.600 .991900 .00042

7.650 .995700 .00043

7.700 .999100 .00044

7.751 .003280 .00045

7.801 .007030 .00044

7.851 .011290 .00043

7.901 .015010 .00045

7.951 .020050 .00043

8.001 .024290 .00044
118

6.500 .917420 .00045

6.550 .922640 .00044

6.600 .926670 .00046

6.650 .931080 .00043

6.700 .936380 .00044

6.750 .940810 .00046

6.800 .944870 .00045

6.850 .950510 .00044

6.900 .954580 .00044

6.950 .958240 .00046

7.000 .963630 .00044

7.050 .967290 .00044

7.100 .972830 .00045

7.150 .976240 .00046

7.200 .981160 .00045

7.250 .985860 .00045

7.300 .989600 .00045

7.350 .994690 .00045

7.400 .997550 .00045

7.451 .002580 .00045

7.501 .005620 .00044

7.551 .010610 .00045

7.601 .014210 .00044

7.651 .019230 .00045

7.701 .022300 .00044
150

6.200 .914550 .00047 6.250 .919690 .00045 6.300 .924890 .00046 6.350 .929780 .00046 6.400 .934980 .00045 6.450 .940070 .00045 6.500 .944110 .00045
200

6.000 .917680 .00046 6.050 .922830 .00044 6.100 .928440 .00045 6.150 .932150 .00046 6.200 .938010 .00045 6.250 .942810 .00045 6.300 .947160 .00047 
6.750 .953270 .00044 6.800 .957200 .00046 6.850 .961930 .00045 6.900 .965950 .00044 6.950 .970920 .00045 7.000 .975090 .00044 7.050 .979780 .00045 7.100 .984520 .00045 7.150 .988460 .00044 7.200 .992780 .00045 7.250 .997620 .00045 7.301 .001380 .00046 7.351 .005650 .00044 7.401 .010670 .00044 7.451 .014240 .00044 7.501 .017910 .00046

235

5.900 .916070 .00046 5.950 .920960 .00047 6.000 .926290 .00046 6.050 .931610 .00046 6.100 .936560 .00046 6.150 .942200 .00047 6.200 .945850 .00046 6.250 .951630 .00046 6.300 .957380 .00047 6.350 .961790 .00047 6.400 .966260 .00047 6.450 .970580 .00046 6.500 .975330 .00046 6.550 .980410 .00046 6.600 .985310 .00046 6.650 .989720 .00047 6.700 .993840 .00045 6.750 .998770 .00047 6.801 .003990 .00046 6.851 .007660 .00047 6.901 .013090 .00047 6.951 .016130 .00047 7.001 .021550 .00045
6.550 .949240 .00046

6.600 .953670 .00045

6.650 .958450 .00045

6.700 .963060 .00045

6.750 .967530 .00046

6.800 .972730 .00046

6.850 .976290 .00047

6.900 .982050 .00046

6.950 .986520 .00046

7.000 .990420 .00046

7.050 .994950 .00046

7.100 .999240 .00045

7.151 .003750 .00045

7.201 .007860 .00045

7.251 .012530 .00046

7.301 .017060 .00047

7.351 .020560 .00046

7.401 .026010 .00046
6.350 .952460 .00047

6.400 .957440 .00046 6.450 .963020 .00047 6.500 .966920 .00045 6.550 .971730 .00046 6.600 .975850 .00047 6.650 .982160 .00047 6.700 .985520 .00048 6.750 .990090 .00047 6.800 .994880 .00047 6.850 .999680 .00046 6.901 .003900 .00048 6.951 .009460 .00047 7.001 .014240 .00046 7.051 .017550 .00047 7.101 .021480 .00047
250

5.900 .917970 .00048 5.950 .924240 .00047 6.000 .928870 .00046 6.050 .933870 .00046 6.100 .939170 .00047 6.150 .944040 .00047 6.200 .948770 .00047 6.250 .953490 .00047 6.300 .958040 .00047 6.350 .963540 .00047 6.400 .968040 .00047 6.450 .973160 .00046 6.500 .977920 .00047 6.550 .982840 .00047 6.600 .987440 .00049 6.650 .992000 .00045 6.700 .996860 .00048 6.751 .001170 .00047 6.801 .005460 .00047 6.851 .009770 .00048 6.901 .014720 .00048 6.951 .018260 .00048 7.001 .023480 .00046
353

5.900 .924060 .00047 5.950 .928540 .00046 6.000 .934300 .00047 6.050 .938730 .00047 6.100 .943740 .00046 6.150 .948770 .00047 6.200 .954360 .00047 6.250 .958700 .00048 6.300 .963190 .00046 6.350 .967840 .00048 6.400 .971710 .00047 6.450 .977520 .00047 6.500 .982470 .00046 6.550 .986050 .00048 6.600 .990630 .00048 6.650 .994970 .00048 6.700 .999750 .00048 6.751 .004090 .00048 6.801 .009350 .00047 6.851 .013620 .00046 6.901 .018410 .00046 6.951 .022230 .00047 7.001 .026590 .00048 
470

5.900 .915580 .00046 5.950 .920660 .00046 6.000 .925860 .00045 6.050 .930400 .00046 6.100 .935660 .00047 6.150 .939820 .00046 6.200 .944240 .00046 6.250 .950140 .00047 6.300 .953770 .00046 6.350 .959380 .00046 6.400 .962860 .00048 6.450 .968540 .00047 6.500 .972590 .00048 6.550 .976690 .00047 6.600 .981170 .00048 6.650 .986180 .00048 6.700 .989640 .00047 6.750 .993730 .00047 6.800 .998720 .00047 6.851 .003890 .00047 6.901 .007450 .00048 6.951 .012230 .00048 7.001 .016530 .00047 7.051 .019800 .00047 7.101 .023900 .00047

486

6.000 .924670 .00045 6.050 .929070 .00046 6.100 .934310 .00045 6.150 .938570 .00047 6.200 .942400 .00046 6.250 .948110 .00046 6.300 .953260 .00046 6.350 .956950 .00047 6.400 .962140 .00047 6.450 .966690 .00047 6.500 .970450 .00048 6.550 .975390 .00047 6.600 .979300 .00047 6.650 .983880 .00048 6.700 .988280 .00049 6.750 .992770 .00047 6.800 .997670 .00048 6.851 .000980 .00047 6.901 .005370 .00047 6.951 .009570 .00046 7.001 .014340 .00047 7.051 .018460 .00048 7.101 .021620 .00047
475

5.900 .916430 .00048

5.950 .920640 .00044

6.000 .925270 .00046

6.050 .929410 .00046

6.100 .935270 .00048

6.150 .939520 .00048

6.200 .943820 .00047

6.250 .948700 .00048

6.300 .952920 .00046

6.350 .959260 .00046

6.400 .963300 .00047

6.450 .967330 .00047

6.500 .972080 .00047

6.550 .976590 .00046

6.600 .980750 .00046

6.650 .985320 .00047

6.700 .989920 .00046

6.750 .994200 .00048

6.800 .998540 .00047

6.851 .002340 .00047

6.901 .006990 .00046

6.951 .011210 .00048

7.001 .014990 .00046

7.051 .019250 .00047

7.101 .022420 .00047
480

6.000 .924350 .00046

6.050 .928460 .00047

6.100 .935000 .00047

6.150 .938690 .00048

6.200 .943870 .00046

6.250 .948310 .00047

6.300 .952550 .00047

6.350 .958030 .00047

6.400 .961600 .00046

6.450 .966530 .00047

6.500 .971550 .00047

6.550 .975580 .00047

6.600 .980480 .00048

6.650 .984660 .00047

6.700 .988760 .00046

6.750 .992730 .00047

6.800 .997470 .00047

6.851 .001500 .00048

6.901 .005680 .00047

6.951 .009670 .00048

7.001 .014430 .00047

7.051 .018610 .00047

7.101 .022470 .00047

\section{0}


U5o2N20-icyl-0935-00

12

23.500 .817540 .00021

25.100 .832090 .00021

26.700 .843770 .00020

28.300 .854650 .00019

29.900 .864600 .00019

31.500 .871950 .00019

33.100 .879110 .00018

34.700 .885290 .00017

36.300 .891240 .00017

37.900 .896020 .00017

39.500 .900390 .00017

41.100 .903960 .00016

42.700 .907780 .00016

44.300 .911460 .00016

45.900 .914050 .00015

47.500 .916640 .00015

49.100 .919300 .00014

50.700 .921170 .00015

52.300 .923400 .00014

53.900 .925270 .00014

55.500 .926720 .00014

55.700 .927420 .00014
15

24.500 .927470 .00023

25.700 .938210 .00023

26.900 .948210 .00022

28.100 .956180 .00022

29.300 .964650 .00021

30.500 .971560 .00020

31.700 .977760 .00021

32.900 .983830 .00020

34.100 .989860 .00019

35.300 .994020 .00019

36.500 .998560 .00019

37.701 .002540 .00019

38.901 .006390 .00018

40.101 .009800 .00018

41.301 .013050 .00017

42.501 .016150 .00017

43.701 .018970 .00017

44.901 .021830 .00017

46.101 .024040 .00017

47.301 .025550 .00017

48.401 .027550 .00016
25

13.900 .921660 .00035

14.000 .924910 .00034

14.100 .929160 .00034

14.200 .931570 .00034

14.300 .936670 .00035

14.400 .939750 .00034

14.500 .944060 .00034

14.600 .946540 .00034

14.700 .950250 .00033

14.800 .953970 .00035

14.900 .957850 .00033

15.000 .960930 .00035

15.100 .964030 .00034

15.200 .967850 .00035

15.300 .970180 .00034

15.400 .974320 .00034

15.500 .977740 .00035

15.600 .980110 .00034

15.700 .983430 .00034

15.800 .986290 .00034
30

12.600 .925390 .00085

12.700 .930660 .00083

12.800 .934520 .00082

12.900 .938810 .00089

13.000 .942140 .00079

13.100 .948810 .00081

13.200 .952840 .00080

13.300 .957510 .00080

13.400 .962070 .00082

13.500 .965710 .00077

13.600 .970050 .00076

13.700 .974970 .00082

13.800 .979260 .00077

13.900 .984660 .00081

14.000 .986880 .00082

14.100 .990980 .00082

14.200 .993980 .00078

14.301 .000180 .00084

14.401 .002260 .00080

14.501 .005380 .00079
20

16.600 .923180 .00031

16.800 .927660 .00030

17.000 .932870 .00030

17.200 .937680 .00029

17.400 .942840 .00030

17.600 .946760 .00030

17.800 .952270 .00029

18.000 .956470 .00030

18.200 .960410 .00029

18.400 .965000 .00029

18.600 .968710 .00029

18.800 .972230 .00029

19.000 .976040 .00029

19.200 .980240 .00029

19.400 .983270 .00030

19.600 .987330 .00028

19.800 .990870 .00028

20.000 .994560 .00029

20.200 .996910 .00028

20.401 .001560 .00028

20.601 .003870 .00028

20.801 .007130 .00028

21.001 .010680 .00028

21.201 .013420 .00027

\section{2}

12.100 .919590 .00084

12.200 .923740 .00081

12.300 .931580 .00081

12.400 .936570 .00087

12.500 .940380 .00085

12.600 .946000 .00082

12.700 .952540 .00084

12.800 .956500 .00089

12.900 .958990 .00080

13.000 .963910 .00083

13.100 .968540 .00088

13.200 .973170 .00085

13.300 .977660 .00086

13.400 .983290 .00084

13.500 .987580 .00083

13.600 .991740 .00076

13.700 .995080 .00082

13.800 .998240 .00082

13.901 .003640 .00082

14.001 .009150 .00079 
15.900 .989240 .00033 16.000 .992990 .00033 16.100 .995070 .00033 16.200 .998240 .00033 16.301 .000690 .00033 16.401 .004420 .00033 16.501 .006110 .00033 16.601 .008910 .00033 16.701 .011620 .00034 16.801 .015270 .00033
14.601 .009430 .00078 14.701 .012870 .00079 14.801 .019100 .00074
14.101 .012080 .00084 14.201 .018010 .00086 14.301 .021430 .00081
35

11.600 .920710 .00090 11.700 .925680 .00088 11.800 .931200 .00088 11.900 .935690 .00094 12.000 .941810 .00085 12.100 .945430 .00091 12.200 .951150 .00084 12.300 .957890 .00088 12.400 .962010 .00085 12.500 .967420 .00085 12.600 .973670 .00084 12.700 .977630 .00086 12.800 .983440 .00087 12.900 .987570 .00088 13.000 .994040 .00090 13.100 .997050 .00084 13.201 .000870 .00083 13.301 .007390 .00086 13.401 .009770 .00086 13.501 .015130 .00087 13.601 .020120 .00085
40 11.000 .920410 .00089 11.100 .925480 .00089 11.200 .931460 .00089 11.300 .936870 .00089 11.400 .944930 .00084 11.500 .950180 .00088 11.600 .957900 .00089 11.700 .961540 .00090 11.800 .968700 .00090 11.900 .973620 .00086 12.000 .979590 .00093 12.100 .984350 .00094 12.200 .989420 .00093 12.300 .995460 .00086 12.401 .000830 .00085 12.501 .006730 .00091 12.601 .013220 .00095 12.701 .015020 .00094 12.801 .020680 .00094
50

10.200 .917630 .00099

10.250 .920010 .00100

10.300 .925540 .00097

10.350 .929780 .00094

10.400 .932080 .00089

10.450 .934600 .00097

10.500 .939010 .00099

10.550 .942550 .00094 10.600 .946360 .00092 10.650 .950750 .00094 10.700 .955010 .00098 10.750 .957630 .00094 10.800 .959810 .00099 10.850 .963440 .00093 10.900 .968460 .00099 10.950 .971500 .00104 11.000 .976430 .00094 11.050 .980220 .00094 11.100 .981510 .00096 11.150 .984560 .00099 11.200 .990510 .00093 11.250 .991910 .00094 11.300 .995690 .00090 11.350 .997800 .00103 11.400 .999890 .00095 11.451 .004600 .00090 11.501 .008150 .00096 11.551 .011650 .00094 11.601 .012970 .00096 11.651 .015790 .00091 11.701 .020420 .00095 
60

9.700 .916240 .00104

9.750 .922420 .00102

9.800 .925990 .00107

9.850 .926720 .00102

9.900 .933670 .00100

9.950 .937850 .00100

10.000 .942970 .00100

10.050 .946290 .00096

10.100 .949180 .00097

10.150 .953610 .00107

10.200 .957970 .00100

10.250 .961900 .00108

10.300 .966370 .00104

10.350 .970050 .00103

10.400 .972230 .00107

10.450 .976530 .00095

10.500 .979790 .00096

10.550 .983340 .00094

10.600 .987210 .00100

10.650 .992760 .00098

10.700 .995220 .00098

10.750 .996190 .00104

10.801 .001950 .00100

10.851 .003980 .00098

10.901 .009620 .00098

10.951 .012860 .00105

11.001 .015750 .00103

11.051 .017930 .00103

11.101 .023750 .00099
70

9.400 .919660 .00107

9.450 .924640 .00105

9.500 .930500 .00109

9.550 .932930 .00106

9.600 .938080 .00101

9.650 .941730 .00102

9.700 .947100 .00099

9.750 .951560 .00102

9.800 .954070 .00108

9.850 .958570 .00102

9.900 .964680 .00103

9.950 .965730 .00101

10.000 .969550 .00096

10.050 .974000 .00101

10.100 .979190 .00107

10.150 .984910 .00104

10.200 .984760 .00101

10.250 .990510 .00107

10.300 .994840 .00103

10.350 .998180 .00107

10.401 .003750 .00108

10.451 .005820 .00106

10.501 .011640 .00101

10.551 .015250 .00102

10.601 .015820 .00105

10.651 .020160 .00098

10.701 .023990 .00097
80

9.100 .914890 .00106

9.150 .918960 .00103

9.200 .924130 .00104

9.250 .929280 .00105

9.300 .933270 .00105

9.350 .938070 .00102

9.400 .942450 .00116

9.450 .947840 .00103

9.500 .951300 .00101

9.550 .956990 .00101

9.600 .960280 .00107

9.650 .963710 .00104

9.700 .970910 .00100

9.750 .974370 .00107

9.800 .979450 .00111

9.850 .982840 .00104

9.900 .985830 .00102

9.950 .991830 .00107

10.000 .993050 .00106

10.050 .999060 .00101

10.101 .000250 .00114

10.151 .004640 .00110

10.201 .010300 .00109

10.251 .014590 .00106

10.301 .018420 .00109

10.351 .022360 .00108

10.401 .026610 .00111
90

8.900 .913700 .00104

8.950 .917750 .00107

9.000 .923860 .00107

9.050 .928440 .00106

9.100 .933190 .00099

9.150 .936230 .00103

9.200 .942050 .00099

9.250 .946410 .00111

9.300 .952770 .00111

9.350 .955110 .00108

9.400 .959930 .00112

9.450 .964490 .00105

9.500 .968390 .00101

9.550 .972960 .00109

9.600 .976520 .00108

9.650 .983930 .00109

9.700 .988480 .00111
100

8.800 .916270 .00105

8.850 .921620 .00108

8.900 .926580 .00107

8.950 .931180 .00112

9.000 .937660 .00110

9.050 .942610 .00114

9.100 .947780 .00116

9.150 .951800 .00100

9.200 .955810 .00113

9.250 .960340 .00101

9.300 .964940 .00113

9.350 .969600 .00107

9.400 .974000 .00109

9.450 .978360 .00115

9.500 .984470 .00105

9.550 .988520 .00102

9.600 .993410 .00105
118

8.600 .915580 .00105

8.650 .920950 .00112

8.700 .928030 .00109

8.750 .932300 .00109

8.800 .935600 .00112

8.850 .944040 .00108

8.900 .947800 .00116

8.950 .951730 .00115

9.000 .956590 .00107

9.050 .961860 .00109

9.100 .965860 .00112

9.150 .970480 .00112

9.200 .978880 .00110

9.250 .980950 .00109

9.300 .985230 .00110

9.350 .990880 .00101

9.400 .995120 .00113 
9.750 .992300 .00105 9.800 .995650 .00108 9.851 .001000 .00104 9.901 .004320 .00111 9.951 .008100 .00110 10.001 .013060 .00108 10.051 .015350 .00103 10.101 .022080 .00109
9.650 .996370 .00100

9.701 .000630 .00115

9.751 .005940 .00108

9.801 .009920 .00117

9.851 .011930 .00111

9.901 .019100 .00104

9.951 .024820 .00107 10.001 .028390 .00114
9.451 .000870 .00115 9.501 .005490 .00118 9.551 .008610 .00116 9.601 .012690 .00110 9.651 .018080 .00114 9.701 .021810 .00111
130

8.500 .918150 .00106 8.550 .920490 .00117 8.600 .926580 .00110 8.650 .931280 .00114 8.700 .938550 .00106 8.750 .942460 .00113 8.800 .945920 .00113 8.850 .953090 .00111 8.900 .956260 .00112 8.950 .963520 .00107 9.000 .966960 .00111 9.050 .971250 .00110 9.100 .976790 .00110 9.150 .982820 .00111 9.200 .985950 .00116 9.250 .991350 .00112 9.300 .996350 .00117 9.351 .001580 .00118 9.401 .002680 .00113 9.451 .011020 .00119 9.501 .014680 .00116 9.551 .021350 .00112 9.601 .023760 .00112

\section{5}

8.200 .921090 .00108 8.250 .927520 .00118 8.300 .932460 .00117 8.350 .940930 .00117 8.400 .944170 .00118 8.450 .947760 .00119 8.500 .956780 .00115 8.550 .961210 .00106 8.600 .966140 .00112 8.650 .969030 .00110 8.700 .974730 .00117 8.750 .980960 .00118
150

8.400 .920530 .00116

8.450 .922990 .00121

8.500 .929140 .00113

8.550 .936320 .00116

8.600 .941180 .00114

8.700 .949240 .00111

8.750 .954810 .00112

8.800 .960080 .00120

8.850 .965120 .00110

8.900 .970780 .00115

8.950 .974920 .00113

9.000 .979740 .00110

9.050 .986060 .00108

9.100 .990050 .00120

9.150 .994350 .00110

9.200 .999360 .00124

9.251 .003080 .00110

9.301 .007440 .00115

9.351 .014490 .00117

9.401 .020000 .00111

9.451 .022870 .00113

9.501 .028860 .00108

250

8.100 .913770 .00116

8.150 .919390 .00115

8.200 .923210 .00114

8.250 .929690 .00111

8.300 .933850 .00120

8.350 .939720 .00120

8.400 .945670 .00115

8.450 .950260 .00120

8.500 .956580 .00120

8.550 .961670 .00119

8.600 .968090 .00121

8.650 .970220 .00120
200

8.200 .915650 .00114 8.250 .917860 .00112 8.300 .927410 .00119 8.350 .933000 .00118 8.400 .936940 .00122 8.450 .942130 .00114 8.500 .946930 .00116 8.550 .954420 .00115 8.600 .957590 .00114 8.650 .964150 .00114 8.700 .969850 .00112 8.750 .974130 .00117 8.800 .980330 .00103 8.850 .983780 .00111 8.900 .990030 .00116 8.950 .993420 .00108 9.001 .000030 .00115 9.051 .004410 .00120 9.101 .011820 .00114 9.151 .014760 .00119 9.201 .017450 .00116 9.251 .025380 .00114 9.301 .029130 .00122

353

8.100 .913840 .00122 8.150 .923650 .00119 8.200 .925230 .00115 8.250 .932600 .00116 8.300 .937870 .00110 8.350 .944260 .00114 8.400 .946120 .00109 8.450 .953790 .00115 8.500 .961000 .00115 8.550 .962980 .00111 8.600 .969280 .00118 8.650 .974220 .00112 
8.800 .986240 .00116 8.850 .990640 .00118 8.900 .996980 .00118 8.951 .000470 .00116 9.001 .005470 .00117 9.051 .011730 .00116 9.101 .015430 .00115 9.151 .021560 .00114 9.201 .024710 .00119

470

8.200 .922500 .00116 8.250 .924690 .00113 8.300 .931370 .00120 8.350 .935270 .00113 8.400 .941710 .00113 8.450 .947370 .00115 8.500 .952030 .00117 8.550 .958250 .00117 8.600 .962970 .00118 8.650 .966470 .00119 8.700 .975430 .00122 8.750 .979490 .00121 8.800 .981950 .00124 8.850 .987330 .00115 8.900 .993230 .00105 8.950 .997460 .00114 9.001 .003800 .00107 9.051 .008990 .00115 9.101 .012740 .00119 9.151 .017890 .00112 9.201 .020600 .00115

\section{5}

8.300 .916260 .00108 8.350 .922610 .00111 8.400 .926320 .00117 8.450 .930410 .00117 8.500 .937090 .00122 8.550 .942490 .00117 8.600 .946740 .00112 8.650 .952390 .00115 8.700 .956030 .00113
8.700 .977250 .00120 8.750 .981810 .00127 8.800 .986630 .00121 8.850 .992480 .00115 8.900 .998550 .00121 8.951 .002490 .00116 9.001 .006980 .00119 9.051 .014040 .00113 9.101 .018650 .00118 9.151 .023290 .00115 9.201 .026830 .00115

500

8.200 .917190 .00107 8.250 .922100 .00118 8.300 .927770 .00118 8.350 .932570 .00112 8.400 .937110 .00122 8.450 .943760 .00123 8.500 .947210 .00115 8.550 .954270 .00114 8.600 .960030 .00108 8.650 .964890 .00113 8.700 .969510 .00115 8.750 .973410 .00110 8.800 .979170 .00118 8.850 .983120 .00118 8.900 .989740 .00111 8.950 .994440 .00117 9.001 .000090 .00119 9.051 .005350 .00114 9.101 .009310 .00110 9.151 .011730 .00124 9.201 .017420 .00112 9.251 .022520 .00112 9.301 .029260 .00125

$$
610
$$

8.300 .917170 .00112 8.350 .920130 .00120 8.400 .924930 .00113 8.450 .930310 .00106 8.500 .936060 .00117 8.550 .940340 .00114 8.600 .945770 .00125 8.650 .951170 .00114 8.700 .953870 .00106
8.700 .980530 .00114 8.750 .985400 .00120 8.800 .989650 .00112 8.850 .995080 .00122 8.901 .000900 .00113 8.951 .006250 .00116 9.001 .012600 .00120 9.051 .016600 .00110 9.101 .019300 .00109

\section{F-502}


8.750 .961570 .00112

8.800 .966100 .00113

8.850 .972360 .00113

8.900 .977870 .00117

8.950 .981730 .00118

9.000 .987740 .00120

9.050 .990170 .00115

9.100 .997230 .00119

9.150 .999440 .00119

9.201 .006100 .00116

9.251 .011660 .00119

9.301 .015020 .00113

9.351 .018970 .00114

9.401 .023300 .00109

620

8.300 .913360 .00107

8.350 .919290 .00107

8.400 .924900 .00112

8.450 .930290 .00115

8.500 .933500 .00111

8.550 .938640 .00114

8.600 .945400 .00118

8.650 .949110 .00111

8.700 .955130 .00114

8.750 .959630 .00113

8.800 .964510 .00116

8.850 .971200 .00109

8.900 .973320 .00112

8.950 .978980 .00116

9.000 .983880 .00113

9.050 .989190 .00113

9.100 .993300 .00118

9.150 .997550 .00115

9.201 .004270 .00114

9.251 .008820 .00119

9.301 .011570 .00113

9.351 .013680 .00117

9.401 .024410 .00121
8.750 .960740 .00123

8.800 .965370 .00113

8.850 .968590 .00119

8.900 .978870 .00119

8.950 .981180 .00113

9.000 .984530 .00111

9.050 .992080 .00126

9.100 .996020 .00111

9.151 .001040 .00128

9.201 .004010 .00122

9.251 .009730 .00114

9.301 .014540 .00122

9.351 .020590 .00119

9.401 .022870 .00117
8.750 .960070 .00113

8.800 .964660 .00112

8.850 .970050 .00119

8.900 .975280 .00115

8.950 .980470 .00112

9.000 .984550 .00112 9.050 .989420 .00111 9.100 .994900 .00113 9.150 .999400 .00109 9.201 .006360 .00114 9.251 .008130 .00118 9.301 .012920 .00112 9.351 .016490 .00120 9.401 .021960 .00109

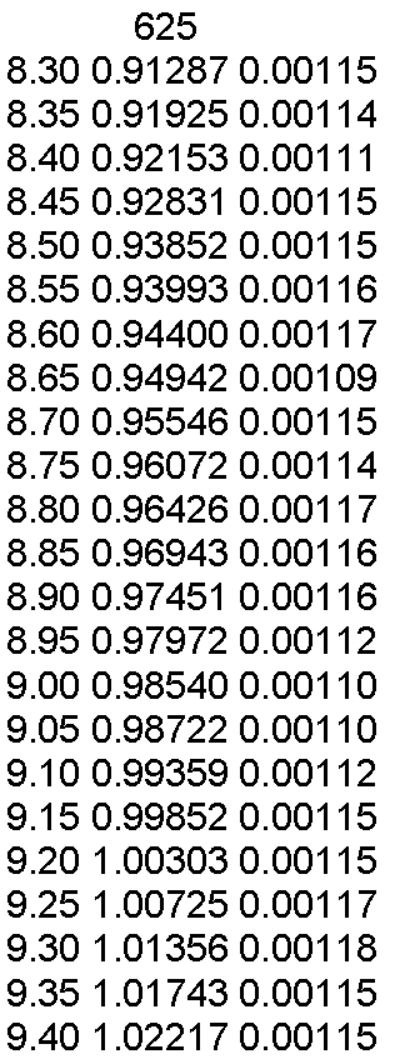

629

8.300 .911210 .00114 8.350 .916940 .00113 8.400 .924180 .00118 8.450 .928130 .00122 8.500 .931410 .00121 8.550 .937120 .00114 8.600 .943800 .00118 8.650 .948390 .00115 8.700 .953320 .00117 8.750 .959700 .00117 8.800 .962920 .00112 8.850 .968820 .00114 8.900 .972100 .00119 8.950 .977820 .00108 9.000 .982970 .00117 9.050 .986050 .00121 9.100 .992830 .00107 9.150 .996360 .00115 9.201 .001400 .00113 9.251 .005080 .00119 9.301 .010170 .00117 9.351 .016980 .00114 9.401 .020040 .00114 9.451 .025600 .00111 9.501 .029190 .00111 
U5o2N20-icyl-0935-01

12

25.000 .842690 .00020

25.300 .845410 .00019

25.600 .847500 .00019

25.900 .849220 .00019

26.200 .851210 .00019

26.500 .852880 .00019

26.800 .855180 .00019

27.100 .857050 .00019

27.400 .858400 .00019

27.700 .860030 .00019

28.000 .861850 .00019

28.300 .863430 .00019

28.600 .865420 .00019

28.900 .866550 .00018

29.200 .867990 .00018

29.500 .869720 .00019

29.800 .871410 .00018

30.100 .872570 .00018

30.400 .873850 .00019

30.700 .875890 .00018

31.000 .877140 .00017

31.300 .878190 .00017
15

22.900 .926900 .00022 23.600 .932930 .00023

24.300 .939800 .00022

25.000 .945280 .00021

25.700 .950400 .00022

26.400 .955380 .00021

27.100 .959870 .00021

27.800 .964970 .00021

28.500 .968790 .00021

29.200 .973240 .00020

29.900 .976730 .00020

30.600 .979950 .00020

31.300 .983740 .00019

32.000 .987070 .00019

32.700 .990280 .00019

33.400 .992560 .00019

34.100 .995400 .00019

34.800 .997770 .00018

35.501 .000440 .00018

36.201 .002650 .00018

36.801 .005080 .00018
25

12.400 .921420 .00034

12.500 .925760 .00033

12.600 .929140 .00033

12.700 .932810 .00034

12.800 .936830 .00033

12.900 .940370 .00032

13.000 .943890 .00033

13.100 .947460 .00034

13.200 .951670 .00033

13.300 .954960 .00033

13.500 .961440 .00034

13.600 .964620 .00032

13.700 .967980 .00033

13.800 .971040 .00033

13.900 .974630 .00032

14.000 .978580 .00032

14.100 .981190 .00033

14.200 .983890 .00032

14.300 .986670 .00032

14.400 .989610 .00032
30

11.000 .921060 .00074

11.100 .925230 .00079

11.200 .930790 .00077

11.300 .935210 .00078

11.400 .939530 .00083

11.500 .945260 .00082

11.600 .948200 .00080

11.700 .954870 .00078

11.800 .957850 .00086

11.900 .962940 .00076

12.000 .966150 .00076

12.100 .971300 .00079

12.200 .974920 .00075

12.300 .979870 .00078

12.400 .983140 .00079

12.500 .987470 .00079

12.600 .993090 .00078

12.700 .995080 .00079

12.800 .998200 .00080

12.901 .004410 .00076
20

15.100 .923160 .00030

15.300 .928820 .00029

15.500 .933700 .00029

15.700 .937700 .00030

15.900 .943470 .00029

16.100 .947960 .00029

16.300 .951870 .00028

16.500 .957130 .00028

16.700 .960880 .00028

16.900 .964700 .00028

17.100 .968520 .00028

17.300 .973170 .00028

17.500 .976840 .00028

17.700 .980090 .00028

17.900 .984520 .00028

18.100 .987450 .00028

18.300 .991640 .00027

18.500 .994710 .00028

18.700 .998330 .00027

18.901 .001730 .00027

19.101 .005440 .00027

19.301 .008120 .00027

19.501 .011070 .00026

19.701 .014320 .00027

32

10.600 .922400 .00078

10.700 .925490 .00078

10.800 .932220 .00081

10.900 .934980 .00083

11.000 .940760 .00081

11.100 .947390 .00082

11.200 .951360 .00081

11.300 .956230 .00079

11.400 .959690 .00081

11.500 .965150 .00081

11.600 .968880 .00080

11.700 .974350 .00077

11.800 .979910 .00080 11.900 .982880 .00081 12.000 .988770 .00078 12.100 .991960 .00078 12.200 .997400 .00080 12.300 .999940 .00076 12.401 .004860 .00082 12.501 .009880 .00082 
14.500 .992770 .00032 14.600 .995950 .00033 14.700 .998400 .00032 14.801 .001570 .00033 14.901 .004640 .00032 15.001 .007310 .00032 15.101 .009870 .00032 15.201 .012540 .00031 15.301 .014710 .00032 15.401 .018060 .00032

35

10.100 .919010 .00083 10.200 .926640 .00089 10.300 .930210 .00082 10.400 .936870 .00082 10.500 .941370 .00084 10.600 .947370 .00080 10.700 .955710 .00078 10.800 .958670 .00082 10.900 .963680 .00080 11.000 .968800 .00081 11.100 .972300 .00085 11.200 .977760 .00084 11.300 .985030 .00082 11.400 .988580 .00084 11.500 .992770 .00082 11.600 .998920 .00081 11.701 .003650 .00076 11.801 .007350 .00084 11.901 .011680 .00079 12.001 .016330 .00089
13.001 .007420 .00076 13.101 .010400 .00077 13.201 .013550 .00077 13.301 .019310 .00079
12.601 .014930 .00077 12.701 .017210 .00081 12.801 .020800 .00078
40

9.500 .920200 .00092 9.550 .922820 .00085 9.600 .927640 .00091 9.650 .931290 .00088 9.700 .931670 .00085 9.750 .937270 .00082 9.800 .939760 .00092 9.850 .940730 .00081 9.900 .945850 .00089 9.950 .948950 .00085 10.000 .951490 .00088 10.050 .955100 .00092 10.100 .957390 .00089 10.150 .961870 .00089 10.200 .963490 .00085 10.250 .964900 .00086 10.300 .970700 .00092 10.350 .971620 .00084 10.400 .974340 .00085 10.450 .976920 .00085 10.500 .978920 .00088 10.550 .984420 .00087 10.600 .985810 .00086 10.650 .988990 .00094 10.700 .991520 .00084 10.750 .994900 .00090 10.800 .995700 .00091 10.850 .998760 .00093 10.901 .001760 .00087 10.951 .005350 .00089 11.001 .009870 .00093 11.051 .012520 .00089 11.101 .014270 .00086 11.151 .015760 .00084 11.201 .017900 .00086
50

8.700 .920160 .00093

8.750 .921420 .00093

8.800 .926560 .00096

8.850 .930440 .00094

8.900 .933410 .00089

8.950 .935390 .00094 9.000 .939870 .00091 9.050 .944580 .00097 9.100 .949690 .00091 9.150 .953290 .00089 9.200 .955560 .00093 9.250 .957420 .00092 9.300 .963060 .00099 9.350 .965640 .00092 9.400 .969920 .00093 9.450 .971720 .00089 9.500 .976050 .00089 9.550 .980520 .00091 9.600 .982520 .00091 9.650 .985760 .00094 9.700 .987900 .00093 9.750 .992550 .00094 9.800 .995120 .00088 9.850 .998810 .00093 9.901 .002720 .00092 9.951 .005580 .00091 10.001 .008920 .00093 10.051 .013780 .00085 10.101 .015270 .00088 10.151 .017770 .00094 10.201 .021900 .00093 
60

8.200 .918790 .00096 8.250 .924250 .00097 8.300 .927220 .00094 8.350 .929520 .00098 8.400 .937600 .00100 8.450 .940370 .00098 8.500 .943010 .00096 8.550 .946680 .00102 8.600 .952090 .00097 8.650 .956830 .00098 8.700 .959670 .00095 8.750 .965670 .00094 8.800 .967680 .00103 8.850 .970430 .00096 8.900 .972770 .00093 8.950 .977060 .00099 9.000 .981170 .00095 9.050 .985940 .00098 9.100 .990480 .00098 9.150 .993000 .00098 9.200 .996070 .00102 9.251 .001280 .00099 9.301 .003500 .00094 9.351 .007710 .00102 9.401 .011390 .00096 9.451 .013720 .00093 9.501 .017690 .00093 9.551 .021520 .00093 9.601 .024880 .00089

90

7.500 .925030 .00104 7.550 .930630 .00099 7.600 .937020 .00100 7.650 .941340 .00103 7.700 .944640 .00102 7.750 .948190 .00103 7.800 .953620 .00103 7.850 .958120 .00104 7.900 .963300 .00098 7.950 .966520 .00104 8.000 .970140 .00102 8.050 .977140 .00102 8.100 .983110 .00105 8.150 .986230 .00103 8.200 .991050 .00103 8.250 .993340 .00110
70

7.900 .921640 .00103

7.950 .927440 .00096

8.000 .930380 .00105

8.050 .935410 .00099

8.100 .939710 .00097

8.150 .945250 .00103

8.200 .949920 .00094

8.250 .951880 .00101

8.300 .958000 .00098

8.350 .960380 .00101

8.400 .964890 .00103

8.450 .968810 .00102

8.500 .973960 .00105

8.550 .978750 .00095

8.600 .981020 .00093

8.650 .984290 .00107

8.700 .988310 .00108

8.750 .991280 .00099

8.800 .996260 .00091

8.851 .002500 .00103

8.901 .005110 .00101

8.951 .009060 .00103

9.001 .012030 .00098

9.051 .014260 .00091

9.101 .020100 .00103
80

7.700 .927790 .00098

7.750 .929730 .00107

7.800 .937170 .00103

7.850 .940520 .00108

7.900 .943300 .00095

7.950 .951470 .00105

8.000 .954160 .00102

8.050 .959020 .00103

8.100 .961210 .00101

8.150 .966530 .00107

8.200 .972280 .00105

8.250 .975050 .00099

8.300 .980070 .00100

8.350 .982200 .00097

8.400 .989270 .00095

8.450 .994040 .00104

8.500 .997200 .00109

8.550 .999520 .00101

8.601 .005980 .00104 8.651 .008850 .00103 8.701 .013400 .00099 8.751 .016650 .00108 8.801 .023350 .00103
7.300 .921020 .00108

7.350 .926620 .00100

7.400 .931130 .00106

7.450 .935830 .00107

7.500 .940800 .00104

7.550 .944650 .00101

7.600 .950480 .00105

7.650 .954430 .00105

7.700 .959330 .00104

7.750 .961710 .00103

7.800 .969080 .00110

7.850 .973050 .00103

7.900 .979980 .00108

7.950 .982170 .00108

8.000 .989200 .00100

8.050 .992510 .00105
118

7.100 .921600 .00104

7.150 .925960 .00103

7.200 .931060 .00108

7.250 .936060 .00104

7.300 .939480 .00108

7.350 .944740 .00109

7.400 .950360 .00102

7.450 .956200 .00107

7.500 .960320 .00105

7.550 .963710 .00109

7.600 .970290 .00103

7.650 .972690 .00109

7.700 .980540 .00104

7.750 .986310 .00111

7.800 .991460 .00102

7.850 .994630 .00108 
8.300 .999660 .00101

8.351 .003560 .00104 8.401 .007770 .00105 8.451 .010030 .00099 8.501 .015730 .00101 8.551 .020610 .00107 8.601 .023760 .00103
8.100 .996900 .00109

8.151 .000840 .00105

8.201 .004580 .00107

8.251 .010900 .00104

8.301 .015840 .00106

8.351 .016570 .00109

8.401 .021550 .00099
7.900 .998440 .00106

7.951 .004180 .00109 8.001 .007360 .00108 8.051 .013080 .00104 8.101 .016560 .00107 8.151 .022240 .00108 8.201 .027250 .00104
130

7.000 .919170 .00105 7.050 .927100 .00105 7.100 .927300 .00109 7.150 .934890 .00099 7.200 .940920 .00112 7.250 .947180 .00102 7.300 .953340 .00110 7.350 .956580 .00114 7.400 .962070 .00105 7.450 .965840 .00109 7.500 .972320 .00109 7.550 .977640 .00107 7.600 .982270 .00109 7.650 .985270 .00109 7.700 .990790 .00111 7.750 .993520 .00117 7.800 .999900 .00105 7.851 .007280 .00103 7.901 .009670 .00109 7.951 .016830 .00108 8.001 .019040 .00110

235

6.600 .917110 .00112 6.650 .922690 .00113 6.700 .927450 .00110 6.750 .932310 .00115 6.800 .939710 .00115 6.850 .945550 .00107 6.900 .951220 .00105 6.950 .955160 .00105 7.000 .960510 .00112 7.050 .963350 .00107 7.100 .970780 .00111 7.150 .977210 .00112 7.200 .982720 .00107 7.250 .984970 .00119 7.300 .992920 .00113
150

6.900 .922690 .00109 6.950 .927290 .00104 7.000 .933030 .00107 7.050 .939640 .00105 7.100 .943140 .00115 7.150 .949650 .00108 7.200 .954110 .00111 7.250 .958470 .00106 7.300 .962690 .00105 7.350 .968910 .00105 7.400 .975880 .00106 7.450 .978580 .00116 7.500 .983710 .00105 7.550 .989320 .00111 7.600 .992240 .00110 7.650 .999390 .00106 7.701 .005340 .00112 7.751 .005590 .00113 7.801 .013040 .00106 7.851 .019000 .00109 7.901 .024030 .00113
200

6.700 .921180 .00115 6.750 .926200 .00110 6.800 .930620 .00110 6.850 .935020 .00106 6.950 .946040 .00115 7.000 .953850 .00105 7.050 .958570 .00109 7.100 .962790 .00109 7.150 .968700 .00111 7.200 .974480 .00107 7.250 .978510 .00106 7.300 .983660 .00110 7.350 .990220 .00112 7.400 .993930 .00099 7.450 .999440 .00113 7.501 .003020 .00118 7.551 .008930 .00110 7.601 .015340 .00112 7.651 .018530 .00115 7.701 .025040 .00114
6.600 .918330 .00114 6.650 .923210 .00114 6.700 .929840 .00117 6.750 .932940 .00110 6.800 .939350 .00107 6.850 .945590 .00109 6.900 .950850 .00114 6.950 .956540 .00108 7.000 .963590 .00113 7.050 .967790 .00105 7.100 .974410 .00108 7.150 .976540 .00117 7.200 .983350 .00115 7.250 .987140 .00115 7.300 .992830 .00116
353

6.600 .923060 .00108 6.650 .927280 .00112 6.700 .931280 .00114 6.750 .938070 .00111 6.800 .946110 .00112 6.850 .949260 .00110 6.900 .954260 .00104 6.950 .960610 .00116 7.000 .968050 .00114 7.050 .971870 .00110 7.100 .976900 .00114 7.150 .981450 .00113 7.200 .986650 .00113 7.250 .991330 .00119 7.300 .998450 .00111 
7.350 .995950 .00109 7.401 .001280 .00113 7.451 .009140 .00110 7.501 .011400 .00105 7.551 .016080 .00107 7.601 .022210 .00114
7.350 .997330 .00116

7.401 .002520 .00107

7.451 .009590 .00109

7.501 .013420 .00118

7.551 .016560 .00116

7.601 .022420 .00110
7.351 .002090 .00105

7.401 .007900 .00109

7.451 .010860 .00116

7.501 .018810 .00111

7.551 .019900 .00115

7.601 .025710 .00117
470

6.600 .915290 .00110 6.650 .923120 .00110 6.700 .928330 .00118 6.750 .933610 .00106 6.800 .937580 .00110 6.850 .945780 .00116 6.900 .948050 .00112 6.950 .952420 .00111 7.000 .960610 .00110 7.050 .965190 .00117 7.100 .968620 .00108 7.150 .975770 .00117 7.200 .979730 .00106 7.250 .984190 .00109 7.300 .991500 .00111 7.350 .994450 .00106 7.400 .999990 .00110 7.451 .003850 .00106 7.501 .011340 .00105 7.551 .015730 .00118 7.601 .020680 .00115 7.651 .023770 .00110 7.701 .029520 .00119

\section{5}

6.700 .913910 .00113 6.750 .919910 .00113 6.800 .923830 .00110 6.850 .930150 .00117 6.900 .935440 .00106 6.950 .941360 .00111 7.000 .946010 .00105 7.050 .951370 .00110 7.100 .956480 .00105 7.150 .959040 .00116 7.200 .965150 .00113 7.250 .970380 .00110 7.300 .976630 .00114 7.350 .981820 .00112

\section{0}

6.600 .914300 .00109 6.650 .918280 .00107

6.700 .924980 .00113

6.750 .929310 .00109

6.800 .936130 .00108

6.850 .941520 .00111

6.900 .946060 .00112

6.950 .950570 .00118

7.000 .957280 .00102

7.050 .961160 .00112

7.100 .965440 .00112

7.150 .971270 .00109

7.200 .977190 .00109

7.250 .981940 .00108

7.300 .987540 .00119

7.350 .992360 .00112

7.400 .995960 .00113

7.451 .003420 .00109

7.501 .006980 .00113

7.551 .010840 .00113

7.601 .016250 .00113

7.651 .021930 .00112

7.701 .025690 .00111
600

6.700 .913960 .00109 6.750 .922040 .00108 6.800 .926790 .00110 6.850 .930710 .00107 6.900 .935550 .00117 6.950 .940590 .00117 7.000 .946830 .00113 7.050 .951960 .00110 7.100 .956280 .00110 7.150 .960120 .00108 7.200 .965780 .00109 7.250 .971560 .00110 7.300 .976670 .00109 7.350 .980940 .00111 7.400 .987600 .00107 7.450 .990050 .00115 7.500 .994450 .00113 7.551 .000910 .00114 7.601 .004380 .00109 7.651 .010160 .00116 7.701 .013500 .00113 7.751 .018680 .00109 7.801 .021850 .00113
$610 \quad 615$

6.700 .914550 .00107

6.750 .919470 .00112

6.800 .925560 .00119

6.850 .931880 .00114

6.900 .934910 .00108

6.950 .939400 .00113

7.000 .944620 .00113

7.050 .949630 .00108

7.100 .956370 .00109

7.150 .960230 .00112

7.200 .966480 .00107

7.250 .969440 .00110

7.300 .975430 .00113

7.350 .981110 .00108
6.800 .924060 .00109

6.850 .929850 .00116

6.900 .933150 .00118 6.950 .937680 .00108 7.000 .943180 .00110 7.050 .949190 .00110 7.100 .953730 .00112 7.150 .958120 .00112 7.200 .964230 .00114 7.250 .969440 .00110 7.300 .974280 .00114 7.350 .978420 .00118 7.400 .984160 .00110 7.450 .988980 .00115 
7.400 .983280 .00111

7.450 .988880 .00111

7.500 .994590 .00116

7.551 .000260 .00118

7.601 .004990 .00121

7.651 .008270 .00116

7.701 .013690 .00115

7.751 .018360 .00108

7.801 .022910 .00116
7.400 .986600 .00112

7.450 .992020 .00111

7.500 .995620 .00113

7.550 .997140 .00117

7.601 .004610 .00103

7.651 .011380 .00115

7.701 .012440 .00111

7.751 .016820 .00114

7.801 .019080 .00115
7.500 .992780 .00111

7.550 .996680 .00112

7.601 .002360 .00113

7.651 .005480 .00111

7.701 .011300 .00113

7.751 .015920 .00107

7.801 .022130 .00119
620

6.800 .923030 .00114 6.850 .926810 .00109

6.900 .933710 .00110

6.950 .938290 .00109 7.000 .943440 .00114 7.050 .948230 .00108 7.100 .954720 .00110 7.150 .958390 .00114 7.200 .965220 .00110 7.250 .967790 .00119 7.300 .976480 .00116 7.350 .978810 .00104 7.400 .984800 .00110 7.450 .987470 .00113 7.500 .993380 .00107 7.550 .996340 .00108 7.601 .002550 .00114 7.651 .005830 .00115 7.701 .010820 .00113 7.751 .015240 .00114 7.801 .018560 .00116
625

6.800 .923310 .00110 6.850 .927670 .00107 6.900 .933910 .00118 6.950 .938690 .00108 7.000 .943550 .00113 7.050 .946910 .00112 7.100 .953990 .00107 7.150 .959860 .00115 7.200 .961980 .00109 7.250 .969370 .00106 7.300 .973840 .00113 7.350 .978580 .00117 7.400 .983820 .00110 7.450 .988220 .00108 7.500 .992530 .00114 7.550 .996340 .00102 7.601 .002270 .00112 7.651 .006300 .00112 7.701 .011850 .00114 7.751 .016400 .00108 7.801 .021490 .00113
629

6.800 .921470 .00116 6.850 .925790 .00113 6.900 .932210 .00116 6.950 .938400 .00111 7.000 .941200 .00111 7.050 .946690 .00112 7.100 .953820 .00119 7.150 .957030 .00109 7.200 .962640 .00115 7.250 .966200 .00118 7.300 .972210 .00113 7.350 .975120 .00116 7.400 .982650 .00113 7.450 .985290 .00108 7.500 .992820 .00105 7.550 .995730 .00112 7.601 .001900 .00115 7.651 .004490 .00113 7.701 .009420 .00119 7.751 .015880 .00107 7.801 .021990 .00115 7.851 .025660 .00112 7.901 .028410 .00111 
U5o2N20-icyl-0935-10

12

31.700 .883570 .00017 32.900 .888760 .00017

34.100 .892270 .00016

35.300 .895810 .00016

36.500 .898800 .00016

37.700 .902360 .00016

38.900 .905420 .00015

40.100 .907650 .00016

41.300 .910440 .00015

42.500 .912480 .00015

43.700 .914770 .00014

44.900 .916630 .00014

46.100 .918670 .00015

47.300 .920220 .00014

48.500 .921760 .00014

49.700 .923700 .00014

50.900 .924460 .00014

52.100 .926060 .00014

53.300 .927050 .00014

54.500 .928920 .00014

55.700 .930290 .00013

56.900 .930620 .00013

57.100 .930990 .00013

25

11.300 .921060 .00032

11.400 .925220 .00031

11.500 .929690 .00031

11.600 .932060 .00032

11.700 .936550 .00031

11.800 .940100 .00032

11.900 .943090 .00031

12.000 .947850 .00032

12.100 .951370 .00030

12.200 .954180 .00031

12.300 .958180 .00031

12.400 .961510 .00031

12.500 .964810 .00032

12.600 .967900 .00032

12.700 .970960 .00032

12.800 .974070 .00030

12.900 .978060 .00030

13.000 .980940 .00031

13.100 .984250 .00030

13.200 .986980 .00030

13.300 .990460 .00031

13.400 .993330 .00030
15

21.800 .926340 .00022

22.500 .933010 .00022

23.200 .939100 .00021

23.900 .944670 .00021

24.600 .950330 .00020

25.300 .955610 .00021

26.000 .960670 .00020

26.700 .964620 .00020

27.400 .969120 .00019

28.100 .972830 .00019

28.800 .976370 .00019

29.500 .980730 .00019

30.200 .983890 .00019

30.900 .986760 .00019

31.600 .989810 .00018

32.300 .993080 .00018

33.000 .995500 .00019

33.700 .997600 .00018

34.401 .000740 .00017

35.101 .002720 .00018

35.801 .005010 .00017

36.501 .007670 .00017

36.701 .007980 .00018

30

9.900 .921300 .00034

10.000 .926020 .00035

10.200 .935460 .00034

10.300 .940770 .00033

10.400 .945440 .00033

10.500 .949420 .00033

10.600 .954650 .00034

10.700 .958600 .00034

10.800 .962990 .00033

10.900 .967430 .00035

11.000 .972080 .00033

11.100 .975820 .00034

11.200 .979440 .00034

11.300 .984330 .00032

11.400 .988510 .00033

11.500 .992090 .00033

11.600 .995990 .00034

11.701 .000320 .00034

11.801 .004460 .00033

11.901 .008010 .00031

12.001 .011750 .00033

12.101 .015900 .00033
20

14.300 .930370 .00027

14.500 .935530 .00028

14.700 .940730 .00027

14.900 .944840 .00027

15.100 .949820 .00028

15.300 .954080 .00028

15.500 .958300 .00026

15.700 .962950 .00026

15.900 .966770 .00027

16.100 .971130 .00027

16.300 .974490 .00027

16.500 .978750 .00027

16.700 .982580 .00026

16.900 .985880 .00025

17.100 .989730 .00026

17.300 .992870 .00026

17.500 .996530 .00026

17.701 .000110 .00026

17.901 .003520 .00026

18.101 .006050 .00026

18.301 .009650 .00025

18.501 .013050 .00025

18.601 .013770 .00026

32

9.600 .926850 .00034

9.700 .931340 .00035

9.800 .936900 .00033

9.900 .941870 .00035

10.000 .947090 .00034

10.100 .952020 .00034

10.200 .957150 .00033

10.300 .961400 .00035

10.400 .966740 .00034

10.500 .970930 .00034

10.600 .975690 .00034

10.700 .980010 .00034

10.800 .984410 .00035

10.900 .989380 .00034

11.000 .992820 .00035

11.100 .998380 .00035

11.201 .002090 .00034

11.301 .006070 .00034

11.401 .009800 .00035

11.501 .014710 .00035

11.601 .019070 .00034 
13.500 .996070 .00031

13.600 .998500 .00031

13.701 .002190 .00031

13.801 .004960 .00030

13.901 .007580 .00030

14.001 .010330 .00030
35

9.000 .920200 .00035

9.100 .926110 .00035

9.200 .931410 .00036

9.300 .936870 .00037

9.400 .942480 .00036

9.500 .948260 .00035

9.600 .954410 .00036

9.700 .959630 .00036

9.800 .964320 .00035

9.900 .969970 .00036

10.000 .974940 .00036

10.100 .980740 .00036

10.200 .984590 .00035

10.300 .989910 .00034

10.400 .994790 .00035

10.500 .998770 .00035

10.601 .003940 .00035

10.701 .009330 .00036

10.801 .013930 .00035

10.901 .018030 .00035
40

$8.400 .920570 .00037 \quad 7.600 .921430 .00040$

$\begin{array}{lll}8.450 .92354 & 0.00038 & 7.650 .925050 .00038\end{array}$

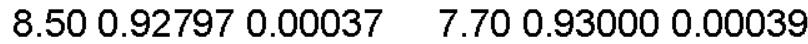

$\begin{array}{lll}8.550 .930150 .00037 & 7.750 .933300 .00040\end{array}$

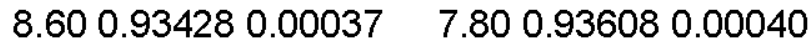

$\begin{array}{lll}8.650 .937530 .00038 & 7.850 .940430 .00039\end{array}$

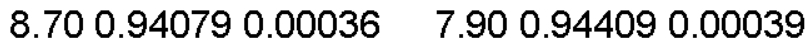

$\begin{array}{lll}8.750 .943420 .00037 & 7.950 .948280 .00039\end{array}$

$\begin{array}{lll}8.80 & 0.946450 .00037 & 8.000 .950750 .00039\end{array}$

$\begin{array}{lll}8.850 .94970 & 0.00036 & 8.050 .955150 .00040\end{array}$

$8.900 .952890 .00037 \quad 8.100 .958680 .00039$

$\begin{array}{lll}8.95 & 0.956090 .00037 & 8.150 .961980 .00039\end{array}$

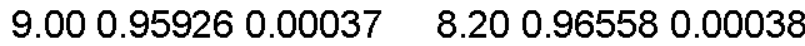

9.050 .962930 .00037
8.250 .969870 .00039

$\begin{array}{lll}9.10 & 0.964440 .00037 & 8.300 .972780 .00040\end{array}$

9.150 .968430 .00037
8.350 .977060 .00040

$9.200 .970250 .00037 \quad 8.400 .979670 .00040$

$9.250 .974420 .00037 \quad 8.450 .983920 .00039$

$9.300 .976470 .00038 \quad 8.500 .986010 .00040$

$9.350 .979670 .00037 \quad 8.550 .990410 .00039$

$9.400 .982940 .00037 \quad 8.600 .993050 .00040$

$9.450 .985710 .00036 \quad 8.650 .996670 .00038$

$9.500 .987860 .00037 \quad 8.701 .000320 .00040$

9.550 .991260 .00037
8.751 .002890 .00039

$9.600 .993060 .00037 \quad 8.801 .006220 .00039$

$9.650 .996730 .00038 \quad 8.851 .008680 .00040$

$9.700 .999710 .00037 \quad 8.901 .013010 .00039$

$9.751 .001580 .00037 \quad 8.951 .016080 .00039$

$9.801 .004600 .00037 \quad 9.001 .019500 .00040$
9.851 .007090 .00037

9.901 .009660 .00036

9.951 .012180 .00036

10.001 .015400 .00037

10.051 .017660 .00037

10.101 .020900 .00037 
60

7.100 .921510 .00040

7.150 .927370 .00041

7.200 .930320 .00042

7.250 .935530 .00042

7.300 .939760 .00040

7.350 .943620 .00040

7.400 .946950 .00040

7.450 .952140 .00040

7.500 .955600 .00042

7.550 .960190 .00043

7.600 .963250 .00041

7.650 .967930 .00040

7.700 .971240 .00040

7.750 .976360 .00040

7.800 .979120 .00041

7.850 .982190 .00041

7.900 .987080 .00041

7.950 .990340 .00040

8.000 .994660 .00040

8.050 .998000 .00041

8.101 .002280 .00042

8.151 .005680 .00041

8.201 .009340 .00041

8.251 .012620 .00041

8.301 .016150 .00042

8.351 .019580 .00040

8.401 .023580 .00041
70

6.700 .918540 .00042

6.750 .922730 .00042

6.800 .927010 .00042

6.850 .932090 .00042

6.900 .936700 .00042

6.950 .940530 .00043

7.000 .945090 .00043

7.050 .949500 .00042

7.100 .953720 .00042

7.150 .957220 .00042

7.200 .962500 .00042

7.250 .966410 .00042

7.300 .971420 .00042

7.350 .976420 .00042

7.400 .979320 .00043

7.450 .982880 .00042

7.500 .987280 .00041

7.550 .991560 .00042

7.600 .995390 .00044

7.650 .999690 .00043

7.701 .004000 .00043

7.751 .007470 .00042

7.801 .011420 .00041

7.851 .015920 .00042

7.901 .019820 .00042
80

6.400 .914380 .00043

6.450 .920240 .00041

6.500 .925330 .00042

6.550 .928660 .00042

6.600 .933940 .00044

6.650 .938040 .00042

6.700 .942870 .00043

6.750 .947960 .00043

6.800 .952500 .00043

6.850 .957550 .00043

6.900 .961540 .00042

6.950 .966200 .00042

7.000 .969550 .00044

7.050 .975360 .00044

7.100 .979290 .00042

7.150 .983810 .00044

7.200 .988210 .00043

7.250 .992710 .00042

7.300 .997360 .00043

7.351 .001240 .00045

7.401 .005760 .00042

7.451 .009590 .00043

7.501 .012770 .00044

7.551 .018090 .00042

7.601 .021540 .00043
90

6.200 .913860 .00043

6.250 .919200 .00044

6.300 .924290 .00044

6.350 .929730 .00042

6.400 .934390 .00045

6.450 .938660 .00044

6.500 .944610 .00044

6.550 .948560 .00044

6.600 .954170 .00043

6.650 .959060 .00042

6.700 .962790 .00042

6.750 .967080 .00042

6.800 .972760 .00044

6.850 .977090 .00042

6.900 .981260 .00043

6.950 .986520 .00044

7.000 .991200 .00044

7.050 .995920 .00044

7.100 .999100 .00045
100

6.100 .920680 .00045

6.150 .925660 .00044

6.200 .931000 .00044

6.250 .935460 .00044

6.300 .940710 .00044

6.350 .946090 .00044

6.400 .950000 .00044

6.450 .955270 .00043

6.500 .960450 .00045

6.550 .964690 .00044

6.600 .970470 .00045

6.650 .973820 .00044

6.700 .980380 .00044

6.750 .984130 .00045

6.800 .989620 .00046

6.850 .992620 .00044

6.900 .997790 .00044

6.951 .001770 .00043

7.001 .006660 .00044
118

5.900 .921550 .00046

5.950 .926870 .00045

6.000 .932580 .00044

6.050 .938150 .00045

6.100 .942960 .00045

6.150 .947640 .00046

6.200 .953540 .00045

6.250 .957300 .00044

6.300 .963510 .00046

6.350 .969600 .00045

6.400 .973080 .00045

6.450 .978100 .00045

6.500 .983120 .00046

6.550 .988000 .00045

6.600 .993820 .00043

6.650 .998150 .00046

6.701 .002700 .00045

6.751 .007140 .00046

6.801 .011550 .00046 
7.151 .003860 .00043 7.201 .008380 .00043 7.251 .012900 .00044 7.301 .017020 .00044 7.351 .021200 .00044 7.401 .025920 .00044

130

5.700 .911950 .00046 5.750 .917490 .00045 5.800 .922650 .00045 5.850 .928550 .00045 5.900 .934030 .00045 5.950 .939200 .00045 6.000 .944550 .00045 6.050 .949700 .00045 6.100 .954830 .00046 6.150 .959480 .00045 6.200 .965640 .00045 6.250 .970000 .00044 6.300 .975150 .00045 6.350 .980040 .00046 6.400 .985540 .00046 6.450 .990970 .00045 6.500 .996230 .00045 6.551 .001330 .00045 6.601 .006040 .00045 6.651 .010440 .00045 6.701 .014200 .00046 6.751 .019720 .00046 6.801 .024800 .00045

235

5.300 .917160 .00047 5.350 .922620 .00047 5.400 .928330 .00047 5.450 .933860 .00047 5.500 .939340 .00045 5.550 .946550 .00047 5.600 .952320 .00047 5.650 .957410 .00047 5.700 .962140 .00048 5.750 .968690 .00049 5.800 .973810 .00047 5.850 .978910 .00047 5.900 .984490 .00048 $\begin{array}{ll}5.95 & 0.989720 .00047\end{array}$
7.051 .012710 .00046

7.101 .016880 .00045

7.151 .020900 .00045

7.201 .024910 .00044
6.851 .017500 .00044 6.901 .021230 .00044
150

5.600 .915930 .00046 5.650 .921080 .00046 5.700 .927290 .00045 5.750 .932830 .00046 5.800 .938270 .00046 5.850 .943160 .00046 5.900 .948800 .00046 5.950 .953920 .00046 6.000 .959730 .00046 6.050 .965420 .00046 6.100 .970460 .00046 6.150 .975140 .00046 6.200 .980280 .00046 6.250 .986460 .00046 6.300 .990580 .00045 6.350 .995780 .00045 6.401 .001030 .00047 6.451 .006080 .00046 6.501 .010390 .00045 6.551 .015470 .00047 6.601 .019550 .00047
200

5.400 .918800 .00048 5.450 .924350 .00046 5.500 .930100 .00047 5.550 .935970 .00046 5.600 .941440 .00046 5.650 .946780 .00047 5.700 .952670 .00046 5.750 .957450 .00047 5.800 .963170 .00047 5.850 .968760 .00048 5.900 .974340 .00049 5.950 .979950 .00047 6.000 .985180 .00047 6.050 .989210 .00044 6.100 .995640 .00046 6.151 .000950 .00047 6.201 .006080 .00049 6.251 .010460 .00047 6.301 .016380 .00048 6.351 .020700 .00048 6.401 .026070 .00048
5.300 .920500 .00047

5.350 .926840 .00047

5.400 .931880 .00048

5.450 .936930 .00046

5.500 .942800 .00047

5.550 .948720 .00047

5.600 .955450 .00048 5.650 .960930 .00047 5.700 .965530 .00047 5.750 .971620 .00046 5.800 .976790 .00046 5.850 .981620 .00048 5.900 .986700 .00048 5.950 .992950 .00048
353

5.200 .920060 .00047 5.250 .926150 .00047 5.300 .931730 .00047 5.350 .938080 .00047 5.400 .943220 .00047 5.450 .948620 .00046 5.500 .954760 .00048 5.550 .960400 .00048 5.600 .966170 .00046 5.650 .970540 .00047 5.700 .977410 .00049 5.750 .982200 .00048 5.850 .992370 .00046 5.900 .997650 .00048 
6.000 .995130 .00048 6.051 .000040 .00047 6.101 .005470 .00047 6.151 .010540 .00047 6.201 .014870 .00048 6.251 .020590 .00047 6.301 .025700 .00047
6.000 .997580 .00047 6.051 .003700 .00047 6.101 .008150 .00048 6.151 .013200 .00047 6.201 .019700 .00047
5.951 .004250 .00048 6.001 .008200 .00049 6.051 .013490 .00047 6.101 .017890 .00048
470

5.200 .923220 .00047 5.250 .927980 .00047 5.300 .933770 .00048 5.350 .939020 .00047 5.400 .944970 .00046 5.450 .950660 .00049 5.500 .954830 .00047 5.550 .961530 .00047 5.600 .967330 .00047 5.650 .972330 .00047 5.700 .977060 .00047 5.750 .982700 .00047 5.800 .987660 .00047 5.850 .992740 .00048 5.900 .998250 .00049 5.951 .002590 .00049 6.001 .007740 .00047 6.051 .012900 .00048 6.101 .019040 .00049

605

5.200 .915890 .00048 5.250 .922460 .00048 5.300 .927370 .00047 5.350 .932390 .00047 5.400 .937410 .00047 5.450 .943550 .00048 5.500 .948260 .00048 5.550 .954590 .00048 5.600 .959170 .00046 5.650 .964290 .00047 5.700 .970050 .00048 5.750 .974810 .00048 5.800 .979710 .00047 5.850 .984350 .00048 5.900 .989350 .00047
500

5.200 .920630 .00048

5.250 .926590 .00046

5.300 .933170 .00047

5.350 .937560 .00048

5.400 .943690 .00048

5.450 .948630 .00049

5.500 .954170 .00047

5.550 .959760 .00047

5.600 .964350 .00047

5.650 .970130 .00047

5.700 .975510 .00046

5.750 .980130 .00048

5.800 .986260 .00048

5.850 .990840 .00047

5.900 .995900 .00048

5.951 .001460 .00048

6.001 .006240 .00047

6.051 .010480 .00049

6.101 .015660 .00048

6.151 .020380 .00048

6.201 .026280 .00048

610

5.200 .915550 .00047

5.250 .922200 .00048

5.300 .926920 .00047

5.350 .932430 .00047

5.400 .938100 .00046

5.450 .942970 .00046

5.500 .949480 .00047

5.550 .953240 .00046

5.600 .959930 .00047

5.650 .964820 .00047

5.700 .968950 .00047

5.750 .974190 .00048

5.800 .979760 .00049

5.850 .984210 .00047

5.900 .988750 .00048
600

5.200 .916350 .00047 5.250 .922390 .00046 5.300 .927730 .00047 5.350 .933170 .00048 5.400 .938000 .00047 5.450 .944180 .00045 5.500 .949100 .00048 5.550 .953690 .00048 5.600 .959240 .00047 5.650 .965050 .00046 5.700 .969680 .00047 5.750 .974960 .00048 5.800 .979610 .00047 5.850 .985390 .00047 5.900 .990170 .00047 5.950 .995110 .00047 6.000 .999490 .00048 6.051 .003910 .00047 6.101 .010740 .00046 6.151 .015080 .00047 6.201 .018480 .00048 
5.950 .994930 .00048 6.000 .999850 .00047 6.051 .004300 .00048 6.101 .008880 .00048 6.151 .013130 .00047 6.201 .018480 .00047

\section{0}

5.200 .914800 .00047 5.250 .920530 .00046 5.300 .926330 .00046 5.350 .932190 .00045 5.400 .936250 .00047 5.450 .942150 .00048 5.500 .947610 .00046 5.550 .952870 .00046 5.600 .958330 .00047 5.650 .962310 .00047 5.700 .968060 .00047 5.750 .973820 .00049 5.800 .978820 .00046 5.850 .983520 .00047 5.900 .988040 .00047 5.950 .993730 .00047 6.000 .998430 .00048 6.051 .003890 .00049 6.101 .008410 .00048 6.151 .013070 .00047 6.201 .017550 .00047

U5o2N23-icyl-0935-00

12

52.100 .875380 .00015 52.900 .875930 .00015 53.700 .877010 .00014 54.500 .878240 .00014 55.300 .878920 .00014 56.100 .879740 .00014 56.900 .881010 .00014 57.700 .881300 .00014 58.500 .881520 .00014 59.300 .882760 .00014 60.100 .883410 .00014 60.900 .883730 .00014
5.950 .994430 .00048 6.000 .998820 .00046 6.051 .004540 .00047 6.101 .008990 .00047 6.151 .014550 .00048 6.201 .018430 .00046
5.950 .993720 .00047 6.000 .998500 .00049 6.051 .003040 .00047 6.101 .009570 .00048 6.151 .012810 .00049 6.201 .018210 .00048

\section{5}

5.200 .914050 .00046 5.250 .920890 .00048 5.300 .925630 .00046 5.350 .931410 .00048 5.400 .935600 .00045 5.450 .941650 .00047 5.500 .947090 .00047 5.550 .952650 .00047 5.600 .957320 .00046 5.650 .963190 .00047 5.700 .967670 .00046 5.750 .973340 .00047 5.800 .978180 .00048 5.850 .983600 .00048 5.900 .988830 .00046 5.950 .993940 .00048 6.000 .997490 .00047 6.051 .002760 .00047 6.101 .007850 .00049 6.151 .013240 .00047 6.201 .017820 .00047
629

5.200 .915720 .00048 5.250 .920480 .00047 5.300 .925310 .00047 5.350 .931060 .00046 5.400 .936170 .00048 5.450 .941160 .00048 5.500 .947000 .00047 5.550 .952590 .00046 5.600 .957490 .00046 5.650 .963060 .00046 5.700 .967850 .00047 5.750 .971750 .00048 5.800 .977560 .00048 5.850 .981800 .00047 5.900 .988590 .00048 5.950 .993580 .00048 6.000 .997720 .00046 6.051 .003120 .00048 6.101 .006680 .00047 6.151 .011240 .00048 6.201 .016600 .00047 6.251 .021090 .00047 6.301 .024940 .00048
15

29.900 .920650 .00021 30.300 .922290 .00021 30.700 .924560 .00021 31.100 .926490 .00021 31.500 .929010 .00020 31.900 .931110 .00020 32.300 .932840 .00020 32.700 .935060 .00020 33.100 .936390 .00020 33.500 .938600 .00020 33.900 .939850 .00020 34.300 .941960 .00020

\begin{abstract}
20
18.800 .925130 .00029 19.100 .930970 .00028 19.400 .936700 .00029 19.700 .941930 .00028 20.000 .946770 .00028 20.300 .951600 .00028 20.600 .956940 .00027 20.900 .961030 .00027 21.200 .965990 .00027 21.500 .970520 .00028 21.800 .974310 .00027 22.100 .978460 .00027
\end{abstract}


61.700 .884860 .00014 62.500 .885330 .00014 63.300 .886580 .00013 64.100 .886190 .00014 64.900 .886590 .00014 65.700 .887140 .00014 66.500 .888100 .00014 67.300 .888750 .00013 68.100 .889040 .00013 68.900 .889540 .00014 69.500 .890030 .00013
34.700 .943840 .00019 35.100 .944940 .00019 35.500 .946460 .00019 35.900 .948020 .00019 36.300 .949400 .00019 36.700 .951190 .00018 37.100 .952110 .00019 37.500 .953610 .00018 37.900 .955230 .00019 38.000 .955570 .00019
22.400 .982390 .00027 22.700 .986320 .00028 23.000 .990320 .00025 23.300 .993780 .00026 23.600 .997490 .00026 23.901 .001230 .00026 24.201 .004180 .00025 24.501 .007730 .00025 24.801 .011090 .00026 25.001 .013190 .00025
25

15.300 .923260 .00033 15.400 .926610 .00033 15.500 .930770 .00032 15.600 .932950 .00033 15.700 .935770 .00034 15.800 .939190 .00033 15.900 .941300 .00032 16.000 .945750 .00033 16.100 .947250 .00033 16.200 .950630 .00033 16.300 .953320 .00033 16.400 .956060 .00032 16.500 .959110 .00032 16.600 .962530 .00033 16.700 .964850 .00032 16.800 .967130 .00033 16.900 .970000 .00032 17.000 .972570 .00033 17.100 .975560 .00033 17.200 .977820 .00032 17.300 .980310 .00032 17.400 .982780 .00032 17.500 .985680 .00032 17.600 .986660 .00032 17.700 .989910 .00032 17.800 .992480 .00032 17.900 .994820 .00031 18.000 .996960 .00032 18.100 .999180 .00031 18.201 .001290 .00032 18.301 .004010 .00032 18.401 .006130 .00032 18.501 .008020 .00031 18.601 .009810 .00032 18.701 .012680 .00031 18.801 .014550 .00032
30

13.600 .923490 .00036 13.700 .927830 .00036 13.800 .931990 .00036 13.900 .936450 .00036 14.000 .941000 .00036 14.100 .944090 .00037 14.200 .948670 .00036 14.300 .951980 .00036 14.400 .955510 .00036 14.500 .959160 .00036 14.600 .963330 .00035 14.700 .966960 .00036 14.800 .970960 .00036 14.900 .974260 .00036 15.000 .979030 .00036 15.100 .981690 .00034 15.200 .984940 .00035 15.300 .988070 .00036 15.400 .991000 .00035 15.500 .995020 .00035 15.600 .997690 .00035 15.701 .002110 .00035 15.801 .003950 .00036 15.901 .008020 .00034 16.001 .010640 .00035 16.101 .014080 .00036 16.201 .017290 .00035
32

13.100 .923190 .00037 13.200 .927190 .00037 13.300 .932130 .00037 13.400 .936480 .00038 13.500 .941000 .00037 13.600 .945080 .00037 13.700 .949250 .00038 13.800 .952900 .00038 13.900 .956910 .00037 14.000 .961090 .00037 14.100 .965740 .00037 14.200 .970160 .00036 14.300 .973680 .00036 14.400 .978040 .00036 14.500 .981040 .00037 14.600 .985050 .00037 14.700 .989130 .00037 14.800 .992130 .00036 14.900 .996170 .00036 15.000 .999380 .00036 15.101 .003120 .00035 15.201 .007170 .00037 15.301 .009740 .00035 15.401 .014080 .00036 15.501 .017470 .00036 
35

12.500 .921100 .00039

12.600 .927110 .00039

12.700 .932050 .00038

12.800 .936860 .00038

12.900 .940780 .00040

13.000 .946060 .00040

13.100 .950880 .00038

13.200 .955810 .00038

13.300 .959100 .00039

13.400 .964540 .00039

13.500 .968780 .00038

13.600 .973610 .00039

13.700 .978280 .00037

13.800 .982230 .00038

13.900 .985820 .00037

14.000 .990260 .00039

14.100 .994760 .00038

14.200 .998590 .00038

14.301 .003180 .00038

14.401 .006660 .00037

14.501 .010120 .00039

14.601 .014070 .00038

14.701 .018540 .00038
40

11.800 .922350 .00042

11.900 .928010 .00041

12.000 .933570 .00042

12.100 .938780 .00041

12.200 .944400 .00040

12.300 .949770 .00041

12.400 .955530 .00040

12.500 .960420 .00041

12.600 .965920 .00040

12.700 .971710 .00041

12.800 .975660 .00041

12.900 .981430 .00041

13.000 .986340 .00040

13.100 .990770 .00039

13.200 .995730 .00039

13.301 .000310 .00041

13.401 .004940 .00039

13.501 .009880 .00039

13.601 .014080 .00039

13.701 .019100 .00039
50

10.900 .922480 .00042 10.950 .926010 .00043 11.000 .929330 .00042 11.050 .932410 .00044 11.100 .936320 .00043 11.150 .939350 .00045 11.200 .943350 .00044 11.250 .946680 .00043 11.300 .949760 .00043 11.350 .952780 .00044 11.400 .955850 .00044 11.450 .958220 .00043 11.500 .962100 .00043 11.550 .965460 .00044 11.600 .968290 .00043 11.650 .971650 .00043 11.700 .974650 .00043 11.750 .977290 .00042 11.800 .980520 .00043 11.850 .983410 .00044 11.900 .986400 .00044 11.950 .989440 .00043 12.000 .992550 .00043 12.050 .994710 .00042 12.100 .998090 .00042 12.151 .002640 .00043 12.201 .004600 .00044 12.251 .006330 .00042 12.301 .009150 .00043 12.351 .012540 .00042 12.401 .015530 .00042 12.451 .017560 .00043 12.501 .020310 .00043

60

10.300 .920550 .00046 10.350 .922730 .00046 10.400 .927220 .00044 10.450 .931070 .00045 10.500 .935010 .00045 10.550 .937920 .00044 10.600 .941530 .00045 10.650 .945240 .00045 10.700 .949380 .00046 10.750 .952770 .00045
70

9.900 .917840 .00047

9.950 .922520 .00048

10.000 .926590 .00047

10.050 .929830 .00047

10.100 .933310 .00047

10.150 .937320 .00047

10.200 .941490 .00046

10.250 .947240 .00046

10.300 .949550 .00045

10.350 .953850 .00047
80

9.600 .914680 .00046 9.650 .918730 .00048

9.700 .923740 .00049

9.750 .928460 .00048 9.800 .933300 .00048 9.850 .936020 .00049 9.900 .939870 .00048 9.950 .945720 .00049 10.000 .949160 .00049 10.050 .952580 .00048 
10.800 .956030 .00045 10.850 .960490 .00044 10.900 .963360 .00046 10.950 .967600 .00045 11.000 .970950 .00046 11.050 .974320 .00045 11.100 .977450 .00045 11.150 .981000 .00045 11.200 .984320 .00044 11.250 .987280 .00046 11.300 .990840 .00047 11.350 .994370 .00044 11.400 .997240 .00045 11.451 .001200 .00044 11.501 .003230 .00046 11.551 .007990 .00044 11.601 .010740 .00046 11.651 .013660 .00046 11.701 .017500 .00045
10.400 .957660 .00047 10.450 .961950 .00048 10.500 .964310 .00048 10.550 .968610 .00046 10.600 .972930 .00046 10.650 .975670 .00048 10.700 .980590 .00048 10.750 .984310 .00048 10.800 .987690 .00046 10.850 .991470 .00047 10.900 .994610 .00047 10.950 .998300 .00047 11.001 .001290 .00046 11.051 .005750 .00047 11.101 .009080 .00047 11.151 .011670 .00047 11.201 .015510 .00046 11.251 .018980 .00046 11.301 .022820 .00047
10.150 .962020 .00048 10.200 .964990 .00048 10.250 .969860 .00048 10.300 .973040 .00047 10.350 .977040 .00047 10.400 .981030 .00048 10.450 .984740 .00047 10.500 .988890 .00047 10.550 .992610 .00048 10.600 .996470 .00048 10.651 .000260 .00046 10.701 .004750 .00047 10.751 .008210 .00048 10.801 .012200 .00047 10.851 .015620 .00048 10.901 .018870 .00047
90

9.400 .915790 .00049

9.450 .920150 .00048

9.500 .924790 .00048

9.550 .929240 .00047

9.600 .934590 .00049

9.650 .938350 .00050

9.700 .942350 .00049

9.750 .947190 .00049

9.800 .952160 .00048

9.850 .956190 .00049

9.900 .959240 .00049

9.950 .963800 .00050

10.000 .967960 .00049

10.050 .972810 .00049

10.100 .976650 .00048

10.150 .981510 .00049

10.200 .984720 .00050

10.250 .989590 .00048

10.300 .993140 .00048

10.350 .997340 .00048

10.401 .001060 .00047

10.451 .004170 .00048

10.501 .009130 .00050

10.551 .013470 .00050

10.601 .016200 .00048

10.651 .020210 .00049

10.701 .023520 .00049
100

9.300 .921500 .00050

9.350 .926040 .00049

9.400 .930590 .00049

9.450 .934360 .00049

9.500 .939470 .00050

9.550 .944350 .00050

9.600 .949040 .00049

9.650 .952010 .00050

9.700 .957820 .00050

9.750 .961610 .00050

9.800 .966080 .00050

9.850 .970930 .00049

9.900 .974480 .00051

9.950 .978770 .00049

10.000 .983730 .00049

10.050 .987470 .00048

10.100 .991240 .00051

10.150 .995850 .00049

10.201 .000050 .00049

10.251 .002970 .00049

10.301 .008460 .00049

10.351 .011980 .00048

10.401 .016550 .00050

10.451 .019600 .00050

10.501 .023400 .00049
118

9.100 .921690 .00053

9.150 .925740 .00050

9.200 .931390 .00050

9.250 .935620 .00050

9.300 .940820 .00049

9.350 .945030 .00052

9.400 .949520 .00050

9.450 .954510 .00051

9.500 .959260 .00051

9.550 .963500 .00050

9.600 .968040 .00050

9.650 .972600 .00049

9.700 .977560 .00049

9.750 .981610 .00049

9.800 .986910 .00051

9.850 .991010 .00050

9.900 .995050 .00051

9.950 .998630 .00051

10.001 .004490 .00051

10.051 .007740 .00051

10.101 .012380 .00051

10.151 .016600 .00050

10.201 .020400 .00051 
130

8.900 .911850 .00053

8.950 .915960 .00050

9.000 .922000 .00050

9.050 .926740 .00051

9.100 .930810 .00051

9.150 .936590 .00053

9.200 .941620 .00051

9.250 .946310 .00050

9.300 .950990 .00051

9.350 .955770 .00052

9.400 .960190 .00052

9.450 .964490 .00051

9.500 .969750 .00050

9.550 .974040 .00053

9.600 .979890 .00051

9.650 .983270 .00051

9.700 .987490 .00051

9.750 .991250 .00051

9.800 .996420 .00052

9.851 .000680 .00051

9.901 .005290 .00053

9.951 .009690 .00051

10.001 .014310 .00051

10.051 .018480 .00050

10.101 .022120 .00052

235

8.600 .918290 .00053

8.650 .923000 .00053

8.700 .928670 .00054

8.750 .933410 .00054

8.800 .938280 .00053

8.850 .944090 .00053

8.900 .947760 .00053

8.950 .954380 .00052

9.000 .959150 .00052

9.050 .964440 .00053

9.100 .968120 .00054

9.150 .973050 .00053

9.200 .978770 .00055

9.250 .982920 .00052

9.300 .988580 .00052

9.350 .993270 .00054

9.400 .997500 .00054

9.451 .002350 .00053

9.501 .007110 .00052
150

8.800 .914230 .00051

8.850 .919120 .00051

8.900 .924150 .00050

8.950 .928480 .00051

9.000 .934180 .00052

9.050 .939550 .00052

9.100 .943760 .00051

9.150 .948720 .00051

9.200 .953140 .00052

9.250 .958870 .00053

9.300 .963600 .00052

9.350 .968580 .00052

9.400 .973190 .00053

9.450 .978340 .00053

9.500 .982020 .00053

9.550 .986920 .00052

9.600 .991040 .00052

9.650 .995610 .00051

9.701 .000730 .00051

9.751 .004600 .00052

9.801 .009110 .00052

9.851 .013500 .00052

9.901 .018680 .00053

9.951 .022930 .00052

10.001 .026410 .00051
200

8.700 .921120 .00054

8.750 .926880 .00052

8.800 .931330 .00053

8.850 .935410 .00054

8.900 .942440 .00053

8.950 .946680 .00053

9.000 .952680 .00053

9.050 .957450 .00052

9.100 .961970 .00053

9.150 .967150 .00053

9.200 .972690 .00052

9.250 .976740 .00053

9.300 .982100 .00053

9.350 .986130 .00052

9.400 .991700 .00053

9.450 .995740 .00053

9.501 .000950 .00053

9.551 .004930 .00053

9.601 .010020 .00054

9.651 .015260 .00053

9.701 .019280 .00054

9.751 .023900 .00053

9.801 .027670 .00052
250
8.600 .918070 .00053
$\begin{array}{ccc} & 353 \\ 8.60 & 0.91854 & 0.00054 \\ 8.65 & 0.92244 & 0.00052 \\ 8.70 & 0.92785 & 0.00053 \\ 8.75 & 0.93376 & 0.00053 \\ 8.80 & 0.93828 & 0.00053 \\ 8.85 & 0.94307 & 0.00055 \\ 8.90 & 0.94904 & 0.00053 \\ 8.95 & 0.95360 & 0.00054 \\ 9.00 & 0.95909 & 0.00053 \\ 9.05 & 0.96370 & 0.00054 \\ 9.10 & 0.96883 & 0.00053 \\ 9.15 & 0.97280 & 0.00053 \\ 9.20 & 0.97832 & 0.00054 \\ 9.25 & 0.98306 & 0.00053 \\ 9.30 & 0.98745 & 0.00053 \\ 9.35 & 0.99292 & 0.00053 \\ 9.40 & 0.99624 & 0.00052 \\ 9.45 & 1.00109 & 0.00053 \\ 9.50 & 1.00619 & 0.00053\end{array}$
8.650 .923290 .00053
8.700 .929280 .00052
8.750 .933760 .00051
8.800 .938390 .00052
8.850 .943060 .00054
8.900 .950230 .00055
8.950 .953810 .00052
9.000 .959320 .00053
9.050 .964750 .00054
9.100 .969220 .00054
9.150 .974150 .00053
9.200 .978900 .00053
9.250 .983810 .00053
9.300 .988740 .00054
9.350 .993430 .00052
9.400 .998040 .00052
9.451 .003440 .00054
9.501 .008310 .00053 
9.551 .011610 .00053 9.601 .016450 .00053 9.651 .020700 .00053 9.701 .025130 .00053

470

8.700 .915410 .00053 8.750 .921270 .00053 8.800 .926350 .00053 8.850 .930770 .00052 8.900 .936480 .00054 8.950 .941760 .00051 9.000 .945390 .00051 9.050 .949480 .00054 9.100 .955310 .00052 9.150 .960540 .00054 9.250 .969500 .00051 9.300 .975030 .00052 9.350 .980030 .00052 9.400 .983870 .00052 9.450 .989070 .00053 9.500 .992750 .00052 9.550 .998660 .00053 9.601 .001610 .00054 9.651 .007120 .00052 9.701 .010530 .00052 9.751 .015300 .00052 9.801 .019900 .00054

\section{0}

8.800 .917820 .00052 8.850 .921220 .00052 8.900 .926830 .00052 8.950 .932620 .00052 9.000 .938230 .00053 9.050 .940770 .00053 9.100 .946890 .00052 9.150 .950910 .00052 9.200 .956230 .00055 9.250 .960350 .00052 9.300 .965530 .00051 9.350 .970050 .00052 9.400 .974150 .00053 9.450 .980270 .00051
9.551 .011500 .00053 9.601 .017320 .00055 9.651 .020950 .00053 9.701 .026390 .00053
9.551 .012050 .00053 9.601 .015760 .00055 9.651 .020630 .00054 9.701 .024240 .00054
500

8.800 .920940 .00051 8.850 .924880 .00052 8.900 .929690 .00053 8.950 .934630 .00053 9.000 .940360 .00053 9.050 .944560 .00052 9.100 .949530 .00053 9.150 .955210 .00053 9.200 .959490 .00053 9.250 .964170 .00052 9.300 .968220 .00052 9.350 .973700 .00053 9.400 .978670 .00053 9.450 .983460 .00053 9.500 .987030 .00054 9.550 .992740 .00052 9.600 .995660 .00052 9.651 .000720 .00052 9.701 .005630 .00052 9.751 .009740 .00053 9.801 .014350 .00054 9.851 .018490 .00052 9.901 .023500 .00054
510

8.800 .919130 .00054 8.850 .923060 .00052 8.900 .928340 .00052 8.950 .932820 .00053 9.000 .939030 .00053 9.050 .943150 .00053 9.100 .948360 .00052 9.150 .953070 .00052 9.200 .957600 .00054 9.250 .963300 .00053 9.300 .967620 .00051 9.350 .972210 .00053 9.400 .976170 .00053 9.450 .980640 .00055 9.500 .985520 .00054 9.550 .989360 .00055 9.600 .995170 .00053 9.650 .998690 .00053 9.701 .004050 .00053 9.751 .008080 .00053 9.801 .012780 .00054 9.851 .016740 .00054 9.901 .021040 .00053 9.951 .025980 .00052 10.001 .029910 .00053

530

8.800 .914690 .00052 8.850 .920930 .00052 8.900 .924740 .00053 8.950 .930300 .00053 9.000 .935070 .00053 9.050 .940160 .00051 9.100 .944540 .00053 9.150 .949960 .00053 9.200 .954710 .00051 9.250 .958370 .00053 9.300 .964180 .00053 9.350 .967590 .00052 9.400 .973550 .00053 9.450 .977160 .00052
540

8.800 .913360 .00052 8.850 .918410 .00052 8.900 .923330 .00052 8.950 .928500 .00051 9.000 .932520 .00053 9.050 .937910 .00052 9.100 .942570 .00051 9.150 .947360 .00052 9.200 .952120 .00051 9.250 .956400 .00052 9.300 .962810 .00052 9.350 .966120 .00052 9.400 .971090 .00052 9.450 .975230 .00054 
9.500 .984030 .00054

9.550 .988400 .00053

9.600 .992260 .00052

9.650 .996960 .00054

9.701 .001100 .00052

9.751 .006840 .00054

9.801 .010590 .00054

9.851 .014730 .00053

9.901 .020100 .00053

9.951 .023310 .00053

10.001 .027790 .00054
9.500 .982100 .00052

9.550 .987000 .00052

9.600 .991470 .00053

9.650 .995360 .00054

9.701 .000280 .00052

9.751 .005230 .00052

9.801 .008820 .00054

9.851 .012960 .00051

9.901 .016480 .00053

9.951 .022030 .00054

10.001 .026700 .00053
9.500 .980020 .00053

9.550 .985540 .00054

9.600 .989040 .00052

9.650 .993170 .00052

9.700 .998080 .00052

9.751 .002200 .00052

9.801 .006910 .00052

9.851 .010090 .00053

9.901 .015880 .00052

9.951 .019620 .00055

10.001 .024310 .00052

550

8.900 .920570 .00052

8.950 .926270 .00052

9.000 .931710 .00054

9.050 .936500 .00052

9.100 .941120 .00051

9.150 .945800 .00054

9.200 .950080 .00051

9.250 .955240 .00053

9.300 .959970 .00052

9.350 .964090 .00052

9.400 .968820 .00053

9.450 .972770 .00052

9.500 .978680 .00053

9.550 .983430 .00052

9.600 .987310 .00053

9.650 .992680 .00054

9.700 .996010 .00053

9.751 .000770 .00053

9.801 .005230 .00052

9.851 .009890 .00052

9.901 .014740 .00051

9.951 .018490 .00053

10.001 .022180 .00052

560

8.900 .920010 .00052

8.950 .924210 .00053

9.000 .930050 .00052

9.050 .934400 .00053

9.100 .938910 .00054

9.150 .944300 .00053

9.200 .949740 .00052

9.250 .954500 .00052

9.300 .957680 .00055

9.350 .963560 .00052

9.400 .967630 .00053

9.450 .972230 .00052

9.500 .976470 .00052

9.550 .980720 .00053

9.600 .984900 .00051

9.650 .989740 .00053

9.700 .994040 .00054

9.750 .998730 .00053

9.801 .003240 .00052

9.851 .006340 .00052

9.901 .012390 .00053

9.951 .016420 .00053

10.001 .020920 .00054 
U5o2N23-icyl-0935-01

12

38.500 .854480 .00016 40.200 .858250 .00016 41.900 .861980 .00016 43.600 .865070 .00015 45.300 .867570 .00015 47.000 .870440 .00015 48.700 .872930 .00015 50.400 .875140 .00015 52.100 .876970 .00014 53.800 .879070 .00014 55.500 .880750 .00014 57.200 .882040 .00014 58.900 .883470 .00014 60.600 .884580 .00013 62.300 .886240 .00013 64.000 .887800 .00013 65.700 .888260 .00013 67.400 .889380 .00013 69.100 .890260 .00013 70.800 .891100 .00013 72.500 .892340 .00013 73.100 .891960 .00013

25

13.800 .925260 .00032 13.900 .928170 .00031 14.000 .931330 .00032 14.100 .934870 .00032 14.200 .937840 .00032 14.300 .941240 .00032 14.400 .943210 .00031 14.500 .946030 .00031 14.600 .948990 .00031 14.700 .952090 .00031 14.800 .954880 .00032
15

28.900 .923530 .00020 29.000 .923480 .00020 29.100 .924670 .00020 29.200 .925320 .00020 29.300 .925420 .00021 29.400 .926040 .00020 29.500 .926310 .00020 29.600 .927420 .00021 29.700 .927300 .00020 29.800 .928560 .00020 29.900 .929240 .00020 30.000 .929450 .00019 30.100 .929630 .00020 30.200 .930330 .00020 30.300 .930820 .00020 30.400 .930980 .00019 30.500 .931590 .00020 30.600 .932440 .00020 30.700 .932840 .00021 30.800 .933620 .00021 30.900 .933380 .00020 31.000 .934300 .00020 31.100 .934760 .00020 31.200 .934790 .00019 31.300 .935800 .00019 31.400 .936610 .00019 31.500 .936940 .00020 31.600 .937000 .00019 31.700 .937450 .00020 31.800 .937950 .00019 31.900 .938500 .00020 32.000 .939260 .00020 32.100 .938820 .00020

30

12.000 .921950 .00036 12.100 .927000 .00035 12.200 .930960 .00035 12.300 .933990 .00036 12.400 .938580 .00036 12.500 .942470 .00035 12.600 .946670 .00035 12.700 .950350 .00035 12.800 .954830 .00035 12.900 .958050 .00036 13.000 .961490 .00034
20

17.200 .923790 .00029 17.500 .929720 .00028 17.800 .935250 .00027 18.100 .941150 .00027 18.400 .945660 .00027 18.700 .950600 .00027 19.000 .956030 .00027 19.300 .960500 .00027 19.600 .965520 .00027 19.900 .969420 .00027 20.200 .973600 .00026 20.800 .982140 .00026 21.100 .986010 .00026 21.400 .990540 .00026 21.700 .993070 .00026 22.000 .997770 .00025 22.301 .000360 .00026 22.601 .003580 .00025 22.901 .007690 .00025 23.201 .010170 .00025 23.501 .013390 .00024

$$
\text { F-522 }
$$


14.900 .957830 .00033 15.000 .960030 .00032 15.100 .962660 .00032 15.200 .966000 .00032 15.300 .968530 .00031 15.400 .971170 .00031 15.500 .974130 .00032 15.600 .976770 .00031 15.700 .979470 .00031 15.800 .981980 .00031 15.900 .984210 .00032 16.000 .987060 .00031 16.100 .987970 .00032 16.200 .992100 .00031 16.300 .993840 .00030 16.400 .996120 .00030 16.500 .997870 .00031 16.601 .000770 .00030 16.801 .004710 .00032 16.901 .007070 .00031 17.001 .009210 .00030 17.101 .012060 .00030 17.201 .013470 .00030
13.100 .965170 .00035 13.200 .968720 .00036 13.300 .972150 .00034 13.400 .975700 .00033 13.500 .979730 .00034 13.600 .983360 .00034 13.700 .986360 .00034 13.800 .990130 .00034 13.900 .993280 .00035 14.000 .996390 .00035 14.100 .999880 .00034 14.201 .002840 .00034 14.301 .005980 .00034 14.401 .009860 .00034 14.501 .012320 .00034 14.601 .015850 .00033
12.600 .968010 .00035 12.700 .971980 .00035 12.800 .976610 .00036 12.900 .980120 .00036 13.000 .983560 .00035 13.100 .987250 .00036 13.200 .990980 .00036 13.300 .994720 .00036 13.400 .998350 .00036 13.501 .002030 .00035 13.601 .005070 .00036 13.701 .008640 .00035 13.801 .012430 .00034 13.901 .015420 .00035 14.001 .019490 .00035
35

10.900 .918970 .00038 11.000 .923730 .00037 11.100 .928870 .00039 11.200 .933960 .00038 11.300 .938940 .00037 11.400 .944230 .00038 11.500 .949100 .00037 11.600 .953490 .00037 11.700 .957990 .00036 11.800 .962210 .00038 11.900 .967060 .00037 12.000 .972020 .00036 12.100 .975410 .00037 12.200 .979710 .00036 12.300 .984590 .00037 12.400 .989110 .00037 12.500 .991740 .00036 12.600 .996870 .00036 12.701 .000740 .00037 12.801 .006090 .00036 12.901 .008950 .00037 13.001 .012280 .00035 13.101 .016660 .00037
40

10.300 .925130 .00040 10.400 .930600 .00039 10.500 .936380 .00040 10.600 .942820 .00040 10.700 .947660 .00040 10.800 .952660 .00039 10.900 .958810 .00039 11.000 .963530 .00039 11.100 .968350 .00038 11.200 .973500 .00039 11.300 .978620 .00040 11.400 .983880 .00039 11.500 .989080 .00039 11.600 .993010 .00039 11.700 .997710 .00037 11.801 .003050 .00039 11.901 .007720 .00039 12.001 .013300 .00039 12.101 .016750 .00039
50

9.300 .919450 .00043 9.350 .922850 .00042 9.400 .926910 .00043 9.450 .930570 .00041 9.500 .933480 .00042 9.550 .936740 .00042 9.600 .939430 .00041 9.650 .943110 .00041 9.700 .946790 .00042 9.750 .949790 .00042 9.800 .952810 .00041 9.850 .956630 .00041 9.900 .959660 .00043 9.950 .963340 .00042 10.000 .966460 .00042 10.050 .969100 .00041 10.100 .971590 .00042 10.150 .974760 .00042 10.200 .977580 .00041 10.250 .980510 .00041 10.300 .984550 .00041 10.350 .987000 .00041 10.400 .990560 .00042 10.450 .993330 .00041 
10.500 .995680 .00041

10.550 .998770 .00042

10.601 .002410 .00043

10.651 .004910 .00041

10.701 .007770 .00040

10.751 .009880 .00041

10.801 .013100 .00041

10.851 .015640 .00042

10.901 .018850 .00041

60

8.700 .917310 .00044

8.750 .919920 .00044

8.800 .924750 .00045

8.850 .927820 .00044

8.900 .931830 .00044

8.950 .935430 .00044

9.000 .938570 .00043

9.050 .942290 .00043

9.100 .945840 .00044

9.150 .950280 .00044

9.200 .953700 .00044

9.250 .957580 .00045

9.300 .961210 .00044

9.350 .965080 .00042

9.400 .968360 .00043

9.450 .971520 .00044

9.500 .974870 .00043

9.550 .978550 .00043

9.600 .981780 .00042

9.650 .984800 .00046

9.700 .989450 .00044

9.750 .991590 .00044

9.800 .995240 .00043

9.850 .999380 .00043

9.901 .001670 .00044

9.951 .005350 .00044

10.001 .008160 .00044

10.051 .010930 .00042

10.101 .015230 .00045

10.151 .017820 .00044

10.201 .020410 .00043
70

8.400 .922830 .00045

8.450 .926340 .00045

8.500 .931340 .00044

8.550 .935120 .00044

8.600 .939600 .00045

8.650 .943320 .00045

8.750 .951550 .00046

8.800 .955870 .00044

8.850 .958830 .00045

8.900 .962100 .00044

8.950 .967220 .00044

9.000 .970780 .00046

9.050 .974260 .00046

9.100 .976760 .00045

9.150 .981190 .00044

9.200 .985710 .00045

9.250 .988490 .00045

9.300 .992010 .00044

9.350 .995470 .00045

9.400 .999240 .00045

9.451 .002930 .00046

9.501 .007540 .00044

9.551 .010620 .00045

9.601 .013600 .00046

9.651 .017340 .00045

9.701 .020740 .00045
80

8.100 .921330 .00045

8.150 .924600 .00046

8.200 .929480 .00046

8.250 .933980 .00045

8.300 .938070 .00046

8.350 .942280 .00046

8.400 .946580 .00046

8.450 .950570 .00046

8.500 .954400 .00045

8.550 .958230 .00046

8.600 .962220 .00046

8.650 .967390 .00046

8.700 .971740 .00046

8.750 .974600 .00046

8.800 .978620 .00046

8.850 .983020 .00044

8.900 .986220 .00047

8.950 .991080 .00047

9.000 .994810 .00045

9.050 .998190 .00046

9.101 .002650 .00046

9.151 .005520 .00048

9.201 .010170 .00045

9.251 .014760 .00046

9.301 .016520 .00047

9.351 .019990 .00045

9.401 .023860 .00046
90

7.900 .921740 .00046 7.950 .926300 .00047 8.000 .930900 .00047
100

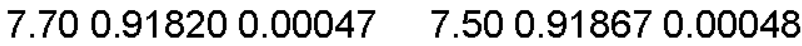

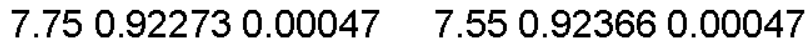

$\begin{array}{lll}7.80 & 0.927390 .00047 \quad 7.600 .928870 .00048\end{array}$ 
8.050 .936030 .00047

8.100 .939070 .00046

8.150 .943950 .00047

8.200 .949410 .00047

8.250 .952950 .00048

8.300 .957320 .00047

8.350 .961690 .00047

8.400 .965730 .00047

8.450 .969630 .00047

8.500 .974510 .00047

8.550 .978180 .00047

8.600 .982630 .00046

8.650 .985740 .00046

8.700 .990770 .00047

8.750 .995340 .00047

8.800 .998520 .00046

8.851 .001980 .00047

8.901 .006250 .00048

8.951 .010040 .00048

9.001 .014140 .00046

9.051 .017640 .00046

9.101 .022430 .00048

130

7.400 .919920 .00049

7.450 .924250 .00049

7.500 .929150 .00048

7.550 .934230 .00050

7.600 .939200 .00048

7.650 .944280 .00048

7.700 .948460 .00048

7.750 .953670 .00050

7.800 .959070 .00049

7.850 .962770 .00048

7.900 .967320 .00049

7.950 .971610 .00050

8.000 .976270 .00049

8.050 .981020 .00047

8.100 .985690 .00048

8.150 .990630 .00049

8.200 .994600 .00050

8.250 .999640 .00048

8.301 .003630 .00049

8.351 .007980 .00049

8.401 .012410 .00049

8.451 .017030 .00049

8.501 .021600 .00048
7.850 .932430 .00048

7.900 .936820 .00046

7.950 .941020 .00046

8.000 .945530 .00046

8.050 .949630 .00048

8.100 .955640 .00047

8.150 .959000 .00046

8.200 .964330 .00048

8.250 .968400 .00048

8.300 .972060 .00049

8.350 .977060 .00048

8.400 .980330 .00048

8.450 .985390 .00047

8.500 .989480 .00047

8.550 .994140 .00049

8.600 .998150 .00048

8.651 .002580 .00050

8.701 .006360 .00047

8.751 .009680 .00046

8.801 .014300 .00049

8.851 .018370 .00047

8.901 .021900 .00046
7.650 .932930 .00048

7.700 .937850 .00047

7.750 .942760 .00049

7.800 .947210 .00050

7.850 .951830 .00048

7.900 .956190 .00049

7.950 .962680 .00048

8.000 .966170 .00047

8.050 .971030 .00048

8.100 .975170 .00049

8.150 .979440 .00048

8.200 .984130 .00048

8.250 .988610 .00048

8.300 .992630 .00050

8.350 .998170 .00049

8.401 .001730 .00048

8.451 .006080 .00050

8.501 .009640 .00049

8.551 .014260 .00049

8.601 .018620 .00048

8.651 .021950 .00048

8.701 .026590 .00048
7.300 .922430 .00050

7.350 .927280 .00049

7.400 .933530 .00050

7.450 .937290 .00051

7.500 .943540 .00050

7.550 .947490 .00050

7.600 .952250 .00052

7.650 .957370 .00049

7.700 .961930 .00048

7.750 .966290 .00049

7.800 .971520 .00050

7.850 .975550 .00049

7.900 .980980 .00049

7.950 .984100 .00050

8.000 .990370 .00051

8.050 .994870 .00051

8.100 .999490 .00050

8.151 .003790 .00051

8.201 .007530 .00051

8.251 .012980 .00049

8.301 .016750 .00050

8.351 .021410 .00049

8.401 .026490 .00050
200

7.100 .920500 .00051

7.150 .926050 .00051

7.200 .931310 .00051

7.250 .936730 .00051

7.300 .942870 .00052

7.350 .945960 .00050

7.400 .951820 .00051

7.450 .956470 .00052

7.500 .961370 .00051

7.550 .966370 .00050

7.600 .971520 .00052

7.650 .976350 .00049

7.700 .981510 .00052

7.750 .985760 .00051

7.800 .991620 .00050

7.850 .995200 .00052

7.900 .999980 .00051

7.951 .004910 .00050

8.001 .010160 .00051

8.051 .014210 .00050

8.101 .018650 .00051 
235

7.000 .917210 .00052

7.050 .922760 .00048

7.100 .927760 .00051

7.150 .932970 .00051

7.200 .937500 .00052

7.250 .943130 .00052

7.300 .948600 .00051

7.350 .953940 .00051

7.400 .958610 .00051

7.450 .964210 .00051

7.500 .967920 .00052

7.550 .974110 .00052

7.600 .978780 .00051

7.650 .983070 .00050

7.700 .987730 .00051

7.750 .992520 .00051

7.800 .996730 .00051

7.851 .002410 .00051

7.901 .006960 .00050

7.951 .011980 .00051

8.001 .016820 .00053

8.051 .021980 .00050

8.101 .024760 .00051

\section{0}

7.100 .919950 .00051

7.150 .925670 .00051

7.200 .929740 .00052

7.250 .935360 .00052

7.300 .940190 .00052

7.350 .944960 .00050

7.400 .950140 .00051

7.450 .953850 .00051

7.500 .959120 .00052

7.550 .964500 .00053

7.600 .969040 .00052

7.650 .974540 .00050

7.700 .978810 .00051

7.750 .983180 .00052

7.800 .988220 .00051

7.850 .992230 .00051

7.900 .996310 .00052

7.951 .000630 .00053

8.001 .005780 .00052

8.051 .011130 .00051

8.101 .014880 .00051

8.151 .019960 .00053

8.201 .024600 .00051
250

7.000 .918080 .00051

7.050 .922730 .00052

7.100 .929070 .00050

7.150 .933930 .00050

7.200 .940030 .00049

7.250 .943620 .00052

7.300 .950330 .00051

7.350 .954390 .00051

7.400 .959090 .00051

7.450 .964630 .00050

7.500 .970020 .00052

7.550 .974460 .00050

7.600 .979460 .00051

7.650 .984580 .00050

7.700 .989570 .00051

7.750 .994450 .00052

7.800 .998890 .00051

7.851 .002680 .00052

7.901 .008660 .00053

7.951 .012620 .00052

8.051 .022250 .00050

8.101 .026730 .00053

500

7.100 .915150 .00050

7.150 .920770 .00050

7.200 .925540 .00052

7.250 .930210 .00053

7.300 .935160 .00051

7.350 .939440 .00051

7.400 .943580 .00051

7.450 .948530 .00054

7.500 .954600 .00051

7.550 .959670 .00050

7.600 .963510 .00052

7.650 .967910 .00051

7.700 .973890 .00051

7.750 .977300 .00051

7.800 .982980 .00051

7.850 .987570 .00051

7.900 .992150 .00051

7.950 .995520 .00051

8.001 .000930 .00054

8.051 .005380 .00052

8.101 .009720 .00052

8.151 .013990 .00053

8.201 .018360 .00051
353

7.000 .920180 .00050

7.050 .924430 .00050

7.100 .930550 .00051

7.150 .935200 .00051

7.200 .941700 .00053

7.300 .950270 .00053

7.350 .955660 .00051

7.400 .960320 .00053

7.450 .964950 .00051

7.500 .970830 .00052

7.550 .976030 .00051

7.600 .980380 .00053

7.650 .984000 .00050

7.700 .989480 .00051

7.750 .994010 .00051

7.800 .999190 .00052

7.851 .004440 .00050

7.901 .009140 .00052

7.951 .012590 .00054

8.001 .018950 .00053

8.051 .021860 .00053

8.101 .027170 .00051

510

7.100 .912240 .00051

7.150 .917970 .00050

7.200 .923320 .00050

7.250 .929460 .00052

7.300 .933440 .00053

7.350 .938420 .00051

7.400 .943480 .00051

7.450 .947730 .00051

7.500 .953720 .00051

7.550 .957040 .00052

7.600 .962260 .00050

7.650 .966500 .00052

7.700 .972790 .00052

7.750 .977370 .00051

7.800 .980770 .00052

7.850 .985890 .00051

7.900 .990020 .00053

7.950 .995760 .00052

8.000 .998530 .00052

8.051 .002960 .00051

8.101 .007520 .00052

8.151 .012970 .00049

8.201 .016620 .00051

8.251 .021200 .00050

8.301 .024910 .00051 
520

7.200 .921510 .00051

7.250 .926490 .00051

7.300 .931260 .00051

7.350 .936400 .00052

7.400 .941420 .00051

7.450 .945660 .00051

7.500 .951280 .00052

7.550 .956200 .00052

7.600 .961200 .00049

7.650 .964980 .00052

7.700 .970480 .00053

7.750 .974030 .00051

7.800 .980130 .00051

7.850 .984150 .00051

7.900 .989150 .00050

7.950 .992790 .00052

8.000 .996960 .00051

8.051 .002010 .00050

8.101 .006800 .00052

8.151 .010690 .00051

8.201 .015200 .00051

8.251 .019300 .00052

8.301 .023360 .00051
530

7.200 .920990 .00051

7.550 .954850 .00051

7.600 .959370 .00052

7.650 .963620 .00051

7.700 .968820 .00050

7.750 .973160 .00051

7.800 .977380 .00051

7.850 .982120 .00049

7.900 .987220 .00053

7.950 .991570 .00051

8.000 .995740 .00050

8.051 .000110 .00050

8.101 .005010 .00051

8.151 .008980 .00051

8.201 .013730 .00052

8.251 .018840 .00050

8.301 .022370 .00050
550

7.200 .916600 .00052

7.250 .922050 .00050

7.300 .927560 .00051

7.350 .932230 .00050

7.400 .937140 .00051

7.450 .942270 .00051

7.500 .946100 .00050

7.550 .951250 .00051

7.600 .956410 .00051

7.650 .959730 .00052

7.700 .966180 .00051

7.750 .970300 .00051

7.800 .975080 .00051

7.850 .978850 .00052

7.900 .983590 .00052

7.950 .988260 .00051

8.000 .992380 .00052 8.050 .997630 .00052

8.101 .002180 .00050

8.151 .005860 .00050

\section{0}

7.200 .915950 .00051

7.250 .920540 .00052

7.300 .925410 .00051

7.350 .931260 .00051

7.400 .935130 .00052

7.450 .941100 .00049

7.500 .944310 .00051

7.600 .955640 .00053

7.650 .959320 .00051

7.700 .963640 .00051

7.750 .968690 .00050

7.800 .972670 .00052

7.850 .977280 .00050

7.900 .983380 .00051

8.000 .991570 .00051

8.050 .994980 .00052

8.101 .000020 .00052

8.151 .004860 .00050

8.201 .008460 .00052

8.251 .012900 .00053
540

7.200 .919380 .00050

7.250 .924000 .00051

7.300 .928450 .00051

7.350 .933230 .00050

7.400 .938610 .00050

7.450 .943900 .00050

7.500 .947560 .00052

7.550 .952980 .00052

7.600 .957360 .00052

7.650 .962080 .00051

7.700 .967980 .00050

7.750 .970980 .00053

7.800 .976050 .00051

7.850 .980600 .00051

7.900 .985150 .00051

7.950 .988850 .00052

8.000 .994960 .00052

8.050 .998640 .00053

8.101 .003850 .00052

8.151 .007920 .00051

8.201 .011770 .00051

8.251 .015720 .00052

8.301 .020620 .00051 
8.201 .009380 .00052

8.251 .014360 .00051

8.301 .018380 .00051

U5०2N23-icyl-0935-10

12

42.800 .865610 .00015

44.600 .868470 .00015

46.400 .871430 .00015

48.200 .874260 .00014

50.000 .876330 .00014

51.800 .878070 .00014

53.600 .880040 .00014

55.400 .881990 .00013

57.200 .883370 .00014

59.000 .884860 .00014

60.800 .885760 .00013

62.600 .887460 .00013

64.400 .888110 .00013

66.200 .889000 .00013

68.000 .889930 .00013

69.800 .891080 .00013

71.600 .891610 .00013

73.400 .892670 .00013

75.200 .893710 .00012

77.000 .894450 .00013

78.800 .894890 .00013

79.000 .895310 .00012

25

12.600 .922850 .00030

12.700 .926880 .00031

12.800 .930040 .00030

12.900 .932560 .00031

13.000 .935920 .00030

13.100 .938920 .00030

13.200 .941880 .00030

13.300 .945300 .00032

13.400 .947850 .00030

13.500 .950630 .00029

13.600 .953810 .00030

13.700 .956560 .00030

13.800 .958940 .00029

13.900 .961890 .00030

14.000 .964400 .00029

14.100 .967420 .00030

14.200 .970280 .00029
8.301 .017300 .00051

8.351 .021230 .00050

8.401 .025700 .00051
15

28.700 .928310 .00020 30.900 .938930 .00019 33.100 .947670 .00018 35.300 .955480 .00018 37.500 .962000 .00017 39.700 .967260 .00017 41.900 .972680 .00017 44.100 .976320 .00017 46.300 .980180 .00016 48.500 .983510 .00016 50.700 .986750 .00016 52.900 .989190 .00015 55.100 .991690 .00015 57.300 .993630 .00015 59.500 .995480 .00015 61.700 .997480 .00014 63.900 .998940 .00014 66.101 .000270 .00014 68.301 .001430 .00014 70.501 .003170 .00014 72.701 .003650 .00013 74.201 .004710 .00013
20

16.200 .926740 .00027 16.500 .932490 .00026 16.800 .937340 .00027 17.100 .943320 .00026 17.400 .948230 .00026 17.700 .953630 .00026 18.000 .957760 .00026 18.300 .963050 .00026 18.600 .967490 .00025 18.900 .972200 .00025 19.200 .976110 .00026 19.500 .980400 .00025 19.800 .983470 .00025 20.100 .987610 .00025 20.400 .991450 .00024 20.700 .995320 .00024 21.000 .998790 .00025 21.301 .002160 .00024 21.601 .005840 .00024 21.901 .008610 .00024 22.201 .012360 .00024
30

10.900 .924210 .00034 11.000 .928790 .00033 11.100 .932610 .00034 11.200 .936450 .00034 11.300 .941040 .00034 11.400 .945240 .00032 11.500 .948140 .00033 11.600 .953340 .00033 11.700 .956570 .00032 11.800 .960530 .00034 11.900 .964280 .00033 12.000 .967360 .00032 12.100 .971390 .00033 12.200 .974410 .00033 12.300 .978360 .00033 12.400 .981600 .00032 12.500 .985670 .00033
32

10.400 .923310 .00034 10.500 .927950 .00034 10.600 .932230 .00034 10.700 .936830 .00035 10.800 .941390 .00034 10.900 .945650 .00033 11.000 .950240 .00034 11.100 .954500 .00034 11.200 .958360 .00034 11.300 .962590 .00034 11.400 .966770 .00034 11.500 .970930 .00034 11.600 .974880 .00033 11.700 .978630 .00034 11.800 .981780 .00034 11.900 .986570 .00032 12.000 .990380 .00033

$$
\text { F-528 }
$$


14.300 .973040 .00029 14.400 .975750 .00029 14.500 .978010 .00030 14.600 .981000 .00029 14.700 .983200 .00029 14.800 .985950 .00030 14.900 .988280 .00029 15.000 .990580 .00029 15.100 .992520 .00029 15.200 .995130 .00030 15.300 .997740 .00029 15.401 .000630 .00030 15.501 .001850 .00029 15.601 .004280 .00030 15.701 .006330 .00029 15.801 .009130 .00029 15.901 .010300 .00029 16.001 .013030 .00029 16.101 .014840 .00029

\section{5}

9.800 .922300 .00034 9.900 .927770 .00035 10.000 .932690 .00035 10.100 .937800 .00035 10.200 .942420 .00035 10.300 .947530 .00035 10.400 .951750 .00034 10.500 .956670 .00034 10.600 .961830 .00034 10.700 .966390 .00035 10.800 .969920 .00034 10.900 .974390 .00035 11.000 .978900 .00035 11.100 .983330 .00035 11.200 .987630 .00034 11.300 .992580 .00035 11.400 .996140 .00034 11.501 .000420 .00035 11.601 .004330 .00034 11.701 .007860 .00034 11.801 .012630 .00035 11.901 .016240 .00034
12.600 .989020 .00033

12.700 .992600 .00032

12.800 .995990 .00032

12.900 .998950 .00032

13.001 .002290 .00031

13.101 .006190 .00032

13.201 .008410 .00032

13.301 .012060 .00031

13.401 .014570 .00033
12.100 .993730 .00034

12.200 .997410 .00035

12.301 .001450 .00033

12.401 .004020 .00033

12.501 .008000 .00033

12.601 .011780 .00033

12.701 .015010 .00032
40

9.100 .923360 .00037

9.200 .929230 .00035

9.300 .935640 .00037

9.400 .940120 .00037

9.500 .946030 .00037

9.600 .952360 .00037

9.700 .957410 .00037

9.800 .962750 .00037

9.900 .967920 .00037

10.000 .972870 .00036

10.100 .978940 .00036

10.200 .982620 .00037

10.300 .988220 .00036

10.400 .993020 .00036

10.500 .998030 .00036

10.601 .002650 .00035

10.701 .007390 .00036

10.801 .011520 .00035

10.901 .016410 .00036
50

8.100 .919010 .00041

8.150 .922320 .00039

8.200 .926290 .00038

8.250 .929200 .00039

8.300 .932430 .00039

8.350 .935750 .00039

8.400 .939060 .00040

8.450 .942290 .00039

8.500 .946090 .00038

8.550 .950020 .00038

8.600 .952700 .00037

8.650 .955460 .00038

8.700 .960070 .00039

8.750 .962530 .00038

8.800 .966050 .00039

8.850 .969320 .00039

8.900 .972800 .00039

8.950 .975060 .00039

9.000 .978110 .00039

9.050 .980960 .00040

9.100 .984900 .00039

9.150 .987090 .00038

9.200 .990580 .00039

9.250 .994050 .00038

9.300 .996060 .00039

9.350 .998440 .00038

9.401 .002360 .00038

9.451 .005660 .00037 
9.501 .008210 .00039

9.551 .010330 .00038

9.601 .013580 .00038

9.651 .017460 .00039

9.701 .019370 .00040

60

7.500 .916470 .00039

7.550 .921060 .00041

7.600 .925170 .00041

7.650 .929080 .00039

7.700 .933000 .00040

7.750 .935040 .00039

7.800 .940650 .00040

7.850 .943980 .00040

7.900 .947430 .00040

7.950 .951090 .00039

8.000 .955330 .00041

8.050 .958690 .00041

8.100 .961260 .00040

8.150 .965650 .00040

8.200 .969470 .00041

8.250 .972310 .00039

8.300 .976270 .00041

8.350 .980100 .00040

8.400 .983240 .00041

8.450 .986900 .00041

8.500 .990380 .00041

8.550 .992920 .00039

8.600 .997220 .00040

8.651 .000650 .00041

8.701 .003620 .00041

8.751 .006920 .00040

8.801 .009550 .00040

8.851 .013460 .00040

8.901 .016710 .00040

8.951 .019440 .00040

9.001 .022920 .00040
70

7.100 .916370 .00042

7.150 .920920 .00042

7.200 .924770 .00041

7.250 .928680 .00040

7.300 .932860 .00042

7.350 .936460 .00041

7.400 .941170 .00042

7.450 .944970 .00042

7.500 .949190 .00041

7.550 .952870 .00042

7.600 .957120 .00042

7.650 .960540 .00042

7.700 .965500 .00041

7.750 .968500 .00042

7.800 .972620 .00040

7.850 .976930 .00042

7.900 .980150 .00042

7.950 .984310 .00043

8.000 .988490 .00041

8.050 .991650 .00042

8.100 .995080 .00041

8.201 .002710 .00042

8.251 .005840 .00041

8.351 .013010 .00042

8.401 .016560 .00042
80

6.900 .923700 .00042

6.950 .928870 .00043

7.000 .933150 .00043

7.050 .937110 .00042

7.100 .941790 .00042

7.150 .945750 .00042

7.200 .949140 .00042

7.250 .954780 .00044

7.300 .958380 .00043

7.350 .962370 .00041

7.400 .966360 .00042

7.450 .970960 .00043

7.500 .974680 .00042

7.550 .978600 .00043

7.600 .983700 .00042

7.650 .986480 .00043

7.700 .991750 .00042

7.750 .994280 .00042

7.800 .999020 .00043

7.851 .002810 .00043

7.901 .006670 .00043

7.951 .010550 .00043

8.001 .014190 .00041

8.051 .017290 .00042

8.101 .021220 .00042
90

6.600 .917030 .00045 6.650 .921280 .00043

6.700 .926590 .00043 6.750 .930570 .00044 6.800 .935330 .00041 6.850 .939310 .00043 6.900 .944790 .00044
100

6.400 .914310 .00044

6.450 .919880 .00044

6.500 .923370 .00043

6.550 .929310 .00044

6.600 .933380 .00044

6.650 .937710 .00044

6.700 .942820 .00043
118

6.200 .917730 .00045

6.250 .922870 .00044

6.300 .927060 .00045

6.350 .931650 .00045

6.400 .936780 .00045

6.450 .942390 .00045

6.500 .946820 .00044 
6.950 .948450 .00043

7.000 .953110 .00043

7.050 .958020 .00042

7.100 .961860 .00042

7.150 .966830 .00043

7.200 .969760 .00042

7.250 .975370 .00044

7.300 .978670 .00042

7.350 .983590 .00043

7.400 .987060 .00044

7.450 .991920 .00043

7.500 .996060 .00044

7.550 .999940 .00043

7.601 .003400 .00044

7.651 .008040 .00043

7.701 .012800 .00043

7.751 .016320 .00042

7.801 .020000 .00045

130

6.100 .919410 .00044

6.150 .923680 .00044

6.200 .930110 .00045

6.250 .933550 .00045

6.300 .939290 .00045

6.350 .943870 .00045

6.400 .949390 .00046

6.450 .953900 .00045

6.500 .958530 .00045

6.550 .963370 .00047

6.600 .968860 .00046

6.650 .973540 .00045

6.700 .977920 .00046

6.800 .987550 .00045

6.850 .991330 .00045

6.900 .996080 .00045

6.951 .000890 .00046

7.001 .005420 .00046

7.051 .009050 .00045

7.101 .013940 .00045

7.151 .018620 .00045

7.201 .022810 .00047
6.750 .946870 .00044

6.800 .952020 .00043

6.850 .957160 .00044

6.900 .960980 .00043

6.950 .965530 .00045

7.000 .969820 .00044

7.050 .974840 .00044

7.100 .978670 .00043

7.150 .982890 .00044

7.200 .987480 .00044

7.250 .991910 .00045

7.300 .995870 .00044

7.351 .000630 .00044

7.401 .004460 .00045

7.451 .009160 .00045

7.501 .013520 .00044

7.551 .017230 .00044

7.601 .019500 .00043
6.550 .951910 .00044

6.600 .955840 .00044

6.650 .960390 .00044

6.700 .964960 .00046

6.750 .969890 .00045

6.800 .974820 .00044

6.850 .979100 .00045

6.900 .984550 .00043

6.950 .987900 .00044

7.000 .993180 .00044

7.050 .997150 .00044

7.101 .001900 .00045

7.151 .006320 .00044

7.201 .009580 .00045

7.251 .015370 .00045

7.301 .018510 .00045
150

5.900 .913790 .00045

5.950 .918920 .00046

6.000 .924020 .00045

6.050 .928800 .00046

6.100 .935110 .00045

6.150 .939270 .00044

6.200 .945120 .00045

6.250 .950060 .00045

6.300 .954600 .00047

6.350 .959530 .00047

6.400 .965210 .00045

6.450 .969640 .00046

6.500 .974660 .00046

6.550 .978900 .00047

6.600 .984080 .00046

6.650 .989340 .00045

6.700 .993050 .00045

6.750 .998250 .00046

6.801 .003770 .00046

6.851 .006530 .00046

6.901 .010930 .00045

6.951 .016300 .00044

7.001 .020430 .00045
200

5.700 .916870 .00046 5.750 .922460 .00046 5.800 .927540 .00045 5.850 .931850 .00046 5.900 .938020 .00046 5.950 .943430 .00046 6.000 .947970 .00046 6.050 .953530 .00047 6.100 .957890 .00046 6.150 .963860 .00046 6.200 .969100 .00047 6.250 .974790 .00048 6.300 .978760 .00046 6.350 .983380 .00047 6.400 .989470 .00047 6.450 .994160 .00046 6.500 .998090 .00046 6.551 .002760 .00047 6.601 .008080 .00046 6.651 .013030 .00047 6.701 .018110 .00047 
235

5.600 .915940 .00047

5.650 .921930 .00044

5.700 .926950 .00046

5.750 .932410 .00046

5.800 .937130 .00047

5.850 .943240 .00047

5.900 .948710 .00046

5.950 .952470 .00046

6.000 .959080 .00046

6.050 .964280 .00048

6.100 .969100 .00047

6.150 .974530 .00046

6.200 .978530 .00047

6.250 .983820 .00047

6.300 .988450 .00047

6.350 .993710 .00047

6.400 .998340 .00047

6.451 .003790 .00046

6.501 .007510 .00046

6.551 .013250 .00046

6.601 .017720 .00046

470

5.500 .915990 .00047

5.550 .921340 .00046

5.650 .930380 .00046

5.700 .936800 .00048

5.750 .941500 .00046

5.800 .946570 .00049

5.850 .951000 .00048

5.900 .957980 .00047

5.950 .960670 .00046

6.000 .966850 .00047

6.050 .971180 .00047

6.100 .976350 .00046

6.150 .981600 .00047

6.200 .985550 .00046

6.250 .991020 .00047

6.300 .996560 .00047

6.351 .000880 .00047

6.401 .005400 .00047

6.451 .009140 .00048

6.501 .014490 .00047

6.551 .018470 .00049

6.601 .023010 .00048
250

5.600 .918970 .00048

5.650 .924320 .00046

5.700 .929380 .00046

5.750 .935580 .00046

5.800 .940120 .00047

5.850 .945460 .00047

5.900 .950400 .00047

5.950 .955830 .00045

6.000 .961680 .00046

6.050 .966270 .00047

6.100 .971080 .00046

6.150 .976600 .00047

6.200 .981160 .00047

6.250 .986380 .00046

6.300 .991930 .00048

6.350 .996600 .00047

6.401 .001100 .00047

6.451 .005980 .00046

6.501 .011310 .00046

6.551 .016250 .00047

6.601 .020180 .00046
353

5.500 .917720 .00047

5.550 .922550 .00047

5.600 .927840 .00046

5.650 .933190 .00046

5.700 .938910 .00047

5.750 .944200 .00047

5.800 .948640 .00048

5.850 .955210 .00048

5.900 .959550 .00046

5.950 .965610 .00048

6.000 .969550 .00047

6.050 .976200 .00047

6.100 .980240 .00047

6.150 .984430 .00047

6.200 .990160 .00047

6.250 .994860 .00046

6.300 .999800 .00046

6.401 .009460 .00050

6.451 .013100 .00048

6.501 .018380 .00048
5.500 .913410 .00047

5.550 .919090 .00047

5.600 .923210 .00046

5.650 .929680 .00048

5.700 .934620 .00046

5.750 .938310 .00046

5.800 .943520 .00046

5.850 .949390 .00047

5.900 .954030 .00047

5.950 .958700 .00046

6.000 .964350 .00047

6.050 .968640 .00047

6.100 .973600 .00047

6.150 .978540 .00046

6.200 .984110 .00047

6.250 .988130 .00048

6.300 .993050 .00047

6.350 .997010 .00045

6.401 .000910 .00047

6.451 .006030 .00050

6.501 .010250 .00047

6.551 .014620 .00048

6.601 .019830 .00048
510

5.600 .923250 .00047

5.650 .928210 .00048

5.700 .932670 .00046

5.750 .938550 .00046

5.800 .943250 .00047

5.850 .948330 .00047

5.900 .953110 .00047

5.950 .958400 .00047

6.000 .962880 .00047

6.050 .968280 .00046

6.100 .973710 .00046

6.150 .977670 .00047

6.200 .982320 .00048

6.250 .987090 .00047

6.300 .990890 .00047

6.350 .995900 .00047

6.401 .000990 .00047

6.451 .005370 .00048

6.501 .009940 .00048

6.551 .015260 .00048

6.601 .019810 .00045 
520

5.600 .922030 .00046 5.650 .927410 .00046 5.700 .932430 .00048 5.750 .937620 .00046 5.800 .943350 .00047 5.850 .947930 .00047 5.900 .952770 .00046 5.950 .957400 .00047 6.000 .962120 .00048 6.050 .967410 .00047 6.100 .972470 .00046 6.150 .976460 .00048 6.200 .981130 .00047 6.250 .985860 .00046 6.300 .990570 .00047 6.350 .995320 .00047 6.401 .000080 .00048 6.451 .004640 .00047 6.501 .009150 .00047 6.551 .013630 .00048 6.601 .018550 .00048

550

5.600 .919650 .00047 5.650 .925710 .00048 5.700 .929520 .00045 5.750 .934860 .00045 5.800 .939840 .00047 5.850 .945140 .00046 5.900 .949520 .00047 5.950 .954730 .00047 6.000 .959780 .00048 6.050 .964550 .00046 6.100 .968990 .00046 6.150 .974080 .00047 6.200 .978930 .00047 6.250 .982930 .00046 6.300 .988330 .00048 6.350 .991650 .00047 6.400 .996650 .00048 6.451 .002220 .00047 6.501 .006340 .00048 6.551 .011170 .00048 6.601 .015530 .00047 6.651 .019370 .00047 6.701 .023010 .00049
530

5.600 .921690 .00048

5.650 .927500 .00046

5.700 .932050 .00046

5.750 .936560 .00046

5.800 .942120 .00047

5.850 .946990 .00047

5.900 .950890 .00048

5.950 .956870 .00047

6.000 .961860 .00047

6.050 .966470 .00047

6.100 .971060 .00047

6.150 .975830 .00047

6.200 .979990 .00047

6.250 .986550 .00048

6.300 .989880 .00048

6.350 .994600 .00047

6.400 .998430 .00048

6.451 .003780 .00046

6.501 .007390 .00048

6.551 .012840 .00048

6.601 .017560 .00048

6.651 .021220 .00047

6.701 .025400 .00049

560

5.600 .919350 .00046 5.650 .923230 .00046 5.700 .929470 .00046 5.750 .933750 .00048 5.800 .938470 .00046 5.850 .944050 .00047 5.900 .948470 .00048 5.950 .954740 .00047 6.000 .958390 .00047 6.050 .963480 .00046 6.100 .967810 .00046 6.150 .973090 .00046 6.200 .978160 .00047 6.250 .982230 .00048 6.300 .986370 .00047 6.350 .990810 .00047 6.400 .996280 .00048 6.451 .000870 .00048 6.501 .004710 .00046 6.551 .009260 .00048 6.651 .019520 .00049 6.701 .022620 .00048
540

5.600 .921140 .00046

5.650 .925040 .00046

5.700 .930710 .00048

5.750 .936600 .00047

5.800 .940810 .00047

5.850 .945770 .00047

6.250 .985100 .00048

6.300 .989020 .00048

6.350 .993940 .00047

6.400 .997980 .00047

6.451 .002800 .00046

6.501 .005990 .00048

6.551 .012320 .00047

6.601 .015750 .00047

6.651 .020150 .00047

6.701 .024380 .00048 
U5o2N26-icyl-0935-00

12

63.800 .845490 .00014 64.700 .846430 .00014 65.600 .846640 .00014 66.500 .847410 .00014 67.400 .848160 .00014 68.300 .848430 .00014 69.200 .848960 .00014 70.100 .849500 .00014 71.000 .849990 .00013 71.900 .850510 .00013 72.800 .851270 .00013 74.600 .852190 .00013 75.500 .851890 .00013 76.400 .853120 .00013 77.300 .852830 .00013 78.200 .853810 .00013 79.100 .853350 .00013 80.000 .854070 .00013 80.900 .854270 .00013 81.800 .854690 .00013 82.700 .855080 .00013 83.400 .855670 .00012
15

26.500 .853630 .00022 27.600 .861690 .00022 28.700 .869670 .00021 29.800 .876340 .00021 30.900 .882680 .00021 33.100 .893450 .00020 34.200 .898760 .00019 35.300 .902960 .00019 36.400 .907360 .00019 37.500 .910830 .00019 38.600 .914670 .00019 39.700 .918110 .00018 40.800 .920720 .00018 41.900 .923640 .00018 43.000 .926080 .00018 44.100 .928720 .00017 45.200 .931470 .00018 46.300 .933550 .00017 47.400 .935440 .00017 48.500 .937480 .00017 49.600 .938680 .00017 50.100 .939900 .00017
25

17.000 .925250 .00033 17.200 .929840 .00033 17.400 .935320 .00032 17.600 .940410 .00031 17.800 .945590 .00031 18.200 .954930 .00031 18.400 .959100 .00031 18.600 .962680 .00033 18.800 .967670 .00031 19.000 .971860 .00030 19.200 .975810 .00030 19.400 .979990 .00031 19.600 .984260 .00030 19.800 .988160 .00030 20.200 .995590 .00030 20.601 .002410 .00030 21.001 .009680 .00030 21.201 .012280 .00029 21.301 .014250 .00029
30

14.800 .921640 .00035 14.900 .925190 .00036 15.000 .929540 .00035 15.100 .933020 .00035 15.200 .936160 .00035 15.300 .939460 .00035 15.400 .943360 .00035 15.500 .946570 .00035 15.600 .949500 .00035 15.700 .952370 .00036 15.800 .956090 .00035 15.900 .959040 .00034 16.000 .962380 .00036 16.100 .966040 .00035 16.200 .968870 .00035 16.300 .971150 .00034 16.400 .974720 .00035 16.500 .977540 .00034 16.600 .980100 .00034
20

21.600 .926190 .00028 22.000 .932000 .00027 22.400 .936630 .00028 22.800 .942900 .00026 23.200 .948120 .00027 23.600 .952220 .00026 24.000 .956960 .00026 24.400 .961590 .00026 24.800 .965650 .00026 25.200 .970500 .00026 25.600 .974350 .00025 26.000 .977620 .00024 26.400 .981420 .00025 26.800 .985050 .00025 27.200 .988660 .00025 27.600 .992180 .00024 28.000 .995500 .00024 28.400 .998450 .00024 28.801 .000900 .00024 29.201 .004400 .00023 29.601 .006880 .00023 30.001 .010020 .00024 30.401 .012610 .00023 30.601 .013760 .00023

32

14.200 .920690 .00037 14.300 .924880 .00036 14.400 .928840 .00037 14.500 .932580 .00036 14.600 .936230 .00036 14.700 .940130 .00036 14.900 .947980 .00036 15.000 .951180 .00036 15.100 .954900 .00036 15.200 .957830 .00035 15.300 .961990 .00036 15.400 .965850 .00037 15.500 .968390 .00036 15.600 .972520 .00035 15.700 .975140 .00036 15.800 .978730 .00035 15.900 .982200 .00036 16.000 .984740 .00036 16.100 .988450 .00034 
16.700 .983320 .00035 16.800 .985910 .00034 17.000 .991360 .00034 17.200 .997000 .00033 17.301 .000030 .00033 17.401 .003130 .00034 17.501 .005490 .00035 17.701 .010270 .00033 17.801 .012870 .00034 17.901 .015790 .00034

35

13.600 .923720 .00036 13.700 .928080 .00037 13.900 .936730 .00039 14.000 .940180 .00037 14.100 .944540 .00038 14.200 .949060 .00036 14.300 .952940 .00038 14.400 .956990 .00038 14.500 .960900 .00038 14.600 .965570 .00037 14.700 .969760 .00038 14.800 .973040 .00037 14.900 .976840 .00038 15.000 .980150 .00037 15.100 .984070 .00038 15.200 .988330 .00037 15.300 .991080 .00037 15.400 .994440 .00036 15.601 .000970 .00037 15.701 .005310 .00037 15.801 .008920 .00037 15.901 .011530 .00036 16.001 .015320 .00036

60 11.300 .939350 .00045 11.350 .943740 .00045 11.400 .946230 .00044 11.450 .949050 .00044 11.500 .952740 .00047 11.550 .956280 .00044 11.600 .958560 .00045 11.650 .962310 .00045 11.700 .966030 .00044 11.750 .968900 .00046
12.700 .920870 .00040 12.800 .926670 .00039 12.900 .930520 .00040 13.000 .935780 .00040 13.100 .940540 .00041 13.200 .946270 .00040 13.300 .950000 .00041 13.400 .955290 .00039 13.500 .959490 .00039 13.600 .963880 .00040 13.700 .968790 .00039 13.800 .973460 .00040 13.900 .977600 .00039 14.000 .981810 .00040 14.100 .986050 .00039 14.200 .991460 .00039 14.300 .994720 .00039 14.401 .000270 .00040 14.501 .003130 .00038 14.601 .007960 .00039 14.701 .010870 .00041 14.801 .014900 .00039
10.600 .921380 .00048 10.650 .924310 .00046 11.150 .960630 .00047 11.200 .963330 .00047 11.250 .967500 .00045 11.300 .971400 .00047 11.350 .974800 .00047 11.400 .978600 .00046 11.500 .984090 .00046 11.550 .988490 .00047
16.200 .991030 .00035 16.300 .994320 .00035 16.400 .998110 .00035 16.501 .000440 .00036 16.801 .009940 .00035 16.901 .013170 .00036 17.001 .015280 .00036
50

11.800 .929480 .00044 11.900 .935410 .00041 12.000 .941130 .00043 12.100 .946660 .00042 12.200 .952600 .00042 12.300 .958760 .00043 12.400 .965000 .00042 12.500 .969810 .00044 12.600 .975490 .00042 12.700 .980820 .00042 12.900 .991550 .00042 13.000 .997830 .00043 13.201 .007410 .00041 13.301 .011870 .00042 13.401 .016350 .00042
80

10.300 .919120 .00047 10.350 .923730 .00048 10.450 .931570 .00046 10.500 .936300 .00047 10.550 .939260 .00049 10.600 .943350 .00047 10.650 .947830 .00047 10.700 .951580 .00048 10.750 .954270 .00048 10.800 .958590 .00047 
11.800 .972780 .00045 11.850 .974630 .00044 11.900 .977710 .00045 11.950 .981020 .00045 12.000 .985000 .00044 12.050 .988060 .00045 12.100 .990660 .00044 12.200 .996270 .00045 12.250 .999650 .00043 12.301 .002620 .00045 12.351 .005610 .00045 12.401 .006890 .00046 12.451 .010740 .00045 12.501 .014200 .00044 12.551 .016870 .00044 12.601 .018790 .00044

90

10.100 .922710 .00048 10.150 .927180 .00048 10.200 .930430 .00050 10.250 .934260 .00047 10.300 .938270 .00048 10.350 .942230 .00048 10.400 .947110 .00048 10.450 .950580 .00049 10.500 .954910 .00049 10.550 .958590 .00048 10.600 .961510 .00050 10.650 .966680 .00048 10.700 .971030 .00050 10.750 .975700 .00050 10.800 .977890 .00048 10.850 .980990 .00049 10.900 .985360 .00049 10.950 .988960 .00049 11.000 .992450 .00048 11.050 .996410 .00048 11.101 .000890 .00048 11.151 .004120 .00049 11.201 .007010 .00047 11.251 .010330 .00050 11.301 .014130 .00049 11.351 .017260 .00048 11.401 .021860 .00049
11.600 .991120 .00046 11.650 .994090 .00046 11.700 .998430 .00046 11.751 .000290 .00046 11.801 .004010 .00046 11.851 .007730 .00045 12.001 .016370 .00045 12.101 .022870 .00047

100

9.900 .920310 .00049 9.950 .924150 .00049 10.000 .927810 .00049 10.100 .937480 .00049 10.200 .945280 .00049 10.250 .949630 .00050 10.400 .961060 .00050 10.450 .966210 .00049 10.500 .970810 .00049 10.550 .972950 .00049 10.600 .977410 .00048 10.650 .981450 .00049 10.700 .985290 .00049 10.750 .989350 .00049 10.800 .993460 .00050 10.850 .997120 .00050 10.901 .001180 .00048 10.951 .004620 .00049 11.001 .008130 .00050 11.051 .012730 .00050 11.101 .016180 .00049 11.151 .018660 .00049 11.201 .022580 .00051
10.850 .961660 .00047 10.900 .965370 .00047 10.950 .970840 .00047 11.000 .973880 .00048 11.050 .976500 .00047 11.100 .980580 .00048 11.150 .984260 .00048 11.200 .988180 .00048 11.250 .991520 .00046 11.300 .995570 .00048 11.350 .998840 .00048 11.451 .005240 .00047 11.501 .009230 .00047 11.551 .012870 .00047 11.601 .015690 .00047 11.701 .021680 .00048

$$
118
$$

9.700 .922900 .00050 9.750 .926610 .00049 9.800 .930960 .00051 9.850 .934890 .00051 9.900 .940850 .00050 9.950 .943770 .00050 10.000 .948230 .00051 10.050 .953270 .00050 10.150 .961960 .00051 10.200 .965820 .00048 10.250 .969950 .00049 10.300 .974600 .00049 10.350 .977900 .00050 10.400 .981860 .00050 10.450 .986140 .00049 10.500 .990210 .00051 10.550 .994270 .00050 10.600 .999080 .00050 10.651 .002660 .00051 10.701 .007090 .00051 10.751 .009720 .00050 10.801 .013910 .00048 10.851 .017170 .00050 
130

9.500 .914860 .00051

9.550 .918750 .00049

9.650 .927960 .00051

9.700 .932760 .00051

9.750 .937540 .00052

9.800 .942000 .00052

9.850 .945920 .00052

9.900 .950520 .00051

9.950 .955730 .00050

10.000 .959840 .00050

10.050 .963590 .00052

10.100 .967860 .00049

10.150 .972430 .00051

10.200 .976370 .00051

10.250 .980730 .00051

10.300 .985750 .00051

10.350 .988030 .00052

10.400 .993260 .00051

10.450 .997140 .00051

10.501 .001440 .00051

10.551 .006030 .00051

10.601 .009790 .00052

10.651 .012520 .00051

10.701 .016720 .00052

10.751 .021390 .00050

10.801 .024540 .00050
150

9.400 .918950 .00052

9.450 .922160 .00051

9.550 .931190 .00052

9.600 .935210 .00051

9.650 .940490 .00051

9.700 .945200 .00052

9.750 .950000 .00052

9.800 .953660 .00052

9.850 .958430 .00051

9.900 .962000 .00052

10.050 .977110 .00050

10.100 .980920 .00051

10.150 .985070 .00051

10.200 .989370 .00051

10.250 .993680 .00051

10.300 .998110 .00052

10.351 .000390 .00051

10.451 .009860 .00051

10.551 .017480 .00051
200

9.250 .918570 .00053

9.300 .923620 .00053

9.400 .933410 .00051

9.450 .937820 .00052

9.550 .947760 .00054

9.600 .951730 .00053

9.650 .957820 .00052

9.700 .961450 .00053

9.750 .966450 .00052

9.800 .970770 .00052

9.900 .979060 .00052

9.950 .983350 .00052

10.000 .987940 .00053

10.050 .991780 .00052

10.100 .996980 .00053

10.201 .005460 .00054

10.251 .010420 .00054

10.301 .013790 .00053

10.351 .018690 .00052

10.401 .023710 .00053
235

9.250 .923450 .00051

9.300 .927800 .00053

9.350 .932400 .00052

9.400 .937600 .00053

9.450 .941530 .00053

9.500 .946430 .00052

9.600 .956840 .00054

9.650 .960840 .00053

9.700 .965620 .00052

9.750 .970550 .00052

9.800 .974420 .00054

9.850 .979850 .00054

9.900 .984200 .00054

9.950 .988590 .00053

10.000 .992070 .00051

10.050 .996650 .00054

10.101 .001560 .00055

10.251 .014050 .00053

10.301 .018200 .00055

10.401 .026620 .00054

\begin{abstract}
250
9.200 .917900 .00053

9.300 .927650 .00052

9.400 .938140 .00053

9.450 .942330 .00053

9.500 .947760 .00053

9.550 .952980 .00051

9.600 .956760 .00052

9.650 .961450 .00054

9.750 .970090 .00052

9.850 .979550 .00053

9.950 .988540 .00053

10.000 .993510 .00052

10.050 .997660 .00054

10.101 .000940 .00053

10.151 .006510 .00053

10.201 .010950 .00053

10.251 .014400 .00053

10.301 .019440 .00053

10.351 .023740 .00052

10.401 .027870 .00054
\end{abstract}

353

9.300 .921250 .00054

9.350 .926040 .00053

9.400 .930850 .00053

9.450 .934630 .00052

9.500 .940660 .00052

9.550 .944440 .00053

9.600 .948720 .00053

9.650 .954030 .00052

9.700 .957680 .00052

9.800 .967140 .00053

9.850 .972100 .00053

9.950 .980730 .00053

10.050 .990210 .00053

10.100 .993310 .00052

10.150 .998850 .00053

10.201 .002420 .00054

10.251 .006530 .00054

10.301 .011190 .00053

10.351 .015390 .00054

10.401 .020210 .00052 
10.451 .023200 .00054

10.501 .027960 .00053

470

9.550 .919980 .00053 9.600 .925910 .00052

9.650 .930450 .00053

9.700 .934070 .00051

9.750 .939510 .00051

9.800 .943480 .00054

9.850 .947220 .00054

9.900 .951570 .00052

9.950 .956780 .00053

10.050 .965430 .00053

10.100 .969280 .00051

10.250 .981650 .00052

10.300 .986800 .00054

10.350 .990750 .00053

10.450 .999100 .00053

10.501 .003860 .00054

10.551 .006510 .00053

10.651 .015220 .00053

10.701 .018540 .00054

10.751 .023850 .00052

10.801 .027940 .00053

486

9.600 .920120 .00051

9.650 .924010 .00052

9.800 .938990 .00053

10.000 .955690 .00053

10.300 .981570 .00053

10.350 .985860 .00052

10.500 .998370 .00052

10.551 .001370 .00053

10.601 .005910 .00053

10.651 .010480 .00053

10.701 .015220 .00054

10.751 .018420 .00052

10.801 .022070 .00054
475

9.500 .914530 .00053

9.550 .919760 .00052

9.600 .924420 .00052

9.650 .928760 .00052

9.700 .932840 .00053

9.750 .937270 .00051

9.800 .942480 .00053

9.850 .946870 .00053

9.900 .950700 .00052

9.950 .955120 .00053

10.050 .963300 .00054

10.150 .972150 .00053

10.250 .981180 .00053

10.300 .985060 .00053

10.350 .989060 .00054

10.400 .993640 .00053

10.450 .996890 .00053

10.551 .006190 .00052

10.601 .009180 .00051

10.701 .017150 .00053

10.751 .021780 .00053

10.801 .025570 .00052
480

9.500 .913490 .00052

9.600 .920930 .00052

9.650 .927220 .00052

9.700 .931640 .00052

9.750 .936140 .00050

9.800 .940570 .00052

9.850 .945390 .00052

9.900 .949170 .00053

10.000 .957700 .00052

10.050 .962580 .00053

10.100 .966680 .00052

10.200 .976440 .00051

10.250 .979310 .00052

10.300 .983840 .00052

10.400 .992370 .00051

10.450 .996130 .00053

10.500 .999950 .00052

10.551 .004480 .00054

10.651 .012030 .00053

10.701 .015790 .00052

\section{0}

(n)

.


U5o2N26-icyl-0935-01

12

68.800 .849790 .00014 69.900 .850450 .00013 71.000 .850900 .00013 72.100 .851600 .00013 73.200 .851980 .00013 74.300 .852350 .00014 75.400 .852780 .00013 76.500 .853440 .00013 77.600 .853880 .00013 79.800 .854260 .00013 80.900 .854830 .00013 82.000 .855430 .00013 83.100 .855430 .00013 84.200 .856140 .00012 85.300 .856170 .00012 86.400 .856800 .00013 88.600 .857160 .00012 89.700 .857620 .00013 90.800 .857790 .00013 91.900 .858270 .00012 92.300 .857930 .00013

25

15.400 .925040 .00031 15.800 .935030 .00030 16.000 .940910 .00030 16.200 .944850 .00031 16.600 .954250 .00030 16.800 .958830 .00031 17.000 .963850 .00030 17.200 .967800 .00030 17.400 .972420 .00029 17.600 .976120 .00029 17.800 .980490 .00030 18.000 .983310 .00030 18.200 .988230 .00030 18.600 .995030 .00029 19.001 .002290 .00029 19.201 .005920 .00029 19.401 .009470 .00029 19.601 .012760 .00029 19.701 .014840 .00028
15

50.400 .942980 .00016 50.500 .943290 .00016 50.550 .942450 .00017 50.600 .942740 .00016 50.650 .943080 .00016 50.700 .943310 .00016 50.750 .943070 .00017 50.800 .943460 .00016 50.850 .943140 .00016 50.950 .943490 .00016 51.000 .943210 .00016 51.050 .943480 .00016 51.100 .943900 .00016 51.150 .943590 .00017 51.200 .943880 .00016

\section{0}

13.200 .922400 .00034 13.300 .926240 .00035 13.400 .929190 .00034 13.500 .932910 .00033 13.600 .936210 .00035 13.700 .939930 .00034 13.800 .942740 .00033 13.900 .946000 .00033 14.000 .949840 .00034 14.100 .953300 .00034 14.200 .956010 .00036 14.300 .959750 .00033 14.400 .962050 .00033 14.500 .965950 .00033 14.600 .968790 .00034 14.700 .971630 .00034 14.800 .974570 .00034 14.900 .978230 .00033 15.100 .983940 .00032 15.300 .989250 .00033 15.400 .992350 .00033 15.500 .995060 .00034 15.600 .997710 .00033
20

20.100 .928190 .00026 20.500 .933530 .00026 20.900 .938910 .00026 21.300 .943530 .00026 22.100 .953560 .00026 22.500 .957860 .00026 22.900 .962990 .00026 23.300 .966910 .00025 23.700 .971450 .00024 24.100 .975330 .00024 24.500 .978810 .00025 24.900 .983010 .00025 25.300 .986100 .00024 25.700 .989470 .00024 26.100 .993010 .00024 26.500 .995950 .00023 26.900 .998860 .00023 27.701 .005170 .00024 28.101 .008230 .00023 28.501 .010840 .00022 28.701 .011890 .00023

32

12.700 .924740 .00035 12.800 .928950 .00036 12.900 .932060 .00036 13.000 .936060 .00035 13.100 .940860 .00035 13.200 .944050 .00037 13.300 .947860 .00035 13.400 .951560 .00034 13.500 .955150 .00036 13.600 .958780 .00035 13.700 .962610 .00034 13.800 .965870 .00035 13.900 .969280 .00035 14.000 .972360 .00035 14.100 .976250 .00034 14.200 .979490 .00034 14.300 .981670 .00034 14.500 .988140 .00034 14.600 .991840 .00034 14.700 .994510 .00035 14.800 .998510 .00034 14.901 .000510 .00034 15.001 .003970 .00034 
15.701 .000270 .00035 15.901 .005400 .00033 16.001 .008260 .00033 16.101 .010500 .00033 16.201 .013380 .00033 16.301 .015930 .00032

35

12.000 .924600 .00036

12.100 .928990 .00037

12.200 .933100 .00037

12.300 .936790 .00036

12.400 .941260 .00037

12.500 .944860 .00037

12.600 .949300 .00037

12.700 .953660 .00036

12.800 .957190 .00034

12.900 .961960 .00036

13.000 .966230 .00035

13.200 .973220 .00037

13.300 .976490 .00036

13.400 .980560 .00037

13.500 .984270 .00036

13.600 .988000 .00037

13.700 .991160 .00036

13.800 .994310 .00037

13.900 .998280 .00036

14.001 .002150 .00036

14.101 .005190 .00037

14.201 .008830 .00036

14.301 .011710 .00036

14.401 .015370 .00035
40

11.100 .921310 .00038

11.200 .926800 .00038

11.300 .931250 .00038

11.400 .936970 .00038

11.500 .941260 .00038

11.600 .946270 .00038

11.700 .951860 .00039

11.800 .956100 .00040

11.900 .959890 .00038

12.000 .965240 .00038

12.200 .973840 .00037

12.300 .978280 .00038

12.400 .983320 .00038

12.600 .992070 .00038

12.700 .995580 .00038

12.800 .999560 .00037

12.901 .003590 .00037

13.001 .008390 .00039

13.101 .011590 .00038

13.201 .016010 .00037
15.101 .006750 .00034

15.301 .012730 .00034

15.401 .015800 .00034

15.501 .018160 .00034
50

10.000 .917520 .00041

10.100 .924170 .00040

10.200 .930360 .00042

10.400 .941680 .00041

10.600 .954080 .00041

10.700 .959180 .00042

10.800 .964770 .00041

11.601 .007310 .00041

11.701 .013460 .00039

11.801 .018070 .00041
60

9.400 .919950 .00043

9.500 .926740 .00043

9.550 .930290 .00043

9.600 .933710 .00044

9.650 .936870 .00042

9.700 .941020 .00044

9.750 .943940 .00043

9.800 .947170 .00043

9.850 .950360 .00042

9.900 .953580 .00043

9.950 .957060 .00044

10.000 .960060 .00043

10.050 .963240 .00043
70

9.050 .925150 .00045

9.150 .932880 .00044

9.200 .937270 .00045

9.300 .944740 .00045

9.400 .951180 .00046

9.450 .954840 .00044

9.500 .959210 .00044

9.550 .961940 .00044

9.650 .969040 .00044

9.750 .975480 .00045

9.800 .979200 .00044

9.850 .982310 .00043

9.950 .989410 .00044

\section{0}

8.700 .922670 .00047

8.750 .925960 .00046

8.800 .930640 .00046

8.850 .934690 .00045

8.900 .937730 .00047

8.950 .942190 .00044

9.000 .945460 .00046

9.050 .949450 .00046

9.100 .953390 .00046

9.150 .956810 .00046

9.200 .960020 .00045

9.250 .963880 .00045

9.300 .968280 .00045 
10.100 .966930 .00043 10.200 .973110 .00042 10.250 .974950 .00044 10.300 .978550 .00043 10.350 .982570 .00043 10.400 .985020 .00044 10.450 .988150 .00043 10.951 .017650 .00043 11.001 .020180 .00043
10.000 .991790 .00045 10.050 .995630 .00044 10.100 .997970 .00044 10.151 .001610 .00044 10.201 .005860 .00045 10.251 .008760 .00042 10.301 .012240 .00044 10.351 .015240 .00045

9.400 .975760 .00045 9.450 .978620 .00045 9.500 .982930 .00046 9.550 .986280 .00046 9.600 .989820 .00046 9.650 .994280 .00046 9.700 .996060 .00046 9.751 .000970 .00045 9.801 .003330 .00047 9.851 .007370 .00045 9.901 .010630 .00045 9.951 .014040 .00046 10.001 .016940 .00046 10.051 .020700 .00045 10.101 .024070 .00046

\section{8}

8.300 .922060 .00048

8.350 .927530 .00048

8.400 .931310 .00046

8.450 .935910 .00048

8.500 .939330 .00047

8.550 .944220 .00047

8.600 .948230 .00047

8.650 .953000 .00047

8.700 .956280 .00047

8.750 .960230 .00048

8.800 .965220 .00048

8.850 .967930 .00046

8.900 .971390 .00048

8.950 .976720 .00047

9.000 .980230 .00047

9.050 .984540 .00048

9.100 .988270 .00048

9.150 .991540 .00046

9.200 .996260 .00048

9.250 .999170 .00048

9.301 .003350 .00047

9.351 .006880 .00046

9.401 .010710 .00047

9.451 .014110 .00047

9.501 .017900 .00046
8.100 .925160 .00047

8.200 .935060 .00047

8.250 .938400 .00048

8.350 .947190 .00049

8.400 .951900 .00047

8.450 .956410 .00047

8.500 .959740 .00049

8.550 .964220 .00048

8.600 .969210 .00047

8.650 .972480 .00048

8.700 .977160 .00049

8.750 .981060 .00047

8.800 .985750 .00048

8.850 .989220 .00048

8.900 .993640 .00047

8.950 .997470 .00050

9.001 .001980 .00048

9.051 .005410 .00049

9.101 .008950 .00047

9.151 .013310 .00047

9.201 .016480 .00047

9.251 .020720 .00047

9.301 .024740 .00047
8.000 .916220 .00048 
130

7.900 .917470 .00048

7.950 .923580 .00047

8.000 .926610 .00048

8.050 .931180 .00049

8.100 .935680 .00047

8.150 .940660 .00049

8.200 .945030 .00049

8.250 .949820 .00048

8.300 .953790 .00049

8.350 .957290 .00050

8.400 .962120 .00048

8.450 .966620 .00049

8.500 .972400 .00048

8.550 .975610 .00050

8.600 .979780 .00048

8.700 .988380 .00048

8.750 .992080 .00050

8.800 .996450 .00049

8.851 .000730 .00048

8.901 .003800 .00049

8.951 .008230 .00049

9.001 .012530 .00048

9.051 .015940 .00046

9.101 .019610 .00048

235

7.500 .914590 .00050

7.550 .920200 .00051

7.600 .924060 .00051

7.650 .929470 .00051

7.700 .935030 .00050

7.800 .943830 .00050

7.850 .947610 .00050

7.900 .952550 .00050

7.950 .957850 .00050

8.050 .967940 .00050

8.100 .972130 .00053

8.150 .976180 .00052

8.200 .980080 .00052

8.250 .985800 .00051

8.350 .993360 .00051

8.400 .998640 .00052

8.551 .011910 .00051

8.601 .015940 .00053

8.651 .020200 .00051

8.701 .024480 .00050
150

7.850 .926240 .00048

7.900 .930690 .00048

7.950 .934820 .00049

8.000 .939750 .00050

8.100 .949780 .00049

8.150 .953660 .00049

8.200 .957860 .00049

8.250 .962010 .00049

8.350 .971740 .00049

8.400 .976080 .00050

8.450 .980630 .00050

8.500 .985150 .00050

8.550 .988070 .00051

8.600 .992360 .00049

8.650 .997740 .00050

8.701 .001590 .00050

8.751 .005470 .00049

8.801 .010460 .00049

8.901 .018080 .00051
200

7.600 .919380 .00051

7.650 .923080 .00051

7.700 .929350 .00050

7.750 .933100 .00050

7.800 .938620 .00050

7.900 .947020 .00051

7.950 .953070 .00051

8.000 .955920 .00049

8.050 .961310 .00048

8.100 .966790 .00051

8.150 .971000 .00050

8.200 .974870 .00051

8.250 .979900 .00051

8.300 .984010 .00049

8.350 .989270 .00051

8.400 .992880 .00050

8.450 .997540 .00050

8.501 .002530 .00050

8.551 .006770 .00050

8.601 .010970 .00050

8.651 .014340 .00050

8.701 .018660 .00051
7.500 .915720 .00051

7.550 .921560 .00051

7.600 .926800 .00051

7.650 .931000 .00052

7.700 .936240 .00051

7.750 .940140 .00050

7.800 .945490 .00051

7.850 .949640 .00051

7.900 .954650 .00051

7.950 .959020 .00051

8.000 .963580 .00052

8.050 .967830 .00051

8.100 .972100 .00052

8.150 .977220 .00052

8.250 .985870 .00050

8.300 .989660 .00050

8.451 .004240 .00050

8.501 .007290 .00051

8.551 .012500 .00052

8.601 .017140 .00053

8.701 .025540 .00050
353

7.600 .921590 .00051

7.650 .925670 .00050

7.700 .930280 .00053

7.750 .935540 .00052

7.800 .941250 .00050

7.850 .944660 .00051

7.950 .953950 .00051

8.000 .958630 .00050

8.050 .963140 .00053

8.100 .968340 .00051

8.150 .971760 .00050

8.200 .977480 .00050

8.300 .985050 .00052

8.350 .989190 .00052

8.450 .998700 .00052

8.501 .002180 .00051

8.551 .007210 .00051

8.601 .010620 .00052

8.701 .019810 .00051 
470

7.800 .920940 .00051

7.850 .925090 .00049

7.950 .933550 .00051

8.000 .939400 .00050

8.050 .943090 .00051

8.100 .947010 .00050

8.150 .951920 .00052

8.200 .955280 .00051

8.250 .960250 .00051

8.300 .964380 .00050

8.400 .974060 .00051

8.450 .977360 .00051

8.500 .981750 .00052

8.550 .986150 .00051

8.600 .990250 .00050

8.700 .998170 .00051

8.801 .005740 .00051

8.901 .014450 .00052

8.951 .018630 .00052

9.001 .022570 .00050

486

7.950 .929830 .00051

8.000 .934180 .00051

8.050 .938980 .00050

8.100 .942750 .00052

8.150 .947580 .00049

8.200 .952320 .00051

8.250 .957170 .00052

8.300 .960480 .00051

8.350 .964550 .00050

8.400 .969010 .00051

8.450 .973630 .00052

8.550 .981390 .00051

8.600 .985380 .00051

8.650 .989580 .00050

8.700 .993910 .00050

8.750 .998170 .00051

8.801 .002080 .00051

8.851 .005680 .00050

8.901 .009990 .00051

8.951 .014430 .00051

9.001 .018310 .00052

9.051 .021810 .00051

9.101 .024990 .00050
475

7.800 .918980 .00051

7.900 .927680 .00050

7.950 .932800 .00050

8.000 .936510 .00051

8.050 .941920 .00051

8.100 .946670 .00051

8.150 .949970 .00051

8.200 .954610 .00051

8.250 .958770 .00051

8.350 .968410 .00052

8.400 .972170 .00051

8.450 .977050 .00051

8.500 .981130 .00053

8.550 .984210 .00052

8.600 .989030 .00051

8.650 .992550 .00051

8.751 .000430 .00051

8.801 .005400 .00052

8.851 .009550 .00051

8.901 .012560 .00050

8.951 .017300 .00051

9.001 .020880 .00050
480

7.800 .917590 .00052

7.900 .926890 .00052

7.950 .931760 .00050

8.000 .936070 .00050

8.050 .940630 .00050

8.100 .944280 .00051

8.150 .948830 .00050

8.200 .954500 .00049

8.300 .962660 .00051

8.350 .966390 .00052

8.700 .996160 .00051

9.001 .019960 .00051

\section{0}


U5o2N26-icyl-0935-10

12

73.700 .852450 .00013 75.000 .853050 .00013 76.300 .853970 .00013 77.600 .854010 .00013 78.900 .854580 .00013 80.200 .854740 .00013 81.500 .855760 .00012 82.800 .855860 .00012 84.100 .855800 .00013 86.700 .857280 .00012 88.000 .857690 .00012 89.300 .857760 .00012 90.600 .858170 .00012 91.900 .858170 .00012 93.200 .858480 .00012 94.500 .858630 .00012 97.100 .859580 .00012 98.400 .859690 .00012 99.700 .859840 .00012 101.000 .860160 .00012
15

2.610 .155890 .00014 7.810 .511600 .00024 10.410 .620600 .00024 13.010 .697810 .00024 15.610 .753750 .00023 18.210 .795170 .00022 20.810 .826690 .00022 23.410 .851180 .00021 26.010 .870090 .00020 28.610 .885810 .00019 31.210 .897730 .00020 33.810 .908420 .00018 36.410 .915990 .00018 39.010 .923520 .00017 41.610 .929850 .00017 44.210 .934770 .00016 46.810 .939240 .00016 49.410 .943200 .00016 52.010 .946060 .00016 52.600 .946650 .00016
25

14.200 .925210 .00029 14.600 .936030 .00029 14.800 .941000 .00030 15.000 .945880 .00028 15.200 .949820 .00029 15.400 .955120 .00028 15.600 .959900 .00029 15.800 .963810 .00028 16.000 .968480 .00028 16.400 .977070 .00028 16.800 .985000 .00028 17.000 .988490 .00028 17.200 .992510 .00028 17.400 .995800 .00028 17.601 .000070 .00029 17.801 .003030 .00028 18.001 .006290 .00028 18.201 .009470 .00028 18.401 .013390 .00027 18.501 .015200 .00027
30

12.000 .923280 .00032

12.100 .926540 .00032

12.200 .930380 .00032

12.300 .934130 .00032

12.400 .937670 .00032

12.500 .940920 .00032

12.600 .943510 .00032

12.700 .947840 .00032

12.800 .950220 .00031

12.900 .953200 .00031

13.000 .956870 .00031

13.100 .960420 .00032

13.200 .963520 .00031

13.300 .966960 .00032

13.500 .972320 .00032

13.700 .978690 .00031

13.800 .981470 .00031

13.900 .984050 .00031

14.000 .987490 .00031

14.100 .990760 .00033

14.200 .992850 .00032
20

18.800 .926230 .00026 19.200 .932260 .00025 19.600 .937200 .00025 20.000 .942640 .00025 20.400 .947820 .00025 20.800 .952220 .00025 21.200 .957350 .00024 21.600 .961750 .00024 22.400 .970500 .00024 22.800 .973990 .00023 23.200 .978580 .00024 23.600 .981820 .00023 24.000 .985000 .00023 24.400 .989300 .00023 24.800 .992050 .00023 25.200 .995380 .00022 25.600 .998920 .00022 26.001 .001990 .00023 26.401 .004630 .00022 26.801 .006910 .00022 27.201 .010120 .00022

\section{2}

11.400 .921950 .00032 11.500 .926080 .00033 11.600 .930090 .00032 11.700 .933310 .00033 11.800 .938230 .00033 11.900 .941740 .00033 12.000 .945730 .00032 12.100 .948790 .00033 12.200 .952190 .00033 12.300 .956290 .00032 12.400 .960320 .00033 12.500 .964030 .00032 12.600 .966680 .00031 12.700 .970050 .00032 12.800 .973480 .00032 13.100 .983390 .00032 13.200 .986420 .00032 13.300 .990460 .00033 13.400 .992450 .00032 13.500 .995840 .00033 13.701 .002040 .00033 
14.300 .995660 .00031 14.400 .998840 .00032 14.501 .000600 .00032 14.601 .004220 .00031 14.701 .006610 .00032 14.801 .009170 .00031 14.901 .012100 .00031 15.101 .017090 .00031
13.801 .005160 .00032 13.901 .008440 .00032 14.001 .011320 .00032 14.101 .013340 .00032 14.201 .016860 .00032
35

10.700 .921260 .00034 10.800 .925970 .00035 10.900 .930780 .00033 11.000 .934370 .00034 11.100 .938970 .00034 11.200 .943270 .00034 11.300 .947180 .00033 11.400 .951200 .00033 11.500 .955050 .00035 11.700 .964570 .00034 11.800 .967500 .00033 11.900 .971440 .00035 12.000 .975630 .00034 12.100 .978870 .00034 12.300 .985840 .00033 12.400 .989570 .00034 12.500 .993270 .00034 12.600 .996890 .00033 12.701 .001130 .00033 12.801 .003850 .00033 12.901 .007690 .00032 13.001 .010480 .00033 13.101 .014450 .00034 13.201 .017170 .00033
40

9.900 .923100 .00035 10.000 .928380 .00036 10.100 .933900 .00036 10.200 .938460 .00035 10.300 .943810 .00036 10.400 .948590 .00035 10.500 .953670 .00035 10.600 .958200 .00035 10.700 .962880 .00036 10.900 .971970 .00037 11.000 .976800 .00035 11.100 .981570 .00035 11.200 .983920 .00035 11.300 .990030 .00036 11.400 .994150 .00035 11.500 .997810 .00035 11.601 .002280 .00036 11.701 .006440 .00035 11.801 .010330 .00035 12.001 .018390 .00034
60

8.500 .946610 .00041 8.550 .949510 .00040 8.600 .953800 .00040 8.650 .956680 .00040 8.750 .963610 .00039 8.800 .966530 .00040 8.900 .972880 .00040 8.950 .976500 .00041 9.050 .982030 .00040 9.100 .985630 .00040
70

7.700 .921650 .00041

7.750 .926460 .00041

7.800 .930300 .00041

7.850 .933240 .00042

7.900 .937340 .00041

7.950 .940680 .00042

8.000 .944850 .00040

8.050 .948170 .00041

8.100 .952570 .00041

8.150 .955610 .00041
50

8.850 .924950 .00039 8.900 .928400 .00038 8.950 .930690 .00038 9.350 .955850 .00038 9.400 .958160 .00038 9.450 .960240 .00038 9.500 .964230 .00039 9.550 .967040 .00040 9.600 .969860 .00039 9.650 .972840 .00038 9.700 .974560 .00037 9.750 .977560 .00039 9.800 .980850 .00038 9.850 .982890 .00038 9.950 .988130 .00038 10.000 .990620 .00037 10.050 .993850 .00039 10.100 .996530 .00037 10.150 .999800 .00038 10.201 .002340 .00038 10.251 .004880 .00037 10.301 .007700 .00038 10.351 .008920 .00039 10.401 .012270 .00037 10.451 .014960 .00038

80

7.400 .923310 .00043 7.450 .927040 .00041 7.500 .932380 .00042 7.550 .936080 .00041 7.600 .939860 .00041 7.650 .943780 .00043 7.700 .947990 .00042 7.750 .951670 .00043 7.800 .955190 .00042 7.850 .959170 .00043 
9.150 .988510 .00041

9.200 .991590 .00040

9.300 .997560 .00039

9.401 .003410 .00040

9.451 .006980 .00039

9.501 .009530 .00039

9.601 .015000 .00039

9.651 .018320 .00040
8.200 .959290 .00041

8.250 .962530 .00041

8.300 .966380 .00041

8.350 .969550 .00041

8.400 .972940 .00040

8.450 .977230 .00042

8.550 .983920 .00042

8.600 .986900 .00041

8.650 .990990 .00041

8.700 .993830 .00042

8.801 .000570 .00042

8.851 .003770 .00041

8.901 .007400 .00039

8.951 .010640 .00042

9.001 .012740 .00040

9.051 .016700 .00042

9.101 .019750 .00042
7.900 .962590 .00042

7.950 .966500 .00042

8.000 .970460 .00043

8.050 .974810 .00043

8.100 .977490 .00043

8.150 .981490 .00042

8.200 .984670 .00041

8.250 .988950 .00043

8.300 .992330 .00042

8.350 .996450 .00043

8.400 .999630 .00042

8.451 .002540 .00042

8.501 .006350 .00043

8.651 .017840 .00041

8.701 .020940 .00042
90

7.250 .931720 .00042

7.300 .935990 .00042

7.350 .939950 .00043

7.400 .944720 .00043

7.450 .947830 .00044

7.500 .951750 .00042

7.550 .956140 .00042

7.600 .960550 .00044

7.650 .963940 .00042

7.700 .968180 .00043

7.750 .972050 .00043

7.800 .975870 .00042

7.850 .980610 .00043

7.900 .983660 .00042

8.000 .991560 .00043

8.050 .995220 .00044

8.100 .998770 .00043

8.151 .002380 .00043

8.201 .005470 .00042

8.251 .009220 .00043

8.301 .012950 .00042

8.351 .017550 .00042

8.401 .020600 .00042
100

6.950 .922900 .00043

7.000 .927880 .00043

7.050 .931690 .00041

7.100 .936110 .00042

7.150 .940660 .00044

7.200 .943800 .00043

7.250 .948600 .00044

7.300 .953680 .00044

7.350 .957630 .00043

7.400 .960930 .00043

7.450 .965290 .00043

7.500 .969330 .00042

7.600 .976360 .00044

7.650 .982690 .00044

7.750 .989480 .00043

7.800 .993550 .00043

7.850 .996990 .00044

7.901 .001750 .00042

7.951 .004070 .00042

8.001 .007890 .00043

8.051 .011920 .00045

8.101 .015450 .00045

8.151 .019850 .00044

8.201 .023040 .00043
118

6.700 .923030 .00044

6.750 .928480 .00045

6.800 .932250 .00044

6.850 .936860 .00044

6.900 .941670 .00045

6.950 .945910 .00044

7.000 .951060 .00044

7.050 .955190 .00044

7.100 .959350 .00044

7.150 .963020 .00043

7.200 .968320 .00043

7.250 .971940 .00045

7.300 .976040 .00044

7.350 .980800 .00045

7.400 .984680 .00043

7.450 .989220 .00044

7.500 .992640 .00044

7.550 .996830 .00045

7.601 .001060 .00044

7.651 .004980 .00044

7.701 .009280 .00045

7.801 .017900 .00044 
130

6.500 .916840 .00044

6.550 .921290 .00044

6.600 .926150 .00044

6.650 .930160 .00044

6.700 .935500 .00044

6.750 .940080 .00045

6.800 .945380 .00045

6.900 .953980 .00044

6.950 .958310 .00046

7.000 .961930 .00046

7.150 .975270 .00044

7.200 .979350 .00045

7.250 .985170 .00045

7.300 .987930 .00044

7.350 .992990 .00046

7.451 .000970 .00046

7.501 .005360 .00045

7.551 .009390 .00044

7.601 .013210 .00044

7.651 .017360 .00045

7.701 .021580 .00044
150

6.450 .927650 .00045

6.500 .932160 .00045

6.550 .937390 .00045

6.600 .942490 .00047

6.700 .951110 .00045

6.750 .955240 .00046

6.800 .960190 .00045

6.850 .964990 .00045

6.900 .969410 .00045

6.950 .973910 .00045

7.000 .978140 .00046

7.100 .986250 .00045

7.150 .989910 .00045

7.200 .995690 .00046

7.250 .999490 .00046

7.301 .004230 .00045

7.351 .008370 .00045

7.451 .016150 .00044

7.501 .020260 .00046
200

6.100 .916490 .00046

6.150 .921630 .00046

6.200 .926560 .00045

6.250 .931190 .00045

6.300 .935620 .00047

6.350 .941260 .00047

6.400 .946160 .00046

6.450 .951180 .00046

6.500 .956490 .00046

6.550 .961620 .00044

6.600 .965110 .00047

6.650 .970430 .00047

6.700 .973490 .00047

6.800 .983200 .00048

6.850 .987680 .00047

6.900 .991980 .00047

7.051 .005520 .00045

7.101 .010480 .00046

7.151 .013750 .00047

7.201 .019050 .00046
235

6.000 .915720 .00046

6.100 .924850 .00046

6.150 .930350 .00045

6.200 .935350 .00048

6.300 .946810 .00045

6.350 .950020 .00046

6.450 .960070 .00047

6.500 .964640 .00046

6.550 .968720 .00047

6.600 .973480 .00046

6.650 .977740 .00047

6.700 .983790 .00046

6.750 .987360 .00047

6.800 .992450 .00046

6.850 .996870 .00047

6.901 .000840 .00046

6.951 .005340 .00047

7.001 .010010 .00047

7.051 .014070 .00046

7.101 .017980 .00046
250
353
6.000 .917850 .00046
6.050 .922520 .00045
6.100 .927540 .00045
6.250 .943040 .00045
6.300 .947460 .00047
6.350 .952390 .00047
6.400 .957120 .00046
6.450 .961900 .00045
6.500 .967100 .00046
6.600 .976110 .00047
6.650 .979770 .00049
6.700 .985110 .00046
6.750 .989980 .00046
6.800 .994030 .00047
6.850 .998520 .00046
6.901 .003360 .00046
7.001 .011590 .00047
7.051 .016440 .00047
7.101 .020850 .00047 
470

6.000 .915680 .00046 6.050 .920620 .00046 6.100 .925250 .00047 6.150 .929990 .00046 6.200 .933430 .00046 6.250 .937710 .00047 6.300 .942810 .00048 6.350 .947840 .00047 6.500 .961090 .00048 6.600 .968940 .00047 6.650 .973300 .00047 6.700 .978660 .00047 6.750 .982260 .00047 6.850 .991340 .00046 6.900 .996160 .00047 7.001 .003870 .00047 7.051 .007860 .00047 7.101 .012050 .00048 7.151 .016280 .00046

486

6.100 .922130 .00046 6.150 .927050 .00046 6.250 .935980 .00047 6.300 .940500 .00045 6.350 .944450 .00046 6.400 .949840 .00046 6.450 .953860 .00047 6.550 .962940 .00047 6.600 .967370 .00046 6.650 .970560 .00045 6.700 .975920 .00047 6.750 .980580 .00048 6.800 .984810 .00047 6.850 .988830 .00048 6.900 .992400 .00047 6.950 .996860 .00045 7.001 .001180 .00046 7.051 .004420 .00047 7.101 .009840 .00047 7.201 .016090 .00047
475

6.100 .924140 .00047

6.150 .929280 .00047

6.200 .933130 .00046

6.250 .937080 .00047

6.300 .942390 .00045

6.350 .947280 .00047

6.400 .951380 .00047

6.500 .959600 .00048

6.550 .963920 .00045

6.600 .968870 .00047

6.700 .977230 .00048

6.800 .986130 .00047

6.900 .993590 .00047

6.950 .998980 .00047

7.001 .003020 .00047

7.051 .006610 .00047

7.101 .011540 .00047

7.201 .018790 .00047
480

6.150 .928070 .00047

6.200 .932180 .00046

6.500 .959200 .00048

6.850 .989690 .00047

6.950 .997590 .00049

7.001 .001570 .00048

7.051 .006700 .00047

7.101 .010570 .00048

7.151 .015310 .00047

7.201 .017860 .00048

\section{0}


U5o2N20-pl-1000-00

12

24.000 .929200 .00017 25.700 .936780 .00016 27.400 .942950 .00016 29.100 .947690 .00015 30.800 .952260 .00015 32.500 .956180 .00014 34.200 .959520 .00014 35.900 .962150 .00013 37.600 .964970 .00014 39.300 .966850 .00014 41.000 .969090 .00013 42.700 .970740 .00013 44.400 .972400 .00013 46.100 .974020 .00012 47.800 .974890 .00012 49.500 .976510 .00012 51.200 .977440 .00012 52.900 .978050 .00012 54.600 .978850 .00012 56.300 .979940 .00012 58.000 .980530 .00012 59.200 .981180 .00012

\section{5}

8.100 .920900 .00035 8.150 .923780 .00035 8.200 .927300 .00035 8.250 .930740 .00034 8.300 .933830 .00034 8.350 .936320 .00033 8.400 .940000 .00034 8.450 .942240 .00034 8.500 .946730 .00033 8.550 .950160 .00034 8.600 .952690 .00034 8.650 .955210 .00033 8.700 .958600 .00034 8.750 .961430 .00033 8.800 .964170 .00033 8.850 .967110 .00033 8.900 .969800 .00034 8.950 .972620 .00033
15

13.500 .925860 .00024

13.800 .931970 .00023

14.100 .937570 .00024

14.400 .943040 .00022

14.700 .947920 .00023

15.000 .952940 .00023

15.300 .957660 .00022

15.600 .962670 .00022

15.900 .967130 .00022

16.200 .970790 .00022

16.500 .974880 .00022

16.800 .978950 .00021

17.100 .982190 .00022

17.400 .985870 .00022

17.700 .988900 .00021

18.000 .992110 .00021

18.300 .995370 .00021

18.600 .998550 .00021

18.901 .001470 .00021

19.201 .004170 .00020

19.501 .007270 .00020

19.801 .009410 .00020

20.101 .011940 .00020

20.201 .012470 .00020

30

7.300 .919130 .00037

7.350 .923820 .00036

7.400 .927720 .00038

7.450 .931520 .00037

7.500 .935480 .00036

7.550 .939330 .00036

7.600 .943870 .00037

7.650 .948050 .00037

7.700 .951570 .00036

7.750 .955860 .00037

7.800 .959380 .00037

7.850 .962720 .00036

7.900 .966530 .00036

7.950 .970340 .00036

8.000 .974030 .00037

8.050 .977370 .00036

8.100 .980840 .00036

8.150 .984300 .00036
20

9.600 .923160 .00030 9.700 .927660 .00030 9.800 .932200 .00030 9.900 .937030 .00030 10.000 .941620 .00030 10.100 .945510 .00029 10.200 .949530 .00030 10.300 .953400 .00030 10.400 .957520 .00030 10.500 .961240 .00029 10.600 .964540 .00029 10.700 .969750 .00029 10.800 .972540 .00029 10.900 .976260 .00029 11.000 .979790 .00029 11.100 .983410 .00029 11.200 .986650 .00028 11.300 .990100 .00029 11.400 .993750 .00029 11.500 .996850 .00029 11.600 .999820 .00028 11.701 .002630 .00029 11.801 .005880 .00028 11.901 .009260 .00028 12.001 .012170 .00027 12.101 .014980 .00028

32

7.100 .921560 .00037

7.150 .925560 .00037

7.200 .930520 .00037

7.250 .935070 .00037

7.300 .938890 .00037

7.350 .943240 .00037

7.400 .947470 .00038

7.450 .951020 .00038

7.500 .955920 .00037

7.550 .960110 .00038

7.600 .963670 .00038

7.650 .967560 .00037

7.700 .971550 .00037

7.750 .974680 .00036

7.800 .979780 .00038

7.850 .983120 .00038

7.900 .987000 .00036

7.950 .990870 .00037 
9.000 .975490 .00033 9.050 .978840 .00033 9.100 .981320 .00033 9.150 .984040 .00033 9.200 .985840 .00034 9.250 .988910 .00034 9.300 .991560 .00034 9.350 .994060 .00033 9.400 .997020 .00032 9.451 .000030 .00033 9.501 .002560 .00033 9.551 .004600 .00032 9.601 .007000 .00033 9.651 .009600 .00032 9.701 .011600 .00032 9.751 .014990 .00032 9.801 .016390 .00032

\section{5}

6.800 .919940 .00038 6.850 .924080 .00040 6.900 .929560 .00039 6.950 .933940 .00038 7.000 .938960 .00038 7.050 .943230 .00038 7.100 .948040 .00039 7.150 .952890 .00039 7.200 .956730 .00038 7.250 .961310 .00038 7.300 .964990 .00038 7.350 .969660 .00039 7.400 .974350 .00039 7.450 .978450 .00038 7.500 .982060 .00038 7.550 .987420 .00039 7.600 .991100 .00038 7.650 .994460 .00037 7.700 .998900 .00040 7.751 .002840 .00040 7.801 .006240 .00037 7.851 .009540 .00038 7.901 .014300 .00038 7.951 .017800 .00038 8.001 .022010 .00038
8.200 .987740 .00036

8.250 .991430 .00036

8.300 .993880 .00035

8.350 .997770 .00037

8.401 .001110 .00036

8.451 .004410 .00035

8.501 .008250 .00036

8.551 .011220 .00036

8.601 .014200 .00035

8.651 .017390 .00035

8.701 .020470 .00036
8.000 .994020 .00037

8.050 .998060 .00037

8.101 .001270 .00037

8.151 .005360 .00036

8.201 .007950 .00037

8.251 .011990 .00037

8.301 .015640 .00036

8.351 .018250 .00036

8.401 .021980 .00036
40
6.400 .914750 .00041

6.450 .919800 .00039

6.500 .925340 .00040

6.550 .930320 .00041

6.600 .936490 .00041

6.650 .940350 .00040

6.700 .947290 .00040

6.750 .951650 .00040

6.800 .956590 .00040

6.850 .961310 .00041

6.900 .966120 .00041

6.950 .971640 .00040

7.000 .976030 .00040

7.050 .980390 .00040

7.100 .985560 .00039

7.150 .990100 .00040

7.200 .995200 .00040

7.250 .999130 .00039

7.301 .003910 .00040

7.351 .008140 .00040

7.401 .012840 .00040

7.451 .016680 .00039

7.501 .021630 .00040
50

6.000 .921670 .00044 6.050 .926850 .00042 6.100 .934150 .00043 6.150 .939200 .00042 6.200 .945600 .00043 6.250 .951800 .00043 6.300 .957630 .00042 6.350 .963380 .00043 6.400 .969540 .00042 6.450 .974740 .00042 6.500 .980030 .00042 6.550 .985720 .00042 6.600 .991520 .00042 6.650 .996450 .00043 6.701 .002260 .00043 6.751 .007680 .00042 6.801 .012090 .00043 6.851 .017530 .00043 6.901 .022130 .00039 
60

5.700 .920880 .00044 5.750 .928130 .00042 5.800 .934260 .00045 5.850 .941460 .00043 5.900 .947970 .00044 5.950 .954130 .00045 6.000 .960670 .00045 6.050 .966760 .00045 6.100 .973770 .00044 6.150 .979490 .00045 6.200 .985850 .00044 6.250 .991070 .00043 6.300 .997010 .00043 6.351 .003750 .00045 6.401 .009460 .00044 6.451 .014160 .00046 6.501 .020860 .00043

90

5.200 .915180 .00048 5.250 .923050 .00047 5.300 .931030 .00048 5.350 .938550 .00048 5.400 .945930 .00047 5.450 .954540 .00048 5.500 .961890 .00046 5.550 .968650 .00048 5.600 .976980 .00048 5.650 .984470 .00048 5.700 .990660 .00048 5.750 .997270 .00046 5.801 .004500 .00047 5.851 .010920 .00047 5.901 .019150 .00047 5.951 .025920 .00047 6.001 .031350 .00048

130 4.900 .907960 .00049 4.950 .917690 .00049 5.000 .926850 .00047 5.050 .934360 .00048 5.100 .942990 .00050 5.150 .951420 .00050 5.200 .959670 .00050 5.250 .967340 .00050
70

5.500 .920970 .00046

5.550 .928790 .00046

5.600 .934790 .00046

5.650 .943020 .00046

5.700 .949970 .00046

5.750 .956270 .00046

5.800 .963570 .00045

5.850 .970800 .00046

5.900 .976880 .00046

5.950 .983790 .00045

6.000 .989700 .00046

6.050 .995940 .00046

6.101 .002760 .00044

6.151 .008930 .00045

6.201 .015240 .00046

6.251 .021790 .00047

6.301 .028090 .00048
80

5.300 .913170 .00046

5.350 .920840 .00046

5.400 .928520 .00047

5.450 .936430 .00047

5.500 .943290 .00047

5.550 .951000 .00047

5.600 .958630 .00047

5.650 .965050 .00046

5.700 .972770 .00045

5.750 .979760 .00047

5.800 .986600 .00047

5.850 .993390 .00046

5.901 .000390 .00047

5.951 .006700 .00046

6.001 .013940 .00046

6.051 .020570 .00046

6.101 .027120 .00046
100

5.100 .911980 .00050

5.150 .920540 .00048

5.200 .929400 .00049

5.250 .937410 .00048

5.300 .945030 .00048

5.350 .953290 .00048

5.400 .961010 .00048

5.450 .968410 .00048

5.500 .975610 .00049

5.550 .983290 .00050

5.600 .990630 .00048

5.650 .998040 .00048

5.701 .005290 .00048

5.751 .011730 .00049

5.801 .020260 .00048

5.851 .025660 .00047

5.901 .032870 .00047
118

5.000 .915350 .00047 5.050 .924710 .00049 5.100 .933230 .00050 5.150 .940110 .00049 5.200 .947840 .00049 5.250 .957860 .00049 5.300 .964410 .00050 5.350 .972480 .00049 5.400 .980710 .00049 5.450 .987510 .00049 5.500 .995900 .00049 5.551 .003760 .00049 5.601 .011470 .00047 5.651 .018440 .00049 5.701 .026400 .00049
150

4.800 .904620 .00050 4.850 .913660 .00050 4.900 .922310 .00050 4.950 .930900 .00050 5.000 .939910 .00049 5.050 .947980 .00049 5.100 .956580 .00051 5.150 .964900 .00050
200

4.700 .906600 .00051 4.750 .914580 .00049 4.800 .924090 .00050 4.850 .933300 .00052 4.900 .941410 .00050 4.950 .951180 .00052 5.000 .958430 .00050 5.050 .967640 .00051 
5.300 .976160 .00049 5.350 .983240 .00050 5.400 .991160 .00049 5.450 .999800 .00048 5.501 .007650 .00049 5.551 .014640 .00048 5.601 .022810 .00050

235

4.700 .913180 .00051 4.750 .922140 .00052 4.800 .931760 .00051 4.850 .939880 .00051 4.900 .949630 .00051 4.950 .958060 .00051 5.000 .966230 .00052 5.050 .975810 .00052 5.100 .983950 .00052 5.150 .992800 .00050 5.201 .000210 .00051 5.251 .008820 .00052 5.301 .016410 .00050 5.351 .023830 .00050 5.401 .032550 .00050

470

4.700 .916070 .00052 4.750 .925400 .00049 4.800 .934230 .00049 4.850 .944370 .00051 4.900 .951690 .00051 4.950 .959820 .00049 5.000 .968490 .00051 5.050 .976330 .00052 5.100 .985180 .00052 5.150 .994240 .00051 5.201 .000460 .00051 5.251 .009970 .00052 5.301 .016910 .00051 5.351 .025770 .00051 5.401 .032570 .00050
5.200 .973290 .00050 5.250 .981000 .00051 5.300 .989330 .00049 5.350 .996700 .00049 5.401 .005150 .00049 5.451 .011930 .00051 5.501 .020260 .00051 5.551 .028440 .00050 5.601 .035700 .00051

250

4.700 .915390 .00052 4.750 .924420 .00051 4.800 .933320 .00050 4.850 .942890 .00052 4.900 .951660 .00051 4.950 .960240 .00050 5.000 .968260 .00051 5.050 .976970 .00050 5.100 .986180 .00052 5.150 .994050 .00051 5.201 .002540 .00050 5.251 .010720 .00053 5.301 .018400 .00051 5.351 .027000 .00053 5.401 .034390 .00050
5.100 .976170 .00050 5.150 .984100 .00050 5.200 .992750 .00050 5.251 .001070 .00049 5.301 .009820 .00050 5.351 .017300 .00050 5.401 .025020 .00050
353

4.700 .919620 .00052 4.750 .928770 .00051 4.800 .938470 .00051 4.850 .946840 .00050 4.900 .955500 .00053 4.950 .964190 .00051 5.000 .973260 .00051 5.050 .980930 .00053 5.100 .990090 .00053 5.150 .998900 .00051 5.201 .006460 .00052 5.251 .015720 .00051 5.301 .022360 .00052
500
4.700 .914470 .00050
4.750 .923890 .00050
4.800 .932210 .00050
4.850 .941410 .00051
4.900 .949120 .00051
4.950 .957860 .00051
5.000 .966400 .00051
5.050 .975070 .00051
5.100 .982790 .00051
5.150 .990640 .00050
5.200 .999760 .00051
5.251 .007240 .00051
5.301 .014980 .00051
5.351 .022580 .00051
5.401 .030560 .00052

600

4.700 .904810 .00051

4.750 .912890 .00050 4.800 .922130 .00049 4.850 .930740 .00050 4.900 .939260 .00051 4.950 .947120 .00051 5.000 .956620 .00050 5.050 .963540 .00050 5.100 .972240 .00051 5.150 .980530 .00051 5.200 .987880 .00050 5.250 .995450 .00051 5.301 .004400 .00050 5.351 .011120 .00050 5.401 .018760 .00051 5.451 .026340 .00050 5.501 .033620 .00051 
605

4.700 .904370 .00051

4.750 .913270 .00052

4.800 .920790 .00050

4.850 .931120 .00051

4.900 .938850 .00049

4.950 .947830 .00051

5.000 .955460 .00051

5.050 .963490 .00051

5.100 .972580 .00050

5.150 .980640 .00051

5.200 .988150 .00051

5.250 .995650 .00050

5.301 .003200 .00051

5.351 .010700 .00051

5.401 .018810 .00051

5.451 .026210 .00050

5.501 .033160 .00050
610

4.800 .921680 .00050

4.850 .929990 .00051

4.900 .937910 .00049

4.950 .946870 .00051

5.000 .955550 .00050

5.050 .963540 .00050

5.100 .971060 .00050

5.150 .980050 .00050

5.200 .986470 .00052

5.250 .995080 .00051

5.301 .004220 .00051

5.351 .009880 .00050

5.401 .018390 .00050

5.451 .025530 .00050

5.501 .033540 .00051
615

4.800 .921010 .00049

4.850 .929210 .00049

4.900 .937450 .00050

4.950 .946690 .00050

5.000 .954020 .00049

5.050 .962950 .00051

5.100 .971080 .00050

5.150 .978650 .00050

5.200 .986310 .00050

5.250 .994710 .00052

5.301 .003190 .00051

5.351 .009590 .00049

5.401 .018060 .00050

5.451 .025670 .00050

5.501 .032220 .00050
620

4.800 .920470 .00050

4.850 .928080 .00050

4.900 .937120 .00049

4.950 .945700 .00051

5.000 .953810 .00050

5.050 .961410 .00050

5.100 .969440 .00050

5.150 .977560 .00050

5.200 .986010 .00050

5.250 .993670 .00051

5.301 .002160 .00050

5.351 .009160 .00049

5.401 .015860 .00051

5.451 .023570 .00051

5.501 .031800 .00050
625

4.800 .919200 .00051

4.850 .928340 .00050

4.900 .935890 .00050

4.950 .944810 .00052

5.000 .952730 .00050

5.050 .961610 .00049

5.100 .969800 .00050

5.150 .977790 .00050

5.200 .985770 .00050

5.250 .993890 .00051

5.301 .001400 .00051

5.351 .008380 .00051

5.401 .015830 .00050

5.451 .023690 .00051

5.501 .030730 .00051
629

4.800 .917860 .00051

4.850 .926980 .00050

4.900 .935740 .00050

4.950 .944080 .00051

5.000 .952020 .00049

5.050 .960280 .00051

5.100 .968310 .00051

5.150 .976810 .00050

5.200 .984020 .00049

5.250 .992620 .00051

5.301 .000060 .00051

5.351 .008230 .00051

5.401 .016060 .00051

5.451 .023350 .00051

5.501 .030830 .00051 
U5o2N20-pl-1000-01

12

22.400 .928720 .00017

24.700 .938610 .00016

27.000 .946370 .00015

29.300 .952600 .00015

31.600 .957450 .00014

33.900 .961730 .00014

36.200 .964960 .00013

38.500 .968140 .00013

40.800 .970370 .00013

43.100 .972570 .00013

45.400 .974300 .00012

47.700 .975940 .00012

50.000 .977400 .00012

52.300 .979090 .00012

54.600 .980490 .00011

56.900 .980710 .00012

59.200 .982020 .00011

61.500 .982610 .00011

63.800 .983270 .00011

66.100 .983640 .00011

68.400 .984780 .00011

69.400 .984570 .00011
15

12.000 .926650 .00023

12.300 .932590 .00023

12.600 .938150 .00023

12.900 .943650 .00023

13.200 .948890 .00022

13.500 .953790 .00022

13.800 .958410 .00022

14.100 .962910 .00021

14.400 .967190 .00021

14.700 .971550 .00022

15.000 .975370 .00021

15.300 .979000 .00021

15.600 .982550 .00021

15.900 .986110 .00021

16.200 .989990 .00021

16.500 .992580 .00021

16.800 .995800 .00020

17.100 .998850 .00020

17.401 .001520 .00020

17.701 .004410 .00020

18.001 .007380 .00019

18.301 .008770 .00020

18.601 .012210 .00019
20

8.100 .924090 .00029

8.200 .928880 .00029

8.300 .934110 .00029

8.400 .938280 .00029

8.500 .942170 .00028

8.600 .946070 .00028

8.700 .950450 .00029

8.800 .954730 .00029

8.900 .958460 .00029

9.000 .962590 .00028

9.100 .966900 .00028

9.200 .969960 .00028

9.300 .973880 .00028

9.400 .977360 .00028

9.500 .981180 .00028

9.600 .984600 .00028

9.700 .987420 .00027

9.800 .991410 .00029

9.900 .994520 .00027

10.000 .997950 .00028

10.101 .000630 .00027

10.201 .004100 .00028

10.301 .007250 .00027

10.401 .009480 .00027

10.501 .012990 .00027

10.601 .016040 .00026

\section{2}

5.800 .922160 .00036

5.850 .925310 .00035

5.900 .929330 .00036

5.950 .933490 .00035

6.000 .938180 .00035

6.050 .942190 .00036

6.100 .946310 .00035

6.150 .949630 .00034

6.200 .954110 .00035

6.250 .958020 .00036

6.300 .960520 .00035

6.350 .965420 .00034

6.400 .969150 .00035

6.450 .972040 .00036

6.500 .976370 .00035

6.550 .979280 .00034

6.600 .983060 .00035

6.650 .986900 .00034
5.500 .914490 .00036

5.550 .919730 .00036

5.600 .924170 .00036

5.650 .928450 .00037

5.700 .932830 .00037

5.750 .938070 .00036

5.800 .941270 .00036

5.850 .945660 .00037

5.900 .949740 .00037

5.950 .954200 .00037

6.000 .958270 .00037

6.050 .962310 .00036

6.100 .965580 .00035

6.150 .970310 .00037

6.200 .974000 .00036

6.250 .977960 .00036

6.300 .982370 .00036

6.350 .985530 .00035 
7.500 .977070 .00032

7.550 .979910 .00032

7.600 .982990 .00032

7.650 .985840 .00031

7.700 .988000 .00031

7.750 .990460 .00032

7.800 .992930 .00032

7.850 .995760 .00031

7.900 .999020 .00032

7.951 .001190 .00032

8.001 .003810 .00033

8.051 .006410 .00031

8.101 .008590 .00032

8.151 .011770 .00031

8.201 .012590 .00030

8.251 .015860 .00031

8.301 .018550 .00031
6.700 .990290 .00033

6.750 .992910 .00034

6.800 .996570 .00035

6.851 .000520 .00035

6.901 .003610 .00036

6.951 .007150 .00034

7.001 .010070 .00035

7.051 .013070 .00036

7.101 .016000 .00035
6.400 .989270 .00036

6.450 .993360 .00036

6.500 .996530 .00035

6.551 .000250 .00035

6.601 .004150 .00035

6.651 .006910 .00036

6.701 .011470 .00035

6.751 .014740 .00035

6.801 .017880 .00035
35

5.300 .922250 .00038

5.350 .927200 .00038

5.400 .931460 .00037

5.450 .937180 .00038

5.500 .941070 .00037

5.550 .946700 .00038

5.600 .950330 .00038

5.650 .955500 .00037

5.700 .960330 .00037

5.750 .964920 .00037

5.800 .968320 .00038

5.850 .972690 .00038

5.900 .977190 .00037

5.950 .981190 .00037

6.000 .986000 .00037

6.050 .989320 .00037

6.100 .993050 .00037

6.150 .997490 .00036

6.201 .001090 .00037

6.251 .005300 .00036

6.301 .009700 .00037

6.351 .013220 .00037

6.401 .017640 .00037
40

4.900 .918520 .00038

4.950 .923820 .00038

5.000 .929070 .00039

5.050 .934150 .00038

5.100 .938900 .00039

5.150 .944570 .00039

5.200 .949650 .00038

5.250 .955680 .00038

5.300 .960040 .00040

5.350 .964900 .00038

5.400 .969110 .00038

5.450 .974670 .00038

5.500 .979420 .00038

5.550 .983590 .00038

5.600 .988660 .00039

5.650 .993670 .00038

5.700 .996970 .00038

5.751 .002440 .00039

5.801 .006190 .00038

5.851 .011110 .00039

5.901 .015870 .00038

5.951 .019970 .00039

6.001 .023940 .00038
50

4.500 .925600 .00042 4.550 .931400 .00040 4.600 .938310 .00042 4.650 .944540 .00040 4.700 .949720 .00041 4.750 .956290 .00041 4.800 .961710 .00040 4.850 .967980 .00041 4.900 .972860 .00040 4.950 .978750 .00042 5.000 .984790 .00040 5.050 .990280 .00041 5.100 .996340 .00041 5.151 .000540 .00041 5.201 .006090 .00042 5.251 .011870 .00041 5.301 .016350 .00040
60

4.100 .912090 .00043 4.150 .918670 .00044 4.200 .926320 .00042
70

3.900 .912880 .00044 3.950 .919890 .00044 4.000 .927430 .00045
80

3.800 .919840 .00045 3.850 .928560 .00045 3.900 .935760 .00045 
4.250 .933470 .00043 4.300 .939250 .00042 4.350 .946280 .00043 4.400 .952480 .00044 4.450 .960120 .00043 4.500 .966030 .00043 4.550 .971980 .00043 4.600 .979080 .00043 4.650 .983800 .00041 4.700 .990910 .00044 4.750 .995590 .00043 4.801 .002950 .00042 4.851 .009020 .00042 4.901 .014430 .00043 4.951 .020880 .00043 5.001 .025620 .00042

90 3.700 .922350 .00047 3.750 .930190 .00046 3.800 .938250 .00046 3.850 .946390 .00045 3.900 .953050 .00045 3.950 .962010 .00045 4.000 .969320 .00047 4.050 .976230 .00046 4.100 .982820 .00045 4.150 .991020 .00047 4.200 .997100 .00046 4.251 .004790 .00047 4.301 .012090 .00047 4.351 .018250 .00045 4.401 .025760 .00046
4.050 .934240 .00043

4.100 .941570 .00044

4.150 .948520 .00044 4.200 .955430 .00043 4.250 .962060 .00044 4.300 .969040 .00045 4.350 .975740 .00045 4.400 .983370 .00044 4.450 .989550 .00044 4.500 .996380 .00044 4.551 .002420 .00044 4.601 .008650 .00043 4.651 .014960 .00044 4.701 .021400 .00043
3.950 .942910 .00047

4.000 .950560 .00045

4.050 .957240 .00044

4.100 .965530 .00045

4.150 .971820 .00045

4.200 .979220 .00044

4.250 .986860 .00044

4.300 .992740 .00045

4.351 .000180 .00044

4.401 .006710 .00046

4.451 .013250 .00045 4.501 .019870 .00044
100

2.900 .794740 .00044

2.920 .798600 .00045

2.940 .802510 .00045

2.960 .806290 .00047

2.980 .809450 .00046

3.000 .813490 .00046

3.020 .817960 .00046

3.040 .822130 .00046

3.060 .825050 .00046

3.080 .828980 .00045

3.100 .832540 .00045

3.120 .836570 .00045

3.140 .840800 .00046

3.160 .844420 .00045

3.180 .847150 .00046

3.200 .851670 .00046

3.220 .855350 .00047

3.240 .857830 .00046

3.260 .862190 .00046

3.280 .865580 .00045

3.300 .869550 .00047

3.320 .872370 .00045

3.340 .876140 .00047

3.360 .879740 .00046

3.380 .883050 .00046

3.400 .887250 .00046
118

2.600 .752590 .00046

2.620 .757450 .00046

2.640 .760980 .00047

2.660 .765570 .00047

2.680 .770140 .00045

2.700 .773160 .00047

2.720 .777840 .00046

2.740 .782120 .00046

2.760 .785460 .00046

2.780 .790060 .00047

2.800 .794270 .00047

2.820 .797930 .00045

2.840 .802480 .00046

2.860 .806610 .00046

2.880 .810230 .00045

2.900 .813810 .00046

2.920 .818990 .00046

2.940 .822060 .00046

2.960 .826830 .00047

2.980 .829880 .00047

3.000 .833960 .00047 
130

2.200 .674210 .00045

2.220 .678700 .00044

2.240 .683060 .00044

2.260 .688350 .00045

2.280 .692720 .00044

2.300 .697050 .00046

2.320 .701970 .00045

2.340 .706210 .00045

2.360 .710500 .00045

2.380 .715210 .00046

2.400 .719760 .00045

2.420 .724100 .00046

2.440 .728390 .00045

2.460 .733390 .00047

2.480 .737240 .00047

2.500 .742020 .00046
150

3.300 .914520 .00049

3.350 .923480 .00048

3.400 .931790 .00049

3.450 .940610 .00048

3.500 .949640 .00048

3.550 .958130 .00048

3.600 .967530 .00048

3.650 .974380 .00048

3.700 .983520 .00047

3.750 .991320 .00049

3.800 .999630 .00048

3.851 .007180 .00048

3.901 .014130 .00047

3.951 .022570 .00047

4.001 .028910 .00048
200

3.200 .918200 .00050

3.250 .927600 .00050

3.300 .936150 .00049

3.350 .945150 .00049

3.400 .954060 .00049

3.450 .962510 .00048

3.500 .970850 .00049

3.550 .978970 .00050

3.600 .987670 .00049

3.650 .996370 .00049

3.701 .005170 .00048

3.751 .012230 .00048

3.801 .020210 .00048
235

3.100 .908030 .00050

3.150 .917970 .00049

3.200 .926680 .00048

3.250 .935400 .00048

3.300 .944230 .00049

3.350 .953470 .00050

3.400 .962960 .00047

3.450 .969830 .00049

3.500 .979960 .00049

3.550 .988350 .00050

3.600 .996310 .00050

3.651 .004390 .00050

3.701 .012030 .00049

3.751 .020720 .00049

3.801 .029680 .00050
250

3.100 .911070 .00050

3.150 .919550 .00050

3.200 .928380 .00048

3.250 .938470 .00047

3.300 .947010 .00049

3.350 .956360 .00049

3.400 .965240 .00050

3.450 .973170 .00050

3.500 .982280 .00049

3.550 .990370 .00050

3.600 .997750 .00048

3.651 .005900 .00049

3.701 .015550 .00049

3.751 .023290 .00051

3.801 .030960 .00049
353

3.100 .917060 .00051

3.150 .927960 .00050

3.200 .935960 .00049

3.250 .944940 .00049

3.300 .953360 .00051

3.350 .962340 .00050

3.400 .971040 .00049

3.450 .980050 .00050

3.500 .987310 .00049

3.550 .996470 .00050

3.601 .004440 .00050

3.651 .012600 .00050

3.701 .020450 .00049
470

3.100 .916700 .00049

3.150 .924850 .00049

3.200 .934190 .00050

3.250 .943050 .00049

3.300 .951240 .00050

3.350 .960390 .00049

3.400 .968230 .00049

3.450 .976870 .00049

3.500 .985800 .00049

3.550 .993900 .00050

3.601 .002230 .00049
500

3.100 .914830 .00049

3.150 .923820 .00048

3.200 .932970 .00051

3.250 .942520 .00049

3.300 .951140 .00049

3.350 .958900 .00049

3.400 .966680 .00050

3.450 .975770 .00049

3.500 .983910 .00050

3.550 .992490 .00050

3.601 .000880 .00050
600

3.200 .924410 .00049

3.250 .933070 .00048

3.300 .941240 .00050

3.350 .950200 .00049

3.400 .959190 .00050

3.450 .968090 .00048

3.500 .974420 .00050

3.550 .982870 .00050

3.600 .991360 .00048

3.650 .998090 .00050

3.701 .006890 .00049 
3.651 .010340 .00051

3.701 .018310 .00050

3.751 .024900 .00050

3.801 .032640 .00050
3.651 .007580 .00049

3.701 .015650 .00050

3.751 .023400 .00050

3.801 .030660 .00050
3.751 .013770 .00049

3.801 .022110 .00049
605

3.200 .925490 .00049 3.250 .933460 .00049 3.300 .941740 .00048 3.350 .950500 .00049 3.400 .958210 .00049 3.450 .967500 .00048 3.500 .974130 .00050 3.550 .982750 .00050 3.600 .990780 .00049 3.650 .997520 .00048 3.701 .005840 .00049 3.751 .014210 .00049 3.801 .021730 .00052

620

3.200 .923880 .00050 3.250 .931650 .00050 3.300 .940900 .00050 3.350 .948980 .00049 3.400 .957700 .00050 3.450 .965090 .00049 3.500 .972570 .00049 3.550 .981280 .00049 3.600 .989280 .00050 3.650 .996660 .00048 3.701 .004230 .00048 3.751 .011760 .00050 3.801 .019040 .00049
610

3.200 .924640 .00049 3.250 .933610 .00049 3.300 .941920 .00050 3.350 .950100 .00051 3.400 .957870 .00051 3.450 .966720 .00050 3.500 .973700 .00048 3.550 .983460 .00050 3.600 .990430 .00049 3.650 .997990 .00050 3.701 .005900 .00049 3.751 .013260 .00050 3.801 .021110 .00049
615

3.200 .924200 .00049 3.250 .932990 .00049 3.300 .940570 .00049 3.350 .950110 .00049 3.400 .958930 .00048 3.450 .965730 .00048 3.500 .973770 .00049 3.550 .982500 .00051 3.600 .990090 .00049 3.650 .997720 .00050 3.701 .005230 .00050 3.751 .012440 .00050 3.801 .020770 .00049
3.200 .922810 .00049

3.250 .931250 .00049 3.300 .940420 .00048 3.350 .947750 .00050 3.400 .957400 .00050 3.450 .964410 .00050 3.500 .972280 .00049 3.550 .980740 .00050 3.600 .987450 .00050 3.650 .995580 .00049 3.701 .004170 .00049 3.751 .011630 .00049 3.801 .019310 .00049
629
3.200 .923160 .00049
3.250 .931530 .00050
3.300 .938720 .00048
3.350 .947380 .00050
3.400 .956470 .00050
3.450 .963990 .00050
3.500 .971160 .00049
3.550 .979270 .00050
3.600 .987910 .00049
3.650 .995960 .00050
3.701 .002660 .00049
3.751 .011370 .00051
3.801 .018730 .00050
3.851 .025380 .00048
3.901 .033010 .00049 
U5o2N20-pl-1000-10

12

21.200 .928370 .00016 26.300 .947030 .00015 31.400 .959440 .00014 36.500 .967220 .00013 41.600 .972590 .00013 46.700 .976340 .00012 51.800 .978880 .00012 56.900 .981380 .00011 62.000 .983130 .00011 67.100 .984450 .00011 72.200 .985530 .00010 77.300 .986350 .00011 82.400 .987260 .00010 87.500 .988040 .00010 92.600 .988180 .00010 97.700 .988980 .00010 102.800 .989140 .00010 107.900 .989880 .00010 113.000 .989700 .00010 118.100 .990430 .00010 123.200 .990340 .00010 123.600 .990750 .00010
10.900 .926100 .00022

11.200 .932430 .00022 11.500 .937640 .00021 11.800 .943680 .00021 12.100 .948260 .00021 12.400 .953600 .00020 12.700 .958540 .00020 13.000 .962710 .00021 13.300 .966910 .00021 13.600 .971560 .00020 13.900 .975120 .00020 14.200 .979130 .00020 14.500 .982840 .00020 14.800 .986360 .00019 15.100 .989520 .00020 15.400 .992700 .00019 15.700 .995870 .00019 16.000 .998980 .00019 16.301 .001650 .00019 16.601 .004640 .00019 16.901 .006910 .00019 17.201 .009910 .00019 17.401 .011030 .00019
25

5.500 .922540 .00030 5.550 .926110 .00030 5.600 .928790 .00031 5.650 .932670 .00031 5.700 .935570 .00030 5.750 .938420 .00030 5.800 .942450 .00030 5.850 .945680 .00030 5.900 .949020 .00031 5.950 .951560 .00030 6.000 .954820 .00029 6.050 .957370 .00030 6.100 .960980 .00030 6.150 .963720 .00030 6.200 .967080 .00030 6.250 .969090 .00029 6.300 .973080 .00029 6.350 .975960 .00030

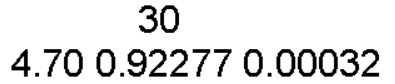
4.750 .926370 .00032
4.800 .931060 .00033
4.850 .934880 .00032
4.900 .939120 .00033
4.950 .943380 .00033
5.000 .947260 .00032
5.050 .950890 .00033
5.100 .955530 .00032
5.150 .959020 .00033
5.200 .962970 .00032
5.250 .966650 .00033
5.300 .970420 .00033
5.350 .973990 .00032
5.400 .977530 .00031
5.450 .981400 .00031
5.500 .984680 .00032
5.550 .987750 .00032

20

7.000 .924280 .00027

7.100 .928460 .00027

7.200 .933830 .00027

7.300 .938230 .00027

7.400 .942300 .00027

7.500 .946280 .00027

7.600 .950580 .00027

7.700 .954590 .00027

7.800 .958700 .00028

7.900 .962260 .00027

8.000 .966360 .00027

8.100 .970370 .00027

8.200 .974060 .00027

8.300 .977460 .00026

8.400 .981450 .00026

8.500 .985140 .00026

8.600 .987630 .00025

8.700 .991530 .00027

8.800 .994800 .00026

8.900 .997760 .00026

9.001 .001190 .00025

9.101 .004300 .00026

9.201 .006960 .00025

9.301 .010690 .00025

9.401 .013600 .00025

9.501 .015900 .00026 
6.400 .978070 .00029 6.450 .980750 .00029 6.500 .983710 .00030 6.550 .986880 .00029 6.600 .989040 .00030 6.650 .991800 .00030 6.700 .994540 .00029 6.750 .996820 .00029 6.800 .999890 .00028 6.851 .001690 .00029 6.901 .004250 .00030 6.951 .006950 .00029 7.001 .009460 .00029 7.051 .012380 .00029 7.101 .014620 .00029

35

4.200 .925010 .00035 4.250 .929240 .00034 4.300 .933920 .00034 4.350 .938870 .00036 4.400 .943410 .00034 4.450 .948290 .00036 4.500 .952890 .00035 4.550 .957290 .00035 4.600 .962060 .00034 4.650 .966580 .00034 4.700 .970990 .00034 4.750 .976100 .00034 4.800 .980050 .00033 4.850 .984250 .00033 4.900 .988310 .00034 4.950 .992670 .00034 5.000 .996450 .00034 5.051 .001220 .00034 5.101 .005250 .00034 5.151 .008900 .00033 5.201 .013420 .00034 5.251 .016590 .00033 5.301 .020570 .00033

\section{0}

3.000 .920710 .00040 3.050 .927710 .00039 3.100 .934780 .00039 3.150 .941830 .00039 3.200 .948710 .00039
5.600 .992250 .00032 5.650 .995540 .00032 5.700 .999500 .00031 5.751 .001970 .00032 5.801 .005670 .00032 5.851 .009030 .00031 5.901 .012410 .00032 5.951 .015570 .00032 6.001 .018270 .00032
5.400 .999490 .00033 5.451 .002430 .00033 5.501 .006250 .00032 5.551 .010420 .00032 5.601 .014070 .00033 5.651 .017040 .00032 5.701 .020700 .00032
40

3.800 .920690 .00036

3.850 .926490 .00035

3.900 .931570 .00037

3.950 .938020 .00037

4.000 .943360 .00037

4.050 .948740 .00036

4.100 .952780 .00035

4.150 .958220 .00036

4.200 .964060 .00035

4.250 .968660 .00035

4.300 .973770 .00035

4.350 .978660 .00036

4.400 .983700 .00035

4.450 .988340 .00035

4.500 .993220 .00036

4.550 .997950 .00035

4.601 .001980 .00035

4.651 .006980 .00034

4.701 .011530 .00036

4.751 .015770 .00035

4.801 .020710 .00035
50

3.300 .918900 .00037

3.350 .925560 .00037

3.400 .932360 .00037

3.450 .937930 .00037

3.500 .944250 .00038

3.550 .950840 .00037

3.600 .956560 .00037

3.650 .963300 .00037

3.700 .968480 .00037

3.750 .975210 .00037

3.800 .980140 .00038

3.850 .985890 .00037

3.900 .991590 .00038

3.950 .997290 .00037

4.001 .002440 .00039

4.051 .008640 .00038

4.101 .013450 .00038

4.151 .019380 .00037

4.201 .023870 .00038
70

2.800 .923820 .00040

2.850 .931500 .00039

2.900 .938600 .00040

2.950 .946990 .00039

3.000 .954160 .00040
80

2.600 .918310 .00040 2.650 .926900 .00041 2.700 .934830 .00040 2.750 .942270 .00040 2.800 .950630 .00042 
3.250 .955380 .00039

3.300 .961600 .00039

3.350 .968760 .00039

3.400 .975670 .00039

3.450 .981880 .00039

3.500 .987980 .00039

3.550 .994910 .00040

3.601 .000820 .00040

3.651 .006470 .00039

3.701 .012510 .00038

3.751 .018720 .00038

3.801 .023930 .00039
3.050 .960620 .00041

3.100 .967600 .00040

3.150 .975160 .00039

3.200 .982020 .00040

3.250 .988780 .00041

3.300 .995250 .00040

3.351 .001590 .00041

3.401 .009130 .00041

3.451 .015300 .00040

3.501 .021800 .00040
2.850 .958240 .00041

2.900 .965430 .00041

2.950 .972910 .00042

3.000 .979890 .00040

3.050 .988110 .00041

3.100 .994680 .00041

3.151 .001550 .00040

3.201 .008850 .00041

3.251 .015550 .00041

3.301 .022120 .00041
90

2.500 .923100 .00041

2.550 .931680 .00041

2.600 .940500 .00042

2.650 .948050 .00042

2.700 .955590 .00042

2.750 .963390 .00042

2.800 .972010 .00041

2.850 .978850 .00042

2.900 .986660 .00041

2.950 .993930 .00041

3.001 .001310 .00041

3.051 .009260 .00041

3.101 .016500 .00041
100

2.400 .923370 .00042

2.450 .932700 .00042

2.500 .941280 .00041

2.550 .948510 .00041

2.600 .956830 .00042

2.650 .965610 .00042

2.700 .973840 .00043

2.750 .981210 .00042

2.800 .989760 .00041

2.850 .996440 .00041

2.901 .004170 .00042

2.951 .012300 .00042

3.001 .018910 .00043
118

2.200 .913570 .00042

2.250 .922420 .00043

2.300 .931430 .00044

2.350 .940450 .00042

2.400 .948880 .00042

2.450 .958030 .00042

2.500 .966540 .00042

2.550 .974090 .00042

2.600 .982060 .00041

2.650 .990900 .00043

2.700 .999030 .00043

2.751 .006270 .00043

2.801 .014320 .00044

2.851 .022460 .00041

2.901 .029440 .00044

150

2.000 .908230 .00044

2.050 .917100 .00043

2.100 .926250 .00044

2.150 .936400 .00044

2.200 .945560 .00043

2.250 .954090 .00042

2.300 .963100 .00043

2.350 .972250 .00044

2.400 .980920 .00044

2.450 .989620 .00043

2.500 .997760 .00043

2.551 .006260 .00043

2.601 .014480 .00045

2.651 .021900 .00045

2.701 .029620 .00044
200

1.900 .920090 .00043

1.950 .929520 .00043

2.000 .938990 .00043

2.050 .948160 .00044

2.100 .957760 .00044

2.150 .967120 .00044

2.200 .976040 .00044

2.250 .984740 .00045

2.300 .993230 .00044

2.351 .001140 .00044

2.401 .009580 .00044

2.451 .018710 .00044

2.501 .026940 .00045 
235

1.800 .914780 .00045

1.850 .923880 .00044

1.900 .933380 .00044

1.950 .943990 .00044

2.000 .952900 .00043

2.050 .961940 .00043

2.100 .971150 .00044

2.150 .980020 .00044

2.200 .990360 .00045

2.250 .998510 .00045

2.301 .006560 .00044

2.351 .014280 .00044

2.401 .023100 .00044

470

1.600 .911960 .00046

1.650 .921810 .00042

1.700 .931490 .00045

1.750 .941190 .00045

1.800 .950450 .00044

1.850 .958980 .00046

1.900 .968690 .00046

1.950 .977340 .00046

2.000 .985950 .00046

2.050 .993730 .00044

2.101 .002910 .00045

2.151 .010640 .00046

2.201 .018880 .00045

605

1.600 .917810 .00044

1.650 .926450 .00045

1.700 .935520 .00044

1.750 .944750 .00044

1.800 .953280 .00044

1.850 .962140 .00045

1.900 .970490 .00046

1.950 .978430 .00045

2.000 .986540 .00045

2.050 .994880 .00045

2.101 .003490 .00046

2.151 .011990 .00045

2.201 .019030 .00045
250

1.800 .919560 .00044

1.850 .928970 .00045

1.900 .938450 .00046

1.950 .947560 .00043

2.000 .957000 .00045

2.050 .966340 .00045

2.100 .976070 .00045

2.150 .984400 .00045

2.200 .994260 .00045

2.251 .001250 .00046

2.301 .010990 .00044

2.351 .019730 .00045

2.401 .027570 .00044
353

1.700 .920460 .00045

1.750 .930940 .00045

1.800 .939690 .00043

1.850 .949560 .00044

1.900 .958740 .00045

1.950 .967570 .00044

2.000 .976730 .00045

2.050 .986300 .00046

2.100 .994530 .00043

2.151 .003140 .00046

2.201 .011960 .00044

2.251 .020510 .00045

2.301 .028260 .00046

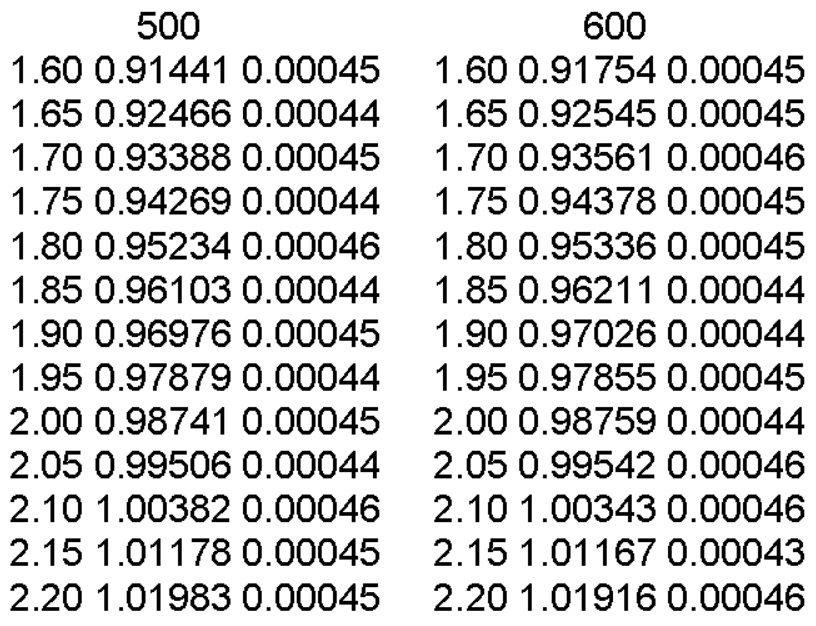

610

1.600 .917400 .00044

1.650 .926660 .00045

1.700 .934640 .00045

1.750 .944520 .00044

1.800 .953520 .00045

1.850 .961330 .00045

1.900 .970360 .00045

1.950 .978370 .00043

2.000 .986860 .00043

2.050 .995710 .00045

2.101 .003880 .00045

2.151 .011230 .00046

2.201 .019640 .00045
615

1.600 .917670 .00045

1.650 .926510 .00045

1.700 .935870 .00045

1.750 .944500 .00045

1.800 .953230 .00044

1.850 .962260 .00045

1.900 .970900 .00045

1.950 .979600 .00045

2.000 .987690 .00045

2.050 .995530 .00045

2.101 .003770 .00045

2.151 .012110 .00045

2.201 .018950 .00046 


\section{0}

625

629

$\begin{array}{lllll}1.60 & 0.917970 .00044 & 1.60 & 0.917480 .00044 & 1.600 .917670 .00044\end{array}$

$\begin{array}{lll}1.650 .925920 .00044 & 1.650 .926790 .00045 & 1.650 .925830 .00045\end{array}$

$1.700 .935300 .00045 \quad 1.700 .935850 .00045$

$1.750 .943940 .00046 \quad 1.750 .943960 .00045$

$1.800 .953370 .00045 \quad 1.800 .952840 .00045$

$1.850 .961090 .00045 \quad 1.850 .962050 .00044$

$1.900 .971010 .00044 \quad 1.900 .969440 .00045$

$1.950 .978440 .00046 \quad 1.950 .978690 .00045$

$2.000 .987430 .00045 \quad 2.000 .987200 .00044$

$2.050 .994310 .00046 \quad 2.050 .995610 .00046$

$2.101 .003100 .00044 \quad 2.101 .003190 .00045$

$2.151 .010960 .00046 \quad 2.151 .010850 .00045$

$2.201 .018900 .00044 \quad 2.201 .018570 .00045$

1.700 .935190 .00045

1.750 .944440 .00045

1.800 .952820 .00045

1.850 .961750 .00045

1.900 .970500 .00046

1.950 .978640 .00046

2.000 .987420 .00045

2.050 .995150 .00046

2.101 .003030 .00046

2.151 .010940 .00045

2.201 .019290 .00045 
U5o2N23-pl-1000-00

12

33.700 .910220 .00014

35.200 .913160 .00014

36.700 .915030 .00014

38.200 .917360 .00014

39.700 .919340 .00014

41.200 .920990 .00013

42.700 .922650 .00013

44.200 .924240 .00013

45.700 .925100 .00013

47.200 .926390 .00014

48.700 .927640 .00013

50.200 .928610 .00013

51.700 .929400 .00013

53.200 .930310 .00012

54.700 .930920 .00012

56.200 .931980 .00013

57.700 .932360 .00013

59.200 .932840 .00012

60.700 .933770 .00012

62.200 .933730 .00012

63.700 .934480 .00012

64.800 .934970 .00012
15

16.500 .926380 .00021

17.100 .934520 .00021

17.700 .941050 .00021

18.300 .947650 .00021

18.900 .952820 .00020

19.500 .958200 .00020

20.100 .963460 .00020

20.700 .968020 .00019

21.300 .972210 .00020

21.900 .976130 .00019

22.500 .980090 .00019

23.100 .983420 .00018

23.700 .986550 .00019

24.300 .989730 .00018

24.900 .992490 .00018

25.500 .995020 .00018

26.100 .997620 .00017

26.701 .000290 .00017

27.301 .002290 .00017

27.901 .004790 .00017

28.501 .006930 .00017

29.101 .008480 .00017

29.701 .010200 .00017

29.901 .010600 .00016
20

10.800 .925010 .00028

10.900 .928970 .00028

11.000 .932200 .00028

11.100 .935780 .00028

11.200 .938950 .00028

11.300 .942520 .00028

11.400 .946240 .00027

11.500 .949060 .00027

11.600 .952260 .00028

11.700 .955380 .00028

11.800 .958620 .00027

11.900 .961370 .00029

12.000 .964160 .00029

12.100 .966780 .00027

12.200 .970140 .00028

12.300 .973230 .00027

12.400 .975570 .00027

12.500 .977890 .00027

12.600 .980820 .00027

12.700 .983520 .00027

12.800 .986170 .00027

12.900 .988640 .00027

13.000 .990760 .00027

13.100 .993350 .00026

13.200 .995830 .00028

13.300 .998680 .00027

13.400 .999970 .00026

13.501 .003100 .00026

13.601 .004880 .00027

13.701 .007820 .00027

13.801 .009130 .00026

13.901 .011560 .00025

14.001 .013470 .00026
25

8.900 .923060 .00033

9.000 .929180 .00033

9.100 .934120 .00032

9.200 .939650 .00032

9.300 .944890 .00032

9.400 .950080 .00032

9.500 .954750 .00033

9.600 .959610 .00031

9.700 .964520 .00032

9.800 .969730 .00031

9.900 .973870 .00031
30

7.900 .920160 .00034

7.950 .923630 .00036

8.000 .927250 .00036

8.050 .930310 .00036

8.100 .934240 .00035

8.150 .937690 .00036

8.200 .941040 .00035

8.250 .944840 .00036

8.300 .948510 .00035

8.350 .951350 .00034

8.400 .954530 .00036
32

7.600 .917570 .00037

7.650 .920760 .00037

7.700 .925300 .00037

7.750 .928820 .00036

7.800 .932490 .00038

7.850 .936660 .00036

7.900 .940020 .00036

7.950 .944250 .00037

8.000 .947940 .00036

8.050 .951530 .00036

8.100 .954230 .00036 
10.000 .979060 .00031 10.100 .982750 .00031 10.200 .987690 .00031 10.300 .992060 .00031 10.400 .996320 .00032 10.501 .000660 .00031 10.601 .004180 .00032 10.701 .008400 .00031 10.801 .012110 .00030 10.901 .016450 .00032

\section{5}

7.300 .919090 .00037

7.350 .923660 .00038

7.400 .928000 .00037

7.450 .932670 .00038

7.500 .936620 .00037

7.550 .940640 .00037

7.600 .943750 .00038

7.650 .948480 .00038

7.700 .953460 .00038

7.750 .956690 .00039

7.800 .960490 .00037

7.850 .964650 .00037

7.900 .967830 .00036

7.950 .972280 .00037

8.000 .975600 .00037

8.050 .979350 .00036

8.100 .983420 .00037

8.150 .986670 .00036

8.200 .989910 .00038

8.250 .994010 .00037

8.300 .996970 .00037

8.351 .000150 .00037

8.401 .003430 .00036

8.451 .007690 .00038

8.501 .011120 .00037

8.551 .014420 .00037

8.601 .017580 .00036
8.450 .958290 .00035

8.500 .961250 .00035

8.550 .963930 .00035

8.600 .967870 .00035

8.650 .970720 .00035

8.700 .973860 .00035

8.750 .976800 .00035

8.800 .979940 .00034

8.850 .983180 .00035

8.900 .985550 .00035

8.950 .988780 .00035

9.000 .991040 .00034

9.050 .994770 .00034

9.100 .997890 .00036

9.151 .000320 .00035

9.201 .003340 .00034

9.251 .006220 .00034

9.301 .008590 .00035

9.351 .010790 .00035

9.401 .014300 .00035

9.451 .016610 .00035

9.501 .019160 .00034

\section{0}

6.900 .920090 .00038

6.950 .925820 .00040

7.000 .930020 .00039

7.050 .934390 .00038

7.100 .939700 .00040

7.150 .943870 .00040

7.200 .948520 .00039

7.250 .953460 .00040

7.300 .957860 .00040

7.350 .962180 .00039

7.400 .966320 .00040

7.450 .970700 .00039

7.500 .974600 .00040

7.550 .978930 .00037

7.600 .983730 .00039

7.650 .988220 .00038

7.700 .991420 .00039

7.750 .995870 .00039

7.800 .999380 .00039

7.851 .003980 .00039

7.901 .007590 .00039

7.951 .011810 .00038

8.001 .016200 .00038

8.051 .018850 .00039

8.101 .023000 .00039
8.150 .958760 .00036

8.200 .962270 .00036

8.250 .964970 .00036

8.300 .969320 .00036

8.350 .972460 .00037

8.400 .976030 .00036

8.450 .978690 .00037

8.500 .981890 .00037

8.550 .985650 .00035

8.600 .988440 .00035

8.650 .991370 .00037

8.700 .994740 .00037

8.750 .998330 .00037

8.801 .001350 .00036

8.851 .003960 .00035

8.901 .007640 .00036

8.951 .009830 .00035

9.001 .013680 .00035

9.051 .016810 .00035

9.101 .019150 .00036

\section{0}

6.300 .912780 .00042

6.350 .918480 .00041

6.400 .923870 .00042

6.450 .929180 .00042

6.500 .934930 .00041

6.550 .939840 .00042

6.600 .945790 .00042

6.650 .952120 .00042

6.700 .956730 .00043

6.750 .961460 .00044

6.800 .966760 .00042

6.850 .972690 .00043

6.900 .978550 .00042

6.950 .981770 .00042

7.000 .986820 .00042

7.050 .992090 .00042

7.100 .997060 .00044

7.151 .002400 .00042

7.201 .006600 .00042

7.251 .011290 .00043

7.301 .015910 .00041

7.351 .020470 .00041

7.401 .025030 .00042 
60

6.000 .914630 .00043 6.050 .920980 .00044 6.100 .927430 .00044 6.150 .933780 .00043 6.200 .939960 .00045 6.250 .946380 .00044 6.300 .951930 .00043 6.350 .957580 .00043 6.400 .964320 .00043 6.450 .969650 .00043 6.500 .975730 .00044 6.550 .981000 .00044 6.600 .986400 .00043 6.650 .991910 .00044 6.700 .997730 .00042 6.751 .002520 .00044 6.801 .008630 .00044 6.851 .013430 .00043 6.901 .018610 .00042

90

5.500 .916260 .00046 5.550 .924400 .00047 5.600 .931530 .00047 5.650 .937330 .00048 5.700 .946110 .00046 5.750 .953360 .00046 5.800 .960360 .00048 5.850 .966920 .00047 5.900 .973360 .00047 5.950 .979450 .00047 6.000 .987230 .00045 6.050 .992720 .00046 6.101 .000230 .00045 6.151 .005400 .00047 6.201 .013790 .00046 6.251 .019610 .00046 6.301 .025540 .00046

130 5.200 .913680 .00049 5.250 .921690 .00049 5.300 .929540 .00050
70

5.800 .918190 .00045

5.850 .924620 .00046

5.900 .931430 .00046

5.950 .938110 .00045

6.000 .944280 .00044

6.050 .950500 .00044

6.100 .958130 .00044

6.150 .963720 .00045

6.200 .970120 .00044

6.250 .975830 .00046

6.300 .982190 .00045

6.350 .988970 .00045

6.400 .993790 .00045

6.451 .000460 .00046

6.501 .005440 .00045

6.551 .011460 .00046

6.601 .017630 .00045

6.651 .023590 .00045

6.701 .028470 .00044

100

5.400 .915010 .00048

5.450 .922870 .00048

5.500 .931390 .00048

5.550 .937950 .00048

5.600 .945570 .00046

5.650 .952700 .00047

5.700 .960630 .00048

5.750 .967180 .00049

5.800 .974350 .00049

5.850 .982170 .00048

5.900 .988690 .00048

5.950 .995100 .00048

6.001 .002580 .00047

6.051 .008500 .00047

6.101 .015460 .00046

6.151 .021580 .00048

6.201 .027990 .00048
80

5.600 .911820 .00048 5.650 .919390 .00046 5.700 .926840 .00046 5.750 .934620 .00046 5.800 .941030 .00047 5.850 .947540 .00047 5.900 .954700 .00045 5.950 .960940 .00048 6.000 .968090 .00046 6.050 .975050 .00046 6.100 .981420 .00045 6.150 .987340 .00048 6.200 .994240 .00046 6.250 .998900 .00046 6.301 .006340 .00046 6.351 .012270 .00045 6.401 .018530 .00046 6.451 .024180 .00046 6.501 .029580 .00047

118

5.300 .918460 .00049 5.350 .927790 .00047 5.400 .935520 .00049 5.450 .943180 .00048 5.500 .949890 .00049 5.550 .957950 .00050 5.600 .966440 .00049 5.650 .972390 .00048 5.700 .979870 .00048 5.750 .987430 .00048 5.800 .994540 .00048 5.851 .001600 .00048 5.901 .008480 .00049 5.951 .015710 .00049 6.001 .022280 .00050
150

5.100 .909380 .00049 5.150 .918050 .00049 5.200 .926930 .00049
200

5.000 .911200 .00049 5.050 .919100 .00050 5.100 .928200 .00050 
5.350 .937510 .00050 5.400 .945060 .00048 5.450 .953020 .00050 5.500 .960460 .00049 5.550 .968180 .00049 5.600 .975760 .00048 5.650 .982970 .00048 5.700 .990610 .00050 5.750 .998360 .00049 5.801 .005070 .00049 5.851 .012040 .00050 5.901 .019110 .00049 5.951 .025410 .00050 6.001 .032510 .00048

235 5.000 .918170 .00051 5.050 .925810 .00052 5.100 .935180 .00050 5.150 .943700 .00049 5.200 .951390 .00048 5.250 .960380 .00052 5.300 .968190 .00051 5.350 .976070 .00051 5.400 .983360 .00051 5.450 .991790 .00050 5.500 .999230 .00051 5.551 .007020 .00051 5.601 .014960 .00052 5.651 .022480 .00050 5.701 .029690 .00051

\section{0}

5.000 .910870 .00050 5.050 .919240 .00049 5.100 .927220 .00050 5.150 .935830 .00050 5.200 .944800 .00050 5.250 .951540 .00051 5.300 .959790 .00053 5.350 .967820 .00051 5.400 .975730 .00050 5.450 .982930 .00051 5.500 .990960 .00051 5.550 .997890 .00050 5.601 .005830 .00051 5.651 .013000 .00051
5.250 .934750 .00048

5.300 .942280 .00049

5.350 .949510 .00050

5.400 .958220 .00049

5.450 .966550 .00049

5.500 .973410 .00051

5.550 .981880 .00048

5.600 .989700 .00049

5.650 .996600 .00049

5.701 .003220 .00049

5.751 .010620 .00048

5.801 .017730 .00051

5.851 .025660 .00050

5.901 .032290 .00049
5.150 .937490 .00051 5.200 .944780 .00050 5.250 .952830 .00052 5.300 .961400 .00052 5.350 .969080 .00051 5.400 .976370 .00052 5.450 .985020 .00050 5.500 .993060 .00051 5.551 .000810 .00051 5.601 .008260 .00051 5.651 .016300 .00050 5.701 .022470 .00050
250

5.000 .919790 .00051

5.050 .927690 .00050

5.100 .936570 .00049

5.150 .945040 .00052

5.200 .953480 .00051

5.250 .961610 .00050

5.300 .969080 .00050

5.350 .977930 .00049

5.400 .985480 .00049

5.450 .993280 .00052

5.501 .001420 .00050

5.551 .008470 .00051

5.601 .015910 .00050

5.651 .023850 .00051

5.701 .031340 .00049
353

5.000 .921280 .00053 5.050 .929110 .00050 5.100 .937550 .00050 5.150 .946830 .00050 5.200 .954090 .00050 5.250 .962390 .00052 5.300 .970430 .00051 5.350 .978410 .00051 5.400 .985700 .00051 5.450 .994080 .00051 5.501 .001590 .00051 5.551 .008340 .00050 5.601 .016690 .00051 5.651 .024260 .00053 5.701 .030920 .00051
5.000 .906210 .00050 5.050 .915130 .00051 5.100 .923370 .00050 5.150 .931770 .00050 5.200 .940630 .00049 5.250 .948170 .00050 5.300 .955210 .00050 5.350 .963350 .00050 5.400 .970500 .00050 5.450 .979640 .00051 5.500 .986560 .00051 5.550 .993600 .00051 5.601 .000890 .00051 5.651 .007600 .00051
510

5.000 .904900 .00050 5.050 .914560 .00050 5.100 .922490 .00051 5.150 .930730 .00050 5.200 .938560 .00051 5.250 .945830 .00051 5.300 .953880 .00050 5.350 .961710 .00049 5.400 .970210 .00050 5.450 .977620 .00049 5.500 .984550 .00051 5.550 .992190 .00052 5.600 .999730 .00052 5.651 .007140 .00050 


\subsection{1 .020410 .00051}

520

5.100 .921090 .00049

5.150 .929230 .00051

5.200 .936990 .00049

5.250 .944550 .00050

5.300 .952840 .00050

5.350 .960300 .00051

5.400 .968980 .00050

5.450 .976100 .00051

5.500 .982910 .00050

5.550 .989910 .00050

5.600 .997850 .00051

5.651 .004930 .00051

5.701 .012400 .00051

5.751 .020260 .00051

5.801 .027100 .00051

550

5.100 .915580 .00052

5.150 .923750 .00050

5.200 .931970 .00051

5.250 .940240 .00051

5.300 .947070 .00049

5.350 .954950 .00049

5.400 .963220 .00051

5.450 .971200 .00051

5.500 .978060 .00050

5.550 .986710 .00049

5.600 .992920 .00051

5.651 .000730 .00051

5.701 .008140 .00052

5.751 .014700 .00051

5.801 .021990 .00051
560
5.100 .914500 .00050
5.150 .922690 .00049
5.200 .931540 .00050
5.250 .938470 .00050
5.300 .945710 .00050
5.350 .953610 .00050
5.400 .961030 .00051
5.450 .969820 .00051
5.500 .977340 .00053
5.550 .984400 .00051
5.600 .991310 .00052
5.650 .998800 .00051
5.701 .005420 .00050
5.751 .013290 .00051
5.801 .020000 .00050
5.851 .027240 .00051
5.901 .033930 .00052

5.701 .015790 .00050

5.751 .023310 .00052

5.801 .029730 .00050

5.701 .014010 .00051

5.751 .021490 .00051 5.801 .028090 .00051

530

5.100 .918870 .00051

5.150 .927270 .00051

5.200 .934550 .00049

5.250 .943410 .00049

5.300 .950590 .00050

5.350 .958120 .00051

5.400 .967300 .00052

5.450 .973990 .00050

5.500 .981230 .00049

5.550 .988940 .00050

5.600 .996110 .00052

5.651 .003090 .00051

5.701 .011790 .00051

5.751 .017970 .00050

5.801 .025050 .00051

540

5.100 .918080 .00051

5.150 .925960 .00050

5.200 .934390 .00051

5.250 .941690 .00052

5.300 .948620 .00050

5.350 .956640 .00050

5.400 .964580 .00050

5.450 .972320 .00051

5.500 .979770 .00052

5.550 .986960 .00051

5.600 .995100 .00051

5.651 .001340 .00050

5.701 .009900 .00053

5.751 .016410 .00050

5.801 .022920 .00049 
U5o2N23-pl-1000-01

12

33.900 .913360 .00014

34.400 .914180 .00014

34.900 .915300 .00014

35.400 .915830 .00014

35.900 .916530 .00014

36.400 .916970 .00014

36.900 .917760 .00014

37.400 .918240 .00014

37.900 .919320 .00014

38.400 .919840 .00014

38.900 .920320 .00013

39.400 .921030 .00013

39.900 .921340 .00013

40.400 .921580 .00013

40.900 .922410 .00013

41.400 .922890 .00013

41.900 .923130 .00013

42.400 .924060 .00013

42.900 .924230 .00013

43.400 .924800 .00013

43.900 .925340 .00013

44.400 .925670 .00013

44.900 .925990 .00013
15

15.000 .927540 .00021

15.600 .935030 .00021

16.200 .941650 .00021

16.800 .947990 .00020

17.400 .953520 .00020

18.000 .958370 .00020

18.600 .963690 .00020

19.200 .968370 .00019

19.800 .972810 .00019

20.400 .976880 .00019

21.000 .979950 .00018

21.600 .983480 .00018

22.200 .986540 .00019

22.800 .989850 .00018

23.400 .993000 .00017

24.000 .995160 .00018

24.600 .997680 .00017

25.201 .000090 .00017

25.801 .002380 .00017

26.401 .005020 .00016

27.001 .006670 .00017

27.601 .008520 .00017

28.201 .010390 .00016

28.401 .010730 .00016
20

9.200 .923550 .00027

9.300 .927680 .00028

9.400 .930870 .00027

9.500 .934380 .00027

9.600 .937650 .00028

9.700 .941010 .00027

9.800 .944650 .00028

9.900 .948070 .00027

10.000 .950510 .00027

10.100 .953860 .00027

10.200 .956600 .00026

10.300 .959880 .00027

10.400 .963280 .00027

10.500 .966460 .00027

10.600 .968860 .00027

10.700 .971550 .00027

10.800 .973950 .00026

10.900 .976710 .00027

11.000 .979980 .00026

11.100 .982530 .00026

11.200 .984570 .00027

11.300 .987480 .00026

11.400 .989920 .00026

11.500 .992520 .00026

11.600 .994900 .00026

11.700 .997070 .00025

11.800 .999800 .00026

11.901 .001880 .00025

12.001 .003830 .00027

12.101 .006660 .00026

12.201 .008900 .00026

12.301 .010470 .00025

12.401 .012630 .00024
25

7.300 .920540 .00032

7.400 .926530 .00031

7.500 .931600 .00032

7.600 .937800 .00032

7.700 .943290 .00033

7.800 .948010 .00031

7.900 .952740 .00031

8.000 .958340 .00031

8.100 .962970 .00031

8.200 .968040 .00031

8.300 .972900 .00031
30

6.400 .924460 .00034

6.450 .928680 .00034

6.500 .931320 .00034

6.550 .935110 .00034

6.600 .938420 .00035

6.650 .942410 .00034

6.700 .945290 .00034

6.750 .948720 .00034

6.800 .952270 .00036

6.850 .955640 .00034

6.900 .958690 .00035
32

6.100 .922650 .00035

6.150 .926800 .00036

6.200 .930190 .00036

6.250 .933400 .00035

6.300 .937530 .00034

6.350 .941330 .00035

6.400 .945370 .00035

6.450 .948810 .00035

6.500 .951910 .00035

6.550 .956690 .00036

6.600 .959350 .00036 
8.400 .977290 .00032

8.500 .982210 .00030

8.600 .986330 .00031

8.700 .990600 .00031

8.800 .994080 .00031

8.900 .999270 .00031

9.001 .003020 .00030

9.101 .006920 .00030

9.201 .011030 .00030

9.301 .014520 .00031
6.950 .962490 .00034

7.000 .965650 .00034

7.050 .968560 .00034

7.100 .971510 .00034

7.150 .974480 .00035

7.200 .977810 .00034

7.250 .980820 .00034

7.300 .983900 .00035

7.350 .986840 .00033

7.400 .990170 .00034

7.450 .992430 .00035

7.500 .995020 .00033

7.550 .998800 .00033

7.601 .000680 .00034

7.651 .004440 .00033

7.701 .007390 .00033

7.751 .009890 .00033

7.801 .012450 .00033

7.851 .015210 .00033

7.901 .017580 .00033
6.650 .962440 .00034

6.700 .966360 .00036

6.750 .969880 .00035

6.800 .973220 .00035

6.850 .977180 .00035

6.900 .980310 .00035

6.950 .983630 .00034

7.000 .986690 .00034

7.050 .989850 .00035

7.100 .993140 .00035

7.150 .996110 .00035

7.200 .998980 .00035

7.251 .001810 .00034

7.301 .005150 .00034

7.351 .008380 .00033

7.401 .011310 .00035

7.451 .014610 .00034

7.501 .017050 .00034
35

5.800 .925320 .00037

5.850 .929290 .00037

5.900 .933310 .00038

5.950 .937980 .00037

6.000 .941020 .00036

6.050 .946290 .00036

6.100 .949910 .00037

6.150 .953820 .00036

6.200 .957420 .00036

6.250 .961670 .00036

6.300 .965360 .00035

6.350 .969340 .00036

6.400 .972970 .00035

6.450 .977420 .00036

6.500 .980410 .00036

6.550 .983780 .00036

6.600 .988010 .00036

6.650 .991420 .00036

6.700 .994310 .00036

6.750 .998740 .00036

6.801 .002480 .00036

6.851 .005580 .00035

6.901 .009050 .00036

6.951 .012410 .00035

7.001 .015800 .00035
40

5.300 .916410 .00038

5.350 .922850 .00038

5.400 .927650 .00038

5.450 .931180 .00039

5.500 .935840 .00038

5.550 .940950 .00037

5.600 .945370 .00038

5.650 .950660 .00038

5.700 .954200 .00037

5.750 .959520 .00038

5.800 .964090 .00037

5.850 .968310 .00038

5.900 .971970 .00038

5.950 .977090 .00038

6.000 .981360 .00037

6.050 .985310 .00039

6.100 .989250 .00037

6.150 .993530 .00037

6.200 .997950 .00037

6.251 .001550 .00038

6.301 .005120 .00039

6.351 .009130 .00038

6.401 .013070 .00038

6.451 .017320 .00037

6.501 .020620 .00037
50

4.800 .920420 .00040 4.850 .925480 .00041 4.900 .931310 .00041 4.950 .937240 .00041 5.000 .942780 .00040 5.050 .948580 .00038 5.100 .954120 .00041 5.150 .958690 .00041 5.200 .964150 .00040 5.250 .969030 .00041 5.300 .973820 .00041 5.350 .979740 .00041 5.400 .985100 .00040 5.450 .989740 .00039 5.500 .994250 .00041 5.550 .999250 .00040 5.601 .004150 .00041 5.651 .008880 .00040 5.701 .013360 .00040 5.751 .018170 .00040 5.801 .023280 .00040 
60

4.400 .911790 .00042 4.450 .918210 .00043 4.500 .924820 .00042 4.550 .930440 .00042 4.600 .937860 .00043 4.650 .943410 .00042 4.700 .948850 .00042 4.750 .955250 .00041 4.800 .961070 .00043 4.850 .966620 .00041 4.900 .973390 .00042 4.950 .978260 .00042 5.000 .983490 .00041 5.050 .990360 .00043 5.100 .995410 .00043 5.151 .000270 .00041 5.201 .005830 .00041 5.251 .010650 .00040 5.301 .016080 .00042 5.351 .022130 .00041 5.401 .026490 .00042

90

3.900 .913960 .00045 3.950 .921070 .00044 4.000 .929260 .00045 4.050 .936580 .00044 4.100 .943630 .00046 4.150 .949880 .00045 4.200 .957700 .00045 4.250 .965490 .00045 4.300 .972030 .00046 4.350 .978600 .00045 4.400 .985500 .00044 4.450 .991940 .00046 4.500 .998140 .00047 4.551 .004390 .00045 4.601 .011810 .00046 4.651 .017400 .00046 4.701 .023930 .00045

130

3.600 .912940 .00047 3.650 .920350 .00046 3.700 .929450 .00046
70

4.200 .915330 .00043

4.250 .921380 .00044

4.300 .928670 .00042

4.350 .935380 .00044

4.400 .942090 .00043

4.450 .948650 .00045

4.500 .954820 .00043

4.550 .961490 .00043

4.600 .967500 .00043

4.650 .973880 .00042

4.700 .980160 .00043

4.750 .985740 .00044

4.800 .991420 .00044

4.850 .997870 .00044

4.901 .003980 .00044

4.951 .010290 .00043

5.001 .015960 .00043

5.051 .021270 .00044

5.101 .026600 .00043
80

4.100 .924470 .00044

4.150 .931360 .00045

4.200 .937970 .00045

4.250 .945850 .00044

4.300 .953210 .00044

4.350 .959360 .00044

4.400 .965110 .00046

4.450 .972690 .00045

4.500 .978690 .00044

4.550 .985410 .00044

4.600 .992070 .00044

4.650 .998180 .00044

4.701 .003900 .00044

4.751 .010440 .00043

4.801 .016300 .00044

4.851 .022330 .00045

4.901 .028220 .00045
3.800 .913670 .00046

3.850 .921250 .00045

3.900 .928910 .00044

3.950 .936410 .00045

4.000 .944400 .00047

4.050 .951530 .00046

4.100 .959660 .00046

4.150 .966160 .00046

4.200 .972380 .00046

4.250 .979140 .00046

4.300 .986950 .00044

4.350 .993660 .00047

4.401 .000470 .00046

4.451 .007590 .00046

4.501 .013670 .00046

4.551 .021440 .00046

4.601 .027420 .00045
118

3.700 .918050 .00046

3.750 .926650 .00046

3.800 .934820 .00046

3.850 .941410 .00046

3.900 .949760 .00047

3.950 .957050 .00046

4.000 .964150 .00047

4.050 .972040 .00047

4.100 .979720 .00046

4.150 .987020 .00047

4.200 .993620 .00048

4.251 .000950 .00046

4.301 .008010 .00047

4.351 .014490 .00047

4.401 .021910 .00047
150

2.800 .781020 .00046

2.820 .784820 .00046

2.840 .788950 .00046
200

2.400 .719050 .00047 2.420 .723430 .00047 2.440 .727390 .00046 
3.750 .938140 .00047 3.800 .945610 .00047 3.850 .953360 .00047 3.900 .960840 .00048 3.950 .968120 .00047 4.000 .975210 .00048 4.050 .982680 .00048 4.100 .990630 .00046 4.150 .998080 .00047 4.201 .004810 .00047 4.251 .012500 .00046 4.301 .019220 .00047
2.860 .793470 .00047

2.880 .797460 .00046

2.900 .801610 .00047

2.920 .804380 .00046

2.940 .809030 .00047

2.960 .811600 .00047

2.980 .816560 .00047

3.000 .820440 .00047

3.020 .824740 .00048

3.040 .827290 .00046

3.060 .831130 .00047

3.080 .835390 .00047

3.100 .839280 .00047

3.120 .843100 .00047

3.140 .845640 .00046

3.160 .850040 .00047

3.180 .853800 .00048

3.200 .857270 .00047

3.220 .860920 .00047

3.240 .864850 .00047

3.260 .868960 .00048

3.280 .871590 .00048

3.300 .875340 .00048
2.460 .731600 .00045

2.480 .735710 .00046

2.500 .739940 .00047

2.520 .744150 .00046

2.540 .748080 .00047

2.560 .753340 .00047

2.580 .757340 .00046

2.600 .761450 .00047

2.620 .765070 .00047

2.640 .769910 .00047

2.660 .773270 .00047

2.680 .777670 .00046

2.700 .781200 .00048

2.720 .785710 .00048

2.740 .789000 .00047

2.760 .794380 .00047

2.780 .797950 .00048

2.800 .802390 .00047
235

1.900 .613420 .00045 1.920 .618120 .00044 1.940 .623160 .00044 1.960 .627190 .00047 1.980 .631810 .00045 2.000 .637440 .00046 2.020 .641330 .00047 2.040 .645970 .00044 2.060 .651200 .00045 2.080 .656060 .00046 2.100 .660570 .00046 2.120 .665050 .00045 2.140 .669720 .00046 2.160 .673510 .00046 2.180 .678280 .00045 2.200 .683440 .00046 2.220 .686930 .00045 2.240 .691860 .00046 2.260 .696940 .00045 2.280 .700880 .00047 2.300 .705120 .00045
250
2.000 .639730 .00045
2.020 .644210 .00047
2.040 .649610 .00046
2.060 .653800 .00044
2.080 .658910 .00045
2.100 .662740 .00047
2.120 .667900 .00047
2.140 .671500 .00046
2.160 .676610 .00045
2.180 .681040 .00046
2.200 .686100 .00047
2.220 .689950 .00046
2.240 .694550 .00046
2.260 .699240 .00047
2.280 .703860 .00047
2.300 .708510 .00046
2.320 .712800 .00045
2.340 .716710 .00047
2.360 .720910 .00047
2.380 .725190 .00046
2.400 .730780 .00047 
470

2.300 .715410 .00048

2.320 .719790 .00046

2.340 .724120 .00048

2.360 .728530 .00048

2.380 .731870 .00047

2.400 .735610 .00046

2.420 .740220 .00045

2.440 .744450 .00048

2.460 .748100 .00046

2.480 .753380 .00047

2.500 .756990 .00049

2.520 .760610 .00048

2.540 .764810 .00048

2.560 .768400 .00046

2.580 .772970 .00048

2.600 .777640 .00047
520

2.200 .691820 .00045

2.220 .696180 .00047

2.240 .699620 .00046

2.260 .704200 .00047

2.280 .709160 .00046

2.300 .713210 .00046

2.320 .717160 .00046

2.340 .721050 .00046

2.360 .725500 .00047

2.380 .729200 .00047

2.400 .732970 .00047

2.420 .738040 .00047

2.440 .741060 .00047

2.460 .745700 .00047

2.480 .749200 .00046

2.500 .753740 .00048
500

2.100 .671730 .00046

2.120 .675430 .00047

2.140 .679880 .00046

2.160 .684570 .00047

2.180 .688980 .00046

2.200 .692760 .00045

2.220 .697670 .00046

2.240 .702000 .00047

2.260 .706000 .00046

2.280 .710010 .00046

2.300 .714210 .00048

2.320 .718410 .00047

2.340 .722900 .00047

2.360 .726740 .00047

2.380 .730610 .00046

2.400 .734570 .00046

2.420 .738000 .00047

2.440 .742590 .00048

2.460 .747410 .00049

2.480 .751420 .00048

2.500 .755070 .00048
510

2.200 .692290 .00047

2.220 .696460 .00046

2.240 .701670 .00047

2.260 .705390 .00047

2.280 .709540 .00048

2.300 .713650 .00047

2.320 .717990 .00046

2.340 .721440 .00046

2.360 .725340 .00046

2.380 .729660 .00046

2.400 .734880 .00047

2.420 .739020 .00046

2.440 .742360 .00046

2.460 .746220 .00047

2.480 .749980 .00047

2.500 .754990 .00047

2.520 .758360 .00048

2.540 .762840 .00047

2.560 .765980 .00048

2.580 .770260 .00046

2.600 .774180 .00048

540

2.600 .772290 .00047

2.620 .776580 .00048

2.640 .780800 .00047

2.660 .784880 .00047

2.680 .787780 .00048

2.700 .791340 .00048

2.720 .796940 .00047

2.740 .799080 .00047

2.760 .804010 .00049

2.780 .806790 .00049

2.800 .810490 .00047

2.820 .814520 .00047

2.840 .818430 .00049

2.860 .821900 .00049

2.880 .825460 .00048

2.900 .829040 .00049

2.920 .832530 .00049

2.940 .837180 .00049

2.960 .840700 .00048

2.980 .845060 .00048

3.000 .846820 .00049
2.300 .711940 .00047

2.320 .715690 .00046

2.340 .719880 .00047

2.360 .724200 .00046

2.380 .727680 .00047

2.400 .731800 .00047

2.420 .736130 .00047

2.440 .739390 .00047

2.460 .744590 .00048

2.480 .748130 .00047

2.500 .751400 .00046

2.520 .756060 .00046

2.540 .759430 .00047

2.560 .764180 .00047

2.580 .768140 .00048

2.600 .771490 .00047 
550 560

$2.200 .689840 .00046 \quad 2.400 .730590 .00047$

$2.220 .694160 .00047 \quad 2.420 .734010 .00047$

$\begin{array}{lll}2.24 & 0.698030 .00047 & 2.440 .738210 .00047\end{array}$

$2.260 .702870 .00047 \quad 2.460 .742210 .00046$

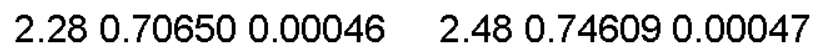

$2.300 .711760 .00046 \quad 2.500 .750180 .00048$

$\begin{array}{lll}2.320 .714420 .00047 & 2.520 .754620 .00048\end{array}$

$\begin{array}{lll}2.340 .718990 .00047 & 2.540 .758930 .00047\end{array}$

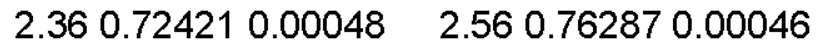

$2.380 .727360 .00047 \quad 2.580 .765750 .00047$

$2.400 .731440 .00047 \quad 2.600 .769690 .00048$

$2.420 .735280 .00046 \quad 2.620 .773670 .00047$

$\begin{array}{ll}2.440 .739340 .00048 & 2.640 .778170 .00047\end{array}$

$2.460 .743830 .00047 \quad 2.660 .781670 .00048$

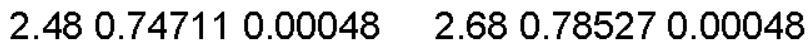

$2.500 .750560 .00047 \quad 2.700 .789330 .00046$

$\begin{array}{lll}2.520 .75490 & 0.00048 & 2.720 .792570 .00047\end{array}$

$\begin{array}{lll}2.540 .759290 .00047 & 2.740 .797140 .00047\end{array}$

$\begin{array}{lll}2.560 .764050 .00047 & 2.760 .799630 .00048\end{array}$

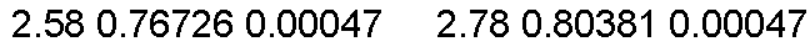

$2.600 .770490 .00047 \quad 2.800 .808820 .00048$ 
U5o2N23-pl-1000-10

12

25.200 .896020 .00015 30.500 .909140 .00014 35.800 .918010 .00013 41.100 .923780 .00013 46.400 .927940 .00012 51.700 .931060 .00012 57.000 .933270 .00012 62.300 .935110 .00012 67.600 .936170 .00012 72.900 .937630 .00011 78.200 .938440 .00011 83.500 .939320 .00011 88.800 .939760 .00011 94.100 .940330 .00011 99.400 .941020 .00011 104.700 .941260 .00011 110.000 .941720 .00011 115.300 .941910 .00011 120.600 .942250 .00011 125.900 .942520 .00011 131.200 .942780 .00011 132.200 .942590 .00011
15

13.900 .928360 .00020 14.500 .935470 .00020 15.100 .942190 .00020 15.700 .948320 .00019 16.300 .954000 .00019 16.900 .959420 .00019 17.500 .964250 .00018 18.100 .968600 .00019 18.700 .972670 .00018 19.300 .977370 .00018 19.900 .980490 .00018 20.500 .984090 .00017 21.100 .986970 .00017 21.700 .990310 .00017 22.300 .992920 .00017 22.900 .996110 .00017 23.500 .998360 .00017 24.101 .000700 .00017 24.701 .003140 .00016 25.301 .004920 .00016 25.901 .006630 .00016 26.501 .008560 .00016 26.901 .009890 .00016
25

6.200 .924000 .00030 6.300 .929230 .00029 6.400 .935030 .00030 6.500 .940740 .00028 6.600 .945570 .00029 6.700 .951470 .00029 6.800 .956370 .00029 6.900 .961110 .00029 7.000 .966400 .00029 7.100 .970890 .00029 7.200 .975690 .00028
30

5.200 .921230 .00032

5.250 .925230 .00031

5.300 .929780 .00032

5.350 .932830 .00031

5.400 .936510 .00032

5.450 .940490 .00031

5.500 .942960 .00031

5.550 .947120 .00031

5.600 .950290 .00031

5.650 .953730 .00031

5.700 .957230 .00032
20

8.100 .925410 .00026 8.200 .929150 .00026 8.300 .932650 .00026 8.400 .936370 .00027 8.500 .939060 .00026 8.600 .942770 .00026 8.700 .945900 .00026 8.800 .949230 .00026 8.900 .952850 .00025 9.000 .955940 .00026 9.100 .958330 .00025 9.200 .961510 .00026 9.300 .964750 .00026 9.400 .967150 .00026 9.500 .970080 .00025 9.600 .972740 .00025 9.700 .975590 .00025 9.800 .979340 .00026 9.900 .981650 .00025 10.000 .983820 .00024 10.100 .986430 .00025 10.200 .988770 .00025 10.300 .991520 .00024 10.400 .993750 .00025 10.500 .996240 .00025 10.600 .999040 .00024 10.701 .000530 .00025 10.801 .003440 .00025 10.901 .005420 .00024 11.001 .007450 .00024 11.101 .010320 .00024 11.201 .011830 .00024 11.301 .014180 .00024

32

4.900 .918930 .00033 4.950 .923680 .00033 5.000 .927830 .00032 5.050 .931770 .00033 5.100 .935410 .00033 5.150 .939650 .00032 5.200 .943440 .00033 5.250 .946980 .00033 5.300 .951020 .00032 5.350 .954010 .00032 5.400 .957940 .00032 
7.300 .980120 .00030 7.400 .984780 .00028 7.500 .989090 .00029 7.600 .993180 .00028 7.700 .997550 .00028 7.801 .002050 .00028 7.901 .005500 .00029 8.001 .009500 .00028 8.101 .013770 .00028 8.201 .017590 .00028

\section{5}

4.600 .922710 .00033 4.650 .927130 .00034 4.700 .931010 .00033 4.750 .935460 .00033 4.800 .939890 .00034 4.850 .943980 .00033 4.900 .948590 .00033 4.950 .951990 .00033 5.000 .955900 .00033 5.050 .960520 .00033 5.100 .963880 .00033 5.150 .968400 .00033 5.200 .972200 .00033 5.250 .975080 .00033 5.300 .980070 .00033 5.350 .983540 .00033 5.400 .986610 .00033 5.450 .990720 .00033 5.500 .993370 .00034 5.550 .997020 .00034 5.601 .001240 .00034 5.651 .005560 .00033 5.701 .008070 .00034 5.751 .011960 .00033 5.801 .014850 .00033
5.750 .960570 .00032

5.800 .963370 .00031

5.850 .966970 .00032

5.900 .970160 .00032

5.950 .972840 .00031

6.000 .976630 .00031

6.050 .978880 .00032

6.100 .982090 .00033

6.150 .985450 .00031

6.200 .988200 .00032

6.250 .992000 .00030

6.300 .994240 .00030

6.350 .997420 .00031

6.400 .999930 .00031

6.451 .003040 .00031

6.501 .005860 .00031

6.551 .008310 .00031

6.601 .010860 .00030

6.651 .014310 .00030

6.701 .017190 .00030
5.450 .961860 .00032

5.500 .964770 .00032

5.550 .968500 .00032

5.600 .972470 .00033

5.650 .975450 .00032

5.700 .978790 .00033

5.750 .981830 .00032

5.800 .985600 .00033

5.850 .989170 .00032

5.900 .991720 .00032

5.950 .995110 .00032

6.000 .998080 .00032

6.051 .001350 .00032

6.101 .004550 .00033

6.151 .007230 .00033

6.201 .010800 .00032

6.251 .014120 .00031

6.301 .017140 .00032
40

4.200 .924890 .00035

4.250 .930810 .00036

4.300 .934810 .00035

4.350 .939870 .00035

4.400 .944860 .00035

4.450 .950000 .00035

4.500 .954400 .00034

4.550 .958650 .00035

4.600 .963330 .00034

4.650 .967510 .00034

4.700 .971770 .00035

4.750 .975340 .00035

4.800 .980570 .00035

4.850 .985020 .00035

4.900 .988970 .00034

4.950 .993360 .00034

5.000 .998000 .00034

5.051 .002110 .00035

5.101 .005730 .00034

5.151 .009420 .00036

5.201 .013000 .00034
50

3.600 .919870 .00037

3.650 .926490 .00037

3.700 .932480 .00035

3.750 .937680 .00037

3.800 .943410 .00037

3.850 .948650 .00037

3.900 .954030 .00037

3.950 .960210 .00037

4.000 .965220 .00037

4.050 .970870 .00036

4.100 .975760 .00036

4.150 .980390 .00037

4.200 .986580 .00037

4.250 .991440 .00037

4.300 .996550 .00037

4.351 .001400 .00037

4.401 .005940 .00038

4.451 .010850 .00037

4.501 .015870 .00038 
60

3.200 .913850 .00039

3.250 .921030 .00037

3.300 .928080 .00038

3.350 .934120 .00039

3.400 .940130 .00039

3.450 .946560 .00039

3.500 .952670 .00039

3.550 .958640 .00039

3.600 .965450 .00038

3.650 .970700 .00040

3.700 .976780 .00039

3.750 .983010 .00038

3.800 .988270 .00037

3.850 .994440 .00037

3.900 .999800 .00038

3.951 .004520 .00038

4.001 .010160 .00038

4.051 .015820 .00039

4.101 .021760 .00038
70

3.000 .920460 .00041

3.050 .927290 .00039

3.100 .934320 .00039

3.150 .941330 .00039

3.200 .947740 .00039

3.250 .954370 .00040

3.300 .961220 .00039

3.350 .966780 .00039

3.400 .974130 .00039

3.450 .980720 .00039

3.500 .985890 .00040

3.550 .993080 .00039

3.600 .998720 .00040

3.651 .004450 .00039

3.701 .011530 .00040

3.751 .015820 .00039

3.801 .022790 .00040
80

2.800 .917990 .00041

2.850 .924930 .00040

2.900 .932360 .00041

2.950 .939390 .00040

3.000 .946400 .00040

3.050 .953930 .00041

3.100 .960790 .00040

3.150 .967160 .00040

3.200 .974240 .00041

3.250 .980790 .00041

3.300 .988770 .00040

3.350 .994080 .00040

3.401 .000870 .00042

3.451 .007210 .00040

3.501 .013830 .00040

3.551 .019830 .00041

3.601 .025920 .00040
90

2.700 .923170 .00040

2.800 .938830 .00040

2.850 .946610 .00042

2.900 .953770 .00040

2.950 .961350 .00040

3.000 .969650 .00040

3.050 .976030 .00042

3.100 .982610 .00040

3.150 .989440 .00041

3.200 .995820 .00043

3.251 .003120 .00041

3.301 .009550 .00041

3.351 .016070 .00041

3.401 .023150 .00040
130

2.300 .915300 .00043

2.350 .924520 .00041

2.400 .932570 .00042

2.450 .941530 .00042

2.500 .949530 .00042

2.550 .956780 .00043

2.600 .964440 .00042
100

2.600 .926710 .00041

2.650 .933860 .00043

2.700 .941130 .00042

2.750 .949600 .00041

2.800 .957900 .00041

2.850 .963930 .00042

2.900 .972280 .00041

2.950 .979180 .00041

3.000 .986500 .00041

3.050 .993780 .00041

3.101 .000940 .00041

3.151 .007960 .00041

3.201 .014350 .00042

3.251 .021120 .00041

3.301 .027660 .00041
118

2.400 .919180 .00042

2.450 .927500 .00042

2.500 .935650 .00042

2.550 .944100 .00042

2.600 .951460 .00042

2.650 .959850 .00043

2.700 .967250 .00042

2.750 .974730 .00042

2.800 .982430 .00042

2.850 .989740 .00041

2.900 .996670 .00043

2.951 .004840 .00043

3.001 .011900 .00042

3.051 .018380 .00042

3.101 .026230 .00042
150

2.200 .916200 .00042

2.250 .925060 .00043

2.300 .934270 .00043

2.350 .943050 .00043

2.400 .950990 .00043

2.450 .959410 .00044

2.500 .967010 .00042
200

2.000 .911170 .00043

2.050 .920780 .00043

2.100 .929210 .00044

2.150 .938640 .00044

2.200 .946580 .00044

2.250 .955450 .00044

2.300 .964540 .00044 
2.650 .974050 .00042

2.700 .981460 .00043

2.750 .987730 .00043

2.800 .995900 .00044

2.851 .003420 .00042

2.901 .011070 .00044

2.951 .017860 .00042

3.001 .024970 .00044
2.550 .975650 .00042

2.600 .982800 .00043

2.650 .990560 .00043

2.700 .998960 .00044

2.751 .006440 .00045

2.801 .013210 .00043

2.851 .021490 .00043

2.901 .028520 .00045
2.350 .971830 .00044

2.400 .980290 .00044

2.450 .988970 .00044

2.500 .996690 .00044

2.551 .004430 .00044

2.601 .011590 .00045

2.651 .020110 .00044

2.701 .028050 .00045
235

2.000 .924340 .00044

2.050 .934500 .00044

2.100 .943230 .00043

2.150 .951090 .00044

2.200 .960740 .00043

2.250 .968440 .00045

2.300 .976960 .00045

2.350 .985300 .00044

2.400 .992950 .00044

2.451 .000750 .00044

2.501 .009400 .00044

2.551 .016600 .00045

2.601 .025280 .00044

470

1.800 .920860 .00045

1.850 .929940 .00045

1.900 .938200 .00045

1.950 .946450 .00045

2.000 .955510 .00045

2.050 .963510 .00045

2.100 .972340 .00045

2.150 .979490 .00045

2.200 .987500 .00045

2.250 .995220 .00045

2.301 .003740 .00045

2.351 .010670 .00044

2.401 .018240 .00046
250

1.900 .911970 .00045

1.950 .920320 .00045

2.000 .930000 .00045

2.050 .938950 .00044

2.100 .947860 .00045

2.150 .955580 .00045

2.200 .964620 .00043

2.250 .972760 .00044

2.300 .980920 .00046

2.350 .990000 .00044

2.400 .998330 .00043

2.451 .005110 .00044

2.501 .013560 .00046

2.551 .021750 .00045

2.601 .029050 .00045
353

1.800 .912580 .00046

1.850 .922100 .00044

1.900 .931140 .00044

1.950 .939830 .00044

2.000 .949060 .00045

2.050 .956930 .00046

2.100 .965320 .00044

2.150 .973400 .00046

2.200 .981510 .00044

2.250 .990090 .00045

2.300 .998210 .00045

2.351 .005400 .00045

2.401 .013150 .00043

2.451 .021800 .00044

2.501 .028860 .00045
520

1.800 .923060 .00046 1.850 .930420 .00044 1.900 .938940 .00045
530

1.800 .921710 .00044

1.850 .930400 .00044

1.900 .939060 .00044
510

1.800 .922550 .00045

1.850 .931140 .00044

1.900 .939850 .00044

1.950 .948480 .00044

2.000 .956010 .00045

2.050 .963980 .00046

2.100 .972950 .00045

2.150 .979650 .00045

2.200 .988710 .00044

2.250 .995390 .00046

2.301 .002550 .00045

2.351 .009540 .00045

2.401 .017880 .00046 
1.950 .947280 .00046 2.000 .955740 .00045 2.050 .964390 .00045 2.100 .972690 .00045 2.150 .978730 .00045 2.200 .987150 .00044 2.250 .994890 .00044 2.301 .002430 .00045 2.351 .009870 .00044 2.401 .017260 .00045
1.950 .947560 .00044 2.000 .955930 .00044 2.050 .963580 .00045 2.100 .972310 .00044 2.150 .979340 .00045 2.200 .987060 .00045 2.250 .995360 .00045 2.301 .002420 .00045 2.351 .009310 .00046 2.401 .017160 .00045
1.950 .947380 .00044 2.000 .955920 .00045 2.050 .963340 .00045 2.100 .971790 .00046 2.150 .979550 .00045 2.200 .986720 .00046 2.250 .994520 .00045 2.301 .002930 .00045 2.351 .009920 .00047 2.401 .017280 .00045

550 560

$1.800 .922560 .00044 \quad 1.800 .922270 .00045$ $1.850 .931170 .00044 \quad 1.850 .930530 .00045$ $1.900 .939420 .00046 \quad 1.900 .940100 .00045$ $\begin{array}{lll}1.95 & 0.948020 .00045 & 1.950 .947450 .00045\end{array}$ $2.000 .955140 .00044 \quad 2.000 .955800 .00045$ $2.050 .963310 .00045 \quad 2.050 .963020 .00045$ $2.100 .971990 .00044 \quad 2.100 .971190 .00045$ $2.150 .978960 .00045 \quad 2.150 .979480 .00044$ $\begin{array}{lll}2.20 & 0.987400 .00045 & 2.200 .986910 .00045\end{array}$ $2.250 .995160 .00046 \quad 2.250 .994230 .00046$ $2.301 .001590 .00045 \quad 2.301 .001560 .00044$ $2.351 .010220 .00045 \quad 2.351 .008470 .00046$ $2.401 .016910 .00045 \quad 2.401 .015840 .00047$ 
U5o2N26-pl-1000-00

12

44.600 .883820 .00014 46.300 .885050 .00013 48.000 .886730 .00013 49.700 .887250 .00013 51.400 .888470 .00013 53.100 .889000 .00013 54.800 .889990 .00013 56.500 .891320 .00013 58.200 .891730 .00013 59.900 .892580 .00013 61.600 .893130 .00012 63.300 .893780 .00013 65.000 .894240 .00013 66.700 .895050 .00013 68.400 .895330 .00012 70.100 .895750 .00012 71.800 .896160 .00012 73.500 .896240 .00012 75.200 .897010 .00012 76.900 .897220 .00012 78.600 .897560 .00012 80.000 .897750 .00012
15

21.300 .929170 .00020 23.000 .940010 .00019 24.700 .948660 .00018 26.400 .956090 .00018 28.100 .962630 .00018 29.800 .968190 .00017 31.500 .972690 .00017 33.200 .976550 .00016 34.900 .980440 .00016 36.600 .983500 .00016 38.300 .986150 .00015 40.000 .988980 .00015 41.700 .990700 .00015 43.400 .992410 .00015 45.100 .994470 .00014 46.800 .995920 .00014 48.500 .997130 .00014 50.200 .998170 .00014 51.900 .999350 .00014 53.601 .000570 .00014 55.201 .001640 .00013
25

9.800 .922220 .00032 9.900 .926930 .00031 10.000 .931130 .00032 10.100 .935560 .00031 10.200 .940560 .00031 10.300 .944410 .00031 10.400 .949060 .00030 10.500 .953030 .00030 10.600 .957680 .00032 10.700 .961120 .00031 10.800 .964700 .00031 10.900 .969120 .00031 11.000 .973020 .00030 11.100 .976720 .00031 11.200 .980760 .00030 11.300 .983790 .00031 11.400 .987480 .00031 11.500 .991130 .00030 11.600 .994520 .00030 11.700 .998120 .00030 11.801 .001140 .00030
8.700 .924930 .00034 8.750 .928180 .00034 8.800 .931180 .00035 8.850 .934260 .00034 8.900 .937150 .00035 8.950 .940180 .00035 9.000 .943540 .00035 9.050 .946400 .00034 9.100 .948790 .00034 9.150 .951730 .00034 9.200 .955240 .00035 9.250 .957440 .00033 9.300 .959950 .00034 9.350 .962810 .00034 9.400 .966220 .00035 9.450 .968370 .00034 9.500 .970990 .00034 9.550 .973710 .00034 9.600 .976360 .00035 9.650 .979240 .00034 9.700 .981230 .00034

20

12.200 .923930 .00027 12.400 .929650 .00027 12.600 .934530 .00028 12.800 .940730 .00027 13.000 .944450 .00027 13.200 .949960 .00026 13.400 .955060 .00026 13.600 .959430 .00026 13.800 .964050 .00026 14.000 .968600 .00026 14.200 .971950 .00026 14.400 .976250 .00026 14.600 .979930 .00026 14.800 .984530 .00026 15.000 .987490 .00025 15.200 .991100 .00025 15.400 .994850 .00025 15.600 .997820 .00026 15.801 .001280 .00025 16.001 .004330 .00025 16.201 .008050 .00024 16.401 .010600 .00025 16.501 .011850 .00025
32
8.300 .920010 .00036 8.350 .923020 .00036 8.400 .926890 .00035 8.450 .929480 .00036 8.500 .933500 .00035 8.550 .936850 .00036 8.600 .939830 .00035 8.650 .943090 .00036 8.700 .946210 .00036 8.750 .949790 .00036 8.800 .952880 .00036 8.850 .956480 .00034 8.900 .958570 .00035 8.950 .962060 .00034 9.000 .964710 .00035 9.050 .967380 .00035 9.100 .970440 .00035 9.150 .974060 .00035 9.200 .976550 .00036 9.250 .979200 .00036 9.300 .982170 .00036 
11.901 .004630 .00031

12.001 .008130 .00030

12.101 .011590 .00029

12.201 .013760 .00029
9.750 .984070 .00034

9.800 .986870 .00033

9.850 .989730 .00034

9.900 .991260 .00034

9.950 .994130 .00034

10.000 .996470 .00035

10.050 .999100 .00034

10.101 .001690 .00033

10.151 .003560 .00032

10.201 .005620 .00034

10.251 .007940 .00032

10.301 .010560 .00033

10.351 .013160 .00034

10.401 .015080 .00033
9.350 .985060 .00034

9.400 .987770 .00035

9.450 .990310 .00034

9.500 .993590 .00035

9.550 .995790 .00035

9.600 .998640 .00034

9.651 .001210 .00035

9.701 .003570 .00035

9.751 .007000 .00034

9.801 .008520 .00035

9.851 .011320 .00036

9.901 .014110 .00035

9.951 .016410 .00034

10.001 .019220 .00035
35

7.900 .918330 .00038

7.950 .921810 .00037

8.000 .926270 .00037

8.050 .929870 .00038

8.100 .933540 .00037

8.150 .936780 .00036

8.200 .940050 .00037

8.250 .944180 .00037

8.300 .947550 .00036

8.350 .950810 .00036

8.400 .954910 .00038

8.450 .957820 .00036

8.500 .961570 .00037

8.550 .964620 .00036

8.600 .967720 .00036

8.650 .971970 .00036

8.700 .974150 .00038

8.750 .978020 .00038

8.800 .981800 .00036

8.850 .985210 .00037

8.900 .987350 .00037

8.950 .990370 .00036

9.000 .994090 .00036

9.050 .996000 .00036

9.100 .999810 .00036

9.151 .002840 .00036

9.201 .005750 .00035

9.251 .008430 .00036

9.301 .011380 .00037

9.351 .013940 .00036

9.401 .016790 .00036
40

7.400 .915470 .00038

7.450 .920980 .00040

7.500 .924940 .00039

7.550 .929160 .00038

7.600 .933340 .00039

7.650 .938060 .00039

7.700 .940950 .00039

7.750 .946300 .00039

7.800 .949380 .00038

7.850 .953070 .00038

7.900 .957570 .00039

7.950 .962070 .00038

8.000 .965320 .00038

8.050 .969330 .00038

8.100 .973240 .00038

8.150 .977250 .00038

8.200 .980400 .00039

8.250 .984390 .00038

8.300 .988170 .00039

8.350 .991910 .00038

8.400 .995080 .00038

8.450 .998240 .00038

8.501 .002080 .00038

8.551 .005530 .00037

8.601 .009300 .00039

8.651 .012200 .00038

8.701 .016000 .00038

8.751 .019690 .00038

8.801 .022870 .00037
50

6.800 .916060 .00041

6.850 .920920 .00041

6.900 .926240 .00041

6.950 .930800 .00041

7.000 .936040 .00042

7.050 .941180 .00042

7.100 .946520 .00042

7.150 .950950 .00041

7.200 .956060 .00042

7.250 .960360 .00041

7.300 .965120 .00041

7.350 .970710 .00042

7.400 .974110 .00042

7.450 .978770 .00040

7.500 .983740 .00041

7.550 .987780 .00042

7.600 .992900 .00040

7.650 .997320 .00041

7.701 .000510 .00041

7.751 .005720 .00041

7.801 .009070 .00040

7.851 .013510 .00042

7.901 .017770 .00042 
60

6.400 .911950 .00043 6.450 .917670 .00043 6.500 .923520 .00044 6.550 .929630 .00043 6.600 .934960 .00044 6.650 .940620 .00043 6.700 .945940 .00044 6.750 .952220 .00042 6.800 .956670 .00043 6.850 .961910 .00043 6.900 .967340 .00043 6.950 .972810 .00043 7.000 .977670 .00043 7.050 .983270 .00044 7.100 .987730 .00043 7.150 .992890 .00044 7.200 .998220 .00042 7.251 .003370 .00044 7.301 .008290 .00043 7.351 .012880 .00043 7.401 .017550 .00043

90

5.900 .919360 .00046 5.950 .926050 .00046 6.000 .933170 .00046 6.050 .939640 .00046 6.100 .946330 .00047 6.150 .953140 .00047 6.200 .959110 .00046 6.250 .966150 .00046 6.300 .972240 .00046 6.350 .977670 .00047 6.400 .984560 .00048 6.450 .990370 .00045 6.500 .997000 .00046 6.551 .002950 .00047 6.601 .008150 .00046 6.651 .014320 .00047 6.701 .020380 .00047

130

5.600 .921620 .00047 5.650 .928680 .00049 5.700 .935270 .00048
70

6.200 .918190 .00045

6.250 .924710 .00046

6.300 .930000 .00044

6.350 .936170 .00044

6.400 .942560 .00044

6.450 .947740 .00045

6.500 .953760 .00046

6.550 .959700 .00044

6.600 .966010 .00045

6.650 .971120 .00046

6.700 .977380 .00043

6.750 .982910 .00044

6.800 .987980 .00045

6.850 .993290 .00045

6.900 .999550 .00045

6.951 .004390 .00045

7.001 .009710 .00045

7.051 .014760 .00044

7.101 .019910 .00045
80

6.000 .914810 .00046

6.050 .921690 .00047

6.100 .928080 .00047

6.150 .934150 .00046

6.200 .940480 .00046

6.250 .946830 .00046

6.300 .953480 .00046

6.350 .959460 .00045

6.400 .965630 .00045

6.450 .971090 .00047

6.500 .977590 .00045

6.550 .983660 .00045

6.600 .989190 .00046

6.650 .995280 .00046

6.701 .000650 .00045

6.751 .006180 .00045

6.801 .011670 .00046

6.851 .016900 .00046

6.901 .022560 .00046
5.800 .919750 .00048

5.850 .927100 .00048

5.900 .934670 .00047

5.950 .941720 .00046

6.000 .947640 .00047

6.050 .954860 .00048

6.100 .961630 .00047

6.150 .967770 .00047

6.200 .973770 .00047

6.250 .980840 .00048

6.300 .986460 .00047

6.350 .993260 .00047

6.400 .999150 .00048

6.451 .006780 .00046

6.501 .011990 .00047

6.551 .017420 .00048

6.601 .024030 .00047
118

5.600 .909450 .00049

5.650 .918180 .00048

5.700 .925400 .00047

5.750 .932050 .00049

5.800 .939580 .00049

5.850 .947330 .00048

5.900 .954900 .00048

5.950 .961500 .00046

6.000 .968030 .00048

6.050 .974900 .00048

6.100 .981160 .00048

6.150 .987950 .00049

6.200 .994400 .00048

6.251 .001570 .00048

6.301 .007900 .00047

6.351 .014480 .00049

6.401 .020390 .00048
150

5.500 .918130 .00050

5.550 .926680 .00049

5.600 .933520 .00049
200

5.400 .919310 .00051

5.450 .927600 .00051

5.500 .935080 .00049 
5.750 .943460 .00049 5.800 .951120 .00048 5.850 .957300 .00049 5.900 .964320 .00048 5.950 .972480 .00049 6.000 .979480 .00048 6.050 .986130 .00050 6.100 .992770 .00049 6.150 .999350 .00048 6.201 .005630 .00048 6.251 .011780 .00047 6.301 .019560 .00049 6.351 .023750 .00049 6.401 .031270 .00048

235

5.300 .908620 .00051 5.350 .917150 .00050 5.400 .924480 .00048 5.450 .932890 .00050 5.500 .939960 .00050 5.550 .948320 .00050 5.600 .955200 .00051 5.650 .963050 .00049 5.700 .971900 .00048 5.750 .978940 .00052 5.800 .985070 .00050 5.850 .992950 .00051 5.900 .999800 .00050 5.951 .006580 .00051 6.001 .014310 .00051 6.051 .020770 .00051 6.101 .027310 .00051

\section{0}

5.500 .914400 .00049 5.550 .922620 .00051 5.600 .929540 .00049 5.650 .937580 .00052 5.700 .944910 .00049 5.750 .952150 .00050 5.800 .959190 .00051 5.850 .966310 .00051 5.900 .973070 .00051 5.950 .980190 .00051 6.000 .987090 .00049 6.050 .994870 .00048
5.650 .940830 .00049

5.700 .947660 .00048

5.750 .955740 .00050

5.800 .963630 .00049

5.850 .970760 .00050

5.900 .977290 .00049

5.950 .984400 .00049

6.000 .991250 .00049

6.050 .998240 .00049

6.101 .005780 .00048

6.151 .011520 .00048

6.201 .018080 .00051

6.251 .025280 .00051

6.301 .032110 .00049
5.550 .943430 .00050 5.600 .950430 .00051 5.650 .958620 .00050 5.700 .965900 .00050 5.750 .972260 .00050 5.800 .981080 .00049 5.850 .987300 .00049 5.900 .995690 .00051 5.951 .001870 .00051 6.001 .009210 .00050 6.051 .016290 .00051 6.101 .022790 .00051
250

5.300 .908720 .00050

5.350 .917300 .00051

5.400 .925580 .00049

5.450 .933020 .00051

5.500 .940550 .00049

5.550 .949460 .00051

5.600 .956300 .00051

5.650 .965330 .00048

5.700 .970830 .00050

5.750 .978150 .00050

5.800 .985910 .00051

5.850 .993810 .00052

5.901 .001150 .00051

5.951 .007570 .00050

6.001 .014490 .00052

6.051 .021540 .00051

6.101 .029140 .00051
353

5.400 .919380 .00050 5.450 .927640 .00051 5.500 .936240 .00050 5.550 .942880 .00050 5.600 .950950 .00050 5.650 .958290 .00050 5.700 .965940 .00050 5.750 .973030 .00050 5.800 .980240 .00051 5.850 .988010 .00051 5.900 .994350 .00051 5.951 .001160 .00050 6.001 .007920 .00051 6.051 .015620 .00051 6.101 .021740 .00051
475

5.500 .913020 .00050

5.550 .921200 .00049

5.600 .928340 .00051

5.650 .935760 .00049

5.700 .943530 .00051

5.750 .949660 .00050 5.800 .956810 .00051 5.850 .965570 .00051 5.900 .971830 .00051 5.950 .978420 .00051 6.000 .984860 .00050 6.050 .992770 .00051
480

5.500 .911370 .00050 5.550 .920080 .00051 5.600 .927760 .00050 5.650 .934510 .00050 5.700 .941420 .00050 5.750 .949530 .00051 5.800 .956970 .00050 5.850 .964140 .00050 5.900 .970320 .00049 5.950 .977940 .00051 6.000 .983960 .00051 6.050 .990940 .00050 
6.101 .000400 .00049

6.151 .007050 .00049

6.201 .013760 .00051

6.251 .019830 .00049

6.301 .026020 .00051

486

5.500 .910130 .00050

5.550 .919160 .00050

5.600 .927270 .00050

5.650 .933880 .00049

5.700 .941150 .00050

5.750 .947900 .00050

5.800 .955520 .00050

5.850 .962640 .00049

5.900 .970030 .00050

5.950 .976740 .00051

6.000 .983760 .00051

6.050 .989880 .00051

6.100 .998130 .00050

6.151 .003780 .00050

6.201 .010500 .00050

6.251 .017310 .00051

6.301 .023100 .00051
6.100 .999100 .00051

6.151 .007850 .00052

6.201 .012240 .00050

6.251 .019000 .00050

6.301 .025460 .00051
6.100 .997840 .00049

6.151 .004060 .00051

6.201 .011620 .00051

6.251 .017370 .00051

6.301 .024440 .00051

\section{0}


U5o2N26-pl-1000-01

12

51.800 .889880 .00013

53.300 .890610 .00013

54.800 .891160 .00013

56.300 .892160 .00013

57.800 .892460 .00012

59.300 .892920 .00013

60.800 .893270 .00013

62.300 .893770 .00012

63.800 .894470 .00013

65.300 .894740 .00012

66.800 .895170 .00012

68.300 .895810 .00012

69.800 .896280 .00012

71.300 .896520 .00012

72.800 .896780 .00012

74.300 .896960 .00012

75.800 .897510 .00012

77.300 .897280 .00012

78.800 .897630 .00012

80.300 .897870 .00012

81.800 .897990 .00012

82.100 .898160 .00012
15

19.500 .927810 .00019

21.400 .939710 .00019

23.300 .950010 .00018

25.200 .957620 .00017

27.100 .964840 .00017

29.000 .970520 .00017

30.900 .974680 .00016

32.800 .979220 .00015

34.700 .982880 .00016

36.600 .985970 .00015

38.500 .988890 .00015

40.400 .991140 .00015

42.300 .992910 .00015

44.200 .995210 .00015

46.100 .996540 .00014

48.000 .997960 .00014

49.900 .998990 .00014

51.801 .000840 .00014

53.701 .001150 .00015

55.601 .002470 .00013

57.501 .003600 .00014

57.801 .003610 .00013
20

10.600 .924360 .00027

10.800 .929670 .00026

11.000 .935490 .00026

11.200 .940480 .00026

11.400 .945560 .00026

11.600 .949810 .00026

11.800 .954780 .00026

12.000 .959730 .00026

12.200 .964050 .00026

12.400 .967950 .00025

12.600 .972290 .00026

12.800 .976800 .00025

13.000 .980340 .00024

13.200 .984740 .00025

13.400 .988180 .00025

13.600 .991780 .00024

13.800 .994880 .00025

14.000 .998050 .00024

14.201 .000980 .00024

14.401 .004560 .00024

14.601 .007930 .00024

14.801 .010420 .00024

15.001 .013740 .00024

\section{5}

8.200 .922150 .00031

8.300 .927620 .00031

8.400 .932020 .00030

8.500 .936580 .00031

8.600 .940950 .00031

8.700 .944810 .00030

8.800 .950140 .00030

8.900 .953450 .00030

9.000 .957830 .00031

9.100 .961350 .00030

9.200 .965480 .00030

9.300 .968970 .00030

9.400 .972260 .00030

9.500 .977000 .00029

9.600 .980220 .00030

9.700 .983870 .00030

9.800 .987870 .00029

9.900 .991780 .00029

10.000 .995120 .00029

10.100 .997970 .00030

10.201 .001730 .00030
30

7.000 .919240 .00034

7.100 .926310 .00035

7.200 .931750 .00033

7.300 .938370 .00034

7.400 .943870 .00033

7.500 .949450 .00034

7.600 .955410 .00032

7.700 .961160 .00033

7.800 .966480 .00034

7.900 .971690 .00033

8.000 .976570 .00032

8.100 .981920 .00033

8.200 .987250 .00033

8.300 .992030 .00032

8.400 .997470 .00033

8.501 .001670 .00032

8.601 .006650 .00032

8.701 .011460 .00032

8.801 .015870 .00032
32

6.700 .920970 .00034

6.750 .924250 .00033

6.800 .927500 .00035

6.850 .930700 .00035

6.900 .933850 .00035

6.950 .936820 .00035

7.000 .941040 .00035

7.050 .944140 .00034

7.100 .947040 .00033

7.150 .950460 .00034

7.200 .953270 .00034

7.250 .956550 .00033

7.300 .959680 .00034

7.350 .962380 .00034

7.400 .966210 .00034

7.450 .968610 .00034

7.500 .971210 .00034

7.550 .974920 .00033

7.600 .976870 .00033

7.650 .979530 .00034

7.700 .982780 .00034 
10.301 .004520 .00029

10.401 .008120 .00029

10.501 .011210 .00028

10.601 .014170 .00029

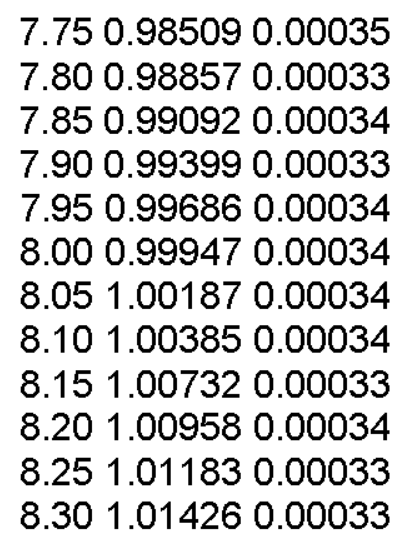

50

35

40

5.800 .916940 .00037

5.850 .922040 .00036

5.900 .926850 .00038

5.950 .930430 .00038

6.000 .934800 .00038

6.050 .938890 .00038

6.100 .942610 .00037

6.150 .947080 .00036

6.200 .951080 .00037

6.250 .954700 .00037

6.300 .959330 .00037

$\begin{array}{ll}6.35 & 0.963220 .00037\end{array}$

6.400 .967050 .00037

6.450 .969980 .00037

6.500 .975120 .00038

6.550 .978380 .00037

6.600 .981670 .00038

6.650 .985560 .00037

6.700 .988900 .00037

6.750 .992850 .00037

6.800 .996200 .00037

6.851 .000410 .00036

6.901 .003960 .00037

6.951 .006770 .00037

7.001 .010850 .00036

7.051 .013980 .00036

7.101 .017620 .00037
5.200 .917600 .00039

5.250 .923860 .00040

5.300 .928630 .00040

5.350 .933160 .00039

5.400 .938070 .00040

5.450 .943680 .00040

5.500 .949430 .00041

5.550 .953160 .00039

5.600 .957820 .00041

5.650 .962220 .00040

5.700 .968180 .00039

5.750 .971850 .00041

5.800 .976950 .00040

5.850 .981280 .00039

5.900 .986000 .00040

5.950 .990320 .00040

6.000 .994540 .00040

6.050 .998690 .00039

6.101 .003210 .00039

6.151 .007730 .00039

6.201 .011370 .00039

6.251 .015550 .00040

6.301 .019520 .00038 
60

4.800 .914970 .00042 4.850 .921100 .00041 4.900 .927050 .00041 4.950 .932920 .00041 5.000 .937720 .00042 5.050 .944330 .00042 5.100 .948370 .00041 5.150 .954210 .00041 5.200 .960220 .00042 5.250 .965290 .00042 5.300 .970900 .00042 5.350 .975460 .00041 5.400 .981330 .00042 5.450 .985840 .00042 5.500 .991100 .00041 5.550 .996360 .00041 5.601 .001310 .00043 5.651 .005650 .00041 5.751 .014640 .00041 5.801 .020050 .00040

90

4.200 .911060 .00045 4.250 .918520 .00045 4.300 .925210 .00045 4.350 .932940 .00044 4.400 .938460 .00045 4.450 .944860 .00046 4.500 .952330 .00045 4.550 .958460 .00045 4.600 .964110 .00046 4.650 .971530 .00045 4.700 .977160 .00043 4.750 .983120 .00045 4.800 .988990 .00045 4.850 .995610 .00044 4.901 .001670 .00045 4.951 .006710 .00045 5.001 .012570 .00044 5.051 .019990 .00044 5.101 .024100 .00045

130

3.900 .914160 .00047 3.950 .921890 .00046 4.000 .929160 .00047
70

4.600 .921170 .00043

4.650 .927890 .00042

4.700 .934120 .00044

4.750 .940500 .00043

4.800 .945760 .00042

4.850 .951710 .00043

4.900 .957980 .00044

4.950 .963500 .00044

5.000 .970060 .00043

5.050 .974560 .00043

5.100 .980480 .00044

5.150 .985700 .00044

5.200 .991850 .00043

5.250 .997780 .00043

5.301 .002170 .00042

5.351 .008330 .00043

5.401 .013210 .00043

5.451 .017870 .00043

5.501 .023820 .00044
80

4.400 .919030 .00043

4.450 .925580 .00045

4.500 .931800 .00046

4.550 .939820 .00043

4.600 .944910 .00043

4.650 .952250 .00043

4.700 .958040 .00044

4.750 .964540 .00043

4.800 .970350 .00043

4.850 .976440 .00044

4.900 .982040 .00044

4.950 .987570 .00043

5.000 .993290 .00045

5.050 .999030 .00044

5.101 .005100 .00043

5.151 .010780 .00044

5.201 .015870 .00045

5.251 .021200 .00045

5.301 .026860 .00044
100

4.100 .912610 .00046

4.150 .919610 .00046

4.200 .926340 .00045

4.250 .933170 .00045

4.300 .940220 .00046

4.350 .946920 .00045

4.400 .953970 .00046

4.450 .960210 .00044

4.500 .966970 .00045

4.550 .973780 .00045

4.600 .979870 .00045

4.650 .986990 .00045

4.700 .992280 .00046

4.750 .999090 .00045

4.801 .005220 .00045

4.851 .011550 .00046

4.901 .016830 .00045
118

4.000 .918870 .00045

4.050 .926090 .00047

4.100 .932420 .00046

4.150 .940290 .00047

4.200 .948020 .00047

4.250 .954000 .00046

4.300 .961320 .00046

4.350 .968430 .00045

4.400 .975430 .00048

4.450 .981460 .00047

4.500 .987780 .00046

4.550 .994910 .00046

4.601 .001590 .00044

4.651 .007970 .00047

4.701 .014610 .00047

4.751 .020220 .00046

4.801 .026890 .00046
150

3.800 .912040 .00047

3.850 .920690 .00048

3.900 .927810 .00047
200

3.700 .915940 .00048

3.750 .924070 .00047

3.800 .932180 .00049 
4.050 .937180 .00048

4.100 .943640 .00047

4.150 .951410 .00047

4.200 .958430 .00046

4.250 .965820 .00048

4.300 .972020 .00046

4.350 .978890 .00047

4.400 .986430 .00047

4.450 .992440 .00046

4.500 .998930 .00047

4.551 .005490 .00047

4.601 .012820 .00048

4.651 .018980 .00046

4.701 .025120 .00048

235

3.700 .922680 .00048

3.750 .931090 .00048

3.800 .938770 .00049

3.850 .946420 .00048

3.900 .953640 .00047

3.950 .960750 .00049

4.000 .968500 .00049

4.050 .976290 .00049

4.100 .983820 .00048

4.150 .990750 .00049

4.200 .997650 .00049

4.251 .005700 .00050

4.301 .011360 .00049

4.351 .019160 .00049

4.401 .026060 .00050

470

3.700 .908510 .00047

3.750 .916900 .00049

3.800 .924540 .00048

3.850 .932540 .00050

3.900 .938810 .00050

3.950 .946200 .00048

4.000 .953160 .00049

4.050 .960840 .00050

4.100 .968340 .00047

4.150 .974920 .00048

4.200 .981590 .00049

4.250 .989070 .00050
3.950 .935460 .00047

4.000 .943540 .00048

4.050 .950730 .00047

4.100 .957390 .00047

4.150 .964650 .00047

4.200 .972640 .00048

4.250 .979130 .00048

4.300 .985840 .00047

4.350 .992890 .00048

4.401 .000630 .00047

4.451 .006940 .00047

4.501 .013320 .00048

4.551 .019830 .00049

4.601 .026390 .00048
3.850 .939990 .00047

3.900 .946890 .00049

3.950 .955060 .00048

4.000 .962520 .00049

4.050 .969930 .00047

4.100 .977260 .00047

4.150 .984750 .00049

4.200 .991800 .00047

4.250 .998450 .00047

4.301 .006130 .00048

4.351 .012110 .00048

4.401 .019420 .00048
250

3.600 .908240 .00049

3.650 .916590 .00049

3.700 .924830 .00049

3.750 .932530 .00049

3.800 .939780 .00049

3.850 .947360 .00047

3.900 .955420 .00049

3.950 .962910 .00049

4.000 .970610 .00049

4.050 .977450 .00050

4.100 .984780 .00049

4.150 .991890 .00049

4.200 .999380 .00049

4.251 .006220 .00050

4.301 .012840 .00049

4.351 .020000 .00049

4.401 .026860 .00049
353

3.700 .924150 .00048

3.750 .930690 .00049

3.800 .939040 .00048

3.850 .946350 .00049

3.900 .954570 .00050

3.950 .961680 .00047

4.000 .968150 .00048

4.050 .975920 .00049

4.100 .983400 .00050

4.150 .990860 .00049

4.200 .997430 .00048

4.251 .005070 .00050

4.301 .011180 .00049

4.351 .018300 .00051

4.401 .024450 .00050
475

3.800 .922920 .00048

3.850 .930610 .00048

3.900 .937740 .00048

3.950 .945550 .00050

4.000 .952980 .00049

4.050 .960360 .00049

4.100 .965540 .00049

4.150 .974420 .00050

4.200 .980430 .00049

4.250 .987850 .00050

4.300 .993900 .00050

4.351 .000910 .00048
480

3.800 .922770 .00049 3.850 .929800 .00049 3.900 .936790 .00048 3.950 .944370 .00049 4.000 .952430 .00049 4.050 .958480 .00050 4.100 .965380 .00049 4.150 .972840 .00049 4.200 .978820 .00050 4.250 .986220 .00049 4.300 .992370 .00050 4.351 .000100 .00048 
4.300 .995180 .00052

4.401 .008120 .00050

4.451 .014410 .00050

4.501 .021270 .00050

486

3.800 .921210 .00048

3.850 .928850 .00049

3.900 .936380 .00049

3.950 .943890 .00048

4.000 .950350 .00049

4.050 .958590 .00048

4.100 .964730 .00049

4.150 .972030 .00050

4.200 .979160 .00047

4.250 .985170 .00049

4.300 .992060 .00050

4.350 .998730 .00050

4.401 .005670 .00050

4.451 .012580 .00050

4.501 .018540 .00049
4.401 .008230 .00051

4.451 .013390 .00049

4.501 .020660 .00050
4.401 .006610 .00051

4.451 .012650 .00049

4.501 .019610 .00049

\section{0}

.

.


U5o2N26-pl-1000-10

12

69.100 .896330 .00012

70.100 .896380 .00012

71.100 .896590 .00012

72.100 .897060 .00012

73.100 .896760 .00012

74.100 .897390 .00012

75.100 .897520 .00012

76.100 .897550 .00012

77.100 .897980 .00012

78.100 .897870 .00012

79.100 .898110 .00012

80.100 .898140 .00012

81.100 .898260 .00012

82.100 .898750 .00012

83.100 .898780 .00012

84.100 .898770 .00012

85.100 .899350 .00011

86.100 .899170 .00011

87.100 .899240 .00012

88.100 .899320 .00011

89.100 .899540 .00012

90.100 .899650 .00012

90.600 .899450 .00012
15

18.300 .927390 .00019

20.000 .938540 .00018

21.700 .947860 .00017

23.400 .955430 .00017

25.100 .961710 .00016

26.800 .967350 .00017

28.500 .972320 .00016

30.200 .976490 .00016

31.900 .979810 .00016

33.600 .982920 .00015

35.300 .985870 .00015

37.000 .988110 .00015

38.700 .990390 .00015

40.400 .992410 .00014

42.100 .994170 .00015

43.800 .995050 .00014

45.500 .997150 .00014

47.200 .998010 .00014

48.900 .999470 .00014

50.601 .000580 .00013

52.301 .001480 .00013

53.301 .001910 .00013
20

9.500 .926820 .00025

9.700 .933020 .00026

9.900 .938130 .00025

10.100 .943080 .00026

10.300 .948770 .00024

10.500 .952910 .00025

10.700 .957330 .00024

10.900 .961840 .00025

11.100 .966890 .00024

11.300 .971280 .00024

11.500 .975070 .00024

11.700 .979110 .00024

11.900 .982910 .00024

12.100 .986320 .00024

12.300 .989930 .00024

12.500 .993710 .00023

12.700 .996890 .00024

12.901 .000250 .00023

13.101 .003550 .00023

13.301 .006560 .00023

13.501 .009820 .00023

13.701 .013110 .00022
25

7.000 .923690 .00028

7.100 .928230 .00028

7.200 .932630 .00029

7.300 .937390 .00028

7.400 .941430 .00028

7.500 .945810 .00028

7.600 .950190 .00028

7.700 .954050 .00029

7.800 .958460 .00029

7.900 .962400 .00028

8.000 .966860 .00027

8.100 .970520 .00028

8.200 .974250 .00028

8.300 .978190 .00028

8.400 .982090 .00027

8.500 .985660 .00027

8.600 .988890 .00028

8.700 .992380 .00028

8.800 .995900 .00028

8.900 .999500 .00028

9.001 .002910 .00027
30

5.800 .921210 .00032

5.850 .924730 .00031

5.900 .927960 .00031

5.950 .931130 .00031

6.000 .934300 .00031

6.050 .937160 .00031

6.100 .940680 .00031

6.150 .943690 .00030

6.200 .945920 .00031

6.250 .949400 .00031

6.300 .952240 .00030

6.350 .955280 .00031

6.400 .957580 .00030

6.450 .960180 .00030

6.500 .963270 .00031

6.550 .965520 .00030

6.600 .969000 .00031

6.650 .971590 .00031

6.700 .974710 .00031

6.750 .976640 .00031

6.800 .979200 .00031
32

5.500 .923010 .00032

5.550 .927070 .00031

5.600 .930510 .00032

5.650 .932920 .00032

5.700 .936310 .00031

5.750 .939740 .00032

5.800 .943160 .00031

5.850 .946970 .00032

5.900 .950040 .00032

5.950 .953210 .00031

6.000 .956150 .00032

6.050 .959950 .00031

6.100 .962350 .00032

6.150 .965300 .00032

6.200 .968890 .00032

6.250 .971190 .00031

6.300 .973960 .00032

6.350 .976690 .00031

6.400 .980310 .00032

6.450 .982750 .00032

6.500 .985540 .00032 
9.101 .005400 .00028 9.201 .008960 .00027 9.301 .012940 .00028 9.401 .015510 .00028

35

5.100 .922720 .00033 5.150 .926160 .00034 5.200 .930470 .00034 5.250 .934700 .00033 5.300 .937630 .00034 5.350 .941770 .00033 5.400 .945610 .00033 5.450 .948850 .00033 5.500 .952630 .00032 5.550 .955980 .00032 5.600 .959700 .00032 5.650 .962850 .00033 5.700 .966110 .00033 5.750 .970000 .00033 5.800 .972550 .00032 5.850 .976160 .00033 5.900 .979350 .00032 5.950 .982430 .00033 6.000 .986470 .00034 6.050 .989210 .00033 6.100 .992580 .00032 6.150 .994880 .00033 6.200 .998730 .00032 6.251 .001000 .00033 6.301 .004520 .00032 6.351 .007420 .00032 6.401 .010430 .00033 6.451 .013080 .00032 6.501 .015900 .00032
6.850 .982400 .00030

6.900 .984590 .00029

6.950 .986910 .00030

7.000 .989330 .00031

7.050 .992410 .00030

7.100 .994590 .00031

7.150 .997420 .00030

7.200 .999210 .00031

7.251 .001510 .00031

7.301 .004420 .00030

7.351 .006140 .00030

7.401 .008710 .00030

7.451 .010940 .00031

7.501 .013780 .00030
6.550 .987580 .00031 6.600 .991340 .00031 6.650 .993770 .00032 6.700 .997030 .00032 6.750 .999390 .00030 6.801 .002660 .00031 6.851 .004640 .00031 6.901 .007580 .00031 6.951 .009880 .00030 7.001 .012290 .00031 7.051 .015000 .00032 7.101 .017770 .00031
40
4.600 .922180 .00034

4.650 .927320 .00034

4.700 .931800 .00034

4.750 .935140 .00034

4.800 .940060 .00033

4.850 .944060 .00035 4.900 .948510 .00035 4.950 .951850 .00034 5.000 .956130 .00035 5.050 .960010 .00033 5.100 .963580 .00034 5.150 .968050 .00034 5.200 .971890 .00034 5.250 .976570 .00034 5.300 .980020 .00034 5.350 .983150 .00035 5.400 .986420 .00035 5.450 .990620 .00034 5.500 .993690 .00034 5.550 .997910 .00034 5.601 .001740 .00034 5.651 .005100 .00034 5.701 .008780 .00033 5.751 .011560 .00033 5.801 .015080 .00034
50

3.900 .915210 .00037 3.950 .921130 .00037 4.000 .925670 .00036 4.050 .931790 .00037 4.100 .935980 .00037 4.150 .941220 .00036 4.200 .946570 .00037 4.250 .952120 .00037 4.300 .955930 .00037 4.350 .961530 .00036 4.400 .965850 .00037 4.450 .970550 .00037 4.500 .975530 .00036 4.550 .980220 .00037 4.600 .984850 .00037 4.650 .988690 .00037 4.700 .993280 .00036 4.750 .998490 .00036 4.801 .002470 .00036 4.851 .006870 .00037 4.901 .010710 .00037 4.951 .015290 .00036 5.001 .019440 .00037 
60

3.600 .927360 .00039

3.650 .931600 .00039

3.700 .938050 .00038

3.750 .943330 .00039

3.800 .949390 .00038

3.850 .955470 .00039

3.900 .960840 .00038

3.950 .965820 .00037

4.000 .970660 .00038

4.050 .976600 .00038

4.100 .981760 .00038

4.150 .987060 .00038

4.200 .992320 .00038

4.250 .997190 .00038

4.301 .001410 .00038

4.351 .006510 .00038

4.401 .012290 .00039

4.451 .017560 .00039

4.501 .021470 .00038
70

3.300 .924060 .00040

3.350 .930090 .00039

3.400 .936960 .00039

3.450 .943010 .00038

3.500 .948990 .00040

3.550 .954590 .00039

3.600 .961010 .00040

3.650 .966810 .00038

3.700 .973490 .00039

3.750 .978310 .00040

3.800 .983420 .00039

3.850 .989350 .00039

3.900 .995650 .00040

3.951 .000940 .00040

4.001 .006170 .00039

4.051 .011510 .00039

4.101 .016800 .00039
80

3.100 .923950 .00040

3.150 .931130 .00040

3.200 .937420 .00040

3.250 .944570 .00039

3.300 .950370 .00039

3.350 .957010 .00040

3.400 .963140 .00040

3.450 .969350 .00040

3.500 .975050 .00040

3.550 .982000 .00040

3.600 .987450 .00041

3.650 .993630 .00040

3.700 .999020 .00040

3.751 .005600 .00039

3.801 .011090 .00039

3.851 .016840 .00040

3.901 .023100 .00040
90

2.900 .918690 .00040

2.950 .925570 .00041

3.000 .931540 .00041

3.050 .939600 .00039

3.100 .945850 .00041

3.150 .952320 .00040

3.200 .959940 .00041

3.250 .965940 .00040

3.300 .972310 .00041

3.350 .978620 .00040

3.400 .985720 .00041

3.450 .991730 .00040

3.500 .997170 .00040

3.551 .004450 .00040

3.601 .010340 .00040

3.651 .016120 .00039

3.701 .021870 .00041
100

2.800 .921550 .00042

2.850 .928540 .00041

2.900 .935870 .00041

2.950 .943450 .00041

3.000 .950510 .00040

3.050 .957320 .00042

3.100 .964080 .00041

3.150 .970530 .00041

3.200 .977330 .00041

3.250 .984230 .00041

3.300 .990830 .00043

3.350 .996990 .00043

3.401 .003140 .00040

3.451 .010030 .00041

3.501 .015050 .00041
130

2.500 .914940 .00042

2.550 .923090 .00043

2.600 .931380 .00042

2.650 .938690 .00043

2.700 .945850 .00043

2.750 .954540 .00043
150

2.400 .917340 .00043

2.450 .925470 .00042

2.500 .933180 .00041

2.550 .940800 .00043

2.600 .948550 .00043

2.650 .956500 .00043
118

2.600 .916810 .00040

2.650 .924900 .00041

2.700 .933450 .00042

2.750 .940420 .00042

2.800 .946180 .00041

2.850 .954720 .00043

2.900 .961530 .00042

2.950 .968330 .00042

3.000 .975650 .00042

3.050 .982860 .00041

3.100 .989460 .00042

3.150 .996770 .00042

3.201 .003260 .00044

3.251 .009730 .00042

3.301 .016380 .00040

3.351 .022650 .00043

3.401 .029240 .00042 
2.800 .961460 .00042 2.850 .968330 .00042 2.900 .975810 .00043 2.950 .981430 .00042 3.000 .988650 .00042 3.050 .995940 .00042 3.101 .002780 .00042 3.151 .009690 .00042 3.201 .015970 .00042

235

2.100 .910640 .00043 2.150 .919410 .00044 2.200 .927250 .00043 2.250 .934940 .00045 2.300 .943320 .00044 2.350 .950530 .00043 2.400 .959240 .00043 2.450 .966720 .00043 2.500 .974350 .00043 2.550 .982690 .00044 2.600 .989780 .00045 2.650 .996680 .00044 2.701 .004110 .00044 2.751 .011220 .00044 2.801 .018750 .00043

470

2.000 .919250 .00045 2.050 .926530 .00044 2.100 .933930 .00045 2.150 .941660 .00044 2.200 .949280 .00044 2.250 .957350 .00043 2.300 .963610 .00044 2.350 .970850 .00044 2.400 .978870 .00045 2.450 .984720 .00045 2.500 .992800 .00045 2.550 .999100 .00044 2.601 .005800 .00044 2.651 .012490 .00045 2.701 .019360 .00044
2.700 .963500 .00043

2.750 .970890 .00043

2.800 .978430 .00044

2.850 .985940 .00042

2.900 .993360 .00043

2.950 .999700 .00043

3.001 .006440 .00043

3.051 .014460 .00042

3.101 .020700 .00043
2.500 .962540 .00044

2.550 .969500 .00044

2.600 .977510 .00044

2.650 .985040 .00045

2.700 .992590 .00043

2.750 .999830 .00044

2.801 .006540 .00045 2.851 .013230 .00045 2.901 .020930 .00044
250

2.100 .914140 .00044

2.150 .922150 .00044

2.200 .931360 .00044

2.250 .939200 .00044

2.300 .947190 .00046

2.350 .955030 .00044

2.400 .963250 .00044

2.450 .971200 .00045

2.500 .978220 .00044

2.550 .985420 .00043

2.600 .993430 .00044

2.651 .000940 .00043

2.701 .007670 .00045

2.751 .015440 .00046

2.801 .022360 .00044
353

2.000 .915130 .00043 2.050 .922880 .00045 2.100 .931250 .00045 2.150 .938880 .00043 2.200 .946940 .00045 2.250 .954230 .00044 2.300 .962090 .00044 2.350 .970000 .00044 2.400 .977250 .00043 2.450 .984120 .00043 2.500 .992760 .00045 2.550 .999590 .00045 2.601 .005520 .00044 2.651 .013600 .00045 2.701 .020590 .00045
475
480
2.000 .919160 .00045
2.050 .927140 .00043
2.100 .934780 .00044
2.150 .942000 .00044
2.200 .949420 .00046
2.250 .956310 .00045
2.300 .964300 .00047
2.350 .970700 .00044
2.400 .977090 .00046
2.450 .984640 .00045
2.500 .991910 .00044
2.550 .998780 .00045
2.601 .005460 .00045
2.651 .012520 .00044
2.701 .019210 .00045

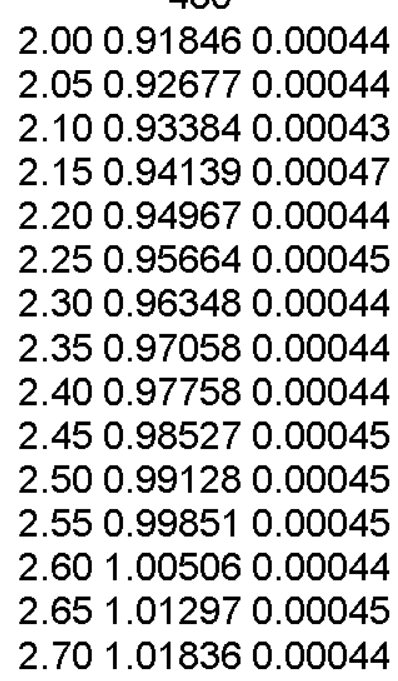


CHPRC-01552, Revision 0

486

2.000 .919360 .00044

2.050 .926920 .00043

2.100 .934370 .00046

2.150 .941740 .00043

2.200 .949100 .00043

2.250 .956350 .00044

2.300 .963940 .00045

2.350 .970370 .00044

2.400 .978400 .00044

2.450 .985070 .00045

2.500 .992000 .00045

2.550 .998070 .00045

2.601 .005440 .00044

2.651 .011460 .00045

2.701 .017890 .00045 
U5o2N20-pl-0935-00

12

32.500 .921680 .00014 33.400 .923240 .00014 34.300 .925130 .00014 35.200 .926460 .00014 36.100 .927820 .00014 37.000 .928950 .00013 37.900 .930490 .00014 38.800 .931260 .00014 39.700 .932440 .00013 40.600 .933810 .00013 41.500 .934210 .00013 42.400 .935190 .00013 43.300 .936570 .00013 44.200 .937220 .00012 45.100 .938260 .00013 46.000 .938620 .00012 46.900 .939170 .00013 47.800 .940210 .00012 48.700 .940600 .00012 49.600 .941140 .00012 50.500 .942020 .00012 50.600 .941840 .00012
15

15.300 .926940 .00022 15.800 .934010 .00021 16.300 .940790 .00021 16.800 .946810 .00021 17.300 .952600 .00021 17.800 .957830 .00020 18.300 .963260 .00020 18.800 .967960 .00019 19.300 .972150 .00020 19.800 .976570 .00020 20.300 .980410 .00019 20.800 .984240 .00019 21.300 .987320 .00019 21.800 .991120 .00019 22.300 .993440 .00018 22.800 .996630 .00018 23.300 .999330 .00018 23.801 .002360 .00017 24.301 .004780 .00018 24.801 .006950 .00018 25.301 .009330 .00017 25.801 .011450 .00017
25

8.500 .921530 .00033

8.600 .927250 .00033

8.700 .933610 .00033

8.800 .938850 .00032

8.900 .944070 .00033

9.000 .950350 .00033

9.100 .955480 .00033

9.200 .960970 .00031

9.300 .966270 .00032

9.400 .971860 .00032

9.500 .976040 .00031

9.600 .980610 .00032

9.700 .985750 .00032

9.800 .990160 .00032

9.900 .995250 .00031

10.000 .999500 .00031

\begin{abstract}
30
7.600 .921220 .00037

7.650 .924420 .00036

7.700 .928070 .00035

7.750 .932400 .00036

7.800 .935750 .00035

7.850 .939790 .00035

7.900 .943100 .00034

7.950 .947280 .00034

8.000 .950010 .00035

8.050 .953760 .00035

8.100 .957540 .00036

8.150 .960990 .00035

8.200 .964470 .00035

8.250 .967600 .00035

8.300 .971100 .00036

8.350 .974310 .00035
\end{abstract}

20

10.300 .925520 .00029

10.400 .929780 .00029

10.500 .933780 .00028

10.600 .936870 .00029

10.700 .941240 .00028

10.800 .944910 .00028

10.900 .947500 .00028

11.000 .951370 .00027

11.100 .954860 .00029

11.200 .958250 .00028

11.300 .961470 .00028

11.400 .964990 .00028

11.500 .967940 .00027

11.600 .971100 .00027

11.700 .973990 .00028

11.800 .977660 .00028

11.900 .979960 .00027

12.100 .984780 .00027

12.300 .991060 .00027

12.400 .993760 .00026

12.500 .996640 .00026

12.600 .999520 .00027

12.701 .001090 .00027

12.801 .004350 .00026

12.901 .007200 .00026

13.001 .008530 .00026

13.101 .011990 .00027

13.201 .014030 .00026

32

7.300 .916380 .00037

7.350 .921650 .00036

7.400 .925720 .00036

7.450 .929500 .00037

7.500 .933080 .00037

7.550 .937380 .00037

7.600 .941400 .00036

7.650 .944700 .00035

7.700 .949230 .00037

7.750 .953250 .00035

7.800 .956670 .00036

7.850 .960840 .00036

7.900 .964310 .00036

7.950 .967430 .00036

8.000 .971580 .00036

8.050 .974130 .00036 
10.101 .003970 .00031 10.201 .008140 .00031 10.301 .012030 .00031 10.401 .016480 .00031

35

7.000 .916890 .00039 7.050 .922290 .00038 7.100 .927320 .00036 7.150 .931090 .00037 7.200 .935750 .00037 7.250 .940500 .00039 7.300 .944600 .00037 7.350 .948330 .00037 7.400 .952800 .00036 7.450 .956310 .00038 7.500 .960300 .00037 7.550 .965240 .00038 7.600 .969440 .00037 7.650 .972870 .00038 7.700 .977140 .00037 7.750 .980640 .00038 7.800 .984590 .00036 7.850 .988620 .00038 7.900 .992080 .00038 7.950 .995460 .00038 8.000 .999200 .00038 8.051 .002930 .00037 8.101 .007340 .00036 8.151 .010550 .00037 8.201 .014080 .00037 8.251 .018070 .00037 8.301 .020630 .00037

60 5.800 .918150 .00042 5.850 .924970 .00044 5.900 .932040 .00045
8.400 .977530 .00035 8.450 .980890 .00035 8.500 .983980 .00035 8.550 .986990 .00034 8.600 .990270 .00035 8.650 .992730 .00034 8.700 .996050 .00034 8.801 .002640 .00034 8.851 .005660 .00035 8.901 .008780 .00035 9.001 .013570 .00034 9.051 .016350 .00034 9.101 .019820 .00034
8.100 .978760 .00036 8.150 .981850 .00037 8.200 .985730 .00036 8.250 .988830 .00036 8.300 .992260 .00035 8.350 .996150 .00037 8.400 .999240 .00037 8.451 .002270 .00036 8.501 .005130 .00035 8.551 .009280 .00036 8.601 .011930 .00035 8.651 .015410 .00036 8.701 .018620 .00035
40

6.600 .916610 .00040

6.650 .921180 .00040

6.700 .926060 .00038

6.750 .931560 .00040

6.800 .937180 .00040

6.850 .941080 .00039

6.900 .946140 .00040

6.950 .951020 .00040

7.000 .956020 .00038

7.050 .960730 .00038

7.100 .964860 .00039

7.150 .969240 .00039

7.200 .974150 .00040

7.250 .978370 .00039

7.400 .991020 .00040

7.450 .995830 .00039

7.501 .000130 .00039

7.551 .003720 .00040

7.601 .008090 .00039

7.651 .012620 .00040

7.701 .016860 .00039

7.751 .020660 .00037

7.801 .024370 .00039
50

6.100 .916610 .00043

6.150 .921940 .00042

6.200 .928460 .00042

6.250 .934140 .00041

6.300 .940140 .00042

6.350 .945210 .00042

6.400 .951200 .00042

6.450 .956660 .00043

6.500 .962420 .00042

6.550 .968480 .00042

6.600 .973230 .00043

6.650 .978680 .00042

6.700 .983520 .00042

6.750 .988270 .00042

6.800 .993360 .00042

6.850 .999150 .00041

6.901 .003660 .00042

6.951 .009270 .00042

7.001 .013800 .00042

7.051 .018960 .00041

7.101 .023630 .00041
70

5.600 .920270 .00045

5.650 .927680 .00045

5.700 .934400 .00043
80

5.400 .915100 .00045 5.450 .923140 .00046 5.500 .929960 .00044 
5.950 .937730 .00043 6.000 .944330 .00043 6.050 .950980 .00045 6.100 .956570 .00043 6.150 .962970 .00045 6.200 .968780 .00044 6.250 .974940 .00044 6.300 .981640 .00043 6.350 .986590 .00044 6.400 .992930 .00043 6.450 .998700 .00045 6.501 .003980 .00043 6.551 .009270 .00044 6.601 .014720 .00043 6.651 .020070 .00044 6.701 .025490 .00043

90

5.300 .918150 .00047 5.350 .925690 .00047 5.400 .933400 .00046 5.450 .940910 .00046 5.500 .948060 .00047 5.550 .955500 .00046 5.600 .962220 .00047 5.650 .970000 .00047 5.700 .977480 .00047 5.800 .990740 .00046 5.850 .997510 .00048 5.901 .004860 .00047 5.951 .011010 .00046 6.001 .017420 .00046 6.051 .024490 .00046 6.101 .030500 .00047

130

5.000 .914950 .00048 5.050 .923540 .00050 5.100 .931980 .00049 5.150 .941250 .00047 5.350 .971550 .00049 5.400 .979960 .00049 5.450 .987400 .00050 5.500 .994530 .00048 5.551 .002380 .00048 5.601 .010000 .00049 5.701 .025240 .00049
5.750 .941760 .00045 5.800 .947980 .00045 5.850 .955070 .00045 5.900 .961350 .00044 5.950 .967680 .00046 6.000 .974910 .00045 6.050 .980690 .00045 6.100 .987010 .00046 6.150 .993630 .00045 6.200 .999780 .00045 6.251 .005740 .00045 6.301 .012040 .00046 6.351 .018680 .00046 6.401 .023870 .00047
5.550 .936240 .00046 5.600 .944540 .00045 5.650 .951630 .00046 5.700 .958700 .00045 5.750 .965680 .00046 5.800 .972810 .00046 5.850 .979470 .00045 5.900 .986390 .00046 5.950 .992660 .00048 6.000 .999350 .00047 6.051 .005770 .00046 6.101 .012450 .00046 6.151 .018170 .00045 6.201 .024030 .00047
100

5.200 .915950 .00048

5.250 .924430 .00047

5.300 .931840 .00047

5.350 .940030 .00048

5.400 .947850 .00049

5.450 .955680 .00048

5.500 .963380 .00047

5.550 .970270 .00048

5.600 .977460 .00047

5.650 .984910 .00047

5.700 .990840 .00047

5.750 .999170 .00048

5.801 .005750 .00049

5.851 .013360 .00048

5.901 .019610 .00047
118

5.100 .919860 .00048 5.150 .929300 .00049 5.200 .937500 .00048 5.250 .945150 .00049 5.300 .952710 .00047 5.350 .961020 .00048 5.400 .968090 .00047 5.450 .977000 .00048 5.500 .983550 .00049 5.550 .991720 .00049 5.600 .998370 .00050 5.651 .005470 .00049 5.701 .012930 .00049 5.751 .020730 .00048 5.801 .027480 .00050
150

4.900 .910440 .00049 4.950 .919000 .00049 5.000 .926700 .00049 5.050 .936120 .00049 5.100 .943860 .00051 5.150 .953220 .00048 5.200 .960860 .00050 5.250 .970000 .00050 5.300 .977030 .00049 5.350 .983930 .00051 5.400 .992810 .00050
200

4.800 .912650 .00050 4.850 .922110 .00051 4.900 .931960 .00050 4.950 .941260 .00049 5.000 .947920 .00050 5.050 .957840 .00051 5.100 .965420 .00052 5.150 .974450 .00051 5.200 .982370 .00051 5.250 .991030 .00050 5.300 .998210 .00050 
5.450 .999920 .00050

5.501 .007520 .00051

5.551 .015470 .00049

5.601 .023320 .00051
5.351 .006470 .00050 5.401 .013790 .00051 5.451 .021640 .00052 5.501 .028720 .00050
235

4.800 .920850 .00051 4.850 .929100 .00051 4.900 .937820 .00050 4.950 .947240 .00050 5.000 .956500 .00050 5.050 .964260 .00050 5.100 .972660 .00051 5.150 .980900 .00051 5.200 .988430 .00050 5.250 .997110 .00052 5.301 .004990 .00051 5.351 .012710 .00052 5.401 .021670 .00051

470

4.800 .922570 .00052 4.850 .930830 .00052 4.900 .939980 .00051 4.950 .947730 .00051 5.000 .956980 .00051 5.050 .964960 .00051 5.100 .971820 .00051 5.150 .981800 .00049 5.200 .989360 .00051 5.250 .997390 .00051 5.301 .004860 .00051 5.351 .012470 .00050 5.401 .019960 .00050 5.451 .027160 .00050 5.501 .035620 .00050
250

4.700 .904880 .00050

4.750 .913740 .00050

4.800 .922680 .00050

4.850 .931930 .00052

4.900 .939850 .00051

4.950 .949680 .00051

5.000 .958260 .00051

5.050 .965660 .00051

5.100 .973470 .00051

5.150 .982850 .00050

5.200 .992530 .00051

5.250 .999610 .00052

5.301 .007220 .00051

5.351 .014680 .00051

5.401 .023280 .00053
353

4.700 .909600 .00051

4.750 .919100 .00051

4.800 .927380 .00052

4.850 .935830 .00050

4.900 .945320 .00051

4.950 .954300 .00050

5.000 .962130 .00051

5.050 .969960 .00049

5.100 .978930 .00052

5.150 .986700 .00050

5.200 .994900 .00050

5.251 .003870 .00052

5.301 .011090 .00051

5.351 .018990 .00051

5.401 .026900 .00050

\begin{abstract}
500
4.800 .919010 .00051

4.850 .927520 .00050

4.900 .935460 .00051

4.950 .944320 .00051

5.000 .952520 .00050

5.050 .961450 .00050

5.100 .969230 .00052

5.150 .976900 .00049

5.200 .984480 .00051

5.250 .992490 .00051

5.301 .001580 .00051

5.351 .007950 .00052

5.401 .015980 .00050

5.451 .022720 .00051

5.501 .031620 .00051
\end{abstract}

\begin{tabular}{ccc}
\multicolumn{1}{c}{600} \\
4.80 & 0.90881 & 0.00050 \\
4.85 & 0.91636 & 0.00050 \\
4.90 & 0.92510 & 0.00050 \\
4.95 & 0.93345 & 0.00050 \\
5.00 & 0.94068 & 0.00049 \\
5.05 & 0.94949 & 0.00050 \\
5.15 & 0.96623 & 0.00051 \\
5.20 & 0.97338 & 0.00050 \\
5.25 & 0.98091 & 0.00052 \\
5.30 & 0.98891 & 0.00050 \\
5.35 & 0.99663 & 0.00049 \\
5.40 & 1.00427 & 0.00051 \\
5.45 & 1.01164 & 0.00051 \\
5.50 & 1.01873 & 0.00051 \\
5.55 & 1.02644 & 0.00050 \\
5.60 & 1.03326 & 0.00052
\end{tabular}

615

4.800 .906280 .00050

4.850 .913830 .00050

4.900 .923610 .00049

4.950 .931800 .00051 
5.000 .941880 .00051

5.050 .948810 .00051

5.100 .958190 .00051

5.150 .964720 .00052

5.200 .973430 .00051

5.250 .981200 .00050

5.300 .988930 .00050

5.350 .996450 .00050

5.401 .003930 .00053

5.451 .011470 .00051

5.501 .018620 .00051

5.551 .025730 .00050

5.601 .033050 .00051
5.000 .941180 .00049

5.050 .949630 .00050

5.100 .956900 .00052

5.150 .965010 .00050

5.200 .973140 .00051

5.250 .980520 .00052

5.300 .988850 .00050

5.350 .995710 .00050

5.401 .004240 .00050

5.451 .009860 .00051

5.501 .018350 .00051

5.551 .025090 .00050

5.601 .032200 .00050
5.000 .938800 .00050

5.050 .947930 .00051

5.100 .955390 .00049

5.150 .964120 .00051

5.200 .971450 .00050

5.250 .979960 .00051

5.300 .987090 .00052

5.350 .994290 .00051

5.401 .001940 .00050

5.451 .008600 .00049

5.501 .016230 .00051

5.551 .024120 .00050

5.601 .031330 .00050
620

4.800 .906110 .00050 4.850 .914110 .00050 4.900 .923050 .00051 4.950 .931050 .00048 5.000 .938530 .00050 5.050 .946940 .00051 5.100 .955000 .00050 5.150 .964090 .00048 5.200 .971760 .00050 5.250 .978290 .00051 5.300 .986450 .00050 5.350 .994520 .00051 5.401 .001420 .00052 5.451 .009930 .00051 5.501 .016260 .00050 5.551 .023950 .00052 5.601 .030680 .00052
625

4.800 .905960 .00050

4.850 .914680 .00050

4.900 .922640 .00051

4.950 .931070 .00051

5.000 .939090 .00051

5.050 .946880 .00050

5.100 .955030 .00050

5.150 .963520 .00051

5.200 .971320 .00052

5.250 .979240 .00051

5.300 .985940 .00051

5.350 .993160 .00051

5.401 .000460 .00051

5.451 .009000 .00051

5.501 .015930 .00050

5.551 .022860 .00051

5.601 .030260 .00051
629

4.900 .921170 .00049 4.950 .929400 .00050 5.000 .939020 .00051 5.050 .946060 .00050 5.100 .955030 .00050 5.150 .961900 .00049 5.200 .969870 .00051 5.250 .977270 .00050 5.300 .984880 .00052 5.350 .993260 .00050 5.400 .999650 .00050 5.451 .007360 .00051 5.501 .014550 .00050 5.551 .022310 .00050 5.601 .029230 .00051 
U5o2N20-pl-0935-01

12

39.200 .933330 .00013 43.200 .937590 .00012 47.200 .940450 .00013 51.200 .942990 .00011 55.200 .945000 .00012 59.200 .946130 .00011 63.200 .947520 .00010 67.200 .948630 .00011 71.200 .949490 .00011 75.200 .950580 .00011 79.200 .951430 .00010 83.200 .951420 .00010 87.200 .952080 .00011 91.200 .952680 .00010 95.200 .953290 .00010 99.200 .953090 .00010 103.200 .953650 .00010 107.200 .954040 .00010 111.200 .954290 .00010 115.200 .954630 .00010 119.200 .954620 .00010 120.900 .955100 .00010
15

13.800 .927210 .00021 14.300 .934420 .00021 14.800 .940690 .00021 15.300 .947200 .00020 15.800 .952820 .00020 16.300 .958480 .00020 16.800 .963250 .00020 17.300 .968170 .00019 17.800 .972390 .00019 18.300 .977160 .00019 18.800 .980840 .00019 19.300 .984420 .00018 19.800 .987850 .00018 20.300 .991010 .00018 20.800 .994010 .00018 21.300 .996890 .00018 21.800 .999630 .00018 22.301 .002280 .00017 22.801 .004640 .00017 23.301 .007330 .00017 23.801 .009490 .00017 24.301 .011220 .00017

\begin{abstract}
20
8.700 .922970 .00028 8.800 .926510 .00028 8.900 .930480 .00027 9.000 .935140 .00028 9.100 .937680 .00028 9.200 .941840 .00028 9.300 .945540 .00027 9.400 .948950 .00026 9.500 .952170 .00027 9.600 .955810 .00027 9.700 .958870 .00026 9.800 .962950 .00028 9.900 .965570 .00027 10.000 .968970 .00027 10.100 .971770 .00027 10.200 .974480 .00027 10.300 .978290 .00026 10.400 .980720 .00027 10.500 .983830 .00026 10.600 .986880 .00026 10.700 .989320 .00026 10.800 .992550 .00026 11.000 .997520 .00026 11.201 .002580 .00025 11.301 .005160 .00026 11.401 .007730 .00026 11.501 .010010 .00025 11.601 .012390 .00026 11.701 .014170 .00025
\end{abstract}

32

5.800 .919720 .00035 5.850 .923190 .00036 5.900 .927860 .00035 6.000 .935810 .00036 6.050 .940900 .00034 6.100 .943860 .00035 6.250 .955550 .00035 6.300 .959610 .00034 6.350 .962630 .00035 6.400 .966370 .00035 6.450 .970420 .00036 6.500 .973750 .00035 6.550 .977230 .00035 6.600 .980450 .00034 6.650 .984490 .00035 
8.501 .000900 .00031

8.601 .005200 .00030

8.701 .009660 .00030

8.801 .013920 .00029

8.901 .018280 .00030
35

5.500 .919930 .00036

5.550 .924550 .00036

5.600 .928970 .00036

5.650 .933650 .00037

5.700 .938120 .00036

5.750 .942650 .00037

5.800 .946510 .00035

5.850 .951410 .00037

5.900 .955600 .00036

5.950 .959660 .00036

6.000 .963550 .00036

6.050 .967790 .00037

6.100 .971630 .00037

6.150 .975240 .00037

6.200 .979570 .00036

6.250 .983140 .00036

6.300 .987170 .00035

6.350 .991210 .00036

6.400 .994930 .00037

6.450 .998380 .00035

6.501 .002330 .00035

6.551 .005990 .00036

6.601 .009670 .00036

6.651 .013190 .00036

6.701 .016190 .00036
6.850 .976450 .00034

6.900 .978740 .00033

6.950 .983460 .00033

7.000 .986350 .00034

7.050 .989240 .00033

7.100 .992430 .00034

7.150 .995300 .00033

7.200 .998970 .00033

7.251 .000950 .00033

7.301 .004590 .00033

7.351 .007450 .00033

7.401 .009850 .00033

7.451 .013270 .00033

7.501 .016270 .00034
6.700 .987820 .00036

6.750 .991290 .00035

6.800 .994520 .00035

6.850 .997810 .00035

6.901 .001580 .00034

6.951 .004240 .00035

7.001 .008260 .00034

7.051 .010940 .00034

7.101 .014300 .00035

7.151 .017060 .00034

7.201 .020630 .00034
40

5.100 .919540 .00039

5.150 .924580 .00037

5.200 .929140 .00037

5.250 .934770 .00038

5.300 .939130 .00038

5.350 .944140 .00037

5.400 .949550 .00036

5.450 .954590 .00038

5.500 .959130 .00038

5.550 .963120 .00038

5.600 .967440 .00037

5.650 .973060 .00038

5.700 .976940 .00038

5.750 .981340 .00038

5.800 .986210 .00037

5.850 .990750 .00037

5.900 .994460 .00038

5.950 .998720 .00037

6.001 .002600 .00037

6.051 .007380 .00039

6.101 .011220 .00038

6.151 .014740 .00036

6.201 .019820 .00038
50

4.600 .920090 .00041

4.650 .926230 .00040

4.700 .931600 .00041

4.750 .937760 .00040

4.800 .944370 .00041

4.850 .949820 .00040

4.900 .955040 .00041

4.950 .960730 .00040

5.000 .966380 .00041

5.050 .971450 .00041

5.100 .977060 .00041

5.150 .982390 .00041

5.200 .987540 .00040

5.250 .993080 .00040

5.451 .012860 .00041

5.501 .017490 .00041
60

4.300 .922820 .00041

4.350 .929880 .00041

4.400 .936650 .00042

4.450 .942610 .00043
70

4.000 .912100 .00043 4.050 .919270 .00044

4.100 .926190 .00043

4.150 .933760 .00043
80

3.900 .921800 .00044 3.950 .929410 .00043 4.000 .935780 .00045 4.050 .943320 .00045 
4.500 .948710 .00042 4.550 .956230 .00042 4.600 .961940 .00042 4.650 .968130 .00042 4.700 .974010 .00043 4.750 .980600 .00042 4.800 .986150 .00043 4.850 .991840 .00042 4.900 .997020 .00042 4.951 .003390 .00042 5.001 .008660 .00042 5.051 .014650 .00042 5.101 .019340 .00042

90

3.800 .924360 .00043 3.850 .932600 .00044 3.900 .941000 .00045 3.950 .948650 .00046 4.000 .954970 .00045 4.050 .962850 .00046 4.100 .969680 .00047 4.150 .977480 .00045 4.200 .983480 .00045 4.250 .990710 .00046 4.300 .997530 .00045 4.351 .004710 .00045 4.401 .011210 .00045 4.451 .017870 .00046 4.501 .025300 .00045

130

3.400 .906920 .00046 3.450 .915390 .00047 3.500 .924420 .00047 3.550 .933010 .00047 3.600 .940650 .00047 3.650 .949900 .00046 3.700 .956780 .00047 3.750 .965770 .00047 3.800 .973290 .00047 3.850 .980760 .00047 3.900 .987960 .00047
4.200 .939740 .00043

4.250 .946960 .00044

4.300 .954080 .00044

4.350 .960160 .00043

4.400 .967690 .00043

4.450 .974140 .00044

4.500 .980480 .00043

4.550 .986600 .00044

4.600 .992250 .00043

4.650 .999460 .00044

4.701 .005490 .00043

4.751 .010970 .00043

4.801 .016600 .00042

4.851 .022570 .00043

4.901 .029130 .00044

100

3.600 .907800 .00046

3.650 .916230 .00045

3.700 .924010 .00046

3.750 .931760 .00045

3.800 .940170 .00046

3.850 .947270 .00045

3.900 .955150 .00046

3.950 .962840 .00048

4.000 .971060 .00045

4.050 .979300 .00047

4.100 .985150 .00045

4.150 .992190 .00047

4.200 .999170 .00046

4.251 .006130 .00046

4.301 .013260 .00046

4.351 .020130 .00045

4.401 .027350 .00046

150

3.400 .920500 .00047

3.450 .929330 .00047

3.500 .937840 .00048

3.550 .945760 .00049

3.600 .954660 .00045

3.650 .963000 .00047

3.700 .972340 .00047

3.750 .978990 .00048

3.800 .986440 .00048

3.850 .995570 .00048

3.901 .003570 .00048
4.100 .951960 .00045

4.150 .957550 .00045

4.200 .964810 .00044

4.250 .971360 .00043

4.300 .978900 .00045

4.350 .985280 .00046

4.400 .992280 .00044

4.450 .998510 .00044

4.501 .005420 .00044

4.551 .011930 .00042

4.601 .017580 .00046

4.651 .023800 .00044

4.701 .030390 .00043
118

3.500 .912760 .00047

3.550 .921510 .00046

3.600 .929370 .00046

3.650 .937200 .00046

3.700 .945480 .00045

3.750 .953710 .00046

3.800 .961530 .00046

3.850 .969630 .00047

3.900 .977040 .00046

3.950 .984260 .00046

4.000 .991330 .00047

4.051 .000290 .00047

4.101 .007260 .00046

4.151 .014560 .00046

4.201 .021360 .00047 
3.950 .996800 .00049 4.001 .003680 .00048 4.051 .010940 .00048 4.101 .019400 .00047

235

3.200 .916990 .00049 3.250 .924680 .00047 3.300 .933800 .00049 3.350 .943120 .00050 3.550 .977860 .00050 3.600 .985870 .00050 3.650 .993890 .00047 3.701 .001220 .00050 3.751 .010350 .00049 3.801 .017160 .00050 3.851 .025590 .00050 3.901 .032650 .00051

470

3.200 .922820 .00049 3.250 .932350 .00050 3.300 .940370 .00049 3.350 .949310 .00049 3.400 .957410 .00049 3.450 .965010 .00050 3.500 .973790 .00049 3.550 .983050 .00048 3.600 .989920 .00050 3.650 .997940 .00048 3.701 .005900 .00049 3.751 .013730 .00048 3.801 .021170 .00050

605

3.200 .911730 .00048 3.250 .920810 .00048 3.300 .929430 .00048 3.350 .937860 .00049 3.400 .946080 .00049
3.951 .010130 .00048 4.001 .018150 .00050

250

3.200 .918970 .00048

3.250 .927810 .00048

3.300 .936490 .00049

3.350 .945900 .00047

3.400 .953590 .00048

3.450 .962600 .00048

3.500 .970880 .00049

3.550 .979220 .00050

3.600 .988050 .00048

3.650 .996410 .00049

3.701 .003210 .00049

3.751 .012630 .00049 3.801 .019970 .00048

500

3.200 .920500 .00049

3.250 .929640 .00050

3.300 .938390 .00049

3.350 .945740 .00048

3.400 .954970 .00050

3.450 .962400 .00049

3.500 .970520 .00050

3.550 .978750 .00050

3.600 .986460 .00050

3.650 .994950 .00049

3.701 .002630 .00050

3.751 .009490 .00050

3.801 .017450 .00051

3.851 .025510 .00049

3.901 .032570 .00049
3.751 .001820 .00049

3.801 .009540 .00049

3.851 .018070 .00049

3.901 .025390 .00048

353

3.100 .908310 .00049

3.150 .916590 .00049

3.200 .926570 .00049

3.250 .934780 .00049

3.300 .943880 .00049

3.350 .952480 .00049

3.400 .960700 .00048

3.450 .968960 .00049

3.500 .977540 .00050

3.550 .985840 .00049

3.600 .994090 .00048

3.651 .002950 .00050

3.701 .010040 .00050

3.751 .017290 .00050

3.801 .025990 .00049

600

3.200 .912700 .00049

3.250 .921200 .00050

3.300 .929020 .00049

3.350 .937250 .00051

3.400 .945210 .00049

3.450 .953520 .00049

3.500 .962730 .00048

3.550 .970540 .00050

3.600 .977930 .00047

3.650 .985940 .00049

3.700 .993240 .00049

3.750 .999600 .00050

3.801 .007980 .00050

3.851 .014870 .00049

3.901 .022880 .00050
3.200 .911340 .00048

3.250 .920450 .00050

3.300 .928760 .00049

3.350 .936940 .00049

3.400 .944890 .00049
615

3.200 .910960 .00050 3.250 .918890 .00049 3.300 .927430 .00050 3.350 .935920 .00048 3.400 .943930 .00048 
3.450 .953870 .00048 3.500 .960950 .00050 3.550 .969680 .00049 3.600 .976470 .00049 3.650 .985130 .00049 3.700 .992450 .00048 3.751 .000180 .00049 3.801 .006760 .00050 3.851 .014590 .00050 3.901 .021750 .00049

620

3.200 .909930 .00049 3.250 .918840 .00048 3.300 .927280 .00048 3.350 .935780 .00050 3.400 .944230 .00048 3.450 .952510 .00050 3.500 .959490 .00050 3.550 .967250 .00050 3.600 .976250 .00049 3.650 .983700 .00049 3.750 .997970 .00049 3.801 .005230 .00049 3.851 .013100 .00049 3.901 .020680 .00049
3.450 .953380 .00051

3.500 .961290 .00049

3.550 .969390 .00050 3.600 .977030 .00050 3.650 .984750 .00049 3.700 .992780 .00049 3.750 .999940 .00051 3.801 .006860 .00051 3.851 .013980 .00049 3.901 .021450 .00051
3.450 .951920 .00049

3.500 .960230 .00050

3.550 .968080 .00049

3.600 .976320 .00049 3.650 .983820 .00050 3.700 .991680 .00049 3.750 .998740 .00049 3.801 .005670 .00050 3.851 .012840 .00049 3.901 .020600 .00049
625
3.200 .910870 .00051

3.250 .918080 .00048

3.300 .926840 .00049

3.350 .935180 .00049

3.400 .944380 .00048

3.450 .951740 .00049

3.500 .959110 .00051

3.550 .967640 .00050 3.600 .975880 .00049 3.650 .982970 .00049 3.700 .990990 .00050 3.750 .998060 .00047 3.801 .004770 .00050 3.851 .012590 .00050 3.901 .019780 .00050
629

3.200 .910000 .00048 3.250 .917620 .00049 3.300 .926250 .00049 3.350 .935130 .00048 3.400 .942480 .00050 3.450 .951250 .00049 3.500 .959400 .00049 3.550 .966320 .00048 3.600 .975090 .00049 3.650 .982760 .00050 3.700 .989530 .00049 3.750 .997650 .00050 3.801 .004530 .00050 3.851 .011850 .00049 3.901 .019430 .00049 
U5o2N20-pl-0935-10

12

34.500 .929580 .00013 39.200 .935070 .00012 43.900 .939180 .00012 48.600 .942330 .00011 53.300 .944800 .00011 58.000 .946480 .00011 62.700 .948090 .00011 67.400 .949270 .00011 72.100 .950220 .00010 76.800 .950850 .00010 81.500 .951760 .00010 86.200 .952270 .00010 90.900 .952830 .00010 95.600 .953300 .00010 100.300 .953660 .00010 105.000 .953560 .00010 109.700 .954310 .00010 114.400 .954550 .00010 119.100 .955090 .00010 123.800 .955000 .00010 128.500 .954980 .00010 129.500 .955290 .00010
15

12.700 .926720 .00020 13.200 .934290 .00021 13.700 .940930 .00020 14.200 .947670 .00019 14.700 .953310 .00019 15.200 .958390 .00019 15.700 .963170 .00019 16.200 .967890 .00019 16.700 .972520 .00018 17.200 .976590 .00018 17.700 .980540 .00018 18.200 .984090 .00018 18.700 .987970 .00017 19.200 .991140 .00018 19.700 .994280 .00018 20.200 .997070 .00017 20.700 .999760 .00017 21.201 .002300 .00017 21.701 .004820 .00017 22.201 .007350 .00016 22.701 .009690 .00017 23.201 .011140 .00016
20

7.700 .926290 .00025 7.800 .930450 .00027 7.900 .934410 .00026 8.000 .938240 .00026 8.100 .942020 .00026 8.200 .945450 .00025 8.300 .948790 .00026 8.400 .952520 .00026 8.500 .955380 .00026 8.600 .959220 .00025 8.700 .962820 .00025 8.800 .966170 .00025 9.000 .971960 .00025 9.200 .978220 .00025 9.300 .981560 .00025 9.400 .983960 .00026 9.500 .986890 .00025 9.600 .989160 .00025 9.700 .992630 .00024 9.800 .994870 .00024 9.900 .998130 .00024 10.001 .000120 .00024 10.101 .002490 .00024 10.201 .005540 .00024 10.301 .007510 .00025 10.401 .010600 .00024 10.501 .012950 .00024

\section{5}

5.900 .923440 .00030 6.000 .929330 .00029 6.100 .935680 .00029 6.200 .941060 .00029 6.300 .946180 .00029 6.400 .952280 .00029 6.500 .957590 .00030 6.600 .962740 .00029 6.700 .968360 .00029 6.800 .972920 .00029 6.900 .978110 .00029 7.000 .983240 .00028 7.100 .987660 .00029 7.200 .992930 .00029 7.300 .997190 .00028 7.401 .002400 .00029 7.501 .006310 .00028
30

5.000 .923830 .00032

5.050 .927910 .00031

5.100 .931450 .00032

5.150 .935060 .00032

5.200 .938910 .00031

5.250 .942940 .00032

5.300 .945840 .00031

5.350 .950440 .00032

5.400 .953890 .00031

5.450 .956610 .00031

5.500 .960590 .00032

5.550 .964370 .00031

5.600 .967260 .00032

5.650 .970860 .00032

5.700 .974450 .00032

5.750 .977550 .00032

5.800 .981320 .00032
32

4.700 .920270 .00033

4.750 .924840 .00033

4.800 .929010 .00033

4.850 .932560 .00032

4.900 .936910 .00032

4.950 .940440 .00032

5.000 .945240 .00033

5.050 .948490 .00033

5.100 .953010 .00033

5.150 .957470 .00033

5.200 .961080 .00033

5.250 .964460 .00033

5.300 .967880 .00032

5.350 .972070 .00032

5.400 .975660 .00033

5.450 .978970 .00033

5.500 .983240 .00032 
7.601 .011170 .00028 7.701 .014740 .00028

35

4.400 .921170 .00033 4.450 .926000 .00034 4.500 .931390 .00033 4.550 .935710 .00034 4.600 .940210 .00033 4.650 .944320 .00034 4.700 .948470 .00033 4.750 .953620 .00033 4.800 .957040 .00033 4.850 .961470 .00033 4.900 .965930 .00034 4.950 .970270 .00034 5.000 .973590 .00034 5.050 .977830 .00033 5.100 .982160 .00034 5.150 .985960 .00033 5.200 .989760 .00033 5.250 .993430 .00034 5.300 .997230 .00033 5.351 .001120 .00033 5.401 .005240 .00033 5.451 .008570 .00032 5.501 .012170 .00033 5.551 .015580 .00032 5.601 .019670 .00032

60

3.100 .917830 .00039 3.150 .924530 .00038 3.200 .931580 .00038 3.250 .938150 .00038 3.300 .944530 .00037 3.350 .951430 .00039 3.400 .957590 .00038 3.450 .964770 .00038
5.850 .984890 .00033

5.900 .987630 .00032

6.000 .993610 .00031

6.050 .996600 .00031

6.100 .999550 .00032

6.251 .009220 .00030

6.301 .011610 .00031

6.351 .014990 .00031

6.401 .017750 .00031

40

4.000 .921820 .00035

4.050 .926990 .00035

4.100 .932270 .00035

4.150 .936860 .00035

4.200 .942230 .00034

4.250 .947380 .00035

4.300 .952540 .00035

4.350 .957320 .00036

4.400 .962090 .00035

4.450 .967360 .00034

4.500 .971860 .00036

4.550 .975690 .00035

4.600 .980980 .00034

4.650 .985310 .00035

4.700 .989900 .00034

4.750 .994470 .00035

4.800 .998490 .00035

5.001 .015790 .00034
5.550 .986550 .00032 5.600 .989400 .00032 5.650 .993090 .00031 5.700 .997130 .00032 5.751 .000280 .00032 5.801 .003080 .00032 5.851 .006240 .00032 5.901 .010040 .00032 5.951 .013330 .00032 6.001 .016130 .00031

50

3.500 .925260 .00037 3.550 .931720 .00036 3.600 .937560 .00037 3.650 .943860 .00038 3.700 .949380 .00037 3.750 .955240 .00037 3.800 .961530 .00036 3.850 .966720 .00037 3.900 .972420 .00038 3.950 .977810 .00038 4.000 .983750 .00036 4.050 .988920 .00038 4.100 .993900 .00037 4.150 .999160 .00037 4.201 .004220 .00037 4.251 .009870 .00036 4.301 .014290 .00038 4.351 .020190 .00037 4.401 .024380 .00037
2.900 .922290 .00038 2.950 .929510 .00039 3.000 .937060 .00039 3.050 .944340 .00040 3.100 .951400 .00039 3.150 .959040 .00039 3.200 .965680 .00039 3.250 .972860 .00039
80

2.700 .919610 .00039 2.750 .926910 .00040 2.800 .934860 .00040 2.850 .942630 .00041 2.900 .950290 .00040 2.950 .957410 .00041 3.000 .965330 .00040 3.050 .971710 .00039 
3.500 .970680 .00039 3.550 .976510 .00037 3.600 .982670 .00039 3.650 .988410 .00038 3.700 .994770 .00038 3.751 .000800 .00038 3.801 .007630 .00039 3.851 .012440 .00039 3.901 .018370 .00039

90

2.500 .908060 .00039 2.550 .916730 .00039 2.600 .924850 .00040 2.650 .933450 .00041 2.700 .940760 .00041 2.750 .949430 .00041 2.800 .956790 .00041 2.850 .964270 .00041 2.900 .971990 .00042 2.950 .979660 .00040 3.000 .986750 .00042 3.050 .993800 .00040 3.101 .000990 .00041 3.151 .007170 .00042 3.201 .015650 .00041 3.251 .021910 .00041 3.301 .029290 .00041

130

2.200 .915130 .00042 2.250 .923780 .00043 2.300 .932090 .00044 2.350 .941670 .00042 2.400 .949590 .00043 2.450 .958990 .00043 2.500 .966890 .00043 2.550 .975080 .00042 2.600 .983530 .00041 2.650 .990710 .00042 2.700 .999080 .00042 2.751 .007470 .00043 2.801 .014770 .00042 2.851 .021430 .00043 2.901 .030620 .00042
3.300 .979240 .00040

3.350 .986210 .00040

3.400 .992090 .00040

3.450 .998590 .00039

3.501 .004660 .00039

3.551 .011570 .00040 3.601 .017820 .00040
3.100 .978740 .00040 3.150 .986530 .00041 3.200 .992530 .00040 3.251 .000130 .00041 3.301 .006480 .00041 3.351 .013760 .00041 3.401 .019920 .00039
100

2.400 .909690 .00040

2.450 .918460 .00042

2.500 .926340 .00042

2.550 .935210 .00041

2.600 .942620 .00042

2.650 .951440 .00042

2.700 .960200 .00042

2.750 .966900 .00041

2.800 .975010 .00040

2.850 .983260 .00042

2.900 .990580 .00042

2.950 .997930 .00043

3.001 .005440 .00042

3.051 .012200 .00042

3.101 .018840 .00042
118

2.300 .919060 .00042 2.350 .926950 .00043 2.400 .935650 .00041 2.450 .944360 .00041 2.500 .953010 .00043 2.550 .961770 .00042 2.600 .970090 .00042 2.650 .977190 .00043 2.700 .985530 .00043 2.750 .992850 .00043 2.801 .001020 .00043 2.851 .008840 .00043 2.901 .015470 .00042 2.951 .024410 .00042 3.001 .031860 .00043
2.250 .943080 .00044
2.300 .950480 .00043
2.350 .960010 .00042
2.400 .968510 .00044
2.450 .977280 .00044
2.500 .985520 .00043
2.550 .993540 .00044
2.601 .001230 .00044
2.651 .009390 .00043
2.701 .017530 .00043
200
1.900 .909770 .00043
1.950 .919410 .00042
2.000 .927810 .00043
2.050 .937760 .00044
2.100 .946780 .00044
2.150 .955680 .00044
2.200 .964160 .00044
2.250 .973330 .00044
2.300 .982190 .00044
2.350 .991170 .00045
2.400 .999860 .00045
2.451 .008160 .00045
2.501 .016230 .00044 
235

1.900 .923110 .00044 1.950 .933150 .00045 2.000 .942410 .00044 2.050 .951170 .00044 2.100 .960560 .00044 2.150 .969880 .00045 2.200 .978410 .00045 2.250 .987300 .00045 2.300 .995710 .00045 2.351 .003650 .00044 2.401 .012200 .00043 2.451 .020410 .00044 2.501 .028390 .00043

470

1.700 .922650 .00044 1.750 .930940 .00045 1.800 .940490 .00045 1.850 .949460 .00044 1.900 .957650 .00045 1.950 .967220 .00045 2.000 .975100 .00045 2.050 .983810 .00046 2.100 .992470 .00046 2.151 .000830 .00045 2.201 .009110 .00046 2.251 .016510 .00045 2.301 .024500 .00045

605

1.700 .925050 .00044 1.750 .934040 .00045 1.800 .941390 .00045 1.850 .951060 .00045 1.900 .959100 .00046 1.950 .967340 .00044 2.000 .976190 .00047 2.050 .983810 .00045 2.100 .992570 .00045 2.150 .999500 .00046 2.201 .008020 .00045 2.251 .016090 .00045 2.301 .023250 .00046
250

1.800 .908970 .00044

1.850 .917720 .00044

1.900 .928820 .00045

1.950 .937540 .00044

2.000 .946460 .00045

2.050 .955970 .00045

2.100 .964640 .00044

2.150 .973690 .00045

2.200 .983060 .00045

2.250 .991170 .00044

2.301 .000220 .00045

2.351 .008730 .00044

2.401 .017260 .00044
353

1.700 .911060 .00045

1.750 .921140 .00044

1.800 .929870 .00044

1.850 .939360 .00045

1.900 .948450 .00044

1.950 .957580 .00045

2.000 .966710 .00046

2.050 .976270 .00045

2.100 .984660 .00045

2.150 .993320 .00046

2.201 .001630 .00045

2.251 .010650 .00044

2.301 .018140 .00045
500

1.700 .923100 .00044

1.750 .931490 .00044

1.800 .940850 .00044

1.850 .949500 .00046

1.900 .958790 .00044

1.950 .967570 .00044

2.000 .975580 .00044

2.050 .984170 .00045

2.100 .992860 .00045

2.151 .000280 .00045

2.201 .009160 .00045

2.251 .016740 .00045

2.301 .023950 .00047
600

1.700 .925400 .00044

1.750 .933590 .00045

1.800 .942190 .00044

1.850 .950780 .00045

1.900 .958910 .00044

1.950 .967720 .00044

2.000 .975890 .00046

2.050 .983900 .00044

2.100 .992690 .00045

2.150 .999740 .00045

2.201 .007800 .00044

2.251 .015350 .00046

2.301 .024060 .00044
610

1.700 .924850 .00043

1.750 .933880 .00045

1.850 .952370 .00045

1.900 .958920 .00044

1.950 .967510 .00044

2.000 .975400 .00044

2.050 .984640 .00044

2.100 .992830 .00045

2.151 .000640 .00045

2.201 .008490 .00045

2.251 .016420 .00045

2.301 .022890 .00047
615

1.700 .925400 .00046

1.750 .933340 .00045

1.800 .942660 .00047

1.850 .950580 .00045

1.900 .959840 .00046

1.950 .967710 .00045

2.000 .975710 .00044

2.050 .984520 .00045

2.100 .991480 .00045

2.150 .999790 .00046

2.201 .007280 .00044

2.251 .015520 .00046

2.301 .022340 .00046 
620

625

629

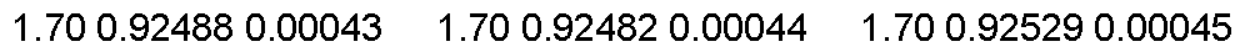

$\begin{array}{llll}1.750 .93433 & 0.00046 & 1.750 .933570 .00045 & 1.750 .932960 .00045\end{array}$

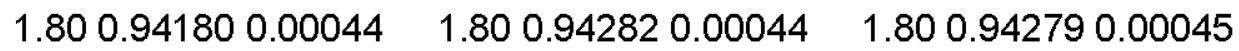

$\begin{array}{lll}1.850 .951160 .00044 & 1.850 .951290 .00046 & 1.850 .950980 .00046\end{array}$

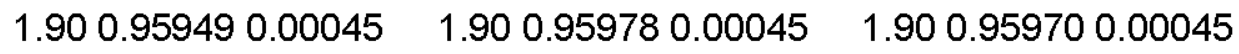

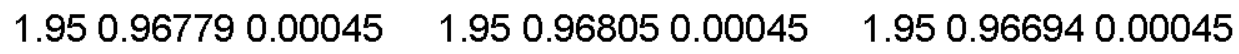

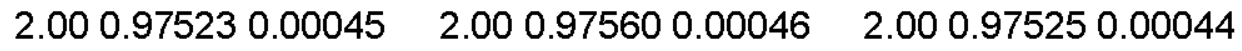

$\begin{array}{llll}2.050 .98349 & 0.00045 & 2.050 .983960 .00045 & 2.050 .983180 .00045\end{array}$

$\begin{array}{llll}2.100 .99174 & 0.00045 & 2.100 .991360 .00044 & 2.100 .992020 .00046\end{array}$

$\begin{array}{llll}2.150 .99927 & 0.00044 & 2.150 .999540 .00046 & 2.150 .999420 .00046\end{array}$

$\begin{array}{llll}2.201 .007150 .00045 & 2.201 .007210 .00045 & 2.201 .006870 .00043\end{array}$

$\begin{array}{llll}2.251 .014640 .00047 & 2.251 .014850 .00044 & 2.251 .015270 .00044\end{array}$

$\begin{array}{lllll}2.301 .022370 .00046 & 2.301 .022540 .00046 & 2.301 .022090 .00044\end{array}$ 